\title{
ilass EUROPE
}

\section{TH EUROPEAN CONFERENCE On}

LIQUID ATOMIZATION and SPRAY SYSTEMS

6TH·8TH SEPTEMBER 2017 VALENCIA SPAIN
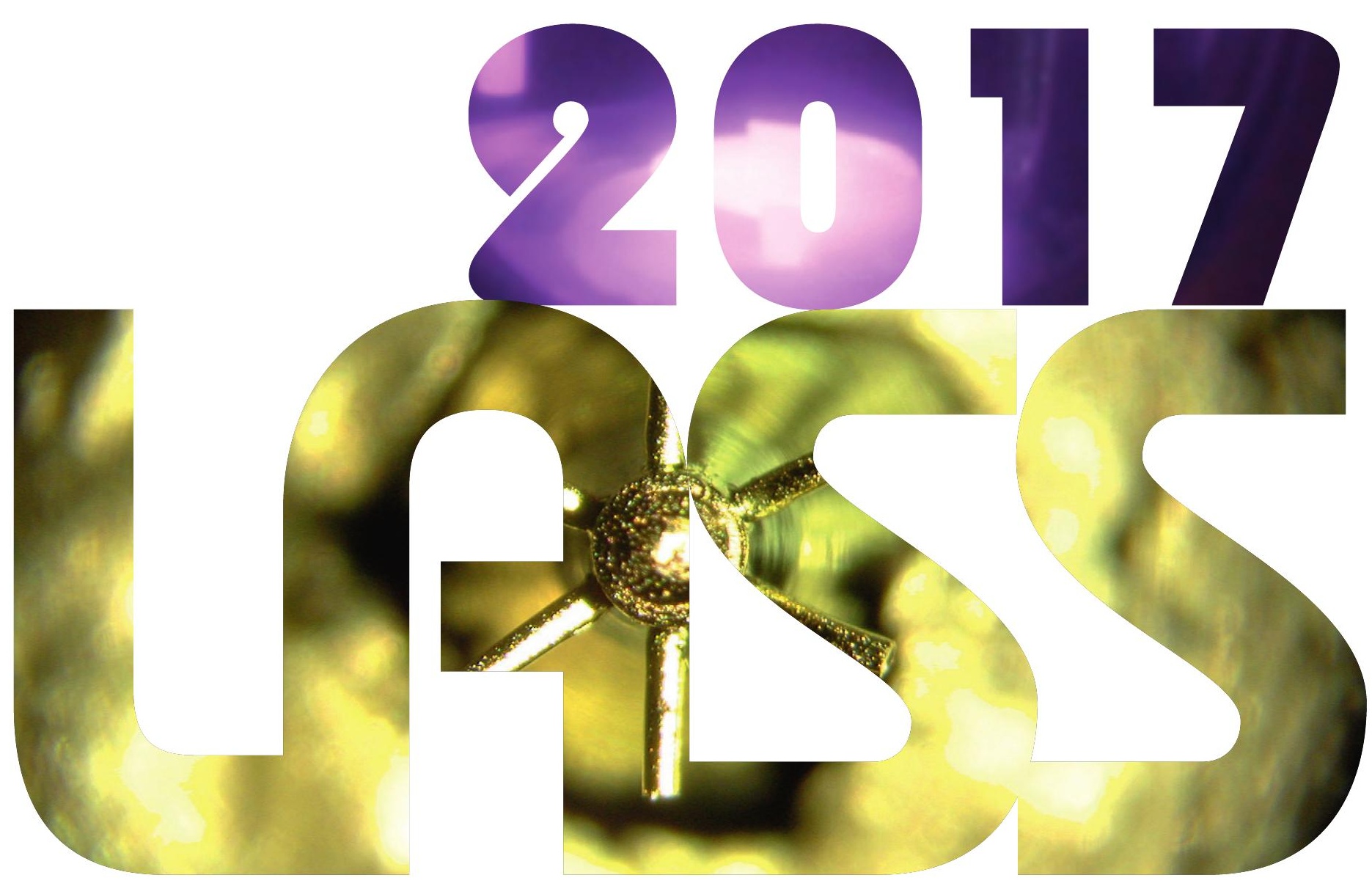
Congress UPV

$28^{\text {th }}$ European Conference ILASS 2017

The contents of this publication have been evaluated by the Scientific Committee which it relates and the procedure set out http://ocs.editorial.upv.es/index.php/ILASS/ILASS2017

\section{Scientific Editors \\ Raul Payri \\ Xandra Margot}

\section{Publisher}

Editorial Universitat Politècnica de València, 2017

www.lalibreria.upv.es / Ref.: 2075_05_01_01

ISBN: 978-84-9048-580-4

DOI: http://dx.doi.org/10.4995/LLASS2017.2017.6912

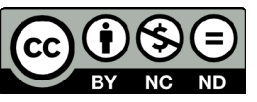

$28^{\text {th }}$ European Conference ILASS 2017

This book is licensed under a Creative Commons Atribution-NonCommercial-NonDetivates-4.0 International Licensed Editorial Universitat Politècnica de Valencia http://www.lalibreria.upv.es/portalEd/UpvGEStore/products/p_2075-5-1 


\section{ILASS-Europe 2017 \\ 28th Conference on Liquid Atomization and Spray Systems}

The Universitat Politècnica de València (UPV), Spain, is honored to host the $28^{\text {th }}$ edition of the European Conference on Liquid Atomization and Spray Systems from the $5^{\text {th }}$ to the $8^{\text {th }}$ September 2017, organized by CMT-Motores Térmicos and ILASS Europe.

Our University is located on the east coast of Spain in the beautiful city of Valencia with very good connections to the rest of Spain. It hosts about 36000 students, who attend the courses given by it's over 2800 academic staff. It has reached international renown, attracting every year over 2000 Erasmus students, which ranks UPV as the $6^{\text {th }}$ hosting institution in Europe.

Now, let me briefly present the main organizer of this conference, CMT Motores Térmicos. It is an internationally well-known research institute of the Universitat Politècnica de València dedicated to research on thermo-and fluid dynamics processes in direct injection engines. Its staff comprises over 120 people, with 41 faculty members and about 60 research assistants (mainly Ph.D students). Our institute has a total annual budget of $16 \mathrm{M} €$ coming from projects with private companies and public administrations. Two visits to our CMT laboratories have been scheduled during the ILASS conference, and participants will be welcome to discover our state of the art facilities, which comprise 16 engine test cells and another 20 specific test installations. Among these, 8 test benches dedicated to sprays, including a high pressure and high temperature vessel, a rapid compression machine, a transparent engine and several hydraulic test benches to measure injection rate and spray momentum.

With three keynote lectures and a total of 135 oral presentations distributed in 28 sessions, the ILASS 2017 Conference program reflects the success of this edition, in line with previous ones. There are three main topics: Automotive Sprays, Atomizers, and Atomization \&Droplets, with about 30 papers each. The rest of the papers are grouped in four smaller topics: internal nozzle flow, combustion, experimental techniques, and atmospheric \& medical sprays. A closer look at the program reveals the extensive participation of both industry and academia, with the common objective of sharing the latest scientific advances to help improve knowledge about the atomization processes for different applications.

Last but not least, I would like to convey our warmest welcome to all the guests attending the conference, wishing them a fruitful conference and enjoyable stay in Valencia.

Prof. Raul Payri

ILASS 2017 Conference Chairman 


\section{CONFERENCE CHAIRMAN}

\author{
Prof. Raúl Payri
}

CMT. Universitat Politècnica de València

(SPAIN)

\section{CONFERENCE \\ COORDINATOR}

\author{
Dr. Xandra Margot
}

CMT. Universitat Politècnica de València

(SPAIN)

\section{Organizing and Scientific Committee}

Dr. Lydia Achelis / Universität Bremen - Germany

Ass. Prof. Lucio Araneo / Politecnico di Milano - Italy

Dr. Gabriela Bracho / Universitat Politècnica de Valencia - Spain

Prof. Günter Brenn / TU Graz - Austria

Dr. Joaquín de la Morena / Universitat Politècnica de Valencia - Spain

Prof. Joachim Domnick / Esslingen University of Applied Sciences - Germany

Dr. Christophe Dumouchel / Université et INSA de Rouen - France

Prof. Udo Fritsching / Universität Bremen - Germany

Dr. Antonio García / Universitat Politècnica de Valencia - Spain

Prof. Manolis Gavaises / City University - UK

Prof. Eva Gutheil / Universität Heidelberg - Germany

Dr. Chawki Habchi / IFP Energies nouvelles - France

Dr. Jérôme Hélie / Continental Automotive - France

Ass. Prof. Jan Jedelsky / Brno University of Technology - Czech Republic

Dr. Grazia Lamanna / Universität Stuttgart - Germany

Dr. Antonio Lozano / Universidad de Zaragoza - Spain

Prof. Marco Marengo / University of Brighton - UK

Dr. Xandra Margot / Universitat Politècnica de Valencia - Spain

Dr. Ana Moita / Instituto Superior Técnico Lisboa - Portugal

Dr. Ricardo Novella / Universitat Politècnica de Valencia - Spain

Dr. Pablo Olmeda / Universitat Politècnica de Valencia - Spain

Dr. José Manuel Pastor / Universitat Politècnica de Valencia - Spain

Dr. Raffaele Ragucci / Istituto di Ricerche sulla Combustione - CNR - Italy

Dr. Simona Tonini / Università degli Studi di Bergamo - Italy

Dr. Qiaoyan Ye / Fraunhofer Institut - Germany 


\section{Session Index}

\section{B Automotive 1 G. Bracho UNIVERSITAT POLITĖCNICA DE VALÈNCIA (SPAIN)}

A study of the controlling parameters of fuel air mixture formation for ECN Spray A

K. Vogiatzaki, C. Crua, R. Morgan, M. Heikal UNIVERSITY OF BRIGHTON (UK)

Evidence of vortex driven primary breakup in high pressure fuel injection

J. Shi, P. Aguado Lopez, E. Gomez Santos, N. Guerrassi, G. Dober DELPHI (LUXEMBOURG) - W. Bauer ANSYS (GERMANY) - M.C. Lai WAYNE STATE UNIVERSITY (USA) - J. Wang ARGONNE NATIONAL LABORATORY (USA)

CFD simulations of the diesel jet primary atomization from a multihole injector

C. Chasos FREDERICK UNIVERSITY (CYPRUS)

Large eddy simulations of atomisation and sprays: application to a high pressure multihole injector

P. Chausserie-Laprée, J. Hélie CONTINENTAL AUTOMOTIVE (FRANCE) - J. Chesnel ALTRAN, (FRANCE)

- F.X. Demoulin CORIA (FRANCE)

\section{R Atomizers $1 \quad$ L. Achelis INSTITUT FÜR WERKSTOFFTECHNIK}

Influence of liquid properties on ultrasonic atomization

A. Lozano, J.A. García, J. Alconchel, F. Barreras, E. Calvo UNIVERSIDAD DE ZARAGOZA / CSIC (SPAIN) - J.L. Santolaya UNIVERSIDAD DE ZARAGOZA (SPAIN)

Effect of excitation on gas centered swirl coaxial injectors

G. Park, S. Oh, J. Bae, Y. Yoon SEOUL NATIONAL UNIVERSITY (KOREA)

Effect of liquid viscosity on the aerodynamic breakup of non-spherical droplets

K. Bergeles, G. Charalampous, Y. Hardalupas, A. Taylor IMPERIAL COLLEGE LONDON (UK)

Numerical Investigation of Droplet Impact on Smooth Surfaces with Different Wettability Characteristics: Implementation of a dynamic contact angle treatment in OpenFOAM.

K. Vontas, M. Andredaki, A. Georgoulas UNIVERSITY OF BRIGHTON (UK) - K. Nikas PIRAEUS UNIVERSITY (GREECE) - M. Marengo UNIVERSITY OF BRIGHTON (UK)

\section{$1 Y$ Droplet $1 \quad$ S.S. Sazhin UNIVERSITY OF BRIGHTON (UK)}

A model for mono- and multi-component droplet heating and evaporation and its implementation into ANSYS Fluent.

L. Poulton, O. Rybdylova, S. Sazhin, C. Crua UNIVERSITY OF BRIGHTON (UK) - M. al Qubeissi COVENTRY UNIVERSITY (UK) - A.E. Elwardany ALEXANDRIA UNIVERSITY (EGYPT)

Modelling of spheroidal drop evaporation with non-uniform temperature conditions

G. Cossali, S. Ravasio, S. Tonini UNIVERSITY OF BERGAMO (ITALY)

Numerical studies of turbulent particle-laden jets using spatial approach of one-dimensional turbulence M. Fistler CHALMERS UNIVERSITY OF TECHNOLOGY (SWEDEN) - D. Lignell BRIGHAM YOUNG UNIVERSITY (USA) - A. Kerstein, M. Oevermann CHALMERS UNIVERSITY OF TECHNOLOGY (SWEDEN) Energy Aspects in Spray Formation by Homogenous Flash Boiling Process 
Energy Aspects in Spray Formation by Homogenous Flash Boiling Process

Y. Moshkovich, Y. Levy TECHNION-ISRAEL INSTITUTE OF TECHNOLOGY (ISRAEL) - I. Sher

CRANFIELD UNIVERSITY (UK) - E. Sher TECHNION- ISRAEL INSTITUTE OF TECHNOLOGY (ISRAEL)

\section{B Automotive 2 J.Domnik UNIVERSITY OF APPLIED SCIENCES ESSLINGEN (GERMANY)}

Modelling and validation of near-field Diesel spray CFD simulations based on the $\Sigma-Y$ model J.M. Desantes, J.M. García-Oliver, J.M. Pastor UNIVERSITAT POLITĖCNICA DE VALĖNCIA (SPAIN) - A. Pandal UNIVERSIDAD DE OVIEDO (SPAIN) - B. Naud CIEMAT (SPAIN) - K. Matusik, D. Duke, A. Kastengren, C. Powell ARGONNE NATIONAL LABORATORY (USA) - D.P. Schmidt UNIVERSITY OF MASSACHUSETTS-AMHERST (USA)

The liquid penetration of diesel substitutes

S. Riess, L. Weiss, J. Rezaei, A. Peter, M. Wensing UNIVERSITÄT ERLANGEN-NÜRNBERG (GERMANY)

Numerical and Experimental Investigations on rotary Bell Atomizers with predominant Air Flow Rates

N. Guettler, S. Paustian, Q. Ye, O. Tiedje FRAUNHOFER INSTITUTE (GERMANY)

Investigations on nano- and submicron-particle generation by spray painting processes

Q. Ye, O. Tiedje, S.R. Srinivas FRAUNHOFER INSTITUTE (GERMANY) - T. Noest, U. Uhrner GRAZ UNIVERSITY OF TECHNOLOGY (AUSTRIA)

Experimental and Numerical Investigation of Phase Separation due to Multi-Component Mixing at HighPressure Conditions

C. Traxinger, H. Müller, M. Pfitzner BUNDESWEHR UNIVERSITY MUNICH (GERMANY) - S. Baab, G. Lamanna, B. Weigand UNIVERSITÄT STUTTGART (GERMANY) - J. Matheis, C. Stemmer, N. Adams TECHNICAL UNIVERSITY OF MUNICH (GERMANY) - S. Hickel DELFT UNIVERSITY OF TECHNOLOGY (NETHERLANDS)

Spreading model for wall films generated by high-pressure sprays

Q. Lamiel, N. Lamarque, J. Hélie CONTINENTAL AUTOMOTIVE (FRANCE) - D. Legendre INSTITUT DE MÉCANIQUE DES FLUIDES DE TOULOUSE (FRANCE)

\section{R Atomizers 2 U. Fritsching IWT / UNIVERSITY BREMEN (GERMANY)}

Application of dry-ice for transient spray cooling

M. Panao, J. Costa, M. Bernardo UNIVERSIDADE DE COIMBRA (PORTUGAL)

Effect of Prefilmer Edge Thickness on Breakup Phenomena of Liquid Film in Prefilming Airblast Atomizer

T. Okabe, N. Katagata, T. Sakaki, T. Inamura, K. Fumoto HIROSAKI UNIVERSITY (JAPAN)

Spray Conditioning for the Preparation of Spray Dried Submicron Particles

R. Gorny, G. Schaldach, P. Walzel, M. Thommes TU DORTMUND (GERMANY)

Multiple Impinging Jet Air-Assisted Atomization

M. Costa, B. Pizziol UNIVERSIDADE DE LISBOA (PORTUGAL) - M. Panao UNIVERSIDADE DE COIMBRA (PORTUGAL) - A. Silva UNIVERSIDADE DE BEIRA INTERIOR (PORTUGAL)

Investigation on the role of atomization air mixing on the spray characteristics of air assisted atomizer N. Kawaharada, M. Höltermann, J. Wichmar, F. Dinkelacker UNIVERSITY OF HANOVER (GERMANY)

Effects of Injection Nozzle Geometry on Spray Characteristics for Direct Injection Diesel Engines

N. Tamaki, T. Harada KINDAI UNIVERSITY (JAPAN) 


\section{B Automotive $3 \quad$ P. Olmeda UNIVERSITAT POLITĖCNICA DE VALÈNCIA (SPAIN)}

Fuel spray vapour distribution correlations for a high pressure diesel fuel spray cases for different injector nozzle geometries.

D. Njere, N. Emekwuru COVENTRY UNIVERSITY (UK)

Spray/wall interaction analysis on an ECN single-hole injector at diesel-like conditions through Schlieren visualization.

R. Payri, J. Gimeno, J. Peraza UNIVERSITAT POLITÈCNICA DE VALÈNCIA (SPAIN) - T. Bazyn CATERPILLAR (USA)

An Experimental Study of Diesel Spray Impingement on a Flat Plate: Effects of Injection Conditions

X. Zhu, L. Zhao, Z. Zhao, N. Ahuja, J. Naber, S.Y. Lee MICHIGAN TECHNOLOGICAL UNIVERSITY (USA)

Quantification of diesel injector dribble using 3D reconstruction from x-ray and DBI imaging

V. Sechenyh, J. Turner, D. Sykes UNIVERSITY OF BRIGHTON (UK) - D. Duke, A. Swantek, K. Matusik, A. Kastengren, C. Powell ARGONNE NATIONAL LABORATORY (USA) - A. Viera, R. Payri UNIVERSITAT POLITĖCNICA DE VALÈNCIA (SPAIN) - C. Crua UNIVERSITY OF BRIGHTON (UK)

Zooming into primary breakup mechanisms of high-pressure automotive sprays

V. Kirsch, M. Reddemann, J. Palmer, R. Kneer RWTH AACHEN UNIVERSITY (GERMANY)

\section{R Atomizers 3 C. Dumouchel CORIA (FRANCE)}

Internal flow and air core dynamics in simplex and spill-return pressure-swirl atomizers

M. Maly, L. Janáčková, J. Jedelský BRNO UNIVERSITY OF TECHNOLOGY (CZECH REPUBLIC) - J. Sláma PROVYKO S.R.O (CZECH REPUBLIC) - M. Sapík BRNO UNIVERSITY OF TECHNOLOGY (CZECH REPUBLIC) G. Wigley LOUGHBOROUGH UNIVERSITY (UK)

Behaviour of free falling viscoelastic liquid jets

C. Tirel, M.C. Renoult, C. Dumouchel, J.B. Blaisot CORIA (FRANCE)

Detailed simulation of air-assisted spray atomization: effect of numerical scheme at intermediate Weber number

G. Tretola IMPERIAL COLLEGE LONDON (UK) - K. Vogiatzaki UNIVERSITY OF BRIGHTON (UK) - S. Navarro-Martinez IMPERIAL COLLEGE LONDON (UK)

Towards Resolving the Atomization Process of an Idealized Fire Sprinkler with VOF Modeling

K. Meredith, X. Zhou, Y. Wang FM GLOBAL (USA)

Numerical Simulation of Like and Unlike Impinging Jets

H. Dolatkhahi, G. Oliaee, A. Kebriaee SHARIF UNIVERSITY OF TECHNOLOGY (IRAN)

\section{Y Droplet $2 \quad$ Q. Ye FRAUNHOFER INSTITUTE}

Time resolved thermographic analysis of droplet impacts onto heated surfaces under extreme wetting scenarios

P. Pontes, E. Teodori, A. Moita, A. Moreira UNIVERSIDADE DE LISBOA (PORTUGAL)

Instantaneous heat transfers at the impact of a droplet onto a hot surface in the film boiling regime W. Chaze, G. Castanet, O. Caballina, D. Maillet, J.F. Pierson, F. Lemoine UNIVERSITÉ DE LORRAINE (FRANCE) 
Experimental study on the effect of initial liquid droplet size on the evaporation in a heterogeneous droplet

K.H. Sung CHUNG-ANG UNIVERSITY (KOREA) - J.S. Nam KOREA FIRE INSTITUTE (KOREA) - H.S. Ryou CHUNG-ANG UNIVERSITY (KOREA)

Selected Results of the Collaborative Research Center "Droplet Dynamics under Extreme Ambient Conditions" SFB/TRR 75

C. Tropea TECHNISCHE UNIVERSITÄT DARMSTADT (GERMANY) - B. Weigand, K. Schulte UNIVERSITÄT STUTTGART (GERMANY)

Experimental and numerical study on sensible heat transfer at droplet/wall interactions

E. Teodori, P. Pontes, A. Moita, A. Moreira UNIVERSIDADE DE LISBOA (PORTUGAL) - A. Georgoulas, M. Marengo UNIVERSITY OF BRIGHTON (UK)

\section{B Automotive 4 L. Araneo POLITECNICO DI MILANO (ITALY)}

A study on the relationship between internal nozzle geometry and injected mass distribution of eight ECN Spray G nozzles.

K. Matusik, D. Duke, N. Sovis, A. Swantek, C. Powell ARGONNE NATIONAL LABORATORY (USA) - R. Payri, D. Vaquerizo, S. Giraldo-Valderrama UNIVERSITAT POLITĖCNICA DE VALĖNCIA (SPAIN) - A. Kastengren ARGONNE NATIONAL LABORATORY (USA)

Flashboiling atomization in nozzles for GDI engines

S. Bornschlegel, C. Conrad, L. Eichhorn, M. Wensing UNIVERSITÄT ERLANGEN-NÜRNBERG (GERMANY)

Measurement of a Direct Water-Gasoline-Emulsion-Injection

T. Wagner, H. Rottengruber, F. Beyrau, P. Dragomirov, M. Schaub OTTO-VON-GUERICKE-UNIVERSITY MAGDEBURG (GERMANY)

High speed visualization of gasoline pump injector (GPI)

N. Balasubramanian, T. Iwaszkiewicz, J. Sethuraman STANADYNE (INDIA)

Oxygenated fuels properties and its relationship with engine performance in port fuel injection engines.

U. Gonzalez, I. Schifter INSTITUTO MEXICANO DEL PETRÓLEO (MEXICO)

\section{R Atomizers $4 \quad$ M. Marengo UNIVERSITY OF BRIGHTON (UK)}

Primary breakup of liquids using a high-speed rotary bell atomizer for spray painting processes B. Shen UNIVERSITY OF APPLIED SCIENCES ESSLINGEN (GERMANY) - Q. Ye, O. Tiedje FRAUNHOFER INSTITUTE (GERMANY) - J. Domnick UNIVERSITY OF APPLIED SCIENCES ESSLINGEN (GERMANY)

Mechanisms of Liquid Stream Breakup: Vorticity and Time and Length Scales

A. Zandian, W. Sirignano UNIVERSITY OF CALIFORNIA (USA) - F. Hussain TEXAS TECH UNIVERSITY (USA)

Direct numerical simulation of an atomizing biodiesel jet: Impact of fuel properties on atomization characteristics

Y. Ling, G. Legros, S. Popinet, S. Zaleski UPMC (FRANCE) 
Experimental Characterization of Spray generated by a Rotary Atomizer Wheel

M. Kuhnhenn, M. Luh TECHNISCHE UNIVERSITÄT DARMSTADT (GERMANY) - T. Joensen GEA PROCESS ENGINEERING (DENMARK) - I. Roisman, C. Tropea TECHNISCHE UNIVERSITÄT DARMSTADT (GERMANY)

Subgrid Liquid Flux and interface modelling for LES of Atomization

J. Anez, A. Ahmed CORIA (FRANCE) - S. Puggelli UNIVERSITY OF FLORENCE (ITALY) - J. Reveillon, J.C. Brändle de Motta, F.X. Demoulin CORIA (FRANCE)

\section{$4 Y$ Droplet $3 \quad$ A. Moita UNIVERSIDADE DE LISBOA (PORTUGAL)}

Theoretical and Experimental Study of Grouping Effects on Droplet Streams

N. Roth, H. Gomaa UNIVERSITÄT STUTTGART (GERMANY) - A. Livne, D. Katoshevski BEN-GURION UNIVERSITY (ISRAEL) - B. Weigand UNIVERSITÄT STUTTGART (GERMANY)

Comparison between numerical and experimental water-in-oil dispersion in a microchannel

P. Desjonquères, T. Ménard CORIA (FRANCE) - D. Tarlet, J. Bellettre UNIVERSITÉ DE NANTES (FRANCE)

Simulation of droplet spreading on micro-CT reconstructed 3D real porous media using the volume-offluid method

M. Aboukhedr, N. Mitroglou CITY UNIVERSITY OF LONDON (UK) - A. Georgoulas, M. Marengo, K. Vogiatzaki UNIVERSITY OF BRIGHTON (UK)

Numerical analysis of sprays with an advanced collision model

M. Sommerfeld OTTO-VON-GUERICKE-UNIVERSITY MAGDEBURG (GERMANY) - S. Lain UNIVERSIDAD AUTONOMA DE OCCIDENTE (COLOMBIA)

Microexplosion and Puffing of an Emulsion Fuel Droplet

J. Xia BRUNEL UNIVERSITY LONDON (UK) - J. Shinjo SHIMANE UNIVERSITY (JAPAN)

\section{B Automotive 5 J.M. Pastor UNIVERSITAT POLITÈCNICA DE VALÈNCIA (SPAIN)}

Influence of Stokes Number on Collisional Interfacial Area Production Terms within the $\Sigma$-Y Eulerian Spray Atomization Model

D. Eichler, P. Pischke, R. Kneer RWTH AACHEN UNIVERSITY (GERMANY)

A new approach to modelling the two way coupling for momentum transfer in a hollow-cone spray

A. Papoutsakis, S. Sazhin, S. Begg UNIVERSITY OF BRIGHTON (UK) - I. Danaila, F. Luddens UNIVERSITÉ DE ROUEN (FRANCE)

Mathematical models for turbulent round jets based on "ideal" and "lossy" conservation of mass and energy

F. Franco OSAKA UNIVERSITY (JAPAN) - Y. Fukumoto KYUSHU UNIVERSITY (JAPAN)

Maximum entropy method for biodiesel spray droplet distribution

R. Kolodnytska, S. Skurativskyi, P. Moskvin ZHYTOMYR STATE TECHNOLOGICAL UNIVERSITY (UKRAINE) 


\section{R Atomizers $5 \quad$ M. Gavaises CITY UNIVERSITY OF LONDON (UK)}

Experimental Investigation on the Early Stage Spray Characteristics with Biodiesel and Diesel S. Yu, B. Yin, S. Wen, X. Li, H. Jia JIANGSU UNIVERSITY (CHINA) - J. Yu WUXI FUEL INJECTION EQUIPMENT RESEARCH INSTITUTE (CHINA)

Properties of Fuel Spray Obtained by Electrohydrodynamic Atomization M. Daaboul, N. Saba, J. Rishmany UNIVERSITY OF BALAMAND (LEBANON) - C. Louste UNIVERSITY OF POITIERS (FRANCE)

Characterization of Liquid Impinging Jet Injector Sprays for Bi-Propellant Space Propulsion: Comparison of PDI and High-Magnification Shadowgraphy

B. Boust, Q. Michalski, A. Claverie, C. Indiana, M. Bellenoue PPRIME INSTITUTE (FRANCE)

Fundamental analysis of liquid breakup mechanism in a rotary atomizer with square discharge orifice M. Ghorbanhoseini, S. Rezayat, M. Farshchi SHARIF UNIVERSITY OF TECHNOLOGY (IRAN)

\section{Y Atmospheric and medical sprays J. Jedelský BRNO UNIVERSITY OF TECHNOLOGY (CZECH REPUBLIC)}

Coupled study of film and spray on a basic annular prefiming airblast atomizer

V. Gosselin, B. Ferret, R. Bazile INSTITUT DE MÉCANIQUE DES FLUIDES DE TOULOUSE (FRANCE)

Influence of droplet clustering in sprays on liquid deposition rate on spherical targets

P. Andrade, G. Charalampous, Y. Hardalupas IMPERIAL COLLEGE LONDON (UK)

Viability of coaxial atomization for disintegration of cell solutions in cell spray applications

M. Bieber, S. Menzel, A. Thiebes, C. Cornelissen, S. Jockenhoevel, R. Kneer, M. Reddemann RWTH AACHEN UNIVERSITY (GERMANY)

Numerical Simulation of the Dispersion and Deposition of a Spray Carried by a Pulsating Airflow in a Patient-Specific Human Nasal Cavity

A. Farnoud HEIDELBERG UNIVERSITY (GERMANY) - X.G. CUi LAWRENCE BERKELEY NATIONAL LABORATORY (USA) - I. Baumann, E. Gutheil HEIDELBERG UNIVERSITY (GERMANY)

\section{B Automotive 6 A. García UNIVERSITAT POLITĖCNICA DE VALÈNCIA (SPAIN)}

Analysis of PDA measurements in double injection GDI sprays

L. Araneo POLITECNICO DI MILANO (ITALY) - R. Dondè CNR-ICMATE (ITALY) - L. Postrioti, A. Cavicchi UNIVERSITÁ DI PERUGIA (ITALY)

Imaging of gasoline-like sprays with planar laser-induced exciplex fluorescence using a stereoscopic imaging system

M. Andersson CHALMERS UNIVERSITY OF TECHNOLOGY (SWEDEN) - A. Yamaguchi DENSO (JAPAN) H. Wang DANTEC DYNAMICS (DENMARK)

Transcritical mixing of sprays for multi-component fuel mixtures

J. Manin SANDIA NATIONAL LABORATORIES (USA) - C. Crua UNIVERSITY OF BRIGHTON (UK) - L.M. Pickett SANDIA NATIONAL LABORATORIES (USA)

Influence of the Fuel Properties on the Injection Process and Spray Development in a large ship diesel Engine

I. Najar, B. Buchholz, B. Stengel, C. Fink, E. Hassel UNIVERSITY OF ROSTOCK (GERMANY) 
Characteristics of Control Piston Motion and Pressure Inside of a Common Rail Diesel Injector

S. Schuckert, G. Wachtmeister TECHNICAL UNIVERSITY OF MUNICH (GERMANY)

LIF/Mie Droplet Sizing of Water Sprays from SCR System Injector using Structured Illumination

Ł. Kapusta WARSAW UNIVERSITY OF TECHNOLOGY (POLAND)

\section{R Atomizers 6 M. Carreres UNIVERSITAT POLITĖCNICA DE VALÈNCIA (SPAIN)}

Numerical simulation of superheated jets using an Eulerian method

K. Lyras, S. Dembele KINGSTON UNIVERSITY LONDON (UK) - C. Madhav Rao Vendra, J. Wen UNIVERSITY OF WARWICK (UK)

Supercritical and transcritical real-fluid mixing using the PC-SAFT EOS

C. Rodriguez, A. Vidal, P. Koukouvinis, M. Gavaises CITY UNIVERSITY OF LONDON (UK)

Where does the drop size distribution come from?

R. Canu, C. Dumouchel, B. Duret CORIA (FRANCE) - M. Essadki IFP ENERGIES NOUVELLES (FRANCE) M. Massot ECOLE CENTRALE PARIS (FRANCE) - T. Ménard CORIA (FRANCE) - S. Puggelli UNIVERSITY OF FLORENCE (ITALY) - J. Reveillon, F.X. Demoulin CORIA (FRANCE)

Weakly nonlinear instability of a viscous liquid jet

I. Mutabazi UNIVERSITÉ DU HAVRE (FRANCE) - M.C. Renoult CORIA (FRANCE) - G. Brenn GRAZ UNIVERSITY OF TECHNOLOGY (AUSTRIA)

Flexible Piezoelectric Drop-On-Demand Droplet Generation

N. Riefler, T. Wriedt, U. Fritsching IWT / UNIVERSITY BREMEN (GERMANY)

A Comprehensive Predictive Model to Predict Droplets Size Distribution in Pressure Swirl Atomizer

S. Jafari, A. Kebriaee SHARIF UNIVERSITY OF TECHNOLOGY (IRAN) - S. Sohrabi UNIVERSITAT POLITĖCNICA DE CATALUNYA (SPAIN)

\section{B Experimental techniques 1 J.M. García-Oliver UNIVERSITAT POLITĖCNICA DE VALĖNCIA (SPAIN)}

Determination of Heat and Flow Characteristics in Spray Cooling with Rectangular Finned Heat Sink

A. Karabey YUZUNCU YIL UNIVERSITY (TURKEY) - K. Yakut ATATÜRK UNIVERSITY (TURKEY)

Liquid-Liquid Secondary Fragmentation with Solidification

M. Hadj-Achour, N. Rimbert, M. Gradeck UNIVERSITÉ DE LORRAINE (FRANCE)

Planar droplet sizing: Application to a spray of Jet A1 kerosene

P. Doublet, C. Lempereur, V. Bodoc, M. Orain, P. Gajan ONERA (FRANCE)

Development steps of 2-color laser-induced fluorescence with MDR-enhanced energy transfer for instantaneous planar temperature measurement of micro-droplets and sprays

J. Palmer, M. Reddemann, V. Kirsch, R. Kneer RWTH AACHEN UNIVERSITY (GERMANY)

High Speed X-Ray Imaging for Nozzle Exit Velocity and Density Distribution Measurements of GDI Nozzles R. Distler, C. Hamann, M. Krämer, E. Kull CONTINENTAL AUTOMOTIVE (GERMANY) - M. Wensing UNIVERSITÄT ERLANGEN-NÜRNBERG (GERMANY) - Z. Li, Y. Gao, J. Wang ARGONNE NATIONAL LABORATORY (USA) 


\section{R Combustion $1 \quad$ E. Gutheil HEIDELBERG UNIVERSITY (GERMANY)}

Influence of the chemical mechanism in the frame of diesel-like CFD reacting spray simulations using a presumed PDF flamelet-based combustion model

F. Payri, J.M. García-Oliver, R. Novella, E.J. Pérez-Sánchez UNIVERSITAT POLITÈCNICA DE VALÈNCIA (SPAIN)

Analytical / Computational Approach to Liquid Spray Heating and Vaporization at Supercritical Pressures A. Jorda Juanos, W. Sirignano UNIVERSITY OF CALIFORNIA (USA)

Theory of Stagnation-Point Spray Flame Ignition

G. Kats, J.B. Greenberg TECHNION- ISRAEL INSTITUTE OF TECHNOLOGY (ISRAEL)

Polydisperse spray flames in vortex flows

Y. Dagan TECHNION- ISRAEL INSTITUTE OF TECHNOLOGY (ISRAEL) - D. Katoshevski BEN-GURION UNIVERSITY (ISRAEL) - J.B. Greenberg TECHNION- ISRAEL INSTITUTE OF TECHNOLOGY (ISRAEL)

\section{Y Droplet $4 \quad$ P. Walzel TU DORTMUND (GERMANY)}

Experimental investigation of size effects in colliding droplet

L. Pasternak, M. Sommerfeld OTTO-VON-GUERICKE-UNIVERSITY MAGDEBURG (GERMANY)

Splashing of a very viscous liquid drop impacting onto a solid wall wetted by another liquid

H. Kittel, I. Roisman, C. Tropea TECHNISCHE UNIVERSITÄT DARMSTADT (GERMANY)

Drop Stream - Immiscible Jet Collisions: Regimes and Fragmentation Mechanisms

C. Planchette, H. Hinterbichler, G. Brenn GRAZ UNIVERSITY OF TECHNOLOGY (AUSTRIA)

Generalized analysis of the deposition/splashing limit for one- and two-component droplet impacts upon thin films

R. Bernard, P. Foltyn, A. Geppert, G. Lamanna, B. Weigand UNIVERSITÄT STUTTGART (GERMANY)

Experimental study of liquid-vapor mass transfer in non-reacting and reacting droplet chains

M. Stöhr, S. Werner, W. Meier GERMAN AEROSPACE CENTER (DLR) (GERMANY)

\section{B Experimental techniques 2 G. Brenn GRAZ UNIVERSITY OF TECHNOLOGY (AUSTRIA)}

Vapor phase penetration measurements with both single and double-pass Schlieren for the same injection event

R. Payri, F. Salvador, G. Bracho, A. Viera UNIVERSITAT POLITÈCNICA DE VALÈNCIA (SPAIN)

X-ray diagnostics of dodecane jet in spray A conditions using the new one shot engine (NOSE)

I. Chiboub, H. Ajrouche, O. Nilaphai, S. Dozias, B. Moreau, C. Hespel, F. Foucher, C. MounaïmRousselle, J.M. Pouvelse, E. Robert UNIVERSITÉ D'ORLÉANS (FRANCE)

A quantitative analysis of nozzle surface bound fuel for diesel injectors

J. Turner, D. Sykes, G. De Sercey, V. Stetsyuk UNIVERSITY OF BRIGHTON (UK) - M. Gold, R. Pearson BRITISH PETROLEUM (UK) - C. Crua UNIVERSITY OF BRIGHTON (UK) 
Design and experiments to investigate spray and impingement characteristics of a common rail type lubrication system

M. Stark WINGD (SWITZERLAND) - A. de Risi, M. Giangreco UNIVERSITY OF SALENTO (ITALY) - S. Diggelmann WINGD (SWITZERLAND)

\section{R Combustion $2 \quad$ R. Ragucci IRC-CNR (ITALY)}

Multi-scale Eulerian-Lagrangian simulation of a liquid jet in cross-flow under acoustic perturbations S. Thuillet, D. Zuzio, O. Rouzaud, P. Gajan ONERA (FRANCE)

Investigation of the Influence of Microexplosions on a Laminar Premixed Water-in-Fuel Emulsion Spray Flame

N. Yokev, J.B. Greenberg TECHNION- ISRAEL INSTITUTE OF TECHNOLOGY (ISRAEL)

Spray and Combustion Characterizations of Acetone-Butanol-Ethanol (ABE) blend at High-Pressure and High-Temperature Conditions

O. Nilaphai, H. Ajrouche, C. Hespel, B. Moreau UNIVERSITÉ D'ORLÉANS (FRANCE) - C. Somchai KING MONGKUT'S UNIVERSITY OF TECHNOLOGY THONBURI (THAILAND) - F. Foucher, C. Mounaïm-Rousselle UNIVERSITÉ D'ORLÉANS (FRANCE)

Computational Prediction of Primary Breakup in Fuel Spray Nozzles for Aero-Engine Combustors

T. Dauch, S. Braun, L. Wieth, G. Chaussonnet, M. Keller, R. Koch, H.J. Bauer KARLSRUHER INSTITUT FÜR TECHNOLOGIE (GERMANY)

\section{Y Droplet 5 G. Lamanna UNIVERSITÄTSTUTTGART (GERMANY)}

Experimental and Computational Investigation of binary drop collisions under elevated pressure L. Reitter, M. Liu, J. Breitenbach TECHNISCHE UNIVERSITÄT DARMSTADT (GERMANY) - K.L. Huang NATIONAL TAIWAN UNIVERSITY (TAIWAN) - D. Bothe TECHNISCHE UNIVERSITÄT DARMSTADT (GERMANY) - G. Brenn GRAZ UNIVERSITY OF TECHNOLOGY (AUSTRIA) - K.L. Pan NATIONAL TAIWAN UNIVERSITY (TAIWAN) - I. Roisman, C. Tropea TECHNISCHE UNIVERSITÄT DARMSTADT (GERMANY)

Experimental investigation on puffing and micro-explosion occurrence of water in rapeseed oil emulsions droplets. Effect of the surfactant concentration.

E.A. Melo-Espinosa CUJAE (CUBA) - J. Bellettre, D. Tarlet UNIVERSITÉ DE NANTES (FRANCE) - R. Piloto-Rodriguez CUJAE (CUBA) - S. Verhelst GHENT UNIVERSITY (BELGIUM)

Experimental Investigation of Droplet Injections in the Vicinity of the Critical Point: A comparison of different model approaches

C. Steinhausen, G. Lamanna, B. Weigand, R. Stierle, J. Groß UNIVERSITÄT STUTTGART (GERMANY) -

A. Preusche, A. Dreizler TECHNISCHE UNIVERSITÄT DARMSTADT (GERMANY)

Break up length on Urea Water Solution jet in hot cross flow

P. Senthilkumar, B. Shamit, T.N.C. Anand INDIAN INSTITUTE OF TECHNOLOGY MADRAS (INDIA)

\section{B Combustion 3 R. Novella UNIVERSITAT POLITĖCNICA DE VALÈNCIA (SPAIN)}

Spray ignition and local flow properties in a swirled confined spray-jet burner: experimental analysis J. Marrero Santiago, A. Verdier, A. Vandel, G. Godard, G. Cabot, B. Renou CORIA (FRANCE) 
Visualization of pilot flame of an optically-accessible coaxially-staged aero-engine lean-burn fuel injector K. Matsuura JAPAN AEROSPACE EXPLORATION AGENCY (JAPAN) - S. Uesaka, T. Iwasaki HOSEI UNIVERSITY (JAPAN) - Y. Kurosawa, H. Yamada, T. Yamamoto JAPAN AEROSPACE EXPLORATION AGENCY (JAPAN) - S. Hayashi HOSEI UNIVERSITY (JAPAN)

Spray Combustion Chamber: History and Future of a Unique Test Facility A. Schmid, N. Yamada WINGD (SWITZERLAND)

Study of the soot quantification for two-stage injection of hydrogenated catalytic biodiesel in a constantvolume combustion chamber

J. Cao, Z. He, B. Li, W. Zhong, M. Wang JIANGSU UNIVERSITY (CHINA)

\section{R Internal flow 1 J. de la Morena UNIVERSITAT POLITĖCNICA DE VALÈNCIA (SPAIN)}

Towards design optimization of high-pressure gasoline injectors using Genetic Algorithm coupled with Computational Fluid Dynamics (CFD)

R. Hellmann, P. Jochmann, K.G. Stapf, E. Schuenemann ROBERT BOSCH GMBH (GERMANY) - L. Daróczy, D. Thévenin OTTO-VON-GUERICKE-UNIVERSITY MAGDEBURG (GERMANY)

Investigation of the effects of fluid properties representation and boundary condition selection in numerical simulations of micro scale flows with phase change

D. Pearce IMPERIAL COLLEGE LONDON (UK) - K. Vogiatzaki UNIVERSITY OF BRIGHTON (UK) - A. Taylor, Y. Hardalupas IMPERIAL COLLEGE LONDON (UK)

Numerical simulation of compressible cavitating two-phase flows with a pressure-based solver M. Cristofaro, W. Edelbauer AVL (AUSTRIA) - M. Gavaises, P. Koukouvinis CITY UNIVERSITY OF LONDON (UK)

Role of heat transfer in bubble dynamics neglecting phase change. A numerical study

S.R. Fostiropoulos, I. Malgarinos CITY UNIVERSITY OF LONDON (UK) - G. Strotos PIRAEUS UNIVERSITY (GREECE) - N. Nikolopoulos, E. Kakaras CENTRE FOR RESEARCH AND TECHNOLOGY HELLAS (GREECE) P. Koukouvinis, M. Gavaises CITY UNIVERSITY OF LONDON (UK)

\section{Y Droplet 6 P. Martí UNIVERSITAT POLITĖCNICA DE VALĖNCIA (SPAIN)}

Evaluation of the combustion characteristics of isolated droplets of Jet $A$ blended with ethanol and butanol

Á. Muelas, P. Remacha, J. Ballester UNIVERSIDAD DE ZARAGOZA / CSIC (SPAIN)

Experimental and Numerical Investigation of Dispersed and Continuous Liquid Film under Boiling conditions

C. Habchi IFP ENERGIES NOUVELLES (FRANCE) - N. Lamarque, J. Hélie CONTINENTAL AUTOMOTIVE (FRANCE) - S. Jay IFP ENERGIES NOUVELLES (FRANCE)

Development of an evaporation model for the dense spray region in Eulerian-Eulerian multiphase flow simulations

S. Puggelli, L. Palanti, A. Andreini UNIVERSITY OF FLORENCE (ITALY) - F.X. Demoulin CORIA (FRANCE)

Drop Impact onto a Metallic Porous Layer: Effect of Liquid Viscosity and Air Entrapment

C. Boscariol, D. Sarker, B. Kang, C. Crua, M. Marengo UNIVERSITY OF BRIGHTON (UK) 


\section{B Experimental techniques 3 J.Monsalve UNIVERSITAT POLITÈCNICA DE VALÈNCIA (SPAIN)}

Visualisation and quantitative analysis of the near nozzle formation and structure of a high pressure water jet in air and water.

S. Jasper BOCHUM UNIVERSITY OF APPLIED SCIENCES (GERMANY) - J. Hussong RUHR-UNIVERSITY BOCHUM (GERMANY) - R. Lindken BOCHUM UNIVERSITY OF APPLIED SCIENCES (GERMANY)

Experimental investigation of Ethane and Propane injection under sub- and super-critical conditions N. Vallee, G. Ribert, J.B. Blaisot, D. Lisiecki CORIA (FRANCE)

An improved image processing method for particle characterization by shadowgraphy

H. Wang DANTEC DYNAMICS (DENMARK) - F. Felis, S. Tomas IRSTEA (FRANCE) - F. Anselmet, $M$. Amielh IRPHE (FRANCE)

Measurement of polymeric time scales from linear drop oscillations

G. Plohl, G. Brenn GRAZ UNIVERSITY OF TECHNOLOGY (AUSTRIA)

Visualization of water droplet deformation and breakup in a continuously accelerating flow field

A. Garcia-Magariño ISDEFE (SPAIN) - S. Sor INTA (SPAIN) - A. Velazquez UNIVERSIDAD POLITÉCNICA DE MADRID (SPAIN)

Analysis of Heat and Flow Characteristics on Spray Cooling Heat Transfer with an Optimized Hexagonal Finned Heat Sink

F. Yesildal AGRI IBRAHIM CECEN UNIVERSITY (TURKEY) - K. Yakut ATATÜRK UNIVERSITY (TURKEY)

\section{R Internal flow 2 C. Habchi IFP ENERGIES NOUVELLES (FRANCE)}

Statistical methods of images analysis as an essential tool in the assessment process of computer methods intended for numerical simulations of cavitating flows

A. Niedźwiedzka, S. Lipiński UNIVERSITY OF WARMIA AND MAZURY IN OLSZTYN (POLAND)

Effect of geometry of real-size transparent nozzles on cavitation and on the atomizing jet in the near field Y. Cao, S. Idlahcen, J.B. Blaisot, C. Rozé CORIA (FRANCE) - L. Méès ÉCOLE CENTRALE DE LYON (FRANCE) - D. Maligne DELPHI (FRANCE)

VOF Simulation of The Cavitating Flow in High Pressure GDI Injectors

F. Giussani, A. Montorfano, F. Piscaglia, A. Onorati POLITECNICO DI MILANO (ITALY) - J. Hélie CONTINENTAL AUTOMOTIVE (FRANCE)

High-speed X-Ray Phase Contrast Imaging of String Cavitation in a Diesel Injector Orifice

I. Karathanassis, P. Koukouvinis, M. Lorrenzi, E. Kontolatis CITY UNIVERSITY OF LONDON (UK) - Z. Li, J. Wang ARGONNE NATIONAL LABORATORY (USA) - N. Mitroglou, M. Gavaises CITY UNIVERSITY OF LONDON (UK)

High Speed Shadowgraphy of Transparent Nozzles as an Evaluation Tool for In-Nozzle Cavitation Behavior of GDI Injectors

D. Mamaikin, T. Knorsch, P. Rogler, P. Leick ROBERT BOSCH GMBH (GERMANY) - M. Wensing UNIVERSITÄT ERLANGEN-NÜRNBERG (GERMANY)

Binary cavitation in a transparent three hole GDI nozzle

H. Chaves, S. Donath TECHNICAL UNIVERSITY OF FREIBERG (GERMANY) 
10Y Droplet 7 F. Salvador UNIVERSITAT POLITÈCNICA DE VALÈNCIA (SPAIN)

Models for automotive fuel droplets heating and evaporation M. al Qubeissi COVENTRY UNIVERSITY (UK) - S. Sazhin UNIVERSITY OF BRIGHTON (UK) - N. Al-Esawi COVENTRY UNIVERSITY (UK)

Numerical investigation of the aerodynamic breakup of diesel droplets under various gas pressures D. Stefanitsis, I. Malgarinos CITY UNIVERSITY OF LONDON (UK) - G. Strotos PIRAEUS UNIVERSITY (GREECE) - N. Nikolopoulos, E. Kakaras CENTRE FOR RESEARCH AND TECHNOLOGY HELLAS (GREECE) M. Gavaises CITY UNIVERSITY OF LONDON (UK)

Droplets heating and evaporation: an application to diesel-biodiesel fuel mixtures M. al Qubeissi, N. Al-Esawi COVENTRY UNIVERSITY (UK) - S. Sazhin UNIVERSITY OF BRIGHTON (UK)

Expansion rates of bubble clusters in superheated liquids

D. Dietzel, T. Hitz, C.D. Munz, A. Kronenburg UNIVERSITÄT STUTTGART (GERMANY)

Kinetic and MD modelling of automotive fuel droplets heating and evaporation: recent results S. Sazhin UNIVERSITY OF BRIGHTON (UK) - I. Shishkova MOSCOW POWER ENGINEERING INSTITUTE (RUSSIA)

Dilute polymer solution drops impacting on heated surfaces: New impact morphologies and impact regime maps

V. Bertola, C. Lakhanpal UNIVERSITY OF LIVERPOOL (UK) 


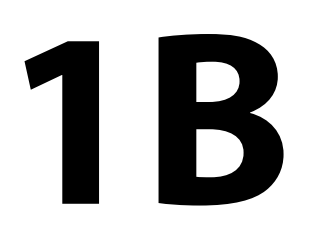

Automotive 1 


\title{
A study of the controlling parameters of fuel air mixture formation for ECN Spray A
}

\author{
K. Vogiatzaki ${ }^{1 *}$, C. Crua ${ }^{1}$, R. Morgan ${ }^{1}$, M.R. Heikal ${ }^{1}$ \\ ${ }^{1}$ Advanced Engineering Centre, University of Brighton, Lewes road, Brighton BN2 4GJ, UK \\ ${ }^{*}$ Corresponding author: k.vogiatzaki@brighton.ac.uk
}

\begin{abstract}
Designing future ultra-high efficiency, ultra-low emission engines requires an in depth understanding of the multiscale, multi-phase phenomena taking place in the combustion chamber. The performance of the fuel delivery system is key in the air fuel mixture formation and hence the combustion characteristics, however in most spray modelling approaches is not considered directly. Thus, it is important to understand how the selection of models that mimic injection process affect predictions. In this paper we present an Eulerian-Lagrangian framework based on OpenFOAM libraries to model spray injection dynamics. The framework accounts for primary droplet formation (based on a parcel method with predefined initial droplet size distribution), secondary droplet breakup, evaporation and heat transfer. In order to account for the interaction of droplets with turbulence, simulations were performed within the LES context with two different turbulence models. A systematic variation of the key injection parameters (parcel number, parcel size distribution) of the parcel method as well as the grid size was considered. Varying the parcel number affects the initial droplet size distribution which in turn, depending on the selection of the turbulence and the evaporation sub-models, affects: spray dispersion; spray penetration; and subsequent droplet size distribution. Results were validated against the baseline experimental data for evaporating ECN Spray A with n-dodecane chosen as a surrogate for Diesel fuel.
\end{abstract}

\section{Keywords}

ECN Spray A, Eulerian-Lagrangian, LES, OpenFOAM

\section{Introduction}

In both Diesel and spark ignition engines fuel is injected into the combustion chamber at elevated pressures. Nowadays realistic injection pressures for common-rail Diesel reach up to 3500 bars. The reason behind the need for such extreme pressures is to promote primary and secondary atomisation until a combustible mixture is formed. The higher the injection pressure the higher the shear between the static air within the combustion cylinder and the liquid. At extreme ambient pressures and temperatures the fuel can even exhibit supercritical behaviour that causes a reduction in its surface tension. Combustion occurs in a lifted, turbulent diffusion flame mode. Numerous studies indicate that the combustion and emissions in such engines are strongly influenced by the lifted flame characteristics, which are in turn determined by fuel and air mixing in the upstream region of the lifted flame, and consequently by the liquid breakup and spray development processes $[1,19,14,5,6]$. These processes clearly play a critical role in determining the engine combustion and emission characteristics.

From a numerical standpoint simulating spray combustion in modern engines involves a number of challenges mostly associated with the multiphase, multi-scale nature of the phenomenon. Scales vary from the molecular level (reactions) to microns (droplets) to mm (turbulence) and to meters (combustor dimensions). Thus, 'all componentall scale' analysis with direct numerical simulations (DNS) is prohibitive with the current computational capabilities for real size combustors. Only a very limited number of studies have been performed in engines with DNS [18]. Reynolds Averaged Navier Stokes (RANS) [17] and more recently Large Eddy Simulation (LES) based approaches are typically employed for engine simulations [7, 3, 22, 12]. RANS, which is the industrial standard approach is based on ensemble averaged governing equations. Although numerically efficient and relatively accurate in predicting the qualitative behaviour of the sprays, RANS cannot predict the local unsteadiness in the mixing flow field. This is a considerable drawback considering that ultra-high injection pressures promote supersonic behaviour of the jet and locally the creation of shock waves that further promote unsteadiness [15]. Moreover, RANS does not allow the study of cycle-to-cycle variation phenomena relevant to the spray evolution that currently is a subject that attracts considerable interest, and is also linked to spray spatial and temporal variations. The LES approach, which is based on spatially filtered governing equations, can capture the large scale flow structures based on the filter size. However, the unresolved small-scale structures are still modelled, which makes LES dependent on the sub grid scale models used and the grid resolution. Since LES can capture local unsteadiness and is computationally more attractive than a DNS based approach, it has received significant attention in the past decade, especially for simulation of internal combustion engines.

An additional difficulty in the modelling of turbulent combustion in realistic engine geometries is that in the effort to reduce computational cost and grid complexity, in many of the existent approaches the injector is not simulated directly and the effects of the in-nozzle flow and primary atomisation are modelled indirectly. The existent models based on the so-called parcel method have considerable weaknesses, the most important of which is the fact that 
the injected (initial) droplet size distribution needs to be selected a-priory. If experimental data are not available then fine tuning is required, making the final results dependent on the degree of tuning. Moreover, these models are mostly tailored to intermediate injection pressures of equipment of the past as well as to the RANS context. Extending these models to the LES context, understanding the resulting challenges and suggesting modifications is an area of active research. The sensitivity of the predictions to the injection model also undermines efforts for better evaporation and combustion models since in sprays all the phenomena present are interlinked and the injection part is a key controlling parameter of the initial mixture formation. For instance, even if the evaporation model predicts accurately the droplet size reduction because of heat transfer, if the parcels initially injected represent droplets with sizes considerably smaller than the real ones, simulations of liquid penetration will fail because the droplets will evaporate considerably quicker.

In recent years, various studies were performed in both experimental $[9,14]$ and numerical front $[21,20,22,23]$ in an effort to better understand spray dynamics at ultra-high pressures. Within the experimental results several institutions have provided high-fidelity measurements of macroscopic spray parameters such as spray penetration, liquid length and vapor penetration as well combustion related parameters such as ignition delay, lift-off length, and soot emissions for a range of fuels, ambient and injection conditions. Such datasets can be accessed through the Engine Combustion Network [11].

\section{Experimental data}

In the current work we use experimental data from Sandia National Laboratories at operating conditions known as ECN 'Spray A' (see Table 1). A constant-volume, quiescent, pre-burn-type combustion vessel is used to generate high-temperature and high-pressure gases. A premixed combustible mixture is spark-ignited. The combustion products are cooled until they reach the desired pressure and temperature. Then the diesel fuel injector is triggered and fuel injection occurs. The conditions for $n$-dodecante used as a surrogate of diesel fuel. In Fig 1 the experimental pictures of Spray A at three differnt time instances obtained with different techniques is presetned. In pictures from both techniques the light blue line indicates the liquid penetration vs time [11]. The time instances were selected to correspond to the time instances that numerical results will be rpesented in the following secitons.

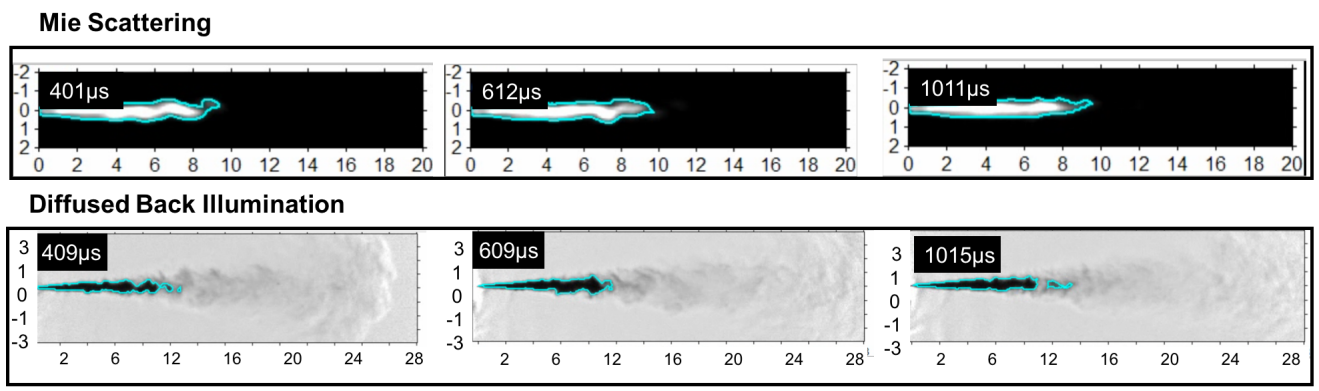

Figure 1. Experimental images of Spray $A$ at three different time instances obtained with different techniques. In pictures from both techniques the light blue line indicates the liquid penetration vs time [11].

\section{Numerical setup}

The numerical simulation of the two-phase flows is performed in the framework of an Eulerian-Lagrangian approach within an Open Source code (OpenFOAM) [8]. The governing equations were solved on two different grid sizes. For

Table 1. Summary of experimental conditions [11].

\begin{tabular}{c|c}
\hline Experimental conditions & n-dodecane \\
\hline \hline Ambient temperature $(\mathrm{K})$ & 900 \\
Ambient density $\left(\mathrm{kg} / \mathrm{m}^{2}\right)$ & 22.8 \\
Composition & $100 \% \mathrm{~N}_{2}$ \\
Injection pressure $(\mathrm{bar})$ & 1500 \\
Fuel temperature $(\mathrm{K})$ & 363 \\
Nozzle diameter $(\mu \mathrm{m})$ & 90 \\
Duration of injection $(\mathrm{ms})$ & 1.5 \\
Total mass injected $(\mathrm{mg})$ & 3.5 \\
Fuel density $\left(\mathrm{kg} / \mathrm{m}^{3}\right)$ & 750 \\
\hline
\end{tabular}


the rest of the paper we use the notation "Grid 1" for the coarser grid with average cell size of $0.5 \mathrm{~mm}$ and "Grid 2" for a finer grid with average cell size of $0.3 \mathrm{~mm}$. The time step is calculated based on the Courant number criterion: Comax $_{\max }=\frac{u \Delta t}{\Delta x}$ where $\Delta x$ is the grid size while $\Delta t$ is the time step. For the calculations presented in the results
section the $\mathrm{Co}_{\max }=0.1$.

\section{Turbulence model}

LES is based on the idea of computing the large, energy-containing eddy structures (filtered quantities) which are resolved on the computational grid, whereas the smaller, more isotropic, sub-grid structures (SGS) are modelled. The filter width is taken as the cube root of the local grid cell volume. The effect of the small scales is obtained through the sub-grid scale stress term $\left(\tau_{i j}^{s g s}=\widetilde{u_{i} u_{j}}-\widetilde{u}_{i} \widetilde{u}_{j}\right)$ that must be modelled. There are two popular types of turbulence models: a) algebraic eddy viscosity models in which the stress tensor $\tau_{i j}^{\text {sgs }}$ is related to the resolved strain rate tensor $\widetilde{S}_{i j}$ by means of a scalar eddy viscosity given by an algebraic equation; b) one-equation eddy viscosity models. Both model groups are based on the Boussinesq hypothesis associating $\tau_{i j}^{s g s}$ with a SGS turbulent viscosity $\mu_{T}$. However their main difference is that one-equation SGS models overcome the deficiency of local balance assumption between the SGS energy production and dissipation adopted in algebraic models. Such a phenomenon may occur in high Reynolds number flows and/or in the cases of coarse grid resolutions. In this paper we assess two models (one of each group): The Wall-Adapting Local Eddy-viscosity model (WALE) [13] which is an algebraic eddy viscosity models and the Kinetic Energy Model (KEM) [24] that belongs to the category of oneequation eddy viscosity models. The main difference between the proposed WALE model in comparison to other models of this group, is that the SGS viscosity is dynamically computed with the square of the velocity gradient tensor rather than the resolved strain rate used in Smagorinsky-type models that have been tested in previous work [21] for Spray A. This velocity tensor can not only account for the effects of both strain and rotation rate of the smallest resolved turbulence fluctuations, but also recover the proper near-wall scaling for the eddy viscosity without requiring dynamic procedure. Moreover, the WALE model is invariant to any coordinate translation or rotation and no test-filtering operation is needed, it is therefore considered well suitable for LES in complex geometries [13] as the ones in IC engines.

\section{Injection model}

As mentioned in the introduction one of the greatest challenges associated with the Eulerian-Lagrangian approach is modelling the near-nozzle flow. In this region a liquid core forms from the liquid fuel being injected through the injector. Ligaments are separated from this liquid core and form droplets that evaporate and mix with the ambient gas. When a combined Eulerian-Lagrangian framework is used then the fuel spray is treated as a dispersed liquid phase, which moves and interacts with the surrounding continuous gas phase. The spray is represented by an ensemble of discrete "parcels". Each parcel contains a number of droplets with the same size, velocity and temperature. Droplets in a parcel are considered as spherical, which is a rather strong assumption especially for regions close to the nozzle where ligaments instead of droplets are expected to be formed. The droplet parcels are tracked in a Lagrangian fashion as they move through the gas phase, exchanging mass, momentum and energy. The effect of the droplet parcels on the continuous phase due to drag, heat and mass transfer is implemented via source terms in the gas phase conservation equations.

Figure 2 shows a schematic of the injection process modelled in our current calculations. The injection model is a solid-cone injection model. The user supplies a drop diameter probability density function (PDF) with parameters. In our work we have examined two different models a) a Rosin-Rammler (RR) [2] with spreading parameter $n=3$ and mean diameter $\bar{d}=50 \mu \mathrm{m}$. The RR distribution function is based on the assumption that an exponential relationship exists between the droplet diameter, $d$, and the mass fraction $Y_{d}$ of droplets with diameter greater than $d: Y_{d}=e^{(d / \bar{d})^{n}}$ b) A fixed value distribution with mean droplet size $\bar{d}=90 \mu \mathrm{m}$. In this method all droplets injected have the same size and their size only changes as they move through the domain because of evaporation and secondary break up. One point that should be made is that for sprays with low initial velocity, the droplets can retain their sizes for quite a long period after the primary breakup. It is, therefore, essential to provide a correct droplet size distribution for fuel sprays of low injection velocity, such as the pressure-swirl type of gasoline injector. For diesel sprays of high initial velocity, the droplet size distribution is not expected to be as important to the final droplet distribution if an appropriate model for the secondary breakup is applied. The higher the initial velocity of the jet, the sooner the secondary breakup occurs and the lower the dependence of the final droplet sizes on the droplet size distribution of the primary breakup.

The velocity of the injected parcel is calculated as $u_{d}=\frac{\dot{m}}{C_{d} \rho A}$ where $\mathrm{A}$ is the area of the injection (defined by the diameter of the nozzle), $C_{d}$ is the discharge coefficient (=0.9) and $m \dot{a} s s$ is the mass flow rate. Within this model the velocity vector direction is defined by a random angle size within a limit (in our case $10^{\circ}$ ) which is a user-defined constant and does not depend on the droplet size which might lead to inaccuracies. It should be underlined that the mass (or volume) given initially to each parcel depends on the mass flow rate profile of the injector while the mean droplet size characterising the parcel depends on the initial distribution. This means that the number of droplets in each parcel is a statistical number which can vary from a fraction of a droplet to thousands of droplets depending also on the number of parcels used. The higher the number of parcels used the lower the number of droplets each 
parcel will contain.

It becomes evident that one of the most important parameters in this injection model is the number of parcels injected per second (PPS). The higher the PPS the more accurate is the representation of the initial pre-selected distribution. For the case of a fixed droplet size this is not particularly important since even a small size of parcels is enough for the statistical representation of the injection process while for the case of the RR the number of parcels is more important to faithfully reproduce the droplet size distribution.

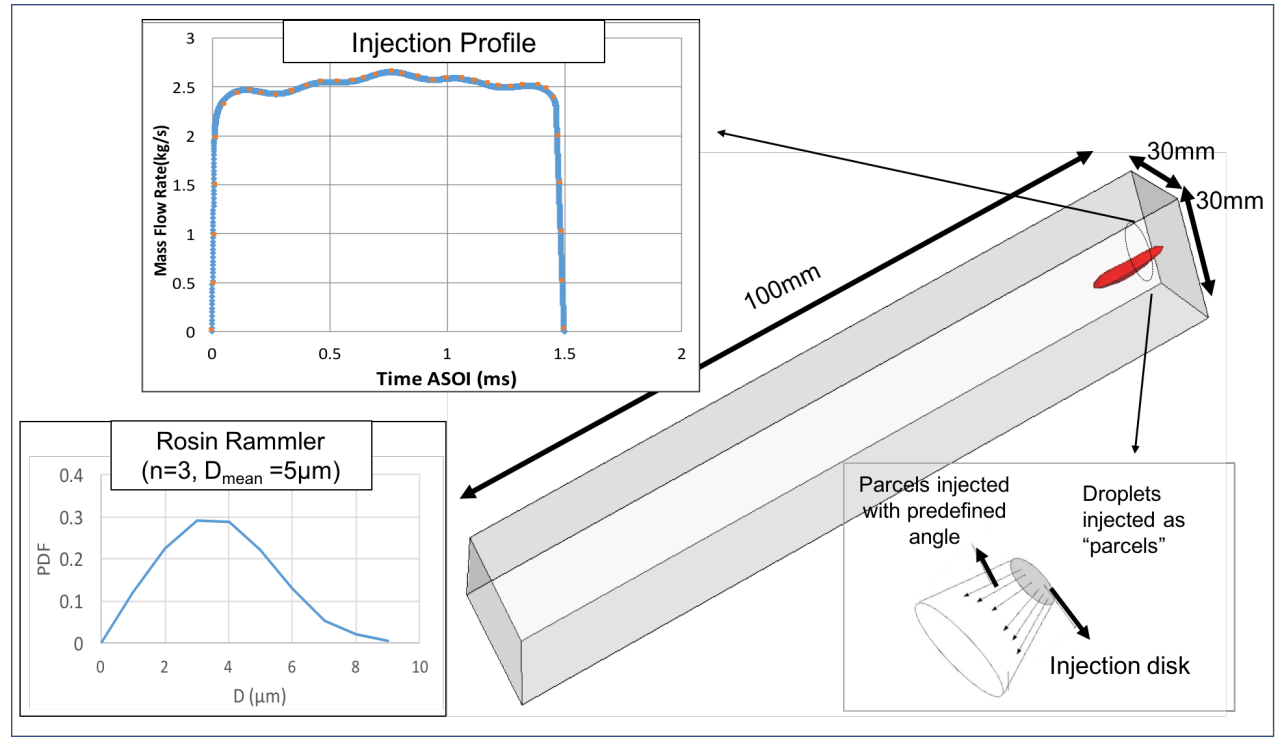

Figure 2. Simulation details of the injection process including the domain size

\section{Parcel Tracking}

In Lagrangian spray simulations, the particles representing the liquid are moving in a fixed Eulerian framework as described above. Tracking them and defining the cells they go through are clearly important issues. In OpenFOAM the approach used is the face-to-face tracking. The process can be described in four steps [10]: 1) Initially the parcel is moved until it reaches a cell boundary or the entire time step if it remains in the same cell; 2) Then a check is performed to evaluate if the parcel changes cell; 3) The time it took to move out of the first cell is calculated, and the parcel properties are updates; 4) Following the momentum change to the cell that the parcel has been in are added. If the parcel still has time left to move we go back the the first step of the algorithm. Parcels tracked by face-to-face tracking cannot 'skip' cells, which improves the predictions of transfer of mass, momentum and energy.

\section{Secondary breakup}

The breakup model used is the Kelvin-Helmholtz-Rayleigh-Taylor (KHRT) model [16]. This model, along with the TAB model, is one of the most widely used in Lagrangian spray simulations today. The KHRT model was chosen here since previous studies (within the RANS context) have indicated its superior performance under Diesel conditions [4]. It should be mentioned that it is also possible to use the TAB model, but often in conjunction with some form of primary atomisation model. The TAB model tends to break up the droplets very rapidly. The KHRT model includes two modes of breakup: KH breakup, accounting for unstable waves growing on the liquid jet due to differences in velocity between the gas and liquid; and RT breakup, accounting for waves growing on the droplets' surface due to acceleration normal to the droplet-gas interface. The relative performance of the two models within the LES context needs to be also examined in a future study.

\section{Summary of Test Cases}

In Table 2 a summary of all the cases considered in this paper is provided.

\section{Results and discussion}

Figure 3 shows predicted and measured liquid spray penetration at different times after start of injection (ASOI) under non-reacting conditions for n-dodecane at an ambient temperature of $900 \mathrm{~K}$. Liquid penetration is defined as the axial location encompassing $97 \%$ of the injected mass at that instant in time. The first observation is that for Cases 1-3 that the RR is used as initial distribution the results show great sensitivity to the particle number. Case 3 (with the lower number of particles and thus the less accurate representation of the RR) shows closer agreement with the experiments. This can be considered an indication that the RR with the selected parameters might not be 
the optimum distribution for this spray condition. Moving to a finer grid it can be seen that rather surprisingly for both Cases 4 and 5, irrespectively of the number of parcels used a considerably higher liquid penetration is predicted in comparison to the coarser mesh. Also the predictions are less dependent on the PPS number. Differences can only be noticed when a considerably low number of PPS is used as in Case 6. Using though 50.000 PPS is not a reasonable number to represent any spray statistics and thus it is not considered in the liquid penetration predictions. An additional interesting point is that the turbulence model appears to play a considerable role. Case 1 and 3 are run with the same number of parcels as Case 7 and 8 as well as the same grid however the liquid penetration predicted is different. For Case 7 and 8 regardless of the number of parcels the predictions agree with the experiments while for Case 1 and 3 the predictions depend on the number of parcels. An additional case (Case 9 ) is run with the KEM model and with 20 million PPS. It can be seen that for this case since the initial distribution is different (a fixed mean diameter value for all the injected droplets is chosen) the predictions are slightly different than Cases 7 and 8 although still in reasonably good agreement with the experiments. Finally it should be mentioned that looking at pictures in Fig 1 for all cases the spray disperses less than in experiments and potentially an even higher grid resolution with modifications to the particle model is required.

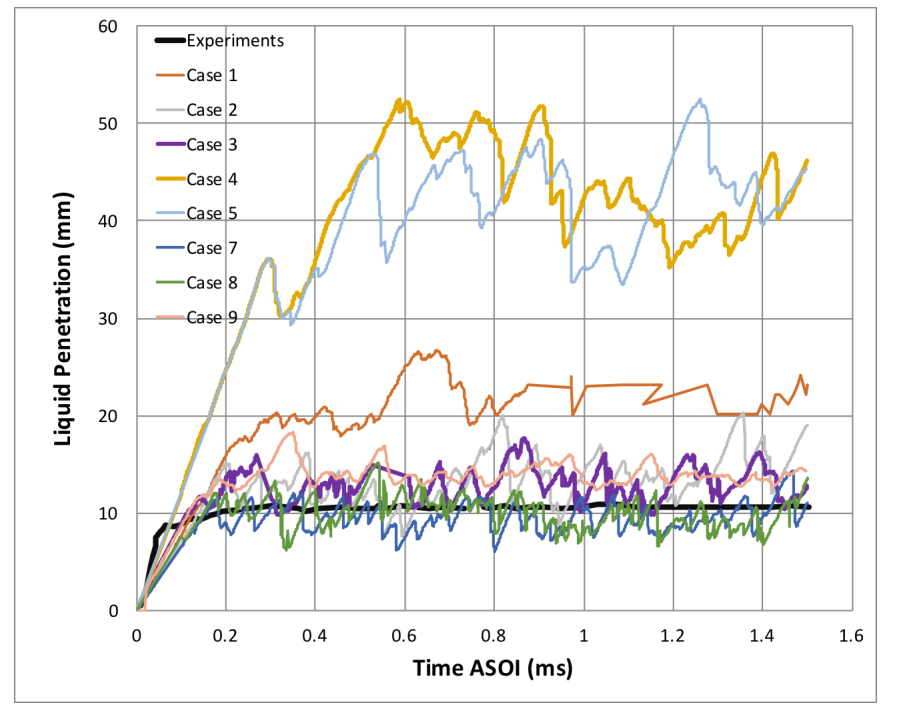

Figure 3. Measured [11] and predicted liquid penetration vs. time for the cases of Table 2

In order to get a better understanding of the conditions leading to these differences we include in our analysis Figs 4-6 that demonstrate the $\mathrm{C}_{12} \mathrm{H}_{26}$ (vapour) contour at two different time instances. We can see that in Fig 4 for the cases that use a rather large number of parcels $(20,000,000$ and 2,000,000) the results are similar. The length of the spray is similar while we can see that the vapour (indicated by the bright red areas of high $\mathrm{C}_{12} \mathrm{H}_{26}$ ) starts being formed even very close to the injection point. The behaviour is different in Fig 5 in which a higher grid resolution is used. The vapour diffuses less while it penetrates more. However the areas of high vapour concentration are at the tip of the spray and not close to the injection point. This behaviour is not compatible to what has been reported in the literature when different codes are used (see for example [21]). It should be underlined that the different grid sizes, apart from the direct effect they have on the turbulence resolution, also affect the parcel injection method because for the current calculations the time step is adjusted based on the Courant number. The average time step for Grid $1 \Delta t=2.5 \times 10^{-7} \mathrm{~s}$ while for Grid $2 \Delta t=1.3 \times 10^{-7} \mathrm{~s}$. The difference in the time step means that even for the cases that the same number of parcels per second is injected (for example Case 1 and 4 ) in reality a different number of parcels is injected per time step leading to a different representation of the initial droplet distribution PDF.

Table 2. Summary of numerical test cases.

\begin{tabular}{c|rccc}
\hline \hline Test Case & PPS & Grid Size $(\mathrm{mm})$ & Turbulence Model & Initial Distribution \\
\hline \hline Case 1 & $20,000,000$ & 0.5 & Wale & RR \\
Case 2 & $2,000,000$ & 0.5 & Wale & RR \\
Case 3 & 200,000 & 0.5 & Wale & RR \\
Case 4 & $20,000,000$ & 0.3 & Wale & RR \\
Case 5 & $2,000,000$ & 0.3 & Wale & RR \\
Case 6 & 50,000 & 0.3 & Wale & RR \\
Case 7 & $2,000,000$ & 0.5 & KEM & RR \\
Case 8 & 200,000 & 0.5 & KEM & RR \\
Case 9 & $20,000,000$ & 0.5 & KEM & Fixed Value \\
Case 10 & $20,000,000$ & 0.5 & KEM & RR \\
\hline
\end{tabular}


Moreover, the size of the time step is linked to the time that the particles interact with the underlying gas properties as explained in the Section "Parcel Tracking". For Grid 1 the average local Courant number is 0.0005 while for Grid 2 is 0.0009 . The difference in the local Courant number results because of the turbulence resolution locally which leads to a different local velocity. This implies that in reality for Grid 2 the particles remain less time in the cell, and thus they have less time to interact with the underlying Eulerian velocity field and exchange momentum which might explain why for the Grid 2 the spray penetrates much more than the other cases. Looking at Fig 6 we can see that when KEM turbulence model is used less sensitivity to the other parameters is noticed (PPS and initial distribution). The vapour penetration for all cases is similar however the distribution of the vapour is different. High vapour concentration (bright red areas) is seen through the spray for Case 7 while some isolated dense regions are noticed for Case 8. For Case 9 the behaviour is closer to Case 7 although a more uniform dispersion is noticed which might be attributed to the fact that less randomness is introduced in the inlet since all droplets have the same diameter.

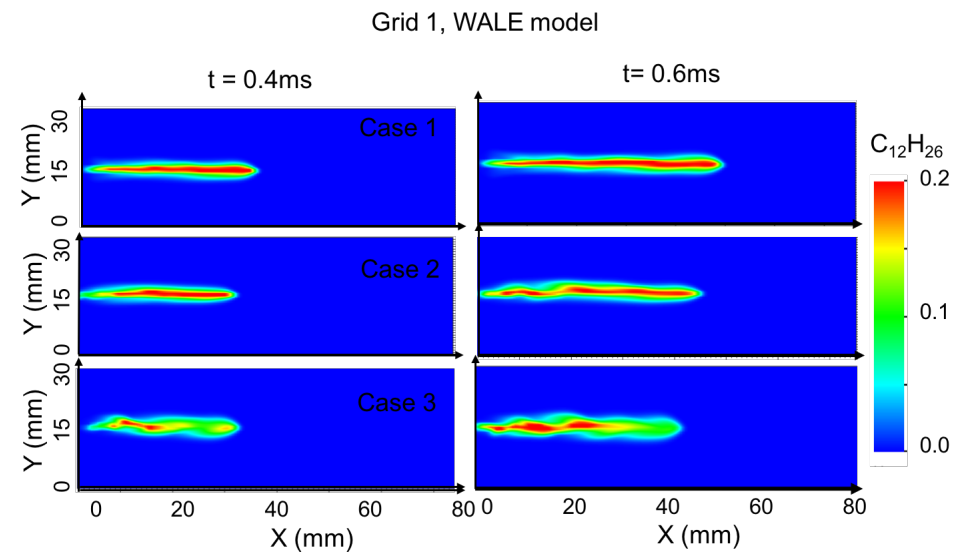

Figure 4. $\mathrm{C}_{12} \mathrm{H}_{26}$ contour plots for two different time instances using Grid 1 and WALE turbulence model.

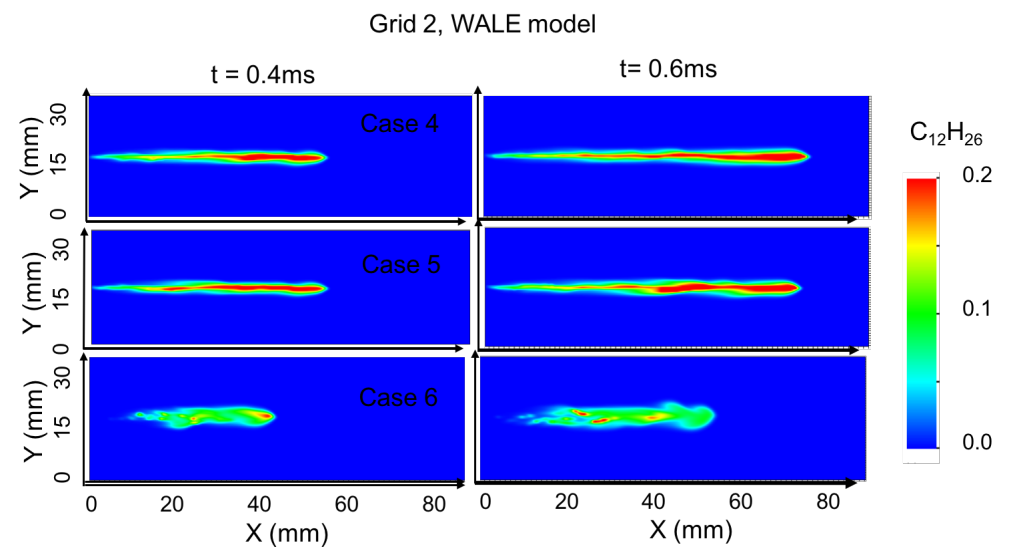

Figure 5. $\mathrm{C}_{12} \mathrm{H}_{26}$ contour plots for two different time instances using Grid 2 and WALE turbulence model.

Figure 7 shows the scatter plot of droplet diameter versus velocity magnitude (coloured by temperature) for the total number of the droplets in the domain at $t=3 \mathrm{~ms}$ for three different simulation cases. In all cases the PPS is 20 million. We will use this figure in order to get a better insight into the links between the predictions of the droplet size, the droplet velocity and the droplet temperature. For all cases the droplet size is mostly clustered in the range of 1-4 $\mu \mathrm{m}$ and only fe parcels have diameters above $10 \mu \mathrm{m}$. Also we can see that in all cases droplets with smaller diameters have lower velocities while the droplets with larger diameters have higher velocities in some cases reaching up to $500 \mathrm{~m} / \mathrm{s}$. For Cases 1 and 4 the behaviour is similar although we can see that droplets with similar diameter for Case 1 have considerable lower velocity. For Case 4 there is a greater variation of the droplet velocity even for droplets with similar sizes. Droplets with small diameter $2-4 \mu \mathrm{m}$ have velocities ranging $200 \mathrm{~m} / \mathrm{s}-$ $300 \mathrm{~m} / \mathrm{s}$ and the temperature depends on the velocity. Droplets with higher velocities have also lower temperature since they have les tile to interact with the underlying flow field. For case 9 the behaviour is different and we can see a more linear relation between droplet size and velocity. Also we can see that for droplets in the range of 2-4 $\mu \mathrm{m}$ the temperatures are higher (above 550K)

Figure 8 shows the number of droplets vs droplet sizes for tho axila locations. Top raw is at $x=0.003 \mathrm{~m}$ (close to the injector) ant bottom raw at $x=0.09 \mathrm{~m}$ (close to the tip of the spray). In all cases the PPS is 20 million 
Grid 1, KEM model

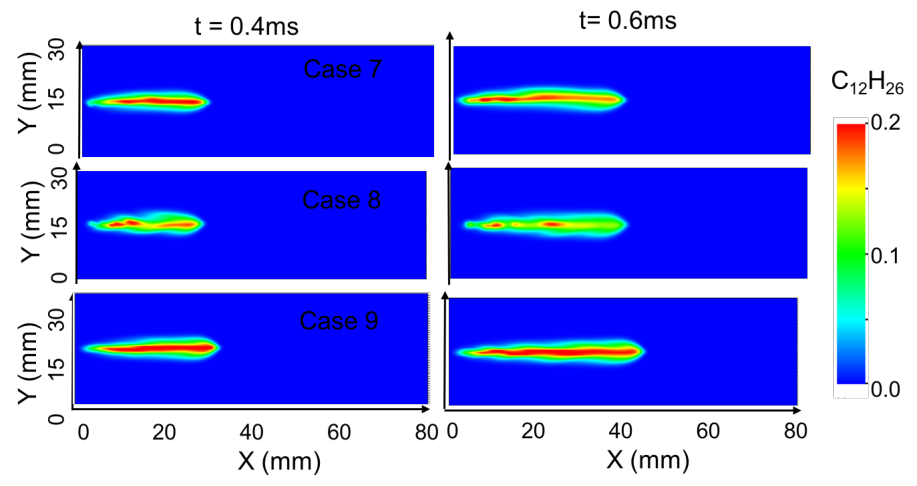

Figure 6. $\mathrm{C}_{12} \mathrm{H}_{26}$ contour plots for two different time instances using Grid 1 and KEM turbulence model.
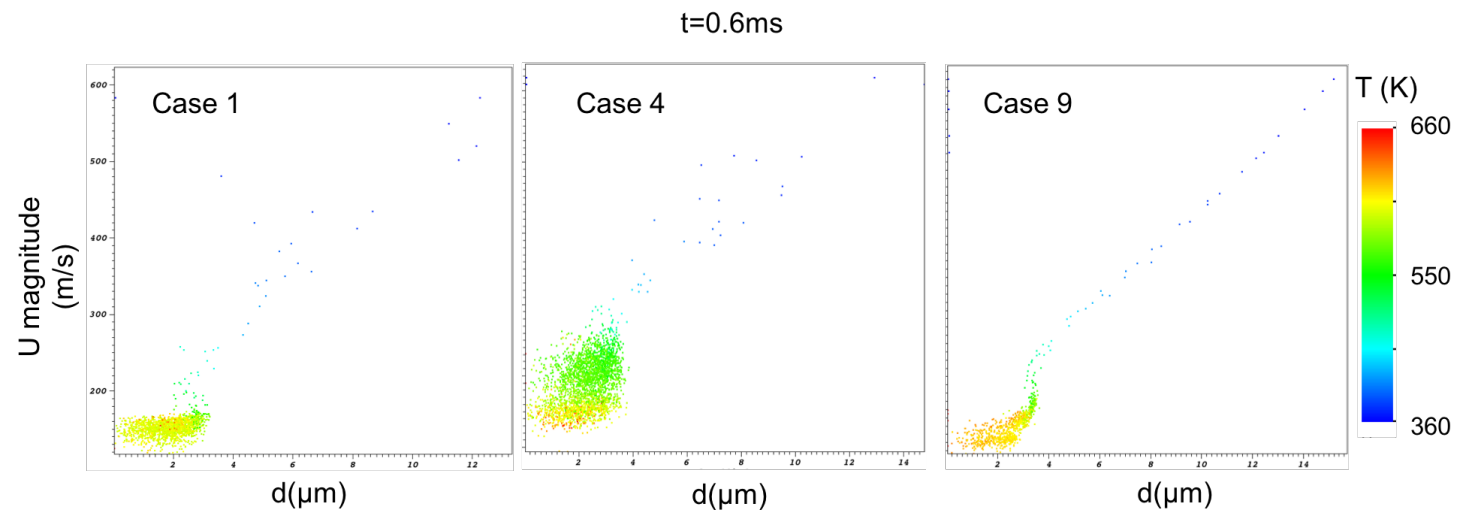

Figure 7. Scatter plot of droplet diameter versus velocity magnitude (coloured by temperature) for the total number of the droplets in the domain at $\mathrm{t}=3 \mathrm{~ms}$ for three different cases. In all cases the PPS is 20 million.

and the samples were taken for $t=0.6 \mathrm{~ms}$. It can be seen that close to the injector the droplet distribution as expected is different depending the selection of the initial distribution. When the RR distribution is used regardless of the turbulence selection model (for Case 1 Wale and for Case $10 \mathrm{KEM}$ ) the droplet distribution is similar. Much narrower distribution around droplet sizes of $3 \mu \mathrm{m}$ is noticed when a fixed value distribution is used (Case 9). Also it can be noticed that in both case the initial droplet size reduces rapidly. For example for Case 9 all droplets are injected with an average diameter of $90 \mu \mathrm{m}$ and after $3 \mathrm{~mm}$ their size has already reduced to $3 \mu \mathrm{m}$. Further downstream as expected the effect of the initial distribution reduces and all three cases predict an average droplet size of $2 \mu \mathrm{m}$. Moving from $3 \mathrm{~mm}$ to $9 \mathrm{~mm}$ the droplet radius reduction rate is smaller.

\section{Conclusions}

In this work we present an LES Eulerian-Lagrangian framework within OpenFOAM for the modelling of high pressure injection dynamics of ECN Spray A conditions. The framework accounts for primary droplet formation (based on a parcel method), secondary droplet breakup, evaporation and heat transfer. The sensitivity of the framework to different parameters that affect the predictions of the local mixture formation during breakup and evaporation is considered. Initially a systematic variation of the key injection parameters (parcel number, parcel size distribution) of the parcel method as well as the grid size is presented. Varying the parcel number affects the accuracy of the representation of the initial droplet size distributions, which in turn, depending on the selection of the initial droplet PDF, turbulence model and the evaporation model, affects a) spray dispersion b) spray penetration and c) downstream droplet size distribution. Moreover, two different turbulence models are considered. The selection of the turbulence model appears to be of high importance. For the cases considered the KEM model results are less sensitive to the other parameters (PPS, initial droplet PDF). Finally a rather unexpected discrepancy between the predictions of the coarse and the fine grid is noticed which is attributed to the algorithm for the parcel method and the adjustable time step used for the calculations. More detailed examination of the droplet diameter statistics including analysis of their radial distribution will be the subject of future work.

\section{Acknowledgements}

This work was supported by the UK's Engineering and Physical Science Research Council [grants EP/P012744/1 and EP/K020528/1]. 
20 million PPS, $\mathrm{t}=0.6 \mathrm{~ms}$
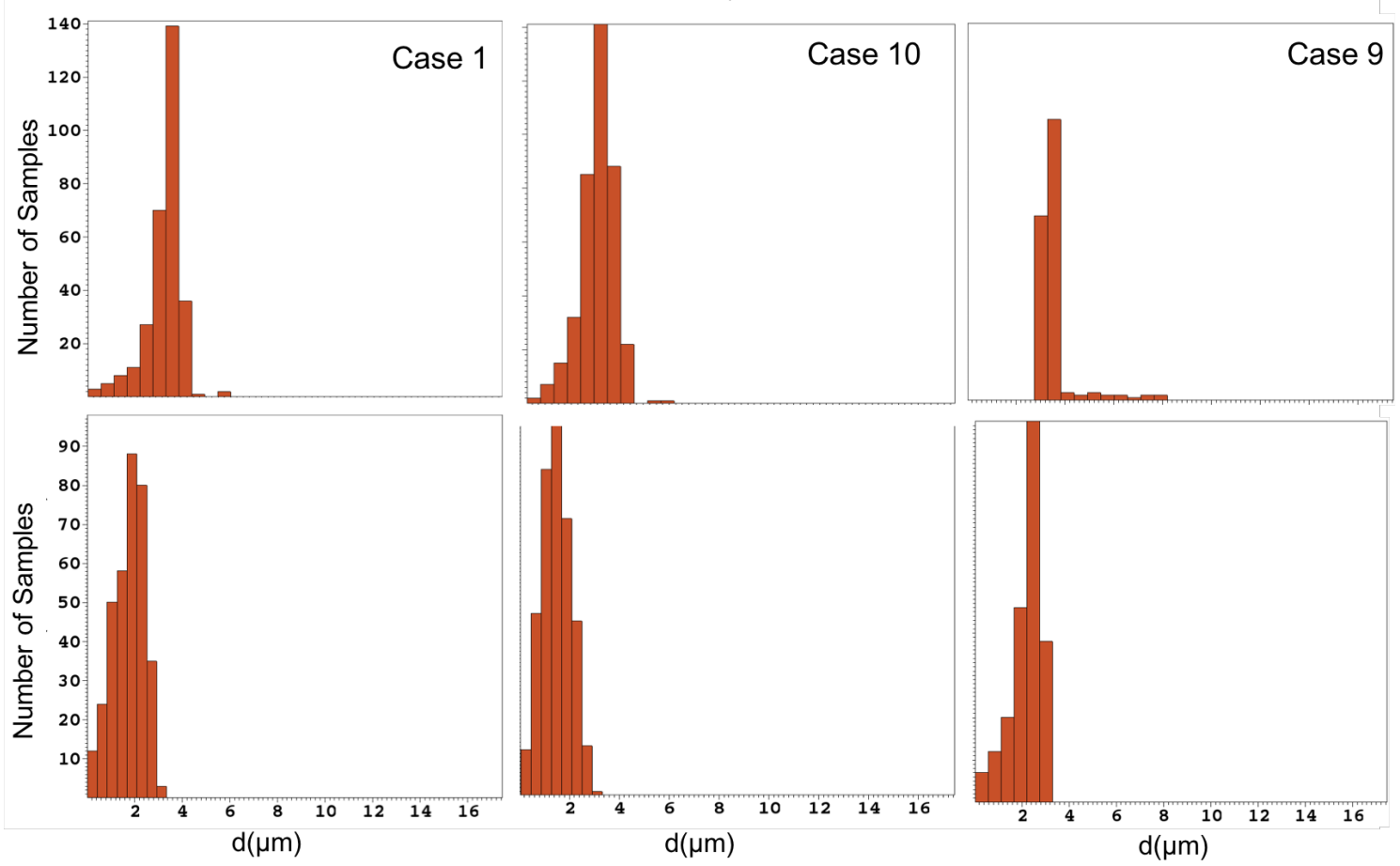

Figure 8. Number of droplets vs droplet sizes for two axial locations. Top raw is at $x=0.003 \mathrm{~m}$ (near-nozzle region) ant bottom row at $x=0.09 \mathrm{~m}$ (close to the tip of the spray). In all cases the PPS is 20 million and the samples were taken for $t=0.6 \mathrm{~ms}$.

\section{References}

[1] U. Azimov, N. Kawahara, and K. Tsuboi E. Tomita. Journal of Thermal Science and Technology, 5:238-251, 2010.

[2] A.G. Bailey, W. Balachandran, and T.J. Williams. Journal of Aerosol Science, 14(1):39 - 46, 1983.

[3] A. Banaeizadeh, A. Afshari A., H. Schock H., and F. Jaberi. International Journal of Heat and Mass Transfer, 60:781 - 796, 2013.

[4] G.M. Bianchi and P. Pelloni. SAE Technical Paper, 031999.

[5] C. Crua, S. Shoba, M. Heikal, M. Gold, and C. Higham. SAE paper 2010-01-2247, 2010.

[6] C. Cyril, R. Morgan, M. Heikal, and M.R. Gold. Fuel, 157:140 - 150, 2015.

[7] V. Granet, O. Vermorel, C. Lacour, B. Enaux, V. Dugue, and T. Poinsot. Combustion and Flame, 159(4):1562 1575, 2012.

[8] C.W Hirt and B.D Nichols. Journal of Computational Physics, 39(1):201 - 225, 1981.

[9] C.A. Idicheria and L. M. Pickett. SAE Technical Paper, 042007.

[10] F.P. Kärrholm. Numerical modelling of diesel spray injection, turbulence interaction and combustion. PhD thesis, Mech Eng, Goteborg, 2008.

[11] Sandia Laboratories. Engine Combustion Network, 2017 (accessed April 2017).

[12] T. M. Nguyen, F. Proch, I. Wlokas, and A. M. Kempf. Flow, Turbulence and Combustion, 97(1):191-230, 2016.

[13] F. Nicoud and F. Ducros. Flow, Turbulence and Combustion, 62(3):183-200, 1999.

[14] L.M. Pickett, J. Manin, C. L. Genzale, D.L. Siebers, M.P.B. Musculus, and C.A. Idicheria. SAE Int. J. Engines, 4:764-799, 042011.

[15] S. Quan, M. Dai, E. Pomraning, P.K. Senecal, K. Richards, S. Som, S. Skeen, J. Manin, and L. M. Pickett. SAE Int. J. Engines, 7:1054-1060, 042014.

[16] R.D. Reitz. Atomisation Spray Technology, 3:309-337, 1987.

[17] R. Scarcelli, K. Richards, E. Pomraning, P. K. Senecal, T. Wallner, and J. Sevik. SAE Technical Paper, 04 2016.

[18] M. Schmitt, C. E. Frouzakis, A. G. Tomboulides, Y. M. Wright, and K. Boulouchos. Proceedings of the Combustion Institute, 35(3):3069 - 3077, 2015.

[19] S.N. Soid and Z. Zainal. Energy, 36(2):724-741, 2011.

[20] S. Som, G. D'Errico, D. Longman, and T. Lucchini. SAE paper 2012-01-1263, apr 2012.

[21] S. Som, P.K. Senecal, and E. Pomraning. Comparison of rans and les turbulence models against constant volume diesel experiments. In ILASS, pages 1-10, 2012.

[22] Q. Xue, S. Som, P. K. Senecal, and E. Pomraning. Atomization and Sprays, 23(10):925-955, 2013.

[23] S. Som Y. Pei, B. Hu. J. Energy Resour. Technol., 138(4):032205-032205-10, 2016.

[24] A. Yoshizawa and K. Horiuti. Journal of the Physics Society Japan, 54(8):2834-2839, 1985. 


\title{
Evidence of vortex driven primary breakup in high pressure fuel injection
}

\author{
Junmei Shi*1, Pablo Aguado Lopez ${ }^{1}$, Eduardo Gomez Santos ${ }^{1}$, Noureddine \\ Guerrassi ${ }^{1}$, Gavin Dober ${ }^{1}$, Wolfgang Bauer ${ }^{2}$, Ming-Chia Lai ${ }^{3}$, Jin Wang ${ }^{4}$ \\ ${ }^{1}$ Delphi Automotive Systems, Bascharage, Luxembourg \\ ${ }^{2}$ ANSYS Germany GmbH, Otterfing, Germany \\ ${ }^{3}$ Wayne State University, USA \\ 4Argonne National Laboratory, USA
}

*Corresponding author: Junmei.shi@delphi.com

\begin{abstract}
This paper is to present a detailed case study on how the nozzle flow dynamics influences the primary breakup in the spray formation process of diesel injection. The investigation was based on a 3-hole real-application nozzle with highly tapered injection holes using a URANS-LES (Large Eddy Simulation) hybrid approach in combination with the coupled Volume of Fluid (VOF) and Level Set method. High resolution LES was applied to simultaneously resolve the multi-scale nozzle flow dynamics downstream of the needle seat and the primary breakup process in the near-nozzle spray. Phase Contrast X-ray imaging (PCX) was applied to characterize the liquid-gas interfaces in the near-nozzle spray for validation purposes. The results provide detailed information on how the vortex shedding and vortex interactions in the injection hole drives the jet deformation, ligament and droplet formation in the primary breakup process.
\end{abstract}

\section{Keywords}

Primary breakup, Fuel injection, Vortex dynamics, LES, Phase Contrast X-ray imaging

\section{Introduction}

Clean internal combustion engine technology improvement requires the capability to control and optimise the fuelgas mixing, ignition, and combustion process. However, how to transfer the individual engine requirements on the spray to a specific nozzle design still remains a challenging engineering task. One blocking point is the lack of detailed understanding on the fundamental physics of the primary breakup process. This process involves highly complex multi-phase and multi-scale fluid dynamics phenomena, including turbulence, cavitation and their interaction. A significant number of investigations have been dedicated to the cavitation phenomenon over the last 30 years. As for turbulence, the scales and dynamics of the vortex structures in the nozzle flow need to be understood. Two experimental investigations have reported vortex phenomena in injection nozzles. One is the cavitation visualisation of (1) in a real-size VCO nozzle. The vapour distribution in the injection holes indicated the occurrence of strong swirling vortex structures and vortex shedding. Though the investigation was focused on the in-nozzle flow, the authors proposed that the vortex shedding can impact the jet breakup downstream of the injection hole exit. Another is the string cavitation characterization in a scale-up nozzle (2), which demonstrated that string cavitation is caused by large-scale vortex strings in the sac and injection holes and has a correlation with the fluctuation of the spray dispersion angle. Nevertheless, the vortex structures are expected to be much more complex and have richer scales in real applications due to much higher velocity gradients. It is almost impossible to make detailed experimental characterization of field turbulence and vortex dynamics inside a realsize nozzle due to the small dimensions and high speed of the problem. CFD simulation is advantageous over measurement techniques to gain insight into the nozzle flow dynamics and vortex structures and their impact on the spray as shown in (3), (4). In order to resolve the involved multi scale and dynamic phenomena, ScaleResolved Simulation approaches (SRS), such as LES, are needed.

For the primary breakup diagnostic, several effective visualization techniques have been developed in the current century. It is worth mentioning the high resolution PCX imaging developed at Argonne National Lab (5), and the recent application of Transmitted Light Microscopy to the near-nozzle spray visualization (6). Both tools are useful for the characterization of the liquid-gas interface in the primary breakup process having different strengths. From the simulation point of view, interface tracking techniques like the Level-set method have been successfully applied to resolve the liquid-gas interface in the ligament and droplet formation process (7) (8). In order to obtain 
detailed information on how the fluid dynamic instabilities in the nozzle flow trigger ligament and droplet formation and how the nozzle geometry influences those processes and consequently the spray structure, techniques allowing for simultaneous diagnostic of the nozzle flow and the near-nozzle spray are needed. Considering the limitation of measurement techniques for the characterization of field turbulence in a real-size fuel injection nozzle, Scale-Resolved Simulation is a more feasible tool for this purpose. The main issue for simulation is how to deal with the cavitation phenomenon using an interface tracking technique, which naturally requires applying Direct Numerical Simulation (DNS) and is still beyond the capability of most available CFD codes and computational power. An alternative is to treat cavitation by using the Volume of Fluid (VOF) approach, which is a naturally conservative method tracking the volume fraction of a particular phase in each cell rather than the interface itself, being effective for the in-nozzle flow analysis but at the expense of having an excessive numerical diffusion for the jet breakup prediction. This approach might be useful for predicting the liquid jet fragmentation and fuel distribution in the breakup process, but might not be able to provide details for the droplet formation process.

Considering the strength and limitations of both measurement and simulation techniques, the authors have adopted a correlation based approach for years to work out understanding on how nozzle design and operating conditions influence on the spray behaviour. This approach involved the application of simulation for the nozzle flow and measurement techniques for the near-nozzle spray characterization and identifying links between both (4), (9), (10), (11). These successful studies have given the authors confidence in the simulation tools (ANSYS CFX and Fluent) for the nozzle flow diagnostic. In this work we present a detailed case study on the primary breakup of Diesel fuel jet injected from a so-called High Performance atomization (HP) hole nozzle (12). The HP hole uses very high hole taper (Kfactor $=($ Dout-Din $) / 10[\mu \mathrm{m}]=5$, see Figure 1$)$ to increase the hydraulic efficiency and the spray momentum rate. The target is to make a direct investigation on how the nozzle flow dynamic impacts the primary breakup and to reveal the flow dynamic processes in detail. Since the high hole taper prevents the occurrence of cavitation, the coupled VOF-Level Set LES method (13) can be applied simultaneously to resolve the nozzle flow and the liquid jet primary breakup. In order to ensure the numerical quality, the influence of grid resolution on the simulation results has been carefully analysed. Phase Contrast $X$ ray imaging $(P C X)$ was applied to visualize the liquid-gas interface structures for the near-nozzle spray to support the simulation analysis. The experimental investigation was carried out for injection pressures from 400bar to 2000 bar using a spray chamber at atmospheric pressure. The simulation case study was carried out for the injection pressure of 800 bar.

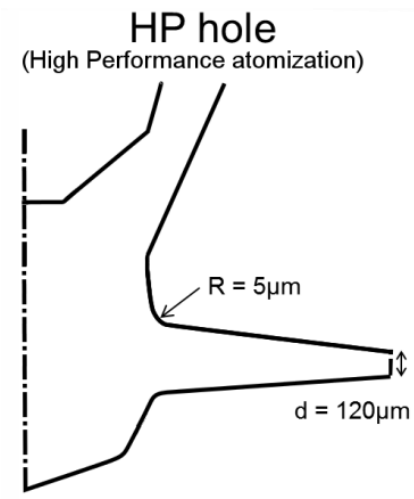

Figure 1. Schematic illustration of injection nozzle geometry

\section{Simulation setup}

The simulation was performed with the software ANSYS Fluent 16.2 adopting a hybrid URANS-LES approach and using a 120-degree sector nozzle model. The computational domain was divided into two subdomains as shown in Figure 2. The sub-domain upstream of the seat sealing was solved with URANS using a tetrahedral mesh and the sub-domain downstream of the seat sealing, including the near-nozzle spray region, with LES using high-quality hexahedral cells. A careful best practice study was carried out to ensure the quality of the simulation results. It was verified that the location of the URANS-LES interface is far enough from the region of interest to avoid causing a distortion of the results. At the URANS-LES interface, only the pressure and velocity fields were interpolated without introducing any artificial disturbance to the LES flow. Regarding the numerical setup, a VOF Level Set method (13) was applied to simultaneously resolve the nozzle flow and jet breakup. A standard k- $\omega$ SST turbulence model was used in the URANS domain and the Wall-Adapting Local Eddy (WALE) sub-grid scale (SGS) viscosity model (14) in the LES domain owing to its ability to correctly predict the near-wall eddy viscosity. 
A fully implicit, 2nd-order time-accurate scheme was used together with a 2nd-order scheme for spatial discretization. In order to ensure sufficient numerical resolution, the following criteria were used: local SGS eddy viscosity ratio below 1 , local CFL number below 2 , and $\mathrm{y}^{+}<1.0$.

Two different meshes were utilized in the discharge volume in order to assess the effects of mesh resolution. A coarser mesh (mesh 1) was defined with $\sim 15$ million cells and an average cell size of $\sim 5.7 \mu \mathrm{m}$, and a finer mesh (mesh 2) with $\sim 31$ million cells and an average cell size of $\sim 3 \mu \mathrm{m}$ for the domain outside of the nozzle. The timestep for mesh 1 was $\Delta t=5 \times 10^{-9} \mathrm{~s}$ and for mesh 2 was $\Delta t=2.5 \times 10^{-9} \mathrm{~s}$ to ensure numerical stability. Each simulation was first run with URANS for $100 \mu$ s and then switched to LES for $50 \mu$ s for initialization purpose to ensure proper development of LES flow. After initialization, the simulation ran further for $100 \mu$ so provide sufficient data for statistical sampling and analysis.

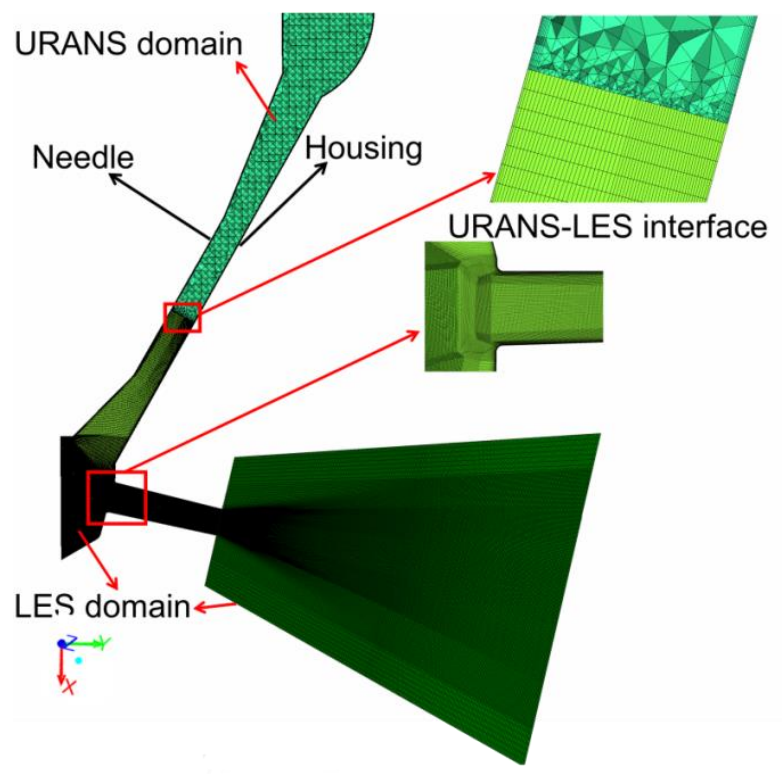

Figure 2: Computational domain decomposition and Hybrid LES mesh for the nozzle tip.

\section{Experimental setup}

The PCX imaging was performed at the XOR 7ID beamline in the Advanced Photon Source (APS) to visualize the near-nozzle spray. The third generation synchrotron $x$-ray beam can produce ultra-short x-ray pulses and weak interaction with the object materials. With these features and the high transmittance of the $x$-ray in dense materials, it becomes possible to capture the instantaneous liquid-gas interface structures in the near-nozzle spray. After passing thought the spray, the x-ray beam forms a phase-contrasted image on a scintillator crystal CCD camera. The field of view of the camera was $1.734 \mathrm{~mm} \times 1.310 \mathrm{~mm}$ with a pixel resolution of $0.66 \mu \mathrm{m} / \mathrm{pixel}$ when a 20 times objective lens was used. The imaging frequency is $50 \mathrm{kHz}$, or $20 \mu \mathrm{s}$ per image. A detailed description of the experimental setup can be found in (9).

\section{Results and discussion}

\section{Liquid-gas interface structures: mesh resolution effect}

Similar liquid core interface structures and jet breakup patterns were predicted on both meshes, but the higher resolution of mesh 2 captured much more small droplets. Ideally, a proper post-processing tool for scale separation and calculation should be developed and used to assess the minimum droplet size which can be captured by each mesh. As this tool was not available a concept of interface diffusion thickness is used instead. This value is calculated using the Level Set function $(\varphi)$ and liquid volume fraction, and is introduced to help estimate the mesh resolution effect on the diffusion of the liquid-gas interface structures. The interface diffusion is a result of mesh resolution and diffusion caused by numerical schemes. Therefore, this method is also useful for a coarse estimation of the smallest droplet resolution as will be explained. The process of the interface smearing due to numerical diffusion is schematically plotted in Figure 3 a) for a single droplet. The Level set function is exactly zero at the interface and has a value equal to the distance to the interface (with a positive or negative sign 
according to the convention for each phase) for any other points (13). Initially, the droplet is bounded by a sharp interface $\varphi=0$ where the liquid volume fraction jumps from $\alpha_{l}=0$ outside the droplet to $\alpha_{l}=1$ inside the droplet. After a number of time steps the interface smears, leading to a smooth volume fraction variation across the interface. In this new state, the region where $\alpha_{l}=1$ is restricted to some cells in the center of the droplet, bounded by a surface where $\varphi=0$. Taking $\alpha_{l}=0.01$ as the threshold of the interface diffusion, the thickness of the diffused interface $d_{1 \%}$ can be estimated using the distance between the volume fraction iso-surface, $\alpha_{l}=0.01$ and the iso-surface $\varphi=0$. Under a symmetric interface diffusion assumption the diameter $d$ of a spherical droplet is $2 r \leq d \leq 2 r+2 d_{1 \%}$, where $r$ is the distance from the droplet core center to the undiffused droplet core interface $\varphi=0$. The smallest spherical droplets near the intact liquid core $\left(\alpha_{l}=1\right)$ are only distributed over one or a few cells, leading to $r \sim 0$. In such cases, $d \sim 2 d_{1 \%}$ is a reasonable estimate of the actual characteristic droplet size.

An instantaneous near-nozzle spray visualisation is presented in Figure 3 b) for both meshes using the instantaneous liquid volume fraction iso-surfaces 0.01 colored by $d_{1 \%}$. The colour scale threshold chosen here is aimed at separating the smallest droplets $d \sim 2 d_{1 \%}<10 \mu \mathrm{m}$ from the larger droplets and the liquid core interface. It is observed that Mesh 1 only captured a few droplets with $d \sim 2 d_{1 \%}<10 \mu \mathrm{m}$ very close to the liquid core during initiation of breakup. Further downstream, only larger droplets and ligaments are resolved with increasing diffusion due to mesh coarsening. In contrast, mesh 2 is fine enough to capture droplets smaller than $10 \mu m$ over the entire primary breakup region modelled. In addition, it is noted that the diffusion thickness is low for the liquid core obtained based on both meshes. Therefore, it is safe to say that the mesh resolution is unlikely to influence the numerical observations of the liquid jet deformation and ligament formation phenomena in the primary breakup process.

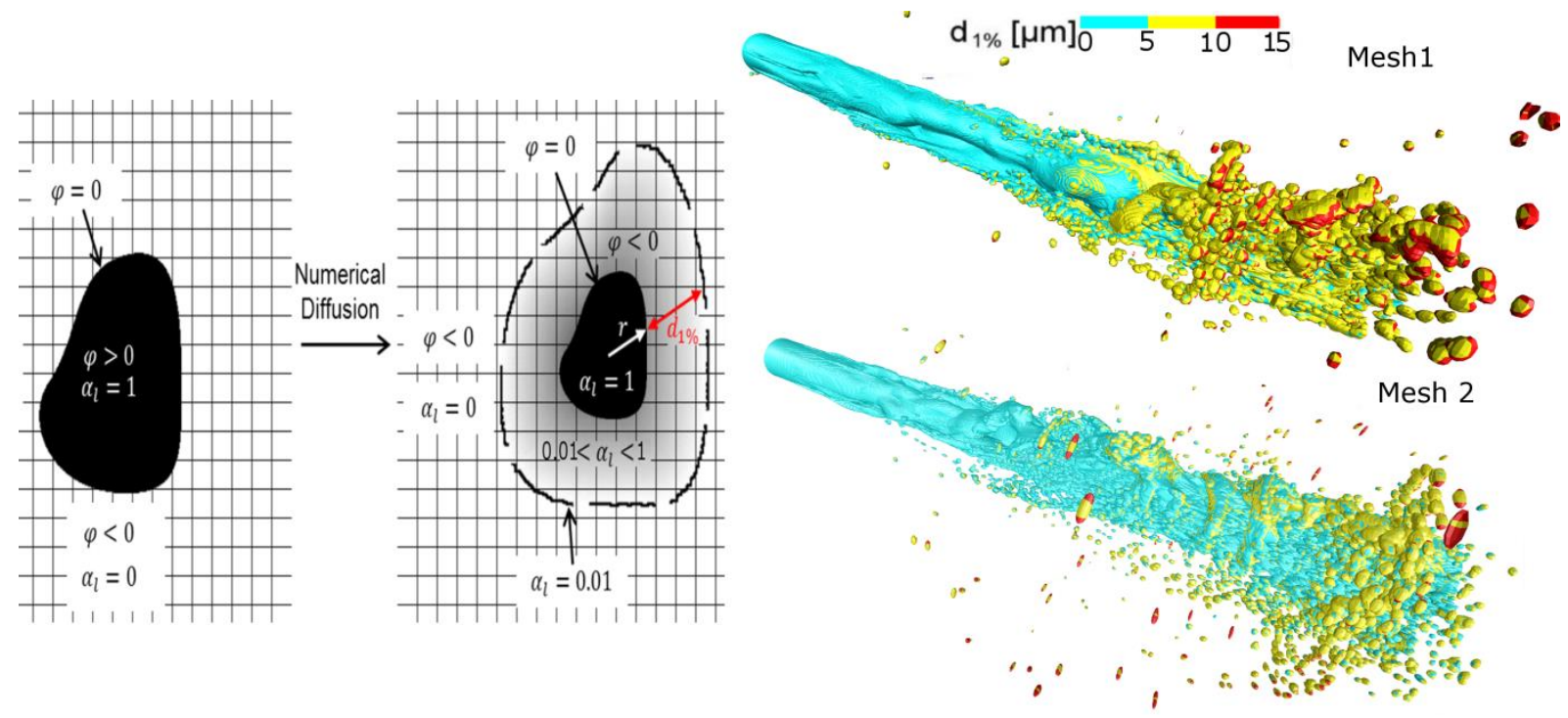

a) Sketch of interface smearing due to numerical diffusion. Sharp interface (left) and diffused interface right.

b) Estimation of droplet size based on $d_{1 \%}$. Liquid volume fraction iso-surface 0.01 colored by $d_{1 \%}$.

Figure 3: Assessment on mesh resolution effect on small droplets

\section{Liquid-gas interface structures: LES solution vs. PCX spray images}

Comparison for the liquid-gas interface structures between LES solution and PCX spray images should be based on a statistical approach. This is hindered by the differences in the physical time durations and the time resolution between simulation and measurement. Spray imaging was performed for an injection duration of $1 \mathrm{~ms}$ at full needle lift and at a time interval of $20 \mu \mathrm{s}$ per image (50 images in total). The physical time in simulation was $100 \mu \mathrm{s}$ for both meshes. Flow visualisation images were stored every $5 \times 10^{-8} \mathrm{~s}$. Obviously, the time resolution and the image number in the measurements were not sufficient in the sense of a rigorous statistical analysis of the nearnozzle spray structures, while the physical time duration in the simulation is too short as it is limited by the available computational resources. Under these limitations, effort was made to identify similarities between the instantaneous spray morphologies captured by PCX imaging and by the simulations. 
The LES results for mesh 1 and mesh 2 are treated as independent time series, considering the fact that the initialization has an influence on the flow development. The mesh 1 solution of the near nozzle spray was found to have a close correlation with 7 out of the 50 PCX spray images recorded over the open needle operation time interval. Sample results are presented in Figure 4, where the LES near-nozzle sprays are represented by using the iso-surface of 0.1 liquid volume fraction. The predicted undisturbed liquid core before the initiation of jet breakup is obviously longer than the measurement, but the simulation is able to capture some features observed in the PCX spray images. For example, a linear streak crossing the liquid core very close to the nozzle exit can be recognized from both the predicted and measured spray (Figure 4, left). In particular, a close similarity between both is observed at the lower side of the spray, where the wavy structures begin to break up into smaller structures and droplets. Figure 4 right shows another example. The wavy structures with higher local breakup intensity on the upper side of the spray as recorded by the PCX imaging are also captured in the simulation. In addition, a "horizontal boundary" between the continuous un-atomized liquid core (lower part of the spray) and the upper spray regions with small structures can be well noted both from the PCX spray image and the LES solution.

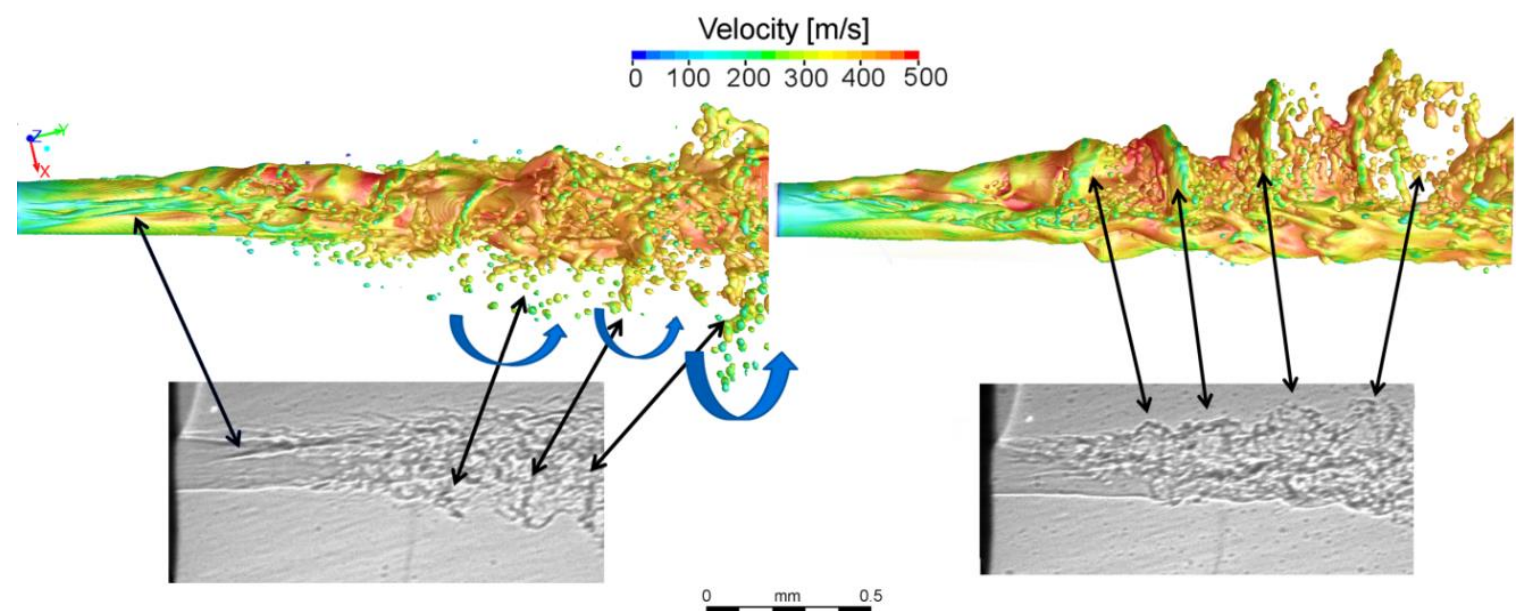

Figure 4: Instantaneous LES liquid volume fraction iso-surfaces (value=0.1) on mesh 1 (top) vs. PCX images (bottom)

The LES solution on mesh 2 was found to produce similar spray features with 8 out of the 50 PCX images. Figure 5 shows for the correlations between some example mesh 2 results and PCX spray images. The LES spray in the left image shows a braid-like (helical) structure appearing on the upper side of the very initial jet, which can also be noticed from the PCX spray image. This type of structures are caused by vortex shedding and rotating string vortices occurring in the nozzle flow and will be discussed in detail in the next section. In addition, similar vertical streaks are observed both in simulation and measurement. The LES spray on the right shows a close similarity in terms of breakup patterns and spray shape to the corresponding mesh 1 result shown on the right of Figure 4, As with the mesh 1 result, the wavy structures on the upper side of the spray correlate well with the PCX image.

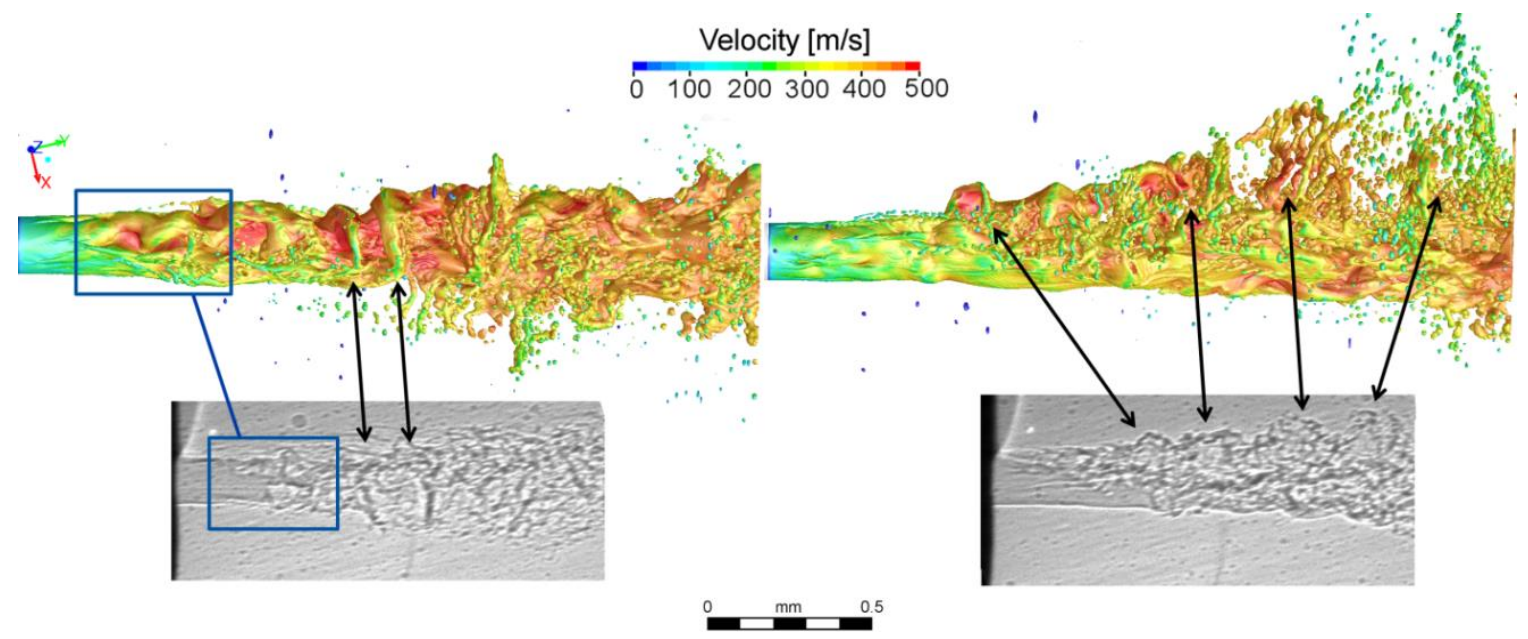

Figure 5: Instantaneous LES liquid volume fraction iso-surfaces (value=0.1) for mesh 2 (top), PCX images (bottom). 
These results show a clear impact of the mesh resolution on the small structures and droplets in the near-nozzle spray. However, both meshes have captured some breakup patterns and morphological features of the spray, which can be recognized in PCX spray images, are therefore valid. Since the mesh 2 results were only very recently obtained in this work, the understanding on the physics of the primary breakup process reported below is mainly derived from the mesh 1 solution.

\section{Vortex driven primary breakup process}

Figure 6 illustrates the correlation between the predicted vortex structures and near-nozzle spray structures obtained on mesh 1 together with a similar PCX spray image. Two type of vortices can be observed in the nozzle flow. Small-scale vortices and vortex shedding occur at the upper lip of injection hole inlet as the flow turns into the hole. At the same time, large-scale string vortices are generated in the bulk flow of weak shear due to flow recirculation in the sac and flow acceleration into the hole. The results indicate that the upper-lip vortex shedding and the interaction between the string vortices and the shed vortices in the nozzle are the triggering mechanism of ligament formation in the primary breakup region. A vortex shedding event produces low momentum vortices and a pulsation in the local flow. The shed vortices interact with the string vortices in the injection hole. As they exit the injection hole they transfer their local instability and their pulsating momentum into the liquid jet, causing its deformation and the development of ligaments.

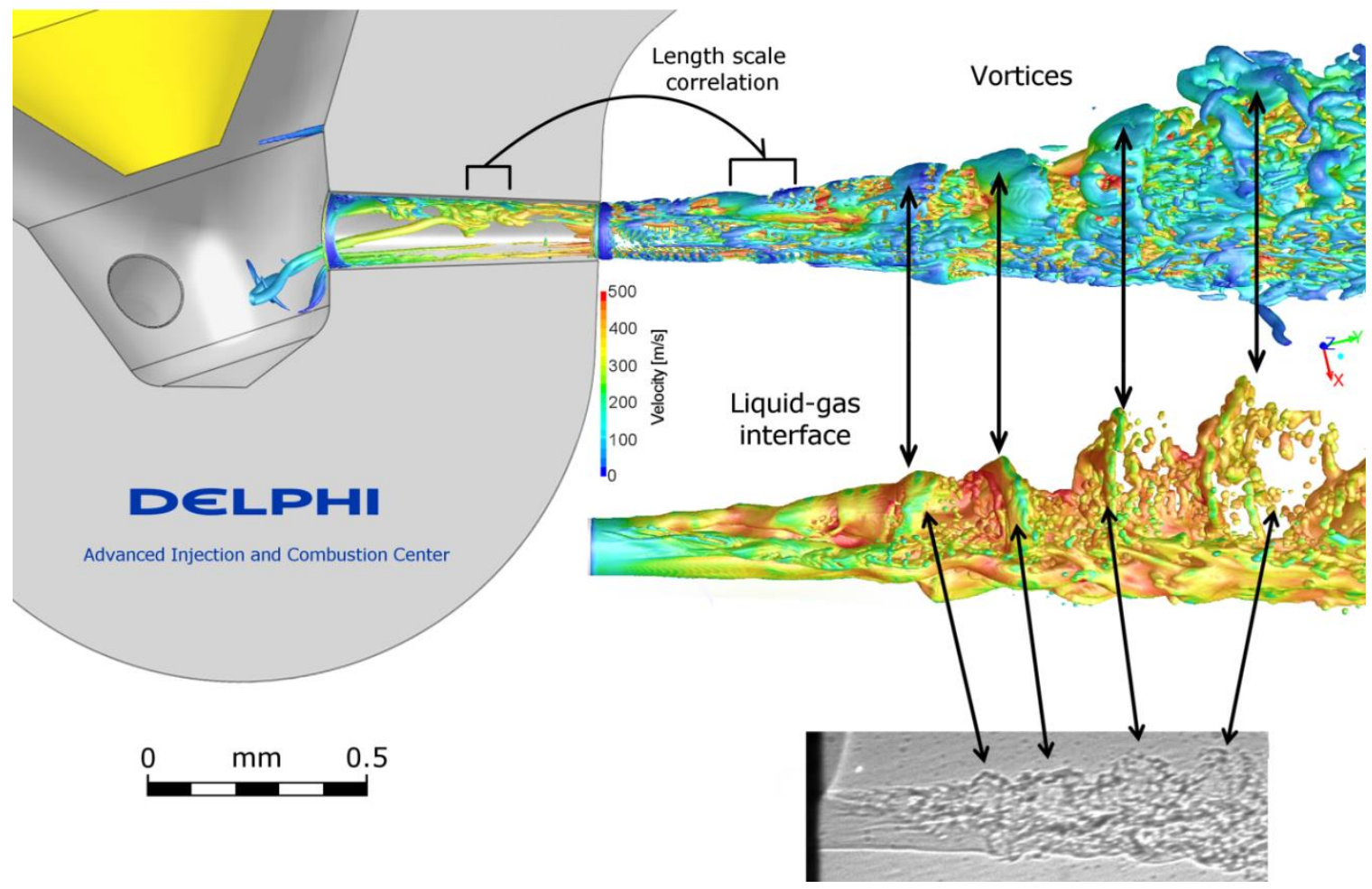

Figure 6: Correlation between vortex shedding in the nozzle and jet breakup: vortex structure $\left(Q=1 \mathrm{e} 13\left[\mathrm{~s}^{-2}\right]\right)$, predicted spray morphology(liquid volume fraction $90 \%$ ), and PCX image (bottom).

This vortex-driven ligament formation and breakup process is illustrated in Figure 7 using a time sequence of instantaneous results for the vortex flow and near-nozzle spray. At a certain time instant $t_{0}$, the string vortices move upwards and interact with the shed vortices close to the hole exit, creating a local flow instability and upward momentum. This pulsating momentum is transported into the near-nozzle flow after a shed vortex leaves the injection hole exit. At $t_{0}+0.45 \mu \mathrm{s}$ the upward moving shed vortex triggers the wavy surface vortex enhanced by the interaction with the surrounding gas. This vortex causes deformation of the liquid jet on the upper side, leading to wavy liquid-gas interface structures. At $t_{0}+2 \mu \mathrm{s}$ the surface vortex gets further developed due to airliquid interaction and the liquid surface deformation continues to grow causing the roll-up of the liquid-gas interface and ligament formation. At $t_{0}+5 \mu \mathrm{s}$ the surface vortices lose their momentum, get separated from the high speed bulk flow and decompose into smaller vortices causing atomization of ligaments and formation of droplets. A detailed video showing this process is available in (15). 


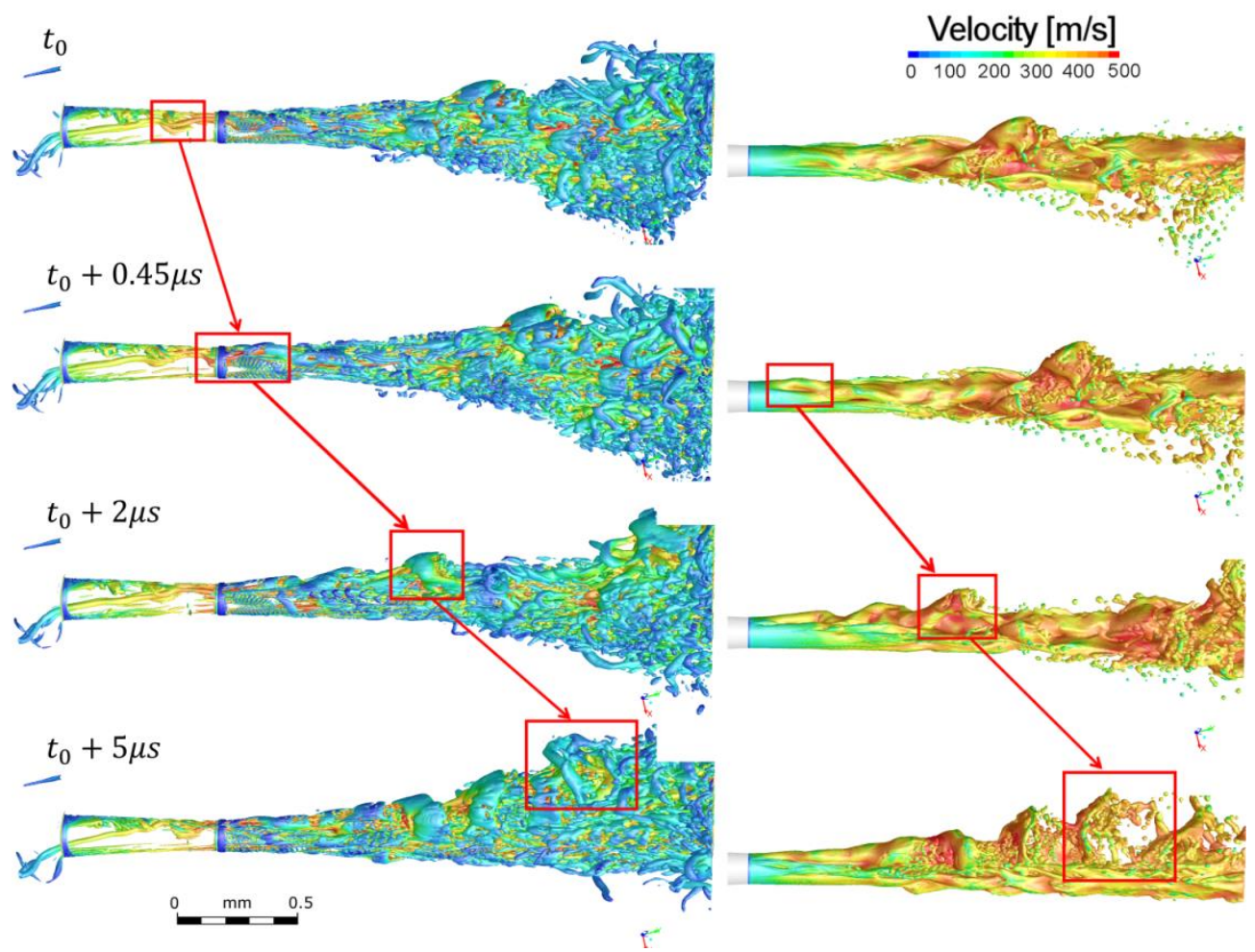

Figure 7: The process of vortex driven ligament formation and jet breakup. Vortex structure $Q=1 \mathrm{e} 13\left[\mathrm{~s}^{-2}\right]$ (left), Liquid-gas interface of the spray using liquid volume fraction (right).

In addition, large scale vortex strings are high energy containing structures. Their morphology, location and motion direction have an important impact on the primary breakup behaviour as is shown in Figure 8 using selected instantaneous results. In case (a), the string vortices move upwards. This triggers liquid core deformation and ligament formation further downstream on the upper side of the jet. The string vortices are pushed downwards by the strong shed vortices at the hole exit in case (b), leading to liquid-core deformation on the lower side. In case (c), the string vortices show an unstable ' $\mathrm{S}$ '-shape motion, causing an earlier jet breakup both on the upper and lower sides. In contrast, relatively undisturbed flow with all large vortices well aligned with the injection hole axis is predicted close to the injection hole exit in (d). Under this situation, weak perturbations on the liquid jet and thus weak jet breakup is observed.
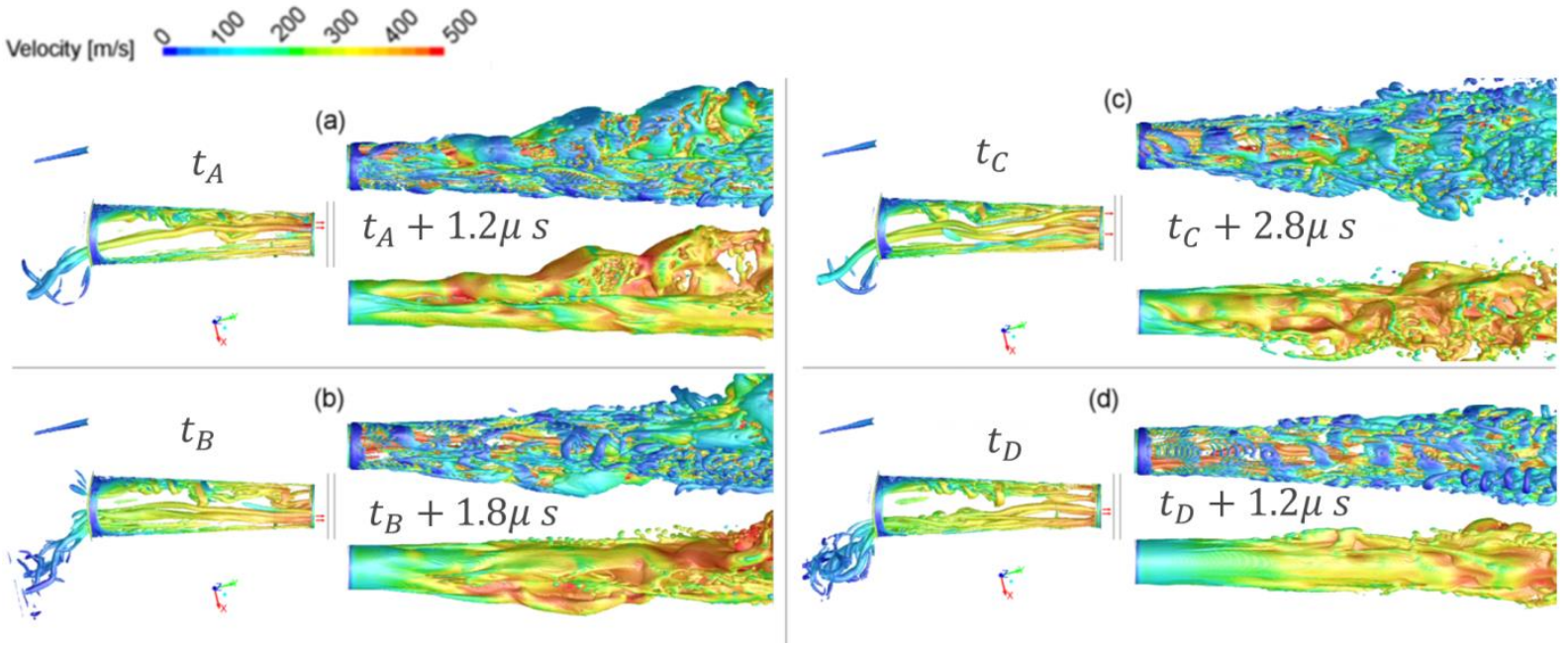

Figure 8: Link between vortex dynamics and liquid-gas interface for different instants. For each case: Vortex structures $\mathrm{Q}=1 \mathrm{e} 13\left[\mathrm{~s}^{-2}\right]$ (left and top-right), Liquid-gas interface of the spray using liquid volume fraction 0.1 (right). Red arrows at the outlet denote the flow direction 


\section{Conclusions}

A detailed case study was carried out on the primary breakup process in high pressure fuel injection based on a production diesel nozzle design. A coupled VOF-Level Set LES simulation methodology was applied to simultaneously resolve the multi-scale flow dynamics in the nozzle and the jet primary breakup process after the hole exit. Phase Contrast X-ray imaging was applied to characterize the liquid-gas interface in the near-nozzle spray. The simulation successfully reproduced many structures of the spray captured by the PCX imaging. It was observed from the simulations that, as the flow is deflected into the nozzle hole, it triggers vortex shedding events, producing high speed, energetic vortex structures and local flow instabilities. These structures continue to develop into the liquid jet and initiate the deformation and ligament formation processes within the primary spray breakup. Additionally, vortex ejection from the nozzle causes small surface vortices at the liquid-gas interface that interact with the surrounding gas and ultimately lead to droplet formation. These results provide evidence for a vortex driven atomization mechanism. With this understanding, fuel injector nozzle designs can be optimized by control and optimization of the vortices.

\section{Acknowledgements}

The FNR funding to the PhD project of Pablo Aguado Lopez (number 588638), the European Union Horizon-2020 Research and Innovation Programme funding to Eduardo Gomez Santos (No 675676), the ANSYS research license grant and the CPU hours grant from Gompute to this work are gratefully acknowledged. The $x$-ray investigation was carried out using the Advanced Photon Source of the Argonne National Laboratory of US Department of Energy. The kind assistance of the scientist of beam line \#32, Dr. Kamel Fezzaa, is highly appreciated by the authors.

\section{References}

1. Cavitation in an asymmetric transparent real-size VCO nozzle. Chaves, Humberto and Schuhbauer, Ingo. 2006. Spray'06, Workshop uber Sprays, Erfassung von Spruhvorgangen und Techniken der Fluidzerstaeubung. 2. Vortex flow and cavitation in diesel injector nozzles. Andriotis A, Gavaises M and Arcoumanis C. 2008, J Fluid Mech, Vol. 10, pp. 195-215.

3. URANS and SAS analysis of flow dynamics in a GDI nozzle. Shi, J.-M.; Wenzlawski, K.; Helie J; Nuglisch, H.; Cousin, J. Brno : s.n., 2010. ILASS-Europe.

4. Using LES and $x$-ray imaging to understand the influence of injection hole geometry on Diesel spray formation. Shi, J.; Aguado Lopez, P.; Dober, G.; Guerrassi, N.; Bauer, B.; Lai, M.-C. Valencia : THIESEL 2016 Conference on Thermo-and Fluid Dynamic Processes in Direct Injection Engines, 2016.

5. X-ray vision of fuel sprays. Wang, Jin. 2, 2005, J Synchrotron Rad., Vol. 12, pp. 197-207.

6. Transmitted light microscopy for visualizing the turbulent primary breakup of a microscale liquid jet. Reddemann, Manuel A. ; Mathieu, Florian ; Kneer, Reinhold. 11, 2013, Experiments of Fluids, Vol. 54, pp. 1-10.

7. On simulating Primary Atomization Using the Refined Level set grid Method. Hermann M. 4, 2011, Atomisation and Sprays, Vol. 21, pp. 283-301.

8. Simulation of liquid jet primary breakup: Dynamics of ligament and droplet formation. Shinjo J. 7, 2010, International Journal of Multiphase Flow, Vol. 36, pp. 513-532.

9. Correlating the Nozzle Flow to Spray and Primary Breakup using Visualization and Multi-phase Simulation. Lai M-C; Wang F; Xie X; Shi J; Dober G; Guerrassi N; Meslem Y. Rouen : s.n., 2014. SIA.

10. Fuel property and fuel temperature effects on internal nozzle flow, atomization and cyclic spray fluctuations of a direct injection spark ignition-injector. Zigan, L, et al., et al. 6, 2013, International Journal of Engine Research, Vol. 14, pp. 543-556.

11. Characterization of Internal flow and Spray of Multihole DI Gasoline Spray using X-ray Imaging and CFD. Ming-Chia Lai, Yi Zheng, Mark Shost, Xingbin Xie, Atsushi Matsumoto, Jin Wang, Xusheng Zhang, Seoksu Moon, Jian Gao, Kamel Fezzaa, Lars Zigan, Ingo Schmitz, Michael Wensing, Alfred Leipertz. 2011. JSAE. Vols. JSAE 20119225, SAE 2011-01-1881.

12. Guerrassi, N.; Doradoux, L.; Garsi, C.; Lesieur, C. Injection nozzle. US 20110215177 A1. 2011.

13. A coupled level set and volume-of-fluid method for computing $3 d$ and axisymmetric incompressible two-phase flows. Sussman, M.; Puckett, E. G. 2, 2000, J. Computational Physics, Vol. 162, pp. 301-337.

14. Subgrid-scale stress modelling based on the square of the velocity gradient tensor flow. Nicoud, F.; Ducros., F. 3, 1999, Turbulence and combustion, Vol. 62, pp. 183-200.

\begin{tabular}{l} 
15. Delphi Automotive Systems Luxembourg. ANSYS Hall of FAME 2017. [Online] \\
\hline
\end{tabular} http://www.ansys.com/other/hall-of-fame. 


\title{
CFD simulations of the diesel jet primary atomization from a multihole injector
}

\author{
C. A. Chasos*1 \\ ${ }^{1}$ Mechanical Engineering Department, Frederick University, Nicosia, Cyprus \\ *Corresponding author: eng.cca@fit.ac.cy
}

\begin{abstract}
High pressure multi-hole diesel injectors are currently used in direct-injection common-rail diesel engines for the improvement of fuel injection and air/fuel mixing, and the overall engine performance. The resulting spray injection characteristics are dictated by the injector geometry and the injection conditions, as well as the ambient conditions into which the liquid is injected. The main objective of the present study was to design a high pressure multi-hole diesel injector and model the two-phase flow using the volume of fluid (VOF) method, in order to predict the initial liquid jet characteristics for various injection conditions. A computer aided design (CAD) software was employed for the design of the three-dimensional geometry of the assembly of the injector and the constant volume chamber into which the liquid jet emerges. A typical six-hole diesel injector geometry was modelled and the holes were symmetrically located around the periphery of the injector tip. The injector nozzle diameter and length were $0.2 \mathrm{~mm}$ and $1 \mathrm{~mm}$, respectively, resulting in a ratio of nozzle orifice length over nozzle diameter $L / D=$ 5. The commercial computational fluid dynamics (CFD) code STAR-CD was used for the generation of the computational mesh and for transient simulations with an Eulerian approach incorporating the VOF model for the two-phase flow and the Rayleigh model for the cavitation phenomenon. Three test cases for increasing injection pressure of diesel injection from the high pressure multi-hole diesel injector into high pressure and high temperature chamber conditions were investigated. From the injector simulations of the test cases, the nozzle exit velocity components were determined, along with the emerging liquid jet breakup length at the nozzle exit. Furthermore, the spray angle was estimated by the average radial displacement of the liquid jet and air mixture at the vicinity of the nozzle exit. The breakup length of the liquid jet and the spray cone angle which were determined from the simulations, were compared with the breakup length and cone angle estimated by empirical equations. From the simulations, it was found that cavitation takes place at the nozzle inlet for all the cases, and affects the fuel and air interaction at the upper area of the spray jet. Furthermore, the spray jet breakup length increases with elapsed time, and when the injection pressure increases both the breakup length and the spray cone angle increase.
\end{abstract}

\section{Keywords}

Diesel injector, VOF, atomization.

\section{Introduction}

The main objective of the present work was to characterize the flow phenomena at the exit of the nozzle of a multi-hole Diesel injector. It was of main interest to examine the behaviour of the emerging two-phase flow spray jet with emphasis in the primary spray jet atomization. The objectives included, first the setup of the CFD model for a typical three-dimensional valve-covered orifice (VCO) sac-less six-hole diesel injector for carrying out the analysis of the initial spray characteristics, namely the liquid breakup length and the spray angle at the nozzle exit. The second objective was the calculation of the primary atomization characteristics with empirical equations and to compare the empirical data with the simulations. The injector which was used had sharp nozzle entry, a nozzle diameter equal to $0.2 \mathrm{~mm}$ and a ratio of nozzle orifice length over nozzle diameter $L / D=5$.

Previous experimental $[1,10,14,15]$ and computational $[1,6,9,11,13]$ studies investigated the internal and external flow of diesel injectors. In some experiments, large scale transparent injectors were used [8], [12], and it was found that cavitation phenomena are present. Experimental studies [10,14] revealed that the emerging liquid jet is affected by both cavitation and the interaction with the surrounding gas flow. Various methodologies were adopted for injector flow simulations, including the VOF method [8] and the large eddy simulation (LES) framework [5]. The simulations from previous studies revealed that strong vortex structures were generated around the liquid jet penetrating in the gas phase and these were the results of velocity relaxation inside the liquid [8]. However, as it was reported in [8], the problem of jet disintegration is complex and not well understood.

The effect of injection pressure on the initial spray atomization characteristics predicted from simulations and comparisons with pertinent data estimated from empirical models, have not been found by the author of the 
present work in published work. Thus, it is required to quantify the effects of the injection pressure on the injected spray jet, as well as compare the primary spray atomization characteristics from simulations with data from empirical equations. In the present work, the adopted CFD methodology is described first. Then, the results for three test cases at low, medium and high injection pressure of the diesel injector into high pressure and high temperature chamber conditions are presented. Finally, conclusions and recommendations are provided.

\section{Methodology}

The CFD methodology along with the simulations setup are described first, followed by the illustration of the empirical equations which were used for the calculation of the liquid jet breakup length and the spray jet angle.

For the CFD simulations, the CFD code STAR-CD [3] was used. The Eulerian modelling methodology employing the VOF method was utilized, which included the mass, momentum and energy conservation equations for the two phases. The interface-capturing method in the VOF method was employed, by computing the convective terms in the volume fraction equations using the High-Resolution Interface-Capturing (HRIC) model [3]. For the two-phase flow modelling, the pressure in the two phases was assumed to be the same. A constant value of surface tension was used, with which the normal force due to the surface tension is treated using the continuum surface force (CSF) model [3], while the tangential force is not accounted. The CSF model provides a source term in the momentum conservation equation. The turbulence was modelled with the k- $\varepsilon$ high Reynolds number RNG model [17], and the boundary layer was handled with the standard wall functions. The MARS [3] differencing scheme was used for the discretization of the conservation equations. Transient simulations were carried out, and the SIMPLE algorithm [3] was employed for the numerical solution of the problem.

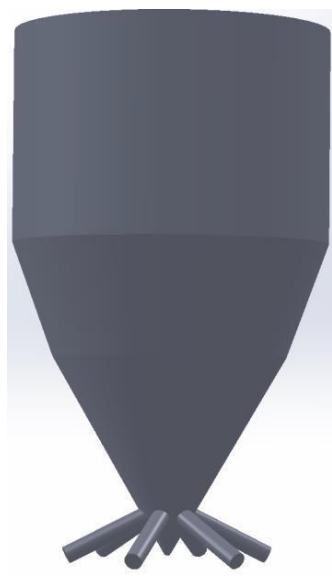

Figure 1. Three-dimensional injector geometry design.

A VCO sac-less six-hole diesel injector and a constant volume chamber were assembled for the computational mesh generation. The injector design was based on typical injector geometries found from literature $([10,11,14$, 15]). The three-dimensional injector which was designed with a CAD software [4] is shown in Figure 1 . The injector design was for a six-hole diesel injector whose nose holes were symmetrically located around the periphery of the injector tip as shown in Figure 1. The nozzle entry was designed with sharp edge at the body of the injector. The injector nozzle diameter and length were $0.2 \mathrm{~mm}$ and $1 \mathrm{~mm}$, respectively, resulting in a ratio of nozzle orifice length over nozzle diameter $L / D=5$. The designed constant volume chamber had length $5 \mathrm{~mm}$ which corresponds to $25 \mathrm{D}$ distance downstream the nozzle, and a square cross-sectional area with side width 1 $\mathrm{mm}$. The constant volume chamber was assembled at the tip of the nozzle exit, and the centreline of the nozzle and the symmetry axis of the chamber coincided. The computational mesh was generated with the automatic mesh generation tool of STAR-CD [3], where prism type cells computational were used. The resulting mesh was composed of around 850000 cells and it is presented in Figure 2. The cell size ranged from 5 to $10 \mu \mathrm{m}$ within the injector and the cell size varied between 10 to $20 \mu \mathrm{m}$ in the constant volume chamber. Figure 2 includes the boundary conditions which were imposed for the simulation setup. Inlet boundary condition was defined at the entry of the injector on the top, shown in dark yellow color in Figure 2. Symmetry plane boundary conditions were imposed on the symmetry sides of the one-sixth segment of the injector, which are presented in violet color in Figure 2. No-slip wall boundary condition was imposed on the four sides of the chamber, which is indicated with orange color in Figure 2. Wall boundary condition was set at the remaining surfaces, including the injector shell, the nozzle and the back plane of the chamber where the nozzle tip was assembled. Pressure boundary condition 
was defined at the chamber front plane. At the pressure boundary, the pressure and temperature were set equal to 42 bar and $1000 \mathrm{~K}$, respectively, which resemble diesel engine conditions during compression stroke.

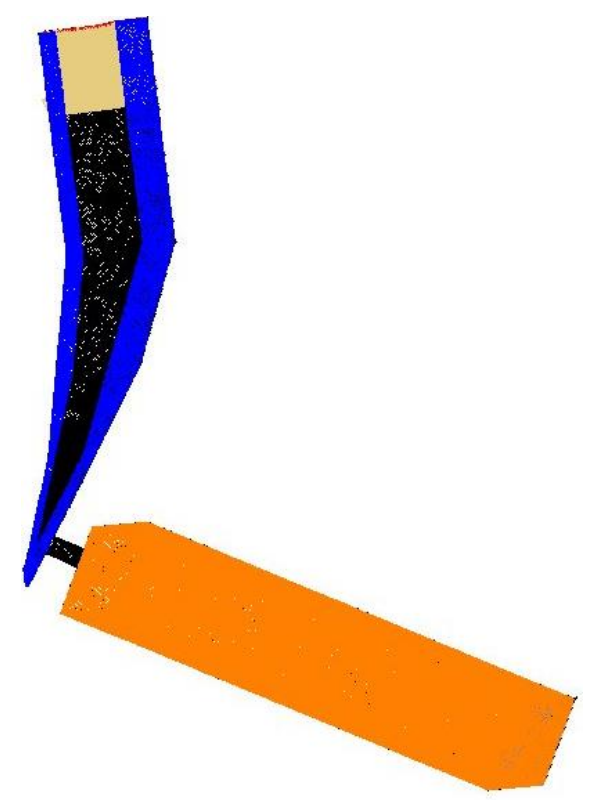

Figure 2. Computational mesh and boundary conditions.

At the inlet boundary, the volume fraction of the fuel was set equal to 1 and uniform inlet velocities for the three test cases were defined. The code uses the inlet velocity and calculates the injection pressure at the inlet. For the simulations, $n$-heptane and air were utilized. The properties of $n$-heptane liquid, $n$-heptane vapour and air from the database of STAR-CD [3] were employed, and are contained in Table 1.

Table 1. Physical properties of the fuel and air.

\begin{tabular}{c|ccc}
\hline \hline Property & Liquid & Vapour & Air \\
& n-heptane & n-heptane & \\
\hline \hline Density $\left(\mathrm{Kg} / \mathrm{m}^{3}\right)$ & 678.3 & 2.48639 & 1.18415 \\
Molecular viscosity $(\mathrm{Kg} / \mathrm{ms})$ & $3.9207310^{-4}$ & $1.0137710^{-5}$ & $1.85510^{-5}$ \\
Surface tension coefficient $(\mathrm{N} / \mathrm{m})$ & 0.0727 & &
\end{tabular}

For the initial conditions of the transient simulations, stagnant air was set in the computational domain. The simulations for the three test cases were performed for an injection duration of $1 \mathrm{~ms}$. The computational time step size was constant and equal to $0.5 \mu \mathrm{s}$. The numerical processing of the simulations was performed on a sequential computer. The simulation results are presented and discussed in the next section.

In the present work, in the absence of experimental data for validation of the simulations, empirical equations were used in order to obtain data for comparison with the simulations. Empirical equations provide the primary atomization characteristics and are usually employed within the atomization modelling setup in diesel engine CFD simulations. From a literature survey, the empirical equations for the calculation of the breakup length and spray angle were adopted from [15] and [2], respectively. The liquid jet breakup length was estimated by the following empirical equation (from [15]),

$$
L=0.39\left(2 \Delta p / \rho_{l}\right)^{1 / 2} t
$$

where $\Delta p$ is the pressure drop along the nozzle, $\rho_{l}$ is the liquid density and $t$ is the elapsed time after the start of injection. The cone angle for diesel jet spray in the atomisation region was calculated from the empirical equation of Arai [2] by,

$$
\theta=0.017\left(\frac{D^{2} \rho_{a} \Delta p}{\mu_{\alpha}^{2}}\right)^{0.25}
$$

where $D$ is the nozzle diameter, $\rho_{a}$ is the density of air in the chamber and $\mu_{\alpha}$ is the molecular viscosity of air. 


\section{Results}

In the present section, the results and discussion from the simulations for the three test cases are presented. For the test cases, the injection pressure was calculated by the code at the inlet boundary. The velocity at the inlet boundary, the resulting injection pressure and the mass flow rate of fuel are included in Table 2. The three cases denoted, low, medium and high injection pressure correspond to modern common rail system injection pressure. As it can be seen in Table 2, the injection pressure was 1124, 1669 and 2245 bar.

Table 1. Test cases conditions.

\begin{tabular}{c|cccc}
\hline \hline Test case & Description & $\begin{array}{c}\text { Inlet velocity } \\
(\mathrm{m} / \mathrm{s})\end{array}$ & $\begin{array}{c}P_{\text {inj }} \\
(\mathrm{bar})\end{array}$ & $\begin{array}{c}\text { Fuel mass flow } \\
\text { rate }(\mathrm{Kg} / \mathrm{s})\end{array}$ \\
\hline \hline 1 & Low injection pressure & 20 & 1124 & 0.0422 \\
2 & Medium injection pressure & 24 & 1669 & 0.0507 \\
3 & High injection pressure & 28 & 2245 & 0.0592
\end{tabular}

First, the evolution of the VOF field from the simulations at the vertical symmetry section plane of the nozzle and the chamber are presented in Figure 3,4 and 5. Then, comparisons of the VOF field and the velocity field between the three cases at $0.25 \mathrm{~ms}$ after start of injection (ASOI) are presented. Finally, the breakup length and spray ject cone angle from the simulations are compared with the empirical data.

For the low injection pressure, Case 1, the evolution of the liquid fuel injection and the propagation of the emerging fuel jet are presented with the VOF flow field in Figure 3. From the simulations of Case 1, it was found that the fuel starts to emerge from the nozzle exit at $0.2 \mathrm{~ms}$ after the start of the simulation. As it can be seen in Figure 3, there is cavitation area which is created at the upper edge of the nozzle inlet. The axial penetration of the spray jet increases with elapsed time, and at $0.3 \mathrm{~ms}$ the spray reaches the chamber front plane. Also, the spanwise spreading of the two-phase spray jet increases with time. A spray jet with VOF higher than 0.5 , indicated with green colour, is present at the nozzle exit, which increases with time. For this case the spray jet slightly bends at an angle of around $10^{\circ}$, which is the effect of the induced gas recirculation at the upper area of the spray jet.

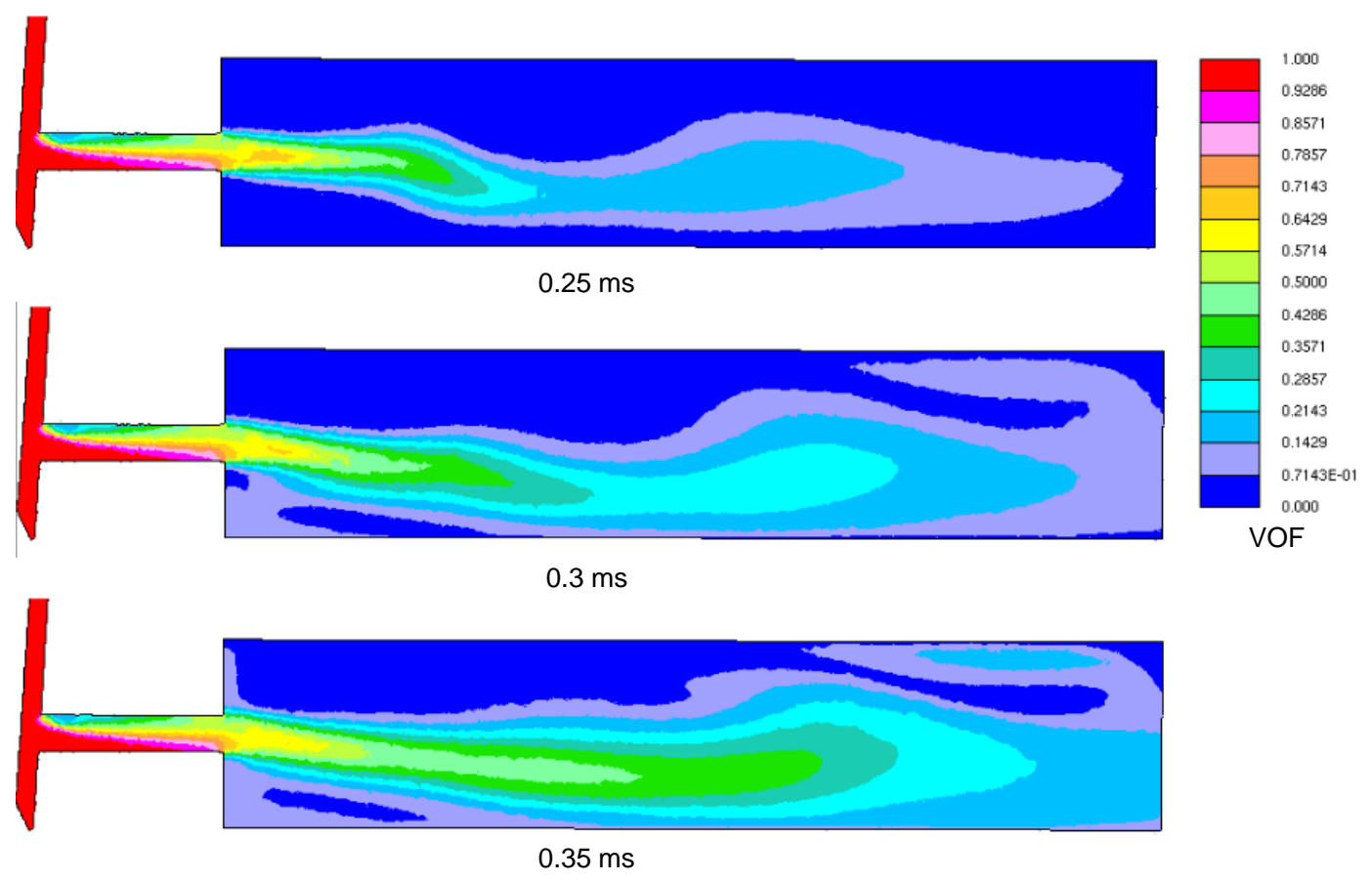

Figure 3. Evolution of the VOF field for Case 1 at $0.2,0.25$ and $0.3 \mathrm{~ms}$ ASOI.

The emerging spray jet, for the medium injection pressure case is presented in Figure 4, at time $0.2,0.25$ and $0.25 \mathrm{ASOI}$. The simulations of the medium pressure case revealed that by increasing the injection pressure for 1124 to 1669 , then the required time for the fuel to emerge from the nozzle exit is $0.18 \mathrm{~ms}$. The cavitation area is present at all times after start of injection and the spray jet recirculates at the upper area of the emerging jet at a 
downstream distance of seven nozzle diameters. The spray jet core with VOF higher than 0.5 , is almost symmetrical and has a length of around five nozzle diameters.

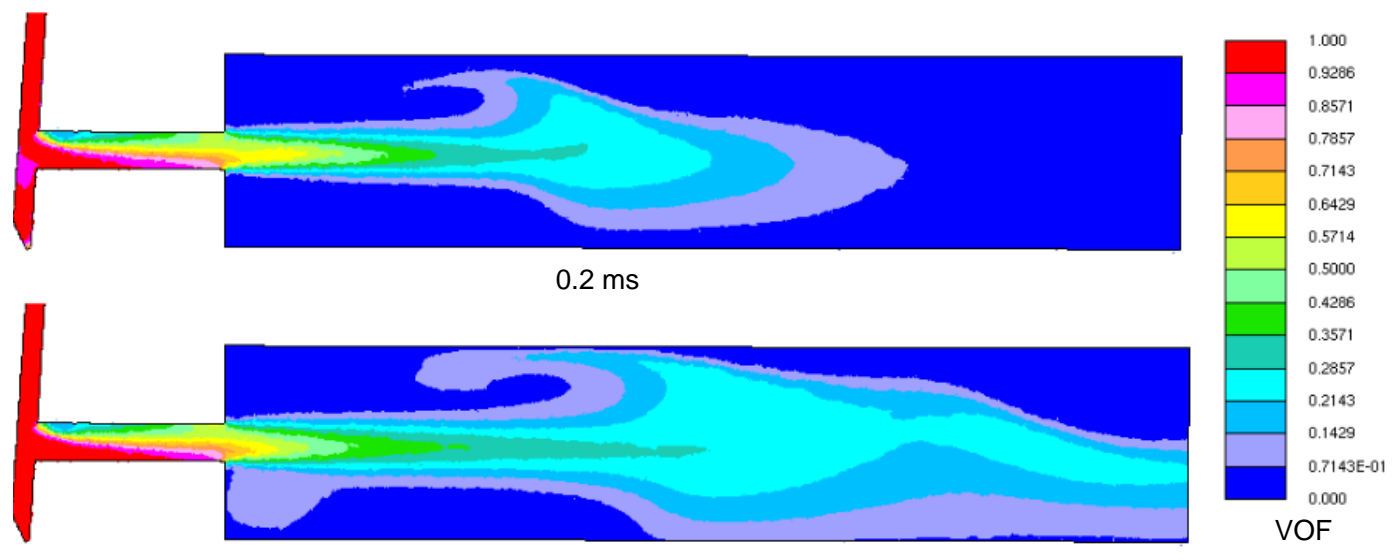

$0.25 \mathrm{~ms}$

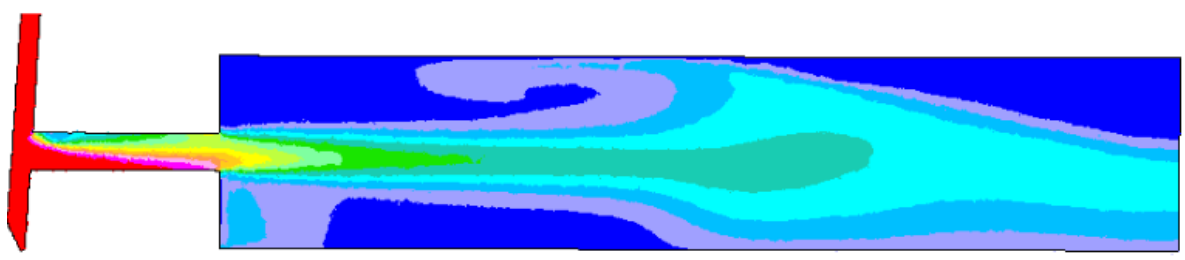

$0.3 \mathrm{~ms}$

Figure 4. Evolution of the VOF field for Case 2 at $0.2,0.25$ and $0.3 \mathrm{~ms}$ ASOI.

The results for Case 3 are included in Figure 5, at $0.15,0.2$ and $0.25 \mathrm{~ms} \mathrm{ASOI.} \mathrm{For} \mathrm{the} \mathrm{high} \mathrm{injection} \mathrm{pressure}$ case, the time needed by the fuel to exit the nozzle was $0.14 \mathrm{~ms}$, and this is lower than the time needed for the low and medium injection pressure cases. Figure 4 shows that the vortex structure at the upper area of the spray becomes stronger and that a small amount of fuel accumulates near the wall at the vicinity of the nozzle. Also, it can be observed that the fuel air mixture travels downstream and accumulates towards the front plane of the chamber at $0.25 \mathrm{~ms}$. The VOF and velocity fields for the three are further discussed and compared below.
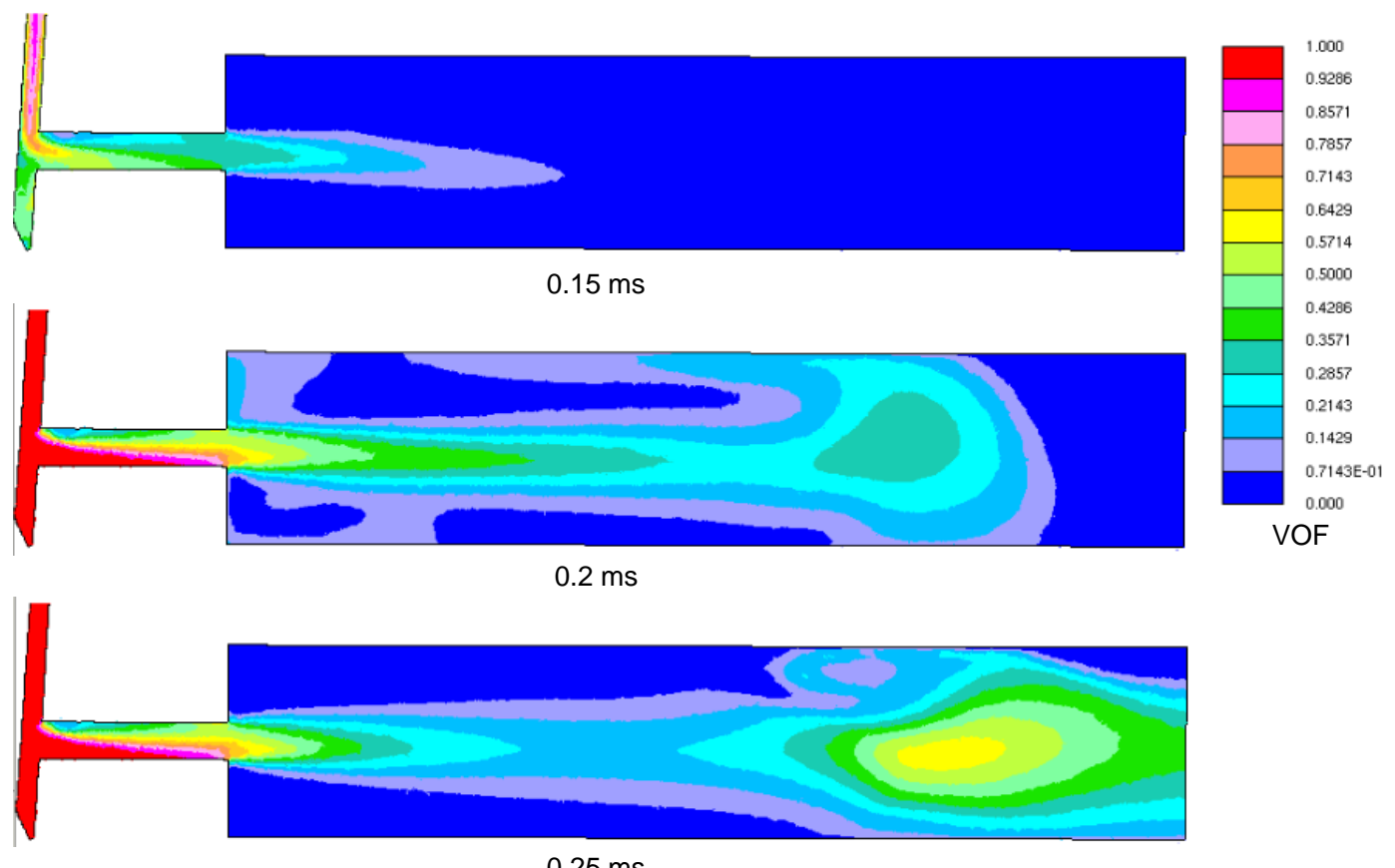

Figure 5. Evolution of the VOF field for Case 3 at $0.15,0.2$ and $0.25 \mathrm{~ms}$ ASOI. 


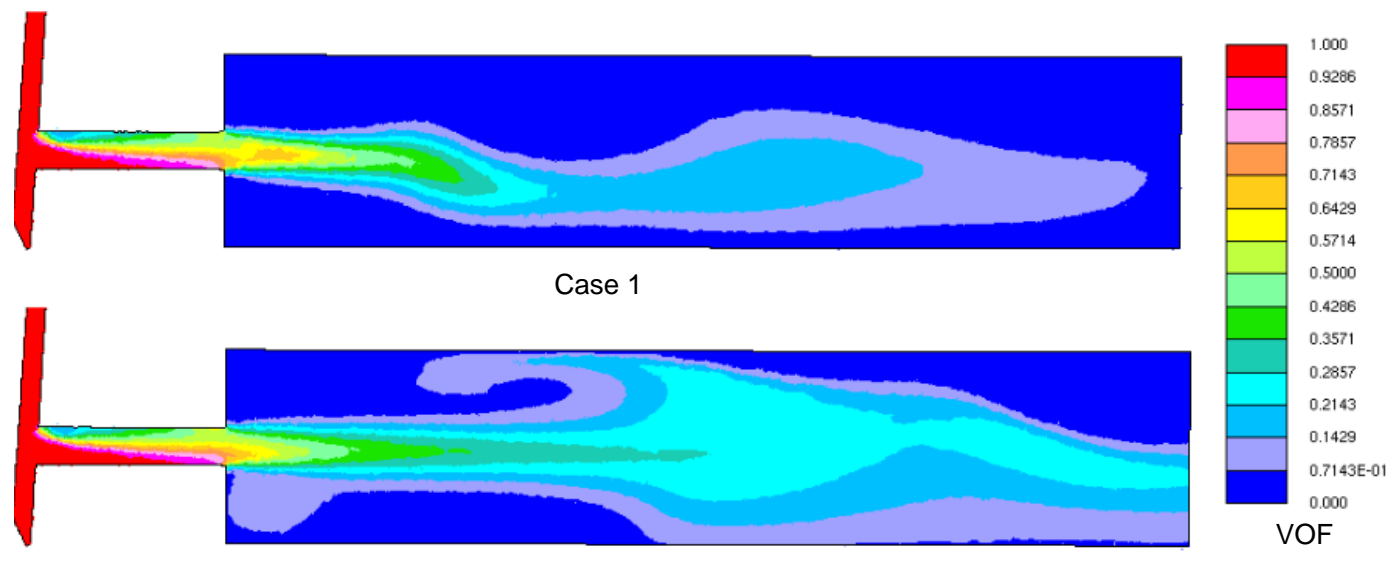

Case 2

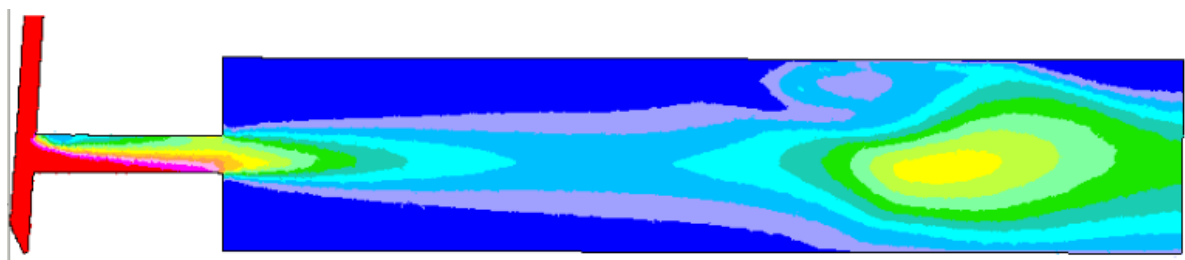

Case 3

Figure 6. Comparison of the VOF field of Case 1,2 and 3 at $0.25 \mathrm{~ms}$ ASOI.

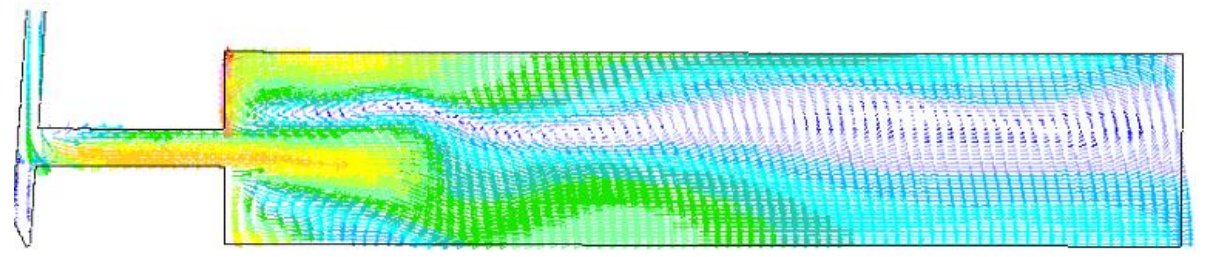

Case 1

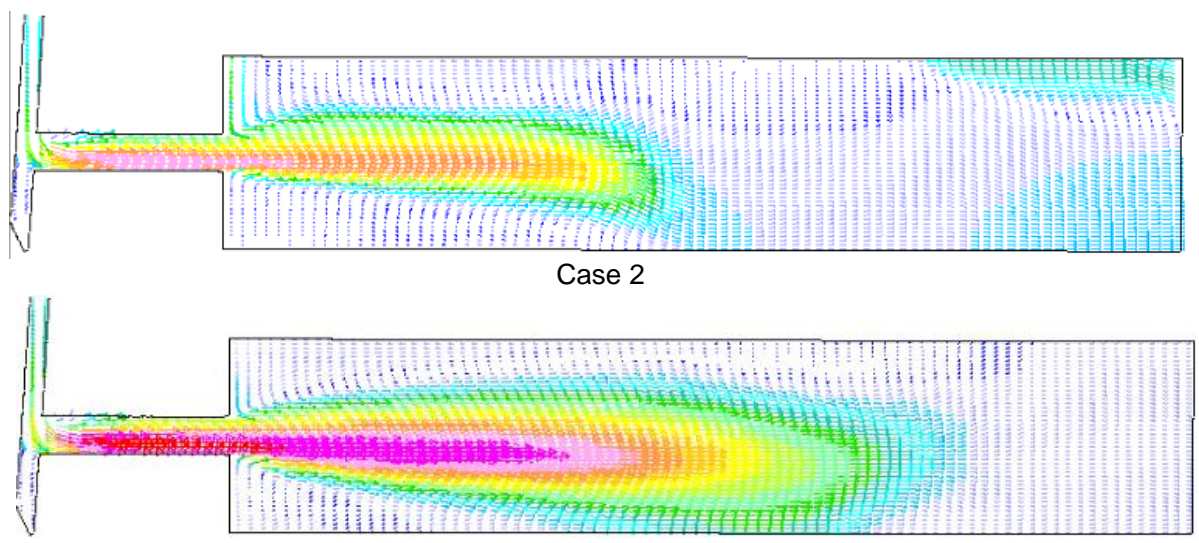

Case 3

Figure 7. Comparison of the velocity field of Case 1,2 and 3 at $0.25 \mathrm{~ms}$ ASOI.

Figure 6 compares the predicted VOF field at $0.25 \mathrm{ASOI}$. It can be observed that the spray jet for the low injection pressure case is narrower that the spray of the higher injection pressure cases. This observation reveals that the spray cone angle increases when the injection pressure increases. Regarding the spray jet, it can be seen that the penetration of the spray core with VOF greater than 0.5 increases when the injection pressure increases. However, for the high injection pressure cases there is a core at the vicinity of the nozzle and an accumulated spray jet towards the front plane of the chamber. Figure 7 illustrates the velocity fields for the three cases. The high velocity pattern is wider and longer for the maximum injection pressure case. The latter observation again reveals that the spray cone angle of the high pressure case is larger than the spray angle of the lower pressures. As it can be observed in Figure 7, for all the cases there is a recirculation at the nozzle entry which is the cavitation zone, and the flow has higher velocities at the lower area of the nozzle. By increasing the injection 
pressure, then the nozzle velocity increases. The increase of the injection velocity induces a recirculation zone at the upper area of the spray. The recirculation zone becomes stronger with increasing injection velocity and this can be also seen in Figure 6, where the spray recirculation zone occurs for the medium and high injection pressure cases. For the low injection pressure case, the injection velocity is not sufficiently high and the recirculation in upper area affects the spray which slightly bends as observed in Figure 6 and 7.

The estimated breakup length from the simulations is compared against the calculated breakup length from the empirical equation in Figure 4. The time of the start of injection for Case 1, 2, and 3 was adjusted with the values of $0.2,0.18$ and $0.14 \mathrm{~ms}$. This was done for the purpose of comparison, and it is considered as the delay time for the emergence of the liquid jet from the nozzle exit. Figure 8 compares the breakup length data estimated from the simulation against the calculated empirical data for each case. From the simulations, the breakup length was estimated at the distance from nozzle where the VOF value was equal and greater than 0.3. For both the simulation and the experimental data, the breakup length increases with time, as it can be observed in Figure 8 . Here, it is noted that the empirical expression does not account for the phenomena of evaporation, while the present simulation was carried out in a chamber with high pressure and temperature conditions and the evaporation was simulated. Thus during the early injection period, for each case, there is slight under prediction of the breakup length and this considered as a very good agreement. However, in Figure 8 it can be seen that for later times the breakup length is substantially underpredicted by the simulation, and this can be explained by the evaporation phenomena which are not considered in the empirical equation.

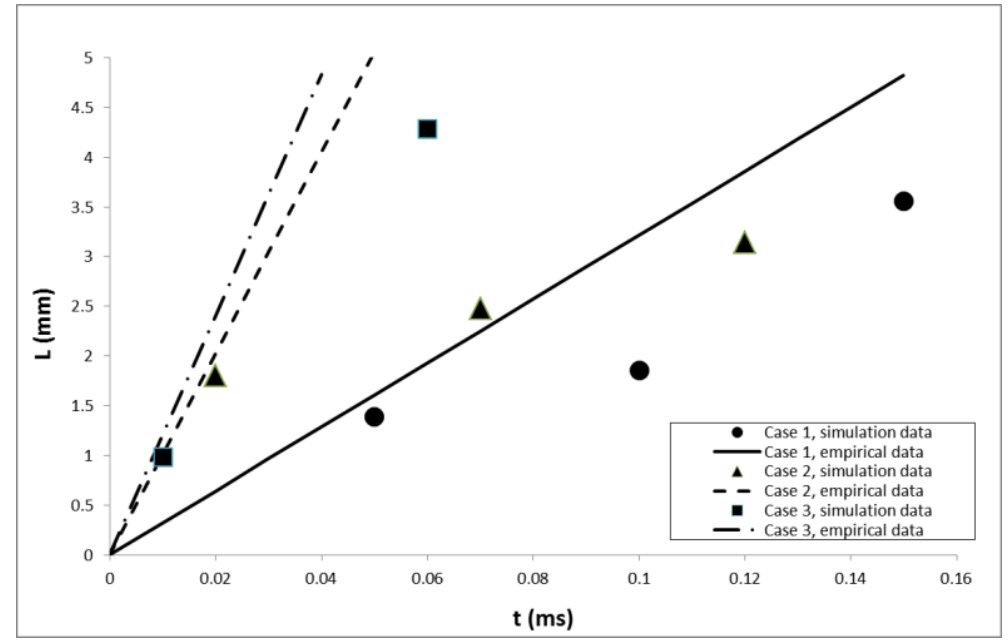

Figure 8. Comparison of the breakup length between simulation and empirical data with elapsed time after the start of injection into the chamber.

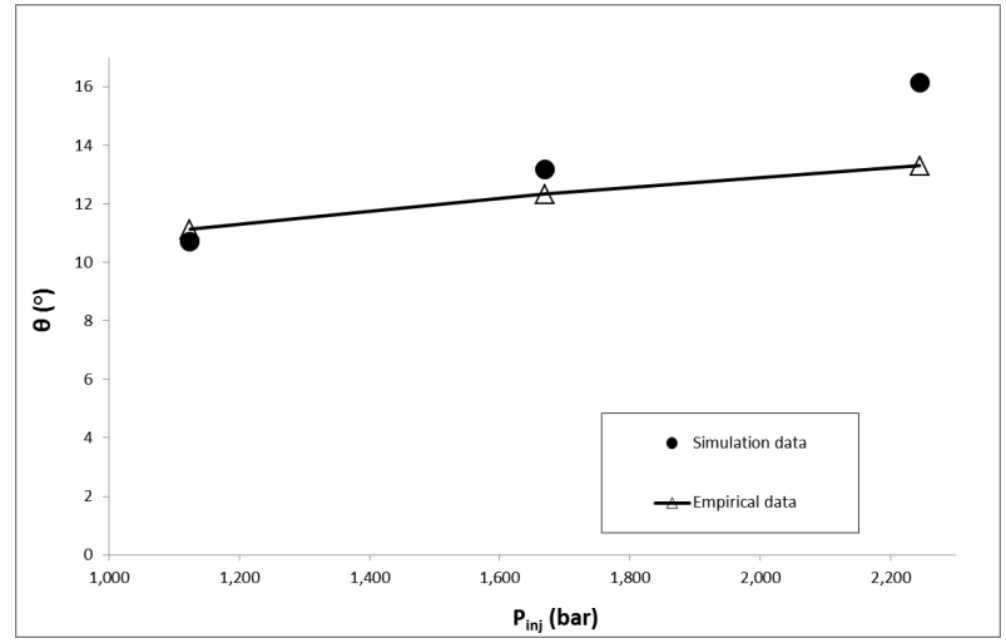

Figure 9. Comparison of the spray jet angle between simulation and empirical data for increasing injection pressure at $0.25 \mathrm{~ms}$ ASOI. 
The spray jet angle from the simulations was estimated at $0.25 \mathrm{~ms}$ ASOI for the three test cases. Tangents from the nozze exit upper and lower edges were drawn to the outer edge of the jet spray at five diameters distance from the nozzle exit, and the average angle for each case was found. The spray cone angle from the simulations for each test case is compared against the empirical data in Figure 9. As it can be seen in Figure 9, there is very good agreement on cone angle for the low injection pressure case, while for the medium and high injection pressure the cone angle is slightly overpredicted. It can be observed that the cone angle increases increases linearly when the injection pressure increases. The differences between the predictions and the empirical data can be explained by the spanwise spreading of the spray jet in the simulation and the interaction with the surrounding gas. However, in order to be able to draw firm conclusions about the overprediction of the cone angle at higher injection pressures, it will be required to carry out further simulation investigation and compare with experimental data. Furthermore, it will be required to assess available empirical data and the conditions under which the empirical expressions can be applied, since in the present work high injection pressures and evaporating conditions were simulated. In the following section, the main conclusions from the present work are described and recommendations for future work are provided.

\section{Conclusions and recommendations}

From the three test cases of increasing injection pressure into high pressure and temperature chamber conditions, it was found that cavitation takes place and affects the fuel and air interaction at the upper area of the spray jet. The predicted jet breakup length increases with elapsed time. When the injection pressure increases, then both the breakup length and the downstream penetration of the spray jet increase. The spray cone angle estimated at the vicinity of nozzle exit increases with increasing injection pressure. When the injection pressure doubled, then the cone angle increased by around 50\%. Comparisons with empirical data revealed that there is very good agreement on the breakup length size during the early stages of injection, for all the test cases. However, when time elapses from the start of injection then the predicted breakup length is underpredicted because of the evaporation phenomena, which are not accounted in the empirical expression.

In future work a bigger constant volume chamber should be used in order to examine the downstream behaviour of the atomized jet and the resulting downstream spray cone angle. It is recommended to use the predicted values of breakup length and spray cone angle from the present simulations in combination with the EulerianLagrangian framework for simulation of direct injection diesel engine sprays, which should be validated against experimental data in order to reach robust conclusions.

\section{Acknowledgements}

The provision of computer facilities by Frederick University is acknowledged.

\section{References}

[1] Andriotis, A, Gavaises, M. and Arcoumanis, C., 2008, J. Fluid Mech., 60, pp. 195-215.

[2] Arai, M., Sep. 2.-6. 2012, 12th Triennial International Conference on Liquid Atomization and Spray Systems.

[3] Computational Dynamics adapco Ltd., 2015, STAR-CD Methodology, Version 4.22. London, England.

[4] Dassault Systèmes SolidWorks Corporation, http://www.solidworks.com.

[5] de Villiers, E., Gosman, A. D. and Weller, H. G, 2004, SAE Technical Paper Series 2004-01-0100.

[6] Giannadakis, E., Gavaises, M. and Arcoumanis, C., 2008, J. Fluid Mech., 616, pp. 153-193.

[7] Heywood, J. B. 1987, "Internal Combustion Engine Fundamentals"

[8] Jameel, A., Bowen, P., and Yokoi, K., 8.-10. Sep., 2014, $26^{\text {th }}$ European Conference on Liquid Atomization and Spray Systems.

[9] Margot, X., Hoyas, S., Fajardo, P. and Patouna, S., 2010, Mathematical and Computer Modelling, 52, pp. 1143-1150.

[10] Oda, T., Ohnishi, K., Sumi, T. and Ohsawa, K., Sep. 2.-6. 2012, 12th Triennial International Conference on Liquid Atomization and Spray Systems.

[11] Payri, F., Payri, R., Salvador, F. J. and Martinez-Lopez, J., 2012, Computers \& Fluids, 58, pp.88-101.

[12] Payri, F., Salvador, F. J., Marti-Aldaravi, M. and Martinez-Lopez, J., 2012, Energy Conversion and Management, 54, pp.90-99.

[13] Salvador, F. J., Martinez-Lopez, J., Romero, J. V. and Rosello, M. D., 2013, Mathematical and Computer Modelling, 57, pp. 1656-1662.

[14] Sou, A., Pratama, R. H., Tomisaka, T. and Kibayashi, Y., 2.-6. 2012, $12^{\text {th }}$ Triennial International Conference on Liquid Atomization and Spray Systems.

[15] Tamaki, N., Nishikawa, K. and Fukamichi, S., 1.-4. Sep.2013, $25^{\text {th }}$ European Conference on Liquid Atomization and Spray Systems.

[16] Varde, K. S., 1985, The Canadian Journal of Chemical engineering 63

[17] Yakhot, V., and Orszag, S.A. 1986, J. Scientific Computing, 1, pp. 1-51. 


\title{
Large eddy simulations of atomisation and sprays: application to a high pressure multihole injector
}

\author{
Chausserie-Laprée Paul ${ }^{1}$, Hélie Jérome ${ }^{1 *}$, Chesnel Jeremy², Demoulin Francois-Xavier ${ }^{3}$ \\ ${ }^{1}$ Continental Automotive, Toulouse, France, \\ ${ }^{2}$ ALTRAN, Toulouse, France \\ ${ }^{3}$ CORIA, Rouen, France, demoulin@coria.fr \\ *Corresponding author: jerome.helie@continental-corporation.com
}

\begin{abstract}
A weak coupling strategy is proposed to simulate the pressurised spray without any empirical readjustment. Volume Of Fluid is used to simulate the nozzle internal flow with cavitation and its primary atomization into ligaments. Lagrangian simulations are then used to get the spray evolution, even temporal. Large Eddy Simulations are used for these two simulations types. The coupling between both is realized by a recording and an analysis of the ligaments with local break up modelling into drops. Two test cases are presented, the second one deals with full, complex geometry, 6-holes Gasoline Direct Injection nozzle. Such approach shows a huge potential for prediction of the final spray from the nozzle geometry.
\end{abstract}

\section{Keywords}

Large Eddy Simulation, spray, multihole, atomization, Volume Of fluid

\section{Introduction}

The present work is driven within the industrial automotive contextual aim of reducing the carbon particles emission of car engines. It has been shown that these emissions can be linked to the liquid wall films generated by the penetration of gasoline sprays (i.e. drop jets generated by pressurized atomizer, without assistance of a coflow) impacting on the engine walls. Therefore a better understanding of these sprays physics through simulations is needed. To do so, we developed an Euler-Lagrange solver, implemented in the OpenFOAM platform which allows us to model the non-evaporating gasoline sprays from high-pressure injectors: Large Eddy Simulation (LES) is used for the carrier phase whereas Lagrangian simulation is used for the dispersed phase. A 2-way coupling between the 2 phases and several subgrid submodels have been investigated [1]. The Lagrangian simulation uses the treated results of a Large Eddy-Volume Of Fluid simulation of the cavitating and atomizing flow within and at the close exit of the nozzle. This strategy is efficient because at least one order of magnitude separates the characteristic times of each type of flow but request an effort in the coupling which is specifically described in the present paper.

\section{Simulation Approach}

Second order numerical schemes are used for spatial and temporal discretisations. The numerical developments are based on OpenFoam ${ }^{8}$ platform. For the Volume OF Fluid (VOF) approach, first-order reconstruction of the interface is used together with the sharpening process of Weller, see for instance [2]. The subgrid models used in this work are the one-equation eddy for the LES-VOF and the dynamic Smagorinski for the lagrangian-LES,. The choice of the first one is due to the presence of walls and flow detachment inside the nozzle, and the choice of the second one is due to the capture of transitional turbulent jet flow that requires a minimum of numerical dissipation. For more details on the lagrangian simulations, the readers are referred to Helie et al. [1].

To carry out the lagrangian computation of the drops, a special attention is required for the spray inlet condition. This is done through an original weak coupling with LES-VOF simulation of the flow in the internal part and closevicinity of the nozzle (see for instance [3]). The liquid instantaneous presence and the associated velocity field are stored to be reused identically as input of the spray. The primary atomization process is then analysed and a modelling approach is proposed in 2 steps: Firstly, a rupture into ligaments, almost bidimensional, and a second step where these ligaments propagate further and atomise into rounded ligaments and then into drop populations. Figure 1 left indicates the sketch of this process for one single ligament, the flow being a set of numerous different ligaments. This paradigm of considering a set of different ligaments that will be individually modeled is, to our knowledge, new. The first step is captured with a limited computational effort in the LES-VOF simulation, whereas the second step will be approached using well-established sheet atomization models from the literature. The first 
step from the LES-VOF results gives the ligament structures at each instant, and the atomization model returns the final drop size population.
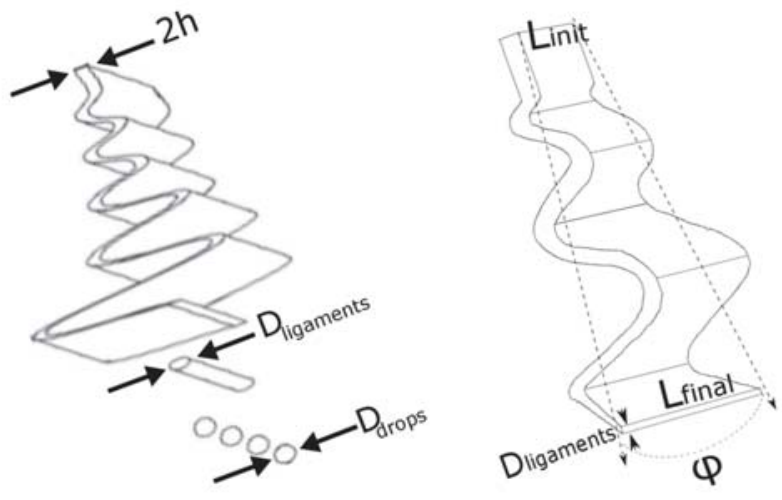

Figure 1 : Left, schematic of the atomisation model for one specific sheet; Right: effect of the sheet stretching

It has to be noted that, at least in this particular case of these nozzles, the result of the primary rupture is to break the circular liquid jet into bidimensional sheets, mostly due to 1) the presence of vapor locations in between the liquid sheets 2) the hydrodynamic in the nozzle that generates an expanding flow at the exit. Therefore the analysis is done on each individual, 2D sheet. Evidence of such sheets will be shown later Figure 1 and 4 . Obviously this is a strong modeling assumption, and a limit of the present approach that should be completed for a more universal model and different nozzle types. The break-up of bidimensional sheets to ligaments is based on the instability theory of thin sheet. The growing rate and therefore the final size with $\Lambda=2 \pi / \mathrm{K}_{\mathrm{S}}$ are chosen according to Senecal et al. [4].

$\omega=-2 v_{l} k^{2}+\sqrt{4 v_{l}^{2} k^{4}+\left(\rho_{l} / \rho_{g}\right) U^{2} k^{2}-\frac{\sigma k^{3}}{\rho_{l}}}$

and

$D_{\text {ligaments }}=\sqrt{\frac{16 h}{\Lambda}}$

The primary ligaments are then submitted to instabilities, and they are subsequently atomized in drops, using simple model [5]:

$K_{\text {ligaments }}=\frac{1}{D_{\text {ligaments }}}\left(0.5+\frac{3 \mu_{l}}{2 \sqrt{\rho_{l} \sigma D_{\text {ligaments }}}}\right)^{-1 / 2}$

allowing to derive :

$D_{\text {drops }}=\left(\frac{3 \pi D_{\text {ligaments }}{ }^{2}}{K_{\text {ligaments }}}\right)^{1 / 3}$

The initial model of Senecal [4] was initially developed for thin sheets as resulting from swirl atomizer. The validity hypothesa are to have a small ratio of density between gas and liquid, which is the case in gasoline engines where the spray is injected in limited counterpressure and to have a Weber number We $>27 / 16$ which is the case in current high pressure injection. In our cases, $\mathrm{h}>2 \mathrm{um}$ and $U_{\text {rel }}>125 \mathrm{~m} / \mathrm{s}$, then $\mathrm{We}>1.79>27 / 16$. The break-up length $L=U_{\text {rel }} \cdot \tau$ with the break-up time $\tau=12 / \omega(\Lambda)$ results typically in our cases $\mathrm{L} \sim \mathrm{O}(\mathrm{mm}) ; \tau \sim \mathrm{O}(5-10$ us $)$ which looks reasonable based on the data available from the literature.

A specificity of our case is the stretching due to the nozzle exit angle $\varphi$ (Figure 1 right). Due to the internal flow, as characterised for instance by the hole exit angle, the liquid films are extended radially. Each sheet can be stretched 
during the break-up time and so by conservation of the mass, its thickness will then diminish. This effect is quantified using the estimation of the flow stretching along the centre of the ligaments and then reintroduced as a correction parameter in the model. An interesting property of the Senecal model equation (1-2) is that instabilities are independent from the sheet thickness. More details of the practical numerical implementation can be found in ref [1]. The typical steps are illustrated Figure 2. The cross stream slice is extracted at one instant at 500um from the hole from the LES-VOF, where the ligaments are separated. Ligaments thickness is extracted using a distance function from centre to boundary (this last one being associated to an arbitrary iso-level of VOF). Then the coefficients due to the stretching but also due to the breakup into drops, equation (4), are computed (bottom left). This local computation is an improvement of the procedure used in ref [1], where the coefficients were estimated only in an average way. All together, it is easy to return the drop population locally (bottom right), the one that will be reinjected stochastically in the lagrangian simulations. Lagrangian computations cannot be described here into detail but are identical to ref [1]. The mesh is identical when comparing different geometries.
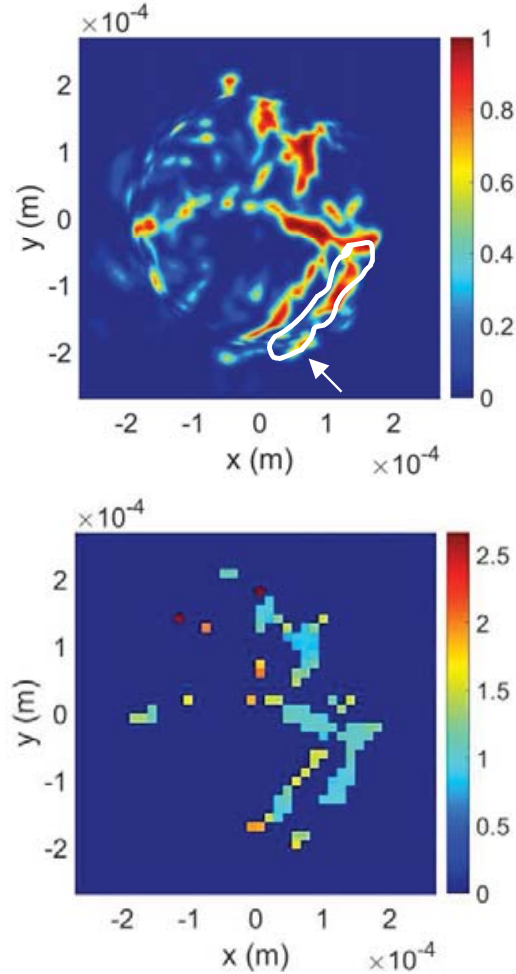

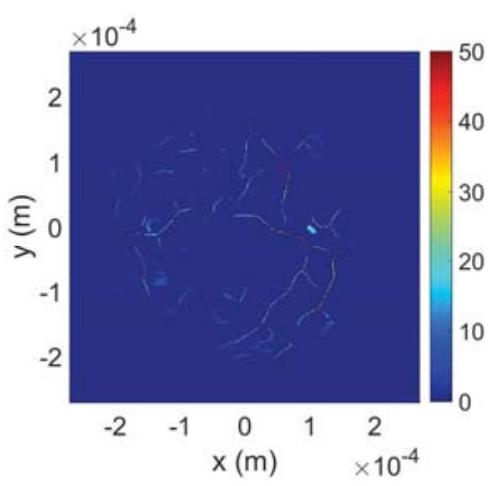

(a)

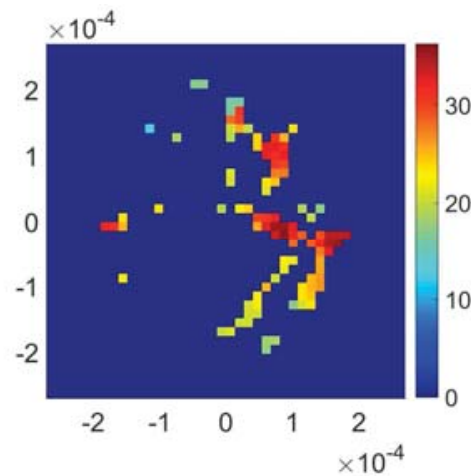

(b)

(d)

Figure 2 : Ligaments analysis principle on an instantaneous slice. (a) Initial alpha field; (b) distance function at the center of the ligaments; (c) coefficient between ligament and drop size (d) final deduced drop size on this field. The slice is taken perpendicularly to the main direction of the hole. One ligament identification, as modelled fig 1, is indicated on (a), white arrow.

\section{Testcases}

The fuel atomizer used here as reference are a special Continental GDI prototype, 3-hole injector (first test, each hole has a different diameter), and 6-hole injector (second test, each hole is inclined differently). The first testcase (Table 1) has holes that are $7 \%$ convergent, their length is 345 micron, the needle lift is $75 \mathrm{um}$. The second testcase (Table 2) has a needle lift of $100 \mathrm{um}$, with different geometries, extracted from the remeasured ones. The hexahedral mesh is around $15 \mathrm{Mo}$ cells for each case. Each hole is followed by a conical external domain that is meshed up to a length of 5 diameters (Figure 3). The simulations are two-phase (not 3-phase, which is a limitation of the present approach). Experimental (fixed) mass flow rate is imposed at the inlet, pressure at the exit.

Table 1. Frst testcase, periodic 3-hole injectors

\begin{tabular}{c|ll}
\hline \hline IHole \# & b angle $\left[^{\circ}\right]$ & L/D \\
\hline \hline 1 & 30 & 1.7 \\
2 & 30 & 2.0
\end{tabular}


Table 2. Second testcase, 6-hole injector

\begin{tabular}{c|cc}
\hline \hline Hole \# & b angle $\left[{ }^{\circ}\right]$ & L/D \\
\hline \hline 1 & 20 & 1.68 \\
2 & 30 & 1.76 \\
3 & 40 & 1.90 \\
4 & 40 & 1.90 \\
5 & 30 & 1.76 \\
6 & 20 & 1.68
\end{tabular}
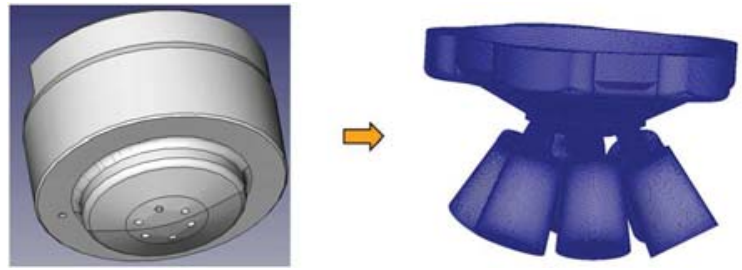

Figure 3 : Left : nozzle CAD ; Right: generated mesh. The 6 cylinders on the bottom represents the external domain (after the nozzle exit) where the atomization process occurs.

\section{Results and discussion, first testcase}

The atomization process that has been mentioned above is clearly illustrated Figure $\mathbf{4}$ for the first testcase. Slices perpendicular to the hole direction are showed in the second part of the hole and in the first part of the atomisation process. The flow development in the hole and at the exit clearly exhibits the breakup into ligaments mostly due to 1) a huge presence of cavitation 2) the ligaments are separated thanks to the radial velocity at the hole exit (as visualised experimentally with the close spray angle at hole exit for instance). A limited hole-to-hole interaction inside the sac volume has been found, due to the high distance between the holes, as only 3-hole are implemented in this prototype test injector. Whatever the hole, shear cavitation is developing largely and reaches the hole exit, even if a small convergence of the hole geometry is present. For holes with the higher diameters, vortex cavitation also develops. Hydraulic flip appears while D is increased. Increasing the hole diameter the primary atomization length is increasing.
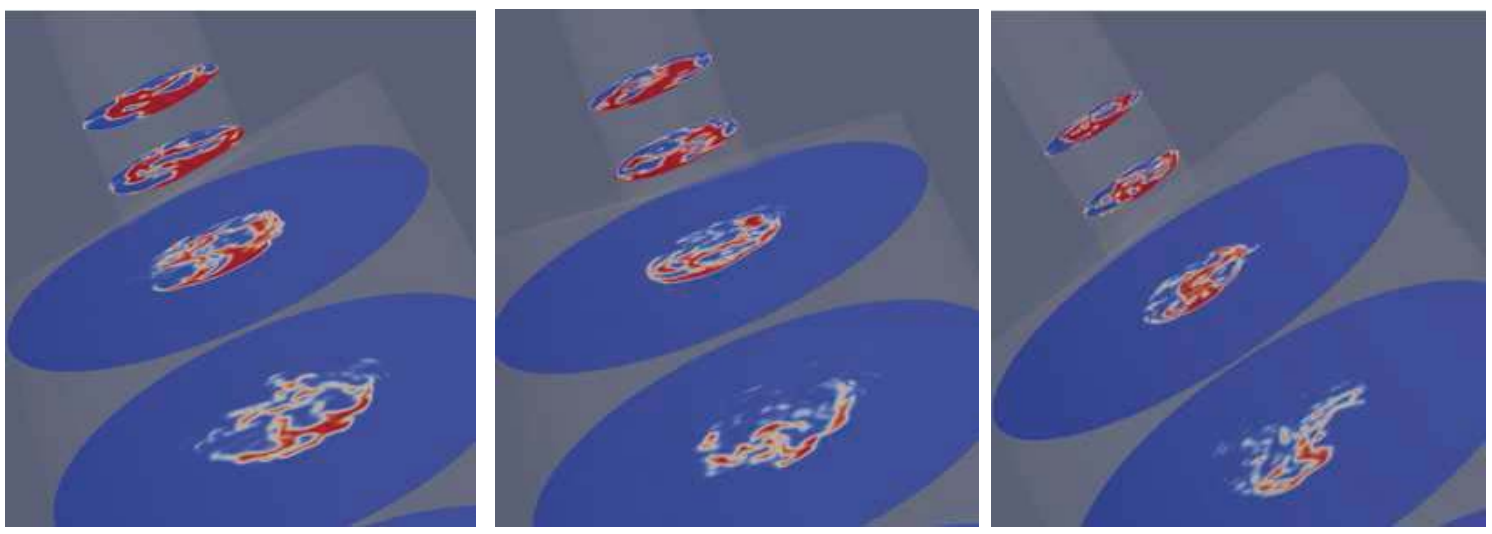

Figure 4 : Instantaneous snapshot of the Volume of Fluid (liquid: red color), 200bar fuel pressure.

Left: large hole diameter ; center: medium hole diameter ; right : small hole diameter. The slices are placed at the hole middle, hole exit, one and two diameters distance after the hole exit (hole length is 345 micron) 
In the first application case, each hole has a different diameter. This characteristic is visible in the development of the plumes, computed separately here, Figure 5. The spray from the first hole is much larger than the one from the last, small, hole. The Q-criterion as an indicator of the centers of the vortices classically defined by $\tilde{Q} \equiv$ $\frac{1}{2}\left(\tilde{S}_{i j} \tilde{S}_{i j}-\widetilde{\Omega}_{i j} \widetilde{\Omega}_{i j}\right)$, where $\tilde{S}_{i j}$ is the strain-rate tensor and $\widetilde{\Omega}_{i j}$ is the rotation-rate tensor $\widetilde{\Omega}_{i j}=\left(\partial \tilde{u}_{i} / \partial x_{j}-\partial \tilde{u}_{j} / \partial x_{i}\right)$, displays, in both cases, roll-up structures close to the injection location which then evolve in helicoildal structures [1]. In the spray from the small hole, this transition to turbulence is longer: indeed, the spray is denser and its exchange with the entrained gas is expected to be reduced.
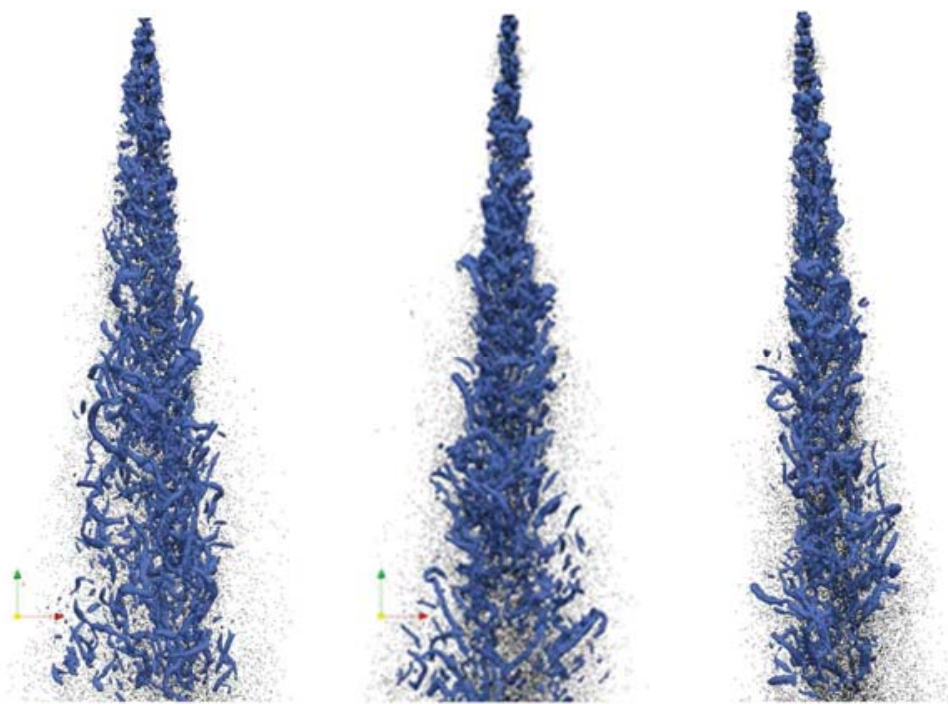

Figure 5 : Instantaneous snapshot of the spray with drop parcels (dots) and isosurface of Q=0.5e9 $\mathrm{s}^{\wedge}(-1)(\mathrm{blue})$, 200bar fuel pressure. Left: large hole diameter ; center: medium hole diameter ; right : small hole diameter.

As expected, the studied spray is not sensitive to the smallest scales $\left(\tau_{p} / \tau_{\eta}>1\right.$ and $\left.D_{p} / \eta>1\right)$. On the other hand, it is sensitive to the sub-grid scale eddies $\left(\tau_{p} / \tau_{s g s}<1\right)$, so a basic sub-grid dispersion model have been introduced in order to take into account this interaction [1]. However, in this dispersion model the main driving factor remains the relative velocity between drops and eddies $\left(\tau_{r e l} / \tau_{s g s}<1\right)$. Fortunately, as the turbulent fluctuations are mostly resolved, $u^{\prime} / \sqrt{2 / 3 k_{s g s}} \cong 0.95$, the influence of the turbulent dispersion model should remain limited.
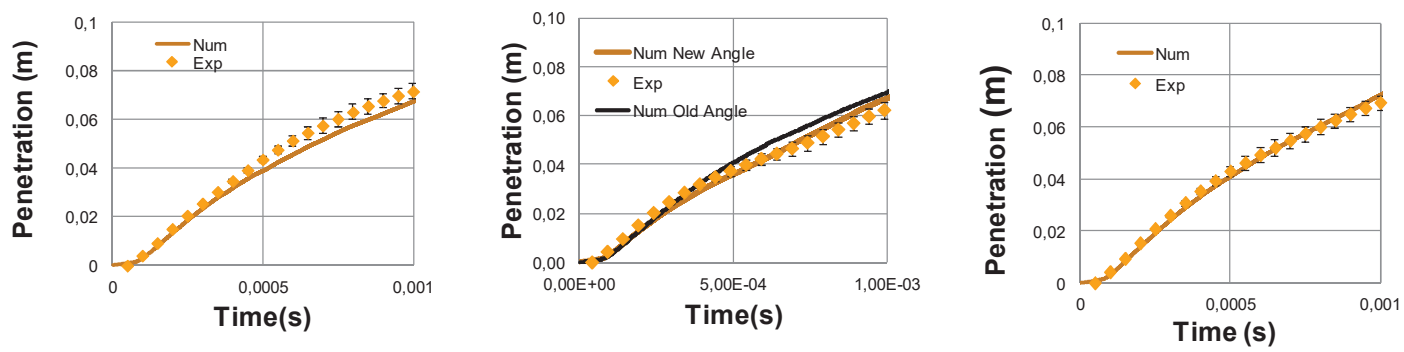

Figure 6 : Penetration curves, ), 200bar fuel pressure.

Left: large hole diameter ; center: medium hole diameter ; right : small hole diameter

To simulate the transient, the single additional input data that is needed is the mass flow rate as a function of time. The specific features at needle opening are initially neglected, using only full lift results, as will be discussed later. Temporal penetration curve in the direction of the spray are compared Figure 6 . The global behaviour is satisfactory, slightly lower than the experimental one for the small hole case. Initially, a large difference was found for the medium-diameter hole. Its reason is now explained. Regarding the transient phenomena, in ref [6] the spray exhibited a flapping behaviour at the needle opening, impacting the spray penetration and which had been reproduced successfully in the simulations. In the present testcase, the transient influence of the needle opening is also highlighted. Indeed, with (only) the medium hole of this prototype injector a spurious behaviour was found experimentally: an important increase of the spray angle at the beginning of the injection: the exit angle is increasing 
from $25^{\circ}$ to $45^{\circ}$ during the period $0.1 \mathrm{~ms}-0.4 \mathrm{~ms}$. When applying this angle increase in our simulation a satisfactory penetration is recovered back, which was not the case without taking this angle increase into account as the slope change was not captured (figure 6). This result confirms that the transient opening phase has to be measured into detail to be re-introduced empirically as in the present work. Otherwise moving mesh has to be used to simulate the opening needle phase as in ref [3].

\section{Results and discussion, second testcase}

Orientation angle B also clearly influences the cavitation behaviour, as seen Figure 8. Flow detachement is increasing with higher angles, and therefore the shear cavitation (right part of the nozzle slice) is progressively visible. For a small angle, a flow reattachment is visible on both sides, without hydraulic flip, and with a strong presence of cavitation vortex. For an intermediate to large angle, strong shear and vortex cavitation developments generate tridimensional effects and, in average, display a typical "smiley" shape.

In addition, the flow is strongly asymmetric and unsteady due to the hole-to-hole interaction in the nozzle flow development. The arrows refer to the vicinity of the next holes. The top arrow is in the direction of 20deg-hole ; and bottom toward the $40 \mathrm{deg}$-hole. This is a particular effect of this mirror geometry. It is of great interest to look into the vicinity of the holes (along the circumferential direction), see table $2 \&$ fig 3 for the geometry. The 20deg-hole is in between 30deg-hole and 40deg-hole. The other holes have one neighbour with the same hole direction, reinforcing the stability of the flow. Only the intermediate hole direction has neighbour with different hole orientation.

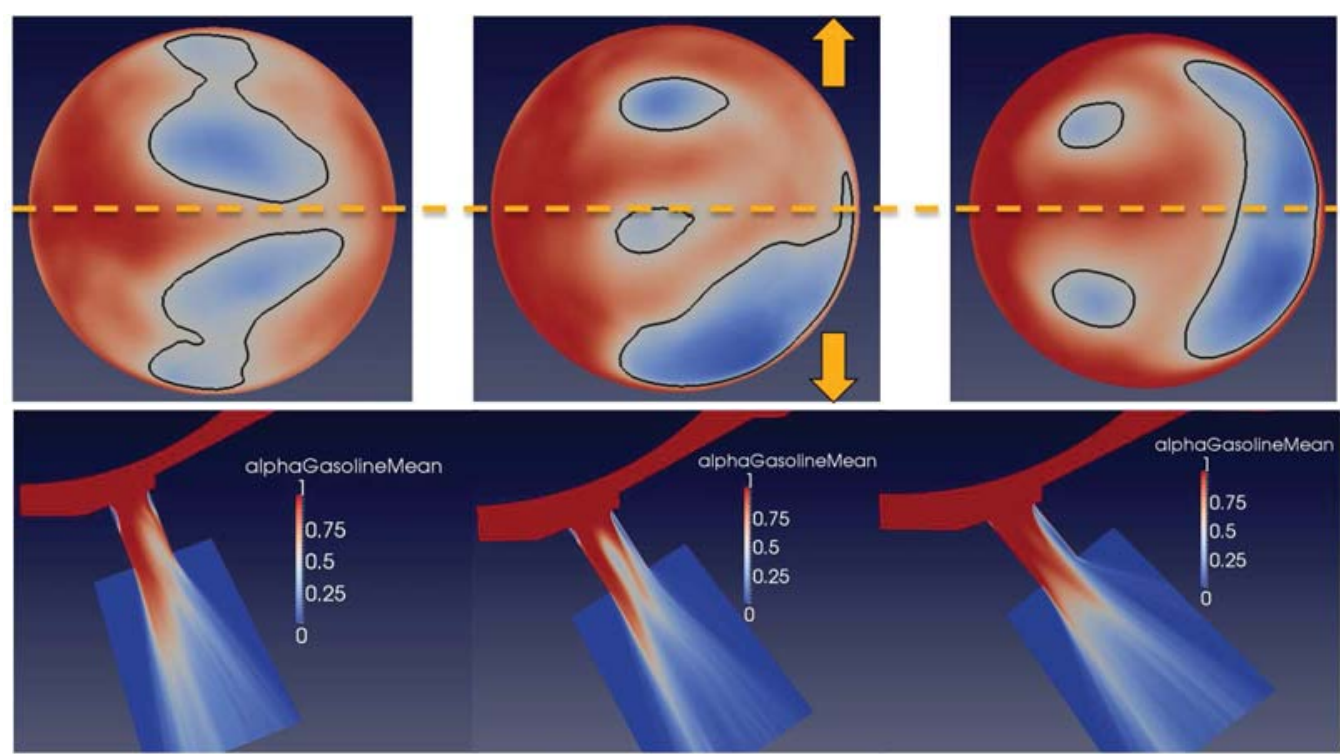

Figure 7 : Time-average of volume of fluid.Red: liquid; blue: vapour. Flow is coming from the right side (needle seat). Top: Slice at the hole exit, perpendicular to the hole direction axis. Bottom: Cut-plan. Left: beta angle $20^{\circ}$; center: beta angle $30^{\circ}$; right : beta angle $40^{\circ}$ Arrow: see text

The large beta angle shows a stable but reduced cavitating double contrarotating vortices. Stable attached shear cavitation is also present. It leads to a typical "smiley shape" that has been already described with a different injector design but still with a large beta angle of $40 \mathrm{deg}$ in ref [1]. Also coherent with this former paper, a side jet appears, due to the reorientation of a liquid sheet in between the two vortices. It can lead to small droplets with high velocity and angle on the side, as has been experimentally confirmed in the former work. Lastly, the higher angles (30deg$40 \mathrm{deg}$ ) angles exhibit a hydraulic flip. As only two phases (vapour/liquid) are considered here, and not three phases (vapour/air/liquid), we can identify in this simplification a clear limit of the numerical model representation that will be overcome in the future works.

It should be also noticed that the geometry has been generated from a complete 6-holes 3D design, and not from a mirror projected 3 hole case. Therefore, small geometrical differences are intended to be introduced from both sides. However this effect can be lower than in reality, for instance in case of some needle non-axisymetric positioning. This effect can also be damped by the limited resolution mesh. Indeed the flow is found quite stable in 
time concerning its pattern, and even comparing the 2 sides. Considering one representative instantaneous flow event, the full picture is provided Figure 8. Differences are obviously observed, but the number and the area of the cavitating structures are almost identical when comparing both sides, even for 20deg-hole. The captured effect on the average mass flow rate is here not found as the main reason (average mass flow rate differs only by $3 \%$, and instantaneous mass flow rate by less than $10 \%$ ), but the average flow direction seems to break the symmetry for this $20 \mathrm{deg}$-hole, as already discussed.

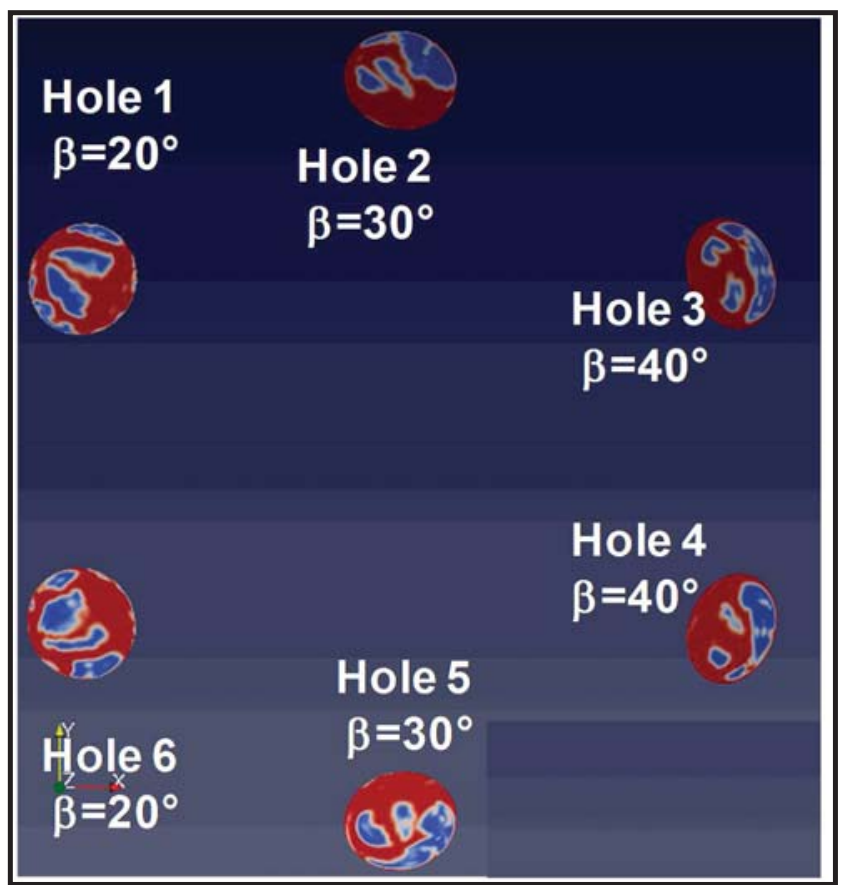

Figure 8 : Instantaneous snapshot of volume of fluid .Red: liquid; blue: vapour.

Cut-plan at the hole exit, projected on a plane perpendicular to the injector central axis.

The spray simulation is now realised with all the plumes injected together. As expected, some jet to jet interaction is observed, especially for the small drops that are entrapped in between the holes. Some turbulent eddies can also connect between the narrowest jets. The general comparison returns a very correct behaviour on Figure 9 . The injector is inclined to get the central hole in the vertical direction at the center of the image. The estimation of the external angle depends on the thresholding level that is used, but the tendency is well recovered despite this simple offset.
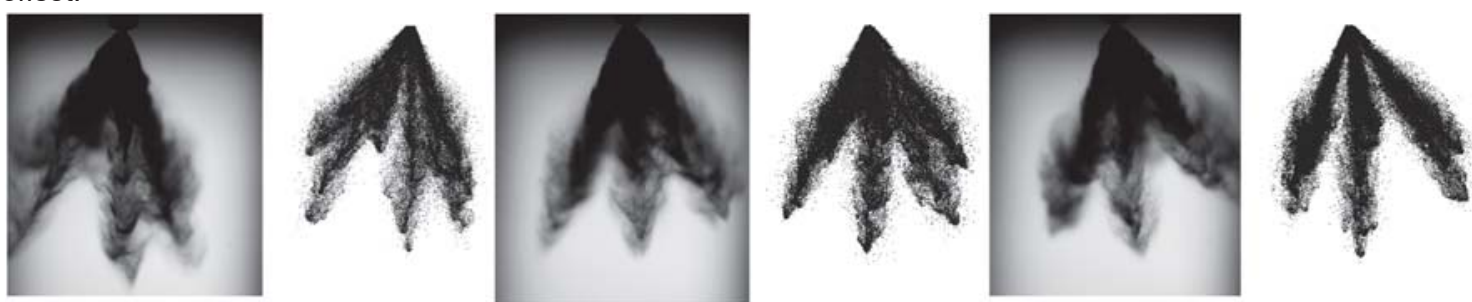

Figure 9 : Instantaneous snaphots of the developed spray a t=1 ms ; experimental shadowgraphy and simulations Left: central hole is beta angle $20^{\circ}$; centre: central hole is beta angle $30^{\circ}$; right : central hole is beta angle $40^{\circ}$

The experimental penetration is also correctly recovered in Figure 9 and Figure 10, even if the case to case difference is really small, below the RMS shot to shot variation. Interestingly, a manual recording of the typical size of the visible large scale structures of drop segregation returns also a good agreement which explains quantitatively the visible agreement between the images, with fish-bones and drop segregation structures. 

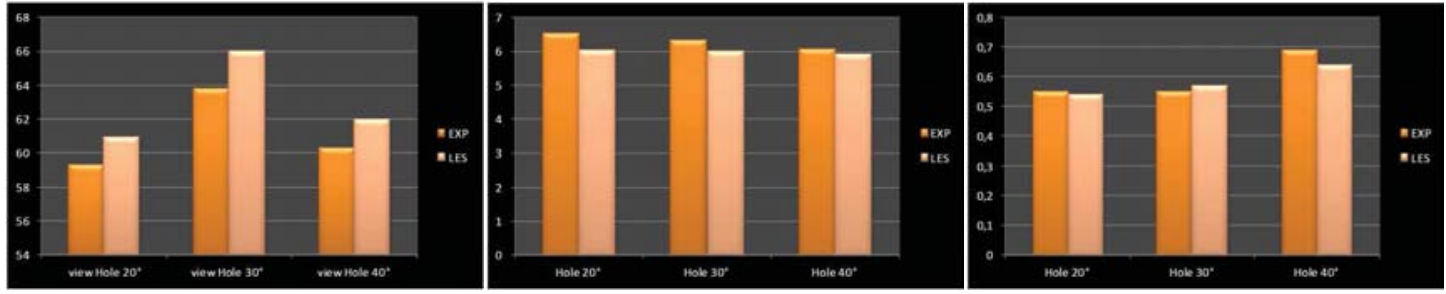

Figure 10 : Resulting comparison of the instantaneous snapshots of the developed spray at $1 \mathrm{~ms}$; from experimental shadowgraphy and simulations. Top left : total external spray angle; Top right : penetration ; Bottom: large scale structures of drops segregation.

\section{Conclusions}

Multijets spray structures, penetrations, and interactions are simulated with large Eddy Simulations. Differences between largely separated holes and narrow holes are depicted, with variations of hole diameters and orientation angles. Comparisons with local quantities will complete this work in the future.

\section{Acknowledgements}

CRIHAN and GENCl supercomputer centers are acknowledged for their support. ANR and ANRT are also funding a part of this activity.

\section{Nomenclature}

\section{$D$}

$\rho_{l}$

$v_{l}$

$\rho_{g}$

$\sigma$

$h$

$\tau_{p}$

$D_{\text {ligaments }}$

$D_{\text {drops }}$

w

$k$

$\Lambda$

K

$U_{\text {rel }}$

$\tau$

$L$

u'

$\tau_{s g s}$

$\tau_{\text {rel }}$

$\tau_{\eta}$

$\eta$

$k_{s g s}$
Hole diameter [m]

Liquid volumetric mass density $\left[\mathrm{kg} / \mathrm{m}^{\wedge} 3\right]$

Liquid viscosity [kg/s.m]

Gas volumetric mass density $\left[\mathrm{kg} / \mathrm{m}^{\wedge} 3\right]$

Surface tension [N/m]

Liquid sheet width [m]

Drop relaxation time [s]

Drop diameter [m]

Drop diameter [m]

Growing rate

Wave number $\left[\mathrm{m}^{\wedge}-1\right]$

Maximum unstable wavelength [m]

Wave number corresponding to the maximum growth rate

Relative velocity between the gas and the liquid $[\mathrm{m} / \mathrm{s}]$

Break-up time [s]

Break-up length $[\mathrm{m}]$

Fluctuating velocity $[\mathrm{m} / \mathrm{s}]$

Sub-grid time scale [s]

Time for a droplet to traverse an eddy in sub-grid scale [s]

Kolmogorov time-scale [s]

Kolmogorov length scale [m]

Turbulent kinetic energy $\left[\mathrm{m}^{\wedge} 2 / \mathrm{s}^{\wedge} 2\right]$

\section{References}

[1] Helie, J., Khan, M.M., Gorokhovski, M., Journal of turbulence, 2016

[2] Deshpande, S.S., Anumolu, L., Trujillo, MF., Comput Sci Discov. 2012;5:014016.

[3] Piscaglia, F., Montorfano A., Helie J., Demoulin F.-X., International Congress Multiphase Flow, Firenze, Italy, 2016

[4] Senecal, P.K. , Schmidt, D.P., Nouar, I. , Rutland, C.J. , Reitz, R.D., Corradini, M.L., International Journal of Multiphase Flow, vol 25, pp 1073-1097, 1999

[5] Dombrowski, N., and Johns, W.R., Vol. 18, pp. 203-214, Chemical Engineering Science, 1963

[6] J. Hélie, Large Eddy Simulation for Atomization and Spray, LES4ICE, Rueil-Malmaison, 2014 


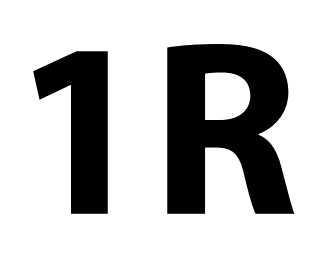

Atomizers 1 


\title{
Influence of liquid properties on ultrasonic atomization
}

\author{
A. Lozano*, J.A. García, J. Alconchel, F. Barreras, E. Calvo, J.L. Santolaya \\ LIFTEC, CSIC - Universidad de Zaragoza, Zaragoza, Spain \\ ${ }^{*}$ Corresponding author: alozano@litec.csic.es
}

\begin{abstract}
Ultrasonic atomization is very convenient because it can generate droplets with diameters of a few microns and with very narrow size distribution. Besides, opposite to twin fluid nozzles, in ultrasonic atomization, droplet generation and transport are decoupled processes. Droplets are ejected from the liquid surface with very low velocities, so driving them is relatively simple. Although this atomization method is now common in some specific applications, for example in household humidifiers, there are still some details about the physics of this process that are not completely understood. Up to date, most of the published results have been limited to experiments with water. However, it has been demonstrated that atomization rates quickly decrease as liquid viscosity increases. This work analyzes the characteristics of ultrasonic atomization of some alternative fluids to determine if there is any influence of other physical properties such as surface tension or vapor pressure. Experiments are performed using a commercial piezoceramic disk with a resonance frequency of $1.65 \mathrm{MHz}$. The disk is excited with a sinusoidal signal with voltage amplitudes that go up to $60 \mathrm{~V}$. Sprays are visually characterized analyzing instantaneous images and high speed video sequences. Besides atomization rates are calculated by measuring the weight loss in a fixed time.
\end{abstract}

\section{Keywords}

Ultrasonic atomization, ultrasound, piezocramic.

\section{Introduction}

Ultrasonic atomization has some unique characteristics that are ideal for many specific applications. Typical examples are most commercial household air humidifiers, or some inhalers for drug delivery to the lungs. In these devices, atomization is achieved by vibration of an ultrasonic transducer submerged in a liquid volume. As a result, droplets can be generated from the liquid surface with diameters of a few microns, with very narrow size distribution, and with low velocity. Opposite to pressure and twin fluid nozzles, in which small droplet diameters are associated to high liquid and gas velocities, in ultrasonic atomization, droplet generation and transport are decoupled processes. Droplets are ejected from the liquid surface with very low velocity, so driving them is relatively simple.

The possibility to generate a cloud of droplets by means of ultrasonic waves was first reported by Wood and Loomis in 1927 [1]. Since then, many theoretical and experimental works have been published to explain the physics controlling this phenomenon. Two main mechanisms are considered to be responsible for the spray formation: cavitation inside the liquid mass and instabilities of standing waves on its free surface. Droplet detachment from wave crests can clearly be observed for low excitation frequencies but cannot be distinguished for frequencies in the $\mathrm{MHz}$ range. The importance of cavitation might be dependent on the forcing frequency or the ultrasonic power, but these extremes have not been demonstrated in a definite way. As confirmed by numerous experiments, it is now generally accepted that, in ultrasonic atomization, spray mean droplet diameter is essentially determined only by the oscillation frequency. The oscillation amplitude controls the spray flow rate but does not have a major influence on drop diameter [2]. When using ultrasonic transducers this amplitude is proportional to the driving signal voltage.

A major part of the published results are limited to water atomization. The influence of fluid physical properties has been studied in a relative low number of papers [3]. Most of them are based on experimental considerations [4,5], although some theoretical analysis can also be found [6]. Furthermore, in some of these works, the ultrasonic frequency is low [7]. Liquid viscosity does not substantially alter the droplet size distribution, but has a dramatic effect on atomization rate [8]. With the specific conditions in the present experiments, efficiency drops dramatically for kinematic viscosity values over $3 \times 10-6 \mathrm{~m}^{2} / \mathrm{s}$. This is a severe limitation that can preclude the use of this atomization method in many processes of industrial interest, for example in surface coating, or to introduce the droplets in a chemical reactor. However mists of viscous liquids can be obtained diluting them first in volatile solvents and atomizing the low viscosity mixture [9]. Once the solvent has evaporated, the result is a mist of a liquid that otherwise could not have been directly nebulized. To facilitate this possibility and advance in the 
understanding of the dependence on different physical properties, this work analyzes the characteristics of ultrasonic atomization of some organic compounds. In particular, several alkanes and allcohols are considered. Results are compared to those obtained atomizing water in the same conditions. Measured parameters include atomization rate and droplet size distribution. Visual aspect of the sprays is also examined, recording instant images and high speed sequences.

\section{Material and methods}

In the experiments here described, atomization was performed using commercially available inexpensive ultrasonic transducers as the one shown in Fig. 2. They consist of lead zirconate titanate PZT-4 piezoceramic disks with a diameter of $20 \mathrm{~mm}$, a thickness of $1.3 \mathrm{~mm}$ and a measured resonance frequency of $1.65 \mathrm{MHz}$.

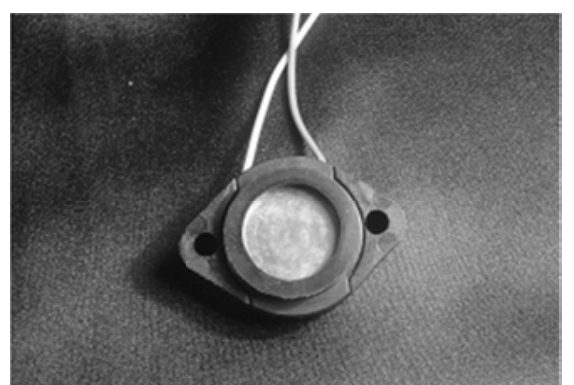

Figure 1. Image of one of the piezoceramic disks used in the present experiments

The disks were excited with a sinusoidal wave coincident with the resonance frequency and variable amplitude. It has to be noted that disks of this type only oscillate with significant amplitude when the excitation wave coincides with the main resonance peak which is quite narrow. The resonant frequency depends on the ceramic type and the disk geometry, and this is the only frequency for which atomization occurs efficiently. A scheme of the oscillator circuit used to force the disks is presented in Fig. 2. It is very important to be sure that the disks operate submerged into the fluid volume, because in this way, the liquid also acts as a cooling medium preventing the disk from overheating. Otherwise, the Curie temperature, defined as the point where the material undergoes a transition from ferromagnetic to paramagnetic, might be reached. In this case, the magnetic moments would become randomly oriented, and the ceramic element would depolarize losing its electrostrictive properties. As the disk heating increases with the applied voltage, the maximum delivered value was limited to $80 \mathrm{~V}$ to avoid damaging the ceramic. Values over $100 \mathrm{~V}$ would also risk the integrity of the power transistor in the electronic oscillator circuit (marked as T in Fig. 2).

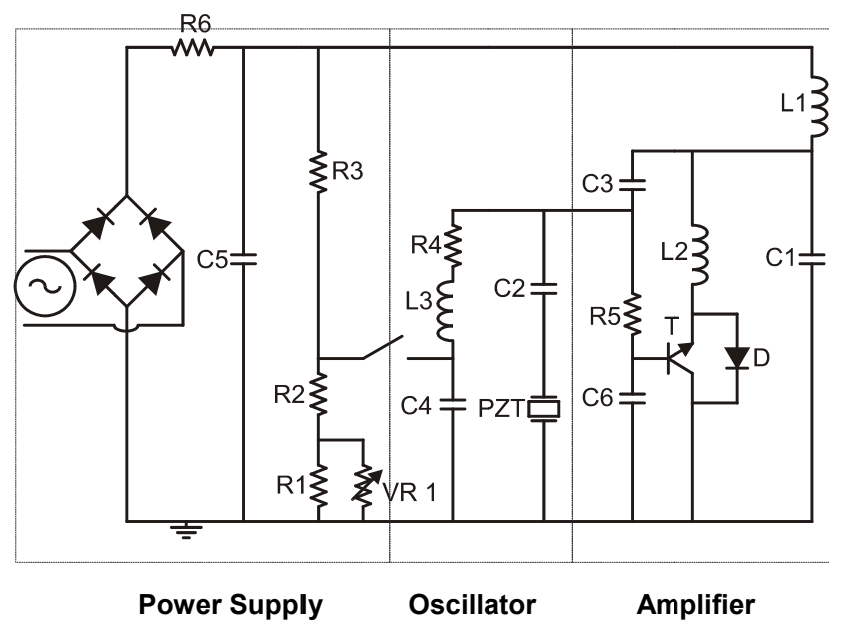

Figure 2. Scheme of the oscillator circuit

The disks were attached to the bottom plate of two different open vessels. The first one has a section of $18 \mathrm{~cm} \mathrm{x}$ $18 \mathrm{~cm}$ and a height of $24 \mathrm{~cm}$. It was used for the atomization rate measurements. A relatively large section was chosen to avoid a noticeable liquid heating from the piezoceramic transducer. The second one is even larger, with dimensions of $36 \mathrm{~cm} \times 36 \mathrm{~cm} \times 24 \mathrm{~cm}$. This one was used for the image recording, to minimize possible interferences from the walls. 
To determine the atomization rates, the decrease in the liquid mass inside the vessel during a minimum time interval of 5 minutes was monitored by weighting it with a Kern FCB scales, capable of measuring a maximum mass of $8 \mathrm{~kg}$ with a precision of $0.1 \mathrm{~g}$. For all the measurements the initial liquid level was identical and equal to 3 $\mathrm{cm}$. Care was taken to evacuate the liquid droplets out of the atomizer to prevent condensation, suctioning them with an extraction fan. This extraction, however, was limited to the small droplets that form the spray mist and not to other larger drops. This group includes, for example, splashing caused by falling of the liquid cone tip onto the pool surface. This is mainly because these drops are not useful for most applications requiring small droplets where ultrasonic atomization could be a first choice. Besides, they can be filtered out in a relatively simple manner. This study will, thus, focus on the micron-sized droplets resulting from the ultrasonic atomization.

Droplet size distributions were measured with a Malvern Mastersizer S laser difractometer equipped with a 300 $\mathrm{mm}$ focal length lens. According to the manufacturer specifications, this lens is suitable to cover a droplet diameter range from $0.5 \mu \mathrm{m}$ to $900 \mu \mathrm{m}$. The maximum obscuration in the Malvern measurements was lower than $25 \%$, with a minimum of $3.6 \%$. The room was darkened to maximize the contrast for low obscuration values. To calculate the droplet size distribution, the polydisperse model of the Malvern software was selected. The small droplets were driven to the laser beam dragging them with an air flow. To visualize the atomization process, instantaneous images were acquired with a Hamamatsu 1,024 x 1,344 pixels 12-bit C4742-95-12 ORCA-ER CCD camera with a Sigma $70-200 \mathrm{~mm}$ zoom lens. Exposure time was set to $10 \mathrm{~ms}$, and the covered field of view was $90 \mathrm{~mm} \times 118 \mathrm{~mm}(87.8 \mu \mathrm{m} / \mathrm{pixel})$. Image sequences were also acquired with a high speed CMOS RedLake Motion Pro HS4 camera, capable of recording 5,000 frames per second (fps) at a maximum image size of $512 \mathrm{x}$ 512 pixels. Two different types of sequences were registered. The first one corresponds to a field of view of 130 $\mathrm{mm} \times 130 \mathrm{~mm}$, recording speed of $5000 \mathrm{fps}$ and exposure time varying between $50 \mu \mathrm{s}$ and $150 \mu \mathrm{s}$. The second configuration corresponds to close ups with a field of view reduced to $4.2 \mathrm{~mm} \times 4.2 \mathrm{~mm}$, recording speed of 3000 fps and exposure time of $330 \mu \mathrm{s}$. To achieve this magnification $(8.2 \mu \mathrm{m} / \mathrm{pixel})$, a Nikon PB6 bellows focusing attachment was placed between the camera and the lens, together with a set of three Kenko extension rings. Two $500 \mathrm{~W}$ halogen lamps were used as illumination source.

Experiments were performed atomizing several organic compounds as well as pure water. Table 1 summarizes the values of several physical properties that can be influential in the atomization process. Liquid selection includes three alkanes and three alcohols. Density and surface tension values are quite similar for all of them, in all cases lower than those for water. Viscosity and vapor pressure cover a wider range, increasing for increasing number of $\mathrm{C}$ atoms.

Table1. Physical properties of the atomized liquids

\begin{tabular}{lcrlrrr}
\hline & $\begin{array}{l}\text { Density } \rho \\
\left(\mathrm{kg} / \mathrm{m}^{3}\right) 20^{\circ} \mathrm{C}\end{array}$ & $\begin{array}{l}\text { Viscosity } v \\
\left(\mathrm{~m}^{2} / \mathrm{s}\right) 20^{\circ} \mathrm{C}\end{array}$ & $\begin{array}{l}\text { Surface } \\
\text { tension } \sigma \\
(\mathrm{N} / \mathrm{m}) 20^{\circ} \mathrm{C}\end{array}$ & $\begin{array}{l}\text { Vapor } \\
\text { pressure } \\
(\mathrm{Pa}) 25^{\circ} \mathrm{C}\end{array}$ & $\begin{array}{l}\text { Compressibility } \\
\text { modulus K }(\mathrm{Pa}) \\
20^{\circ} \mathrm{C}\end{array}$ & $\begin{array}{l}\text { Boiling } \\
\text { Temperature } \\
\left({ }^{\circ} \mathrm{C}\right)\end{array}$ \\
\hline Water & 998.2 & $1 \times 10^{-6}$ & 0.0728 & $3.167 \times 10^{3}$ & $22.0 \times 10^{8}$ & 100 \\
Hexane & 654.8 & $0.294 \times 10^{-6}$ & 0.01843 & $20.4 \times 10^{3}$ & $7.9 \times 10^{8}$ & 69 \\
Heptane & 683.8 & $0.408 \times 10^{-6}$ & 0.0197 & $6.06 \times 10^{3}$ & $9.4 \times 10^{8}$ & 98 \\
Decane & 730.0 & $0.92 \times 10^{-6}$ & 0.02337 & $0.185 \times 10^{3}$ & $11.0 \times 10^{8}$ & 174 \\
Methanol & 781.8 & $0.745 \times 10^{-6}$ & 0.02261 & $16.96 \times 10^{3}$ & $8.23 \times 10^{8}$ & 65 \\
Ethanol & 789.0 & $1.36 \times 10^{-6}$ & 0.0228 & $7.924 \times 10^{3}$ & $8.94 \times 10^{8}$ & 78 \\
2-Propanol & 786.3 & $3.05 \times 10^{-6}$ & 0.022 & $6.02 \times 10^{3}$ & $7.5 \times 10^{8}$ & 82
\end{tabular}

\section{Results and discussion}

Before presenting and discussing the results obtained from the different measurements, it can be interesting to describe the ultrasonic atomization process. It takes place according to the following scheme. The piezoceramic disk submerged below the fluid surface starts vibrating when excited with a $1.65 \mathrm{MHz}$ sinusoidal wave. For low voltages the only noticeable effect is the appearance of some waves on the liquid surface over the disk. As the voltage is increased, this part of the surface assumes a conical shape most likely induced by an acoustic streaming phenomenon [10]. A further increase causes the elongation of the cone that forms a stem with a neck zone. Eventually, the tip of the cone detaches, and falls on the liquid pool forming big droplets due to splashing. When voltage surpasses a determinate value that depends on the liquid to be atomized and its viscosity, superimposed both to the whole mass displacement that produces the conical shape and the interfacial waves, a fine mist of small micron-sized droplets is generated, particularly in the middle part of the cone region. Together with it, some medium sized droplets are also ejected from the cone surface. The amount of this last type of droplets and their detachment velocity clearly increase with increasing forcing voltage. All these steps are 
illustrated in the series of images in Fig. 3, where water has been used as the atomized fluid. Voltages are $20 \mathrm{~V}$, $30 \mathrm{~V}, 45 \mathrm{~V}$ and $55 \mathrm{~V}$.

It has to be remarked that although the response of the CCD camera is linear, to better discern low intensity details, the images are not displayed with a linear look-up table, but with a gamma $\gamma=2$ grey scale according to the expression

$I_{\text {corr }}=I_{\max }\left(\frac{I}{I_{\max }}\right)^{1 / \gamma}$

where $I_{\text {corr }}$ are the intensity corrected values, and $I_{\max }$ is the maximum intensity.
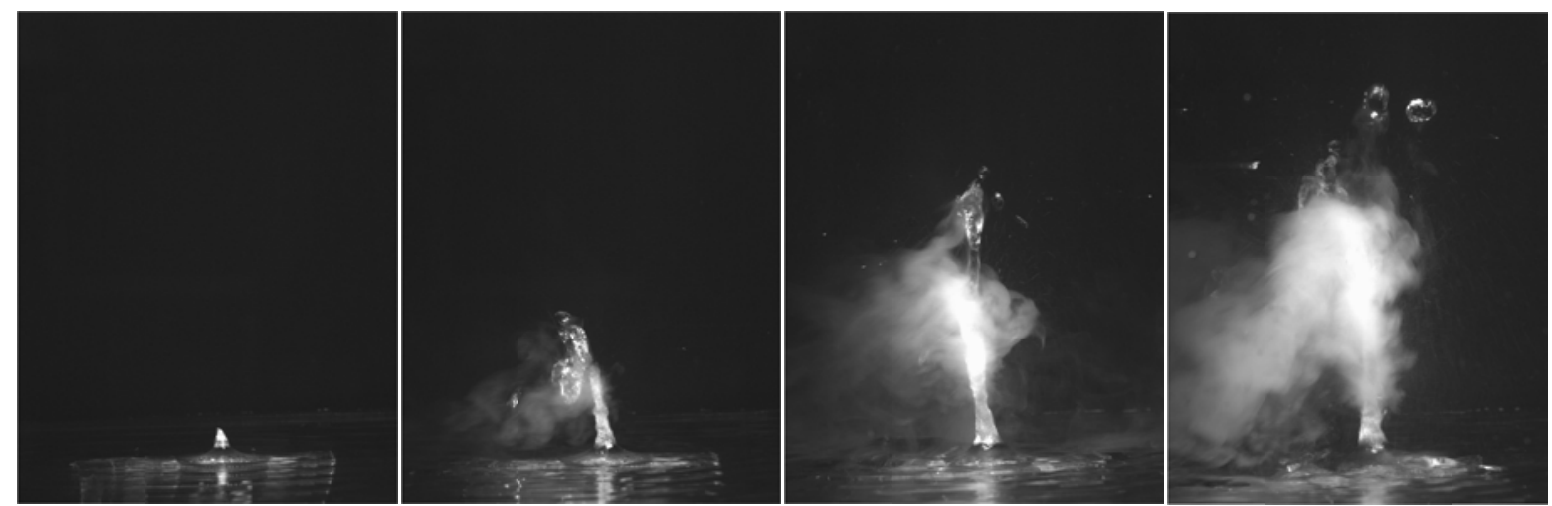

Figure 3. Evolution of the ultrasonic atomization process for increasing voltages. From left to right: $20 \mathrm{~V}, 30 \mathrm{~V}, 45 \mathrm{~V}$ and $55 \mathrm{~V}$. Atomized liquid is water.

\section{Atomization rate measurements}

A compilation of the results of the atomization rate measurements is presented in Fig. 4. Firom it, a first conclusion is noticeable. Water exhibits a trend that differs from the rest of liquids. While water atomization rate keeps increasing with voltage, all the other curves present a maximum for an intermediate voltage value, which varies between $35 \mathrm{~V}$ and $40 \mathrm{~V}$. Although maximum voltage in this plot is limited to $60 \mathrm{~V}$, values up to $80 \mathrm{~V}$ where tested without finding a maximum in the water graph. It is possible that still higher voltages could be required to reach it, but they would be out of the limits set for these experiments, as explained in Section 2. Setting aside water as a special case, the relative atomization rate values for the alkanes and alcohols have an inverse correlation with the kinematic viscosity, as shown in Fig. 5. Here, the maximum atomization rate for each liquid has been plotted as a function of the respective viscosity value. Again, this is in good agreement with the results in [8].

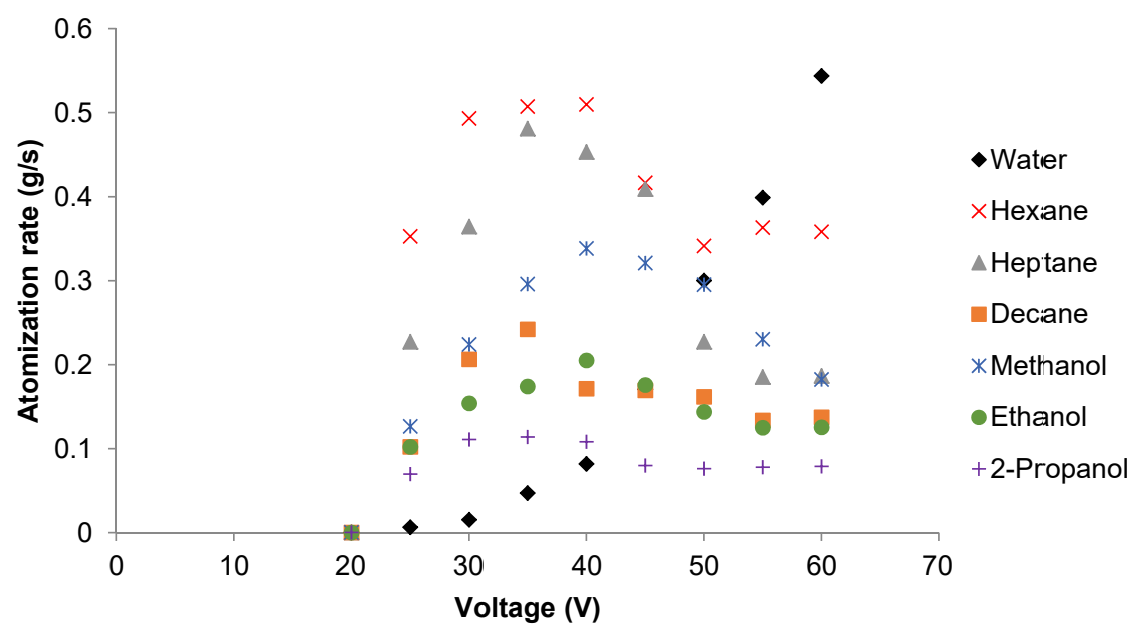

Figure 4. Atomization rate as a function of the forcing voltage for the different working liquids. 


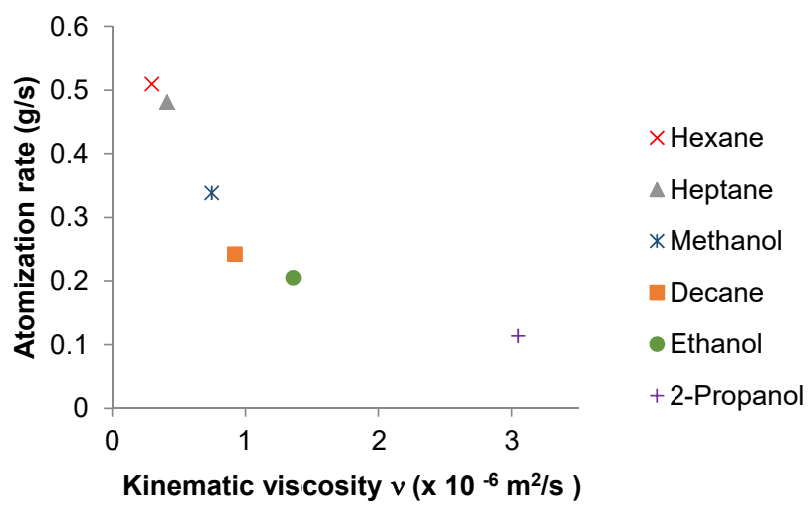

Figure 5. Maximum atomization rate for each one of the liquids as a function of the corresponding kinematic viscosity.

\section{Image analysis}

In order to confirm the results in Fig. 4, an analysis of instantaneous images of the atomization process has been performed. Figure 6 shows the situation for the different liquids at a common voltage of $30 \mathrm{~V}$. As in Fig. 3, images are displayed with a $\gamma=2$ look-up table. While for hexane, heptane and methanol the mist of small droplets is clearly visible, for decane, ethanol and water, this production is still in its initial stages. In the case of 2-propanol these droplets are almost not generated at all. Visual estimation of atomization intensity is in perfect agreement with the atomization rate values plotted in Fig. 4. From analysis of images obtained at different voltages, it can be concluded that production of the micron-sized droplets seems to be independent of the formation of the coneshaped liquid fountain. Fountain height increases with voltage, but mist formation is inhibited for high viscosity values. It is interesting to note that according to the experiments in [8] working with mixtures of glycerol and water, ultrasonic atomization is difficult for kinematic viscosities over $3 \times 10-6 \mathrm{~m}^{2} / \mathrm{s}$. Viscosity of 2-propanol, for which fog production is very low, is slightly above this limit.

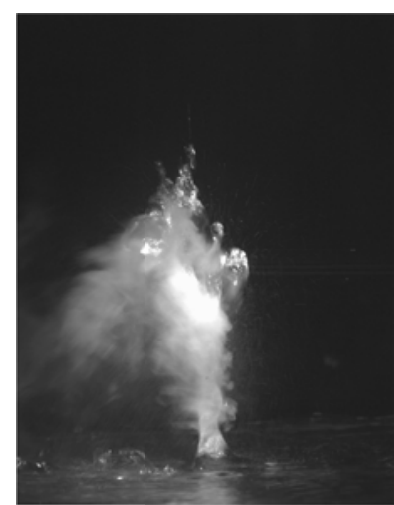

Hexane

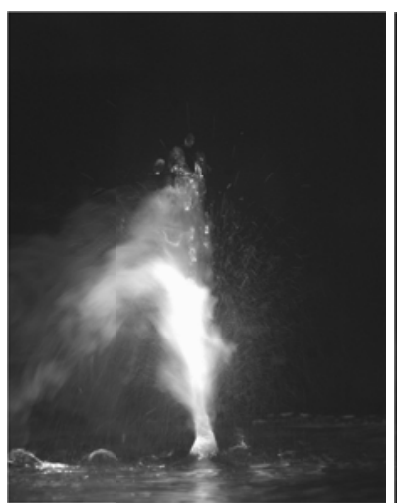

Heptane

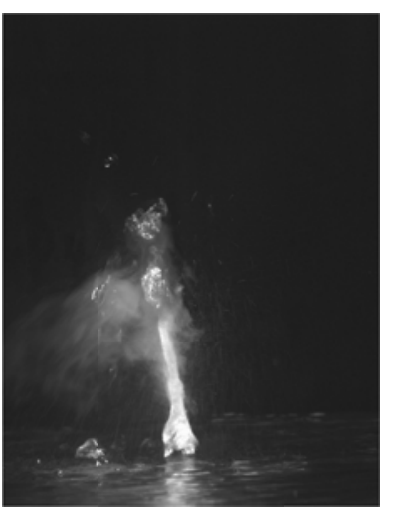

Decane

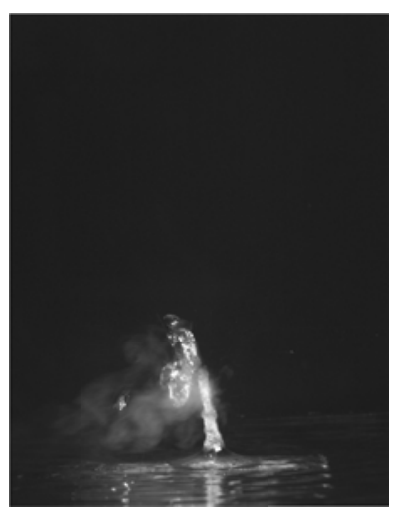

Water

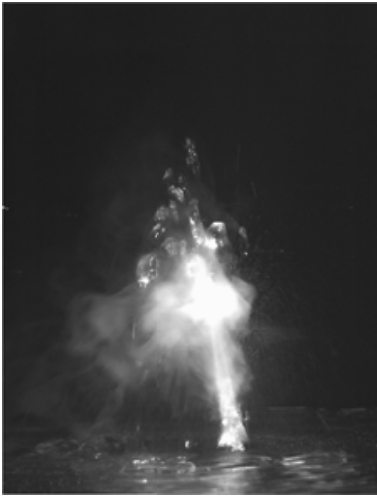

Methanol

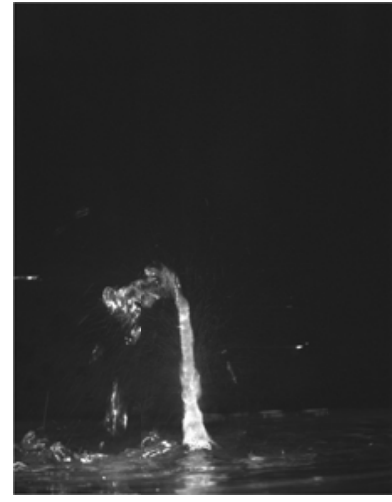

Ethanol

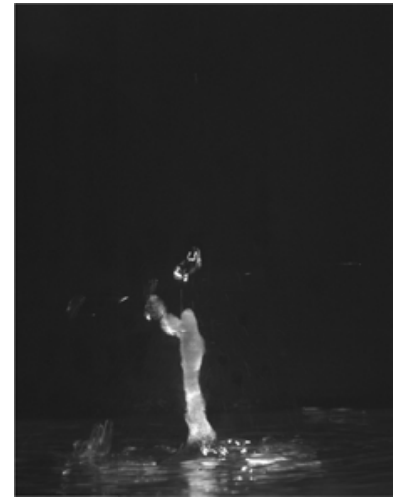

2-Propanol

Figure 6. Ultrasonic atomization of different liquids for a common forcing voltage of $30 \mathrm{~V}$ 
From the physical properties listed in Table 1, it is to be observed that the main difference between water and the rest of the liquids can be found in the values for surface tension and compressibility modulus, which are the highest. This could be a reason for its distinct behavior, but further research is required.

Arguments are still required to explain the presence of the atomization rate maximum for some of the tested liquids. Figure 7 shows some images of heptane atomization for driving voltages of $25 \mathrm{~V}, 30 \mathrm{~V}$ and $55 \mathrm{~V}$. Although for all the recorded images atomization seems to be more violent for higher forcing, what appears to increase in a higher degree is the formation of medium-sized droplets, in detriment of the production of the smallest ones. As in the present experiments the atomization rate measurements are limited to this last group of droplets, this might explain the maximum in the curves in Fig. 4.
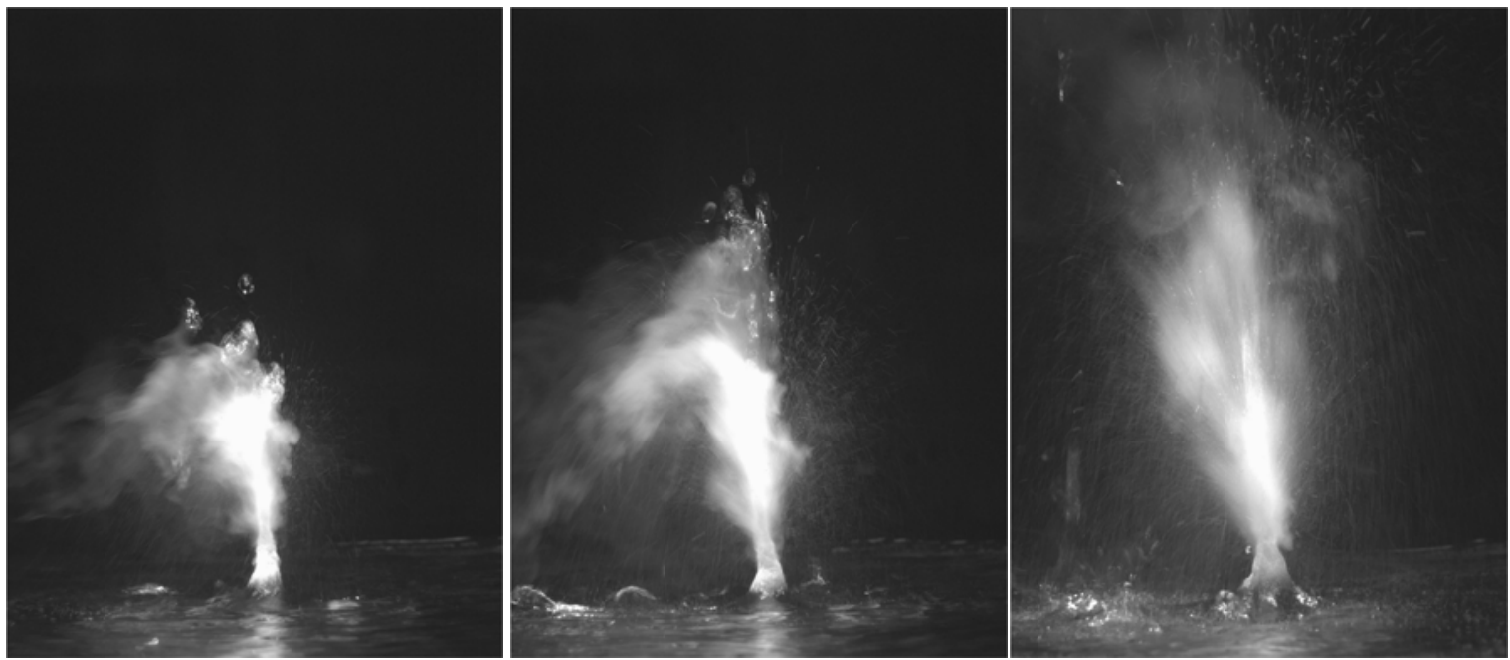

Figure 7. Ultrasonic atomization of heptane for forcing voltage values of $25 \mathrm{~V}, 30 \mathrm{~V}$ and $55 \mathrm{~V}$.

The process of droplet ejection from the cone surface is shown in Fig. 8. It corresponds to methanol atomization for a forcing voltage of $25 \mathrm{~V}$. Time interval between frames is $333 \mu \mathrm{s}$. Relatively large droplets can be seen escaping from the liquid surface. The smallest droplets cannot be discerned individually, and they appear as a fine mist. As a first estimate, this indicates that their size has to be lower than $8 \mu \mathrm{m}$. The droplet size distribution measurements should confirm this extreme.
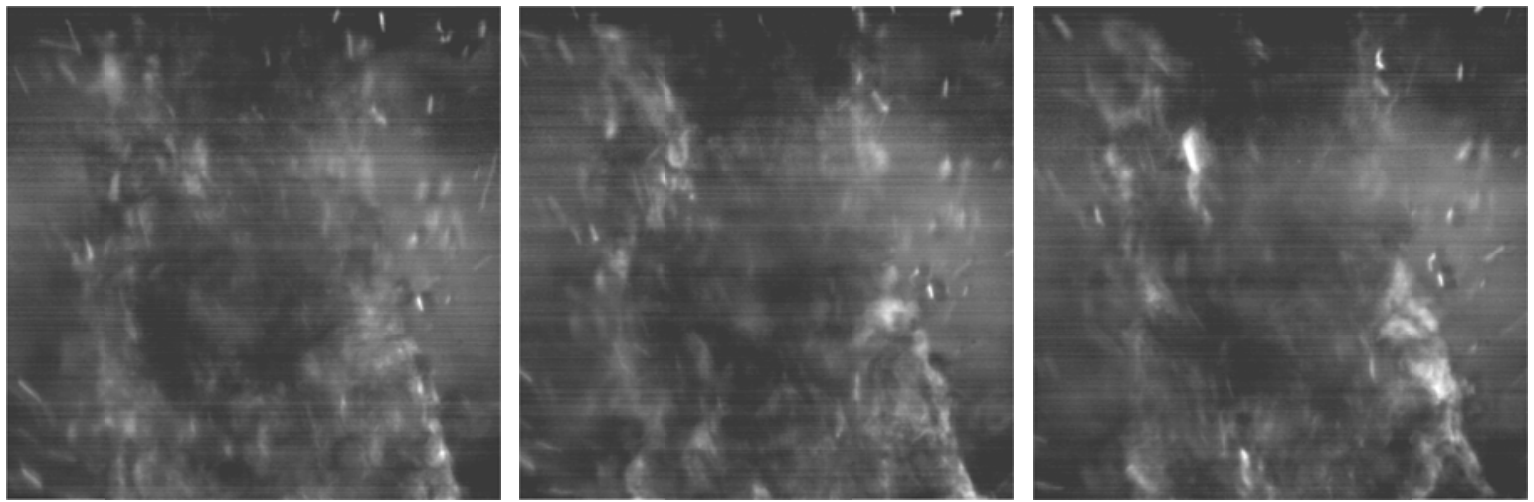

Figure 8. Close up view of ultrasonic atomization of methanol for a forcing voltage of $25 \mathrm{~V}$. Time interval between frames is $333 \mu \mathrm{s}$.

\section{Droplet size distribution measurements}

Similarly to what happened for the atomization rate measurements, the specific experimental set up arranged to determine droplet size distribution only considers the small drops that form the mist. As they are dragged out of the recipient by a slow air flow, large droplets are effectively filtered out by effect of gravity. It is again to be reminded that large droplets are not considered of interest because they can be produced in an efficient way by a variety of atomization methods. It is the generation of micron-sized droplets what is difficult to achieve with other procedures alternative to ultrasonic atomization. 
Measurements for water were already obtained in previous works [2,8]. Some of them have been retaken to ensure repeatability. Main results are compiled in Fig. 9. As expected, it is confirmed that for the liquids in these experiments and for the droplets in the micron range, variation in their physical properties has a weak influence on the size distribution function. For most of the liquids, the shape of the distribution function is nearly identical, with two distinct peaks at $3.3 \mu \mathrm{m}$ and $6.2 \mu \mathrm{m}$. Size distributions for hexane, ethanol and propanol show a slightly different shape. It may be due to the fact that accuracy of these measurements is somewhat lower. The measurements for hexane are inaccurate because they were affected by laser beam steering due to the high concentration of vapors. Non-zero results were obtained flowing the dragging air inside the liquid container with the ultrasonic transducer disconnected. However, we were unable to separate the vapor from the droplets to be measured. Ethanol and 2-Propanol measurements are also inaccurate because droplet concentration was very low and laser obscuration was below the recommended level. Measurements for the rest of the liquids seem to confirm the hypothesis postulated in many previous papers that relates droplet size only to ultrasonic frequency.

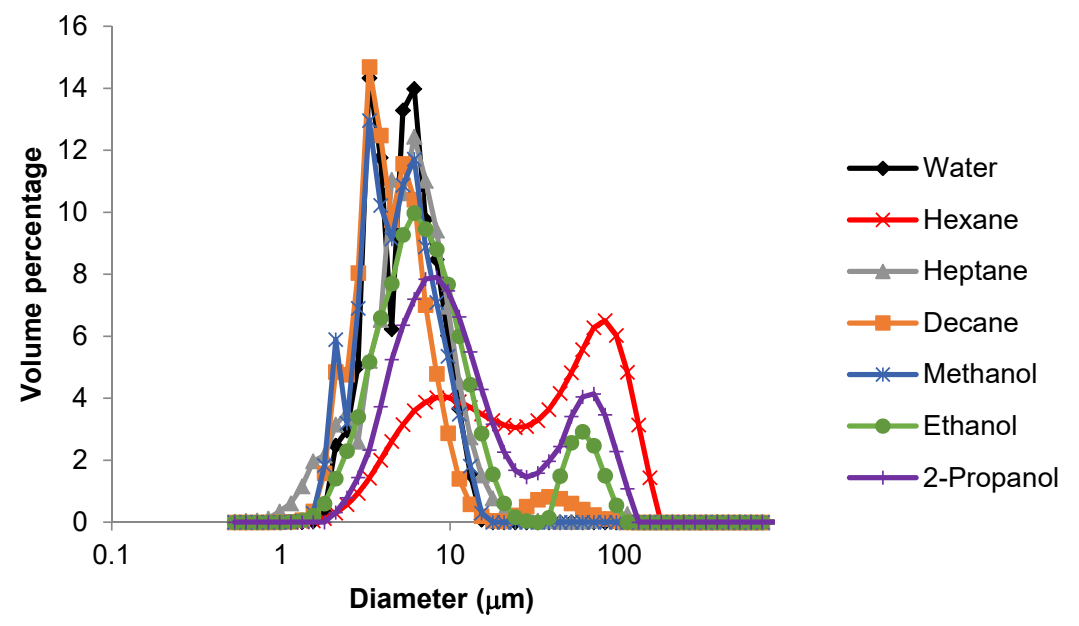

Figure 9. Droplet size distribution functions for the different atomized liquids.

\section{Conclusions}

An experimental study has been conducted to analyze the characteristics of ultrasonic atomization when working with different organic compounds. Three alkanes (hexane, heptane and decane) and three alcohols (methanol, ethanol and 2-propanol) have been considered. Density and surface tension values are quite similar for all of them, in all cases lower than those for water. Viscosity and vapor pressure cover a wider range, increasing for increasing number of $\mathrm{C}$ atoms. Tests operating with water have also been performed for comparison purposes. A commercial piezoceramic disk with a resonance frequency of $1.65 \mathrm{MHz}$, has been used in the experiments. It has been forced with a sinusoidal wave coincident with the resonance frequency and variable amplitude. The maximum applied voltage has been limited to $80 \mathrm{~V}$. In all cases, atomization rates have been measured. Sprays have been characterized analyzing instantaneous images and high speed video sequences, and measuring droplet size distribution functions. It has been observed that in the process of atomization a fine mist of small micron-sized droplets is generated, together with some medium sized droplets that are also ejected from the liquid surface. The amount of this last type of droplets and their detachment velocity clearly increase with increasing forcing voltage. In this study, attention has been only focused on the small drops that form the mist. The large droplets have not been considered of interest because they can be produced in an efficient way by a variety of atomization methods and can be easily filtered out. It is the generation of micron-sized droplets what is difficult to achieve with other procedures alternative to ultrasonic atomization. A somehow unexpected observation is that, contrary to the case of operation with water in which atomization rate always increases with voltage, alkane and alcohol atomization presents a maximum for a certain value, decreasing when voltage is further increased. The presence of this maximum can be attributed to an increase with voltage in the formation of medium-sized droplets, in detriment of the production of the smallest ones. Results also confirm that viscosity strongly conditions the atomization efficiency. On the other hand it has been verified that for a fixed ultrasonic frequency, size of the fog droplets seems to be independent of the liquid nature. 


\section{Acknowledgements}

This work has been partially funded by the Secretariat of State for Research of the Spanish Ministry of Economy and Competitiveness under project DPI2013-45814-P. Support of the Regional Government of Aragon to the Experimental Fluid Dynamics Research Group (T03) is also acknowledged.

\section{References}

[1] Wood, W.R. and Loomis, A.L., (1927), The physical and biological effects of high frequency sound-waves of great intensity, Phil. Mag., vol. 4 (22), 417-437.

[2] Barreras, F., Amaveda, H., Lozano, A., (2002), Transient High-Frequency Ultrasonic Water Atomization, Exp. Fluids, vol. 33 (3), 405-413.

[3] Avvaru, B., Patil, M.N., Gogate, P.R., Pandit, A.B., (2006), Ultrasonic atomization: Effect of liquid phase properties, Ultrasonics, 44, 146-158.

[4] Bassett, J.D. and Bright, A.W., (1976), Observations Concerning the Mechanism of Atomization in an Ultrasonic Fountain, J. Aerosol Sci., vol. 7, (1) 47-51.

[5] Donnelly T.D., Hogan J., Mugler A., Schommer, N., Schubmehl, M., Bernoff, A.J., Forrest, B., (2004), "An experimental study of micron-scale droplet aerosols produced via ultrasonic atomization", Phys. Fluids, vol. 16 (8), 2843-2851.

[6] Sindayihebura, D. and Bolle, L., (1998), Ultrasonic Atomization of Liquid: Stability Analysis of the Viscous Liquid Film Free Surface, Atomization and Sprays, vol. 8, 217-233.

[7] Rajan, R., Pandit, A.B., (2001), Correlations to predict droplets size in ultrasonic atomization, Ultrasonics, 39, 235-255

[8] Lozano, A., García, J.A., Navarro, J.L., Calvo, E., Barreras, F., (2010), Influence of viscosity on droplet size distribution and generation rate in ultrasonic atomization Atomization and Sprays, 20, (11): 923-934.

[9] Lozano, A., García, J.A., Ranz, A., Barreras, F., Santolaya, J.L., (2014), Ultrasonic atomization of mixtures and suspensions, 26th Annual Conference on Liquid Atomization and Spray Systems, Bremen, Germany, 810 Sep. 2014.

[10] Lighthill, J., Acoustic streaming, (1978), Journal of Sound and Vibration, vol. 61 (3), 391-418, 1978. 


\title{
Effect of excitation on gas centered swirl coaxial injectors
}

\author{
Gujeong Park ${ }^{* 1}$, Sukil Oh ${ }^{1}$, Jinhyun Bae ${ }^{1}$, Youngbin Yoon ${ }^{1}$ \\ ${ }^{1}$ Department of Mechanical and Aerospace Engineering, Seoul National University, Republic \\ of Korea \\ *Corresponding author: ybyoon@snu.ac.kr
}

\begin{abstract}
Studies on combustion instability in liquid rocket engines are important in improving combustion efficiency and preventing combustion chamber losses. To prevent combustion instability, methods such as baffles and cavities are used. The injector is located in the middle of the perturbation-propagation process in the rocket engine, so it is important to study the suppression of combustion instability using the design of the injector. Much research has been focused on the study of liquid excitation in a single injector; however, the actual injector used in a liquid rocket engine is a coaxial injector. In this study, the dynamic characteristics of a gas-centred swirl coaxial injector were investigated by varying the gap thickness and momentum-flux ratio. Spray photographs were captured by synchronizing a stroboscope and digital camera, and a high-speed camera and Xenon lamp were also used. To measure the liquid film, a measurement system was implemented using the electrical conductance method. For excitation of the gas, an acoustic speaker was used to impart a frequency to the gas. The gGas velocity and effect of excitation were measured by hot-wire anemometry. A mechanical pulsator was used for liquid flow excitation. Liquid fluctuation was measured by a dynamic pressure sensor. In both gas and liquid excitation cases, the gain increased as the gap thickness decreased and the momentum-flux ratio increased. From these results, it can be concluded that gap thickness and momentum-flux ratio are major factors in suppressing combustion instability.
\end{abstract}

\section{Keywords}

Gas-centred Swirl Coaxial Injector, Excitation, Gain

\section{Introduction}

Research on combustion efficiency and combustion instability is important in the development of a liquid rocket engine. Combustion instability occurs in the combustion chamber when the combustion and the flow of the propellant supply system are combined. This not only reduces combustion efficiency, but also leads to combustion chamber losses in extreme cases. Disturbances that can occur in propellant supply systems ranges from a few $\mathrm{Hz}$ to a few thousand $\mathrm{Hz}$, depending on the causes [1].

One of the causes of combustion instability is perturbations in the flow rate of the propellant to the combustion chamber, which can be caused by various factors. This disturbance of the flow travels through the feed line, the injector, and the combustion chamber, affecting the stability of the combustion. The pressure perturbation resulting from combustion instability can also affect the propellant flow in the feed system. Therefore, the design of an injector located in the middle of this process is of considerable importance. If the injector can serve as a shock absorber to reduce the disturbance from the supply line by optimized design, it would be able to prevent instability of the spray, which could cause combustion instability.

Research into the suppression of combustion instability through injector geometry has been going on since the 1990s. In 1996, Bazarov et al. found that the injector dynamics affected the instability of the liquid rocket engine [2]. In 2007, Soller et al. investigated the combustion instability of an oxidant-feed system and combustion chamber in various gas centred swirl coaxial injectors. They confirmed that the perturbation of the oxidant supply system affects the perturbation of the combustion chamber [3]. Heister et al. proposed several models of selfresonance phenomena of a close-type single swirl injector and analysed it numerically [4]. Fu et al. confirmed the dynamic characteristics of an open-type single swirl injector by changing the injector geometry using a mechanical pulsator.

The previous research only focused on the response characteristics to liquid disturbance in a single swirl injector. The phenomenon of propellant disturbance in a gas-liquid injector used in a liquid rocket engine has not been extensively studied. In this study, the dynamic characteristics of the spray from disturbances of the gas and the liquid were investigated by varying the gap thickness of the gas centred swirl coaxial injector, used mainly in the Russian multi-stage combustion cycle liquid rocket engine, such as the RD-170. 


\section{Material and methods}

The experimental setup is shown in Fig. 1. In this experiment, air and water were used as the experimental fluids to represent oxidant and fuel, respectively. The apparatus comprises a function generator, a data acquisition system (DAQ), an injector, and a high-speed camera. The function generator supplied a constant frequency, and the voltage for the liquid film thickness was measured by the electrodes in the orifice. The method of measuring the liquid film thickness with electrodes, using the electrical conductivity of water, was proposed by Suyari and Lefebvre [5]. Two thin electrodes were placed at the end of the orifice to obtain the voltage generated by the liquid film thickness. The manifold pressure of the injector was measured by a static- and a dynamic-pressure sensor, and was recorded by the DAQ system. The spray image of the injector was obtained using a high-speed camera and a DSLR camera.

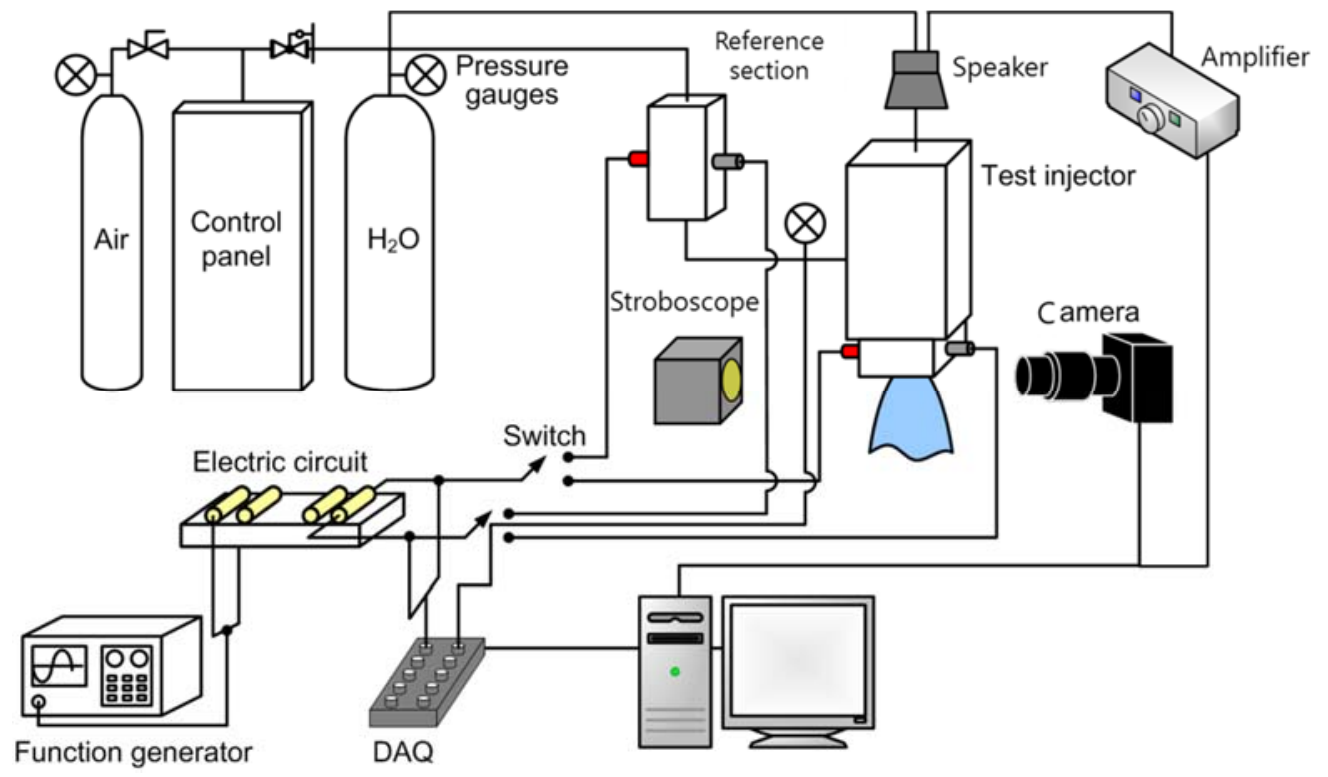

Figure 1. Experimental apparatus

For the gas excitation, a speaker, amplified by an amplifier, was installed in the middle of the gas supply line to generate the perturbation of the gas flow. The influence of the gas velocity perturbation was measured by using hot-wire anemometry. For the liquid excitation, a mechanical pulsator was installed in the middle of the liquid supply line to generate perturbations in the pressure of the supplied liquid. The pulsator was designed to change the area of the flow path by rotating a plate at a predetermined speed, up to $1000 \mathrm{~Hz}$.

The geometry of the gas-centred swirl injector used in the experiment is shown in Fig. 2. The gas orifice is located in the middle of the injector, surrounded by the liquid orifice. The gas is jetted and the liquid is swirled through the tangential inlet and injected. The diameter of the injector orifice was $8 \mathrm{~mm}\left(2 R_{n}\right)$ and the gas orifice diameter $\left(D_{g}\right)$ was $6 \mathrm{~mm}$. The tangential inlet diameter $\left(R_{\text {inlet }}\right)$ was $0.9 \mathrm{~mm}$ for the gas flow perturbation case, and $1.5 \mathrm{~mm}$ for the liquid flow perturbation case. The gap thickness ( $h_{\text {gap }}$ ) was set at $0.3 \mathrm{~mm}, 0.5 \mathrm{~mm}$, and $0.7 \mathrm{~mm}$.

In this experiment, the speaker frequency was varied from $200-1100 \mathrm{~Hz}$ at $100 \mathrm{~Hz}$ intervals to simulate disturbances in the oxidant excess gas. The liquid fuel disturbances were simulated by varying the pulsator frequency from $200-1000 \mathrm{~Hz}$ in $50 \mathrm{~Hz}$ increments. Experiments were carried out by setting the gap thickness, which influences the gas-liquid mixing and the gas-liquid momentum-flux ratio as the main parameter in the gasliquid injector. The momentum flux ratio is defined by Eq. 1, and the experimental conditions are shown in Table 1.

$$
M R=\frac{\rho_{g} U_{g}^{2}}{\rho_{l} U_{a l}^{2}}
$$




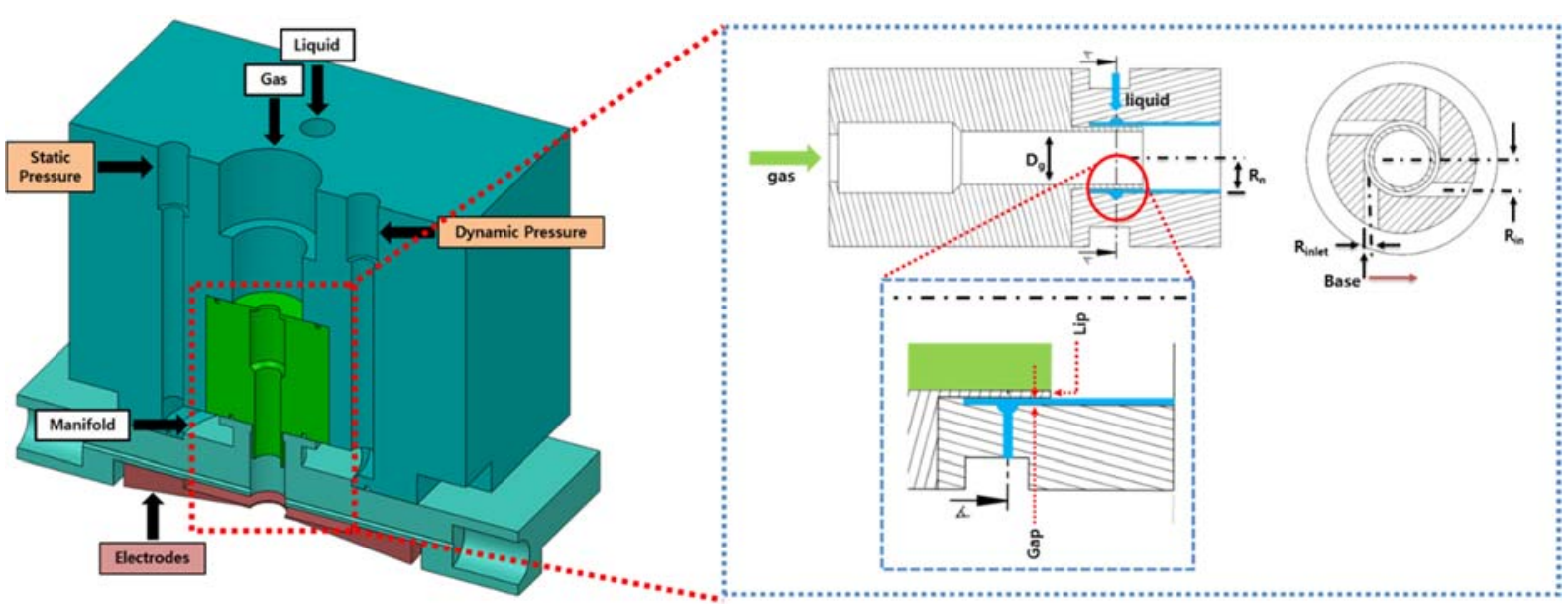

Figure 2. Geometry of gas centred swirl coaxial injector

Table 1. Experimental condition.

\begin{tabular}{c|cc}
\hline \hline & Gas Excitation & Liquid Excitation \\
\hline \hline Gap thickness $[\mathrm{mm}]$ & \multicolumn{2}{|c}{$0.3,0.5,0.7$} \\
Momentum flux ratio & \multicolumn{2}{|c}{$0,0.5,1,2$} \\
Input Frequency $[\mathrm{Hz}]$ & $200-1100$ & $200-1000$ \\
Tangential inlet diameter $[\mathrm{mm}]$ & 0.9 & 1.5
\end{tabular}

\section{Results and discussion}

The thickness of the liquid film at the end of the injector orifice is an important factor influencing the spray angle, breakup length, and droplet size [6]. Therefore, understanding the change in liquid film thickness at the end of the orifice in response to the generated flow disturbances is important in terms of spray- and combustion-instability predictions. In this experiment, the response characteristics of the liquid film thickness to the perturbation during gas excitation and liquid excitation is expressed by the injector transfer function as shown in Eqs 2-4.

$I T F_{\text {gas }}=\frac{\text { output }}{\text { Input }}=\frac{t^{\prime} / \bar{T}}{u^{\prime} / \bar{U}}$

$I T F_{\text {liquid }}=\frac{\text { Output }}{\text { Input }}=\frac{t^{\prime} / \bar{T}}{p^{\prime} / \bar{P}}$

gain $=|\mathrm{ITF}|$

In a gas centred swirl coaxial injector, the gap thickness is a geometric factor that determines the liquid film thickness in a liquid flow. Fig. 3 shows the results of the injector dynamic characteristics for varying gap thickness when the gas is excited while the gas and liquid are sprayed the same time. As the gap thickness decreased, the gain increased. A decrease in the gap thickness means an increase in the lip thickness because the diameter of the gas and liquid orifices is kept constant. Larger lip thickness produces greater flow eddy [7]. Therefore, when the gap is thinner, i.e., when the lip is thicker, a large vortex forms, and the magnitude of the perturbation becomes larger. 


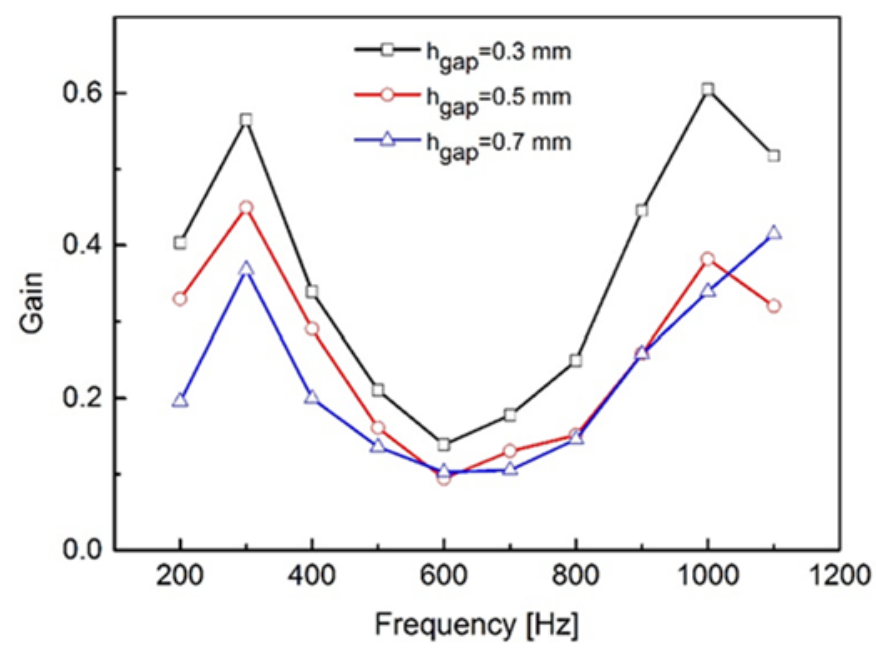

Figure 3. Dynamic characteristics for varying gap thickness with gas flow perturbations

Figure 4 shows the response characteristics of the spray when only the gas is excited, the liquid flow rate is fixed, and the gas-liquid momentum-flux ratio is changed. The increase in the gas-liquid momentum-flux ratio means that the momentum of the gas relative to that of the liquid increases, resulting in a larger momentum transfer for the same mixing-zone length. Therefore, more gas velocity perturbations are transmitted to the liquid, which increases the liquid film tremor at the end of the orifice. However, it can be seen that the increase of the gain due to the increase of the momentum-flux ratio changes with frequency. The effect of the spray pattern on frequency is shown in Fig. 5. When the magnitude of the gain is larger, the liquid film thickness reduces because the effect of the excitation acted on strongly liquid flow. Thus, it can be seen that the spray breaks up quicker and has a qualitatively wider angle due to its earlier breakup.

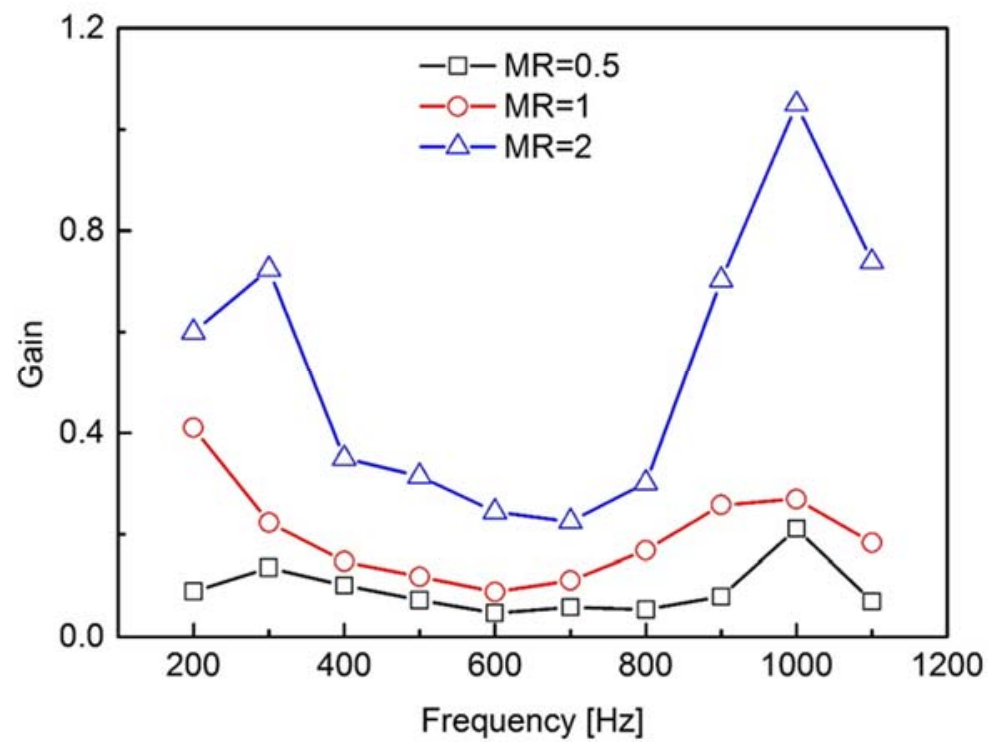

Figure 4. Dynamic characteristics for varying momentum-flux ratios with gas flow perturbations 


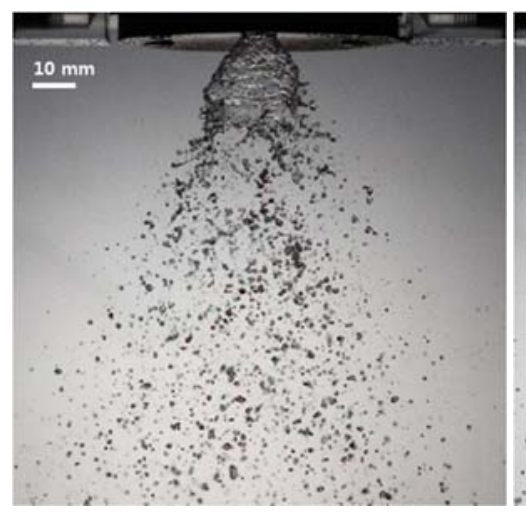

(a)

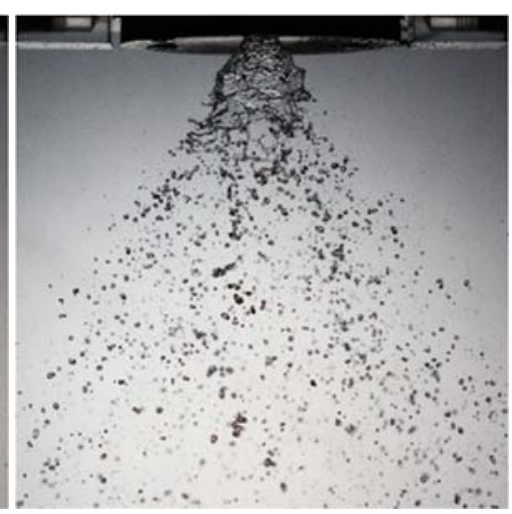

(b)

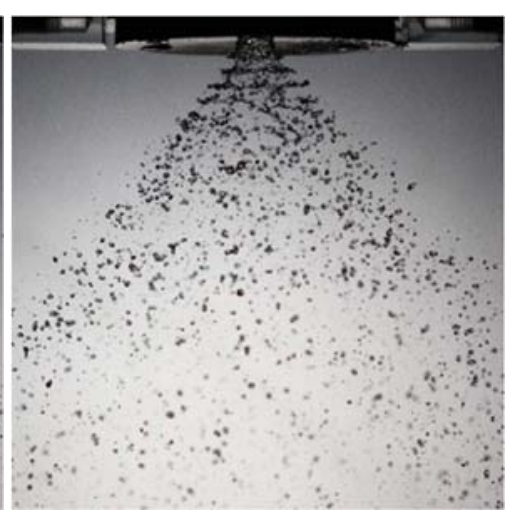

(c)

Figure 5. Spray pattern with gas excitation for $h_{\text {gap }}=0.5 \mathrm{~mm}$ and $M R=2 ;$ (a) $f=0 \mathrm{~Hz}$, (b) $f=600 \mathrm{~Hz}$, and (c) $f=1000 \mathrm{~Hz}$

\section{Liquid Excitation}

Figure 6 shows the dynamic characteristics of the injector for the three gap thicknesses with liquid excitation, while the liquid flow rate is fixed without injecting gas. Gain changes and a phenomenon in which the gain decreased at a specific frequency (sharp drop) can be observed. When the gap thickness was $0.3 \mathrm{~mm}$, the gain was more than twice that of the other cases, which is thought to be due to the liquid film thickness. As shown in Table 2, when the gap thickness was $0.3 \mathrm{~mm}$, the liquid film thickness was approximately half that of the other two gaps. Such a thin liquid film is susceptible to tremors, so that the change in thickness was doubled due to the influence of the excitation. As the gap thickness increased, the frequency at which the gain decreased also decreased. This is because the gap thickness acts as a low pass filter; the fluid passing through the tangential inlet meets the gap, which is a suddenly enlarged space, and only the low-frequency fluid passes through.

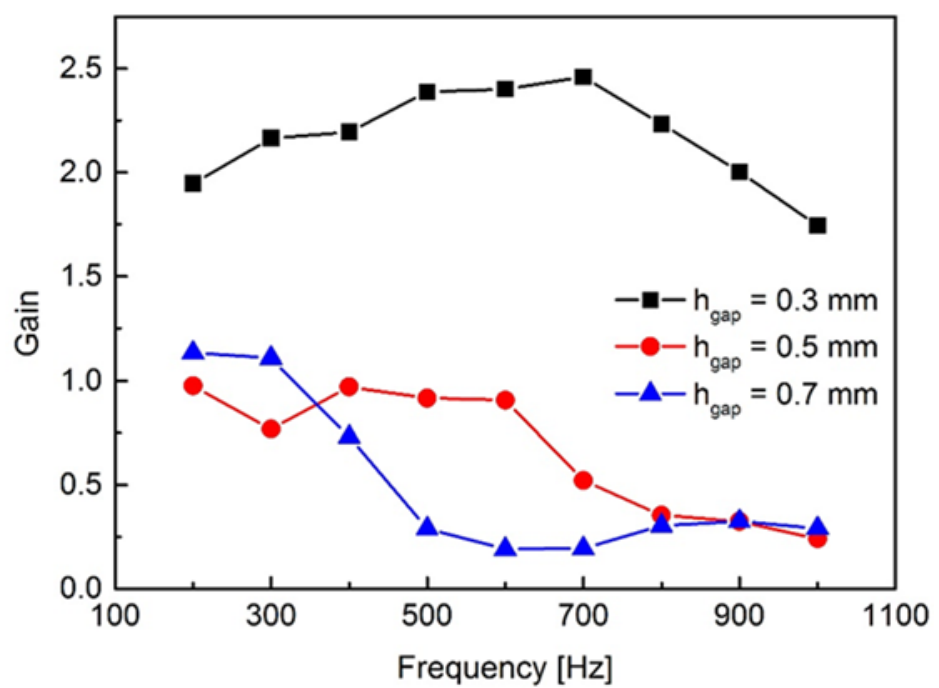

Figure 6. Dynamic characteristics for varying gap thickness with liquid flow perturbations

Table 2. Film thickness and film thickness fluctuation for varying gap thicknesses.

\begin{tabular}{c|c|c}
\hline \hline $\begin{array}{c}\text { Gap thickness } \\
{[\mathrm{mm}]}\end{array}$ & $\begin{array}{c}\text { Film thickness } \\
@ 300 \mathrm{~Hz}[\mu \mathrm{m}]\end{array}$ & $\begin{array}{c}\text { Film thickness fluctuation } \\
@ 300 \mathrm{~Hz}[\mu \mathrm{m}]\end{array}$ \\
\hline \hline 0.3 & 480.70 & 55.84 \\
0.5 & 714.82 & 26.97 \\
0.7 & 887.77 & 29.21
\end{tabular}

Figure 7 shows the results of the dynamic characteristics with different gas-liquid momentum-flux ratios when the gas and the liquid were injected simultaneously, with only the liquid having flow perturbations. An increase in the 
gas-liquid momentum-flux ratio leads to the formation of a thin liquid film thickness that is susceptible to perturbations, thereby increasing the gain. It was also considered that the gas momentum added to the liquid tremor, and increased the gain. Figure 8 shows the spray pattern at three different frequencies. It can be seen that the spray has a clear single layer of the liquid mass concentrated at every excitation period in the case where the gain was larger, similarly to the case of gas flow perturbations.

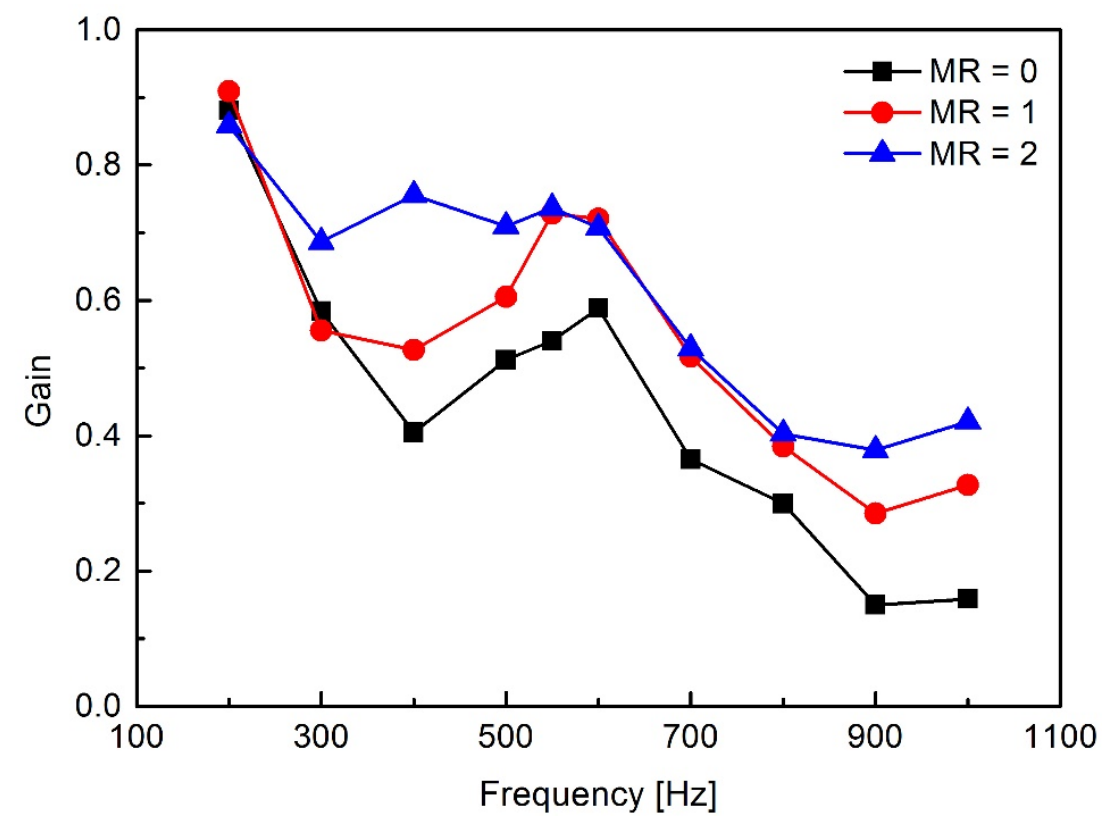

Figure 7. Dynamic characteristics for varying momentum-flux ratios with liquid flow perturbations

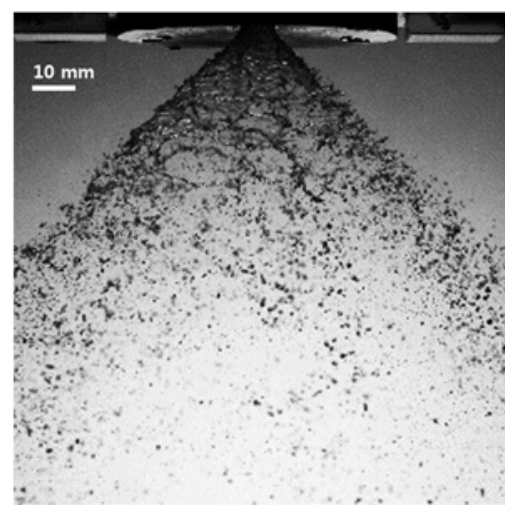

(a)

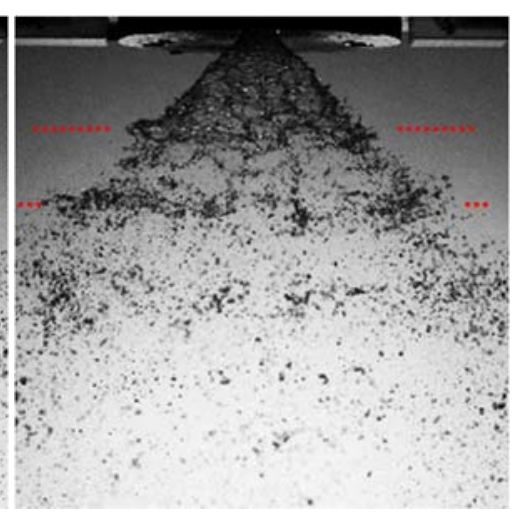

(b)

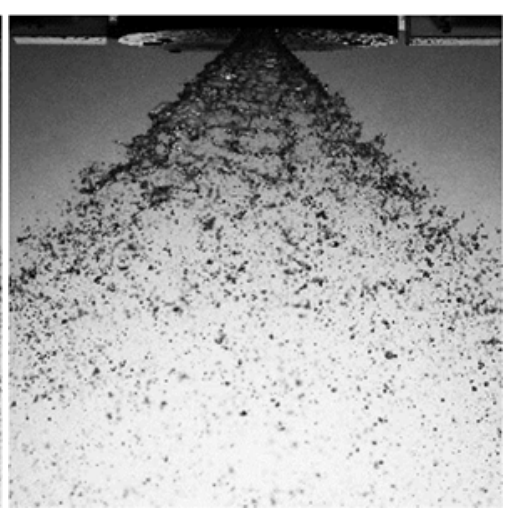

(c)

Figure 8. Spray pattern with liquid excitation for $h_{\text {gap }}=0.5 \mathrm{~mm}$ and $M R=2 ;(a) f=0 \mathrm{~Hz}$, (b) $f=400 \mathrm{~Hz}$, and (c) $f=900 \mathrm{~Hz}$

\section{Conclusions}

To investigate the dynamic characteristics of a gas-centred swirl coaxial injector, which is used mainly in a multistage combustion cycle engine, a speaker and a mechanical pulsator were used to generate gas- and liquid-flow perturbations. The effect of the geometry on the dynamic characteristics was investigated by varying the gap thickness, and the dynamic characteristics of the injector were determined by varying the momentum flux ratio. In the case of gas excitation, the gain increased as the gap thickness decreased. It is considered that as the gap thickness decreased and the lip became thicker, a larger vortex formed, resulting in a larger gain. As the momentum flux ratio increased, the gain increased. This is because more of the gas momentum was transferred to the liquid, as the momentum of the gas relative to that of the liquid increased.

In the case of liquid excitation, the gain increased as the gap thickness decreased. Because of the spatial limitations of the smaller gap thickness, a thin liquid film was formed and was susceptible to tremors, which are caused by the influence of the excitation. As the momentum flux ratio increased, the gain increased. This is thought to be due to the momentum of the gas being transmitted to the liquid. 
From these results, it can be concluded that the gap thickness is considered to be the main geometric factor for suppressing combustion instability, and that the momentum-flux ratio was also a factor for suppressing combustion instability.

\section{Acknowledgements}

This work was supported by the Advanced Research Centre Program (NRF-2013R1A5A1073861) through a National Research Foundation of Korea (NRF) grant, funded by the Korean government (MSIP), and contracted through the Advanced Space Propulsion Research Centre at Seoul National University and was supported by an NRF (National Research Foundation of Korea) Grant funded by the Korean Government (NRF-2016-Fostering Core Leaders of the Future Basic Science Program/Global Ph.D. Fellowship Program).

\section{Nomenclature}

ITF injector transfer function

t' film thickness fluctuation $[\mu \mathrm{m}]$

$\bar{T} \quad$ average of film thickness $[\mu \mathrm{m}]$

p' manifold pressure fluctuation [bar]

$\bar{P} \quad$ average of manifold fluctuation [bar]

MR momentum flux ratio

$\rho_{g} \quad$ density of gas

$\rho_{l} \quad$ density of liquid

u' gas velocity fluctuation $[\mathrm{m} / \mathrm{s}$ ]

$\bar{U} \quad$ average of gas velocity $[\mathrm{m} / \mathrm{s}]$

$U_{g} \quad$ velocity of gas

$U_{a l} \quad$ axial velocity of liquid

$D_{g} \quad$ diameter of the gas orifice

$\mathrm{R}_{\text {in }} \quad$ distance from the centre of the injector orifice to the centre of the tangential inlet

$R_{n} \quad$ radius of the injector orifice

$R_{\text {inlet }} \quad$ radius of the tangential inlet

\section{References}

[1] Harrje, D. T., and Reardon, F. H., 1972, NASA Rept. SP-194.

[2] Bazarov, V. G., and Yang, V., 1998, Journal of Propulsion and Power, 14 (5).

[3] Soller, S., Wagner, R., Kau, H., Martin, P., and Mading, C., 2007, 43rd AIAA/ASME/SAE/ASEE Joint Propulsion

Conference and Exhibit.

[4] Ahn, B., Ismaliov, M., and Heister, S. D., 2009, 45th AIAA/ASME/ASE/ASEE Joint Propulsion Conference \& Exhibit.

[5] Suyari, M., and Lefebvre, A. H., 1986, Journal of Propulsion and Power, 2 (6), pp. 528-533

[6] Bayvel, L., and Orzechowski, Z., 1993, Liquid Atomization.

[7] Tsohas, J., Canino, J. V., and Heister, S. D., 2007, 43rd AIAA/ASME/SAE/ASEE Joint Propulsion Conference and Exhibit. 


\title{
Effect of liquid viscosity on the aerodynamic breakup of non-spherical droplets
}

\author{
Konstantinos Bergeles ${ }^{1, *}$, Georgios Charalampous ${ }^{1}$, Yannis Hardalupas ${ }^{1}$ and Alex M. Taylor ${ }^{1}$ \\ ${ }^{1}$ Mechanical Engineering Department, Imperial College London, UK \\ *Corresponding author: k.bergeles12@imperial.ac.uk
}

\begin{abstract}
This paper studies the effect of liquid viscosity on the atomisation regimes of initially spherical and non-spherical droplets and also kinematic characteristics of non-spherical droplets. The droplets consisted of water-glycerol solutions with viscosities ranging from 6.3 to $697 \mathrm{mPas}$, and the initial aspect ratio was $1<\mathrm{AR}<2$. The range of $W e$ number was from 10 to 200 and of the $O h$ number from 0.01 to approximately 4 . The experimental findings showed that the equivalent $W e_{\mathrm{eq}}$ and $O h_{\mathrm{eq}}$ numbers, proposed in previous work [1] for water droplets, are also applicable to spherical and non-spherical droplets in the range of $O h$ numbers of this study in order to classify the breakup regimes on the existing morphological charts. The kinematic characteristics of the centre of mass for droplets with $A R=1.2$ are evaluated and the role of viscosity examined in the no breakup and bag-stamen regimes.
\end{abstract}

\section{Keywords}

non-spherical, droplet, breakup, aerodynamic

\section{Introduction}

Understanding the physics and nature of liquid droplet-air interaction, investigating the droplet internal and external flow and capturing droplet deformation and breakup is of importance in various scientific fields and technological applications [2]. In some technological applications, such as automotive sprays, the need is to avoid the generation of the largest droplets. Although their number density is low, these sizes carry a disproportionate fraction of the injected fuel, which evaporates slowly, and follow 'ballistic' trajectories resulting in locally overly rich mixtures, associated with undesirable emission characteristics.

The literature on experimental droplet atomisation assumes an initial spherical droplet. In other words, most research classifies and investigates the breakup modes under morphological regimes treating the droplet as a sphere. However, in regions of intense atomisation, as found in the interior of the crankcase, the technological motivator of the present work, droplets are known to have an initial non-spherical shape before the interaction with the flow inside the crankcase.

According to Hinze [3] droplets droplet deformation occurs as the value of $W e$ becomes greater than one $(W e>1)$. The external aerodynamic force acting on the droplet's surface, which scales as $\rho u^{2}$ becomes larger than the counteracting force owing to the surface tension $\sigma / d_{0}$ of the liquid. The deformation evolves to breakup at the critical value of $W e_{\text {crit }}=12$ number. In cases where $W e \approx W e_{\text {crit }}$ number simple breakup mechanism occurs, while for increasing $W e$ number, the disintegration of the droplet becomes a more chaotic atomisation process.

Pilch and Erdman [4] performed experiments to quantify characteristic variables of the breakup process, classifying the breakup modes. For increasing $W e$ number the atomisation regimes found were the vibrational, bag, bagstamen, sheet stripping and catastrophic breakup as shown in Figure 1. The normalised displacement $x_{d} *$ (Eq. (1)), velocity $u_{d}^{*}$ (Eq. (2)) and acceleration $a_{d}^{*}$ (Eq. (3)) of the centre of mass of the droplet were also presented. The study modelled the droplet as a rigid sphere, neglecting mass loss during the atomisation, as also the droplet velocity relative to the flow assuming an average, constant drag coefficient $C_{d}$ throughout the breakup process.

$$
\begin{aligned}
& x_{d}^{*}=\frac{x_{d}}{d_{0}}=\frac{3}{8} C_{d}\left(t^{*}\right)^{2} \\
& u_{d}^{*}=\frac{u_{d}}{u_{g} \cdot \epsilon^{0.5}}=\frac{3}{4} C_{d} t^{*} \\
& \alpha_{d}^{*}=\frac{\alpha_{d} \cdot d_{0}}{u_{g}^{2} \cdot \epsilon}=\frac{3}{4} C_{d}
\end{aligned}
$$

, where $x_{d}, u_{d}, a_{d}$ are the dimensioned displacement, velocity and acceleration of the centre of mass of the droplet. The timescale $t^{*}$ of the atomisation phenomenon (Eq. (4)) equals to:

$$
t^{*}=\frac{t \cdot u_{g} \epsilon^{1 / 2}}{d_{0}},
$$

where $\epsilon$ is the density ratio $\rho_{g} / \rho_{l}, t$ is the dimensioned time and $C_{d}$ is the droplet drag coefficient which remains constant during the atomisation and equal to $C_{d}=2.5$ and $C_{d}=1.7$ for compressible and incompressible flows respectively. 


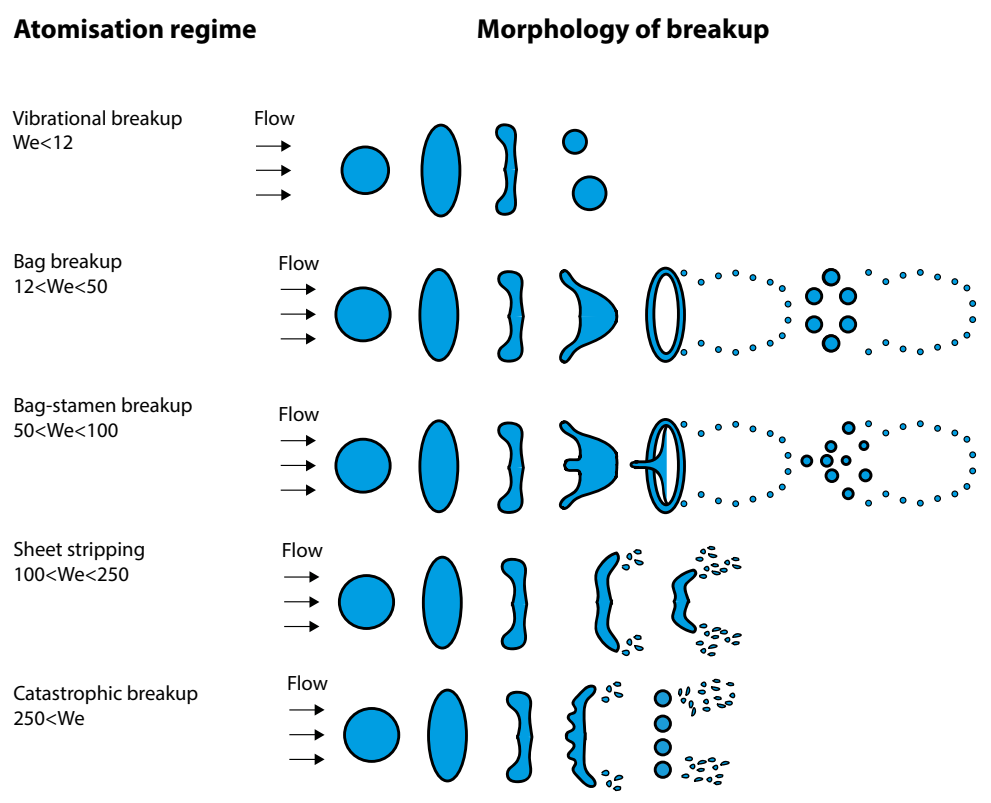

Figure 1. Morphological classification of breakup modes based on $W e$ number as derived by Pilch and Erdman [4]

In addition Pilch and Erdman [4] proposed a third degree polynomial to express the droplet displacement $x_{d} *$ (Eq. (5)), velocity $u_{d}^{*}$ (Eq. (6)) and acceleration $a_{d}^{*}$ (Eq. (7)) using the empirical constant $B$ to provide a correct initial behaviour.

$$
\begin{aligned}
& x_{d}^{*}=\frac{x_{d}}{d_{0}}=\frac{3}{8} C_{d}\left(t^{*}\right)^{2}+B\left(t^{*}\right)^{3} \\
& u_{d}^{*}=\frac{u_{d}}{u_{g} \cdot \epsilon^{0.5}}=\frac{3}{4} C_{d} t^{*}+3 B\left(t^{*}\right)^{2} \\
& \alpha_{d}^{*}=\frac{\alpha_{d} \cdot d_{0}}{u_{g}^{2} \cdot \epsilon}=\frac{3}{4} C_{d}+6 B t^{*}
\end{aligned}
$$

with $C_{\mathrm{d}}=1, B=0.116$ for compressible flow and $C_{d}=0.5, B=0.0758$ for incompressible flow. The correlation assumes a constant drag coefficient $C_{\mathrm{d}}$ of a rigid constant-mass sphere. the change of the frontal area of the droplet due to deformation is not taken into account. Moreover, the equations are valid for the period of time until the velocity of the droplet is $60 \%$ of the velocity of the flow.

The transition between two breakup regimes has been found to be a function of the $W e$ and $O h$ number and independent of the density ratio or the Re number. These transitional $W$ e numbers have been quantified by Brodkey [5] in Eq. (8) and Gelfand [6] in Eq. (9).

$$
\begin{aligned}
& W e_{\text {crit }}=W e_{\text {crit }, 0}\left(1+1.077 O h^{1.6}\right) \\
& W e_{\text {crit }}=W e_{\text {crit }, 0}\left(1+1.5 O h^{0.74}\right)
\end{aligned}
$$

, where $W e_{\text {crit }}$ is the transitional $W e$ number for increased $O h$ number and $W e_{\text {crit }, 0}$ is the transitional $W e$ number for $O h \rightarrow 0$ respectively.

However a gap exists in the literature of the droplet atomisation as all the previous analytical work and interpretation of experimental data assumed initial spherical droplets.

\section{Experimental arrangement and measurement technique}

The experimental arrangement is shown in Figure 2. The main components are a Tec5 ultrasonic levitator which is used to levitate individual droplets and control their sphericity, a Photron CMOS high-speed camera for the imaging of the breakup and a nozzle controlled by a high-speed solenoid valve which produces the cross-flow that atomises the droplet.

Droplet levitation: An ultrasonic wave is emitted from the transducer of the levitator, which is reflected back to its source by a reflector, creating a vertical standing wave. A single droplet with diameter of the order of one millimetre is 


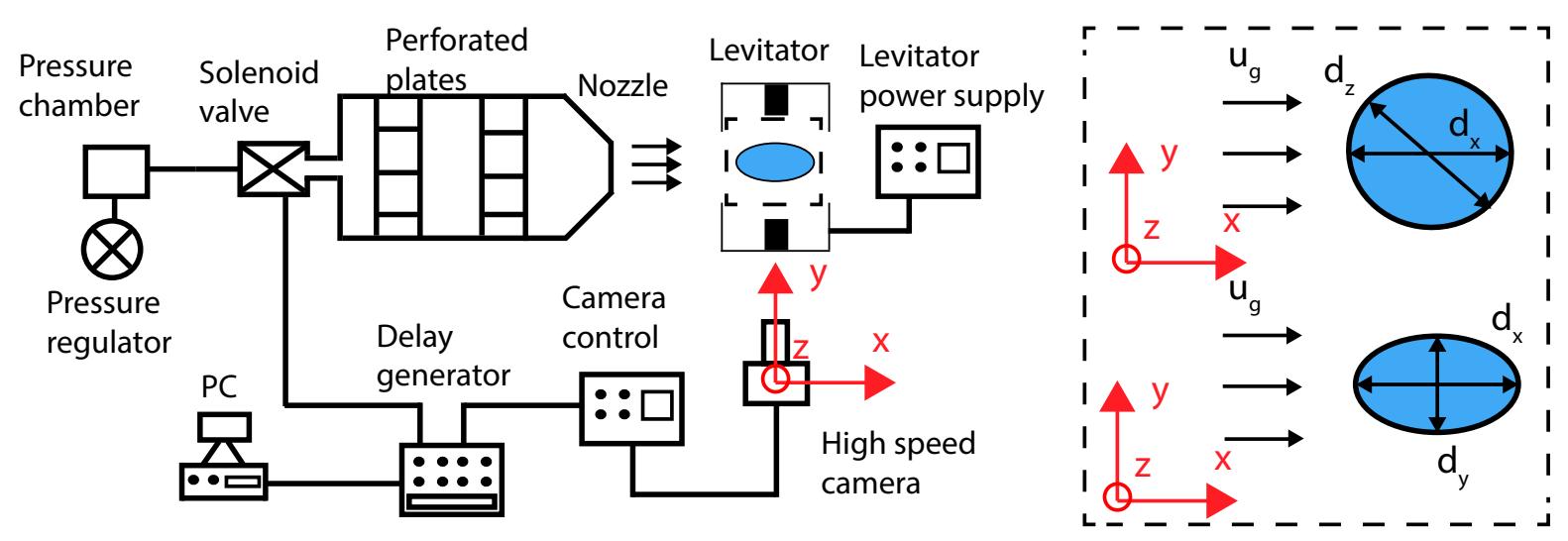

Figure 2. Experimental arrangement of the aerodynamic breakup study of spherical and non-spherical droplets

Table 1. Physical properties of glycerol-water solutions at $20^{\circ} \mathrm{C}$ ambient temperature

\begin{tabular}{lcccc}
\hline $\begin{array}{l}\text { Name } \\
\text { (\% glycerol) }\end{array}$ & $\begin{array}{c}\text { Dynamic viscosity } \\
\mu_{l}[\mathrm{mPa}]\end{array}$ & $\begin{array}{c}\text { Kinematic viscosity } \\
\nu_{l}\left[\mathrm{~mm}^{2} / \mathrm{s}\right]\end{array}$ & $\begin{array}{c}\text { Density } \\
\rho_{l}\left[\mathrm{~kg} / \mathrm{m}^{3}\right]\end{array}$ & $\begin{array}{c}\text { Surface tension } \\
\sigma[\mathrm{mN} / \mathrm{m}]\end{array}$ \\
\hline \hline & & & & \\
Mix-1 (50\%) & 6.386 & 5.674 & 1125.5 & 68.5 \\
Mix-2 (80\%) & 58.96 & 48.839 & 1207.3 & 65.5 \\
Mix-3 (97\%) & 697 & 557.15 & 1251.0 & 63.0 \\
\hline
\end{tabular}

placed at a pressure node and levitated until aerodynamically atomised from a high speed air stream. The sphericity of the droplet is controlled by adjusting the amplitude of the ultrasonic waves, permitting the transformation of a spherical droplet into an oblate spheroidal droplet.

The acoustic pressure induced on the upper and lower pole of the droplet plays a minor role during the deformation and atomisation process. For a millimetre droplet, the lift force generated by the acoustic pressure balances the gravitational force and caused a total pressure within the droplet to increase by approximately $10 \mathrm{~Pa}$. The acoustic pressure is the cause of transformation of the spherical droplet shape into an oblate spheroidal shape, due to surface tension, approximately $70 \mathrm{~Pa}$ for the maximum $A R$. The minimum dynamic pressure of the flow is $500 \mathrm{~Pa}$, therefore the contribution of the maximum acoustic pressure to the droplet atomisation is an order of magnitude lower compared to the minimum dynamic pressure of the flow. As a result, the role of the acoustic pressure on the atomisation is expected to be negligible.

Gas flow: The droplets were impulsively accelerated by a sudden gust of air, which was released from a pressure chamber by a fast solenoid valve. The cross-flow consisted of filtered air at room temperature $20^{\circ} \mathrm{C}$. The air jet flow was ejected through a nozzle with $10 \mathrm{~mm}$ diameter. The velocity at the exit of the nozzle was measured using a Dantec hotwire device to examine the uniformity and repeatability of the velocity profile. The velocity profile of the flow as a function of time is close to a step function with accelerations ranging from 1928 up to $13 \cdot 10^{3} \mathrm{~m} / \mathrm{s}^{2}$ over the range of experiments. The coefficient of variance of the acceleration is lower than $8 \%$ for all operating conditions.

The maximum and mean velocity value of the flow were measured at four distinct points at a radius of $2 \mathrm{~mm}$ and at the centre of the nozzle. The measurements, show an almost uniform velocity profile with a standard deviation well below 3\%, thus the droplet atomisation occurs under a uniform velocity profile. However, for low air velocities, discrepancies in the flow are larger, but still within an acceptable range with maximum standard deviation of around $4.3 \%$.

High-speed imaging: The shadowgraphic technique is employed for the visualisation of the droplet breakup. A Photron high-speed CMOS camera records the droplet breakup at a frame rate of $20 \mathrm{kHz}$. The spatial resolution of the images is $512 \times 256$ pixels with $5 \mu$ s exposure time. A commercial Nikon lens (f-number $f / \#=2.8)$ with appropriate extension tubes and a teleconverter (TELEPLUS MC7 AF 2.0X DGX) are used leading to an image magnification of $12.3 \mu \mathrm{m} /$ pixel. The imaged area of the camera is $6.2 \mathrm{~mm} \times 3.1 \mathrm{~mm}$ in the xy plane as shown in Figure 2 .

Due to the high acquisition rate, the droplet breakup was temporally resolved. The camera was placed perpendicular to the flow direction and it was focused on the symmetry plane of the droplet. The synchronisation of the solenoid valve with the high-speed camera was achieved using a SRS 535 digital delay generator. Finally, a back illumination lamp of $1000 \mathrm{~W}$ was used and uniform illumination was accomplished with ground glass diffusers. The processing of the images was performed in Matlab environment using an in-house code and in ImageJ [7], an image manipulation software.

For the present study three glycerol-water solutions were produced and the viscosity measured using a viscometer. The physical properties were derived for $20^{\circ} \mathrm{C}$ ambient temperature and the characterisation error is within $2 \%$. Table 1 summarises the physical properties of the glycerol-water solutions. 


\section{Morphological classification of droplet breakup}

The breakup modes appearing for increasing $W e$ numbers are the no, bag, bag-stamen and sheet stripping breakup modes. These regimes change for increasing $O h$ numbers and it must be pointed out that the transition between breakup regimes is not a step function but it occurs gradually. Therefore the characterisation of a breakup could be difficult and rather arbitrary, especially between bag-stamen and sheet stripping breakup regimes and also for viscous liquids.

The most commonly used theoretical curves describing this transition of the breakup modes are produced by Brodkey [5] (Eq. (8)) and Gelfand [6] (Eq. (9)). According to these relations, a steep change in the transitional $W e$ numbers appears for higher $O h$ number $(O h>0.1)$. From this $O h$ number and onward the liquid viscous forces become dominant and an exponential change occurs to the transitional $W e$ numbers.

The morphological classification in the $O h-W e$ number plane of the present experimental data is shown in Figure 3. The derivation of the dimensionless numbers was done with the diameter $d_{0}$ based on droplet's volume. Overlapping regions appeared, especially in the bag and bag-stamen breakup regimes for Mix-1 (50\% glycerol) and Mix-2 (80\% glycerol) solutions.

The theoretical curve of Brodkey [5] and Gelfand [6] are also presented in Figure 3. The regime boundaries are approximately flat for $O h<0.1$; Gelfand's relation [6] produces higher transitional $W e$ numbers for lower $O h$ numbers than Brodkey's [5], however the latter one predicts a steeper change in the transitional $W e$ numbers. The theoretical curves of Brodkey [5] and Gelfand [6] predicting the breakup regime boundaries should be taken as a general rule.

For $O h>0.05$ the discrepancies of the two theoretical curves become significant, showing that the transition criteria of breakup regimes for increasing $\mathrm{Oh}$ are not well defined even for initially spherical droplets. The experimental data does not agree with the predicted transitional $W e$ numbers because the initial sphericity of the droplet has not taken into consideration by the theoretical curves.

Figure 4 is the representation of the breakup modes in $W e_{\mathrm{eq}}-O h_{\mathrm{eq}}$ plane. The derivation of the dimensionless numbers was done with the equivalent droplet diameter $d_{\text {eq }}$ (Eq. (11). The equivalent $W e_{e q}$ [1] (Eq. (10)) number for deformed droplet is:

$$
W e_{e q}=\frac{\rho_{g} u_{g}^{2} d_{\mathrm{eq}}}{\sigma}
$$

, where $d_{\text {eq }}$ is the equivalent diameter and defined in Eq. (11)

$$
d_{e q}=\frac{d_{x}^{2}}{d_{y}}
$$

, where $d_{x}, d_{y}$ are the stream-wise and cross-stream diameter of the droplet respectively. In the case of the spherical droplet, the equivalent diameter is equal to the diameter of the droplet $d_{0}$ based on its volume. In the same way the equivalent $O h_{e q}[1]$ is formulated (Eq. (12))

$$
O h_{e q}=\frac{\mu_{l}}{\sqrt{\rho_{l} \sigma d_{\mathrm{eq}}}}
$$

Figure 3 and Figure 4 map the breakup mode of both initially spherical and non-spherical droplets; however the initial sphericity of the droplets is not illustrated in the figures for clarity purposes. The introduction of $d_{\text {eq }}$ to the $O h$ number gives $O h_{\text {eq }}<O h$; in other words the equivalent diameter $d_{\text {eq }}$ "reduces" the effect of liquid viscosity (and as a consequence of the overall $\mathrm{Oh}$ number) to the transition of breakup modes. For the same $W e$ number, higher initial $d_{\text {eq }}$ leads to lower $O h_{\text {eq }}$ compared to a spherical droplet $\left(d_{0}, O h\right)$ resulting to a "higher" breakup regime. The combined effect of the equivalent $O h_{\mathrm{eq}}$ and $W e_{\mathrm{eq}}$ is to translate the experimental data upwards and to the left; meaning that if one is held constant the introduction of $d_{e q}$ leads to "higher" breakup regimes.

For the two least viscous solutions (50\% and $80 \%$ glycerol) the overlapping of the experiential data appears decreased compared to the $\mathrm{We}-\mathrm{Oh}$ plane representation. Additionally, both sets of experimental data are in better agreement with the two theoretical curves (Figure 8 and Figure 9).

The equivalent dimensionless groups $\left(W e_{\text {eq }}\right.$ and $\left.O h_{\text {eq }}\right)$ incorporate the initial non-sphericity of the droplets which lead to a better mapping of the experimental data than the conventional $W e$ and $O h$ numbers. For droplets with $O h>1$ ( $97 \%$ glycerol) the regions where breakup modes overlap remains. As the viscosity effects become dominant, the role of $O h$ number becomes important.

\section{Effect of liquid viscosity to the kinematic characteristics of droplets}

The displacement $x_{d}^{*}$, velocity $u_{d}^{*}$ and acceleration $\alpha_{d}^{*}$ of the centre of droplet's viewable area are presented as a function of dimensionless time $t^{*}$ for water and three glycerol-water solutions $(50 \%, 80 \%$ and $97 \%$ glycerol). The results of the study are split in two sections, for low and high $W e$ numbers, or in other words for no and bag-stamen breakup regimes. The aspect ratio $A R$ chosen for this study is $A R=1.2$. It could be achieved with all liquid with satisfactory repeatability leading to minimisation of the errors regarding the droplet's volume and initial $A R$; both of the errors are below $5 \%$. For the normalisation of the results the diameter $d_{0}$ is used. This is done in order to maintain the physical meaning of the kinematic characteristics. For example, an initially spherical droplet translates faster than an initially non-spherical. When $d_{\mathrm{eq}}$ is employed for the normalisation of the velocity this would not be the case for the non-dimensional velocity $u_{d}^{*}$. However, in the present paper, since the is constant $(A R=1.2)$, diameter $d_{\text {eq }}$ could be used as well as it is the same for all breakup cases presented. 


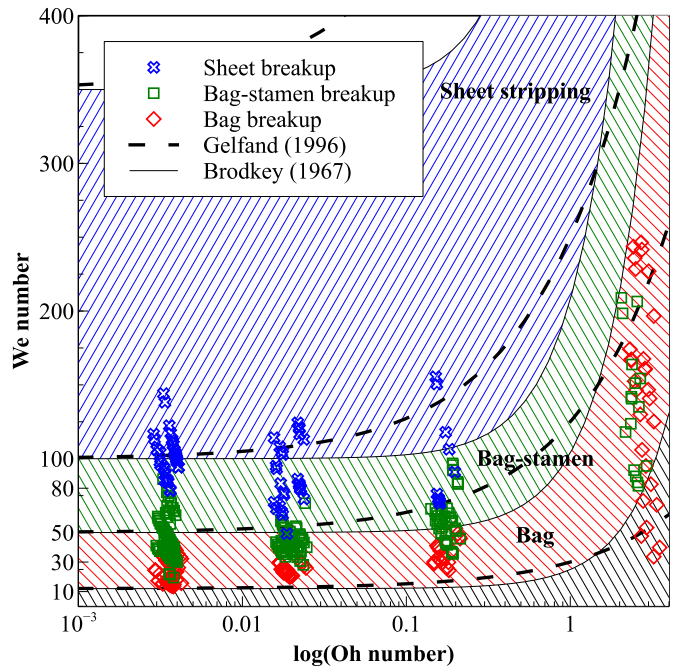

Figure 3. Morphological classification of breakup modes of spherical and non-spherical droplets consist of water and Mix-1, 2 and 3 (50\%, 80\% and 97\% glycerol) in $W e$-Oh plane derived with $d_{0}$

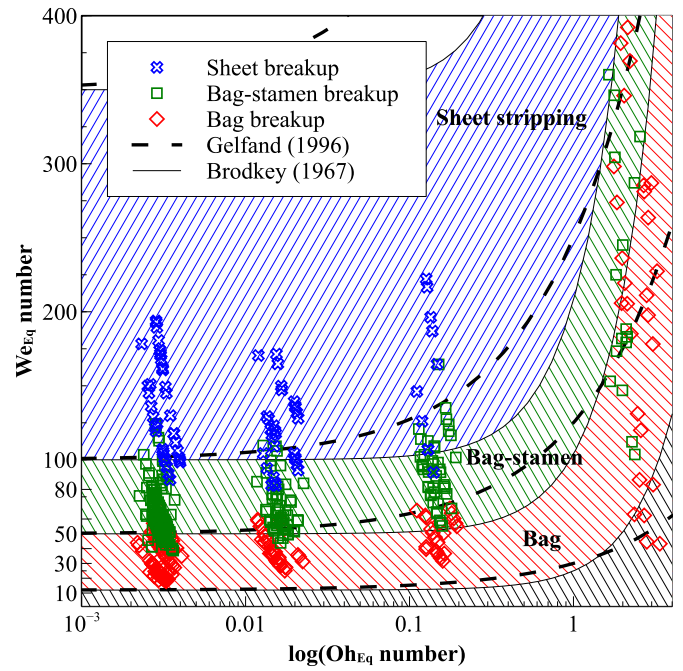

Figure 4. Morphological classification of breakup modes of spherical and non-spherical droplets consist of water and Mix-1, 2 and 3 (50\%, 80\% and $97 \%$ glycerol) in $W e_{\mathrm{eq}}-\mathrm{Oh}$ eq plane derived with $d_{\mathrm{eq}}$

Low $W e$ number - No breakup regime

The $W e$ number ranges from 10 to 13 with the $O h$ number being $3.410^{-3}, 0.02,0.19$ and 2.2 for increasing liquid viscosities. The diameter $d_{0}$ based on the droplet volume is $d_{0}=1.25 \pm 0.05 \mathrm{~mm}$ and the $A R=1.20 \pm 0.02 \mathrm{The}$ variation in the values for $W e$ number occurs due to the difference in the physical properties of the liquids, namely the density and the surface tension.

Figure $5 a$ presents a comparison of the non-dimensional displacement $x_{d}^{*}$ of the centre of viewable area of the droplet with dimensionless time $t^{*}$ for the four liquids (water and $50 \%, 80 \%$ and $97 \%$ glycerol) with the $3^{\text {rd }}$ degree fitting curve of Pilch and Erdman [4] which is independent of viscosity. The fitting curve generally under-predicts the displacement of the droplet and this is mainly due to the increased frontal area of the droplets compared to the spherical droplet assumed by the $3^{\text {rd }}$ degree fitting curve.

Figure $5 \mathrm{~b}$ shows the non-dimensional velocity $u_{d}^{*}$ of the centre of viewable area of the droplet. For glycerol-water solutions, after they achieve their maximum deformation $d_{\max }^{*}$, which occurs at $t_{\max }^{*}=1.18,2.55$ and 2.6 for $50 \%, 80 \%$ and $97 \%$ glycerol solutions respectively, they continue to translate with constant frontal area approximately equal to their maximum deformation.

Regarding the water droplet, its velocity $u_{d}^{*}$ oscillates due to the changes of the frontal area of the droplet. At $t_{\max }^{*}=1.07$ the water droplet achieves its maximum deformation $d_{\max }^{*}=1.42$, however at $t^{*}=2.1$ it becomes nearly spherical again. The reason why the oscillatory behaviour did not appear to that extent for the glycerol-water solutions is because the increased viscosity dampens the droplet oscillations. This oscillatory behaviour is not predicted by the $3^{\text {rd }}$ degree fitting curve. The velocities of the droplet as they exit the imaged area are $u_{d}^{*}=2.6,2.8$, 3.3 and 3.0 for increasing liquid viscosity and are directly related to the droplet frontal area.

The extent of the droplet's oscillatory behaviour decreases with the increase of the liquid viscosity as also does its effect on the translation behaviour of the droplet. Figure $5 c$ shows the temporal evolution of the normalised acceleration of the droplet $\alpha_{d}^{*}$. The data for the acceleration were derived by fitting a $5^{\text {th }}$ degree polynomial to the displacement data of the four liquids and taking the second derivative of the polynomial. A $5^{\text {th }}$ degree polynomial was used so that its second derivation was a third degree, which is able to predict the oscillation; the correlation coefficient is more than 0.95 regarding the displacement data. The bars in Figure $5 \mathrm{c}$ indicate the difference of the fitting curve value from the corresponding value of the experimental data for the acceleration $\alpha_{d}$.

The acceleration provides an overview of the behaviour of the droplet's frontal area with time; in particular the rate of change of the acceleration is related with the rate of change of the frontal area. As expected the water droplet accelerates faster than the rest of the droplets as it deforms faster due to its low viscosity. Additionally, the water and the least viscous glycerol-water solution ( $50 \%$ glycerol) are oscillating; Mix-2 ( $80 \%$ glycerol) oscillates but with a very small amplitude and finally no oscillation occurs for the most viscous case $(97 \%$ glycerol). The two least viscous droplets accelerate faster than the other two more viscous droplets. After that time the frontal area of the two droplet reduces and therefore the acceleration decreases; at time $t^{*}=1.6$ the two more viscous droplets, which did not oscillate, have higher acceleration than the least viscous droplets despite the fact that the latter ones have higher rate of increase of the frontal area. 


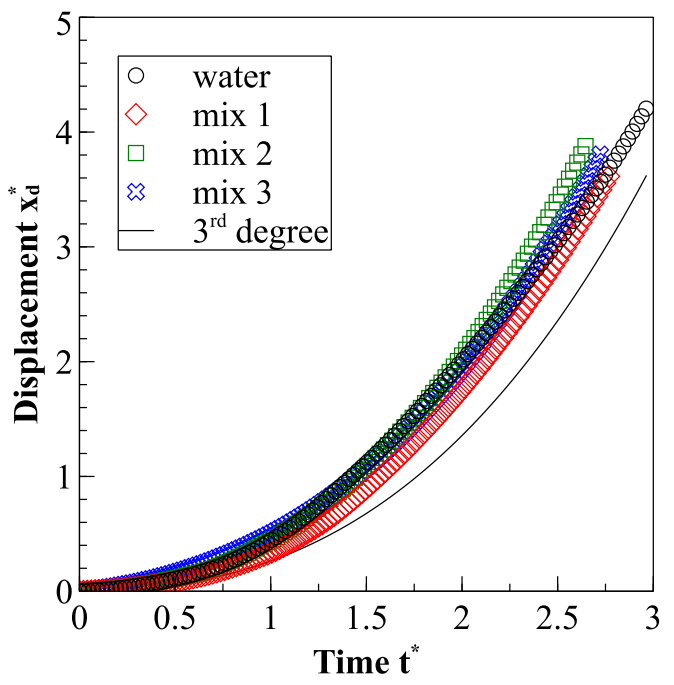

(a) Displacement $x_{d}^{*}$

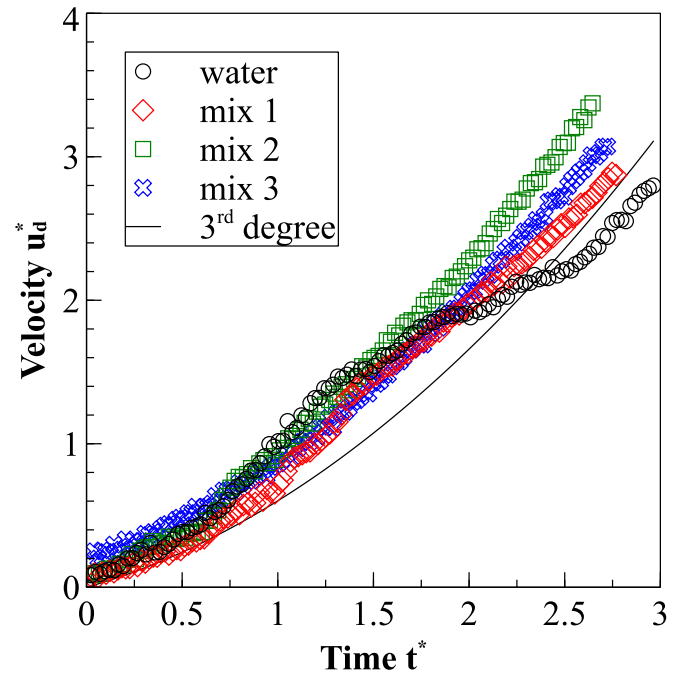

(b) Velocity $u_{d}^{*}$

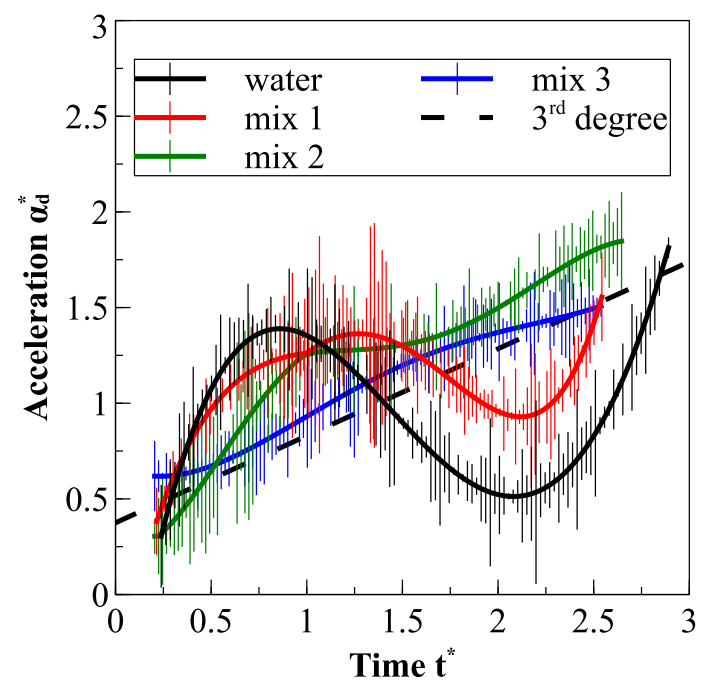

(c) Acceleration $\alpha_{d}^{*}$

Figure 5. Comparison of the temporal evolution of the (a) displacement $x_{d}^{*}$, (b) velocity $u_{d}^{*}$ and (c) acceleration $\alpha_{d}^{*}$ of oblate droplets $(A R=1.2)$ under the no breakup regime for water, $50 \%, 80 \%$ and $97 \%$ glycerol solutions with the $3^{\text {rd }}$ degree fitting curve [4] 


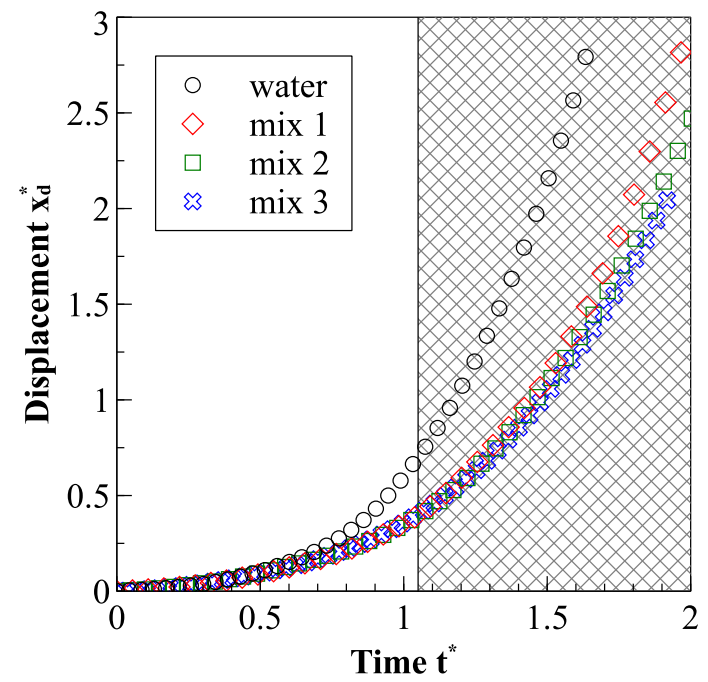

(a) Displacement $x_{d}^{*}$

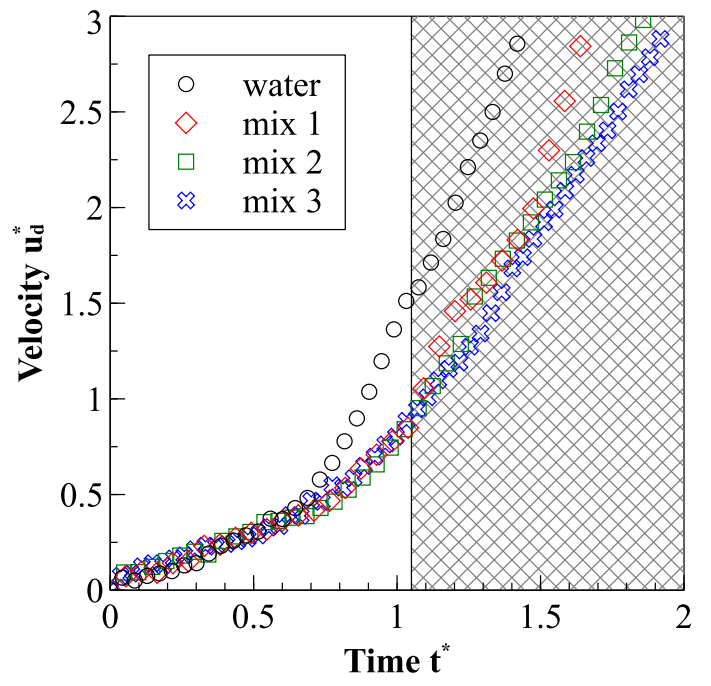

(b) Velocity $u_{d}^{*}$

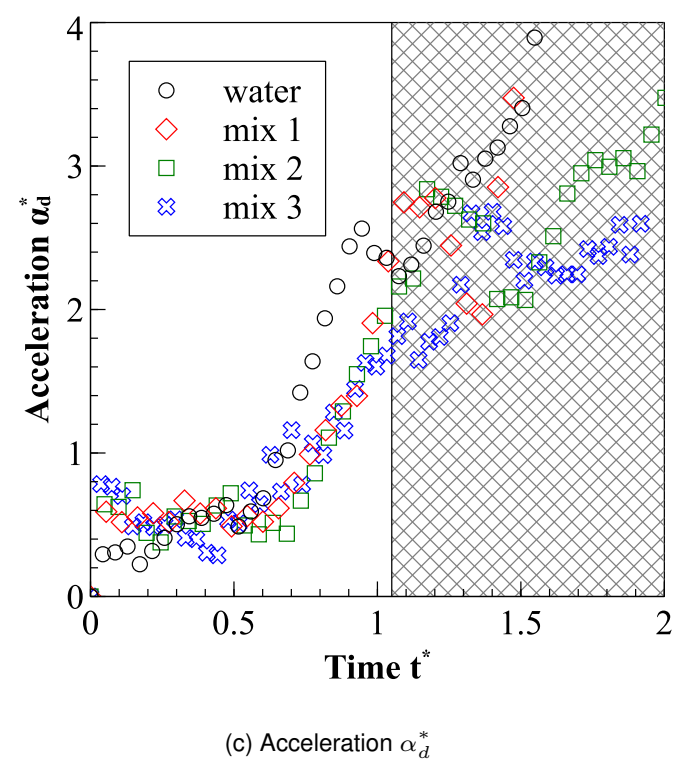

Figure 6. Comparison of the temporal evolution of the (a) displacement $x_{d}^{*}$, (b) velocity $u_{d}^{*}$ and (c) acceleration $\alpha_{d}^{*}$ of the centre of mass of oblate droplets $(A R=1.2)$ under the bag-stamen breakup regime for water, $50 \%, 80 \%$ and $97 \%$ glycerol solutions with the $3^{\text {rd }}$ degree fitting curve [4]

High $W e$ number - Bag-stamen breakup regime

Figure 6 presents the measured displacement $x_{d}^{*}$, and the deduced velocity $u_{d}^{*}$ and acceleration $\alpha_{d}^{*}$ of the droplet centre of mass with time for various liquid viscosities. The gray area of the figures correspond to the breakup initiation time $t_{\text {init }}^{*}$ of the most viscous case and is equal to $t_{\text {init }}^{*}=1.02$; the corresponding breakup initiation time for the least viscous droplet is $t_{\text {init }}^{*}=0.97$.

At short times the effect of increasing viscosity on both displacement and velocity is minor, despite the order of magnitude in the viscosity differences. Detailed differences can be seen in the acceleration values (Figure 6c). The acceleration of the water droplet at time $t^{*}=0$ is smaller than the acceleration for the more viscous droplet due to the higher initial deformation. In later times, due to its higher frontal area the acceleration increases and consequently its velocity increases and its displacement overtake the other droplets. It is of interest to notice that the three more viscous cases have almost the same velocity and displacement and trajectory differentiation starts much latter in time, in fact approximately around the breakup initiation time.

\section{Conclusions}

The role of the initial sphericity of the droplet on the breakup process was experimentally investigated, over a range of $W$ e from 10 to 200 and of the $O h$ number from 0.01 to approximately 4 . It was observed that the breakup modes of initially spherical droplets were in good agreement with the literature but equivalent $W e_{\mathrm{eq}}$ and $O h_{\mathrm{eq}}$ numbers were required to extend the existing morphological classification charts for initially non-spherical as well as spherical 
droplets. For the higher $O h$ numbers $(O h>1)$ not a discernible distinction between breakup modes was possible. The kinematic characteristics of droplets of $A R=1.2$ showed that the liquid viscosity has an important role through the dampening of the internal flow field and two viscosity droplets are accelerated at higher rates due to faster deformation which results in greater frontal area.

\title{
Acknowledgments
}

The authors would like to acknowledge the financial support of Volvo Car Corporation (VCC), Gothenburg, Sweden and EPSRC grant EP/K019732/1.

\author{
Nomenclature \\ $\mathrm{t}$ time [s] \\ $\mathrm{t}^{*}$ dimensionless time [-] \\ AR aspect ratio [-] \\ We Weber number [-] \\ $\mathrm{We}_{e q}$ equivalent Weber number [-] \\ Oh Ohnesorge number [-] \\ $\mathrm{Oh}_{e q}$ equivalent Ohnesorge number [-] \\ $\rho_{g, l} \quad$ gas, liquid density $\left[\mathrm{kg} / \mathrm{m}^{3}\right]$ \\ $u_{g} \quad$ gas velocity $[\mathrm{m} / \mathrm{s}]$ \\ $d_{0} \quad$ volume based droplet diameter $[\mathrm{m}]$ \\ $d_{e q} \quad$ equivalent droplet diameter $[\mathrm{m}]$ \\ $\mu_{g, l} \quad$ gas liquid dynamic viscosity [Pa s] \\ $\sigma \quad$ surface tension $[\mathrm{N} / \mathrm{m}]$ \\ $\epsilon \quad$ density ratio [-] \\ $C_{d} \quad$ drag coefficient [-] \\ $x_{d}^{*} \quad$ dimensionless droplet displacement [-] \\ $u_{d}^{*} \quad$ dimensionless droplet velocity [-] \\ $\alpha_{d}^{*} \quad$ dimensionless droplet acceleration [-]
}

\section{References}

[1] K. Bergeles, G. Charalampous, Y. Hardalupas, and A. M. K. P. Taylor, "Breakup of Non-spherical Droplets," in 27th Annual Conference on Liquid Atomization and Spray Systems, vol. 1, pp. 4-7, 2016.

[2] E. Villermaux, "Fragmentation," Annual Review of Fluid Mechanics, vol. 39, pp. 419-446, jan 2007.

[3] J. O. Hinze, "Fundamentals of the hydrodynamic mechanism of splitting in dispersion processes," AlChE Journal, vol. 1, no. 3, pp. 289-295, 1955.

[4] M. Pilch and C. Erdman, "Use of breakup time data and velocity history data to predict the maximum size of stable fragments for acceleration-induced breakup of a liquid drop," International Journal of Multiphase Flow, vol. 13, no. 6, pp. 741-757, 1987.

[5] R. Brodkey, Formation of drops and bubbles. Addison-Wesley, 1967

[6] B. Gelfand, "Droplet breakup phenomena in flows with velocity lag," Progress in Energy and Combustion Science, vol. 22, no. 96, pp. 201-265, 1996.

[7] C. Schneider, W. Rasband, and K. Eliceiri, "NIH Image to ImageJ: 25 years of image analysis," Nature Methods, vol. 9, no. 7, pp. 671-675, 2012. 


\title{
Numerical Investigation of Droplet Impact on Smooth Surfaces with Different Wettability Characteristics: Implementation of a dynamic contact angle treatment in OpenFOAM
}

\author{
Vontas K. ${ }^{1,2}$., Andredaki M. ${ }^{1}$, Georgoulas A. ${ }^{1}$, Nikas K.-S. ${ }^{2}$, Marengo M. ${ }^{1}$ \\ ${ }^{1}$ Advanced Engineering Centre, School of Computing Engineering and Mathematics, \\ University of Brighton \\ ${ }^{2}$ Mechanical Engineering Department, Piraeus University of Applied Sciences \\ *Corresponding author: A.Georgoulas@brighton.ac.uk
}

\begin{abstract}
The "Direct Numerical Simulations" (DNS) of droplet impact processes is of great interest and importance for a variety of industrial applications, where laboratory experiments might be difficult, costly and time-consuming. Furthermore, in most cases after validated against experimental data, they can be utilised to further explain the experimental measurements or to extend the experimental runs by performing "virtual" numerical experiments. In such "DNS" calculations of the dynamic topology of the interface between the liquid and gas phase, the selected dynamic contact angle treatment is a key parameter for the accurate prediction of the droplet dynamics. In the present paper, droplet impact phenomena on smooth, dry surfaces are simulated using three different contact angle treatments. For this purpose, an enhanced VOF-based model, that accounts for spurious currents reduction, which has been previously implemented in OpenFOAM CFD Toolbox, is utilised and further enhanced. Apart from the already implemented constant and dynamic contact angle treatments in OpenFOAM, the dynamic contact angle model of Kistler, that considers the maximum advancing and minimum receding contact angles, is implemented in the code. The enhanced VOF model predictions are initially compared with literature available experimental data of droplets impacting on smooth surfaces with various wettability characteristics. The constant contact angle treatment of OpenFOAM as well as the Kistler's implementation show good qualitative and quantitative agreement with experimental results up to the point of maximum spreading, when the spreading is inertia dominated. However, only Kistler's model succeeds to accurately predict both the advancing and the recoiling phase of the droplet impact, for a variety of surface wettability characteristics. The dynamic contact angle treatment fails to predict almost all stages of the droplet impact. The optimum version of the model is then applied for 2 additional series of parametric numerical simulations that identify and quantify the effects of surface tension and viscosity, in the droplet impact dynamics.
\end{abstract}

\section{Keywords}

Droplet impact, dynamic contact angle treatment, VOF, OpenFOAM

\section{Introduction}

In the last 20 years, many investigations of droplet impact have taken place experimentally as well as numerically. Wettability constitutes an important controlling parameter in the dynamics of droplet impact, as it can completely alter the impact characteristics and output [1]. The "Direct Numerical Simulations" (DNS) of droplet impact processes is of great interest and importance for a variety of industrial applications, where laboratory experiments might be difficult, costly and time-consuming. Furthermore, in most cases after validated against experimental data, they can be utilised to further explain the experimental measurements or to extend the experimental runs by performing "virtual" numerical experiments. In such "DNS" calculations of the dynamic topology of the interface between the liquid and gas phase, the selected Dynamic Contact Angle (DCA) treatment is a key parameter for the accurate prediction of the droplet dynamics, since it underpins the wettability characteristics of the simulated phenomenon. The droplet impact of water on a flat, solid surface has been studied by Pasandideh-Fard et al. [2]. A numerical solution of the Navier-Stokes equations, using a modified SOLA-VOF method was utilised to model the interface deformation. Yokoi et al. [3] investigated liquid droplet impact behaviour onto a dry and flat surface numerically and compared their results with experimental data, indicating the significant role of the DCA modelling in reproducing the droplet impact behaviour. Their numerical method consists of a Coupled Level Set and VOF framework (CLSVOF), a volume/surface integrated average based multi-moment method, and a continuum surface force model. The experimental work of Antonini et al. [4] is focused in the understanding of the effect of surface wettability on impact characteristics of water drops, onto solid dry surfaces. Their results indicated the role of advancing contact angle and contact angle hysteresis as fundamental wetting parameters. They also found that, generally, if Reynolds $(\mathrm{Re})$ and Weber numbers $(\mathrm{We})$ are high enough, the spreading drop can be 
subdivided into two main regions: a radially spreading lamella and an almost circular rim appearing due to capillary forces and viscosity [4]. A novel numerical implementation for the adhesion of liquid droplets impacting normally on solid dry surfaces was presented by Malgarinos et al. [5]. The benefit of this new approach, compared to most existing models, is that the DCA forming during the surface wetting process is not inserted as a boundary condition, but is derived implicitly by the induced fluid flow characteristics and the adhesion physics of the gas-liquid-solid interface, starting only from the advancing and receding equilibrium contact angles. Zhang et al. [6] investigated the phenomenon of spray impinging on in-cylinder walls, a phenomenon that has important impact on combustion processes as well as harmful emissions for internal combustion engines. Droplet impact with a numerical methodology for modelling contact line motion in a Dual-Grid Level-Set method (DGLS), on hydrophobic and super-hydrophobic surfaces was implemented by Patil et al. [7], using a quasi - DCA model based on experimental inputs. The accuracy of the partially refined DGLS method is close to that of the fine-grid based LS method, at a computation cost which is close to that of the coarse-grid based LS method. Furthermore, the DGLS method is demonstrated as an improved LS method for computational multi-fluid dynamics simulations, involving contact line motion.

In the present paper, droplet impact phenomena on smooth, dry surfaces are simulated utilising and comparing three different numerical treatments for the contact angle at the solid-liquid-gas triple contact line against existing, literature available, experimental measurements. For this purpose, an enhanced VOF-based model that accounts for spurious currents reduction, which has been previously implemented in OpenFOAM CFD Toolbox, and it was validated and applied for the case of adiabatic bubble dynamics [8], is further improved. In more detail, apart from the already implemented Constant Contact Angle (CCA) and DCA treatments in OpenFOAM, the DCA treatment of Kistler, that considers the limiting advancing and receding contact angles, is also implemented in the code. In the first part of the paper, the predictions of the three different contact angle treatments in the utilised enhanced VOF models are compared with literature reported experimental data of droplets impacting on smooth surfaces [2]. The CCA and Kistler's DCA models show good qualitative and quantitative agreement with the experimental results reported by Pasandideh-Fard et al. [2] up to the point of maximum spreading, when the spreading is inertia dominated. However, only Kistler's model succeeds to accurately predict the recoiling phase of the droplet impact. The original DCA model of OpenFOAM fails to predict almost all stages of the simulated droplet impact case. Then Kistler's treatment implementation, as it performs better, is further validated by the numerical reproduction of three additional experiments with varying wettability characteristics [3,7]. The proposed cases vary from hydrophilic to hydrophobic. The optimum version of the proposed numerical framework is then applied for two additional series of parametric numerical simulations (virtual experiments) that isolate, identify and quantify the effects of surface tension and viscosity, in the droplet impact dynamics. The effect of the varied parameters on the droplet spreading factors with time is identified and quantified and comparisons with the theoretical correlation by Roisman for the maximum droplet spreading factor are also conducted [9]. The proposed correlation is given by Equation 1,

$$
\beta_{\max }=0.87 R e^{1 / 5}-0.4 R e^{2 / 5} W e^{-1 / 2}
$$

\section{Numerical Method}

With the VOF approach, the transport equation for the volume fraction, $\alpha$, of the secondary (dispersed) phase is solved simultaneously with a single set of continuity and Navier-Stokes equations for the whole flow field. The corresponding volume fraction of the primary phase is simply calculated as $(1-\alpha)$. The main underlying assumptions are that the two fluids are Newtonian, incompressible, and immiscible. The governing equations can be written as:

$$
\begin{aligned}
& \nabla \cdot \vec{U}=0 \\
& \frac{\partial \rho_{b} \vec{U}}{\partial t}+\nabla \cdot\left(\rho_{b} \vec{U} \vec{U}\right)=-\nabla p+\nabla \cdot \mu_{b}\left(\nabla \vec{U}+\nabla U^{T}\right)+\rho_{b} f+F_{S} \\
& \frac{\partial a}{\partial t}+\nabla \cdot(\alpha \vec{U})-\nabla \cdot\left(\alpha(1-\alpha) U_{r}\right)=0
\end{aligned}
$$

where the bulk fluid properties are calculated as weighted averages of the individual phase properties as follows, 


$$
\begin{aligned}
& \rho_{b}=\rho \alpha+\hat{\rho}(1-\alpha) \\
& \mu_{b}=\mu \alpha+\hat{\mu}+\hat{\mu}(1-\alpha)
\end{aligned}
$$

In the VOF method, $\alpha$ is advected by the velocity field. For the case of incompressible flow, this is equivalent to volume fraction conservation, which makes the method mass conservative. Interface sharpening is very important in simulating two-phase flows of two immiscible fluids. In OpenFOAM the sharpening of the interface is achieved artificially by introducing an extra compression term in the advection equation of $\alpha$ (last term in the left-hand side of Eq. 4). $U_{r}$ is the artificial compression velocity which is calculated from the following relationship,

$$
U_{r}=n_{f} \min \left[C_{\gamma} \frac{|\varphi|}{\left|s_{f}\right|}, \max \left(\frac{|\varphi|}{s_{f}}\right)\right]
$$

Finally, the surface tension force is modelled as a volumetric force using the Continuum Surface Force (CSF) method by Brackbill et al. [10], applying the following equations:

$$
\begin{aligned}
& F_{S}=\gamma \mathrm{K}(\nabla \alpha) \\
& \mathrm{K}=\nabla \cdot\left(\frac{\nabla \tilde{\alpha}}{|\nabla \tilde{\alpha}|}\right)
\end{aligned}
$$

where $\gamma$ is the surface tension coefficient and $\mathrm{k}$ is the curvature of the interface. As mentioned in the introduction section of the present paper the utilized numerical framework constitutes an enhanced version of the original VOF-based solver of OpenFOAM [8], that suppresses numerical artefacts of the original model, known as "spurious currents". The proposed enhancement involves the calculation of the interface curvature $\mathrm{k}$ using the smoothed volume fraction values $\widetilde{\alpha}$, which are obtained from the initially calculated $\alpha$ field, smoothing it over a finite region near the interface. All other equations are using the initially calculated (non-smoothed) volume fraction values of $\alpha$. Further details on the proposed numerical modelling framework can be found in [8].

In OpenFOAM, there are two ways to predict the evolution of the contact angle between the liquid-gas interface and the solid wall boundary. The simplest approach is to assign a constant value equal to the equilibrium contact angle, and therefore neglecting the contact angle hysteresis. This is usually known as static or constant contact angle treatment. A more complicated approach involves the application of a contact angle that varies with respect to the instantaneous flow quantities. Such treatments are known as dynamic contact angle treatments (DCA). The original distribution of OpenFOAM includes both a CCA treatment as well as a DCA treatment.

For the purposes of the present investigation, after the satisfactory predictions in a similar investigation by Criscione et al. [11], the adopted, enhanced, VOF-based solver from the work of Georgoulas et al. [8], is further improved by implementing an additional DCA treatment in the solver. The proposed treatment, is known as Kistler's model [12], and it calculates the DCA, $\theta_{d}$, using the Hoffman function, $f_{\text {Hoff }}$, as follows:

$$
\theta_{d}=f_{\text {Hoff }}\left[C_{a}+f_{H o f f}^{-1}\left(\theta_{\varepsilon}\right)\right]
$$

where $\theta_{\epsilon}$ is the equilibrium contact angle. The capillary number, $C_{a}$, is calculated as $C_{a}=\frac{\mu U_{C L}}{\gamma}$ and $U_{\mathrm{CL}}$, is the spreading velocity of the contact line. $f_{\text {Hoff }}^{-1}$ is the inverse function of "Hoffman's" empirical function which is given in the following form [11].

$$
f_{\text {Hoff }}=\operatorname{acos}\left[1-2 \tanh \left(5.16\left(\frac{x}{1+1.31 x^{0.99}}\right)^{0.706}\right)\right]
$$

\section{Validation of Numerical Method}

All simulations presented in the present paper constitute $2 \mathrm{D}$ axisymmetric runs. The computational domain is a $5^{\circ}$ wedge, with $5 \mathrm{~mm}$ width and $8 \mathrm{~mm}$ height. The computational mesh consists of 1.6 million hexahedral cells $(1000 \times 1600 \times 1)$. The dimensions of the computational domain and the total number of computational cells, were 
selected after an initial sensitivity analysis and a mesh independency study, respectively. The computational domain, mesh and boundary conditions are illustrated in Figure 1. To validate the adopted, enhanced, VOF-based solver for droplet impact cases and compare the numerical predictions with the previously mentioned, contact angle treatments, four different, literature available, experiments on droplet impact are numerically reproduced. In the first case, all three contact angle models are tested, while in the rest three cases only Kistler's dynamic contact angle is used, to reproduce the proposed experimental runs. The initial conditions and the wetting characteristics of the proposed experimental runs are summarized in Table 1.

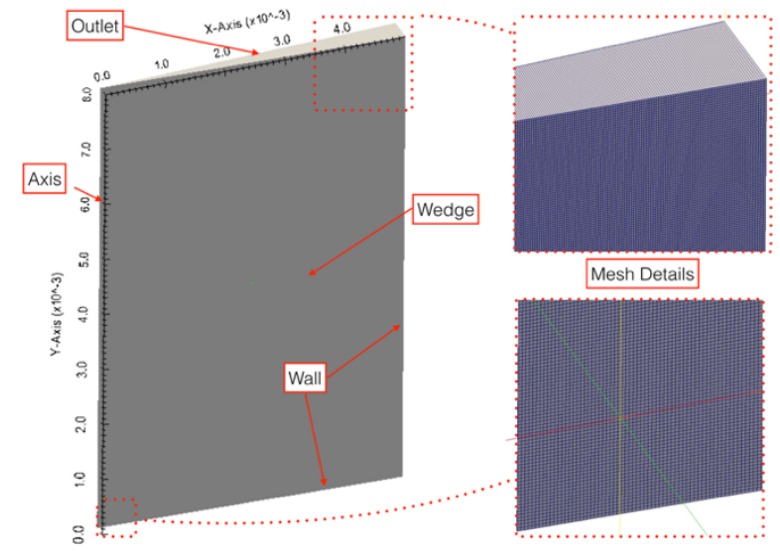

Figure 1. Computational domain, mesh and boundary conditions for numerical simulations

As it can be seen for the axisymmetric simulations of the present paper, a structured computational mesh, consisting of hexahedral and prismatic elements was used, with grid clustering towards the bottom left corner of the computational domain (centre of droplet impact). At the solid walls, a no-slip velocity boundary condition was used with a fixed flux pressure boundary condition for the pressure values and a contact angle condition, according to the selected in each case treatment, for the volume fraction values. These contact angle boundary conditions are used to correct the surface normal vector, and therefore adjust the curvature of the interface near the wall, in relation to the prescribed wettability characteristics. At the outlet, a fixed valued (atmospheric) pressure boundary condition and a zero-gradient boundary condition for the volume fraction were used, while for the velocity values a special (combined) type of boundary condition was used that applies a zero-gradient when the fluid mixture exits the computational domain and a fixed value condition to the tangential velocity component, in cases that fluid enters the domain. Further details regarding the utilised boundary conditions can be found in OpenFOAM Documentation [13].

Table 1. Initial conditions and wetting characteristics of validation experiments

\begin{tabular}{c|ccccccc}
\hline \hline & $\mathrm{D}_{0}[\mathrm{~m}]$ & $\mathrm{U}_{0}[\mathrm{~m} / \mathrm{s}]$ & $\mathrm{We}$ & $\mathrm{Re}$ & $\theta_{\mathrm{e}}\left[^{\mathrm{O}}\right]$ & $\left.\theta_{\mathrm{a}}{ }^{0}\right]$ & $\theta_{\mathrm{r}}\left[^{0}\right]$ \\
\hline \hline Experiment I [2] & 0.002 & 1 & 27 & 2000 & 90 & 110 & 40 \\
Experiment II [3] & 0.00228 & 1 & 32 & 2280 & 90 & 107 & 77 \\
Experiment III [7] & 0.0017 & 0.34 & 2.7 & 578 & 147 & 161 & 132 \\
Experiment IV [7] & 0.002 & 0.44 & 5.3 & 880 & 158 & 165 & 142
\end{tabular}

As it can be observed from Table 1, the selected validation cases constitute experiments with various impact as well as wettability characteristics. Experiments I and II constitute droplet impacts in hydrophilic surfaces, while in experiments III and IV hydrophobic and super-hydrophobic surfaces are used for the impacts, respectively. As mention previously, in the case of Experiment I all three different contact angle treatments are used to numerically reproduce the considered droplet impact. A macroscopic comparison of the numerical predictions for the droplet evolution with the corresponding experimental snapshots at the same time instances, for each treatment, is illustrated in Figure 2. For a more quantitative comparison, the contact diameter of the droplet with the solid surface with respect to time is plotted for each of the three numerical simulations as well as for the experimental measurements, in the diagram of Figure 3. 


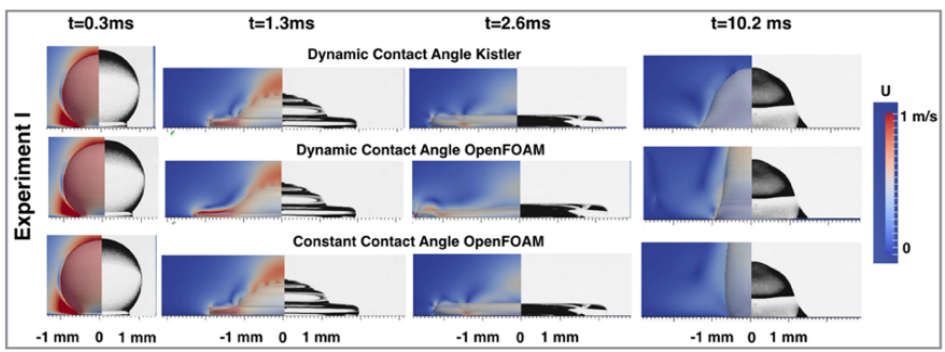

Figure 2. Droplet evolution with time - Comparison of numerical predictions for all three contact angle treatments (present investigation) with corresponding experimental snapshots from [2]. The velocity magnitude field in a central vertical section of the droplet, is also shown in the numerical snapshots.

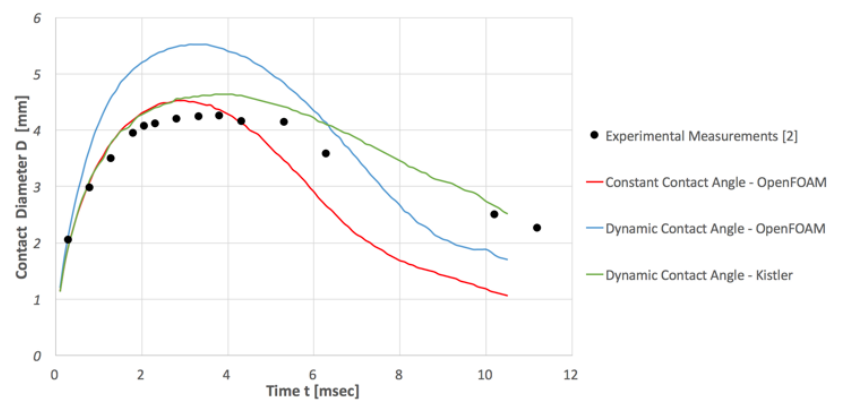

Figure 3. Contact diameter with time - Comparison of numerical predictions (present investigation) for all three contact angle treatments with corresponding experimental data from [2].

After examining Figures 2 and 3, it is obvious that both the CCA and DCA treatments implemented in the original distribution of OpenFOAM fail to predict the experimental data, while Kistler's DCA treatment follows the experimental points well, both before and after the maximum spreading. However, in order to further check the validity of the Kistler's DCA treatment for cases with varying wettability characteristics, three additional, literature available experimental runs that are reported in [3] and [7], are numerically reproduced. In more detail, another hydrophilic (Experiment II), a hydrophobic (Experiment III) and a super-hydrophobic case (Experiment IV) are selected for this purpose. A macroscopic comparison of the numerical predictions for the droplet evolution with the corresponding experimental snapshots, is illustrated in Figure 4. For a more quantitative comparison, the contact diameter of the droplet with the solid surface with respect to time, is plotted for each case in the diagrams of Figure 5.

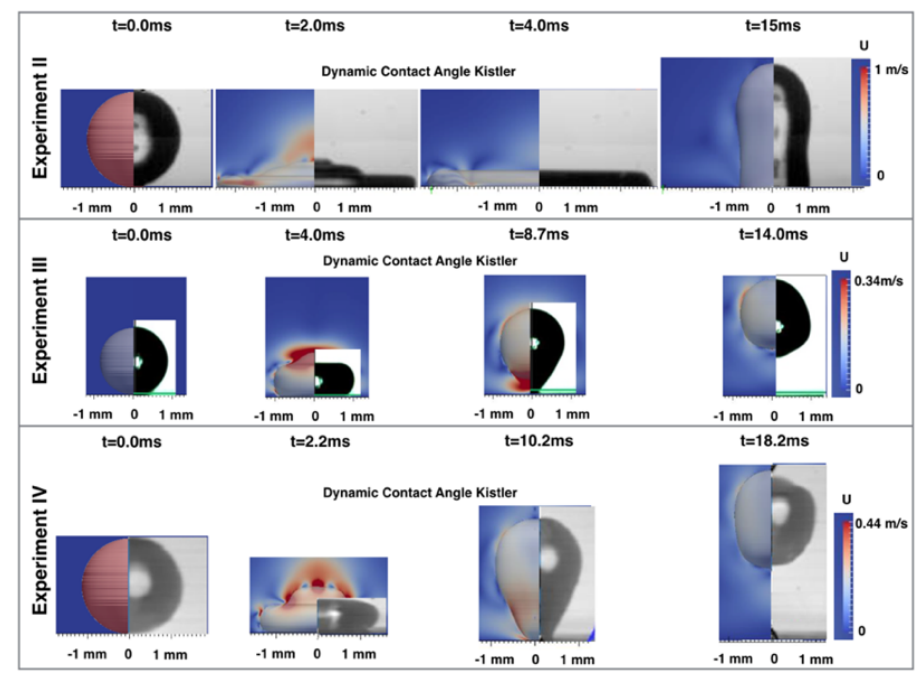

Figure 4. Droplet evolution with time - Comparison of numerical predictions (present investigation) using Kistler's dynamic contact angle treatment with corresponding experimental snapshots reported in [3] (top) and [7] (middle) and (bottom). The velocity magnitude field in a central vertical section of the droplet, is also shown in the numerical snapshots. 


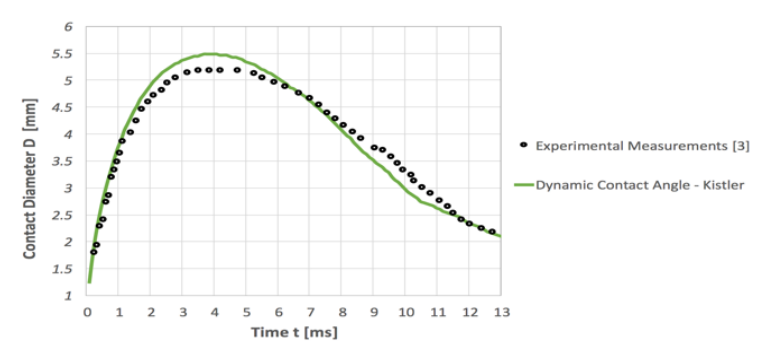

(a)

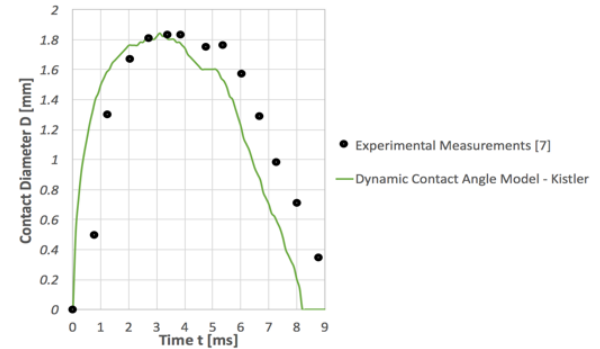

(b)

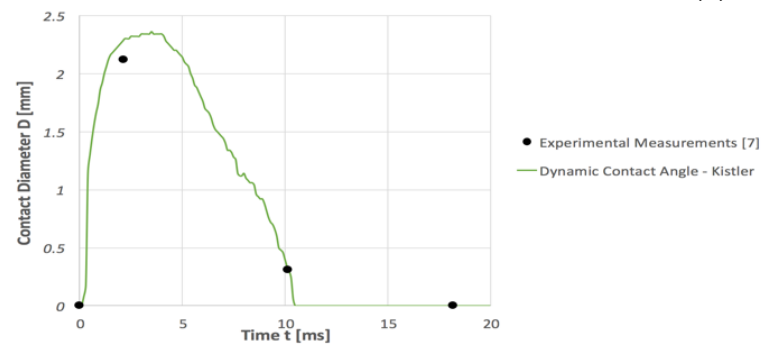

(c)

Figure 5. Contact diameter with respect to time - Comparison of numerical predictions (present investigation) using Kistler's dynamic contact angle treatment with corresponding experimental snapshots reported in [3] (a) and [7] (b) and (c).

As it can be observed, Kistler's DCA treatment successfully predicts the spatial and temporal evolution of the droplet in all stages of the considered impacts. Therefore, it can be concluded that the utilised enhanced VOF framework in conjunction with the implemented DCA treatment of Kistler, can safely be applied for the investigation of droplet impacts through "virtual" numerical experiments, as it successfully predicts the droplet impact stages in hydrophilic, hydrophobic and super-hydrophobic cases.

\section{Parametric Analysis - Effect of fluid viscosity and surface tension}

In the present section of the paper, the optimum version of the VOF-based numerical framework that is presented and validated against experimental data in the previous sections, is further applied for the conduction of two additional series of parametric numerical simulations. The proposed numerical experiments mainly aim to identify and quantify the effects of viscosity and surface tension on the spatial and temporal evolution of the droplets after their impact on a hydrophilic surface. Furthermore, the validity of a widely used theoretical correlation by Roisman [9] (Equation 1) for the case of "virtual" fluids is assessed. For this purpose, the validation case reproducing Experiment II is selected as the base case for the proposed parametric numerical investigations. In the first parametric investigation 5 additional simulations are conducted "virtually" varying only the liquid viscosity, by factors of $0.5,1.5,2.0,3.0$ and 3.5, while keeping all the other properties and impact characteristics constant and equal to the base case. In the second parametric investigation, again 5 additional simulations are conducted virtually varying only the surface tension coefficient, by factors of $0.5,1.5,2.0,3.0$ and 3.5 , while keeping all the other properties and impact characteristics constant and equal to the base case. The evolution of the spreading factor $\beta$ with dimensionless time $t^{*}$ for each case is plotted. The spreading factor is calculated as the contact diameter over the initial droplet diameter $\frac{D}{D_{0}}$ while the dimensionless time $t^{*}$ is calculated as $t \frac{U_{0}}{D_{0}}$. The effects of the variation of viscosity and surface tension on the spreading factor $\beta$ with respect to dimensionless time $t^{*}$ are given in Figures 6(a) and 6(b). In each case the corresponding maximum spreading factor from Equation 1 (Roisman correlation [9]) is plotted for comparison purposes. 


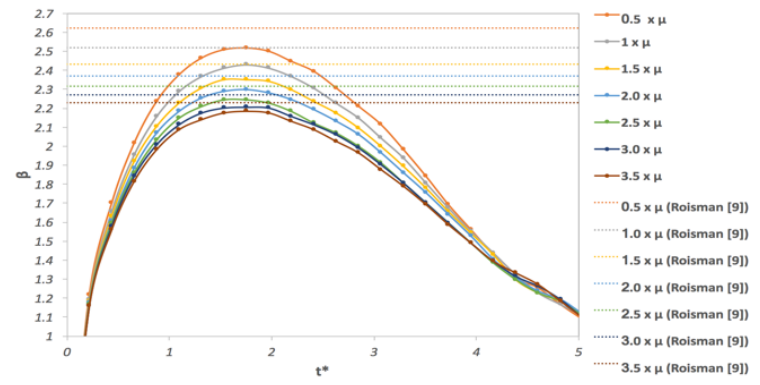

(a)

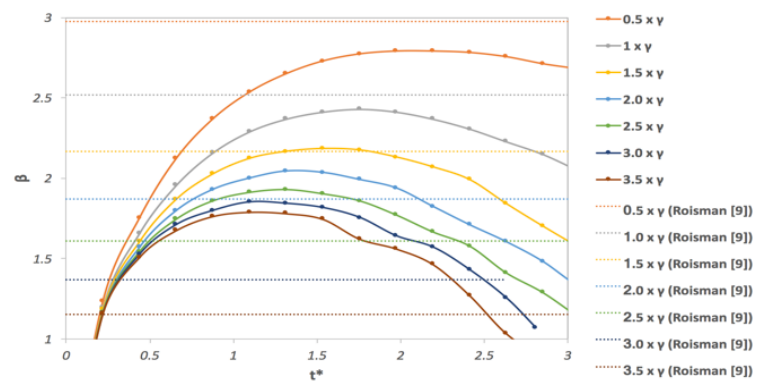

(b)

Figure 6. (a) Spreading factor vs dimensionless time - effect of viscosity variation (b) spreading factor vs dimensionless time effect of surface tension variation. Dotted lines correspond to the maximum spreading factor as predicted using the theoretical correlation of Roisman [9] (Equation 1).

As it can be observed from Figure 6 (a), as viscosity increases both the spreading as well as the recoiling stages of the droplet are dumped. It is characteristic however that the proposed effect, is mor intense around the maximum spreading stage of the drople,t while it appears to be negligible during the initial spreading and the later recoiling stages. It can also be seen that the theoretical correlation of Roisman, in each case slightly over-predicts the resulting maximum spreading factors from the numerical simulations, by $2 \%$ up to $4 \%$. Examining Figure 2 (b), it can be observed that as surface tension increases, the maximum spreading is significantly reduced and the recoiling stage is strengthened. The proposed effect is quite evident from the first stages of the spreading and it progressively increases towards the last stages of the recoiling phase. It is characteristic that a variation of the surface tension coefficient by a factor of just 0.5 can significantly alter the post-impact dynamics of the droplet. As for the comparison with the theoretical correlation of Roisman an increasing deviation from the numerically predicted maximum spreading factor is observed with the corresponding increase of the surface tension. The deviation between the theoretical and numerical maximum spreading factors in this case varies from $0.85 \%$ up to $55 \%$.

The results from the present parametric investigation illustrate that the correlation proposed by Roisman [9] can be safely used for the prediction of the maximum spreading of viscous fluids with viscosities up to 3.5 times higher than water, but it significantly fails to predict the maximum spreading for fluids with surface tension more than two times the surface tension of water, such as metal fluids.

\title{
Conclusions
}

In the present paper, Kistler's DCA model has been implemented in a previously improved version of the VOFbased solver of OpenFOAM. The performance of the proposed contact angle treatment is compared with the existing contact angle treatments of OpenFOAM's original distribution, through comparison with literature reported experimental measurements on water droplets impacting on hydrophilic surfaces. To further test the revealed accuracy of the proposed DCA implementation, three additional experimental runs are numerically reproduced; a second hydrophilic case as well as a hydrophobic and a super-hydrophobic case. In all cases, Kistler's model implementation in the utilised, enhanced VOF-based solver, successfully predicts the spreading, recoiling and rebounding stages of the droplet impact. Further application of the numerical model for the conduction of two additional series of parametric numerical experiments identifies and quantifies the effects of viscosity and surface tension variation in the post-impact stages of the droplet evolution. Comparison of the numerical predictions with a widely accepted theoretical correlation [9], indicate that the proposed correlation can be safely applied to predict the maximum spreading of fluids with higher viscosity than water but not for fluids with more than two times the surface tension of water. Finally, from the overall presentation and analysis of the results it is obvious that the proposed enhanced VOF framework can be safely used to further examine the effects of a variety of important controlling parameters to the post-impact characteristics of droplets impinging on solid surfaces with various wetting characteristics, ranging from hydrophilic to super-hydrophobic cases.

\author{
Nomenclature \\ $\beta_{\max } \quad$ maximum spreading factor [-] (contact droplet diameter / initial droplet diameter) \\ Re Reynolds Number [-] \\ We Weber Number [-] \\ $\vec{U} \quad$ velocity vector $\left[\mathrm{ms}^{-1}\right]$ \\ $\rho_{b} f \quad$ volumetric representation of the gravitational force $\left[\mathrm{kg} / \mathrm{m}^{2} \mathrm{~s}^{2}\right]$ \\ $p \quad$ pressure $[\mathrm{Pa}]$
}




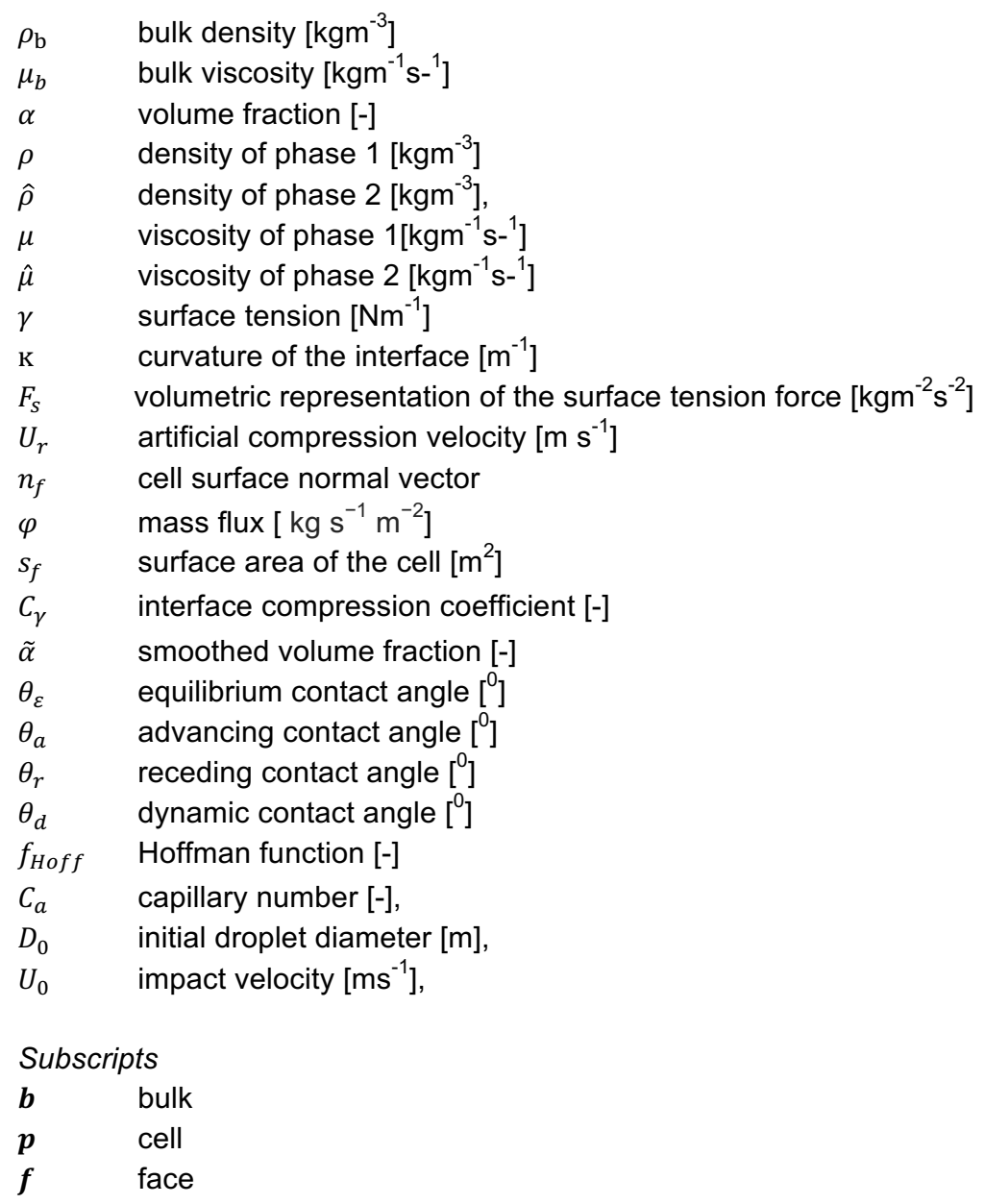

\section{References}

[1] Marengo, M., Antonini, C., Roisman, I. V., and Tropea, C., 2011, "Drop Collisions with Simple and Complex Surfaces," Curr. Opin. Colloid Interface Sci., 16(4), pp. 292-302.

[2] Pasandideh-Fard, M., Y. M. Qiao, Y. M., Chandra, S., and Mostaghimi, J., 1996, "Capillary Effects during Droplet Impact on a Solid Surface," Phys. Fluids, 8(3).

[3] Yokoi, K., Vadillo, D., Hinch, J., and Hutchings, I., 2009, "Numerical Studies of the Influence of the Dynamic Contact Angle on a Droplet Impacting on a Dry surfaceNo Title," Phys. Fluids, 21(7), p. 72102.

[4] Antonini, C., Amirfazli, A., and Marengo, M., 2012, "Drop Impact and Wettability: From Hydrophilic to Superhydrophobic Surfaces," Phys. fluids, 24(10), p. 102104.

[5] Malgarinos, I., Nikolopoulos, N., Marengo, M., Antonini, C., and Gavaises, M., 2014, "VOF Simulations of the Contact Angle Dynamics during the Drop Spreading: Standard Models and a New Wetting Force Model," Adv. Colloid Interface Sci., 212, pp. 1-20.

[6] Zhang, Z., Liu, H., Zhang, F., and Yao, M., 2016, "Numerical Study of Spray Micro-Droplet Impinging on Dry/wet Wall," Appl. Therm. Eng., 95, pp. 1-9.

[7] Patil, N. D., Gada, V. H., Sharma, A., and Bhardwaj, R., 2016, "On Dual-Grid Level-Set Method for Contact Line Modeling during Impact of a Droplet on Hydrophobic and Superhydrophobic Surfaces," Int. J. Multiph. Flow, 81, pp. 54-66.

[8] Georgoulas, A., Koukouvinis, P., Gavaises, M., and Marengo, M., 2015, "Numerical Investigation of Quasi-Static Bubble Growth and Detachment from Submerged Orifices in Isothermal Liquid Pools: The Effect of Varying Fluid Properties and Gravity Levels," Int. J. Multiph. Flow, 74, pp. 59-78.

[9] Roisman, I., 2009, "Inertia Dominated Drop Collisions. II. An Analytical Solution of the Navier-Stokes Equations for a Spreading Viscous Film," Phys. fluids, 21(52104).

[10] Brackbill, J. ., Kothe, D. ., and Zemach, C., 1992, "A Continuum Method for Modeling Surface Tension,” J. Comput. Phys., 100(2), pp. 335-354.

[11] Criscione, A., Rohrig, R., Opfer, L., Roisman, I., and Jakirlic, S., 2011, "Numerical Investigation of Impacting Water Drops in Air Cross-Flow," ILASS-Europe 2011, 24th European Conference on Liquid Atomization and Spray Systems, Estoril, Portugal.

[12] Kistler, S., 1993, "Hydrodynamics of Wetting," Wettability, 6(Marcel Dekker, New York), pp. 311--430.

[13] CFD_Direct, 2013, "The OpenSource CFD Toolbox, User Guide." 


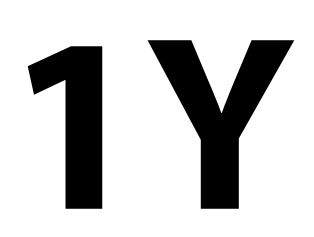

Droplet 1 


\title{
A model for mono- and multi-component droplet heating and evaporation and its implementation into ANSYS Fluent
}

\author{
Luke Poulton ${ }^{1}$, Oyuna Rybdylova ${ }^{1 *}$, Sergei S. Sazhin ${ }^{1}$, Cyril Crua $^{1}$, Mansour Qubeissi², $^{2}$ \\ Ahmed E. Elwardany ${ }^{3}$ \\ ${ }^{1}$ Advanced Engineering Centre, School of Computing, Engineering and Mathematics, University of \\ Brighton, Brighton BN2 4GJ, UK \\ ${ }^{2}$ Centre for Mobility \& Transport, School of Mechanical, Aerospace and Automotive Engineering, \\ Coventry University, Coventry CV1 2JH, UK \\ ${ }^{3}$ Mechanical Engineering Department, Faculty of Engineering, Alexandria University, Alexandria \\ 21544, Egypt \\ *Corresponding author: O.Rybdylova@Brighton.ac.uk
}

\begin{abstract}
A model for heating and evaporation of mono- and multi-component droplets, based on analytical solutions to the heat transfer and species diffusion equations in the liquid phase, is summarised. The implementation of the model into ANSYS Fluent via User-Defined Functions (UDF) is described. The model is applied to the analysis of pure acetone, ethanol, and mixtures of acetone/ethanol droplet heating/cooling and evaporation. The predictions of the customised version of ANSYS Fluent with the newly implemented UDF model are verified against the results predicted by the previously developed in house, one-dimensional code.
\end{abstract}

Keywords: Droplets, multi-component fuel, heating, species diffusion, evaporation.

\section{Introduction}

The problem of modelling droplet heating and evaporation is a longstanding one and has been widely discussed in the literature [1-3]. Modelling of these processes is an integral part of the analysis of many engineering processes, including those which take place during spray combustion in Diesel engines [4]. The models incorporated in Computational Fluid Dynamics (CFD) codes used for the analysis of these processes, are based on a number of assumptions, the applicability of which to practical engineering problems is not at first evident [24]. One of these assumptions is that there is no temperature gradient and/or recirculation inside droplets, which could be justified in the case when liquid thermal conductivity is infinitely large. Liquid thermal conductivity is indeed much larger than that of gas in most cases, and this has been generally considered as a justification of the abovementioned assumption [4]. This approach, however, overlooks the fact that heating and evaporation of droplets in most engineering applications is not a steady state, but transient process, for which heat transfer is characterised by thermal diffusivity rather than thermal conductivity. The thermal diffusivity of liquid, in contrast to thermal conductivity, is much lower than that of gas in most cases. This allows us to question the widely used assumption that temperature gradients inside droplets can be ignored when modelling droplet heating and evaporation. The need to take into account temperature gradient inside droplets was highlighted in many experiments including those discussed in [5] (see also [2]).

The authors of [6] were perhaps the first to describe the preliminary results of implementation of a model for droplet heating and evaporation, taking into account the effects temperature gradient and recirculation inside droplets, into the commercial CFD software ANSYS Fluent. This problem was investigated later in more details in [7]. In the latter paper, the results of the implementation of the model of mono-component droplet heating and evaporation in ANSYS Fluent, using User-Defined Functions (UDF), was described. The predictions of the customised version of ANSYS Fluent were verified against experimental measurements performed at the Combustion Research Facility, Sandia National Laboratories, Livermore, California and results predicted by in-house research code for an $n$ dodecane droplet heated and evaporated in hot air. The main limitation of the model described in [7] was that it is applicable only to mono-component droplets, while most droplets used in engineering, including automotive applications, are multi-component. For the case of multi-component droplets, the process of species diffusion inside droplets needs to be taken into account alongside the heat transfer process [2]. The characteristic times of species diffusion are generally much longer than temperature relaxation times. Thus, taking into account species diffusion inside droplets is expected to be even more important than taking into account temperature gradients.

The main focus of our paper is on the generalisation of the results reported in [7], to the case of multi-component droplets, using the results of preliminary analysis presented in [6]. In contrast to mono-component droplets, the 
modelling of multi-component droplets needs to take into account both heat and species transfer processes inside droplets. The model to be used in our analysis is based on analytical solutions to the heat transfer and species diffusion equations in the liquid phase and is described in detail in [2]. The effect of recirculation in the liquid phase is taken into account based on the Effective Thermal Conductivity (ETC)/ Effective Diffusivity (ED) model [2]. The Abramzon and Sirignano approach [8] is used for modelling the gas phase.

The main ideas of the model used in our analysis are summarised in the following section, based on [2]. Then the details of the implementation of the model into ANSYS Fluent are described. The predictions of the version of ANSYS Fluent, with the new model implemented into it, will be compared with the predictions of the previously developed one-dimensional code for the case of heating/cooling and evaporation of acetone/ethanol droplets. The choice of these droplets was based on the fact that the predictions of the one-dimensional code for them were validated against experimental data [9]. Then the main results of the paper are summarised.

\section{Basic equations and approximations}

The heat transfer inside the droplets is described by the one-dimensional heat transfer equation, assuming that all processes are spherically symmetric. The analytical solution to this equation is presented as [2]:

$T(r, t)=\frac{1}{r} \sum_{n=1}^{\infty}\left\{\left(I_{n}-\frac{R_{d} \sin \lambda_{n}}{\lambda_{n}{ }^{2}} \zeta(0)\right) \frac{\exp \left(-\kappa \lambda_{n}{ }^{2} t\right)}{b_{n}}-\frac{R_{d} \sin \lambda_{n}}{b_{n} \lambda_{n}{ }^{2}} \int_{0}^{t} \frac{d \zeta(t)}{d \tau} \exp \left(-\kappa \lambda_{n}{ }^{2}(t-\right.\right.$

$\tau)) d \tau\} \sin \left[\lambda_{n} \frac{r}{R_{d}}\right]+T_{\mathrm{eff}}(t)$

where $r$ is the distance from the droplet centre, $R_{d}$ is the droplet radius, $\lambda_{n}$ are positive solutions to the eigenvalue equation (numeration starts from the first positive root, the roots are in ascending order):

$\lambda \cos \lambda+h_{0} \sin \lambda=0$,

$b_{n}=\frac{1}{2}\left(1+\frac{h_{0}}{{h_{0}{ }^{2}+\lambda_{n}{ }^{2}}^{2}}\right), I_{n}=\int_{0}^{R_{d}} \frac{r}{R_{d}} T(r, 0) \sin \left[\lambda_{n} \frac{r}{R_{d}}\right] d r, h_{0}=\frac{h R_{d}}{k_{\mathrm{eff}}}-1, h=\frac{k_{g} \mathrm{Nu}}{2 k_{\mathrm{eff}}}$,

$T(r, 0)$ is initial temperature distribution inside the droplet,

$\kappa=\frac{k_{\mathrm{eff}}}{C_{p l} \rho_{l} R_{d}^{2}}, \zeta(t)=\frac{h T_{\mathrm{eff}}(t) R_{d}}{k_{\mathrm{eff}}}, T_{\mathrm{eff}}=T_{g}+\frac{\dot{m}_{d} L}{2 \pi R_{d} \mathrm{Nu} k_{g}}$

$c_{l}$ and $\rho_{l}$ are liquid specific heat capacity and density, respectively, $L$ is specific heat of evaporation, $h$ and Nu are convection heat transfer coefficient and Nusselt number for the gas phase, respectively, $T_{g}$ is the ambient gas temperature, $k_{g}$ is gas thermal conductivity, $\dot{m}_{d}$ is droplet evaporation rate, $k_{\text {eff }}$ is the effective conductivity of the liquid phase:

$k_{\text {eff }}=\left(1.86+0.86 \tanh \left(2.225 \lg \left(\frac{\mathrm{Pe}_{l}}{30}\right)\right)\right) k_{l}$,

$k_{l}$ is the liquid thermal conductivity, $\mathrm{Pe}_{/}=\mathrm{Re}_{/} \mathrm{Pr} /$ is the liquid Peclet number.

The introduction of $k_{\text {eff }}$ was made within the so called the Effective Thermal Conductivity (ETC) model developed to take into account the effects of droplet velocity on the average value of its surface temperature. Obviously, this model cannot adequately describe the distribution of temperature inside droplets due to recirculation triggered by their relative velocities. Assuming that the time dependence of $\zeta$ is weak (this is particularly good approximation when Equation (1) is applied to a short time step (see below)), the terms proportional to the time derivative of $\zeta$ can be considered small. This allows us to simplify Equation (1) to:

$T(r, t)=\frac{1}{r} \sum_{n=1}^{\infty}\left\{\left(I_{n}-\frac{R_{d} \sin \lambda_{n}}{\lambda_{n}^{2}} \zeta(0)\right) \frac{\exp \left(-\kappa \lambda_{n}^{2} t\right)}{b_{n}} \sin \left(\lambda_{n} \frac{r}{R_{d}}\right)\right\}+T_{\mathrm{eff}}(t)$

The droplet evaporation rate $\dot{m}_{d}$ is estimated as:

$\dot{m}_{d}=-2 \pi R_{d} D \rho_{g} \ln \left(1+B_{M}\right) S h^{*}$

This work is licensed under a Creative Commons 4.0 International License(CC BY-NC-ND 4.0). 
where $D$ is the binary diffusion coefficient in the gas phase,

$S h^{*}=2+\frac{\left(1+\mathrm{Re}_{d} \mathrm{Sc}\right)^{1 / 3} \max \left(1, \mathrm{Re}_{\mathrm{d}}{ }^{0.077}\right)-1}{F\left(B_{M}\right)}, F\left(B_{M}\right)=\left(1+B_{M}\right)^{0.7} \frac{\ln \left(1+B_{M}\right)}{B_{M}}$,

$B_{M}$ is the Spalding mass transfer number, $\mathrm{Sc}$ is the Schmidt number. $h$ and $\mathrm{Nu}$ are linked by the equation:

$h=\frac{k_{g} \mathrm{Nu}}{2 R_{d}}$

$\mathrm{Nu}=\frac{\ln \left(1+\mathrm{B}_{T}\right)}{B_{T}} \mathrm{Nu}^{*} \mathrm{Nu}^{*}=2+\frac{\left(1+R e_{d} \mathrm{Pr}\right)^{1 / 3} \max \left(1, R e_{d}^{0.077}\right)-1}{F\left(B_{T}\right)}, F\left(B_{T}\right)=\left(1+B_{T}\right)^{0.7} \frac{\ln \left(1+B_{T}\right)}{B_{M}}$

$\mathrm{Nu}$ is the Nusselt number, $B_{T}$ is the Spalding heat transfer number and $\operatorname{Pr}$ is the Prandtl number for the gas phase.

In the limit of infinitely large liquid thermal conductivity, Equation (1) can be simplified to (see [2] for the details):

$c_{p l} m_{d} \frac{d T}{d t}=N u \frac{k_{g}}{2 R_{d}} A_{d}\left(T_{g}-T_{s}\right)+L \dot{m}_{d}$

Where $m_{d}, A_{d}$ and $T_{s}$ are the droplet mass, surface area and surface temperature, respectively.

The derivation of Equation (1) was based on the assumption that $h$ and $T_{g}$ are constant during the process of droplet heating and evaporation which is not satisfied in most engineering applications (e.g. the reduction of $R_{d}$ during the evaporation process would lead to an increase in $h$ ). That means that neither Equation (1) nor Equation (4) can be used directly during the whole period of droplet heating and evaporation. Both these equations, however, can be used during short time steps $\Delta t$ when the assumption of constant $h$ and $T_{g}$ is valid. In this case, the solution to these equations at the end of the time step can be used as the initial condition for following time step with updated values of all other parameters, if necessary. As in [7] our analysis is based on Equation (4).

As in the case of heat transfer equation inside droplets, we assume that species diffusion inside them is described by the one-dimensional species diffusion equation and all processes are spherically symmetric. The analytical solution to this equation for the mass fractions $Y_{i}$ is presented as [2]:

$$
\begin{aligned}
Y_{i}(r, t)=\varepsilon_{i}+ & \frac{1}{r}\left[\frac{1}{b_{y 0}} \exp \left[D_{\mathrm{eff}}\left[\frac{\lambda_{0}}{R_{d}}\right]^{2} t\right]\left(I_{i 0}+\varepsilon_{i} \frac{1}{\lambda_{0}{ }^{2}}\left(1+h_{Y 0}\right) \sinh \lambda_{0}\right) \sinh \left(\lambda_{0} \frac{r}{R_{d}}\right)+\right. \\
& \left.\sum_{n=1}^{\infty} \frac{1}{b_{Y n}} \exp \left[-D_{\mathrm{eff}}\left(\frac{\lambda_{n}}{R_{d}}\right)^{2} t\right]\left(I_{i n}-\varepsilon_{i} \frac{1}{\lambda_{n}{ }^{2}}\left(1+h_{Y 0}\right) \sin \lambda_{n}\right) \sin \left(\lambda_{n} \frac{r}{R_{d}}\right)\right]
\end{aligned}
$$

where $\lambda_{0}$ and $\lambda_{n}$ are solutions to the eigenvalue equations:

$\lambda_{0} \cosh \lambda_{0}+h_{Y 0} \sinh \lambda_{0}=0, \lambda_{n} \cos \lambda_{n}+h_{Y n} \sin \lambda_{n}=0,(n=1,2, \ldots)$

$\lambda_{n}(n \geq 1)$ are in ascending order, $\varepsilon_{i}=\frac{Y_{v i s}}{\sum_{i} Y_{v i s}}, Y_{v i s}$ is vapour species mass fractions at the surface of the droplet,

$$
\begin{aligned}
& b_{Y 0}=-\frac{R_{d}}{2}\left(1+\frac{h_{Y 0}}{h_{Y o}^{2}-\lambda_{0}^{2}}\right), b_{Y n}=\frac{R_{d}}{2}\left(1+\frac{h_{Y 0}}{h_{Y o}^{2}+\lambda_{n}^{2}}\right), h_{0 Y}=-\left(1+\frac{\alpha R_{d}}{D_{l}}\right), \\
& I_{i 0}=\int_{0}^{R_{d}} \frac{r}{R_{d}} Y_{i}(r, 0) \sinh \left[\lambda_{0} \frac{r}{R_{d}}\right] d r, I_{i n}=\int_{0}^{R_{d}} \frac{r}{R_{d}} Y_{i}(r, 0) \sin \left[\lambda_{n} \frac{r}{R_{d}}\right] d r, n \geq 1,
\end{aligned}
$$

$Y_{i}(r, 0)$ Is the initial ith species mass fraction distribution inside the droplet.

$D_{\text {eff }}$ is the effective species diffusivity in the liquid phase assumed to be the same for all species and defined as [2]

$D_{\mathrm{eff}}=\left(1.86+0.86 \tanh \left(2.225 \log \left(\frac{\mathrm{Pe}_{l}}{30}\right)\right)\right) D_{l}$, 
$D_{l}$ is the liquid diffusivity, $\mathrm{Pe}_{/ Y}=\mathrm{Re}_{/} \mathrm{Sc}_{/}$is the liquid Peclet number referring to species diffusion.

The introduction $D_{\text {eff }}$ was made within the so called Effective Diffusivity (ED) model developed to take into account the effects of droplet velocity on the average values of its surface mass fractions in the liquid phase. This model cannot adequately describe the distribution of species inside droplets due to recirculation triggered by their relative velocities. Vapour molar fractions of species at the surface of the droplets and the corresponding molar fractions in the liquid phase are linked by Rault law [2].

\section{Implementation of the model into ANSYS Fluent}

Equation (7) was implemented into the customised version of ANSYS Fluent alongside with the previously implemented Equation (4). The right-hand sides of these equations were calculated via the User Defined Functions (UDF); thermodynamic and transport parameters were calculated based on the average temperature inside the droplets, using Simpson's method [10]. The droplet volume was discretised into $N_{L}=500$ concentric layers to calculate the series in Equations (4) and (7). The integrals ( $I_{n}$ and $I_{i n}$ ) and average temperatures inside droplets were also calculated using the Simpson's method with 501 points for 500 layers. At each time step temperatures and species distributions were calculated from Equations (4) and (7). Time-steps for calculations were taken equal to $\delta t=10^{-6}$ seconds. The roots of the eigenvalue Equations (2) and (8) were found using the bisection method with accuracy of $10^{-8}$.

\section{Results and discussion}

The newly developed customised version of ANSYS Fluent was applied to the analysis of cooling and evaporation of droplets of ethanol, acetone and mixture of ethanol and acetone, as described in the experiments the results of which are presented in [9]. The transport and thermodynamic properties of acetone and ethanol were taken from [9]. The initial droplet temperatures were in the range 293-296 K, while the temperatures of ambient air were in the range $305-312 \mathrm{~K}$ at atmospheric pressure. Gas parameters in the vicinity of the droplet surface were calculated based on reference temperature $T_{\text {ref }}=\left(2 T_{s}+T_{g}\right) / 3.200$ eigenvalues were used to calculate temperature distribution and 10 eigenvalues were used to calculate species mass fraction distribution. Gas and liquid properties are taken from $[5,11]$.

The results predicted by ANSYS Fluent were verified against the results predicted by the one-dimensional in-house code. The predictions of the latter code, in their turn, were verified against the predictions of the code based on the numerical solutions of the heat transfer and species diffusion equations [9]. This allows us to use the abovementioned code $(\mathrm{IHC})$ as the reference code to verify the results for heating and evaporation of droplets of various compositions predicted by the new customised version of ANSYS Fluent.

The results of the comparison between ANSYS Fluent and in-house code for the temperatures at the centre and surface of the droplet and droplet average temperature are shown in Figs. 1-5 for various droplet and gas parameters and various compositions of droplets: pure acetone (Fig. 1), pure ethanol (Fig. 2), 75\% ethanol and $25 \%$ acetone (Fig. 3), 50\% ethanol and 50\% acetone (Fig. 4), 25\% ethanol and 75\% acetone (Fig. 5). Input parameters used in calculations are summarised in Table 1. As can be seen from these figures, in all cases the agreements between Fluent and IHC results are reasonably good. The percentage errors for the abovementioned three temperatures after $7 \mathrm{~ms}$ is between $1.0-4.0 \%$ for acetone, 6.0-6.1\% for ethanol, between $2.0-3.5 \%$ for the mixture with $25 \%$ of ethanol, between $0.5-1.8 \%$ for the mixture with $50 \%$ of ethanol and between $0.3-1.8 \%$ for the mixture with for $75 \%$ of ethanol. Note that the results shown in Figs. 1 and 2 were obtained without using the new features of the ANSYS Fluent code developed in our work (they could be obtained based on the version of the code developed in [7]). In all cases shown in Figs. 1-5 the changes in droplet radii were small and the corresponding curves are not shown.

Table 1 Droplet and gas temperatures, droplet diameters and approximations of droplet velocities, inferred from [11] and used in calculations.

\begin{tabular}{|l|l|l|l|l|}
\hline Composition & $\begin{array}{l}\text { Droplet } \\
\text { temperature } \\
(\mathrm{K})\end{array}$ & $\begin{array}{l}\text { Droplet } \\
\text { diameter } \\
(\mu \mathrm{m})\end{array}$ & $\begin{array}{l}\text { Gas } \\
\text { temperature } \\
(\mathrm{K})\end{array}$ & $\begin{array}{l}\text { Droplet velocity } \\
\text { approximations in m/s } \\
(\mathrm{t} \text { is in } \mathrm{ms})\end{array}$ \\
\hline acetone & 308.25 & 143.4 & 294.65 & $12.81-0.316 t$ \\
\hline ethanol & 311.15 & 140.8 & 295.15 & $12.30-0.344 t$ \\
\hline$+25 \%$ ethanol & 305.65 & 133.8 & 294.25 & $12.75-0.370 t$ \\
\hline$+50 \%$ ethanol & 310.65 & 142.7 & 293.95 & $12.71-0.488 t$ \\
\hline$+75 \%$ ethanol & 311.75 & 137.1 & 294.75 & $12.28-0.306 t$ \\
\hline
\end{tabular}


As can be seen in Figs. 1-5 the difference between the temperatures at the centre and surface of the droplets and droplet average temperatures is approximately 2-9 K, which cannot be ignored in most engineering applications. This difference was ignored in the conventional version of ANSYS Fluent (e.g. [4]), which cannot be justified in the general case. The agreement between ANSYS Fluent and IHC results gives us confidence in applying the new customised version of ANSYS Fluent to more complex problems that those presented in Figures. 1-5.

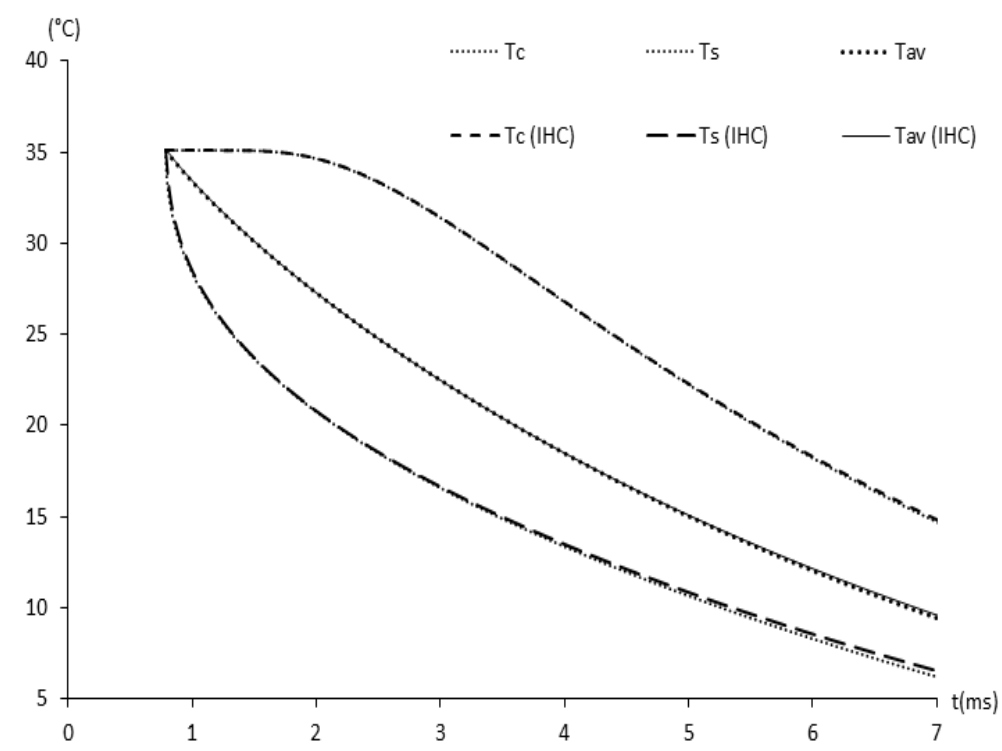

Figure 1. Time evolution of an acetone droplet surface, average and centre temperatures $\left(T_{s}, T_{a v}\right.$ and

$T_{c}$ ) (see Table 1). ANSYS Fluent results (dotted curves) and compared with results of the previously developed In house code (IHC) (dashed and solid curves).

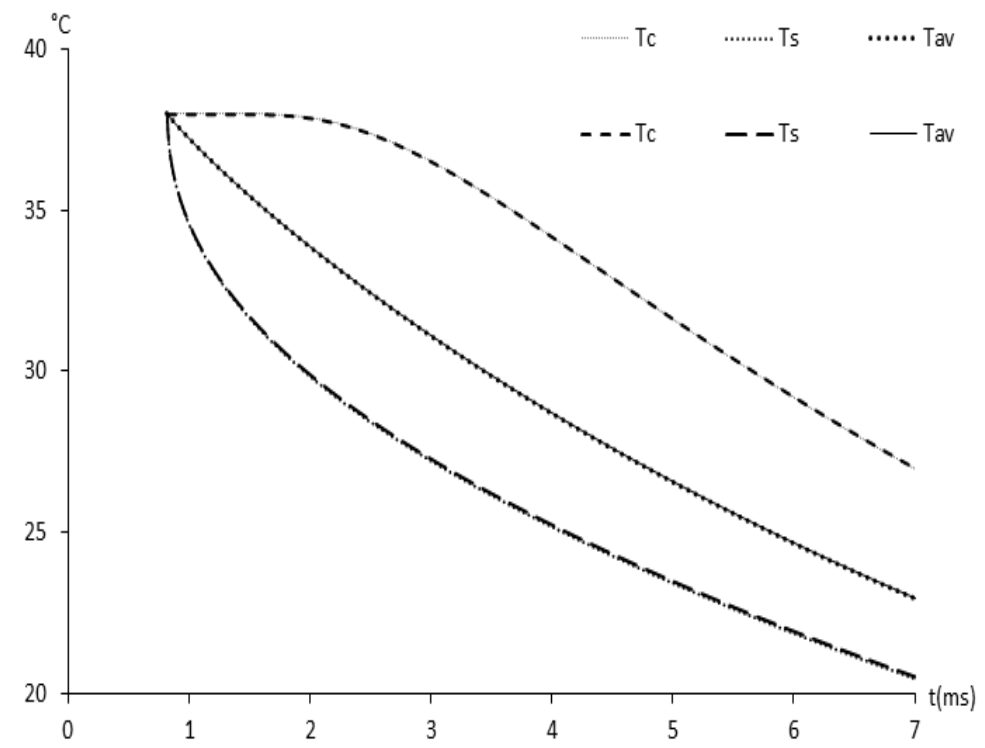

Figure 2. Time evolution of ethanol droplet surface, average and centre temperatures $\left(T_{s}, T_{a v}\right.$ and $\left.T_{c}\right)$ (see Table 1). ANSYS Fluent results (dotted curves) and compared with results of the previously developed In house code (IHC) (dashed and solid curves). 
ILASS - Europe $2017,6^{\text {th }}-8^{\text {th }}$ September, Valencia, Spain.

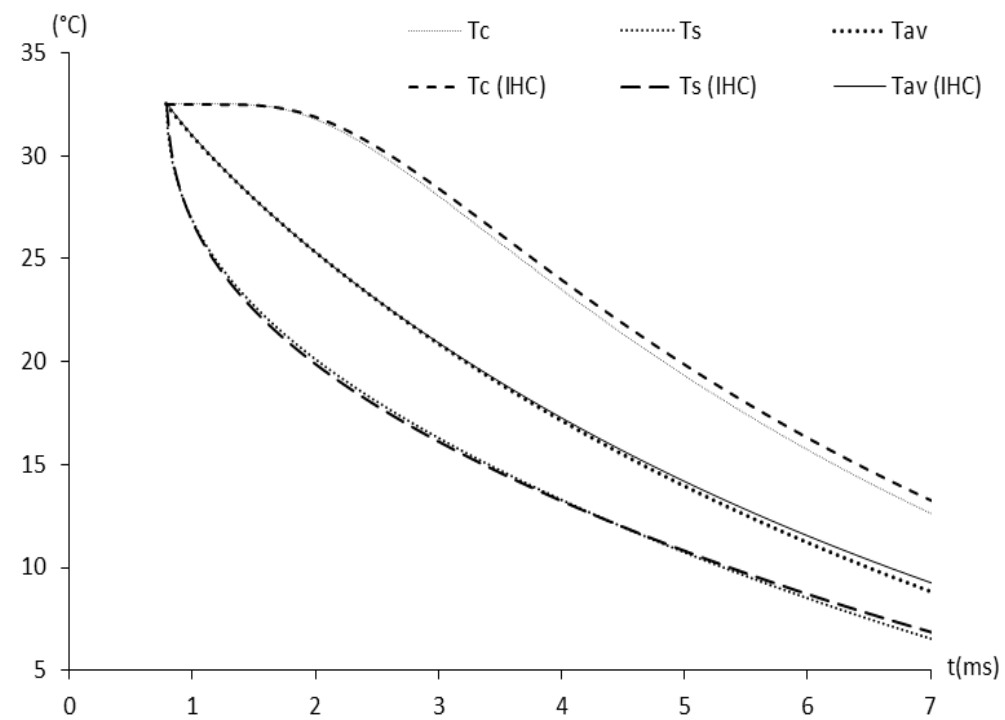

Figure 3. Time evolution of $75 \%$ ethanol and $25 \%$ acetone droplet surface, average and centre temperatures $\left(T_{s}, T_{a v}\right.$ and $T_{c}$ ) (see Table 1). ANSYS Fluent results (dotted curves) and compared with results of the previously developed In house code (IHC) (dashed and solid curves).

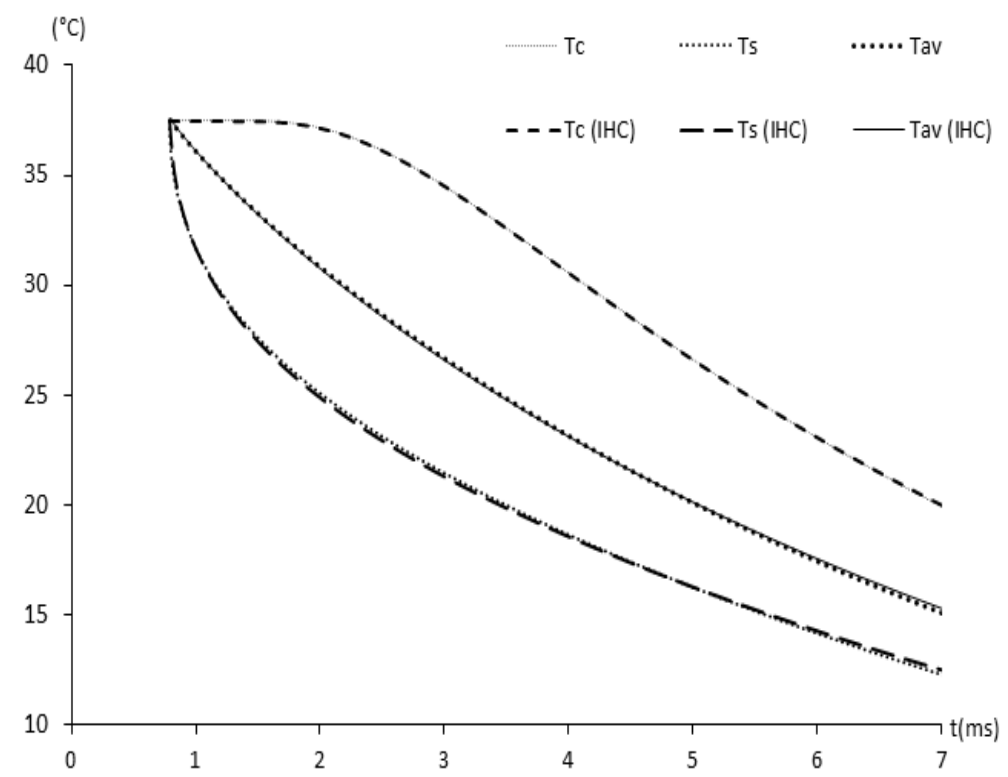

Figure 4. Time evolution of $50 \%$ ethanol $50 \%$ acetone droplet surface, average and centre temperatures $\left(T_{s}, T_{a v}\right.$ and $T_{c}$ ) (see Table 1). ANSYS Fluent results (dotted curves) and compared with results of the previously developed In house code (IHC) (dashed and solid curves). 


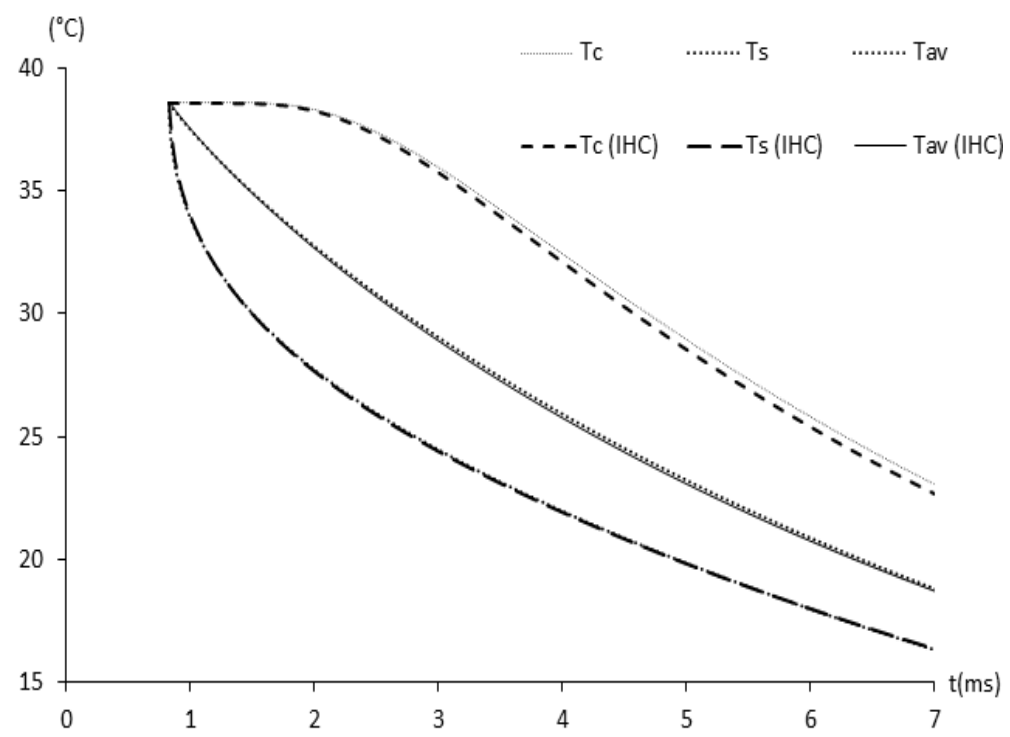

Figure 5. Time evolution of $25 \%$ ethanol $75 \%$ acetone droplet surface, average and centre temperatures ( $T_{s}, T_{a v}$ and $T_{c}$ ) (see Table 1). ANSYS Fluent results (dotted curves) and compared with results of the previously developed In house code (IHC) (dashed and solid curves).

\section{Conclusions}

A model for heating and evaporation of mono- and multi-component droplets, based on analytical solutions to the heat transfer and species diffusion equations in the liquid phase, is summarised. The implementation of the model into ANSYS Fluent via User-Defined Functions (UDF) is described. The model is applied to the analysis of pure acetone, ethanol, and mixtures of acetone/ethanol droplets heating/cooling and evaporation. The predictions of the customised version of ANSYS Fluent with the new model implemented into it are verified against the results predicted by previously developed one-dimensional in-house code based on the analytical solutions to the heat transfer and mass diffusion equations. The latter code in its turn was verified against the predictions of the in-house code developed at the University of Nancy (France) and validated against experimental data obtained at the same university.

The agreement between the predictions of these codes is shown to be reasonably good for mono-component (pure acetone and ethanol) and multi-component droplets comprising acetone with 25, 50 and 75-percentage mass fractions of ethanol, with input parameters used for pervious validation of the in-house code. This gives us confidence in using the new customised version of ANSYS Fluent for the analysis of more complex engineering processes.

\section{Acknowledgements}

The authors would like to recognise that this work was supported by the UK's Engineering and Physical Science Research Council, a studentship to support one of the authors (LP) [EPSRC grant EP/N509607/1; EP/K005758/1; EP/K020528/1; EP/M002608/1] 


\section{Nomenclature.}

\begin{tabular}{|c|c|c|c|}
\hline$B_{M} ; B_{T}$ & Spalding mass and heat transfer numbers & $\operatorname{Re}$ & Reynolds number \\
\hline$b_{n}$ & Parameter introduced in (1) & Sc & Schmidt number \\
\hline$b_{Y n}$ & Parameter introduced in (7) & Sh & Sherwood number \\
\hline$C_{p}$ & Specific heat capacity at constant pressure & $t$ & Time \\
\hline$D$ & Binary diffusivity coefficient of vapour in air & $T$ & Temperature \\
\hline$F$ & Function introduced in $\mathrm{Sh}^{*}$ and $\mathrm{Nu}^{*}$ & $v$ & Velocity \\
\hline$h$ & Convection heat transfer coefficient & Y & Mass fraction \\
\hline$h_{0}$ & Parameter introduced in (2) & $x$ & Molar fraction \\
\hline$h_{Y O}$ & Parameter introduced in (8) & & \\
\hline $\ln$ & Integrals, used in Solutions (1) and (4) & \multicolumn{2}{|c|}{ Greek symbols } \\
\hline$l_{\text {in }}$ & Integrals, used in Solution (7) & $\varepsilon_{\mathrm{i}}$ & Vapour species mass fractions \\
\hline j & Parameter, defined in (3) & $\mathrm{K}$ & Parameter introduced in (1) \\
\hline$k$ & Thermal conductivity & $\lambda_{n}$ & Eigenvalues \\
\hline$L$ & Latent heat of evaporation & $\mu$ & Dynamic viscosity \\
\hline$m$ & Mass & $\rho$ & Density \\
\hline$\dot{m}_{d}$ & Evaporation rate & $\varphi$ & Parameter defined by Equation (4) \\
\hline M & Molar mass & $x$ & Correction function defined by Equation (1c) \\
\hline$N_{L}$ & Number of layers inside a droplet & $\zeta$ & parameter defined by (1c) \\
\hline $\mathrm{Nu}$ & Nusselt number & & \\
\hline $\mathrm{Pe}$ & Peclet number & \multicolumn{2}{|c|}{ Subscripts } \\
\hline$p$ & Pressure & $d$ & Droplet \\
\hline $\operatorname{Pr}$ & Prandtl number & eff & Effective \\
\hline$q$ & Heat flux & $g$ & Gas \\
\hline$r$ & Distance from the centre of the droplet & int & Internal \\
\hline$R_{d}$ & Radius of a droplet & $\mathrm{l}$ & Liquid \\
\hline
\end{tabular}

\section{References}

[1] Sazhin, S.S., 2006, Progress in Energy and Combustion Science 32(2), pp. 162-214.

[2] Sazhin, S.S., 2014, "Droplets and Sprays". Springer.

[3] Sazhin, S.S., 2017, Fuel, 196, pp. 69-101.

[4] Sazhina, E.M., Sazhin, S.S., Heikal, M.R., Babushok, V.I., Johns, R.J.T., 2000, Combustion Science and Technology, 160(1), pp. 317-344.

[5] Maqua, C., 2007, Contribution à la compréhension de l'évaporation de gouttes de combustible bi-composant à l'aide de méthodes optique, PhD Thèse, Nancy-Université, 2007.

[6] Elwardany, A.E., Sazhin, S.S., Castanet, G., Lemoine, F. and Heikal, M.R., 2011, Proceedings of ILASS Europe 2011, 24th European Conference on Liquid Atomization and Spray Systems, Estoril, Portugal, 5-7 September 2011, paper 1018 (CD).

[7] Rybdylova, O., Al Qubeissi, M., Braun, M., Crua, C., Manin, J., Pickett, L.M., de Sercey, G., Sazhina, E.M., Sazhin, S.S, Heikal, M., 2016, International Communications in Heat and Mass Transfer, 76, pp. 265-270.

[8] Abramzon B., Sirignano W.A., 1989, International Journal of Heat and Mass Transfer 32. pp. 1605-1618.

[9] Elwardany, A.E., Gusev, I.G., Castanet, G., Lemoine, F., Sazhin, S.S., 2011, Atomization and Sprays 21, pp. 907-931.

[10] Eagle, W.E., Musculus, M., Oefelein, J.C., Malbec, L.-M., Bruneaux, G., 2014. Measuring transient entrainment rates of a confined vaporizing diesel jet, in: 26th ILASS Americas, Portland (OR), USA, May, 2014.

[11] Sazhin, S.S., Elwardany, A., Krutitskii, P. A., Castanet, G. Lemoine, F., Sazhina, E. M., Heikal, M. R., 2010, International Journal of Heat and Mass Transfer, 53, pp. 4495-4505. 


\title{
Modelling of spheroidal drop evaporation with non-uniform temperature conditions
}

\author{
Gianpietro Elvio Cossali, Stefano Ravasio and Simona Tonini* \\ Department of Engineering and Applied Sciences, University of Bergamo, Italy \\ ${ }^{*}$ Corresponding author: simona.tonini@unibg.it
}

\begin{abstract}
The heating and evaporation of single component spherical and spheroidal drops in gaseous quiescent environment are predicted, accounting for the effect of a non-uniform distribution of the temperature at the drop surface. The analytical solution of the species conservation equations in the proper coordinate system (spherical/spheroidal) is implemented to numerically solve the energy equation in a rectangular domain. The effect of temperature non-uniformity on the local Nusselt number and global heat and evaporation rates is calculated for different species, drop deformation and gaseous temperature.
\end{abstract}

\section{Keywords}

Drop evaporation, spheroidal coordinates, non-uniform Dirichlet Boundary conditions.

\section{Introduction}

Most of the models predicting the drop heating and evaporation to be implemented in CFD codes for dispersed phase applications rely on the assumption that drops are spherical, thus allowing a simpler solution in spherical coordinates of the energy and species conservation equations. However, experimental investigation on liquid drops in multi-particle systems has revealed that they are subject to significant shape deformations while interacting with the carrier phase [1-3], due to the interaction of surface tension and fluid-dynamic stresses on the drop surface [3]. Numerical investigations on oscillating drops [4,5] have shown that the vapour and heat fluxes on the drop surface are not uniform and they were empirically correlated to the local mean curvature of the surface $[1,6]$. Analytical modelling of the heating and evaporation of spheroidal drops have shown that the local vapour and heat flux scale with the fourth root of the Gaussian curvature $[7,8]$ and later the same result was extended to a wider class of drop shapes [9].

When dynamical simulation of droplet heating and evaporation is necessary, uniform drop temperature is often assumed, on the basis of a commonly accepted belief that the internal recirculation would maintain uniform conditions. However a more accurate simulation can be obtained by using the concept of effective conductivity, firstly introduced by [10], to account for the effect of recirculation (see also [11] and [12]) and, although this cannot properly describe the temperature field inside the droplet, it can give a better estimation of the droplet surface temperature [13] .

Recent modelling of heating and evaporation of spheroidal droplets [14] revealed that the uneven distribution of fluxes on the drop surface causes a corresponding uneven distribution of temperature on the drop surface, during most of the drop lifetime. This non-uniform temperature distribution affects the heat and vapour flow fields in a non neglectful way.

The motivation of the work reported here is to investigate, through a combined analytical-numerical solution of the species and energy conservation equations, the effect of non-uniform Dirichlet boundary conditions at the drop surface (for spheroidal liquid drops) on the local heat and mass transfer coefficients.

\section{Mathematical modelling}

The evaporation of a single-component drop under quasi-steady conditions in a quiescent atmosphere, where the Stefan flow characterises the flow field, is described by the species conservation equations coupled with the energy conservation equation:

$\nabla_{j} n_{j}^{(p)}=0 \quad p=0,1$

where:

$n_{j}^{(p)}=\rho U_{j} \chi^{(p)}-\rho D_{10} \nabla_{j} \chi^{(p)} \quad p=0,1$ 
are the mass fluxes, $p=1$ stands for the evaporating component and $p=0$ for the gas. After summation, equations (1) yield the mass conservation equation:

$\nabla_{j} \rho U_{j}=0$

These three equations are then not independent and one of the species conservation can be disregarded. Moreover, assuming that the diffusion of component $p=0$ into the drop can be neglected, the mass flux of the component $p=0$ is nil at drop surface and then is nil everywhere. This last observation, after introducing the new variable: $G=\ln \left(1-\chi^{(1)}\right)$, and assuming constant properties, allows to write the conservation equations under the form:

$\nabla^{2} G=0$

(see also [9] for further details). The evaporation model is coupled with the energy equation, which has the form:

$L e^{-1} \nabla_{j} G \nabla_{j} T-\nabla^{2} T=0$

where $L e=\frac{k}{\rho D_{10} c_{p, v}}$ (see again [9] for further details).

\section{Spherical and spheroidal drops}

Equation (4) and (5) can be used to model heating and evaporation of drops of any shape, since the drop shape enters the problem through the boundary conditions. The case of spherical and spheroidal drops can be more easily treated solving the problem in proper coordinate systems. Using the spherical coordinates:

$x=R_{0} \frac{\sqrt{1-\eta^{2}}}{\zeta} \cos \varphi ; y=R_{0} \frac{\sqrt{1-\eta^{2}}}{\zeta} \sin \varphi ; z=R_{0} \frac{\eta}{\zeta}$

where:

$\zeta=\frac{R_{0}}{r} ; \eta=\cos \theta$

the drop surface of a spherical drop is defined by the equation $\zeta=\zeta_{0}$, and similarly in spheroidal prolate and oblate coordinates:

$$
\begin{aligned}
& x=a \sqrt{\zeta^{2}-1} \sqrt{1-\eta^{2}} \cos \varphi ; y=a \sqrt{\zeta^{2}-1} \sqrt{1-\eta^{2}} \sin \varphi ; z=a \zeta \eta \text { (prolate) } \\
& x=a \sqrt{\zeta^{2}+1} \sqrt{1-\eta^{2}} \cos \varphi ; y=a \sqrt{\zeta^{2}+1} \sqrt{1-\eta^{2}} \sin \varphi ; z=a \zeta \eta \quad \text { (oblate) }
\end{aligned}
$$

the drop surface (a prolate or oblate spheroid, see figure 1 ) is again defined by the equation $\zeta=\zeta_{0}$, but it must be noticed that now the coordinates $\zeta$ and $\eta$ have different definitions.

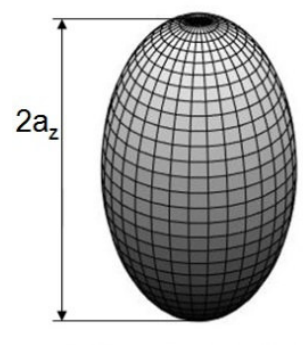

Prolate spheroid

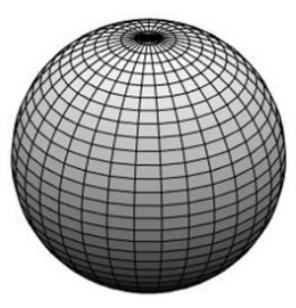

Sphere

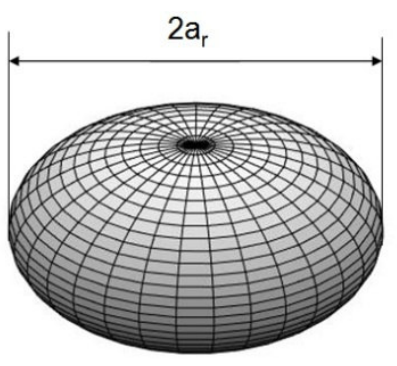

Oblate spheroid

Figure 1. Drop shapes and definition of semi-axes $a_{z}$ and $a_{r}$.

For spheroidal drops, an equivalent radius $R_{0}$ can be defined as the radius of a spherical drop having the same volume, and the constant $a$ in equations (8) can be related to this radius by:

$a=R_{0} \frac{\left|1-\varepsilon^{2}\right|^{1 / 2}}{\varepsilon^{1 / 3}}$

where the eccentricity parameter $\varepsilon$ is defined as the ratio between the axial and radial drop semi-axes (see again figure 1).

An analytical solution of equation (3) has the following form, for the prolate, oblate and spherical drops: 


$$
\begin{array}{ll}
G=b_{0} \ln \frac{\zeta-1}{\zeta+1}+\sum_{n=1}^{\infty} b_{n} P_{n}(\eta) Q_{n}(\zeta)+G_{\infty} & \text { (prolate) } \\
G=c_{0}\left(\arctan (\zeta)-\frac{\pi}{2}\right)+\sum_{n=1}^{\infty} c_{n} P_{n}(\eta) Q_{n}(i \zeta)+G_{\infty} & \text { (oblate) } \\
G=d_{0} \zeta+\sum_{n=1}^{\infty} d_{n} P_{n}(\eta) \zeta^{n+1}+G_{\infty} & \text { (sphere) }
\end{array}
$$

where $P_{n}$ and $Q_{n}$ are the Legendre functions of the first and second kind, respectively. The conditions at drop surface and at infinity:

$G\left(\zeta=\zeta_{0}, \eta\right)=G_{s}(\eta) ; \quad G(\zeta=\infty, \eta)=G_{\infty}$

allow to calculate the coefficients $b_{n}, c_{n}$ and $d_{n}$ and the constant $G_{\infty}$. The value of $G$ at infinity is constant since the vapour mass fraction is considered uniformly distributed at infinite distance from the drop, while on the surface the Dirichlet type conditions will be generally non-uniform and depending on the surface temperature conditions, since the saturation values of the mass fraction are correlated to the drop surface temperature and pressure and then:

$$
G_{s}(\eta)=\ln \left(1-\frac{P_{v, s a t} M m^{(1)}}{P_{v, \text { sat }}\left(M m^{(1)}-M m^{(0)}\right)+P_{T} M m^{(0)}}\right)
$$

The boundary conditions for the energy equation (5), again of Dirichlet type, are:

$T\left(\zeta=\zeta_{0}, \eta\right)=T_{s}(\eta) ; T(\zeta=\infty, \eta)=T_{\infty}$

The temperature distribution along the drop surface is chosen to be a polynomial of even degree (to satisfy the symmetry requirement across $\eta=0$ ). A fourth degree polynomial is the simplest choice to fit the values at $\eta=0$ and $\eta=1$ and to satisfy the further symmetry requirement: $\left.\frac{\partial T}{\partial \eta}\right)_{\eta=1}=0$.

The surface distribution of $G_{s}$ (equation 12) is approximated by a polynomial of even degree (again to satisfy symmetry condition across $\eta=0$ ) and the coefficients are calculated to satisfy the further symmetry condition on $\eta=1$ and to fit the values at $\eta=0$ and $\eta=1$. The choice of a polynomial form for $G_{s}$ allows to reduce the number of terms needed in equation (10). Since a polynomial of degree $p$ can be always written as a sum of the first $p$ Legendre polynomials, only the first $p$ coefficients in the series expansion (12) are different from zero.

\section{Solution of the energy equation}

Currently, to the best knowledge of the Authors, no analytical solution of the energy equation (5) satisfying general non-uniform boundary conditions at the drop surface is available (the simple solution $T=A e^{G(\zeta, \eta) / L e}+B$ of equation (5) cannot satisfy general non-uniform boundary conditions on the drop surface) and the energy problem is solved numerically, implementing a finite difference scheme on the rectangular computational grids $\eta \in(0,1) \times$ $\zeta \in\left(\zeta_{0}, \zeta_{\max }\right)$. Tests were performed to choose a proper value of $\zeta_{\max }$ to make the solution practically independent of it. Grid refinement was performed in the drop surface vicinity introducing a proper stretching function, which was optimised by comparison to the analytical solution of the energy equation available for uniform surface temperature condition (see [9]). Grid independence tests were performed and a final grid of 2000 cells proved to be enough to assure a sufficient grid independence of the solution and particularly of the fluxes on the drop surface.

\section{Results and discussions}

This section reports and comments the results obtained by implementing the model described in the previous paragraph, focusing on the effect of non-uniform temperature boundary conditions for the case of prolate drops. The choice of a prolate drop is related to the fact that for such shape it was shown [14] that the uneven heat flux caused by the variable curvature produces, during drop heating and evaporation, an uneven distribution of temperature over the surface. However, similar results can be obtained for the case of an oblate drop and even for the spherical drop.

The values of the fluid properties are evaluated at reference conditions (temperature and mass fraction) by the "1/3 rule" [15]: 
$T_{\text {ref }}=\frac{2 \bar{T}_{s}+T_{\infty}}{3} ; \chi_{\text {ref }}=\frac{2 \bar{\chi}_{s}+\chi_{\infty}}{3}$

Figure 2(a) shows qualitatively a sample of the vapour field around a prolate water drop with the eccentricity parameter $\varepsilon$ equal to 2.5; the surrounding gas temperature at free stream condition is fixed equal to $700 \mathrm{~K}$, the vapour mass fraction at infinity is taken equal to zero and the average surface temperature of the drop is equal to $340 \mathrm{~K}$, while seven different surface temperature profiles are imposed, defined by the maximum temperature difference at the drop surface as follows:

$\Delta T=T_{s}\left(\zeta_{0}, \eta=1\right)-T_{s}\left(\zeta_{0}, \eta=0\right)$

(a) Prolate drops, $\varepsilon=2.5$

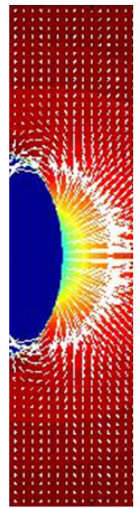

$\Delta T=-40 \mathrm{~K}$

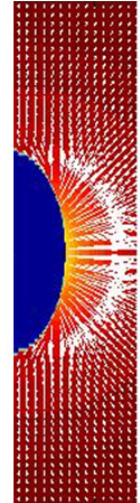

$\Delta T=-20 \mathrm{~K}$

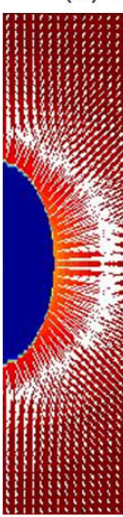

$\Delta T=-10 \mathrm{~K}$

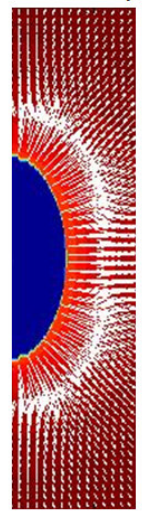

$\Delta T=0 \mathrm{~K}$

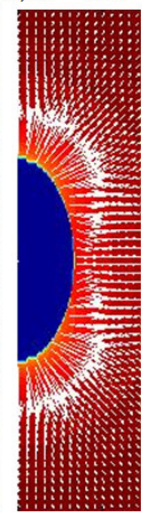

$\Delta T=10 \mathrm{~K}$

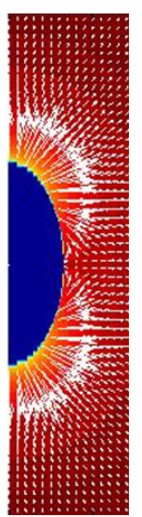

$\Delta T=20 \mathrm{~K}$

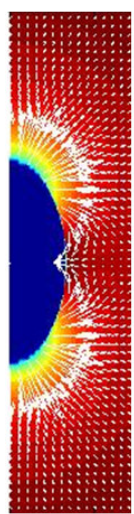

$\Delta T=40 \mathrm{~K}$

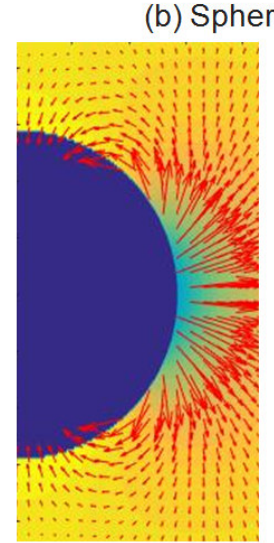

$\Delta T=-40 \mathrm{~K}$

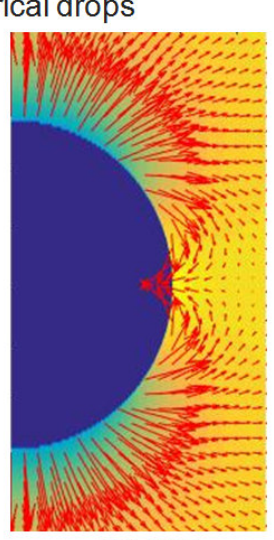

$\Delta T=40 \mathrm{~K}$

Figure 2. $G$-field and vapour flux around (a) prolate $(\varepsilon=2.5)$ and (b) spherical water drops, for different surface temperature profiles; $T_{\infty}=700 \mathrm{~K}, G_{\infty}=0, \bar{T}_{s}=340 \mathrm{~K}$.

The flow field is substantially affected by the non-uniform temperature boundary conditions at the drop surface, since vapour recirculation can be observed when the local surface temperature drops below the dew point value of the surrounding gas. The effect is mainly related to the temperature distribution and it is not peculiar of deformed drops, figure 2(b) shows the vapour distribution and flux around a spherical drop having a non-uniform surface temperature, and similar paths can be observed.

The calculated vapour distribution (equation 10a) is then used to numerically solve equation (5) and the local surface heat flux can be calculated. Figure $3(a)$ shows the heat fluxes, non-dimensionalised by the factor $\frac{2 R_{0}}{\left(\bar{T}_{s}-T_{\infty}\right) k}$ for the case of a prolate drop $(\varepsilon=2.5)$ while figure $3(\mathrm{~b})$ shows the local Nusselt number, defined as:

$$
N u(\eta)=\frac{\varphi(\eta) 2 R_{0}}{\left[T_{s}(\eta)-T_{\infty}\right] k}
$$

along the surface. The case $\Delta T=0$ is the one with uniform surface temperature and for this case the function $N u(\eta)$ can be found analytically (see [14]), showing that the surface curvature itself influences this parameter. The results for $\Delta T \neq 0$ show that the surface heat flux is strongly influenced by the non-uniform temperature distribution (see figure 3a). The local Nusselt number is also influenced by the non-uniform temperature distribution, but to a lesser extent, specially close to the "pole" $(\eta=1)$ where the effect of the different temperature distribution is lower (figure $3 b)$. 
(a)

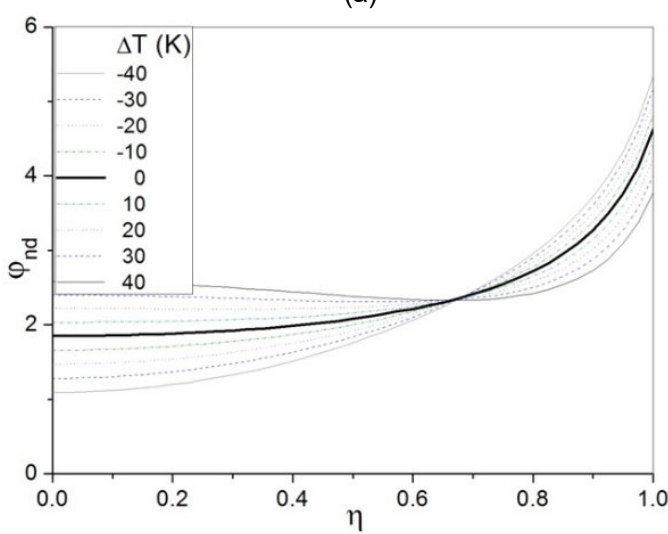

(b)

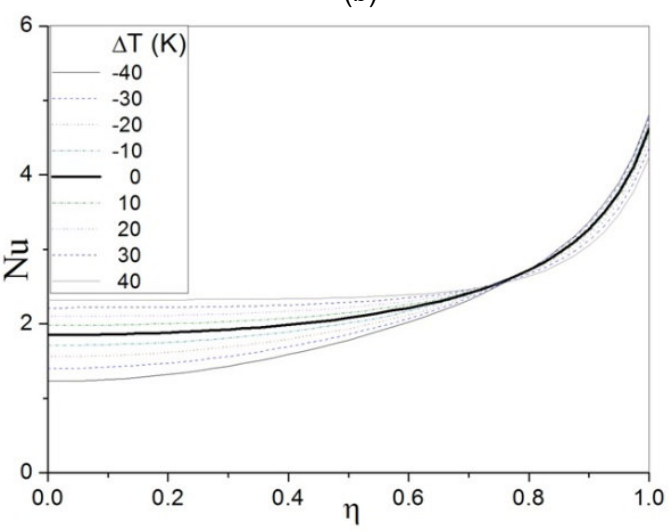

Figure 3. (a) Local vapour flux and (b) local Nusselt number along the drop surface for various surface temperature profiles (water drop, $\bar{T}_{s}=320 \mathrm{~K}, T_{\infty}=500 \mathrm{~K}$ ).

These effects are clearly not peculiar of one particular species but their magnitude depends on the thermophysical characteristics of the species. Calculations were performed selecting five different species, whose properties are reported in Table 1. These species represent some of the most common working fluid used for spray modelling in industrial applications.

\begin{tabular}{l|lll}
\hline \hline Species & $M m(\mathrm{~kg} / \mathrm{kmol})$ & $T_{b, n}(\mathrm{~K})$ & $L\left(T_{b, n}\right)(\mathrm{kJ} / \mathrm{kg})$ \\
\hline \hline water $\mathrm{H}_{2} \mathrm{O}$ & 18.02 & 373.15 & 2257.4 \\
ethanol $\mathrm{C}_{2} \mathrm{H}_{6} \mathrm{O}$ & 46.07 & 351.39 & 850.53 \\
acetone $\mathrm{C}_{3} \mathrm{H}_{6} \mathrm{O}$ & 58.08 & 329.22 & 501.85 \\
n-octane $\mathrm{C}_{8} \mathrm{H}_{18}$ & 114.23 & 398.80 & 301.10 \\
n-dodecane $\mathrm{C}_{12} \mathrm{H}_{26}$ & 179.34 & 489.50 & 256.70
\end{tabular}

Table 1. Molar mass, normal boiling temperature and latent heat of evaporation for the selected liquid species.

The profiles of the local Nusselt number $(\mathrm{Nu})$ and the percentage deviation $\Delta \%$ from the values obtained with a surface temperature uniformly equal to the mean temperature are presented in Fig. 4 for each fluid.

These results were obtained maintaining constant the drop deformation $(\varepsilon=2.5)$ and the gas temperature $\left(T_{\infty}=500 \mathrm{~K}\right)$ and setting the drop mean surface temperature equal to the corresponding quasi-steady (sometimes called plateau or asymptotic [16]) temperature. The deviations from the uniform temperature case are relatively small for water and n-dodecane while they are more relevant for acetone and n-octane. The different behaviour is due to a combination of the effects of boiling temperature and latent heat of vaporisation. These peculiarities should be considered when approximating the heat transfer coefficient from the analytic relation that can be deduced from the uniform surface temperature analysis $[9,14]$.

The drop deformation has an effect on the deviation of the local Nusselt number from the values for the uniform temperature case, as reported in figure 5. The increase of the deformation decreases the deviation, which should be expected since the effect of curvature becomes dominant over the effect of temperature non-uniformity when $\varepsilon$ increases.

Also the increase of the gas temperature has a similar effect (see figure 6), since in this case the relative nonuniformity of the temperature difference between drop and gas decreases, leaving again the curvature as the dominant parameter.

Finally the evaporation rates and the sensible heat rate, calculated integrating the species and heat fluxes over the drop surfaces, are reported in figure 7 as a function of the temperature non-uniformity for the case of $n$ dodecane. The temperature non-uniformity has a non neglectful effect on the evaporation rate, which mainly increases when the temperature becomes non-uniform, reaching relative variation of about $30 \%$ for the case $\Delta T=-40^{\circ} \mathrm{C}$, with a peculiar behaviour for small temperature non-uniformity, which may cause a small decrease of the evaporation rate (around $1 \%$ ) when $\Delta T \approx+10^{\circ} \mathrm{C}$. The heat rate instead increases for positive values of $\Delta T$ and decreases for negative values, but the maximum differences are lower than $8 \%$. 

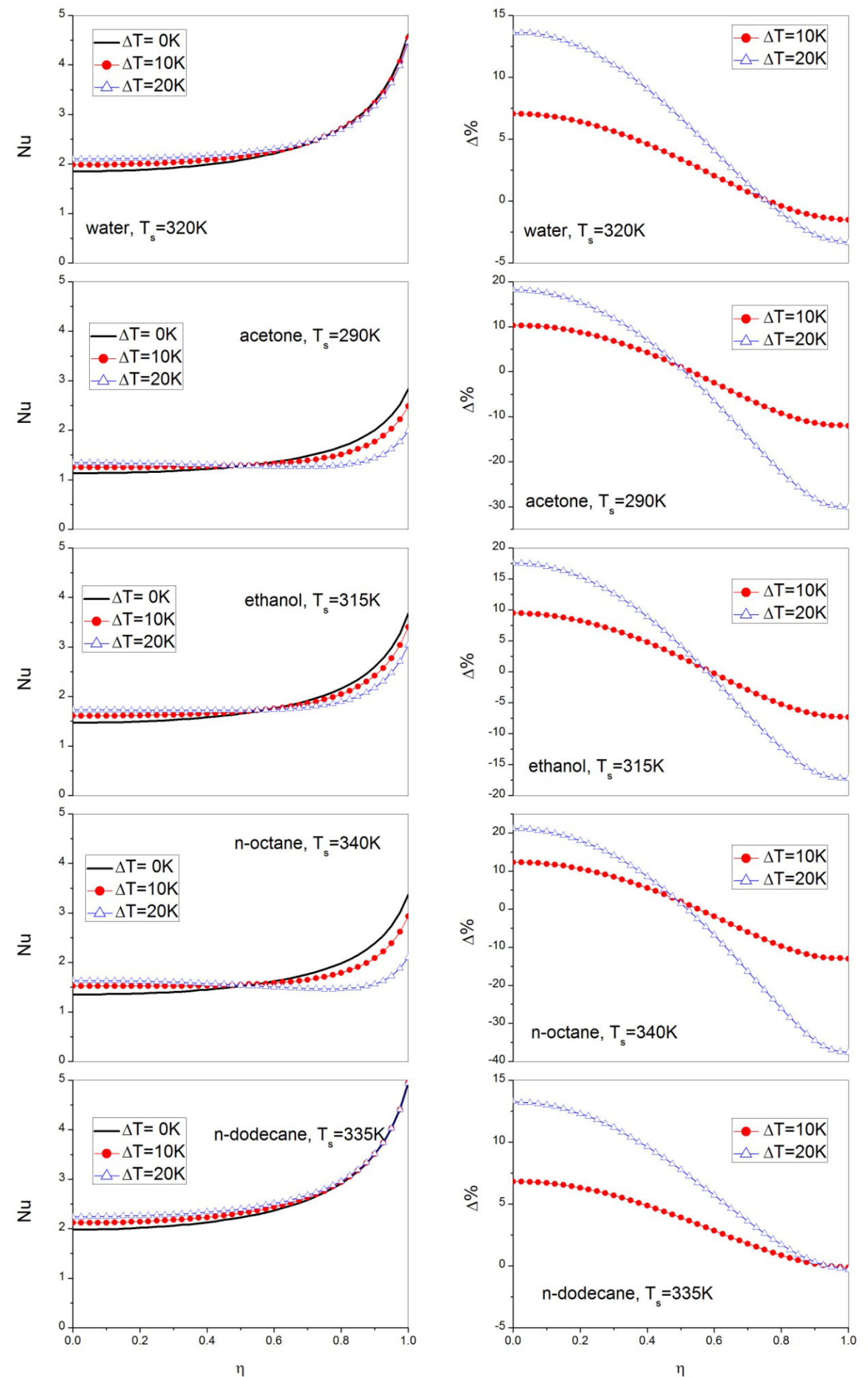

Figure 4. Local Nusselt number $(\mathrm{Nu})$ and percentage deviation $(\Delta \%)$ from the uniform surface temperature case for five species: water, acetone, ethanol, $\mathrm{n}$-octane, $\mathrm{n}$-dodecane, for different $\Delta T\left(\varepsilon=2.5, T_{\infty}=500 \mathrm{~K}, T_{s}=T_{\text {plateuu }}\right)$.

This work is licensed under a Creative Commons 4.0 International License (CC BY-NC-ND 4.0). 
(a)

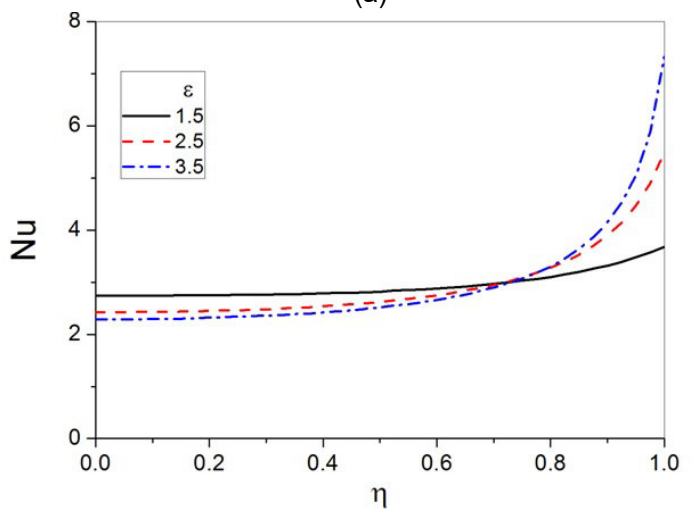

(b)

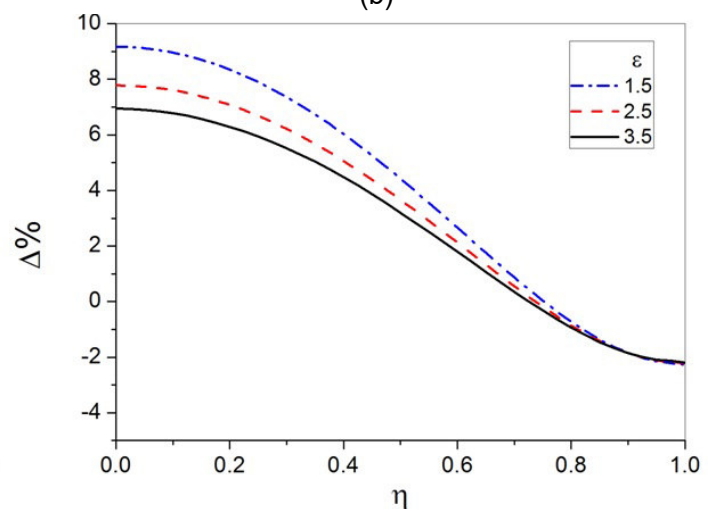

Figure 5. Local Nusselt number $(N u)$ and percentage deviation ( $\triangle \%)$ (figures (a) and (b) respectively) for different values of $\varepsilon(\mathrm{n}$ dodecane, $\left.T_{\infty}=700 \mathrm{~K}, T_{s}=380 \mathrm{~K}, \Delta T=20 \mathrm{~K}, P=10^{5} \mathrm{~Pa}\right)$.

(a)

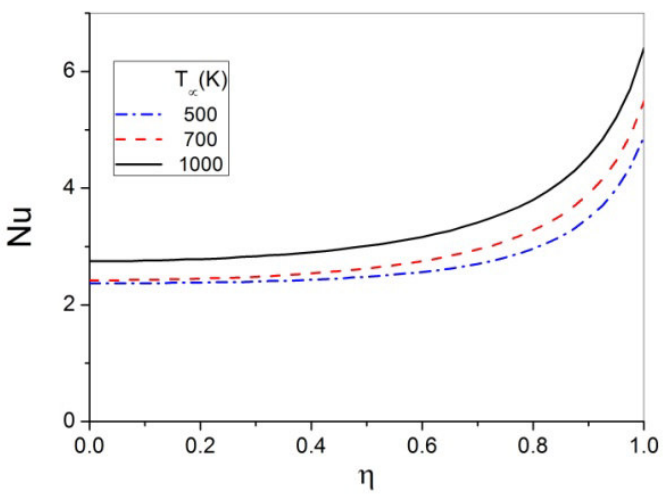

(b)

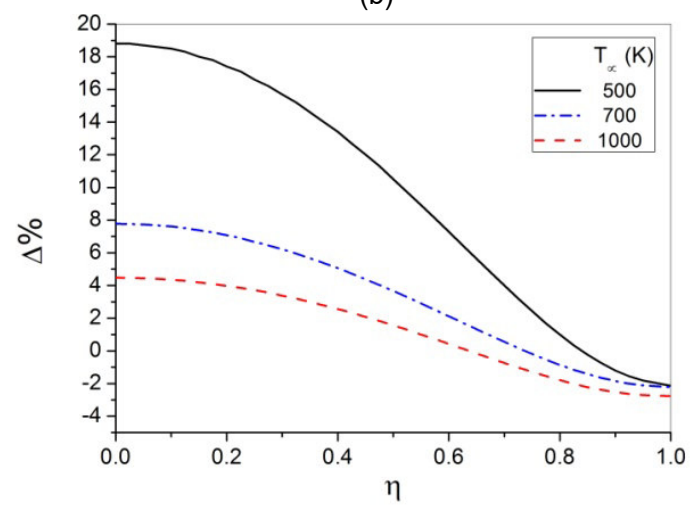

Figure 6. Local Nusselt number $(N u)$ and percentage deviation ( $\Delta \%$ ) (figures (a) and (b) respectively) for different $T_{\infty}(\mathrm{n}$ dodecane, $\left.\varepsilon=2.5, T_{s}=380 \mathrm{~K}, \Delta T=20 \mathrm{~K}, P=10^{5} \mathrm{~Pa}\right)$.

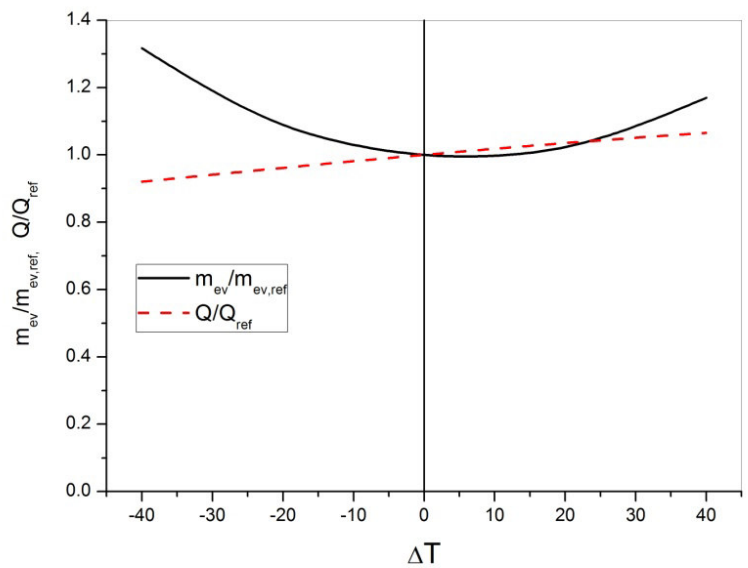

Figure 7. Evaporation rate and heat rate for a n-dodecane prolate drop $(\varepsilon=2.5)$ as a function of the temperature profile; the values are non-dimensionalised by the respective values for the uniform surface temperature case.

\section{Conclusions}

The effect of temperature non-uniformity on the heating and evaporation of spherical and spheroidal drops in a still gaseous environment are investigated analytically and numerically. The species conservation equations are analytically solved in proper coordinate systems (spherical or spheroidal) and the energy equation is numerically solved in the same coordinate system. 
A detailed analysis is reported for the case of a prolate evaporating drop, accounting for different species (water, acetone, n-octane, ethanol, n-dodecane), different gas temperature, drop deformation and temperature nonuniformity.

The effects on the local mass flux can become quite important, with local re-circulations when the temperature differences between the drop pole and equator become relative large $\left(20\right.$ to $\left.40^{\circ} \mathrm{C}\right)$.

The local Nusselt number is found to depend on local curvature and temperature, although for not too large values of the maximum surface temperature variation (less than $20^{\circ} \mathrm{C}$ ) the effect of curvature prevails and the analytical values obtained for the uniform temperature case are still usable with acceptable errors.

The local heat transfer coefficient is less affected by the non-uniformity of surface temperature when the deformation is large and when the gas temperature increases.

The total evaporation rate is affected by the temperature distribution and can increase up to $30 \%$ when drop nonuniformity is increased up to $40^{\circ} \mathrm{C}$, still maintaining the same average surface temperature. The heat rate is less affected showing deviation less than $8 \%$ for temperature difference between drop pole and equator as large as $40^{\circ} \mathrm{C}$.

\section{References}

[1] Mashayek, F., 2001, International Journal of Heat and Mass Transfer, 44(8), pp. 1517-1526.

[2] Haywood, R. J., Renksizbulut, M., and Raithby, G. D., 1994, International Journal of Heat and Mass Transfer, 37(9), pp. 1401-1409.

[3] Loth, E., 2008, International Journal of Multiphase Flow, 34(6), pp. 523-546.

[4] Hase, M., Weigand, B., 2004, Transient heat transfer of deforming droplets at high Reynolds numbers, International Journal of Numerical Methods for Heat and Fluid Flow, 14(1), pp. 85-97.

[5] Schlottke, J., Weigand, B., 2008, Direct numerical simulation of evaporating droplets, Journal of Computational Physics, 227, pp. 5215--5237.

[6] Mashayek, F., 2001, International Journal of Heat and Mass Transfer, 44, pp. 1527-1541.

[7] Tonini, S., Cossali, G.E., 2013, International Journal of Heat and Mass Transfer, 60, pp. 236-240.

[8] Tonini, S., Cossali, G.E., 2014, International Journal of Heat and Mass Transfer, 51, pp. 18-24.

[9] Tonini, S., Cossali, G.E., 2016, International Journal of Heat and Mass Transfer, 97, pp. 301-307.

[10] Abramzon, B., Sirignano, W.A., 1989, International Journal of Heat and Mass Transfer, 32 (9), pp. 16051618.

[11] Abramzon, B., Sazhin, S., 2006, Fuel, 85 (1), pp. 32-46.

[12] Sazhin, S., Krystadi, T., Abdelghaffar, W.A., Heikal, M.R., 2006, Fuel, 85 (12-13), pp. 1613-1630.

[13] Al Quebeissi, M., PhD Thesis, University of Brighton (2015).

[14] Zubkov, V.S., Cossali, G.E., Tonini, S., Rybdylova, O., Crua, C., Heikal, M., Sazhin, S.S., 2017, International Journal of Heat and Mass Transfer, 108, pp. 2181-2190.

[15] Yuen, M.C., Chen, L.W., 1976, Combustion Science and Technology, 14, pp. 147-154.

[16] Tonini, S., Cossali, G.E., 2014, International Journal of Thermal Sciences, 75, pp. 194-203.

\section{Nomenclature}

Roman symbols

a length scale in spheroidal coordinate [m]

$b_{n}, c_{n}, d_{n} \quad$ constants, eqs. (10) [-]

$c_{p, v} \quad$ vapour heat capacity $[\mathrm{J} / \mathrm{kgK}]$

$D_{10} \quad$ mass diffusivity $\left[\mathrm{m}^{2} / \mathrm{s}\right]$

$G \quad$ non-dimensional function [-]

$k \quad$ thermal conductivity [W/mK]

Le Lewis number [-]

$m_{e v} \quad$ evaporation rate $[\mathrm{kg} / \mathrm{s}]$

$M_{m} \quad$ molar mass $[\mathrm{kg} / \mathrm{kmol}]$

$n_{v} \quad$ mass flux $\left[\mathrm{kg} / \mathrm{sm}^{2}\right]$

Greek symbols

$\varepsilon \quad$ drop eccentricity parameter [-]

$\chi \quad$ mass fraction [-]

$\gamma, \eta, \zeta \quad$ spheroidal coordinate system [-]

Subscripts

$k, p \quad$ index [-]

ref reference case [-]
$\mathrm{Nu} \quad$ Nusselt number [-]

$P_{n} \quad$ Legendre function of first kind [-]

$P_{v, s a t} \quad$ saturation vapour pressure [Pa]

$P_{T} \quad$ total pressure [Pa]

$Q_{n} \quad$ Legendre function of second kind [-]

$Q \quad$ heat rate [W]

$R_{0} \quad$ drop radius [m]

$T$ temperature [K]

$U \quad$ Stefan velocity $[\mathrm{m} / \mathrm{s}]$

$x, y, z \quad$ coordinate system [-]

$\varphi \quad$ heat flux $\left[\mathrm{W} / \mathrm{m}^{2}\right]$

$\rho$ density $\left[\mathrm{m}^{3} / \mathrm{kg}\right]$

$v \quad$ vapour [-]

$\infty \quad$ at infinite [-] 


\title{
Numerical studies of turbulent particle-laden jets using spatial approach of one-dimensional turbulence
}

\author{
Marco Fistler*1, David Lignell ${ }^{2}$, Alan Kerstein ${ }^{3}$ and Michael Oevermann ${ }^{1}$ \\ ${ }^{1}$ Division of Combustion, Chalmers University of Technology, Sweden \\ ${ }^{2}$ Brigham Young University, Provo, UT 84602, USA \\ ${ }^{3}$ Consultant, 72 Lomitas Road, Danville, CA 94526, USA \\ ${ }^{*}$ Corresponding author: marco.fistler@chalmers.se
}

\begin{abstract}
To challenge one of the major problems for multiphase flow simulations, namely computational costs, a dimensionreduced model is used with the goal to predict these types of flow more efficiently. One-dimensional turbulence (ODT) is a stochastic model simulating turbulent flow evolution along a notional one-dimensional line of sight by applying instantaneous maps that represent the effect of individual turbulent eddies on property fields. As the particle volume fraction is in an intermediate range above $10^{-5}$ for dilute flows and under $10^{-2}$ for dense ones, turbulence modulation is important and can be sufficiently resolved with a two-way coupling approach, which means the particle phase influences the fluid phase and vice versa. For the coupling mechanism the ODT multiphase model is extended to consider momentum transfer and energy in the deterministic evolution and momentum transfer during the particle-eddy interaction. The changes of the streamwise velocity profiles caused by different solid particle loadings are compared with experimental data as a function of radial position. Additionally, streamwise developments of axial RMS and mean gas velocities along the centerline are evaluated as functions of axial position. To achieve comparable results, the spatial approach of ODT in cylindrical coordinates is used here. The investigated jet configuration features a nozzle diameter of $14.22 \mathrm{~cm}$ and a Reynolds number of 8400 , which leads to a centerline inlet velocity of $11.7 \mathrm{~m} / \mathrm{s}$. The particles used are glass beads with a density of $2500 \mathrm{~kg} / \mathrm{m}^{3}$. Two different particle diameters $(25$ and $70 \mu \mathrm{m})$ were tested for an evaluation of the models capability to capture the impact of a varying Stokes number and also two different particle solid loadings ( 0.5 and 1.0$)$ were evaluated. It is shown that the model is capable of capturing turbulence modulation of particles in a round jet.
\end{abstract}

\section{Keywords}

Jet, particle-laden flows, turbulence modulation, one-dimensional turbulence

\section{Introduction}

Turbulent particle-laden jets play a major role in a wide range of industrial applications and natural phenomena. Especially the effect of particles on the gas-phase, named turbulence modulation, is of particular interest. Several experimental studies, e.g. Schreck and Kleis [11], Geiss et al. [4] and Budilarto [1], have shown the large influence of high particle concentrations on the turbulence level. To investigate this type of flow experimentally and to understand the physical fundamentals is still very challenging, because only a few advanced techniques to obtain spatially resolved unsteady data exist today. CFD (computational fluid dynamics) proved to be a powerful tool to investigate particle-laden flows and to acquire detailed flow information. Many CFD approaches for these flow types have limited predictive capabilities or rely on many assumptions, which affects the accuracy and restrict that generality. Even with access to a high-performance computational infrastructure direct numerical simulation (DNS) studies for particle-laden flow are still limited to academic cases and low particle numbers. On the other hand, the accuracy of large eddy simulations (LES) seems to be very sensitive to its parameters, especially of its subgrid-model, which is required to achieve a good turbulence prediction.

In our previous study [3] we achieved promising results with a stochastic particle dispersion simulation in a turbulent jet with an alternative approach called one-dimensional turbulence (ODT). ODT is a stochastic approach with reduced dimension to resolve the full range of length and time scales as in DNS and introduced in [5] and extended in [6]. ODT has demonstrated to predict topologically simple flows such as boundary layers and jets with large property gradients in one direction very well compared to DNS studies and experimental results. While molecular phenomena like viscous dissipation evolve along that single spatial dimension, the turbulent advection is modeled through a stochastic remapping of the velocity profiles, called eddy events. This and the previous study are a continuation of the work of Schmidt et al. [9] and Sun et al. [12], who extended the ODT model to predict particle-carrier phase interaction excluding particle collision. These models has still issues to convert temporal ODT output data to spatial results, which can be evaluated with spatial experimental results. Therefore, the model is extended by using a spatial approach of ODT in cylindrical coordinates. Also, a new formulation of the particle-eddy interaction is introduced. The following study is based on the same test cases as in [12], which includes experimental results from [1]. 


\section{One-dimensional turbulence}

This section describes the concept of the ODT model, which is used in this study to simulate the carrier gas phase. ODT is a stochastic model to simulate turbulent flows on a one-dimensional domain, which is usually oriented in the direction of the largest expected velocity gradients. In case of turbulent dispersed jet configurations the domain is oriented in radial/lateral direction $r$. By the assumption that a jet flow is axisymmetric, one realization with the radial coordinate $r$ plus the evolving direction $z$ can interpreted as a representation of a 3D unsteady flow field. For a spatial evolution of the ODT line in the Lagrangian framework the governing equations are described by a mass flux and a momentum flux balance (illustrated in Fig. 1). In ODT these equations are only governing the steady solution of the flow and the unsteadiness due to turbulence is captured by a random sequence of eddy events. In fact the model conserves statistically the steady solution of flows but also represents the unsteadiness of turbulence.
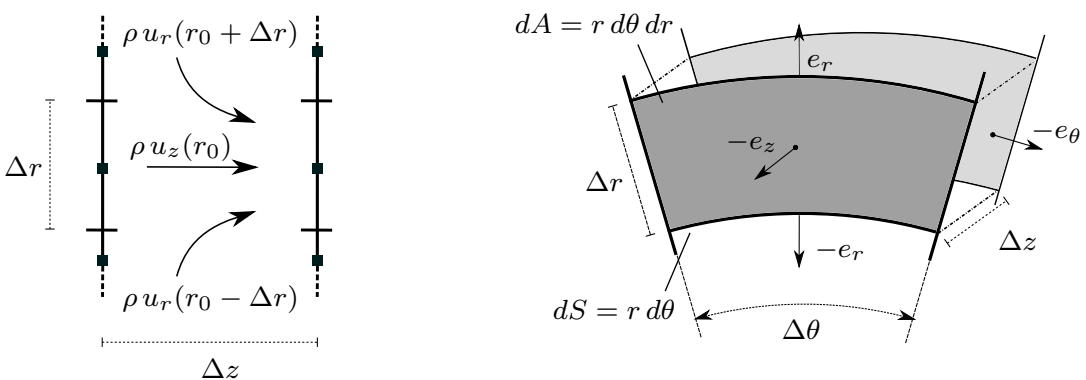

Figure 1. Sketch of mass flux balance and control volume.

\section{GOVERNING EQUATIONS}

Governing equations of spatial ODT evolution with a constant density over the domain are obtained by considering the balances of mass and momentum flux to a control volume (see Fig. 1). We consider an axisymmetric flow without swirl, i.e. $u_{\theta}=0$, and all quantities are independent of $\theta$. Therefore, the integration can be solved explicitly and the angle $\Delta \theta$ drops out. This shows that the mass flux balance is independent of $\theta$ and given as

$$
\int_{\Delta r} \frac{\partial\left(\rho u_{z}(r, z)\right)}{\partial z} r d r+\int_{\Delta z} \frac{\partial\left(\rho u_{r}(r, z)\right)}{\partial r} r d z=d_{\rho u_{z}}
$$

where $d_{\rho u_{z}}$ represents the sudden changes during eddy events in a spatial step $\Delta z$, which are discussed below in more detail. Outside the eddy region the term is zero. The same assumptions as above are applied to the momentum flux balance equation, which is given as

$$
\int_{\Delta r} \frac{\partial\left(\rho u_{z}(r, z) u_{i}(r, z)\right.}{\partial z} r d r+\int_{\Delta z} \frac{\partial\left(\rho u_{r}(r, z) u_{i}(r, z)\right.}{\partial r} r d z=\mu \int_{\Delta z} \frac{\partial\left(u_{i}(r, z)\right)}{\partial r} r d z+d_{\rho u_{z} u_{i}}+s_{p, i}
$$

where the right hand side summarizes all source and sink terms considered. The first represents viscous effects and the second, $d_{\rho u_{z} u_{i}}$, accounts for momentum transfer during eddy events in a spatial step $\Delta z . s_{p, i}$ describes the momentum transfer between dispersed and gas phase and is defined as

$$
s_{p, i}=\frac{1}{\int_{\Delta r} \rho u_{z} r d r} \sum_{j}^{n_{p}} m_{p, j}\left(u_{j, i}\left(r, z_{0}\right)-u_{j, i}\left(r, z_{0}+\Delta z\right)\right) .
$$

For this study the pressure term is omitted due to a zero pressure gradient in the flow configuration.

\section{EDDY EVENTS}

In ODT flow unsteadiness due to turbulence advection is modeled through eddy events, which results in remapping of the velocity profiles over a sampled eddy region $r_{0}<r<r_{0}+l$, characterized by a position $r_{0}$ and a length scale $l$. Both parameters are sampled randomly to mimic the occurrence of eddies in turbulent flow. The mapping method used in ODT is called triplet map [5]. In a planar reference frame it means the original profile is compressed by a factor of three over the eddy region and three copies are filled in. To ensure continuity of the profile the second copy in the middle is inverted. In planar ODT the cell sizes depend only on the length in ODT line direction. However, in a cylindrical framework it depends on the square of the length. As a result, in cylindrical coordinates the mapping process compresses the profiles with respect to the square of the length. The mapping function depending on the post-position for $r_{0} \geq 0$ is given as

$$
f(r)=r_{0}+ \begin{cases}\sqrt{3\left(r-r_{0}\right)^{2}} & \text { if } r_{0} \leq r \leq r_{0}+\sqrt{\frac{l^{2}}{3}} \\ \sqrt{2 l^{2}-3\left(r-r_{0}\right)^{2}} & \text { if } \sqrt{\frac{l^{2}}{3}} \leq r \leq \sqrt{\frac{2 l^{2}}{3}} \\ \sqrt{3\left(r-r_{0}\right)^{2}-2 l^{2}} & \text { if } \sqrt{\frac{2 l^{2}}{3}} \leq r \leq l \\ r-r_{0} & \text { otherwise. }\end{cases}
$$


For the case $r_{0}<0$ the algebraic signs have to be adjusted in consideration of the possibility that $r_{0}+l$ is greater or smaller zero. Examples are illustrated in Fig. 2.

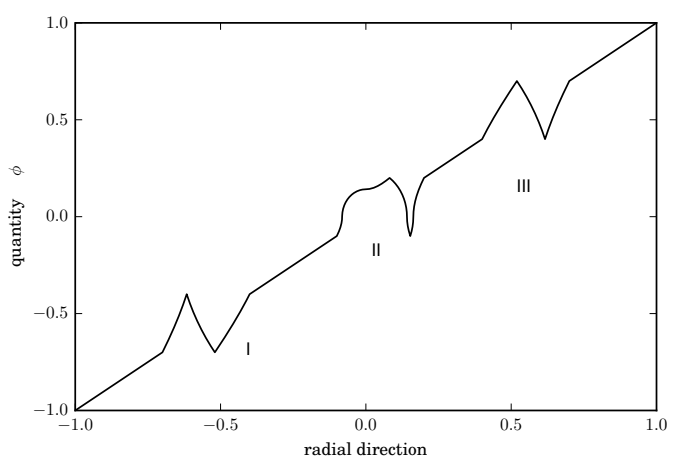

Figure 2. Examples for applying a cylindrical triplet map to a linear profile of quantity $\phi$. I: $r_{0}, r_{0}+l<0, \quad$ II: $r_{0}<0, r_{0}+l>0$, III: $r_{0}, r_{0}+l>0$, where $r_{0}$ and $l$ are eddy position and length, respectively.

An essential part of turbulence is the phenomena of return-to-isotropy, which requires on the ODT modeling side a re-distribution of turbulent kinetic energy among the velocity components. This is achieved by introducing kernel transformations to the mapping function, which gives a profile transformation

$$
u_{i}(y) \rightarrow u_{i}^{\mathrm{TM}}(y)+c_{i} K(y)+b_{i} J(y),
$$

where $u_{i}$ is the velocity in $i$-th direction before and $u_{i}^{\mathrm{TM}}$ after the mapping process. The Kernel $K(y)$ is defined as the fluid displacement profile under the triplet map and integrates to zero over the eddy region. $J(y)$ is the absolute of $K(y)$ and so it does not integrate to zero over region. Thus, it forces momentum change of the profiles if its kernel coefficient $b_{i}$ is non-zero. $c_{i}$ defines the kernel amplitude of $K(y)$. Thus, both kernels are important in the case of particle-gas phase coupling. Due to momentum and energy flux conservation over the sampled eddy region it is required to meet the following equations:

$$
\begin{aligned}
& \int \dot{\rho} u_{i} r d r=\int \dot{\rho}\left(u_{i}^{\mathrm{TM}}+c_{i} K+b_{i} J\right) r d r+S_{\mathrm{p}, i}, \\
& \frac{1}{2} \int \dot{\rho} u_{i}^{2} r d r=\frac{1}{2} \int \dot{\rho}\left(u_{i}^{\mathrm{TM}}+c_{i} K+b_{i} J\right)^{2} r d r-\Delta E_{i}+S_{\mathrm{pE}, i} .
\end{aligned}
$$

$S_{\mathrm{p}, i}$ and $S_{\mathrm{pE}, i}$ represent the sum of momentum and energy flux penalties caused by particles, respectively. $\triangle E_{i}$ stands for the above mentioned re-distribution of energy between velocity components. Under consideration of the measure preserving character of the mapping process itself, which means $\int \rho u_{i} r d r=\int \rho^{\prime} u_{i}^{\mathrm{TM}} r d r$ and $\int \rho u_{i}^{2} r d r=\int \rho^{\prime}\left(u_{i}^{\mathrm{TM}}\right)^{2} r d r, b_{i}$ and $c_{i}$ can be determined and define the new profile. Subsequently, the resultant kinetic energy of the sampled region is used to determine the eddy timescale $t_{\mathrm{e}}\left(l, r_{0}\right)$. Based on the scaling assumption for the kinetic energy $E \sim \rho l^{3} / 2 t_{\mathrm{e}}^{2}$, the eddy time scale is modeled as

$$
\frac{1}{t_{\mathrm{e}}}=C \sqrt{\frac{2}{\rho l^{3}}\left(E_{\mathrm{kin}}-Z E_{\mathrm{vp}}\right)} .
$$

The viscous energy penalty is given as $E_{\mathrm{vp}}=\mu^{2} / 2 \rho l$ and $C$ is the adjustable eddy rate parameter, which scales the overall eddy event frequency. $Z$ is the viscous penalty parameter, which suppresses unphysically small eddies. An equivalent procedure for large eddies exists as well. In this study the elapsed time method is used in which the eddy time can be compared with elapsed time $t$ of the simulation. Eddy events are only allowed if $t \geq \beta t_{\mathrm{e}}$, where $\beta$ is a model parameter.

We assume that the occurrence of eddies of a certain size follows a Poisson process in time with a rate determined by the eddy timescale provided in (8). Technically this is solved by oversampling, i.e. generation of candidate eddies at a much higher rate than requested, and thinning of the Poisson process with an acceptance-rejection method. For details we refer to [7]. 


\section{Lagrangian Particle Model}

The particulate phase is modelled in a Lagrangian way where individual particles are tracked following Newton's second law of motion. Here we consider only drag forces and gravity acting on the particles leading to the following set of equations:

$$
\begin{aligned}
& \frac{d u_{p, i}}{d t}=-\frac{u_{p, i}-u_{g, i}}{\tau_{p}} f+g_{i}, \\
& \frac{d r_{p}}{d t}=u_{p, r} .
\end{aligned}
$$

Here, the subscript $p$ and $g$ represent the particle and gas phase, respectively, and $g_{i}$ is the $i$-th component of the gravity acceleration vector. The particle response time, $\tau_{p}=\rho_{p} d_{p}^{2} / 18 \mu$ based on Stokes flow, is given with consideration of mass $m_{p}$ and density $\rho_{p}$ of the particle and the fluid viscosity $\mu$. Clift et al.[2] suggested a nonlinear correction factor $f$ for a particle slip-velocity Reynolds number $R e=\frac{\rho_{g}\left|\vec{u}_{p}-\vec{u}_{g}\right| d_{p}}{\mu}$ smaller than 200, which is for many practical dilute flow systems the case. The factor $f$ is defined as

$$
f=1+0.15 R e_{p}^{0.687} \text {. }
$$

The drag law (9) is solved by a first-order Euler method. As the ODT line evolves in spatial dimension $(\Delta z)$ the temporal step $\Delta t$ should be transformed. Therefore, a constant particle velocity over $\Delta z$ is assumed, which yields to

$$
\Delta t=\frac{\Delta z}{u_{p, z}} .
$$

In the spatial advancement of the steady-state solution of the underlying flow each particle always has a local gas velocity, which it interacts with. However, an eddy event appears instantaneous in the ODT simulation and so a model is required to capture the displacement in radial direction during a particle-eddy interaction.

\section{PARTICLE-EDDY INTERACTION MODEL}

The particle-eddy interaction (PEI) model is defined as the only effect of particle phase motion in ODT line-direction and so the gas velocity in this direction for the drag law (9) is zero. The PEI model in this study was developed by Schmidt et al. $[9,10]$ as a so-called instantaneous PEI model (noted as type-l) and governs the radial displacement due to an eddy event. Each particle obeys the model if they are located in the sampled eddy region. The main model assumption is that the eddy time scale $t_{\mathrm{e}}$ calculated in (8) defines the time an eddy needs to create the remapped profile. That means before an eddy event the particle motion in (9) is integrated over the eddy time with a radial velocity, which has to be corrected to account for the finite time of an eddy event. Therefore, it is required to define an eddy gas phase velocity in radial direction and an interaction time $\tau_{\mathrm{PEI}}$, which determines the time interval in (9), to correct the integration over the time interval $\tau_{\mathrm{PEI}}$.

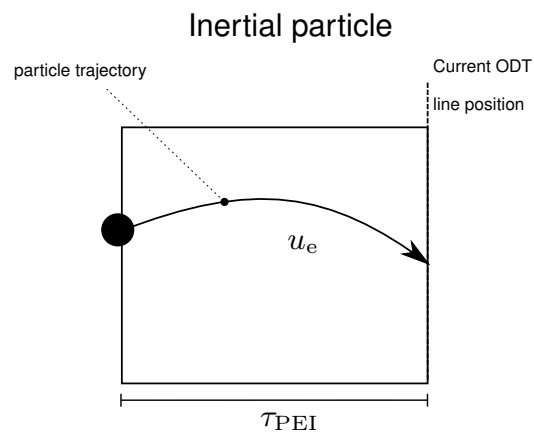

Tracer particle

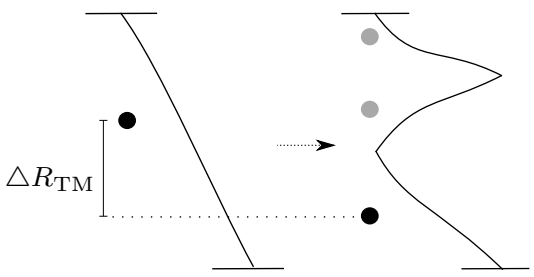

Figure 3. Example of re-integration of drag law (Eq. 9) over particle-eddy interaction time $\tau_{\text {PEI }}$. Eddy velocity $u_{\mathrm{e}}$ is defined as the tracer particle displacement $\triangle R_{\mathrm{TM}}$ by the triplet map divided by the eddy time scale $t_{e}$. For the displacement one (black circle) of three possible positions (grey circles) is chosen randomly.

Determining the radial gas phase velocity $u_{g, r}$ during the eddy time, the concept of the displacement of a mass-less tracer particle governed by the mapping method (4) is used. This method provides three possible tracer particle positions and a unique position is sampled randomly with a uniform distribution from those three possible ones. The final displacement $\triangle R_{T M}$, see Fig. 3, divided by the eddy time scale $t_{\mathrm{e}}$ defines the gas velocity during the PEI. As a next step the integral time scale has to be determine and therefore a so-called eddy box $\left[l \times l \times \beta_{p} t_{\mathrm{e}}\right]$, where $\beta_{p}$ is the model parameter for the PEI, is introduced. The PEI integration time $\tau_{\mathrm{PEI}}$ is given as the time the particle needs to exit the box. Therefore, the Stokes law is modified and analytically solved to find the earliest time when the particle leaves the space-time eddy box. 


\section{Test case}

\section{EXPERIMENTS}

For validation purposes the model is compared with experiments of Budilarto [1], which provide data for a constant air jet with a Reynolds number of 8400 and solid loadings of 0.5 and 1 . Also, different Stokes numbers are investigated by varying the single particle diameter. The jet exit has a diameter $D$ of $0.014224 \mathrm{~m}$. According to the Reynolds number, the jet exit velocity $u_{0}$ is $11.7 \mathrm{~m} / \mathrm{s}$. The particles used are glass beads with a density $\rho_{p}=2500$ $\mathrm{kg} / \mathrm{m}^{3}$ and with a number averaged diameter of $25 \mu \mathrm{m}$ and $75 \mu \mathrm{m}$. Their initial velocity depends on the coordinate framework of the simulation and is discussed in the following.

\section{SIMULATION SETUP}

As the initial jet profile in the ODT domain the following function is used

$$
u_{g, z}(r)=\frac{u_{g, z 0}}{2}\left[\left(1+\tanh \left(\frac{r-L_{1}}{\omega_{l}}\right)\right) \cdot\left(1-\frac{1}{2}\left(1+\tanh \left(\frac{r-L_{2}}{\omega_{l}}\right)\right)\right)\right] .
$$

$L_{1}$ and $L_{2}$ are the middle positions of the transitions and $\omega_{l}$ is the transition boundary layer width. Due to the fact that the axisymmetrical approach used to represent the jet flow configuraton in ODT is independent of the angular direction, the ODT line volume is determined by the square of the domain length. Thus, the solid loading, which is the ratio between particle-phase and gas-phase mass, is computed for the square of the jet diameter $D$. The initial particle positions are exponentially distributed over the nozzle exit. The initial velocities are based on the experimental data in [1]. The initial properties for the particle-phase in the ODT simulation are summarized in Tab. 1. This study uses 512 ODT realizations, which Sun et al. [12] reported as sufficient to capture stationary statistics.

Table 1. Initial particle-phase properties of particle diameter $d_{p}$, solid loading $s l$, axial velocity $u_{p, 0}$ number of parcels (particle clouds) $n_{p}$ and representing particles per parcel $N$, for a particle density of $2500 \mathrm{~kg} / \mathrm{m}^{3}$.

\begin{tabular}{c|cccc}
\hline$d_{p}(\mu \mathrm{m})$ & $s l$ & $u_{p, 0}(\mathrm{~m} / \mathrm{s})$ & $n_{p}$ & $N$ \\
\hline 25 & 0.5 & 11.324 & 310 & 9500 \\
& 1 & 11.205 & 310 & 19000 \\
70 & 0.5 & 9.664 & 300 & 450 \\
& 1 & 9.474 & 300 & 900
\end{tabular}

\section{Results}

SINGLE PHASE FLOW

Considering the assessment of gas-particle interaction, the first important step is to achieve an agreement between ODT simulations and experimental data for the single-phase case without particles. The ODT parameters are equal to the ones reported in [3] due to the same flow configuration, i.e. for the viscous penalty parameter we have $Z=400$ and for the eddy frequency parameter $C=12$. These parameters result in an overall agreement between ODT results and experimental data for the mean axial velocity and the velocity fluctuation along the centerline and for the mean axial velocity in radial direction for $x / D=5,10$ and 15 as shown in Fig. 4a and Fig. 4b, respectively. Axial and radial positions are both normalized by the jet diameter $D$ and axial velocities and fluctuations by the

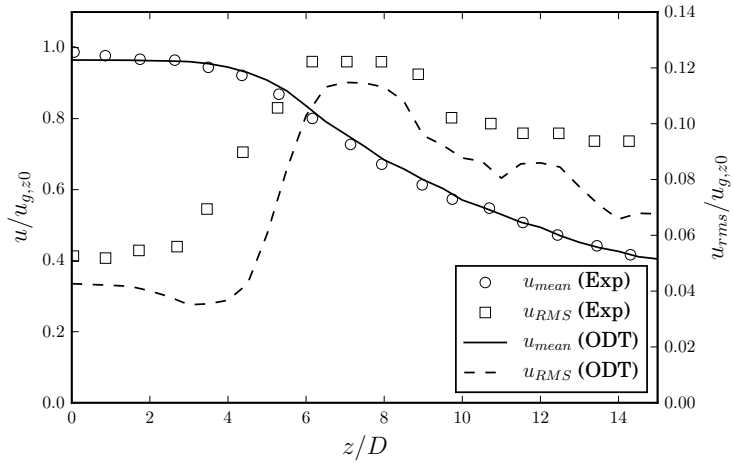

(a) Mean axial velocity along centerline

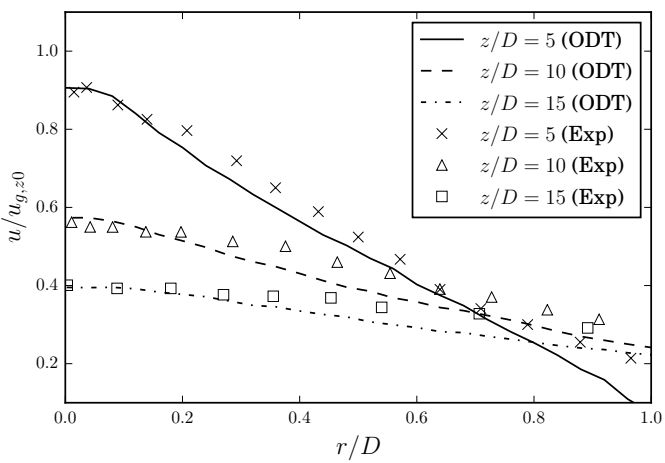

(b) Mean axial velocity profiles in radial direction

Figure 4. Axial velocity profiles of gas phase.

nozzle exit velocity $u_{g, z 0}$. Fig. 4a shows the same axial velocity decay along the centerline as the experimental data. The characteristics of the velocity fluctuations is also well captured. Additionally, the axial velocities in radial direction show good agreement with the experimental data, as shown in Fig. 4b. 
TWO-PHASE FLOW

For the two-phase flow simulations the particle-eddy interaction parameter $\beta_{p}$ is set to 0.008 based on previous parameter studies which are not discussed here. In the following the development of the mean axial centerline velocity and of the corresponding velocity fluctuation along the centerline is presented for different solid loadings in Fig. 5 and Fig. 6, respectively. Axial positions are normalized by the jet diameter $D$ and axial velocities and fluctuations by the nozzle exit velocity $u_{g, z 0}$. Fig. 5 shows that ODT is capturing the trend of the experimental data

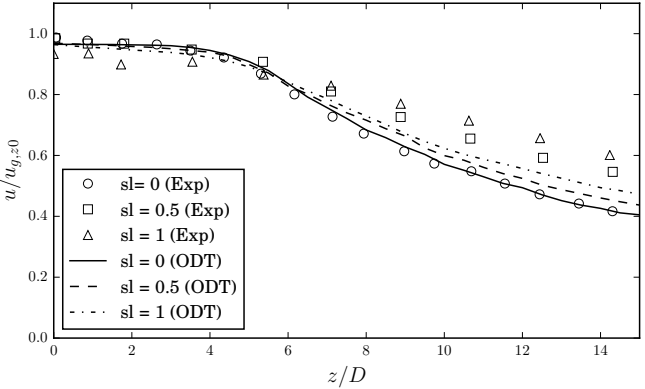

(a) $d_{p}=70 \mu \mathrm{m}$

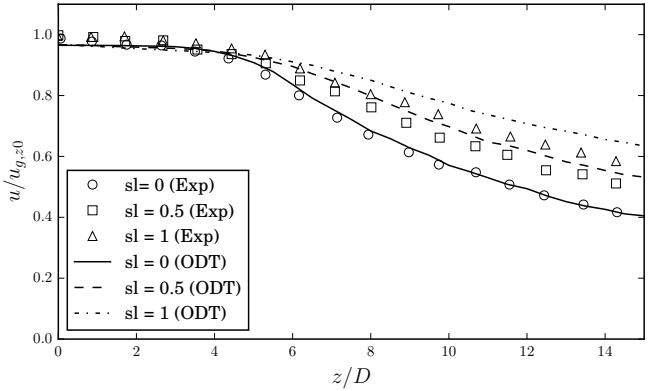

(b) $d_{p}=25 \mu \mathrm{m}$

Figure 5. Mean axial velocity along centerline with different solid loadings.

reasonably well. For a diameter of $70 \mu \mathrm{m}$ the differences in the ODT results for different solid loadings are smaller than observed in the experiments, but ODT is capturing the trend of increasing gas phase velocities/momentum for $z / D>5 D$ with increasing solid loading. Also, the velocity decrease till $z / D \sim 5 D$ caused by the higher momentum penalty for $s l=1$ can be observed. In Fig. $5 \mathrm{~b}$ the deviations between ODT and experiments for the smaller particles with $d_{p}=25 \mu \mathrm{m}$ are smaller compared to the particles with $d_{p}=70 \mu \mathrm{m}$ in Fig. $5 \mathrm{a}$ and a slight over-prediction can be seen.

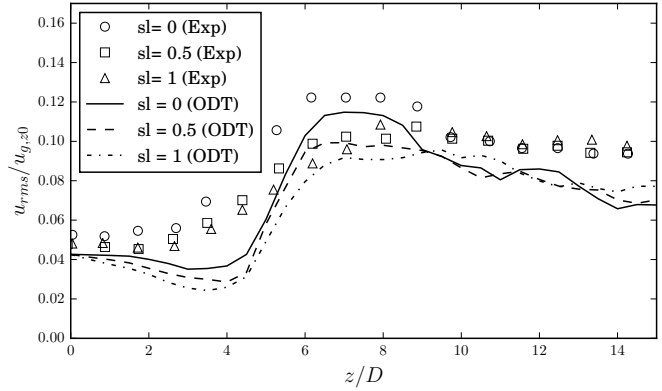

(a) $d_{p}=70 \mu \mathrm{m}$

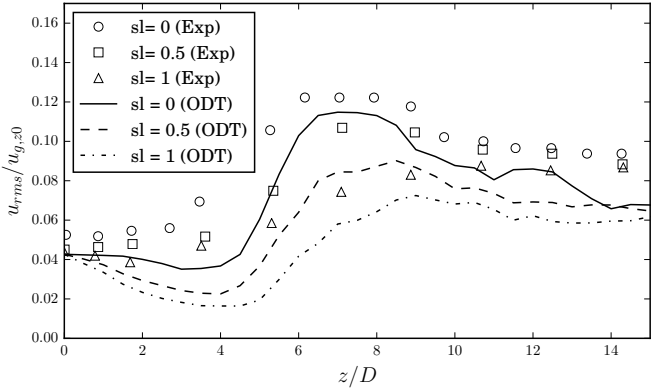

(b) $d_{p}=25 \mu \mathrm{m}$

Figure 6. Mean axial velocity fluctuation along centerline with different solid loadings

Another important indicator for turbulence modulation is the behavior of the turbulent velocity fluctuations for different solid loadings. The effect of turbulence suppression caused by solid particles is well shown in Fig. 6a and Fig. $6 \mathrm{~b}$. Both figures show a decrease of the velocity fluctuations with increasing solid loading and an enhanced damping of turbulence for the smaller particles.

\section{Conclusions}

The objective of this study was to extend the existing ODT model described in [12] by using a spatial approach in cylindrical coordinates. For this purpose the momentum and energy exchange between the particle and gas phase during the deterministic advancement and during the particle-eddy interaction were modified. Also, a new formulation to compute the kernel coefficients were introduced. The presented results show that ODT is capable of capturing the characteristics of a particle-laden jet with respect to variations in the solid loading. Furthermore, the results of Sun [12] could be reproduced without the need to transform the temporal coordinate. Additional extensions, e.g. considering droplet collision and coalescence, are planned for the near future. These model extensions are the base for the future goal to get a better understanding of the underlying physics of particle/dropletturbulence interaction. The scope for ODT will be fundamental investigations in parameter ranges which are not accessible using DNS or high resolution LES. 


\section{Nomenclature}

$d_{p} \quad$ particle diameter [m]

$D \quad$ jet diameter [m]

$m \quad$ mass $[\mathrm{kg}]$

$r \quad$ radial coordinate [m]

$u_{i} \quad$ i-th velocity component [m/s]

$z \quad$ axial coordinate $[\mathrm{m}]$

$\rho \quad$ density $\left[\mathrm{kg} / \mathrm{m}^{3}\right]$

Subscripts

$0 \quad$ initial

e eddy

$g \quad$ gas phase

$p \quad$ particle phase

PEI particle-eddy interaction

TM triplet map

\section{References}

[1] Budilarto, S. G., 2003, PhD thesis, Purdue Univeristy, USA.

[2] Clift, R., Grace, J. R. and Weber, M. E., 1978, Bubbles, drops and paticle, Academic Press, New York.

[3] Fistler, M., Lignell, D. O., Kerstein, A., Oevermann, M., Mai 15.-18. 2017, 29th ILASS-Americas.

[4] Geiss, S., Dreizler, A., Stojanovic, Z., Chrigui, M., Sadiki, A. and Janicka, J., 2004, Experiments in Fluids, 36 (2), pp. 344-354.

[5] Kerstein, A. R., 1999, Journal of Fluid Mechanics, 392, pp.277-334.

[6] Kerstein, A. R. and Ashurst, W. T., 2005, Physics of Fluid, 17-025107, pp.1-26.

[7] Lignell, D. O., Kerstein, A. R., Sun G. and Monson, E. I., 2013, Theoretical and Computational Fluid Dynamics, 27(3-4), pp. 273-295.

[8] Poelma, C., 2004, PhD thesis, TU Delft, Netherlands.

[9] Schmidt, J. R., Wendt, J. O. L., Kerstein, A. R.,2004, IUTAM Symposium on Computational Approaches to Disperse Multiphase Flow.

[10] Schmidt, J. R., Wendt, J. O. L., Kerstein, A. R.,2009, Journal of Statistical Physics, 37, pp. 233-257.

[11] Schreck, S. and Kleis, S., 1993, Journal of Fluid Mechanics, 249, pp. 665-688.

[12] Sun, G., Hewson, J. C., Lignell, D. O., 2017, Submitted to International Journal of Multiphase flow. 


\title{
Energy Aspects in Spray Formation by Homogenous Flash Boiling Process
}

\author{
Yahav Moshkovich*1, Yeshayahou Levy ${ }^{1}$, Ilai Sher ${ }^{2}$, Eran Sher ${ }^{1}$ \\ ${ }^{1}$ Faculty of Aerospace Engineering, Technion - Israel Institute of Technology, Haifa, Israel \\ ${ }^{2}$ Cranfield University, Cranfield, Bedfordshire MK43 0AL, United Kingdom \\ ${ }^{*}$ Corresponding author: yahavmoshko@gmail.com
}

\begin{abstract}
When a pressurized bubbly mixture is driven out through an orifice, the mixture pressure abruptly drops and the bubbles undergo a rapid expansion process, which under some circumstances results in a rapid disintegration of the liquid bulk into small droplets (atomization). Depending on the initial conditions, heterogeneous or homogeneous nucleation of vapor bubbles may occur. For homogeneous nucleation, the vapor bubbles grow rapidly one towards the other, and when they touch each other the bubbles "explode". In this stage, the liquid around the bubbles is teared, and a spray with small and uniform droplets is formed. In the literature, it seems that the efficiency of the homogenous flash boiling process is very low. In this work, we analyse this process and analyse it for possible energy losses.
\end{abstract}

\section{Keywords}

Flash boiling atomization, Homogeneous nucleation and Spray Formation.

\section{Introduction}

Over the years, different methods have been developed in order to obtain suitable sprays for different applications. The more important characteristics of a spray include the drops diameter, droplet size distribution, spray shape, flow velocity and mass flux. Former studies [1]-[7] show that the flash boiling method is one of the most efficient methods to obtain a spray with very small drops and with a uniform distribution. These are very relevant for many applications such as combustion systems, for which higher combustion efficiency and low pollution are important. Today, flash boiling sprays are widely used to generate fine sprays in air refreshers, insect fighting, painting and some pharmaceutical applications. The flash boiling obtained by pressure reduction of compressed liquid bellow the saturation pressure.

The flash boiling spray is generated under well determined specific thermodynamic conditions. Based on the Levy et al. [8] model, the process is divided into three areas. When a liquid having a high vapor pressure, in the container (Fig. 1 area a), is discharged to a low pressure ambient through a orifice, ( $i$-e area). Under these conditions the rapid depressurization, results in a high bubbles nucleation, (point $n$ ). Vapor bubbles with radius $R_{c r}$ are created, and grow one towards another up to the point in which they touch each other (point $t$ ) and tear the liquid around them into small and relatively uniform droplets (area $d$ ).

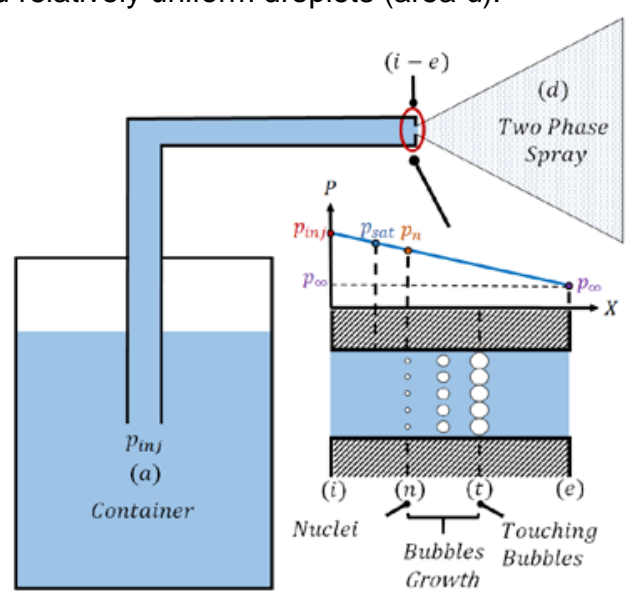

Figure 1. Homogenous flash boiling process sketch. 


\section{Bubbles nucleation}

Bubbles nucleation is one of the most important mechanisms in generation of the flash boiling spray. Depending on the thermodynamic conditions, two types of nucleation may occur. First, the bubbles generated on the orifice wall defined as a heterogeneous nucleation. Second, the bubbles nucleation occurs in the fluid bulk as known as homogeneous nucleation. Under homogeneous regime the bubbles nucleation rate is greater than the heterogeneous rate, and thus, the spray is finer [5], [7]. In addition, under this regime a simple orifice (Fig. 1) is required, therefore it may be applicable for fuel injection systems in engines and combustors.

The desirable homogenous nucleation occurrence depends on two criteria based on extensive experimental work. The Avedisian [9] criterion is on the initial temperature, i.e. the initial temperature is higher than $90 \%$ of the critical temperature, $T_{i n j}>0.9 T_{c r}$. In addition, Hutcherson et al. [10], [11] determined that the depressurization rate limit is $400 \mathrm{MPa} / \mathrm{s}$ for homogenous nucleation.

During the nucleation bubbles are generating in a various sizes. On each bubble two forces are acting. One, causing the bubble to increase, is the pressure difference across the bubble surface. Meaning, between inside bubble pressure, $p_{v}$, and liquid surrounding pressure, $p_{l}$. On the other hand, the bubble surface tension, $\sigma$, is acting to shrink it, when the surface tension is calculated by Sher et al. [12] method. The critical radius is defined by Young-Laplace equation, Eq. 1, and determines the collapsing or bubble spontaneously growing.

$$
R_{c r}=\frac{2 \sigma}{p_{v}-p_{l}}
$$

The nucleation flux density is very important quantity in flash boiling sprays. The homogeneous nucleation flux density suggestion, in seedy state system, depends on the critical size bubbles generation quantity and the bubble grows rate from the critical size. Thus, the expression for number of bubbles formed during a time unit per unit volume is [13].

$$
J=N_{A}^{\frac{3}{2}}\left(\frac{3 \rho_{l}^{2} \sigma}{\pi \bar{M}^{3}}\right)^{1 / 2} \exp \left[-\frac{16 \pi \sigma^{3}}{3 k_{B} T_{l}\left[\gamma p_{s a t}\left(T_{l}\right)-p_{l}\right]^{2}}\right]
$$

where $T_{l}$ is the liquid initial temperature, $p_{s a t}\left(T_{l}\right)$ is the saturation pressure at initial temperature, $\gamma=\exp \left\{\left[p_{l}-p_{\text {sat }}\left(T_{l}\right)\right] / \rho_{l} \bar{R} T_{l}\right\}$ and the pressure difference is obtained by Redlich - Kwong equation of state (EOS).

\section{Bubbles growth}

When stable vapor bubble is formed, it spontaneously grow. The control growth type and the bubbles growth rate influence the spray droplets characteristics. This bubble growth process is very complicated. The momentum and mass conservation are coupled and non-linear. Furthermore, at the bubble wall, between the vapor and the liquid, there is hydrodynamic and thermal interaction. When, the Generalized Rayleigh-Plesset equation of motion describe a spherical vapor bubble growth in spherical coordinates, in infinite liquid pool.

$$
R \frac{d^{2} R}{d t^{2}}+\frac{3}{2}\left(\frac{d R}{d t}\right)^{2}=\frac{1}{\rho_{l}}\left(p_{v}-p_{\infty}-\frac{2 \sigma}{R}-\frac{4 \mu}{R} \frac{d R}{d t}\right)
$$

Where $R, \dot{R}$ and $\ddot{R}$ are radius, velocity and acceleration of the bubble radius growth respectively, and $p_{\infty}$ is the liquid pressure outside the boundary layer. Because it is not possible to solve this equation analytically without assumptions, the process of their growth can be divided into three stages:

1. Inertia control growth.

2. Coupled inertia and thermal-diffusion control growth.

3. Thermal-diffusion control growth.

When a bubble is formed, the temperature inside the bubble is assumed to be equal to the surrounding liquid temperature $\left(T_{v}=T_{\infty}\right)$, and the pressure inside the bubble is the saturation pressure at this temperature $\left(p_{v}=\right.$ $p_{\text {sat }}\left(T_{\infty}\right)$ ). During the first stage of the bubble growth, the limiting factor of the bubbles growth rate is the outward acceleration of the liquid around it. The growth rate of the bubble has been shown by Rayleigh [14] to be at the form of:

$$
R=A t
$$

In the final stage, the bubble becomes bigger, therefore, a larger amount of vapor is needed in order to increase its size. Furthermore, the pressure of the bubble decreases until it is equal to the pressure of the liquid surrounding $\left(p_{v}=p_{\infty}\right)$, and the bubble temperature is according to the saturation temperature at this pressure $\left(T_{v}=T_{\text {sat }}\left(p_{\infty}\right)\right)$. As a result of the bubble growth, this layer of the surrounding liquid cools down and create a temperature difference. This temperature difference drives a thermal diffusion from the liquid into the bubble. Thus, at the third stage, the heat that can be supplied to the bubble walls is the limiting factor of the growth rate of the bubble. This stage of the bubble growth is shown by Plesset and Zwick [15] to be:

$$
R=B \sqrt{t}
$$


In the intermediate stage, the bubble grows and both the temperature and the pressure decreases. The limiting factor of the bubbles growth is the combination between the growth rate by inertia and the thermal diffusion. Mikic et al. [16] showed that the combined effects of the inertia and thermal diffusion determine the growth rate of the bubble:

$$
\begin{aligned}
& R^{*}=\frac{2}{3}\left[\left(t^{*}+1\right)^{\frac{3}{2}}-\left(t^{*}\right)^{\frac{3}{2}}-1\right] \\
& R^{*}=\frac{A}{B^{2}} R, t^{*}=\left(\frac{A}{B}\right)^{2} t \\
& A=\left[\frac{2}{3} \frac{\left(T_{\infty}-T_{\text {sat }}\right) h_{f g} \rho_{v}}{T_{\text {sat }} \rho_{l}}\right]^{\frac{1}{2}}, \quad B=\left[\frac{12}{\pi} J a^{2} \alpha\right]^{\frac{1}{2}} \\
& J a=\frac{\rho_{l_{\text {inj }}} c_{p_{\text {inj }}}\left[T_{\text {inj }}-T_{\text {sat }}\left(p_{\infty}\right)\right]}{\rho_{v_{n}} h_{f g}}
\end{aligned}
$$

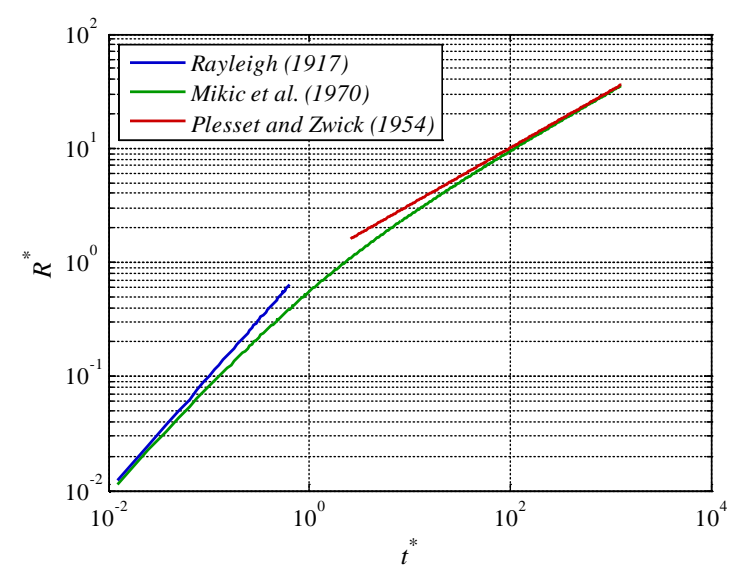

Figure 2. Mikic et al. [16], Plesset \& Zwick [15] and Rayleigh [14] models for predicting bubble growth in superheated R-22.

A better agreement to experiments of the bubble growth rate can be achieved by using numerical models. Robinson and Judd [17] offered a numerical model that can solve the coupling between the three following equations:

1. Generalized Rayleigh-Plesset equation, (Eq. 3).

2. Energy conservation equation of the bubble walls:

$$
h_{f g} \frac{\partial}{\partial t}\left(\frac{4}{3} \pi R^{3} \rho_{v}\right)=\frac{1}{4 \pi R^{2}} \int_{A_{s}} k_{l}\left(\frac{\partial T}{\partial r}\right)_{r=R} d A
$$

3. Energy equation outside the bubble:

$$
\frac{\partial T}{\partial t}+\frac{R^{2}}{r^{2}} \frac{d R}{d t} \frac{\partial T}{\partial r}=\alpha_{l}\left(\frac{\partial^{2} T}{\partial^{2} r}+\frac{2}{r} \frac{\partial T}{\partial r}\right)
$$

Eqs. (3), (7) and (8) are solved using the forth order Runga-Kutta numerical method.
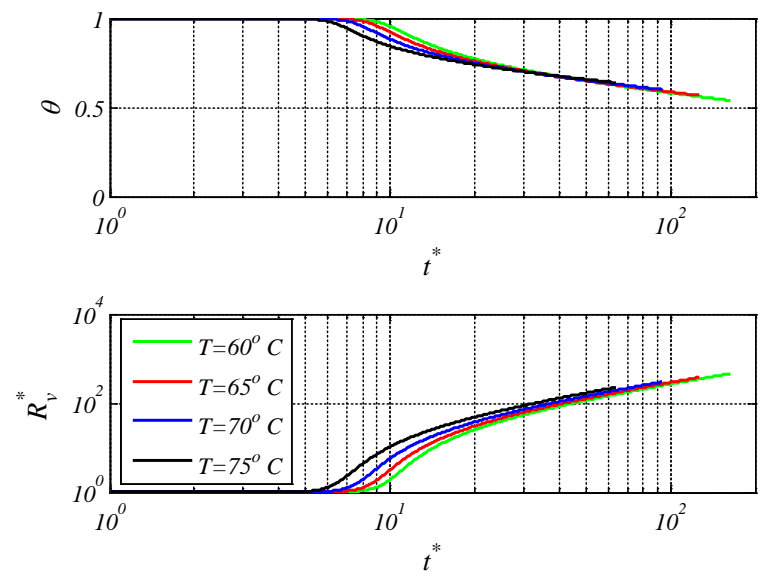

Figure 3. Dimensionless bubble radius and bubble vapor temperature versus dimensionless time, using Robinson and Judd [17] method for a few injection temperatures. 


\section{Energy mechanisms}

During the homogenous flash boiling generation spray process, the compressed liquid energy, in the container, is transformed into a several different energy mechanisms. By the energy partition model investigation, it is possible to examine the effect of the compressed liquid properties on the spray properties. This model is based on the flash boiling generation spray, the homogenous nucleation rate and Robinson and Judd [17] numerical bubble growth model, With the steady state steady flow (SSSF) assumption.

In the initial condition, when the compressed liquid is in the container (Fig. 1 area a). The liquid's energy will be its availability [18]:

$$
\Psi_{\text {inj }}=\dot{m}_{l_{i}}\left[\left(h_{\text {inj }}-h_{0}\right)-T_{0}\left(s_{\text {inj }}-s_{0}\right)\right]
$$

where $h$ and $s$ are the enthalpy and the entropy respectively, the subscripts inj and 0 denotes to compressed liquid (container) and surrounding conditions. The mass flow rate in the orifice is:

$$
\dot{m}_{l}=\rho_{l_{i}} \bar{U}_{i} A_{N}=\dot{\phi}_{l_{i}} A_{N}
$$

Where $A_{N}$ is the orifice cross section area, $\dot{\phi}_{l}$ is the mass flux and $\bar{U}_{l_{i}}$ is the mean flow velocity, under the assumption that the flow isn't chocked.

When the liquid flow throw the orifice, rapid depressurization occurs and linearly pressure decrease is assumed. In consequence of the depressurization, a massive bubble nucleation occurs. To simplify, we assume that the formed bubbles are spherical and with uniform arrangement, Face- Centered Cubic (FCC) (Fig. 4) and stay that way for all of the growth process until they touch each other at point ( $\mathrm{t}$ ) (Fig. 1). Furthermore, we assume that those kernels are formed in a uniform flow cross section, with width unit sell of (a) (Fig. 4). Also, we assume that the flow is adiabatic, meaning that there is no heat transfer from the liquid to the orifice walls and there is no relative flow between the bubbles and the liquid.

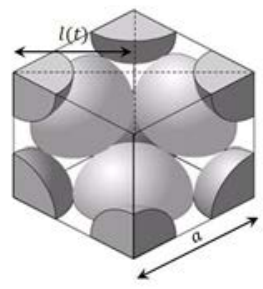

Figure 4. Face-Centred Cubic (FCC) unit cell.

According to those assumptions, the distance between the bubbles, $l$, is constant and can be evaluated the following way.

$$
l=\left(\frac{\bar{U}_{i}}{J}\right)^{1 / 4}
$$

During the process, from the moment the bubbles are formed $(n)$ until the moment they touch each other ( $t$ ) (areas $n$ and $t$ ), the energy can be expressed by using the following mechanisms:

- The flow kinetic energy flux of the orifice entrance:

$$
E_{k}^{\prime \prime}(t)=\dot{\phi}_{l_{i}} \frac{\bar{U}(t)^{2}}{2}
$$

- The evaporation energy flux of the liquid into the bubbles:

$$
E_{h_{n}}^{\prime \prime}=\frac{\dot{N}}{A_{N}}\left[\frac{4}{3} \pi R_{0}^{3} \rho_{v_{n}} h_{f g_{n}}+\int_{0}^{t} \dot{m}_{e v p}(t) h_{f g}(t) d t\right]
$$

- The mechanical work invested when the bubbles push the liquid while growing:

$$
E_{p}^{\prime \prime}(t)=4 \pi \frac{\dot{N}}{A_{N}} \int_{0}^{R(t)} p_{l}(t) r^{2} d r
$$

- The bubbles surface tension energy in the liquid a moment before the explosion:

$$
E_{\sigma}^{\prime \prime}(t)=\dot{N} 4 \pi \sigma R(t)
$$




\section{Spray characteristic}

After the bubbles touch each other, they explode and spread the liquid between them into a little droplets, so the spray is created (area $d$ ). Assuming that the number of bubbles is equal to the number of droplets, by mass conservation at the liquid phase, it is possible to evaluate the average radius of the formed droplets.

The efficiency of the process is defined by the following [1]:

$$
\eta=\frac{E_{\sigma_{d}}}{\Psi_{\text {inj }}-\Psi_{0}}
$$

When the droplets surface tension energy defined by:

$$
E_{\sigma_{d}}^{\prime \prime}=4 \pi \sigma_{d} \sum_{j=1}^{\dot{N}}\left(\frac{D_{j}}{2}\right)^{2}
$$

\section{Results and discussion}

At the following, we can see the dimensionless energy distribution of various mechanisms from the bubbles formation, point $(n)$ until they touch each other, point $(t)$. It is obtained based on the bubbles formation rate and the bubble growth rate model.

At the following figure, we can see the dimensionless energy distribution as function of the dimensionless time, for injection temperature $T_{i n j}=340 \mathrm{~K}$, meaning $93 \%$ of the critical temperature. A large part of the energy is converted into kinetic energy, to move the liquid throw the orifice as a result of the pressure differences.

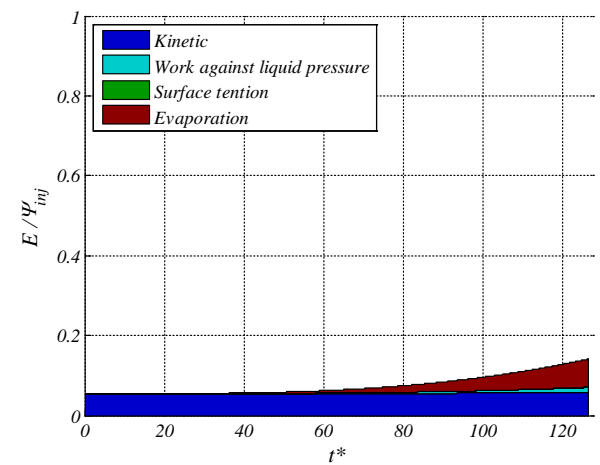

Figure 5. Dimensionless energy distribution as function of dimensionless time, from the bubbles formation, point $(n)$ until they touch each other, point $(t)$, for injection temperature $T_{i n j}=340 \mathrm{~K}$.

The following figures present the dimensionless energy distribution as function of the dimensionless temperature. In the left side (Fig 6.a) at the bubbles formation moment, point (n), and in the right side (Fig 6.b) the moment the bubbles touch each other, point $(t)$.

Most of the energy is converted into evaporation of the bubbles during their growth. Also, a significant amount of energy is wasted when the bubbles push the liquid around them. Furthermore, a small amount of energy is accumulated in the surface tension of the bubbles. Another interesting thing that can be concluded is that the energy of the different mechanisms increases with the liquid injection temperature.

(a)

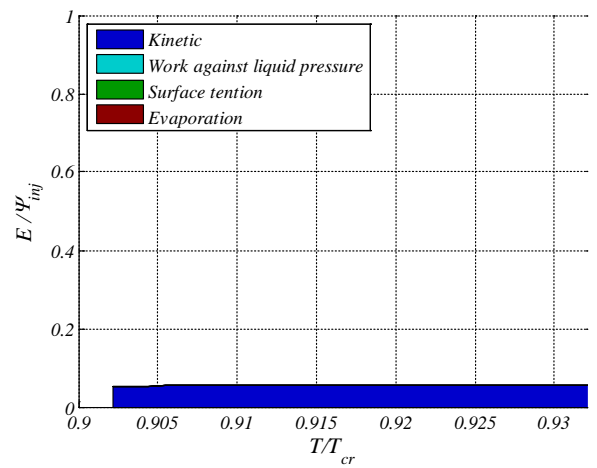

(b)

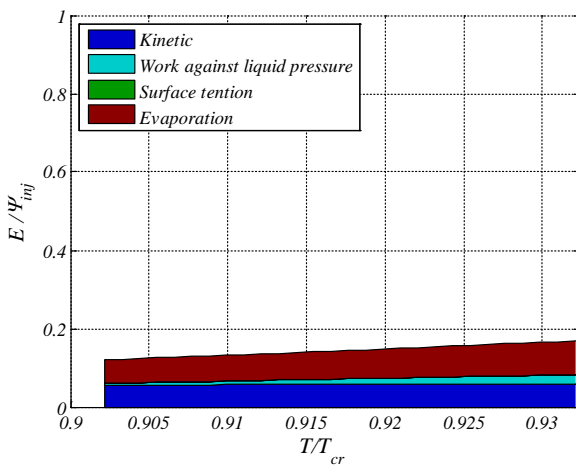

Figure 6. Dimensionless energy distribution as function of the dimensionless temperature. In (a), at the bubbles formation moment and in (b), at the moment the bubbles touch each other. 
Fig. 7.a shoes the average droplets diameter and Fig. 7.b presents the process efficiency as function of the dimensionless temperature for $\mathrm{CHCIF}_{2}$, Chlorodifluoromethane (R-22). It is possible to distinguish that the droplets radius reduces linearly when the temperature increases.

(a)

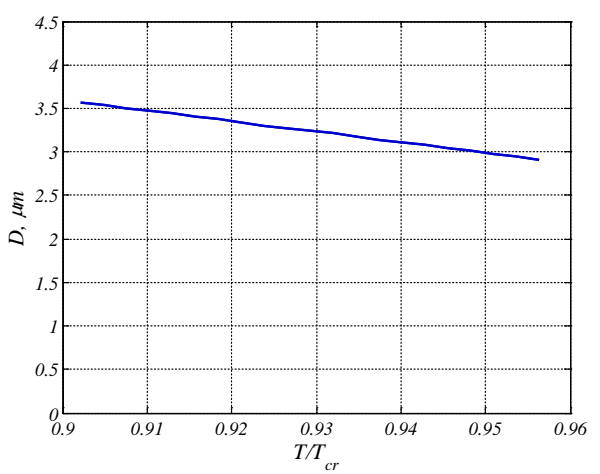

(b)

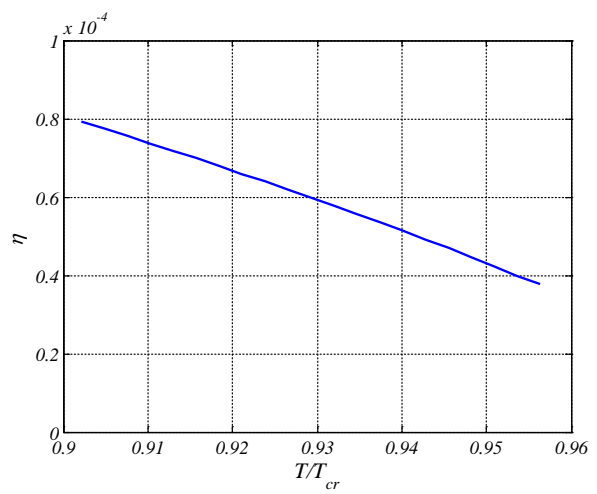

Figure 7. Average droplets diameter (in a) and process efficiency (in b) as function of the dimensionless temperature.

\section{Conclusions}

The current model present the energy distribution along the jet breaking process into a spray using homogenous flesh boiling. The model can evaluate changes of energy as a result of condition changes or the compressed liquid characteristics. Furthermore, the model can evaluate the sprays droplet radius. Using an experiment comparison allows to add a correction factor for the models assumptions. Homogeneous nucleation can be used in injection systems, such as combustors and engines because it can be achieved using a simple atomizer construction

\section{Nomenclature}

$A_{N} \quad$ Orifice cross section $\left[\mathrm{m}^{2}\right]$

D Diameter $[m]$

$h \quad$ Enthalpy $\left[\mathrm{Jg}^{-1}\right]$

$J \quad$ nucleation rate $\left[\mathrm{n} \mathrm{m}^{-3} \mathrm{~s}^{-1}\right]$

$\mathrm{Ja}$ Jacobs number [- ]

$k \quad$ Thermal conduction $\left[W \mathrm{~m}^{-1} K^{-1}\right.$ ]

$k_{b} \quad$ Boltzmann constant $\left[J K^{-1}\right]$

$l \quad$ Length between bubbles $[\mathrm{m}]$

$\dot{m} \quad$ Mass flow rate $\left[\mathrm{kg} \mathrm{s}^{-1}\right]$

$\bar{M} \quad$ Molar mass $\left[\mathrm{kg} \mathrm{mol}^{-1}\right]$

$N_{A} \quad$ Avogadro number [-]

$p \quad$ Pressure $[\mathrm{Pa}]$

$R \quad$ Bubble radius $[\mathrm{m}]$

$\bar{R} \quad$ Gas constant $\left[\mathrm{K} \mathrm{K}^{-1} \mathrm{~kg}^{-1}\right]$

$s \quad$ Entropy $\left[\mathrm{Jg}^{-1} \mathrm{~K}^{-1}\right]$

$T$ Temperature $[K]$

$t \quad$ Time $[s]$

$\bar{U} \quad$ Average velocity $\left[m s^{-1}\right]$

$\theta \quad$ Dimensionless temperature [-]

$\mu \quad$ Dynamic viscosity [Pas $]$

$\rho \quad$ Density $\left[\mathrm{kg} \mathrm{m}^{-3}\right]$

$\sigma \quad$ Surface tension $\left[\mathrm{N} \mathrm{m}^{-1}\right]$

$\dot{\phi} \quad$ mass flux $\left[\mathrm{kg} \mathrm{s}^{-1} \mathrm{~m}^{-2}\right]$

$\Psi \quad$ Availability $[W]$

$\begin{array}{ll}\text { Indices } & \\ c r & \text { Critic } \\ d & \text { Droplets } \\ h_{n} & \text { Evaporation energy } \\ \text { inj } & \text { Injection } \\ k & \text { Kinetic energy } \\ l & \text { Liquid } \\ n & \text { Nucleation } \\ p & \text { Mechanical work } \\ s a t & \text { Saturation } \\ t & \text { Bubble exploding } \\ v & \text { Vapor } \\ & \\ 0 & \text { Surrounding conditions } \\ \infty & \text { Outside of the boundary layer } \\ * & \text { Dimensionless } \\ \sigma & \text { Surface tension energy }\end{array}$




\section{References}

[1] E. Sher and C. Elata, "Spray formation from pressure cans by flashing," Ind. Eng. Chem. Process Des. Dev., vol. 16, no. 2, pp. 237-242, 1977.

[2] T. Bar-Kohany and E. Sher, "Effervescent atomization under sub-sonic and choked conditions-a theoretical approach," Chem. Eng. Sci., vol. 59, no. 24, pp. 5987-5995, Dec. 2004.

[3] T. Bar-Kohany, I. Sher, and E. Sher, "Choked flow of a bubbly mixture through an effervescent and flashboiling atomizer: A theoretical approach," At. Sprays, vol. 17, no. 5, 2007.

[4] E. Sher, T. Bar-Kohany, and A. Rashkovan, "Flash-boiling atomization," Prog. Energy Combust. Sci., vol. 34, no. 4, pp. 417-439, Aug. 2008.

[5] M. Levy, Y. Levy, and E. Sher, "Spray structure as generated under homogeneous flash boiling nucleation regime," Appl. Therm. Eng., vol. 73, no. 1, pp. 416-423, Dec. 2014.

[6] T. Bar-Kohany and M. Levy, "STATE OF THE ART REVIEW OF FLASH-BOILING ATOMIZATION," At. Sprays, vol. 26, no. 12, 2016.

[7] M. Levy, Y. Levy, and E. Sher, "EFFECT OF THE PROPELLANT MASS FRACTION IN A BINARY MIXTURE ON THE SPRAY CHARACTERISTICS AS GENERATED BY HOMOGENEOUS FLASH BOILING," At. Sprays, vol. 26, no. 12, 2016.

[8] M. Levy, Y. Levy, and E. Sher, "Diameter Estimation of Droplets Under Homogeneous Flash Boiling," presented at the ILASS 2016, Brighton, England, 2016.

[9] C. T. Avedisian, "The Homogeneous Nucleation Limits of Liquids," J. Phys. Chem. Ref. Data, vol. 14, no. 3, pp. 695-729, 1985.

[10] M. N. Hutcherson, R. E. Henry, and D. E. Wollersheim, "Two-Phase Vessel Blowdown of an Initially Saturated Liquid-Part 1: Experimental," J. Heat Transf., vol. 105, no. 4, pp. 687-693, 1983.

[11] M. Hutcherson, R. Henry, and D. Wollersheim, "Two-Phase Vessel Blowdown of an Initially Saturated Liquid-Part 2: Analytical," J. Heat Transf., vol. 105, no. 4, pp. 694-699, 1983.

[12] I. Sher, S. Haber, and G. Hetsroni, "A new state model of liquid-vapor interfaces-to yield analytical expression for surface tension," Chem. Eng. Sci., vol. 60, no. 3, pp. 711-716, Feb. 2005.

[13] V. P. Carey, "Liquid-vapor phase-change phenomena," 1992.

[14] Lord Rayleigh, "VIII. On the pressure developed in a liquid during the collapse of a spherical cavity," Lond. Edinb. Dublin Philos. Mag. J. Sci., vol. 34, no. 200, pp. 94-98, 1917.

[15] M. S. Plesset and S. A. Zwick, "The Growth of Vapor Bubbles in Superheated Liquids," J. Appl. Phys., vol. 25, no. 4, p. 493, 1954.

[16] B. Mikic, W. Rohsenow, and P. Griffith, "On bubble growth rates," Int. J. Heat Mass Transf., vol. 13, no. 4, pp. 657-666, 1970.

[17] A. J. Robinson and R. L. Judd, "The dynamics of spherical bubble growth," Int. J. Heat Mass Transf., vol. 47, no. 23, pp. 5101-5113, Nov. 2004.

[18] E. Sher and M. Zeigerson-Katz, "Spray formation by flashing of a binary mixture: An energy balance approach," At. Sprays, vol. 6, no. 4, 1996. 


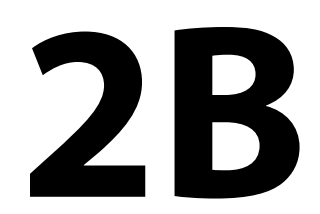

Automotive 2 


\title{
Modelling and validation of near-field Diesel spray CFD simulations based on the $\Sigma-Y$ model
}

\author{
J.M. Desantes ${ }^{1}$, J.M. García-Oliver ${ }^{1}$, J.M. Pastor ${ }^{\star 1}$, A. Pandal², B. Naud ${ }^{3}$, K. Matusik ${ }^{4}$ D. \\ Duke $^{4}$, A. Kastengren ${ }^{4}$, C. Powell ${ }^{4}$, D.P. Schmidt ${ }^{5}$ \\ ${ }^{1}$ CMT Motores Térmicos, Universitat Politècnica de València, Spain \\ 2 Área de Mecánica de Fluidos - Dpto. Energía, Universidad de Oviedo, Spain \\ ${ }^{3}$ CIEMAT, Spain \\ ${ }^{4}$ Argonne National Laboratory, USA \\ 5 University of Massachusetts, Amherst, USA \\ *Corresponding author: jopasen@mot.upv.es
}

\begin{abstract}
Diesel spray modelling still remains a challenge, especially in the dense near-nozzle region. This region is difficult to experimentally access and also to model due to the complex and rapid liquid and gas interaction. Modelling approaches based on Lagrangian particle tracking have struggled in this area, while Eulerian modelling has proven particularly useful. An interesting approach is the single-fluid diffuse interface model known as $\Sigma$-Y, based on scale separation assumptions at high Reynolds and Weber numbers. Liquid dispersion is modelled as turbulent mixing of a variable density flow. The concept of surface area density is used for representing liquid structures, regardless of the complexity of the interface.

In this work, an implementation of the $\Sigma-Y$ model in the OpenFOAM CFD library is applied to simulate the ECN Spray $A$ in the near nozzle region, using both RANS and LES turbulence modelling. Assessment is performed with measurements conducted at the Advanced Photon Source at Argonne National Laboratory (ANL). The ultra-smallangle $x$-ray scattering (USAXS) technique has been used to measure the interfacial surface area, and $x$-ray radiography to measure the fuel dispersion, allowing a direct evaluation of the $\Sigma-Y$ model predictions.
\end{abstract}

\section{Keywords}

Sprays, Diesel, atomization, CFD, OpenFOAM, X-ray

\section{Introduction}

Fuel injection and subsequent spray development are critical factors for charge preparation, combustion development and pollutants formation in engines. The liquid atomization process occurs at extremely small length scales and high speeds in current injection systems, which complicates both the investigation and modelling of spray flow, especially in the near-nozzle region. The lack of optical accessibility, except by means of special diagnostic techniques [1][2], hinders the flow characterization and the development of predictive primary atomization models.

The common spray modelling approaches, based on the representation of the liquid phase using a Lagrangian framework [3], are not well suited to represent this dense region, while fully Eulerian approaches have recently shown their potential to simulate near-nozzle physics [4][5]. Complex modelling techniques devoted to capturing the liquid-gas interface [6][7][8] have been successfully applied to simulate initial spray development, but the computational requirements can make those calculations impractical for spray applications in combustion systems due to high Reynold and Weber numbers.

Under these conditions, one may assume a separation of the large scale flow features, such as mass transport, from the atomization process occurring at smaller scales, as proposed in[9][10]. Then large scale liquid dispersion can be modelled as the turbulent mixing of a variable density fluid. For atomization, the surface density concept is introduced in order to evaluate the mean size of liquid fragments, assuming that interfacial details are smaller than the mesh size. The end result is a diffuse-interface treatment in an Eulerian framework. This framework is naturally extensible to near critical or super-critical regimes [11]. Rather than directly tracking the unresolved interface features, they are modelled in an Eulerian framework.

These diffuse-interface Eulerian spray models have two common elements: a model for the transport of liquid (or gas) and a model for the evolution of the interfacial surface area. The density of interfacial area is typically denoted by Sigma $(\Sigma)$ while the liquid fraction is denoted by $\mathrm{Y}$. Hence, we refer to the strictly Eulerian model as a $\Sigma-Y$ approach, in contrast to ELSA (Eulerian-Lagrangian Spray Atomization), which includes a transition to Lagrangian particle tracking [12][13]. 
The transport of the liquid employs mass-averaged convection along with turbulent mixing. This model is derived from basic Favre averaging or LES filtering [14]. Thus, the accuracy of the liquid fraction transport is largely dependent on the accuracy of the two-phase turbulent modelling. Despite the challenges of such modelling, there is at least an extensive theoretical basis to deal with the unclosed terms, putting the $Y$ transport equation on a much firmer foundation than the interfacial evolution.

However, the model for the interface evolution is somewhat more speculative, with several unclosed terms [15]. There are several interface modelling approaches that have been applied to sprays as researchers have explored competing ideas of how these terms should be treated [10][7][15].

In this paper, the $\Sigma-Y$ model is evaluated in diesel sprays near-nozzle region by comparing its predictions with experiments conducted at Argonne National Laboratory (ANL) within the Engine Combustion Network (ECN) framework [16]. Those experiments provide unique data using x-ray radiography [1] and the recently developed ultra-small-angle x-ray scattering (USAXS) [17].

Previous works [4][18][19] have shown liquid spray dispersion predictions compared to $x$-ray radiography data, and the noticeable effect of the turbulence model in those RANS based simulations. In this paper, a LES approach is also applied and its impact on spray dispersion is evaluated.

The interfacial density predictions have rarely been validated, and these validations have been in the context of downstream drop size [20][21], which is not ideal since we wish to avoid the assumption that the liquid is in the form of droplets. A few prior examples used DNS simulations [7] for validation, and in this paper the validation is performed via USAXS measurements, which directly measures the interfacial surface density. In this work, the formulation proposed by [22] has been assessed and compared with recent modelling results [23].

In summary, we wish to validate the transport of liquid mass and assess when the model for interfacial evolution is accurate.

\section{Experimental methodology}

Two different techniques, developed and performed at the Advanced Photon Source at ANL, have been applied in order to characterize diesel spray structure: X-ray radiography and Ultra-small angle x-ray scattering (USAXS)

The $x$-ray radiography [24] experiments provide a path-length-integrated measure of the fuel density along one beam path through the spray. To measure the spatial distribution of the fuel, a two-dimensional raster-scan approach is used, with each point measured from a different set of spray events. To further improve the signal/noise ratio in the final data, each data point is an average of 128-256 individual spray events. As such, the final data represent the ensemble averaged three-dimensional fuel density projected onto a plane. The fuel distribution data are thus reported as a Projected Mass Density (PMD), providing valuable information concerning liquid spray dispersion.

The USASX technique was used in order to evaluate interfacial surface area [17], due to its ability to interrogate the dense region and provide quantitative information about the complex interface without resorting to the assumption that the liquid is in the form of droplets. In performing these experiments, the Bonse-Hart instrument at the 9-IDbeamline measures the scattering intensity, $\mathrm{I}_{\text {scat }}(q)$, for a wide range of scattering vectors, $q$ [25]. A beam of $\mathrm{x}$-rays at $17.9 \mathrm{keV}$ is first shaped into a $100 \times 500 \mu \mathrm{m} \mathrm{H} \times \mathrm{V}$ spot with a set of high precision $2 \mathrm{D}$ slits. The incoming beam is collimated with a pair of $\mathrm{Si}(220)$ crystals before impinging on the spray, from which the incident $\mathrm{x}$-rays scatter at small angles. The scattered x-rays are filtered downstream with a pair of $\mathrm{Si}(220)$ analyzer crystals, and the scattered photons are measured with a detector. The pair of analyzer crystals is rotated to measure the transmitted beam intensity as a function of scattering vector. The scattering vector was varied between $1 \times 10^{-4} \AA^{-}$ ${ }^{1}<q<1 \times 10^{-2} \AA^{-1}$ with a step size of $1 \times 10^{-5} \AA^{-1}$ at low $q$, with increasing step size for larger $q$. The scattering intensity as a function of $q$ was measured at axial distances ranging from 1 to $20 \mathrm{~mm}$ downstream of the injection nozzle, at the centerline of the spray. Once $I_{\text {scat }}(q)$ is known, the differential cross-section may be calculated, and related to the total shape and surface area per volume of fuel droplets, with post-processing performed using the Irena data analysis package [26].

A solenoid diesel injection nozzle provided by the Engine Combustion Network (ECN); the single-hole Spray A 210675 nozzle, with a nominal hole diameter of $90 \mu \mathrm{m}$, was investigated. The injector was mounted horizontally in a $0.5 \mathrm{~L}$ vessel pressurized with $\mathrm{N} 2$ gas, which also supplied a purge flow of approximately 4 standard L/min in order to inhibit droplet accumulation within the domain during measurements.

In order to provide useful data for comparison with other ECN experiments, the experimental conditions have been matched as closely as possible to the ECN Spray A specification [16].The specified injector, fuel, rail and injections line were used, but ambient gas is at room temperature instead of the prescribed high-temperature (900 K). The current experiments have, however, matched the ambient density of the Spray A specification, at the expense of not matching the ambient pressure; density is expected to be a more critical parameter in fuel-air mixing than 
pressure [27]. In addition, two additional injection pressures and one ambient pressure were also consider for the USAXS experiments, as shown in Table 1.

Table 1. Operating conditions in experiments

\begin{tabular}{|c|c|c|c|c|}
\hline Injector & \multicolumn{3}{|c|}{ Rail Pressure (bar) } & Chamber Pressure (bar) \\
\hline Spray A 210675 & \multicolumn{3}{|c|}{1500} & 6.7 \\
\hline Spray A 210675 & 1500 & 1000 & 500 & 20 \\
\hline
\end{tabular}

\section{Modelling approach}

To track the dispersion of the liquid phase an indicator function is used, taking a value of unity in the liquid phase and zero in the gas phase. The liquid volume fraction is denoted as $\bar{Y}$, and the mass-averaged fraction is defined as $\widetilde{\boldsymbol{Y}}=\overline{\boldsymbol{\rho} Y} / \overline{\boldsymbol{\rho}}$. Favre averaging or filtering the transport equation for the liquid mass fraction yields Eq. (1).

$$
\frac{\partial \bar{\rho} \widetilde{Y}}{\partial t}+\frac{\partial \bar{\rho} \widetilde{u_{\imath}} \widetilde{Y}}{\partial x_{i}}=-\frac{\partial \tau_{y i}}{\partial x_{i}}
$$

The turbulent diffusion liquid flux term $\boldsymbol{\tau}_{\boldsymbol{y} i}$, captures the effect of the relative velocity between the two phases [14]. This term is modelled using a standard turbulent gradient flux model, which successfully worked for Diesel spray compared to DNS results [15].

Under the assumption that the two phases form an immiscible mixture, the mass-averaged value of the indicator function is related to the density by Eq. (2). An equation of state is then assigned to each phase. The gas phase obeys an ideal gas law, that the liquid phase maintains a constant linear compressibility, and that both phases experience the same pressure.

$$
\frac{1}{\bar{\rho}}=\frac{\widetilde{Y}}{\rho_{l}}+\frac{1-\widetilde{Y}}{\rho_{g}}
$$

To close the above system of equations, the temperature is obtained from a bulk mixture enthalpy equation, where $h_{l}$ and $h_{g}$ denote the enthalpy of the liquid and gas phases respectively:

$$
h(T)=\widetilde{Y} \cdot h_{l}(T)+(1-\widetilde{Y}) \cdot h_{g}(T)
$$

The solution of the above equations fully characterizes the large-scale bulk motion of the flow. Conversely, the small scale atomization is modelled by solving a transport equation for the evolution of the interphase surface area density, Sigma $(\Sigma)$. This surface density is based on Vallet and Borghi [9] formulation, which includes source terms to account for generation due to the growth of fluid instabilities (i.e. Kelvin-Helmholtz) and the destruction of surface due to droplet coalescence (in the case of dispersed flow). However, the most common form for the combination of these two source terms is the restoration to an equilibrium value $\left(\bar{\Sigma}_{e q}\right)$ or critical surface density to which the local surface density is driven [7][21]:

$$
\frac{\partial \widetilde{\Sigma}}{\partial t}+\frac{\partial \widetilde{u_{j}} \widetilde{\Sigma}}{\partial x_{j}}-\frac{\partial}{\partial x_{j}}\left(D_{\Sigma} \frac{\partial \widetilde{\Sigma}}{\partial x_{j}}\right)-C_{\Sigma} \widetilde{\Sigma}\left(1-\frac{\widetilde{\Sigma}}{\bar{\Sigma}_{e q}}\right)-S_{\Sigma_{i n i t}}=0
$$

The $\bar{\Sigma}_{e q}$, already mentioned, is computed from an equilibrium Weber number [22], instead of using an equilibrium droplet radius, as in a recent work [23], in order to avoid any kind of assumption of spherical droplets.

$$
\bar{\Sigma}_{e q}=\alpha_{2} \frac{\left(\rho_{l}+\rho_{g}\right) \bar{Y}(1-\bar{Y}) \widetilde{k}}{\sigma}
$$

Then, the coefficient $\boldsymbol{C}_{\boldsymbol{\Sigma}}$ is modeled as the inverse of the turbulent time scale:

$$
C_{\Sigma}=\alpha_{1} \frac{\tilde{\varepsilon}}{\widetilde{k}}
$$

Note the presence of the two modelling constants $\left(\boldsymbol{\alpha}_{1}, \boldsymbol{\alpha}_{2}\right)$, by default equal to 1.0 [15][22], which should be calibrated.

Finally, as can be seen all the source terms that are involved in this equation are proportional to the interface surface density. As a result, there will be no production if there is no interface. Therefore, a proper initialization should be made by means of the term $\boldsymbol{S}_{\boldsymbol{\Sigma}_{\text {init }}}$. For that purpose, another update is presented in this work with respect to [23] in order to prevent any possible grid dependency. In a similar way as in [7], the initialization corresponds to a production due to the liquid/gas mixing process: 


$$
\begin{gathered}
\Sigma_{\text {init }}=2 \bar{\rho} \frac{v_{\mathrm{t}}}{S c} \frac{6 \bar{\rho}}{\rho_{l} \rho_{g} l_{t}} \frac{\partial \widetilde{Y}}{\partial x} \frac{\partial \widetilde{Y}}{\partial x}, \text { if } \widetilde{Y}(1-\widetilde{Y}) \leq 0.001 \\
\Sigma_{\text {init }}=2 \frac{v_{\mathrm{t}}}{S c} \frac{\widetilde{\Sigma}}{\widetilde{Y}(1-\widetilde{Y})} \frac{\partial \widetilde{Y}}{\partial x} \frac{\partial \widetilde{Y}}{\partial x}, \text { otherwise }
\end{gathered}
$$

The previously described model equations have been implemented into a solver [20] constructed by using the OpenFOAM [28] CFD libraries.

\section{Computational model set-up}

The calculations have been performed for the ECN Spray A using a 3-D computational domain with $80 \mathrm{~mm}$ length and $25 \mathrm{~mm}$ in radius. The mesh is structured with non-uniform grid resolution. There are 20 cells along the orifice diameter, keeping an aspect ratio close to one in the near nozzle region and is stretched in axial and radial directions. This mesh construction is the result of different sensitivity studies previously performed, including an evaluation of nozzle resolution [29].

The boundaries included non-slip conditions for the walls and non-reflective conditions for the open-ends. The inlet boundary condition uses the measured mass-flow rate in order to get the bulk injection velocity. LES cases use a specific inlet condition in order to generate turbulent fluctuations with a given statistical profile, based on the proposals by [30][31] and following the method described in [32]. The characteristic correlation length scale is twice the orifice grid resolution in order to resolve the generated eddies. The initial fluctuation level can be imposed over the mean velocity profile. This inlet profile is then rescaled in order to obtain the prescribed bulk injection velocity at each time-step.

The turbulent flow field for RANS is solved by a standard $k-\varepsilon$ model with a round-jet corrected value (1.6) for the $\mathrm{C}_{1 \varepsilon}$ constant [33], which provided good results for spray near [4] and far-field [20]. An eddy-viscosity based sub-grid model, derived from the analysis of the singular values of the resolved velocity gradient tensor [34], is used for LES cases. Time derivative terms are solved by a first order Euler scheme for RANS cases while LES uses a secondorder backward scheme. Divergence terms are solved by a Gamma NVD scheme for both turbulence modelling.

\section{Liquid spray dispersion: Projected mass density results}

Previous work has shown the $\Sigma$-Y RANS predictions compared to x-ray data [18][29]. Figure 1 shows projected mass density (PMD) at different axial locations downstream the nozzle. Note that the experimental profiles have been shifted to be centred at the axis in order to avoid the effect produced by the offset of the orifice outlet with respect to the needle axis in this injector (Serial\# 210675). It is shown in this figure that the $\Sigma-Y$ model is able to predict the shape of the PMD distributions in the near-nozzle region. The model captures the measured PMD data both in peak and radial dispersion at 0.1 and $2 \mathrm{~mm}$ sections but lower accuracy is achieved downstream. At $6 \mathrm{~mm}$ the PMD is over-predicted, however the spray width is well captured.

Two different inlet boundary condition were used in the RANS simulations: a mapped boundary condition, where all flow variables were taken from previous nozzle flow calculations [18], and a constant radially averaged outlet profile, which will be used to compare with LES cases.
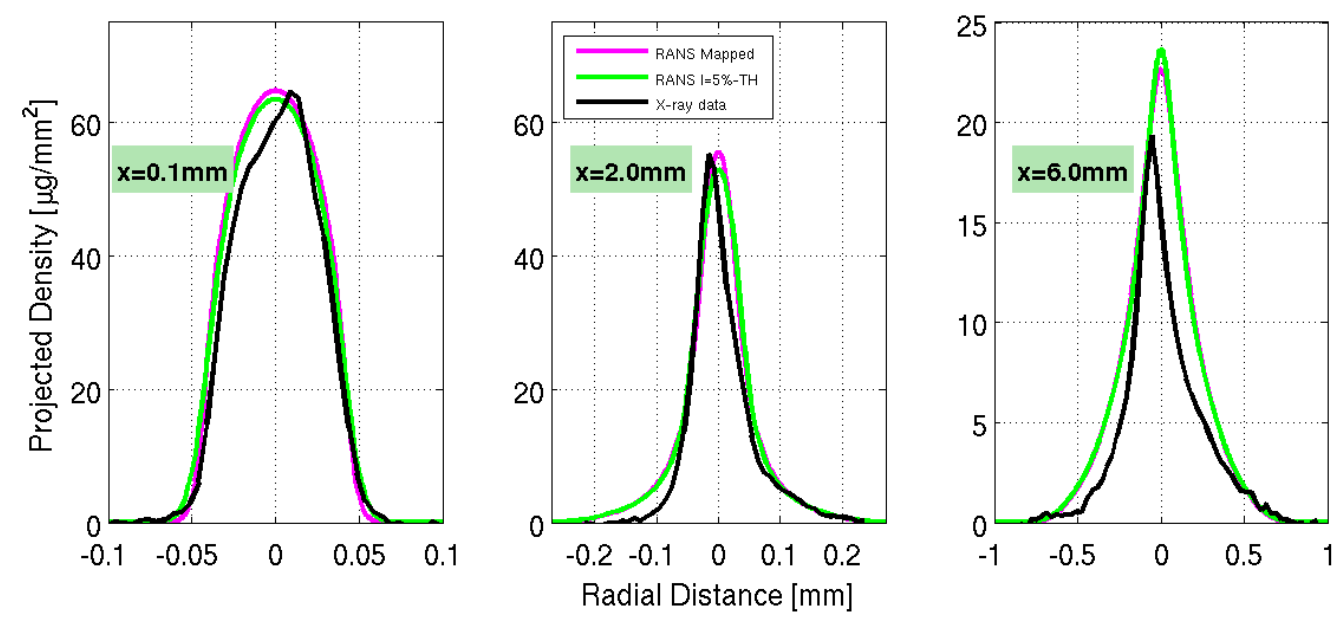

Figure 1. Measured and computed PMD at $0.1,2$ and $6 \mathrm{~mm}$ downstream the nozzle exit. $\mathrm{P}_{\mathrm{inj}}=150 \mathrm{MPa}, \rho_{\mathrm{mmb}}=22.8 \mathrm{~kg} / \mathrm{m}^{3}$ 
LES simulations have been initially performed with a turbulent intensity of 5 -percent and $1 / 7^{\text {th }}$ power law mean velocity profile, as suggested in different LES Diesel spray simulations [11][35]. A single realization was run and time-averaging was performed from $0.5 \mathrm{~ms}$ after the Start of Injection, during the quasi-steady period, as in $\mathrm{x}$-ray experiments [24]. Figure 2 shows the instantaneous and averaged liquid mass fraction. The LES resolution is assessed by the ratio of SGS flow energy over total turbulent kinetic energy (modelled plus resolved). According to the criteria proposed in [36], this ratio (M) should be less than $20 \%$, which is accomplished in the simulations, as shown in RHS picture of Figure 2, within the region of interest for assessment.

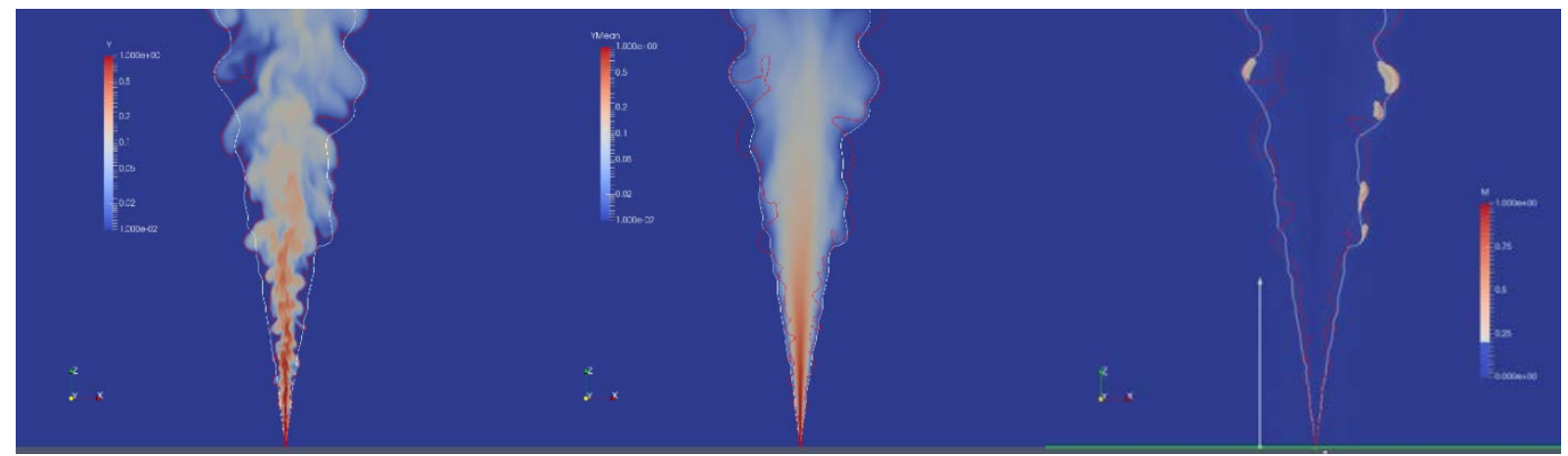

Figure 2. Cut-plane through spray axis showing instantaneous (left) and time-averaged (centre) liquid mass fraction. Modelledto-total flow kinetic energy ratio $M$ (right). Isolines of $1 \% \mathrm{Y}$ (white) and YMean (red). Arrow in right image is $15 \mathrm{~mm}$ length.

The PMD from time-averaged LES data are presented in Figure 3. This figure also includes results using the same mean velocity profile (top-hat) and turbulent intensity for both RANS and LES calculations. It is shown that LES captures PMD at the nozzle exit, but under-predicts at $2 \mathrm{~mm}$ downstream, and better results are obtained at $6 \mathrm{~mm}$, contrary to RANS, independently of the mean exit profile used (i.e. top-hat and power-law). This indicates that spray dispersion is over-predicted in the near-nozzle $(\sim 2 \mathrm{~mm})$ in LES calculations and the effect of the nozzle exit profile is reduced. Notice that the same mass and momentum flux is injected for both cases.

According to this result, the impact of fluctuations intensity was assessed in LES calculations. In this case a 3percent turbulence intensity, which corresponds to the outlet condition of a k- $\omega$ SST RANS nozzle flow simulation [19], has been used. Figure 4 shows a noticeable effect on PMD results when modifying initial fluctuations level, which have not been observed in previous RANS calculations [29]. It is shown that both 2 and $6 \mathrm{~mm}$ profiles predictions are improved with this lower fluctuation level boundary condition.
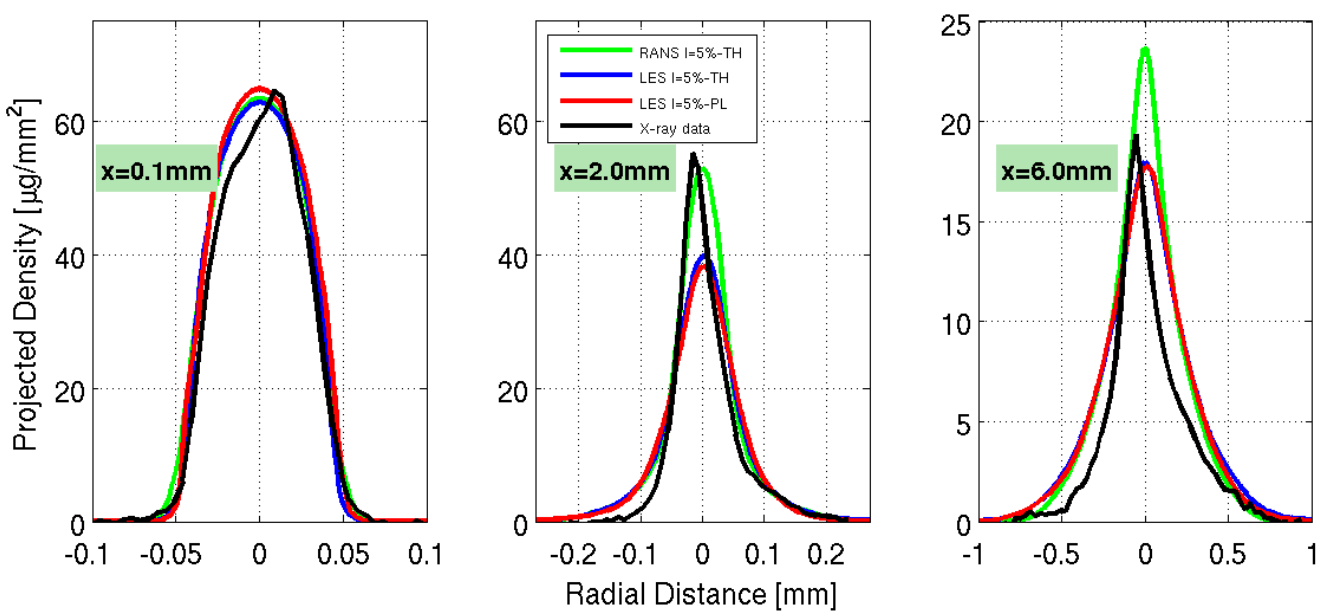

Figure 3. Measured and computed PMD at $0.1,2$ and $6 \mathrm{~mm}$ downstream the nozzle exit for RANS and LES simulations. Pinj $=$ $150 \mathrm{MPa}, \rho \mathrm{amb}=22.8 \mathrm{~kg} / \mathrm{m}^{3}$ 

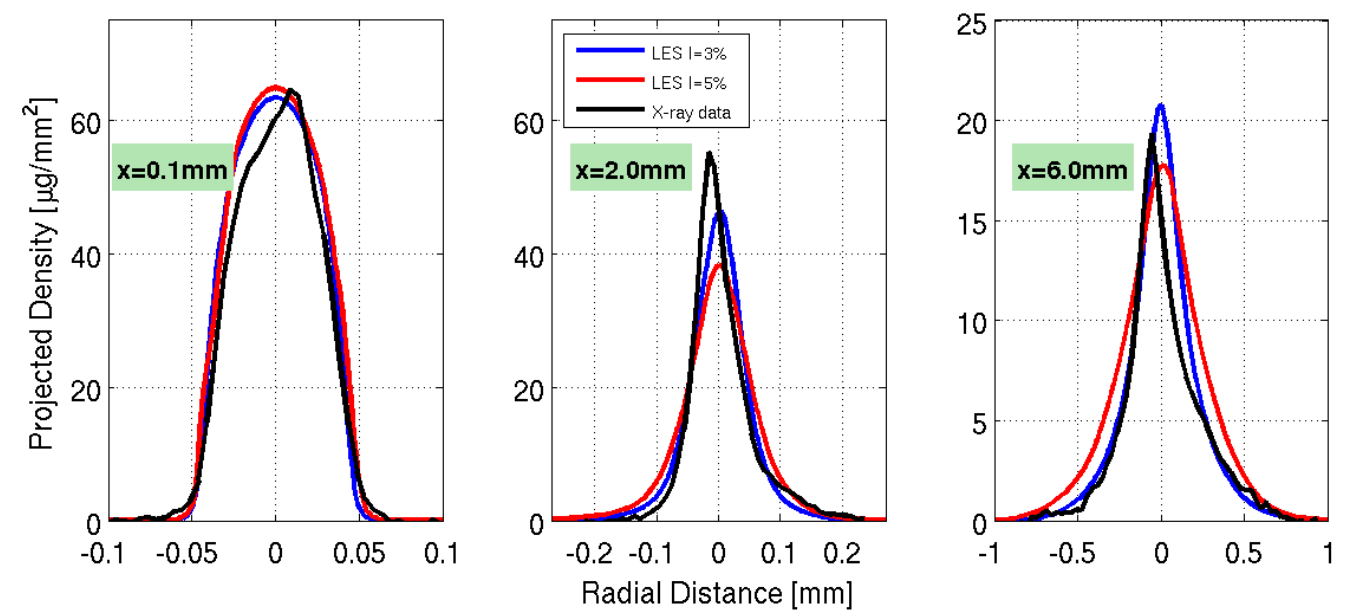

Figure 4. Measured and computed PMD at $0.1,2$ and $6 \mathrm{~mm}$ downstream the nozzle exit. Impact of turbulence intensity I in LES calculations. $P_{\text {inj }}=150 \mathrm{MPa}$, $\rho_{\mathrm{amb}}=22.8 \mathrm{~kg} / \mathrm{m}^{3}$

Figure 5 shows the Transverse Integrated Mass (TIM) which is obtained from the integral of the PMD across the transverse position for each axial location [1]. TIM is related to spray dispersion, so higher TIM indicates faster mixing [18]. It is shown that RANS modelling tends to increasingly over-predict TIM from the nozzle exit and thus spray mixing, while LES calculations are closer to experimental data up to $6 \mathrm{~mm}$. Downstream of this position TIM is over-predicted for both turbulence modelling approaches and also other works [4], but LES modelling has the potential to improve the predictions using lower turbulence intensity boundary conditions.

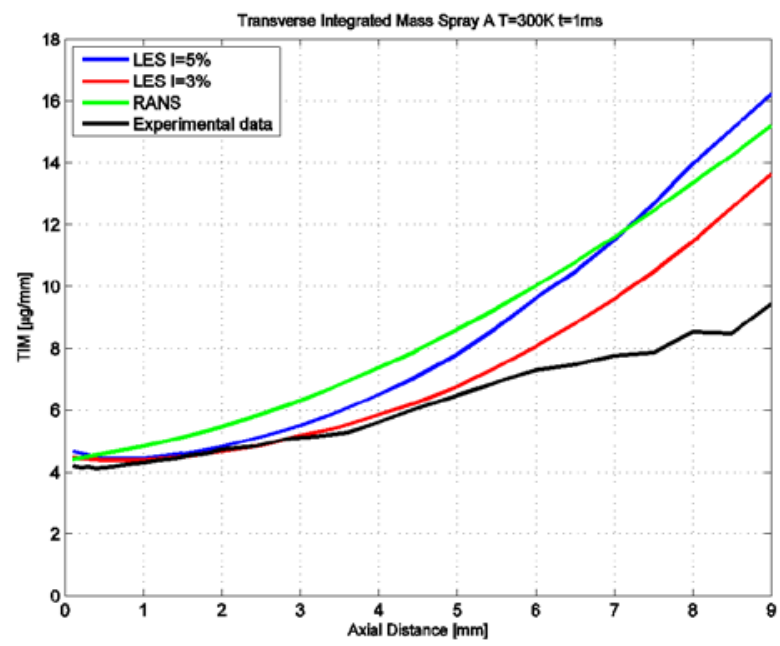

Figure 5. Measured and computed TIM along the spray axis RANS and LES simulations. $P$ inj $=150 \mathrm{MPa}, \rho_{\mathrm{amb}}=22.8 \mathrm{~kg} / \mathrm{m}^{3}$

\section{Spray atomization: Surface density results}

As explained in the modelling approach section, the two modelling constants of the surface density equation should be calibrated. The expertise acquired recently [23] suggests the capital importance of the $\boldsymbol{\alpha}_{2}$ constant while $\boldsymbol{\alpha}_{1}$ effects (with a value in the vicinity of 1.0) could be negligible. Thus, the fist constant has been set to unity and the calibration of $\boldsymbol{\alpha}_{\mathbf{2}}$ parameter has been made. The starting point is an evaluation of the results achieved with the $\boldsymbol{\alpha}_{\mathbf{1}}$ values obtained for limiting conditions of the critical Weber number from [35] $\left(\mathrm{We}_{\mathrm{c}} \sim 6-15\right)$, see Table 2:

Table 2. Reference studies conducted for the interphase surface density equation setup

\begin{tabular}{|c|c|c|}
\hline Case & $\boldsymbol{\alpha}_{1}[-]$ & $\boldsymbol{\alpha}_{2}[-]$ \\
\hline Reference 1 & 1.0 & 0.16 \\
\hline Reference 2 & 1.0 & 0.06 \\
\hline
\end{tabular}


In Figure 6, the results for the two reference cases evaluated are plotted on the left. It is shown both, the CFD surface area (solid line) and the equilibrium value (pointed line) together with the experimental measurements (black dashed line). These configurations over-predict the surface area results and it is clear that a lower $\alpha_{2}$ value is needed to be able to match these measurements. Then, four more simulations are conducted with the following values for the second parameter: $0.04,0.035,0.03$ and 0.02 , these new predictions are depicted in Figure 6 , on the right. It can be seen the great scalability achieved for the surface density predictions as well as the most suitable $\alpha_{2}$ constant value. According to the results, the experimental axial profile is almost completely matched with a parameter value of 0.035 . As a result, it is chosen as the optimum for the following calculations.
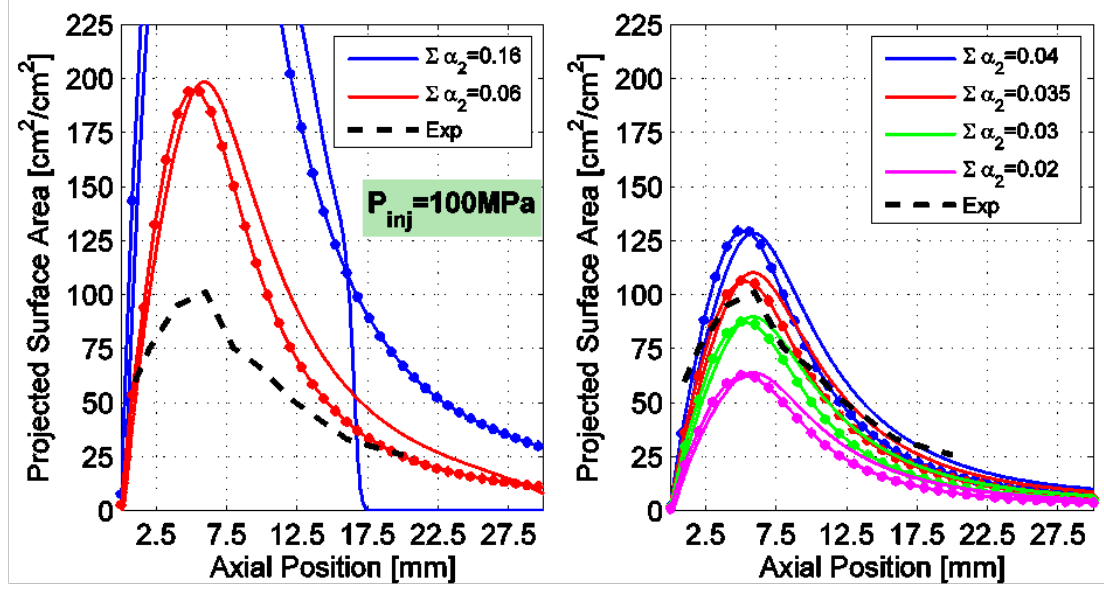

Figure 6. Projected surface area results at $1.5 \mathrm{~ms}$ after $\mathrm{SOI}$ for different values of the second modelling constant. $P_{\mathrm{inj}}=100$ $\mathrm{MPa}, \rho_{\mathrm{amb}}=22.8 \mathrm{~kg} / \mathrm{m} 3$

Finally, the optimum chosen setup is evaluated at the different operating conditions available. These results are depicted again at $1.5 \mathrm{~ms}$ after SOI in Figure 7 . The influence of injection pressure is shown on the left while the back pressure effect, i.e. ambient density, is shown on the right. Experimental trends are well reproduced; decreased injection pressure decreases the surface area profile, as well as the location at which the maximum occurs. However, a little deviation from the experimental data is noticeable for high and low injection pressure conditions. On the other hand, quite remarkable performance is achieved for low ambient density case, which clearly improves recent predictions [23].
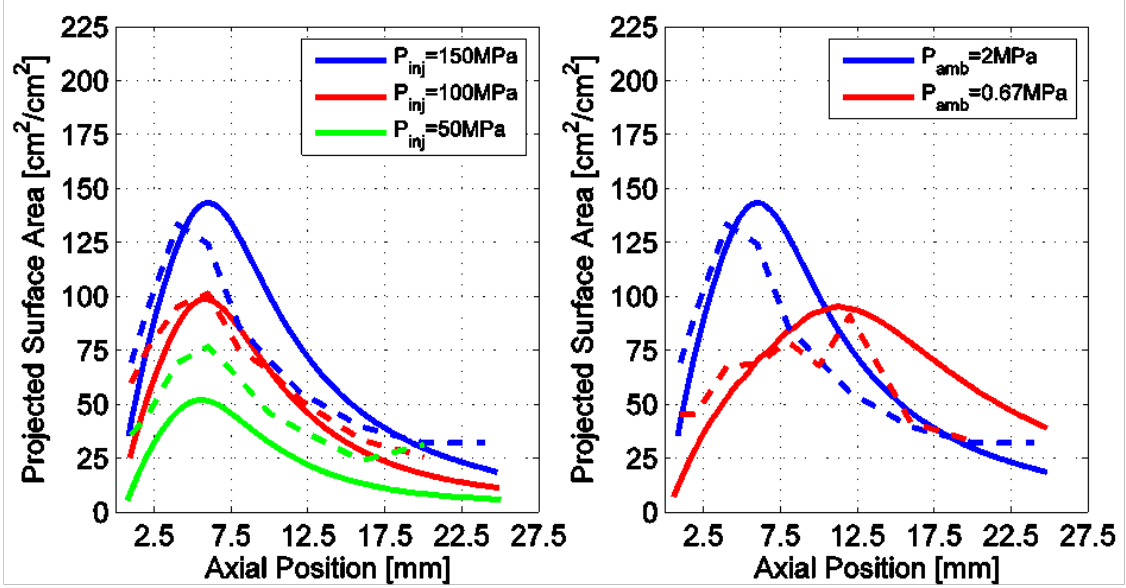

Figure 7. Projected surface area results at $1.5 \mathrm{~ms}$ after $\mathrm{SOI}$ for optimum set-up. The computational predictions are the continuous lines and the experimental measurements are the dashed lines.

\section{Conclusions}

This paper presents an assessment of the $\Sigma-Y$ model in the near-nozzle region of the ECN spray A. Unique experimental techniques performed at Argonne NL, namely x-ray radiography and USAXS, have been used to perform a direct evaluation of the model predictions. 
It is shown that the model is able to capture liquid spray dispersion in the near nozzle region, using previous RANS and newly performed LES calculations. Spray dispersion predictions accuracy can be improved by LES modelling, thought noticeably sensitivity to inflow turbulence intensity levels has been observed, which requires further insight. The interfacial surface evolution model has been directly compared to measurements obtained using USAXS technique. A new formulation for critical surface density has been used and the model was calibrated in a single operation point. Without further adjustment the model was able to fairly predict injection pressure variations, and especially lower ambient density condition, improving previous formulation results.

In order to perform further model evaluation and confirm predictive capabilities, different operating conditions and injectors must be considered.

\section{Acknowledgements}

Authors acknowledge that part of this work was partially funded by the Spanish Ministry of Economy and Competitiveness in the frame of the COMEFF (TRA2014-59483-R) project.

Parts of this research were performed at the 7-BM and 9-ID beam lines of the Advanced Photon Source at Argonne National Laboratory. Use of the APS is supported by the U.S. Department of Energy (DOE) under Contract No. DEAC02-06CH11357. The research was partially funded by DOE's Vehicle Technologies Program, Office of Energy Efficiency and Renewable Energy.

The authors would like to thank Team Leaders Gurpreet Singh and Leo Breton for their support of this work

\section{References}

[1] Kastengren, A.L., Powell, C.F., Wang, Y., Im, K.-S. et al., 2009, Atomization and. Sprays 19 (11), pp. 10311044.

[2] Linne, M., Paciaroni, M., Hall, T. et al., 2006, Exp. Fluids, 40, pp. 836-846.

[3] Dukowicz, J.K., 1980, Journal of Computational Physics 35 (2), pp.229-253.

[4] Xue, Q., Battistoni, M., Powell, C. F., Longman, D. E. et al., 2015, Int. Jou.Multiph. Flow 70 pp. 77-88.

[5] Desantes, J.M., Garcia-Oliver, J.M., Pastor, J.M. and Pandal, A., 2016, Atomization and Sprays 26 (7)

[6] Gorokhovski, M. and Herrmann, M., 2008, Annu. Rev. Fluid Mech. 40 pp. 343-366.

[7] Lebas, R., Menard, T., Beau, P.-A., Berlemont, A. et al., 2009, Int. Jou. Multiph. Flow, 35(3) pp. 247-260.

[8] Arienti, M. and Sussman, M., 2017, Int. Jou. Multiph. Flow 88, pp. 205-221.

[9] Vallet, A. and Borghi, R., 1999, C.R. Acad. Sci, Paris, 327, pp. 1015-1020.

[10] Vallet, A., Burluka, A. and Borghi, R., 2001, Atomization and Sprays 11, pp. 619-642

[11] Lacaze, G., Misdariis, A., Ruiz, A., Oefelein, J. C., 2015, Proceedings of the Combustion Institute 35 (2) pp. 1603-1611.

[12] Blokkeel, G., Barbeau, B., Borghi, R., 2003, SAE Technical Paper 2003-01-005.

[13] Lebas R., Blokkeel, G, Beau, P.A., Demoulin, F.-X., Aug 1-Sep 27, 2006, ICLASS.

[14] Demoulin, F.-X., Beau, P. A., Blokkeel, G., Mura, A. et al., 2007, Atomization and Sprays 17(4).

[15] Demoulin, F.-X., Reveillon, J., Duret, B., Bouali, Z.et al., 2013, Atomization and Sprays 23 (11).

[16] Engine Combustion Network, https://ecn.sandia.gov/ (cit. 2016-12-20).

[17] Kastengren, A., Ilavsky, J., Viera, J., Payri, R. et al., 2017, Int. J. Multiph. Flow (In Press).

[18] Desantes, J., García-Oliver, J., Pastor, J., Pandal, A. et al., 2016, Int. J. Multiph. Flow 80, pp. 89-99.

[19] Salvador, F.J., Gimeno, J, Pastor, J.M., Martí-Aldaraví, P., 2014, Int. J. Multiph. Flow 65, pp. 108-116.

[20] Garcia-Oliver, J.M., Pastor, J.M., Pandal, A., Trask, N. et al.., 2013, Atomization and Sprays 23 (1)

[21] Beheshti, N., Burluka, A., Fairweather, M., 2007, Theoretical and Computational Fluid Dynamics 21 pp. 381 397.

[22] Duret B., Reveillon, J., Menard, T., Demoulin, F.-X, 2013, Int. J. Multiph. Flow 55, pp. 130-137.

[23] Pandal, A., Pastor, J.M, Payri, R., Kastengren et al., 2017, SAE Int. J. Fuels Lubr. 10(2)

[24] Kastengren A.L., Tilocco F.Z., Duke D., Powell C.F. et al., Sep 2-6, 2012, $12^{\text {th }}$ Triennial International Conference on Liquid Atomization and Spray Systems.

[25] Ilavsky, J., Jemian, P. R., Allen, A. J., Zhang, F. et al., J. Appl. Cryst. 42(3), pp. 469-479.

[26] Ilavsky, J. and Jemian, P. R., J. Appl. Cryst. 42(2):347-353.

[27] Naber, J. and Siebers, D., 1996, SAE Technical Paper 960034.

[28] Weller, H. G., Tabor, G., Jasak, H., Fureby, C., 1998, Computers in Physics, 12(6),pp. 620-631.

[29] Pandal, A., Pastor, J.M, García-Oliver, J.M., Schmidt, D.P., 2016, Int. J. Multiph. Flow 83, pp. 162-171.

[30] Kraichan R., 1970, Phys. Fluids 13, pp. 22-31.

[31] Klein M., Sadiki A., Janicka J., 2003, J. Comput. Phys.186, pp. 652-665.

[32] Robert A., Martinez, L., Tillou, J., Richard, S., 2014, Oil \&Gas Science and Technology 69, pp.141-1551.

[33] Pope, S., 1978, AIAA 16, pp. 279-281.

[34] Nicoud, F, Baya-Toda, H., Cabrit, O., Bose, S. et al., 2011, Physic of Fluids, 23, 085106.

[35] Chesnel, J., Reveillon, J., Menard, T., Demoulin, F.-X, 2011, Atomization and Sprays, 21(9), pp. 711-736.

[36] Pope,S.B., 2004, New J. Phys. 6, 35. 


\title{
The liquid penetration of diesel substitutes
}

\author{
Sebastian Riess ${ }^{1,2}$, Lukas Weiss ${ }^{* 1,2}$, Javad Rezaei ${ }^{1}$, Andreas Peter ${ }^{1}$, Michael Wensing ${ }^{1,2}$ \\ ${ }^{1}$ Institute of Engineering Thermodynamics (LTT), FAU Erlangen-Nürnberg, Germany \\ ${ }^{2}$ Erlangen Graduate School in Advanced Optical Technologies (SAOT), \\ FAU Erlangen-Nürnberg, Germany \\ *Corresponding author: lukas.weiss@fau.de
}

\begin{abstract}
Diesel fuel consist of several hundreds of substances on organic basis. Experimental and numerical investigations of this multicomponent fuel are hard to interpret in detail, since the behavior of the multicomponent mixture is complex. Physical and chemical data of this system is not available under engine relevant conditions. Instead, fundamental research substitutes diesel with pure substances, where a big database exists.

Prior work already showed, that overall spray propagation (including vapor phase) is nearly independent on the injected fuel. This is due to the high air entrainment at present diesel engine conditions (very high injection pressure and dense ambient atmosphere). The high air entrainment shortly behind the nozzle exit (within the first $5 \mathrm{~mm}$ penetration) creates a situation where properties of the ambient gas dominate the spray propagation resulting in similar mass and momentum distributions even for different fuels, if the injection conditions are kept constant. On the other hand, the liquid length is clearly different for different fuels, so that location and time of the phase change differ with consequences on the time available for mixture formation in the gas phase. The paper describes the liquid length as a function of the enthalpy necessary for the phase transition (given by the fuel and fuel temperature at injection) and the injection conditions (ambient gas properties, injector design and injection pressure). We compare two different models describing the enthalpy balance. Siebers et al. presented "Model I", where mass transfer dominates the enthalpy transfer and evaporation takes place. In our own "Model II" evaporation is suppressed, resulting in a heat transfer driven enthalpy transfer without mass transport. The calculations are validated with experimental data.

The liquid length is optically accessible by Mie-Scattering imaging techniques, the complete spray evolution by Schlieren technique. The experimental study was carried out in the high-pressure combustion vessel "OptiVeP" at FAU. The data shown in this paper derived from measurements with dodecane injected at 1200 bar into $613 \mathrm{~K}$ ambient. The ambient pressure varies from 1-10 MPa. A Continental research injector with a $115 \mu \mathrm{m}$ hole and L/D of 6.5 was used. Nitrogen atmosphere suppressed ignition.

Increasing the ambient pressure leads to a change in the mechanism in phase transition. It switches from a mass transfer dominated regime to a heat transfer dominated regime at high ambient pressures.
\end{abstract}

\section{Keywords}

Diesel, liquid-penetration, enthalpy

\section{Introduction}

Diesel injection is in focus of research for many years now, but still basic parameters like the liquid length are not physically described yet. One problem is that no fluid data of multicomponent systems under relevant ambient conditions are available. Thus, research often substitutes diesel with pure substances like dodecane.

Dennis Siebers delivered the most comprehensive description of diesel fuel injection in the late 90 s. He summarized his findings in his scaling law [2], which will be reviewed later on. During the injection a momentum transfer from fuel to entrained air occurs. The spray velocity decreases with increasing mass of entrained air. The amount of entrained air is very high. Yue et al. showed with $\mathrm{x}$-ray absorption, that the fuel volumetric fraction on the spray axis decreases to 0.8 at $1 \mathrm{~mm}$ distance to the nozzle and to 0.2 at $6 \mathrm{~mm}$ to the nozzle [3]. Leick et al. confirmed the results with a similar technique under modern diesel relevant ambient conditions $\left(\rho_{a}=21.7 \mathrm{~kg} / \mathrm{m}^{3} ; p_{i n j}=135 \mathrm{MPa}\right)$ [4]. Riess et al. extended this view and showed that the extreme high air entrainment is fuel independent. They investigated different Diesel-Ethanol blends with Raman spectroscopy, where local substance amount ratios can be determined. They found similar high air entrainment ratios and additionally showed that mixture formation is independent from the fuel, which results in similar penetration curves for all blends and pure substances in Schlieren measurements. The maximum liquid penetration from the orifice, though is strongly determined by the fuel [5]. This maximum liquid length is called "liquid length" further on in this paper, see Figure 2. 


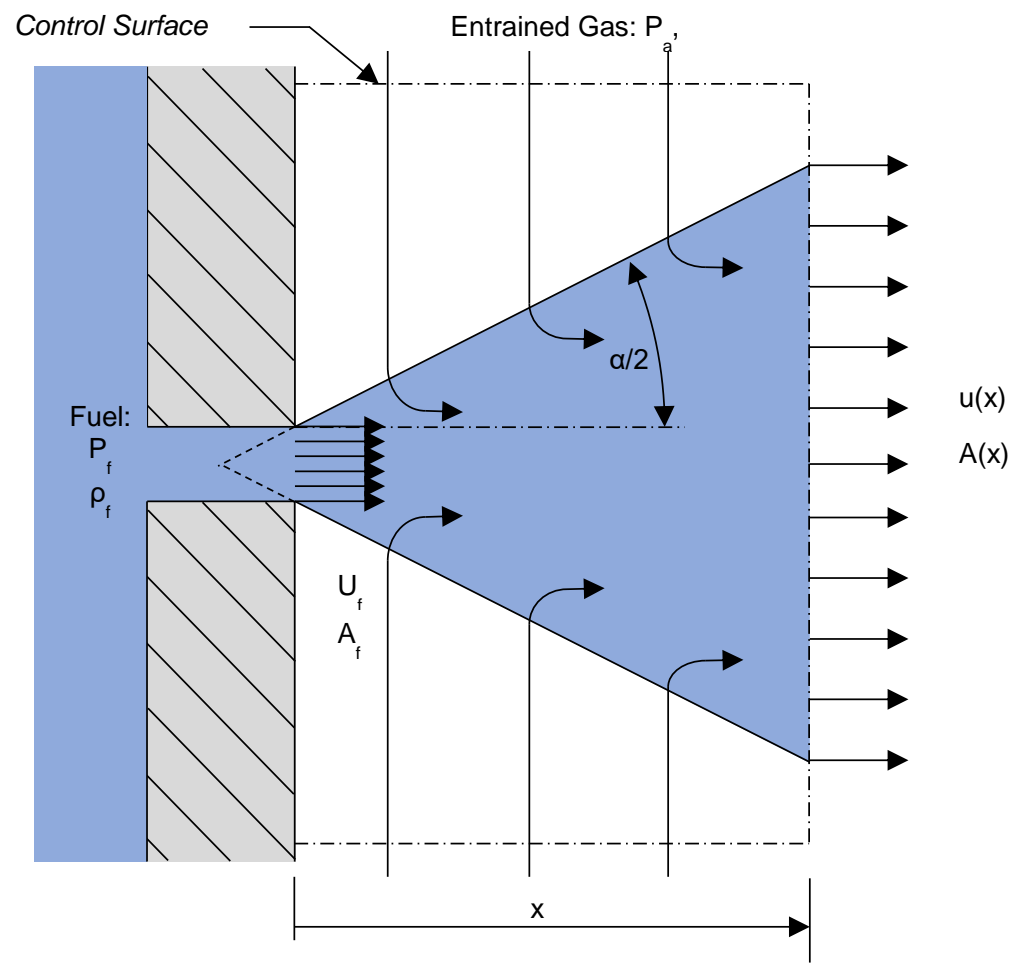

Figure 1. Schematic of the spray model according to Siebers [1]

\section{Volume and momentum balance}

Naber and Siebers formulated a momentum model for a full diesel spray under non-vaporizing conditions, that explains the fuel independency from Riess et al. [1, 5]. His schematic view is drawn in Figure 1. The momentum source is the fuel injection with mass rate $\dot{m}_{f}$ and exit velocity $u_{0}$. The momentum is transferred to the entrained air $\dot{m}_{a}$. The spray velocity $u(x)$ is decreasing with distance to the orifice and thus with increasing entrained air mass.

$\dot{m}_{f} \cdot u_{0}=\left(\dot{m}_{f}+\dot{m}_{a}\right) \cdot u(x)=$ const.

Since the fuel volume in the spray is negligible small, the spray cross section that is covered by fuel $A_{f}$ is also negligible [5]. Thus the complete spray cross section can be assigned to the entrained air and the resulting air mass flow can be estimated from the momentum balance (1) and the continuum constraints (2) and (3).

$$
\begin{aligned}
& \dot{m}_{f}=\rho_{f 0} \cdot A_{0} \cdot u_{0} \\
& \dot{m}_{a}=\rho_{a} \cdot A(x) \cdot u(x)
\end{aligned}
$$

In this model several assumptions were made:

1) $u(x)$ is constant in every cross section (rectangular velocity profile in the spray)

2) air only moves in radial direction outside the spray [6]

3) orifice exit velocity $u_{0}$, spray spreading angle $\alpha$ and nozzle discharge coefficient $C_{a}$ are constant [5]

4) no-slip condition between air and fuel

5) constant ambient density $\rho_{a}$

Siebers et al. defined a dimensionless orifice distance $\tilde{x}$ for better comparability between different ambient conditions (4). In addition, they defined the mass flow ratio B as function of this dimensionless distance (5).

$$
\begin{aligned}
& \tilde{x}=\sqrt{\frac{\rho_{a}}{\rho_{f}}} \cdot \frac{x}{\sqrt{c_{a}} \cdot d} \cdot a \cdot \tan \left(\frac{\alpha}{2}\right) \\
& \frac{\dot{m}_{f}}{\dot{m}_{a}}:=B=\frac{2}{\sqrt{1+16 \tilde{x}}-1}
\end{aligned}
$$


With equation (4) the non-vaporizing spray penetration is described and equation (5) gives the according mass ratios. The equations also show, that the mass ratio in a certain distance $\mathrm{x}$ is independent from the injection pressure. Consequently an increase in injection pressure does not increase the air entrainment. Schlieren measurements from Riess et al. confirm the proposed model even for the full spray with vaporizing conditions [5]. The gaseous spray penetrates steadily independent from the fuels physical state, which again shows the dominating role of the entrained air.

\section{Calculation of the liquid length}

The liquid length $L$ of a diesel spray is the maximum penetration of liquid fuel. Under vaporizing conditions, the liquid length is constant as long as the fuel mass flow is constant, see Figure 2. At this distance, enough ambient gas is mixed in and thus enthalpy is transported into the spray to vaporize all fuel. According to equation (5) this distance is assigned to a certain mass ratio. Two simplified models are considered:

Calculation model l: Mass transport is much faster than heat transfer $\rightarrow$ Fuel droplets evaporate and mass transport is only limited by the saturation of the ambient. The mixture temperature $T_{m i x}$ is a consequence of the evaporation cooling and heating due to the entrained hot ambient gas. (Siebers scaling law)

Calculation model II: Heat transfer is much faster than mass transport $\rightarrow$ Fuel droplets do not evaporate at all until they have reached their phase change temperature (boiling or critical). Enthalpy is provided by entrained hot air.

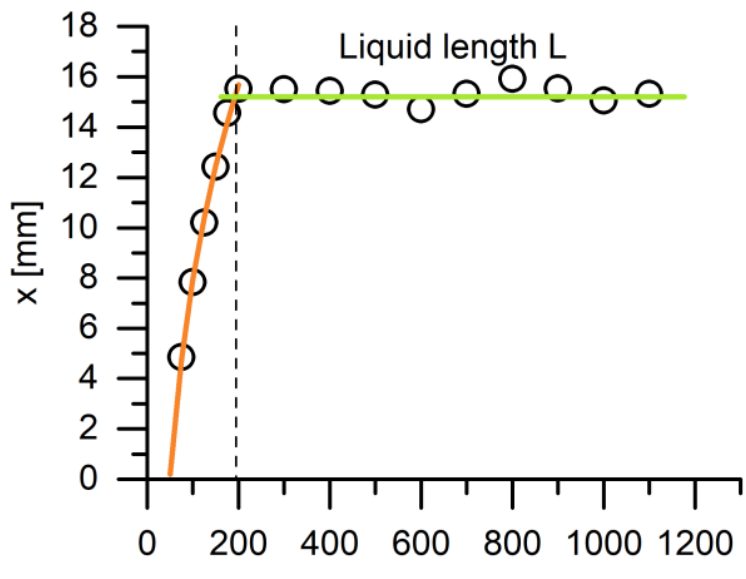

$\mathrm{t}[\mu \mathrm{s}]$

Figure 2. Definition of the liquid length

\section{Calculation model I}

The idea of Siebers' scaling law is to search for the mass ratio at which the enthalpy balance and the resulting partial pressure ratios are satisfied. The general enthalpy balance for an evaporating spray is:

$$
\dot{m}_{a}\left(h_{a}\left(T_{a}, p_{a}\right)-h_{a}\left(T_{a 2}, p_{a 2}\right)\right)=\dot{m}_{f}\left(h_{f}\left(T_{f 2}, p_{f 2}\right)-h_{f}\left(T_{f}, p_{a}\right)\right)
$$

with the thermodynamic equilibrium at:

$$
T_{a 2}=T_{f 2}=T_{m i x}
$$

The equilibrium pressures are equal to the partial pressures $\tilde{p}_{i}$ satisfying Dalton's law (9):

$$
\begin{aligned}
& p_{a}=p_{a 2}+p_{f 2}=\tilde{p}_{a}+\tilde{p}_{f} \\
& \tilde{p}_{i}=\dot{m}_{i} \cdot \frac{z_{i}}{M_{i}} \cdot \frac{R_{m} \cdot T_{m i x}}{\dot{V}}
\end{aligned}
$$


The scaling law assumes a much faster mass transport than heat transfer, which means, that the environment is always saturated of fuel vapor. Thus, the partial pressure of the fuel is equal to the saturation pressure $p_{s}(T)$ at a certain condition:

$$
\begin{aligned}
& \tilde{p}_{f}=p_{s}\left(T_{m i x}\right) \\
& \tilde{p}_{a}=p_{a}-p_{s}\left(T_{\text {mix }}\right)
\end{aligned}
$$

To allow a complete phase change, the enthalpy and the partial pressure balances have to result in the same value for the mass ratio at a certain mixture temperature $T_{\text {mix }}$ and have to be solved iteratively:

$$
\frac{1}{B_{L}}=\frac{\dot{m}_{a}}{\dot{m}_{f}}=\frac{h_{f}\left(T_{m i x}, p_{s}\left(T_{m i x}\right)\right)-h_{f}\left(T_{f}, p_{a}\right)}{h_{a}\left(T_{a}, p_{a}\right)-h_{a}\left(T_{m i x},\left(p_{a}-p_{s}\left(T_{m i x}\right)\right)\right)}=\frac{\left(p_{a}-p_{s}\left(T_{m i x}\right)\right) \cdot M_{a} \cdot z_{f}}{p_{s}\left(T_{m i x}\right) \cdot M_{f} \cdot z_{a}}
$$

With the known mass ratio $\mathrm{B}_{\mathrm{L}}$, equation (5) can be solved for the corresponding dimensionless distance $\tilde{x}\left(B_{L}\right)$, which is defined as the dimensionless liquid length $\tilde{L}$.

\section{Calculation model II}

The second model assumes heat transfer to be much faster than mass transport. Thus no mass transfer to in the vapor phase is taken into account during heating up of the fuel. Enthalpy is transferred from the ambient gas to the liquid fuel until its phase change temperature is reached. The same general enthalpy balance like before is valid, see equation (6), but the equilibrium conditions have changed:

$$
T_{a 2}=T_{f 2}=T_{\text {mix }}=T_{\text {Phase Change }}
$$

The phase change temperature can be the boiling temperature or the critical temperature. Table 1 shows some phase change conditions for dodecane.

Table 1. Phase change conditions for dodecane [7]

\begin{tabular}{|l|c|c|c|}
\hline Pphase change & $1 \mathrm{MPa}$ & $1,5 \mathrm{MPa}$ & $p_{\text {crit }}=1,82 \mathrm{MPa}$ \\
\hline $\mathrm{T}_{\text {phase change }}$ & $614,5 \mathrm{~K}$ & $643 \mathrm{~K}$ & $658,1 \mathrm{~K}$ \\
\hline
\end{tabular}

The necessary mass ration can directly be calculated from equation (6). The according liquid length $\tilde{L}$ is again the solution of equatzion (5) for $\tilde{x}\left(B=B_{L}\right)$.

$$
\frac{1}{B_{L}}=\frac{\dot{m}_{a}}{\dot{m}_{f}}=\frac{h_{f}\left(T_{\text {Phase Change }}, p_{a}\right)-h_{f}\left(T_{f}, p_{a}\right)}{h_{a}\left(T_{a}, p_{a}\right)-h_{a}\left(T_{\text {Phase Change }}, p_{a}\right)}
$$

\section{Experimental methods}

The liquid length is optically accessible by Mie-Scattering imaging techniques, the complete spray evolution by Schlieren technique. The experimental study was carried out in the high-pressure combustion vessel "OptiVeP" at FAU. For more details see Riess et al. [5]. The operation point was constant at $873 \mathrm{~K}$ ambient temperature, $120 \mathrm{MPa}$ injection pressure and $363 \mathrm{~K}$ fuel temperature in nitrogen atmosphere, whereas the ambient pressure was varied from $1 \mathrm{MPa}$ to $9 \mathrm{MPa}$. A Continental 3 hole research injector was used. The spray spreading angles are derived from Schlieren images. The acquired Mie and Schlieren images where processed with a self-developed MATLABß code SprayAnalysis.

\section{Results and discussion}

This section starts with basic predictions by the two models, further the predicted liquid length are compared to the dodecane measurements

\section{Model Prediction - Enthalpy Balance}

The fuel enthalpy developing for different ambient pressures for both calculation models is drawn in Figure 3. With dominating mass transport (calculation model I) drawn in Figure 3a the initial enthalpy of the fuel $h_{f 0}$ is almost constant with rising pressure, whereas the enthalpy needed for evaporation $\Delta h_{f}$ is increasing steadily. A dominating heat transfer (calculation model II) leads to a complete different behavior, see Figure $3 \mathrm{~b}$. Here the enthalpy for the phase change $\Delta h_{\text {liq }}$ increases to a maximum, the decrease flats out forming a peak before a plateau. This 
developing is a direct consequence from the shape of the phase boundary line in the H-S diagram, see Figure 4. Since the critical point is placed in the turning point of the phase boundary line, the phase change enthalpy has a maximum below the critical pressure. Simultaneously, the evaporation enthalpy $\Delta h_{V}$ drops to zero as the critical point is reached. Thus the peak is even emphasized in the overall enthalpy $\Delta h_{\text {ges }}$. A suppressed mass transport demands a higher phase change enthalpy, which is mainly due to the much higher resulting mixing temperatures. In terms of the model assumptions these two models define upper and lower limits for the needed phase change enthalpy.
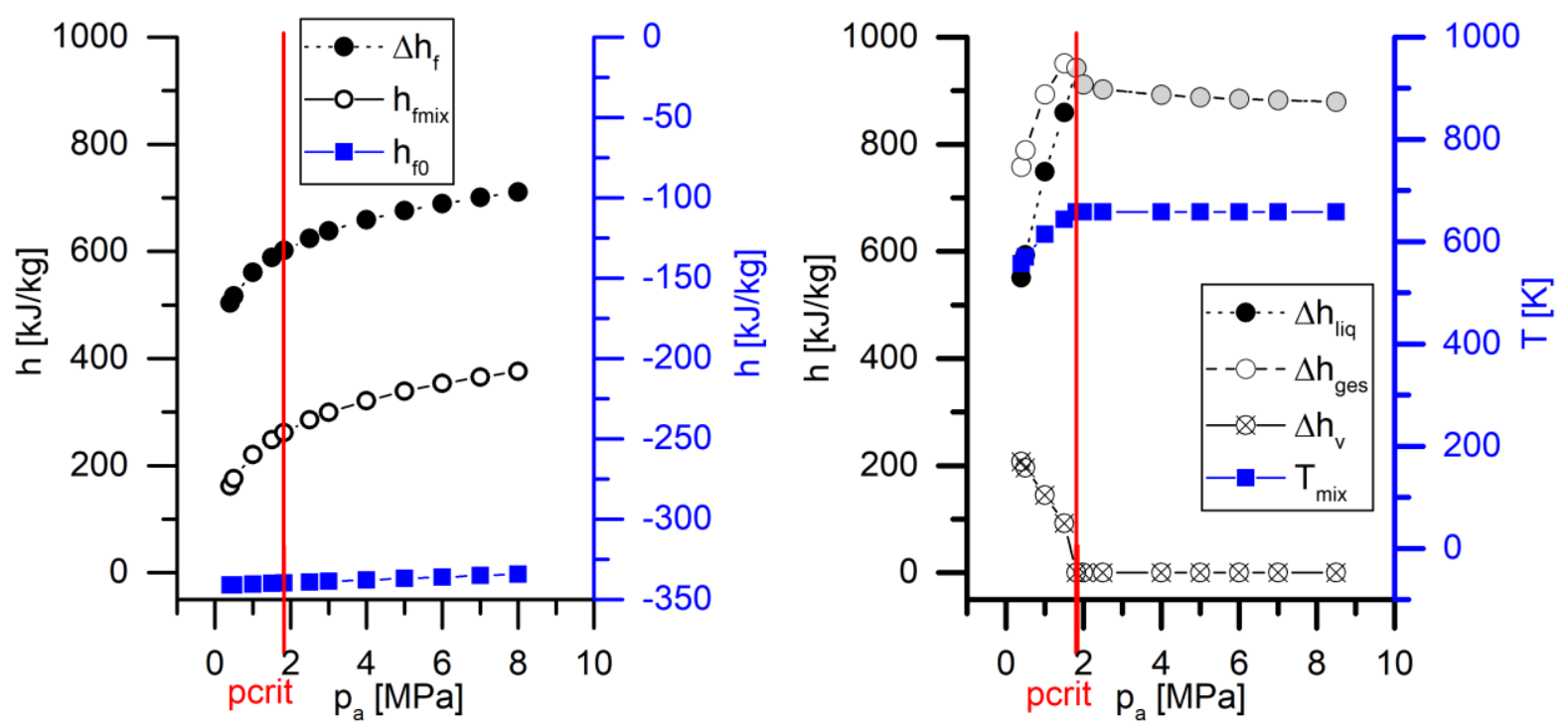

Figure 3. Enthalpy developing over pressure for calculation model I (a) and calculation model II (b)

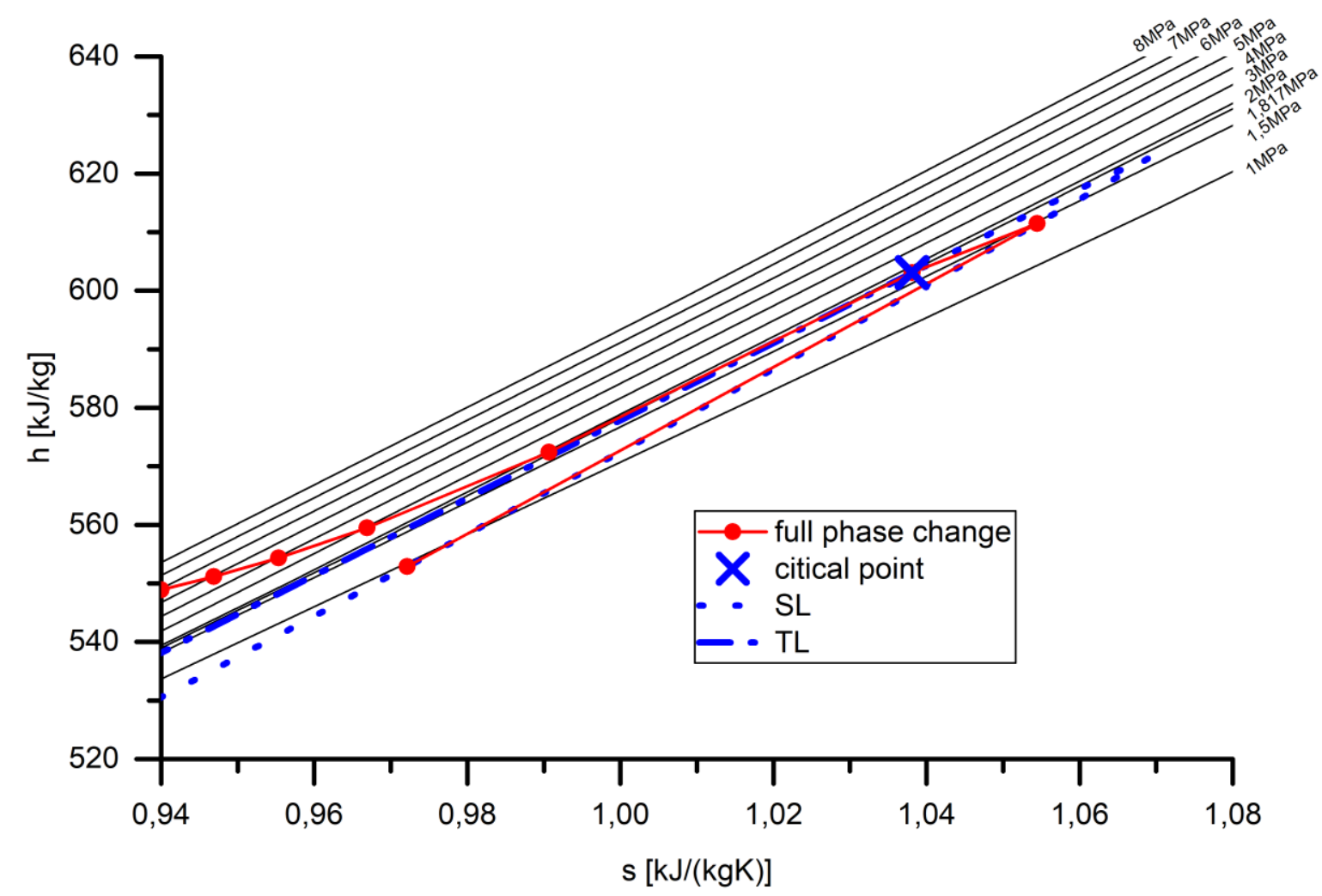

Figure 4. H-S-diagram for dodecane at $873 \mathrm{~K}$ with data from NIST [7] 
The peak in the enthalpy difference from calculation model II consequently results in a peak liquid length, see Figure 5. In contrast, model I predicts a steady decrease of the liquid length over pressure. It can also be seen, that model I underestimates and model II overestimates the liquid length, according to the upper and lower limits, depending on the mechanism of phase change. Siebers already noticed that and multiplied the calculated values with a suitable scaling factor. The best fits to the measured data points are shown in Figure 5. At a pressure of $3 \mathrm{MPa}$ a transition from model I to model II takes places. It even gets clearer in Figure 6, where the dimensionless liquid length is plotted. The peak in the dimensionless liquid length of model II cannot be reproduced experimentally. Instead, model I fits very well here. Above $3 \mathrm{MPa}$ ambient pressure model II predicts a constant dimensionless liquid length, which is confirmed by the experiment. Recalling the assumptions of the calculation models, at low ambient pressures mass transport dominates the liquid length. With increasing ambient pressure, this changes to a heat transfer dominated mechanism. The transition point seems to be fuel dependent, which calculations with different substances suggest. With these two models an experiment can be designed to confirm this conclusion. Measurements with other fuels and additional isothermal rows have to be carried out to clarify the transition between both mechanisms.

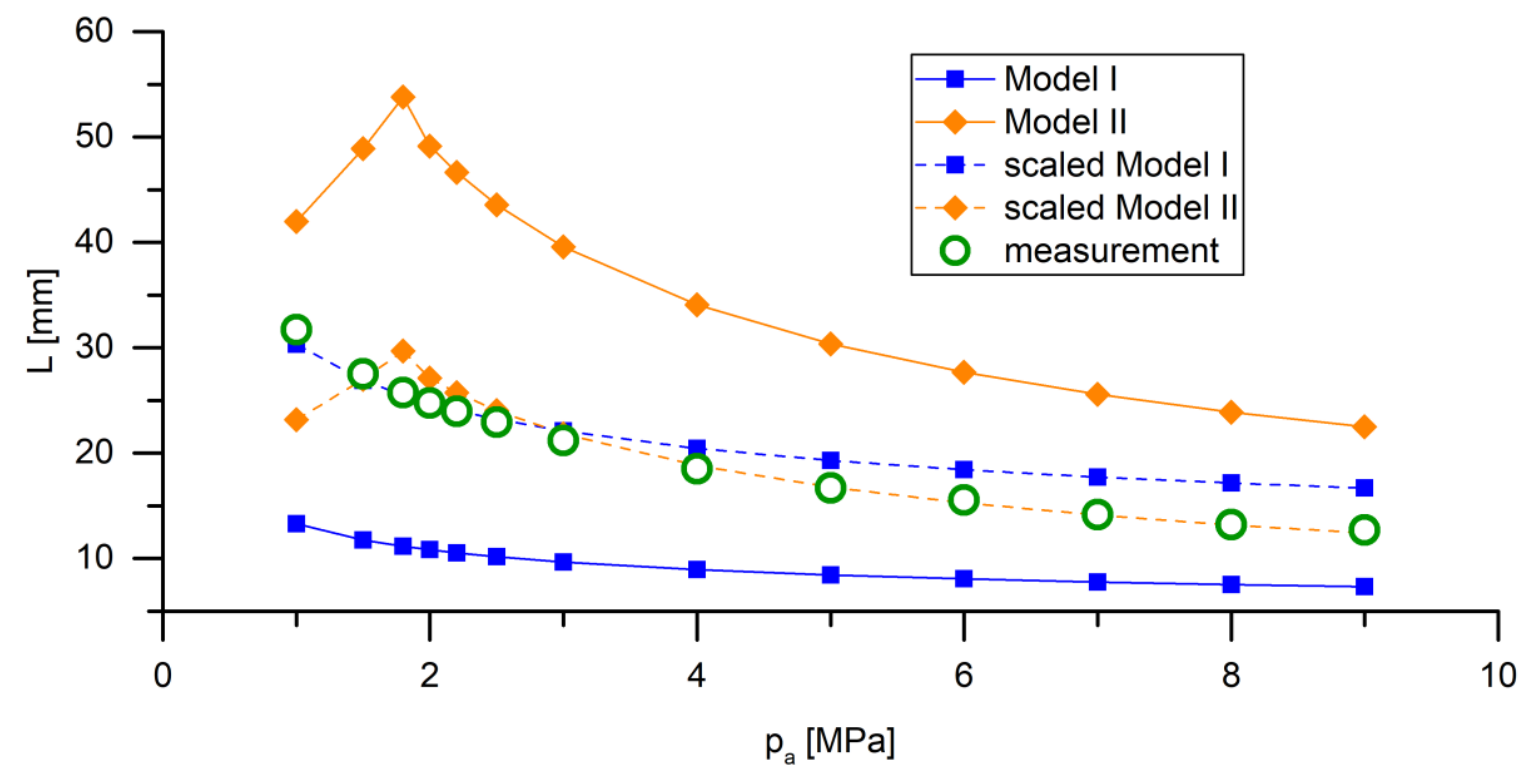

Figure 5. Comparison of the calculated liquid length according to model I and II with measured data of dodecane.

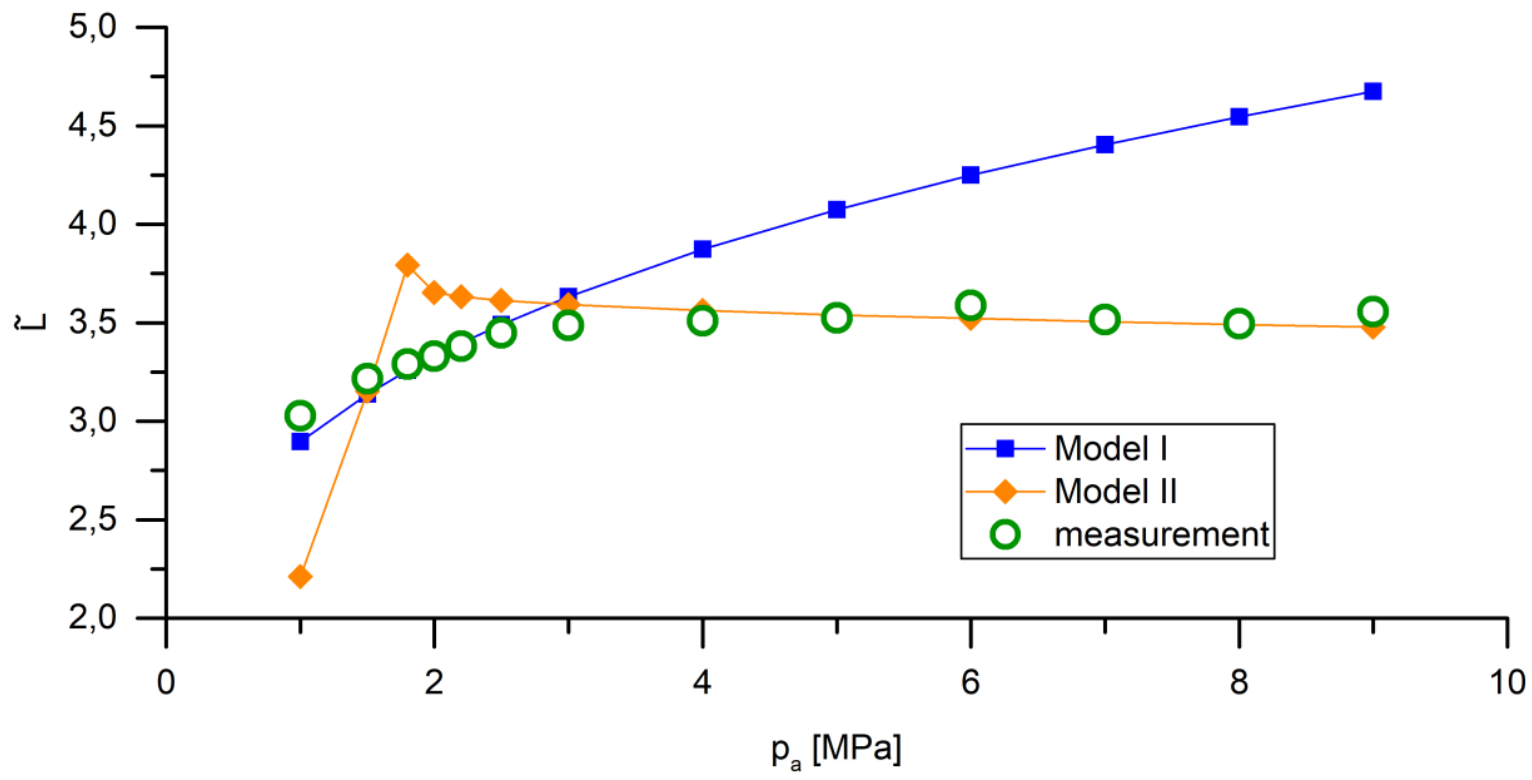

Figure 6. Fitted dimensionless liquid length to measured data of dodecane. 
One example of an additional isothermal row is presented in Figure 7. Dodecane was injected at $613 \mathrm{~K}$ ambient temperature. Model II fails to calculate any liquid length, because the temperature is below any phase change temperature at these pressures. Model I, which is expected to be predictive at low conditions, still promised a constant liquid length. This expectation could be confirmed in the experiment. A liquid length also occurs significantly below the boiling temperature, compare Table 1.

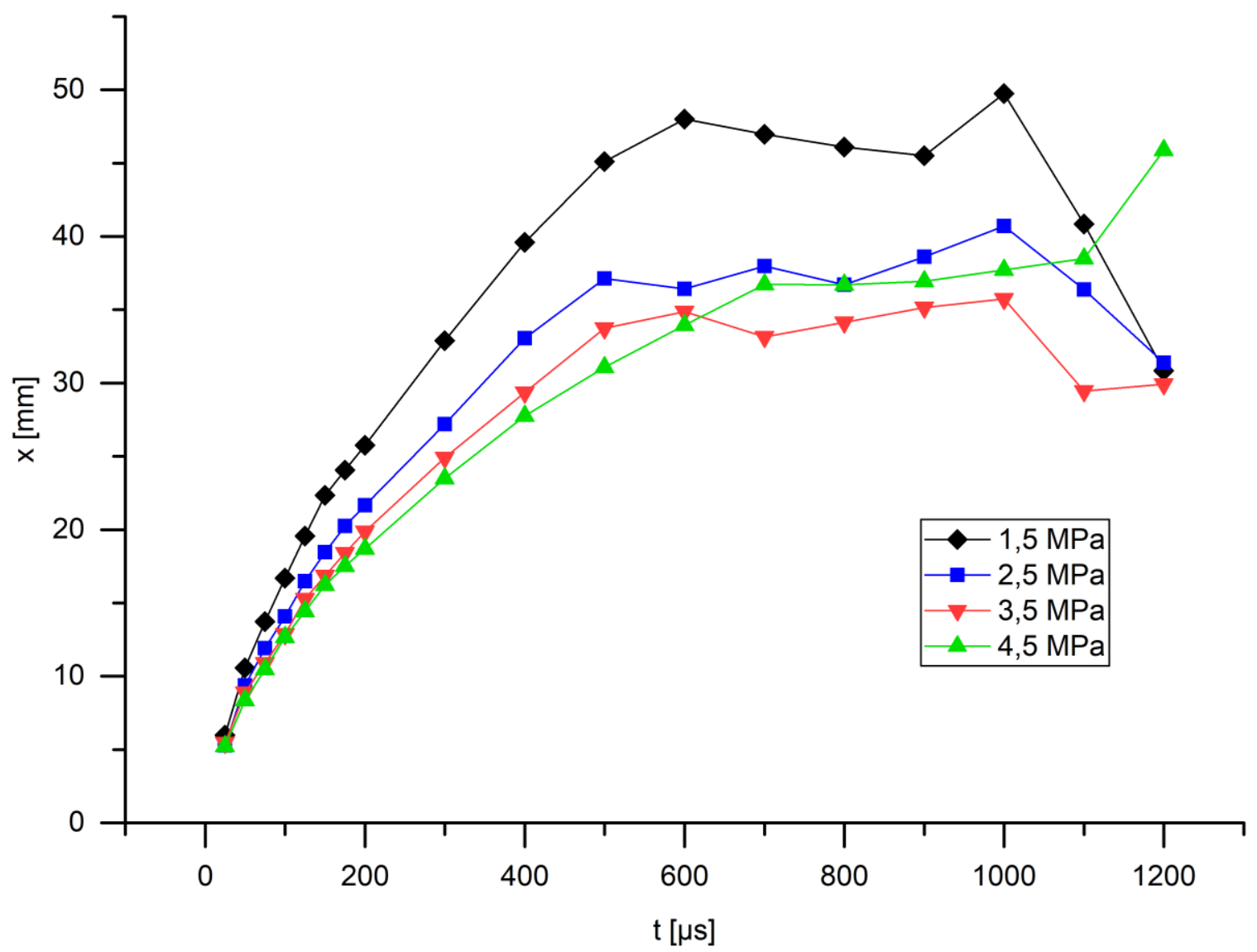

Figure 7. Liquid length of dodecane - pressure variation at $613 \mathrm{~K}$.

\section{Conclusions and outlook}

The full spray penetration under non-vaporizing and vaporizing conditions is described very well with Siebers' momentum balance and is confirmed by several authors. In contrast, the resulting liquid length is still not fully described, although a high effort is made in this research field. Siebers' scaling law, here called calculation model $I$, is a very good approach for low ambient pressures. The formation of a constant liquid length below boiling temperatures can be predicted qualitatively. The introduced calculation model II, in which the heat transfer is much faster than the mass transport, fits qualitatively very well at high ambient pressures. This concludes, that a change in the phase transition mechanism takes place from a mass transport dominated to a heat transfer dominated regime with increasing ambient pressure. The transition point seems to be fuel dependent. Similar experiments where already done with decane, hepatane, hexane, ethanol and rapeseedmethylester. Unfortunately only operation points at high ambient conditions with respect to the fluids phase transition temperatures and pressures where performed, thus a validation of the model results is not possible at that time.

\section{Nomenclature}

$\theta \quad$ spray angle $\left[{ }^{\circ}\right]$

$\rho_{a} \quad$ gas density $\left[\mathrm{kg} / \mathrm{m}^{3}\right]$

$\rho_{f 0} \quad$ fuel density (initial) $\left.\left[\mathrm{kg} / \mathrm{m}^{3}\right]\right]$

$\mathrm{P}_{\text {inj }} \quad$ injection pressure [MPa]

$\dot{m}_{f} \quad$ fuel mass flow [g/s]

$\dot{m}_{a} \quad$ air mass flow $[\mathrm{g} / \mathrm{s}]$ 


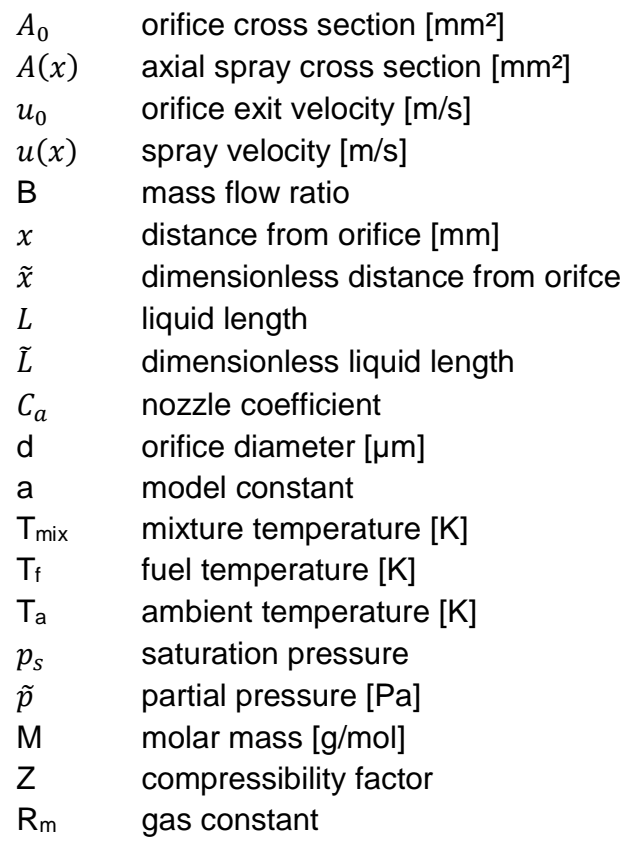

\section{References}

[1] Naber, J., and Siebers, D. L., 1996, "Effects of gas density and vaporization on penetration and dispersion of diesel sprays," No. 0148-7191, SAE technical paper.

[2] Siebers, D. L., 1999, "Scaling liquid-phase fuel penetration in diesel sprays based on mixing-limited vaporization," No. 0148-7191, SAE technical paper.

[3] Yue, Y., Powell, C. F., Poola, R., Wang, J., and Schaller, J. K., 2001, "Quantitative measurements of diesel fuel spray characteristics in the near-nozzle region using X-ray absorption," Atomization and sprays, 11(4).

[4] Leick, P., Riedel, T., Bittlinger, G., Powell, C., Kastengren, A., and Wang, J., "X-ray measurements of the mass distribution in the dense primary break-up region of the spray from a standard multi-hole common-rail diesel injection system," Proc. 21st Annual ILASS-Europe Conference.

[5] Riess, S., Klima, T., Wensing, M., and Braeuer, A., 2016, "Influence of Bio-Diesel and Ethanol on the Diesel Engine Process Chain Investigated by Optical Measurement Techniques," ILASS - Europe Brighton, UK.

[6] Wu, Z., Zhu, Z., and Huang, Z., 2006, "An experimental study on the spray structure of oxygenated fuel using laser-based visualization and particle image velocimetry," Fuel, 85(10-11), pp. 1458-1464.

[7] Linstrom, P. J., and Mallard, W. G., 2001, "The NIST Chemistry WebBook: NIST Standard Reference Database Number 69," Journal of Chemical \& Engineering Data, National Institute of Standards and Technology, Gaithersburg MD, 20899, http://webbook.nist.gov. 


\title{
Numerical and Experimental Investigations on Rotary Bell Atomizers with predominant Air Flow Rates
}

\author{
Nico Guettler ${ }^{1 *}$, Stephan Paustian ${ }^{1}$, Qiaoyan Ye ${ }^{1}$, Oliver Tiedje ${ }^{1}$ \\ ${ }^{1}$ Fraunhofer Institute for Manufacturing Engineering and Automation, Stuttgart, Germany \\ *Corresponding author: Nico.Guettler@ipa.fraunhofer.de
}

\begin{abstract}
For high-quality spray painting of small parts, a rotary bell atomizer with a narrow spray pattern is used in the automotive industry. The required unusual high shaping air flow rate yields in an atomization process predominated by a pneumatic atomization and rather than by a rotary atomization, called hybrid bell atomizer in this article. Numerical and experimental investigation on typical high-speed rotary bell atomizers, with rotation type of high rotational speed $40000-60000 \mathrm{rpm}$ of the bell, were already successful demonstrated. For these high-speed rotary bell atomizer for painting bigger areas the ratio between tangential velocity at the bell edge and axial shaping air velocity at the bell edge is in the range of 0.8 and 4 , depending on the process parameter. At the hybrid bell atomizer (10000-20000 rpm), this ratio is between 0.2 and 0.4 .

The first step of the present study includes the theoretical characterization of spray cone velocity profile using two definitions of swirl-number compared to experimental measurements of particle velocities using Laser-DopplerVelocimetry (LDV). This study was carried out on varying shaping air settings and rotational speeds. The results show that the the swirl of the main airflow field is dominated by the secondary airflow, which is induced coaxial in an angle of $45^{\circ}$. The influence of the circumferential speed of the bell cup on the swirl of the main airflow field plays a subordinate role, so the resulting spray pattern is only weakly influenced by the number of revolutions of the bell-cup.

In the second step, the hybrid bell atomizer was examined numerically. In order to implement the hybrid atomization concept in the simulation correctly, methods for creating droplet initial conditions in the trajectory calculation was developed. The simulation results were verified through comparisons of calculated and measured velocity profiles inside the spray cone and calculated and measured film thickness distributions on the work piece. In the present investigations of the atomizer, it has been demonstrated numerically and experimentally that the airflow field of this hybrid bell atomizer is strongly impacted by the secondary shaping air and both the circumferential speed of the bell cup and the direct electrostatic charge on the bell have only a minor effect on the generated spray pattern and the resulting transfer efficiency.
\end{abstract}

\section{Keywords}

Rotary bell atomizer, Spray painting, Atomization characteristics, Numerical Coating, Swirl-number

\section{Introduction}

High-speed rotary bell-cup atomizers are widely used in automotive painting industry and increasingly replacing the pneumatic atomizers in high-quality coating processes. The application range of rotary bell-cup atomizers includes large-area coating processes, such as hoods and car roofs, as well as areas for detailed coatings, such as door extensions.

Previous studies on atomization technology in the automotive industry have been performed on pneumatic atomizers and high-speed rotary bell atomizers. In particular, numerical studies on electrostatic effects on high-rotary bell-cup atomization have been carried out intensively in recent years [5, 6, 8, 9].

The high-speed rotary bell atomizer investigated in this study is predominantly used for detailed coating processes. In order to produce a narrow spray cone, a high axial velocity of the shaping air, which is defined by an airflow coaxial with the bell-cup, is required. This unusually strong axial shaping air velocity and a small diameter of the bell leads to an rotary atomization process with pneumatic behavior. For this reason, this class of high-speed rotary bell atomizers is referred as hybrid bell atomizers.

In the present study, the effects of the strong axial shaping air are investigated experimentally and numerically and a new method for creating the droplet initial conditions adapted to the strong shaping air is presented and validated. 


\section{Material and methods Experimental setup}

In order to investigate the characteristics of a hybrid atomizer with predominat airflow rates, a detailed study was performed at the Fraunhofer Institute for Manufacturing Engineering and Automation IPA in Stuttgart, Germany. The hybrid bell atomizer investigated in this study is the Dürr ECOBELL 2 HD. The experiments were carried out in a environmentally controlled paint booth with an fixed ambient temperature of $23^{\circ} \mathrm{C}$, a relative humidity of $60 \%$ and a vertical booth airflow of $0.3 \mathrm{~m} \mathrm{~s}^{-1}$.

The Dürr ECOBELL 2 HD hybrid bell atomizer, shown in figure 1, consists of 60 shaping air tubes with a diameter of $d_{S A}=0.6 \mathrm{~mm}$. Thirty shaping air tubes are arranged perpendicular to the bell edge whereas 30 shaping air tubes are arranged with an given angle of $45^{\circ}$. Both the perpendicular, which is referred as shaping air 1 and the angular shaping air tubes (shaping air 2) are arranged at the back of the bell cup in an annular fashion. The bell cup has a diameter of $38 \mathrm{~mm}$ and a serrated section at the edge of the bell cup.

In the present study, two basic shaping air settings, which have the same amount of $600 \mathrm{I}_{\mathrm{s}} / \mathrm{min}$ as defined by ISO2533, were examined. A basecoat with a non-volatile content of $44.4 \mathrm{~m} \%$, a wet density of $\rho_{\text {wet }}=1136.1 \mathrm{~kg} \mathrm{~m}^{-3}$ and a dry density of $\rho_{\text {dry }}=1820 \mathrm{~kg} \mathrm{~m}^{-3}$ was used as coating material.

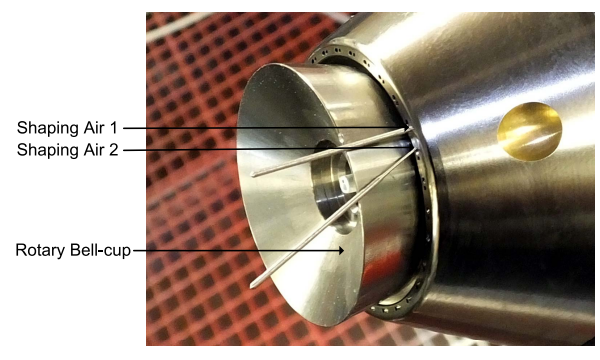

Figure 1. Rotary bell atomizer ECOBELL $2 \mathrm{HD}$ (Dürr AG, Bietigheim-Bissingen), needles in a drilling of shaping air 1 and 2 are used to show the direction of the air outlets

Table 1. Design of Experiment

\begin{tabular}{c|ccccc}
\hline & $\begin{array}{c}\text { shaping air 1 } \\
\mathrm{I}_{\mathrm{s}} / \mathrm{min}\end{array}$ & $\begin{array}{c}\text { shaping air } 2 \\
\mathrm{I}_{\mathrm{s}} / \mathrm{min}\end{array}$ & $\begin{array}{c}\mathrm{rpm} \\
\mathrm{min}^{-1}\end{array}$ & $\begin{array}{c}\text { paint massflow } \\
\mathrm{m} \mathrm{min}^{-1}\end{array}$ & $\begin{array}{c}\text { high voltage } \\
\mathrm{kV}\end{array}$ \\
\hline E01 & 200 & 400 & 10000 & 300 & $0 / 30$ \\
E02 & 200 & 400 & 15000 & 300 & $0 / 30$ \\
E03 & 200 & 400 & 20000 & 300 & $0 / 30$ \\
E04 & 400 & 200 & 10000 & 300 & $0 / 30$ \\
E05 & 400 & 200 & 15000 & 300 & $0 / 30$ \\
E06 & 400 & 200 & 20000 & 300 & $0 / 30$ \\
\hline
\end{tabular}

The characterization of the hybrid bell atomizer and typical quantities for coating applications are determined by means of the particle size distribution, the droplet velocity using Laser-Doppler-Velocitmetry, the coated film thickness profile and the transfer efficiency. The particle size distribution is determined by use of a SPRAYTEC RTS 5001 from Malvern Instruments. The measurement technique of this device relies on Mie-scattering and Fraunhoferdiffraction. The particle size distributions were measured in a horizontal measurement setup (see figure 2a) at a defined distance of $50 \mathrm{~mm}$ to the bell edge.

The droplet velocity in the spray cone is determined by means of 2-dimensional Laser-Doppler-Velocimetry using a laser power of $400 \mathrm{~mW}$. The investigations are based on a rasterized scanning of the spray cone in the $x-$ and $y$-direction of $30 \mathrm{~mm}$ at intervals of $5 \mathrm{~mm}$ and a distance from the bell edge of $z=50 \mathrm{~mm}$ (see figure $2 \mathrm{~b}$ ). The coated spray pattern were recorded at a distance to the bell edge of $z=180 \mathrm{~mm}$, which is a typical distance in industrial coating applications. The hybrid bell atomizer was driven at a speed of motion $v_{\text {robot }}=300 \mathrm{~mm} \mathrm{~s}^{-1}$ (see figure 2c) and the resulting dry film thickness profile was measured in a transverse direction to the motion of atomizer using magnetic inductive measuring equipment. The transfer efficiency is determined as the ratio of the mass of the dry coating material deposited on the test sheet and the mass of the solids contained in the coating material, which is sprayed by the rotary bell atomizer. The speed of motion for determining the transfer efficiency is set to $\mathrm{v}_{\text {robot }}=200 \mathrm{~mm} \mathrm{~s}^{-1}$ according to DIN EN 13966-1.

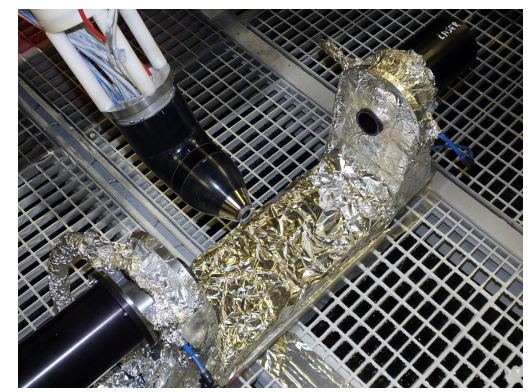

(a) Measurement of particle size distribution

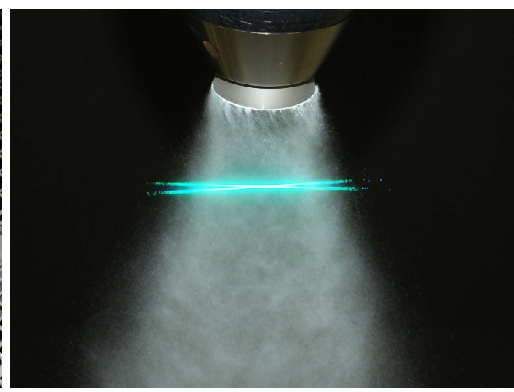

(b) 2-dimensional Laser-Doppler-Velocimetry

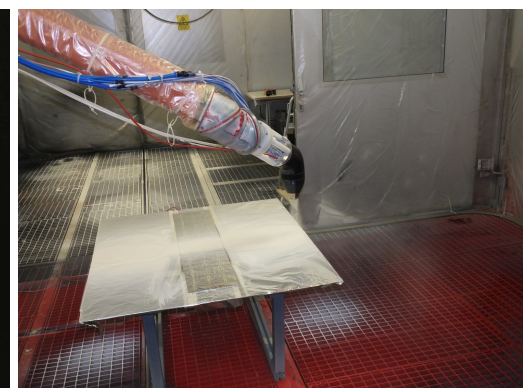

(c) Spray pattern and transfer efficiency

Figure 2. Setup for the experimental investigation the hybrid bell atomizer 


\section{Numerical setup}

The numerical investigation of the hybrid bell atomizer was carried out using the commercial computational fluid dynamics software ANSYSFluent. For this purpose, the hybrid atomizer was positioned in a fluid domain with the dimensions of $240 \mathrm{~mm} \times 1100 \mathrm{~mm} \times 1100 \mathrm{~mm}$ (height $\mathrm{x}$ width $\mathrm{x}$ depth). The distance of the plate to the bell edge is equivalent to the experimental investigations, $z=180 \mathrm{~mm}$. Above the hybrid atomizer, a velocity inlet of $v_{\text {in }}=0.3 \mathrm{~m} \mathrm{~s}^{-1}$ was defined as the inlet boundary condition, which represent the downdraft air velocity in the painting booth. The boundary conditions for the shaping air are defined by a massflow inlet, which can be calculated from the measured volumetric flow rate and the definition of a standard liter according to ISO2533.

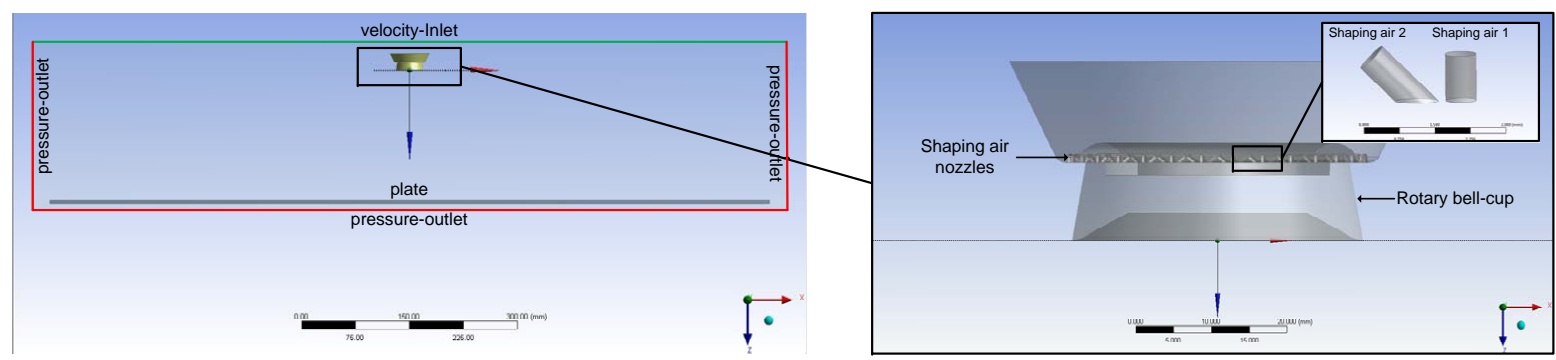

Figure 3. Geometrical model used in the simulation for the Dürr ECOBELL 2 HD

First a grid sensitive study was performed, from which a full structured hexahedral mesh yields in both most stable and most precise results (see figure 4).

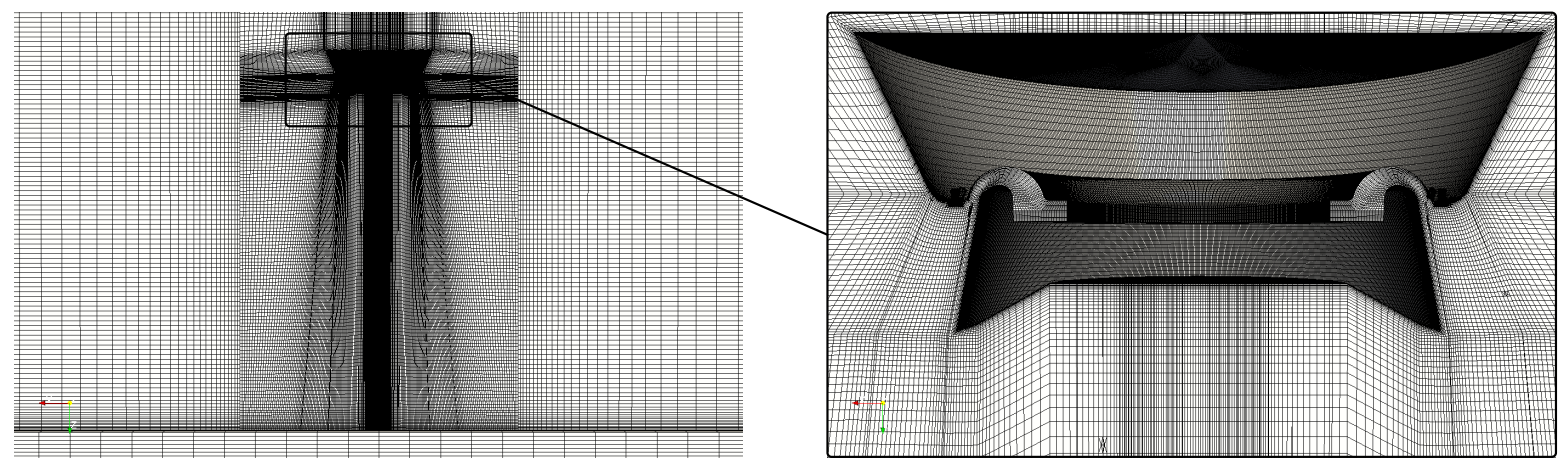

Figure 4. Full structured hexahedral grid with an total amount of $20 \mathrm{M}$ cells

For the calculation of the airflow field at this strong shaping air, turbulence models were compared using a k- $\epsilon$, k- $\omega$ and a Reynolds Stress turbulence model. Scalable wall function was applied - if available at the turbulence model. In addition compressibility effects insight the shaping air tubes were also taken into account through the turbulence models.

The motion of paint droplets are represented by inert particles computed through FLUENT discrete-phase-model. The discrete-phase-model (DPM) is an Lagrangean model for calculating the trajectories of particles through the computational domain. To capture the effects of particle on the flow field, the interaction to the continuous phase was enabled. Turbulence effects on particle trajectories are included by means of stochastic tracking using a randomwalk model. As a result of which the paint mass flow and the particle size distribution are known, the mass flow can be determined at each injection point for each particle size class.

Furthermore, electrostatic effects in the simulation were taken into account by a two-way coupling of both the continuous phase and the motion of particles and electrostatic field. For more details on this topic the reader is referred to Kulkarni et al. and Ye et al. [5, 6].

\section{Results and discussion \\ Investigation of Velocity Profile and Particle Velocity}

At rotary bell atomizers the velocity profile as well as the geometrical profile of the spray cone are controlled via the volume flow rates of the shaping air and the speed of the bell cup. At hybrid bell atomizers a strong axial velocity component prevails at the bell edge, but is supplemented by a tangential velocity component with increasing shaping air 2. Thus, with increasing the angular shaping air the flow field leads into a swirling flow. Due to the design and the manner of function of rotary bell atomizers, a swirl can be caused either by circumferential speed of the bell cup or by angular shaping air. In order to investigate the influence of the swirl generation, two swirl-numbers are defined in this study. First of all, the swirl-number from the rotating bell-cup is examined, whereby the swirl-number $S_{\text {cup }}$ is defined according to Stevenin et al.[1]. The swirl-number $S_{\text {cup }}$ is defined as the ratio of the bell edge tangential velocity $U_{t a n, c u p}$ to a characteristic value of mean velocity in the axial direction $U_{a x, m e a n}[1]$. Based on the results from 
numerical study, the characteristic mean velocity in axial direction was calculated over an area-weighted average in a annular plane at $z=0$ with an approximated flow thickness of $1 \mathrm{~mm}$ at the bell edge.

$$
S_{\text {cup }}=\frac{U_{\text {cup }, \text { tan }}}{U_{a x, \text { mean }}}=\frac{d_{\text {cup }} \pi n}{U_{a x, \text { mean }}}
$$

Due to the predominant axial airflow velocity, the swirl-numbers caused by the circumferential speed of the bell cup are weak to moderate (see table 2). The swirl-numbers are in a range of $0.2<S_{\text {cup }}<0.42$, where the flow field in the vicinity of the axis of symmetry is decelerated, but a vortex breakdown is not achieved. In swirling flows a vortex breakdown is defined as an abrupt change of flow structure, where reversed axial airflow near the axis of symmetry can be observed. In the present study the definitions swirl-number and vortex breakdown are used to describe flow field on rotary bell atomizers with predominant airflow rates.

Table 2. Swirl-number induced by rotational bell-cup

\begin{tabular}{c|c|c|c}
\hline Experiment & Swirl-Number $S_{\text {cup }}$ & Experiment & Swirl-number $S_{\text {cup }}$ \\
\hline E01 & 0.21 & E04 & 0.17 \\
E02 & 0.31 & E05 & 0.25 \\
E03 & 0.42 & E06 & 0.34 \\
\hline
\end{tabular}

However, this type of definition of the swirl-number induced by the bell cup is only a local average of the circumferential and axial speed, which, in addition, does not take account of swirl from the angular shaping air 2. A much more common definition of a global swirl-number, which also includes the influence of tangential shaping air, was provided by Chigier and Beer et al.. They defined the swirl-number as the ratio between the axial flux of the tangential momentum to the axial flux of the axial momentum [2]. In the present study, the swirl-number was investigated numerically and the influence of the swirl formation by the adjustment of the angular shaping air 2 as well as the circumferential speed was investigated. For the determination of the swirl-number at a given distance of $z=50 \mathrm{~mm}$ $\left(z / d_{\text {cup }}=1.3\right)$ to the bell edge, integration over a circular plane with a characteristic length $\mathrm{R}$ is executed [4]. At this given distance, the airflow velocities are below Mach-number $\mathrm{Ma}=0.3$, which is why a constant density $\rho$ of the air can be assumed.

$$
S=\frac{1}{R} \frac{\int_{A} \rho U_{a x} U_{t a n} r d A}{\int_{A} \rho U_{a x}^{2} r d A}
$$

This definition of the swirl-numbers (see table 3) show that the influence of the angular shaping air 2, which causes a strong tangential impulse, clearly dominates the swirl formation. Due to the fact that the bell is rotating against the angular shaping air direction, the swirl-number decreases with increasing rotation.

Table 3. Integral swirl number calculated in the plane $z=50 \mathrm{~mm}$

\begin{tabular}{c|c|c|c}
\hline Experiment & Swirl-Number $S$ & Experiment & Swirl-Number $S$ \\
\hline E01 & 1.006 & E04 & 0.244 \\
E02 & 1.001 & E05 & 0.239 \\
E03 & 0.996 & E06 & 0.234 \\
\hline
\end{tabular}

The comparison of the two tested shaping air settings shows that the critical swirl-number of 0.6 is exceeded in experiments E01 to E03. If the swirl-number exceeds this critical value, a vortex breakdown occurs. The vortex breakdown describes the reversal of the axial flow direction in the vicinity of the symmetry axis due to a prevailing negative axial pressure gradient which is greater than the axial kinetic forces. In order to determine the magnitude of the vortex breakdown and the effect on its flow profile, the spray cone was examined by means of Laser-DopplerVelocimetry (LDV) in the plane $z=50 \mathrm{~mm}$. The seeding required for the LDV are directly used from the sprayed paint material. In this way the measured velocities represent the integral droplet velocities in the spray jet. 

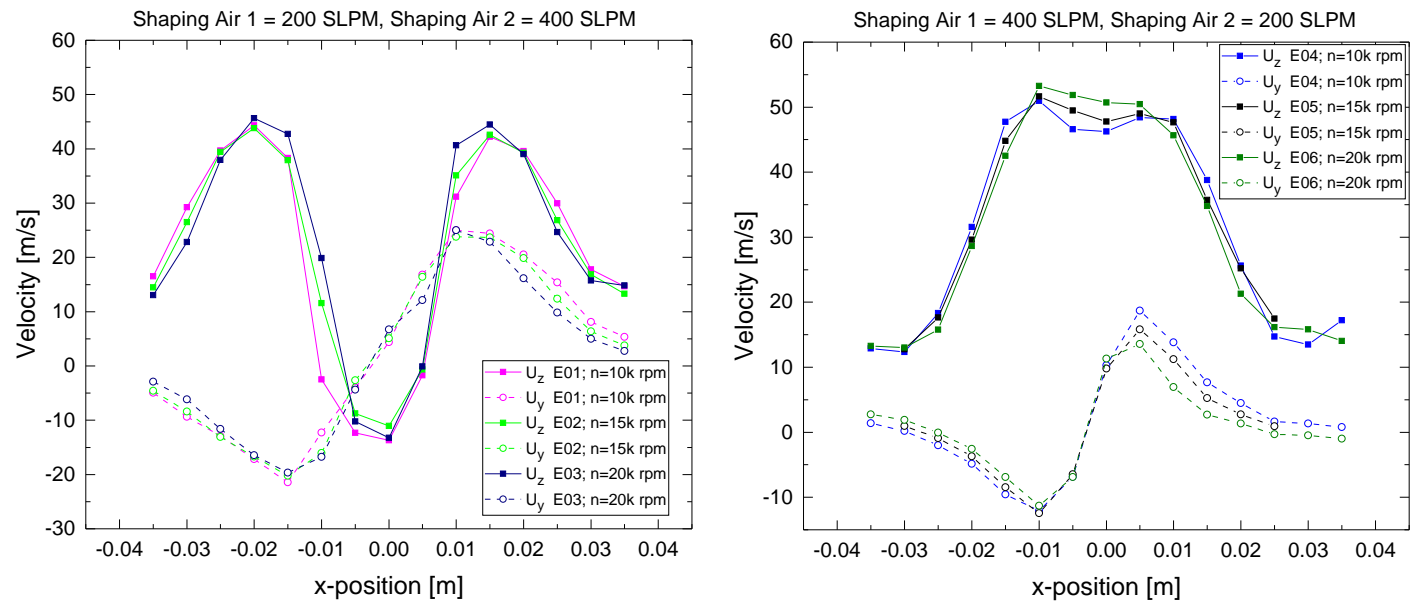

Figure 5. Experimental results of LDV measurements

The particle velocities of the investigated process parameters, as shown in the figure 5, show that the reversal axial flow direction is very pronounced due to the strong swirl in cases E01-E03. In the cases E04-E06 with a calculated swirl-number of approximately $S=0.2$, a slight deceleration occurs in the vicinity of the axis of symmetry. The experimental flow profile shows a good confirmation of the integral swirl-number. The determination of the swirl-number on high-speed rotary bell atomizers is an important dimensionless quantity for the design of shaping air process parameters and can be used for the analysis of the stability of the coating processes.

In the introduction the term "'hybrid bell atomizer"' has been defined, which states that predominant airflow rates have a significant impact on the atomization mechanisms. The dominant axial velocity is demonstrated experimentally and confirmed the characteristics of the particle velocity of a pneumatic behavior.

\section{Numerical Investigation of hybrid bell atomizer}

In the following section, the hybrid bell atomizer is numerically investigated on the basis of the process parameters E01 and E04 and a new approach to calculate the initial particle conditions for the discrete phase model is presented. Previous investigations on high-speed rotary bell atomizers used the $\mathbf{k}-\epsilon$ realizable turbulence model $[5,6]$. The high-speed rotary bell atomizers studied by Kulkarni et al. and Ye et al. were based on a weak axial flow velocity and can not be compared with the flow profiles in this study. As a result a turbulence study was performed using the k- $\epsilon$ realizable, the k- $\omega$ SST and the Reynolds-Stress model baseline were compared. The results of the axial $U_{z}$ and tangential $U_{\tan }$ airflow velocities are compared to the particle velocities from the LDV measurements. The experimental uncertainty is shown in the following figure 6.
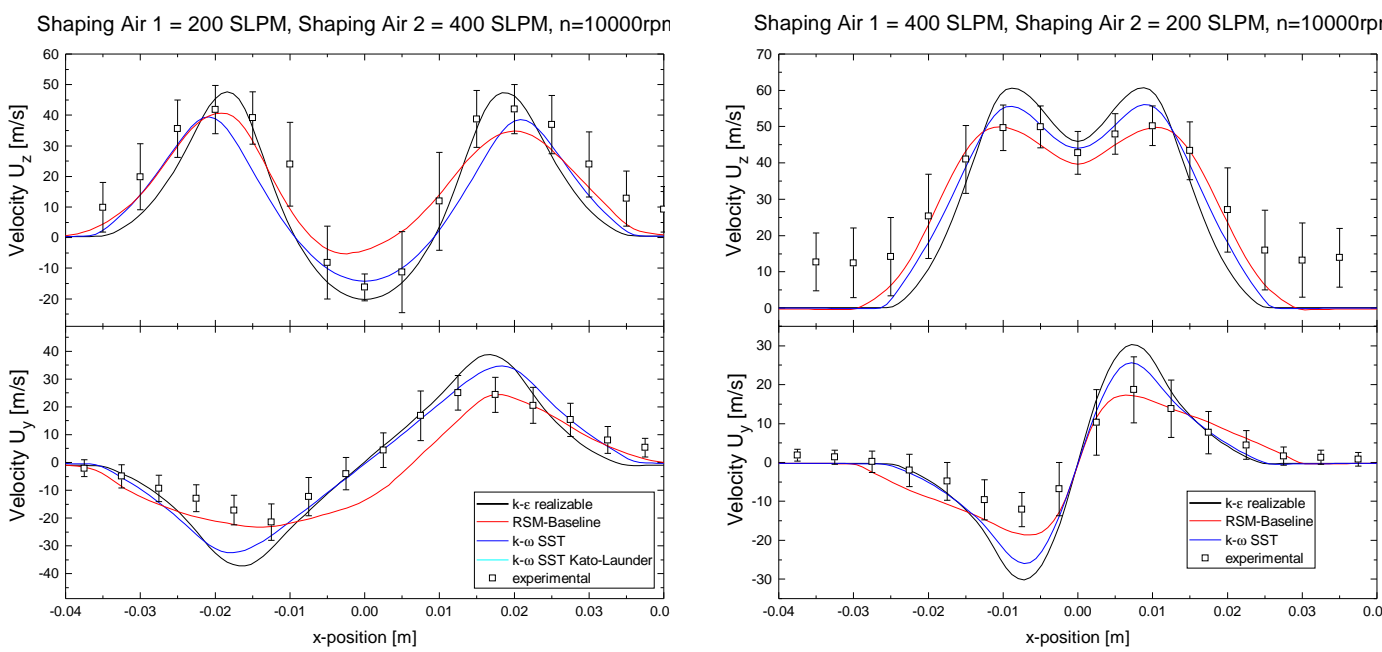

Figure 6. Results of turbulence study compared to the LDV measurement data on the experiment E01 (left) and E04 (right)

The $\mathrm{k}-\epsilon$ realizable turbulence model produces the highest values of axial airflow velocity with the smallest diameter of the spray cone. The Reynolds-Stress-Baseline turbulence model shows very good results in the tangential velocity in the case E04, but no stable solution could be achieved within the case E01. The k- $\omega$ SST turbulence model has very good velocity values, both in the axial as well as the tangential direction. Furthermore the k- $\omega$ SST turbulence 
model shows good stability as well as symmetry of the spray cone, which is why this turbulence model is used for further investigations.

Since the droplet break process at the bell edge is not simulated, experimental data on the particle size distribution (see figure 7) were used in this study. As Husam et al. has already been extensively studied, the resulting spray pattern depends on the number of injection points, the position of the injection points and the initial particle velocity. A obvious approach for the particle injection of high-speed rotary bell atomizers is to set the position of injection points as close as possible to the bell edge. However, this position also depends on the applied grid, which should refined until a very close position can be reached. In this study, 120 injection points with an axial offset of $0.0001 \mathrm{~m}$ and a radial offset of $0.0002 \mathrm{~m}$ were defined in an evenly fashion with respect to the bell edge. In order to implement the strong influence of the shaping air realistically into the particle injection velocities $U_{P_{a x, t a n, r a d}}$, the data of the simulated air velocity in the injection plane were interpolated to the injection points.

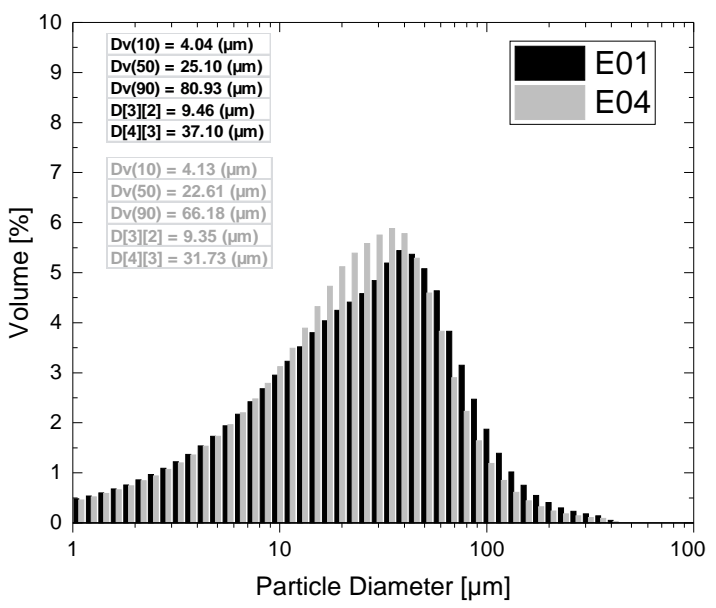

Figure 7. Results of measured particle size distribution at $\mathrm{z}=50 \mathrm{~mm}$

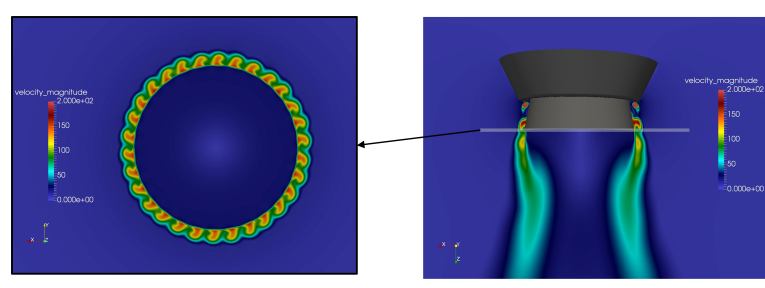

(a) Case E01

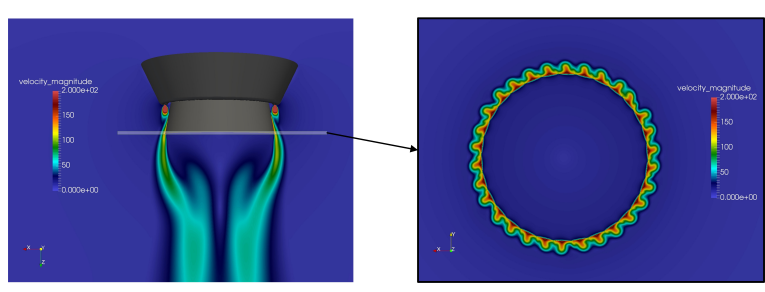

(b) Case E04

Figure 8. Velocity magnitude both at injection plane and at the center of the hybrid bell atomizer

Subsequently, the initial particle velocity $U_{P_{a x}}, U_{P_{t a n}}, U_{P_{\text {rad }}}$ was calculated using the following equation. The tangential initial particle velocity is composed of the tangential air velocity in this equations, as well as the circumferential speed $U_{\text {cup }}$ of of the bell edge.

$$
\left(\begin{array}{c}
U_{P_{a x}} \\
U_{P_{t a n}} \\
U_{P_{\text {rad }}}
\end{array}\right)=\left(\begin{array}{ll}
\alpha_{a x} & U_{a x} \\
\alpha_{t a n} & U_{t a n}+\beta_{t a n} U_{c u p t a n} \\
\alpha_{\text {rad }} & U_{\text {rad }}
\end{array}\right)
$$

Due to the opposing tangential velocities from the swirl-forming shaping air to the circumferential speed of the bell edge and the defined injection particle position close to the bell edge, the tangential airflow velocity component was eliminated. Therefore, the calculation of initial particle conditions is based on the circumferential speed of the bell edge, the axial and the radial airflow velocities.

Table 4. Inlet coefficient, determined by fitting to the spray pattern

\begin{tabular}{l|cccc}
\hline Case & $\alpha_{a x}$ & $\alpha_{\text {tan }}$ & $\alpha_{\text {rad }}$ & $\beta_{\text {tan }}$ \\
\hline E01 & 0.3 & 0 & 0.2 & 0.9 \\
E04 & 0.4 & 0 & 0.15 & 0.9 \\
\hline
\end{tabular}

In order to validate the new approach the experimental data of the particles velocities as well as the spray pattern are used. At the beginning, the numerically iterated air flow velocities were compared to the particle velocities and the k- $\omega$ turbulence model was choosen. Since the impact of the paint droplets on the airflow is known, the velocity of the particles in the simulation and in the experiment are compared. In order to obtain a representative amount of particle streams in the simulation, the measurement area is was set to $25 \mathrm{~mm}^{2}$ with the origin of the LDV measurement points. 

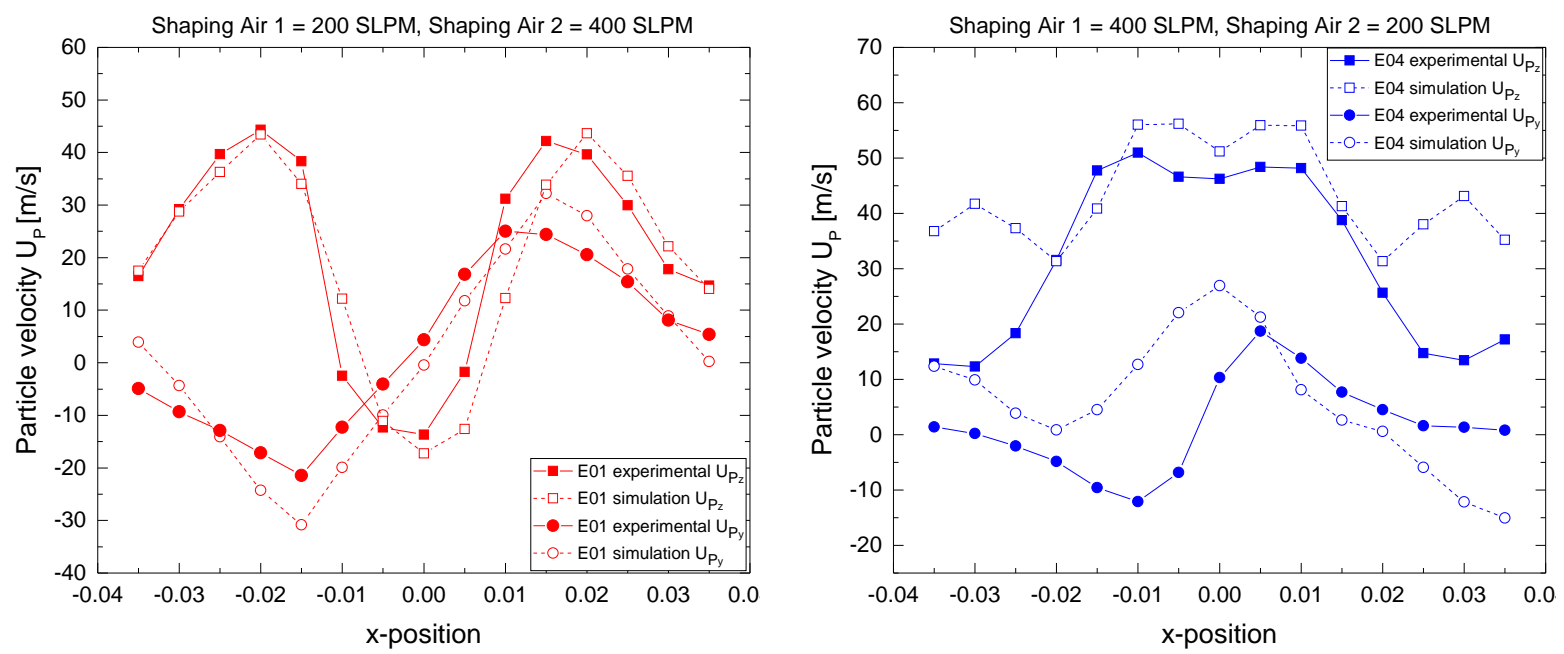

Figure 9. Comparison of simulated and experimental particle velocities

The results from the numerical investigation show very good agreement with the experimental data in experiment E01 and confirms that the selected method of particle injection works very well. In the case of E04, larger deviations in the simulated particle velocity occur, especially in the outer regions of the spray cone. It is known that the large particles concentrate in the outer regions of the spray cone and have higher velocities due to the high momentum. Furthermore, the initial particle velocities leads to a concentration of large particles with an diameter above $d_{p}>100 \mu \mathrm{m}$ in this region which results in a higher simulated particle velocity. Nevertheless, the injection method shows very good results for hybrid atomizers with predominant airflow rates.

\section{Investigation on Application Specific Values}

Important quantities for the characterization of atomization and application processes in the coating technology are the resulting spray patterns (dry film thickness profile) and the magnitude of transfer efficiency (TE). The dry film thickness profile is measured transversely to the direction of motion of the hybrid atomizer and is predominantly used to calculate the overlap respectively the distance between to parallel painting robot paths. The transfer efficiency is defined as the ratio of the paint mass deposited on the object to the sprayed paint mass. The use of rotary bell atomizers for detailed coatings requires a narrow spray pattern, which is realized by the strong axial shaping air. In this case, the film thickness profiles have a structure very similar to the velocities measured by means of LDV. The higher the swirl-number, both the more the spray pattern is enlarging and the deeper the valley in the vicinity of the symmetry axis. The influence of the circumferential speed of the bell cup on the change of the film thickness profile at given shaping air setting is not significant for current coating processes. The use of direct charging support also leads to an insignificant change in the film thickness profile. The simulated spray pattern also shows good agreement with the experimental data in both cases E01 and E04.
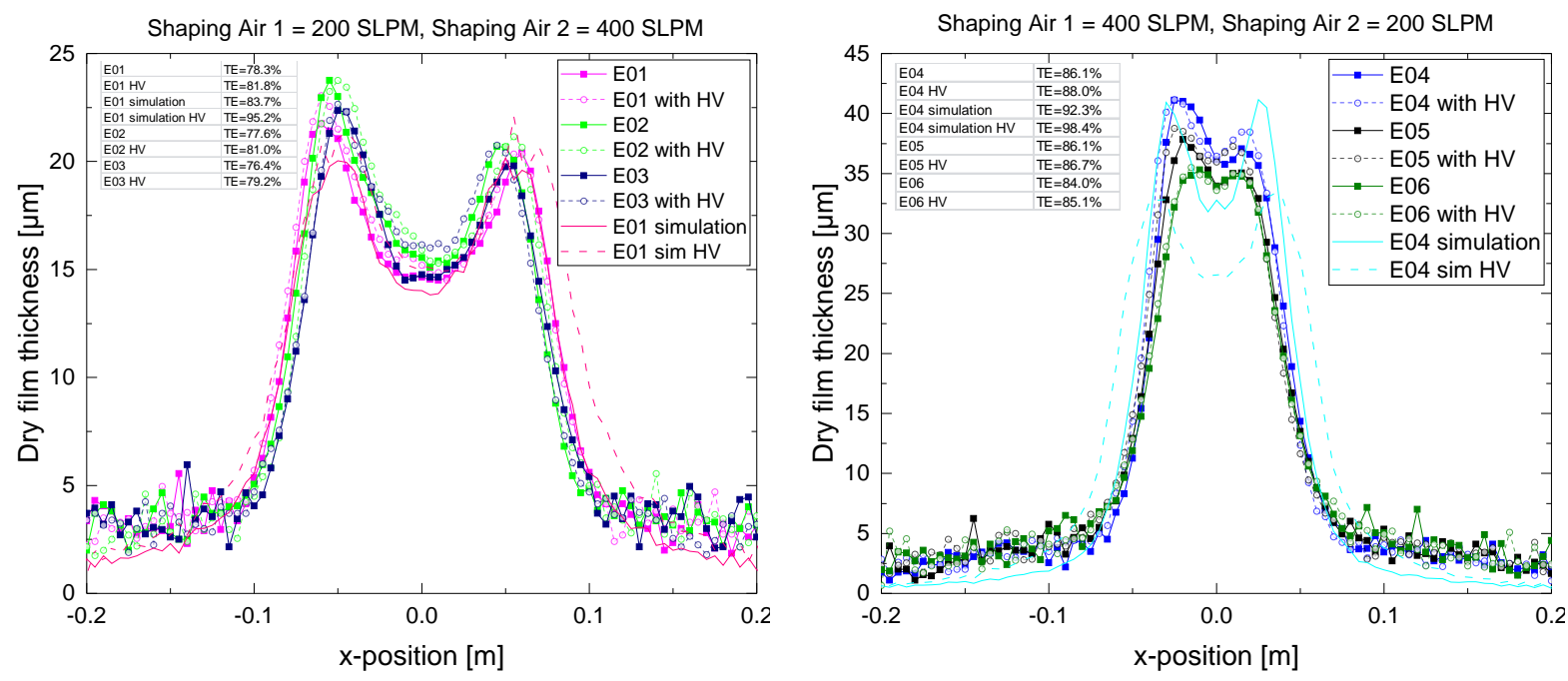

Figure 10. Results of film thickness profile and transfer efficiency 
For the calculation of the film thickness profile using direct charging, the same initial particle velocities were used and a constant initial Rayleigh-limit of $5 \%$ was assumed [5].

The transfer efficiency, shown in the left upper corner of figure 10a and 10b, shows the same dependencies as the spray pattern. Especially the predominant airflow leads to the fact that further process parameters plays only a subordinate role. Particularly in experiments the direct electrostatic charging shows only a small increase in the transfer efficiency of $1-5 \%$.

\title{
Conclusions
}

Rotary bell atomizers for detailed coatings requires an unusually strong axial shaping air velocity. This leads to the fact, that other process parameter, such as the number of revolutions of the bell cup and the direct electrostatic charge plays a only subordinate role. The description of these predominant shaping airflow rates was carried out using two definitions of a swirl-numbers. Very good agreement could be achieved via an integral description of the swirl-number. The vortex breakdown arising from a critical swirl-number of $S=0.6$ was calculated theoretically and measured experimentally. Adapted to the prevailing shaping air, a new method for calculating the initial particle velocities for the discrete-phase model of the commercial CFD code FLUENT was presented. The validation of this injection method shows very good coincidences, both in the particle velocities, as well as the resulting film thickness profiles and transfer efficiencies measured in an industrial paint booth.

\section{Acknowledgements}

This research was supported within the project "SelfPaint« by Fraunhofer-Gesellschaft zur Förderung der angewandten Forschung e.V.

\author{
Nomenclature \\ $S_{\text {geo }} \quad$ geometrical Swirl-Number \\ $S \quad$ integrated Swirl-Number \\ $d_{\text {cup }} \quad$ bell-cup diameter $[\mathrm{m}]$ \\ $d_{P} \quad$ particle diameter $[\mathrm{m}]$ \\ $n \quad$ number of revolutions [rpm] \\ $U \quad$ air velocity $\left[\mathrm{m} \mathrm{s}^{-1}\right]$ \\ $U_{\text {mean }}$ area-weighted average velocity $\left[\mathrm{m} \mathrm{s}^{-1}\right]$ \\ $U_{P} \quad$ initial particle velocity $\left[\mathrm{m} \mathrm{s}^{-1}\right]$
}

\section{References}

[1] Stevenin, Ch., Béreaux, Y., Charmeau, J.-Y., Balcaen, J., 2015, Journal of Fluids Engineering, 137.

[2] Beer, J.M. and Chigier, N.A., 1972 , Combustion Aerodynamics, Applied Science Publishers Ltd.

[3] Sheen, H.J.,Chen, S.Y., Huang T.L., 1996, Experimental Thermal and Fluid Science, (12), 444-451.

[4] Vondál, J., Hájek, J., 2012 Chemical Engineering Transactions, 29, 1069-1074

[5] Kulkarni, J., and Watve, A., May. 2008, 21st Annual Conference on Liquid Atomization and Spray System.

[6] Ye, Q., Pulli, K., Steinhilber, and Scheibe, A., July. 5.-6. 2007, 3rd European Automotive CFD Conference.

[7] Wiedemann, A., 2001 Mehrkomponenten-Laser-Doppler-AnemometerMessungen in einer drallbehafteten Rohr- und Brennkammerströmung

[8] Husam, O., Adamiak, K., Castle P., Fan, H., Simmer, J., Nov. 13.-19. 2015, International Mechanical Engineering Congress and Exposition.

[9] Mark, A.,Andersson, B., Tafuri, S., Engström, K., Söröd, H., Edelvik, F. and Carlson, J.S., 2013, Atomization and Sprays, 23. 


\title{
Investigations on nano- and submicron-particle generation by spray painting processes
}

\author{
Q. Ye ${ }^{1^{*}}$, O. Tiedje ${ }^{1}$, S.R. Srinivas ${ }^{1}$, T. Noest ${ }^{2}$, U. Uhrner ${ }^{2^{*}}$ \\ ${ }^{1}$ Fraunhofer Institute for Manufacturing Engineering and Automation \\ Nobelstr. 12, 70569 Stuttgart, Germany \\ ${ }^{2}$ Institute of Internal Combustion Engines and Thermodynamics Graz University of Technology \\ Inffeldgasse 21a, 8010 Graz, Austria \\ *Corresponding author: qiaoyan.ye@ipa.fraunhofer.de and Uhrner@ivt.tugraz.at
}

\begin{abstract}
This paper presents experimental and numerical studies to determine the particle size distributions (PSD) and concentrations in paint overspray. Two kinds of paint materials, solvent borne and water borne paints, both with and without manufactured nanomaterials (pigments), and an industrial spray gun were used. Different aerosol measuring techniques, namely the Spraytec Fraunhofer type particle sizer for micro-sized droplets in the spray jet and the Scanning Mobility Particle Sizer (SMPS) for nano particles in paint overspray were applied. It was found, that solvent borne clear coats create significantly higher number concentration of nano-sized droplets than the water borneprimers. Only small differences in PSD between paints with and without manufactured nanomaterials were found. Numerical simulations of droplet trajectories within the spray booth, for both micro and nano sized droplets, were carried out. Based on the experimental and numerical results, a representative particle size distribution (smaller than $1 \mu \mathrm{m}$ ) for the given spray gun was obtained. Effects of turbulence models on the particle deposition on targets, especially for submicron particles, have been analysed in detailed.
\end{abstract}

\section{Keywords}

Nano- and submicron-particle, spray coating, aerosol measurement, droplet trajectory calculation

\section{Introduction}

The increasing application of manufactured nanomaterials (MNMs) in industrial products requires more knowledge about environmental and human safety. This is especially true for the spray painting process, which produces a high percentage of paint overspray. During the migration of the overspray droplets, solvent continues to evaporate, resulting in the formation of non-volatile fractions of paint aerosols that consist of paint matrix and the applied MNMs, such as $\mathrm{TiO}_{2}, \mathrm{ZnO}$, carbon black, etc. The MNMs could remain embedded in paint matrix particles or released from the paint matrix. Thus, the characterization of paint overspray aerosol becomes more and more important.

Information about the estimation of worker nanoparticle exposure to paint overspray is limited and particularly the process understanding of nanomaterial release during spray applications. A review of nanoparticle exposure at nanotechnology workplaces has been stated by Kuhlbusch et al. [1]. Carlton and Flynn [2] developed an empirical-conceptual model based on dimensional analysis, to predict breathing zone concentrations of a paint mist during spray painting tasks. Later, they also carried out CFD calculations to simulate production of breathingzone concentration of a paint overspray, for a simple case of the spray painting of a flat plate in a cross-flow ventilated booth [3].

For the risk estimation of exposure to paint overspray, it is also useful to categorize the paint aerosol particles in a spray jet, which can provide important information with regard to the worst case for a given spray atomizer and the applied paint material. Based on the experimental and numerical studies [4-6], paint droplet injection models for different atomizers and corresponding application parameters have been developed, with which the spray transfer efficiency TE (amount of paint reaching the work piece) as well as the paint overspray can be well predicted. However, these studies considered particle dispersion and deposition mainly for droplets larger than $1 \mu \mathrm{m}$. Study on the generation and characterization of nano- and sub-micro paint aerosols for a given atomizer is still quite scarce.

Recently Göhler and Stintz [7] analysed the airborne particle release from spray guns by sampling small particles in a turbulent pipe flow. The results showed that depending upon the material and pray unit used, $5 \times 10^{8}$ to $3 \times 10^{8}$ particles were released per gram of paint ejection, of which around $10 \mathrm{no} \%$ to $60 \mathrm{no} \%$ were finer than $100 \mathrm{~nm}$. These results were later applied to their CFD-modelling of nano particle dispersion for the prediction of worker exposure [8]. 
Concerning to the particle transport in turbulence flows, especially for nano particles, there are many reports [912], in which the effect of different turbulence models, wall treatments and models of turbulent dispersion on particles were investigated using CFD tools. In many of such studies, direct numerical simulation (DNS) data of channel flows were applied to create damping functions for predicting more accurate particle fluctuation velocity in near wall turbulent flow. However, in spray painting applications the spray jet is characterized with turbulent impinging jet. There is no available DNS data for such complicated turbulent near wall flow with high Reynolds number.

Currently, a joint transnational research project (NanoGeCo) has been set up, focusing on detailed characterization of nanoparticles by atomization processes in spray painting. Within this project different stages of experimental and numerical studies have been identified. In this stage of the research, determination of particle size distributions and concentrations in paint overspray were carried out. Two kinds of paint materials, solvent borne and water borne paints, both with and without manufactured nanomaterials (pigments), and an industrial spray gun were used. Different aerosol measuring techniques, namely the Spraytec Fraunhofer type particle sizer for micro-sized droplets in the spray jet and the Scanning Mobility Particle Sizer (SMPS) for nano particles in paint overspray were applied.

Numerical simulations of droplet trajectories within the spray booth for both micro and nano sized droplets were carried out, taking into account, the solvent evaporation in the droplets. Droplet solid fractions in overspray and droplet transfer efficiencies on a target were analysed, especially for nano- and submicron droplets. The effects of turbulence models on the dispersion and deposition of small sized particles were studied using RANS models (Renolds Averaged Navier-Stokes), namely k- $\varepsilon$ and RSM (Reynolds stress model). The simulated number concentrations of nano-sized droplets were compared with the measurements, resulting in an improved injection model for nano sized droplets. Based on the experimental and numerical results, a representative particle size distribution (smaller than $1 \mu \mathrm{m}$ ) for the given spray gun has been obtained, which provides information for further investigations, to assess human exposure to nano particles, in a practical paint booth and for toxicological studies of atomized nano-particles.

\section{Experimental study}

Paint materials, spray gun and spray booth

All measurements were carried out in a practically relevant painting booth with a well-defined homogeneous booth air velocity of $0.3 \mathrm{~m} / \mathrm{s}$. A HVLP-spray gun (High Volume Low Pressure) from SATA was used. A flat plate with size of $200 \times 800 \mathrm{~mm}^{2}$ as droplet deposition target was located horizontally. Painting distance between the gun and the target was $180 \mathrm{~mm}$. Table 1 shows the application parameters. Table 2 summarizes the properties of used paint liquids.

Table 1: Operating parameters

\begin{tabular}{|l|l|}
\hline Liquid flow rate & $150[\mathrm{~g} / \mathrm{min}]$ \\
\hline Gun air flow & $410[\mathrm{NL} / \mathrm{min}]$ \\
\hline Gun pressure & $2[\mathrm{bar}]$ \\
\hline Painting distance & $180 \mathrm{~mm}$ \\
\hline
\end{tabular}

Table 2: Properties of paint materials

\begin{tabular}{|l|l|l|l|}
\hline Name & $\begin{array}{l}\text { Density (wet paint) } \\
\mathrm{kg} / \mathrm{m}^{3}\end{array}$ & $\begin{array}{l}\text { Density (dry) } \\
\mathrm{kg} / \mathrm{m}^{3}\end{array}$ & $\begin{array}{l}\text { Non-volatile } \\
\text { fraction }\end{array}$ \\
\hline Clear coat & 999.2 & 1160 & $53.1 \%$ \\
\hline Primer & 999.0 & 1135 & $13.3 \%$ \\
\hline
\end{tabular}

Basically, two-component, solvent borne clear coat and water borne paint (primer) were used. Paint materials with and without pigments ( $\mathrm{ZnO}$ and carbon black) were applied in measurements. A nanoparticle concentration of approximately $1.5 \mathrm{v} / \mathrm{v} \%$ calculated on solid binder was used, corresponding to the normal coating applications. It was found that there were no significant effects of pigments or MNM on the listed properties of paint liquids and as well as on the rheological behaviours, as shown in Fig. 1. Both liquids shows shear thinning behaviour of viscosity, but it is stronger for the primer. However, the apparent viscosity of the clear coat is significantly lower than that of the primer. The viscosity curves in Fig. 1 can be helpful for understanding the measured droplet size distributions shown later. For clear coat the solvent is composed mainly of Butyl acetate that was used in the droplet trajectory calculation in the numerical study.

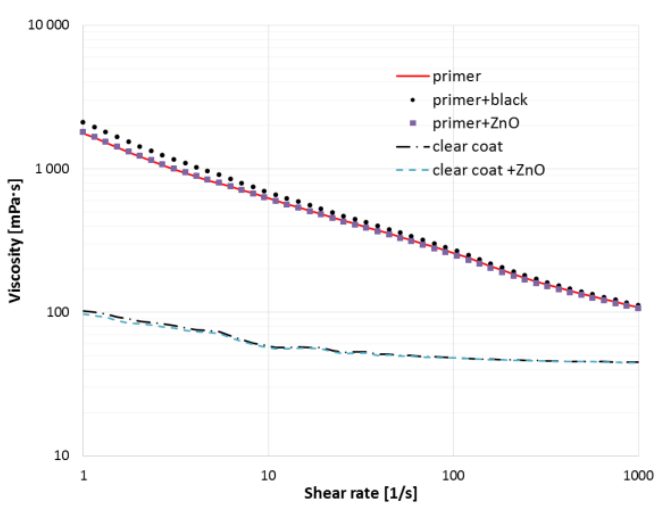

Figure 1. Viscosity measurement (shear curve) of the paint materials 
Micro droplet size measurement in spray jet

In terms of mass, the spray jet air flow is mainly influenced by micro-sized particles and to a far lesser extent by nanoparticles. It is, therefore, necessary to measure micro droplet size distribution in a spray jet, in order to correctly model spray jet turbulent flow using CFD tools. Figure 2 shows the droplet size measurement setup using a Malvern Spraytec Fraunhofer type particle sizer. A distance of $z=50 \mathrm{~mm}$ from the nozzle was chosen and the laser beam of the Malvern Spraytec was oriented along the $x$ axis. The spray gun that was mounted on a robot was traversed along the major axis y of the elliptical spray cone. A similar approach was applied in our previous studies $[5,6]$.

Based on the individual droplet size distributions in the elliptical spray region, the integral distribution of the whole spray region was then calculated and shown in Fig. 3. The corresponding Sauter mean diameter $D_{3,2}$ is $7.11 \mu \mathrm{m}$ for the primer and $6.52 \mu \mathrm{m}$ for the clear coat. In order to show more clearly the difference in size distributions for small particles between the two paint liquids, mean diameter Dv10 (10 percent of particle volume accumulation distribution lies below the Dv10, which characterizes the small particle size) along the elliptical spray cone are depicted in Fig. 4. Clearly, the clear coat creates somewhat finer particles (Dv10) than the primer. Measurements of nanoparticle size distributions created from the paint liquids will be illustrated in the next section.

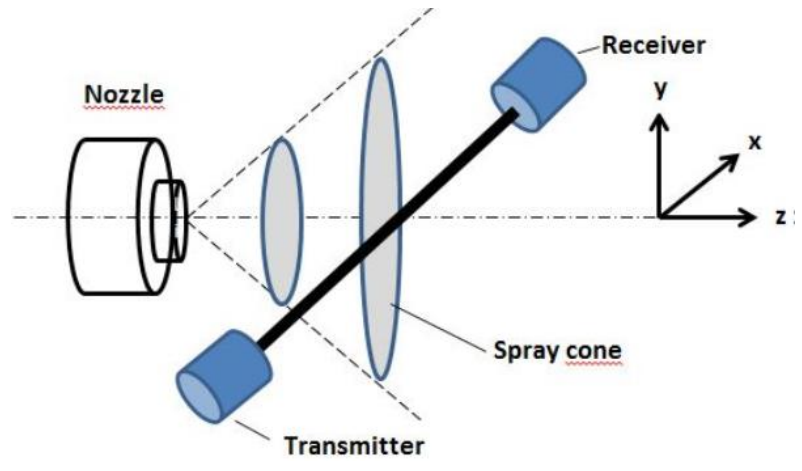

Figure 2. Schematic of the droplet size measurement setup

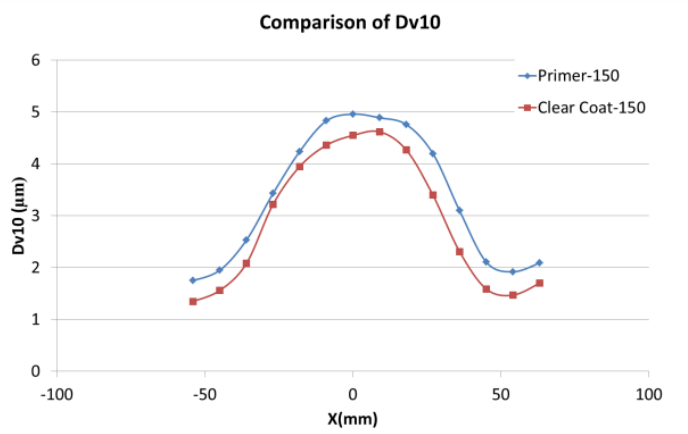

Figure 4. Mean particle size Dv10 distributions along the spray cone

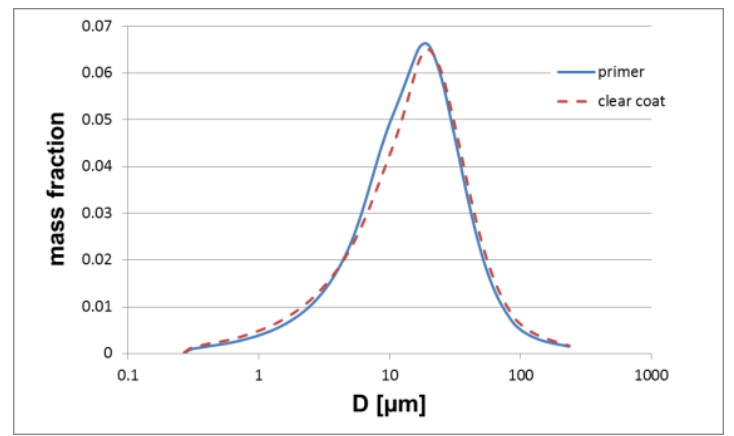

Figure 3. Integral droplet size distributions

\section{Nano and submicron particle concentration in spray overspray}

Particle size distribution (PSD) measurements in the nanometre range require on one hand a certain time interval scanning from a few nanometres to several hundreds of nanometres including an instrument specific reset time (retrace time). Therefore, a varying evaporation speed of droplets in overspray as well as coagulation of droplets and further atomization and dilution processes at the same time makes a representative sampling difficult. The paint aerosol is sticky, which may make the sensor signal quickly messy during data sampling close to overspray with high concentration. With this consideration an experimental arrangement and setup was established, as shown in figure 5. Basically, a target plate was used for depositing large paint droplets. Two measuring points $(\mathrm{L} 50 \mathrm{~cm}$ and $\mathrm{S} 30 \mathrm{~cm})$ were located in the middle of the two plate edges, but far away from the edges and at the same level of the plate $(\mathrm{H} 79.5 \mathrm{~cm})$. The quasi-static spray jet flow allows subsequent scans during atomization 
and facilitates repeatability of measurements. A SMPS (scanning mobility particle sizer) and a CPC (condensation particle counter) were applied to measure nanoparticles of paint overspray. The SMPS/CPC was operated in "batch" mode. An impactor is used as a pre-filter to limit the measurement range to sub-micron particles and to avoid damage due to overflow to the instruments sensitive parts. Cleaning on the impactor had to be carried out frequently, in order to obtain accurate results. Parameters such as sheath air flow and scanning time were set to 3 $1 /$ min and 60 seconds.

Background particle number concentration (PNC) in the spray booth with the booth and gun air flow was always measured before each operation of spray gun and is shown in Figs. $6-8$. In Fig. 6 results are shown for primer and primer with pigments (carbon black). For the water borne paint (Fig. 6) it can be observed that nanoparticle concentrations are higher than those of the background for particles with mobility diameter $D_{p}>200 \mathrm{~nm}$. It can be also seen that number distribution for the primer with carbon black is a little bit lower than that of primer.

Figures 7 to 8 shows results from solvent borne paints (clear coat with and without $\mathrm{ZnO}$ ) for the measuring positions at $L=50 \mathrm{~cm}$ and $S=30 \mathrm{~cm}$. The PNC differences between paint aerosols and the background are generally small in the $40 \mathrm{~nm}$ to $80 \mathrm{~nm}$ range. The difference between clear coat with and without $\mathrm{ZnO}$ is also small. Significant higher PNC can be observed for particles in the range of 100 to $640 \mathrm{~nm}$. The PNC at measuring position $\mathrm{L}=50 \mathrm{~cm}$ is higher than that at $S=30 \mathrm{~cm}$, which delivers important information for the nanoparticle dispersion for the trajectory modelling in the numerical study. Comparing the two paints, PNCs of primer are during the atomization process far lower than those of the solvent borne paints. Primers showed a very "stick character" and we assume that these differences are due to the high viscosity of these paints.

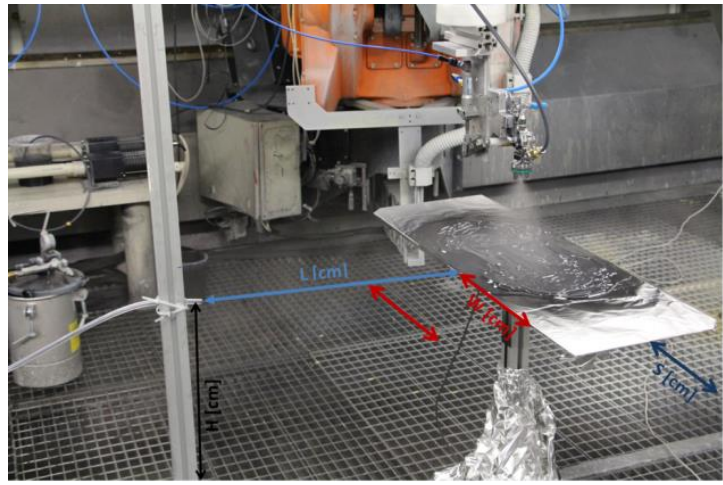

Figure 5. Setup of the spray application and sampling position definition.

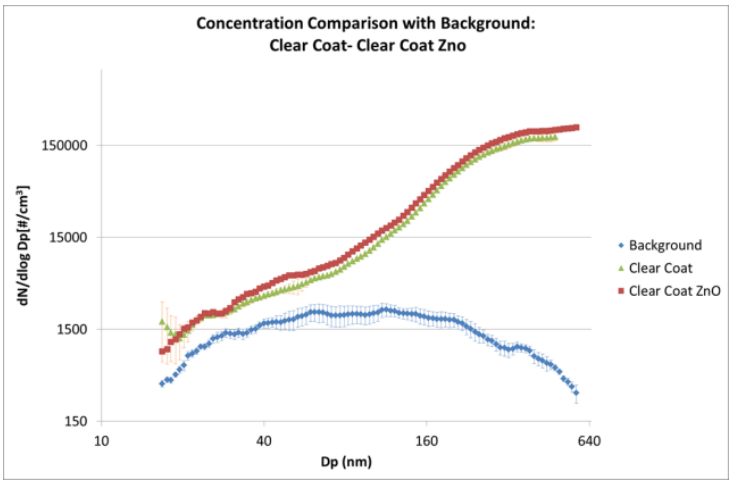

Figure 7. Clear coat particle concentration at the measuring position: $\mathrm{L} 50 \mathrm{~cm}, \mathrm{~W} 0, \mathrm{H} 79.5 \mathrm{~cm}$

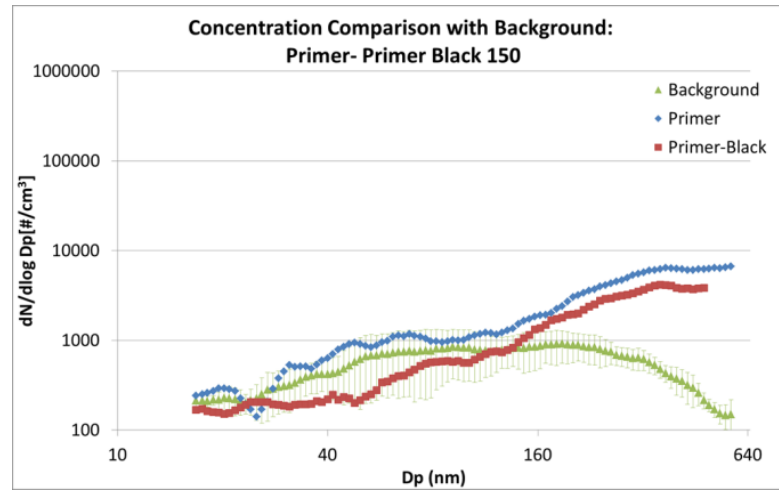

Figure 6. Primer particle concentrations at the measuring position: $\mathrm{L} 50 \mathrm{~cm}$, WO $\mathrm{H} 79.5 \mathrm{~cm}$

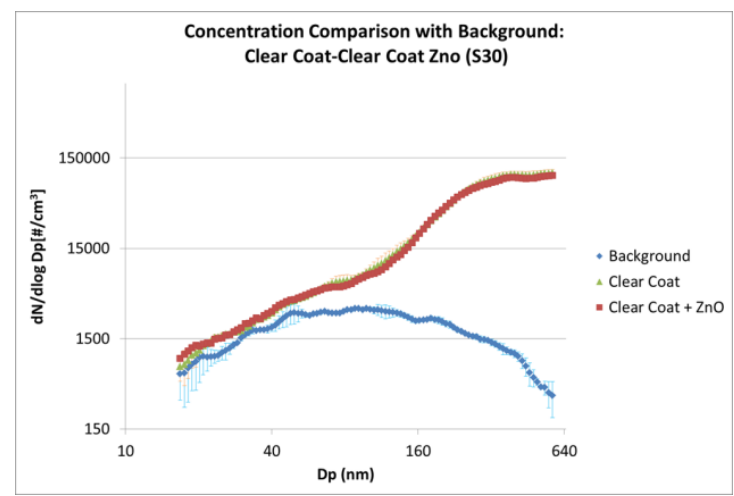

Figure 8. Clear coat particle concentration at the measuring position: $\mathrm{S} 30 \mathrm{~cm}, \mathrm{H} 79.5 \mathrm{~cm}$

\section{Numerical study}

Numerical methods

The commercial CFD code ANSYS-Fluent 17, based on the finite-volume approach, was used for the numerical simulations. The gas phase was modelled using the Eulerian conservation equations of mass, momentum, and energy. The three-dimensional compressible airflow was directly simulated from the nozzle using the coupled solver, as it was found to be more stable than the segregated solver for this specific airflow calculation. As inlet 
boundary conditions, booth air velocity of $0.3 \mathrm{~m} / \mathrm{s}$ and air mass flow rate and stagnation temperature were used at the air nozzles.

An unstructured mesh with 3.3 million cells was used to discretize the computational domain of $3.5 \times 2.5 \times 2.0 \mathrm{~m}^{3}$, including the complicated spray gun geometry. Mesh refinement was carried out. Prism mesh layers on the target wall were created with the first layer thickness of $0.5 \mathrm{~mm}$. For this mesh model the turbulence Realizable k- $\varepsilon$ model with scalable wall function was applied, since the dimensionless wall distance $\mathrm{y}^{+}$ranges from $1-20$ on the plate.

It is well known that the particle deposition is strongly influenced by the used near wall turbulence models, especially for small size particles. An isotropic turbulent flow is basically assumed in two equation RANS model (Reynolds Averaged Navier-Stokes), which results in an over estimation of particle deposition rate if the original eddy interaction model (EIM) in common CFD-codes is used [10-12]. In order to model the near-wall anisotropy flow using k- $\varepsilon$ models, some modifications [9-10] of EIM have been performed previously by using damping functions that were derived using DNS-data of channel flows. However, such approach is not suitable to the present complicated turbulent impinging jet. Instead of using damping functions the Reynold-stress turbulence model (RSM), which can account for anisotropic turbulence, could be applied to particularly study dispersion and deposition of quite small particles. However, the RSM is quite sensitive to the grid quality. The present simulations with RSM using tetrahedral mesh near the atomizer resulted in an unrealistic spray jet. For this reason a computational domain with hexahedral meshes but without atomizer was used to study the near wall nanoparticle deposition and dispersion. The obtained air flow and the particle trajectories in the near-atomizer region based on the $\mathrm{k}-\varepsilon$ model were exported then for the inlet conditions in the hexahedral mesh model. Reasonable results could be obtained, as shown later.

Droplet trajectories were calculated using the Lagrangian particle tracking method (LPT) by integration of the equation of motion,

$\frac{d \mathbf{u}_{\mathrm{p}}}{d t}=f_{D}\left(\mathbf{u}-\mathbf{u}_{\mathrm{p}}\right)+\mathbf{F}_{G}+\mathbf{F}_{L}, \quad \frac{d \mathbf{x}}{d t}=\mathbf{u}_{\mathrm{p}}$,

in which the drag force $f_{D}\left(\mathbf{u}-\mathbf{u}_{\mathrm{p}}\right)$ and the gravity force $\mathbf{F}_{G}$ (force/unit particle mass) are dominant forces for large droplets. For nano- and sub-micro droplets the Saffman's lift force $\mathbf{F}_{L}$ and the Stokes-Cunningham drag laws were taken into account. The stochastic tracking model with random walk and eddy lifetime was applied to calculate the instantaneous air velocity $\mathbf{u}$. For $\mathrm{k}-\varepsilon$ model the fluctuating velocity component is equal to $(2 \mathrm{k} / 3)^{1 / 2}(\mathrm{k}$ is turbulent kinetic energy), whereas anisotropy fluctuating components: (uu $)^{1 / 2}$, (vv) ${ }^{1 / 2}$ and (ww) ${ }^{1 / 2}$ can be obtained using RSM. The other important issue for modelling the turbulence dispersion on the droplet motion is the determination of integral time scale constant that is used for calculation of the particle-eddy interaction time in the EIM,

$T_{L}=C_{L} \frac{k}{\varepsilon}$

In Fluent the integral time scale constant $C_{L}$ is 0.15 by default, which can be modified for the trajectory calculation of small particles as suggested in previous works $[9,16]$. A Larger value $C_{L}=0.8$ was used in current study, in order to achieve better agreement with experimental results of the nanoparticle dispersion. The corresponding effect of $C_{L}$ is shown in the following section.

Droplet size distributions shown in Fig. 3 were used to create injection data for the trajectory calculation of microsized particles. The injection position was located quite close to the atomizer. The initial droplet injection velocities were fitted by using the information from the gas flow field and by matching the film thickness distribution on the flat plate. The detailed approach for creating injection data can be referred to in our previous studies [5, 6]. Water and Butyl acetate were used as solvents in calculation of droplet evaporation for the water borne coat and the clear coat respectively. Species transports in gas phase, such as air- Butyl acetate and air-water-steam, have been performed. Raoult's law was used to calculate the pressure equilibrium at the gas/liquid interface. Detailed physical models are shown in reports $[14,15]$. Two phase coupling was taken into account.

Static film thickness distribution, namely the film growth rate $[\mu \mathrm{m} / \mathrm{s}]$ on the target can be obtained after particle trajectory calculation. The spray gun is static in the present numerical simulation, hence in order to compare with the measured dynamic film thickness distribution, the simulated static film pattern has to be integrated, taking into consideration the robot velocity, the wet as well as the dry density of the paint material. Finally, the simulated two phase bulk flow field was applied to study the dispersion/deposition of nano- and sub-micro particles. 


\section{Simulation results}

Trajectory calculation for spray jet using micro-meter sized particles

At first, air flow using $k-\varepsilon$ turbulence model and trajectory calculations using micro-sized particles were carried out. Figure 9 shows the air velocity close to the atomizer. High velocities about $370 \mathrm{~m} / \mathrm{s}$, can be observed at the air nozzles. Droplets were injected in a circular region with a radius of $2 \mathrm{~mm}, 5 \mathrm{~mm}$ downstream the liquid nozzle and above the cross-section of the shaping air flow jets. The flat spray jet, as shown in Fig. 10, was created because of the shaping air flow jets. To provide a sensible resolution of the entire flow field, the velocity contours are depicted in the range of $0-30 \mathrm{~m} / \mathrm{s}$ in Fig. 10. A quite narrow elliptic flow region is formed with a narrow extension along $z$, which results in a narrow elliptic film pattern on the plate (the so-called static film thickness distribution or static film growth rate $[\mu \mathrm{m} / \mathrm{s}])$. The calculated dynamic dry film thickness profile was compared with the experimental result that was measured by means of magneto-inductive method. A good agreement between measured and predicted film thickness was obtained (Fig. 11). Except the film thickness distribution, non-volatile fractions in droplets for both paint liquids were also analysed. As shown in Fig. 12, solvents were fully evaporated for droplets with diameter smaller than $1 \mu \mathrm{m}$, which delivers important information for the nanoparticle sampling measurement. A re-condensation in paint overspray can be well neglected, since the solvent concentration far away from the spray jet is quite low and the process temperature is constant. The validated numerical simulation results in this section provide the reasonable flow field of spray jet for the further study of dispersion/deposition of nanoparticles.

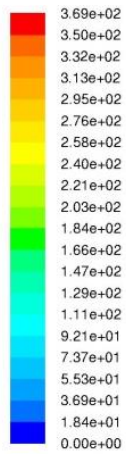

Figure 9. Velocity contours near the atomizer

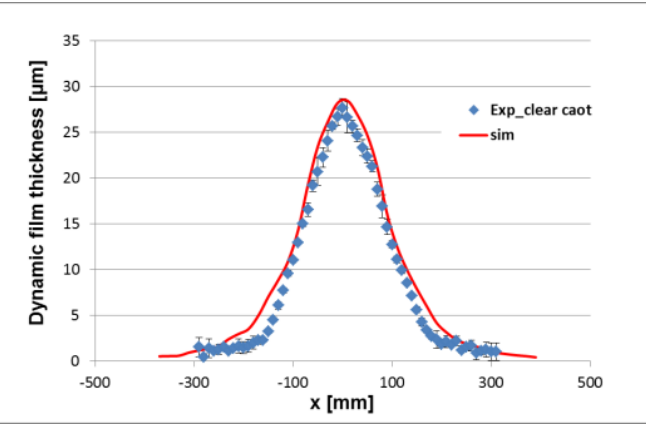

Figure 11. Comparison of measured and calculated dynamic film thickness distributions for clear coat

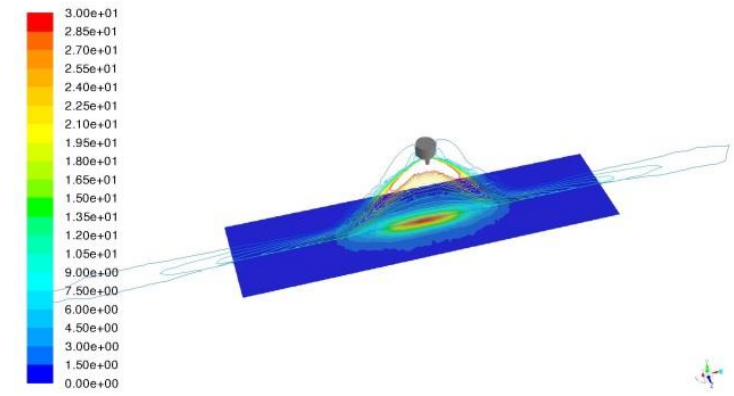

Figure 10. Calculated velocity contours colored by velocity magnitude $[\mathrm{m} / \mathrm{s}]$ in the plane $\mathrm{z}=0$. The static film thickness distribution on the plate is also overlaid.

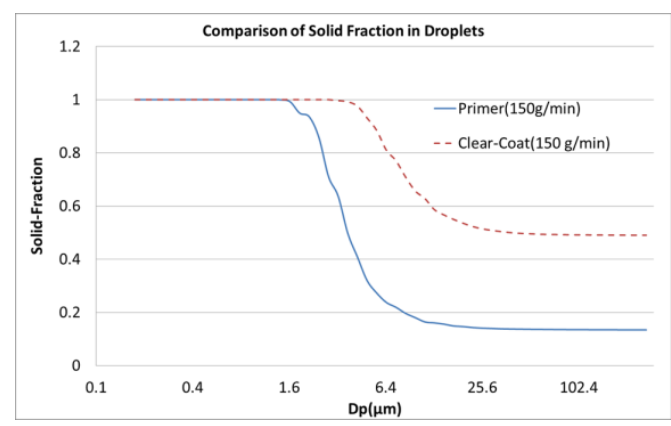

Figure 12. Comparison of solid (non-volatile) fraction in droplets on the target plate

\section{Trajectory calculation with consideration of nanoparticles}

As mentioned above, a computational domain $\left(3.5 \times 0.22 \times 2.0 \mathrm{~m}^{3}\right)$ with a hexahedral mesh model without atomizer was used so that simulations of turbulent flow with RSM could be carried out without any difficulty. In order to study effects of turbulence models on particle deposition, simulation with the k- $\varepsilon$ model was also performed. The air flow field and particle trajectory information downstream from the liquid nozzle in Fig. 10, namely at the crosssection of $y=15 \mathrm{~mm}$, were used as inlet conditions for the current computational domain. The obtained spray jet flow field using RSM (Fig. 13) was compared with that in Fig. 10, which ensured the similar spray jet flow field due to the interpolation of inlet boundary conditions. 
For the analysis of simulation results, more attention was paid to the turbulent near wall flow on the spray target. Distributions of turbulent kinetic energy $k$ and the normal turbulent fluctuation $v^{\prime 2}$ in cells adjacent to the wall along the centre line of the spray pattern were depicted in Fig. 14. The differences between RSM and k- $\varepsilon$ model in the spray pattern centre are quite small, but higher $k$ and the normal turbulent fluctuation can be observed at $x= \pm 0.15 \mathrm{~m}$ for the results using the $k-\varepsilon$ model. However, it is interesting to examine the mean normal velocity component close to the wall. Figure 15 shows negative Uy component in wall cells by using RSM, whereas almost positive values with $\mathrm{k}-\varepsilon$ model. The negative Uy, in wall cells, that is in line with the spray jet direction will enhance the particle deposition.

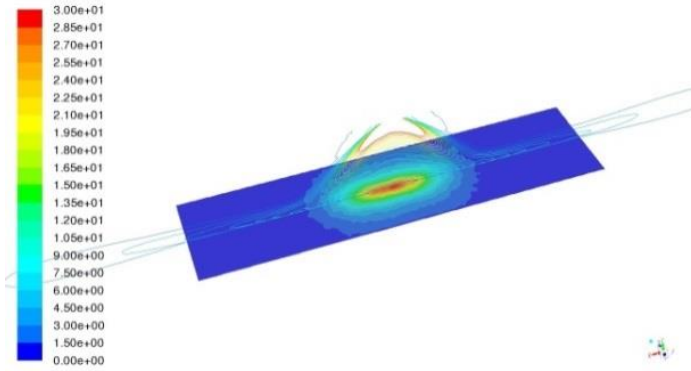

Figure 13. Calculated velocity contours colored by velocity magnitude $[\mathrm{m} / \mathrm{s}]$ in the cross-section $\mathrm{z}=0$ with hexahedral mesh model without atomizer. The static film thickness distribution on the plate is also overlaid.

Particle trajectory calculations were then carried out first using micro-sized particles (Fig. 3) to study the effect of turbulence model on particle deposition rate. The corresponding transfer efficiency TE $=64 \%$ for RSM and $63 \%$ for $\mathrm{k}-\varepsilon$ model were obtained.

The effect of $C_{L}$ on particle dispersion/deposition in EIM with RSM was also studied using $C_{L}=0.15$ and $C_{L}=0.8$. It was found that the influence of $C_{L}$ is quite small for high-inertia particles, i.e. large particles. However, the effect of $C_{L}$ for small particles cannot be neglected. Figure 16 shows sample trajectories of $0.5 \mu \mathrm{m}$ particle by using RSM and two values of $C_{L}$. With $C_{L}=0.15$ most of particles stream along the long axis of the elliptic spray pattern and away from two sides of the wall, which results in a quite dilute particle concentration in the direction of the short axis of the target. In contrast, a higher nanoparticle concentration was measured in the direction of the short target axis (Fig. 7) than that at the longer axis (Fig.8). The particle dispersion with $C_{L}=0.8$ in Fig. 16 shows similar trend as the experiment and is therefore considered to be reasonable. The TE with $C_{L}=0.15$ is $30 \%$ and $40 \%$ with $C_{L}=0.8$ in Fig. 16 .

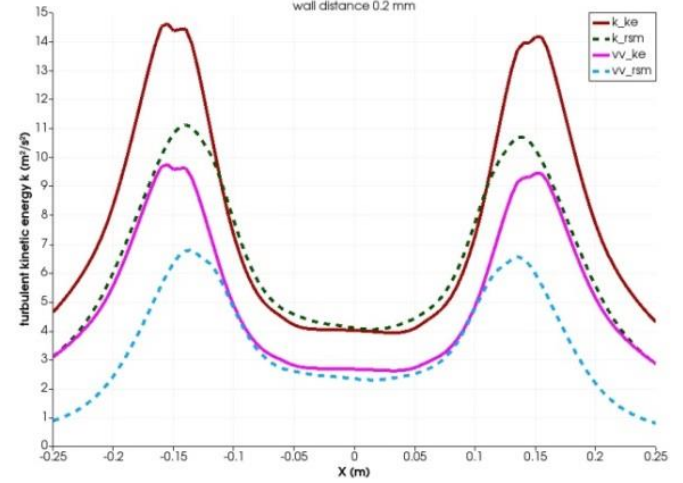

Figure 14. Turbulent kinetic energy and normal fluctuation $v^{\prime 2}$ in the wall cell
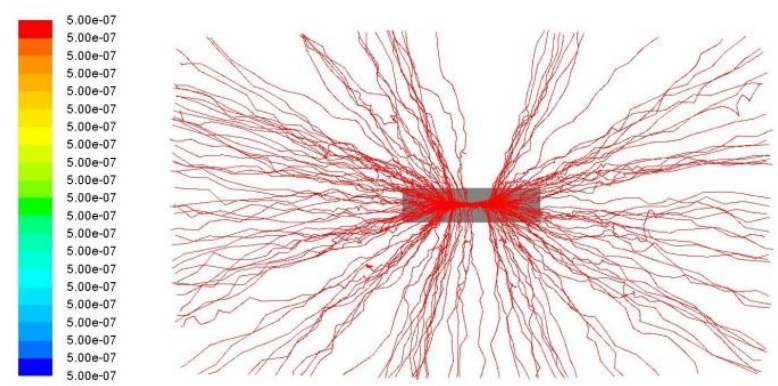

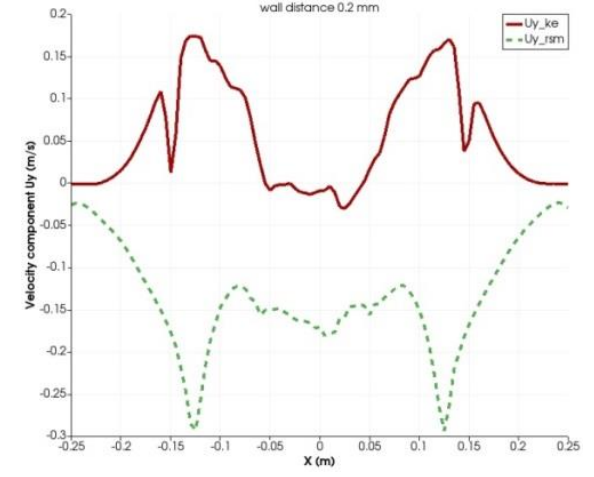

Figure 15. Normal velocity component Uy in the wall cell

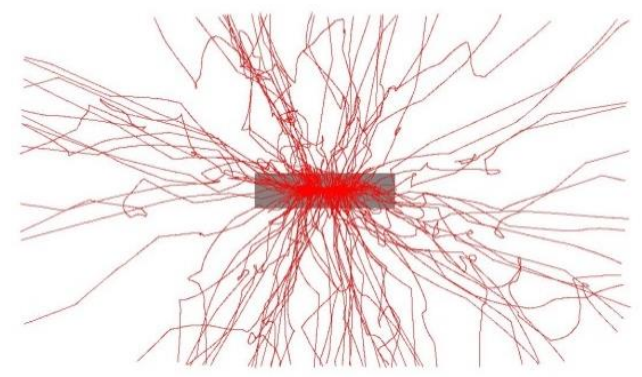

Figure 16. Trajectories of $0.5 \mu \mathrm{m}$ particles, left $C_{L}=0.15$, right $C_{L}=0.8$

Finally, the total nanoparticle distribution for the given operation conditions was fitted by means of the measured aerosol concentrations. The simulated concentrations of small paint aerosol particles were compared with the experiment at the sampling position $(\mathrm{L} 50 \mathrm{~cm}, \mathrm{~W} 0, \mathrm{H} 79.5 \mathrm{~cm})$ and shown in Fig. 17. In the figure, the experimental data was converted to obtain non-normalized particle concentrations, to be able to compare with those obtained from numerical simulations. For the simplification the particle distributions were regrouped and the background 
concentration was subtracted from the measured concentration. Based on the current experimental and numerical simulation results, the total number of nano and sub-micro particles in the range of diameter from $20-550 \mathrm{~nm}$ from the given spray gun is obtained, which is $1.56 \times 10^{7}$ per gram of paint ejection for water borne paint and $5.2 \times 10^{8}$ for clear coat. The results deliver important information for the further investigation of worker exposure to paint overspray.

\section{Conclusions}

Experimental and numerical studies of the determination of particle size distributions and concentrations in paint overspray created by a handcraft spray gun have been carried out. Although the droplet Sauter mean diameter obtained by micro particle size measurement is quite similar between two different paint materials, it was found that the solvent borne paint (clear coat) creates significantly higher number concentration of nano-sized droplets than the water borne paint. The relative low viscosity and strong solvent evaporation of clear coat could be the reason for the resulting atomisation behaviour. There were no significant differences of nano droplet concentrations between paints with and without manufactured nanomaterials, especially for the clear coat.

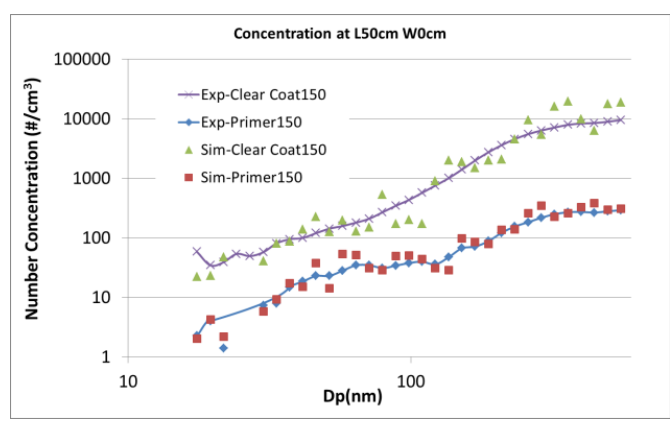

Figure 17. Comparison of simulated and measured nanoparticle concentrations at sampling position $\mathrm{L} 50 \mathrm{~cm}, \mathrm{~W} 0 \mathrm{~cm}$

Numerical simulations of droplet trajectories in spray booth both for micro and nano-sized droplets were carried out. The simulated two phase bulk flow field of a spay jet was validated by using measured film thickness distribution on the target. Particle dispersion and deposition for small-sized particles in turbulent near wall flow were then studied using two different turbulence models. The effect of turbulent time constant in the standard EIM in LPT was analysed. It was found that the default small value of $C_{L}$ yields unrealistic predictions for the sub-micro particle dispersion. Higher $C_{L}$ and RSM for near wall turbulent flow calculation were applied, which results in higher small-sized particle deposition than that using k- $\varepsilon$ model and standard EIM. Finally, representative nano and sub-micro particle distributions for a given spray gun were obtained based on the measured particle concentration in paint overspray, which delivers useful information for the further investigations of human exposure to a paint overspray in a practical paint booth and toxicological studies for evaluating the corresponding potential risk.

\section{Acknowledgements}

The present investigations have been supported by Verkehr, Innovation und Technologie, Austria (BMVIT) and Fraunhofer Society in Germany within a European joint research project NanoGeCo. This support is gratefully acknowledged by the authors.

\section{References}

[1] Kuhlbusch, T., Asbach C., Fissan, H., Göhler, D., and Stintz, M., Particle and Fibre Toxicology 2011, 8:22

[2] Carlton, G. N. \& Flynn, M. R. A, App. Occup. Environ. Hyg. 12, 375-382 (May 1997).

[3] Flynn, M. R. \& Sills, E. D., Annals of Occupational Hygiene 44, 191-202 (Aug. 2000).

[4] Ye, Q. and Domnick, J. Coat. Technol. Res., 14(2) 467-476 2017, DOI 10.1007/s11998-016-9867-4

[5] Ye, Q., Shen, B, Bauernhansl, T \& Dominick, J., Atomization and Sprays 25, 643-656 (2015).

[6] Ye, Q. \& Pulli, K., Coatings 2017, 7, 13.

[7] Göhler, D. \& Stintz, M., Journal of Nanoparticle Research 16, 1-15 (2014).

[8] Stintz, M, Technical Report, TU Dresden, 2016

[9] Wang, Y., \& James, P.W. (1999), International Journal of Multiphase Flow, 25, 551-558.

[10] Dehbi, A. (2008), International Journal of Multiphase Flow, 34, 819-828.

[11] Agnihotri, V., Ghorbaniasl, G., Verbanck, S., Lacor, C., J. of Aerosol Science 47 (2012) 39-47.

[12] Mehel, M., Sagot, B., Tanière, A., and Oesterlé, B., Int. J. Nonlinear Sci. Numer. Simul. 2012; 13(6): 417-425

[13] Stintz, M, Technical Report, TU Dresden, 2016

[14] Domnick, J. Gruseck, D., Pulli, K., Scheibe, A. Ye, Q., and Brinckmann, F., Chemical Engineering and Processing 50 (2011) 495-502

[15] Ye, Q., Tiedje, T. and Shen, B., ILASS - Europe 2014, 26th Annual Conference on Liquid Atomization and Spray Systems, 8-10 Sep. 2014, Bremen, Germany

[16] Graham, D.I., and James, P.W., IMA Journal of Mathematics Applied in Business \& Industry (1996) 7, 149-179 


\title{
Experimental and Numerical Investigation of Phase Separation due to Multi-Component Mixing at High-Pressure Conditions
}

\author{
C. Traxinger ${ }^{1, *}$, H. Müller ${ }^{1, * *}$, M. Pfitzner ${ }^{1}$ \\ ${ }^{1}$ Institute for Thermodynamics, Bundeswehr University Munich, Germany \\ S. Baab², G. Lamanna ${ }^{2}$, B. Weigand ${ }^{2}$ \\ ${ }^{2}$ Institute of Aerospace Thermodynamics, University of Stuttgart, Germany \\ J. Matheis ${ }^{3}$, C. Stemmer ${ }^{3}$, N. A. Adams ${ }^{3}$ \\ ${ }^{3}$ Chair of Aerodynamics and Fluid Mechanics, Technical University of Munich, Germany \\ S. Hickel ${ }^{4}$ \\ ${ }^{4}$ Faculty of Aerospace Engineering, Technische Universiteit Delft, The Netherlands \\ *Corresponding author: christoph.traxinger@unibw.de \\ **Now at: MTU Aero Engines AG, hagen.mueller@mtu.de
}

\begin{abstract}
Experiments and numerical simulations were carried out in order to contribute to a better understanding and prediction of high-pressure injection into a gaseous environment. Specifically, the focus was put on the phase separation processes of an initially supercritical fluid due to the interaction with its surrounding. N-hexane was injected into a chamber filled with pure nitrogen at $5 \mathrm{MPa}$ and $293 \mathrm{~K}$ and three different test cases were selected such that they cover regimes in which the thermodynamic non-idealities, in particular the effects that stem from the potential phase separation, are significant. Simultaneous shadowgraphy and elastic light scattering experiments were conducted to capture both the flow structure as well as the phase separation. In addition, large-eddy simulations with a vaporliquid equilibrium model were performed. Both experimental and numerical results show phase formation for the cases, where the a-priori calculation predicts two-phase flow. Moreover, qualitative characteristics of the formation process agree well between experiments and numerical simulations and the transition behaviour from a dense-gas to a spray-like jet was captured by both.
\end{abstract}

\section{Keywords}

elastic light scattering, shadowgraphy, large-eddy simulation, Peng-Robinson, tangent plane distance

\section{Introduction}

Injection into a high-pressure gaseous environment is a crucial process within energy conversion machines. Nowadays, many fluid flow devices are operated at pressures that exceed the critical pressure $p_{c}$ of the involved pure fluids. The increase in operating pressure in aircraft and car engines mainly stems from the demand for higher engine efficiency and reduced $\mathrm{CO}_{2}$ emissions. The main reason for rising the chamber pressure in liquid rocket engines (LREs) is the proportionality between operating pressure and specific impulse [1]. Typically, the operating pressure in LREs is supercritical with respect to both fuel and oxidizer $\left(p>p_{c}\right)$, whereas the injection temperature may be sub- or supercritical, corresponding to liquid-like or gas-like states. At supercritical pressure, the fluid properties, such as density, enthalpy and viscosity, are highly non-linear functions of temperature and pressure. Furthermore, phase separation due to non-linear interaction of the different components may occur. The phenomenon of phase separation due to mixing at high pressures is well-known in process engineering. Remarkably, up to now, high-pressure fuel injection into a gaseous environment is not completely understood and no commonly accepted theoretical approach exists.

Within the past 20 years, many research groups have focused on understanding the behaviour of jets at high pressures using experimental and numerical methods. Chehroudi et al. [2] injected cryogenic nitrogen into gaseous nitrogen at sub- and supercritical pressures. Based on shadowgraphy visualizations, they observed classical twophase phenomena at subcritical pressure indicated by very fine ligaments and droplets being ejected from the jet. As the pressure exceeds the critical value, surface tension effects diminish and the enthalpy of vaporization disappears. As a consequence, droplets were no longer detected and finger-like structures were observed on the jet surface. Similar phenomena occur in multi-component mixtures. Mayer et al. [3] investigated coaxial $\mathrm{LN}_{2} / \mathrm{GHe}$ injection into $\mathrm{GHe}$ and reported a drastic change of the interfacial structure depending on the chamber pressure. In the low pressure case, a liquid spray with droplets was formed, whereas the breakup seemed to transit into gas-like turbulent mixing for pressures significantly beyond $p_{c}$. Another interesting situation for two-phase disintegration was experimentally investigated by Roy et al. [4]. Here, an initially supercritical fluid was injected into a supercritical pressure environment. It was found that, for sufficiently low ambient temperatures, the jet undergoes phase separation leading to the formation of droplets and ligaments in the jet. As stated above, this mainly stems from the interaction between the injectant and the surrounding gas that, in turn, demands for an accurate thermodynamic framework in order to investigate high-pressure injection numerically. Popular numerical test cases are the jets investigated by Mayer et al. [5], where cold (cryogenic) nitrogen is injected into a warm nitrogen environment at supercritical pressure and thus phase separation is not likely to occur. These jets were numerically investigated by different groups, e.g., Zong et al. [6], Schmitt et al. [7] and Müller et al. [8], using the commonly 
accepted dense-gas approach. A multi-component benchmark case is the Spray-A of the Engine Combustion Network (www. sandia.gov/ECN), where cold n-dodecane is injected into a warm nitrogen atmosphere at a pressure of $6 \mathrm{MPa}$, which exceeds the critical pressure of both components. Different modelling approaches, e.g., Lagrangian particle tracking in Wehrfitz et al. [9], a single-phase dense gas approach in Lacaze et al. [10] and a two-phase approach based on a vapor-liquid equilibrium model in Matheis and Hickel [11], have been used to simulate this test case. Up to now, none of these approaches is commonly accepted because of a common disagreement on the actual state of the fluid or the mixture. This is partially due to the lack of experimental data and well-defined boundary conditions. The latter are highly important as flow phenomena may be very sensitive to thermodynamic (mixture) states.

It is the objective of this study to gain a better understanding of high-pressure injection and mixing. Experiments and numerical simulations were conducted, in which accurate synchronization of the thermodynamic boundary conditions was assured. For these investigations, a multi-component system consisting of $n$-hexane and nitrogen was chosen and a systematic study was carried out at supercritical pressure with respect to the pure components value. The injection temperatures of $n$-hexane were chosen such that they cover regimes in which the thermodynamic non-idealities, in particular, the effects that stem from the potential phase separation, are significant. The objective of this paper is to investigate whether or not a clear transition from a dense-gas to a spray-like jet with droplets can be observed. The study shows that the large-eddy simulation (LES) in combination with a vapor-liquid equilibrium (VLE) model [11] is able to predict the experimental results very well.

\section{Experimental facility and injection system}

The injection experiments of $n$-hexane into nitrogen ( $\geq 99.9990 \%$ purity) have been conducted at the ITLR (University of Stuttgart) using the experimental and optical setup that is sketched in Fig. 1 . The facility consists of a cylindrical constant-volume chamber $\left(V \approx 4 \cdot 10^{-3} \mathrm{~m}^{3}\right)$ that is designed for injections into non-heated ambient gas

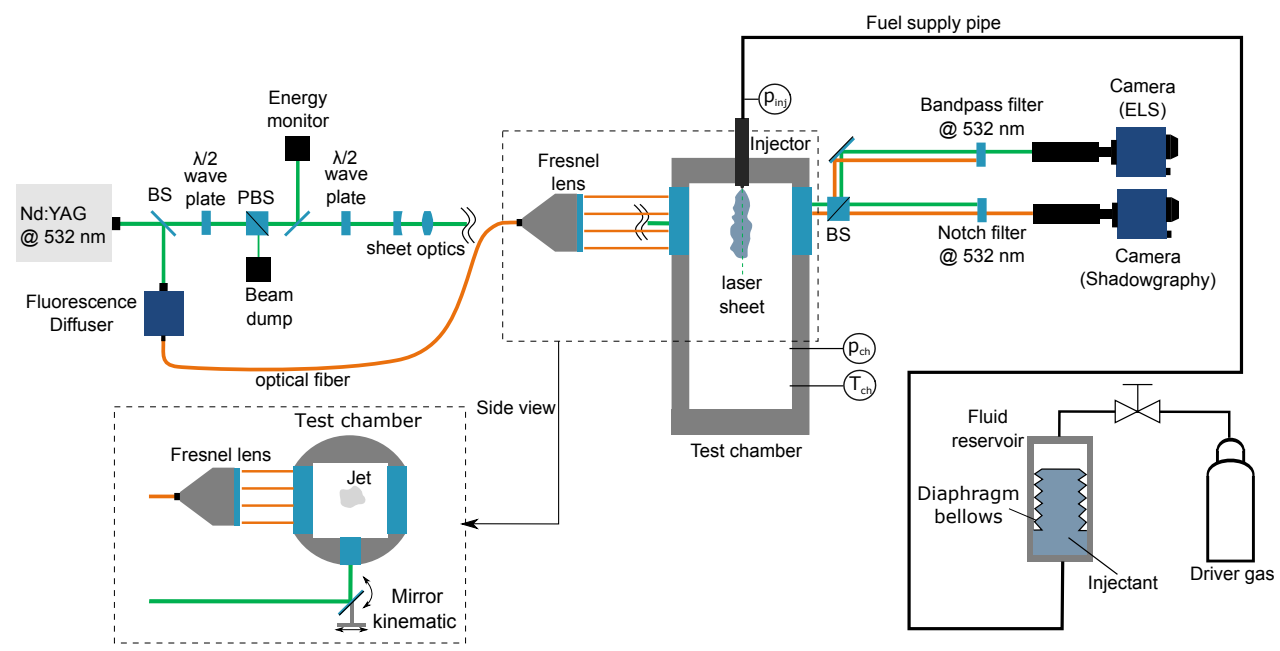

Figure 1. Experimental facility including optical arrangement for simultaneous shadowgraphy/ELS measurements.

with a maximum pressure of up to 60 bar. The chamber pressure $p_{c h}$ was measured with a piezoresistive sensor (Keller PA-21Y, $0.25 \%$ uncertainty), while a resistance thermometer provided the chamber temperature within $\pm 1 \mathrm{~K}$. Three quartz windows (two at the side walls, one at the bottom) enabled optical access into the chamber. A magnetic-valve common-rail injector (distributed by Robert Bosch AG) is mounted to the chamber such that the center axes of the injector nozzle and the cylinder coincide. The injector nozzle is custom-made and features a single straight-hole of diameter $D=0.236 \mathrm{~mm}$ and length $L=0.8 \mathrm{~mm}$ (i.e. $L / D \approx 3.4$ ). It is important to note that the focus of this study was put on jets with low expansion ratios $p_{i n j} / p_{c h}$, which is contrary to conventional Diesel engine applications operating at injection pressures of typically several hundred bar. Consequently, we made adjustments to the needle/spring configuration in order to provide proper needle lift also for comparably low $p_{i n j}$ of $<60$ bar (as required here). $\mathrm{N}$-hexane was thoroughly degasified prior to the campaign and stored in a fluid reservoir that is separated into two sections using a diaphragm bellows. The section that contained the $\mathrm{n}$-hexane was directly connected to the fuel pipe of the injector. The exterior section was filled with a driver gas to establish the injection pressure, which was also measured with a piezoresistive sensor (Keller PA-23, 0.2\% uncertainty). Using this configuration avoids contamination of the injectant with dissolved driver gas, which is of particular importance for near-critical fluid investigations. The injection temperature $T_{i n j}$ was controlled by two heater cartridges with an uncertainty of $\pm 2 \mathrm{~K}$ as detailed in Baab et al. [12].

\section{Optical techniques and data processing}

We used parallel-light direct shadowgraphy in combination with planar 90 degree-elastic light scattering (ELS) for the experimental jet analysis. In several recent studies, this choice has been shown suitable to assess both the geometric jet topology as well as the occurrence of two-phase regions embedded within the dense core region $[13,14]$. Specifically, scattered signal intensities from a fluid jet illuminated by a light source are very sensitive to 

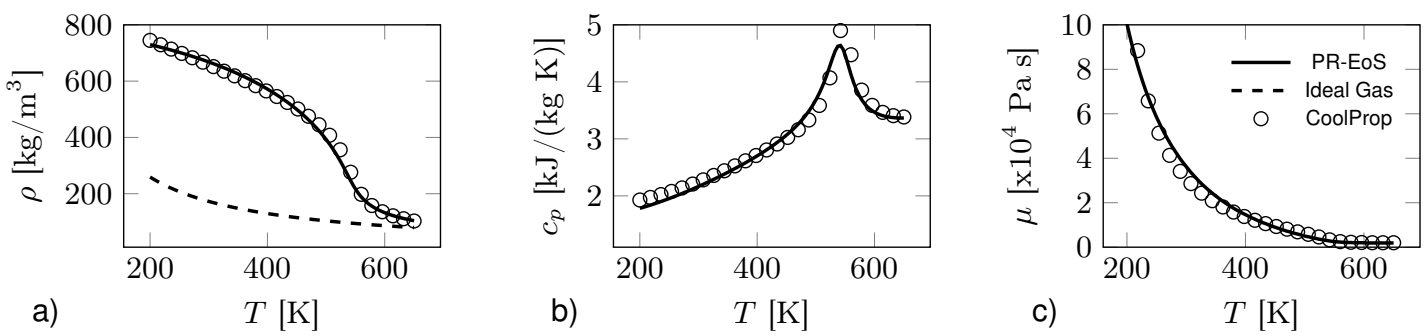

Figure 2. Comparison of PR-EoS and ideal gas EoS with reference data obtained from CoolProp [17] for $\mathrm{n}$-hexane at $p=5 \mathrm{MPa}$; a) density $\rho$, b) specific heat at constant pressure $c_{p}$ and c) dynamic viscosity $\mu$.

the size of present scattering particles. Thus, signal magnitudes vary by several orders of magnitude among singlephase flow (molecular Rayleigh scattering) and two-phase flows with embedded - potentially very small - droplets in the particle Rayleigh or Mie regime, Miles et al. [15]. Proper acquisition and interpretation of the scattered light portion, therefore, allows for a qualitative characterization of multi-phase regions within a fluid flow.

The used optical arrangement resembles the one used in Baab et al. [14] in the sense that a single frequencydoubled pulse from an Nd:YAG laser (Continuum Powerlite DLS 8010, $\lambda=532 \mathrm{~nm}$ ) provided illumination for both the shadowgraphy and ELS measurement. It is important to note that this assures instantaneous and simultaneous acquisition of the experimental data. Moreover, stereoscopic image correction of shadowgram and ELS image enables accurate assignment of two-phase phenomena to the local jet region and specific flow features revealed in the shadowgram (e.g. jet core or mixing layer). Both images were captured with a LaVision sCMOS camera (2560 x 2160 pixels, 16-bit dynamic range or 0-65535 digital counts) and a long-distance microscope (Infinity K2 DistaMax) leading to a resolution in the object plane of around $9 \mu \mathrm{m} /$ pixel. For the ELS measurement, a laser sheet was formed by serial arrangement of a plano-concave and a plano-convex cylindrical lens with focal lengths of -200 and $500 \mathrm{~mm}$, respectively. The laser sheet was reflected into the chamber through the bottom window (see side view in Fig. 1), where the sheet precisely aligned with the nozzle center axis. The excitation intensity could be continuously varied using a $\lambda / 2$ wave plate in combination with a polarizing beam splitter cube and was monitored with a pyroelectric energy head. Shadowgraphic illumination was attained by guiding a fraction of the laser pulse into a fluorescence diffuser connected to a Fresnel lens. As the diffuser red-shifts the laser light to a spectrum of approx. $574-580 \mathrm{~nm}$, the shadowgraphy and ELS signals could be captured independently by spectral filtering. Specifically, a $532 \mathrm{~nm}$-notch filter removed scattered laser light in the shadowgram, whereas a $532 \mathrm{~nm}$-narrow band-pass filter eliminated the diffuser light in the ELS image. Dark-frame subtraction reduced the noise level in the ELS image below 10 counts. We additionally rescaled the noise-corrected camera intensity $I_{\text {cam }}$ (in counts) with the measured excitation intensity $I_{0}$ according to $\tilde{I}=I_{\text {cam }} / I_{0}$ (counts $/ \mathrm{mJ}$ ) to account for inevitable shot-to-shot variability of the laser. Furthermore, ELS experiments at different magnitudes of $I_{0}$ remain comparable (in assumption of a linear scattering response). Note, once again, that scattering intensities are subject to strong variations depending on the two-phase jet properties (e.g. droplet sizes and number densities). Consequently, $I_{0}$ was therefore adjusted to assure proper sensor saturation for all experimental conditions.

\section{Numerical method}

In order to investigate high-pressure injection, large-eddy simulations have been carried out with two different solvers. These solvers have been extensively used for supercritical injection simulations of both pure as well as binary jets, see e.g., [8, 11, 16], whereby one of those solvers is the density-based INCA (www.inca-cfd.com) and the other is a pressure-based version of OpenFOAM (www.openfoam.org). For the reason of clarity and with thermodynamic aspects being the main objective - not the cross-comparison of LES codes - only one set of LES results (INCA) is presented in this paper. Identical thermodynamic models are implemented in both solvers and are discussed in the following.

At supercritical pressures, intermolecular forces become increasingly important. Using the equation of state (EoS) for an ideal gas would lead to severe deviation from the actual fluid properties, see Fig. 2a. Commonly accepted, especially in LES, are cubic EoS and the approximation of real-gas behaviour/effects based on the corresponding state principle. In the present study, the cubic EoS of Peng and Robinson [18] (PR-EoS)

$$
p=\frac{R T}{v-b}-\frac{a}{v^{2}+2 v b-b^{2}}
$$

is used, where $R$ is the universal gas constant, $T$ is the temperature, $v$ is the molar volume and $a$ and $b$ account for the intermolecular attractive and repulsive forces, respectively. For calculating $a$ and $b$, the concept of a one-fluid mixture in combination with mixing rules is used [19]:

$$
a=\sum_{i}^{N_{c}} \sum_{j}^{N_{c}} z_{i} z_{j} a_{i j} \quad \text { and } \quad b=\sum_{i}^{N_{c}} z_{i} b_{i} .
$$


Here, $z_{i}$ is the mole fraction of species $i$, whereby in the following we denote the ofverall mole fraction by $\mathbf{z}=$ $\left\{z_{1}, \ldots, z_{N_{c}}\right\}$ and the liquid and vapor mole fractions by $\mathbf{x}=\left\{x_{1}, \ldots, x_{N_{c}}\right\}$ and $\mathbf{y}=\left\{y \mid \ldots, y_{N_{c}}\right\}$, respectively. The variables $a_{i j}$ and $b_{i}$ in Eq. (2) are calculated based on the corresponding state princip e:

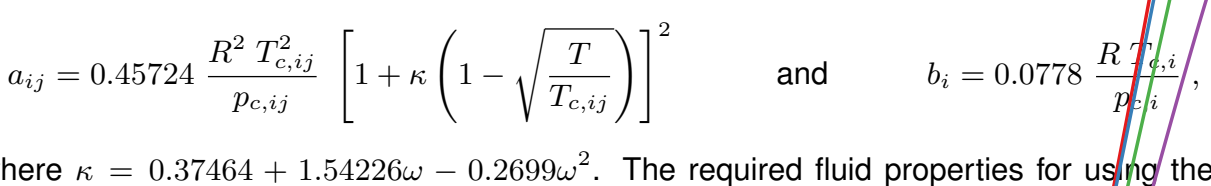

where $\kappa=0.37464+1.54226 \omega-0.2699 \omega^{2}$. The required fluid properties for us $\ln$ the PR-EoS are the critical pressure $p_{c}$, the critical temperature $T_{c}$ and the acentric factor $\omega$. For the off-diagohal elemients of $a_{i j}$, the pseudocritical combination rules are used in this study [20]:

$$
\begin{aligned}
& \omega_{i j}=0.5\left(\omega_{i}+\omega_{j}\right), \quad v_{c, i j}=\frac{1}{8}\left(v_{c, i}^{1 / 3}+v_{c, j}^{1 / 3}\right)^{3}, \quad Z_{c, i j}=0.5\left(Z_{c, i}+Z_{c, j}\right) \\
& T_{c, i j}=\sqrt{T_{c, i} T_{c, j}}\left(1-k_{i j}\right) \quad \text { and } \quad p_{c, i j}=Z_{c, i j} R T_{c, i j} / v_{c, i j} .
\end{aligned}
$$

It has to be noted that in this study the PR-EoS was used in a predictive manner, meaning the binary irteraction parameter $k_{i j}$ in Eq. (4) was set to zero. The comparison with experimental detta of vapor-liquid equilibr a, however, shows good agreement for the investigated pressure around $5 \mathrm{MPa}$, see Fig. $\$$. Especially the dew-point line shows very good agreement, which is of essential importance in this study.
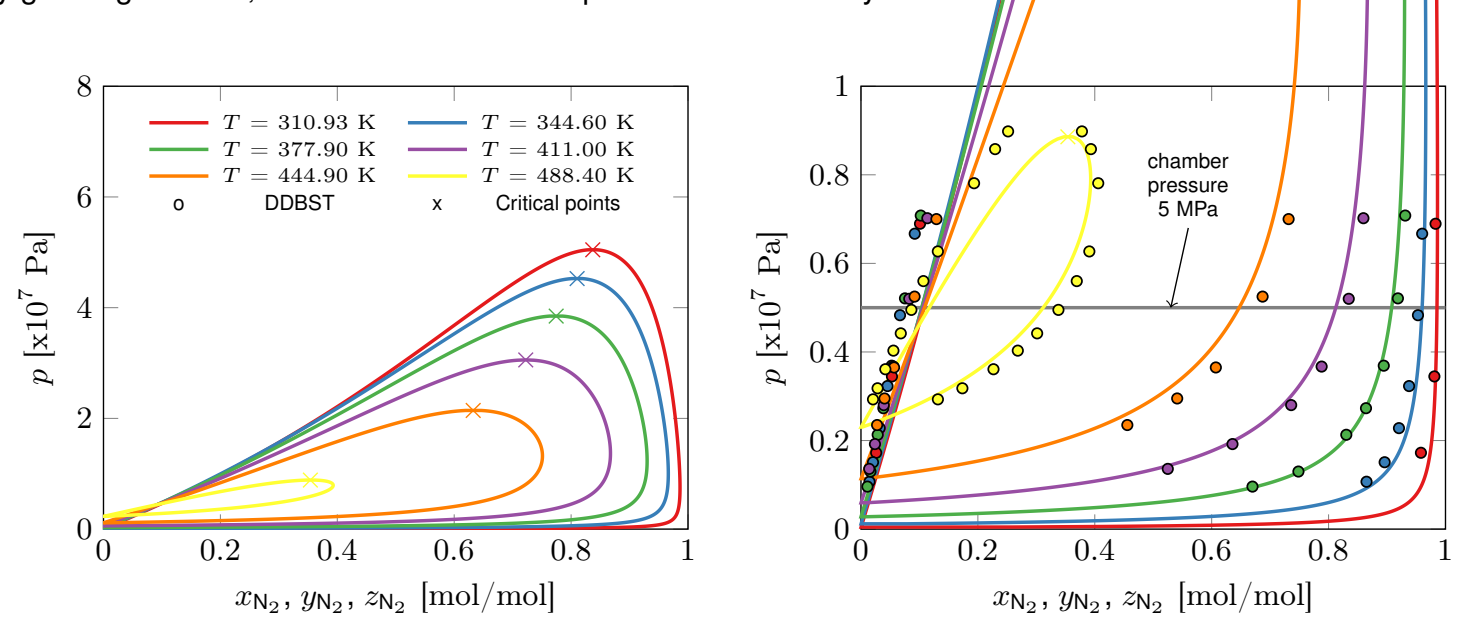

Figure 3. Vapour-liquid equilibria at constant temperatures for the investigated binary $n$-hexane nitrogen mixture; reference data from Dortmund Data Bank Software \& Separation Technology (DDBST), Poston et al. [21] and Eliosa et al. [22].

Caloric properties are computed with the departure function formalism, Poling et al. [19]. The reference condition is determined using the nine-coefficient NASA polynomials proposed by Goos et al. [23]. The empirical correlation of Chung et al. [24] is used for the calculation of the viscosity and the thermal conductivity. For pure n-hexane the present thermodynamic model and the reference data from CoolProp [17] show excellent agreement, see Fig. 2.

In this study, the single-phase dense-gas approach was extended with a vapor-liquid equilibrium model which is inspired by the work of Qiu and Reitz [25]. In this approach, the single-phase solution of the mixture is considered stable if and only if the Gibbs energy is at its global minimum, see, e.g., Michelsen and Mollerup [26]. To check whether a mixture is stable or not, the tangent plane distance (TPD) method of Michelsen [27]

$$
T P D(\mathbf{w})=\sum_{i} w_{i}\left[\ln w_{i}+\ln \varphi_{i}(\mathbf{w})-\ln z_{i}-\ln \varphi_{i}(\mathbf{z})\right]
$$

is used, where $\mathbf{w}=\left\{w_{1}, \ldots, w_{N_{c}}\right\}$ is a trial phase composition and $\varphi_{i}$ is the fugacity coefficient of component $i$ calculated from the PR-EoS. If the TPD-analysis leads to a negative value for any of the trial phase compositions $\mathbf{w}$, the mixture is unstable and a separation in two phases, namely a vapor $(v)$ and a liquid $(l)$ phase, is done yielding a decrease in Gibbs energy. It is assumed that this phase split occurs instantaneously and an adiabatic flash is solved. The solution is characterized by the equality of the fugacities $f$ of each component $i$ in the considered phases at a given temperature, pressure and overall composition, i.e. $f_{i, l}(p, T, \mathbf{x})=f_{i, v}(p, T, \mathbf{y})$. Further details on the implementation can be found in Matheis and Hickel [11]. For details on the numerical discretization of the governing equations and turbulence model we refer to Hickel et al. [28].

\section{Test case definition}

A binary system consisting of $n$-hexane and nitrogen was chosen and a systematic study was carried out at super-

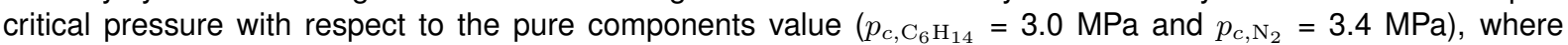
$\mathrm{n}$-hexane is injected into a quiescent nitrogen atmosphere at (cold) ambient temperature $T_{c h}$, see Fig. 1 and 
Table 1. Overview of the experimental conditions and boundary conditions used in the LES.

\begin{tabular}{c|cccccc}
\hline Case & $\begin{array}{c}p_{\text {ch }} \\
{[\mathrm{MPa}]}\end{array}$ & $\begin{array}{c}T_{c h} \\
{[\mathrm{~K}]}\end{array}$ & $\begin{array}{c}T_{t, \mathrm{C}_{6} \mathrm{H}_{14}} \\
{[\mathrm{~K}]}\end{array}$ & $\begin{array}{c}T_{\mathrm{C}_{6} \mathrm{H}_{14}} \\
{[\mathrm{~K}]}\end{array}$ & $\begin{array}{c}u_{\mathrm{C}_{6} \mathrm{H}_{14}} \\
{[\mathrm{~m} / \mathrm{s}]}\end{array}$ & $\begin{array}{c}\rho_{\mathrm{C}_{6} \mathrm{H}_{14}} \\
{\left[\mathrm{~kg} / \mathrm{m}^{3}\right]}\end{array}$ \\
\hline T480 & 5.0 & 293.0 & 480.0 & 479.3 & 51.0 & 443.2 \\
T560 & 5.0 & 293.0 & 560.0 & 554.8 & 72.1 & 202.0 \\
T600 & 5.0 & 293.0 & 600.0 & 595.0 & 90.3 & 136.9
\end{tabular}

Tab. 1. The pressure for all test cases was set to $p_{c h}=5 \mathrm{MPa}$, which correspond to a reduced pressure $p_{r}=$

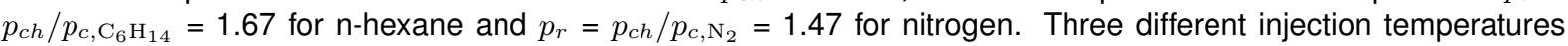

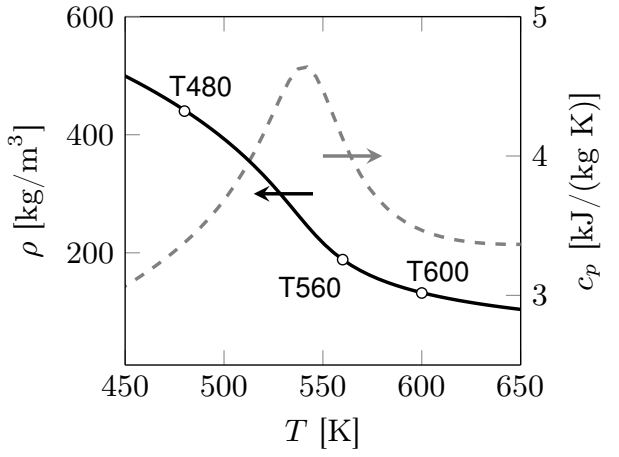

Figure 4. Density $\rho$ and heat capacity $c_{p}$ of $\mathrm{n}$-hexane at $5 \mathrm{MPa}$. Symbols mark injection conditions. were selected for the present study as defined in Tab. 1 and visualized in Fig. 4, based on a-priori calculations of the adiabatic mixture considering two-phase separation; for details see, e.g., Qiu and Reitz [25]. Case T480 shows strong twophase effects, see Fig. 5, and the adiabatic mixture fully penetrates the VLE at $5 \mathrm{MPa}$. For case T560, only minor two-phase effects can be expected, see Fig. 5 detailed view, and the $600 \mathrm{~K}$ case is expected to be a dense-gas jet. In addition to the potential phase separation, n-hexane shows strong real-gas effects in the temperature range between $450 \mathrm{~K}$ and $650 \mathrm{~K}$, see Fig. 4, which can be seen in terms of a strong peak in the heat capacity at around $550 \mathrm{~K}$. This is the point where the fluid crosses its Widom-line, see, e.g., Simeoni et al. [29], and where the fluid undergoes a drastic change from a liquid-like to a gas-like density within a finite temperature interval. The temperatures selected as case names correspond to the total temperature in the injector reservoir $T_{t, \mathrm{C}_{6} \mathrm{H}_{14}}$. The static temperature $T_{\mathrm{C}_{6} \mathrm{H}_{14}}$ as well as the outlet velocity at the nozzle exit $u_{\mathrm{C}_{6} \mathrm{H}_{14}}$ were calculated based on the assumption of an isentropic nozzle flow [14] and were used as boundary condition in the LES.

\section{Results and discussion}

In Fig. 6, experimental single-shot measurements and instantaneous LES results are compared. Snapshots were taken at a time sufficiently large such that the jets are fully developed and can be considered as quasi-stationary. Shadowgram (top frame) and simultaneously taken ELS image superimposed onto the corresponding shadowgram (bottom frame) visualize both the flow structure as well as the thermodynamic state. The intensity $\tilde{I}$ is shown on a logarithmic scale comprising almost three orders of magnitude. Focus shall hence be put on the overall scattering characteristics rather than quantitative evaluation of the measured signals. Values outside the color scale range were cut off to emphasize the distinction between regions of negligible $\tilde{I}$ and two-phase regions within the jet, indicated by high scattering intensities. In the LES, flow structures are visualized by the instantaneous temperature field (top frame). In the bottom frame the vapor volume fraction $\alpha_{v}$ is superimposed to indicate regions of two-phase flow. By doing so, a direct comparison of the phase formation phenomena in the experiment and LES is provided.
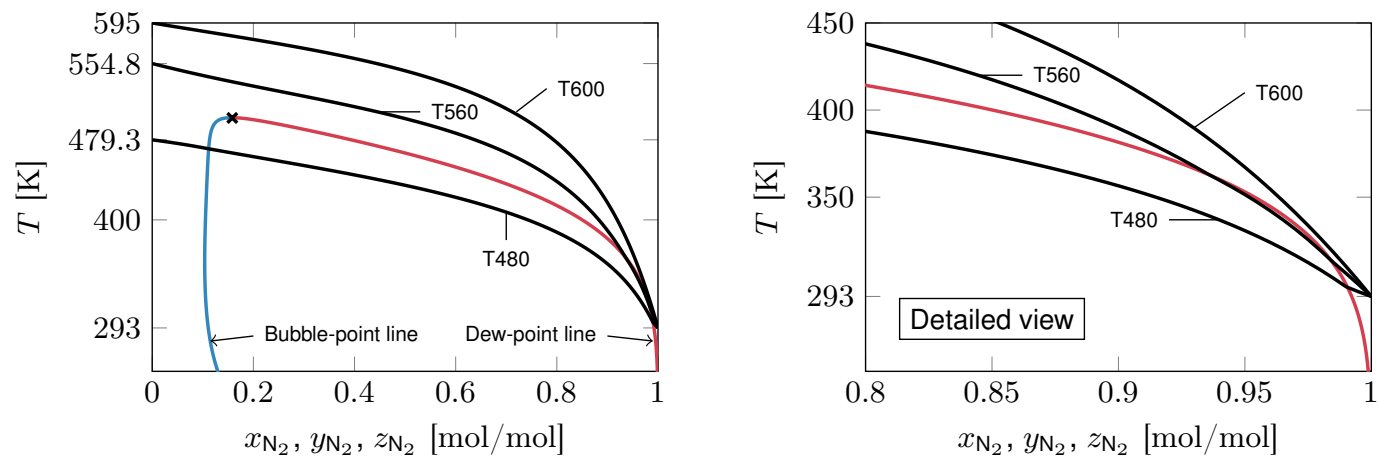

Figure 5. Adiabatic mixtures of $n$-hexane and nitrogen at $5 \mathrm{MPa}$.

The experimental results for Case T600 show a dark core being dissolved in the environment and finger-like structures emerging from the surface of the dark core, see Fig. 6a. No significant (stable) scattering signal is measured and as a result a single-phase state can be deduced. This finding agrees well with both the LES results and the adiabatic mixing line. The thermodynamic model does not predict any thermodynamically unstable states based on the applied tangent plane distance criterion, see Eq. (5). The stability of the single phase in the LES can be seen in more detail in Fig. 7a, where all conditions are presented by means of a scatter plot. All conditions in the LES follow the adiabatic mixture line closely and none of the points lies within the region of the vapor-liquid equilibrium at $5 \mathrm{MPa}$. It is worth mentioning that the jets being investigated are almost isobaric and are therefore not subjected to large fluctuations in pressure. Furthermore, the dew-point line does not show a strong dependency on the pressure close to the investigated pressure of $5 \mathrm{MPa}$, see Fig. 3, underlining the experimental observation that a pronounced 


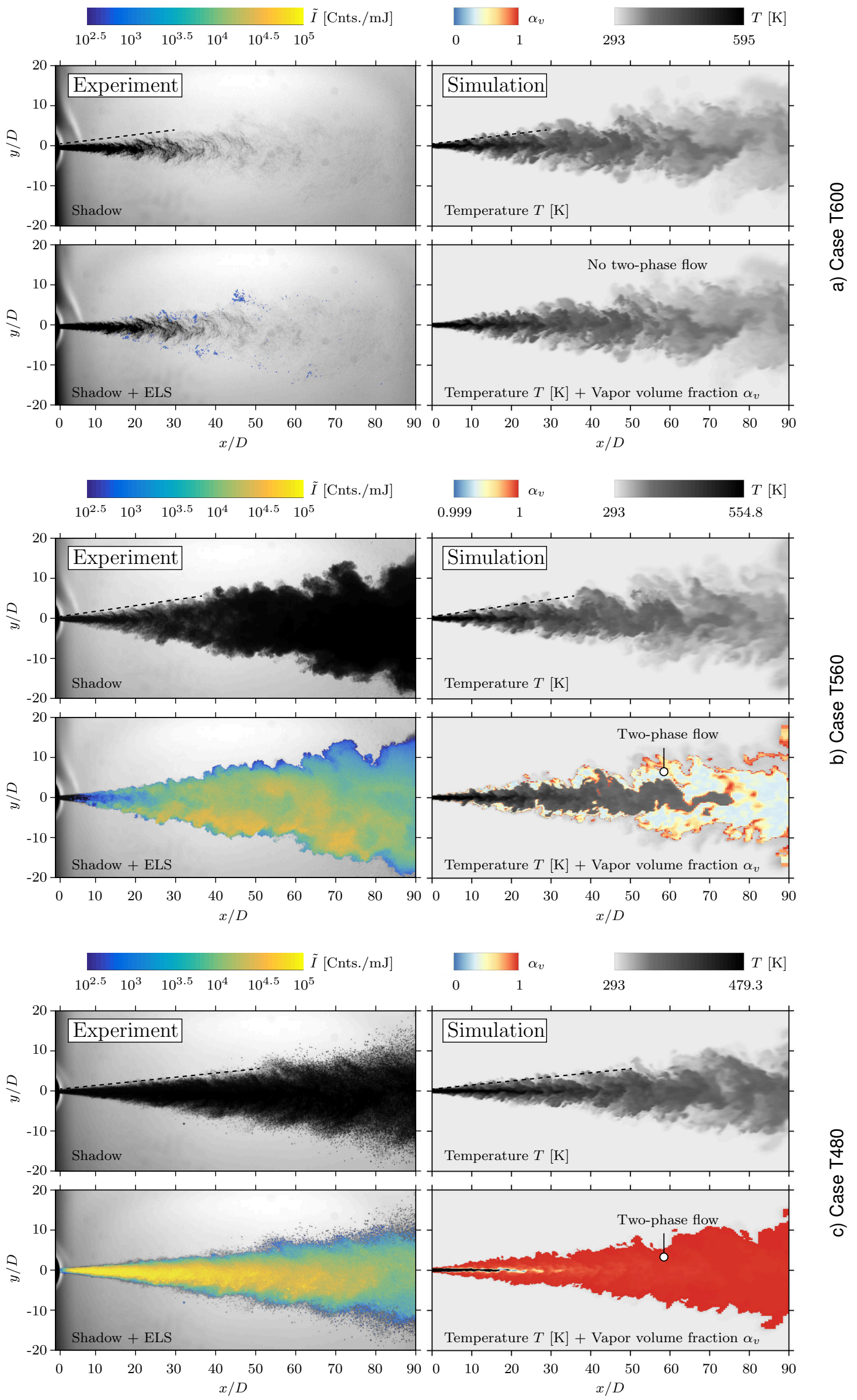

Figure 6. Comparison of experimental and numerical snapshots for the cases T600, T560 and T480. 
two-phase region within the jet does not exist. The minor signals determined in the experiment might stem from spatial fluctuations of thermodynamic properties, which may lead to small areas of phase separation. Downstream of $x / D \gtrsim 10$, these regions form in the outer shear layer but they are highly unstable and collapse instantaneously. Another reason for the detected scattering signal might be a local violation of the adiabatic mixture assumption due to, for instance, heat conduction.

A totally different picture results for case T560, see Fig. 6b. While the shadowgram resembles that for T600 in the near-nozzle region, a strong difference becomes apparent for $x / D \gtrsim 20$. Here, a dark region forms over the entire extension of the jet, which indicates the formation of a dense droplet cloud. This fact is clearly corroborated by a steep increase in the scattering intensity, which is initiated by droplet generation in the mixing layer for $x / D \gtrsim 10$. Further downstream, this flow mixes into the jet core, leading to a pronounced two-phase characteristic throughout the entire jet domain. In fact, this is proved by the LES result of case T560, where a large spatial area of the jet shows two-phase behaviour due to an unstable single-phase mixture determined by the TPD-criterion, see Eq. (5). In agreement with the experimental observation, the LES regions showing two-phase flow are located in the outer shear layer, which merge towards the jet centerline downstream of $x / D \gtrsim 60$. Due to the only minor penetration of the VLE, the vapor volume fraction in the regions with phase separation is very close to unity, see Fig. 7b. In the LES, the phase separation is occurring in the shear layer first where all mixture states are present, forming a layer of two-phase flow around the actual jet. Further downstream, this layer is growing steadily and, as additional nitrogen mixes with the jet, this leads to a progressing dilution and to a cooling of the mixture, see Fig. 6b. For $x / D \gtrsim 60$, this steady mixing and cooling results in a jet where a huge portion exhibits two-phase behaviour, which becomes obvious by comparing the LES snapshot and the $T, z$-diagram in Fig. $7 \mathrm{~b}$. As stated above, this is in very good agreement with the experimental results. The LES vapor-liquid equilibrium model is able to phenomenologically capture the transition from a dense-gas mixture (case T600) to a jet exhibiting phase separation (case T560). In addition, identical to the case T600, all states in the LES group around the adiabatic mixture line as predicted by the a-priori analysis, see Fig. 7b.

a)
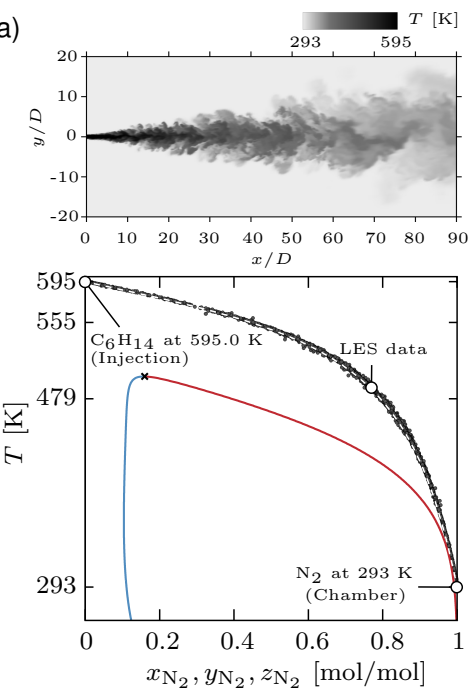

b)
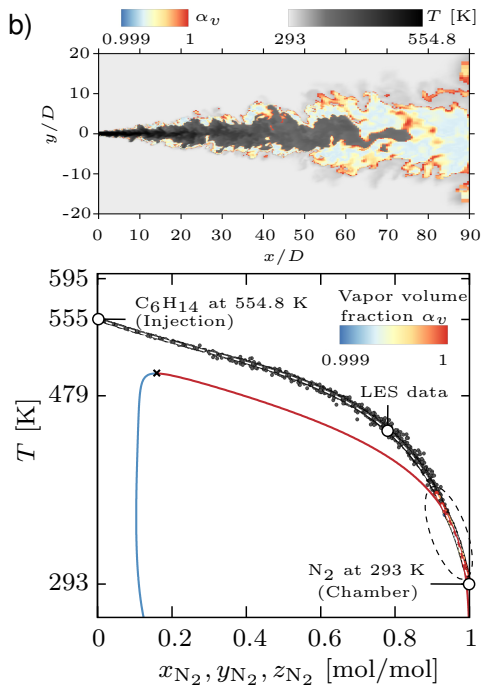

C)
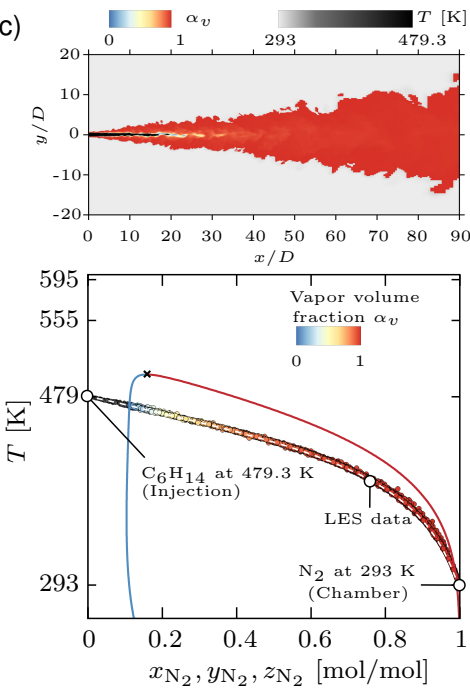

Figure 7. Scatter plots of the cases T600, T560 and T480 showing the thermodynamic state of the LES results together with the vapor-liquid equilibrium at $5 \mathrm{MPa}$ and the adiabatic mixture.

For Case T480, a drastic change in terms of flow phenomena occurs. A spray-like behaviour similar to atomized jets [2, 3] can be seen in Fig. 6c. The corresponding shadowgram shows a constant dark core with distinct droplets visible in the outer jet region for $x / D \gtrsim 15$. This pronounced two-phase characteristic directly reflects in the strong scattering throughout virtually the entire jet domain. As in case T560, the presence of this strong phase separation is confirmed by the LES where nearly the complete jet has entered the VLE, see Fig. 7c. In contrast to the cases T560 and T600, the adiabatic mixture of case T480 crosses both the bubble-point line as well as the dew-point line and the corresponding shadowgram shows a jet exhibiting spray-like behaviour. Due to the Eulerian approach used in the LES, no individual droplets are resolved and, therefore, the spray-like character is represented in terms of the vapor fraction covering the whole range from zero to one. A single-phase state is predicted only in the "dark" core at the injector inlet and in a thin area at the outermost region of the shear layer, see Figs. $6 \mathrm{c}$ and $7 \mathrm{c}$. As soon as the mixture crosses the bubble-point line at $z_{\mathrm{N}_{2}} \approx 0.1$, the jet exhibits two-phase behaviour. Due to the approximate parallelism of the adiabatic mixture line and the dew-point line, high vapor volume fractions larger than 0.5 result from $z_{\mathrm{N}_{2}} \approx 0.3$ on and cause the reddish color in the contour-plot of the jet in Figs. 6c and 7c.

Apart from the good prediction of the LES with respect to the thermodynamic effects, the opening angle of the jet shows a generally good agreement between experiments and numerical simulations for all three cases. For case T480, a smaller opening angle compared to the cases T560 and T600 is observed. For case T600, the LES appears to overestimate the opening angle slightly. It has to be noted, however, that shadowgraphy may be too insensitive to capture the entire jet domain as given by the LES. 


\section{Conclusions}

Experiments and numerical simulations of jet mixing at high pressure were carried out at three different research facilities in Germany in order to contribute to a better understanding and prediction of high-pressure injection and the phase separation processes in initially supercritical fluids. A multi-component system consisting of $n$-hexane and nitrogen was chosen and a systematic study was conducted at supercritical pressure with respect to the pure components value. $\mathrm{N}$-hexane was injected into a chamber filled with pure nitrogen at (cold) ambient temperature. The test case conditions were selected such that they cover regimes in which the thermodynamic non-idealities, in particular the effects that stem from the potential phase separation, are significant. Three different test cases have been presented and discussed in this paper. Simultaneous shadowgraphy and elastic light scattering experiments were conducted in order to capture both the flow structure as well as the phase separation. In addition, numerical simulations were carried out by means of large-eddy simulations with a vapor-liquid equilibrium model. Experimental and numerical results show phase separation and the transition from a dense-gas to a spray-like jet, where the a-priori calculation predicts two-phase flow. Characteristics of the formation process agree well between experiments and numerical simulations. The formation of a two-phase flow is initiated in the mixing layer some distance downstream of the nozzle and eventually mixes into the jet core at large distance.

This study serves as a basis for more thorough investigation of these kind of jets. A more detailed examination of the near injector region as well as a comparison of transient and averaged data from experiments and numerical simulations will be conducted.

\section{Acknowledgements}

The authors gratefully acknowledge the German Research Foundation (Deutsche Forschungsgemeinschaft) for providing financial support in the framework of SFB/TRR 40. Financial support was also provided by Munich Aerospace (www.munich-aerospace.de). Furthermore, the authors thank the Gauss Centre for Supercomputing e.V. (GCS) (www. gauss - centre.eu) for supporting this project by providing computing time on the GCS Supercomputer SuperMUC at Leibniz Supercomputing Centre (www. lrz.de).

\section{References}

[1] Chehroudi, B., 2012. International Journal of Aerospace Engineering, 2012, pp. 1-31.

[2] Chehroudi, B., Talley, D., and Coy, E., 1999. In 37th Aerospace Sciences Meeting and Exhibit, p. 206.

[3] Mayer, W. O. H., Schik, A. H. A., Vielle, B., Chauveau, C., Gökalp, I., Talley, D. G., and Woodward, R. D., 1998. Journal of Propulsion and Power, 14(5), pp. 835-842.

[4] Roy, A., Joly, C., and Segal, C., 2013. Journal of Fluid Mechanics, 717, pp. 193-202.

[5] Mayer, W., Telaar, J., Branam, R., Schneider, G., and Hussong, J., 2003. Heat and Mass Transfer, 39(8-9), pp. 709-719.

[6] Zong, N., Meng, H., Hsieh, S.-Y., and Yang, V., 2004. Physics of Fluids, 16(12), pp. 4248-4261.

[7] Schmitt, T., Selle, L., Cuenot, B., and Poinsot, T., 2009. Comptes Rendus Mécanique, 337(6-7), pp. 528-538.

[8] Müller, H., Niedermeier, C. A., Matheis, J., Pfitzner, M., and Hickel, S., 2016. Physics of Fluids, 28(1), pp. 015102-1015102-28.

[9] Wehrfritz, A., Vuorinen, V., Kaario, O., and Larmi, M., 2013. Atomization and Sprays, 23(5), pp. 419-442.

[10] Lacaze, G., Misdariis, A., Ruiz, A., and Oefelein, J. C., 2015. Proceedings of the Combustion Institute, 35(2), pp. $1603-1611$.

[11] Matheis, J., and Hickel, S., 2016. In Proceedings of the 2016 Summer Program, Center for Turbulence Research, Stanford University, arXiv:1609.08533, pp. 25-34.

[12] Baab, S., Förster, F., Lamanna, G., and Weigand, B., 2016. Experiments in Fluids, 57(11), p. 172.

[13] Lamanna, G., Oldenhof, E., Baab, S., Stotz, I., and Weigand, B., 2012. In 18th AIAA/3AF International Space Planes and Hypersonic Systems and Technologies Conference, p. 5914.

[14] Baab, S., Förster, F., Lamanna, G., and Weigand, B., 2014. In 26th Annual Conference on Liquid Atomization and Spray Systems.

[15] Miles, R. B., Lempert, W. R., and Forkey, J. N., 2001. Measurement Science and Technology, 12(5), p. R33.

[16] Müller, H., Pfitzner, M., Matheis, J., and Hickel, S., 2015. Journal of Propulsion and Power, 32(1), pp. 46-56.

[17] Bell, I. H., Wronski, J., Quoilin, S., and Lemort, V., 2014. Industrial \& Engineering Chemistry Research, 53(6), pp. 24982508.

[18] Peng, D.-Y., and Robinson, D. B., 1976. Ind. Eng. Chem. Fundam, 15(1), pp. 59-64.

[19] Poling, B. E., Prausnitz, J. M., and O'Connell, J. P., 2001. McGraw-Hill, New York.

[20] Reid, R. C., Prausnitz, J. M., and Poling, B. E., 1987. McGraw-Hill, New York.

[21] Poston, R. S., and McKetta, J. J., 1966. Journal of Chemical and Engineering Data, 11(3), pp. 364-365.

[22] Eliosa-Jiménez, G., Silva-Oliver, G., García-Sánchez, F., and de Ita de la Torre, A., 2007. Journal of Chemical \& Engineering Data, 52(2), pp. 395-404.

[23] Goos, E., Burcat, A., and Ruscic, B., 2005. Report ANL 05/20 TAE 960. http://burcat.technion.ac.il/dir.

[24] Chung, T. H., Ajlan, M., Lee, L. L., and Starling, K. E., 1988. Ind. Eng. Chem. Res, 27(4), pp. 671-679.

[25] Qiu, L., and Reitz, R. D., 2015. International Journal of Multiphase Flow, 72, pp. 24-38.

[26] Michelsen, M. L., and Mollerup, J. M., 2007. Tie-Line Publications, Holte.

[27] Michelsen, M. L., 1982. Fluid Phase Equilibria, 9(1), pp. 1-19.

[28] Hickel, S., Egerer, C. P., and Larsson, J., 2014. Physics of Fluids, 26(10), p. 106101.

[29] Simeoni, G., Bryk, T., Gorelli, F., Krisch, M., Ruocco, G., Santoro, M., and Scopigno, T., 2010. Nature Physics, 6(7), pp. 503-507. 


\title{
Spreading Model for Wall Films Generated by High-Pressure Sprays
}

\author{
Q. Lamiel ${ }^{1,2}, \mathrm{~N}$. Lamarque ${ }^{1,{ }^{*}}, \mathrm{~J}$ Hélie $^{1}, \mathrm{D}$. Legendre ${ }^{2}$ \\ ${ }^{1}$ Continental Automotive SAS, 1 avenue Paul Ourliac BP 1149, 31036 Toulouse, France \\ ${ }^{2}$ Institut de Mécanique des Fluides de Toulouse (IMFT) - Université de Toulouse, \\ CNRS-INPT-UPS, Toulouse FRANCE \\ ${ }^{*}$ Corresponding author: nicolas.lamarque@continental-automotive.com
}

\begin{abstract}
This paper presents a new model developed to predict the area of wall films that may develop in gasoline direct injection engines (GDI). In a always more restrictive legislation on gas emissions the injection process in internal combustion (IC) engines has been highlighted as a domain of great concern in order to satisfy these requirements. Many spray wall interactions models exist in literature and are included in different CFD tools. Most often they are based on the sum of single drop-wall impacts. The specificity of the present model lies in its simplicity and the way the film is treated globally. Here its propagation is predicted using a balance between the momentum given by the spray and the viscous shear stress. Jointly with the theoretical model, an experimental set-up has been built up, an optical measurement technique called Refractive Index Matching method is used to follow the development of the wall film.

It has been found that the area of the wall film is proportional to the duration of injection, while the distance between the injector and the wall has not shown many influence on the evolution of area. The influence of the injection pressure has also been identified, when the pressure is doubled the radius of the film is multiplied by $\sqrt[3]{2}$. Eventually the model predicts that film thickness decreases as fuel pressure rises.
\end{abstract}

\section{Keywords}

automotive sprays, spray-wall impingement, refractive index matching method

\section{Introduction}

Fuel impingement in an internal combustion (IC) engine is a critical phenomenon. Despite progresses in gasoline direct injection (GDI) sytem design, fuel spray-wall impingement still happens for certain engine conditions and leads to liquid deposits. These wall films on the piston or the cylinder liner strongly affect mixture formation and entail bad local mixture homogeneity. This has been identified as a major source of pollutant emissions, especially particulate matter $[1,2]$. As a consequence, it is essential to have a good understanding of these spray-wall interactions and identify the key factors to keep them under control.

Drop-wall impingement is a topic widely studied in literature, because many applications (automotive, aeronautics, cooling, fire safety, printing, painting, agrochemicals, phamaceutical to name some) strongly depend on the performances of atomisers and spray systems [3]. Many models have been derived with success to describe the different impact regimes (on cold or hot surfaces) and the spreading characteristics of single droplets $[4,5,6]$. Some of them are commonly used in CFD tools and coupled with a Lagrangian description of the spray [7, 8, 9].

Nevertheless, it has been shown that spray-wall impingement implies many phenomena, which cannot be completly described by single droplet-wall impact models $[5,10]$. Moreover, while available computational power has strongly increased and despite some success, CFD tools sometimes remain expensive to simulate all the different engine conditions, for a first screening or even while optimising injector targeting and injection timings. In the present paper, a simple model based on basic conservation laws is proposed to describe wall film spreading in non- or low-volatile conditions. The global approach used to develop this model makes its originality, as well as the fact that it focuses on the film, rather than the spray dynamics. It is compared and validated with experimental measurements using the refractive index matching method and high-speed video imaging.

\section{Spray-wall interactions and experimental setup}

When a GDI spray impinges upon the wall, the very numerous liquid droplets $\left(10^{9}\right.$ to $\left.10^{10}\right)$ are either deposited or splashed and pushed away because of the high momentum, as depicted on Figure 1. In non-vaporising conditions, the deposited liquid mass quickly generates a continuous wall film. It then propagates under the effect of mass deposition and the spray dynamic pressure. While both the spray and the film can be observed on classical shadowgraph pictures like Figure 1, the film spreading can be better tracked with the refractive index matching (RIM) method.

The experimental setup is presented in Figure 3. It is here composed of a GDI injector (b), fed by a high-pressure pump (a), which generates a spray (c) that impinges upon a transparent roughened quartz plate (e); the impingement and the spreading of the liquid are recorded with a high speed video camera $(\mathrm{g})$. The camera is directed towards a mirror ( $f$ ) placed under the impingement plate in order to observe the phenomena from below. The light source (d) is an optical fiber which illuminates the quartz plate at grazing angle. This method of visualisation is 


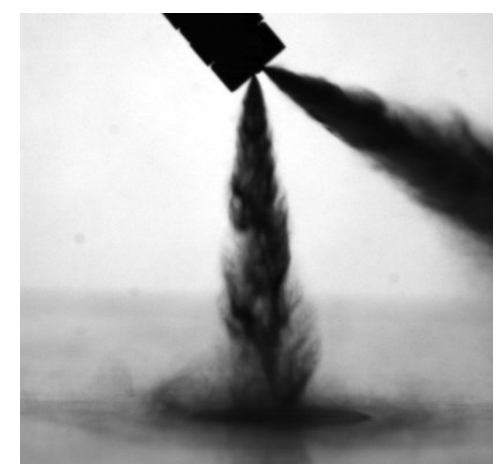

Figure 1. GDI spray impinging upon a smooth plate. Fuel pressure is $100 \mathrm{bar}$.

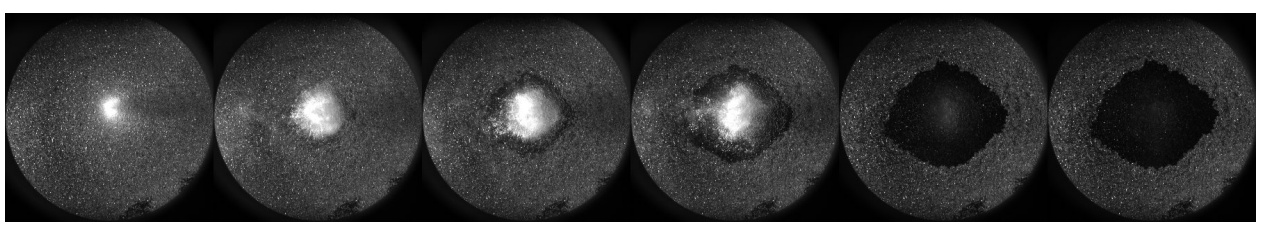

Figure 2. Images of injection as visualised on the RIM setup for $P_{i}=200 \mathrm{bar} T_{i}=5 \mathrm{~ms}$ and $d_{W}=50 \mathrm{~mm}$ images are displayed every $1.5 \mathrm{~ms}$

called Refractive Index Matching method (RIM) and it has originally been introduced by Drake [1] to study a DI engine then used by Yang and Gandhi in a Diesel engine [11]. Finally Maligne and Bruneaux [12] used it in a pressure chamber to compare wall films created by different types of GDI injectors. As the liquid fills in the surface troughs, the illuminated plate scatters less light towards the camera. This enables to clearly identify the wall film footprint and its surface can be evaluated (see Figure 2). After a fine calibration, the wall film thickness is quantified [1, 11, 12]. For the liquid films present in this study the accuracy is $\pm 1 \mu \mathrm{m}$. The injector used for this study has three holes. In this particular case, it is tilted by $30^{\circ}$, hence one of the plumes impacts upon the plate vertically and the spreading is homogeneous in the radial direction. Using a specially developed image post-processing, the edge of the liquid film can be tracked through time. Considering the camera resolution $(1 \mathrm{px}=0.17 \mathrm{~mm})$ the accuracy on the film radius is $1 \mathrm{~mm}$ close to the impinging zone and it becomes better and better as the film is spreading.

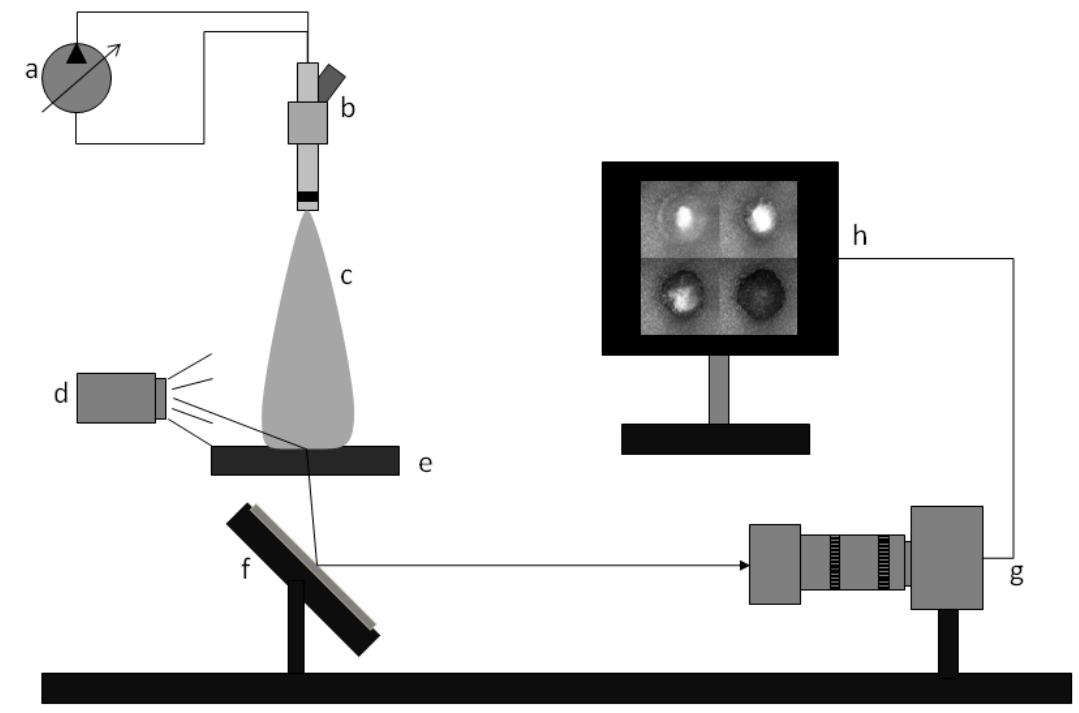

Figure 3. Sketch of the Refractive Index Matching experimental setup 


\section{Wall-film spreading modelling}

As very well underlined in [10], spray-wall impingement is a very complex phenomenon with many interactions between the incoming droplets and the perturbated film surface. Taking into account all the details (especially because the film is strongly perturbated by many simultaneous impacts) would be a tremendous effort. The purpose here is not to develop yet another very fine drop-wall interaction model to be implemented in a CFD tool. In this section is derived a simple global model, which describes the wall film propagation. The first hypothesis is the wall film has a puddle or a pizza shape, with a homogeneous thickness $e$ (see Figure 4). Surface tension effects, which are strong at the wall film edge and create a rim, are here neglected. Only a steady-state balance between a driving (spray pressure / momentum transfer) and a counteracting force in the radial propagation direction is considered.

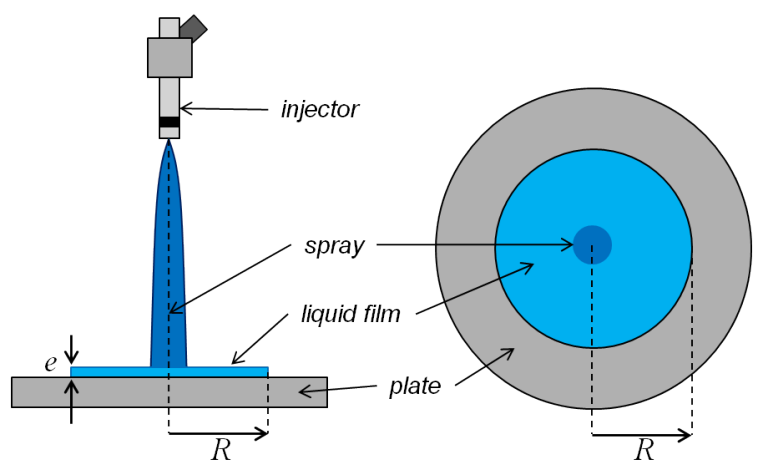

Figure 4. Sketch of the modelled problem. The film thickness is supposed to be homogeneous.

The wall film spreading model relies on the use of the mass and momentum conservation equations, outside the impact zone. Considering the characteristic lengths and times (thickness $e \simeq 10 \mu \mathrm{m}$, radius $R \simeq 10 \mathrm{~mm}$, injection time $T_{i} \simeq 1 \mathrm{~ms}$ ), the thin wall film is supposed to be in the lubrication regime. Considering that the mass is totally $\left(K_{m}=1\right)$ or partially $\left(K_{m} \leq 1\right)$ transferred in the film, the mass balance writes:

$$
\frac{d \pi R^{2} e}{d t}=K_{m} Q
$$

Where $Q$ stands for the injection discharge of the injector, then assuming a constant film thickness and that $Q$ is constant during the injection process:

$$
R=\sqrt{\frac{K_{m} Q t}{\pi e}}
$$

This shows that the radius of the film is proportional to the square root of time, the thickness of the film needs to be derivated to exactly know the evolution of the film.

Now looking at the momentum balance and keeping in mind that the film is in the lubrication regime (i.e. the flow is then parallel and pressure only depends on $r$ ):

$$
\frac{\partial p}{\partial r}=\eta \frac{\partial^{2} v_{r}}{\partial z^{2}}
$$

On the one hand a simple approximation on the pressure gradient gives:

$$
\frac{\partial p}{\partial r} \approx \frac{\Delta P}{R} .
$$

Defining $\Delta P=P_{s}-P_{a}$ and $P_{s}=P_{a}+P_{d}$ then $\Delta P=P_{d}$, which is the dynamic pressure given by the spray or, introducing $K_{p}$ to take into account the fact that the film pressure in the impingement zone may not be equal to the spray total pressure $\Delta P=K_{P} P_{d}$.

On the other hand stress can be approximated as:

$$
\eta \frac{\partial^{2} v_{r}}{\partial z^{2}} \approx \frac{\eta V}{e^{2}}
$$

where $V$ is the depth-averaged radial velocity. It can be used to express the wall film spreading velocity, by writing $V=\dot{R}$. Expressing $e$ with the help of Eq 3 - 5 we get:

$$
e=\sqrt{\frac{\eta V R}{2 \pi K_{P} P_{d}}}
$$


and with $V R \propto Q / e$ we get an expression for the thickness:

$$
e \propto \sqrt[3]{\frac{\eta Q}{P_{d}}}
$$

As the discharge coefficient $Q$ is varying like $P_{i}^{1 / 2}$, a trend for the evolution of $e$ and $R$ is found:

$$
e \propto P_{i}^{-1 / 6} \text { and } R \propto P_{i}^{1 / 3}
$$

For convenience Eq $2 \& 7$ can be rewritten using $K_{P}, K_{m}, \eta, P_{d}$ and $Q$ and it comes:

$$
\begin{aligned}
& e=\sqrt[3]{\frac{\eta K_{m} Q}{2 \pi K_{P} P_{d}}} \\
& R^{6}-R_{0}^{6}=K \frac{P_{d} Q^{2}}{\pi^{2} \eta} t^{3},
\end{aligned}
$$

where $R_{0}$ stands for the impinging zone of the spray, inside this zone the flow is more complex and the accuracy of the model is not guaranteed. And the prefactor $K$ is defined as :

$$
K=2 K_{P} K_{m}^{2},
$$

and takes into account that the spray mass and momentum may only be partially transferred to the liquid film. In what follows, we set $K_{P}=1$ for simplicity, which means that the pressure at the film centre is the spray dynamic pressure. The value of $K_{m}$ will be discussed in the next section.

\section{Results and discussion}

This present paragraph shows the results obtained applying the RIM method and the comparison with the proposed model. Table 1 summarises the different operating points. Fuel pressure $P_{i}$, injection time $T_{i}$ and injector-wall distance $d_{W}$ have been varied within realistic ranges with respect to automotive applications.

Table 1. Operating points tested in the present study

\begin{tabular}{c|cc}
\hline Factor & Unit & Values \\
\hline Fuel pressure $P_{i}$ & bar & $50,100,200$ \\
Injection time $T_{i}$ & $\mathrm{~ms}$ & 1 to 5 \\
Injector-wall distance $d_{W}$ & $\mathrm{~mm}$ & $30,40,50,60,70$
\end{tabular}

Figure 5 shows how the wall film area evolves in time. Whatever the injection time, the area growths collapse and, in the steady-state range are proportional to time. Here, only $d_{W}=50 \mathrm{~mm}$ is displayed, as the injector wall-distance has very little influence on the phenomenon. Hence, it confirms the radius evolution as the square root of time, as shown by Eq 2. It can also be observed that, once the injection is finished, the wall film still has some inertia and continues spreading at a much slower rate; the assumption of a constant thickness is then questionable.

Figure 6 shows the film thickness obtained with the calibration of the RIM method. The gray level is roughly homogeneous on the whole puddle and variations are mainly due to the surface irregularities that can scatter light differently, the profile is made $2 \mathrm{~s}$ after the start of injection. A steady state is reached and it is assumed that, no droplets that could disturb the measure, are present above the film surface. Hence a measurement of the mean film thickness is realised knowing the film surface. A value of $3.7 \mu \mathrm{m}$ is found, with the assumption of constant thickness we can calculate the deposited mass of heptane. It is compared to the injected mass, and it provides the mass ratio $K_{m}=15.11 \%$

A reasonable assumption is to say that a part of the mass deposited at the end of injection has already vaporised after $2 \mathrm{~s}$. After a quick evaluation based on experiments about vaporisation rates of heptane droplets gently deposited on a plate, and the use of a 1D model that predicts lifetime of liquid films [13], we can say that almost twice the mass was present at the end of injection. This gives a new value for $K_{m}=29 \%$, which is in line with results previously obtained in the literature $[11,14,15,16]$. Then, the thickness at the end of injection is obtained using Eq 12:

$$
e_{\left(t=T_{i}\right)}=\frac{e_{(t=2 \mathrm{~s})} A_{(t=2 \mathrm{~s})}}{A_{\left(t=T_{i}\right)}}
$$

The thickness of the film at the end of injection is $14.8 \mu \mathrm{m}$.

On the other hand the model gives with Eq 9 and the slope of Figure 5 a thickness of $16.8 \mu \mathrm{m}$ that is to say a relative error of $12 \%$ of the measured thickness. 


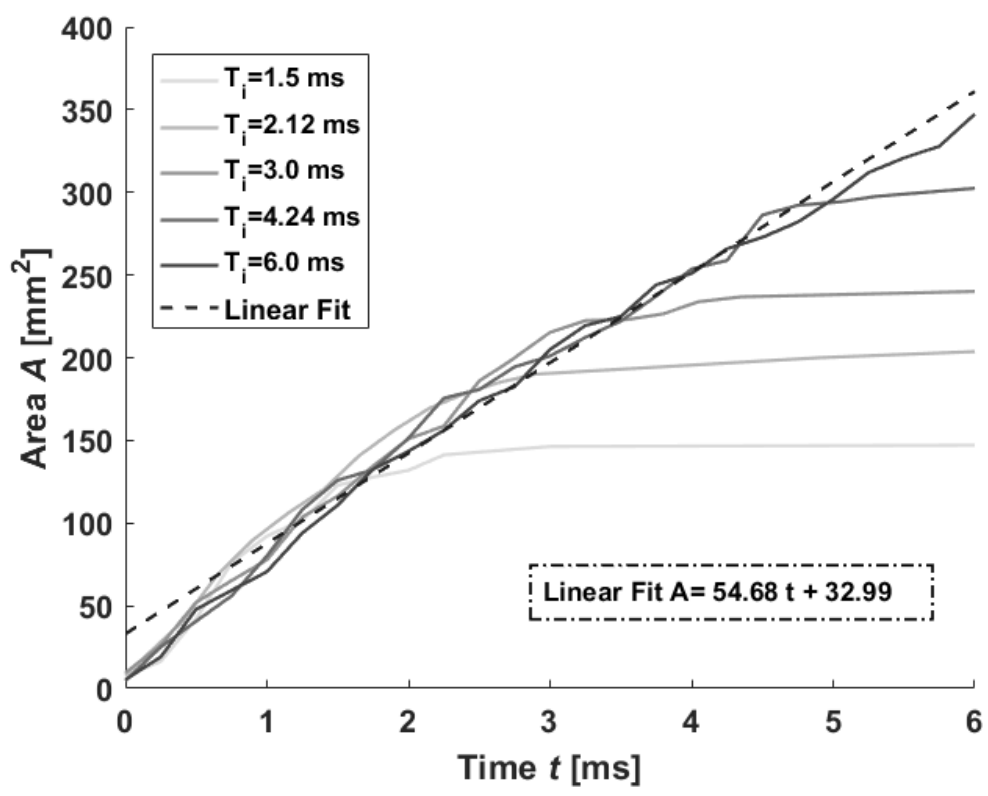

Figure 5. Wall film area time evolution for the operating point $P_{i}=100 \mathrm{bar}, d_{W}=50 \mathrm{~mm}$ and different injection times.

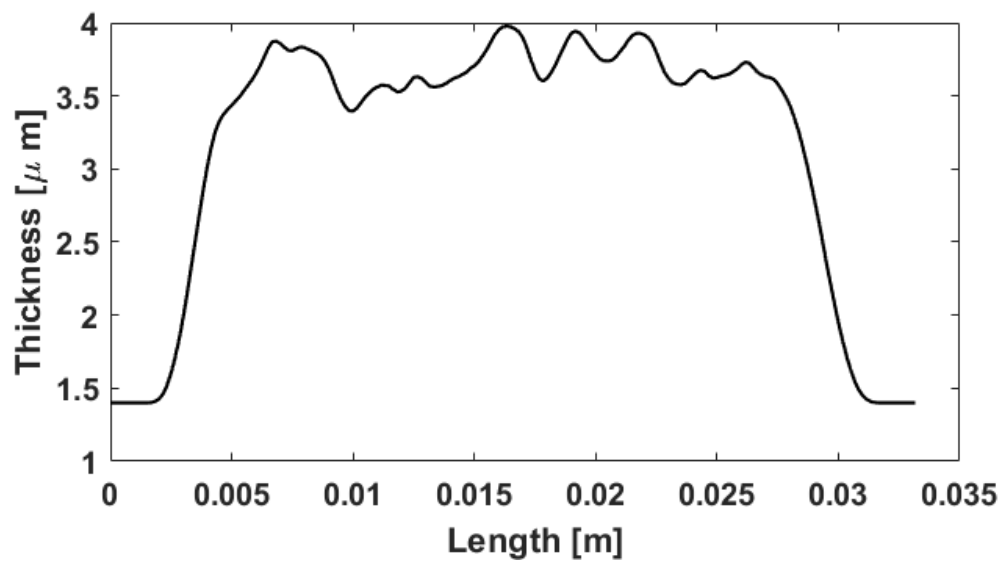

Figure 6. Thickness profile for a film of heptane obtained $2 \mathrm{~s}$ after the end of injection; $P_{i}=100 \mathrm{bar}, d_{W}=60 \mathrm{~mm}, T_{i}=5.0 \mathrm{~ms}$

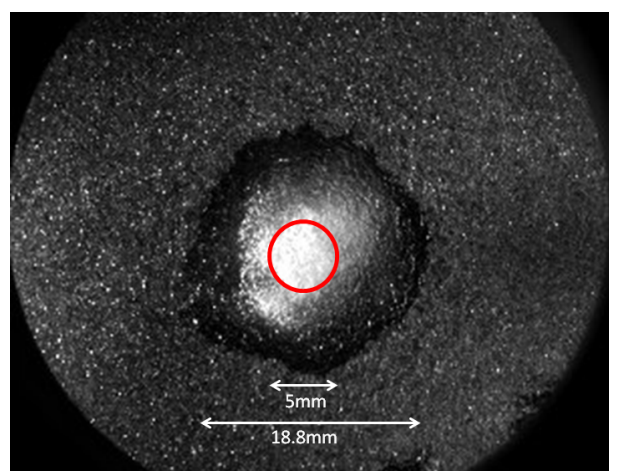

Figure 7. Normal impingement of spray on rough plate; $P_{i}=100 \mathrm{bar} d_{W}=60 \mathrm{~mm} T_{i}=5.0 \mathrm{~ms}$. Red zone highlights the area where momentum is transferred to the film

It has been shown in previous studies that there is less deposited mass when fuel pressure increases [14]. Nevertheless, from 50 to 200bar, changes of $K$ are not expected to be very high. Then, for simplicity, $K_{m}$ is set to 0.29 in what follows which gives a value of 0.58 for $K$. According to Figure 5 and to Eq 10 a value of $12.5 \times 10^{3} \mathrm{~Pa}$ for $P_{d}$ is obtained. To understand the meaning of this value a simple calculation is performed, taking a value of $K_{P}=1$ 
(which is what we assume in the model) means that the momentum is conserved from the injector hole to the impact plate. In other words the pressure force is constant, which can be expressed by:

$$
P_{d} A_{s}=P_{i} A_{o}
$$

where $A_{s}$ is the area where the spray momentum is transferred to the liquid film and $A_{o}$ is the area of the injector hole, then $A_{s}=18.2 \mathrm{~mm}^{2}$ which correspond to a radius of $2.4 \mathrm{~mm}$ which is really close to what we can observe on Figure 7.

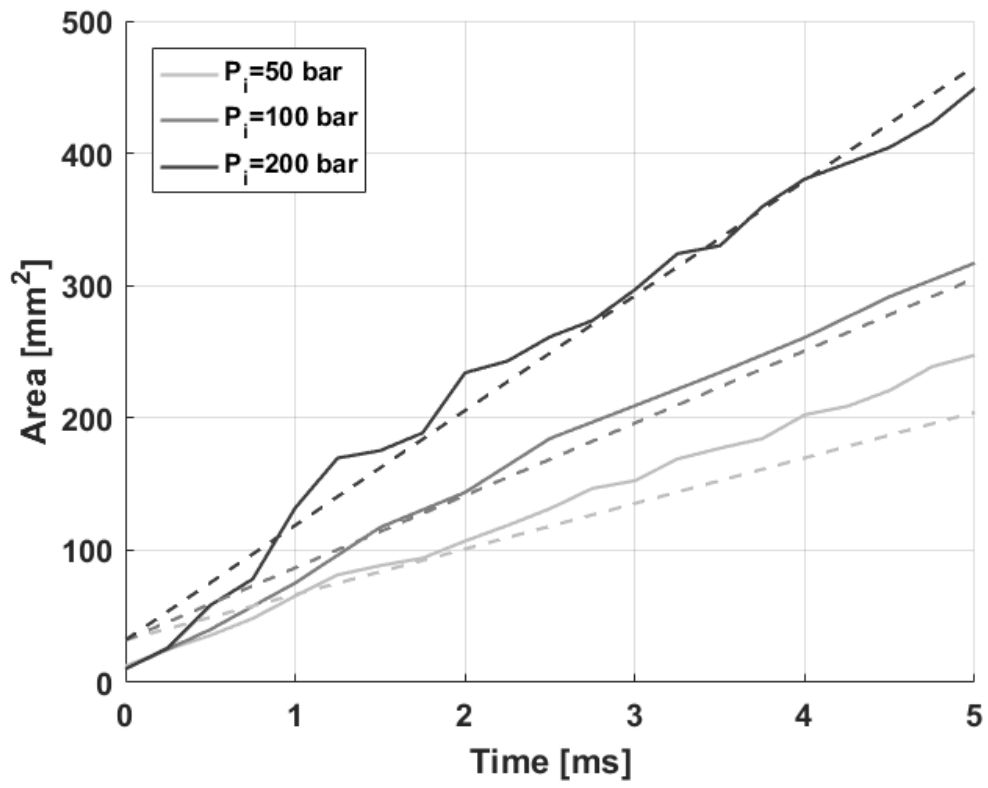

Figure 8. Wall film area time evolution for different fuel pressures $T_{i}=5 \mathrm{~ms}, d_{W}=50 \mathrm{~mm}$.

Figure 8 shows the film area time evolution for different fuel pressures. As expected, area is growing linearly with time. From one fuel pressure to another, the slope of the fitting curve is changing. It is admitted that $P_{d}$ varies linearly with $P_{i}$, while $Q$ varies as $\sqrt{P_{i}}$.

Based on the value of $K$ calculated above with $P_{i}=100 \mathrm{bar}$, one gets for the coefficient in Eq 10, for a fuel pressure $P_{1}$ :

$$
\left(\frac{P_{d} Q^{2}}{\pi^{2} \eta}\right)_{P_{1}}=\left(\frac{P_{1}}{10010^{5}}\right)^{2 / 3}\left(\frac{P_{d} Q^{2}}{\pi^{2} \eta}\right)_{100 \mathrm{bar}} .
$$

Eq 14 then enables to determine the slope of the fitting lines and gives 34.4 and 86.8 for $P_{i}=50$ bar and $P_{i}=200$ bar, respectively they are represented on Figure 8. It is clear that the dependency $R \propto P_{i}^{1 / 3}$ is correctly grasped by the model, however the prediction for the tests at $50 \mathrm{bar}$ is a bit bellow the experimental data. It could come from the value of $K_{m}$. Indeed it has been shown [14] that an increase in pressure causes a decrease in the deposited mass.

Eventually, the comparison between the model, and the experiment can be visualised on Figure 9. Here all the data collected at various pressure, injection duration and wall distance are displayed. The first bisector is represented in plain line to help visualise the quality of the model. Thus, Figure 9 shows the model fits well with the experimental measurements.

\section{Conclusions and perspectives}

The model presented in this paper is based on mass conservation and a steady-state balance between the spray pressure and viscous stresses. The model predictions are compared with experimental results obtained using the refractive index matching method and high-speed video imaging. Despite its simplicity, it clearly shows its ability to fairly predict the time evolution of the film radius $\left(R \propto t^{1 / 2}\right)$, as well as the influence of the fuel injection pressure $\left(R \propto P_{i}^{1 / 3}\right)$. It is also able to predict a wall film thickness with reasonable accuracy $\left(e \propto\left(\eta Q / P_{D}\right)^{1 / 3}\right)$.

The model is currently extended to take into account oblique spray-wall impingement, so as to be more representative of typical impact angles in GDI engines. Heat transfers and phase changes will be then added to include the effects of the strong vaporisation when the wall temperature reaches boiling conditions. Variation of fuels and greater pressure range will be done, in order to validate the evolution of $e$ as $P^{-1 / 6}$. 


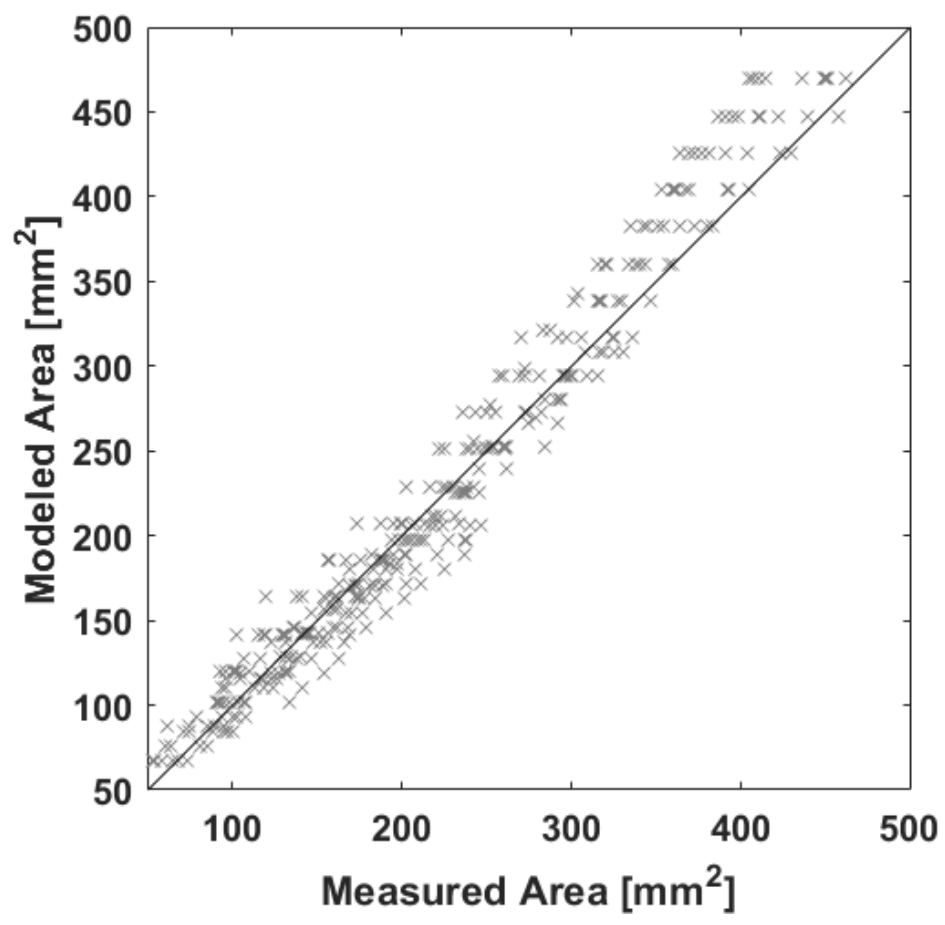

Figure 9. Comparison between measured and modeled area realised at 50, 100 and 200bar with injection time of 5.0ms and variable distance of injection

\section{Acknowledgements}

ANR and ANRT are acknowledged for their financial support.

\section{Nomenclature}

$V \quad$ Depth averaged radial velocity $\left[\mathrm{m} \cdot \mathrm{s}^{-1}\right]$

$v_{r} \quad$ Velocity in the fuel $\left[\mathrm{m} . \mathrm{s}^{-1}\right]$

$e \quad$ Thickness of the liquid film [m]

$P_{d} \quad$ Pressure transmitted by the spray $[\mathrm{Pa}]$

$Q \quad$ Discharge of the injector $\left[\mathrm{m}^{3} . \mathrm{s}^{-1}\right]$

$K_{P} \quad$ Pressure transmission coefficent

$K_{m} \quad$ Deposited mass ration

$K \quad$ Prefactor

$\eta \quad$ Dynamic viscosity [Pa.s]

$R \quad$ Radius of the wall film [m]

$A \quad$ Area of the wall film $\left[\mathrm{m}^{2}\right]$

$A_{s} \quad$ Area of spray impingement $\left[\mathrm{m}^{2}\right]$

$A_{o} \quad$ Area of the injector hole $\left[\mathrm{m}^{2}\right]$

$P_{i} \quad$ Pressure of injection $[\mathrm{Pa}]$

$T_{i} \quad$ Duration of injection [s]

$d_{W} \quad$ Injector-wall distance [m]

\section{References}

[1] Michael C Drake, Todd D Fansler, Arun S Solomon, and GA Szekely. Piston fuel films as a source of smoke and hydrocarbon emissions from a wall-controlled spark-ignited direct-injection engine. Technical report, SAE Technical Paper, 2003.

[2] Florian Steimle, Andre Kulzer, Herwig Richter, Dietmar Schwarzenthal, and Claudia Romberg. Systematic analysis and particle emission reduction of homogeneous direct injection si engines. Technical report, SAE Technical Paper, 2013.

[3] Nasser Ashgriz. Handbook of atomization and sprays: theory and applications. Springer Science \& Business Media, 2011.

[4] AL Yarin. Drop impact dynamics: splashing, spreading, receding, bouncing... Annu. Rev. Fluid Mech., 38:159-192, 2006.

[5] ALN Moreira, AS Moita, and MR Panao. Advances and challenges in explaining fuel spray impingement: How 
much of single droplet impact research is useful? Progress in energy and combustion science, 36(5):554-580, 2010.

[6] Sander Wildeman, Claas Willem Visser, Chao Sun, and Detlef Lohse. On the spreading of impacting drops. Journal of Fluid Mechanics, 805:636-655, 2016.

[7] Donald W Stanton and Christopher J Rutland. Modeling fuel film formation and wall interaction in diesel engines. Technical report, SAE Technical Paper, 1996.

[8] P.J. O'Rourke and A.A. Amsden. A spray/wall interaction submodel for the kiva-3 wall film model. Technical report, SAE Technical Paper, 2000.

[9] CX Bai, H Rusche, and AD Gosman. Modeling of gasoline spray impingement. Atomization and Sprays, 12(1-3), 2002.

[10] Davood Kalantari and Cameron Tropea. Spray impact onto flat and rigid walls: Empirical characterization and modelling. International Journal of Multiphase Flow, 33(5):525-544, 2007.

[11] Bo Yang and Jaal Ghandhi. Measurement of diesel spray impingement and fuel film characteristics using refractive index matching method. Technical report, SAE Technical Paper, 2007.

[12] David Maligne and Gilles Bruneaux. Time-resolved fuel film thickness measurement for direct injection si engines using refractive index matching. Technical report, SAE Technical Paper, 2011.

[13] Yangbing Zeng and Chia-Fon Lee. Multicomponent-fuel film-vaporization model for multidimensional computations. Journal of Propulsion and Power, 16(6):964-973, 2000.

[14] F Schulz, W Samenfink, J Schmidt, and F Beyrau. Systematic lif fuel wall film investigation. Fuel, 172:284292, 2016.

[15] Kyungnam Ko and Masataka Arai. Diesel spray impinging on a flat wall, part i: Characteristics of adhered fuel film in an impingement diesel spray. Atomization and Sprays, 12(5\&6), 2002.

[16] C Weiss. The liquid deposition fraction of sprays impinging vertical walls and flowing films. International journal of multiphase flow, 31(1):115-140, 2005.

The Maximum length of an ILASS 2017 paper should be 8 pages including all sections of the paper (abstract, keywords, introduction, main body, conclusions, acknowledgement, nomenclature and references. Your manuscript must be submitted in PDF file format through the ILASS 2017 webpage. The maximum file size must not exceed 10 MB. (regular text will be Arial 9 with 1.15 line spacing) 


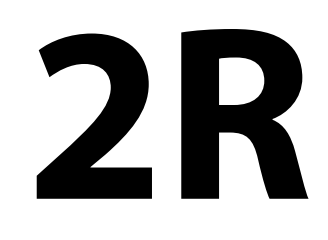

Atomizers 2 


\title{
Application of dry-ice for transient spray cooling
}

\author{
Miguel R. Oliveira Panão*, José J. Costa, Mário Bernardo \\ ADAI-LAETA, Universidade de Coimbra, Portugal \\ ${ }^{*}$ Corresponding author: miguel.panao@dem.uc.pt
}

\begin{abstract}
Spray cooling systems are able to remove large amounts of heat due to phase-change. Although vaporization is the most common phase-change process used in applications requiring thermal management, the use of liquids often implies the presence of a liquid film which is known to mitigate cooling performance. Thus, it is worth exploring other approaches for spray cooling avoiding liquid films. The work presented here explores sublimation using $\mathrm{CO}_{2}$ particles (dry-ice) formed through the Joule-Thomson effect. The application of interest is the molding industry, where reducing the cycle time taking advantage of the time-frame available between the mold opening and closing during the part's extraction, allows a production increase and, consequently, a higher competitive advantage in the market. The purpose of the experiments performed in dry-ice particle spray cooling is to investigate the effect of the impingement distance $(350-450 \mathrm{~mm})$, and injection duration, on the total energy flux removed from the surface, and cooling efficiency, in order to assess the performance of sublimation spray cooling. The results show an evolution of temperature distribution from a more homogeneous pattern with shorter pulses to a heterogeneous one for pulse durations longer than $1 \mathrm{~s}$. This is particularly useful in hotspot cooling. In terms of changing the spray impact distance, the higher particle dispersion achieved with a larger distance led to a decrease in thermal performance, probably due to the saturation of $\mathrm{CO}_{2}$ close to the impact surface. However, the pattern observed for the evolution of the total energy flux removed, with a maximum around an injection duration of $0.5 \mathrm{~s}$, remains unaltered. The maximum cooling efficiency, obtained for the shortest distance, is up to $30 \%$, which is comparable to spray cooling systems based on vaporization.
\end{abstract}

\section{Keywords}

Dry-Ice, spray cooling, molding processes

\section{Introduction}

The optimization of cycle times in molding processes depends on the ability to improve the cooling period. Wire coil inserts inside water channels already contribute to enhance heat transfer. However, the time between the extraction of the plastic molded part and closing for initiating the next cycle presents an opportunity for an additional cooling. Although most phase-change technique involving sprays consider liquid coolants, our purpose is to avoid the formation of liquid films. Therefore, this work explores sublimation phase-change using a dry-ice (solid $\left.\mathrm{CO}_{2}\right)$ particle spray to assess its potential for reducing cycle times in molding processes.

There are few cooling systems using dry-ice particles. To the best of our knowledge, only Linde developed a patented spot cooling system using $\mathrm{CO}_{2}$ to perform thermal management in local hotspots of molded parts [1]. However, the process operates in steady-state and may not apply in short transient cooling.

Most applications using dry-ice sprays aim at surface cleaning due to the solvent properties of $\mathrm{CO}_{2}$ particles [2]. Only a few studies consider the thermal effects of its impact on a surface, and even less consider their application to develop thermal management systems. For low flow rates $(0.236-1.18 \mathrm{l} / \mathrm{s})$, Kim and Lee [3] characterized the heat transfer coefficient $(h)$ in steady-state conditions, measuring values between 1 and $3.5 \mathrm{~kW} \cdot \mathrm{m}^{-2} \mathrm{~K}^{-1}$. For the highest value of $h$, the stagnation temperature varied between $0^{\circ} \mathrm{C}$ and $16^{\circ} \mathrm{C}$ depending on the impingement distance. In fact, increasing the distance from 5 to 20 times with a diameter at nozzle exit of $d=1 \mathrm{~mm}$ led to a non-linear decrease of $h$ by $40 \%$. The authors conclude that dry-ice particles are an advantage in $\mathrm{CO}_{2}$ jet cooling, making it a better suited system for cooling applications. However, there are applications where $\mathrm{CO}_{2}$ particle sprays have a short time frame to cool a heated surface, meaning it is operating in highly transient conditions. In this sense, the analysis of the corresponding transient thermal behaviour is still lacking in the literature and, thus, is the motivation for this work.

Using a mass flow rate of the order of $\sim 10^{2} \mathrm{~g} / \mathrm{s}$, the present investigation focuses on the transient heat transfer in sublimation spray cooling for several distances between the diffuser exit and the impinging heated surface. The experiments devised allow quantifying the cooling potential of dry ice particle sprays, assessing its potential application in the transient thermal management of molds. The purpose is to explore the short time of mold opening for the part's extraction before starting another injection cycle by achieving a significant heat removal from the molding cavity surface.

\section{Experimental Setup}

The experimental setup comprises a $5 \mathrm{~kg} \mathrm{CO}_{2}$ fire extinguisher vessel with two laser pointers assembled in the diffusor to align it with the center of the impinging surface. The pressure inside the vessel is at $57.3 \mathrm{bar}$ at $20^{\circ} \mathrm{C}$ and an inner probe-tube submerged down to the vessel bottom ensures that liquid $\mathrm{CO}_{2}$ arrives at the nozzle exit. 
In each experiment, the weight difference of the reservoir between the start and the end of injection, divided by its duration, provides the average mass flow rate, resulting in a value of $\dot{m}=154 \pm 2.95 \mathrm{~g} / \mathrm{s}$. The rapid expansion of the liquid $\mathrm{CO}_{2}$ through the nozzle and diffuser implies a sudden temperature decrease towards the sublimation value at atmospheric pressure, forming a two-phase (solid-gas) mixture by the Joule-Thomson effect (process a-b in Fig. 1).

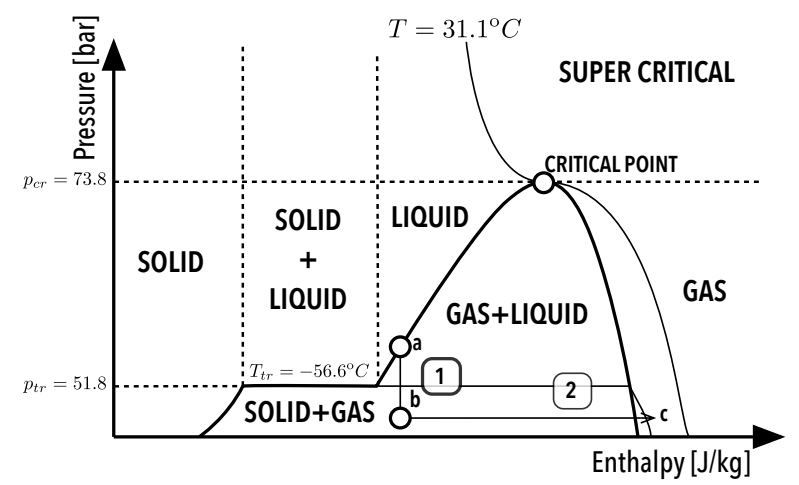

Figure 1. $\mathrm{CO}_{2}$ phase diagram. Process 1 corresponds to the expansion from points a to $\mathrm{b}$ producing a two-phase mixture of gas and $\mathrm{CO}_{2}$ solid particles. Process 2 corresponds to the sublimation associated with surface cooling.

The plate made of AISI 1010 steel has dimensions of $290 \times 290 \times 5[\mathrm{~mm}]$ and its thermophysical properties at $45^{\circ} \mathrm{C}$ (based on the average value measured throughout the experiments) are: thermal conductivity of $k=$ $63 \mathrm{~W} \cdot \mathrm{m}^{-1} \mathrm{~K}^{-1}$, specific heat $c_{p}=443.5 \mathrm{~J} \cdot \mathrm{kg}^{-1} \mathrm{~K}^{-1}$, density $\rho=7832 \mathrm{~kg} / \mathrm{m}^{3}$. Before each test, the plate is preheated with a $200 \mathrm{~W}$ electric resistance up to $100-110^{\circ} \mathrm{C}$, and then placed on a horizontal cork, $40 \mathrm{~mm}$-thick insulation board, its center aligned with the vertical diffuser axis. This configuration ensures a uni-direction internal conduction heat flux inside the steel plate, normal to the impinging surface. The experiments performed consider an initial condition of uniform surface temperature of $90^{\circ} \mathrm{C}$, since this is a common value found in molds upon releasing the molded part. Fig. 2 depicts an overall view of the experimental setup and main flow structures, including the spray dispersion pattern visualized through its angle, the impact region and a toroidal vortex formed by the micro- $\mathrm{CO}_{2}$-particles following the gas flow.
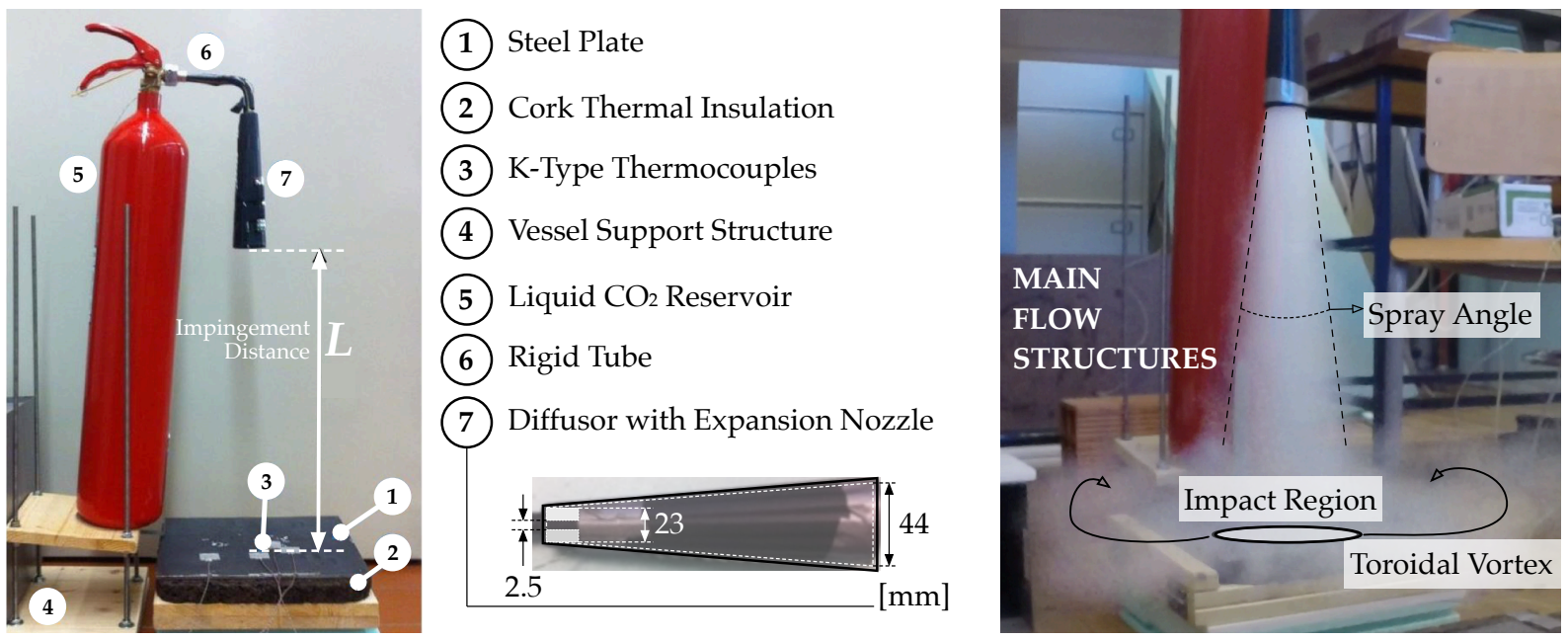

Figure 2. Overall view of experimental setup and indication of main flow characteristics.

The temperature measured by K-type Omega cement-on thermocouples allows characterizing the surface thermal behavior. A thin, highly polished aluminium tape fixes each thermocouple to the impinging surface, with a negligible thermal resistance, and minimizing radiation heat losses. A data logger from Data Translation (DT9828) acquires temperature data at a sampling rate of $120 \mathrm{~Hz}$ in each thermocouple channel. Calculations of the local heat flux from surface temperature measurements allow characterizing the cooling effect at the dry ice particles spray impact. Fig. 3a shows four thermocouples in different radial positions. Preliminary tests confirmed the assumption of an axisymmetric particle flow. All experiments where dry-ice particles accumulated around the thermocouple were not considered. While validating the experimental setup, tests showed that placing thermocouples in different angular positions mitigated this unwanted effect.

An analysis of the $\mathrm{CO}_{2}$ phase diagram shows that, when the liquid expands from 58.8 bar to the atmospheric pressure of $1 \mathrm{bar}, \mathrm{CO}_{2}$ solidifies through the Joule-Thomson effect and forms dry-ice particles at $-78.5^{\circ} \mathrm{C}$. However, the flow expansion within the nozzle causes an adverse pressure gradient and, thus, at nozzle exit, the pressure is likely to be above the atmospheric value. Considering this, since the spray is a two-phase mixture of solid and gas, 

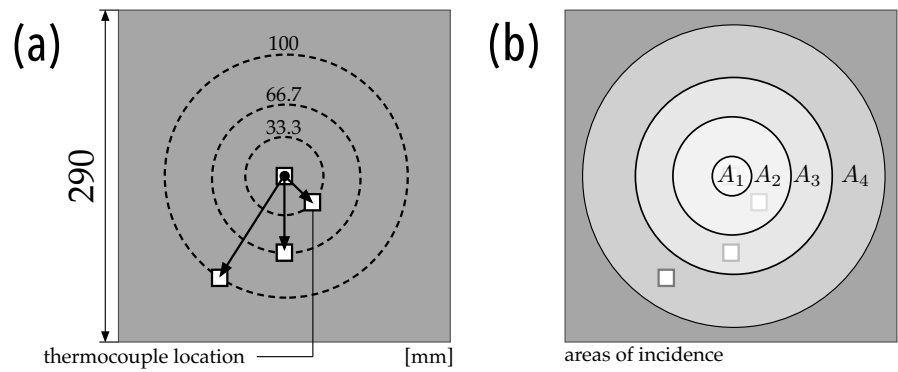

Figure 3. Illustration of thermocouple locations (a) and weighting areas considered in the overall heat transfer analysis.

it would explain why the coolant temperature at nozzle exit stabilizes at a higher value, $T_{c}=-76.8^{\circ} \mathrm{C}$, than $\mathrm{CO}_{2}$ 's sublimation point. This is the value used in the calculations of the heat transfer coefficient.

To investigate the effect of the distance between the spray nozzle and the impinging surface on heat transfer, the values considered are $L=350,400$ and $450 \mathrm{~mm}$.

\section{Measurement Method and Analysis}

Our experiments require a transient heat transfer analysis. The time-frame for applying this cooling technique is up to 6 seconds. This implies calculating the transient $(t)$ heat flux at the impinging surface from local $\left(r_{i}\right)$ temperature measurements for a given impingement distance $(L)$. In Panão and Moreira [4], assuming an adiabatic condition at the bottom surface, the wall heat flux is given by

$$
q^{\prime \prime}\left(t_{n}, r_{i}, L\right)=\frac{2 \beta}{\sqrt{\pi \cdot \delta t}} \sum_{i=1}^{n} \frac{\theta\left(t_{i}, r_{i}, L\right)-\theta\left(t_{i-1}, r_{i}, L\right)}{\sqrt{n-i}+\sqrt{n-i+1}}
$$

where $\beta=\sqrt{\rho k c_{p}}$ corresponds to the thermocouple thermal effusivity, with $\rho, k$ and $c_{p}$ as the density, thermal conductivity and specific heat of the material, respectively. The time interval $\delta t$ depends on temperature data sampling frequency and $\theta\left(t, r_{i}, L\right)=T_{s}\left(t, r_{i}, L\right)-T_{s}\left(0, r_{i}, L\right)$ corresponds to the difference between each instantaneous measurement of the local $\left(r_{i}\right)$ temperature at a given impingement distance $(L)$, and the initial condition at $t=0$. The resultant evolution of surface temperature uses two to three repetitive experiments to ensure reproducibility of the results. The wall heat flux calculated for each temperature profile and, afterwards, used to calculate the spatial-average value for the particle spray impact area,

$$
\left\langle q^{\prime \prime}(t, L)\right\rangle=\frac{1}{A} \sum_{i}\left(q^{\prime \prime}\left(t, r_{i}, L\right) \cdot A_{w}\left(r_{i}\right)\right)
$$

where $A$ corresponds to the total area, $A=\sum_{i} A_{w}\left(r_{i}\right)$, and $A_{w}\left(r_{i}\right)$ is the incidence area associated with each thermocouple, as depicted in Fig. 3b. The positions $r_{i}$ correspond to the thermocouples radial location. An error propagation analysis made for $\left\langle q^{\prime \prime}(t, L)\right\rangle$ resulted in uncertainties starting at $6.5 \%$ and decreasing to a value around $1 \%$ after $100 \mathrm{~ms}$.

Using the temperature measured at the surface $T_{s}\left(t, r_{i}, L\right)$ and the temperature of the two-phase $\mathrm{CO}_{2}$ mixture measured at nozzle exit, $T_{c}$, the spatial-average instantaneous heat convection coefficient $h$ is calculated as

$$
\langle h(t, L)\rangle=\frac{1}{A} \sum_{i}\left(\frac{q^{\prime \prime}\left(t, r_{i}, L\right)}{T_{s}\left(t, r_{i}, L\right)-T_{c}} \cdot A_{w}\left(r_{i}\right)\right)
$$

A final quantity of interest is the total cooling energy removed from the surface,

$$
\left\langle E^{\prime \prime}\right\rangle(\Delta t, L)=\int_{0}^{\Delta t} \frac{1}{A} \sum_{i}\left(q^{\prime \prime}\left(t, r_{i}, L\right) \cdot A_{w}\left(r_{i}\right)\right) \mathrm{dt}
$$

An error propagation analysis made for the spatial average heat transfer coefficient resulted in uncertainties of 2.3-2.7\%, and $0.6-0.8 \%$ for the total energy removed in the cooling process.

\section{Results and discussion}

The first part of the results presented analyzes the surface temperature behavior, and its distribution, to characterize the expected outcome of sublimation spray cooling. Afterwards, the spatial-average evolution of the wall heat flux, heat transfer coefficient and total energy removed in the sublimation process allow characterizing the effect of the impingement distance on the cooling performance. 


\section{Temperature evolution and distribution}

Fig. 4 depicts the average local surface temperature variation for three different impingement distances. The first observation is the significant temperature difference from the plate's center to outer regions in sublimation heat transfer. This is clearer between the axisymmetric center, $r=0 \mathrm{~mm}$ and the first location outwards at $r=33.3 \mathrm{~mm}$ for larger impingement distances, showing the heterogeneous cooling characteristic of impinging jet heat transfer.

Regarding the thermal transient behavior of the surface, $t \approx 1.5 \mathrm{~s}$ appears to set a threshold between a first cooling period with an intense non-linear evolution of surface temperature, and a second period where cooling is less intense, with surface temperature decreasing at a constant and lower rate.

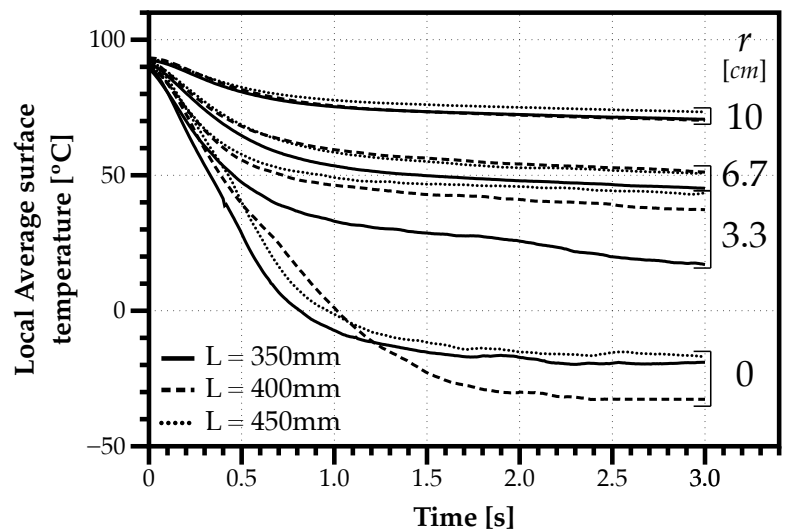

Figure 4. Evolution of local average surface temperature for different impingement distances with an injection duration of $3 \mathrm{~s}$.

Another observation is the range for the surface temperature values $\left(-32^{\circ} \mathrm{C}\right.$ to $\left.-16^{\circ} \mathrm{C}\right)$, which does not reach the sublimation value for $\mathrm{CO}_{2}$ in atmospheric conditions $\left(-78.5^{\circ} \mathrm{C}\right)$. A high-velocity flow of a two-phase (solid-gas) mixture of $\mathrm{CO}_{2}$ impinging on the surface may imply a short residence time of particles in contact with the heated surface for cooling purposes. Compared with cryogen spray cooling, the formation of liquid films allows reaching the saturation temperature (in this case, the boiling temperature, see [5]). However, particles bounce from the surface, justifying the shorter residence time for heat transfer with the surface and, consequently, a higher temperature than the sublimation value.

The experiments performed also considered different durations of injection. Fig. 5 shows the surface temperature decay, $\theta(t)=T_{s}(t)-T_{s}(0)$, for four different durations of injection $(\Delta t=0.5,0.78,1.5,3.75 \mathrm{~s})$ at $r=33.3 \mathrm{~mm}$ and an impingement distance of $400 \mathrm{~mm}$. Since the Start-Of-Injection (SOI), the decay is similar for all pulse durations, and temperature recovery after the end of injection is also similar since it depends on the heat diffusion inside the plate, although the final temperature varies proportionally to the injection duration. Therefore, we assume the wall heat flux evolution for an injection with a longer pulse $\left(\Delta t_{\max }\right)$ can reasonably describe the outcome expected for a shorter pulse, $\Delta t \leq \Delta t_{\max }$. As a fist approximation, this implies considering that the wall heat flux associated with the recovery period has a marginal quantitative effect on the overall outcome. The practical implication is focusing the analysis on the experiments with a longer pulse, assuming that any integration between 0 and $t \leq t_{\max }$ expresses the result expected for the corresponding duration of injection $(\Delta t=t)$ of dry-ice particles onto the heated surface. This is an important consideration in the analysis of the following section on the amount of energy removed from the surface during $\Delta t$, given by Eq. (4).

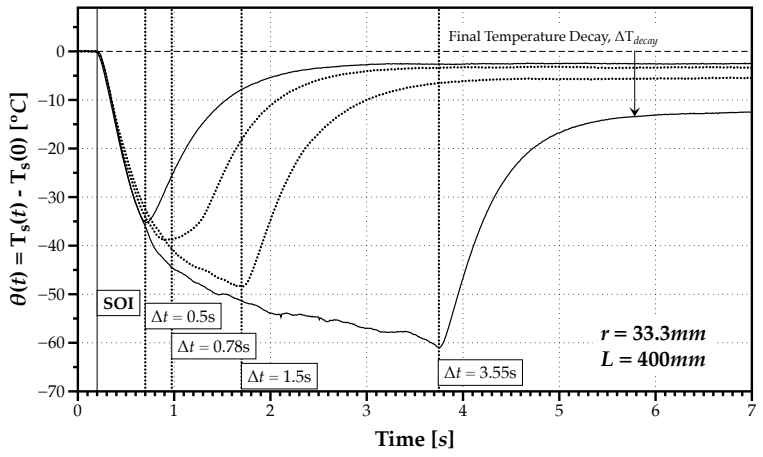

Figure 5. Evolution of the surface temperature decay at $r=33.3 \mathrm{~mm}$ with $L=400 \mathrm{~mm}$ considering different durations of injection $(\Delta t=\{0.5,0.78,1.5,3.75\}[s])$. SOl stands for Start-Of-Injection.

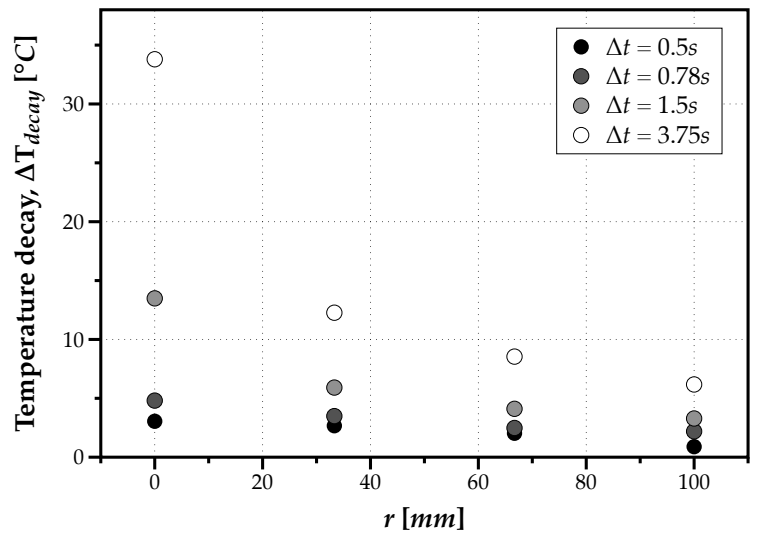

Figure 6. Stabilized final temperature decay after recovery by thermal inertia and within a time window of $6 \mathrm{~s}$.

In Fig. 5, after the end of injection, the final stabilized temperature shows a decay $\left(\Delta T_{\text {decay }}\right)$ produced by the 
impinging dry-ice spray. Fig. 6 depicts the temperature decay in each radial location, considering several durations of injection. A significant growth in temperature heterogeneity within the impact area as the duration of injection increases, particularly in the central region, allows concluding that smaller pulses enable a more uniform cooling in the impingement area, while longer pulses are more adequate for hotspot cooling. The next section focuses on the effect of the impingement distance on sublimation spray cooling heat transfer.

\section{Effect of distance on dry-ice particle spray cooling}

From the surface temperature evolution analyzed in the previous section, Fig. 7a depicts the spatial-average wall heat flux calculated according to Eq. (2). The three curves correspond to different impingement distances and the pattern is similar to the observed in intermittent spray cooling [4], and the magnitude similar to typical values found in cryogen spray cooling [7]. The maximum wall heat flux occurs $0.5-0.6 \mathrm{~s}$ after impact, for all distances $L$, and decreases thereafter, probably due to the $\mathrm{CO}_{2}$ saturation close to the impinging surface, inhibiting sublimation.

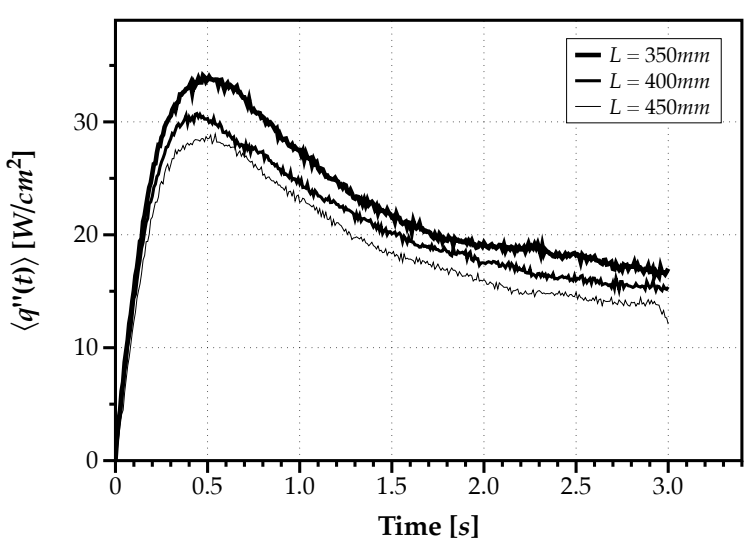

(a) spatial-average wall heat flux

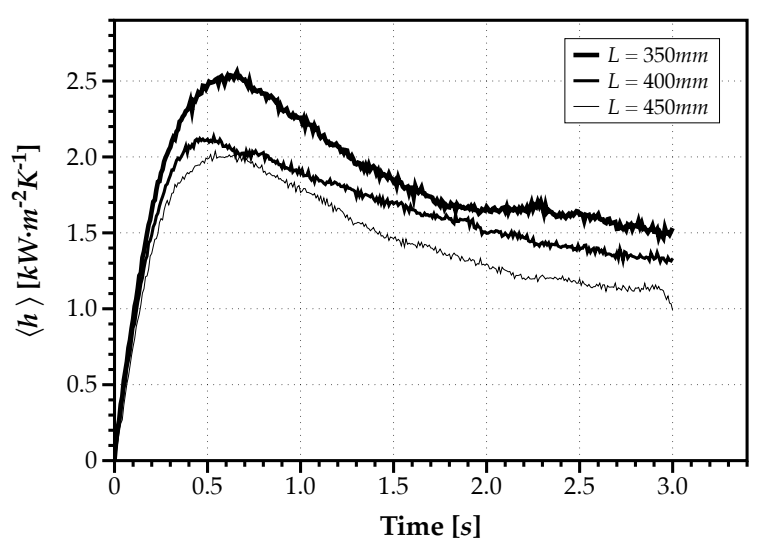

(b) spatial-average heat transfer coefficient

Figure 7. Evolution of the spatial-average heat transfer removed by the dry ice particle spray.

As to the spatial-average heat transfer coefficient, $\langle h\rangle$, Fig. 7b suggests a higher performance for the shorter distance $(L=350 \mathrm{~mm})$, but the behavior is similar for all cases. Moreover, above $t=1 \mathrm{~s}$, $\langle h\rangle$ evolves toward stabilization, suggesting that pulse durations above $3 \mathrm{~s}$ may lead to a steady-state cooling. This is better understood through the analysis of the total energy removed in the cooling process as a function of the duration of injection $(\Delta t)$. Fig. 8 shows a non-linear increase of the energy removed from the surface, and independent of distance, for short pulse durations $\Delta t \leq 0.5 \mathrm{~s}$. Thereafter, the values for $\left\langle E^{\prime \prime}(L, \Delta t)\right\rangle$ increase monotonically with $\Delta t$.

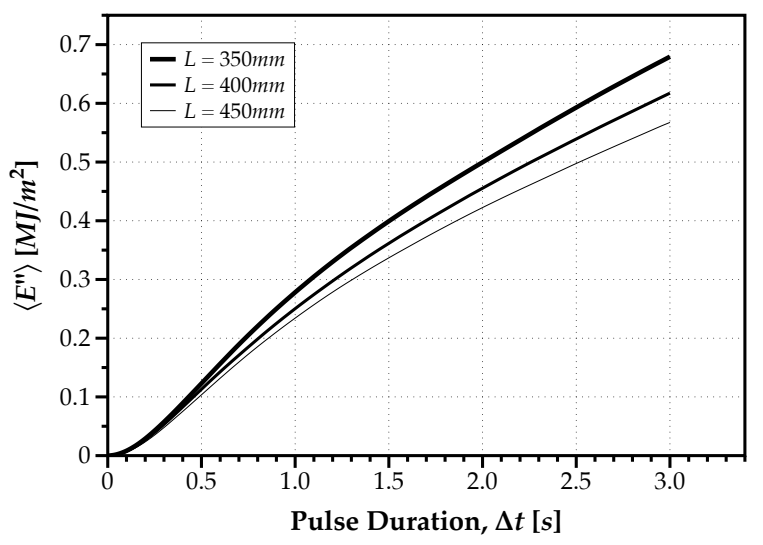

Figure 8. Total cooling energy on the impact of a dry ice spray as a function of the duration of injection.

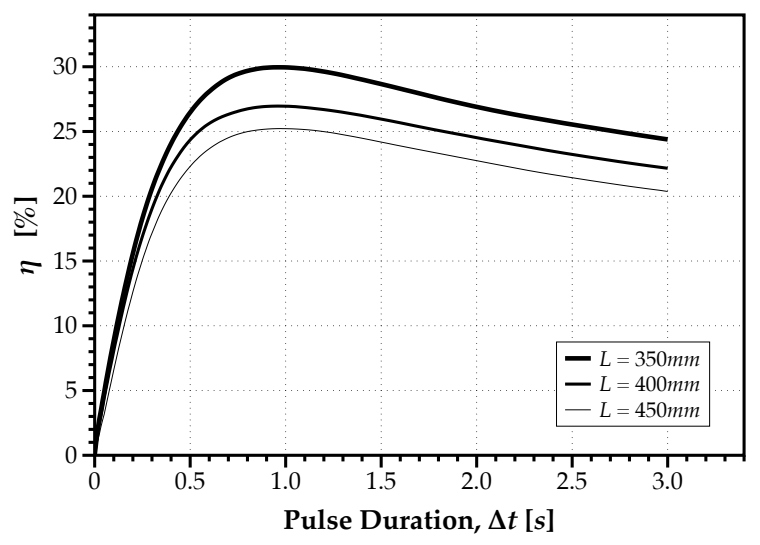

Figure 9. Effect of the impingement distance on the overall cooling efficiency as a function of pulse duration.

Considering the application of this thermal management strategy to mold cooling, the cooling time $(C T)$ in molding is generally proportional to the natural logarithm of a temperature ratio considering the values of injection of material $\left(T_{i n j}\right)$, extraction of molding part $\left(T_{e x t}\right)$, and average mold temperature $\left(T_{m}\right)$,

$$
C T \propto \ln \left(\frac{T_{i n j}-T_{m}}{T_{e x t}-T_{m}}\right)
$$

Thus, if the recurrent use of $\mathrm{CO}_{2}$ would lead to a general decrease of the mold temperature in its cyclic operation, it 
would represent a percentual reduction of the cycle time proportional to the decrease of $T_{m}$ relatively to a reference value without $\mathrm{CO}_{2}$ cooling of the molding cavity surface. This is the research subject of ongoing modeling work. Considering the cooling energy in each injection pulse, and the liquid $\mathrm{CO}_{2}$ quantity expected to form dry ice in order to benefit from sublimation, which is approximately $45 \%$ according to Sherman and Adam [8], the cooling efficiency estimated depends on the pulse duration as

$$
\eta=\frac{\left\langle E^{\prime \prime}(\Delta t, L)\right\rangle}{0.45 \dot{m} h_{s g} \Delta t}
$$

where $\dot{m}$ corresponds to the measured mass flow rate of $\mathrm{CO}_{2}$ and $h_{s g}=573 \mathrm{~kJ} / \mathrm{kg}$ is the sublimation latent heat at atmospheric pressure. The results depicted in Fig. 9 evidence a local maximum around a pulse duration of $\Delta t=1 \mathrm{~s}$. As mentioned before, a longer injection leads to a loss in performance, probably due to the saturation of the mixture flowing environment close to the surface. Although the total cooling energy increases for a longer $\mathrm{CO}_{2}$ injection pulse, Fig. 9 points to a cost in performance. This suggests the possibility of exploring multiple shorter pulses, instead of longer ones, as observed in Panão et al. [9] for cooling with intermittent liquid sprays.

Relatively to the magnitude of cooling efficiency values, compared to other intermittent sprays using liquid [4, 9], the results are similar despite pulse durations in the present experiments are 2-3 orders of magnitude higher. Fig. 9 also points to a performance degradation when injecting the spray farther from the surface. However, this detriment is not significant, since an increase of $30 \%$ of the distance only decreases $\eta$ by $5 \%$.

\section{Conclusions}

In typical thermal management, spray cooling systems often use the injection of liquid onto heated surfaces. However, a liquid film formed after impact mitigates phase-change in most cases and causes losses in cooling performance. Therefore, the present work aims at introducing the application of dry ice particle sprays in the development of thermal management cooling systems. In particular, the work focuses on its transient application within the time frame available for cooling the molding cavity surface in molding processes during its operation time.

The thermal assessment of the technique includes the effect of pulse duration and impingement distance on the temperature distribution, overall heat transfer (in terms of fluxes and heat transfer coefficient), on the total energy removed from the heated surface, and cooling efficiency. Although dry ice sprays are usually associated with cleaning surfaces, the results presented here show their prominent application in transient thermal management cooling systems. Namely,

- Dry ice spray cooling produced different temperature distributions, depending on the duration of injection. With shorter pulses lower than $1 \mathrm{~s}$, the temperature decay is relatively uniform throughout the impact area, while longer pulses produce a more heterogeneous temperature distribution with a higher decay in the central region. This is particularly useful in hotspot cooling.

- The effect of the impingement distance on dry ice spray cooling considers the distances of 350,400 and $450 \mathrm{~mm}$. As expected, a higher impingement distance leads to a higher particle dispersion, leading to a lower thermal performance, thus, lower wall heat flux values. However, it does not affect the thermal behaviour producing a maximum around the instant $0.5 \mathrm{~s}$. This implies an inflection point in the total energy removed by the spray at the injection duration of $0.5 \mathrm{~s}$, distinguishing a correlation for shorter pulses independent on the distance, from another correlation for longer durations depending on the impingement distance parameter.

Given the prominent character of the application of sublimation in thermal management systems, future and ongoing research work includes: the thermal modeling of introducing this additional cooling in molding processes to reduce the cycle time; a more detailed characterization of the dry ice particle spray, with particular emphasis on the impact region; and the effect of the diffusor geometry on the dynamics of dry ice particle and dispersion patterns.

\section{Acknowledgements}

The authors would like to acknowledge to the financial support of project EMCool - Efficient Mold Cooling (POCI01-0247-FEDER-011375).

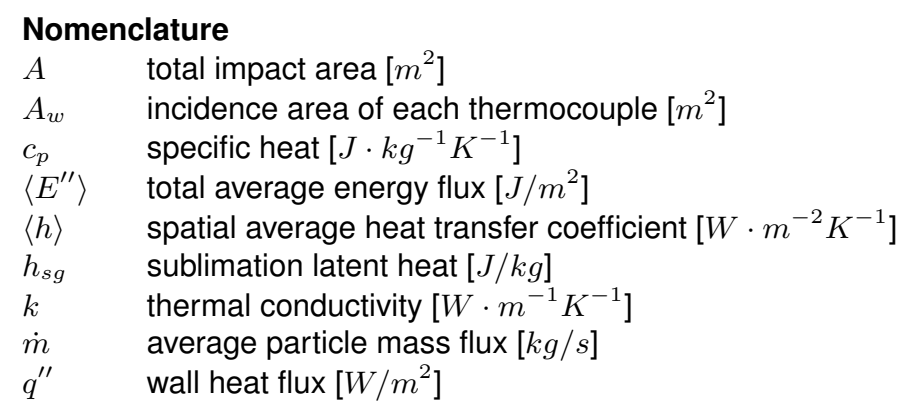




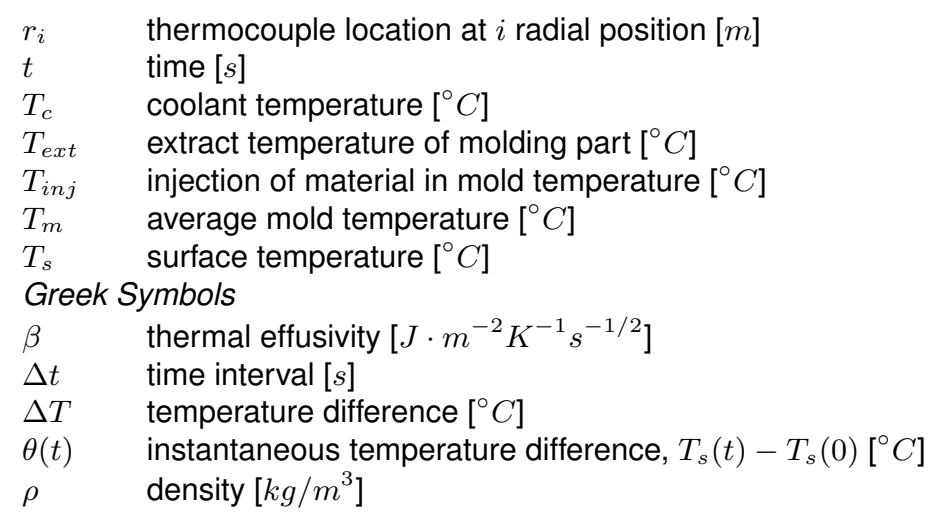

\section{References}

[1] Stanley, J. R., Praller, A., 2015, Spot cooling with $\mathrm{CO}_{2}$, Plastics Engineering, July/August, pp. 20-22.

[2] Sherman, R., Grob, J., Whitlock, W., 1991, Dry surface cleaning using $\mathrm{CO}_{2}$ snow, J. Vac. Sci. Technol. B 9, pp. 1970-1977.

[3] Kim, D., Lee, J., Experimental investigation of $\mathrm{CO}_{2}$ dry-ice assisted jet impingement cooling, Applied Thermal Engineering 107, pp. 927-935.

[4] Panão, M. R. O., Moreira, A. L. N., 2009, Intermittent spray cooling: A new technology for controlling surface temperature, International Journal of Heat and Fluid Flow 30 (1), pp. $117-130$.

[5] Aguilar, G., Wang, G.-X., Nelson, J. S., 2003, Dynamic behavior of cryogen spray cooling: Effects of spurt duration and spray distance, Lasers in Surgery and Medicine 32 (2), pp. 152-159.

[6] Majaron, B., Svaasand, L. O., Aguilar, G., Nelson, J. S., 2002, Intermittent cryogen spray cooling for optimal heat extraction during dermatologic laser treatment, Physics in Medicine and Biology 47 (18), pp. 3275-3288.

[7] Zhou, Z.-F., Wang, R., Chen, B., Yang, T., Wang, G.-X., 2016, Heat transfer characteristics during pulsed spray cooling with R404A at different spray distances and back pressures, Applied Thermal Engineering 102 (C), pp. 813-821.

[8] Sherman, R., Adam, P., Carbon Dioxide Snow Cleaning - The next Generation of Clean, Precision Cleaning '95 Proceedings, pp. 271-300.

[9] Panão, M. R. O., Correia, A. M., Moreira, A. L. N., 2012, High-power electronics thermal management with intermittent multijet sprays, Applied Thermal Engineering 37, pp. 293-301. 


\title{
Effect of Prefilmer Edge Thickness on Breakup Phenomena of Liquid Film in Prefilming Airblast Atomizer
}

\author{
Takahiro Okabe $^{\star 1}$, Naoki Katagata ${ }^{1}$, Toshihiro Sakaki ${ }^{1}$, Takao Inamura ${ }^{1}$, Koji Fumoto ${ }^{1}$ \\ ${ }^{1}$ Graduate School of Science and Technology, Hirosaki University, Japan \\ ${ }^{\star}$ Corresponding author: oka@hirosaki-u.ac.jp
}

\begin{abstract}
This paper describes the investigation of the effect of a prefilmer edge thickness on the breakup phenomena of a liquid film in a prefilmer airblast atomizer. The breakup phenomena of the liquid film at five prefilmer edge thicknesses $(160,500,1250,2000$, and $3000 \mu \mathrm{m})$ under various conditions was observed using a high-speed camera. The breakup length of the liquid film was calculated by an image processing technique developed in this study. In order to quantitatively evaluate the effect of the prefilmer edge thickness on the breakup frequency, the Fast Fourier Transformation (FFT) analysis was conducted based on the time evolution of the breakup length. The results indicated that the breakup length increase and the breakup frequency decreases by increasing prefilmer edge thickness due to a larger volume of a liquid accumulation attaching to the prefilmer edge. The FFT analysis showed that the increase in prefilmer edge thickness causes the transition of the maximal power spectrum to a lower frequency (i.e. less than $100 \mathrm{~Hz}$ ) due to the increase in the liquid accumulation at the edge as well. Finally, a dimensionless correlation has been proposed for the breakup length of a liquid film.
\end{abstract}

\section{Keywords}

Prefilming airblast atomizer, Breakup length, Breakup frequency, Prefilmer edge thickness, FFT analysis

\section{Introduction}

In the recent jet engine, the prefilming airblast atomizer has been widely used for the liquid fuel injection due to a good spray characteristics even at low fuel injection pressure. This type of atomizer uses the high-speed air stream flowing in the combustor for the atomization of a liquid fuel. Thus, the pressure loss of atomizing air in an atomizer is smaller than a conventional atomizer using the air stream, and leads to stable performances of the combustor over the entire range of operating conditions.

The atomization mechanism of a prefilming type of airblast atomizer is well known. After injection, the liquid homogeneously wet the prefilmer surface, and flows down to the prefilmer edge in the form of a liquid film that is sheared by the high-speed air stream. The liquid film accumulates at the prefilmer edge. This accumulation is sheared by air and disintegrated into the bag-shaped liquid framed by a thicker rim. The bag-shaped part of the structure bursts and generates fine droplets. The rim is stretched and fragments into two elongated ligaments that disintegrate into larger droplets.

Many researchers have studied the spray characteristics of a prefilming type of airblast atomizer, and revealed that a liquid film thickness, liquid flowrate, and air velocity are important parameters. Lefebvre reviewed the investigations on airblast atomizers, and pointed out the advantages of airblast atomizers such as lower fuel pressure, low soot formation, constant fuel distribution over the whole range of fuel flow rate, and fine mixing between fuel and air [1]. Inamura et al. experimentally investigated the spray characteristics of a prefilming type of airblast atomizer using the two-dimensional test atomizer with a rectangular cross-section [2]. Furthermore, they proposed new breakup model of a liquid film at a prefilmer edge based on the Kelvin-Helmholtz and Rayleigh-Taylor instability. The predicted wavelength, ligament diameter and mean droplet diameter were compared with the measurements. The predicted SMDs showed good agreements with the measurements at small liquid flowrate, but it showed slightly smaller values than the measurements at large liquid flowrate.

However, little research on the effect of the geometric shape of a prefilmer on the spray characteristics of prefilming airblast atomizer is currently available. Gepperth et al. conducted the experiments to investigate the effect of liquid physical properties, film flow rate, and prefilming configurations on primary atomization of liquid film. Their experimental results showed that mean air velocity and prefilmer edge thickness were found to be the important parameters to the spray characteristics and the breakup phenomena of liquid film [3]. The prefilmer length, liquid physical properties and liquid volume flow rate seem to have only a minor effect on the mean droplet diameter but affect the ligament formation process. They pointed out three important parameters: the prefilmer edge thickness, the surface tension of the liquid, and the momentum flux of the gas. However, they did not investigate the effect of widely varying the prefilmer edge thickness on the breakup phenomena and spray characteristics. 
Furthermore, up to now there is no correlation which contains the effect of the prefilmer edge thickness on the characteristics of the breakup phenomena such as the breakup length and the breakup frequency, etc.

In this study, we focus on the prefilmer edge thickness as an important parameter, and aim to experimentally clarify the effect on the breakup phenomena of a liquid sheet in a prefilming airblast atomizer. In addition, we propose a dimensionless correlation for the breakup length of a liquid film which contains the effect of the prefilmer edge thickness.

\section{Experimental Apparatus}

Figure 1 (a) shows the experimental apparatus. The air generated by the blower is supplied to the contraction nozzle via a honeycomb. The test atomizer is attached to the exit of the contraction nozzle. The water in the pressure tank is pressurized by a high-pressure air from the compressor, and then it is supplied to the prefilmer in the test atomizer through a flow meter. The details of the experimental apparatus are presented in the previous paper [4]. Figure 1 (b) shows the test atomizer used in this study. The test atomizer is two-dimensional for easy observation of the breakup phenomena of a liquid film. The prefilmer is set at the center in the atomizer, and the porous metal was used at a liquid outlet part to uniform the liquid flowrate distribution in the traverse direction. In order to avoid an undesirable change in the air velocity in the test channel, the channel height is fixed to $10 \mathrm{~mm}$ for all prefilmers with different edge thicknesses. The breakup phenomena of a liquid film are observed by the high-speed video camera which is placed above the prefilmer edge. The metal halide lamp is used as the light source. The field of view is appropriately adjusted in accordance with the experimental conditions. The frame rate is also adjusted from 3000 to $10000 \mathrm{fps}$. In this study, tap water was used as the atomized liquid and air as the atomizing gas. The liquid volume flowrate per unit width of a prefilmer was ranged from 0.8 to $2.6 \mathrm{~cm} 3 /(\mathrm{s} \cdot \mathrm{cm})$, and the air velocity at the atomizer exit was ranged from 16.6 to $83.0 \mathrm{~m} / \mathrm{s}$. During the experiments, the film dry-out was not observed upstream the prefilmer edge except the case under $u_{g}=16.6 \mathrm{~m} / \mathrm{s}$. All experiments were conducted under the atmospheric pressure.

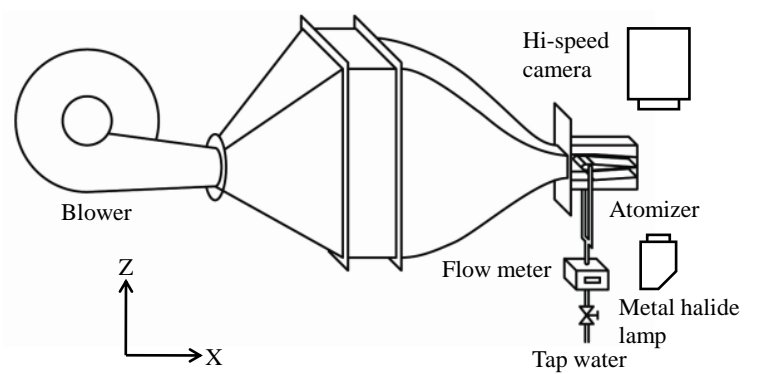

(a) Experimental apparatus
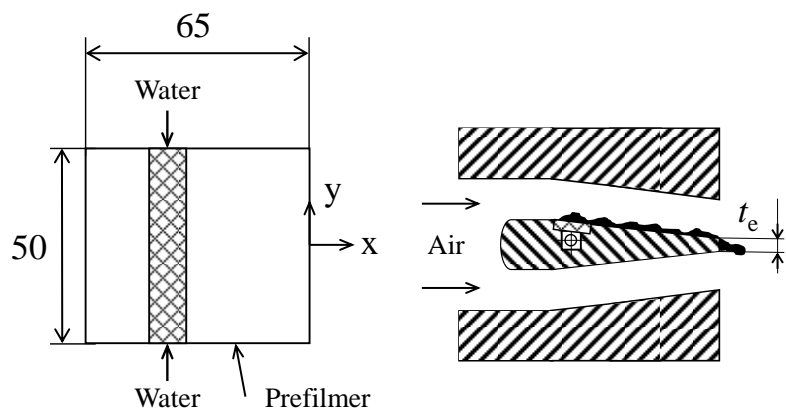

(b) Test atomizer.

Figure 1. Experimental apparatus.

\section{Breakup phenomena of liquid film}

Details of the effects of an air velocity and prefilmer edge thickness on the breakup phenomena of a liquid film at a prefilmer edge under liquid flowrate of $2.6 \mathrm{~cm}^{3} /(\mathrm{s} \cdot \mathrm{cm})$ are illustrated in Figure 2. In this study, five different prefilmer configurations have been tested to investigate the effect of widely varying prefilmer edge thickness $\left(t_{e}=160,500\right.$, $1250,2000$, and $3000 \mu \mathrm{m})$. The air and liquid flow from the bottom to the top in the photographs.

During the experiments, it can be observed that the breakup process basically consists of the three stages. First, a liquid film that was flowing over the prefilmer surface accumulates at the edge and in the wake region of the prefilmer edge. Second, the liquid accumulated at a prefilmer edge is then sheared by air and fragmented into bag-shaped liquid film and ligaments. As soon as this bag-shaped film bursts, fine droplets are generated. The ligaments remain attaching to the prefilmer edge, and disintegrates later into larger droplets.

The visual inspection showed a significant effect of the prefilmer edge thickness on the breakup phenomena of the liquid film such as the breakup length, the size and number of ligaments, and the liquid accumulation attaching to the prefilmer edge. As the prefilmer edge thickness increases, the breakup length of the liquid film and the size of ligaments increases. Due to these geometrical changes, larger droplets are generated. At investigating the liquid accumulation, it was observed that the accumulated volume of liquid clearly depends on the prefilmer edge thickness. It should be noted that, for the case of $t_{e}=160 \mu \mathrm{m}$ under larger air velocity $\left(u_{g}=66.7\right.$ and $\left.83.0 \mathrm{~m} / \mathrm{s}\right)$, no liquid accumulation was observed. Also, at the lower flowrate condition $\left(Q_{l}=0.8 \mathrm{~cm} 3 /(\mathrm{s} \cdot \mathrm{cm})\right)$, there was no liquid accumulation at the prefilmer edge under even a smaller air velocity $\left(u_{g}=32.7\right.$ and $\left.50.0 \mathrm{~m} / \mathrm{s}\right)$. On the other hand, it 
is obvious that increasing air velocity reduces the breakup length of the liquid film. In addition, it can be seen that the number of atomization events per second increases with increasing air velocities.

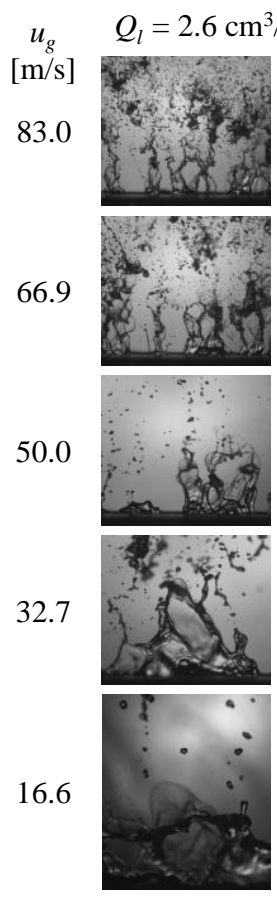

160
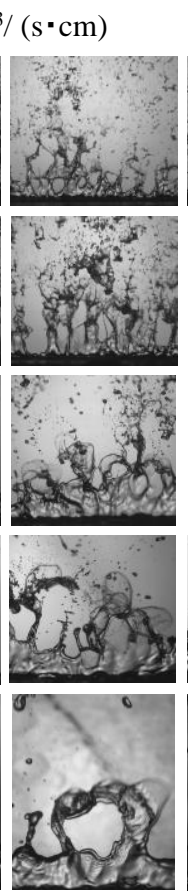

500
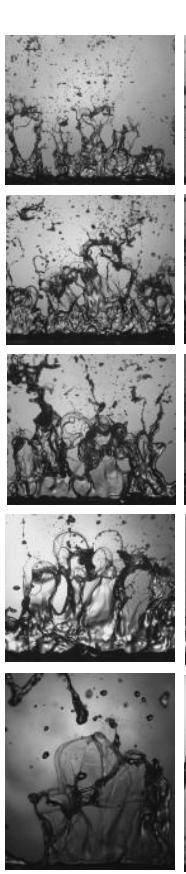

1250
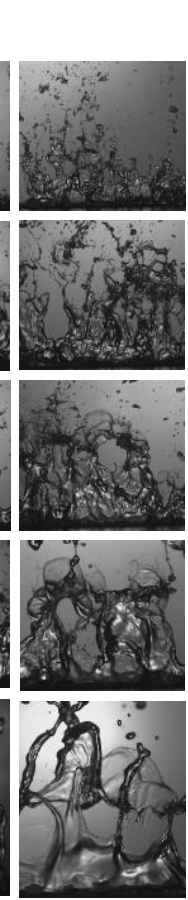

2000

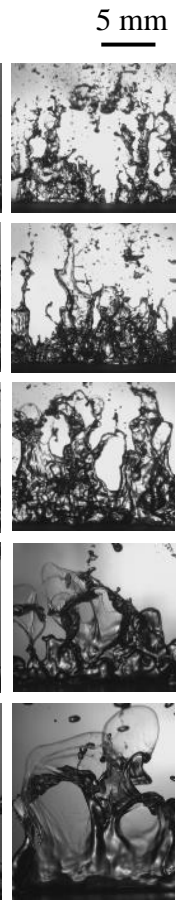

3000 $t_{e}$

Figure 2. Breakup phenomena of liquid film at each prefilmer edge thickness when $Q_{l}=2.6 \mathrm{~cm}^{3} /(\mathrm{s} \cdot \mathrm{cm})$.

\section{Image processing method}

To quantitatively evaluate the effect of the prefilmer edge thickness on the breakup phenomena of a liquid film, the image processing technique to determine the breakup length has been developed using MATLAB (Math Works) in this study.

Figure 3 shows the procedure of an image processing method developed in this study. From the original image at the prefilmer edge (a), a binary image (b) is generated. This binary image processing replaces each pixel in an image with a black pixel if the image intensity is less than the threshold, or a white pixel if it is greater than the threshold. Then, all the pixels framed by a white pixel are filled with white as shown in (c). The parts of droplets and liquid lumps are removed, and only the part of accumulated liquid film remains (d). The area $A_{b}$ is calculated by the number of pixels in the accumulated liquid film. Finally, based on the prefilmer edge width and the calculated area of the liquid film, the breakup length $L_{b}$ can be derived as follows:

$$
L_{b}=A_{b} / w,
$$

where $L_{b}$ is the mean breakup length, $A_{b}$ is the area of the liquid film, and $w$ is the width of the prefilmer edge. The red line in Figure 3 (d) indicates the mean breakup length which is the spatially-averaged value of the entire calculation domain. For each experimental condition, a set of 1000 images was processed using above mentioned method.
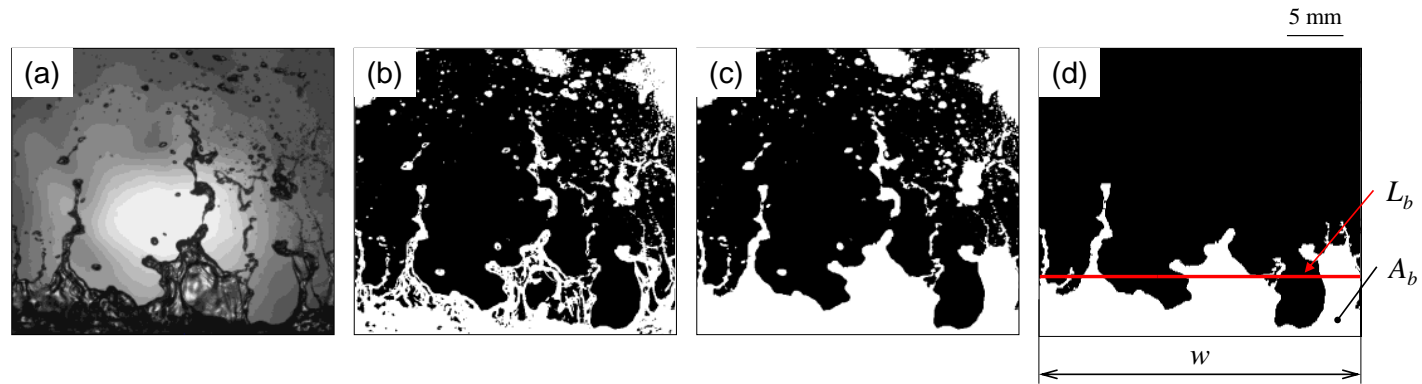

Figure 3. Procedure of image processing for determining breakup length of liquid film $L_{b}$. 


\section{Results and discussion}

Figure 4 shows the time evolution of the breakup length of a liquid film at each prefilmer edge thickness $\left(t_{e}=160\right.$, $500,1250,2000$, and $3000 \mu \mathrm{m}$ ) under (a) $u_{g}=32.7 \mathrm{~m} / \mathrm{s}$ and (b) $u_{g}=66.7 \mathrm{~m} / \mathrm{s}$, at $Q_{l}=2.6 \mathrm{~cm}^{3} /(\mathrm{s} \cdot \mathrm{cm})$. The red line in each graph indicates the time-averaged breakup length for 1000 images. The time variations of the breakup length show a sawtooth-shaped curve. The primary breakup is characterized by a sudden drop of this curve. This is similar to the simulation results by Koch et al. [5].

As the prefilmer edge thickness increases, the mean breakup length increases and the breakup frequency decreases. In conjunction with the visual observations shown in Figure 2, this seems to be due to the accumulated liquid at the prefilmer edge. Obviously, the liquid film was accumulated the atomizer edge before the primary disintegration. The accumulated liquid volume depends on the prefilmer edge thickness. A larger liquid volume is accumulated at a thicker atomizing edge, and results in the elongation of the liquid film attaching to the edge. This is consistent with the previous studies (Gepperth et al. [3], and Koch et al. [5]). In addition, Sattelmayer and Wittig concluded that the characteristics of the wavy liquid film on the prefilmer such as the film velocity, film thickness, and breakup frequency have a weak impact on the breakup process due to a suppression by the liquid accumulation at the prefilmer edge [6]. Also, it is obvious that the maximum breakup length, which means the elongation of the liquid film, became larger as the prefilmer edge thickness increased. For the case of $t_{e}=160 \mu \mathrm{m}$ under a larger air velocity $\left(u_{g}=66.7\right.$ and $\left.83.0 \mathrm{~m} / \mathrm{s}\right)$, no liquid accumulation was observed. At the lower flowrate condition $\left(Q_{l}=0.8\right.$ $\left.\mathrm{cm}^{3} /(\mathrm{s} \cdot \mathrm{cm})\right)$, there was also no liquid accumulation at the prefilmer edge under even smaller air velocity $\left(u_{g}=32.7\right.$ and $50.0 \mathrm{~m} / \mathrm{s}$ ). This leads to decrease of the breakup length and increase of the breakup frequency. Therefore, the breakup length increased and the breakup frequency decreased by increasing prefilmer edge thickness. In addition, the atomization process at $t_{e}=160 \mu \mathrm{m}$ may be separated from those at other thicker edge thicknesses.

On the other hand, it was observed that increasing air velocity reduces the breakup length, and also increases the breakup frequency. An increase of air velocity causes stronger shear force due to a larger air velocity. This force results in the elongated liquid film and makes unstable it faster. Thus, it leads to earlier disintegration which causes increasing the breakup frequency.

The FFT analysis was conducted to quantitatively evaluate the effect of the prefilmer edge thickness on the breakup frequency. Figure 5 shows the power spectrum at each prefilmer edge thickness, when the air velocity is 32.7 or $66.7 \mathrm{~m} / \mathrm{s}$ at $Q_{l}=2.6 \mathrm{~cm}^{3} /(\mathrm{s} \cdot \mathrm{cm})$. For the constant air velocity, the increase of prefilmer edge thickness caused the transition of the maximal power spectrum to a lower frequency. This was also due to the increase of liquid accumulation volume which causes the elongation of the liquid film. Comparing the power spectrums at different air velocities for the same edge thickness, a distinct tendency was found that the breakup frequency increase with increasing air velocity. The peak spectrums for $u_{g}=32.7 \mathrm{~m} / \mathrm{s}$ were less than $100 \mathrm{~Hz}$, while the peak power spectrums for $u_{g}=66.7 \mathrm{~m} / \mathrm{s}$ were ranged between $100-300 \mathrm{~Hz}$.

Figure 6 shows the mean breakup length of the liquid film as function of the prefilmer edge thickness when $Q_{l}=0.8$ and $2.6 \mathrm{~cm}^{3} /(\mathrm{s} \cdot \mathrm{cm})$. As the liquid flowrate increases, the breakup length increases. For both liquid flow rates of 0.8 and $2.6 \mathrm{~cm}^{3} /(\mathrm{s} \cdot \mathrm{cm})$, the breakup length shows a linear increase with the prefilmer edge thickness. This tendency was found for all air velocity conditions. The larger errors for $u_{g}=16.6 \mathrm{~m} / \mathrm{s}$ were due to the elongated liquid film that exceed the field of view. Also, the film dry-out was observed upstream the prefilmer edge, and leads to the larger errors. The increase of liquid flow rate leads to an increase of the breakup length. Especially at larger prefilmer edge thicknesses such as 2000 and $3000 \mu \mathrm{m}$, the effect of the air velocity on the breakup length becomes larger. Due to the increase of the number of atomization events per second and the increase of the accumulated liquid volume, the area of the liquid film attaching to the prefilmer edge in the calculation domain increases at $Q_{l}=2.6$ $\mathrm{cm}^{3} /(\mathrm{s} \cdot \mathrm{cm})$. Thus, the mean breakup length becomes larger than that at $Q_{l}=0.8 \mathrm{~cm}^{3} /(\mathrm{s} \cdot \mathrm{cm})$. 

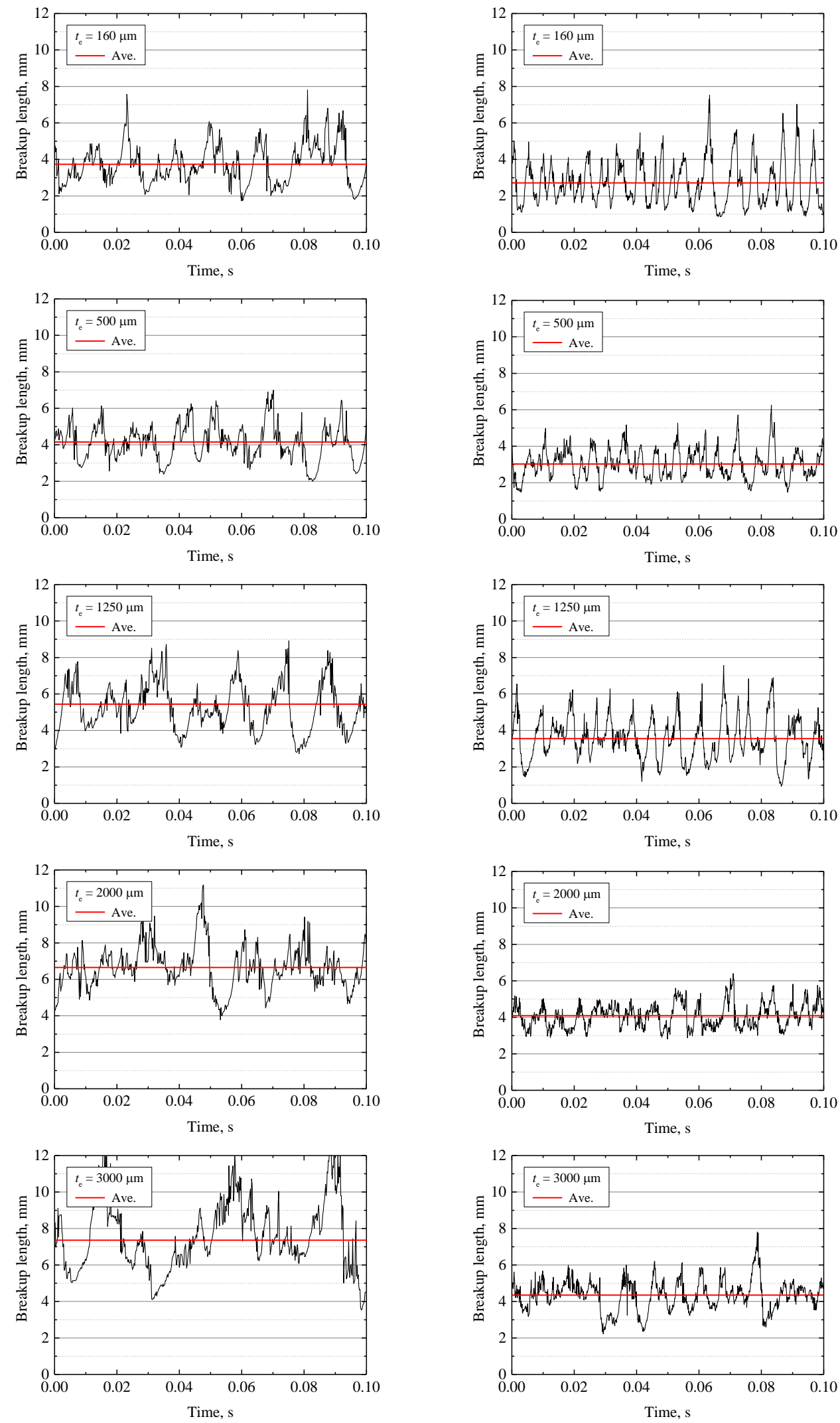

(a) $u_{g}=32.7 \mathrm{~m} / \mathrm{s}$

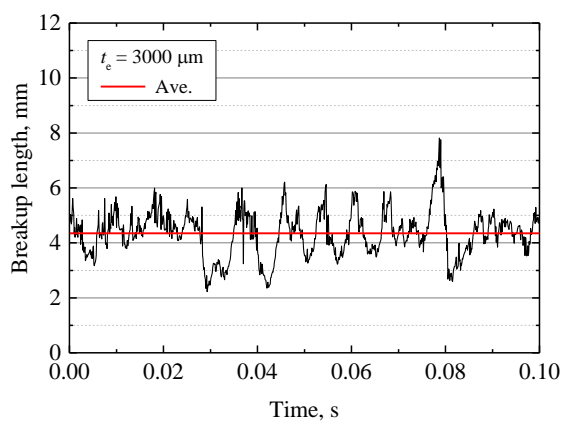

(b) $u_{g}=66.7 \mathrm{~m} / \mathrm{s}$

Figure 4. Time evolution of breakup length of liquid film at each prefilmer edge thickness. 

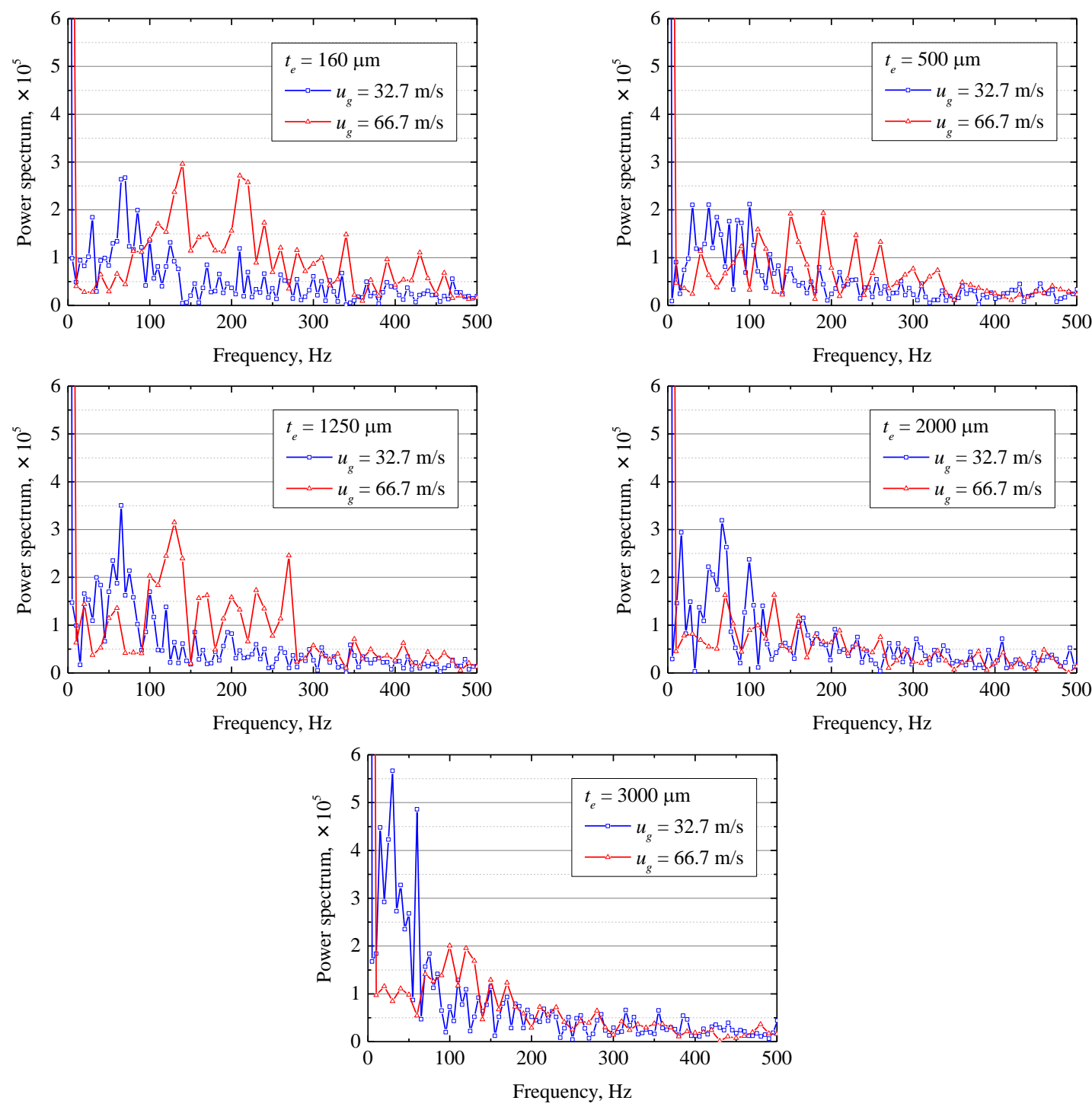

Figure 5. Variations of power spectrum with air velocity at each edge thickness of prefilmer at $Q_{l}=2.6 \mathrm{~cm}^{3} /(\mathrm{s} \cdot \mathrm{cm})$.
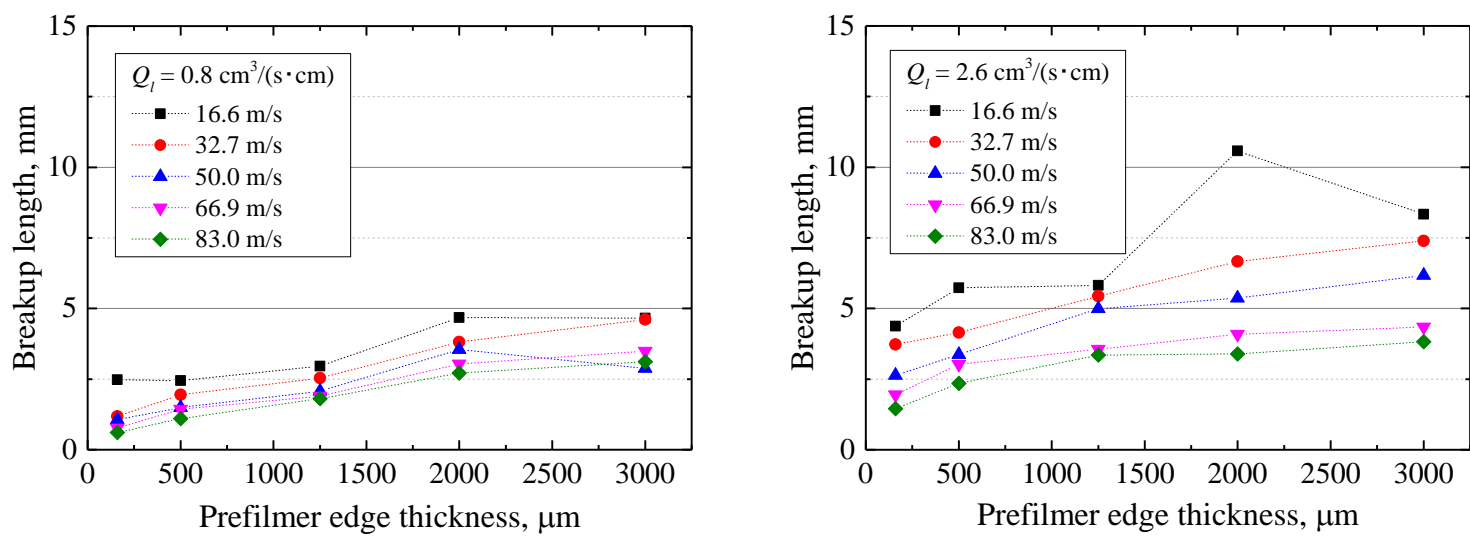

Figure 6. Mean breakup length of liquid film as function of prefilmer edge thickness. 
Fernandez et al. investigated the primary atomization of a liquid film generated by a planar airblast atomizer [7]. They obtained a correlation that the breakup length divided by the liquid film thickness is a function of the momentum flux ratio times the Weber number. To characterize the breakup length at various prefilmer edge thicknesses, we contain the thickness of the accumulated liquid at the prefilmer edge in the correlation as follows:

$$
\begin{aligned}
& L_{b} / t_{l}=\left[\left(u_{l} / u_{g}\right) /\left(M \cdot W e_{t l}\right)\right]^{1 / 3}, \\
& t_{l}=t_{e}+t_{p l}, \\
& W e_{t l}=\left(\rho t_{l} u_{l}\right) / \sigma,
\end{aligned}
$$

where $t_{l}$ is the thickness of the accumulated liquid at the edge, $t_{p l}$ is the liquid film thickness on the prefilmer. The liquid film thickness flowing over the prefilmer, $t_{p l}$, was measured by the contact needle method in our previous study [2]. The measured liquid film thicknesses are ranged from 55 to $600 \mu \mathrm{m}$ at each tested condition, depending on the air velocity. Inamura et al. revealed that the film thickness flowing on the prefilmer decreases as the air velocity increases [2]. At small air velocity, the film thickness increases with the liquid flowrate. At large air velocity, however, the effect of the liquid flowrate on the film thickness becomes smaller.

Figure 7 shows the dimensionless breakup length of the liquid film. The results plotted in Figure 7 show that there was a correlation between the dimensionless breakup length and $\left[\left(u_{l} / u_{g}\right) /\left(M \cdot W e_{t}\right)\right]^{1 / 3}$. However, the data surrounded by a red line strays off the solid line. The solid line in the figure shows the line which was approximated by a linear approximation with the data measured at all experimental conditions except those at a thinner prefilmer edge thickness $\left(t_{e}=160\right.$, and $\left.500 \mu \mathrm{m}\right)$ at $Q_{l}=2.6 \mathrm{~cm}^{3} /(\mathrm{s} \cdot \mathrm{cm})$ surrounded by a red line. This could be due to the ratio of prefilmer thickness to the liquid film thickness flowing over the prefilmer. For the cases when $t_{e}=160$, and $500 \mu \mathrm{m}$, the edge thickness is of the same order of the liquid film thickness flowing over the prefilmer. A sensitivity to the liquid film thickness is relatively large compared to the case of a thicker edge thickness. Thus, the dimensionless breakup length can be easily affected by the experimental error in the liquid film thickness. In fact, the liquid film was measured at $2 \mathrm{~mm}$ upstream from the prefilmer edge, because it is difficult to exactly measure the liquid film thickness of the accumulated liquid film at the edge by the contact needle method used in this study. It may also be that the atomization process is separated from other cases of the prefilmer edge thickness. In fact, no liquid accumulation was only observed at a thinner prefilmer edge thickness. Further research efforts are needed to clarify this issue.

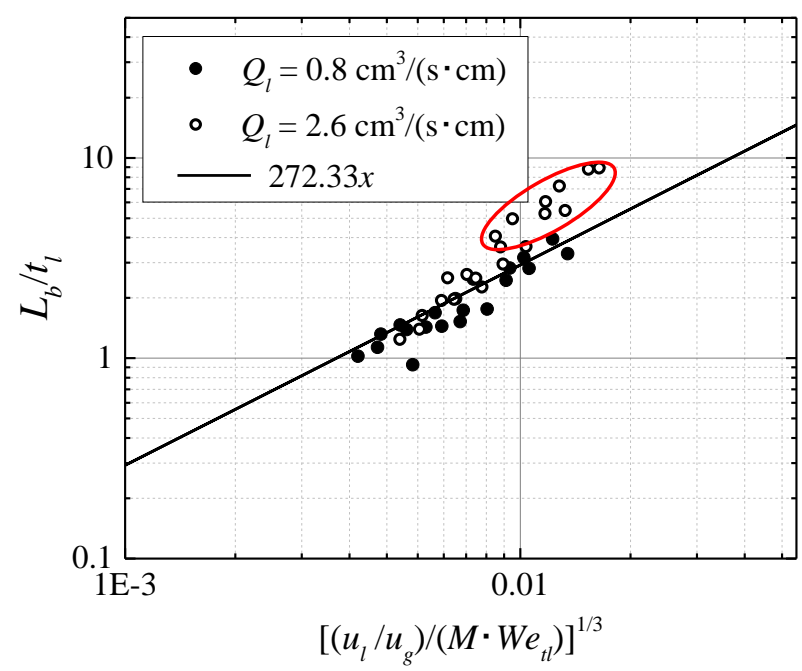

Figure 7. Dimensionless breakup length of liquid film.

\section{Conclusions}

In this study, the effect of widely varying prefilmer edge thickness $(160,500,1250,2000$, and $3000 \mu \mathrm{m})$ on the breakup phenomena of the liquid film in the prefilming airblast atomizer was experimentally investigated. The conclusions obtained in this study are as follows:

1. To quantitatively evaluate complicated breakup process of a liquid film at the prefilmer edge, the image processing method to determine the spatially-averaged breakup length and frequency based on the area of a liquid film and the prefilmer width has been developed. 
2. In a prefilming airblast atomizer, the liquid accumulation attaching to the prefilmer edge has a significant impact on the breakup process of a liquid film. As the prefilmer edge thickness increases, the liquid accumulation volume increases. A larger liquid accumulation causes the increase of the breakup length of the liquid film and the decrease of the breakup frequency.

3. For the case of $t_{e}=160 \mu \mathrm{m}$ at larger air velocity $\left(u_{g}=66.7\right.$ and $\left.83.0 \mathrm{~m} / \mathrm{s}\right)$, no liquid accumulation was observed. Also, at the lower flowrate condition $\left(Q_{l}=0.8 \mathrm{~cm}^{3} /(\mathrm{s} \cdot \mathrm{cm})\right)$, there was no liquid accumulation at the prefilmer edge at even smaller air velocity $\left(u_{g}=32.7\right.$ and $\left.50.0 \mathrm{~m} / \mathrm{s}\right)$. It could be that the breakup process at a thinner prefilmer edge thickness can be separated from that at a thicker one.

4. The FFT analysis indicated that the increase of prefilmer edge thickness causes the transition of the maximal power spectrum to a lower frequency, because of the increase of liquid accumulation volume which causes the elongation of the liquid film. The peak spectrums for $u_{g}=32.7 \mathrm{~m} / \mathrm{s}$ were less than $100 \mathrm{~Hz}$, while the peak power spectrums for $u_{g}=66.7 \mathrm{~m} / \mathrm{s}$ were ranged between $100-300 \mathrm{~Hz}$. These tendency were found in all prefilmer thicknesses.

5. The breakup length of a liquid film is expressed by the following empirical equation which contains the effect of the prefilmer edge thickness:

$$
\frac{L_{b}}{t_{l}}=272.33\left(\frac{u_{l} / u_{g}}{M \cdot W e_{t l}}\right)^{\frac{1}{3}}
$$

\section{Nomenclature}

$A_{b} \quad$ Area of liquid film [mm²]

$f_{b} \quad$ Breakup frequency $[\mathrm{Hz}]$

$L_{b} \quad$ Breakup length $[\mathrm{kg}]$

Le Width of prefilmer edge [mm]

$M \quad$ Momentum flux ratio [-]

Q $\quad$ Liquid flow rate $\left[\mathrm{cm}^{3} /(\mathrm{s} \cdot \mathrm{cm})\right]$

$t_{e} \quad$ Prefilmer edge thickness $[\mu \mathrm{m}]$

$t_{p l} \quad$ Liquid film thickness on prefilmer $[\mu \mathrm{m}]$

tı Accumulated liquid film thickness $[\mu \mathrm{m}]$

\section{References}

[1] Lefebvre, A. H., 1980, Progress in Energy and Combustion Science, 6 (3), pp. 233-261.

[2] Inamura, T., Shirota, M., Tsushima, M., Kato, M., Hamajima, S., and Sato, A., Sep. 2-6, 2012, $12^{\text {th }}$ Triennial International Conference on Liquid Atomization and Spray Systems.

[3] Gepperth, S., Müller, A., Koch, R., and Bauer, H. -J., Sep. 2-6, 2012, $12^{\text {th }}$ Triennial International Conference on Liquid Atomization and Spray Systems.

[4] Tsushima, M., Yamashita, K., Sato, A., Inamura, T., and Shirota, M., Dec. 17-18, 2009, 18th ILASS-Japan Symposium (in Japanese).

[5] Koch, R., Braun, S., Wieth, L., Chaussonnet, G., Dauch, T., and Bauer, H. -J., 2017, European Journal of Mechanics B/Fluids, 61, pp. 271-278.

[6] Sattelmayer, T., and Wittig, S., 1986, Jornal of Engineering for Gas Turbines and Power, 108, pp. 465-472.

[7] Fernandez, V. G., Berthoumie, P., Lavergne, G., 2009, C. R. Mecanique, 337, pp. 481-491. 


\title{
Spray Conditioning for the Preparation of Spray Dried Submicron Particles
}

\author{
Ramona Gorny*, Gerhard Schaldach, Peter Walzel, Markus Thommes \\ Laboratory of Solids Process Engineering, TU Dortmund University, Germany \\ *Corresponding author: ramona.gorny@bci.tu-dortmund.de
}

\begin{abstract}
Particle size reduction down to the submicron range $(0.1-1 \mu \mathrm{m})$ is an effective option to increase the bioavailability of low water soluble active pharmaceutical ingredients. According to the Nernst-Brunner equation, the preparation of submicron sized particles increases the specific surface area, thus increases the dissolution rate. Conventional spray drying devices for submicron particles show certain limitations. The main challenge is the preparation of small and uniform droplets during the atomisation step. In this work, fine droplets were generated combining a nozzle with a droplet separator. Therefore, the aerosol is generated with a pneumatic nozzle and is sprayed into a cyclone droplet separator. Depending on the characteristics of the cyclone, droplets larger than the cut-off-size were separated and returned into the liquid feed. The conditioned aerosol at the top of the cyclone separator can then be introduced into the drying chamber. With this concept the usable part is separated, thus no classification process after drying is necessary. The investigations show that the dependencies during atomisation of the droplets size on the liquid-to-gas mass flow ratio $\mu_{m}$ and the liquid properties (e.g. viscosity) do not apply to the separation step. The conditioned aerosol only depends on the separation characteristics of the cyclone droplet separator. However, the amount of droplets separated is determined by the atomisation step. Hence, the amount of droplets smaller than the cut-off-size can be increased by decreasing the droplet size of the primary aerosol. This is realised by secondary droplet fragmentation. An impact surface causes breakup of the droplets of the primary aerosol before separation. The investigations show an increased amount of droplets $<2 \mu m$.
\end{abstract}

\section{Keywords}

spray drying, low water soluble drugs, submicron particles, droplet fragmentation

\section{Introduction}

The preparation of particles down to the submicron scale $(0.1-1 \mu \mathrm{m})$ obtains much attention in many different application fields, since their chemical or physical behavior differs compared to bulk forms [1]. For pharmaceutical application a high amount of newly identified active pharmaceutical ingredients show low water solubility and therefore exhibit low bioavailability [2]. So far, different strategies were developed (physical or chemical modification) to ensure pharmacological response [3]. This work focuses on physical modification. For low water soluble drugs, the bioavailability may be limited by the dissolution rate, which can be described by the NernstBrunner equation [4,5]:

$\frac{d m}{d t}=A \cdot \frac{D}{h} \cdot\left(c_{s}-c\right)$

The increase in the specific surface area directly increases the dissolution rate $d m / d t$, thus increases the bioavailability. Next to the dissolution rate, for particles down to $1 \mu \mathrm{m}$, solubility is a function of particle size. The relation between particle size and solubility is described by the Ostwald-Freundlich equation [6]:

$c_{s}=c_{s}^{\infty} \cdot \exp \left(\frac{2 \gamma M}{r \rho R T}\right)$

Thus, particle size reduction below $1 \mu \mathrm{m}$ will lead to increased saturation concentration $c_{s}$ for nanosized particles. Up to this point, different submicron particle preparation methods were developed, which can be divided into topdown and bottom-up techniques. For top-down methods, the particle size is reduced by milling or high-pressure homogenization. In bottom-up approaches, the particles were generated by precipitation or evaporation from a bulk solution. Spray drying is a bottom-up technique where the solution is initially atomised into fine droplets in a drying chamber. The dried particles are separated usually by a cyclone. Spray drying enables the generation of particles in a single step, where particle size, morphology and shape can be adjusted by the spray drying conditions. For submicron sized particles, conventional spray drying processes show limitations. This includes the separation of particles with cyclones, since they are not suitable for submicron particles. [3] 
One major challenge is the atomisation step. The particle size is mainly defined by the droplets size, thus small and uniform droplets $d<3 \mu m$ are crucial. With an estimate of limits including small geometric dimensions of the nozzle and water properties, the smallest mean droplet size for conventional pneumatic nozzle is limited at about $5 \mu \mathrm{m}$. [7]

The main concept for a robust process to generate small and uniform droplets includes the separation of the suitable part of the aerosol by using a cyclone droplet separator. A pneumatic nebulizer with internal mixing is used to produce the aerosol. During aerosol conditioning the aerosol is sprayed into a cyclone. The droplet separator was designed with regard to generate a small cut-off size with $d_{\text {cut-off }}=2.6 \mu \mathrm{m}$ during separation. The experimental setup and an image of the designed cyclone [8] are presented in Figure 1.

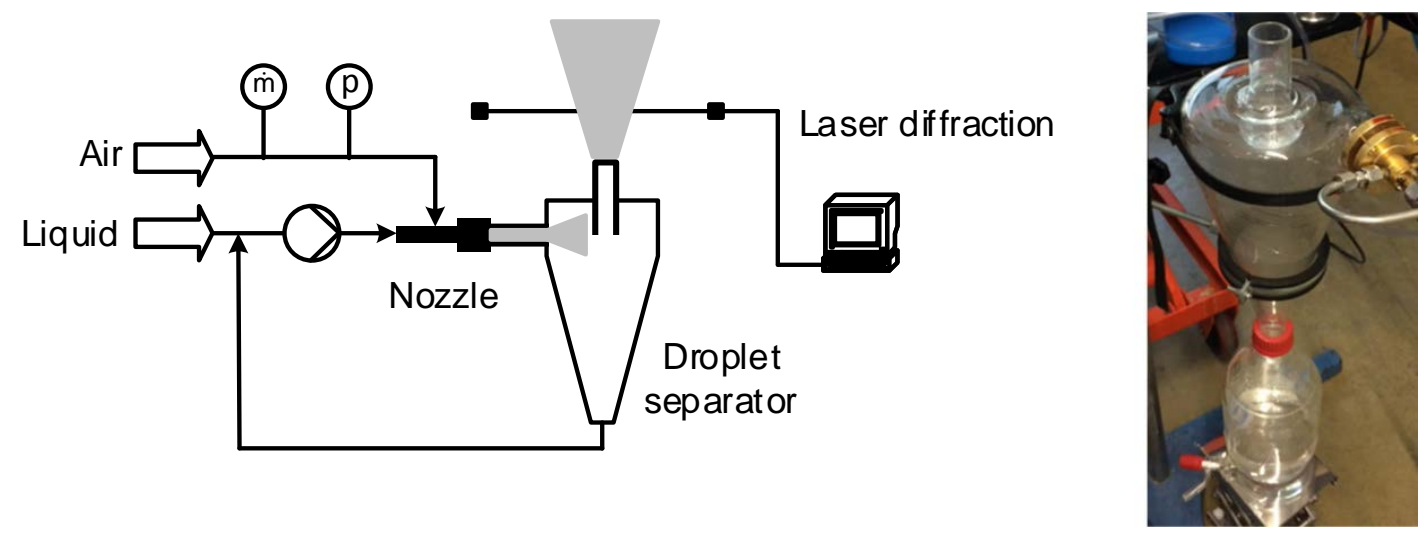

Figure 1: Left: Setup for aerosol conditioning for the generation of small and uniform droplets. Right: Image of the cyclone droplet separator in combination with a pneumatic nozzle

Droplets larger the cut-off size were separated and returned back to the liquid feed. With the designed droplet separator, only the desired part of the aerosol is introduced into the drying chamber. First experiments were carried out with a $10 \mathrm{wt}$ - $\%$ mannitol solution for different liquid-to-gas-mass flow ratios $\mu_{m}=\frac{\dot{m}_{L}}{\dot{m}_{G}}$ and a Povidone $\mathrm{K}$ 30 solution with different viscosities. The conditioned aerosol shows a constant mean droplet size for different liquid-to-gas mass flow ratios and viscosities. Thus, the droplet size of the conditioned aerosol only depends on the separation characteristics of the cyclone with low sensitivity to the flow rate. Hence, this concept is robust regarding high concentrations or viscosities and is able to handle fluctuations of the spraying device. First drying experiments confirm the generation of submicron sized mannitol particles [8].

After drying, the product yield of dried particles is mainly limited by the amount of small droplets, generated by the pneumatic nozzle. With the current setup, the yield of the conditioned aerosol was only about $1 \%$. Therefore, an impact sphere is positioned at a specific distance in front of the nozzle orifice to cause a break up of too large droplets. The collision process of a droplet on a dry or wetted surface is analyzed manifold [9-11]. The required collision velocity for droplet fragmentation with a wetted surface can be estimated according to experimental results [10] with equation 3 :

$v=50 \cdot d_{0}^{-0.6} \cdot \rho^{-0.6} \eta^{0.2} \gamma^{0.4}$

For a droplet size of about $10 \mu \mathrm{m}$ and liquid properties of water an impact velocity of $70 \mathrm{~m} / \mathrm{s}$ is necessary. The droplet size distribution of the aerosol resulting from additional fragmentation will now be discussed.

With a subsequent droplet separator, bigger droplets are removed and a combination with a drying step is possible.

\section{Material and methods}

\section{Aerosol fragmentation with an impact sphere}

Again, the aerosol is firstly generated with a pneumatic internal mixing nozzle. The liquid is dispersed into the gas flow by seven capillaries with an inner capillary diameter of $d_{c a p}=0.5 \mathrm{~mm}$. Deionised water was pumped with a HPLC pump (80P, KNAUER, Berlin, Germany) and sprayed with compressed air. The mass flow of compressed air was measured with a flow meter (Model D10A11, ABB, Göttingen, Germany) and the gas inlet pressure was read on a manometer. For all experiments, the gas inlet pressure was held constant and the self-regulating gas mass flow was measured $\left(\dot{m}_{G}=4.3-4.8 \mathrm{~kg} / \mathrm{h}\right)$. The liquid mass flow rate was varied between 
$\dot{m}_{L}=1.2-3 \mathrm{~kg} / \mathrm{h}\left(\dot{V}_{L}=20-50 \mathrm{~mL} / \mathrm{min}\right)$ and the liquid-to-gas mass flow ratio $\mu_{\mathrm{m}}$ ranged between $0.24-0.70$. The experimental setup for spray fragmentation is presented in Figure 2. For the fragmentation of the aerosol, a sphere with the diameter $d_{s}=7 \mathrm{~mm}$ is located in front of the nozzle orifice $D_{o}=1 \mathrm{~mm}$ at a distance $L=5 \mathrm{~mm}$.

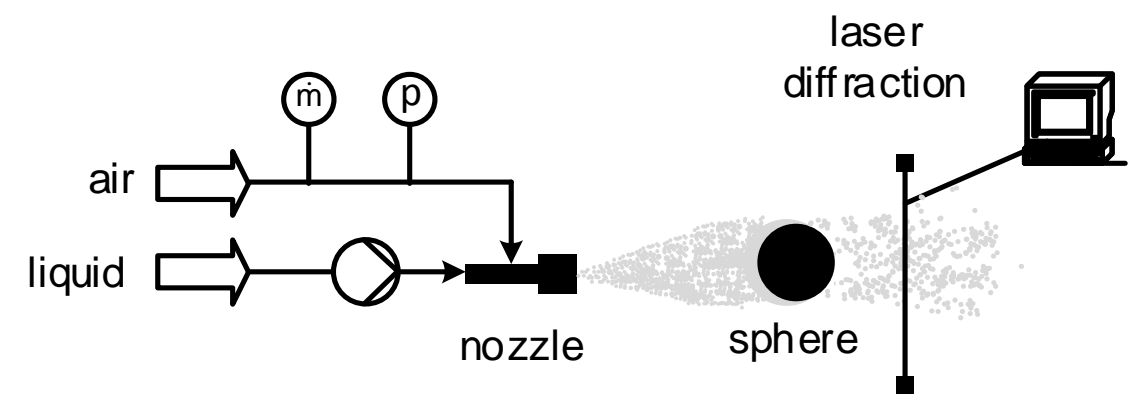

Figure 2: Experimental setup for spray fragmentation

A photograph shows the pneumatic nozzle with the impact sphere:

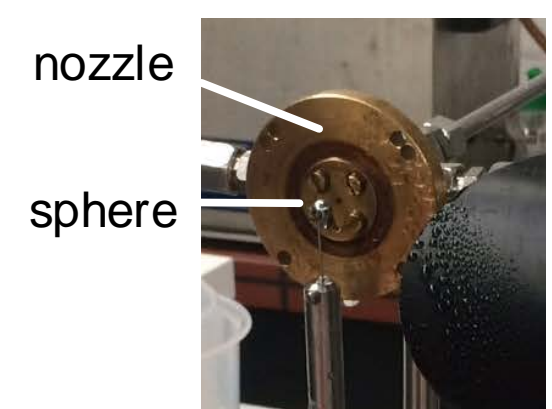

Figure 3: Photograph of the experimental setup for spray fragmentation

The droplet size distribution of the secondary aerosol was measured by laser diffraction technique (Spraytec, Malvern, Malvern, Worcestershire, United Kingdom). A lens focal length of $300 \mathrm{~mm}$ was used allowing for measurements of droplet sizes between 0.1-900 $\mu \mathrm{m}\left(d_{50,3}=0.5-600 \mu \mathrm{m}\right)$. The volumetric droplet size distribution was analysed. The distance between nozzle orifice and the laser beam was held constant at $8 \mathrm{~cm}$.

\section{Results and discussion}

For the primary and the secondary spray, the cumulative volumetric droplet size distribution and the volumetric droplet size distribution density is presented exemplarily for $\mu_{m}=0.25$ in Figure 4.
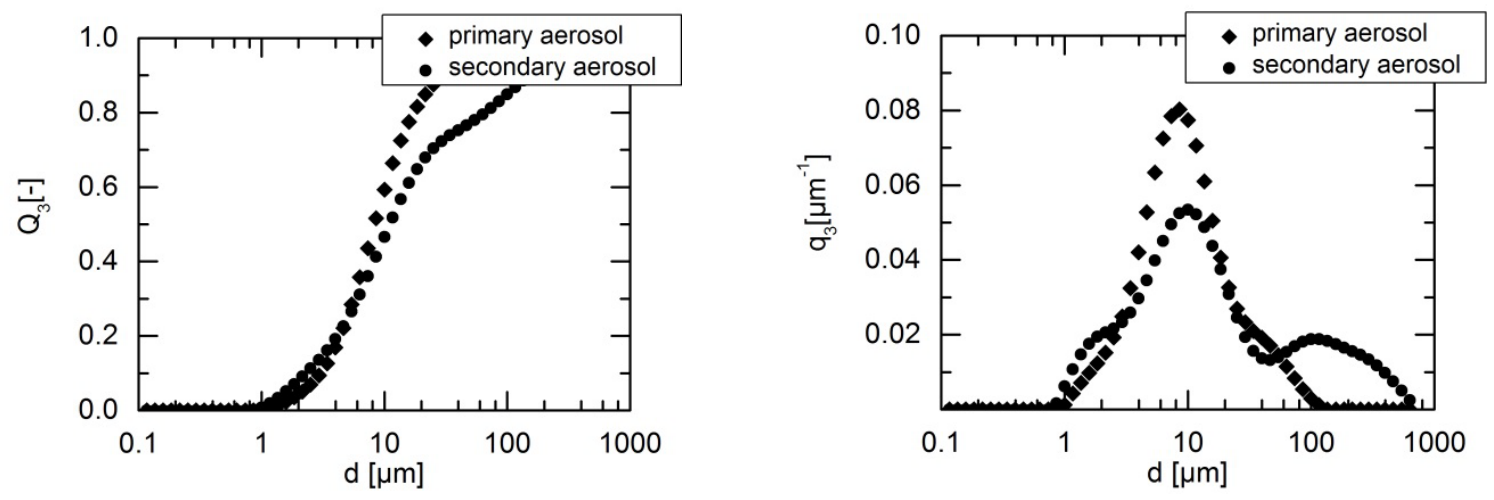

Figure 4: Cumulative (left) volumetric droplet size distribution and the volumetric droplet size distribution density (right) for the primary and the secondary aerosol. $\left(\mu_{m}=0.25\right)$

In Figure 5 the cumulative volumetric droplet size distribution is zoomed in for small droplet sizes.

This work is licensed under a Creative Commons 4.0 International License (CC BY-NC-ND 4.0).

EDITORIAL UNIVERSITAT POLITÈCNICA DE VALÈNCIA 


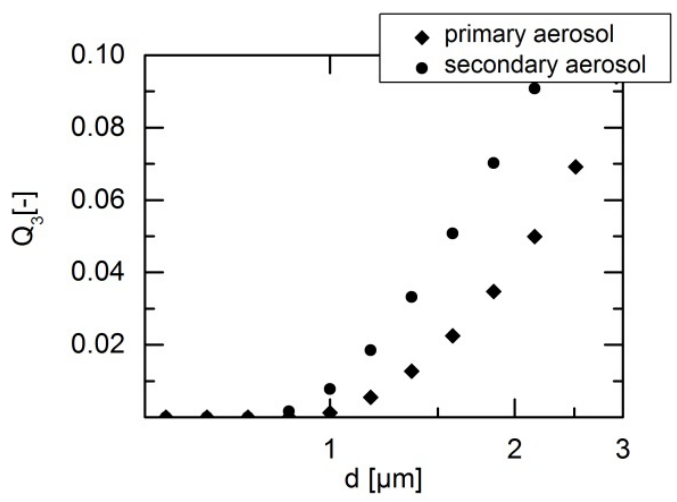

Figure 5: Detail view of the cumulative volumetric droplet size distribution for small droplets $\left(\mu_{m}=0.25\right)$

The secondary aerosol shows a slightly higher width of the distribution, thus generating also bigger droplets. Large droplets detach downward from the sphere and may be entrained by the gas flow. Additionally, a thin liquid layer is formed at the sphere during atomisation. Hence, secondary droplets are also formed from the layer due to the gas stream.

The amount of small droplets is increased upon impact. In Figure 6 the volumetric flowrate of droplets $<2 \mu m$ is presented as a function of the volumetric flowrate in the feed solution. A maximum flowrate of fine droplets was found at $\dot{V}_{\text {feed }}=40 \mathrm{~mL} / \mathrm{min}$ corresponding to a yield of about $2 \%$ of the total liquid flowrate. Further increased liquid flow rate decreases the amount of fines.

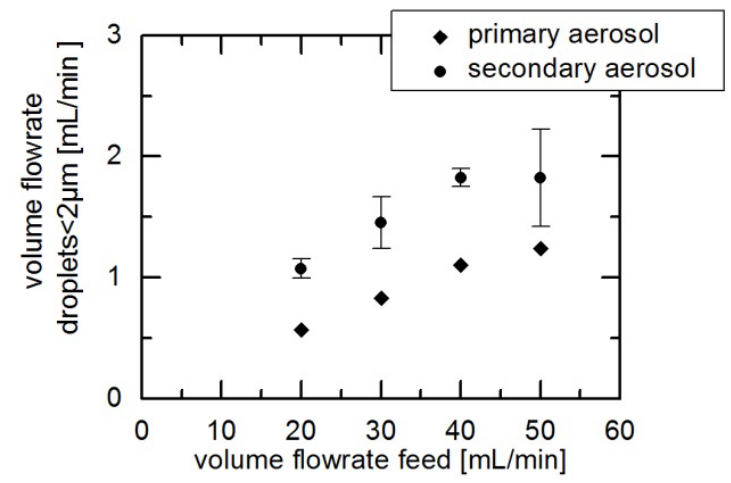

Figure 6:Volumetric flowrate of droplets $<2 \mu \mathrm{m}[\mathrm{mL} / \mathrm{min}]$ depending on the volumetric flowrate in the feed solution for the primary $(n=1)$ and the secondary aerosol $(\bar{x} \pm s d, n=3)$

The volumetric flowrate of droplets $<2 \mu \mathrm{m}$ was calculated from a cut of the cumulative volumetric droplet size distribution at $d=2 \mu m$ and reading the corresponding portion at the $y$-axis. This value is then multiplied with the total feed volumetric flow of the solution.

For the primary aerosol the absolute mass flow of small droplets increases with increasing volumetric feed flow. The amount of small droplets is increased due to fragmentation e.g. for $\dot{V}_{\text {feed }}=20 \mathrm{~mL} / \mathrm{min}$ from 0.5 (primary aerosol) to $1.1 \mathrm{~mL} / \mathrm{min}$ (secondary aerosol).

\section{Conclusions}

With the concept of aerosol conditioning, large droplets were separated from the primary aerosol before drying. The advantages of a robust pneumatic nozzle regarding to high solid concentrations and viscosities of the liquid is combined with a cyclone separator, generating a constant mean droplet size. This combination accomplishes all requirements of a system for industrial application in case of robustness, simplicity and long-term stability.

The volumetric amount of fine droplets separated depends on the characteristics of the pneumatic nozzle, since only $1 \%$ is in the suitable range of the primary aerosol. Therefore, in this work the primary aerosol is fragmented by positioning a sphere in front of the orifice. The geometry of a sphere was used to ensure uniform flow pattern of the spray after fragmentation. The amount of droplets $<2 \mu \mathrm{m}$ is increased for a liquid-to-gas mass flow ratio range of $0.24<\mu_{m}>0.7$ of about $100 \%$ from e.g. 0.5 to $1.1 \mathrm{~mL} / \mathrm{min}$. 
Furthermore, the phenomenon of spray impact on a sphere will be further investigated. Different geometric and fluid parameters will be varied and the effect on the droplet fragmentation will be analysed. During fragmentation, a liquid layer is formed on the sphere. The high gas velocity entrains the secondary droplets and leads to droplet detachment from the liquid film, thus generating also bigger droplets. This will be analysed with regard to the liquid loading.

After selection of the suitable geometric conditions for the smallest droplet size after fragmentation, the pneumatic nozzle with the impact sphere will be introduced into the cyclone droplet separator. The cyclone separation leads to a cut-off of the secondary aerosol, thus generating a narrow droplet size distribution with a small mean droplet size.

\section{Nomenclature}

A surface $\left[\mathrm{m}^{2}\right]$

C concentration $\left[\mathrm{kg} \mathrm{m}^{-3}\right]$

$c_{s} \quad$ saturation solubility nanosized API [ $\left.\mathrm{kg} \mathrm{m}^{-3}\right]$

$C_{S}^{\infty} \quad$ saturation solubility of infinitely large API [ $\left.\mathrm{kg} \mathrm{m}^{-3}\right]$

$D \quad$ diffusion coefficient $\left[\mathrm{m}^{2} \mathrm{~s}^{-1}\right]$

d droplet size [m]

$d_{0} \quad$ droplet diameter before fragmentation [m]

$d_{50,3} \quad 50 \%$-quantile of the volumetric distribution [m]

$d_{\text {cut-off }}$ cut-off diameter of the cyclone [m]

$d_{\text {cap }} \quad$ inner capillary diameter [m]

$D_{0} \quad$ nozzle orifice diameter [m]

$d m / d t \quad$ dissolution rate $\left[\mathrm{kg} \mathrm{s}^{-1}\right]$

$h \quad$ diffusion layer thickness [m]

$M \quad$ molar mass $\left[\mathrm{kg} \mathrm{mol}^{-1}\right]$

$\dot{m}_{G} \quad$ gas mass flow $\left[\mathrm{kg} \mathrm{s}^{-1}\right]$

$\dot{m}_{L} \quad$ liquid mass flow [ $\mathrm{kg} \mathrm{s}^{-1}$ ]

$\Delta p_{G} \quad$ pressure drop of the gas phase [Pa]

$Q_{3} \quad$ volumetric cumulative size distribution [-]

$R \quad$ gas constant $\left[\mathrm{J} \mathrm{mol}^{-1} \mathrm{~K}^{-1}\right.$ ]

$r \quad$ particle radius [m]

$T \quad$ temperature [K]

$\dot{V} \quad$ volume flowrate $\left[\mathrm{m}^{3} \mathrm{~s}^{-1}\right]$

$v \quad$ collision velocity [ $\left.\mathrm{m} \mathrm{s}^{-1}\right]$

$\gamma \quad$ interfacial tension [ $\mathrm{J} \mathrm{m}^{-2}$ ]

$\eta \quad$ viscosity [mPas]

$\mu_{m} \quad$ liquid-to-air mass flow ratio [ $\mathrm{kg}_{\text {liquid }} \mathrm{kg}_{\text {air }}{ }^{-1}$ ]

$\rho \quad$ density $\left[\mathrm{kg} \mathrm{m}^{-3}\right]$

\section{References}

[1] Okuyama, K., Wules Lenggoro, I., 2003, Chemical Engineering Science, 58, pp. 537-547

[2] Keck, C., Müller, R., 2006, European Journal of Pharmaceutics and Biopharmaceutics, 62, pp.3-16.

[3] Sosnik, A., 2015, Advances in Colloid and Interface Science, 223, pp. 40-54.

[4] Nernst W., 1904, Zeitschrift für Physikalische Chemie, 47(1), pp. 52-55.

[5] Brunner, E., 1904, Zeitschrift für Physikalische Chemie, 47(1), pp. 56-102.

[6] Wu, W., Nancollas, G., 1998, Journal of Solution Chemistry, 27(6), pp.521-531.

[7] Walzel, P., 1990, Chemie Ingenieur Technik, 62(12), pp. 983-994.

[8] Gorny, R., Schaldach, G., Walzel, P., Thommes, M., 4-7 Sep. 2016, ILASS - Europe, Brighton, UK

[9] Rein, M., 1993, Fluid Dynamics Research, 12, pp. 61-93.

[10] Walzel, P., 1980, Chemie Ingenieur Technik, 52 (4), pp. 338-339.

[11] Bakshi, S., Roisman, I., Tropea, C., 2007, Physics of Fluids, 19 (3), p. 32102 


\title{
Multiple Impinging Jet Air-Assisted Atomization
}

\author{
Bruno Pizziol ${ }^{1}$, Mário Costa*1, Miguel Oliveira Panão ${ }^{2}$, André Silva ${ }^{3}$ \\ ${ }^{1}$ IDMEC-LAETA, Instituto Superior Técnico, Universidade de Lisboa, Lisboa, Portugal \\ ${ }^{2}$ ADAI-LAETA, Universidade de Coimbra, Coimbra, Portugal \\ ${ }^{3}$ AreoG-LAETA, Universidade da Beira Interior, Covilhã, Portugal \\ ${ }^{*}$ Corresponding author: mcosta@tecnico.ulisboa.pt
}

\begin{abstract}
The growth of the aviation sector triggered the search for alternative fuels and continued improvements in the combustion process. This work addresses the technological challenges associated with spray systems and the concern of mixing biofuels with fossil fuels to produce alternative and more ecological fuels for aviation. This work proposes a new injector design based on sprays produced from the simultaneous impact of multiple jets, using an additional jet of air to assist the atomization process. The results evidence the ability to control the average drop size through the air-mass flow rate. Depending on the air-mass flow rate there is a transition between atomization by hydrodynamic breakup of the liquid sheet formed on the impact point, to an aerodynamic breakup mechanism, as found in the atomization of inclined jets under cross-flow conditions. The aerodynamic shear breakup deteriorates the atomization performance, but within the same order of magnitude. Finally, our experiments show that mixing a biofuel with a fossil fuel does not significantly alter the spray characteristics, regarded as a step further in developing alternative and more ecological fuels for aero-engines.
\end{abstract}

\section{Keywords}

Impinging Jets Atomization, Air-Assisted, Biofuel

\section{Introduction}

In the commercial aviation sector, aircraft fleets operate on single fossil fuel products, and contribute to $3 \%$ of global carbon emissions [1]. Increasing concerns around the environmental impact of the sector, along with its future growth rate - estimated to be $4.7 \%$ every year on fleet basis [2] - raised the interest in improving the efficiency of engine combustion technology to reduce greenhouse gases emissions. One of the most prominent strategies is the use of biofuels based on renewable feedstock toward a more neutral carbon cycle. However, one of the key components in aero-engines is the fuel atomization, and introducing biofuels or mixtures between fossil jet fuels and biofuels requires making sure the differences in thermo-physical properties do not alter spray formation and development, and droplets characteristics.

Alternative fuels allow a faster benefit, since industrial assets of the aeronautical sector do not restrict their development, where high investment costs slow innovation in their production [3]. Using alternative fuels presents further advantages over the use of fossil jet fuel, as a reduced cost fluctuation, a worldwide homogeneous distribution of the feedstock and, depending on the alternative fuel production, better fuel properties [4]. In light of this, the use of alternative jet fuels expects a growth of $30 \%$ until 2030 [5].

Among engine components, the atomizer affects in a great extent the combustion promptness, cleanness, and efficiency. The technological development of this component aims at achieving the best atomization - in terms of evaporation rate and spray penetration - while reducing power consumption for the pressurization of the fuel and, if present, the atomizing fluid.

In this context, this work explores the use of an atomization strategy based on the simultaneous impact of multiple impinging jets $(2,3$ and 4 ) to design a spray system with the ability to have some control over the atomization process. While the use of multiple impinging-jets lowers the liquid supply pressure and produces relatively small droplets (depending on the jet diameter), the assistance of an air-jet is the hypothesis for a more adequate control of the atomization process. To the best of our knowledge, Avulapati and Venkata [6] proposed this strategy for a wide range of experimental conditions, although restricted to the impingement of 2 jets. Besides the injector design, through an analysis of the spray characteristics, we assess the effect of mixing biofuel to a fossil fuel by analysing the atomization efficiency. 


\section{Materials and Methods}

To investigate the effect of the air assisting the atomization process in a multiple jets impingement strategy, the injector prototype developed can collide up to 12 cylindrical jets. Fig. 1 shows a schematic of the injector design for the case of 2-impinging jets. The injector composes an upper part, referred to as injector tail (A), and a lower part, referred to as injector head $(B)$. The two parts connect on a horizontal surface, referred to as connection surface, through bolted joints.

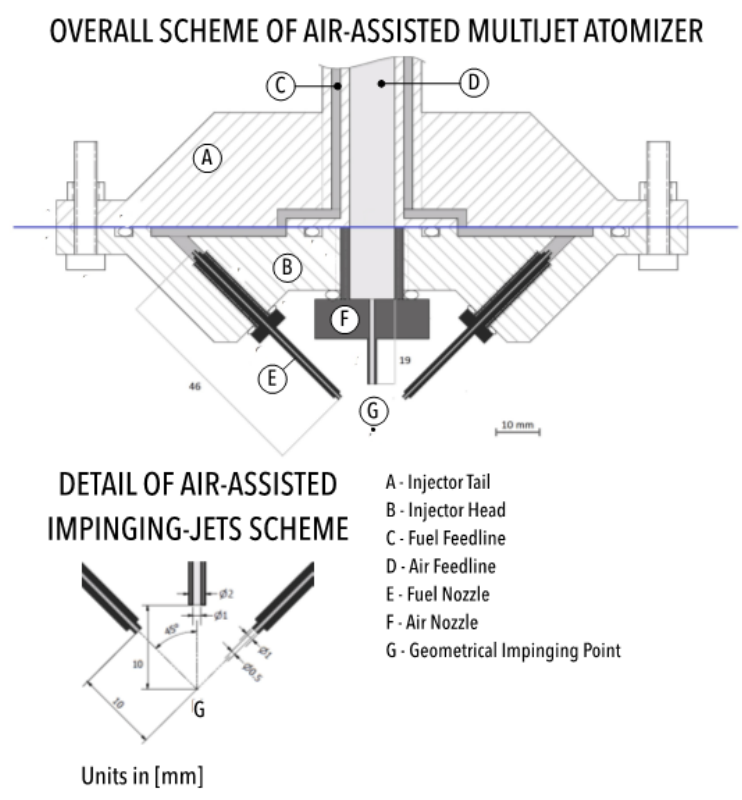

Figure 1. Schematic of the injector and details of the impinging jets configuration.

The injector tail is the vertical segment through which the fuel and the air flow from the connections to the corresponding feed lines ( $C$ and $D$ ) connected with the injector head, which holds the atomizer. The fuel and the air nozzles ( $E$ and $F$ ) are separate components, screwed to the injector head. Interchangeable nozzles allow using the same injector head for the experimental characterization of different atomizer configurations, in terms of nozzle diameter and length, and number.

The fuel path through the injector has three distinct stages. A first stage through the vertical feed line to the bottom of the injector tail, a second stage, where the fuel follows a horizontal path, and a third stage of angled flowing towards the impingement point $\mathrm{G}$.

The injector tail comprises two concentric tubes, defining the axial channels of both air (within the internal tube) and fuel flow (between the internal and external tubes). Its length is $500 \mathrm{~mm}$ for a symmetrical flow development and to compensate any misalignment on the feed-line connection.

In the second stage, the fuel path develops from the inner to the external region of the injector head. As the flow reaches the end of the axial channel, it branches into two radial channels (one for each nozzle block) carved into the injector head connection surface. As for the axial channel, the width of $2 \mathrm{~mm}$ guarantees a correct development of the flow within the section. The fuel leakage through the connection plane, directed towards both the external atmosphere and the internal air path, is avoided using an internal and an external large O-rings.

In the third and final stage, the flow moves from the external end of the radial channel to the impingement point with a fixed impingement angle of $45^{\circ}$. The diameter of the fuel inner tube is $0.5 \mathrm{~mm}$, resulting in a laminar flow for all conditions $(\operatorname{Re}<2300)$. In the laminar regime, the entrance length to ensure a fully developed flow at nozzle exit $\left(\mathrm{x}_{\mathrm{fd}}\right)$ is $x_{f d}=0.05 \mathrm{Re}_{D} \cdot D$. For the highest flow rate, this corresponds to a length of $40 \mathrm{~mm}$, and the tube has $46 \mathrm{~mm}$, thus, every fuel mass flow rate considered is fully developed at nozzle exit. Also in this case, an O-ring between the nozzle screw head and the injector head avoids fuel leakage through the nozzle thread. 
The air flow exits from a vertical $10 \mathrm{~mm}$ tube coaxial with the fuel feed line, through the injector tail until the $1 \mathrm{~mm}$ diameter nozzle. The air flow impingement distance is $10 \mathrm{~mm}$. Fig. 2 shows the injector and the spray produced by the multiple impinging jets atomizer.
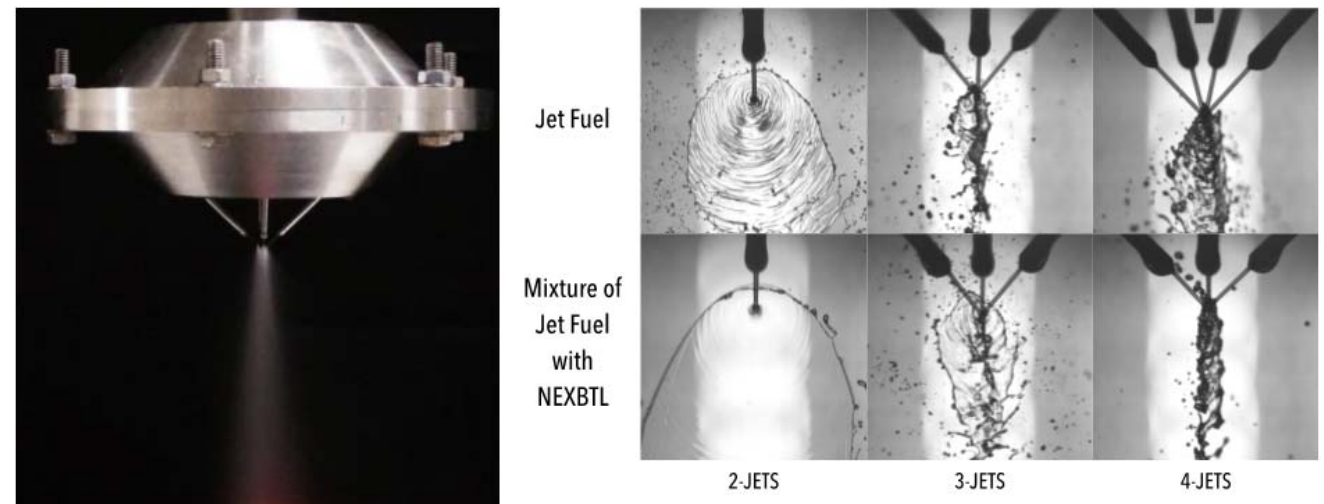

Figure 2. Injector and spray produced by the multiple impinging jets atomizer (left); Spray produced by the 2-4 impinging jets configurations considered without the central air flow rate $\left[\dot{m}_{l}=1.67 \mathrm{~g} / \mathrm{s}-\right.$ Jet Fuel; $\dot{m}_{l}=1.54 \mathrm{~g} / \mathrm{s}-$ Fuel Mixture] (right)

The experiments used two fuels. A commercial Jet Fuel A-1 used in aero-engines and an alternative fuel 50:50 blending the previous Jet Fuel with a Biodiesel known as NEXBTL (Next Generation Biomass-to-Liquid) produced by Neste Oil. Table 1 presents the relevant physical properties for atomization of the two fuels.

Table 1. Physical properties of the fuels.

\begin{tabular}{c|ccc}
\hline \hline & $\begin{array}{c}\text { Density at } 15^{\circ} \mathrm{C} \\
{\left[\mathrm{kg} / \mathrm{m}^{3}\right]}\end{array}$ & $\begin{array}{c}\text { Surface tension at } 20 \\
{ }^{\circ} \mathrm{C}[\mathrm{N} / \mathrm{m}]\end{array}$ & $\begin{array}{c}\text { Dynamic viscosity } \\
\text { at } 20^{\circ} \mathrm{C}\left[\mathrm{Ns} / \mathrm{m}^{2}\right]\end{array}$ \\
\hline \hline Commercial Jet Fuel A-1 & 785.8 & 0.0225 & 0.0044 \\
'Jet Fuel' - 'Biodiesel NEXBTL' (50:50) & 782.0 & 0.0225 & 0.0038
\end{tabular}

Experimental Setup and Diagnostic Techniques

During the experiments, the injector was in the vertical position, with the injector head - air and fuel nozzles - on the bottom and the connections to the air and fuel feed-lines on the top. An air feed-line connects to the injector top from the pressurized line at 7.5 bar. A pressure regulator reduces this value to 2.5 bar before entering the rotameter. In these conditions, the line provided a mass flow rate through the air orifice up to $0.6 \mathrm{~g} / \mathrm{s}$.

The fuel feed-line connected the pressure vessel to the injector top, loading it in the vessel at atmospheric pressure. A high-pressure bottle of gaseous nitrogen pressurized this vessel, but a pressure regulator set between the two, reduced the 200 bar pressure in the bottle to the injection value of 3.2 bar. A rotameter between the vessel and the end of the feed-line controlled the fuel flow. An additional ball valve allowed for prompt closure, reducing the fuel consumption. This pressure reduction to injection values, coupled with injector head configuration, presented the higher losses, and allowed using the rotameter full scale when setting the fuel mass flow rate. Thus, the maximum value available in these conditions is $5.90 \mathrm{~g} / \mathrm{s}$.

A laser diffraction diagnostic technique (Malvern 2600 Particle Size Analyser) was used to measure the droplet size distributions for the various sprays examined as a function of the injector head configuration and position, fuel and air flows. The instrument comprised a low power $5 \mathrm{~mW} H e-N e$ laser transmitter and a receiver detector unit. The collimated $9 \mathrm{~mm}$ diameter beam, produced by the transmitter associated optics, was directed into the spray perpendicularly to its axis. The receiver lens used was a $300 \mathrm{~mm}$ focal length lens, which is able to measure droplets ranging from 5.8 to $564 \mu \mathrm{m}$. In this study, we restricted the droplet size measurements to spray regions where the obscuration, which is a measure of the attenuation of the laser intensity through the spray, was less than 0.5 , so that multiple scattering effects were negligible. Finally, to fit the measured energy distribution we used the model independent (a fifteen parameter function), available in the Malvern software, which provided excellent fits of the experimental data - log errors of around 3. 
In addition, a Phantom V4.2 HSC (High Speed Camera) was used to visualize the atomization mechanisms near the impingement point (see Fig. 2). An exposure time of $1 \mu$ s allowed capturing the ligaments formation and the main structures in the spray pattern.

\section{Results and Discussion}

From the results characterizing drop sizes in air-assisted multijet impinging sprays with $N_{j}=2,3$ and 4 impinging jets, we analyse the effect of the air-mass flow rate on the Sauter mean diameter retrieved from the distribution measured by the laser diffraction diagnostic technique. Subsequently, we develop a definition for the efficiency of atomization and analyse the results.

\section{Effect of Air-Mass Flow Rate on Average Drop Sizes}

A preliminary analysis of the spray characterization evidenced the air-mass flow rate as the main parameter changing the atomization outcome. Figs. 3 and 4 show the effect of that parameter on the Sauter mean diameter.

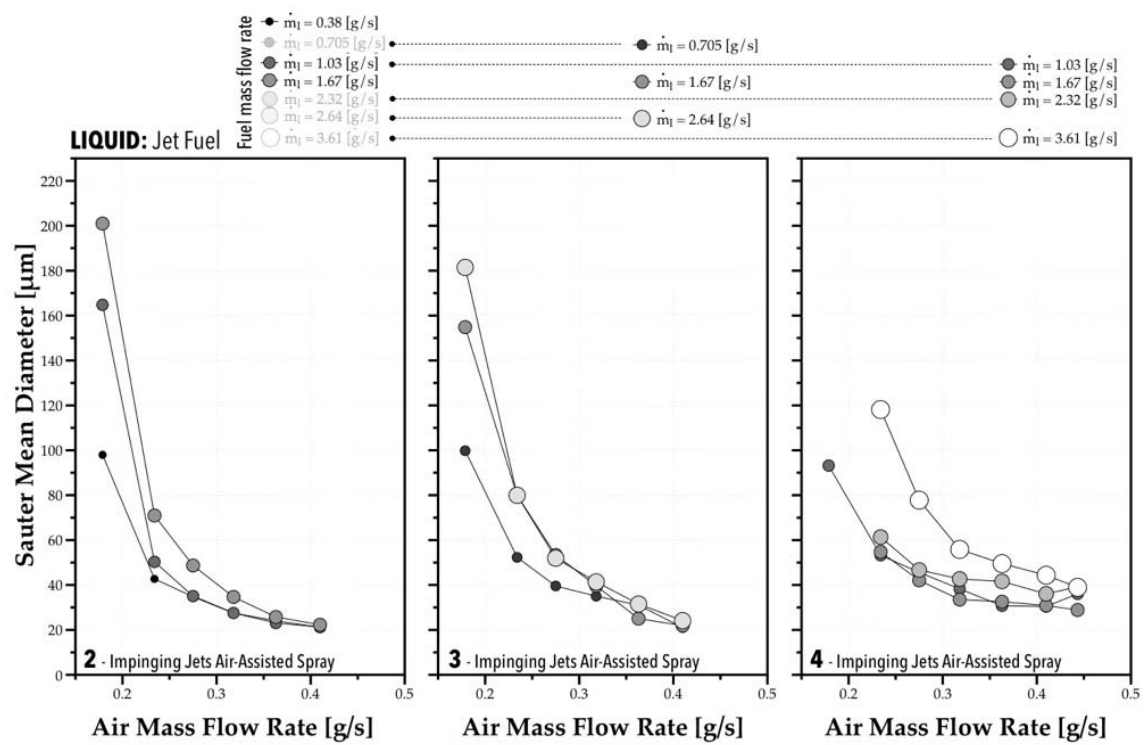

Figure 3. Effect of the air-mass flow rate on the SMD of the Jet Fuel for different impinging-jets configurations (2, 3 and 4 jets) and mass flow rates $\left(\dot{m}_{f}=0.38-3.61 \mathrm{~g} / \mathrm{s}\right)$.

Note that the size of the symbols in Figs. 3 and 4 is proportional to the fuel mass flow rate. While increasing the mass flow rate leads to larger average drop sizes in every configuration, as reported by several other authors, our results point to the fundamental role of the air flow rate in dominating the atomization process, more than the air/fuel ratio. A closer visualization of the impact point region explains why. Fig. 5 shows the effect of the minimum and maximum air-mass flow rate in the deflection of the impinging jet for the case with 3-jets, and the change in the atomization mechanism from hydrodynamic forces to aerodynamic, with a small increase of air-mass flow rate. The figure reveals that there is a threshold above which increasing the air-mass flow rate leads to a deflection of the impinging jets and, instead of producing droplets by hydrodynamic breakup from the liquid sheet formed at the impact point, the aerodynamic forces dominate breakup, like the atomization of inclined jets under cross-flow [7, 8]. The two images on the right of Fig. 5 show the differences in the spray structure with an increase of $15 \%$ in the air-mass flow rate $\left(\dot{m}_{\text {air }}=0.18-0.41 \mathrm{~g} / \mathrm{s}\right)$.

The disruptive effect of the aerodynamic forces intensifies the atomization mechanisms, resulting in smaller drop sizes. Only with the 4-impinging jets does the mass flow rate produce some effect on the average drop size. However, it is noteworthy that the order of magnitude of the average drop sizes is similar for all configurations, which points to the possibility of controlling drop size through the atomizing air flow.

With the 50:50 mixture of Jet Fuel and NEXBTL Biodiesel, the dominating effect of the air-mass flow rate remains. However, while in the conventional jet fuel spray, the mass flow rate induced a slight variability in the results, with the fuel mixture, such effect is negligible. These results suggest that mixing biodiesel with fossil jet fuel does not alter the average size of droplets in the spray. 


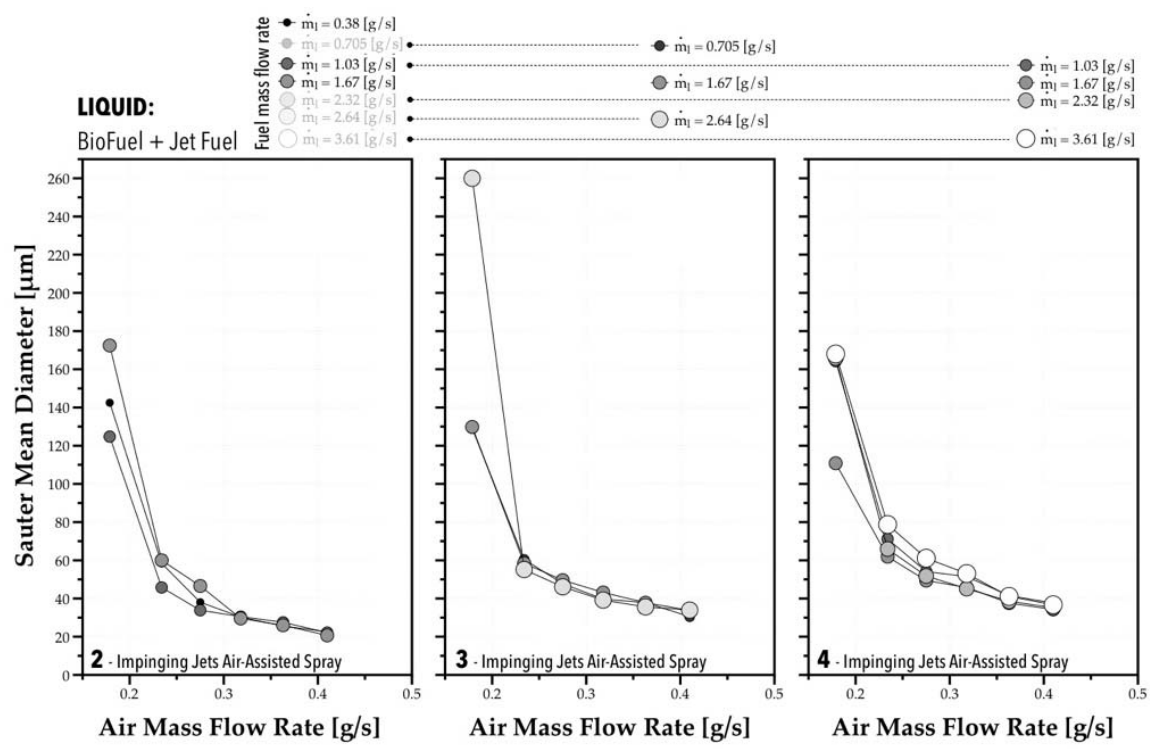

Figure 4. Effect of the air-mass flow rate on the SMD of the Jet Fuel-Biodiesel Mixture for different impinging-jets configurations $(2,3$ and 4 jets $)$ and mass flow rates $\left(\dot{\mathrm{m}}_{\mathrm{f}}=0.38-3.61 \mathrm{~g} / \mathrm{s}\right)$.

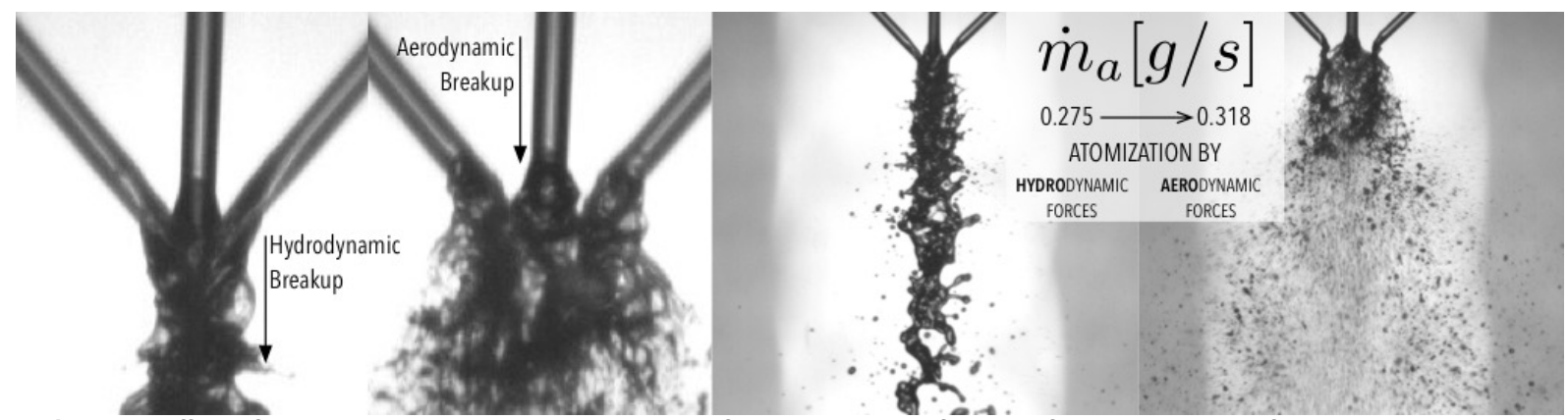

Figure 5. Effect of the minimum and maximum air-mass flow rate in the deflection of the impinging jet for the case with 3-jets (2 images on the left); Change in the atomization mechanism from hydrodynamic forces to aerodynamic, with a small increase of air-mass flow rate (2 images on the right).

In light of this, we suggest an empirical approach to examine the effect of the air-mass flow rate $\left(\dot{m}_{\text {air }}[\mathrm{g} / \mathrm{s}]\right)$ on the Sauter Mean Diameter $\left(D_{32}[\mu m]\right)$. expressed as

$$
D_{32}=D_{32, s}+a \cdot \dot{m}_{\text {air }}^{b}
$$

This expression considers a stabilization of $D_{32}$ as the air-mass flow rate $\left(\dot{m}_{\text {air }}\right)$ increases, represented by $D_{32, s}[\mu \mathrm{m}]$. The empirical coefficient a represents a scale parameter associated with the air flow effect, and $b$ its magnitude on the outcome. Fig. 6 shows the results of the curve fitting in Eq. (1) for the experiments with Jet Fuel (left) and biofuel mixed with Jet Fuel (right).

The empirical correlations in Fig. 6 point to the marginal effect of mixing biodiesel to fossil Jet Fuel in terms of the average size of droplets. Therefore, the mixing does not appear to generate droplets with different characteristics in the experiments reported. However, the increase of exponent $b$ in the correlation with the Jet Fuel-Biodiesel 50:50 mixture suggests an improvement of the air-mass flow rate ability for controlling average drop size. This points to a robust atomization strategy based on air-assisted multiple impinging jets, although requiring an analysis from the point of view of the atomization efficiency, performed below.

The correlations in Fig. 6 are still dimensional. Thus, if we normalize $D_{32}$ by the lower limit to the average drop size $\left(D_{32, s}\right)$, resulting from the effect of the air-mass flow rate,

$$
D_{32}^{*}=\frac{D_{32}}{D_{32, s}}
$$



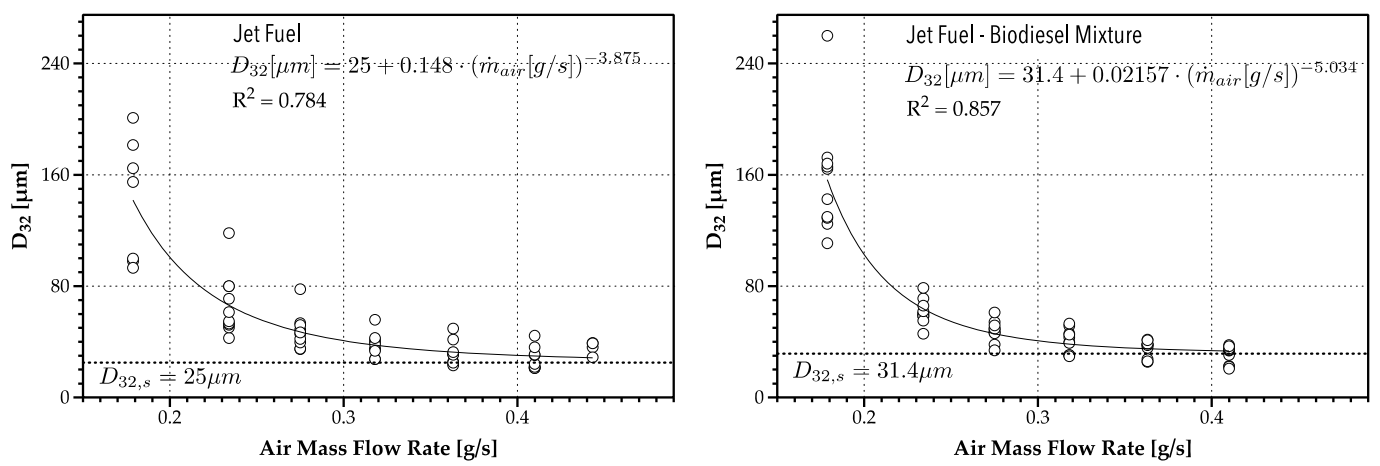

Figure 5. Empirical correlation between the air-mass flow rate on the SMD of the conventional Jet-Fuel and the 50/50 mixture between Jet Fuel and NEXBTL Biodiesel Mixture.

and, instead of the air-mass flow rate, consider the relation between a scale for the volumetric kinetic energy of the air jet,

$$
E_{k, a}^{\prime \prime \prime} \sim \frac{1}{\rho_{\text {air }}}\left(\frac{\dot{m}_{\text {air }}}{d_{\text {air }}^{2}}\right)^{2}
$$

where $\rho_{\text {air }}=\rho_{\infty}\left(p / p_{\infty}\right)$ is the air density, $d_{\text {air }}$ the diameter of the air jet at nozzle exit, relatively to a scale for the volumetric surface energy of the lower limit for the average drop size

$$
E_{s, f}^{\prime \prime \prime} \sim \frac{\sigma_{f}}{D_{32, s}}
$$

we relate both to scale the non-dimensional effect of the air jet as $\frac{E_{k, a}^{\prime \prime \prime}}{E_{s, f}^{\prime \prime \prime}}=\frac{1}{\rho_{\text {air }}}\left(\frac{\dot{m}_{\text {air }}}{d_{\text {air }}^{2}}\right)^{2} \frac{D_{32, s}}{\sigma_{f}}$

The result for both fuels provides the following empirical correlation

$$
D_{32}^{*}=1+79.28 \cdot \Theta^{-3.05}
$$

where $\Theta=\left(E_{k, a}^{\prime \prime \prime} / E_{s, f}^{\prime \prime \prime}\right) \times 10^{5}$. The correlation depicted in Fig. 7 obtained for our results shows a reasonable agreement (see Fig. 8 which includes the standard deviation of the residual value between experimental data and the results obtained from the correlation), including its application with data reported in the literature by Avulapati and Venkata [6].

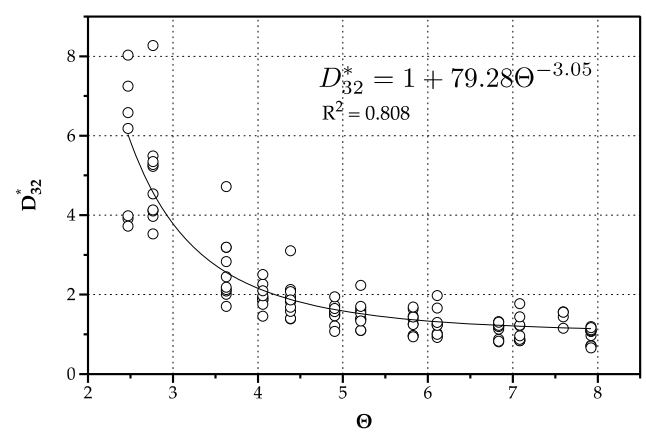

Figure 6. Normalized correlation a normalized $D_{32}$ and a scale parameter relating the volumetric kinetic energy of the jet with the volumetric surface energy of the lower limit

for $D_{32}$. The results plotted include all experimental conditions and both fuels.

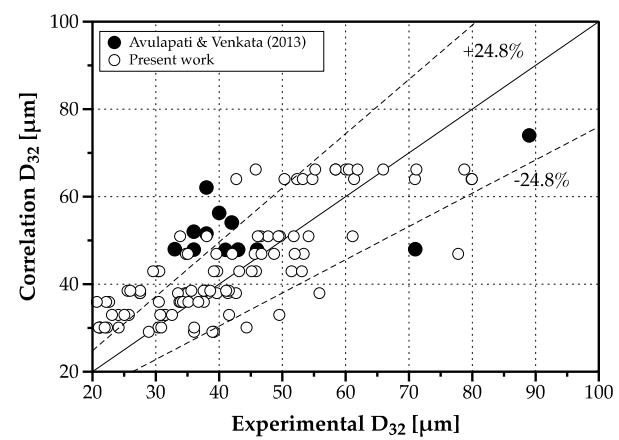

Figure 7. Comparison between experimental results and those provided by the correlation in Eq. (2), including the data from ref. [6] within the validation domain.

A possible explanation for the stabilization of $D_{32}$ might be related to the change of the breakup mechanisms. When aerodynamic forces dominate breakup, a liquid sheet forms at the end of the deflected jet before reaching the impact point, detaching in ligaments by shear breakup further downstream. The scale of shear breakup involves the wavelength of the liquid surface waves, which is of the order of $\lambda_{s} / d_{j} \approx 0.1$ [9]. Therefore, considering 
the size of our impinging jets, this results in $\lambda_{\mathrm{s}} \approx 50 \mu \mathrm{m}$, which is similar to the Sauter mean diameter measured for the highest air-mass flow rate.

\section{Atomization Efficiency}

The definition of the atomization efficiency is not straightforward. Avulapati and Venkata [6] define it for airassisted multiple impinging jets atomization as

a. the difference between the sum of surface energy of all droplets and the jets' initial surface energy;

b. divided by the sum of gas and liquid energy.

The energy in the air jet corresponds to the isothermal compression required to compress the atomizing air. However, this assumption is based on effervescent sprays where the air dissolved in the liquid compose the twophase mixture injected. We argue instead that air-assisting multiple impinging jets relates the final volumetric surface energy of droplets produced by atomization,

$$
E_{s, d}^{\prime \prime \prime}=\frac{n \sigma_{f} \pi D_{32}^{2}}{n \frac{\pi}{6} D_{32}^{3}}=\frac{6 \sigma_{f}}{D_{32}}
$$

with the total energy available from the kinetic component of the air jet $\left(E_{a}^{\prime \prime \prime}\right)$, plus the kinetic and surface components available on the liquid jets $\left(E_{f}^{\prime \prime \prime}\right)$,

$$
\begin{gathered}
E_{a}^{\prime \prime \prime}=\frac{8}{\rho_{\text {air }}}\left(\frac{\dot{m}_{\text {air }}}{\pi d_{\text {air }}^{2}}\right)^{2} \quad E_{f}^{\prime \prime \prime}=N_{j}\left[\frac{8}{\rho_{f}}\left(\frac{\dot{m}_{f}}{\pi d_{f}^{2}}\right)^{2}+\frac{4 \sigma_{f}}{d_{j}}\right] \quad E_{i}^{\prime \prime \prime}=E_{a}^{\prime \prime \prime}+E_{f}^{\prime \prime \prime} \\
\eta_{\text {atom }}=\frac{E_{s, d}^{\prime \prime \prime}}{E_{i}^{\prime \prime \prime}}
\end{gathered}
$$

Therefore, while in effervescent sprays the air assists atomization from inside out, in our case and the case of Avalupati and Venkata [6], the assistance is the opposite. Fig. 9 depicts the results obtained for the atomization efficiency in the present experiments.
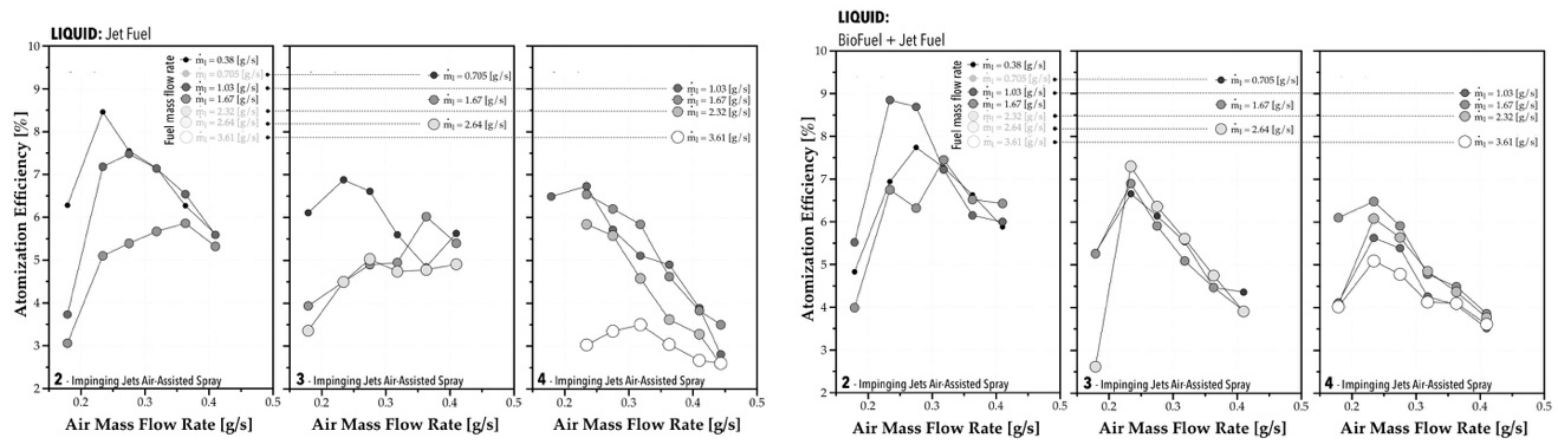

Figure 8. Effect of air-mass flow rate on the spray atomization efficiency for all configurations and operating conditions

$$
\left(\dot{m}_{f}=0.38-3.61 \mathrm{~g} / \mathrm{s}\right) \text {. }
$$

The present efficiency values are larger than those reported by other authors. This could be the result of the definition of the atomization efficiency. Although a higher air-mass flow rate produces smaller droplets due to a change in the atomization mechanism (from hydrodynamic to aerodynamic), this occurs as the cost of performance, since, obtaining droplets of smaller sizes means a lower atomization efficiency because is takes more energy to breakup the liquid into smaller droplets. It is noticeable that the atomization efficiency is more sensitive to the mass flow rate in the case of 4-jets relatively to the remaining configurations. However, when biodiesel is mixed with Jet Fuel, we observe some changes. Namely, (i) the atomization efficiency ceases being affected by the mass flow rate, which is more evident with the 4-jets spray; (ii) the effect of the gas mass flow rate on atomization efficiency is more systematic; and (iii) maximum atomization efficiency obtained between $\dot{m}_{\text {air }}=$ $0.2-0.25[\mathrm{~g} / \mathrm{s}]$ in every configuration.

\section{Concluding Remarks}

The continued growth rate of the aviation sector demands the search of alternative and more ecological fuels. However, this implies optimizing the combustion process for the new fuels and improved spray systems as key 
components. The purpose of this work is twofold. First, we propose a new injector design based on air-assisting multiple impinging jets sprays. Second, we address the concern that mixing biofuel to a fossil fuel, as a first approach to produce alternative fuels, might alter sprays characteristics and the combustion process. The results point to the following conclusions:

- in multiple impinging jets sprays, considering 2, 3 and 4 jets, the air flow rate assisting atomization allows some control over the average drop size;

- a transition occurs between hydrodynamic and aerodynamic atomization breakup mechanisms when the airmass flow rate increases;

- this transition deteriorates the efficiency of the atomization process, but within the same order of magnitude the range of air flow rates considered;

- mixing biofuel with fossil jet fuel does not significantly alter spray characteristics, thus, this alternative should not produce changes in droplets characteristics that would negatively influence the combustion process.

\section{Acknowledgements}

This work was supported by Fundação para a Ciência e Tecnologia (FCT), through IDMEC, under LAETA, project UID/EMS/50022/2013.

\section{Nomenclature}

$d_{\text {air }} \quad$ Diameter of the air nozzle [m]

$D_{32} \quad$ Sauter mean diameter $[\mu \mathrm{m}]$

$D_{32, s} \quad$ Stable Sauter mean diameter $[\mu \mathrm{m}]$

$E_{a}^{\prime \prime \prime} \quad$ Volumetric kinetic energy of air flow $\left[\mathrm{J} / \mathrm{m}^{3}\right]$

$E_{i}^{\prime \prime \prime} \quad$ Volumetric total energy at the jets impact point $\left[\mathrm{J} / \mathrm{m}^{3}\right]$

$E_{f}^{\prime \prime \prime} \quad$ Volumetric surface and kinetic energy of the fuel jet $\left[\mathrm{J} / \mathrm{m}^{3}\right]$

$E_{k, a}^{\prime \prime \prime} \quad$ Scale for the volumetric kinetic energy of the air flow $\left[\mathrm{J} / \mathrm{m}^{3}\right]$

$E_{s, d}^{\prime \prime \prime} \quad$ Volumetric surface energy of droplets based on the $D_{32}\left[\mathrm{~J} / \mathrm{m}^{3}\right]$

$E_{s, f}^{\prime \prime \prime} \quad$ Volumetric surface energy of the fuel jet $\left[\mathrm{J} / \mathrm{m}^{3}\right]$

$\dot{m}_{\text {air }} \quad$ Air-mass flow rate $[\mathrm{g} / \mathrm{s}]$

$\dot{m}_{f} \quad$ Fuel-mass flow rate [g/s]

$N_{j} \quad$ Number of impinging jets [-]

Greek Symbols

$\rho_{\text {air }} \quad$ Air density $\left[\mathrm{kg} / \mathrm{m}^{3}\right]$

$\sigma_{f} \quad$ Liquid fuel surface tension [N/m]

$\Theta \quad$ Non-dimensional scale parameter [-]

\section{References}

[1] Penner, J., Lister, D.H., Griggs, D.J., Dokken, D.J., McFarland, M., Aviation and the Global Atmosphere, 1999, Cambridge University Press.

[2] Wilcox, D.C., 1993, "Turbulence Modeling for CFD", DCW Industries Incorporated.

[3] Parker, R., Lathoud, M., Green aero-engines: Technology to mitigate aviation impact on environment, Proceedings of the Institution of Mechanical Engineers, Part C: Journal of Mechanical Engineering Science, 244 (2010) 529-538.

[4] Hari, T.K., Yaakob, Z., Binitha, N.N., Aviation biofuel from renewable resources: Routes, opportunities and challenges, Renewable and Sustainable Energy Reviews, 42 (2015) 1234-1244.

[5] IATA Sustainable Association Aviation Fuel Roadmap (2015), International Air Transport Association.

[6] Avulapati, M.M. Venkata, R.R., Experimental studies on air-assisted impinging jet atomization, International Journal of Multiphase Flow, 57 (2013) 88 - 101.

[7] Almeida, H., Sousa J.M.M., Costa, M., Effect of the liquid injection angle on the atomization of liquid jets in subsonic crossflows, Atomization and Sprays, 24 (2014) 81-96.

[8] Mashayek, A., and Ashgriz, N., Model or deformation of drops and liquid jets in gaseous crossflows, AIAA Journal, 47 (2009) 303-313.

[9] Sallam, K.A., Aalburg, C., Faeth, G.M., Breakup of round nonturbulent liquid jets in gaseous crossflow, AIAA Journal. 42 (2004) 2529-2540. 


\title{
Investigation on the role of atomization air mixing on the spray characteristics of air assisted atomizer
}

\author{
Noritsune Kawaharada*1, Markus Höltermann ${ }^{1}$, Jan Wichmar ${ }^{1}$, Friedrich Dinkelacker ${ }^{1}$ \\ ${ }^{1}$ Institute of Technical Combustion, Leibniz Universität Hannover, Germany \\ *Corresponding author: kawaharada@itv.uni-hannover.de
}

\begin{abstract}
Air assisted atomizers are widely used for various purposes, for example coating processes, medical processes, and sprinkler. However, the spray development processes, especially the breakup phenomena are not fully understood yet. Generally, the main breakup of the air assisted atomizer depends on whether the mixing is internal or external. The border between these processes is not clear. In order to study changes in the spray configuration due to the way of mixing, the spray was investigated for the transition from internal to external mixing. Therefore, an air assisted atomizer which allows adjusting the distance between injector to cover was used. The atomizer consists of two components, which are a liquid injector and an injector cover with orifice. The atomization air flows through the gap between the injector and the cover. The position of cover orifice is able to be traversed from the position fully attached to the injector to several millimetre distances from the injector nozzle. In this study, the water spray characteristics depending on the air and liquid mixture position were investigated experimentally by two optical measurement techniques.

Imaging techniques were used for taking the spray structure as well as liquid core in near nozzle field. The water mass flow, atomization air mass flow, and the cover position were changed and their effects on the spray were investigated. Phase Doppler Anemometer (PDA) was applied for measurement of velocity and size of droplets in water spray which injected into the atmosphere. The measurement positions were set at planes which located on 20 and $40 \mathrm{~mm}$ downstream from the nozzle orifice. For each downstream position, radial profiles of the spray pattern were measured on one air to water flow rate condition. The cover positions were changed and the differences on the droplet velocity and diameter as a function of radial distance on each nozzle cover positions were discussed. Also, high resolution direct imaging technique has been developed and applied for measurement of size of droplets. The results from these measurement techniques were compared and they correspond well at the high air to water flow rate conditions. At the low air to water flow rate condition, the agreement of the results of these measurements became lower. It might be caused by the bouncing unspherical droplets.
\end{abstract}

\section{Keywords}

Air-assisted-atomizer, Laser diagnostics, Direct imaging techniques, Atomization process, Atmospheric-pressure

\section{Introduction}

The twin fluid atomizer can be applied for various purposes for example coating processes, medical processes, and sprinkler. Most of twin-fluid nozzles were categorized to air assisted or air blast. The main difference is velocity of air flow, and the air velocity of air assisted atomizer is higher than that of air blast atomizer. The effect of mass flow rate of air and water on droplet diameter from air blast atomizer was investigated by Nukiyama etal. [1] in 1939. Lefebvre [2] summarized and explained about factors which influenced to mean droplet size. However, the spray development processes, especially the breakup phenomena in near nozzle are not fully understood yet.

The main breakup of the air assisted atomizer depends on whether the mixing is internal or external. The border between these processes is not clear. In order to study changes in the spray configuration due to the way of mixing, the spray was investigated for the transition from internal to external mixing. Therefore, an air assisted atomizer which allows adjusting the distance between injector to cover was used. The atomizer consists of two components, which are a liquid injector and an injector cover with orifice. The atomization air flows through the gap between the injector and the cover. In this study, the water spray characteristics depending on the air and liquid mixture position were investigated experimentally by two optical measurement techniques.

\section{Material and methods}

In this study, PDA and Direct imaging techniques are used to investigate about the spray breakup. 


\section{Phase Doppler Anemometer}

PDA is an established method to acquire droplet spectra of sprays. Durst et al. [3] showed that the phase shift of the scattered light of laser-Doppler anemometry directly yields the droplet diameter. The phase shift is measured by at least two detectors, which are placed at an off-axis position according to the experimental setup. The receiver angle was set as the recommended value of around $40^{\circ}$ to be higher the signal to noise ratio. The measurement positions are set at $20 \mathrm{~mm}$ and $40 \mathrm{~mm}$ downstream from liquid nozzle exit.

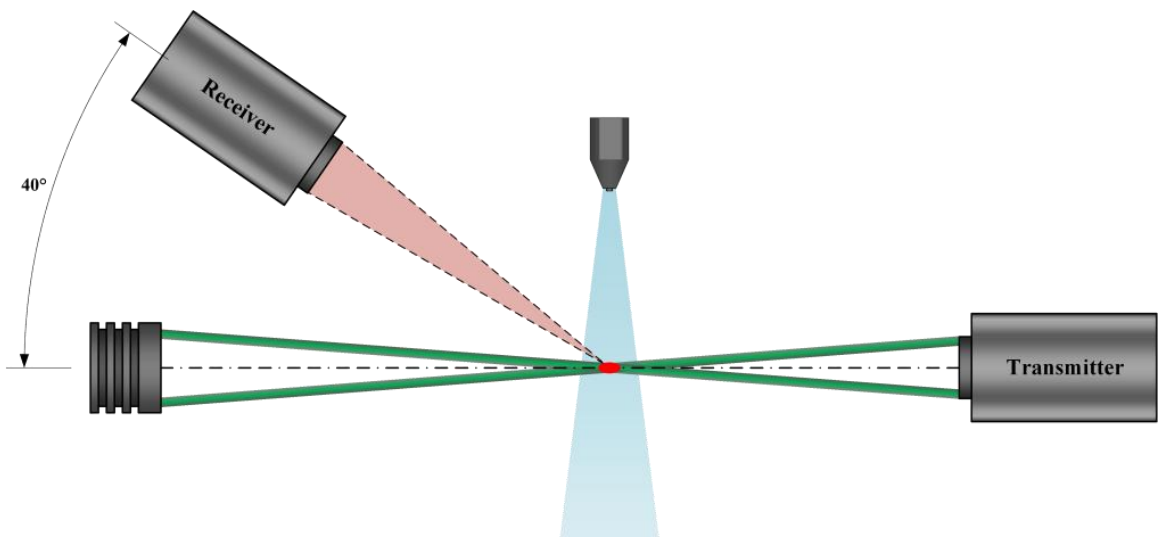

Figure 1. Setup of Phase Doppler Anemometer

\section{Direct droplet imaging}

The direct droplet imaging is based on a shadowgraphy setup and an evaluation algorithm programmed in MATLAB. Kapulla et al. [4] showed by a similar principle in 2007 that droplet imaging is possible for droplets with a diameter down to at least $5 \mu \mathrm{m}$. In order to resolve droplets down to $10 \mu \mathrm{m}$ or even less, magnifications up to 20 and hence strong illumination is needed.

The measurement positions are set at $40 \mathrm{~mm}$ downstream from liquid nozzle exit. Also, same setup was used for investigating the spray structure near nozzle exit with low magnification.

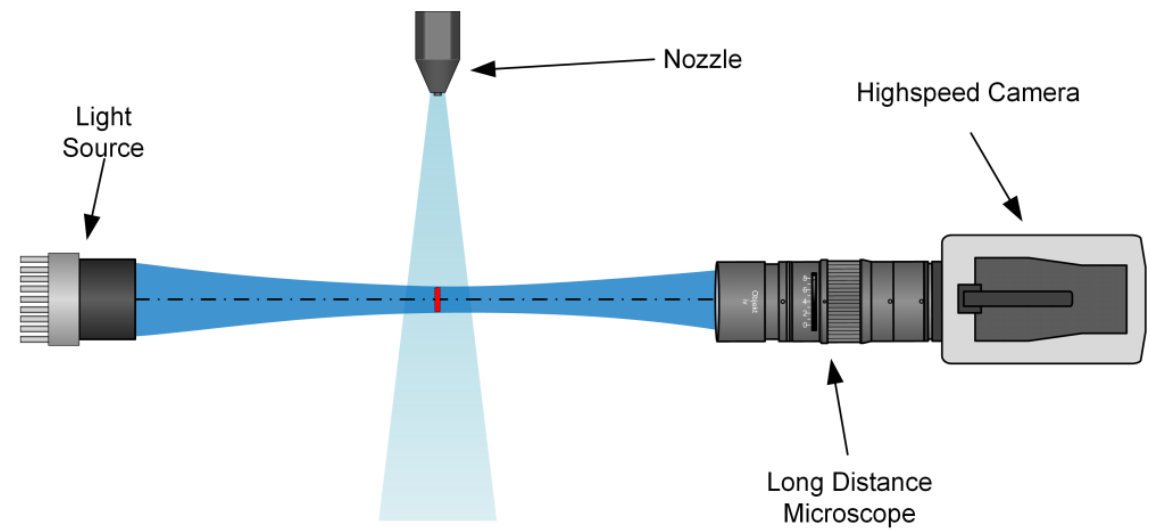

Figure 2. Setup of direct droplet imaging by means of LED light source, long distance microscope and high speed camera

In the setup (Figure 2), light source, measurement volume and camera are located on one axis. A 2.1 W LED is used to illuminate the measurement volume, the shadows of the droplet are captured by a high speed camera (Phantom v711, Vision Research) and focused and enlarged by a long distance microscope (K2-DistaMax, Infinity). The short exposure times of the high speed camera allow grabbing the droplets without unacceptable motion blur. The position and depth of the plane of focus determine which of the droplets are mapped on the sensor of the camera. Therefore the focus adjustment defines the space resolved observation of the spray. Droplets outside the plane of focus either cause a blurred image or only noise if the defocusing is big enough. This relation yields the criterion for recognition of droplets and calculating the diameter. In order to evaluate only the droplets which are within the plane of focus, the local intensity gradient of droplet images is used as criterion for droplet recognition (see Figure 3). 


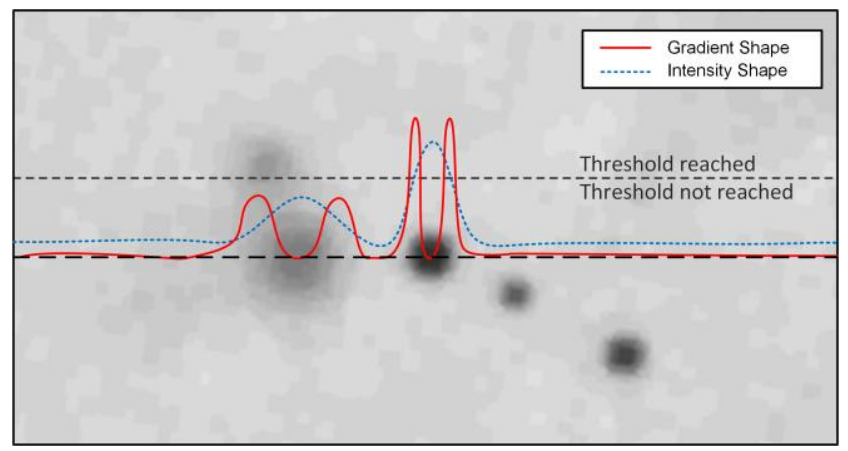

Figure 3. Principle of droplet recognition by threshold values for intensity and intensity gradient

The images are scanned vertically and horizontally for pixels, which fulfil the threshold requirement. Several morphological operations lead to a binary image with potential droplets, which are validated according to circularity and filling factors. Figure 4 refers to a trial measurement of a water spray from same atomizer at $40 \mathrm{~mm}$ downstream from nozzle. Such measurements were used to develop the method. Figure 4 shows the original image (a), the binarized and validated image (b) and the histogram for the results of 1000 images (c).
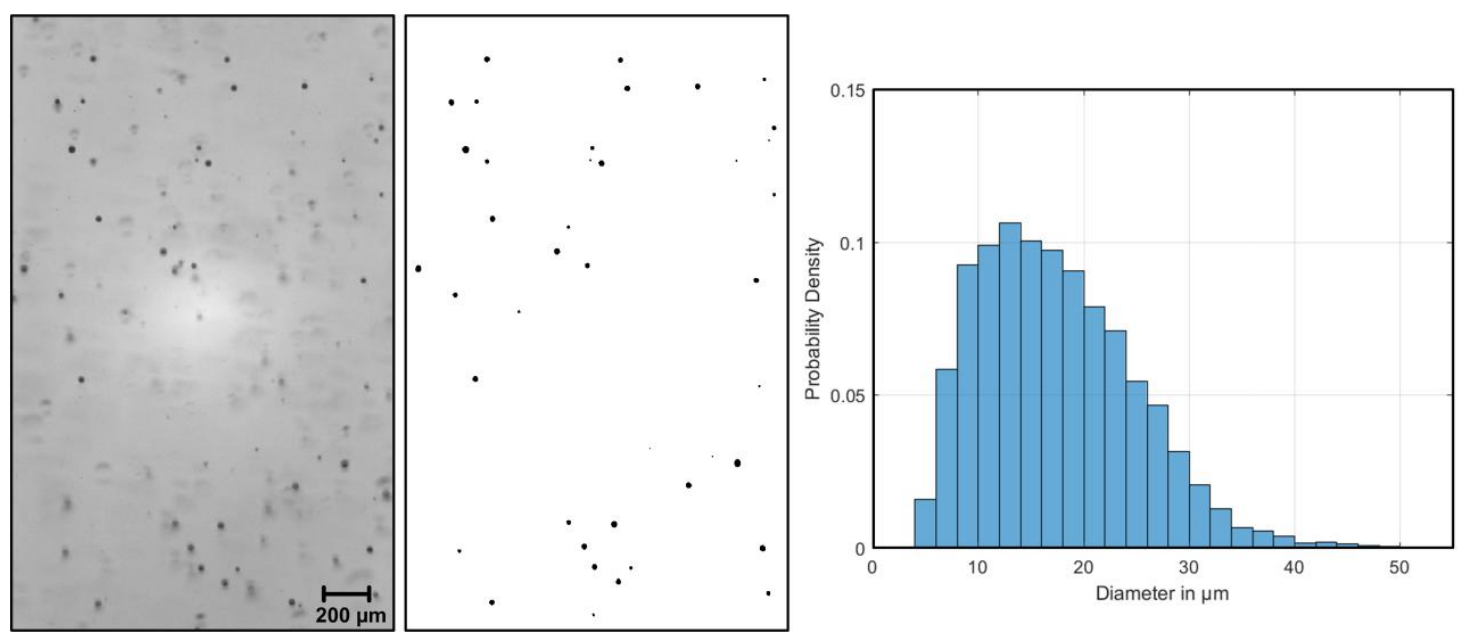

Figure 4. Droplet imaging: raw image (a), binary image (b) and histogram of droplet diameters derived from 1000 images (c)

\section{Air assisted atomizer}

In this study, 3 types of nozzle were used for experiments. Schematic diagram of nozzles are shown in Figure 5. The nozzle has threads for adjusting the distance between injector to cover was used. The atomizer consists of two components, which are a liquid injector and an injector cover with orifice. The atomization air flows through the gap between the injector and the cover. The air channel has swirl generator indicated by a cross in Figure 5 . The position of cover orifice is able to be traversed from the position fully attached to the injector to several millimetre distances from the injector nozzle.

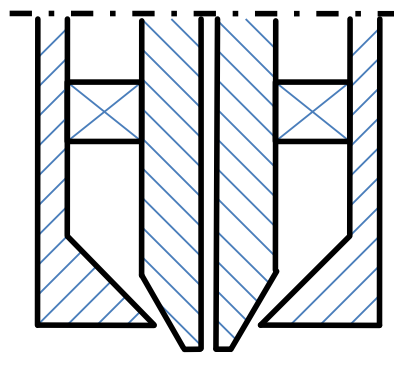

(a) Position 1

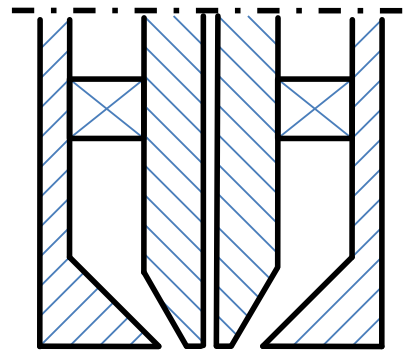

(b) Position 2

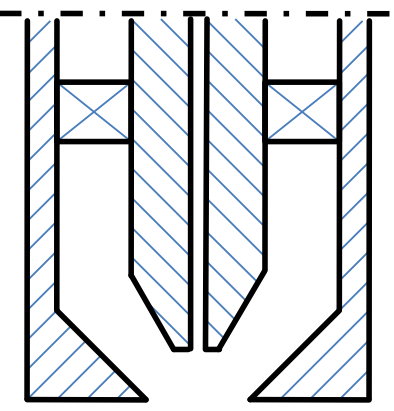

(c) Position 3

Figure 5. Schematic diagram of nozzle cover positions 
The liquid nozzle was fixed and outer cover was adjusted on each measurements. Figure 5(a) shows nozzle cover position 1. Air and liquid are mixed at outside of nozzle (external mixing). Figure 5(b) shows nozzle cover position 2. Exit of air and liquid are in same plane. Figure 5(c) shows nozzle cover position 3. Air and liquid are mixed at inside of outer cover (internal mixing).

\section{Measurement conditions}

Measurements were conducted in room temperature and atmospheric pressure conditions. Two fluids are air and water. 4 constant mass flows of air and water conditions were chosen for operating condition as shown in Table 1. Approximate ratios of air volume flow rate $\left(Q_{a}\right)$ to water volume flow rate $\left(Q_{w}\right)$ are shown in third row.

Table 1. Operating condition of air and water flow rate.

\begin{tabular}{c|ccc}
\hline \hline & Air & Water & $\mathrm{Q}_{\mathrm{a}} / \mathrm{Q}_{\mathrm{w}}$ \\
\hline \hline Condition 1 & $8 \mathrm{~kg} / \mathrm{h}$ & $3 \mathrm{~kg} / \mathrm{h}$ & 2200 \\
Condition 2 & $8 \mathrm{~kg} / \mathrm{h}$ & $6 \mathrm{~kg} / \mathrm{h}$ & 1100 \\
Condition 3 & $14 \mathrm{~kg} / \mathrm{h}$ & $3 \mathrm{~kg} / \mathrm{h}$ & 3800 \\
Condition 4 & $14 \mathrm{~kg} / \mathrm{h}$ & $6 \mathrm{~kg} / \mathrm{h}$ & 1900
\end{tabular}

\section{Results and discussion}

\section{Liquid core and spray structure in near nozzle field}

The liquid core and spray structure in near nozzle field is important information to investigate about breakup phenomena. The high-speed imaging technique was conducted to investigate about the structure. Figure 6 shows the structure in each condition. The field of view was from nozzle exit to around $15 \mathrm{~mm}$ downstream from water nozzle exit. It should be noted that liquid core goes not completely vertical in some conditions are caused by small trajectory error of nozzle when it was fixed.

The liquid core and spray structures depending on the operating conditions on nozzle cover position 1 show relatively small difference compared to that on nozzle cover position 2 . The nozzle cover position 1 has minimum air flow area at nozzle exit. And the velocity at nozzle exit was already reached to subsonic on low mass flow rate of air conditions. Nozzle cover position 2 has larger air flow area at nozzle exit compared to the position 1 and the velocity at nozzle exit did not reached to subsonic at low mass flow rate of air condition. However, the velocity reached to subsonic at high mass flow rate of air conditions. It is understood that the small difference on the liquid core and spray structure of nozzle position 1 was caused by the velocity difference between low mass flow rate of air and high mass flow of that was relatively small compared to the other case.

All pictures on nozzle position 1 and 2 have a liquid core. Despite of condition 2 and 4 , the spray structure on the nozzle cover position 3 have no liquid core on each condition. In the condition 2 and 4, it seems that the liquid core was not completely atomized by swirl air, and the liquid core length on condition 4 was shorter than that of condition 2. The nozzle cover position 3 has largest air flow area at nozzle exit and the predicted velocity at nozzle exits were around $50 \mathrm{~m} / \mathrm{s}$ in condition 2 and around $100 \mathrm{~m} / \mathrm{s}$ in condition 4 . It is suggested that the difference between the liquid core and spray structures on two conditions were caused by the deference between breakups by relative velocity. It seems that the nozzle position 3 had highest sensitivity for $Q_{a} / Q_{w}$ and nozzle position 1 had lowest sensitivity for that.

\section{Spray width against ratio of volume flow rate of air to water}

The spray widths on each condition were compared on measurement plane at 20 and 40mm. The spray width was obtained from averaged image from 1000 pictures on each condition and position. Figure 7 shows the spray width against the conditions. The solid line shows the results of $20 \mathrm{~mm}$ downstream from water nozzle exit, and the dotted line shows the results of $40 \mathrm{~mm}$ downstream from water nozzle exit. Horizontal axis means ratios of air volume flow rate $\left(Q_{a}\right)$ to water volume flow rate $\left(Q_{w}\right)$. The results of position 1 at $20 \mathrm{~mm}$ and $40 \mathrm{~mm}$ show almost same values on the combinations of $Q_{a} / Q_{w}=1100$ and 2200, and $Q_{a} / Q_{w}=1900$ and 3800. Difference between these combinations is air volume flow rate. It seems that the spray width of nozzle cover position 1 was not affected by $Q_{a} / Q_{w}$ but affected by air flow rate. On the results of position 3 at $20 \mathrm{~mm}$ and $40 \mathrm{~mm}$, there were almost positive correlation between $Q_{a} / Q_{w}$ and spray width. Despite of $Q_{a} / Q_{w}=1100$, the spray widths on nozzle position 3 were wider than that on nozzle position 1. Also, spray widths on nozzle position 2 were between them and a little close to that on position 3. On the condition of $Q_{a} / Q_{w}=1100$, the spray width at $20 \mathrm{~mm}$ of each nozzle cover position were almost same value. This similarity was also shown in Fig.6(b). 


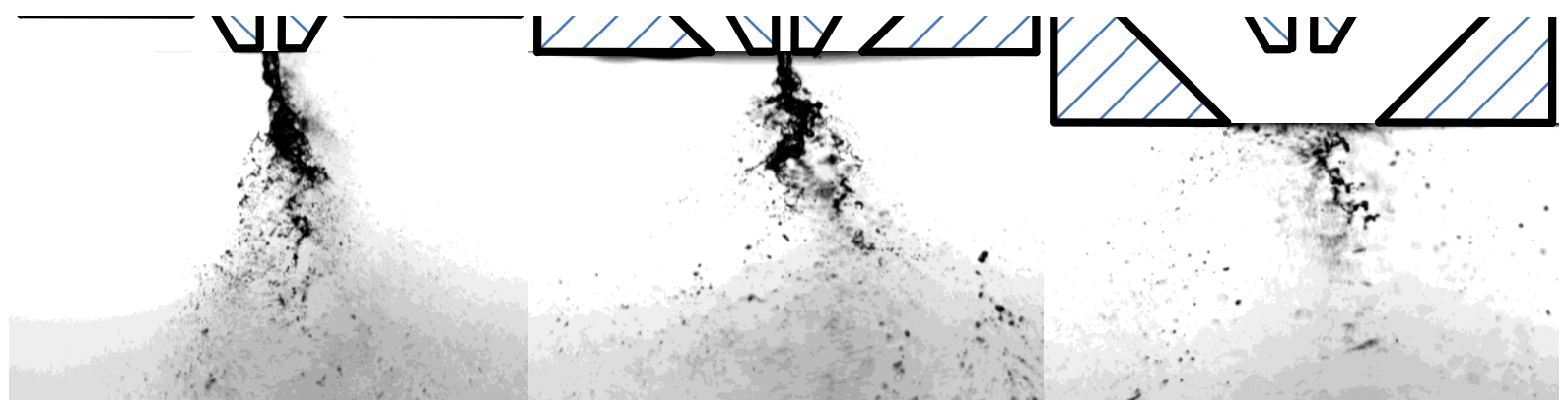

(a) Condition 1: $Q_{a} / Q_{w}=2200$ (Nozzle cover position 1 to 3 from left to right)

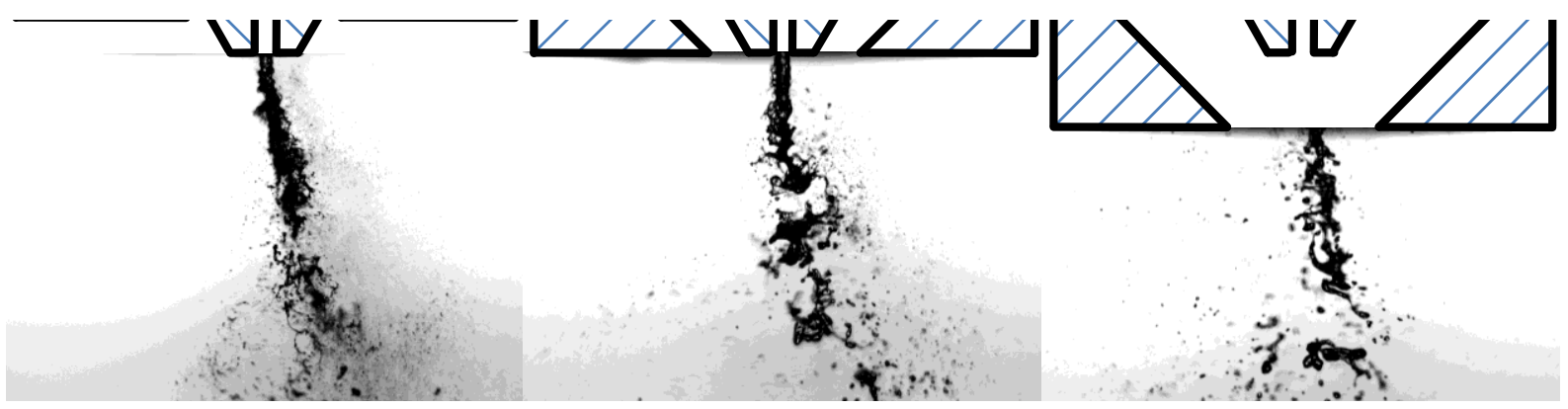

(b) Condition 2: $Q_{a} / Q_{w}=1100$ (Nozzle cover position 1 to 3 from left to right)

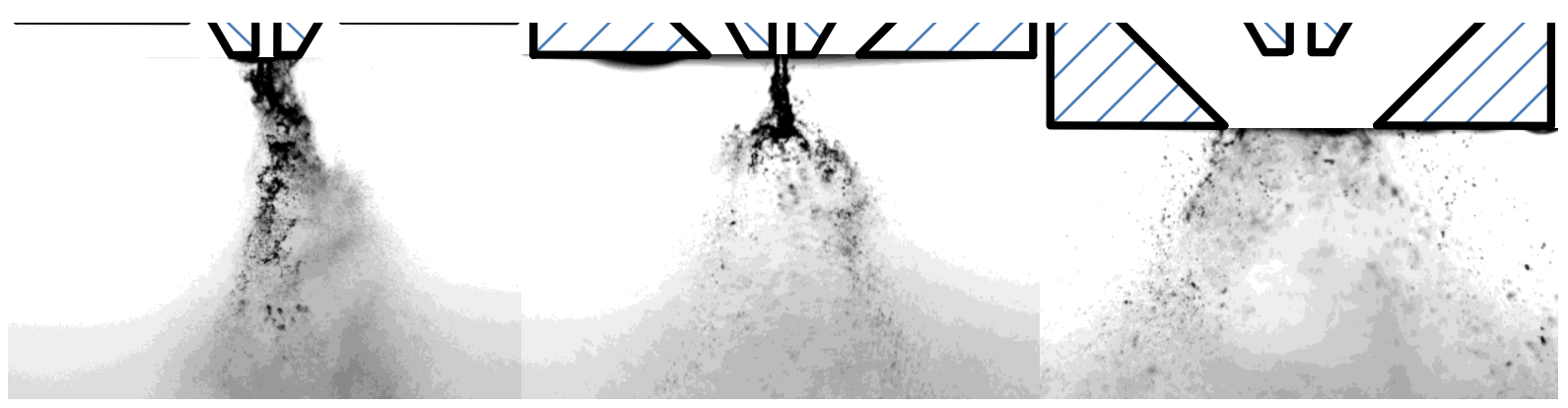

(c) Condition 3: $Q_{a} / Q_{w}=3800$ (Nozzle cover position 1 to 3 from left to right)

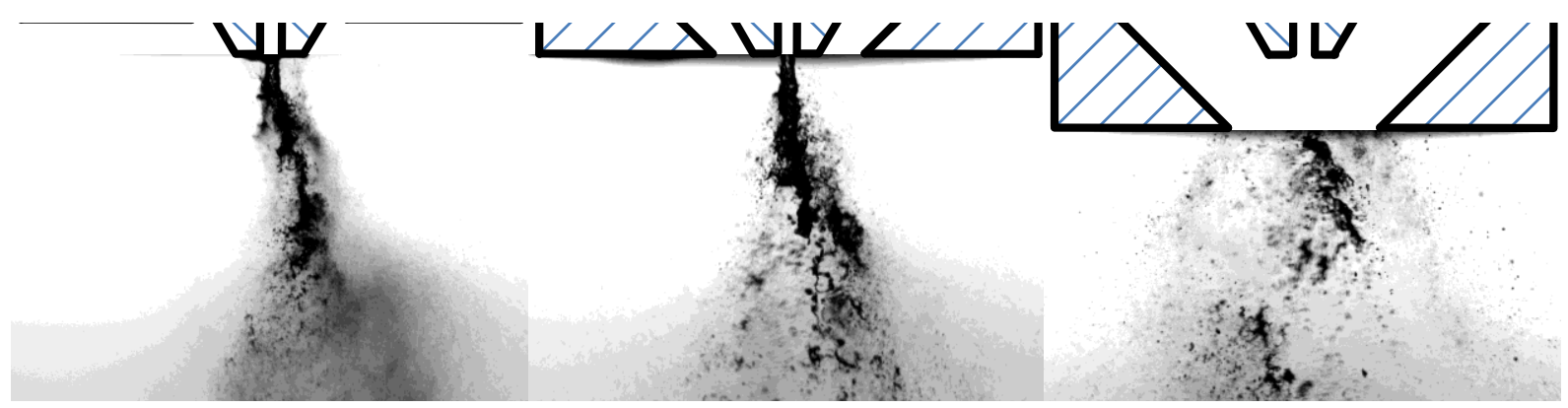

(d) Condition 4: $Q_{a} / Q_{w}=1900$ (Nozzle cover position 1 to 3 from left to right)

Figure 6. Spray structure in near nozzle field. 


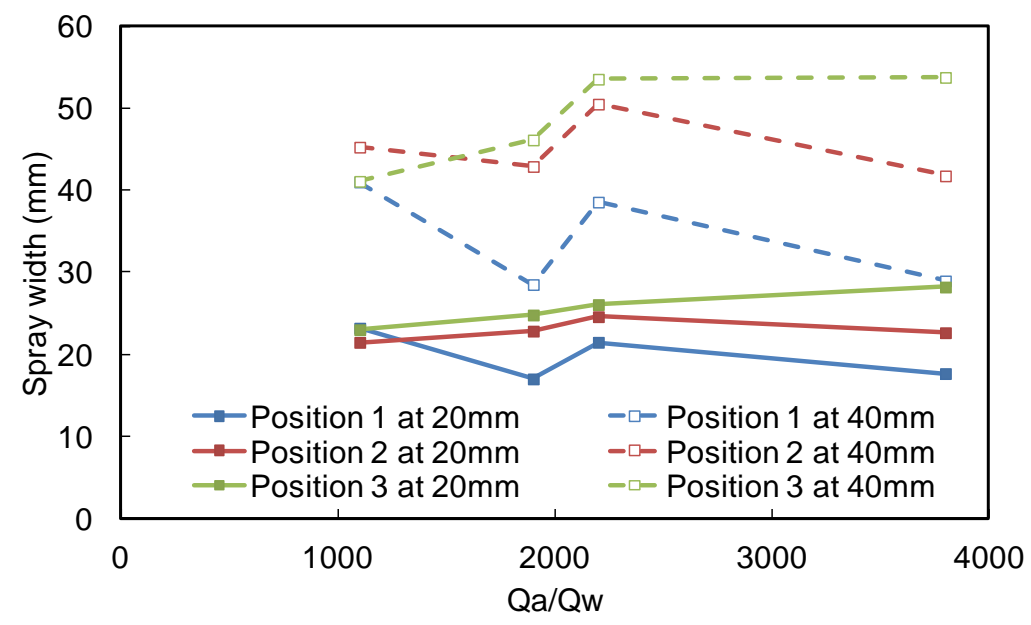

Figure 7. Spray width on each measurement plane

\section{Velocity and diameter of droplets as a function of radial distance}

The arithmetic mean velocity and diameter as a function of radial distance were compared on measurement plane at 20 and $40 \mathrm{~mm}$ on condition 1. These measurements were conducted by PDA around spray centre to middle of centre and periphery to investigate about the breakup phenomena near spray centre. Figure 8(a) shows that the velocities as a function of radial distance on every nozzle cover position. The solid line shows the results of $20 \mathrm{~mm}$ downstream from water nozzle exit, and the dotted line shows the results of $40 \mathrm{~mm}$ downstream from water nozzle exit. Horizontal axis means radial distance from spray centre. The velocities on the nozzle position 1 at $20 \mathrm{~mm}$ downstream shows highest velocity difference between spray centre and $10 \mathrm{~mm}$ radial position. This large velocity difference is also shown on the nozzle position 1 at $40 \mathrm{~mm}$ downstream. As shown in the figure, the velocity distribution on the nozzle position 1 had not so much difference between two measurement planes. The velocities of the nozzle position 2 show the nearly constant value entire the spray at $20 \mathrm{~mm}$ downstream. In case of nozzle position 3 , the velocity decreased around $10 \mathrm{~m} / \mathrm{s}$ near the spray centre with increasing the distance from nozzle exit.

Figure 8 (b) shows that the diameters as a function of radial distance on every nozzle cover position. The droplet diameters were minimum value on spray centre on each position, and the droplet diameters were increased with increasing the distance from spray centre. The droplet diameters decreased on every nozzle cover position with increasing the distance from nozzle, despite of spray centre on the nozzle cover position 1 and 3 . The largest difference between measurement planes on the droplet diameter was occurred on nozzle cover position 2 .

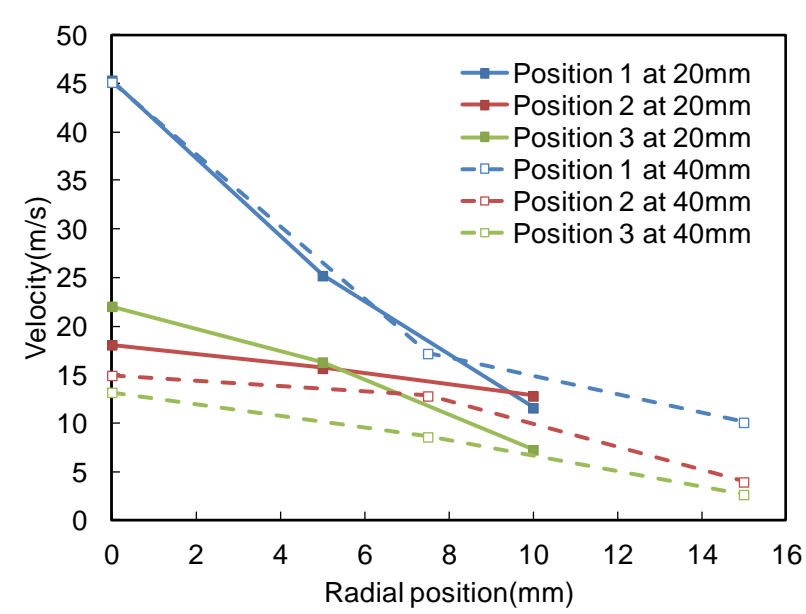

(a) Velocity

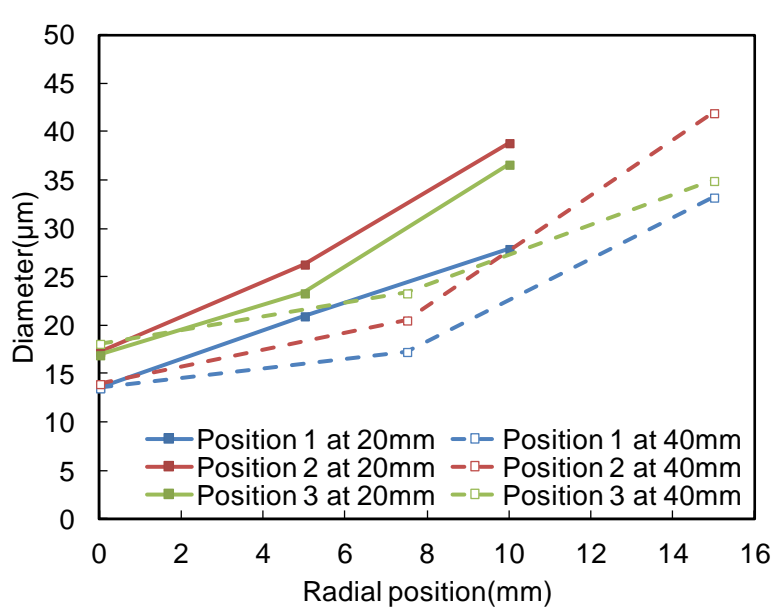

(b) Diameter

Figure 8. Droplet velocity and diameter as a function of radial distance on condition 1 
Table 2. PDA valid rate and RMSD on each measurement position

\begin{tabular}{|c|c|c|c|c|c|c|c|c|c|c|}
\hline \multicolumn{2}{|c|}{ Distance $(\mathrm{mm})$} & \multicolumn{3}{c|}{ PDA valid rate(\%) } & \multicolumn{2}{c|}{ RMSD on velocity $(\mathrm{m} / \mathrm{s})$} & \multicolumn{3}{c|}{ RMSD on diameter $(\mu \mathrm{m})$} \\
\cline { 2 - 11 } & \multicolumn{2}{|c|}{ Nozzle cover position } & \multicolumn{2}{c|}{ Nozzle cover position } & \multicolumn{3}{c|}{ Nozzle cover position } \\
\hline from nozzle & from spray centre & 1 & 2 & 3 & 1 & 2 & 3 & 1 & 2 & 3 \\
\hline \multirow{3}{*}{20} & 0 & 87.81 & 96.70 & 95.31 & 12.04 & 6.63 & 8.48 & 14.36 & 18.46 & 20.97 \\
\cline { 2 - 11 } & 5 & 91.61 & 95.38 & 93.68 & 15.70 & 7.80 & 7.79 & 16.78 & 31.89 & 25.80 \\
\cline { 2 - 11 } & 10 & 96.43 & 92.52 & 88.59 & 10.01 & 7.87 & 5.58 & 16.82 & 39.21 & 32.54 \\
\hline \multirow{3}{*}{40} & 0 & 83.95 & 97.32 & 96.99 & 10.00 & 4.09 & 4.24 & 7.41 & 8.69 & 13.02 \\
\cline { 2 - 11 } & 7.5 & 85.02 & 97.67 & 96.10 & 7.84 & 5.21 & 4.08 & 9.02 & 14.47 & 14.38 \\
\cline { 2 - 11 } & 15 & 87.42 & 85.63 & 89.05 & 8.93 & 3.08 & 2.24 & 16.84 & 26.28 & 24.17 \\
\hline
\end{tabular}

Table 2 shows PDA valid rate and root mean square deviation (RMSD) on each measurement position. In case of nozzle cover position 1, minimum PDA valid rate in same plane were obtained on measurement at spray centre. On the other nozzle cover positions, those were obtained on most outside measurement position. RMSD shows the magnitude of dispersion of the measured data. In this study, RMSD was calculated by following equation.

$$
R M S D=\sqrt{\frac{1}{n} \sum_{i=1}^{n}\left(x_{i}-\bar{x}\right)^{2}}
$$

Here, $n$ means number of measurement data, $x_{i}$ means one of the measurement data, and $\bar{x}$ means averaged value of the data. Relatively large RMSD on velocity were obtained on the measurement of nozzle cover position 1 at $20 \mathrm{~mm}$ from nozzle plane, because of the high arithmetic mean value. Relatively large RMSD on diameter were obtained on the measurement of nozzle cover position 2 at $20 \mathrm{~mm}$ from nozzle plane. The largest RMSD was obtained at the most outside measurement position, and it was almost same as the arithmetic mean diameter of the measurement position. It might be caused by large diameter droplets which were small number but existing as shown in the centre picture of Figure $6(a)$.

\section{Probability density of droplet diameter}

The probability densities of droplet diameter were investigated around spray centre at $40 \mathrm{~mm}$ downstream from nozzle exit by using PDA and direct imaging techniques. These diagnostics performed on difference spray sampling methods, PDA is temporal sampling and direct imaging techniques is spatial sampling. Tropea et al reviewed and explained the several optical measurement techniques[5]. According to the literature, it seems that the differences between two diagnostics are obtained when there are positive or negative relation between droplet velocity and diameter. In this study, the chosen conditions are 2, 3, and 4 of nozzle position 3 , because the structure of spray on these conditions has clear difference between conditions as shown in Figure 6 . In these conditions, there are almost no relation between velocity and diameter of droplets.

Figure 9 shows the probability density distribution of droplet diameter around spray centre. The solid line shows the results from PDA measurement, and the dotted line shows the results of direct imaging techniques. Horizontal axis means diameter. The PDA and direct imaging results on condition 3 have a peak at the same diameter and almost same value. Also, the distribution curve was nearly fit to the others. The PDA results on condition 4 shows slightly different to direct imaging results. On the condition 2, there are relatively high difference between PDA results and direct imaging. As shown in Figure 6, there are huge droplets or ligaments on condition 2, and also there are almost no huge droplets in near spray centre on condition 3 . The diameter measurement principle of PDA is depending on the surface curvature. Therefore, it is understood that the difference between the results of PDA and direct imaging were be caused by the incorrect measurement of bouncing unspherical droplets. 


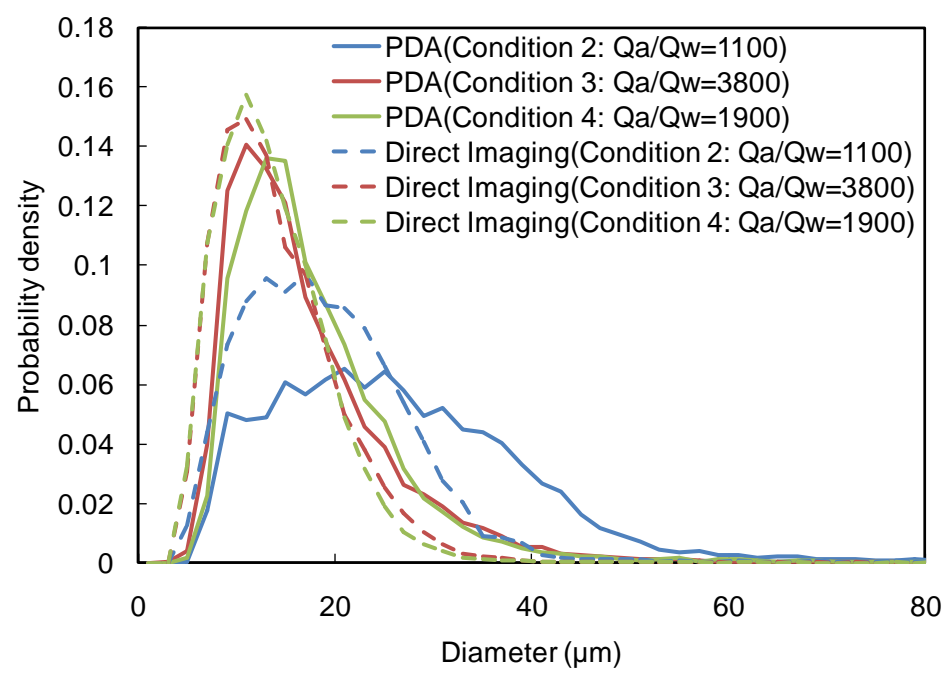

Figure 9. Probability density of droplet diameter around spray centre on nozzle cover position 3

\section{Conclusions}

Imaging techniques were used for taking the spray structure as well as liquid core in near nozzle field. The water mass flow, atomization air mass flow, and the cover position were changed, and their effects on the spray core structure and spray width were investigated. From these investigations, it seems that the nozzle position 3 had highest sensitivity for $Q_{a} / Q_{w}$ and nozzle position 1 had lowest sensitivity for that on spray core structure. And the spray width of nozzle cover position 1 was not affected by $Q_{a} / Q_{w}$ but affected by air flow rate.

Phase Doppler Anemometer (PDA) was applied for measurement of velocity and size of droplets in water spray which injected into the atmosphere. The measurement positions were set at planes which located on 20 and $40 \mathrm{~mm}$ downstream from the nozzle orifice. For each downstream position, radial profiles of the spray pattern were measured on one air to water flow rate condition. The cover positions were changed and the differences on the droplet velocity and diameter as a function of radial distance on each nozzle cover position were discussed. The velocity distribution on the nozzle position 1 had not so much difference between two measurement planes. The droplet diameters were minimum value on spray centre on each nozzle cover position, and the droplet diameters were increased with increasing the distance from spray centre.

Also, high resolution direct imaging technique has been developed and applied for measurement of diameter of droplets. The results from these measurement techniques were compared and they correspond well at the high air to water flow rate conditions. At the low air to water flow rate condition, the agreement of the results of these measurements became lower. From spray picture, there were huge droplets or ligaments. The diameter measurement principle of PDA is depending on the surface curvature. Therefore, it might be caused by the incorrect measurement of bouncing unspherical droplets.

\section{References}

[1] Nukiyama, S., Tanasawa, Y., "An Experiment on the Atomization of Liquid by Means of an Air Stream(the $2^{\text {nd }}$ Report)", Transaction of JSME Vol.4 (15),1939, pp. 138-145.

[2] Lefebvre, A. H., "Twin-fluid atomization: Factors influencing mean drop size", 1991, Proceedings of ICLASS91, pp. 49-64.

[3] Durst, F., Zare, M., "Laser Doppler Measurements in Two-Phase Flow", Proceedings of the LDA Symposium Copenhagen 1975, pp.403-429.

[4] Kapulla, R., Trautmann, M., Hernandez Sanchez, A., Calvo Zaragoza, S., Hofstetter, S., Häfeli, C., Güntay , S., "Droplet size distribution measurements using phase-Doppler anemometry and shadowgraphy: Quantitative comparison", 15. GALA Fachtagung Lasermethoden in der Strömungsmesstechnik 2007.

[5] Tropea, C., "Optical Particle Characterization in Flows", 2011, Annu. Rev. Fluid Mech., 43, pp. 399-426. 


\title{
Effects of Injection Nozzle Geometry on Spray Characteristics for Direct Injection Diesel Engines
}

\author{
Nobushige Tamaki ${ }^{* 1}$, Takato Harada ${ }^{2}$ \\ ${ }^{1}$ Technical Research Institute of Kindai University, Japan \\ ${ }^{2}$ Graduate School of Kindai University, Japan \\ *Corresponding author: tamaki@hiro.kindai.ac.jp
}

\begin{abstract}
The purpose of this study is to improve the spray characteristics of a direct injection Diesel nozzle. Spray atomization of the multi-hole atomization enhancement nozzle, which was invented in previous studies, is explored. The aim is to improve and obtain excellent spray characteristics. The effects of different nozzle geometries and dimensions on spray characteristics are investigated, including chamfered and rounded nozzle hole inlets and a multi-hole atomization enhancement nozzles. It is found that the multi-hole atomization enhancement nozzle with a rounded chamfered inlet is effective in achieving a high-dispersion spray, a large spray angle and small droplets. However, it should be noted that the spray is significantly atomized and a large number of small droplets as suspended as a mist. The photographic images of the spray are taken against a white background and the boundaries between the ejected spray and the background are indistinct. Furthermore, the breakup length is short at $3 \mathrm{~mm}$ for a hole diameter of $0.3 \mathrm{~mm}$, the spray angle is about $70^{\circ}$ and the Sauter mean diameter decreases dramatically to $15 \mu \mathrm{m}$ at a low injection pressure of $10 \mathrm{MPa}$.
\end{abstract}

Keywords: Atomization, Diesel Engine, Spray Characteristics, Injection Nozzle, Cavitation

\section{Introduction}

It is necessary to reduce carbon dioxide $\left(\mathrm{CO}_{2}\right)$ emissions to control its effect on global warming. To this end, a direct injection (D.I.) Diesel engine offers the highest thermal efficiency and a low fuel consumption rate. In order to improve combustion efficiency and exhaust gas characteristics and to further reduce the fuel consumption rate of the D.I. Diesel engine, the aim of this research is to improve the spray characteristics of the fuel spray. In recent years, automobile manufacturers have demanded the development of a Diesel injection nozzle that makes leanburn combustion possible. The final objectives of this study include improving the combustion characteristics of the D.I. Diesel engine, reducing exhaust gas emissions, and improving the engine's thermal efficiency and fuel consumption rate by developing the desired injection nozzle and its spray characteristics.

In previous studies conducted by the authors, it was found that the proposed atomization enhancement nozzle, in which cavitation occurs in the nozzle hole and a strong disturbance is produced in the liquid flow in the nozzle hole, yielded excellent spray characteristics. It was also found that cavitation phenomena in the nozzle hole considerably affected the atomization of the spray [1]-[7].

This study focuses on improving the atomization of the multi-hole nozzle and improving the spray characteristics of the D.I. Diesel nozzle. The engine's nozzle hole inlet is chamfered and rounded to improve flow characteristics. However, it is well known that, although this leads to a larger discharge coefficient, the spray characteristics become poor unless the injection pressure is increased significantly. Therefore, the effects of different nozzle geometries and dimensions on spray characteristics were investigated.

This includes chamfered and rounded nozzle hole inlets and multi-hole nozzles, which were invented in previous studies. By using a multi-hole nozzle to separate one nozzle hole into four nozzle holes, an atomization enhancement nozzle can be achieved, with a high-dispersion and high-efficiency spray and improved spray and flow characteristics [8], [9].

It is found that for the case where the multi-hole atomization enhancement nozzle inlet was chamfered and rounded, the breakup length is reduced by $10 \%$ and the spray angle increases by $20 \%$ compared to a sharp edged nozzle inlet. The spray angle for the rounded chamfered inlet is about $70^{\circ}$ and the Sauter mean diameter is decreased to $15 \mu \mathrm{m}$ at a low injection pressure of $10 \mathrm{MPa}$.

\section{Apparatus and methods}

A schematic of the experimental apparatus is shown in Fig.1. The equipment consisted of a high-pressure pump that was controlled by an air compressor, a spark light source for photographing the spray, a screen detector for measuring the breakup length and an LDSA particle analyser for measuring the droplet size and its distribution. 
Room-temperature water, which was pressurized by a high-pressure pump, was continuously injected under atmospheric pressure conditions. The maximum injection pressure was $10 \mathrm{MPa}$, and experimental data is discussed at spray region. Based on the results of previous studies, the injection pressure inside the spray region is estimated to be over about $5 \mathrm{MPa}$ for a hole diameter of $0.3 \mathrm{~mm}$.

In this work, the maximum injection pressure is $10 \mathrm{MPa}$, the ambient pressure is atmospheric pressure condition of $0.1 \mathrm{MPa}$, steady state injection and test liquid is water, they are quite far from an actual Diesel application. These reasons are as follows. In the previous studies [2], [4]-[7], the spray of the nozzles, which were designed and invented in this study, atomizes significantly and excellent spray characteristics i.e. the spray angle is large, the breakup length is short and the Sauter mean diameter is small, were obtained at the low injection pressure of 15 $\mathrm{MPa}$ and $20 \mathrm{MPa}$ independent of the injection pressure, the ambient pressure, kinematic viscosity and injection style. Spread of the spray at the injection pressure of $10 \mathrm{MPa}$ was wide compared with one of the injection pressure of $200 \mathrm{MPa}$ [4]. Moreover, the results and tendencies obtained at steady state injection were almost same at intermittent injection [7]. Furthermore, excellent spray characteristics were obtained, even though high-viscosity liquid i.e. correspond to the heavy oil was used [6]. Therefore, on a basis of these results, backgrounds and possibility of easy measurement, the authors studied these conditions [See Appendix Figs. 1 - 7].

The disintegration behavior of the spray was studied by photographing the scattered light using the stroboscope. The breakup length of a liquid core, defined as the distance from the exit point of the nozzle to the breakup point of the liquid core, was measured using an electrical resistance method. The spray angle was defined as the angle of the outer edge of the spray. Droplet size distributions were measured $100 \mathrm{~mm}$ downstream from the exit point of the nozzle using the LDSA particle analyzer based on a narrow-angle forward-scattering technique.

Where, an aspect of the LDSA particle analyzer is shown in Fig.2, and a schematic of the narrow-angle forwardscattering technique is shown in Fig.3. Mean droplets diameter of the spray was calculated automatically by

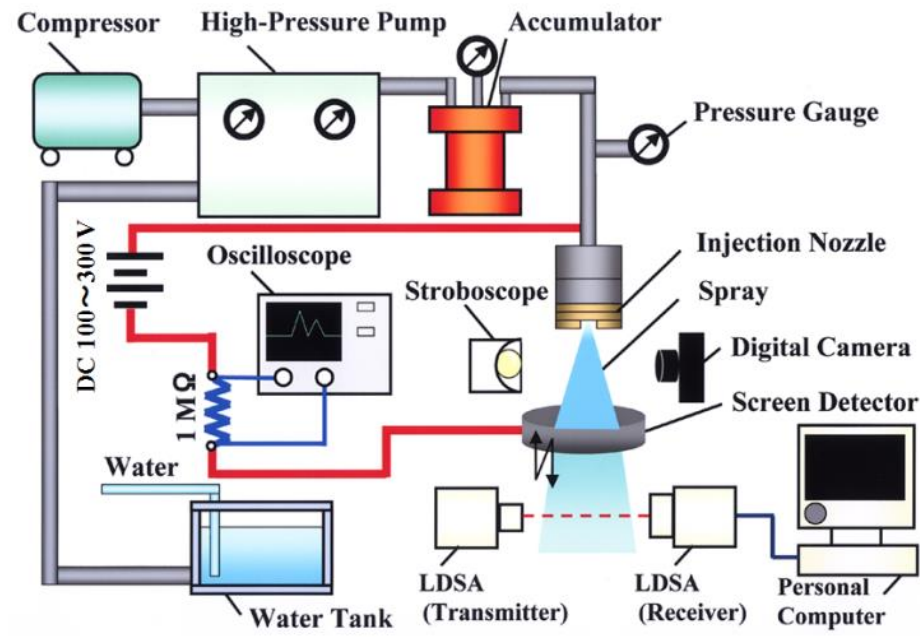

Figure 1. Schematic of experimental apparatus.

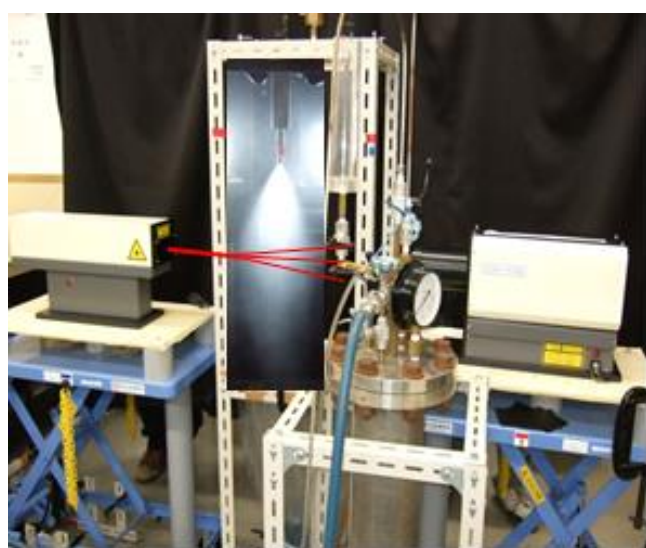

Figure 2. Aspect of LDSA particle analyzer.
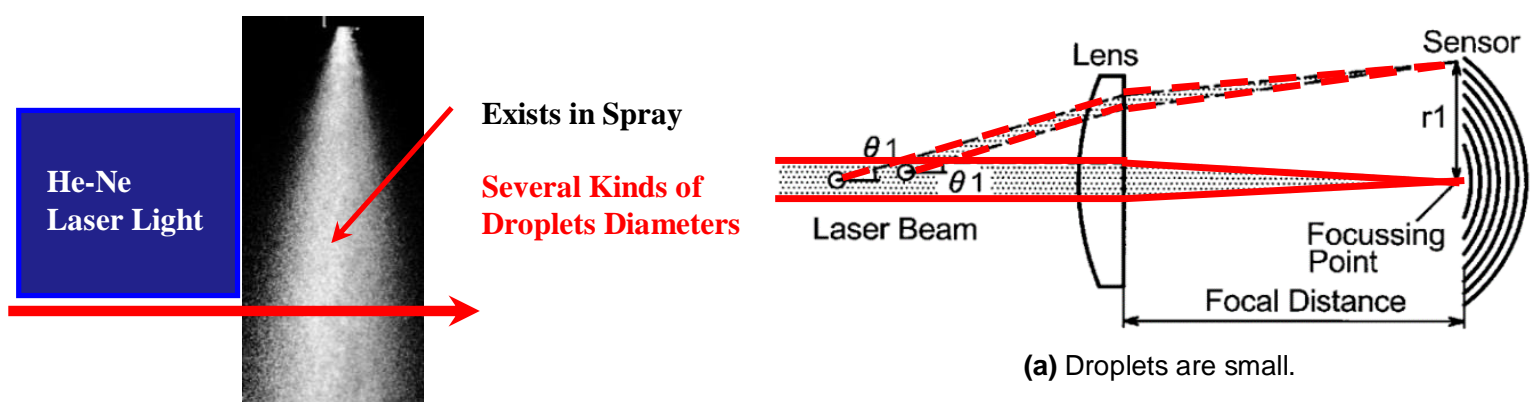

(a) Droplets are small.
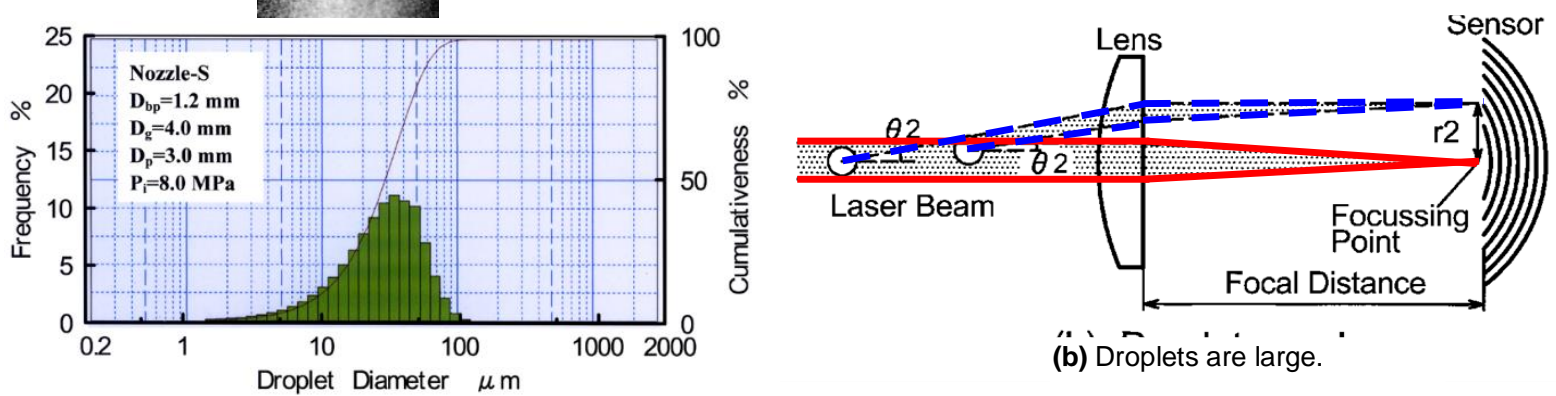

(b) Droplets' are large.

Figure 3. Schematic of narrow-angle forward-scattering technique.

This work is licensed under a Creative Commons 4.0 International License (CC BY-NC-ND 4.0). 
analyzing software, which is installed at the LDSA. It gives the Sauter mean diameter $\mathrm{D}_{32}$ that is spatially averaged along a line through the spray. There are several kinds of droplets in the spray. When He-Ne laser light was radiated the small droplet, it was refracted to large angle [Fig.3 (a)]. To the contrary, when laser light was radiated the large droplet, it was refracted to small angle. Radiation light from the spray droplets condensed at a receiver of the LDSA. The Sauter mean diameter $\mathrm{D}_{32}$, which is important for fields of spray combustion and which is defined as ratio of volume of droplets to surface area of its, were calculated by the LDSA particle analyzer.

The appearance and a schematic of an injector for the D.I. Diesel engine are shown in Figs. 4 and 5, respectively. The atomization enhancement nozzle consists of a bypass, a gap and nozzle holes. The gap is located in the middle of the nozzle hole. The bypass is a hole, connected between the upstream chamber, which corresponds to the sac chamber of an actual Diesel injector and the gap. The role of the bypass is to increase the pressure in the gap to cause the collapse of cavitation bubbles. The swirling flow is believed to be caused by the liquid flow in the bypass.

Three-dimensional images and a schematic of a test nozzle are shown in Fig.6. The test nozzles used included a nozzle with a sharp edged inlet (called Nozzle-S, S), nozzles with a rounded chamfered inlet or outlet (called Nozzle-

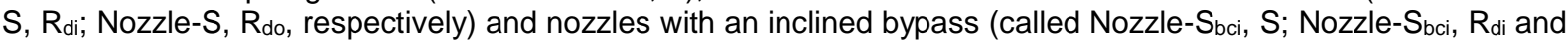
Nozzle-Sbci, Rdo, respectively).

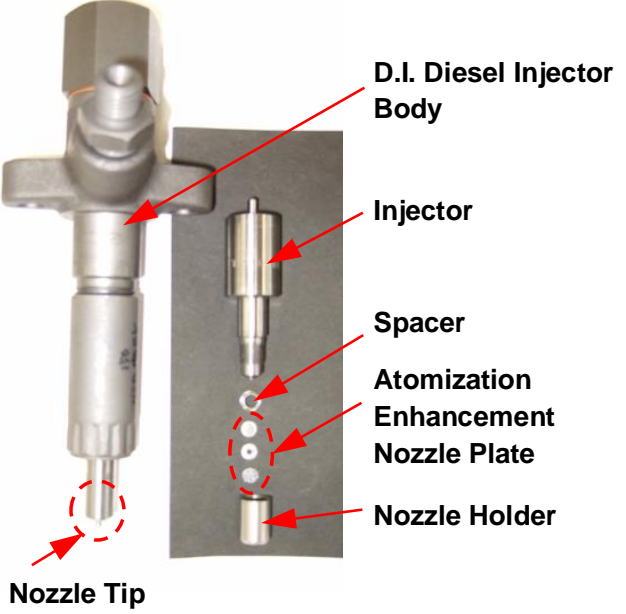

Figure 4. Appearance of injector for D.I. Diesel engine.

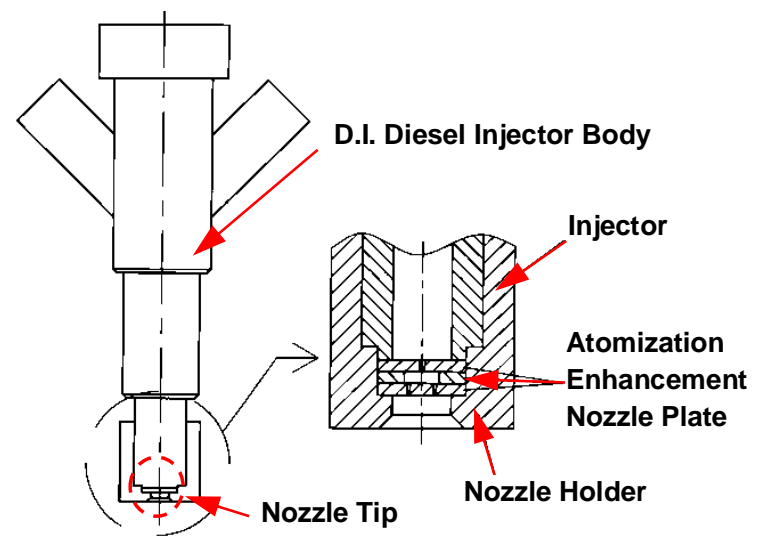

Figure 5. Schematic of injector for D.I. Diesel engine.

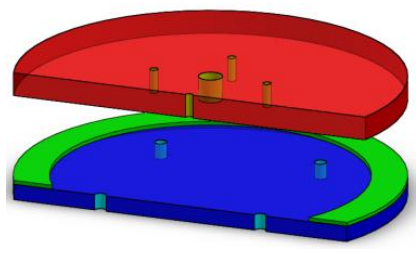

(a) Nozzle-S, S

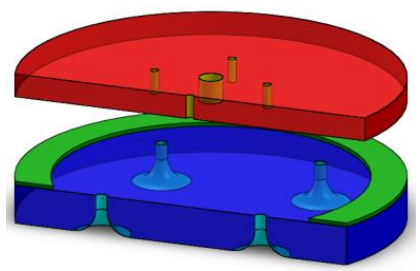

(c) Nozzle-S, $R_{\text {do }}$

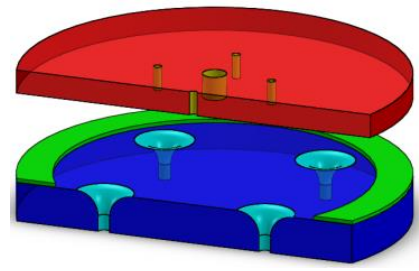

(b) Nozzle-S, $R_{\mathrm{di}}$

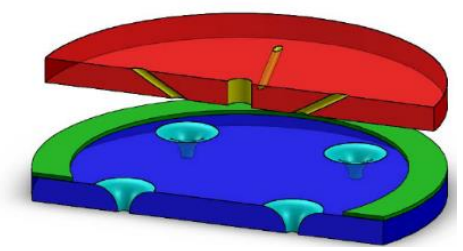

(d) Nozzle-S $S_{b c i}, R_{d i}$

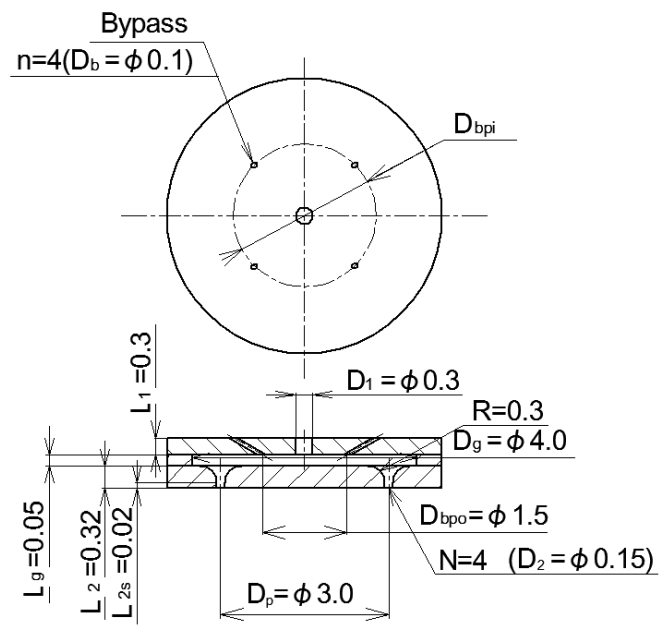

chematic of test nozzle. 


\section{Results and discussion}

Effects of nozzle hole inlet and outlet shapes on atomization characteristics

The effects of the nozzle hole inlet and outlet shapes on the disintegration behavior of the spray are presented in Fig.7. Since the spray of Nozzle-S, $R_{d i}$ atomizes significantly, a large number of small droplets are suspended and form a mist at the surroundings of the ejected spray. The photographic images of the spray are taken against a white background and the boundaries between the ejected spray and the background are indistinct. The spread of the spray of Nozzle-S, $R_{d i}$ is larger than that of Nozzle-S, $S$ and Nozzle-S, $R_{d o}$.

The effects of the nozzle hole inlet and outlet shapes on breakup length and spray angle are shown in Figs. 8 and 9 , respectively. It is well known that when the breakup length is short and the spray angle is large, excellent atomization characteristics are obtained. As shown in Fig.8, the breakup length of Nozzle-S, $R_{\text {di }}$ is the shortest. Although the breakup lengths of Nozzle-S, Rdi and Nozzle-S, S only differ by $0.5 \mathrm{~mm}$, it is apparent that the breakup length is shortest when the spray is injected from a small hole diameter of $0.15 \mathrm{~mm}$. Figure 7 shows that as injection pressure is increased, the spray angle increases independent of the nozzle hole geometry. The spray angle of Nozzle-S, Rdi becomes approximately twice that of the other nozzles for the overall injection pressure.

\section{Effect of including bypass inclination on spray characteristics}

The effect of the inclusion of an inclined bypass on the disintegration behavior of the spray is shown in Fig.10. The spread of the spray of Nozzle-S $S_{b c i}, R_{d i}$, which has the inclined bypass, is wider compared to that of Nozzle-S, $R_{d i}$, which comprises the bypass that is not inclined. It is over $70^{\circ}$ at the maximum injection pressure of $\mathrm{P}_{\mathrm{i} \text { max. }}=10 \mathrm{MPa}$. The effects of the inclusion of the inclined bypass on the spray angle and the Sauter mean diameter are shown in Figs. 11 and 12, respectively. As seen in Fig.11, the spray angle monotonically increases with an increasing injection pressure. The spray angle of Nozzle-S $\mathrm{dci}, \mathrm{R}_{\mathrm{di}}$, for which the bypass was inclined, is $50 \%$ larger than that of Nozzle$S, R_{d i}$ for any arbitrary injection pressure value. Figure 12 shows that for Nozzle-S, $R_{d i}$, as the injection pressure is increased to the maximum value of $10 \mathrm{MPa}$, the Sauter mean diameter decreases monotonically, whereas for

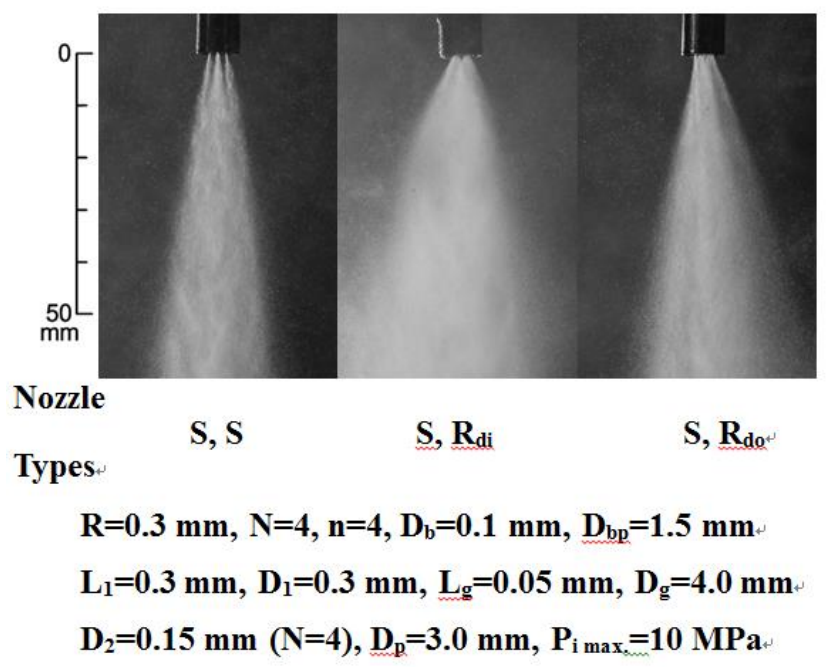

Figure 7. Effects of nozzle hole inlet and outlet shapes on disintegration behavior of spray.

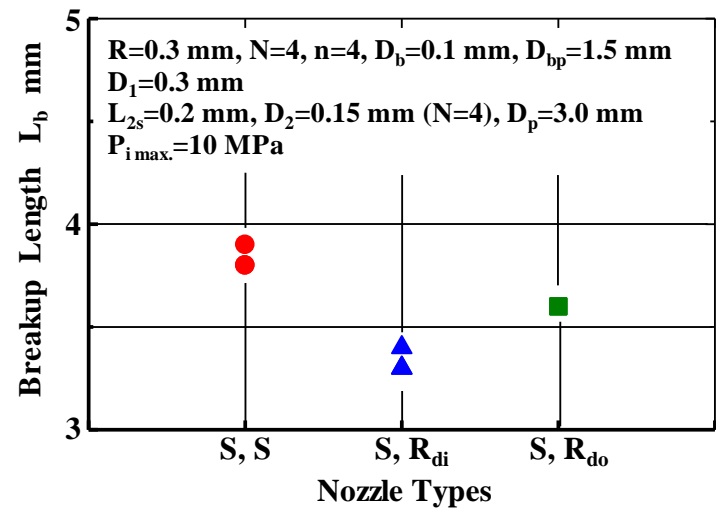

Figure 8. Effects of nozzle hole inlet and outlet shapes on breakup length.

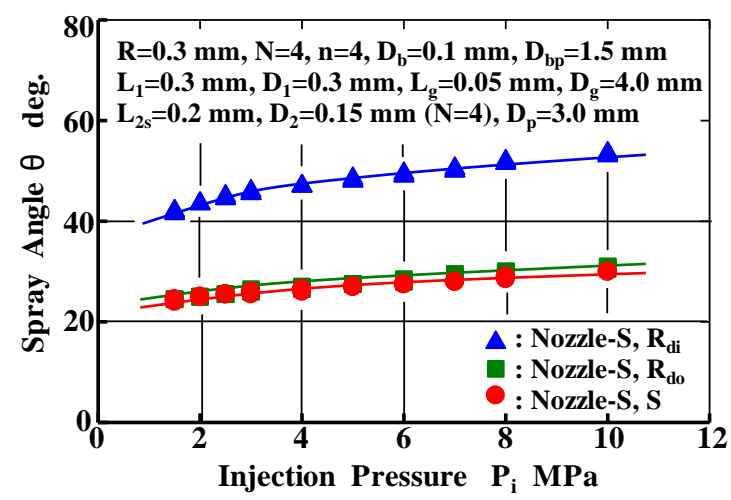

Figure 9. Effects of nozzle hole inlet and outlet shapes on spray angle. 

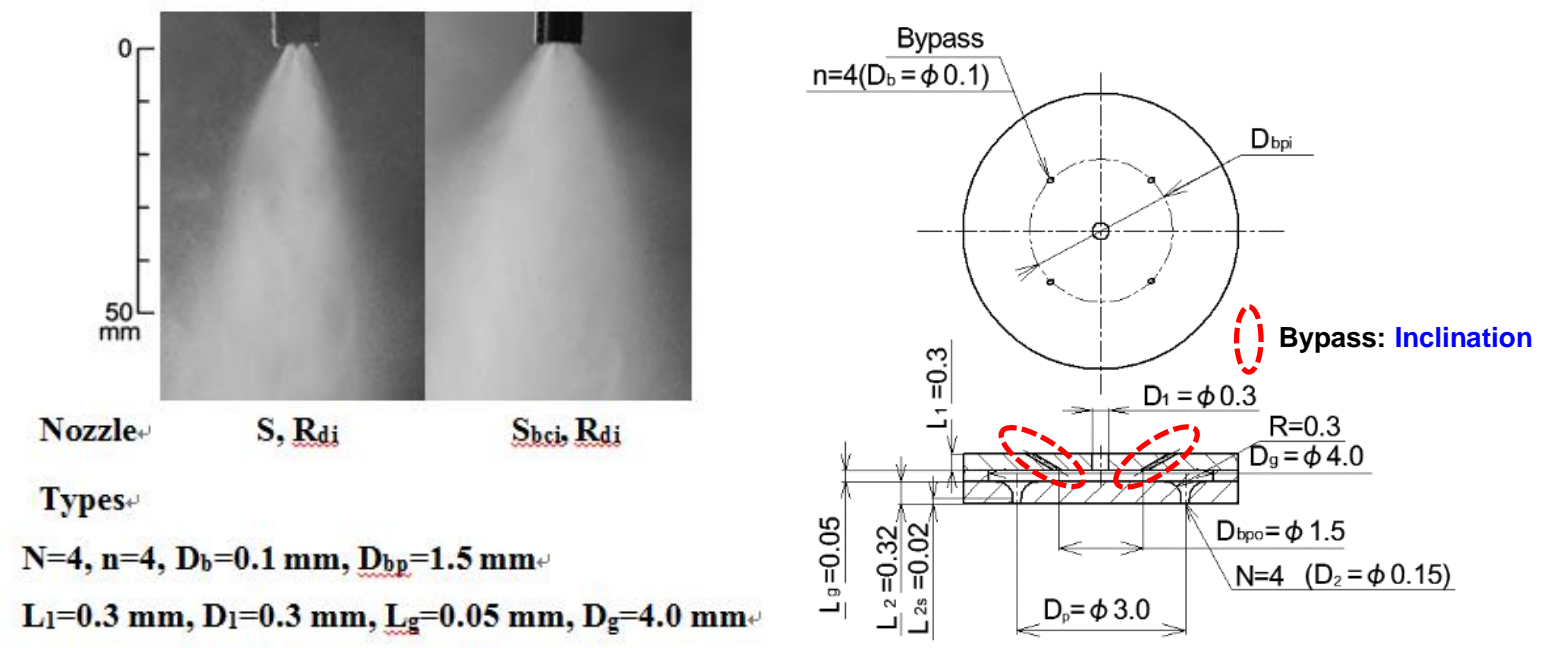

Types

$\mathrm{N}=4, \mathrm{n}=4, \mathrm{D}_{\mathrm{b}}=0.1 \mathrm{~mm}, \mathrm{D}_{\mathrm{bp}}=1.5 \mathrm{~mm}$

$\mathrm{L}_{\mathrm{l}}=0.3 \mathrm{~mm}, \mathrm{D}_{\mathrm{l}}=0.3 \mathrm{~mm}, \mathrm{~L}_{\mathrm{g}}=0.05 \mathrm{~mm}, \mathrm{D}_{\mathrm{g}}=4.0 \mathrm{~mm}$

$\mathrm{L}_{2}=0.3 \mathrm{~mm}, \mathrm{D}_{2}=0.15 \mathrm{~mm}(\mathrm{~N}=4), \mathrm{D}_{\mathrm{p}}=3.0 \mathrm{~mm}$

$P_{\text {imax. }}=10 \mathrm{MPa}$

Figure 10. Effect of inclusion of inclined bypass on disintegration behavior of spray.

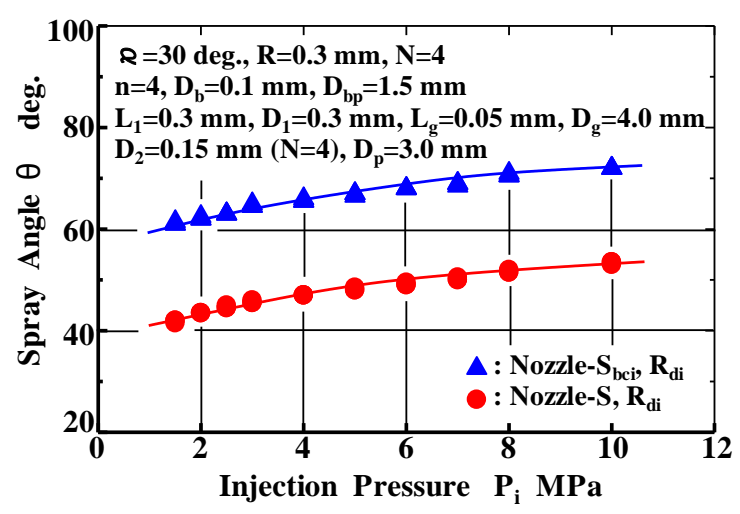

Figure 11. Effects of inclusion of inclined bypass on spray angle.

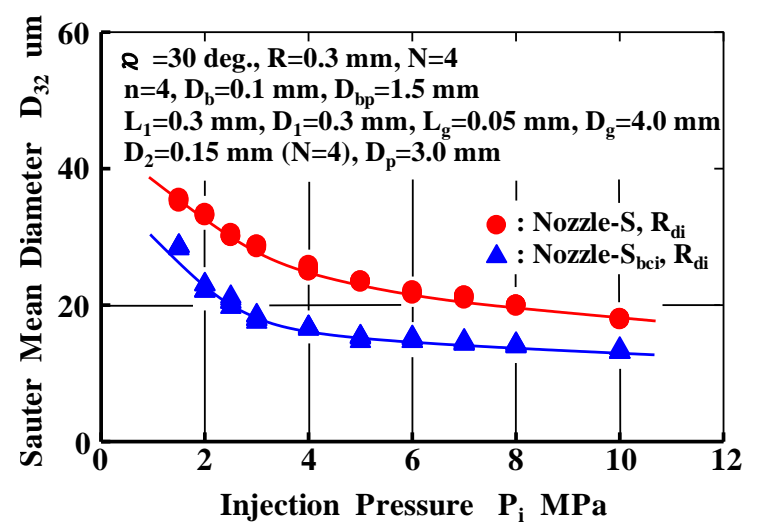

Figure 12. Effects of inclusion of inclined bypass on Sauter mean diameter.

Nozzle-S $S_{b c i}, R_{d i}$, the Sauter mean diameter decreases with increasing injection pressure only until $5 \mathrm{MPa}$, after which it plateaus. Therefore, any further increases in injection pressure, up until a maximum of $10 \mathrm{MPa}$, causes little to no changes in the Sauter mean diameter of Nozzle-Sbci, $R_{d i}$. For the pressure regions tested, the Sauter mean diameter of Nozzle-S bci, $_{\text {di }}$ was smaller than that of Nozzle-S, $R_{d i}$. Hence, it can be concluded that an inclined bypass is an effective method for enhancing spray atomization and improving spray characteristics. A possible explanation for this is that when the bypass is inclined towards the radial direction, it is possible that strong disturbance like the collapse of cavitation bubbles and strong swirling flow occurs inside the gap and four nozzle holes. Moreover, it is expected that momentum towards the radial direction is retained, owing to the swirling flow. Therefore, Nozzle-Sbci, $R_{d i}$ can be used to provide improved spray characteristics.

\section{Effect of bypass inclination on spray characteristics}

The effects of different bypass inclination angles on the disintegration behavior of the spray are presented in Fig.13. The spread of the spray was the widest for the bypass inclination angle of $\alpha=30^{\circ}$ while for $\alpha=60^{\circ}$, the spread was the narrowest. For $\alpha=30^{\circ}$, a large number of small droplets forming a mist were observed within and around the spray. It can be seen that a homogeneous spray is obtained.

Figure 14 shows the effect of the bypass inclination angle on breakup length. The breakup length for the bypass inclination angle of $\alpha=30^{\circ}$ was relatively short. As the inclination angle was increased, the breakup length also increased from a microscopic point of view. 


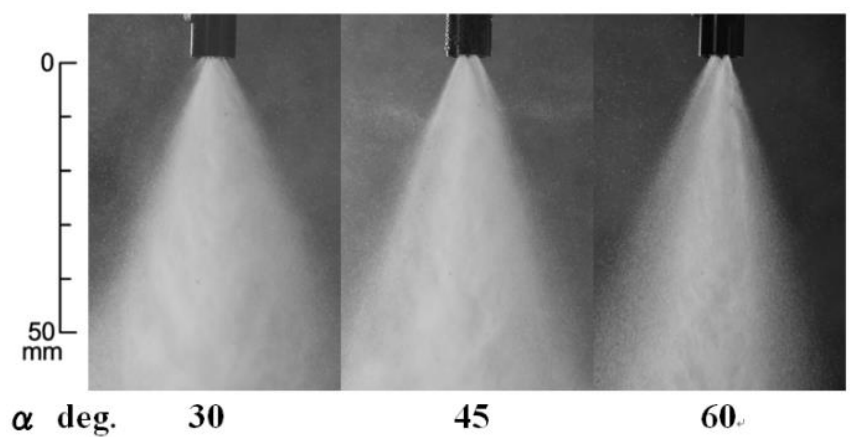

Nozzle- $S_{b c i}, S, N=4, n=4, D_{b}=0.1 \mathrm{~mm}, D_{b p}=1.5 \mathrm{~mm}$

$\mathrm{L}_{1}=0.3 \mathrm{~mm}, \mathrm{D}_{1}=0.3 \mathrm{~mm}, \mathrm{~L}_{\mathrm{g}}=0.05 \mathrm{~mm}, \mathrm{D}_{\mathrm{g}}=4.0 \mathrm{~mm}$

$\mathrm{L}_{2}=0.2 \mathrm{~mm}, \mathrm{D}_{2}=0.15 \mathrm{~mm}(\mathrm{~N}=4), \mathrm{D}_{\mathrm{p}}=3.0 \mathrm{~mm}$

$\mathrm{P}_{\mathrm{imax}}=10 \mathrm{MPa}$

Figure 13. Effects of different bypass inclination angles on disintegration behavior of spray.

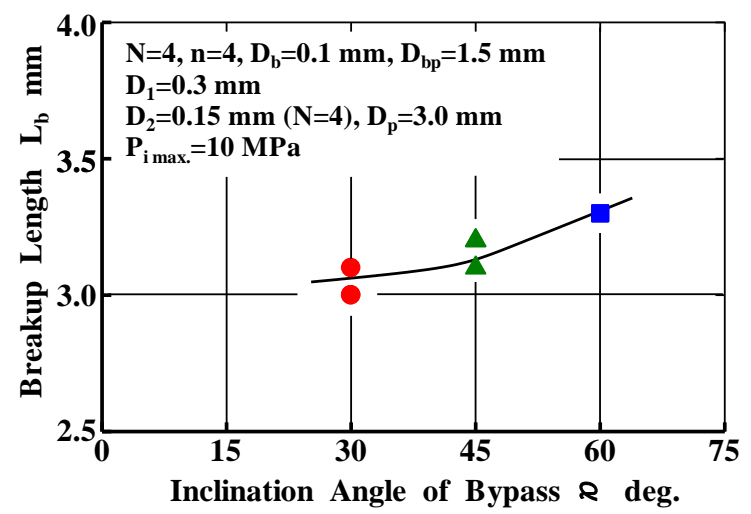

Figure 14. Effect of bypass inclination angle on breakup length.

\section{Conclusions}

[1] The spray angle of Nozzle-S, $R_{\mathrm{di}}$, which comprised a rounded nozzle hole inlet, was approximately twice the spray angles of Nozzle-S, S and Nozzle-S, Rdo.

[2] An inclined bypass strongly affected the spray characteristics of the nozzle. The spray angle for Nozzle-Sdci, $R_{d i}$, which consisted of the inclined bypass, was approximately $50 \%$ larger than that for Nozzle-S, Rdi, which did not include an inclined bypass. The Sauter mean diameter of Nozzle-Sbci, $R_{d i}$ was smaller than that of Nozzle-S, Rdi.

[3] For the bypass inclination angle of $\alpha=30^{\circ}$, the spread of the spray was the widest obtained. A large number of small droplets forming a mist were observed and a homogeneous spray was obtained.

\section{Acknowledgements}

This research was partly supported by the Japan Society for the Promotion of Science (JSPS) KAKENHI (C) Grant Number 15K05847 and also partly supported by the Ministry of Education, Culture, Sports, Science and Technology (MEWT) MEXT-Supported Program for the Strategic Research Foundation at Private Universities. The authors wish to express their thanks for the supporting for this research.

\section{Nomenclature}

$D_{1} \quad$ hole diameter upstream from gap [mm]

$\mathrm{D}_{2} \quad$ hole diameter downstream from gap [mm]

$D_{32} \quad$ Sauter mean diameter $[\mu \mathrm{m}]$

$\mathrm{D}_{\mathrm{b}} \quad$ bypass diameter $[\mathrm{mm}]$

$D_{b p} \quad$ pitch circle diameter of bypass $[\mathrm{mm}]$

$D_{\text {bpi }} \quad$ pitch circle diameter of bypass at inlet of nozzle hole [mm]

$D_{\mathrm{bpo}} \quad$ pitch circle diameter of bypass at outlet of nozzle hole [mm]

$\mathrm{D}_{\mathrm{g}} \quad$ gap diameter [mm]

$D_{p} \quad$ pitch circle diameter of nozzle hole [mm]

$\mathrm{L}_{1} \quad$ hole length upstream from gap [mm]

$\mathrm{L}_{2} \quad$ hole length downstream from gap [mm]

$\mathrm{L}_{2 \mathrm{~s}} \quad$ straight pipe length downstream from gap [mm]

$\mathrm{Lb}_{\mathrm{b}} \quad$ breakup length [mm]

$\mathrm{Lg} \quad$ gap length [mm]

$\mathrm{n}$ bypass number [number]

$\mathrm{N}$ hole number [number]

$\mathrm{Pi}_{\mathrm{i}} \quad$ injection pressure [MPa]

$\mathrm{Pimax}_{\mathrm{m}} \quad$ maximum injection pressure $[\mathrm{MPa}]$

$\mathrm{R} \quad$ round chamfer [mm]

$R_{\mathrm{di}} \quad$ inlet shape of nozzle hole downstream from gap (round chamfer)

Rdo outlet shape of nozzle hole downstream from gap (round chamfer)

$\mathrm{S} \quad$ inlet and outlet shapes of nozzle hole downstream from gap (sharp edged)

$\alpha \quad$ inclination angle of bypass [deg.] 


\section{Superscripts}

$\begin{array}{ll}1 & \text { upstream } \\ 2 & \text { downstream } \\ 2 s & \text { straight pipe length } \\ 32 & \text { volume / surface area } \\ \text { b } & \text { bypass, breakup } \\ \text { bp } & \text { pitch circle diameter of bypass } \\ \text { bpi } & \text { pitch circle diameter of bypass at inlet } \\ \text { bpo } & \text { pitch circle diameter of bypass at outlet } \\ \text { di } & \text { inlet shape of nozzle hole } \\ \text { do } & \text { outlet shape of nozzle hole } \\ \text { g } & \text { gap } \\ \text { i } & \text { injection } \\ \text { i max. } & \text { maximum injection pressure }\end{array}$

\section{References}

[1] Chaves, H, Knapp, M., Kubitzek, A., Obermeier, F. and Schneider, T., 1995, SAE Technical Paper: No. 950290, pp.645-657.

[2] Tamaki, N., Nishida, K., Shimizu, M. and Hiroyasu, H., 1998, Atomization and Sprays, 8 (2), pp.179-197.

[3] Arcoumanis, C. and Gavaises, M., July 6-8, 1998, Proceedings 14th European Conference on Liquid Atomization and Spray Systems.

[4] Tamaki, N., Shimizu, M. and Hiroyasu, H., July 16-20, 2000, Proceedings Eighth Internal Conference on Liquid Atomization and Spray Systems.

[5] Tamaki, N., Shimizu, M. and Hiroyasu, H., 2001, Atomization and Sprays, 11 (2), pp.125-137.

[6] Tamaki, N. and Shimizu, M., September 9-11, 2002, Proceedings 18th European Conference on Liquid Atomization and Spray Systems.

[7] Tamaki, N., July 26-30, 2009, Proceedings 11th Internal Conference on Liquid Atomization and Spray Systems.

[8] Tamaki, N., Kato, A., Kato, K. and Imano, K., Sept. 6-8, 2010, Proceedings 23rd European Conference on Liquid Atomization and Spray Systems.

[9] Tamaki, N. and Katakami, K., Sept. 2-6, 2012, Proceedings 12th Internal Conference on Liquid Atomization and Spray Systems.

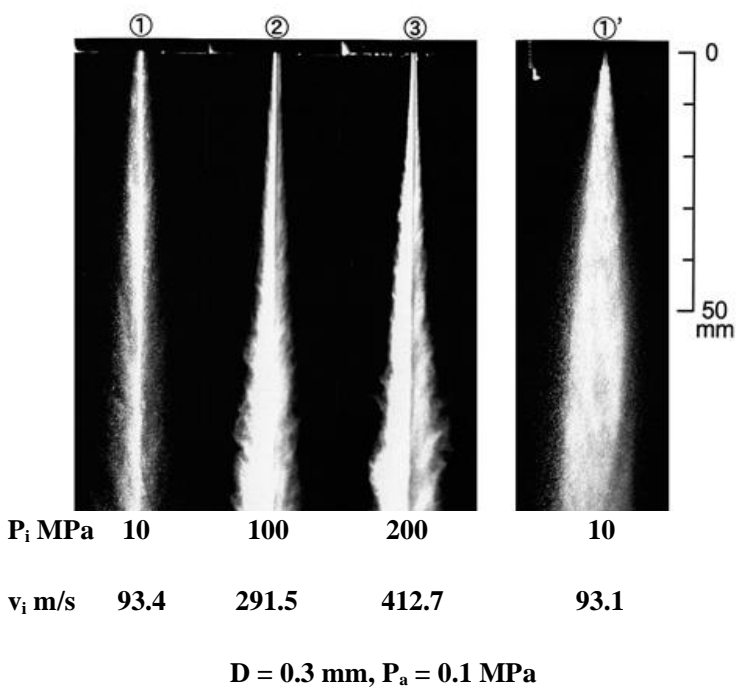

(a) Single Hole Nozzle.

(b) Atomization Enhancement Nozzle.

Appendix Figure 1. Disintegration behavior of spray at super-high injection pressure [4].

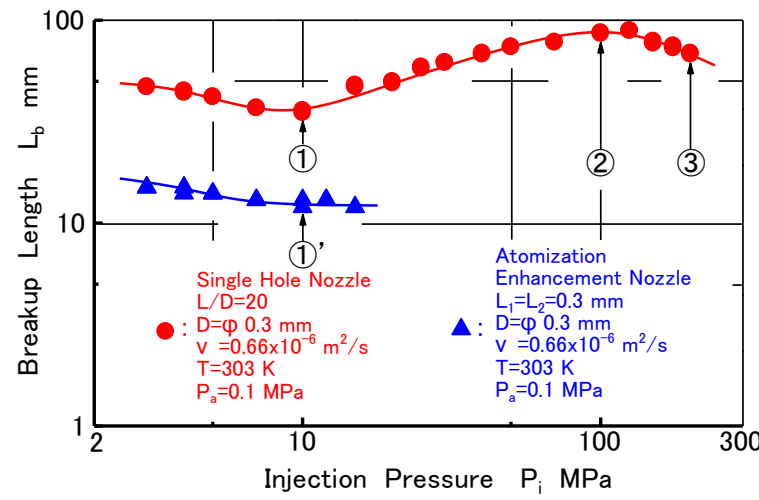

Appendix Figure 2. Comparison of breakup length [4]

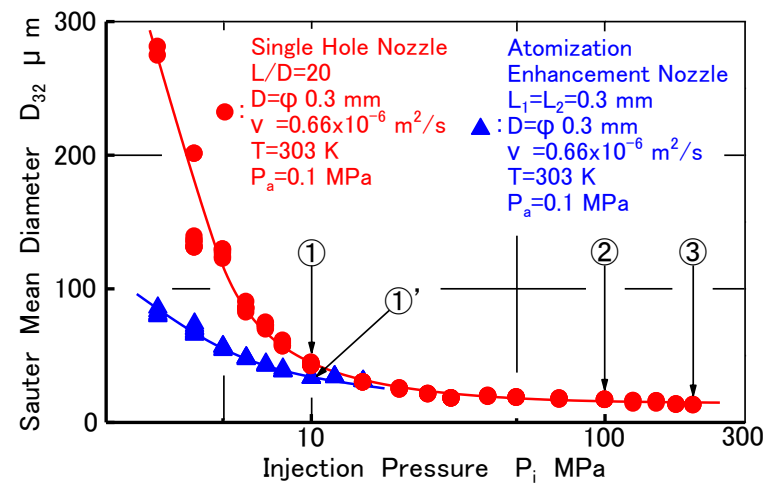

Appendix Figure 3. Comparison of Sauter mean diameter [4]. 


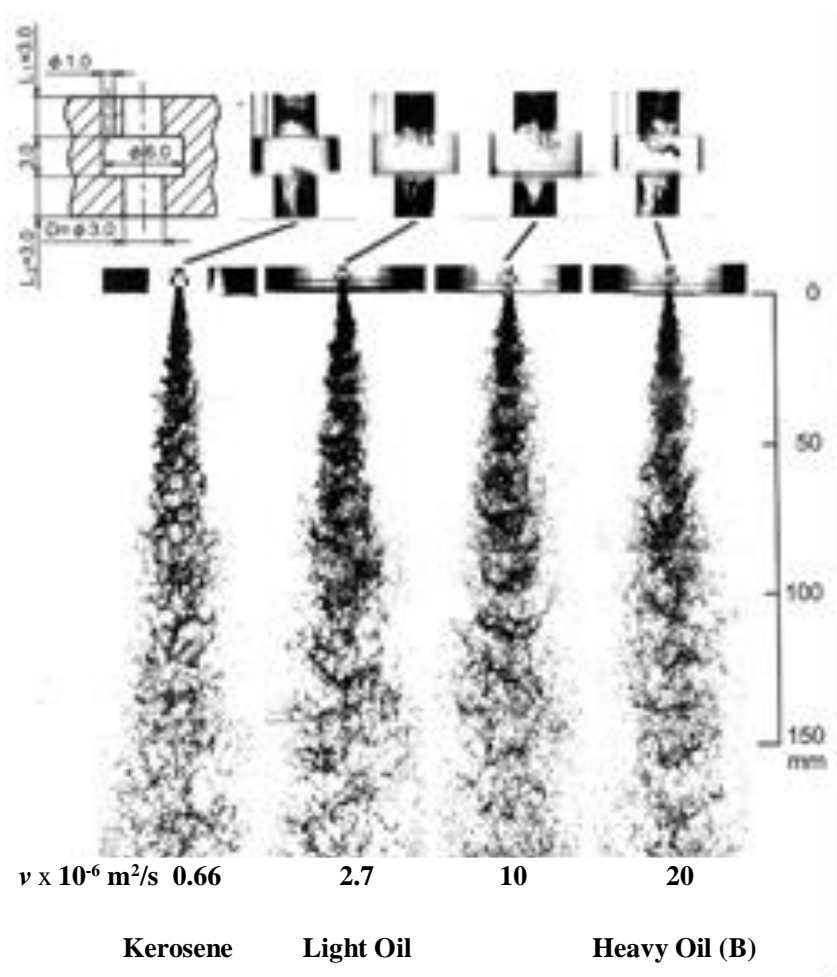

$\mathrm{D}=3.0 \mathrm{~mm}, \mathrm{P}_{\mathrm{i}}=1.5 \mathrm{MPa}, \mathrm{T}_{\mathrm{a}}=313 \mathrm{~K}, \mathrm{P}_{\mathrm{a}}=0.1 \mathrm{MPa}$

Appendix Figure 4. Effect of kinematic viscosity of liquid on disintegration behaviour of spray (Magnified nozzle) [6].

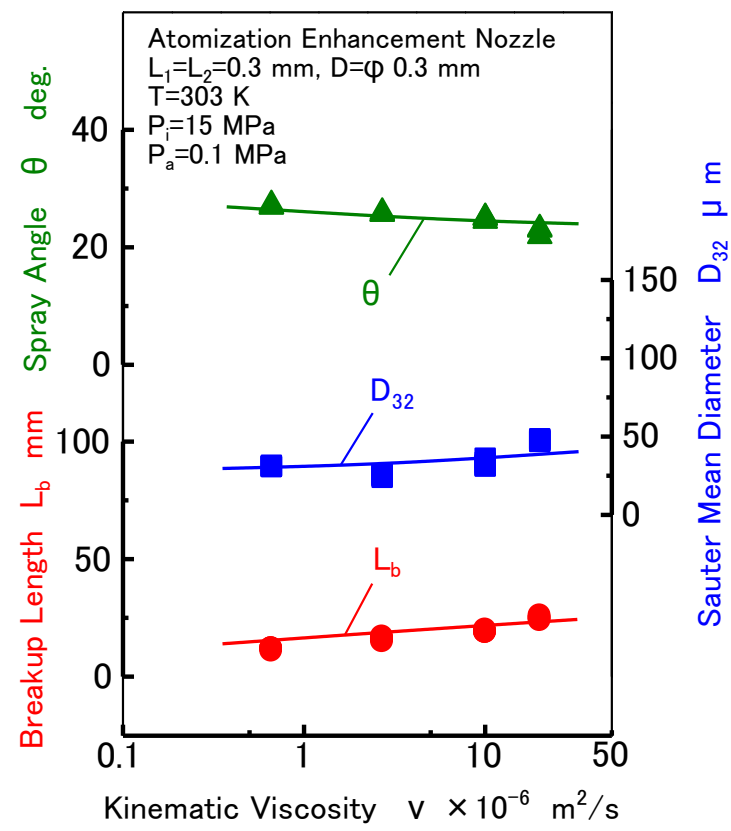

Appendix Figure 6. Effect of kinematic viscosity of liquid on spray characteristics (Actual size nozzle) [6].

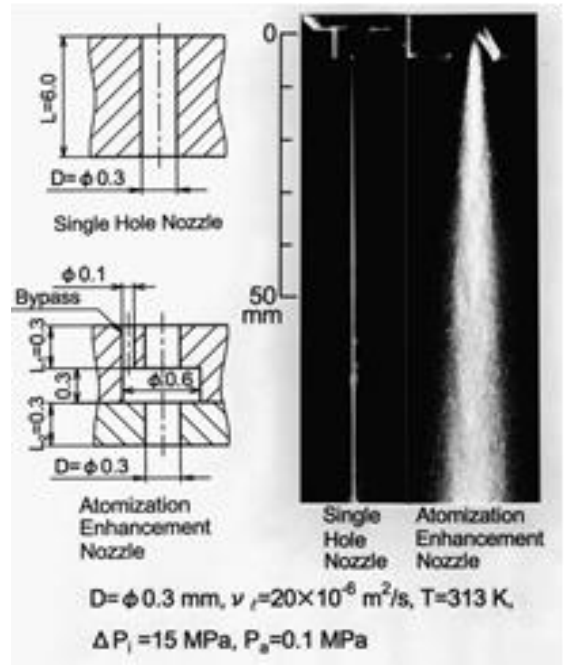

Appendix Figure 5. Atomization of high-viscosity liquid (Heavy Oil B) (Actual size nozzle) [6]

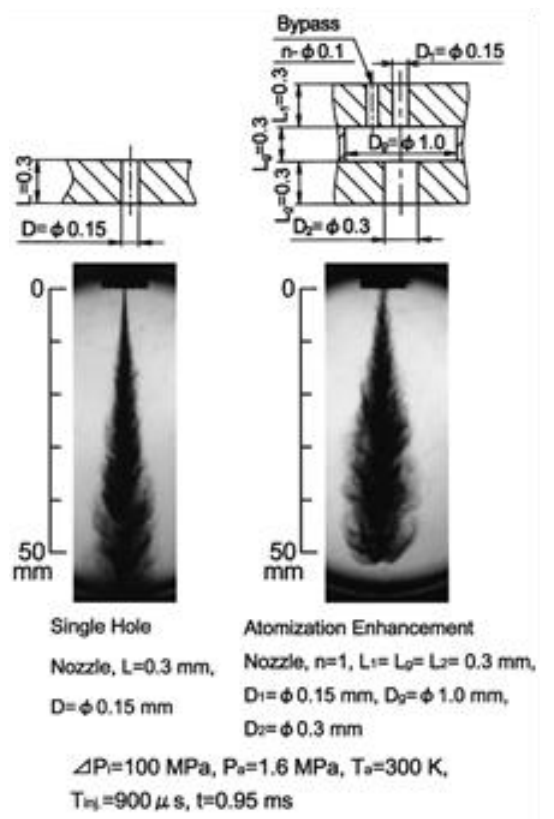

Appendix Figure 7. Atomization of intermittent spray (Light Oil) (Actual size nozzle) [7] 


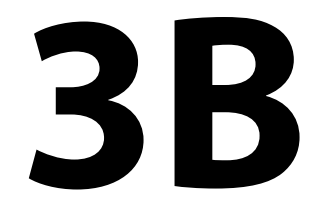

Automotive 3 


\title{
Fuel spray vapour distribution for high pressure diesel fuel spray cases for different injector nozzle geometries.
}

\author{
Darlington Njere*, Nwabueze Emekwuru \\ Centre for Mobility and Transport, Faculty of Engineering, Environment and Computing, \\ Coventry University, Coventry CV12JH, UK \\ *Corresponding author: darlington.njere@coventry.ac.uk
}

\begin{abstract}
The evolution of diesel fuel injection technology, to facilitate strong correlations of in-cylinder spray propagation with injection conditions and injector geometry, is crucial in facing emission challenges. More observations of spray propagation are, therefore, required to provide valuable information on how to ensure that all the injected fuel has maximum contact with the available air, to promote complete combustion and reduce emissions. In this study, high pressure diesel fuel sprays are injected into a constant-volume chamber at injection and ambient pressure values typical of current diesel engines. For these types of sprays the maximum fuel liquid phase penetration is different and reached sooner than the maximum fuel vapour phase penetration. Thus, the vapour fuel could reach the combustion chamber wall and could be convected and deflected by swirling air. In hot combustion chambers this impingement can be acceptable but this might be less so in larger combustion chambers with cold walls. The fuel-ambient mixture in vapourized fuel spray jets is essential to the efficient performance of these engines. For this work, the fuel vapour penetration values are presented for fuel injectors of different k-factors. The results indicate that the geometry of fuel injectors based on the k-factors appear to affect the vapour phase penetration more than the liquid phase penetration. This is a consequence of the effects of the injector types on the exit velocity of the fuel droplets.
\end{abstract}

\section{Keyword}

Vapour, spray, k-factor, shadowgraph.

\section{Introduction}

Spray formation occurs with the introduction of liquid into a gaseous environment through an orifice such that the liquid breaks-up into droplets by interacting with the surrounding gases and causing its own unsteadiness [3]. For diesel engines, spray characteristics (liquid/vapour penetration and distribution) significantly affect the combustion and emission processes. By optimizing these characteristics, the tailpipe emissions, mainly oxides of Nitrogen $\left(\mathrm{NO}_{\mathrm{x}}\right)$ and partciculate matter (PM), can be minimized [2]. Spray penetration, which is usually analysed macroscopically, considers the development of the liquid and vapour components. It is desirable to achieve optimal travel of these spray components to avoid the adverse effects of impingement caused by under/overpenetration of liquid spray [19,20]. Advances in fuel injection system, with the introduction of the common rail technology, have provided increased controllability of the injection event.

The analyses of injection system development have been presented from several viewpoints. Nozzle geometry has been studied for the influence on the internal flow and spray characteristics with respect to: atomization [4,5], mixing processes [6,7], emission [9,10] and cavitation [8, 13]. Different injection strategies have been investigated to show the effect on pollutant emissions [2,3]. Specific studies have also been conducted with conical and cylindrical nozzles [11, 12], and to develop more understanding on the effects of nozzle holes [14-16] on spray characteristics. Many works in the literature also focused on parameters that affected spray penetration, whilst developing useful correlations [21, 22]. From these studies, it appears that the influence of nozzle geometry on spray characteristics has been established [7, 28]. Despite the comprehensive nature of these investigations, conducted using experimental/numerical methods under evaporative/non-evaporative conditions and often with the aid of optical techniques, the understanding is by no means complete and challenges not dismissed. The physics of the effects of nozzle geometry on in-cylinder spray development and combustion is still of interest to the research community and the auto-industry. For example, the survey in this work [7, 28], supported by other investigations [23, 24], clearly showed the significant effects of nozzle flow characteristics on spray development. This contrasts with other works $[25,26]$ which suggested negligible influence of nozzle flow characteristics over spray formation. Clearly, the true extent of the effect of nozzle geometry over a wide range of operating conditions and response variables need to be fully understood. Part of this uncertainty derives from unclear details about vaporisation, which is crucial for the injection process [27]. Since the vapor fuel continues to penetrate downstream while the liquid-phase fuel penetration stays at the liquid length, there is a correlation between the vapour penetration rate, total air entrainment rate and fuel-air mixing. Thus, depending on the injector geometry and the in-cylinder conditions, a higher vapour penetration may cause better air utilization, 
which affects the combustion process.

It is common to specify injector geometry based on the k-factor, this places emphasis on the hole diameter and its profound effect on fuel injection. Most injection characteristics change with the hole diameter. The k-factor can have a negative, zero or positive value. Spray hole (or orifice) with a negative conical shape factor exhibit an orifice with increasing diameter towards the nozzle exit. With a positive value of the conical shape factor, the orifice diameter decreases towards the exit (Figure 1). These two configurations implement a conical (or tapering) profile. A zero value for $\mathrm{k}$ (i.e. no conicity) indicates a cylindrical orifice since the diameters are equal. In this study, optical technique is applied to visualize in-cylinder spray penetration in the absence of a piston bowl, by implementing: high speed liquid spray visualization (Mie scattering technique) and vapour spray visualization using high-speed laser shadowgraph. Two injectors with different k-factors are utilized via a standard common rail system, and a low sulphur automotive diesel as the test fuel. It is expected that the result will be useful for vapour phase penetration modeling and for use in the validation of spray models.

$K=\frac{\left(D_{\text {inlet }}-D_{\text {outlet }}\right)}{10}$

Where $\mathrm{K}$ is the $\mathrm{k}$-factor, a measure of conicity; $\mathrm{Di}_{\text {nlet }}$ and $\mathrm{D}_{\text {outlet }}$ are inlet and outlet orifice diameters in micrometres $(\mu \mathrm{m})$, respectively.

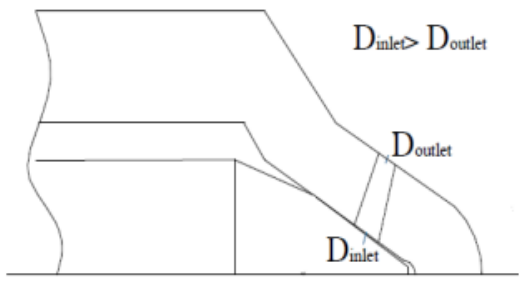

Figure 1: Nozzle configuration (Inlet/outlet diameter)

\section{Material and methods}

The liquid and vapour spray measurement systems were based on an optical engine, the Ricardo Proteus rig, installed at the University of Brighton, UK, where the tests were conducted under thermodynamic conditions similar to those found in a direct injection (DI) diesel engine. Optical diagnostics using high speed image acquisition equipment were developed and optimised around the Proteus, to visualise in-cylinder spray penetration. The Proteus is a 2-stroke, liner ported, single cylinder reciprocating rapid compression machine (RCM), with a specially designed head (top-hat shape and optical chamber with windows) for optical access. For the current study, steady in-cylinder (non-combusting) conditions were maintained all through the tests.

The injection system for this study was based on the high pressure (HP) common-rail system (CRS) fuel injection equipment on the Proteus rig using different injectors with key specifications summarised in Table 1. The Injectors have same hole diameters but different k-factors. Both injector types are classified by the k-factor parameter defined in equation (1). Only one, out of the four common rail injector outlets, was used at any given time, so the other unused three were fitted with plugs.

Table 1: Injector specifications

\begin{tabular}{|lrl|r|r|r|r|}
\cline { 3 - 7 } \multicolumn{1}{c|}{} & $\begin{array}{l}\text { Flow } \\
\text { (cc/min) }\end{array}$ & Holes & $\begin{array}{l}\text { Hole size } \\
(\mathbf{m m})\end{array}$ & $\begin{array}{l}\text { Cone angle } \\
\text { (deq.) }\end{array}$ & $\begin{array}{l}\text { k- } \\
\text { factor }\end{array}$ \\
\hline $\begin{array}{l}\text { Injector } \\
\text { 3601) } \\
\text { Injector } \\
\text { 3603) }\end{array}$ & 960 & 8 & & 0.137 & 155 & 1.3 \\
\hline
\end{tabular}

The optical arrangements were varied slightly between the liquid and vapour spray experiments, but similar steps were applied in setting-up. Engine logs were recorded with AVL Indiset high speed data acquisition system for fast logs (crank angle resolved), and EmTronics data logger for slow logs. For each regime of test, a minimum of fifty (50) videos were acquired with the laser/camera combination. The videos were post-processed with a Matlab software and analysed. In addition, the engine was stopped at intervals, during the tests, to clean the optical windows.

The liquid spray was visualised using the Mie scattering technique as shown in Figure 2. Here, the laser beam 
was aligned to pass through the vertical plane of the fuel spray and cause elastic scattering of the laser light by the liquid fuel droplets as soon as fuel was injected into the chamber. The high-speed camera was positioned perpendicular to the laser illumination to collect the scattered light. This visualised the liquid fuel distribution and showed the liquid spray penetration within the spray. The laser light was collimated before entering the optical engine, at reduced intensity via neutral density filters, by carefully placing the filter between the laser and a collimating lens. A slightly varied optical arrangement in Figure 3 implemented the shadowgraph technique for vapour spray visualisation

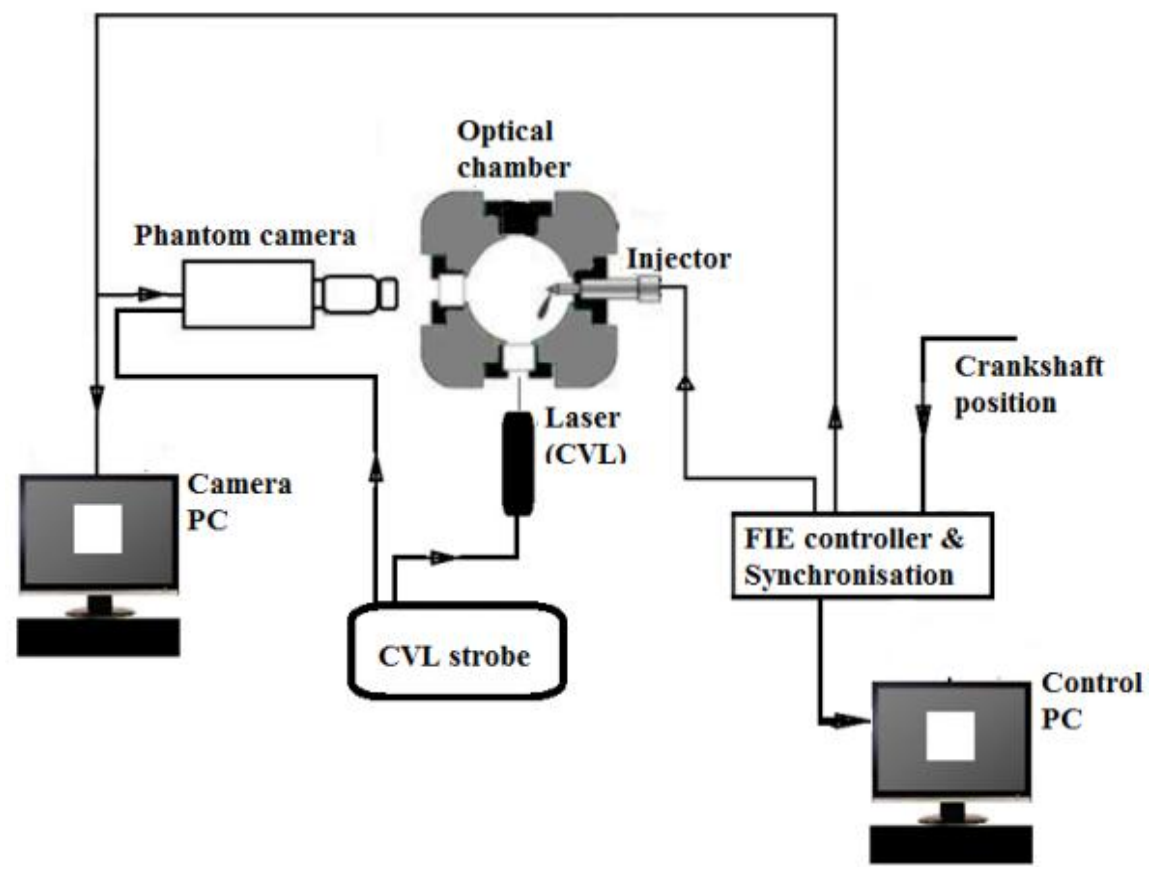

Figure 2: Schematic for Liquid spray visualisation

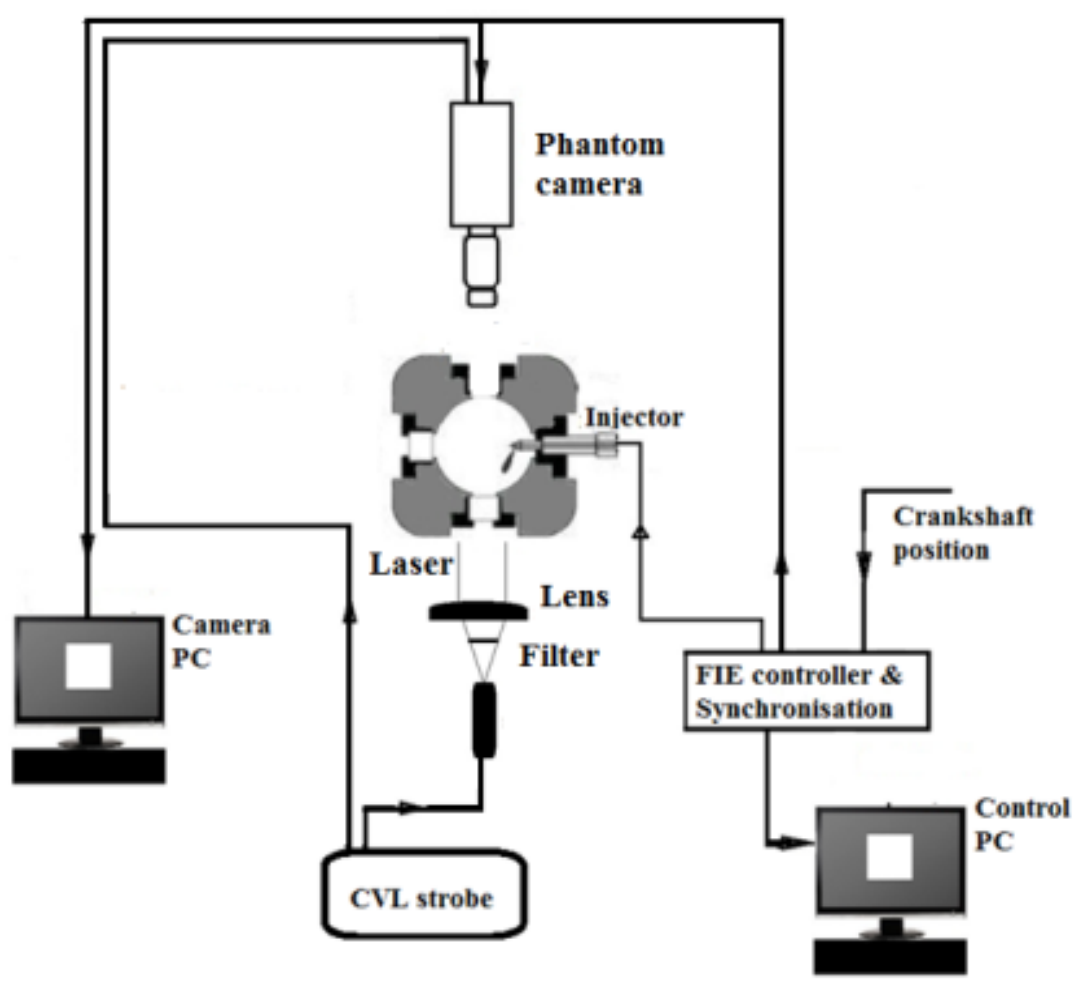

Figure 3: Set-up for vapour visualisation

Liquid and vapour spray penetrations were investigated with Injectors C and D respectively. The test conditions 
were selected from the matrix in Table 2, for investigations across injection pressure ranges (high, medium and low). While the test fuel used was Carcal RF06-08-B5 (density; $833.2 \mathrm{~kg} / \mathrm{m}^{3}$ ). Normal injection was timed at top dead centre (TDC). The actual intake air temperature was determined by the intake manifold temperature (TMAN), and hence varied significantly (less than $100^{\circ} \mathrm{C}$ ). Spray parameters were quantified with respect to time after start of injection (ASOI) for every spray plume.

Table 2: Test Matrix

\begin{tabular}{|r|r|r|r|r|}
\hline $\begin{array}{l}\text { Test } \\
\text { point } \\
(\text { TP) }\end{array}$ & $\begin{array}{c}\text { Intake air } \\
\text { temperature } \\
\text { TMAN }\left({ }^{\circ} \mathrm{C}\right)\end{array}$ & $\begin{array}{c}\text { Fuel } \\
\text { Peak in-cylinder } \\
\text { pressure ICP (bar) }\end{array}$ & $\begin{array}{c}\text { Injection } \\
\text { quantity } \\
\text { pressure } \\
\text { P (bar) }\end{array}$ & $\begin{array}{c}\left.\mathrm{mm}^{3}\right) @ 38 \\
{ }^{\circ} \mathrm{C}\end{array}$ \\
\hline 1 & 100 & 84 & 2000 & 65 \\
2 & 100 & 84 & 1600 & 36.31 \\
3 & 100 & 84 & 1400 & 36.71 \\
4 & 100 & 84 & 1000 & 35.31 \\
5 & 100 & 84 & 600 & 34.86 \\
6 & 100 & 50 & 2000 & 45 \\
7 & 100 & 50 & 1600 & 45 \\
8 & 100 & 50 & 1400 & 45 \\
9 & 100 & 50 & 1000 & 25 \\
10 & 100 & 66 & 2000 & 65 \\
11 & 100 & 66 & 1400 & 45 \\
12 & 100 & 66 & 1000 & 45 \\
13 & 100 & 79 & 1800 & 60 \\
14 & 100 & 73 & 1600 & 50 \\
15 & 100 & 40 & 600 & 20 \\
\hline
\end{tabular}

\section{Result and discussion}

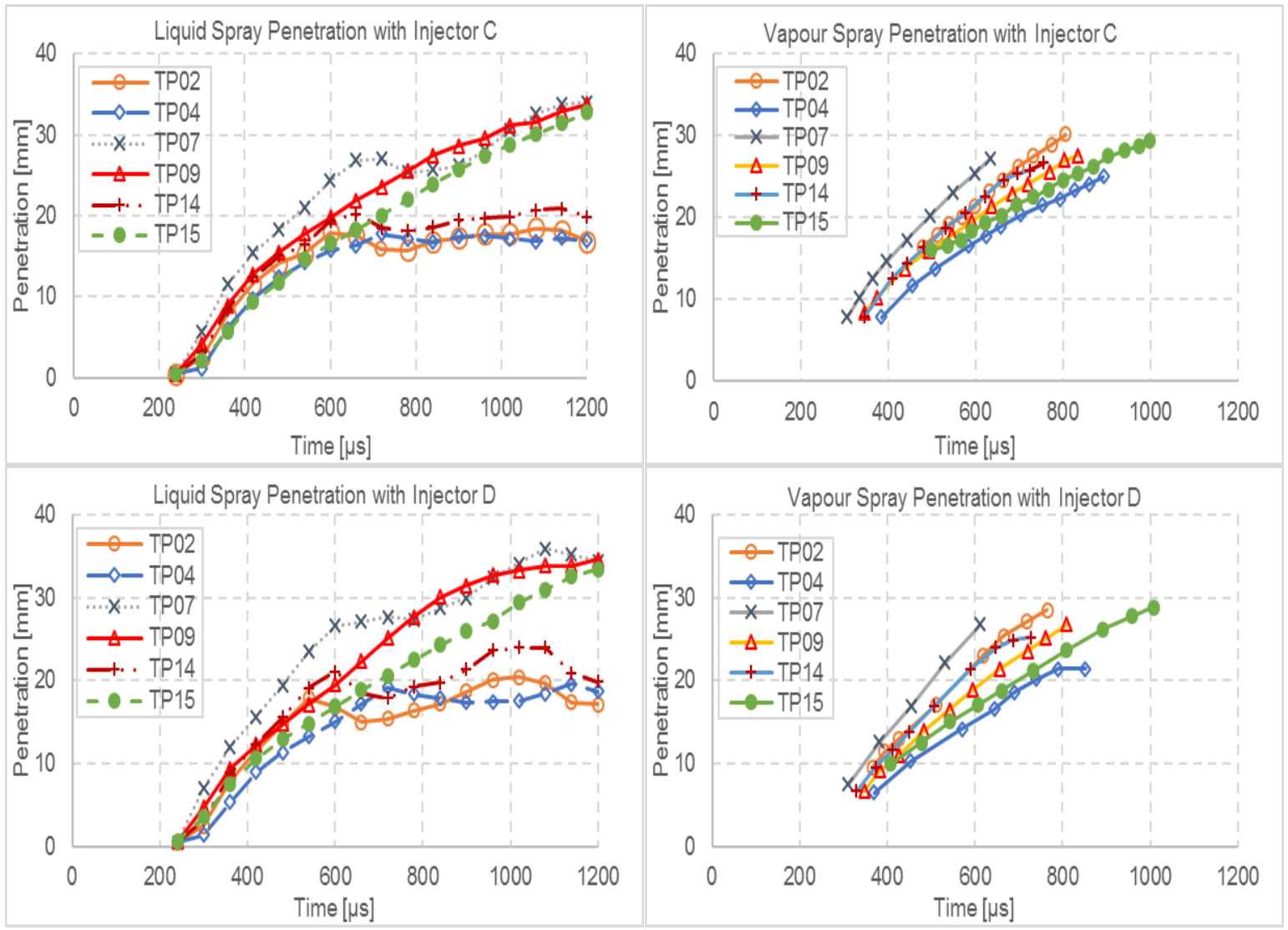

Figure 4: Comparison between injectors $C$ and $D$ spray (liquid and vapour) penetration measurements 

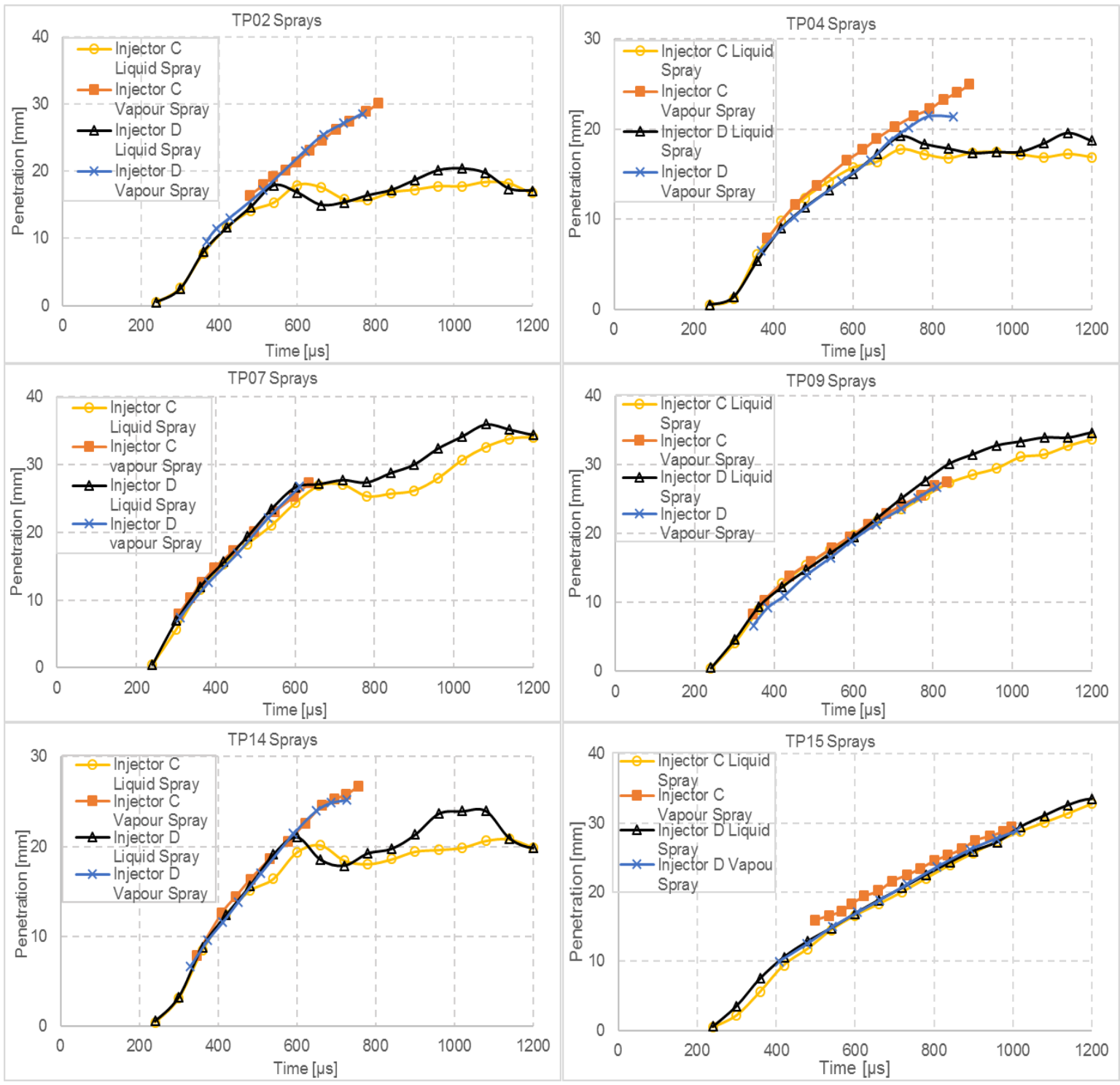

Figure 5: Comparison between injectors $C$ and $D$ spray (liquid and vapour) penetration measurements

Injector C

Injector D

TP14

TP15
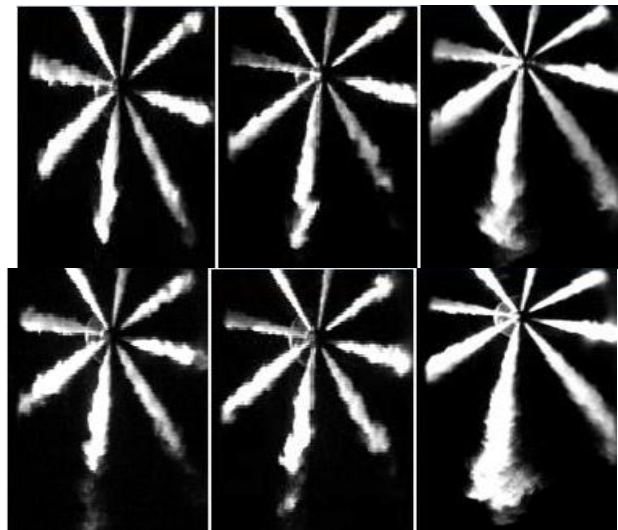

Figure 6: Image sequence for selected liquid spray penetration from all the injectors

Figures 4 and 5 show the results for the spray (liquid and vapour) penetration tests conducted with injectors $C$ and $D$ at Test Points selected from the matrix, for different injection pressures (low to high). Excerpt from the 
image sequence of liquid spray penetration from the injectors are presented in Figure 6.

In Table 1, the k-factor for injectors $C$ and $D$ were specified as: 1.3 and 3.5 respectively. Results from the plots and observations of the image sequence, which agree with literature, showed that nozzles with higher k-factor caused higher spray (liquid and vapour) penetration. It was also observed that the nozzle exit velocity and density increased with increase in conicity $[29,30]$ The results also showed that these behaviours were more evident under high (injection) pressures, as shown in Figures 4 and 5 for all the test points, except TP15 (with low pressures).

From the results, the effects of in-cylinder pressure on liquid spray penetration agree with previous investigations [21]. Increasing the ambient pressure increases the ambient temperature which causes the spray to evaporate faster and travel less. This trend is manifest even with increased injection pressure, especially for liquid spray penetration across the injectors. From the point of view of fluid dynamics, the evaporation of droplets involves simultaneous heat and mass transfer processes. The heat of evaporation is transferred from surrounding hot gases to the drop surface by conduction and convection, while vapour is transferred to the surrounding by convection and diffusion. The evaporation rate is dependent on the thermo-physical properties of the fuel and the surrounding air. For very high in-cylinder pressure and temperature, evaporation is quick since the latent heat drastically decreases for high temperatures. As the hot entrained air initiates evaporation of the spray, the fuel cools and contracts. These processes significantly reduce droplet dispersion and hence liquid spray penetration $[1,31]$. The effect of increased injection pressure, which increased spray velocity/momentum [21], is more noticeable in the transient part of the liquid spray penetration than the quasi steady stage. The slight positive slope observed at the steady period in some of the liquid penetration curves in Figures 4 and 5 can be attributed to two factors $[8,17]$. Firstly, the temperature of the fuel at the beginning of the injection is higher because the injector sac is always in direct contact with the high temperature gas of the combustion chamber; during the injection, the fuel flowing from upstream of the sac cools down the injector tip. This causes the temperature of the fuel at the orifice outlet to decrease during the injection, which impacts on liquid length [8]. Secondly, for long injections, low temperature fuel-air mix is re-entrained in the spray causing a decrease in the effective ambient temperature and an increment in liquid length. Taking the large size of the chamber into account as well as the tendency of the slope to decrease at the end of the injection, the first fact was generally considered to be responsible for the observed phenomenon.

The effect of increased injection pressure is clearer in the vapour penetration, which is further than the liquid spray travel [21]. Vapour penetration profiles have been shown to depend on both injection pressure and incylinder density [18]. It was further suggested that the mechanism for the vapour transport was the gas motion induced by the liquid phase momentum exchange, from the droplets to the gas phase. To support these facts, increased penetration of the vapour was observed at higher injection pressures and lower gas densities, which was when the liquid phase had a higher momentum. The momentum effect is felt more, across the injectors as the k-factor increases. The vapour spray penetrated more, at higher injection pressures (and higher ambient pressure), because the consequent level of turbulence was such that increased the hydrodynamic interaction of the spray. This process was facilitated by smaller nozzle exit as the k-factor increased. Primary breakup was accelerated leading to smaller droplets and reduced liquid spray penetration. The resulting increase in dispersion increases the vaporization rate and fuel air mixing.

Knowing that the k-factor could significantly affect vapour spray penetration is important for high speed direct injection (HSDI) diesel engine. Increase in vapour penetration improves mixing, results in greater premix burning and faster combustion, which may cause $\mathrm{NO}_{x}$ emission to increase, but reduce PM emissions.

\section{Conclusion}

The liquid and vapour phases of diesel fuel spray were characterised in this work, using optical techniques based on visualization. Mie-Scaterred and shadowgraph images were continuously recorded to expose liquid and vapour penetrations respectively, under real engine conditions. The injectors used were essentially different in terms of the k-factors. From the results, the significant influence of injector design (k-factor) on HSDI diesel engine spray development was confirmed. It was observed that the geometry of fuel injectors based on the kfactors affected the vapour phase penetration more than the liquid phase penetration. This was a consequence of the effects of the injector types on the exit velocity of the fuel droplets.

Further work could be done by replicating the characterisation experiments with piston bowl for impingement studies. The understanding from the present work will provide the necessary guidance and basis for comparison. The comparison of the data with commonly used correlations would provide further confidence on its use for spray model validations

\section{Acknowledgements}

Support for this work was provided by Ricardo UK, at Sir Harry Ricardo Laboratory in University of Brighton. Gratitude is also extended to: Prof. C. Crua, Dr. R. Morgan and Dr. G. de Sercey. 


\begin{tabular}{ll}
\multicolumn{2}{l}{ Nomenclature } \\
ASOI & After start of injection \\
CRS & Common-rail system \\
CVL & Copper vapour laser \\
D & Nozzle diameter $[\mu \mathrm{m}]$ \\
DI & Direct injection \\
HP & High pressure \\
HSDI & High speed direct injection \\
k & k-factor \\
NOx & Oxides of Nitrogen \\
PC & Personal computer (Dedicated desktop) \\
PM & Particulate matter \\
RCM & Rapid compression machine \\
TDC & Top dead centre \\
TMAN & Intake manifold temperature \\
TP & Test point
\end{tabular}

\section{References}

[1] Arcoumanis, C., C. Bae, R. Crookes and E. Kinoshita (2008). "The potential of di-methyl ether (DME) as an alternative fuel for compression-ignition engines: a review." Fuel 87: 1014-1030.

[2] Arrègle J, Pastor JV, López JJ, Garcı 'a A. (20028). Insights on post injection-associated soot emissions in direct injection diesel engines. Combust Flame154, 448-61.

[3] Suh HK, Yoon SH, Lee CS. (2010). Effect of multiple injection strategies on the spray atomization and reduction of exhaust emissions in a compression ignition engine fueled with dimethyl ether. Energy Fuels 24,1323-32.

[4] Yi Y, Reitz RD. (2003). Modeling the effect of primary atomization on diesel engine emissions. SAE Trans Sect 3 J Engines (SAE Paper 2003-01-1041)112, 1311-30.

[5] Som S, Aggarwal SK. (2010). Effects of primary breakup modeling on spray and combustion characteristics of compression ignition engines. Combust Flame 157,1087-96.

[6] Kampmann S, Dittus B, Mattes P, Kirner M. (1996). The influence of hydro grinding at VCO nozzles on the mixture preparation in a DI diesel engine. SAE Paper 960867

[7] Payri R, Salvador FJ, Gimeno J, Zapata DL. (2008). Diesel nozzle geometry influence on spray liquid-phase fuel penetration in evaporative conditions. Fuel 87, 1165-76.

[8] Payri F, Payri R, Salvador FJ, Martı 'nez-López J. (2012). A contribution to the understanding of cavitation effects in diesel injector nozzles through a combined experimental and computational investigation. Comput Fluids 58, 88-101

[9] Karra PK, Kong SC. (2010). Experimental study on effects of nozzle hole geometry on achieving low diesel engine emissions. J Eng Gas Turbine Power 132, 022802-11.

[10] Som S, Aggarwal SK, El-Hannouny EM, Longman DE. (2010). Investigation of nozzle flow and cavitation characteristics in a diesel injector. J Eng Gas Turbines Power 132, 2802-14

[11] Han J. S., Lu P-S., Xie X. B., Lai M. C. and Henein N. A. (2002). Investigation of Diesel Spray Primary Break-up and Development for Different Nozzle Geometries, SAE Paper 2002-01-2775

[12] Benajes J, Pastor JV, Payri R, Plazas AH. (2004). Analysis of the influence of diesel nozzle geometry in the injection rate characteristics. Journal of Fluids Eng, 126, 63-71.

[13] Payri R, Salvador F, Gimeo J, Venegas O. (2012). Study of Cavitation phenomenon using different fuels in a transparent nozzle by hydraulic characterization and visualisation. Experimental Thermal and Fluid Science 44, 235-244

[14] Zhang W, Nishida K, Gao J, Miura D. (2008). An experimental study on flat-wall impinging spray of microhole nozzles under ultra-high injection pressures, Proc. IMechE Part D J. Auto. Eng. 222 (9) 1731-41.

[15] Suh HK, Park SW, Lee CS. (2009). Effect of grouped-hole nozzle geometry on the improvement of biodiesel fuel atomization characteristics in a compression ignition engine, Proc. IMechE Part D J. Auto. Eng. 223 1587-1600.

[16] Karra PK, Kong SC. (2009). Experimental study on effects of nozzle hole geometry on achieving low diesel engine emissions, J. Eng. Gas Turbines Power 132 (2)

[17] Pickett, L. M., C. L. Genzale, G. Bruneaux, L.-M. Malbec, L. Hermant, C. Christiansen and J. Schramm (2010). Comparison of Diesel Spray Combustion in Different High- Temperature, High-Pressure Facilities. SAE Technical Paper 2010-01-2106.

[18] Crua, C. (2002). Combustion processes in a diesel engine. SCEM/CAE. University of Brighton, Brighton. Ph.D.: 271.

[19] Stanton DW, Lippert AM, Reitz RD, Rutland CJ. (1998). Influence of spray-wall interaction and fuel films on 
cold starting in direct injection diesel engines (SAE Paper 982584). SAE Trans J Fuels Lubric 107,1540-63

[20] Kashdan JT, Mendez S, Bruneaux G. (2007). On the origin of unburned hydrocarbon emissions in a wallguided, low NOx diesel combustion system (SAE 2007-01-1836). SAE Trans J Fuels Lubric 116, 234-57

[21] Naber JD and S. DL (1996). Effects of gas density and vaporization on penetration and dispersion of diesel sprays. SAE Paper 960034.

[22] Siebers DL. (1999). Scaling liquid-phase fuel penetration in diesel sprays based on mixing-limited vaporization (SAE Paper 1999-01-0528). SAE Trans J Engines 108, 703-28

[23] Morgan R, Wray J, Kennaird DA, Crua C, Heikal MR. (2001). The Influence of Injector Parameters on the Formation and break-Up of a Diesel Spray, SAE Technical Paper 2001-01-0529

[24] Zhang A, Montanaro A, Allocca L, Naber J, Lee S-Y. (2014). Measurement of Diesel Spray Formation and Combustion upon Different Nozzle Geometry using Hybrid Imaging Technique, SAE Paper 2014-01-1410

[25] Badock C, Wirth R, Tropea C. (1999). The influence of hydro grinding on cavitation inside a diesel injection nozzle and primary break-up under unsteady pressure conditions, in: Proc. 15th ILASS-Europe 99, Toulouse, July 5-7, Toulouse (France), 1999.

[26] Ganippa LC, Andersson S, Chomiak J, Matsson J. (2003). Combustion characteristics of diesel sprays from equivalent nozzles with sharp and rounded inlet geometries, Combustion Science and Technology 175 (6) 1015-32

[27] Lefebvre, A. H., (1989). Atomization and Sprays. Taylor \& Francis, ISBN 0-891116-603-3

[28] Som S, Ram'irez AI, Longman DE, Aggarwal SK. (2011). Effect of nozzle orifice geometry on spray, combustion, and emission characteristics under diesel engine conditions, Fuel 90 (3) (2011) 1267-76

[29] Petkar, R. M., C. A. Kardile, P. V. Deshpande, R. Isenburg and R. Soorajith (2004). Influence of Increased Diesel Fuel Spray Velocities and Improved Spray Penetration in DI Engines. SAE Paper 2004-010031.

[30] Montanaro, A., Allocca L, Johnson J, Lee S-Y, Naber J, Zhang A. (2013). Influence of the Nozzle Geometry of a Diesel Single-Hole Injector on Liquid and Vapor Phase Distributions at Engine-Like Conditions. SAE paper 2013-24-0038.

[31] Bougie, Tulej BM, Dreier T, Dam N, Meulen JT. Gerber T. (2005). "Optical diagnostics of diesel spray injections and combustion in a high-pressure high- temperature cell." Appl Phys B 80, 1039-1045. 


\title{
Spray/wall interaction analysis on an ECN single-hole injector at diesel-like conditions through Schlieren visualization
}

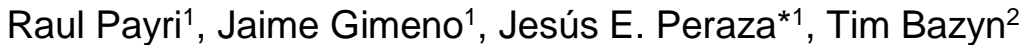 \\ ${ }^{1}$ CMT Motores Térmicos. Universitat Politècnica de València, Spain \\ ${ }^{2}$ Caterpillar Inc. Peoria, Illinois, USA \\ *Corresponding author: jepeav@mot.upv.es
}

\begin{abstract}
To continuously improve CFD models which simulate spray evolution, breakup and evaporation mechanisms, it is helpful to validate them with results obtained by experimental research. In the present study, a mono-orifice target nozzle from Engine Combustion Network, referred to as Spray D, was investigated at conditions of spray-wall interaction, which actually is a real situation in internal combustion engines that is not frequently analyzed by visualization. A Photron SA-X2 high-speed camera was employed to record the vapor phase development of the spray in an inert atmosphere using a Schlieren imaging single-pass setup. The experiments show that the spreading of the spray along the wall has a behavior fairly similar to penetration at free-jet situations, especially regarding to its susceptibility to the operating conditions and its proportionality to the square root of time once the spray reaches a steady regime interacting with the wall. Furthermore, the spray film thickness was measured at three distances from the spray-wall impact point during the injection event, thereby characterizing that parameter both spatially and temporally. The tests were carried out in a constant pressure-flow facility able to reproduce pressure and temperature conditions, similar to those seen into a diesel engine. In order to observe the behavior of the spray colliding with a wall within this test rig, a system capable to being fitted into it and to holding a fused quartz wall at different injector tip-wall distances and impingement angle configurations, was designed and employed.
\end{abstract}

\section{Introduction}

At present, the study of the spray-wall interactions into the field of transportation systems is increasingly relevant in a world aimed at reducing the displacement per cylinder of the internal combustion engines. This phenomenon plays a fundamental role in the mixing and evaporation of fuel[1]-[3], combustion behaviour and the formation of pollutant emissions[4]-[6]. Despite the growing interest around this field and the various efforts in its research [1][6], the impact of spray with a wall is a subject of difficult analysis both theoretically and experimentally due to the highly transient nature of the spray and the high spatio-temporal variability of the event. Because of this, its effects on the engine performance are not entirely known. On the one hand, the incidence and accumulation of fuel in the cylinder walls can lead to the formation of a fuel film that worsens combustion, promotes the emission of carbon monoxide and unburned hydrocarbons and involves energy losses given the increase of heat transfer [7]. On the other hand, the impact of the spray with a surface tends to improve the mixing of the fuel in the air due to both the impact and the subsequent expansion of the front of the jet. The complexity of the jet-wall impact phenomenon has to be added to the overall complexity of the injection-combustion processes, which, although they have been extensively studied in 'free jet' conditions [7]-[9] remain an active area of research.

In the literature there are a large number of experimental studies aimed at understanding physics in the spray-wall collision, in applications which are extrapolable to diesel engines. The distance from the tip of the nozzle to the surface affects the spray-wall interaction [10], since the impact conditions depend on the spray momentum and the energy exchange between the jet and the surrounding air until it hits the wall. Also, the orientation of the jet, or angle of incidence with respect to the wall also modifies the interaction mechanisms, as it changes the balance of forces during impact [11], [12]. High injection pressures increase the collision velocity, which makes the droplets smaller and improves atomization after the impact [13]. The experimental analysis of surface jet impact has been accompanied in recent years, by the development of models oriented to study both the macroscopic behavior of the jet-wall impact and the simulation of the microscopic interaction of isolated droplets [10], [12], [14]. However, there is a large number of processes that are not sufficiently known to achieve certain accurate numerical predictions, such as evaporation of the fuel film [14].

The Engine Combustion Network (ECN) is an international open group which promotes the collaboration and sharing of high quality data between scientists and institutions involved to the engines research field [15]. In order to provide a coherent and comparable database, nominally identical injectors are commonly tested at determined target conditions. This investigation attempts to conduct an experimental study to better understand the spray-wall interaction at high injection pressures under real engine conditions, employing the ECN Bosch injector referred to 
as 'Spray D'. The work characterizes the geometrical evolution of a spray after the impact with a quartz flat plate in terms of the penetration along the wall and the thickness of the spray with respect to the wall. Those parameters were obtained on the vapor phase of the spray, through the lateral view of the collision by the Schlieren optical technique. The study was conducted at several operating conditions and wall location-inclination arrangements.

\section{Material and methods}

\section{Experimental Vessel}

The experiment were performed in a high temperature and high pressure chamber which can be defined as a constant-pressure flow facility. The test section has three large windows to allow optical access to the injection process. Furthermore, the chamber is able to reproduce the in-cylinder thermo-dynamic conditions present in a Diesel engine, up to a maximum pressure of $15 \mathrm{MPa}$ and a maximum ambient temperature of $1000 \mathrm{~K}$. Although there are several test rigs over the world capable of performing analogously [8], [16], nearly quiescent and steady thermodynamic conditions can be obtained within this particular chamber. A detailed description of the vessel can be found in [7], [9], [17]. In this test rig it is possible to study the spray development either in a standard air atmosphere or with $\mathrm{O}_{2} / \mathrm{N}_{2}$ mixtures with different proportions, enabling to simulate exhaust gas recirculation conditions by decreasing the oxygen concentration of the charge. In this study, the vessel was operated at inert atmosphere conditions, filling it totally with nitrogen which flows in a closed loop to guarantee the evaporative but non-reacting desired scenario.

\section{Injection System}

The injector employed to carry out this study is referred to as Spray D \#209103 according to the Engine Combustion Network dataset. It is an axial single-hole Bosch 3-22 injector with an outlet diameter of $192 \mu \mathrm{m}$ [18], a k-factor of 1.5 whose rounded nozzle entrance and a convergent shape is oriented to avoid the cavitation phenomenon. The geometrical features of the Spray D can be found on the ECN webpage [15] and it has been hydraulically characterized in previous studies [9], [19], [20]. The injection setup consisted of a conventional Common-Rail system, which is mainly constituted by a standard rail with pressure regulator and a high pressure volumetric Bosch CP3 pump which is driven by an electric motor. The injector was kept at $363 \mathrm{~K}$ using an injector holder engineered to have a continuous ethylene glycol flow at a constant and controlled temperature running in a parallel circuit in contact with the injector during all the testing time. The injector was inserted in the test rig by this holder and connected to the common rail with a high pressure line. Moreover, the injection system is controlled by the ECU directly and all parameters are digitally inputted.

\section{Wall Supporting System}

Several 'free-jet' experiments have been carried out previously in the test rig [7]-[9], [19]. However, for this type of tests involving a wall obstructing the free path of the jet, it was necessary to design a system to reliably support the wall at determined angles and distances from the injector tip achieved with high accuracy. It was required a modification on the conventional injector protective cap (number 1 in Figure 1-left) of the test rig so that the wall supporting system could be screwed to it. Two folded sheets (2) are attached to the protective cap and also are holding two fixed ' $U$ ' shaped structures (3) which are responsible to bear the wall arrangement at its determined distance-angle configuration. Those different configuration are possible due to six pairs of exchangeable lateral frames (4). Each set of those frames is used depending on the desired distance-angle arrangement. They are screwed to a wall holder (5) which protrudes slightly on the bottom to support the JGS1 fused quartz wall (6). The support is further held in place by four claws (7) made of bent sheet that press the wall against the holder by its corners. The impact available surface of the wall is of $60 \times 100 \mathrm{~mm}^{2}$ and its thickness is $10 \mathrm{~mm}$.
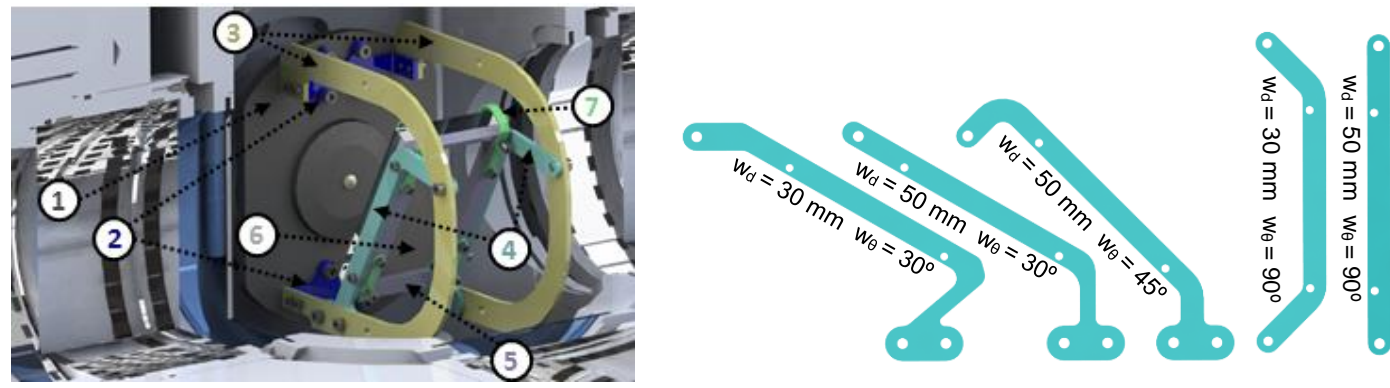

Figure 1. Right: Parts of the wall mounting system. Left: Lateral frames geometries. 


\section{Test Matrix}

Test conditions can be found summarized in Table 1. Most of them are common target conditions of the ECN [15], along with other parametric values combinations in order to embrace a wide range of test points and have a better comprehension of the parameter variation effects. Regarding to the wall position, the tests were performed with different configurations of distance from the injector tip to the plate $(\mathrm{Wd})$ and wall angle to the horizontal $(\mathrm{w} \theta)$, also detailed in Table 1. Those configurations were possible by changing the pair of frames (indicated with the number 4 in Figure 1-left), whose geometry depends on the desired configuration as shown in Figure 1-right.

\begin{tabular}{lll}
\multicolumn{3}{c}{ Table 1. Test conditions of the experiment } \\
\hline \hline Parameter & Values & Units \\
\hline \hline Fuel & Diesel & - \\
Energizing time & 2.5 & $\mathrm{~ms}$ \\
Injector tip temperature & 363 & $\mathrm{~K}$ \\
Ambient temperature $\left(T_{\text {amb }}\right)$ & $800-900$ & $\mathrm{~K}$ \\
Ambient density $\left(\rho_{\text {amb }}\right)$ & $22.8-35$ & $\mathrm{~kg} / \mathrm{m}^{3}$ \\
Injection pressure $\left(p_{\text {rail }}\right)$ & $50-100-150-200$ & $\mathrm{MPa}$ \\
Injector-wall distance $\left(w_{d}\right)$ & $30-50$ & $\mathrm{~mm}$ \\
Wall horizontal angle $\left(w_{\theta}\right)$ & $30-45^{\star}-90$ & $\mathrm{deg}\left(^{\circ}\right)$ \\
\hline
\end{tabular}

\section{Optical Setup}

In the present work, the experiments were performed using a Schlieren single-pass setup. Schlieren imaging technique consists of directing a beam of parallel light rays through a region of interest, which in this case is limited by the $128 \mathrm{~mm}$ vessel windows diameter. As these rays pass through a medium with density gradients, some of them are deflected. By collecting the resulting beam and directing it to a camera, a shadowgraph is obtained, in which the different pixel intensities are representative of different refractive indexes in the region.

A scheme of the optical setup is shown in Figure 2, where can be seen the entire light beam path from its source to the high-speed camera. The light source is a white-light $150 \mathrm{~W}$ halogen lamp with an incorporated diaphragm. The lamp is placed at the focal length of a collimating mirror that reflect the light rays making them parallel to each other. To reduce beam straightening, the incident angle of the lamp respect to the mirror was minimized. Subsequently, the beam is directed to the high pressure and high temperature vessel, where it is deflected as a result of the different densities of the interest region. After the chamber, the beam is collected by a $150 \mathrm{~mm}$ collimating lens, at whose focal distance is placed an adjustable Fourier filtering diaphragm, just before the Photron SA-X2 camera. The Fourier diaphragm is employed to filter or discard how much light may to go to the camera, controlling the sensitivity of the technique.

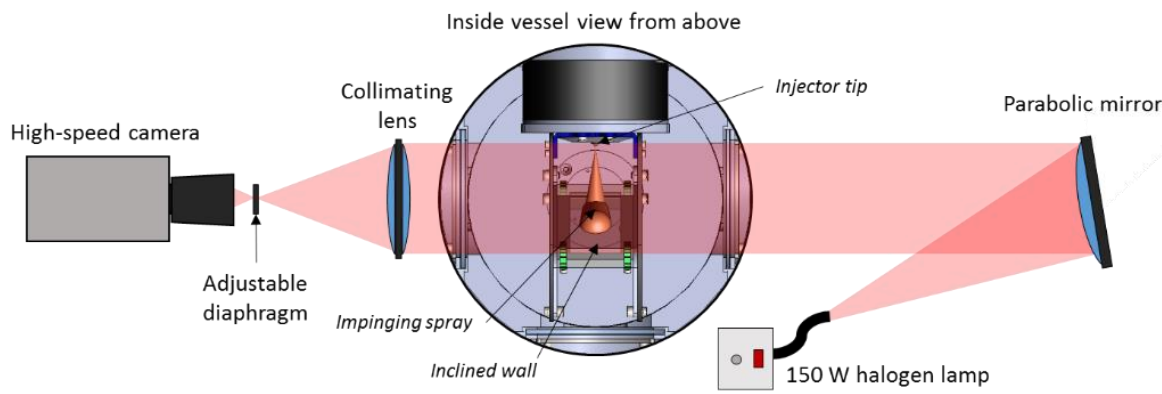

Figure 2. Scheme of the Schlieren optical setup

Image Recording and Processing

The Photron SA-X2 camera was configured to record at an acquisition speed of 40000 frames per second, which represent a time gap between images of $24 \mu \mathrm{s}$. This slight step allows detection of penetrations near to $0.4 \mathrm{~mm}$ on average, which represent good resolution to observe the spray development with time and the capability of estimate precisely the start of the injection with respect to the energizing moment given by the signal of the trigger. This recording speed could be achieved thanks to the camera's own capacity using a shutter time of $1.01 \mu$ s (time selected to provide a good compromise between sensitivity and margin of safety to avoid saturation and excessive image noise) and a spatial resolution of $512 \times 584$ pixels, which was enough to cover the spray path from the injector tip to near the wall limits without sacrificing too much camera speed. Since the pixel-per-mm relation of the images was equal to 5.88, this resolution represents an approximated image size of $87 \times 100 \mathrm{~mm}$.

This work is licensed under a Creative Commons 4.0 International License (CC BY-NC-ND 4.0). 
After recording, the information about spray evolution was extracted applying a contour-detecting processing methodology employing in-house software. First of all, it is important to highlight that the image background captured through the Schlieren technique is very irregular as seen in the leftmost image in Figure 3 , due to the heterogeneities in the temperature (i.e. density) of the nitrogen that fills the chamber, and which continuously enters and exits from it. In tests of the 'free-jet' kind, the background correction is more or less easy by taking it as the image acquired before the start of injection, and subtracting it, arithmetically, from spray images. This method is based on the assumption that, despite the background is constantly moving, this movement is slow contrasted to the spray velocities, so the background can be considered steady in the time scale of an injection event. However, the wall system inside the chamber is just after the nitrogen entry location in the chamber, and this makes the gas flow pattern more turbulent and disordered. This amplifies considerably the background noise and makes that its inhomogeneities due to the gas density gradients in the gas cannot be considered always as static between two consecutive images. The solution adopted for this issue was to average the images of all repetitions ( 8 for each condition) of the injection event, removing largely the variation of the background. Figure 3 center and right images show a comparison of an averaged image and a single shot and how the background noise affects the detected contour.
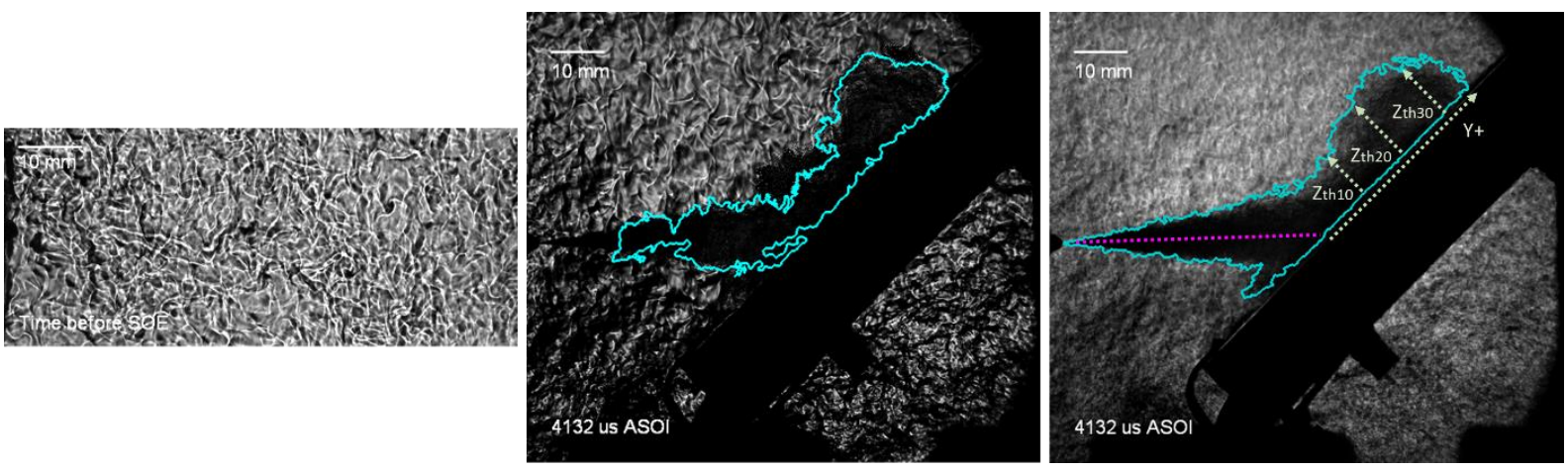

Figure 3. Comparison of backgrounds and contours at $T_{\text {amb }}=900 \mathrm{~K} ; \rho_{a m b}=35 \mathrm{~kg} / \mathrm{m}^{3} ; p_{\text {rail }}=100 \mathrm{MPa} ; w_{d}=50 \mathrm{~mm} ; w_{\theta}=45^{\circ}$. Left: Free-jet test sample before start of energizing. Center: Single-shot image of one repetition. Right: Rep-averaged image.

(Both spray contours were obtained keeping the same processing configuration)

In the following step, the repetition-averaged image is masked in the region back to the wall impact surface, in order to only get the spray development region of interest and reduce calculation time. Next, the image is inverted to have the spray as the high intensity area and the threshold is calculated as a certain percentage of the dynamic range of the image [9]. Then, some small areas which appear as result of the background noise are erased by erodingdilating filtering methods according to spray connectivity criteria. Once the spray contour has been determined, its characteristics are obtained by its analysis. In this work, the criteria followed to calculate the geometrical parameters of the spray were:

- $\quad$ Free spray penetration (S): The penetration of the spray before its impact with the wall is calculated as the position of furthest point of the contour respect to the nozzle exit.

- $\quad$ Spray spreading along the wall $\left(Y_{+}\right)$: The spray penetration along the wall is calculated as the distance between the 'collision point' and the furthest contour point in the direction of the top of the wall (considering as 'collision point' the interception between the axial spray axis and the wall plane, and not as necessarily the first point of the wall that is reached for the spray, which ideally differs of the first definition depending on the wall angle). In Figure 3 right image is shown this dimension as $Y_{+}$.

- $\quad$ Spray film thickness $\left(Z_{\text {th }}\right)$ : The thickness of the spray was measured at three consecutive places from the collision point with a distance of $10 \mathrm{~mm}$ between them. At those measuring points, the film thickness is calculated as the normal distance between the wall and the furthest contour point as seen in Figure 3.

\section{Results and discussion}

Free Penetration and Spreading onto the Plate

Figure 4 top row shows the free spray penetration and spray-wall spreading evolution with time since the start of injection changing all variables but the wall configuration, which is in this case perpendicular to the spray axis. It can be observed that the spray behavior along the wall is quite analogous to the free penetration pattern and it is affected equivalently by the parametric variations. Ambient temperature does not seems to represent a significant This work is licensed under a Creative Commons 4.0 International License (CC BY-NC-ND 4.0). 
influence on both spray advancements. On the other hand, the density has a strong effect, which seems to become higher while the spray goes over the plate. Also, the injection pressure has, as expected, a positive effect on momentum, making the spray penetrate and spread onto the wall more. However, the ASOI (after start of injection) temporal reference does not show those spray-wall spreading trends are independent of the free penetration, since the sooner the spray-wall collision, the earlier the start of the $Y_{+}$development.
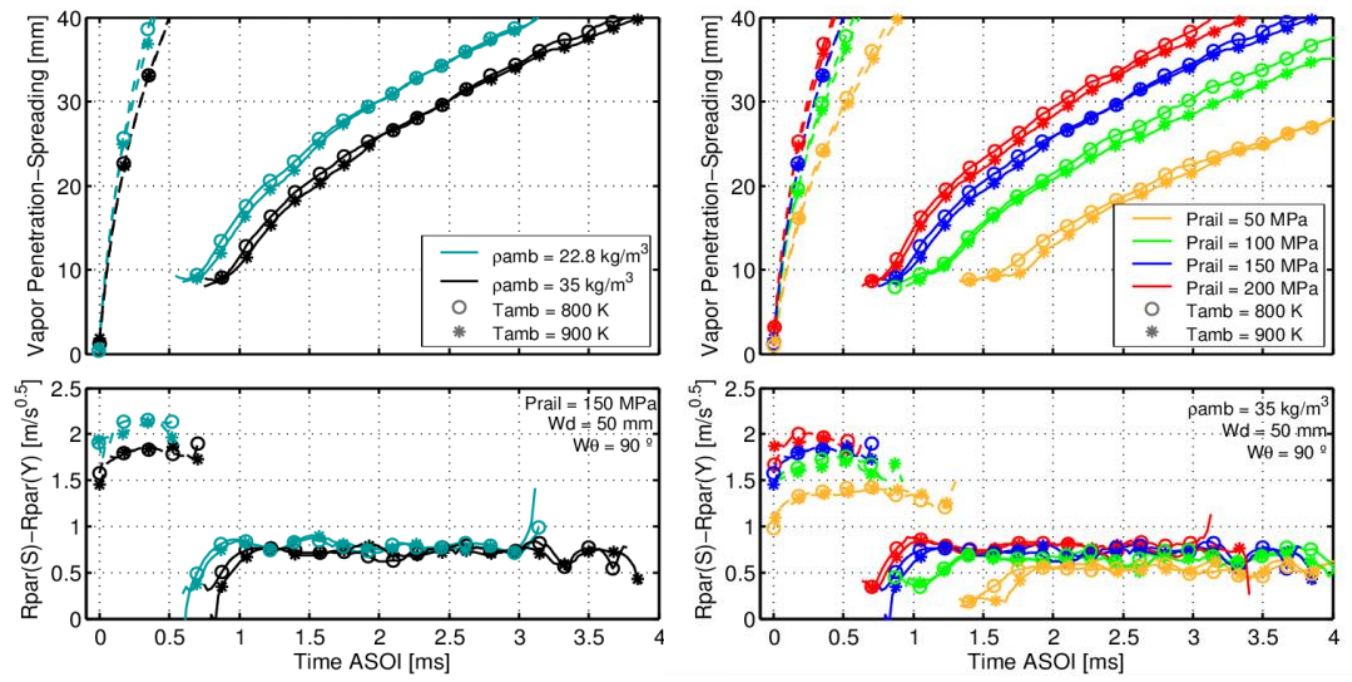

Figure 4. Free penetration (striped lines) and spreading along the wall (solid lines) together with their R-parameters at different gas and injection conditions and $w_{d}=50 \mathrm{~mm} ; w_{\theta}=90^{\circ}$. Left column: Ambient density and temperature comparison at $p_{\text {rail }}=150$ $\mathrm{MPa}$. Right column: Injection pressure and temperature contrast at $\rho_{\mathrm{amb}}=35 \mathrm{~kg} / \mathrm{m}^{3}$.

A way to express the spreading in a time-independent form and to compare its behavior with the well-known one of the free penetration, is to convert it in terms of its time squared root derivative $\partial S(t) / \partial \sqrt{ } t$, as have been made in previous works with the free penetration under the name of $R$-parameter [9], [19]. In those works, was demonstrated how, accordingly with literature, the steady spray vapor penetration of an inert spray is proportional to the time raised to the power of 0.5 , making possible to obtain a constant value referred to as $R$-parameter, independent of the time and of the penetration evolution. In Figure 4 bottom row, the squared root derivative of free penetration $\operatorname{Rpar}(S)$ and of spray-wall spreading $\operatorname{Rpar}(Y)$ are plotted. As is shown, $\operatorname{Rpar}(Y)$ also seems to reach a constant value at its stable stage. This also occurs for $\operatorname{Rpar}(S)$ although it is difficult to observe given the interruption in the evolution of the free jet by the plate. However, the trends are clear. The velocities reached by the free jet are much higher than the impinging spray velocities due to the loss of kinetic energy when hitting the wall and the spreading friction. Also, the effects of density, temperature and injection pressure seems to be the same on these timeindependent parameters for direct free jet penetration and spray-wall spreading.

On the other hand, Figure 5 shows how those same parameters are affected by the wall configuration and, again, by the density of the surroundings. As the left column plots show, the spreading onto the wall is higher for shorter distances between the injector and the plate. However, the earlier impact in the $30 \mathrm{~mm}$ case is the most important factor, so studying the $\operatorname{Rpar}(Y)$ is an appropriate approach to determine fundamental differences. Observing this parameter, it can be seen that the $R$-parameter is quite similar for different injector-wall distances, meaning that, even when the spreading and its velocity (the spreading curve slope) seem to differ varying wd in the left-top graph, it is a product of the different collision moments and free velocities of the spray before that instant (considering that at shorter distance from the injector tip, the spray must impact with a higher velocity). Figure 5 right column presents the effect of the wall inclination. The first remarkable thing which does not happen in the inclined plates, is that the spreading of the $90^{\circ}$ case starts in a determined value and with an inflecting behavior (also seen in Figure 4). This is because of the very width of the free jet, which makes that, at the moment of the impact of the spray tip, a short part of the wall has been already covered by the spray. This is supported by the fact that this 'initial value' given by the width seems to be larger for higher densities. This effect of the spray width is mitigated in the direction of $Y$ for the inclined plates. Furthermore, the spray-wall spreading and its $R$-parameter are strongly affected by the inclination. For the normal wall, the momentum is distributed in all directions, resulting in a spreading and an $\operatorname{Rpar}(Y)$ considerably smaller than for inclined walls. Between $30^{\circ}$ and $45^{\circ}$ the differences are not as great, because for both cases the spray momentum has as predominant direction the same as $Y_{+}$. However, observing the last and more stabilized values of $\operatorname{Rpar}(Y)$ also show a slight trend to be higher for the lower angle accordingly with the last 
deduction. It is not necessary to mention that the free-spray parameters in this cases are not affected by the wall configuration.
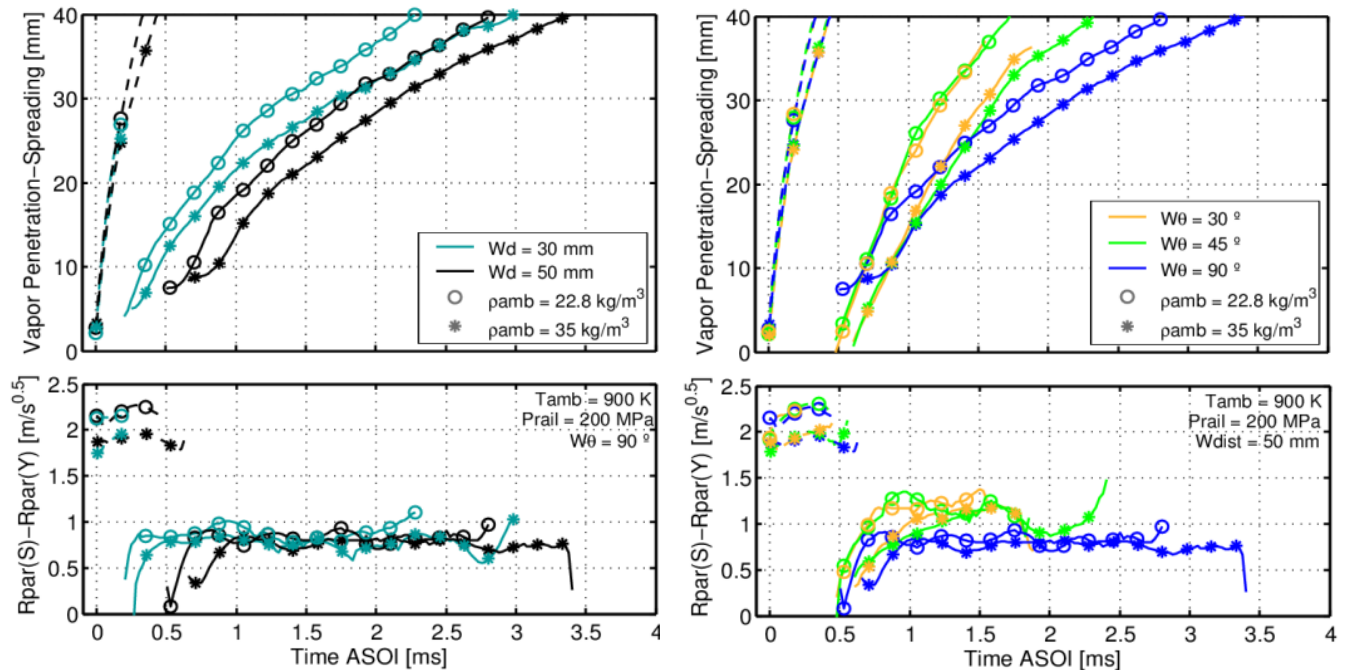

Figure 5. Free penetration (striped lines) and spreading along the wall (solid lines) together with their $R$-parameters at different wall configurations at $T_{a m b}=900 \mathrm{~K} ; p_{\text {rail }}=200 \mathrm{MPa}$. Left column: Ambient density and wall distance contrast at $w_{\theta}=90^{\circ}$. Right column: Ambient density and wall angle comparison at $\mathrm{w}_{\mathrm{d}}=50 \mathrm{~mm}$.

\section{Spray Film Thickness}

After reviewing the spreading results, the $w_{d}=30 \mathrm{~mm}-w_{\theta}=30^{\circ}$ configuration was considered by the authors as the most likely to quickly achieve steadiness in the flow onto the wall since the spray reaches the plate earlier and the transient is less abrupt when deflecting the jet at the lower angles. Figure 6 shows the spray thickness development measured at 10; 20 and $30 \mathrm{~mm}$ from the 'collision point', varying different operating conditions (in columns from left to right: density, injection pressure and gas temperature). For this wall configuration, it can be seen how the general tendency is to start growing once the spray reaches the measuring point to a maximum due to the spray front vortex, and then to slightly stabilize in a constant value. Observing the left column, it can be appreciated that, similar to the spray angle in free-jet conditions, but in a not so strong way, the density seems to widen the spray thickness as a result of the enhancement in gas entrainment into the spray. For higher densities, also the 'bump' in the thickness curves seems to last longer, however the stable final value is quite similar. With respect to the different injection pressure column of Figure 6, it shows that the thicknesses measured near the impact point, tends to the same value; but when measured further, the lower the injection pressure, the thicker the spray is. Nevertheless, in general, and similar to what happens for the spreading, the gas temperature seems to have a negligible influence on the film thickness, independently of how far it is measured. These effects on spray thickness also shown to be stronger the further they are measured, with the $Z_{\text {th10 }}$ being the distance in which the
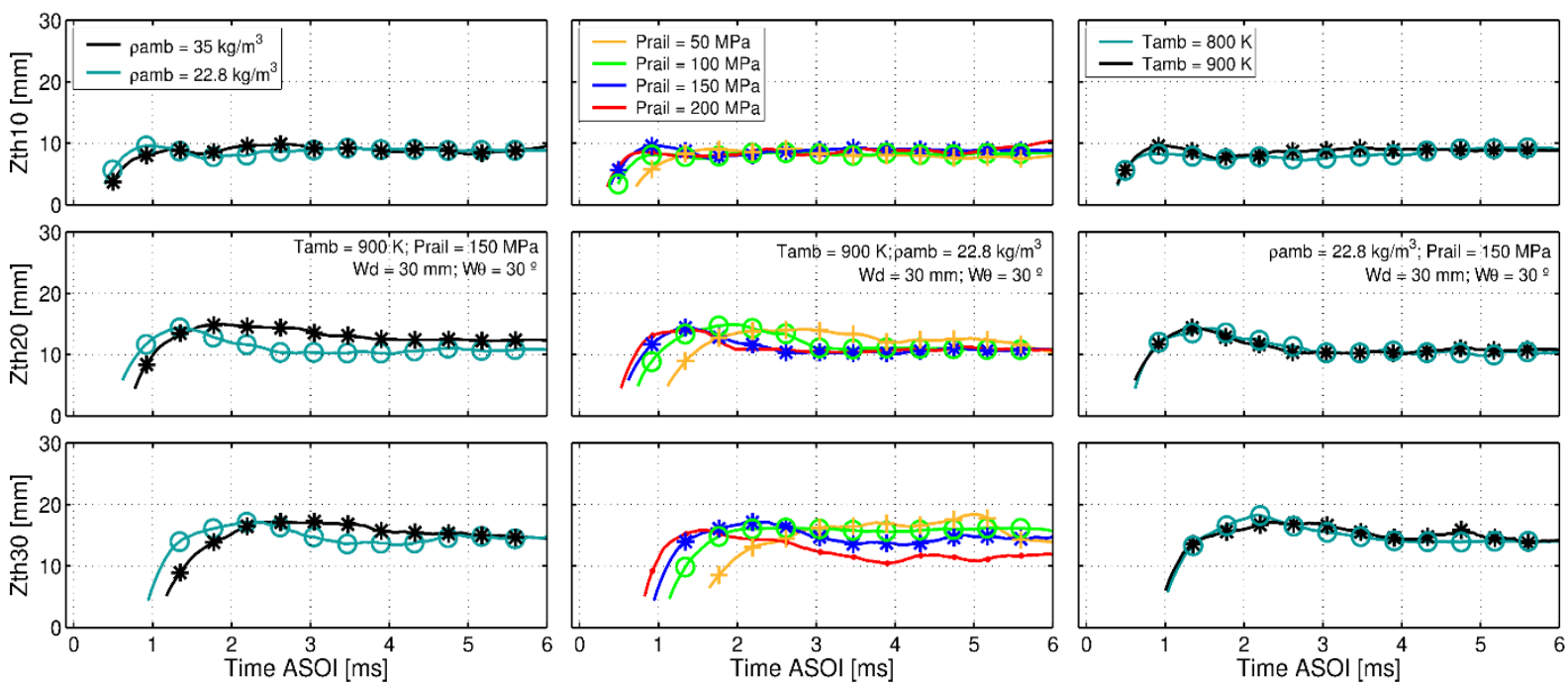

Figure 6. Spray thicknesses $Z_{\text {th } 10}, Z_{\text {th20 }}$ and $Z_{\text {th } 30}$ at a fixed wall configuration $\left(w_{d}=30 \mathrm{~mm} ; w_{\theta}=30^{\circ}\right)$. Left column: Varying ambient density. Center column: Changing injection pressure. Right column: At different ambient temperatures.

This work is licensed under a Creative Commons 4.0 International License (CC BY-NC-ND 4.0). 
operating conditions seems to affect the least. It has to be considered that before the stabilization of the impinging spray, the differences in $Z_{\text {th }}$ with the conditions, are also influenced by the difference in the moments when the spray collides with the wall and the time that takes to become steady. This effect is incremented considering the time that the spray takes to reach the measuring point. For instance, lower prail sprays not only take longer to impact with the plate, but also take longer to reach the measurement point, and this is more noticeable for the further locations as the one of $Z_{\text {th }}$.

Figure 7 shows the influence of the wall positioning on the film thickness. In the left set of plots, can be seen how despite the earlier impact at of the $30 \mathrm{~mm}$ configuration, the spray thickness takes longer to stabilize. This could be explained by the higher velocity of collision of the injection with the nearer wall, which makes the impact and spreading processes more turbulent and initially more unstable. On the other hand, Figure 7 right column shows different angle configurations, where it can be seen how the steady value of the thickness is pretty similar for all of them, but it is reached differently by each. The perpendicular wall case, exhibits a slow stabilization while the inclined ones seems to be significantly faster to stabilize. Even between $30^{\circ}$ and $45^{\circ}$, it can be noted how the $30^{\circ}$ case stabilizes faster, probably because the deviation of the flow is less and causes the least disturbances in the spray evolution. The greater the angle, the more transitory the behavior of the spray-wall impact is.
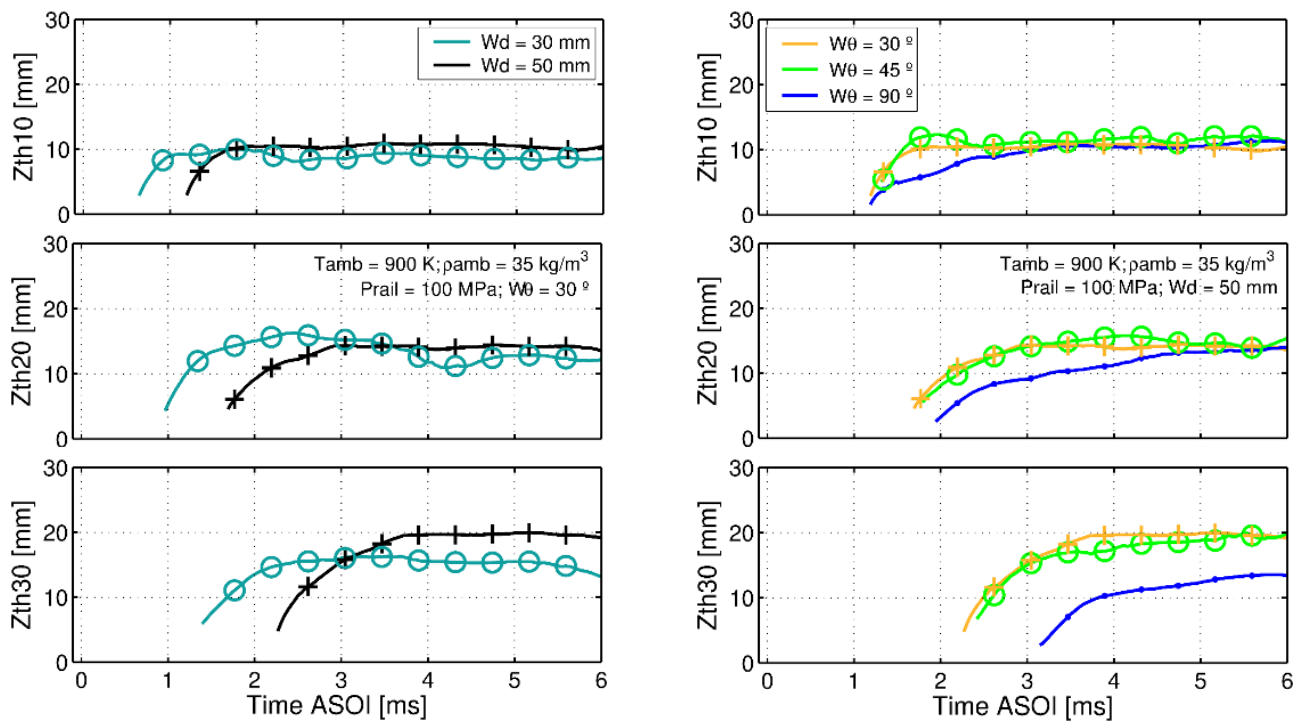

Figure 7. Spray thicknesses $Z_{\text {th10 }}, Z_{\text {th20 }}$ and $Z_{\text {th30 }}$ at a determined ambient and injection condition $\left(T_{\text {amb }}=900 \mathrm{~K} ; \rho_{\text {amb }}=35 \mathrm{~kg} / \mathrm{m}^{3}\right.$; $p_{\text {rail }}=100 \mathrm{MPa}$ ). Left column: Changing injector-wall distance. Right column: Varying wall angle.

\section{Conclusions}

The effects of ambient and injection conditions and wall positioning were analysed. Results show that the spreading along the wall and the 'free' penetration are both affected similarly by gas density, temperature and injection pressure. Since a unique time start reference does not define the wall interaction phenomenon as easily and accurately as the ASOI for the free-jet stage, those parametric behaviors were also studied employing the definition of $R$-parameter. The wall spreading is also strongly affected by the wall angle, due to change in the spray momentum deviation. Injector-wall distance seems not to have an important influence on spreading. The thickness of the spray along the wall was measured at different locations and inclinations, determining that, broadly speaking, it can be described by an initial thick region followed by stabilization at a thinner value. This steady value is higher at further measuring distances due to its progressive expansion. This metric was studied under several parametric changes, determining how it is affected by different variables.

Although a flat wall is a simplified geometry compared with a typical piston-bowl into a reciprocating engine, the possibility of making these kinds of experiments at real-engine conditions is an opportunity to study a fundamental factor on combustion such as the mixing process, in terms of the vapor spray geometry; considering the existing phenomenon of spray-wall interaction. This study represents a huge potential to improve the understanding, not only experimentally through big test rigs, but numerically also as a next step, of the processes that concern the diesel engines research.

\section{Acknowledgements}

The authors would like to thank Caterpillar Inc. for their cooperation during the project, as well as the support provided by the "Ministerio de Economía y Competitividad" of the Spanish Government in the frame of the project This work is licensed under a Creative Commons 4.0 International License (CC BY-NC-ND 4.0). 
TRA2015-67679-c2-1-R. Furthermore, the collaboration of Alberto Viera ${ }^{1}$ and Borja Hurtado ${ }^{1}$ in the measurements and setup of the experiment is thanked.

\section{Nomenclature}

ASOI after start of injection

CFD Computational Fluid Dynamics

ECN Engine Combustion Network

Prail injection pressure

$R \operatorname{Rar}(X)$ squared root of ' $X$ respect of time $\left[\mathrm{m} \mathrm{s}^{-0.5}\right]$

$S \quad$ free-jet penetration [mm]

Tamb ambient temperature $[\mathrm{K}]$ $\mathrm{t} \quad$ time

$\mathrm{W}_{\mathrm{d}} \quad$ injector tip-wall axial distance [mm]

$W_{\theta} \quad$ wall angle respect to the injector axis [deg]

$Y_{+} \quad$ spray spreading along the wall [mm]

$Z_{\text {th }} \quad$ spray thickness respect to the plate $[\mathrm{mm}]$

$\rho_{a m b} \quad$ ambient density $\left[\mathrm{kg} \mathrm{m}^{-3}\right]$

\section{References}

[1] G. E. Cossali, A. Coghe, and G. Brunello, "Effect of Spray-Wall Interaction on Air Entrainment in a Transient Diesel Spray," in SAE Technical Paper, 1993.

[2] K. Li, P. Dong, T. Matsuo, B. Shi, Y. Ogata, and K. Nishida, "Characteristics of Diesel Spray Flame under Flat Wall Impinging Condition --LAS, $\mathrm{OH}^{*}$ Chemiluminescence and Two Color Pyrometry Results," in SAE Technical Paper, 2014.

[3] J. Senda, Y. Tanabe, H. Fujimoto, and Y. Fukami, "Vizualization of Evaporative Diesel Spray Impinging Upon Wall Surface by Exciplex Fluorescence Method," in SAE Technical Paper, 1992.

[4] A. Mohammadi, Y. Kidoguchi, and K. Miwa, "Effect of Injection Parameters and Wall-Impingement on Atomization and Gas Entrainment Processes in Diesel Sprays," in SAE Technical Paper, 2002.

[5] T. Tomonaga, K. Murai, T. Takano, and H. Sami, "A Study on Combustion Behavior of a Diesel Fuel Spray Impinging on a Wall," in SAE Technical Paper, 1996.

[6] J. E. Dec and D. R. Tree, "Diffusion-Flame / Wall Interactions in a Heavy-Duty DI Diesel Engine," in $S A E$ Technical Paper, 2001.

[7] R. Payri, J. Gimeno, M. Bardi, and A. H. Plazas, "Study liquid length penetration results obtained with a direct acting piezo electric injector," Appl. Energy, vol. 106, no. JUNE, pp. 152-162, 2013.

[8] M. Bardi, R. Payri, L.-M. Malbec, G. Bruneaux, L. M. Pickett, J. Manin, T. Bazyn, and C. L. Genzale, "Engine Combustion Network: Comparison of Spray Development, Vaporization, and Combustion in Different Combustion Vessels," At. Sprays, vol. 22, no. 10, pp. 807-842, 2012.

[9] J. Gimeno, G. Bracho, P. Martí-Aldaraví, and J. E. Peraza, "Experimental study of the injection conditions influence over n-dodecane and diesel sprays with two ECN single-hole nozzles. Part I: Inert atmosphere," Energy Convers. Manag., vol. 126, pp. 1146-1156, 2016.

[10] K. Su and S. C. Yao, "Numerical studies of sprays impacting normally on an infinite plate," in $X V$ Congresso Brassileiro de Engenharia Mecânica, 1999.

[11] D. F. Durão, A. L. N. Moreira, and M. R. O. Panão, "The effect of a cross-flow on secondary atomization in multipoint fuel injection systems," 13th Int Symp Appl. Laser Tech. to Fluid Mech., no. January, pp. 112, 2006.

[12] J. Senda, M. Ohnishi, T. Takahashi, H. Fujimoto, A. Utsunomiya, and M. Wakatabe, "Measurement and Modeling on Wall Wetted Fuel Film Profile and Mixture Preparation in Intake Port of SI Engine," in SAE Technical Paper, 1999.

[13] U. Meingast, M. Staudt, L. Reichelt, and U. Renz, "Analysis of Spray / Wall Interaction Under Diesel Engine Conditions," SAE Tech. Pap. 2000-01-0272, no. 724, pp. 1-15, 2000.

[14] F. Birkhold, U. Meingast, P. Wassermann, and O. Deutschmann, "Analysis of the Injection of Urea-WaterSolution for Automotive SCR DeNOx-Systems: Modeling of Two-Phase Flow and Spray/Wall-Interaction," in SAE Technical Paper, 2006.

[15] ECN, "Engine Combustion Network," 2010. [Online]. Available: www.sandia.gov/ecn/.

[16] L. M. Pickett, C. L. Genzale, G. Bruneaux, L.-M. Malbec, and C. Christiansen, "Comparison of diesel spray combustion in different high-temperature, high-pressure facilities," SAE Int. J. Engines, vol. 3, pp. 156-181, 2010.

[17] R. Payri, J. M. Garcia-Oliver, M. Bardi, and J. Manin, "Fuel temperature influence on diesel sprays in inert and reacting conditions," Appl. Therm. Eng., vol. 35, no. March, pp. 185-195, Mar. 2012.

[18] R. Payri, J. Gimeno, J. Cuisano, and J. Arco, "Hydraulic characterization of diesel engine single-hole injectors," Fuel, vol. 180, pp. 357-366, 2016.

[19] R. Payri, F. J. Salvador, J. Gimeno, and J. E. Peraza, "Experimental study of the injection conditions influence over n-dodecane and diesel sprays with two ECN single-hole nozzles. Part II: Reactive atmosphere," Energy Convers. Manag., vol. 126, pp. 1157-1167, 2016.

[20] F. R. Westlye, M. Battistoni, S. A. Skeen, J. Manin, L. M. Pickett, and A. Ivarsson, "Penetration and combustion characterization of cavitating and non-cavitating fuel injectors under diesel engine conditions," SAE Tech. Pap. 2016-01-0860, p. 15, 2016. 


\title{
An Experimental Study of Diesel Spray Impingement on a Flat Plate: Effects of Injection Conditions
}

\author{
Xiucheng Zhu, Le Zhao, Zhihao Zhao, Nitisha Ahuja, \\ Jeffrey Naber, Seong-Young Lee* \\ Michigan Technological University, Houghton, MI, USA \\ *Corresponding author: sylee@mtu.edu
}

\begin{abstract}
Advanced injection strategies for internal combustion engines have been extensively studied although there still exists a significant fundamental knowledge gap on the mechanism for high-pressure spray interaction with the piston surface and chamber wall in the internal combustion engine. The current study focuses on providing qualitative and quantitative information on spray-wall impingement and its characteristics by expanding the range of operating parameters under engine-like conditions. Parameters considered in the experiment are ambient gas and fuel injection conditions. The test included the non-vaporizing spray at the different ambient density $(14.8,22.8$ and $30 \mathrm{~kg} / \mathrm{m}^{3}$ ) and injection pressure (1200, 1500 and $1800 \mathrm{bar}$ ) with the isothermal condition (ambient, and plate temperatures of $423 \mathrm{~K}$ ). The test was conducted in the constant-volume vessel with the 60 -degree impinging spray angle relative to the plate. The free spray and impinged spray properties were qualitatively analysed based on Mie and schlieren images. The results showed that the lower ambient density and higher injection pressure tended to result in relatively higher impinged spray height. The expanding shape of the impinged spray on the wall showed the oval shape.
\end{abstract}

\section{Keywords}

free spray; impinged spray; wall-impinged expanding spray; flat plate

\section{Introduction}

Internal combustion engines (ICEs) have been the dominant power supply for automobiles since the $20^{\text {th }}$ century and will keep playing an important role in transportation sectors in the coming decades. Increasingly stringent fuel consumption and emission standards are driving automotive research. Advanced injection strategies such as increasing injection pressure, multiple injections, injection timing control, and many others can enhance fuel efficiency in the application of direct injection spark ignition (DISI) engines and direct injection (DI) diesel engines. The DISI and DI diesel engines are able to achieve such higher efficiencies by means of better spray atomization and air-fuel mixing. Besides injection parameters mentioned above, the spray-wall interaction plays a critical role in fuel spray dispersion and subsequent combustion event [1-3]. The fuels used in ICEs undergo the vaporization process and mix with air before combustion. However, the fuel spray may impinge on the engine surfaces before it is fully vaporized [4]. The non-vaporizing spray possibly impinges on the cylinder head or liner in DI engines and even on the inlet valves in port fuel injected (PFI) engines. The spray-wall impingement usually forms a liquid film on the wall, which is referred to wall-wetting phenomenon [5]. The fuel deposition on the wall may survive during the combustion phase, resulting in producing unburned hydrocarbon $(\mathrm{HC})$ and particulate matter (PM) emissions [6-8]. Especially in cold operation (such as cold start and warm-up), the wall wetting in PFI engines becomes dominant mechanism for engine-out HC emissions [9-13]. When the spray droplets hit the wall, they may undergo the following scenarios: stick, rebound, and splash, depending on the Weber number and droplet impact dynamics [14]. It was also reported that the impinging process could enhance the air-fuel mixing [15]. In addition, a heated plate may also lead to the heating or secondary evaporation of the impinged droplets.

Early work in an optically accessible engine has characterized the spray-wall interaction phenomena and measured the after-collision spray properties such as droplet sizes and film thickness on wall. Siewert et al. [16] performed experiments in a 103-mm bore DI single cylinder diesel engine (reentrant-shaped piston bowl) to explore the effect of the calculated amount of liquid spray that misses the piston bowl. By changing spray impacting angle and injection timing, a different amount of liquid spray that misses the piston bowl was acquired in their study, which was highly linked to the $\mathrm{HC}, \mathrm{CO}$, and smoke formation. Stanglmaier et al. [5] experimentally investigated the effect of wallwetting on the $\mathrm{HC}$ emissions in an optical gasoline-fuelled SI engines. A spark-plug mounted directional injection probe was used to study the impingement effects on $\mathrm{HC}$ emissions independent from all other $\mathrm{HC}$ sources, by operating engine on pre-mixed liquefied petroleum gas (LPG). Their results argued the importance of the impingement location, which significantly influenced the $\mathrm{HC}$ emission generation regardless of the injection timing and coolant temperature.

Besides engine spray-wetting test, well-controlled constant volume chambers have been utilized to acquire a higher level of visualization access and identify precise boundary conditions near the wall surface such as film thickness 
and heat flux. Akop et al. [17] captured the entire injection event using high-speed shadowgraph and weighted the film mass on the wall to explore the adhesion characteristics of the diesel impinging spray. They found that the adhered mass kept constant by changing disk diameter from $25 \mathrm{~mm}$ to $50 \mathrm{~mm}$, and the thickness of the adhesion liquid became lower with increasing injection pressure. Furthermore, the ratio of adhered fuel mass to total injected mass tends to decrease with an increase of the disk inclination. Lindgren et al. [18] studied the spray-wall interactions in a spray chamber with simulated engine conditions using an AVL VisiScope. The images indicated that most of the reflected drops were carried away from the impinging point by means of a wall jet and very few drops were bounced away with a large reflection angle. Meanwhile, Phase Doppler Anemometry (PDA) was also employed to measure the size and velocity of droplets reflected from a wall. They observed a secondary break-up at the wall, resulting in smaller drops. A PDA and high-speed imaging methods were used by Montorsi et al. [19] in a high pressure and temperature spray rig to measure the surface temperature with three coaxial thermocouples. The measured temperatures were used to calculate heat fluxes using a one-dimensional transient heat conduction model. Four different breakup models (i.e. Reitz-Diwakar, Hsiang-Faeth, Pilch-Erdman, and Kelvin-Helmholtz (KH)Rayleigh-Taylor (RT), see Table 3 in Ref. [19]) were compared with their test results and showed sufficient agreement with experimental results except for the overestimation in liquid penetration.

Film formation is one of the important properties as a result of spray-wall impingement. Senda et al. [20] applied laser-induced fluorescence (LIF) to quantitatively measure the adhered fuel film on a flat glass under atmospheric pressure and room temperature. The adhered fuel measured by LIF showed almost $40 \%$ of the total injection quantity at $10 \mathrm{~ms}$ after the end of injection while the adhered fuel is relatively insensitive to injection duration. They also classified the radial spreading velocity into three stages: early constant velocity stage, gradually decreasing stage, and fully stop stage. Another optical method of measuring the spatial and temporal thickness of the wall liquid film is the Refractive Index Matching (RIM) technique. RIM utilizes the similar refractive indexes between glass/quartz and fuel to characterize the impingement deposition on the wall. The thickness of the wetted area on a roughed glass surface has a relationship with the scattering light intensity. Drake et al [6]. have used this method to measure the film thickness of the impinged spray and the total mass injected on a piston top in an optical engine to successfully establish a correlation between $\mathrm{HC}$ emissions/PM and the film thickness variation.

Montanaro et al. [21] studied the effects of the injection pressure and wall temperature on the atomization and vaporization processes in their constant volume chamber. High-speed Mie-scattering and schlieren diagnostics were used to capture the impinged liquid and vapor widths (maximum radial penetration) and maximum heights. They found that the impinged spray widths showed a proportional increase with wall temperature, and the heights for both impinged liquid and vapor were proportional to the injection pressure.

The objective of the current study is to experimentally explore the effects of the injection pressures and ambient densities on the spray-wall impingement characteristics including free spray penetration, spray angle, impinged spray height, impinged spray radius, and expansion rate. The spray-wall test in the current work was carried out in a high pressure-temperature constant volume vessel with a wide range of diesel engine conditions. High-speed visualization diagnostics including Mie-scattering and schlieren techniques were used to characterize the rebound/impinged spray formation and spray wall interactions under different injection pressures and ambient conditions. Injection pressures were varied from 1200 bar to 1800 bar and ambient densities changed from 14.8 $\mathrm{kg} / \mathrm{m}^{3}$ to $30 \mathrm{~kg} / \mathrm{m}^{3}$. The detailed experimental results obtained in the study will support the validation of the existing spray-combustion CFD codes in future.

\section{Experimental setup}

This spray-wall impingement experiment was carried out in a high-pressure, high-temperature constant volume vessel (CV) as shown in Figure 1. The CV is $1.1 \mathrm{~L}$ cubic chamber with a good optical access. Three types of port windows were installed on all the six surfaces of the cube: $101 \mathrm{~mm}$ diameter transparent window (sapphire), blank metal window, and injector window. The unobstructed orthogonal optical access is coupled with high-speed imaging techniques to study spray development. The impinging plate was mounted on the bottom window, and the injector was installed on one of the side windows. Three ports on the eight vertices of the chamber are used for the intake/ exhaust of chamber ambient gas, and a mounted dynamic pressure transducer. A Kistler 6001 piezo-electric dynamic pressure transducer coupled with a Kistler 5044a charge amplifier was used to measure the CV pressure and detailed vessel information is available in Ref. [14].

A single-hole diesel injector (off-axis injector nozzle) was mounted in the middle of the injector window, which resulted in the vertical distance $(40 \mathrm{~mm}$ for metal window and $52 \mathrm{~mm}$ for transparent window) between the injector tip and impinging window surface. For both the front view and side view spray measurement (see the left-top corner in Figure 1), the optical arrangement of simultaneous Mie scattering for the front view and schlieren for the side view is shown in Figure 1. A high-intensity pulsed LED1 with a pin-hole aperture is the light source for schlieren while additional LED2 and LED3 for the volumetric Mie scattering imaging are placed in front of the optical access 
of the chamber. For the bottom view and front view measurement, camera 1 is placed below the vessel with a 45 degree reflector. The test conditions and fuel properties are listed in Table 1.

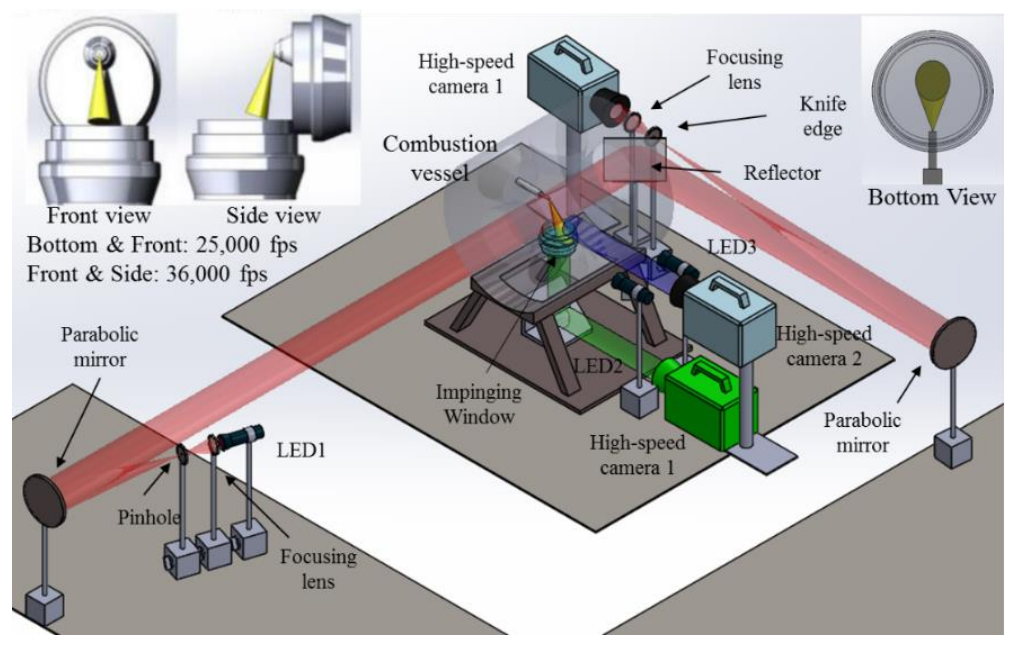

Figure 1. Optical setup for Mie and schlieren diagnostics with front/side/bottom view.

Table 1. Test conditions and fuel properties

\begin{tabular}{|c|c|c|c|}
\hline \multicolumn{2}{|c|}{ Injector conditions } & \multicolumn{2}{c|}{ Ambient and boundary conditions } \\
\hline Nozzle outlet diameter & $200 \mu \mathrm{m}$ & Ambient gas temperature & $423 \mathrm{~K}$ \\
\hline Nozzle number & single-hole & Ambient gas density & $14.8-30 \mathrm{~kg} / \mathrm{m}^{3}$ \\
\hline Nozzle K factor & 0 & Ambient gas & $\mathrm{N}_{2}$ \\
\hline $\begin{array}{c}\text { Orifice orientation } \\
\text { relative to injector axis }\end{array}$ & $\begin{array}{c}\alpha=60 \text {-degree (inclined } \\
\text { angle: } 120 \text {-degree) }\end{array}$ & $\begin{array}{c}\text { Distance from injector tip } \\
\text { to plate }\end{array}$ & $\begin{array}{l}\text { Metal: } 40 \mathrm{~mm} \\
\text { Transparent: } 52 \mathrm{~mm}\end{array}$ \\
\hline Injection duration & $2 \mathrm{~ms}$ & Wall temperature & $423 \mathrm{~K}$ \\
\hline \multicolumn{2}{|c|}{ Fuel properties and conditions } & $8 \mathrm{ppm}$ \\
\hline Fuel & ULSD & Sulfur & 47.2 \\
\hline Density & $848 \mathrm{~kg} / \mathrm{m}^{3}$ & Cetane Index & $42.83 \mathrm{MJ} / \mathrm{kg}$ \\
\hline Viscosity & $2.6 \mathrm{cSt}$ & Temperature at nozzle & $363 \mathrm{~K}$ \\
\hline Carbon (wt \%) & 86.8 & Fuel injection pressure & $1200,1500,1800 \mathrm{bar}$ \\
\hline Hydrogen (wt \%) & 13.2 & \multicolumn{2}{|}{}
\end{tabular}

\section{Image processing}

Spray images for the front, side, and bottom views were processed with an in-house MATLAB code to obtain key spray properties such as spray tip penetration, impinged spray radii and height. First, the procedure starts with the background subtraction and images enhancement in order to achieve a better boundary detection of the spray. During the boundary detection, Otsu's threshold method [22], which provides a default threshold value, is used to convert the grayscale image into the binary image. A sensitivity analysis to the threshold has been performed in the previous study [20] to obtain the accurate spray boundary. Unnecessary shapes obtained in the binary image are filtered using morphological tools. Finally, the largest connected area obtained is considered for exact contouring of the spray boundary. After the boundary detection, the analytical parameters of the free and impinged spray properties are measured by extracting the extreme points of the binary Image. Figure 2 (top) shows the sample image processing for the front, side, and bottom views.

A schematic of spray boundaries along with nomenclatures is shown in Figure 2 (bottom). The free spray penetration $(Z)$ defines the extent of spray into the chamber before impinging on the wall. The spray angle $(\beta)$ is also measured based on the front view high-speed Mie-scattering images. The impinged spray characteristics include the impinged spray radius $(R)$ and impinged spray height $(H)$. For the front and side images, impinged spray radius is the maximum spread of the spray in the horizontal direction with respect to the impinging surface while impinged spray height is the maximum height formed after impingement with respect to the impinging surface.

The imaging analysis procedure from the bottom view image is quite different from the front and side impinging surface imaging analysis procedure. First, the precise location of the first impinging spray on the surface is identified as a reference origin to calculate the boundary of the wall-impinged expanding spray (WIES) front as shown in Figure 2 (bottom right). A 'centroid' method in the Matlab which is the mean position of all the points in the impinging 
spray was used to identify the first impinging origin. The boundary of the WIES front is traced by the same method used in the front and side view images. WIES radii in both the axial $\left(R_{S}\right)$ and radial $\left(R_{f}\right)$ directions are then defined as the distance between the origin and WIES front. In the WIES radius calculation for both the radial and axial directions, because the WIES front is highly wrinkled as it propagates on the surface of the wall, an additional procedure was taken for estimating the averaged radius over the arc sector. The final arc central angle is determined by varying the arc angle for the least sensitivity to the radius variation. It was found 30-degree for the least averaged radius variation over the entire impinging spray lifetime. In addition, the corrugation ratio can also be calculated by comparing the actual WIES front chord length over the corresponding smooth elliptic arc chord length, indicating the degree of the corrugated WIES front.
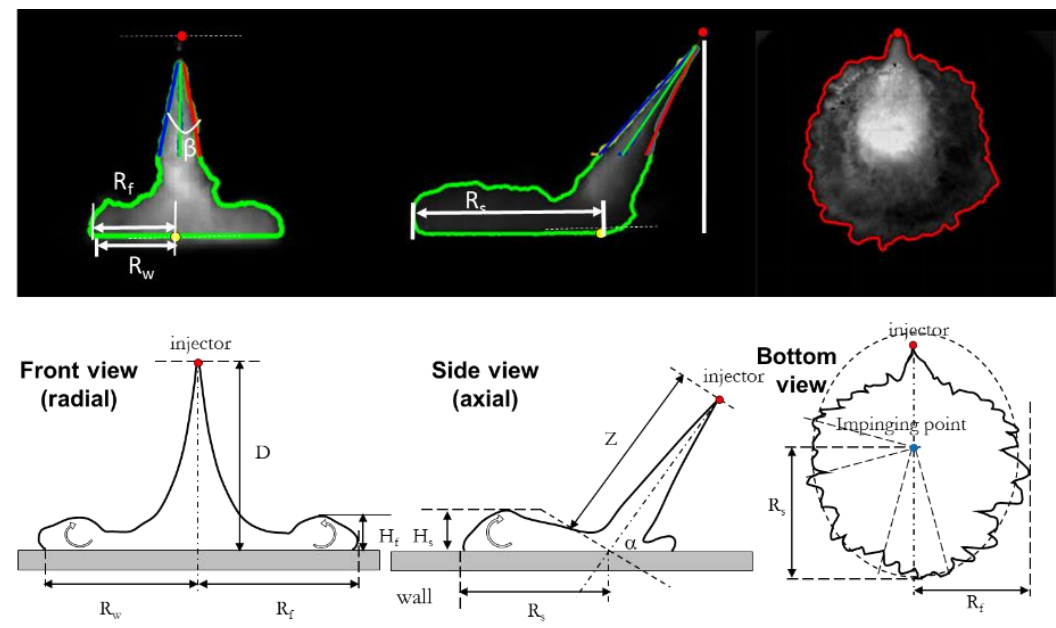

Figure 2. Images processing steps (top) and schematics of free and impinged spray (bottom). Nomenclature: $Z$ =free spray penetration; $R=$ =impinged spray radius; $H=$ =impinged spray height; $D=$ distance between injector and wall; $\beta=$ free spray dispersion angle; $\alpha=$ spray impacting angle. Subscript: $f=$ front; $s=$ side; $w=$ wall.

\section{Results and Discussion}

In general, fuel enters the chamber from the injector and spray experiences the normal free spray process before impinging on the wall. Liquid fuel disperses, breaks up, vaporizes, and mixes with air. The free spray contains two regions: main-jet region (particles with large velocities, momentum, and densities) and mixing flow region (air mixing expands the spray spatially). The main jet portion refers to the core region of the spray, and the mixing flow region stays outside the spray surrounding the main-jet region. The turbulence is generated because of air mixing, and particles in this region lose momentum in axial spray direction. Therefore, the core portion of the free spray has a higher velocity than the outer part. Then, all the droplets impinge on the wall with a certain angle (half of the included angle). Initially, the spray impinges on a dry wall, and the following interaction occurs between the spray and a wet wall. After impingement, the spray spreads on the plate around the impinging point and moves parallel to the plate. Droplets distributed near the plate show higher velocity and momentum than those farther from the plate due to the droplets near the plate come from the free spray core, which causes the velocity gradient and generates a vortex around the leading edge of spray. As a result, the rolling up motion enhances the air entrainment, lifts the lower momentum particles higher from the plate surface, and increases the height of impinged spray (see Figure 2 (bottom)). It is also found that the leading edge of the impinged spray is not along the wall but slightly higher away from the wall. The complicated turbulence environment and the distribution of droplets after impingement provide the possibility of collisions among droplets.

In this study, the injection rate was obtained by measuring the pressure wave generated when fuel is injected using Bosch rate of injection (ROI) meter [23]. ROI profile for energizing injection time of $2 \mathrm{~ms}$ at the injection pressure of 1500 bar is shown in Figure 3 (left), which was also selected as the experimental baseline condition. The corresponding injection duration is approximately $2.45 \mathrm{~ms}$. The total injected mass measured at ambient temperature is $28.39 \mathrm{mg}$ based on 3 repeats and the discharge coefficient is approximately 0.79 at the steady state. During spray-wall impingement, the velocity, momentum, and distribution of the spray before impingement are critical to the impinging behaviors. Injection pressure and ambient density influence the free spray penetration as well as the spray dispersion angle. The free penetration at an injection pressure of 1500 bar and different ambient densities are presented in Figure 3 (middle). The ambient density was varied from $14.8 \mathrm{~kg} / \mathrm{m}^{3}$ to $30 \mathrm{~kg} / \mathrm{m}^{3}$ and the experimental results are averaged from 5 runs. It can be observed that the penetration decreases 
as the ambient density increases at a given time. At the ambient density of $14.8 \mathrm{~kg} / \mathrm{m}^{3}$, spray impinges on the wall at the after the start of injection (ASOI) of $0.42 \mathrm{~ms}$, which is earlier than those at the higher ambient density. Figure 3 (right) provides the effect of injection pressure $\left(1200,1500\right.$, and $1800 \mathrm{bar}$ ) at the $22.8 \mathrm{~kg} / \mathrm{m}^{3}$ density. The penetration at the higher injection pressure shows the similar trend with the observation at the various densities at a fixed injection pressure, showing longer penetration than those at lower injection pressure. It is noted that the penetrations at the injection pressures of 1500 bar and 1800 bar show less difference than those at the injection pressures of 1200 bar and 1500 bar. At ASOI of $0.3 \mathrm{~ms}$, the free spray penetration at 1200 bar reaches $34 \mathrm{~mm}$, but the penetration at 1500 bar is around $37.5 \mathrm{~mm}$ which is close to the one at 1800 bar $(39 \mathrm{~mm})$. This would imply that the effect of injection pressure on the impinged spray becomes, to some extent, less as injection pressure increases. The penetration suggested by Naber et al. [24] is proportional to the $\left(P_{\mathrm{f}}-P_{\mathrm{a}}\right)^{1 / 4}$, where $P_{\mathrm{f}}$ is the injection pressure and $P_{\mathrm{a}}$ the ambient pressure. Assumed all the parameters remain constant, the only variable is $P_{\mathrm{f}}$ with three different cases: 1200 bar, 1500 bar, and 1800 bar. $P_{a}$ is 27.6 bar when the ambient density is $22.8 \mathrm{~kg} / \mathrm{m}^{3}$. $S_{1800 b a r}$ should be 1.04 times of $S_{1500 \mathrm{bar}}$ and $S_{1500 \mathrm{bar}}$ should be 1.06 times of $S_{1200 \mathrm{bar}}$, which confirms the experimental results.
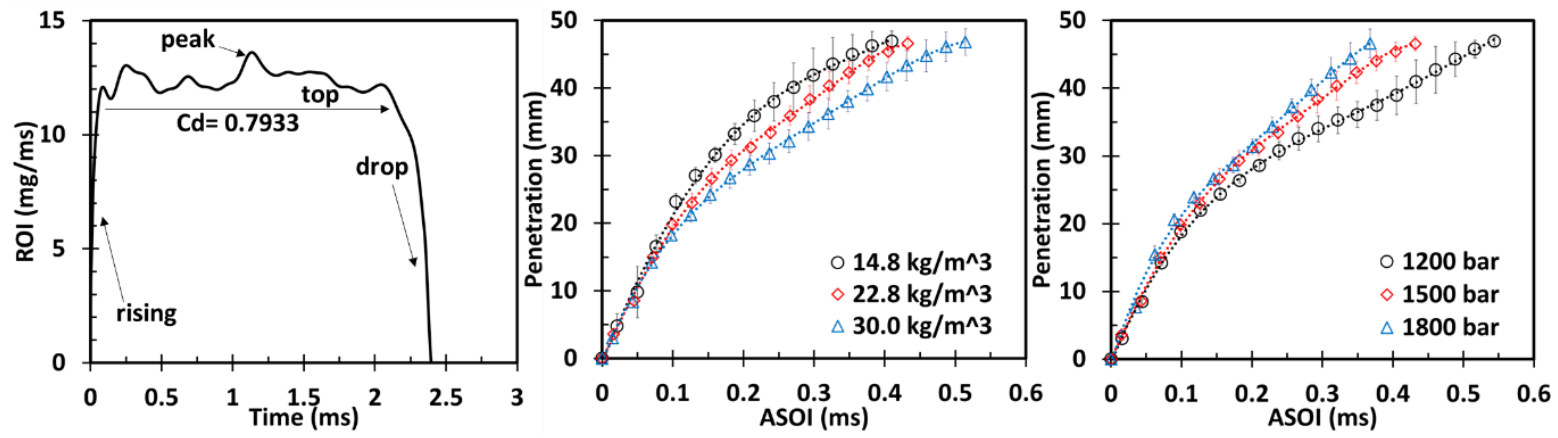

Figure 3. ROI profile at 1500 bar (left) and free spray penetrations at different ambient density with 1500 bar injection pressure (middle) and different injection pressure with $22.8 \mathrm{~kg} / \mathrm{m}^{3}$ density (right).

Figure 4 shows the effects of the ambient density (left) and injection pressure (right) on the spray dispersion angle. At the early stage of the injection, dispersion angle rises to a high value, and then a relative steady angle ( 23 degrees) establishes rapidly later. The large uncertainties underline the turbulent nature of the air entrainment process. From Figure 4 (left), the dispersion angle increases with the ambient density increase. In Figure 4 (right), however, it does not show a monotonic trend by the injection pressure. Before ASOI $1.3 \mathrm{~ms}$, dispersion angle at 1200 bar is the largest, next is the one at 1800 bar, and finally 1500 bar, implying no specific trend. After ASOI of $1.3 \mathrm{~ms}$, dispersion angles from all the conditions show the very small difference. Here, the larger dispersion angle indicates the higher level of air entrainment, since the entrainment is proportional to ambient air density, orifice diameter, fuel velocity, and spray dispersion angle [22]. Spray dispersion angle is a global parameter that describes the droplet distribution. Since in fact that the spray impingement obeys the stagnation flow model, the distribution of droplets before impacting affects their distribution after impingement.
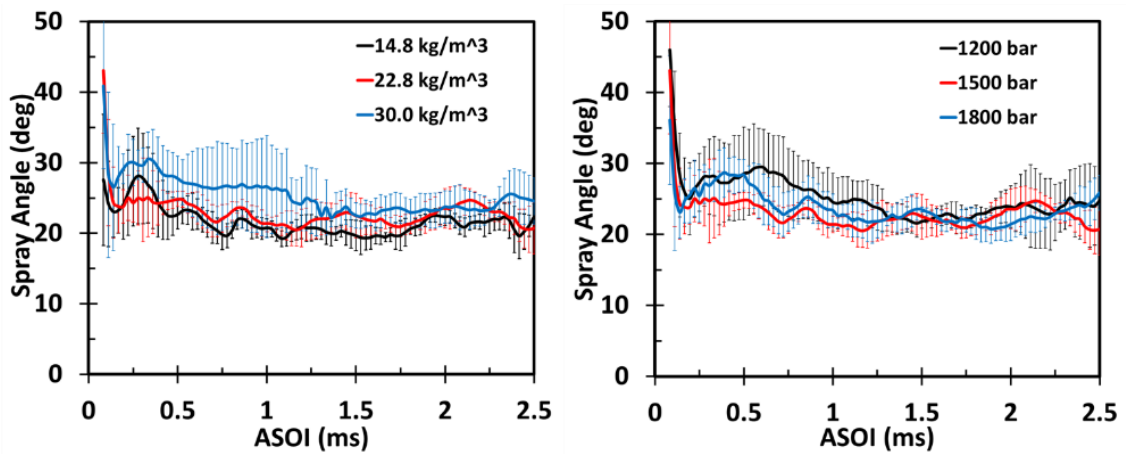

Figure 4. Spray dispersion angles at different ambient density with 1500 bar injection pressure (left) and different injection pressure with $22.8 \mathrm{~kg} / \mathrm{m}^{3}$ density (right).

Figure 5 (left) shows the effect of the ambient density on the WIES front radius for both axial and radial directions along with the time mark of the first impingement. The radius information was acquired from the bottom view image. Both directional radii start increasing after the first impingement, where the $14.8 \mathrm{~kg} / \mathrm{m}^{3}$ case impinges on the surface earlier than the other two ambient cases. Both radii increase until the end of injection where the actual rate of 
injection (ROI) ends at around ASOI $2.45 \mathrm{~ms}$, which is a similar observation of a stop of liquid film spread after the supply of the droplet to the film stopped [19]. The current test data did not catch this stop in radius changing due to the limitation of the chamber size. All the radii started with an initial value around $10 \mathrm{~mm}$ due to the overlap of impinged spray and free spray which causes the failure of the impinged radius calculation in earlier impinging time, the impinging time, however, is marked for different conditions in Figure 5.

Additionally, at the time of ASOI $2.5 \mathrm{~ms}$, axial radii reached $51.9 \mathrm{~mm}, 44.6 \mathrm{~mm}$, and $39.3 \mathrm{~mm}$ for ambient densities of $14.8,22.8$, and $30 \mathrm{~kg} / \mathrm{m}^{3}$, respectively. Both WIES radii tend to increase with the ambient density at the fixed time while the axial radius propagates faster than the radial radius regardless of the ambient density. The ratios of the axial and radial radii at the ASOI $2.5 \mathrm{~ms}$ are $1.5,1.44$ and 1.4 for the $14.8,22.8$, and $30 \mathrm{~kg} / \mathrm{m}^{3}$ cases, respectively, which results in oval shape impinged spray. This is partly attributed by higher axial velocity $(\sim 50 \%$ higher than the radial velocity) along the inclined plate (c.f. impact angle is 60-degree) when spray impinging, which elongates the axial impinged spray front than that in the radial direction. Figure 5 (right) reports the axial impinged height with various ambient densities. The height was measured by the side view schlieren images. Since the earlier impinged spray (i.e. around ASOI of $0.5 \mathrm{~ms}$ ) is highly complicated and mixed with the on-coming free spray, leading to a higher uncertainty. Initially, the impinged spray height increases fast, at the later time, the increasing rate slows down gradually and the impinged height reaches a maximum value. Similarly, the impinged height decreases as the ambient density raises.
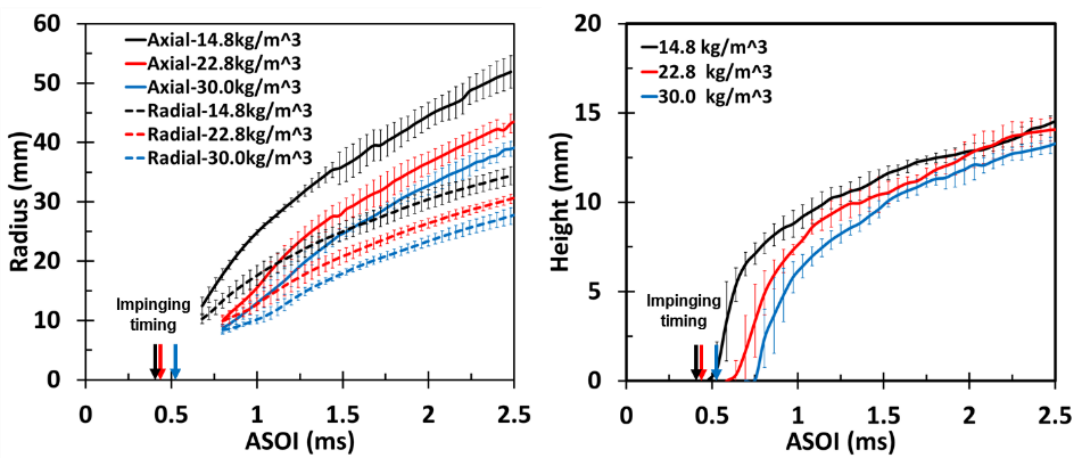

Figure 5. Impinged radii (left) and axial impinged heights (right) at different ambient density: $14.8,22.8$, and $30 \mathrm{~kg} / \mathrm{m}^{3}$.

Figure 6 provides the effect of injection pressure (1200, 1500, and 1800 bar) on WIES radius (left) and the WIES height (right) at the ambient density of $22.8 \mathrm{~kg} / \mathrm{m}^{3}$. Both axial and radial radii start increasing after the first impingement, where the 1800 bar case impinges on the surface earlier than the other two cases. The radii continue increasing up to the end of the injection around $2.45 \mathrm{~ms}$. Radial WIES radius at different injection pressures rises at a nearly same rate, but the axial radii increase with a steeper slope due to the higher velocity occurred in the axial direction as mentioned earlier. The ratios of the axial and radial radii at the ASOI $2.5 \mathrm{~ms}$ are $1.37,1.43$ and 1.41 for 1200, 1500, and 1800 bar cases, respectively, causing an oval shape of the impinged spray.

The directional radii and the WIES height tend to increase with the injection pressure at a fixed time. Because high injection pressure accelerates the free spray and thus causes the increase of impinged spray axial/radial expansion rates, and the WIES height. The proportionality between the impinged spray properties and the injection pressures are quite similar to the effect of ambient density but a sort of saturation appears at the 1800 bar in WIES height. This saturation behaves the same way as it is in the free spray penetration.
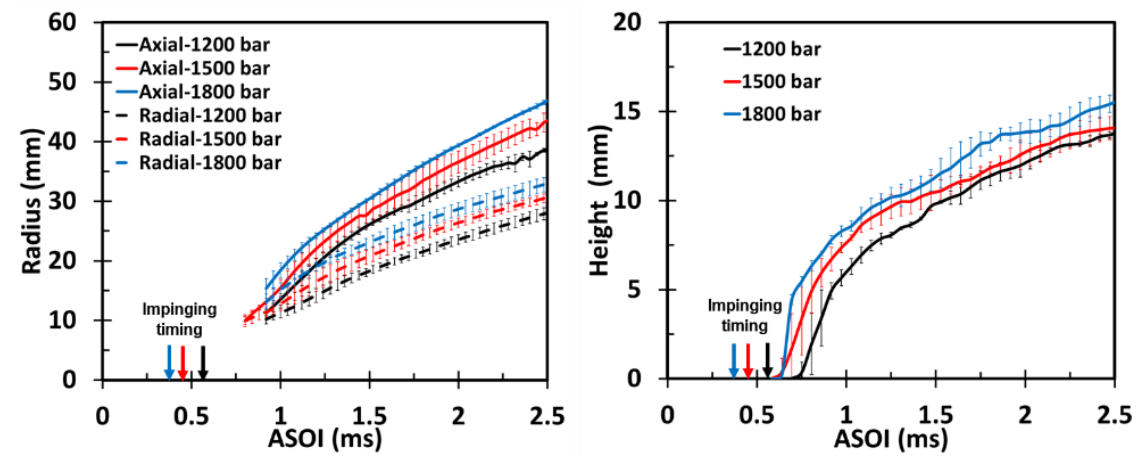

Figure 6. Impinged radii (left) and axial impinged heights (right) at different injection pressure: 1200, 1500, and 1800 bar. 
The temporal and spatial evolution of the WIES outer boundary and the corresponding axial WISE front speed profiles are shown in Figure 7. These boundaries were obtained from the bottom view imaging at a time interval of $40 \mu \mathrm{s}$ at the injection pressure of 1500 bar and $22.8 \mathrm{~kg} / \mathrm{m} 3$. The blank region in the center is the free spray projection on the plate, around which the actual boundaries of impinged spray are imposed. The origin is the impinging point. First, the WIES boundary displays a highly corrugated and complex front with the time increase, indicating the turbulent nature of the expanding spray interacted with the plate surface and surrounding ambient gas. The space between the two adjacent boundaries indicates the WIES expansion rate or speed that the larger the space the faster the spray expansion. The WIES expansion rate becomes smaller with time such that the boundaries away from the impinging point are much denser than those close to the impacting point. A substantial number of spikes (highly wrinkled boundaries) with time increase are randomly formed instead of staying at the same location, partly due to the injected flow rate fluctuation as seen in Figure 3 (left). There are spikes appeared after the first impingement, and then the subsequent spray, which generated more spikes at random locations, moved and merged with the previous spray continually. The interaction between the impinging sprays at the time shows a complicated WIES boundary development.

The axial expansion rates are also shown in Figure 7 after impingement. Since the impinging spray starts expanding parallel to the surface, the interaction between the impinging spray and wall/air retard the horizontal propagation across the surface. Expansion rate decreases from $40 \mathrm{~m} / \mathrm{s}$ to $10 \mathrm{~m} / \mathrm{s}$ within $2.5 \mathrm{~ms}$ and the similar spray-leading rate is shown regardless of ambient density (middle) and injection pressure (right). The power-law curve-fit equations show the similar amplitude and power for both injection pressure and density cases. Based on these equations, the WIES expansion process is divided into four stages: rapidly decelerated stage (before ASOI $1.2 \mathrm{~ms}$ ), slowly decreasing stage (ASOI 1.2 to $2 \mathrm{~ms}$ ), relatively constant stage (after ASOI $2 \mathrm{~ms}$ ), and expanding termination stage (not shown in Figure 7).
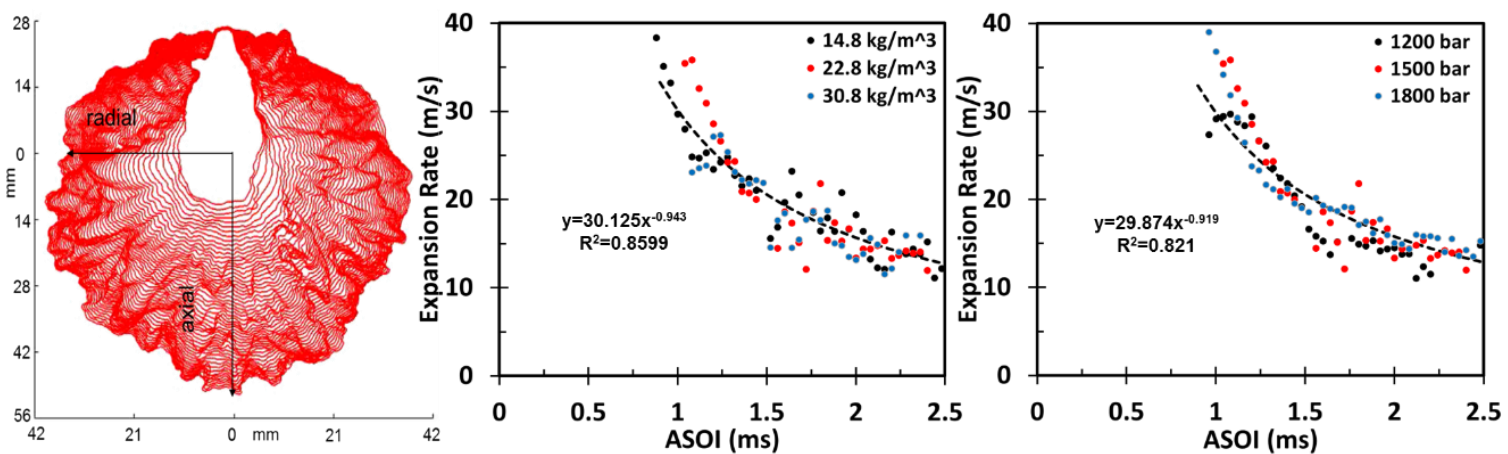

Figure 7. Evolution of the WIES outer boundary (left) and the expansion rate of WIES after impingement at different ambient densities (middle); at different injection pressures (right).

\section{Summary}

The spray impingement test was performed in a well-controlled vessel using a single-hole injector (60-degree impact angle) to characterize the impinged spray on a flat plate with the combined condition of injection pressures $(1200 / 1500 / 1800 \mathrm{bar})$ and ambient densities $\left(14.8 / 22.8 / 30 \mathrm{~kg} / \mathrm{m}^{3}\right)$ at isothermal conditions. The simultaneous Mie scattering and schlieren images were acquired for the liquid spray development and spray-wall interaction. An inhouse Matlab code for image processing was developed to extract the free and impinged spray properties.

Several observations can be made from the experiment. (1) The WIES expansion process is divided into four stages: rapidly decelerated stage, slowly decreasing rate stage, relatively constant rate stage, and expanding termination stage. (2) The WIES front has complicated, highly wrinkled structures as it propagates on the plate surface. The evolution of the WIES showed the boundary spikes which are formed at random locations. (3) The averaged radius of WIES was calculated over a 30-degree angle arc which was found having the least averaged radius variation. This arc-angle based radius method compensates for the wrinkled effect of the WIES front. (4) The effect of ambient density and injection pressure on the free and impinged spray were explored. Both lower ambient density and higher injection pressure intensify the free spray dispersion, impinged spray expansion, and lead to higher impinged spray height. Free spray angle increases with ambient density but is not significantly affected by injection pressure. Similarly, the WIES expansion rates on the wall showed no obvious dependence of the ambient density and the injection pressure. (5) The magnitude of the temporal axial radius is larger than that of the radial radius because of the higher momentum in the axial direction when spray impinges on the wall. The relative ratios of the axial radius to the radial radius are around 1.4 , resulting in the oval impinged spray. 


\section{Acknowledgements}

This material is based upon work supported by the Department of Energy, Office of Energy Efficiency and Renewable Energy (EERE) and the Department of Defense, Tank and Automotive Research, Development, and Engineering Center (TARDEC), under Award Number DE-EE0007292.

\section{References}

[1] Nauwerck, A., Pfeil, J., Velji, A., Spicher, U., and Richter, B., 2005, "A basic experimental study of gasoline direct injection at significantly high injection pressures," No. 0148-7191, SAE Technical Paper.

[2] Abart, M., Schmidt, S., Schoegl, O., Trattner, A., Kirchberger, R., Eichlseder, H., and Jajcevic, D., 2010, "Basic Investigations on the Prediction of Spray-Wall and Spray-Fluid Interaction for a GDI Combustion Process," No. 0148-7191, SAE Technical Paper.

[3] Alfuso, S., Allocca, L., Greco, M., Montanaro, A., and Valentino, G., 2008, "Time-and Space Characterization of Multi-hole GDI Sprays for IC Engines by Images Processing and PDA Techniques," Jets, 20, p. 21.

[4] Stanton, D. W., and Rutland, C. J., 1996, "Modeling fuel film formation and wall interaction in diesel engines," No. 0148-7191, SAE Technical Paper.

[5] Stanglmaier, R. H., Li, J., and Matthews, R. D., 1999, "The effect of in-cylinder wall wetting location on the HC emissions from SI engines," No. 0148-7191, SAE Technical Paper.

[6] Drake, M. C., Fansler, T. D., Solomon, A. S., and Szekely, G., 2003, "Piston fuel films as a source of smoke and hydrocarbon emissions from a wall-controlled spark-ignited direct-injection engine," No. 0148-7191, SAE Technical Paper.

[7] Stevens, E., and Steeper, R., 2001, "Piston wetting in an optical DISI engine: fuel films, pool fires, and soot generation," No. 0148-7191, SAE Technical Paper.

[8] Sandquist, H., Lindgren, R., and Denbratt, I., 2000, "Sources of hydrocarbon emissions from a direct injection stratified charge spark ignition engine," No. 0148-7191, SAE Technical Paper.

[9] Cheng, W. K., Hamrin, D., Heywood, J. B., Hochgreb, S., Min, K., and Norris, M., 1993, "An overview of hydrocarbon emissions mechanisms in spark-ignition engines," No. 0148-7191, SAE Technical Paper.

[10] Alkidas, A. C., 1994, "The effects of fuel preparation on hydrocarbon emissions of a SI engine operating under steady-state conditions," No. 0148-7191, SAE Technical Paper.

[11] Alkidas, A., and Drews, R., 1996, "Effects of mixture preparation on HC emissions of a si engine operating under steady-state cold conditions," No. 0148-7191, SAE Technical Paper.

[12] Fox, J., Min, K. D., Cheng, W., and Heywood, J. B., 1992, "Mixture preparation in a SI engine with port fuel injection during starting and warm-up," No. 0148-7191, SAE Technical Paper.

[13] Fulcher, S. K., Gajdeczko, B., Felton, P., and Bracco, F., 1995, "The effects of fuel atomization, vaporization, and mixing on the cold-start UHC emissions of a contemporary SI engine with intake-manifold injection," No. 01487191, SAE Technical Paper.

[14] Zhao, L., Torelli, R., Zhu, X., Scarcelli, R., Som, S., Schmidt, H., Naber, J., and Lee, S.-Y., 2017, "An experimental and numerical study of diesel spray impingement on a flat plate," SAE International Journal of Fuels and Lubricants, 10(2017-01-0854).

[15] Zhang, Y., Jia, M., Liu, H., Xie, M., Wang, T., and Zhou, L., 2014, "Development of a new spray/wall interaction model for diesel spray under PCCl-engine relevant conditions," Atomization and Sprays, 24(1).

[16] Siewert, R. M., 2007, "Spray angle and rail pressure study for low NOx diesel combustion," No. 0148-7191, SAE Technical Paper.

[17] Akop, M. Z., Zama, Y., Furuhata, T., and Arai, M., 2013, "Characteristics of adhesion of diesel fuel on impingement disk wall. Part 1: effect of impingement area and inclination angle of disk," Atomization and Sprays, 23(8).

[18] Lindagren, R., and Denbratt, I., 2004, "Influence of wall properties on the characteristics of a gasoline spray after wall impingement," No. 0148-7191, SAE Technical Paper.

[19] Montorsi, L., Magnusson, A., and Andersson, S., 2006, "A numerical and experimental study of diesel fuel sprays impinging on a temperature controlled wall," No. 0148-7191, SAE Technical Paper.

[20] Senda, J., Ohnishi, M., Takahashi, T., Fujimoto, H., Utsunomiya, A., and Wakatabe, M., 1999, "Measurement and modeling on wall wetted fuel film profile and mixture preparation in intake port of SI engine," No. 0148-7191, SAE Technical Paper.

[21] Montanaro, A., Allocca, L., Meccariello, G., and Lazzaro, M., 2015, "Schlieren and Mie Scattering Imaging System to Evaluate Liquid and Vapor Contours of a Gasoline Spray Impacting on a Heated Wall," No. 0148-7191, SAE Technical Paper.

[22] Otsu, N., 1975, "A threshold selection method from gray-level histograms," Automatica, 11(285-296), pp. 2327.

[23] Bower, G. R., and Foster, D. E., 1991, "A comparison of the Bosch and Zuech rate of injection meters," No. 0148-7191, SAE Technical Paper.

[24] Naber, J. D., and Siebers, D. L., 1996, "Effects of gas density and vaporization on penetration and dispersion of diesel sprays," No. 0148-7191, SAE technical paper. 


\title{
Quantification of diesel injector dribble using 3D reconstruction from $x$-ray and $D B I$ imaging
}

\author{
Vitaliy Sechenyh ${ }^{1}$, Jack Turner ${ }^{1}$, Dan Sykes ${ }^{1}$, Daniel J. Duke ${ }^{2}$, Andrew B. Swantek ${ }^{2}$, \\ Katarzyna E. Matusik ${ }^{2}$, Alan L. Kastengren ${ }^{2}$, Christopher F. Powell ${ }^{2}$, Alberto Viera ${ }^{3}$, Raul \\ Payri $^{3}$, Cyril Crua ${ }^{1, *}$ \\ ${ }^{1}$ Advanced Engineering Centre, University of Brighton, Brighton, UK \\ ${ }^{2}$ Argonne National Laboratory, USA \\ ${ }^{3}$ CMT Motores Térmicos - Universitat Politècnica de València, Spain \\ *corresponding author: c.crua@brighton.ac.uk
}

\begin{abstract}
Post-injection dribble is known to lead to incomplete atomisation and combustion due to the release of slow moving, and often surface-bound, liquid fuel after the end of the injection event. This can have a negative effect on engine emissions, performance, and injector durability. To better quantify this phenomenon we present a new image processing approach to quantify the volume and surface area of ligaments produced during the end of injection, for an ECN 'Spray B' 3-hole injector. Circular approximation for cross-sections was used to estimate three-dimensional parameters of droplets and ligaments. The image processing consisted in three stages: edge detection, morphological reconstruction, and 3D reconstruction. For the last stage of 3D reconstruction, smooth surfaces were obtained by computation of the alpha shape which represents a bounding volume enveloping a set of 3D points. The object model was verified by calculation of surface area and volume from 2D images of figures with well-known shapes. We show that the object model fits non-spherical droplets and pseudo-cylindrical ligaments reasonably well. We applied our processing approach to datasets generated by different research groups to decouple the effect of gas temperature and pressure on the fuel dribble process. High-speed X-ray phase-contrast images obtained at room temperature conditions (297 K) at the 7-ID beamline of the Advanced Photon Source at Argonne National Laboratory, together with diffused back-illumination (DBI) images captured at a wide range of temperature conditions (293-900 K) by CMT Motores Térmicos, were analysed and compared quantitatively.
\end{abstract}

\section{Keywords}

Diesel injector; dribble; ligament; droplet shape; atomisation.

\section{Introduction}

The end-of-injection (EOI) fuel dribble causes a formation of unburned hydrocarbons and decreases the performance of internal combustion engines in a variety of ways. For example, deposits lead to an increase in air pollutant emissions [1, 2], a decrease in quality of injection [2, 3, 4] and further coking of the nozzle [5]. Understanding of the fuel dribbling process is particularly important for the development of a strategy for optimal use of fuels. However, observing the transient end-of-injection processes is particularly challenging due to the extreme operating conditions and the microscopic scales involved. Consequently, there is a lack of quantitative information on the fuel dribble events and the parameters that affect them.

Recently published studies [6-10] demonstrate different aspects of the EOI fuel dribble based on a qualitative and quantitative analysis of experimental images of the injection process. The following important factors affecting the mechanism of the fuel dribble were studied: peak injection velocity [7], needle closing speed [7], in-cylinder pressure [6, 7], injection pressure [6], fuel mass expulsion [9], bubble ingestion at the EOI [10], liquid length recession at the EOI [8] and different flow characteristics at the EOI [11].

The present study is dedicated to a quantitative analysis of the fuel dribble with the focus on dribble volumes estimated by processing of images from high-speed X-ray phase-contrast and diffused back-illumination (DBI) imaging techniques. The 3D reconstruction algorithm was developed to analyse volumes of the liquid when the fuel emerges from the orifice of Engine Combustion Network (ECN) "Spray B" injector. The main motivation of this investigation is to provide a better understanding of the parameters that influence the uncontrolled release of low velocity fuel at the end of injection. 


\section{D reconstruction algorithm}

The extraction of size and shape from two-dimensional images of droplets, particles, liquid ligaments or tree-like structures is used in a number of industrial applications such as medical imaging [12-15], soot formation in combustion [16], etc. Similar to previously published algorithms [17-19], the present analysis is based on the estimation of object cross-sections from a line-based structure of an initial image followed by smoothing of 3D shape. The present study is based on the object model demonstrated in Fig. 1a. A circular assumption for object cross-sections allowed to reconstruct the $3 D$ shape using the limited information available from a single $2 \mathrm{D}$ image.

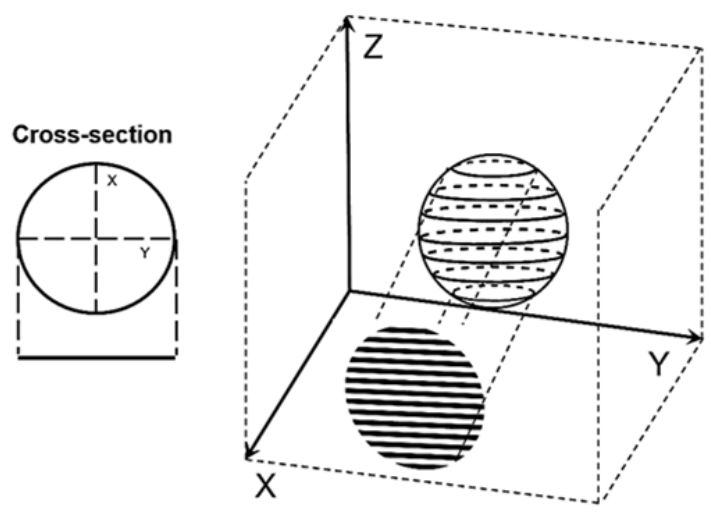

(a)

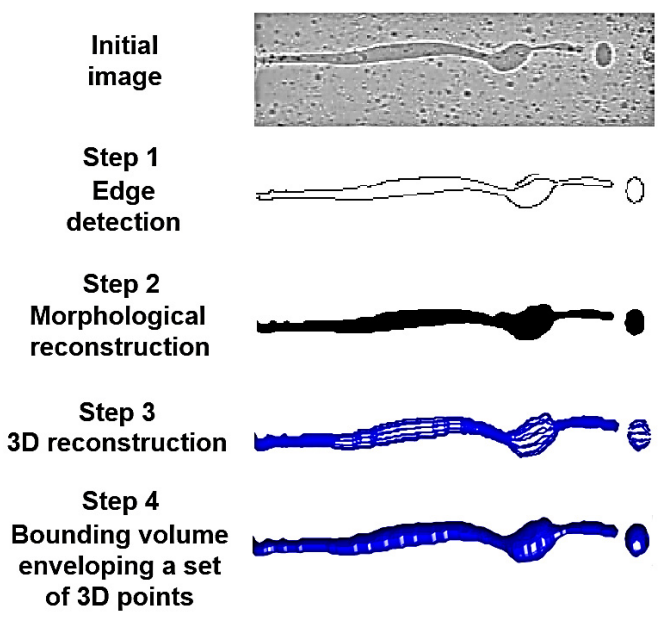

(b)

Figure 1. Schematic of the object model (a) and main steps of the image processing algorithm (b).

The image processing algorithm consisted of the following stages (see Fig. 1b):

- $\quad$ Step 1. Edge detection coupled with conversion from grey scale to binary image.

- Step 2. Morphological reconstruction of contours for all detected objects. Intermediate stage: Line-based structure of binary image is used to calculate characteristics of object cross-sections.

- Step 3. 3D reconstruction. Coordinates of 3D points are calculated based on centres and radii of crosssections.

- $\quad$ Step 4. Computation of the bounding volume which envelops a set of 3D points - alpha shape.

The present study applies this image processing approach to two different experimental techniques: high-speed $\mathrm{x}$-ray phase contrast and diffused back-illumination (DBI). Three different edge detection methods were used to remove background and noise from experimental images: Canny algorithm [20], wavelet filter and morphological opening.

A choice of the Canny edge detector is motivated by its excellent performance in the presence of flickering background and boundaries which are caused by a combination of X-ray absorption and phase contrast effects $[21,22]$. Since detected edges usually have breaks, a Delaunay triangulation algorithm [23] was applied for twodimensional reconstruction of contours of multiple objects. Compared to the X-ray imaging, the DBI method provides similar light intensity values for each pixel which belongs to the liquid phase [24]. DBI pictures were processed with help of a transformation which consists of a convolution of the image with a wavelet filter, followed by a thresholding. The wavelet filter detects concavity and convexity of the grey level variation in the image [25]. Edges from DBI images were also detected by morphological opening with erosion followed by a dilation, using the same structuring element for both operations. Details related to the application of morphological operations to greyscale images were discussed elsewhere in the literature $[25,26]$. Following recommendations in the literature $[25,26,28,29,30]$, the adjustable parameters for each edge detection method were chosen depending on a number factors: size and number of objects, type of a background noise, movements of the image background and image contrast.

The intermediate stage of the image processing algorithm uses line-based structure of binary images with contours of objects. The detection of grouped pixels in single lines of images was used to determine diameters and coordinates of centres for all detected circles. A middle point and a number of grouped pixels were accepted as centre and diameter of circles, respectively. 
Previously determined characteristics together with a parametric equation for a circle are used to build a 3D point cloud for each cross-section (slice) of the object, in a plane orthogonal to the image plane. Merging all available "slices" generates a 3D array which represents the shape of the object. At the last stage of 3D reconstruction, a smooth surface is obtained by computation of the bounding volume enveloping the 3D point cloud - the alpha shape. The definition of the alpha shape in three-dimensional space was discussed in the literature by Edelsbrunner et al. [31].

There are three main parameters necessary to build the alpha shape: alpha radius, region threshold and holes threshold. Depending on a value of the alpha radius, the alpha shape can transform from concave into convex object. Larger values of alpha radius usually result in convex objects. Lafarge et al. [32] provide recommendations for the choice of the alpha radius which are necessary for correct representation of the 3D shape. The region threshold allows the maximum number of objects in the 3D array to be determined. Finally, holes threshold reduces the number of defects and holes in the alpha shape.

\section{Verification of the 3D reconstruction algorithm}

The 3D reconstruction algorithm was verified by calculation of surface area $(S)$ and volume $(V)$ from $2 \mathrm{D}$ images of figures with well-known shapes: sphere, cylinder, and spheroid. The average relative error was chosen as a measure of accuracy in calculation of $S$ and $V$. The description of test shapes and errors are summarized in Table 1. A cylinder, spheres, and prolate and oblate spheroids were considered as models for liquid ligaments and droplets. Two half-spheres were attached to the cylinder in order to model a typical shape of the ligament.

Average relative errors in calculations of $V$ and $S$ for spherical objects are $4.7 \%$ and $3.4 \%$, respectively. Inclined spheroids were chosen to simulate the rotation of ligaments in images of the fuel dribble. As is seen in Table 1 , the largest relative error in calculations of $V$ and $S$ is observed in cases when the inclination angle of the major axis of the spheroid $(A)$ equals 129 degrees. It should be mentioned that the most accurate reconstruction of the 3D shape was observed for the object with a vertically oriented major axis - a cylinder with two hemispherical caps.

Two spheres with different distances between their centres were chosen as models of the pinch-off effect. Current analysis showed that the change of the distance between two spheres produces errors which equal $19 \%$ and $5.9 \%$ for $V$ and $S$, respectively. In addition, a combination of prolate spheroid and sphere was also considered as the model for the initial stage of the pinch-off effect.

Table 1. Average relative error in calculations of volume and surface area for different test shapes

\begin{tabular}{c|c|c}
\hline \hline Description of test shapes & $\begin{array}{c}\text { Volume error } \\
(\%)\end{array}$ & $\begin{array}{c}\text { Surface area error } \\
(\%)\end{array}$ \\
\hline \hline Sphere & 4.7 & 3.4 \\
Prolate spheroid. $A=0^{\circ}$ & 2.7 & 1.9 \\
Prolate spheroid. $A=1^{\circ}$ & 6.5 & 4.3 \\
Prolate spheroid. $A=5^{\circ}$ & 7.6 & 4.9 \\
Prolate spheroid. $A=12^{\circ}$ & 11 & 7.6 \\
Prolate spheroid. $A=21^{\circ}$ & 13 & 10 \\
Prolate spheroid. $A=29^{\circ}$ & 30 & 24 \\
Oblate spheroid. $A=90^{\circ}$ & 8.3 & 45 \\
Prolate spheroid and sphere & 16 & 6.4 \\
Cylinder with two hemispherical caps & 0.6 & 0.1 \\
Two spheres separated by $0.05 D$ & 14 & 0.8 \\
Two spheres separated by $0.15 D$ & 14 & 1.6 \\
Two spheres separated by $0.2 D$ & 19 & 5.9
\end{tabular}

For this initial approach at modelling the 3D morphology of microscopic droplets and ligaments we have made two simplifying assumptions: the liquid structures are axisymmetric, and aligned with the pixel array. While these assumptions preclude the 3D modelling of completely arbitrary shapes, they can be justified by the experimental configurations used in this particular study. 
The assumption of axisymmetry can be justified by the fact that the experiments were performed with the spray axis aligned with the image plane, and both surface tension and momentum limit the formation of asymmetric liquid structures in the plane orthogonal to the spray axis. This is particularly the case when liquid velocities are small, which is to be expected for our experiments performed during the end of injection. Although this assumption cannot be easily verified since measurements for the depth of the fuel structures were not available, we note that this should be a reasonable hypothesis for the study of slow-moving ligaments and droplets.

The assumption that liquid structures are aligned with the pixel array was made to simplify the slice by slice reconstruction process. This assumption was satisfied by ensuring that the spray axis was aligned with the pixel array, either during the image acquisition or by rotation of the acquired images. The effect of rotation of spheroid structures with regard to the pixel array is quantified in Table 1, and as expected larger errors are obtained for angles that deviate from the images axes. It should be mentioned that such large errors are expected to be rare in this study, as the vast majority of ligaments were well aligned with the image (i.e. spray) axis.

\section{Results and discussion}

As mentioned previously, the present analysis uses pictures of EOI fuel dribble obtained by X-ray imaging and DBI. Both experimental techniques were already reported extensively [9, 10, 11, 21, 22, 24, 33], hence for conciseness the technical details of the experimental setup will not be repeated here. The main experimental parameters relevant to the study of the fuel dribble process are listed in Table 2.

Table 2. Summary of experimental conditions for datasets used for analysis of the EOI fuel dribble

\begin{tabular}{c|c|c}
\hline \hline $\begin{array}{c}\text { Experimental } \\
\text { conditions }\end{array}$ & CMT & Argonne \\
\hline \hline Injected liquid & n-dodecane & n-dodecane \\
Injection pressure $P_{\text {inj; }}[\mathrm{bar}]$ & $500 ; 1000 ; 1500$ & $500 ; 1000 ; 1500$ \\
Ambient gas & $100 \% \mathrm{~N}_{2}$ & $100 \% \mathrm{~N}_{2} ;$ \\
Gas temperature $T_{g},[\mathrm{~K}]$ & $293-900$ & $82 \% \mathrm{~N}_{2}+18 \% \mathrm{He}$ (by mass) \\
Gas pressure $P_{g},[\mathrm{bar}]$ & $6.7-62$ & 297 \\
Ambient gas density, $\left[\mathrm{kg} / \mathrm{m}^{3}\right]$ & $7.6-30$ & $14.4 ; 30$ \\
& & $16.5 ; 34$
\end{tabular}

X-ray imaging of the fuel injection process was done at room temperature conditions at the 7-ID beamline of the Advanced Photon Source at Argonne National Laboratory (US). The exposure time and the pixel size were $1.473 \cdot 10^{-5} \mathrm{~s}$ and $5 \cdot 10^{-6} \mathrm{~m}$ square, respectively. A raw image and the result of the $3 \mathrm{D}$ reconstruction is shown in Fig. 2. The nozzle orifice is located above the top part of both pictures.
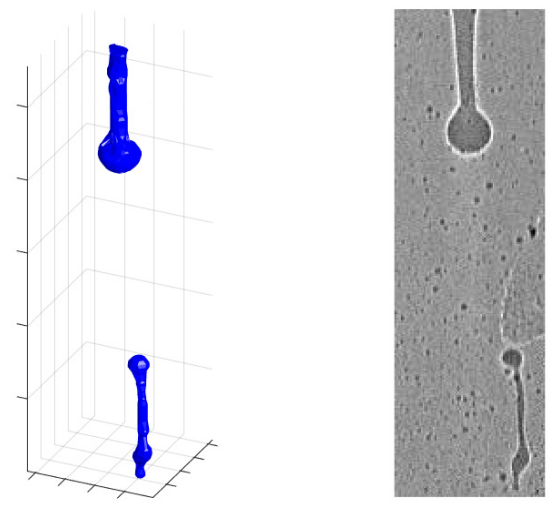

Figure 2. The raw image from $X-$ Ray phase contrast technique (right image) and the result of 3D reconstruction (left image). Experimental conditions: $P_{i n j}=500 \mathrm{bar}, P_{g}=30$ bar and $T_{g}=297 \mathrm{~K}$.

DBI images were captured at the CMT Motores Térmicos (Spain) in two different sets of experimental measurements: room temperature $(293 \mathrm{~K})$ and high temperatures $(473-900 \mathrm{~K})$ conditions. The first image set was carried out with a LED pulse length (exposure time) of $1 \cdot 10^{-7} \mathrm{~s}$ and pixel sizes of $1.35 \cdot 10^{-5} \mathrm{~m}$ or $1.99 \cdot 10^{-5} \mathrm{~m}$, depending on the optical magnification. An example of 3D reconstructed image is shown in Fig. 3. Similar to Fig. 2 , the nozzle orifice is located just above the top of Fig. 3. 

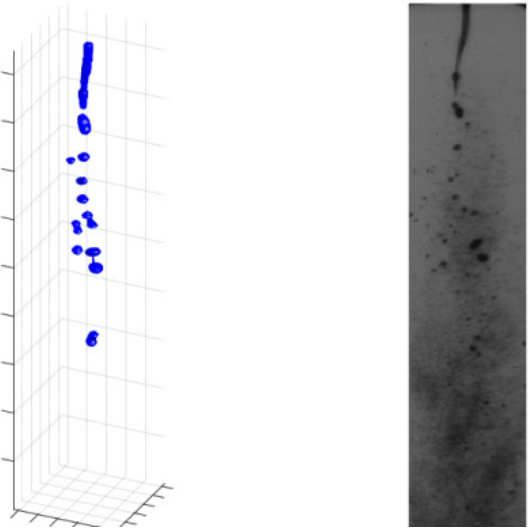

Figure 3. Room temperature DBI image (right image) and the result of 3D reconstruction (left image).

Experimental conditions: $P_{i n j}=500$ bar, $P_{g}=27$ bar and $T_{g}=293 \mathrm{~K}$.

The present analysis required the separation of the dribble events from the main injection stage, when a finely atomised spray is formed. As can be seen in Fig. 4, the liquid volume present in the region of interest increases rapidly at the beginning of the injection stage, and reduces almost to zero when the needle of the diesel injector closes (at $4.5 \mathrm{~ms}$ in Fig. 4). The volume of liquid present in the region of interest then increases again due to the dribble process. The image analysis shows that the relative duration of dribble events varied from 5 to $23 \%$ of the time between opening and closing of the injector needle.

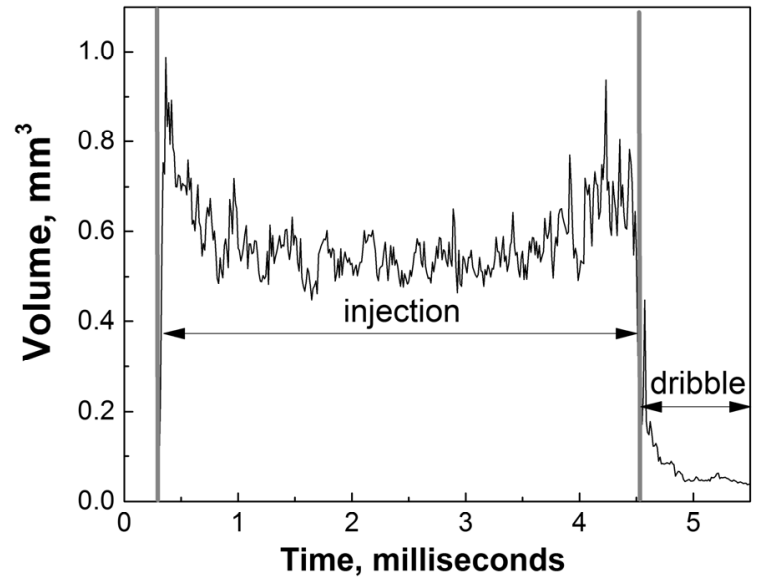

Figure 4. Time dependence of the liquid volume during the injection process for: $P_{i n j}=1500$ bar, $T_{g}=900 \mathrm{~K}$ and $P_{g}=20$ bar.

Experimental images were provided by Argonne.

As is seen in Fig. 5, both the volume $(V)$ and external surface area $(S)$ of the liquid structures present in the region of interest increase rapidly to a maximum value before gradually decreasing to zero. Solid and dashed lines were included in Fig. 5 in order to provide a graphical representation of time evolution for $V$ and $S$. All dribble events showed similar behaviour with respect to changes in liquid volume and surface area. The initial increases in V and $S$ relate to the fuel being released from the orifice after the end of the main injection event. The decrease in these quantities is related to the disappearance of the structures from the region of interest, as they move out of the field of view of the imaging systems. Since all dribble image sequences demonstrated this behaviour, the maximum dribble volume was considered as a characteristic parameter of the process.

Firstly, we can note that the values for the maximum dribble volume obtained from the very different experiments performed by CMT and Argonne are in good quantitative agreement (see data at 293 - $297 \mathrm{~K}$ in Fig. 6), suggesting that the fuel volumes estimated from our morphological reconstructions are consistent. In agreement with previously published studies [6,7], this investigation revealed a high cycle-to-cycle deviation in the quantity of fuel delivered after the end of injection. No clear dependence of the fuel dribble on injection pressure was observed (Fig. 6), although the lowest injection pressure tested (500 bar) seemed to generate relatively more dribble at room temperature conditions. As is seen in Fig. 6, the volume measurements for high gas temperature are less consistent, and as a result it is more difficult to identify a clear overall dependence of the fuel dribble on injection pressure. One may argue that fuel injection pressure should be expected to have no direct effect on the 
release of fuel after the nozzle has been closed. At the same time, we should consider that higher injection pressures are known to lead to higher gas volume fractions in the nozzle (through cavitation), which in turn should reduce the volume of liquid trapped inside the nozzle and orifices after needle closure.

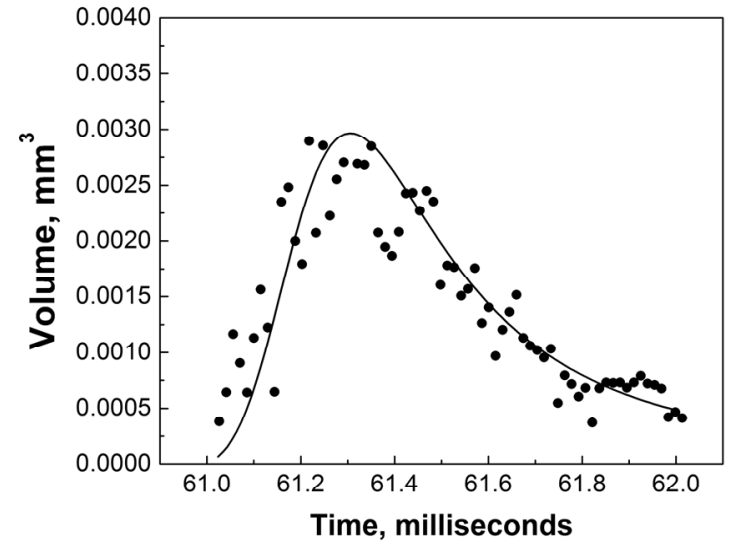

(a)

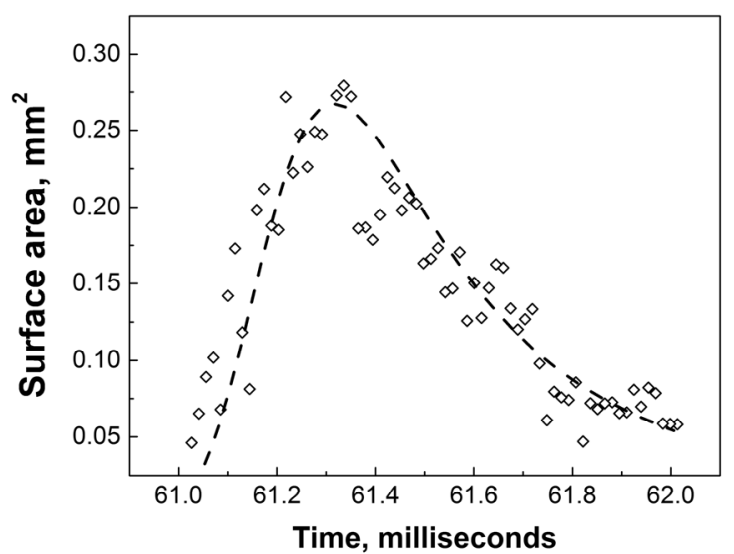

(b)

Figure 5. Dribble volume (left image) and surface area (right image) versus time. Both diagrams obtained by processing of images which correspond to following conditions: $P_{i n j}=1000$ bar, $T_{g}=297 \mathrm{~K}$ and $P_{g}=30$ bar. Experimental images were provided by Argonne.

Our analysis shows that gas temperature has a significant impact on the volume of fuel dribble (Fig. 6). Measurements performed at elevated temperatures $(473-900 \mathrm{~K})$ were found to generate between 2 and 4 times more liquid after the end of the main injection event, for all injection pressures (Fig. 6). Interestingly, the largest volume of fuel dribble was recorded for mid-range temperatures $(473 \mathrm{~K})$. This may suggest that at room temperature the high viscosity of the liquid prevents some of the fuel from dribbling out of the nozzle. As the gas temperature is increased (and the viscosity and surface tension of the liquid reduced) a larger volume of fuel is able to flow out of the orifice. Since the dribble process is affected by momentum, it can also be expected that the lower gas densities obtained at high gas temperatures (for a fixed gas pressure) should lead to more fuel being released from the nozzle. As temperature is further increased to $820-900 \mathrm{~K}$, the trend reverses and the measured dribble volume somewhat reduces (Figs. 6 and 7a), indicating that other parameters are affecting the process. We note that the apparent reduction in measured dribble volume at high temperatures could be related to increased evaporation at these conditions, rather than a net reduction in released liquid volume.

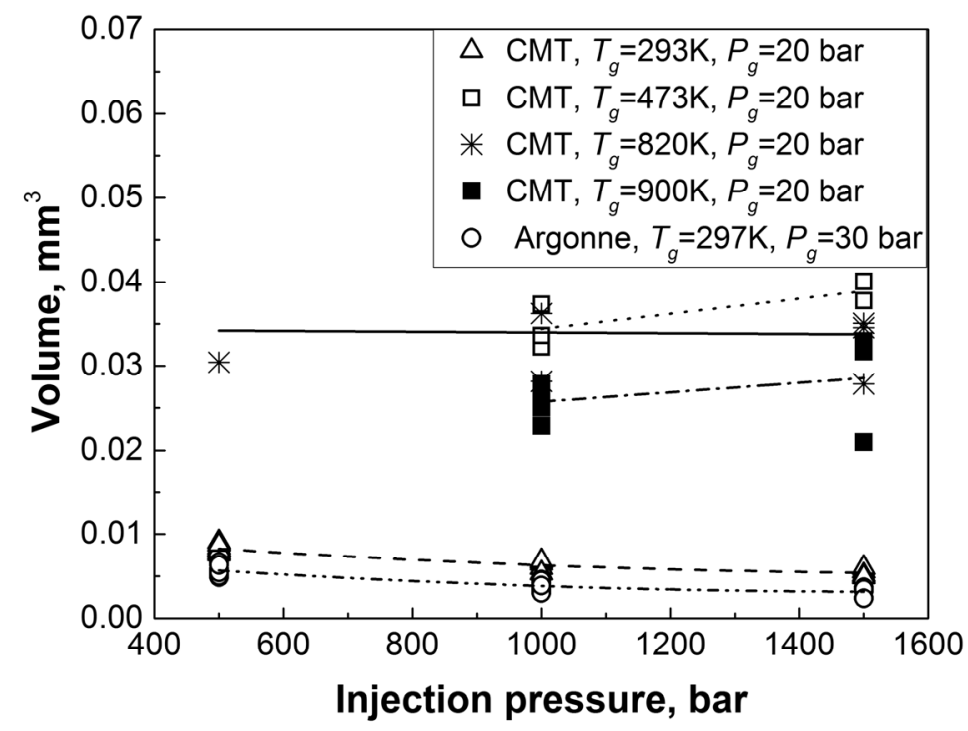

Figure 6. Dependence of maximum dribble volume on injection pressure $P_{i n j}$.

The investigation shows that an increase in gas pressure reduces the volume of dribble (Fig. 7b). Again this behaviour is expected to be related to a reduction in gas density, providing less resistance to the flow of fuel out of the injector's orifices. 


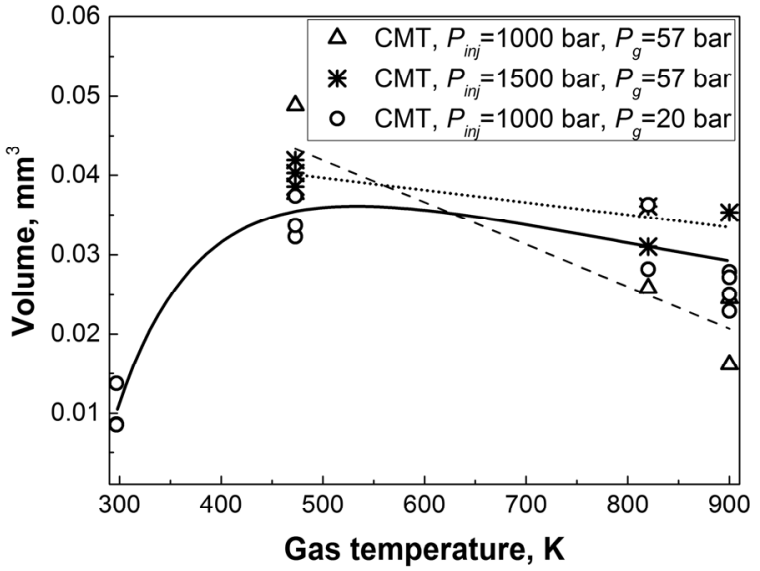

(a)

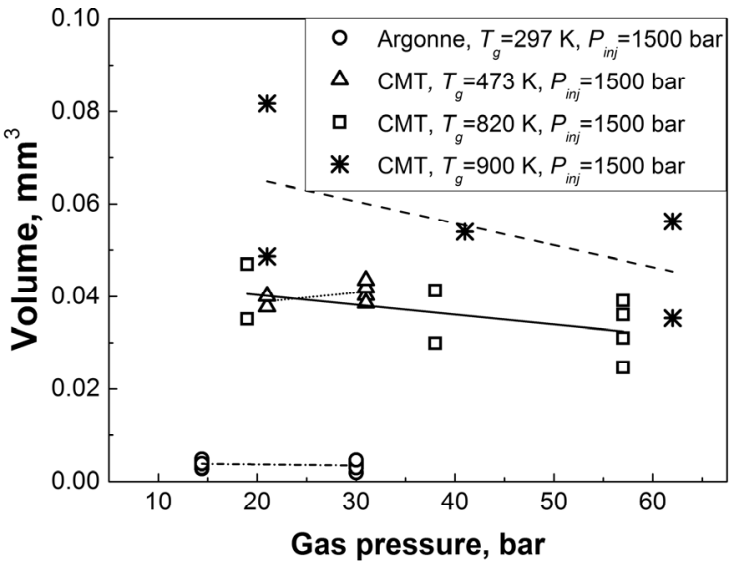

(b)

Figure 7. Dependence of the maximum dribble volume on gas temperature $T_{g}(\mathrm{a})$ and pressure $P_{g}(\mathrm{~b})$.

\section{Conclusions}

We performed a quantitative investigation of fuel injector dribble using 3D morphological reconstruction of liquid structures using high-speed videos recorded at Argonne (X-ray phase-contrast) and CMT Motores Térmicos (diffused back-illumination). This allowed us to estimate the volume of liquid released from the orifice of an ECN "Spray B" injector after the end of injection. Our analysis showed that the duration of dribble events varied from 5 to $23 \%$ of the injection time. We found that a larger volume of fuel dribble occurred at low injection pressures, mid-range gas temperatures $(\sim 73 \mathrm{~K})$ and low gas pressures. Although a more detailed analysis would be needed to confirm these trends, it is interesting to note that these conditions are generally found at idle engine conditions.

\section{Acknowledgements}

This work was supported by the UK's Engineering and Physical Science Research Council [grants EP/K020528/1 and EP/M009424/1], and BP Formulated Products Technology.

The authors acknowledge the support of this work from CMT Motores Térmicos (Universitat Politècnica de València, Spain). Parts of this research were performed at the 7-ID beam line of the Advanced Photon Source at Argonne National Laboratory. Use of the APS is supported by the U.S. Department of Energy (DOE) under Contract No. DEAC02-06CH11357. This research was partially funded by DOE's Vehicle Technologies Program, Office of Energy Efficiency and Renewable Energy. The authors would like to thank Team Leaders Gurpreet Singh and Leo Breton for their support of this work

\section{Nomenclature}

$T \quad$ temperature $[\mathrm{K}]$

$P \quad$ pressure [bar]

$V \quad$ volume $\left[\mathrm{m}^{3}\right]$

$S \quad$ surface area $\left[\mathrm{m}^{2}\right]$

$D \quad$ diameter [m]

$A \quad$ angle [degrees]

\section{References}

[1] Sandquist, H., Denbratt, I., Owrang, F., Olsson, J., Influence of Fuel Parameters on Deposit Formation and Emissions in a Direct Injection Stratified Charge SI Engine, 2001, SAE Paper 2001-01-2028.

[2] Caprotti R., Fowler W.J., Diesel additive technology effects on injector hole erosion/corrosion, injector fouling and particulate traps, 1993, SAE Paper 932739.

[3] Lepperhoff G., Houben, M., Mechanisms of Deposit Formation in Internal Combustion Engines and Heat Exchangers, 1993, SAE Paper 931032.

[4] Birgel, A., Ladommatos, N., Aleiferis, P., Zülch, S., Milovanovic, N., Lafon, V., Orlovic, A., Lacey, P., Deposit Formation in the Holes of Diesel Injector Nozzles: A Critical Review, SAE Paper 2008-01-2383. 
[5] Argueyrolles, B., Dehoux, S., Gastaldi, P., Grosjean, L., Levy, F., Michel, A., Passerel, D., Influence of injector nozzle design and cavitation on coking phenomenon, SAE Paper 2007-01-1896.

[6] Turner, J.E., Stetsyuk, V., Crua, C., Pearson, R.J. Gold, M.R., August 23-27 2015, 13th International Conference on Liquid Atomization and Spray Systems, Tainan, Taiwan.

[7] Moon, S., Huang, W., Li Z., Wang, J., 2016, Applied Energy, 179, pp.7-16.

[8] Kook, S., Pickett, L., Musculus, M., Influence of diesel injection parameters on end-of-injection liquid length recession, 2009, SAE Int. J. Engines, 2, pp. 1194-210.

[9] Swantek, A. B., Duke, D. J., Tilocco, F. Z., Sovis, N., Powell, C., Kastengren, A. L., 2014, ILASS-Americas 26th Annual Conference on Liquid Atomization and Spray Systems, Portland, Oregon

[10] Swantek, A. B., Kastengren, A. L., Duke, D. J., Tilocco, F., Sovis, N., Powell, C. F., 8-10 September, 2014, ILASS Europe, 26th Annual Conference on Liquid Atomization and Spray Systems, Bremen, Germany.

[11] Kastengren, A., Powell, C.F., Tilocco, F.Z., Liu, Z., Moon, S., Zhang, X., Gao, J., 2012, J. Eng. Gas Turbines Power, 134, p. 094501.

[12] Fessler, J. A., Macovski, A., 1991, IEEE Transactions on Medical Imaging 10, pp. 25-39.

[13] Kumar, S.S, Amutha, R., March 30-31, 2012, IEEE-International Conference On Advances In Engineering, Science And Management (ICAESM -2012).

[14] Cárdenes, R., Alexey, N., Julian, G., Rod H., Alejandro, F.F., 2012, IEEE International Symposium on Biomedical Imaging.

[15] Goyal, M., Prakash A., Yang, J., 2013. 3D Reconstruction of Coronary Arteries from Angiographic Images: A Survey.

[16] Sommer, H.J., Kao, C.C., Turns, S.R., 1990, 13th Annual Energy-Sources Technology Conference. Petroleum Division, The American Society of Mechanical Engineers, New York

[17] Kitamura K., Tobis J. M., Sklansky J., 1988, IEEE Truns. Med. Imaging, 7, pp. 173-187.

[18] Hoffmann K.R., Doi K., Chan H., Chua K., 1987, Proc. SPIE 767, Med. Imaging, pp. 449-453.

[19] Colombo C., Bimbo A., Pernici F., 2005, IEEE transactions on pattern analysis and machine intelligence, 27 , 1, pp.99-144.

[20] Canny, J., A Computational Approach to Edge Detection, 1986, IEEE Transactions on Pattern Analysis and Machine Intelligence, PAMI-8, 6, pp. 679-698.

[21] Duke, D. J., Swantek, A., Tilocco, Z., Kastengren, A., Fezzaa, K., Neroorkar, K., et al., 2014, SAE Int. J. Engines, 7(2), pp. 1003-1016.

[22] Kastengren, A. L., Tilocco, F., Duke, D. J., Powell, C., Zhang, X., \& Moon, S., 2014, Atomization and Sprays, 24, 3, pp. 251-272.

[23] Delaunay, B., 1934, Bulletin de l'Académie des Sciences de l'URSS, Classe des sciences mathématiques et naturelles, 6, pp. 793-800.

[24] Pauri, R., Bracho, G., Gomes-Aldaravi, P.M., Viera, A., 2017, Atomization and Sprays, DOI: 10.1615/AtomizSpr.2017017949.

[25] Nicolas Fdida, Développement d'un système de granulométrie par imagerie: application aux sprays larges et hétérogènes, 2008, pp. 71-76.

[26] Serra, J., 1983, Image Analysis and Mathematical Morphology. Academic Press, Inc., Orlando, FL, USA.

[27] Dougherty, E.R., An introduction to morphological image processing, 1992, SPIE Optical Engineering Press.

[28] Kumar S.S., Amutha R., March 30-31, 2012, IEEE international conference on advances in engineering, science and management.

[29] Igbinosa I. E., 2013, International Journal of Information Technology and Electrical Engineering, 2, pp. 25-29.

[30] Shrivakshan G. T., Chandrasekar C., 2012, International Journal of Computer Science, 9, 5, pp. 269-276.

[31] Edelsbrunner H., Mucke E.P., 1994, ACM Trans. Graph., 13, 1, pp. 43-72.

[32] Lafarge T., Pateiro-López B., Possolo A., Dunkers J., 2014, Journal of Statistical Software, 56, 4, pp. 1-17.

[33] Manin, J., Bardi, M., Pickett, L. M., and Payri, R., 2016, International Journal of Multiphase Flow, 83, pp. 267-

278, DOI: 10.1016/j.ijmultiphaseflow.2015.12.001 


\title{
Zooming into primary breakup mechanisms of high-pressure automotive sprays
}

\author{
Valeri Kirsch*, Manuel A. Reddemann, Johannes Palmer, Reinhold Kneer \\ Institute of Heat and Mass Transfer, RWTH Aachen University, Augustinerbach 6, 52056 \\ Aachen, Germany \\ ${ }^{*}$ Corresponding author: kirsch@wsa.rwth-aachen.de
}

\begin{abstract}
In-cylinder mixture formation and combustion are highly influenced by primary breakup of injected fuel. Experimental investigation of this phenomenon directly at the outlet of a diesel injector requires a specialized transmitted light microscopy technique combined with a constant-pressure flow microscopy vessel. The method allows verification of the existence of an intact jet core for various states of injection and different fuels. The jet core is dominated by axisymmetric surface waves during the initial injection phase. By quantification of the wavelengths and comparison with existing breakup theories, boundary layer instabilities are identified as origin of surface waves. Boundary layer wavelengths are found to be larger for a higher fuel viscosity. An occasionally appearing non-cylindrical helical jet shape is visible during the injector's opening and closing phase. The helical jet shape is directly resulting from the nozzle outlet flow. Inner nozzle effects are found to be responsible for generation of the helical structure. A fuel dependence of the helical structure formation and its breakup could not be proved. Results also prove that fuel is exiting the nozzle even after the injector needle is closed, while air is simultaneously moving into the nozzle orifice.
\end{abstract}

\section{Keywords}

automotive spray, primary breakup, microscopy, boundary layer instability

\section{Introduction}

Underlying physics of primary jet breakup into poly-disperse sprays at engine-relevant conditions has been an important research topic for decades. Engine-relevant conditions require a complex experimental setup. Such a complex setup usually allows only limited optical accessibility of the region of interest. In addition, optical measurements of the liquid breakup of automotive sprays are difficult, because of the phenomenon itself: Injection pressures up to $300 \mathrm{MPa}$ generate billions of liquid structures (jet, ligaments, droplets) with high velocities (up to $800 \mathrm{~m} / \mathrm{s}$ ) and microscopic scales (up to $1 \mu \mathrm{m}$ ). Multiply scattered light at the gas-liquid interface of these structures increase the optical density of the spray by such a degree that almost no information is gained in the near-field of the nozzle based on classical visualization techniques [1]. As a consequence, scaled generic nozzles are used for fundamental primary breakup investigations. Though, based on generic nozzles, engine-relevant dimensionless numbers and nozzle effects (such as cavitation) cannot be comprehensively reproduced. Therefore, automotive nozzles must be investigated for a complete primary breakup description.

To enable optical investigation of primary breakup even for engine-relevant injection conditions, several experimental methods are specifically developed, e.g. Ballistic Imaging [2] or X-Ray Absorption [3]. Besides these advanced methods, conventional microscopy with optimized optics and adjusted illumination sources have shown great capability for detailed investigations of primary breakup directly at the nozzle orifice. Reddemann et al. [4, 5] investigated primary breakup of diesel jets under atmospheric conditions with a double-frame transmitted light microscope (DF-TLM). Further publications also show microscopic investigations of the primary breakup for increased ambient conditions $[6,7,8]$.

In this work, a DF-TLM system is used for microscopic imaging of the primary breakup at cold engine-like ambient densities. Images are recorded directly at the nozzle outlet of a diesel injector. Focus of the analysis lies on the transient moments of the injection (the opening and closing phase). Three different fuels (diesel, 1-octanol, di-n-butyl ether) are utilized, to investigate the effect of a wide range of dimensionless numbers (Ohnesorge, Reynolds) on the jet breakup. Recorded images deliver highly resolved information of the breakup structures, which are evaluated in size, shape and velocity. These values are used for comparison with physical theories that could explain the observed breakup mechanisms.

\section{Material and methods}

An upgraded version of the DF-TLM system and the constant-pressure flow microscopy (CPF-M) vessel presented by Reddemann et al. [4, 7] are used for primary breakup investigations directly at the nozzle orifice (figure 1). The optical system of TLM is adjusted for automotive sprays, covering a numerical aperture of NA=0.16 and a resolution of $2 \mu \mathrm{m}$ (Abbe criterion [9]). This configuration leads to a depth of field of DOF=70 $\mu \mathrm{m}$ (airy disk diameter as criterion for circle of confusion). The CPF-M vessel enables investigations at ambient pressures up to $5 \mathrm{MPa}$ at atmospheric temperature $(\mathrm{T}=293 \mathrm{~K})$. The illumination source, a pulsed diode laser (Cavilux Smart), produces almost incoherent light pulses ${ }^{1}$ with a duration of $10 \mathrm{~ns}$ and a wavelength of $640 \mathrm{~nm}$. The light source suppresses speckles,

${ }^{1}$ The light source has a coherence length of about $80 \mu \mathrm{m}$. Thus, this light source can be treated as incoherent for most observed structures in this work. 


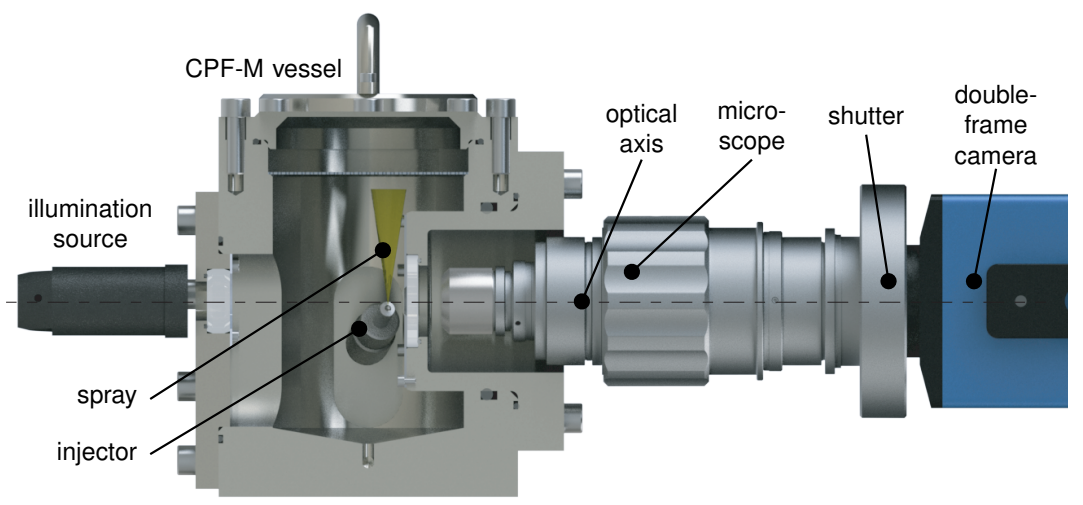

Figure 1. Combined setup of the Double Frame Transmitted Light Microscope (DF-TLM) and the Constant-Pressure Flow Microscopy (CPF-M) chamber.

chromatic aberrations and motion blur up to a spray velocity of $100 \mathrm{~m} / \mathrm{s}^{2}$. A double-frame CCD-Camera (LaVision Flowmaster) delivers not only shapes and sizes at the nozzle near-field of the primary breakup structures, but also offers possibility to measure displacements of the structures during an inter-framing time of $1 \mu \mathrm{s}$. Double-frame images allow velocity determination under consideration of the magnification factor of the optical system $(M=6.7)$. Central element of the experimental setup is a piezo-injector with a 3-hole nozzle $(D=140 \mu \mathrm{m}$, length-to-diameter ratio $=7.32$, inlet to outlet ratio $=1.1$, midi sac-hole). Alignment of injector and optical axis is shown in figure 1 . An injection pressure of $100 \mathrm{MPa}$ and an energizing duration of $1800 \mu \mathrm{s}$ are chosen for the investigation. Compared to real engine applications, this long energizing duration ensures a steady-state injection phase. 1-octanol, di-n-buthyl ether (DnBE) as alternative fuels and diesel are used to cover a wide range of Reynolds and Ohnesorge numbers. Physical properties, dimensionless numbers and operating conditions (OC) are shown in table 1.

Table 1. Fuel properties at $293 \mathrm{~K}$ and dimensionless numbers at investigated operating conditions (OC).

\begin{tabular}{|c|c|c|c|c|c|c|c|c|}
\hline & OC & $\begin{array}{c}\rho \\
\mathrm{kg} / \mathrm{m}^{3}\end{array}$ & $\begin{array}{c}\eta \\
\mathrm{mPas}\end{array}$ & $\begin{array}{c}\sigma \\
\mathrm{mN} / \mathrm{m}\end{array}$ & $\begin{array}{c}p_{v} \\
\mathrm{kPa}\end{array}$ & $\begin{array}{c}\rho^{*} \\
-\end{array}$ & $\begin{array}{c}R e \\
-\end{array}$ & $\begin{array}{c}O h \\
-\end{array}$ \\
\hline $\begin{array}{l}\text { diesel } \\
\text { EN590 }\end{array}$ & $\begin{array}{l}d i 1 \\
d i 2\end{array}$ & 833 & 3 & 20.5 & $<1$ & $\begin{array}{c}2.9 \mathrm{e}-3 \\
11.5 \mathrm{e}-3\end{array}$ & $\approx 19000$ & 0.061 \\
\hline 1-octanol & $\begin{array}{l}o c 1 \\
o c 2\end{array}$ & 826 & 8.6 & 26.6 & 0.01 & $\begin{array}{c}2.9 \mathrm{e}-3 \\
11.6 \mathrm{e}-3\end{array}$ & $\approx 6600$ & 0.155 \\
\hline DnBE & $\begin{array}{l}d n 1 \\
d n 2\end{array}$ & 768 & 0.69 & 22.6 & 0.82 & $\begin{array}{c}3.1 \mathrm{e}-3 \\
12.5 \mathrm{e}-3\end{array}$ & $\approx 79500$ & 0.014 \\
\hline
\end{tabular}

\section{Results and discussion}

An exemplary time series of an 1-octanol injection is shown in figure 2. Regardless of the injection phase, a connected jet core is visible directly at the nozzle orifice. The injection process can be subdivided into five characteristic phases: I) start of injection (needle opening) shortly after start of energizing ( $t=295 \mu \mathrm{s})$, II) acceleration during needle lifting $(\mathrm{t}=500 \mu \mathrm{s})$, III) steady-state $(\mathrm{t}=2500 \mu \mathrm{s})$, IV) deceleration during needle subsiding $(\mathrm{t}=4500 \mu \mathrm{s})$ and $\mathrm{V})$ end of injection after the needle is closed ${ }^{3}(\mathrm{t}=4750 \mu \mathrm{s})$. Injector opening and closing moments vary slightly, depending on the utilized fuel. The needle response depends strongly on viscosity of injected fuel and can be determined by injection rate measurements. For the low viscosity fuel $\mathrm{DnBE}$, needle closing occurs $\mathrm{t}=4250 \mu$ s and for high viscosity

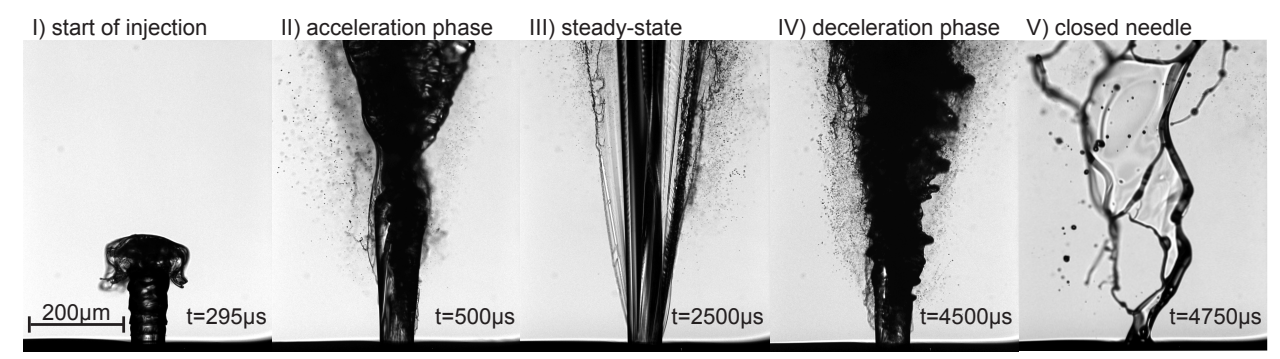

Figure 2. Exemplary images of the primary breakup process at different injection phases. Injected fuel: 1-octanol, injection pressure: $100 \mathrm{MPa}$, ambient density: $2.4 \mathrm{~kg} / \mathrm{m}^{3}$ and ambient temperature: $293 \mathrm{~K}$.

\footnotetext{
${ }^{2}$ Based on the criterion of one pixel displacement on the image plane.

${ }^{3}$ Additional injection rate measurements performed with a Bosch-type method rate meter [10] for the same injection and ambient conditions, proof needle closing at a time of $t \approx 4600 \mu$ s after start of energizing.
} 
fuel 1 -octanol needle closing is reached later at $t=4600 \mu \mathrm{s}$. In the following, breakup mechanisms of the injection phases are described and analyzed in detail, exemplary for 1-octanol.

\section{I) Start of injection:}

Figure 3 shows images of the start of injection for three fuels (1-octanol, diesel and DnBE) for two different ambient densities $\left(\rho=2.4\right.$ and $\left.9.6 \mathrm{~kg} / \mathrm{m}^{3}\right)$. Even if time after energizing is kept constant, the jet penetration is not identical but varies from 300 to $450 \mu \mathrm{m}$ ). The typical jet tip shape during start of injection is a spheroidal cap and is basically shown for all images of figure 3. However, in case of DnBE at low ambient density, the spheroidal cap is covered with a pre-ligament. Just below the spheroidal tip axisymmetric surface waves are also visible in all shown images. Wavelengths of those disturbances are manually measured and illustrated in figure 3 . Measured wavelengths are fuel-dependent and decrease in length from 1-octanol to diesel to DnBE. Occurring wavelengths of each fuel are independent of ambient density. Tip velocities are determined with double-frame images and are illustrated also in figure 3. Calculated velocities for the higher ambient density $(\mathrm{v} \approx 35-61 \mathrm{~m} / \mathrm{s})$ are lower compared to the velocities at the lower ambient density $(\mathrm{v} \approx 64-68 \mathrm{~m} / \mathrm{s})$. It is important to note for later wavelength evaluation that for an ambient density of $\rho=2.4 \mathrm{~kg} / \mathrm{m}^{3}$ tip velocities are almost the same. However, images presented in figure 3 are selected, because they give insight into the process of a more or less laminar jet propagation during start of injection. The same injection conditions may also produce a turbulent jet tip with many small droplet and ligaments, but neither a spheroidal cap nor axisymmetric surface waves. Turbulent jet tips require further information for interpretation and are discussed in the section of the closed needle phase.
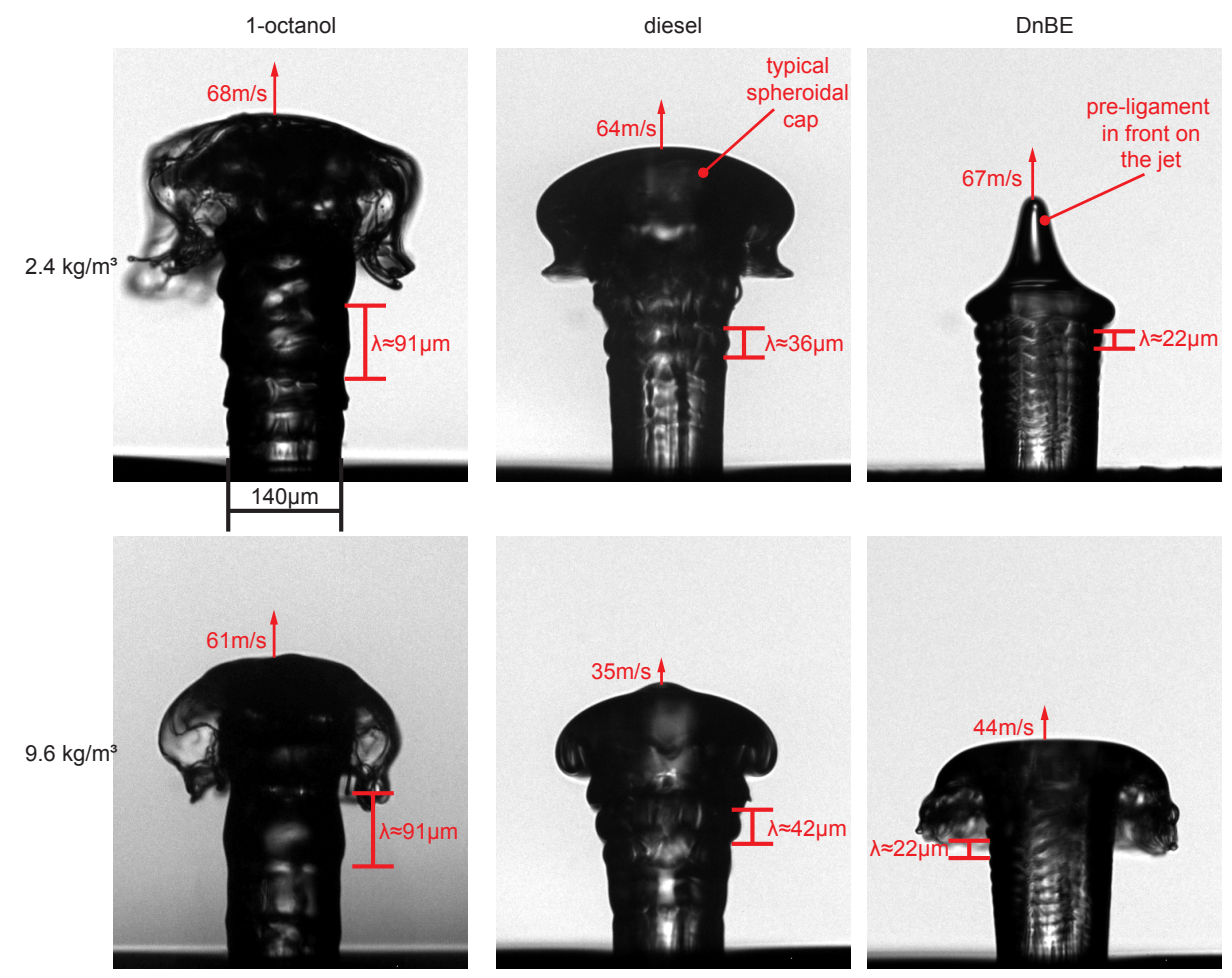

Figure 3. Images of the start of jet penetration of the three fuels 1-octanol, diesel and DnBE for two ambient densities $2.4 \mathrm{~kg} / \mathrm{m}^{3}$ and $9.6 \mathrm{~kg} / \mathrm{m}^{3}$. Axisymmetric waves are present at almost the same axial nozzle distance, but with different wavelengths. Time after energizing: 285 - $295 \mu \mathrm{s}$, injection pressure: $100 \mathrm{MPa}$, ambient temperature: $293 \mathrm{~K}$.

The spheroidal cap at the jet tip, with and without pre-ligaments, are also observed and described for laminar jet tips by other authors, e.g. Crua et al. [11], Badock and Wirth [12], Busch [13], Liu et al. [14], Hult et al. [15] or Schugger [16]. Crua et al. [11] explains the existence of a spheroidal cap at the jet tip by an interaction of adhesion forces at the nozzle surface and rapidly increasing axial jet velocity: fresh fuel from inside the nozzle hole pushes the jet forwards into the atmosphere until inertia of the jet tip compensates the adhesion force and the spheroidal cap detaches from the nozzle surface. Pre-ligaments in front of the spheroidal cap are explained by a slipstream effect, which propels the central pre-ligament ahead of the jet into the atmosphere [11].

Occurrence of axisymmetric surface waves during jet formation have been rarely discussed for automotive sprays so far. In a more general context of low Reynolds and Weber number, formation of axisymmetric surface disturbances are explained by two possible reasons: i) Kelvin-Helmholtz instabilities or ii) boundary layer instabilities [17]. It is commonly known that wavelengths that occur due to Kelvin-Helmholtz instabilities behave inversely proportional to changes in ambient density $[18,17]$. Results presented in figure 3 are in contradiction to this theory. Therefore, axisymmetric surface waves cannot be caused by Kelvin-Helmholtz instabilities. Instead, bounrary layer instabilities could be an explanation for the origin of the surface waves. Boundary-layer instabilities are developed, due to jet velocity profile relaxation [17]. According to Brennen's analytic boundary layer theory, the ratio between mean jet velocity $u$ and instability frequency $f$ can be expressed as function of boundary layer momentum thickness $\delta_{2}$ and 
dimensionless frequency $\gamma$, which in turn can be approximated as boundary layer instability wavelength $\lambda_{B L}$ [19]:

$$
\frac{u}{f}=\frac{2 \cdot \pi \cdot \delta_{2}}{\gamma} \approx \lambda_{\mathrm{BL}}
$$

For a laminar case, dimensionless frequency $\gamma$ is given with a maximum growth rate of 0.175 . Under the assumption of a Blasius's solution for laminar flow fields [17], boundary layer momentum thickness depends on fuel properties (density and viscosity) and jet velocity (injection pressure), only. These influences are usually taken into account on the basis of the steady-state Reynolds number. Such an assumption is not allowed in the current case due to the transient behavior of the jet (increasing velocity, uncompleted velocity profile relaxation). Nevertheless, analyzing the illustrated images in figure 3 at the ambient density of $\rho=2.4 \mathrm{~kg} / \mathrm{m}^{3}$ show an almost constant jet tip velocity. Thus, boundary layer momentum thickness and boundary layer instability wavelengths depend on fuel properties, only. Fuel viscosity must have a major influence on the wavelengths, because viscosity differences between the three fuels are much larger compared to differences in density (compare table 1). If boundary layer instabilities and wavelengths are only depending on the fuel viscosity, a smaller boundary layer momentum thickness is expected and a small boundary layer wavelength for a lower viscosity should be visible in the recorded images (compare equation 1). This is clearly the case (see figure 3). As a result, observed surface waves are in in good agreement with boundary layer instability theory.

\section{II and IV) Acceleration and deceleration phase:}

Figure 4 shows the acceleration phase during needle lifting. A rough jet surface with many surface disturbances and structures (sheets, ligaments and droplets) around the jet core can be observed in both double-frame images (top). Directly at the nozzle outlet, the jet core has glare lines on its surface (see magnification). Glare lines run on the jet's surface in an axial-tangential direction. Further downstream, optical density is increased due to surface disturbances and structures around the jet in such a degree that almost no details about the inner jet core are visible. The outer shape of the jet shows frequent constrictions and expansion along the flow direction. Same observations are found for the deceleration phase of the jet during needle subsiding (not shown in the figure). In the following, both phases are analyzed simultaneously, due to their physical similarity.

Cavitation is assumed to be responsible for the high amount of surface disturbances during this injection phases. Turbulence is generated by cavitation, which in turn disturbs the jet surface [20]. Near nozzle glare lines observed on the jet surface indicate a tangential velocity share in the jet flow field. Proximity of the glare lines to the nozzle hole suggests that the tangential flow is caused by a vortex flow inside the nozzle hole. This vortex flow can be caused by string cavitation inside the sac hole [21, 22] or by an off-centered needle position [23]. Soteriou et al. [24] showed that centrifugal forces caused by such a vortex flow is able to shape the jet core into a helical shape. Such a three-dimensional helical shape cannot be fully proven just by analyzing the in this work taken two dimensional double-frame images. Nevertheless, the hypothesis of such a helical structure is reasonable for the observed jet properties. A schematically illustration of a here maybe observed left-handed helical jet shape is shown at the right bottom of figure 4. Main constriction and expansion of a hypothetical helix along the flow direction can be approximated as shown in the top left image of figure 4. The mode of such a helix cannot be determined with the given limited informations. However, as shown by Kubitschek and Weidman [25] a helical shaped jet is accompanied by formation of liquid sheet structures. Sheet structures start to form at the outer curvature of the hypothetical helix due to centrifugal forces. The same centrifugal forces cause a subsequent thinning of the sheet structures. The curvature of the helix is thus decreased for a larger distance from nozzle outlet. Thinning of the sheet structures finally ends in breakup and generation of ligaments and droplets with a flight path in radial-axial direction. Similar effects can be observed at both double-frame images in figure 4. The only difference is that breakup of in this work observed sheet structures is most likely not only caused by centrifugal and surface tension forces but also by aerodynamic forces. These aerodynamic forces disturb the sheet surface until the sheet structure cannot be hold together by cohesion forces anymore and break up in ligaments and droplets.

\section{III) Steady-state of the injection:}

The steady-state phase of the injection has already been analyzed in our previous publication [26] and is therefore not topic of this work. For the same fuels, injection and ambient conditions, a rippled structure and transversal disturbances on the jet surface are found. Rippled structures occurring directly at the nozzle hole are independent of ambient conditions and fuel properties. The jet cross-section is shaped by this rippled structures into a star-like polygon. An explanation for transversal disturbances on the jet surface have not been found so far, but will be investigated in a future work.

\section{V) Closed needle:}

Figure 5 shows double-frame images of the injection shortly after needle closing. At this injection state, fuel in the vessel is connected to the nozzle hole. Mostly large connected liquid structures are visible across the entire image. These connected structures consist of large ligaments (diameters up to $100 \mu \mathrm{m}$ ), which are often connected by sheets (sheet thickness $\leq 3 \mu \mathrm{m})^{4}$. On most of these sheet structures possible interference patterns are visible. Scattered big droplets $(d \approx 5-35 \mu \mathrm{m})$ around the structures and some bubbles $\left(d_{b} \approx 35 \mu \mathrm{m}\right)$ inside the structures ${ }^{5}$

\footnotetext{
${ }^{4}$ The sheet thickness can be approximated by measuring the size of the smallest ligament connected to the sheet with a diameter of approximately $3 \mu \mathrm{m}$. The ligament has to be larger in diameter as the sheet thickness due to surface tension forces.

${ }^{5}$ Three reasons justify the conclusion that the structures inside the large ligaments have to be bubbles: 1) If those round structures are considered to be droplets, some of the droplets have to be formed inside the nozzle hole. Since some of them are really close to the nozzle hole. It is very
} 


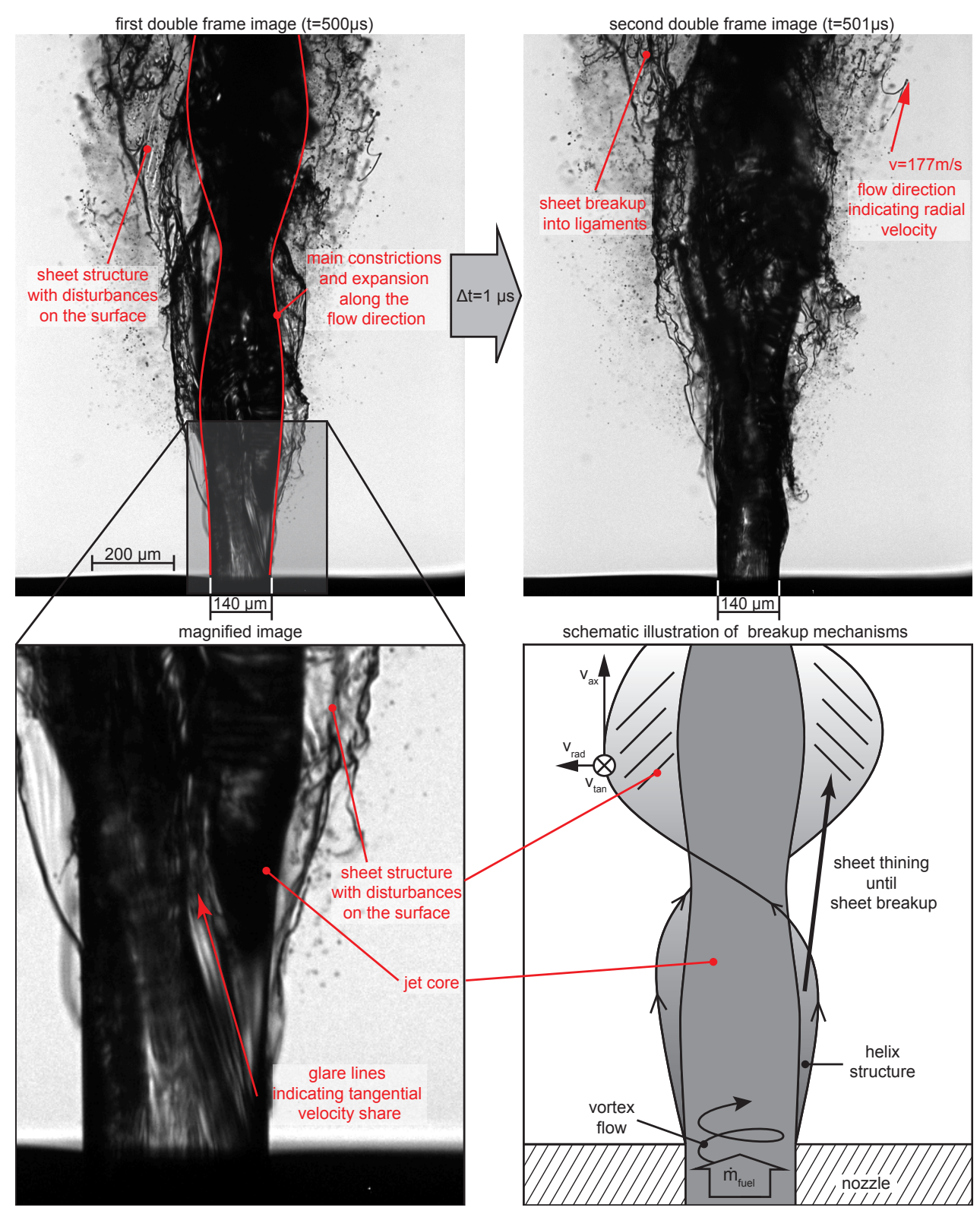

Figure 4. Double-frame images of the phase during the needle opening with a schematic illustration of observed mechanisms. Time after energizing: $500 \mu \mathrm{s}$, Injected fuel: 1 -octanol, injection pressure: $100 \mathrm{MPa}$, ambient density: $2.4 \mathrm{~kg} / \mathrm{m}^{3}$ and ambient temperature: $293 \mathrm{~K}$.

are present as well. A high amount of small droplets $(d \approx 1-4 \mu \mathrm{m})$ can be observed in some finite areas (magnification in figure 5). By analyzing double-frame images, a tangential velocity share and maximum velocities up to $v \leqq 22 \mathrm{~m} / \mathrm{s}$ can be calculated. With further nozzle distance, velocities are increasing. Almost no fluid velocity is measurable directly at the nozzle orifice. The orifice is not fully covered with fuel, but the surface around the orifice is partly covered with liquid.

The uninterrupted flow of fuel after needle closing may be explained by the remaining injection inertia. The inertia propels fuel out of the nozzle hole until friction forces fully dissipate the inertia force inside the nozzle hole (balance of forces in figure 5, bottom right). Once this state is reached, structures close to the nozzle hole are thinned due to surface tension forces. Eventually, the liquid structure will tear apart at this thinned position. Structures downstream the tear-off edge separate from remaining fuel and move further into the vessel. Fuel upstream the tear-off edge remains attached to the nozzle and stays there until the next injection starts. Fuel attaches to the nozzle due to adhesion forces.

The remaining radial and tangential inertia of liquid structures moving inside the vessel tears the structure apart into many separate secondary ligaments. Connecting sheets in between the structures are thinning until they fragment into small ligaments and droplets due to the increasing distance between the sheet surrounding ligament

unlikely that droplets will form inside the nozzle and fly into ambiance without sticking to other structures. 2) The observed round structures follow the ligaments the entire observed time. This is only possible if they are included into the ligaments. 3) Some of the big round structures are constantly deformed to a longitudinal shape. The only possible reason for such a deformation even at the observed small velocities is surface tension forces caused by an enclosing surface of the bigger sized ligaments. 


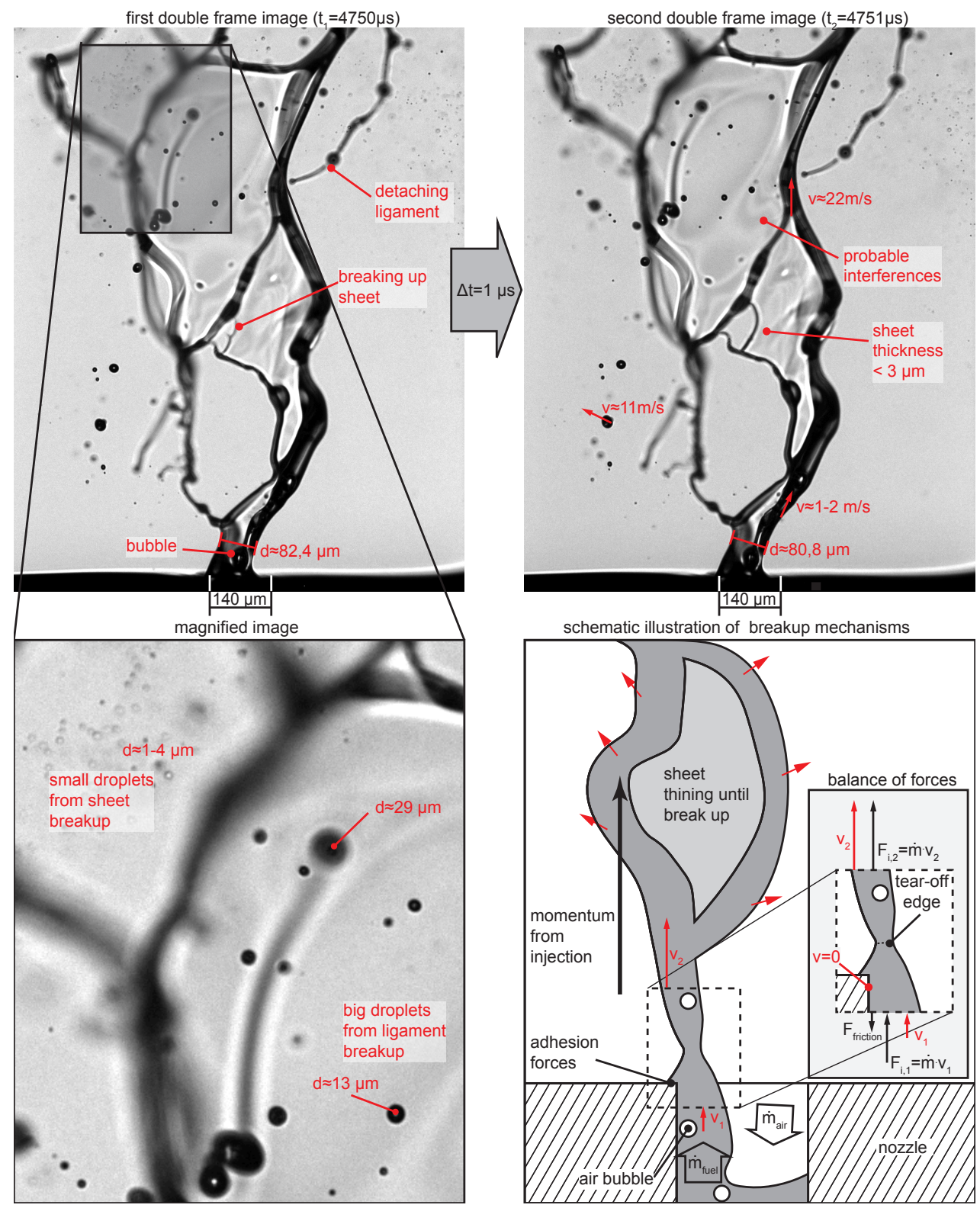

Figure 5. Double-frame images of the phase shortly after the needle closed with a schematic illustration of observed mechanisms. Time after energizing: $4750 \mu \mathrm{s}$, Injected fuel: 1 -octanol, injection pressure: $100 \mathrm{MPa}$, ambient density: $2.4 \mathrm{~kg} / \mathrm{m}^{3}$ and ambient temperature: $293 \mathrm{~K}$.

structures. Size of those developing small ligaments and droplets has to be in the same order of the sheet thickness. This means that a high amount of small droplets and ligaments $(d<4 \mu \mathrm{m})$ develop in a finite area by sheet breakup. Big droplets $(d>4 \mu \mathrm{m})$ in turn, develop by fragmentation of large ligaments. Most of the observed fuel mass at this phase remains as big droplets or big sized ligaments inside the vessel.

A not fully covered nozzle hole can be explained as follows: As soon as the injector needle is closed, fuel from inside the injector cannot flow into the nozzle. As a result, air moves inside the nozzle hole as compensation for fuel that has left the nozzle after needle closing. Once inertia is too small to propel further fuel into the vessel, air inside the nozzle is trapped until start of a consecutive injection. Similar observations where are observed by Blessing [20] and Ghiji et al. [27].

Observed bubbles inside liquid structures must consist either of i) fuel vapor or ii) ambient gas. Fuel vapor inside the nozzle hole usually develops at automotive injections by cavitation. Cavitation is caused by high fuel velocities with a hydrostatic pressure below the vapor pressure of the injected fuel [28]. For a closed needle, the total pressure inside the nozzle hole can be approximated with the ambient pressure of $0.2 \mathrm{MPa}$. Considering this fact, velocity inside the nozzle hole has to be at least $22 \mathrm{~m} / \mathrm{s}$ for cavitation to set in (approximated with Bernoulli equation). As shown in figure 5, fuel velocity decreases with lower nozzle distance even below $2 \mathrm{~m} / \mathrm{s}$ until no movement is measurable anymore directly at the nozzle hole. Over time, inertia inside the nozzle hole is dissipated by friction forces. As a result, velocity inside the nozzle hole has to be smaller than the velocity outside the nozzle hole. With this low fluid velocity observed, bubbles inside liquid structures cannot be caused by cavitation. As a result, bubbles must consist 
of air that has just moved into the nozzle.

Air that moved into the nozzle, may exit the nozzle again immediately shaped as air bubbles. Air bubbles trapped in liquid structures are streaming out the nozzle as long as inertia of the injection is not completely dissipated. Remaining air inside the nozzle is most likely responsible for the varying initial jet shape of a subsequent injection. Air bubbles trapped inside the liquid fuel are probably compressed during the fuel acceleration due to inertia forces. As soon as air bubbles leave the nozzle hole, pressure drops significantly and bubbles abruptly decompress and disturb the jet. This mechanism was analysed in detail by Ghiji et al. [27]. However, this latter mechanism has not been shown in this work.

\section{Summary}

High resolution images of the near nozzle primary breakup region are shown for increased ambient densities. Two alternative fuels (1-octanol, DnBE) and diesel are investigated. Images are recorded by a double-frame transmitted light microscope, optimized just for the investigation of the primary breakup region of automotive jets. Five typical injection phases are resolved and described in detail: start of injection (needle opening), acceleration phase during during needle rising, steady-state, deceleration phase during needle subsiding and end of injection after the needle is closed. A spheroidal jet tip and axisymmetric surface waves are often visible during the initial injection phase. The analysis of the surface wavelengths suggests that those waves are caused by boundary layer instabilities. During needle opening and closing, a vortex flow caused by string cavitation or an off-centered needle position shapes the jet into a helical structure. The steady-state of the injection is described in a previous work and is therefore not a topic in this work. After the injector needle is closed, fuel still leaves the nozzle hole due to the not fully dissipated momentum of the injection. As compensation for fuel that still exits the nozzle hole, air is streaming into the nozzle hole. This mechanism is most likely responsible for the varying jet shape during the initial state of a consecutive injection. Fuel influences are found with high certainty for the axysmmetric jet waves only. For other phenomena, such as the helical jet shape ligament and sheet-breakup after needle closing, reproducibility is low and no fuel influences are identified.

\section{Acknowledgement}

This work was performed as part of the Cluster of Excellence "Tailor-Made Fuels from Biomass", which was funded by the Excellence Initiative of the German federal and state governments to promote science and research at German universities.

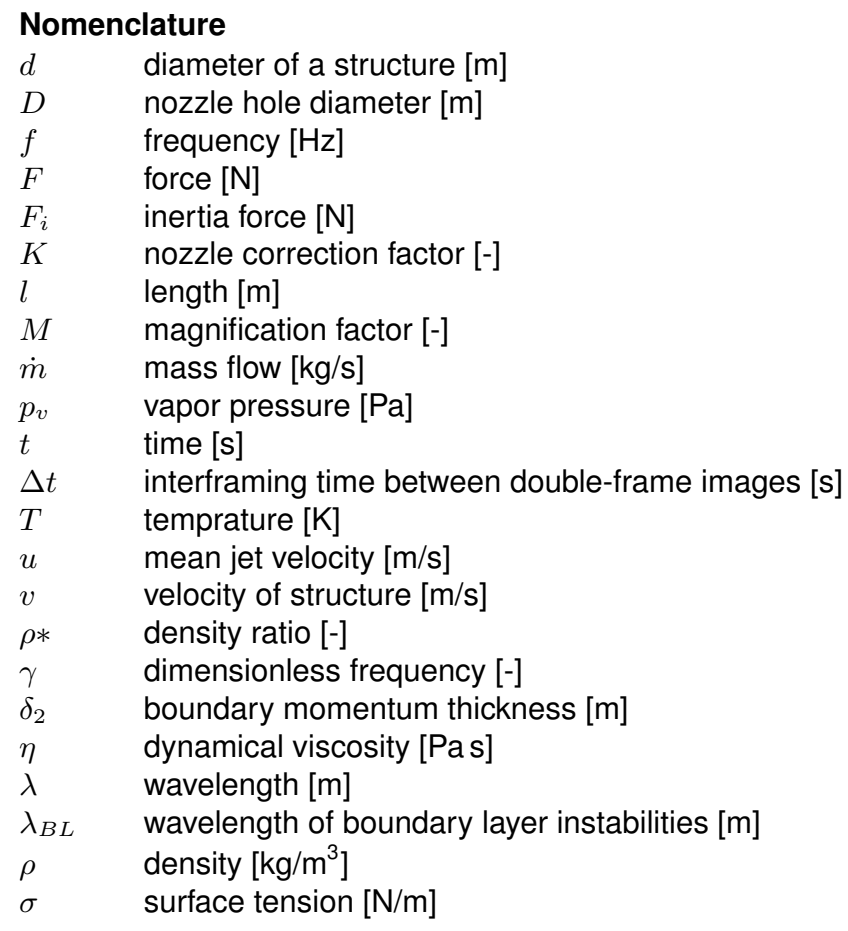

\section{References}

[1] Linne, M., 2013. "Imaging in the optically dense regions of a spray: A review of developing techniques". Progress in Energy and Combustion Science, 39(5), pp. 403-440.

[2] Linne, M., Paciaroni, M., Hall, T., and Parker, T., 2006. "Ballistic imaging of the near field in a diesel spray". Experiments in Fluids, 40(6), pp. 836-846. 
[3] Yue, Y., Powell, C. F., Poola, R., Wang, J., Lai, M.-C., and Parrish, S. E., 2001. "Quantitative Measurements of Direct-Injection Gasoline Fuel Sprays in Near-Nozzle Region Using Synchrotron X-Ray". In SAE 2001 World Congress, SAE Technical Paper Series.

[4] Reddemann, M. A., Mathieu, F., and Kneer, R., 2013. "Transmitted light microscopy for visualizing the turbulent primary breakup of a microscale liquid jet". Experiments in Fluids, 54(11).

[5] Reddemann, M. A., Mathieu, F., and Kneer, R., 2013. "Zooming into the primary breakup region of enginerelated sprays". In ILASS - Europe 2013, 25th European Conference on Liquid Atomization and Spray Systems, Chania, Greece, 1-4 September 2013.

[6] Crua, C., Shoba, T., Heikal, M., Gold, M., and Higham, C., 2010. "High-Speed Microscopic Imaging of the Initial Stage of Diesel Spray Formation and Primary Breakup". In SAE 2010 World Congress \& Exhibition, SAE Technical Paper Series.

[7] Reddemann, M. A., Kirsch, V., and Kneer, R., 2014. "Transmitted light microscopy of primary breakup for engine-relevant ambient densities". In ILASS - Europe 2014, 26th Annual Conference on Liquid Atomization and Spray Systems, 8-10 Sep. 2014, Bremen, Germany.

[8] Shoba, T., Crua, C., Heikal, M. R., and Gold, M., 2011. "Optical Characterisation of Diesel, RME and Kerosene Sprays by Microscopic Imaging". In ILASS - Europe 2011, 24th European Conference on Liquid Atomization and Spray Systems, Estoril, Portugal, September 2011.

[9] Abbe, E., 1873. "Beiträge zur Theorie des Mikroskops und der mikroskopischen Wahrnehmung". Archiv für mikroskopische Anatomie, 9(1), pp. 413-418.

[10] W. Bosch, 1964. "Der Einspritzgesetz-Indikator, ein neues Meßgerät zur direkten Bestimmung des Einspritzgesetzes von Einzeleinspritzungen". MTZ - Motortechnische Zeitschrift, 25(7), pp. 268-282.

[11] Crua, C., Heikal, M. R., and Gold, M. R., 2015. "Microscopic imaging of the initial stage of diesel spray formation". Fuel, 157, pp. 140-150.

[12] Badock, C., Wirth, R., Fath, A., and Leipertz, A., 1999. "Investigation of cavitation in real size diesel injection nozzles". International Journal of Heat and Fluid Flow, 20(5), pp. 538-544.

[13] Busch, R., 2001. "Untersuchung von Kavitationsphänomenen in Dieseleinspritzdüsen". PhD thesis, Hannover, Hannover.

[14] Liu, Z., Im, K.-S., Wang, Y., Fezzaa, K., Xie, X.-B., Lai, M.-C., and Wang, J., 2010. "Near-Nozzle Structure of Diesel Sprays Affected by Internal Geometry of Injector Nozzle: Visualized by Single-Shot X-ray Imaging". In SAE 2010 World Congress \& Exhibition, SAE Technical Paper Series.

[15] Hult, J., Simmank, P., Matlok, S., Mayer, S., Falgout, Z., and Linne, M., 2016. "Interior flow and near-nozzle spray development in a marine-engine diesel fuel injector". Experiments in Fluids, 57(4), p. 195.

[16] C. Schugger, 2007. "Experimentelle Untersuchung des primären Strahlzerfalls bei der motorischen Hochdruckeinspritzung". PhD thesis, Rheinisch Westfälische Technische Hochschule Aachen, Aachen.

[17] Yoon, S. S., and Heister, S. D., 2003. "Categorizing linear theories for atomizing round jets". Atomization and Sprays, 13(5-6), pp. 499-516.

[18] Reitz, R. D., and Bracco, F. V., 1982. "Mechanism of atomization of a liquid jet". Physics of Fluids, 25(10), p. 1730.

[19] Brennen, C., 1970. "Cavity surface wave patterns and general appearance". Journal of Fluid Mechanics, 44(01), p. 33.

[20] Blessing, M., 2004. "Untersuchung und Charakterisierung von Zerstäubung, Strahlausbreitung und Gemischbildung aktueller Dieseldirekteinspritzsysteme". PhD thesis, Universität Stuttgart.

[21] Gavaises, M., and Andriotis, A., 2006. "Cavitation Inside Multi-hole Injectors for Large Diesel Engines and Its Effect on the Near-nozzle Spray Structure". In 2006 SAE World Congress, SAE Technical Paper Series.

[22] Papoulias, D., Giannadakis, E., Mitroglou, N., Gavaises, M., and Theodorakakos, A., 2007. "Cavitation in Fuel Injection Systems for Spray-Guided Direct Injection Gasoline Engines". In 2007 World Congress, SAE Technical Paper Series.

[23] Oda, T., Iwatani, T., Takahashi, N., Sumi, T., and Ohsawa, K., eds., 2014. Behavior of Internal cavitating flow and primary spray break-up of a large scaled VCO Diesel nozzle with a small-lifted and eccentric needle.

[24] Soteriou, C., Andrews, R., Torres, N., Smith, M., and Kunkulagunta, R., 2001. "Through the diesel nozzle hole: A journey of discovery II". In ILASS - Europe 2001, European Conference on Liquid Atomization and Spray Systems, 2 -6 September 2001, Zürich, Switzerland, pp. 297-305.

[25] Kubitschek, J. P., and Weidman, P. D., 2007. "Helical instability of a rotating viscous liquid jet". Physics of Fluids, 19(11), p. 114108.

[26] Kirsch, V., Reddemann, M., Thalheim, B., Palmer, J., and Kneer, R., 2016. "Laser correlation velocity measurements in the near field of a diesel injector for variable ambient densities". In 4th TMFB International Conference, Aachen, Germany, 21-23 June 2016.

[27] Ghiji, M., Goldsworthy, L., Brandner, P. A., Garaniya, V., and Hield, P., 2016. "Numerical and experimental investigation of early stage diesel sprays". Fuel, 175, pp. 274-286.

[28] Lefebvre, A. H., 1989. Atomization and sprays. Taylor \& Francis, New York. 


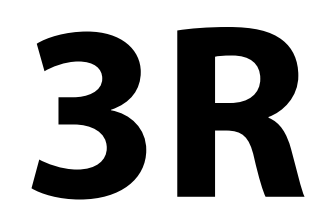

Atomizers 1 


\title{
Internal flow and air core dynamics in Simplex and Spill-return pressure-swirl atomizers
}

\author{
Milan Malý¹, Lada Janáčková1, Jan Jedelský1, Jaroslav Sláma², Marcel Sapík, \\ Graham Wigley ${ }^{3}$ \\ ${ }^{1}$ Faculty of Mechanical Engineering, Brno University of Technology, Czech Republic \\ 2 Provyko s.r.o, Czech Republic \\ ${ }^{3}$ Loughborough University, United Kingdom \\ ${ }^{*}$ Corresponding author: milan.maly@vutbr.cz
}

\begin{abstract}
It is well known that the spray characteristics of pressure-swirl atomizers are strongly linked to the internal flow and that an unstable air core may cause instabilities in the spray. In this paper, a 10:1 scale transparent Plexiglas (PMMA) model of a pressure-swirl atomizer as used in a small gas turbine is introduced. The internal flow was examined using high-speed imaging, laser-Doppler anemometry and computational fluid dynamics tools. The experimental and numerical results were analysed and compared in terms of the air core morphology and its temporal stability. Two different liquids were used, Kerosene-type Jet A-1 represented a commonly used fuel while p-Cymene (4-Isopropyltoluene) matched the refractive index of the Plexiglas atomizer body. The internal flow characteristics were set using dimensionless numbers i.e. the Reynolds number and Froude number. The flow test conditions were limited to inlet Reynolds numbers from 750 to 1750. Two atomizers were examined to represent a Simplex and Spill-return (SR) geometries. In a comparative manner, the SR atomizer features a central passage in the rear wall of the swirl chamber. The main advantage of this concept is that the fuel is always supplied to the swirl chamber at a high pressure therefore providing good atomization over a wide range of the injection flow rate. However, the presence of the spill orifice strongly affects the internal flow even if the spill-line is closed. The air core in the Simplex atomizer was found fully developed and stable. The SR atomizer behaved differently, the air core did not form at all, and the spray was therefore unstable.
\end{abstract}

\section{Keywords}

Internal flow dynamics, Pressure-swirl, transparent nozzle, CFD

\section{Introduction}

Pressure-swirl (PS) atomizers are used in many applications where a large surface area of droplets is needed or a surface must by coated by a liquid e.g. combustion, fire suspension or air conditioning. PS atomizers are easy to manufacture, reliable and provide good atomization quality. They convert the pressure energy of the pumped liquid into kinetic and surface energy of the resulting droplets. The pumped liquid is injected via tangential ports into a swirl chamber, where it gains a swirl motion, under which it leaves the exit orifice as a conical liquid sheet. The centrifugal motion of the swirling liquid creates a low-pressure zone in the centre of the swirl chamber and generate an air core along the centreline. The flow inside the atomizer is rather complex; it is two-phase with secondary flow effects. There is a strong link between internal flow conditions and the resulting spray characteristics, however, not all aspects of the internal flow are well understood.

Before the advent of computational fluid dynamics, a number of authors attempted to describe the internal flow by relatively simple analytical approaches. One of the first was presented by Taylor [1] which focused on an inviscid analysis using Bernoulli's equation and the principle of maximal flow. Taylor derived an equation for the discharge coefficient $\left(C_{D}\right)$ and the spray cone angle (SCA) solely dependent on the atomizer constant $k=2 \cdot A_{p} /\left(\pi \cdot d_{o} \cdot d_{s}\right)$, where $A_{p}$ is the area of the inlet ports, $d_{o}$ and $d_{s}$ are defined in Figure 1. Similar results were found independently by other authors and these works have been compared and reviewed by Chinn [2, 3]. Results obtained by the inviscid theory are not generally in good agreement with experiments. However, findings from the inviscid theory may be used as a base for design improvements.

The experimental correlations for $C_{D}$ were found to be more complex than the inviscid theory predicted. Rizk and Lefebvre [4] derived a semi-empirical correlation, where, beside the constant $k$, the ratio $d_{s} / d_{0}$ has a strong influence. Jones [5] found a weak dependence of $C_{D}$ on the length of the swirl chamber and exit orifice and liquid viscosity. Ballester [6] added a dependence on the inlet pressure. Benjamin [7] followed the work of Jones [5] and found 
inverse trends for the some parameters. Wimmer and Brenn [8] theoretically uncovered a relatively strong effect of the liquid viscosity on $C_{D}$, which was experimentally confirmed later by Maly et al. [9].

The internal flow characteristics, especially the air core stability, were investigated by a few authors. Halder [10] investigated the air core shape in 21 different transparent atomizers at various inlet mass flow rates of water. Two limiting values of Reynolds number $(\mathrm{Re})$ were conducted for the inception of the air core for each atomizer. Below the lower limit, the air core was not formed at all, while above the upper, it was always found stable. He observed that the limiting Re decreases with an increase in $d_{0} / d_{s}$ and a decrease in $A_{p} / d_{s}$. The stable air core had a cylindrical shape, and for large Re values, it was almost constant in diameter. For Re values close to the limiting value the diameter of the air core sharply increased with increasing Re. A similar concept of limiting values of $R e$ was introduced by Lee et al. [11]. In their experimental work, they used a transparent atomizer with diesel and kerosene for a range of inlet pressures and temperatures. They deduced that the air core stability was a function of Re related to the exit orifice, $R_{e}$. It was stable for $R e>3300$, at lower values it became unstable until for $\mathrm{Re}_{\circ}$ below 2400 , there was no air core at all due to insufficient centrifugal forces and the spray fluctuated strongly. Kim et al. [12] investigated the influence of diameter and length of the swirl chamber on the air core stability. Atomizers with ratios of $h_{s} / d_{s}$ higher than 1.27 demonstrated an unstable air core. The authors [12] described the unstable air core as having a rotating and double helical structure. Moon [13] found a limiting value of the swirl number $S_{0}=0.6$, which ensured a stable air core.

SR atomizers have rarely been studied while the effect of the spill arrangement on the internal flow is not at all clear. The liquid return can be realized by a single axial orifice, by several off-axis orifices or by an annular slot. The simplest designs use a single, axially placed spill orifice but the problems with spray stability were reported [14, 15] especially under operating regimes with a closed spill-line.

\section{Tested atomizers and liquids}

Both Simplex and SR atomizers were tested together with their geometrically identical transparent copies in 10:1 scale. The dimensions of the original atomizer are documented in Figure 1. It was not possible to manufacture and examine such small atomizers in the transparent version, therefore scaled models had to be used. The scaled atomizers have a modular construction (Figure 2, left). The assembly consists of three parts, each made from Plexiglas. The bottom part contains the swirl chamber with the exit orifice, the central one forms the tangential inlet ports, while the top part is a plain wall in the case of simplex atomizer or contains the spill orifice in the case of the SR atomizer. The modular construction allows each part to be replaced by another of a different geometry or shape. The surfaces of each part were ground and polished to achieve the transparency necessary for optical access.

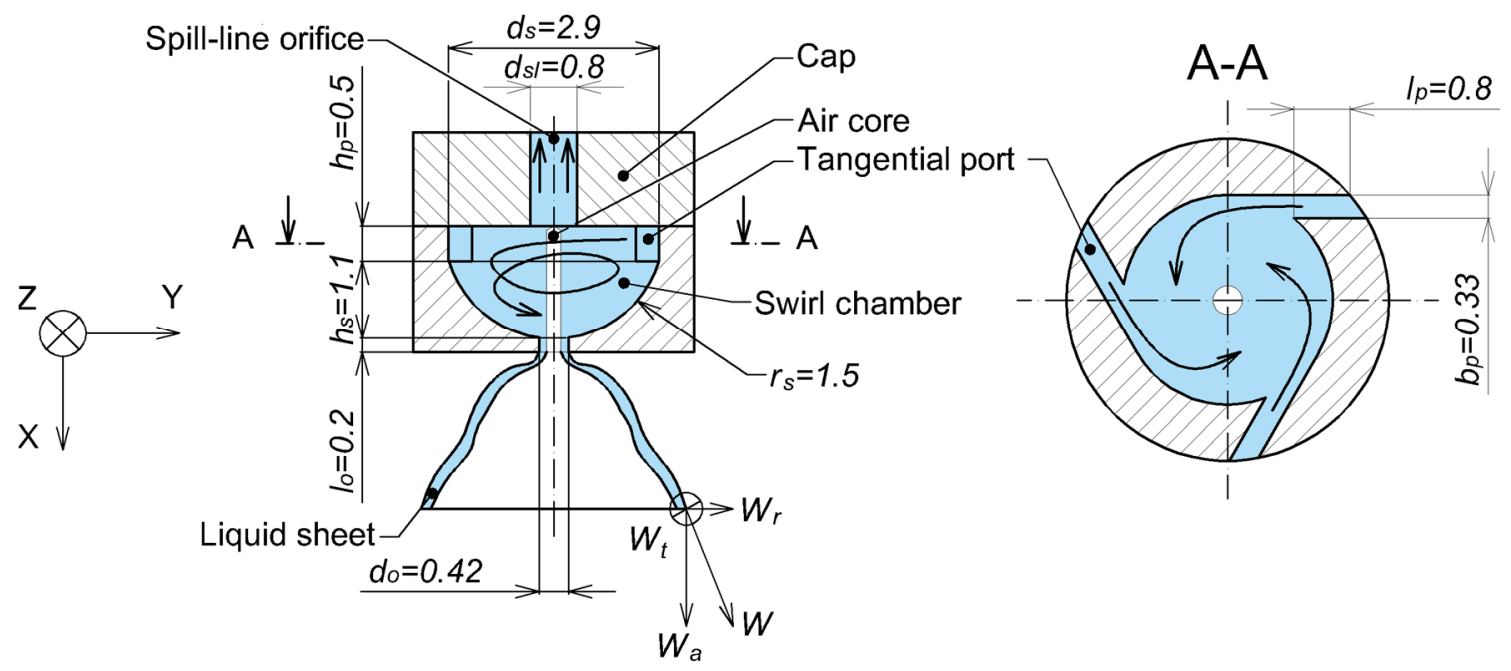

Figure 1. Sketch of the original SR atomizer with the main dimensions in millimetres. The simplex atomizer has no spill-line. The transparent atomizer has all dimensions 10 times larger.

It is necessary to match the flow of the real and scaled atomizers so the relevant dimensionless numbers must be considered. $\mathrm{Re}$ is defined as the ratio of inertial force to the viscous force. In the case of the swirl atomizer, the most common definition of $\mathrm{Re}$ is related to the inlet ports [16] as $\operatorname{Re}=w_{p} d_{p} / v$, where $w_{p}$ is mean velocity in the inlet ports, calculated as a volumetric flow rate divided by the total cross-section of inlet ports, $v$ is liquid kinematic viscosity, and $d_{p}$ is the hydraulic diameter of inlet ports $d_{p}=2 h_{p} b_{p} /\left(h_{p}+b_{p}\right)$, see figure 1 . The Re values for 
the scaled model must match those of the original to keep the same internal flow character. $S_{0}$ is useful in determining the ratio of the angular momentum to the axial momentum. It can be derived as a function of internal geometry [16]: $S_{0}=\pi R r_{o} / A_{p}$ where $R$ is a radius of flow entry to the swirl chamber. It is evident that the swirl numbers for the real and sized atomizers are identical. The Froude number (Fr) shows the effect of gravity in comparison with the energy of the bulk flow and can be defined as:

$$
F r=\frac{Q}{2 \pi\left(r_{o}^{2}-r_{o a c}^{2}\right) \sqrt{r_{o} g}},
$$

where $Q$ is the volume flow rate and $r_{o a c}$ is the radius of the air core in the exit orifice. To minimize the effect of gravity, it is necessary to keep $\mathrm{Fr} \gg 1$, as is applicable in the real atomizer case. Spray related dimensionless numbers such as Weber number and Ohnesorge number differ between the real and scaled atomizers by an order of magnitude and thus the spray parameters were not investigated except for the spray cone angle, SCA, close to the exit orifice.

Table 1 lists the experimental regimes with their dimensionless numbers. The operating regimes were derived from those in a previous work [14]. The main control parameter was the inlet pressure of the real atomizer and consequently its mass flow rate, from which the Re was calculated. The SR atomizer was also evaluated with closed spill-line to simulate the maximum injection rate. Two liquids were involved in this analysis with kerosene type Jet A-1 representing the commonly used fuel. However, its refraction index differs from that of the Plexiglas nozzle which complicates the optical measurement inside the transparent atomizer. A liquid with the refraction index very close to the Plexiglas should be used to overcome this problem. For this purpose, several different liquids and mixtures were evaluated to determine the most suitable one. Paracymene (p-cymene or 1-Methyl-4-(propan-2yl)benzene) was chosen. It is a colourless, transparent organic compound with refraction index differing from the Plexiglas by less than 0.001 at $660 \mathrm{~nm}$ wavelength and $25^{\circ} \mathrm{C}$. It also has a relatively low aggressivity to Plexiglas, however, after a few measurement hours it caused cracks in those parts where increased internal stresses may be anticipated, i.e. in the vicinity of bolts and threads. The physical properties of Jet A-1 are $\sigma=0.029 \mathrm{~kg} / \mathrm{s}^{2}$, $\mu_{l}=0.0016 \mathrm{~kg} /(\mathrm{m} \cdot \mathrm{s}), \rho_{l}=795 \mathrm{~kg} / \mathrm{m}^{3}$ and p-cymene: $\sigma=0.028 \mathrm{~kg} / \mathrm{s}^{2}, \mu_{l}=8 \times 10^{-4} \mathrm{~kg} /(\mathrm{m} \cdot \mathrm{s}), \rho_{l}=850 \mathrm{~kg} / \mathrm{m}^{3}$.

Table 1 Operating regimes

\begin{tabular}{|c|c|c|c|c|c|c|c|c|c|}
\hline \multirow{2}{*}{} & \multicolumn{4}{|l|}{ Real atomizer } & \multicolumn{2}{l|}{ Transparent atomizer } \\
\cline { 2 - 10 } & $\mathbf{R e}$ & $\boldsymbol{\Delta} \boldsymbol{p}$ & $\boldsymbol{m}_{\boldsymbol{I}}$ & $\mathbf{F r}$ & $\boldsymbol{C}_{\boldsymbol{D}}$ & $\boldsymbol{m}_{\boldsymbol{I}}$ & $\mathbf{F r}$ & $\boldsymbol{m}_{\boldsymbol{I}}$ & $\mathbf{F r}$ \\
\cline { 2 - 10 } & {$[-]$} & {$[\mathbf{M P a}]$} & {$[\mathbf{k g} / \mathbf{h}]$} & {$[-]$} & {$[-]$} & {$[\mathbf{k g} / \mathbf{h}]$} & {$[-]$} & {$[\mathbf{k g} / \mathbf{h}]$} & {$[-]$} \\
\hline Simplex & 755 & $\mathbf{0 . 5}$ & 5.41 & 137.4 & 0.387 & 54.1 & 6.9 & 29.0 & 3.4 \\
\hline Simplex & 1021 & $\mathbf{1}$ & 7.31 & 293.2 & 0.369 & 73.1 & 9.3 & 39.2 & 4.7 \\
\hline Simplex & 1252 & $\mathbf{1 . 5}$ & 8.97 & 359.8 & 0.365 & 89.7 & 11.4 & 48.1 & 5.7 \\
\hline SR & 1075 & $\mathbf{0 . 5}$ & 7.7 & 308.8 & 0.542 & 77 & 9.8 & 41.3 & 4.9 \\
\hline SR & 1431 & $\mathbf{1}$ & 10.25 & 411.1 & 0.519 & 102.5 & 13.0 & 55.0 & 6.5 \\
\hline SR & 1731 & $\mathbf{1 . 5}$ & 12.4 & 497.3 & 0.510 & 124 & 15.7 & 66.5 & 7.9 \\
\hline
\end{tabular}

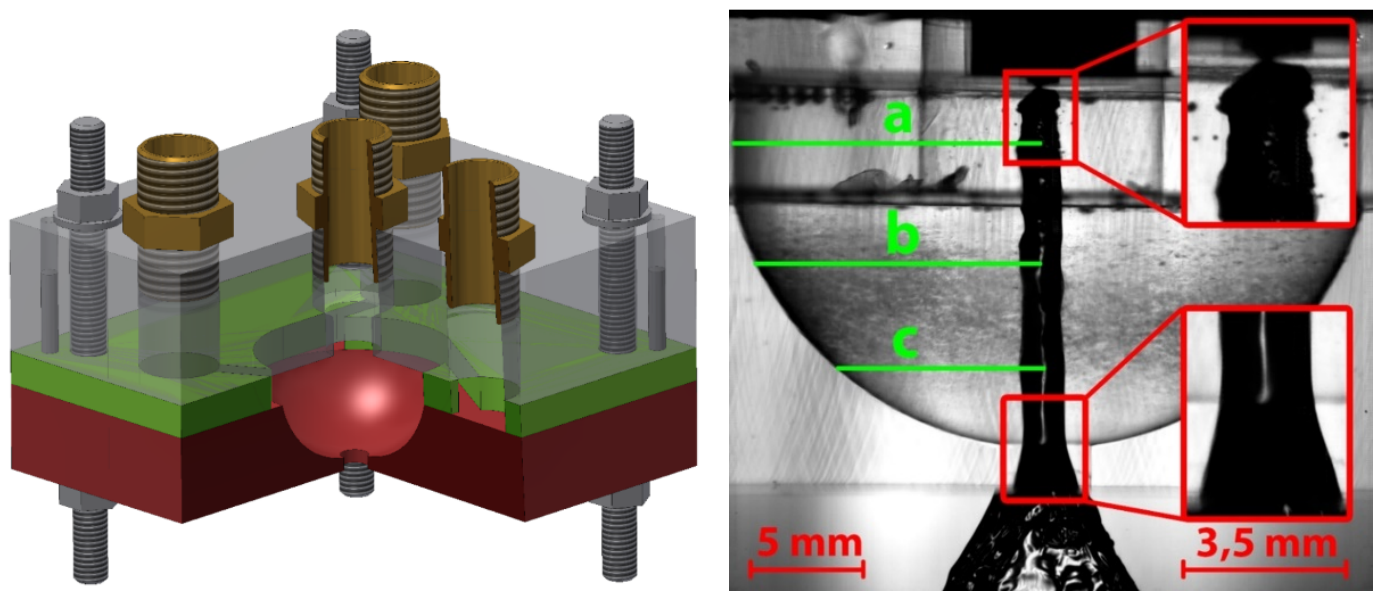

Figure 2. Left: Model of the scaled transparent atomizer. Right: High-speed visualization, p-cymene, $1 \mathrm{MPa}$, simplex. 


\section{Experimental setup}

The experiments were performed on the cold test bench at room temperature. A Photron SA-Z high-speed camera was used to document the spatial and temporal behaviour of the air core. The atomizer was illuminated by a background light using an LED panel. Three records were acquired at each operating regime; a general image showing the whole atomizer while the other two observe the exit orifice and the top of the swirl chamber in close up, see Figure 2 right. The camera frame rate was 20,000 fps for the general image; the resolution was $1024 \times 1024 \mathrm{px}$ and the shutter speed was set to 20 us. The close up records used a frame rate of $28,000 \mathrm{fps}$, resolution $768 \times 904 \mathrm{px}$ and a shutter time of $10 \mu \mathrm{s}$. The air core fluctuations were processed using a Fast Fourier Transform (FFT) applied to the average pixel intensities over rectangle $3 \times 3 \mathrm{px}$.

The laser-Doppler anemometer (LDA), a FlowExplorer (Dantec Dynamics A/S), was employed for the point-wise measurement of the swirl velocity in three cross-sections across the swirl chamber (see Figure 2 right). The axial distances from the top of the swirl chamber were $2.5,8$ and $13 \mathrm{~mm}$ for cross-sections $\mathrm{a}, \mathrm{b}$ and $\mathrm{c}$ respectively. The LDA was configured in the backscatter mode. The measuring volume position, relative to the LDA traverse system has to be corrected due to the different refraction index of the atomizer body and the liquid. Moreover, in the case of kerosene, it was also necessary to correct the measured velocity. The flow tracer particles were SL75 e-spheres with a mean diameter of $45 \mu \mathrm{m}$. The Stokes number, based on the swirl velocity and diameter of the swirl chamber, was less than 0.01 for each regime, which should ensure a sufficiently small flow traceability error.

\section{Numerical setup}

Conservation of mass (continuity) and conservation of momentum (Navier-Stokes) equations were solved numerically using Ansys Fluent 17.2. The flow simulation was conducted as a transient 2D axisymmetric model. A Volume of Fluid (VOF) model with the geo-reconstruct scheme was used to capture the boundary of the air core. The 3D inlet boundary condition was set to conserve the mass flow rate in the radial direction and ensure the same angular momentum in the tangential direction. The pressure outlet boundary condition was applied on the outer boundaries with no-slip conditions applied on the wall boundaries. Laminar flow was assumed due to the low Re values inside the inlet ports, and also due to the fact that inside the swirl chamber, the radial forces of the swirl tend to laminarise the flow [16]. The simulations were performed for both real and scaled atomizers.

The all quad structured mesh with an average skewness 0.058 and average aspect ratio 1.18 was created (Figure 3 ) and the grid independency test was carried out for four different element base sizes in terms of $C_{D}$, SCA and air core diameter $\left(d_{a}\right)$ at the end of the exit orifice $\left(d_{0}\right)$ in a dimensionless form as $d_{a} / d_{0}$ (see Table 2). There was a huge difference between the meshes of 11,684 and 22,669 elements. These differences decreased with further increase in the number of elements, and the mesh with 46,765 elements was chosen as a good compromise between accuracy and calculation speed. Two sizes of the outflow area were also tested, but the difference was found to be negligible.

Table 2. Grid independency test

\begin{tabular}{|c|c|c|c|}
\hline \hline Number of elements & $\boldsymbol{C}_{\boldsymbol{D}}$ & $\boldsymbol{d}_{\boldsymbol{a}} / \boldsymbol{d}_{\boldsymbol{o}}[-]$ & SCA [deg] \\
\hline \hline 11,684 & 0.392 & 0.655 & 58 \\
\hline 22,669 & 0.365 & 0.707 & 58 \\
\hline 46,765 & 0.358 & 0.710 & 57 \\
\hline $68,610^{*}$ & 0.359 & 0.710 & 57 \\
\hline 90,684 & 0.356 & 0.711 & 56 \\
\hline
\end{tabular}

*The base size of elements was the same as in the case of 46,765 element. The outflow area was four times larger.

\section{Results and discussion}

Air core shape

The air core was fully developed in the case of all the Simplex atomizers, as shown in Figure 4. It was cylindrically shaped and larger in diameter inside the exit orifice. Such behaviour has been described by other authors [10, 11, 17]. The dimensionless diameter of the air core in the exit orifice was $d_{a} / d_{0}=0.74 \pm 0.02$ for all the inlet pressures and both liquids with no evident correlations to Re. Inside the swirl chamber $d_{a} / d_{o}=0.47 \pm 0.03$ and was also almost independent of Re. Both findings are in accordance with several authors [10, 18, 19], who reported independent air core size for regimes of high Re. Instabilities, in the form of air core fluctuations, both in the axial and radial direction (Figure 5) were observed at the top of the swirl chamber. These fluctuations are linked with the wavy structure on the air core surface. The frequency of the surface waves $f=32 \pm 4 \mathrm{~Hz}$ was estimated using the FFT analysis of images for the Simplex atomizer with $\mathrm{p}$-cymene at $\mathrm{Re}=1021$. A similar evaluation was reported by Sumer et al. [20] who used a similarly sized atomizer, but with the velocity in the inlet ports approximately ten times higher they found wave frequencies of $f=273 \mathrm{~Hz}$. 


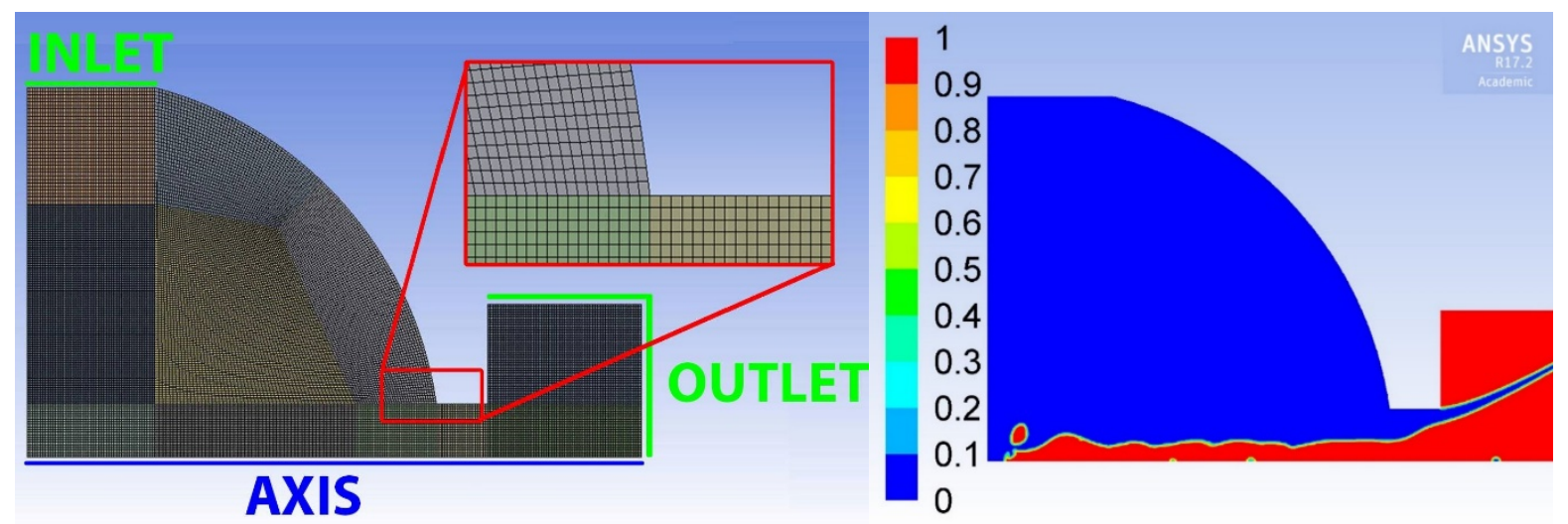

Figure 3. Left: Numerical domain and its mesh. Right: Typical results obtained with the wavy surface of the air core.
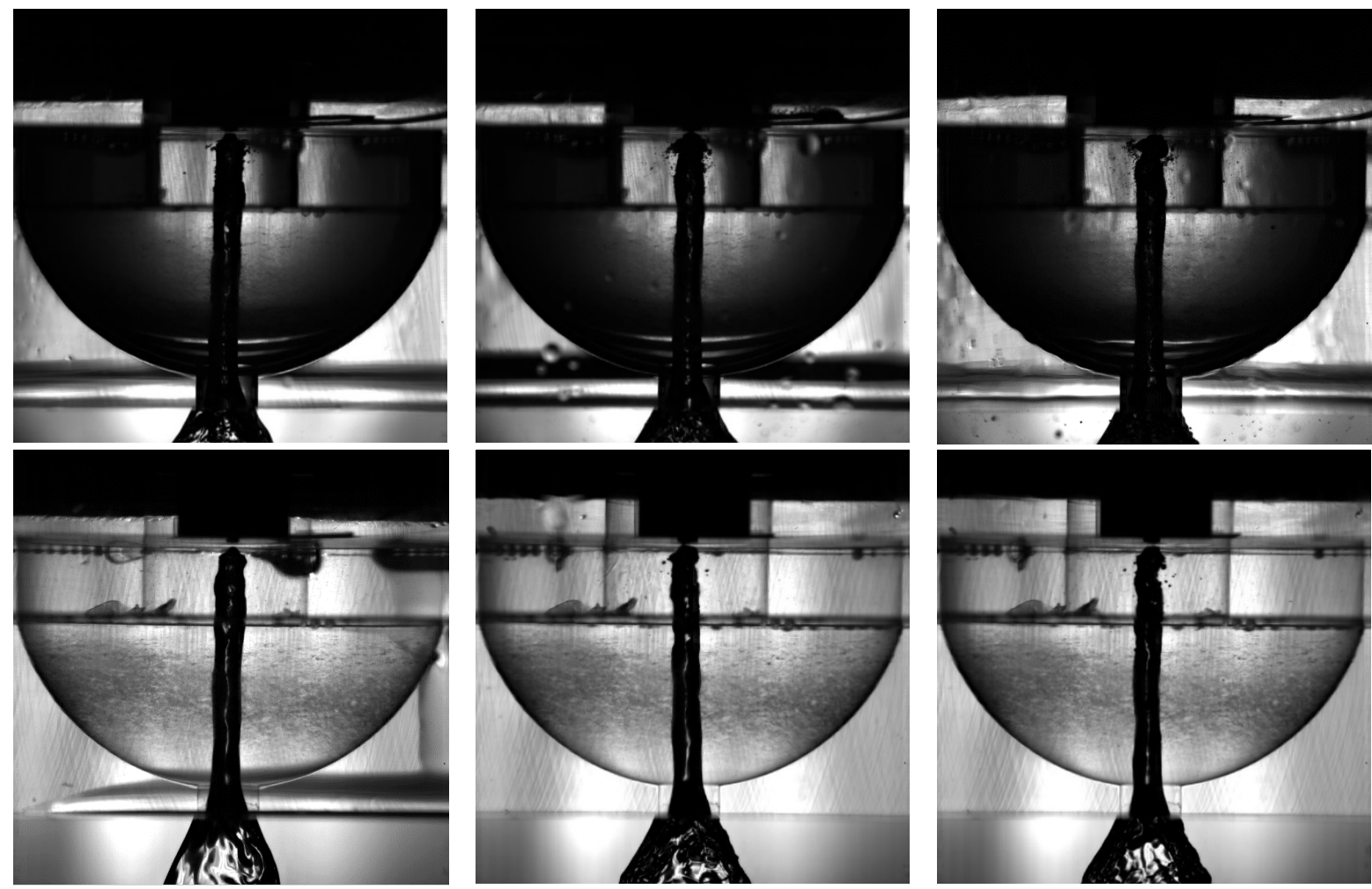

Figure 4. Simplex atomizer, Top: Kerosene, Bottom: $\mathrm{P}$-cymene. From left: equivalent $0.5 \mathrm{MPa}(\mathrm{Re}=755)$, equivalent $1 \mathrm{MPa}$ $(\operatorname{Re}=1021)$, equivalent $1.5 \mathrm{MPa}(\operatorname{Re}=1252)$.
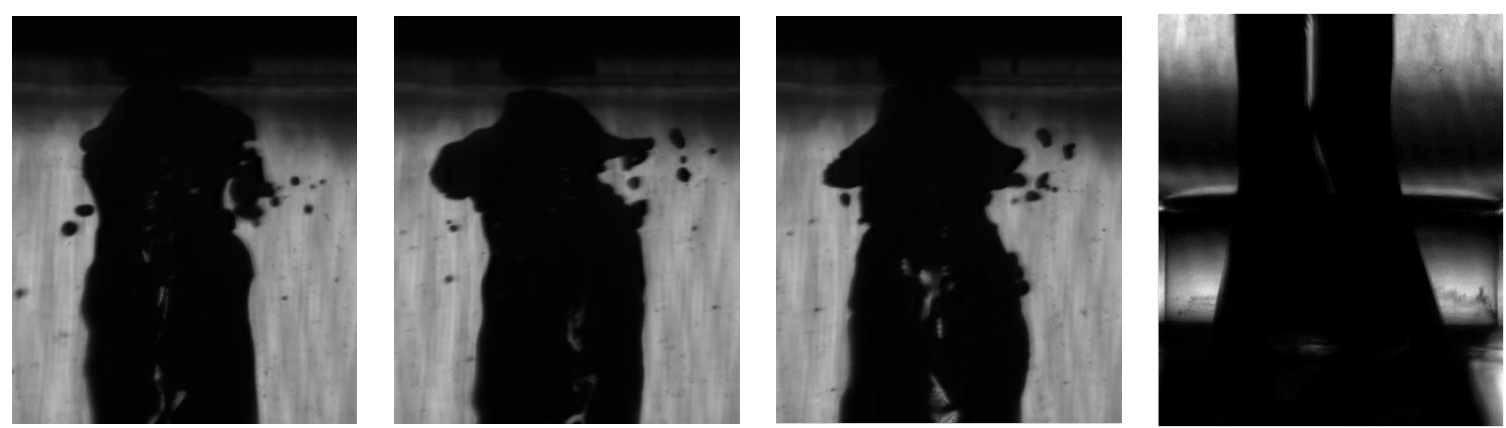

Figure 5. Simplex, p-cymene, $R e=1021$. The detail on fluctuating end of the air core. Right: the detail on the exit orifice. 

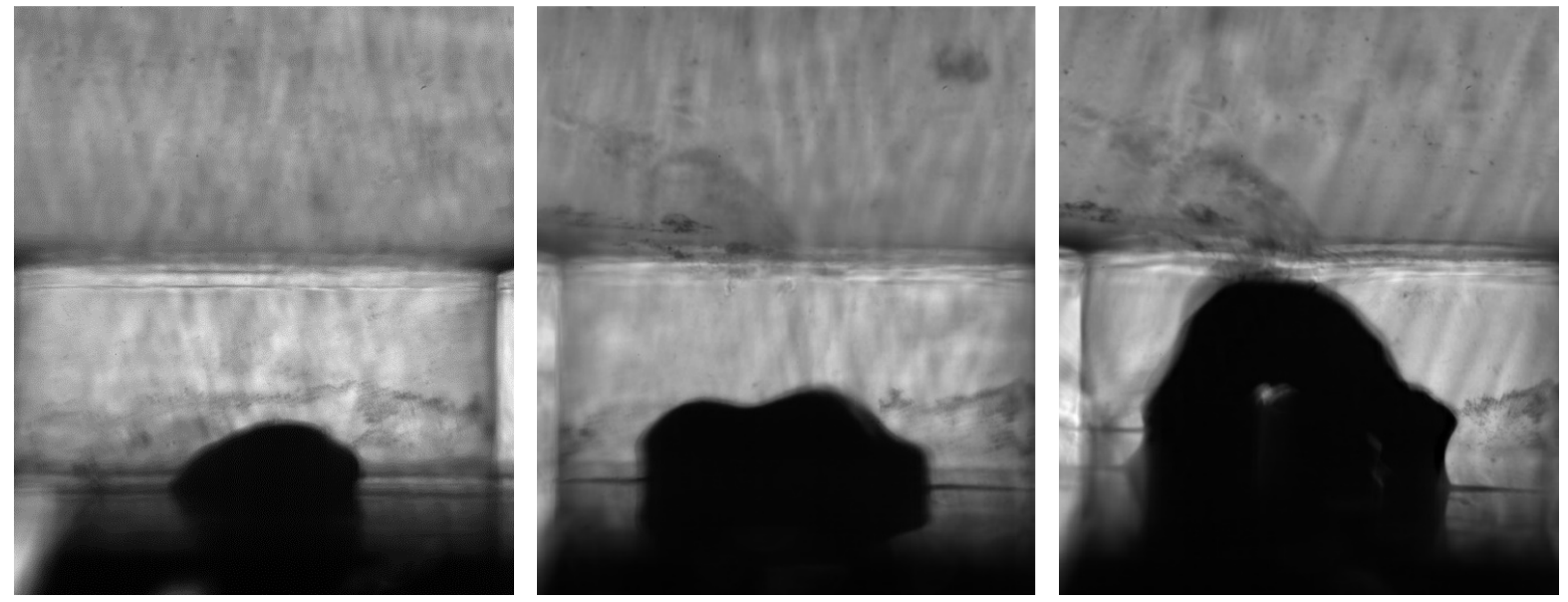

Figure 6. The exit orifice in detail. $\mathrm{SR}$ atomizer, equivalent $0.5 \mathrm{MPa}(\mathrm{Re}=1075)$, equivalent $1 \mathrm{MPa}(\mathrm{Re}=1431)$, equivalent $1.5 \mathrm{MPa}(\mathrm{Re}=1731)$

The situation changes dramatically when the SR atomizer was used. The air core is no longer present in the swirl chamber and the spray becomes unstable, even for the highest Re values. Only fragments of the core are visible inside the exit orifice - see Figure 6. The air core was unstable and strongly fluctuating and even occasionally disappeared. This behaviour was observed for all regimes studied.

It shows that our former hypothesis, as regards a periodically decaying air core as based on the external observation of the spray for the real atomizer [14], was misleading; the air core is not formed at all. The liquid, contained in the spill-line, is probably sucked back into the swirl chamber due to the low-pressure regime in the swirl chamber. That agrees with a fact that atomizers with the spill-orifice placed off-axis have a stable spray under all regimes [14].

\section{Velocity profile inside the swirl chamber}

The measured profiles of the swirl velocity (Figure 7, left) show a disparity between the Simplex and SR atomizers. The velocity profile for the Simplex version features a relatively sharp maximum near the air core interface while the SR atomizer shows a flatter peak with lower velocity maximum at similar Re values. As no air core is formed this behaviour would be expected.

\section{Numerical results}

The numerical results, when compared with the experiments in terms of global characteristics $\left(C_{D}\right.$ and $\left.S C A\right)$ and, give a very good agreement (Table 3). The most significant difference was found in the case of low Re values, but it was still less than $5 \%$.

In all the numerical simulations of the Simplex atomizers, the wavy interface between the liquid and gas phases was unsteady, see Figure 3, right. The frequency of the surface wave in the centre of the swirl chamber was, in the case of $p$-cymene, for $R e=1021$ about $25 \pm 4 \mathrm{~Hz}$, which is quite comparable to $f=32 \pm 4 \mathrm{~Hz}$ as in the experiment. The unstable behaviour of the SR atomizer was well captured by the simulation. The air core was limited to the exit orifice area in the similar way as it was in the experiments. The SCA fluctuated between 56 and 86 deg.

The swirl velocities are almost identical for all axial distances in both experiment and simulation (see Figure 7, right). This is in agreement with the inviscid theory where the swirl velocity is depended on the inlet velocity and radial distance from the axis of the swirl chamber out to the mid-point of inlet ports. The trends in the swirl velocity profiles were equivalent between experiments and simulations even the local minima are well captured. However, the simulation underestimates the velocity magnitude at the higher radius. The simulated swirl velocity underestimates by a factor of 2 compared with the experiments for higher radial positions. However, the difference is getting less near the axis of the swirl chamber.

Hansen and Madsen [21, 22] performed both experimental and 3D computational studies of a large-scale PS atomizer. Their earlier study [21] also showed a similar underestimation in the swirl velocity magnitude. In their following work [22], the numerical grid was modified and the inlet tangential ports were properly modelled which reduced the differences between the experimental and numerical velocity magnitude. It may be concluded that it is essential to make sure that the inlet boundary conditions are representative. 
Table 3 Comparison of numerical and experimental results of real simplex atomizer

\begin{tabular}{|c|c|c|c|c|c|c|c|c|}
\hline \multirow{2}{*}{} & \multicolumn{2}{|c|}{$\mathbf{R e}=\mathbf{7 5 5}$} & \multicolumn{2}{c|}{$\mathbf{R e}=\mathbf{1 0 2 1}$} & \multicolumn{2}{c|}{$\mathbf{R e}=\mathbf{1 2 5 2}$} & \multicolumn{2}{c|}{ SR, Re = 1431 } \\
\cline { 2 - 9 } & Num. & Exp. & Num. & Exp. & Num. & Exp. & Num. & Exp. \\
\hline $\boldsymbol{C}_{\boldsymbol{D}}[-]$ & 0.410 & 0.387 & 0.359 & 0.369 & 0.366 & 0.365 & 0.491 & 0.519 \\
\hline SCA [deg] & 56.7 & 58.6 & 58 & 61.5 & 58.7 & 62.3 & 72 & 73 \\
\hline $\boldsymbol{d}_{\mathbf{a}} / \boldsymbol{d}_{\boldsymbol{o}}[-]$ & 0.623 & 0.698 & 0.710 & 0.708 & 0.711 & 0.712 & N/A & N/A \\
\hline
\end{tabular}
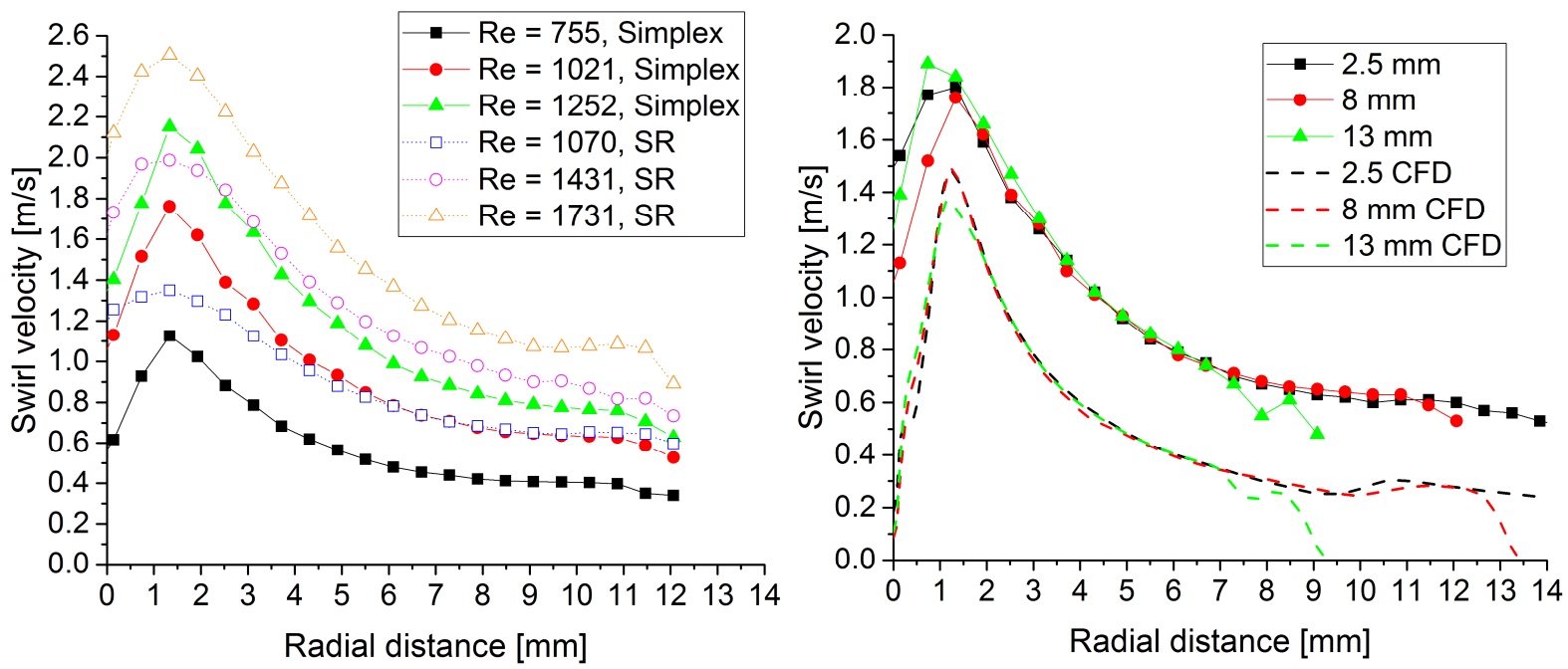

Figure 7. Swirl velocity profiles from various atomizers, left: $p$-cymene, cross-section $b$ ( $8 \mathrm{~mm}$ from the top), right: comparison of numerical and experimental profiles of swirl velocity, $\mathrm{p}$-cymene, simplex, $\mathrm{Re}=1021$.

\section{Conclusions}

The internal flow characteristics of a Simplex and an SR atomizer, with a central SR orifice, were examined both experimentally and numerically. The numerical results were verified against the results obtained from the images and point wise LDA measurements.

The Simplex atomizer featured a stable, cylindrically shaped air core. Its diameter was found independent of $\mathrm{Re}$ under the measured range of operation conditions.

The numerical simulation, assuming laminar flow, was able to predict the global characteristics $\left(C_{D}, S C A\right)$ closely. The trends in mean swirl velocity were captured, but the velocity magnitude was underestimated. Unstable waves were observed on the surface of the air core using high speed imagining and were also predicted by the numerical simulation.

The SR atomizer produced an internal flow without the air core and the spray therefore fluctuated strongly. The velocity profiles showed lower and flatter peak values in comparison to the simplex atomizer at similar Re values. This study forms the groundwork for an analysis into the internal flow of SR atomizers. Further investigations including a more realistic 3D computational model, several different arrangements of the spill orifice and a range of spill to feed ratios will follow.

\section{Acknowledgements}

This work has been supported by the project No. GA15-09040S funded by the Czech Science Foundation and the project LO1202 NETME CENTRE PLUS with the financial support from the Ministry of Education, Youth and Sports of the Czech Republic under the "National Sustainability Programme I". 


\section{Nomenclature}

$\begin{array}{ll}A & \left.\text { area [mm }{ }^{2}\right] \\ b & \text { width [mm] } \\ C_{D} & \text { discharge coefficient [-] } \\ \mathrm{d} & \text { diameter [m] } \\ f & \text { frequency [Hz] } \\ \mathrm{Fr} & \text { Froude number [-] } \\ h & \text { height [mm] } \\ \dot{m} & \text { mass flow rate }[\mathrm{kg} / \mathrm{s}] \\ Q & \text { Volumetric flow rate }\left[\mathrm{m}^{3} / \mathrm{s}\right] \\ r & \text { radial distance }[\mathrm{mm}] \\ \mathrm{Re} & \text { Reynolds number }[-] \\ \mathrm{SCA} & \text { spray cone angle }[\mathrm{deg}]\end{array}$

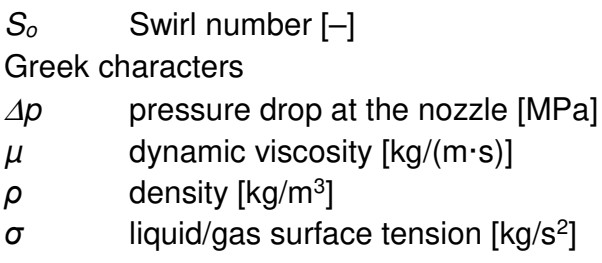

$\begin{array}{ll}\begin{array}{l}\text { Subscripts and Superscripts } \\ I\end{array} & \text { atomized liquid } \\ o & \text { exit orifice } \\ s & \text { swirl chamber } \\ p & \text { inlet port } \\ a & \text { air core }\end{array}$

\section{References}

[1] Taylor, G. I., 1948, "The Mechanics of Swirl Atomizers," International Congress of Applied MechanicsLondon. [2] Chinn, J. J., 2009, "An appraisal of swirl atomizer inviscid flow analysis, Part 1: The principle of maximum flow for a swirl atomizer and its use in the exposition and comparison of early flow analyses," Atomization and Sprays, 19(3).

[3] Chinn, J. J., 2009, "An appraisal of swirl atomizer inviscid flow analysis, part 2: inviscid spray cone angle analysis and comparison of inviscid methods with experimental results for discharge coefficient, air core radius, and spray cone angle," Atomization and Sprays, 19(3).

[4] Rizk, N. K., and Lefebvre, A. H., 1985, "Internal flow characteristics of simplex swirl atomizers," Journal of Propulsion and Power, 1(3), pp. 193-199.

[5] Jones, A., 1982, "Design optimization of a large pressure-jet atomizer for power plant," Proc. Proceedings of the Second International Conference on Liquid Atomization and Spray Systems, p. 181.

[6] Ballester, J., and Dopazo, C., 1994, "Discharge coefficient and spray angle measurements for small pressureswirl nozzles," Atomization and sprays, 4(3).

[7] Benjamin, M., Mansour, A., Samant, U., Jha, S., Liao, Y., Harris, T., and Jeng, S., "Film thickness, droplet size measurements and correlations for large pressure-swirl atomizers," Proc. ASME 1998 International Gas Turbine and Aeroengine Congress and Exhibition, American Society of Mechanical Engineers, pp. V003T006A050V003T006A050.

[8] Wimmer, E., and Brenn, G., 2013, "Viscous flow through the swirl chamber of a pressure-swirl atomizer," International Journal of Multiphase Flow.

[9] Malý, M., Janáčková, L., Jedelský, J., Jícha, M., 2016, "Impact of alternative fuel rheology on spraying process of small pressure-swirl atomizer," Proc. AIP Conference Proceedings, AIP Publishing, p. 020031.

[10] Halder, M., Dash, S., and Som, S., 2002, "Initiation of air core in a simplex nozzle and the effects of operating and geometrical parameters on its shape and size," Experimental thermal and fluid science, 26(8), pp. 871-878.

[11] Lee, E. J., Oh, S. Y., Kim, H. Y., James, S. C., and Yoon, S. S., 2010, "Measuring air core characteristics of a pressure-swirl atomizer via a transparent acrylic nozzle at various Reynolds numbers," Experimental thermal and fluid science, 34(8), pp. 1475-1483.

[12] Kim, S., Khil, T., Kim, D., and Yoon, Y., 2009, "Effect of geometric parameters on the liquid film thickness and air core formation in a swirl injector," Measurement Science and Technology, 20(1), p. 015403.

[13] Moon, S., Abo-Serie, E., and Bae, C., 2009, "Air flow and pressure inside a pressure-swirl spray and their effects on spray development," Experimental Thermal and Fluid Science, 33(2), pp. 222-231.

[14] Jedelsky, J., Malý, M., Janáčková, L., and Jícha, M., 2016 "Effect of Geometric Factors on Spray Characteristics and Stability for Small Spill-Return Pressure-Swirl Atomizers," ILASS 2016 Brighton, p. 12.

[15] Khavkin, Y., 2004, The Theory and Practice of Swirl Atomizers, Taylor \& Francis.

[16] Chinn, J. J., 2008, "The numerics of the swirl atomizer."

[17] Amini, G., 2016, "Liquid flow in a simplex swirl nozzle," International Journal of Multiphase Flow, 79, pp. 225235.

[18] Datta, A., and Som, S., 2000, "Numerical prediction of air core diameter, coefficient of discharge and spray cone angle of a swirl spray pressure nozzle," International journal of heat and fluid flow, 21(4), pp. 412-419.

[19] Moon, S., Abo-Serie, E., and Bae, C., 2010, "Liquid film thickness inside the high pressure swirl injectors: Real scale measurement and evaluation of analytical equations," Experimental Thermal and Fluid Science, 34(2), pp. 113-121.

[20] Sumer, B., Erkan, N., Uzol, O., and Tuncer, I., 2012, "Experimental and Numerical Investigation of a Pressure Swirl Atomizer."

[21] Hansen, K., Madsen, J., Trinh, C., Ibsen, C., Solberg, T., and Hjertager, B., 2002, "A computational and experimental study of the internal flow in a scaled pressure-swirl atomizer," Zaragoza, 9, p. 11.

[22] Madsen, J., Hjertager, B. H., and Solberg, T., 2004, "Numerical simulation of internal flow in a large-scale pressure-swirl atomizer," Proc. ILASS-Europe 2004, pp. 183-188. 


\title{
Behaviour of free falling viscoelastic liquid jets.
}

\author{
Christophe Tirel, Marie-Charlotte Renoult*, Christophe Dumouchel, Jean-Bernard Blaisot \\ Normandie Université, Université et INSA de Rouen, CNRS - CORIA \\ 76801 Saint-Etienne du Rouvray, France \\ *Corresponding author: renoultm@coria.fr
}

\begin{abstract}
In a recent work, a protocol to measure the relaxation time of dilute polymer solutions, known to be challenging, has been established [1]. This protocol is based on a 2D multi-scale description of free-falling low velocity viscoelastic liquid jets. Although the relaxation time reached an asymptotic value for high jet velocities, a significant dependence with the jet velocity is observed for low velocities. The present work reconsiders these previous experimental data using a 3D multi-scale analysis in order to identify the origin of the dependence between the relaxation time and the jet velocity. The 3D analysis demonstrates the importance of a velocity-dependent coalescence mechanism in the jet behaviour. Thanks to a simple model of jet deformation it is demonstrated that this coalescence mechanism prevents the elasto-capillary contraction of the smallest scales from occurring when the jet velocity is reduced.
\end{abstract}

\section{Keywords}

Dilute polymer solutions, Viscoelasticity, Jet break-up, Multi-scale analysis

\section{Introduction}

Contrary to Newtonian fluids, the atomisation of a cylindrical jet of a viscoelastic solution presents long life time cylindrical ligaments connecting spherical beads, the so called beads-on-a-string (BOAS) structures [2]. These structures are observed at times prior jet breakup. It is well known that the thinning evolution of the ligaments diameter is controlled by the elasticity of the liquid [3]. The decrease is exponential, with a relaxation time depending only on the physical properties of the liquid. The measurement of this relaxation time is also known to be particularly challenging for dilute polymer solutions [4] for which the relaxation time is very short (micro-second or under). Previous studies [5-8] have investigated the possibility to use a cylindrical jet to measure this quantity, a method that could be suitable to probe very short times [4]. Yet, it was shown that the relaxation time obtained with this method depends on the jet operating conditions, i.e. the jet velocity, the amplitude and the frequency of the initial disturbance [6-8], thus limiting the development of this experimental method. Until recently, new results [4] suggest that an operating domain where these dependences vanish may exist.

In this context, a multi-scale analysis of the behaviour of free falling jets of a dilute viscoelastic solution was performed, allowing measurements of the relaxation time [1]. The dynamics of the smaller scales was monitored and an exponential decrease was observed from which a relaxation time was extracted. It was found that the measured relaxation time decreases with increasing jet velocity, reaching a plateau for sufficiently high velocities. This behaviour was related to the influence of the initial amplitude disturbance as reported in [6-8]. The higher jet velocity was imposed, the larger initial amplitude was observed on jet images and the lower relaxation time was measured in agreement with [6-8]. To date, the dependence of the relaxation time with the jet velocity (or the initial amplitude disturbance) remains unexplained. The objective of the present paper is thus to complete this previous work [1] by performing a 3D multi-scale analysis for these free falling jets of dilute viscoelastic solutions in order to gain a deeper insight into the jet velocity dependence. A model of jet deformation is used in complement to enrich the $3 \mathrm{D}$ analysis. Section 2 summarizes the previous investigation and describes the 3D multi-scale analysis and the model. Section 3 presents the results and the application of the model. The article ends with a conclusion.

\section{Jet images: acquisition and analysis}

This section summarizes the experimental work and the 2D multi-scale analysis that are presented in detail in [1]. It also introduces the 3D multi-scale analysis and the model of jet deformation which constitute the new aspect of the present study.

The experimental setup produces free falling jets of a low-viscosity viscoelastic polymer solution containing $5 \mathrm{ppm}$ of Poly(ethylene oxide) (PEO) dissolved into a solvent (density $\rho=989 \mathrm{~kg} / \mathrm{m}^{3}$; shear viscosity $\mu=1.34 \mathrm{mPa}$.s; surface tension $\sigma=48.9 \mathrm{mN} / \mathrm{m}$ ). The liquid was kept in a pressurized tank and fed an injector with a cylindrical nozzle whose diameter is $D_{o r}=200 \mu \mathrm{m}$. Images of the free falling jets were obtained with a high spatial resolution camera and a light source of short duration (20 ns) used in a diffused backlight illumination optical arrangement. 
The field of view covered by the images was $6.56 \mathrm{~mm} \times 5.54 \mathrm{~mm}$ and the spatial resolution was equal to $2.56 \mu \mathrm{m} /$ pixel. A 3D displacement system was used to translate the injector to be able to visualize the jets on their full length, i.e., from the nozzle exit down to the breakup point.

The parameter of the experiment was the injection pressure that ranged from 0.05 to 1 bar. The mean jet velocity $V_{q}$ was obtained by dividing the measured volume flow rate by the jet section area $\pi D_{j}^{2} / 4$ where the mean jet diameter $D_{j}$ was measured on the jet within the three first millimeters after the nozzle exit. The mean jet velocity ranged from $1.9 \mathrm{~m} / \mathrm{s}$ to $10 \mathrm{~m} / \mathrm{s}$. For each injection pressure and for each position of the injector, 204 images decorrelated in time were recorded and the subsequent analysis was statistical. A reconstructed full jet is shown in Fig. 1. The jet issuing from the nozzle is slightly deformed. At around $16 \mathrm{~mm}$ a sinusoidal disturbance appears. This disturbance grows and rearranges to the expected beads-on-a-string pattern (BOAS) which remains visible until breakup occurs.

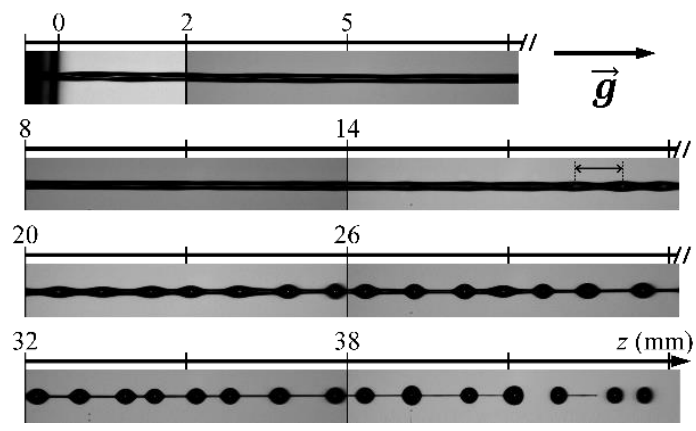

Figure 1. Reconstructed jet (Fluid: polymer solution, Mean jet velocity $V_{q}=8.58 \mathrm{~m} / \mathrm{s}$ )

The analysis of these images used a 2D multi-scale description of the jet. This description consisted in measuring the surface-based cumulative scale function $E_{2}(d, z)$ as a function of the position $z$ (see [1]). First, image treatments were applied to provide two-level images where the liquid appeared as black pixels on a white background. Second, a rectangular analyzing window delimiting a portion of the jet was positioned at a distance $z$ from the nozzle exit. Third, the surface-based cumulative scale function $E_{2}(d, z)$ of the jet portion delimited by the analyzing window was measured. Introduced in several previous works (see [9-11] for instance), the measurement of this function is inspired from the Euclidean Distance Mapping method to determine fractal dimension [12] and consists in applying successive erosion operations at a scale $d$ ranging from 0 to infinity. The erosion operation consists in removing a strip of width $d / 2$ around the whole system. The cumulative scale function $E_{2}(d, z)$ is the ratio of surface area of the removed strip on the total surface area of the local jet portion. For the scale $d=0$, the surface area removed by erosion is equal to zero and $E_{2}(0, z)=0$. For a sufficiently large scale, the surface area removed by erosion is equal to the total surface area of the local jet portion and $E_{2}(d, z)=1$. Between these two scales, $E_{2}(d, z)$ monotonously increases. A more detailed definition of the cumulative scale function is available in [9-11]. At each $z$ position, the measurement was performed on 204 images and an average cumulative function was calculated. The spatial evolution of the average cumulative scale function was obtained by sliding the analyzing window from the top of the jet (just under the nozzle) down to the bottom (in the spray region). The size of the analyzing window was chosen of the order of the length of the BOAS pattern i.e. 390 pixels for every velocity. Furthermore, 390 pixels appeared also appropriate for the shift of the analyzing window along the $z$ axis. The spatial series of local cumulative scale functions in space was transformed as a temporal series using the equivalent time $t$ defined as:

$$
t=\frac{\sqrt{V_{q}^{2}+2 g z}-V_{q}}{g}
$$

where $g$ is the gravitational acceleration. When $V_{q}^{2}>>2 g z$ (Froude number $>>1$ ), Eq. (1) simplifies as $t=z / V_{q}$. The 2D multi-scale analysis concentrated on the 2D scale-distribution $e_{2}(d, t)$ which is the first derivative of the cumulative function in the scale space: $e_{2}(d, t)=d E_{2}(d, t) / d d$. This distribution is equal to the ratio of the perimeter of the eroded local system at scale $d$ on twice the total surface area of the local system. The temporal evolution of the scale distribution $e_{2}(d, t)$ gives information on the physical mechanisms involved in the shape evolution of the jet. To illustrate this, one can consider the temporal derivative of the scale distribution as shown for instance in Fig. 2 $\left(V_{q}=1.9 \mathrm{~m} / \mathrm{s}, t=6.3 \mathrm{~ms}\right.$, BOAS pattern). This derivative is equal to zero for three characteristic scales noted $d_{1}, d_{2}$ and $d_{3}$. The $d_{3}$ scale is the maximum scale $d_{\max }$ of the system. The scale $d_{4}$ for which the derivative is a minimum is a characteristic scale. It has been demonstrated ([11] for instance) that the specific behavior of the derivative in the small scale range $\left(d<d_{1}\right)$ in Fig. 2 denotes the presence of a thinning cylindrical ligament. The scale $d_{1}$ appears 
as a characteristic scale of the dynamics of the ligament. The temporal evolution of $d_{1}$ for the free viscoelastic jets reported a decrease according to three regimes [1]. The second one is an exponential regime which corresponds to an elastic-controlled capillary thinning behavior [1], i.e.:

$$
d_{1} \propto \exp \left(-\frac{t}{3 t_{r}}\right)
$$

where $t_{r}$ is the relaxation time of the viscoelastic solution. The relaxation time was measured from Eq. (2) as a function of the mean jet velocity $V_{q}[7]$. Figure 3 reports the results by plotting the Deborah number $D_{e}=t_{r} / t_{\sigma}$ as a function of the square root of the jet Weber number We where:

$$
t_{\sigma}=\sqrt{\frac{\rho D_{j}^{3}}{8 \sigma}} \quad W e=\frac{\rho V_{q}^{2} D_{j}}{\sigma}
$$

Figure 3 shows a dependence between $t_{r}$ and the mean jet velocity. Nevertheless, we note that an asymptotic value is reached when We increases. This value is $t_{r}=0.44 \mathrm{~ms}$ and is believed to be the relaxation time of the solution. In order to obtain a better understanding of the behaviour of low-velocity free-falling viscoelastic jets, we suggest completing the previous analysis by a 3D multi-scale approach. This is the purpose of the present work.

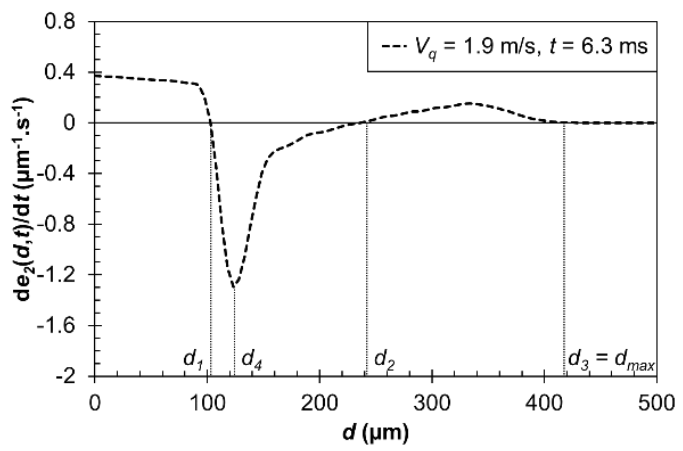

Figure 2. Temporal derivative of the scale distribution. Introduction of characteristic scales

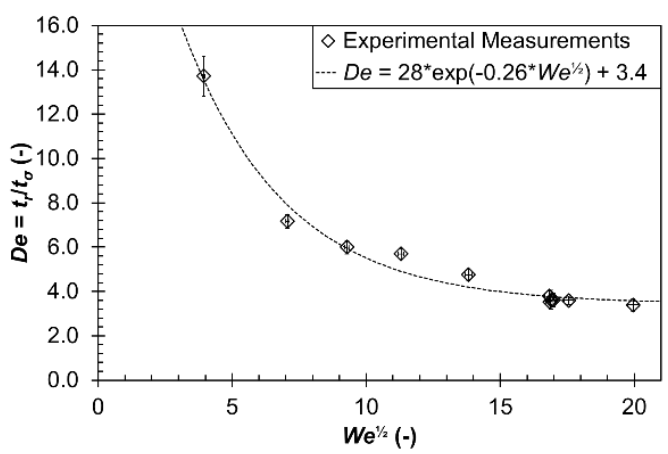

Figure 3. Deborah number $D_{e}$ as a function of the Weber number (from [7])

In the case where 3D system information is available, the 3D generalization of the 2D multi-scale description summarized above is straightforward (see $[11,13]$ for applications). The system is characterized by its volume and the erosion operation removes the peripheral volume of a $d / 2$ thickness from the system boundary. The cumulative distribution $E_{3}(d, t)$ is the ratio of the volume removed by the erosion at scale $d$ on the total volume of the system. The scale distribution $e_{3}(d, t)$ defined as the first derivative of the cumulative distribution $E_{3}(d, t)$ in the scale space is equal to the ratio of the surface area of the eroded system at scale $d$ on twice the total volume of the system. Therefore, $e_{3}(0, t)$ is directly proportional to the specific-surface-area (surface of the interface per unit volume) introduced by Evers [14].

In the present work, the studied free liquid jets are assumed axisymmetric. This assumption seems reasonable since the capillary instability is known as an axisymmetric mechanism. For each jet portion delimited by the analyzing window, this hypothesis allows to calculate the jet volume and surface by integration for each successive values of the scale $d$. Then, the 3D multi-scale description uses these two pieces of information to calculate the scale distribution $e_{3}(d, t)$. After the analysis of all the images, we determine $V(t)$ which is the local average volume of the jet portion delimited by the analyzing window, and $e_{3}(d, t)$ which is the local average volume-based scale distribution.

One of the main objectives of the 3D multi-scale approach is to describe the behavior of the jet when it is shaped as a BOAS pattern. In this case, the jet can be described as the composition of two simple systems: a cylinder of diameter $D_{1}(t)$ and volume $V_{1}(t)$ (system 1 ) and a sphere of diameter $D_{2}(t)$ (volume $V_{2}(t)$, system 2). The volumebased scale distributions for these two systems are:

$$
\text { System } 1 \quad e_{3}(d, t)=\frac{2}{D_{1}(t)}\left(1-\frac{d}{D_{1}(t)}\right) \quad \text { for } d<D_{1}(t)
$$


System $2 \quad e_{3}(d, t)=\frac{3}{D_{2}(t)}\left(1-\frac{d}{D_{2}(t)}\right)^{2} \quad$ for $d<D_{2}(t)$

The similarity between these two expressions must be noted. Furthermore, the scale distribution of a cylinder shows a first order dependence with the scale and a sphere shows a second order one. This observation suggests that a system whose shape is between a cylinder and a sphere would report a scale dependence order between 1 and 2 . Therefore, since the free falling jet evolves from a cylindrical system to an arrangement of a cylinder and a sphere (see Fig. 1), its scale distribution can be modelled as:

$$
\begin{aligned}
& e_{3}(d, t)=\alpha(t) \frac{2}{D_{1}(t)}\left(1-\frac{d}{D_{1}(t)}\right)+(1-\alpha(t)) \frac{n}{D_{2}(t)}\left(1-\frac{d}{D_{2}(t)}\right)^{n-1} \quad \text { for } d<D_{1}(t) \\
& e_{3}(d, t)=(1-\alpha(t)) \frac{n}{D_{2}(t)}\left(1-\frac{d}{D_{2}(t)}\right)^{n-1} \quad \text { for } D_{1}(t)<d<D_{2}(t)
\end{aligned}
$$

In Eq.(6), the parameter $n$ varies from 2 to 3 as time increases. At early times the jet is cylindrical and $n=2$. Then, when a part of the jet swells up, $n$ increases. It then approaches 3 when the swollen part is almost a sphere. In Eq.(6), the parameter $\alpha(t)$ represents the relative volume of the ligament part of the jet: $\alpha(t)=V_{1}(t) /\left(V_{1}(t)+V_{2}(t)\right)$. The knowledge of this parameter may provide a better characterization of the behaviour of the ligaments in the BOAS pattern. In particular, if the evolution of the BOAS is due to a capillary contraction of the string controlled by the viscoelasticity of the solution, the volume $V_{1}(t)+V_{2}(t)$ should be constant, the diameter of the string should decrease as Eq. (2) and therefore, the dynamic of the parameter $\alpha(t)$ is expected to be:

$$
\alpha(t) \propto \exp \left(-\frac{2 t}{3 t_{r}}\right)
$$

Therefore the model expressed by Eq. (6) can bring precious information about the analysis of the 3D multi-scale description of the free falling viscoelastic liquid jets.

\section{Results}

Figure 4 presents the temporal evolution of $V(t)$, the average volume of liquid delimited by the analysing window, for three jet velocities. $\left(V(t)\right.$ is divided by $V\left(t_{i}\right)$ where the initial time ti corresponds to the top position of the analysing window.) The time is made dimensionless by using the breakup time $t_{B \cup}$ whose average value has been determined for every jet velocity. We see that $V(t)$ is rather constant during time for each velocity. This shows that 204 images are enough to perform statistical analysis. Note however that for $V_{q}=4.5 \mathrm{~m} / \mathrm{s}$ and $9.0 \mathrm{~m} / \mathrm{s}, V(t)$ slightly decreases first before becoming constant. This evolution results from the fact that the assumption of axisymmetry is not fully satisfied at the beginning for these jets that are initially subjected to a slight deformation. This remains insignificant for the subsequent analysis.

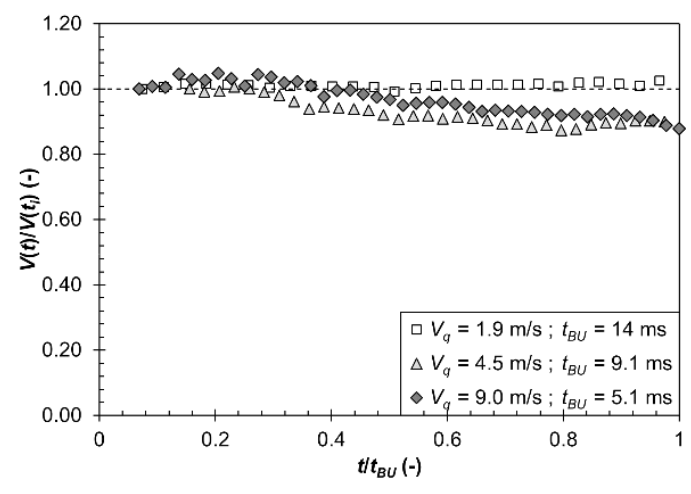

Figure 4. Temporal evolution of the ratio $V(t) / V\left(t_{i}\right)$ as a function of the mean jet velocity $V_{q}\left(t_{B U}\right.$ is the breakup time)

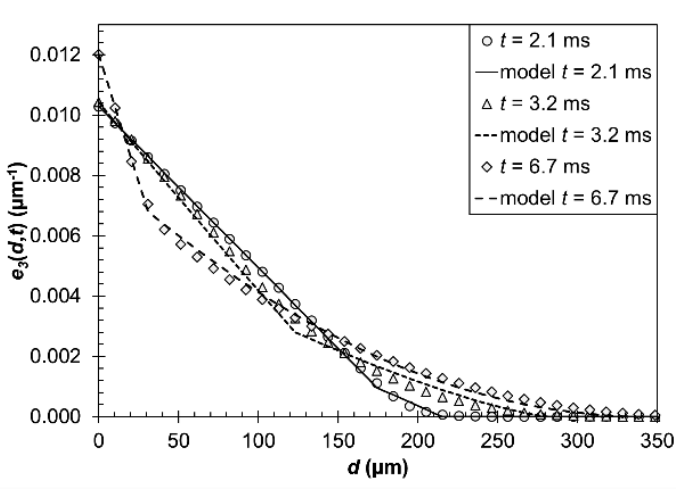

Figure 5. Volume-based scale distribution $e_{3}(d, t)$ as a function of time $\left(V_{q}=5.7 \mathrm{~m} / \mathrm{s}, t_{B U}=7.2 \mathrm{~ms}\right.$, dots: measurements; lines: model)

Figure 5 shows an example of the temporal evolution of the volume-based scale distribution $e_{3}(d, t)\left(V_{q}=5.7 \mathrm{~m} / \mathrm{s}\right)$. At $t=2.1 \mathrm{~ms}$, the scale distribution is linear which, according to Eq. (4), signifies that the jet is still a cylinder. As times goes, the scale distribution loses this linearity indicating that the jet experiences a deformation. In the large scale region, we note a continuous increase of the maximum scale $d_{\max }$. This illustrates the presence of a thickening 
mechanism that is due to the growth of swells along the jet. In the small scale region, the distribution remains linear with the scale and the absolute value of the slope increases with time. These two behaviours reveal the presence of a thinning cylindrical ligament.

As mentioned in the previous section, $e_{3}(0, t)$ is equivalent to the specific-surface-area of the system, i.e., the interface area per unit volume. The temporal evolution of this quantity appears to be very much dependent on the mean jet velocity. This is illustrated in Fig. 6 -a where the product $e_{3}(0, t) D_{j} / 2$ is plotted as a function of the ratio $t / t_{B U}$ for several mean jet velocities. At early times, since the jets are cylindrical for every velocity, $e_{3}(0, t) D_{j} / 2$ should be equal to 1 (see Eq. (4)). Figure 6-a reports this value for all cases. As time increases, two types of evolution are identified. For the high velocities $(5.7 \mathrm{~m} / \mathrm{s}$ to $9 \mathrm{~m} / \mathrm{s}$ in Fig. $6-\mathrm{a}), e_{3}(0, t) D_{j} / 2$ increases up to a local maximum at around $t / t_{B U}=0.5$ and then decreases before increasing again when the breakup is approached. The first increase corresponds to the deformation of the cylindrical jet caused by the growth of the swells (as for the Newtonian case [11]) and the subsequent decrease occurs during the evolution of the BOAS pattern. This indicates that the evolution of the BOAS is an interface reduction mechanism. The last increase before the breakup is moderate for $7.0 \mathrm{~m} / \mathrm{s}$ and $9.0 \mathrm{~m} / \mathrm{s}$ but is much pronounced for $5.7 \mathrm{~m} / \mathrm{s}$.

For the other velocities, the evolution of $e_{3}(0, t) D_{j} / 2$ is different: it shows a continuous increase until the breakup is reached. Furthermore, the variations of $e_{3}(0, t) D_{j} / 2$ are much higher than for the highest velocities. Such an increase of the specific-surface-area suggests that the system is very much deformed, i.e., it is far less spherical.

This is verified by considering the product $S P=e_{3}(0, t) d_{\max }(t) / 2$. $S P$ is a shape parameter. It is equal to 1 for a cylinder (Eq. 4) and to 1.5 for a sphere (Eq. 5). For a system composed of a cylinder of diameter $D_{1}(t)$ plus a sphere of diameter $d_{\max }(t)$ (with $d_{\max }>D_{1}(t)$ ), the parameter $S P$ is equal to (see Eq. (6)):

$$
S P=\alpha(t) \frac{d_{\max }(t)}{D_{1}(t)}+\frac{3}{2}(1-\alpha(t))
$$

Figure 6-b shows the temporal evolution of the shape parameter $S P$ for the same velocities as in Fig. 6-a. The horizontal dash lines indicate $S P$ for a cylinder and for a sphere. For all velocities, $S P=1$ at the initial time. Then, the increase of $S P$ until the breakup is approached $\left(t / t_{B U}=1\right)$ depends on the mean jet velocity. For the high velocities $(5.7 \mathrm{~m} / \mathrm{s}$ to $9 \mathrm{~m} / \mathrm{s}$ in Fig. 6-b), we note an inflection of $S P$ when the value 1.5 is exceeded. Then, $S P$ moderately increases until the breakup is reached. (Once again, we note that the jet with $V_{q}=5.7 \mathrm{~m} / \mathrm{s}$ shows a different behaviour near the breakup with a more pronounced increase than for the two other velocities.) The fact that $S P$ remains close to 1.5 at breakup means that the surface-area of the system is mainly brought by the beads that are close to spheres. In other words, the amount of surface-area brought by the strings is almost negligible. For the low velocities $(1.9 \mathrm{~m} / \mathrm{s}$ to $4.5 \mathrm{~m} / \mathrm{s}$ in Fig. 6-b), the inflection after $S P=1.5$ is far less pronounced than for higher velocities and the subsequent increase of $S P$ is far more pronounced. Equation (8) indicates that high values of $S P$ are due to a combination of a high $d_{\max } / D_{1}$ ratio and a non-negligible parameter $\alpha(t)$. Therefore, contrary to the previous case, the surface-area of the system at breakup is not brought by spherical beads only and the contribution of the string is not negligible anymore.

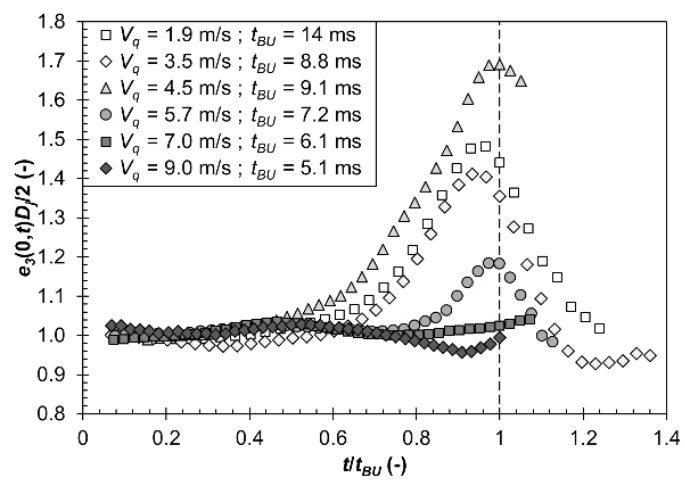

Figure 6-a. Temporal evolution of the specific-surface-area. Influence of the mean jet velocity

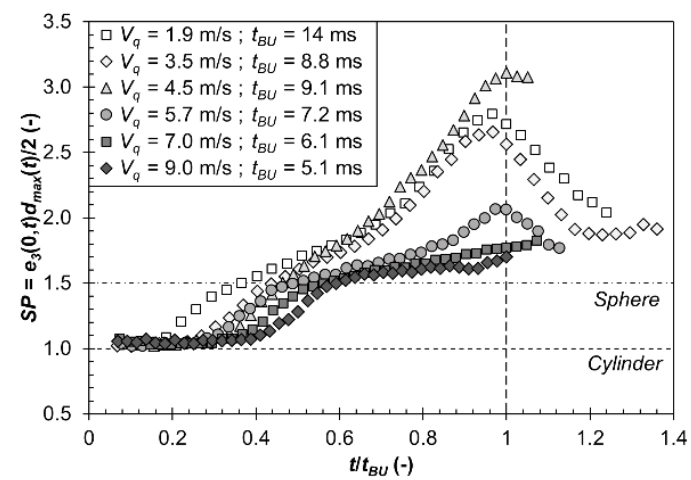

Figure 6-b. Temporal evolution of the shape parameter $S P$. Influence of the mean jet velocity

As a complement of the results shown in Fig. 6-a and 6-b, Fig. 7 presents the pdf (probability density function) of the maximum scale $d_{\max }$ of the drops visible after the breakup. We clearly see in this figure that the pdf of $d_{\text {max }}$ depends on the mean jet velocity: it is shifted to larger $d_{\max }$ as the velocity decreases. According to Eq. (8) this could contribute to the high values of $S P$. Furthermore, since at low velocity the beads are larger than at high velocity, they could be also more deformed and could explain the high specific-surface-area reported in Fig. 6. 
A close examination of the images revealed the presence of a mechanism of coalescence of consecutive swells along the jet. This mechanism is visible for each velocity. It is illustrated in Fig. $8\left(V_{q}=1.9 \mathrm{~m} / \mathrm{s}\right.$, several positions). The coalescence mechanism increases the size of the beads and therefore the scale $d_{\text {max }}$. The shift of the $d_{\text {max }}$ pdf when $V_{q}$ decreases reported in Fig. 7 suggests an increase of the number and degree of coalescence events. We believe that the symmetry loss of the pdf in Fig. 7 is an illustration of this point.

The application of the model given by Eq. (6) may help understanding the evolution of $S P$ and identifying the coalescence mechanism. This model represents one unit pattern of the BOAS, i.e., one string plus one bead, the latter being more or less spherical according to the value of the parameter $n$. If the evolution of this unit pattern is only due to the capillary contraction of the string controlled by the viscoelastic forces, a part of the string volume will transfer in the bead but the volume of the unit pattern will remain constant. Thus, the parameter $\alpha(t)$ will decrease according to Eq. (7). However, if a coalescence event occurs, the volume of the unit pattern increases and the parameter $\alpha(t)$ should decrease more rapidly than in the previous case. Furthermore, the parameter $n(t)$ of the model gives an information on the shape of the beads and should indicate whether they are more or less spherical according to the velocity. Thus, the model given by Eq. (6) is applied in order to examine the variation of the parameters $\alpha(t)$ and $n(t)$.

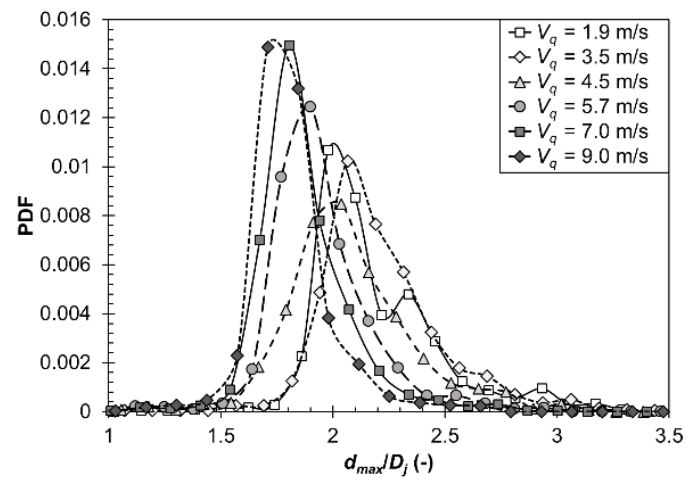

Figure 7. PDF of $d_{\max } / D_{j}$ of the droplets. Influence of the velocity

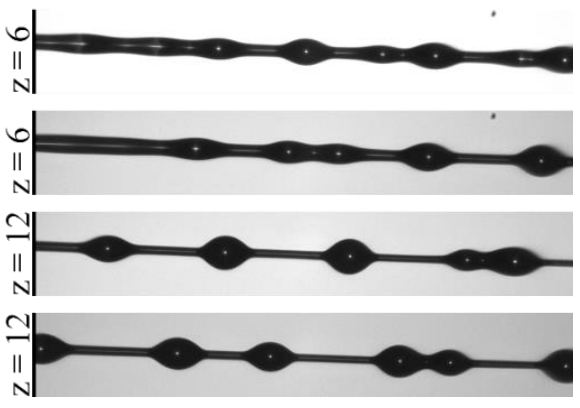

Figure 8. Illustrations of the coalescence mechanism $\left(V_{q}=1.9 \mathrm{~m} / \mathrm{s}\right)$

The application of the model was performed as follows. Equation (6) is used to express $e_{3}(0, t)$ and $e_{3}{ }^{\prime}(0, t)$. (The prime indicates a derivative in the scale space.) It comes:

$$
\left\{\begin{array}{c}
e_{3}(0, t)=\alpha(t) \frac{2}{D_{1}(t)}+(1-\alpha(t)) \frac{n(t)}{D_{2}(t)} \\
e_{3}^{\prime}(0, t)=-\alpha(t) \frac{2}{D_{1}(t)^{2}}-(1-\alpha(t)) \frac{n(t)(n(t)-1)}{D_{2}(t)^{2}}
\end{array}\right.
$$

The quantities $e_{3}(0, t)$ and $e_{3}{ }^{\prime}(0, t)$ are given by the experiments. The parameter $D_{2}(t)$ that represents the maximum scale is taken equal to $d_{\max }(t)$. Finally, the parameter $D_{1}(t)$ is taken equal to the characteristic scale $d_{4}$ that is introduced in Fig. 2. The reason for this choice is that this scale allows us to obtain very good fits. The parameters $\alpha(t)$ and $n(t)$ are found by solving the system Eq. (9). At each time we paid attention that $0 \leq \alpha(t) \leq 1$ and $2 \leq$ $n(t) \leq 3$. The agreement between the model and the experiments is satisfactory as illustrated in Fig. 5 .

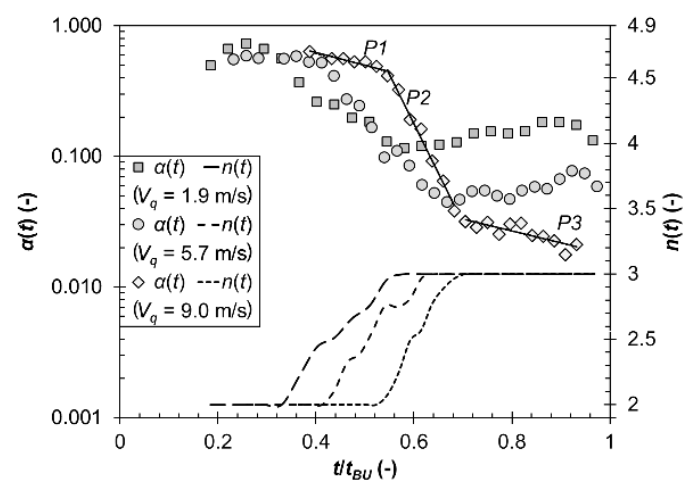


Figure 9. Parameters $\alpha(t)$ and $n(t)$ versus $t$ from the beginning of the elasto-capillary regime to the breakup time. Influence of the velocity. (The symbols correspond to the parameter $\alpha(t)$ and the dashed lines to the parameter $n(t)$ )

Figure 9 presents the parameters $\alpha(t)$ and $n(t)$ for three velocity as a function of the ratio $t / t_{B U}$. For all velocities, the parameter $\alpha(t)$ shows a sharp exponential decrease (P2 in Fig. 9) whose slope depends on the velocity. The characteristic time $t_{2}$ of this decrease $\left(=-t_{B U} /(\right.$ slope P2)) are given in Table 1 for each velocity. These times can be compared with $3 t_{r} / 2$ also given in Table 1 where $t_{r}$ is the characteristic time obtained from the analysis of the scale $d_{1}$ and shown in Fig. 3 (see previous section). For the high velocities (form $7 \mathrm{~m} / \mathrm{s}$ to $10 \mathrm{~m} / \mathrm{s}$ ) we see that $t_{2}<1.5 t_{r}$. This says that the behaviour P2 is the region where the mechanism of coalescence is the more active. This mechanism is fast and produces strings on which the capillary contraction takes place. For the other velocities $(1.9 \mathrm{~m} / \mathrm{s}$ to $5.7 \mathrm{~m} / \mathrm{s}), t_{2}$ is more of the order of $1.5 t_{r}$. This says that the $t_{r}$ measured for these velocities is a characteristic time of the coalescence mechanism and not of the extensional relaxation time as expected. The coalescence is slow and produces strings that are not subject to the elasto-capillary regime of contraction.

The behaviour P2 is preceded by a P1 behaviour (not found for $1.9 \mathrm{~m} / \mathrm{s}$ ). For high velocities, we see in Table 1 that the corresponding characteristic time $t_{1}$ is of the order of $1.5 t_{r}$. The mechanism found here corresponds to the elasto-capillary regime of contraction. However, for low velocities, $t_{1}>>1.5 t_{r}$ : the string is not in the elasto-capillary regime of contraction.

Table 1. Characteristic times of the linear behaviours in Fig. 9 (all velocities)

\begin{tabular}{c|c|c|c|c}
\hline \hline$V_{q}(\mathrm{~m} / \mathrm{s})$ & $t_{r}\left(3 t_{r} / 2\right)(\mathrm{ms})$ & $t_{1}(\mathrm{~ms})$ & $t_{2}(\mathrm{~ms})$ & $t_{3}(\mathrm{~ms})$ \\
\hline \hline 1.9 & $2.18(3.27)$ & - & 2.3 & - \\
3.5 & $1.07(1.61)$ & 7.14 & 0.75 & - \\
4.5 & $0.91(1.37)$ & 3.86 & 0.89 & - \\
5.7 & $0.79(1.19)$ & 5.65 & 0.71 & - \\
7.0 & $0.64(0.96)$ & 0.88 & 0.26 & 29 \\
9.0 & $0.47(0.71)$ & 1.40 & 0.29 & 2.4 \\
10. & $0.43(0.65)$ & 0.96 & 0.16 & 2.4
\end{tabular}

Finally, after the behaviour P2, two situations occur. For the low velocities, $\alpha(t)$ becomes almost constant: the coalescence has stopped and the volume of the ligament is rather constant. Therefore, the continuous thinning of the ligament reported by the scale $d_{1}$ [7] corresponds to an elongation mechanism probably due to gravity effects. For the high velocities, the behaviour P3 is a third exponential decrease whose characteristic time $t_{3}>1.5 t_{r}$ (see Table 1). This mechanism is not the elasto-capillary regime of contraction anymore.

The temporal evolution of $n(\mathrm{t})$ (Fig. 9) shows that it increases from 2 to 3 during the coalescence mechanism. In other words, for every velocity, $n(t)=3$ when the coalescence is over saying that the beads are reasonably spherical. Therefore, the high values of the parameter $S P$ reported in Fig. 6 -b for the small velocity $V_{q}$ is due to a non-negligible contribution of the string to this quantity. This is confirmed by the fact that the string at small velocity contain more liquid than at high velocity (see Fig. 9). Images confirm that strings at small velocity are longer than those at high velocity.

\section{Conclusions}

This work completes a previous experimental study of the behaviour of free-falling liquid jet of dilute viscoelastic solutions [1]. This study considered the dynamic at small scales that was detected by applying a 2D multi-scale description of the jets and identified an exponential decreasing regime from which the viscoelastic relaxation time of the solution could be measured. The objective of the present complement is to understand why this relaxation time, which is expected to be a constant physical property of the viscoelastic solution, shows a sharp increase at small jet velocities. To achieve this, the $2 \mathrm{D}$ multi-scale description is extended to a $3 \mathrm{D}$ multi-scale one. This extension is possible because the capillary instability that develops on a cylindrical liquid jet is an axisymmetric mechanism. This characteristic has been validated here despite a slight initial deformation of the jets mainly observed at high velocity. Among other results, the 3D analysis reports the temporal evolution of the specificsurface-area (interface area per unit volume) of the jet and highlights a deep variation of this evolution when the jet velocity decreases. This evolution is found to be accompanied by an increasing deviation from sphericity of the system. On the other hand, a mechanism of swell-coalescence, whose intensity increases for small velocities, has been identified. The interpretation of these results has been conducted from the application of a simple model of the jet deformation, i.e., of the 3D scale distribution. The model demonstrates that the dynamic of coalescence is very much velocity dependent and that, at low velocity, this mechanism prevents the elasto-capillary contraction at 
small scales from occurring. Furthermore, at low velocity it is found that after the coalescence mechanism, a pure elongation mechanism takes place at small scales that conserves the volume of the strings. In conclusion, the volume of the strings when the breakup is approached is much higher at low velocity explaining the corresponding high values of the specific-surface-area for these cases. These conclusions constitute another demonstration of the practical interest of the multi-scale description and analysis for free liquid flows. The next step for this work is to investigate the coalescence mechanism on free-falling jets of dilute polymer solutions in order to control it when the viscoelastic relaxation time wants to be measured.

\section{Acknowledgments}

The authors acknowledge the financial support from the Frend National Research Agency (ANR) through the program Investissement d'Avenir (ANR-10 LABX-09-01), LABEX EMC3.

\section{References}

[1] Tirel, C., Renoult, M.C., Dumouchel, C., Lisiecki, D., Crumeyrolle, O., Mutabazi, I., (2017) Journal of NonNewtonian Fluid Mechanics (under revision).

[2] Goldin M., Yerushalmi J., Pfeffer R., Shinnar R., (1969) Journal of Fluid Mechanics, 38, pp. 689-711

[3] M. Stelter, G. Brenn, A.L. Yarin, R.P. Singh, F. Durst, (2000) Journal of Rheology, 44, pp. 595-616.

[4] Keshavarz, B., Sharma, V., Houze, E. C., Koerner, M. R., Moore, J. R., Cotts, P. M., Threlfall Holmes, P., McKinley, G. H., (2015) Journal of Non-Newtonian Fluid Mechanics, 222, pp. 171-189.

[5] Christanti, Y., Walker, L.M., (2001) Journal of Non-Newtonian Fluid Mechanics 100(1), pp. 9-26.

[6] Schümmer, P., Tebel, K.H., (1982) Journal of Rheology, 26(1), pp. 77-78.

[7] Schümmer, P., Tebel, K.H., (1982) Rheologica Acta, 21(4-5), pp. 514-516.

[8] Schümmer, P., Tebel, K.H., (1983) Journal of Non-Newtonian Fluid Mechanics, 12(3), pp. 331-347.

[9] Dumouchel, C., Grout, S., (2009) International Journal of Multiphase Flow 35, pp. 952-962.

[10] Dumouchel, C., Aniszewski, W., Ménard, T., Vu, T.T, August 23-27. 2015, $13^{\text {th }}$ ICLASS Conference, Tainan (Taiwan).

[11] Dumouchel, C., Aniszewski, W., Vu, T.T, Ménard, T., (2017) International Journal of Multiphase Flow, 92, pp. 181-192.

[12] Bérubé, J., Jébrak, M., (1999) Comput. Geoscie. 25, pp. 1059-1071.

[13] Dumouchel, C., (2017) ASME - FEDSM, Fluids Engineering Summer Conference, FEDSM2017-69590

[14] Evers, L.W., SAE Technical Series (1994) Paper n`940190. 


\title{
Detailed simulation of air-assisted spray atomization: effect of numerical scheme at intermediate Weber number
}

\author{
G. Tretola ${ }^{1 *}$, K. Vogiatzaki ${ }^{2}$, S. Navarro-Martinez ${ }^{1}$ \\ ${ }^{1}$ Department of Mechanical Engineering, Imperial College, London SW7 2AZ, UK \\ ${ }^{2}$ School of Engineering and Mathematics, University of Brighton, Brighton, BN2 4AT, UK \\ *Corresponding author: g.tretola16@imperial.ac.uk
}

\begin{abstract}
Numerical simulations are often used to understand spray atomisation and estimate the size of the liquid fragments. Several techniques (Level Set, Volume of Fluid, Smooth Particle Hydrodynamics, among others) exist to compute multiphase flows and potentially represent liquid-break-up. However, the complexity of the breakup process and the wide range of scales prevents the use of an unified approach to simulate the complete spray. Numerical techniques face different challenges depending on the spray characteristics. The incorrect representation of surface forces in capillary dominated flows, creates large parasitic currents that distort and in some cases destroy the interface. Methods that perform well in the capillary regime aim to capture the interface directly and the surface radius curvature is therefore larger than the mesh size. However, this creates large constrains on the mesh resolution and limits its applications to low Weber number flows, when there is no extensive atomization. Methods that simulate large Weber number flows (typical of industrial injectors) do not resolve the interface directly and the mesh is larger than the smallest radius of curvature. These models often have numerical or artificial diffusion that destroys small scale structures and alters the break-up. However, even at large Weber flows, the spray formation can be affected by errors due to the local imbalance between pressure and surface tension forces and interface curvature errors. Numerical schemes work around these problems by adjusting the amount of numerical diffusion of the scheme depending on the spray application. Intermediate Weber number sprays are well suited to study the performance of numerical methods as they exhibit hybrid behaviour between capillary flows and full atomization. In the present work an intermediate gas Weber of a laboratory air-blast atomiser is investigated using a volume of fluid approach. The amount of numerical diffusion is controlled by a compressive factor in the volume of fluid transport equation. The effect of the compressive term on spray atomization and droplet size distribution is explored. The results suggest that the optimal amount of diffusion depends on the local Weber number.
\end{abstract}

\section{Keywords}

VOF, OpenFOAM, Atomisation, intermediate Weber.

\section{Introduction}

Liquid atomization is a complex phenomenon present in many engineering processes. The atomization process depends on complex interactions between aerodynamic and capillary forces. Liquid structures are shed from the dense spray core, forming ligaments that pinch-off and form droplets. The droplet break-up pattern itself is also very complex, a droplet may break into few large droplets (for example: vibrational break-up) or a myriad of small droplets (bag break-up), among other. For detailed reviews regarding the physics of the atomisation, see [4] and [5].

The atomisation process involves a wide range of length scales: from the nozzle diameter to the smallest droplets. The smallest scale in atomisation is not still characterised and this size can be three orders of magnitude smaller than the large-scale diameter depending on the Weber number. In order to achieve efficient atomization, two strategies are often used: either the liquid is accelerated to high velocities in a near-quiescent gas, pressurised injection; or the liquid fuel is injected at low speed, surrounded by a coflow high-velocity gas, air-assisted injection. When multiphase flows are solved numerically, the different phases need to be identified. Two major approaches exist: one is the two-fluid model [1] where each phase is governed by an individual set of transport equations for mass, momentum, and total energy. These methods have been implemented either in a pure Eulerian way or most commonly in an Eulerian/Lagrangian framework. An alternative approach is the one-fluid model, where the two phases are treated as one, with a single mixture velocity but a variable density and viscosity. The amount of volume that each phase occupies in the mixture fluid results from a marker function (hereafter $\alpha$ ), also called a "color" function, which follows a simple advection transport equation. The popular volume of fluid (VOF) approach [6] belongs to this category; where the marker function is the fractional volume occupied by one fluid, in liquid-gas simulations often taken as the liquid. There are more approaches to model multiphase flows, however, a detailed review is out of the scope of this work and the reader is referred to [7].

VOF methods are widely spread and have been implemented in many commercial and open-source CFD software. In low Weber number flows, VOF-techniques aim to solve the interface directly as it is possible to have enough computational points within a radius of curvature. Methods in this regime use low-diffusion or compressive numerical schemes to transport the marker $\alpha$ and maintain a sharp interface. However, "classical" VOF requires an interface reconstruction to evaluate the interface curvature to compute surface forces. The accuracy of the reconstruction is based on the smoothness of the marker function and errors create parasitic currents that disturb the interface [14]. 
Numerical VOF methods at large Weber (typical of industrial injectors) do not have a completely resolved interface and they posses large numerical diffusion (as the interface is thickened over several cells). This "artificial" diffusion, destroys small scale structures and alters the break-up process. However, even at large Weber numbers, the local Weber can be relatively small and the spray formation affected by error in the local imbalance between pressure and capillary forces.

The present work aims to investigate the effect of the numerical scheme on the spray characteristics, mainly the droplet size distribution and spray core length. The test case is an air-assisted laboratory spray at intermediate Weber, where VOF-techniques will face challenges corresponding to both Weber regimes. By varying the "compression" of the numerical scheme (and therefore its numerical diffusion) the numerical scheme effects on atomisation can be shown.

The goal is to find a local parameter that can be used to adapt the numerical scheme to the local requirements.

The paper is organised as follows: First, the mathematical model for the VOF scheme and its implementation are presented. Followed by the results section; where the effects of the numerical scheme are presented through snapshots of the marker and the droplet size distributions.

\section{Mathematical model}

The incompressible one-fluid Navier-Stokes equations neglecting body forces together with the $\alpha$ transport equation are:

$$
\begin{aligned}
& \nabla \cdot \mathbf{u}=0 \\
& \frac{\partial \rho \mathbf{u}}{\partial t}+\nabla \cdot(\rho \mathbf{u u})=-\nabla p+\nabla \cdot \mathbf{T}+\rho \mathbf{f}_{\sigma} \\
& \frac{\partial \alpha}{\partial t}+\nabla \cdot(\mathbf{u} \alpha)=0
\end{aligned}
$$

where $\mathbf{u}$ represent the mixture velocity and the stress tensor is $\mathbf{T}=\mu\left[\nabla \mathbf{u}+(\nabla \mathbf{u})^{T}\right]$. The surface forces assuming a constant surface tension are

$$
\mathbf{f}_{\sigma}=\sigma \kappa \mathbf{n} \cdot \delta_{S}
$$

where the curvature of the surface is $\kappa=\nabla \cdot \mathbf{n}$ In the VOF method, the marker $\alpha$ is used to compute the surface normals $\mathbf{n}=\nabla \alpha$ and the surface force is implemented as a body force using the continuum surface force (CSF) model [8].

Ideally, the interface between the two phases should be massless since it represents a sharp discontinuity and $\alpha$ should be a discontinuous function which takes the value 0 for gas and 1 for liquid. However in a finite volume formulation, intermediate values of $\alpha$ exist in cells where the interface is present. In practice due to the numerical diffusion of Eqn.(3) intermediate value of $\alpha$ are present in a "thick" region around the interface. The physical properties are calculated as weighted averages based on $\alpha$, viz.

$$
\begin{gathered}
\rho=\alpha \rho_{l}+(1-\alpha) \rho_{g} \\
\mu=\alpha \mu_{l}+(1-\alpha) \mu_{g}
\end{gathered}
$$

Equation (3) can be modified by assuming that the mixture velocity can be defined as a weighted average of the phase velocities (see [10])

$$
\mathbf{u}=\alpha \mathbf{u}_{l}+(1-\alpha) \mathbf{u}_{g}
$$

Replacing the velocity by the above definition into the transport equation for $\alpha$ :

$$
\frac{\partial \alpha}{\partial t}+\nabla \cdot\left\{\left[\alpha \mathbf{u}_{l}+(1-\alpha) \mathbf{u}_{g}\right] \alpha\right\}=0
$$

From the definition of the relative velocity $\mathbf{u}_{r}=\mathbf{u}_{l}-\mathbf{u}_{g}$, is possible to isolate the gas velocity from the above equation, and rearranging as:

$$
\frac{\partial \alpha}{\partial t}+\nabla \cdot\left(\mathbf{u}_{l} \alpha\right)-\nabla \cdot\left[(1-\alpha) \alpha \mathbf{u}_{r}\right]=0
$$

The first two terms represent the transport of $\alpha$ in the liquid phase, which in incompressible flows is $0 \partial \alpha / \partial t+$ $\mathbf{u}_{l} \nabla \alpha=0$. Therefore,

$$
\nabla \cdot\left[(1-\alpha) \alpha \mathbf{u}_{r}\right]=0
$$

The term can therefore be added to Eqn. (3), obtaining

$$
\frac{\partial \alpha}{\partial t}+\nabla \cdot(\mathbf{u} \alpha)-\nabla \cdot\left[(1-\alpha) \alpha \mathbf{u}_{r}\right]=0
$$


The last term in the above equation is the compression contribution, which is active only at the interface region and has no physical meaning in the continuum formulation. However, in a discrete formulation it acts as an anti-diffusion term and provides a sharper interface compared to the classical VOF formulations, The model does not alter mass conservation of the scheme, however a closure is needed for the relative velocity, $\mathbf{u}_{r}$, as the phase velocities are not available in an one-fluid formulation.

\section{Numerical Implementation}

To solve Eqns. (1), (2) and (11) the unstructured finite volume solver interFoam [11] is used, which is implemented into OpenFOAM [9] The compression term is evaluated using the divergence theorem and the corresponding relative velocity at the cell faces $u_{r, f}=\mathbf{u}_{r, f} \cdot \mathbf{n}_{f}$ is modelled proportional to face normal flow velocity

$$
u_{r, f}=C_{\alpha} u_{f}^{*}
$$

where the subscript $f$ indicates the corresponding cell face. As reported and highlighted in [11], $u_{f}^{*}$ is not evaluated by face interpolation of the velocity, but is obtained from the conservative volume flux from the pressure-velocity coupling algorithm to maintain $\nabla \cdot \mathbf{u}=0$.

The model is based on the definition of relative velocity in Eqn. (7) and suggest an dependence of both $\alpha$ and flow across the face. The constant $C_{\alpha}$ controls the magnitude of the compression term and therefore the diffusion of the numerical scheme. $C_{\alpha}$ is a user-specified value, which in capillary flow is often case specific [12]. Small values of $C_{\alpha}$, provide moderate compression and numerically diffusive schemes (small relative velocity), while large compression, $C_{\alpha} \geq 1$ provide sharper interfaces.

\section{Results and discussion Numerical Set-Up}

The selected test-case is an air-assisted atomiser, presented and studied by [3] and [2]. The injector geometry consists of a straight jet of diameter $d_{1}=1.3335 \cdot 10^{-3} \mathrm{~m}$, where $\mathrm{n}$-dodecane flows. The pipe is surrounded by a co-flow of inner diameter $d_{2}=1.5875 \cdot 10^{-3} \mathrm{~m}$ and thickness $h=2.8575 \cdot 10^{-4} \mathrm{~m}$, with a faster nitrogen stream. The main properties with the Reynolds and Weber numbers are presented in Table 1. The gas-to-liquid momentum ratio is $M=2.53$, which suggest a length core of 3.9 jet diameters (based on experimental correlations [15]) The simplicity of the geometry is well suited for numerical modelling and validation. Desjardins et al. [3] performed Direct Numerical Simulation using an advanced level-set method of the same configuration, with a mesh of $512 \times 256 \times 256$ on a domain of $16 d_{1} \times 8 d_{1} \times 8 d_{1}$. The atomization DNS was performed on 1024 processors [3]. In the present work, the grid size is increased approximately three times in each direction respect to the base DNS, using a mesh of $180 \times 90 \times 90$ grid cells and the simulations were performed in a single workstation. The computational domain is the same as the DNS and a constant CFL number below 0.9 is used throughout the simulation. The code used is the VOF solver interFoam present in OpenFOAM 3.0. Due to the relative low Reynolds number at the tubes outlet, turbulence modelling is neglected.

Table 1. Liquid and gas properties and flow properties for the test case

\begin{tabular}{c|cccccc}
\hline & $\rho\left[\mathrm{kg} / \mathrm{m}^{3}\right]$ & $\mu[\mathrm{kg} / \mathrm{ms}]$ & $\sigma[\mathrm{N} / \mathrm{m}]$ & $U[\mathrm{~m} / \mathrm{s}]$ & $R e[-]$ & $W e[-]$ \\
\hline Liquid & 746 & $1.36 \cdot 10^{-3}$ & $2.535 \cdot 10^{-2}$ & 1.8 & 1336 & 127 \\
Gas & 1.25 & $1.718 \cdot 10^{-5}$ & $2.535 \cdot 10^{-2}$ & 69.89 & 1453 & 321
\end{tabular}

\section{Results}

Figure 1 shows the interface for three different values of the compression factor $C_{\alpha}: 0.1,1$ and 4 . The original DNS [3] showed the formation of the instabilities along the liquid core, which develop into liquid ligaments and then droplets. The present results show similar overall characteristics but with a large dependency of the compression ratio.
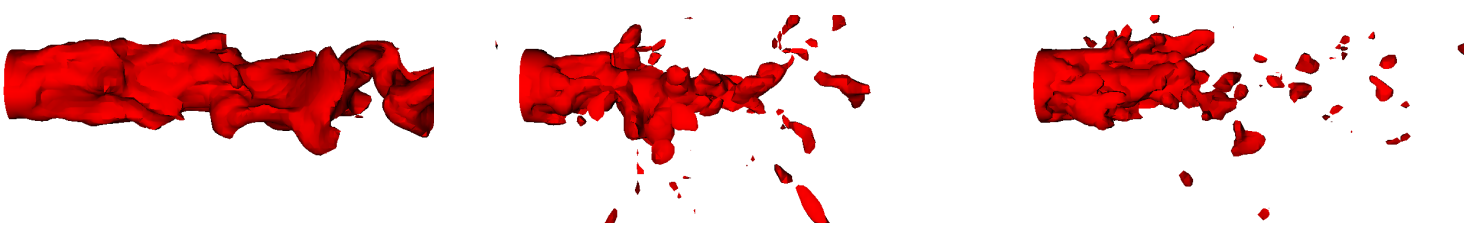

Figure 1. Iso-surface of $\alpha=0.05$ close to the injector. From left to right, $C_{\alpha}=0.01,1$ and 4 , respectively.

Figure 2, shows different iso-contours of $\alpha$ at different compression factors. The distance between the minimum and maximum iso-levels of $\alpha=0.01$ and 0.7 can be understood as a measure of the artificial thickness of the interface. 
The value of 0.7 to represent the core jet is arbitrary and in principle any value of $\alpha$ grater then 0.5 can be used. Nevertheless, in the present simulation the iso-levels greater than 0.5 are very close together (see Fig 2 ) and the precise choice of iso-contour does not affect the discussion."

In the break-up region, the results with the $C_{\alpha}=0.01$ shows an interface thickness of more than 10 cells. In this case, the liquid core does not break but follows a mixing profile typical of gaseous jets. Assuming the dense core is determined by the iso-line of $\alpha=0.7$, both $C_{\alpha}=1$ and $C_{\alpha}=4$ simulations have similar core lengths of $L_{c} / d_{1} \approx 4$, which agree well with empirical correlations $L_{c} / d_{1} \approx 6 / \sqrt{M}$. However, the structure after break-up is significantly different.

For $C_{\alpha}=0.01$, regions with low volume fraction are pushed towards the side, forming ligaments, while large volume fraction regions remain near the centre line of the injector. The results are that only a small fraction of liquid goes into ligaments therefore preventing the break-up. Large liquid structure detaches from the core carried on along the centre by the gas phase. At large compression factors, the thickness of the interface is reduced and dense ligaments form. Then these ligaments break into droplets.
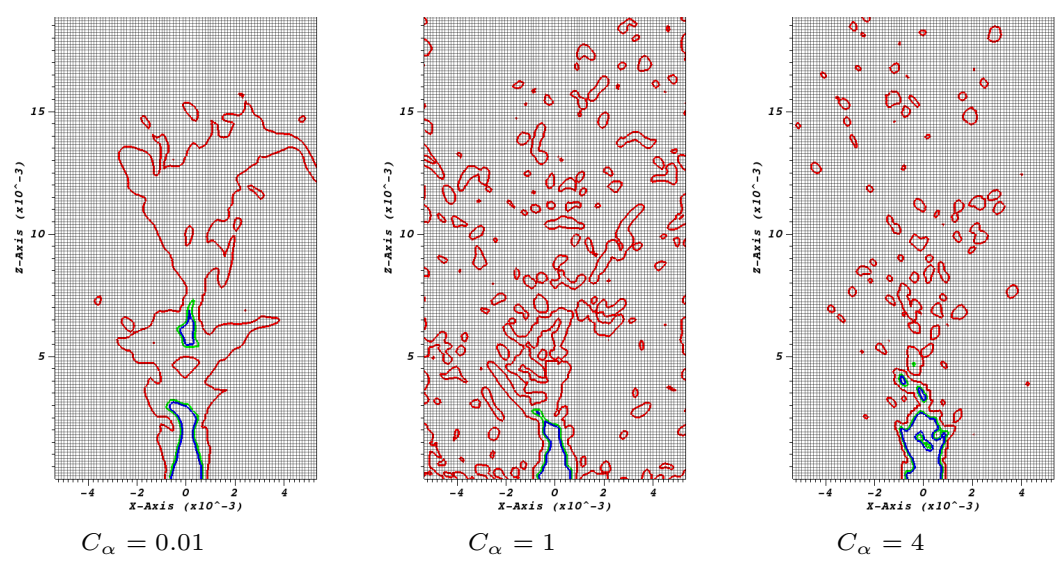

Figure 2. Iso-contours of $\alpha=0.01$ (red), 0.5 (green) and 0.7 (blue) for different compression factor.

Figures 3 and 4 show the local Weber number distributions Two different reference lengths are used: in one case the grid size, $W e_{\Delta}=\rho U^{2} \Delta / \sigma$, and in the other the local mean curvature, $W e_{\kappa}=\rho U^{2} /(\sigma \kappa)$. These choices are used to see the influence of the numerical parameters (the grid size ) and the effect of the error on the volume fraction estimate (which is translated into an error for the mean curvature), on the interface and on the breakup mechanism. As regards $W e_{\Delta}$ it is expected to be of the order of one to be resolved accurately [16] .

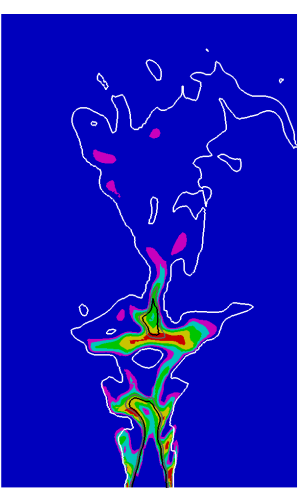

$C_{\alpha}=0.01$

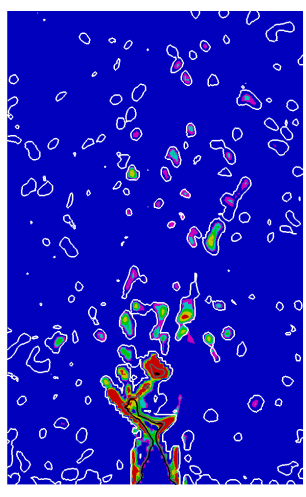

$C_{\alpha}=1$
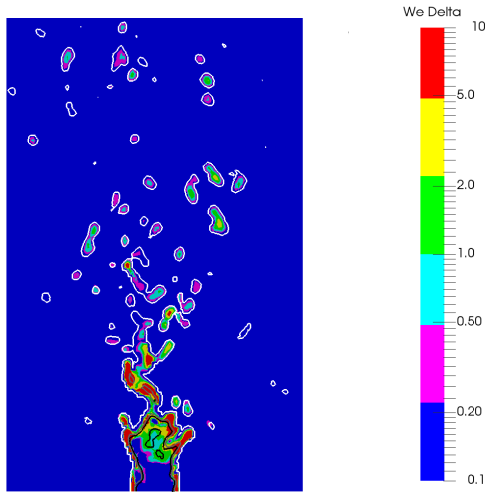

$C_{\alpha}=4$

Figure 3. Local Weber distribution, $W e_{\Delta}$, based on cell size as reference length

The more diffusive scheme, creates very smooth surfaces, with low values of density. Paradoxically, the more the diffuse the interface, the better the capillary effects are resolved. Increasing the compression constant, the local Weber $W e_{\Delta}$ increase along the ligaments and the resolution is inadequate with $W e_{\Delta}>>1$. Further downstream, some droplets are still characterised by a Weber greater than unity. However, most of the droplets are below 2 , which suggest that capillary effects are quasi-resolved.

Figure 4 shows the local Weber based on curvature. The large compression factors, show droplets with $W e_{\kappa}<10$, which suggest that they will not further break [17] as critical Weber is around 10. In the ligament region $W e_{\kappa} \sim 100$, 
which exceeds critical Weber and droplets are expected to appear. However, at $C_{\alpha}=0.01, W e_{\kappa}<10$ nearly everywhere, which prevents filaments to break into droplets. The shear layer between the gas and the air presents the strongest instabilities and large variations of $W e_{\kappa}$.

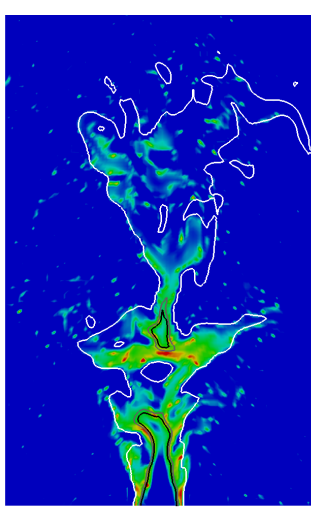

$C_{\alpha}=0.01$

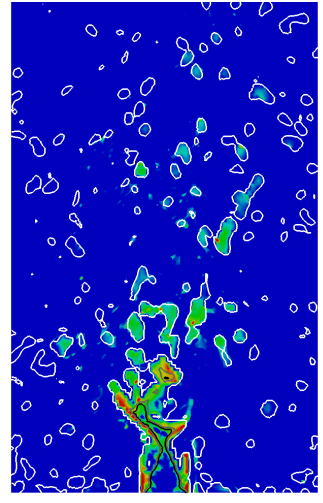

$C_{\alpha}=1$
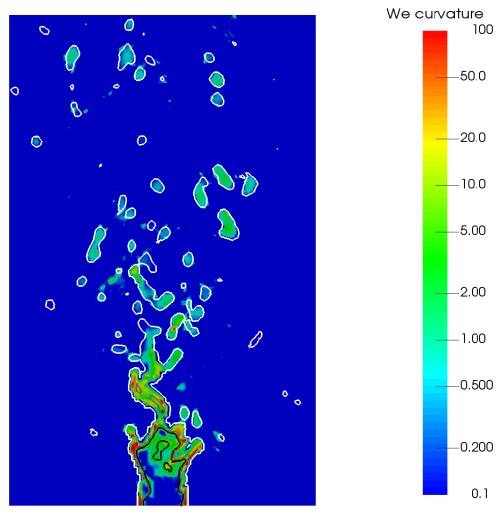

$C_{\alpha}=4$

Figure 4. Local Weber distribution, $W e_{\kappa}$, based on curvature radius as reference length

Figure 5 shows the droplet distribution of the present simulations, compared to the experimental and DNS results presented in [3]. To present fair comparison a diameter normalisation $d^{*}=\left(d-d_{\min }\right) /\left(d_{\max }-d_{\min }\right)$ has been performed. Table 2 reports the peak values $\left(d_{\text {peak }}^{*}\right)$ and mean $\left(\bar{d}^{*}\right)$ of these distributions with both the one obtained by [3]. For all the compression constant, distributions present at least a local peak close the experimental and DNS results. Due to the higher resolution, the DNS captures more and smaller droplets. Also if the the lower compression factor has a $d_{\text {peak }}^{*}$ close to the validated results, it shows slower decay than DNS and experimental, with more uniform droplets along the number, as indicated by $\bar{d}^{*}$.

The results for $C_{\alpha}=1$, show two peaks, the first one near the experimental data. However an unrealistic second peak appears at large values. The case $C_{\alpha}=4$ shows the best agreement with experimental and DNS data on the mean $d^{*}$. as well as the exponential decay at large diameter.

Table 2. Peak Values $\left(d_{\text {peak }}^{*}\right)$ and mean $\left(\bar{d}^{*}\right)$ of droplets distributions

\begin{tabular}{c|cc}
\hline & $d_{\text {peak }}^{*}$ & $\bar{d}^{*}$ \\
\hline DNS & 0.12 & 0.158 \\
Experiment & 0.04 & 0.125 \\
$C_{\alpha}=0.01$ & 0.16 & 0.408 \\
$C_{\alpha}=1$ & 0.48 & 0.409 \\
$C_{\alpha}=4$ & 0.25 & 0.208
\end{tabular}
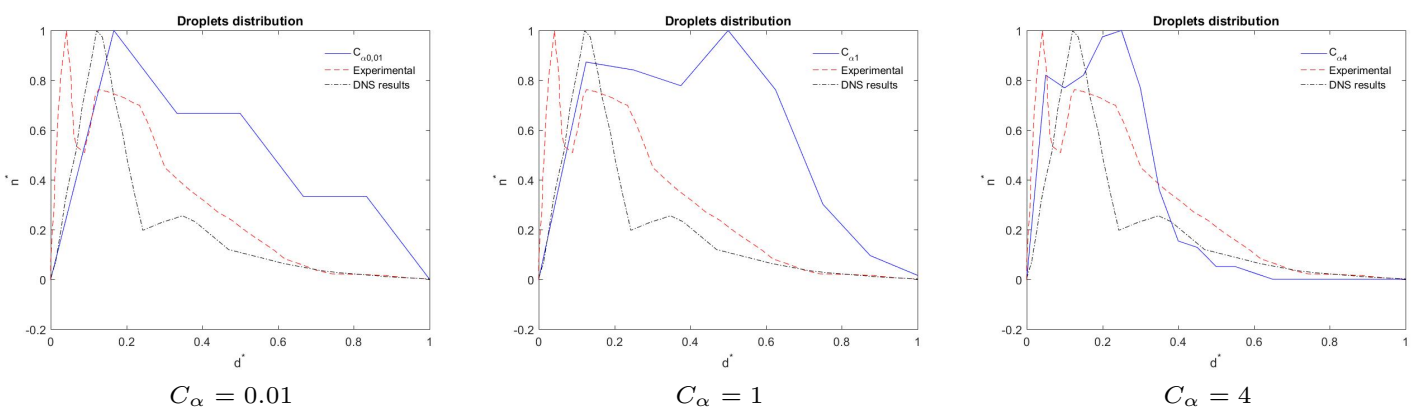

Figure 5. Normalised droplet size distribution with $d^{*}=\left(d-d_{\min }\right) /\left(d_{\max }-d_{\min }\right)$

In this sections, the same simulations set-up are proposed, but a filter process is applied to $\alpha$ in the interface region using a Laplacian filter [13], viz.

$$
\tilde{\alpha}_{P}=\frac{\sum_{f=1}^{n} \alpha_{f} S_{f}}{\sum_{f=1}^{n} S_{f}}
$$


where the subscripts $P$ indicates the cell index. The smoothed volume fraction obtained by (13) is used to compute the interface mean curvature $\kappa$ and the surface normal $\mathbf{n}$.

The results show large difference. Figure 6 shows the results at low compression factor. The liquid detachment of the liquid structure from the dense core is much clearer now and consist of big structures $\sim d_{1}$ that break further downstream. However, no small droplets are present and the results are not realistic. Increasing interface compression, (Figure 7 ), there is no detachment of liquid structure and there is the formation of ligaments of liquid, which breaks producing droplets which size at least one order of magnitude smaller than liquid inlet diameter. The smoothing of the $\alpha$ marker, creates a larger spray angle and some filament-like structures.

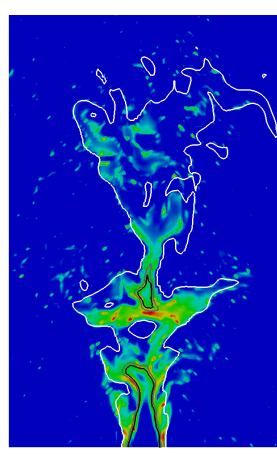

$\alpha$

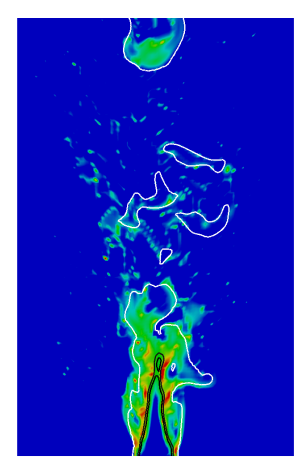

$\tilde{\alpha}$

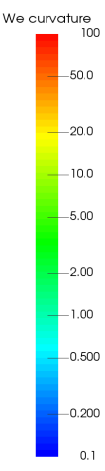

Figure 6. Local Weber distribution, $W e_{\kappa}$, based on curvature radius as reference length for $C_{\alpha}=0.01$. Original $\alpha$ (left), smooth $\tilde{\alpha}$ (right)

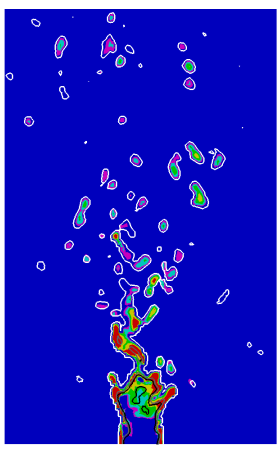

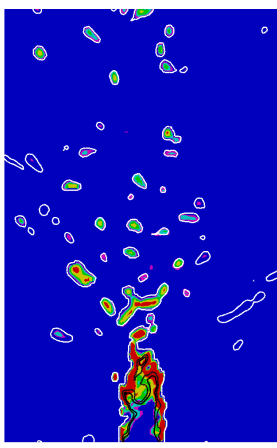

$\tilde{\alpha}$

Figure 7. Local Weber distribution, $W e_{\Delta}$, based on cell size as reference length for $C_{\alpha}=4$. Original $\alpha$ (left), smooth $\tilde{\alpha}$ (right)

Table 3. Peak Values $\left(d_{\text {peak }}^{*}\right)$ and mean $\left(\bar{d}^{*}\right)$ of droplets distributions for smooth $\tilde{\alpha}$.

\begin{tabular}{c|cc}
\hline & $d_{\text {peak }}^{*}$ & $\bar{d}^{*}$ \\
\hline DNS & 0.12 & 0.158 \\
Experiment & 0.04 & 0.125 \\
$C_{\alpha}=0.01$ & 0.4 & 0.508 \\
$C_{\alpha}=1$ & 0.55 & 0.40 \\
$C_{\alpha}=4$ & 0.19 & 0.164
\end{tabular}

Although the trend is the same as non smoothing case, looking at Table 3 the results drift from the experimental data for low and intermediate compression. For the $C_{\alpha}=4$, Figure $8 \mathrm{c}$, both $d_{\text {peak }}^{*}$ and $\bar{d}^{*}$ are close to the DNS results, showing the best agreement for the droplet distribution.

\section{Conclusions}

The simulation of an air-assisted atomiser at intermediate Weber numbers were carried out in this work. The effects of the compression factor were investigated. The description of the liquid core, as the core length or the 

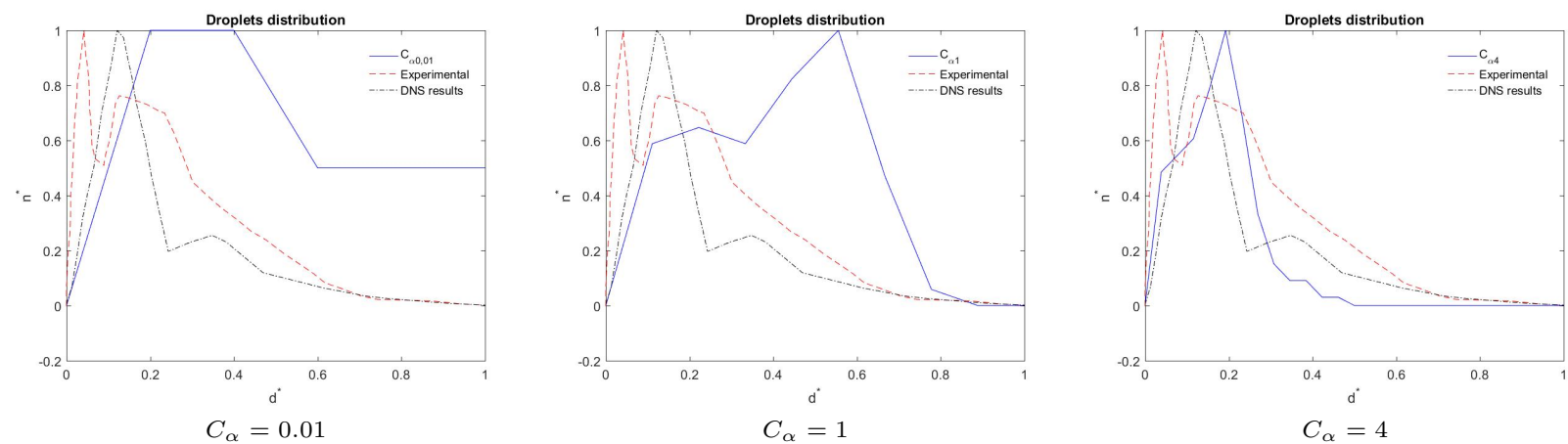

Figure 8. Normalised droplet size distribution with $d^{*}=\left(d-d_{\min }\right) /\left(d_{\max }-d_{\min }\right)$ for smooth $\tilde{\alpha}$.

interface shape, are better obtained by compression factors below unity. However downstream the injector, the low compression factors barely produced any droplets and only the largest compression factors produce similar droplet size distribution than DNS and experimental data. The results show that for the atomisation process at intermediate Weber, an unique compression factor is not useful.

At the injection a small compression factor is needed. However, downstream the injector, at the top of the dense core, more compression is needed to allow the formation of ligaments and create droplets. Increasing the compression factor augments the density, and therefore the value of the local $W e_{\Delta}$, reducing the quality of the capillary/inertial forces interaction. Smoothing the marker function, does not improve the overall results although it greatly affects the break-up shape at low compression factors, however it reduces the amount of small droplets produced. For low compression and without smoothing, the error for both the curvature and the normal interface is too large. Increasing the compression the interface is sharper improving the curvature but giving worse results for the normal vectors. Introducing the smoothing, the estimate of these interface parameters is improved, giving a better estimate for the surface force that will define the breakup and so the particle distributions. The results suggest that a curvature-based Weber, $W e_{\kappa}$, could be used as a marker for the compression factor in a form $C_{\alpha} \propto 1 / W e_{\kappa}$. Close to the nozzle, $W e_{\kappa}$ is large and therefore low compression is needed to accurately capture the propagation of instabilities. However this is reversed as the break-up approaches and more compression is needed. This will be subject of future work.

\section{Acknowledgements}

This work is part of the HAoS project, which is supported by the EU as part of the Horizon 2020 program.

\section{Nomenclature}

$\alpha \quad$ volume fraction [-]

$\tilde{\alpha} \quad$ smoothed volume fraction [-]

$\mu \quad$ dynamic viscosity $\left[\mathrm{kg} \mathrm{m}^{-1} \mathrm{~s}^{-1}\right]$

$\sigma \quad$ surface tension $\left[\mathrm{N} \mathrm{m}^{-1}\right]$

$\kappa \quad$ mean curvature $\left[\mathrm{m}^{-1}\right]$

$\rho \quad$ density $\left[\mathrm{kg} \mathrm{m}^{-3}\right]$

$p \quad$ pressure $\left[\mathrm{kg} \cdot \mathrm{m}^{-1} \mathrm{~s}^{-2}\right]$

$\mathrm{u} \quad$ velocity $\left[\mathrm{m} \mathrm{s}^{-1}\right]$

f force [N]

$\mathbf{S}_{f} \quad$ cell face area [-]

$C_{\alpha} \quad$ compression factor constant [-]

$d \quad$ droplet diameter [mm]

$n$ number of droplet [-]

\section{References}

[1] Ishi, M, 1975, "Thermo-fluid Dynamic Theory of Two-phase Flow", Eyrolles, Paris/Scientific and Medical Publications of France.

[2] Marmottant, P., and Villermaux, E., 2004, Journal of fluid mechanics, 498 , pp. 73-111.

[3] Desjardins, O., McCaslin, J., Owkes, M., Brady, P., 2013, Atomization and Sprays, 23 (11), pp. 1001-1048.

[4] Sirignano, W.A. and Mehring, C., 2000, Progress in Energy and Combustion Science, 26 (4), pp. 609-655.

[5] Eggers, J., and Villermaux, E., 2008, Reports on progress in physics, 71 (3), pp. 1-19.

[6] Hirt, C.W.; Nichols, B.D. ,1981, Journal of Computational Physics, 39 , 201-225

[7] Tryggvason, G., Scardovelli, R, Zaleski, S., 2011, "Direct numerical simulations of gas-liquid multiphase flows". 
Cambridge University Press.

[8] Brackbill, JU., Kothe, D., Zemach, C., 1992, Journal of computational physics, 100 (2), pp. 335-354.

[9] Weller, H.G., Tabor, G., Jasak, H., Fureby, C., 1998, Computer in physics, 12 (6), pp. 620-631.

[10] OpenCFD, Technical Report No. TR/HGW/02, 2005 (unpublished).

[11] Damián, S. M., 2013 Ph.D. thesis , An extended mixture model for the simultaneous treatment of short and long scale interfaces.

[12] Aboukhedr, M., Gavaises, M., Georgoulas, A., Marengo, M., Vogiatzaki, K., 27th Annual Conference on Liquid Atomization and Spray Systems .

[13] Lafaurie, B., and Nardone, C., and Scardovelli, R., and Zaleski, S., and Zanetti, G., 1994, Journal of Computational Physics, 113 (1), pp. 134-147.

[14] Aubin, J., Abadie, T. and Legendre, D., 2016 Journal of computational physics, 297, pp. 611-636.

[15] Lasheras, J. and Hopfinger, H., 2000 Annual Review of Fluid Mechanics, 32, pp. 275-308.

[16] Menard, T., Tanguy, S. and Berlemont, A., 2007, Int. J. Multiphase Flow, 33, pp 510-524

[17] Desjardins, O and Pitsch, H, 2009 J. Comp. Phys., pp. 1658-1677. 


\title{
Towards Resolving the Atomization Process of an Idealized Fire Sprinkler with VOF Modeling
}

\author{
K. V. Meredith*, X. Zhou, and Y. Wang \\ Research Division, FM Global, USA \\ *Corresponding author: karl.meredith@fmglobal.com
}

\begin{abstract}
Fire suppression modeling depends on accurate characterization of the atomization processes of fire sprinklers. Numerical modeling, particularly the volume of fluid (VOF) method, has been applied to understanding the atomization behavior of an idealized sprinkler geometry consisting of a $9.5 \mathrm{~mm}$ inner-diameter cylindrical nozzle and a flat, $25.4 \mathrm{~mm}$ diameter disk with a liquid flow rate of $0.87 \mathrm{~L} / \mathrm{s}$. The simulations have been performed with an OpenFOAM based VOF solver, using the isoAdvector scheme for interfacial reconstruction. The sheet breakup distance and film thickness were calculated and compared with measurements from a previous study. A mesh refinement study identified the sensitivities in the predicted quantities to mesh resolution. This study enables further application of the model to simulation of the fully atomized spray.
\end{abstract}

\section{Keywords}

Fire suppression, primary atomization, VOF, sheet breakup distance, film thickness, sprinkler patternation

\section{Introduction}

The atomization of water by a fire sprinkler is of great interest for fire suppression research. The resulting droplet velocity, diameter, and liquid volume flux largely determine the suppression effectiveness. For example, large droplets can easily penetrate through a fire plume, while small droplets tend to be easily evaporated or carried away with the fire plume and have difficulty reaching the burning surfaces.

Traditionally, atomization in fire sprinklers has been studied experimentally [1]. A great amount of effort has been invested in measuring the sprinkler droplet diameter distribution, droplet velocities, and mass flux profiles [1-7]. These measurements have been used to better understand the atomization process and to develop spray injection models for use within fire suppression modeling [8].

Since the appearance of the first VOF model [9], computational methods associated with VOF have greatly improved in the areas of fidelity and usability [10-13]. VOF simulations provide insight into the underlying processes critical for primary and secondary atomization. With modeling, sprinkler geometry and operating conditions can potentially be varied in a parametric fashion to better understand sprinkler operation. Once the model has been adequately validated, the resulting predictions for droplet diameter, velocity, and liquid volume flux can be directly used in fire suppression CFD simulations as an injection profile derived from first principles.

The goal of this work is to demonstrate the feasibility of using VOF modeling to adequately capture key aspects of sprinkler atomization in an idealized sprinkler geometry, and to provide a path forward to subsequently simulate realistic sprinkler geometries. Accurately resolving the key flow features in the near-field, such as film thickness and sheet breakup length, is critical to enable eventual simulation of the fully atomized spray. Comparisons of the predicted flow features are made with a previously characterized idealized fire sprinkler geometry [14]. A mesh refinement study is performed for better understanding of the required resolution necessary to enable accurate representation of the liquid surface and the details of the subsequent breakup.

\section{Technical Approach}

\section{Experimental Configuration}

The experiment of Zhou and Yu [14] was used for model comparison and validation. The idealized sprinkler used in this experiment consists of a horizontal disk placed beneath a vertical, cylindrical nozzle. Being idealized, there are no slots, tines, frame arms, or boss elements that typically are present in a realistic sprinkler. Three disk diameters were considered: $25.4 \mathrm{~mm}, 38.1 \mathrm{~mm}$, and $50.8 \mathrm{~mm}$. The nozzle was placed $20 \mathrm{~mm}$ above the disk top surface and had an inner diameter of $9.5 \mathrm{~mm}$. 

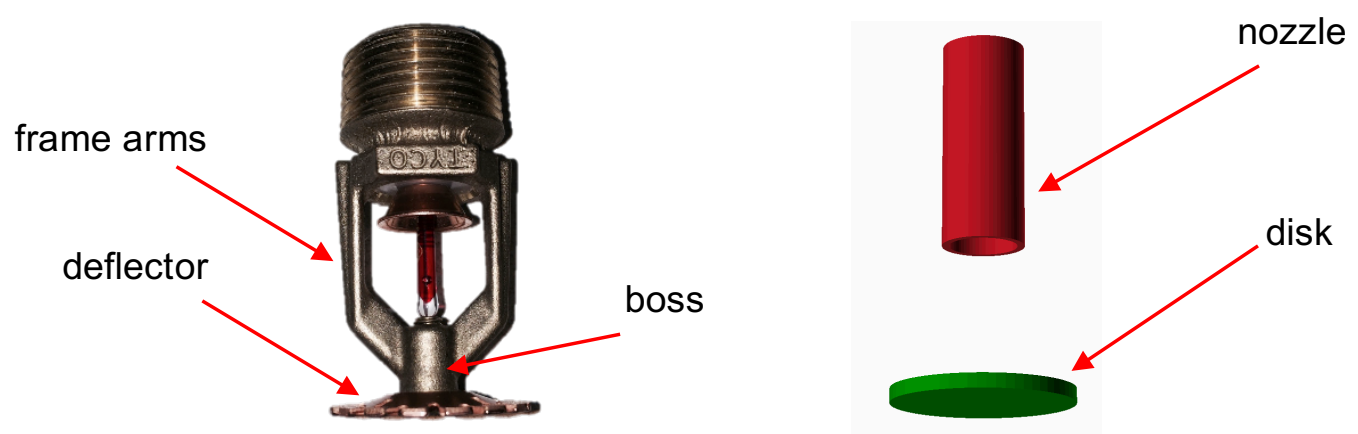

Figure 1. Comparison of a) typical sprinkler geometry showing the frame arms, the boss, and deflector; and b) with idealized sprinkler geometry showing the nozzle and disk.

Water discharge pressures ranging from 0.034 bar to 0.83 bar were used to investigate the spray formation as affected by sprinkler geometry and operating pressure using a laser-based shadow imaging system. The water film thickness (only measured for the lower end of the tested range of discharge pressures), sheet breakup distance, and drop size distributions were measured. An example of the measurements is shown in Figure 2. Refer to Ref. [14] for further details.

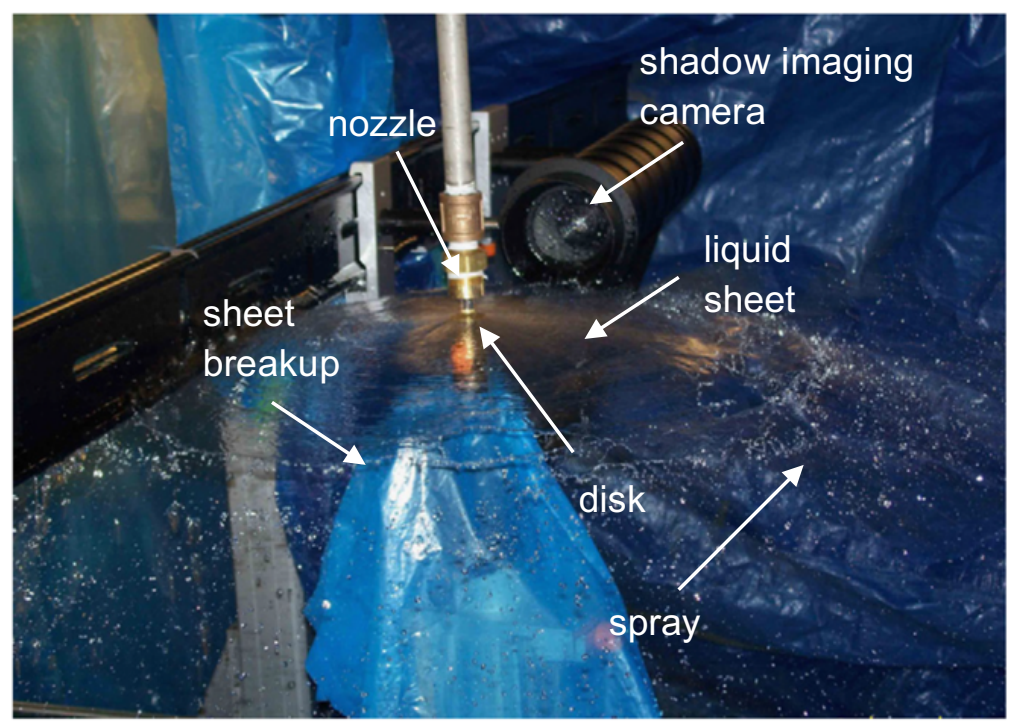

Figure 2. Experimental measurements of atomization process for an idealized fire sprinkler [14].

\section{Numerical Model}

The sprinkler simulations utilized a VOF solver, navalFoam, implemented in foam-extend [15], a community driven fork of the OpenFOAM [16] CFD software. The equations solved in navalFoam have been adequately documented elsewhere [11-13], and portions of the model are reproduced here for reference purposes only. Equations (1-3) represent the continuity, momentum, and phase volume fraction transport equations, respectively,

$$
\boldsymbol{\nabla} \cdot \mathbf{U}=0
$$

$$
\begin{aligned}
\frac{\partial(\rho \mathbf{U})}{\partial t}+\boldsymbol{\nabla} \cdot(\rho \mathbf{U U})-\boldsymbol{\nabla} \cdot\left(\mu_{e f f} \boldsymbol{\nabla} \mathbf{U}\right) \\
=-\boldsymbol{\nabla} p_{d}-\mathbf{g} \cdot \mathbf{x} \boldsymbol{\nabla} \rho+\boldsymbol{\nabla} \mathbf{U} \cdot \boldsymbol{\nabla} \mu_{e f f}+\sigma \kappa \boldsymbol{\nabla} \alpha \\
\frac{\partial \alpha}{\partial t}+\boldsymbol{\nabla} \cdot(\mathbf{U} \alpha)=0
\end{aligned}
$$


where $\mathbf{U}$ represents the velocity vector, $\alpha$ represents the phase fraction, $\rho$ is the density (assumes a combination of phase densities weighted by respective phase fractions), $\mathbf{g}$ is the gravity vector, and $\mu_{e f f}$ is the effective dynamic viscosity from turbulence modeling. The phase fraction, $\alpha$, will only have values between 0 and 1 over the few cells spanning the interface between fluids.

The r.h.s. of Equation 2 represents the pressure body force, the gravity body force, the variation of dynamic viscosity across the interface, and the interfacial surface tension effects. The dynamic pressure is represented as

$$
p_{d}=p-\rho \mathbf{g} \cdot \mathbf{x}
$$

where $\mathbf{x}$ represents the position vector. For the surface tension effect, $\sigma$ represents the liquid surface tension and $\kappa$ represents the mean curvature of the free surface.

$$
\kappa=-\boldsymbol{\nabla} \cdot\left(\frac{\nabla \gamma}{|\nabla \gamma|}\right)
$$

The surface tension force has a finite value only at the interface between phases [17]. Rather than using typical interface compression schemes [18] to try to maintain a sharp interface, navalFoam includes the isoAdvector scheme [11] for approximating a geometric reconstruction of the interface. This scheme explicitly reconstructs a phase interface in each computational cell where $0<\alpha<1$ (i.e., at the intersection between the two phases). This interface is advected through the cell. When calculating fluxes through cell faces, the interface information is used to determine the relative amounts of phase 1 or phase 2 to be advected out of the cell. This approach results in the ability to strongly limit numerical diffusion of the interface. A detailed description of the isoAdvector scheme is beyond the scope of this work, but additional details can be found in Ref. [11].

Large eddy simulation (LES) was used to treat turbulence, and the one-equation eddy model was used for simulating the turbulent kinetic energy. Additional details of the navalFoam model can be found in Refs. [12, 13].

\section{Simulated Geometry}

The simulated geometry, shown in Figure 4, consists of a cylindrical nozzle, $r=4.25 \mathrm{~mm}$ and $l=28 \mathrm{~mm}$, where $r$ is the inner radius and $l$ is the length. The pipe flow inside the nozzle is simulated, having the nozzle inlet at the top of the domain. A disk with diameter $d=12.7 \mathrm{~mm}$ is placed $20 \mathrm{~mm}$ below the nozzle outlet. The disk thickness is set to $2 \mathrm{~mm}$. The overall domain bounds are $x=[-36 \mathrm{~mm}, 288 \mathrm{~mm}], y=[-36 \mathrm{~mm}, 36 \mathrm{~mm}]$, and $z=$ $[-24 \mathrm{~mm}, 48 \mathrm{~mm}]$. The reason for the asymmetry in the $x$-direction is to allow for simulation of the breakup length of the liquid sheet, which for the flow rates studied in Ref. [14], ranges from $153 \mathrm{~mm}$ to $223 \mathrm{~mm}$ for this disk size. Simulating the sheet breakup distance in only one direction allows for a minimization of the required computational cells, and should not affect the flow to any significant extent due to the supercritical nature of the flow (i.e., Froude number $\gg 1$ ). The top, bottom, and sides of the domain are open to the ambient.

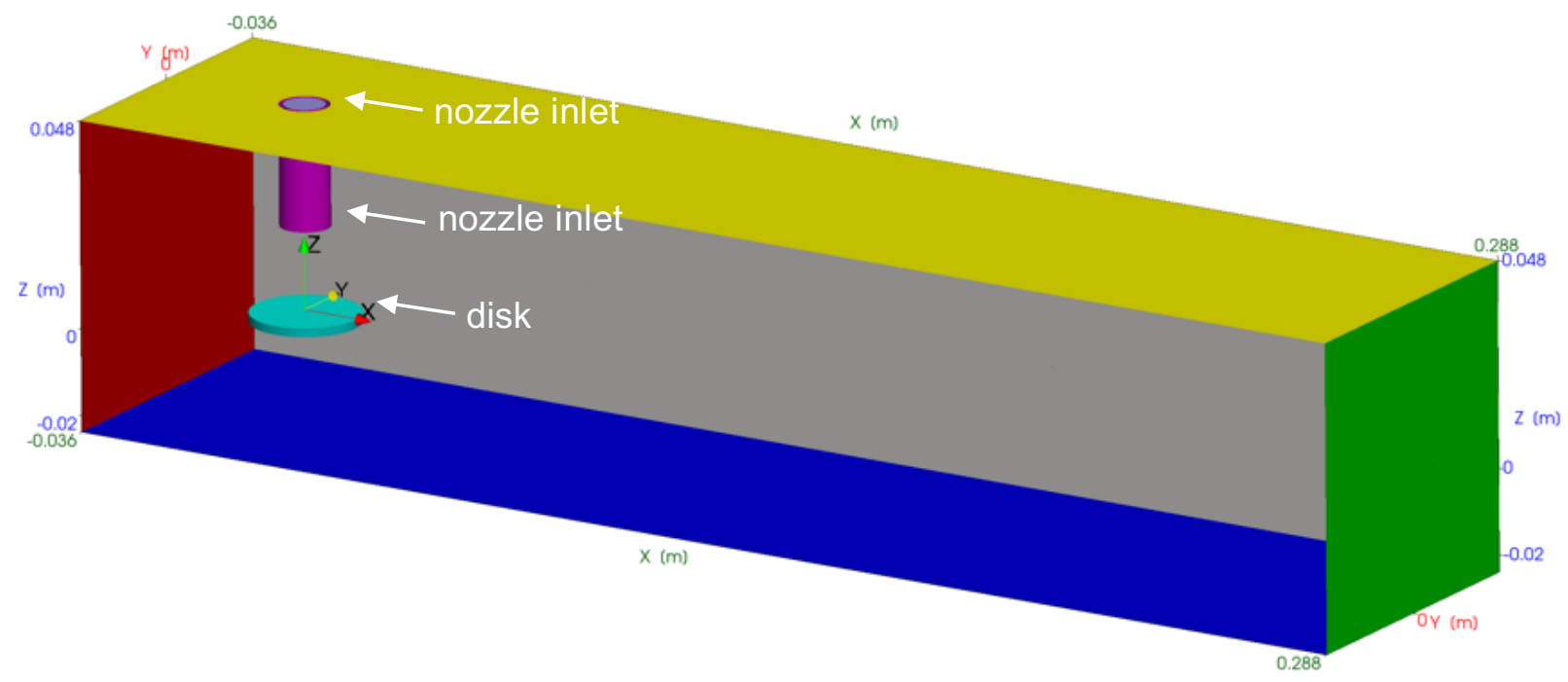

Figure 3. Simulated geometry showing the cylindrical nozzle oriented above the cylindrical disk with inlet, top, side, and bottom boundaries shown. 
The boundary conditions employed in the simulation are listed in Table 1, and correspond to the boundary patches shown in Figure 3. To improve stability in the simulation, the inlet velocity was ramped over time from $U=0 \mathrm{~m} / \mathrm{s}$ at $t=0 \mathrm{sec}$ to $U=12.2 \mathrm{~m} / \mathrm{s}$ at $t=5 \mathrm{~ms}$ and then held constant. This final velocity corresponds to a volumetric flow rate of $52 \mathrm{lpm}$. The inlet was set to a phase fraction of one, and represents the only source of water inflow into the domain. The nozzle and disk wall boundaries consisted of a no-slip velocity condition and a Spallart Allmaras wall function treatment for the subgrid-scale viscosity [19]. The initial conditions were set to quiescent flow and a zero liquid volume fraction. For this study, no turbulent fluctuations were specified at the inlet boundary. This assumption warrants further investigation in future studies, as an operating sprinkler will likely have large fluctuations at the inlet due to pump frequencies, turbulent pipe flow, and flow turning effects from the feed line (typically oriented perpendicular to the nozzle).

Table 1. Simulated boundary conditions.

Inlet

Sides/top/bottom Nozzle/disk

\begin{tabular}{r|l|l|l|} 
Liquid phase fraction & Fixed value $(1)$ & Inlet/outlet & Zero gradient \\
\cline { 2 - 4 } Velocity & Time varying fixed value & No-slip & Pressure inlet/outlet \\
\cline { 2 - 4 } Turbulent kinetic energy & Fixed value $\left(1 \times 10^{-5}\right)$ & Inlet/outlet & Fixed value $(0)$ \\
\cline { 2 - 4 } Pressure & Zero gradient & Total pressure & Zero gradient \\
\cline { 2 - 4 } Subgrid-scale viscosity & Zero gradient & Zero gradient & Spallart Allmaras wall function [19] \\
\cline { 2 - 4 } & &
\end{tabular}

\section{Computational Mesh}

The grid requirements for a VOF simulation to be able to accurately resolve drop sizes is typically on the order of 5 computational cells across the targeted droplet diameter or liquid sheet thickness. For fire sprinkler atomization, the volume mean diameter is approximately $1 \mathrm{~mm}$, with the smallest drop size of interest being on the order of 0.1 $\mathrm{mm}$ diameter. Thus, to resolve these droplets a minimum grid resolution of $\sim 20 \mu \mathrm{m}$ would be necessary. For a uniform grid spacing the number of cells is directly proportional to $\Delta x^{-3}$, and therefore has the potential for resulting in a requirement for a very large number of computational cells. One of the main goals of this research was to identify the minimum grid spacing to resolve the sheet breakup length and most of the breakup processes. Ultimately, adaptive mesh refinement will be required to capture the finest resolutions of interest.

VOF has the potential to predict the liquid volume-flux with a high degree of certainty without necessarily fully resolving each individual droplet. The reason for this is two-fold: 1) VOF accurately conserves the mass of the liquid, and 2) the spatial distribution of liquid volumetric flux is largely dictated by interaction of the solid-core liquid jet emerging from the nozzle with the macroscopic geometry of the idealized sprinkler. Thus, for practicality, minimum mesh resolutions in this study ranged from $1 \mathrm{~mm}$ to $0.25 \mathrm{~mm}$. While these mesh resolutions will certainly not be adequate to capture the finest features of the atomization process (e.g., drop sizes $\approx 0.2 \mathrm{~mm}$ and less), larger features of the flow such as sheet thickness $(\approx 0.8$ to $1.0 \mathrm{~mm})$, ligaments, and the droplets carrying most the liquid volume $\left(D_{v 50} \approx 1 \mathrm{~mm}\right)$ for this condition [14] should be able to be resolved, especially with the finest mesh resolution.

The computational mesh was created by the standard OpenFOAM mesh generation software, snappyHexMesh. The bounds of the simulated domain are shown in Figure 4. One of the goals of this work is to identify the sensitivity of the solution to the mesh refinement. To that end, three mesh sizes were considered. Localized mesh refinement was used to resolve the expected regions of the liquid flow and minimize cell count. The local refinement consisted of three regions: 1) a cylindrical region extending from the bottom of the nozzle to the top of the disk with $r=8 \mathrm{~mm}$, and 2) another cylindrical region with a radius extending beyond the bounds of the computational mesh, with the top and bottom of the cylinder being positioned at $x=-4 \mathrm{~mm}$ and $x=5 \mathrm{~mm}$, respectively. The resolution in these refinement zones was set to values of $\Delta x_{\min }=1 \mathrm{~mm}, \Delta x_{\min }=0.5 \mathrm{~mm}$, and $\Delta x_{\min }=0.25 \mathrm{~mm}$ for the three meshes. The background mesh for each simulation was $\Delta x=4 \mathrm{~mm}$. 
a)

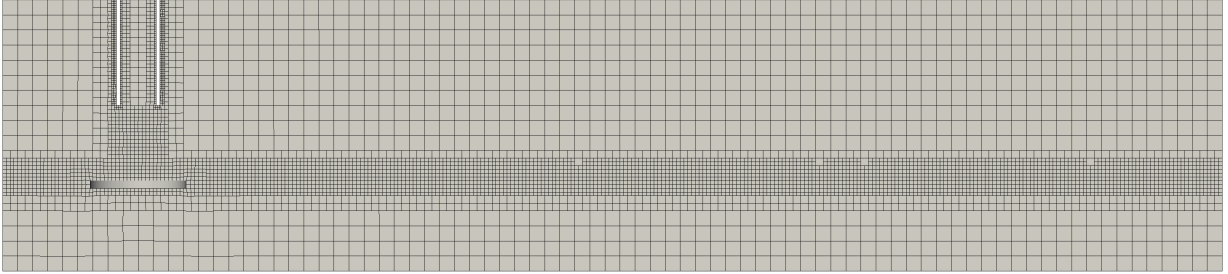

b)

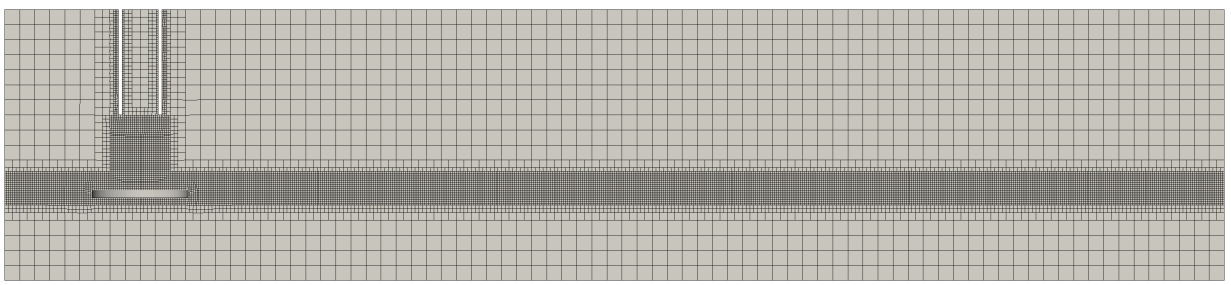

c)

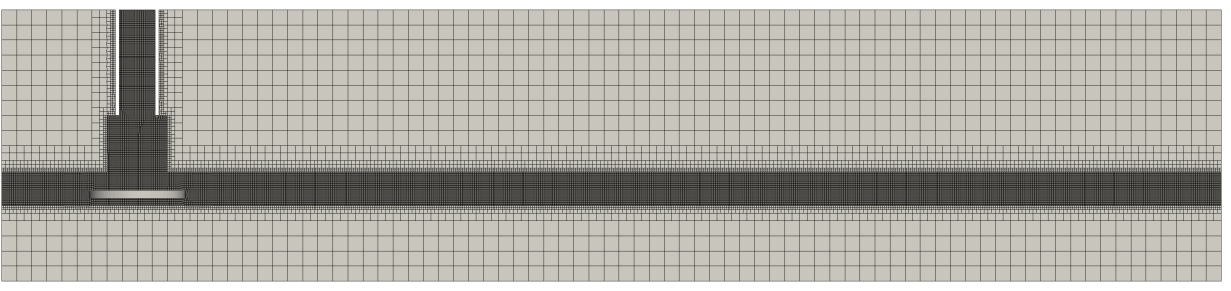

Figure 4. Depictions of the three levels of mesh refinements for the idealized sprinkler geometry: a) $\Delta x_{\min }=1 \mathrm{~mm}$, b) $\Delta x_{\min }=$ $0.5 \mathrm{~mm}$, and c) $\Delta x_{\min }=0.25 \mathrm{~mm}$.

The resulting mesh sizes were $0.3 \mathrm{M}, 1.8 \mathrm{M}$, and $14.1 \mathrm{M}$ respectively. The finest mesh size was also refined within the nozzle region, as this allowed greater stability of the solution while the interface was transiting through this region. The meshes consists of mainly hexahedral cells ( $>98 \%$ of the total cell count) with only a small number of tetrahedral and prismatic cells needed to accurately represent the curvature of the nozzle and disk.

\section{Results}

Each mesh resolution was simulated for a flow rate of $0.87 \mathrm{~L} / \mathrm{s}$ entering the nozzle inlet. This corresponds to the upper range of flow rates tested in Ref. [14]. The $1 \mathrm{~mm}$ and $0.5 \mathrm{~mm}$ resolutions were computed on in-house on a cluster with Intel ${ }^{\circledR} \mathrm{Xeon}^{\circledR}$ CPU E5-2630 v3 @ 2.40GHz processors and using 60 cores each, requiring approximate wall-clock time of $2.5 \mathrm{hr}, 14.1 \mathrm{hr}$, respectively for a simulation time of $100 \mathrm{~ms}$. The finest mesh resolution was simulated with on a Cray XC30 cluster with Intel ${ }^{\circledR}$ Xeon ${ }^{\circledR}$ CPU E5-2670 @ 2.60GHz processors using 600 cores and required $25 \mathrm{hr}$ for $100 \mathrm{~ms}$ of simulation time.

A series of iso-surfaces of the liquid volume fraction at a value of $\alpha=0.5$ are shown in Figure 5 for a range of times starting from $6 \mathrm{~ms}$ to $20 \mathrm{~ms}$ for the $\Delta x=0.25 \mathrm{~mm}$. After $6 \mathrm{~ms}$ the liquid jet has emerged from the nozzle but not yet impinged on the disk. After $7 \mathrm{~ms}$, the jet impinges on the disk and forms a high velocity liquid film on the surface of the disk as pressure forces the flow outwards. At $8 \mathrm{~ms}$ the liquid film leaves the surface of the disk and forms a liquid sheet. At the leading edge of the sheet ligaments and droplets are formed and subsequently shed due to the high velocity film entering a stagnant gas-phase flow. The liquid sheet continues to spread outwards, shedding droplets along the way. By $13 \mathrm{~ms}$, the high velocities induced by the initial jet impingement (and observed at $9 \mathrm{~ms}$ ) have resided. By $20 \mathrm{~ms}$ the liquid sheet has reached a radial distance of $\sim 70 \mathrm{~mm}$ from the center of the disk.

As the sheet transports radially outward, the sheet velocity decreases due to momentum exchange with the surrounding air. Eventually, due to the sheet thinning as it expands radially and due to instabilities in the flow, the sheet breaks up. Experimental observation suggests that for this flow rate and disk/nozzle configuration, the sheet transitions into ligaments and droplets at a radius of $\sim 165 \mathrm{~mm}$ from the disk center [14]. In the simulations, the sheet breakup occurs at a radius of about $25 \mathrm{~mm}, 160 \mathrm{~mm}$, and $155 \mathrm{~mm}$ for the three mesh resolution results shown in Figure 6. The $1 \mathrm{~mm}$ mesh resolution case clearly under predicts the sheet breakup distance, due to a lack of refinement necessary to sufficiently resolve the phase interface. Refining the mesh to $0.5 \mathrm{~mm}$ and subsequently to $0.25 \mathrm{~mm}$ results in a breakup distance that closely matches the experimental observation to within $10 \mathrm{~mm}$. For these cases, the sheet falls below the local refinement zone at a radial distance between 150 and $200 \mathrm{~mm}$. 

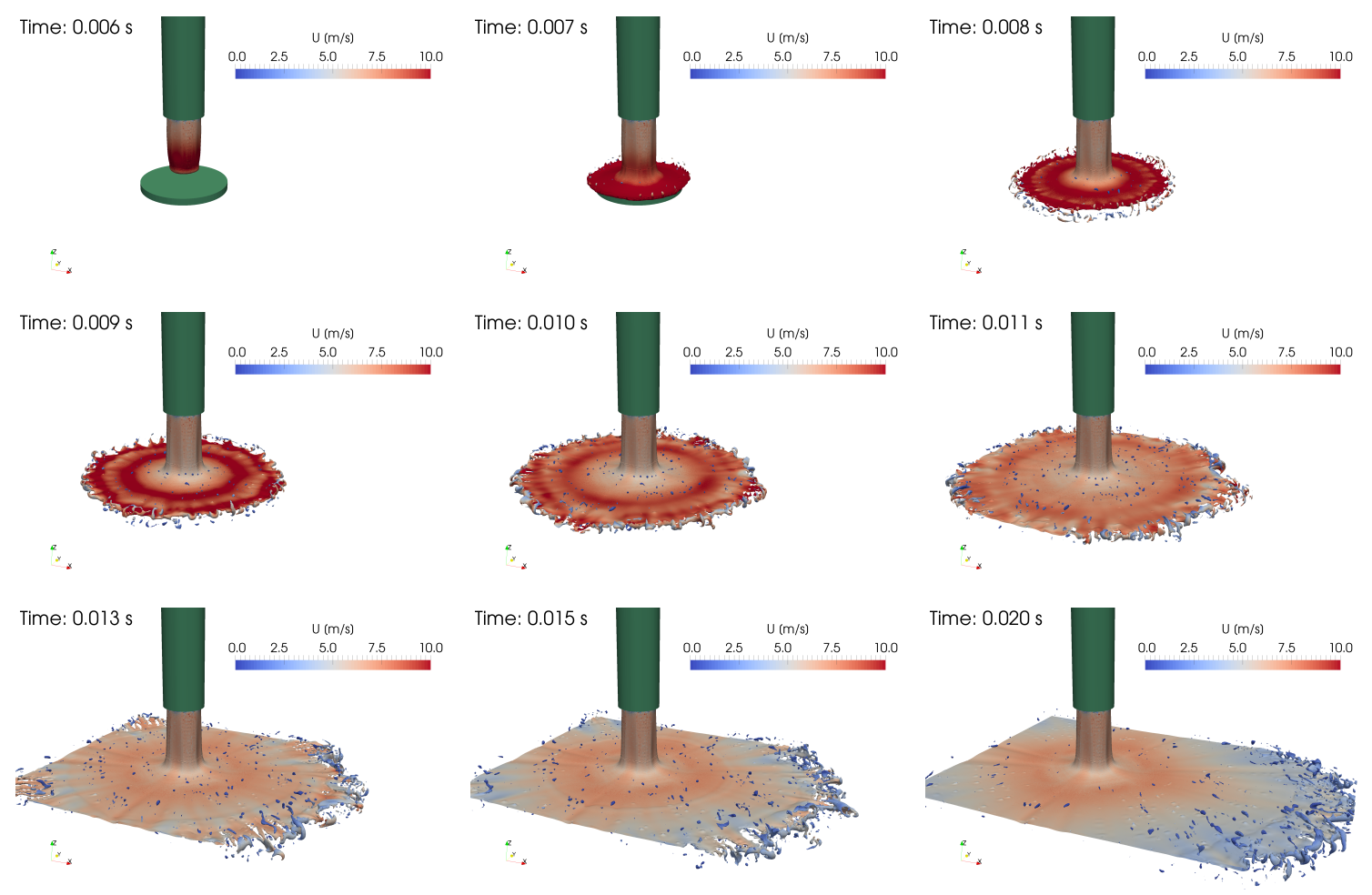

Figure 5. Iso-contours for liquid phase fraction $\alpha=0.5$ showing the phase interface at a series of times ranging from $6 \mathrm{~ms}$ to 20 $\mathrm{ms}$ for the $\Delta x=0.25 \mathrm{~mm}$ resolution case. Iso-contours are colored by velocity magnitude.

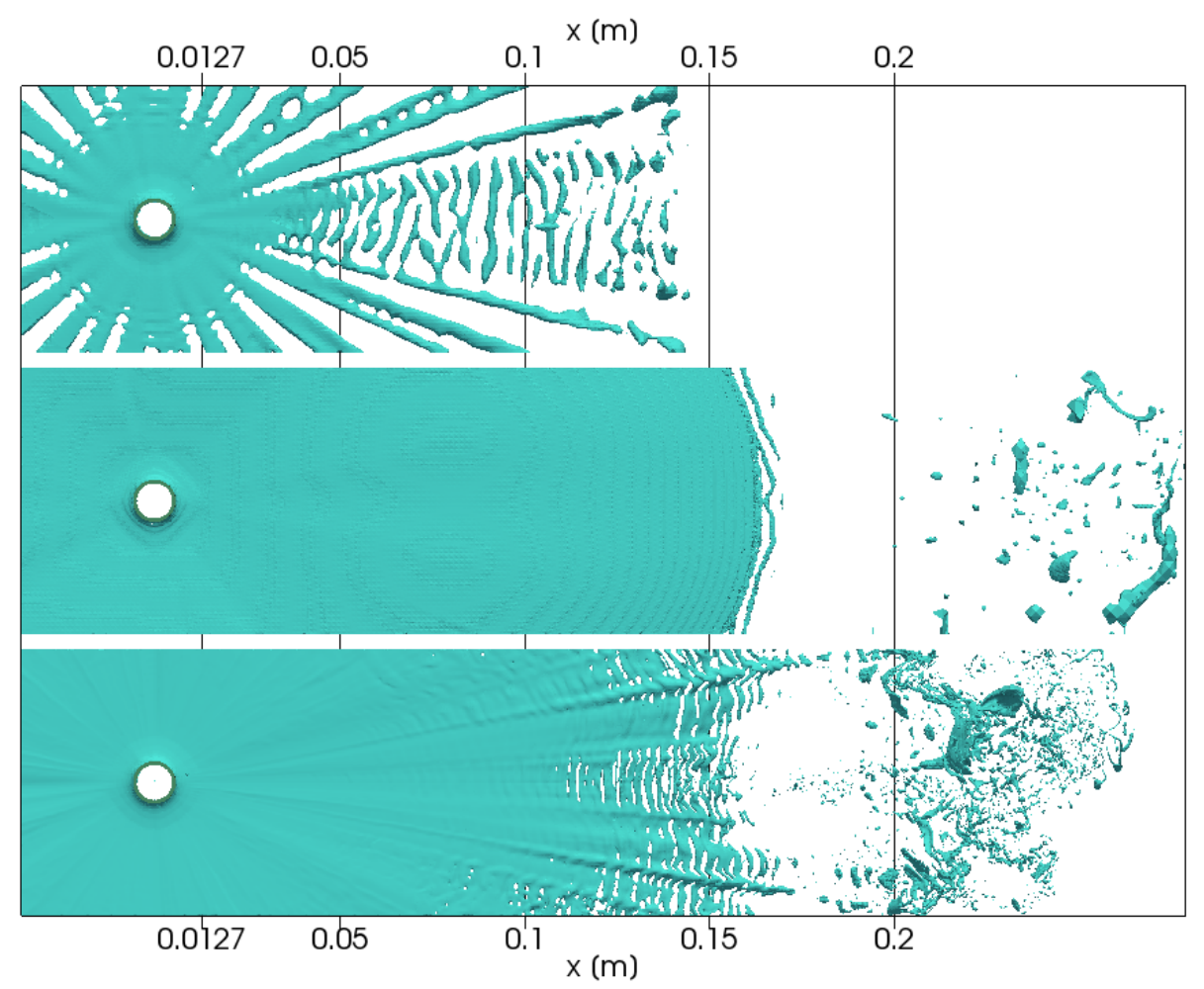

Figure 6. View in the $-\boldsymbol{z}$ direction of liquid surface iso-contours ( $\alpha=0.5)$ demonstrating the sheet breakup distances for the three mesh resolutions: a) $\Delta x=1 \mathrm{~mm}$, b) $\Delta x=0.5 \mathrm{~mm}$, and c) $\Delta x=0.25 \mathrm{~mm}$. 

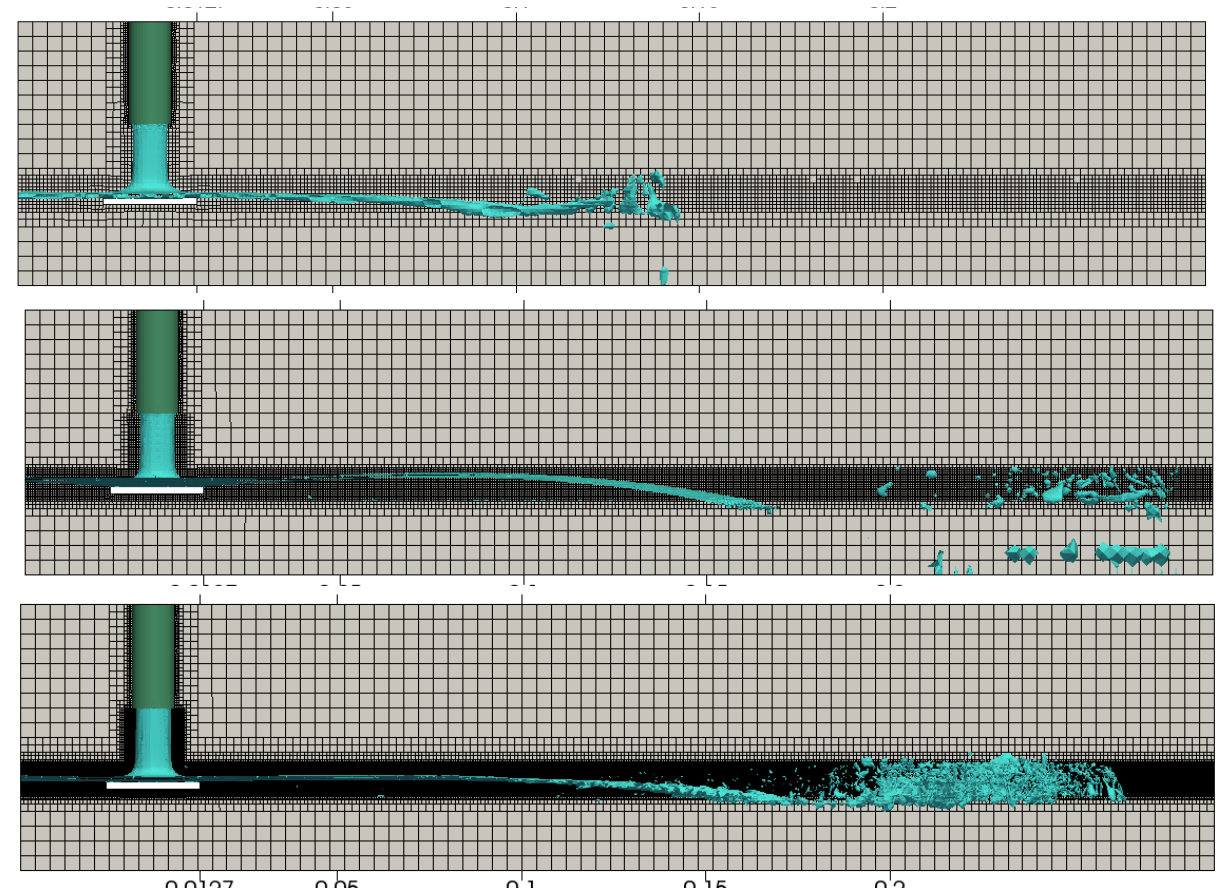

$0.0127 \quad 0.05$

0.1

$x(m)$

Figure 7. View in the $-y$ direction of the liquid surface $(\alpha=0.5)$ demonstrating the extent of the sheet breakup distance in relation to the mesh refinement regions.

A representation of the film flow on the surface of the disk is shown in Figure 8 . The thickness of the liquid film as it exits the disk is of interest, as this sheet thickness influences the size distribution of droplets formed upon breakup. Experimentally, while direct measurements of the sheet thickness for this flow rate are not available, estimates for the film thickness at the edge of the disk based on analytical models place the value at $\sim 1.0$ to $1.2 \mathrm{~mm}$ [14]. The film thickness from the simulations, determined as the distance above the disk at which the liquid phase fraction drops to 0.5 , is shown in Table 2 . The simulated values are approximately in the expected range.

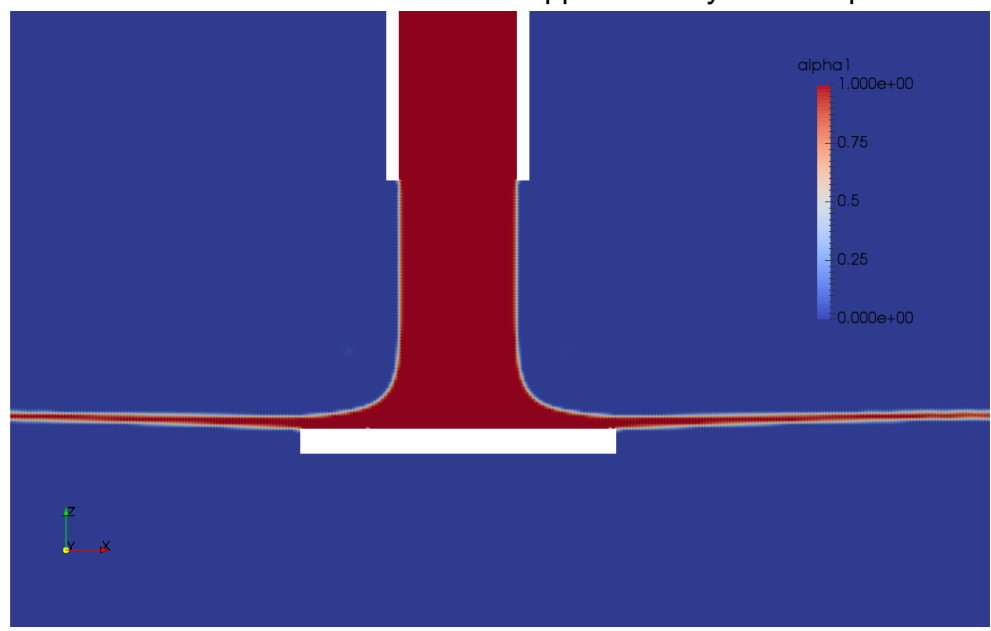

Figure 8. Representation of the liquid volume fraction in the near field of the nozzle and deflector for $\Delta x=0.25 \mathrm{~mm}$.

Table 2. Film thickness sampled at the edge of the disk for three mesh refinements.

\begin{tabular}{l|l|l|l|}
\multicolumn{2}{c}{$\Delta x=\mathbf{1 ~ m m}$} & \multicolumn{1}{c}{$\Delta x=\mathbf{0 . 5} \mathbf{m m}$} & $\mathbf{0 . 2 5} \mathbf{m m}$ \\
\hline Film thickness $[\mathrm{mm}]$ & 1.33 & 1.29 & 1.03
\end{tabular}

\section{Summary and Conclusions}

Simulating the atomization processes of a sprinkler has the potential to allow for enhanced insight into the key physics and controlling parameters. This approach also has the potential to be used as a predictive tool to estimate 
the spray injection profiles. In this study, the atomization of an idealized sprinkler geometry was simulated and comparisons were made to experimental data. An innovative isoAdvector scheme allowed for maintaining a sharp interface even with relatively coarse mesh resolution. A mesh refinement study was used to identify requirements for simulating sheet breakup distance. Mesh resolutions of $1 \mathrm{~mm}$ provided insufficient refinement, as the sheet breakup distance was greatly under predicted due to numerical diffusion of the interface, while a mesh resolution of $0.5 \mathrm{~mm}$ closely matched the experimentally determined value. A final mesh resolution of $0.25 \mathrm{~mm}$ was also simulated, resulting in finer detail in the region of the flow following sheet breakup. Ultimately, a mesh resolution of less than $0.25 \mathrm{~mm}$ will be required to resolve the flow features necessary to accurately predict atomization processes. Comparisons for film thickness predictions were also made, showing the simulated values for the finest mesh resolution closely resemble the values estimated from analytical models.

This study establishes the feasibility of using VOF modeling to adequately obtain injection patterns. The numerical model can subsequently be used to provide insight into sprinkler geometry effects on spray characteristics and to eventually lead to simulating realistic sprinkler geometries to obtain the atomization results necessary to initialize the sprinkler spray in fire suppression simulations.

\section{Acknowledgements}

This work was funded by FM Global as part of the Strategic Research Program for Fire and Suppression Modeling. Drs. Hrv Jasak and Vuko Vukčević of Wikki Ltd. are gratefully acknowledged for their guidance in using the isoAdvector and ghost-fluid-method schemes. For part of the simulations, this research used resources of the Oak Ridge Leadership Computing Facility at the Oak Ridge National Laboratory, which is supported by the Office of Science of the U.S. Department of Energy under Contract No. DE-AC05-00OR22725.

\section{References}

[1] Ren, N., Baum, H. R., and Marshall, A. W., 2011, "A comprehensive methodology for characterizing sprinkler sprays," Proceedings of the Combustion Institute, 33(2), pp. 2547-2554.

[2] Yu, H. Z., 1986, "Investigation of Spray Patterns of Selected Sprinklers with the FMRC Drop Size Measuring System," Proceedings of the 1st International Symposium on Fire Safety Science, pp. 1165-1176.

[3] Chan, T. S., Kung, H. C., Yu, H. Z., and Brown, W. R., 1994, "Experimental Study of Actual Delivered Density for Rack-Storage Fires," Proc. of the 4th International Symposium on Fire Safety Science, pp. 913-924.

[4] Widmann, J. F., 2001, "Phase Doppler Interferometry Measurements in Water Spray Produced by Residential Fire Sprinklers," Fire Safety Journal, 36, pp. 545-567.

[5] Sheppard, D. T., 2002, "Spray Characteristics of Fire Sprinklers," National Institute of Standards and Technology.

[6] Zhou, X., D'Aniello, S., and Yu, H. Z., 2010, "Spray Pattern Measurements of Selected Fire Sprinklers," Proceedings of the 12th Fire Science and Engineering Conference, INTERFLAM, Nottingham, UK, pp. 177-188.

[7] Zhou, X., D'Aniello, S. P., and Yu, H. Z., 2012, "Spray characterization measurements of a pendent fire sprinkler," Fire Safety Journal, 54(0), pp. 36-48.

[8] Wang, Y., Meredith, K. V., Zhou, X., Chatterjee, P., Xin, Y., Chaos, M., Ren, N., and Dorofeev, S. B., 2013, "Numerical Simulation of Sprinkler Suppression of Rack Storage Fires," Fire Safety Science.

[9] Hirt, C. W., and Nichols, B. D., 1981, "Volume of fluid (VOF) method for the dynamics of free boundaries," Journal of Computational Physics, 39(1), pp. 201-225.

[10] Jasak, H., and Weller, H. G., 1995, "Interface Tracking Capabilities of the Inter-Gamma Differencing Scheme," Technical Report, Imperial College.

[11] Roenby, J., Bredmose, H., and Jasak, H., 2016, "A computational method for sharp interface advection," Royal Society Publishing open sci, 3(160405).

[12] Vukčević, V., Jasak, H., and Malenica, S. i., 2016, "Decomposition model for naval hydrodynamic applications, Part I: Computational method," Ocean Engineering, 121, pp. 37-46.

[13] Vukčević, V., Jasak, H., and Malenica, S. i., 2016, "Decomposition model for naval hydrodynamic applications, Part II: Verification and validation," Ocean Engineering, 121, pp. 76-88.

[14] Zhou, X., and Yu, H.-Z., 2011, "Experimental investigation of spray formation as affected by sprinkler geometry," Fire Safety Journal, 46, pp. 140-150.

[15] 2016, "foam-extend," http://www.sourceforge.net/projects/foam-extend.

[16] Weller, H. G., Tabor, G., Jasak, H., and Fureby, C., 1998, "A tensorial approach to computational continuum mechanics using object-oriented techniques," Computers in Physics, 12(6), pp. 620-631.

[17] Brackbill, J. U., Kothe, D. B., and Zemach, C., 1992, "A continuum method for modeling surface tension," Journal of Computational Physics, 100(2), pp. 335-354.

[18] Suraj, S. D., Lakshman, A., and Mario, F. T., 2012, "Evaluating the performance of the two-phase flow solver interFoam," Computational Science \& Discovery, 5(1), p. 014016.

[19] Spalart, P. R., and Allmaras, S. R., 1992, "A One-Equation Turbulence Model for Aerodynamic Flows," AIAA Paper 92-0439. 


\title{
Numerical Simulation of Like and Unlike Impinging Jets Hamed Dolatkhahi', Ghader Oliaee ${ }^{1,}$ Azadeh Kebriaee*1 $^{* 1}$ \\ ${ }^{1}$ Aerospace Engineering Department, Tehran, Iran
}

\begin{abstract}
In the present study, using the open source OpenFOAM code, a numerical simulation is performed taking the adaptive mesh refinement (AMR) technique during solution. Formation of liquid sheet after the impact of two identical cylindrical jets at various conditions is studied. Since the flow pattern depends upon the Reynolds and Weber numbers, numerical tests are conducted at a variety of flow velocities and Reynolds numbers to demonstrate the effect of these parameters on the sheet formation. It is then concluded that at various conditions, different instabilities occur in the flow; hence, different sheet formations a flow patterns happen.

In this study, impact of two dissimilar cylindrical fluid jets is successfully simulated for the first time in literature. Actually, water and oil jets are taken into account and their impact behavior is studied. In the presence of the surrounding air, an unstable sheet will form after impact due to the high injection speed of the jets. As depicted in the results, since the inertia and other physical characteristics of the two fluids are dissimilar, different phases are more intensely diffused.
\end{abstract}

Keywords: Two phase flow, Injector, Impingement jet, numerical solution, Dynamic mesh.

\section{Introduction}

Atomization is defined as a process of producing a large number of droplet from a liquid spot [1]. In the atomization process, liquid jet or a sheet of liquid are broken apart due to high kinetic energy content, exposure to high velocity air/gas or the mechanical energy transferred to the system in the form of rotational movements of vibrations. Due to random characteristic of atomization, the produced spray consists a large spectrum of various size of diameters. More recently, on account for a wide range of spray utility, liquid atomization has been turned out to be a fascinating subject. The spray produced in the Impingement jet injectors has a variety of industrial applications. Impingement injectors are among the most common fuel injectors in turbine engines. Such injectors are also utilized to control droplets in combustion, recreational fountains and also to provide uniform painting/coating [2]. Impingement jet injectors are widely used in liquid propellant and solid propellant motors due to their simplicity in manufacturing as well as high efficiency and excellent atomization characteristics. They have also been recently in the center of attention due to their low cost and high efficiency. Combustion performance of liquid rocket engines highly depend on mixture uniformity which is created by impingement jet injector. Impingement jet injector is an appropriate method to control droplet size as well as spray distribution which are highly applicable in industry. This injector is used in liquid rocket jet where reactants are produced by impinged fuel and oxidizer jets [3].

In such injectors, impact of two cylindrical jets leads to liquid sheets. Overall shape and thickness of the sheet is dependent upon the impacting angle between two jets, diameter of the jets, velocity and physical characteristics of the jets [4]. A variety of impingement jet injectors exist, which are used for different fluids (liquid, gas or gel) and are based on different parameters such as motor conditions, cooling of the walls in the combustor and its length, mixture ratio and the operating pressure [5].

Although studies on Impingement jet injectors date back to a long time ago, simulations of such injectors are so scarce in literature due to complexities inherent in multiscale two-phase flows and the computational difficulties in such simulations. In a first simulation, Inoue et al. [6] presented a numerical study to provide characteristics of the impingement jet injectors. Their results were then compared with experimental findings and analytical solutions. Despite the shape of liquid sheet being periodic, their simulation was incapable of providing accurate measures for ligaments and droplets during collapse due to coarse mesh and inadequate mesh studies.

In 2013 Arienty et al. [7] investigated the effect of mesh refinement on the calculations of the jet atomization. In this research, interfacial surface is analyzed by an appropriate combination of VOF and Level Set, using impingement jet atomization by means of CLSVOF formulation. Doing this so, collapse of the produced sheet by means of two jets with a high velocities at various levels of a refined mesh was investigated and compared with each other.

Dong-Jun $\mathrm{Ma}$ et al. [8] have also used numerical simulations to study the behavior of impingement injectors and atomization patterns. In this simulation, they used refined adaptive mesh to study initial atomization. They also studied the non-Newtonian impingement jets and obtained two distinct flow patterns. In the most recent study and simulation, $X$. Chen et al. [9] investigated the flow using VOF method along with the refined adaptive mesh. They also provided the details of the flow behavior in a vast range of Reynolds and Weber numbers and compared their findings with the experimental data.

In the present study, the open source solver (Open-FOAM) is utilized to provide a numerical simulation for the flow of Impingement jet injectors in a variety of conditions. For the models under study, refined dynamic meshes are utilized which can significantly minimize the computational cost and provide a reliable solution for two phase flows with different liquid flow sizes after collapse (comprising droplets and ligaments). Moreover, in this study, impact of two jets with distinct physical properties is simulated for the first time. 
Flow Physics and Governing Equations

Studies show that when two jets collide, a sheet is formed perpendicular to the colliding plane, as shown in figure (1).

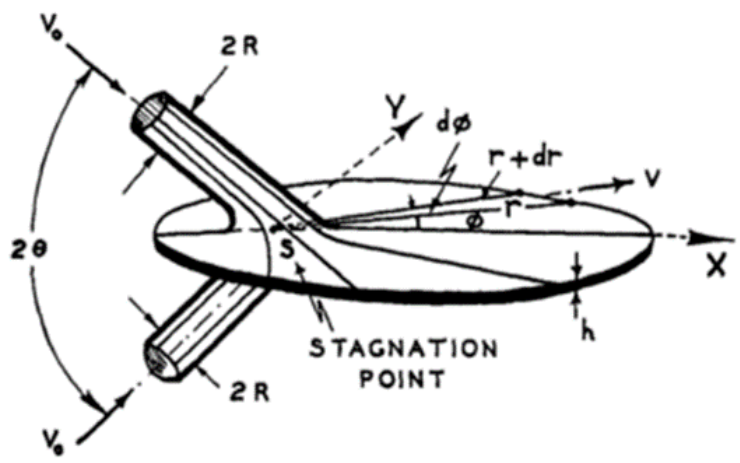

Figure 1. Schematic collapse of two impingement jets [3].

If the two jets are concentric, the resulting sheet will be circular otherwise the sheet will have an oval or leaf shape. To express the spray characteristics properly, a variety of parameters such as collision angle, jet velocity, jet radius, radial position and sheet thickness must be taken into account. After formation of the sheet, waves generate over the sheet and develop until the sheet is transformed into droplets. The resulting sheet of fluid has an oval cross section and its principal axis lies in the plane of impingement. The size of droplets in an impingement jet injectors is directly proportional to the sheet's thickness. The sheet thickness on the other hand is inversely dependent upon the radial distance to the collision point and also some other miscellaneous parameters of the flow.

Actually development the flow in the atomization can be divided to four categories as follows: jet impingement, sheet oscillation, ligament formation, and droplet formation. Impingement waves grow due to hydrodynamic instability, and hence form disturbances with high amplitude. As a result, sheet is broken up. After impingement of two jets, a sheet is produced which is oriented from the impingement point, where the oscillated sheet breaks up and becomes instable. This is because, the waves are created on this sheet and spread on them radially. As a result, capillary instabilities are formed and converted in to the droplet by means of Rayleigh instabilities [10].

Injectors have usually simple configurations but the two phase flow and the contact of distinct phases in the injector is highly complicated. Flow is usually transient with some parts being turbulent and rotational. Considering a generic control volume comprising two sub-control volumes with a common interface, the governing equations and stress tensor for each incompressible phase are described as Eqs. (1-3)

$$
\begin{aligned}
& \frac{D\left(\rho_{i} u_{i}\right)}{D t}=\nabla \cdot \Pi_{i}+\rho_{i} g \\
& \nabla \cdot u_{i}=0 \\
& \Pi_{\mathrm{i}}=-\mathrm{p}_{\mathrm{i}} \mathrm{I}+\mu_{\mathrm{i}}\left(\nabla \mathrm{u}_{\mathrm{i}}+\left(\nabla \mathrm{u}_{\mathrm{i}}\right)^{\mathrm{T}}\right)
\end{aligned}
$$

Where the subscripts $i$ denotes each of the first or second phases of the fluid. Also, $\rho$ and $\mu$ represent the density and viscosity, respectively. For the interface of liquid-gas $\Gamma$ which is observable in the atomization process, impermeability condition holds and there is no mass transfer between the two phases, hence the continuity equation yields

$$
u_{1}=u_{2} \text { for } x \in \Gamma
$$

Variations of the normal stress over the interface is balanced with surface tensions. Taking the coefficient of surface tension to be constant, Laplace-Young boundary condition for conservation of momentum over the interface is defined as

$$
\Pi_{1}-\Pi_{2}=\sigma k n \text { for } x \in \Gamma
$$

Where $k$ is the curvature radius of the interface and $n$ is the unit vector perpendicular to the interface as well as $\sigma$ is surface tension coefficient. To provide a numerical simulation of the flow model, interface of fluid and gas should be determined accurately. The motion of interface is described with Eq. (6) where scalars are defined differently according to following the interface.

$$
\frac{\partial C}{\partial t}+u \cdot \nabla C=0
$$

In the VOF method, $\mathrm{C}$ is the volume fraction. Moreover, physical quantities such as density, viscosity, normal function and curvature are defined for the VOF method as Eqs. (7-10)

$$
\rho=\rho_{2}+\left(\rho_{1}-\rho_{2}\right) C
$$




$$
\begin{aligned}
& \mu=\mu_{2}+\left(\mu_{1}-\mu_{2}\right) C \\
& n=\Delta C /|\Delta C| \\
& k=-\nabla . n
\end{aligned}
$$

\section{Solution Method}

The main purpose of this numerical solution is to simulate the flow of two liquid jets impingement under different conditions. The simulation is carried out in OpenFOAM. In OpenFOAM, there are different solvers for simulation of multiphase flows in which one of the most well-known solvers is the InterDyMFoam used to simulate two phase, isothermal and incompressible based on VOF method. Energy equation is not utilized in this solver.

In simulations of colliding jets, the two liquid jets collide and a thin sheet is formed in the vicinity of the two jets, Figure (2).

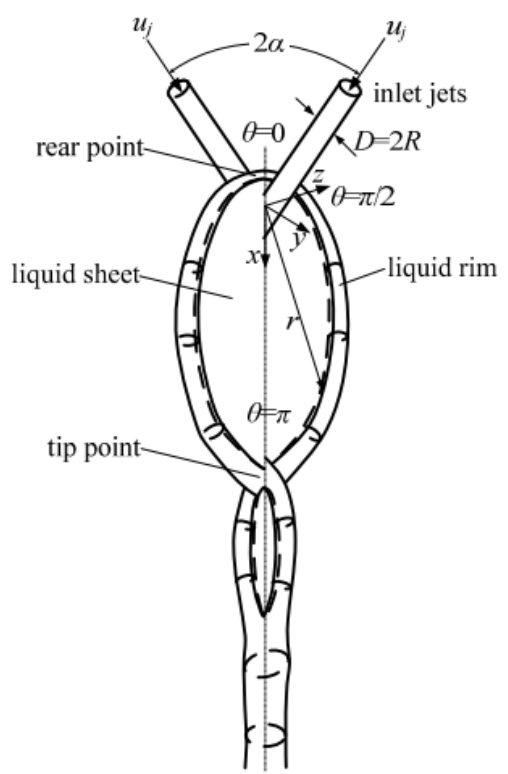

Figure 2. Schematic collision of two jets [10]

The domain for the present study is a cube purely air-filled domain as the initial condition. In order to eliminate the boundary condition effects, the volume is considered with the dimension $50 d \times 30 d \times 25 d$, where $d$ is jet radius. The fluid used in this study is Newtonian at standard temperature and pressure conditions. Gravity force is neglected due to negligible implications.

In accordance to atomization characteristics and two phase flow simulations where there are various length scale, mesh refinement modeling is carried out. All domain is solved with coarse size of mesh. In addition, the implemented method for refining the mesh is carried out at different stages. In this simulation the main areas which requires refinement mesh are as follows: 1- impingement area with high curvature 2- area of liquid sheet formation 3- area of rim at surrounding of the liquid sheet 4- areas with small size of droplet. Meanwhile, the size of cells at the rest of areas (where there is no liquid and are located at a specific distance from the impingement area), is constant. The schematic of the solution domain is displayed at Figure (3).

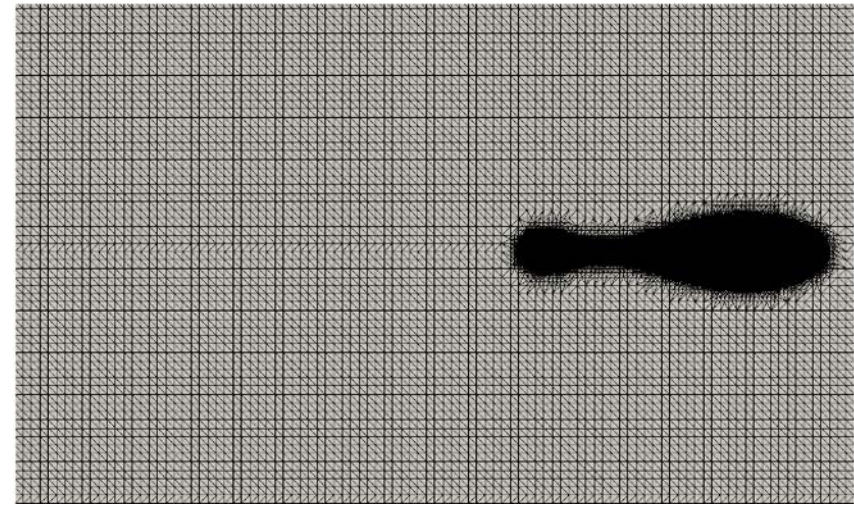

a) two dimensional view of domain with different sizes of mesh adapted in the region of liquid sheet formation

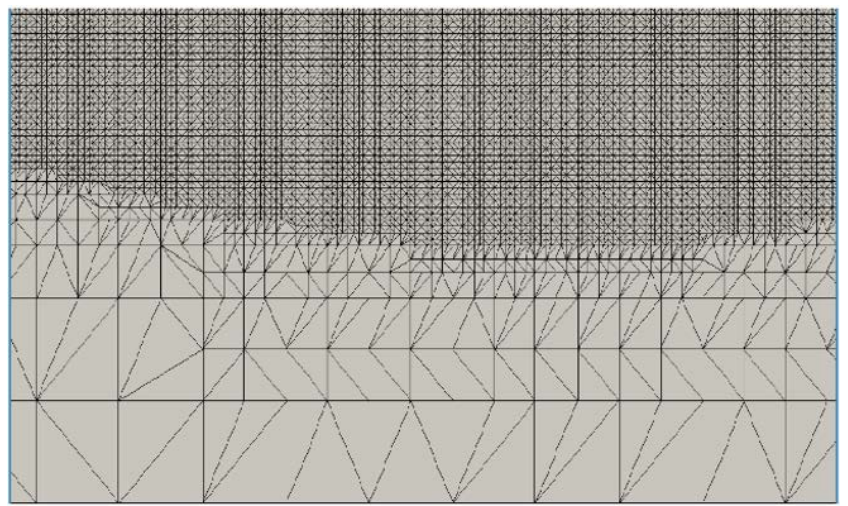

b) magnified view near the liquid sheet edge with different size of grid mesh

Figure 3. Schematic of the solution domain 
According to the physics and conditions of the problem, two jets are considered on the upper edge as the input with an velocity boundary condition. The nozzle has a circular geometry and the liquid jet is pumped into the domain with a volume fraction of unity during the solution. In other boundaries, far field conditions hold which are zero pressure gradient, zero velocity gradient and zero volume fraction. If the flow direction is inward, the tangential component would take a constant value.

\section{Results}

As a result, the sheet breaks up into ligaments and these ligaments further will be breaks into droplets. In Figure 4, verification of an example model with the analytical and numerical ones presented in Refs. [11-12] are provided. As can be seen, the length and shape of the formed sheet is in a very good agreement with experimental results.

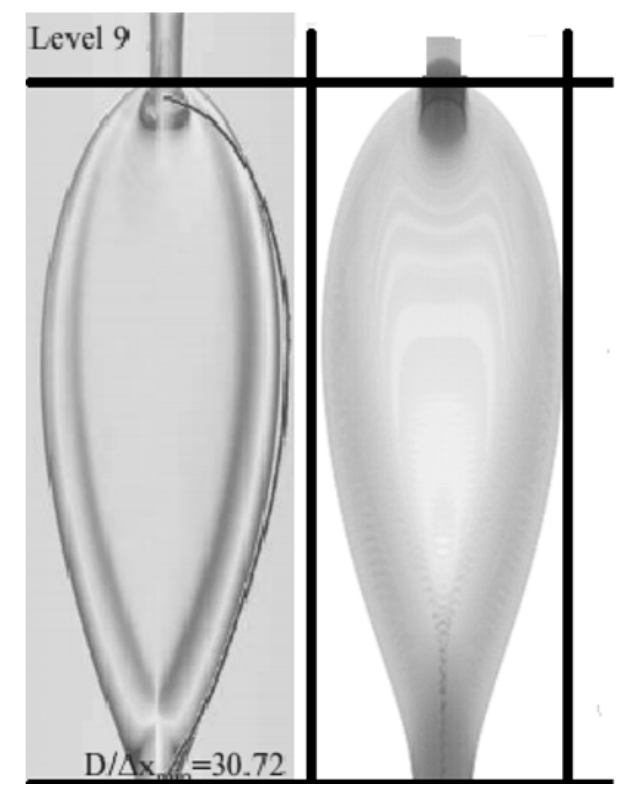

Figure 4. Validation of one model with numerical and analytical simulations.

Experimental and simulation conditions for collision of the jets at low velocities are taken as the simulations performed by Bremond and Villermaux [11]. The injected liquid is water-glycerin with a diameter and jet velocity of 400 micro meters and 3.3 meters per second, respectively. Collision angle of the two jets is also taken to be 89 degrees. According to the experimental conditions, Reynolds and Weber numbers of the flow are 40.4 and 58.8, respectively. Based on the physics of the flow, a stable sheet is formed according to the balance of surface tension forces, centrifugal force and inertial force at the edge of the sheet. For such conditions, when the two jets with low velocities collide, they will finally form a single jet after formation of the sheet, if surface tension force and inertial force are balanced.
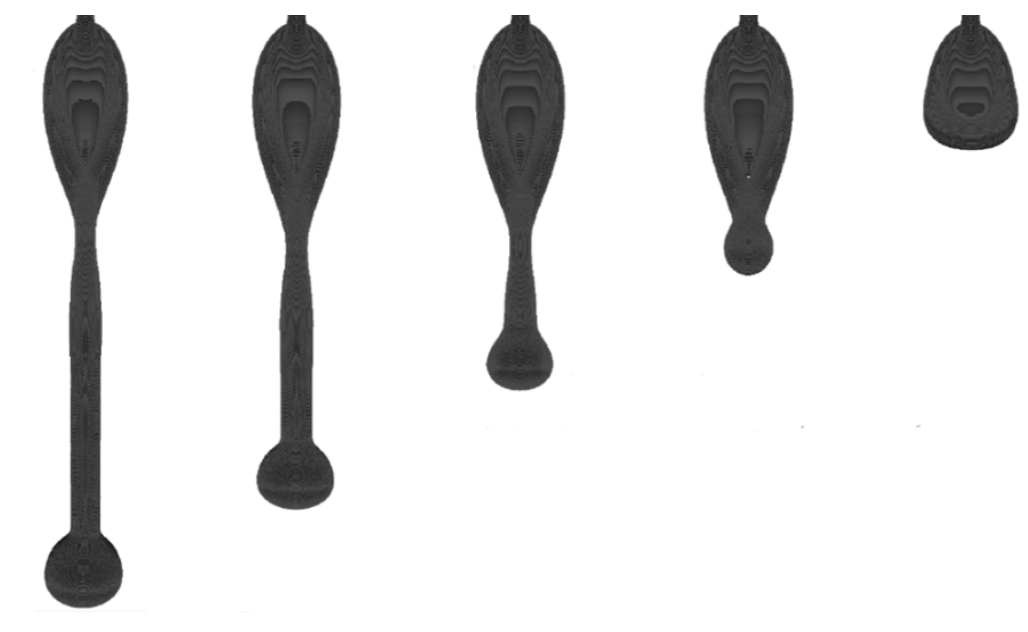

$$
t=10 \mathrm{~ms}
$$

$$
t=8 m s
$$

$$
t=6 m s
$$

$t=4 m s$

$t=2 m s$

Figure 5. Numerical solution of collision of the two jets for the $2 \alpha=89^{\circ}, W e=58.8, \operatorname{Re}=40.4, u_{j}=3.3 \frac{\mathrm{m}}{\mathrm{s}}$ 


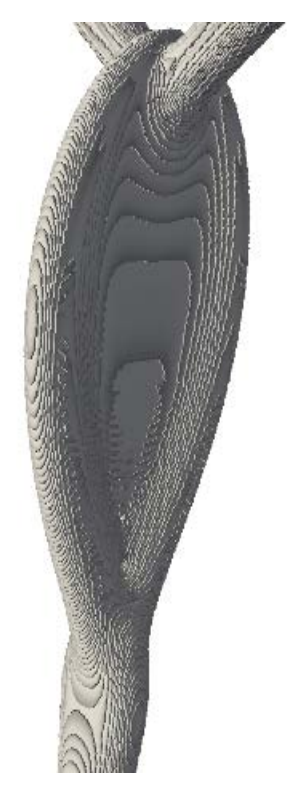

Figure 6. Close-up view of the collision of two jets with condition $2 \alpha=89^{\circ}, W e=58.8, \operatorname{Re}=40.4, u_{j}=3.3 \frac{\mathrm{m}}{\mathrm{s}}$.

At the peak point of the sheet and after a unit jet formation, the prescribed jet is ultimately break into a large droplets, where this breakup is consistent with capillary instability. The results of the simulation is illustrated in Figures 5 and 6 for development of liquid sheet bounded by a rim.

In low velocities and high viscosities, the fluid can be followed by segregated form of the sheet by means of two impingement jets. After impingement, whole fluid is bounded by an edge surrounding the sheet. Injected fluid flows along of the surrounding edge of the sheet. After that, two fluids are impinged until a jet or a secondary liquid film will be formed at the lower peak point of the sheet. Increasing of the velocity in this case will cause small grains at the surrounding edges, caused by small disturbances, and thus the sheet is disintegrated. In another simulation with an enhanced flow rate and Reynolds number, results are depicted in Figure 7. In this simulation the nozzle diameter is $D=400 \mu \mathrm{m}$ and collision angle of the two jets is 60 degrees. The jet velocity under this condition is $18.5 \mathrm{~m} / \mathrm{s}$ and the Weber and Reynolds numbers are 2987 and 11724 , respectively.
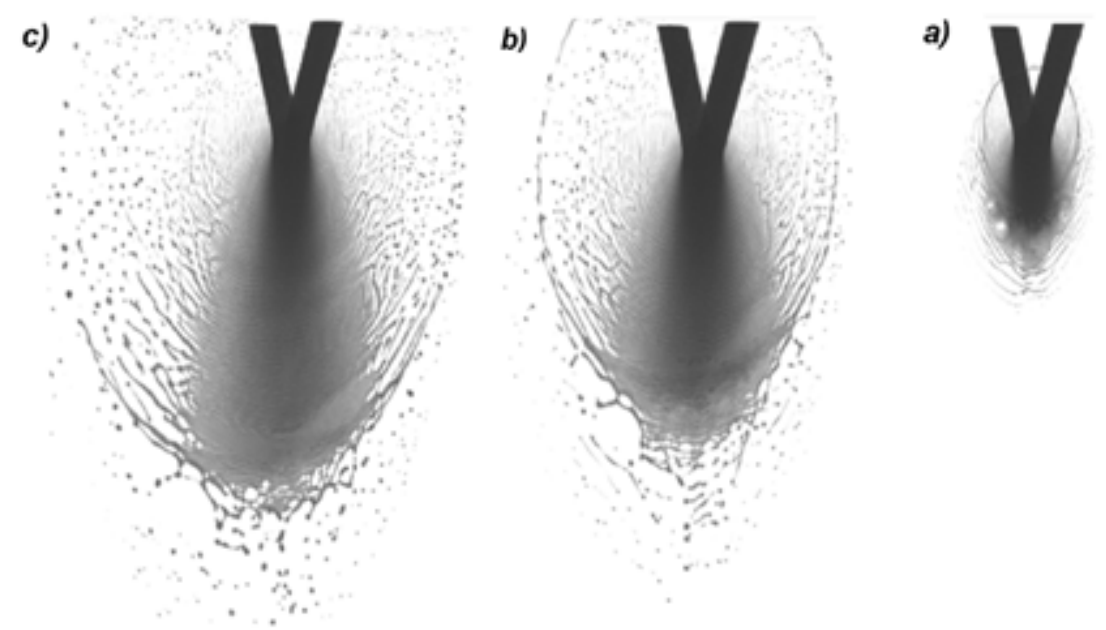

Figure 7. Numerical solution to collision of two jets for the $2 \alpha=60^{\circ}, W e=2987, \operatorname{Re}=11724, u_{j}=18.5 \frac{\mathrm{m}}{\mathrm{s}}$, a) 0.2 msecond b) 0.3 msecond, c) 0.46 msecond

Large amplitude hydrodynamic instabilities which is typically known as the collision wave, can be observed on the sheets of Fig.7. These waves have high frequency which are dominant in sheet collapse for high velocities and high collision angles. In addition, advancing the sheet through the time ( 0.2 millisecond -0.46 millisecond $)$ and forming the final shape of the sheet can be followed in Fig. 7.a - Fig. 7.c.

One of the most significant advantages of the present study is the refined mesh algorithm. To make it more clear, we may compare the number of computational cells for the present algorithm which is 5408549 with the traditional and uniform mesh (with the smallest cell being defined according to the smallest cell in the dynamic simulation) which is 491520000 (almost 91 times larger). 


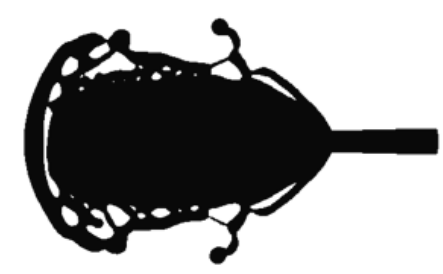

(a) $\operatorname{Re}=294, W e=152$

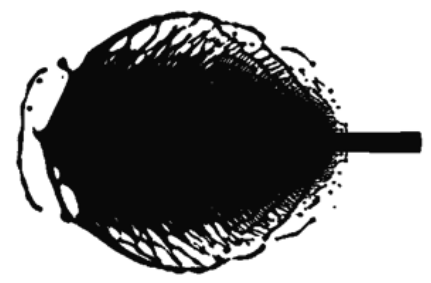

(b) $\mathrm{Re}=3536, \mathrm{We}=353.5$

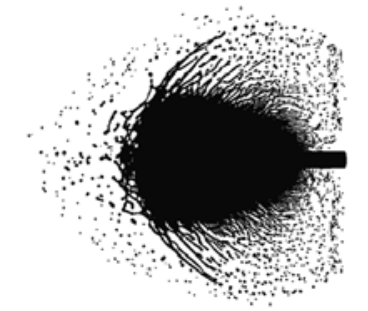

(c) $\operatorname{Re}=11724, W e=11724$

Figure 8. Sheet formed as a result of a 60 degree collision with different Reynolds and Weber numbers

In Figure 8, three different formation modes of the sheet are depicted for a variety of Reynolds numbers. As the jet velocity increases, if the film of liquid grows sufficiently, some random holes would appear in the sheet. For further increments of the liquid velocity, gas inertia may lead to formation of some waves over the surface of the sheet and creates a rugged surface. These perturbations also create asymmetric waves with increasing radial amplitudes. In general, the overall flow pattern is dependent upon the Weber and Reynolds numbers. As mentioned earlier, increasing the jet velocity or the Reynolds number make liquid sheet unstable. In this case, by increasing of the velocity, the properties of the sheet will highly depend on the jet velocity and liquid properties. Increasing velocity also disappear the rim of the sheet, and hence collapse is seen at near lower peak point. The main reason for this instability is the direct interaction of the sheet with gaseous media surrounding the sheet. In this way, the rapid growth of the wave on the sheet would be occurred, and when the wave is reached to its critical value the collapse will be happened. As it is pointed out in the beginning of the simulation, two liquid jets at low Weber and Reynolds numbers will form leaf like sheet bounded by a rim. Increasing of the velocity may create small satellite droplets at the surrounding of the edges caused by small disturbances which may result in the sheet breakup. Formation of the droplets can be formulated following by the Rayleigh of capillary instabilities. In this case, a liquid droplet will be formed which its size will be increased as the velocity increases.

In order to simulate heterogeneous fluid impacts, the multiphaselnterFoam solver which is a typical in OpenFOAM, is utilized. This solver employs the same equations as the InterFoam solver and uses the same sets of codes to simulate incompressible and isothermal two phase flows by means of VOF method. Energy equation is not included in this solver. The only distinction of the multiphaselnterFoam solver with InterFoam is that the former is not restricted for two phase flows and can be used for multiphase flow simulations as well.

For this solver all phases are considered to be incompressible. Moreover, a fluid must be taken as the base fluid which is usually the air for the most applications. The rest of present phases with a phase ratio between zero to one are compared with the base fluid. Also, according to the number of fluids used, a separate surface stress must be defined between any two phases of the flow.

Iterative loops are commonly used in this solver to determine the physical quantities such as density and viscosity. The loop for determination of the surface tension is different. The surface tension is indeed calculated between two phases and is defined as Eq. 11

$$
F_{S}=\sigma\left(\nabla \cdot\left(\frac{\nabla \alpha}{|\nabla \alpha|}\right)\right)(\nabla \alpha)
$$

Where $\nabla \alpha$ is the normal vector to the interface of the two phases and $\sigma$ is the surface stress. 
In this simulation, two oil and water liquid jets which have different properties are impinged with each other. In this case, by considering of air, a multi-phase flow will be formed which an instable sheet would be formed due to high velocity of the injected jets. As it will be shown in the results section, since the inertial and properties of two fluid are not equal, advancing each fluid on the surface of the other is different. It means the extension of each fluid in the gaseous medium is different and balancing between inertia force, viscosity and surface tension is a crucial role in the shape of unlike impinging jets.

(a) Side view of two unlike jets

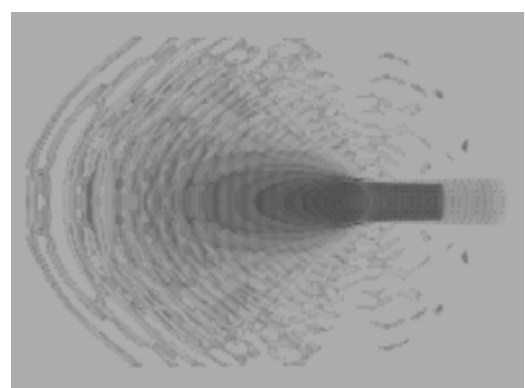

(b) Top view of two unlike jets

Figure 9. Unstable sheet formed by the collision of two jets of water and oil

\section{Conclusion}

In this study, the behavior and physics of formation of a sheet as a result of collision of two cylindrical jets in the laboratory condition is investigated. According to the flow physics and injection conditions, collision of the two jets can cause a variety of instabilities in the sheet. To this aim, using the open sourced OPENFOAM code, numerical simulation is performed with adaptive refined mesh to characterize the formation of the flow after collision of two cylindrical jets of liquid. Results of the present study are also verified with analytical, experimental and other numerical simulations. In the numerical simulations performed, different experimental conditions are studied for a variety of Reynolds and Weber numbers. It is found that in different conditions due to the variety of instability conditions that can occur, the shape of the sheet and flow is different.

Dynamic mesh refinement makes it possible to start the solution with coarse meshes and refine the mesh as required in the critical and sensitive regions. This will finally reduce the computational costs and make it possible to provide solutions of two-phase flows, i.e. sprays with different dimensions, which require very tiny mesh sizes.

In addition, simulation of two unlike impinging jets was done by a refined mesh for water and petroleum. The shape of the sheet and breakup mechanism of sheet in unlike fluid are generally similar to like fluid and penetration of each liquid in to another can be observed in the simulations.

\section{References}

[1] A. H. Lefebvre, Atomization and Spray. 1989.

[2] J. C. P. Huang, “The Break-Up of Axisymmetric Liquid Sheets,” J. Fluid Mech., Vol. 43, No. 2, P. 305, Aug. 1970.

[3] D. Hasson And R. E. Peck, “Thickness Distribution in A Sheet Formed by Impinging Jets,” Aiche J., Vol. 10, No. 5, Pp. 752-754, Sep. 1964.

[4] G. Taylor, “Formation of Thin Flat Sheets of Water,” Proc. R. Soc. Lond. A. Math. Phys. Sci., Vol. 259, No. 1296, Pp. 1-17, 1960.

[5] N. Ashgriz, Ed., Handbook of Atomization and Sprays. Boston, Ma: Springer Us, 2011.

[6] C. Inoue, T. Watanabe, and T. Himeno, "Study on Atomization Process of Liquid Sheet Formed by Impinging Jets,” In 44th Aiaa/Asme/Sae/Asee Joint Propulsion Conference \&Amp; Exhibit, 2008.

[7] M. Arienti, X. Li, M. C. Soteriou, C. A. Eckett, M. Sussman, And R. J. Jensen, “Coupled Level-Set/Volumeof-Fluid Method for Simulation of Injector Atomization,” J. Propuls. Power, Vol. 29, No. 1, Pp. 147-157, Jan. 2013.

[8] D.-J. Ma, X.-D. Chen, P. Khare, And V. Yang, “Atomization Patterns and Breakup Characteristics of Liquid 
Sheets Formed by Two Impinging Jets,” In 49th Aiaa Aerospace Sciences Meeting Including The New Horizons Forum And Aerospace Exposition, 2011.

[9] X. Chen And V. Yang, "Thickness-Based Adaptive Mesh Refinement Methods for Multi-Phase Flow Simulations with Thin Regions,” J. Comput. Phys., Vol. 269, Pp. 22-39, Jul. 2014.

[10] X. Li, M. Arienti, M. Soteriou, And M. Sussman, “Towards an Efficient, High-Fidelity Methodology for Liquid Jet Atomization Computations,” In 48th Aiaa Aerospace Sciences Meeting Including The New Horizons Forum and Aerospace Exposition, 2010.

[11] N. Bremond And E. Villermaux, “Atomization by Jet Impact,” J. Fluid Mech., Vol. 549, No. 1, P. 273, Feb. 2006.

[12] X. Chen, D. Ma, V. Yang, And S. Popinet, “High-Fidelity Simulations of Impinging Jet Atomization,” At. Sprays, vol. 23, no. 12, pp. 1079-1101, 2013. 


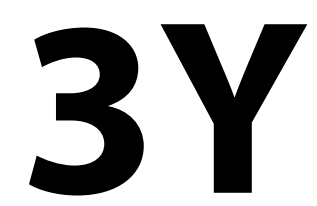

Droplet 2 


\title{
Time Resolved Infrared Analysis of Droplet Impacts onto Heated Surfaces Under Extreme Wetting Scenarios
}

\author{
P. Pontes, E. Teodori ${ }^{1}$, A. S. Moita*1, A. L. N. Moreira ${ }^{1}$ \\ ${ }^{1} \mathrm{IN}+$ - Center for Innovation, Technology and Policy Research, Instituto Superior Técnico, \\ Universidade de Lisboa, Lisbon, Portugal \\ ${ }^{*}$ Corresponding author: anamoita@tecnico.ulisboa.pt
}

\begin{abstract}
The present paper explores the use of time resolved infrared IR thermography combined with high-speed imaging to describe the liquid-surface interfacial heat transfer phenomena occurring at droplet/wall interactions. Custom made calibration and post-processing methods are proposed and discussed. The results show that the methodology proposed captures very well particular details on droplet dynamics and heat transfer, allowing to identify air bubble trapping at the impact region as well as the temperature variations at the formation of the rim. Furthermore, the calibration proposed here allowed amending some physically incorrect results that were often obtained with the IR camera's default calibration. The combined analysis of droplet dynamics (e.g. the spreading factor) with the radial temperature profiles, heat flux and cooling effectiveness computation allowed establishing qualitative and quantitative trends on the effect of various parameters on the heat transfer occurring at droplet/wall interactions. Particularly, the effect of the initial surface temperature is observed to play a minor role, as long as it is low enough to prevent the occurrence of boiling. On the other hand, extreme wetting scenarios, such as superhydrophobicity limit the heat transfer between the spreading droplet and the surface. However, the thermal analysis reveals that a major reason for this is not related to the reduced contact time of the droplet on the surface (due to rebound) or air entrapment, but is rather associated to the reduced wetted area caused by the high contact angles.
\end{abstract}

\section{Keywords}

Droplet impact, heated surfaces, wettability, superhydrophobicity, Infrared high-speed thermography.

\section{Introduction}

Transport phenomena occurring at liquid-solid interfaces run a wide range of processes in various numerous applications. For instance, cooling systems are vital in different applications and at diverse temporal and spatial scales, such as electronics cooling, nuclear and chemical reactors, refrigeration systems, thermal generation of electricity or even in the food industry. Focusing on electronics cooling, the thermal management of microprocessors is argued to be the largest limitation to the development of new processors in the near future, which demands for innovative cooling strategies. Popular strategies include liquid cooling with phase change, such as pool boiling and spray cooling and often address surface modification to enhance the heat transfer processes, e.g. [1-2]. In any of these cases, the heat transfer is mostly governed by the mechanisms occurring at the liquid-(modified) surface interface. Despite being studied for many years, an accurate description of these processes is not provided yet, as it demands for experimental data obtained under demanding conditions, with high spatial and temporal resolutions. A clear example of these limitations is the study of droplet wall/interactions. Despite being studied for more than a century by many researchers as reviewed for instance in [3-4], the complex relation between droplet dynamics and heat transfer is far to be understood. The current knowledge on droplet/wall interactions recognizes wettability as playing a fundamental role in droplet dynamics and heat transfer processes. However, the accurate description of its effect is also still far from being completely described. Particularly, rudimentary knowledge is still achieved when complex surfaces with modified topography and/or chemistry are used [5]. This knowledge is even sparser when the complexity of the surfaces includes hierarchical structures, such as those present in biomimetic surfaces, which requires demanding static and dynamic wetting characterization methodologies, as recently reported by [6]. Infrared (IR) thermography has been recently explored as a high potential alternative to the intrusive measuring methods often used, based on thermocouples, which can provide important information on droplet impact under such particular boundary conditions [7-9]. However, many of these studies are focused on sessile droplets [10] or in particular boiling regimes such as the film boiling [11] where wettability plays a secondary role. Furthermore, care must be taken in the calibration and post-processing methods to obtain reliable measurements. Within this scope, the present paper explores the use of time resolved infrared thermography with high spatial resolution, combined with high-speed imaging to describe 
the droplet-surface interfacial heat transfer phenomena occurring during droplet impact onto heated surfaces. Custom made calibration and post-processing methods are proposed and discussed. The experimental impact conditions consider the effect of different parameters such as impact velocity and fluid properties. Emphasis is given to the effect of wettability (hydrophilic vs superhydrophobic surfaces).

\section{Material and methods}

Experimental set-up and working conditions

Droplets are generated at the tip of a hypodermic needle and fall by action of gravity on the heated surface. Water and ethanol are chosen as working fluids to address the effect of surface tension (partial wetting vs well wetting fluid) on the heat transfer processes occurring at the liquid-surface interface. The main physico-chemical properties of the working fluids is summarized in Table 1.

Table 1. Thermo-physical properties of the fluids used in the present work.

\begin{tabular}{l|cc}
\hline \hline Properties & Water & Ethanol \\
\hline \hline Saturation Temperature $-\mathrm{T}_{\text {sat }}\left[{ }^{\circ} \mathrm{C}\right]$ & 100 & 78.3 \\
Density $-\rho\left[\mathrm{kg} / \mathrm{m}^{3}\right]$ & 1000 & 757 \\
Dynamic viscosity $-\mu \times 10^{-3}\left[\mathrm{Ns} / \mathrm{m}^{2}\right]$ & 1.05 & 1.19 \\
Surface tension $-\sigma \times 10^{-3}[10-3 \mathrm{~N} / \mathrm{m}]$ & 72.88 & 22.8
\end{tabular}

Droplet initial diameters $D_{0}$ range between $2.4 \mathrm{~mm}<D_{0}<3 \mathrm{~mm} \pm 0.2 \mathrm{~mm}$ and the impact velocities $V_{0}$ are varied between $0.8 \mathrm{~m} / \mathrm{s}$ and $2 \mathrm{~m} / \mathrm{s}$. The impact surface is a stainless steel electrically heated foil, with $20 \mu \mathrm{m}$ thick, $20 \mathrm{~mm}$ wide and $100 \mathrm{~mm}$ long. The heating assembly consists in copper electrodes clamped on the top of the stainless steel foil, which is then glued on the top of an insulating thermal glass. This entire assembly is then placed on a stainless steel support for an easier positioning. The bottom side of the stainless steel foil used for IR thermography is black matt painted to increase the emissivity $(\varepsilon r=0.95)$. The surface is considered to be smooth, even after application of a chemical coating to turn it superhdrophobic. (variations in the average and in the peakto-valley roughness, measured with a profilemeter Dektak 3 from Veeco are smaller than 20nm). This commercial coating called Glaco® is mainly a perfluoroalkyltrichlorosilane combined with perfluoropolyether carboxylic acid and a fluorinated solvent.

Wettability is characterized measuring the quasi-static advancing and receding and the static contact angles, using an optical tensiometer (THETA from Attention). The static contact angle, measured by the sessile drop

method was $\theta=81.7^{\circ} \pm 1^{\circ}$ for the uncoated surface and $\theta=162.5^{\circ}$ for the coated surface. The quasi-static advancing and receding angles were used to evaluate the hysteresis of the foil (i.e. the difference between the quasi-static angles), which was always larger than $20^{\circ} \pm 1^{\circ}$ for the hydrophilic foils tested here and always lower than $10^{\circ}$ for the coated superhydrophobic surfaces. A detailed description of the experimental procedure taken to characterize the wettability of the surfaces can be found in [6]

The topography and wettability of each foil are carefully evaluated before and after droplet impact to assure consistent boundary conditions for each impact event. Furthermore, care was taken to assure that the initial surface temperature was reproducible before each new droplet impact.

An infrared IR-high speed camera (ONCA-MWIR-InSb from Xenics - ONCA 4696 series) is placed below the heated target, while a high-speed camera (Phantom v4.2) is mounted to take side views of the droplets. During impact, simultaneously but not synchronized high-speed video and high-speed thermographic images are taken to record the dynamic behavior of the droplets. The acquisition frequency and resolution are $2200 \mathrm{fps}$, $512 \times 512 \mathrm{px}^{2}$ and $1000 \mathrm{fps}$ and $150 \times 150 \mathrm{px}^{2}$ for the high-speed video and high-speed thermographic camera, respectively. For each experimental condition considered here, five tests were performed to assure reproducibility of the experiments.

\section{Post-processing methods}

Image post-processing is used to obtain the initial diameter $D_{0}$, the impact velocity $V_{0}$, the spreading diameter $D(t)$ and the spreading factor $\beta=D(t) / D_{0}$. The curves are averaged from at least 3 events taken at similar conditions.

The radial temperature profiles were obtained after post processing the IR images using a homemade MatLab code which allowed converting the raw IR images to temperature data. For the calibration method, a custom- 
made cavity based blackbody radiator device was designed and assembled. For each imposed temperature at the cavity and after achieving a stable condition for which the temperature is homogeneous in the cavity, the measured temperatures are converted into radiated energy flux $\left(\mathrm{W} / \mathrm{m}^{2}\right)$ performing an energy balance and plotted against the received intensity signal in ADU's (Analogic to Digital Units). Then a polynomial curve is fitted to these data. This calibration is performed considering a pixel by pixel approach. As for the post-processing procedure, a number of steps must be performed and a sequence of filters was developed and applied, as follows:

\section{1) Background removal filter}

To remove background noise from the the image, obtaining real temperature variations requires removing the image background. While a common background filter removes the background (the grey scale value) leaving visible changes in the following frames, the custom-made filter designed here considers the temperature variations to be relative to the background temperature. The developed filter is applied to each pixel as given in equation 1:

$\operatorname{vid}^{*}\left(x_{p}, y_{p}, t_{n}\right)=\left(\frac{\operatorname{vid}\left(x_{p}, y_{p}, t_{n}\right)-v i d\left(x_{p}, y_{p}, 1\right)}{\operatorname{vid}\left(x_{p}, y_{p}, 1\right)}\right) \operatorname{avTemp}+\operatorname{avTemp}$

where vid is a $3 d$ matrix with the temperatures for each pixel and for different timesteps, vid* is the resulting matrix, $x_{p}$ and $y_{p}$ are the Cartesian coordinates for the pixel, $t_{n}$ the timestep and avTemp is the average background temperature.

\section{2) Noise removal filter}

A median filter was used to perform noise removal. This filter has the potential to remove random bad pixel noise from the picture. It uses a MATLAB function that outputs the median of a 3-by-3 neighbourhood of the input pixel.

\section{Measurement uncertainties}

The main uncertainties associated to droplet dynamics are summarized in Table 2, while Table 3 depicts the uncertainties associated to the main parameters used to describe the heat transfer process at droplet/wall interaction.

Table 2. Uncertainties of the main parameters used to describe droplet dynamics.

\begin{tabular}{l|l}
\hline \hline Parameter & Uncertainties (rel. or abs) \\
\hline $\begin{array}{l}\text { Droplet diameter before impact } D_{o} \\
{[\mathrm{~mm}]} \\
\text { Spreading diameter } D[\mathrm{~mm}]\end{array}$ & $U_{D}= \pm 160 \mu \mathrm{m}$ \\
& $U_{D_{o}}= \pm 160 \mu \mathrm{m}$ \\
Spreading factor $\beta=D / D_{o}[-]$ & $u_{D^{\prime} D_{o}} \max = \pm 37 \%$ at $\left(D / D_{o}=0.17\right)$ \\
& $u D / D_{o} \operatorname{mim}= \pm 7 \%$ at $\left(D / D_{o}=3.86\right)$ \\
Impact velocity $V_{0}[\mathrm{~m} / \mathrm{s}]$ & $U_{V_{o}}=0.08[\mathrm{~m} / \mathrm{s}]$
\end{tabular}

Table 3. Uncertainties of the main parameters used to describe the heat transfer process during droplet spreading.

\begin{tabular}{l|l}
\hline \hline Parameter & Uncertainties U (rel. or abs) \\
\hline \hline Temperature $\mathrm{T}[\mathrm{K}]$ & $U_{T}= \pm 1 \mathrm{~K}$ \\
Temperature difference $\Delta \mathrm{T}[\mathrm{K}]$ & $U_{\Delta T}= \pm 1.4 \mathrm{~K}$ \\
& $u_{\Delta T} \max = \pm 14 \%$ at $(\Delta T=10 \mathrm{~K})$ \\
& $u_{\Delta T} \min = \pm 1.7 \%$ at $(\Delta T=78 \mathrm{~K})$ \\
Non-dimensional temperature $T^{*}[-]$ & $U_{T^{*} \max = \pm 0.04 \text { at }\left(T^{*}=1\right)}$
\end{tabular}


Imposed volumetric heat flux $q^{\prime \prime \prime}\left[W / m^{3}\right]$

Radial distance $r[\mathrm{~mm}]$

$$
\begin{aligned}
& U_{T^{*}} \min = \pm 0.02 \text { at }\left(T^{*}=0.3\right) \\
& u_{T^{*}} \max = \pm 6 \% \text { at }\left(T^{*}=0.3\right) \\
& u_{T^{*}} \max = \pm 4 \% \text { at }\left(T^{*}=1\right) \\
& u q^{\prime \prime \prime} \max = \pm 12 \% \text { at }\left(q^{\prime \prime \prime}=6.5 \cdot 10^{6}\left[\mathrm{~W} / \mathrm{m}^{3}\right]\right) \\
& U_{r}= \pm 200 \mu m
\end{aligned}
$$

\section{Results and discussion}

Simultaneous analysis of droplet dynamics and thermal processes

Although the IR and the high-speed cameras were not perfectly synchronized, the simultaneous images captured during droplet impact and spreading allow understanding the qualitative relation between droplet dynamics and the heat transfer processes occurring during the spreading. Hence, Figure 1 depicts the impact of a water droplet $\left(D_{0}=2.6 \mathrm{~mm}, V_{0}=0.8 \mathrm{~m} / \mathrm{s}\right)$ on the smooth uncoated (i.e. hydrophilic) stainless steel foil, initially heated at $T_{w 0}=80^{\circ} \mathrm{C}$. The figure shows the side view of the impacting droplet and the corresponding thermal images taken on the heated foil (bottom view) during impact and spreading. The corresponding temperature profiles, from the center of the droplet $(r=0 \mathrm{~mm})$ to the rim of the lamella are shown at the bottom images. The figure clearly shows the sudden temperature decrease at $r=0 \mathrm{~mm}$, at the point of impact, which quickly recovers to the rim, at the early instants after impact. A steeper temperature difference between the center of the droplet and the rim is then observed at later instants after impact, near the maximum spreading ( $t=6 m s)$, which remains for later stages, during recoiling, for the wetted area, while heat is removed at the liquid-solid interface. The rim is perfectly identified in the thermal images, which can be related to the temperature profiles, by the slight bump, associated to the change in the thickness of the lamella.

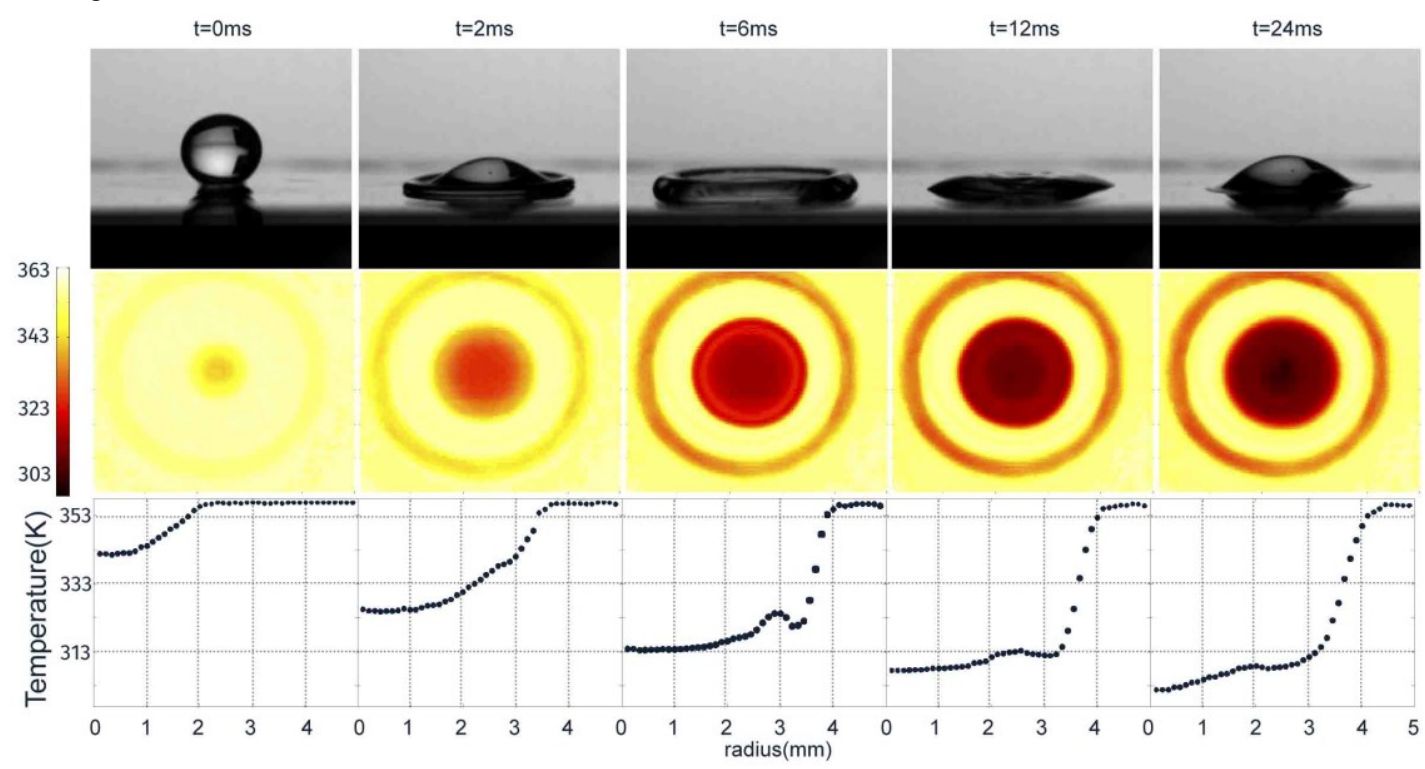

Figure 1. Correspondence between the high-speed images taken to the droplet (dynamic behaviour) and the IR thermal images taken to the surface in contact with the spreading droplet (heat transfer process) and to the resulting temperature profiles taken at various instants after impact. The water droplet $D_{0}=2.6 \mathrm{~mm}$ impacts the smooth stainless steel surface at $V_{0}=0.8 \mathrm{~m} / \mathrm{s}$. Initial surface temperature $T_{w 0}=80^{\circ} \mathrm{C}$.

\section{Evaluating the calibration method}

To infer on the efficacy of our calibration method, some preliminary images were taken using the camera's default calibration, which were then compared to those taken using the custom-made calibration. For illustrative purposes the result of applying our calibration process is discussed for the impact of water droplets at $2 \mathrm{~m} / \mathrm{s}$ and $0.8 \mathrm{~m} / \mathrm{s}$, for initial foil temperatures of $60^{\circ} \mathrm{C}, 100^{\circ} \mathrm{C}$ and $110^{\circ} \mathrm{C}$. Real working temperature values is provided, so small variations can be observed around these reference values. 
Figure 2 depicts the temperature variation on the foil, as a function of the spreading radius, of a water droplet $\left(D_{0}=2.6 \mathrm{~mm}\right)$ impacting on the smooth and hydrophilic stainless steel foil at $V_{0}=0.8 \mathrm{~m} / \mathrm{s}$ at the initial foil temperature $T_{w 0}=107^{\circ} \mathrm{C}$ (Figure 2a) and $T_{w_{0}}=117^{\circ} \mathrm{C}$ (Figure 2b). Particularly, this Figure compares the results obtained using the camera's default calibration (Figure 2a) with those attained after applying our calibration and post-processing method (Figure $2 b$ ). The different curves in each plot correspond to different time instants after impact. Hence, at the time instant immediately after impact $($ e.g. $t=1 \mathrm{~ms})$ the temperature decrease observed on the impact region of the foil (central region of the droplet) due to the contact with the cold liquid is small and swiftly recovers for the small spreading factor that is still observed at that instant. This trend contrasts with the higher temperature drops at later time instants after impact. Despite the small variations that occurred in the initial impact conditions, which could not be entirely controlled, the Figure clearly shows that during the spreading phase (all the points before $t=13 \mathrm{~ms}$ ), the calibration and post-processing method proposed here are able of capturing the effect of air entrapment air in the stagnation point (the point of droplet impact) often referred in the literature, e.g. [7], which is related to the small temperature decrease that is observed from the point of impact $(r=0 \mathrm{~mm})$ to the immediately subsequent points.

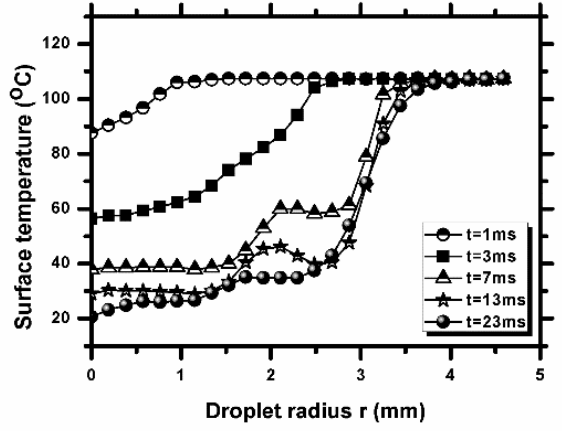

a)

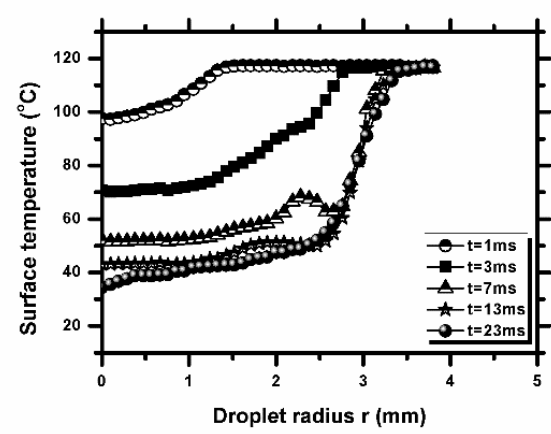

b)

Figure 2. Temperature variation on the stainless steel foil (smooth and hydrophilic, with an equilibrium angle $\theta=87.1^{\circ} \mathrm{I}$ ) during impact and spreading of a water droplet $\left(D_{0}=3 \mathrm{~mm}, U_{0}=0.8 \mathrm{~m} / \mathrm{s}\right)$, for an initial foil temperature of: a) $T_{w 0}=107^{\circ} \mathrm{C}$. The results were obtained from the thermal images after applying the camera's default calibration. These results were taken for an ambient temperature $T_{a m b}=21^{\circ} \mathrm{C}$; b) $T_{w o}=117^{\circ} \mathrm{C}$. The results were obtained after applying our custom-made calibration and postprocessing method. The ambient temperature in this case was $T_{a m b}=24^{\circ} \mathrm{C}$.

The calibration and post-processing procedures proposed here also allowed amending some physically incorrect results that were often obtained with the camera's default calibration. For instance, in Figure 2a, for the curve obtained at $t=23 \mathrm{~ms}$ after impact, the foil temperature at the impact region $(r=0 \mathrm{~mm})$ is slightly lower than the ambient temperature, which is not possible, being corrected in our custom-made method.

As the temperature profiles alone are not enough to characterize the heat transfer process, further analysis can be performed by computing the heat flux and the so-called cooling effectiveness $\varepsilon$, as defined by [7]. Considering an axisymmetry condition at the center of the droplet, the heat flux removed by the droplet from the foil $q$ " can be written as:

$q^{\prime \prime}=q_{0}^{\prime \prime}+k_{\delta} \frac{\partial^{2} T}{\partial r^{2}}-\rho C_{p} \delta \frac{\delta T}{\delta t}$

Here, $k_{\delta}, \rho, C_{p}$ and $\delta$ are the thermal conductivity, density, heat capacity and thickness of the heated foil, respectively. Assuming that there is no phase change of the liquid during droplet impact and spreading, [7] define as cooling efficiency $\varepsilon$, the ratio between the actual sensitive heat removed by the droplet and the maximum sensitive heat that can be theoretically removed:

$\varepsilon=\frac{\int_{t} \int_{A} q^{\prime \prime} d A d t}{\left(m C_{p} \Delta T\right)_{\text {water }}}$

These quantities were used to evaluate the effect of various parameters in droplet dynamics and heat transfer. The results were analysed to describe the heat transfer processes coupled with the fluid flow and dynamic characteristics during droplet impact.

Effect of the initial surface temperature $T_{\text {wo }}$

Although the detailed results cannot be shown here, due to paper length constrains, the analysis of the temperature profiles, the heat fluxes and the cooling effectiveness shows a negligible effect of the initial surface temperature, as all the curves obtained at different initial surface temperatures tend to collapse. Exception was 
made to the curve obtained at $T_{w 0}=110^{\circ} \mathrm{C}$, which tends to converge for higher values of the cooling effectiveness, which may be attributed to the beginning of the liquid phase change and to an incipient boiling.

\section{Effect of liquid surface tension}

To infer on how good our method captures the physics governing the fluid dynamics and heat transfer processes at droplet/wall interactions, the effect of the liquid surface tension is addressed here comparing the thermal processes occurring at the impact of water and ethanol droplets for initial surface temperatures below saturation. Given the lower surface tension of ethanol, the spreading factor is much larger than that of water, so the lamella is also much thinner. Furthermore, after spreading, good wetting fluids like ethanol tend to proceed the spreading for very long time intervals after impact, without recoiling, in a regime controlled by capillary forces [12-13], often aided by the formation of a precursor film [12]. This reduced thickness of the lamella of the ethanol droplet became an obstacle when performing the experiments, since for initial foil temperatures above saturation, the applied electrical current is very high deforming the stainless-steel foil. These deformations are not relevant for the spreading of the water droplets but are enough to cause the ethanol lamella to slip away from the measurement area, being impossible to capture IR images under those conditions. Hence, measurements could be performed for ethanol only at the lowest impact velocity $\left(V_{0}=0.8 \mathrm{~m} / \mathrm{s}\right)$ and for initial foil temperatures of $40^{\circ} \mathrm{C}$ and $60^{\circ} \mathrm{C}$. The spreading factor $\beta=D(t) / D_{0}$ obtained for water and ethanol droplets is depicted in Figure 3 . The maximum spreading factor is reached at $7 \mathrm{~ms}$ after impact for ethanol and $5 \mathrm{~ms}$ after impact for water. Naturally, the wetted area of the spreading ethanol droplet is larger than that of water.

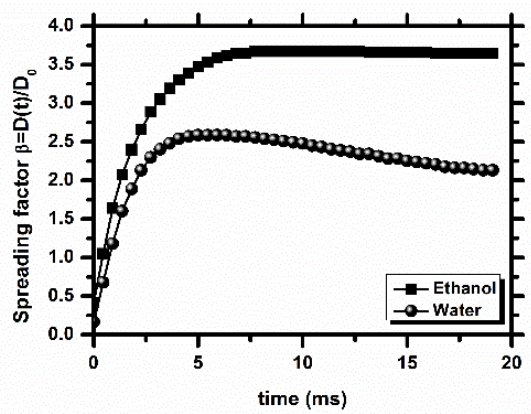

Figure 3. Temporal evolution of the spreading factor for a water and an ethanol droplet impacting on the smooth and uncoated hydrophilic stainless steel surface at $V_{0}=0.8 \mathrm{~m} / \mathrm{s}$. $T_{w 0}=60^{\circ} \mathrm{C}$.

Comparing the heat flux removed by the water and the ethanol droplet, as depicted in Figure 4, the heat flux peak is more accentuated for the water droplet. This is due to the the thicker lamella edge, caused by a higher surface tension and naturally to the higher values of the thermal properties of the water. So, overall the heat flux removed by the water is about twice that removed by the ethanol. The fact that the temperature difference to the saturation temperature is different in both liquids seems not to have a significant effect in this case. The radius is made nondimensional since the initial diameter of the ethanol droplets is slightly smaller than that of the water droplets.

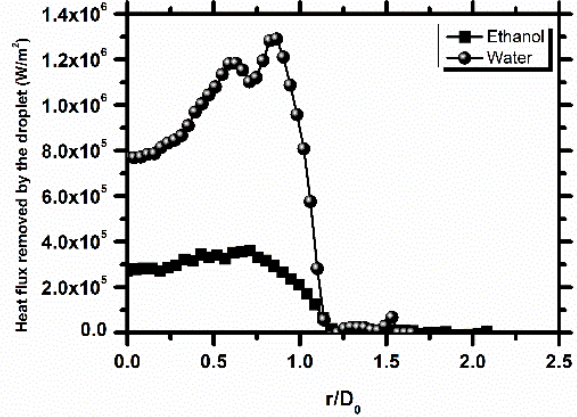

a) $t=2 m s$ after impact

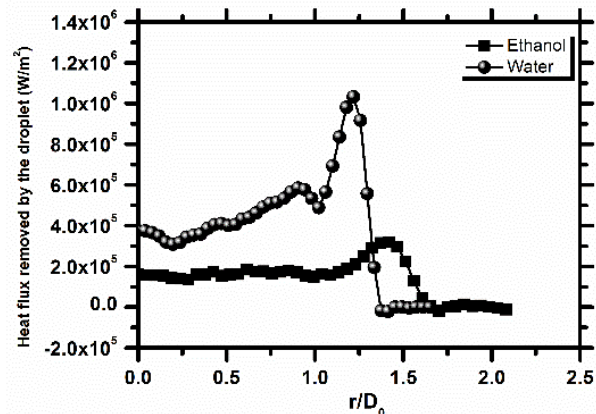

b) $\mathrm{t}=4 \mathrm{~ms}$ after impact

Figure 4. Heat flux along the radius for or a water and an ethanol droplet impacting on the smooth and uncoated hydrophilic stainless steel surface at $V_{0}=0.8 \mathrm{~m} / \mathrm{s}$. $T_{W 0}=60^{\circ} \mathrm{C}$. 
Although the heat flux is difficult to compare between the water and the ethanol droplets, the cooling effectiveness can be easily compared for both liquids, as it considers the effect of both the droplet's initial diameter and thermal properties. The cooling effectiveness computed as a function of the non-dimensional time $t^{*}=t V_{0} / D_{0}$, for the ethanol and water droplets is depicted in Figure 5, which clearly shows a higher cooling effectiveness for ethanol.

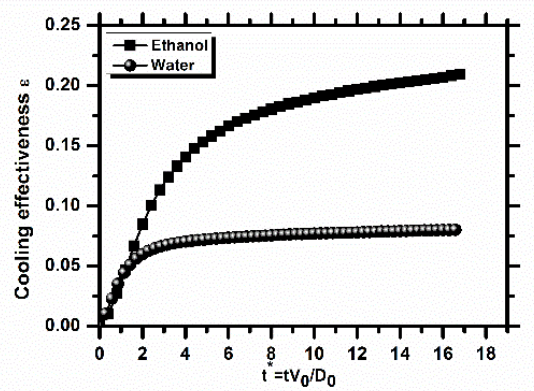

Figure 5. Comparison between the cooling effectiveness of a water and an ethanol droplet impacting on the smooth and uncoated hydrophilic) stainless steel surface at $V_{0}=0.8 \mathrm{~m} / \mathrm{s}$. $T_{w 0}=60^{\circ} \mathrm{C}$.

This result may seem odd, following the previous analysis of the heat flux. Nevertheless, considering the definition of the cooling effectiveness (the total heat removed is divided by the maximum possible heat removed) this result is actually plausible. Since ethanol has worse thermal properties, the maximum possible heat removed is also lower than that of water, so in the end the cooling effectiveness considering the improved wetted area is actually higher for ethanol. It is worth noting, however, that this better cooling effectiveness is not associated to a better cooling performance, as one must evaluate all the parameters and effectively access the heat that is removed from the surface, which is naturally larger for water.

\section{Effect of surface wettability}

To infer on the effect of wettability, the heat flux (Figure 6) and cooling effectiveness (Figure 7) are compared for the impact of a water droplet $\left(D_{0}=2.6 \mathrm{~mm}, V_{0}=0.8 \mathrm{~m} / \mathrm{s}\right)$ on the stainless steel foil, with and without the Glaco $尺$ coating. As aforementioned, the coated foil becomes superhydrophobic, depicting equilibrium angles with water of $\theta=162.5^{\circ}$ with a hysteresis always lower than $10^{\circ}$. Although the spreading factor is similar for both droplets, the shape of the rim is quite different. Hence, while in the hydrophilic case, the droplet spreads and wets the surface, in the superhydrophobic case, the shape of the rim reduces the true wetted area, thus reducing the liquid-solid contact area. The superhydrophobic surface also promotes the complete rebound of the droplet, which occurs approximately at $t=20 \mathrm{~ms}$ after impact. Consequently, the heat flux depicted in Figure 6 is considerably smaller for the superhydrophobic surface. The heat flux peaks at the contact edge are also smaller. The peak observed at $t=4 \mathrm{~ms}$ is associated to the maximum spreading diameter which occurs at that time instant (Figure 6b).

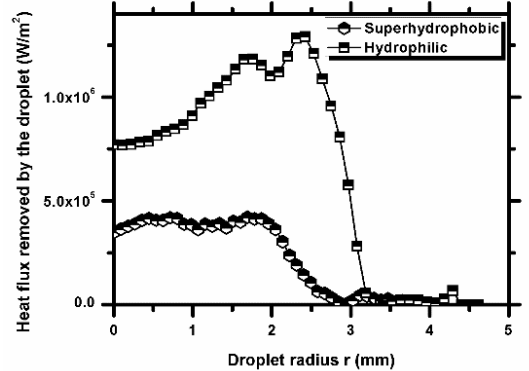

a) $t=2 m s$ after impact

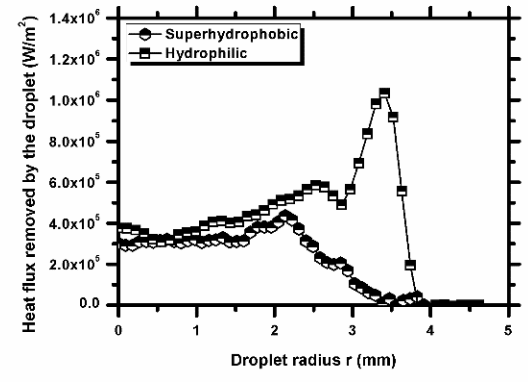

b) $t=4 \mathrm{~ms}$ after impact

Figure 6. Heat flux during the spreading of a water droplet on a hydrophilic vs superhydrophobic surface $\left(D_{0}=2.6 \mathrm{~mm}\right.$, $V_{0}=0.8 \mathrm{~m} / \mathrm{s}, T_{w_{0}}=100^{\circ} \mathrm{C}$ ).

The lower cooling effectiveness computed as a function of the non-dimensional time $t^{*}=t V_{0} / D_{0}$ for the superhydrophobic surface is therefore not only related to the reduced contact time between the droplet and the surface due to droplet rebound, but also due to the reduced wetting contact area resulting from the high contact angles. Despite the spatial resolution does not allow the clear detection of very thin layers of air, it is good enough to detect bubble entrapment, as discussed in Figure 1. From this perspective, the presence of an air layer precluding droplet contact with the superhydrophobic surface is not evident, except at the contact line edges 
where an important part of the heat transfer takes part. Hence, the reduced contact area due to the shape of the droplet visualization seems to play a major role in the heat transfer process, although a more detailed analysis and relative quantification is required in the near future to confirm these trends.

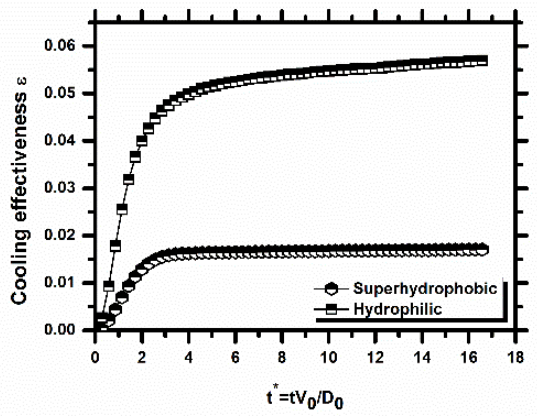

Figure 7. Cooling effectiveness as a function of the non-dimensional time $t^{*}$, for a water droplet spreading on a hydrophilic vs superhydrophobic surface $\left(D_{0}=2.6 \mathrm{~mm}, V_{0}=0.8 \mathrm{~m} / \mathrm{s}, T_{w_{0}}=100^{\circ} \mathrm{C}\right)$.

\section{Conclusions}

The present paper analyses the liquid-surface interfacial heat transfer phenomena occurring at droplet/wall interactions combining the use of time resolved infrared thermography with high-speed imaging. The results show that the methodology proposed captures well particular details on droplet dynamics and heat transfer, allowing to identify air bubble trapping at the impact region as well as the temperature variations at the formation of the rim. The combined analysis of droplet dynamics (e.g. the spreading factor) with the radial temperature profiles, heat flux and cooling effectiveness computation allowed establishing qualitative and quantitative trends on the effect of various parameters on the heat transfer occurring at droplet/wall interactions. Hence, the effect of the initial surface temperature is observed to play a minor role, as long as it is low enough to prevent the occurrence of boiling. The physico-chemical properties of the working fluids naturally play a vital role in the heat transfer process, so the heat fluxes obtained during the spreading of a water droplet are much larger than those obtained during the spreading of an ethanol droplet (up to two times higher). However, the cooling effectiveness of the ethanol droplet is actually higher than that of the water droplet given its larger spreading area, which is accounted in the effectiveness, together with the properties of the fluids. The superhydrophobic wetting regime limits the heat transfer between the spreading droplet and the surface, as expected, given the particular dynamic behaviour of the droplets within this regime (limited droplet-surface contact due to air entrapment and occurrence of rebound). However, the thermal analysis reveals that a major reason for this is not related to the reduced contact time of the droplet on the surface (due to rebound) or air entrapment, but is rather associated to the reduced wetted area caused by the high contact angles.

\section{Acknowledgements}

The authors are grateful to Fundação para a Ciência e Tecnologia (FCT) for partially financing the research under the framework of the project RECI/EMS-SIS/0147/2012 and for supporting P. Pontes with a research fellowship. A. S. Moita acknowledges FCT for financing her contract through the IF 2015 recruitment program (IF 008102015) and E. acknowledges FCT for supporting his PhD fellowship (SFRH/BD/88102/2012).

\section{References}

[1] Panão, M., Guerreiro, J. and Moreira, A.L.N., 2012, Int. J. Heat Mass Transf. 55, pp. 2854-2863.

[2] Moura, M., Teodori, E., Moita, A.S. and Moreira, A.L.N., 2016, Proc. IEEE ITherm Conference, The Intersociety Conference on Thermal and Thermomechanical Phenomena in Electronic Systems, pp. 378-387.

[3] Moreira, A.L.N., Moita, A.S. and Panão, M.R., 2010, Prog Energy Comb Sci., 36, pp. 554-580.

[4] Liang, G., and Mudawar, I., 2017, Int. J. Heat Mass Transf., 106, pp. 103-126.

[5] Marengo, M., Antonini, C., Roisman, I.V. and Tropea, C., 2011, Curr. Op. Coll. Interface Sci., 16, pp. $292-302$.

[6] Pereira P., Moita, A. S., Monteiro, G. and Prazeres, D.M.F., 2014, J. Bionic Eng., 11(3), pp. 346-359.

[7] Pasandideh-Fard, M., Aziz, S., Chandra, S. and Mostaghimi, J., 2001, Int. J. Heat Fluid Flow, 22, pp. 201-210.

[8]Girard, F., Antoni, M. and Sefiane, K. 2010, Langmuir, 26, pp. 4576-4580.

[9] Kim, H. and Bungiorno, J., 2011, Int. J. Multiphase Flow, 37, pp. 166-172.

[10] Tartarini, P., Corticelli, M.A. and Tarozzi, L., 2009, Appl. Thermal Eng., 29, pp. 1391-1397.

[11] Gradeck, M., Seiler, N., Ruyer, P. and Maillet, D., 2013, Exp. Thermal Fluid Sci., 47, pp. 14-25.

[12] Moita, A.S. and Moreira, A.L.N., 2003, $9^{\text {th }}$ ICLASS 2003, Sorrento, Italy.

[13] Rioboo, R., Marengo. M. and Tropea, C., 2002, Exp. Fluids, 33(1), pp. 112-124. 


\title{
Instantaneous heat transfers at the impact of a droplet onto a hot surfaces in the film boiling regime
}

\author{
William Chaze ${ }^{1,2}$, Guillaume Castanet ${ }^{*, 1,2}$, Ophélie Caballina ${ }^{1,2}$, Denis Maillet ${ }^{1,2}$, \\ Jean-François Pierson ${ }^{3}$, Fabrice Lemoine ${ }^{1,2}$ \\ ${ }^{1}$ LEMTA, UMR 7563, Université de Lorraine, Vandœuvre-Lès-Nancy, France \\ ${ }^{2}$ LEMTA, UMR 7563, CNRS, Vandœuvre-Lès-Nancy, France \\ ${ }^{3}$ Institut Jean Lamour, UMR CNRS 7198, Université de Lorraine, Nancy, France \\ ${ }^{\star}$ Corresponding author: guillaume.castanet@univ-lorraine.fr
}

\begin{abstract}
Heat and mass transfers at the impact of a droplet onto a hot solid surface are investigated experimentally. Millimetersized water droplets impinges onto a perfectly flat sapphire surface heated at $600^{\circ} \mathrm{C}$. The temperature of the liquid inside the droplet is measured using the two-color laser-induced fluorescence (2cLIF) technique. Water is seeded with a temperature-sensitive fluorescent dye, while a nanosecond pulsed laser is used for the excitation of the fluorescence. The ratio of fluorescence signal detected in two appropriate spectral bands allows to determine the liquid temperature. One advantage of this non-intrusive optical technique is that it eliminates adverse effects associated with signal variations caused by droplet shape during its impact. In parallel, the temperature of the solid surface is characterized using infrared thermography. The latter measurements are made possible by the deposition of a nanosize coating of titanium aluminium nitride (TiAIN) on the upper surface of the sapphire window. Thanks to the high frame rate of the IR camera, the time evolution of the heat flux distribution at the solid surface can be reconstructed. A comparison of IR and 2cLIF techniques enable to correlate the heating of the liquid with the cooling of the wall. This reveals that most of the heat removed from the solid surface is devoted to the heating of the liquid, the energy used for liquid vaporization being significantly lower.
\end{abstract}

\section{Introduction}

Many industrial applications require a rapid cooling of surfaces from high temperatures. Among the cooling technologies, spray cooling is certainly one of the most attractive for the thermal management of high heat flux systems. Compared to jet impingement, it has the capability of cooling a relatively wider surface area with a single nozzle. It also has an unrivalled cooling efficiency, meaning that significant quantities of coolant liquid can be saved to remove the same amount of heat. These features explain why it is widely employed in many industrial applications, especially in metal production and processing industry. However, while it is applied for decades, its integration remains a complex and cumbersome process because of still incomplete knowledge of the fluid flow and heat transfer characteristics. In particular, scientific investigations focused on individual droplets are still required to understand the underlying physics behind the interactions between droplets and a hot solid surface. When a drop impacts a hot wall, different behaviours are observed. The drop can spread over the solid surface and remain attached to it due to wettability forces. It can splash and creates several smaller secondary droplets or simply rebounds. Extensive experimental investigations were carried out in the past to characterize the parameters influencing the drop behavior at the impact. Among them, some can be related to the dynamic of the impacting droplets (velocity, diameter, etc.), the physical properties of the liquid (viscosity, surface tension, etc.), and the solid surface (temperature, roughness, thermal effusivity, etc.) [1]. Descriptions of an impact are usually made on the basis of correlations with dimensionless numbers characterizing the relative magnitude of the forces acting on the impinging droplet, i.e. Reynolds, Weber and Ohnesorge numbers.

Regarding heat transfers, almost all the studies were focused around the heat removal from the solid surface, whose temperature was monitored using either thermocouples embedded in the wall thickness [2] and more recently by IR thermography $[3,4]$. Coupled with an inverse model, these measurements made quantification of the heat removed from the wall and the cooling efficiency [4] possible .Recently, Jung et al. [3] used IR thermography to characterize the temporal evolution of the heat flux at the wall during the impact of millimeter-size water droplets. A sapphire window, transparent in the IR up to $5 \mu \mathrm{m}$, is coated with a nanometer layer of platinum on the impact surface. Direct observation of the platinimum deposit through the sapphire allows to reconstruct the heat flux evolution with almost no limitation in temporal resolution other than the frame rate of the IR camera. Even though platinum is very reflective and therefore not very emissive $(\varepsilon \approx 0.04)$, accurate measurements of the surface temperature could be achieved.

The contributions of liquid evaporation and liquid heating to the overall heat exchange between the droplet and the wall are not obvious to evaluate if measurements are restricted to the wall heat flux. The droplet heating can be characterized using the two-color laser-induced fluorescence thermometry (2cLIF) which is one of the few proven techniques available to measure the temperature of droplets [5]. The liquid (in this case water) is seeded by a temperature-dependent fluorescent dye. The capabilities of the technique for studying the drop impact was initially demonstrated on mono-sized droplet streams impinging obliquely a heated wall [6]. Dunand et al. [7] made use of a laser sheet and two cameras in order to visualize the temperature of the impacting droplets. However, using a $\mathrm{CW}$ laser, measurements suffer from serious limitations. Light intensity within the laser sheet was not sufficient to 
shorten the exposure time of the cameras enough to obtain instantaneous images of the impinging droplets. More recently, several improvements to the 2cLIF technique were made by [8] to achieve single shot measurements of the temperature using nanosecond pulse laser.

The present study relies on these previous works for the development of optical diagnostics adapted to the characterization of heat and mass transfers at the drop impact on heated solid surfaces. The focus is put on the film boiling regime, in which a thin vapor film at the interface between the droplet and the solid surface prevents the deposition of liquid on the wall surface. Ultimately, the experimental results will help understanding the coupling between the droplet deformation at the impact and the heat and mass transfers. They will also be useful benchmarks to validate detailed numerical simulations which are taking into account phase change and heat transfers.

\section{Experimental set-up}

The experimental setup is presented in Figure 1. Millimeter-sized drops are generated in a very reproducible manner by a syringe. When the drop pending to the end of the needle exceeds a certain size, it detaches itself. A $400 \mu \mathrm{m}$ base diameter needle is used to produce water drops with a diameter of $2.5 \mathrm{~mm}$. The frequency of the droplet detachment is controlled by means of a syringe drivers which allows delivering a constant liquid flow rate. The free-falling droplets impinge a sapphire window (1" in diameter and $5 \mathrm{~mm}$ thick) placed on a steel holder which is heated at $600^{\circ} \mathrm{C}$ using cartridges heaters $(4 \times 250 \mathrm{~W})$. Owing to the large thermal conductivity of sapphire (about $40 \mathrm{~W} / \mathrm{m} / \mathrm{K}$ ), the temperature of the solid surface is uniform and and nearly equal to that of the steel holder. A thermal shield is necessary to prevent heating of the liquid inside the needle caused by the hot air plume rising from the sapphire window. The tip of the needle is placed into a cavity of a few millimeters arranged inside a metallic plate which is cooled down by a water circulation. Moreover, the fluorescent solution passes into a heat exchanger before entering the needle. A thermocouple, placed at

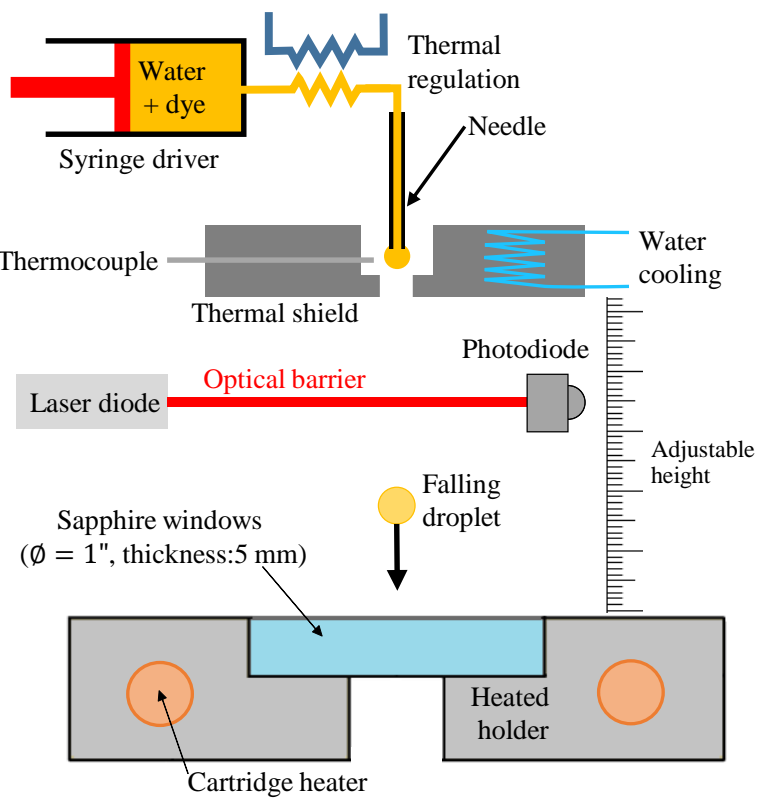

Figure 1. The experimental setup a short distance from the droplet in the cavity, is used to control the ambient temperature. This provides a good approximation for the temperature of the pendant droplet, because the same setpoint is imposed in the thermal regulations with the heat exchanger and the thermal shield. The needle is moved up and down to modify the impact velocity. Finally, an aperture managed in the steel holder allows using a sapphire window for a backside illumination and/or visualization of the impinging droplet.

\section{Droplet temperature measurements}

A pulsed Nd: YAG laser at $532 \mathrm{~nm}$ (Quantel Brillant B, pulse energy $E=450 \mathrm{~mJ}$, pulse duration $d t=5 \mathrm{~ns}$ ) is utilized for the excitation of the fluorescence. The laser beam is much larger than the droplets, which yields an illumination of the whole liquid volume during the droplet deformation. The repetition rate of the pulsed laser $(10 \mathrm{~Hz})$ is by far too low to resolve temporally the impact process. However, a time reconstruction is possible owing to the fact that droplets can be produced repeatedly with exactly the same size. An optical barrier consisting of a laser diode and a photodiode, placed a few millimeters above the sapphire surface, makes the detection of the fall of a droplet possible. The time delay between the detection by the barrier and the triggering of the laser pulse is varied by a small increment in order to explore, droplet after droplet, the whole period of an impact. As displayed in Figure 2, droplets are observed by means of two CCD cameras (Allied Vision Tech Prosilica GT3300 B/C GigE Camera $3296 \times 2472,12$ bits, $5.5 \mu \mathrm{m}$ ) each one equipped with an interference filter for the detection of the fluorescence in a specific spectral band. The optical system also includes an objective lens (SIGMA APO MACRO $150 \mathrm{~mm}$ F2.8 EX DG OS HSM and its teleconverter $\times 2$ ) and a beamsplitter mounted in front of the cameras. A high pass filter $(\lambda>542 \mathrm{~nm})$ is added between the objective lens and the beamsplitter to eliminate more efficiently the laser light. Examples of temperature measurements based on 2cLIF can be found in the literature. Application are related to single-phase liquid flows [8], monosized droplet streams [7] and sprays [5]. In most of these applications, a CW laser was used to induce fluorescence. However, for the purpose of instantaneous imaging, it is very advantageous using pulsed laser sources, which allow delivering a considerable amount of energy over a very short period of time (typically a few ns). According to Chaze et al.[8], the fluorescence $F_{\lambda}$ signal emitted at a wavelength $\lambda$ in an elementary volume $V$ of liquid can be expressed by:

$$
F_{\lambda}=\eta \frac{\Omega}{4 \pi} \varepsilon_{0} \phi_{\lambda} \frac{I_{0}}{\left(1+I_{0} / I_{\text {sat }}\right)} C V .
$$

In this expression, $I_{0}$ corresponds to the laser intensity, $C$ is the molar concentration of the dye molecules. $\eta$ is the transmission efficiency of the fluorescence light to the detector. $\Omega$ denotes the solid angle of the collection. $\varepsilon_{0}$ is the molar extinction coefficient of the fluorescent molecules at the excitation wavelength of the laser beam. $\phi_{\lambda}$ is 


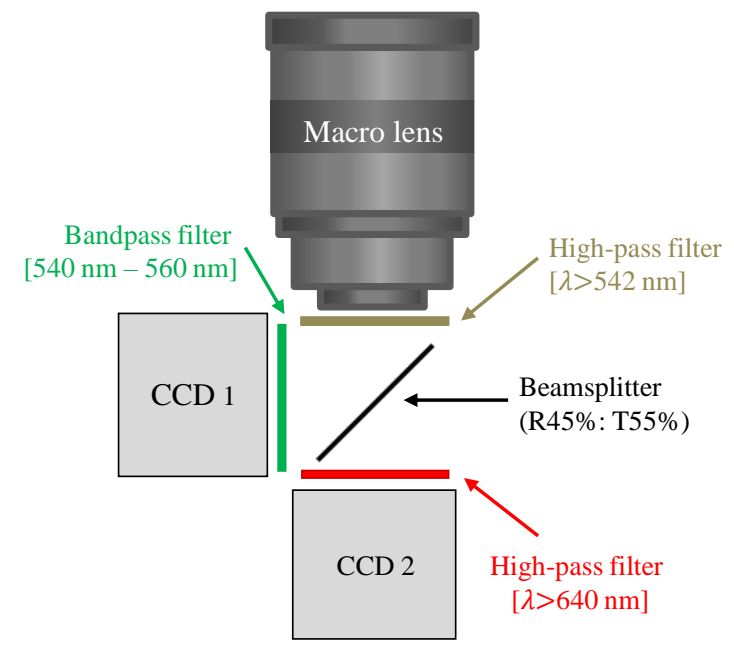

Figure 2. Arrangement of the optical detection system of the 2cLIF technique

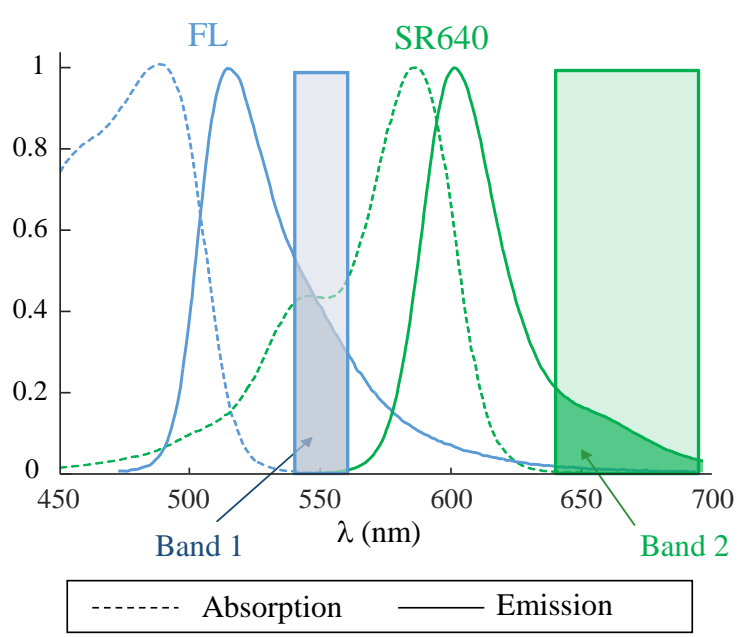

Figure 3. The two detection bands selected for the application of the 2cLIF technique using the couple of dyes

to the fluorescence quantum yield. $I_{s a t}$ is the saturation intensity of the fluorescent dye (typically a few $\mathrm{MW} / \mathrm{cm}^{2}$ [8]). In Eq.(1), parameters $\varepsilon_{0}, \phi_{\lambda}$ and $I_{s a t}$ are temperature dependent. While $\phi_{\lambda}$ decreases with temperature due to collisional quenching, the saturation intensity $I_{\text {sat }}$ follows an inverse trend [8]. As a result, fluorescent dyes can lose some of their temperature sensitivity at high laser irradiance. In the following, fluorescein disodium (FL) is used as its temperature sensivity arises exclusively from $\varepsilon_{0}$ and therefore it retains a high and unchanged temperature sensitivity regardless of the laser irradiance [8]. FL is mixed with sulforhodamine 640 (SR640) whose fluorescent emission does not vary with temperature. Figure 3 shows the detection bands selected for the experiments. In the region [ $540 \mathrm{~nm}-560 \mathrm{~nm}$ ], only FL has a contribution to the signal while the emission of SR640 is predominant above $640 \mathrm{~nm}$. Taking the ratio of the signals detected in the two emission bands allows to eliminate the dependence on the droplet shape, since the fluorescence emitted in the two detection bands is affected almost identically by light dispersion at the droplet surface. The ratio $R$ of the signals in the detection bands can be determined by:

$$
R=R_{0} \frac{\varepsilon_{0, \mathrm{FL}}(T)}{\varepsilon_{0, \mathrm{FL}}\left(T_{0}\right)}
$$

where $R_{0}$ is a reference ratio measured at a known temperature $T_{0}$. The fluorescence ratio $R$ calculated by dividing the images of the two cameras is converted into temperature using a calibration curve. The calibration was carried out in a glass cell in which the fluorescent solution was heated progressively from $20^{\circ} \mathrm{C}$ to $80^{\circ} \mathrm{C}$. Images were recorded in the two detection bands and the fluorescence ratio was determined. For a mixture $C_{\mathrm{SR} 640}=0.7 .10^{-6} \mathrm{M}$ and $C_{\mathrm{FL}}=2.10^{-4} \mathrm{M}$ (used later to investigate the drop impact), the temperature sensitivity of the fluorescence ratio $R$ can be valuated at $2.7 \% /{ }^{\circ} \mathrm{C}$, meaning that $R / R_{0} \approx \exp \left\{-0.027\left(T-T_{0}\right)\right\}$. An offset of a few pixels between the images of the two cameras may remain despite a careful alignment of the beamsplitter. A polynomial transform is created to obtain a perfect matching between the coordinates of the pixels in the images of the two cameras. This polynomial function is determined with the help of a dotted grid placed in front of the detection system. In Eq.(2), $T_{0}$ is assimilated to the temperature measured by the thermocouple inserted in the thermal shield (Figure 1). It is assumed that the droplets do not heat up significantly when they travel in the hot air plume above the sapphire window.

\section{Measurements of the temperature and heat flux at the solid surface}

The temperature of the impact surface is characterized by means of an IR camera (FLIR ORION SC7000) which incorporates a cooled InSb detector that operates in the 1.5- to 5.5- $\mu \mathrm{m}$ waveband. The camera is capable of capturing up to $400 \mathrm{fps}$ at full resolution (320 x 240 pixels). Windowing makes acquiring the images at a few thousands fps possible, however at the expense of the number of pixels. Bottom view images of the impact surface can be recorded thanks to the transparency of the sapphire substrate below $5 \mu \mathrm{m}$. The IR camera is equipped with a high-magnification lens with a large numerical aperture. The acquisition sequence is triggered by the passage of the droplet through the optical barrier. The top face of the sapphire window is coated with a thin film of TiAIN (typical thickness: $300 \mathrm{~nm}$ ) which is resistant to the oxidation and stress encountered at high temperature. Furthermore, this coating has a high emissivity in the detection band of the camera $(\epsilon=0.926)$ which is almost independent of temperature. A first benefit of a high emissivity is that the radiative emission from the surface is sufficiently large to shorten the integration time of the camera to a few tens of $\mu s$ and thus to resolve temporally the impact process. Because of the high surface emissivity, reflections of environment radiations (especially those coming from the heated holder) are very limited and can be safely ignored in the processing of the images. The level of the random noise does not exceed $0.1 \%$ of the signal which yields an error of about $0.5^{\circ} \mathrm{C}$ on the evaluation of the temperature. This error remains acceptable in comparison to the temperature variations of the solid surface observed during an impact, which are typically of the order of a few tens of degree. 
Experimentally, a windowing is used to increase the acquisition frame rate of the camera. A windows of $60 \times 80$ pixels was chosen allowing a frame rate of $3.5 \mathrm{kfps}$. In a first test, a non-uniformity correction (NUC) is performed in order to obtain a flat field without any dispersion of the numerical level when there is no droplet and that the conditions are perfectly stationary. A good approximation of the variation in temperature of the wall surface for each pixel $i$ in the image can be expressed by:

$$
\Delta T_{i}(t)=T_{i}\left(t=0^{-}\right)\left[\Delta N_{i}(t) / N_{i}\left(t=0^{-}\right)\right]^{1 / 4}
$$

This expression implies that the camera is linear in flux and $\epsilon$ remains constant with time but knowing the value of $\epsilon$ is not necessary given that the temperature before impact $T_{i}\left(t=0^{-}\right)$is known (the wall temperature being regulated at $600^{\circ} \mathrm{C}$ ). The wall heat flux is deduced from the wall temperature measurement thanks to an inversion of an axisymmetric analytical heat conduction problem. The geometry of the problem is presented in Figure 4. Under the hypothesis of axysymmetry, the transient heat transfer equation can be written:

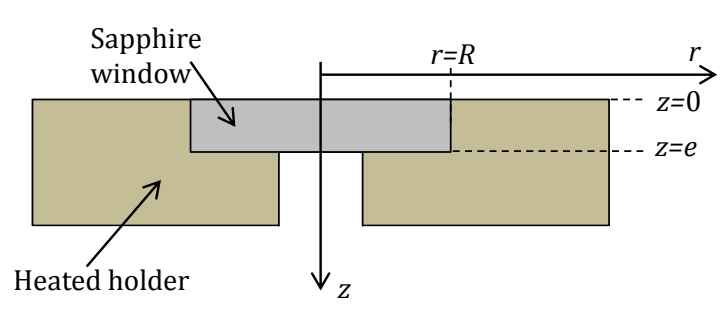

Figure 4. : Geometry of the system

$$
\frac{\partial^{2} T}{\partial r^{2}}+\frac{1}{r} \frac{\partial T}{\partial r}+\frac{\partial^{2} T}{\partial z^{2}}=\frac{1}{a} \frac{\partial T}{\partial t}
$$

With the following boundary conditions :

$$
\begin{array}{r}
T=T_{\text {holder }}, \text { at } r=R, \\
\frac{\partial T}{\partial r}=0, \text { at } r=0, \\
T=T_{\text {holder }}, \text { at } z=e, \\
\lambda \frac{\partial T}{\partial z}=q(r, z=0, t), \text { at } z=0
\end{array}
$$

The heat flux $q(r, z=0, t)$ is assumed to be the contribution of the droplet only and it is therefore the target of the inversion. Indeed, the heat flux associated with natural convection is negligible in comparison with the heat flux induced by the impact of the droplet (typically on the order of a few $\mathrm{MW} / \mathrm{m}^{2}$ ). For the inversion, the reduced variable $\theta=T-T_{\text {holder }}$ is introduced then a Hankel transform in space is applied $(\widetilde{*})$ [9]. This yields:

$$
\widetilde{\theta_{n}}(z=0, t)=\widetilde{Z_{n}}(t) \otimes \widetilde{q_{n}}(z=0, t)
$$

This equation establishes a relationship between the $n^{t h}$ mode of the temperature at the front face $\widetilde{\theta_{n}}(z=0)$ and the $n^{t h}$ mode of the heat flux induced by the drop impact $\widetilde{q_{n}}(z=0)$ in the Hankel transform. $\widetilde{Z_{n}}$ denotes the thermal impedance, which depends on the thermal properties and the geometry of the wall. It can be expressed analytically using in addition to the hankel transform, a Laplace transform in time [9].The number of modes to be considered for the resolution of Eq.(4) is generally chosen using a trial and improvement method. It has to be adapted to the geometry and to the transient period. Presently, 70 modes are considered in the computations. Radial profiles of temperature are extracted from the images assuming a radial symmetry of the problem and the Hankel transformation is applied to them, which allows obtaining $\widetilde{\theta_{n}}(z=0, t)$. Eq.(9) is put in a matrix form for the inversion:

$$
\left[\widetilde{q_{n}}\right]=\left[\widetilde{Z_{n}}\right]^{-1}\left[\widetilde{\theta_{n}}\right] \text { with }\left[\widetilde{\theta_{n}}\right]=\left[\widetilde{\theta_{n}}\left(t_{1}\right), \ldots, \widetilde{\theta_{n}}\left(t_{i}\right), \ldots, \widetilde{\theta_{n}}\left(t_{\text {end }}\right)\right]
$$

Here, $\left[\widetilde{\theta_{n}}\right]$ is the vector of the temperatures measured at all discrete time steps $t_{1}, \ldots, t_{i}, \ldots, t_{\text {end }}$ of the acquired sequence of images. The last step of the inversion consists in applying the Hankel reverse transformation to get the estimated heat flux in the real space.

\section{Heating of the impacting droplets}

The experiments consisted in varying the falling height of the droplet and thus its impact velocity $V$. The Weber number $W e=\rho V^{2} d / \gamma$, which compares the kinetic energy of the droplet $\left(\sim \rho V^{2}\right)$ to its surface energy $(\sim \gamma / d)$, ranges from 10 to 140 . The wall temperature is set to $600^{\circ} \mathrm{C}$, while the initial droplet temperature is maintained at $20^{\circ} \mathrm{C}$. Figures 5 to 8 show instantaneous images of the temperature taken at different times during the impact. Side and bottom views are recorded for each impact condition. To interpret these images, it should be kept in mind that the imaging system does not provide optical sectioning of the droplet. The images roughly correspond to an average of the temperature in the depth of the droplet given that the depth of field of the cameras is several millimetres. In the following description, the spreading, the recoiling and the fragmentation of the droplet are considered separately.

Spreading: The spreading phase lasts about 3-4 ms. Even if the bulk of the droplet maintains its initial temperature in the early stage of the spreading, there is a thin liquid layer close to the interface with the vapor film, where the liquid temperature quickly reaches about $100^{\circ} \mathrm{C}$. However, this liquid layer is too thin to be observed on the side view images. Its contribution to the bottom views is all the more important than the liquid thickness is small. In the case of $W e=10.2$, the heating is rather moderate during the spreading. In Figure 5, it is difficult to point out a region where the heating is more intense. At higher Weber numbers (Figures 6-8), bottom views show a higher temperature band around the edge of the droplet at $t=1 \mathrm{~ms}$ and $2 \mathrm{~ms}$. At these times, the ejected lamella is 
much thinner than the central region of the droplet, which helps observing a heating of the liquid in the bottom views. Provided a sufficiently large impact velocity, the lamella rapidly takes a gaussian shape surrounded by an annular rim, which is growing due to the deceleration by the surface force opposed to the spreading [10]. Liquid is progressively heated while flowing along the hot wall from the core of the lamella in the direction of the rim at the edge of the droplet. In Figure 8, the liquid temperature apparently reaches almost $80^{\circ} \mathrm{C}$ in the region of minimum thickness just before the entrance of the rim. Afterwards, in the rim, the hot liquid coming from the lamella rapidly mixes with colder liquid already accumulated there. Disturbances on the rim of the spreading drop can be easily observed for $W e=92$ at the early stage of the impact. These disturbances increase with time leading to a spatter with characteristics fingers. Disturbances of smaller amplitudes also develop for lower Weber numbers but much more slowly. Even for $W e=10.2$, the droplet does not keep a perfectly circular shape in the bottom views. It was proposed that fingering is caused by the Rayleigh-Taylor instability because a heavier fluid liquid rim is decelerated with respect to a lighter one air during spreading [11, 12]. The magnitude of the initial deceleration determines the number of fingers and their growth rate. At $t=4 \mathrm{~ms}$ and $5 \mathrm{~ms}$, it can be seen for $W e=92$, both on the side and bottom images, that the fingers are colder than the thinner regions in the rim.

Fragmentation of the rim and splashing: For $W e=92$, the previously mentioned fingers detach from the rest of the rim causing the fragmentation of the rim and ultimately the splashing of the droplet. The temperature of the secondary droplets resulting from the rim fragmentation evolves very little with time. It is of the order of $55^{\circ} \mathrm{C}-60^{\circ} \mathrm{C}$. In parallel, the lamella becomes so thin at the end of the spreading that it breaks. When this occurs, the temperature of the thin liquid sheet (about $80^{\circ} \mathrm{C}$ ) is much larger than that of the surrounding liquid in the rim. Holes usually open in the vicinity of the rim where the lamella is thinner. The holes rapidly expands following the Taylor-Culick theory [13]. During this process, hot liquid ligaments are created because of the opening and expansion of several holes at the same time.

Recoiling and bouncing: The recoiling of the droplet is initiated by the surface forces acting on the rim edge. The case $W e=64.7$ has some similarities with $W e=92$. Here also, several holes open the lamella at the end of the spreading phase. However, the rim does not break up and the empty space replacing the lamella narrows during the recoiling phase. For $W e=10.2$ and $W e=30.6$, the temperature is not uniform when the droplet takes off from the solid surface at about $t=12 \mathrm{~ms}$. For $W e=30.6$, the temperature is lower at the top end where a satellite droplet nearly detaches. In Figure 5, mixing currents are visible at $t=9 \mathrm{~ms}$ and $t=12 \mathrm{~ms}$, while the droplet is about to leave the wall. Hence, the flow induced by the droplet deformation is not capable of a full mixing of the liquid when the impact velocity is weak in the bouncing regime, typically for $W e \leq 30$.

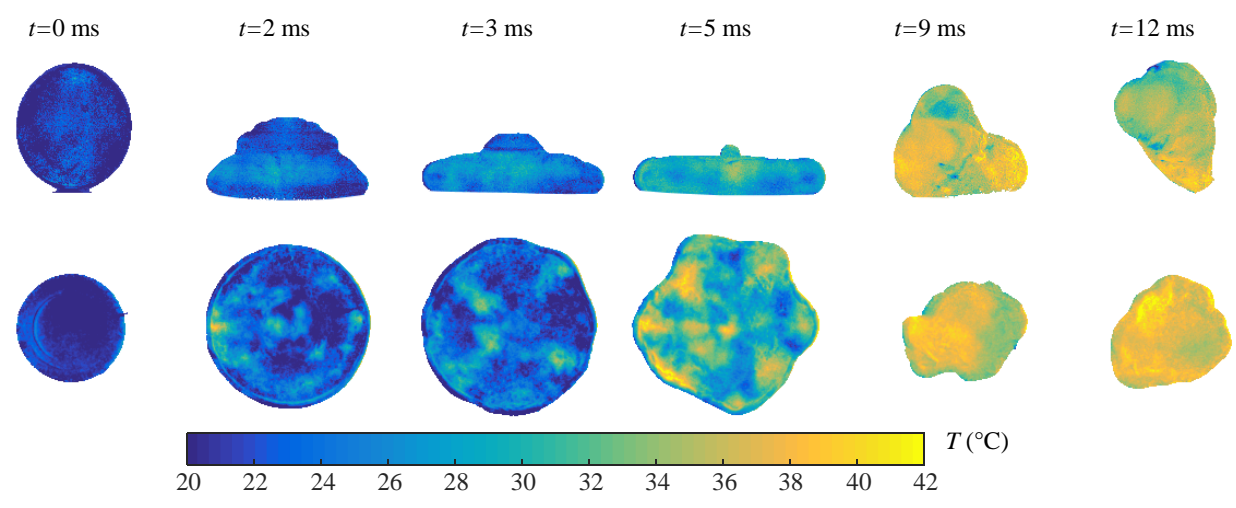

Figure 5. Side and bottom views of the temperature field within an impacting droplet at $W e=10.2$

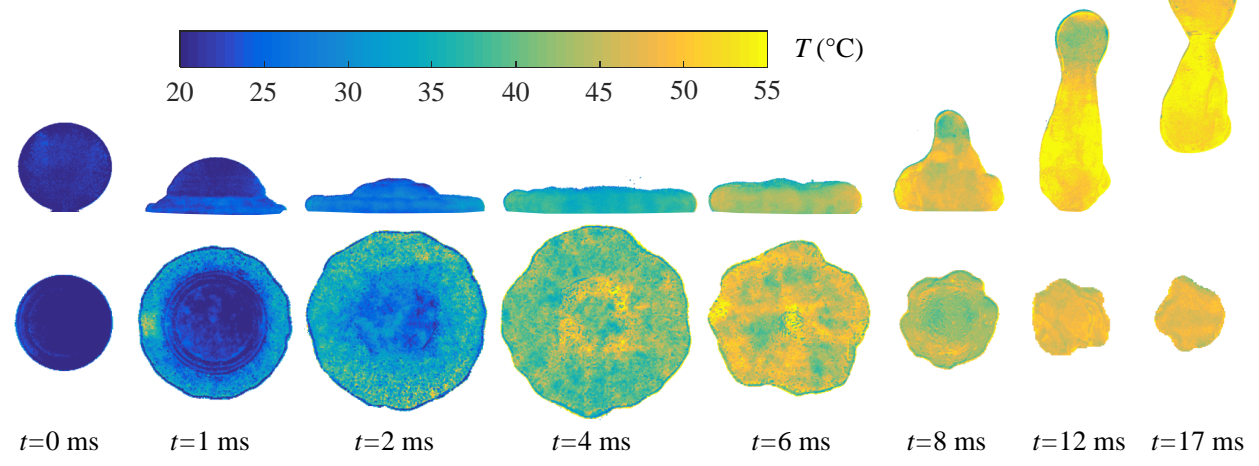

Figure 6. Side and bottom views of the temperature field within an impacting droplet at $W e=30.6$ 


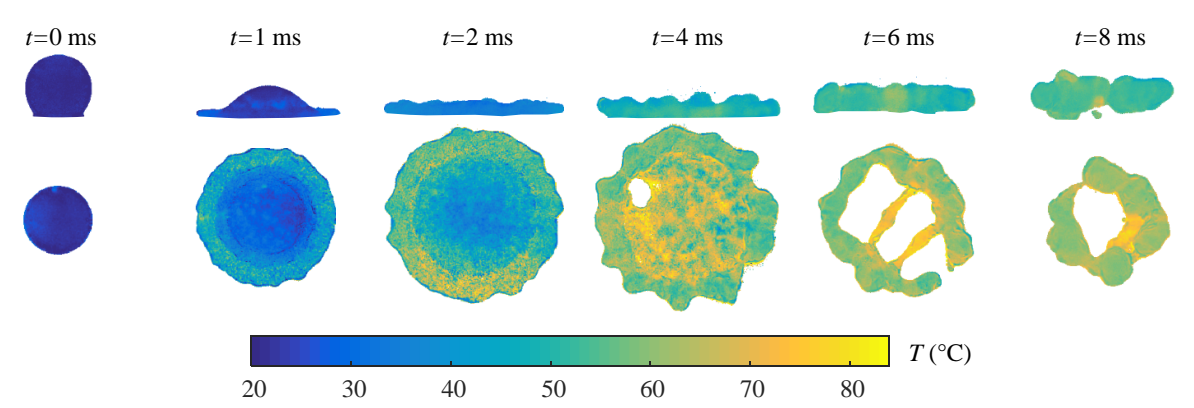

Figure 7. Side and bottom views of the temperature field within an impacting droplet at $W e=64.7$

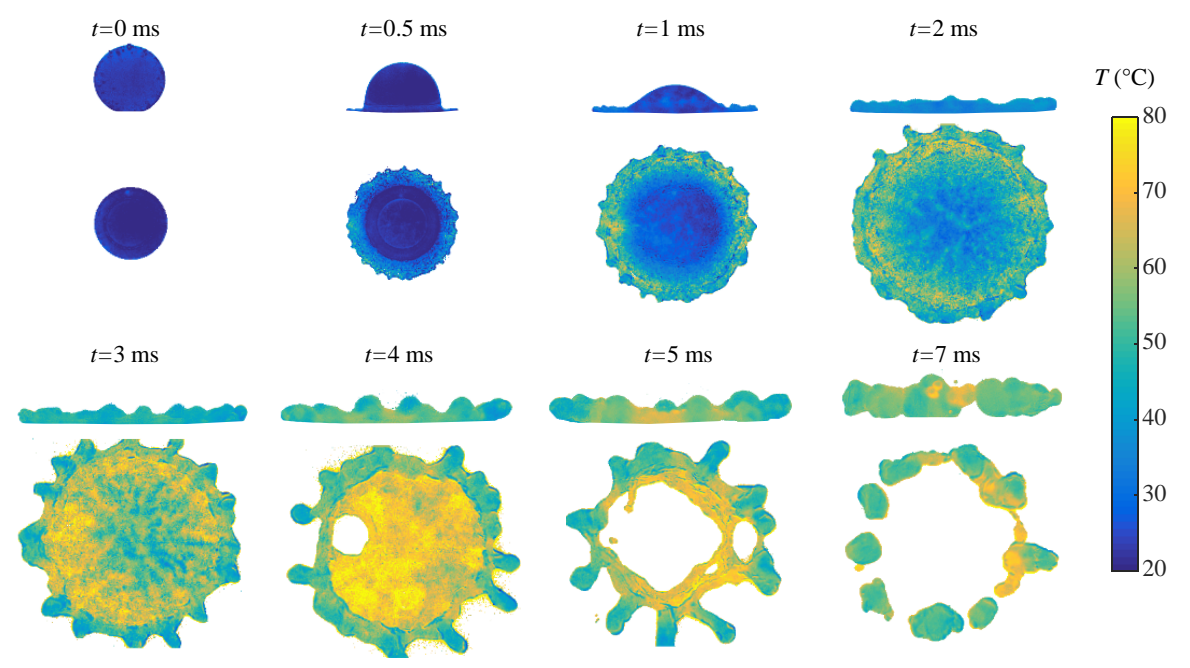

Figure 8. Side and bottom views of the temperature field within an impacting droplet at $W e=92$

\section{Evolution of the heat flux removed at the solid surface}

Figure 9 displays the evolution of the solid surface temperature measured during the impact of a droplet at $W e=$ 140. Measurements situated at the same distance from the droplet center in these images are first averaged. Then, the obtained radial temperature profile is used in the inversion to respect the axial symmetry of the model (Eq.4). The estimated heat flux densities $q(r, z=0)$ is also presented in figure 9 . The heat flux density is maximal shortly before the end of the spreading phase and vanishes progressively thereafter. The obtained values for the heat flux, a few $\mathrm{MW} / \mathrm{m}^{2}$, compare with the literature[3, 4]. The images of the solid surface temperature show some finger-like structures. There are important similarities with the fingering patterns reported by Khavari et al.[14] in the transition boiling regime for the contact area between the droplet and the solid wall. Thus, it may be a reminiscence of the same phenomenon at higher wall temperatures in the film boiling regime.

\section{Influence of the impact velocity}

Figure 10 shows the temporal evolution of the droplet heating for all the tested impact velocities. The reported values are calculated from the spatial averages of the side and bottom images presented in Figures 5-8. Whatever the Weber number, it is observed that the heating evaluated from the bottomview images is systematically larger than that determined from the sideview images. Obviously, both meet at the end of the impingement. Median evolutions, corresponding to the solid curves in Figure 10, are assumed to be closer to the true volumic heating of the droplet. The heating increases with $W e$, but there is a saturation, meaning that $W e$ has almost not influence on the final liquid temperature in the splashing regime contrary to the bouncing regime. This trend has already been observed by Castanet et al.[6] and Dunand et al.[4], who also measured an heating of about $40^{\circ} \mathrm{C}$ in the case of splashing droplets with an initial temperature of $20^{\circ} \mathrm{C}$. The duration of the heating decreases with $W e$. At low impact velocity, the heating period covers both the spreading and the recoiling phases. For $W e=10.2$, the heating is about one third of its final value at the end of the spreading. On the contrary, most of the heating takes place during the spreading for $W e \geq 64.7$. For the high Weber numbers, the heating has reached (or is about to reach) its final value just before that the fragmentation of the rim takes place. Hence, measurements reveal that the fragmentation of the droplet in itself has little effect on the final heating. For comparison, the energy removed from the wall was also calculated by time and space integration of the heat flux determined by the inverse model. The results, shown in Figure 11, are very similar to those presented before for the droplet heating in Figure 10. For instance, the same stagnation of the energy and the same reduction of the period of intense heat exchange can be observed at the high Weber numbers. Both IR and 2cLIF measurements indicate that the heat exchange with the wall is greatly enhanced by the creation of surface under the droplet that takes place during the spreading phase. The creation of surface seems to be the one of the main reason for the differences observed among the experimental cases. In 
addition, the thickness of the vapor film is also influenced by the impact velocity of the droplet as explained in the following.

a)
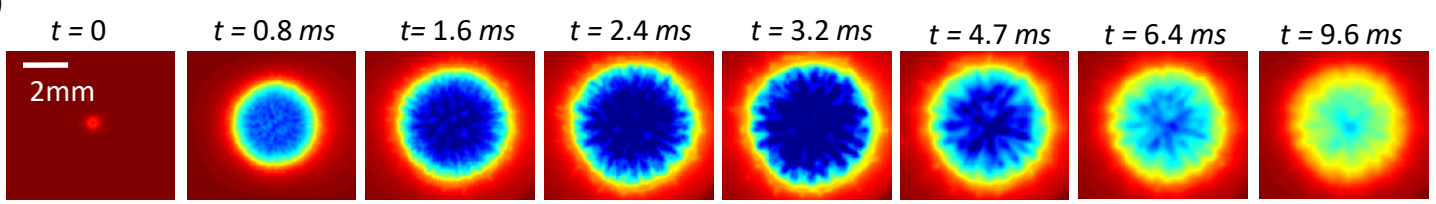

b)
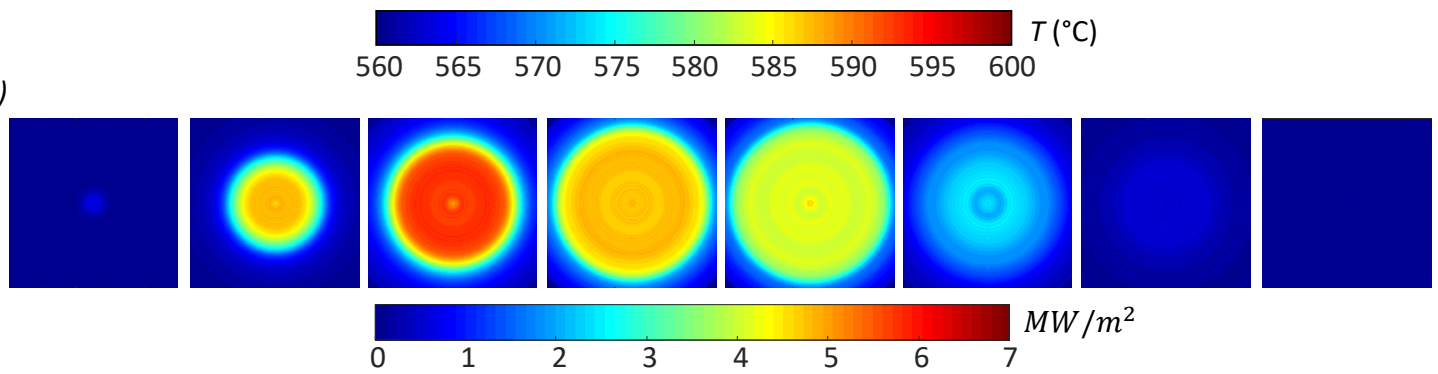

Figure 9. Results of the IR thermography in the case $W e=140$ (a: temperature distribution at the solid surface, $b$ : heat flux density $q(r, z=0)$ derived from the inverse model).

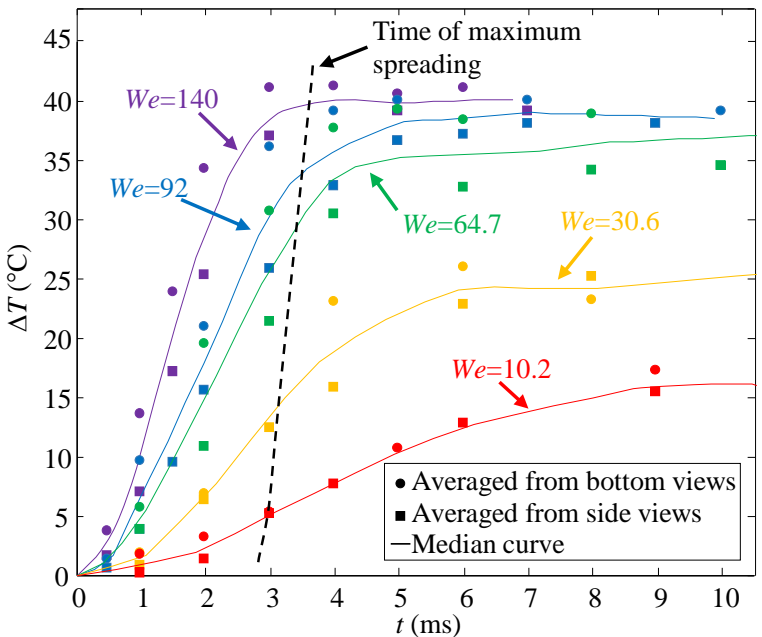

Figure 10. Temporal evolution of the spatially averaged droplet heating

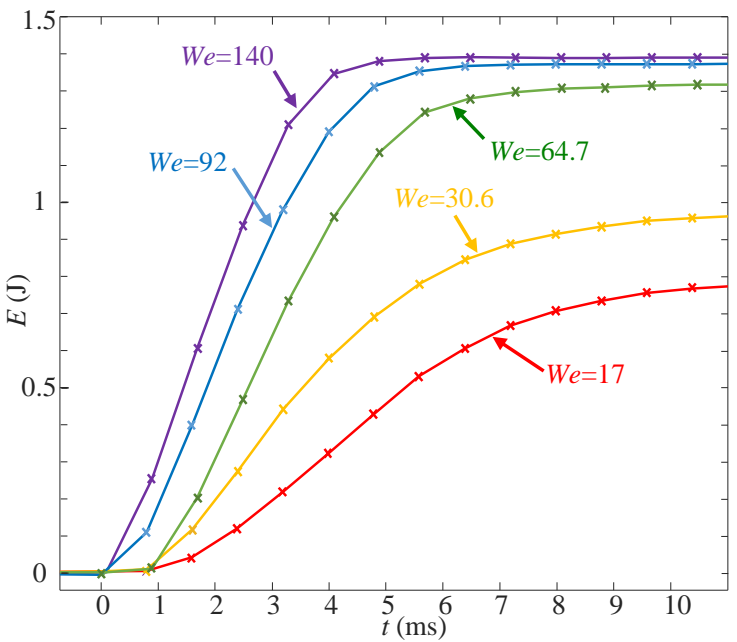

Figure 11. Temporal evolution of the energy removed from the solid surface

The wall heat flux estimated from the IR measurements can be used to evaluate the thickness of the vapor film $h$. Assuming that $h$ is small, even if vapor has a low thermal conductivity, the heat transfer through the vapor film is largely dominated by heat conduction. It can be stated therefore that:

$$
q(r, z=0) \approx \lambda_{v}\left(T_{W}-T_{S A T}\right) / h
$$

where $q(r, z=0)$ is the heat flux extracted at the solid surface, $T_{W}$ the temperature of the solid surface and $T_{S A T}$ the saturation temperature of the liquid equal to $100^{\circ} \mathrm{C}$. The thickness of the vapor film varies spatially and temporally. The thickness of the vapor film $h$ varies in time and space. It takes a minimum value when the heat flux density is maximum. This occurs shortly before the end of the spreading as seen in Figure 9. Figure 12 shows the influence of the Weber number on the minimum thickness of the vapor film $h_{\min }$. As expected, $h_{\min }$ decreases with the impact velocity. The thickness of the vapor film is about 1-2 $\mu \mathrm{m}$, which is typically one order of magnitude lower than the vapor film thickness reported in the literature for sessile droplets (ie. at thermal equilibrium and for $W e=0$ ) [15]. For the high $W e$, the thickness of the vapor film still decreases with $W e$, but very moderately.

Finally, the emphasis was placed onto the comparison between the contributions to the energy heat balance. The heat removed from the wall $E_{\text {solid }}$ and the sensible heat transferred to the liquid $E_{\text {liquid }}$ are determined at the end of the impacts based on the measurements. The difference between these two quantities is related to the energy $E_{\text {vapor }}$ used to evaporate a mass flow $\dot{m}$ of liquid during the impact:

$$
E_{\text {vapor }}=L_{v} \int \dot{m} d t=\frac{1}{1+J a}\left(E_{\text {solid }}-E_{\text {liquid }}\right)
$$

where $J a$ is the Jakob number defined by $J a=H\left(T_{f i l m}\right) / L\left(T_{S A T}\right), H(T)=\rho_{v} C p_{v} T$ et $L(T)=\rho_{v}\left(L_{v}+C p_{v} T\right)$ and $T_{\text {film }}=\left(T_{W}+T_{S A T}\right) / 2$. Here, $J a$ is about equal to 0.35 . In Figure $13, E_{\text {solid }}, E_{\text {liquid }}$ et $E_{\text {vapor }}$ are displayed 
as a function of the Weber number. The value of $E_{\text {solid }}$ and $E_{\text {liquid }}$ are very close whatever the Weber number. Hence, almost all of the energy removed from the wall is used to heat up the liquid. Evaporation typically accounts for less than $10 \%$ of the heat taken from the solid wall. The variations observed for $E_{\text {vapor }}$ in Figure 13 are not really significant because $E_{\text {vapor }}$ compares to the experimental uncertainty estimated at about $0.1 \mathrm{~J}$.

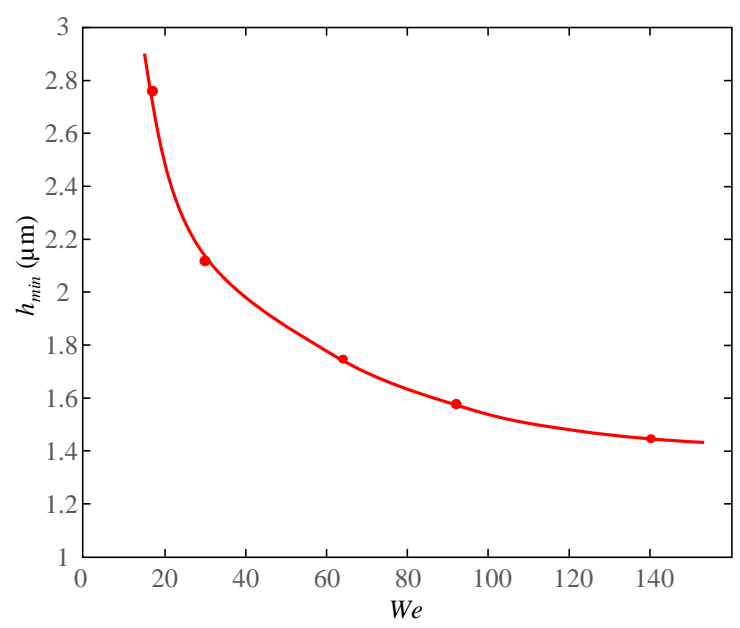

Figure 12. Influence of the Weber number on the minimum thickness of the vapor film

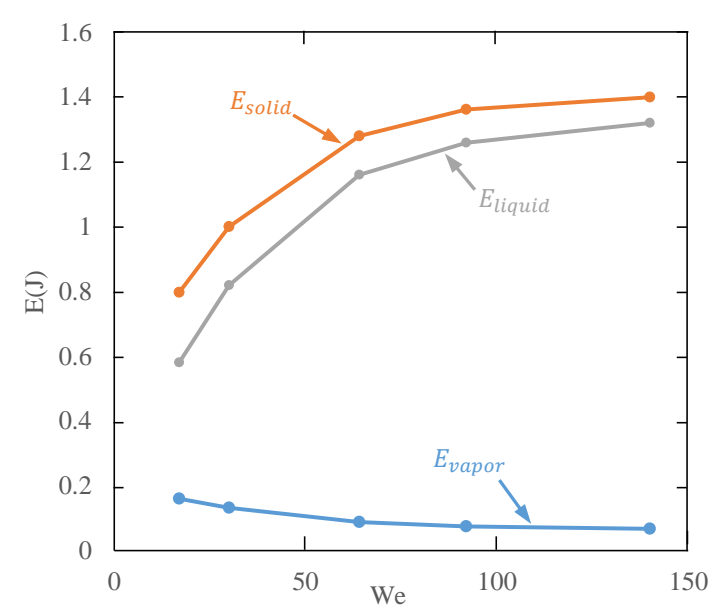

Figure 13. Influence of the Weber number on the heat exchanged between the solid surface, the liquid droplet and the vapor layer

\section{Conclusions}

The heating of the impacting droplet and the cooling of the solid surface in the film boiling regime were quantified accurately using two non-intrusive optical techniques. Measurements show an important effect of the Weber number on the heat transfers. As the Weber number increases, the exchange surface increases and the thickness of the vapor film decreases, which induces an increase in the heat removed from the wall and in the heating of the liquid. However, when increasing the Weber number, the influence of the impact velocity becomes less and less noticeable. There is almost a saturation of the heat extracted from the solid surface in the splashing regime of impact. In the case of the millimeter-sized droplets considered in this study, almost all the cooling of the solid wall results from the sensible heating of the liquid. This shows the great interest in modelling the deformation and the thermal convection within the impacting droplets to predict the intensity of the wall cooling.

\section{Acknowledgements}

The authors acknowledge the financial support of the Lorraine region through the CPER ENERBATIN projet.

\section{References}

[1] Liang, G., and Mudawar, I., 2017. International Journal of Heat and Mass Transfer, 106, pp. 103 - 126.

[2] Bernardin, J. D., and Mudawar, I., 1997. International Journal of Heat and Mass Transfer, 40(11), pp. 2579 2593.

[3] Jung, J., Jeong, S., and Kim, H., 2016. International Journal of Heat and Mass Transfer, 92, pp. $774-783$.

[4] Dunand, P., Castanet, G., Gradeck, M., Maillet, D., and Lemoine, F., 2013. International Journal of Heat and Fluid Flow, 44, pp. 170-180.

[5] Lemoine, F., and Castanet, G., 2013. Experiments in Fluids, 54(7).

[6] Castanet, G., Lienart, T., and Lemoine, F., 2009. International Journal of Heat and Mass Transfer, 52(3-4), pp. 670-679.

[7] Dunand, P., Castanet, G., and Lemoine, F., 2012. Experiments in Fluids, 52(4), pp. 843-856.

[8] Chaze, W., Caballina, O., Castanet, G., and Lemoine, F., 2016. Experiments in Fluids, 57(4), p. 58.

[9] Gradeck, M., Ouattara, J., Rémy, B., and Maillet, D., 2012. Experimental Thermal and Fluid Science, 36, pp. $56-64$.

[10] Castanet, G., Caballina, O., and Lemoine, F., 2015. Physics of Fluids, 27(6), p. 063302.

[11] Thoroddsen, S. T., and Sakakibara, J., 1998. Physics of Fluids, 10(6), pp. 1359-1374.

[12] Mehdizadeh, N. Z., Chandra, S., and Mostaghimi, J., 2004. Journal of Fluid Mechanics, 510, pp. $353-373$.

[13] Culick, F. E. C., 1960. Journal of Applied Physics, 31(6), pp. 1128-1129.

[14] Khavari, M., Sun, C., Lohse, D., and Tran, T., 2015. Soft Matter, 11, pp. 3298-3303.

[15] Celestini, F., Frisch, T., Cohen, A., Raufaste, C., Duchemin, L., and Pomeau, Y., 2014. Physics of Fluids, 26(3), p. 032103. 


\title{
Experimental Study on The Effect of Initial Liquid Droplet Size on The Evaporation in a Heterogeneous Droplet
}

\author{
Kun Hyuk Sung ${ }^{1}$, Jun Seok Nam², Gi-Bae Hong ${ }^{3}$, Hong Sun Ryou*1 \\ ${ }^{1}$ Department of Mechanical Engineering, Chung-ang University, Seoul, South Korea \\ ${ }^{2}$ Research and Developed Laboratory, Korea Fire Institute, Kyounggi-Do, South Korea \\ ${ }^{3}$ School of Mechanical, Automotive and Aeronautical Engineering, \\ Korea National University of Transportation, Chung-ju, South Korea \\ ${ }^{*}$ Corresponding author: cfdmec@cau.ac.kr
}

\begin{abstract}
In the present work, we experimentally investigated the effect of initial liquid droplet size on the evaporation in the heterogeneous droplet. Spherical carbon and water were used for particle and liquid droplet comprising the heterogeneous droplet. four initial droplet volumes of 1,2, 3 and $4 \mu \mathrm{l}$ were considered when the diameter of the particle was $5 \mathrm{~mm}$. The heterogeneous droplet was suspended with a rod at $20 \mathrm{~cm}$ away from the radiator which surface temperature was fixed to $473 \mathrm{~K}$. Ambient temperature and relative humidity remained $296 \mathrm{~K}$ and $40 \%$, respectively, during the experiment.

As the results, the evaporation rate of $4 \mu \mathrm{l}$ case increased about 1.8 times compared with that of $1 \mu \mathrm{l}$ case. The evaporation rate increased almost linearly with the volume ratio, and that is related closely with the contact surface between particle and water droplet. Contact surface area remained almost constantly with time, whereas it increased with the initial volume of water droplet. The energy from radiator can be accumulated at the contact surface at the side of particle, thereby intensifying the evaporation of water droplet because more heat transfers from particle to droplet through the contact surface. Consequently, the initial volume of liquid droplet is one of the influence factors on the evaporation rate in the heterogenous droplet.
\end{abstract}

\section{Keywords}

Contact surface area, Evaporation rate, Heat transfer, Heterogeneous droplet

\section{Introduction}

Drop evaporation is an important topic of interest because it plays a crucial role in many engineering applications such as spray drying, fuel injection into combustion engines, medical care, controlling the deposition of particles on solid surfaces, rapid cooling by drop wise heat exchange and also occurs in natural processes such as rain, fog, dew, snow formation and is used in meteorological estimates [1]. Nowadays, one of the most important directions of development in the field of fire extinguishing technologies is the improvement of heat exchange between extinguishing liquid and combustion products in flame zone and in its close vicinity [2]. Firstly, sprinkler systems are intended to either control the fire or to suppress the fire. For instances, sprinklers are used to control the heat release rate of the fire to prevent building structure collapse, and pre-wet the surrounding combustibles to prevent fire spread. Lastly, water curtain, which is a thermal and smoke control system in a fire hazard, is mainly used to diminish radiative heat transfer from fire and prevent spreading of smoke. In the development and deployment of practical systems, it is very important to understand how the flame extinction characteristics depend on droplet diameter, number density, and overall water loading.

The traditional approach of droplet breakup when spraying extinguishing liquid received a lot of criticisms. In real practice the droplets of extinguishing liquid coalesce during their motion through flames. Moreover, soot particles in fire plume or ceiling jet flow have potential for adhering to the surface of water drops released from a nozzle at ceiling. When size of soot is comparable with that of drop, which is called as a heterogeneous droplet [3], evaporation rate of the droplet steeply increases, then it can cause explosive breakup. These effects complicate the problem of selection of initial droplet sizes and their control during the motion to absorb the fire energy as well as to expel both the oxidant and flammable combustion products. Piskunov, Shcherbinina and Strizhak [4] investigated the features of evaporation, boiling and explosive breakup of water droplets containing a nontransparent inclusion when immersed in gaseous environment at high temperatures corresponding to typical fires. They conducted experiments with droplets containing $1 \mathrm{~mm}$ size non-transparent and non-metallic inclusions. The previous researches $[3,4]$ were mainly focused on the explosive breakup phenomena of the droplet and showed that shape and size of inclusions have an influence on evaporation rate of droplet associated with total evaporation 
time or explosive breakup time in only high temperature range near film boiling regime. In practical engineering applications, it is very important in predicting the beginning and the end of nucleate boiling or film boiling regime at the interface between solid and liquid as well as instantaneous droplet sizes. This is because nucleate vapors on the surface of inclusion dramatically change heat transfer characteristics. For example, in the high temperature or film boiling regime, the quench proceeds rather slowly as liquid-solid contact is minimized by the rapid formation of an insulating vapor blanket at the droplet-solid interface [5]. Therefore, it is firstly necessary for understanding heat transfer phenomena in heterogenous droplet to develop a practical engineering model for evaporation including nucleate/transition/film boiling.

In the present work, we experimentally investigated the effect of initial liquid droplet size on the evaporation in the heterogeneous droplet before nucleate boiling regime as a basic study. Carbon and water were used for particle and liquid droplet comprising the heterogeneous droplet. four initial droplet volumes of $1,2,3$, and $4 \mu$ l were considered when the diameter of the particle was $5 \mathrm{~mm}$. The heterogeneous droplet was suspended with a rod at $20 \mathrm{~cm}$ away from the radiator which surface temperature was fixed to $473 \mathrm{~K}$. Ambient temperature and relative humidity remained $296 \mathrm{~K}$ and $40 \%$, respectively, during the experiment.

\section{Material and methods}

To measure the volume change of the droplet, the experimental system consists of a telocentric lens (NAVITAR) combining two CMOS cameras (SONY, 5 Mega-pixels, $1 \mathrm{fps}$ and SONY, 8 Mega-pixels, 15fps) and multi-arrayed LED lamp (100W) as shown in Figure 1. All captured images were converted the gray scale of pixel intensities to determine the interface among multiphase materials such as water, air and particle as shown in Figure 2. With the captured images, the droplet volume of water volume was calculated by Image-J software using the LB-ADSA (lowbond axisymmetric drop shape analysis) method [6] under the assumption of axisymmetric droplet.

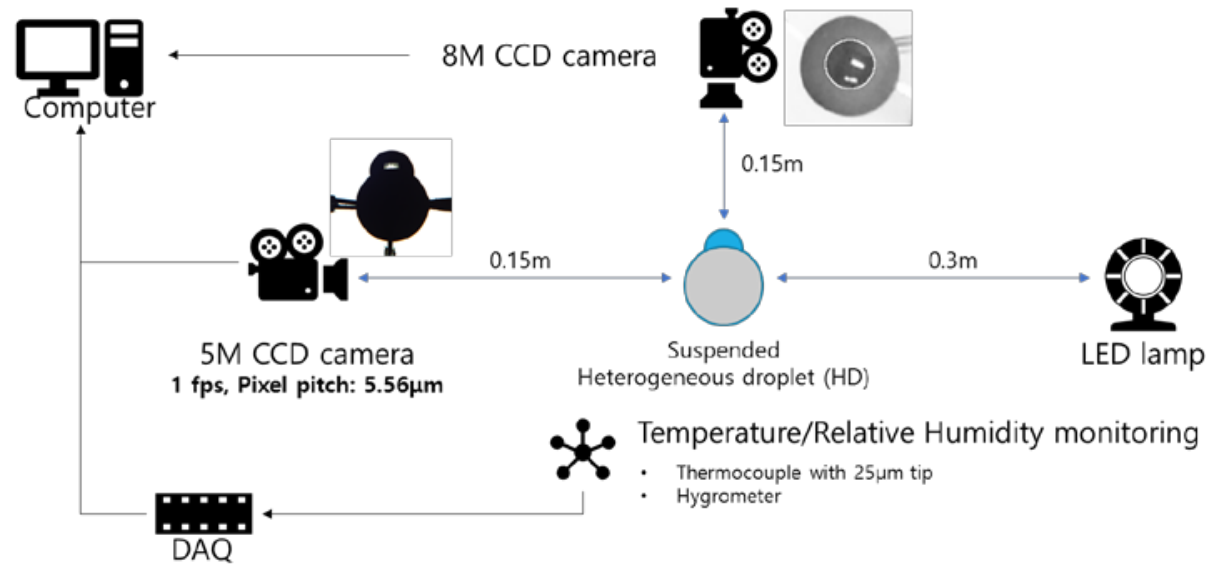

Figure 1. Schematic and picture of experimental apparatus.

Heterogeneous droplet consisted of a particle and a deionized(DI) water droplet. To investigate the effect of initial water droplet size, the diameter of particle was fixed to $5 \mathrm{~mm}$ whereas the volume of water droplet was changed from $1 \mu \mathrm{l}$ and $4 \mu \mathrm{l}$. The spherical particle was manufactured with by a micro milling machine and was made up of graphite. The heterogenous droplet was suspended with a thin rod. Very narrow holes, which diameter and depth are about $0.5 \mathrm{~mm}$, were drilled on the surface of the particle for inserting tips of the rod and thermocouples. Two Ktype thermocouples (measurement range: $200 \sim 1250 \mathrm{~K}$, error $0.4 \%, \mathrm{OMEGA}^{\circledR}$ ) were used to measure the surface temperature of particle and the diameter of tip was $25 \mu \mathrm{m}$. The particle was bonded with the rod by using an adhesive had very lower conductivity not to influence the heat and mass transfer related with evaporation. The radiator consisted of quartz plate (W $\times \mathrm{H} \times \mathrm{D}: 230 \mathrm{~mm} \times 85 \mathrm{~mm} \times 10 \mathrm{~mm}$ ) and resistance coils and was used to heat the heterogenous droplet and it was $20 \mathrm{~cm}$ away from the droplet at the opposite site of the rod. The quartz surface temperature of radiator was adjusted by electrical power controller (Model: MT4Y, Autonics ${ }^{\circledR}$ ) to remain 473 $\mathrm{K}$. The spatial and temporal deviations in the surface were within $\pm 10 \mathrm{~K}$ and $\pm 1 \mathrm{~K}$, respectively, in the steady state. The dosing system of water droplet consisted of pump, syringe and needle. As shown in Figure 2 , the water droplet was placed on the top of the particle as much as a setting volume by using a syringe pump (LSP01-1A, Longerpump $\left.{ }^{\circledR}\right)$ with a syringe (1001TLL, Hamilton $\left.{ }^{\circledR}\right)$. The internal diameter of needle was $0.3 \mathrm{~mm}$. Ambient temperature and relative humidity remained $290 \mathrm{~K}$ and $40 \%$, respectively, during the experiment. For each case, 
the experiments were carried out with five replicates and three data samples were used for the analysis of evaporation after the image processing.

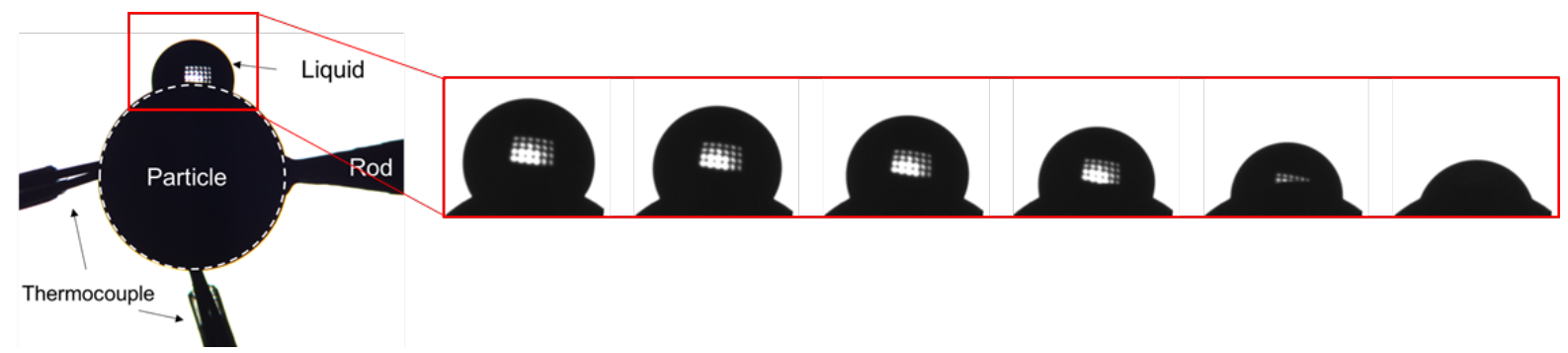

Figure 2. Heterogenous droplet suspended with a rod and converted images for image processing over time.

\section{Results and discussion}

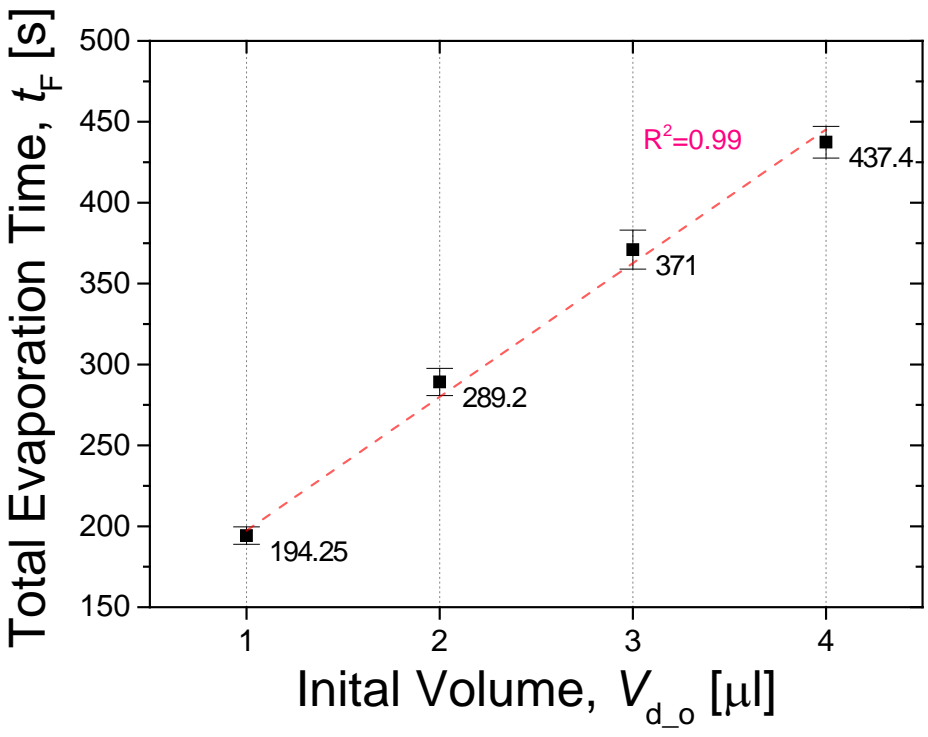

Figure 3. Total evaporation time with the initial volume of water droplet.

Figure 3 shows total evaporation time with the initial volume of water droplet. Total evaporation time $\left(t_{\mathrm{F}}\right)$ linearly increased with the initial volume of water droplet $\left(V_{\mathrm{d}_{\mathrm{o}} \mathrm{o}}\right)$ as shown in Figure 3 . The evaporation time of $4 \mu \mathrm{l}$ case increased about 2.3 times compared with that of $1 \mu$ l case. Also, Figure 4 shows the variation of volume of water droplet with normalized time $\left(t_{\mathrm{n}}\right)$ which was defined as the ratio of any instantaneous time $(\mathrm{t})$ to the total evaporation time $\left(t_{\mathrm{F}}\right)$ in each case. In each point of line in Figure 4. The volume of water droplet linearly decreases after at least $t_{n}=0.2$ in all cases, that is, evaporation process reached a quasi-steady state. 


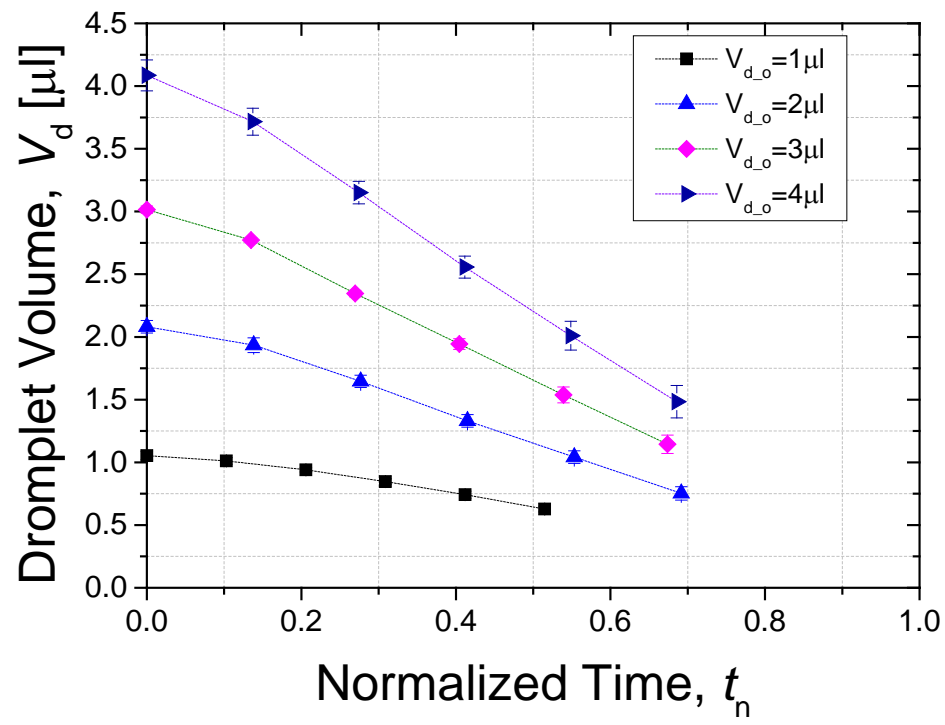

Figure 4. Evolution of volume of water droplet.

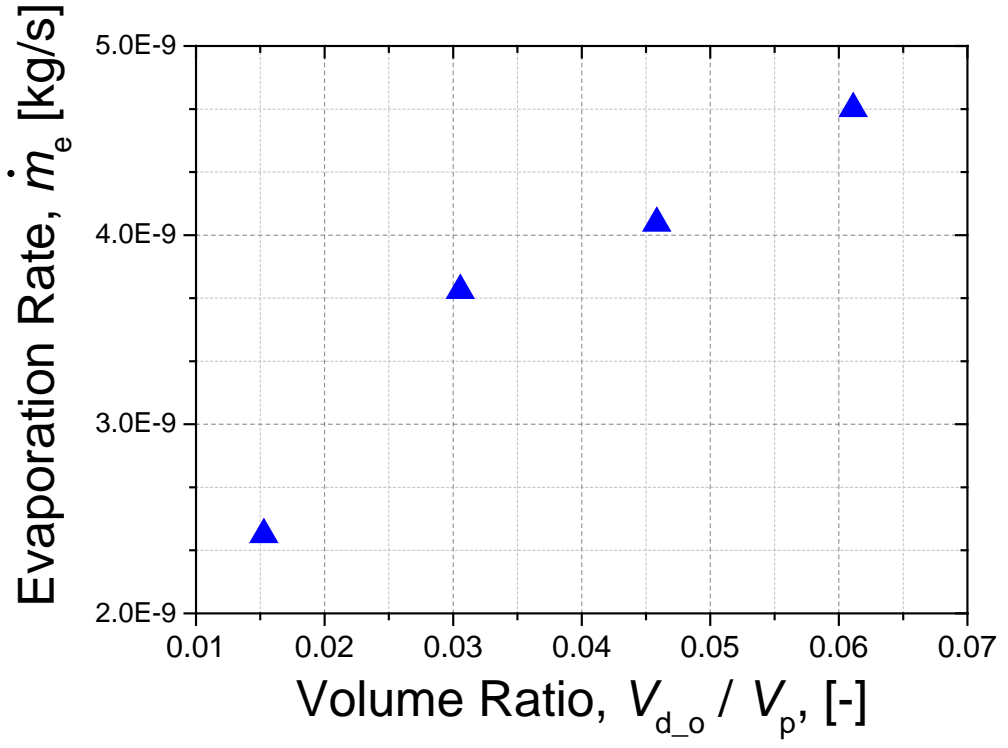

Figure 5. Evaporation rate during the quasi-steady state evaporation.

Figure 5 shows evaporation rate with the volume ratio of the initial volume of water droplet $\left(V_{\mathrm{d}_{\mathrm{O}} \mathrm{o}}\right)$ to the volume of particle $\left(V_{\mathrm{p}}\right)$ during the period of quasi-steady state evaporation. Here, the evaporation rate, $\dot{m}_{e}[\mathrm{~kg} / \mathrm{s}]$, is the product of water density and the decrease rate of droplet volume during the quasi-steady evaporation, i.e., $t_{\mathrm{n}}>0.2$ in Figure 4. The evaporation rate of $4 \mu \mathrm{l}$ case increased about 1.8 times compared with that of $1 \mu \mathrm{l}$ case. The evaporation rate increased almost linearly with the volume ratio, and that is associated with the contact surface area between the droplet and the particle illustrated in Figure 6. In terms of thermal-physical property, the particle is an opaque and non-evaporating material, and it has larger thermal conductivity in comparison with water. The energy from radiator, therefore, can be accumulated at the contact surface at the side of particle, thereby intensifying the evaporation of water droplet.

In this study, contact surface area was calculated by image processing and Figure 7 shows the evolution of contact surface area with normalized time. Contact surface area remained almost constantly with time, whereas it increased with the initial volume of water droplet. Therefore, the initial volume of liquid droplet is one of the influence factors on the evaporation rate in the heterogenous droplet. 


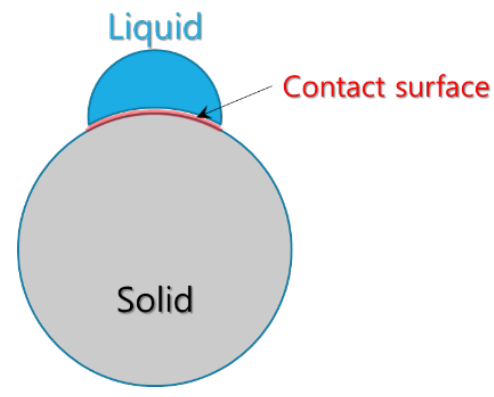

Figure 6. Schematic of the heterogeneous droplet.

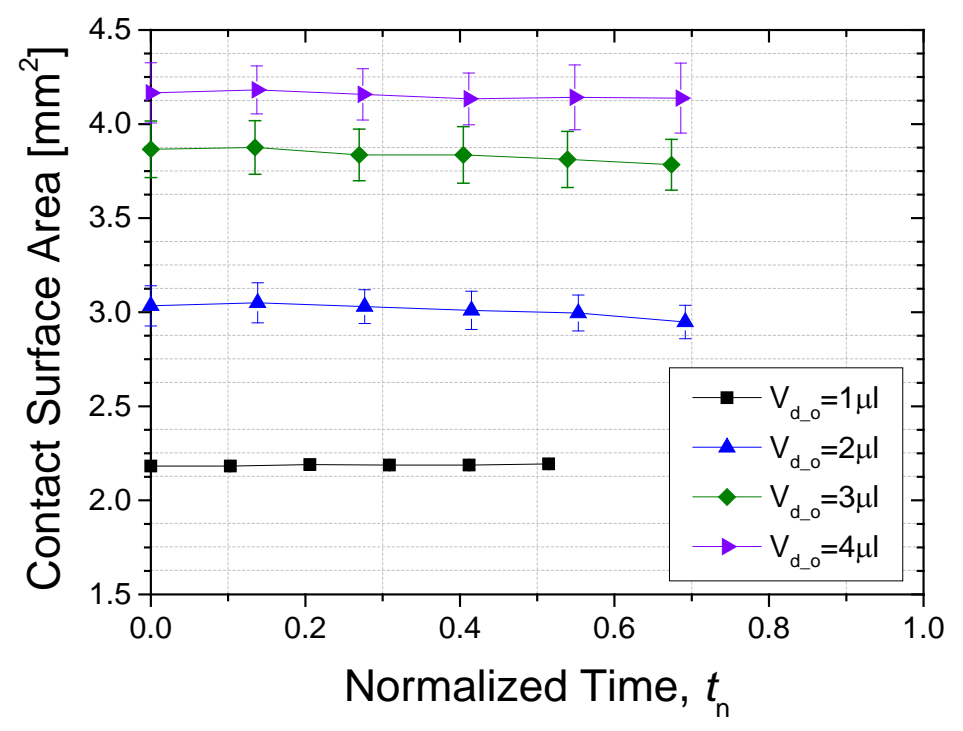

Figure 7. Evolution of contact surface area with normalized time.

\section{Conclusions}

In the present work, we experimentally investigated the effect of initial liquid droplet size on the evaporation in the heterogeneous droplet as a basic study.

- Total evaporation time linearly increased with the initial volume of water droplet. The evaporation time of $4 \mu \mathrm{l}$ case increased about 2.3 times compared with that of $1 \mu \mathrm{l}$ case.

- The evaporation rate of $4 \mu \mathrm{l}$ case increased about 1.8 times compared with that of $1 \mu$ case. The evaporation rate increased almost linearly with the volume ratio.

- $\quad$ Contact surface area remained almost constantly with time, whereas it increased with the initial volume of water droplet. The energy from radiator can be accumulated at the contact surface at the side of particle, thereby intensifying the evaporation of water droplet because more heat transfers from particle to droplet through the contact surface.

Consequently, the initial volume of liquid droplet is one of the influence factors on the evaporation rate in the heterogenous droplet.

\section{Acknowledgements}

This research was supported by the Fire Fighting Safety \& 119 Rescue Technology Research and Development Program funded by the Ministry of Public Safety and Security (NEMA-NG-2014-46) and Research Project of Air Sampling Detector funded by Alllitelife co. Inc.. 


\section{Nomenclature}

$\dot{m}_{e} \quad$ evaporation rate $[\mathrm{kg} / \mathrm{s}]$

$t_{\mathrm{F}} \quad$ total evaporation time [s]

$t_{\mathrm{n}} \quad$ normalized time [-]

$V_{\mathrm{d}} \quad$ volume of water droplet at any instantaneous time $[\mu \mathrm{l}]$

$V_{\mathrm{d}_{-} \mathrm{o}} \quad$ Initial volume of water droplet $[\mu \mathrm{l}]$

$V_{\mathrm{p}} \quad$ volume of particle $[\mu \mathrm{l}]$

\section{References}

[1] Erbil, H. Y., 2012, "Evaporation of pure liquid sessile and spherical suspended drops: a review," Adv Colloid Interface Sci, 170(1-2), pp. 67-86.

[2] Zhou, X., D'Aniello, S. P., and Yu, H.-Z., 2012, "Spray characterization measurements of a pendent fire sprinkler," Fire Safety Journal, 54, pp. 36-48.

[3] Kuznetsov, G. V., Piskunov, M. V., and Strizhak, P. A., 2016, "Evaporation, boiling and explosive breakup of heterogeneous droplet in a high-temperature gas," International Journal of Heat and Mass Transfer, 92, pp. 360369.

[4] Piskunov, M., Shcherbinina, A., and Strizhak, P., 2015, "Analysis of the Water Droplet Evaporation Features with a Solid Nontransparent Inclusion in High-Temperature Gas Environment as a Part of University Research Work," Procedia - Social and Behavioral Sciences, 206, pp. 315-320.

[5] Baumeister, K. J., 1964, "Heat transfer to water droplets on a flat plate in the film boiling regime."

[6] Stalder, A. F., Melchior, T., Müller, M., Sage, D., Blu, T., and Unser, M., 2010, "Low-bond axisymmetric drop shape analysis for surface tension and contact angle measurements of sessile drops," Colloids and Surfaces A: Physicochemical and Engineering Aspects, 364(1), pp. 72-81. 


\title{
Selected Results of the Collaborative Research Center "Droplet Dynamics under Extreme Ambient Conditions" SFB/TRR 75
}

\author{
Cameron Tropea*1, Bernhard Weigand ${ }^{2}$, Kathrin Schulte ${ }^{2}$ \\ ${ }^{2}$ Institute of Aerospace Thermodynamics, University of Stuttgart, Germany \\ ${ }^{1}$ Institute of Fluid Mechanics and Aerodynamics, Techn. Universität Darmstadt, Germany \\ *Corresponding author: ctropea@sla.tu-darmstadt.de
}

\begin{abstract}
The Collaborative Research Center (CRC) SFB-TRR 75 was established in January 2010 to focus on the dynamics of basic drop processes, and in particular on processes involving extreme boundary conditions, for example, near thermodynamic critical conditions, very low temperatures, under strong electric fields or in situations involving extremely large gradients. The CRC is a joint initiative of the University of Stuttgart, the TU Darmstadt and the German Aerospace Center (DLR) in Lampoldshausen, operating with 17 projects structured into three main research areas and involving researchers from numerous faculties: Mathematics, Chemistry, Electrical Engineering, Aerospace Engineering, Mechanical Engineering, Informatics and Computer Sciences. Some of the topics pursued at the CRC include
\end{abstract}

- The behaviour of supercooled and potentially electrified droplets in clouds

- $\quad$ The impact of Supercooled Large Droplets (SLD) on aircraft icing

- The behaviour of strongly electrified drops on insulator surfaces, which can be found on high voltage power lines, affecting the partial discharge behaviour and performance and durability of the insulator.

- Trans-critical injection conditions of fuel with flash boiling in rocket combustion chambers

- Atomization and vaporization of droplets at high pressures and temperature, as occurring in future combustion systems

This article provides an overview of the projects being carried out at the SFB-TRR 75 and highlights scientific results from selected subprojects. The main purpose of the paper is to familiarize colleagues with this extensive and dedicated research effort in the area of drop dynamics and to motivate and initiate future collaboration with others in this field.

Keywords: drop dynamics, extreme boundary conditions, supercooled large droplets, spray combustion, rocket engine sprays, flash boiling.

\section{Introduction}

Drops and drop dynamics are ubiquitous, in nature, e.g. in clouds, fog, rain, or spindrift, and in many engineering systems e.g. in gasoline or Diesel engines, gas turbines, aero-engines, rocket engines, spray cooling processes, chemical reactors, or agricultural sprays. Despite the fact that interest and research into the behaviour of droplets has begun very early, e.g. (Plateau, 1873) (Rayleigh, 1878) many fundamental aspects of droplet behaviour and drop dynamics remain unclear, especially under extreme ambient conditions. Under such extreme ambient conditions, the prediction and simulation of drop behaviour is often unreliable, moreover, standard literature on the topic of drop dynamics, e.g. (Lefebvre, 1989) (Pruppacher \& Klett, 1978) (Frohn \& Roth, 2000) often treat drops under only moderate ambient conditions. Therefore, in the particular area of drops under extreme ambient conditions different topics were identified for further basic research, with the aim to improve our understanding and predictive capabilities of both naturally occurring and engineering systems involving droplets.

Following the insight that complex droplet dynamic processes are determined by the interaction of very fundamental processes, the first 4-year period of the SFB-TRR 75 was dedicated to the behaviour of single droplets. In the current period, which runs from 2014-2017, the physical understanding and the methodology gained in the first phase are applied to small clusters of droplets to better understand their interaction. In the final period of the CRC in the years $2018-2021$, the research will focus on ensembles of drops, or sprays.

The SFB-TRR75 is structured into three main research areas comprising 17 subprojects, listed below together with the responsible researcher(s):

\section{Research Area A: Methods and Fundamentals}

- TP-A1 Interactive visualization of droplet dynamic processes (T. Ertl / F. Sadlo)

- TP-A2 Development of numerical methods for the simulation of compressible droplet dynamic processes under extreme conditions (C.-D. Munz) 
- TP-A3 Analysis and numerics of front and phase field models for droplet dynamics (C. Rohde / V. Schleper)

- TP-A4 Molecular dynamics simulations of droplet evaporation in the non-linear response regime (F. MüllerPlathe)

- TP-A5 Simulation of the mechanical deformation and movement of droplets under the influence of high electric fields (T. Weiland / E. Gjonaj)

- TP-A6 Development and application of new thermodynamic models for interfaces based on classical density functional theory (J. Groß)

- TP-A7 Modelling and simulation of droplet collisions and explosions of droplets at high pressure and far from equilibrium (D. Bothe)

\section{Research Area B: Free Droplets}

- TP-B1 Investigation of the behaviour of supercooled droplets concerning evaporation, condensation and freezing at different boundary conditions (B. Weigand / N. Roth)

- TP-B2 Experimental investigation of droplet evaporation under extreme conditions by temporally highly resolved laser diagnostic methods (G. Lamanna / A. Dreizler)

- TP-B3 Modelling and simulation of droplet evaporation in different gas environments under supercritical conditions (A. Sadiki / J. Janicka)

- TP-B4 Experimental investigation of transient injection phenomena in rocket combusters at vacuum with flash evaporation (M. Oschwald / C. Manfletti)

- TP-B5 Modelling and simulation of the flash evaporation of cryogenic liquids (A. Kronenburg)

\section{Research Area C: Droplets with Wall-Interactions}

- TP-C1 Numerical simulation of the transport processes during drop impingement onto heated walls with special consideration of the evaporating three-phase contact line (T. Gambaryan-Roisman / P. Stephan)

- TP-C2 High resolution measurements of heat transfer during drop impingement onto a heated wall with particularly consideration of evaporation at the three phase contact line (P. Stephan / T. Gambaryan-Roisman)

- TP-C3 Impact of supercooled droplets onto cold surfaces (S. Jakirlic / C. Tropea)

- TP-C4 Interaction of a single drop with a heated wall at high ambient pressures (I. Roisman / C. Tropea)

- TP-C5 Mechanical and electrical phenomena of droplets under the influence of high electric fields (V. Hinrichsen)

In Research Area A numerical and analytical methods are developed which are pre-requisites for conducting the work in the other research areas. Methods for visualization are developed in TP-A1. TP-A2 and TP-A3 are concerned with the development of numerical methods. In TP-A4 basic droplet dynamic processes are investigated by molecular thermodynamics. In TP-A5 simulations of the mechanical deformation and movement of droplets on polymer insulation surfaces with strong electric fields are investigated. TP-A6 investigates the nonequilibrium thermodynamics of interfaces. Here the classical density functional theory is applied to mixtures of polar substances. In TP-A7 the focus is set on droplet collision processes.

In Research Area B droplets free of solid boundaries are investigated. In TP-B1 droplet dynamics problems involving supercooled droplets in clouds and the formation of ice crystals in clouds are investigated. TP-B2 investigates experimentally the evaporation of droplets under extreme thermodynamic conditions. The modelling and simulation of droplet evaporation in a foreign-gas environment under transcritical conditions are investigated in TP-B3, whereas the project TP-B4 investigates flash evaporation for the transient injection of propellant in a rocket combustion chamber. Project TP-B5 focuses on the numerical simulation of flash evaporation processes in strong cooperation with TP-B4.

In Research Area C droplet-wall interactions are investigated. In TP-C1 and TP-C2 special focus is set on the three-phase contact line during droplet-wall interaction. In TP-C3 droplet-wall interactions are investigated for supercooled droplets on cold surfaces and in TP-C4 droplet-wall interaction is investigated for hot surfaces under high ambient pressures.

Results from this CRC have been reported previously in (Weigand \& Tropea 2014, 2015, 2016); the present article presents further results and progress in selected subprojects. 


\section{Electrohydrodyamic simulation of electrically controlled droplet generation (Cooperation TP-A5, TP-B1, TP-B2, TP-C3) Dreizler, Gjonaj, De Gersem, Lamanna, Ouedraogo, Preusch, Steinhausen, Weigand, Weiland}

In this study, the generation of drops from an electrically driven droplet generator is experimentally realized and numerically simulated. An electro hydrodynamic model for the simulation of droplet formation, detachment and motion in the droplet generator is introduced. The numerical approach is based on the coupled solution of the multiphase flow problem with the charge continuity equation. For the latter, a modified convection-conduction model is applied, taking into account conductive, capacitive as well as convective electrical currents in the fluid. This allows for a proper description of charge relaxation phenomena in the moving fluid. In particular, the charge received by the droplet after detachment is an important parameter influencing the droplet dynamics in the test chamber. Simulation results are shown for highly conductive acetone droplets and for low conductivity pentane droplets, respectively. The operation characteristic of the droplet generator is investigated by computing droplet sizes and detachment times with respect to the applied voltage. Further details of this work, in particular about the solved equations and numerical solution procedure, can be found in Ouedraogo et al., 2017. The presentation here will focus only on selected results.

Electric fields can be used for controlled droplet generation, allowing for a reliable injection of liquid samples into a test chamber, even under extreme atmospheric conditions (Weckenmann et al., 2011). The strong electric field applied to a suspended droplet at the tip of a capillary produces the necessary momentum kick, causing the droplet to detach with a given volume and at a well-defined time instant. However, the process of droplet formation, its detachment from the tip of the capillary and, later, the droplet motion in the chamber represent a highly nonlinear and coupled problem, which can be described accurately only by means of numerical simulation. Such a detailed characterization of the droplet dynamics is necessary, in particular, for the prediction of device behaviour for different applied voltages, liquid types, and chamber atmospheric conditions.

(a)

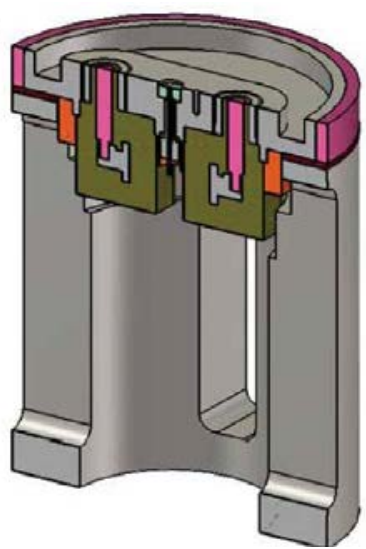

(b)

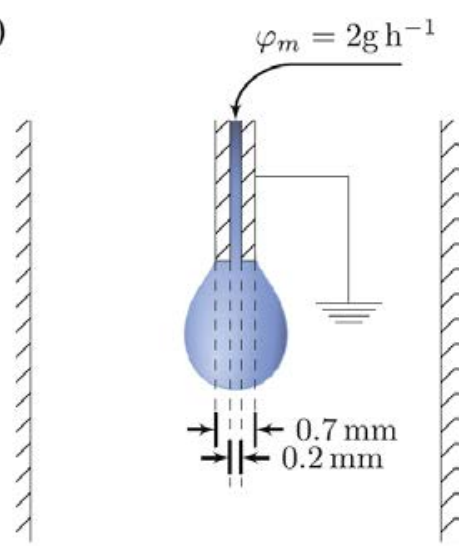

Figure 1: (a) Full model of the droplet generator including capillary, electrodes and test chamber. (b) Schematic view and main parameters of the simulation model

The complete generator setup is shown in Figure 1(a). Its main components are a metallic capillary tube and two high voltage electrodes. The capillary is kept at ground potential, whereas the electrodes are connected to a highvoltage source. In order to prevent electric breakdown, the latter are embedded in two large insulator blocks. Both, the capillary and the electrodes are installed in a high-pressure test chamber capable of sustaining a high pressure, high temperature gaseous environment. The geometry of the droplet generator is not quite axisymmetric. Nevertheless, the electric field is nearly axisymmetric near the capillary tip. This is why a $2 \mathrm{D}$-simulation approach is used.

Observations show that droplet detachment time and charging behaviour, as well as the flow dynamics after detachment depend strongly on the electrical properties of the liquid phase. This is due to the modification of the local electric field pattern by the droplet. This is illustrated in Figure 2 , where the electric field and force distribution is shown for a highly conductive acetone and for a low conductivity pentane droplet. In the low conductivity case, electric fields penetrate much deeper into the liquid, featuring a clear field singularity at the tip of the capillary. Contrary to this, the electric field within conductive acetone droplets nearly vanishes. Furthermore, the different electric properties of the two liquids result in a substantially different distribution of the electric force density on the droplet surface. In order to emphasize the different droplet detachment and charging behaviour these two cases were analysed by separate numerical experiments. In the following, only the results of the n-pentane drop generation will be presented. 

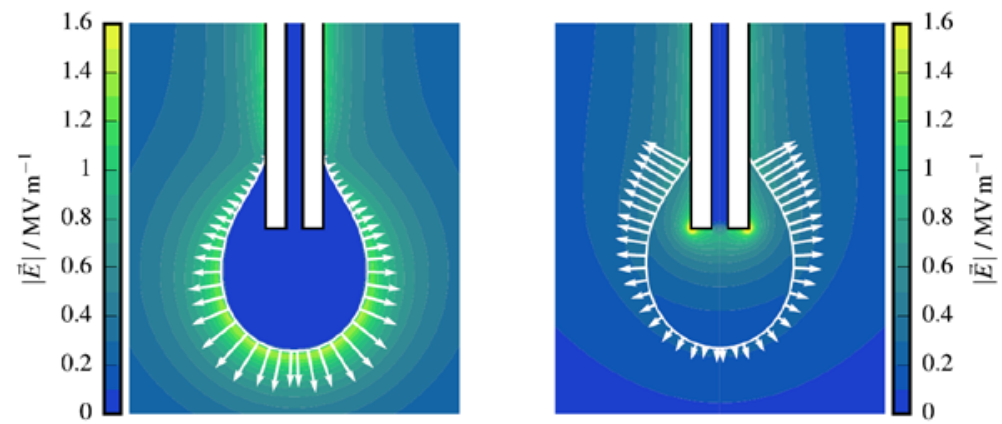

Figure 2: (a) Electric field and surface force distributions for pending (static) droplets assuming different electric properties of the liquid. (a): acetone (high conductivity) droplet. (b): n-pentane (low conductivity) droplet.

The assumed electric conductivity of $n$-pentane is $20 \mathrm{pS} / \mathrm{m}$, corresponding to a charge relaxation time of $\sim 1 \mathrm{~s}$. Since this relaxation time is much larger than a typical voltage pulse duration of $\sim 30 \mathrm{~ms}$, pentane droplets are always electrically charged. The results of the pentane droplet simulation are shown in Figure 3 . The test chamber is at temperature $27^{\circ} \mathrm{C}$ and pressure 1 bar. A voltage pulse of $2.5 \mathrm{kV}$ and duration $26.5 \mathrm{~ms}$ is applied. The static contact angles were estimated to $\theta_{\mathrm{adv}}=10^{\circ}$ and $\theta_{\mathrm{rec}}=20^{\circ}$, respectively. As seen in Figure 3, the agreement between simulation and experiment is nearly perfect. In particular, the liquid thread developing at detachment time (last frame in Figure 3) very closely resembles the experimentally recorded image. Furthermore, the simulation reveals a different behaviour in the n-pentane case compared to the acetone one. As seen in frames 3 to 4 of the figure (at times $40 \mathrm{~ms}$ to $75 \mathrm{~ms}$ ), the droplet is initially pushed up in the direction of the capillary when the external voltage is applied. When the voltage is switched off, the uncompensated weight of the droplet causes it to move down again and, eventually, detach. This is a completely different detachment mechanism than observed in the case of acetone droplets. It can be explained with the low electrical conductivity of $n$-pentane. In this case, electric fields penetrate deep into the liquid phase (see also Figure 2). Thus, electric charges are induced on the droplet surface as well as within the droplet and on the capillary tip. With the electric fields being concentrated close to the capillary tip in the upper half of the droplet, the effective electric polarization force points upwards, causing the droplet to ascend. This situation, however, may change again when the droplet bottom approaches the capillary tip. Thus, as long as the external voltage is not switched off, the droplet will perform oscillations around the capillary tip and may never detach.

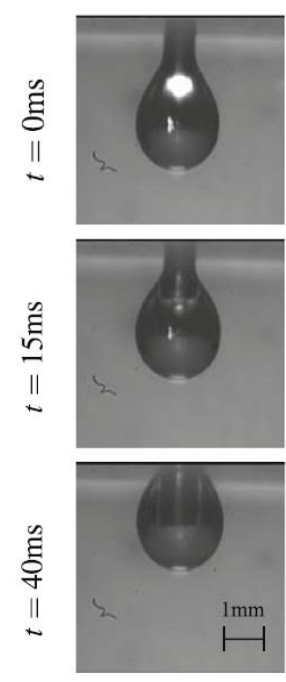

experiment
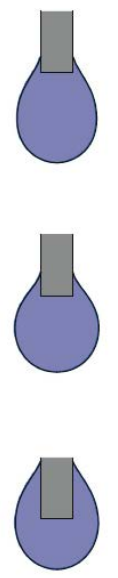

simulation
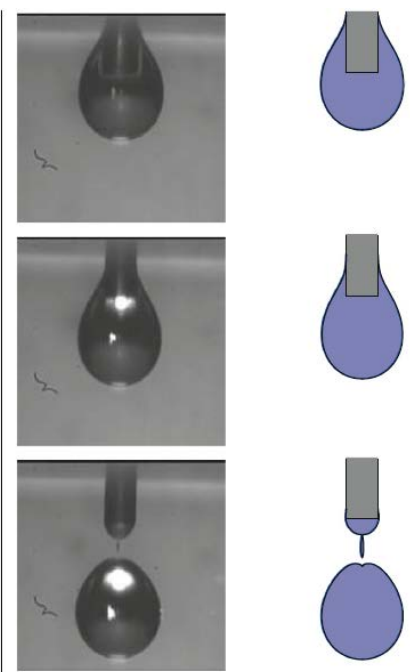

experiment
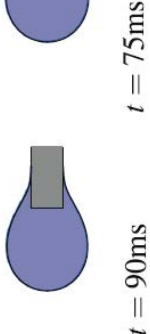

$\frac{2}{n}$

ฏู

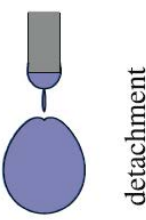

simulation

Figure 3: Pentane droplet dynamics: comparison between simulation and experiment for the droplet shape in the generator at different time instants during the detachment process.

In summary, the droplet charging effect is demonstrated for pentane droplets as well as for acetone droplets under long voltage pulses. This investigation shows that due to the very different relaxation times, the charging behaviour of the two liquids is very different. This behaviour is closely related to the drop dynamics in the generator. In particular, the detachment mechanisms for acetone and pentane droplets are completely different due to their electric properties. For low conductivity (pentane) droplets, the detachment is primarily due to the uncompensated droplet weight, which dominates droplet dynamics only after the generator voltage is switched off. 


\section{Effect of nano-textured heater surfaces on evaporation at a single meniscus (TP-C1, TP-C2) Fischer, Gambaryan-Roisman, Sahu, Sinha-Ray, Stephan, Yarin}

Nanofiber coatings have shown a unique potential for heat transfer enhancement during drop impact cooling, nucleate boiling and flow boiling. In order to gain insight into the mechanisms of heat transfer enhancement invoked by the nanofiber coating, the evaporation of liquid in the vicinity of an apparent contact line of a single meniscus, where the liquid-vapor interface meets the nanotextured substrate, has been investigated. Experiments have been performed for stationary, advancing and receding menisci. It has been found that the local heat flux near a stationary apparent contact line increases by approximately $60 \%$ in the presence of nanofiber coating. The receding meniscus leaves behind an extended region, in which the pores within the nano-textured mat are fully or partially filled with evaporating liquid, contributing significantly to heat transfer enhancement. These phenomena can be attributed to very strong capillary forces acting within the porous media and retaining the cooling liquid in contact with the heater surface. Further details of this work can be found in Fischer et al., 2017. The presentation here will focus only on selected results.

Increasing heat release from electronic devices and miniaturization of such devices pose serious challenges for heat removal from the heated surfaces. One of the promising and low-cost methods of surface modification is coating of a high-powered substrate with electrospun nanofibers, which has shown a significant increase in cooling rate during drop impact cooling, pool boiling and flow boiling in minichannels. The nanostructured surfaces are also capable of suppressing the Leidenfrost effect when coolant drops impact onto a high-temperature surface. The observed heat transfer enhancement during drop impact onto nanofiber mats has been attributed to two distinct phenomena: to hydrodynamic focusing,which prevents the liquid splashing after drop impact, and to imbibition of the liquid into the pores after the initial spreading phase. The imbibition of liquid into the porous structure leads to a two-order of magnitude increase of the substrate area available for evaporative cooling in comparison to the drop impact onto an uncoated substrate.

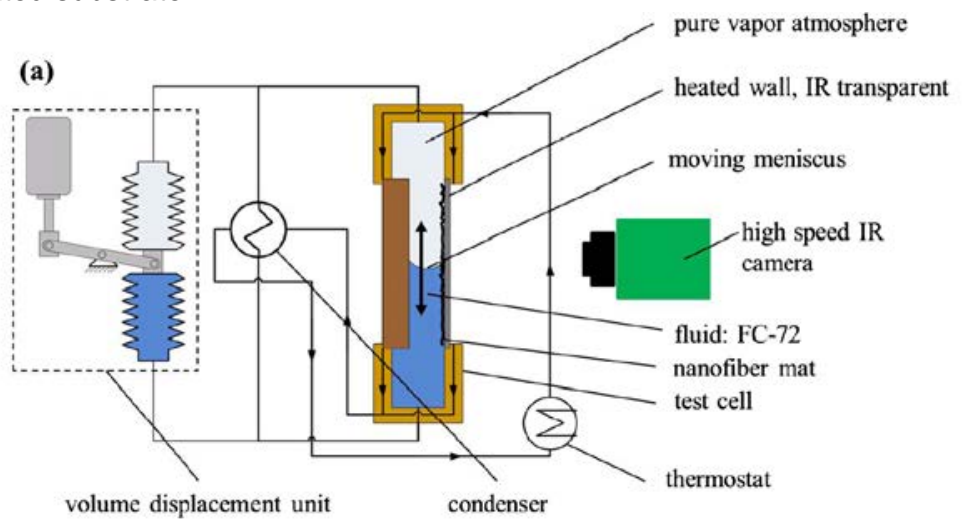

(b)

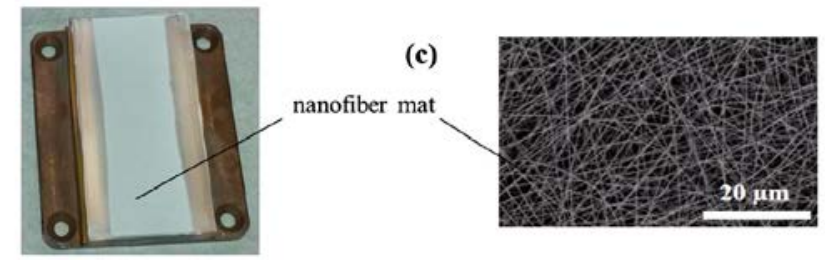

Figure 4: Experimental setup. (a) capillary slot apparatus. (b) target surface. (c) nanofiber mat.

A series of experiments has been performed using the experimental setup described in Fischer et al. (2015). The experimental setup is schematically shown in Figure 4a. A single capillary slot with a width of $1.4 \mathrm{~mm}$ is formed between a polished copper wall kept at a constant temperature and a heater wall, which consists of a $4 \mathrm{~mm}$ thick infrared-transparent $\mathrm{CaF}_{2}$ glass substrate with a three-layer coating. The first coating layer is a CrN black layer, enhancing the surface emissivity, and the second layer is a pure chromium layer acting as a resistance heater. Each of these two layers has a thickness of approximately $400 \mathrm{~nm}$. The third layer consists of nanofibers formed by electrospinning of $9 \mathrm{wt} \%$ Polyacrylonitrile solution (PAN; Mw = $150 \mathrm{kDa}$ ) in N,N-dimethylformamide (DMF). The nanofibers were electrospun for $60 \mathrm{~s}$ onto the chromium-coated substrate (see Figure $4 \mathrm{~b}$ ). The nanofiber mat thickness was 50-70 $\mu \mathrm{m}$, and the fiber diameter varied in the range 200-300 nm. The SEM image of the nanofiber mat deposited over the glass substrate is depicted in Figure 4c. To improve the adhesion of the nanofiber mat to the glass substrate and prevent its delamination, the edges of the nanofiber mat were wetted with ethanol at the sidewalls. 
The temporal evolution of temperature at the backside of the black layer was recorded by an infrared (IR) camera with a frame rate of $1000 \mathrm{~Hz}$, spatial resolution of $29.27 \mu \mathrm{m} /$ pixel and a field of view of 224 pixel x 224 pixel. The IR camera was calibrated in situ. The two-dimensional temperature distribution was then used for computation of the time-dependent distribution of the heat flux transferred from the heater surface to the fluid (liquid or vapour). The calibration process and the method for determination of the local heat flux are described in Fischer et al. (2015). The quantitative difference in the heat flux distribution for a non-moving meniscus is quantified in Figure 5, in which the heat flux line profiles for non-moving menisci for the plain chromium surface and chromium surface with nanofibers are shown as functions of the vertical coordinate. In this diagram, the coordinate origin has been placed at the position of the maximal heat flux. The data have been spatially averaged over 184 pixel rows and time-averaged over 250 frames. The maximal heat flux on the nanofiber-coated surface exceeds the maximal heat flux on the plain chromium surface by approximately $60 \%$. In addition, the region of the higher heat flux is about $8 \%$ wider in the case of the coated surface than for plain chromium surfaces. Both results indicate a significant heat transfer enhancement due to the nano-textured heater surface.

(a)

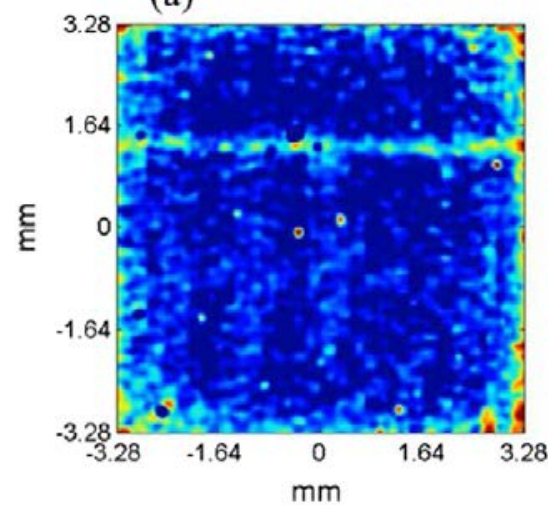

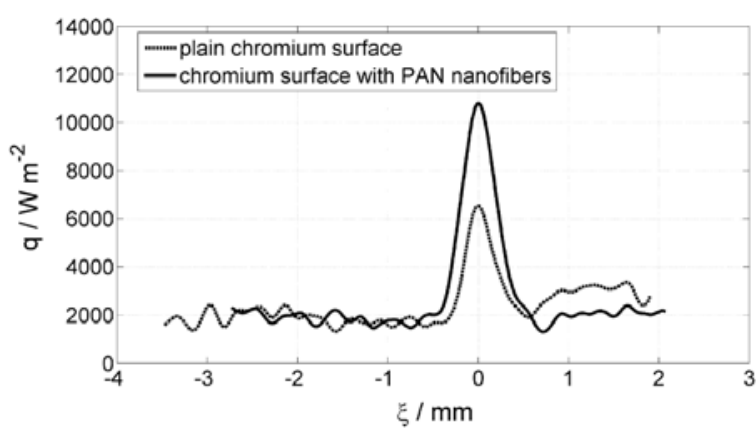

Figure 5: Heat flux profiles for a non-moving liquid-vapour interface at the saturation temperature $T_{s}=53.6 \pm 0.6^{\circ} \mathrm{C}$ and the average wall superheat $\Delta T_{w}=4.4 \pm 0.2 \mathrm{~K}$ (b)

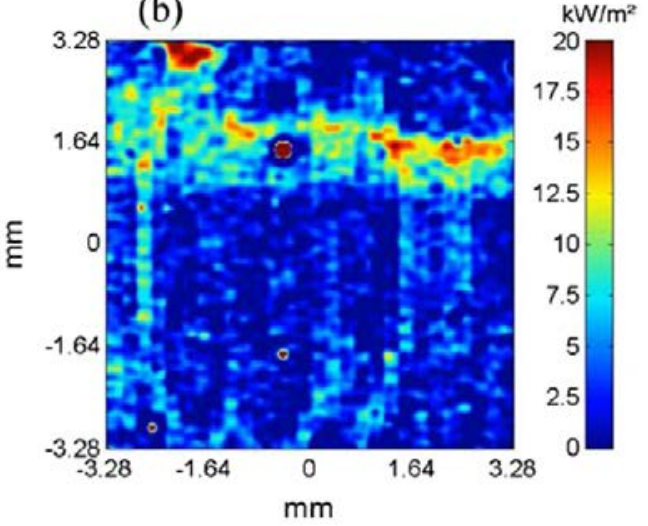

Figure 6: Heat flux fields for a receding meniscus. (a) Plain chromium surface; (b) chromium surface coated with PAN nanofiber mat. The meniscus receding velocity in both cases is $10 \mathrm{~mm} / \mathrm{s}$. The saturation temperature is $T_{s}=$ $52.9 \pm 0.6^{\circ} \mathrm{C}$ and the average wall superheat $\Delta T_{w}=3.6 \pm 0.2 \mathrm{~K}$.

The two-dimensional heat flux distribution at the solid-fluid interface for the receding meniscus is shown in Figure 6. If the substrate is not coated with nanofibers, the region of high heat flux is sharply localized around the apparent threephase contact line, showing qualitatively the same behaviour as for the non-moving meniscus. In contrast, if the substrate is coated, the region of high heat flux is distributed over an extended area. This effect is illustrated in Figure 7, showing the heat flux profiles at different time instants. In this figure, the data for each time instant have been averaged over 184 pixel rows. It is clearly seen that the region of high heat flux having a length of about 1-1.5 mm moves with approximately constant velocity in the direction of

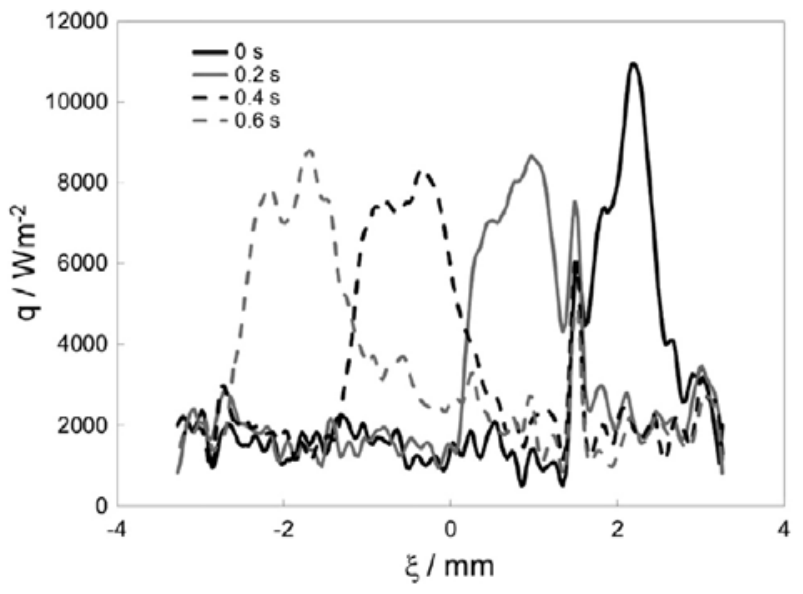

Figure 7: Heat flux profiles at different time instances for a receding meniscus on a chromium surface coated with PAN nanofiber mat. The saturation temperature $T_{s}=52.9 \pm 0.6^{\circ} \mathrm{C}$ and the average wall superheat $\Delta T_{w}=3.6 \pm 0.2 \mathrm{~K}$. The meniscus receding velocity is $10 \mathrm{~mm} / \mathrm{s}$. 
receding motion of the meniscus. It can be suggested that the receding meniscus leaves behind a region, in which the pores within the mat are fully or partially filled with the evaporating liquid. This effect is caused by strong capillary forces exerted by the porous structure and by the viscous forces decelerating the dewetting flow.

Evaporation of liquid at single non-moving, receding and advancing menisci on a nanofiber-coated heater surface has been investigated experimentally. The heat flux distribution has been determined from the temperature field measured by infrared thermography. We have shown that the evaporation near a non-moving meniscus in the presence of nanofibers results in the enhancement of maximal heat flux transferred from the heater by about $60 \%$ and in an increased length of high heat flux area by about $8 \%$. We have confirmed experimentally that the receding meniscus on a coated heater surface leaves behind a broad wetted region. The deposited liquid layer evaporates and thus significantly contributes to higher heat transfer rate. The behaviour of a single evaporating meniscus formed near a heater with nanofiber coating explains the mechanisms of heat transfer enhancement recently observed for two-phase flows on heated nanofiber-coated surfaces.

\section{Transient effects in ice nucleation of a water drop impacting onto a cold substrate [13] (TP-C3) Schremb, Roisman, Tropea}

The impact of supercooled water drops and subsequent icing of the impact surface is a severe hazard for transportation such as air traffic, shipping and road traffic, but is also a frequent problem for power supply systems and wind turbines. It is a highly complex process comprising several physical mechanisms from hydrodynamics and thermodynamics. Drop impact may be accompanied by heat transfer due to non-isothermal drop impact [14], is followed by nucleation of the liquid [13] and finally results in solidification of the liquid which is potentially influenced by the solid wall [15]. Due to its stochastic nature based on Brownian motion of molecules, nucleation is the most complex process involved, and is influenced by many different parameters such as the liquid temperature, the impact conditions, fluid flow and heat transfer during impact, the impact surface morphology, chemical properties of the impinging drop, etc.

So far, nucleation of supercooled water has only been investigated for simple configurations such as a liquid at rest, or in a defined shear flow or temperature gradient. Within the CRC, we've examined the stochastic nature of nucleation for the first time in the context of the complexity of a drop impact. Therefore, impact experiments have been performed numerous times under constant conditions to allow statistical analysis. It has been shown that in contrast to the nucleation rate of sessile drops, the nucleation rate during drop impact is not constant in time. It is the highest directly after impact and decreases with time. Based on a statistical nucleation model derived from Poisson statistics, the average number of nucleation sites per unit area on the wetted surface has been calculated. As shown in Fig. 8 for the case of inclined drop impact onto polished aluminum (8a) and normal impact onto sandblasted glass $(9 \mathrm{~b})$, the number of nucleation sites per unit area of the wetted surface $\left(\lambda_{s}\right)$ increases with time. However, the rate of increase of the nucleation rate decreases with time, although classical nucleation theory predicts constant nucleation rates for constant conditions.

a)

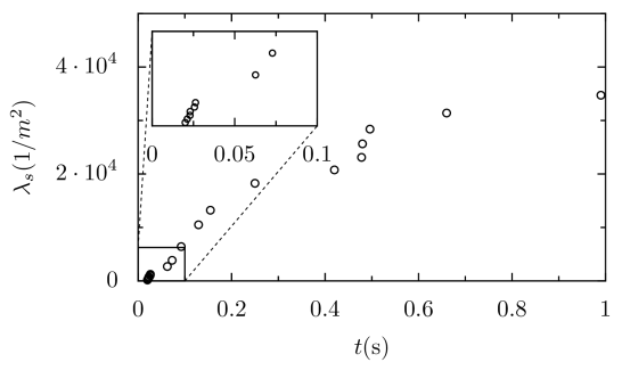

b)

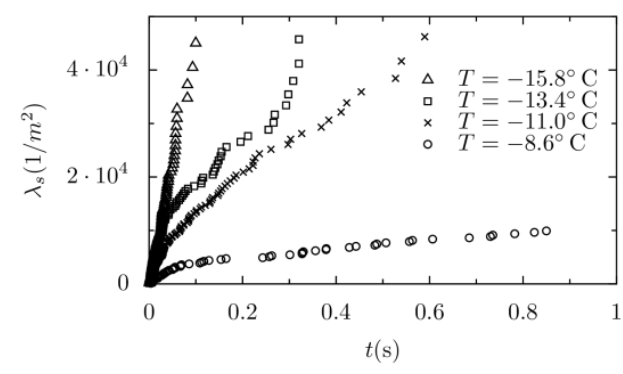

Figure 10. Evolution of the average number of nucleation sites per unit area, $\lambda_{s}$, during drop impact. a) Impact of water drops at $14.3^{\circ} \mathrm{C}$ onto polished aluminum at $-17.0^{\circ} \mathrm{C}$. The drop diameter, impact velocity and impact angle are $3.09 \mathrm{~mm}, 4.09 \mathrm{~m} / \mathrm{s}$ and $30^{\circ}$, respectively. b) Normal impact of supercooled water drops onto a sandblasted glass surface at the same temperature as the drops. The drop diameter and impact velocity are $3.2 \mathrm{~mm}$ and $2.2 \mathrm{~m} / \mathrm{s}$, respectively.

The reason for this transient behavior of nucleation has been found by a comparison of the time scales of the physical processes involved. While all other processes take place on a time scale much smaller than the time during which nucleation significantly changes, only the dissolving of air bubbles, which are commonly known to serve as nucleation sites, may be identified as relevant for a change of the nucleation rate. These air bubbles are entrapped during spreading over the surface and their size is of the order of the size of the surface roughness elements. To proof this hypothesis, experiments have been repeated with degassed water drops. In the case of an impact of degassed water drops, air bubbles entrapped during impact are dissolved much faster than in the case of nondegassed drops, as shown in Fig. 9 a) and b) for spread drops 40 ms after normal impact onto sandblasted glass. Therefore, less nucleation sites are available in the case of degassed water, resulting in a lower nucleation rate and 
a slower decrease of the relative number of liquid drops over time, which is the average probability of a drop to be liquid, as shown in Fig. 9 c).
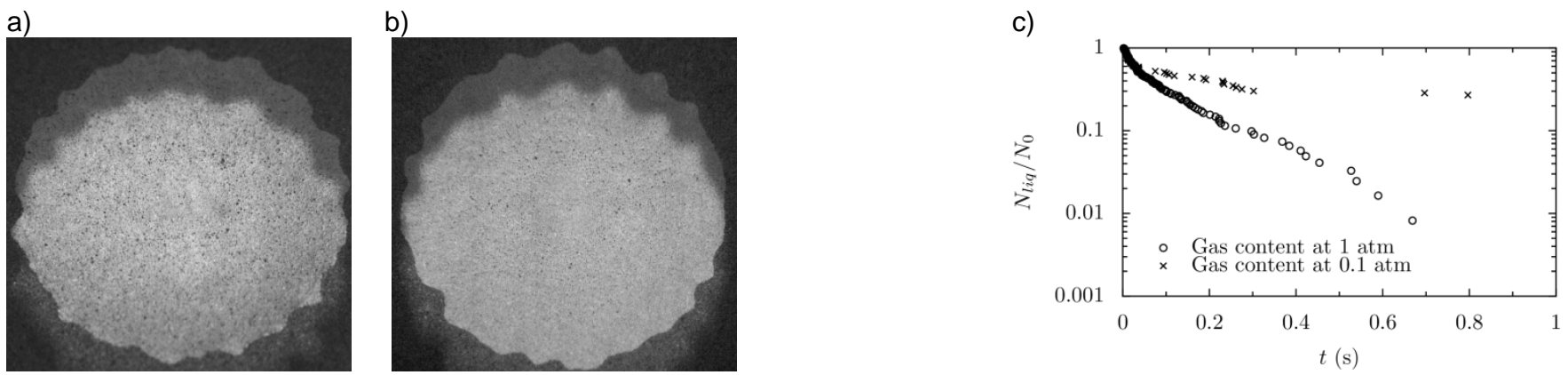

Figure 11. Influence of the liquid's gas content on nucleation of supercooled water drops after normal impact onto a sandblasted glass surface. The drop diameter is $3.2 \mathrm{~mm}$, the impact velocity is $2.2 \mathrm{~m} / \mathrm{s}$ and both the drops and the surface are at $-11^{\circ} \mathrm{C}$. a) Gas bubbles entrapped during drop spreading over the rough surface. Gas content according to saturation at $1 \mathrm{~atm}$. b) Less gas bubbles due to increased dissolving of bubbles in the case of degassed drops. Gas content according to saturation at $0.1 \mathrm{~atm}$. c) Temporal evolution of the relative number of liquid drops $N_{\text {liq }} / N_{0}$.

\section{Conclusions}

This paper has shown the structure of and the work carried out in the Collaborative Research Center SFB-TRR 75: "Droplet Dynamics under Extreme Ambient Conditions". The work in the current funding period of this CRC focuses on single drops and small groups of drops. Future work will consider large number of drops and in many instances the behaviour and application of sprays under extreme ambient conditions. Also further projects involving industrial participation are planned.

\section{Acknowledgements}

The members of the SFB-TRR 75 would like to thank the Deutsche Forschungsgemeinschaft (DFG) for the financial support of the SFB-TRR 75 .

\section{References}

[1] Plateau, J.A.F., Statique. Gauthier \& Villars, Paris (1873).

[2] Rayleigh, Lord J.S.W., Proc. London Math. Soc. 10, pp. 4-13 (1878).

[3] Pruppacher, H.R. and Klett, J.D., Microphysics of Clouds and Precipitation. Reidel Publ. , Dordrecht (1978).

[4] Lefebvre, A.H., Atomization and Sprays. Combustion: An International Series. Hemisphere Publishing Corporation, New York (1989).

[5] A. Frohn, N. Roth., Dynamics of Drops. Springer-Verlag, Heidelberg (2000).

[6] Weigand, B. Tropea, C., and Birkefeld, A., ILASS-Europe 2014, Bremen, Germany (2014)

[7] Tropea, C., and Weigand, B., ICLASS 2015, 13th Int. Conf. on Liquid Atomization and Spray Systems, Tainan, Taiwan (2015)

[8] Weigand, B., Tropea, C. and Schulte, K., ILASS-Europe, Brighton, UK (2016)

[9] Ouedraogo, Y., Gjonaj, E., Weiland, T., De Gersem, H., Steinhausen, C., Lamanna, G., Weigand, B., Preusche, A., Dreizler, A. and Schremb, M. Int. J. Heat and Fluid Flow 64:120-128 (2017).

[10] Weckenmann, E., Bork, B., Oldenhof, E., Lamanna, G., Weigand, B., Boehm, B., Dreizler, A. Zeitschrift für Phys. Chemie 225:1417-1431 (2011).

[11] Fischer, S., Sahu, R.P., Sinha-Ray, S., Yarin, A.L., Gambaryan-Roisman, T. and Stephan, P. Int. J. Heat and Mass Transfer 108:2444-2450 (2017).

[12] Fischer, S., Gambaryan-Roisman, T. and Stephan, P. Int. J. Heat Mass Transfer 88: 346-356 (2015)

[13] Schremb, M., Roisman, I. V. and Tropea, C. Phys Rev E 95: 022805 (2017).

[14] Schremb, M., Borchert, S., Berberovic, E., Jakirlic, S., Roisman, I.V. and Tropea, C. Int. J. Heat and Mass Transfer to appear (2017).

[15] Schremb, M. and Tropea, C. Phys Rev E 94: 052804 (2016). 


\title{
Experimental and Numerical Study on Sensible Heat Transfer at Droplet/Wall Interactions
}

\author{
E. Teodori ${ }^{1}$, P. Pontes ${ }^{1}$, A. S. Moita*1, A. L. N. Moreira ${ }^{1}$, A. Georgoulas ${ }^{2}$, M. Marengo ${ }^{2}$ \\ ${ }^{1} \mathrm{IN}+$ - Center for Innovation, Technology and Policy Research, Instituto Superior Técnico, \\ Universidade de Lisboa, Lisbon, Portugal \\ ${ }^{2}$ Advanced Engineering Centre, School of Computing, Engineering and Mathematics, \\ Cockcroft Building, Lewes Road, University of Brighton, Brighton, UK \\ *Corresponding author: anamoita@tecnico.ulisboa.pt
}

\begin{abstract}
The present study addresses a detailed experimental and numerical investigation on the impact of water droplets on smooth heated surfaces. High-speed infrared thermography is combined with high-speed imaging to couple the heat transfer and fluid dynamic processes occurring at droplet impact. Droplet spreading (e.g. spreading ratio) and detailed surface temperature fields are then evaluated in time and compared with the numerically predicted results. The numerical reproduction of the phenomena was conducted using an enhanced version of a VOFbased solver of OpenFOAM previously developed, which was further modified to account for conjugate heat transfer between the solid and fluid domains, focusing only on the sensible heat removed during droplet spreading. An excellent agreement is observed between the temporal evolution of the experimentally measured and the numerically predicted spreading factors (differences between the experimental and numerical values were always lower than $3.4 \%$ ). The numerical and experimental dimensionless surface temperature profiles along the droplet radius were also in good agreement, depicting a maximum difference of 0.19 . Deeper analysis coupling fluid dynamics and heat transfer processes was also performed, evidencing a strong correlation between maximum and minimum temperature values and heat transfer coefficients with the vorticity fields in the lamella, which lead to particular mixing processes in the boundary layer region. The correlation between the resulted temperature fields and the droplet dynamics was obtained by assuming a relation between the vorticity and the local heat transfer coefficient, in the first fluid cell i.e. near the liquid-solid interface. The two measured fields revealed that local maxima and minima in the vorticity corresponded to spatially shifted local minima and maxima in the heat transfer coefficient, at all stages of the droplet spreading. This was particularly clear in the rim region, which therefore should be considered in future droplet spreading models.
\end{abstract}

\section{Keywords}

Droplet impact, smooth heated surface, high-speed infrared thermography, VOF, vorticity.

\section{Introduction}

Understanding the fluid dynamic and heat transfer mechanisms of droplet impact on heated surfaces is relevant for a wide range of applications, from fire sprinklers to cooling applications. A popular solution for microprocessors cooling is based on spray impingement [1-2]. The elementary representation of a spray composed by arrays of single droplets impacting onto a heated surface is not straightforward in many of the aforementioned applications, but the complexity of the observed phenomena relays on the study of single droplet impacts to understand the basic governing processes. Such approximation is not so far from the real systems for microelectronics cooling, which actually deal with single droplets or with very sparse sprays $[1,3]$. In many of these applications, liquid phase change is promoted to take advantage of the latent heat of evaporation. However, efficient cooling can be obtained only from sensible heat [4]. Although droplet spreading on heated surfaces has been extensively studied in the literature [4-6], the complex coupling between the fluid dynamics and the heat transfer processes has not yet been completely described. An important limitation in many of the aforementioned studies is that they quantify the heat transfer mainly based on surface temperature measurements at the centre of the impact to the surface, using thermocouples. An alternative approach has been followed more recently by some authors who characterize the spatial and temporal evolution of the temperature field on the surface and/or in the droplet during spreading [7-9]. The resulting data, when carefully processed provide unique information, important to validate more complex numerical models of the droplet/wall interaction process.

In fact, following the pioneering work of [10], who proposed the "Marker and Cell" (MAC) finite difference method to solve the Navier-Stokes equations, other methods have been used to solve numerically the problem of drop impact onto heated surfaces such as the Lagrangian approach [11], the immersed boundary method (IBM) [12] and the Level Set (LS) [13]. In [13], the authors highlight different regions of heat flux along the radial direction of the impacting surface, which can be related with the flow in the lamella. They report that a dip in the heat flux was 
noticed close to the droplet rim when the film becomes thinner and thus unable to remove as much heat as the other regions of the spreading droplet. More recently in [6], a VOF based approach is used. The authors were able to solve the conjugate problem of fluid flow and heat transfer during the impact of water droplets onto a heated surface, at surface temperatures low enough to prevent boiling. Liquid properties were a function of local temperature. In [6] a region of high heat flux close to the contact line is reported. The authors related the presence of this region with higher evaporation rates occurring in the contact line.

Despite the completeness of the different models proposed so far in literature, their validation was as aforementioned mostly based on the comparison only with experimental measurements of the surface temperature at the centre of droplet impact. Instead, the present study addresses a more detailed validation process, in which the entire calculated temperature field of the surface in contact with the spreading droplet is compared with the temperature fields obtained experimentally, for different time instants during droplet impact and spreading. The experimental data were gathered combining high-speed IR thermography with high-speed visualization. Main emphasis is put on the sensible heat removed by the droplet and in how the flow field of the droplet can be related with heat and mass transfer phenomena.

\section{Material and methods}

Experimental arrangement and methodology

Water droplets formed at the tip of a hypodermic needle and fed by a syringe pump impact on the heated surface by action of gravity. The impact velocity of the droplets is varied by adjusting the height of the needle. The initial droplet diameter is fixed at $D_{0}=2.6 \pm 0.1 \mathrm{~mm}$. The impact Weber numbers, $W e=\rho V_{0}^{2} D_{0} / \sigma$, where $\rho$ is the liquid density, $\sigma$ the surface tension and $D_{0}$ and $V_{0}$ the initial droplet diameter and impact velocity, respectively, range between 24 and 151. The initial surface temperature $\mathrm{T}_{\mathrm{W}(\mathrm{in})}$ was varied between $333.15 \mathrm{~K}$ and $373.15 \mathrm{~K}$ and the ambient temperature was kept at $T_{a m b}=293 \pm 2 K$. The heated surface is a stainless steel foil electrically heated by copper electrodes clamped on its top. The foil is glued on the top of an insulating thermal glass and the whole assembly is then fixed on a stainless-steel support for an easier positioning. The bottom side of the foil which is used for infrared (IR) thermography is black matt painted to increase the emissivity $(\varepsilon=0.95)$. The foil is $20 \mu \mathrm{m}$ thick, $20 \mathrm{~mm}$ wide and $100 \mathrm{~mm}$ long.

Wettability is characterized measuring the quasi-static advancing and receding and the static contact angles, using an optical tensiometer (THETA from Attention). The static contact angle, measured by the sessile drop method was $\theta=81.7^{\circ} \pm 1^{\circ}$. The quasi-static advancing and receding angles were used to evaluate the hysteresis of the foil (i.e. the difference between the quasi-static angles), which was always larger than $20^{\circ} \pm 1^{\circ}$ for the hydrophilic foils tested here. The surface is considered to be smooth, with a mean roughness amplitude of the order of $0 \mu \mathrm{m} \pm 0.02 \mu \mathrm{m}$, as evaluated by a Dektak 3 profile meter (Veeco). A detailed description of the experimental procedure taken to characterize the wettability of the surfaces can be found in [14].

An infrared IR-high speed camera (MWIR-InSb from Xenics 179 - ONCA 4696 series) and a high-speed camera (Phantom v4.2) are placed bellow the heated surface and on the side, respectively, to capture simultaneous (but not synchronized) thermal and dynamics images of the impacting droplet.

The IR images were taken at $1000 \mathrm{fps}$ and $150 \times 150 \mathrm{px}^{2}$, while the high-speed images were taken at $2200 \mathrm{fps}$ and $512 \times 512 \mathrm{px}^{2}$. The measurements taken for each experimental condition are averaged from five events, to assure reproducibility of the observed phenomena. Care was taken to assure that the initial surface temperature and wetting conditions were reproducible before each new droplet impact. The impact velocity is evaluated by image post processing, as the vertical displacement of the droplet before impact divided by the time elapsed (i.e. three successive frames of the high-speed video). The initial $D_{0}$ and spreading $D$ droplet diameters are also evaluated from image post-processing, with a home-made routine, as in [15], being then used to determine the spreading ratio $D / D_{0}$. The radial temperature profiles were obtained after post processing the IR images using a home-made MatLab code, which converts the raw IR images to temperature data.

To eliminate the effect of the initial surface temperature, which is varied within the experimental campaign, a nondimensional temperature is considered, which is defined as $\mathrm{T}^{*}$ :

$T^{*}=\frac{\left[T_{w}(r, t)-T_{a m b}\right]}{\left[T_{w}(i n)-T_{a m b}\right]}$

where $T w(r, t)$ is the temperature of the bottom of the foil. at time $t$ after droplet impact and at a distance $r$ from the center of the droplet (point of impact). Temperature profiles are obtained by plotting $T^{*}$ as a function of the non-dimensional radial distance $r / D 0$.

The imposed volumetric heat flux $q^{\prime \prime \prime}$ is evaluated as:

$q^{\prime \prime \prime}=\frac{V \cdot I}{e \cdot L \cdot W}$ 
being $V$ and $I$ the applied voltage and resulting current passing through the stainless steel foil. $e, L$ and $W$ are the thickness, length and width of the foil, respectively.

\section{Measurement uncertainties}

The main uncertainties associated to droplet dynamics are summarized in Table 1, while Table 2 depicts the uncertainties associated to the main parameters used to describe the heat transfer process at droplet/wall interaction. These uncertainties are evaluated following Abernethy et al. [16]. For the quantities taken from image analysis, the uncertainties depend on the definition of the boundaries of the droplet in pixels ( \pm 2 pixels in the worst case scenario). More details can be found in Valente et al. [17].

Table 1. Uncertainties of the main parameters used to describe droplet dynamics.

\begin{tabular}{l|l}
\hline \hline Parameter & Uncertainties (rel. or abs) \\
\hline \hline $\begin{array}{l}\text { Droplet diameter before impact } D_{o} \\
{[\mathrm{~mm}]}\end{array}$ & $U_{D}= \pm 160 \mu \mathrm{m}$ \\
Spreading diameter $D[\mathrm{~mm}]$ & $U_{D_{o}}= \pm 160 \mu \mathrm{m}$ \\
& \\
Spreading ratio $D / D_{o}[-]$ & $u_{D / D_{o}} \max = \pm 37 \%$ at $\left(D / D_{o}=0.17\right)$ \\
& $u D / D_{o} \operatorname{mim}= \pm 7 \%$ at $\left(D / D_{o}=3.86\right)$ \\
Impact velocity $V_{0}[\mathrm{~m} / \mathrm{s}]$ & $U_{V_{o}}=0.08[\mathrm{~m} / \mathrm{s}]$ \\
Weber number $\mathrm{We}[-]$ & $u_{W e} \max = \pm 15 \%$ at $(W e=24)$ \\
& $u_{W e} \operatorname{mim}= \pm 8 \%$ at $(\mathrm{We}=151)$
\end{tabular}

Table 2. Uncertainties of the main parameters used to describe the heat transfer process during droplet spreading.

\begin{tabular}{|c|c|}
\hline Parameter & Uncertainties U (rel. or abs) \\
\hline Temperature T[K] & $\bar{~} U_{T}= \pm 1 K$ \\
\hline Temperature difference $\Delta \mathrm{T}[\mathrm{K}]$ & $\begin{array}{l}U_{\Delta T}= \pm 1.4 \mathrm{~K} \\
u_{\Delta T} \max = \pm 14 \% \text { at }(\Delta T=10 \mathrm{~K}) \\
u_{\Delta T} \min = \pm 1.7 \% \text { at }(\Delta T=78 \mathrm{~K})\end{array}$ \\
\hline Non-dimensional temperature $\mathrm{T}^{*}[-]$ & $\begin{array}{l}U_{T^{*}} \max = \pm 0.04 \text { at }\left(T^{*}=1\right) \\
U_{T^{*}} \min = \pm 0.02 \text { at }\left(T^{*}=0.3\right) \\
u_{T^{*}} \max = \pm 6 \% \text { at }\left(T^{*}=0.3\right) \\
u_{T^{*}} \max = \pm 4 \% \text { at }\left(T^{*}=1\right)\end{array}$ \\
\hline $\begin{array}{l}\text { Imposed volumetric heat flux } \\
q^{\prime \prime \prime}\left[\mathrm{W} / \mathrm{m}^{3}\right]\end{array}$ & $u q^{\prime \prime \prime} \max = \pm 12 \%$ at $\left(q^{\prime \prime \prime}=6.5 \cdot 10^{6}\left[\mathrm{~W} / \mathrm{m}^{3}\right]\right)$ \\
\hline Radial distance $r$ [mm] & $U_{r}= \pm 200 \mu m$ \\
\hline
\end{tabular}




\section{Numerical methodology}

A VOF-based approach was used for interface capturing, with an enhanced VOF model implemented in OpenFOAM CFD Toolbox [18]. This enhanced model is coupled with the energy equation that accounts for twophase heat transfer in the liquid domain and with transient heat conduction in a solid domain. In the VOF method, a volume fraction field $\alpha$ identifies the volume of liquid within a cell. The volume of the gaseous phase is therefore given as (1- $\alpha$ ). The value of $\alpha$ is 1 inside the pure liquid cells, 0 in the pure gas cells and between 0 and 1 in the cells containing the interface area. This procedure allows using a single set of continuity and momentum equations for the entire flow domain:

Continuity equation

$\Delta \cdot \vec{U}=0$

Momentum equation

$\frac{\partial \rho_{b}}{\partial t}+\nabla \cdot\left(\rho_{b} \vec{U} \vec{U}\right)=-\nabla p+\nabla \cdot \mu_{b}\left(\nabla \vec{U}+\nabla \vec{U}^{T}\right)+\rho_{b} f+F_{s}$

Interface advection

$\frac{\partial \alpha}{\partial t}+\nabla \cdot(\alpha \vec{U})=0$

Energy equation

$\rho_{b} c_{p_{b}} \frac{\partial T}{\partial t}+\nabla \cdot\left(\vec{U} \rho_{b} c_{p_{b}} T\right)=\nabla \cdot\left(k_{b} \nabla T\right)$

Here, $\vec{U}$ stands for the velocity of the liquid, $p$ the pressure and $T$ the temperature. Gravitational forces are represented as $f$ while $F_{s}$ represents the volumetric surface tension forces. $k_{b}, \rho_{b}, \mu_{b}$ and $c_{p_{b}}$ are the thermal conductivity, the density, the dynamic viscosity and the heat capacity of the bulk liquid, respectively. These are calculated as:

$\gamma_{b}=\gamma \alpha+\check{\gamma}(1-\alpha)$

The energy equation for the liquid (3) does not account for evaporation or diffusion of the liquid phase in the gas phase. This approximation is considered valid in the relatively small time scale that is investigated here. This is in agreement with the results reported, for instance by [12-13, 19-20]. The Continuum Surface Force (CFS) method proposed in [21] was used to model the surface tension as a volumetric force. The following equations stand:

$F_{S}=\sigma \kappa(\nabla \alpha)$

$\kappa=\nabla\left(\frac{\nabla \alpha}{|\nabla \alpha|}\right)$

where $\kappa$ is the curvature of the interface and $\sigma$ is the tension of the interface. Heat is transported only by conduction in the solid. The governing energy equation is therefore given as:

$\rho_{s} c_{v_{s}} \frac{\partial T_{s}}{\partial t}-\nabla \cdot\left(k_{s} \nabla T_{s}\right)=q_{V}^{\prime \prime \prime}$

where the subscript " $\mathrm{s}$ " indicates that the properties are of the solid only. The volumetric heat source is represented as $q_{V}^{\prime \prime \prime}\left[\mathrm{W} / \mathrm{m}^{3}\right]$ and is homogenously distributed in the solid domain.

\section{Numerical domain and boundary conditions}

The set of equations described above is solved in an axisymmetric domain, represented by a $5^{\circ}$. An $8 \times 8 \mathrm{~mm}^{2}$ fluid domain in the $X-Y$ plane was chosen to avoid the influence of the boundaries in the fluid flow. The dimensions of

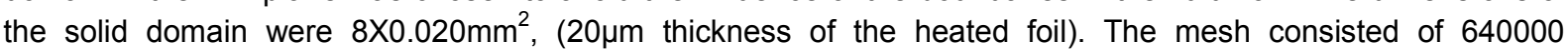
hexahedral cells in the fluid domain and 4000 in the solid domain. In the fluid domain, the mesh progressively coarsens away from the initial droplet position by a grading factor of 5 in both $X$ and $Y$ directions (last to first cell dimension in each direction is equal to 5). This leads to a minimum cell size of $4 \mu \mathrm{m}$ and a maximum cell size of $20 \mu \mathrm{m}$. These cell dimensions assure the solution to be mesh independent. Before each simulation, an arbitrary thermal boundary layer was patched in the domain to facilitate the initial convergence of the coupling between the solid and liquid temperatures. A droplet with the same diameter and velocity as in the experimental conditions was patched as well, at the time instant just before it contacts with the heated surface. The solid was considered as a volumetric heat source. Constant contact angle was assumed between the fluid and the solid with a value of $\theta=81.7^{\circ}$, following the experimentally measured value.

The PISO algorithm is used for pressure-velocity coupling, considering an Eulerian scheme for the time derivative and a Gauss linear for the gradient divergence as well as for the Laplacian terms. The flow field is assumed to be laminar. The conjugate heat transfer problem is solved by iteratively coupling of the temperature field and the heat flux between the solid and liquid domains, following a procedure similar to that reported in [22]. The simulations 
were run with a variable calculation time step to assure a constant Courant number of 0.2. Numerical reproduction of some of the experimental results was performed to benchmark the code. An overview of the conducted simulations is reported in Table 3.

Table 3 Thermo-physical properties of the fluids and solid used in the performed simulations. $\rho, C_{p}, k_{l}, v$ and $\sigma$ and the liquid density, specific heat, thermal conductivity, kinematic viscosity and surface tension, respectively.

\begin{tabular}{l|lllll}
\hline \hline & $\rho\left[\mathrm{kg} / \mathrm{m}^{3}\right]$ & $c_{p}[\mathrm{~J} / \mathrm{kgK}]$ & $k_{l}[\mathrm{~W} / \mathrm{mK}]$ & $v\left[\mathrm{~m}^{2} / \mathrm{s}\right]$ & $\sigma[\mathrm{N} / \mathrm{m}]$ \\
\hline \hline Air & 1 & 1006.4 & 0.025874 & 0.0000148 & - \\
Water & 1000 & 4184 & 0.59844 & 0.000001 & 0.007 \\
Stainless Steel & 7880 & 477 & 18 & - & -
\end{tabular}

\section{Results and discussion}

For comparative purposes between the experimental data and the numerical results, Figure 1 depicts a sequence of high-speed images, the corresponding numerical simulations (side view of the droplet) and IR images of the surface (bottom view) taken during the spreading of a water droplet at We=24 on the stainless steel foil, initially heated at $T_{W(i n)}=353.15 \mathrm{~K}$. The figure anticipates a good agreement between the experimental data and the numerical predictions, regarding the shape of the spreading droplet and the temperature field. A quantitative comparison is shown in Figure 2, which depicts the temporal evolution of the calculated and measured spreading factors.

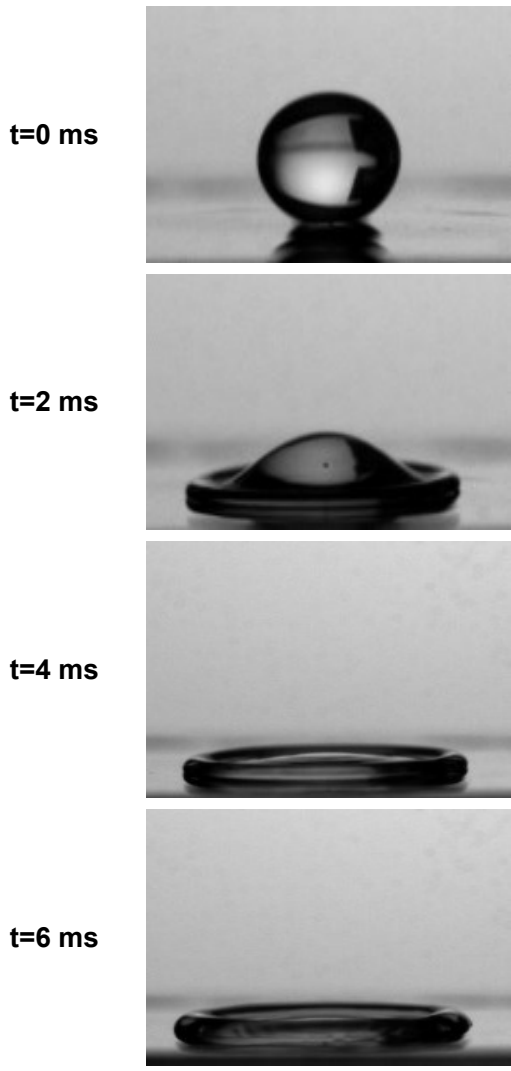

$6 \pm 0.2 \mathrm{~mm}$
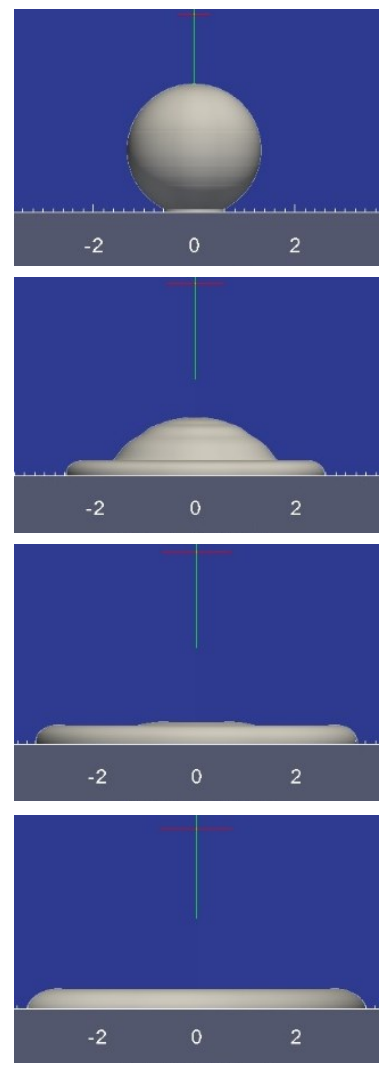

$6 \mathrm{~mm}$

Num. droplet shape
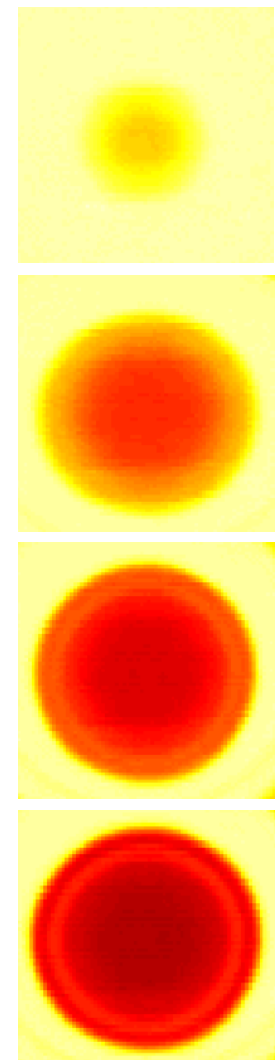

$\begin{array}{lllllll}303 & 313 & 323 & 333 & 343 & 353 & 363\end{array}$

IR Tw field [K]

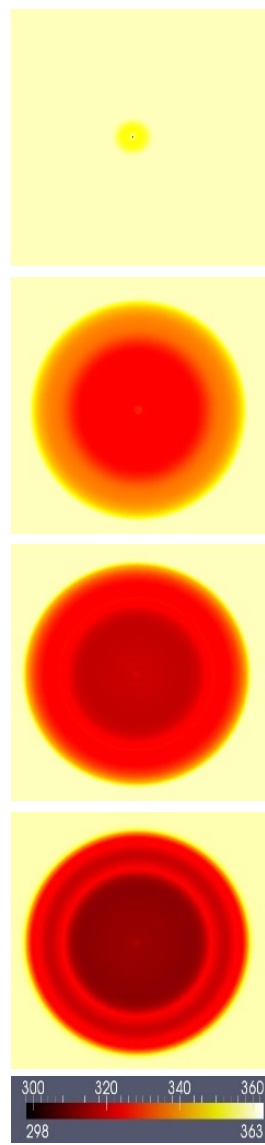

Num. Tw field[K]

Figure 1. Qualitative comparison between high-speed images, the corresponding numerical simulations (side view of the droplet), IR images of the surface (bottom view) and the corresponding calculated temperature field. The comparative analysis is performed for various time instants after the impact of a water droplet at $\mathrm{We}=24$ on the stainless steel foil initially heated at $T_{\mathrm{w}(\mathrm{in})}=353.15 \mathrm{~K}$.

Figure 2 supports the excellent agreement between the numerical and the experimental results. At the first stage of spreading $(\mathrm{t}<2 \mathrm{~ms})$ the observed discrepancies are mostly due to the uncertainty 
associated to the definition of the instant of impact, $t=0 \mathrm{~ms}$. Considering the temporal resolution of the high-speed recordings, the actual impact can occur up to $0.5 \mathrm{~ms}$ before or after the frame chosen to establish the instant of impact. In the numerical simulation, on the other hand, the time resolution of the post-processed data is $0.1 \mathrm{~ms}$. Hence, particularly at these early stages after impact, the differences between the numerical and the experimental results can be considerable. However, as the spreading factor increases and, therefore the time after impact increases, the difference between numerical and experimental results reduces. At the maximum spreading diameter, the maximum difference between the experimentally measured and the numerically predicted spreading factor is $3.4 \%$.

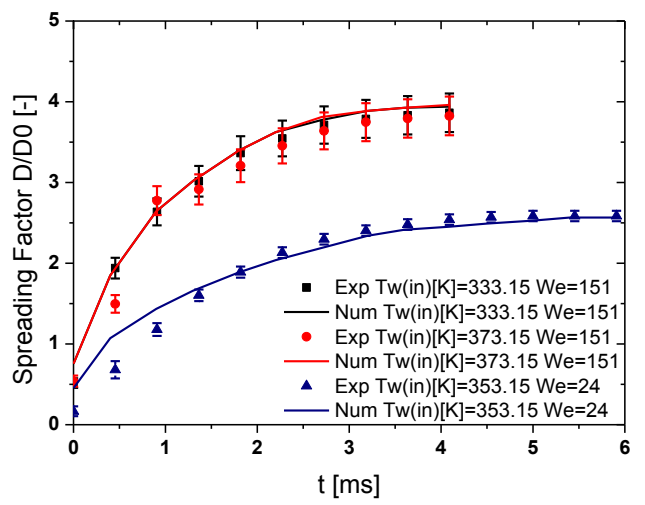

Figure 2. Temporal evolution of the calculated and measured spreading factors for the impact conditions of Figure 1.

A preliminary relation can be established between droplet dynamics and the heat transfer processes, comparing dimensionless surface temperature profiles $T^{*}$, as defined in equation (1), along the dimensionless radial distance $r / D_{0}$ at different instants after impact. Such profiles are depicted in Figure 3 for two different Weber numbers, $\mathrm{We}=24$ and $\mathrm{We}=151$ and for three different initial surface temperatures, namely $\mathrm{T}_{\mathrm{W}(\mathrm{in})}=333.15,353.15$ and $373.15 \mathrm{~K}$
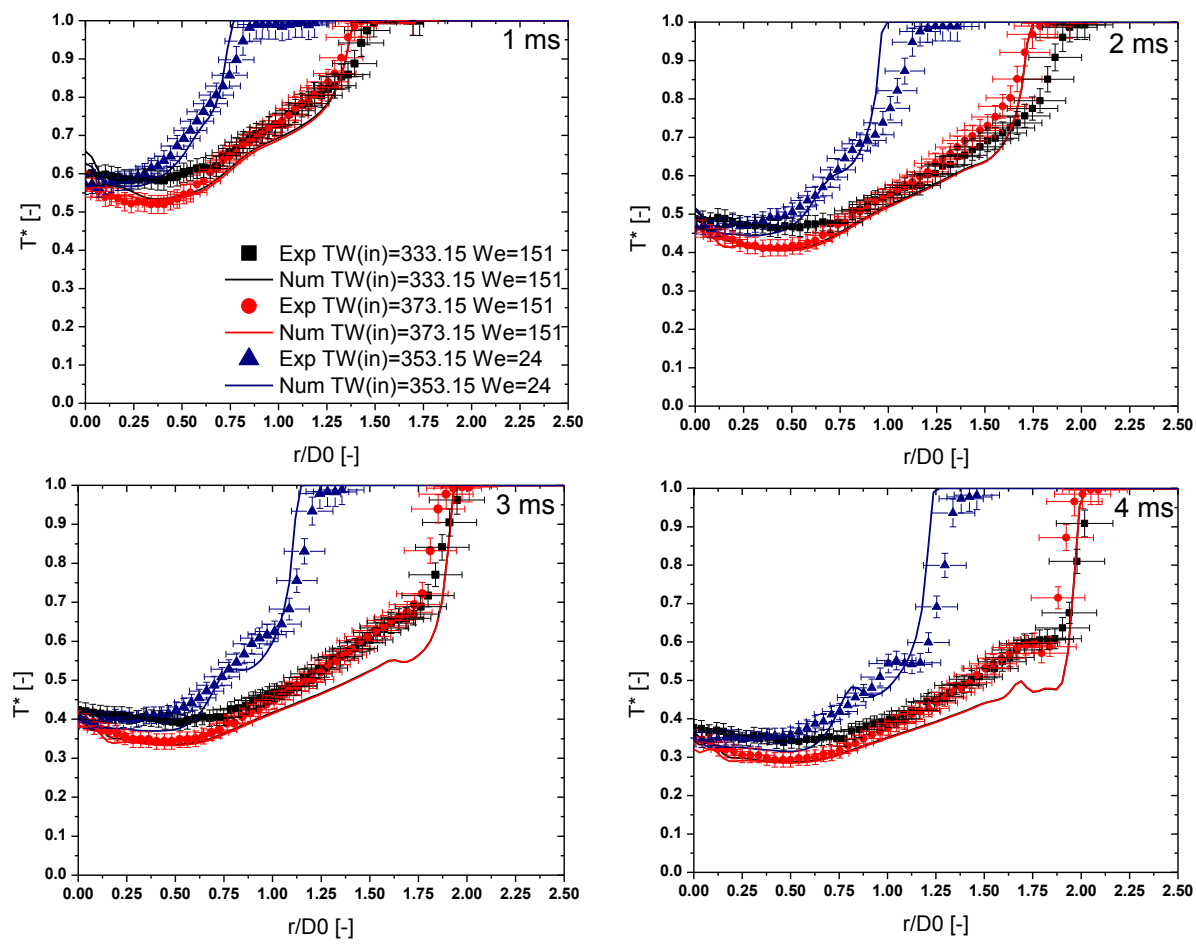

Figure 3. Dimensionless temperature profiles along the dimensionless radial distance for $1,2,3$ and $4 \mathrm{~ms}$ after impact, for $\mathrm{We}=24$ and $\mathrm{We}=151$ and for three different initial surface temperatures, namely $T_{\mathrm{W}(\mathrm{in})}=333.15,353.15$ and $373.15 \mathrm{~K}$. 
Given that the maximum spreading diameter for $W e=151$ is reached $4 \mathrm{~ms}$ after impact, the results are only presented and discussed up to this time instant. The figure clearly evidences a very good agreement between the experimental results and the numerical predictions, particularly towards the center of the impact $\left(r / D_{0} \approx 0\right)$, for all the conditions tested. For instance, for $\mathrm{We}=151$, in the range of $r / D 0<1.2$ the maximum difference between the numerical and experimental profiles $T^{*}$ is $11 \%$. For $W e=24$ in the range $r / D_{0}<0.5$, the maximum difference in $T^{*}$ is $5 \%$. These differences are negligible, being within the uncertainty of the experimental measurements. Some discrepancies are however noticeable at higher values of $r / D_{0}$, in the region of the rim and of the gas-liquid-solid contact line. The contact line can be identified at the maximum value of $r / D 0$ before $T^{*}=1$. $T^{*}=1$ means that any temperature variation occurs on the surface, i.e. the surface is not wetted yet by the droplet. On the other hand, close to the contact line, a steep temperature variation occurs. Consequently, any small difference between the measured and the calculated position of the contact line can lead to substantial differences between the measured and the numerically predicted $\mathrm{T}^{*}$ (up to $30 \%$ of the experimentally measured value). The rim is identified by the non-monotonic decrease in the temperature along $r / D_{0}$. Both experimental and numerical results show that the temperature does not decrease with increasing $r / D_{0}$, but local maxima and minima occur instead. This is particularly evident at 3 and $4 \mathrm{~ms}$ after impact, for all the We numbers tested. Here, the maximum difference between the experimental and the numerical values of $T^{*}$ is $16 \%$ for $W e=151$ at $4 \mathrm{~ms}$ after impact and at $r / D_{0}=1.75$ and $11 \%$ for We $=24$ at $4 \mathrm{~ms}$ after impact and at $r / D_{0} \approx 1$. Within the rim, the various heat transfer mechanisms occur in extremely small temporal and spatial scales. Hence, matching the experimental and the numerically calculated temperatures strongly depends on the relation between the instrumental and the numerical resolution. In the numerical simulations, the mesh size varies between $4 \mu \mathrm{m}$ up to $20 \mu \mathrm{m}$ and the reported numerical profiles were sampled with a sampling size of $80 \mu \mathrm{m}$. The temporal resolution was $0.1 \mathrm{~ms}$. On the other hand, the spatial resolution of the IR camera was of $110 \mu \mathrm{m} / \mathrm{px}$, the temporal resolution of 483 the IR camera was $1 \mathrm{~ms}$ and the integration time was $200 \mu \mathrm{s}$. This means that the temperature variation captured by the IR camera could be integrated within a larger temporal-spatial domain and thus the resulting values can be spatially damped or temporally delayed up to a certain amount. Despite these limitations, the trend of the temperature profiles is very well captured by the numerical simulations.

Three typical characteristic regions can be identified in the temperature profile: 1) The first is a steep increase in temperature at the entrance to the rim, 2) the second is the appearance of a temperature local minimum in the region within the rim and 3 ) the third is a steep decrease in temperature, observed near the contact line (Figure 4).

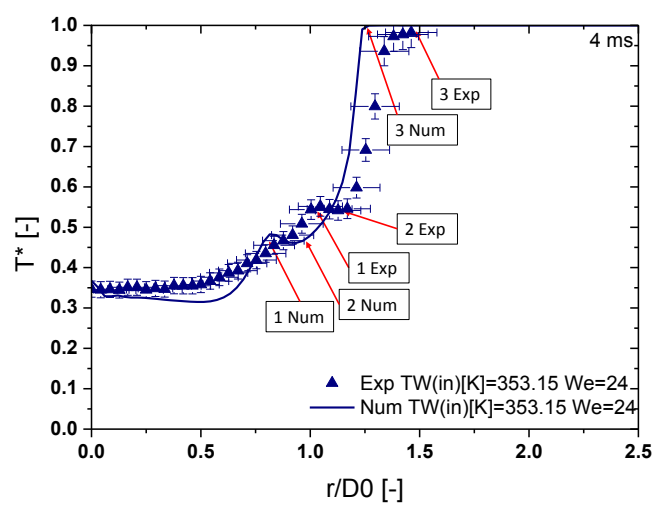

Figure 4. Measured and calculated temperature distribution along the dimensioless radial distance for a droplet impacting at $\mathrm{We}=24$ on the surface initially heated at TW8in $)=353.15 \mathrm{~K} .1,2$ and 3 identify the 3 characteristic regions of the temperature profile.

These trends differ from those reported by for e.g. [20], who measured a constant temperature increase along the droplet radius due to the decreased convective heat transfer in the outward fluid direction. However, numerically, a temperature distribution similar to that obtained here was reported for instance by [13]. These discrepancies arising from experimental and numerical studies can be explained by further detailing the fluid dynamics within the droplet, specifically at the lamella and within the rim, in the attempt to relate the fluid dynamic behaviour with the heat transfer process. Such detailed study was performed for the impact of a droplet at We $=24$ and $T_{W(i n)}=353.14 \mathrm{~K}$. The analysis performed, which is not presented here due to paper length constrains clearly evidenced large local temperature variations, particularly within the rim, where adverse flow can occur. In fact, there is a complex structure of vortices within the rim and in the lamella, which can be related with the local heat 
transfer coefficients and with the aforementioned temperature variations. These vortex structures promote the mixing in the thermal boundary layer, which affects particularly the heat transfer process at the liquid-solid interface. In line with this, the vorticity was evaluated and correlated with the heat transfer coefficient at the centre of the first layer of fluid cells i.e. at a vertical distance of $2 \mu \mathrm{m}$ from the liquid-solid interface. The correlation between the resulted temperature field and the droplet dynamics was obtained by assuming a relation between the vorticity and the local heat transfer coefficient, in the first fluid cell i.e. near the liquid-solid interface. The two measured fields revealed that local maxima and minima in the vorticity correspond to spatially shifted local minima and maxima in the heat transfer coefficients, at all stages of the droplet spreading. This was particularly clear in the rim region.

\section{Conclusions}

This work concerns a detailed analysis of the spreading process of a water droplet on a heated smooth surface, coupling the fluid dynamics with the heat transfer processes. The experimental analysis comprises the combination of high-speed infrared thermography with high-speed imaging evaluate the spreading behaviour (e.g. the spreading ratio) together with detailed surface temperature fields along the spreading radius, during the spreading. The numerical simulations were conducted using an enhanced version of a VOF-based solver of OpenFOAM previously developed, which was further modified to account for conjugate heat transfer between the solid and fluid domains, focusing only on the sensible heat removed during droplet spreading.

An excellent agreement is observed between the temporal evolution of the experimentally measured and the numerically predicted spreading factors. The numerical and experimental dimensionless surface temperature profiles along the droplet radius were also in good agreement. The temperature fields revealed a nonhomogenous cooling of the surface which was related with droplet dynamics. Hence, the coupled analysis of the fluid dynamics and heat transfer processes within the lamella evidenced a strong correlation between maximum and minimum temperature values and heat transfer coefficients with the vorticity fields in the lamella, which lead to particular mixing processes in the boundary layer region, at all stages of droplet spreading. This was particularly clear in the rim region, which therefore should be always accounted for in droplet spreading models.

\section{Acknowledgements}

The authors are grateful to Fundação para a Ciência e Tecnologia (FCT) for partially financing the research under the framework of the project RECI/EMS-SIS/0147/2012 and for supporting P. Pontes with a research fellowship. A. S. Moita acknowledges FCT for financing her contract through the IF 2015recruitment program (IF 008102015) and E. acknowledges FCT for supporting his PhD fellowship (SFRH/BD/88102/2012).

\section{References}

[1] Kim., J., 2007, Int. J. Heat Fluid Flow, 28, pp. 253-267.

[2] Panão, M., Guerreiro, J. and Moreira, A.L.N., 2012, Int. J. Heat Mass Transf. 55, pp. 2854-2863.

[3] Moita, A.S. and Moreira, A.L.N., 2012, Experiments in Fluids, 52, pp. 679-695.

[4] Pasandideh-Fard, M., Aziz, S., Chandra, S. and Mostaghimi, J., 2001, Int. J. Heat Fluid Flow, 22, pp. 201-210.

[5] Haley W., Hartley, J. and Abdel-Khalik, S., 2001, Int. J. Heat Mass Transf., 44, pp. 3869-3881, 2001.

[6] Strotos G., Aleksis G., Gavaises M., Nikas, K.-S., Nikolopoulos, N. and Theodorakakos, A., 2011, Int. J. Thermal Sci., 50, pp. 698-711.

[7] Tartarini, P., Corticelli, M.A. and Tarozzi, L., 2009, Appl. Thermal Eng., 29, pp. 1391-1397.

[8] Girard, F., Antoni, M. and Sefiane, K., 2010, Langmuir, 26, pp. 4576-4580.

[9] Gradeck, M., Seiler, N., Ruyer, P. and Maillet, D., 2013, Exp. Thermal Fluid Sci., 47, pp. 14-25.

[10] Harlow, F. H. and Shannon, J. P., 1967, J. Appl. Phys., 38, pp. 3855-3866.

[11] Zhao, Z., Poulikakos, D. and Fukai, J., 1996, Int. J. Heat Mass Transf., 39, pp. 2771-2789.

[12] Francois, M. and Shyy, W., 2003, Numer. Heat Transf. Part B Fundam., 44, pp. 101-118.

[13] Healy, W.M., Hartley, J.G. and Abdel-Khalik, S.I., 2001, Int. J. Heat Mass Transf., 44, pp. 3869-3881.

[14] Pereira P., Moita, A. S., Monteiro, G. and Prazeres, D.M.F., 2014, J. Bionic Eng., 11(3), pp. 346-359.

[15] Moita, A.S., Herrmann, D. and Moreira, A.L.N., 2015, Appl. Thermal Eng., 88, pp. 33-46.

[16] Abernethy, R., Benedict, R. and Dowdell, R., 1985, J. Fluids Eng. 107, pp. 161-164.

[17] Teodori, E., Valente, T., Malavasi, I., Moita, A.S., Marengo, M. And Moreira, A.L.N., 2017, Applied Thermal Eng., 115, pp. 1424-1437.

[18] Georgoulas, A., Andredaki, M. and Marengo, M., 2017, Energies, 10(3), pp. 1-35. ISSN 1996-1073.

[19] Francois, M. and Shyy, W., 2003, Numer. Heat Transf. Part B Fundam., 44, pp. 119-143.

[20] Jackson, R.G., Kahani, M., Karwa, N., Wu, A., Lamb, R., Taylor, R. and Rosengarten, G. 2014, J. Phys. Conf. Ser., 525, p. 12024.

[21] Brackbill, J., Kothe, D., and Zemach, C., 1992, J. Comput. Phys., 100, pp. 335-354.

[22] Kunkelmann, C. Numerical Modeling and Investigation of Boiling Phenomena, Technische Universitaet Darmstadt, 2011. 


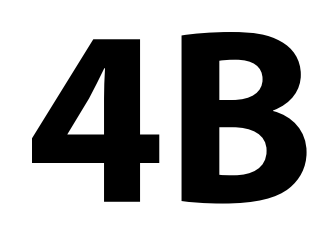

Automotive 4 


\title{
A study on the relationship between internal nozzle geometry and injected mass distribution of eight ECN Spray G nozzles
}

\author{
K.E. Matusik ${ }^{1, *}$, D.J. Duke ${ }^{1,2}$, N. Sovis ${ }^{1}$, A.B. Swantek ${ }^{1}$, C.F. Powell ${ }^{1}$, R. Payri ${ }^{3}$, D. Vaquerizo ${ }^{3}$, \\ S. Giraldo-Valderrama ${ }^{3}$, A.L. Kastengren ${ }^{4}$ \\ ${ }^{1}$ Energy Systems Division, Argonne National Laboratory, Lemont, IL, USA \\ ${ }^{2}$ Laboratory for Turbulence Research in Aerospace \& Combustion, Department of Mechanical \\ \& Aerospace Engineering, Monash University, Australia \\ ${ }^{3} \mathrm{CMT}$ Motores Termicos, Universitat Politècnica de València, València, Spain \\ ${ }^{4}$ X-Ray Science Division, Argonne National Laboratory, Lemont, IL, USA \\ *Corresponding author: kmatusik@anl.gov
}

\begin{abstract}
Gasoline direct injection (GDI) nozzles are manufactured to meet geometric specifications with length scales on the order of a few hundred microns. The machining tolerances of these nominal dimensions are not always known due to the difficulty in accurately measuring such small length scales in a nonintrusive fashion. To gain insight into the variability of the machined dimensions as well as any effects that this variability may have on the fuel spray behavior, a series of measurements of the internal geometry and fuel mass distribution were performed on a set of eight nominally duplicate GDI "Spray G" nozzles provided by the Engine Combustion Network. The key dimensions of each of the eight nozzle holes were measured with micron resolution using full spectrum $x$-ray tomographic imaging at the 7-BM beamline of the Advanced Photon Source at Argonne National Laboratory. Fuel density distributions at $2 \mathrm{~mm}$ downstream of the nozzle tips were obtained by performing $x$-ray radiography measurements for many lines of sight. The density measurements reveal nozzle-to-nozzle as well as hole-to-hole density variations. The combination of high-resolution geometry and fuel distribution datasets allows spray phenomena to be linked to specific geometric characteristics of the nozzle, such as variability in the hole lengths and counterbore diameters, and the hole inlet corner radii. This analysis provides important insight into which geometrical characteristics of the nozzles may have the greatest importance in the development of the injected sprays, and to what degree these geometric variations might account for the total spray variability. The goal of this work is then to further the understanding of the relationship between internal nozzle geometry and fuel injection, provide input to improve computational models, and ultimately aid in optimizing injector design for higher fuel efficiency and lower emissions engines.
\end{abstract}

\section{Keywords}

GDI, nozzle geometry, fuel spray density, fuel injector, DISI, Spray G, ECN, gasoline

\section{Introduction}

To fully realize the benefits of a gasoline direct injection (GDI) engine, precise control over the amount of injected fuel and the fuel-to-air mixing ratio is necessary. Any variations to the fuel spray distribution can affect the combustion process, and by extension the fuel efficiency and emissions levels. Modern multi-hole GDI nozzles generally feature a relatively complex step-hole geometry in which each hole might require knowledge of at least nine dimensions to accurately model. Due to the importance of the injection process on the output metrics of a GDI engine, a subset of internal combustion engine research has been dedicated to investigating the effects of nozzle geometry on the corresponding fuel spray distribution. X-ray spray tomography measurements of three six-hole GDI nozzles with varying hole patterns explored the effects of geometric asymmetries on the spray structure [1]. The authors found that the emitted fuel spray distribution varied between jet-like, hollow-coned, or crescent-shaped, depending on the nozzle hole pattern. A study using a single-cylinder optical engine reported higher soot emissions for a laser-drilled GDI injector in comparison to one manufactured using electrical discharge machining [2]. Research has also focused on elucidating the relationship between nozzle shape and cavitation, whose presence can significantly perturb fuel spray characteristics such as the discharge coefficient, outlet velocity, spray angle, and atomization behavior [3]. A converging diameter profile and a sufficiently rounded hole inlet corner were found to decrease or eliminate geometric cavitation in comparison to a sharp-cornered, cylindrical geometry $[4,5]$. In addition, local asymmetries at the hole inlet have been shown to generate correspondingly asymmetric cavitation, forming at the location where the fuel flow experiences the largest change in direction [5].

Research in the same vein has been carried out in collaboration with the Engine Combustion Network (ECN). The ECN coordinates the sharing of well-characterized gasoline and diesel injection nozzles and standardized hardware with experimental facilities worldwide. These facilities perform various diagnostics at established conditions and share their findings publicly, with the intent of building a comprehensive database of experimental and computational measurements of the fuel injection and combustion processes. The ECN community's target injector for GDI studies is "Spray G", an eight-hole solenoid-activated valve-covered orifice injector with a step-hole geometry [6]. A number of nominally duplicate Spray G injectors, as identified by the last two digits of their respective serial numbers, 
have been in circulation at multiple facilities for experimental diagnostics. Nozzle geometry measurements of Spray G \#28 exposed 7\% variability in hole area and a factor of two variability in the inlet corner radius. Complementary spray tomography measurements showed significant plume-to-plume mass flux variations [7]. Simulation results for Spray G using nominal geometry have revealed strong asymmetric transients in the internal flow structure [8], which are expected to respond to geometric asymmetries such as an azimuthally-varying inlet hole corner radius. These findings support an inextricable link between the internal nozzle geometry and the fuel spray distribution, and as such, the need to expose which geometric features are responsible for spray variability. In addition, an understanding of spray sensitivity to geometric variations can provide more educated tolerances to manufacturing designers.

A number of experimental measurements have been executed using Spray G hardware. Optical techniques were implemented to investigate fuel spray characteristics [9]. Schlieren and diffused back illumination were used to characterize the vapor and liquid penetration [10], shadowgraphy and Mie scattering have provided spray visualization [11], and phase Doppler anemometry has been used to measure fuel droplet sizes and velocities [11, 12], to name a few. Intrusive techniques, such as patternation, can provide a footprint of the spray mass distribution [13]. Rate of injection and rate of momentum measurements have also been successfully demonstrated on Spray G \#26 [14].

To isolate hole-to-hole spray variations, measurements must be made in a noninvasive manner and near to the nozzle exit before plume interactions significantly influence the density field. These dense regions of the spray do not lend themselves well to optical techniques on account of multiple scattering which causes short optical depths. At low energies, $x$-rays are only weakly scattered by the spray, with photoelectric absorption instead being their main mode of interaction with the fuel [15]. As such, x-rays generated by a benchtop or synchrotron source are useful in obtaining quantitative density measurements in multiphase flows [16, 17]. A benchtop x-ray system coupled with polycapillary optics has been previously used to obtain density measurements of the fuel spray emitted by a six-hole GDI injector [18]. In general, the inherent divergence of the x-ray source limits the spatial resolution, and the lower flux limits the temporal resolution and signal-to-noise ratio. The high photon flux of a third-generation synchrotron source, such as the Advanced Photon Source (APS) at Argonne National Laboratory, provides the sub-microsecond temporal and micron spatial resolution that is desired for investigating fuel sprays. The 7-BM beamline at the APS is tailored for such experiments, including time-resolved measurements of the ensemble-averaged projected fuel density with micron spatial resolution [19], as well as shot-to-shot statistics [20]. Measuring the projected density at a few lines of sight is useful for modeling the density field of simple sprays such as those from a single-hole nozzle [21]. However, for a more detailed quantification of the density distribution of a more complicated spray structure such as one from a multi-hole injector, a full tomographic reconstruction can be implemented [1, 7, 22, 23].

There exist various methods for measuring the internal nozzle geometry. Depending on the shape, the dimensions of a silicone mold of the hole can measured with a scanning electron microscope [24]. Single-micron resolution of geometric features is possible using optical microscopy, but the technique is limited by the depth of focus and location of the minimum hole diameter, past which measurements cannot be made [25]. Computed tomography (CT) can be implemented to obtain the nozzle geometry by making use of $x$-rays and scanning the object for a wide range of angles. Tomographically reconstructing the projection images provides a full three-dimensional representation of the scanned section of the nozzle. A benchtop x-ray source may be used to generate the requisite $x$-rays, but, once again, the angular divergence of the beam limits the spatial resolution. Sub-micron resolution with a benchtop source is possible, but is generally reserved for low-density materials [26]. Synchrotron sources, such as the APS and the European Synchrotron Radiation Facility, for example, are particularly well suited for CT measurements due to their highly brilliant $\mathrm{x}$-ray sources, and each maintains a facility that specializes in x-ray microtomography measurements of low-density samples $[27,28]$. Within the last few years, a new instrument has been added to the 7-BM beamline at the APS that allows for high-resolution CT imaging of injection nozzles. With this complementary capability, the 7-BM facility now supports measurements of the nozzle geometry as well as the density field with minor modifications to the experimental setup.

The following sections outline the experimental setup for performing nozzle tomography and spray tomography experiments at the 7-BM beamline. We summarize the methodology followed to obtain the dimensions of eight Spray G nozzles, as well as the time-resolved density field at $2 \mathrm{~mm}$ downstream of the nozzle tip. We then provide results from multiple linear regression analysis that couples the effects of geometric features on the steady-state density field. These findings highlight the key players that may be responsible for spray variability, and provide a prioritized list of geometric features that may be critical for accurate representation in future computational efforts, as well as machining with high precision during the injector manufacturing process.

\section{Material and methods}

All experiments were performed at the 7-BM beamline of the APS [29]. The eight Spray G injectors that contributed to this study are numbers $12,17,18,21,22,25,26$, and 29 . The coordinate system that is used to describe the nozzle geometry is consistent with that provided on the ECN website [6]. Specifically, the origin is located at the tip of the nozzle. The $z$-coordinate is positive in the direction of the spray, the $x$-coordinate is positive in the direction of the electrical connector, and the $y$-coordinate is transverse to the $x$-coordinate.

For nozzle tomography measurements, the beamline operated in "white beam" mode, which enables access to the unfiltered broadband (white) beam from the bending magnet source. This mode is necessary to image the internal nozzle geometry because the white beam provides increased flux at higher photon energies that are capable of penetrating through the dense steel. Figure 1 schematizes the experimental setup. Each Spray G nozzle 


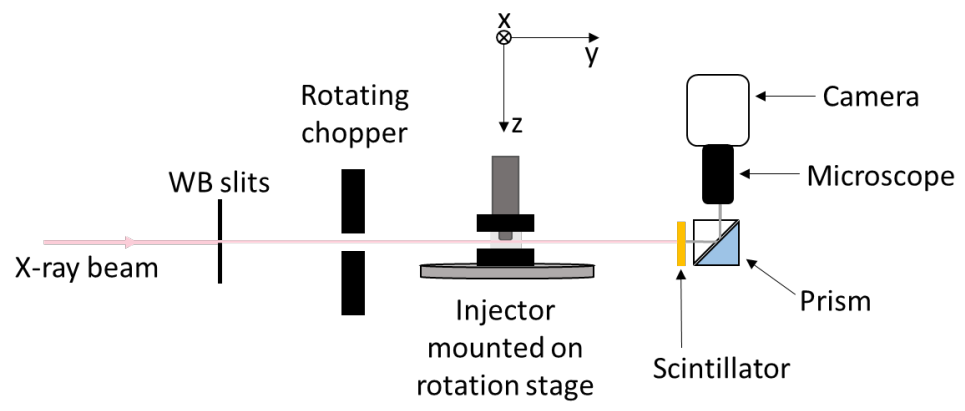

Figure 1. Schematic of the experimental setup for nozzle tomography (not to scale).

was mounted vertically in a specifically designed V-block holder that ensured nozzle stability during rotation. The holder was secured to two orthogonal stages that provided adjustment of the center of rotation. The pair of linear stages were mounted on an air-bearing rotation stage. From the APS storage ring, the polychromatic $x$-ray beam passes through a set of white beam slits that delimit the footprint of the x-ray source. Downstream of the slits, two metal filters, a $250 \mu \mathrm{m}$ copper and subsequent $25 \mu \mathrm{m}$ molybdenum filter, are placed in the beam path to reduce heat load on the nozzle. A rotating chopper downstream of the filters further minimizes the heat load by limiting beam exposure to $10 \%$ duty cycle, synchronized with the camera's frame rate. As the beam passes through the nozzle tip, x-ray photons are absorbed by the metal, creating spatial contrast in the beam cross-section. The outgoing $x$-ray photons that penetrated through the nozzle are absorbed by a lutetium aluminum garnet (LuAg) scintillator, and re-emitted as visible light. The propagation distance between the nozzle and scintillator was reduced to $48 \pm$ $1 \mathrm{~mm}$ in order mitigate phase effects that would otherwise increase the uncertainty in the geometry measurements by blurring the air/nozzle interface. The output image from the scintillator is magnified with a $5 x$ long-distance microscope to a field of view (FOV) of $2.25 \times 1.4 \mathrm{~mm}$ and pixel resolution of $1.17 \mu \mathrm{m}$. The diffraction-limited resolution of the raw images, determined by imaging an Xradia resolution target in the same tomography setup, is $1.5 \mu \mathrm{m}$. For nozzle tomography measurements, $\theta=0^{\circ}$ corresponds to the electrical connector pointing in the $+x$ direction. Each nozzle was rotated from $0^{\circ}$ to $180^{\circ}$ at $0.1^{\circ}$ steps, with 5 images recorded and subsequently averaged at each angle to increase the image contrast. Additional information with regard to the optimization of the imaging process may be found in previous work [30]. In brief, the process has been iteratively improved in order to minimize image manipulation during post-processing, and consequently preserve as much as possible the spatial resolution of the raw images. The resulting image stack undergoes tomographic reconstruction to transform the nozzle projections into $x-y$ slices through the nozzle body. The software that was used to perform the reconstruction is TomoPy, an open-source Python code developed for synchrotron tomography [31]. The reconstruction algorithm is a direct Fourier-based method called Gridrec [32]. The spatial uncertainty associated with the full imaging and post-processing procedure is $1.8 \mu \mathrm{m}$, determined from calibration of three steel pin gauges ranging in diameters between approximately 100 and $250 \mu \mathrm{m}$.

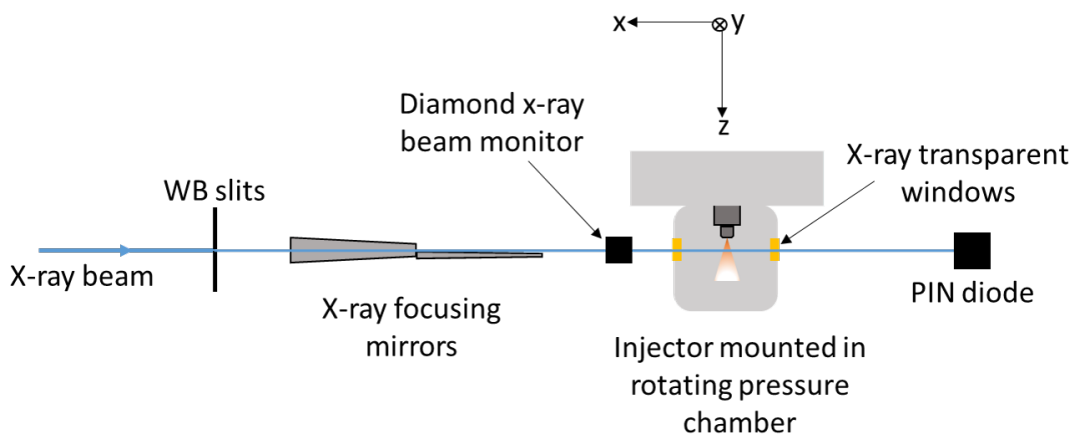

Figure 2. Schematic of the experimental setup for spray tomography (not to scale). The pressure chamber is shown as being transparent to visualize the location of the spray and $x$-ray transparent windows.

Spray tomography measurements were carried out using a monochromatic beam at $8 \mathrm{keV}$ energy. The working fuel was a gasoline surrogate, Viscor 1487, doped with 3.1 wt\% Rhodia DPX9 cerium additive to increase the signalto-noise ratio. The density and viscosity of the fuel, measured by a third party laboratory, are $810.1 \mu \mathrm{gm}^{-3}$ and $1.240 \mathrm{~mm}^{2} \mathrm{~s}^{-1}$ at standard temperature and pressure.

A schematic of the experimental setup is shown in Figure 2. Each injector was mounted vertically, spraying down, in a steel pressure chamber that allows for the injector's full rotation. The injection pressure was maintained at approximately $19 \mathrm{MPa}$ by a piston accumulator system. The back pressure was held constant at $0.3 \mathrm{MPa}$, and the 
electronic injection duration was set to $680 \mu \mathrm{s}$. The injector fired at $3 \mathrm{~Hz}$ into an ambient temperature environment that was pressurized with $\mathrm{N}_{2}$, which also provided a constant purge flow of 4 standard $L$ min $^{-1}$ in order to inhibit fuel droplet formation on the windows during data acquisition. The x-ray source was focused to a beam size of 5 $\times 6 \mu \mathrm{m}$. A transverse scan was carried out $2 \mathrm{~mm}$ downstream from the nozzle tip for $0^{\circ} \leq \theta \leq 180^{\circ}$ at $2^{\circ}$ steps. A total of 113 points spaced $80 \mu \mathrm{m}$ apart were measured at each rotation angle with a temporal resolution of 3.68 $\mu \mathrm{s}$. The outgoing beam intensity was averaged for 16 spray events at each measured position. The upstream beam intensity, $I_{0}$, was measured using a diamond x-ray beam monitor. As the beam passes through the spray, it becomes attenuated to an intensity $I$ by the fuel in the beam path on account of photoelectric absorption. The attenuation of the beam is related to the amount of fuel in the beam path via the Lambert-Beer law, which provides a measure of the projected density, $M$, of fuel at the measured location [15],

$$
\mathrm{M}\left(\mu \mathrm{g} \mathrm{mm}^{-2}\right)=\frac{1}{\mu} \log \left(\frac{I_{0}}{I}\right),
$$

where $\mu\left(\mathrm{mm}^{2} \mu \mathrm{g}^{-1}\right)$ is the mass absorption coefficient, found through calibration of the working fluid. The projected density measurements at the recorded 90 lines of sight are reconstructed using TomoPy's penalized maximum likelihood algorithm with weighted linear and quadratic penalties [33]. The result of the reconstruction is a time-resolved ensemble-average density field, $\rho(x, y, t)$, at $z=2 \mathrm{~mm}$ downstream of the nozzle tip. It should be noted that in this experiment setup, $\theta=0^{\circ}$ corresponded to the electrical connector pointing upstream to the beam propagation direction, as opposed to the nozzle tomography setup, in which the electrical connector was orthogonal to the beam direction. The inconsistency in the $\theta=0^{\circ}$ position relative to the beam between the spray and nozzle tomography experiments was accounted for in the analysis to ensure that the nozzle holes were properly indexed between the two measurement techniques.

\section{Data Analysis}

To remove background noise from the density field measurements, values that were less than $10 \%$ of the maximum density were masked at all time steps and all measured locations. In general, this threshold omitted approximately $2.5 \%$ of the total mass in the field from the analysis. In order to measure the fuel density corresponding to each hole, local minima in the density field were determined between holes by azimuthally resolving the steady-state density field. Wedge-shaped bins extending from the spray's steady-state center of mass through the local density minimum were used to isolate the density field associated with each nozzle hole (see Figure 4). The binning procedure is collectively exhaustive in $\theta$. The density field in each bin was integrated in both the $x$ - and $y$ - coordinate to arrive at the planar integrated mass (PIM),

$$
\operatorname{PIM}\left(\mu \mathrm{g} \mathrm{mm}^{-1}\right)=\int_{A} \rho(x, y, t) \mathrm{d} A \text {. }
$$

Ten dimensions of interest were measured per each nozzle hole. Figure 3 displays the relevant dimensions superposed on the isosurface of a Spray $G$ injector to illustrate the high spatial resolution of the measurements. The measured radii of curvature, lengths, diameters, and the drill angle are denoted by prefixes "R", "L", "D", and "A", respectively. The numbering scheme of the dimensions starts at 1 from the sac exit and successively increases in the direction of flow. The drill angle is calculated by tracking the centroid through the hole, and calculating the angle between the line through the center of mass (dashed in Figure 3) and the $z$-axis.

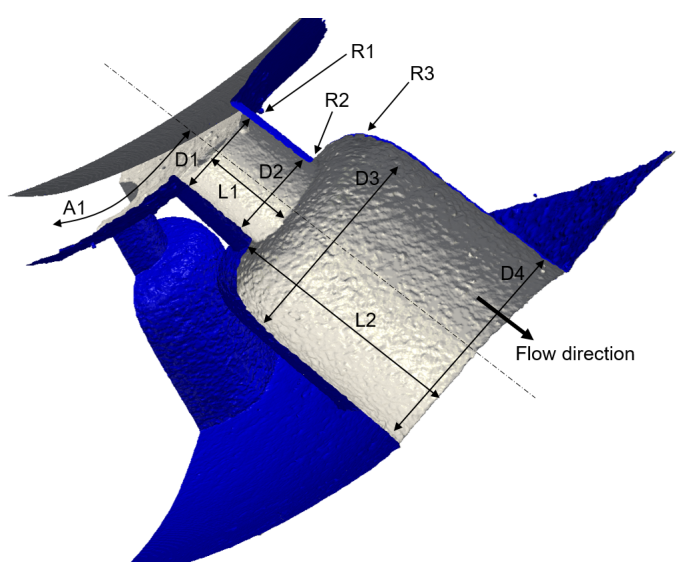

\begin{tabular}{l|l} 
ID & Feature $(\boldsymbol{\mu m})$ \\
\hline D1 & Hole inlet diameter \\
\hline D2 & Hole outlet diameter \\
\hline R1 & Hole inlet corner radius \\
\hline R2 & Hole outlet corner radius \\
\hline L1 & Hole length \\
\hline A1 & Drill angle \\
\hline D3 & Counterbore upstream diameter \\
\hline D4 & Counterbore downstream diameter \\
\hline R3 & Counterbore fillet \\
\hline L2 & Counterbore length
\end{tabular}

Figure 3. Measured hole dimensions of Spray G from x-ray nozzle tomography results.

In order to measure the dimensions summarized in Figure 3, each hole was individually rotated so that its horizontal axis aligned with the $x$-axis. A canny edge detector with a built-in Gaussian filter of specified standard deviation $\sigma=3$ was used to locate the hole cross-section at each slice along the $z$-plane [34]. A least-squares 
ellipse fitting algorithm $[35,36]$ was then used to measure the hole diameter and eccentricity. In general, because the eccentricity of the holes was typically less than the spatial resolution, an average of the diameters along the major and minor axes is quoted as the hole diameter. Any pixel location where the root-mean-square (RMS) error of the elliptic fitting exceeded 20 was removed from the analysis. The total uncertainty in the hole diameter measurements includes the spatial resolution, the RMS error associated with the elliptic fitting, and the error associated with approximating the cross-section as a perfect circle of 0 eccentricity. This error was typically dominated by the spatial resolution of $1.8 \mu \mathrm{m}$. In order to locate the hole inlet, a data point near the center of the hole was chosen as a starting point for a running average of the diameter, moving in the direction of the hole inlet. At each pixel location, the running average was updated, and the location of the hole inlet was defined as the point at which the diameter exceeded $3 \%$ of the running mean. A similar procedure was used to locate the hole and counterbore outlets. This technique proved successful in capturing the hole inlet and outlet locations because of the relatively cylindrical diameter profiles of both the hole and counterbore. The $3 \%$ threshold was chosen due to its ability to qualitatively capture the actual inlet and outlet locations. The hole outlet radius of curvature as well as the counterbore fillet were determined by fitting a 5th order polynomial to the hole corner profile and fillet, respectively. The radius of curvature was calculated using,

$$
C(\mu \mathrm{m})=\frac{\left(1+y^{\prime 2}\right)^{3 / 2}}{y^{\prime \prime}}
$$

where $y^{\prime}$ and $y^{\prime \prime}$ are the first and second derivatives of the fitted polynomial, respectively [29]. The radius of curvature corresponding to the corner was taken to be equal to $C_{m i n}$. Because it more accurately captured the curvature, a 6th order polynomial fit was used to model the hole inlet corner profile. Even though nominal dimensions are quoted in Table 1, it should be made clear that this work does not make an effort to compare measured to ideal dimensions, on account of the fact that the thresholds used to obtain the latter are unknown. For this reason, comparison between measured and nominal dimensions remains a subject for future work. The important point to note is that the same thresholds and methods of calculating nozzle dimensions are applied to all eight nozzles in order to maintain any errors associated with the data analysis as systematic errors, which would in turn not affect the relative trends in geometric variability.

Multiple linear regression analysis was used to fit a linear relationship between the steady-state PIM and the measured nozzle dimensions. Each nozzle hole was treated as a sample point, allowing statistics to be carried out on $n=64$ observations. The PIM was averaged during the steady portion of the spray, between $0.5<t<0.7 \mathrm{~ms}$. The general model for multiple linear regression, given $n$ observations, is,

$$
y_{i}=\beta_{0}+\beta_{1} x_{i, 1}+\beta_{2} x_{i, 2}+\ldots \beta_{m} x_{i}+\epsilon_{i} \text { for } i=1,2, \ldots n \text {, }
$$

where $y_{i}$ is the predictor variable (the average PIM), $x_{1} \ldots x_{m}$ are the explanatory variables (the dimensions listed in Figure 3), $\beta_{0} \ldots \beta_{i}$ are the coefficients, and $\epsilon$ is a normally distributed random variable to account for any noise in the data. To solve for the coefficients, a least-squares approach is used wherein the linear regression model attempts to minimize the sum of squared residuals between the observed and predicted values. In essence, this model attempts to describe the average PIM as a linear weighted function of the nozzle dimensions. The value of the adjusted coefficient of determination, $R^{2}$, is used to assess goodness of fit as well as to indicate whether or not the addition of a geometric feature improves the overall predictability of the model.

\section{Results and discussion}

To illustrate typical analysis results of spray tomography measurements as well as the binning procedure, Figure 4 plots the mean steady-state density field, $\bar{\rho}(x, y, t)$, for Spray $\mathrm{G} \# 12$, with and without masking density values that fall below a specified $10 \%$ threshold. All measured Spray G nozzles feature a well-atomized fuel spray at $2 \mathrm{~mm}$ downstream from the nozzle tip, with typical maximum liquid volume fractions of approximately 0.03 , as observed in previous work [7].

Figure 5a plots the PIM for all eight Spray G nozzles, in which the time of commanded start of injection occurs at $t=0 \mathrm{~ms}$. There is some variability in the apparent start and end of injection, as evidenced by the temporal spread of the PIM profiles. The apparent start of injection and the injection slope during the needle opening stage are not a strong function of injection pressure in GDI nozzles [37]. Aside from geometric variability, this may potentially be attributed to variable needle lift behavior. Needle motion measurements using x-ray phase contrast imaging at the APS have been previously obtained for Spray G \#28 [8]. It would be instructive to perform such measurements on the current injector set; this endeavor remains a topic for future work. In addition to the temporal spread, the PIM of Spray G \#22 is noticeably lower than that of the other injectors. Nevertheless, the variability in the steady-state PIM across all injectors is $2.4 \%$, which seems minor. Both Spray G \#12 and \#17 exhibited repeatable injector dribble after the end of injection. Figure $5 \mathrm{~b}$ displays this injector dribble, as well as hole-to-hole mass variations, for Spray G \#17. The hole numbering is consistent with that provided on the ECN website [6]. The mean variability in the hole-to-hole PIM for all eight injectors is $4.2 \%$. Table 1 provides a summary of the statistics for each measured dimension, calculated by treating each nozzle hole as an independent observation, i.e. with $n=64$ samples. In order to compare the variation from the mean value, we define the relative standard deviation (RSD) as,

$$
\mathrm{RSD}=\sigma / \mu,
$$




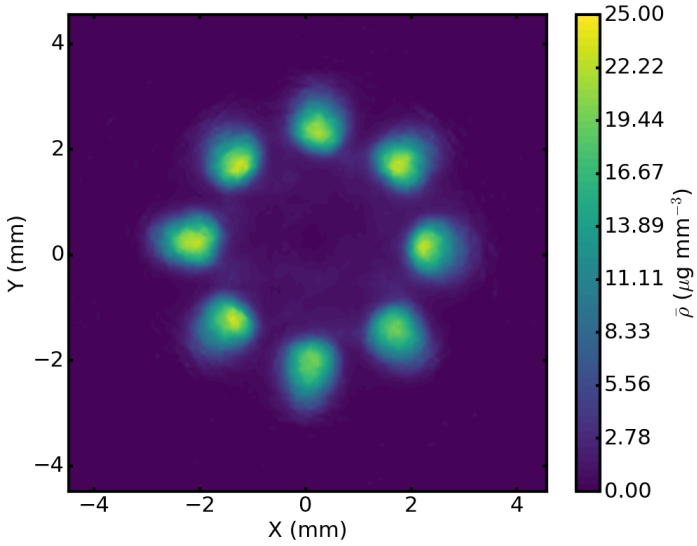

(a) Unmasked mean density field.

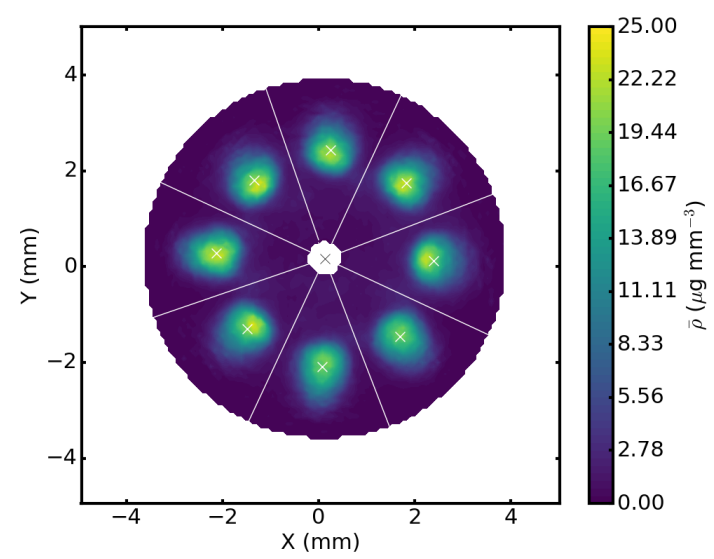

(b) Masked mean density field (mask is depicted by the white region). The crosses mark the center of mass of the holes (white) and the bulk spray (black). The data are binned into eight collectively exhaustive bins depicted by the white lines emanating radially from the center.

Figure 4. The mean density field for Spray G \#12, temporally averaged between 0.5 and $0.7 \mathrm{~ms}$.

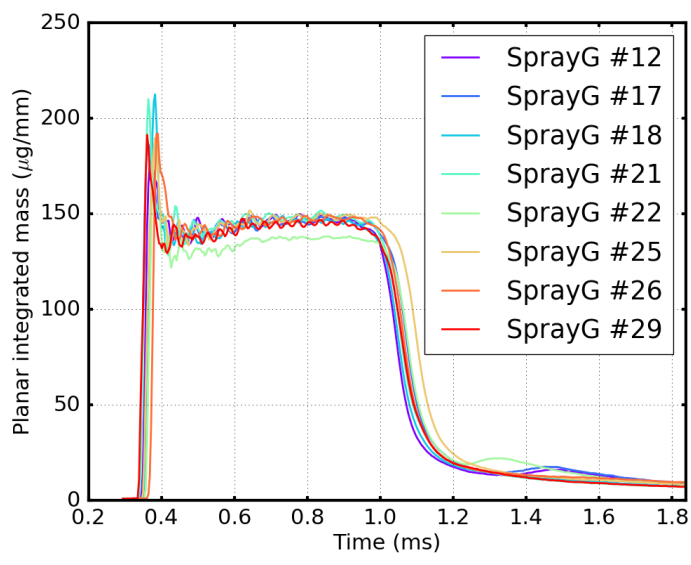

(a)

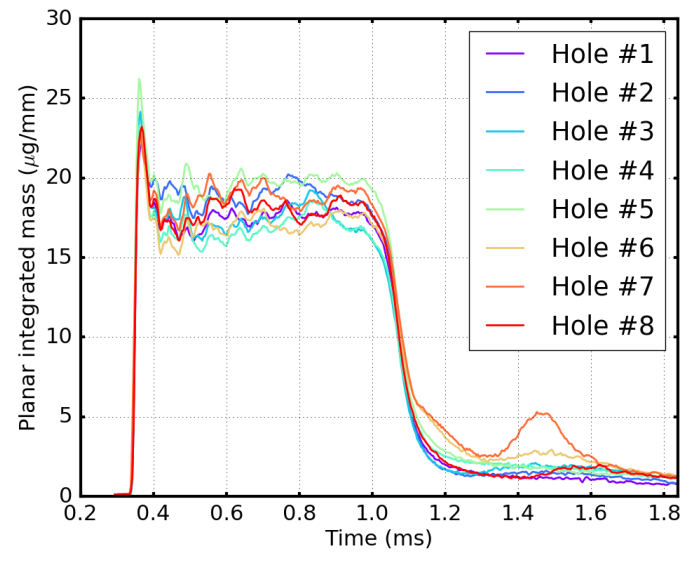

(b)

Figure 5. Planar integrated mass as a function of time for (a) all eight Spray G nozzles and (b) Spray G \#17.

where $\mu$ and $\sigma$ are the sample mean and standard deviation, respectively. This parameter shows the variability in relation to the mean, allowing for comparison among nozzle dimensions that vary in magnitude.

Generally, the largest variability is among the hole inlet and outlet corner radii. Table 2 lists the results of the multiple linear regression analysis, as well as the standard errors. $33.2 \%$ of the variability in the mean mass can be explained by variations in the inlet hole corner radius, the hole length, and the counterbore upstream diameter. The hole inlet corner radius has the strongest correlation with PIM variation. Because the coefficient is positive, the PIM is predicted to increase with increasing inlet hole radius. Simulations using the nominal geometry showed that the inlet corners can produce a pressure drop high enough for vapor formation [8]. However, the nominal geometry mesh features very sharp corners, and it is unknown whether this effect would still occur using a modified mesh with the prescribed dimensions listed in Table 1. In addition, inspection of select isosurfaces reveals voids at the hole inlet as large as $10 \mu \mathrm{m}$ in diameter, which can perturb the internal flow as the fuel enters the hole [38]. Increasing the inlet hole corner radius is expected to decrease the propensity for cavitation formation, which would in turn increase the discharge through the hole, in agreement with the regression analysis. The existence of vapor in the holes may also explain the temporal fluctuations in the PIM during steady injection, as shown in Figure 5 [8]. Time-resolved measurements of the needle motion and injection rate would provide further insight into this notion. The regression model also predicts that a larger counterbore upstream diameter decreases the mean steady-state mass. A potential reason for this may be increased area for mixing of fuel with ingested ambient gas inside the counterbore. Lastly, hole length is positively correlated with the mean PIM. An increase in hole length results in a larger ratio of length to diameter (L/D), which has been previously shown to suppress atomization [39]. 
Table 1. Hole dimensions measured by $\mathrm{x}$-ray nozzle tomography, as well as nominal dimensions from the manufacturer.

\begin{tabular}{c|cccc}
\hline Feature ID & Mean $(\mu \mathrm{m})$ & Standard deviation $(\mu \mathrm{m})$ & RSD $(\%)$ & Nominal $(\mu \mathrm{m})$ \\
\hline D1 & 175 & 1.14 & 0.65 & 165 \\
D2 & 176 & 1.00 & 0.57 & 165 \\
R1 & 4.93 & 0.88 & 18 & 0 \\
R2 & 2.54 & 0.47 & 18 & 0 \\
L1 & 150 & 10.50 & 7.0 & 170 \\
A1 & 38 & 0.47 & 1.3 & 37 \\
D3 & 394 & 1.29 & 0.33 & 388 \\
D4 & 394 & 1.27 & 0.32 & 388 \\
R3 & 65 & 3.30 & 5.1 & 40 \\
L2 & 402 & 11.18 & 2.8 & 470 \\
\hline
\end{tabular}

Table 2. Regression coefficients and standard errors.

\begin{tabular}{c|cc}
\hline Predictor Variable & Value & Standard error \\
\hline Constant, $\beta_{0}$ & 17.90 & 0.095 \\
R1, Hole inlet corner radius & 0.43 & 0.110 \\
L1, Hole length & 0.020 & 0.009 \\
D3, Counterbore upstream diameter & -0.21 & 0.074 \\
\hline
\end{tabular}

\section{Conclusions}

Measurements of the time-resolved density field at $z=2 \mathrm{~mm}$ downstream of the nozzle tip were obtained for eight nominally duplicate eight-hole Spray G nozzles using x-ray spray tomography. In addition, critical hole dimensions were retrieved from geometry measurements obtained by x-ray nozzle tomography. Both experiments were carried out at the 7-BM beamline of the APS at Argonne. Analysis of the PIM of each nozzle revealed subtle variations in the apparent start and end of injection timing among the injectors, as well as injector dribble from a few holes for two of the injectors. A multiple linear regression model was implemented to model the variability in the mean hole mass as a function of the hole's geometric features. Approximately $33 \%$ of the variation in the steady-state mass can be attributed to variations in the hole inlet radius of curvature, hole length, and counterbore upstream diameter. The remaining measured dimensions did not improve the predictability of the model. In general, the variability in the nozzle dimensions was low except for the hole inlet and outlet corner radii.

There are a variety of avenues to explore with regard to the future direction of this analysis. The strength of the statistical model may be improved by the addition of more Spray G nozzle holes, specifically targeting outlier injectors that may be expected to feature large variability. Integrating rate of injection and needle motion measurements into the analysis is also possible. The effect of nozzle upstream conditions on specific holes should be investigated by running an analysis that considers the spatial location of each hole, rather than treating all holes as independent observations. Considering the maximum and minimum dimensions in the analysis, as well as azimuthally resolving the inlet and outlet corner radii, may also prove useful in explaining more of the mass variability. In addition, quantifying surface roughness and the frequency of voids should be considered. Further data acquisition and data mining could explore such considerations in the future. At present, the analysis helps highlight which dimensions may need to be treated more carefully in both computational modeling efforts as well as in manufacturing practices, and conversely, which ones do not seem to strongly affect the ejected mass. Lastly, it should be noted that the linear regression model can only predict correlation, and not a cause-and-effect relationship. As such, the model findings will require further experimental and numerical investigation to prove causality between the relevant nozzle dimensions and fuel spray characteristics.

\section{Acknowledgments}

This research was performed at the 7-BM beamline of the APS at Argonne National Laboratory. Use of the APS is supported by the U.S. Department of Energy (DOE) under Contract No. DE-AC02-06CH11357. We gratefully acknowledge the computing resources provided on Blues, a high-performance computing cluster operated by the Laboratory Computing Resource Center at Argonne National Laboratory. We thank Dr. Doga Gürsoy for the use of TomoPy and corresponding user support, as well as Dr. Xianghui Xiao at the APS 2-BM beamline for technical guidance in performing x-ray tomography. Argonne's x-ray fuel injection research is sponsored by the DOE Vehicle Technologies Program under the direction of Gurpreet Singh and Leo Breton. 


\section{References}

[1] Duke, D.J., Swantek, A.B., Sovis, N., Tilocco, F.Z., Powell, C.F., Kastengren, A.L., Gürsoy, D. and Biçer, T., 2016, SAE Int. J. Engines, 9(1), pp. 143-152.

[2] Zhang, M., Drake, M.C., and Peterson, K., 2013, Proceedings of the ASME 2013 Internal Combustion Engine Division Fall Technical Conference.

[3] Sou, A., Minami, S., Prasetya, R.H., Moon, S., Wada, Y., and Yokohata, H., Aug 2015, 13th Triennial International Conference on Liquid Atomization and Spray Systems.

[4] Payri, R., Viera, J.P., Gopalakrishnan, V., and Szymkowicz, P.G., 2016, Fuel, 183, pp.20-33.

[5] Chaves, H. and Ludwig, Ch., 2005, Proceedings of the 20th ILASS Europe Meeting.

[6] Sandia National Laboratories. Engine Combustion Network "Spray G" injector plume orientation, https://ecn.sandia.gov/gasoline-spray-combustion/target-condition/spray-g-plume-orientation/ ([cit. 2017-03-07]).

[7] Strek, P., Duke, D., Swantek, A., Kastengren A., Powell, C.F., and Schmidt, D.P., 2016, SAE Technical Paper, 2016-01-0858.

[8] Baldwin, E.T., Grover Jr., R.O., Parrish, S.E., Duke, D.J., Matusik, K.E., Powell, C.F., Kastengren, A.L., and Schmidt, D.P., 2016, Int. J. Multiphase Flow, 87, pp. 90-101.

[9] Linne, M., 2013, Prog. in Energy and Comb. Sci., 39, pp. 403-440.

[10] Paryi, R., Salvador, F.J., Martí-Aldaravií, P., and Vaquerizo, D., 2017, Appl. Therm. Eng., 112, pp. 304-316.

[11] Aleiferis, P.G., Serras-Pereira, J., van Romunde, Z., Caine, J., and Wirth, M., 2010, Comb. and Flame, 157, pp.735-756.

[12] Mitroglou, N., Nouri, J.M., Gavaises, M., and Arcoumanis, C., 2005, Int. J. Engine Res. 7, pp. 255-270.

[13] Das, S., Chang, S.-I., and Kirwan, J., 2009, SAE Technical paper, 2009-01-1488.

[14] Payri, R., Gimeno, J., Marti-Aldaravi, P., and Vaquerizo, D., 2015, SAE Technical Paper, 2015-01-1893.

[15] Als-Nielsen, J. and McMorrow, D., 2011, "Elements of Modern X-Ray Physics". John Wily \& Sons.

[16] Lee, J.S., Weon, B.M.,and Je, J.H., 2013, J. Phys. D: Appl. Phys., 46:494006.

[17] Kastengren, A.L., and Powell, C.F., 2014, Exp. Fluids, 55(3): 1686.

[18] Marchitto, L., Hampai, D., Dabagov, S.B., Allocca, L., Alfuso, S., Polese, C., and Liedl, A., 2015, Int. J. Multiphase Flow, 70, pp. 15-21.

[19] Bartolucci, L., Scarcelli, R., Wallner, T., Swantek, A., Powell, C.F., Kastengren, A., and Duke, D., 2016, SAE Technical Paper, 2016-01-0850.

[20] Swantek, A., Kastengren, A. Duke, D., Tilocco, Z. Sovis, N., and Powell, C.F., 2015, SAE Int. J. Fuels Lubr., 8(1), pp.160-166.

[21] Kastengren, A.L., Powell, C.F., Zunping, L., and Wang, J., 2009, SAE Technical Paper, 2009-01-0840.

[22] Cai, W., Powell, C.F., Yue, Y., Narayan, S., Wang, J., Tate, M.W., Renzi, M.J., Ercan, A., Fontes, E., Gruner, S.M., 2003, Appl. Phys. Lett. , 83(8), pp. 1671-1673.

[23] Liu, X., Cheong, S.-K., Powell, C.F., Wang, J., Hung, D.L.S., Winkelman, J.R., Tate, M.W., Ercan, A., Schuette, D.R., Koerner, L., Gruner, S.M., May 2005, 18th Annual Conference on Liquid Atomization and Spray Systems.

[24] Macian, V., Bermúdez, V., Payri, R., and Gimeno, J., 2003, Experimental Techniques, 27(2), pp.39-43.

[25] Kastengren, A.L., Tilocco, F.Z., Powell, C.F., Manin, J., Pickett, L.M., Payri, R. and Bazyn, T., 2012, Atomization and Sprays, 22(12), pp. 1011-1052.

[26] Feser, M., Gelb, J., Chang, H., Cui, H., Duewer, F., Lau, S.H., Tkachuk, A., and Yun, W., 2008, Meas. Sci. Technol., 19:094001.

[27] Wang, Y., De Carlo, F., Mancini, D.C., McNulty, I., Tieman, B., Bresnahan, J., Foster, I., Insley, J., Lane, P., von Laszewki, G., Kesselman, C., Su, MH., and Thiebaux, M., 2001, Rev. of Sci. Instr. 72(4), pp. 2062-2068.

[28] Pateyron, M., Peyrin, F., Laval-Jeantet, A.M., Spanne, P., Cloetens, P., and Peix, G., 1996, SPIE Med. Imag. 2708, pp.417-426.

[29] Kastengren, A., Powell, C.F., Arms, D., Dufresne, E.M., Gibson, H., and Wang, J., 2012, Journal of Synchrotron Radiation, 19(4), pp. 654-657.

[30] Matusik, K.E., Duke, D.J., Kastengren, A.L., Sovis, N., Swantek, A.B., and Powell, C.F., 2017, Int. J. Engine Res., submitted.

[31] Gürsoy, D., De Carlo, F., Xianghui, X., and Jacobsen, C., 2014, Journal of Synchrotron Radiation, 21(5), pp. 1188-1193.

[32] Marone, F. and Stampanoni, M., 2012, Journal of Synchrotron Radiation, 19(6), pp. 1029-1037.

[33] Chang, J.-H., Anderson, J.M.M., and Votaw, J.T., 2004, Medical Imaging, IEEE Transactions on, 23(9), pp.1165-1175.

[34] Canny, J., 1986, IEEE Trans. Pattern Analysis and Mach. Intel., 8, pp. 679-714.

[35] Fitzgibbon, A.W., Pilu, M., and Fischer, R.B., 1996, Proceedings of the 13th International Conference on Pattern Recognition, pp. 253-257.

[36] "Fitting an ellipse to a set of data points", http://nicky.vanforeest.com/misc/fitEllipse/fitEllipse.html ([cit. 2017-03-07]).

[37] Payri, R., Gimeno, J., Marti-Aldaravi, P., Vaquerizo, D., 2016, Atomization and Sprays, 26(9), pp. 889-919.

[38] Duke, D., Finney, C., Kastengren, A., Matusik, K., Sovis, N., Santodonato, L., Bilheux H., Schmidt, D., Powell, C., and Toops, T., 2017, SAE Technical Paper, 2017-01-0824.

[39] 2012, "Fuel Systems for IC Engines", edited by IMechE, Woodhead Publishing. 


\title{
Flashboiling atomization in nozzles for GDI engines
}

\author{
Sebastian Bornschlegel $^{\star 12}$, Chris Conrad ${ }^{12}$, Lisa Eichhorn ${ }^{12}$, Michael Wensing ${ }^{12}$ \\ ${ }^{1}$ Institute of Engineering Thermodynamics (LTT), FAU Erlangen-Nürnberg, Germany \\ ${ }^{2}$ Erlangen Graduate School in Advanced Optical Technologies (SAOT), \\ FAU Erlangen-Nürnberg, Germany \\ *Corresponding author: sebastian.bornschlegel@fau.de
}

\begin{abstract}
Flashboiling denotes the phenomenon of rapid evaporation and atomization at nozzles, which occurs when fluids are injected into ambient pressure below their own vapor pressure. It happens in gasoline direct injection (GDI) engines at low loads, when the cylinder pressure is low during injection due to the closed throttle valve. The fuel temperature at the same time approaches cylinder head coolant temperature due to the longer dwell time of the fuel inside the injector. Flash boiling is mainly beneficial for atomization quality, since it produces small droplet sizes and relative broad and homogenous droplet distributions within the spray. Coherently, the penetration depth normally decreases due to the increased aerodynamic drag. Therefore the thermal properties of injectors are often designed to reach flash boiling conditions as early as possible. At the same time, flash boiling significantly increases the risk of undesired spray collapsing. In this case, neighbouring jets converge and form a single jet. Due to the now concentrated mass, penetration depth is enhanced again and can lead to liner or piston wetting in addition to the overall diminished mixture formation.

In order to understand the underlying physics, it is important to study the occurring phenomena flashboiling and jet-to-jet interacting i.e. spray collapsing separately. To this end, single hole injectors are built up to allow for an isolated investigation of flashboiling. The rapid expansion at the nozzle outlet is visualized with a microscopic high speed setup and the forces that lead to the characteristic spray expansion are discussed. Moreover, the results on the macroscopic spray in terms of penetration, cone angles and vapor phase are shown with a high speed Schlieren setup. Resulting droplet diameters and velocities are measured using LDA/PDA.

As a result, we find a comprehensive picture of flash boiling. The underlying physics can be described and discussed for the specific case of high pressure injection at engine relevant nozzle geometries and conditions, but independently from neighbouring jets. These findings provide the basis to understand and investigate flashboiling and jet-to-jet interaction as distinct, but interacting subjects rather than a combined phenomenon.
\end{abstract}

\section{Keywords}

Flashboiling, GDI, Single-Hole-Injector, High-Speed, LDA/PDA

\section{Introduction}

With the introduction of gasoline direct injection (GDI) systems in significant shares in the worldwide market, new challenges for spray and combustion layout were generated, originating from the increased injection pressure as well as the hotter injector and fuel temperatures due to the changed injector mounting position.

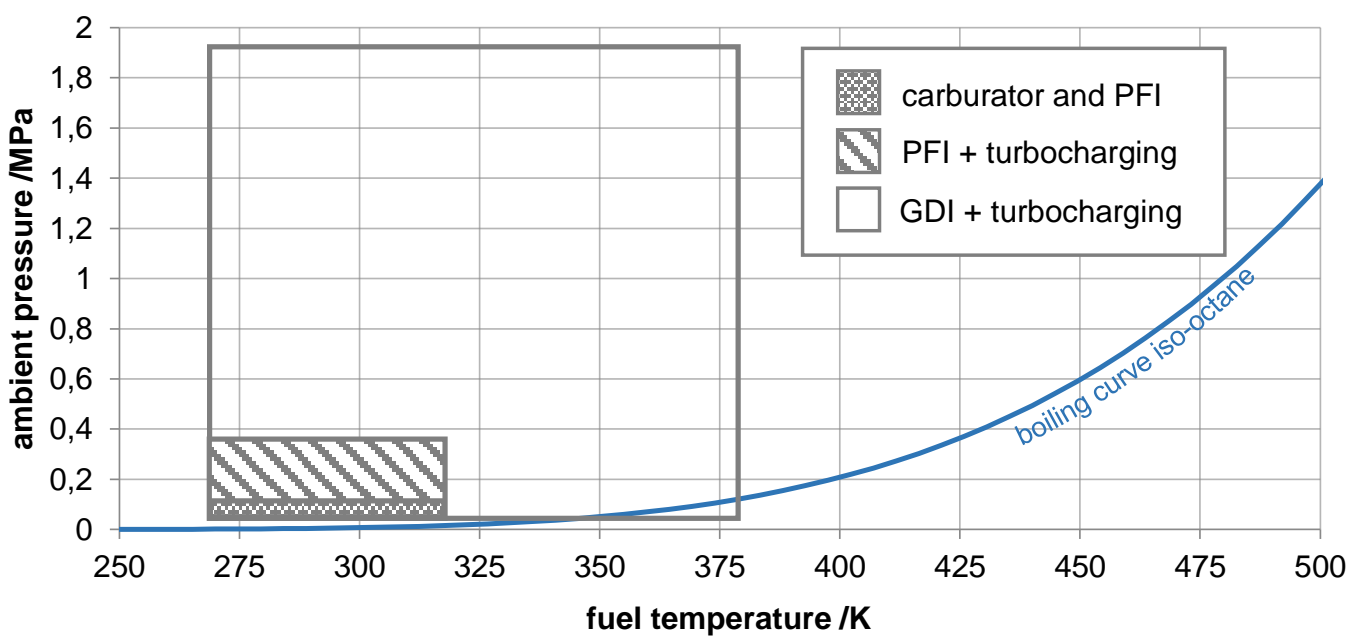

Figure 1. $p$-T diagram of iso-octane with possible fuel conditions for different injection systems 
Figure 1 shows estimated possible injection conditions relative to the boiling curve of the fuel surrogate iso-octane for different injection systems, particularly. One effect that is in focus ever since GDI entered the market is flashboiling. It denotes the phenomenon of rapid evaporation and atomization at nozzles, which occurs when fluids are injected into ambient pressure below their own vapor pressure and correlates to the lower right corner in Figure 1. In GDI engines, this happens at low loads, when the cylinder pressure is low due to closed throttle valves. At the same time, the dwell time of the fuel inside the injector increases due to low injected mass and low engine revolutions, causing the fuel to approach cylinder head coolant temperature. During injection the pressure then drops below the fuels vapor pressure as indicated in Figure 2 for an exemplary fuel pressure of $15 \mathrm{MPa}$ and the fuel-surrogate iso-octane, where the vertical arrow displays an isenthalpic throttling from in-injector conditions to chamber conditions. The horizontal arrows mark the separation of liquid and vapor phase due to superheating, with the respective arrow length representing vapor and liquid fractions. Real gasoline fuel in contrast to iso-octan is a multi-component mixture. This means that the boiling curve is no well-defined line but the mixture evaporates in a certain range instead. This means for real fuel, a small fraction of the mixture is superheated under nearly all conditions. Iso-octane however is a good average for the components in real fuel and shows comparable behaviour.

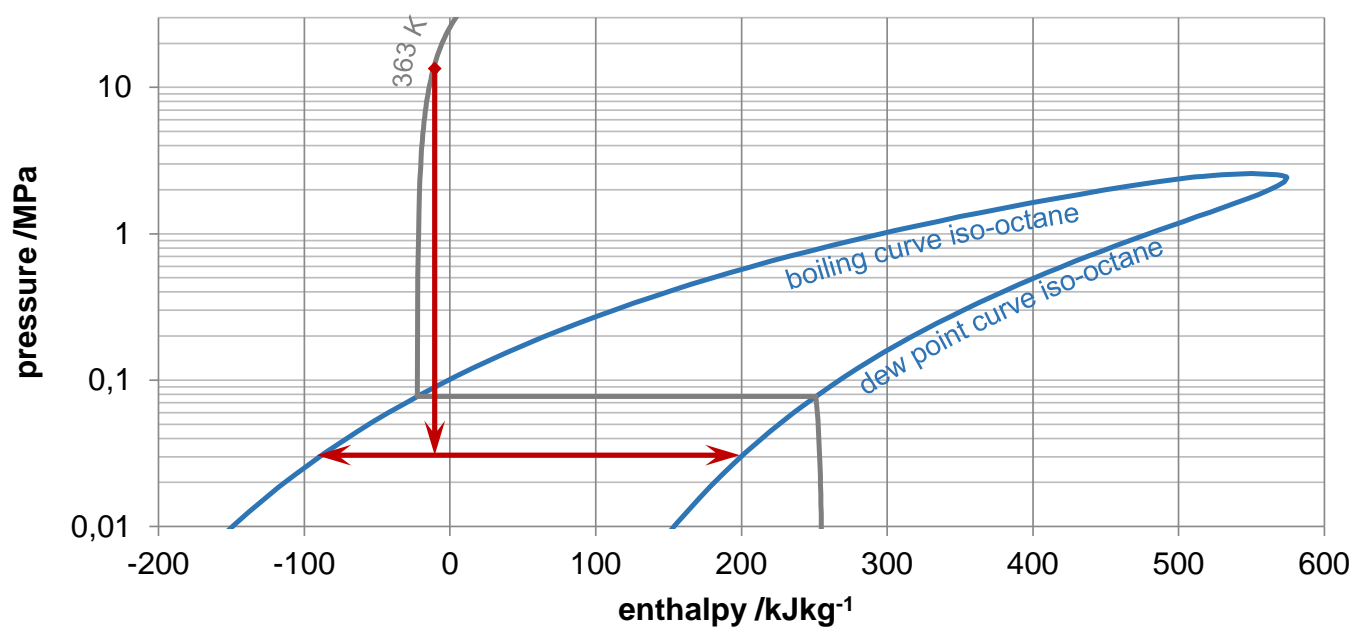

Figure 2. p-h-diagramm of iso-octane with an exemplary injection from $15 \mathrm{MPa}$ fuel pressure to $0.03 \mathrm{MPa}$ ambient pressure at $363 \mathrm{~K}$ fuel temperature

Flashboiling is usually described as a three step process [1],[2]. When the degree of superheating is so high that enthalpy cannot be emitted by heat transfer and evaporation at the free surface, evaporation starts within the fluid volume, causing the formation of gas bubbles. This effect is referred to as nucleation. Those nuclei grow during the next step which is referred to as bubble growth, till they reach an instable size. In the last state of flashboiling they collapse, which leads to the creation of new free surface and consequently boosts atomization [3]. Wood shows a decrease in the Sauter-Mean diameter $D_{32}$ of $42 \%$ for a flash boiling spray [4]. The spray used however is a six-hole spray, that shows considerable collapsing under flash boiling conditions. In this case, neighbouring jets converge and form a single jet. Due to the now concentrated mass, penetration depth is enhanced again and can lead to liner or piston wetting in addition to the overall diminished mixture formation. Zeng shows this behaviour for an eight-hole injector [5]. For low degrees of superheating, spray width is enhanced and penetration depth decreased. For higher degrees of superheating the spray collapses and the spray penetration increases again while the spray width decreases. Krämer points out that flashboiling and jet-to-jet interaction are separate phenomena that however influence each other [6]. In order to understand the underlying physics of both effects, it is important to study the occurring phenomena flashboiling and jet-to-jet interacting i.e. spray collapsing separately. To this end, in this paper single hole injectors are used to allow for an isolated investigation of flashboiling.

\section{Material and methods}

The injector used is a common GDI solenoid injector which however has just one single hole. To keep the inflow conditions as realistic as possible, the hole is not placed centrally but on a usual reference circle, resulting in a jet height-angle of $30^{\circ}$ from injector axis. Both hole diameter and L/D ratio are unchanged from the original multi-hole design. The injectors are investigated with three different measurement techniques and results from four 
operation points (OP) are shown in this paper as depicted in Table 1. Each OP is defined by fuel pressure, fuel temperature, chamber pressure, chamber temperature and the injection duration $t_{i}$.

Table 1. Operation Points

\begin{tabular}{|c|c|c|c|c|c|c|c|}
\hline OP & $\bar{T}_{\text {fuel }}$ & $p_{\text {fuel }}$ & $\overline{T_{\text {chamber }}}$ & $\mathbf{p}_{\text {chamber }}$ & $\mathbf{t}_{\mathbf{i}}$ & $\mathrm{DS}_{\mathrm{p}}$ & $\mathrm{DS}_{\mathrm{h}}$ \\
\hline 1 & \multirow{2}{*}{$303 \mathrm{~K}$} & \multirow{4}{*}{$15 \mathrm{MPa}$} & \multirow{4}{*}{$298 \mathrm{~K}$} & $0.1 \mathrm{MPa}$ & \multirow{4}{*}{$1.5 \mathrm{~ms}$} & 0,08 & -0,53 \\
\hline 2 & & & & $0.03 \mathrm{MPa}$ & & 0,28 & $-0,19$ \\
\hline 3 & \multirow{2}{*}{$363 \mathrm{~K}$} & & & $0.1 \mathrm{MPa}$ & & 0,77 & $-0,03$ \\
\hline 4 & & & & $0.03 \mathrm{MPa}$ & & 2,58 & 0,27 \\
\hline
\end{tabular}

All measurements are performed with iso-octane as a fuel surrogate, due to its well-known properties. The degree of superheating generally can be represented by two expressions, the ratio of vapor pressure to ambient pressure as shown in equation (1) or the ratio of inherent enthalpy of the fluid to the enthalpy of phase transition as shown in (2). The latter is partly similar to the dimensionless Jacob-number that however often is defined in a volumespecific way [7].

$$
\begin{aligned}
& D S_{p}=\frac{p_{\text {sat }}\left(T_{i n j}\right)}{p_{a}} \\
& D S_{h}=\frac{h_{i n j}-h_{l}}{h_{v}-h_{l}}
\end{aligned}
$$

Index "sat" refers to saturation conditions at the state point on the boiling curve defined by the thermodynamic quantity given in brackets. Index "inj" refers to the conditions inside the injector, while index "a" refers to ambient i.e. chamber conditions. $\mathrm{h}$ is the enthalpy and indices "l" and " $\mathrm{v}$ " refer to the liquid and vapor phase. While the first definition is common in technical applications, because the necessary values are gained more easily also for real multi-component fuels, the enthalpy-based definition provides more information. A negative value means the fluid is not superheated while values between 0 and 1 give the amount of fuel that can be evaporated solely by the inherent enthalpy of the fuel, and a value above 1 means that full phase transition can be achieved during flashboiling. The values from both definitions are given in Table 1. In this case, only OP 4 provides real superheated conditions, while OP 2 and 3 show the effects of reducing chamber pressure and increasing fuel temperature respectively, without crossing the boiling curve of iso-octane.

Three measurement techniques are used to examine the spray. The full spray behaviour is visualized by high speed Schlieren imaging. To this end a folded Z-type Schlieren setup with 6 inch parabolic field mirrors is used. The light source is a high-power LED array in cw-mode, the camera a Photron SA-Z high-speed-camera that is used at a repetition rate of $20 \mathrm{kHz}$. The images are used to determine macroscopic spray paramters e.g. penetration depth and cone angle. Due to its sensitivity towards gradients in the refractive index, the Schlieren measurement technique is sensitive towards both liquid and vapor phase. To verify the obtained results, they were compared to results from two different integral spray measurement techniques, Mie scattering and shadowgraphy. It can be shown that for the operation points considered in this publication, the Schlieren measurement technique shows the same results as the other two techniques that are only sensitive towards the liquid phase due to their measurement principle. This means that a separation of liquid and vapor phase does not occur in the spray on a macroscopic scale and that the results are therefore not depending on the sensitivity of the measurement technique towards gaseous fuel. This however is only true for operation points with comparably slow heat transfer from the surrounding gas phase and therefore low evaporation rates.

To provide microscopic spray parameters i.e. droplet sizes and velocities, a commercial Dantec fibre PDA system is used. In total 119 measurement positions are investigated for each operation point, with a repeat count of 60 injections for each position. All measurement positions lie in a common plane through both injector and jet axis as depicted in Figure 6.

Finally the nozzle near region is investigated by line of sight close up measurements in ultra-high-speed with framerates of 5 and 2 million frames per second to investigate the initial process of flashboiling itself. To this end a SI-AD 500 high power flash lamp and a Kirana camera were used, both provided by Specialised Imaging, in combination with a Questar QM-100 long distance microscope as well as a Navitar 12x Zoom Lense.

\section{The effect of flashboiling on macroscopic spray parameters}

A jet usually is described by two basic macroscopic parameters, its penetration depth and its jet cone angle. For the latter, a large number of definitions are in use, depending of the particular application. In this paper, a 
definition proposed in [8] is used. In addition, the jet volume is calculated under the assumption of an axissymmetrical spray-shape. To give a visual impression of the effect of flashboiling on the spray shape, in Figure 3 raw images for each OP are compared at a similar time after actuating of the injector i.e. after electrical start of injection (eSOI).
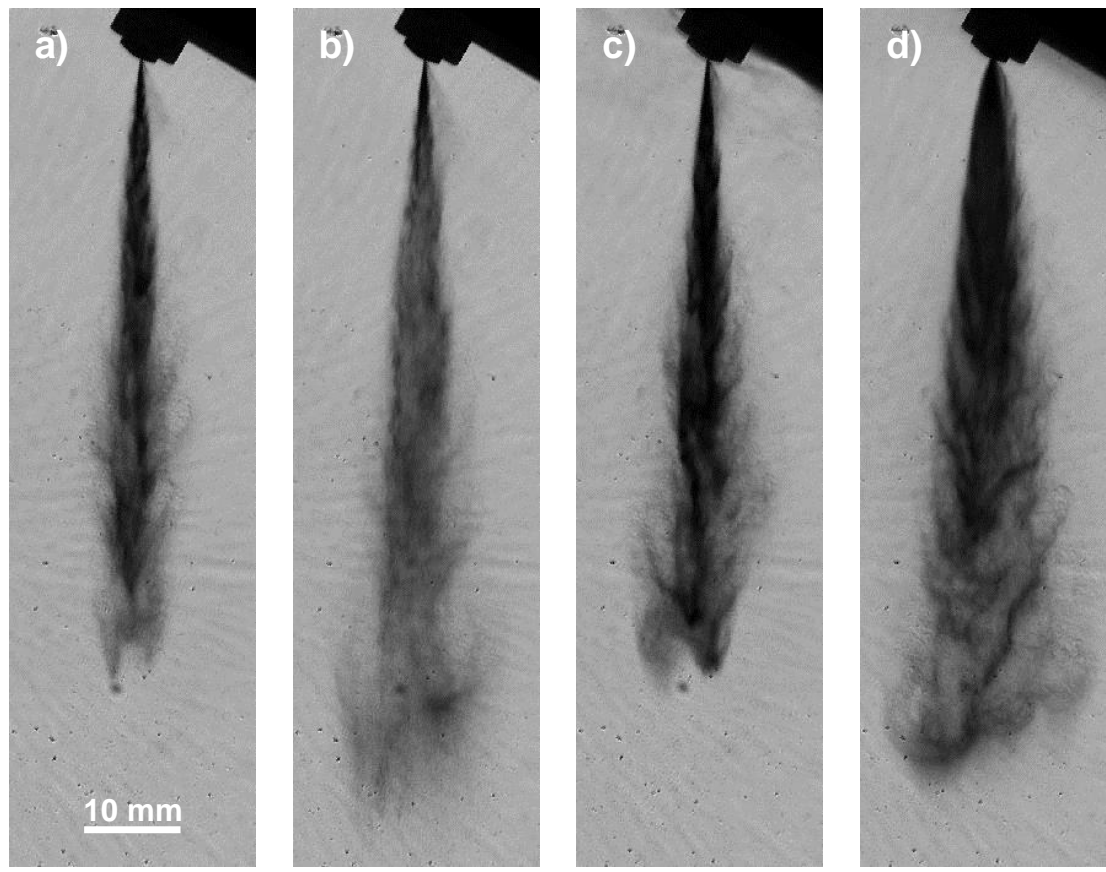

Figure 3. Raw Schlieren images $1000 \mu \mathrm{s}$ after eSOI for a) OP1 (303 K / $0.1 \mathrm{MPa})$, b) OP2 (303 K / $0.03 \mathrm{MPa})$, c) OP3 (363 K/ $0.1 \mathrm{MPa})$ and d) OP4 (363 K / $0.03 \mathrm{MPa})$

Even though injection pressure and duration are kept constant, the spray form changes significantly between the four OPs. On the upper side of the spray, for each OP a small fog of droplets is visible, that is most likely caused by the changed inflow of the single hole nozzle. This however can be neglected in evaluation and the jet itself behaves comparable to a jet from a multi hole injector. The different spray forms are also visible in the temporal evaluation of the high speed images.
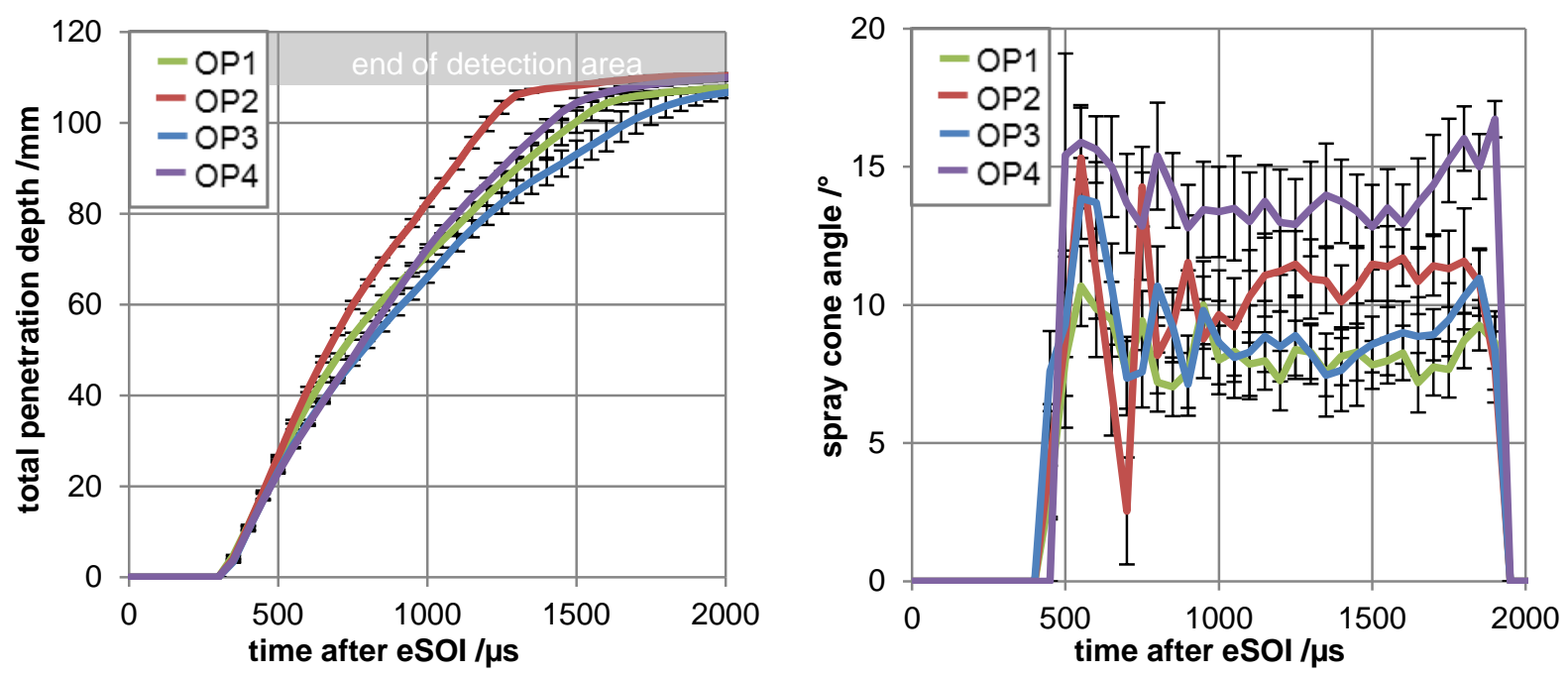

Figure 4. Total penetration depth and spray cone angle relative to time after eSOI for OP1 ( $303 \mathrm{~K} / 0.1 \mathrm{MPa}), \mathrm{OP} 2(303 \mathrm{~K} / 0.03$ $\mathrm{MPa})$, OP3 (363 K / $0.1 \mathrm{MPa})$ and OP4 (363 K/ $0.03 \mathrm{MPa})$

Figure 4 on the left shows the temporal development of the total spray penetration depth (absolute distance between spray front and nozzle outlet). Note that at around $105 \mathrm{~mm}$ the end of the optical access and therefore 
the maximum possible penetration depth is reached. Generally an increase in penetration depth can be found for OPs with lower ambient density and therefore lower gas density. Higher fuel temperature in contrast reduces the penetration depth. Both effects are found for the non-superheated OPs as well as for the superheated one, even though the effect of heated fuel is stronger between OP2 and OP4 than between OP1 and OP3.

Figure 4 on the right shows the spray cone angle. While both OPs at $0.1 \mathrm{MPa}$ ambient pressure show similar spay cone angles of around $8^{\circ}$ in the stationary spray phase, a decrease in ambient pressure results in a wider spray angle for both hot and cold fuel conditions. For the superheated spray however, this effect is significantly stronger with a spray cone angle of around $14^{\circ}$ in the stationary phase. This effect is also visible in Figure 3 , where the spray for OP4 is noticeable broader than the other OPs.

Since the spray penetration depth only significantly diverges in regions more than $20 \mathrm{~mm}$ away from the nozzle outlet the differences have to be caused by aerodynamic drag. While the reduction of aerodynamic drag with decreasing pressure is due to the decreasing ambient density, the increase with temperature is due to larger spray front cross section that can also be obtained in the increasing spray cone angle.
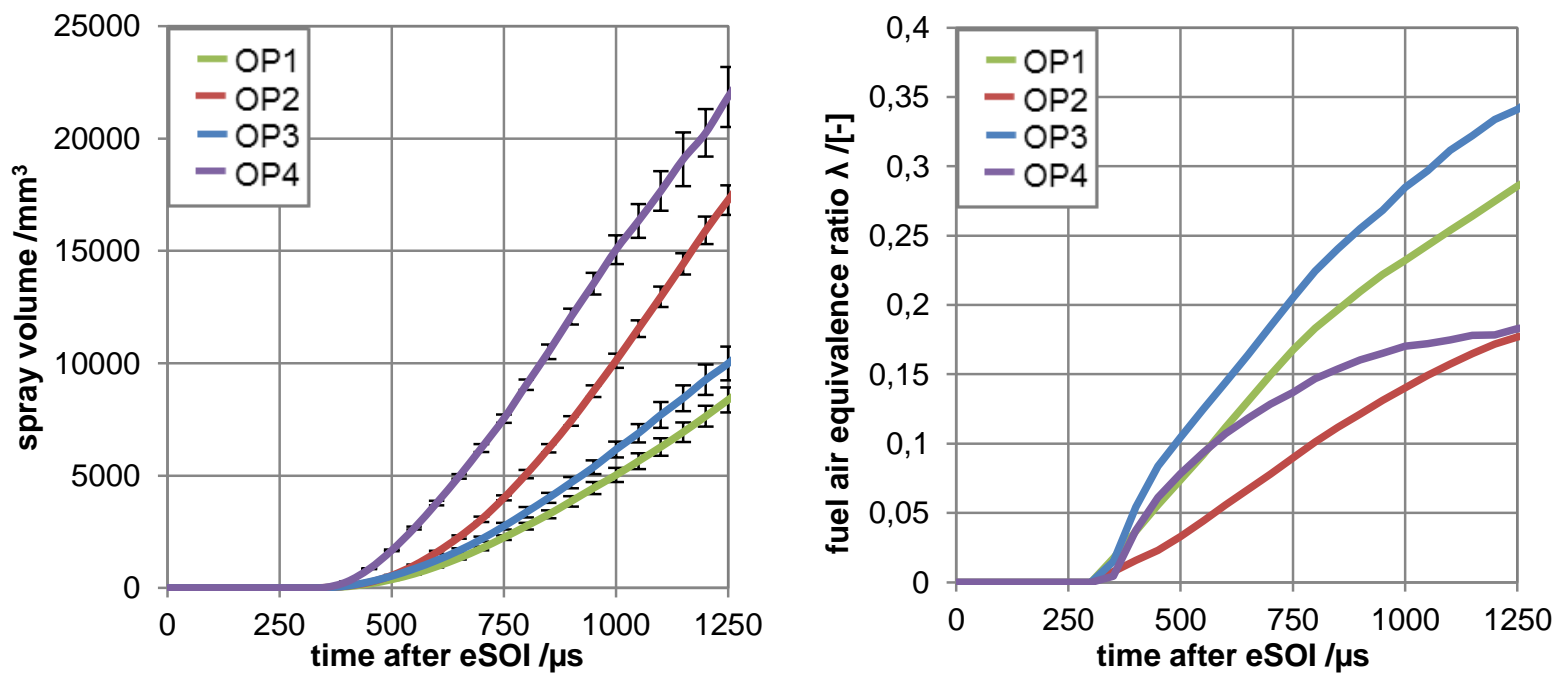

Figure 5. Spray volume and calculated fuel-air ratio relative to time after eSOI for OP1 ( $303 \mathrm{~K} / 0.1 \mathrm{MPa})$, OP2 ( $303 \mathrm{~K} / 0.03$ $\mathrm{MPa})$, OP3 (363 K / $0.1 \mathrm{MPa})$ and OP4 (363 K / $0.03 \mathrm{MPa})$

Under the assumption of an axis symmetrical jet, the spray volume can be calculated from the detected spray area. Its temporal development is given in Figure 5 on the left side. Both, an increase in fuel temperature and a decrease in ambient pressure result in an increased spray volume. While the non-superheated OPs start on the same level and diverge over time, the flash boiling spray shows a significantly increased spray volume right from the visible start of injection ( $\mathrm{vSOI}$ ).

Based on the spray volume and the injected fuel mass known from injection rate measurements, the global fuelair ratio within the spray can be estimated. This is based on the two assumption that the injected fuel mass is constant for all 4 OPs and that the liquid-vapor fraction of the fuel can be estimated assuming isenthalpe throttling. Both assumptions are simplifications, as a change in mass-flow due to changed fuel properties is neglected as well as enthalpy that adds to energy forms different than heat of evaporation, such as free surface energy. Nevertheless, an estimation of the fuel-air ratio is possible as the neglected terms are sufficiently small. The assumptions given lead to the following mathematical expression:

$$
\lambda=\frac{n_{O_{2}}}{n_{O_{2}, \text { min }}}=\frac{\frac{V_{\text {spray }}-\left(m_{\text {fuel }} \cdot \rho_{\text {fuel }}\right)}{V_{\text {mol }}}}{m_{\text {fuel }} \cdot A_{\text {st }}}
$$

The minimum stoichiometric oxygen amount $\mathrm{n}_{\mathrm{O} 2 \text {, min }}$ can be calculated with the known fuel mass $\mathrm{m}_{\text {fuel }}$ and the stoichiometric air consumption $\mathrm{A}_{\mathrm{st}}$ of iso-octane. The actual oxygen amount results from the difference between spray volume and estimated fuel volume, converted by the molar volume $\mathrm{V}_{\mathrm{mol}}$. The temporal development of the calculated fuel-air equivalence ratio is given in Figure 5 on the right. It shows that even though the flashboiling OP 4 has significantly more spray volume than the other OPs, the amount of air inside the spray is lower than for the $0.1 \mathrm{MPa}$ OPs. This effect is caused by the lower air density at $0.03 \mathrm{MPa}$. Compared to the cold fuel at 
reduced ambient pressure (OP2) however, the flashboiling OP shows a significantly increased air capture in the inertial spray phase. During late injection the curves for both OPs converge. This means that the rapid inertial expansion leads to an enhanced air capture, while air entrainment might even be weakened due to air displacement by fuel vapor.

\section{The effect of flashboiling on droplet size and velocity}

The quality of atomization can be described by the sizes of the individual spray droplets. To determine those, the spray is analysed by PDA/LDA. The investigated measurement points are visualized in Figure 6 with the measurement results for each point displayed in the corresponding schlieren image. To compare the OPs individually, the values in the nozzle distance of $29 \mathrm{~mm}$ are chosen as indicated by the green line. This distance is chosen for two reasons. The first is its relevance for the application, since compared to bore and stroke of modern DI engines this is often roughly the distance between nozzle tip and the centre of the combustion chamber. The second reason is the optical density of the spray closer to the nozzle tip. In order to minimize the influence of potential measuring gaps, $29 \mathrm{~mm}$ represents a distance with a sufficient high data rate
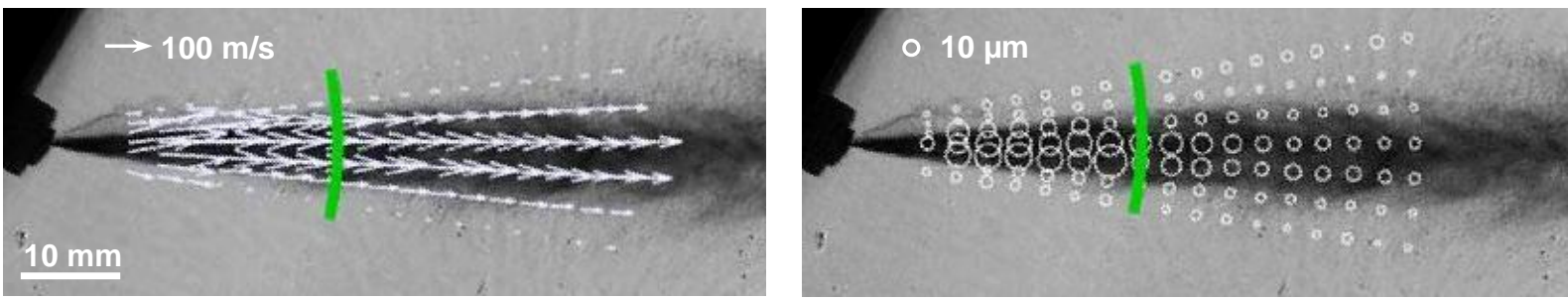

Figure 6. Mean droplet sizes (right) and velocities (left) for each of the 119 measurement points displayed in the corresponding schlieren image, all data for $1500 \mu \mathrm{s}$ after eSOI
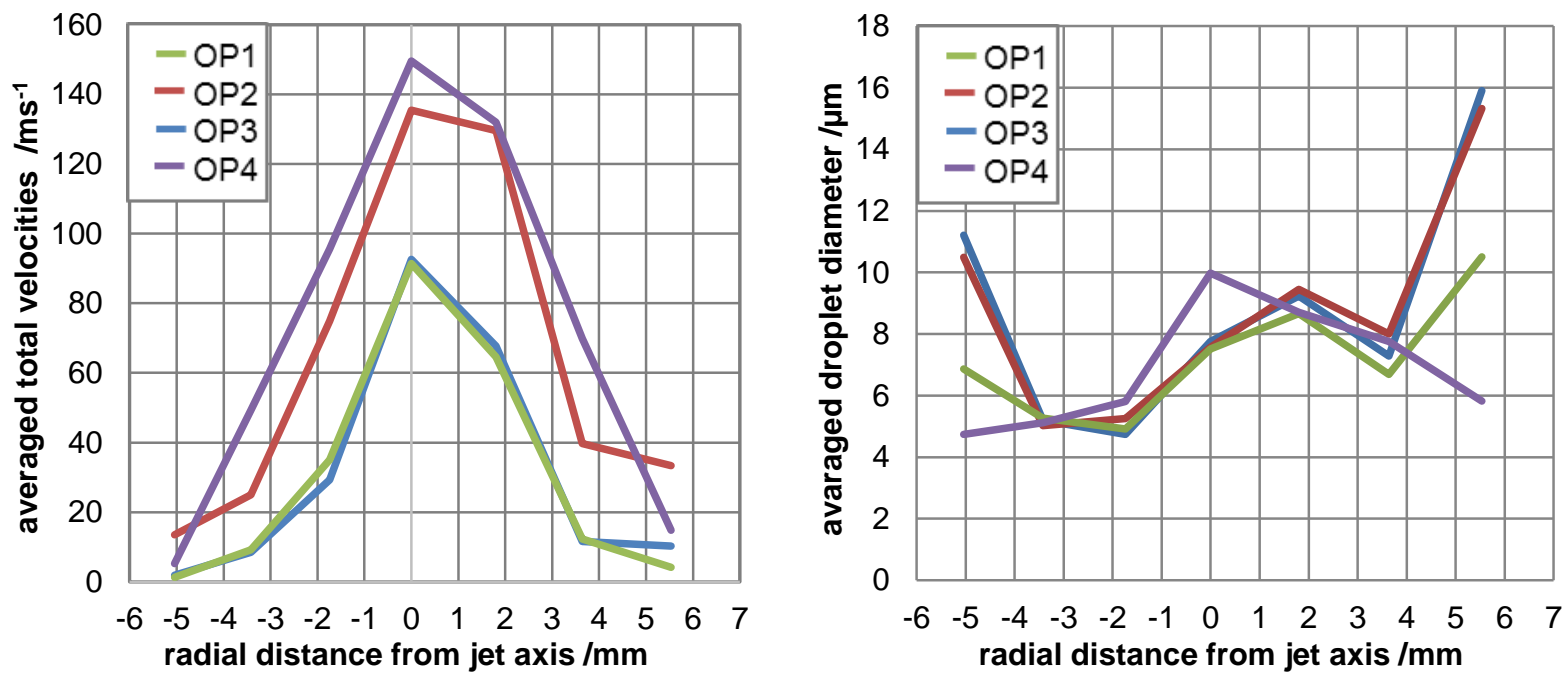

Figure 7. Radial distributions of averaged total droplet velocities (left) and droplet diamteres (right) in $29 \mathrm{~mm}$ nozzle distance for OP1 (303 K / 0.1 MPa), OP2 (303 K / $0.03 \mathrm{MPa})$, OP3 (363 K / $0.1 \mathrm{MPa})$ and OP4 (363 K / $0.03 \mathrm{MPa})$

Figure 7 shows radial droplet size and velocity distributions for the given distance. The velocities on the left show a significant dependency from the ambient pressure, while for the $0.1 \mathrm{MPa}$ OPs an influence of the fuel temperature is not detectable. For the $0.03 \mathrm{MPa}$ OPs, the superheated hot fuel OP 4 shows higher velocities than the cold fuel point. This is contrary to the macroscopic spray behaviour shown in Figure 4 . The difference is that the penetration depth is the integrated spray front velocity, while PDA measures individual droplet velocities in the spray front but also in the bulk spray phase. This means that the spray front is decelerated by increased aerodynamic drag due to the increased spray front cross section while the aerodynamic drag on droplets in the spray bulk phase is even decreased by air displacement due to the increased fuel vapor volume.

\section{Visualization of the primary atomization with and without flashboiling}

Since flashboiling is a very fast process, which takes place at the direct vicinity of the nozzle tip respectively partially inside the nozzle, a very high temporal and spatial resolution is required to visualize the process. With the 
setup described above a temporal resolution of $5 \mathrm{MHz}$ and a spatial resolution of about $4.8 \times 4 \mathrm{~mm}$ can be achieved. Since the camera is limited to 180 frames, only the opening process is analysed. For the stationary spray phase the Schlieren results show sufficient details anyway. Figure 8 shows the opening process for the reference OP 1 and the superheated OP 4 . For a clear arrangement, only every $20^{\text {th }}$ frame is shown here, which correlates to a step width of $4 \mu \mathrm{s}$ between each image, starting from vSOI. The definition of vSOI is however somewhat challenging, since for the hot fuel OPs fuel starts to leak from the injector early during needle lift due to the lower viscosity, creating a pre-jet with very little momentum. That is why in Figure 8 for the superheated OP fuel is visible outside the nozzle already at vSOI. While for the cold OP 1 spray structures and ligaments are clearly visible. For the superheated fuel only a fuel cloud is visible, which the very early stage shows very high radial expansion velocities. Only from $16 \mu \mathrm{s}$ after vSOI a contraction of the spray root and a convergence towards the later spray cone angle is visible. Even though the spray penetration depth for this OP is significantly higher than for the cold reference OP 1, as shown in Figure 9, evidence for a significant axial expansion of the spray due to flashboiling cannot be found as the comparison with the cold $0.03 \mathrm{MPa}$ OP 2 shows. This evaluation however has to be treated with care since as mentioned before, the definition of $\mathrm{vSOI}$ is challenging. An evaluation relative to eSOI on the other hand is not possible, since the camera is only able to cover a very limited timespan. In addition, the evaluation is only based on two injections per OP making shot-to-shot variation a significant source of errors.
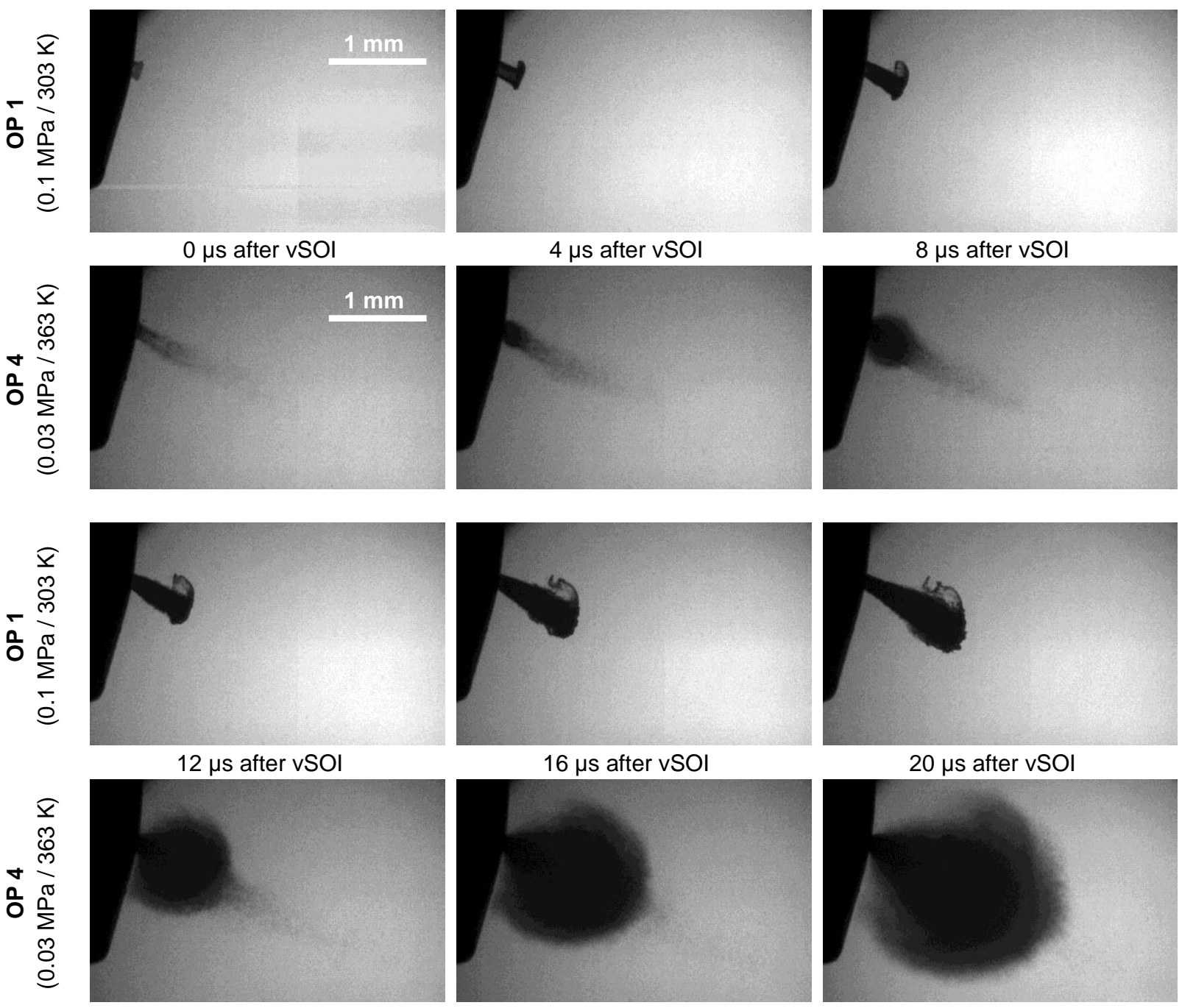

Figure 8. Visualization of the injector opening under non-superheated (OP $1-0.1 \mathrm{MPa} / 303 \mathrm{~K}-$ top row) and superheated (OP 4 - $0.03 \mathrm{MPa} / 303 \mathrm{~K}$ - bottom row) conditions 


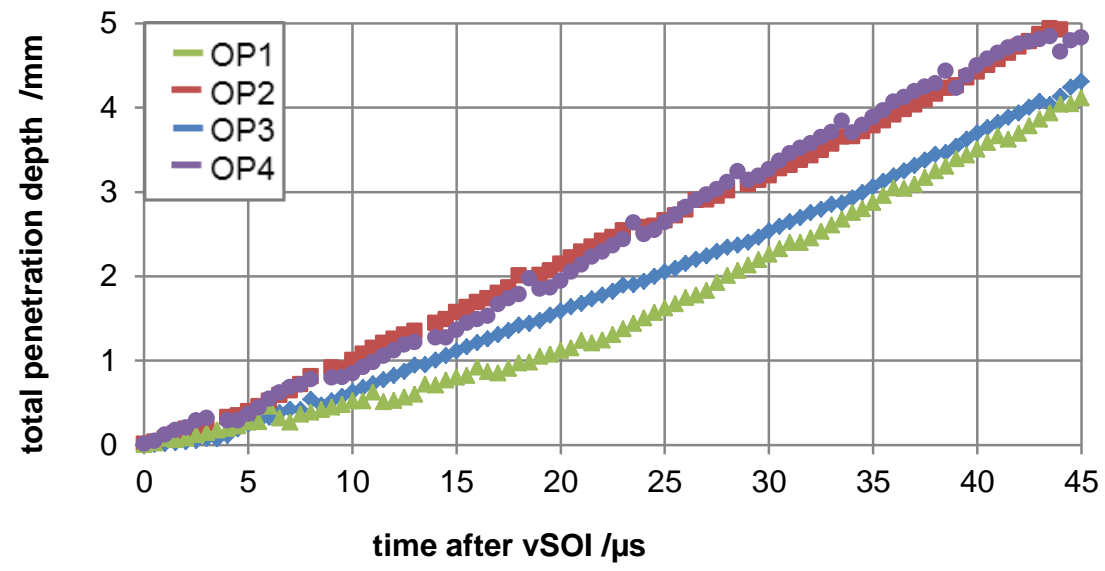

Figure 9. Total penetration depth relative to time after vSOI, obtained from close up ultra-high-speed images distance for OP1 (303 K / 0.1 MPa), OP2 (303 K / $0.03 \mathrm{MPa})$, OP3 (363 K/ $0.1 \mathrm{MPa})$ and OP4 (363 K / $0.03 \mathrm{MPa})$

\section{Conclusions}

For single hole injectors, the effects of flashboiling on the spray behavior are less significant than expected. The macroscopic spray behaviour is changed in terms of penetration depth, cone angle and spray volume, but the tendencies are already visible for the changes in fuel temperature respectively ambient pressure without superheating. Also, the enhanced primary atomization due to flashboiling has no detectable influence on the droplet size distribution in application relevant nozzle distances. This of course does not mean that flashboiling has no benefits for the GDI spray, since the evaporation of fuel is faster under those conditions, which then boosts the mixture formation in the engine.

What is noticeable in the spray however, is the behaviour in direct vicinity of the nozzle outlet where a strong radial acceleration of the spray is detectable. This leads to a significantly increased spray root width and an early increase in spray volume. The volume balance in addition hinds towards a changed and potentially extenuated air entrainment due to the significant increase of spray vapor volume. Therefore, to understand the effect of flashboiling on multi hole sprays, the interconnection to Jet-to-Jet interaction and finally spray collapsing, the flashboiling induced flow field in the surrounding air and the jet overlap in the initial spray phase, before the flow field is fully developed have to be investigated more closely.

\section{Acknowledgement}

The authors would like to thank Continental for providing the experimental injectors used in this paper and Specialised Imaging for providing parts of the equipment used for the shown measurements.

Additionally the authors gratefully acknowledge the financial support for parts of their work from the Erlangen Graduate School in Advanced Optical Technologies (SAOT) within the framework of the German Excellence Initiative by the German Research Foundation (DFG).

\section{References}

[1] Martin Krämer, E. K., Michael Wensing, 2016, "Flashboiling-induced targeting changes in gasoline direct injection sprays," international $\mathrm{J}$ of Engine Research, 17(1), p. 10.

[2] RD Oza, J. S., 1983, "An experimental and analytical study of flash-boiling fuel injection," SAE technical paper 830590.

[3] Min Xu, Y. Z., Wei Zeng, Gaoming Zhang and Ming Zhang, 2013, "Flash Boiling: Easy and Better Way to Generate Ideal Sprays than the High Injection Pressure," SAE Int. J. Fuels Lubr., 6(1).

[4] Andrew Wood, G. W., Jerome Helie, 2014, "Flash Boiling Sprays produced by a 6-hole GDI Injector," 17th International Symposium on Applications of Laser Techniques to Fluid MechanicsLisbon.

[5] Wei Zeng, M. X., Gaoming Zhang, Yuyin Zhang, David J. Cleary, 2012, "Atomization and vaporization for flash-boiling multi-hole sprays with alcohol fuels," Fuel, 95, p. 11.

[6] Krämer, M., 2016, Einfluss motorischer Randbedingungen auf die Spraycharakteristik bei direkteinspritzenden Ottoverfahren. 
[7] Günther, A., 2016, Zerstäubung überhitzter Fluide - Einfluss der Durchflusscharakteristik auf das Spray, Friedrich-Alexander-Universität Erlangen-Nürnberg (FAU).

[8] Hung, D., Harrington, D., Gandhi, A., Markle, L. et al., 2009, "Gasoline Fuel Injector Spray Measurement and Characterization - A New SAE J2715 Recommended Practice," SAE Int. J. Fuels Lubr., 1(1), p. 14. 


\title{
Measurement of a Direct Gasoline-Water-Emulsion-Injection
}

\author{
Dipl.-Ing. Thilo Wagner, Prof. Dr.-Ing. Hermann Rottengruber \\ Prof. Dr.-Ing. Frank Beyrau*, Dr.-Ing. Plamen Dragomirov*, M. Sc. Maximilian Schaub \\ Institute of Mobile Systems, Otto von Guericke University, Magdeburg, Germany \\ *Institute of Fluid Dynamics \& Thermodynamics, Otto von Guericke University, Magdeburg, Germany \\ Corresponding author: thilo.wagner@ovgu.de
}

\begin{abstract}
The investigation focus for combustion engines is on reduction of emissions as well as fuel consumption. By introducing the gasoline direct injection combined with downsizing the efficiency and the fuel consumption of gasoline engines has been optimized. An additional potential to the previous solutions provides the water injection. The article include the results of the fundamental research of water injection for combustion engines, it shows the influence of the amount of water in a water-gasoline-emulsion on the spray in a high pressure injection chamber. Therefor the spray of a gasoline direct injection injector is visualized by a high speed camera using the shadowgraph technique.
\end{abstract}

\section{Keywords}

Direct Injection System, Gasoline-Water-Emulsion, Spray Measurement, Spray angle and Penetration

\section{Introduction - The water injection}

Water injection was introduced very early in the development of combustion engines, especially for aircrafts and race cars. However it could not make its way to large-scale serial applications. In the first place the waterinjection benefits from its high evaporation enthalpy. It can be used for the reduction of the intake air temperature to increase the engine power, to increase the efficiency and reduce the emissions.

\section{Mode of action and influence on combustion}

Water has a six times higher evaporation enthalpy and a doubled higher heat capacity as gasoline. Both leads to a temperature reduction. On the one hand, based on the evaporation, the temperature level before the actual combustion is lower and on the other hand, the evaporated water acts like an inert gas to lower the combustion temperature by increasing the global heat capacity. Therefore full load enrichment is no longer needed. The effects of water injection on the combustion are shown in Figure [1].

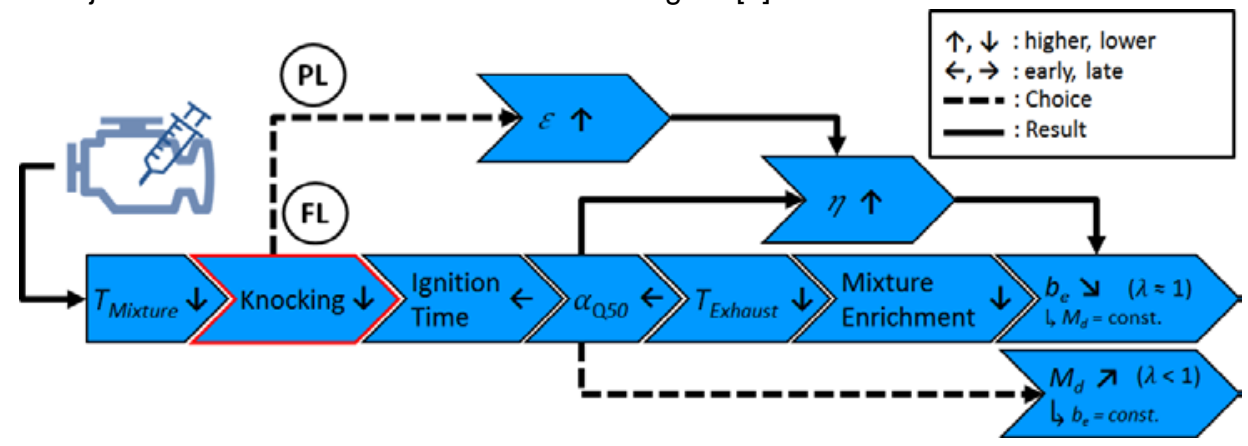

Figure 1. Influence of water injection on combustion

Durst [1] compared the various systems in his publication. He investigated in an intake water injection system, a mixture injection system and a water direct injection system. Each system was able to increase the proportion of water up to $50 \%$ without any kind of spark failure or cycle fluctuations [1]. Additional, all investigated methods reduce fuel consumption, emissions (particle, $\mathrm{HC}, \mathrm{CO}$ ) and lead to an optimized mass fraction burned $50 \%$. At full load with $40 \%$ of water it was possible to reduce the gas mixture temperature by $40 \mathrm{~K}$. Also the point of mass fraction burned $50 \%$ was relocated around $6^{\circ}$ crank angle towards the ignition TDC (top dead center). The biggest advantage of water injection for efficiency and emissions (particle, HC, CO) could be seen in high RPM and high load. Except the NOx emissions, which increase in conditions of high RPM and high load. At their maximum they reach a 4 times higher level with water injection [1]. The reason is the higher level of oxygen compared to full load operation point with fuel enrichment. The higher level of oxygen is promoting NOx formation reactions. However the 3-Way-Catalytic converter works best with lambda $=1$, so the NOx emissions can be converted. 


\section{Methods of water injection}

There are different ways to add water to the combustion process. Figure 2 shows four different methods. The systems differ in costs, in the application effort and in the influence on the combustion.

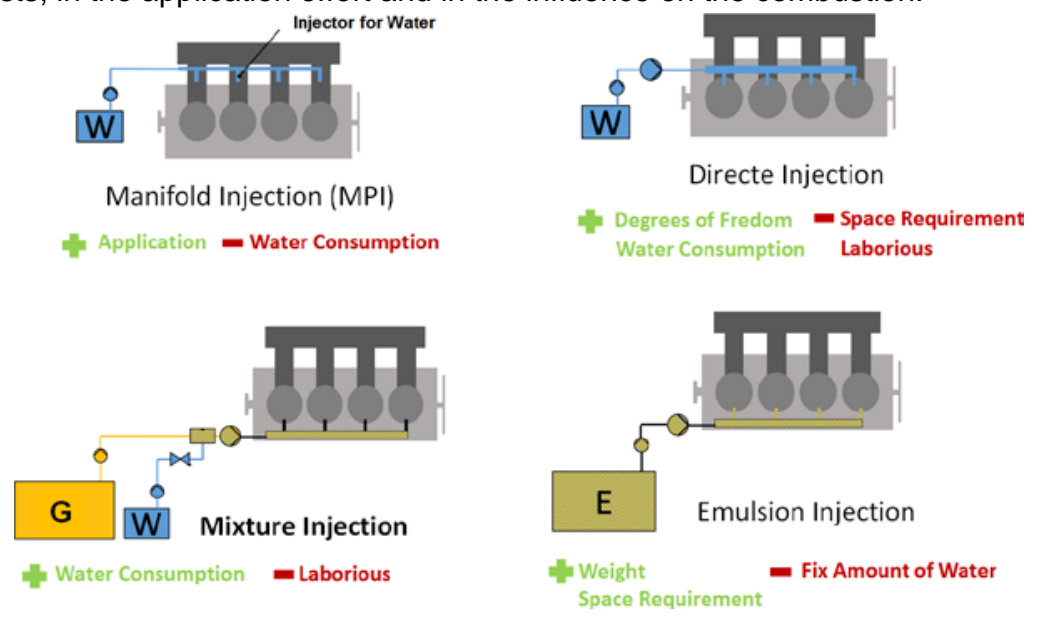

Figure 2. Methods of water injection

\section{Airbox Injection}

The manifold injection is characterized by the injection of water with one main injector or cylinder selective injectors in the intake system after the intercooler. The main advantage is the easy continuous injection of water, without synchronization to the crank angle [1].

\section{Manifold Injection}

The water injection takes place near to the intake valve. It is individual for every cylinder and needs to be synchronized to the crank angle. The advantage is a higher amount of water which enters the cylinder liquidly. But it is still more water necessary to have the same effects as the direct injection

\section{Mixture and Emulsion Injection}

The fuel need to be mixed before injected into the cylinder. Gasoline and water can be mixed by three different systems. First is a mixture injection, without producing a real emulsion. This could be done by using the high pressure pump (HDP5) [3] [4]. Therefor it is mandatory to bring both fluids together in front of the pump. Second way is the creation of a macro emulsion with an onboard system with/without using surfactant. The emulsion is created shortly before injection and isn't stored. Disadvantages of the system are the delay on the water injection, because of the production and flow time and the high amount of energy to create the emulsion [5]. Third option to add water to the combustion is to fuel the car with a micro emulsion. The micro water gasoline emulsion is thermodynamic stable, nano-disperse, temperature invariant, non-corrosive and can be store unlimited [2]. Two disadvantages are the fix amount of water in the emulsion and the complex micro emulsion production [6].

\section{Separate Water injection}

The water is injected by a separate independent high pressure injector. This system offers most flexibility for the injection timing and for the amount of water, but the system is very complex [1].

On Injector Mixture Injection

For this kind of water injection a special injector is necessary where water and gasoline are mixed direct in the injector and injected together through one nozzle. Some research was done for stationary Diesel engines [7] [8]. A disadvantage is the need for a separate high pressure water system.

\section{Scientific Basis}

An emulsion is a drop shaped distribution of at least two not mixable fluids [9] [10]. To generate an emulsion you need an inner phase (disperse phase) from fine particles and a liquid outer phase which enclose the fine particles of the first liquid [10] [11]. The average droplet diameter is from 100 nanometers to one millimeter. The larger the average droplet diameter the more intensive is the white color of the emulsion. With help of the droplet diameter emulsions are divided in macro- and micro emulsions. Micro emulsions have a smaller droplet diameter, are optical clear and are formed spontaneous. Macro emulsions are always thermodynamic unstable, which leads in a segregation process [11]. It's possible to stabilize the macro emulsion, but it is only possible to extend the time of segregate. Emulsions tend to minimize their surface free energy $\Delta G$, which is the product of interfacial tension $\gamma$ and the change in size of the interfaces $\Delta \mathrm{A}$.

$\Delta G=\gamma \cdot \Delta A$ 
The balance of a stable condition is reached, when the surface free energy equals $\Delta \mathrm{G}=0$ [9]. This can be reached by reducing the interfacial tension $\gamma$ (adding a surfactant) or by reducing the interfaces $\Delta \mathrm{A}$ (segregation of the fluids). But due to the fact, the interfacial tension $\gamma$ couldn't be zero, a macro emulsion can never be stable. Consequently, the smaller the droplets the more unstable the emulsion [9] is without protection.

\section{Instability Phenomena}

The stability of an emulsion is defined by the time in which it is useable without the phenomena of segregation [12]. Typical phenomena of segregation are sedimentation, flocculation, Ostwald-Maturation, coalescence and phase inversion. Sedimentation and flocculation are reversible by simply mix the emulsion by shaking or stirring to undo both stability phenomena. Ostwald-Maturation and coalescence are irreversible phenomena which end in the segregation of the emulsion.

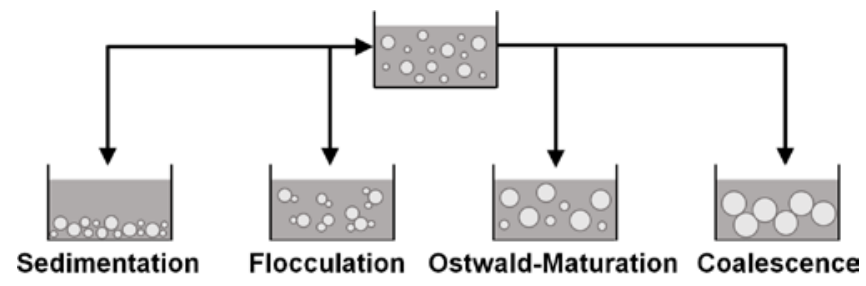

Figure 3. Instability Phenomena

Sedimentation is the subsidence of water caused by the density difference [13]. The kinetic stability of the emulsion can be raised by a smaller droplet diameter. Flocculation is the loose accumulation of droplets, without losing its phase boundary. These process can be reduced by steric, electrostatic or electro steric mechanisms [12]. Ostwald-Maturation describes the irreversible growing of big droplets at the expense of small droplets [13]. Coalescence is the irreversible merge of small droplets to big droplets by reducing the interface and surface energy. The reason for coalescence is the strive for an energy minimum and for a minimal surface volume ratio [10]. Another instability phenomenon is phase inversion, which leads in an inversion from W/O-emulsion to O/Wemulsion or reversed. It can be caused by energy input, temperature changes or changes in the composition of the components [14].

\section{Stabilization of an emulsion}

Steric stabilization of an emulsion is meant to add a surfactant with a large space extension. The surfactant gets absorbed on the boundaries of the water drops and prevents the accumulation of further water drops [12]. Stabilization is also termed as kinetic stabilization and is different from thermodynamic stabilization. Macro emulsions are not stable due to the fact of minimization of boundary surfaces and free energy. With a surfactant this process can be slowed down to a level where the emulsion is stable for weeks or even years. This condition is termed as metastable, inert or kinetic stable [16]. All influences that reduce the movement of the droplets increase the stability of the emulsion [15].

\section{Water-Gasoline-Emulsion as passenger car fuel}

The fluids water and gasoline are not mixable because of their chemical molecular structure. But it is possible to create a metastable macro emulsion with surfactant and emulsifying apparatus. The product is a water-oilemulsion. The use of a surfactant causes the investigation of emissions produced by the combustion of the surfactant. Experiments with a test engine using water proportions from 0 to $50 \%$ show that most benefits are reached with a water proportion of $40-50 \%$. Even with $50 \%$ proportion of water the cycle variations are very low [1]. It is not known if a homogenous emulsion is necessary for a good combustion. Experiments by Durst [1] and Böhm [3] show that it is sufficient to mix water and gasoline in front of the high pressure pump [3]. But it is not known what kind of mixture is produced by this system of Durst [1] and Böhm [3]. Literature research indicates that the use of emulsified fuel with surfactant cause additional emissions after the combustion. Faced with a large number of different surfactants it is difficult to conclude a general statement, but it could be assumed that it is appropriate to use surfactants with elements which are already part of the fuel mixture like carbon, hydrogen, oxygen and nitrogen. All surfactant containing phosphorus and sulphur should not been used. Most of the surfactants are long chain molecules with a slow and incomplete combustion which results in particle emissions. This fact was proven in studies on a diesel-water-emulsion [4].

\section{Measuring System and Optical Evaluation}

\section{Test Bench and Parameter}

The performed studies are aimed to homogenous turbo charged gasoline engines with direct injection often used in small and mid-range cars. The injection well as the chamber pressure, the chamber temperature and the injection time are based on that conditions. 
State of the art for injection pressure of modern passenger gasoline direct injection engines is 200 bar. Next generations aim is 350 bar. In low load operation points the injection pressure drops to 80 bar. For measurement 50,125 and 200 bar was defined. These measurement points should give a general statement for the influence of the injection pressure.

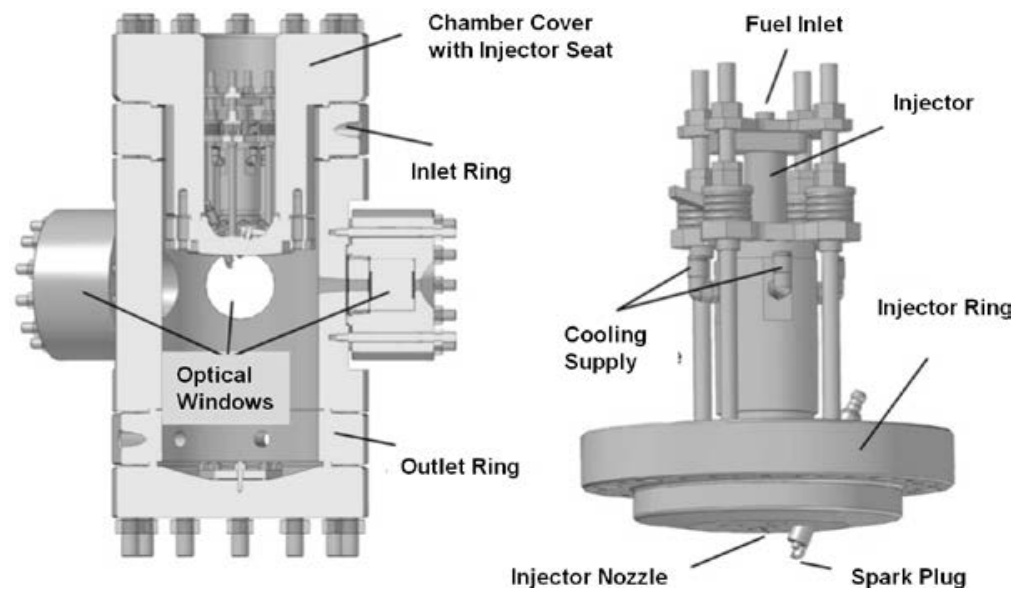

Figure 4. The Pressure Chamber for Injection Tests [17]

The injection time for homogeneous direct injection gasoline engines is during the induction stroke. For turbo charged SI-engines the cylinder pressure during the induction stroke is nearly the boost pressure. The chamber pressure is defined to 1 and 2 bar, for two engine load scenarios.

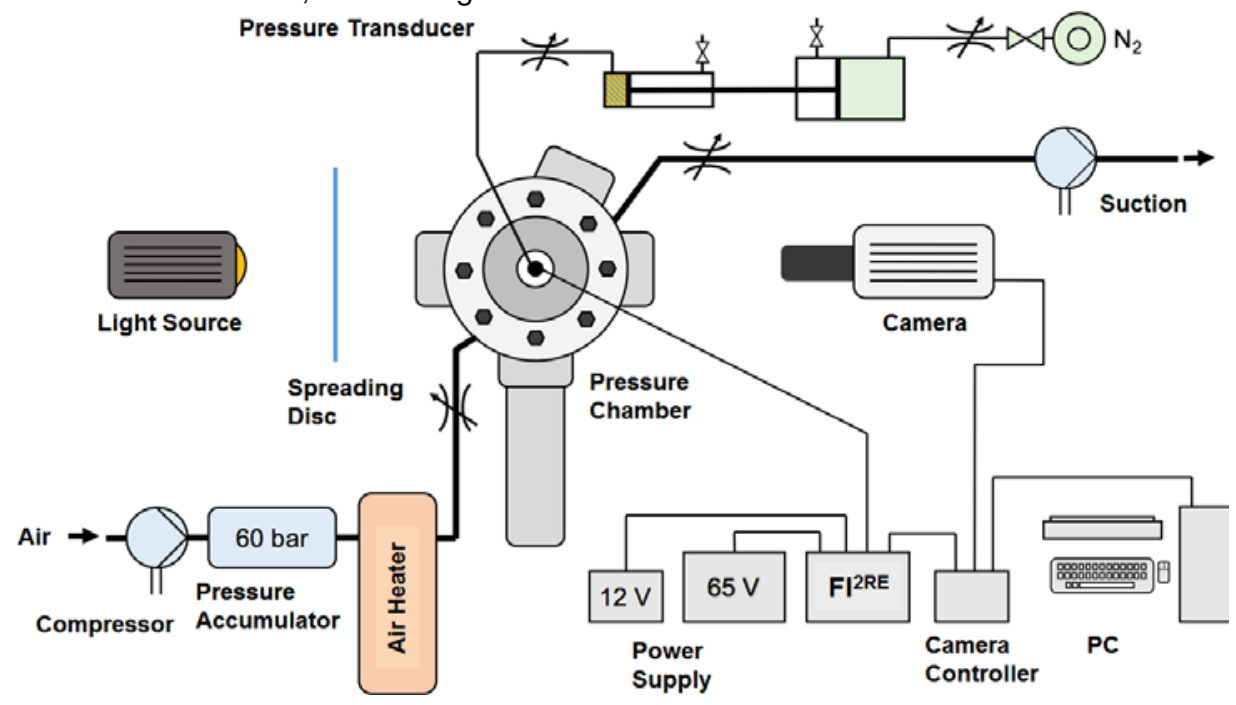

Figure 5. Schematic Test Bench Structure

The pressure chamber is for the optical measurements and investigation of the injector spray. The pressure of the chamber can be set from $p_{\mathrm{dk}}=1$ to $60 \mathrm{bar}$ and the temperature can be adjusted from $20^{\circ} \mathrm{C}$ up to $200{ }^{\circ} \mathrm{C}$. The volume of the chamber is 13 liters and the shape is cylindrical with four $80 \mathrm{~mm}$ windows.

The temperature of the fresh air in the cylinder depends on the boost air temperature and the amount of thermal energy transferred from the cylinder head, cylinder wall, residual gas and piston surface. Following the temperatures of a modern turbo charged engine with $65 \mathrm{~kW}$ per liter as an example the boost air temperature at $21^{\circ} \mathrm{C}$ atmosphere temperature rises from 18 to $52{ }^{\circ} \mathrm{C}$. The engine temperature in running condition is between 83 ${ }^{\circ} \mathrm{C}$ and $93^{\circ} \mathrm{C}$. The surface temperature of the piston is around $300^{\circ} \mathrm{C}$, depending on the engine load [26]. Based on this the temperature for the pressure chamber was set to $t_{\mathrm{dk}}=100^{\circ} \mathrm{C}$.

The used injector [HDEV 5) is a six-hole solenoid direct injection injector with central installation position and a maximal injection pressure of 200 bar. For the control of the injector a FI ${ }^{2 E R}$ control unit of IAV Ltd. was used.

The used measurement is shadowgraph techniques. The injected spray is illuminated with an intense light source and recorded with a high speed camera. The data from the measurements were analysed by the software DaVis 7.2 from LaVision. The algorithm is automatically detecting the spray boundaries, the penetration and the spray angle [15].For every injection 100 pictures (Frames) with a frequency of $10 \mathrm{kHz}$ was taken. 

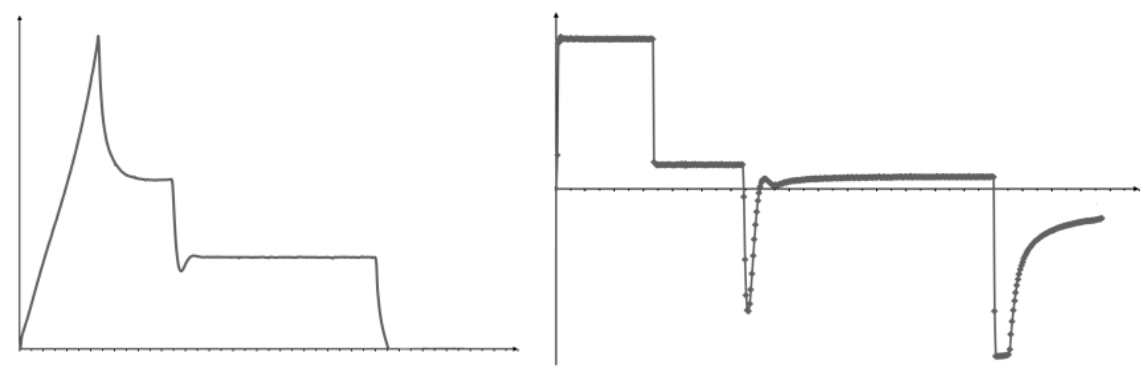

Figure 6. Control Parameters for the Injector (left power, right voltage) [18]

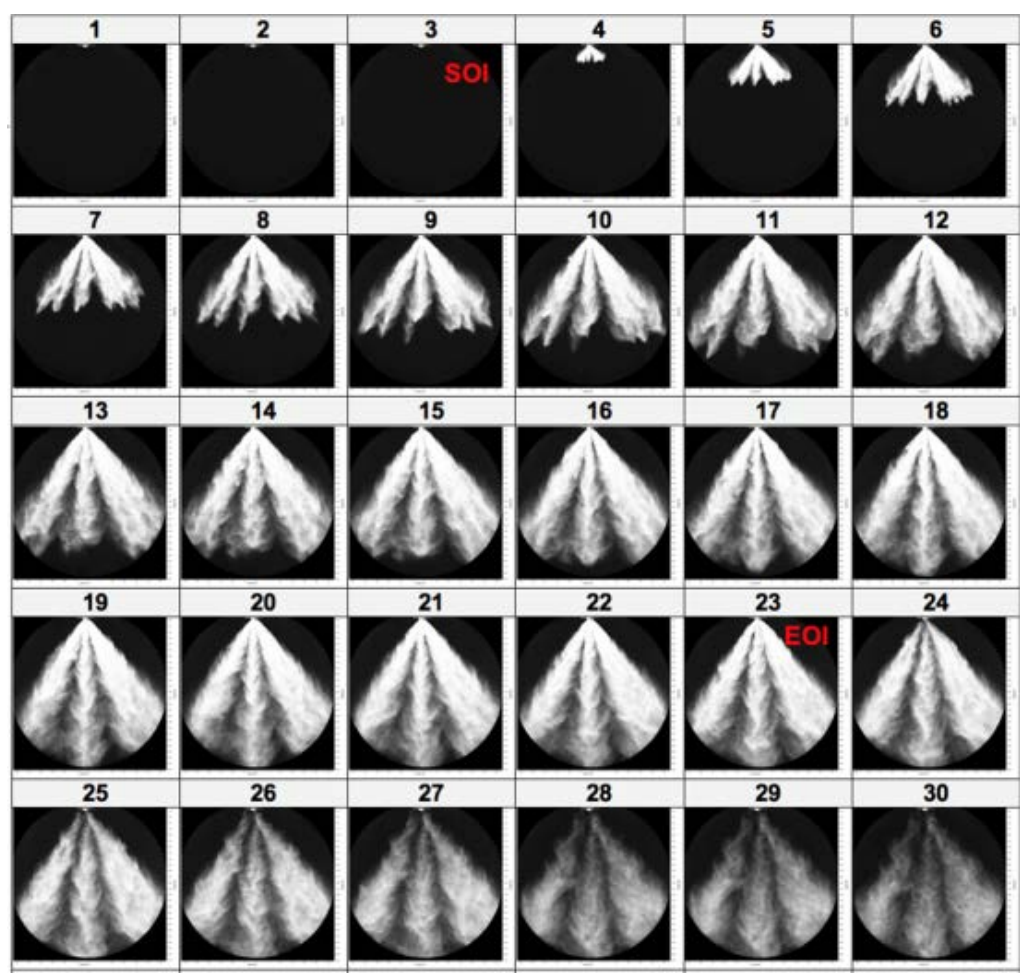

Figure 7. Spray evolution over injection time (water proportion $\Delta \mathrm{w}=0 \%, \mathrm{p}_{\mathrm{dk}}=2$ bar, $\mathrm{p}_{\mathrm{inj}}=200$ bar)

The measurements were taken as shown in table 1. The injection duration is $2 \mathrm{~ms}$. Both, the activation of the injection and of the camera are trigger by the controller for an accurate timing. Every measurement was repeated twenty times.

Table 1. Measurement Plan

\begin{tabular}{|c|c|c|c|c|c|c|c|}
\hline \multirow{2}{*}{$\begin{array}{l}\text { Chamber } \\
\text { Temperature }\end{array}$} & \multirow{2}{*}{$\begin{array}{l}\text { Chamber } \\
\text { Pressure }\end{array}$} & \multirow{2}{*}{$\begin{array}{l}\text { Injection } \\
\text { Pressure }\end{array}$} & \multicolumn{5}{|c|}{ Amount of Water } \\
\hline & & & $0 \%$ & $25 \%$ & $50 \%$ & $75 \%$ & $100 \%$ \\
\hline \multirow{6}{*}{$100^{\circ} \mathrm{C}$} & \multirow{3}{*}{2 bar } & 50 bar & $20 x$ & $20 x$ & $20 x$ & $20 x$ & $20 x$ \\
\hline & & $125 \mathrm{bar}$ & $20 x$ & $20 x$ & $20 x$ & $20 x$ & $20 x$ \\
\hline & & 200 bar & $20 x$ & $20 x$ & $20 x$ & $20 x$ & $20 x$ \\
\hline & \multirow{3}{*}{1 bar } & 50 bar & $20 x$ & $20 x$ & $20 x$ & $20 x$ & $20 x$ \\
\hline & & 125 bar & $20 x$ & $20 x$ & $20 x$ & $20 x$ & $20 x$ \\
\hline & & 200 bar & $20 x$ & $20 x$ & $20 x$ & $20 x$ & $20 x$ \\
\hline
\end{tabular}

\section{The Spray Records}

Figure 7 shows the chronology of an injection with pure gasoline fuel at 2 bar chamber and 200 bar injection pressure. It is obvious that the start of injection (SOI) is in frame 3 and ends (EOI) is in frame 23 due to $2 \mathrm{~ms}$ injection time. The penetration length of the spray exceeds the windows of the chamber at frame 12, which means it only can be analyzed up to $0.9 \mathrm{~ms}$ after SOI. The control power for the injector indicates that it is opening up to frame 10. Consequently most of the analyzed measurements are during the opening phase of the nozzle, which has to be considered because of cavitation effects in the needle seat. 


\section{Influence of the Proportion of Water \\ Penetration}

The spray measurements in Figure 8 show that with an increasing amount of water the penetration is decreasing, this becomes obvious especially for $p_{i n j}=125$ bar and $p_{i n j}=200$ bar. With $p_{i n j}=50$ bar the penetration remains nearly constant, but nevertheless the peripheral spray cones have a slightly lower penetration.

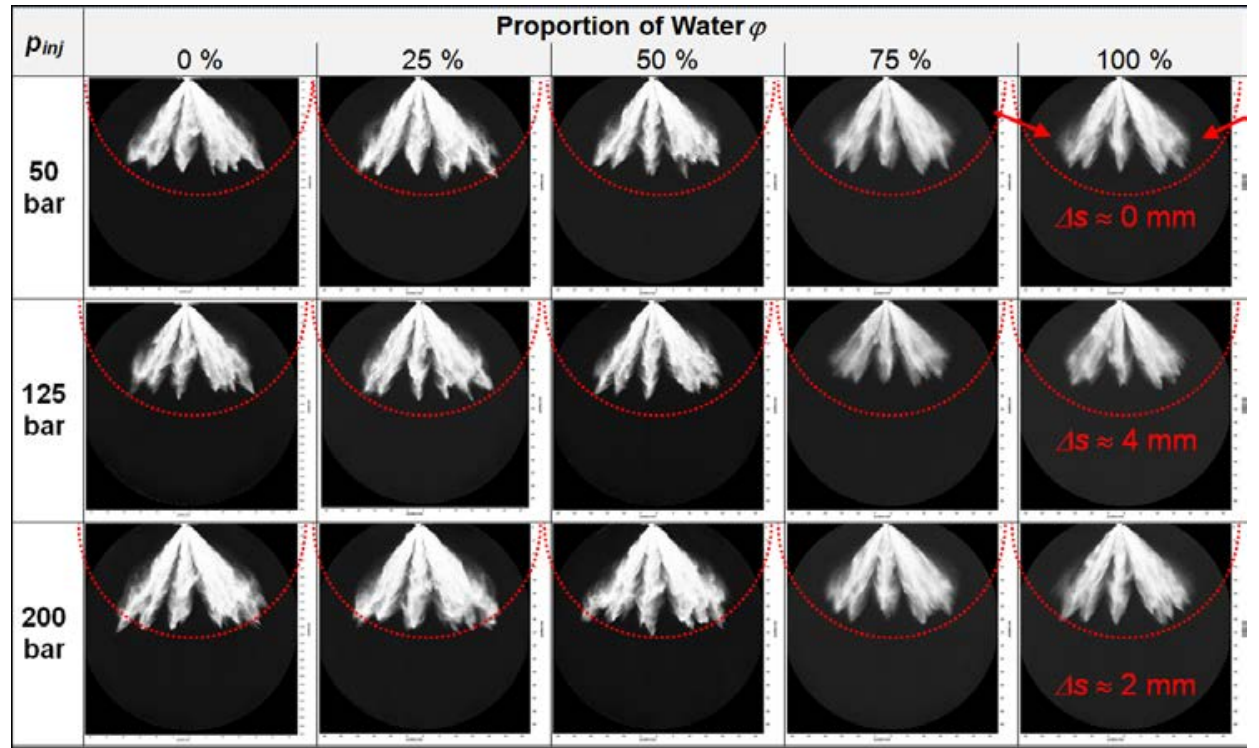

Figure 8. Spray images at 2 bar Chamber Pressure from 0 to $100 \%$ water

The properties of water and gasoline are shown in table 2 . The values show that water has a $30 \%$ higher density and a magnificent higher viscosity. The consequence of equation 2 is that the outlet velocity vout and subsequently the penetration length (s) are smaller with a higher density, subsequently with a higher amount of water.

$$
s(t) \sim \sqrt[2]{v_{\text {out }}} \sim \frac{1}{\sqrt[2]{\rho_{f}}}
$$

\section{Quality of atomization}

Figure 8 shows that the spray cones gets wider with less proportion of water. This is an indication for a better atomization. The quality of atomization is higher with an increased Reynolds- and Ohnesorge-umber. Both parameters are influenced by the fluid density $\rho f$, the outlet velocity $v_{\text {out }}$, droplet size $d_{T r}$, the dynamic fluid viscosity $\eta_{f}$ and the surface tension $\sigma_{f}$. As you can see in Table 2 water have a $33 \%$ higher density, a $67 \%$ higher dynamic viscosity and a $222 \%$ higher surface tension than gasoline. Also the outlet velocity is decreasing with higher amount of water. The equation for the Re- and Oh- number shows clearly that the influence parameters on the quality of atomization are opposed. In conclusion from the equations and spray measurements it can be said that the quality of atomization is higher with a lower proportion of water in the emulsion. Furthermore in table 2 the Re-, the We- and the Oh - number are calculated assuming that the outlet velocity and the droplet size are equal. Both assumptions don't fit the reality so the effects would be higher because of lower $\mathrm{Re}-$, We- and Oh- numbers. Another influence on the quality of atomization is the boiling temperature of water $\left(100^{\circ} \mathrm{C}\right)$ and the boiling range of gasoline $95 \mathrm{E} 5\left(25-215^{\circ} \mathrm{C}\right)[18]$.

Table 2. Comparison of Properties of Gasoline 95 Octane and Water [18]

\begin{tabular}{c|c|c|c|c|c|c}
\hline & $\begin{array}{c}\rho f \\
\left.\text { (at } 15{ }^{\circ} \mathrm{C}\right)\end{array}$ & $\begin{array}{c}\eta f \\
\left.\text { (at } 20^{\circ} \mathrm{C}\right)\end{array}$ & $\begin{array}{c}\sigma f \\
\left(\text { at } 20^{\circ} \mathrm{C}\right)\end{array}$ & $\boldsymbol{R e}^{*}$ & $W^{*}$ & Oh $^{*}$ \\
\hline $\begin{array}{c}\text { Gasoline 95 } \\
\text { Octane E5 }\end{array}$ & $\begin{array}{c}100 \% \\
\left(750 \mathrm{~kg} / \mathrm{m}^{3}\right)\end{array}$ & $\begin{array}{c}100 \% \\
\left(0,6 \times 10^{-3} \mathrm{~Pa} \mathrm{~s}\right)\end{array}$ & $\begin{array}{c}100 \% \\
\left(22,6 \times 10^{-3} \mathrm{~N} / \mathrm{m}\right)\end{array}$ & $100 \%$ & $100 \%$ & $100 \%$ \\
\hline Water & $\begin{array}{c}133 \% \\
\left(1000 \mathrm{~kg} / \mathrm{m}^{3}\right)\end{array}$ & $\begin{array}{c}167 \% \\
\left(1 \times 10^{-3} \mathrm{~Pa} \mathrm{~s}\right)\end{array}$ & $\begin{array}{c}322 \% \\
\left(72,75 \times 10^{-3} \mathrm{~N} / \mathrm{m}\right)\end{array}$ & $80 \%$ & $41 \%$ & $80 \%$ \\
\hline
\end{tabular}

\section{Volume of Injection}

The spray picture of Figure 8 shows that a spray of pure gasoline has a higher grey level dynamic as a spray of pure water. This indicates that the gasoline spray has a higher density. In conclusion the volume of injection is higher with less amount of water in the emulsion. This fact can be confirmed with the equation of outflow volume. 


$$
\dot{V}_{K}=\mu \cdot A_{\text {noz }} \cdot \sqrt{\frac{2 \cdot \Delta p}{\rho_{K}}}=\mu \cdot A_{\text {noz }} \cdot v_{\text {out }}
$$

A higher proportion of water within the emulsion leads to a higher density of the fluid injected. This results in a lower flow speed inside the nozzle and therefore to a reduced penetration.

\section{Spray Angle}

The pictures of the injection in Figure 8 also show that the spray angle is increased with higher amount of water. The spray gets wider and the tip of the spray is thinning out.

\section{Influence of the Injection Pressure}

In Figure 9 the chronological sequences of injection with $\Delta \mathrm{w}=50 \%$ proportion of water, a chamber pressure of $p_{d k}=2$ bar and injection pressures from $p_{i n j}=50$ to 200 bar are shown.

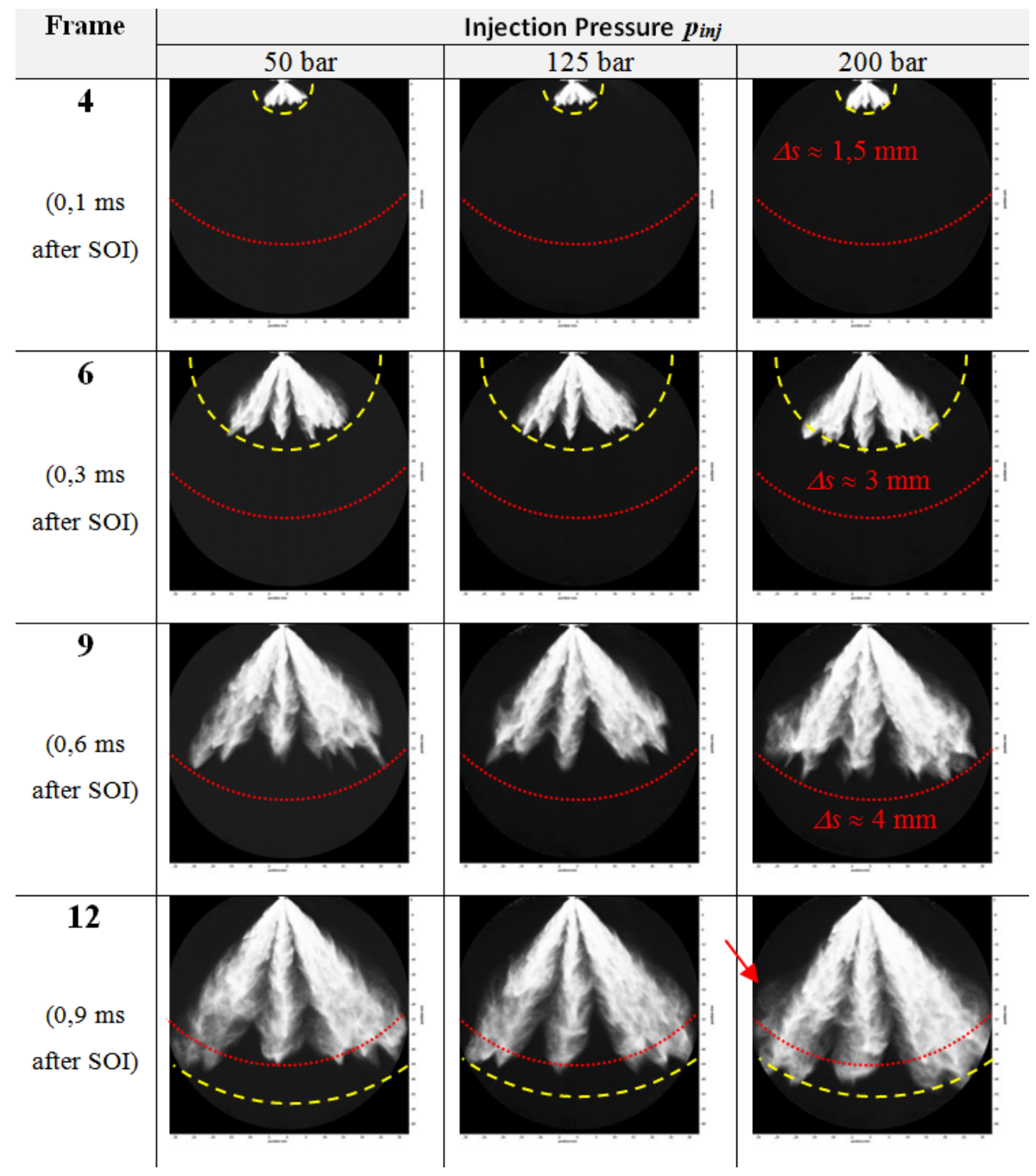

\section{Penetration}

Figure 9. Chronological sequences of injection (proportion of water $\Delta \mathrm{w}=50 \%, \mathrm{p}_{\mathrm{dk}}=2$ bar)

The measurements show that the spray penetration is higher with rising injection pressure. That is the result of a higher nozzle velocity caused by the higher injection pressure. It is valid that the injection outlet speed and the spray penetration length are proportional to the square root of the injection pressure which theory is consistent with all empiric models in the literature [18][19][20].

Quality of atomization

It is striking that the sides of the spray are wider with higher injection pressure. That is an indication for a better quality of atomization. It is valid, that the atomization gets better with increased Reynolds- and Ohnesorgenumber. The Reynolds-number becomes higher if the outlet velocity is rising and the outlet velocity is higher with 
a high injection pressure. In conclusion the quality of atomization gets better if the injection pressure-level is higher.

\section{Spray Angle}

As you can see in Figure 9 the injection pressure has no significant influence on the spray angle near the nozzle. But in a higher distance to the nozzle the spray interacts with the gas in the chamber and gets thinner/lighter. The result of the rising evaporation with higher injection pressure is a smaller spray angle in distance to the nozzle.

\section{Influence of the chamber pressure}

\section{Penetration}

The comparison between 1 and 2 bar chamber pressure in Figure 10 shows the influence on the length of penetration. The higher the chamber pressure the less the penetration length. It takes $0.4 \mathrm{~ms}$ after SOI before the effect is visible.

\section{Quality of Atomization}

The boundary areas of the spray are wider with 1 bar chamber pressure compared to 2 bar. The logical conclusion is that the quality of atomization is higher with higher pressure, because of the higher gas density in the chamber. It is also visible that the spray is less dense with higher chamber pressure which is an indication for a better evaporation.
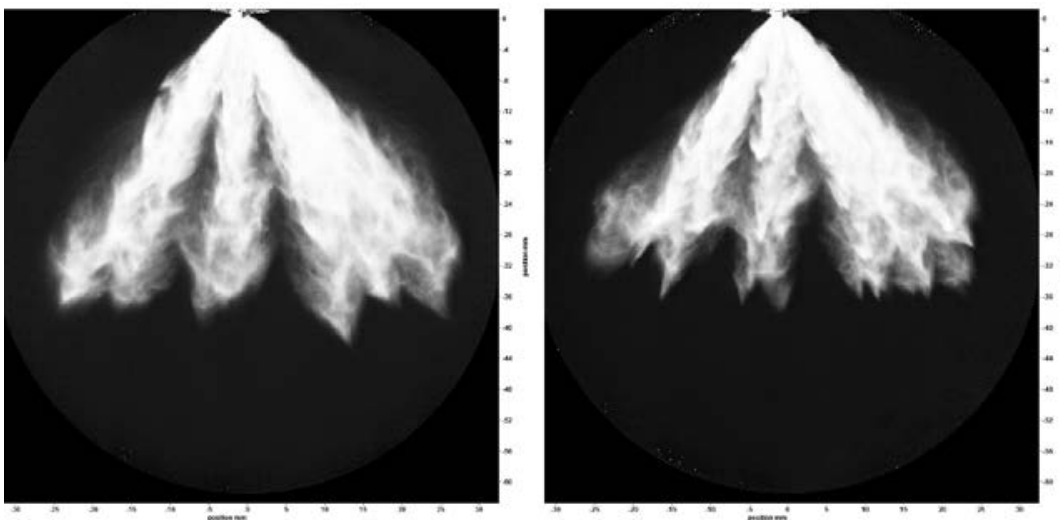

Figure 10. Picture of the Spray at 1 (left) and 2 bar (right) chamber pressure (proportion of water $\Delta w=50 \%$, Injection Pressure $\mathrm{p}_{\mathrm{inj}}=200$ bar)

\section{Spray Angle}

The influence of the chamber pressure on the spray angle is insignificant small which is visible in Figure 10. With 1 bar chamber pressure the spray angle is slightly wider then with 2 bar chamber pressure.

\section{Conclusions}

It is not possible to create a kinetic stable water-gasoline-emulsion without using a surfactant. With the use of the two surfactants it was possible to create a kinetic stable macro emulsion with various proportions of water. Studies have shown that the current penetration length is less with rising proportion of water. The reason is the lower outlet velocity of the spray with higher proportion of water, because of the higher density. As a reaction the spray evade to the sides, which makes the spray angle bigger and the spray looks wider. It was shown with fluid mechanical equations that the high surface tension and the high viscosity of water reduce the quality of atomization for water-gasoline-emulsions with rising proportion of water.

\section{Acknowledgements}

It has to be clarified if a real emulsion is necessary for a combustion with low cyclic variations, with low emissions and a good efficiency. It must be investigated how the injection volume behave with rising proportion of water to ensure a constant engine power. From the authors point of view it will make sense to do more optical investigations with different measuring methods to validate the result of this study. Furthermore the injector has to be observed and analyzed for damages caused by the -emulsion injected.

\section{References}

[1] B. Durst, G. Unterweger, A. Witt, M. Böhm: „Thermody. Wirkung der Wassereinspritzung beim Ottomotor - ein Konzeptvergleich," in 15th Conference „The Working Process of the Internal Combustion Engine“, Graz, 2015.

[2] C. Simon et al.: „Erzeugung und Einspritzung von Diesel-Wasser-Emulsionen,“ MTZ - Motortechnische Zeitschrift, Bd. 71, pp. 5126-523, Juli 2010. 
[3] M. Böhm et al.: „Funktionale Integration einer Wassereinspritzung in den Ottomotor,“ MTZ, Januar 2016.

[4] C. Bartsch: „Mit Wasser gegen Rußemissionen,“ MTZ - Motortechnische Zeitschrift, Bd. 75, Oktober 2014.

[5] L. Bemert und R. Strey: „Diesel-Mikroemulsionen als alternativer Kraftstoff," in 5. FAD-Konferenz „Herausforderung Abgasnachbehandlung für Dieselmotoren“, Dresden, 2007.

[6] P. Dittmann et al: „Einfluss von Mikroemulsionen auf die dieselmotorische Verbrennung,“ MTZ Motortechnische Zeitschrift, Bd. 76, pp. 60-67, April 2015.

[7] H. Dörksen: „Vorgelagerte Kraftstoffmenge bei geschichteter Diesel- Wasser-Einspritzung,“ MTZ Motortechnische Zeitschrift, pp. 58-64, Januar 2007.

[8] R. Pauls, C. Simon: „Einfluss der geschichteten Wassereinspritzung,“ MTZ, Januar 2004.

[9] H. Mollet, A. Grubenmann: Formulierungstechnik - Emulsionen, Suspensionen, Feste Formen, Weinheim: WILEY-VCH Verlag, 2000.

[10] M. J. Schwuger: Lehrbuch der Grenzflächenchemie, Stuttgart: Georg Thieme Verlag, 1996.

[11] H.-D. Dörfler: Grenzflächen- u. Kolloidchemie, Weinheim: VCH Verlag, 1994.

[12] K. Petersen: „Emulsionsstabilisierung durch Marine Polysaccharide,“ Dissertation, Kiel, 2013.

[13] K. Köhler: Emulgiertechnik: Grundlagen, Verfahren und Anwendungen, Hamburg: Behr's Verlag, 2012.

[14] K. Kosswig, H. Stache: Die Tenside, Wien: Carl Hanser Verlag, 1993.

[15] La Vision, Product-Manual for DaVis 7.2, Gottingen: LaVision GmbH, 2009.

[16] A. Hofacker: „Abgasnorm u. Wirklichkeit - Eine Annäherung“, MTZ 76, 2/2015

[17] T. Breuninger et al.: „Einfluss der Spraycharakteristik einer Piezo-A-Düse auf die Entflammung beim strahlgeführten Brennverfahren“, in 7. Tagung Diesel- und Benzindirekteinspritzung, Magdeburg, 2010.

[18] Lehrstuhl Strömungsmechanik \& Strömungstechnik, Arbeitsheft IV - Stoffwerte - Flüssigkeiten, Magdeburg: Otto-von-Guericke-Universität Magdeburg

[19] H. Hiroyasu, T. Kadota, M. Arai: „Development and Use of a Spray Combustion Modeling to Predict Diesel Engine Efficiency and Pollutant Emissions“, Bulletin of the JSME, Bd. 26, Nr. 214, pp. 569-575, April 1983.

[20] J. K. Dukowicz: „A Particle-Fluid Numerical Model for Liquid Sprays“, Journal of Computational Physics, Nr. 35, pp. 229-253, 1980. 


\title{
High speed visualization of gasoline pump injector (GPI)
}

\author{
Balasubramanian Nallannan ${ }^{1 *}$, Titus Iwaszkiewicz ${ }^{2}$, Jayabalan Sethuraman ${ }^{3}$ \\ 13 Stanadyne India Private Ltd, Thiruvallur 602025, India \\ ${ }^{2}$ Stanadyne LLC, 92 Deerfield Road,Windsor, CT 06095. USA \\ *Corresponding author: nbalasubramanian@stanadyne.com
}

\begin{abstract}
Two-wheeler engines still use carburetor as a fuelling system in many Asian countries, owing to its low cost and less maintenance. The usage of carburetor to handle the upcoming stringent emission norms gets difficult, due to the absence of a closed-loop fuel correction. An electronic fuel injector (EFI), on the other hand, with the help of an electronic control unit (ECU), can correct the fuel quantity and set the air-fuel mixture close to stoichiometric, based on the feedback obtained from the oxygen sensor placed in the exhaust pipe. In this context, an innovative injection system has been developed, that can be applied for such electronic fuel injection in two-wheelers. In this design, the pump and injector are integrated into a single unit, making the system, simple, compact and less expensive. The integrated injector uses a solenoid and spring arrangement, for pressurizing the fuel in a small chamber, and the pressurized fuel is then injected through orifices to produce spray in the intake port. Two-wheeler engines can operate in the order of $10,000 \mathrm{rpm}$ and it poses a big challenge in such injector designs, and therefore the time response of the mechanical and magnetic components of the injector become critical. High-speed back-lit imaging helps in understanding the time response of such injector, by visualizing the spray, while injecting continuously over a period of time. This paper presents the results of high-speed images, obtained from the spray of this new-concept gasoline pump injector (GPI). This exercise, demonstrated that this injector can work at a frequency as high as 83 $\mathrm{Hz}$ and also consistently. The spray pattern was found to be very unique and different from the conventional PFI injection sprays.
\end{abstract}

\section{Keywords}

Port fuel injection, Spray visualization, solenoid pump injector

\section{Introduction}

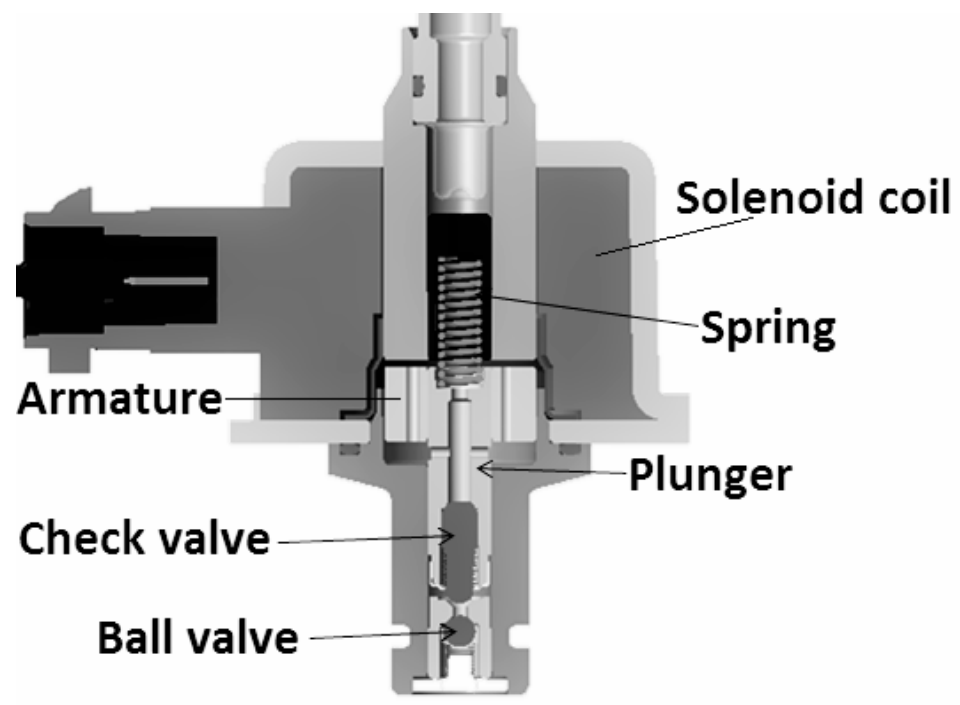

Figure 1. Figure showing the construction of gasoline pump injector (GPI).

The conventional port fuel injector (PFI) receives pressurized fuel, through a pipeline, from an electric rotary pump placed externally. The injector injects the pressurized fuel as and when the electronic control unit (ECU) demands. The pressure of the pump is maintained using an external pressure regulator. The injected fuel quantity is adjusted using pulse width control, to maintain the exhaust lambda values, close to stoichiometric, required for optimum catalytic converter efficiency. This system consumes power continuously as its pump runs continuously and also occupies more space including that of fuel tank's, due to the presence of multiple components discussed. Ways to simplify the injection system have been tried out through several techniques over decades, and one such technique which uses an electromagnetic reciprocating pump to directly displace the fuel is discussed by Heimberg et al.[1] 


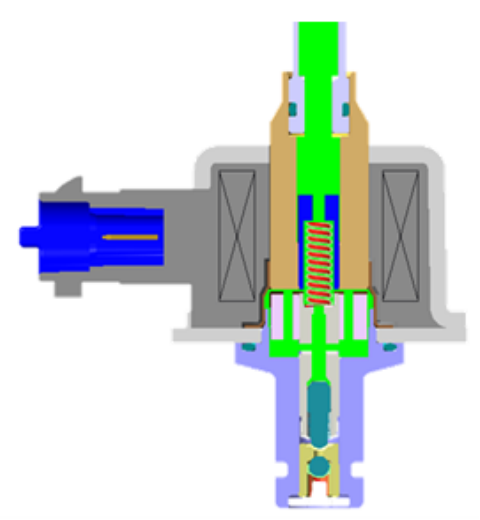

Suction

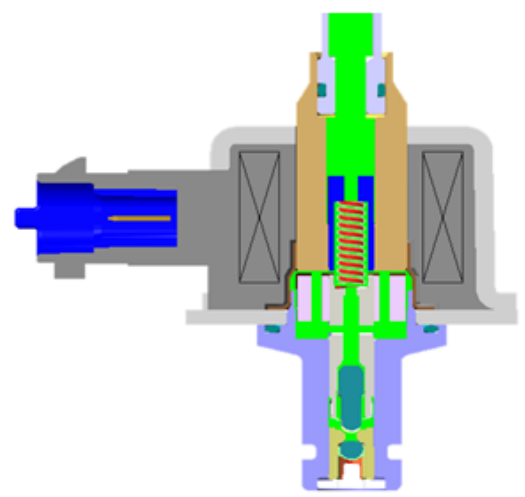

Filling

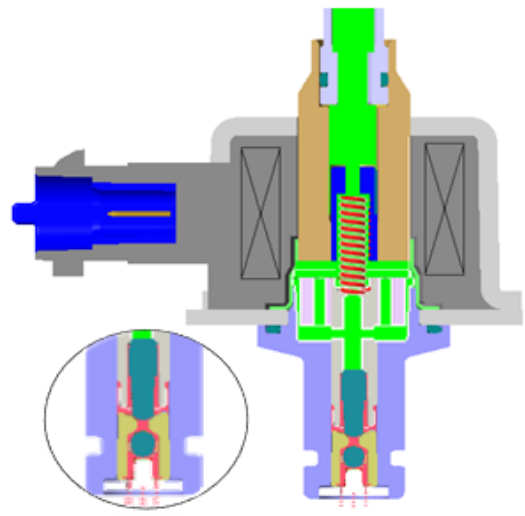

Pumping

Figure 2. Figure showing the working of GPI at various stages.

A similar pump-integrated design has also been invented for after treatment solutions, authored by our colleagues Cavanagh et al.[2] [3], and the work presented in this paper is an extension of that work, to apply the pump-integrated injector on a two-wheeler engine for port fuel injection (PFI). More specifically, this paper studies the time response of the GPI injector, which is very critical as its application on two-wheeler engine, demands faster injector response, owing to shorter cycle times at higher engine rpms.

The construction details of the gasoline pump injector (GPI) are shown in Fig. 1. The GPI is a reciprocating pump and is driven by an electromagnetic solenoid. Upon energizing the solenoid, a magnetic flux is generated around the coil, which pulls the armature up against the spring force. A plunger attached to the armature also moves along with it. A check valve assembly is placed below the plunger also moves along with the plunger. The check valve assembly consists of a check valve loaded by a spring, both placed below the plunger. The check valve moves with a lag, owing to the low pressure produced in the chamber, below the check valve. This low pressure is caused by the increase in the volume produced between the plunger bottom and the ball valve top, when the plunger moves upward, which is shown as 'Suction' in Fig. 2. The effective volume available between the bottom of the check valve and the ball valve top is essentially the 'pumping chamber' of the injector. The low pressure generated in the pumping volume allows the fuel to pass through the clearance between the plunger and the check valve, during the upward motion of the plunger, which is shown as 'Filling' in Fig. 2. When the solenoid is de-energized, the plunger moves down owing to the spring force acting on it. The check valve connected to the plunger gets closed and pressure is generated in the pumping volume, which is shown as 'Pumping' in Fig. 2. Balasubramanian et al. [4] has studied and reported the hydraulic characteristics of this injector in detail.

\section{Methodology}

The spray from the injector was investigated using high speed imaging technique and the test arrangements used are shown in Fig. 3 and Fig. 4. The fuel is fed from a fuel tank, by gravity, to the injector through a filter, as shown in Fig. 3. The injector is placed inside the visualization chamber. The spray zone is back lit using a halogen lamp. A high speed camera of Photron make captures the images from the front side. The injector is controlled by National Instruments' Calview software as shown in Fig. 4, where in the current profile and injection timings are set. The camera is controlled using camera manufacturer's (Photron's) software itself. Necessary pixel-size calibration is done prior to the spray imaging using a syringe-needle arrangement, whose dimensions are known. Upon calibration, the image is seen to occupy 15.9 pixels per $\mathrm{mm}$ of length. The size of the images obtained were of 256 x 472 pixels. The obtained images are post processed using Matlab code, where the images are cropped, background subtracted and finally converted to binary images using Otsu's algorithm. 


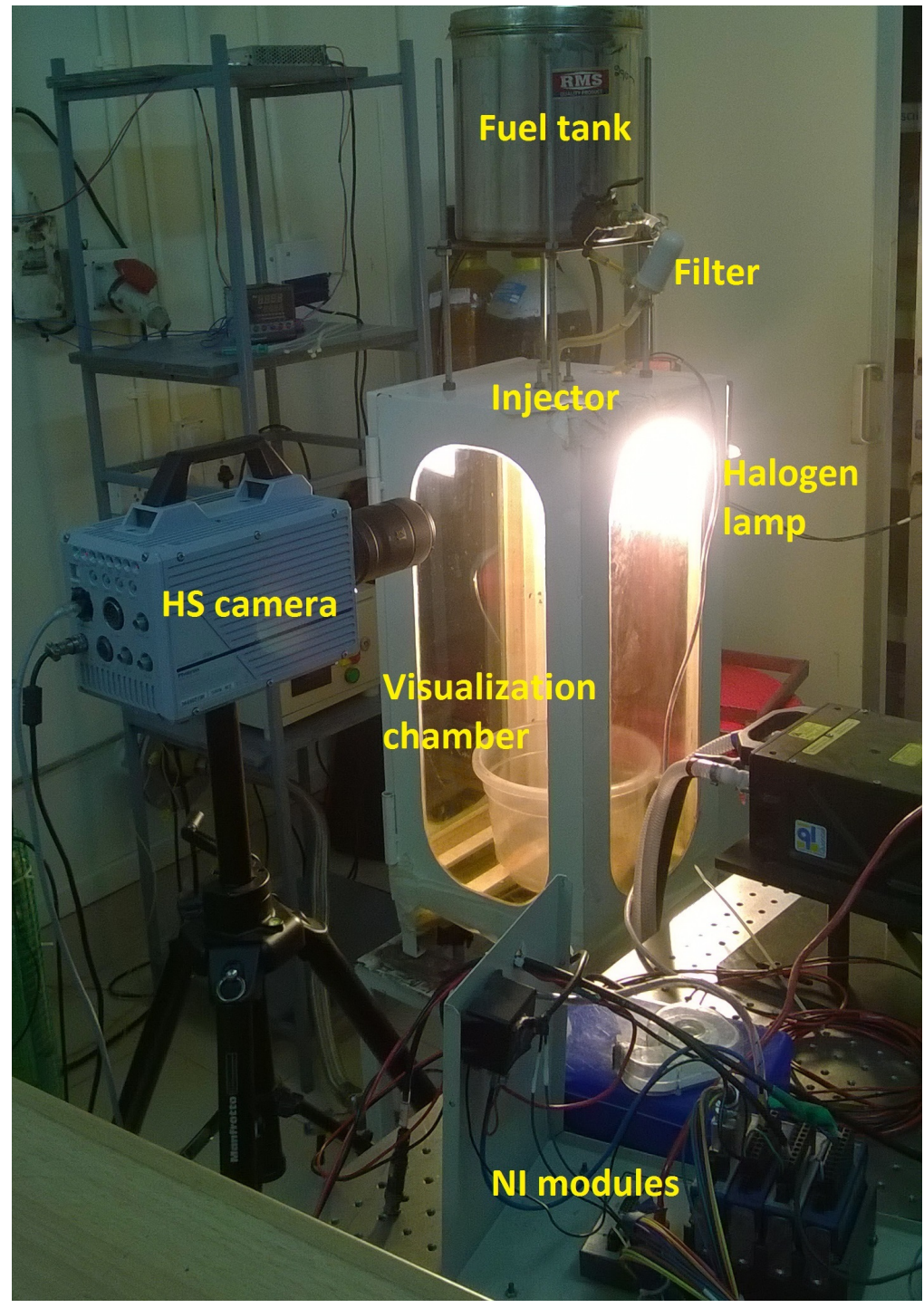

Figure 3. Photograph of the high speed imaging setup

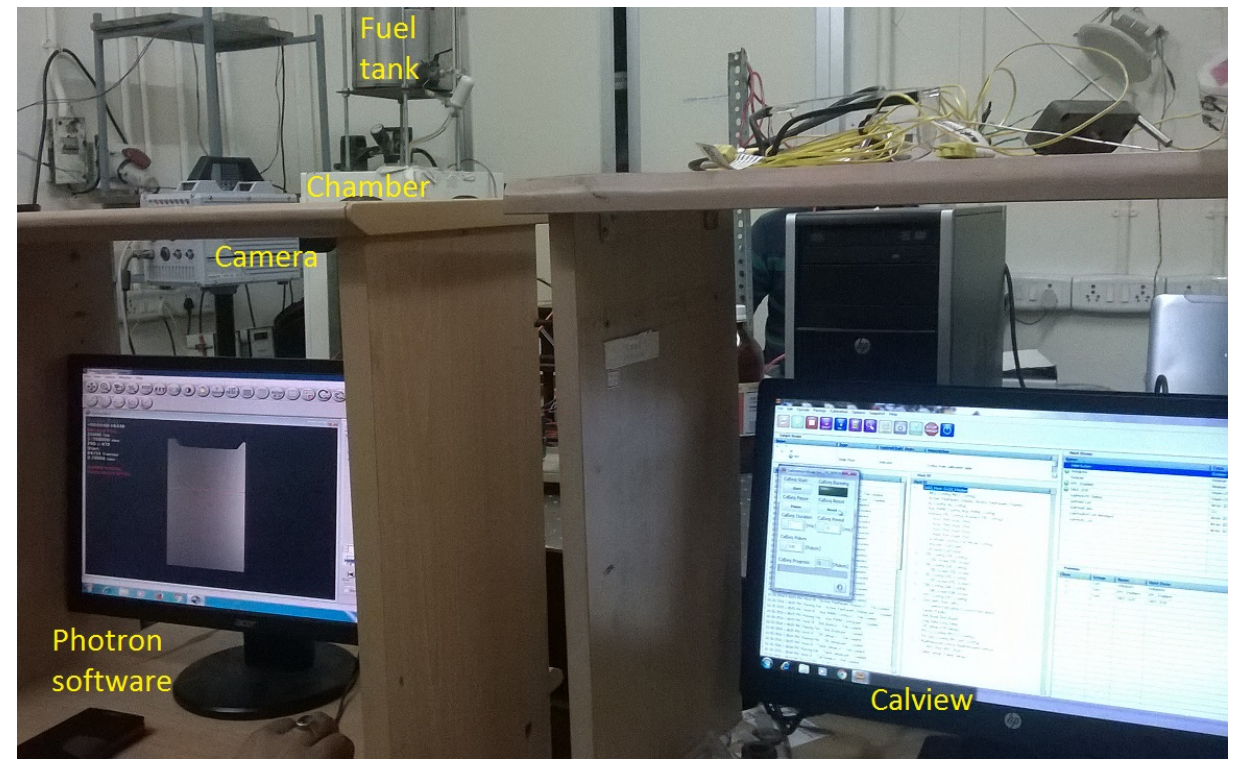

Figure 4. Photograph showing the computer control arrangements for camera and injector 


\section{Results and discussion}

The high speed images were acquired at the rate of $25000 \mathrm{fps}$ (at a time step of $40 \mu \mathrm{s}$ ). The images were continuously taken for two seconds. The injector was triggered at a frequency of $83 \mathrm{~Hz}$ (corresponding to cycles of 10,000 rpm of a 4 stroke engine). All the images obtained during these two seconds were analyzed individually, and confirmed that the injection events were consistent without missing of any cycle. The images captured in consecutive cycles at every $12 \mathrm{~ms}$ time (cycle time)gap are shown in Fig. 5. These images of eight consecutive cycles also correspond to $1.36 \mathrm{~ms}$ after the start of injection(SOI).
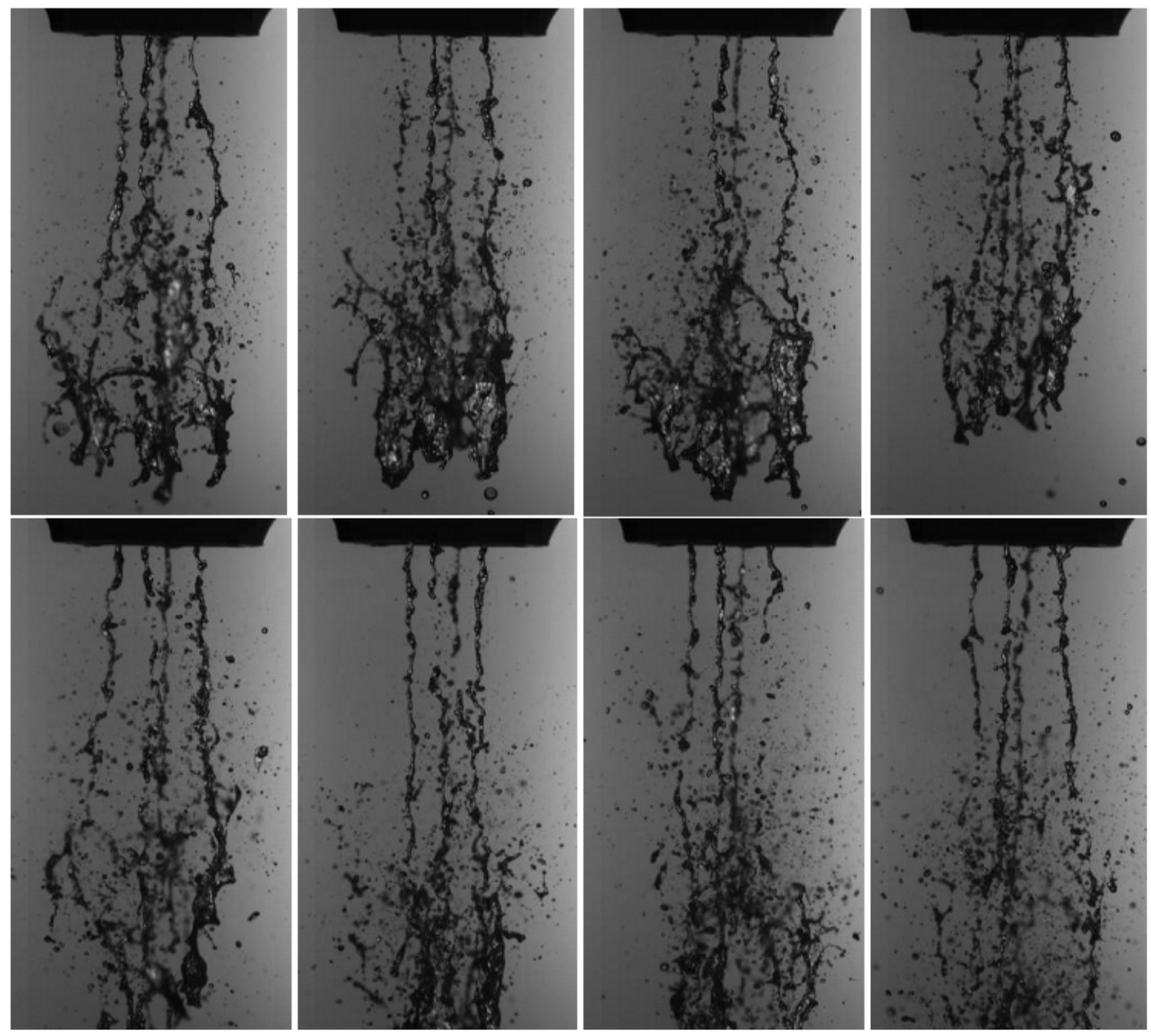

Figure 5. Raw high-speed images of GPI spray acquired continuously at every $12 \mathrm{~ms}$ time interval and at $1.36 \mathrm{~ms}$ after SOI

This visualization work becomes more important and critical, as it assesses the mechanical spring's performance in consistently pumping the fuel at such a high frequency. Continuously captured images corresponding to a single injection event are shown in Fig. 6 . The first and third row show the sequential raw images obtained at $40 \mu$ s time step. The second and fourth rows show the corresponding post processed images.

The summary of the the GPI spray evolution is shown in Table 1. The row titled 'Abs. time' refers to the beginning of acquisition as zero and the row titled 'Rel. time' refers to the relative time taking the start of injection (SOI) as zero. After the SOI, the ligaments are formed in the spray and sustain till $3.36 \mathrm{~ms}$. Thereafter ligaments are not seen and only droplets are seen to come out till $7.56 \mathrm{~ms}$. There are no droplets seen after 7.56 and the GPI waits for the next injection event.

Table 1. Spray events at an injection pulse frequency of $83 \mathrm{HZ}$, corresponding to $10,000 \mathrm{rpm}$ of engine.

\begin{tabular}{c|cccc}
\hline & SOI [ms] & End of ligaments [ms] & End of droplets [ms] & Next injection [ms] \\
\hline Abs. time & 9.28 & 12.64 & 16.84 & 21.24 \\
Rel. time & 0 & 3.36 & 7.56 & 12
\end{tabular}




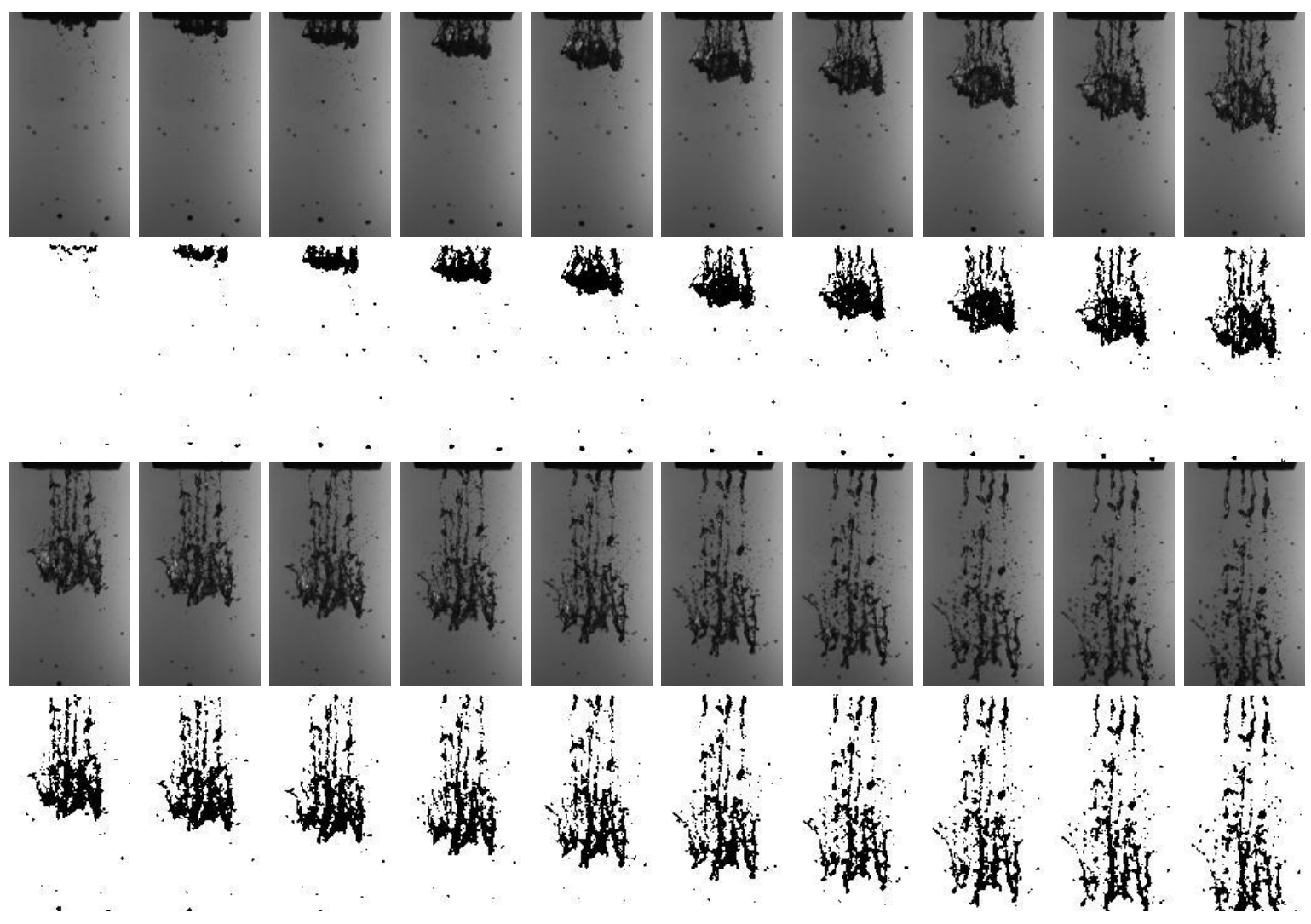

Figure 6. Unprocessed and processed high-speed images of GPI spray acquired at the rate of $25000 \mathrm{fps}$

As the injection is driven by an impulse from the plunger, the spray is seen to eject as a parcel initially, and continues to inject as a jet later, and finally ends with droplets ejection. The later part of the injection event (droplets ejection), is an outcome of the ball valve bouncing back to its home position, due to drop in the fluid pressure above, and assisted by the upwardly-acting spring force below. As the ball valve is closed, the fluid remaining in the injector volume below the ball, exits the nozzle due to gravity, and without pressure assistance from the pump above.

\section{Conclusions}

High-speed back-lit imaging work was successfully carried out on the new-concept gasoline pump injector (GPI). This exercise, demonstrated that this injector can work at a frequency as high as $83 \mathrm{~Hz}$ consistently, and can comply to the demands of its application on a two-wheeler engine (of higher rpms). The spray pattern was found to be very unique as it evolves in two stages, namely ligament stage and droplet stage. The major portion of the injection quantity is seen to get over in less than $4 \mathrm{~ms}$ in the ligament stage and the total injection is seen to get over in less than $8 \mathrm{~ms}$. More than $4 \mathrm{~ms}$ of time is seen to be available, for the preparation of next injection event. The outcome of this visualization work gives enough confidence to apply the GPI on a two-wheeler engine.

\section{Acknowledgements}

This high speed imaging work was carried in the spray research facility of internal combustion engines laboratory at the Indian Institute of Technology Madras (IIT Madras) and we thank Dr.Anand T.N.C. for kindly allowing us to carry out this work there. Manas kumar pal, Keerthi Ganesh and Nithin Tito are acknowledged for their kind help in conducting the experiments at IIT Madras. Our colleagues Sanjay Chadda, Arvind S., Karthick Durairaj, Mark Cavanagh, Rajat Dhara and Mike Plouffe are also acknowledged for their kind support in this project. 


\author{
Abbreviation \\ EFI Electronic fuel injection \\ FPS Frames per second \\ $G P I \quad$ Gasoline pump injector \\ $P F I \quad$ Port fuel injection \\ SOI Start of injection
}

\title{
References
}

[1] Wolfgang Heimberg, Ebersberg; Wolfram Hellmich, Munich; Franz Kogl, Kaufbeuren, all of Germany; Paul Malatinszky, Bulle, Switzerland "Fuel injection device according to the solid-state energy storage principle for internal combustion engines" United states patent US5469828, Date of Patent: Nov. 28, 1995

[2] Mark Cavanagh, Robert Lucas and Yevgeniy Norkin., "Integrated pump and injector for exhaust after treatment," US patent No.US 8,225,602 B2, Date of patent: July 24, 2012.

[3] Mark Cavanagh, Robert Lucas and Yevgeniy Norkin., "Integrated pump and injector for exhaust after treatment," US patent No.US 20100313553A1, Date of patent: Dec 16, 2010.

[4] Balasubramanian Nallannan, Karthick Durairaj, Jayabalan Sethuraman "New-concept Gasoline Pump Injector (GPI) for Motorcycle" SAE Technical Paper 2017-01-0861. 


\title{
Oxygenated fuels properties and relationship with engine performance in port fuel injection engines.
}

\author{
Uriel Gonzalez*, Isaac Schifter. \\ Gerencia de Sustentabilidad Energética, \\ Instituto Mexicano del Petróleo, México \\ *Corresponding author: ugonzale@imp.mx
}

\begin{abstract}
Gasoline oxygenating agents (alcohols, ethers and a carbonate) were used to formulate gasoline at different oxygen contents up to $20 \mathrm{wt} . \%$ and compared with commercial Premium gasoline.

The performance of each fuel was investigated in a port fuel injected, single cylinder, spark-ignited engine at different stages i.e. air fuel mixture preparation, combustion behavior and exhaust emissions. In all cases, the intake cooling effect (related mainly to fuel properties like latent heat of vaporization and Reid Vapor Pressure), shows an important relationship with engine performance and emissions, probably due to reductions in heat losses associated with decreases in charge temperature at compression stroke before ignition. This results was confirmed by means of vehicle FTP-75 test.

The high RVP promotes high intake manifold evaporation rate, and the high HoV is related to important cooling effect as the fuel absorbs heat during evaporation. If the fuel evaporates faster upstream intake valves, the advantages of high HoV as a way to reduce compression work and heat transfer fallen.

The quantification of the charge cooling effect was done by means of precision intake air temperature control and the instrumentation of a temperature downstream the injector at intake port and as close as possible to the intake valves.

The use of oxygenates reduce the hydrogen and carbon fuel contents as a result of fuel dilution. For a given level of oxygenation as lower is the molecular oxygen content in the additive, higher will be the fuel dilution.

For 10 wt.\% oxygen and more, fuel performance in port engines depends mainly on oxygenate contents and its relationship with HoV and RVP. For oxygenated gasolines, fuel sensitivity have a direct relationship with latent heat of vaporization, because charge cooling is one of the way alcohols increase RON. In the other hand, MON is almost insensible to high heat of vaporization, because the intake air is heated to $159 \mathrm{C}$ as a test requirement.
\end{abstract}

\section{Keywords}

Oxygenated gasoline, charge cooling, sensitivity, combustion, emissions

\section{Introduction}

Air pollution is becoming a serious problem in many urban cities of the world and it can have a severe effect on both health and the environment. All kind of internal combustion engines i.e. Spark Ignition (SI) and Compression Ignition $(\mathrm{Cl})$ are equally responsible for the emission as carbon monoxide (CO), nitrogen oxides (NOx), hydrocarbons (HC), and particles pollutants [1]

Since $\mathrm{CO}_{2}$ emissions cause global warming, which is one of the major environmental problems today; it is assessed as a pollutant. In the occurrence of harmful substances in exhaust gas emissions, when type of fuel is taken into consideration, it is possible to lower these emissions by using different fuels. The use of oxygenated fuels in gasoline provides more oxygen in the combustion chamber, allows the use of higher compression ratios, and has the potential to reduce emissions [2].

Additives are integral part of today's fuel. Together with carefully formulated base fuel composition, they contribute to combustion efficiency and engine long life. They are chemicals, which are added in small quantities either to enhance fuel performance, or to correct a deficiency as desired by the current legislation. They can have surprisingly large effects even when added in little amount [3].

Replacing one or other additive means that the specifications and volume of the petroleum base fuels will have to be adapted in order to meet vapor pressure, octane numbers specification and other requirements for the entire gasoline blend sold at refilling stations. This in turn has an effect on the composition and on other specifications of the petroleum base fuels [4].

Although experimental studies have shown that oxygenated fuels burn cleaner than unleaded gasoline and produce lesser emission, there is limited information regarding the comparison among the oxygenated fuels as gasoline additive in spark-ignited engines. Among various renewable fuels, alcohols, such as ethanol, methanol, isobutanol, are the most popular fuels utilized in internal combustion engines.

Schifter et al. [5] used gasoline and gasoline-ethanol blends at the ratios of $0-20 \%$ ethanol in a single cylinder engine, and showed that the combustion rate, efficiency and fuel consumption increased with the blend ratio. In addition, with the increasing of the ethanol ratio in the blend, the $\mathrm{HC}$ and $\mathrm{CO}$ emissions have decreased, but $\mathrm{NOx}$ emission has increased.

In order to satisfy the requirements for incorporation of fuel renewable compounds, in USA and other countries, the use of ethanol as an oxygenate agent was promoted. In places like Brazil, the use of ethanol as a fuel in Otto 
engines is more related to energetic diversification and economics reasons than to environmental aspects. From the comparison of the properties of fuels it is apparent than the amount of injected fuel has to be increased when alcohol is added to maintain the desired - typically stoichiometric - air to fuel ratio, which is necessary for the proper operation of the engine including its exhaust gas after treatment devices [6].

Jung et al [7] noted that light-duty vehicles with direct injection (DI) gasoline engine have been in commercial production since the late 1990's, and it is widely accepted that smaller displacement engines will replace larger ones as a way to meet higher fuel economy regulations. Turbocharged GDI engines can be more fuel-efficient and offer a performance benefit due to the higher volumetric efficiency at high load. Direct injection Otto engines are more efficient than port fuel injection ones mainly because the fuel is injected inside of the combustion chamber allowing different combustion modes in order to diminish emissions and fuel consumption. At the same time DI engines can have approximately one additional unit in compression ratio i.e.11.5:1 instead of 10.5:1 in port fuel injected engines.

Production ECUs (engine control units) generally have the capability of some degree of short-term and long-term adjustment of the fuel injection pulse width, intended to serve as a compensation for variations in the composition and fuel properties and in general working of the engine at any load, limited to full load. Such capacity has not, intended to cover, for example, a rather substantial increase in the fuel delivery rates when switching from gasoline to ethanol [6].

Currently, ethanol blended into gasoline at various concentrations is the most utilized alternative for spark ignition engines in order to incorporate non fossil components and for energetic diversification. Unfortunately, ethanol exhibits some negative attributes, such as significantly lower energy content, hygroscopic behavior and chemical toxicity [8]. In addition, broader consequences of fuel production need to be considered, as the current ethanol feedstock crops may compete with food production and be negatives in terms of cost-benefit.

One promising alternative to ethanol are ethers like ethyl-tert butyl ether (ETBE), used in Asia and some European regions not only as a manner to improve octane numbers in a cost-effective way, but also as an approach to reduce air pollution. The main advantages of ethers like ETBE over alcohols (mostly ethanol and methanol) are related with mixture stability, water tolerance, corrosion, and the achievement of low Reid Vapour Pressure (RVP) standards to reduce fugitive emissions [8].

In order to satisfy fuel environmental requirements in Europe, ETBE is used, because is derived from ethanol obtained from biomass and is considered as a biofuel, nevertheless, the required catalytic reaction to produce ETBE required isobutylene, usually obtained from fossil sources from either refining or from natural gas [9].

For an ethanol 30\%volume/70 \% gasoline blend, Wang et al. found in direct injection engines a the positive effect of charging cooling was reflected in the more advanced combustion phasing, higher engine thermal efficiency, and lower unburned gas temperature at Top Dead Center. The high heat of vaporization and low stoichiometric air/fuel ratio of ethanol blends both contributed to a better charge cooling effect [10].

Fuel spray properties play an important role in direct injection engines, nevertheless, in port injection engines, the air fuel mixture is prepared in the intake manifold, before intake valves with enough time to achieve uniform mixture between air and fuel. Nevertheless with the incorporation of high latent heat of vaporization compounds like alcohols in gasoline, the fuel properties change, modifying the temperature of the blend as the fuel gets evaporated.

The more often octane numbers used as a way to quantify the capacity of a fuel to avoid auto ignition and suppress engine knock, are Research Octane Number (RON) introduced in 1928 and the Motor Octane Number (MON). This properties are obtained by means of a machine named Cooperative Fuel Research (CFR) engine. The MON test is considered more aggressive than the RON test, i.e. the MON is lower than the RON.The main difference between the RON and MON methods are the engine regime and the ignition time. In general, MON can be more representative of high load conditions and have higher engine speed (900 rpm vs. $600 \mathrm{rpm}$ ) and a higher intake temperature of $149^{\circ} \mathrm{C}$ downstream of the carburetor vs. $52{ }^{\circ} \mathrm{C}$ upstream of the carburetor in RON test. To accomplish manifold temperature in MON test uses heater, cancelling any effect associated with fuel heat of vaporization.

This research is focused in the determination of relationships between fuel formulation, engine performance and its exhaust emissions for different oxygenated gasolines, taking in to account base fuel composition, oxygenating level and agent, considering in deep physicochemical analysis, fuel oxygen contents form $3.5 \mathrm{wt} \%$ to $20 \mathrm{wt}$. $\%$ and more and three different functional groups (i.e. ethers, alcohols and one carbonate).

\section{Material and methods}

Test Fuels

A baseline fuel was formulated without oxygen content from refinery-typical blending streams. The inspection data for the gasoline components was performed following American Society for Testing Materials procedures [11]. The test fuels were prepared gravimetrically to the oxygen target value using a precision balance with a repeatability of $0.001 \mathrm{~g}$. by "splashing" the oxygenate compound with the base gasoline following prescriptions reported by Pahl and McNally [12]. In splash blending, the oxygenate compound is added to a conventional gasoline without much consideration for the final properties of the fuel.

Further details on the properties, composition and main characteristics of the blends are reported in Table 1 


\begin{tabular}{|c|c|c|c|c|c|c|c|}
\hline Property - Fuel ID & 1 & 2 & 3 & 4 & 5 & 6 & Commercial \\
\hline Oxygenated compound & ETBE & $\mathrm{EtOH}$ & $\mathrm{EtOH}$ & DMC & $\mathrm{EtOH}$ & $\mathrm{MeOH}$ & MTBE \\
\hline Oxygen in gasoline, wt.\% & 3.5 & 3.5 & 10 & 20 & 20 & 20 & 1.8 \\
\hline Volume in gasoline, vol.\% & 22 & 9.4 & 27.1 & 29.2 & 55.5 & 38.1 & 9.7 \\
\hline Paraffins, wt.\% & 9.25 & 10.53 & 7.19 & 7.55 & 5.15 & 7.27 & 8.2 \\
\hline Isoparaffins, wt.\% & 29.61 & 33.71 & 22.98 & 23.91 & 16.33 & 23.03 & 48 \\
\hline Olefins, wt.\% & 6.62 & 7.44 & 4.89 & 5.51 & 3.77 & 5.31 & 8.20 \\
\hline Napthenes, wt.\% & 3.43 & 5.04 & 3.60 & 3.63 & 2.48 & 3.50 & 2.90 \\
\hline Aromatics, wt.\% & 27.67 & 31.03 & 22.06 & 21.71 & 14.83 & 20.91 & 23.00 \\
\hline Specific gravity & 0.735 & 0.741 & 0.749 & 0.783 & 0.760 & 0.750 & 0.731 \\
\hline LHV, MJ/kg & 41.59 & 41.48 & 38.49 & 32.86 & 33.85 & 33.94 & 42.8 \\
\hline RVP, psi & 6.53 & 8.46 & 8.17 & 7.61 & 6.38 & 10.34 & 7.65 \\
\hline C, wt.\% & 82.5 & 82.3 & 73.7 & 68.2 & 66.2 & 66.2 & 86.2 \\
\hline $\mathrm{H}$, wt.\% & 13.7 & 13.6 & 13.1 & 9.9 & 12.6 & 12.1 & 11.8 \\
\hline $\mathrm{H} / \mathrm{C}, \mathrm{mol}$ & 2.0 & 2.0 & 2.1 & 1.7 & 2.6 & 2.3 & 1.9 \\
\hline Air-to-fuel ratio, wt./wt.\% & 14.17 & 14.2 & 12.53 & 10.70 & 11.33 & 11.23 & 14.15 \\
\hline RON & 94.6 & 94.0 & 101.9 & 98.0 & 121.8 & 109.0 & 97.0 \\
\hline MON & 84.3 & 83.0 & 87.0 & 89.2 & 87.2 & 86.4 & 87.0 \\
\hline $\mathrm{AKI},(\mathrm{RON}+\mathrm{MON}) / 2$ & 89.4 & 88.5 & 94.4 & 93.6 & 104.5 & 97.7 & 92.0 \\
\hline $\mathrm{HoV}, \mathrm{KJ} / \mathrm{kg}$ & 342 & 406 & 512 & 356 & 678 & 647 & 312 \\
\hline $\mathrm{IBP},{ }^{\circ} \mathrm{C}$ & 42.1 & 39.8 & 43.3 & 40.1 & 50.0 & 40.0 & 37.0 \\
\hline $\mathrm{T}_{10},{ }^{\circ} \mathrm{C}$ & 64.2 & 53.2 & 56.2 & 58.2 & 67.5 & 49.9 & 58.1 \\
\hline $\mathrm{T}_{50},{ }^{\circ} \mathrm{C}$ & 89.4 & 95.4 & 73.6 & 89.2 & 78.6 & 62.2 & 98.7 \\
\hline $\mathrm{T}_{90},{ }^{\circ} \mathrm{C}$ & 158.5 & 162.3 & 160.7 & 161.7 & 80.7 & 153.5 & 163.5 \\
\hline End point, ${ }^{\circ} \mathrm{C}$ & 208.9 & 211.6 & 211.8 & 207.8 & 174.1 & 205.1 & 205.3 \\
\hline
\end{tabular}

Table 1. Physical and chemical properties of the fuels

Single cylinder engine tests

The experiments were performed on an AVL 5401 SI single cylinder engine electronically controlled, capable of 25 $\mathrm{KW}$ at $6000 \mathrm{rpm}$. The engine load is absorbed by an asynchronous dynamometer controlling the engine speed with $\pm 1 \mathrm{rpm}$ accuracy. The supply system comprises an AVL-735C fuel temperature conditioner, and an AVL-735 consumption meter based on Coriolis principle.

The system utilizes a stand-alone fuel management system model brand General Engine Management Systems allowing to modify injection and spark ignition timing independently, and with an AVL-619 Indimeter in order to monitor and acquired the combustion related camera pressure information .

The crank angle position and combustion chamber pressure is monitored with a $0.1^{\circ}$ of turn resolution. The engine is controlled by AVL Puma Open 7.1 software and data acquisition, integrating the dynamometer, delivery systems, fuel conditioning, and measurement, as well as emissions analysis.

The load was selected as a partial load condition at mid-range speed to avoid the engine to become chocked during the inlet flow in the admission process at high speeds that may reduce the volumetric efficiency.

On the other hand, avoiding slow engine speeds prone to higher heat transfer through the walls, lowering the average combustion gas temperature and pressure, and reducing the work per cycle transferred to the piston. In Table 2, the main engine characteristics and operating conditions are showed. 


\begin{tabular}{lc}
\hline \hline Type & spark ignition, double overhead cam, 4V \\
Bore and stroke & $86 \mathrm{~mm}$ \\
Displacement & $0.5 \mathrm{~L}$ \\
Compression ratio & $10.5: 1$ \\
Fuel delivery & port fuel injection \\
Regime & $2,400 \mathrm{rpm}$ \\
IMEP & $6.5 \mathrm{Bar}$ \\
Spark advance & $12.42{ }^{\circ} \mathrm{BTDC}$ \\
Admitted air mass & $24.5 \mathrm{~kg} / \mathrm{hr}$ \\
Intake air pressure & $1035 \mathrm{mBar}$ \\
Intake air temperature & $48{ }^{\circ} \mathrm{C}$ \\
Intake air humidity & $<5 \%$ \\
fuel temperature & $40^{\circ} \mathrm{C}$ \\
Coolant temperature & $90^{\circ} \mathrm{C}$ \\
Oil temperature & $95^{\circ} \mathrm{C}$ \\
\hline
\end{tabular}

Table 2. Engine characteristics and operating conditions

During test development with different fuels, the indicated mean effective pressure (IMEP) was keeping as constant as possible and the fuel was adjusted to keep the stoichiometric air-fuel ratio and engine load, therefore the IMEP was kept almost constant $( \pm 0.5 \%)$.

In order to compare fuels with different oxygenate contents, it becomes necessary the use of a dimensionless parameter that allows comparisons at the same air-fuel ratio for every fuel composition. This is accomplished by Brettschneider equation [13] which provides the lambda value based on exhaust emissions provided by AVL-CEB II gas analyser and fuel composition, including $\mathrm{H} / \mathrm{C}$ and $\mathrm{O} / \mathrm{C}$ ratios.

To insure the reproducibility of the measurements for different fuels and operating conditions, experimental data were averaged over consecutive 100 cycles, and its dispersion analysed by means of descriptive statistics common methods. The mean and the median value of each data set was compared in order to determine the nature of distribution obtained, considering for each variable a $95 \%$ statistical confidence level.

Chassis dynamometer tests

We employed the FTP-75 light-duty driving cycle in order to test the emissions of a Nissan TIIDA 2013 gasoline vehicle, designed to comply with United States Tier 1 limits of emissions when new $\left(C O, 2.1 \mathrm{~g} \mathrm{~km}^{-1}\right.$; non-methane hydrocarbons (NMHC), $0.156 \mathrm{~g} \mathrm{~km}^{-1}$; NOx, $0.25 \mathrm{~g} \mathrm{~km}^{-1}$ ). The vehicle was equipped with air-fuel ratio (AFR) control systems mainly supported in lambda sensor signal.

The FTP test is used in México and another countries to certify the emissions of new vehicles. This cycle has three separate phases: a cold-start (505-second) phase known as Bag 1, a hot-transient (864-second) phase known as Bag 2 , and a hot-start (505-second) phase known as Bag 3. In each phase, an aliquoted sample diluted with air by means of a constant volume sampler (CVS) is collected in one different bag, and analyzed individually.

The total test time for the FTP-75 is 1874 seconds, the average speed is $34.1 \mathrm{~km} \mathrm{~h}^{-1}$, and the travel distance is 17.8 $\mathrm{km}$ [14]. Before the tests, the vehicle was conditioned on the road over a course designed to simulate a portion of the FTP-75 cycle in terms of typical speeds, as well as number of stops, and a sequence of engine off and idles were performed.

The experiments were carried out in our facilities on a standardized dynamometer system. Emission gas samples were collected into Tedlar bags during each phase of the FTP-75 cycle, and the vehicles operated on a Horiba-ECDM-48 single-roll electric dynamometer coupled to a constant-volume sampler unit (Horiba CVS-45).

Total HCs, NMHCs, CO, NOx, and $\mathrm{CO}_{2}$ concentrations were measured using a Horiba MEXA-9200D-gas analyzer system [14]. The test program was examined using the statistical methods described in previous works [14].

The criterion to determine whether a repeat-test was necessary was performed when FTP emissions ratio between the larger value divided by the smaller value, was greater than 1.17 for THC, 1.33 for CO, and 1.40 for NOx [15]. The absolute excess cold start emissions is defined as the additional emission value obtained under cold conditions compared to the emission value that could have been recorded for the same period (cycle) under hot conditions. The absolute excess cold start emissions can be calculated by subtraction of the hot soak Bag 3 emissions from the cold start Bag 1. 


\section{Results and discussion}

\section{About Fuel Properties}

As oxygen content in the fuel increases, the fuel carbon and hydrogen contents diminish as a result of fuel dilution. i.e. for a given oxygenating level, the effect is inversely proportional to the molecular oxygen content in the additive, in spite of oxygenant functional group. The fuel added with DMC have the lowest hydrogen contents, associated mainly to the $\mathrm{DMC}$ lowest $\mathrm{H} / \mathrm{C}$ ratio respect to the other oxygenates

Fuel composition depends mainly of oxygenation level (how much oxygen by weight have the gasoline) and oxygenated compound added (how much oxygen have the oxygenated molecule) In figure 1. Are presented the experimental results for low heating value for fuels with different levels of oxygenation by means of different oxygenated compounds i.e. ethers (ETBE, MTBE), alcohols (EtOH, $\mathrm{MeOH}, \mathrm{IBuOH})$ and DMC. The labels identify the fuel included in the test matrix in Table 1. In spite of the fuel composition, base gasoline used, or oxygenate agent, the LHV reduce linearly with weight oxygen content. The R squared of the linear adjust showed is 0.99 . The abundance of samples with $3.5 \mathrm{wt}$ \% oxygen is related to Mexico actual specification. This result have relevance because make easy to stablish similar test conditions for very different test fuels. I.e. if the admitted air mass and the Lambda value (air-fuel ratio) is constant, the results can be compared back-to back for every fuel.

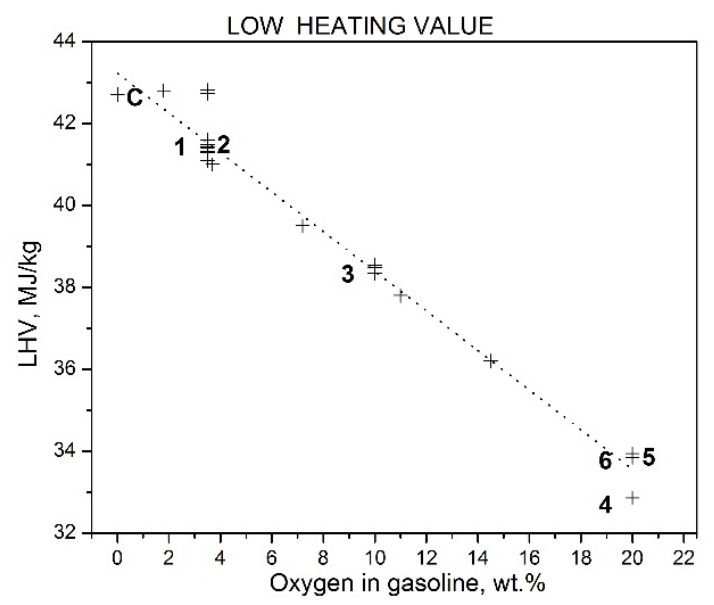

Figure 1. LHV as a function of fuel oxygen content

In figure 2, shows a survey of different fuels tested in single cylinder engine at similar conditions to the ones described in Table 2. In abscissa, the heat of vaporization and in ordinate, the Reid Vapor Pressure. The charge cooling (i.e. intake air temperature upstream injector minus blend temperature at intake port is included as a thermal scale i.e. cold colors for the highest cooling effect. The stream cooling in the manifold depends strongly on RVP i.e. the fuel mass evaporated in the intake manifold depend on how fast the fuel evaporates before intake valves. HoV determines how much energy is absorbed by the air-fuel blend during evaporation. i.e. fuel with alcohols have the highest HoV and the ones with ethers (ETBE and MTBE) and carbonate (DMC) the lowest ones, as shown the labels that identifies the oxygenated used in each fuel. The number in the left side of the points identifies the fuel on Table 1. As the HoV increases and RVP diminishes, the conditions to take advantage of fuel cooling effect are better, because more fuel is getting evaporated inside of combustion chamber, reducing the compression work and heat transfer to engine refrigerant circuit. This result suggest intake manifold insulation, geometries to reduce the mixture residence time in the manifold and use of low RVP fuel with high HoV (added with alcohols) as a strategies to improve engine efficiency. In accordance with this criteria, fuel 5 can be identified as the best fuel in the matrix. 


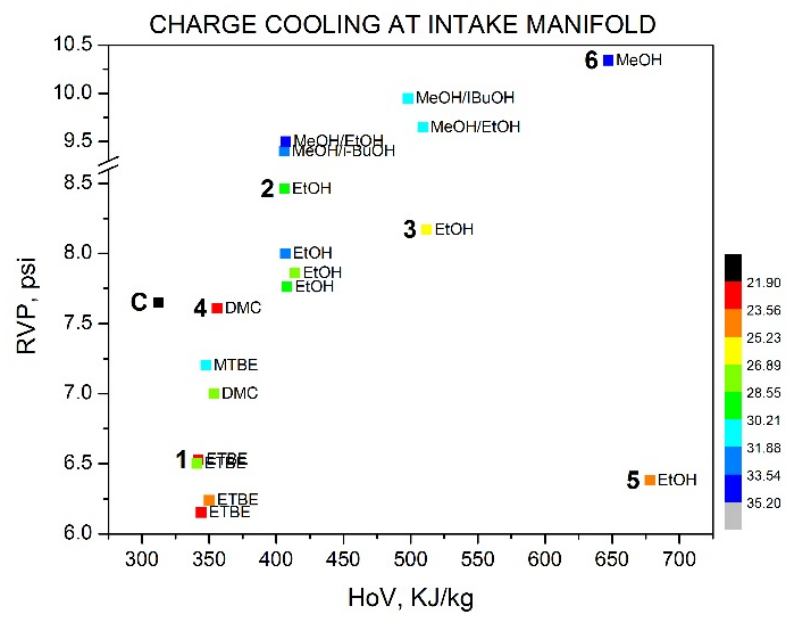

Figure 2. RVP and HoV Charge cooling for selected fuels. Cooling effect in thermal scale

In figure 3 the fuel sensitivity vs. HoV is presented for different fuels, including the ones from matrix 1 . In spite of fuel formulation or base fuel octane index, the fuel sensitivity strongly depends on HoV and this is governed by oxygen content and oxygenant functional group (ethers and carbonates < alcohols). As mentioned before, in MON test, the air-fuel blend must be heated to 149 Centigrade, hiding all the fuel improvement in knock resistance associated with charge cooling effect. This result is highly relevant, in actual port and direct injection engines the manufacturers try to avoid intake manifold high temperature reducing the heat transfer from the engine head and from the surroundings using nonmetallic materials ad specific geometries, contributing to the low specificity of MON test as a way to avoid knocking for in use and new vehicles.

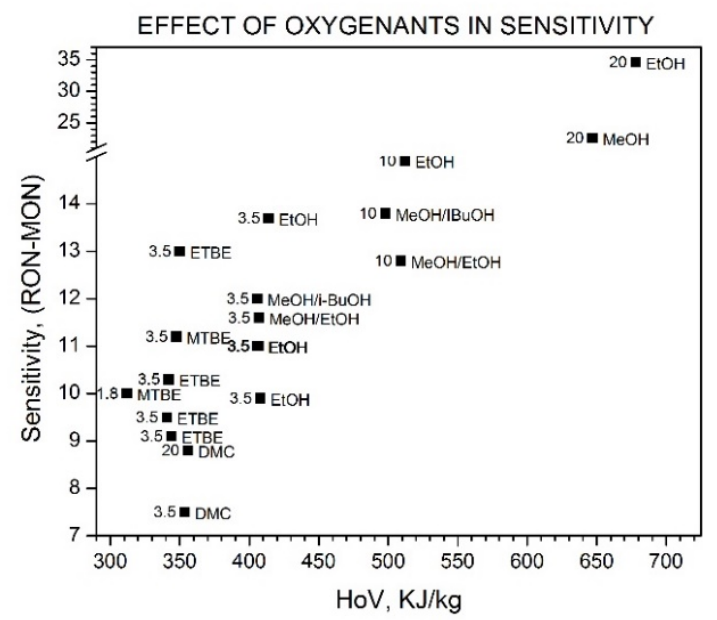

Figure 3.Fuel sensitivity as a function of HoV. In labels, oxygen content in wt.\% and oxygenant

In figure 4 , for $50 \%$ mass burned fraction crank angle i.e. combustion centre of gravity, the ignition advance was optimized for commercial fuel, formulated with MTBE, then, all the fuels was tested with the same ignition spark angle in order to detect changes related to speed of combustion. For the fuels formulated with low oxygenation level (3.5 wt.\%), the combustion delays respect to the Commercial fuel. Never the less, for fuels formulated with alcohols at high concentrations, were latent heat of vaporization depends on oxygen contents, the combustion gets faster. The fuel formulated with DMC (low HoV) the combustion was the one with the slowest combustion. 


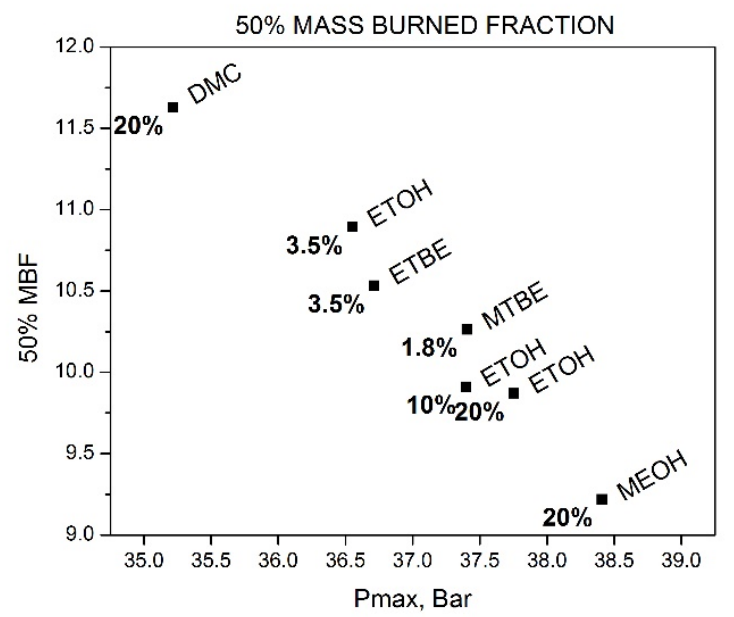

Figure 4.Combustion centre of gravity for tested Fuels. In labels, oxygen content in wt.\%

The fuel properties-engine experimental results matrix was examined by Factor analysis. In Figure 5, Factor 1 explains $47.4 \%$ of the total variability and Factor 2 explains $18.7 \%$ of total variability. Several gasoline and engine variables were included with high factor loadings, indicating stronger associations. In this analysis, also are included different baseline gasoline formulations.

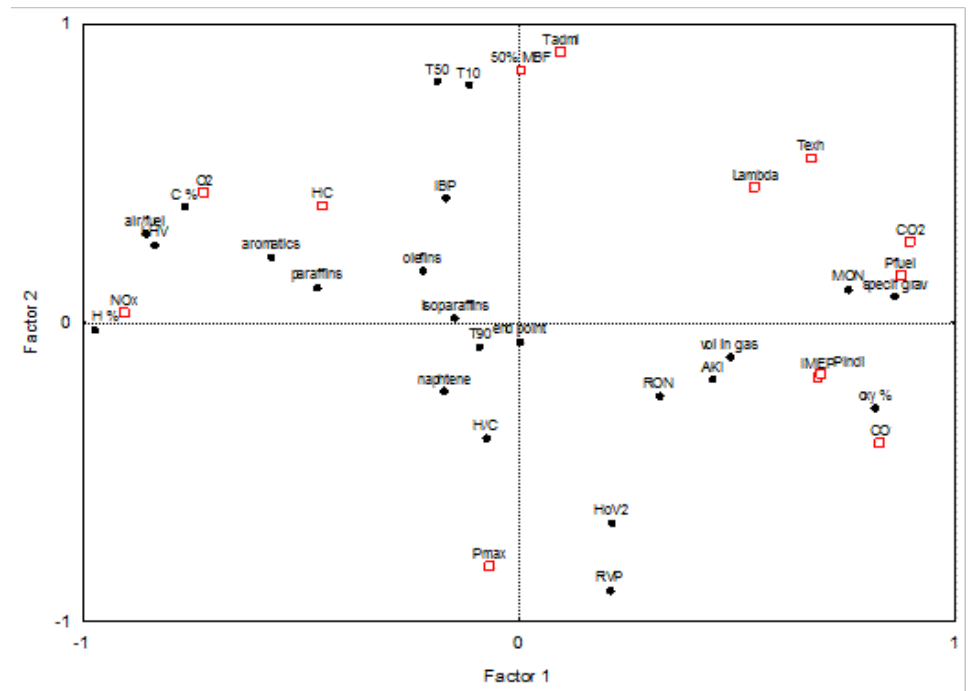

Figure 5. Factor analysis for tested fuels. Fuel properties in black points and engine results in red squares

In accordance with this analysis, the fuel oxygen content (oxy\%) and specific gravity (spec grav) are positively associated with $\mathrm{CO}$ and $\mathrm{CO} 2$ emissions and negatively associated with NOx. Properties like LHV, fuel hydrogen wt.\% $(\mathrm{H} \%)$ are positively associated with NOX emissions. NOX and CO2 are negatively correlated between them (i.e. inversely proportional).

Again in Figure 5, in ordinate axis Factor 2 (which explains 18.7\% of total variability) is presented. Properties related to fuel evaporation like ASTM D-86 temperatures for $10 \%$ and $50 \%$ distillation (T10, T50) and air fuel temperature downstream injector (Tadmi), have a proportional association with $50 \% \mathrm{MBF}$ and inversely relationship with Pmax. At the same time, Heat of vaporization and RVP (related to fuel evaporation to) correlates directly with Pmax and inversely with $50 \%$ MBF. Factor analysis clearly confirm the importance of properties related with fuel evaporation in combustion centre of gravity.

Factors like, T90, final boiling point, isoparaffins and naphtenic hydrocarbons contents or base gasoline show marginal effects in engine operation or emissions. About base fuel composition, only hydrogen, carbon and aromatics contents looks relevant and this properties are more related to fuel dilution than to base fuel formulation. Finally, in order to test results in full vehicle following the FTP-75 chassis dynamometer cycle, blends with 0, 10, 20,45 and 85 wt.\% ethanol was prepared by splash blend from the same base fuel. The vehicle was instrumented 
for data acquisition form ECU, including throttle position (TPS), engine rpm and load, and intake air and coolant temperatures. In figure 8 , the pondered emissions results are showed.

The weighted results shows emissions below the United States Tier 1 limits for 0,10 and 20 wt. \% ethanol in fuel, Nevertheless, for $45 \%$ and $85 \%$, the NOx emissions are above the limit of $0.25 \mathrm{~g} / \mathrm{Km}$, as a result of enleanment. In spite of the used fuel, the hydrocarbon emissions are one order of magnitude higher for bag 1 (cold start) than form bag 3 (hot start). As ethanol contents increases in the fuel, the $\mathrm{CO}$ emissions gets lower, never the less, hydrocarbons increases, showing opposite effect to the enleanment, probably as a result of wall quenching phenomena related to lower cylinder wall temperature (because high $\mathrm{HoV}$ ) specially during the first seconds since cold start.

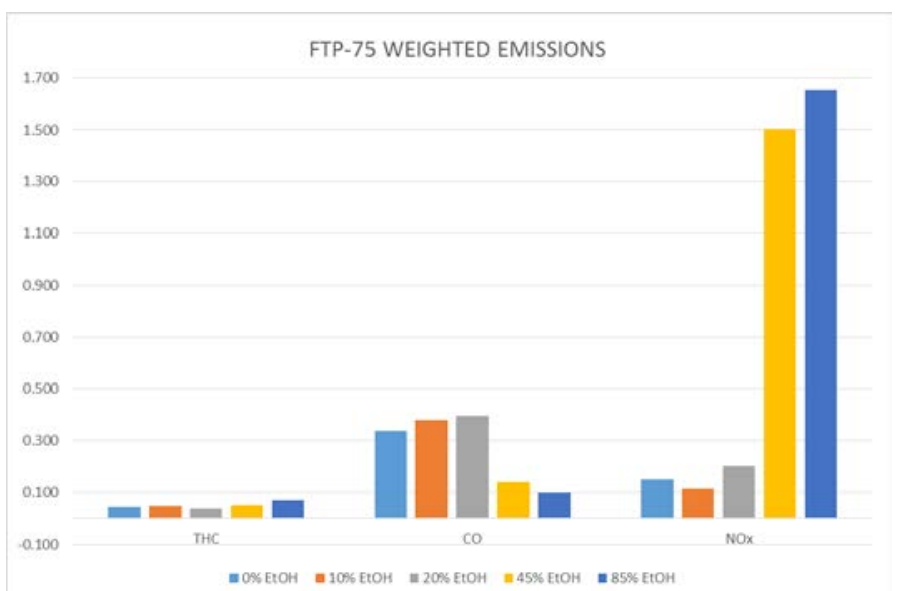

Figure 8. FTP 75 weighted emissions for tested fuels up to $85 \%$ ethanol

In figure 9, in the right side, the evolution of engine speed during the first minute since cold start is showed, is possible to see some erratic operation for fuel with $85 \% \mathrm{EtOH}$ during the first 25 seconds, the fuel with $45 \% \mathrm{EtOH}$ show higher rpm at idle than the rest of the fuels. Next, in the right side, the coolant temperature evolution during the first 505 seconds is presented. For the coolant (left side), at the end of the 505 test, the difference between the highest temperature (fuel with $10 \% \mathrm{EtOH}$ ) and the lowest one (fuel with $85 \% \mathrm{EtOH}$ ) are 6 Centigrade, opposite, the intake air lowest temperature corresponds to fuel with $10 \% \mathrm{EtOH}$ and the highest to the one with $85 \% \mathrm{EtOH}$. Clearly this performance is connected with RVP and HoV. As the HoV gets higher i.e. more $\mathrm{EtOH}$, the engine gets cooled because the heat is absorbed during fuel evaporation and delays the coolant temperature increase. In the other hand, the fuels with 10 and $20 \mathrm{wt} . \% \mathrm{EtOH}$ have higher RVP than the gasoline without EtOH and gets evaporated early in the intake manifold, reducing air temperature. As the ethanol content increases and the RVP increases to, the fuel delays to evaporate absorbing heat from the combustion chamber and not from the intake manifold, increasing the intake air temperature.
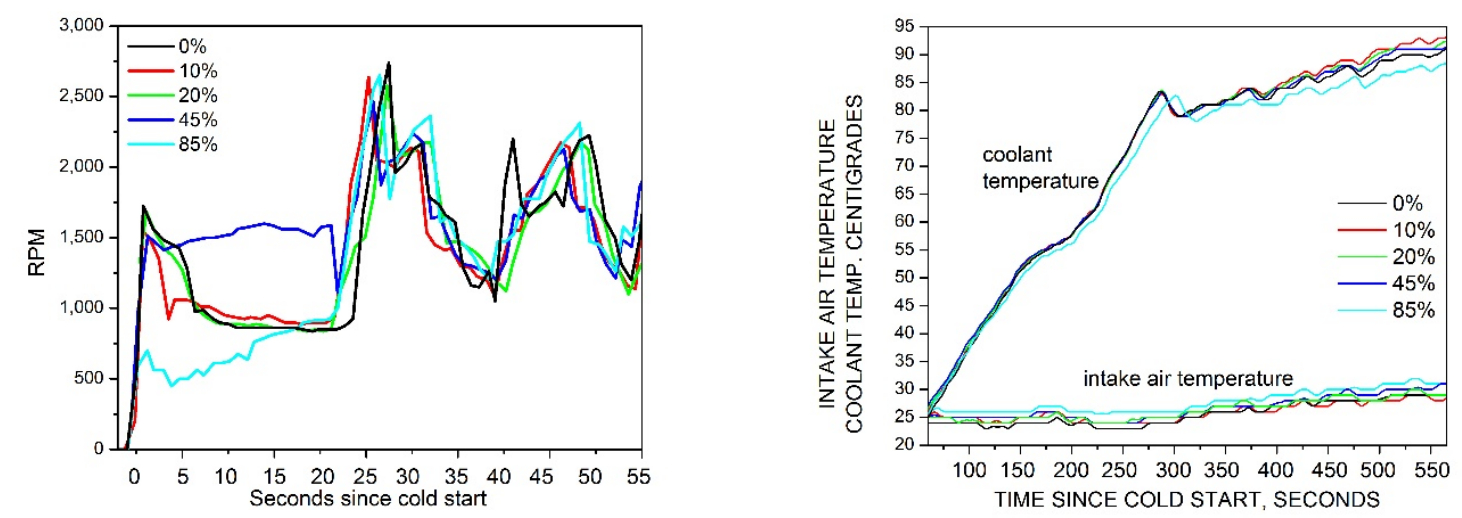

Figure 9. RPM during the first minute of FTP 75 (right side). Intake air temperature and coolant during the first 550 seconds in the same cycle (left side). 


\section{Conclusions}

Relationships between fuel formulation, engine performance and emissions for port fuel injection engines was identified for gasolines with oxygen contents up to $20 \mathrm{wt} . \%$.

The use of oxygenates reduce the hydrogen and carbon fuel contents as a result of fuel dilution. For a given level of oxygenation as lower is the molecular oxygen content in the additive, higher will be the fuel dilution. The calorific power diminish linearly with oxygen content by weight, in spite of the oxygenant and base fuel used.

As higher is the heat of vaporization i.e. higher alcohol content, higher is the charge cooling effect, but in order to take advantage of the high $\mathrm{HoV}$ as a way to reduce the compression work and the heat losses before ignition, a low Reid vapour pressure is necessary in order to absorb more heat from the air inside of combustion chamber and less form the intake manifold.

For oxygenated gasolines, fuel sensitivity have a direct relationship with latent heat of vaporization, because charge cooling effect is one of the way alcohols increase RON. In the other hand, MON is almost insensible to high heat of vaporization, because the intake air is heated to $159 \mathrm{C}$ as a test requirement.

Highly oxygenated fuels with alcohols (i.e. 10wt.\% oxygen and more) burn faster than other fuels and this effect is related to alcohols high heat of vaporization. In counterpart, highly oxygenated fuels with low HoV burns slow. For low oxygenated fuels (3.5 wt.\%) no matter which oxygenant is used, the combustion speed is similar and depends more on fuel base formulation.

Evaporative fuel properties like T10, T50, HoV and RVP have important effects in combustion centre of gravity. Factors like, T90, final boiling point, isoparaffins and naphthenic contents or base gasoline formulation, have marginal effects in highly oxygenated fuel performance and exhaust emissions.

As the ethanol content increases in the fuel, some enleanment take place, producing high NOx emissions. During the FTP-75 cold start phase, with 10wt.\% ethanol fuel, the intake air temperature in lower than for baseline fuel without ethanol as a result of cooling charge at intake manifold associated with high RVP. As the ethanol contents increases, the intake air temperature increases again as a result of RVP reduction, decreasing cooling charge at the manifold. For $85 \%$ ethanol, the intake air temperature is 3 centigrade higher than for $10 \%$. At the end of FTP75 cold start phase the coolant temperature is 6 centigrade lower for $85 \%$ ethanol fuel than for the reference without ethanol.

\section{References}

[1] Varol,Y., Öner, C., Öztop, H. F., Altun, S., 2014, Comparison of Methanol, Ethanol, or n-Butanol Blending with Unleaded Gasoline on Exhaust Emissions of an SI Engine, Energy Sources, Part A, 36,pp 938-948.

[2]. Balki,M.K., Sayin,C., Canakci, M., 2014, The effect of different alcohol fuels on the performance, emission and combustion characteristics of a gasoline engine, Fuel ,pp.901-906.

[3]. Balaji,D., Govindarajan,P., Venkatesan,J., 2010, Influence of isobutanol blend in spark ignition engine Performance and emissions operated with gasoline and Ethanol, International Journal of Engineering Science and Technology, 2 (7), pp. 28592868

[4] Croezen, H.J., Kampman, B.E., van de Vreede, G., Sevenster, M.N., 2007, ETBE and ethanol: A comparison of CO2-savings CE publication number 07.4226.42. Delf, NE.

http://www.cedelft.eu/publicatie/etbe and ethanol\%3A a comparison of co2 savings/716.

[5] Schifter, I., Diaz, L., Rodriguez, R., Gómez, J.P., Gonzalez, U., 2011. Combustion and emissions behavior for ethanol-gasoline blends in a single cylinder engine. Fuel, 90 pp. 3586-3592.

[6] Fenkl, M., Pechout, M., Vojtisek, M, N-butanol and isobutanol as alternatives to gasoline: Comparison of port fuel injector characteristics, 2016, EPJ Web of Conferences 114, 02021.DOI 10.1051/epjconf/201611402021.

[7]. Jung, H., Shelby, M., Newman, C., Stein, R., 2013. Effect of ethanol on part load thermal efficiency and $\mathrm{CO}_{2}$ emissions of $\mathrm{SI}$ engines. SAE Int. J. Engines, 6 pp. 456-469.

[8]. Jones, B., Mead, G., Steevens, P., 2008, The Effects of E20 on Plastic Automotive Fuel System Components,

Tech. Rep. 20082/21/2008, Minnesota Center for Automotive Research,

[9]. Rodríguez-Antón, L.M., Hernández-Campos, M., Sanz-Pérez, F. 2013, Experimental determination of some physical properties of gasoline, ethanol and ETBE blends. Fuel, 112, pp 178-

[10]. Wang, C., Janssen, A., Prakash, A., Cracknell, R., Xu, H,(2017), Splash blended ethanol in a spark ignition engine - Effect of RON, octane sensitivity and charge cooling. Fuel 196 pp. 21-31 84 .

[11].American Society for Testing Materials, Standard Specification for Spark-Ignition Engine Fuel, D-4814-01a. PA, USA 2001.

[12]. Pahl R.H and McNally MJ. Fuel blending and analysis for the auto/oil air quality improvement research program; Paper No. 902098; Society of Automotive Engineers; Warrendale, PA. USA 1990.

[13]. Brettschneider, J., 1997, Extension of the equation for calculation of the air-fuel equivalence ratio. SAE technical paper 972989, DOI: 10.4271/972989.

[14]. Schifter, I., González, U., González-Macías, C., 2016, Effects of ethanol, ethyl-tert-butyl ether and dimethyl-carbonate blends with gasoline on SI engine. Fuel, 183, pp.253-261

[15]. Painter, L., Rutherford, J.A. (1992). Statistical design and analysis methods for the auto/oil air quality research program. In Auto/Oil Quality Improvement Research Program; SAE Technical Paper No. 920319 PA, USA. Society of Automotive Engineers 


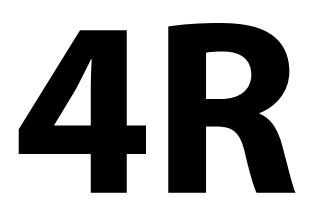

Atomizers 4 


\title{
Primary breakup of liquids using a high-speed rotary bell atomizer for spray painting processes
}

\author{
Bo Shen ${ }^{1}$, Q. Ye ${ }^{2}$, O. Tiedje ${ }^{2}$, J. Domnick*1 \\ ${ }^{1}$ University of Applied Sciences Esslingen, Germany \\ ${ }^{2}$ Fraunhofer Institute for Manufacturing Engineering and Automation, \\ Stuttgart, Germany \\ ${ }^{*}$ Corresponding author: joachim.domnick@hs-esslingen.de
}

\begin{abstract}
The present contribution deals with numerical studies of the primary liquid breakup process using a commercial high-speed rotary bell atomizer. The first part of the investigations focuses on the film formation on the distributor disk and the inner surface of the rotary bell. Numerical simulations using the volume of fluid (VOF) approach were carried out. A non-Newtonian liquid is used to investigate the effect of the viscosity on the initial wetting, the film formation process and the film thickness distribution on the bell. The Herschel-Bulkley model is considered to describe the shear-thinning (pseudoplastic) behavior of the fluid. A non-homogeneous film structure is found on the inside surface of the rotary bell. This is also observed in experimental investigations using a high-speed camera.

The following disintegration process is studied by applying an appropriately fine computational mesh. The VOF method and Reynolds stress model are used to calculate the disintegration process and the two-phase flow field. The numerical results are compared with images which are recorded by means of a high-speed camera.
\end{abstract}

\section{Keywords}

Numerical simulation, rotary bell atomizer, film formation, liquid disintegration

\section{Introduction}

High-speed rotary bell atomizers are widely used in automated painting processes. They provide excellent paint film quality as well as high transfer efficiencies due to electrostatic support. Compared to other rotary atomizers for other applications, e.g., spray drying or powder production, the atomizers for painting are characterized by a small bell diameter varying between 20 and $70 \mathrm{~mm}$ and very high rotary speeds of the bell of up to $70,000 \mathrm{~min}^{-1}$.
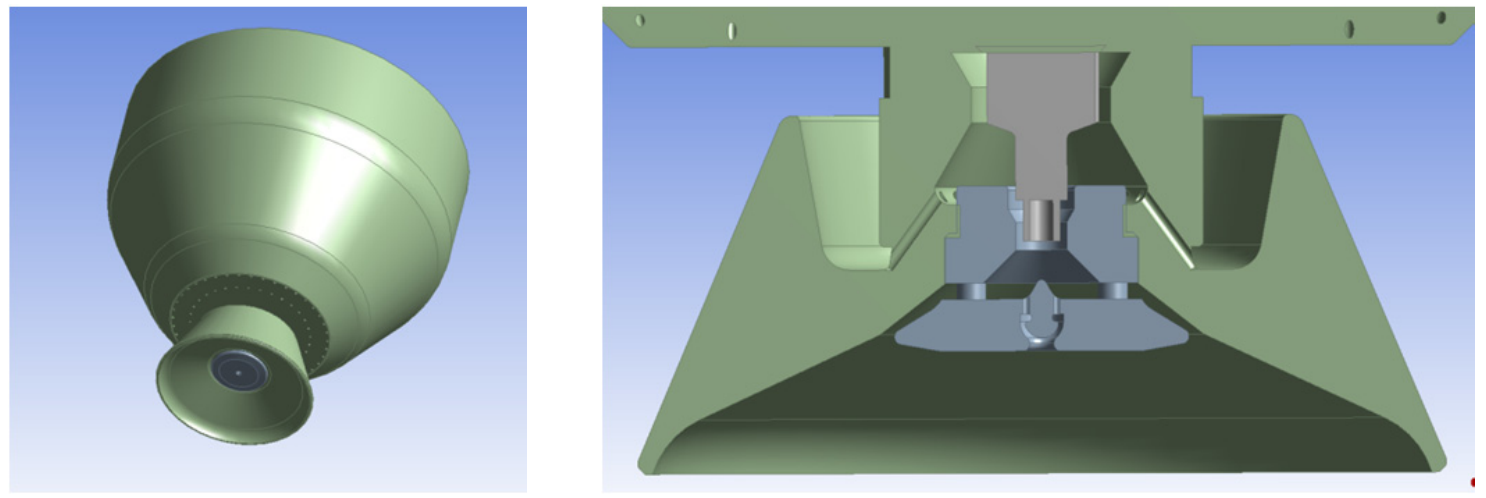

Figure 1. CAD model of the rotary bell atomizer (left) and cross section of the bell (right)

In general, some research works have already been done to investigate the atomization of rotary atomizers (e.g. [1-3]). There are also a few publications presenting results from the experimental works [4] dealing with highspeed rotary bells used in the painting processes. However, the experimental investigations on the film formation on the distributor disk and the bell surface are very difficult due to the complexity of the liquid flow emerging from the nozzle and hitting the surface of the distributor disk. Hence, numerical simulations provide an appropriate alternative. Domnick et al. [5] presented the numerical investigations aiming to calculate the two phase flow field inside the manifold of a rotary bell atomizer and the free film on the bell surface. However, the cell resolution of 
the computational domain was quite limited due to the previously available computational capacity. The viscosity of the paint used in their investigation was chosen to be constant, and the non-Newtonian behavior which real paints can show was not considered. The present contribution summarizes numerical investigations using a significantly finer mesh and a real paint having the shear-thinning behavior. Further, the result of numerical studies on the disintegration of the paint at the bell edge is discussed in this paper.

\section{Characteristics of the atomizer}

The investigations presented in this paper were carried out with a high-speed rotary bell atomizer by EISENMANN LacTec GmbH, which is used in automated paint applications. Figure 1 shows the CAD model of the atomizer and a cross section of the bell. Paint liquid is injected through an orifice which has a narrow diameter of $1.6 \mathrm{~mm}$. This paint supply nozzle is surrounded by an annular gap with a width of $0.5 \mathrm{~mm}$, through which ambient air is sucked down. This gas flow helps to form an initial stable fluid film on the bell and to prevent the formation of ligaments on the bell surface, so that the atomization quality could be improved [6]. Downstream of the paint supply nozzle, there is an inner distributor disk with a diameter of $20 \mathrm{~mm}$. The distributor disk and the bell are assembled together using three bolts. During application they rotate at high speed, while the paint supply nozzle remains static. The paint is injected onto the distributor disk and builds a paint film on it. The paint film is further radially accelerated by centrifugal forces and eventually becomes unstable at a certain distance from the rotating axis. Finally, the paint becomes distributed on the distributor disk surface as well as the inner surface of the bell.

\section{Rheological properties of the paint}

For the investigations shown here, a real paint used in automated painting processes was chosen, whose experimentally measured viscosity is plotted as a function of the shear rate in Figure 2 . The paint shows shearthinning behavior, i.e., the viscosity decreases with increasing shear rate. This kind of non-Newtonian behavior was applied in the simulations shown in this work. The correlation between the viscosity and the shear rate is described by using a power law model, which is known as the Herschel-Bulkley model, and also plotted in Figure 2 .

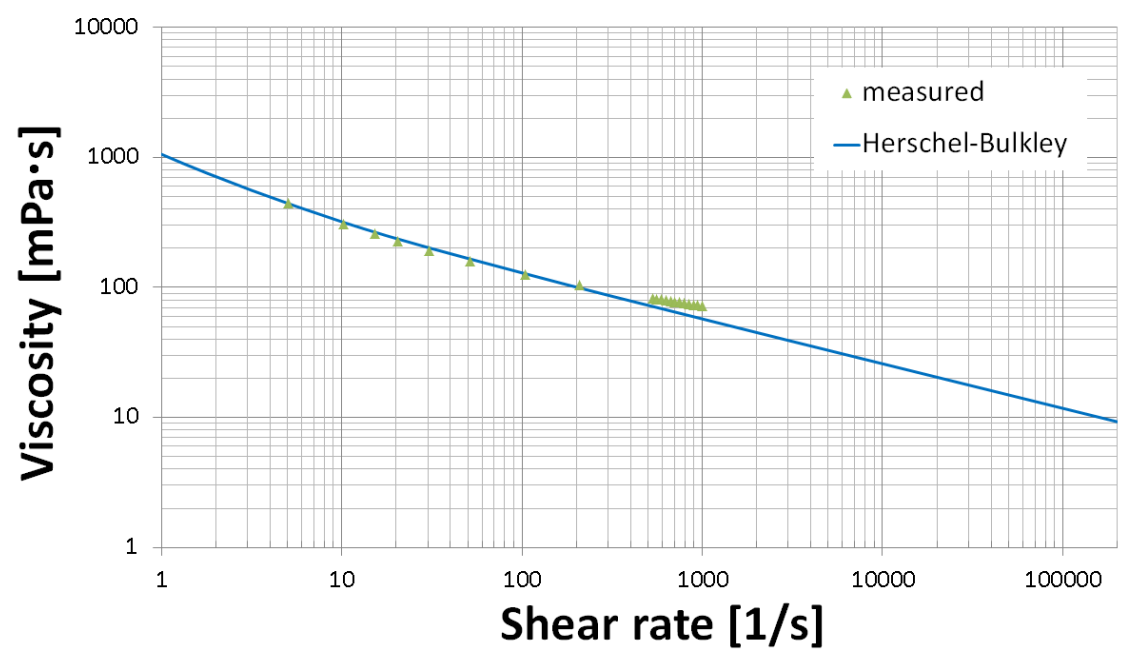

Figure 2. Correlation between viscosity and shear rate of the paint used in the investigations

\section{Numerical method}

A cross-section of the three-dimensional computational domain used in the present investigations is shown in Figure 3. During the generation of the grid, the whole domain was sliced into two parts. Due to the three bolts between the distributor disk and the bell the region above the horizontal surface of the distributor disk is not symmetrical. Thus the middle part of the computational domain had to be built using an unstructured / tetra grid, while in the rest of the domain a structured mesh could be generated. Prism layers were created near the bell as well as near the distributor disk. The height of the first prism layer to the bell was $5 \mu \mathrm{m}$, which provides the necessary resolution for the calculation of the film thickness on the bell. The growth rate of the prism layers is 1.2. The overall mesh cell count was about 8.2 million.

The numerical simulations were carried out using the commercial CFD code FLUENT based on the finite-volume approach. The code was used to solve the unsteady, three-dimensional Reynolds stress transport equations. The 
VOF model was used to calculate the two-phase field and the gas-liquid interface inside the rotary bell. For timedependent VOF calculations, variable time stepping was used in order to automatically change the time step when an interface is moving through dense cells or if the interface velocity is high. The time step was varied in a range between 0.1 and $1 \mu$ s based on the preconfigured maximum global Courant number of 2, which ensures the computational stability of the numerical simulations. The simulations were carried out on a CRAY Cascade Supercomputer at the High-Performance Computing Center of the University of Stuttgart.

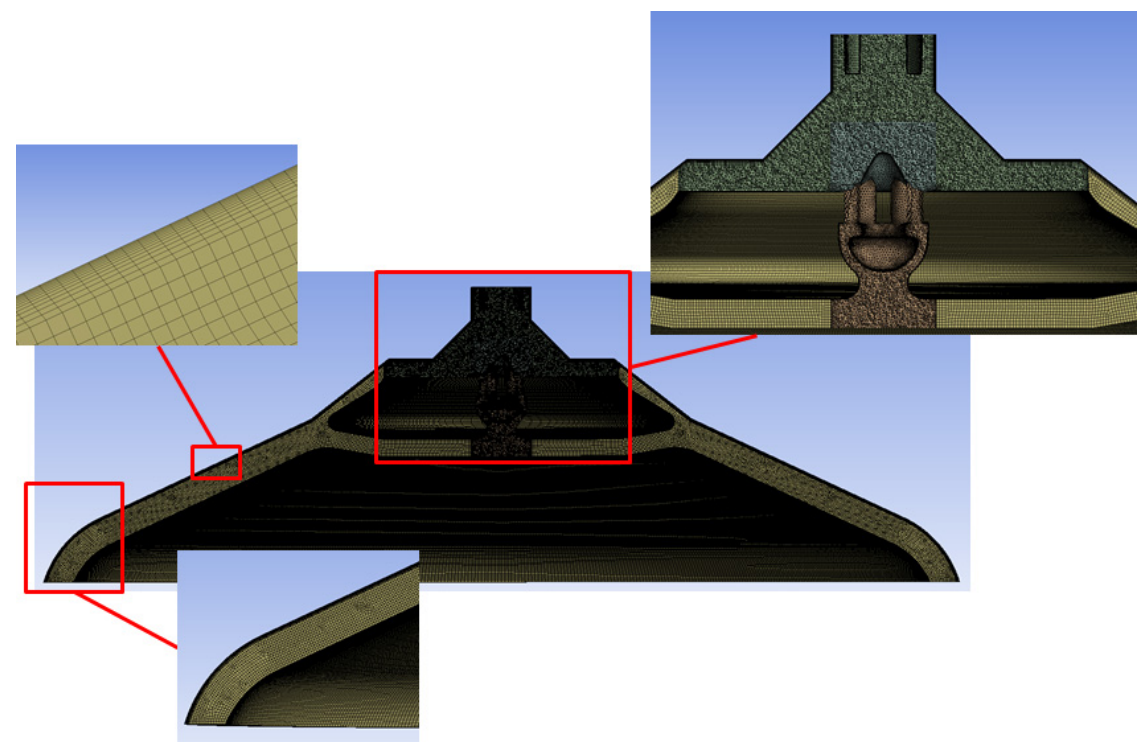

Figure 3. Cross-section of the computational domain

\section{Wetting process on the bell}

The surface wetting on the distributor disk in the quasi-stable state is depicted in Figure 4. The red color indicates that computational cells are completely filled with paint liquid, whereas blue cells contain no paint. The cells in other colors have a volume fraction of paint between 0 and 1 . The left figure shows the case with a paint volume flow rate of $250 \mathrm{ml} / \mathrm{min}$. The horizontal surface of the distributor disk is wetted up to the edge of the three bolts. On the outer inclined surface only several "paint stripes" can be seen. In the case with a larger paint flow rate of $500 \mathrm{ml} / \mathrm{min}$, more areas are wetted. The horizontal surface is almost completely wetted. Due to the rotation of the disk and the relative movement of paint around the bolts, small wake flow regions are generated which are not wetted.
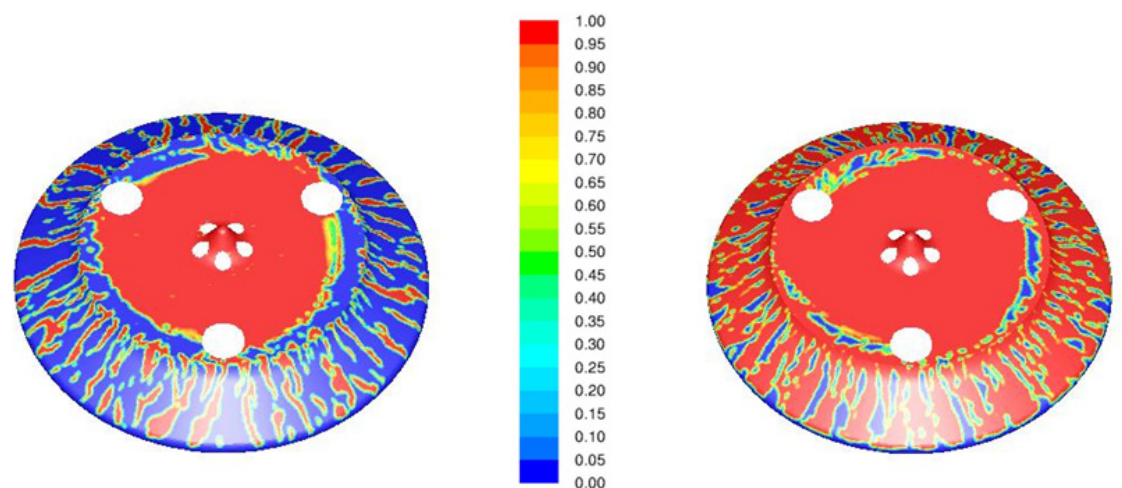

Figure 4. Contour of the paint volume fraction on the distributor disk at a paint volume flow rate of $250 \mathrm{ml} / \mathrm{min}$ (left) and $500 \mathrm{ml} / \mathrm{min}$ (right)

Figure 5 is a snapshot of the contour of the paint volume fraction in the region between the distributor disk and the bell surface from an unsteady simulation, where already a quasi-stable state has been reached. The picture shows the momentary phase distribution in this region. Above the horizontal surface of the distributor disk quite a few drops of liquid can be observed. This indicates that some fraction of the paint material detaches already 
before it reaches the edge of the horizontal surface. This may be caused by a blocking effect of the bolts and the centrifugal force.

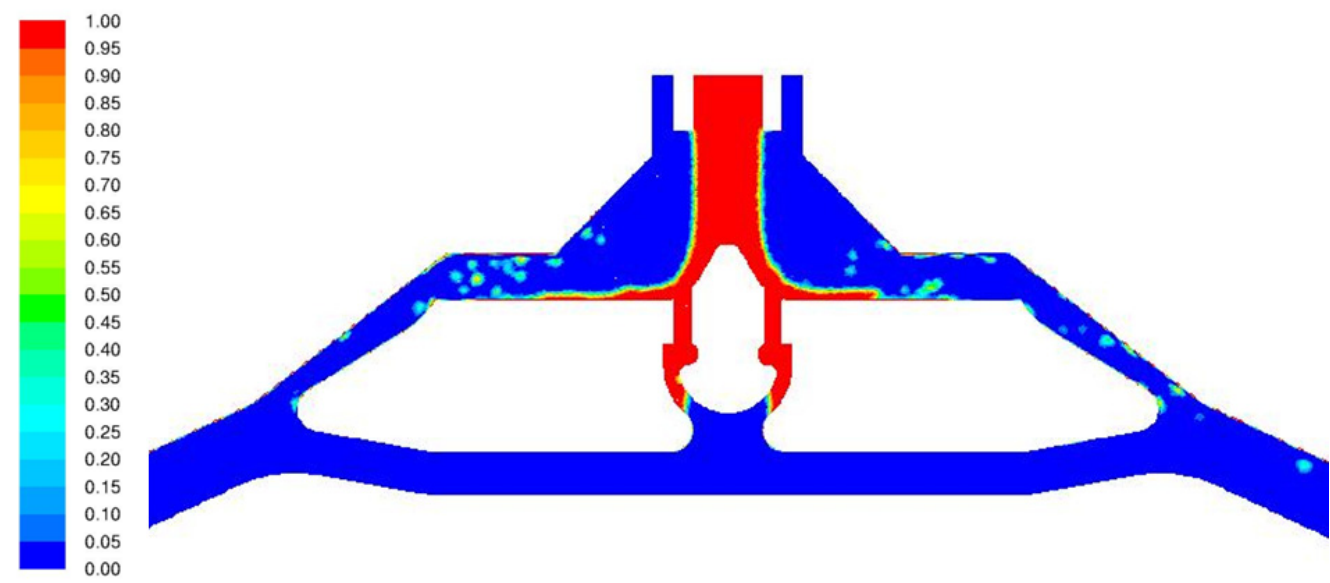

Figure 5. Contour of the paint volume fraction in the region between the distributor disk and the bell surface

The film formation process on the bell surface is shown in Figure 6. In contrast to the distributor disk, the inner surface of the bell is completely wetted after a certain time, e.g., after about $30 \mathrm{~ms}$ for the case with a paint flux of $250 \mathrm{ml} / \mathrm{min}$ and after approx. $20 \mathrm{~ms}$ for $500 \mathrm{ml} / \mathrm{min}$ at the same rotary speed of $40,000 \mathrm{~min}^{-1}$, which is in reasonable agreement with the duration of the wetting process on the bell experimentally measured by means of a high-speed camera.

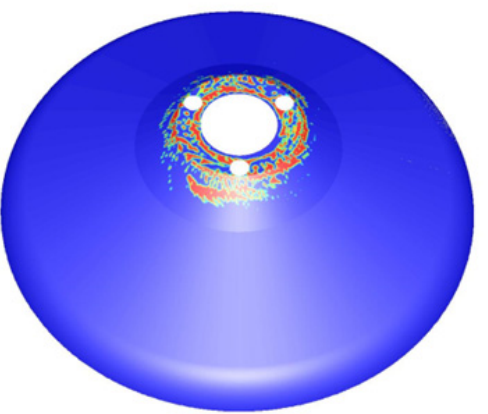

$\mathrm{t}=5.3 \mathrm{~ms}$

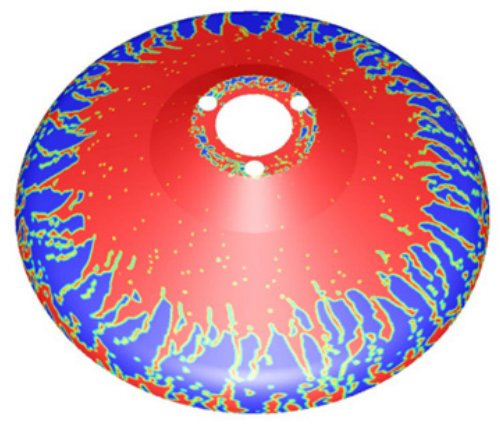

$\mathrm{t}=10.6 \mathrm{~ms}$
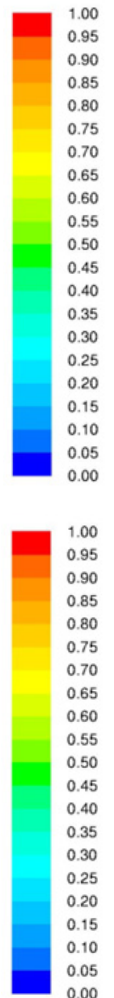

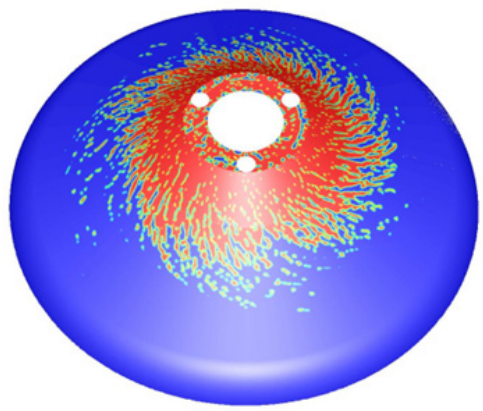

$\mathrm{t}=7.1 \mathrm{~ms}$

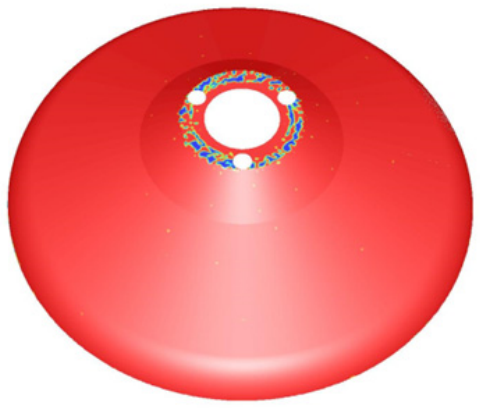

$\mathrm{t}=21.3 \mathrm{~ms}$

Figure 6. Visualization of the film development on the inner bell surface for the case with a paint flux of $500 \mathrm{ml} / \mathrm{min}$ at a rotary speed of $40,000 \mathrm{~min}^{-1}$ 
After the bell surface has been completely wetted, a quasi-stable state is reached. In order to illustrate the paint distribution on the bell surface, an iso-surface positioned at a constant normal distance of $11 \mu \mathrm{m}$ from the inner bell surface was created. The contour of the paint volume fraction on this surface as shown in Figure 7 indicates a non-homogeneous paint distribution. The film thickness in the middle of the bell is relatively high and decreases along the flow direction of the paint. At the edge of the bell the paint film becomes thicker again. In addition, small ripples and a quasi-spiral pattern are obtained on the surface of the paint film. These phenomena can also be observed in experiment using a high-speed camera.
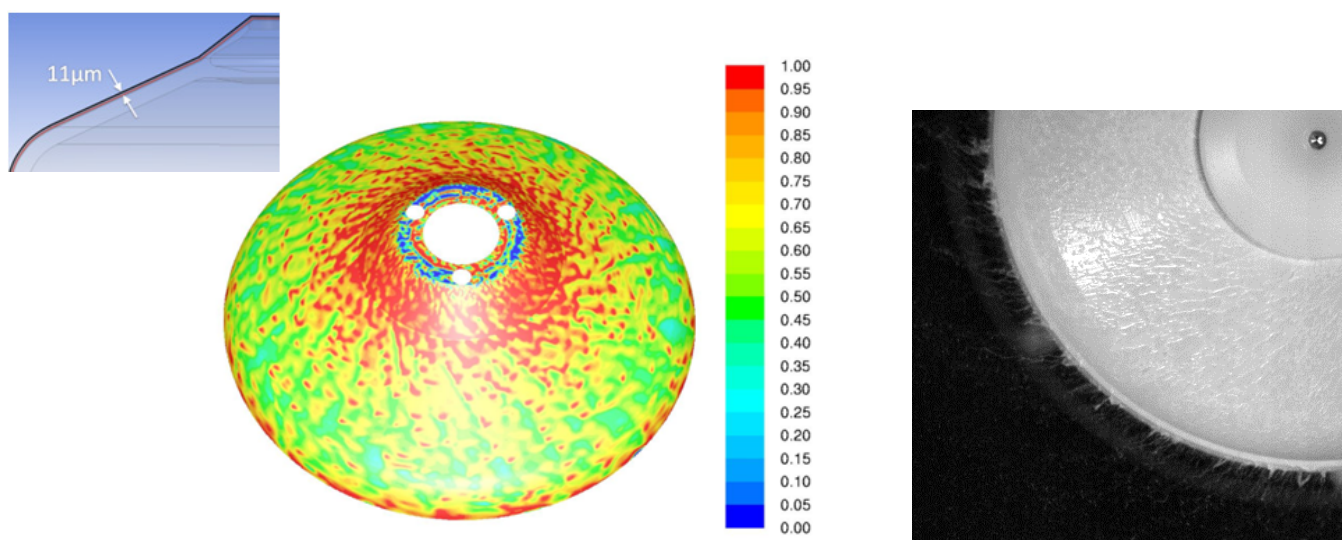

Figure 7. Structure of paint film surface observed in the simulation (left) and in the experiment (right)

A closer look onto the region near the inner bell surface is provided by Figure 8 . The film thickness can be easily obtained from the contour of the volume fraction of the paint. With the thickness of the first prism layer of $5 \mu \mathrm{m}$, the second of $6 \mu \mathrm{m}$, and the third of $7.2 \mu \mathrm{m}$, multiplied by the paint volume fractions in the layers of 1,1 and 0.75 respectively, the thickness of the paint film shown in Figure 8 is calculated to be approximately $16.4 \mu \mathrm{m}$.
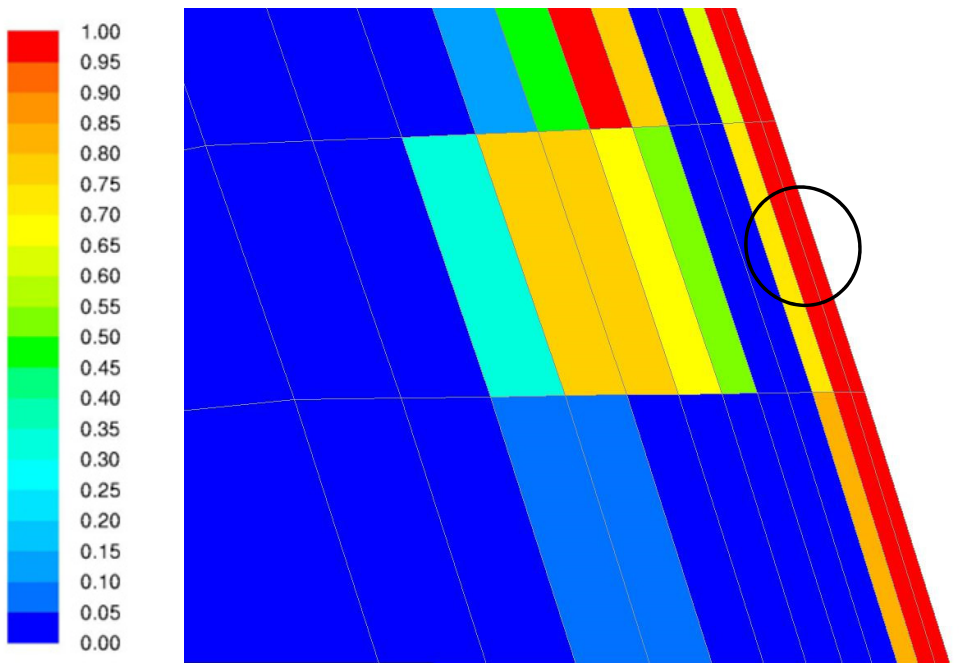

Figure 8. Contour of the paint volume fraction near the inner surface of the bell

Ten measuring points were chosen on the bell surface (Figure 9). The film thickness at each point is calculated using the same method as shown above. The distribution of the film thickness along the outer contour line of the bell is plotted in Figure 9. The tendency shown here is similar to that observed in Figure 7. The film thickness at the bell edge, where subsequent atomization might occur, is determined to be $15 \mu \mathrm{m}$ for the case with a paint flux of $250 \mathrm{ml} / \mathrm{min}$ at the rotary speed of $40,000 \mathrm{~min}^{-1}$. 


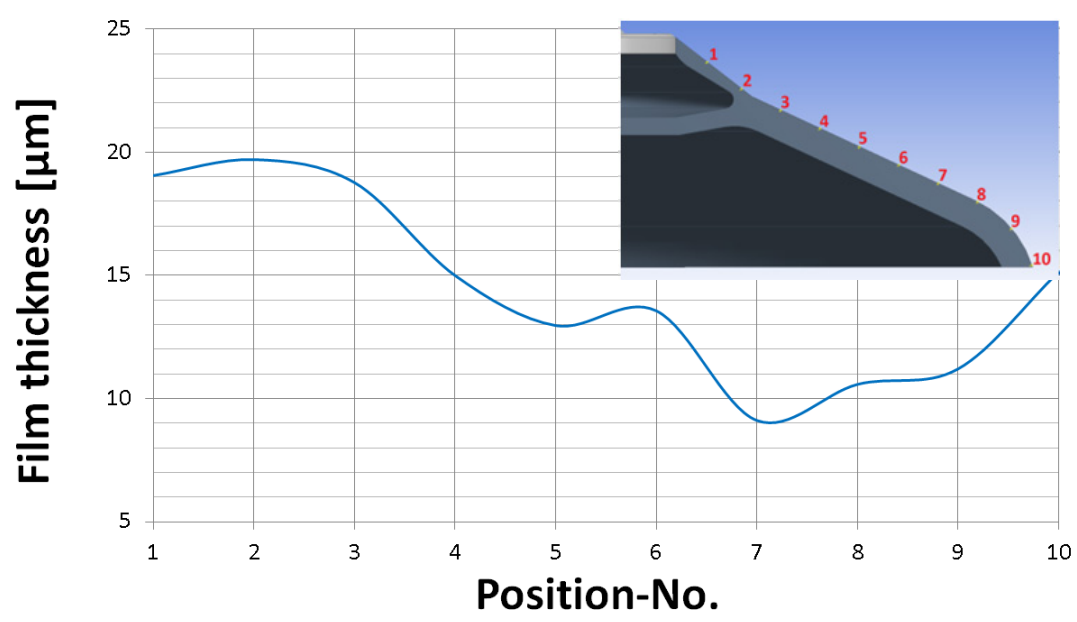

Figure 9. Distribution of film thickness along the flowing direction of the paint

\section{Disintegration process at the bell edge}

Numerical studies on the atomization at the bell edge need a significantly finer mesh in this region. The computational domain as shown in Figure 10 contains only 1/8 of the bell without bolts. The wall boundary of the bell is extended to the fillet on the outer bell surface. The domain is enlarged laterally by $4 \mathrm{~mm}$ and downwards by $2 \mathrm{~mm}$. The total of cell elements in the domain amounts to 17.5 million.
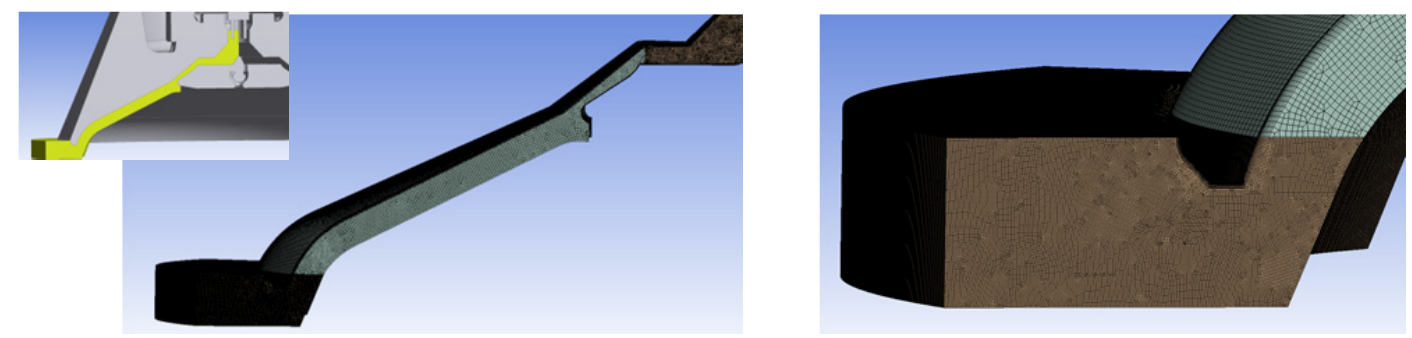

Figure 10. Computational domain for the simulation of atomization

In the simulation for the case with a paint flow rate of $500 \mathrm{ml} / \mathrm{min}$ at a rotary speed of $60,000 \mathrm{~min}^{-1}$, turbulent disintegration is found, because no liquid jet emerging from the bell edge can be observed. This coincides well with previous experimental work by Domnick et al. [4], in which an equation defining the separation between jet and transition disintegration at the rotary bell edge was derived. It is also observed in the simulation that the major paint fraction leaves the bell not directly at the bottom edge of the bell. The paint flows over the bottom surface of the bell, builds a film on the surfaces and reaches the lower edge of the fillet on the outer bell surface, where drops and ligaments are obtained as the paint detaches the bell. This phenomenon can also be observed by means of a high-speed camera.
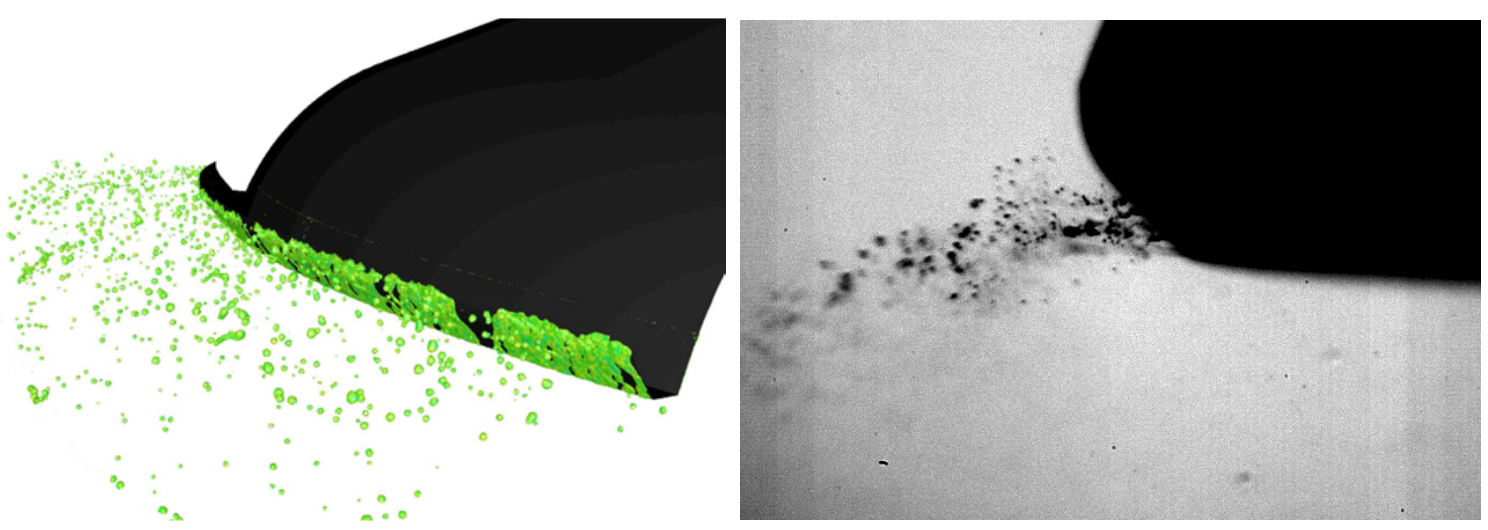

Figure 11. Liquid disintegration at the bell edge observed in the simulation (left) and in the experiment (right) 


\section{Conclusions}

As presented in this contribution, numerical simulations based on the VOF method and Reynolds stress model were carried out to investigate the film formation process of the paint on the distributor disk and the bell itself. The propagating process of the paint from the disk to the bell could be observed. The blocking effect of the bolts, with which the distributor disk and the bell are assembled together, plays an important role in this process. The paint leaves the distributor disk in form of drops or ligaments, reaches the inner surface of the bell and builds a film on it. The paint flows further along the inner surface towards the bottom edge of the bell. After a certain time, the inner surface of the bell is completely wetted by the paint. However, the distribution of the film thickness is not homogeneous. The major part of the paint material does not leave the bell at the bottom edge of the inner bell. Instead, the paint flows further over the bottom surface of the bell in form of a film and reaches the fillet on the outer surface of the bell. There, the paint disintegrates into a chain of drops or ligaments.

For the further atomization study, numerical simulation will be carried out using finer mesh around the bell edge. The computational domain will be limited quite close to the bell edge, in order to save the computational consumption. The corresponding boundary conditions, such as velocity and film thickness distributions, can be obtained from the present numerical simulation results of the film formation on the bell.

\section{Acknowledgements}

The present investigations were supported by AiF Arbeitsgemeinschaft industrieller Forschungsvereinigungen. We thank our colleagues from the Fraunhofer Institute for Manufacturing Engineering and Automation in Stuttgart who provided the results from the experimental work that greatly assisted the present research. We also thank Anyang Wang for his assistance in producing the results used in this paper.

\section{References}

[1] Lefebvre, A. H., 1989, "Liquid Atomization."

[2] Walzel, P., Zerstäuben von Flüssigkeiten, 1990, Chemie Ingenieur Technik, vol. 62, pp. S983-S994

[3] Bayvel, L., Orzechowski, Z., 1993, "Liquid Atomization."

[4] Domnick, J., Thieme, M., 2006, Atomization and Sprays, vol. 16, pp. 857-874

[5] Domnick, J., Yang, Z., Ye, Q., Sep. 8.-10. 2008, 22nd European Conference on Liquid Atomization and Spray Systems

[6] Mehrhardt, E., 1978, Ph.D. thesis, Technical University of Berlin 


\title{
Mechanisms of Liquid Stream Breakup: Vortices and Time and Length Scales
}

\author{
A. Zandian ${ }^{1}$, W. A. Sirignano*1, F. Hussain ${ }^{2}$ \\ ${ }^{1}$ Department of Mechanical and Aerospace Engineering, University of California, Irvine, USA \\ ${ }^{2}$ Department of Mechanical Engineering, Texas Tech University, Lubbock, USA \\ *Corresponding author: sirignan@uci.edu
}

\begin{abstract}
The 3D, temporal instabilities on a planar liquid jet are studied using DNS with level-set and VoF interface-capturing methods. The $\lambda_{2}$ method has been used to relate the vortex dynamics to the surface dynamics at different stages of the jet breakup. The breakup character depends on the Ohnesorge number $(O h)$ and gas-to-liquid density ratio. At high Reynolds number $(R e)$ and high $O h$, hairpin vortices form on the braid and overlap with the lobe hairpins, thinning the lobes, which then puncture creating holes and bridges. The bridges break, creating ligaments that stretch and break into droplets by capillary action. At low $O h$ and high $R e$, lobe stretching and thinning is hindered by high surface tension and splitting of the original Kelvin-Helmholtz vortex, preventing early hole formation. Corrugations form on the lobe edges, influenced by the split vortices, and stretch to form ligaments. Both mechanisms are present in a transitional region in the $W e$-Re map. At lower $R e$ and not-too-large Weber number $(W e)$, lobe stretching occurs but with longer and larger ligaments in this third domain which has a hyperbolic transition to the hole formation domain as $W e$ increases. The three domains with differing breakup behaviors each occupy distinct portions of a plot of $W e$ based on gas density versus $R e$ based on liquid properties. Characteristic times for the hole formation, as well as the lobe and ligament stretching are different - the former depending on the surface tension and the latter on liquid viscosity. In the transitional region, both times are of the same order.
\end{abstract}

\section{Keywords}

Gas/liquid flow, primary atomization, breakup mechanism, hydrodynamic instability, vortex dynamics.

\section{Introduction}

Earlier computational works on the breakup of liquid streams at higher Weber number $(W e)$ and Reynolds number $(R e)$ focused on the surface dynamics using either volume-of-fluid or level-set methods [1-3]. More recently, Jarrahbashi and Sirignano [4] and Jarrahbashi et al. [5] numerically simulated the temporal behavior of round jets with additional data analysis that related the vorticity dynamics to the surface dynamics. Jarrahbahsi et al. [5] showed that important spray characteristics, e.g. droplet size and spray angle, differed in different ranges of $W e, R e$, and density ratio. Therefore, further studies of the breakup mechanisms are needed to fully understand the causes of these differences. Consequently, there are remaining questions to be addressed in this paper: What are the details of the liquid dynamics in each breakup domain? What causes the difference in the breakup cascade? What are the roles of surface tension, liquid viscosity, and gas density? The answers to these questions would be crucial in understanding and controlling the droplet size distribution in primary atomization of liquid jets.

Vortex dynamics concepts can clearly explain surface deformation of a liquid jet in the primary atomization process. The Kelvin-Helmholtz $(\mathrm{KH})$ instability promotes the formation of spanwise vorticity waves growing into coherent vortices. These vortices evolve into hairpins with counter-rotating streamwise vortices [6]. The streamwise and spanwise vortical waves combine to produce different surface structures, e.g. lobes, bridges, and ligaments, which eventually break up into droplets. The link between the vortex dynamics and surface dynamics in primary atomization is important, but rarely explored and poorly understood, and this study is an attempt to fill that gap.

There have been several studies in the field of jet instabilities from the vortex dynamics perspective. Most of them however do not consider density and viscosity discontinuities. The main focus of these studies has been on understanding and relating vortex stretching [7], vortex tilting [8], and baroclinic effects [9] to the appearance of three-dimensionality in liquid jet instabilities. Earlier experimental studies in this field [6, 8-14] have been followed and reproduced in more detail by numerical computations [1, 4, 5, 15-21].

In the first studies of the role of streamwise vorticity for round liquid jets flowing into a gas, Jarrahbashi and Sirignano [4] and Jarrahbashi et al. [5] showed how the mechanisms of the formation of lobes and ligaments are related to the growth of streamwise vorticity. For control of spray character, it is very valuable to understand the details in the cascade processes following liquid injection for each of the various atomization domains that have been identified [5]. While we do not address control and optimization in this study, it gives justification for the detailed exploration and the behavioral characterizations reported here.

Our objectives for the planar jet are to explain (i) cascade of structures on the liquid surface with time, including lobe, ligament, and droplet formations; (ii) the breakup mechanisms at different flow conditions; (iii) proper definition of the time scale of each of the breakup mechanisms, which would help predict the dominant mechanism at different flow conditions; and (iv) the mechanisms for surface deformation and breakup in different domains using more sophisticated data analysis for the vortex dynamics (i.e. the $\lambda_{2}$ method). 


\section{Numerical Modeling}

The three-dimensional Navier-Stokes (NS) with level-set (LS) and volume-of-fluid (VoF) interface-capturing methods yield computational results for the liquid-segment which captures the liquid-gas interface deformations with time. The incompressible continuity and Navier-Stokes equations follow:

$$
\nabla \cdot \mathbf{u}=0, \quad \frac{\partial(\rho \mathbf{u})}{\partial t}+\nabla \cdot(\rho \mathbf{u u})=-\nabla p+\nabla \cdot(2 \mu \mathbf{D})-\sigma \kappa \delta(d) \overrightarrow{\mathbf{n}},
$$

where $\mathbf{D}$ is the rate of deformation tensor, and $\mathbf{u}$ is the velocity field; $p, \rho$, and $\mu$ are the pressure, density and dynamic viscosity of the fluid, respectively. The last term in the NS equation is the surface tension force per unit volume, where $\sigma$ is the surface tension coefficient, $\kappa$ is the surface curvature, $\delta(d)$ is the Dirac delta function and $\overrightarrow{\mathbf{n}}$ is the unit vector normal to the liquid/gas interface.

Direct numerical simulation is done by using an unsteady three-dimensional finite-volume solver for the NS equations for the planar incompressible liquid sheet segment (initially stagnant), which is subject to instabilities due to a gas stream that flows past it on both sides. A uniform staggered grid is used with the mesh size of $2.5 \mu \mathrm{m}$ and a time step of $5 \mathrm{~ns}$. Third-order accurate QUICK scheme is used for spatial discretization and the Crank-Nicolson scheme for time marching. The continuity and momentum equations are coupled through the SIMPLE algorithm. The LS method developed by Osher and his coworkers [23-25] captures the liquid-gas interface. The level set $\phi$ is a distance function which is advected by the velocity field:

$$
\frac{\partial \phi}{\partial t}+\mathbf{u} \cdot \nabla \phi=0
$$

For detailed descriptions for this interface capturing see [24].

At low density ratios, a transport equation similar to equation (2) is used for the volume fraction $f$, also called the VoF-variable, in order to describe the temporal and spatial evolution of the two-phase flow [26]. The VoF-variable $f$, introduced by Hirt and Nichols [26], represents the volume of (liquid phase) fluid fraction at each cell.

The computational domain, shown in Figure 1, consists of a cube, which is discretized into uniform-sized cells. The liquid segment, which is a sheet of thickness $h(h=200 \mu \mathrm{m})$ is located at the center of the box and is stationary in the beginning. The domain size in terms of the sheet thickness is $4 h \times 4 h \times 8 h$, in the $x, y$ and $z$ directions, respectively. The liquid segment is surrounded by the gas zones on top and bottom. The gas moves in the positive $x$-direction (streamwise direction), with a constant velocity $(U=100 \mathrm{~m} / \mathrm{s})$ at the top and bottom boundaries, and its velocity diminishes to the interface velocity with a hyperbolic tangent profile. The velocity decays exponentially to zero at the center of the liquid sheet. For more detailed description of the initial conditions, see Zandian et al. [22] Grid independency and domain-size independency tests have also been performed [4, 5, 22].

The liquid-gas interface is initially perturbed symmetrically on both sides with a sinusoidal profile and predefined wavelength and amplitude obtained from 2D full-jet simulations [22]. Both streamwise ( $x$-direction) and spanwise ( $y$-direction) perturbations are considered in this study. Periodic boundary condition for all components of velocity as well as the level-set/VoF variable is imposed on the four sides of the computational domain.

The most important dimensionless groupings in this study are the Reynolds number $(R e)$, the Weber number $(W e)$, density ratio $(\hat{\rho})$, viscosity ratio $(\hat{\mu})$, and the initial wavelength-to-sheet-thickness ratio $(\Lambda)$, as defined below.

$$
R e=\frac{\rho_{l} U h}{\mu_{l}}, \quad W e=\frac{\rho_{l} U^{2} h}{\sigma}, \quad \hat{\rho}=\frac{\rho_{g}}{\rho_{l}}, \quad \hat{\mu}=\frac{\mu_{g}}{\mu_{l}}, \quad \Lambda=\frac{\lambda}{h} .
$$

The sheet thickness $h$ and the velocity of the far field gas $U$ are considered as the characteristic length and velocity. The subscripts $l$ and $g$ refer to the liquid and gas, respectively.

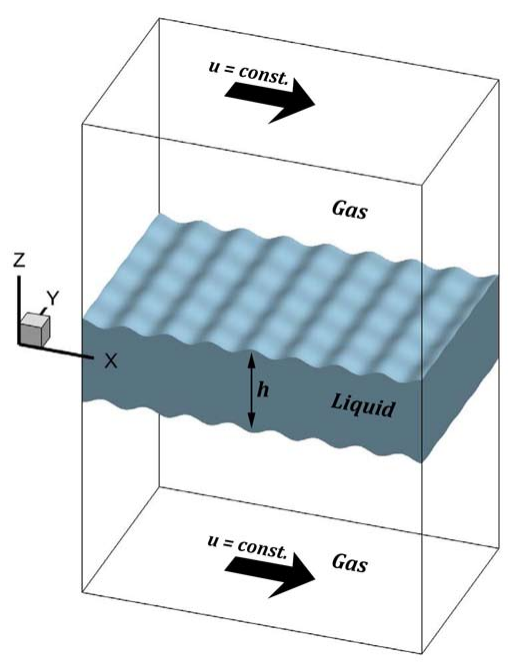

Figure 1. The computational domain with the initial liquid and gas zones. 
Our goal is to study the vorticity dynamics as well as the liquid surface dynamics in order to understand breakup mechanisms at different flow conditions. To this end, $\lambda_{2}$ criterion introduced by Jeong and Hussain [27] is used to define a vortex.

\section{Results and discussion}

Three mechanisms for liquid sheet surface deformation and breakup are identified, which are well categorized on a gas Weber number $\left(W e_{g}\right)$ versus liquid Reynolds number $\left(R e_{l}\right)$ map, shown in Figure 2. At high $R e_{l}$, the liquid sheet breakup characteristics change based on a modified Ohnesorge number, $O h_{m} \equiv \sqrt{W e_{g}} / R e_{l}$, as follows: (i) at high $O h_{m}$ and high $W e_{g}$, the lobes become thin and puncture, creating holes and bridges. Bridges break as perforations expand and create ligaments. Ligaments then stretch and break into droplets by capillary action; see Figure 3. This domain is indicated as Atomization Domain II in Figure 2, and its mechanism is called LoHBrLiD, based on the cascade of structures that are seen in this domain ( $L o \equiv$ Lobe, $H \equiv$ Hole, $B r \equiv$ Bridge, $L i \equiv$ Ligament, and $D \equiv$ Droplet); (ii) at low $O h_{m}$ and high $R e_{l}$, holes are not seen at early times; instead, many corrugations form on the lobe front edge and then stretch into ligaments. This so called LoCLiD mechanism ( $C \equiv$ Corrugation) occurs in Atomization Domain III (see Figure 2) and results in ligaments and droplets without having the hole and bridge formation steps; see Figure 4. The third mechanism follows a LoLiD process and occurs at low $R e_{l}$ and low $W e_{g}$ (Atomization Domain I in Figure 2), but with some difference in details from the LoCLiD process. The main difference between the two ligament formation mechanisms at high and low $R e_{l}$ 's is that at higher $R e_{l}$ the lobes become corrugated before stretching into ligaments. Hence, each lobe may produce multiple ligaments, which are typically thinner and shorter than those in the lower $R e_{l}$. At low $R e_{l}$, on the other hand, because of the higher viscosity, the entire lobe stretches into one thick, usually long ligament; see Figure 5 . The structures seen in all these breakup mechanisms are sketched in Figure 6. Recent microscopic visualization approaches can deliver high-resolution images, which could be used for the validation of the three main breakup mechanisms introduced. There is also a transitional region in which both lobe/ligament stretching and hole-formation mechanisms are seen simultaneously. The transitional region at low $R e_{l}$ follows a hyperbolic relation, i.e. $W e_{g}=A / R e_{l}$, shown by the dash-dotted line in Figure 2; while at high $R e_{l}$ limit, it follows a parabolic curve, i.e. $W e_{g}=B^{2} R e_{l}^{2}$, shown by the dashed line in Figure 2. The constant $B$ is a critical $O h_{m}$ at high $R e_{l}, B \approx 0.021$. The two borderlines can be combined into a single function with some extra constants for a better fit as follows

$$
W e_{g}=\frac{A}{R e_{l}+\epsilon}+B^{2} \operatorname{Re}_{l}^{2}+C,
$$

where $A, B$ and $C$ are empirical constants, and $\epsilon$ is a small parameter for better curve fitting. As $R e_{l}$ gets very large, we would retain the parabolic function (second term) with the constant $B$ being the product of critical $O h$ and $\sqrt{\hat{\rho}}$. In the limit where $R e_{l}$ gets very small, the hyperbolic function (first term) dominates and gives the asymptote. There are two different characteristic times for the formation of holes and the stretching of lobes and ligaments. At the same $R e_{l}$, as surface tension increases (decreasing $O h_{m}$ and $W e_{g}$ ), the characteristic time for hole formation becomes larger, hence delaying the hole formation. Thus, for lower $O h_{m}$ (or $W e_{g}$ ) most of the earlier ligaments are formed due to direct stretching of the lobes and/or corrugations, while the hole formation is inhibited. On the other hand, at relatively large $\operatorname{Re}_{l}(>3000)$, as the liquid viscosity is increased (decreasing $R e_{l}$ and increasing $\left.O h_{m}\right)$, at the same $W e_{g}$, the ligament-stretching time gets larger. In this case, hole formation prevails compared

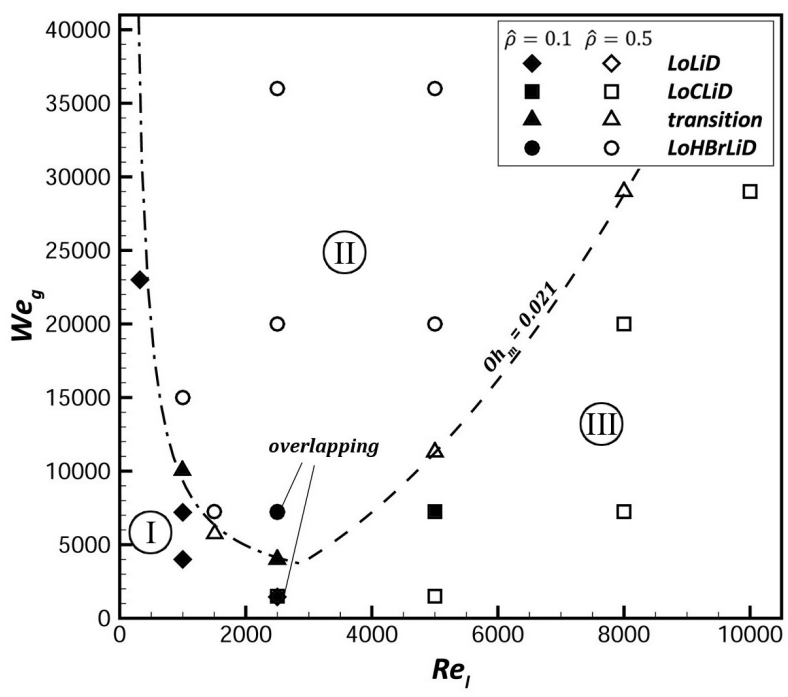

Figure 2. The breakup characteristics based on $W e_{g}$ and $R e_{l}$, showing the LoLiD mechanism denoted by diamonds (Atomization Domain I), the $\mathrm{LoHBrLiD}$ mechanism denoted by circles (Atomization Domain II), the LoCLiD mechanism denoted by squares (Atomization Domain III), and the transitional region denoted by triangles. The $\hat{\rho}=0.1$ cases are shaded. The low and high density ratio cases that overlap at the same point on this diagram have been marked; $-\cdot-\cdot-$, transitional boundary at low $R e_{l}$; and---, transitional boundary at high $R e_{l}$. 


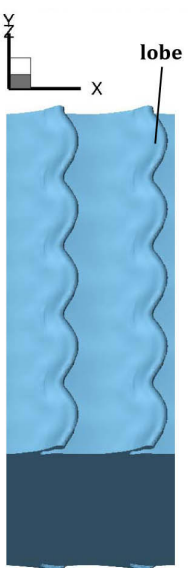

(a)

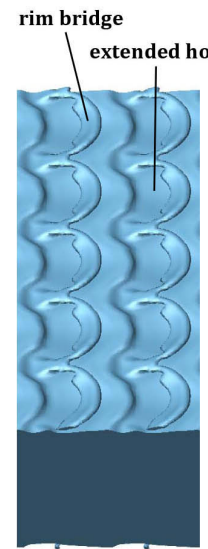

(b)

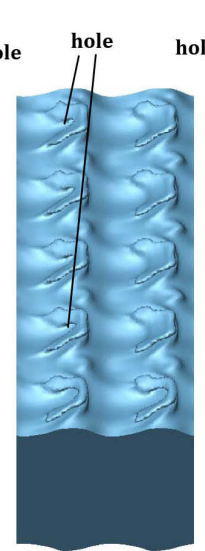

(c)

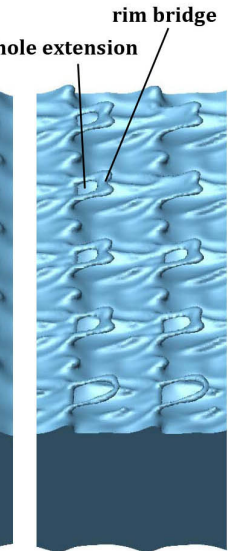

(d)

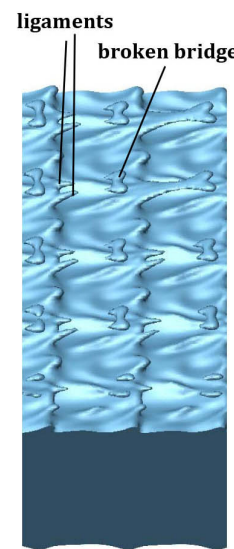

(e)

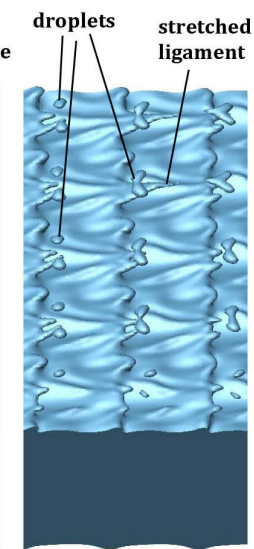

(f)

Figure 3. Liquid surface deformation in the $\mathrm{LoHBr} L i D$ mechanism (Domain II); $R e_{l}=320, W e_{g}=115,000, \hat{\rho}=0.5$, and $\hat{\mu}=0.0022$, at $t=18 \mu \mathrm{s}(a), 22 \mu \mathrm{s}(b), 26 \mu \mathrm{s}(c), 28 \mu \mathrm{s}(d), 30 \mu \mathrm{s}(e)$, and $32 \mu \mathrm{s}(f)$. Gas flows from left to right.

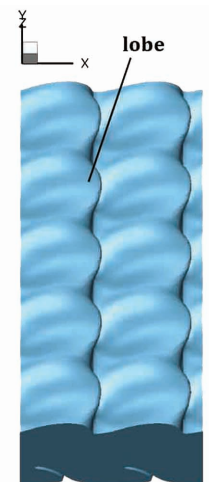

(a)

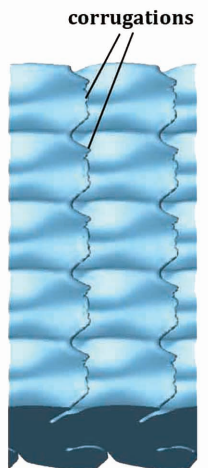

(b)

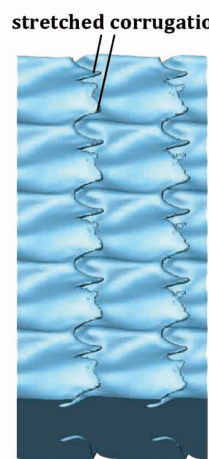

(c)

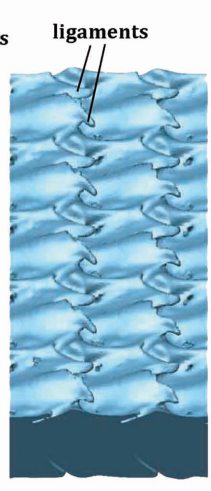

(d)

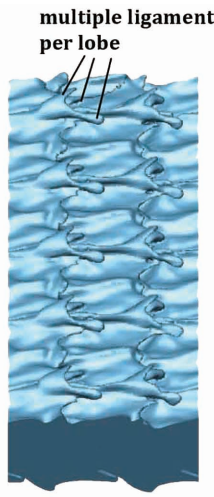

(e)

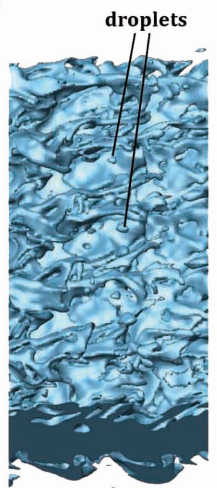

(f)

Figure 4. Liquid surface deformation in the $L o C L i D$ mechanism (Domain III); $R e_{l}=5000, W e_{g}=7200, \hat{\rho}=0.5$, and $\hat{\mu}=0.0066$, at $t=44 \mu(a), 48 \mu \mathrm{s}(b), 50 \mu \mathrm{s}(c), 52 \mu \mathrm{s}(d), 56 \mu \mathrm{s}(e)$, and $60 \mu \mathrm{s}(f)$. Gas flows from left to right.

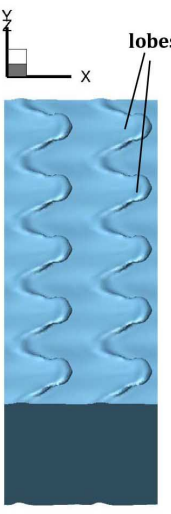

(a)

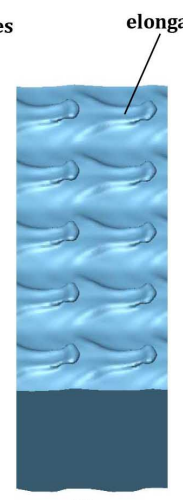

(b)

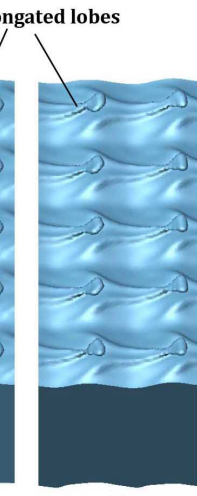

(c)

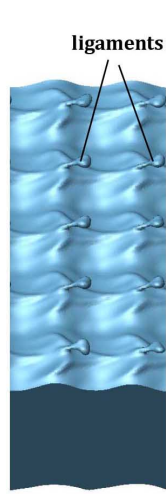

(d)

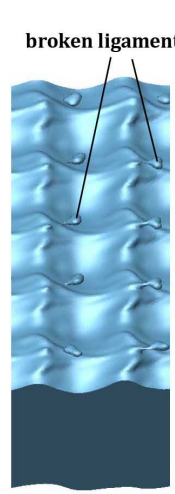

(e)

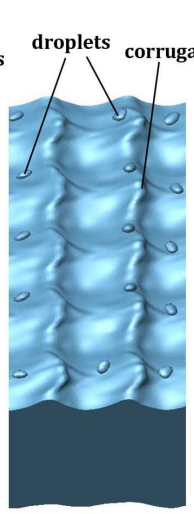

$(f)$ stretching corrugations

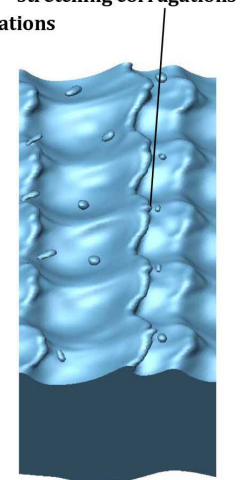

$(g)$

Figure 5. Liquid surface showing the $L o L i D$ mechanism (Domain I) at low $\operatorname{Re}_{l} ; \operatorname{Re}_{l}=320, W e_{g}=23,000, \hat{\rho}=0.1$ and $\hat{\mu}=0.0022$, at $t=26 \mu \mathrm{s}(a), 36 \mu \mathrm{s}(b), 40 \mu \mathrm{s}(c), 44 \mu \mathrm{s}(d), 46 \mu \mathrm{s}(e), 48 \mu \mathrm{s}(f)$, and $52 \mu \mathrm{s}(g)$. Gas flows from left to right.

to the ligament stretching mechanism, resulting in more holes on the liquid lobes. As $W e_{g}$ increases, the time at which the first holes form decreases. This indicates that the hole formation characteristic time should be inversely proportional to $W e_{g}$. At low $R_{l}(<3000)$, the liquid viscosity has an opposite effect on the hole formation and ligament stretching. As shown in Figure 2, near the left boundary, the time scale of the stretching becomes relatively smaller than the hole-formation time scale as $R e_{l}$ is reduced at a constant $W e_{g}$. Hence, there is a move back to ligament stretching from hole formation with decreasing $R e_{l}$ at a fixed $W e_{g}$. Keeping all these effects in mind, the following two nondimensional characteristic times are proposed for these mechanisms:

$$
\frac{U \tau_{h}}{h} \propto \frac{\sigma}{\rho_{g} U^{2} h}\left(1+\frac{k}{R e_{l}}\right)=\frac{1}{W e_{g}}\left(1+\frac{k}{R e_{l}}\right), \quad \frac{U \tau_{s}}{h} \propto \frac{\mu_{l}}{\rho_{l} U h}=\frac{1}{R e_{l}},
$$



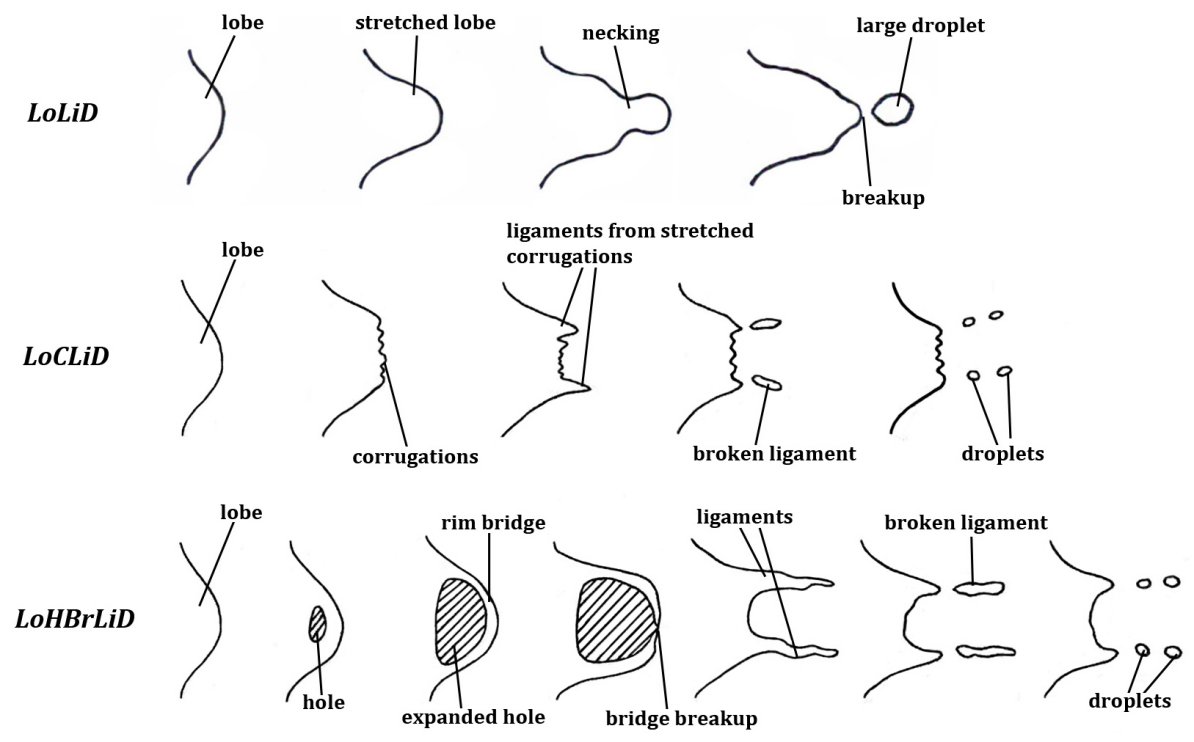

Figure 6. Sketch showing the cascade of structures on a liquid lobe from a top view for the LoLiD (top), LoCLiD (center), and LoH BrLiD (bottom) mechanisms. The gas flows from left to right and time increases to the right.

where $\tau_{h}$ and $\tau_{s}$ are the dimensional characteristic times for hole formation and ligament stretching, respectively, and $k$ is a nondimensional constant.

Combination of the above two equations yields a relation between the two time scales involving $O h_{m}$.

$$
\frac{U \tau_{h}}{h} \propto\left(\frac{U \tau_{s} / h}{O h_{m}}\right)^{2}\left(1+\frac{k}{R e_{l}}\right) .
$$

So, at low $R e_{l}$, the hole formation and the ligament stretching time scales are not related only via $O h_{m} ; R e_{l}$ also has a significant role in the breakup mechanism at lower $R e_{l}$. At high $R e_{l}$ however, the term in the second parenthesis in equation (6) approaches unity, and the two time scales relate via $O h_{m}$ only. In the transitional region, near the boundary, where the two characteristic times are of the same order, both hole formation and corrugation stretching appear at different parts of the liquid sheet.

The breakup mechanisms are functions of $R e_{l}$ and $W e_{g}$ only. The qualitative behavior is not much affected by the viscosity ratio (thus the gas $R e$ ); the influence of the density ratio appears only through $W e_{g}$, and the effect of the sheet thickness appears only through $R e_{l}$ and $W e_{g}$.

\section{Vortex dynamics in the LoHBrLiD mechanism}

The vortex structures in the $\mathrm{LoHBrLiD}$ breakup mechanism are sketched in Figure 7. This figure shows the liquid surface (a lobe) and also the qualitative location of the nearby vortices some time after the start of injection. Hairpin vortices form on the braids, between two consecutive $\mathrm{KH}$ vortices, where the maximum strain rate occurs [8]. The hairpins closer to the streamwise $\mathrm{KH}$ wave crest are stretched downstream towards the $\mathrm{KH}$ vortex, and the hairpins

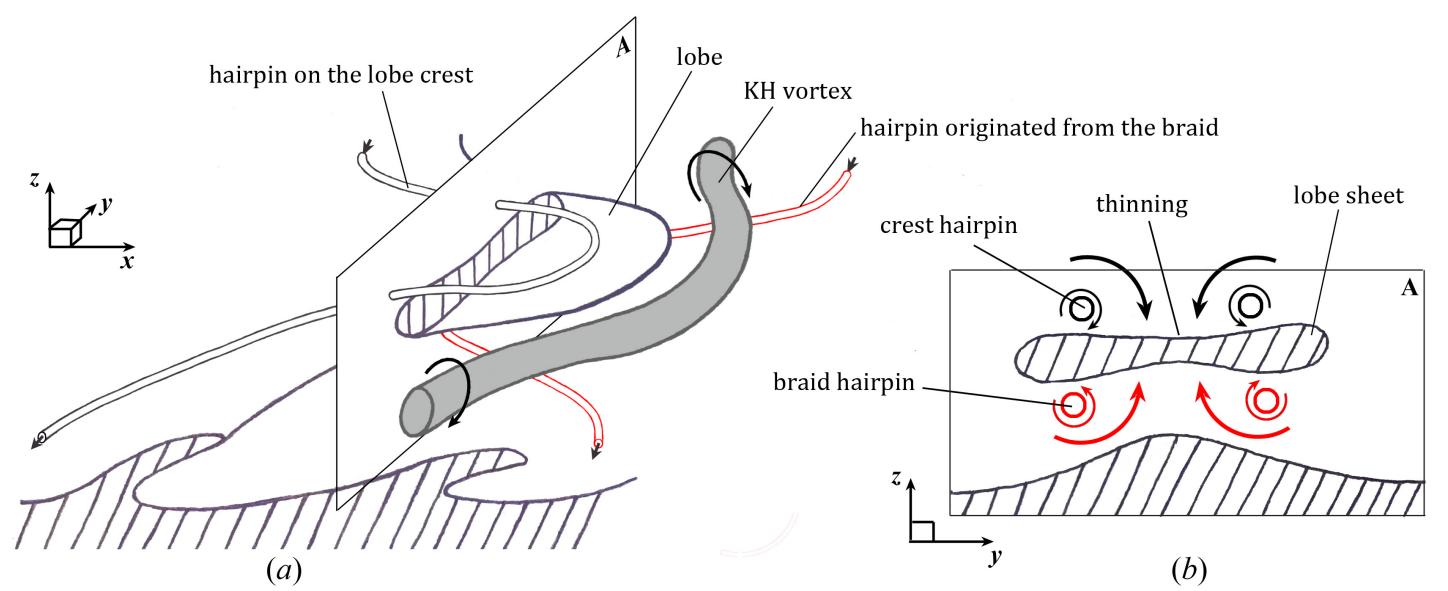

Figure 7. 3D Schematics showing the overlapping of the two hairpin vortices - one from the lobe crest (black tube) and the other from the braid (red tube) $(a)$ - $A$ is the plane in which $(b)$ is drawn; cross-sectional view of the $A$-plane showing the thinning of the lobe sheet due to the hairpin overlapping $(b)$. The vortex schematics are meant to be periodic in $x$ - and $y$-directions. 
near the streamwise trough are stretched upstream due to the pull from the upstream $\mathrm{KH}$ roller. The hairpins that are stretched downstream are rolled over the $\mathrm{KH}$ vortex tube, while the hairpins that are stretched upstream are pulled under the $\mathrm{KH}$ vortex tube.

Later, the $\mathrm{KH}$ vortices deflect more under the influence of the hairpin vortex filaments. The $\mathrm{KH}$ vortex pulls the lobes over itself and lies underneath the surface of the lobe at later times. Pulling of the upstream and downstream hairpins from the outer and inner sides of the interface by the $\mathrm{KH}$ roller causes these two hairpin groups to collect together and overlap on the outer side of the lobe, i.e. on the streamwise wave crest, and its inner side; i.e. on the streamwise wave trough. As shown in Figure 7 and also described by Jarrahbashi et al. [5], the liquid sheet between a pair of these overlapping hairpins becomes thinner. Figure 7(a) schematically depicts two neighboring hairpin vortices in the liquid-gas interface region - one originating from the lobe crest and stretching downstream (shown by the slender black tube) and the other originating from the braid and stretching upstream (shown by the red tube). The $\mathrm{KH}$ vortex is shown by the thicker gray tube in this figure. Figure $7(b)$ shows a cross-sectional view of the vortex structure along with the liquid sheet located between the two hairpin vortices on the $A$-plane of Figure $7(a)$. The induced velocities of the overlapping hairpin vortices (see the qualitative streamlines shown by the black and red arrows in Figure $7 b$ ) push the top surface of the liquid lobe downward and the bottom surface upward, causing the liquid lobe to become thinner in the middle and thus vulnerable to puncture at that region.

Whether the liquid sheet subject to these conditions punctures or not depends on other flow conditions, particularly the surface tension. At high $W e_{g}$ (high $O h_{m}$ ), the hole formation prevails and occurs at the predicted locations. As these overlapping hairpin filaments continue to stretch, the holes stretch and expand with them, creating even larger perforations and thinner bridges. If $W e_{g}$ is not large enough, the liquid sheet in the overlapping region can recover instead. In this case, hole formation is inhibited, and the lobes stretch directly, but more slowly, into ligaments via LoLiD or LoCLiD mechanisms (Domains I and III), as will be discussed in the next sections.

\section{Vortex dynamics in the LoCLiD mechanism}

The role of vortices on the liquid surface deformation at high $R e_{l}$ and low $W e_{g}$ is summarized schematically in Figure 8. At early times $t_{1}$, the spanwise vortices on the braid deflect due to the pull from the neighboring $\mathrm{KH}$ rollers in both the upstream and downstream directions, creating the hairpin vortex structures with a wavelength equal to the spanwise perturbation wavelength. The deflected hairpin filaments form the lobes as they are stretched by the $\mathrm{KH}$ roller. So far, the process is similar to the $\mathrm{LoHBrLiD}$ mechanism.

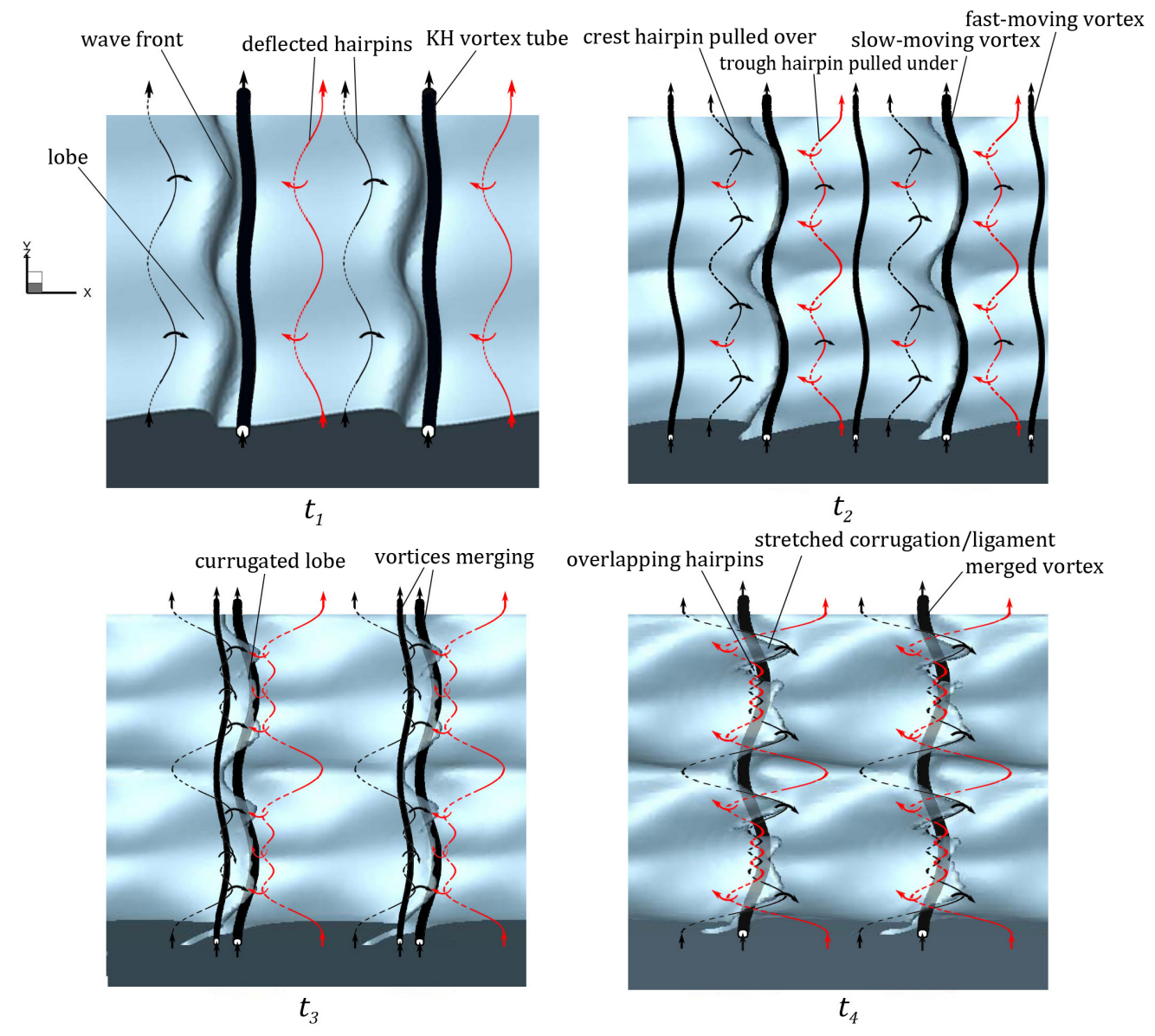

Figure 8. Schematics of the $\mathrm{LoCLiD}$ mechanism at four consecutive times. The liquid surface is shown in blue, and the the $\mathrm{KH}$ vortex in black. The red and black lines are the hairpin vortices near the trough and crest of the KH wave, respectively. The solid and dashed lines show where the hairpins are stretched upstream and inward, or downstream and outward, respectively. 
At high $R e_{l}$, the inertial effects dominate the viscous forces. Consequently, the higher velocity of the gas phase compared to the liquid phase causes the $\mathrm{KH}$ roller to split into two eddies at $t_{2}$, as shown in Figure 8 . The low liquid viscosity does not allow sufficient resistance to keep the $\mathrm{KH}$ vortex bound near the interface; hence, the outer part of the $\mathrm{KH}$ roller, which resides in the fast-moving gas layer, separates from the part of the $\mathrm{KH}$ vortex that is closer to the liquid surface at lower speed.

As demonstrated in Figure 8, the part of the $\mathrm{KH}$ vortex that resides outside of the liquid advects faster than the slowmoving eddy in the liquid. The slow-moving eddy advects with the interface velocity and remains stationary relative to the liquid surface. This vortex separation has two significant effects. (i) The slow-moving eddy downstream of the $\mathrm{KH}$ wave is not strong enough to curl the $\mathrm{KH}$ wave and pull the lobe downstream over itself. Consequently, the outer hairpin filaments on the lobe do not overlap with the inner braid hairpins as in the $\operatorname{LoHBrLiD}$ process, and hole formation at early times is inhibited. (ii) The fast-moving eddy gets closer to the downstream hairpins as it moves farther from the upstream hairpins near the trough. The hairpin filaments become less orderly due to the time-variant pull from this fast-moving eddy in opposite directions. As the fast-moving vortex passes over the trough hairpin (the red line), it pulls the trough hairpin in the downstream direction and creates a new bend on the hairpin, as shown in Figure 8 at $t_{2}$. Similarly, the crest hairpin filament (the black line) is pulled in the upstream direction by the fast-moving vortex tube, causing the hairpin vortices to undergo more undulations with smaller wavelengths, and also prevents further streamwise stretch in the original hairpins. Consequently, the lobes are less stretched and more blunt compared to the LoHBrLiD mechanism.

When the fast-moving eddy passes over the crest hairpin (black hairpin) at $t_{3}$, it creates yet another turn in the hairpin vortex, as shown in Figure 8. The liquid surface approximately follows the hairpin structures with some delay at this high $R e_{l}$ range - as the vortex lines are nearly material lines. Because of these shorter hairpin wavelengths, corrugations with length scales comparable to the hairpin wavelengths are formed on the front-most edge of the lobes, as shown in Figure 8 at $t_{3}$. Both experimental observations [8,14] and numerical simulations [20,21] show that the number of corrugations (lobes) increases by increasing the jet Reynolds number.

Upon creation of a stronger eddy downstream of the $\mathrm{KH}$ waves at $t_{4}$ - after amalgamation of the fast- and slowmoving eddies - the new KH roller (now a thicker tube) has enough strength to stretch the hairpin vortices. The hairpin filaments on the outer and inner sides of the lobe overlap as illustrated in Figure 8 at $t_{4}$. In this figure, the dashed lines represent the hairpins stretching upstream and under the liquid lobes, i.e. inner surface of the gas lobe, while the solid lines are parts of the hairpin filament that are stretched downstream on the inner side of the liquid lobe; i.e outer surface of the gas lobe.

The flow induced by the hairpin vortices creates undulations on both the bottom surface of the lobe and the trough surface. The gap filled by the gas layer gets closed, i.e. the lobe collapses on the jet core, as the bottom surface of the liquid lobe descends and the trough surface ascends. The counter-rotating pairs of streamwise vortices on the liquid lobe create the ligaments on the two sides of the lobe at $t_{4}$ (see Figure 8 ). The corrugations also stretch into ligaments but at a slower growth rate. The ligaments stretch downstream and break into droplets as they undergo capillary instabilities later. In the meantime, the eddies separate from the interface and advect outward into the gas phase.

\section{Vortex dynamics in the LoLiD mechanism}

Similar to the previous cases, the vortex field starts with a large $\mathrm{KH}$ roller downstream of the $\mathrm{KH}$ wave, hairpin filaments on the braid, and a stronger hairpin filament on the $\mathrm{KH}$ wave crest. Later, the entire $\mathrm{KH}$ roller departs from the liquid surface and moves downstream into the gas zone. The $\mathrm{KH}$ roller gains higher velocity as it moves away from the interface, while the rest of the hairpin vortices are almost stationary with respect to the interface.

While the $\mathrm{KH}$ roller reaches the neighboring downstream crest hairpin filament, the braid hairpins overlap with the crest hairpins constraining the lobe sheet in between. So far, the vortex dynamics manifest the conditions required for both hole formation and corrugation formation. However, none of these structures are seen on the lobe. The

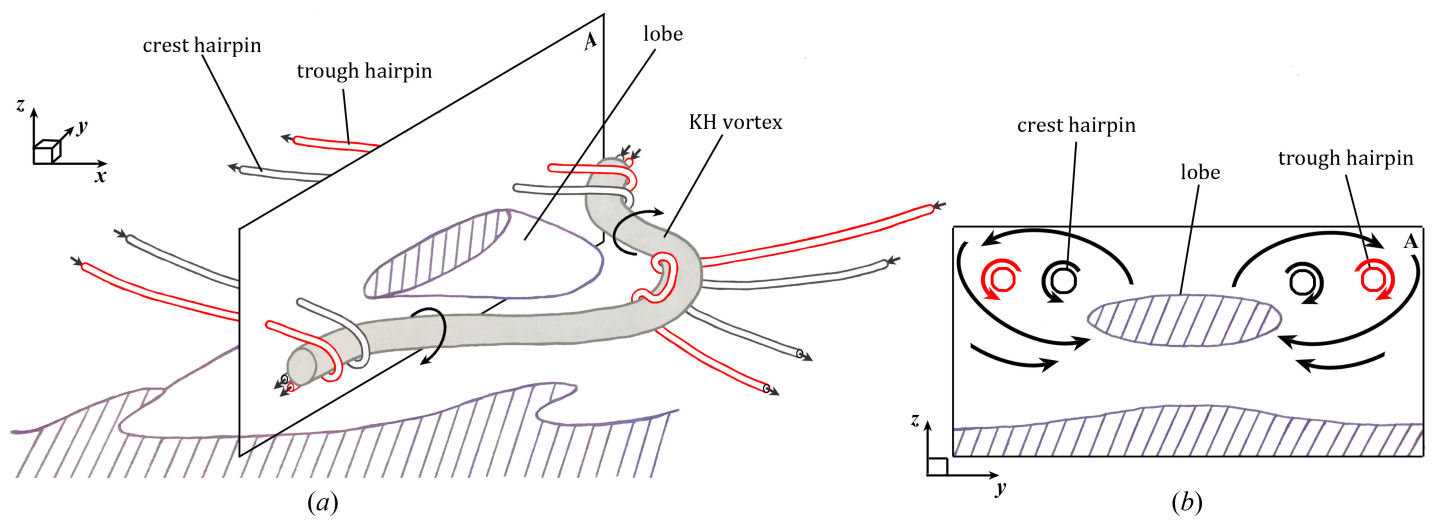

Figure 9. 3D Schematics showing the vortex structures in the LoLiD mechanism of Domain I $(a), A$ is the plane in which $(b)$ is drawn; cross-sectional view of the $A$-plane showing the spanwise squeezing of the lobe by the hairpin vortices $(b)$. The vortex schematics are meant to be periodic in $x$ - and $y$-directions. 
inhibition of the hole formation is due to the high surface tension. In such low $W e_{g}$, the inertial and viscous forces are not strong enough to overcome the surface tension force to thin and stretch the lobe; hence, the lobe perforation is inhibited. Also, because of the high liquid viscosity at such low $R e_{l}$, the liquid surface response to the vortex structures is retarded (vortex lines and material lines are not identical). Hence, the lobe edges are not corrugated following the hairpin filaments as with higher $R e_{l}$. Moreover, the $\mathrm{KH}$ roller is more distant from the interface in this case compared to the LoCLiD mechanism, which means that it has a much weaker influence on the hairpin filaments, resulting in fewer bends and less undulation on the hairpins.

The schematics of the vortex structures in the LoLiD mechanism of Domain I are illustrated in Figure 9. The $\mathrm{KH}$ vortex has a higher undulation in this domain compared to the other two domains and is also farther away from the interface in the gas zone. Because of the high strain rate at the braid, the hairpin vortices become streamwise near the braid region in both spanwise crest and trough. Two pairs of counter-rotating hairpins - one on the lobe crest (shown by the black tube) and the other on the trough (shown by the red tube) - stretch and wrap around the KH vortex; see Figure $9(a)$. As shown in the cross-sectional view of the $A$-plane passing through the lobe (Figure $9 b$ ), both the black and the red hairpins are located slightly above and on the sides of the lobe at this moment. While the flow induced by the $\mathrm{KH}$ vortex creates a streamwise flow on the two sides of the lobe, the gas flow induced by these two counter-rotating hairpins (shown by the curly arrows is Figure $9 b$ ) generates a spanwise flow towards the lobe. Consequently, the lobe is both squeezed in the spanwise direction - via the hairpins effects - and stretched in the streamwise direction by the induced flow of the $\mathrm{KH}$ vortex. The gas flow also lifts the lobe in the normal direction. The flow induced by the streamwise hairpins transforms the lobe into a thick ligament as the strong shear near the lobe sides deforms the liquid surface.

\section{Conclusions}

The temporal development of surface waves and their breakup into droplets are studied numerically. Three main breakup mechanisms are identified. The breakup characteristics are well categorized on a parameter space of gas Weber number $\left(W e_{g}\right)$ versus liquid Reynolds number $\left(R e_{l}\right)$. The atomization regime is now separated as three sub-domains.

The vortex dynamics is able to explain the hairpins formation, and the interaction between the hairpin vortices and the $\mathrm{KH}$ vortex explains the perforation of the lobes at moderate $R e_{l}$ and high $W e_{g}$, which is attributed to the overlapping of the two pairs of hairpin vortices on top and bottom of the lobe. The formation of corrugations on the lobe front edge at high $R e_{l}$ is also explained by the structure of the hairpins and the splitting of the $\mathrm{KH}$ vortex. At low $R e_{l}$ and low $W e_{g}$, on the other hand, the lobe perforation and corrugation formation are inhibited due to high surface tension and high liquid viscosity. The hairpin vortices stretch in the normal direction while wrapping around the $\mathrm{KH}$ vortex. The induced gas flow squeezes the lobe from the sides and forms a thick and long ligament.

\section{References}

[1] Shinjo, J., and Umemura, A., 2010, International Journal of Multiphase Flow, 36, pp. 513-532.

[2] Desjardins, O., and Pitsch, H., 2010, Atomization and Sprays, 20 (4), pp. 311-336.

[3] Herrmann, M., 2011, Atomization and Sprays, 21 (4), pp. 283-301.

[4] Jarrahbashi, D., and Sirignano, W. A., 2014, Physics of Fluids, 26 (10), p. 73.

[5] Jarrahbashi, D., Sirignano, W. A., Popov, P., and Hussain, F., 2016, Journal of Fluid Mechanics, 792, pp. 186-231.

[6] Bernal, L. P., and Roshko, A., 1986, Journal of Fluid Mechanics, 170, pp. 499-525.

[7] Pope, S. B., 1978, AIAA Journal, 16 (3), pp. 279-281.

[8] Lasheras, J. C., and Choi, H., 1988, Journal of Fluid Mechanics, 189, pp. 53-86.

[9] Schowalter, D. G., Van Atta, C. W., and Lasheras, J. C., 1994, Meccanica, 29 (4), pp. 361-371.

[10] Widnall, S. E., and Sullivan, J. P., 1973, Proceedings of the Royal Society of London A: Mathematical, Physical and Engineering Sciences, 332 (1590), pp. 335-353.

[11] Widnall, S. E., Bliss, D. B., and Tsai, C. Y., 1974, Journal of Fluid Mechanics, 66 (1), pp. 35-47.

[12] Breidenthal, R. E., 1981, Journal of Fluid Mechanics, 109, pp. 1-24.

[13] Jimenez, J., 1983, Journal of Fluid Mechanics, 132, pp. 319-336.

[14] Liepmann, D., and Gharib, M., 1992, Journal of Fluid Mechanics, 245, pp. 643-668.

[15] Ashurst, W. T., and Meiburg, E., 1988, Journal of Fluid Mechanics, 189, pp. 87-116.

[16] Martin, J. E., and Meiburg, E., 1991, Journal of Fluid Mechanics, 230, pp. 271-318.

[17] Comte, P., Lesieur, M., and Lamballais, E., 1992, Physics of Fluids A: Fluid Dynamics, 4 (12), pp. $2761-2778$.

[18] Collis, S. S., Lele, S. K., Moser, R. D., and Rogers, M. M., 1994, Physics of Fluids, 6 (1), pp. 381-396.

[19] Schoppa, W., Hussain, F., and Metcalfe, R. W., 1995, Journal of Fluid Mechanics, 298, pp. 23-80.

[20] Brancher, P., Chomaz, J. M., and Huerre, P., 1993, Physics of Fluids, 6 (5), pp. 1768-1774.

[21] Danaila, I., Dusek, J., and Anselmet, F., 1997, Physics of Fluids, 9 (11), pp. 3323-3342.

[22] Zandian, A., Sirignano, W. A., and Hussain, F., 2016, 54th AIAA Aerospace Sciences Meeting, p. 1593.

[23] Zhao, H. K., Chan, B., Merriman, B., and Osher, S., 1996, Journal of computational physics, 127 (1), pp. 179-195.

[24] Sussman, M., Fatemi, E., Smereka, P., and Osher, S., 1998, Computers and Fluids, 27 (5), pp. 663-680.

[25] Osher, S., and Fedkiw, R. P., 2001, Journal of computational physics, 169 (2), pp. 463-502.

[26] Hirt, C. W. and Nichols, B. D., 1981, Journal of computational physics, 39 (1), pp. 201-225.

[27] Jeong, J., and Hussain, F., 1995, Journal of Fluid Mechanics, 285, pp. 69-94. 


\title{
Direct numerical simulation of an atomizing biodiesel jet: Impact of fuel properties on atomization characteristics
}

\author{
Yue Ling ${ }^{1 *}$, Guillaume Legros ${ }^{2}$, Stéphane Popinet ${ }^{2}$, Stéphane Zaleski \\ ${ }^{1}$ Department of Mechanical Engineering, Baylor University, Texas, USA \\ ${ }^{2}$ Sorbonne Universités, UPMC, Universitris 06, CNRS, UMR 7190, Institut Jean Le Rond \\ d'Alembert, F-75005, Paris, France \\ ${ }^{*}$ Corresponding author: stanley_ling@baylor.edu
}

\begin{abstract}
The utilization of biodiesel is an effective approach to reduce pollution from internal combustion engines and thus has attracted steadily increasing interest in the recent years. As the viscosity of biodiesel is much higher than that of standard diesel, the atomization characteristics of a biodiesel jet can significantly deviate from those of a standard diesel jet under identical injection conditions. Since atomization of the injected fuel has a strong impact on fuel-air mixing and the following combustion processes, it is important to investigate the atomization of biodiesel and in particular to understand how the fuel properties affect the atomization process and the resulting spray characteristics. In the present study, three-dimensional direct numerical simulations are conducted to investigate atomizing biodiesel and diesel jets. The novel adaptive multiphase solver Basilisk is used for simulations. The statistics of droplets formed in the biodiesel jet is compared to the diesel jet under identical injection conditions.
\end{abstract}

\section{Keywords}

Biodiesel, atomization, direct numerical simulation, Volume-of-fluid method

\section{Introduction}

The utilization of biodiesel is an effective approach to reduce pollution from internal combustion engines and thus has attracted ever increasing interest in recent years. As the material properties of biodiesel are different from those of the standard diesel, the atomization characteristics of a biodiesel jet can significantly deviate from those of a standard diesel jet under identical injection conditions. Since atomization of the injected fuel has a strong impact on the fuel-air mixing and following combustion processes, it is important to investigate the atomization of biodiesel and in particular to understand how the fuel properties affect the atomization process and the resulting spray characteristics.

Atomization of a liquid jet is a process of enormous complexity involving a wide range of spatial scales. As a result, numerical simulation of atomization is very challenging. In the present work, direct numerical simulations of atomizing jets are performed with a novel adaptive multiphase solver, Basilisk [1]. The solver uses the Volume-Of-Fluid (VOF) method to capture the liquid-gas interface. A balanced-force discretization is employed for surface-tension calculation [2]. An octree spatial discretization is utilized, which gives a very important flexibility allowing dynamic grid refinement into user-defined regions. Finally, massively parallel simulations are conducted on a supercomputer using up to 2048 processors.

\section{Material and methods}

In the present study, we simulate a round liquid fuel jet into a chamber containing pressurized stationary gas. Two different fuels are considered, a standard European diesel and a biodiesel of soybean Methyl Ester. The fuel properties are given by Battistoni and Grimaldi [3]. Three different injection conditions, i.e., the jet radius and injection velocity, are investigated for each fuel. Therefore, in total six cases are simulated. The parameters are summarized in Table 1. The case names " $D$ " and "B" represent diesel and biodiesel fuels, respectively, where " $\mathrm{n}=1,2,3$ " indicates different injection conditions.

Table 1. Fuel properties and injection conditions of simulation cases.

\begin{tabular}{c|ccccc}
\hline Cases & $\begin{array}{c}\text { Fuel Density } \\
\rho_{l}\left(\mathrm{~kg} / \mathrm{m}^{3}\right)\end{array}$ & $\begin{array}{c}\text { Fuel Viscosity } \\
\mu_{l}(\mathrm{Pas})\end{array}$ & $\begin{array}{c}\text { Surface Tension } \\
\sigma(\mathrm{N} / \mathrm{m})\end{array}$ & $\begin{array}{c}\text { Jet Radius } \\
R_{\text {inj }}(\mathrm{m})\end{array}$ & $\begin{array}{c}\text { Injection Velocity } \\
U_{\text {inj }}(\mathrm{m} / \mathrm{s})\end{array}$ \\
\hline D1 & 825 & $2.10 \times 10^{-3}$ & 0.024 & $6.50 \times 10^{-5}$ & 70 \\
D2 & 825 & $2.10 \times 10^{-3}$ & 0.024 & $4.00 \times 10^{-5}$ & 50 \\
D3 & 825 & $2.10 \times 10^{-3}$ & 0.024 & $6.50 \times 10^{-5}$ & 50 \\
B1 & 870 & $3.90 \times 10^{-3}$ & 0.028 & $6.50 \times 10^{-5}$ & 70 \\
B2 & 870 & $3.90 \times 10^{-3}$ & 0.028 & $4.00 \times 10^{-5}$ & 50 \\
B3 & 870 & $3.90 \times 10^{-3}$ & 0.028 & $6.50 \times 10^{-5}$ & 50
\end{tabular}

The gas in the chamber is taken to be air under the pressure of $10^{6} \mathrm{~Pa}$ and the temperature $313.15 \mathrm{~K}$. The density and viscosity of the gas are $11.13 \mathrm{~kg} / \mathrm{m}^{3}$ and $\left.1.46 \times 10^{5} \mathrm{~Pa} \mathrm{~s}\right)$, respectively. If the gas density, the jet radius, 
and the injection velocity are chosen to be reference scales, the key dimensionless parameters of problem can be obtained, which are shown in Table 2. The Reynolds and Weber numbers of the liquid jet are defined as $\operatorname{Re}_{l}=\rho_{l}\left(2 R_{i n j}\right) U_{i n j} / \mu_{l}$ and $\mathrm{We}_{l}=\rho_{l}\left(2 R_{i n j}\right) U_{i n j}^{2} / \sigma$, respectively, which measure the inertial effect compared to the viscous and surface tension forces. When $\mathrm{Re}_{l}$ and $\mathrm{We}_{l}$ are large, the viscous and surface tension forces are insufficient to hold the injected fuel as a bulk liquid, and the liquid fuel will be atomized into small droplets. As the injected liquid will also induce flow motion of the gas in the chamber, the Reynolds number based on the gas properties, $\operatorname{Re}_{g}=\rho_{g}\left(2 R_{i n j}\right) U_{i n j} / \mu_{g}$, is to characterize the induced gas flow. When $\operatorname{Re}_{g}$ is large the gas flow can become turbulent. Finally, the liquid-to-gas density ratio is represented by $r$. Other dimensionless parameters can be computed based on these four parameters, for example, the liquid-to-gas viscosity ratio is equal to $r \operatorname{Re}_{g} / \operatorname{Re}_{l}$.

Table 2. Fuel properties and injection conditions of simulation cases.

\begin{tabular}{c|cccc}
\hline Cases & $\operatorname{Re}_{l}=\rho_{l}\left(2 R_{i n j}\right) U_{i n j} / \mu_{l}$ & $\mathrm{We}_{l}=\rho_{l}\left(2 R_{i n j}\right) U_{i n j}^{2} / \sigma$ & $r=\rho_{l} / \rho_{g}$ & $\operatorname{Re}_{g}=\rho_{g}\left(2 R_{i n j}\right) U_{i n j} / \mu_{g}$ \\
\hline D1 & $3.58 \times 10^{2}$ & $2.19 \times 10^{4}$ & 74.1 & $6.94 \times 10^{3}$ \\
D2 & $1.57 \times 10^{3}$ & $6.88 \times 10^{3}$ & 74.1 & $3.05 \times 10^{3}$ \\
D3 & $2.55 \times 10^{3}$ & $1.12 \times 10^{4}$ & 74.1 & $4.95 \times 10^{3}$ \\
B1 & $2.03 \times 10^{3}$ & $1.98 \times 10^{4}$ & 78.2 & $6.94 \times 10^{3}$ \\
B2 & $8.92 \times 10^{2}$ & $6.21 \times 10^{3}$ & 78.2 & $3.05 \times 10^{3}$ \\
B3 & $1.45 \times 10^{3}$ & $1.01 \times 10^{4}$ & 78.2 & $4.95 \times 10^{3}$
\end{tabular}

It can be observed from Tables 1 and 2 that the main difference between the diesel and biodiesel fuels lies at the fuel viscosity. The density and surface tension of the two fuels are quite similar. As a result, for a given injection condition, the diesel and biodiesel jets (such as $\mathrm{D} 1$ and $\mathrm{B} 1$ ) have similar $\mathrm{We}_{l}, \mathrm{Re}_{g}$, and $r$; while $\mathrm{Re}_{l}$ for the diesel is about two times of that for the biodiesel. It is expected a large liquid viscosity can damp small scale instability and thus weaken the atomization process [4]. It is the goal of the present study to investigate in detail the impact of the larger viscosity of the biodiesel to the atomization characteristics.

Thanks to the octree discretization of Basilisk, we can use a large domain for the present simulation so that the effects of boundaries to the atomizing jet are negligible. The computational domain for the atomizing jet is shown in Fig. 1. The fuel is injected into a cubic domain along the $z$ direction from the left boundary. A single mode perturbation is added to the inflow velocity as $U_{l, \text { inflow }}=U_{i n j}(1+\xi \sin (2 \pi t / \eta))$ to accelerate the development of the Kelvin-Helmholtz instability at the liquid-gas interface, where $\xi=0.05$ and $\eta=2.56 R_{i n j} / U_{i n j}$. The outflow boundary condition is invoked at the right boundary. All the rest boundaries of the domain are then taken as symmetric boundaries. The edge length of the domain is $96 R_{i n j}$. The minimum cell size of the adaptive mesh used is indicated by $\Delta_{\min }$. For cases D1, D2, B1, and B2, $\Delta_{\min }=R_{i n j} / 43$ and for cases D3 and B3, $\Delta_{\min }=R_{i n j} / 85$. A snapshot of the atomizing jet and the corresponding adaptive mesh are shown in Fig. 1. It can be seen that high mesh resolution is used to resolve the complex liquid-gas interface while the mesh away from the jet is coarsen to reduce computational costs. The total number of cells increases in time as more liquid is injected into the domain. The mesh shown in Fig. 1, which is a snapshot corresponding to $t U_{i n j} / R_{i n j}=49$, consist of about 50 million cells. It should be mentioned here that the liquid injection velocity used in the present study is lower than typical injection velocity in diesel engines. The usage of smaller injection velocity is simply to constrain the Reynolds number in the regime where a DNS is feasible with current computational resources. For practical injection velocity which can be 10 times of the present ones, the total number of cells would exceed 3 billion even with an adaptive mesh. Finally, the simulation data generated are visualized and analyzed by the open source package Vis/t [7].

\section{Results and discussion General behavior}

A snapshot of the atomizing liquid jet for the case 3 and $\Delta_{\min }=R_{i n j} / 85$ at $t U_{i n j} / R_{i n j}=47$ is shown in Fig. 2. When the liquid fuel is injected into the domain with a high speed, the impingement with the pressurized gas causes the liquid to spread laterally forming a liquid sheet. The liquid sheet moves backward so eventually the head of the liquid jet takes a mushroom shape, a closeup of which is given in Fig. 2. The liquid sheet of the mushroom head flaps and breaks violently. Ligaments are formed near the edge of the liquid sheet, which then in turn break into a large number of droplets. Different formation mechanisms of liquid ligaments have been discussed in previous works $[5,6]$. These mechanisms including the expansion of holes-in-liquid-sheets and the Rayleigh-Plateau instability of rims are also observed in the present simulation. The shapes of the liquid ligaments display strong variability and thus when they break the size of formed droplets is spread over a wide range. When the ligaments and droplets detach from the bulk liquid jet, their streamwise velocity decreases and they fall behind the mushroom head, forming a spray around the bulk liquid column.

Beyond the mushroom head, the interfacial waves on the bulk liquid column also produce droplets. Due to the shear between the moving liquid and the stagnant gas, a Kelvin-Helmholtz $(\mathrm{KH})$ instability develops. The $\mathrm{KH}$ interfacial waves are initially small and axisymmetric. As they are advected downstream, they grow in amplitude and roll backward like umbrellas. Lateral instability then show up and azimuthal variations are observed at the umbrella edge. When the waves propagate further downstream, holes forms in the liquid sheet and expansion of which finally causes the umbrella-shape waves break into ligaments, similar to the mushroom head but in a less violent manner. The orientations of the formed ligaments vary in a large extent. The ones more perpendicular to the jet 


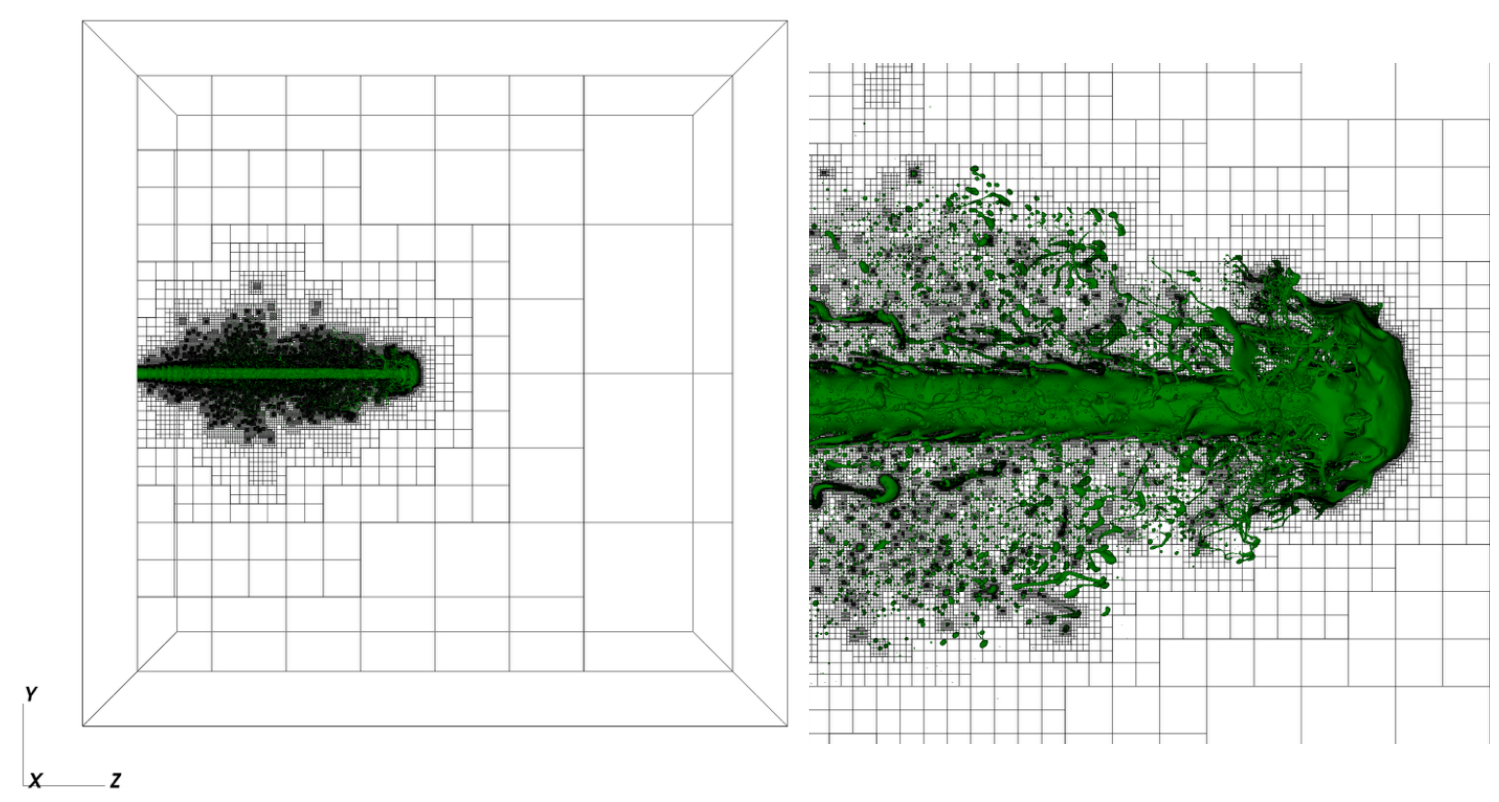

Figure 1. Simulation setup for an atomizing liquid jet and the adaptive mesh utilized.

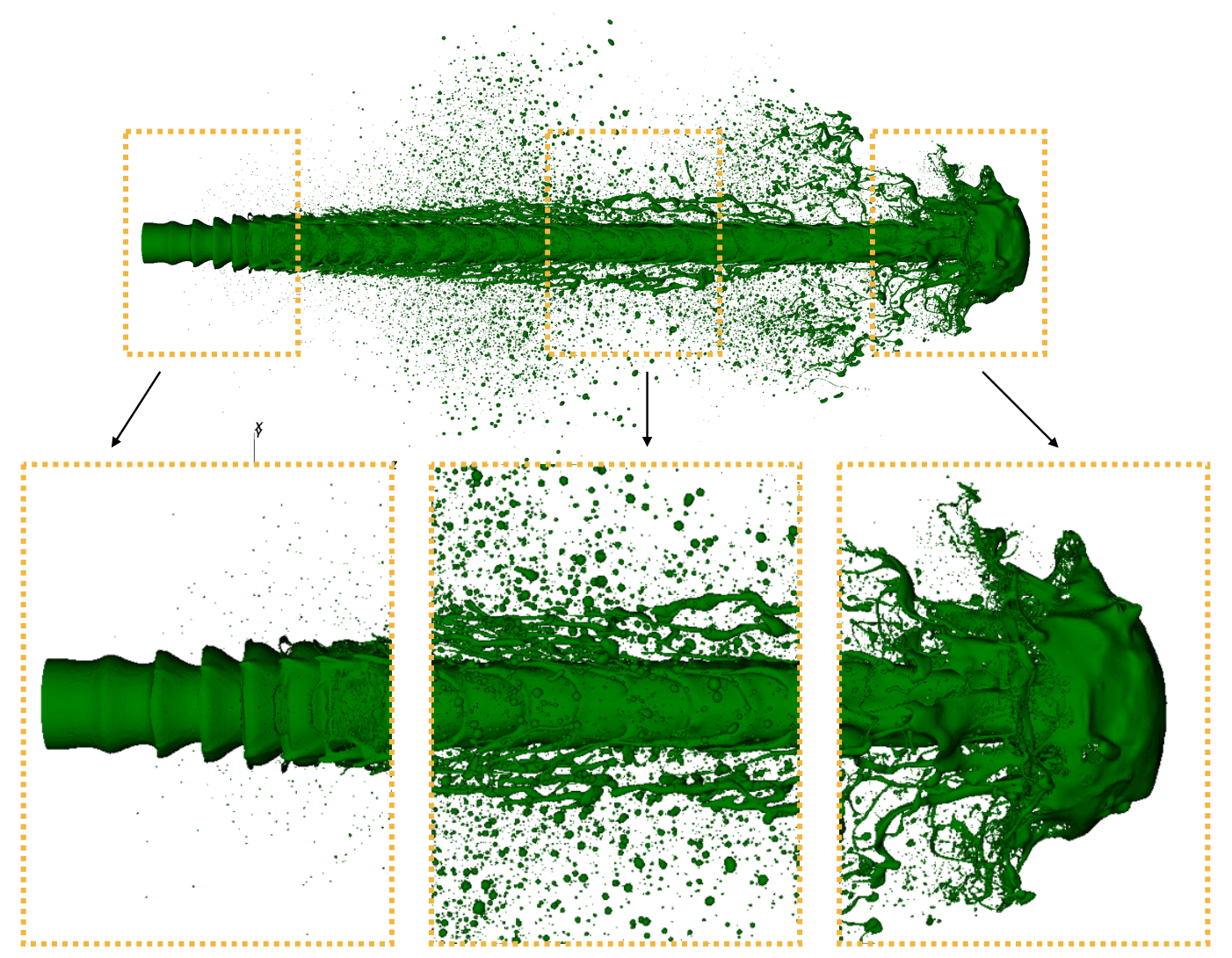

Figure 2. The atomizing jet for the case D3 at $t U_{i n j} / R_{i n j}=47$. The minimum cell size of the adaptive mesh is $\Delta_{\text {min }}=R_{\text {inj }} / 85$. 
breaks into droplets faster; while those more aligned to the jet tend to last for a longer time. Compared to the droplets formed at the mushroom head, the ones formed at the interfacial waves stay closer to the liquid jet and are less spread.

\section{Effect of grid resolution}

In order to verify whether the mesh resolution is sufficient to resolve the atomization process, the minimum cell size $\Delta_{\text {min }}$ is varied from $R_{i n j} / 21$ to $R_{i n j} / 85$ for cases D3 and B3. The results of the grid-refinement study are shown in Fig. 3. It can be observed that the results for the coarse mesh $\Delta_{\min }=R_{i n j} / 21$ are substantially different from the results for the other two meshes: the number of droplets produced is much lower and the ligaments formed at the interfacial waves are completely missed. The results for the intermediate and fine meshes $\Delta_{\min }=R_{\text {inj }} / 43$ and $R_{i n j} / 85$ are similar, although the ligaments formed at the edge of the mushroom head tends to break earlier for the intermediate mesh.

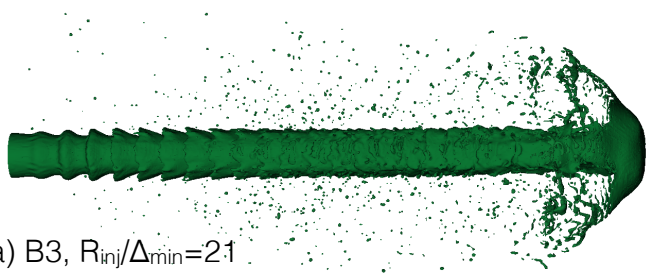

(a) B3, R Rinj $/ \Delta_{\min }=21$
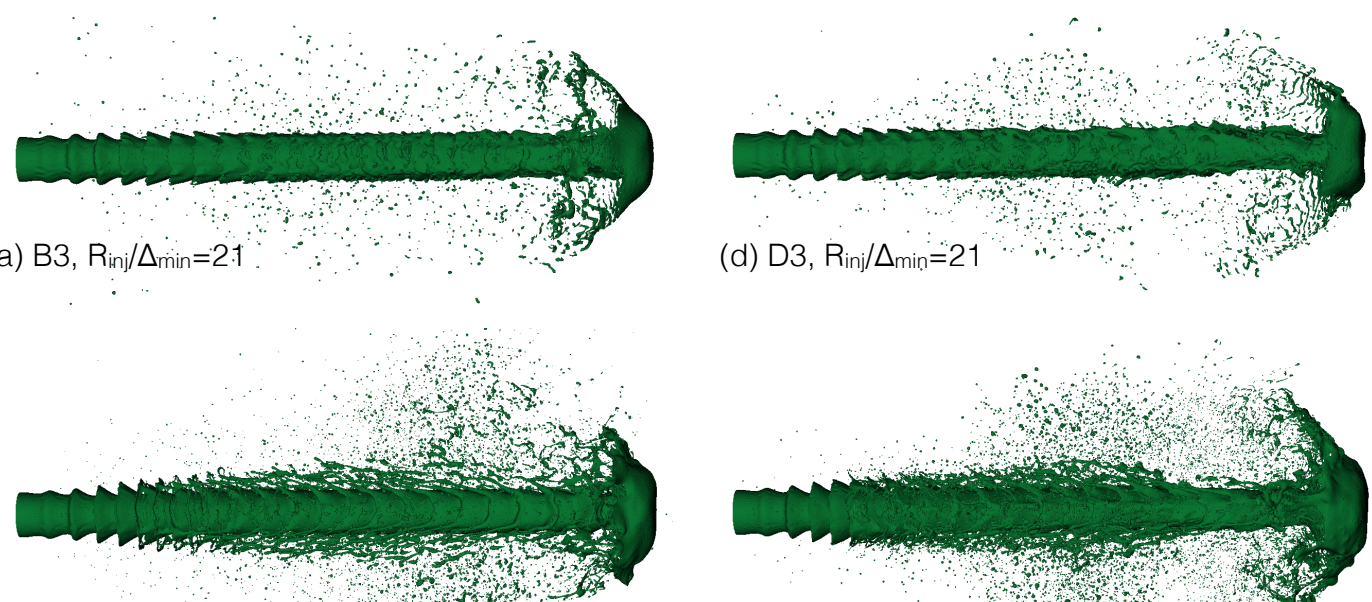

(b) $B 3, R_{\text {inj }} / \Delta_{\min }=43$
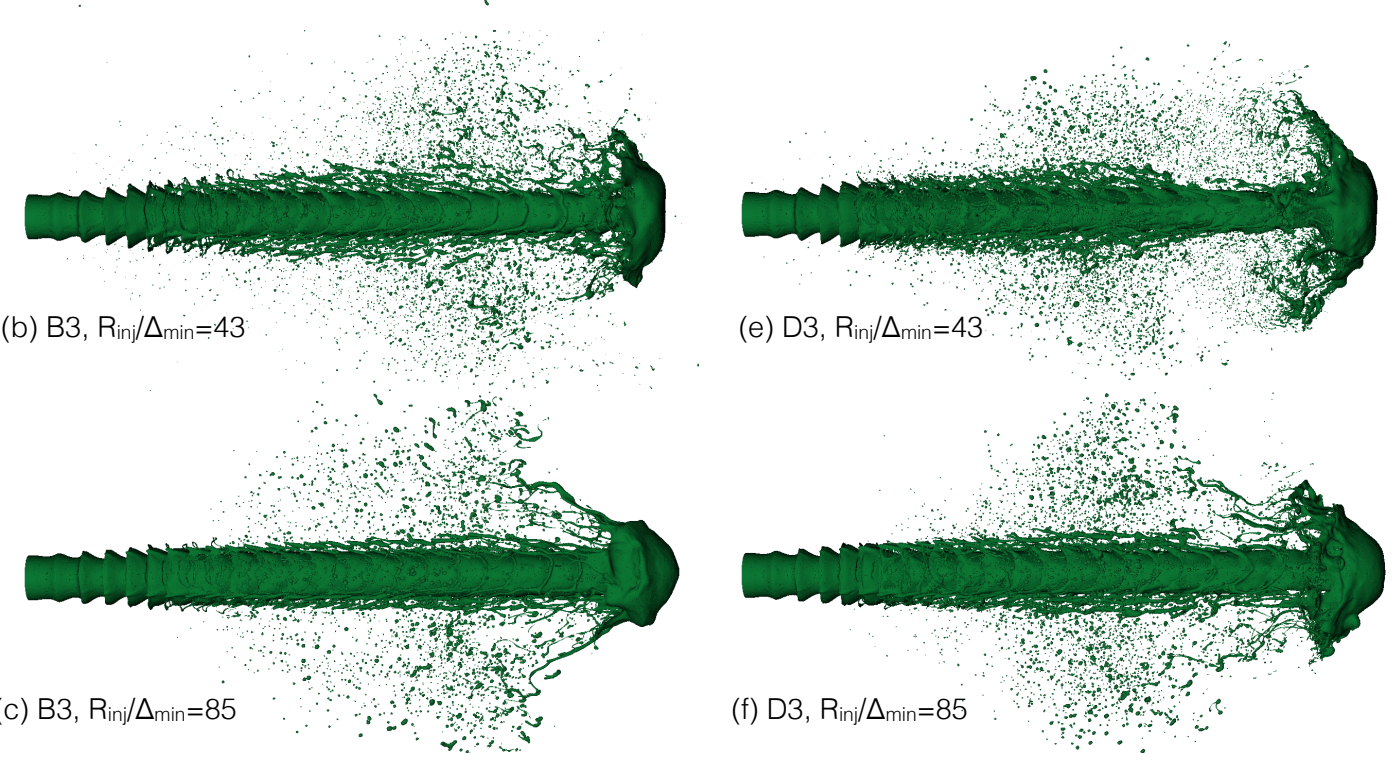

Figure 3. Snapshots of the atomizing biodiesel and diesel jets (B3 and D3) for different mesh resolutions: $R_{i n j} / \Delta_{\min }=21,43$, and 85 .

As $\operatorname{Re}_{g}$ is high for both cases B3 and D3, the gas flow around the liquid jet becomes turbulent. Figure 4 show the vortical structures by the $\lambda_{2}$ vortex-identification criterion proposed by Jeong and Hussain [8]. The turbulent vortical structures are most profound near the head of the liquid jet. When the mesh is refined, smaller vortical structures are captured. It is interesting to observe that actually less vortical structures are observed in the fine mesh results than in the coarse ones. As the cell size in simulations behaves like a cut-off scale that control the breakup of liquid sheets and ligaments. A coarse mesh will result in an earlier and more violent breakup, which thus will produce more vortical structures near the small liquid pieces. Up to the maximum simulation time in the present study, the liquid jet is in a transient process, toward being fully atomized. Therefore, the results here are insufficient to estimate the turbulence kinetic energy dissipation and the Kolmogorov length scale. Nevertheless as shown in Fig. 4, the simulation with the finest mesh is quite effective in capturing the small vortices generated.

\section{Effect of fuel viscosity to atomization}

From Fig. 3 it can also be observed that under the same injection condition, the biodiesel jet (B3) tends to produce thicker filaments and fewer droplets than the standard diesel (D3). The higher viscosity of the biodiesel is believed to be responsible for the discrepancy.

A more detailed comparison between the biodiesel and diesel atomizing jets is shown in Fig. 5. The two jets shown here correspond to the injection condition 3, i.e., $R_{i n j}=65 \mu \mathrm{m}$ and $U_{i n j}=50 \mathrm{~m} / \mathrm{s}$, see Tables 1 and 2 . While $\mathrm{We}_{l}, \mathrm{Re}_{g}$, and $r$ for the two cases are similar, $\operatorname{Re}_{l}=1450$ for the biodiesel is $43 \%$ lower than that for the diesel, I.e., $\operatorname{Re}_{l}=2550$. Several observations on the difference between the two cases can be made here. First, it is observed 
(a) $B 3, R_{\text {inj }} / \Delta_{\min }=21$

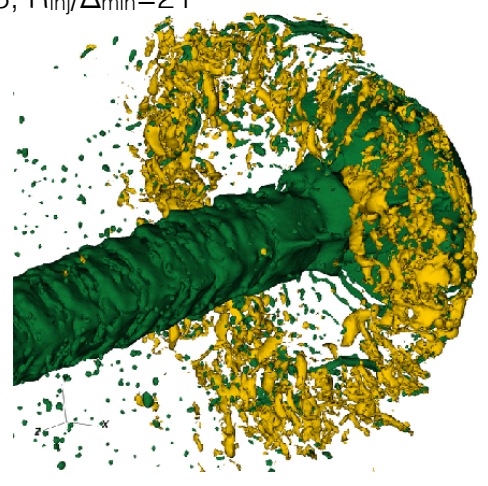

(b) B3, Rinj/ $\Delta_{\min }=43$

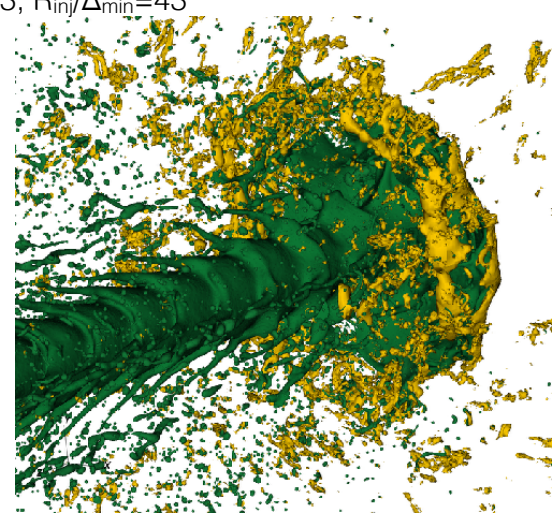

(c) $B 3, R_{\text {in }} / \Delta_{\min }=85$

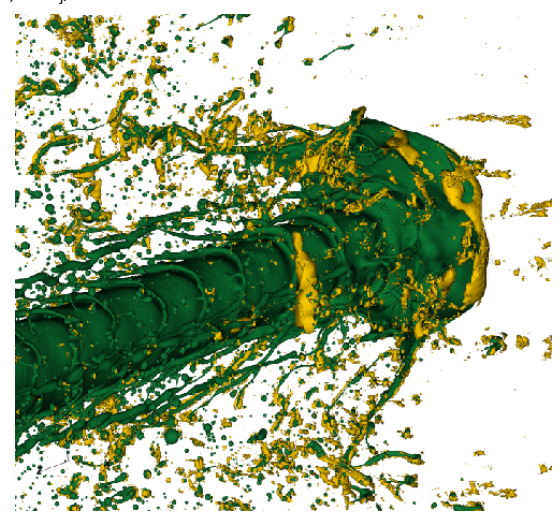

(d) $D 3, R_{\text {inj }} / \Delta_{\min }=21$

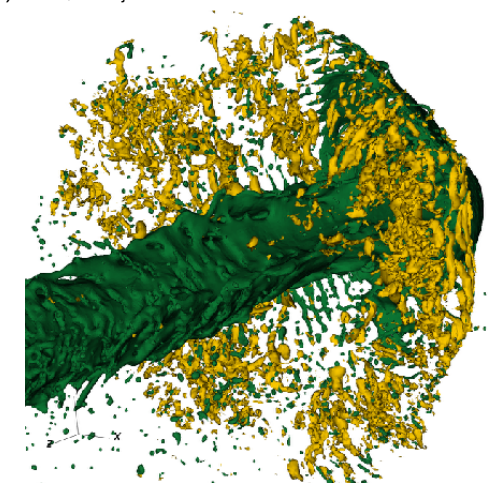

(e) D3, $R_{\text {in }} / \Delta_{\min }=43$

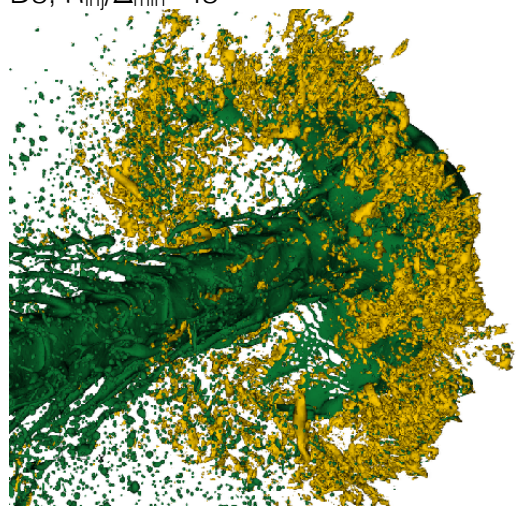

(f) $\mathrm{D} 3, \mathrm{R}_{\mathrm{in} j} / \Delta_{\min }=85$

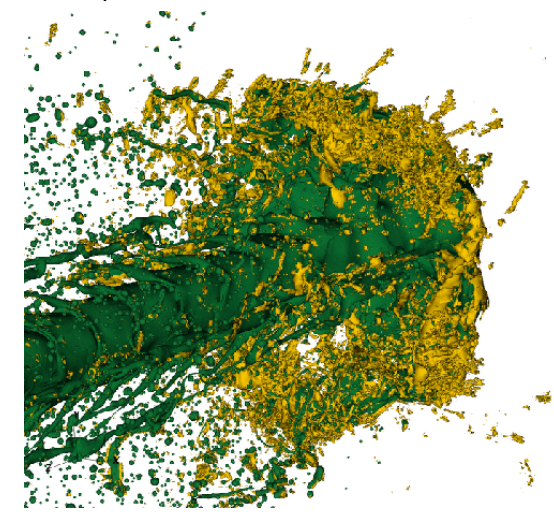

Figure 4. Snapshots of the vortical structures near heads of the biodiesel and diesel jets (B3 and D3) for different mesh resolutions: $R_{i n j} / \Delta_{\min }=21,43$, and 85 . 
that the holes in the interfacial waves on the bulk liquid column appear later for the biodiesel than the diesel. Holes are first observed on the fourth wave (counting from the left) for the diesel jet; while similar holes are not seen until the fifth wave for the biodiesel. Second, the ligaments formed in the biodiesel jet are thicker and longer for the biodiesel. The biodiesel ligaments also seem to last for longer before they break into droplets, see the second row of Fig. 5, indicating that the higher viscosity of the biodiesel is effective in damping small scale instability and delaying ligament breakup. Third, the lateral radius of the mushroom head is smaller for the biodiesel and the roll-up sheets sustain longer and less holes are observed. Compared to the diesel analog, much more ligaments attaching to the edge of the mushroom head are observed for the biodiesel. These ligaments are also longer and thicker. As a result of the delay of sheet and ligament breakups, much less droplets are formed behind the mushroom head for the biodiesel than the diesel.
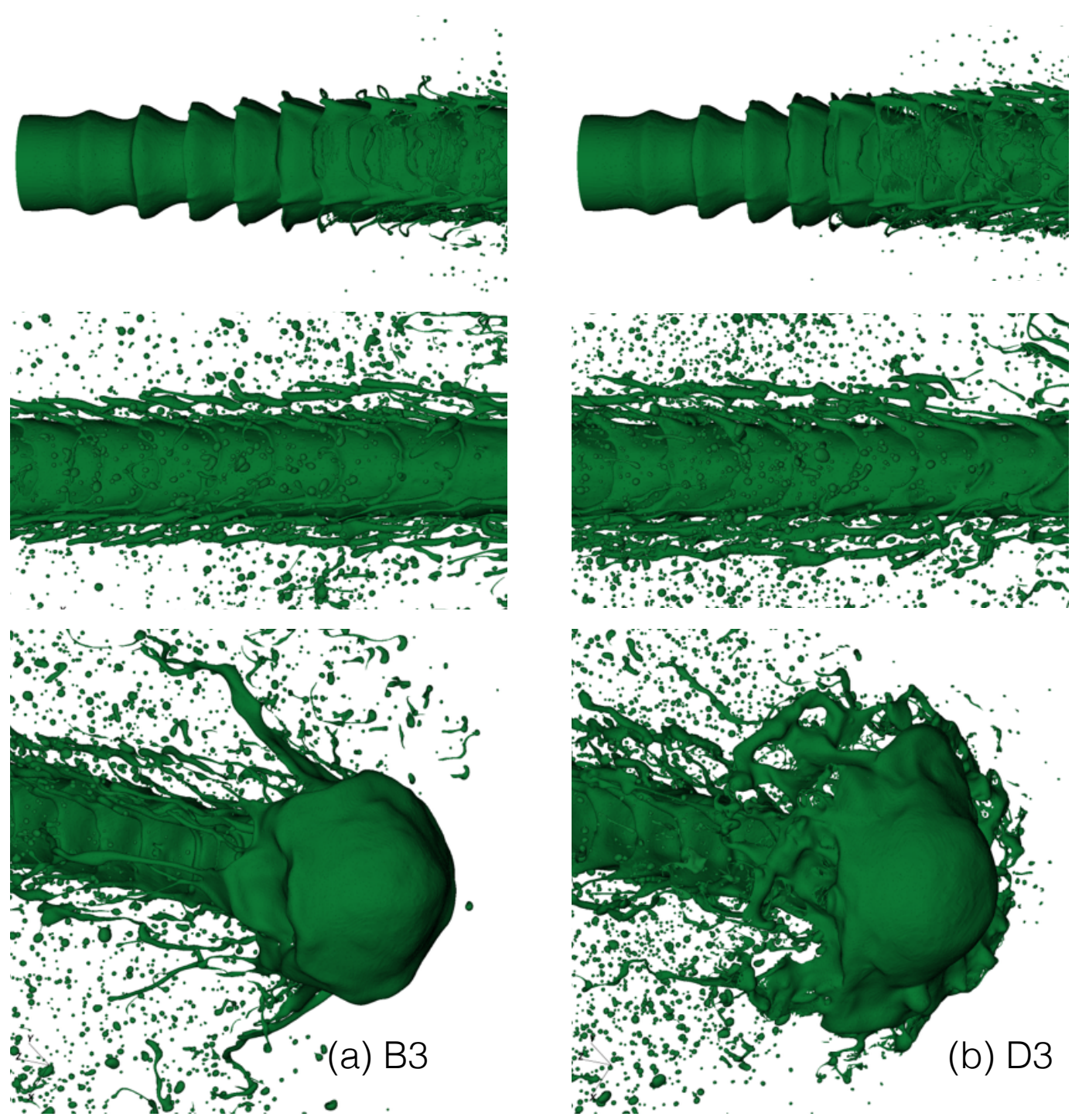

Figure 5. Comparison of biodiesel and diesel jets under identical injection conditions $\left(R_{i n j}=65 \mu \mathrm{m}\right.$ and $\left.U_{i n j}=50 \mathrm{~m} / \mathrm{s}\right)$.

\section{Droplet size distribution}

The statistics of the droplets formed are also investigated since which is an important to many atomization applications. Figure 6 shows the size distribution of droplet number $N_{p}$ for cases B3 and D3 at $t U_{i n j} / R_{i n j}=47$. The droplet diameter is calculated as $d_{v}=\left(6 V_{p} / \pi\right)^{1 / 3}$, where $V_{p}$ is the droplet volume and which is in turn computed by tagging the volume-fraction function and identifying the connected liquid structures. The histogram plots shown in Figs. 6(a)-(b) are for B3 and different mesh resolutions varying from $R_{i n j} / \Delta_{\min }=21$ to 85 . The bin width is 0.75 $\mu \mathrm{m}$, which is about the minimum cell size of the fine mesh. It is shown that more small droplets are produced when the mesh is refined. However, as the droplets with diameter similar to the cell size are affected by the numerical error (such as fake breakup due to lack of mesh resolution) and thus the accuracy of results for these small droplets are less trustworthy. If these small droplets $\left(d_{v}<3 \mu \mathrm{m}\right)$ are ignored, then it is found that the intermediate and fine meshes actually yield similar results, see Figs. $6(d)$ and $(e)$, which means that we have obtained converged results 
for the droplet size distribution with $R_{i n j} / \Delta_{\min }=85$.

For $d_{v}>3 \mu \mathrm{m}, N_{p}$ decreases with $d_{v}$ and the profile matches well with the exponential function $\exp \left(-d_{v} / \lambda\right)$ for both cases B3 and D3, where $\lambda$ is a length scale characterizing the shape of PDF. When $\lambda$ decreases, the decay of $N_{d}$ with increasing $d_{v}$ is faster, indicating the droplet size distribution inclines more toward smaller droplets, or in other words the liquid jet is "more seriously atomized". It is fitted by the data for the finest mesh that, $\lambda$ is about 2.7 $\mu \mathrm{m}$ for the biodiesel and about $2.2 \mu \mathrm{m}$ for the diesel, see Fig. 6(f). The larger $\lambda$ for the biodiesel is consistent with the previous observation of the less "intense" atomization of biodiesel due to its higher viscosity.

Under the same injection conditions, significantly fewer smaller droplets are formed in the biodiesel jet. Note that $N_{d}$ in Fig. 6 is plotted in the logarithmic scale, the difference of droplet number between the two fuels may look small, but is indeed very large. As an example, for droplet diameter between 4.5 and $5.25 \mu \mathrm{m}, N_{p}=517$ for B3 and $N_{p}=897$ for D3. Namely, the biodiesel jet produces $42 \%$ less droplets for this size range $4.5-5.25 \mu \mathrm{m}$ compared to the standard diesel fuel.
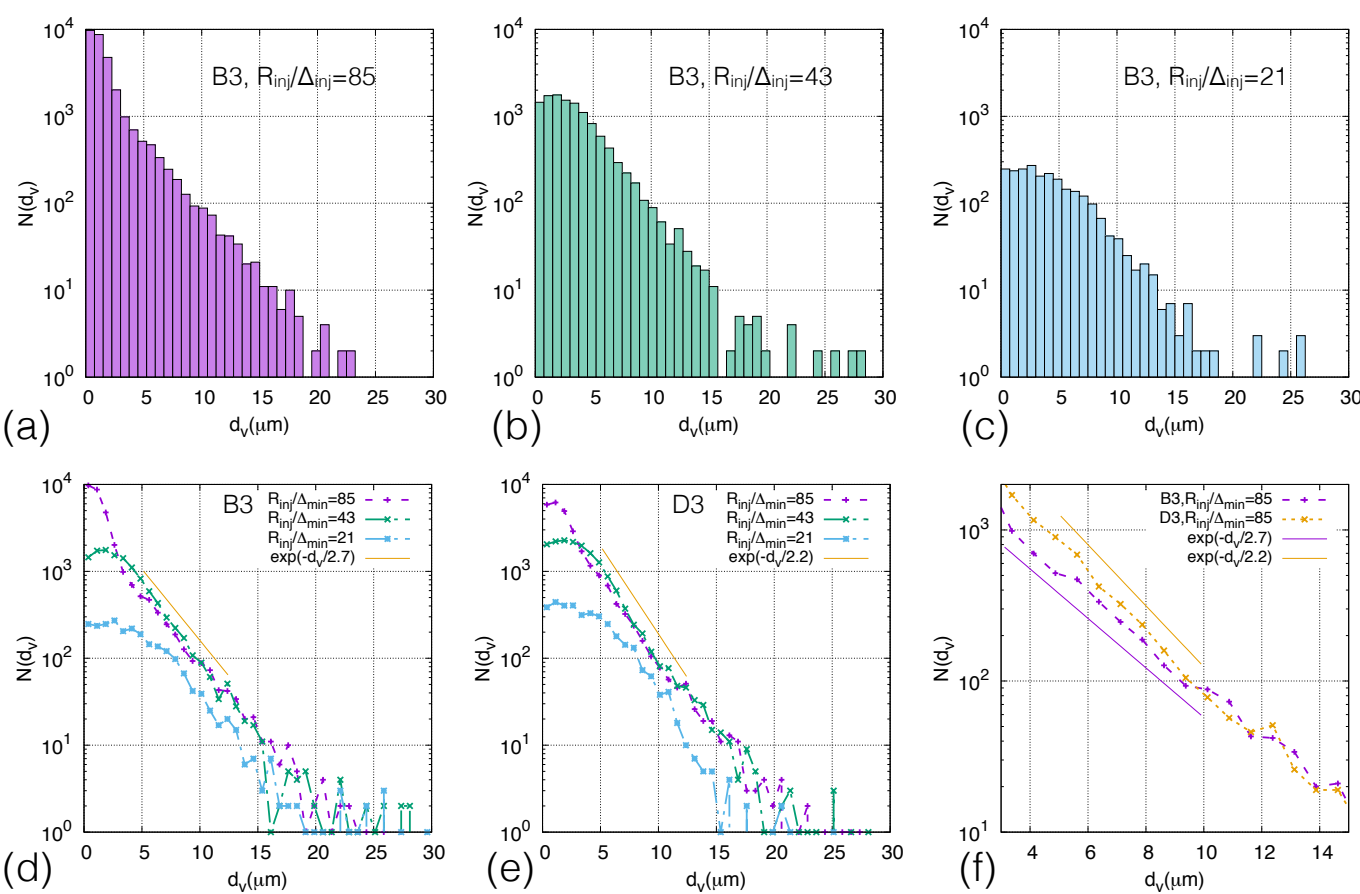

Figure 6. Droplet size distribution for B3 and D3 at $t U_{i n j} / R_{i n j}=47$. (a)-(c) are for B3 with different $\Delta_{\min }$.

\section{Effect of injection conditions}

Finally, we examine the effect of the fuel viscosity on atomization for different injection conditions. Compared to $\mathrm{B} 3 / \mathrm{D} 3$, the injection velocity for B1/D1 is higher and as a result $\mathrm{Re}_{l}$ and $\mathrm{We}_{l}$ are larger, see Table 2. In contrast, the jet radius $R_{i n j}$ for $\mathrm{B} 2 / \mathrm{D} 2$ is smaller than $\mathrm{B} 3 / \mathrm{D} 3$, resulting smaller $\mathrm{Re}_{l}$ and $\mathrm{We}_{l}$. The size distribution of droplet number for these three injection conditions are shown in Fig. 7. The mesh resolution is the same for all cases shown here, i.e., $\Delta_{\min }=R_{\text {inj }} / 43$. When $\mathrm{Re}_{l}$ and $\mathrm{We}_{l}$ decrease in the order of B1/D1, B3/D3, and B2/D2, the number of droplets generated decreases accordingly. The discrepancy of $N_{p}$ between the biodiesel and diesel jets becomes more profound when $\mathrm{Re}_{l}$ and $\mathrm{We}_{l}$ decrease. For $d_{p}>10 \mu \mathrm{m}$, the difference of $N_{p}$ between D1 and B1 is barely seen. In contrast, the difference between D2 and B2 is much more profound. This seems to indicate that for sufficiently large $\mathrm{Re}_{l}$ and $\mathrm{We}_{l}$, the liquid jet is atomized violently and the liquid viscosity has little effect on the size distribution of formed droplets. For that case, the difference of the droplet statistics between the diesel and the biodiesel is small. On the other hand, when $\mathrm{Re}_{l}$ and $\mathrm{We}_{l}$ are small, the atomization of the liquid jet and the resulting droplet statistics are more sensitive to the liquid viscosity. Then replacing the standard diesel with the biodiesel in fuel injection can introduce a significant impact to the droplet size distribution.

\section{Conclusions}

Three dimensional direct numerical simulations are performed in the present work to investigate the effect of fuel properties on the atomization characteristics. Atomizing liquid jets for both the standard diesel and the biodiesel under different injection conditions are considered. Grid-dependent study is conducted, showing that the present mesh resolution is sufficient to capture droplets larger than $6 \mu \mathrm{m}$. Generally, a lower number of droplets are formed in a biodiesel jet compared to those in the diesel jet under the same injection condition due to the higher viscosity of the biodiesel. The size distribution of droplet number is also presented. The decay of droplet number with droplet diameter follows the exponential function $\exp \left(-d_{v} / \lambda\right)$ for both the biodiesel and diesel fuels. The $\lambda$ is a length scale that characterizes the shape of distribution function and $\lambda$ for the biodiesel is larger than that for the diesel under the 
(a)

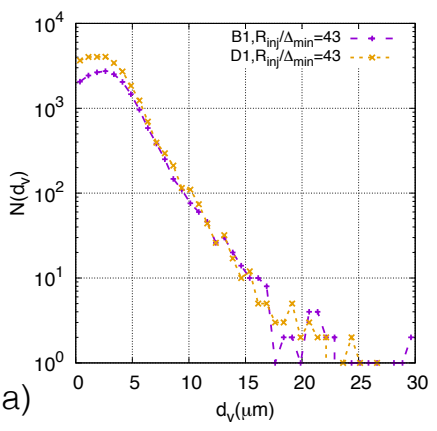

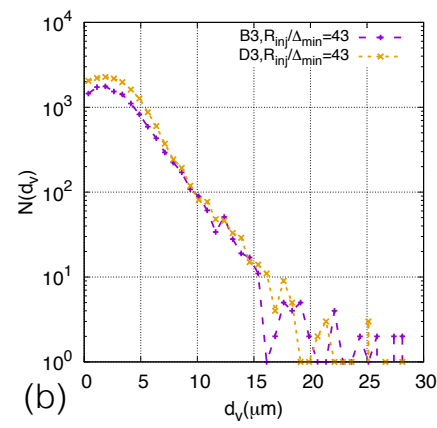

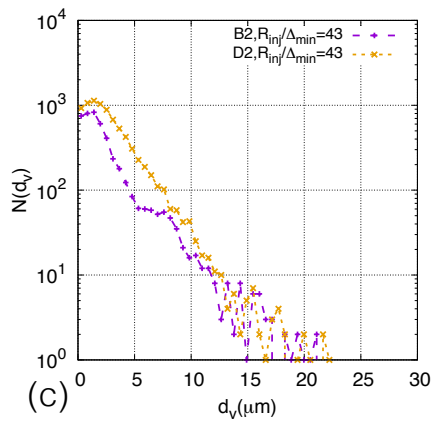

Figure 7. Droplet size distribution for B1-3 and D1-3 at $t U_{i n j} / R_{i n j}=47$ for $\Delta_{\min }=R_{i n j} / 43$.

same injection condition. Finally, the effect of injection condition is also studied, showing that the difference of the droplet statistics between the biodiesel and the diesel decreases with the liquid jet Reynolds and Weber numbers.

\section{Acknowledgements}

This project has been supported by the ANR MODEMI project (ANR-11-MONU-0011) program. This work was granted access to the HPC resources of TGCC-CURIE under the allocations x20152b7325, x20162b7325 and t20162b7760 made by GENCl. We would also acknowledge support from the Academic and Research Computing Services at the Baylor University.

\section{References}

[1] Popinet S., The Basilisk code, Available from http://basilisk.fr/.

[2] Popinet, S., 2009, J. Comput. Phys., 228, pp. 5838-5866.

[3] Battistoni, M. and Grimaldi, C. N., 2012, Appl. Energy, 97, pp. 656-666.

[4] Müller, T., Sänger, A., Habisreuther, P., Jakobs, T., Trimis, D., Kolb, T. and Zarzalis, N., 2016, Int. J. Multiphase Flow, 87, pp. 212-228.

[5] Shinjo, J. and Umemura, A., 2010, Int. J. Multiphase Flow, 36, pp. 513-532.

[6] Ling, Y. and Fuster, D. and Zaleski, S. and Tryggvasson, G., 2017, Phys. Rev. Fluids, 2, pp. 014005.

[7] Childs H., Brugger, E., Whitlock, B., Meredith J., Ahern S., Pugmire D., Biagas K., Miller, M., Harrison, C., Weber, G. H., Krishnan, Hari, Fogal, T. , Sanderson, A. , Garth, C., Bethel, E. W., Camp, D. , Rübel, O., Durant,M., Favre, J. M. and Navrátil P., 2012, Vislt: An End-User Tool For Visualizing and Analyzing Very Large Data, High Performance Visualization-Enabling Extreme-Scale Scientific Insight, pp. 357-372.

[8] Jeong, J. and Hussain, F., 1995, J. Fluid Mech., 285, pp. 69-94. 


\title{
Experimental Characterization of Spray generated by a Rotary Atomizer Wheel
}

\author{
Maximilian Kuhnhenn*1, Max Frederik Luh" ${ }^{1}$, Tórstein V. Joensen², Ilia V. Roisman ${ }^{1}$, Cameron \\ Tropea $^{1}$ \\ ${ }^{1}$ Institute for Fluid Mechanics and Aerodynamics, Technische Universität Darmstadt, \\ Darmstadt, Germany \\ ${ }^{2}$ GEA Process Engineering A/S, Soeborg, Denmark \\ ${ }^{\star}$ Corresponding author: Kuhnhenn@sla.tu-darmstadt.de
}

\begin{abstract}
Rotary atomizers are widely used for spray drying processes to produce powders in various industrial applications. The atomization regime and therefore the droplet size distribution have a substantial impact on the process outcome. Depending on the intended use of the dried powder particles, various droplet size distributions are desired, which should be adjustable by the main operating parameters (e.g. mass flow rate and wheel speed). In this study the spray of a rotary atomizer wheel, operated under normal ambient conditions, is characterized for different mass flow rates, wheel speeds and viscosities with the help of a phase Doppler measurement system. Visual investigations of the spray with a high resolution camera system help to get a better understanding of the atomization process, as well as the thickness of the liquid jets exiting the atomizer.
\end{abstract}

\section{Keywords}

rotary atomizer, spray drying, phase Doppler analyser

\section{Introduction}

The intended use of the dried powder obtained with a rotary atomizer in a spray drying process [1] or for flue gas cleaning [2] determines the desired size distribution of the powder particles, their morphology and porosity. It is important to understand the influencing parameters on the droplet size distribution for a given geometry of an atomizer to be able to change them to achieve a desired droplet size distribution.

In rotary atomizers the liquid is first supplied to the internal atomizer reservoir, flows from there into several (ceramic-) inserts (mainly driven by the centrifugal force due to the atomizer rotation) and is then ejected into the surrounding air. The jets of the processed liquid then breakup and atomize into drops with a certain size distribution. Besides the spray drying industry $[3,4,5]$ rotary atomizers $[6,7]$ and ligament based atomization is also used in numerous other applications, e.g. painting, coating, and generation of agricultural sprays (spreading of pesticides) [8]. The primary atomization process for laminar operating rotary atomizers has already been expressed in terms of dimensionless numbers [9, 10], but such atomizers operate at much lower rotational speeds and mass flow rates compared to the atomizers investigated in this study.

\section{Experimental Method}

The experiments in this study are conducted with two different experimental setups, as shown in Fig. 1 . In both setups a rotary atomizer wheel, a liquid supply system and a spray trap, to catch the atomized liquid, are used. The atomizer is operated at $10,5001 / \mathrm{min}$ to $29,0001 / \mathrm{min}$ and the feed rate is varied between $50 \mathrm{~kg} / \mathrm{h}$ to $450 \mathrm{~kg} / \mathrm{h}$. The processed liquids are water (viscosity $\eta=1 \mathrm{mPas}$ ) and two different aqueous glycerol water solutions (viscosity $\eta=20 \mathrm{mPa}$ s and $\eta=50 \mathrm{mPa}$ ). The first experimental setup (cf. Fig. 1(a)) comprises a high resolution imaging system to observe the atomization process and to (optically) measure the thickness of the liquid jet exiting the atomizer wheel. For further investigations of the atomization a phase Doppler (PD) system is used, as shown in Fig. $1(b)$

The high resolution imaging system consists of a pco-edge 5.5 camera (resolution: $2560 \mathrm{px} \times 2160 \mathrm{px}, 30 \mathrm{~Hz}$ ), a Nikon AF Micro Nikkor lens with $105 \mathrm{~mm}$ focal length, a DualPower 200-15 laser system with 6 ns pulse length and a ShadowStrobe optics to illuminate the diffusing plate by using a mirror. The laser is capable of generating laser pulses at $15 \mathrm{~Hz}$ per cavity and therefore only every 6th to 16th revolution can be captured. Nevertheless, this low frequency is not an obstacle for the collection of a large number of images to achieve statistical significance. The camera is operated in global shutter mode and, due to the short illumination time (6 ns), no motion blur is observed, although the speed of the wheel can reach up to $150 \mathrm{~m} / \mathrm{s}$. The field of view (FOV) is $17.5 \mathrm{~mm} \times 14.8 \mathrm{~mm}$, resulting in a resolution of $6.85 \mu \mathrm{m} / \mathrm{px}$.

The PD system (cf. Fig. 1(b)) is operated in the dual-mode configuration to measure droplet size and velocity distributions of the resulting spray [11]. By adjusting the orientation of transmitting and receiving probe the tangential and radial velocity of the spray exiting the atomizer wheel can be measured. The gravitational influence and the resulting velocity component is neglected, as the spray is oriented horizontally. The PD system can be moved laterally in a range of $30 \mathrm{~mm}$ to $190 \mathrm{~mm}$ distance to the wheel, to be able to capture different stages of the atomization process. The main settings of the system, used to acquire the data presented in this study, are summarized in Table 1. As the atomizer wheel has 4 inserts that pass by the measurement volume of the PD system, the measured spray is 
(a)

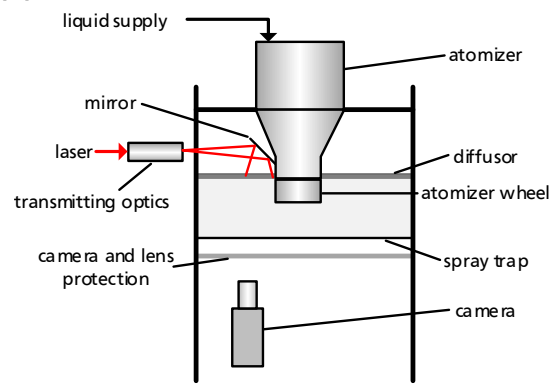

(b)

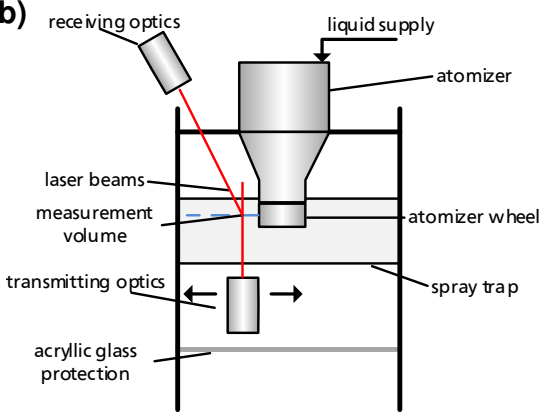

Figure 1. (a) Schematic of experimental setup including: liquid supply system, atomizer and high-resolution imaging system. (b) Alternative configuration with the laser spray diagnostic system (PD) to measure droplet size and velocity. The PD system can be moved laterally using a traversing system to perform measurements at different positions.

highly unsteady. An encoder signal from the atomizer (one signal per revolution) is used as a reference signal for the PD measurements. The detected droplets are assigned to the reference signal in time, which makes it possible to conduct angular resolved measurements (phase averaged with $0.5^{\circ}$ resolution).

Table 1. Settings of the phase Doppler measurement system

\begin{tabular}{lr}
\hline Parameter & Setting \\
\hline Laser Power & $60 \mathrm{~mW}$ \\
Wavelength 1 & $488 \mathrm{~nm}$ \\
Wavelength 2 & $514.5 \mathrm{~nm}$ \\
Transmitter focal length & $600 \mathrm{~mm}$ \\
Receiver focal length & $500 \mathrm{~mm}$ \\
Scattering angle & $30^{\circ}$ \\
Refractive index water & 1.3330 \\
Refractive index 20 mPa s & 1.4255 \\
Refractive index 50 mPa s & 1.4401 \\
Aperture mask & Mask A \\
Maximum particle diameter & $215.2 \mu \mathrm{m}$ \\
Phase validation ratio & $15 \%$ \\
Slit width & $100 \mu \mathrm{m}$
\end{tabular}

\section{Mechanisms of observed Atomization}

The atomization is observed with the high resolution imaging system (cf. Fig. 1(a)) and shown exemplary for one operating point and different viscosities in Fig. 2. The angular velocity and feed rate are kept constant from Fig. 2(a) to 2(c). The processed liquid exits the atomizer wheel through the ceramic insert in a jet type flow, where most of the liquid is accumulating on the trailing edge. This effect is caused by the Coriolis force acting on the liquid. The Coriolis force is acting perpendicular to the main (radial) jet velocity and, by considering the vector of rotation, the liquid is forced towards the trailing edge of the insert (cf. Fig. 2). In addition to the main jet at the trailing edge, the liquid exits the insert in an annular film flow, wetting the hole periphery of the insert. At a viscosity of $\eta=1 \mathrm{mPa}$ s the thin annular film breaks up immediately after the exit (cf. Fig. 2(a)), whereas an increasing viscosity provokes small fingers, protruding from the thin annular film (cf. Fig. 2(b)). These small fingers get longer with a further increase in viscosity from $\eta=20 \mathrm{mPa}$ s to $50 \mathrm{mPa}$ s, as shown in Fig. 2(c). Increasing viscosity leads to a higher mass flow through the thin annular film and a decreasing mass flow through the main jet, while the total mass flow remains constant. Based on these initial photographic images of the atomization process, it is evident that the atomization changes with different viscosities.

\section{Airflow characterization}

To better understand the mechanisms of atomization the velocity field around the wheel has to be characterized in detail. As the wheel is rotating with a high angular velocity and the ambient air in the far field is quiescent, a boundary layer develops. The tangential velocity of the wheel can be calculated by

$$
\hat{u}_{t}=\omega R_{a} .
$$

The tangential velocity of the wheel varies from $55 \mathrm{~m} / \mathrm{s}$ to $150 \mathrm{~m} / \mathrm{s}$, depending on the angular velocity of the wheel. As the wheel has radial openings and is not encapsulated from the surroundings, air is accelerated through the radial openings, based on the centrifugal force (even without water). Pipe friction causes a slight reduction of the 

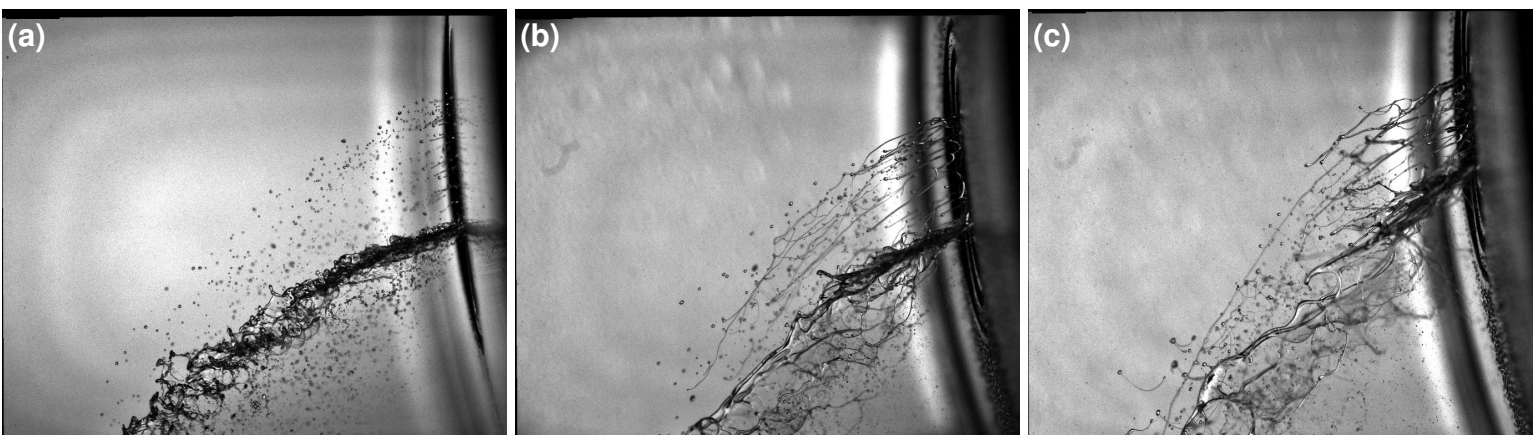

Figure 2. Photographic images of the liquid exiting the atomizer in a jet shape. The speed of the wheel is $10,5001 / \mathrm{min}$ and the feed rate is $100 \mathrm{~kg} / \mathrm{h}$. (a) Viscosity $\eta=1 \mathrm{mPa}$, (b) Viscosity $\eta=20 \mathrm{mPa} \mathrm{s}$, (c) Viscosity $\eta=50 \mathrm{mPa}$ s

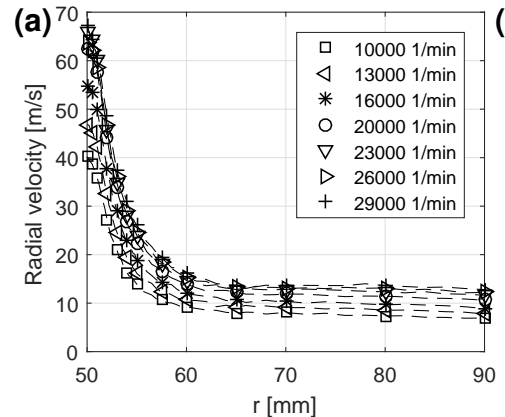

Figure 3. Results of hot-wire measurements.
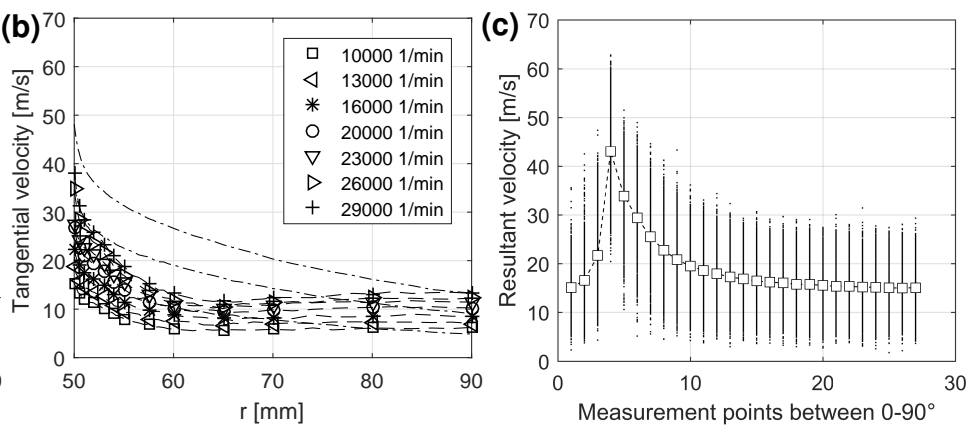
(a) Radial air velocity. (b) Tangenti
with scatter band for $10,5001 / \mathrm{min}$.

(c) Angular resolved air velocity

resulting air velocity. The radial air velocity can be estimated as:

$$
u_{r}=\sqrt{2 \omega^{2} R_{a} l-\frac{\lambda u_{r}^{2} l}{d}}
$$

This boundary-layer flow and the radial air velocity have a significant impact on the atomization of the resulting spray, which is why further knowledge of the airflow is required. Hot-wire measurements are conducted with a single wire boundary-layer probe (55P14), to capture the evolution of the boundary layer of the pure air flow. The experiments are conducted with a StreamLine Pro CTA System from Dantec Dynamics, $20 \mathrm{kHz}$ sampling rate, a 15 point calibration between $2 \mathrm{~m} / \mathrm{s}$ to $60 \mathrm{~m} / \mathrm{s}$ and 84 measurement positions from $0.5 \mathrm{~mm}$ to $101 \mathrm{~mm}$. The measured air velocity is shown in Figure 3, already split into radial (Fig. 3(a)) and tangential (Fig. 3(b)) velocity components. The decomposition of the raw hot-wire signal was done with the help of Figure 3(c), showing the mean trend of 50,000 acquired samples for one distance and angular velocity, split into orthogonal components, as the flow is periodic due to the four inserts. Figure 3(c) shows the resultant air velocity: the tangential air velocity is represented by the lower part and the peak shows the vector addition of the radial and tangential velocities. Figure 3(a) shows the strong deceleration of the radial air velocity within $10 \mathrm{~mm}$ from the wheel outer surface. Different angular velocities have a high impact on the exit velocity, but with increasing distance this impact rapidly reduces. The decay of the tangential velocity is shown in Figure $3(\mathrm{~b})$. Additional measurements are conducted $10 \mathrm{~mm}$ below the inserts in order to show the influence of the airflow coming out of the inserts, represented by the dashed-dotted lines. In contrast to the tangential velocity in front of the inserts, these results illustrate a different behaviour - the airflow prevents the proper evolution of a boundary layer. In the spray drying process, the airflow around the wheel is influenced by the atomized liquid, but nevertheless these measurements demonstrate the thinness of the boundary layer around the wheel. Even $0.5 \mathrm{~mm}$ away from the wheel the tangential air velocity is far away from the tangential velocity of the wheel itself (cf. Eq. (1)). Therefore a large relative velocity between droplets and the surrounding air is established, leading to a strong deceleration of the atomized liquid.

Figure 4 shows the comparison between the experimentally (CTA) and analytically obtained air velocities (Eq. (1) and (2)). Obviously the theoretical prediction of the tangential velocity is much higher compared to the experiments, showing that already $0.5 \mathrm{~mm}$ away from the wheel the air is strongly decelerated. The deviation of the radial air velocity is much smaller, but increasing with the wheel speed. While theoretical prediction and experiments of $u_{\mathrm{r}}$ match quite well at $10,5001 / \mathrm{min}$, they deviate from each other up to a factor of two at $29,0001 / \mathrm{min}$. This effect can be explained by the insufficient temporal resolution of the experimentally obtained radial insert (air) velocity $u_{\mathrm{r}}$. For example at $29,0001 / \mathrm{min}$ only 10 measurement points can be acquired for $90^{\circ}$, due to the $20 \mathrm{kHz}$ sampling rate (cf. Fig. 3(c)). Furthermore this can lead to an underestimation of the actual air velocity. 


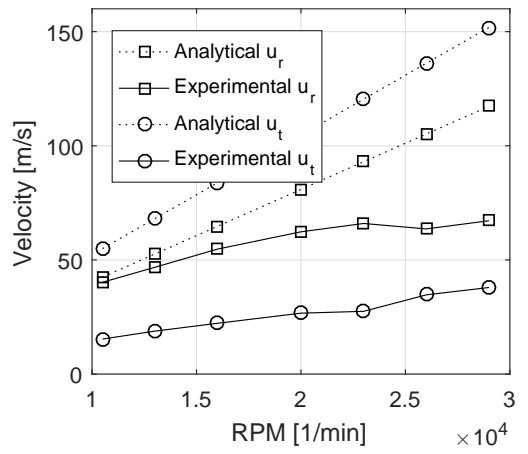

Figure 4. Comparison of analytical and experimental values for radial $\left(u_{\mathrm{r}}\right)$ and tangential $\left(u_{\mathrm{t}}\right)$ air velocities.

\section{Spray characterization}

To characterize the atomization process and the resulting spray, PD measurements are conducted. As the PD system in dual-mode configuration can only measure almost spherical droplets and not a liquid jet, the measurements are conducted beyond the primary breakup zone. From a point $30 \mathrm{~mm}$ radially from the wheel, data is acquired every further $20 \mathrm{~mm}$. Either 50,000 samples or at least $60 \mathrm{~s}$ of measurement time are recorded. Due to the inhomogeneous spray and the liquid jets in the vicinity of the wheel, the validation is poorer compared to the validation in the homogeneous spray, as shown in Fig. 5. With increasing distance, the validation increases and reaches up to $90 \%$. Higher viscosities hinder the measurements in the vicinity of the wheel, as longer ligaments are produced (cf. Fig. 2(c)). Furthermore, it is difficult to measure operating points with $50 \mathrm{~kg} / \mathrm{h}$ mass flow rate, due to the distribution of low volume of atomized liquid in the observation zone and the resulting low data rate combined with high noise levels. The spherical validation (cf. Fig. $5(\mathrm{~b})$ ) is above $70 \%$ in all cases.
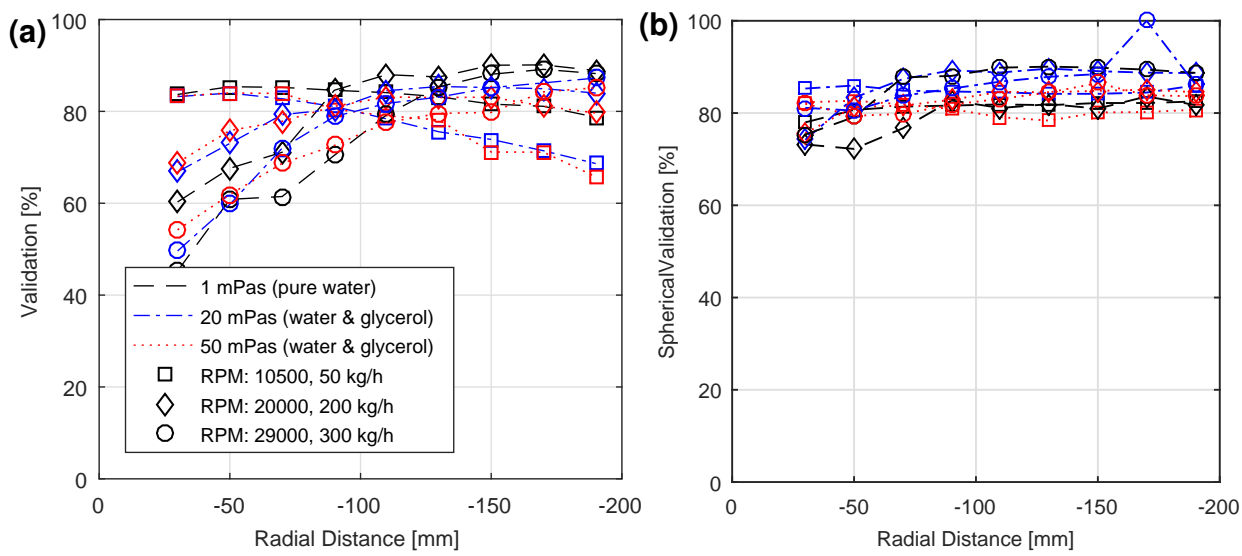

Figure 5. Validation of the conducted PD measurements, depending on the measurement position. (a) Different mass flow rates, viscosities and positions are compared. (b) In all cases the spherical validation is above $70 \%$.

Figure 6 shows the angular resolved velocity components (tangential and radial) of the spray at certain distances from the wheel. Beginning in the vicinity of the wheel, four distinct velocity peaks are visible, reflecting the four inserts of the wheel. As expected, the velocity components decrease with an increase in distance to the wheel (Fig. 6 left to right) due to aerodynamic drag. The tangential velocity component flattens out faster than the radial component, as the initial tangential velocity is lower compared to the radial (also compare to CTA measurements in Fig. 3). As the initial tangential velocity $\hat{u}_{t}$ of the liquid at the exit of the wheel has to match the speed of the wheel, we observe a drastic decrease in tangential velocity, as already seen before. This again shows, that the boundary layer around the rotating wheel must be rather thin.

Figure 7 illustrates the corresponding angular resolved droplet sizes for different radial distances. The mean diameter per angle bin is highlighted by the white markers. This plot indicates, that one mean diameter per measurement point drastically underestimates the droplet size of the jets exiting the wheel. Obviously the droplets produced by the four jets are much bigger compared to the annular thin film, and secondary droplets that are already broken up. With increasing distance, the spray becomes more and more homogeneous and the mean diameter per angle bin increases. Combined with the strong velocity size correlation, this is either a sign of coalescence or of ongoing breakup of filaments that are not recognized by the PD system in the vicinity of the wheel.

To illustrate the influence of wheel speed and mass flow rate on the resulting spray, the Sauter mean diameter $D_{32}$ is plotted in Figure 8 for different viscosities at $110 \mathrm{~mm}$ distance. The influence of the mass flow rate on the $D_{32}$ is rather small, compared to the wheel speed. This behaviour is very interesting for the spray drying industry, as 

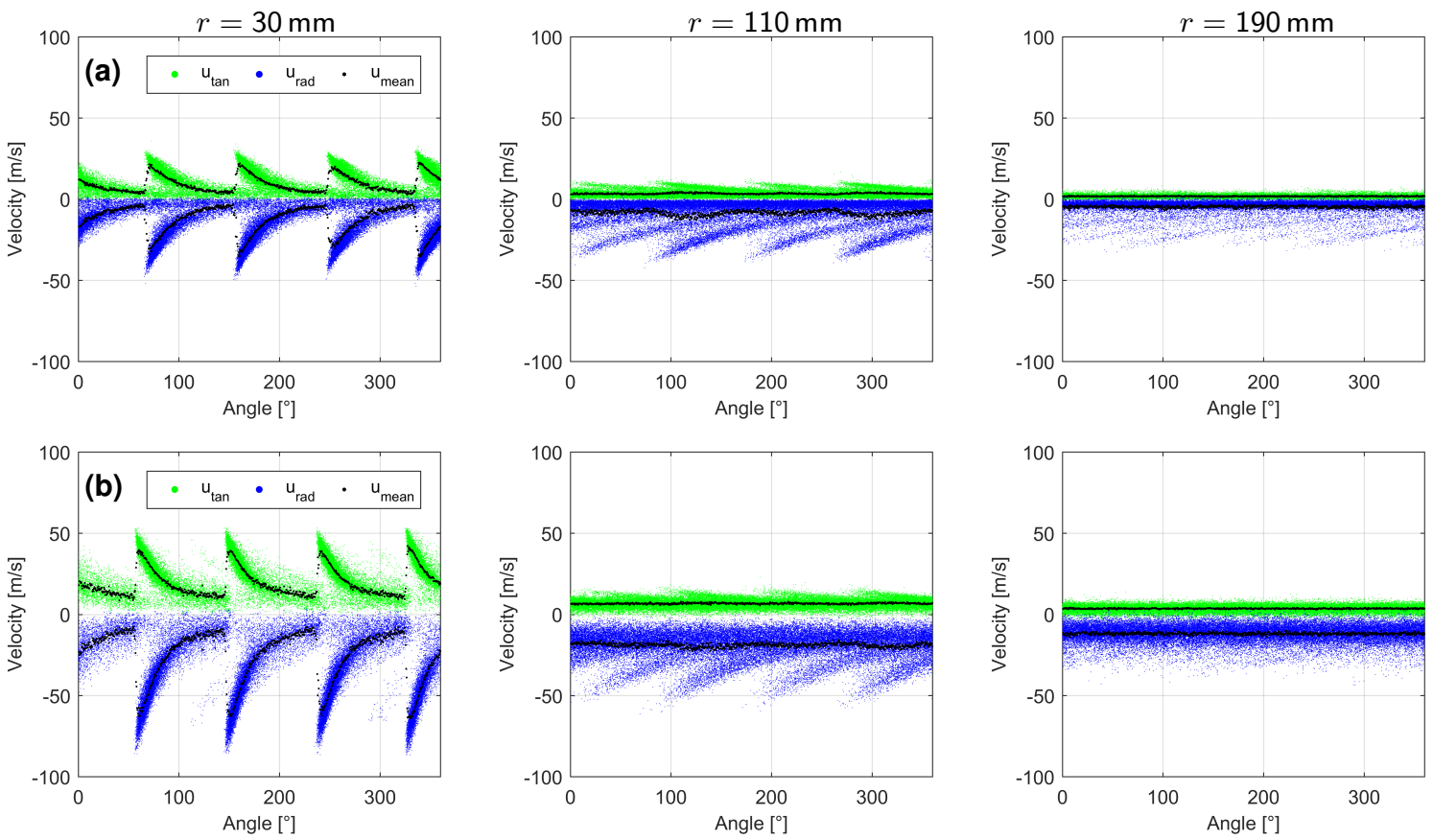

Figure 6. Angular resolved velocity of the resulting spray at different distances to the atomizer exit. The wheel is running with (a) $10,5001 / \mathrm{min}, 50 \mathrm{~kg} / \mathrm{h}$ and $\eta=1 \mathrm{mPa}$; (b) $20,0001 / \mathrm{min}, 200 \mathrm{~kg} / \mathrm{h}$ and $\eta=1 \mathrm{mPa}$ s.
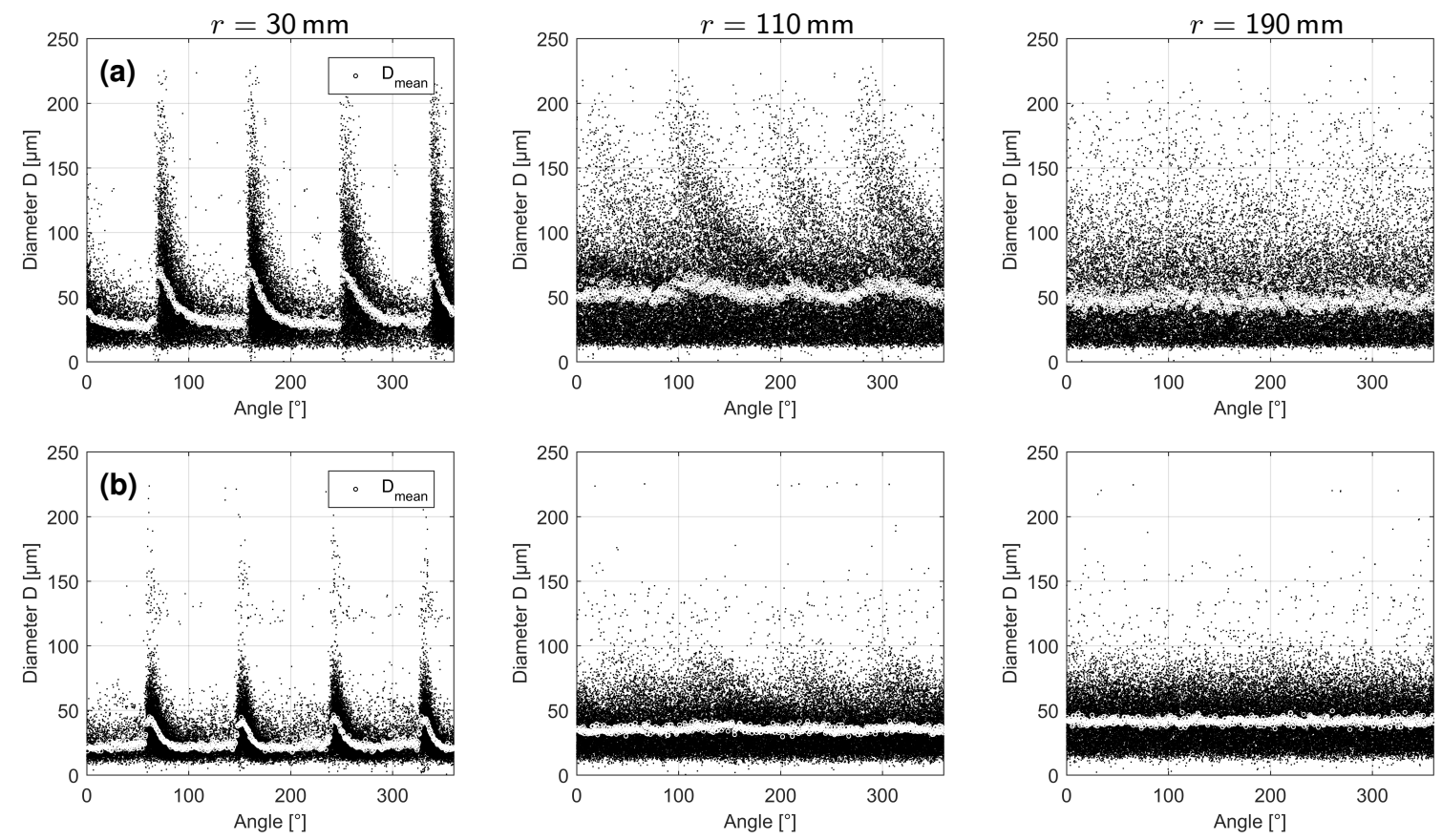

Figure 7. Angular resolved droplet size of the resulting spray at different distances to the atomizer exit. Mean values per angle bin are highlighted by the white markers. The wheel is running with (a) $10,5001 / \mathrm{min}, 50 \mathrm{~kg} / \mathrm{h}$ and $\eta=1 \mathrm{mPa} \mathrm{s}$; (b) $20,0001 / \mathrm{min}$, $200 \mathrm{~kg} / \mathrm{h}$ and $\eta=1 \mathrm{mPa}$. 

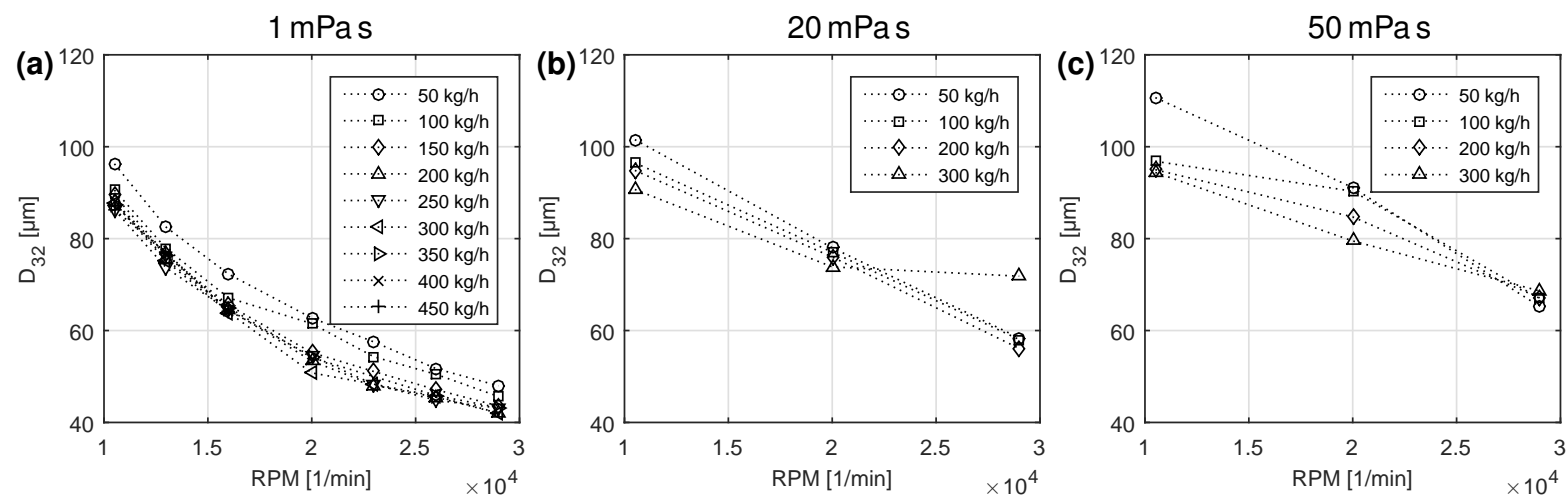

Figure 8. Resulting $D_{32}$ of the spray at a distance of $r=110 \mathrm{~mm}$ to the wheel, where the spray is homogeneous (cf. Figure 7). (a) $\eta=1 \mathrm{mPa}$, (b) $\eta=20 \mathrm{mPas}$, (c) $\eta=50 \mathrm{mPas}$

a change in mass flow rate has a rather small impact on the resulting mean powder size. The main influencing parameter is the speed of the wheel, which can be changed very easily. With increasing viscosity (Fig. 8(a) to 8(c)) the $D_{32}$ is increasing. The negligibly small influence of the mass flow rate still persists.

To further characterize the spray, the width of the volume distribution can be described by the relative span $\Delta$. The relative span $\Delta$ is a dimensionless value for the dispersion of drop sizes, where $\Delta \ll 1$ describes a mono-disperse spray:

$$
\Delta=\frac{D_{0.9}-D_{0.1}}{D_{0.5}}
$$

with the volume mean diameter $D_{0.5} . D_{0.9}$ and $D_{0.1}$ describe the drop size, where $90 \%$ of the drop volume, and $10 \%$ respectively, are contained in droplets of smaller diameters. Figure 9 illustrates the evolution of the relative span $\Delta$ for different wheel speeds (divided into zones of constant speed by the vertical black lines). Within one zone the mass flow rate increases from left to right and several distances are shown for clarity. With increasing wheel speed the relative span $\Delta$ decreases, demonstrating a more uniform droplet size distribution (width of the droplet size distribution histogram). For higher speeds (zones to the right) the relative span $\Delta$ increases with mass flow rate for distances further away, showing a broader distribution. For low mass flow rates and high wheel speeds (e.g. MP 37) the span $\Delta$ is rather broad, showing a change in uniformity with increasing distance - the spray is homogeneous further away. Figure 9 tries to capture several parameters and its influence on the width of the droplet size distribution. It is rather difficult to characterize a spray with only two single numbers $\left(D_{32}\right.$ and $\Delta$ ), but it is evident that the main influencing parameters are the speed of the wheel and the distance from the wheel.

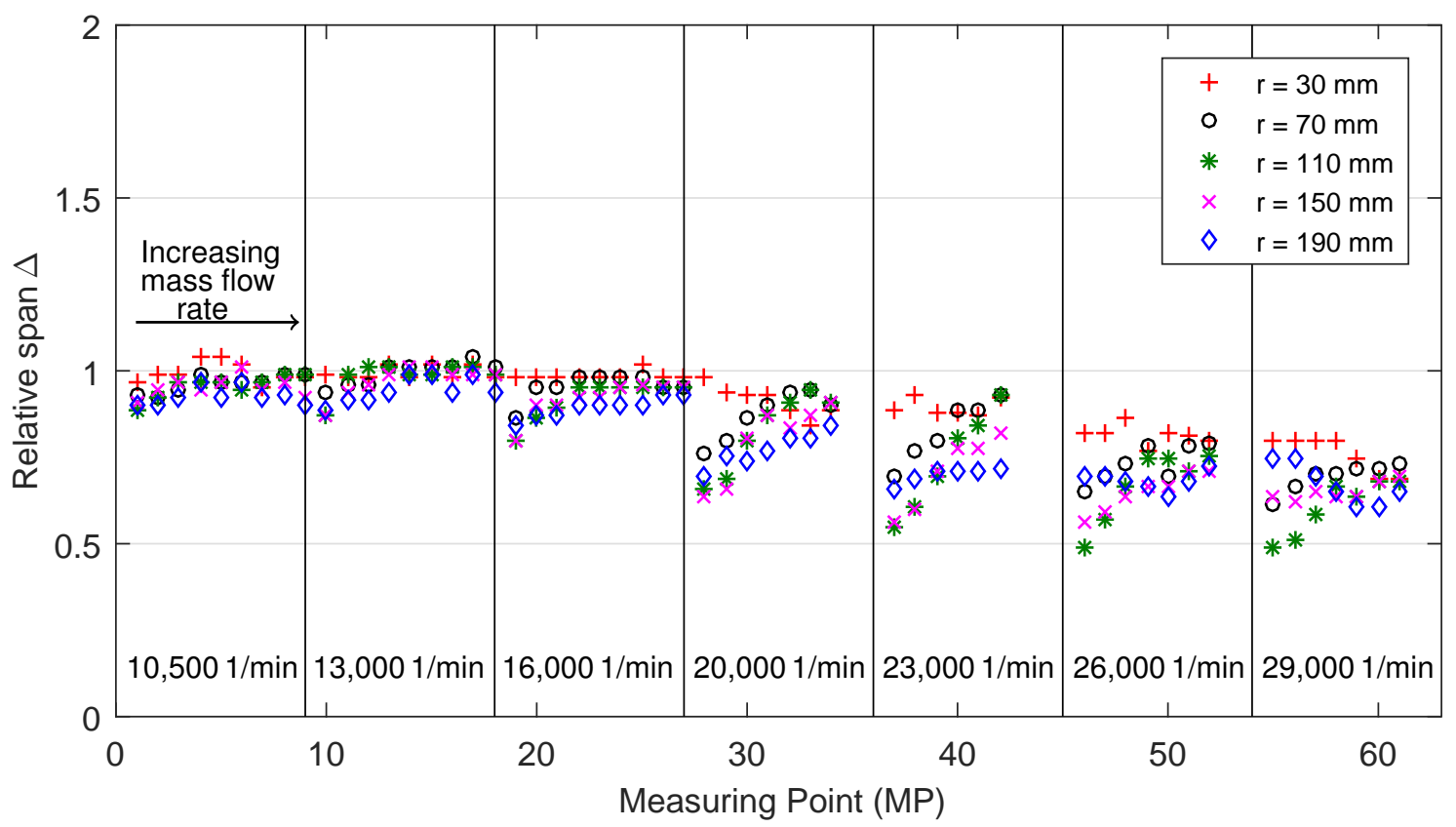

Figure 9. Relative span $\Delta$ (cf. Eq. (3)) for different speeds, mass flow rates and distances to the atomizer exit. The vertical lines divide the diagram into zones of constant speed $(10,5001 / \mathrm{min}$ to $29,0001 / \mathrm{min}$ from left to right). Within one zone the mass flow rate is increasing from $50 \mathrm{~kg} / \mathrm{h}$ to $450 \mathrm{~kg} / \mathrm{h}$ (left to right) in steps of $50 \mathrm{~kg} / \mathrm{h}$. Furthermore different distances to the atomizer wheel are shown to illustrate the ongoing atomization process. 


\title{
Conclusions
}

The ejection of processed liquid and the atomization process have been experimentally investigated in this study. High resolution images of the atomization process clearly show the influence of the viscosity on the formation of ligaments. Furthermore, the importance of the Coriolis force and the resulting jet shape flow of the ejected liquid is observed. Together with PD measurements, the atomization process can be characterized. In the vicinity of the wheel: the boundary layer around the wheel and the resulting strong velocity gradients have a strong impact on the atomization, as high relative velocities act on the drops. The radial oriented inserts in the atomizer wheel produce a radial velocity component, which cannot be neglected. This radial velocity component protrudes further into the surrounding air, than the tangential component, as also shown by the PD measurements. The tangential homogeneity of the spray (in terms of velocity) is reached much faster (closer to the wheel), compared to the radial homogeneity. Although the mean velocities per angle bin already show a homogeneous behaviour at e.g. $110 \mathrm{~mm}$ (cf. Fig.6), the raw values indicate that the mean value doesn't represent the behaviour of the entire spray. Furthermore, measurements conducted without the encoder, leading to one single mean velocity or diameter per measurement point, would lead to a complete missinterpretation of the results - the strong influence of the single jets would be neglected. The variation of diameters near to the wheel is comparably high (e.g. $0 \mu \mathrm{m}$ to $215 \mu \mathrm{m}$ ), but flattens out - depending on the wheel speed and mass flow rate. Additionally, signs of coalescence could be observed, as the mean diameter increases with increasing distance to the wheel, together with a strong deceleration of the flow. The main influencing parameter was found to be the speed of the wheel and not the mass flow rate, as the resulting $D_{32}$ changes drastically with the speed. The influence of viscosity of the processed liquid is - in agreement with the expectations after analysing the initial pictures of the atomization - relatively strong. An increase in viscosity leads to an increase in the resulting mean droplet diameter. The weak influence of the mass flow rate persists. The uniformity of the droplet size distribution was also analysed and it could be shown, that the relative span $\Delta$ only deviates for high wheel speeds. Low mass flow rates lead to a more uniform droplet size distribution, which could not have be seen from the analysis of $D_{32}$ alone.

\author{
Nomenclature \\ $\hat{u}_{t} \quad$ tangential velocity [m/s] \\ $u_{r} \quad$ radial velocity $[\mathrm{m} / \mathrm{s}]$ \\ $\omega \quad$ angular velocity $[1 / \mathrm{s}]$ \\ $R_{a} \quad$ radius of the atomizer wheel [mm] \\ $l \quad$ length of insert [mm] \\ $d \quad$ diameter of insert [mm] \\ $\lambda \quad$ Darcy friction factor [-]
}

\section{References}

[1] GEA Process Engineering A/S, 2016. GEA Niro Spray Drying Absorption - The easy way to clean the flue gas from waste incinerators. http://www.gea.com/global/en/binaries/GEA\%20Spray $\% 20 \mathrm{drying} \%$ 20absorption\%20Waste\%20Incinerator_tcm11-24419.pdf ([online]. [cit. 2016-01-29]).

[2] GEA Process Engineering A/S, 2016. http://www.gea.com ([online]. [cit. 2016-01-29]).

[3] Mujumdar, A. S., 2014. "Handbook of Industrial Drying". CRC Press, Boca Raton.

[4] Baker, C., 1997. "Industrial Drying of Foods". Springer Science \& Business Media, Heidelberg.

[5] Masters, K., 1979. "Spray Drying Handbook". G. Godwin ; Halsted Press, London; New York, 3. ed.

[6] Ashgriz, N., 2011. "Handbook of Atomization and Sprays: Theory and Applications". Springer US, New York.

[7] Liu, H., 2000. "Science and Engineering of Droplets: Fundamentals and Applications". Noyes Publications Andrew, Park Ridge, NJ Norwich, NY.

[8] Domnick, J., 2012. Sep. 2.-6. 2012, 12th Triennial International Conference on Liquid Atomization and Spray Systems, Heidelberg, Germany.

[9] Walzel, P., Schaldach, G., and Wiggers, H., 2008. Sep. 8.-10. 2008, 22nd European Conference on Liquid Atomization and Spray Systems, Como Lake, Italy.

[10] Mescher, A. and Walzel, P., 2010. Sep. 6.-8. 2010, 23rd Annual Conference on Liquid Atomization and Spray Systems, Brno, Czech Republic.

[11] Tropea, C., Xu, T.-H., Onofri, F., Géhan, G., Haugen, P., and StiegImeier, M., 1996. Particle \& Particle Systems Characterization, 13(2), pp. 165-170. URL http://dx.doi.org/10.1002/ppsc.19960130216. 


\title{
Subgrid liquid flux and interface modelling for LES of atomization
} Javier Anez ${ }^{1}$, Aqeel Ahmed ${ }^{1}$, Stefano Puggelli², Julien Réveillon ${ }^{1}$, Jorge Brandle de Motta1, F.X. Demoulin*1

${ }^{1}$ CNRS CORIA UMR 6614, University of Rouen, Technopôle du Madrillet, B.P. 12, 76801 Saint-Etienne-du-Rouvray Cedex, France

${ }^{2}$ Department of Industrial Engineering, University of Florence, Via di Santa Marta 3, 50139 Florence, Italy

${ }^{*}$ Corresponding author: demoulin@coria.fr

\begin{abstract}
Traditional Discrete Particle Methods (DPM) such as the Euler-Lagrange approaches for modelling atomization, even if widely used in technical literature, are not suitable in the near injector region. Indeed, the first step of atomization process is to separate the continuous liquid phase in a set of individual liquid parcels, the so-called primary break-up. Describing two-phase flow by DPM is to define a carrier phase and a discrete phase, hence they cannot be used for primary breakup. On the other hand, full scale simulations (direct simulation of the dynamic DNS, and interface capturing method ICM) are powerful numerical tools to study atomization, however, computational costs limit their application to academic cases for understanding and complementing partial experimental data. In an industrial environment, models that are computationally cheap and still accurate enough are required to meet new challenges of fuel consumption and pollutant reduction. Application of DNS-ICM methods without fairly enough resolution to solve all length scales are currently used for industrial purpose. Nevertheless, effects of unresolved scales are generally cast aside. The Euler-Lagrange Spray Atomization model family (namely, ELSA, also call, $\Sigma-Y$ or $\Omega-Y$ ) developed by Vallet and Borghi pioneering work [1], and [2], at the contrary aims to model those unresolved terms. This approach is actually complementary to DNS-ICM method since the importance of the unresolved term depends directly on mesh resolution. For full interface resolution the unclosed terms are negligible, except in the far-field spray when the unresolved terms become dominant. Depending on the complexity of the flow and the available computational resources, a Large Eddy Simulation (LES) formalism could be employed as modelling approach. This work focus on the two main terms that drive these different modelling approaches namely the subgrid turbulent liquid flux and the resolved interface. Thanks to the open source library OpenFoam ${ }^{\circledR}$ this work is an attempt to review and to release an adapted modelling strategy depending on the available mesh resolution. For validation, these solvers are tested against realistic experimental data to see the overall effect of each model proposal.
\end{abstract}

\section{Keywords}

Euler-Lagrange Spray Atomization model (ELSA), VOF, Sub-grid scales, QME.

\section{Introduction}

From the initial work of Vallet and Borghi [1], different models based on Eulerian modelling for atomization have been studied. Later on, Blokkeel et al. [3] working with the same team completed the original approach by a Lagrangian description of the spray once the primary break-up is achieved. In addition, they proposed to call this approach ELSA for Eulerian-Lagrangian Spray Atomisation model to simplify its denomination, but other names are still in used such as $\Sigma-Y, \Omega-Y$ or $E S A$, depending on which variable has been retained or whether the Lagrangian phase has been activated or not. Moreover, a new model has been attached to this approach such as Quasi-Multiphase Euler flow [4] approach and LES approach [5]. All in all, they belong to the ELSA family of models that try to consider and to model the two main terms that drive atomization process for non-fully resolved cases: the subgrid/unresolved turbulent liquid flux $R_{l i}$ and the unresolved liquid gas interface that will be characterised in this work by $\Sigma$ (area per unit of volume). For brevity the interested reader is referred to [18]. The purpose of this work is to release the model thanks to the open library OpenFoam ${ }^{\circledR}[6]$. Thus a mathematical description is proposed, the solver released will allow to check the numerical methods that must be adapted depending on the modelling proposal. Following the important efforts that have been provided to get data for validation purpose in the ECN workshop framework [7]-[9], the test case designated Spray-A is considered.

\section{Modelling Approaches}

The key point of the proposed ELSA model is the analogy between atomization and turbulent mixing of a jet with large density difference with the ambient medium [1], [2]. This single-phase approach allows the choice of both 
carrier and discrete phases to be avoided [5]. Therefore, the two-phase flow is studied as a single-phase turbulent flow composed of two species with highly variable density. Several features are considered: large-scales properties such as liquid penetration and small-scales characteristics (mean droplet diameter and their size distribution). In this section, starting from this complete approach, governing equations are presented:

$$
\begin{aligned}
& \frac{\partial \rho}{\partial t}+\frac{\partial \rho u_{j}}{\partial x_{j}}=0 \\
& \frac{\partial \rho u_{i}}{\partial t}+\frac{\partial \rho u_{j} u_{i}}{\partial x_{j}}=-\frac{\partial p}{\partial x_{i}}+\frac{\partial}{\partial x_{j}}\left(\rho v\left(\frac{\partial u_{i}}{\partial x_{j}}+\frac{\partial u_{j}}{\partial x_{i}}-\frac{2}{3} \delta_{i j} \frac{\partial u_{k}}{\partial x_{k}}\right)\right)+\rho g_{i}+F_{\sigma}
\end{aligned}
$$

Those equations have to be completed by equation of state and jump relation across the phases [13]. $F_{\sigma}$, is the additional force due to the surface tension depending on the local curvature of the interface. To compute this force and to apply the jump of any variable, the most accurate ICM-DNS code applies direct numerical schemes based on interface reconstruction, along with the numerical mesh characteristics. For instance, the ARCHER code [14] is based on couplet VOF-Level set method for interface reconstruction together with a ghost-fluid approach to represent accurately the discontinuity of variables such as density, pressure and viscosity at the interface. This reconstruction process generally depends on the mesh geometry, hence body-fitted methods based on unstructured mesh are used to address complex geometry. For such complex meshes there is not yet available allpurpose reconstruction methods and lower order approach are applied instead. As for instance, diffusive methods are designed to smear the interface over several mesh cells to recover a continuous behaviour of any variable. Therefore, to reconstruct the field and to localize the interface a phase indicator is used. Among the possible indicator the liquid volume fraction $\left(\alpha_{l}\right)$ has been used mostly due to its conservative form and can be written allowing to ensure the complete conservation of the total amount of liquid during the atomization process. The equation, for constant liquid and gas density reads:

$\frac{\partial \alpha_{l}}{\partial t}+\frac{\partial u_{j} \alpha_{l}}{\partial x_{j}}=0$

Unresolved scales are considered by filtering equation 3 under LES or averaged under RANS framework or simply because the mesh is not fine enough to capture all the fluctuations (i.e., When values integrated over a cell are smeared while discretizing with finite volume method). The accuracy of different RANS turbulence models when applied to two-phase flow was previously studied [23]. All these operators when applied to the previous equation will produce additional terms. First let's consider the LES filtered operation for liquid volume fraction, then equation 3 becomes

$\frac{\partial \overline{\alpha_{l}}}{\partial t}+\frac{\partial \overline{u_{j}} \overline{\alpha_{l}}}{\partial x_{j}}=\frac{\partial R_{l j}}{\partial x_{j}}=\frac{\partial \overline{u_{j} \alpha_{l}}-\overline{u_{j}} \overline{\alpha_{l}}}{\partial x_{j}}=\frac{\left.\partial \overline{u_{\jmath}}\right|_{l} \overline{\alpha_{l}}-\left(\left.\overline{\alpha_{l}} \overline{u_{\jmath}}\right|_{l}+\left.\overline{\alpha_{g}} \overline{u_{\jmath}}\right|_{g}\right) \overline{\alpha_{l}}}{\partial x_{j}}=\frac{\partial \overline{\alpha_{l}}\left(1-\overline{\alpha_{l}}\right)\left(\left.\overline{u_{j}}\right|_{l}-\left.\overline{u_{\jmath}}\right|_{g}\right)}{\partial x_{j}}$

Where the liquid flux, $R_{l j}$ appears (first term on RHS) and is related to the unresolved part of the velocity that is known to produce additional diffusion. This part can be modeled by a turbulent diffusion process [22]. Another feature of this term appears on the last term, where the slip velocity $\left(\left.\bar{u}_{j}\right|_{l}-\left.\bar{u}_{J}\right|_{g}\right)$ is clearly related to $R_{l j}$. This characteristic of multi-phase flow is well known, see for example the work of Simonin et al. [16]. He defined the drift and slip velocity between two phases. For atomization processes this effect might be of some importance, thus two modelling approaches should be developed, namely the basic ELSA approach (only drift is considered) and the Quasi Multiphase Eulerian method, QME [4]. Therefore, the turbulent diffusion liquid flux rewrites:

$\frac{\partial R_{l j}}{\partial x_{j}}=\frac{\partial \overline{\alpha_{l}}\left(1-\overline{\alpha_{l}}\right)\left(\left.\overline{u_{j}}\right|_{l}-\left.\overline{u_{j}}\right|_{g}\right)}{\partial x_{j}}=\frac{\partial \overline{\alpha_{l}}\left(1-\overline{\alpha_{l}}\right) \bar{V}_{r l g j}}{\partial x_{j}}=\frac{\partial \overline{\alpha_{l}}\left(1-\overline{\alpha_{l}}\right)\left(\bar{u}_{\text {slip } j}-\bar{v}_{d r i f t}\right)}{\partial x_{j}}$

\section{Remarks on averaging}

Considering the simple case of constant gas and liquid density, $\rho_{g}$ and $\rho_{l}$, respectively, the filtered density, $\bar{\rho}=$ $\rho_{l} \bar{\alpha}_{l}+\left(1-\bar{\alpha}_{l}\right) \rho_{g}$, is far for being constant and some correlations should be addressed. To reduce closure issues Favre averaging/filtering is employed, which defines for any variable $\tilde{\varphi}=\frac{\bar{\rho} \varphi}{\bar{\rho}}$. This approximation is used widely for single-phase flow with variable density, hence it can be applied for two-phase flow following the modeling approach explained above. Indeed in their original work, Vallet and Borghi [1] have used the Favre averaging. Additionally, the resulting Favre velocity fluctuation is usually related to the turbulent liquid mass flux [22]. However, two 
difficulties arise from this approach. Firstly, any Favre-averaged variable is pondered by the density. Figure 1 shows the Favre-averaged velocity mixture with respect to the liquid volume fraction $\overline{\alpha_{l}}$, for constant liquid and gas velocity, $u_{l}$, and $u_{g}$, respectively. The nonlinear relation of the Favre averaging, namely $\tilde{u}$, is enhanced as the density ratio grows, $r$. For instance, when $r=100$, and the mixture is composed by $90 \%$ of gas $\left(\bar{\alpha}_{l}=0.1\right)$, the Favre-averaged velocity mixture is still mainly related to the liquid velocity. On the other hand, Reynolds-averaged mixture velocity exhibits linear behavior between the limiting cases (pure liquid or pure gas). Secondly, the Reynolds-averaged velocity field is by definition divergence free, on the contrary for the Favre-averaged velocity field, as shown below:

$\rho_{l}=c s t, \rho_{g}=c s t \rightarrow \frac{\partial u_{j}}{\partial x_{j}}=0 \rightarrow \frac{\partial \overline{u_{j}}}{\partial x_{j}}=0 \rightarrow \frac{\partial \widetilde{u_{j}}}{\partial x_{j}}=-\frac{1}{\bar{\rho}} \frac{D \bar{\rho}}{D t}$

These two Favre averaging drawbacks compel us to consider directly Reynolds averaging formulation together with liquid volume fraction (volume formulation) field, instead of liquid mass fraction (mass formulation). The motivation is also to get closer to VOF approaches. Our goals are twofold, firstly to compare how volume formulation matches with well-established results based on mass formulation [22], and secondly to develop an ELSA model compatible with ICM filtered by LES turbulence model.

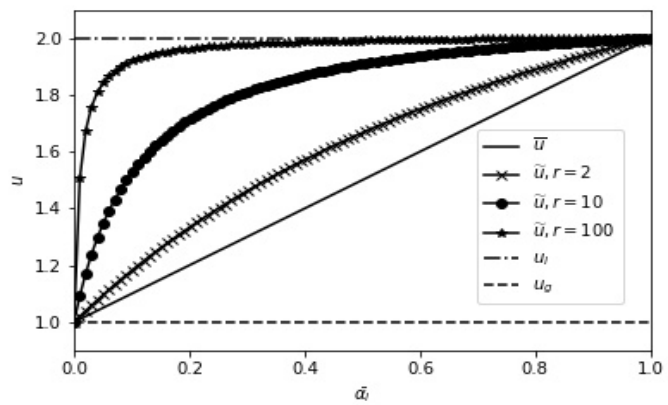

Figure 1: Plot of Favre-averaged velocity and Reynolds-averaged velocity for different density ratio $r=\frac{\rho_{l}}{\rho_{g}}$ as a function of the liquid volume fraction $\left(u_{l}=2, u_{g}=1\right)$.

LES formulation compatible with ICM.

For full scale resolution, ICM method aims at keeping a sharp interface, thus a discontinuous profile of $\alpha_{l}$ across the phases exits in particular during the convection process. This property is either directly included in the numerical scheme (VOF, Level-Set, ghost-fluid, among others) or obtained by additional correction designed to prevent numerical diffusion that could smear the $\alpha_{l}$ profile. The interFoam solver of OpenFOAM® is based on this last technique, where Weller [6] proposed to use an additional flux of liquid directed toward the interface proportional to the local velocity magnitude $\left(u_{r}\right)$ and located only where a mixture of liquid and gas exist (i.e. $\alpha_{l} \in[0,1]$ ). This method is often referred as the VOF method, even if there is no real reconstruction of the interface. See equation 6 , last term on the left. This equation when is solved using Direct Numerical Simulations (DNS) all fluctuations scales are solved up to the grid level and no averaged filtering is required. On the other hand, following the modelling approach in this study, LES filtering or averaged under RANS framework is used, which in either case, the turbulent diffusion liquid flux is set to zero:

$\frac{\partial \overline{\alpha_{l}}}{\partial t}+\frac{\partial \overline{u_{j}} \overline{\alpha_{l}}}{\partial x_{j}}+\frac{\partial u_{r} \overline{\alpha_{l}}\left(1-\overline{\alpha_{l}}\right)}{\partial x_{j}}=0$

Notice that several proposals exist in OpenFOAM® to improve this point in particular the isoAdvector approach [15]. There are many successful examples in the literature of these fully resolved approaches combining ICM method with DNS using mesh resolution high enough to compute all the flow scales. It is important to emphasize that the drift behavior of the unresolved liquid flux $R_{l j}$ is not compatible to the ICM method since the latter assumes the $\alpha_{l}$ profile to be discontinuous. Considering the shortcomings of diffusive interface approaches in the dense spray region and in order to develop a model suitable also in the dilute spray region, a coupling technique between ELSA and the ICM method is proposed, where the turbulent diffusion liquid flux is no longer set to zero. Hence, starting from equations 4,5 and 6 , the liquid volume fraction equation has been modified considering, $C_{\alpha}$ as a pondering coefficient between ELSA-base and an ICM approach. For a complete definition of $C_{\alpha}$, the interested reader is addressed to [17]. Clearly, $R_{l j}$ can be modeled using drift and slip velocity between the phases as explained above. The final assembled model for all scales is presented below: 
$\frac{\partial \overline{\alpha_{l}}}{\partial t}+\frac{\partial \overline{u_{j}} \overline{\alpha_{l}}}{\partial x_{j}}+\frac{\partial C_{\alpha} u_{r} \overline{\alpha_{l}}\left(1-\overline{\alpha_{l}}\right)}{\partial x_{j}}=\left(1-C_{\alpha}\right) \frac{\partial \overline{\alpha_{l}}\left(1-\overline{\alpha_{l}}\right)\left(\bar{u}_{\text {slip } j}-\bar{v}_{d r i f t}\right)}{\partial x_{j}}$

The advantages of the proposed model is to determine a resolution of the interface with ICM in a limited region, whereas $R_{l j}$ prevails when the fluctuations between phases become significant at subgrid-scale.

\section{Numerical model}

Regarding the geometry and the inflow condition several options may be considered, full 3D simulation with in injector flow and needle movement [10] up to a simple 2D axisymmetric configuration [11]. On both cases it has been possible to show that even with the less resolved configuration (RANS) the essential features of the injection can be captured with the ELSA family. An advantage of this test case is the possibility to simulate also the flow inside the injector. A simple geometry consisting of only spray domain is also studied in addition to full injector flow modelling, to visualize more clearly different aspects of spray models. An example of the mesh used for this simulation are presented on Figure 2. The 1D axial profile of injector (210675) is taken from ECN website [9] (red line on the figure). Using GMSH [12] the geometry was extended to include injector sac and needle. Then a structured mesh, consisting of 17168 hexahedral cell types was obtained with 10 cells at the exit of the injector along the radial direction. Finally an axisymmetric case was setup. Another simple spray chamber geometry is also tested (see Figure 1, bottom). For 3D simulation either the geometry is rotational extruded from the axisymmetric case, or the full 3D surface available from ECN website is used. In this latter case because of uncertainties on the real geometry of the injector, and despite several measurements, the shape profile of the $2 \mathrm{D}$ axisymmetric injector pipe is preserved up to 10 diameters of the orifice.

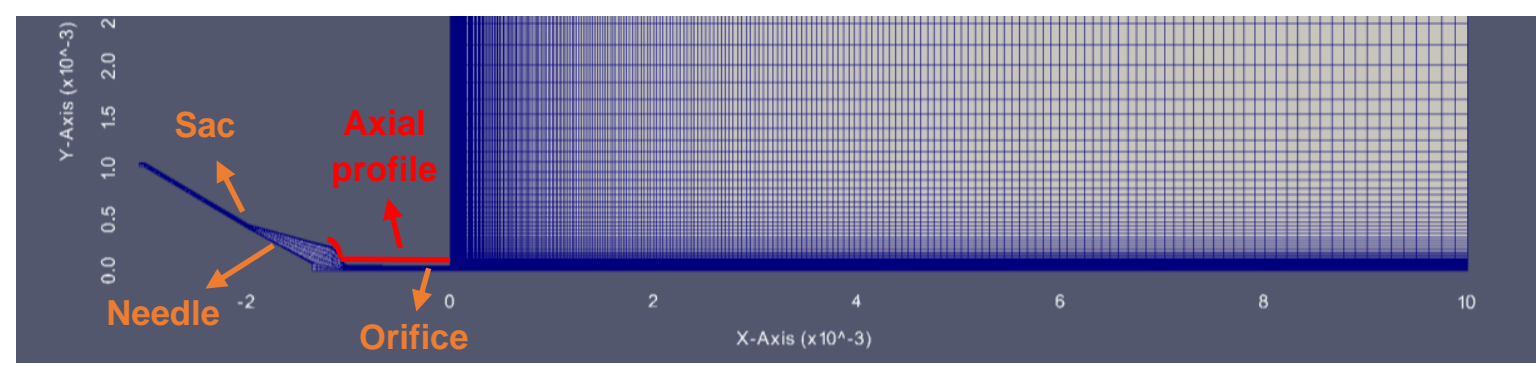

Figure 2. Structured mesh with in injector pipe.

In reality, the injector includes also an axial displacement of the needle, however, despite its potential effect on the flow behaviour it has been chosen not to be considered in this study. By using a transient mass flow rate allows to reproduce partly the effect of the needle motion. Nonetheless, the established injection jet can be obtained directly with a constant mass flow rate. The first comparison will be based on this simplified test case. Accordingly, operating conditions are reported in Table 1.

Table 1. Conditions for non-evaporating ECN Spray-A [9]

\begin{tabular}{ll}
\hline Fuel & $\mathrm{n}$-Dodecane \\
Ambient composition & $100 \% \mathrm{~N} 2$ \\
Injection pressure $[\mathrm{MPa}]$ & 150 \\
Ambient temperature $[\mathrm{K}]$ & 303 \\
Ambient density $\left[\mathrm{kg} / \mathrm{m}^{3}\right]$ & 22.8 \\
Fuel injection temperature $[\mathrm{K}]$ & 343 \\
\hline
\end{tabular}

\section{Results and discussion}

A series of modifications is proposed, based on the physics to be modelled. Whether the interface is resolved or sub-grid scales dominate or relative velocity between the phases occurs, such as the one encountered in flow separation. Firstly elsaBase is presented, which solves the turbulent diffusion liquid flux term $\left(R_{l j}\right)$ including only the drift component, and with an additional scalar transport equation for the liquid-gas surface interface $(\Sigma)$. Secondly, elsaBaseQme, which solves the same equations as ElsaBase with the added slip component of the turbulent diffusion liquid flux $\left(\bar{u}_{\text {slip }}-\bar{u}_{\text {drift }}\right)$. Thirdly, interRansElsa, which is an adapted version of the equation 7 , mainly to be used for 2D axisymmetric geometries, and without the slip part of the diffusion term. Finally, 
interLESElsaBase, which solves equation 7 for 3D complex geometries. Different elsa models are summarized in Table 2.

Table 2. Different ELSA models

\begin{tabular}{lcccc} 
Solver & $\begin{array}{c}\text { Turbulent } \\
\text { diffusion flux }\end{array}$ & $\begin{array}{c}\text { Slip } \\
\text { velocity }\end{array}$ & $\begin{array}{c}\text { Interface } \\
\text { compression term }\end{array}$ & $\begin{array}{c}\text { LES } \\
\text { filtered }\end{array}$ \\
\hline interFoam & & & $\checkmark$ & \\
elsaBase & $\checkmark$ & & & \\
elsaBaseQme & $\checkmark$ & $\checkmark$ & & \\
interRansElsa & $\checkmark$ & & $\checkmark$ & $\checkmark$ \\
elsaBaseLes & $\checkmark$ & & &
\end{tabular}

\section{Validation}

A comparison process has been made against experimental and numerical data available from the Engine Combustion Network (ECN) in order to validate the proposed ELSA model. The "Spray-A" non evaporating configuration has been selected, with exact aforementioned fluid properties. The experimental data used for validation include the Projected Mass Density of the fuel (PMD), which was obtained by x-ray radiography measurement techniques [19, 20], and the Transverse Integrated Mass (TIM), which was acquired from the integral of the projected density across a transverse position at a particular axial location [18]. For the numerical data in the present study a fixed needle motion was modeled using a rate of injection generator for Spray-A, http://www.cmt.upv.es/ECN09.aspx. Figure 3 shows the comparison in terms of the PMD and TIM between experimental and numerical data for the first 5 cases described in table 2. Figure 3 on the left displays both limiting cases such as interFoam (interface resolved) and elsaBase (interface diffused), overpredicting and underpredicting liquid penetration, respectively. On the other hand, both elsaBaseLes (with LES filtered) and elsaBase with the round jet correction are able to fairly match the experimental results. Moreover, small differences can be found when the nozzle is added. However, it is believed these differences come from higher mesh resolution used in the nozzle region. Looking closer to the PMD (Figure 3 on the right), based on the radial profile, all models accurately predict the liquid core at $0.1 \mathrm{~mm}$ from the liquid inlet. At this axial position the turbulent instabilities have not yet been developed while using only the chamber without the nozzle, therefore all models should colapse and match interFoam and the case with the nozzle, as shown. On the latter case, the behavior is expected based on the already establishment of the flow inside the nozzle. The differences observed between 0.05 and $0.06 \mathrm{~mm}$ in the radial PMD for all other cases are mainly due to poor mesh resolution which introduces additional numerical errors. On the other hand, at $2 \mathrm{~mm}$ from the inlet, even though RANS models generally tend to overpredict the fuel dispersion, interface instabilities downstream of the flow are comparable with experiments by showing less radial liquid dispersion and higher liquid penetration than previous aforementioned cases when the round-jet correction is used, and even equivalent results are matchable compared with LES case. It is true that LES does not require any turbulent modelling adjustment as the one made with RANS, nevertheless, both models deal the turbulence as incompressible, which is not completely accurate. Another parameter to measure radial dispersion is the TIM which is shown on the right of the figure. ElsaBase clearly represents the model with the highest flow dispersion. While elsaBase filtered by LES, shows the least diffusivity of all, especially downstream of the flow (near $3 \mathrm{~mm}$ ) where the $3 \mathrm{D}$ turbulent instabilities begin to play a role. It is the turbulent modelling the driven mechanism which dominates the diffusion in this area. Besides the mesh resolution, a proper turbulent modelling is imperative to exactly match the experimental results, therefore a compressible approach is a reasonable choice. For instance, the turbulent kinetic energy should be accounted differently between the phases. Being the liquid heavier than the gas, the turbulent should be diffused more rapidly on the gas phase. Such modelling approach is outside the scope of this paper, since these models are based on the mixture properties. The purpose of the paper is to present different approaches based on ELSA developed so far, the majority of which are able, with minimal computational efforts by using RANS, to offer comparable experimental results within the near nozzle region.

In figure 4 results are shown using 2D axisymmetric geometry with the nozzle for all ELSA versions. Liquid volume fraction and velocity fields are displayed. At the top row of images, interFoam displays a continuous liquid penetration all the way to the exit of the chamber, showing no diffusion as anticipated, which clearly does not correspond with experiments. Hence the need of an alternative, diffusive model to accurately model the liquid dispersion demonstrated with $\mathrm{x}$-ray radiography. 
$\mathrm{PMD} \mu \mathrm{g} / \mathrm{mm}^{2}$
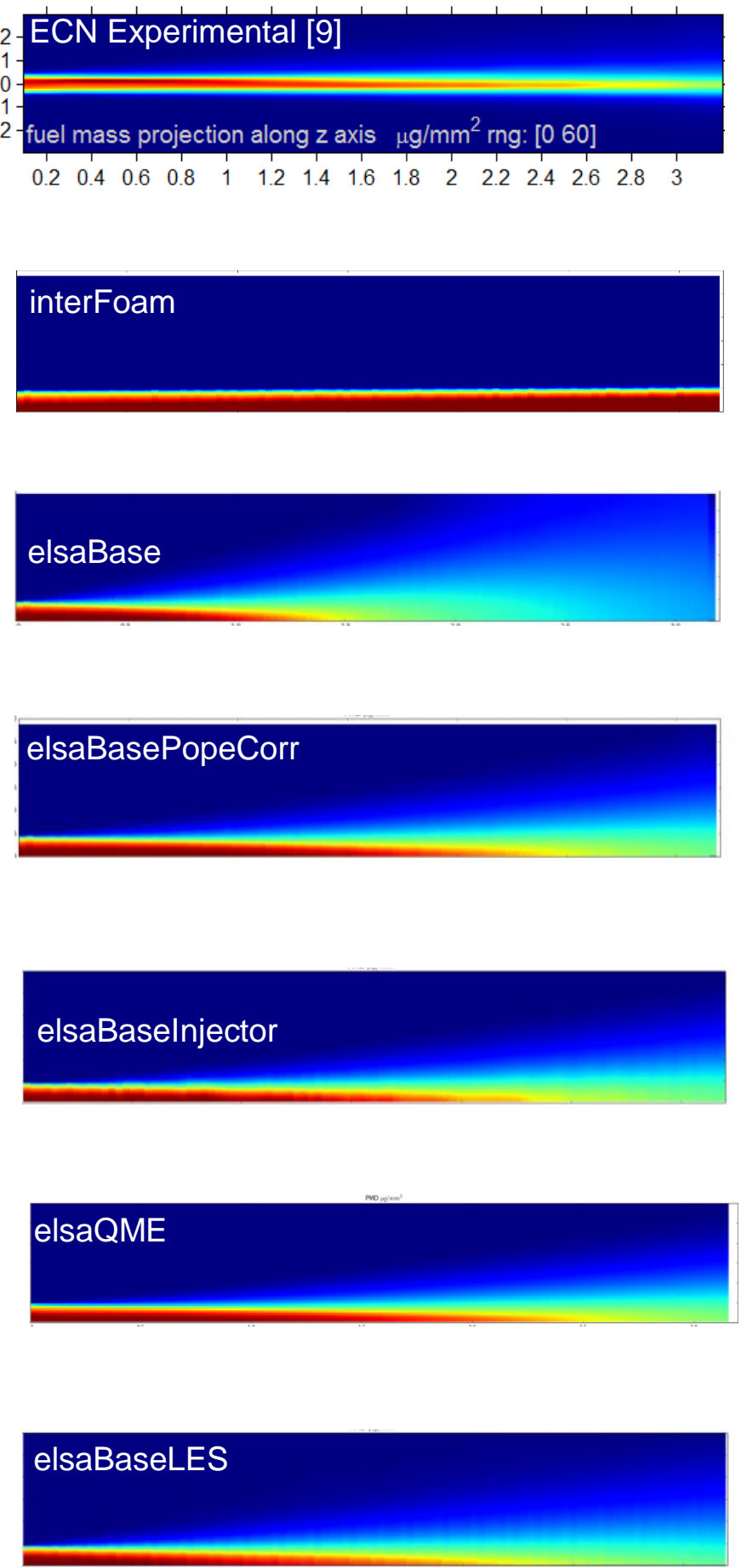
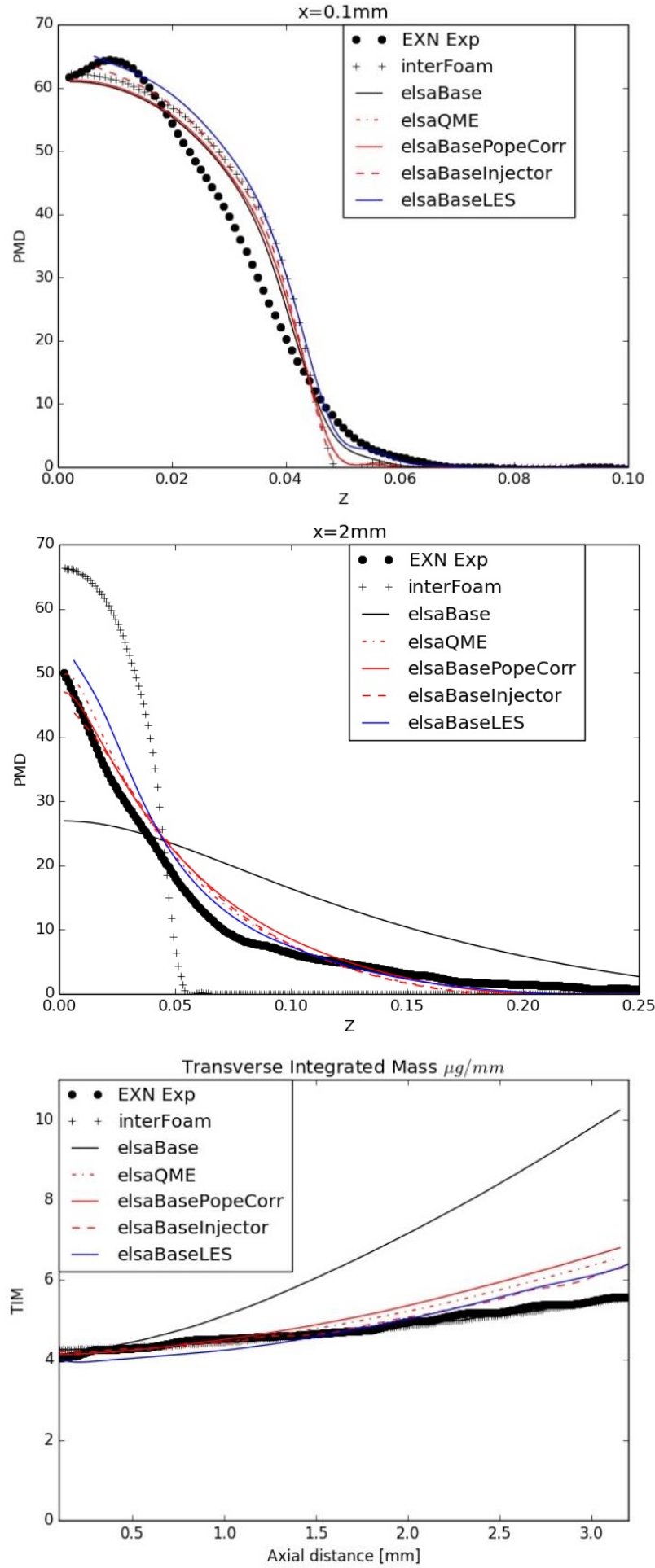

Figure 3. Experimental and numerical Projected Mass Density fields at $0.1 \mathrm{~ms}$ after start of injection (elsaBaselnjector was at $0.5 \mathrm{~ms}$ ) (left). PMD radial profiles at axial location $0.1 \mathrm{~mm}, 2 \mathrm{~mm}$ and TIM (right).

Then, elsaBase and elsaBaseQme model, second and third row of images, respectively, show as expected high diffusivity of the interface, but falls short reproducing the liquid core penetration, with just a little difference between both models on the radial liquid dispersion hardly detectable at the local map span, and especially at the velocity fields. ElsaBase ( $\mathrm{k}-\epsilon$ modified) shows an increase of liquid penetration due to lower diffusivity tuned by the corrected parameter, however, the diffusion is still overpredicted by the lack of appropriate ICM. What is more, an attempt to 
unify both interface resolution physics has been achieved by using, interRansElsa. However, results illustrate no significance difference between ElsaBase, elsaBaseQme, and interRansElsa, mainly due to inappropriate mesh resolution. It is believed, improvements of mesh quality would arise to better liquid penetration, and dispersion to match experimental results by properly capture the interface at the vicinity of nozzle exit, especially before instabilities on the liquid surface due to the turbulent interactions with the gas generates the breakup process. Finally, a refinement meshing process is being held along with 3D Spray-A model reconstruction, both are estimated to be shown and compared at the conference.
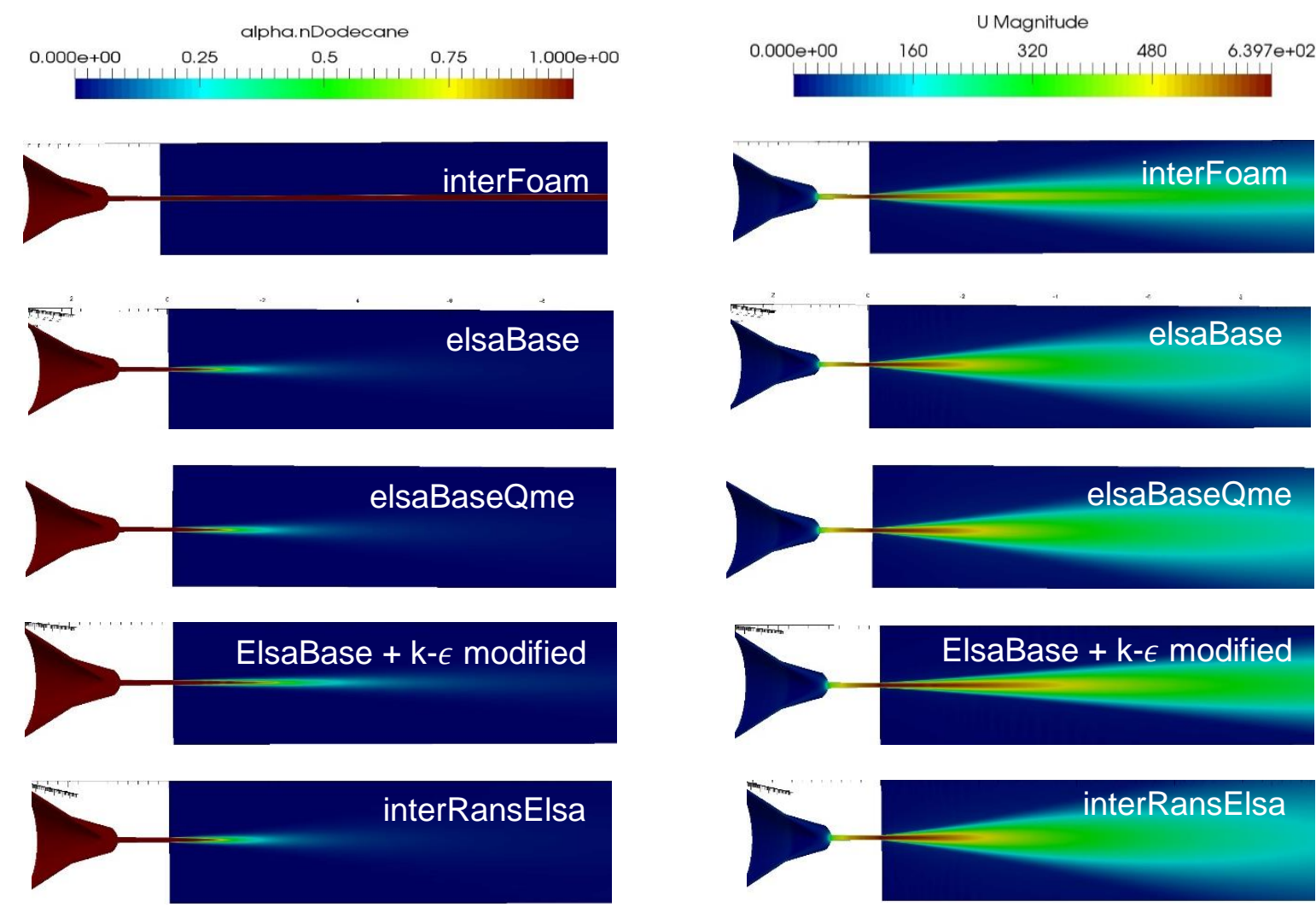

Figure 4. Liquid volume fraction and velocity fields for different ELSA family models, and interFoam, namely, ElsaBase, interRansElsa, elsaBaseQME, and eslaBase with additional modification.

Using second order closure, a slip velocity between the high velocity liquid jet and the quiescent ambient is generated with elsaBaseQME. The relative velocity between the two phases is shown in Figure 5 . It takes its maximum in the near injection region, where the liquid core is still not affected by the breakup process: locally, the liquid presents a liquid volume fraction between 0.6-0.8 and tends to accelerate the gas phase and, due to the air inertia, to generate a relative flux. Even if such values of slip velocity are small with respect to the liquid velocity, a reduction of turbulent diffusion in radial direction with respect to elsaBase can be clearly appreciated. It is interesting to point out that including these effects, the results obtained with elsaBaseQME and interRansElsa are really similar. Going further downstream, the slip velocity is progressively reduced by the drag effects, which, based on the local dynamic relaxation time, lead to similar values of gas and liquid velocity. Clearly, such evolution of the relative flux is directly related to the atomization process, which is shown in Figure 6 for the three ELSA solvers.

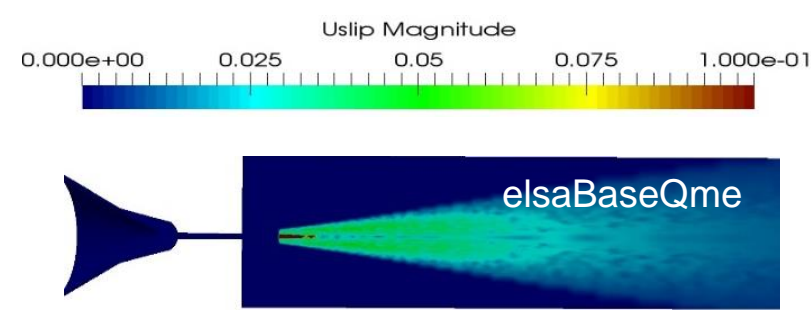

Figure 5. Relative velocity field from elsaBaseQme. 
It is worth pointing out that for interFoam has no information about the evolution of the interface, which is no more available as long as the interface is not sharply resolved. All the proposed versions of ELSA leads to a similar characterization of the atomization process. The liquid jet, due to its high Weber and Reynolds numbers, undergoes a quick atomization process, which is pointed out by the zone where the production of $\Sigma$ is really high. Such violent atomization is related to the growth of instabilities on the liquid surface due to the turbulent interactions with the gas phase in the near injection region. A spray is therefore generated and immediately relaxed to the gas velocity.

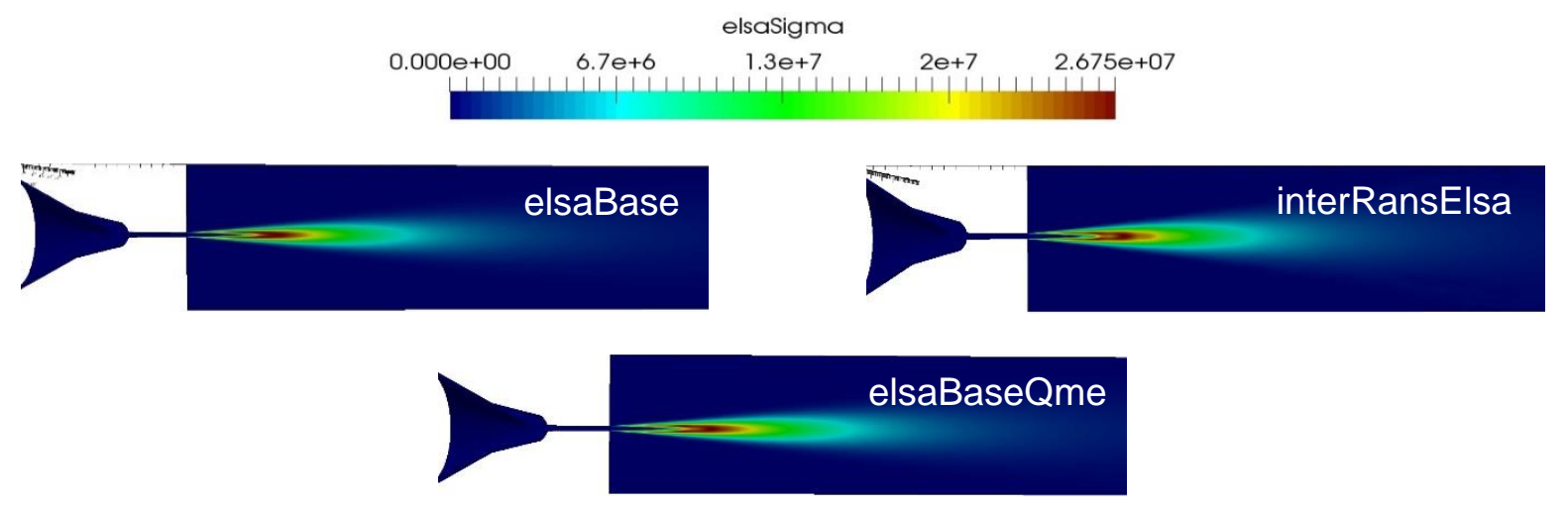

Figure 6. Liquid Gas interface density fields for different ELSA family models, namely, ElsaBase, interRansElsa, and elsaBaseQME.

\section{Conclusions}

The main aim of the present study was to propose a review of the capabilities of several modelling strategies for atomization processes belonging to the ELSA framework. Here, the two-phase flow is studied as a single-phase turbulent flow composed of two species with highly variable density. Starting from the basic approach (i.e. elsaBase), two different extensions based on including the slip velocity effects (i.e. elsaBaseQME) and in dealing with the dense spray region through a ICM (i.e. interRansElsa) are presented. All these approaches have been tested on an experimental test case representative of diesel injection (namely, Spray-A non-evaporating conditions) directly taken from the Engine Combustion Network (ECN). Results obtained on a 2D geometry have been here reported, even if the real $3 \mathrm{D}$ geometry directly taken from the $\mathrm{ECN}$ website is now under investigation. A preliminary comparison both with experimental data and with available numerical results is firstly shown in order to prove that an overall agreement has been achieved. Considering the modelling hypothesis here used, the LES-ELSA approach seems capable of properly reproducing the main features of the analysed test case. Then, a comparison of the performances of the different version of ELSA is reported in order to show the impact of the inclusion of a slip velocity between phases and of an ICM for the dense spray region. Finally, a low-cost atomization modelling approach has been developed suitable from in-flow nozzle injector to far-field spray. What is more, adaptable based on interface resolution, thus avoiding the need to define a carrier phase and a discrete phase. Another important result is the use of RANS modelling instead of LES, can give the same amount of numerical accuracy.

\section{Acknowledgements}

This work was partly supported European Union's Horizon 2020 research and innovation program under the Sklodowska-Curie grant agreement No. 675676. Simulations were carried out at TGCC (The Curie supercomputer, owned by GENCl and operated into the TGCC by CEA), and at CRIHAN (Centre de Ressources de Haute Normandie).

\section{References}

[1] A. Vallet and R. Borghi, "Modélisation Eulerienne de L'atomisation d'un Jet Liquide," C R Acad Sci Paris Sér II B, vol. 327, pp. 1015-1020, 1999.

[2] A. Vallet, A. A. Burluka, and R. Borghi, "Development of a Eulerian model for the 'Atomization' of a liquid jet," At. Sprays, vol. 11, no. 6, pp. 619-642, 2001.

[3] G. Blokkeel, R. Borghi, and B. Barbeau, "A 3d Eulerian model to improve the primary breakup of atomizing jet," SAE Tech. Pap., vol. 2003-01-0005, 2003.

[4] A. Andreini, C. Bianchini, S. Puggelli, and F. X. Demoulin, "Development of a turbulent liquid flux model for Eulerian-Eulerian multiphase flow simulations," Int. J. Multiph. Flow. 
[5] J. Chesnel, J. Reveillon, T. Menard, and F. X. Demoulin, "Large eddy simulation of liquid jet atomization," At. Sprays, vol. 21, no. 9, pp. 711-736, 2011.

[6] H. G. Weller, H. Jasak, and C. Fureby, "A tensorial approach to computational continuum mechanics using object-oriented techniques," Comput. Phys., vol. 12, no. 6, 1998.

[7] A. L. Kastengren et al., "ENGINE COMBUSTION NETWORK (ECN): MEASUREMENTS OF NOZZLE GEOMETRY AND HYDRAULIC BEHAVIOR,” vol. 22, no. 12, pp. 1011-1052, Apr. 2013.

[8] M. Meijer et al., "ENGINE COMBUSTION NETWORK (ECN): CHARACTERIZATION AND COMPARISON OF BOUNDARY CONDITIONS FOR DIFFERENT COMBUSTION VESSELS," vol. 22, no. 9, pp. 777-806, Jan. 2013.

[9] Engine Combustion Network.

[10] Q. Xue et al., "An Eulerian CFD model and X-ray radiography for coupled nozzle flow and spray in internal combustion engines," Int. J. Multiph. Flow, vol. 70, no. 0, pp. 77-88, Apr. 2015.

[11] J. M. Desantes, J. M. García-Oliver, J. Pastor, A. Pandal, E. Baldwin, and D. Schmidt, "Coupled/decoupled spray simulation comparison of the ECN spray a condition with the Sigma- Y eulerian atomization model," Int. J. Multiph. Flow, vol. 80, pp. 89-99, 2016.

[12] C. Geuzaine and J.-F. Remacle, "Gmsh: A 3-D finite element mesh generator with built-in pre-and postprocessing facilities," Int. J. Numer. Methods Eng., vol. 79, no. 11, pp. 1309-1331, 2009.

[13] M. Ishii, Thermo-fluid dynamic theory of two-phase flow. Paris: Eyrolles, 1975.

[14] T. Menard, S. Tanguy, and A. Berlemont, "Coupling level set/VOF/ghost fluid methods: Validation and application to 3D simulation of the primary break-up of a liquid jet," Int. J. Multiph. Flow, vol. 33, no. 5, pp. 510524, 2007.

[15] J. Roenby, H. Bredmose, and H. Jasak, "A computational method for sharp interface advection," R. Soc. Open Sci., vol. 3, no. 11, Nov. 2016.

[16] O. Simonin, "Statistical and continuum modelling of turbulent reactive particulate flows," Lect. Ser. 1996-02 Von Karman Inst. Fluid Dyn., 2000.

[17] Hecht N (2014) Simulation aux grandes 'echelles des 'ecoulements liquide-gaz: application 'a l'atomisation. $\mathrm{PhD}$ thesis, University of Rouen.

[18] Kastengren, Alan L., et al. "X-ray radiography measurements of diesel spray structure at engine-like ambient density." Atomization and Sprays 19.11 (2009)..

[19] Kastengren, A. L., et al. "Time-resolved X-ray radiography of diesel injectors from the engine combustion network." ICLASS Paper 1369 (2012).

[20] Pickett, Lyle M., et al. "Comparison of near-field structure and growth of a diesel spray using light-based optical microscopy and x-ray radiography."SAE International Journal of Engines 7.2014-01-1412 (2014): 1044-1053.

[21] Pope, S. B. "An explanation of the turbulent round-jet/plane-jet anomaly."AIAA journal 16.3 (1978): 279-281.

[22] Lebas, Romain, et al. "Numerical simulation of primary break-up and atomization: DNS and modelling study." International Journal of Multiphase Flow 35.3 (2009): 247-260.

[23] Demoulin, Francois-Xavier, et al. "A new model for turbulent flows with large density fluctuations: Application to liquid atomization." Atomization and Sprays 17.4 (2007). 


\section{$4 Y$ \\ Droplet 3}




\title{
Theoretical and Experimental Study of Grouping Effects on Droplet Streams
}

\author{
Norbert Roth $^{1 *}$, Hassan Gomaa ${ }^{1}$, Alon Livne ${ }^{2}$, David Katoshevski², Bernhard Weigand ${ }^{1}$ \\ ${ }^{1}$ Institute of Aerospace Thermodynamics (ITLR), Universität Stuttgart, Germany \\ ${ }^{2}$ Faculty of Engineering Sciences, Ben-Gurion University of the Negev, Israel \\ *Corresponding author: norbert.roth@ittr.uni-stuttgart.de
}

\begin{abstract}
Grouping of droplets was studied in monodisperse droplet streams. This very controllable system allows to study basic effects. In experiments droplet streams with monodisperse droplets were generated, however, with initially two different inter droplet spacing. A larger inter droplet spacing is followed by a little bit smaller one, which is followed by a larger one and so on. Due to this initial boundary condition groups of two droplets form, which approach each other and finally coagulate. It was found, that the velocity of the droplet approach is linearly dependent on the spacing between the droplets. This process was simulated by direct numerical simulation using the in-house code FS3D. The results of the simulations show the same linear behaviour. For larger computational domains the numerical results approach the experimental results.
\end{abstract}

\section{Keywords}

Droplet streams, Grouping effects, Shadowgraphy, Direct Numerical Simulation

\begin{abstract}
Introduction
In spray systems a tendency to form groups can be observed [1, 2]. That has an influence on their evaporation, the drag force applied on them, their motion, and on their final settling point. This tendency has ramifications in energy and transportation systems as well as inhalation systems, paint and domestic sprays [3]. The better one understand the phenomenon of droplet grouping, the better one can control it and manipulate it tailored for each application, for instance to reduce fine particle emissions [4]. For better understanding the physics of droplet grouping processes, a well defined system of droplets is studied numerically and experimentally. An advantage of such systems is, that in the experiments the initial and boundary conditions are well known and can be controlled precisely. This allows a direct comparison between numerical calculations and experimental results. For this reason in a first approach monodisperse droplet streams are studied, which are produced by a droplet stream generator; the operation characteristics are described in [5]. Due to drag forces the velocity of the droplets in such a stream decrease as described below. Very small irregular disturbances in the initial inter droplet spacing $a$ increase downstream until irregular groups form and finally droplets collide. In order to study basic grouping effects in detail it would be desirable to produce droplet streams with initially small, however, regular differences in inter droplet spacing. This is possible in modulation of the excitation signal of the droplet stream generator. Orme and Muntz suggested and described an amplitude-modulated excitation [6]. A theoretical description is given by Hilbing and Heister [7]. A detailed experimental and theoretical study of amplitude- and frequency-modulated excitation of droplet stream generators has been performed by Brenn und Lackermeier [8]. In this study here two frequency generators are used resulting in a frequency-modulated excitation. The details are described in the next section.
\end{abstract}

\section{Material and methods}

In Fig. 1 a schematic view of the experimental setup is shown including a photograph of the droplet stream generator used. In all experiments iso-propanol was used and the diameter of the orifice was $r_{\text {orifice }} \approx 60 \mu \mathrm{m}$. In Fig 2 on the left hand side a so-called normal monodisperse droplet stream is shown schematically together with the indication of the parameters, which characterize the droplet stream. On the right hand side shadowgraphs of a normal droplet stream are presented, which show the building process of the monodisperse droplet stream. Using an appropriate excitation frequency $f_{s}$ monodisperse droplet streams are obtained. In a first step an optimum frequency $f_{\text {opt }}=f_{s}=49,800 \mathrm{~Hz}$ for the present experimental setup was determined using the method of selfstabilization presented on the DIPSI 2014 workshop in Bergamo [9]. In a second step the optimum of the initial droplet spacing $\lambda_{\text {opt }} \approx 294 \mu \mathrm{m}$ was calculated according to the theory of Lord Rayleigh taking the properties of the droplet liquid, in this case iso-propanol, into account [10].In a third step the droplet generator was operated at the frequency $f_{s}$, the driving pressure was chosen to obtain an initial droplet spacing $a_{0}$ close to $\lambda_{\text {opt }}$. It is expected, that for this configuration an optimum in the regularity of the monodisperse droplet stream is obtained. In ongoing work tests concerning this topic are performed. Results for this normal droplet stream are presented below. Grouping effects were studied for the cases, when groups consisting of two droplets were generated. This was possible using two frequency generators. In the case $n$ droplets were in one group the first frequency generator was running at the frequency $f_{1}=f_{s} / n$. With this frequency $f 1$ the second frequency generator running in a pulse mode was triggered. For each trigger pulse the second frequency generator sent $n$ pulses at a frequency $f_{2}>f_{1}$ to the droplet generator. Good results were obtained for $f_{2}=51,600 \mathrm{~Hz}$. The droplet generator produced then droplets of a temporal distance of $1 / f_{2}$ seconds within a group and a temporal distance between the groups of $1 / f_{1}=n / f_{s}$ 


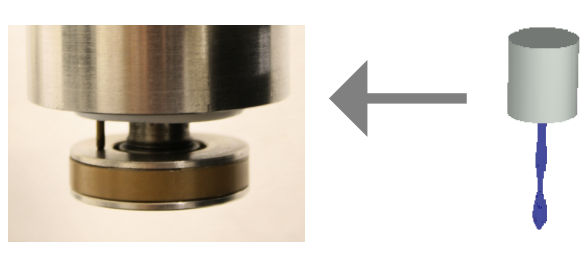

Droplet stream

generator

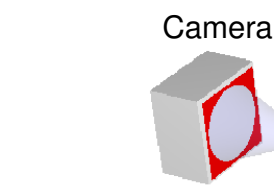

Objective
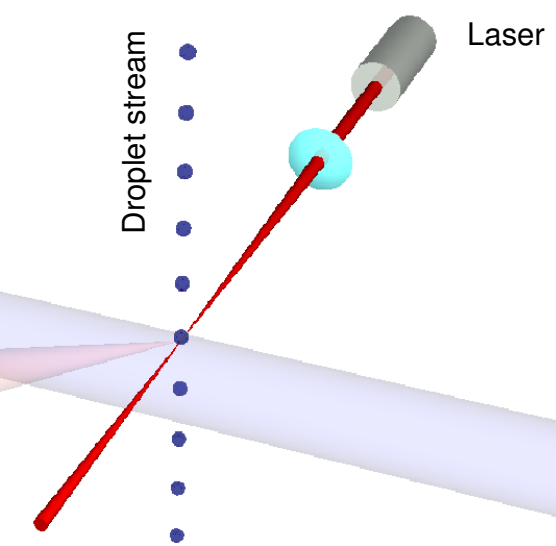

Sensor
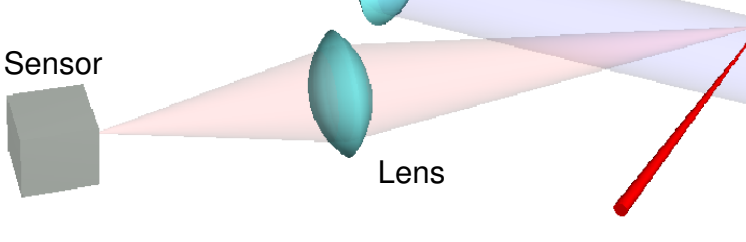

Flash light

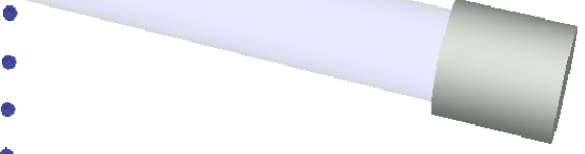

Figure 1. Schematic view of the experimental setup.

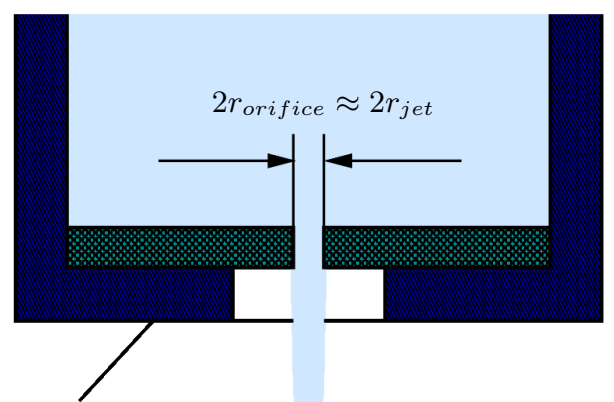

Orifice plate
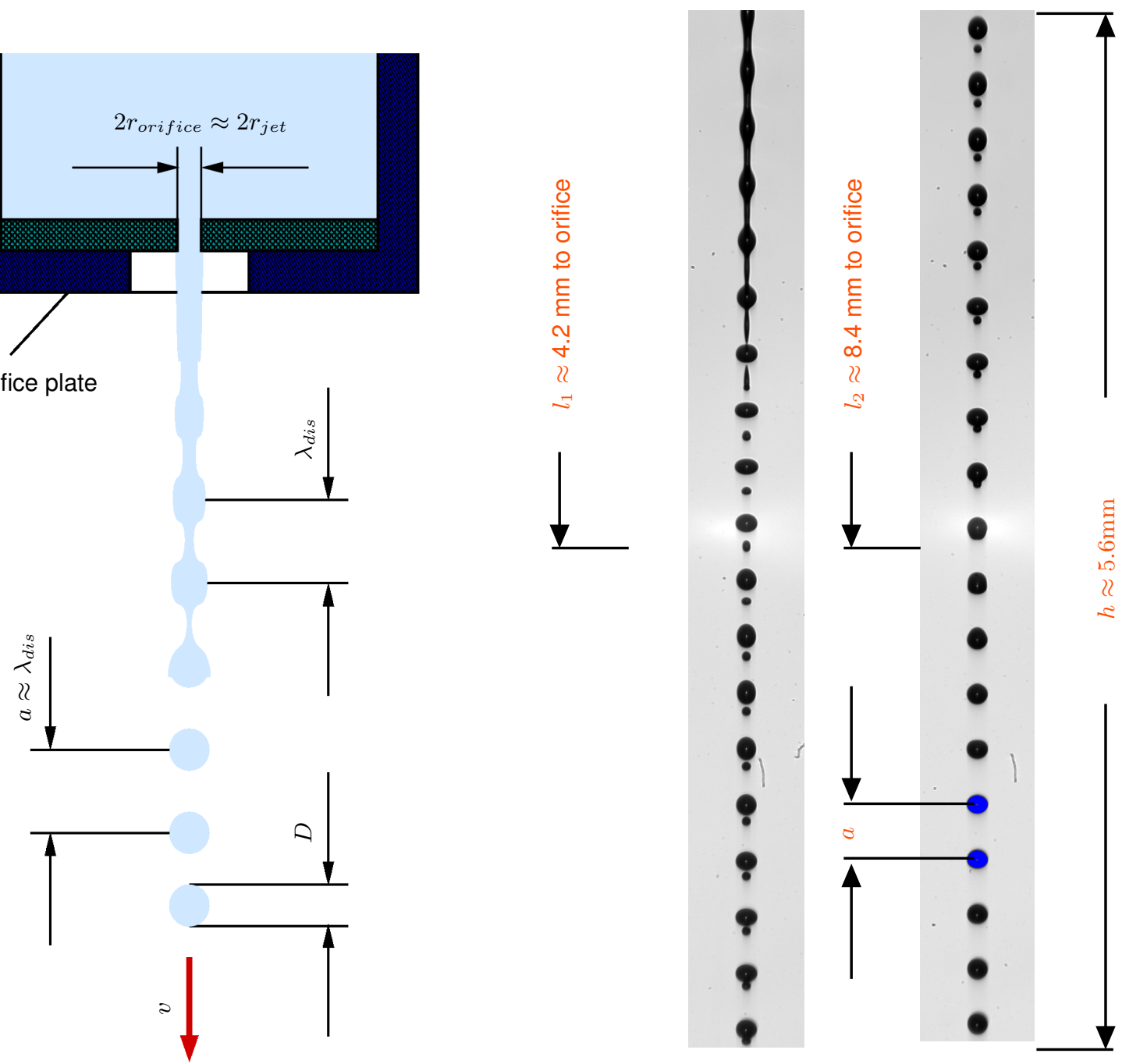

Figure 2. Schematic view of droplet generation indicating the parameters of a normal monodisperse droplet stream on the left hand side. Shadowgraphs of the normal droplet stream at two different distances $l_{i}$ to the orifice plate on the right hand side. 
seconds. Results for pairs $(n=2)$ of droplets are presented below. Each pair is forming a group. The spacing between the groups is denoted by $g$, the spacing between the droplets of the pair by $a_{2}$, and the spacing between the last droplet of the pair and the first droplet of the following pair by $a_{1}$. Therefore one obtains $g=a_{1}+a_{2}$. The velocity of the droplet is obtained by $v=g f_{1}$. The droplet generator can be moved vertically in order to observe different positions $l$ below the orifice plate. From shadowgraphs of the droplet stream taken by the camera the droplet size and the spacing between the droplets are determined. The sensor and the laser beam are used to monitor the passing by of each droplet and to help to adjust the droplet stream correctly. In all cases presented here the droplet diameter was $D \approx 112 \mu \mathrm{m}$. Evaporation processes can be neglected. The initial values for the droplet distances are obtained from shadowgraphs taken at a position $l$, at which the droplets have formed completely. For the initial velocity the value $v_{0} \approx 15.1 \mathrm{~m} / \mathrm{s}$ was obtained. In the experiments the velocity of the droplets is decreasing along the droplet stream due to drag forces resulting in a decrease of the group distance $g$ as the frequency $f_{1}$ of the group production remains constant.

If pairs of droplets are produced the droplets of a pair will collide further downstream. The colliding process was simulated numerically using an in-house program code named Free Surface 3D (FS3D). This code solves the incompressible Navier-Stokes equations. In order to take into account for the liquid phase the Volume-Of-Fluid (VOF) method is used. The free surface of the liquid phase is reconstructed by the Piecewise-Linear-InterfaceConstruction (PLIC) method. Further details can be found in [11]. For the simulation of the droplet stream periodic boundary conditions in the direction of motion were used. Four droplets have been simulated forming two pairs of droplets. Two slightly different spacing between the droplets were initialized in order to take into account of the two pairs of droplets. Using the data from the experiments a length of the computational domain in the direction of motion or $x$-direction of $d_{x}=2 g_{0}=1,211 \mu \mathrm{m}$ was used. Two different widths of the computational domain were used, a smaller domain with $d_{y A}=d_{z A}=300 \mu \mathrm{m}$ (Case A: $512 \times 128 \times 128$ grid cells) and a wider domain with $d_{y B}=d_{z B}=600 \mu \mathrm{m}$ (Case B: $512 \times 256 \times 256$ grid cells). The first tests were performed with continuous boundary conditions at the side borders of the computational domain. This resulted in a drift of the droplet towards the side boundaries with finally leaving of the computational domain. Probably this was caused by an accumulation of the drift due to the periodic boundary conditions in the direction of motion. The droplets could be stabilized using walls with no-slip condition at the side boundaries. This condition, which is in contrast to the experiment, however, changed the behaviour of the droplets dramatically. An approach to the results of the experiments could be realized in widening the computational domain (case B). In analysing velocity profiles it can be seen, that the wider computional domain results in profiles, which are similar to simulations without walls. Results of the experiments and of the numerical simulations are presented in the following section. The numerical simulations with the wider domain have four times more grid cells resulting in a much longer CPU time.

\section{Results and discussion}

First a normal droplet stream with no initial formation of groups was studied. Using shadowgraphs the development of the spacing $a$ between neighbouring droplets along the droplet stream was determined. The result is shown in Fig. 3. Due to drag forces the spacing $a$ is decreasing along the droplet stream. Initial small irregularities in the spacing a increase along the droplet stream. If two droplet initially have a smaller spacing and the spacing to the neighbouring droplets is larger these droplet will approach each other and finally collide. This leads to an increase of the irregularities. The measurements were performed from top to bottom with gaps between the shadowgraphs taken. Afterwards the missing shadowgraphs were taken from bottom to top in order to check if changes occurred during the measurement. Due to the sensitivity to the boundary condition a small difference between both measurement series is observed. For the evaluation of the shadowgraphs the image processing tool ImageJ was used [12].

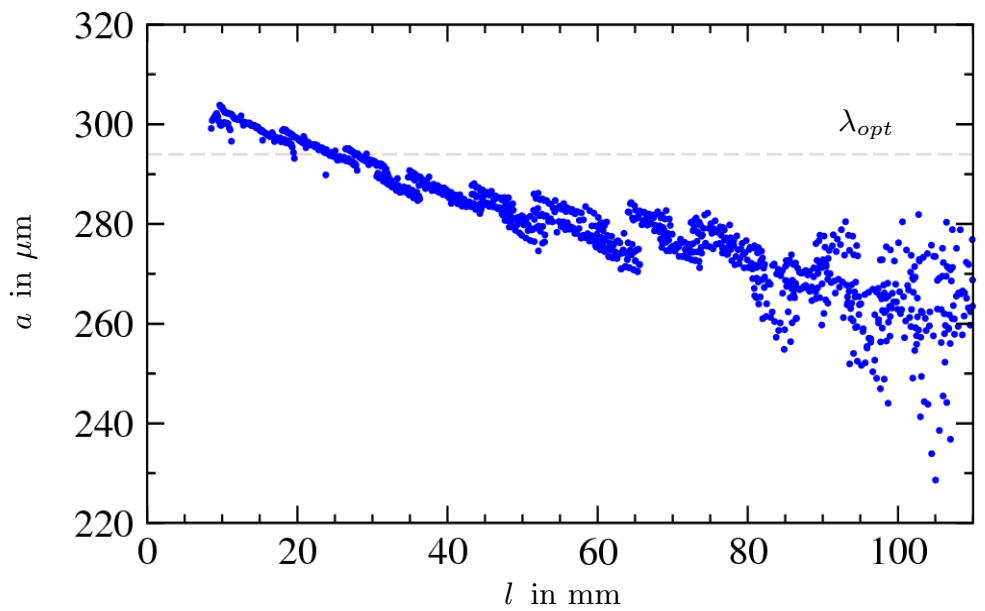

Figure 3. Space $a$ between neighbouring droplets as function of distance $l$ to the orifice plate of the droplet generator. The initial distance $a_{0} \approx 300 \mu \mathrm{m}$ is chosen to be close to the optimum $\lambda_{\text {opt }}$ indicated by the grey dashed line. With an excitation frequency of $f_{s}=49,800 \mathrm{~Hz}$ an initial droplet velocity $v_{0}=a_{0} f_{s} \approx 14.9 \mathrm{~m} / \mathrm{s}$ is obtained. 


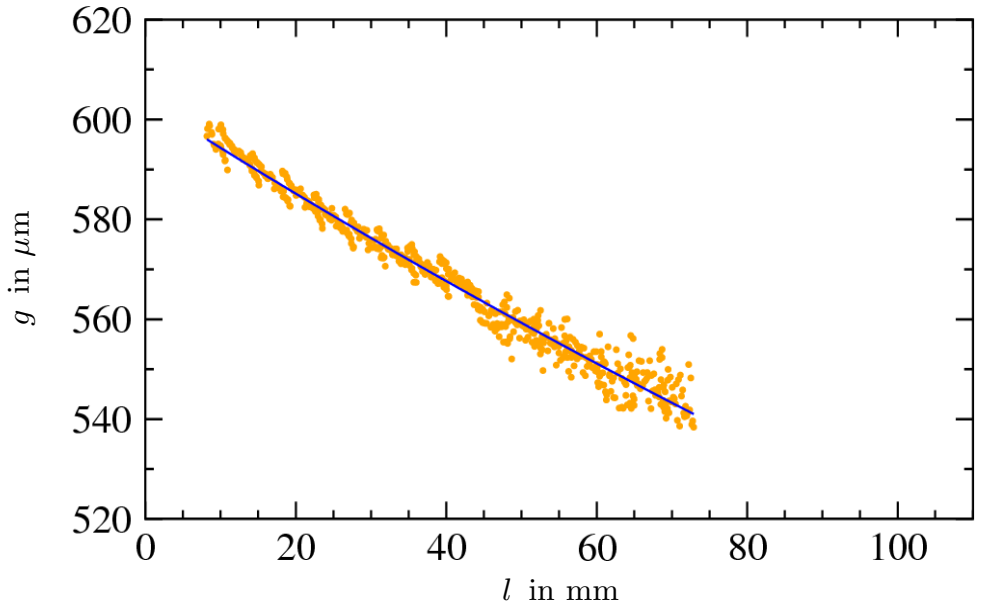

Figure 4. Spacing $g$ between the droplet groups consisting of two droplets as function of distance $l$ to the orifice of the droplet generator. The development of the droplet velocity $\mathrm{v}$ can be obtained by multiplying the spacing $g$ by the frequency $f_{1}$ the groups are generated. The regression to the data is shown as solid line in the diagram.

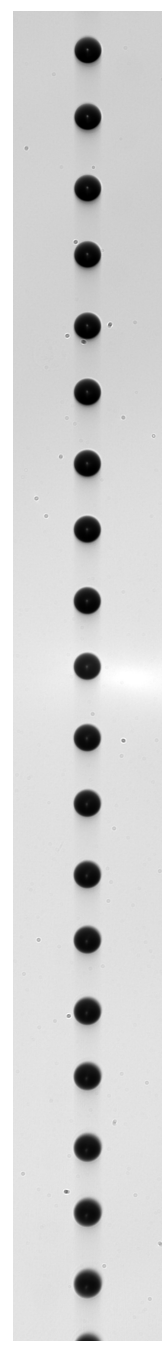

$l_{6}$

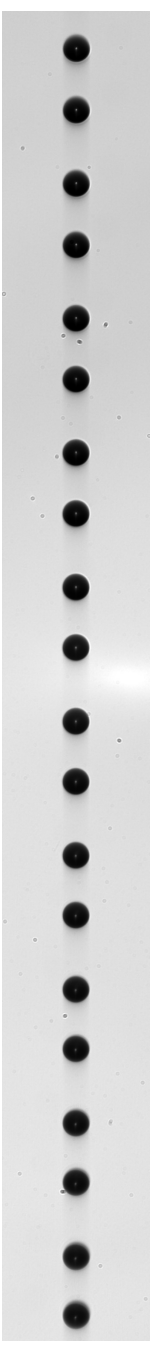

$l_{10}$

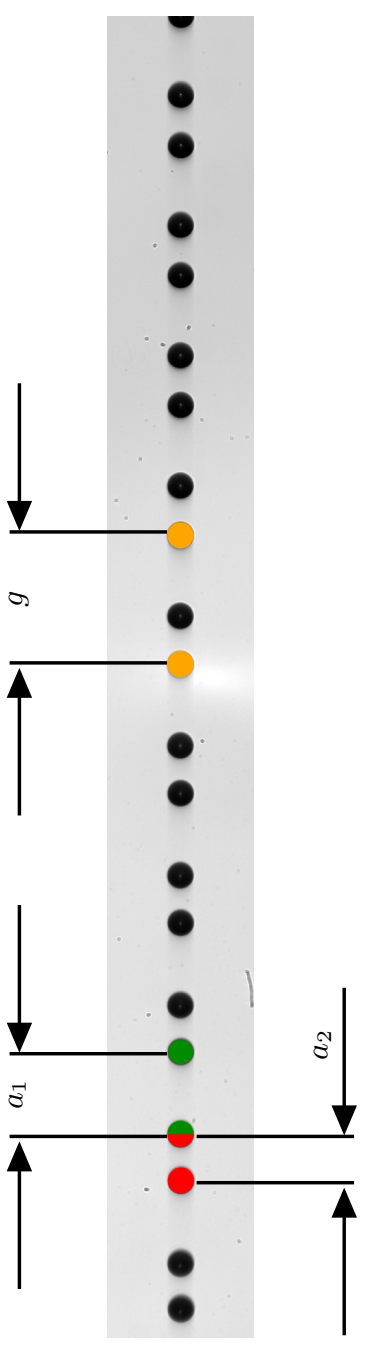

$l_{14}=58.5 \mathrm{~mm}$
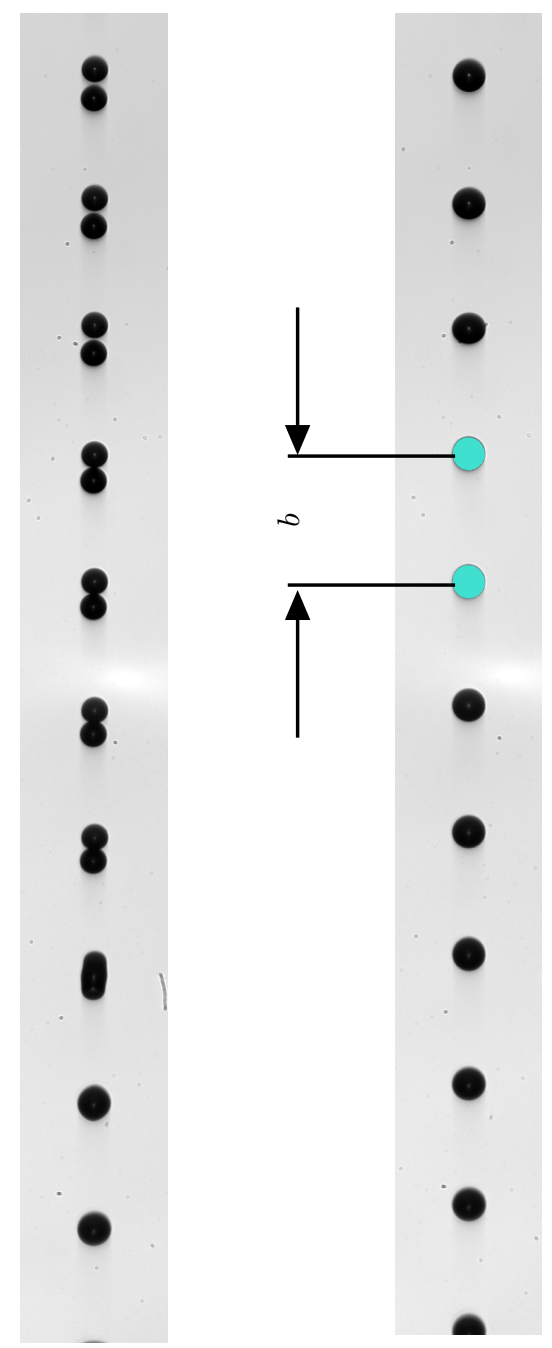

$l_{18}$

$l_{22}=92 \mathrm{~mm}$

Figure 5. Shadowgraphs of the droplet stream at different distances $l_{i}$ to the orifice plate. The distance $l$ increases from left to right by $16.8 \mathrm{~mm}$ from image to image. For a better understanding of Fig. 6 the spacing $a_{i}$ between neighbouring droplets before the coagulation of the two droplets of a group are indicated. The spacing $b$ indicates the inter droplet spacing after the collision

The spacing $g$ indicates the spacing between neighbouring groups (group length). 
The following measurements were performed on droplet streams with pairs of droplet generated initially as described above. The spacing $g$ between the groups consisting of two droplets along the droplet stream is shown in Fig. 4 . Again the shadowgraphs were evaluated as far as possible automatically using scripts developed using the image processing tool ImageJ. The behaviour of $g$ is similar to the development of the spacing $a$ in Fig. 3 . The data obtained can be approximated by a regression function according to $v=1 /\left(p_{3}+p_{4} l\right)$ where $p_{3}$ and $p_{4}$ denote constants. Integration gives the time $t$ a droplet needs to reach the distance $l$ from the orifice plate. Shadowgraphs of the droplet stream at different distances to the orifice are shown in Fig. 5. For clarification all important spacing are indicated. These shadowgraphs give an overview of the approaching and collision process. In Fig. 6 the spacing $a_{i}$ are plotted as a function of time $t$. The spacing seem to change exponentially. A regression with an exponential function $a_{i}=p_{5, i} \exp \left(p_{6, i} t\right)+p_{7, i}$ gives very good results with the constants $p_{j, i}$ taking into account the different inter droplet spacing. However, the data of $a_{1}$ and $a_{2}$ are not symmetrical to a horizontal line at $\left(a_{0,1}+a_{0,2}\right) / 2$ indicated by a dashed grey line in the figure. This is due to the decrease of the group spacing $g$ shown in Fig. 4. If the spacing $a_{i}$ are related to the actual inter group spacing $g$ the values of the relative spacing $a_{1} / g$ and $a_{2} / g$ are symmetrical as can be seen from Fig. 7 .

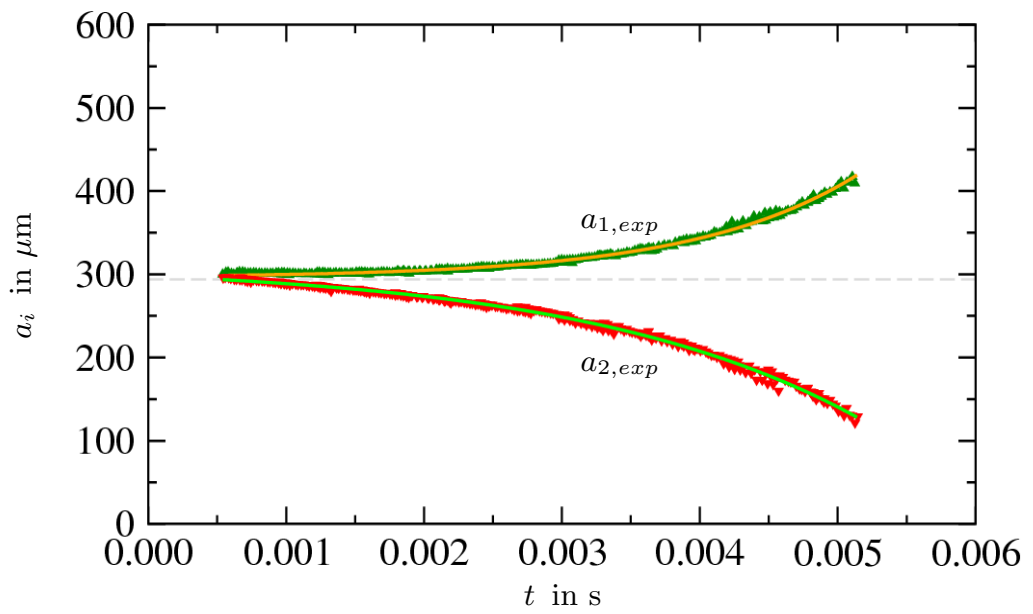

Figure 6. Spacing $a_{i}$ between neighbouring droplets as a function of time $t$. The distance $l$ was converted to the corresponding time $t$. The solid lines represent the exponential regressions to the data.

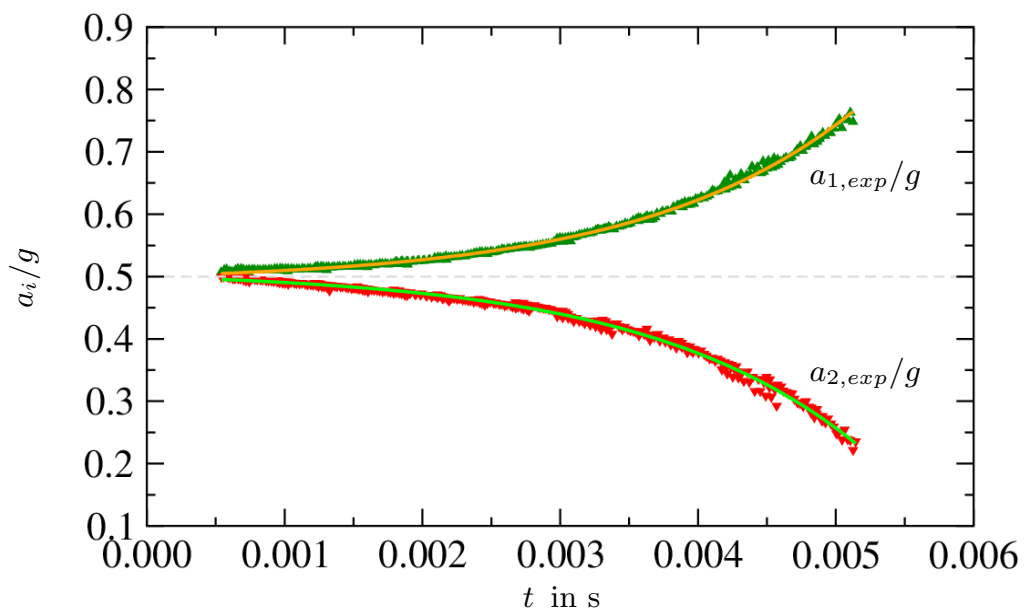

Figure 7. Relative spacing $a_{i} / g$ as a function of time $t$. The solid lines represent the exponential regressions of the data.

Numerical simulations were performed of the grouping process as described above. Results for two different sizes (cases $A$ and $B$ ) of the computational domain are presented in Fig. 8. The results are obtained in evaluating the results from FS3D with a MatLab code developed. Shown are the relative spacing $a_{i} / g$ as a function of time $t$ for both cases. In both cases the data can be approximated like the experimental data by an exponential function, which is represented by the black solid lines. A comparison of the numerical with the experimental results are shown in Fig. 9. Shown are the exponential regression of the data presented in Figs. 7 and 8 . The numerical results approach the experimental results for a wider computational domain. Differentiating the exponential function $a_{i} / g=$ $p_{8, i} \exp \left(p_{9, i} t\right)+p_{10, i}$ one obtaines the velocities $w_{i}=\partial\left(a_{i} / g\right) / \partial t=p_{9, i} p_{8, i} \exp \left(p_{9, i} t\right)$. These velocities indicate how fast the inter droplet spacing are changing. The velocities $w_{i}$ as a function of time are shown in Fig. 10. Here too, the numerical results with a wider computational domain are closer to the experimental results. Substituting the time $t$ by the relative distances $a_{i} / g$ straight lines are obtained, which can easily be shown. This means that the 


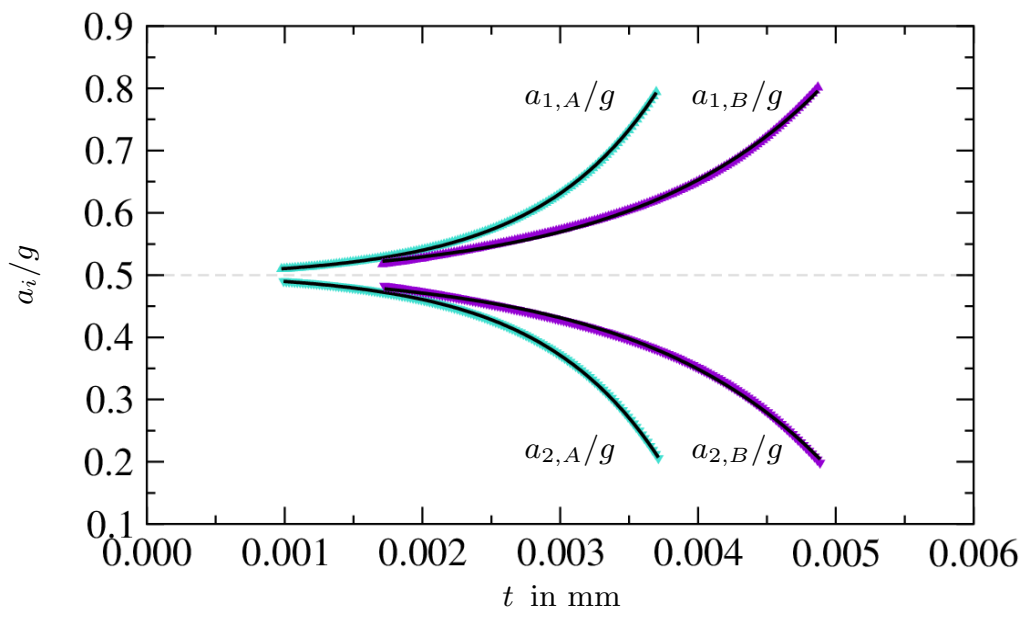

Figure 8. Relative spacing $a_{i} / g$ as a function of time $t$. Shown are results of numerical simulations for different sizes of the computational domain (Cases $A$ and $B$ ). The solid black lines represent the exponential regressions of the data.

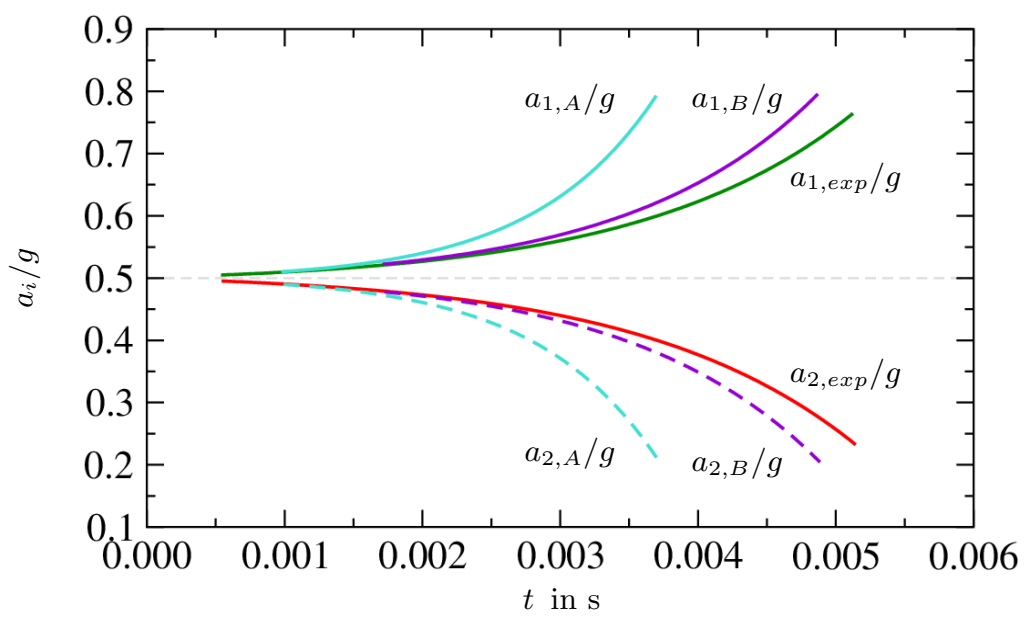

Figure 9. Relative spacing $a_{i} / g$ as a function of time $t$. Shown are results of numerical simulations for different sizes of the computational domain (Cases $A$ and $B$ ) in comparison with the experimental results (Index exp). All lines represent the exponential regressions of the data.

velocity $w_{i}$ two droplets are approaching is a linear function of the relative spacing $a_{i} / g$ between these two droplets. The results of this substitution is shown in Fig. 11. The comparison of numerical with experimental results shows the same trend, that for a wider computational domain the results of the numerical simulations are closer to the

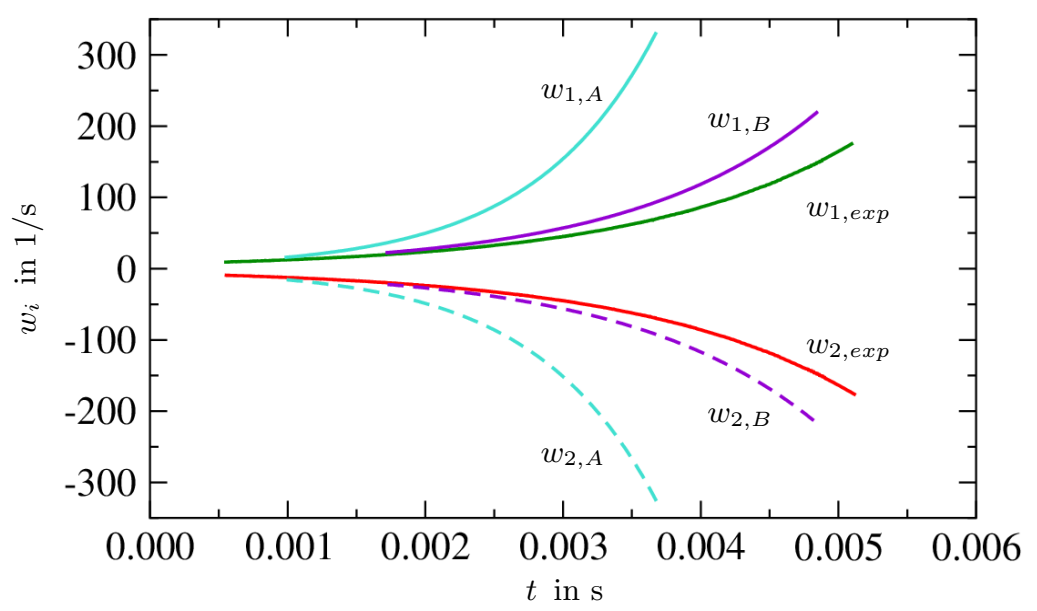

Figure 10. Velocities $w_{i}$ as a function of time $t$. Shown are results of numerical simulations for different sizes of the computational domain (Cases $A$ and $B$ ) in comparison with the experimental results (Index exp). All lines represent derivations of the exponential regressions to the data. 
experimental results.

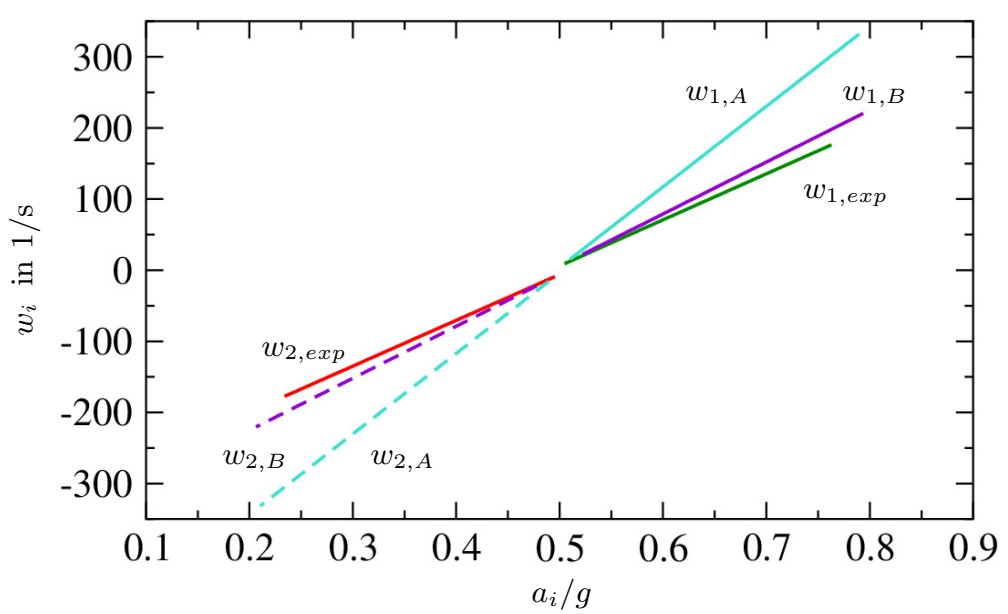

Figure 11. Velocities $w_{i}$ as a function of time relative spacing $a_{i} / g$. Shown are results of numerical simulations for different sizes of the computational domain (Cases $A$ and $B$ ) in comparison with the experimental results (Index exp). All lines represent derivations of the exponential regressions to the data.

\section{Conclusions}

Experiments and Direct Numerical Simulations were performed to study grouping effects in monodisperse droplet streams. It was described, how droplet streams with groups of two droplets can be generated experimentally. The setup for the simulation was presented. It was found, that the behaviour, how droplets within a group approach each other, can be approximated by an exponential law. Assuming such an exponential behaviour the velocity the droplet approach is a linear function of the actual distance between the droplets. This was found in the experiments as well as in the numerical simulations. In comparison of the simulations with the experimental results a wider computational domain show results, which are closer to the experimental results.

\section{Acknowledgements}

\section{Nomenclature}

$D \quad$ droplet diameter [m]

a inter droplet spacing [m]

$b \quad$ inter droplet spacing after coagulation [m]

$f_{1} \quad$ frequency of the group generation $[\mathrm{Hz}]$

$f_{2} \quad$ frequency of the droplet generation within a group [Hz]

$f_{\text {opt }} \quad$ optimum wave frequency at Rayleigh disintegration $[\mathrm{Hz}]$

$f_{s} \quad$ excitation frequency $[\mathrm{Hz}]$

$g \quad$ spacing between the droplet groups [m]

$d_{i} \quad$ dimensions of computational domain with $i=x, y, z[\mathrm{~m}]$

$l, l_{i} \quad$ distance to the orifice of the droplet stream generator [m]

$n \quad$ number of droplets in a group [-]

$p_{3}, p_{4} \quad$ parameters for the approximation of the velocity (various dimensions)

$p_{j, i} \quad$ parameters for the exponential approximations, $j=1$ and $2, i=1$ to 5 (various dimensions)

$r \quad$ radius [m]

$v \quad$ droplet velocity $[\mathrm{m} / \mathrm{s}]$

$w \quad$ velocity the relative inter droplet spacing change [1/s]

Greek symbols

$\lambda \quad$ optimum wave length at Rayleigh disintegration [m]

Indices

$m \quad$ mass $[\mathrm{kg}]$

a acceleration $\left[\mathrm{m} \mathrm{s}^{2}\right]$

$F \quad$ force [N]

$m \quad$ mass $[\mathrm{kg}]$

\section{References}

[1] J. Heinlin and U. Fritsching. Droplet clustering in sprays. Experiments in Fluids, Vol. 40, pp. 464-472, 2006. 
[2] D. Katoshevski. Characteristics of spray grouping/non-grouping behavior. Aerosol Air Quality Research, Vol. 6, No. 10, pp. 54-66, 2006.

[3] D. Katoshevski, T. Shakked, S.S. Sazhin, C. Crua, and M.R. Heikal. Grouping and trapping of evaporating droplets in an oscillating flow. International Journal Heat Fluid Flow, Vol. 29, pp. 415-426, 2008.

[4] J. Gallego-Juaarez, E. Riera-Franco de Sarbia, G. Rodriguez-Corral, T.L. Hoffmann, and J. Gaalvez-Moraleda. Application of acoustic agglomeration to reduce fine particle emissions from coal combustion plants. Environ. Sci. Technol, Vol. 33, pp. 3843-3849, 1999.

[5] K. Anders, N. Roth, and A. Frohn. Operation characteristics of vibrating-orifice generators: The coherence length. Part. Part. Syst. Charact., Vol. 9, pp. 40-43, 1992.

[6] M. Orme and E. P. Muntz. The manipulation of capillary stream breakup using amplitude-modulated disturbances: A pictorial and quantitative representation. Physics of Fluids, Vol. 2, pp. 1124-1140, 1990.

[7] J. H. Hilbing and S. D. Heister. Droplet size control in liquid jet breakup. Physics of Fluids, Vol. 8, pp. 1574$1581,1996$.

[8] G. Brenn and U. Lackermeier. Drop formation from a vibrating orifice generator driven by modulated electrical signals. Physics of Fluids, Vol. 9, pp. 3658-3669, 1997.

[9] N. Roth, H. Gomaa, and B. Weigand. Self-stabilization phenomena in the operation of droplet stream generators. DIPSI Workshop 2010 on Droplet Impact Phenomena \& Spray Investigation, 2014.

[10] Lord J.S.W. Rayleigh F.R.S. On the instability of a cylinder of viscous liquid under capillary force. Philos. Mag., Vol. 34, pp. 145-154, 1892.

[11] K. Eisenschmidt, M. Ertl, H. Gomaa, C. Kieffer-Roth, C. Meister, P. Rauschenberger, M. Reitzle, K. Schlottke, and B. Weigand. Direct numerical simulations for multiphase flows: An overview of the multiphase code fs $3 \mathrm{~d}$. J. of Applied Math, Vol. 272, pp. 508-517, 2016.

[12] C.A. Schneider, W.S. Rasband, and K.W. Eliceiri. Nih image to imagej: 25 years of image analysis. Nature Methods, Vol. 9, pp. 671-675, 2012. 


\title{
Comparison between numerical and experimental water-in-oil dispersion in a microchannel
}

\author{
Philippe Desjonquères ${ }^{1}$, Thibault Ménard ${ }^{1}$, Dominique Tarlet $^{* 2}$, Jérôme Bellettre ${ }^{2}$ \\ ${ }^{1}$ UMR 6614-CORIA, Technopôle du Madrillet, B.P. 12, Avenue de l'Université, 76801 Saint \\ Etienne du Rouvray Cedex, France \\ ${ }^{2}$ Laboratoire de Thermique et Energie de Nantes, LTEN UMR CNRS 6607,BP 50609, 1 rue \\ Christian Pauc, 44306 Nantes cedex 3, France \\ ${ }^{*}$ Corresponding author: dominique.tarlet@univ-nantes.fr
}

\begin{abstract}
The dispersion of water inside a flow of oil is investigated in a microfluidic device, producing a water-in-oil emulsion. The liquid-liquid flow mainly differs from those presented in existing literature through its high capillary number (between 3 and 14), and in the head-on collision between water and oil streams. By comparing with experimental data, numerical simulations can provide more information about the topology of the flow. A coupled Volume of Fluid and Level Set method (CLSVOF) is used to treat the interface between both phases and incompressible Navier-Stokes equations are solved. Three set of parameters, close to those in the experimental setup, are investigated to compare experimental and numerical results. The comparison between experiments and simulation provides a precise knowledge of the liquid-liquid dispersion process and the overall flow pattern within the microfluidic device.
\end{abstract}

\section{Keywords}

Microchannel, water-in-oil dispersion, liquid-liquid flow

\section{Introduction}

Liquid-liquid dispersion within microfluidic devices has become an important issue over the last decade [1]. An emulsion is defined as the temporarily stable dispersion of a liquid into another one that is not miscible [2]. When the scale of the liquid-liquid flow is smaller than its capillary length [3], interfacial tension dominates over shear stress and gravity [4], making the dispersion highly reproducible in slow conditions [5]. These slow conditions ensure a highly monodisperse emulsion [6], that is usually appropriate for targeted applications like microreaction synthesis [7]. However, other application like high flow-rate biofuel production [8] benefit from the considerably increased surface-to-volume ratio $[9,10]$ of microfluidic liquid-liquid dispersion.

In order to better understand the physics of microfluidic in high flow-rate liquid-liquid dispersion, experimental results of the obtained mean diameter and liquid-liquid flow photographies [8] are compared to numerical results. At the present stage, a quantitative validation of the model is not obtained, but we present a qualitative comparison of the liquid-liquid flow pattern. In a first part, experimental material and methods are presented, then numerical methods used to investigate such flows are briefly detailed. Finally, first comparisons are discussed.

\section{Material and methods}

The experimental facility is designed to produce high flow-rate water-in-oil flow within a microfluidic device. The capillary number $\mathrm{Ca}(\mathrm{Eq} .1)$ represents the ratio between shear stress $(\mu \mathrm{V} / \mathrm{L})$ and interfacial tension $\sigma_{\mathrm{mo} / \mathrm{L} .} \mu$ and $\mathrm{V}$ are the viscosity and the superficial velocity of the continuous phase, e.g. filtered sunflower oil in thiscase. It reaches values between 2 and 14, which is three orders of magnitude higher than most of the situations investigated in scientific literature [11].

$$
\mathrm{Ca}=\mu \mathrm{V} / \sigma_{\text {wo }}
$$

The microfluidic device shown Fig. 1 enables a finely dispersed emulsion due to three main reasons. First, streams of oil and water are faced in a head-on collision. This maximizes the energy available to fractionate the water stream, since kinetic energies are added in the collision. Secondly, the viscosity $\mu$ is very high (up to 52.2 $\mathrm{mPa}$.s) to maximize shear stress from the continuous phase. Thirdly, the water inlet is smaller than the oil inlet to produce a swirling flow downstream the impinging zone. Such a swirling flow enhances curvature of streamlines, and in turn shear-induced break-up. The oil inlet and the emulsion outlets are squared cross-sections, with 600 $\mu \mathrm{m}$ per side. The water inlet is also squared, with $300 \mu \mathrm{m}$ per side. All connections between pumps and mini- 
channel are made using Fluoropolymer (FEP) tubing with an internal diameter of $1.55 \mathrm{~mm}$. The outlets are at the atmospheric pressure.

The microchannel is made of two PMMA slabs screwed together to avoid leakage at high injection pressure up to 7 bar. The hydrophobic PMMA material [12] is widely used in the field of two-phase microfluidics, to avoid destruction of the flow structure due to wettability effects on the walls [1]. The average surface roughness on the etched surface at the bottom of the channel is $460 \mathrm{~nm}$, measured by means of an ALICONA optical profilometer. This roughness is very small, when compared with the size of the channels (between 200 and $600 \mu \mathrm{m}$ ), and cannot significantly disturb the flow. The interfacial tension was measured using a KRÜSS tensiometer K-12, since $\sigma_{\mathrm{wo}}$ is a crucial property to investigate the physics of shear induced breakup [11]. The temperature of oil and water is maintained at $25^{\circ} \mathrm{C}$ in a LAUDA thermostated bath, and measured by a thermocouple in the beakers and in the microfluidic device. The size of dispersed water droplets is measured in a sample of emulsion coming out from the microfluidic device using a ZEISS optical microscope. The sufficient size of the sample is determined to calculate the mean diameter of water droplets.

Two piston pumps (ARMENAPF-100-25-1) are used for supplying water and filtered sunflower oil at a high pressure (up to 5 bars). Flow rate measurements are performed using weighing scales (Sartorius-MSE $2203,1 \mathrm{~Hz}$ sampling) connected to a computer. The weighing scales have a measurement accuracy of $10^{-3} \mathrm{~g}$, which results in an uncertainty of $5 \%$ of the flow rate. The flow pattern is observed with a fast CCD camera LAVision HighSpeed Star 6 equipped with the macro objective, necessary to resolve the millimeter scale. The focus was made in the channel depth. The light source consists of a $50 \mathrm{~W}$ halogen spot located in the line of sight, beyond the transparent microfluidic device.

All the details about conception of the microchannel and the experimental set-up, including measurement uncertainties can be found in [8].
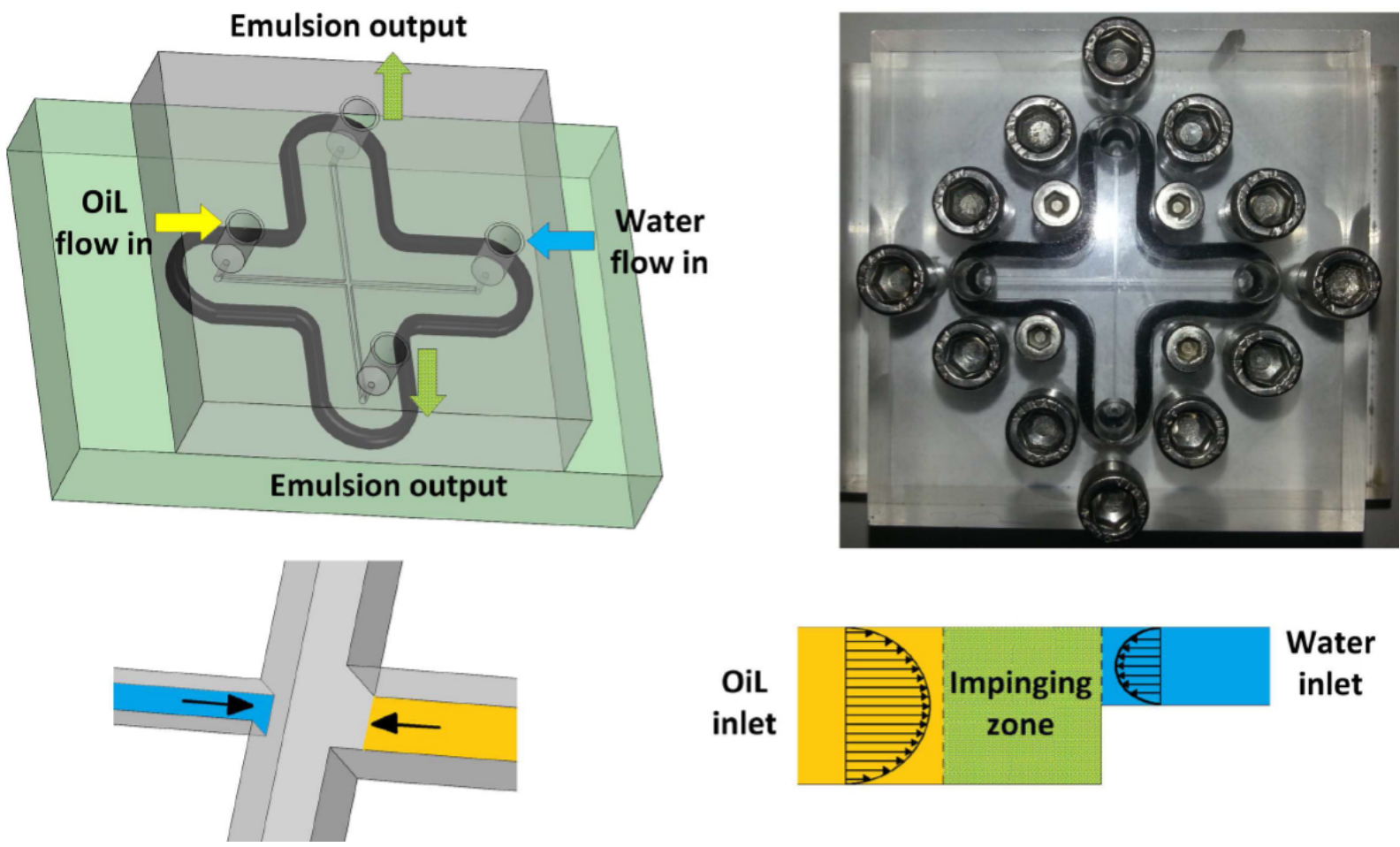

Figure 1.Microfluidic device : water and oil streams are faced in a head-on collision within the impinging zone.

The used fluids are tap water and oil with additives to decrease viscosity $\mu$ and interfacial tension $\sigma_{\text {wo }}$ : Butanol, and surfactant SPAN 83. Tab. 1 shows the three cases chosen for the present study : A, B and C with physical properties at $25^{\circ} \mathrm{C}$, as it was maintained in the experimental set-up. The capillary length $L_{c}$ is also mentioned : it is always larger than the channel side so that interfacial tension dominates [4]. The obtained mean diameter $D_{d}$ shown in Tab. 1 is similar (between 20 and $21 \mu \mathrm{m}$ ) for cases $(A)$ and $(B)$, it is more than $50 \% \operatorname{larger}(32.48 \mu \mathrm{m})$ in case $(C)$. The effect of a lower viscosity of the continuous phase is a decreased shear stress exerted on the water phase, resulting in a larger mean diameter of water droplets. 
Table 1.Characteristics of the three cases chosen for study.

\begin{tabular}{|c|c|c|c|}
\hline Cases & A & B & C \\
\hline $\begin{array}{c}\text { Continuous phase } \\
\text { at } 25^{\circ} \mathrm{C}\end{array}$ & Pure oil & $\begin{array}{c}\text { Oil + 0.3\% wt. } \\
\text { SPAN 83 }\end{array}$ & Oil + 5\% wt. Butanol \\
\hline Qo $\left[\mathrm{mL} . \mathrm{min}^{-1}\right]$ & 72.55 & 62.84 & 76.23 \\
\hline Qw [mL.min $\left.{ }^{-1}\right]$ & 6.94 & 9.06 & 13.85 \\
\hline$\mu[\mathrm{mPa} . \mathrm{s}]$ & 52.2 & 52.2 & 40.4 \\
\hline$\sigma_{\mathrm{wo}}\left[\mathrm{mN} \cdot \mathrm{m}^{-1}\right]$ & 27.6 & 11.7 & 32.48 \\
\hline $\mathrm{D}_{\mathrm{d}}[\mu \mathrm{m}]$ & 20.78 & 20.46 & 3.13 \\
\hline $\mathrm{L}_{\mathrm{c}}[\mathrm{mm}]$ & 4.6 & 2.99 & \\
\hline
\end{tabular}

\section{Numerical methods}

To simulate such flow, we use an in-house code generally applied for the study of liquid jet atomization [13]. The following incompressible Navier-Stokes equations are solved thanks to a projection method and coupled with interface transport equation performed by a CLSVOF method [14,13]:

$$
\frac{\partial \rho \boldsymbol{U}}{\partial t}+\boldsymbol{\nabla} \cdot(\rho \boldsymbol{U} \boldsymbol{U})=-\boldsymbol{\nabla} p+\boldsymbol{\nabla} \cdot(2 \mu \boldsymbol{D})+\sigma \kappa \boldsymbol{n} \delta_{\Gamma}
$$

Surface tension force is treated as a jump condition for the pressure through a Ghost Fluid method [15]. The convective term is computed in a mass/momentum conserving framework [16,17], where one part comes from mass fluxes deduced by the VOF advection and the second part comes from a WENO5 interpolation. The diffusive term is computed thanks to the method developed by Sussman [18] and physical properties are expressed by the VOF or Level Set functions.

The main idea of the CLSVOF method is to benefit of both geometrical computations (normal and curvature of interface) from Level Set, and mass conservation from the VOF. The transport of the VOF is ensured by the method developed by Weymouth [19].

Due to the symmetry of the flow, only half of microchannel is computed and symmetric boundary condition is imposed to mimic the other part. Then, inlet boundary conditions for the water and oil are prescribed using a velocity profile of square channel and flow rates of experimental setup are imposed. Finally, no specific treatment is dedicated for the dynamic of triple line on wall boundary condition. Numerical domain is presented on the figure 2. This equivalent size is $(1800 \times 1800 \times 600) \mu \mathrm{m}^{3}$, that means water and oil inlet channel have a length of $600 \mu \mathrm{m}$, and the equivalent mesh size is $(192 \times 192 \times 64)$. 

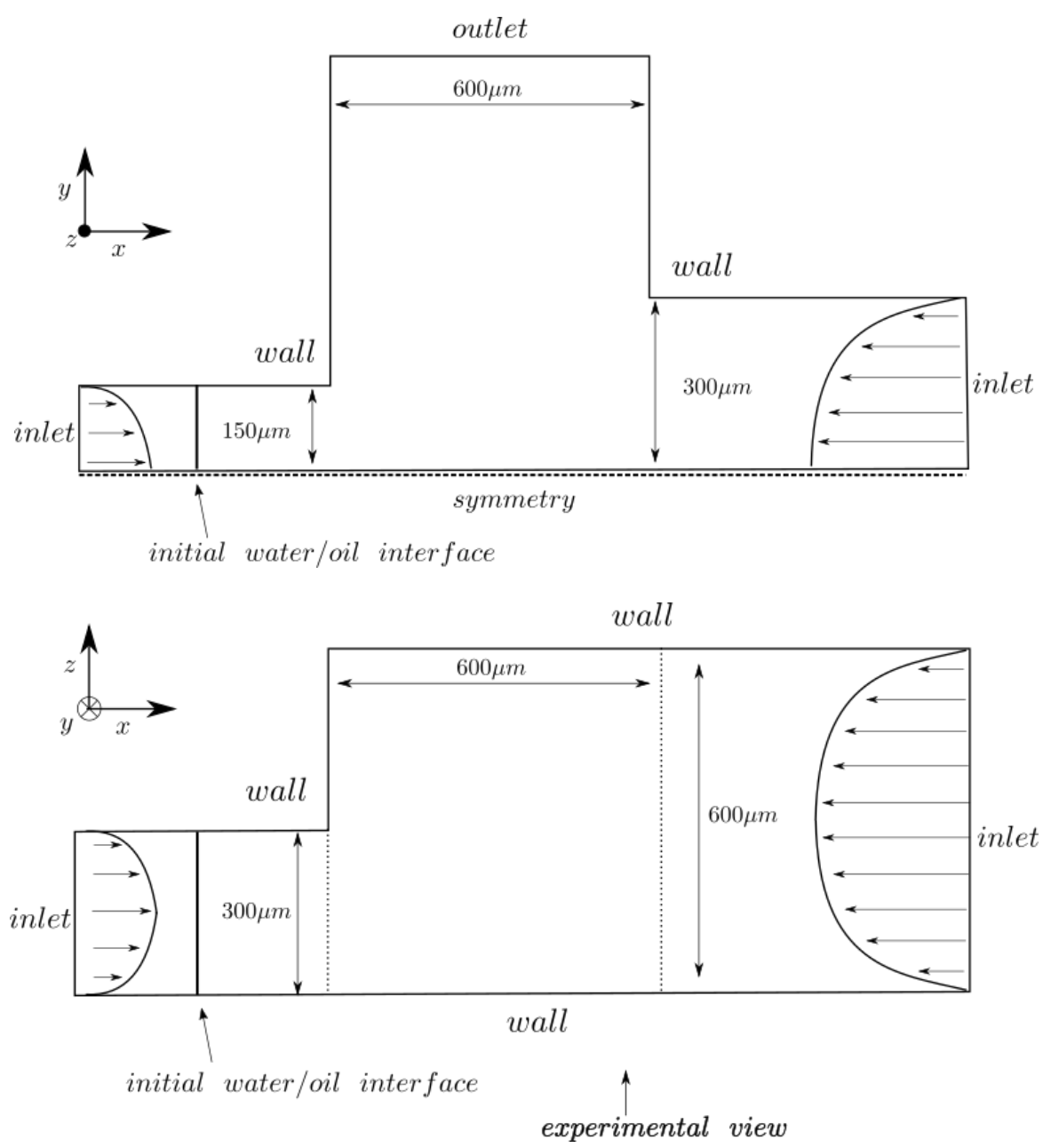

Figure 2.Computational domain.

\section{Results and discussion}

Fig. 3 shows the images from the CCD camera, within the impinging zone. In cases $(A)$ and $(B)$, where viscosity of the continuous phase is high $(\mu=52.2 \mathrm{mPa} . \mathrm{s})$, the water stream is limited in space. Whereas in case $(C)$, which has lower viscosity ( $\mu=40.4 \mathrm{mPa}$.s), the water phase invades nearly all the available space of the channel. In all cases, a symmetry of the liquid-liquid flow can be noticed downstream the impinging zone. A swirling flow can be seen, enhanced by the higher position of the water inlet.

On the Fig. 4 are reported numerical results of cases A, B and C in the same view of experimental images. We can observe that computations present some attached structures on the microchannel walls. Some of them are not observed in the experiment as shown in the case B (Fig. 4). Nevertheless the attached structure with the arc shape seems to be similar to what is observed experimentally (case A and B), with different stretching (especially for the case B).

In general experimental images show smooth shapes compared to numerical pictures, except for the case $\mathrm{C}$ where numerical computation reproduces perturbed two phase flows.

In the three cases, the water/oil interface reaches the oil microchannel, and goes inside for the case $\mathrm{C}$, on the contrary to experimental behaviour. All differences observed in the three cases reveal that the numerical computation is too coarse and probably needs a model to capture the dynamic of the triple line. 


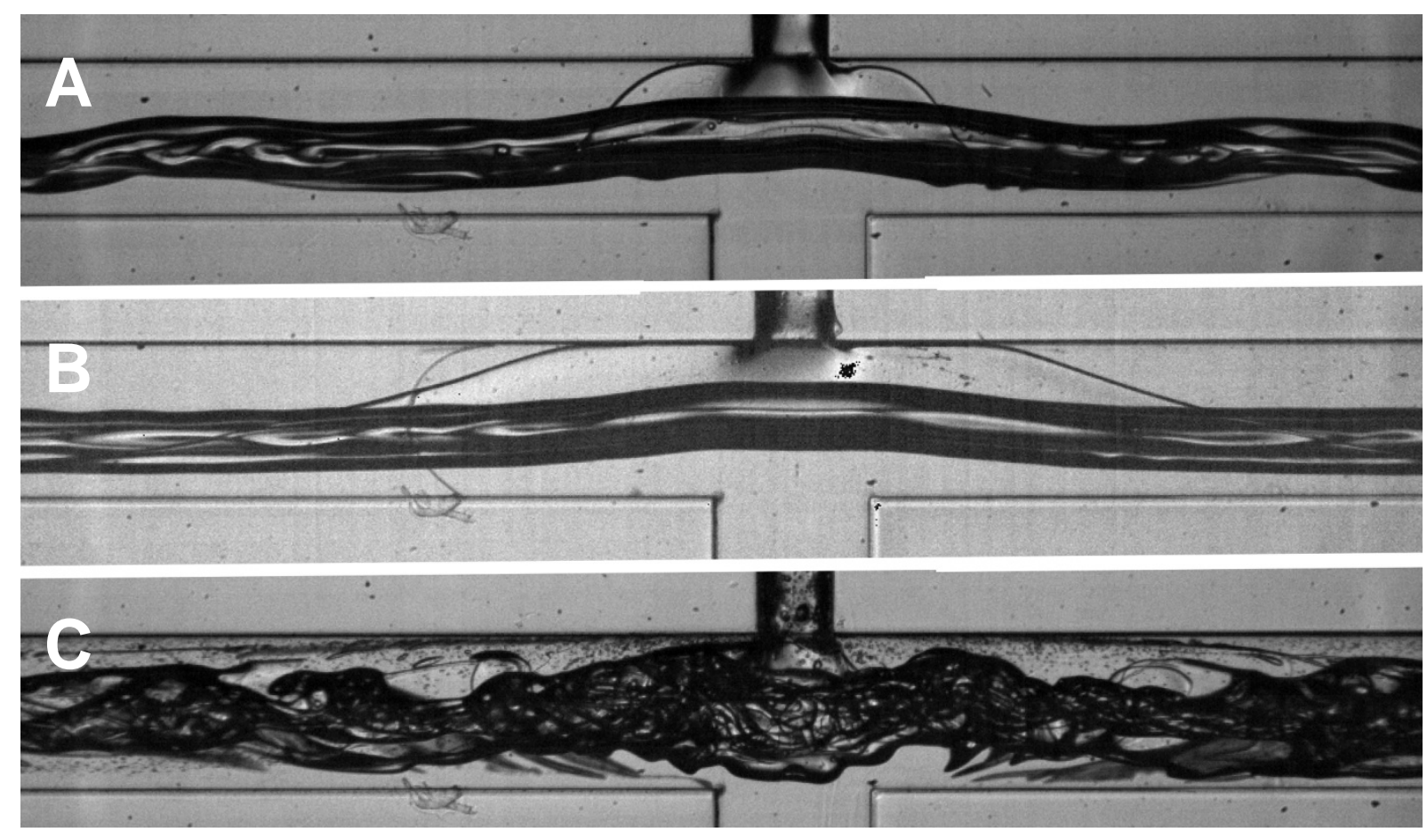

Figure 3.Image of the CCD camera in the impinging zone : Case A (top), B (middle) and C (bottom) - channel width : $600 \mu \mathrm{m}$.

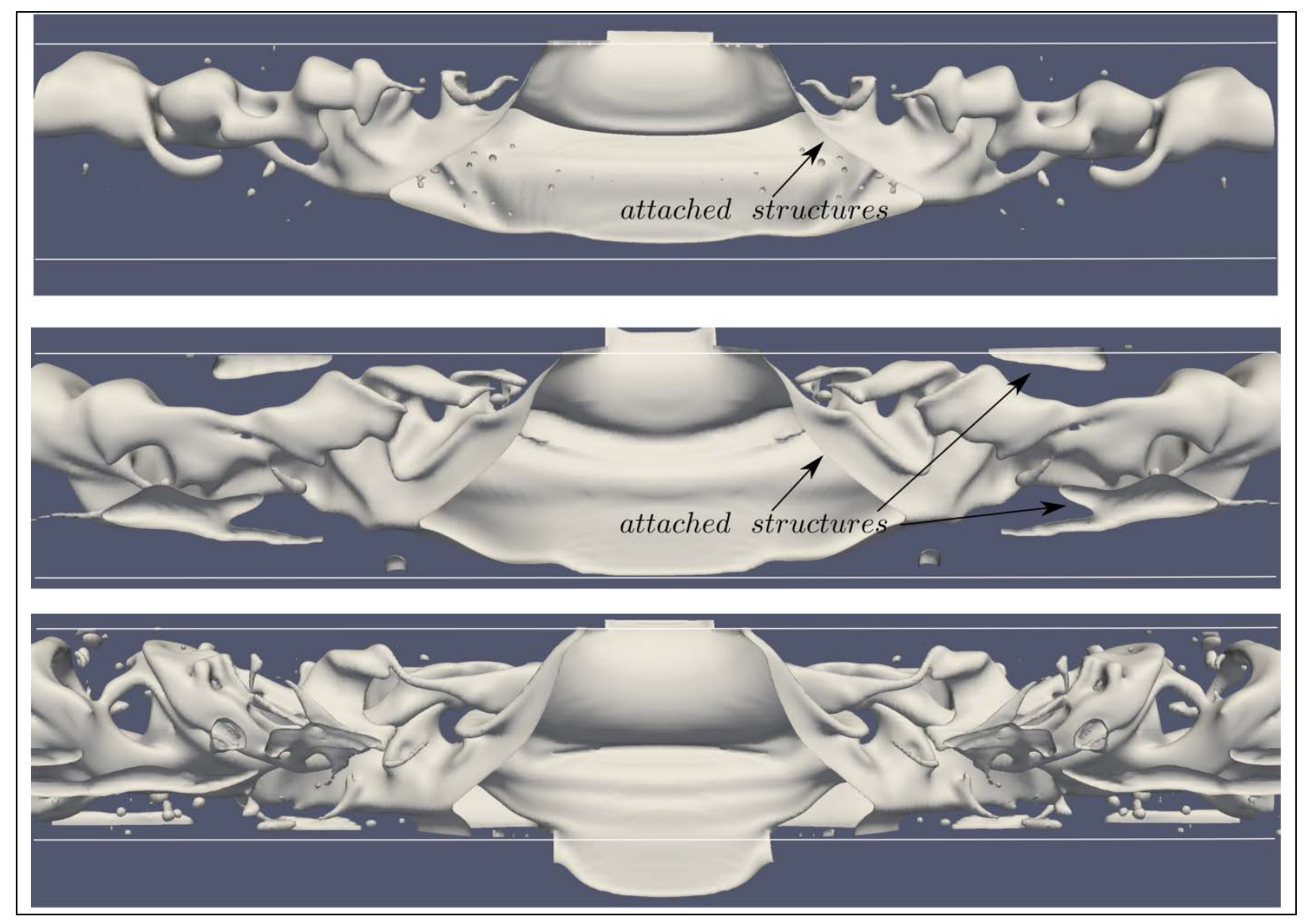

Figure 4.Experimental view of numerical results. (Top to bottom : cases A, B and C) 
Figure 5 reproduces some slices of case $A$ to show the structure of the flow in the impact zone and its consequences in the rest of the microchannel. We can see that the vortex formed by the impact of water/oil flow, subsist on the microchannel and then rolls up the interface. This behaviour, present in all cases, is responsible for the formation of very thin structures which are not well captured by these coarse computations. These structures will probably influence the drop size distribution observed in the experiment at the end of the microchannel.
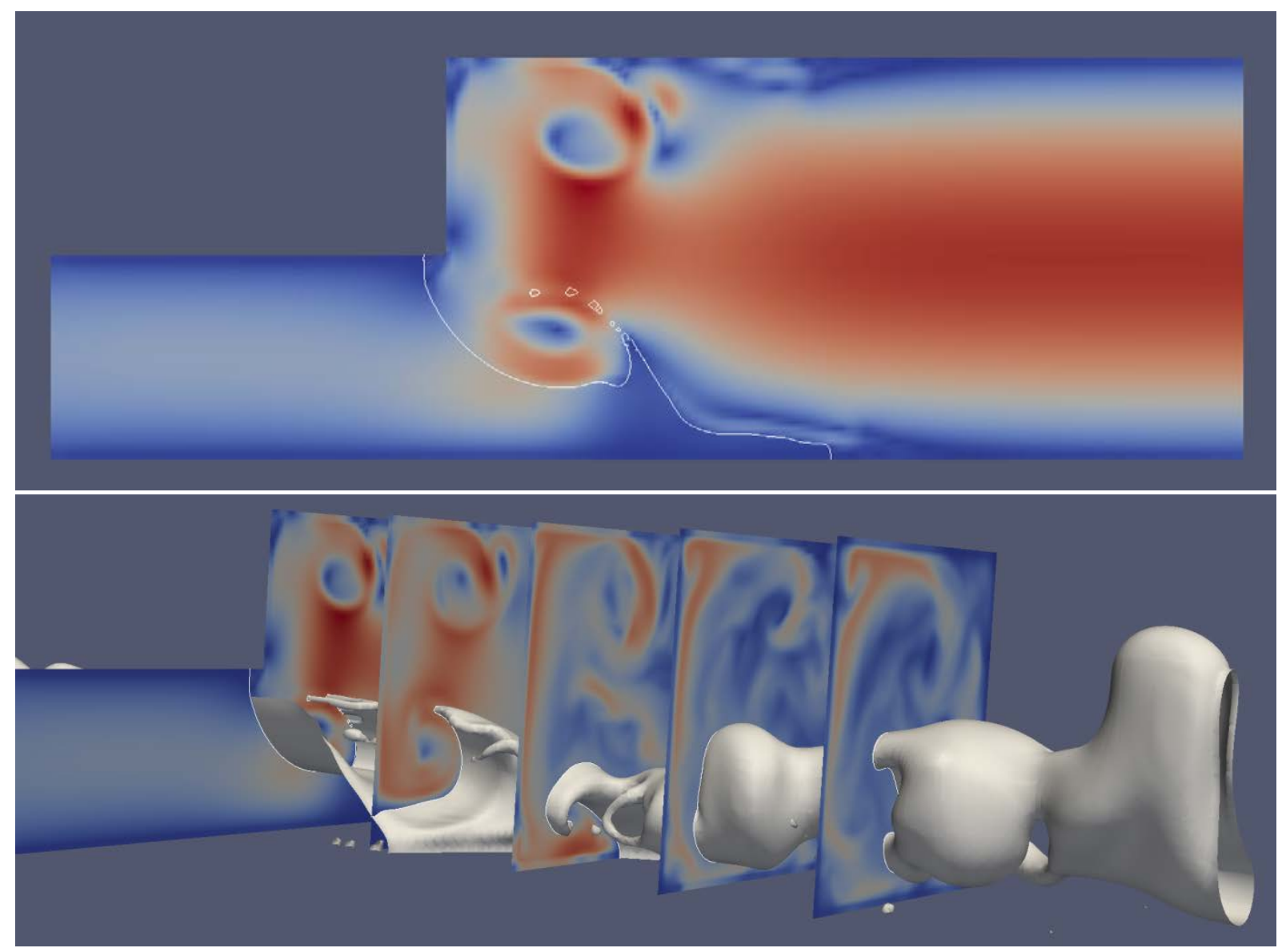

Figure 5.Structure of the flow on the impact zone and at different heights of the micro-channel coloured by velocity, from blue (slowest), to red (fastest).

\section{Conclusions}

The comparison between experimental water-in-oil dispersion within a microchannel and numerical simulation are carried out qualitatively at this stage. Despite the observed differences, these results show the capabilities of numerical computation to capture and reproduce the main structure of the flow. They should easily be improved with a finest mesh. The triple line and contact angle dynamics is probably another parameter which can influence the numerical results.

Finally, to investigate the droplet size distribution, a longer computation domain has to be simulated.

\section{Acknowledgements}

Computations have been carried out in CRIANN (Centre Régional Informatique et d'Applications Numériques de Normandie). 


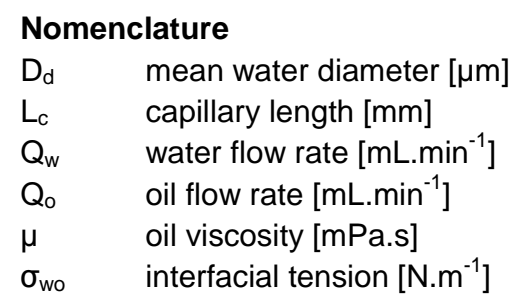

\section{References}

[1] Zhao, C.-X., Middelberg, A.P.J., 2011, Chemical Engineering Science, 66, pp. 1394-1411.

[2] Engl, W., Backov, R., Panizza, P., 2008. Controlled production of emulsions and particles by milli- and microfluidic techniques. Current Opinion inColloid \& Interface Science 13 (13), pp. 206-216.

[3] Aarts, D., 2005. Capillary length in a fluid-fluid demixed colloid-polymermixture. J. Phys. Chem. B, 109, pp. 7407-7411.

[4] de Gennes, P.-G., Brochard-Wyart, F., Quéré, D., 2005. "Gouttes, bulles, perleset ondes". Belin.

[5] Engl, W., Backov, R., Panizza, P., 2008. Controlled production of emulsions and particles by milli- and microuidic techniques. Current Opinion in Colloid \& Interface Science 13 (13), pp. 206 -216.

[6] Sugiura, S., Nakajima, M., Iwamoto, S., Seki, M., 2001. Interfacial tensiondriven monodispersed droplet formation from microfabricated channel array. Langmuir 17 (1), pp. 5562-5566.

[7] Meng, Z.-J., Wang, W., Liang, X., Zheng, W.-C., Deng, N.-N., Xie, R., Ju, X.-J., Liu, Z., Chu, L.-Y., 2015. Plugand-play microfluidic systems from flexible assembly of glass-based flow-control modules. Lab Chip 15, pp. 18691878.

[8] Belkadi, A., Tarlet, D., Montillet, A., Bellettre, J., Massoli, P., 2015.Water-in-oil emulsification in a microfluidic impinging flow at highcapillary numbers. International Journal of Multiphase Flow 72, pp. 11-23.

[9] Burns, J. R., Ramshaw, C., 2001. The intensification of rapid reactions inmultiphase systems using slug flow in capillaries. Lab Chip 1, 10-15.

[10] Song, H., Tice, J., Ismagilov, R. F., 2003. A microfluidic system for controllingreaction networks in time. Angewandte Chemie International Edition 42 (7), pp. 768-772.

[11] Tice, J., Lyon, A., Ismaglov, R., 2004. Effects of viscosity on droplet formationand mixing in microfluidic channels. Analytica Chimica Acta 507, pp.73-77.

[12] Nisisako, T., Torii, T., Higuchi, T., 2002. Droplet formation in a microchannelnetwork. Lab on a chip 2 (1), $24-$ 6.

[13]T. Ménard, S. Tanguy, and A. Berlemont. Coupling level set/vof/ghost fluid methods: Validation and application to 3dsimulation of the primary break-up of a liquid jet. International Journal of Multiphase Flow, 33(5):510 - 524, 2007.

[14]Mark Sussman and Elbridge Gerry Puckett. A coupled level set and volume-of-fluid method for computing 3d andaxisymmetric incompressible two-phase flows. Journal of Computational Physics, 162(2):301 - 337, 2000.

[15]Ronald P Fedkiw, Tariq Aslam, Barry Merriman, and Stanley Osher. A non-oscillatory eulerian approach to interfaces inmultimaterial flows (the ghost fluid method). Journal of Computational Physics, 152(2):457 - 492, 1999.

[16]Murray Rudman. A volume-tracking method for incompressible multifluid flows with large density variations.International Journal for Numerical Methods in Fluids, 28(2):357-378, 1998.

[17]G. Vaudor, T. Ménard, M. Doring, W. Aniszewski, A. Berlemont.A consistent mass and momentum flux computation method for two phaseflows. Application to atomization process.Computer and Fluids (submitted) [18]M. Sussman, K.M. Smith, M.Y. Hussaini, M. Ohta, and R. Zhi-Wei. A sharp interface method for incompressible two-phaseflows. Journal of Computational Physics, 221(2):469 - 505, 2007. 


\title{
Simulation of droplet spreading on micro-CT reconstructed 3D real porous media using the volume-of-fluid method
}

\author{
M. Aboukhedr ${ }^{1 *}$, N. Mitroglou ${ }^{1}$, A. Georgoulas ${ }^{2}$, M. Marengo $^{2}$, K. Vogiatzaki ${ }^{2}$ \\ ${ }^{1}$ Department of Mechanical Engineering, City University of London, UK \\ ${ }^{2}$ Advanced Engineering Centre, University of Brighton, Brighton, UK \\ ${ }^{*}$ Corresponding author: mahmoud.aboukhedr.1@city.ac.uk
}

\begin{abstract}
Droplet impact on porous media has a broad range of applications such as material processing, drug delivery and ink injection etc. The simulation studies of such processes are rather limited. To represent the spreading and absorption process of the droplet on porous materials, robust numerical schemes capable of accurately representing wettability as well as capillary effects need to be established. The current work, presents one of the first studies of droplet impact on a real porous media geometry model extracted from a micro-CT scan. The process involves processing of CT image and subsequent threshold based on the structures segmentation. The porous geometry is extracted in the form of a STL (STereoLithography) model, which, with the aid of dedicated software like ANSA and SnappyHexMesh, is converted to an unstructured mesh for successful discretization of the flow domain. The solution algorithm is developed within the open source CFD toolbox OpenFOAM. The numerical framework to track the droplet interface during the impact and the absorption phases is based on previous work [1, 2]. The volume-of-fluid (VOF) method is used to capture the location of the interface, combined with additional sharpening and smoothing algorithms to minimise spurious velocities developed at the capillary dominated part of the phenomenon (droplet recession and penetration). A systematic variation of the main factors that affect this process are considered, i.e. wettability, porous size, impact velocity. To investigate the influence of porous structures on droplet spreading, the average porosity of the media is varied between $18.5 \%$ and $23.3 \%$. From these numerical experiments, we can conclude that the droplet imbibition mainly depends on the porous wettability and secondly that the recoiling phase can be observed in the hydrophobic case but not in the hydrophilic case.
\end{abstract}

Keywords; Droplet Spreading, Droplet Absorption, Porous media, 3D micro-Topography, VOF

\section{Introduction}

Micro-scale fluid phenomena are involved in various applications and research areas [3]. Understanding the behaviour of droplet spreading on porous media is important for a variety of industrial applications, such as ink jet printing, raindrops on textile, spray paint on wood, 3D-printing, penetration of rain drops into building walls, needle less injection, coating of porous materials, irrigation, cooling of electronic devices etc. Droplet spreading on solid flat surfaces has been the subject of numerous experimental and numerical studies over the last few decades $[5,6]$. However, droplet impact on porous media is still far from being understood. Studies of such micro-scale fluid phenomena need careful and combined consideration of droplet dynamics and porous media characteristics. Generally, this phenomenon is controlled by two main counter-acting processes: droplet spreading on porous surfaces and imbibition inside the porous media [8]. As the droplet spreads on the surface it also fills the voids of the porous material due to capillary action. The spreading behaviour of the impinging droplet on the surface is known to depend on the liquid properties, i.e. density, viscosity and surface tension, impact conditions such as drop size and impact velocity, and surface wettability [9]. Absorption, on the other hand, is governed by both, the liquid and the porous medium properties, like porosity, pore size and pore wettability [10]. Droplet spreading on implicit or of non-realistic shapes porous media using numerical methods has been investigated in a limited number of studies $[11,14]$. Moreover, limited CFD have been published in the literature regarding the effect of porous media heterogeneity on imbibition using explicit porous geometry. Davis and Hocking [15] models were developed to study the time evolution of drop spreading, the position of contact line and liquid motion inside the porous media. In [16] three-dimensional simulations were carried out to study the effect of impact velocity and surface roughness on the spreading of droplets on to a substrate consisting of randomly placed and orientated freely penetrating disks using the lattice-Boltzmann method. The same methodology was used in [17] to simulate the pore-level droplet spreading on a porous surface and investigated the power-law time-evolution of the wetted zone radius.

The present study attempts to numerically analyse the characteristics of a droplet interacting with a real porous surface, using a 3D porous media that is presented in [18]. A direct comparison of simulation versus experimental results found in [19] is presented, alongside an investigation of time evolution of impinging droplet shape as well as velocity. Finally, the effect of permeability is examined through the parametric study.

\section{Micro-CT porous geometry}

This section discusses procedure followed to extract a computational mesh from micro-CT scan data. As a nondestructive technique, X-ray micro-CT provides the required insight into opaque objects eliminating the need of sectioning/partitioning. A micro-CT scanner uses X-rays that penetrate a 3D object and create density-based attenuated two-dimensional projections, which are collected at small angular steps, preferably, around 360 degrees. 
These projections are later synthesised, using a variety of techniques, and the complete volume of the object is reconstructed, in terms of two-dimensional image slices. The computed stack of 2D slices usually share a resolution of a few microns and their pixel values are inextricably bound to material density-based attenuation.

Table 1. Properties of porous stones and mesh quality.

\begin{tabular}{c|cccc}
\hline & Berea B1 & Carbonate C1 & Savonnières(Exp) & Meule(Exp) \\
\hline Size (mm) & $7.4^{3}$ & $4.5^{3}$ & $20^{3}$ & $20^{3}$ \\
Average Porosity & $18.5 \%$ & $23.3 \%$ & $26.9 \%$ & $16.6 \%$ \\
Average Pore Size (microns) & 11.9 & 20.7 & 10.3 & 9.1 \\
\hline Max aspect ratio & 11.9796 & 7.54027 & & \\
Number of cells & 5689735 & 3777008 & & \\
Max non-orthogonality (degrees) & 78.2862 & 76.1127 & & \\
\hline
\end{tabular}

Segmentation of the volume stack of X-ray micro-CT slices was performed using Retomo, a new software package from BETA CAE Systems, which is used to define the rock/air interface contours for the entire volume. The same software was used to then export the interface iso-surface as a STereoLithography (STL) type mesh with connectivity information between the generated triangles. The latter ensures a minimum (if not zero) number of free-hanging elements that could complicate the definition of computational domain. The generated surface mesh was of high quality; however, further mesh operations were conducted in the ANSA pre-processor in order to ensure that the pore and throat representations of the rock were kept intact. The produced surface was then used to define the boundary of the flow domain, which is discretized using an unstructured type mesh. During volume mesh operation, a coarse background Cartesian mesh is generated first, which is then refined around the surface boundary; this approach reduces the overall number of elements. Computational domain dimensions and meshing quality parameters are defined in Table 1. Two different specimens were used. The average pore sizes of Berea and Carbonate sandstone is 11.9 and 20.7 microns, respectively. In the present study, the numerical average porosity of media is fixed $(B 1=18.5 \%$ and $C 1=23.3 \%)$ and surface porosity varies with pore size. The computational domain was divided into two main refinement zones at the area of impact as shown in Fig. 1 (right side)
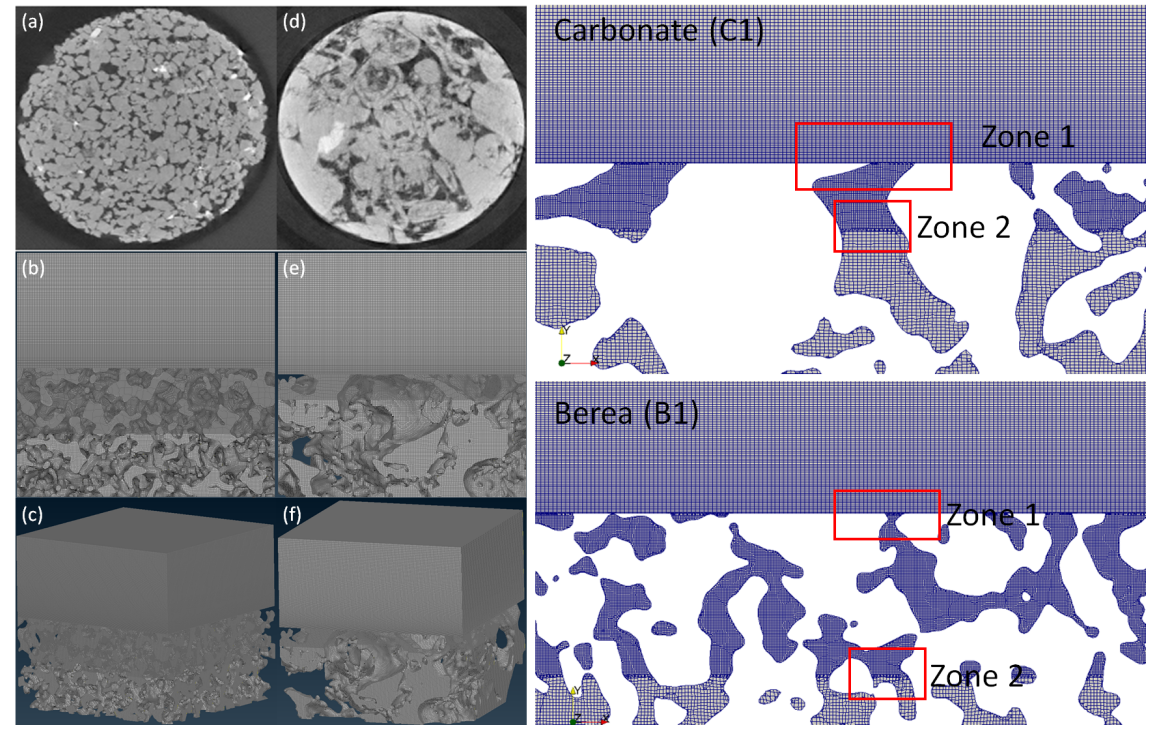

Figure 1. Numerical model for Berea (B1) and Carbonate (C1) porous media. (a,d) represent the original Mico CT for the two different porous media. ( $b$ and $c$ ) represent the computational mesh for the Bera sandstone. (e and f) represent the Carbonate.

On the right-hand side, a section inside the porous media is taken to show the mesh refinement zones

\section{Numerical set-up}

The computational model is implemented within the open source CFD toolkit OpenFOAM and is described in this section. An incompressible two-phase flow with constant phase densities $\rho_{1}$ and $\rho_{2}$ and viscosities $\mu_{1}$ and $\mu_{2}$ is considered. A single set of governing equations are solved in the entire computational domain (shared by both phases) in conjunction with a transport equation for the volume fraction of one of the phases $-\alpha-$. In the proposed numerical method, the Continuum Surface Force (CSF) description of Brackbill et al. [20] is used to represent the surface tension forces in the following form:

$$
f_{s}=\sigma \kappa \delta_{s}
$$


where the term $\kappa=-\nabla \cdot\left(\eta_{s}\right)$ represents the interface curvature, $\delta_{s}$ is a delta function concentrated on the interface and $\eta_{s}$ is the normal vector to the interface that is calculated by the following equation:

$$
\eta_{s}=\frac{\nabla \alpha_{\text {smooth }}}{\left|\nabla \alpha_{\text {smooth }}\right|}
$$

where $\alpha_{\text {smooth }}$ is the artificial smoothed indicator-function, $\delta_{s}$ is the sharpened indicator-function. In the VOF method, the indicator function $\alpha$ represents the volume fraction of one of the fluid phases in each computational cell. The indicator function evolves spatially and temporally according to an advection transport equation of the following general form:

$$
\frac{\partial \alpha}{\partial t}+\nabla \cdot(\alpha \mathbf{u})=0
$$

Ideally, the interface between both phases should be massless since it represents a sharp discontinuity. However, within VOF formulation the value of $\alpha$, at the interface, varies between 0 and 1 due to numerical diffusion. The framework described above, reflects the generalised framework of VOF methods and can be used in a large range of two-phase flow problems with various adjustments. In the following sub-sections, an enhanced version of this basic VOF framework that addresses the previously mentioned challenges, is presented in detail.

\section{Adaptive Compression Scheme (Implicit)}

To deal with the problem of numerical diffusion of $\alpha$, an extra compression term is used in OpenFOAM to control the thickness of the interface as shown below;

$$
\frac{\partial \alpha}{\partial t}+\nabla \cdot(u \alpha) \underbrace{-\nabla \cdot\left\{u_{r} \alpha((1-\alpha))\right\}}_{\text {compression term }}=0
$$

where $\left(u_{r}\right)$ is the compression velocity given by Eq. 5 . It represents the vector of relative velocity and is formulated based on the maximum velocity magnitude at the interface region and its direction, which is determined from the gradient of the phase fraction as follows:

$$
u_{r}=\min \left(C_{c} \frac{\left|\phi_{f}\right|}{|S f|}, \max \left[\frac{\left|\phi_{f}\right|}{|S f|}\right]\right)\left(\eta_{f} \cdot S_{f}\right)
$$

The term $\phi_{f}$ is the mass flux and $S f$ is the cell surface area. The compressive term is taken into consideration only at the interface region and it is calculated in the normal direction to the interface to avoid any dispersion. The maximum operation in Eq. 5 is performed over the entire domain, while the minimum operation is done locally on each face. The constant $\left(C_{c}\right)$ is a user-specified value, which serves as a parameter to obtain different cell results in different levels of compression. In most of the simulations presented here $\left(C_{c}\right)$ is taken as unity, after initial trial simulations. Higher values than unity in this case may lead to unphysical results. To make the compression methodology more general and overcome the need for a priori tuning, an adaptive algorithm has been implemented to the solver based on the following relationship:

$$
C_{a d p}=p o s\left(-\frac{U_{n} \cdot \nabla \alpha}{\left|U_{n}\right||\nabla \alpha|}, C_{\alpha}\right)
$$

where $C_{a d p}$ is the adaptive compression coefficient that acts in the volume fraction field, The constant $\left(C_{\alpha}\right)$ is a user-specified value. In our simulation $\left(C_{\alpha}\right)$ is set to be zero. The term $U_{n}$ represents each phase normal to the interface velocity. It is expressed as;

$$
U_{n}=\left(U \cdot n_{s}\right) *\left(n_{s}\right) * \operatorname{pos}(\alpha-0.01) * \operatorname{pos}(0.99-\alpha)
$$

The concept of using $U_{n}$ is shown in Fig. 2. When the profile of the colour function becomes diffusive $C_{a d p}$ value is increased in order to sharpen the interface relevant to the local diffusion field. When the profile is already sharp and additional compression is not necessary, $C_{a d p}$ will go to zero.

\section{Numerical Treatment of the Indicator Function}

After solving Eq. 4 with the additional compression term, the indicator function is updated at the cell centres. Afterwards the indicator function is obtained at the cell boundaries using a linear extrapolation from the cell centres in order to proceed with the calculation of $\eta$ and $k$. At this stage the value of the indicator function sharply changes over a thin region (as a result of the compression step). This abrupt change of the indicator function creates errors in calculating the normal vectors and the curvature of the interface, which will be used to evaluate the interfacial forces. These errors induce non-physical parasitic currents in the interfacial region. A commonly followed approach to the literature to suppress these artifacts is to compute the interface curvature from a smoothed function $\alpha_{\text {smooth }}$, which is calculated by the smoother proposed by Georgoulas et al. [22]. It should be noticed that the number of 

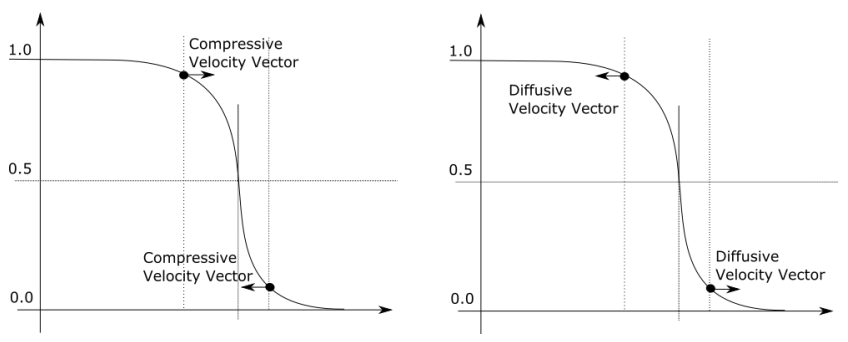

Figure 2. Schematic to represent the adaptive compression $C_{A d p}$ selection criteria

Table 2. Properties of water droplet and impact conditions for drop test.

\begin{tabular}{c|c|c|c|c}
\hline & $\rho\left(K g / m^{3}\right)$ & $\mu(K g / m s)$ & $\sigma(N / m)$ & \\
\hline Water Properties at $25^{\circ} \mathrm{C}$ & 998 & 0.001 & 0.07 & \\
\hline & $R_{0}(\mathrm{~mm})$ & $V_{i}(\mathrm{~m} / \mathrm{s})$ & We & $\operatorname{Re}$ \\
\hline Impact conditions & 1 & $0.5-1$ & $10-20$ & $498-1000$ \\
\hline
\end{tabular}

times that the proposed filter is applied should be handled with care in the calculations since there is a fine balance between smoothing the interface" and altering the curvature, especially in high curvature interfacial regions. For the presented simulations based on sensitivity analysis we repeat the smoothing loop 4 times. Following, capillary forces are calculated at the face centres based on Eq. 1. For the calculation of curvature $k$ we used the smoothing operation as described above. However special care needs to be taken for the calculation interface delta function $\delta_{s}$. In order to maintain its sharpness we also introduce a sharp function $\left(\alpha_{\text {sharp }}\right)$ modelling the capillary pressure in the case of flows through porous media. Then $\delta_{s}$ is calculated based on the $\alpha_{\text {sharp }}$ rather than the $\alpha_{\text {smooth }}$. In the final step non-physical velocities that are parallel to the fluid interface are filtered, so that the term $f_{c}-\nabla p_{c}$ that appears in the Eq 8 finally converges to zero.

\section{Capillary Pressure Jump Modelling and Filtering Numerical Errors}

At the pore scale, the momentum balance equation for incompressible fluids is used to relate the pressure gradient, inertial, capillary, gravity and viscous forces. Knowing the pressure gradient, capillary and gravity forces, the momentum balance equation can be used to update the velocity field at any time-step;

$$
\frac{D}{D t}(\rho u)-\nabla \cdot T=-\nabla p_{d}+\rho g+f_{s}-\nabla p_{c}
$$

where, $\rho g$ is the gravity force and $f_{s}$ is the capillary force. $p_{d}$ is the dynamic pressure and it reads: $p_{d}=p-p_{c}$. where $\mathrm{p}$ is the total (physical) pressure and $\mathrm{pc}$ is a potential field called the microscopic capillary pressure. $P_{c}$ is obtained from the solution of the Poisson's equation:

$$
\nabla \cdot \nabla P_{c}=\nabla \cdot f_{s}
$$

This approach includes explicitly the effect of capillary forces in the Navier-Stokes equations, and therefore allows for the filtering of the numerical errors related to the inaccurate calculation of $f_{c}$. The non-physical velocities that are parallel to the fluid interface are filtered, so that the term $f_{c}-\nabla p_{c}$ that appears in the Eq 8 finally converges to zero as discussed in [2].

\section{Wettability Modelling}

For the present calculations we implemented the Kistler model [24] in the standard and developed solver in order to be able to accurately simulate the contact line movement. The Kistler model calculates the dynamic contact angle, using the Hoffman function, $f_{H o f}$, as $\Theta_{d y n}=f_{H o f}\left[C a+f_{H o f}^{-1}\left(\Theta_{e}\right)\right]$ where $\Theta_{e}$ is the equilibrium contact angle and $\mathrm{Ca}$ the capillary number calculated based on an imposed spreading velocity.

\section{Case set-up}

Three-dimensional simulations were performed. The computational domain, the grid as well as the applied boundary conditions are shown in Fig. 3. The initial conditions for the simulated geometry are illustrated in Fig. 3, while the material properties and the initial conditions for the impacting liquid (water) are summarised in Table 2. Different values for the wetting conditions are illustrated in Table 3, where ten numerical simulations were performed to show the influence of wetting on spreading behaviour at different porous media. In each case, initial droplet radius is set to $\mathrm{R}=1 \mathrm{~mm}$. At the first time step, a spherical liquid drop is patched inside the numerical domain in such a way where the liquid phase just on top the porous surface. 


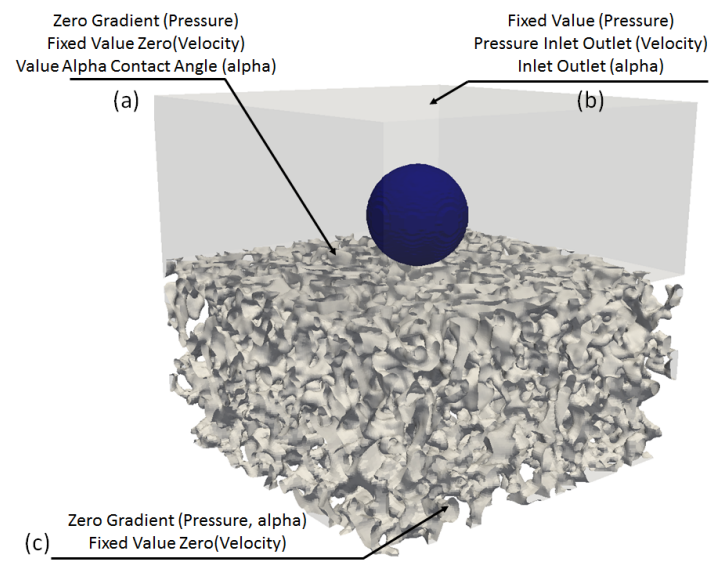

Figure 3. Three-dimensional computational domain, mesh and boundary conditions

\section{Results and discussion}

In the following sections, numerical simulations are presented for one case that assesses the performance of the proposed model. Then the influence of porous wettability on spreading radius dynamics using two different porous media is investigated. Also, the droplet penetration and effect of wettability on inertial spreading stage and the viscous spreading stage are observed. Calculations with the standard VOF-based solver of OpenFOAM (interFoam) are also included for comparison purposes. Additionally, the droplet radius at the time of impact was measured using a custom Matlab code to enable comparison of the results to the given experimental data.

\section{Validation of numerical method}

The test case used to validate the numerical model is of a droplet impacting a Berea sandstone segment with impact velocity $V_{i}=1 \mathrm{~m} / \mathrm{s}(\mathrm{We}=20, \mathrm{Re}=1000)$. The numerical simulation is compared against experiments reported in $[8,19]$. The experimental porous media namely -Meule- is described in Table 1. Although our numerical model and the one that experimental data are taken are not the same materials (they do not share the same porous structures) they share similar average porosity and average pore sizes. Depending on how close are predictions with experimental data, conclusions can be extracted regarding the importance of these two parameters (porosity and average pore sizes) to the evolution of the spreading. One Should note that careful treatment should be taken while modelling the wetting conditions as they may dominate the spreading behaviour. As reported in the experiments $[8,19]$ the advancing and receding contact angles are $\left(125^{\circ}\right.$ and $\left.25^{\circ}\right)$, which is used as initial conditions for our Kistler wetting model in order to guarantee that the numerical and experimental model have the same wetting behavior. Figure 4 shows the normalised time evolution $(t / \tau)$ of the droplet spreading $(r / R)$, where $\mathrm{R}$ is the initial droplet radius, and $r$ is the calculated droplet radius over the porous media during spreading. Spreading radius $(r)$ over the porous media is found to be irregular compared to the normal droplet spreading over a flat surface (not a perfect circular shape). To determine the spreading radius ( $r$ ), we use our numerical data to calculate the wetted area of the irregular spreading shape, and extract an equivalent radius based on the centre of gravity, as seen in Fig. 4, where the black and white picture represents the droplet shape over the porous media while the blue line represents the fitted circle determined from post-processing. The normalised time is defined as the inertial time scale of the first stage of wetting, where $\tau=\left(\frac{\rho R^{3}}{\sigma}\right)^{0.5}$ [25]. As we can see the phenomenon -based on the experimental data for the droplet diameter- is characterised by the following phases: Initially, the droplet diameter is increased (spreading phase) reaching to a maximum length and then stabilises. Although for the first phase the same increase is noticed in the phenomena of droplet spreading over solid surfaces, the second part is different. When droplet impacts on a porous medium we can notice that the diameter remains almost constant implying that the imbibition phase has started (for this case at $(t / \tau)=0.6$ ) [26]. These phases are well captured by our new solver (interPore) while the standard solver fails to predict the correct transition to the imbibition phase. Also, it can be seen that for the imbibition phase our code (interPore) has a very close match with the experiments. It is also an indication that porosity and average pore diameter are the two most important controlling parameters since as mentioned above simulations and experimental data share porosity and average pore diameter but not pore structure. Finally, we notice that for the spreading phase there are some differences between experimental and numerical curve indicating a slower spreading process of the droplet of the numerical model in comparison to experimental one. This can potentially indicate that average pore size might not be enough to describe the spreading process and more information for the pore distribution are necessary. Using the Kistler boundary condition is a key element in capturing the correct physical behaviour for the droplet spending. Nevertheless, by imposing the Kistler boundary condition to the standard solver (interFoam), we noticed an overestimation for droplet size during the spreading phase. The reason comes from the wrong calculated contact line velocity during the inertial phase caused by the parasitic currents. The overestimated spreading is not reflected in our simulation using the modified solver (interPore) due to the parasitic current filter used. Based on [25] we also plotted the theoretical power-law $(r / R)$ as 


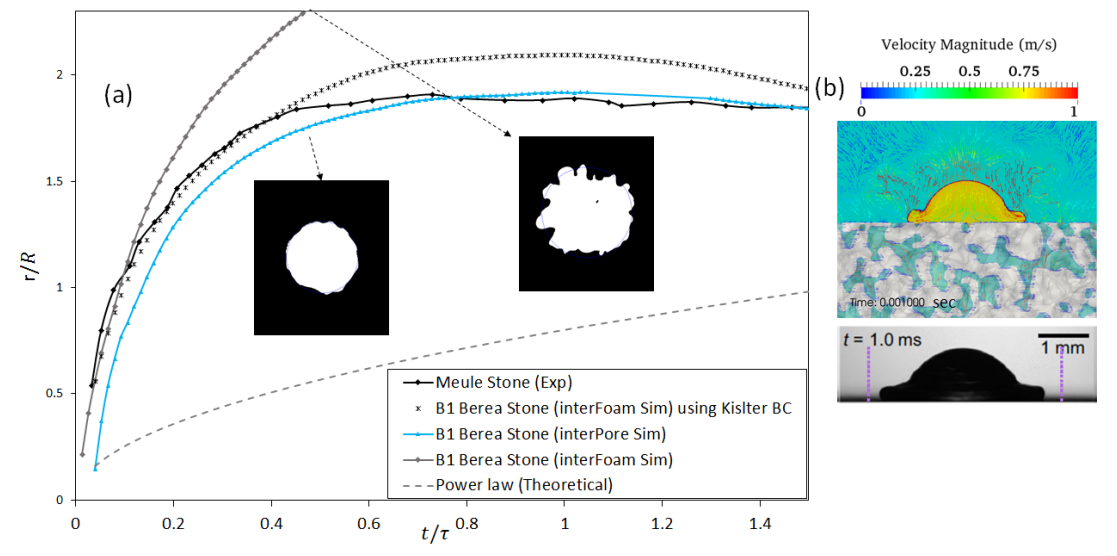

Figure 4. Comparison of drop impacts for $D_{0}=2.0 \mathrm{~mm}$ and $V_{i}=1.0 \mathrm{~m} / \mathrm{s}$ on Berea stone (numerical) and Meule stone (experimental). (a) is a normalised wetted zone width $\mathrm{r} / \mathrm{R}$ evolution for water droplet at $\mathrm{We}=28.5$. Developed solver (interPore) at wetting conditions of $\theta_{A}=125^{\circ}, \theta_{R}=25^{\circ}$ at the porous surface and porous media, Standard solver (interFoam) at wetting conditions of $\theta_{e q}=25^{\circ}$ at the porous surface and porous media. (b) Snapshots of drop impact on porous materials at time $=1$ ms

$\frac{r}{R}=0.8\left(\frac{t}{\tau}\right)^{0.5}$. The power law fails to estimate the correct spreading behaviour due to the unaccounted influence of the porous structure and wettability effect.

\section{Influence of porous wettability on radius dynamics}

To provide further insight into the local phenomena which drive capillary imbibition of the droplet inside porous media, two different material are presented here with different wetting behaviour The contact angle values used to observe the influence of wettability and porosity are shown in Table 3. Figure (5) shows the droplet behaviour (represented by the iso-surface of the droplet volume fraction at 0.5 ) at two different porous wetting conditions (hydrophobic porous surfaces and hydrophilic porous surfaces) for two different porous media (Carbonate and Berea). Two features can be identified in both porous cases; firstly, the droplet invasion mainly depends on the porous wettability regardless the change of the porosity. Secondly, the recoiling phase can be observed in the hydrophobic case but not in the hydrophilic case for both porous structures. At hydrophobic conditions of the pore shows less liquid absorption with a recoiling phase, yet it is still characterised by pinning behaviour. At the hydrophilic porous wetting, regardless of imposing a dynamic wetting conditions on the porous surface in our simulations, no recoiling phase is observed. However, the droplet edge is seen to undergo same pinning with much more absorption, behaviour which limits its spreading. Spreading on the solid surfaces between the holes depend mainly on the pore wettability for same solid surface wetting conditions. The interface shape was captured over time as shown in Fig. 5. The hydrophobic case shows small liquid fingers in the initial phase, which tends to be receding from the pore space again during the recoiling phase. While for the hydrophilic cases, the fingering effect after the invasion and during the initial phase tends to increase by time. This leads to a decrease in droplet size in the recoil phase, yet keeping same spreading diameter. Figure 6 shows the normalised droplet spreading at two different porosities and a wide range of pore wettability. For Figure 6a Case 1 and Case 2 undergo same surface spreading with different penetration behaviour. While Case 3 shows mush less penetration associated with surface spreading. While $6 \mathrm{~b}$ Case 6 and Case 8 undergo maximum penetration duo to the high porosity value. Case 8 shows an increase in surface spreading similar to Case 4.

\section{Conclusions}

In this work, a framework initially developed to capture droplet interface dynamics at low capillary numbers is extended in order to be used in the study of the impact of liquid droplets on a real porous material reconstructed

\begin{tabular}{c|cccc}
\hline Case & Porous model & Surface contact angle & Pore Contact Angle & Vel $(\mathrm{m} / \mathrm{s})$ \\
\hline Case 1 & B1 & $\theta_{A}=125^{\circ}, \theta_{R}=25^{\circ}$ & $\theta_{A}=125^{\circ}, \theta_{R}=25^{\circ}$ & 0.5 \\
Case 2 & B1 & $\theta_{A}=125^{\circ}, \theta_{R}=25^{\circ}$ & $\theta_{e q}=0^{\circ}$ & 0.5 \\
Case 3 & B1 & $\theta_{A}=125^{\circ}, \theta_{R}=25^{\circ}$ & $\theta_{e q}=180^{\circ}$ & 0.5 \\
Case 4 & B1 & $\theta_{A}=60^{\circ}, \theta_{R}=22^{\circ}$ & $\theta_{A}=60^{\circ}, \theta_{R}=22^{\circ}$ & 0.5 \\
Case 5 & C1 & $\theta_{A}=125^{\circ}, \theta_{R}=25^{\circ}$ & $\theta_{A}=125^{\circ}, \theta_{R}=25^{\circ}$ & 0.5 \\
Case 6 & C1 & $\theta_{A}=125^{\circ}, \theta_{R}=25^{\circ}$ & $\theta_{e q}=0^{\circ}$ & 0.5 \\
Case 7 & C1 & $\theta_{A}=125^{\circ}, \theta_{R}=25^{\circ}$ & $\theta_{e q}=180^{\circ}$ & 0.5 \\
Case 8 & C1 & $\theta_{A}=60^{\circ}, \theta_{R}=22^{\circ}$ & $\theta_{A}=60^{\circ}, \theta_{R}=22^{\circ}$ & 0.5 \\
\hline
\end{tabular}

Table 3. Imposed contact angle for porous media surface (using Kistler, dynamic contact angle model) with different assumptions concerning the contact angle of the pore surfaces (varies from fully hydrophilic to fully hydrophobic) 


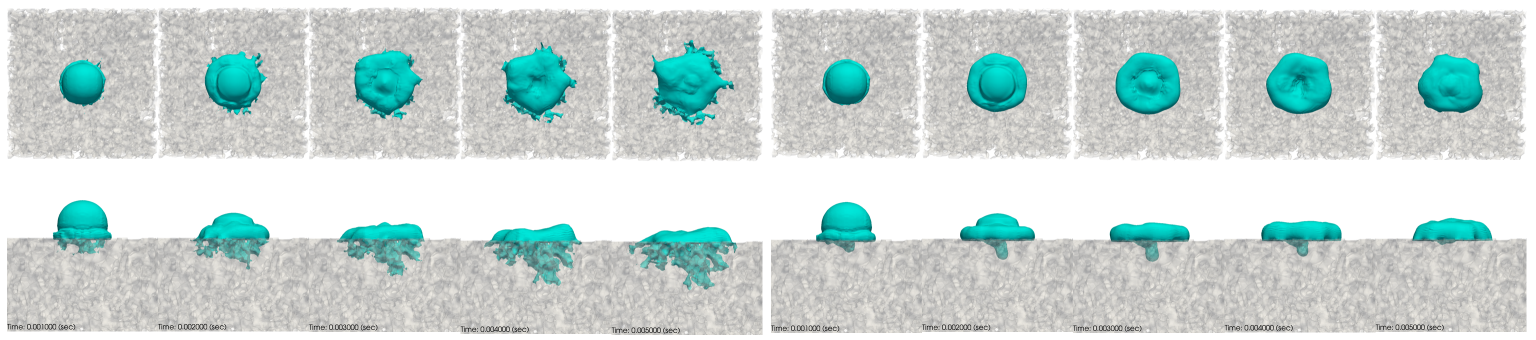

(a) Case 2 represents fully hydrophobic conditions for Berea (b) Case 3 represents fully hydrophilic conditions for Berea sandstone (B1)

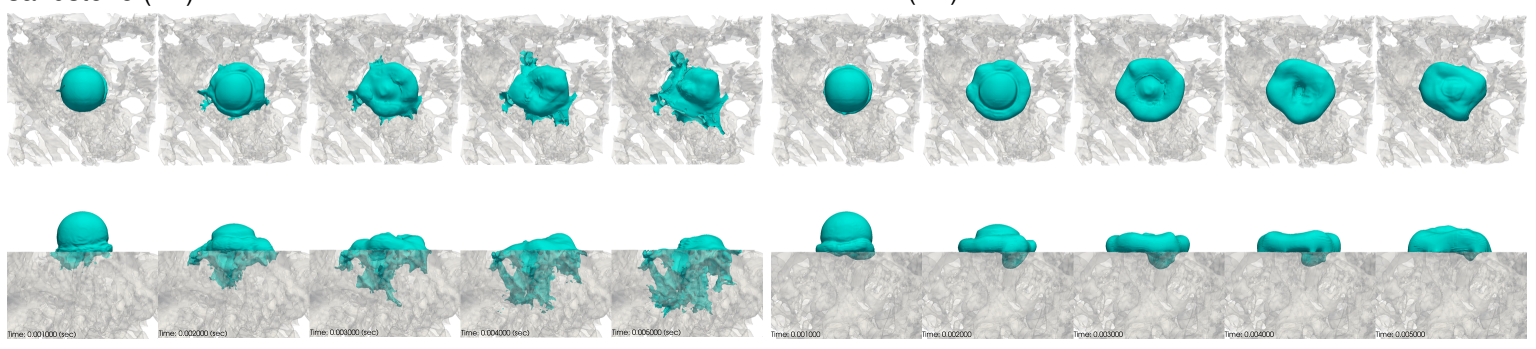

(c) Case 6 represents fully hydrophobic conditions for Carbon- (d) Case 7 represents fully hydrophilic conditions for Carbonate $(\mathrm{C} 1)$ ate $(\mathrm{C} 1)$

Figure 5. The early stage of capillary imbibition of pore after Droplet impacting the solid surfaces. Four views are provided at each five-time steps. The figures show two different wetting conditions ranges from fully hydrophobic to fully hydrophilic.

from micro-CT scanned pictures. We initially test our framework against experimental data from the Meule stone. The Meule stone and the material used for our simulations share the same porosity and average pore size but not the same pore structure. We observe that regardless of this difference both spreading and imbibition phase are well captured by the model which indicates a) that our framework is well suited to represent a complicated phenomenon like this and $b$ ) that the phenomenon as such is governed only by the two parameters. Moreover, we extend our study to the effect of wettability. Two different porous wetting conditions (hydrophobic porous surfaces and hydrophilic porous surfaces) for two different porous media (Carbonate and Berea). From these numerical experiments, it can be concluded that the droplet imbibition mainly depends on the porous wettability regardless the change of the porosity and secondly that the recoiling phase can be observed in the hydrophobic case but not in the hydrophilic case.

\section{References}

[1] M. Abokhedr, K. Vogiatzaki, N. Mitroglou, and E. Gavaises. A numerical simulation of single and two-phase flow in porous media: A pore sale observation of effective microscopic forces. 6th BETA CAE International Conference, 2, 2015.

[2] M Aboukhedr, M Gavaises, A Georgoulas, M Marengo, and K Vogiatzaki. Numerical investigation of droplet spreading on porous and non-porous surfaces. ILASS Europe, 27th Annual Conference on Liquid Atomization and Spray Systems, Brighton, UK, 2016.

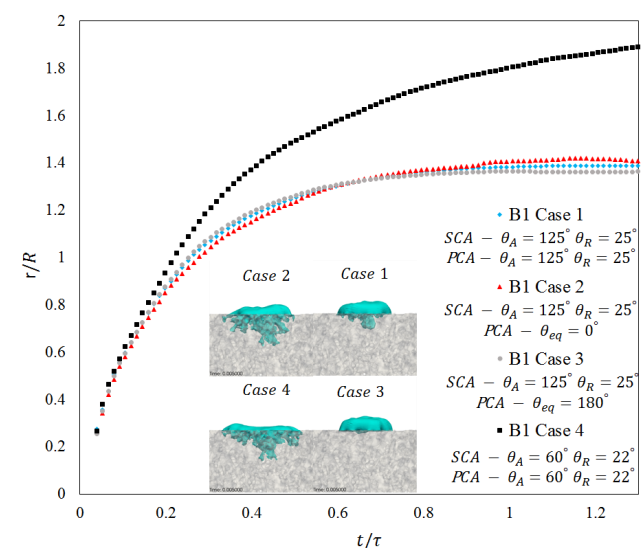

(a) B1

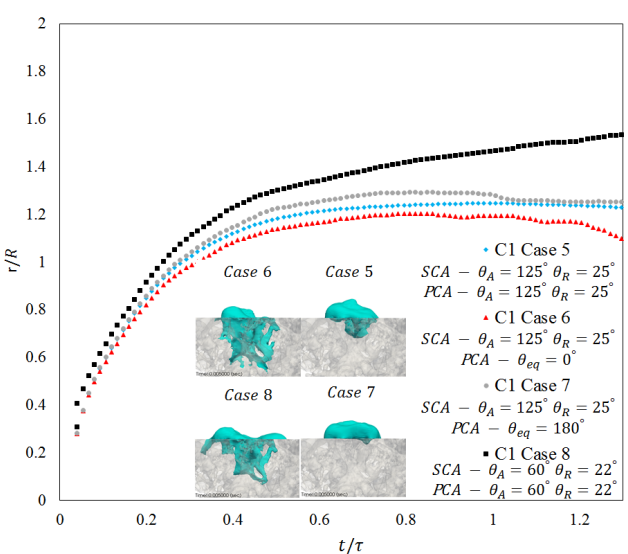

(b) $\mathrm{C} 1$

Figure 6. Normalised radius $r / R$ with different surface and pore contact angle vs. normalised time $t / \tau$ for two different porous media Carbonate (C1) and Berea sandstone (B1). Supporting images are for time $t=0.005$ (sec) 
[3] Tatiana Gambaryan-Roisman. Liquids on porous layers: wetting, imbibition and transport processes. Current Opinion in Colloid \& Interface Science, 19(4):320-335, 2014.

[4] Š Šikalo, H-D Wilhelm, IV Roisman, S Jakirlić, and C Tropea. Dynamic contact angle of spreading droplets: Experiments and simulations. Physics of Fluids, 17(6):062103, 2005.

[5] Kensuke Yokoi, Damien Vadillo, John Hinch, and lan Hutchings. Numerical studies of the influence of the dynamic contact angle on a droplet impacting on a dry surface. Physics of Fluids, 21(7):072102, 2009.

[6] R Rioboo, M Marengo, and C Tropea. Time evolution of liquid drop impact onto solid, dry surfaces. Experiments in Fluids, 33(1):112-124, 2002.

[7] Martin Rein. Phenomena of liquid drop impact on solid and liquid surfaces. Fluid Dynamics Research, 12(2): 61-93, 1993.

[8] JB Lee, AI Radu, P Vontobel, D Derome, and J Carmeliet. Absorption of impinging water droplet in porous stones. Journal of colloid and interface science, 471:59-70, 2016.

[9] AL Yarin. Drop impact dynamics: splashing, spreading, receding, bouncing.... Annu. Rev. Fluid Mech., 38: 159-192, 2006.

[10] Karen P Hapgood, James D Litster, Simon R Biggs, and Tony Howes. Drop penetration into porous powder beds. Journal of Colloid and Interface Science, 253(2):353-366, 2002.

[11] Homayun K Navaz, Bojan Markicevic, Ali R Zand, Yuri Sikorski, Ewen Chan, Matthew Sanders, and Terrence G D'Onofrio. Sessile droplet spread into porous substrates-determination of capillary pressure using a continuum approach. Journal of colloid and interface science, 325(2):440-446, 2008.

[12] NC Reis, RF Griffiths, MD Mantle, and LF Gladden. Investigation of the evaporation of embedded liquid droplets from porous surfaces using magnetic resonance imaging. International Journal of Heat and Mass Transfer, 46 (7):1279-1292, 2003.

[13] Neyval C Reis, Richard F Griffiths, and Jane M Santos. Numerical simulation of the impact of liquid droplets on porous surfaces. Journal of Computational Physics, 198(2):747-770, 2004.

[14] Neyval C Reis, Richard F Griffiths, and Jane Méri Santos. Parametric study of liquid droplets impinging on porous surfaces. Applied Mathematical Modelling, 32(3):341-361, 2008.

[15] SH Davis and LM Hocking. Spreading and imbibition of viscous liquid on a porous base. Physics of Fluids (1994-present), 11(1):48-57, 1999.

[16] VM Starov, SA Zhdanov, and MG Velarde. Spreading of liquid drops over thick porous layers: complete wetting case. Langmuir, 18(25):9744-9750, 2002.

[17] X Frank and Patrick Perre. Droplet spreading on a porous surface: A lattice boltzmann study. Physics of Fluids (1994-present), 24(4):042101, 2012.

[18] Hu Dong and Martin J Blunt. Pore-network extraction from micro-computerized-tomography images. Physical review E, 80(3):036307, 2009.

[19] JB Lee, D Derome, and J Carmeliet. Drop impact on natural porous stones. Journal of colloid and interface science, 469:147-156, 2016.

[20] J.U Brackbill, D.B Kothe, and C Zemach. A continuum method for modeling surface tension. Journal of Computational Physics, 100(2):335 - 354, 1992. .

[21] B. Lafaurie, C. Nardone, R. Scardovelli, S. Zaleski, and G. Zanetti. Modelling merging and fragmentation in multiphase flows with surfer. Journal of Computational Physics, 113(1):134 - 147, 1994. .

[22] A. Georgoulas, P. Koukouvinis, M. Gavaises, and M. Marengo. Numerical investigation of quasi-static bubble growth and detachment from submerged orifices in isothermal liquid pools: The effect of varying fluid properties and gravity levels. International Journal of Multiphase Flow, 74:59 - 78, 2015.

[23] A. Q. Raeini, M. J. Blunt, and B. Bijeljic. Modelling two-phase flow in porous media at the pore scale using the volume-of-fluid method. Journal of Computational Physics, 231(17):5653 - 5668, 2012.

[24] S.F. Kistler. Hydrodynamics of wetting. Marcel Dekker, New York, 1993.

[25] Anne-Laure Biance, Christophe Clanet, and David Quéré. First steps in the spreading of a liquid droplet. Physical Review E, 69(1):016301, 2004.

[26] J.B. Lee, A.I. Radu, P. Vontobel, D. Derome, and J. Carmeliet. Absorption of impinging water droplet in porous stones. Journal of Colloid and Interface Science, 471:59 - 70, 2016. ISSN 0021-9797. . 


\title{
Numerical analysis of sprays with an advanced collision model
}

\author{
M. Sommerfeld ${ }^{* 1,2}$ and S. Lain ${ }^{2}$ \\ ${ }^{1}$ Institut Verfahrenstechnik, Otto-von-Guericke-University Magdeburg, \\ Zeppelinstraße 1, D-06130 Halle (Saale), Germany \\ ${ }^{2}$ Department Mechanical Engineering, Universidad Autónoma de Occidente, Cali, Colombia \\ *Corresponding author: martin.sommerfeld@ovgu.de
}

\begin{abstract}
Modelling of collisions between liquid droplets in the frame of a Lagrangian spray simulation has still many open issues, especially when considering higher viscous droplets and if colliding droplets have a large size difference. A generalisation of the collision maps is attempted based on the behaviour of characteristic points, namely the triple point where bouncing, coalescence and stretching separation coincide and the critical Weber-number where reflexive separation first occurs in head-on collisions. This is done by correlating experimental data with respect to the Capillary number with the Ohnesorge-number for the triple point and the critical Weber-number is also well described by a correlation the Ohnesorge-number. Based on these results the boundary line between stretching separation and coalescence is found by adapting the Jiang et al. (1992) correlation. For the upper boundary of reflexive separation the shifted Ashgriz and Poo (1990) correlation is used. It was however so far not possible to predict the lower bouncing boundary through the Estrade et al. (1999) boundary line correctly. The proposed boundary-line models were validated for various liquid, however still considering only a size ratio of one. With the developed three-line boundary model Euler/Lagrange numerical calculations for a simple spray system were conducted and the droplet collisions were analysed with respect to their occurrence. Droplet collision modelling is performed on the basis of the stochastic droplet collision model, also considering the influence of impact efficiency, which so far was neglected for most spray simulations. The comparison with measurements showed reasonable good agreement for all properties.
\end{abstract}

\section{Keywords}

Droplet collisions, stochastic collision model, impact efficiency, collision outcomes, Euler/Lagrange calculations, spray simulations.

\section{Introduction}

In numerous technical and industrial spraying systems higher viscous liquids, suspensions or solutions are being atomised. Examples are liquid fuels (including bio-fluids) in combustion systems, liquid melts or other mineral melts in the field of materiel science and spray drying of foodstuff or pharmaceutical materials. Therefore, in a numerical calculation of such spraying systems, mostly done with an Euler/Lagrange approach, the resulting different liquid properties have to be accounted for. Here the focus is related to droplet collision modelling. Hence, established droplet collision models have to be generalized regarding liquid properties, mainly viscosity and surface tension. Since colliding droplets may have considerable different size also the droplet size ratio is an important parameter to be considered in droplet collision modelling.

In order to model droplet collisions in the frame of the Lagrangian droplet parcel concept, where only pointmasses are tracked, applied to spraying systems several elementary processes have to be considered (Sommerfeld and Kuschel 2016). The first step is the detection of possible collisions between two droplets. In order to insure that during a Lagrangian tracking time step only binary collisions can occur, the Lagrangian time step needs to be smaller than the reciprocal of the collision frequency (see e.g. Sommerfeld et al. 2008, Crowe et al. 2012). The collision detection may be done in three ways (Sommerfeld 2017), a fully deterministic and geometrical reconstruction of collision trajectories (e.g. Sundaram and Collins 1996), a deterministic-stochastic parcel collision model which requires to identify the probability of collision between any pair of parcels within an computational cell (O'Rourke 1981) and the fully stochastic droplet collision model (Sommerfeld 2001). This stochastic model does not require any knowledge about neighbouring droplets, but creates a fictitious collision partner out of the local droplet population. At this level these collision detection approaches assume $100 \%$ impact efficiency. However in a spray with a wide droplet size distribution, which also may be modified by coalescence, the smaller droplets might move around the larger ones with the relative velocity. This impact efficiency is the area from where the droplets are coming and hit the larger droplet, called collector, divided by the collector crosssection. Thereby the effective collision frequency is drastically reduced. When only considering inertial impact, the 
impact efficiency may be easily obtained through correlations depending on the relative Stokes number and the collector droplet Reynolds number (Ho and Sommerfeld 2002, Sommerfeld and Lain 2009). Resulting from that, the impact point of the small on the surface of the large droplet has to be determined giving the impact parameter B. With the known instantaneous relative velocity between colliding droplets also the collision Weber-number is determined. The next step is the identification of the collision scenario and the outcome on the basis of collision maps (Figure 1). The decision about which type of collision is occurring (i.e. bouncing, coalescence or stretching and reflexive separation), is based on analytical or theoretical derived boundary lines (see Figure 1). The properties of the droplets formed depend on the collision type at hand and have to be calculated based on mass and momentum balances (i.e. number of droplets, droplet sizes and their new velocities).

Very often spray computations are done by only considering one boundary line between coalescence and grazing collision, which involves bouncing and stretching separation (Figure 1). This approach was first suggested by O`Rourke (1981) using the boundary line derived theoretically by Brazier-Smith et al. (1972). The Euler/Lagrange approach was applied to spray calculations using this simplified collision map in various studies (e.g. Gavaises et al. 1996, Rüger et al. 2000, Guo et al. 2004, Nijdam et al. 2006). Composite collision maps applied to spray simulations, including also diesel sprays (Post and Abraham 2002, Mannannur and Reitz 2007), consider three boundary lines; namely the lower boundary of bouncing derived by Estrade et al. (1999), boundary between coalescence and stretching separation based on Brazier-Smith et al. (1972) and the boundary between reflexive separation and coalescence suggested by Ashgriz and Poo (1990). However these boundary lines cannot reflect the regimes observed for Diesel droplet collisions (Chen et al. 2016). This again shows the need for more general boundary lines which may be applied to different liquids.

In our previous studies an attempt was made to develop models which describe the collision outcome based on collision maps with boundary lines accounting for viscosity variations (Sommerfeld 2016). These new correlations could be developed by thorough experiments (Kuschel and Sommerfeld 2013, Sommerfeld and Kuschel 2016) and use the shift of the triple point (Coincidence of bouncing, coalescence and stretching separation) and the critical We-number (beginning of reflexive separation for head-on collisions) with variation of viscosity. The motion of the triple point showed a relative clear correlation with the Ohnesorge-number, which includes only droplet size and liquid properties. The boundary line coalescence-stretching separation was fitted by the Jiang et al. (1992) correlation, adapting the two involved model constants. These again could be well correlated with the Ohnesorgenumber for pure fluids and solutions separately (Sommerfeld 2016). In this contribution the data base for constructing the correlations for the triple point location and the critical We-number is extended based on recent studies and experiments (see also Sommerfeld 2017).

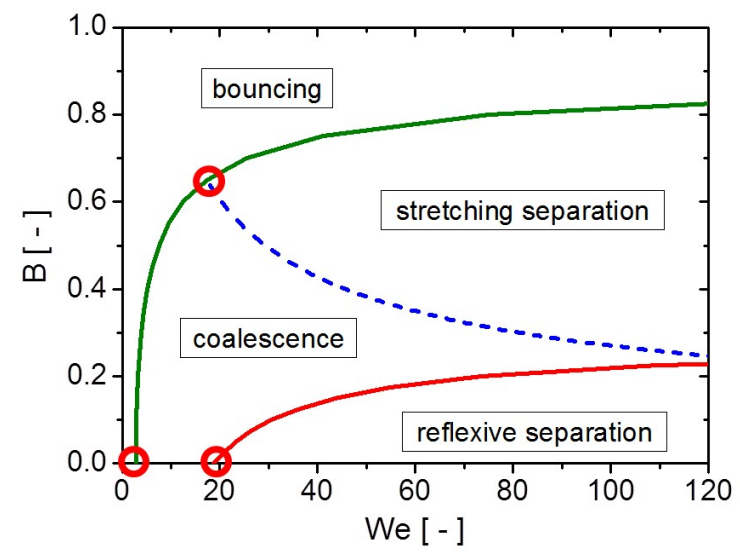

Figure 1: Typical droplet collision map where the non-dimensional impact parameter $B$ is plotted versus collision Weber-number We including boundary lines between collision outcomes and three characteristic points.

The resulting adaptive three-boundary line collision maps are first compared with collision maps obtained from experiments for different droplet liquids. Then the performance of the three boundary line collision maps is analysed for numerical calculation of turbulent sprays using the Euler/Lagrange approach. A comparison with the experiments of Rüger et al. (2000) is also shown for validation.

\section{Theoretical boundary lines}

Based on an energy balance Estrade et al. (1999) developed the lower boundary of bouncing. For that it was assumed that the initial droplet kinetic energy of the deformation region does not exceed the surface energy difference to produce a certain deformation. This derivation neglects any dissipation effects but includes the 
influence of droplet size ratio. Moreover, a shape factor is included in the correlation, depending on the degree of deformation during the bouncing process. Hence the shape factor should be depending on liquid dynamic viscosity. The correlation of Estrade et al. (1999) is given by:

$W e=\frac{\Delta\left(1+\Delta^{2}\right)(4 \Psi-12)}{\chi(\cos (\arcsin B))^{2}}$

$$
\begin{array}{ll}
\chi=1-0.25(2-\tau)^{2}(1+\tau) & \text { if }: \tau>1 \\
\chi=0.25 \tau^{2}(3-\tau) & \text { if }: \tau \leq 1 \\
\tau=(1-B)(1-\Delta) &
\end{array}
$$

The specified value of the shape factor was $\Psi=3.351$ fitting the data for ethanol at two size ratios. In previous studies it was aimed to adapt the bouncing boundary through the shape factor, which is somehow related to viscosity effects (Sommerfeld and Kuschel 2016). Such an adaptation was successful for higher Weber-numbers, but not below the triple point. The variation of the bouncing boundary in dependence of the size ratio is shown in Figure 2 a). With decreasing size ratio $\Delta=d_{S} / d_{L}$ the boundary line is slightly shifted upward and to the left in accordance with the measurements presented by Estrade et al. (1999). However, the question is whether the proposed correlation is also valid for small size ratios which are likely to be found in spraying systems as will be shown below.

Also the effect of the shape factor on the boundary line was analysed showing a rather strong dependence (Figure 2 b). A small value of $\Psi$ implies also a smaller deformation and hence lower shape factors. This is in accordance with measurements of Sommerfeld and Kuschel (2016) where with increasing viscosity, also associated with reduced deformation, the bouncing boundary is shifted upward.
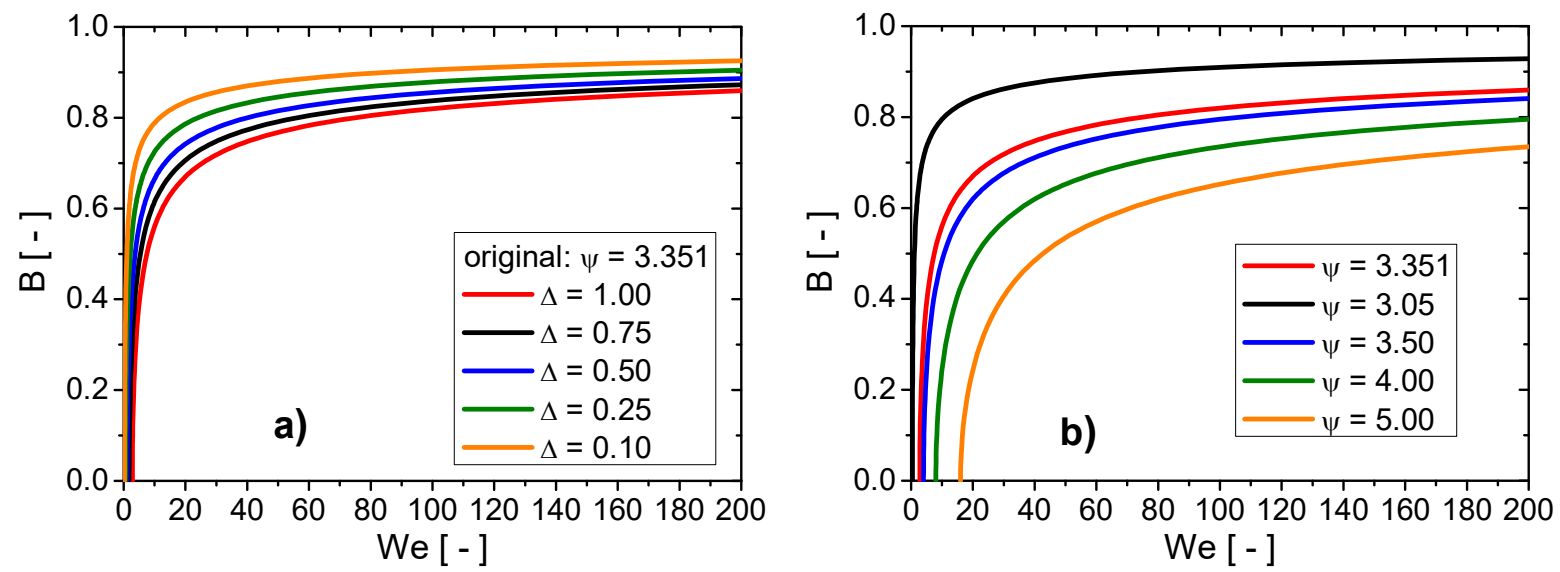

Figure 2: Collision map with Estrade et al. (1990) bouncing boundary lines showing the effect of (a) droplet size ratio $\Delta=d_{S} / d_{L}$ (shape factor $\psi=3.351$ ) and the influence of $(b)$ the shape factor.

The boundary line between coalescence and stretching separation was derived in a number of studies, namely Brazier-Smith et al. (1972), Ashgriz and Poo (1990) and Jiang et al. (1992). Brazier-Smith et al. (1972) assumed that if the rotational energy exceeds the surface energy required to reform two new droplets from the coalesced droplets, separation will occur. Also in this correlation the size ratio $\Delta$ was considered. The Brazier-Smith et al. (1972) correlation is very often used in spray calculations, just separating coalescence and grazing collisions (O'Rourke 1981), which is called here single line boundary model. The correlation of Brazier-Smith et al. (1972) for the boundary line is given by:

$W e=\frac{4.8}{B^{2}}\left(\left(\frac{1}{\Delta}\right)^{3}-2.4\left(\frac{1}{\Delta}\right)^{2}+2.7\left(\frac{1}{\Delta}\right)\right)$

The energy balance of Ashgriz and Poo (1990) assumes that stretching separation occurs if the total effective stretching energy is larger than the surface energy of the small interaction region. Also in this correlation the size ratio $\Delta$ was included and validated by experiments for water.

$W e=\frac{4\left(1-\Delta^{3}\right)^{2}\left\{3(1+\Delta)(1-B)\left(\Delta^{3} \phi_{S}+\phi_{L}\right)\right\}^{1 / 2}}{\Delta^{2}\left\{\left(1+\Delta^{3}\right)-\left(1-B^{2}\right)\left(\phi_{S}+\Delta^{3} \phi_{L}\right)\right\}}$ 


$$
\begin{aligned}
& \phi_{S}=\left\{\begin{array}{ll}
1-\frac{1}{4 \Delta^{3}}(2 \Delta-\tau)^{2}(\Delta+\tau) & \text { for } h>0.5 d_{S} \\
\frac{\tau^{2}}{4 \Delta^{3}}(3 \Delta-\tau) & \text { for } h<0.5 d_{S}
\end{array} \quad \phi_{L}= \begin{cases}1-\frac{1}{4}(2-\tau)^{2}(1+\tau) & \text { for } h>0.5 d_{L} \\
\frac{\tau^{2}}{4}(3-\tau) & \text { for } h<0.5 d_{L}\end{cases} \right. \\
& \mathrm{h}=\frac{1}{2}\left(\mathrm{~d}_{\mathrm{L}}+\mathrm{d}_{\mathrm{S}}\right)(1-\mathrm{B}) ; \quad \tau=(1-\mathrm{B})(1+\Delta)
\end{aligned}
$$

Finally the boundary line developed by Jiang et al. (1992) is based on a momentum balance and therefore includes dissipation effect. Hence, this is the only correlation which may be used to consider viscosity effects, as for example done by Gotaas et al. (2007) and Sommerfeld and Kuschel (2016).

$$
B=\frac{C_{a}}{W e^{1 / 2}}\left[1+C_{b} \frac{\mu}{\sigma}\left(\frac{\rho d_{1}}{\sigma}\right)^{1 / 2}\right]
$$

The analysis of Gotaas et al. (2007) suggested to use for the involved constants the values $C_{a}=2.33$ and $C_{b}=$ $0.41\left(\mathrm{~m}^{2} / \mathrm{s}^{2}\right)$. Since the correlation of Jiang et al. (1992) includes properties of the liquid (i.e. dynamic viscosity, surface tension and density) as well as the small droplet diameter it could be successfully used for liquids with increasing viscosity with the above set of model constants (Gotaas et al. 2007). In the work of Sommerfeld and Kuschel (2016) it could be shown that the model constants may be correlated reasonably well with the relaxation velocity $U_{\text {relax }}=\sigma / \mu$. The Jiang et al. (1992) correlation does not include the droplet size ratio, but the diameter of the small droplets. However, changing the droplet size over an order of magnitude in a relevant size range did not show a large variation of the boundary line.

The different coalescence/stretching boundary lines are compared in Figure 3. The correlations of Brazier-Smith et al. (1972) with size ratio one and Jiang et al. (1992) do not show any large difference. Larger are the deviations to the Ashgriz and Poo (1990) correlation for We smaller than 20, since it approaches the point We $=0$ and $\mathrm{B}=$ 1.0. Decreasing the size ratio in the Ashgriz and Poo (1990) correlation, results in a turning of the line around this point, yielding higher impact parameters $B$ for stretching separation to occur (Figure 3). This is not quite the trend observed in experiments where an upward and right shift of the boundary line is observed. On the other hand the Ashgriz and Poo (1990) correlation has never been validated for size ratios below $\Delta=0.5$. Here also the correlation shows some obscure behaviour for larger We.

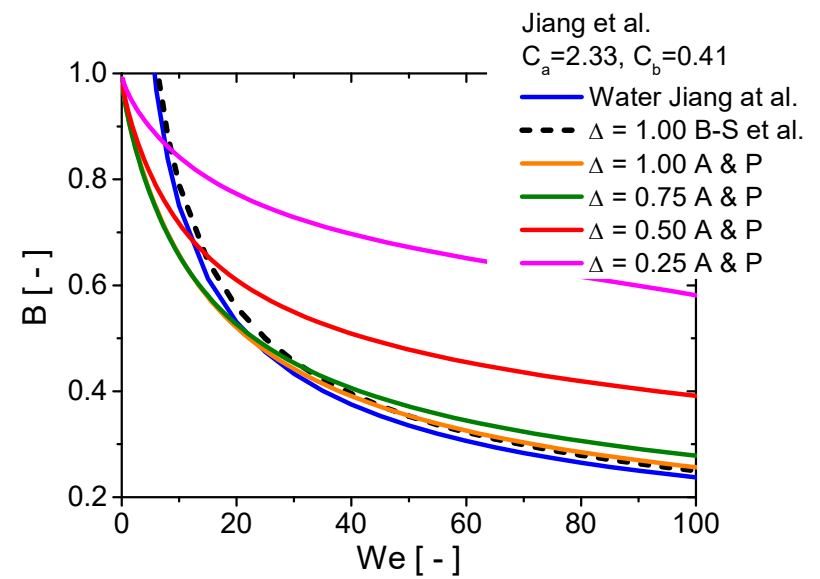

Figure 3: Different boundary lines for stretching coalescence transition in the collision map; Brazier-Smith et al. (1972) model with $\Delta=1.0$, Jiang et al. (1992) model for water with standard constants and Ashgriz and Poo (1990) model with decreasing droplet size ratio $\Delta$ (droplet size $750 \mu \mathrm{m}$ ).

Finally the boundary line between coalescence and reflexive separation is considered. In spray computations mostly the correlation also proposed by Ashgriz and Poo (1990) is used, given below:

$W e_{C}=3\left[7\left(1+\Delta^{3}\right)^{2 / 3}-4\left(1+\Delta^{2}\right)\right] \frac{\Delta\left(1+\Delta^{3}\right)^{2}}{\Delta^{6} \eta_{S}+\eta_{L}}$ 


$$
\begin{aligned}
& \eta_{\mathrm{S}}=2(1-\xi)^{2}\left(1-\xi^{2}\right)^{1 / 2}-1 \\
& \eta_{\mathrm{L}}=2(\Delta-\xi)^{2}\left(\Delta^{2}-\xi^{2}\right)^{1 / 2}-\Delta^{3} \\
& \xi=(1 / 2) \mathrm{B}(1+\Delta)
\end{aligned}
$$

This correlation also neglects viscosity but includes droplet size ratio. For $\Delta=1.0$ the critical Weber-number is about 18.7 as determined from many experimental studies for water. With decreasing size ratio the boundary line is shifted remarkably to the right, to higher We. However, this is not in accordance with the studies of Tang et al. (2012) where $\Delta=0.4$ results in a shift to $W_{e_{c}} \sim 40$. A shift of this boundary line to the right was also observed by Kuschel and Sommerfeld (2013) and Sommerfeld and Kuschel (2016) when increasing the dynamic viscosity of the liquid, similar to the work of Gotaas et al. (2007). This shift of the critical Weber number could be quite well correlated in dependence of the Ohnesorge number as well as the capillary number (Sommerfeld and Kuschel 2016).

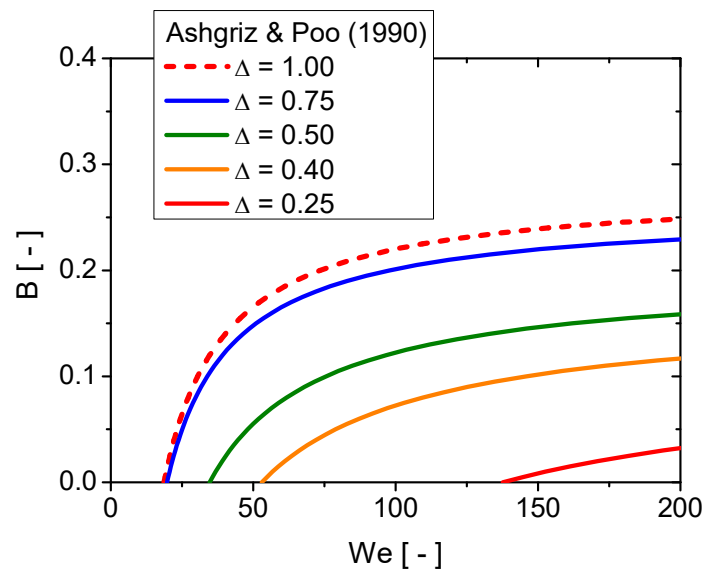

Figure 4: Boundary lines for coalescence/reflexive separation according to Ashgriz and Poo (1990) with decreasing size ratio $\Delta$.

\section{Euler/Lagrange approach}

The numerical scheme adopted to simulate the dispersed two-phase flow developing in a spraying system is the fully coupled stationary and three-dimensional Euler/Lagrange approach (Rüger et al. 2000, Lain and Sommerfeld 2008). The fluid flow was calculated based on the Euler approach by solving the Reynolds-averaged conservation equations on a fixed grid in connection with the standard $k-\varepsilon$ turbulence model equations. The effect of the dispersed phase on the fluid flow was accounted for by appropriate source terms in the momentum equations as well as the conservation equations for turbulent kinetic energy and dissipation rate, i.e., full two-way coupling.

The dispersed droplet phase is simulated by the Lagrangian approach, which implies that the spray is generated by tracking a huge number of droplet parcels through the beforehand calculated flow field. This requires the solution of the equations of the motion for each computational particle or parcel, being a representative of a number of real droplets with the same properties. These equations include the droplet inertia, drag and gravitybuoyancy. The Basset history term, the added mass and the fluid inertia are negligible for high ratios of droplet to gas density. The drag coefficient used in droplet tracking is that of Schiller and Naumann (1933).

For solving the partial differential equations of droplet motion a dynamic time step is used which is automatically adjusted according to the local (droplet location within the grid) governing time scales such as particle response time, integral time scale of turbulence and inter-particle collision time. The maximum tracking time step is selected to be $20 \%$ of the minimum of these time scales.

In order to account for turbulence effects on droplet motion, the instantaneous fluid velocity components along the particle trajectory are determined from the local mean fluid velocity interpolated from the neighbouring grid points and a fluctuating component generated by a single-step isotropic Langevin model described by Sommerfeld et al. (2008) and thoroughly validated in Sommerfeld (1996). In this model the fluctuation velocity is composed of a correlated part from the previous time step and a random component drawn from a Gaussian distribution function. The degree of correlation depends on the turbulent particle Stokes number $\mathrm{St}_{\mathrm{T}}$ and is calculated using appropriate time and length scales of turbulence estimated from the k- $\varepsilon$ turbulence model.

As mentioned above, two-way coupling considers the momentum transfer from the dispersed phase to the continuous phase and vice versa through appropriate source terms in the momentum equations and the conservation equations of turbulent kinetic energy and the dissipation rate. The source terms are accumulated for each control volume during the Lagrangian tracking procedure applying ensemble and temporal averaging 
(Sommerfeld 2017). The momentum sources are obtained from the change of particle velocity during its path through a control volume (Sommerfeld et al. 2008). An under-relaxation approach is used when introducing the source terms in the conservation equations of the fluid flow (for details see Kohnen et al. 1994, Lain and Sommerfeld 2012). Hence, a sequential calculation of fluid flow and particle phase is performed until the coupled hybrid system has converged. For the present calculations typically about 25 to 55 coupling iterations with an under-relaxation factor between 0.1 and 0.5 were necessary in order to yield convergence of the Euler-Lagrange coupling. This convergence behaviour was clearly demonstrated by Lain and Sommerfeld (2012).

\section{Stochastic droplet collision model}

For modelling droplet collisions in a spray the numerically efficient stochastic collision model (Sommerfeld 2001) combined with the impact efficiency (Ho and Sommerfeld 2002) is used. This model does not require searching for collision partners among surrounding droplets, rather during the tracking of the considered parcel a fictitious collision partner is drawn randomly from the local population in each time step (Sommerfeld 2001). This however requires that for each computational cell the droplet phase properties have to be made available, namely, concentration, droplet size distribution, and droplet size-velocity correlations with mean- and rms-values, droplet size-temperature correlations and so forth. From these distributions the properties of the fictitious droplet are drawn with size, velocity and temperature, by a stochastic process. The fictitious droplet velocity fluctuation needs however to be obtained through a pair-correlation with the real particle which depends on the particle Stokes number (i.e. the ratio of particle response time to the integral time scale of turbulence). This accounts for the effect that the velocities of responsive droplets are correlated through turbulence, whereas inertial particles have completely uncorrelated velocities (Sommerfeld 2001, Sommerfeld et al. 2008). Having all information about the collision partners the collision probability can be calculated:

$P=\frac{\pi}{6}\left(d_{S}+d_{L}\right)^{2}\left|\overrightarrow{u_{S}}-\overrightarrow{u_{L}}\right| n_{d} \Delta t_{L}$

If a random number is smaller than this probability a collision occurs. It should be mentioned again that the mean droplet collision time is an additional criterion for limiting the Lagrangian time step $\Delta \mathrm{t}_{\mathrm{L}}$ in order to insure only binary collisions, i.e. the collision probability is smaller than one. The collision itself is then calculated in a coordinate system where the fictitious particle is stationary. Thereby the collision point on the particle surfaces can be randomly determined (Sommerfeld 2001) and the lateral displacement $b$ of the collision partners is known. Such a stochastic model is numerically very efficient and the algorithm can be easily parallelised.

An essential element which has to be integrated in the collision detection is the impact efficiency which is relevant for a collision between small and large droplets (Ho and Sommerfeld 2002). Up to now the reduction of the collision rate in sprays through the impact efficiency has never been considered. In such a situation the smaller droplet might move around the larger one with the relative velocity field, which is a quite frequent phenomenon in sprays due to the wide size spectrum. Effectively, this results in a reduction of collision cross-section and hence collision rate, since often larger droplets will capture smaller droplets due to the existence of a high relative velocity (see Figure 5). This phenomenon may be described in a coordinate system where the larger collector droplet is stationary. Considering only inertial effects, the impact efficiency is then defined as the ratio of the circular cross-section from which the small droplets just will hit the larger droplet to the effective collector crosssection. Accounting also for the so-called blocking effect this cross-section diameter is the sum of the large and small droplet diameters (Figure 5). In the work of Schuch and Löffler (1978) the impact efficiency for inertial effects was calculated in dependence of the relative Stokes number which is the Stokesian relaxation time of the small droplet to the time it needs to pass the collector droplet with the relative velocity (Ho and Sommerfeld 2002):

$S t_{\text {rel }}=\frac{\tau_{P}}{T_{\text {Pass }}}=\frac{\rho_{S} d_{S}^{2} u_{r e l}}{18 \mu d_{L}}$

here the $d_{S}$ and $d_{L}$ refer to the diameters of the small and large droplet, respectively, $\rho$ is the small droplet density and $u_{r e l}$ is the instantaneous relative velocity.

The impact efficiency, as defined below, is related to the relative Stokes number using the two parameters a and $b$, which depend on the collector droplet Reynolds number (Schuch and Löffler 1978):

$\eta=\left(\frac{2 Y_{C}}{d_{S}+d_{L}}\right)^{2}=\left(\frac{S t_{r e l}}{s t_{r e l}+a}\right)^{b}$ 


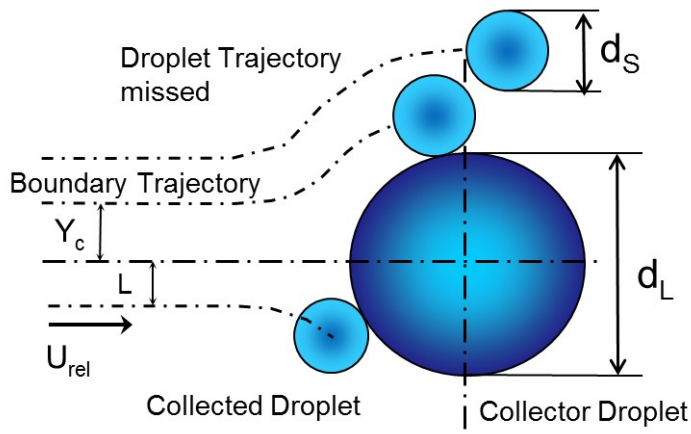

Figure 5: Illustration of inertial impact efficiency for the collision of a small droplet with a larger collector droplet.

In the work of Schuch and Löffler (1978) it was shown that the impact efficiency for inertial impaction is depending on the large droplet Reynolds-number determined with the relative velocity. This is caused by the fact that with increasing collector Reynolds number the flow deflection is increasingly compresses towards the collector. The resulting different values for the constants $a$ and $b$ are provided in Table 1 . The results of the different correlations are given in Figure 6 together with own simulations which agree fairly well. For a given relative Stokes-number the increase in Reynolds-number yields growing impact efficiency (Sommerfeld and Lain 2009) due the raising small particle inertia with respect to the stronger flow deflection. Eventually, a collision is only occurring if the randomly drawn lateral displacement $L$ (see Sommerfeld 2001) is smaller than the value of $\left(Y_{C}+d_{L}\right)$. Note that the lateral displacement $L$ is identical to the impact parameter $B$.

\begin{tabular}{|c|c|c|}
\hline $\mathrm{Re}_{\mathrm{c}}$ & $\mathrm{a}$ & $\mathrm{b}$ \\
\hline$>1$ & 0.25 & 2.0 \\
\hline $60-80$ & 0.506 & 1.84 \\
\hline 40 & 1.03 & 2.07 \\
\hline $10-20$ & 1.24 & 1.95 \\
\hline$<1$ & 0.65 & 3.7 \\
\hline
\end{tabular}

Table 1: Constants for the correlation proposed by Schuch and Löffler (1978) for the impact efficiency (Eq. (8).

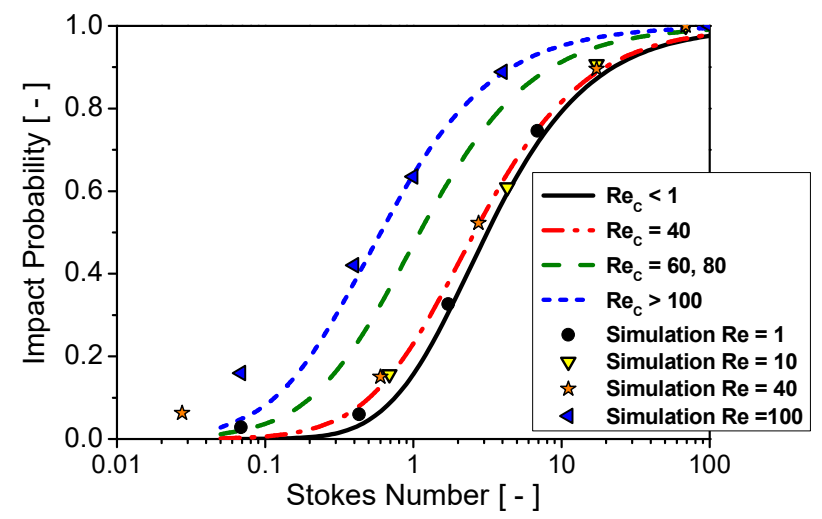

Figure 6: Impact efficiency as a function of relative Stokes number, lines: correlations proposed by Schuch and Löffler (1978); symbols: present numerical calculations.

The above model for considering the impact efficiency may be only applied to turbulent flows if the collision complex is much smaller than the smallest turbulent eddies, namely the Kolmogorov length scale. Otherwise of course the impact efficiency is still very important, but the collision geometry is much more complex. In such a situation the trajectories of the small colliding droplets are affected by turbulent transport, so that even small droplets moving initially outside the collision cross-section might hit the collector. Thereby the impact efficiency will rise again when reducing the Stokes number. This will also happen if the colliding droplets are very small so that their motion is affected by Brownian dispersion. 
The final step in the droplet collision model is the determination of the collision outcome, based on the collision maps $B=f(\mathrm{We})$ as it was described above (Figure 1$)$ and the theoretically derived boundary lines. As indicated in Figure 1 three characteristic points are identified to allow a generalisation of the collision map with regard to droplet fluid properties, mainly viscosity and size ratio $\Delta$.

\section{Characteristic points in the collision maps}

From the collision map in Figure 1 it is obvious that there are three characteristic points which could be used for describing the change of the collision maps for example with droplet size ratio or increasing fluid viscosity. The first point is the We-number at $B=0$ for the transition from coalescence to reflexive separation. The second is the triple point where bouncing, stretching separation and coalescence meet. This point was identified by Sommerfeld and Kuschel (2016) and Sommerfeld (2016) to have a characteristic movement with increasing viscosity. The third characteristic point results from the lower boundary of bouncing intercepting with the horizontal axis (Estrade et al. 1999). In some studies and for some liquids also a coalescence region for very low We over a wide range of impact parameters was found (Qian and Law 1997, Chen 2007 and Huang and Pan 2015), which however will not considered here for the moment.

For collisions of identical sized droplets the critical Weber-number $W e_{C}$ has been quite often identified to shift to the right with increasing Ohnesorge-number and Qian and Law (1997) found a good correlation with minor scatter considering also various literature data (e.g. Jiang et al. 1991):

$W e_{C}=\sqrt{2} 480 \mathrm{Oh}+15$

Also the experiments of Huang and Pan (2015) for different alkanes followed this correlation for both 300 and 600 $\mu \mathrm{m}$ droplets. Actually, there is no difference for the location of the critical Weber-number when plotting the data versus the droplet Ohnesorge-number. Very high viscous droplets up to $58 \mathrm{mPa} \cdot \mathrm{s}$ were considered by Gotaas et al. (2007), namely monoethylenglycol (MEG), diethylenglycol (DEG) and triethylenglycol (TEG), yielding Ohnesorge numbers up to 0.34 . For the low Oh-regime they proposed a similar correlation as above:

$W e_{C}=643 \mathrm{Oh}+14.8$

Moreover, they suggested an additional correlation for Ohnesorge-numbers larger than about 0.045 using also experimental data from Willis and Orme (2003):

$W e_{C}=9309 \mathrm{Oh}^{1.706}$

In the droplet collision studies of Kuschel and Sommerfeld (2013) several solutions were used as liquid with viscosities between 1 and $50 \mathrm{mPa} \cdot \mathrm{s}$, yielding up to $\mathrm{Oh}=0.37$. However, since here the maximum We-number was about 100, not for all cases the critical Weber-number could be identified. Hence the non-linear correlation proposed by Gotaas et al. (2007) fits the high-viscosity data reasonably well (Figure 7 a) including silicon oils, MEG and DEG and a VOF (volume of fluid) simulation of Xia and Hu (2015) for $10 \mu \mathrm{m}$ liquid aluminium droplets $(\mathrm{Oh}=0.115)$. The critical Weber-number for mixtures of Diesel/Biodiesel and Diesel/water emulsions (Chen et al. 2016) are however remarkably over-predicted by this correlation, similar to solution droplets of Kuschel and Sommerfeld (2013).

Now we turn to the low Oh-regime wherefore numerous data are available (Figure $7 \mathrm{~b}$ ). Here only the initial correlation proposed by Qian and Law (1997) is shown for comparison. Most of the data for the critical Webernumber are lying around and along this correlation. However some are a bit more off the line, but it is not possible to specify the reason for that at the moment. Here further experiments are needed for clarifying this deviation. Two data point for the reference oil FVA1 at low temperature (Sommerfeld and Kuschel 2016) give almost double the value as resulting from the correlation. Similarly higher viscous alcohols are lightly above the line (Sommerfeld and Kuschel 2016). Interestingly, all VOF simulations for water droplets (Saroka et al. 2012) of different size are clustered above the correlation, which indicates that some phenomena are not captured properly by these simulations. One result of Rabe et al. (2010) for different sized water droplets is also found in this region. This point is just included for illustrating the fact that decreasing size ratio yields a shift of the triple point as well as $W e_{c}$ to the right (see Tang et al. 2012). The data points for the Diesel/water emulsion droplets are initially above and for higher water content, and hence larger viscosity, below the correlation, which suggests a slightly lower slope of such correlation (Chen et al. 2017). Consequently, the correlation proposed by Qian and Law (1997), Eq. 9 , is still a very good approximation to describe the shift of the critical We-number with changing fluid properties up to $\mathrm{Oh} \approx 0.1$. It seems that higher viscous liquids may be described better by the Gotaas et al. (2007)correlation.

It was shown previously that the adapted correlation of Ashgriz and Poo (1990) predicts the lateral extent of reflexive separation (i.e. the boundary line separating reflexive separation and coalescence) very well for many 
cases (Sommerfeld and Kuschel 2016). Hence the original correlation (Eq. 5) is just shifted by the critical Wenumber:

$W e=\left(W e_{C}-W e_{C, w}\right)+3\left[7\left(1+\Delta^{3}\right)^{2 / 3}-4\left(1+\Delta^{2}\right)\right] \frac{\Delta\left(1+\Delta^{3}\right)^{2}}{\Delta^{6} \eta_{S}+\eta_{L}}$

It is known from various studies summarised above, that the critical Weber-number also is shifted to higher Weber-numbers when the size ratio $d_{S} / d_{L}$ is decreasing. Theoretical correlations for $W_{C}$ in dependence of size ratio also considering different liquids were provided by Tang et al. (2012).
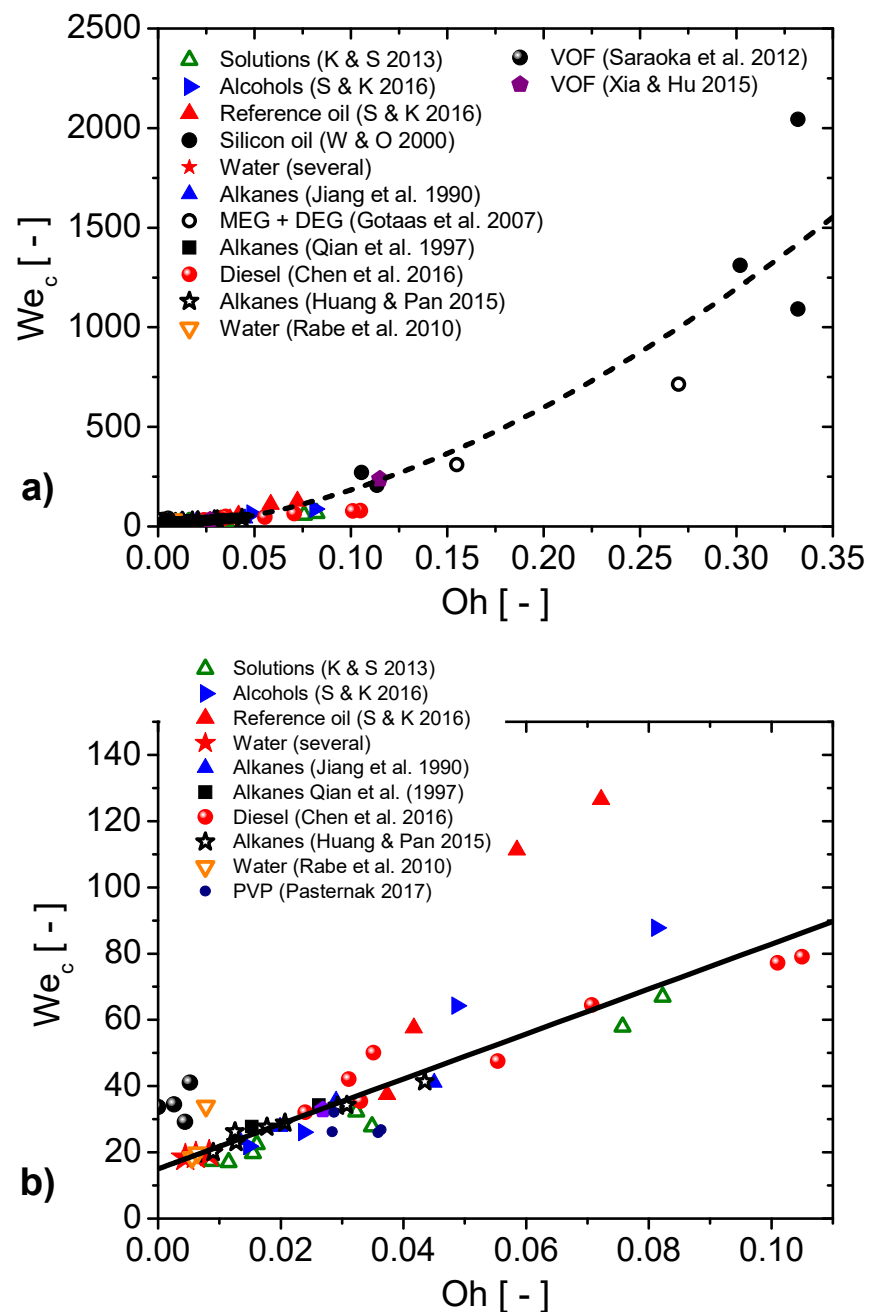

Figure 7: Summary of the critical Weber-number for the full (a) and small (b) regime of Ohnesorge-numbers obtained from different experimental studies using a range of different liquids (K \& S 2013: Kuschel and Sommerfeld (2013); S \& K 2016: Sommerfeld and Kuschel (2016); W \& O 2000: Willis and Orme (2000)), dashed line correlation Eq. (11), closed line correlation Eq. (9).

The next characteristic point is the triple point location determined by the Weber-number $W e_{T}$. In the study of Sommerfeld (2016) the own measurements regarding the location of the triple point showed with growing viscosity and hence Ohnesorge-number first an increase of We followed by a decrease beyond about $\mathrm{Oh}=0.1$. $\mathrm{A}$ suitable correlation between $W e_{T}$ and $\mathrm{Oh}$ could be elaborated based on a very good coherence of the data in a $\mathrm{Ca}=\mathrm{f}(\mathrm{Oh})$ diagram.

$\mathrm{Ca}=-0.0088+5.4246 \mathrm{Oh}-2.2036 \mathrm{Oh}^{2}$

The available experimental results together with the proposed correlation regarding the triple point location are summarized in Figure 8 for the lower Oh-regime. Here the capillary number is obtained from the available data on We and Oh through $\mathrm{Ca}=\mathrm{Oh} \sqrt{W e}$. Most of the data are nicely scattered around the correlation line except one for Diesel with $60 \%$ of Biodiesel (blue star at Oh $=0.11$ ). 


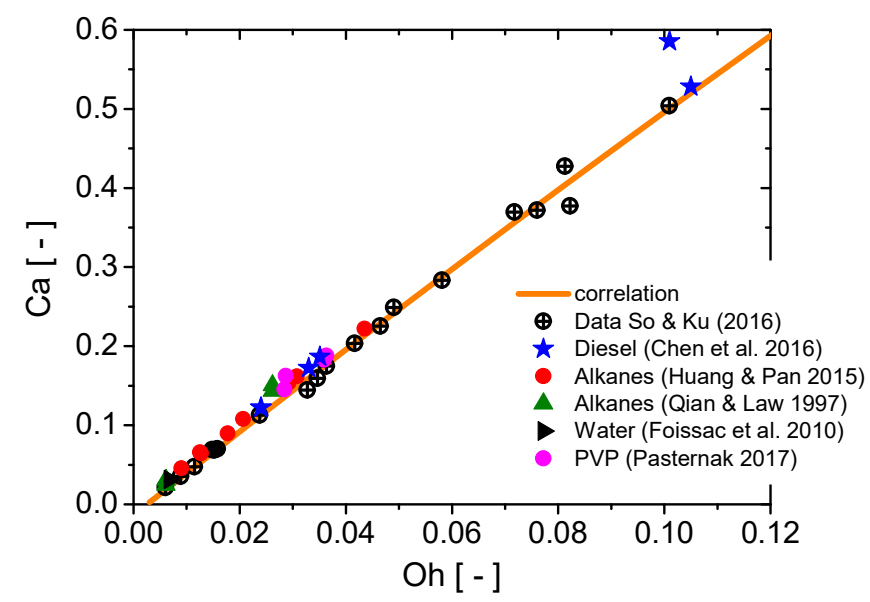

Figure 8: Triple point location from different experimental data (So \& Ku (2016) includes all data presented by Sommerfeld and Kuschel 2016) shown as $\mathrm{Ca}=\mathrm{f}(\mathrm{Oh})$ with best fit correlation given in Eq. (13).

Having now a correlation for the triple point Weber-number in dependence of the Oh-number, which only depends on the material properties of the droplets, it is possible to adapt a boundary line model (in this case the Jiang et al. (1992) model) in such a way that it goes though the triple point ( $B_{T}$ and $W e_{T}$ need to be known from experiments) and thereby matches the experimentally observed boundary between coalescence and stretching separation. If a bouncing region is observed left of the stretching separation region, which was reported by many measurements for alcohols, oils, alkanes and solutions, the triple point is also the starting point of the boundary line. An exception is water where this line extends to $B=1$ at $W e=0$ separating bouncing and coalescence (see e.g. Kuschel and Sommerfeld 2013). This matching procedure was successfully achieved with the Jiang et al. (1997) model which accounts for the effect of viscous dissipation (Sommerfeld 2016). By setting the model constant $C_{b}=1$ (this value was obtained for higher relaxation velocities $u_{\text {relax }}=\sigma / \mu$ by Sommerfeld and Kuschel 2016) it was found that the model parameter $\mathrm{C}_{a}$ could be very well correlated with the Ohnesorge number for pure fluids and solution droplets. For pure fluids the following correlation was found:

$\mathrm{C}_{\mathrm{a}}=2.3-11.12 \cdot \mathrm{Oh}+23.74 \cdot \mathrm{Oh}^{2}-18.2 \cdot \mathrm{Oh}^{3}$

A validation of this modelling approach was presented for higher viscous liquids by Sommerfeld (2017) and Diesel as well as solution droplets by Sommerfeld (2016).

\section{Spray calculations}

For analysing the effect of impact efficiency and the occurring droplet collision outcomes the experimentally studied single spray of Rüger et al. (2000) was considered using first water as liquid. In the measurements a hollow cone spray nozzle with a spread angle of 45 degree was centrally mounted into the upper part of a 400 $\mathrm{mm}$ diameter pipe. The test section had a length of about 1 meter (Figure 9). For avoiding any recirculation region within the test section, a low airflow was forced through the pipe by a suction blower. Above the spray nozzle a honeycomb was installed in order to homogenise the flow. The nozzle was operated at a water flow rate of 0.135 $\mathrm{I} / \mathrm{min}$. Air and droplet velocity profiles at the inlet plane, which was located $25 \mathrm{~mm}$ downstream of the nozzle exit, were measured by a two-component phase-Doppler anemometer. Here droplets with a size less than $2.5 \mu \mathrm{m}$ were identified as tracer particles for the air phase velocities over the entire inlet cross-section. Of course droplets were only present just underneath the spray nozzle exit. Here local droplet size distributions were measured from the centre location of $z=0 \mathrm{~mm}$ up to a radial location of $z=30 \mathrm{~mm}$ at intervals of $1 \mathrm{~mm}$. Therefore, as the inlet condition for the droplet phase besides the droplet size distribution also size-velocity correlations were available for the axial and radial components. The mean tangential velocity component was close to zero. Hence, for each injected parcel a size was drawn from the local cumulative distribution function by a random process. The sizevelocity correlations provided the droplet mean velocities. From the correlation between the size and rms-values the standard deviations for each velocity component was obtained. The instantaneous droplet fluctuating velocities were then randomly drawn from the corresponding normal distribution functions. 

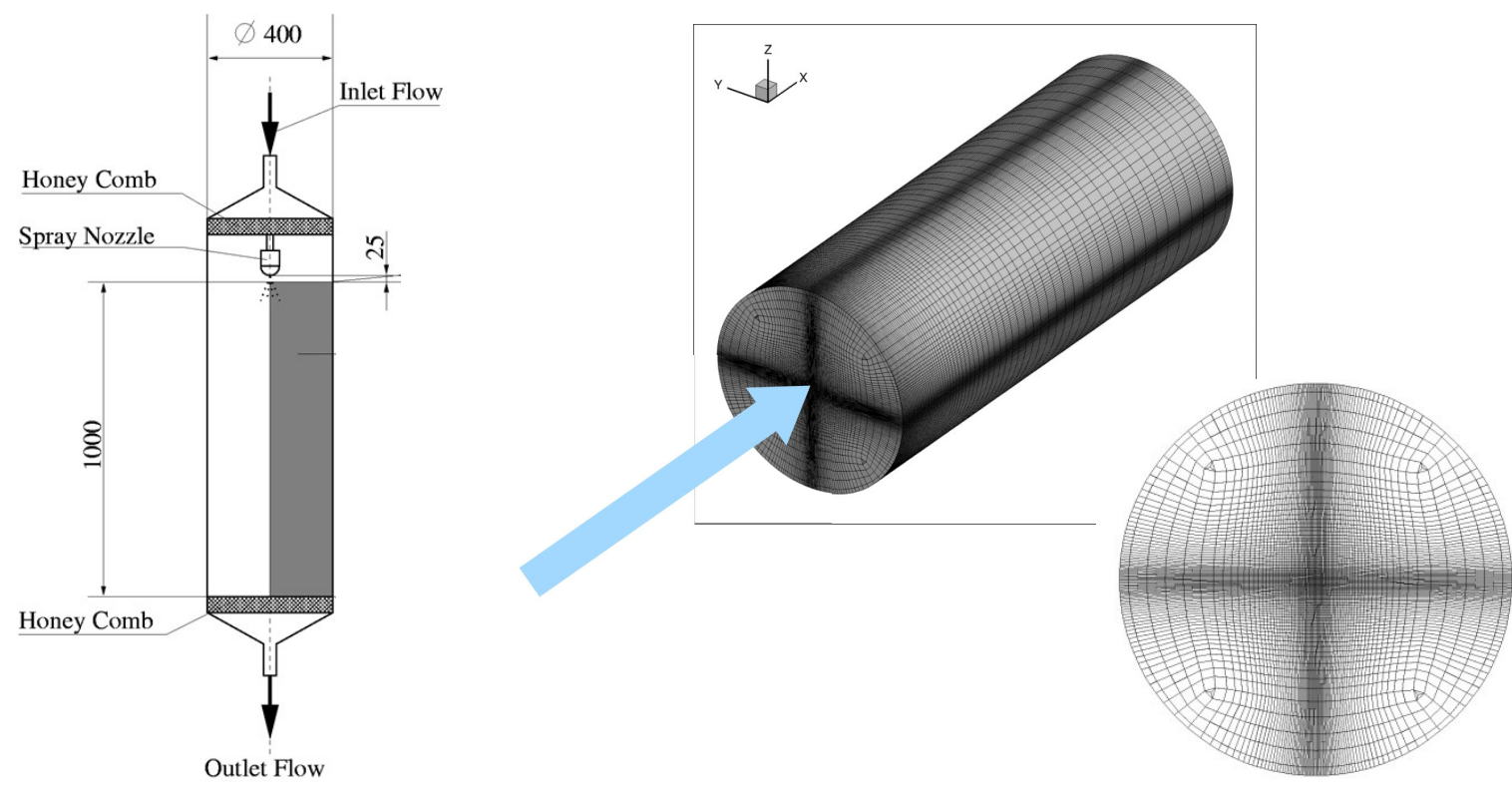

Figure 9: Illustration of the spray facility with major dimensions and spray nozzle location (left) and sketch of the numerical grid used for the computations with about 700,000 control volumes (middle) as well as a cross-sectional view of the grid (right).

The numerical grid used for the fully three-dimensional calculations is also shown in Figure 9 (middle) with the direction of spray injection. The employed grid was a structured O-grid divided in 5 blocks with in total 700,000 control volumes covering the region from the inlet plane $25 \mathrm{~mm}$ downstream the nozzle $(x=0 \mathrm{~mm})$ up to the outlet $1025 \mathrm{~mm}$ downstream the nozzle exit $(x=1000 \mathrm{~mm})$. In order to resolve the spray nozzle injection region sufficiently the grid was remarkably refined (i.e. indicated by the cross-like pattern in Figure 9 right). The numerical computations were done for a steady-state mode where after reaching a converged fluid flow solution 372,000 parcels were sequentially injected at the inlet and tracked through the flow field. Thereafter, the obtained source terms were coupled back to the fluid flow calculations with under-relaxation which then was recalculated until convergence was reached. Eventually about 35 coupling iterations were required to obtain a coupled converged result of the spray.

Since the calculations were first conducted for water according to the measurements, the three boundary line model was used for separating the collision outcome regimes. The boundary line reflexive separation/coalescence was used according to Ashgriz and Poo (1990) given in Eq. (5). As it best fits for water, also the stretching separation/coalescence boundary was that of Ashgriz and Poo (1990) provided in Eq. (3). Finally the lower boundary of bouncing uses the Estrade et al. (1999) correlation (Eq. 1) with the shape factor of $\Psi=3.351$. This boundary line ended of course at the intersection with the stretching/coalescence boundary. Although in the spray simulations collisions occur with a wide range of size ratios, all correlations were used with a fixed size ration of $\Delta=1.0$ for clarity.

As a first step the simulations were validated against the experiments and the agreement of all gas and droplet phase properties was found quite well similar to the comparison published by Rüger et al. (2000). The numerical calculations with droplet collisions were conducted by storing all the collision events occurring. In Figure 10 the total collision events are summarized and also the numbers for bouncing, coalescence and stretching as well as reflexive separation are given. The total collision number is 2.45 million when assuming $100 \%$ impact efficiency. Considering reduced impact efficiency through the motion of small droplet around larger ones upon interaction, only 1.66 million collisions are counted. This demonstrates that the collision rate is over-predicted by $48 \%$ when not considering impact efficiency, which is of course a huge error.

Looking at the number of collisions with respect to collision type, it is clear that coalescence is dominating with about $88 \%$ of the total collisions in both cases, i.e. without and with impact efficiency (Figure 10). Coalescence events occur for a relatively wide region near the nozzle exit, but continue to happen up to the end of the computational domain (Figure $11 \mathrm{~b}$ ) but with reduced probability. The reduction of the collision probability along the spray is of course associated with a decrease of relative velocity and local droplet concentration. Droplet size distributions only slightly change along the spray although coalescence is happening. The second probable collision event is bouncing (Figure 10) occurring mainly in the initial region of the spray (Figure 11). Stretching separation happens of course only if the We-number is exceeding the triple point value, hence can only take 
place close to the nozzle where the relative velocity is still high (Figure 11). The probability of reflexive separation is even lower and also occurs only near the nozzle exit.

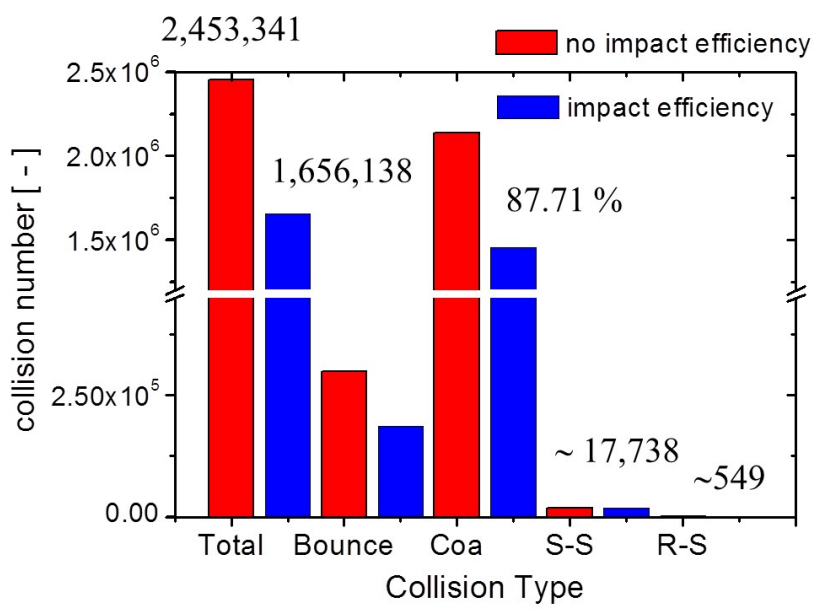

Figure 10: Droplet collision counts throughout the entire spray, comparing a calculation without and with impact efficiency and showing the numbers of collisions resulting in bouncing, coalescence and stretching as well as reflexive separation.

a)

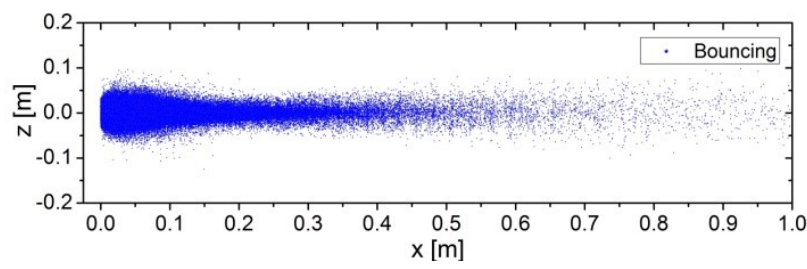

b)

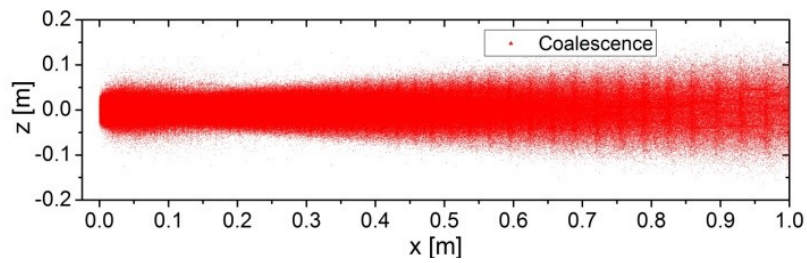

c)

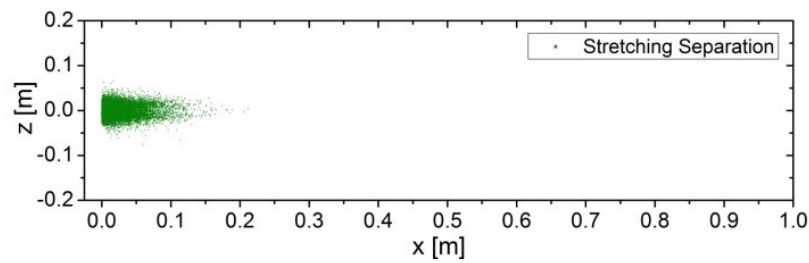

Figure 11: Scatter plot of the location of collision events throughout the spray, a) locations of bouncing, b) locations of coalescence, c) locations of stretching separation.

From the simulations with impact efficiency also the droplet size ratio upon collision was recorded along the spray for different cross-sections. This result shows that preferentially small and large droplets collide due to the higher relative velocity in a spray (Figure 12). Hence, the maximum in the PDF of the size ratio has initially a peak around 0.18 which is further downstream spread to values between 0.2 and 0.3 . It should be noted that such small size ratios have never been analysed experimentally for confirming the above introduced boundary lines.

\section{Conclusions}

An attempt was made for generalising the collision maps describing the outcome of binary collisions between droplets with respect to liquid properties. The triple point location was obtained through a correlation between $\mathrm{Ca}$ $=\mathrm{f}(\mathrm{Oh})$ based on numerous available experiments. As a boundary line to capture viscosity effects, the Jiang et al. (1992) correlation was used. The involved parameters could be adapted properly through a dependence on the Ohnesorge number for pure liquids and solutions. The upper boundary of reflexive separation was demarked through the Ashgriz and Poo (1990) correlation. With increasing viscosity the critical We-number is shifted to the right which could be also obtained with a correlation on the Ohnesorge-number. The lower boundary of bouncing 
Estrade et al. 1999) could not yet be properly adapted with respect to viscosity effects. The three-line boundary model was applied in Euler/Lagrange calculations of a turbulent water spray. It was shown that neglecting the impact efficiency yields an over-prediction of the collision rate by about $48 \%$. The analysis of the collision events showed that coalescence and then bouncing were the most frequent outcome. Moreover it was shown that the most frequent size ratio upon collision has a value of about $\Delta \approx 0.2$. Experimental studies with such a size ratio have not yet been performed. Therefore, it is believed that the discussed boundary lines cannot cover such small size ratios.

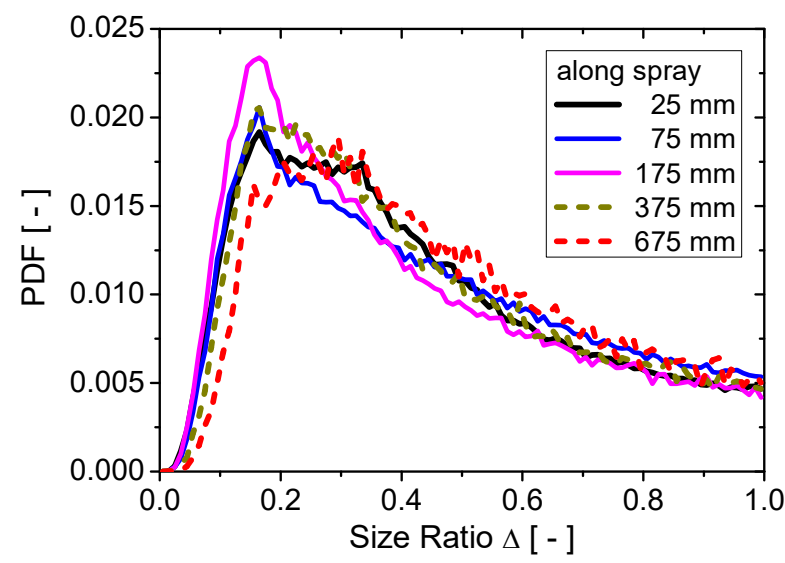

Figure 12: Cross-sectional averages of the PDF (probability density function) for the droplet size ratio upon occurrence of a collision along the simulated spray.

\section{Acknowledgements}

The authors acknowledge the financial support of this research project by the Deutsche Forschungsgemeinschaft (DFG) under contract SO 204/35-1 to 3.

\section{References}

Ashgriz, N.; Poo, J.Y.: Coalescence and separation in binary collisions of liquid drops. Journal of Fluid Mechanics, Vol. 221, $183-204$ (1990)

Brazier-Smith, P. R., Jennings, S. G. and Latham, J.: The interaction of falling water drops: coalescence. Proc. R. Soc. Lond. A, Vol. 326, 393 - 408 (1972)

Chen, R.-H.: Diesel-diesel and diesel-ethanol drop collisions. Applied Thermal Engineering, Vol. 27, 604 - 610 (2007)

Chen, R.-H., Wang, W.-C. and Chen, Y.-W.: Like-drop collisions of biodiesel and emulsion diesel. European Journal of Mechanics B/Fluids, Vol. 60, 62 - 69 (2016)

Crowe, C.T., Schwarzkopf, J.D., Sommerfeld, M. and Tsuji, Y.: Multiphase Flows with Droplets and Particles. $2^{\text {nd }}$ Edition, CRC Press, Boca Raton, U.S.A. (2012), ISBN 978-1-4398-4050-4.

Estrade, J.-P., Carentz, H., Lavergne, G., Biscos, Y.: Experimental investigation of dynamic binary collision of ethanol droplets - a model for droplet coalescence and bouncing. International Journal of Heat and Fluid Flow, Vol. 20, 486 - 491 (1999)

Gavaises, T.L. Theodorakakos, A. Bergerles, G. and Brenn, G.: Evaluation of the effect of droplet collisions on spray mixing. Proc. Inst. Mechanical Engineers, Vol. 210, 465 - 465 (1996)

Gotaas, C., Havelka, P. Jakobson, H. A. and Svendsen H.F, Hase, M., Roth, N., and Weigand, B.: Effect of viscosity on droplet-droplet collision outcome Experimental study and numerical comparison. Physics of Fluids, Vol. 19, 102106 (2007 a)

Guo, B. Fletcher, D.F. and Langrish, T.A.G.: Simulation of the agglomeration in a spray using Lagrangian particle tracking. Applied Mathematical Modelling, Vol. 28, 273 - 290 (2004)

Ho, C.A. and Sommerfeld, M. (2002) Modelling of micro-particle agglomeration in turbulent flow. Chem. Eng. Sci., Vol. 57, $3073-3084$.

Huang, K.L. and Pan, K.L.: Size effect on the transition from coalescence to bouncing regime in binary droplet collision. ICLASS 2015, $13^{\text {th }}$ Triennial International Conference on Liquid Atomization and Spray Systems, Tainan, Taiwan, August 23-27, 2015

Jiang, Y. J., Umemura, A. and Law, C.K.: An experimental investigation on the collision behavior of hydrocarbon droplets. Journal of Fluid Mechanics, Vol. 234, 171 - 190 (1992) 
Kohnen, G., Rüger, M. and Sommerfeld, M.: Convergence behaviour for numerical calculations by the Euler/Lagrange method for strongly coupled phases. In: "Numerical Methods in Multiphase Flows 1994, (Eds. C.T. Crowe et al.), ASME, New York, FED-Vol. 185, 191-202 (1994)

Kuschel, M. and Sommerfeld, M.: Investigation of droplet collisions for solutions with different solids content. Experiments in Fluids, Vol. 54, 1440 (2013)

Laín, S. and Sommerfeld, M.: Euler/Lagrange computations of pneumatic conveying in a horizontal channel with different wall roughness, Powder Technology, Vol. 184, 76-88 (2008)

Lain, S. and Sommerfeld, M.: Numerical calculation of pneumatic conveying in horizontal channels and pipes: Detailed analysis of conveying behaviour. International Journal of Multiphase Flow, Vol. 39, 105-120 (2012)

Munnannur, A. and Reitz, R. D.: A new predictive model for fragmenting and non-fragmenting binary droplet collisions. Int. J. Multiphase Flow, Vol. 33, 873 - 896 (2007)

Nijdam, J.J., Guo, B., Fletcher, D.F. and Langrish, T.A.G.: Lagrangian and Eulerian models for simulating turbulent dispersion and coalescence of droplets within a spray. Applied Mathematical Modelling, Vol. 30, 1196 $1211(2006)$

O’Rourke P.J.: Collective drop effects on vaporizing liquid sprays. Dissertation, Los Alamos National Laboratory, New Mexico (1981)

Post, S. L. and Abraham, J.: Modeling the outcome of drop-drop collisions in Diesel sprays. International Journal of Multiphase Flow, Vol. 28, 997 - 1019 (2002)

Qian, J. and Law, C.K.: Regimes of coalescence and separation in droplet collision. Journal of Fluid Mechanics, Vol. 331, 59 - 80 (1997)

Rabe, C., Malet, J. and Feuillebois: Experimental investigation of water droplet binary collisions and description of outcomes with a symmetric Weber number. Physics of Fluids, Vol. 22, 047101 (2010)

Rüger, M., Hohmann, S., Sommerfeld, M. and Kohnen, G. Euler/Lagrange calculations of turbulent sprays: The effect of droplet collisions and coalescence. Atomization and Sprays, Vol. 10, $47-81$ (2000)

Saroka, M.D. Ashgriz, N. and Movassat, M: Numerical Investigation of Head-on Binary Drop Collisions in a Dynamically Inert Environment. Journal of Applied Fluid Mechanics, Vol. 5, 23-37 (2012)

Schuch, G., Löffler, F.: Über die Abscheidewahrscheinlichkeit von Feststoffpartikeln an Tropfen in einer Gasströmung durch Trägheitseffekte. Verfahrenstechnik Vol. 12, 302-306 (1978)

Schiller, L., and Naumann, A. (1933) Uber die grundegenden Berchnungen bei der Schwerkraftaufbereitung, $Z$. Ver. Deut. Ing., 77, 318-320.

Sommerfeld, M.: Modellierung und numerische Berechnung von partikelbeladenen turbulenten Strömungen mit Hilfe des Euler/Lagrange-Verfahrens. Habilitationsschrift, Universität Erlangen-Nürnberg, Shaker Verlag, Aachen (1996)

Sommerfeld, M. (2001) Validation of a stochastic Lagrangian modelling approach for inter-particle collisions in homogeneous isotropic turbulence". Int. J. of Multiphase Flows, Vol. 27, 1828-1858.

Sommerfeld, M., van Wachem, B. \& Oliemans, R. (2008 a) Best Practice Guidelines for Computational Fluid Dynamics of Dispersed Multiphase Flows. ERCOFTAC, ISBN 978-91-633-3564-8.

Sommerfeld, M. and Lain, S.: From elementary processes to the numerical prediction of industrial particle-laden flows. Multiphase Science and Technology, Vol. 21, 123 - 140 (2009)

Sommerfeld, M. and Kuschel, M.: Modelling droplet collision outcomes for different substances and viscosities. Experiments in Fluids, Vol. 57, 187 (2016)

Sommerfeld, M.: Modelling separation processes of colliding droplets. ILASS - Europe 2016, 27 $7^{\text {th }}$ Annual Conference on Liquid Atomization and Spray Systems, 5. - 7. Sep. 2016, Brighton, UK

Sommerfeld, M. (2017) Lagrangian Modelling of Droplet Collisions in Spraying Systems. Proceedings of the European Combustion Meeting, Dubrovnik, 18. - 21. April 2017

Sundaram, S., and Collins, L. R.: Numerical Considerations in Simulating a Turbulent Suspension of FiniteVolume Particles. J. Comp. Phys. Vol. 124, 337-350 (1996).

Tang, C.G., Zhang, P. and Law, C.K.: Bouncing, coalescence, and separation in head-on collision of unequal-size droplets. Physics of Fluids, Vol. 24, Paper 022101 (2012)

Willis, K. and Orme, M.: Binary droplet collisions in a vacuum environment: an experimental investigation of the role of viscosity. Experiments in Fluids, Vol. 34, $28-41$ (2003)

Xia, S.-Y. and Hu, C.-B.: Numerical investigation of head-on binary collision of alumina droplets. Journal of Propulsion and Power, Vol. 31, 416-428 (2015) 


\title{
Microexplosion and Puffing of an Emulsion Fuel Droplet
}

\author{
J. Xia*, J. Shinjo ${ }^{2}$ \\ ${ }^{1}$ Department of Mechanical, Aerospace and Civil Engineering \& Institute of Energy Futures, \\ Brunel University London, Uxbridge UB8 3PH, UK \\ 2 Department of Mechanical, Electrical and Electronic Engineering, \\ Shimane University, 1060 Nishikawatsu, Matsue 690-8504, Japan \\ *Corresponding author: jun.xia@brunel.ac.uk
}

\begin{abstract}
Microexplosion is rapid disintegration of an emulsion droplet caused by explosive boiling of embedded liquid subdroplets with a lower boiling point. Microexplosion and puffing (partial microexplosion) are potentially beneficial to achieving enhanced secondary atomisation. These eruptive secondary atomisation mechanisms will help to meet conflicting requirements for an atomising fuel spray with regard to penetration achieved by large droplets and evaporation/mixing achieved by small droplets.

Although with great interest, our understanding of microexplosion is still limited and therefore optimising and controlling microexplosion is not feasible yet. This paper reviews our recent research outcome on microexplosion and puffing of an emulsion fuel droplet from high-fidelity interface-capturing simulation study, which directly resolves the dynamics of boiling and evaporating liquid/gas interfaces, immiscible liquid/liquid interfaces with jump conditions for mass, momentum and heat transfer across a resolved interface.

We first unveiled microexplosion and puffing dynamics of an emulsion fuel droplet in a quiescent ambient. Since convective heating has important effects on temperature distribution inside a fuel droplet in realistic engine conditions, which determines the initial nucleation location and thus the microexplosion outcome, a model has been proposed to approximate the temperature distribution inside a droplet, for which momentum and heat transport was found to be only moderately correlated under typical engine conditions. With this model in place that allows for saving considerable computational cost on setting up initial conditions for microexplosion simulation, puffing effects on fuel/air mixing is then investigated, which can be quantified by the scalar dissipation rate (SDR) of the primary fuel decane, the SDR of the secondary fuel ethanol and the cross SDR. We then further extended our simulation studies towards reacting conditions and investigate puffing effects on a droplet wake flame.

The series of high-fidelity simulation studies is believed to have considerably improved our understanding of microexplosion dynamics and impact on local fuel/air mixing and combustion. Based on the improved knowledge, microexplosion induced secondary droplet breakup models can be developed and incorporated into hybrid highfidelity simulation of spray atomisation and combustion enhanced by microexplosion.
\end{abstract}

\section{Keywords}

Microexplosion; Puffing; Emulsion droplet; Mixing; Droplet combustion.

\section{Introduction}

Liquid-fuel spray atomisation and combustion is widely used in combustion engines. For engines, fuel efficiency is critical and emission regulations are more and more stringent. Using emulsion fuels, which can be water-in-oil or biofuel-in-fossil-fuel emulsions, can be an effective way to enhance fuel efficiency and reduce emissions. Emulsion fuel is an immiscible liquid mixture with sub-droplets dispersing in the primary liquid fuel. If physical properties such as the boiling point are distinctly different between the two immiscible liquids, emulsion fuels may show particular physical phenomenon, e.g. microexplosion, when injected into the combustion chamber. Microexplosion can be another important secondary breakup mechanism and play an important role in accelerating spray atomisation.

After injected into the combustion chamber, sprayed emulsion droplets will be heated by the high-temperature ambient. Take water-in-oil emulsions as an example. Since the boiling point of oil is typically higher than that of water, embedded water sub-droplets can be superheated, i.e. the water temperature will become higher than its boiling temperature. This state is physically metastable. With little disturbance, water starts to boil explosively. Within a short time period, explosive boiling can result in violent breakup of the parent droplet. Therefore microexplosion is rapid disintegration of an emulsion droplet caused by explosive boiling of embedded sub-droplets with a lower boiling point $[1,2,3]$. If the degree of breakup is limited, it is often termed as puffing.

Microexplosion and puffing have a great potential for enhancing secondary atomisation. These eruptive secondary atomisation mechanisms will help to meet conflicting requirements for an atomising fuel spray with regard to a deeper penetration achieved by large droplets and more efficient evaporation/mixing achieved by small droplets.

Although with a great potential, our understanding of secondary atomisation induced by microexplosion and puffing is still far from the stage at which they can be controlled and optimised. It is therefore vitally important to better understand the physics of microexplosion and puffing and their impact on fuel/air mixing and combustion, which is the aim of this paper.

To achieve this goal, we have performed a series of high-fidelity simulation study on a puffing emulsion droplet, which directly resolves boiling or evaporating liquid/gas interfaces and immiscible liquid/liquid interfaces. Interface 
capturing is based on a combined level-set and volume-of-fluid approach. The numerical method is based on the CIP (constrained interpolation profile) method [4,5], which is advantageous in many aspects for the simulation cases considered in the present study. Based on the knowledge gained, microexplosion induced secondary droplet breakup models can be developed and incorporated into hybrid high-fidelity simulation of spray atomisation and combustion enhanced by microexplosion.

\section{Methodology \\ Governing Equations}

The Navier-Stokes equations

$$
\dot{\mathbf{f}}+(\mathbf{u} \cdot \nabla) \mathbf{f}=\mathbf{S}
$$

are solved for the primitive variables $\mathbf{f}=\left[\rho, \mathbf{u}, T, Y_{i}\right]^{\mathrm{T}}$, where $\dot{\mathbf{f}}=\partial \mathbf{f} / \partial t$, and $\rho, \mathbf{u}, T$ and $Y_{i}$ denote density, velocity, temperature and the mass fraction of the $i$-th species, respectively. In addition to other general terms in NavierStokes excluding convection, $\mathbf{S}$ also includes the source terms due to surface tension, boiling and evaporation, and combustion in a reacting case. Level set functions are used to capture interfaces [6]. The level-set approach is combined with the MARS (Multi-interface Advection and Reconstruction Solver) [7] to improve mass conservation. Surface tension is modelled by the CSF (Continuum Surface Force) method [8]. Jump conditions [9, 10] are used to compute the heat, momentum and mass transfer across an interface. The details can be found in $[11,12,13,14]$.

\section{Numerical Procedures}

The governing equations are solved by a fractional step approach, which comprises two solving phases. In the advection phase, $\dot{\mathbf{f}}+(\mathbf{u} \cdot \nabla) \mathbf{f}=\mathbf{0}$ is solved using the CIP method. In the non-advection phases, $\dot{\mathbf{f}}=\mathbf{S}$ is solved using the CCUP (CIP-combined and unified procedure) method, which uses a pressure-based algorithm.

In the solver, pressure is first solved using a Poisson equation,

$$
\nabla \cdot\left(\frac{\nabla p^{*}}{\rho^{n}}\right)=\frac{p^{*}-p^{n}}{\rho^{n} c_{s}^{2} \triangle t^{2}}+\frac{\nabla \cdot \mathbf{u}^{\mathbf{n}}}{\triangle t}
$$

where the superscripts $n$ and $*$ indicate the the current time step and the time stage after the non-advective phase, respectively. $c_{s}$ is the adiabatic speed of sound. $\mathbf{u}^{*}, \rho^{*}, T^{*}$ and $Y_{i}^{*}$ can then obtained through $\dot{\mathbf{f}}=\mathbf{S}$ via the approximation $\mathbf{f}^{*}=\mathbf{f}^{n}+\triangle \mathbf{f}=\mathbf{f}^{n}+\mathbf{S} \triangle t$.

In the CIP method, the Lagrangian invariant $f(\mathbf{x}, t)=f(\mathbf{x}-\Delta \mathbf{x}, t-\triangle t)$ is employed. The "constraint" is set up by the first derivative of $f$, which follow an advection equation derived from $\dot{f}+(\mathbf{u} \cdot \nabla) f=0$. It is now well known that if a constraint is independent of the original governing equation, it will not help to retrieve the profile inside a grid cell [5]. Knowing $f$ and its derivative $f^{\prime}$ only at two grid points comprising a grid cell, a cubic polynomial is used to approximate the subgrid or subcell solution of $f$ and $f^{\prime}$ by enforcing their continuities at all the grid points $[4,5]$.

The numerical scheme therefore features compact support and subgrid resolution. It can preserves the sharpness of an interface where large gradients of density exist and does not need an adaptive grid. It is free from the CFL condition that is restricted by a high sound speed. With the two-stage fractional step approach, it can solve incompressible flow in the framework of compressible fluid, e.g. the interaction between a compressible gas and incompressible liquid or solid.

\section{Results and discussion}

Puffing Dynamics [11]

Direct Simulation of Puffing Figures 1 and 2 present the initial puffing and subsequent after-puffing dynamics of two representative two-dimensional cases, in which the ratio of the radius of the low-boiling-point sub-droplet to that of the high-boiling-point parent droplet is $R_{\mathrm{sub}} / R_{\mathrm{p}}=0.25$ in Fig. 1 and 0.35 in Fig. 2, respectively. The other initial parameters are identical for the two cases. Due to the availability of published data on water-in-oil emulsions, in this subsection we use hexadecane and water properties for the parent and sub droplets, respectively. The ambient air pressure is $3 \mathrm{MPa}$, and the initial temperature is $553 \mathrm{~K}$. Since the water boiling temperature is $503 \mathrm{~K}$, the superheat degree is $50 \mathrm{~K}$. The initial velocity is 0 . The initial diameter of the parent droplet is $D_{\mathrm{p}}=30 \mu \mathrm{m}$. The computational domain size is $4.2 D_{\mathrm{p}} \times 4.2 D_{\mathrm{p}}$ discretised with $381 \times 381$ grid points. The minimum grid spacing is $0.26 \mu m$. Open boundaries are used.

Initially, the vapour bubble near the water/oil interface grows due to boiling. Since the liquid oil "wall" between the boiled vapour and the parent droplet surface is thin, the rapidly growing bubble will push the wall strongly, leading to rupture of the parent droplet, puffing of boiled vapour and ejection of liquid oil fragments [Figs. 1(a) and 2(a)]. After puffing, the boiling surface continues to eject vapour, which exerts a thrust force on the sub-droplet itself.

In the confined region where the newly formed water/vapour liquid/gas interface interacts with the water/oil liquid/liquid interface as indicated by blue arrows in Figs. 1(b) and 2(b), defined herein as an "edge", the water/vapour interface becomes unstable due to the Landau-Darrieus instability and the troughs therefore deepen. The edge regression proceeds, so that boiled vapour wrap up the sub-droplet, which finally detaches itself from the parent droplet. After the detachment, the sub-droplet stops affecting the parent droplet and the puffing-induced breakup process mostly ends.

The major difference between the two cases is the time period for the detachment of the whole sub-droplet from the parent droplet after puffing occurs is longer in Fig. 2 than in Fig. 1. It was found that for a big sub-droplet, its 


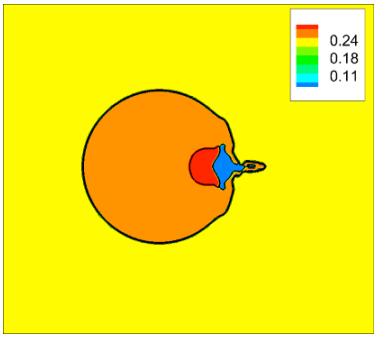

(a) $t=0.4 \mu \mathrm{s}$

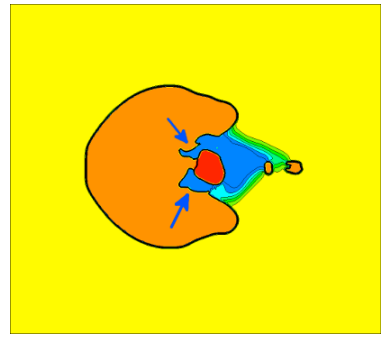

(b) $t=1.4 \mu \mathrm{s}$

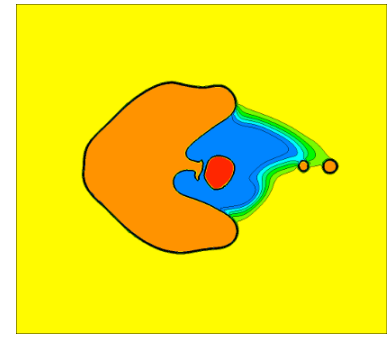

(c) $t=2.5 \mu \mathrm{s}$

Figure 1. Puffing and after-puffing due to boiling of a small sub-droplet $\left(R_{\mathrm{sub}} / R_{\mathrm{p}}=0.25\right)$ [11], where $R_{\mathrm{sub}}$ and $R_{\mathrm{p}}$ are the radius of the sub- and parent droplets, respectively. Orange: parent droplet with a higher boiling point. Red: sub-droplet with a lower boiling point. Gas-phase colour contours: mass fraction of oxygen $Y_{\mathrm{O}_{2}}$. Accordingly, blue: boiled vapour \& yellow: air.

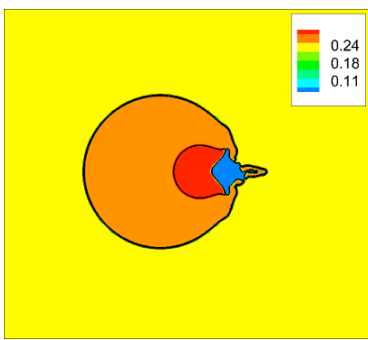

(a) $t=0.4 \mu \mathrm{s}$

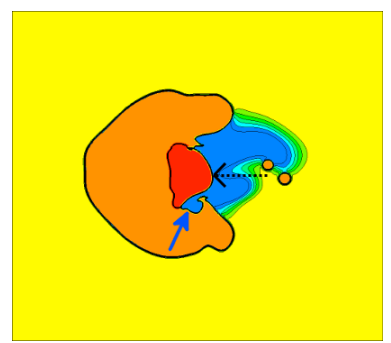

(b) $t=2.2 \mu \mathrm{s}$

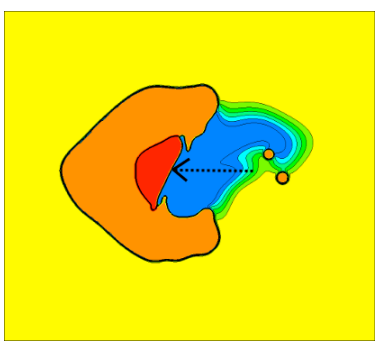

(c) $t=3.2 \mu \mathrm{s}$

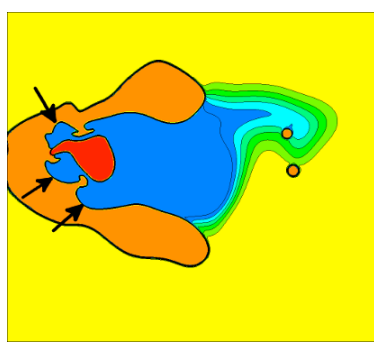

(d) $t=5.5 \mu \mathrm{s}$

Figure 2. Puffing and after-puffing with a big sub-droplet $\left(R_{\mathrm{sub}} / R_{\mathrm{p}}=0.35\right)$ [11]. See Fig. 1 for colour and variable definitions.

boiling surface oscillates strongly and its shape varies accordingly. As shown in Fig. 2(b) and 2(c) by a dashed arrow, the shape of the boiling surface is flattened due to the oscillation and some detached part of the sub-droplet remerges with the parent droplet. The interface edge regression is thus suspended and then restarts. For a smaller sub-droplet in Fig. 1, no remerging was seen and therefore the time period of the sub-droplet detachment is shorter.

Edge Regression A boiling surface exhibits the Landau-Darrieus (LD) instability due to evaporating mass flux at the interface [15] (Fig. 3). Due to the evaporating mass flux, the streamlines are bent at the liquid troughs and crests, working to enhance the interface shape deformation. Even if no vorticity exists in the liquid phase, vorticity will be generated in the gas phase, as illustrated by the dashed arrows in Fig. 3. Vorticity generation in the gas phase has been found to play an important role in the instability development and the detachment of a sub-droplet from the parent droplet after puffing occurs.

Figure 4 shows the vorticity component normal to the paper before and after puffing, with positive and negative vorticities indicating counterclockwise and clockwise rotations, respectively. At $t=0.2 \mu \mathrm{s}$ when puffing will soon occur, the vapour bubble is pushing the thin liquid "wall" towards the right direction. The observed strong symmetric vorticity is due to this outward jet-like flow but not the LD instability. The boiling surface of the sub-droplet is also pulled towards the same direction by the jet flow. As a consequence, the concave shape of the boiling surface becomes convex after puffing occurs, as indicated by the red arrow in Fig. 4(a) and 4(b). This induces oscillation of the sub-droplet.

Vorticity structures near the edges in a trough shape are indicated by small solid black arrows. An "edge" is formed at the intersection among the oil/water liquid/liquid interface, the non-boiling, inert oil/air liquid/gas interface and the boiling water/vapour liquid/gas interface. At $t=0.8 \mu \mathrm{s}$ and $t=1.6 \mu \mathrm{s}$, positive and negative vorticity pairs are observed in the concave regions near an edge. The vorticity generation is due to the local evaporating mass flux from the boiling surface. Note in the immediate vicinity the oil/vapour interface is non-boiling and inert, and therefore does not contribute to vorticity generation. The vorticity pairs work to deepen the trough, as shown in Figs. 4 and 5. Therefore, the initiation of edge regression is due to the LD instability.

After edge regression is initiated, the boiling surface of the sub-droplet gradually extends and regresses towards inside. During this regression process, bubble-like structures grow in the edge regions and a wavy pattern forms on the detached inert interface between the boiled vapour and the liquid species (oil in this case) of the parent droplet, as indicated by bold black arrows in Fig. 4(b) and 4(c), also illustrated in Fig. 5 and can be seen in Fig. 2(d). This is induced by the Rayleigh-Taylor (RT) instability, the mechanism of which is due to density difference and surface acceleration. In the neck regions created by the RT instability, the local ejecting vapour is accelerated by the "nozzle" effect and the vorticity magnitude is large. The vapour flow also pushes the developed RT "plumes" 


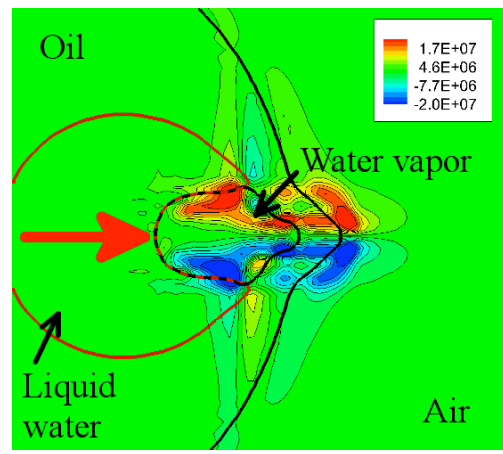

(a) $t=0.2 \mu \mathrm{s}$

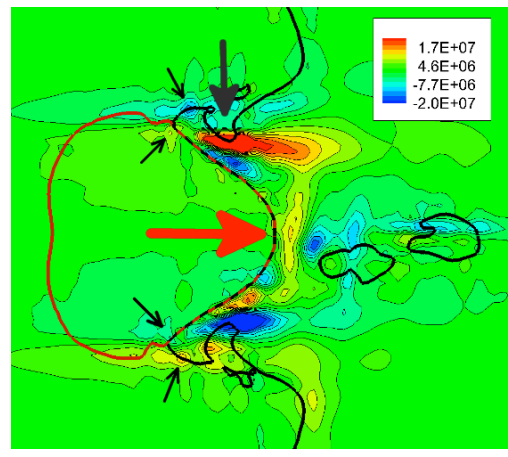

(b) $t=0.8 \mu \mathrm{s}$

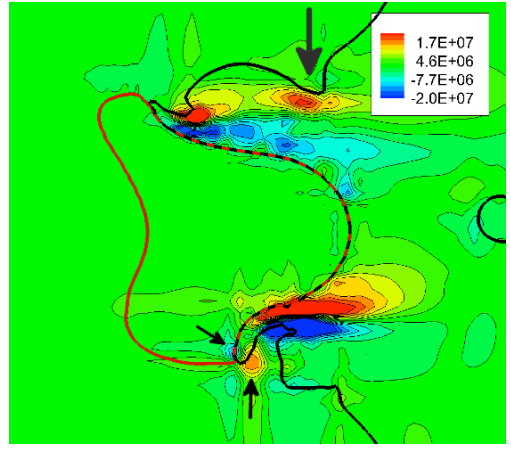

(c) $t=1.6 \mu \mathrm{s}$

Figure 4. Vorticity generation due to Landau-Darrieus instability: an important mechanism for edge regression [11]. Colour contours: vorticity $\left(s^{-1}\right)$.

outward. Following these physical processes, the edge regression proceeds and the sub-droplet will eventually detach itself from the parent droplet.

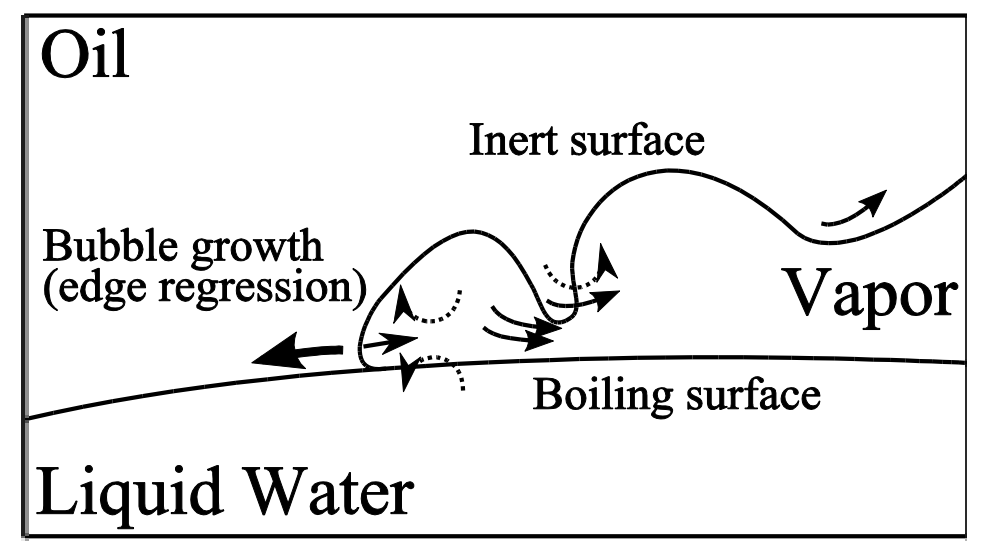

Figure 5. Schematic of edge regression mechanism [11] according to Fig. 4. The Landau-Darrieus instability develops due to the boiling surface. The Rayleigh-Taylor instability develops on the inert surface. Their interactions lead to edge regression.

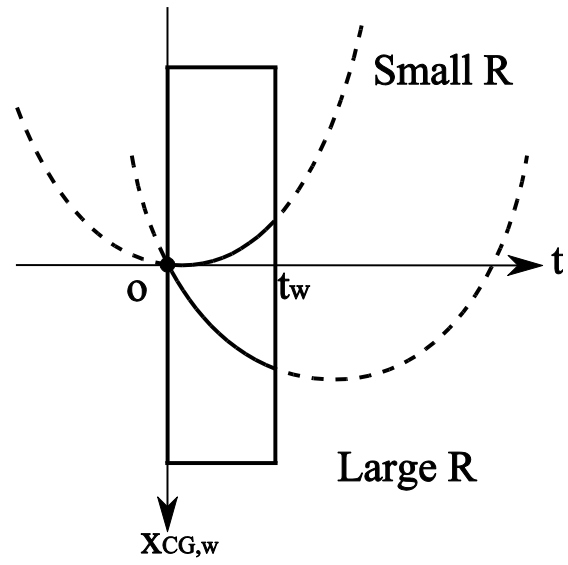

Figure 6. Centre of gravity $x_{\mathrm{CG}, \mathrm{w}}$ of a small and large sub-droplet, indicating whether a one-stage detachment or a two-stage penetration/detachment occurs for the sub-droplet following puffing [11]. At $t_{\mathrm{w}}$, edge regression completes and the entire sub-droplet detaches from the parent droplet.

One-Stage or Two-Stage Detachment An approximate quantitative analysis has been attempted in [11] to investigate how the distance $d_{\mathrm{ow}}$ between the centre of gravity of the parent droplet $\left(x_{\mathrm{CG}, \mathrm{o}}\right)$ and that of the sub-droplet $\left(x_{\mathrm{CG}, \mathrm{w}}\right)$ varies after puffing occurs. Neglecting the movement of the parent droplet, $d_{\mathrm{ow}}=x_{\mathrm{CG}, \mathrm{o}}-x_{\mathrm{CG}, \mathrm{w}}=$ const. $-x_{\mathrm{CG}, \mathrm{w}}$, so $d_{\mathrm{ow}}$ is determined by $x_{\mathrm{CG}, \mathrm{w}}$. The analysis included effects of the inertia of the bubble burst (or the pulling effect) and the thrust force generated by boiling, but excluded sub-droplet shape oscillation. It was found that $x_{\mathrm{CG}, \mathrm{w}}$ can be approximately predicted by $x_{\mathrm{CG}, \mathrm{w}}=-A t^{2}+B(t-C)$, where $A, B$ and $C$ are constants, and $t$ is time. If the sub-droplet size $R_{\text {sub }}$ is small, the first term $-A t^{2}$ due to the pulling effect will be dominant, and thus the sub-droplet tends to move away from the parent droplet, so the detachment process proceeds by edge regression. On the other hand if $R_{\text {sub }}$ is big, the second term $B(t-C)$ due to the thrust will play the dominant role and the subdroplet tends to penetrate towards the parent droplet, leading to partial remerging of the sub- and parent droplets, temporary suspension of edge regression and thus a longer time period for the sub-droplet detachment from the parent droplet. Figure 6 summarises the above findings. It shows that $x_{\mathrm{CG}, \mathrm{w}}$ can have opposite signs for a small and large sub-droplet.

Therefore the direction of the sub-droplet motion determines whether a one-stage detachment or a two-stage penetration/detachment occurs for the sub-droplet following puffing. In either case, the sub-droplet starts shape oscillation after puffing. Only if penetration occurs when the thrust effect is dominant, the oscillating boiling surface will be flattened [see Fig. 2(b) and 2(c)] and partial remerging of the sub- and parent droplets will cancel out part of the already generated boiling surface, leading to a decrease and fluctuation of the boiling surface area $S_{\text {boil }}$ (not shown). Since the edge instability mechanisms exists as long as boiling continues, the surface regression restarts after the remerging until the sub-droplet detaches completely from the parent droplet and the puffinginduced breakup ends. 


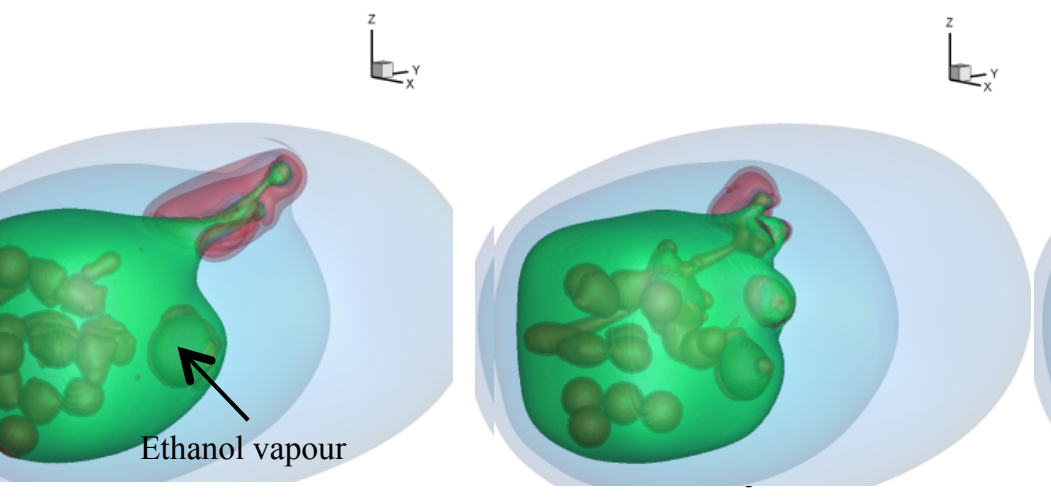

(a) $N_{\text {nuclei }}=2$

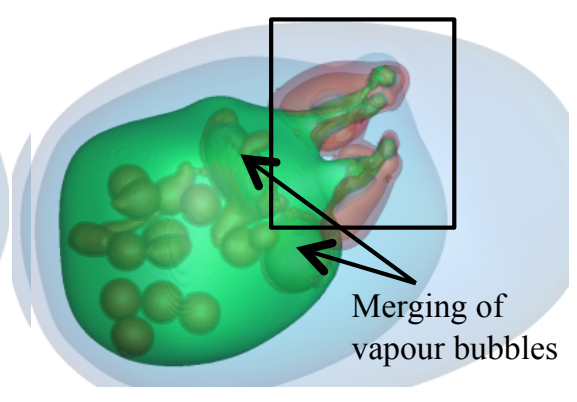

(b) $N_{\text {nuclei }}=4$

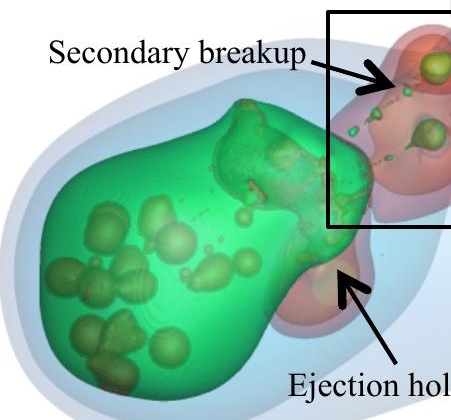

Ejection hol

Figure 7. Puffing of an ethanol-in-decane emulsion droplet under convective heating [13]. $N_{\text {nuclei }}$ is the number of initial nuclei. Vapour of the primary fuel decane is produced at the surface of the parent droplet through evaporation modelled by the Clapeyron-Clausius law. Vapour of the secondary fuel ethanol is produced by sub-droplet boiling and participates in fuel/air mixing after puffing occurs. The two blue iso-surfaces indicate $Y_{\text {decane }}=Y_{1}=0.1$ and 0.2 , and the two red iso-surfaces $Y_{\text {ethanol }}=Y_{2}=0.05$ and 0.1 . The rectangles shows the regions used for analysis of (a) weak puffing and (b) strong puffing accompanying ejections of liquid-fuel ligaments.

(a)

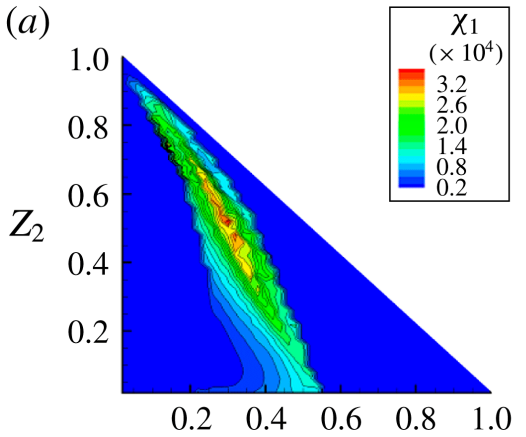

(b)

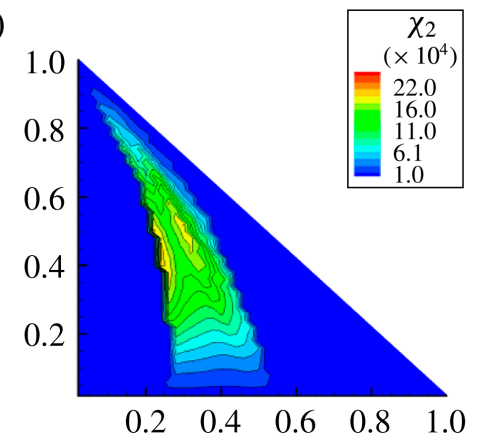

$(c)$

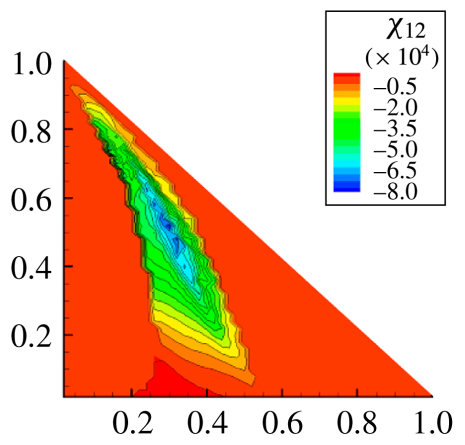

Figure 8. Doubly conditioned mean scalar dissipation rate $\chi$ in weak puffing with no ejection of liquid-fuel ligaments [13]: (a) $\left\langle\chi_{1} \mid Z_{1}, Z_{2}\right\rangle$; (b) $\left\langle\chi_{2} \mid Z_{1}, Z_{2}\right\rangle$; (c) $\left\langle\chi_{12} \mid Z_{1}, Z_{2}\right\rangle$. Z: mixture fraction. Subscript 1: primary fuel decane. Subscript 2: secondary fuel ethanol. Analysis region: rectangle indicated in Fig. $7(\mathrm{a})$. It should be stressed that the contour legends are different.

(a)

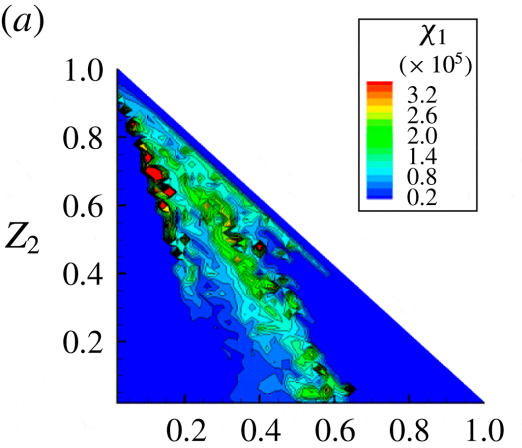

(b)

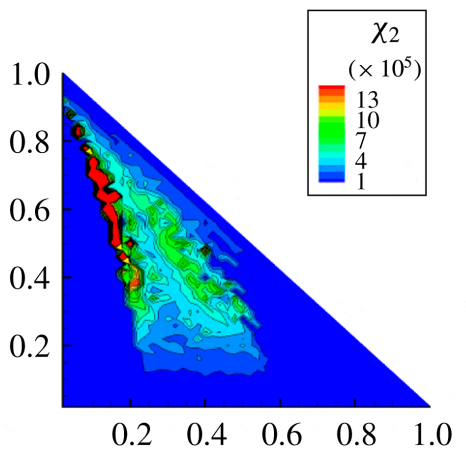

(c)

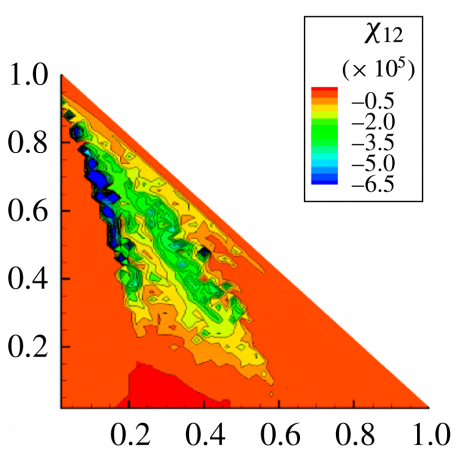

Figure 9. Doubly conditioned mean scalar dissipation rate $\chi$ in strong puffing with ejections of liquid-fuel ligaments [13]: (a) $\left\langle\chi_{1} \mid Z_{1}, Z_{2}\right\rangle$; (b) $\left\langle\chi_{2} \mid Z_{1}, Z_{2}\right\rangle$; (c) $\left\langle\chi_{12} \mid Z_{1}, Z_{2}\right\rangle$. See Fig. 8 for variable definitions. Analysis region: rectangle indicated in Fig. 7(b).

\section{Puffing-Enhanced Fuel/Air Mixing [13]}

Figure 7 presents puffing of an ethanol-in-decane emulsion droplet under convective heating. The only difference between the two cases is the number of initial nuclei $N_{\text {nuclei }}$. With more initial nuclei, ethanol vapour puffing is expected to be more intense. To better understand the effects of puffing on fuel/air mixing, we classify puffing into weak and strong puffing, in which cases liquid-fuel ligaments do not and do accompany the puffing, respectively. Therefore the region indicated by the rectangle in Fig. 7(a) and Fig. 7(b) has been used for analysis of weak and strong puffing.

The diameters of the parent decane droplet and the ethanol sub-droplet are $D_{\mathrm{p}}=30 \mu \mathrm{m}$ and $D_{\text {sub }}=4.6 \mu \mathrm{m}$, respectively. The number of ethanol sub-droplets in a parent droplet is 19 , and therefore the volume fraction of ethanol is $7.4 \%$. The free-stream air velocity is $U_{\mathrm{g}}=10 \mathrm{~m} / \mathrm{s}$, the air temperature $T_{\mathrm{g}}=900 \mathrm{~K}$, and the ambient air pressure $p_{\mathrm{g}}=1 M P a$. The droplet Reynolds number is $R e_{\mathrm{d}}=30$. At $1 \mathrm{MPa}$, the boiling temperature of 

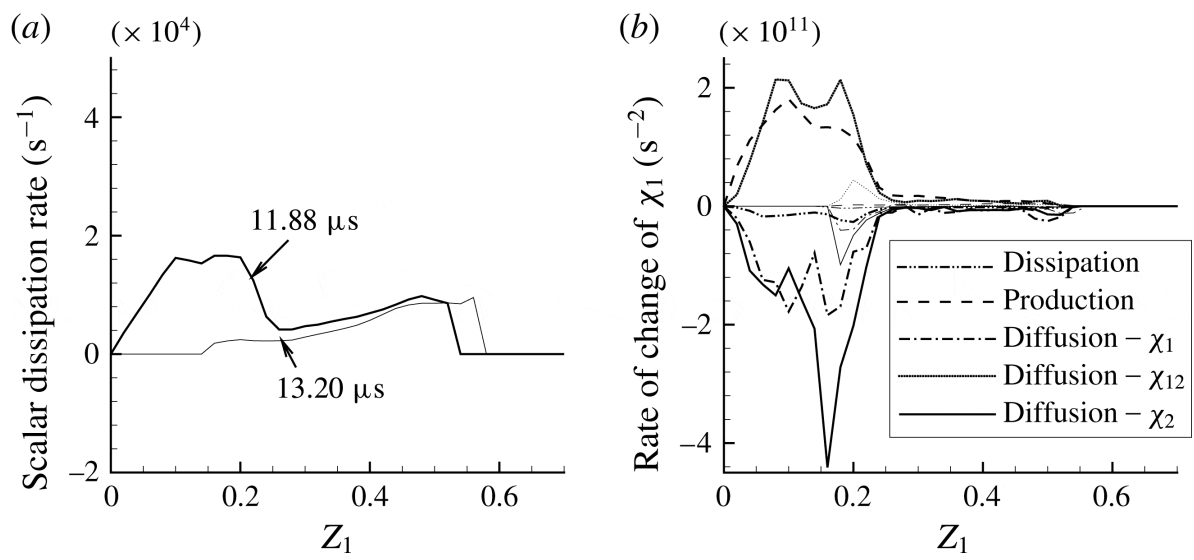

Figure 10. Puffing-enhanced micro-mixing [13]. (a) $\left\langle\chi_{1} \mid Z_{1}\right\rangle$; (b) budget analysis on $\left\langle\chi_{1} \mid Z_{1}\right\rangle$ using Eq. 3.

decane and ethanol is $565 \mathrm{~K}$ and $425 \mathrm{~K}$, respectively. The superheat limit of ethanol is $477 \mathrm{~K}$. The grid spacing is $\Delta x=\Delta y=\Delta z=0.19 \mu \mathrm{m}$. The total number of grid points is 243 million.

The droplet heating process prior to puffing is much slower than puffing. Under convective heating, internal circulation is developed inside an (emulsion) droplet. It was found that under typical flow conditions inside a combustion chamber $\left(R e_{\mathrm{d}} \sim 30\right)$, the liquid Péclet number is in a transitional regime $\left(100<P e_{\mathrm{L}}<500\right)$. Therefore models developed for inner-droplet velocity distribution $[16,17]$ cannot be borrowed to approximate temperature distribution. A new model was therefore developed [12] to approximate the inner-droplet temperature distribution and used here to save computational cost on droplet heating.

A mixture fraction, $Z_{i}=\left(Y_{i}-Y_{i, \mathrm{FAR}}\right) /\left(Y_{i, \mathrm{CTR}}-Y_{i, \mathrm{FAR}}\right)$ [18], has been used to quantify the fuel-vapour/air mixing without combustion, where $i=1$ and 2 indicates the primary fuel decane and the secondary fuel ethanol, respectively. Setting $Y_{i, \mathrm{FAR}}=0$ in the far field and $Y_{i, \mathrm{CTR}}=1$ at the droplet centre, $Z_{i}$ is equivalent to $Y_{i}$. The scalar dissipation rate, $\chi$, is used to quantify the micro-mixing and defined as $\chi_{i j}=2 D_{\text {diffu }} \nabla Z_{i} \cdot \nabla Z_{j}$, where $D_{\text {diffu }}$ is the diffusion coefficient. Therefore $\chi_{1}, \chi_{2}$ and $\chi_{12}$ quantify the primary-fuel diffusion, secondary-fuel diffusion and cross-diffusion of the two fuels in the gas phase.

Figures 8 and 9 present the SDRs doubly conditioned on $Z_{1}$ and $Z_{2}$, i.e. $\left\langle\chi_{1} \mid Z_{1}, Z_{2}\right\rangle$ and $\left\langle\chi_{2} \mid Z_{1}, Z_{2}\right\rangle$, and the cross-SDR $\left\langle\chi_{12} \mid Z_{1}, Z_{2}\right\rangle$ at the initial stage of puffing for weak [Fig. 7(a)] and strong [Fig. 7(b)] puffing, respectively. It should be noted that the upper limit of the mixture fractions in the figures is bounded by $Z_{1}+Z_{2}=1$ by definition. Immediately after the ethanol vapour ejection starts, $\chi_{1}$ and $\chi_{2}$ increase. It is also interesting to see that $\chi_{2}$ is one order of magnitude bigger than $\chi_{1}$, indicating that strong stratification of ethanol vapour exists in the gas phase once puffing occurs. Cross-mixing of the decane and ethanol fuel vapour is also evident, as $\chi_{12}$ is negative. The SDRs in the region of large values of $Z_{2}$ are due to ethanol vapour ejection, and those in the region of large valued of $Z_{1}$ are due to decane evaporation at the parent droplet surface. It should be noted that the upper limit of $Y_{1}$ and $Y_{2}$ is the local saturated vapour pressure dictated by the the Clapeyron-Clausius model and 1 due to boiling, respectively. For strong puffing shown in Fig. 9, the magnitudes of SDRs are larger, and the SDR values are more strongly disturbed than in Fig. 8 due to multiple ejections and ejected liquid fragments.

Since the primary fuel is decane, puffing effects on $\chi_{1}$ and $Z_{1}$ are of primary interest. Assuming that the mixing is dominant in the compressive strain direction [19], the transport equation for $\chi_{1}$ in the mixture fraction space can be written as

$$
\frac{\partial \chi_{1}}{\partial t}=\underbrace{2 a \chi_{1}}_{\text {Production }}-\underbrace{\frac{1}{4}\left(\frac{\partial \chi_{1}}{\partial Z_{1}}\right)^{2}}_{\text {Dissipation }}+\underbrace{\frac{\chi_{1}}{2} \frac{\partial^{2} \chi_{1}}{\partial Z_{1}^{2}}}_{\begin{array}{c}
\text { Primary-fuel } \\
\text { diffusion }
\end{array}}+\underbrace{\chi_{12} \frac{\partial^{2} \chi_{1}}{\partial Z_{1} \partial Z_{2}}}_{\text {Cross-diffusion }}+\underbrace{\frac{\chi_{2}}{2} \frac{\partial^{2} \chi_{1}}{\partial Z_{2}^{2}}}_{\begin{array}{c}
\text { Seondary-fuel } \\
\text { diffusion }
\end{array}}+\underbrace{S_{\chi_{1}}}_{\begin{array}{c}
\text { Evaporation } \\
\text { source }
\end{array}},
$$

where $a$ is the dominant compressive strain rate. The compressive strain rate makes the local gradients steeper, which contributes positively to the rate of change of the SDR. The dissipation term is always negative. The effect of the evaporation source term $S_{\chi_{1}}$ is confined in the vicinity of an evaporating/boiling surface. It is clear from Eq. 3 that the additional terms due to the dissipation rate $\chi_{2}$ and cross-SDR $\chi_{12}$ quantify the direct puffing effects on the mixing and micro-mixing of the vapour of the primary fuel decane with air.

Figure 10(a) presents the conditional mean SDR $\left\langle\chi_{1} \mid Z_{1}\right\rangle$ for the weak puffing case. At $t=11.88 \mu s$ [Fig. 7(a)], enhanced mixing due to the ejected ethanol vapour is evident in $Z_{1}=0.1-0.25$. The other peak in the regions of large values of $Z_{1}$ is due to decane evaporation from the parent droplet. At a later time $t=13.20 \mu \mathrm{s}$, the puffing jet has already stopped and $\chi_{1}$ becomes much smaller in $Z_{1}=0.1-0.25$, which indicates that puffing-induced mixing has weakened. Therefore, puffing enhances mixing in a short period of the ethanol vapour ejection.

The budget analysis in Fig. 10(b) shows the contributions of each conditionally averaged right-hand-side term in Eq. 3 to $\partial\left\langle\chi_{1} \mid Z_{1}\right\rangle / \partial t$. The evaporation source term is not included in the analysis. The production term $2 a \chi_{1}$ is approximated by $2 S \chi_{1}$, where $S$ is the norm of the strain rate tensor $S_{i j}=0.5\left(\partial u_{i} / \partial x_{j}+\partial u_{j} / \partial x_{i}\right)$.

It can be seen that the dissipation term is a sink by definition and its magnitude is small. A leading source is the production term due to the strong strain rate generated by the ejecting ethanol vapour jet. It is interesting to 
note that the cross-diffusion due to $\chi_{12}$ is another leading source that is comparable to the production term. This indicates that puffing-induced cross-diffusion of the primary and secondary fuels works to increase the magnitude of $\chi_{1}$ in the full spectrum of $Z_{1}$. The other two diffusion terms are negative, which represents a generic diffusion process. At $t=11.88 \mu \mathrm{s}$, puffing is intense with mixing in progress. The net contribution of all the right-hand-side terms is negative, which decreases $\chi_{1}$. At a later time $t=13.20 \mu \mathrm{s}$, the magnitudes of these terms are smaller, indicating that the puffing jet has stopped and the fuel/air mixing has progressed further.

The same budget analysis was also performed for strong puffing [Fig. 7(b)] (not shown). An important difference from Fig. $10(\mathrm{~b})$ is that the cross-scalar diffusion due to $\chi_{12}$ now takes the dominant role in enhancing $\left\langle\chi_{1} \mid Z_{1}\right\rangle$, whereas in the weak puffing case the production and cross-scalar diffusion are comparable.

Through the above detailed analysis, it is clear that ejection of boiled ethanol vapour initially generates a strong stratification in the reactive gaseous mixture of decane vapour and air, followed by mixing that homogenises the stratification. Intermittent ethanol vapour puffing therefore modifies local mixing characteristics around the emulsion droplet both in time and in space.

\section{Droplet Combustion under Puffing [14]}

Figure 11 shows a snapshot of puffing and puffing-induced interaction between ejected ethanol vapour and a decane-droplet wake flame at $t=9.2 \mu \mathrm{s}$. Combustion of decane and ethanol is modelled by a global one-step chemistry mechanism. Six species, i.e. decane $\left(\mathrm{C}_{10} \mathrm{H}_{22}\right)$, ethanol $\left(\mathrm{C}_{2} \mathrm{H}_{5} \mathrm{OH}\right), \mathrm{O}_{2}, \mathrm{~N}_{2}, \mathrm{CO}_{2}$ and $\mathrm{H}_{2} \mathrm{O}$, are considered. The other simulation parameters are the same as in the previous subsection. Ethanol vapour ejection starts at $t=5.5 \mu \mathrm{s}$ following bubble nucleation and growth. The ejected ethanol vapour reaches the flame surface and interactions between the flame and ethanol vapour start shortly before $t=7.8 \mu \mathrm{s}$. At $t=9.2 \mu \mathrm{s}$, the flame shape is locally stretched and distorted due to the flame/vapour interaction underway.

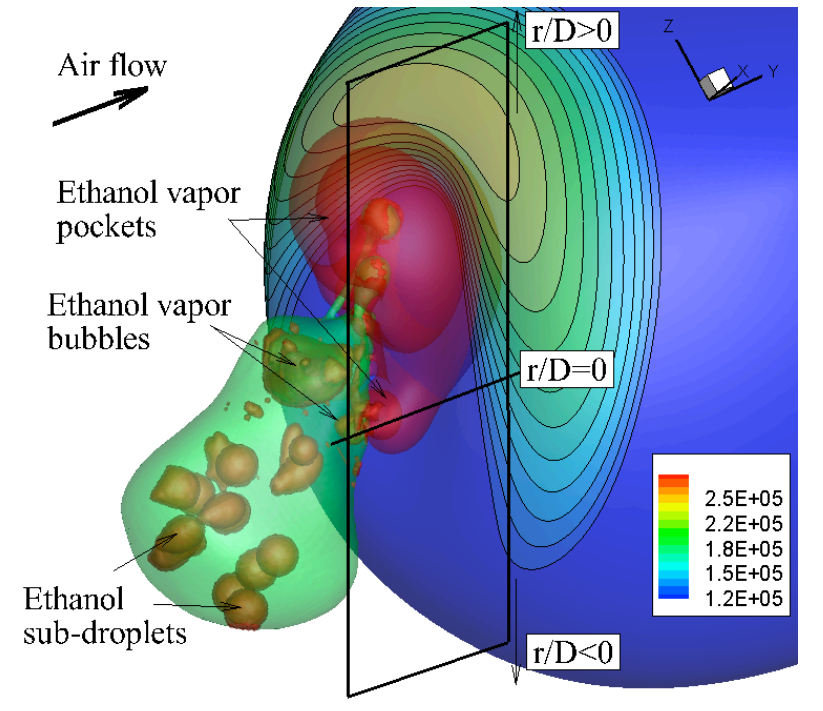

Figure 11. Puffing and puffing-induced interaction between ejected ethanol vapour and the decane-droplet wake flame at $t=9.2 \mu s$ [14]. Red iso-surfaces: $Y_{2}=Y_{\text {ethanol }}=0.05$ and 0.1 . Green iso-surface: parent-droplet surface. Dark green iso-surfaces: sub-droplet surfaces. Blue iso-surface:

$T_{\mathrm{g}}=1,800 \mathrm{~K}$, illustrating the droplet wake flame. Colour contours: ethanol consumption rate $\left(\mathrm{mol} / \mathrm{m}^{3} / \mathrm{s}\right)$.

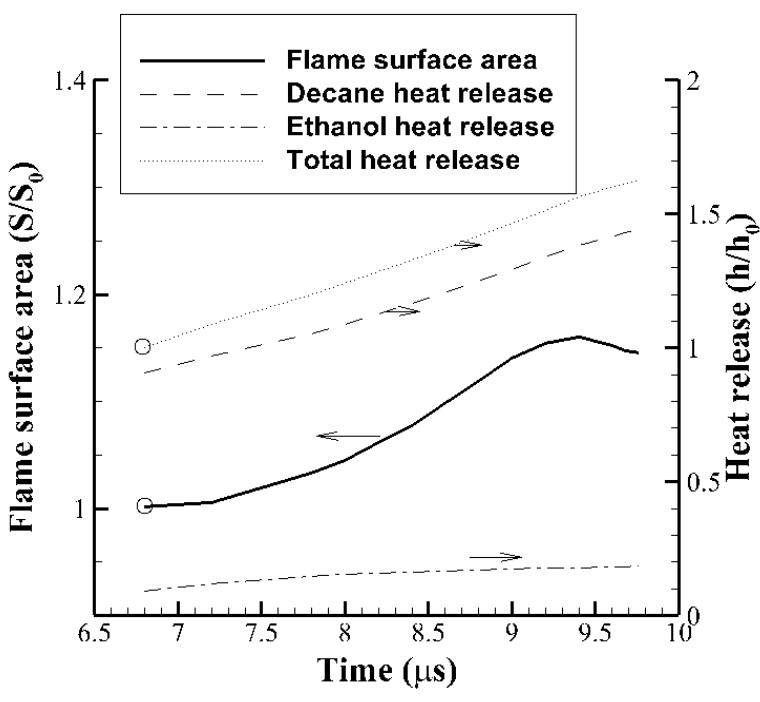

Figure 12. Temporal traces of flame surface area $S$, heat release rate of decane $h_{1}$ and ethanol $h_{2}$, and total heat release rate $h$ [14]. $S_{0}$ and $h_{0}$, indicated by the circles, are used for normalisation.

The global characteristics of the interaction between the puffing and the droplet flame is presented in Fig. 12, which shows the temporal traces of the normalised flame surface area $S / S_{0}$, the normalised heat release rates of the primary fuel decane and the secondary fuel ethanol, and their sum - the normalised total heat release rate. It is clear that the puffing transiently increases the flame surface area and also the total heat release rate. As diffusion progresses, the transient effect becomes weaker. The flame surface area reaches a maximum value at $t \approx 9.4 \mu s$ and decreases thereafter until the next ethanol vapour pocket arrives. The heat release rate reaches a maximum value at a later time than $t=9.5 \mu \mathrm{s}$ due to puffing-enhanced-mixing effects on combustion.

A mixture fraction is defined for fuel-droplet evaporation with combustion [18] as $Z_{i}=\left[Y_{i}+\left(Y_{\mathrm{O}_{2}}, \mathrm{FAR}-Y_{\mathrm{O}_{2}}\right) / s_{i}\right]$ $/\left(1+Y_{\mathrm{O}_{2}}, \mathrm{FAR} / s_{i}\right)$ for a one-step global reaction $\mathrm{F}_{i}+s_{i} \mathrm{O} \rightarrow\left(1+s_{i}\right) \mathrm{P}_{i}$, where $i=1$ and $i=2$ denote the primary fuel decane and the secondary fuel ethanol, respectively. $\mathrm{F}_{i}, \mathrm{O}$ and $\mathrm{P}_{i}$ stand for fuel, oxidiser and product, respectively. $s_{i}$ is the ratio of the mass of oxygen consumed per mass of fuel in a stoichiometric reaction. The budget analysis on the conditional mean scalar dissipation rate $\left\langle\chi_{1} \mid Z_{1}\right\rangle$ has been performed for the droplet wake flame under puffing using Eq. 3, and a qualitatively similar trend has been identified to the strong puffing case (without combustion) as shown in Fig. 7(b). 


\section{Conclusions}

This paper reviews our recent work on high-fidelity simulation study on microexplosion and puffing of an emulsion fuel droplet, including puffing dynamics and impact of puffing on local fuel/air mixing and emulsion-droplet combustion. A combined level-set and volume-of-fluid approach has been used for interface capturing, with jump conditions describing heat, momentum and mass transfer across an interface. The CIP method [4,5] was found to be efficient and effective to solve incompressible flow in the framework of compressible fluid, in our case the interaction between a compressible gas and incompressible liquid. Therefore boiling and evaporating droplet surfaces, liquid/liquid interfaces are directly resolved without the use of an adaptive grid.

Microexplosion and puffing dynamics are examined by direct simulation on a water-in-oil (approximated by hexadecane) emulsion droplet in a quiescent ambient. The growth of a single bubble, initiated at the oil/water interface, leads to puffing. The bubble burst and the sub-droplet dynamics after puffing jointly determine the oil-droplet breakup outcome.

After puffing, whether the detachment of a sub-droplet from the parent droplet is in one stage or two stages is determined by 3 competing mechanisms: (1) shape oscillation of the sub-droplet, (2) thrust generated by boiling, and (3) pulling effects due to the inertia of the bubble burst. If the sub-droplet is small, the pulling effect is dominant. The sub-droplet quickly detaches from the parent droplet due to edge regression, and the extent of the droplet breakup is therefore limited. If the sub-droplet is large, it penetrates into the parent droplet by the thrust. The mutual interaction between the sub- and parent droplets thus takes a longer time and the degree of breakup is larger.

In both one- and two-stage detachment, edge regression is a key mechanism for the sub-droplet detachment from the parent droplet. Through edge regression, the boiling surface of the sub-droplet gradually extends until boiling occurs at the whole oil/water interface, when the sub-droplet detaches itself from the parent droplet. The dominant mechanism is the Landau-Darrieus instability, which generates closely attached vorticity pairs with opposite signs due to the evaporating mass flux at the boiling surface. These vorticities then works to enlarge the vapour bubble area in the vicinity of the edge, which can then extend further towards the oil/water interface.

Puffing effects on fuel/air mixing are quantitatively analysed for weak puffing and strong puffing accompanying ejections of liquid-fuel ligaments in a convective heating flow. The transport equation for the scalar dissipation rate of the primary fuel decane is used. The two additional terms in the equation due to the dissipation rate $\chi_{2}$ of the secondary fuel ethanol and cross diffusion $\chi_{12}$ of the two fuels quantify the direct puffing effects on the mixing and micro-mixing of the vapour of the primary fuel decane with air. The two leading sources are found to be (1) the production terms due to the strong strain rate generated by ejecting ethanol vapour and (2) the cross-diffusion, which is much stronger than the production in strong puffing than in weak puffing. The detailed analysis demonstrates that ejection of boiled ethanol vapour initially generates a strong stratification in the reactive gaseous mixture of decane vapour and air, followed by mixing that homogenises the stratification. Intermittent ethanol vapour puffing therefore modifies local mixing characteristics around the emulsion droplet both in time and in space.

Using a global one-step chemistry mechanism, puffing effects on a droplet wake flame are investigated. The flame surface area and heat release rate are increased due to impact of ejected ethanol fuel vapour on the droplet flame. Budget analysis is also performed on the transport equation for the scalar dissipation rate $\chi_{1}$, and a similar trend is found to the above non-reacting strong puffing case.

\section{Acknowledgements}

The authors are grateful for the financial support of the Engineering and Physical Sciences Research Council (EPSRC) of the UK under Grant No. EP/J018023/1.

\section{References}

[1] Dryer, F. L., 1977, Symposium (International) on Combustion, 16 (1), pp. 279-295.

[2] Law, C. K., 1982, Progress in Energy and Combustion Science, 8 (3), pp. 171-201.

[3] Kadota, T., Yamasaki, H., 2002, Progress in Energy and Combustion Science, 28 (5), pp. 385-404.

[4] Takewaki, H., Nishiguchi, A., Yabe, T., 1985, Journal of Computational Physics, 61 (2), pp. 261-268.

[5] Yabe, T., Xiao, F., Utsumi, T., 2001, Journal of Computational Physics, 169, pp. 556-593.

[6] Sussman, M., Smereka, P., Osher, S., 1994, Journal of Computational Physics, 114 (1), pp. 146-159.

[7] Kunigi, T., 1997, Transactions of the Japan Society of Mechanical Engineers Series B, 63, pp. 1576-1584.

[8] Brackbill, J. U., Kothe, D. B., Zemach, C., 1992, Journal of Computational Physics, 100 (2), pp. 335-354.

[9] Tanguy, S., Ménard, T., Berlemont, A., 2007, Journal of Computational Physics, 221 (2), pp. 837-853.

[10] Juric, D., Tryggvason, G., 1998, International Journal of Multiphase Flow, 24 (3), pp. 387-410.

[11] Shinjo, J., Xia, J., Ganippa, L. C., Megaritis, A., 2014, Physics of Fluids, 26, article no. 103302.

[12] Shinjo, J., Xia, J., Megaritis, A., Ganippa, L. C., Cracknell, R. F., 2016, Atomization and Sprays, 26 (6), pp. 551-583.

[13] Shinjo, J., Xia, J., Ganippa, L. C., Megaritis, A., 2016, Journal of Fluid Mechanics, 793, pp. 444-476.

[14] Shinjo, J., Xia, J., 2017, Proceedings of the Combustion Institute, 36 (2), pp. 2513-2521.

[15] Prosperetti, A., Plesset, M. S., 1984, Physics of Fluids, 27 (7), pp. 1590-1602.

[16] Sirignano, W. A., 1983, Progress in Energy and Combustion Science, 9 (4), pp. 291-322.

[17] Abramzon, B., Sirignano, W. A., 1989, International Journal of Heat and Mass Transfer, 32 (9), pp. 1605-1618.

[18] Bilger, R. W., 2011, Combustion and Flame, 158 (2), pp. 191-202.

[19] Hasse, C., Peters, N., 2005, Proceedings of the Combustion Institute, 30 (2), pp. 2755-2762. 


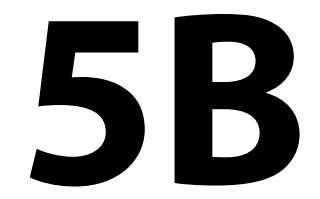

Automotive 5 


\title{
Influence of Stokes Number on Collisional Interfacial Area Production Terms within the $\Sigma-Y$ Eulerian Spray Atomization Model
}

\author{
Dominik Eichler*, Philipp Pischke, Reinhold Kneer \\ Institute of Heat and Mass Transfer, RWTH Aachen University \\ *Corresponding author: eichler@wsa.rwth-aachen.de
}

\begin{abstract}
The present study shows the effects of Stokes number on the modeling of collisional interfacial area production terms within the $\Sigma-Y$ model. This model can be employed for CFD simulations of high Weber and Reynolds number sprays using a RANS turbulence modeling. Within the model production of interfacial area is assumed to result from turbulent stretching and turbulent droplet collisions. The modeling of collisional processes requires the calculation of a characteristic turbulent collision velocity. In the present work this velocity was determined under consideration of Stokes number effects leading to turbulent droplet velocity fluctuations attenuated with respect to the gas phase fluctuations and including partial correlation between the velocities. The influence of this new modeling approach is tested within a 2D spray simulation by comparing the Sauter mean diameters observed to the ones obtained by employing the modeling approaches proposed in the literature which do not consider any Stokes number effects. The reduced collision velocites in the new modeling lead to higher values for Sauter mean diameters in the spray.
\end{abstract}

\section{Keywords}

CFD, eulerian spray modeling, stochastic collision modeling

\section{Introduction}

High Weber and Reynolds number sprays, as to be found in Diesel fuel injection or rocket fuel injection, can be modeled by the $\Sigma-Y$ Eulerian spray atomization model, originally proposed by Vallet et al. [1]. Here, a transport equation for the liquid mass fraction $Y$ (instead of a transport equation for liquid volume fraction) is employed and another transport equation for $\Sigma$ describing the evolution of volumetric interfacial area (dimension $1 / \mathrm{m}$ ). Liquid and gas are assumed to have identical velocities for the bulk flow. Thus, only one set of momentum equations is sufficient to describe the two-phase flow. Mixing of liquid and gas is performed by turbulent diffusion only. Considering sprays with high Weber and Reynolds numbers, the large scales are assumed to be independent of small scales. Thus, the momentum equations do not feature any effects of surface tension nor laminar viscosity. In the $\Sigma$ - $Y$ model, source and sink terms within the $\Sigma$-equation are assumed to arise from turbulent fluctuations of the flow, which lead to turbulent breakup, turbulent droplet collisions leading to fragmentation or coalescence, or turbulent stretching. Employing a RANS eddy viscosity model, as proposed as one alternative by various authors, see for example [1, 2, 3], the Favre averaged transport equations for liquid mass, momentum and interfacial area can be stated as follows:

$$
\begin{aligned}
& \frac{\partial \bar{\rho} \widetilde{Y}}{\partial t}+\operatorname{div}(\bar{\rho} \widetilde{Y} \widetilde{\mathbf{u}})=\operatorname{div}\left(\bar{\rho} \frac{\nu_{\mathrm{t}}}{\operatorname{Sc}_{\mathrm{t}}} \operatorname{grad}(\widetilde{Y})\right) \\
& \frac{\partial \bar{\rho} \widetilde{u}_{i}}{\partial t}+\operatorname{div}\left(\bar{\rho} \widetilde{u}_{i} \widetilde{\mathbf{u}}\right)=\operatorname{div}\left(\bar{\rho} \nu_{\mathrm{t}} \operatorname{grad}\left(\widetilde{u}_{i}\right)\right) \\
& \frac{\partial \bar{\Sigma}}{\partial t}+\operatorname{div}(\bar{\Sigma} \widetilde{\mathbf{u}})=\operatorname{div}\left(\frac{\nu_{\mathrm{t}}}{\operatorname{Sc}_{\mathrm{t}}} \operatorname{grad}(\bar{\Sigma})\right)+\dot{\Sigma}_{\mathrm{P}}-\dot{\Sigma}_{\mathrm{D}}
\end{aligned}
$$

Here, $\mathrm{Sc}_{\mathrm{t}}$ is the turbulent Schmidt number, and $\dot{\Sigma}_{\mathrm{P}}$ and $\dot{\Sigma}_{\mathrm{D}}$ describe the production and destruction terms for the $\Sigma$ equation, respectively. According to Vallet et al. [1], production and destruction of $\Sigma$ are comprised of two mechanisms:

$$
\begin{aligned}
& \dot{\Sigma}_{\mathrm{P}, \mathrm{TS}}=A \Sigma \\
& \dot{\Sigma}_{\mathrm{P}, \text { Coll }}=\frac{\Sigma}{\tau_{\text {coll }}}\left(1-\frac{\Sigma}{\Sigma_{\text {eq }}}\right)
\end{aligned}
$$

The first mechanism, see equation (4), describes the turbulent stretching of the interface. $A$ is a production rate, proportional to the local velocity gradient. The second one, see equation (5), represents the combined production and destruction of interfacial area by droplet collisions. Here, $\tau_{\text {coll }}$ is a characteristic collisional production time, depending on the relative velocity and spacing between droplets, and $\Sigma_{\text {eq }}$ is the equilibrium value for interfacial area. The mathematical form in equation (5) has been adopted in several publications of other authors, see for example [2 3, 4, 5, 6, 7, 8]. However, different formulations for the modeling of $\tau_{\text {coll }}$ and $\Sigma_{\text {eq }}$ exist.

Within the present study, a discussion about the modeling of the collisional time $\tau_{\text {coll }}$ and equilibrium value $\Sigma_{\text {eq }}$ for the application in the $\Sigma$ - $Y$ Eulerian model is given, concerning the velocity scale to be chosen. 


\section{Modeling of collisional time scales and equilibrium values: state of the art}

\section{Characteristic collision time}

Vallet et al. propose in [1] the following value:

$$
\tau_{\text {coll, Vallet }}=C_{\tau, \text { Vallet }} \frac{n^{-\frac{1}{3}}}{\Delta u} \text {. }
$$

In equation (6), $n$ is the local number density of droplets. It can be derived under the assumption that the interfacial area is composed of identical droplets. Their correspdonding Sauter mean diameter can be obtained in the following way:

$$
d_{32}=\frac{6 \bar{\rho} Y}{\rho_{\text {liq }} \Sigma}
$$

For further details, see [1]. $n^{-\frac{1}{3}}$ is the local mean distance of two droplets. $\Delta u$ is a characteristic collision velocity, defined as

$$
\Delta u=\left(\varepsilon n^{-\frac{1}{3}}\right)^{\frac{1}{3}} .
$$

The collision velocity is thus represented by the gas phase velocity fluctuations of a turbulent eddy of size $n^{-\frac{1}{3}}$. $C_{\tau, \text { Vallet }}$ is a constant. Vallet et al. propose in [1] a value of $C_{\tau, \text { Vallet }}=1.0 \ldots 1.35$, whereas Demoulin et al. [6] use a value of $C_{\tau, \text { vallet }}=0.3$, see [6].

Beau et al. 5 propose another formulation for the characteristic collisional time inspired by stochastic droplet collision modeling. The collision velocity is set to the rms value of turbulent gas phase fluctuations:

$$
\tau_{\text {coll, Beau }}=\frac{1}{\sqrt{\Delta u} \Sigma} \text { and } \Delta u=\sqrt{\frac{2}{3} k} .
$$

\section{Equilbrium value of interfacial area}

Vallet et al. [1] derive an expression for an equilibrium diameter by evaluation of an energy balance of two identical colliding droplets. It is assumed that all kinetic energy with respect to the center of mass of the system of colliding droplets is converted into surface energy after collision. Thus, an equilibrium diameter can be obtained, which can be transformed into an equilibrium value of interfacial area by equation (7). This finally leads to

$$
d_{\text {eq }}=C_{\text {eq }, \text { Vallet }} \frac{2 \sigma^{\frac{3}{5}} l_{\mathrm{t}}^{\frac{2}{5}}}{k^{\frac{3}{5}}} \frac{(\bar{\rho} Y)^{\frac{2}{15}}}{\rho_{\text {liq }}^{\frac{11}{15}}}
$$

According to [1], the value of the constant is $C_{\text {eq, Vallet }}=1.2$. In [6] one can find $C_{\text {eq, Vallet }}=1.0$ and in $[3]$ a value of $C_{\text {eq, vallet }}=2.5$ is chosen.

Beau et al. [5] propose another formulation for the equilibrium value of interfacial area. They assume that an equilibrium diameter can be determined according to an equilibrium collisional Weber number, defined as

$$
\mathrm{We}_{\mathrm{eq}}=\frac{\rho_{\mathrm{liq}} \Delta u^{2} d_{32}}{\sigma} \text { and } \Delta u^{2}=\frac{2}{3} k
$$

A value for the equilibrium Weber number is not proposed in [5], but Lebas et al. [2] propose a value of $\mathrm{We}_{\mathrm{eq}}=12$ using the same modeling approach.

A confirmation that a drop size distribution undergoing turbulent collisions tends to an equilibrium Weber number was given in $[9]$.

\section{Modeling of collisional time scale and equilibrium value: present approach}

Within the modeling approaches presented above, effects of particle inertia are not considered, which may have an influence on the turbulent fluctutations of the droplet phase and the relative velocity between droplets: Particles dispersed in a turbulent flow react to turbulent velocity fluctuations of the surrounding fluid on different time scales, depending on their turbulent Stokes number. The latter is defined as the ratio of particle relaxation time and a turbulent time scale. Under assumption of an exponential spatial or temporal correlation function for the fluid velocity, Levins and Glastonbury (temporal) [10] and Williams and Crane (spatial) [11] could derive an expression for the mean square turbulent velocity fluctuation of component $i$ of a particle depending on the corresponding velocity fluctuation of the surrounding fluid and the particle's turbulent Stokes number:

$$
\frac{\overline{u_{\mathrm{p}, i}^{2}}}{\overline{\overline{u_{\mathrm{f}, i}^{2}}}}=\frac{1}{1+\mathrm{St}_{\mathrm{t}}}
$$

with

$$
\mathrm{St}_{\mathrm{t}}=\frac{\tau_{\mathrm{p}}}{T_{\mathrm{t}}} \text { and } \tau_{\mathrm{p}}=\frac{\rho_{\mathrm{p}} d_{\mathrm{p}}^{2}}{18 \mu} \text { (assuming Stokes drag) }
$$


Since the particles will be influenced mainly by the most energetic large turbulent structures, $T_{\mathrm{t}}$ is chosen as the integral turbulent time scale. Considering equations 12 and 13 , small particles tend to follow the turbulent fluctuations ideally, whereas large particles are not affected by turbulent fluctuations. The collisional velocity needed is the relative velocity between droplets, calculated as the absolute value of the velocity difference vectors of two droplets:

$$
\begin{aligned}
\overline{\Delta u_{\mathrm{p}}^{2}} & =\sum_{i=1}^{3} \overline{\left(u_{\mathrm{p}, 1, i}-u_{\mathrm{p}, 2, i}\right)^{2}} \\
& =\sum_{i=1}^{3}[\overbrace{\overline{u_{\mathrm{p}, 1, i}^{2}}+\overline{u_{\mathrm{p}, 2, i}^{2}}}^{\text {uncorrel. }}-2 \overbrace{\overline{u_{\mathrm{p}, 1, i} u_{\mathrm{p}, 2, i}}}^{\text {correl. }}]
\end{aligned}
$$

Thus, the velocity difference between two particles consists of an uncorrelated and a correlated part. Taking into account the definition of turbulent kinetic energy, the uncorrelated part can be specified as

$$
\left(\overline{\Delta u_{\mathrm{p}}^{2}}\right)_{\text {uncorrel. }}=\frac{4 k}{\left(1+\mathrm{St}_{\mathrm{t}, 1}\right)\left(1+\mathrm{St}_{\mathrm{t}, 2}\right)} .
$$

The correlation term is evaluated for a high density ratio between particle and gas phase and the application of a suitable form for the covariance of the fluid velocity surrounding the particle, see [11]. Williams and Crane finally calculate the following universal expression for the ratio of the mean square of particle relative velocity to the mean square of fluid velocity fluctuations, applicable to the whole Stokes number range:

$$
\frac{\overline{\Delta u_{\mathrm{p}, i}^{2}}}{\overline{u_{\mathrm{f}, i}^{2}}}=\frac{\left(\mathrm{St}_{1}+\mathrm{St}_{2}\right)^{2}-4 \mathrm{St}_{1} \mathrm{St}_{2}\left[\frac{1+\mathrm{St}_{1}+\mathrm{St}_{2}}{\left(1+\mathrm{St}_{1}\right)\left(1+\mathrm{St}_{2}\right)}\right]^{0.5}}{\left(\mathrm{St}_{1}+\mathrm{St}_{2}\right)\left(1+\mathrm{St}_{1}\right)\left(1+\mathrm{St}_{2}\right)}
$$

A droplet distribution is not known within the $\Sigma-Y$ model, but a local Sauter mean diameter. Thus, the turbulent Stokes numbers are set to be equal for both droplets. Furthermore, individual droplet velociy components are not known and isotropic turbulence is assumed leading to the following absolute value for the mean square of particle relative velocity:

$$
\overline{\Delta u_{\mathrm{p}}^{2}}=\frac{2 \mathrm{St}}{1+\mathrm{St}^{2}}\left(1-\frac{(1+2 \mathrm{St})^{0.5}}{1+\mathrm{St}}\right) \cdot 2 k
$$

In Figure 1 a graphical representation of the mathematical relationship between Stokes number and particle velocity fluctuation for the case of identical droplets is shown representing equation (17). It can be seen that small Stokes numbers lead to well correlated velocities between droplets since they simply adjust to the gas phase fluctuations. With increasing Stokes numbers the velocity correlation diminishes leading to higher relative velocites between droplets. A maximum value of $\overline{\Delta u_{\mathrm{p}}^{2}} / 2 k \approx 0.13$ is reached at about $\mathrm{St}=3.5$. For higher values of the Stokes number the influence of turbulent gas phase fluctuations on the motion of particles decreases, which reduces the velocity fluctuations of the droplets and hence the relative velocitv between them.

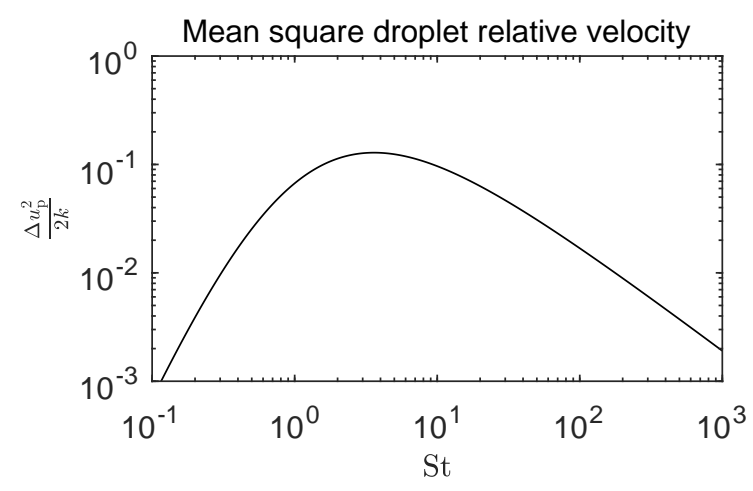

Figure 1. Dependency of particle relative velocity on turbulent Stokes number for identical droplets [11]

Using the stochastic modeling approach for the turbulent collision process within $\Sigma-Y$ model, as proposed in [5], the characteristic collisional time can finally be stated as

$$
\tau_{\text {coll, present }}=\frac{1}{\sqrt{\overline{\Delta u_{\mathrm{p}}^{2}}} \Sigma} .
$$


The equlibrium value for interfacial area is modeled by the definition of an equilibrium collisional Weber number, similar to [5]. But in the new approach presented here the velocity is set according to equation [18):

$$
\mathrm{We}_{\text {eq, present }}=\frac{\rho_{\mathrm{p}} \overline{\Delta u_{\mathrm{p}}^{2}} d_{32}}{\sigma}
$$

In the following sections of this paper the results of CFD simulations for the different modeling approaches introduced above are presented. Attention is given to the influence of the velocity scale on the resulting droplet diameters.

\section{Set-up of the CFD spray simulations}

The $\mathrm{C}_{++}$library OpenFOAM v2.3.0 is used to solve the discretized partial differential equations describing the governing equations for the spray. The dimensions of the computational domain are adopted from [3]. Both, liquid fuel and gas are modeled as incrompressible fluids since the investigation of effects of compressibility are not part of this study. Evaporation is not considered. A $k-\varepsilon$ turbulence model is used with standard values for all constants. Simulations of spray injections for the three different modeling approaches of Vallet et al. [1], Beau et al. [5] and the present one including Stokes number effects are performed. The constants for the collisional production rate and equilibrium value within Vallet et al.'s modeling are set to $C_{\tau, \text { Vallet }}=0.3$ and $C_{\text {eq,Vallet }}=2.5$, respectively. These values are similar to the ones used in [3]. Concerning the transport equation for interfacial area $\Sigma$ only the collisional interfacial area production terms are investigated. All other production terms are switched off. The liquid is injected with a homogeneous velocity (block profile) of $v_{\text {inj }}=350 \mathrm{~m} / \mathrm{s}$ over 6 cells, which corresponds to a nozzle diameter of $d_{\text {nozzle }}=112 \mu \mathrm{m}$.

\section{Results and discussion}

The results obtained for the different modeling approaches of collisional surface production terms are presented. In all figures, the data shown correspond to a time of $t=1000 \mu$ s after start of injection.
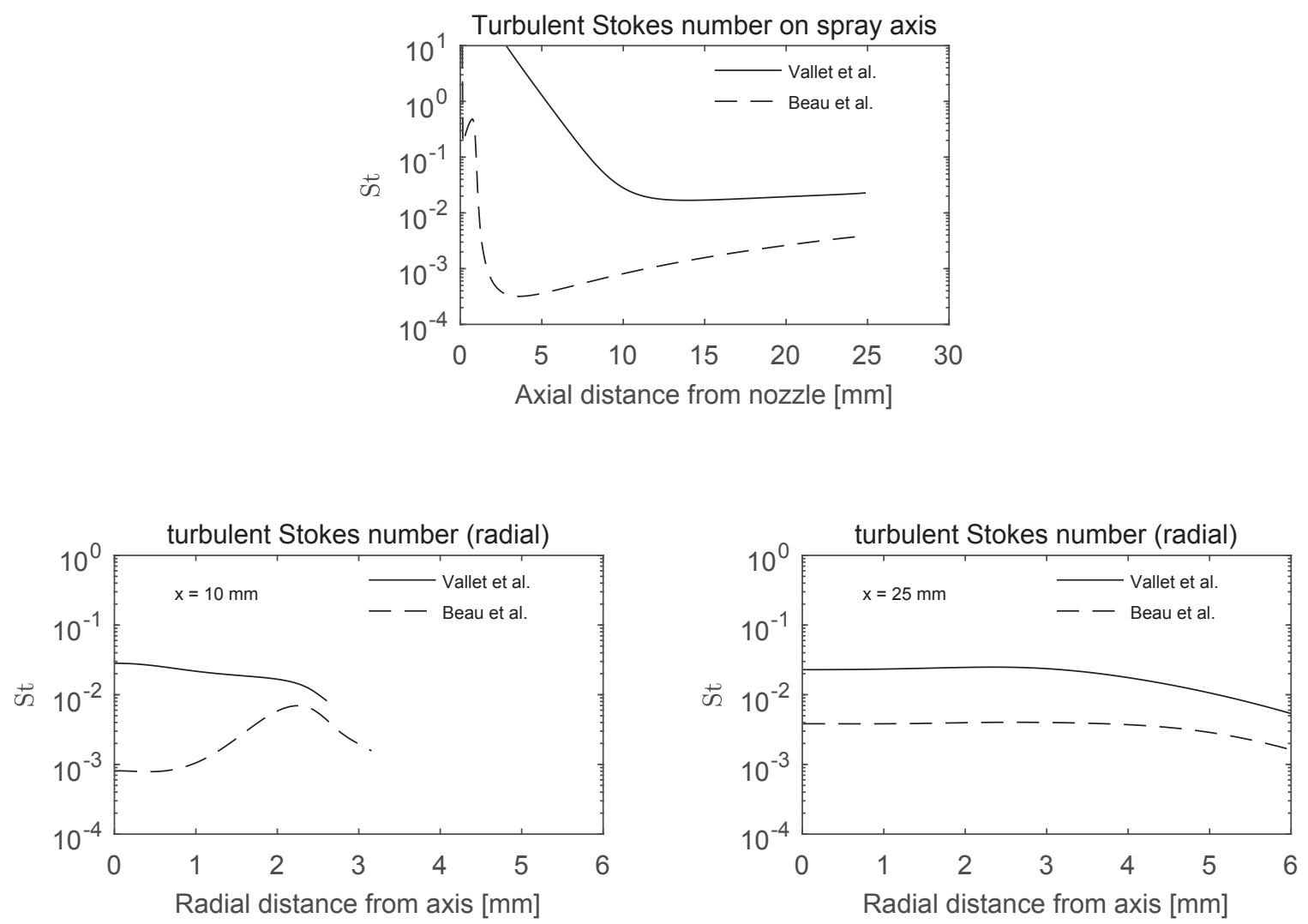

Figure 2. Results of CFD spray simulation showing the axial (top) and radial (bottom) distribution of turbulent Stokes numbers if the collisional surface production terms proposed in Vallet et al. [1] and Beau et al. [5] are used.

\section{Stokes number distribution within the spray}

Taking into account Stokes number effects for the modeling of collisional surface production terms within $\Sigma$-Y model leads to a modification of the corresponding collisional velocity scale. For example, the maximum possible ratio of the particle relative velocity used in the present modeling and the one proposed by Beau et al. [5] is $\Delta u_{\mathrm{p}, \text { present }} / \Delta u_{\mathrm{p}, \text { Beau }} \approx 0.63$. 
In order to show the range of Stokes numbers present in a spray, the distribution of turbulent Stokes number along the spray axis and the radial distribution of Stokes number at two different axial positions are presented in Figure 2 The modeling approaches of Vallet et al. and Beau et al. are applied, which do not consider any Stokes number effects. The simulations show that Stokes numbers in most of the spray reach from St $\approx 3 \cdot 10^{-4} \ldots 2 \cdot 10^{-2}$. Hence, the consideration of Stokes number effects would lead to significantly decreased collisional velocities, see Figure 1 or equation 18. The impact of these effects on the Sauter mean diameter distribution within a spray is investigated in the following paragraph.

\section{Evolution of Sauter mean diameter}

The reduction of collisional relative velocities between particles, if Stokes number effects are considered (new approach presented here), leads to increased collisional time scales, see equation (19), and increased equilibrium droplet diameters, see equation [20, in comparison to the modeling approach by Beau et al. [5]. This behavior can be observed in Figure 3 which presents the evolution of Sauter mean diameter along the spray axis (left) and the radial distribution of Sauter mean diameter at an axial distance of $x=25 \mathrm{~mm}$ from the nozzle (right) for the three different modeling approaches. The results show that at $x=0 \mathrm{~mm}$ the Sauter mean diameter for all modeling approaches is identical $\left(d_{32} \approx 60 \mu \mathrm{m}\right)$ which satisfies the boundary value set for $\Sigma$ at the nozzle exit. The modeling of Beau et al. [5] features the lowest values for $d_{32}$ concerning both the axial and radial distribution. Regarding the values on the spray axis it can be observed that after an initial shrinking of $d_{32}$ to a value of about $d_{32} \approx 0.2 \mu \mathrm{m}$ near the nozzle, Sauter mean diameters are increasing to values of about $d_{32} \approx 3 \mu \mathrm{m}$. Qualitatively, the same trend is obtained for the present modeling approach but at significantly higher values of $d_{32}$ : After a shrinking to $d_{32} \approx 4 \mu \mathrm{m}$, droplet diameters increase to $d_{32} \approx 40 \mu \mathrm{m}$. The evolution of Sauter mean diameter observed on the axis for both modeling approaches is a result of decreasing values of turbulent kinetic energy with increasing axial distance from the nozzle. Collisional velocities used in both modeling approaches and hence equilibrium droplet diameters are mainly determined by this turbulent quantity, cf. equation [11, 18) and 20). The shift between the modeling of Beau et al. and the one presented here stems from the same fact: Since the approach of Beau et al. features lower collisional velocities than the new one, equilibrium droplet diameters calculated are lower for Beau et al.'s modeling than for the new approach presented here.

Sauter mean diameters obtained by Vallet et al.'s modeling [1], which features a quite different calculation of collisional velocity scale and equilibrium value for $\Sigma$ and $d_{32}$ respectively, lie in between the values obtained with the modeling of Beau et al. and the new approach presented here. Droplet diameters initially decrease to a value of $d_{32} \approx 4 \mu \mathrm{m}$ at $x \approx 12 \mathrm{~mm}$ but then increase to a value of $d_{32} \approx 10 \mu \mathrm{m}$ showing the same trend already described for the other modeling approaches.
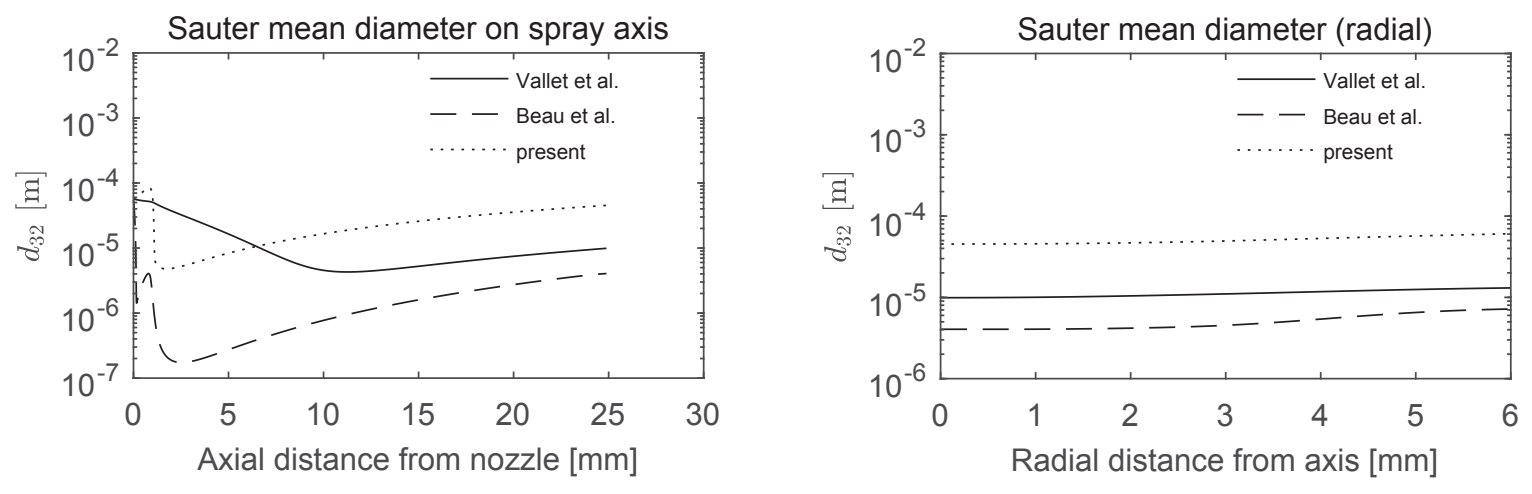

Figure 3. Evolution of Sauter mean diameter along spray axis (left) and radial distribution of Sauter mean diameter at $x=25 \mathrm{~mm}$ (right)

Concerning the radial profile Sauter mean diameters slightly increase when leaving the spray axis. Within Vallet et al's approach this behavior corresponds to decreasing values of turbulent dissipation rate. For the modeling approaches of Beau et al. and the one presented here decreasing values of turbulent kinetic energy are reasonable. The shift in Sauter mean diameters goes along with a shift in turbulent Stokes number, which is presented in Figure 4 The Stokes numbers observed with the new modeling approach are higher than for the modeling approaches of Vallet et al. and Beau et al., cf. Figure 2 in most of the spray. However, Stokes numbers are between St $\approx 0.3 \ldots 0.5$ which still allows the assumption of turbulence induced collisions and hence the application of the present modeling approach to the $\Sigma-Y$ model.

\section{Conclusions}

In the present study, the modeling of collisional velocities applicable to the collisional interfacial area production terms within the $\Sigma-Y$ model was investigated. Two different modeling approaches proposed in the literature were compared against an alternative modeling approach presented here, which includes the influence of Stokes number 


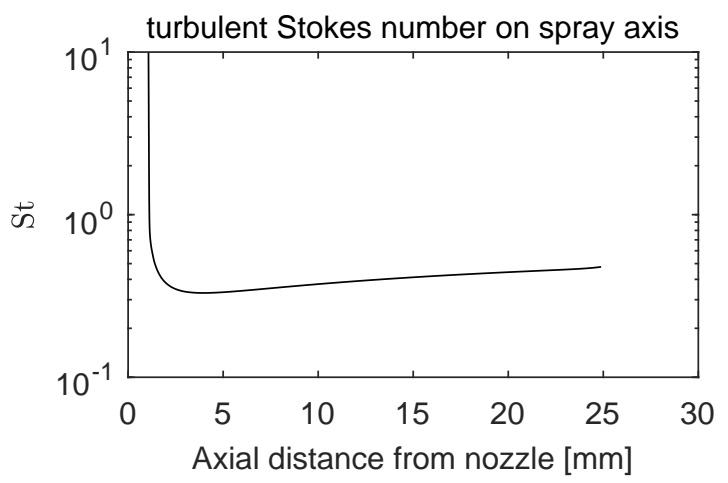

Figure 4. Turbulent Stokes number on the spray axis for the new modeling approach considering Stokes number effects

on the collisional velocity in contrast to the other approaches. It was found that Sauter mean diameters obtained wiith the new modeling approach were larger than the ones calculated if the modeling approaches proposed in the literature were employed.

The modeling approach of Beau et al. and the one presented here are physically similar but differ in the calculation of the actual value for the collisional velocity. The reduced collisional velocities obtained with the new approach (including Stokes number effects) lead to increased values of collisional time scales and equilibrium values for Sauter mean diameters. This again results in increased values for the actual Sauter mean diameters, which are only slightly different from the equilibrium.

Vallet et al. assume a different approach for calculating the collisional velocity and equilibrium Sauter mean diameter. Thus, their approach and the new one presented by the authors was not compared in terms of a superior applicability to the $\Sigma-Y$ model. However, diameters obtained with the new approach roughly have the same order of magnitude as the ones calculated by Vallet et al.'s approach, which has already been successfully applied to spray simulations, see for example [3, 12]. Therefore, an indication of the general applicability of the new modeling for the collisional interfacial area production within the $\Sigma-Y$ model is given.

The new modeling introduced here is supposed to represent an alternative to the modeling approaches of Vallet et al. and Beau et al. But further work is needed to achieve a validation of the modeling proposal, which will be conducted in a continuing study.

\section{Nomenclature}

Roman letters

$A \quad$ production rate of interfacial area by turbulent stretching $\left[\mathrm{s}^{-1}\right]$

$C \quad$ numerical constant [-]

$d_{32} \quad$ Sauter mean diameter [m]

$k \quad$ turbulent kinetic energy $\left[\mathrm{m}^{2} \mathrm{~s}^{-2}\right.$ ]

$l \quad$ length [m]

$n \quad$ droplet number density $\left[\mathrm{m}^{-3}\right]$

Sc Schmidt number [-]

$T \quad$ time scale [s]

$t \quad$ time [s]

$u \quad$ velocity $\left[\mathrm{m} \mathrm{s}^{-1}\right]$

$Y \quad$ liquid mass fraction [-]

\section{Greek letters}

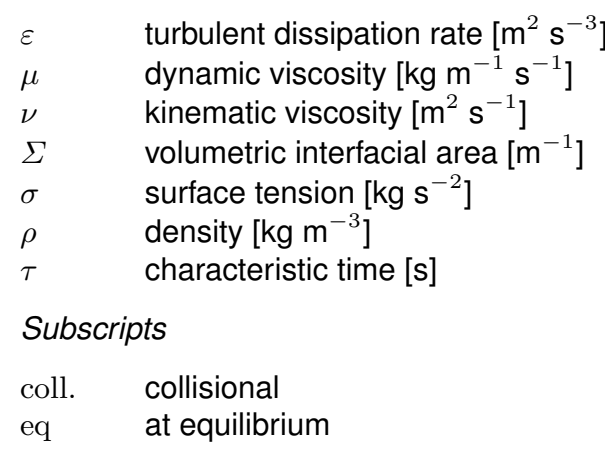




$\begin{array}{ll}\mathrm{f} & \text { fluid } \\ \text { inj } & \text { injection } \\ \text { liq } & \text { liquid } \\ i & \text { in direction } i \\ \text { noz. } & \text { nozzle } \\ \mathrm{P} & \text { production } \\ \mathrm{p} & \text { particle } \\ \mathrm{D} & \text { destruction } \\ t & \text { turbulent }\end{array}$

\section{References}

[1] Vallet A., Burluka, A.A. and Borghi, R., 2001, Development of a Eulerian Model for the "Atomization" of a Liquid Jet, Atomization and Sprays, 11 (6), pp. 619-642.

[2] Lebas, R., Menard, T., Beau, P.A., Berlemont, A., Demoulin, F.X., 2009, Numerical simulation of primary breakup and atomization: DNS and modelling study, Int. J. Multiphase Flow, 35, pp. 247-260.

[3] Garcia-Olíver, J.M., Pastor, J.M., Pandal, A., Trask, N., Baldwin, E. and Schmidt, D.P., 2013, Diesel Spray CFD Simulation based on the $\Sigma-Y$ Eulerian Atomization Model, Atomization and Sprays, 23 (1), pp. 71-95.

[4] Lebas, R., Blokeel, G., Beau, P.-A. and Demoulin, F.X., 2005, Coupling Vaporization Model With the EulerianLagrangian Spray Atomization (ELSA) Model in Diesel Engine Conditions, SAE Technical Paper 2005-01-0213.

[5] Beau, P.A., Menard, T., Lebas, R., Berlemont, A., Tanguy, S. and Demoulin, F.X., July 17.-20. 2006, Numerical Jet Atomization. Part II: Modeling Information and Comparison with DNS Results, FEDSM2006 ASME Joint U.S. - European Fluids Engineering Summer Meeting, Miami, Florida.

[6] Demoulin, F.X., Beau, P.A. Blokeel, G., Arnaud, M. and Borghi, R., 2007, A New Model for Turbulent Flows with Large Density Fluctuations: Application to Liquid Atomization. Atomization and Sprays, 17, pp. 315-345.

[7] Desantes, J.M., Garcia-Olíver, J.M., Pasot, J.M., Pandal, A., Baldwin, E. and Schmidt, D.P., 2016, Coupled/decoupled spray simulation comparison of the ECN spray a condition with the $\Sigma-Y$ Eulerian Atomization Model, Int. J. Multiphase Flow, 80, pp. 89-99.

[8] Pandal, A., Pastor, J.M., Garcia-Olíver, J.M., Baldwin, E. and Schmidt, D.P., 2016, Coupled/decoupled spray simulation comparison of the ECN spray a condition with the $\Sigma-Y$ Eulerian Atomization Model, Int. J. Multiphase Flow, 83, pp. 162-171.

[9] Pischke, P. and Kneer, R., Aug. 23.-27. 2015,, Droplet Collisions and the Equilibrium Drop Size Distribution in Turbulent Dispersions, 13th Triennial International Conference on Liquid Atomization and Spray Systems, Tainan, Taiwan.

[10] Levins, D.M. and Glastonbury, J.R., 1972, Particle-Liquid Hydrodynamics ans Mass Transfer in a Stirred Vessel Part I Particle-Liquid Motion Trans. Instn. Chem. Engrs., 50, pp. 32-41.

[11] Williams, J.J.E. and Crane, R.I., 1983, Particle Collision Rate in Turbulent Flow, Int. J. Multiphase Flow, 9, pp. 421-435.

[12] Blokeel, G., Barbeau, B. and Borghi, R., 2003, A 3D Eulerian Model to Improve the Primary Breakup of Atomizing Jet, SAE Tech. Pap. 2003-01-0005. 


\title{
A new approach to modelling the two way coupling for momentum transfer in a hollow-cone spray
}

\author{
Andreas Papoutsakis ${ }^{1, *}$, Sergei S. Sazhin ${ }^{1}$, Steven Begg ${ }^{1}$, Ionut Danaila ${ }^{2}$, Francky Luddens ${ }^{2}$ \\ ${ }^{1}$ Advanced Engineering Centre, School of Computing, Engineering and Mathematics, \\ University of Brighton, Brighton, BN24GJ, UK \\ ${ }^{2}$ Laboratoire de Mathématiques Raphaël Salem, Université de Rouen Normandie, F-76801, \\ Saint-Étienne-du-Rouvray, France \\ ${ }^{*}$ Corresponding author: a.papoutsakis@brighton.ac.uk
}

\begin{abstract}
A new approach to modelling the interaction between droplets and the carrier phase is suggested. The new model is applied to the analysis of a spray injected into a chamber of quiescent air, using an Eulerian-Lagrangian approach. The conservative formulation of the equations for mass, momentum and energy transport is used for the analysis of the carrier phase. The dispersed phase is modelled using the Lagrangian approach with droplets represented by individual parcels.

The implementation of the Discontinuous Galerkin method (ForestDG), based on a topological representation of the computational mesh by a hierarchical structure consisting of oct- quad- and binary trees, is used in our analysis. Adaptive mesh refinement (h-refinement) enables us to increase the spatial resolution for the computational mesh in the vicinity of the points of interest such as interfaces, geometrical features, or flow discontinuities. The local increase in the expansion order (p-refinement) at areas of high strain rates or vorticity magnitude results in an increase of the order of the accuracy of discretisation of shear layers and vortices.

The initial domain consists of a graph of unitarian-trees representing hexahedral, prismatic and tetrahedral elements. The ancestral elements of the mesh can be split into self-similar elements allowing each tree to grow branches to an arbitrary level of refinement. The connectivity of the elements, their genealogy and their partitioning are described by linked lists of pointers. These are attached to the tree data structure which facilitates the on-the-fly splitting, merging and repartitioning of the computational mesh by rearranging the links of each node of the tree. This enables us to refine the computational mesh in the vicinity of the droplet parcels aiming to accurately resolve the coupling between the two phases.
\end{abstract}

\section{Keywords}

Droplets, Sprays, Vortex Rings, Discontinuous Galerkin, Adaptive Mesh Refinement.

\section{Introduction}

The need to accurately model the interaction between droplets and carrier phase (coupling) in various engineering applications is well known [1]. Although various approaches to modelling this coupling have been suggested [1, 2, $3,4,5]$, this problem is far from being solved. The main focus of this paper is on the description of the new approach to this coupling and the application of this approach to modelling realistic sprays in gasoline-engine-like conditions. This new approach is based on the application of the adaptive mesh refinement in the vicinity of the droplet parcels. The Discontinuous Galerkin (DG) method $[6,7,8,9]$ is used for solving the equations for the carrier phase. The latter method combines high order accuracy with the ability to handle complex geometries described by hybrid unstructured meshes by incorporating a minimal computational stencil. However, the computational efficiency of this method (alongside the spectral volume $[8,10]$ and spectral difference $[8,11,12]$ methods is generally believed to be inferior to more commonly used methods as the Finite Differences (FD) and the Finite Volume (FV)[13, 14] methods.

Solution adaptive refinement strategies of $h / p$-type can reduce the computational time for high resolution simulations of complex flows, using various methods, including the DG method, without compromising numerical accuracy $[15,16,17,18,19,20]$. These refinement strategies result in irregular meshes with hanging nodes and polynomial approximations of different orders across the elements [6]. These issues can be effectively dealt with by the DG method. This makes this method ideal for handling irregular meshes with hanging nodes.

In this paper a new mesh adaptive implementation of the Discontinuous Galerkin methodology is suggested. This implementation allows on the fly local $h / p$ refinement and de-refinement of the computational mesh [21]. The development of a new computational code (ForestDG) based on a hierarchical representation of a Forest of binary, quad- and oct- trees, is described. The accuracy and performance of the new code are assessed. The preliminary results of its application to modelling gasoline fuel sprays are described.

\section{Governing equations}

The Eulerian-Lagrangian approach is used for the description of the two phases. The droplets are suspended in the carrier gas phase and are modelled using the Lagrangian approach. The carrier phase is modelled as an Eulerian flow field, described by the state vector $\mathbf{U}(\mathbf{x}, t)$, which contains the values of density, momentum and energy at each position of the computational domain $\mathrm{x}$ at time $t$. The model is developed for a general case of compressible 
flow and the effects of turbulence are taken into account using the standard LES approach. The Favre averaging operator $\widetilde{(\cdot)}=\overline{\rho(\cdot)} / \bar{\rho}$ is used for the separation of the small turbulent fluctuations from the large ones. The state vector for the Favre averaged velocity $\mathbf{u}$ and specific energy $e$ is defined as $\widetilde{\mathbf{U}}(\mathbf{x}, t)=\left(\bar{\rho}, \bar{\rho} \widetilde{u}_{1}, \bar{\rho} \widetilde{u}_{2}, \bar{\rho} \widetilde{u}_{3}, \bar{\rho} \widetilde{e}\right)$. The conservation of mass, momentum and energy [22] provides the set of the governing equations for the turbulent compressible flow of the carrier phase, in the following strong conservative form for $\widetilde{\mathbf{U}}$ :

$$
\frac{\partial \widetilde{\mathbf{U}}}{d t}+\nabla \cdot \mathbf{f}_{i n v}(\widetilde{\mathbf{U}})-\frac{1}{\operatorname{Re}} \nabla \cdot \mathbf{f}_{v i s}(\widetilde{\mathbf{U}}, \widetilde{\boldsymbol{\Theta}})=\mathbf{w}_{d}(\widetilde{\mathbf{U}}), \widetilde{\boldsymbol{\Theta}}=\nabla \mathbf{f}_{a u x}(\widetilde{\mathbf{U}}),
$$

where $\mathbf{w}_{d}$ is the vector of the source terms stemming from the two way coupling for the momentum and energy transfer between the carrier and the discrete phase, $\mathbf{f}_{i n v}$ is the $5 \times 3$ tensor of the inviscid fluxes and $\mathbf{f}_{v i s}$ is the $5 \times 3$ tensor for the viscous fluxes, defined as:

$$
\mathbf{f}_{i n v}=\left[\begin{array}{c}
\bar{\rho} \widetilde{u}_{j} \\
\bar{\rho} \widetilde{u}_{i} \widetilde{u}_{j}+\bar{p} \delta_{i, j} \\
(\bar{\rho} \widetilde{e}+\bar{p}) \widetilde{u}_{j}
\end{array}\right], \mathbf{f}_{v i s}=\left[\begin{array}{c}
0 \\
2\left(\mu+\mu_{t}\right) S_{i, j}^{*} \\
2\left(\mu+\mu_{t}\right) \widetilde{u}_{i} S_{i, j}^{*}
\end{array}\right], \mathbf{w}_{d}=-\left[\begin{array}{c}
0 \\
n_{d} f_{d_{i}} \\
n_{d} f_{d_{j}} \widetilde{u}_{j}
\end{array}\right], \mathbf{f}_{a u x}=\left[\begin{array}{c}
0 \\
\widetilde{u}_{i} \\
\widetilde{e}
\end{array}\right] .
$$

$S_{i j}^{*}=\frac{1}{2}\left(\frac{\partial \widetilde{u}_{j}}{\partial x_{i}}+\frac{\partial \widetilde{u}_{i}}{\partial x_{j}}\right)-\frac{1}{3} \delta_{i j} \frac{\partial \widetilde{u}_{k}}{\partial x_{k}}$ is the traceless rate of strain tensor related to the viscous stress tensor $\tau_{i j}=$ $2 \mu S_{i j}^{*}$, with $\mu$ being the local non-dimensional viscosity of the gas face. The viscous fluxes are evaluated from $\widetilde{\boldsymbol{\Theta}}$ which contains the spatial gradients of $\widetilde{\mathbf{U}}$ as $\widetilde{\Theta}_{i j}=\frac{\partial \widetilde{U}_{i}}{\partial x_{j}}$. $\widetilde{\boldsymbol{\Theta}}$ is the divergence of the state vector $\widetilde{\boldsymbol{\Theta}}=\nabla \widetilde{\mathbf{U}}$ and constitutes a set of auxiliary variables which is solved separately. Equations (1) are the coupled form of the governing equation for $\widetilde{\mathbf{X}}=[\widetilde{\mathbf{U}}, \widetilde{\mathbf{\Theta}}]$.

The contribution of the sub-grid scales, not accounted by the spatially filtered state vector, is taken into account by the turbulent viscosity term $\mu_{t}$ in the definition of the viscous fluxes in Equation (2). This term is approximated using the standard Smagorinsky model:

$$
\mu_{t}=\widetilde{\rho}\left(c_{s} \Delta\right)^{2}\left|\widetilde{S_{i j}}\right|
$$

where $c_{s}$ is the Smagorinsky constant assumed equal to $0.1, \Delta=V_{m}^{1 / 3}$ is the filter width assumed equal to the characteristic length of the computational element, and $\left|\widetilde{S_{i}}\right|=\sqrt{2 \widetilde{S}_{i j} \widetilde{S}_{i j}}$ is the magnitude of the resolved rate of strain tensor. Equation (1) is non-dimensionalised over the characteristic length of the flow, gas dynamic viscosity $\mu_{g}$ at the ambient conditions. The Reynolds number of the flow is estimated as $\operatorname{Re}=\rho_{g} c L / \mu_{g}$, where $c$ is the velocity of sound.

The discrete phase is modelled as parcels of droplets with diameters $d_{d}$ and velocities $\mathbf{v}_{\mathbf{d}}$. The effect of the dispersed phase on the energy and momentum of the carrier phase is modelled as the source term $\mathbf{w}_{d}$ in Equation (2). $n_{d}$ is the droplet number density. The term $f_{i}$ in Equation (2) is the force acting on each individual droplet in the parcel. Assuming that the flow is Stokesian, the expression for the drag force can be presented as:

$$
n_{d} \mathbf{f}_{d}=n_{d} \frac{3 \pi d_{d} \mu}{\operatorname{Re}}\left(\widetilde{\mathbf{u}}-\mathbf{v}_{d}\right)
$$

Note that in some cases considered later $\operatorname{Re}_{d}>1$ when the Stokesian approximation is not strictly valid. In these cases the results of our analysis can be used for qualitative but not quantitative description of the process.

The trajectories of droplets are described by the following equations:

$$
\frac{d \mathbf{x}_{d}}{d t}=\mathbf{v}_{d}, \quad d \mathbf{v}_{d}=\mathbf{f}_{d} d t+\left(\frac{k_{t}}{\tau_{t}}\right)^{1 / 2} d \mathbf{W}_{t}, \quad \mathbf{f}_{d}=\frac{18 \mu}{\operatorname{Re} d_{d}^{2} \rho_{l}}\left(\widetilde{\mathbf{u}}-\mathbf{v}_{d}\right) .
$$

The term $d \mathbf{W}_{t}$ represents the increment of the Wiener process, which introduces the stochastic component for the motion of the dispersed phase to model the effect of the unresolved turbulent scales to the droplet trajectories. In the case of equilibrium, the subgrid turbulent kinetic energy term $k_{t}$ is evaluated from the 'production equals dissipation' assumption as $k_{t}=2 \Delta^{2} c_{s}^{4 / 3} \widetilde{S_{i j}} \widetilde{S_{i j}}$. The term $\tau_{t}$ is the time scale of the interaction between the droplet and the turbulent motions modelled as $\tau_{t}=\tau_{d}^{1.6}\left(\frac{\sqrt{k_{t}}}{\Delta}\right)^{0.6}$

\section{Discretisation of the equations}

We consider the discretisation of the computational domain $\Omega$ into $N$ elements $E_{m}\left(\Omega=\cup E_{m}\right)$. A weak formulation of the governing equations is derived by multiplying the conservative forms of these equations with a test function $w(\mathbf{x})$ and integrating them over the element. In the Galerkin context, the test function is taken from the same set of polynomial basis functions as used for the interpolation of the state vector $\widetilde{\mathbf{X}}$. The interpolated distribution $\mathbf{X}_{h}^{m}$ for $\widetilde{\mathbf{X}}$ is defined for each element $E_{m}$ as the weighted sum of $N_{p}$ polynomial basis functions:

$$
\mathbf{X}_{h}^{m}=\sum_{i=1}^{N_{p}} \mathbf{c}_{i}^{m}(t) b_{i}(\mathbf{x}), \text { for } m=1, N_{p},
$$


where $p$ is the maximum degree of the basis functions. In this expansion, the solution coefficients $\mathbf{c}_{i}(t)$ are the degrees of freedom. $b_{i}(\mathbf{x})$ is the tensor product of the Legendre polynomial basis functions in the three spatial dimensions. The integral formulation of Equations (1) is expressed as:

$$
\begin{aligned}
& \int_{E_{m}} b_{i} \frac{\partial \mathbf{U}_{h}^{m}}{\partial t} d E+\oint_{S_{m}} b_{i} \mathbf{f}\left(\mathbf{U}_{h}^{m}\right) \cdot \mathbf{n} d S-\int_{E_{m}} \nabla b_{i} \cdot \mathbf{f}\left(\mathbf{X}_{h}^{m}\right) d E=\int_{E_{m}} b_{i} \mathbf{w}_{d}^{m} d E \quad \text { for } i=1, N_{p}, m=1, N, \\
& \int_{E_{m}} b_{i} \Theta_{h}^{m} d E=\oint_{S_{m}} b_{i} \mathbf{f}_{a u x}\left(\mathbf{U}_{h}^{m}\right) \cdot \mathbf{n} d S-\int_{E_{m}} \nabla b_{i} \cdot \mathbf{f}_{a u x}\left(\mathbf{X}_{h}^{m}\right) d E \quad \text { for } \quad i=1, N_{p}, m=1, N,
\end{aligned}
$$

providing a set of $N_{p} \times N$ equations for $\mathbf{c}_{i}^{m}(t)$.

The surface integrals are defined on the surface of the element $S_{m}=\partial E_{m}$ with $\mathbf{n}$ defined as the outward normal unit vector. In the weak formulation presented in Equation (7), the flux $\mathrm{f}$ represents both the viscous and inviscid fluxes in Equation (1) as $\mathbf{f}=\mathbf{f}_{i n v}-(1 / \mathrm{Re}) \mathbf{f}_{v i s}$; the flux $\mathbf{f}_{a u x}$ was defined in Equation (2). In the DG context we do not require the continuity of the interpolated variables across the element faces. Thus the values of $\mathbf{U}_{h}^{m}$ and $\Theta_{h}^{m}$ on the faces $S_{m}$ are defined twice. The conservation of fluxes at the boundaries of the elements infers from the approach to the evaluation of the surface integrals used in our analysis. In the Local Lax-Friedrichs (LLF) scheme for the evaluation of viscous fluxes the signs of surface sides of the elements (refered to as minus $(-)$ or plus $(+)$ ) are introduced.

Calculating the fluxes from one of the two sides for the adjoin elements guarantees the conservation properties of the scheme. Specifically for the LLF scheme the surface integrals for $\mathbf{f}_{v i s}$ and $\mathbf{f}_{a u x}$ in Equation (1) are evaluated from the opposite sides, enhancing the stability of the scheme, and reducing the stiffness of the problem since:

$$
\mathbf{f}_{v i s}=\mathbf{f}_{v i s}\left(\mathbf{U}_{h}^{-m}, \boldsymbol{\Theta}_{h}^{-m}\right), \mathbf{f}_{a u x}=\mathbf{f}_{a u x}\left(\mathbf{U}_{h}^{+m}\right) \text {. }
$$

The inviscid flux $\mathbf{f}_{i n v}$ is evaluated from the mean value of the variables on the two face sides $\left\{\mathbf{U}_{h}^{m}\right\}$, where an artificial diffusion term, proportional to the jump of the fluxes on the bounding surface $\left[\left[\mathbf{U}_{h}^{m}\right]\right]$, is introduced via the equation:

$$
\mathbf{f}_{i n v}=\mathbf{f}_{i n v}\left(\left\{\mathbf{U}_{h}^{m}\right\}\right)+\frac{c}{2}\left[\left[\mathbf{U}_{h}^{m}\right]\right],
$$

where $c$ is defined as $c_{i}=\max \left(\left|f_{\text {inv }}^{\prime}\left(U_{i}^{-}\right)\right|,\left|f_{\text {inv }}^{\prime}\left(U_{i}^{+}\right)\right|\right)$in the local Lax-Friedrichs scheme.

The volume and surface integrals in Equations (7) and (8) are defined in the physical space. The integrals are evaluated in the transformed domain for the canonical elements using the Legendre-Gauss quadrature rule and the Jacobian of the transformation [24].

\section{Mesh representation}

Unstructured, conforming grids are represented using a finite serial addressing of the cells with each cell determined by the address of it's vertices. The connectivity of the cells is then defined by appointing the numerical addresses of the neighbouring cells to each face. In our case, the serial array of the cells is substituted by a graph of elements forming a forest of nodes.

Each node refered to as tree node contains all the pointers needed to define a cell by its relative relations rather than its address in a global addressing system. This scheme provides the versatility of adding or removing nodes and manipulating the relations between the nodes by maintaining the relative relations of the node without interfering to the addressing scheme of the remaining nodes. A node is accessed by a dynamic linked list structure, naming an arbitrary node as the "first" node of the mesh. By assigning a "next" node for every cell we can go through all cells of the grid. This is achieved by advancing to the "next" node each time starting from the "first" as shown in Figure 1 (Left). The tree in our implementation is a data structure that contains all necessary information needed for the
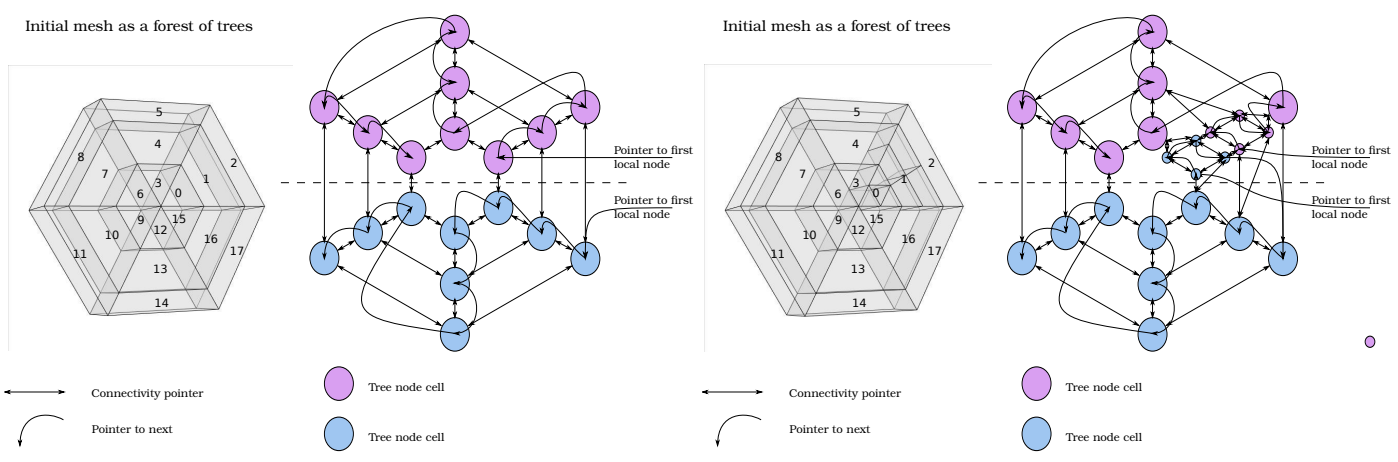

Figure 1. Left: Example of hybrid unstructured mesh and the corresponding graph. Right: Prismatic and hexahedral cells are split into four cells; the nodes of the adapted mesh are repartitioned by introducing the new nodes to the local element lists and the connectivity pointers are re-defined.

definition of the relative relations of the cell and also its geometrical characteristics, i.e. the nodes, the edges and 
the faces. A node of the graph can be split furnishing a tree of nodes while a single node is perceived as a unitarian tree. An example of a developed forest is shown in the figure 2.

The actual solution vector $\mathbf{X}$ is stored at a special data structure of the tree node, named leaf. The leaf contains the characteristic coefficients of the basis functions used for the description of the conservative variables, the element Jacobian matrix, the mass matrix and the matrices used for the calculation of the derivative and basis values at the face and volume quadrature points. Eventually, the leaf data structure contains all the memory consuming information that describes the actual field. In the ForestDG implementation, the initial unrefined mesh is transformed

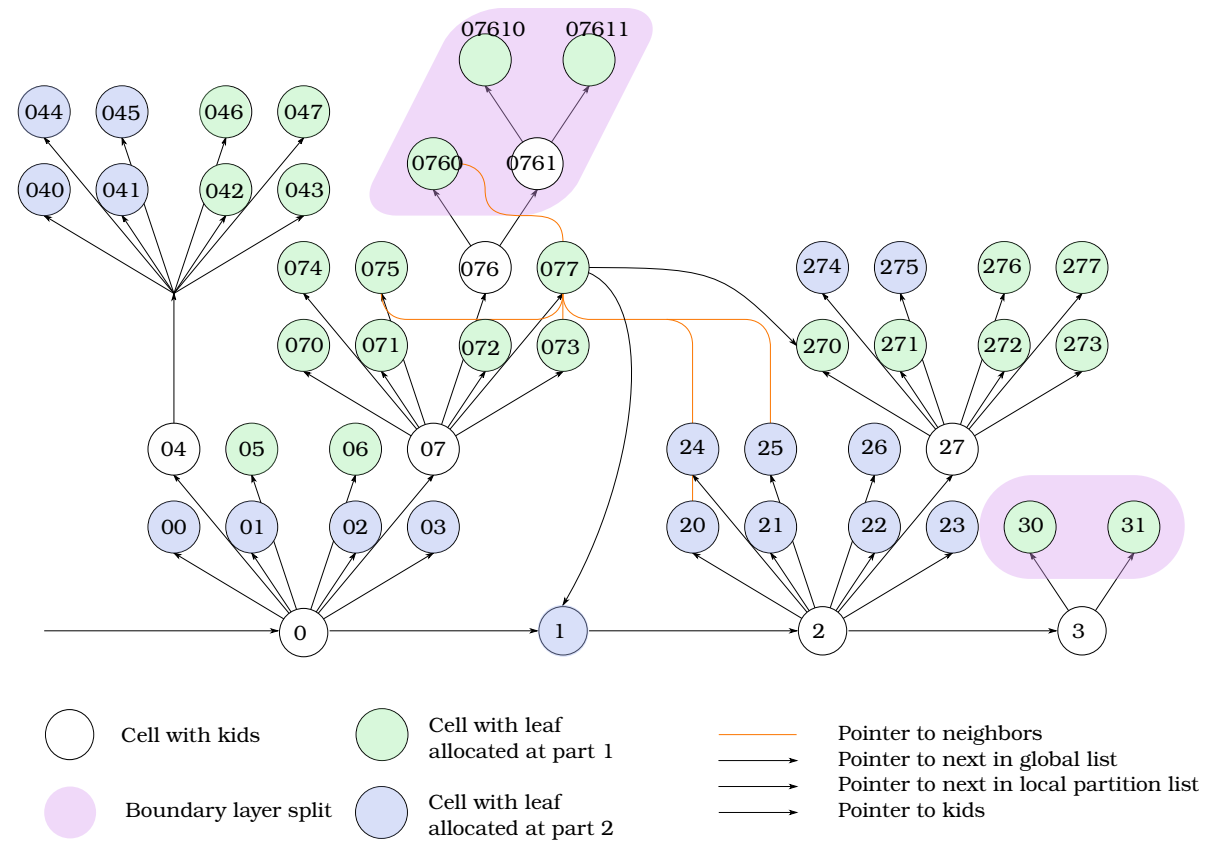

Figure 2. A forest of oct-trees representing a tree dimensional adapted topology.

into a graph representation and the nodes are partitioned using the METIS [25] graph domain decomposition library. In the event of splitting a tree, a number of new nodes, allocated by the kids [ikid] pointer array, are created
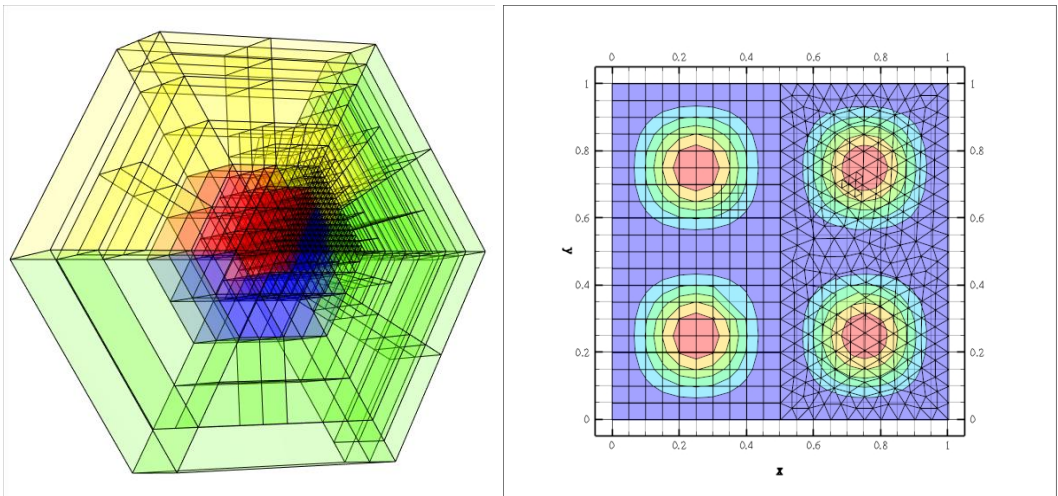

Figure 3. Left: Oct tree splitting of a hybrid unstructured mesh. Node 0 is split to level 5 resulting in a 4736 element grid starting from 18 initial nodes; blue, green, yellow and red represent the mapping of the four different partitions. Right: Examples of projecting the solution during merging and spliting cells for the manufactured solution used for testing the order of accuracy of the discretisation.

depending of the type of splitting maintaining the hierarchical structure of the forest of trees. A quad tree type of splitting results in four children, an oct-tree splitting results in eight children. The parent node is removed from the linked list that controls the accessing of the cells and replaced by the children nodes. The parent node is removed from the linked list and replaced by the kids.

When a cell is split into a number of kids or when a set of kids merge to a bigger cell, the solution has to be projected to the new cells. In order to achieve this, the position of the existing quadrature points must be identified in the new geometry as shown in the Figure 4 (Left). Since the basis of the interpolation is evaluated in the computational space which spans from -1 to 1 , the coordinates for the quadrature points need to be transformed in the computational space. The transformation of the $i$ th canonical coordinate for each kid $\eta_{i}^{k i d}$ to the canonical coordinates of the parent $\eta_{i}$ has the general form:

$$
\eta_{i}=a_{1,2,3}^{i, k i d} \eta_{1}^{k i d} \eta_{2}^{k i d} \eta_{3}^{k i d}+b_{1,2}^{i, k i d} \eta_{1}^{k i d} \eta_{2}^{k i d}+b_{1,3}^{i, k i d} \eta_{1}^{k i d} \eta_{3}^{k i d}+b_{2,3}^{i, k i d} \eta_{2}^{k i d} \eta_{3}^{k i d}+c_{1}^{i, k i d} \eta_{1}^{k i d}+c_{2}^{i, k i d} \eta_{2}^{k i d}+c_{3}^{i, k i d} \eta_{3}^{i, k i d}+d^{i, k i d}
$$


where the coefficients $a^{k i d}, b^{k i d}, c^{k i d}$ and $d^{k i d}$ are calculated for each kid depending on its position and shape in the canonical space as shown in the left column of the Figure 4 (Left). For prismatic cels the above expression simplifies to:

$$
\eta_{1}=b_{1,2}^{1, k i d} \eta_{1}^{k i d} \eta_{2}^{k i d}+c_{1}^{1, k i d} \eta_{1}^{k i d}+c_{2}^{1, k i d} \eta_{2}^{k i d}+d^{1, k i d}, \eta_{2}=c_{2}^{2, k i d} \eta_{2}^{k i d}+d^{2, k i d}, \eta_{3}=c_{3}^{3, k i d} \eta_{3}^{k i d}+d^{3, k i d},
$$

and for hexahedral cells is expressed as:

$$
\eta_{1}=c_{1}^{1, k i d} \eta_{1}^{k i d}+d^{1, k i d}, \quad \eta_{2}=c_{2}^{2, k i d} \eta_{2}^{k i d}+d^{2, k i d}, \quad \eta_{3}=c_{3}^{3, k i d} \eta_{3}^{k i d}+d^{3, k i d} .
$$

The $3 \times 3$ system in Equation (12) is linear quadratic while Equations (13) are linear. Both systems can be reversed analyticaly and we can obtain the reverse transformation. The Newton-Raphson method is used for reversing the system in Equation (11) for the tetrahedral elements shown in Figure 4 (Left). The forward transformation is used for projecting the solution of a parent to the quadrature points of a kid and the reverse transformation is used for projecting the solution of a parent at the quadrature points of a kid. The above transformations are also used for the evaluation of the fluxes for non-conformal faces. Non-conformalities arise due to changes of the expansion order because of $p$-refinement or due to hanging nodes from h-refinement or form a combination of both as shown in Figure 4 (Right). A smoothing pass assures either 2:1 or 1:2 non-conformalities, (1:4 in three dimensions). No such restriction is applied for the change of the order $p$.
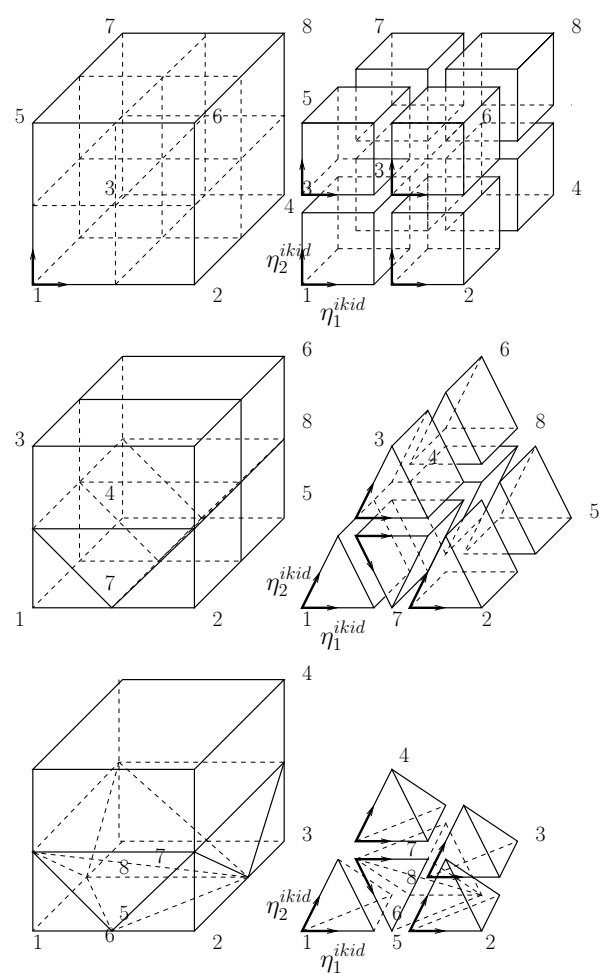

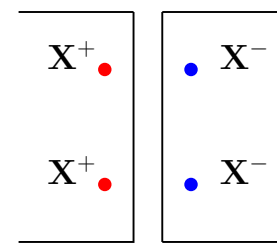

conforming case

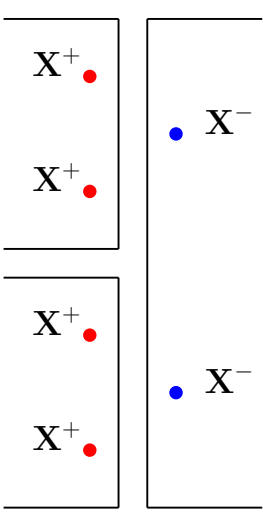

$2: 1$

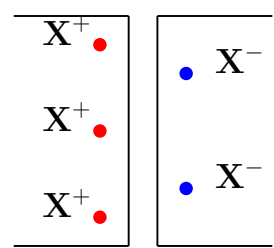

p2:p1

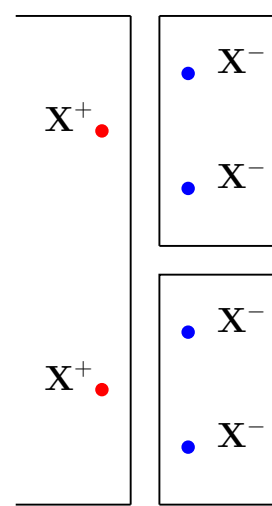

$1: 2$

Figure 4. Left: Canonical space transformations for hexahedral, prismatic and tetrahedral elements. Right: Types of non-conformalities encountered in $\mathrm{h} / \mathrm{p}$ adaptive cases.

Hexahedral elements are split into eight self similar children so that one node is positioned on the vertex of the higher level cell, three nodes are positioned at the midpoints of the adjacent edges, three nodes are positioned on the tree adjacent faces centroids and a final node is positioned at the higher level hexahedral centroid as shown in the Figure 5(Left). The numbering of the children follows the numbering of the higher level cell vertices so that the 1st kid is adjacent to the 1st vertex of the cell an so on. For prismatic elements, splitting leads to self similar elements and the cell numbering follows the vertex numbering of the higher level cell with the exception of the 7-th and 8-th children which are placed along the core of the prism with reversed orientations, as shown in the Figure 5 (Right). Having split the cells to the required resolution, the connectivity of the cells needs to be remapped. This is achieved by tracking the neighbouring cells, neighbouring faces and the orientation of these faces. An example of connectivity tracking for two high level adjacent cells $A$ and $B$ is shown in the Figure 5 , in which cells $A$ and $B$ have the same orientation. The relative orientation of two cells is characterized by two parameters: the face numbers and the relative angle between the faces. For the $\mathrm{i}$-th kid of the higher level cell $\mathrm{A}$ the neighbouring cell is opposite to the $\mathrm{i}$-th cell $\mathrm{B}$, which is described by the following pointer assignment:

$$
A->\operatorname{kids}[i]->\operatorname{neig}\left[i f c_{A}\right]=B->\operatorname{kids}\left[O p\left[i, i f c_{A}\right]\right],
$$

where if $c_{A}$ is the face of the element A that is shared with $\mathrm{B}$. The array $O p$ is a transformation operator that provides the kid opposite to kid $i$ relatively to face $i f c_{A}$. In the general case, however, the neighbouring ancestral cells do 


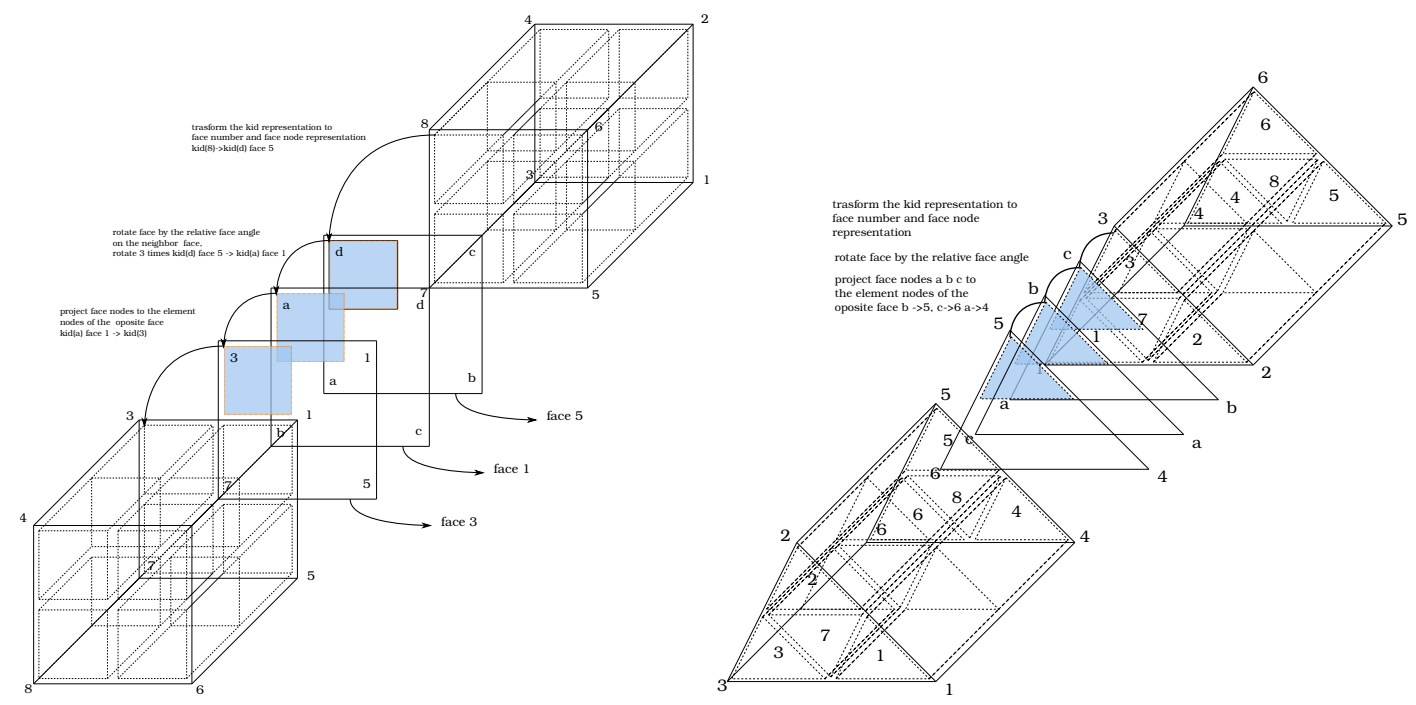

Figure 5. Left: Tracking of a neighbouring element and neighbouring face at a hexahedral to hexahedral connectivity instance. Right: Tracking of a neighbouring element and neighbouring face at a prism to prism connectivity instance.

not have the same orientation. As a result, the connectivity is calculated in steps of transformations shown by the following pointer assignment:

$$
A->\operatorname{kids}[i]->\operatorname{neig}\left[i f c_{A}\right]=B->\operatorname{kids}\left[\operatorname{Op}\left[\operatorname{Tr}\left[i, i f c_{B}, \operatorname{ang}_{B}\right], i f c_{A}\right]\right],
$$

In this expression, operator $\operatorname{Tr}$ remaps the kid address $i$ for element $\mathrm{B}$ into a coordinate system parallel to the coordinate system of element $A$. This transformation aims to align the orientation of the neighbouring cells.

\section{Assessment of the accuracy of the discretisation}

The method of manufactured solutions is used for the investigation of the order of accuracy of the DG discretisation. A two-dimensional manufactured solution shown in Figure 3 (Right) is used. The flow is assumed to be unidirectional in the z-axis and is described as:

$$
\rho=1.0 ; \quad p=1.0 ; \quad T=1.0 ; \quad u=0, v=0, w(\mathbf{x}, t)=w_{0}(1-\cos (4 \pi x))(1-\cos (4 \pi y)) .
$$

Introducing this flow field into the governing equations we obtain analytical expressions for the residuals. These residuals are introduced as source terms in the governing equations to sustain the manufactured solution. The error of the numerical solution, compared with the exact solution in Equation (16), is expressed by the $L_{2}$ norm which is computed as [26]:

$$
L_{2}=\left(\frac{1}{V_{\Omega}} \sum_{m=1}^{N} \int_{E_{m}}\left(\mathbf{u}-\mathbf{u}^{\text {exact }}\right)^{2} d V\right)^{1 / 2} .
$$

The maximum order of accuracy is $p+1$ for basis functions which are polynomials of degree $p$.

As can be seen in Figures 6 , the $L_{2}$ norm of the error between the manufactured solution and analytical expression in Equation (16) reduces to the second order of the mesh resolution. Results similar to those shown in Figures 6 , but for the third and fourth order of accuracy of discretisation when bases with $p=2$ and $p=3$ basis are used, are presented in Figure 7. As follows from this figure, the expected order of accuracy is achieved in this case.

\section{Application to gasoline fuel spray}

The implementation of this modelling approach has been applied to the case of the simulation of a high-pressure, hollow-cone, gasoline, fuel spray utilised in a modern spray-guided combustion system. An accurate prediction of the spray and gas phase characteristics, prior to ignition of the mixture, plays a key role in determining the optimal engine operating conditions for maximum combustion efficiency in highly lean, and stratified mixtures. Experimental observations of the fuel spray were conducted in a quiescent chamber of fixed volume. The ambient gas conditions were $20 \mathrm{deg} \mathrm{C}$ and 1 bar. The piezoelectric fuel injector was mounted in a vertical position at the top of the chamber. Measurements of the spray shape (geometry and thickness of plume) and its droplet size and velocity distributions were carried out using high-speed photography and Phase Doppler Anemometry, respectively. The experimental set-up and measurement procedure have been described in [5].

The fuel used in our analysis is iso-octane injected at a pressure of 100 bar for a duration of 1 msec. Mass flow rate $\dot{m}$ (measured experimentally using a rate tube) increased linearly from zero up to a maximum value of $\dot{m}=30 \mathrm{~g} / \mathrm{s}$ in $0.1 \mathrm{msec}$. During the following $0.8 \mathrm{msec}$ the mass flow rate remains constant and decreases to zero during the last $0.1 \mathrm{msec}$. Every 10 time steps of the simulation 10 droplet parcels are released at an angle of $42.5^{\circ}$ relative to the axis of symmetry with a spread (divergence of wall thickness) of $6^{\circ}$. Droplet velocities were inferred from the mass 

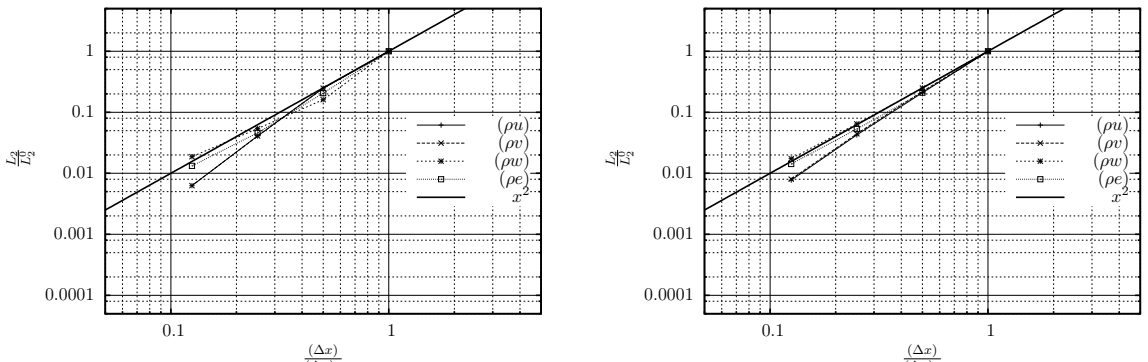

$\frac{(\Delta x)}{[\Delta x)_{0}}$

$\frac{(\Delta x)}{(\Delta x)_{0}}$

Figure 6. Relative $L_{2}$ error for the momentum components and the energy, versus mesh resolution (h) for a second order accurate discretisation $(p=1)$. Left: Hexahedral elements. Right: Prismatic elements.
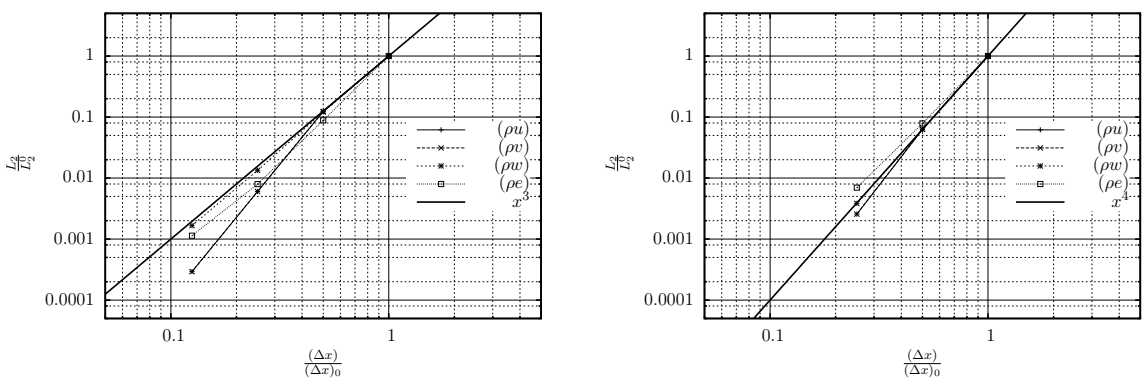

Figure 7. Relative $L_{2}$ error for the momentum components and the energy, versus mesh resolution for hexahedral elements.

Left: Third order accurate discretisation $(p=2)$. Right: Fourth order accurate discretisation $(p=3)$.

flow rate of the injected fuel and prelimanary PDA measurements. The number of individual droplets in the parcel was calculated from the same mass flow rate assuming that the droplet diameters are equal to $d_{d}$. These diameters for different parcels were in the range $3 \mu \mathrm{m}$ to $9 \mu \mathrm{m}$. The most probable droplet diameter, used in our simulations $(6 \mu \mathrm{m})$ corresponded to typical $d_{d}$ observed in the experiments. The preliminary results of the application of the new code to the analysis of sprays in gasoline engine-like conditions are shown in Figure 8 . The results presented in this figure agree with the results of experimental observations of these sprays [5]. Depending on the distance from the orifice, the computational mesh is refined 2 to 3 times at a sector around the spray, 3 to 4 times at areas of high strain rate and 4 to 5 times for cells that contain droplets. The order is increased to p2 (3rd order) in areas of high vorticity.

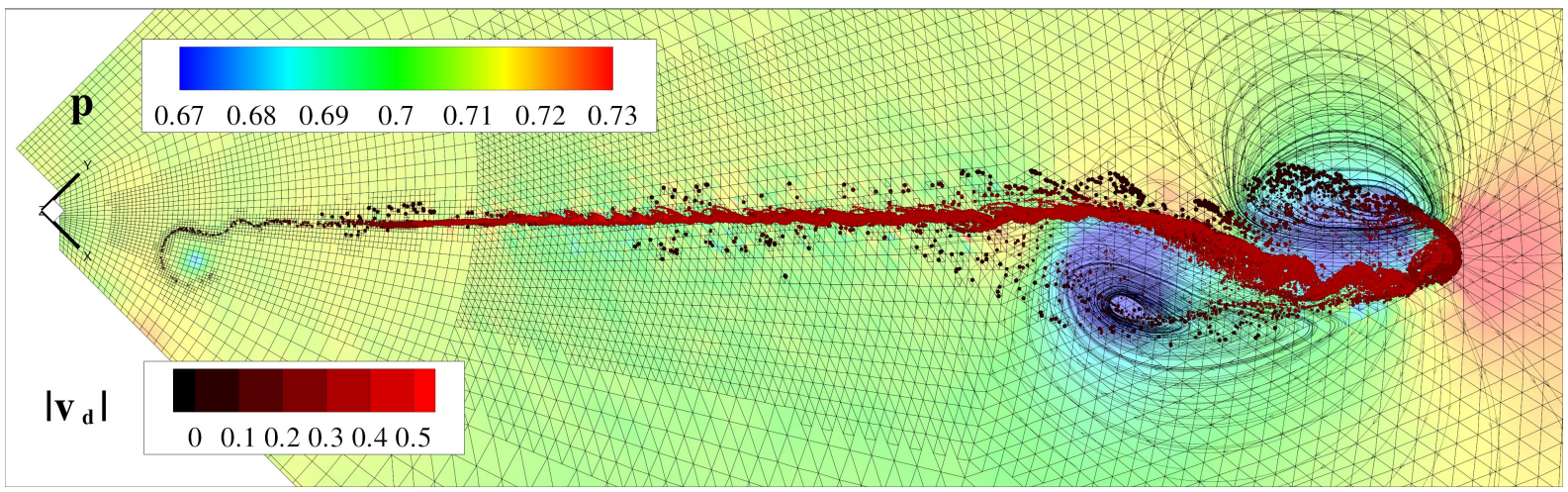

Figure 8. Droplet distribution at $t=1.10 \mathrm{msec}$. A uniform initial distribution of droplets with diameters from 0.003 to $0.009 \mathrm{~mm}$ was assumed. Size of the circles corresponds to droplet sizes and their colours correspond to the non-dimensional droplet velocities $\left|\mathbf{v}_{\mathbf{d}}\right|$ normalised by the speed of sound). Contour colour shows the distribution of non-dimensional pressure (normalised by $\rho c^{2}$ ).

\section{Conclusions}

Preliminary results of the development of a new model for the analysis of the interaction between droplets and the carrier phase are described. This model uses the implementation of the Discontinuous Galerkin method (ForestDG), based on a topological representation of the computational mesh by a hierarchical structure consisting of oct- quadand binary trees. Adaptive mesh refinement (h-refinement) used in the analysis enables us to increase the spatial resolution for the computational mesh in the vicinity of the points of interest such as interfaces, geometrical features, or flow discontinuities. The local increase in the expansion order ( $p$-refinement) at areas of high strain rates or vorticity magnitude results in an increase of the order of the accuracy of discretisation of shear layers and vortices. In our analysis, the initial domain consists of a graph of unitarian-trees representing hexahedral, prismatic and tetrahedral elements. The ancestral elements of the mesh are split into self-similar elements allowing each tree to grow branches to an arbitrary level of refinement. The connectivity of the elements, their genealogy and their 
partitioning are described by linked lists of pointers. These are attached to the tree data structure which facilitates the on-the-fly splitting, merging, and repartitioning of the computational mesh by rearranging the links of each node of the tree. This enables us to refine the computational mesh in the vicinity of the droplet parcels aiming to accurately resolve the coupling between the two phases.

The accuracy of the new code is assessed and preliminary results of the implementation of the code to the analysis of a hollow-cone spray in gasoline engine-like conditions are presented.

\section{Acknowledgements}

The authors are grateful to EPSRC (grants EP/K005758/1 and EP/M002608/1) for financial support

\section{Nomenclature}

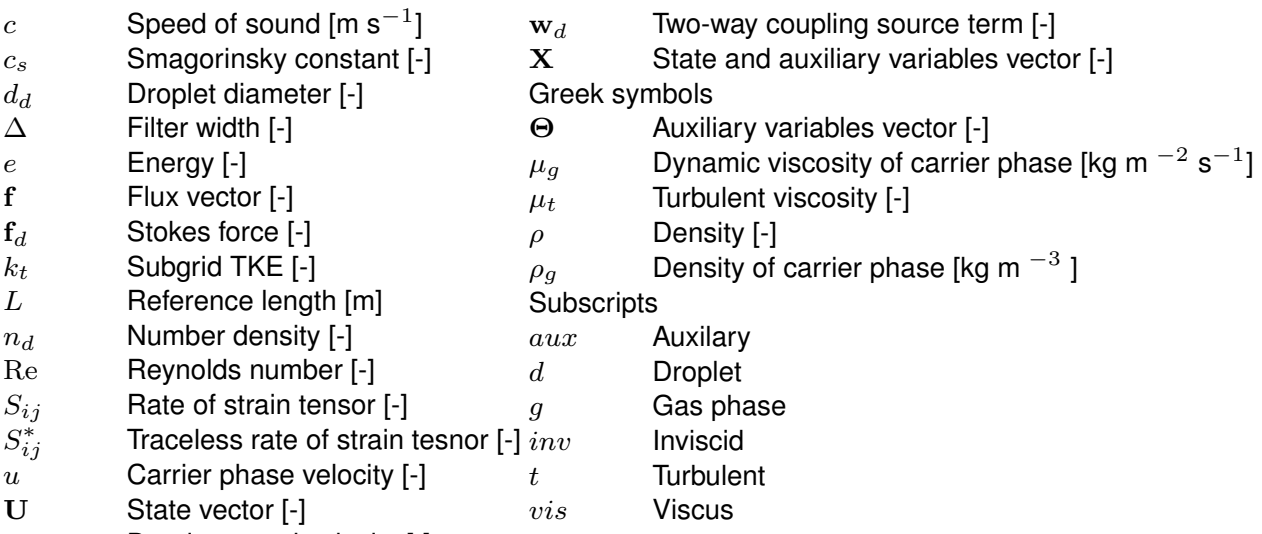

$v \quad$ Droplet parcel velocity [-]

\section{References}

[1] Sazhin, S. S. Droplets and Sprays, p. 345. Springer, London, 2014.

[2] Sazhina, E. M., Sazhin, S. S., Heikal, M.R., Babushok, V. I., and Johns, R. J. R., 2000, Combustion Science and Technology, 160(1):317-344.

[3] Rybdylova, O., Osiptsov, A. N., Sazhin, S. S., Begg, S., and Heikal, M., 2015, International Communications in Heat and Mass Transfer, 15(1):685-686.

[4] Rybdylova, O., Qubeissi, M. A., Braun, M., Crua, C., Manin, J., Pickett, L. M., de Sercey, G., Sazhina, E. M., Sazhin, S. S., and Heikal, M., 2016, International Communications in Heat and Mass Transfer, 76:265-270.

[5] Zaripov, T. S., Gilfanov, A. K., Begg, S. M., Rybdylova, O., Sazhin, S. S., and Heikal, M. R., 2017, In Press, Atomization and Sprays.

[6] Cockburn, B., Karniadakis, G. E., and Shu, C. W., 2000, Lecture Notes in Computational Science and Engineering, (11):3-50.

[7] Ekaterinaris, J.A., 2005, Progress in Aerospace Sciences, 41(3-4):192-300.

[8] Wang, Z., 2007, Progress in Aerospace Sciences, 43(3-4):1-41.

[9] Cockburn, B. and Shu, C.-W., 1998, J. Comput. Phys., 141(2):199-224.

[10] Liu, Y., Vinokur, M., and Wang, Z., 2006, J. Comput. Phys., 212(2):454-472.

[11] Liu, Y., Vinokur, M., and Wang, Z., 2006, J. Comput. Phys, 216(2):780-801.

[12] Yu, M., Wang, Z.J., and Liu, Y., 2014, J. Comput. Phys., 259(15):70-95.

[13] Cheng, J. and Shu, C. W., 2007, J. Comput. Phys., 227(2):567-1596.

[14] Jiang, G. S. and Shu, C. W., 1996, J. Comput. Phys., 126:202-228.

[15] Berger, M. J. and Colella, P., 1989, J. Comput. Phys., 82(2):64-84.

[16] Djaffar, A. Y., Wagdi, G., and Habashi, W. G., 1997, AIAA Journal, 35(5):1294-1302.

[17] Flaherty, J.E., Krivodonova, L., Remachle, J. F., and Shepard, M. S., 2002, Finite Elements in Analysis and Design, 38(10):889-908.

[18] Yelash, L., Müller, A., Lukáčová-Medvid'ová, M. Giraldo, F.X., and Wirth, V., 2014, J. Comput. Phys., 268(1).

[19] van der Vegt, J. J. W. and van der Ven, H., 1998, Journal of Computational Physics, 141:46-77.

[20] van der Vegt, J. J. W. and van der Ven, H., 2002, Journal of Computational Physics, 182:546-58.

[21] Papoutsakis, A., Panourgias, K. T., and Ekaterinaris, J. A., 2014, AlAA Paper 2014-0068, Aerospace Sciences Meeting, AIAA SciTech Forum.

[22] Anderson, J. Computational Fluid Dynamics. Computational Fluid Dynamics: The Basics with Applications. McGraw-Hill Education, 1995.

[23] Jones, W.P., Marquis, A.J., and Vogiatzaki, K., 2014, Combustion and Flame, 161(1):222 - 239.

[24] Karniadakis, G.E. and Sherwin, S. Spectral/hp Element Methods for CFD. Oxford University Press, 2nd edition, 2003.

[25] Karypis, G. and Kumar, V., 2009 MeTis: Unstructured Graph Partitioning and Sparse Matrix Ordering System, Version 4.0. http://www.cs.umn. edu/ 〜metis.

[26] Wang, Z.J., Fidkowski, K., Abgrall, R., Bassi, F., Caraeni, D., Cary, A., Deconinck, H., Hartmann, R., Hillewaert, K., Huynh, H. T., Kroll, N., May, G., Persson, P., Leer, B.van, and Visbal, M., 2013, International Journal for Numerical Methods in Fluids, 72(8):811-845. 


\title{
Mathematical models for turbulent round jets based on "ideal" and "lossy" conservation of mass and energy
}

\author{
Fermin Franco ${ }^{1,2, *}$, Yasuhide Fukumoto ${ }^{3}$ \\ ${ }^{1}$ Center for Mathematical Modeling and Data Science, Osaka University, Osaka, Japan \\ ${ }^{2}$ Graduate School of Medicine, Osaka University, Osaka, Japan \\ ${ }^{3}$ Institute of Mathematics for Industry, Kyushu University, Fukuoka, Japan \\ ${ }^{*}$ Corresponding author: franco@sigmath.es.osaka-u.ac.jp
}

\begin{abstract}
We propose mathematical models for turbulent round atomized liquid jets that describe its dynamics in a simple but comprehensive manner with the apex angle of the cone being the main disposable parameter. The basic assumptions are that (i) the jet is statistically stationary and that (ii) it can be approximated by a mixture of two fluids with the phases in local dynamic equilibrium, or so-called locally homogeneous flow (LHF). The models differ in their particular balance of explanatory capability and precision. To derive them we impose partial conservation of the initial mass and energy fluxes, introducing loss factors again as disposable parameters. Depending on each model, the equations admit explicit or implicit analytical solutions or a numerical solution in the discretized model case. The described variables are the the two-phase fluid's composite density and velocity, both as functions of the distance from the nozzle, from which the dynamic pressure is calculated.
\end{abstract}

\section{Keywords}

Mathematical Modeling, Two Phase Fluid, Locally Homogeneous Flow, Statistically Stationary State

\section{Introduction}

The range of applications involving atomizing liquid jets forming two-phase fluid flows is still large. The complexity of the atomizing process, involving numerous physical phenomena and many variables, ranging from the conditions inside the nozzle (or some generating source) to the interaction between the atomization process and the environment into which the jet is penetrating, all account for numerous challenges in physical and mathematical modeling. Notwithstanding, several such models have been attempted to describe different aspects of the jets in this regime. For example, differential equations for a fuel jet's tip penetration distance as a function of time [1, 2, 3]; models for the gas entrainment rate in a full-cone spray [4]; and a one-dimensional model for the induced air velocity in sprays [5]. None of these models is sufficient by itself as explained below.

In this study we propose three original related 1D mathematical models, so-called "energy jet models", for the macroscopic dynamics of a turbulent round jet ensuing from a circular nozzle into a stagnant fluid. This kind of jets serves as a basis for many industrial processes in modern manufacturing industry [6]. An advantage of our models over other analytical 1D models is that the simplest case of the "ideal energy jet" has a single experimentally measurable parameter (the jet half-angle $\theta$ ) while it maintains reasonable predictive power and gives theoretical understanding that allows it to analytically calculate other physical quantities of interest. Moreover, the herein reported other extended models class, the "lossy energy jet" models, apply an energy conservation approach with simple turbulence and energy dissipation models, resulting in increased accuracy. Compared to the present study, past models either lack a description of the density or liquid fraction of the spray, make unrealistic assumptions or introduce parameters unavailable experimentally. There are also widely used numerical models based on turbulence modelling for jets, like the one by [7] used in CFD programs like Star-CD, KIVA-3V, Ansys Fluent, as well as open source codes like OpenFOAM [8]. The present work is mainly concerned with developing an analytical or nearly analytical model with the advantage of producing closed expressions and/or fast calculations.

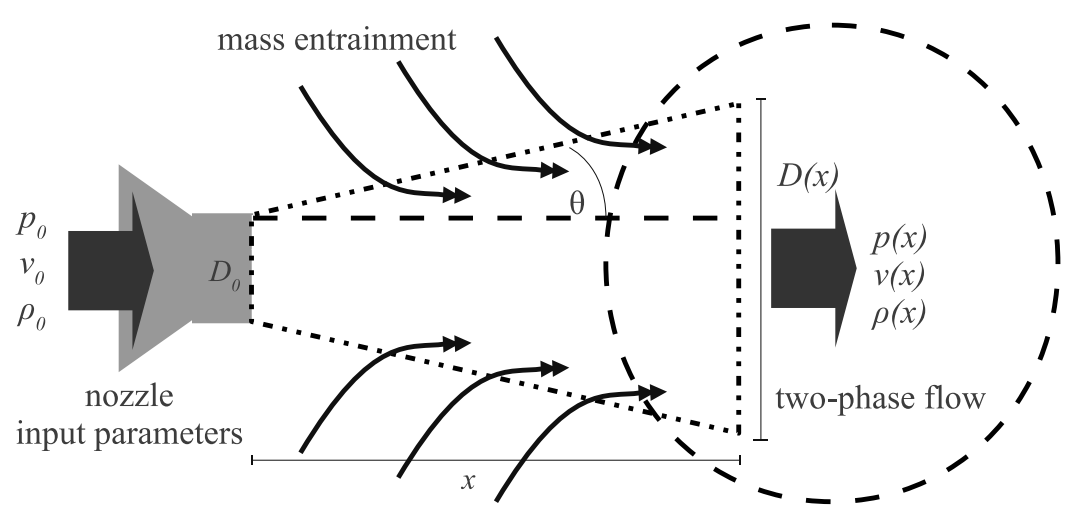

Figure 1. Diagram of the mass entrainment process in a jet and the relevant physical variables. 


\section{The ideal energy jet model}

Consider the statistically stationary state of a full-cone turbulent pressure-atomized liquid jet exiting a single-hole circular nozzle and penetrating into an ambient gas, with constant input liquid gauge pressure and a small conical jet spread angle. We then wish to calculate the dynamical properties of this jet at some axial distance from the nozzle. The relevant variables and parameters are depicted in Figure 1.

\section{Initial power}

The kinetic energy of a flat disc of liquid of infinitesimal width exiting the circular nozzle is

$$
d T=\frac{1}{2} m_{0} v_{0}^{2},
$$

where the mass of the flat disc is $m_{0}=1 / 4 \rho_{0} \pi D_{0}^{2} d z$. Note that $d z=v_{0} d t$. This velocity may be calculated from the input gauge pressure, $p_{0}$, by Bernoulli's theorem, neglecting the dynamic pressure inside the nozzle and assuming that the static pressure is totally converted to the jet's dynamic pressure just outside the nozzle. Thus $p_{0} \approx 1 / 2 \rho_{0} v_{0}^{2}$ implies

$$
v_{0}=\sqrt{\frac{2 p_{0}}{\rho_{0}}} .
$$

Substituting $m_{0}$ and $v_{0}$ from (2) into equation (1) we get

$$
\dot{T}_{0}=\left.\frac{d T}{d t}\right|_{z=0}=\frac{1}{8} \pi \rho_{0} D_{0}^{2} v_{0}^{3}=\frac{1}{2} \pi D_{0}^{2}\left(\frac{p_{0}^{3}}{2 \rho_{0}}\right)^{\frac{1}{2}},
$$

which is the total power (energy flux per unit time) coming out of the nozzle as a result of the input gauge pressure inside the nozzle.

\section{Conservation of power}

We assume that the fluid at a distance $z$ is a two-phase fluid of mixed liquid droplets and gas. The present model has been termed "ideal energy" to distinguish it from the "ideal momentum" developed by the present authors in a previous article $[9,10]$ and the "lossy" models including energy and/or mass loss parameters to be described in sections below. Accordingly, as a first approximation we assume conservation of power, i.e. the energy flux of the two-phase fluid is solely that coming from the original input pressure. The droplets transfer kinetic energy to the initially static gas by drag forces $[11,12]$ and they reach local dynamic equilibrium in such a way that both gas and liquid droplets move at the same speed $v$ inside the jet [13]; this is also the main assumption under the wide class of "Locally Homogeneous Flows" (LHF) $[14,15,16]$. We assume that the latter process occurs so fast immediately outside the nozzle's exit that we may neglect the non-equilibrium zone near the nozzle. The latter assumption is reasonable for high-Reynolds-number pressure-atomized jets, e.g. like the ones used in real life diesel engines [2]. This effectively allows us to treat the two-phase flow as a single fluid with a composite density $\rho$ and a single velocity $v$, both depending on the distance from the nozzle, $z$. Analogous to the calculations in subsection, the kinetic energy of a flat disc of fluid of infinitesimal width at an axial distance $z$ from the nozzle's exit is $d T=1 / 2 v^{2}$, where $m=1 / 4 \rho \pi D^{2} d z$ and $d z=v d t$, and thus

$$
\dot{T}(z)=\left.\frac{d T}{d t}\right|_{z}=\frac{1}{8} \pi \rho D^{2} v^{3},
$$

which is the total power at a cross-section of the two-phase fluid jet at a distance $z$ from the nozzle. Equating (3) and (4) by the idealized assumption of conservation of the energy contained between the two planes $z=0$ and at an axial distance $z$ in steady state, and solving for $v$ we get

$$
v^{3}=\frac{1}{\rho}\left(\frac{D_{0}}{D}\right)^{2} \sqrt{\frac{8 p_{0}^{3}}{\rho_{0}}} .
$$

This can be written in simpler form by using dimensionless units as $\hat{v}^{3}=\hat{\rho}^{-1} \hat{D}^{-2}$, where $v, D$ and $\rho$ have been scaled respectively by $v_{0}, D_{0}$ and $\rho_{0}$ (the quantities at the nozzle's exit) and denoted by a hat symbol.

\section{Volume of gas in the two-phase fluid}

The total fluid volume of the thin disc at the target distance is $d V=d V_{0}+d V_{g}$, where the subscript "g" denotes the quantities related to the "gas", i.e. the total volume $d V$ of the two-phase fluid spray is just the sum of the volume of original quantity of liquid coming out of the nozzle $d V_{0}$ plus the added volume of entrained gas in dynamic equilibrium $d V_{g}$. The volume of liquid is already known since it is just the original volume of liquid. Also, the total volume of the spray at the target distance is straightforward to calculate from the geometry. Then $d V_{g}=d V-d V_{0}$ from where we can calculate a volumetric flow rate of the gas entrainment:

$$
\frac{d V_{g}}{d t}=\frac{1}{4} \pi\left(D^{2} v-D_{0}^{2} v_{0}\right)
$$

Here we can substitute for $v_{0}$ from equation (2) and $D=D_{0}+2 z \tan \theta$. 


\section{Density of the two-phase fluid}

The mean density of the two-phase fluid spray thin-disc element is just the total mass over the total volume:

$$
\rho=\frac{m}{V}=\frac{d m_{0}+d m_{g}}{d V_{0}+d V_{g}}=\frac{\rho_{0} d V_{0}+\rho_{g} d V_{g}}{d V_{0}+d V_{g}}
$$

After substituting $d V_{g}$ from (6), and the initial volume element $d V_{0}$ from analogous calculations, simplifying and solving for $\rho$ we obtain

$$
\rho=\rho_{g}+\frac{D_{0}^{2} v_{0}}{D^{2} v}\left(\rho_{0}-\rho_{g}\right), \quad \text { or } \quad \hat{\rho}=\rho_{*}+\left(1-\rho_{*}\right) / \hat{D}^{2} \hat{v}
$$

the density of the two-phase fluid spray at a distance $z$ from the nozzle. The second equation in (8) is the dimensionless form, where $\rho_{*}=\rho_{g} / \rho_{0}$. Fortunately, it depends on $v$ which makes the dependency circular as we can see from equation (5).

We implicitly assume, by calculating the mean composite density of the two-phase fluid in Equation (7), that the droplets distribution throughout the disc two-phase fluid element does not differ greatly from a uniform distribution. Notice that we approximate the front of the jet by a planar front of equal density, i.e. a "top-hat" radial distribution. In reality this is not true, since the front should be spherical in the first order, and it is then in a spherical shell within the jet's cone that we should consider $\rho$ to be approximately constant, not in a plane. However, for small half-angles $\theta$ and short distances $z$ a plane should suffice as a first order approximation. The same could be said of the front's velocity $v$. Overall, we may take the above considerations as utilizing "top-hat" velocity and density distributions as a first approximation. Note that slicing the spherical jet front with a $z$-normal plane provides a non-constant $\rho$ density distribution in this plane. This distribution should, however, be similar to a two-dimensional Gaussian distribution centered around the $z$-axis, i.e. the jet's "centerline". There are some models $[5,3,17,18,13]$ which apply Gaussian velocity distributions as initial assumptions; however, this calculation will be included in a later work since we anticipate that it would not lead to a major refinement of the axial centreline quantities.

\section{Non-linear equations for the axial velocity and composite density of the spray}

From equations (5) and (8) we identify a system of two non-linear equations with two unknowns, $v$ and $\rho$. We can eliminate $\rho$ to from the system and write in dimensionless form

$$
\rho_{*} \hat{D}^{2} \hat{v}^{3}+\left(1-\rho_{*}\right) \hat{v}^{2}-1=0,
$$

which is a cubic polynomial equation in $\hat{v}$, and which can be solved numerically.

Analogously we can get a non-linear equation for $\hat{\rho}$ eliminating $\hat{v}$ from the same described system and we obtain:

$$
\left(\frac{\hat{\rho}-\rho_{*}}{1-\rho_{*}}\right)^{3}=\frac{\hat{\rho}}{\hat{D}^{4}}
$$

which we can again solve numerically. With both $\hat{\rho}$ and $\hat{v}$ calculated, the dynamic pressure of the two-phase fluid, which accounts for the total pressure at some target axial distance $z$ from the nozzle exit, may be calculated as

$$
\hat{p}=\hat{\rho} \hat{v}^{2} \text {, }
$$

where $\hat{p}=p / p_{0}$, and $p_{0}=\rho_{0} v_{0}^{2} / 2$ is the initial gauge pressure as in (2).

\section{Energy loss}

In this section we will generalize the above model and, instead of the idealized assumption of perfect conservation of power (energy flux), which is not realistic due to multiple energy loss processes detailed below, we will assume instead that only some proportion of the energy is conserved. Consider a two-phase fluid parcel travelling from the nozzle's exit outward in an axial direction. Physically, we can identify the causes of energy loss of the fluid parcel as at least the following. (i) Droplets' surface energy: taken away by the droplets' surface at jet breakup (due to surface tension). (ii) Droplets' oscillations: the internal elastic motion and deformation of the droplets' surface (which may be approximated by the normal modes of oscillation of a sphere). (iii) Droplets' rotation: produced by some net torque at detachment during breakup. (iv) Turbulence: turbulent motion and energy of created vorticity in the two-phase fluid mainly due to gas entrainment at jet breakup and the turbulent wakes behind the travelling droplets. (v) Escaping droplets: kinetic energy taken away by escaping droplets expelled from the jet's bounded conical geometry by the stochastic breakup process and turbulence (notice this also produces mass loss, although this will be addressed until next section). (vi) Thermal dissipation: some of the kinetic energy is transformed into heat due to the skin friction between the droplets and viscosity.

As stated, it is clear that there are multiple sources of energy loss, but they depend mostly on the following two factors: speed and travel time. Alternatively, travel time at certain speed can be transformed into distance.

Therefore, it is reasonable to assume that the proportion of energy loss, due to at least the above listed factors, should depend on both the speed and travel time of each fluid element (disc). Consider two contiguous fluid elements, i.e. two discs of infinitesimal width, then the energy of the second disc, $d T_{2}$, should depend on the energy of the previous disc as $d T_{2}=d T_{1} d \mathcal{L}_{e}\left(v_{1}, \Delta z\right)$, where $0 \leq \mathcal{L}_{e} \leq 1$ is the proportion of energy that is conserved after travelling a distance $\Delta z$ at a speed $v_{1}$, where in turn $v_{1}$ is the velocity of the first disc. Taking the above into consideration, the overall picture is that energy loss should be greater for greater velocities and travel distances, for each fluid disc element. 


\section{Damping with constant velocity}

The simplest model which reproduces the above qualitative behaviour is

$$
d \mathcal{L}_{e}(v, \Delta z)=\frac{1}{1+v \Delta z / H_{e}}=\frac{1}{1+\hat{v} \Delta \hat{z} / \hat{H}_{e}},
$$

where $H_{e}$ is some characteristic "energy half-loss" parameter at which half of the energy is lost for the given system, and $\hat{H}_{e}=H_{e} / v_{0} D_{0}=H_{e} / \nu_{0} \operatorname{Re}_{0}$, where $\nu_{0}$ and $\operatorname{Re}_{0}$ are the kinematic viscosity and Reynolds number of the liquid at the nozzle's exit, respectively. We can think of $H_{e}$ as the necessary distance traveled at a certain fixed speed for the fluid element to lose half its energy. Notice that $H_{e}$ has viscosity units, so we may consider it to be an energy dissipation parameter, similar to a turbulent viscosity. In this sense, the model hereof derived using (12) may be considered to include a simple turbulence model. Notice that, the greater the velocity $v$, the greater the energy loss; this is what we call "damping". The factor $d \mathcal{L}_{e}$ must be considered over the disc's whole trajectory to obtain the total loss. Consider a total flight distance $z$ from the nozzle exit until the target distance. Then there will be $z / \Delta z \equiv n$ total distance intervals and their corresponding time intervals, $\Delta t$, along the jet's trajectory. If the proportion of energy preserved after one such interval is $d \mathcal{L}_{e}$, then $\left(d \mathcal{L}_{e}\right)^{2}$ is the proportion of energy preserved after two such intervals, considering a constant speed $v_{0}$. Then the proportion of the initial energy preserved after travelling an axial distance $z$ from the nozzle exit is

$$
d \mathcal{L}_{e}^{n}=\left(\frac{1}{1+H_{e}^{-1} v_{0} z / n}\right)^{n}
$$

Proceeding to the infinitesimal limit when the disc width $\Delta z \rightarrow 0$, equivalent to $n \rightarrow \infty$, we get

$$
\lim _{n \rightarrow \infty} d \mathcal{L}_{e}^{n}=\lim _{n \rightarrow \infty} \frac{1}{\left(1+H_{e}^{-1} \frac{v_{0} z}{n}\right)^{n}}=\exp \left(-v_{0} z / H_{e}\right):=\mathcal{L}_{e}\left(v_{0}, z\right) .
$$

Using this function $\mathcal{L}_{e}(z)$ as a proportion factor we can rewrite our theory introducing energy loss as a function of axial distance from the nozzle exit. In dimensionless units, $\mathcal{L}_{e}(\hat{z})=\exp \left(-\hat{z} / \hat{H}_{e}\right)$. With this modification, (9) turns into

$$
\rho_{*} \hat{D}^{2} \hat{v}^{3}+\left(1-\rho_{*}\right) \hat{v}^{2}-\exp \left(-\hat{z} / \hat{H}_{e}\right)=0,
$$

and (10) into

$$
\left(\frac{\hat{\rho}-\rho_{*}}{1-\rho_{*}}\right)^{3}=\frac{\hat{\rho}}{\hat{D}^{4}} \exp \left(\hat{z} / \hat{H}_{e}\right) .
$$

We must remark that (13), (14), (15) and (16) hold approximately only for nearly constant axial speed along the jet's axis.

\section{Damping with varying velocity}

The assumption of constant axial speed proved to be fairly accurate when no energy loss factor like (12) was included. However, with the inclusion of energy loss, the velocity along the trajectory starts to considerably decrease with increasing energy loss, i.e. decreasing parameter $H_{e}$. This forces us to further rewrite the above equations into some recurrence formulas by which we can update the velocity value along the trajectory of the fluid element. This can be done by adapting (5) to depend on the immediately previous value. As for the density equation, it may be kept unmodified because the amount of liquid is calculated from the original state without gas entrainment and geometric considerations. Following a procedure analogous as in previous calculations, we obtain the velocity recurrence equation

$$
\rho_{g} v^{3}+\left(\frac{D_{0}}{D}\right)^{2} v_{0}\left(\rho_{0}-\rho_{g}\right) v^{2}-v_{i-1}^{3}\left(\frac{D_{i-1}}{D}\right)^{2} \frac{\rho_{i-1}}{1+v_{i-1} \Delta z H_{e}^{-1}}=0
$$

Notice that (17) reduces to (9) if $i=1$ and $H_{e} \rightarrow \infty$. For completeness, the equivalent of the non-linear equation for the density (10) as a recurrence equation is

$$
\left(\frac{\rho-\rho_{g}}{\rho_{0}-\rho_{g}}\right)^{3}=\frac{\rho v_{0}^{3} D_{0}^{6}\left(1+v_{i-1} \Delta z \gamma^{-1}\right)}{\rho_{i-1} v_{i-1}^{3} D_{i-1}^{2} D^{4}} .
$$

Notice that (18) also reduces to (10) if $i=1, v_{1}=v$ and $H_{e} \rightarrow \infty$.

\section{Mass loss}

We further generalize the above model and assume that only some proportion of the mass is conserved. We can identify the causes of mass loss as at least the following. (i) Escaping droplets: some droplets are expelled from the jet due to the breakup and atomization processes, taking with them mass. (ii) Evaporation: if the surrounding gas is not saturated in liquid vapour, evaporation will take place and, if this vapour escapes it will reduce the mass of the moving jet, albeit slowly. Both these causes also produce energy loss. The mass loss factors are affected by velocity and travel time/distance in the following ways. (i) Escaping droplets: droplets escape rate should be 
dependent on vorticity present and also on instability growth rates which produce jet breakup; both of these factors increase with velocity. (ii) Evaporation: may occur both from the jet's continuous part (if any) and from the droplets. Evaporation turns liquid into vapour which is taken away by the surrounding gas and eventually may escape the jet. For constant temperature and gas humidity, evaporation is "advection-driven" and depends also on the velocity. It is only natural then that the proportion of mass loss, due to at least the above listed factors, should depend on both the velocity and travel time/distance of each fluid element (disc).

\section{Mass loss with constant velocity}

Using an analogous loss factor $\mathcal{L}_{m}(z)$ we can rewrite our theory introducing mass loss. With this modification the velocity equation turns into

$$
\rho_{*} \hat{v}^{3}+\hat{D}^{-2}\left[\left(1-\rho_{*}\right) \exp \left(-\hat{z} / \hat{H}_{m}\right) \hat{v}^{2}-\exp \left(-\hat{z} / \hat{H}_{e}\right)\right]=0 .
$$

Notice that this reduces to the no mass loss case when $H_{m} \rightarrow \infty$. And the density equation turns into

$$
\left(\frac{\hat{\rho}-\rho_{*}}{1-\rho_{*}}\right)^{3}=\hat{\rho} \hat{D}^{-4} \exp \left[\hat{x}\left(\frac{1}{\hat{H}_{e}}-\frac{3}{\hat{H}_{m}}\right)\right] \text {. }
$$

Notice that this also reduces to the no mass loss case when $H_{m} \rightarrow \infty$. We must remark again that this calculation holds only for constant or nearly constant velocity along the jet flight.

\section{Mass loss with varying velocity}

As stated before, the assumption of constant velocity proved to be fairly accurate when no energy loss nor mass loss factors were included. However, with the inclusion of loss factors, the velocity along the trajectory starts to vary depending on the combination of the parameters, $H_{e}$ and $H_{m}$. This forces us to further rewrite the above equations into some recurrence formulas by which we can update the value of the velocity along the trajectory of the fluid element. This is done by adapting the equations to depend on an immediately previous value. Following a procedure analogous as in previous calculations, we obtain the velocity recurrence equation

$$
\rho_{*} \hat{v}_{i}^{3}+\left(\frac{\hat{D}_{i-1}}{\hat{D}_{i}}\right)^{2} \hat{v}_{i-1}\left(\frac{\hat{\rho}_{i-1}}{1+\hat{v}_{i-1} \Delta \hat{z} \hat{H}_{m}^{-1}}-\rho_{*}\right) \hat{v}_{i}^{2}-\hat{v}_{i-1}^{3}\left(\frac{\hat{D}_{i-1}}{\hat{D}_{i}}\right)^{2} \frac{\hat{\rho}_{i-1}}{1+\hat{v}_{i-1} \Delta \hat{z} \hat{H}_{e}^{-1}}=0 .
$$

Notice that (21) also reduces to (9) without loss factors if $H_{m}, H_{e} \rightarrow \infty$ and $i=1$. As for the density, we find that

$$
\left(\frac{\hat{\rho}_{i}-\rho_{*}}{\frac{\hat{\rho}_{i-1}}{1+\hat{v}_{i-1} \Delta \hat{z} \hat{H}_{m}^{-1}}-\rho_{*}}\right)^{3}=\quad\left(\frac{\hat{D}_{i-1}}{\hat{D}_{i}}\right)^{4} \frac{\hat{\rho}_{i}\left(1+\hat{v}_{i-1} \Delta \hat{z} \hat{H}_{e}^{-1}\right)}{\hat{\rho}_{i-1}} .
$$

Notice that (22) also reduces to (10) without any loss if $i=1$ and $H_{m}, H_{e} \rightarrow \infty$.

\section{Liquid-only mass loss}

In the previous modeling of the mass loss factor we implicitly considered mass loss to happen from both phases of the jet, i.e. losing mass from both liquid and gas. The latter is not realistic, since we expect that the mass-loss mechanisms affect only the liquid phase, as was described in the enlisted physical mechanisms above. An ambient gas does not lose any mass as it simply occupies any space not occupied by the liquid. Therefore, we may restrict the mass-loss to happen only at the liquid phase to get a different set of equations. First notice that the volume can be calculated as

$$
V_{j}^{g}=V_{j}-V_{j}^{l}=V_{j}-V_{j-1}^{l} d \mathcal{L}_{m}\left(v_{j-1}, \Delta z\right)
$$

where the superscripts "g" and "l" refer to the quantities for gas and liquid, respectively; and, if no superscript is written, the quantity refers to the composite two-phase fluid. Notice that $V_{j}^{l}$ cannot be calculated as the volume of a cylinder as with $V_{0}^{l}$ or $V_{j}$, since the shape of the distributed droplets inside the jet is irregular. However, we may calculate $V_{j}^{l}$ recursively, since we always know the total volume, $V_{j}=1 / 4 \pi D^{2} \Delta z$, we also know the initial liquid volume $V_{0}^{l}=1 / 4 \pi D_{0}^{2} \Delta z$ and the relationship $V_{j}^{l}=V_{j-1}^{l} d \mathcal{L}_{m}$. Now, separating mass loss to affect only the liquid phase we may calculate again the mean composite density as:

$$
\rho_{j}=\frac{\rho_{0} V_{j}^{l}+\rho_{g} V_{j}^{g}}{V_{j}},
$$

where the subscript " $j$ " indicates the " $j$-th step" position. We may write after simplification

$$
\rho_{j}=\rho_{g}+\frac{V_{j}^{l}}{V_{j}}\left(\rho_{0}-\rho_{g}\right)
$$

Notice that $\rho_{j}$ can be calculated directly from this formula using the previously calculated values of $V_{j}$ and $V_{j}^{l}$. 
As in the case when we introduced the energy loss with varying velocity, we should have the same equation for the velocity:

$$
v_{j}^{3}=\left(\frac{D_{j-1}}{D_{j}}\right)^{2} \frac{\rho_{j-1}}{\rho_{j}} v_{j-1}^{3} d \mathcal{L}_{e} .
$$

Eliminating $\rho_{j}$ from (24) and (25):

$$
v_{j}=\left(\frac{D_{j-1}}{D_{j}}\right)^{2 / 3}\left(\rho_{j-1} d \mathcal{L}_{e}\right)^{1 / 3} \rho_{j} v_{j-1}
$$

From (26) we can calculate $v_{j}$ directly having previously calculated $\rho_{j-1}$ and $\rho_{j}$. Notice that the velocity recurrence relation (26) can be written as

$$
v_{j}^{2}=\frac{\frac{1}{4} \pi D_{j-1}^{2} v_{j-1} d t \rho_{j-1}}{\frac{1}{4} \pi D_{j}^{2} v_{j} d t \rho_{j}} v_{j-1}^{2} d \mathcal{L}_{e}=\frac{V_{j-1} \rho_{j-1}}{V_{j} \rho_{j}} v_{j-1}^{2} d \mathcal{L}_{e}
$$

and then, rearranging terms,

$$
\frac{1}{2} V_{j} \rho_{j} v_{j}^{2}=\frac{1}{2} V_{v-1} \rho_{j-1} v_{j-1}^{2} d \mathcal{L}_{e}
$$

i.e. $K_{j}=K_{j-1} d \mathcal{L}_{e}$, which is just the kinetic energy conservation equation times the energy-loss factor. Both equations reduce to the original ones when $\Delta z \rightarrow 0$.

\section{Limiting and special cases}

Let us now calculate some special or limiting cases of the previous mathematical model. It is worth remembering for the following analysis that $\hat{D}=1+2 \hat{z} \tan \theta$ is the diameter of the jet.

An atomizing jet in a very thin atmosphere, $\rho_{g} \ll \rho_{0}$

With this assumption, which physically means a liquid jet inside a very thin atmosphere, from Equation (9) we see, after simplification, that

$$
v=\sqrt{\frac{2 p_{0}}{\rho_{0}}} \equiv v_{0}
$$

i.e. the velocity remains constant at the same initial value, which is consistent with the physical interpretation since the liquid droplets would not loose any energy to the surrounding vacuum since there would be no drag forces present. Now from Equation (10):

$$
\rho=\rho_{0}\left(\frac{D_{0}}{D_{0}+2 z \tan \theta}\right)^{2}
$$

where we can see that when $z \rightarrow 0$ then $\rho \rightarrow \rho_{0}$, accordingly since at the nozzle we have only liquid. As $z \rightarrow+\infty$ then $\rho \rightarrow 0$, the density of vacuum. Since in this special case both $v$ and $\rho$ are given in closed form from Equations (29) and (30), respectively, we can also calculate a closed form expression for the pressure from (11) as

$$
p=p_{0}\left(\frac{D_{0}}{D_{0}+2 z \tan \theta}\right)^{2} .
$$

The square factor in (31) is just the ratio of the diameters at the nozzle, $D_{0}$, and at the impact zone, $D=D_{0}+$ $2 z \tan \theta$, from where we can see that at the nozzle $p=p_{0}$ and $p \rightarrow 0$ as $z \rightarrow+\infty$.

The submerged jet, $\rho_{g}=\rho_{0}$

From (10) we have that $\rho=\rho_{g} \equiv \rho_{0}$, as expected so the density is just that of liquid for all distances. From (9) we get

$$
v=\left(\frac{D_{0}}{D_{0}+2 z \tan \theta}\right)^{2 / 3} v_{0}
$$

which still gives $v=v_{0}$ for $z=0$, i.e. at the nozzle exit, and $v \rightarrow 0$ as $z \rightarrow+\infty$, intuitively correct. Notice that in this case density remains constant and velocity decays with distance whilst for the case in vacuum we had the converse, viz. velocity remained constant and density decayed. While equation (32) appears to be inconsistent with the classical result for the velocity decay of a submerged jet, $z^{-1}$, e.g. [20, p. 150], the introduction of the energy loss factor as in (12) provides for adjusting of the decay to a faster rate. A similar approach as used in this paper by the same authors but imposing momentum conservation gave the well known result [9]. 


\title{
Comparison with experiments and discussion
}

Figure 2 shows the centreline velocity fits to experimental data for a single-phase air jet as reported in [9]. Each of the three curves represents the theoretical results of a different model. Both the continuous line and the dashed line belong to the ideal momentum jet model family reported in the aforementioned work. The dot-dashed line is given by the discrete model with liquid-only mass loss, for the particular case of the "submerged jet" (air jet) as considered in the corresponding experiment. We can thus see that this discrete model gives the closes fit among these models, increasing the precision, at the cost of losing the analytical solutions.

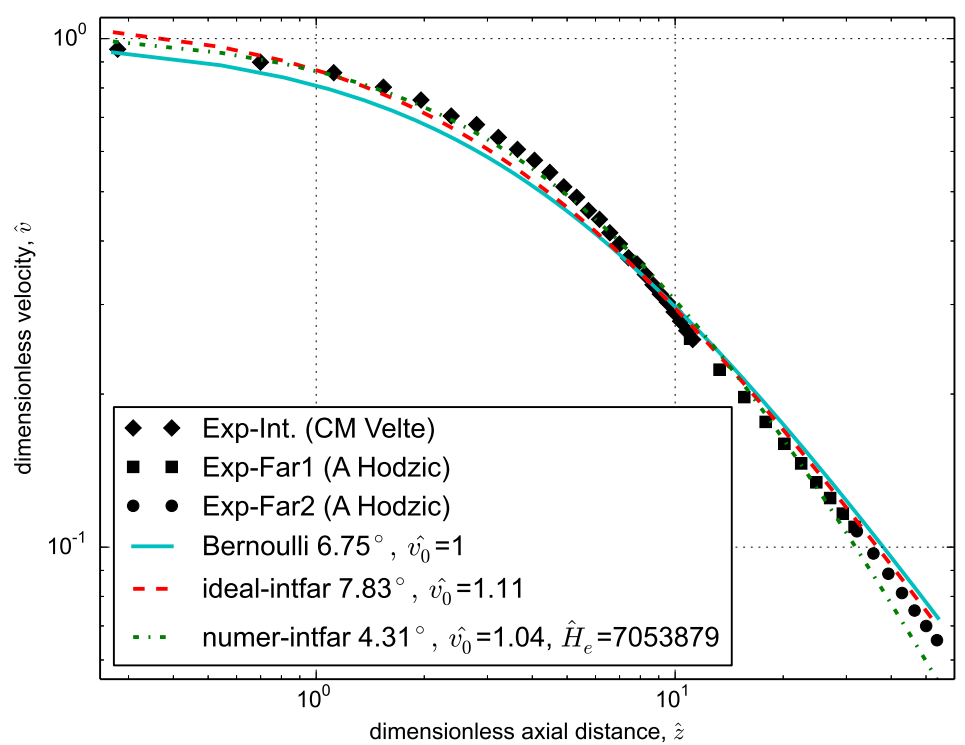

Figure 2. Centerline velocity fits to experimental data for a single-phase air jet as reported in [9].

\section{Conclusions}

We have presented three related one-dimensional mathematical models applicable to the dynamics of a wide class of turbulent atomizing jets. The models' main assumptions include the so-called Locally Homogeneous Flow (LHF) for a two-phase flow. The models are based on conservation laws of mass and energy, and describe the dynamical quantities, viz. density, velocity and dynamic pressure, along the jet's axis. The main advantages of the models over others in the literature are that the solutions can range from implicit to explicit analytical, that they contain a single main free parameter, viz. the jet's angle, and the fact that this angle can be approximated from experimental measurements with a method proposed in Reference [9]. We consider as special cases an atomizing liquid jet in a very thin atmosphere and a submerged jet. For the submerged jet, we carried out our own experiments with turbulent air jets, reported in [9], showing excellent agreement with the centerline velocity decay in the intermediate and far fields.

\section{Acknowledgements}

FFM was in part supported by the Mexican Council of Science and Technology (CONACYT), the Bank of Mexico (BANXICO)'s FIDERH program and KUMIAY Internacional, Co. Ltd.

\author{
Nomenclature \\ $z \quad$ Axial distance from the nozzle's exit \\ $D \quad$ Diameter of the jet \\ $\rho \quad$ Density \\ $m \quad$ mass \\ $v \quad$ Velocity \\ $p \quad$ Pressure \\ $\rho \quad$ Density \\ $\theta \quad$ Jet's cone apex angle \\ $H_{e} \quad$ Energy half loss parameter \\ $H_{m} \quad$ Mass half loss parameter \\ Subscripts and superscripts \\ $e \quad$ Entrainment or energy \\ 0 Quantity at the nozzle's exit position
}


$l \quad$ Liquid

$g \quad$ Gas

Dimensionless quantity

[1] Wakuri, Y., Fujii, M., Amitani, T., and Tsuneya, R., 1960. "Studies on the penetration of fuel spray in a diesel engine". Bull. Japan Soc. Mech. Eng., 3(900475), pp. 123-130. 1

[2] Sazhin, S. S., Feng, G., and Heikal, M. R., 2001. "A model for fuel spray penetration". Fuel, 80, pp. 2171-2180. 1,2

[3] Desantes, J. M., Payri, R., Salvador, F. J., and Gil, A., 2005. "Development and validation of a theoretical model for diesel spray penetration". Fuel, 85(2006), pp. 910-917. 1, 3

[4] Cossali, G. E., 2001. "An integral model for gas entrainment into full cone sprays". J. Fluid Mech., 439, pp. 353-366. 1

[5] Ghosh, S., and Hunt, J. C. R., 1994. "Induced air velocity within droplet driven sprays". Proc. R. Soc. London, Ser. A, 444(1920), Jan., pp. 105-127. 1, 3

[6] Jiang, X., Siamas, G. A., Jagus, K., and Karayiannis, T. G., 2010. "Physical modelling and advanced simulations of gas-liquid two-phase jet flows in atomization and sprays". Prog. Energy Combust. Sci., 36, pp. 131-167. 1

[7] Vallet, A., Burluka, A. A., and Borghi, R., 2001. "Development of an Eulerian model for the atomization of a liquid jet". Atomization and Sprays, 11(6). 1

[8] Stevenin, C., Vallet, A., Tomas, S., Amielh, M., and Anselmet, F., 2016. "Eulerian atomization modeling of a pressure-atomized spray for sprinkler irrigation”. Int. J. Heat and Fluid Flow, 57, pp. 142-149. 1

[9] Franco Medrano, F., Fukumoto, Y., Velte, C. M., and Hodzic, A., 2017. "Mass entrainment rate of an ideal momentum turbulent round jet". J. Phys. Soc. Japan, 86(034401), mar. 2, 6, 7

[10] Franco Medrano, F., 2016. "Dynamics of a full-cone atomizing liquid jet". PhD thesis, Graduate School of Mathematics, Fukuoka, Japan, jun. 2

[11] Sazhin, S., 2006. "Multiple scales in spray modelling". J. Phys. Conf., 55, pp. 191-202. 2

[12] Fuchimoto, T., Yanase, S., Mizushima, J., and Senda, J., 2009. "Dynamics of vortex rings in the spray from a swirl injector". Fluid Dyn. Res., 41(4), May, p. 045503. 2

[13] Desantes, J. M., Salvador, F. J., López, J. J., and De La Morena, J., 2011. "Study of mass and momentum transfer in diesel sprays based on X-ray mass distribution measurements and on a theoretical derivation". Exp. Fluids, 2011(50), pp. 233-246. 2, 3

[14] Faeth, G. M., 1983. "Evaporation and Combustion of Sprays". Prog. Energy Combust. Sci., 9, pp. 1-76. 2

[15] Faeth, G. M., 1987. "Mixing, Transport and Combustion in Sprays". Prog. Energy Combust. Sci., 13, pp. 293345. 2

[16] Faeth, G. M., Hsiang, L.-P., and Wu, P.-K., 1995. "Structure and Breakup Properties of Sprays". Int. J. Multiphase Flows, 21, pp. 99-127. 2

[17] Rabadi, S. A., Friedel, L., and Surma, R., 2007. "Prediction of Droplet Velocities and Rain out in Horizontal Isothermal Free Jet Flows of Air and Viscous Liquid in Stagnant Ambient Air". Chem. Eng. Tech., 30(11), pp. 1546-1563. 3

[18] Pastor, J. V., López, J. J., García, J. M., and Pastor, J. M., 2008. "A 1D model for the description of mixingcontrolled inert diesel sprays". Fuel, 87(13), pp. 2871-2885. 3

[19] Drazin, P. G., and Reid, W. H., 2004. Hydrodynamic Stability, 2nd ed. Cambridge University Press.

[20] Landau, L. D., and Lifshitz, E. M., 1987. Fluid Mechanics, 2nd ed., Vol. 6 of Course of Theoretical Physics. Butterworth Heinemann. 6

[21] Vekstein, G. E., 1998. "Landau resonance mechanism for plasma and wind-generated water waves". Am. J. Physics, 66, p. 886. 


\title{
Maximum entropy method for biodiesel spray droplet distribution
}

\author{
Ruslana Kolodnytska*, Sergii Skurativskyi, Pavel Moskvin \\ Zhytomyr State Technological University, Zhytomyr, 10005, Ukraine \\ *Corresponding author: ruslanakolod@gmail.com
}

\begin{abstract}
The efficiency of combustion process in diesel engine depends on the spray characteristics. The most important of them are droplet size and velocity distributions. There are four methods which are used for describing the droplet size distributions: empirical, maximum entropy formalism (MEF), discrete probability function (DPF) and stochastic method. The MEF assumes that spray formation is a random process that can be described using the principle of maximum entropy. DPF method is a combination of random and non-random processes when the drop-size distribution appears from fluctuations in the initial conditions. Under the DPF approach the spray formation is divided into following steps: liquid breakup, ligaments separation, breakup of ligaments into fragments, fragment breakup into droplets. The stochastic breakup model assumes that the probability of formation of daughter droplet breakup size is independent of its parent size (a fractal scaling of breakup has been identified). This paper presents an investigation into the application of MEF model for distribution of biodiesel droplets. We used the model approach with the constraints: normalization, mass conservation, momentum conservation and surface energy conservation. The resulting probability density function (PDF) for velocity and droplet size is obtained by maximizing the Shannon entropy. We also used the new numerical algorithm to improve the model accuracy. The PDF for droplets diameters with different Weber numbers were calculated for both diesel and biodiesel fuels. The MEF predictions were compared against the experimental data for diesel and biodiesel droplet distribution with different injection pressure. According to the maximum entropy method, the influence of fuel thermodynamic properties on the parameters of drop-size and velocity distribution function for fuel sprays has been analysed.
\end{abstract}

\section{Keywords}

Maximum entropy method, distribution function, spray, biofuel, biodiesel.

\section{Introduction}

The efficiency of combustion process in diesel engine depends on the spray characteristics. The most important of them are droplet size and velocity distributions [1]. There are four methods which are used for describing the droplet size distributions: maximum entropy formalism (MEF), discrete probability function (DPF), stochastic and empirical methods [2]. The MEF assumes that spray formation is a random process that can be described using the principle of maximum entropy [3,4]. This method is useful for processes where the secondary atomization were dominated. DPF method is a combination of random and non-random processes when "the drop size distribution arises from fluctuations in the initial conditions" [2]. Under the DPF approach the spray formation is divided into following steps: liquid breakup, ligaments separation, breakup of ligaments into fragments, fragment breakup into droplets [2]. Sovani et al. [3] showed that the fluid physical properties (density, viscosity and surface tension) have a negligible effect on the droplet distribution in contrast to the velocity fluctuations which has a bigger impact [2]. The stochastic breakup model assumes that the size of daughter droplets formed by the breakup of its parents is stochastically independent of the parent droplets size. Using this approach fractal scaling of breakup has been observed by several authors [5].

At the same time, the thermodynamic description of the fuel sprays can be achieved on the basis of more simple models for spray formations, so-called equilibrium models. The advantage of equilibrium counterparts for spray modelling is the possibility to reduce the number of models' parameters in comparison with the kinetic models. Moreover, the smaller number of governing parameters simplifies the interpretation of the results and identification of main thermodynamic parameters determining the final state of sprays.Among the equilibrium models describing the spray formation it is worth to distinguish the models derived within the framework of maximum entropy method. The natural constraints supporting the existence of local maximum for the entropy of droplet system are the conservation laws for mass, momentum, and energy. As a result, the application of maximum entropy method gives us the drop-size and velocity distribution which obeys the conservation laws and provides the maximum value of system's entropy.

The classical methods to predict the drop-size and velocity distribution are empirical ones that include log-normal, Rosin-Rammler, Nukiyama-Tanasawa distributions, etc. [4]. Nonetheless, some limitations in using these models have to be considered even though attempts have been made by adding more parameters [6]. We have been 
trying to use Rosin-Rammler approximation as well as others mentioned, to fit the experimental data $[7,8]$ for the biodiesel droplet diameter distribution but we have not got any good satisfying results. That is why we have decided to switch to using the MEF method following the assumption stated in [6]: "...former studies indicate that the MEF shows good prediction ability“.

Biofuels have been developed in order to reduce the dependence on crude oil as an alternative to the diesel fuel. The most prominent among fuels that derived from biological sources is biodiesel [9]. But the major problem of using biodiesel is their higher viscosity which leads to "larger drops when atomized and hence lower combustion performance" [10]. To create new type of biodiesel (for example, hemp biodiesel [11, 12]) we need to know not only the value of mean droplet diameters but also the spray droplet distribution.

This paper presents an investigation of the application of MEF model for distribution of diesel, biofuels (vegetable oil and diesel blends) and biodiesel droplets. We used the model from [4] with the following constraints: normalization, mass conservation, momentum conservation and surface energy conservation. Supposing that these processes are essentially characterized with the drop-size and velocity distribution of droplets, we are going to analyse the influence of thermodynamic parameters of fuel sprays on the sizes and velocities of droplets in the sprays.

\section{Material and methods}

\subsection{Model of maximum entropy}

According to the maximum entropy method, the process of spray formation is considered as the transition from one equilibrium state to another. To identify the initial state of the system, let us choose the state of liquid on the exit of injector, whereas the final equilibrium state corresponds to the state when the whole accessible volume is filled with the droplets of the spray.

Thus, within the chosen approach and using the drop-size and velocity probability distribution function $f(\bar{D}, \bar{u})$ for droplets, the equations for conservation of mass (1), momentum (2), and energy (3) can be written in the following dimensionless form [1, 4]:

$$
\begin{aligned}
& \iint f \bar{D}^{3} d \bar{u} d \bar{D}=1+\bar{G}_{m}, \\
& \iint f \bar{D}^{3} \bar{u} d \bar{u} d \bar{D}=1+\bar{G}_{m}, \\
& \iint f\left(\bar{D}^{3} \bar{u}^{2}+B \bar{D}^{2}\right) d \bar{u} d \bar{D}=1+\bar{G}_{e}, \\
& \iint f d \bar{u} d \bar{D}=1,
\end{aligned}
$$

where $W e=\frac{\rho U_{0}^{2} \cdot D_{30}}{\sigma}$ is the Weber number, $D_{30}$ is mass mean diameter of droplets,

$$
B=\frac{12}{W e}, \bar{G}_{m}=\frac{G_{m}}{\dot{m}_{o}}, \bar{G}_{m v}=\frac{G_{m v}}{\dot{J}_{0}}, \bar{G}_{e}=\frac{G_{e}}{\dot{E}_{o}}, \bar{D}=D / D_{30}, \dot{E}_{o}=\dot{m}_{o} U_{0}^{2}, \bar{V}=\frac{V}{V_{m}}, \bar{u}=\frac{u}{U_{0}}, V_{m}=\frac{\dot{m}_{O}}{\rho \dot{n}} \text {. }
$$

Note that the function $f(\bar{D}, \bar{u})$ is the probability of finding a droplet with the volume $V$ and velocity $u$ and it satisfies the normalization condition (4). Equation (3) shows that the droplet of spherical form possesses the kinetic and surface energies. The latter is proportional to the area of droplet surface and surface tension.

According to the classic dependence between entropy $S$ of the system and the probability $t$ of microstates $[1,4]$, the following relation holds:

$$
S=-k_{B} \cdot \iint f \cdot \ln (f) d \bar{V} d \bar{u}
$$


We thus arrive to the mathematical problem of finding the maximal value of the entropy $S$ for droplet system when the auxiliary constraints (1) - (4) are fulfilled. This problem is solved by the Lagrangian multiplier method, application of which gives the following form for the probability density function $t$ :

$$
\left.f(\bar{D}, \bar{u})=3 \bar{D}^{2} \exp -\alpha_{0}-\alpha_{1} \cdot \bar{D}^{3}-\alpha_{2} \cdot \bar{D}^{3} \cdot \bar{u}-\alpha_{3}\left(\bar{D}^{3} \cdot \bar{u}^{2}+B \cdot \bar{D}^{2}\right)\right\rfloor
$$

Therefore, our studies are reduced to the analysis of equations (1) - (6), while the input data for them are the sets of thermodynamic parameters of different fuels and injector operating conditions.

\subsection{Thermodynamic parameters of spray systems}

Let us first to do some analysis of the equations above and choose the thermodynamic parameters of fuels we are interested in. We also neglect the reducing of liquid mass due to evaporation. In this case it is possible to assume that in equation (1) the quantity $\bar{G}_{m}$ vanishes: $\bar{G}_{m}=0$. Similar situation concerning the additional momentum and energy sources in a spray is observed.

Note also that the momentum and energy sources in equations (2) and (3) are caused by the effects of interaction between phases. In this paper we take into account the processes of transferring between liquid and gas, for instance, the frictional effects. From equations (1) - (3) also it follows that the mechanism of liquid atomization is not very important, whereas the energy and momentum losses play a crucial role.

The arguments presented above allow us to develop an informative and efficient approach by reducing the number of governing parameters in the problem. The system (1) - (4) was analysed with different values of parameters $\bar{G}_{m}$ and $\bar{G}_{e}$. These parameters can be regarded as the adjustable parameters derived during the matching the theoretical and experimental data. It is also safe to assume that $\bar{G}_{m N}=\bar{G}_{e}$ in specific calculations. Hence, system (1) - (4) possesses only one parameter, i.e. the Weber number defining the type of system's solution and, consequently, the type of distribution.

We also used the new numerical algorithm to improve the model accuracy. Efficiency of our mathematical and numerical models was checked by means of comparison with the results presented in [4].These results are concerned with the process of water spray formation when the Weber number is fixed at $W e=311$. For clarity, both our calculations and experimental data from [4] are given in Fig. 1. Next we are going to study the profiles of probability density function derived at different values of parameters $W e, \bar{G}_{m}$ and $\bar{G}_{e}$.

\section{Results and discussion}

\subsection{PDF for spray with different We numbers}

The main results of the spray modelling are presented in Figures 1-3. In particular, the profiles for probability density function of normalized diameter of droplets are plotted in Fig. 1, when the Weber number is varied. During these calculations, the energy and momentum losses due to the friction are fixed as follows: $\bar{G}_{e}=0$ and $\bar{G}_{m N}=-0.017$, respectively.

As follows from Fig. 1, the process of fuel spray formation, when the Weber number increases from 100 to 1000 , causes the displacement of profiles for the probability density function. The direction of this deviation corresponds to the conditions of the formation of larger droplets.

We also found some specifics in the drop-size distributions at We $>1000$. It turned out in Fig. 1 that when increasing the Weber number of more than 1000 that would not have any significant effect on the profiles of the probability density function. It means that increasing the mean nozzle exit velocity $U_{O}$ influences on the geometric parameters of droplet system up to certain threshold of Weber number (in Fig. 1 We is less than 800). Therefore, we do not expect the essential variation of droplets sizes due to an increase of the spraying velocity at We $>1000$. As it follows from Fig. 1, the position of the maximum of the probability density function, i.e. the most probable size of droplet in the system, depends on the Weber number essentially. Moreover, when the Weber number increases, we observe the distinct displacement of the maximum in the domain of larger droplets.

Under the typical conditions of spray formation at low injection pressure [7], the fuels spray with the compositions varying from the diesel to biodiesel will have the Weber numbers less 1000 . Note, that in this work we calculated the Weber number based on $D_{30}$ rather than based on the nozzle diameter as in [7]. Fig. 1 also shows the increase of the most probable diameter of droplets from $0.25 \cdot \bar{D}$ to $0.5 \cdot \bar{D}$ when we use biodiesel instead diesel. 
This theoretical result is confirmed by the experimental evidences. It is known [13] that the mean size of droplets for the biodiesel spray is larger than for the diesel fuel spray.

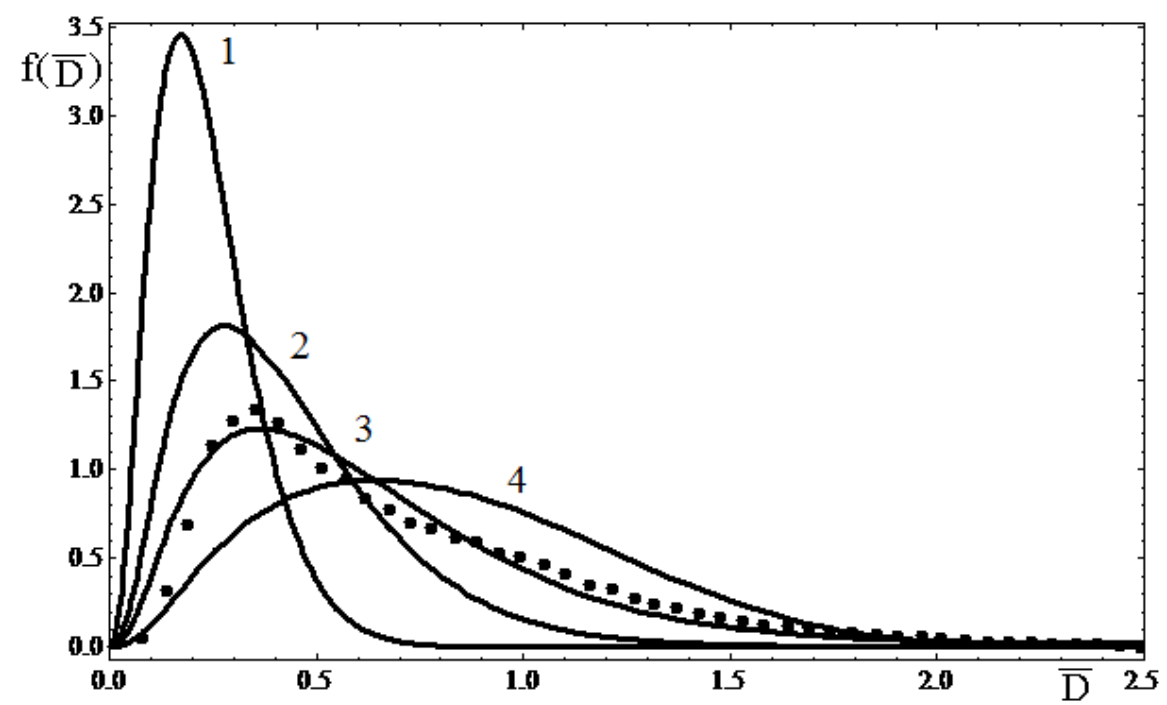

Figure 1. Probability density distribution for normalized droplet diameters at different Weber numbers: $1-$ We $=150$

$2-W e=220 ; 3-W e=311 ; 4-W e=1000$. Experimental data is taken from [13] for the water spray.

\subsection{PDF for biofuels spray (diesel and vegetable oil blends)}

The theoretic curves and experimental results [13] in the form of distributions of droplet diameters for the sprays from the blends of diesel fuel with rapeseed oil are presented in Fig. 2.

To process the experimental data [13] we need to know the fuel density and the surface tension for fuel blends. To estimate them, the mixture rules from [12] are applied to initial components of fuel blends. We also need to know the Weber numbers and the parameters $\bar{G}_{m}, \bar{G}_{e}$ corresponding to the momentum and energy sources.

Note that the mean nozzle exit velocity $U_{0}$ gives the essential uncertainty in the final value of Weber number. It is natural that this velocity depends on the nozzle design, its operational parameters (e.g. injection pressure), and the thermodynamic properties of fuels. However in the case of diesel, the fuel density is the only fuel property that is included in the injection velocity calculation. To increase accuracy the instantaneous injection velocity was defined from transient experimental mass profile [14]. Due to the significant amount of factors defining the biofuels injection, it is difficult to make a reliable theoretical estimation of the considered parameter for the experimental equipment [13].

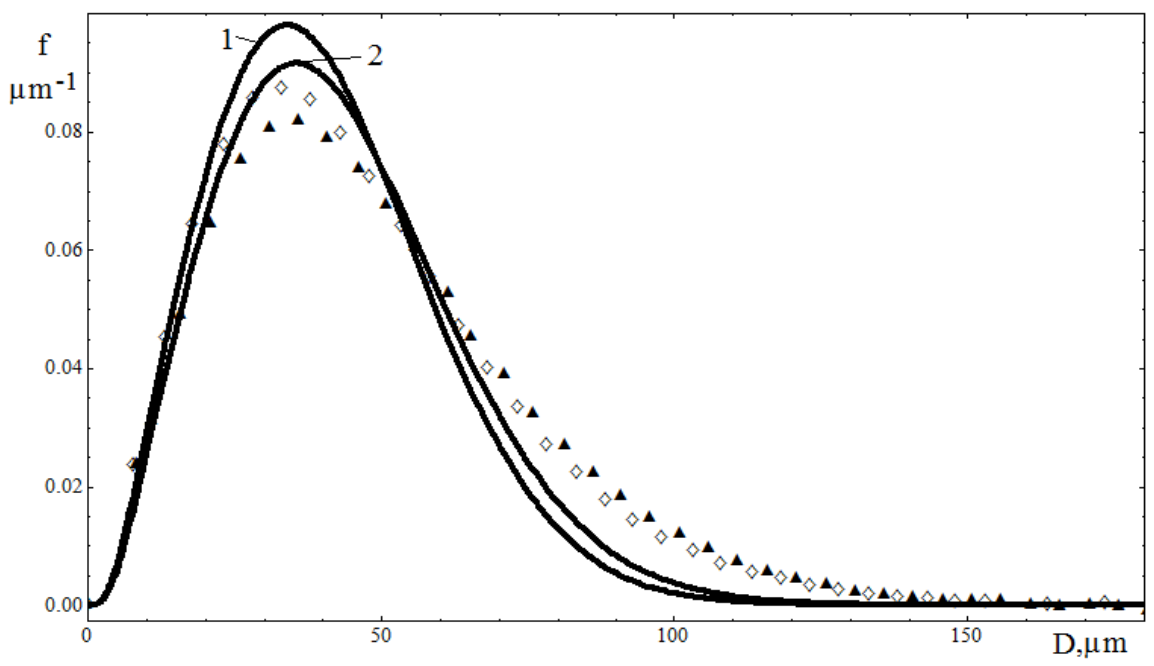

a) 


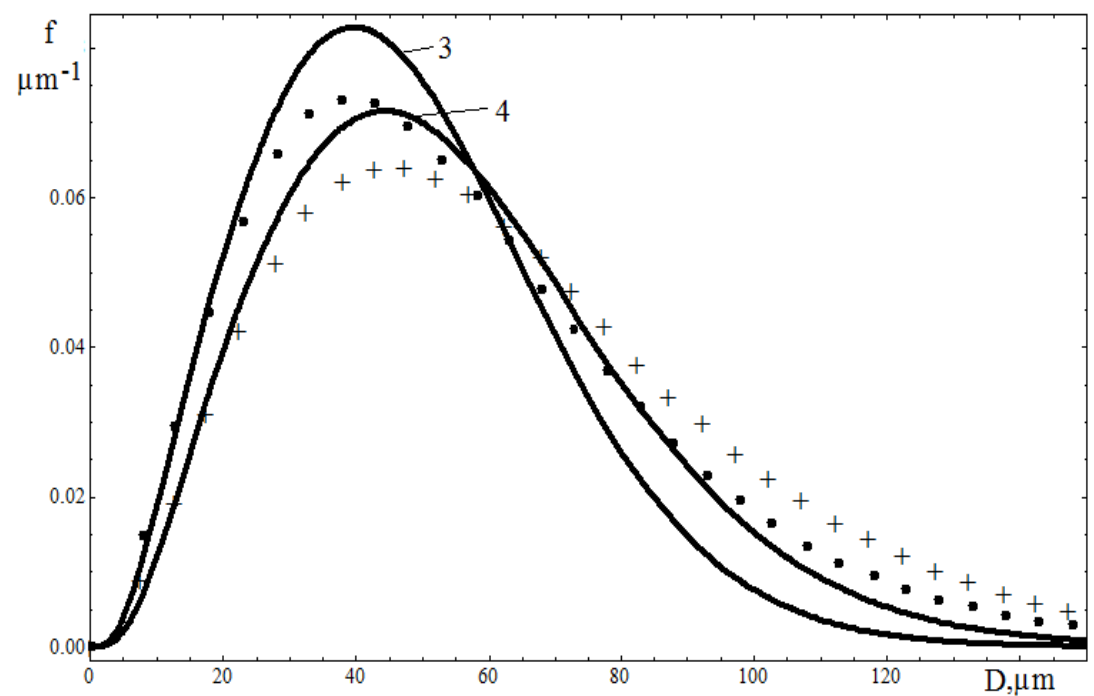

b)

Figure 2. Probability density function for the droplet diameters for diesel fuel and its blends with rapeseed oil (RO)

a) 1 - diesel fuel (DF): $W e=900, \bar{G}_{m v}=\bar{G}_{e}=-0.017$;

$2-20 \% \mathrm{RO}$ and $80 \% \mathrm{DF}: W e=825, \bar{G}_{m v}=\bar{G}_{e}=-0.018$;

$\diamond, \boldsymbol{\Lambda}$ - experimental data is taken from [13] for different compositions of fuel 1 and 2 , respectively.

b) $3-30 \% \mathrm{RO}$ and $70 \% \mathrm{DF}: \mathrm{We}=750, \overline{\mathrm{G}}_{\mathrm{mv}}=\overline{\mathrm{G}}_{\mathrm{e}}=-0.020$;

$4-40 \% \mathrm{RO}$ and $60 \% \mathrm{DF}: \mathrm{We}=450, \overline{\mathrm{G}}_{\mathrm{mv}}=\overline{\mathrm{G}}_{\mathrm{e}}=-0.032$;

,$+ \boldsymbol{\square}$ - experimental data is taken from [13] for different compositions of fuel 3 and 4 , respectively.

At the same time it is well-known that a neat vegetable oil has a higher viscosity (usually $28-40 \mathrm{~mm}^{2} / \mathrm{s}$ ) than diesel which leads to an injector coking due to poorer atomization [9]. Transesterification of the oil reduces the viscosity; biodiesel viscosity (usually $4-5 \mathrm{~mm}^{2} / \mathrm{s}$ ) is closer to diesel one [9]. According to an experimental study at Brighton University, UK, the diesel fuel exit velocity was $43.7 \mathrm{~m} / \mathrm{s}$ and the leading edge velocity of biodiesel jet was 28.3 $\mathrm{m} / \mathrm{s}$ at injection pressure $40 \mathrm{MPa}$ [7]. The same tendency was observed in another study of biodiesel - see [15], when at the start of the injection the first part of the spray (before break-up, near the orifice exit) with biodiesel seems to travel slower than the diesel fuel [15]. It was found that viscosity of the biodiesel has a strong effect on the drop size distribution and droplet mean diameters [16]. So, the viscosity and surface tension of biodiesel appear to play a dominant role on frictional losses in the nozzle [7, 15].

Thus we can assume that the mean nozzle exit velocity in case of biodiesel fuels will be less than of diesel fuel and the Weber numbers would decrease for biodiesel comparing with diesel fuels. We used this statement for low injection pressure biodiesel spray (see in Fig. 1). Also, we can assume that the Weber numbers should decrease when the fraction of vegetable oil increases (see in Fig. 2).

Increasing the fuel viscosity when the rapeseed oil is added affects the general momentum and energy consumption. It means that the parameters corresponding to the momentum and energy sources, $\bar{G}_{m}, \bar{G}_{e}$, should also depends on types of fuels. In this case one can state that the amount of losses of momentum and energy grows when the fraction of vegetable oil in fuel blend increases. From this it follows that $\bar{G}_{m}, \bar{G}_{e}$ are negative quantities the absolute values of which increase when the viscosity of fuel mixture grows. Thus, the assertions presented above allow us to justify the following approach for the theoretical description of experimental dependences from [13].

We are going to estimate the Weber numbers $(\mathrm{We})$, momentum and energy source terms $\left(\bar{G}_{m}, \bar{G}_{e}\right)$ satisfying the proper conditions for the description of experimental data [13]. Using these quantities and solving the system (1) - (4), the identification of probability density function (6) is carried out. The profiles of these functions are plotted in Fig. 2 for different fuel blends.

Analysing the results, we elucidated that among $\bar{G}_{m}, \bar{G}_{e}$, the parameter $\bar{G}_{m}$ impacts the most significantly on the profiles of probability density functions. So, we put $\bar{G}_{m v}=\bar{G}_{e}$ in the final analysis. The following numerical analysis showed that this assumption does not lead to the loss of generality, but reduces the number of adjustable parameters and simplifies the interpretation of obtained results. 
It is worth also to note that there is a satisfactory matching between theoretical and experimental data in Fig. 2 . It is important to emphasize that the values of Weber numbers and source terms for momentum and energy derived during the numerical procedure and presented in the legends in Fig. 2 are physically acceptable and satisfy the requirements stated above.

\subsection{PDF for biodiesel spray}

Fig. 3 shows the drop-size probability density for diesel and biodiesel fuels when experimental data [8] were fitted by the MEF method using equations (1)-(6). This case is close to realistic diesel engine conditions when the injection pressure is $100 \mathrm{MPa}$. In this case diesel fuel has emerged from the nozzle orifice with a velocity of 100 $\mathrm{m} / \mathrm{s}$ as ultra-high-speed videos showed [7]. These conditions indicate that the flow is both turbulent and in atomisation regime [7]. We have analysed two cases for the droplet size distribution for both diesel and biodiesel: 1) at distance of $10 \mathrm{~mm}$ from nozzle exit (see [8] for experimental data), and 2) at distance of $15 \mathrm{~mm}$ from nozzle exit (Fig. 3). We assumed that biodiesel has lower mean injection velocity $U_{0}$ than diesel but this difference is compensated by the higher value of $D_{30}$ for biodiesel. As a result, this leads to the same values of Weber number (We $=2500$ ) for diesel and biodiesel.

At distance of $15 \mathrm{~mm}$ we found that the better agreements against the experimental data were $\bar{G}_{m v}=-0.0035$, $\bar{G}_{e}=0$ for diesel; and $\bar{G}_{m v}=-0.00375, \bar{G}_{e}=0$ for biodiesel (Fig. 3). At distance of $10 \mathrm{~mm}$ our calculations show the same result as for diesel at distance of $15 \mathrm{~mm}$. This case wasn't presented in Fig.3. The difference in momentum sources $\bar{G}_{m v}$ between diesel and biodiesel may be related to different viscosity of fuels.

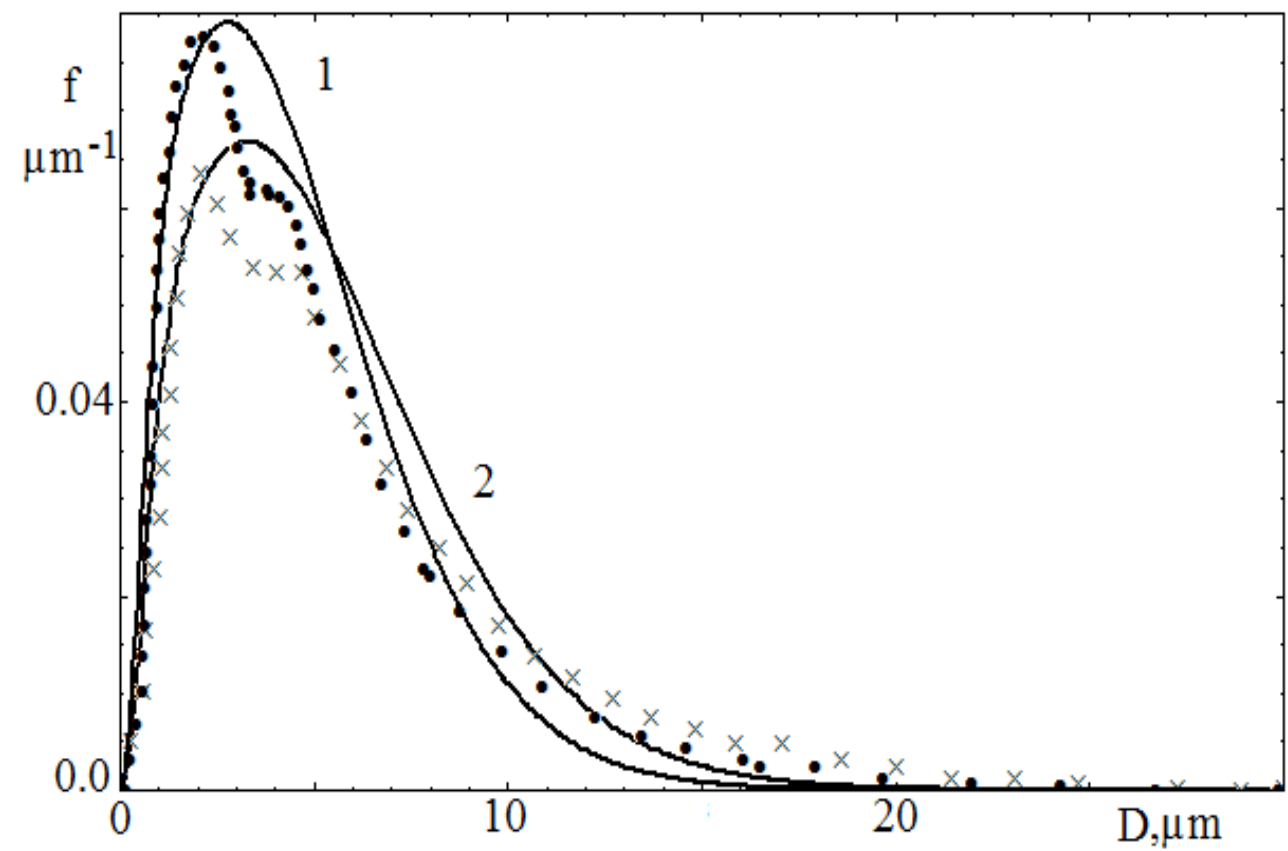

Figure 3. Probability density functions of the droplet diameters at distance of $15 \mathrm{~mm}$ from nozzle exit at We=2500:

1 - for diesel fuel $\bar{G}_{m v}=-0.0035, \bar{G}_{e}=0$

2 - for biodiesel fuel $\bar{G}_{m v}=-0.00375, \bar{G}_{e}=0$

Experimental data for diesel $(\bullet)$ and biodiesel $(x)$ are taken from [8]

At $10 \mathrm{~mm}$ from the nozzle exit, the intersection of experimental droplets size distribution of biodiesel and diesel occurred at the $8 \mu \mathrm{m}$ bin (see Fig. 7a, [8]). At $15 \mathrm{~mm}$ away from nozzle the experimental droplet distribution for RME was more skewed to the right comparing with the diesel with intersection at $5 \mu \mathrm{m}$ bin (see Fig. 7b, [8]).

Almost the same tendency for biodiesel was observed in experiments [10] with a solid-cone pressure-swirl atomizer at $30 \mathrm{~mm}$ from the nozzle exit when biodiesel PDF also was skewed to the right. At $70 \mathrm{~mm}$ from nozzle exit, along injector axis, the drop size distributions of biodiesel were not significantly different to that of diesel fuel [10]. But a big difference between diesel and biodiesel in the area between the core and the edge of spray at the radial position of $1 \mathrm{~cm}$ from the centre has been found in [10].

So, as expected from the physical properties of diesel and biodiesel fuels, both the theoretical and the experimental droplet size distributions show that biodiesel produces a higher number of larger droplets than diesel 
fuel [8]. This tendency is more profound in the sections far from the nozzle and in the area between the core and the edge of spray.

It is also important to notice that for realistic diesel condition when evaporation has place both the spray phenomena and mean nozzle exit velocity of fuel depend on the cavitation process. The detailed velocity research for biodiesel in this case was carried out in [17]. In case of cavitation, when the pressure at the vena contracta was lower than the vapour pressure new discharge coefficient was proposed [17]. If the flow at the nozzle exit is turbulent, the injection velocity is calculated using the volumetric mean velocity and the initial droplet size is equal to the nozzle hole diameter. In case of the super cavitation in the nozzle flow, the cross-sectional area of the initial blob was more diminished in size than the diameter of nozzle exit [17].

\section{Conclusions}

The maximum entropy method was applied for the droplet distribution of diesel, biodiesel and biofuel (vegetable oil and diesel blends) sprays in conditions close to diesel combustion engine. The dependency of the probability density distribution of droplet diameters on Weber number was analysed at momentum and energy losses $\bar{G}_{e}=0$ and $\bar{G}_{m v}=-0.017$ correspondently. The distribution of droplet diameters in biofuel was calculated at $\bar{G}_{e}=\bar{G}_{m v}$. The theoretical distributions show that, as a rule, the biodiesel produces a higher number of larger droplets than diesel fuel. In general the model is matching experimental data with reasonable accuracy.

\section{Acknowledgements}

The authors gratefully acknowledge the contribution of Ministry of Education of Ukraine to this research project by providing financial support. Also we would like to thank Prof. Christophe Dumouchel for his support in providing information about maximum entropy method.

\section{Nomenclature}

$D \quad$ droplet diameter $[\mu \mathrm{m}]$

$t \quad$ probability density function $\left[\mu \mathrm{m}^{-1}\right]$

We Weber number [-]

$\dot{n} \quad$ droplet generation rate $\left[\mathrm{s}^{-1}\right]$

$\dot{m}_{O} \quad$ mass flow rate $\left[\mathrm{kg} \mathrm{s}^{-1}\right]$

$j_{O}$ momentum $\left[\mathrm{kg} \mathrm{m} \mathrm{s}^{-2}\right]$

$\dot{E}_{O} \quad$ energy source introducing into a system $\left[\mathrm{J} \mathrm{s}^{-1}\right]$

$\bar{G}_{m}, \bar{G}_{m}, \bar{G}_{e}$ dimensionless sources for mass, momentum and energy [-]

$\sigma \quad$ surface tension $\left[\mathrm{N} \mathrm{m}^{-1}\right]$

$\rho \quad$ density of liquid phase $\left[\mathrm{kg} \mathrm{m}^{-3}\right]$

$\bar{V} \quad$ dimensionless droplet volume

$\bar{u} \quad$ dimensionless droplet velocity

$U_{0} \quad$ mean nozzle exit velocity $\left[\mathrm{m} \mathrm{s}^{-1}\right]$

$V_{m} \quad$ mean volume of droplets $\left[\mathrm{m}^{3}\right]$

$k_{B} \quad$ Boltzmann constant

$\alpha_{1,2,3}$ Lagrange multipliers

\section{References}

[1] Dumouhel, C. 2009, Entropy, 11, pp.713-747.

[2] "Handbook of Atomization and Sprays. Theory and Applications", 2011, edited by N. Ashgriz, Springer.

[3] Sovani, SD., Sojka, PE., Sivathanu, YR. 1999, Atomization Sprays, 9, pp. 113-152.

[4] Movahednejad, E., Ommi, F., Hosseinalipour, SM., 2010, Entropy, 12, pp.1484-1498.

[5] Sazhin, S., 2014, "Droplets and Spray". Springer.

[6] Yan, K., Ning, Z., Lü, M., Sun, C. 2015, Entropy, 17, pp. 580-593.

[7] Crua, C, Heikal, MR., Gold, MR., 2015, Fuel, 157, pp.140-150. 
[8] Crua, C., de Sercey, G, Heikal M.R., 2012, Sep. 2.-6., ICLASS, 12th Triennial International conference on Liquid Atomization and Spray System.

[9] Knothe, G., 2010, Progress in Energy and Combustion Science, 36, pp. 364-373.

[10] Yongyingsakthavorn, P., Vallikul, P., Fungtammasan, B., Dumouchel, C., Joulain, P., Dec. 1.-3., 2004, The Joint International Conference on "Sustainable Energy and Environment (SEE)".

[11] Kolodnytska, R., Al Qubeissi, M., Sazhin, S.S., 2013, ILASS, 25th European Conference on Liquid Atomization and Spray Systems.

[12] Kolodnytska, R.V. 2010, Visnik SNU, 6 (148), pp. 41-46.

[13] Ivashchenko A.V., Goryachkin V.N., 2011, Internal combustion engines, 2(41), pp. 41-46.

[14] Kolodnytska, R.V., Karimi, K., Crua, C., Heikal M.R., Sazhina, E.M. ,2008, Internal combustion engines, 1. pp. 42-46.

[15] Desantes, J.M., Payri, R., Garcıa, A., Manin, J., 2009, Energy \& Fuels, 23, pp. 3227-3235.

[16] Kamrak, J., Lorturn, P., Grehan, G., Saengkaew, S., Charinpanitkul, T., 2007, Aug 26.-29. The 5th Asian Aerosol Conference.

[17] Park, S.H., Kim H.J., Suh H. K., Lee C. S., 2009, International Journal of Heat and Fluid Flow, 30, pp. 960-970. 


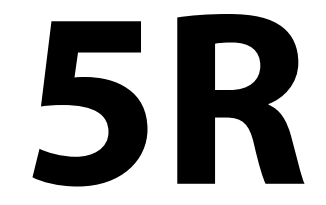

Atomizers 5 


\title{
Experimental Investigation on the Early Stage Spray Characteristics with Biodiesel and Diesel
}

\author{
ShenghaoYu ${ }^{1}$, Bifeng. Yin ${ }^{1}$, Shuai Wen ${ }^{1}$, Xifeng Li ${ }^{1}$, Hekun. Jia ${ }^{1}$, Jianda. Yu ${ }^{2}$ \\ ${ }^{1}$ School of Automotive and Traffic Engineering, Jiangsu University, Zhenjiang 212013, China \\ ${ }^{2}$ Wuxi Fuel Injection Equipment Research Institute, Wuxi 214063, China \\ *Corresponding author: yshujs@163.com
}

\begin{abstract}
The early stage spray characteristics have a great impact on the secondary atomization progress, and thus affect the engine combustion and emission performances. The experimental investigation of the early stage spray behaviors with biodiesel and diesel was carried out by employing a laser-based Mie-scattering method. The results show that the spray tip penetration for biodiesel is higher than that for diesel at the early stage spray under the same injection pressure. Moreover, the early stage spray tip penetration can be longer under high injection pressures for two fuels. Besides, the early stage spray cone angle for biodiesel is narrower than that for diesel, and the spray cone angle is especially higher than biodiesel by $25.8 \%$ after start of injection time of $0.01 \mathrm{~ms}$. Furthermore, under the same injection condition, the difference of early stage spray area between diesel and biodiesel is not obvious, while the spray volume for biodiesel is larger than that for diesel, and also the spray volume can be enlarged by increasing injection pressure for both fuels.
\end{abstract}

\section{Keywords}

Spray characteristics; Mie-scattering; Biodiesel; Diesel

\section{Introduction}

For modern diesel engines, soot and nitrogen oxide are the two main emissions which are mainly influenced by mixture quality of air and fuel, and the emission characteristics are largely governed by atomization and spray processes [1-4]. Biodiesel, derived from the waste cooking oil and a kind of renewable energy, has been widely used as an alternative fuel for diesel engines. It is clear that the use of biodiesel in diesel engine can improve favorable emissions performance [5-9]. Although the importance of biodiesel in reducing diesel engine emission performance has been acknowledged, the research on early stage spray characteristics of biodiesel is still far from sufficient. The early stage spray dominates the secondary spray progress and the mixture quality of fuel and air, and thus affects the atomization quality of biodiesel. Therefore, it is important to define the effects of biodiesel at the early stage spray characteristics.

Wang et al. $[10,11]$ studied the near-field primary spray characteristics of different split injection strategies by using ultra-high speed imaging method. Their results indicated that the second split injection cause smaller spray area and shorten penetration during the early stage spray, and also the larger late injection stage can increase the spray area and the penetration. Moreover, they found that the strong primary collision can be caused by improving the injection pressure and shortening dwell split injection, thus the spray area and the spray cone angle increase, while the spray tip penetration decreases at the same time. And they also found that the long duration of the first injection enhances the primary collision. Wang et al.[12] also studied the spray behaviors of emulsified diesel blended with water through schlieren technique. They concluded that the penetration increases as the magnitude of water increases, while the spray cone angle, spray volume and area decrease. Mo et al. [13] carried out an experimental research on the spray and atomization behaviors with soybean biodiesel and $20 \% \mathrm{n}$-butanol biodiesel blend respectively by schlieren and particle diameter image analysis techniques (PDIA) techniques. Their results showed that the penetration and droplet diameters of BD100 are higher than that of BD80. $\mathrm{Li}$ and Xu [14] used an ultra-high speed camera to study the influence of injection and ambient pressure on the spray behaviors, such as spray penetration and tip velocity. They concluded that the spray peak velocity is related to the change of two-stages of spray. Ding et al.[15] investigated the near nozzle spray patterns with four different fuels through experimental method, and the results suggested that the hydraulic flipping regime is extremely different for four fuels under different injection pressures. Xie et al.[16]experimented on the spray characteristics for different biodiesels and their blends with diesel under different injection conditions. They observed that the penetration increases as the blend ratio of biodiesel increases, while the spray cone angle becomes narrower than that of diesel. 
The literature review mainly focused on the initial breakup with traditional diesel or the secondary spray and atomization characteristics with biodiesel or its blends. However, the research on the early stage spray characteristics when using biodiesel is still insufficient. In addition, the research on the early stage spray with biodiesel is of great importance for better understanding of biodiesel spray characteristics, because the early stage spray dominates the secondary breakup and air/fuel mixture quality, and also can provide helpful basis for improving the atomization quality of biodiesel. In consequence, due to the poorer quality of biodiesel sprays in comparison with diesel, the impact of biodiesel on the early stage spray characteristics requires an in-depth study. Therefore, our research mainly focuses on the early stage spray characteristics of biodiesel and diesel under the condition of different injection pressures $(50 \mathrm{MPa}, 90 \mathrm{MPa})$ and the fixed ambient pressure of $0.1 \mathrm{MPa}$. The early stage spray characteristics, containing spray tip penetration, spray cone angle, spray evolution images at the early stage spray, spray area and spray volume, were employed respectively to investigate the early stage spray characteristics of diesel and biodiesel. It is of great importance to better understand biodiesel spray characteristics at the early stage spray, which can help improve the quality of secondary atomization for biodiesel effectively. In order to analyze the early stage spray characteristics, a laser-based Mie-scatting method was adopted by using Nd:YAG laser beam visualization system and a CCD camera.

\section{Material and methods}

In this research, all the experimental ambient temperatures were set to300K. Figure 1 shows the experimental equipment diagram for studying the early stage diesel and biodiesel spray characteristics. The experimental equipment diagram consists of a 2D image recorder system, a common rail injection system and a laser beam system (Nd:YAG Laser). 2D image recorder system consists of a CCD camera (500 fps), and image acquisitionprocessing system. The early stage macro spray images (the resoltion of the camera is $1600 \times 1200$ pixels) were captured by using the CCD camera with a camera filter. The common rail injection system consists of a high injection pump, high pressure pipes, electromotor, common rail and a sac single diesel nozzle. The high injection pump was driven by electromotor to provide different high injection pressures. Furthermore, the delay time among the laser beam, CCD camera and injector nozzle were set by a delay controller.

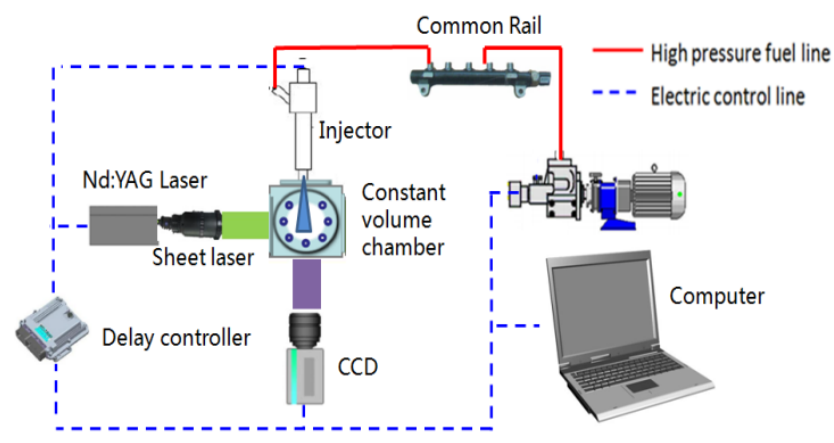

Figure 1. Experimental equipment diagram

In order to study the early stage spray characteristics for diesel and biodiesel, two fuels were selected in the experiment, biodiesel (B100) was derived from waste cooking oil, and conventional diesel (B0) were used as experimental fuels. B0 corresponds to $0 \%$ biodiesel - 100\% diesel; and B100, 100\% biodiesel - $0 \%$ diesel, respectively. The main physical properties of $\mathrm{B} 0$ and $\mathrm{B} 100$ are listed in Table1. It can be found in Table1that the physical properties of $\mathrm{B} 100$ are all higher than those of B0.

Table1 Physical properties for diesel and biodiesel

\begin{tabular}{llllll}
\hline Fuel type & $\begin{array}{l}\text { Density(kg/ } \\
\mathrm{m} 3)\end{array}$ & $\begin{array}{l}\text { Dynamic } \\
\text { viscosity(pa s) }\end{array}$ & $\begin{array}{l}\text { surface } \\
\text { tension(N/m) }\end{array}$ & $\begin{array}{l}\text { Boiling range/ } \\
{ }^{\circ} \mathrm{C}(\mathrm{T} 10)\end{array}$ \\
\hline Diesel(B0) & 830 & 0.0021 & 0.024 & 220 \\
Biodiesel(B100) & 870 & 0.0039 & 0.028 & 330
\end{tabular}

Table 2 represents the experimental working conditions, a single sac nozzle was selected in this study, and two different fuels were adopted to study the early stage spray characteristics. Injection pressure was set as 50MPa and $90 \mathrm{MPa}$ respectively; the ambient pressure was fixed as $0.1 \mathrm{MPa}$; and the ambient temperature was set as $300 \mathrm{~K}$. The present research mainly focused on the early stage spray behaviors, so the after start of injection (ASOI) time changed from $0 \mathrm{~ms}$ to $0.1 \mathrm{~ms}$, and the injection duration was set as $2 \mathrm{~ms}$.

Table2 Experimental working conditions 


\begin{tabular}{ll}
\hline Experimental system & Parameters \\
\hline Fuel & Diesel (B0), Biodiesel(B100) \\
Nozzle type & Sac-nozzle \\
Diameter of nozzle hole & $0.2 \mathrm{~mm}$ \\
Injection pressure & $50 \mathrm{MPa} .90 \mathrm{MPa}$ \\
Ambient pressure & $0.1 \mathrm{MPa}$ \\
Ambient temperature & $300 \mathrm{~K}$ \\
Injection duration & $2 \mathrm{~ms}$ \\
After start of injection (ASOI) & $0 \mathrm{~ms} \sim 0.1 \mathrm{~ms}$ \\
\hline
\end{tabular}

\section{Results and discussion}

This study included comparison of early stage spray images obtained from the spray test platform with a laserbased Mie-scattering method under different injection pressures (50MPa, 90MPa) with diesel (B0) and biodiesel (B100). The results in Figure 2 show the evolution of the early stage spray images under injection pressures of $50 \mathrm{MPa}$ and $90 \mathrm{MPa}$ respectively, and the ambient pressure was fixed at $0.1 \mathrm{MPa}$. The captured images were used to analyze the early stage macroscopic spray characteristics. All the recorded macro spray images were processed by MATLAB software (binary processing) to obtain the macro spray characteristics. In order to improve the measurement accuracy, eight spray images are taken for each given moment, and the spray characteristics are the average of those of the eight times. In addition, the fundamental macro spray characteristics of diesel (B0) and biodiesel (B100) were analyzed and compared in terms of spray tip penetration[10], the cone angle which is formed by the spray considering $60 \%$ of the penetration, spray area and spray volume.

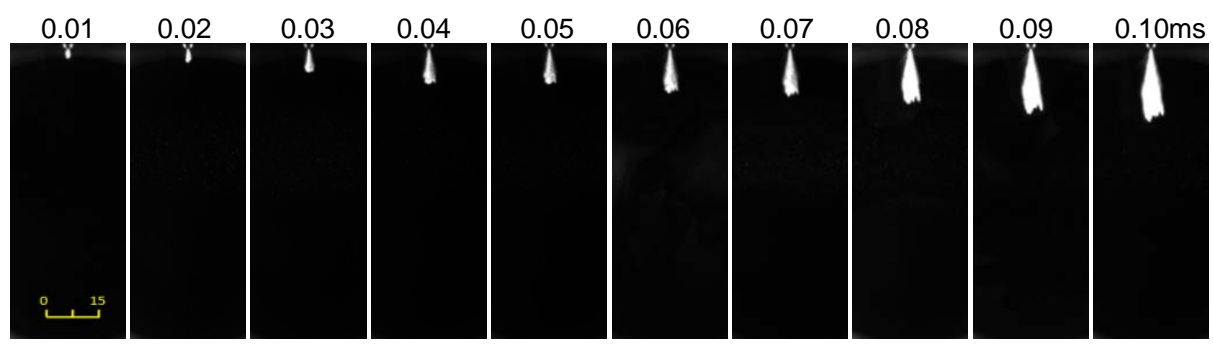

(B0)

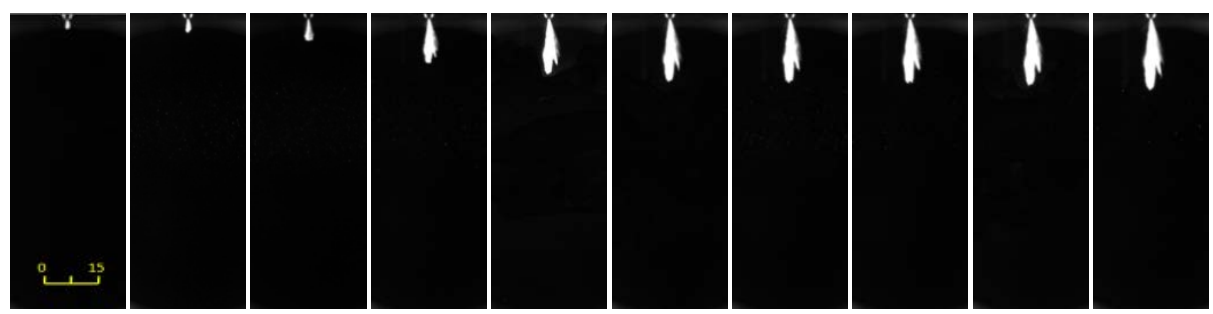

(B100)

$50 \mathrm{Mpa}$

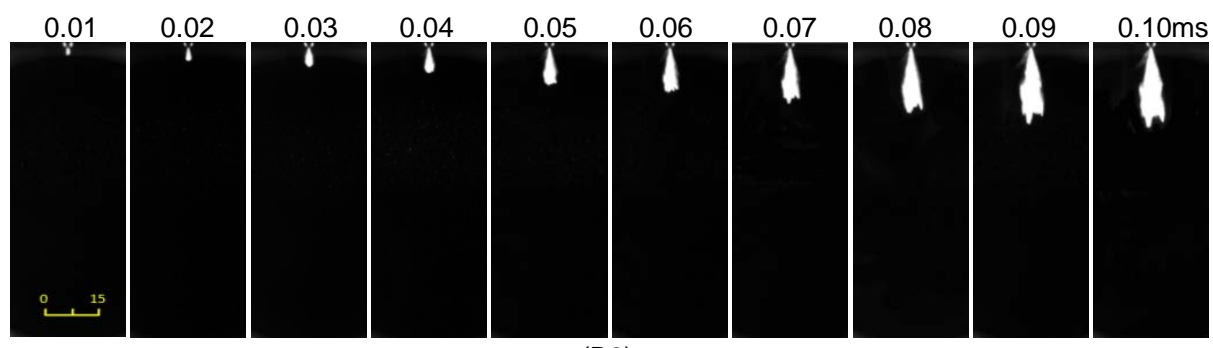

(B0) 

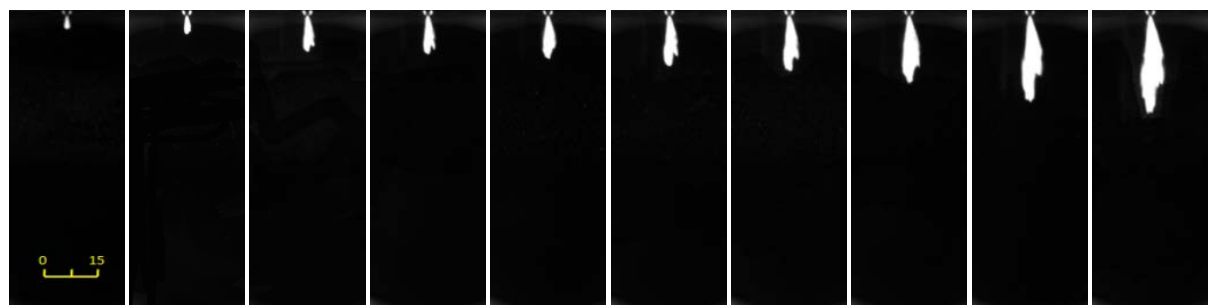

(B100)

$90 \mathrm{MPa}$

Figure 2. Spray morphology (time in ms ASOI) for diesel (B0) and biodiesel (B100) under different injection pressures Spray tip penetration

The results in Figure 3 show the evolution of the early stage spray tip penetration for diesel and biodiesel under different injection pressures $(50 \mathrm{MPa}, 90 \mathrm{MPa})$ and the fixed ambient pressure of $0.1 \mathrm{MPa}$. The results in Figure 3 indicate that the spray tip penetration for biodiesel is higher than that for diesel under the same injection pressure. It is mainly due to the larger surface tension and viscosity of biodiesel, which can inhibit the progress of the early stage spray and atomization, and thus result in large injection momentum and velocity for biodiesel at the nozzle exit. It is favorable for increasing the early stage spray tip penetration. Moreover, the biodiesel in the injector nozzle can promote the friction between the fuel and the inner wall surface of injector orifice, as a result of the higher viscosity of biodiesel. In addition, it can also be found that the early stage spray tip penetration is extended by increasing the injection pressure, because high injection pressure can increase the injection velocity at the nozzle exit.

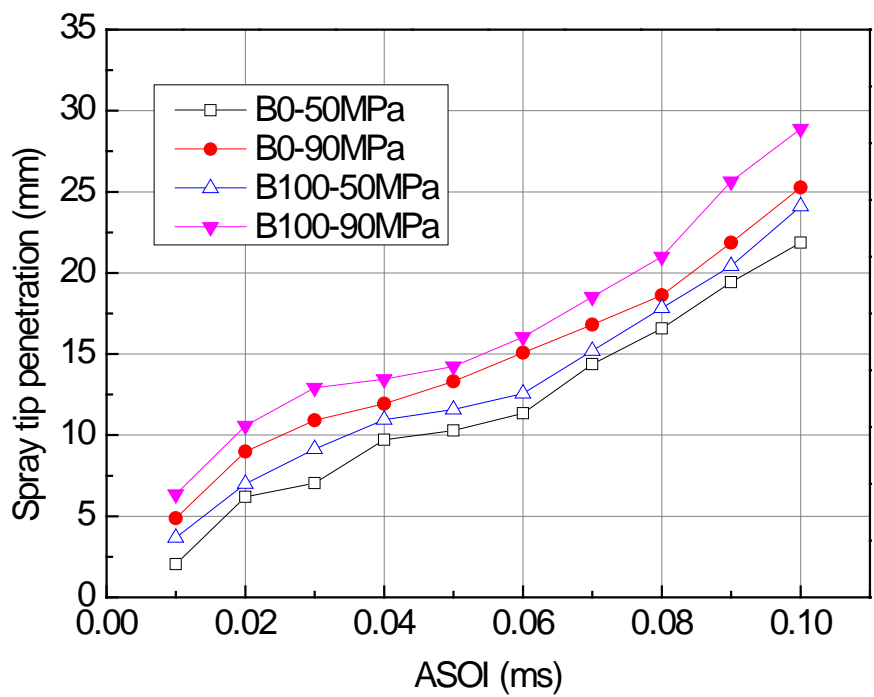

Figure 3. Spray tip penetration for diesel and biodiesel under different injection pressures Spray cone angle

The results in Figure 4 show the evolution of the early stage spray cone angle for diesel and biodiesel under different injection conditions and the fixed ambient pressure of $0.1 \mathrm{MPa}$. It can be found that the spray cone angle was wider for B0 when compared to B100. Moreover, the big difference for spray cone angle is at the beginning of injection. For instance, the spray cone angle for B0 is higher than that forB100 by $25.8 \%$ after start of injection time of $0.01 \mathrm{~ms}$ under the injection pressure of $50 \mathrm{MPa}$. It is mainly due to the higher surface tension for biodiesel, which can suppress the increase of radial velocity of the jet at the nozzle exit and the collapse of spray for biodiesel. As a result, the early stage spray cone angle of B100 is narrower than that of B0. Furthermore, the larger early stage spray cone angle of diesel means strong interaction between fuel and air at the early stage spray, which can improve the quality of primary spray and atomization greatly. By contrast, the quality of primary spray for biodiesel is poor. Finally, it can also be found that the difference of the early stage spray cone angle is not obvious for the same fuel under different injection pressures. 


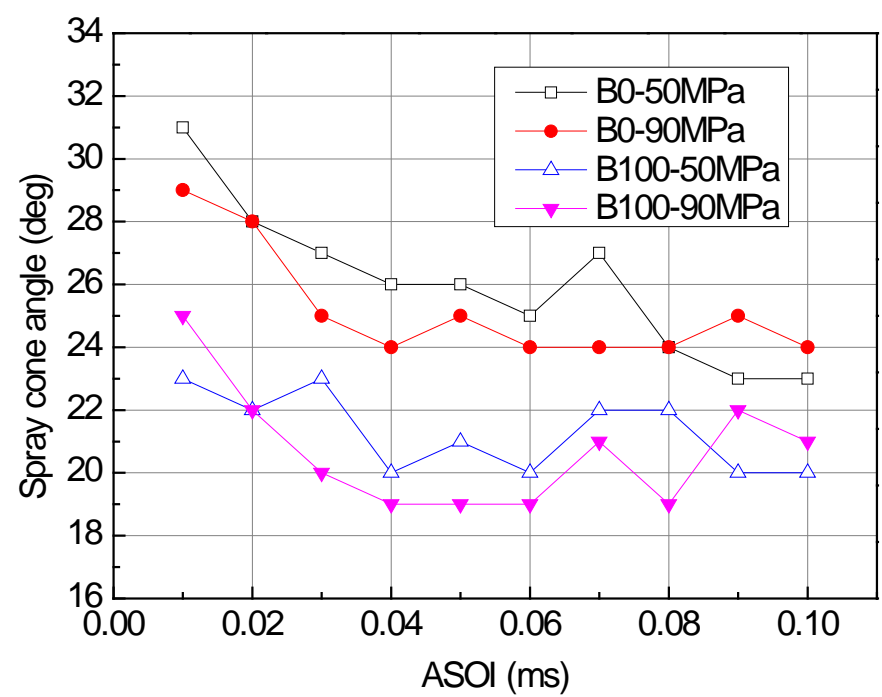

Spray area

Figure 4. Spray cone angle for diesel and biodiesel under different injection pressures

The spray area was used to represent the mixing quality between fuel and air [17]. In the present research, MATLAB software was employed to count the total effective pixels of spray image so as to represent the spray area. Figure 5 shows the values of spray area for diesel and biodiesel under different injection pressures. The results demonstrate that the spray area increases with the increase of ASOI, because the spray tip penetration becomes longer as stated in Figure 3. By comparing diesel and biodiesel under the same injection pressure, it can be found that the difference of spray areas between diesel and biodiesel is not obvious, because the lower surface tension and viscosity for diesel are good for enlarging the spray cone angle, but the diesel holds a shorter spray tip penetration. In addition, for the same fuel, the spray area increases as the injection pressure increases, because the continuously increasing injection pressure improves the development of spray tip penetration for diesel and biodiesel.

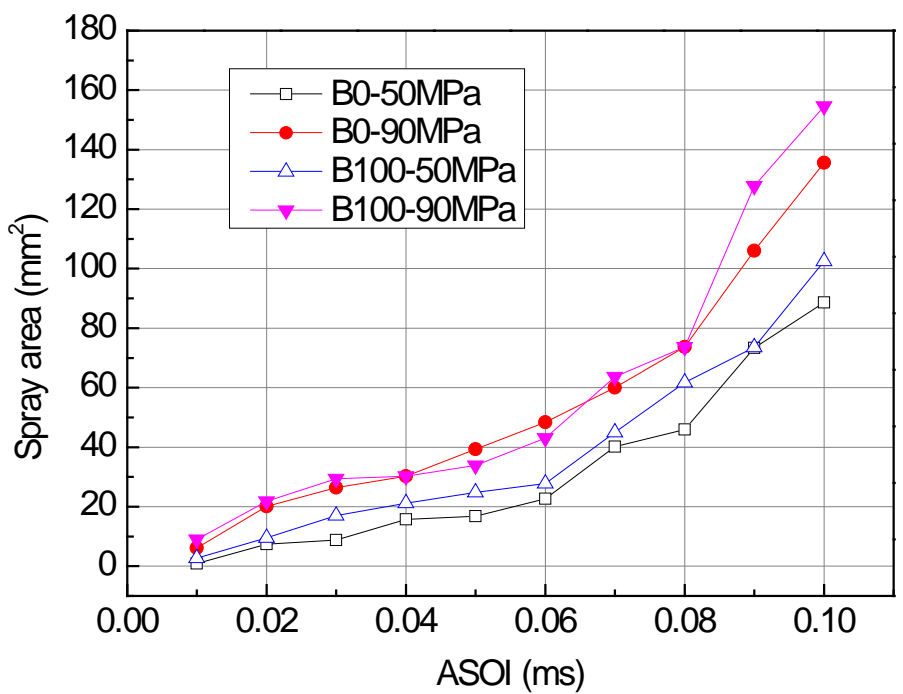

Figure 5. Spray areas for diesel and biodiesel at various injection pressures

Spray volume

The spray volume was of great importance in assessing the air entrainment mixing quality, and it can be calculated by using the following equation[18]:

$$
\mathrm{V}=(\pi / 3) S^{3}\left[\tan ^{2}(\theta / 2)\right] \frac{1+2 \tan (\theta / 2)}{[1+\tan (\theta / 2)]^{3}}
$$

(1)

In the equation, $\mathrm{S}$ represents the spray tip penetration and $\theta$ the spray cone angle respectively. Figure 6 shows the spray volumes for diesel and biodiesel under different injection pressures. It can be found in Figure 6, the spray volume increases slowly before $0.08 \mathrm{~ms}$ (ASOI) for both fuels, and then increases dramatically after $0.08 \mathrm{~ms}$ 
(ASOI).Moreover, the spray volume of the biodiesel is higher than that of diesel at the injection pressure of $90 \mathrm{MPa}$, because higher density and viscosity of biodiesel can lead to higher inertia and fuel injection quantity. Thus, as the spray tip penetration increase as shown in Figure 3, the spray volume, in turn, increases. In addition, the spray volume can be enlarged by increasing the injection pressure at the same ASOI. Because the higher injection pressure can improve the jet momentum as well as strengthen the velocity at the nozzle exit, and result in the longer spray tip penetration. Furthermore, it can also enhance the interaction between fuel and air and thus further increase the spray volume.

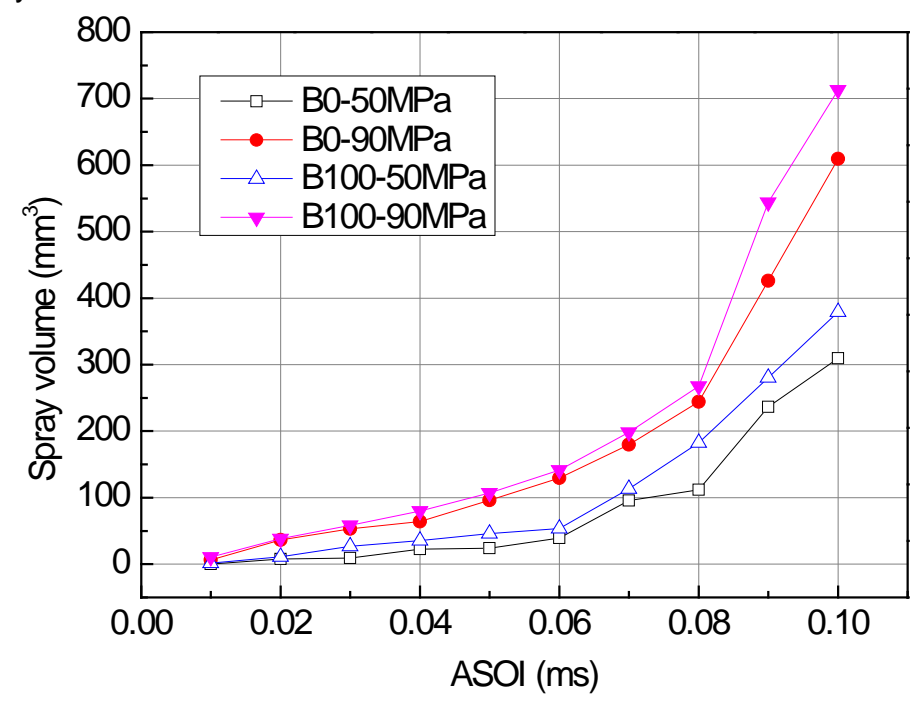

Figure 6. Spray volumes for diesel and biodiesel under different injection pressures

\section{Conclusions}

In this research, the early stage spray characteristics with diesel and biodiesel were investigated in depth under different injection conditions with a laser-based Mie-scattering method in a constant volume chamber. Main conclusions in our research are as follows:

(1) The spray tip penetration for biodiesel is longer than that for diesel at the early stage spray under the same injection pressure. Because the viscosity and surface tension of biodiesel is higher than that of diesel, which caused higher momentum at the nozzle exit as well as the higher friction between the fuel and orifice inner wall surface. In addition, the high injection pressure can enlarge the early stage spray tip penetration for both fuels.

(2) In comparison, the early stage spray cone angle for biodiesel is narrower than that for diesel. The early stage spray cone angle is especially higher than biodiesel by $25.8 \%$ after start of injection time of $0.01 \mathrm{~ms}$ under the injection pressure of $50 \mathrm{MPa}$, because the higher surface tension and viscosity of biodiesel will inhibit the collapse of spray at the initial stage spray, and the internal cavitation flow also play an important role on the spray cone angle.

(3) The difference of early stage spray area between diesel and biodiesel is not obvious under the same injection pressure. The low surface tension and viscosity of diesel can enlarge spray cone angle and hold short spray tip penetration.

(4) Because of higher injection quantity and spray tip penetration for biodiesel, the spray volume for biodiesel is larger than that for diesel under the same injection pressure. In addition, the spray volume can be enlarged by increasing the injection pressure for diesel and biodiesel.

\section{Acknowledgements}

This work was supported by the contribution of China postdoctoral fund projects [grant number2013M530236]; The projects of 'Six talent peak' [grant number 2014-ZBZZ-014]; Research start-up found projects of Jiangsu university [grant number 13JDG104]; Natural Science Foundation of Jiangsu Province of China [grant number BK20150520]; The Priority Academic Program Development of Jiangsu Higher Education Institutions [PAPD].

\section{References}

References should be indicated in the text by full sized numbers enclosed within square brackets. Use different formats for journals[19], books[20], symposium proceedings[21]and web pages[22] as illustrated in the examples below. You may use "ASME" citation style in Endnote. 
[1] Payri, R., Salvador, F., Gimeno, J., and De la Morena, J., 2009, "Effects of nozzle geometry on direct injection diesel engine combustion process," Applied Thermal Engineering, 29(10), pp. 2051-2060.

[2] Jung, D., Wang, W., Knafl, A., Jacobs, T., Hu, S. J., and Assanis, D., 2008, "Experimental investigation of abrasive flow machining effects on injector nozzle geometries, engine performance, and emissions in a DI diesel engine," International Journal of Automotive Technology, 9(1), pp. 9-15.

[3] Payri, R., Salvador, F., Gimeno, J., and Viera, J., 2015, "Experimental analysis on the influence of nozzle geometry over the dispersion of liquid n-dodecane sprays. Front," Mech. Eng, 1, p. 13.

[4] Yaliwal, V., Banapurmath, N., Gireesh, N., Hosmath, R., Donateo, T., and Tewari, P., 2016, "Effect of nozzle and combustion chamber geometry on the performance of a diesel engine operated on dual fuel mode using renewable fuels," Renewable Energy, 93, pp. 483-501.

[5] Lahane, S., and Subramanian, K., 2015, "Effect of different percentages of biodiesel-diesel blends on injection, spray, combustion, performance, and emission characteristics of a diesel engine," Fuel, 139, pp. 537545.

[6] Özener, O., Yüksek, L., Ergenç, A. T., and Özkan, M., 2014, "Effects of soybean biodiesel on a DI diesel engine performance, emission and combustion characteristics," Fuel, 115, pp. 875-883.

[7] Yilmaz, N., Vigil, F. M., Benalil, K., Davis, S. M., and Calva, A., 2014, "Effect of biodiesel-butanol fuel blends on emissions and performance characteristics of a diesel engine," Fuel, 135, pp. 46-50.

[8] Chauhan, B. S., Kumar, N., and Cho, H. M., 2012, "A study on the performance and emission of a diesel engine fueled with Jatropha biodiesel oil and its blends," Fuel \& Energy Abstracts, 37(1), pp. 616-622.

[9] Sanli, H., Canakci, M., Alptekin, E., Turkcan, A., and Ozsezen, A. N., 2015, "Effects of waste frying oil based methyl and ethyl ester biodiesel fuels on the performance, combustion and emission characteristics of a DI diesel engine," Fuel, 159, pp. 179-187.

[10] Wang, Z. M., Xu, H. M., Jiang, C. Z., and Wyszynski, M. L., 2016, "Experimental study on microscopic and macroscopic characteristics of diesel spray with split injection," Fuel, 174, pp. 140-152.

[11] Wang, Z. M., Ding, H. C., Ma, X., Xu, H. M., and Wyszynski, M. L., 2016, "Ultra-high speed imaging study of the diesel spray close to the injector tip at the initial opening stage with single injection," Applied Energy, 165, pp. 335-344.

[12] Wang, Z. W., Chen, X., Vuilleumier, D., Huang, S., and Tang, J., 2016, "Experimental Study on Spray Characteristics Of Emulsified Diesel Blending with Water In a Constant Volume Chamber," Atomization And Sprays, 26(6), pp. 513-533.

[13] Mo, J., Tang, C. L., Li, J. G., Guan, L., and Huang, Z. H., 2016, "Experimental investigation on the effect of nbutanol blending on spray characteristics of soybean biodiesel in a common-rail fuel injection system," Fuel, 182, pp. 391-401.

[14] Li, Y. F., and Xu, H. M., 2016, "Experimental study of temporal evolution of initial stage diesel spray under varied conditions," Fuel, 171, pp. 44-53.

[15] Ding, H. C., Wang, Z. M., Li, Y. F., Xu, H. M., and Zuo, C. J., 2016, "Initial dynamic development of fuel spray analyzed by ultra high speed imaging," Fuel, 169, pp. 99-110.

[16] Xie, H. Z., Song, L. B., Xie, Y. Z., Pi, D., Shao, C. Y., and Lin, Q. Z., 2015, "An Experimental Study on the Macroscopic Spray Characteristics of Biodiesel and Diesel in a Constant Volume Chamber," Energies, 8(6), pp. 5952-5972.

[17] Yuan, W., Hansen, A., and Zhang, Q., 2009, "Predicting the temperature dependent viscosity of biodiesel fuels," Fuel, 88(6), pp. 1120-1126.

[18] Delacourt, E., Desmet, B., and Besson, B., 2005, "Characterisation of very high pressure diesel sprays using digital imaging techniques," Fuel, 84(7), pp. 859-867.

[19] Lee, C. H., and Reitz, R. D., 1999, Atomization and Sprays, 9, pp. 497-517.

[20] Wilcox, D. C., 1993, "Turbulence Modeling for CFD."

[21] Lampa, A., and Fritsching, U., Sep. 5.-7. 2011, 24th European Conference on Liquid Atomization and Spray Systems.

[22] Kastengren, A. L., Powell, C. F., Wang, Y., Im, K.-S., and Wang, J., 2009, "X-ray radiography measurements of diesel spray structure at engine-like ambient density," Atomization and Sprays, 19(11). 


\title{
Properties of Fuel Spray Obtained by Electrohydrodynamic Atomization
}

\author{
Michel Daaboul*1, Nicolas Saba ${ }^{1}$, Jihad Rishmany ${ }^{1}$, Christophe Louste ${ }^{2}$ \\ ${ }^{1}$ Mechanical Engineering Department, University of Balamand, Lebanon \\ ${ }^{2}$ EHD Team, PPRIME Institute, CNRS - University of Poitiers - ISAE-ENSMA, France \\ ${ }^{*}$ Corresponding author: michel.daaboul@balamand.edu.lb
}

\begin{abstract}
Airblast atomization is commonly used to atomize fuel in aircraft engines. An annular liquid sheet is atomized by the shear forces exerted by the co-flowing air stream. Nevertheless, this technique is less effective in some specific cases, e.g. when the external air flow velocity is relatively low. Electrohydrodynamic (EHD) atomization can constitute a solution in these cases. It consists of applying an electric field between two electrodes and electricallycharging the passing carburant. This phenomenon will create instabilities within the liquid, provoking therefore its atomization. The main objective is therefore to electrically atomize a liquid sheet without the application of an external air flow like in airblast atomizers.

This paper presents a novel actuator, based on dielectric barrier injection, used to induce instabilities within a plane liquid sheet of fuel similar to the annular sheet in aircraft engines. The behaviour of this atomizer was described in previous works. Several modes were observed, sometimes leading to a complete atomization, or just inducing instabilities and oscillating the liquid sheet. In the present study, only the cases where the liquid sheet is completely atomized are investigated. Images were recorded with the help of a high speed camera. Primary atomization is only studied, secondaryatomization being neglected. The properties of the spray obtained by EHD atomization are investigated thoroughly, namely the breakup length, the mesh size, the droplet diameter, the droplet count, etc.
\end{abstract}

\section{Keywords}

Atomization, Atomizers, Dielectric Barrier Injection, Electrohydrodynamics, Liquid Sheet.

\section{Introduction}

The phenomenon of atomization [1],[2] occurs through the interaction of a liquid in motion with respect to a surrounding gas. This occurrence results in the disintegration of the liquid in the form of droplets or fragments. Previous works [3] showed the importance of developing instabilities at the gas/liquid interface and thus leading to the liquid fragmentation.

Understanding the atomization of a liquid consists of understanding the processes that cause the conversion of a compact volume of liquid to a set of droplets [4]. The resulting spray is characterized by a distribution of diameters of the drops that compose it. There are many methods for disintegrating a liquid [5]. Atomizing a liquid sheet is a very efficient technique to obtain such sprays. The sheet thickness plays a major role during its atomization.

Whether it is from a jet [6],[7] or a sheet [8], a droplet is the ultimate step in the liquid fragmentation. The process involves several steps. When the liquid is initiallyin the form of a sheet, a sheet-ligament transition precedes the ligament-drops transition. The formation and the geometry of the ligaments depend on several parameters such as the thickness of the sheet or the speed of the surrounding flow.

At low speed, for example, aerodynamic forces exerted on the sheet produce longitudinal surface waves of great amplitude causing the sheet to beat like a flag. These instabilities lead to the formation of drops which size is of the order of magnitude of that of the sheet edge. The origin of these waves, which correspond to a phase displacement of the two interfaces, results from a Kelvin-Helmholtz type shear instability.

At higher speeds, the amplitude of the longitudinal waves becomes smaller and their frequency greater. The appearance of transverse ripples induces transverse fragmentation of the sheet in form of filaments, which leads to the formation of smaller drops.

The atomization of a liquid conventionally comprises two steps: the primary atomization [9] which corresponds to the formation of spherical or non-spherical liquid fragments, and the secondary atomization which refers to the additional fracture of the liquid fragments, resulting from the primary atomization, into finer droplets. The most cited model investigating secondaryatomization is that of Pilch and Erdman [10]. They present a model based on Ohnesorge number and Weber number values. In the current study, only primary atomization is investigated. 


\section{Material and Methods}

The current work presents experimental results obtained on a plane sheet of fuel. The experimental setup is shown in Figure 1.

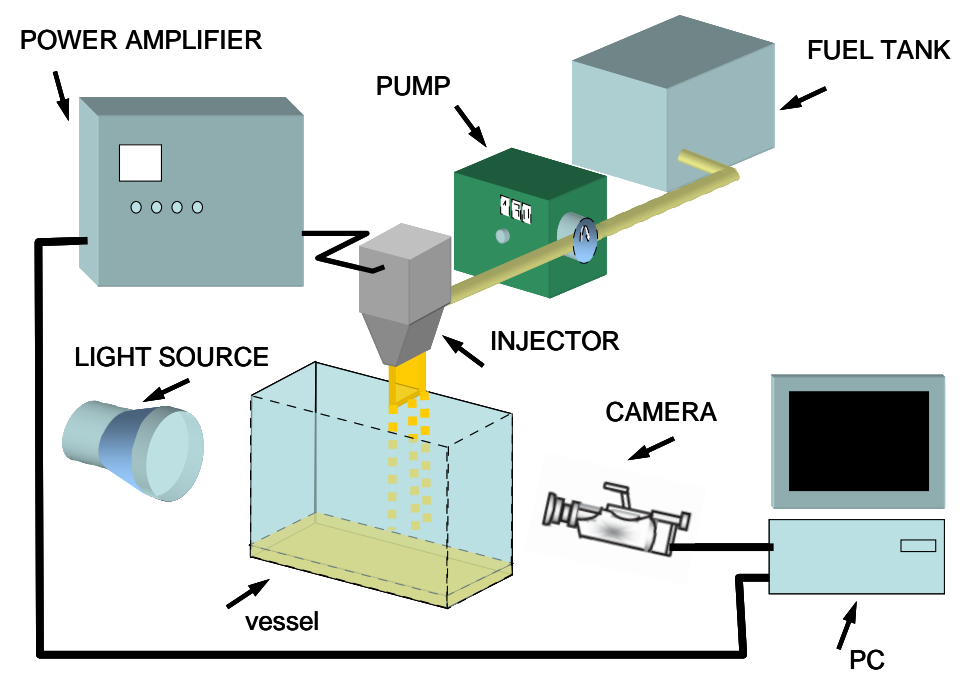

Figure 1. Experimental Setup.

The fuel (Diesel oil) is sucked in through a gear pump and then sent to the injector. The flow is controlled by the pump and adjusted bya manual table. The sheet velocity is therefore regulated by the fuel flow rate. The spray is collected in a vessel placed under the injector. It is also equipped with an anti-splash system (not shown in figure) which prevents the drops created by impact on the bottom of the container from disturbing the measurements. A high voltage square AC signal is provided by a power amplifier connected to the injecting electrode of the fuel injector. Electric signal frequency varies between $1 \mathrm{~Hz}$ and $2000 \mathrm{~Hz}$, and voltage goes up to $30 \mathrm{kV}$ in amplitude. The liquid sheet is filmed as it leaves the injector. A shadow ombroscopy measurement system is installed in order to be able to take instantaneous images of the sheet and study its behaviour with the application of the electric signal. A high speed camera takes up to 5000 images per second of the fuel sheet at a resolution of $640 \times 480$ pixels. The camera resolution could be increased to $1280 \times 960$ pixels but with lower image rate. The captured images are analysed and investigated on a pc.

The fuel injector shown in Figure 2 mainlyconsists of the dielectric material (1). Fuel (2) enters a surge chamber (3) to smooth out turbulence before entering a rectangular slit (4). The blades (5-6) are installed on the tip and allow obtaining a plane sheet (7), having a thickness of $300 \mu \mathrm{m}$ and a width of $62 \mathrm{~mm}$ into the paper. The velocity of the liquid sheet leaving the nozzle ranges between $0.6 \mathrm{~m} / \mathrm{s}$ (which is the minimum speed obtained with the pump) and $2 \mathrm{~m} / \mathrm{s}$. The counter electrode of the electrohydrodynamic actuator (8) is placed inside the injector. Electric charges are injected into the sheet at the level of the electrodes of the actuator.

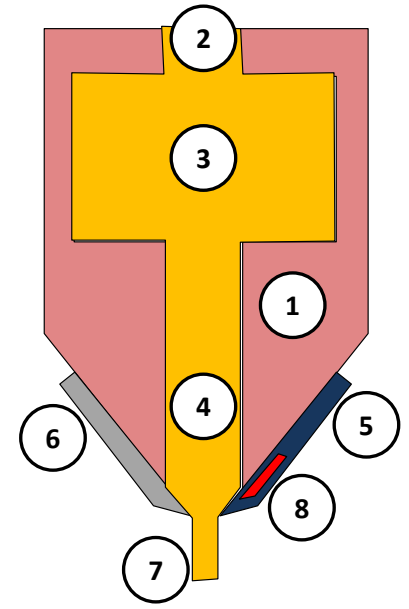

Figure 2. Schematic view of the fuel injector. 
Because such actuators are mainlyused in aircraft engine, kerosene would be the ideal fluid for this experiment. However, a fluid with close properties to kerosene ones is used in the experiments. It is commercial Diesel oil, a slightly conductive dielectric liquid. Its characteristics at a temperature of $20^{\circ} \mathrm{C}$ are presented in Table 1.

Table 1. Typical Characteristics of the Diesel Oil at $20^{\circ} \mathrm{C}$.

\begin{tabular}{|l|l|}
\hline Mass density & 850 \\
\hline Kinematic viscosity & $4.3 \cdot 10^{-}$ \\
\hline Electrical conductivity & $1.15 \cdot 10^{-9}$ \\
\hline Relative permittivity & 2.2 \\
\hline
\end{tabular}

\section{Experimental Results}

When the fuel pump is running, a plane liquid sheet leaves the injector at a width of $62 \mathrm{~mm}$. This width does not remain constant because of surface tension effects. At the boundaries of the liquid sheet, two thick fragments grow as the sheet width decreases. It is shown on the left image of Figure 3. When an appropriate electric voltage is applied on the electrodes, instabilities are generated within the liquid and the sheet is atomized. It is visible on the right image of Figure 3. The development of the flow shows four regions until reaching a spray. First, primary oscillations of high frequency occur in the vicinity of the electrodes. The frequency of these oscillations is probably equal to the electric signal frequency. The second region is characterized by several local perforations of the sheet. Holes bounded by rims appear within the liquid. As these holes grow in size and in number, ligaments are produced and this third region is very close to a net or a mesh. In the lastregion, the ligaments break into droplets of different diameters. The distance from the injector lips until the start of ligaments breakup is defined as the breakup length. Detailed description and investigation on the processes leading to and occurring during atomization were presented in a previous work [11].
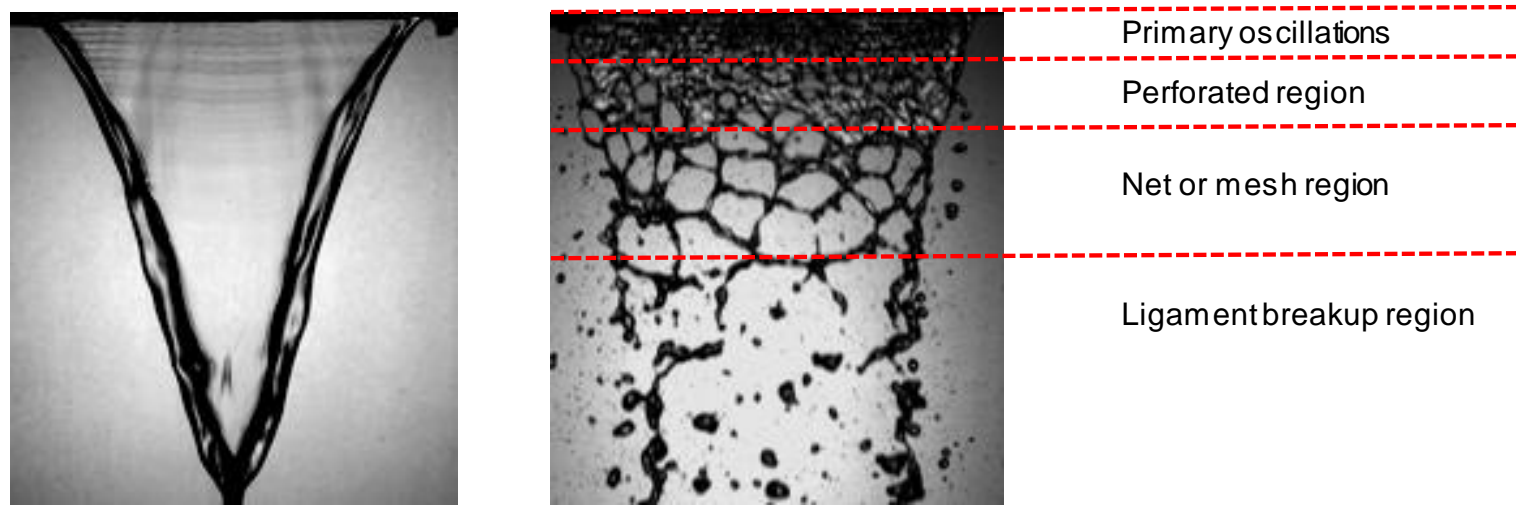

Figure 3. Shadow images of the plane sheet: left image $w$ ith no signal, right image $w$ hen atomized. (actual image size: $66 \mathrm{~mm}$ width, $73 \mathrm{~mm}$ height)

Figure 4 shows images of the liquid sheet for various electric signal frequencies and several liquid velocities. In the absence of electric signal, two edges are visible in black on each side of the liquid sheet. They are formed of a filament of liquid of large diameter. The edges of the sheet meet at few centimetres from the injector (seen in some images). Further downstream (outside the image frame) the sheet quickly turns into a cylindrical jet.

There are numerous physical parameters which can influence the spraying of the liquid sheet: liquid velocity, viscosity, density, air velocity, sheet thickness, etc. To all these mechanical parameters, add the set of electrical parameters: difference of potential, signal shape, signal frequency, polarity, electrode geometry, etc. It is nearly impossible to studyall the interactions resulting from the variations of all these parameters. To limit the number of parameters to be studied, the work presented here is conducted on a single sheet thickness ( $300 \mu \mathrm{m}$ at the exit of the injector) and just one electrode geometry is tested. All tests are carried out without external air flow, which means that atomization is a result of only electrohydrodynamic phenomena. Despite these limitations, the number of parameters and interactions to be studied remains very important. At this stage, it is preferred to conduct a qualitative study rather than a quantitative study [12]. Note that many other frequency values were tested but their corresponding images are not presented in this paper. 

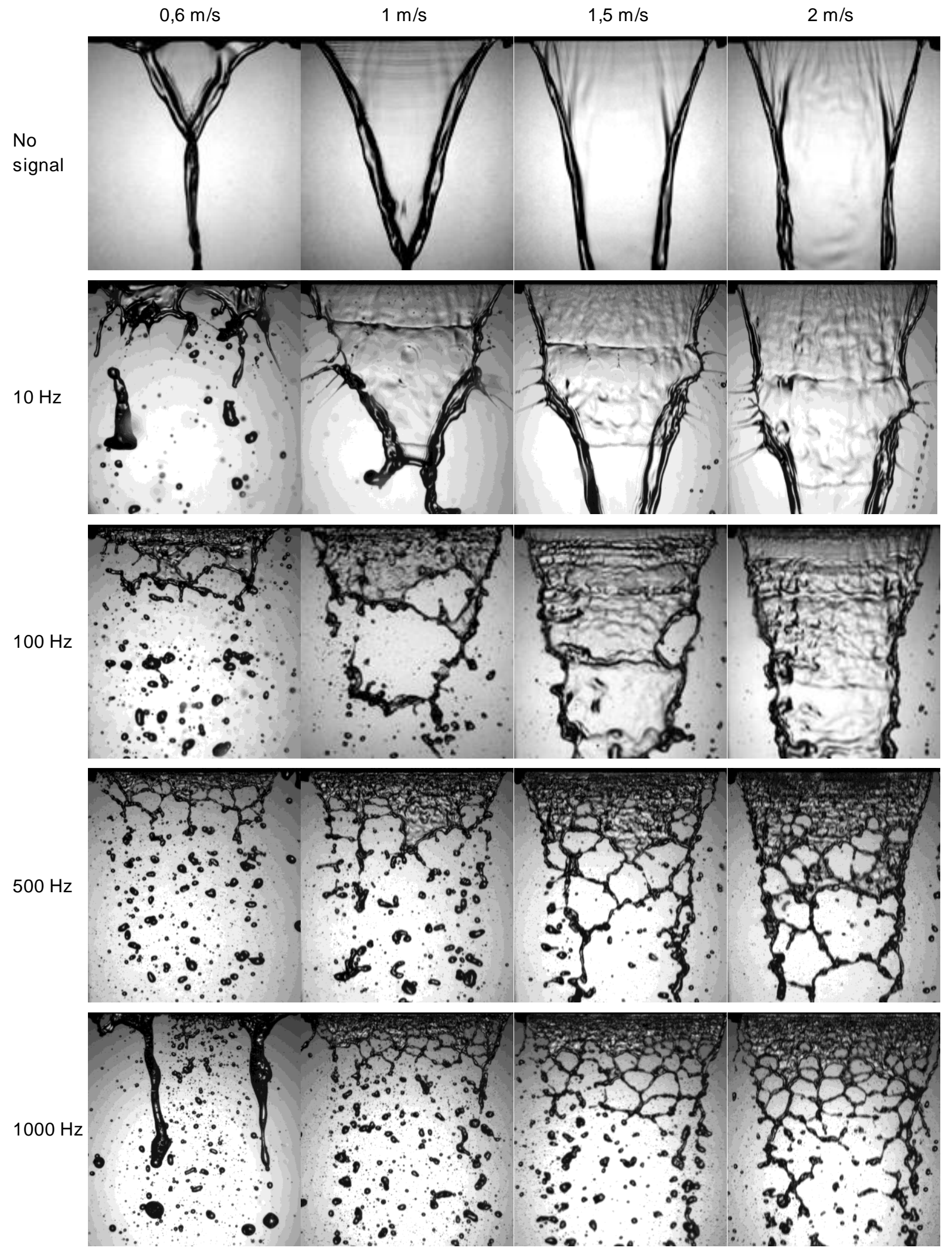

Figure 4. Experimental images of the liquid sheet behaviour $w$ hen exposed to various electric signal frequencies, and for various liquid velocities. Square AC signal, applied voltage $U= \pm 30 \mathrm{kV}$. (actual image size: $66 \mathrm{~mm} w$ idth, $73 \mathrm{~mm}$ height)

This w ork is licensed under a Creative Commons 4.0 International License (CC BY-NC-ND 4.0).

EDITORIAL UNIVERSITAT POLITÈCNICA DE VALÈNCIA 


\section{Interaction between Liquid Sheet Velocity and Electric Signal Frequency}

In previous works [11],[13], three modes of disturbance were observed: a flapping mode where the sheet oscillates like a flag, a disturbed mode where surface vibrations are visible, and a perforated mode where the sheet is pierced so that a mesh and/or ligaments are seen. The last mode only results in an atomization of the sheet.

The spraying mechanisms appear to be different depending on the frequency of the signal. A low frequency behaviour (frequency less than or equal to $100 \mathrm{~Hz}$ ) and a high frequency behaviour (above $300 \mathrm{~Hz}$ ) are distinguished. The transition between the two modes of operation is not immediate but ranges from $100 \mathrm{~Hz}$ to $300 \mathrm{~Hz}$, in which both behaviours can be observed.

At low frequency: The zone of primary oscillation is very extensive. In this zone a longitudinal wave with a frequency identical to that of the signal appears. This wave leads to the appearance on the sheet of thick transverse beads (two per period). The distance between the beads is halved when the frequency is doubled. Their thickness also decreases sharplywhen the frequency increases. The mesh area is practically non-existent at low sheet velocity. The breakup of the sheet occurs by piercing the membrane which connects the beads. They then become filaments. The collapse of these filaments produces a set of drops of large diameter.

At high frequency: The primary zone is reduced considerably, the oscillation takes a much more chaotic aspect and the beads are no longer visible. The mesh area becomes visible. The mesh size is then different according to the form of applied signal. Several shapes of AC electric signals were tested (square, triangle, sine, step) but only square signal results are presented in this paper. It is also observed that the mesh size decreases as the frequency increases.

\section{Study of the Breakup Length}

The breakup length is an important parameter when studying the behaviour of the liquid sheet. It can be easily measured on the previous images. Despite the high measurement uncertainty (especially at low frequency), very clear trends can be deducted. A set of 1000 images was captured for each case, and then the average value is presented in this work. In Figure 5, the behaviour of the breakup length as a function of the electric signal frequency is presented first (left chart). Then the behaviour is plotted versus the liquid sheet velocity (right chart). First, in the absence of electric signal, the sheet closes more or less rapidly. The lower the sheet velocity, the faster it closes.
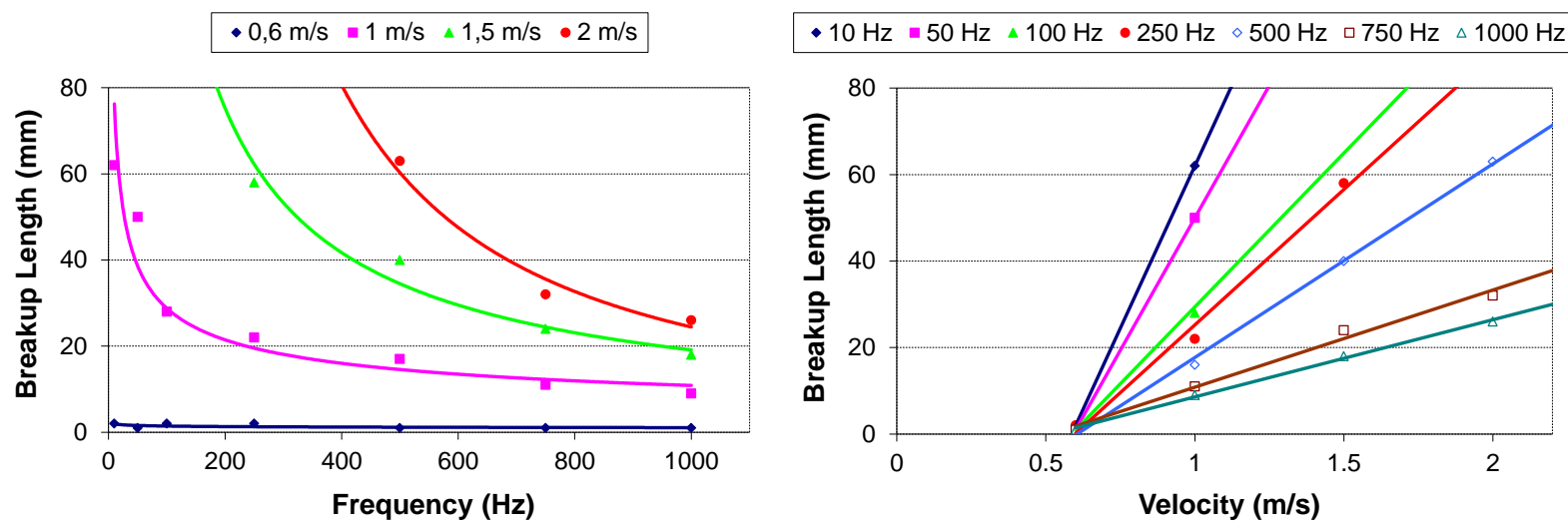

Figure 5. Breakup length variations versus electric signal frequency (left) and versus liquid sheet velocity (right).

A first observation is that the breakup length of the fuel sheet decreases as the electric signal frequency increases. This is due to the instabilities created within the sheet by EHD. At a frequency of $50 \mathrm{~Hz}$, the sheet is not always perforated. It is from the $100 \mathrm{~Hz}$ value that a breakup begins to be observed for the low sheet velocities. The breakup length decreases with increasing the frequency to a value of $7 \mathrm{~mm}$ at $1000 \mathrm{~Hz}$, at $1 \mathrm{~m} / \mathrm{s}$ sheet velocity for example. The decrease is asymptotic, and shorter breakup lengths could be obtained for frequencies over $1000 \mathrm{~Hz}$. The start of the perforation process depends strongly on the sheet velocity.

On the second chart, although curves are plotted with few points, the behaviour of the breakup length appears to be linear with the liquid velocity. Note that the breakup of the sheet occurs very close to the injector lips when the sheet velocity is lowest $(0.6 \mathrm{~m} / \mathrm{s})$. The dynamics of the mechanisms that lead to the piercing of the liquid sheet appear to be independent of the sheet velocity. Similar behaviour was also observed for the mesh size. 


\section{Granulometric Study}

Figure 6 presents zoomed images of front views and side views of the liquid sheet when it is fully atomized at frequencies above $100 \mathrm{~Hz}$. The objective here is to conduct a granulometric studyon the droplet size and count. It is clearly visible on images that the frequency affects significantly the atomization, even for fully atomized sheets.

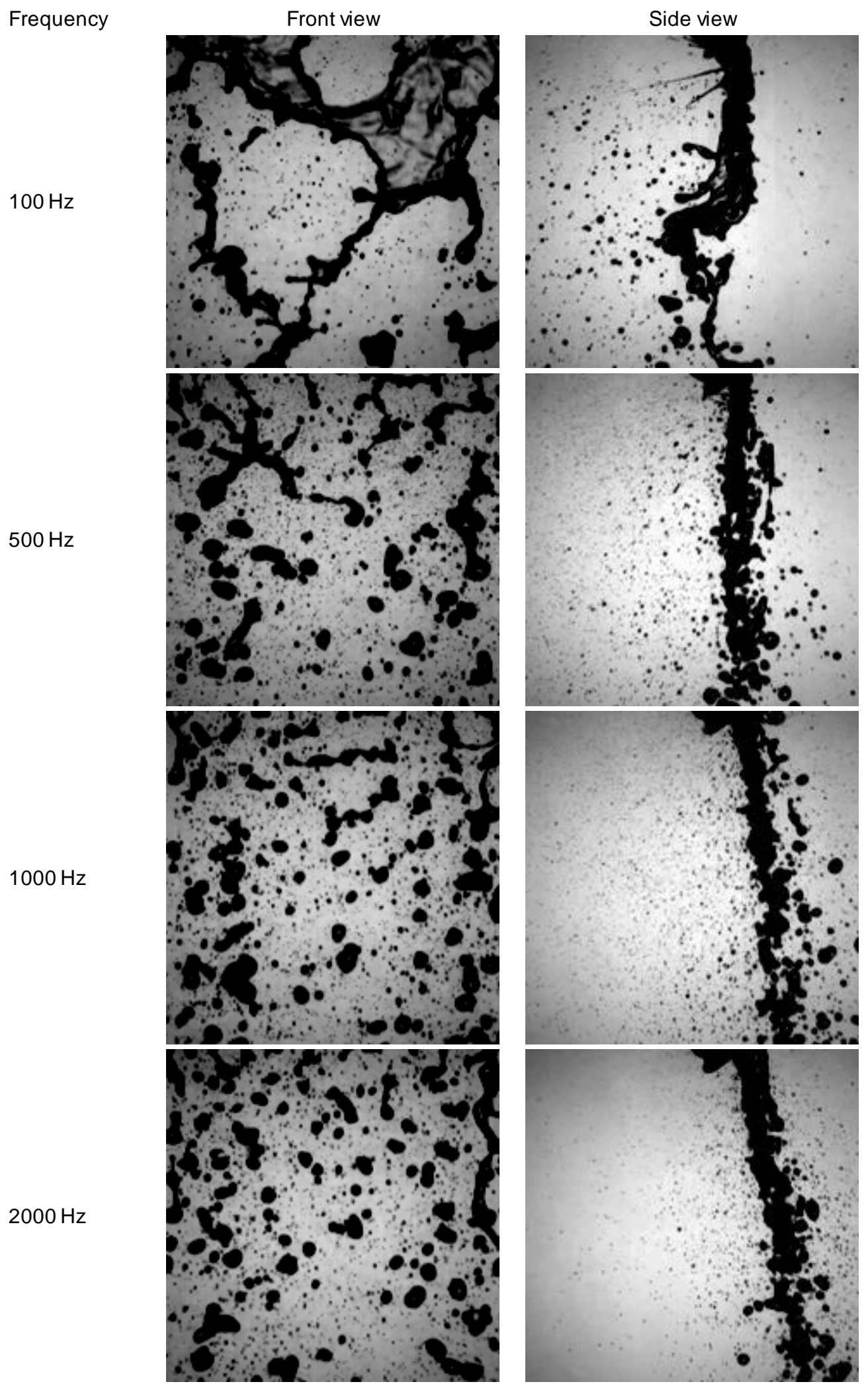

Figure 6. Zoomed images of the atomized sheet at various signal frequencies. (actual image size: $50 \mathrm{~mm}$ width, $50 \mathrm{~mm}$ height) 
If a higher zoom is applied on the images, the smallest drops will appear. A moderate frequency of $100 \mathrm{~Hz}$ leads to the formation of large structures as seen in Figure 6. Between these large structures, smaller drops are clearly visible. As the frequency increases, these drops become smaller and more numerous.

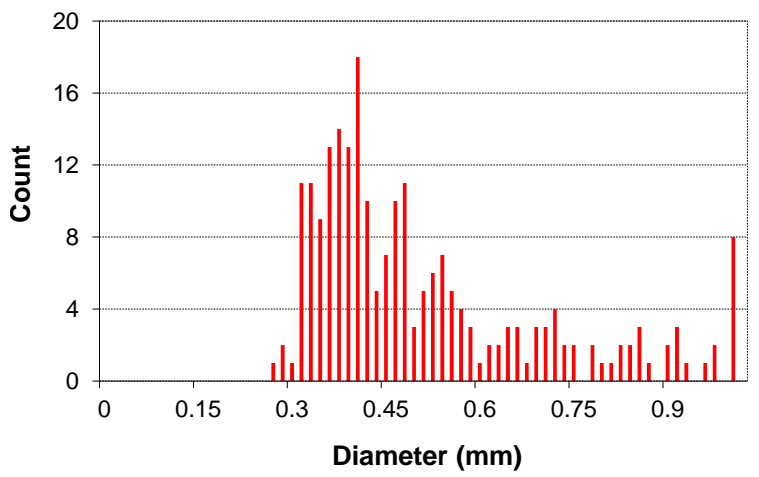

a. $100 \mathrm{~Hz}$

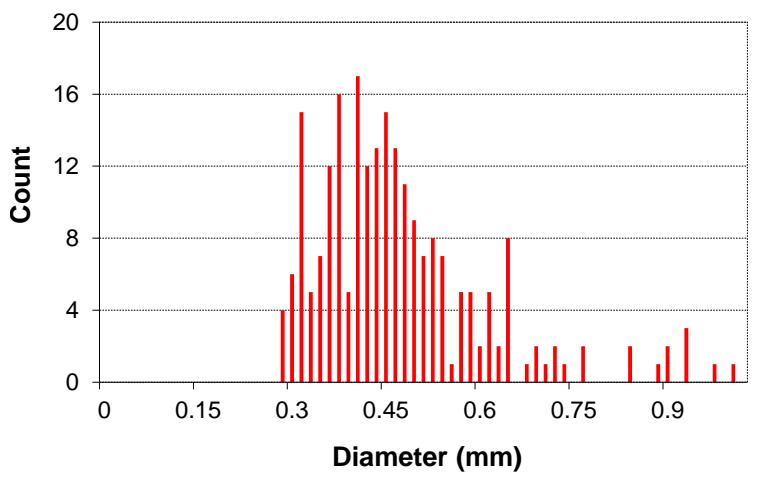

c. $1000 \mathrm{~Hz}$

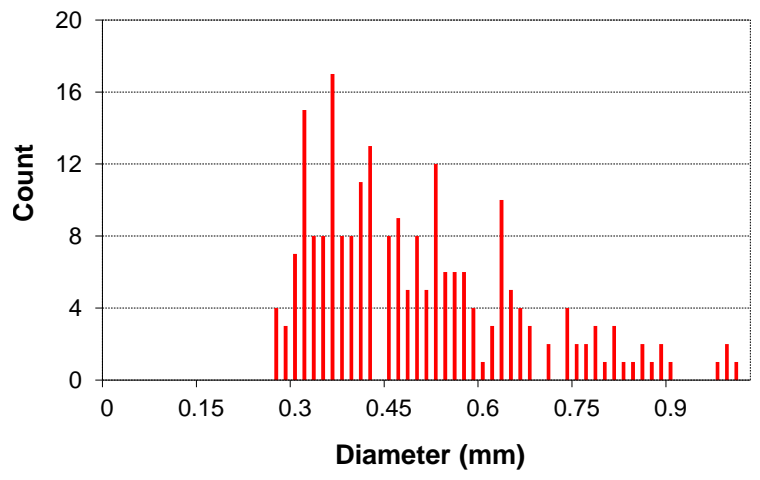

b. $500 \mathrm{~Hz}$

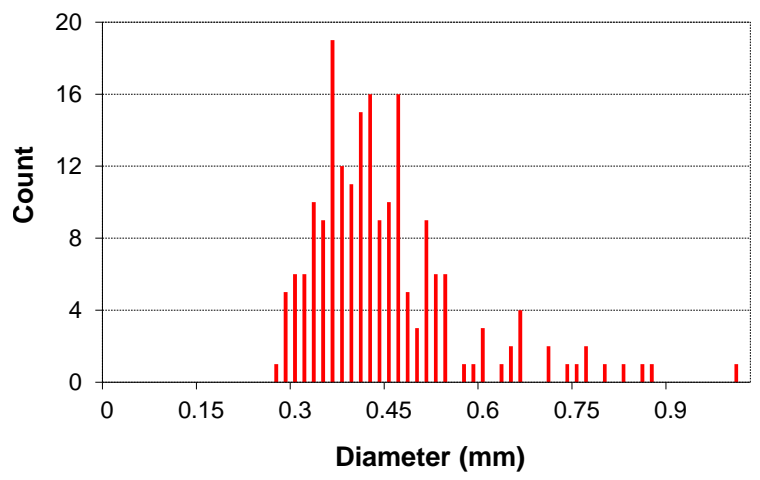

d. $2000 \mathrm{~Hz}$

Figure 7. Histograms of the count of droplet size for various frequencies.

Figure 7 presents the histograms of droplet count having various diameters, and for different frequencies of the electric signal. The software used for this treatment is Davis 7.0 software which is mainly used in Particle Image Velocimetry (PIV) measurements. Unfortunately, because of the dimensions of the images, the software did not allow us to detect drops whose diameter is less than $300 \mu \mathrm{m}$. Drops with a diameter greater than $1 \mathrm{~mm}$ are not rounded. In the case presented here, the liquid sheet is plane and large drops mostly result from the two rims bounding the sheet. On the contrary, in aircraft engines, fuel sheets are cylindrical and this problem does not occur. Therefore these large drops are not counted either.

The granulometric study is imperfect because manydrops are not detected by the software. Nevertheless, as the procedure executed on the images is exactly the same, the trends emerge. At $100 \mathrm{~Hz}$, the distribution of the drops is between 0.3 and $1 \mathrm{~mm}$. When the frequency increases, drops between 0.6 and $1 \mathrm{~mm}$ almost disappear. Therefore, the increase in frequencyseems to reduce the size of the drops. This study must be reconducted with another software appropriate for granulometry. In addition, a more thorough study on the Sauter mean diameter $D_{32}$ [14] should be carried out in order to obtain a more comprehensible behaviour of the atomization.

\section{Conclusions}

In this study, an EHD actuator was used to destabilize a sheet of Diesel fuel and lead to its atomization. The sheet was $62 \mathrm{~mm}$ wide and $300 \mu \mathrm{m}$ thick. Velocities ranging from $0.6 \mathrm{~m} / \mathrm{s}$ to $2 \mathrm{~m} / \mathrm{s}$ and electric signal frequencies ranging from $1 \mathrm{~Hz}$ to $2000 \mathrm{~Hz}$ were tested. A square $\mathrm{AC}$ electric signal with an amplitude of $\pm 30 \mathrm{kV}$ was applied between the electrodes to induce the liquid sheet atomization.

The possibilities offered by the EHD system have proved particularly rich. Two mechanisms of primary atomization seem to exist: a fast mechanism that pierces the sheet and creates a first set of small drops and then a slower mechanism, which corresponds to the disintegration of the mesh-ligaments and drops of millimetre size. 
It was observed that the frequency of the applied electric signal is an important parameter affecting the atomization process. High frequencies result in much better atomization. In addition, the liquid sheet velocity has an influence on the disintegration of the sheet. Higher liquid velocities require greater instabilities to completely atomize the liquid sheet.

The analysis of the images using an ombroscopy software allowed us to conduct a coarse granulometric study. The particle size study of EHD sprays, although carried out in a very fragmentary way, shows a clear tendency for the large drops to disappear when the frequency of the signal increases. A more appropriate granulometry software must be used in order to get the full distribution of droplet sizes.

The work carried out here aimed at demonstrating the efficiency of the process and delimiting the fields of operation. A more precise study must be carried out now to quantify more accurately the influence of each of the parameters governing the atomization process.

Finally, after comparison of the obtained results to those of air-blast atomization, it would be interesting to investigate the combined effect of electrohydrodynamic and air-blast atomizations in future experiments.

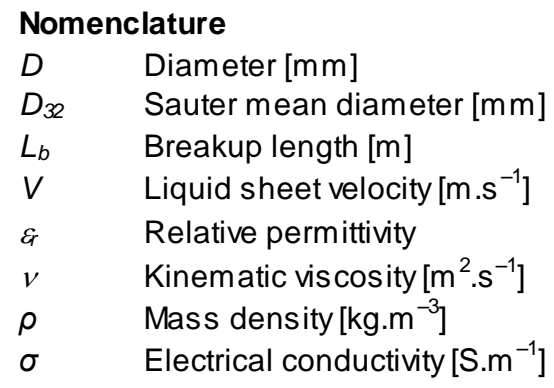

\section{References}

[1] Mansour, A., Chigier, N., Disintegration of liquid sheets, 1990, Physics of Fluids A, 3, pp. 2971-2980.

[2] Mansour, A., Chigier, N., Dynamic behavior of liquid sheets, 1991, Physics of Fluids A, 2, pp. 706-719.

[3] Berthoumieu, P., Carentz, H., Experimental study of a thin planar liquid sheet disintegration, July 16-20, 2000, $8^{\text {th }}$ International Conference on Liquid Atomization and Spray Systems, ICLASS 2000, Pasadena, CA, USA.

[4] Berthoumieu, P., Lavergne, G., Video techniques applied to the characterization of liquid sheet breakup, 2001, Journal of Visualization, 4 (3), pp. 267-275.

[5] Larricq-Fourcade, C., Lavergne, G., Berthoumieu, P., Thivet, F., Improvement of airblast injectors with electro-aerodynamic actuators, October $3-7,2005,2^{\text {nd }}$ International Symposium on Nonequilibrium Processes, Plasma, Combustion and Atmospheric Phenomena, NEPCAP 2005, Sochi, Russia.

[6] Shrimpton, J.S., Electrohydrodynamics of charge injection atomization: Regimes and fundamental limits, 2003, Atomization and Sprays, 13 (2\&3).

[7] Rigit, A.R.H., Shrimpton, J., Electrical performance of charge injection electrostatic atomizers, 2006, Atomization and Sprays, 16 (4), pp. 401-420.

[8] Bremond, N., Clanet, C., Villermaux, E., Atomization of undulating liquid sheets, 2007, Journal of Fluid Mechanics, 585, pp. 421-456.

[9] Dumouchel, C., On the experimental investigation on primary atomization of liquid streams, 2008, Experiments in Fluids, 45, pp. 371-422.

[10] Pilch, M., Erdman, C.A., Use of the breakup time data and velocity history data to predict the maximum size of stable fragments for acceleration induced breakup of a liquid drop, 1987, International Journal of Multiphase Flow, 13, pp. 741-757.

[11] Louste, C., Daaboul, M., Traore, Ph., Romat, H., Experimental Study of electrical primary break-up of a thin sheet of dielectric liquid controlled by an electrohydrodynamic actuator, September 6-9 2010, 23 ${ }^{\text {rd }}$ European Conference on Liquid Atomization and Spray Systems, ILASS-Europe 2010, Brno, Czech Republic.

[12] Lastow, O., Balachandran, W., Numerical simulation of electrohydrodynamic (EHD) atomization, 2006, Journal of Electrostatics, 64 (12), pp. 850-859.

[13] Louste, C., Daaboul, M., Traoré, P., Romat, H., Influence of signal properties on electrohydrodynamic primary break-up of thin sheets of dielectric liquid, September 2-6, 2012, $12^{\text {th }}$ International Conference on Liquid Atomization and Spray Systems, ICLASS 2012, Heidelberg, Germany.

[14] Sauter, J., Die Grössenbestimmung der im Gemischnebel von Verbrennungskraftmaschinen vohrhandenen Brennstoffteilchen, 1926, Mitteilung aus dem Laboratorium für Technische Physik der Technischen Hochschule München, VDI-Verlag. 


\title{
Characterization of Liquid Impinging Jet Injector Sprays for Bi- Propellant Space Propulsion: Comparison of PDI and High- Magnification Shadowgraphy
}

\author{
Bastien Boust ${ }^{\star 1}$, Quentin Michalski ${ }^{1}$, Alain Claverie ${ }^{1}$, Clément Indiana ${ }^{1}$, Marc Bellenoue ${ }^{1}$ \\ ${ }^{1}$ Institut PPRIME, CNRS/ISAE-ENSMA/Université de Poitiers, France \\ *Corresponding author: bastien.boust@ensma.fr
}

\begin{abstract}
Impinging jet sprays are investigated in the reference case of like-doublet injector, for application to bi-propellant combustion. Green propellants are considered, namely ethanol as a fuel and hydrogen peroxide as an oxidizer, that is well represented by water. This study reports original comparisons between standard spray characterization (PDI) and high-magnification shadowgraphy of the spray $(2.5 \times 3.2 \mathrm{~mm}, 2.5 \mu \mathrm{m}$ per pixel) based on short laser backlight illumination ( $5 \mathrm{~ns}$ ). Shadowgraphy images describe accurately the inner spray structure and provide the size and velocity of droplets. This diagnostic is used to analyse the influence of jet momentum (driven by injection pressure) on impinging jet atomization, as well as the evolution of spray topology, drop size distribution and average diameter along the spray centreline. The application of shadowgraphy to the dense region of water and ethanol sprays shows the different atomization behaviour of these two fluids with respect to their surface tension. Elliptical droplets are characterized inside the spray, which confirms the interest of a direct visualization of droplets in such dense sprays.
\end{abstract}

\section{Keywords}

Liquid impinging jet injector, Atomization, Shadowgraphy, Droplet sizing.

\section{Introduction}

Impinging jet injectors are used in most storable bi-propellant space engines. Current storable propellants such as hydrazine and nitrogen tetroxide usually burn after hypergolic ignition, which is allowed by their chemical properties. However, these toxic propellants are to be replaced by "green" propellants, e.g. hydrogen peroxide and ethanol, which atomization, ignition and combustion deserve additional knowledge. Particularly, compared to current storable propellants, the atomization of green propellants may be less prone: i. to generate small droplets due to their surface tension, ii. to generate a gaseous phase to their low volatility (e.g. hydrogen peroxide), iii. to ignite and burn efficiently, as a result. Therefore, attention must be paid to the atomization process of these new "green" propellants, particularly in the physical conditions encountered in combustion chambers i.e. subcritical at high temperature and pressure.

In practice, liquid propellants atomization is usually based on jet impingement, which is a common way to generate droplets with moderate levels of pressure (e.g. a few MPa). In this study, the like-doublet configuration is considered as a reference case, even though real engines injectors may also feature unlike jet impingement (e.g. fuel on oxidizer) such as doublets or triplets etc. In the literature, numerous studies already address this like-doublet configuration in the spraying regime related to engine conditions, i.e. at elevated Reynolds and Weber numbers $\left(10^{3}-10^{4}\right)$ leading to ligaments and fully developed breakup; a review of such spray phenomena can be drawn from detailed experiments [1]. Like-impinging doublets are investigated through theoretical studies [2] and numerical simulation [3]-[4], highlighting the dynamics of the liquid sheet breakup that generates wavy ligaments and then droplets. However in this complex situation, the reference knowledge concerning the spray shape, the distribution of droplet size and velocity, is provided by experiments [5]-[8]; for this purpose, most studies are based on PhaseDoppler Interferometer (PDI) and direct visualization of the spray. In these fundamental experiments the droplet distribution is well characterized downstream the dense part of the spray (i.e. downstream the liquid sheet), but in real engine conditions the flame affects the spray even from the dense zone [8], so that the spray properties cannot be inferred directly from inert condition measurements. This makes spray combustion all the more complex because drop size influences combustion performance and induction length, as an example. Moreover, the Sauter Mean Diameter (SMD) measured with PDI is found to increase with increasing distance from the impingement point [5][6], whereas it is found to decrease in another study combining experiment and simulation [3]. This contradiction shows that impinging jet sprays deserve a specific study, in order to discuss the methods used to analyse the structure of such sprays. 
Thus, the specific objective of this work is to perform original comparisons between standard spray characterization based on PDI, and high-magnification shadowgraphy of the spray. This last diagnostic is known to provide better results in dense sprays [9]. Thanks to this diagnostic, a direct analysis of the spray structure is expected and may provide additional information to the classical PDI analysis. The influence of jet velocity is investigated through the influence of injection pressure, as well as the effect of fluid properties (water or ethanol), for elevated Reynolds and Weber numbers $\left(10^{3}-10^{4}\right)$ leading to a "ligament-structure" or "fully-developed" spray.

\section{Experiment and diagnostics}

Experimental apparatus

The liquids of interest are sprayed using a classical setup for impinging jets under inert gas pressurization, as described in former studies [2],[5]. The two jets of the like-doublet are generated from 2 symmetrical orifices manufactured in a single stainless steel injector designed for combustion experiments; they are drilled with a diameter $d_{0}=0.3 \mathrm{~mm}$, a length $L / d_{0}=20$, and an angle of impingement $60^{\circ}$, that provides good momentum while preventing from backsplash and erosion of the injector. The velocity of the jets is governed by the pressure drop $\Delta P$ across the injector through Bernoulli equation: $V=\sqrt{2 \Delta P / \rho}$.
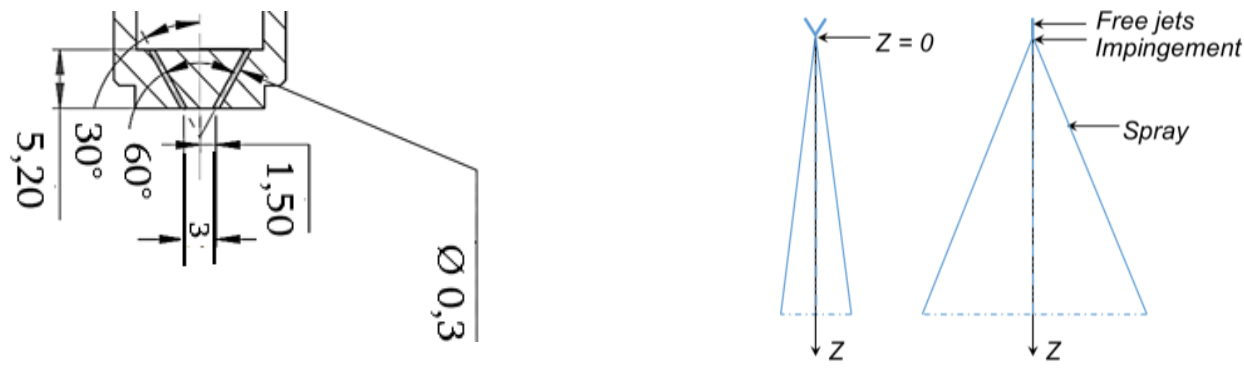

Figure 1. Experimental injector geometry.

The study focuses on green storable propellants, such as ethanol and hydrogen peroxide. Spraying conditions are characterized by the Reynolds (1), Weber (2) and Ohnesorge (3) dimensionless numbers. For safety purposes, deionized water is used to represent the atomization behaviour of hydrogen peroxide, since they have similar We and Oh numbers (see Table 1). In this table, High Test Peroxide (HTP) of mass concentration $87.5 \%$ is reported but not used experimentally. According to the behaviour of impinging jet sprays [1], the magnitude of $R e$ and $W e$ numbers settle our experiments in the "ligament-structure" or "fully-developed" atomization regimes. For this purpose, the relevant injection conditions are reached by controlling the jet momentum thanks to the pressure drop across the injector $\Delta P=0.9-3.0 \mathrm{MPa}$.

$$
\begin{aligned}
& \operatorname{Re}=\frac{\sqrt{2 \rho \Delta P} \times d_{0}}{\mu} \\
& W e=\frac{2 \Delta P \times d_{0}}{\sigma} \\
& O h=W e^{1 / 2} \operatorname{Re}^{-1}=\frac{\mu}{\sqrt{\rho \sigma d_{0}}}
\end{aligned}
$$

\begin{tabular}{|c|c|c|c|c|c|c|}
\hline & \multicolumn{2}{|c|}{ Water } & \multicolumn{2}{|c|}{ Ethanol } & \multicolumn{2}{|c|}{ HTP } \\
\hline Density $\rho(\mathrm{kg} / \mathrm{m} 3)$ & \multicolumn{2}{|c|}{998} & \multicolumn{2}{|c|}{792} & \multicolumn{2}{|c|}{1379} \\
\hline Surface tension $\sigma(\mathrm{N} / \mathrm{m})$ & \multicolumn{2}{|c|}{0.073} & \multicolumn{2}{|c|}{0.022} & \multicolumn{2}{|c|}{0.079} \\
\hline Dynamic viscosity $\mu(\mathrm{kg} / \mathrm{m} / \mathrm{s})$ & \multicolumn{2}{|c|}{$1.002 \mathrm{E}-3$} & \multicolumn{2}{|c|}{1.272 E-3 } & \multicolumn{2}{|c|}{$1.260 \mathrm{E}-3$} \\
\hline $\mathrm{Oh}$ & \multicolumn{2}{|c|}{0.00678} & \multicolumn{2}{|c|}{0.01759} & \multicolumn{2}{|c|}{0.00698} \\
\hline$\overline{\Delta P(\mathrm{MPa})}$ & Re & We & 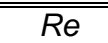 & We & $R e$ & We \\
\hline 0.9 & 12690 & 7400 & 8900 & 24550 & 11860 & 6840 \\
\hline 1.5 & 16380 & 12330 & 11500 & 40910 & 15310 & 11400 \\
\hline 3.0 & 23170 & 24660 & 16260 & 81820 & 21660 & 22790 \\
\hline
\end{tabular}

Table 1. Fluid properties and experimental conditions for $d_{0}=0.30 \mathrm{~mm}-$ HTP is not used experimentally. 
Spray analysis based on Phase-Doppler Interferometer

Following the initial development of Bachalo, the Phase-Doppler Interferometer (PDI) has proved to stand as a powerful standard laser-based diagnostic instrument for simultaneous and accurate measurements of the size and velocity of individual spherical particles in polydisperse particle flow environment [2],[5]-[6].

A modular PDI system (Artium 200 MD) is used for the real-time, non-intrusive measurement of individual drop size and 2 velocity components in this application. The diode-pumped solid-state lasers used in the transmitter provide $532 \mathrm{~nm}$ and $473 \mathrm{~nm}$ wavelengths. The distances from transmitter to probe volume, and from receiver to probe volume, are set to $500 \mathrm{~mm}$ allowing $4.33 \mu \mathrm{m}$ fringe spacing and a range of diameters between $1.5 \mu \mathrm{m}$ and $160 \mu \mathrm{m}$ when used with a light scattering angle set to $40^{\circ}$. The probe volume dimensions are characterized by a quasicircular cross-section of diameter $0.34 \mathrm{~mm}$, and a length of $5.6 \mathrm{~mm}$. PDI data processing includes the classical "probe-volume correction" (PVC) that compensates for the effect of varying sample volume on drop size.

\section{Spray analysis based on high-magnification shadowgraphy}

High-magnification shadowgraphy is recorded with pulsed backlight illumination, that is independent of the shape and material of the particles (either transparent or opaque) and allows for the investigation of drop size down to a few microns per pixel. Based on a PIV system, this technique is expected to provide information on the droplets shape coupled with size distribution and velocity.

As illustrated (see Figure 2), a double-pulse Nd:YAG laser (Continuum Minilite II, $2^{*} 25 \mathrm{~mJ} /$ pulse) is combined with a double-frame camera (LaVision Flowmaster 3S, $1280 \times 1024$ px, $6.35 \mu \mathrm{m} / \mathrm{px}$ ) equipped with a long-distance microscope (Questar QM1) of $60 \mathrm{~cm}$ working distance. A diffusor optics with a wavelength shifting fluorescing plate is mounted to the laser beam outlet, which yields a homogenous speckle-free backlight illumination of wavelength around $590 \mathrm{~nm}$. The measurement volume is defined by the focal plane and the depth of field of the imaging system. The image size is $2.5 \times 3.2 \mathrm{~mm}$, and image pixel size is $2.5 \mu \mathrm{m}$. Given a f-number for the optics of 9.3 , the effective spatial resolution based on Rayleigh criterion is $5.6 \mu \mathrm{m}$. The short laser pulsewidth (between 3 and $5 \mathrm{~ns}$ ) ensures the motion freezing of all imaged droplets. Conventional corrections are applied to the drop size distributions using statistical weighting functions, in order to compensate for the so-called "border" and "depth-of-field" effects [9].

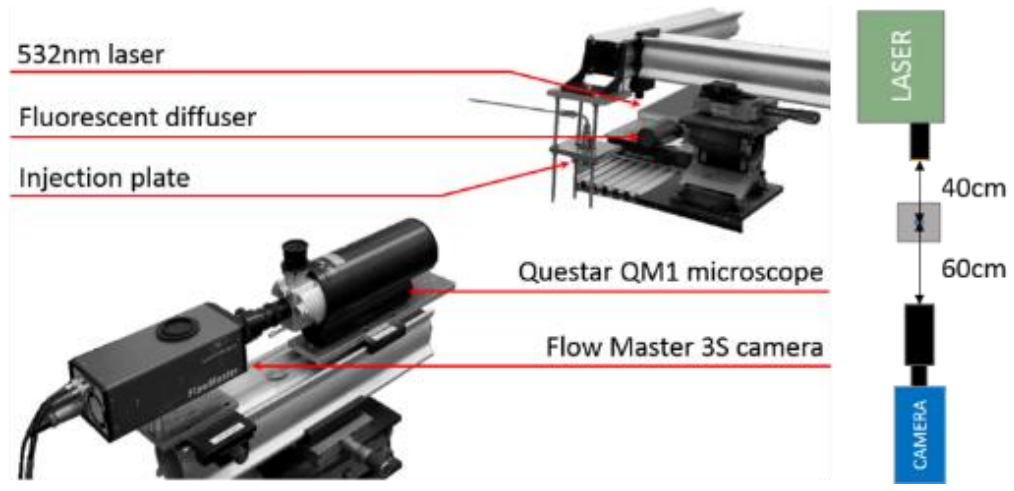

Figure 2. Experimental setup for high-magnification shadowgraphy.

Images are analysed using commercial software (ParticleMaster Shadow, Lavision) that includes several parameters for droplet detection (see Figure 3). The first one is the kernel width of a sliding maximum filter, which is used to ensure background homogeneity for droplets detection prior to the first segmentation: it is set to $100 \mathrm{px}$ $(250 \mu \mathrm{m})$. The binarisation threshold for the first segmentation is a global threshold relative to the maximum intensity found on the image: it is set to $50 \%$. A second segmentation computes a droplet diameter based on an average between the 40\%- and 60\%-threshold relative to the local maximum on the droplet. Blurry particles are rejected based on a minimum slope criterion, locally defined as the relative intensity decrease at the particle rim: it is set to $20 \%$. Given the intensity difference between small and large droplets, the admissible depth of field depends on drop size. Based on the slope criterion, it is comprised between $50 \mu \mathrm{m}$ for the smallest droplets, and $330 \mu \mathrm{m}$ for the biggest ones. Droplet velocity is computed from each doublet of images, provided the droplet is identified in both frames. The identification is based on a diameter variation inferior to $15 \%$, and to a vertical velocity comprised between -10 and $50 \mathrm{~m} / \mathrm{s}$. For each condition, 20 doublets of images are recorded at $2 \mathrm{~Hz}$ with $2 \mu \mathrm{s}$ delay between frames, which yields up to 50 validated droplets per frame. 

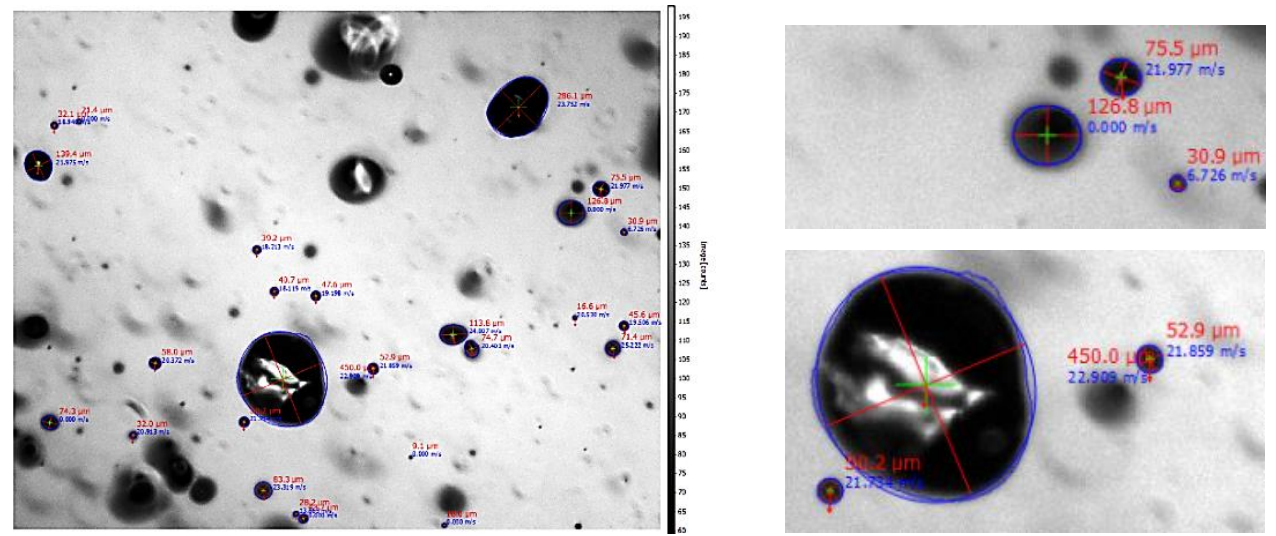

Figure 3. Example of particle detection obtained by the software - Water spray, $0.9 \mathrm{MPa}, Z / \mathrm{d}_{0}=100$.

Even though shadowgraphy is far less sensitive to droplet shape for detection, it may detect overlapping droplets or ligaments to which is attributed an elliptical cross-section (see Figure 4). In order to avoid such erroneous particles, images are computed using a second exclusion criterion, based on the ratio between the elliptical interpolation perimeter and the perimeter of the actual droplet: an optimal value of 0.875 is found for this criterion. This method is especially useful to exclude the erroneous ligaments for which the large extrapolated diameter is not representative of a real diameter.
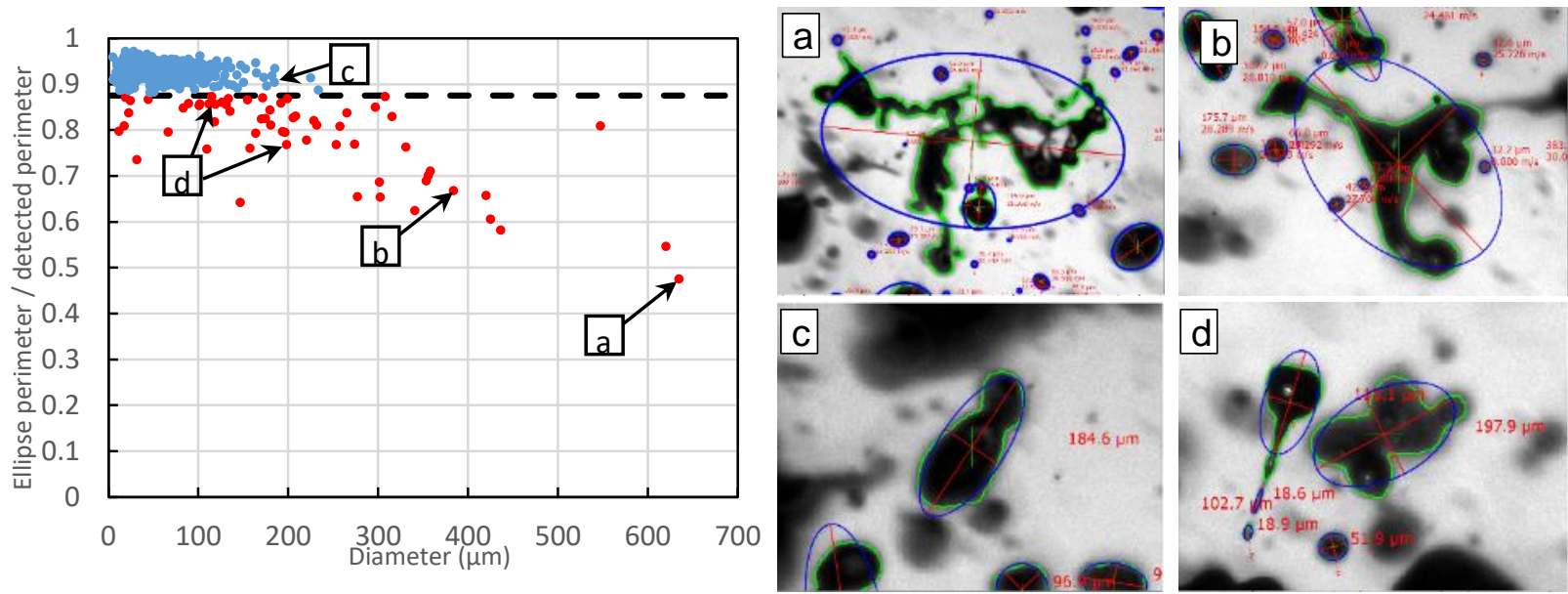

Figure 4. Example of particles excluded (red dots) or included (blue dots) in the statistics. Perimeter detected (green overlay), perimeter of the ellipse (blue overlay). In this case 63 particles are excluded (about 10\%) - Water spray, $0.9 \mathrm{MPa}, Z / d_{0}=50$.

The spray is studied in steady operation. Its wavy behaviour is reported in the literature [2],[6] (see Figure 5 for visualization) and the ligament wavelength is less than $1.0 \mathrm{~mm}$, hence a characteristic frequency about $10 \mathrm{kHz}$ for a typical velocity of $10 \mathrm{~m} / \mathrm{s}$. Our diagnostics are compatible with these space and time scales: the shadowgraphy field of view $(2.5 \times 3.2 \mathrm{~mm})$ corresponds to several wavelengths of the spray (2-3 wavelengths), while PDI is performed over a large number of time-periods $(6 \mathrm{kHz}$ in average during $1-2 \mathrm{~s})$. Thus both diagnostics allow a correct sampling.

Besides, the characteristic dimension of the PDI probe volume cross-section $(0.34 \mathrm{~mm})$ represents around $10-15 \%$ of the shadowgraphy field-of-view. Moreover, shadowgraphy provides uniform results (same drop size distribution and droplet number) whatever the investigation region. Therefore, shadowgraphy and PDI results can be compared in these spray conditions.

\section{Results and discussion}

Topology of the spray

The spray is investigated by high-magnification shadowgraphy in the conditions of interest (see Table 1), for water and ethanol. For each spray condition, the measurement point is displaced from the impingement point of the jets, along the spray centreline coordinate $Z$ (see Figure 5). The corresponding shadowgraphy pictures provide an original view of the inner spray structure. Although the topology of such sprays has been thoroughly described in 
past studies, the present visualizations offer a detailed description of the primary atomization process (sheet breakup from $Z=3 d o$ ), and of the secondary atomization process (ligament breakup from $Z=20 d$ ). These breakup lengths and wavelengths have been discussed in our previous work concerning like-doublets [6].

Additional information is given by the shape of ligaments and droplets. The dynamics of ligaments has a wavy behaviour that generates elongate blobs and droplets from $Z=20-30 d_{0}$, and these structures exhibit a relaxation process towards a stable shape (i.e. spherical). However, pictures at $Z=100-150 d_{0}$ show that small droplets reach a round shape, whereas the numerous big droplets that remain in the spray still have an elliptical or concave shape. As a result, the biggest droplets may be detected well by shadowgraphy, but hardly by PDI.

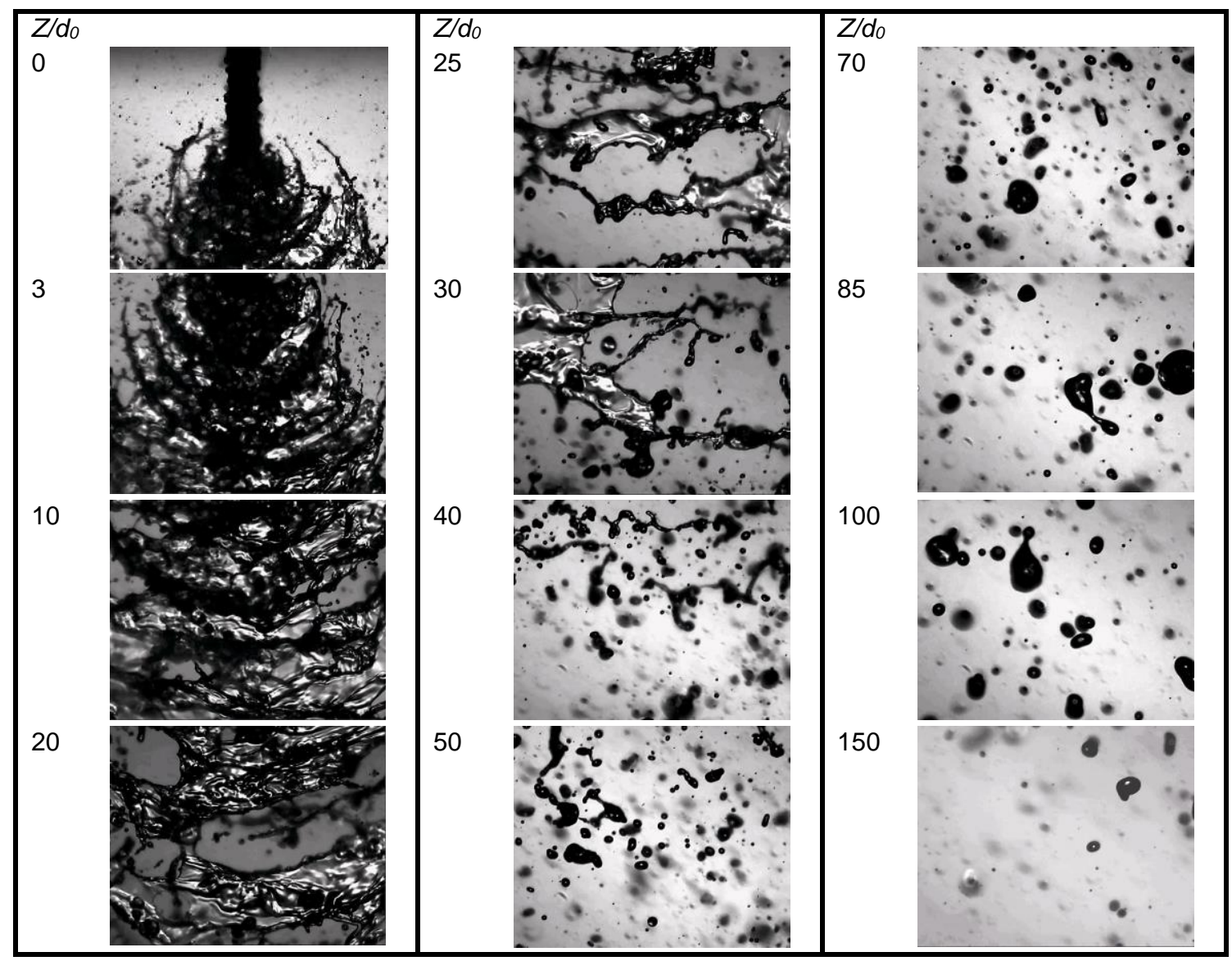

Figure 5. Shadowgraphy of the spray from the impingement point - Water, $\Delta P=0.9 \mathrm{MPa}$, illumination $5 \mathrm{~ns}, 2.5 \times 3.2 \mathrm{~mm}$.

\section{Drop size distributions of the sprays}

The above shadowgraphy pictures reveal the structure of the spray as a function of the operating conditions and measurement location. Additional spray pictures highlight the influence of pressure $(\Delta P)$ that drives the Reynolds and Weber numbers through jet momentum. As $\Delta P$ increases, the spray generates smaller droplets because of this increase in impingement force, which is a common result in like-doublets sprays [2],[5],[7]: this effect is evidenced by PDI and shadowgraphy measurements performed on the same water spray (see Figure 6a).

Both diagnostics show that the drop size distribution is mainly composed of small droplets $\left(D<0.20 d_{0}\right)$; this is due to the measurement location situated far from the impingement point $\left(Z / d_{0}=100\right)$ i.e. after secondary breakup. It should be noticed that, in the case of shadowgraphy, the diameter of maximum probability decreases when $\Delta P$ increases, while in the case of PDI measurements this diameter is the same but with increasing probability. The same comparison is performed on ethanol spray for the same measurement location, $Z / d_{0}=100$ (see Figure $6 \mathrm{~b}$ ): shadowgraphy and PDI lead to a similar drop size distribution mainly composed of smaller droplets $\left(D<0.10 d_{0}\right)$ than water distribution. The drop size distribution is smaller for ethanol than for water mainly due to the lower surface tension of ethanol, and subsequently to its higher Weber number (see Table 1). As a conclusion, PDI and shadowgraphy provide similar drop size distributions, with small quantitative differences.

This result is in agreement with comparable work performed on a steady atomizer [9], that also led to quantitative differences between the PDI and shadowgraphy results. A difference was found in the PDF distribution of drop size (albeit normalized in that work [9]), and differences were also reported in the statistical diameters. 


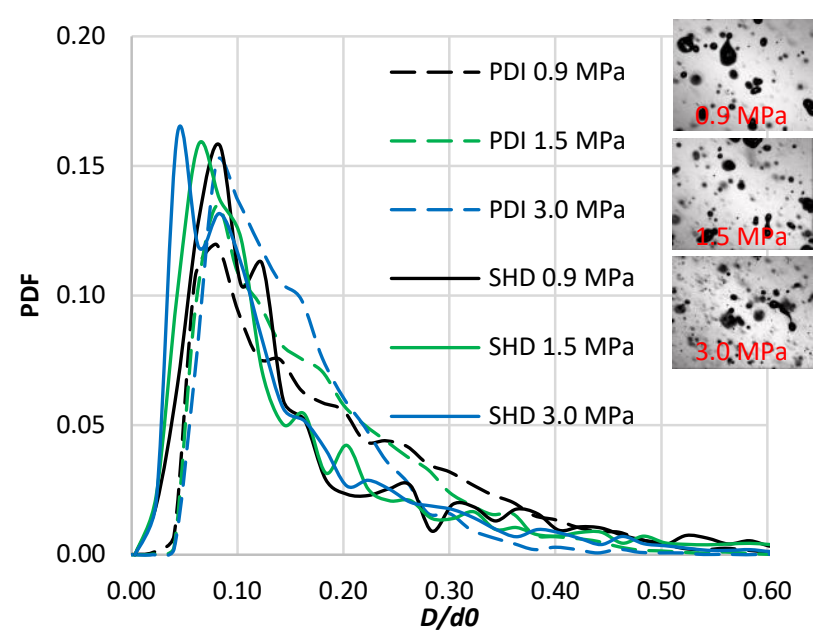

a)

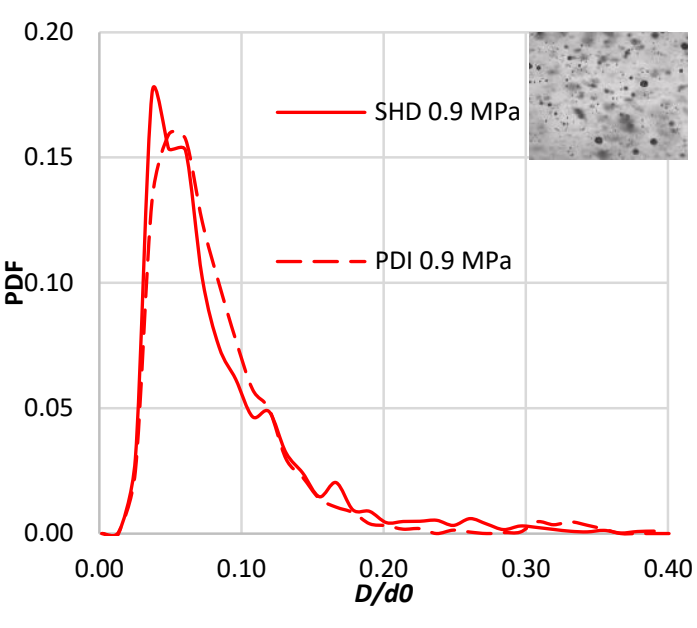

b)

Figure 6. PDF of drop size distribution comparison: a) shadowgraphy vs. PDI, with increasing momentum (water, $d_{0}=0.30 \mathrm{~mm}$, $\left.Z / d_{0}=100\right) ;$ b) shadowgraphy $\left(d_{0}=0.30 \mathrm{~mm}\right)$ vs. PDI $\left(d_{0}=0.51 \mathrm{~mm}\right)-$ Ethanol, $Z / d_{0}=170, \Delta P=0.9 \mathrm{MPa}$.

\section{Effect of distance from the impingement point}

The evolution of this distribution is investigated at different measurement locations by shadowgraphy (see Figure 7a): as distance $Z$ increases from the impingement point, the distribution exhibits droplets of smaller size $\left(D<0.05 d_{0}\right)$. And yet, as $Z$ increases, the characteristic diameter computed from the drop size distribution has distinct behaviours in other work: SMD increases [5]-[6] or decreases [3] with increasing distance from the impingement point. Therefore, a characteristic diameter should be evaluated to quantify this effect. For this purpose, the average diameter $D 10$ is chosen because it represents fairly the whole drop size distribution, taking care to the geometrical drop size that is detected directly by shadowgraphy.

The average diameter $D 10$ is computed from PDI and shadowgraphy measurements on ethanol and water sprays at $\Delta P=0.9 \mathrm{MPa}$ (see Figure $7 \mathrm{~b}$ ). The $D 10$ computed with shadowgraphy is roughly constant for water, and decreases slightly with increasing distance $Z$ in the case of ethanol. The $D 10$ for water measured with PDI is higher, which is consistent with the drop size distribution (see Figure 7a), and decreases slightly with increasing distance $Z$, while the validation rate of the PDI instrument increases up to $80 \%$. In the meantime, the corresponding shadowgraphy pictures (see Figure 5) demonstrate that water droplets turn overall from ligaments and concave droplets $\left(Z / d_{0}=50\right)$, to convex droplets $\left(Z / d_{0}=150\right)$ which cross-section is not perfectly circular. This may explain the difference between the PDI measurement, that is based on the spherical assumption, and the shadowgraphy measurement that encompasses any droplet shape.

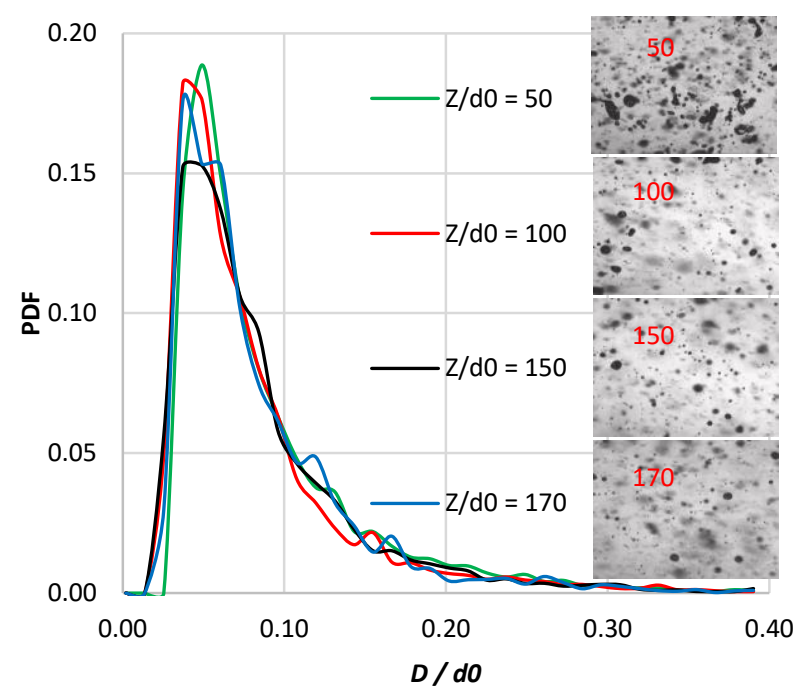

a)

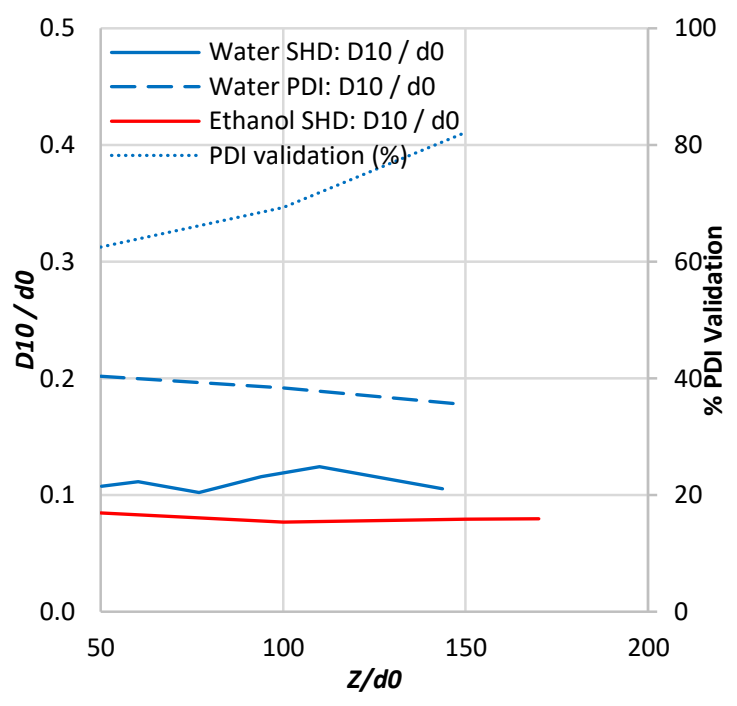

b)

Figure 7. Effect of distance $Z$ from the impingement point $\left(d_{0}=0.30 \mathrm{~mm}, \Delta P=0.9 \mathrm{MPa}\right)$ : a) PDF of ethanol drop size distribution by shadowgraphy; b) average diameter D10 computed from PDI or shadowgraphy. 


\section{Distribution of droplet shapes}

From the above comparison, we can assume that the shadowgraphy measurements are less restrictive than PDI, as far as droplet shape is concerned. In order to confirm this assumption, the droplet shapes detected by the abovementioned shadowgraphy method are further analysed. For this, the centricity distribution of elliptical droplets cross-section is reported for water and ethanol sprays at $Z / d_{0}=50$ and $150, \Delta P=0.9 \mathrm{MPa}$ (see Figure 8a); the ellipse centricity is defined as the ratio of its short axis to long axis. For both fluids, the centricity distribution is closer to unity in the dilute spray region $\left(Z / d_{0}=150\right)$ than in the dense region $\left(Z / d_{0}=50\right)$, thus confirming that some time is required after breakup to generate round droplets. In contrast, the PDI diagnostic may detect only the droplets of centricity close to unity. This causes a bias in the spray characterization because droplets of centricity lower than 0.80 still represent a significant part of the distribution in our case: $10-20 \%$ in the dilute spray $\left(Z / d_{0}=150\right)$, but $30-$ $40 \%$ in the dense region $\left(Z / d_{0}=50\right)$. It is necessary to assess which class of diameter is affected by this bias.

The centricity of maximum probability is reported for each class of diameter, for water and ethanol sprays at $Z / d_{0}=150, \Delta P=0.9 \mathrm{MPa}$ (see Figure 8b); the velocity associated to each droplet is also reported. Most spherical droplets (of centricity close to 1) can be found in the range of diameter 30-100 $\mu \mathrm{m}$, while the other droplets are of lesser centricity. The smallest droplets $(D<30 \mu \mathrm{m})$ are less spherical, and their velocity is much lower than bigger droplets. This may be due to the effect of the aerodynamic drag, that affects significantly this class of small droplets. Under this effect, the unstable droplet shape oscillates due to the surface tension that tends to restore its round shape, hence a periodic, elliptic deformation [10]. The biggest, elongate elliptical droplets $(D>100 \mu \mathrm{m})$ may proceed from ligament parts (see Figure 8a). These large elliptical droplets may be difficult to probe with PDI considering the stringent tolerance in the maximum phase difference between pairs of detectors, but they carry a significant amount of liquid with poor evaporation surface. Consequently, they deserve appropriate diagnostics such as shadowgraphy. Their importance is superior in the dense region $\left(Z / d_{0}=50\right)$; downstream, in the dilute region $\left(Z / d_{0}=150\right)$, largest droplets seem to relax towards spherical shape.

This effect is observed either on water or ethanol, but ethanol seems to produce more elliptical droplets. This is consistent with its lower surface tension compared to water, hence a higher sensitivity to initial conditions (ligament breakup) and a lesser cohesion prone to the oscillating deformation of ethanol droplet.

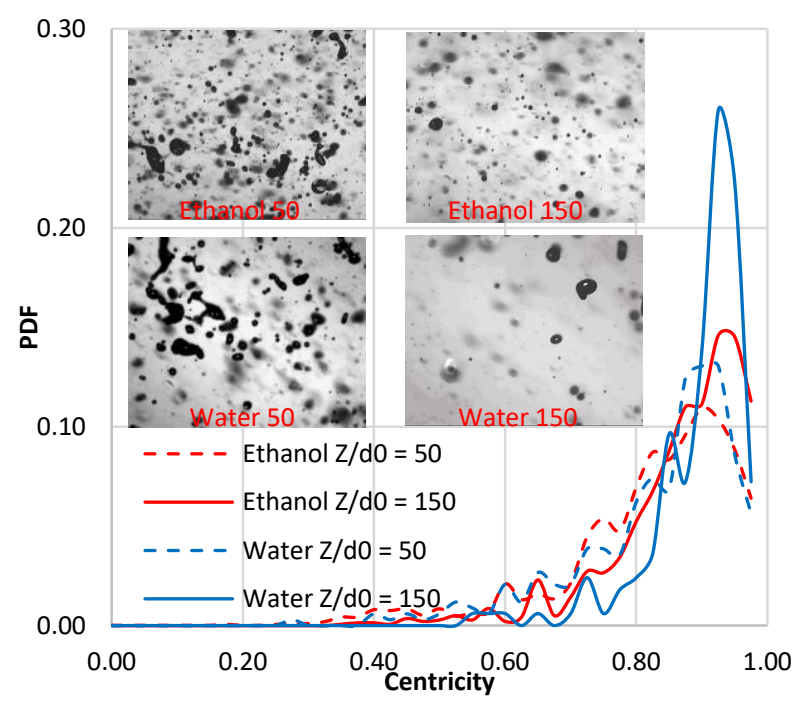

a)

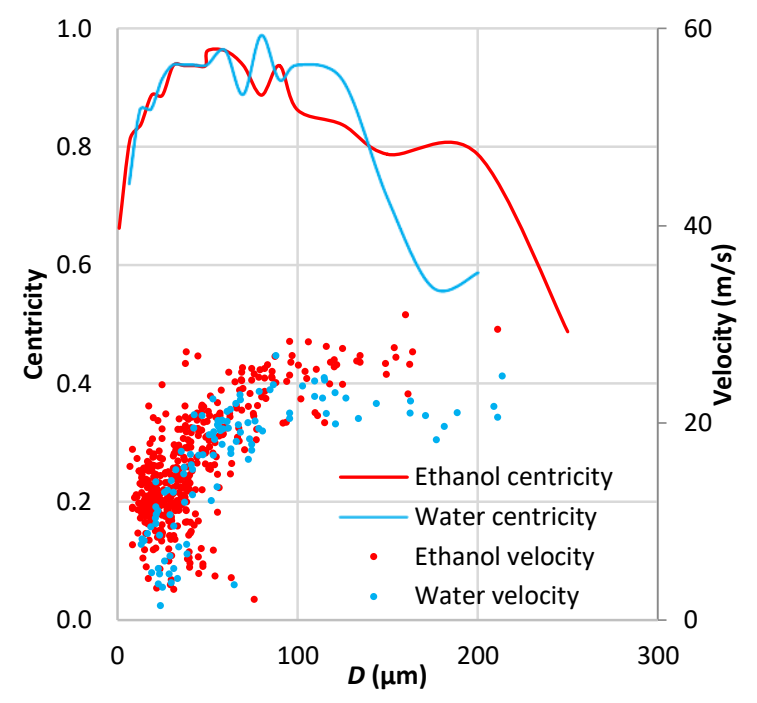

b)

Figure 8. Centricity of elliptical droplets computed from shadowgraphy pictures of water and ethanol sprays $\left(d_{0}=0.30 \mathrm{~mm}\right.$, $\Delta P=0.9 \mathrm{MPa})$ : a) distribution of centricity; b) evolution of centricity and velocity vs. droplet diameter at $Z / d_{0}=150$.

\section{Conclusions}

This study demonstrates the interest of high-magnification shadowgraphy for the study of impinging jet injector. It allows to visualize directly the atomization process, and proves to be particularly useful for direct measurements of drop size, velocity and shape inside dense sprays. Indeed, this atomization process generates various shapes of drops and ligaments, especially non-spherical droplets, most of which can be detected by an elliptical interpolation. It is noticeable that most of the largest droplets generated by this injector are characterized by an elliptical shape, and consequently may be rejected by PDI. 
In the present study, shadowgraphy provides original data concerning the spray topology, as well as droplet morphology, diameter and velocity. The distribution of droplet size has been recorded along the spray centreline, highlighting the effect of jet momentum (driven by injection pressure) and the evolution versus distance from the impingement point. Overall, it is clear that ethanol is prone to generate quickly smaller droplets sprays than water (thus hydrogen peroxide), thanks to its lower surface tension. This effect is quantified clearly by the drop size distributions and mean droplet diameters.

The fluids investigated in this work stand for green storable bi-propellants, such as ethanol (fuel) and hydrogen peroxide (represented by water). Experimental conditions led to ligament- or fully-developed breakup atomization regimes, representative of some engine injector conditions. As far as engine combustion is concerned, the elevated pressure and temperature conditions may modify the spray structure, and combustion may interact with the spray so that the evaporation time allowed to the propellants is limited. In this context, the influence of large droplets is paramount and should be taken into account from the injector characterization.

\section{Acknowledgements}

The authors wish to acknowledge the support of CNES (French Space Agency).

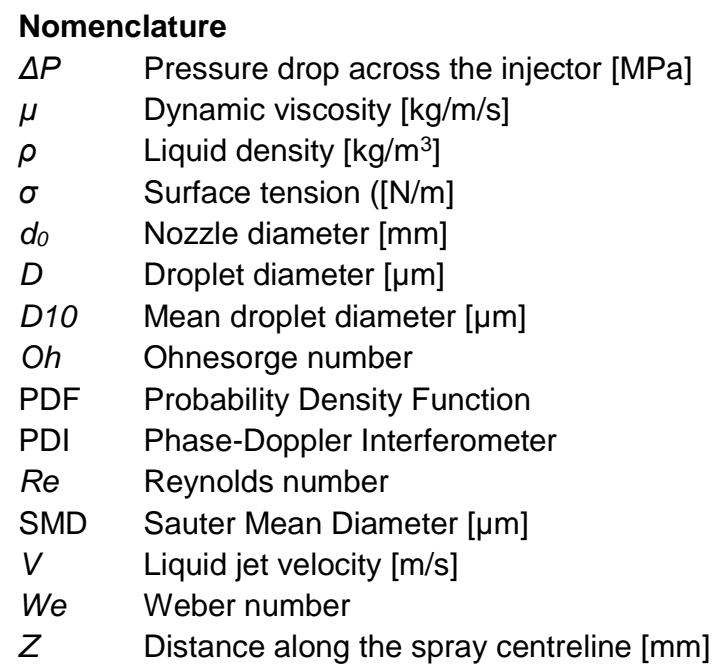

\section{References}

[1] Bailardi, G., Negri, M. and Ciezki, H. K., "Several aspects of the atomization behavior of various newtonian fluids with a like-on-like impinging jet injector", ILASS-Europe 2010, 23rd Annual Conference on Liquid Atomization and Spray Systems, Brno, Czech Republic, 2010.

[2] Ryan, H. M., Anderson, W. E. and Pal, S., "Atomization characteristics of impinging liquid jets", J. of Propulsion and Power, 11 (1), pp. 135-145, 1995.

[3] Zheng, G., Nie, W., Feng, S., Wu, G., "Numerical simulation of the atomization process of a like-doublet impinging rocket injector", Procedia Engineering 99, pp. 930-938, 2015.

[4] Chen, X., Ma, D., Yang, V., Popinet, S., "High-fidelity simulations of impinging jet atomization", Atomization and Sprays, 23 (12), pp. 1079-1101, 2013.

[5] Sakisaka, R., Hayashi, J., Daimon, Y, Yamanishi, N., Akamatsu, F., "Experimental measurements of impinging jet atomization at the vicinity of liquid fan", ICLASS 2012, 12th Triennial International Conference on Liquid Atomization and Spray Systems, Sep. 2-6, 2012.

[6] Indiana, C., Bellenoue, M., Boust, B., "Experimental investigations of drop size distributions with impinging liquid jets using phase-Doppler anemometer", International Journal of Energetic Materials and Chemical Propulsion 14 (3), pp. 241-264, 2015.

[7] Ramamurthi, K., Nandakumar, K. and Patnaik, R. K., "Characteristics of sprays formed by impingement of a pair of liquid jets", J. of Propulsion and Power, 20 (1), pp. 76-82, 2004.

[8] Indiana, C., Bellenoue, M., Boust, B., Petitot, S., "Experimental combustion investigations from likeimpingement sprays of green propellants", 52nd AIAA/SAE/ASEE Joint Propulsion Conference, Salt Lake City (USA), 25-27 July 2016.

[9] Berg, T., Deppe, J., Michaelis, D., Voges, H., Wissel, S., "Comparison of particle size and velocity investigations in sprays carried out by means of different measurement techniques", ICLASS 2006, International Conference on Liquid Atomization and Spray Systems, Aug. 27 - Sep. 1, 2006.

[10] Suh, Y., Lee, C., "A numerical method for the calculation of drag and lift of a deformable droplet in shear flow", Journal of Computational Physics 241 (15) pp. 35-57, 2013. 


\title{
Fundamental analysis of liquid breakup mechanism in a rotary atomizer with square discharge orifice
}

\author{
M. Ghorbanhosseini, S. Rezayat, M. Farshchi* \\ Department of Aerospace Engineering, Sharif University of Technology, Tehran, Iran \\ ${ }^{*}$ Corresponding author: Farshchi@shrarif.edu
}

\begin{abstract}
An experimental investigation of breakup mechanism in a rotary atomizer with square shape discharge orifice at ambient condition has been performed. The effects of a high aspect ratio noncircular discharge channels, particularly a square shape discharge channel, are considered. The motivation of this study is the use of this type of orifice in some small gas turbine engines as well as non-existing observation in literature concerning about high aspect ratio of discharge channel. Visualization experiments are conducted by high speed shadowgraph imaging technique with pulsed light illumination for the first time. The effects of rotational speed and volume flow rate are studied on the breakup structure. The visualizations indicates that the liquid film formed along the channel is pushed to one side of it due to Coriolis force which is dominant in this type of atomizer. Accordingly a crescent shaped liquid film is formed at the square channel exit covering two corners of the square, resulting the combination of Coriolis induced stream mode and surface tension induced stream mode breakup. Observations of the breakup process for different volume flow rates and rotational speeds indicate that the breakup of liquid film stream is dependent on injection conditions and the corresponding cross flow velocity created by atomizer rotation. The breakup regime map is provided as a function of weber number and momentum flux ratio. Four distinct regimes are identified: Rayleigh breakup, bag breakup, multimode breakup, and shear breakup. The present results leads to understanding atomization performance and creating some idea to improved spray quality in this type of atomizer.
\end{abstract}

\section{Keywords}

Rotary atomizer, square shape injection orifice, high aspect ratio, discharge channel, Breakup regimes

\section{Introduction}

Rotary atomizer is one type of fuel injection which is used in small gas turbine engines. In big and popular gas turbine engines are used pressure atomized with a high-pressure fuel pump. The idea of using this type of fuel injectors system was raised to avoid the using of large and heavy pumps in small gas turbine engines. Higher rotational speed at which small gas turbine engines operation allow rotary atomizer to be used to create the fuel spray, replacing the high-pressure fuel pump. Actually, centrifugal force causes the fluid to be thrown from the discharge elements with high velocity. As the injection liquid film is introduced to environment, it gets converted into ligaments and sheets that eventually break into fine droplets. In this injectors, depending on design and application, the orifices may be arranged in a single row, multiple rows or other new arrangements. Simple design, light weight, low cost, decreasing engine size, high performance in spay atomization with high uniformity, and eliminating the high pressure pump are specifications of rotary atomization in small gas turbine engines. Other users also are conceivable for this injectors such as spray drying, spray coating, and etc. Which can be studied to operationalizing them.

There have been a little studies reported in the literature for rotary atomizers and since there is not existed the strong fundamental and theoretical base for this type of injectors yet to explains completely breaks up mechanism or design map. Design map is the important point to design and optimization for different scale of rotary atomizer in various operation conditions and this allows to sizing the combustor of small gas turbine engines or other conceivable application. There are parameters that must be examined such as size, shape, environment specification, fuel properties and holes injector geometry and dimension.

In this type of injectors, the fuel passes from the shaft and enters to the chamber, then discharges from the nozzles which are drilled on the fuel chamber. Dahm et al. [1] indicated that a thin liquid film is formed on the discharge channel wall and flows along it. Also Dahm proposed three injection modes including the regular film mode, irregular film mode, and the Coriolis-induced stream mode in liquid atomization. If this thin film flow can be maintained until it issues from the periphery of each channel, then the atomizer operates in the film-mode liquid breakup. In this type of injection mode, two phenomena can be observed: if the thin liquid film maintains its integrity to the channel exit and gets separated from the orifice edge, the atomizer demonstrates regular film mode injection. On the other hand, if the thin liquid film issues forth from the periphery of the hole pools on the atomizer surface rather than getting 
separated at the hole edge, the atomizer operates in the irregular film mode injection. The third injection mode, namely the Coriolis-induced stream mode, can occur when sufficiently large Coriolis forces act on the liquid flow within the channel and causes the liquid film to pull in to a side. Dahm in this study, attempted to introduces a basically theory for spray mechanism in the rotary atomization. Dahm et al. [2] provided flow visualization results with experimental setup to characterize the liquid breakup regimes involved in fuel rotary atomization over a range of hole geometries, hole sizes, and rotation rates. He was the only person in literature that is used the square hole shape to optimization of rotary atomization. Dahm observed the regular and irregular film mode injection in round radial discharge orifices. The visualization results indicate that in a regular film mode injection, the liquid film breaks up as three regimes subcritical, transition, and supercritical film mode breakups due to the competition between surface tension and inertia effects. In the subcritical regime, the liquid film is drawn together by surface tension to form a single liquid ligament that subsequently undergoes the classical Rayleigh breakup. If the thin liquid film breaks up into small ligaments and droplets soon after being introduced to the air, the atomizer operates in the supercritical film-mode breakup. They have also provided the proper length scale for correlating the atomization performance of the rotary atomizer with injection orifices operating in the subcritical and supercritical film-mode breakup regime [2]. For the rotary atomizer used in these researches, the length of the discharge channel is not long enough to make the Coriolis effect important. Therefore, Dahm no observation has been made for the Coriolisinduced stream mode injection for any shapes hole. In the following years, Choi et al. [3] elucidated the ignition and combustion characteristics of the slinger combustor with a rotating fuel injection system. They also provided the spray visualization of a rotary atomizer with round radial orifices and a clear correlation with drop size and ignition performance with rotational speed. They classified the breakup regimes to subcritical, transition, and supercritical modes without mentioning the Coriolis effect. Choi et al. [4] have measured the spray characteristics of a rotary atomizer with a radial-axial discharge channel by applying the Phase Doppler Particle Analyzer (PDPA) laser diagnostic technique. By using this system, the droplet size (SMD), velocity distribution, and spray pattern were measured for the first time. In this study, the breakup regimes were classified just like previous research studies. To determine the effect of the orifice injection diameter and the number of orifices, Choi et al. [5] constructed a test rig and measured droplet sizes as well as spray pattern visualization under various rotational speeds and liquid flow rates. They observed three spray patterns: ligament spray, regular spray, and irregular spray pattern; they found that there is an optimum orifice size for obtaining a minimum droplet size. No classification of breakup regimes was performed in the ligament spray pattern. This is equivalent to the Coriolis-induced stream mode breakup in this type of rotary atomizer. Most studies on the spray characteristics of the rotary atomizer have focused on droplet size measurement at relatively high rotational speeds. Sescu et al. [6] provided droplet size and volume distribution at a low rotational speed to analyze atomization performance in start-up phase conditions of small gas turbine engines with the rotary atomizer. The following year, Choi et al. studied the effect of rotational speed and air cross-flow velocity on droplet sizes [7] and spatial droplet distribution [8] around a rotary atomizer with round radial discharge orifices. They found that the cross-flow influences the Sauter mean diameter directly. However, the rotational speed has a large effect on the spray at slower cross-flow conditions. Paquet et al. [9] recently reviewed the rotary atomizer to find suitable correlations for an adequate spray distribution.

In this study is investigated breakup mechanism in a rotary atomizer with square shape discharge orifice and the breakup regime map is provided as a function of weber number and momentum flux ratio. The present investigation is used the one operational slinger rotary atomizer which the channel is long enough to observing the Coriolisinduced stream mode injection. Visualization experiments are conducted by high speed shadowgraph imaging technique with pulsed light illumination for the first time and also is observed the exact details from breakup regimes for the first time. Observations of the breakup process for different volume flow rates and rotational speeds indicate that the breakup of liquid film stream is dependent on injection conditions and the corresponding cross flow velocity created by atomizer rotation. Four distinct regimes are identified: Rayleigh breakup, bag breakup, multimode breakup, and shear breakup. The present results leads to understanding atomization performance and creating some idea to improved spray quality in this type of atomizer. The motivation of this study is the use of this type of orifice in some small gas turbine engines as well as non-existing observation in literature concerning about high aspect ratio of discharge channel. Dahm was the only person in literature that is used the square hole shape in rotary atomization which cannot shows Coriolis-induced stream mode injection and because of his experimental methods cannot shows the details of breakups regimes.

\section{Material and methods}

The experimental setup used for the current study was built at the SRMF optical laboratory of Sharif University of Technology. This setup has been designed to capture the spray behavior of a rotary atomizer in high rotational speeds. To provide realistic results, the current rotary atomizer is designed and built like the atomizers used in small gas turbine engines. All experimental tests were performed under ambient pressure and temperature $\left(20^{\circ} \mathrm{C}\right)$ using 
water as the test liquid in place of the aviation fuel to enhance safety. The liquid flow rate and the rotational speed are changed from $30 \mathrm{LPH}$ to $120 \mathrm{LPH}$ and $2,000 \mathrm{rpm}$ to $25,000 \mathrm{rpm}$, respectively. A schematic of the experimental setup and the rotary atomizer is shown in Figs. 1 and 2, respectively.

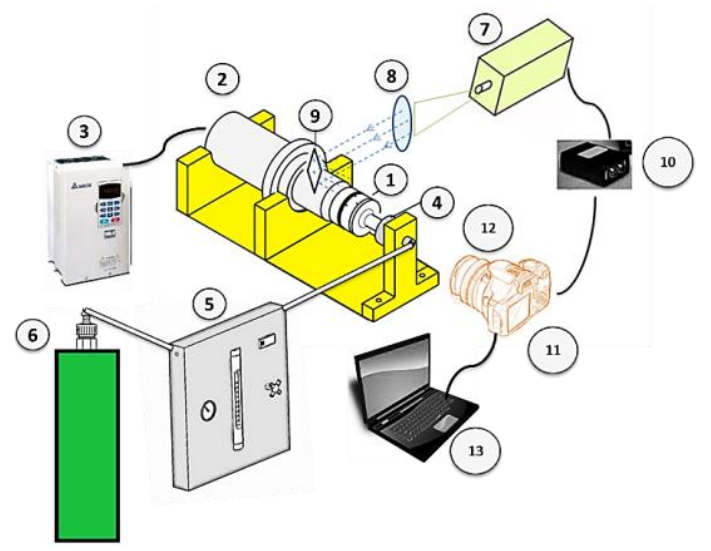

Figure 1: Components of the experimental setup: (1) rotary atomizer, (2) spindle motor, (3) variable-frequency drive, (4) bearing and housing, (5) flow control panel, (6) pressurized water tank, (7) pulse light, (8) Collimating lens, (9) mirror, (10) trigger, (11) camera, (12) macro lens, (13) laptop.

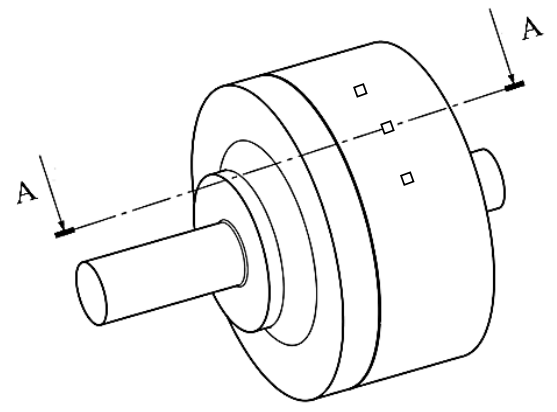

Isometric view

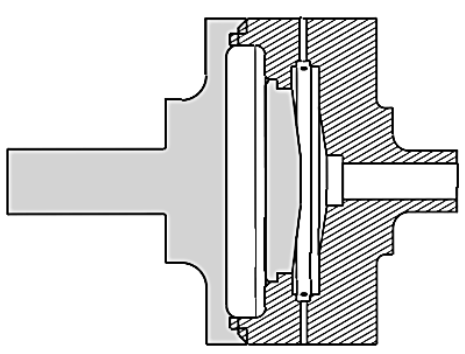

Section view A-A

Figure 2. Schematic of the rotary atomizer.

The experimental setup includes a high-speed spindle motor with $3.5 \mathrm{KW}$ power. The rotary atomizer is directly connected to this motor and rotates with a rotational speed up to $25,000 \mathrm{rpm}$. To adjust the frequency and rotational speed of spindle motor, a variable frequency drive of $5.5 \mathrm{KW}$ power is used. A high-speed angular contact-sealed bearing is mounted to the atomizer shaft. The liquid is fed to the atomizer disk through the hollow shaft designed with a labyrinth seal on the peripheral surface. As the liquid reaches the inner surface of atomizer, it spreads circumferentially and radially before being placed in the maximum radius of the atomizer cavity. The liquid film is discharged into the radial direction through the orifices and followed by its interaction with external air to form the ligaments or sheets that break into fine droplets. The rotary atomizer used in the current study consists of six square shapes injection orifices with width of $1.5 \mathrm{~mm}$. The atomizer was manufactured by CNC machining. The outer and inner diameters of the atomizer disk are $77.5 \mathrm{~mm}$ and $59 \mathrm{~mm}$, respectively and the cannels length are $9.25 \mathrm{~mm}$.

The flow control panel consists of the rotameter, pressure gage, and needle valve. The rotameter measures the flow rate of liquid within a range of $10 \mathrm{LPH}$ to $120 \mathrm{LPH}$ with an accuracy of $\pm 2 \mathrm{LPH}$. A needle valve was used in this panel to adjust the flow rate accurately. Before the needle valve is opened, the spindle motor runs and the atomizer speed reaches the steady speed. To avoid the variation of the volume flow rate during a test, water-pressurized tanks were used at a pressure of 3 bar to ensure that the upstream pressure of the needle valve is kept approximately constant. All the experimental results are taken after the flow rate of the liquid is kept fixed with fluctuations of less than $2 \mathrm{LPH}$. The pressure gauge with a range of $0.1-1$ bar was located upstream closer to the atomizer inlet to measure its pressure loss.

The optical setup consists of pulsed light illumination, collimating lens, mirror, trigger, camera, and macro lens. The pulse light is a high-power LED-based illuminator, which can produce green light pulses with a minimum duration of about $125 \mathrm{~ns}$. The pulse duration was set to $875 \mathrm{~ns}$ in the current work in both spray visualization and 
droplet size measurement. The collimating lens with a focal length of $40 \mathrm{~cm}$ was used to parallel the light beams emitted from the pulse light and to adjust the field of view. The mirror was located on the spindle front surface to change the direction of the light beams. As a result, the light beams align with the atomizer rotation axis and backilluminates the liquid jet emerging from atomizer orifices. Two HDSLR cameras, Nikon D3300 and D7100 are used to capture the breakup structure and droplets sampling, respectively. A trigger provides the possibility of synchronizing the emitted pulse with a camera shutter speed. The camera was operated with an open shutter in a dark room so that exposure times could be controlled by the duration of the light pulses. This pulse duration of 875 $\mathrm{ns}$ is sufficient to freeze the motion of the liquid jet structure in the high-speed rotation of the atomizer. The HDSLR cameras capture a high-resolution image with $6000 \times 4000$ pixels. The Nikon $200 \mathrm{~mm}$ f/4 ED macro lens with a working distance of about $26 \mathrm{~cm}$ was used to ensure the capture of small breakup structures and ligaments.

The shadowgraph technique with pulse back illumination is used for spray visualization. In this technique, a light source illuminates the liquid injection domain. The injected liquid obstructs the light beam and creates a shadow effect. By focusing the camera lens on the shadow plane, the resulting pattern is the picture of a fluid shadow whose contrast is recognizable from the background light. The camera focused at the plane of symmetry above the atomizer periphery surface in which the liquid jet emerges from injection orifices. The field of view is limited to $24 \mathrm{x}$ $36 \mathrm{~mm}$ and a reproduction ratio of 1:1 for spray visualization. This optical recording technique features low-cost low-power consumption and simple optical configurations compared to those of laser-based systems.

\section{Results and discussion}

As mentioned, in this present study is used the pulsed shadowgraph photography technique to Extracts the high resolution results in all range of operation in a rotary atomizer with square shape discharge orifice. The results of observations are unique and perfect. The effects of rotational speed and volume flow rate are studied on the breakup structure. As it is evident from the literature review, there is only one non-dimensional parameter (We) that describes the behavior of liquid jets and sprays by the rotary atomizer. The Weber number plays a significant role in multiphase flows, especially in case of drop formation. This non-dimensional parameter may be defined as the ratio of the inertial force to the surface tension force of the fluid. In the current study, the liquid to air momentum flux ratio, $q$, and the aerodynamic Weber number, Wed, are considered for the analysis. This is because the breakup mechanism primarily depends on the liquid injection velocity and the air cross-flow velocity. The liquid injection velocity can be varied by changing the mass flow rate in a constant rotational speed. Hence, it is not possible to carry out the analysis only by means of Wed. The liquid film thickness formed at the channel exit of slinger orifices is one of the key parameters relevant to its atomization properties. Dahm et al. [1] provided the following equation to calculate the liquid film thickness:

$\mathrm{t}=\left(\frac{3}{\pi}\right)^{1 / 3}\left(\frac{\mu_{\mathrm{L}} \mathrm{Q} / N}{\rho_{\mathrm{L}} R \Omega^{2} \mathrm{~d}}\right)^{1 / 3}$

Where $\mu_{\mathrm{L}}$ is the liquid viscosity, $\mathrm{Q}$ is the total volume flow, $N$ is the number of injection orifices, $\rho_{\mathrm{L}}$ is the liquid density, $R$ is the outer radius of atomizer disk, $\Omega$ is the rotational speed, and $\mathrm{d}$ is the injection orifice diameter. The liquid injection velocity and the air cross-flow velocity are obtained by Equations (2) and (3), respectively.

$\mathrm{V}_{\mathrm{a}}=\mathrm{R} \Omega$,

$\mathrm{V}_{\mathrm{l}}=\frac{\mathrm{Q}}{\mathrm{N} \pi \mathrm{dt}}$.

These velocities are then used for obtaining $\mathrm{q}\left(\rho_{\mathrm{L}} \mathrm{V}_{\mathrm{l}}^{2} / \rho_{\mathrm{a}} \mathrm{V}_{\mathrm{a}}^{2}\right)$. The aerodynamic Weber number based on the orifice diameter and the density and velocity of cross-flowing air, $V_{a}$, is defined as $W e_{d}=\rho_{a} V_{a}^{2} d / \sigma$. The liquid to air momentum flux ratio, q, and the aerodynamic Weber number, Wed, play a major role in primary and secondary atomization. The range of experimental boundary conditions and non-dimensional parameters used in the present work are listed in Table 1.

Table 1: Range of operating conditions and derived parameters used

\begin{tabular}{c|c} 
Rotational speed $(\Omega)$ & $2000-25000 \mathrm{rpm}$ \\
\hline Liquid volume flow rate $(\mathrm{Q})$ & $30-120 \mathrm{LPH}$ \\
Cross flow velocity $\left(V_{a}\right)$ & $12-101 \mathrm{~m} / \mathrm{s}$ \\
Liquid injection velocity $\left(V_{l}\right)$ & $4-45 \mathrm{~m} / \mathrm{s}$ \\
Aerodynamic Weber number $(\mathrm{We})$ & $2-170$ \\
Liquid to air momentum flux ratio $(\mathrm{q})$ & $25-674$ \\
Liquid film thickness (t) & $16-140 \mu \mathrm{m}$
\end{tabular}




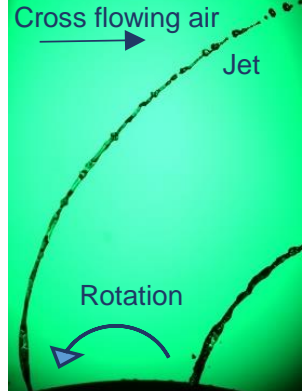

2000 rpm-30 LPH

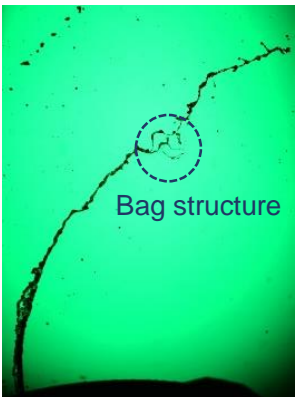

6000 rpm-30 LPH
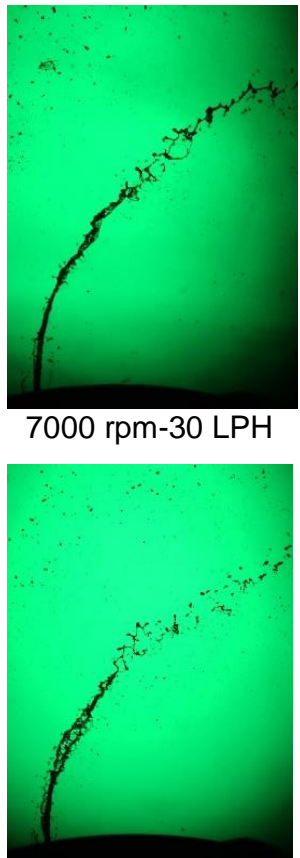

8000 rpm-30 LPH

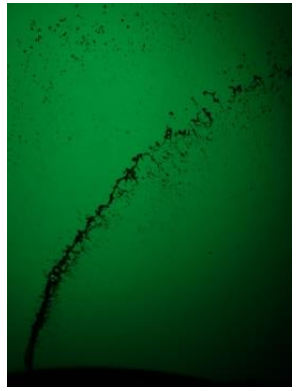

18000 rpm-30 LPH
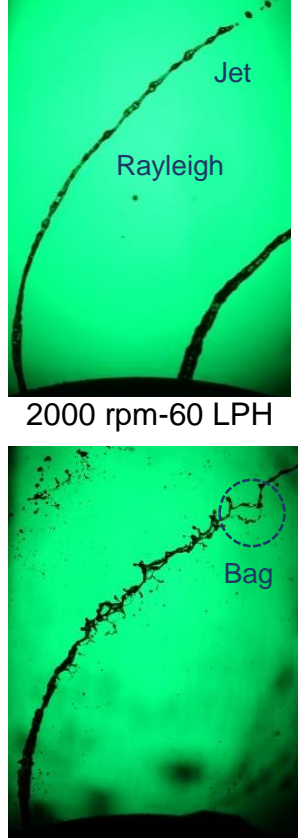

6000 rpm-60 LPH

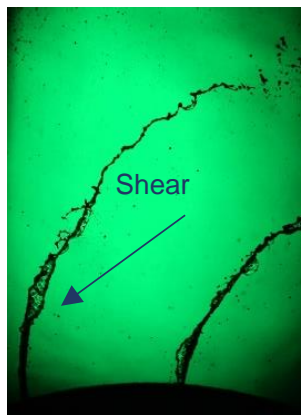

7000 rpm-30 LPH
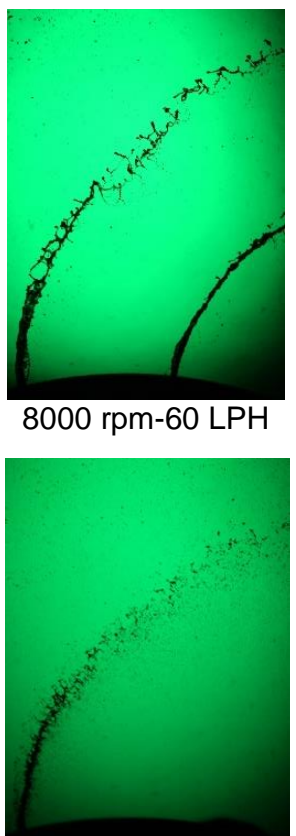

18000 rpm-60 LPH

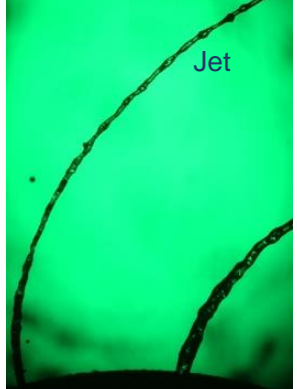

2000 rpm-90 LPH

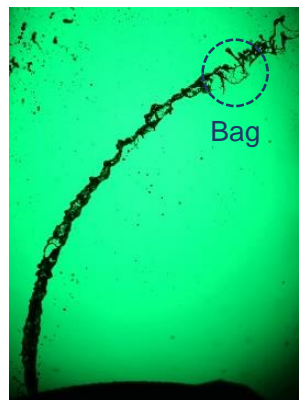

6000 rpm-90 LPH

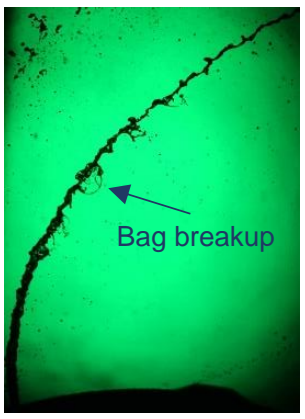

7000 rpm-30 LPH

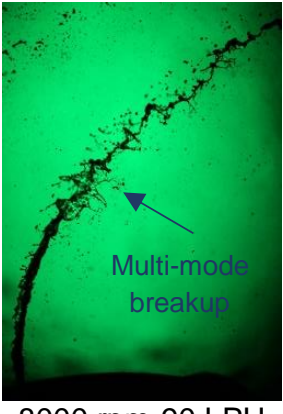

8000 rpm-90 LPH

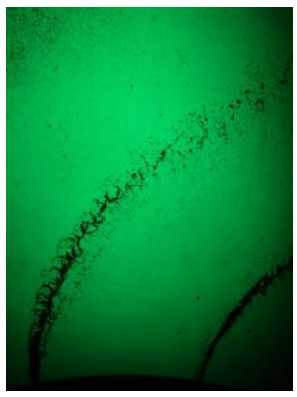

18000 rpm-90 LPH

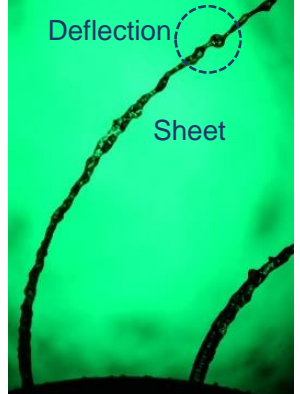

2000 rpm-120 LPH

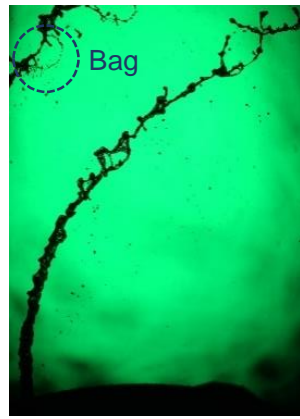

6000 rpm-120 LPH

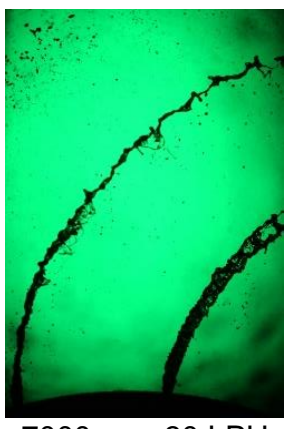

7000 rpm-30 LPH

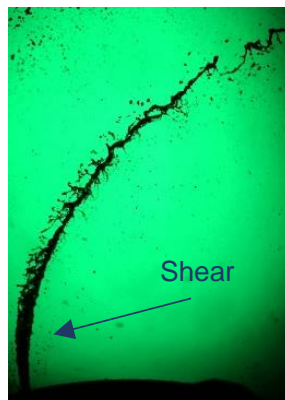

8000 rpm-120 LPH

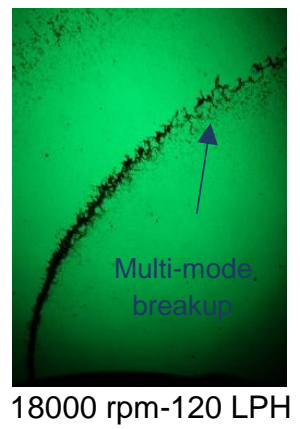

Figure 3. Spray visualization in rotational speed of $2,000,6,000,7,000,8,000$ and $18,000 \mathrm{rpm}$.

This work is licensed under a Creative Commons 4.0 International License (CC BY-NC-ND 4.0). 
For all cases considers in the present experiments, the liquid flow rate is kept constant at 30-120 LPH for six holes, so each holes liquid flow rate is $5-20 \mathrm{LPH}$ which is representative of practical fuel flow rates in small gas turbines and speeds ranging are 2000 to $25,000 \mathrm{rpm}$. Fig. 3 shows shadowgraph photos from side view of rotary atomizer and shows clear trends in breakup patterns as the rotational speed increases from 2000 to $18000 \mathrm{rpm}$ in 30 to 120 LPM. As mentioned, the rotational speed of rotary atomization equivalent of the cross flow velocity and the liquid fuel of rotary atomizer sprays in contras of cross flow velocity. Fig. 4 shows four distinct regimes are identified: Rayleigh breakup, bag breakup, multimode breakup, and shear breakup. First, the injected liquid stream at low rotational speed $(2,000 \mathrm{rpm})$ in all range of volume flow rates shows that the liquid jet is broken up in accordance with the Rayleigh mechanism. The Rayleigh jet breakup is identified by the growth of symmetric and asymmetric oscillations of the jet surface due to a lower inertial force. With increasing rotational speed and inertial force, the wave length of disturbance decreases. If the wave length of the initial disturbance is greater than the minimum wave length of disturbance, the surface tension force increases the disturbances and accordingly leads to disintegration of the liquid jet. Approximately the size of the droplets produced in this breakup regime are the same as that of the jet diameter.

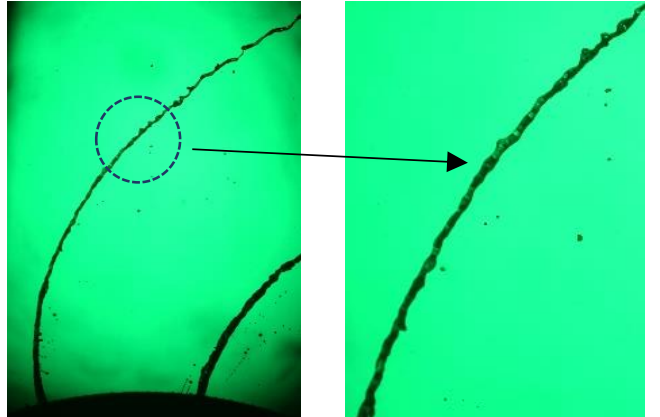

A) Rayleigh breakup regime (4000 rpm-90 LPH)

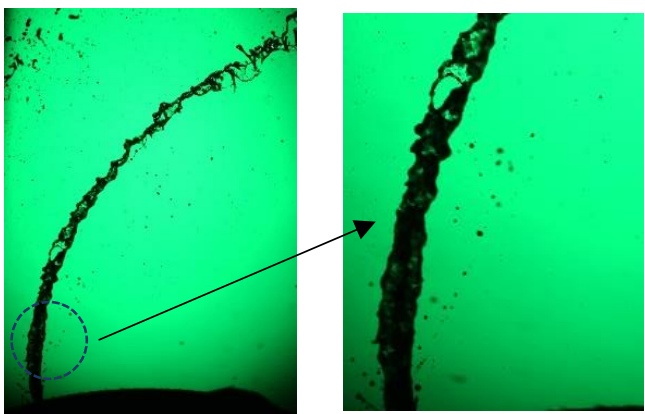

C) sheet breakup regime $(6000 \mathrm{rpm}-90 \mathrm{LPH})$

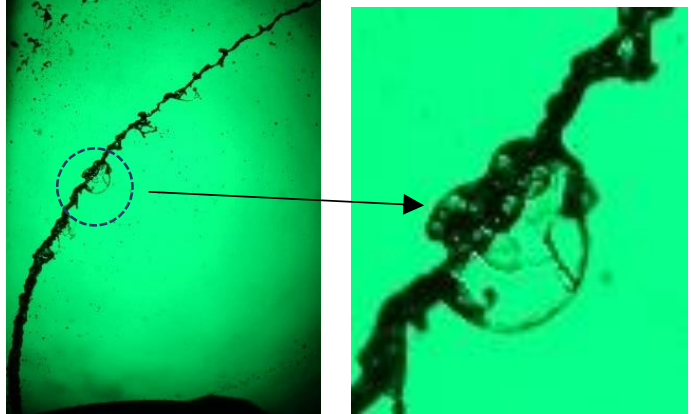

B) Bag breakup regime (7000 rpm-30 LPH)

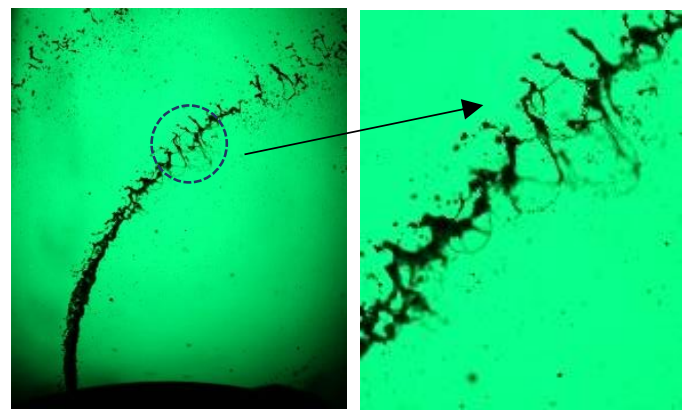

D) multimode breakup regime (6000 rpm-30 LPH)

Figure 4. Four distinct regimes are identified: Rayleigh breakup, bag breakup, and shear breakup, multimode breakup.

Second, next breakup mechanism, which was observed at a rotational speed of 6,000 rpm with a volume flow rate of $30 \mathrm{LPH}$, is bag breakup (fig.3). The bag structures were also observed in other operating conditions such as 7,000 and $8,000 \mathrm{rpm}$ with volume flow rates of $90 \mathrm{LPH}$, among others. The bag structures are formed by increasing the distance between the nodes to allow the gas to be permeated into the spacing between them and mixed with the liquid. The reason of forming these structures on the liquid stream surface is that the liquid film issued from the atomizer orifice deflects in the stream-wise direction, and the timescale of the crossflow velocity is lower than that of the liquid injection. Hence, the crossflowing air has enough time to permeate into the liquid between nodes and to create bag structures.

Third, the other phenomenon, which can be observed in a volume flow rate of $120 \mathrm{LPH}$ at different rotational speeds, is the thin liquid sheet that is formed after the liquid film is injected into the environment (fig.3). This behavior can be explained by comparing two timescales. If the timescale of surface tension is lower than that of the liquid injection inertial force, then the liquid film collapses to a single liquid column as a form of liquid jet. Otherwise, the surface tension has not enough time to exert any effect on the liquid film and hence the film is spread into the spanwise direction as a form of the thin liquid sheet.

Fourth, the last breakup mechanism is multimode regime which can be observed in volume flow rate of $30 \mathrm{LPH}$ at $6000 \mathrm{rpm}$, volume flow rate of $60 \mathrm{LPH}$ at $8000 \mathrm{rpm}$ and etc. (fig.3). 
Dahm et al. [2] introduced the classification for all range of rotary atomizer operation. The visualization results indicate that the liquid film breaks up as three regimes subcritical, transition, and supercritical film mode breakups due to the competition between surface tension and inertia effects. In the subcritical regime, the liquid film is drawn together by surface tension to form a single liquid ligament that subsequently undergoes the classical Rayleigh breakup. If the thin liquid film breaks up into small ligaments and droplets soon after being introduced to the air, the atomizer operates in the supercritical film-mode breakup. They have also provided the proper length scale for correlating the atomization performance of the rotary atomizer with injection orifices operating in the subcritical and supercritical film-mode breakup regime [2]. In this study by definition is provided Dahm, the breakup regime map is provided as a function of weber number and momentum flux ratio in for the first time (fig. 5).

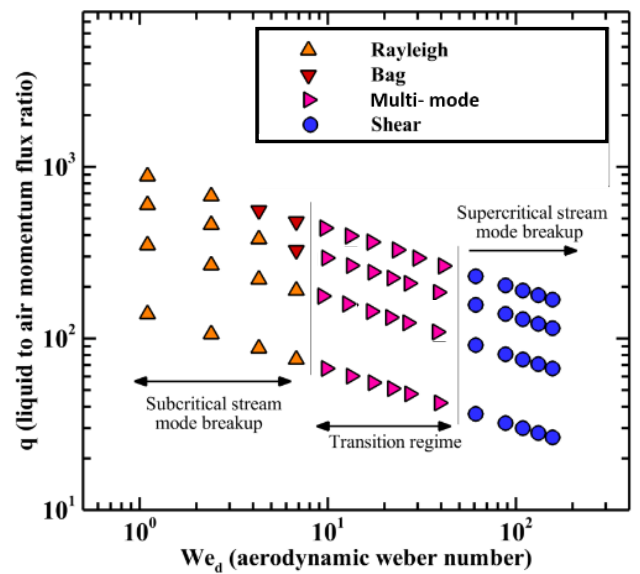

Figure 5. The breakup regime map for the rotary atomizer with the square discharge orifice

Dahm et al. [1] indicated that a thin liquid film is formed on the discharge channel wall and flows along it. Also Dahm proposed three injection modes including the regular film mode, irregular film mode, and the Coriolis-induced stream mode in liquid atomization. In Dahm study, the length of the discharge channel is not long enough to make the Coriolis effect important. Therefore, Dahm no observation has been made for the Coriolis-induced stream mode injection for any shapes hole. In this study, the effects of a high aspect ratio noncircular discharge channels are considered. The motivation of this study is the use of this type of orifice in some small gas turbine engines as well as non-existing observation in literature concerning about high aspect ratio of discharge channel. The visualizations indicates that the liquid film formed along the channel is pushed to one side of it due to Coriolis force which is dominant in this type of atomizer. The liquid film is accumulated on one side of the discharge channel and emerges from the atomizer orifices as a crescent-shaped form. Fig. 6 shows an enlarged view of the liquid film formation at the end of the channel. The Coriolis force acts on the liquid film and it is pushed to one side of the channel opposing the rotation. This observation shows the Coriolis force is stronger than surface tension force which was created by corners of square orifice.

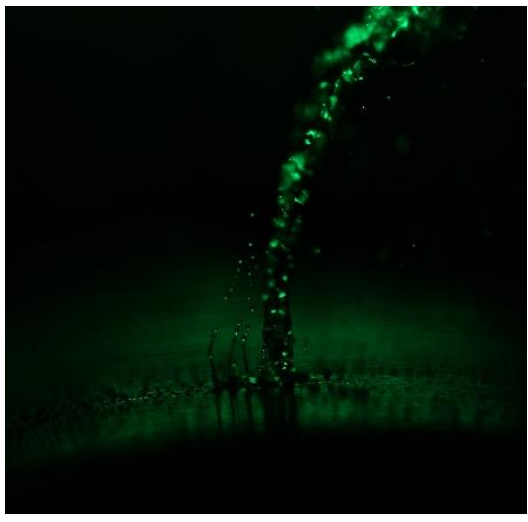

A) liquid injection in square hole's root (1000 rpm-10 LPH)

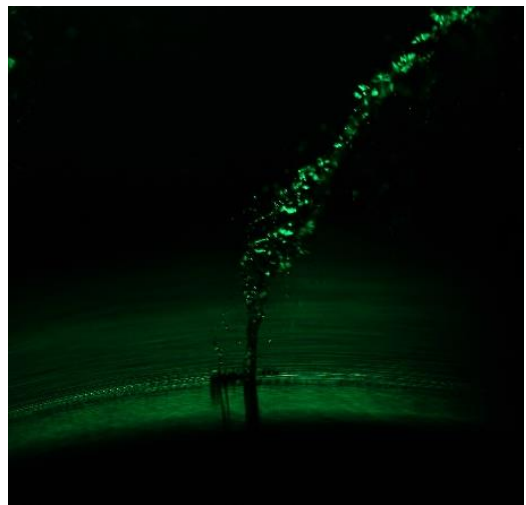

B) liquid injection in square hole's root (2000 rpm-30 LPH)

Figure 6. Liquid injection in square hole's root 


\section{Conclusions}

An experimental investigation of breakup mechanism in a rotary atomizer with square shape discharge orifice operating in the Coriolis-induced stream mode injection over a range of rotational speeds and volume flow rates. The dynamics of the interaction between the air cross-flow and the thin liquid film is explained through flow visualization experiments. Four different regimes of spray breakup are identified: Rayleigh breakup, bag breakup, multimode breakup, and shear breakup. A breakup regime map is presented at We on abscissa and q on ordinate. The results suggest qualitative similarities between the primary breakups of the thin liquid film in this type of atomizer, the primary breakup of the non-turbulent liquid jet, and the fanlike liquid sheet in a cross flow. This observation shows the Coriolis force is stronger than surface tension force and the liquid film formed along the channel is pushed to one side of it due to Coriolis force which is dominant in this type of atomizer. The present results leads to understanding atomization performance and creating some idea to improved spray quality in this type of atomizer.

\section{References}

[1] Dahm, W. J. A., Patel, P. R \& Lerg, B. H., Analysis of liquid breakup regimes in fuel slinger atomization, Atomization and Sprays, vol. 16, no. 8, pp. 945-962, 2006.

[2] Dahm, W. J. A., Patel, P. R \& Lerg, B. H., Experimental visualizations of liquid breakup regimes in fuel slinger atomization, Atomization and Sprays, vol. 16, no. 8, pp. 933-944, 2006.

[3] Choi, S., Lee, D. \& Park, J., Ignition and combustion characteristics of the gas turbine slinger combustor, J. Mech. Sci. Technol., vol. 22, no. 3, pp. 538-544, Mar. 2008.

[4] Choi, S. M., Jang, S. H., Lee, D. H. \& You, G. W., Spray characteristics of the rotating fuel injection system of a micro-jet engine, J. Mech. Sci. Technol., vol. 24, no. 2, pp. 551-558, Feb. 2010.

[5] Jang, S. H. \& Choi, S. M., Spray behavior of the rotary atomizer with In-line Injection Orifices, Atomization and Sprays, vol. 20, no. 10, pp. 863-875, 2010.

[6] Sescu, C., Kucinschi, B. R., Afjeh, A. A. \& Masiulaniec, K. C., Experimental test rig with results on liquid atomization by Slinger injectors, Journal of Engineering for Gas Turbines and Power, vol. 133, no. 11, p. 114505, 2011.

[7] Choi, S. M., Yun, S., Jeong, H. J. \& Corber, A., Spray in cross flow of a rotary atomizer, Atomization and Sprays, vol. 22, no. 2, pp. 143-161, 2012.

[8] Choi, S. M., Yun, S., Jeong, H. J. \& Corber, A., Spatial drop behavior of a rotary atomizer in a cross flow, Atomization and Sprays, vol. 22, no. 12, pp. 1077-1095, 2012.

[9] Paquet, B., de Champlain, A. \& Kalla, S., Review of fuel spray distributions to predict performance of rotary atomizers in a slinger gas turbine combustor, Atomization and Sprays, vol. 26, no. 5, pp. 483-511, 2016. 


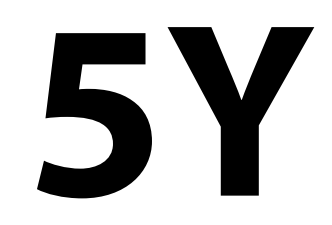

Atmospheric and medical sprays 


\title{
Coupled study of the film and spray on a basic annular prefilming airblast atomizer

\author{
V. Gosselin*1, B. Ferret ${ }^{1}$, R. Bazile ${ }^{1}$ \\ ${ }^{1}$ Instiute de mécanique des fluides de Toulouse, France \\ *Corresponding author: valentingregoire.gosselin@imft.fr
}

\begin{abstract}
One way to increase efficiency and reduce pollution in transport and energetic domain is designing fuel injectors with better atomization. In this work, experiments were performed on a prefilming airblast atomizer often used in gas turbine engines. For this purpose, a new injector was designed to visualize the prefilming zone and the primary atomization together. The flow configuration corresponds to an annular liquid film sheared by inner high velocity airflows. High speed Shadowgraphy was used to observe film and spray, liquid film frequency, wave velocity and wave deformation, primary breakup regime. Finally, a link between liquid film and the primary atomization are shown first qualitatively and after quantitatively.
\end{abstract}

\section{Keywords}

Atomization, liquid film, annular geometry, airblast, prefilming

\section{Introduction}

One way to increase efficiency and reduce pollution in transport and energetic domains is designing fuel injectors with better atomization properties [1]. Prefilming airblast atomizers often used in gas turbine engines, offer advantages in terms of atomization quality over a wide range of fuel flow rates. Nevertheless, spray formation mechanisms due to the disintegration of a liquid film are still not completely known.

This type of injector is often studied in a planar geometry [1] [2] [3] because this is the simplest way to analyze the mechanisms of film dynamics and atomization experimentally. At the same time, studies on industrial configurations [4] have shown that the internal geometry of the injector influences strongly the process of atomization. To make experimental observations in the annular geometry is an enormous challenge. For that reason researchers often study liquid film [5] and the spray [6][7] separately. In this context, we propose an experimental study on the liquid film and the spray for a basic annular prefilming airblast.

The paper starts by presenting the experimental set-up designed at IMFT (Institut de Mécanique des Fluides de Toulouse). The destabilization and the atomization of the liquid film have been studied with high speed shadowgraphy method. Then the liquid film is characterized with its different regimes, frequencies of waves, wave velocities and wave deformations. Furthermore observations are made on the regimes of sprays, the possible dependence between the liquid regime and breakup modes. And to finish the preservation between conditions of film near the injector exit and the initial conditions about the primary atomization.

\section{Experimental setup and metrology}

The set up designed at IMFT is shown in figure 1. The geometry of the atomizer is cylindrical $(D=34.5 \mathrm{~mm})$. The velocity of the air flow generated by a blower can reach $50 \mathrm{~m} . \mathrm{s}^{-1} \pm 0.5 \mathrm{~m} . \mathrm{s}^{-1} \quad\left(\operatorname{Re}_{\mathrm{D}}=100.000\right)$. The liquid (water) passes inside a thin annular duct $(\delta=500 \mu \mathrm{m} \pm 20 \mu \mathrm{m})$ located close to the wall of the cylinder (figure 1.b). A coordinate measure machine was used to verify tolerance on the thickness of the liquid duct. The velocity of the liquid is in the range $\left[1.2-2.2 \mathrm{~m} . \mathrm{s}^{-1}\right]\left( \pm 0.03 \mathrm{~m} . \mathrm{s}^{-1}\right)$ corresponding to low Reynold numbers $\left[600<\operatorname{Re}_{\delta}<1120\right]$.

The length of the prefilming zone is $48 \mathrm{~mm}$ and the atomizer is equipped with optical access (a glass cylinder) that allows the visualization of the liquid film (figure 1.b). To reduce turbulence 2 honeycombs are put before and after the convergent. After the honeycombs, a long tube $(\mathrm{d}=30 \mathrm{~mm}, \mathrm{~L}=90 \mathrm{~cm})$ is used to establish the air flow profile. The important parameters are resumed in table 1.

In order to characterize the mean air flow, a Pitot probe has been used to measure velocity profiles in four sections (figure 1.a): at a distance of $10 \mathrm{~cm}$ before the injector (S1), at the injector inlet (S2), at the beginning of the prefilming zone (S3) and at the end (S4). Whatever the section, the velocity profile is quite similar (figure 2). This result confirms that the tube is long enough for establishing the air flow. Hot wire measures $(d=5 \mu m)$ have been performed in section S4 (dots in figure 2) to obtain the mean turbulence ratio (6\%). LDV measures are planned to complete information about turbulence. Their measures have been made without the liquid flow.

The destabilization and the atomization of the liquid film have been studied with a high-speed camera $(10 \mathrm{kHz})$ Phantom V20 (1280x800pixels). A continuous LED panel $(2000 \mathrm{~mA}, 10 \times 10 \mathrm{~cm})$ put in the background of the camera axis has been used for the backlight. 
For the study two fields of view have been used. The first one, (field 1, red rectangle in figure 1.b) allows to observe the film destabilization). The size of field1 is $L_{x}=35.5 \mathrm{~mm}(27.8 \mu \mathrm{m} / \mathrm{pixel})$ and $L_{Y}=20.7 \mathrm{~mm}(25.9 \mu \mathrm{m} / \mathrm{pixel})$. It is located on the cylinder axis $8 \mathrm{~mm}$ after the liquid injection.

The second field of view (Field 2, blue rectangle in figure1.b) is used for the simultaneous visualization of the end of the liquid film and the beginning of the atomization area. The size of Field2 is the same as Field1 but it can be noted that the spatial resolution will change slightly at the end of the film (the deformation induced by the glass tube disappears).
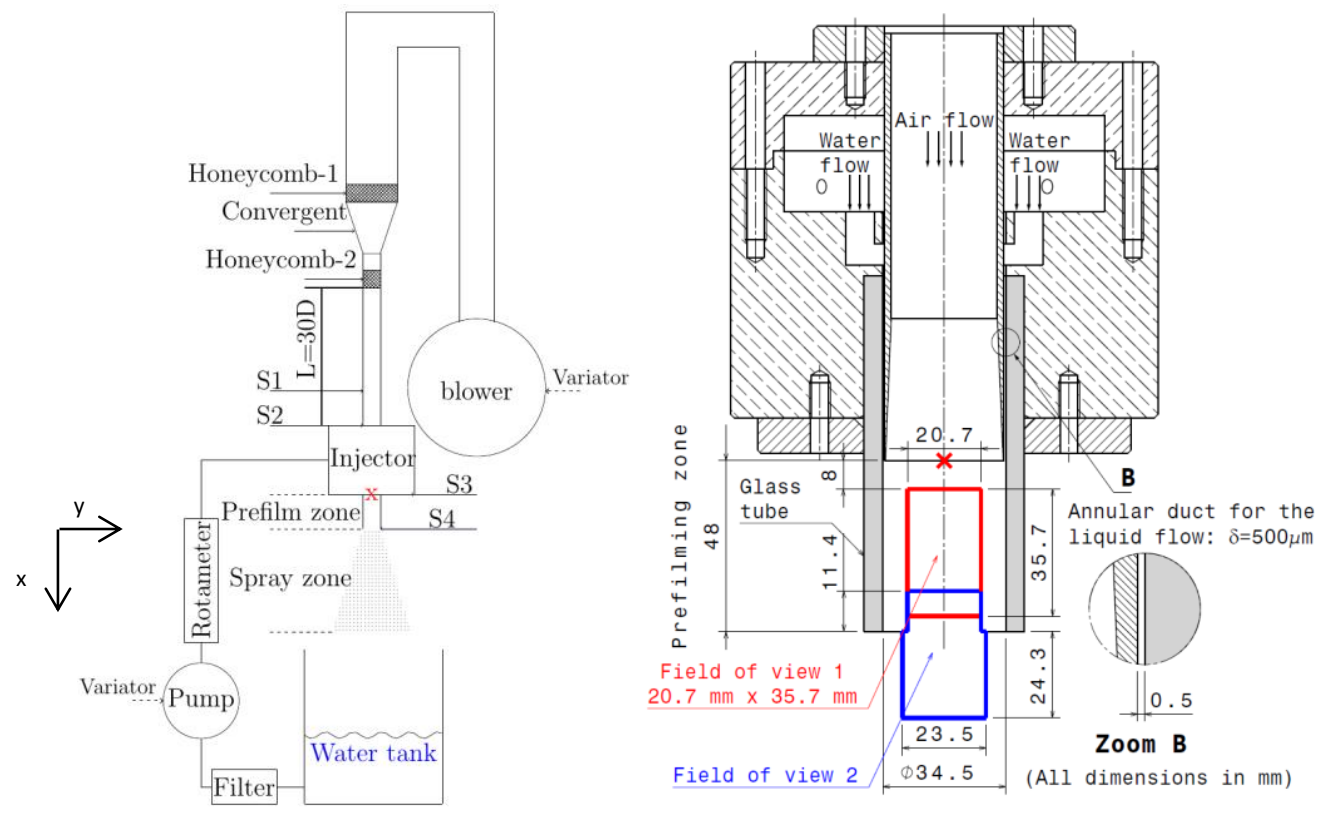

Figure 1. a) Experimental setup .b) Schema of the injector. Air velocity measurements are performed in four sections (S1, S2, S3, S4), the red $x$ is the origin $(x=0, y=0)$.

Table 1 Main injector's characteristics.

\begin{tabular}{c|cc}
\hline \hline & Dimension & nomination \\
\hline \hline Tube diameter & $34.5 \mathrm{~mm}$ & $\mathrm{D}$ \\
Liquid film thickness & $500 \mu \mathrm{m}$ & $\delta$ \\
Prefilming length & $48 \mathrm{~mm}$ & $\mathrm{~L}_{p}$ \\
Injector exit thickness & $5.2 \mathrm{~mm}$ & $\mathrm{~L}_{\mathrm{A}}$ \\
The splitter separating the two phases & $150 \mu \mathrm{m}$ & $\mathrm{L}_{\mathrm{s}}$
\end{tabular}

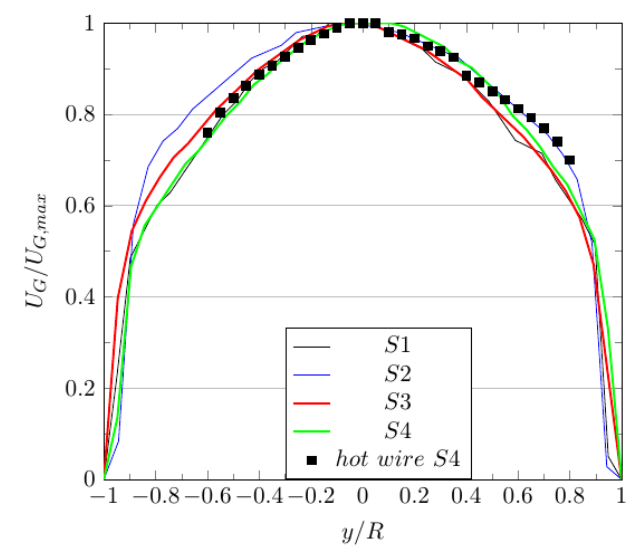

Figure 2. Normalized mean velocity profiles without liquid flow. in sections S1,S2, S3,.S4 (figure 1.a)

\section{Liquid film characteristics}

The analysis of the film deformation was performed in the first field of view (Field1). Its purpose is to study the temporal and spatial evolution of the film structure along the prefilming zone $(8 \mathrm{~mm}$ after the liquid injection up to the end of the glass cylinder). More precisely the high velocity difference between the interface of the liquid film 
and the main air flow generates surface waves that propagate and evolve (with time and space) along the cylinder.

The high-speed images allow the detection of the deformation of the liquid film interface. The more these deformations are important, the more the light of the LED panel will be deviated and the transmission will be attenuated. In the case of an important interface deformation (big surface waves) the transmission of the backlight vanishes and black zones appear in the images.

Figure 3 shows an instantaneous image of the liquid film obtained by shadowgraphy $\left(\operatorname{Re}_{\delta}=600, U_{G}=40 \mathrm{~m} . \mathrm{s}^{-1}\right)$. It can be noted that no film dry-out was occurring during the experiments (i.e. in the range of $\operatorname{Re}_{\delta}$ and $\operatorname{Re}_{D}$ mentioned in experimental setup section). Three regimes of wave structure can be observed. The first one called "initial", close to the liquid injection, reveals wide and quasi-periodic 2D waves. In this regime, the waves have little deformation. The third regime called "disrupted" near the injector exit, develops without specific spatial frequency and waves are 3D. The "transition zone" is observed when 2D waves become unstable and produce 3D waves with smaller scales (figure 3 ).

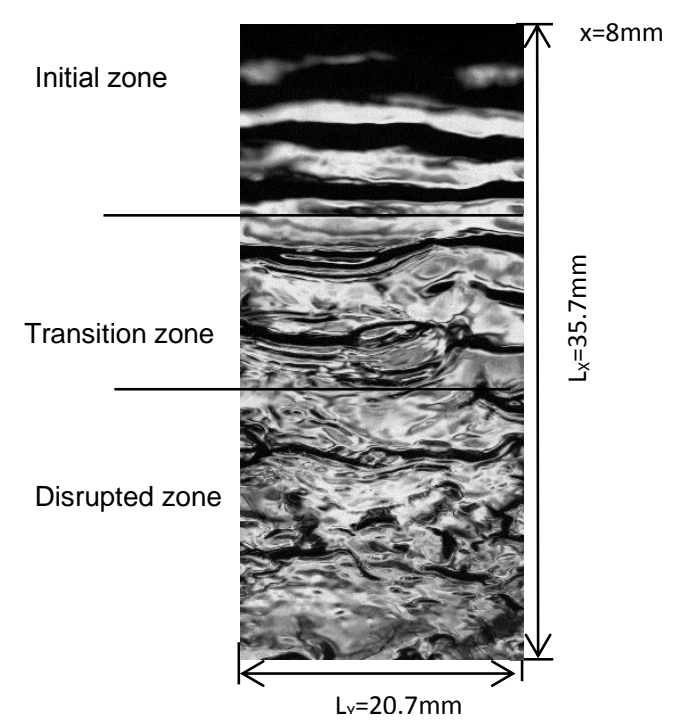

Figure 3 Instantaneous images of the liquid film (Field1) for $R_{\delta}=600$ and $U_{G}=40 m \cdot s^{-1}\left(R_{D}=87,000\right)$

To obtain the wave frequencies and wave velocities along the prefilming zone, the image processing is the same. A window centered on the axis of the cylinder is selected $(-2 m m<y<2 m m ; 8 m m<x<43.7 m m)$. The pixel values are averaged over the $y$-axis to obtain an axial mean profile of gray levels, $P(x)$, on the total length of the Field1. 10,000 successive images ( $1 \mathrm{~s}$ ) have been used to make sure that the statistics are converged. For $x<20 \mathrm{~mm}, \mathrm{a}$ quasi-periodic zone can be identified on $\mathrm{P}(\mathrm{x})$ (figure4).

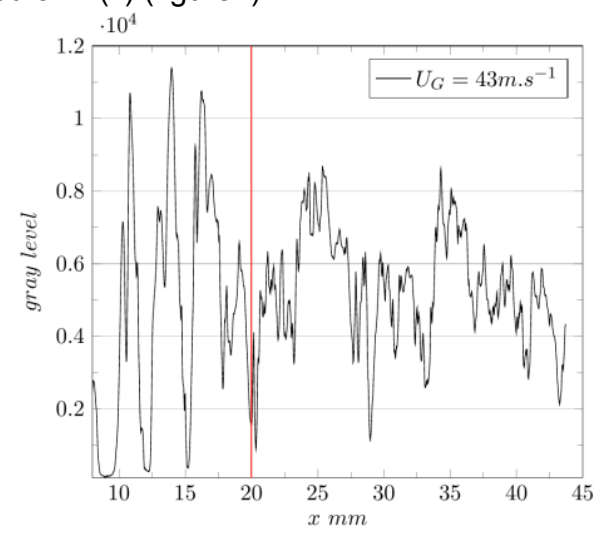

Figure 4 Axial evolution of the gray level in the prefilming zone for $U_{G}=43 m \cdot s^{-1}, R_{\delta}=600$. The red line delimits the quasi-periodic zone $(x<20 \mathrm{~mm})$.

The first post processing of the profiles, based on spectra analysis, was developed to study the wave frequencies. At a given location $x$, fast Fourier transforms are applied to the temporal evolution of $P(x)(10000$ values) and gives the power spectral density (PSD) (256 frequency values with step of $20 \mathrm{~Hz}$ ). For $U_{G}=43 m . s^{-1}$ and $R_{\delta}=600$, figure 5 shows the evolution of the power spectra for three axial locations $(x=10.7,23.5,35.7 \mathrm{~mm})$. 


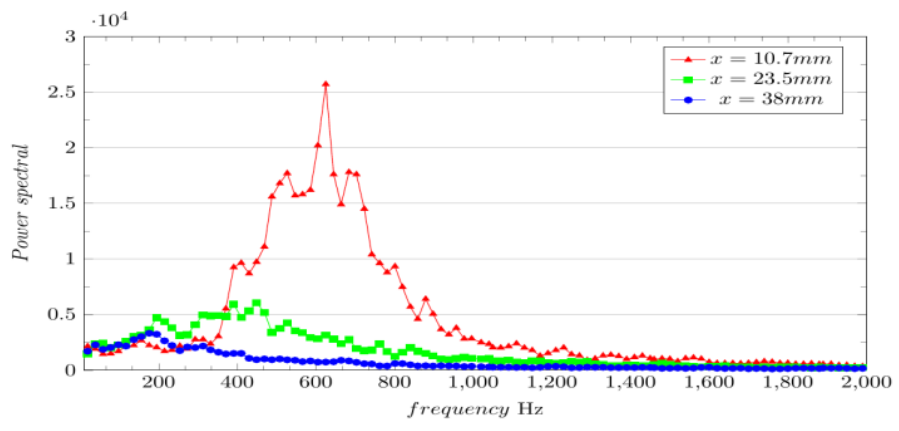

Figure 5 Power Spectra Density for $U_{G}=43 \mathrm{~m} . \mathrm{s}^{-1}$ and $\mathrm{Re}_{\delta}=600$ for 3 axial locations $\mathrm{X}=10.7,23.5,35.7 \mathrm{~mm}$.

In the "initial zone" $(x=10.7 \mathrm{~mm})$ a well-pronounced peak appears around $F_{\max }=600 \mathrm{~Hz}$ and characterizes the quasi-periodic structure detected on the images (see figure 4). Downstream $(x=23.5 \mathrm{~mm}, x=35.7 \mathrm{~mm})$ the initial structure of the liquid film is progressively lost and no significant peak can be detected. One may suppose that the initial waves correspond to the waves of maximum growth rate appearing due to Kelvin-Helmholtz instability on film surface. In the configuration studied by Alekseenko et al [5], a second peak has been observed in the power spectra, due to the presence of "disturbance waves" (not observed in the present study) $F_{\max }$ increases quasi linearly with $U_{G}$ (table 2). This result is in satisfactory agreement with the study of Alekseenko et al [5] carried out with a lower liquid Reynold number $\left(\operatorname{Re}_{\delta}=400\right)$. Therefore the values of $F_{\max }$ cannot be compared directly but the comparison between both studies shows clearly the increase in $F_{\max }$ with $\operatorname{Re}_{\delta}$ and $\mathrm{Re}_{\mathrm{D}}$ as Alekseenko et al [5] observed. At this stage of the study, it is difficult to present the results in terms of Strouhal number (1).

$S t=F_{\max } L / U_{G}$

Indeed, the choice of a length scale, L, is not obvious and different points of view can be found in the literature. Nevertheless, the results summarized in table 2 can be written in the form: St 14L, where $L$ does not seem to depend on the air velocity $U_{G}$, but depends probably of numerous parameters (liquid velocity [5], liquid properties and injector geometry [8]).

Table 2 Maximal frequencies in the initial zone for different air velocities. Comparison with the results of Alekseenko et al [5]

\begin{tabular}{c|cc}
\hline \hline Air velocity $\left({\mathrm{m} . \mathrm{s}^{-1}}^{-1}\right.$ & $\mathrm{F}_{\max }\left(\mathrm{Re}_{\delta}=600\right)(\mathrm{Hz})$ & $\begin{array}{c}\mathrm{F}_{\max } \text { in Alekseenko et al [5] } \\
\left(\operatorname{Re}_{\delta}=400\right)(\mathrm{Hz})\end{array}$ \\
\hline \hline 48 & 675 & 550 \\
43 & 625 & 460 \\
40 & 550 & 410 \\
33 & 507 & 320 \\
27 & 390 & 270
\end{tabular}

The second post processing of the profiles, based on correlation analysis, was developed to calculate the liquid wave's velocity. The axial profile $P(x)$ was divided in several intervals of $5 \mathrm{~mm}$. For each interval, 1D correlations were calculated between time $t$ and time $t+\Delta t(\Delta t=0.2 m s)$ to obtain the mean wave's velocity $U_{\text {wave }}(x)$ (figure 6). Small deformations of the liquid film interface for the case $U_{G}=33 \mathrm{~m} \cdot \mathrm{s}^{-1}$, limit the domain of measure to $x=18 \mathrm{~mm}$ $43 \mathrm{~mm}$.

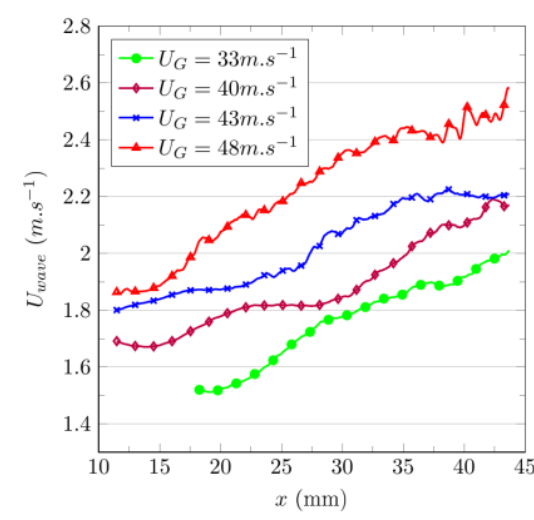

Figure 6 Axial evolution of wave velocity in the prefilming zone for different air velocities, $\mathrm{Re}_{\delta}=600$.

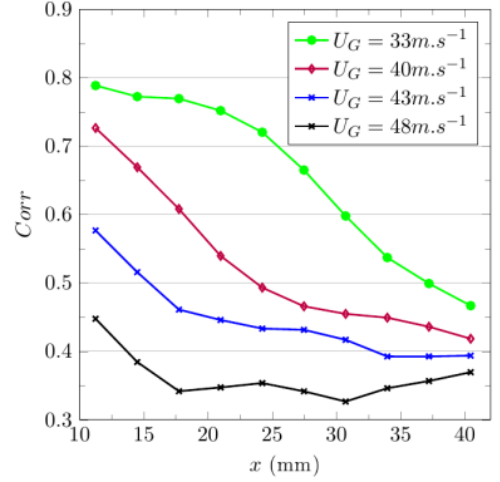

Figure 7 axial evolution of the correlation coefficient (Corr) in the prefilming zone for different air velocities, $\left(\operatorname{Re}_{\delta}=600\right)$ 


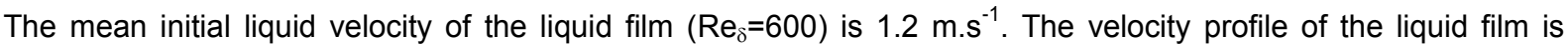
strongly affected by the air velocity and after the $12 \mathrm{~mm}$ liquid injection the waves' velocity can reaches $1.9 \mathrm{~m} . \mathrm{s}^{-1}$ for the highest air velocity. Moreover and whatever the air velocity, the waves' velocity increases with the axial distance. Indeed the main air flow accelerates the liquid film continuously from the liquid inlet up to the injector exit. Along the perpendicular direction to the glass tube, the local velocity of the liquid is probably affected by the air flow and the mean height of the liquid film could decrease with the axial distance, (liquid mass flow rate remaining constant).

Lastly, to quantify the progressive disintegration of the waves, a specific image processing based on spatial correlations was developed. From a first image (time $\mathrm{t}$ ), a small window called $\mathrm{W}_{1}(\mathrm{x}, \mathrm{t}) \quad\left(\Delta \mathrm{x}=1 \mathrm{~mm} ; \Delta_{\mathrm{Y}}=20.7 \mathrm{~mm}\right)$ was extracted at a given axial location $x$. On the next image (time $t+\Delta t ; \Delta t=1.5 \mathrm{~ms}$ ) a new window called $\mathrm{W}_{2}\left(\mathrm{x}+\Delta \mathrm{X}_{\text {corr }}, \mathrm{t}+\Delta \mathrm{t}\right)$ was extracted at location $\mathrm{x}+\Delta \mathrm{X}_{\text {corr }}, \Delta \mathrm{X}_{\text {corr }}$ being given by (2):

$\Delta X_{\text {corr }}=U_{\text {wave }}(X)^{*} \Delta t$.

For every pair of images, the maximum value of the spatial correlation between $W_{1}(x, t)$ and $W_{2}\left(x+\Delta X_{\text {corr }}, t+\Delta t\right)$ is calculated. 1000 pairs of images are used to obtain the mean correlation coefficient Corr(x). Because of weak luminosity near the liquid inlet $\operatorname{Corr}(\mathrm{x})$ is only calculated between $11 \mathrm{~mm}$ and $40 \mathrm{~mm}$.

Figure 7 shows the axial evolution of the correlation coefficient. The results must be interpreted in connection with the three different regimes of the liquid film presented in figure 3. For low air velocity $\left(33 \mathrm{~m} . \mathrm{s}^{-1}\right)$, stable waves are detected in the initial zone and the value of $\operatorname{Corr}(\mathrm{x})$ is high $(0.77)$ and almost constant up to $\mathrm{x}=22 \mathrm{~mm}$. An increase in the air velocity leads to a decrease in the spatial correlation coefficient, insofar as waves deform more quickly. When the air velocity increases from $40 \mathrm{~m} . \mathrm{s}^{-1}$ to $48 \mathrm{~m} . \mathrm{s}^{-1}$, the initial 2D periodic waves disappear more quickly. For $U_{G}=33 \mathrm{~m}_{\mathrm{s}} \mathrm{s}^{-1}$, the "transition zone" begins around $\mathrm{x}=20 \mathrm{~mm}$ and the coefficient Corr $(\mathrm{x})$ decreases progressively from 0.8 to 0.45 . A moderate increase in the air velocity $\left(40 \mathrm{~m} . \mathrm{s}^{-1}\right)$, leads to a significant decrease of Corr(x). Indeed, the "transition zone" is located closer to the injection of the liquid film. Moreover Corr $(x)$ drops sharply and takes the previous value $(0.45)$ for $x=27 \mathrm{~mm}$. Compared to the case $U_{G}=33 \mathrm{~m} \cdot \mathrm{s}^{-1} \operatorname{Corr}(x)$ reaches this value later $(x=43 \mathrm{~mm})$. This result shows that a small increase in air velocity strongly modifies the structure of the liquid film. The "transition zone" and the "disrupted zone" become shorter and are moved upstream.

For the highest velocity $\left(48 \mathrm{~m} \cdot \mathrm{s}^{-1}\right)$, the curve's evolution is a slightly different. Corr(x) reaches a lower value $(0.32$ for $x=32 \mathrm{~mm}$ ) but the value of the coefficient goes back to 0.4 near the injector exit. According to the work of Alekseenko et al [5] this could be due to the formation of "disturbance waves". Additional measures will be necessary to confirm this hypothesis.

\section{Atomization of the liquid film}

Figure 8 shows instantaneous images (Field2) for several air velocities $\left(U_{G}=27 \mathrm{~m} . \mathrm{s}^{-1}, 33 \mathrm{~m} \cdot \mathrm{s}^{-1}, 36 \mathrm{~m} \cdot \mathrm{s}^{-1}, 40 \mathrm{~m} \cdot \mathrm{s}^{-1}\right.$, $\left.43 \mathrm{~m} \cdot \mathrm{s}^{-1}, 48 \mathrm{~m} \cdot \mathrm{s}^{-1}\right)$, corresponding to $\operatorname{Re}_{\mathrm{D}}$ in the $\left.[60,000-100,000]\right)$ and for the same liquid velocity $\left(\mathrm{U}_{\mathrm{L}}=1.2 \mathrm{~m} \cdot \mathrm{s}^{-1}\right.$, $\left.\mathrm{Re}_{\delta}=600\right)$.

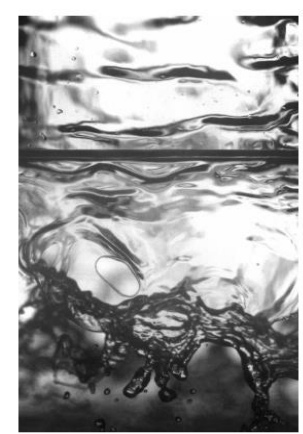

(a) $U_{G}=27 \mathrm{~m} \cdot \mathrm{s}^{-1}$

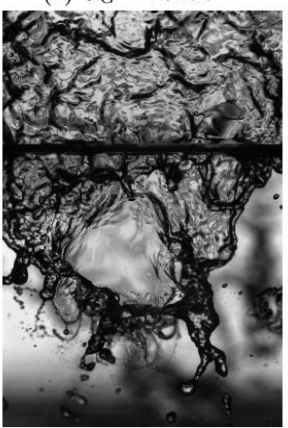

(d) $U_{G}=40 m \cdot s^{-1}$

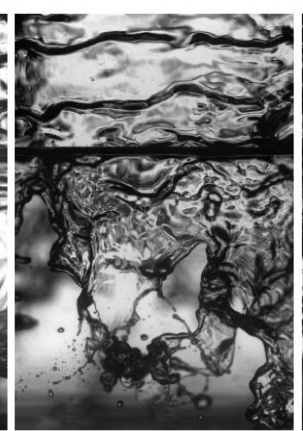

(b) $U_{G}=33 \mathrm{~m} \cdot \mathrm{s}^{-1}$

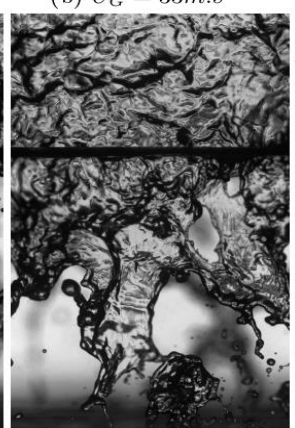

(e) $U_{G}=43 \mathrm{~m} \cdot \mathrm{s}^{-1}$

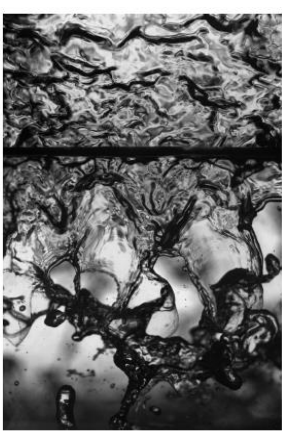

(c) $U_{G}=36 \mathrm{~m} \cdot \mathrm{s}^{-1}$

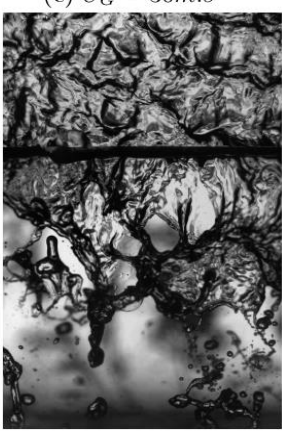

(f) $U_{G}=48 \mathrm{~m} \cdot \mathrm{s}^{-1}$

Figure 8 Instantaneous images of the liquid film and the liquid sheet (Field2) for different air velocities and $\mathrm{Re}_{\delta}=600$ 
The dark zones visible in the background are due to the atomization of the liquid sheet on the other side of the injector. Specific post-treatments will be developed to remove these gray levels. But at this moment, only a qualitative analysis can be made on the primary atomization.

Numerous studies concerning the primary atomization of a liquid sheet without prefilming zone can be found in the literature ([1], [9], [10]) and with prefilming zone ([2], [11]). The experimental results concerning prefilming injectors are limited and the cylindrical configuration has been little studied.

Different breakup regimes can be observed. In the case of a planar liquid sheet, the first observations of the primary atomization regime and its physical analysis can be found in Stapper et al [12] and Park et al [13].

When air velocity is low (27-33 m.s $\left.\mathrm{s}^{-1}\right)$, cellular breakup is detected with membranes and spanwise ligaments. For higher air velocities (from $U_{G}=36 \mathrm{~m} . \mathrm{s}^{-1}$ to $47 \mathrm{~m} . \mathrm{s}^{-1}$ ), stretched ligament breakup is observed with bag-like structure (figure 9) and longitudinal ligaments. In the configuration of the present study, the viscous effects are weak (Ohnesorge number is in the order of 0.005). On the other hand, in the investigated range of air velocity, the Weber number (3) based on the initial thickness of the liquid film is between 5 and 18 .

$W e=\rho_{G} . \delta \cdot\left(U_{G}-U_{L}\right)^{2} / \sigma$

Therefore, the process of secondary breakup is expected to appear after the destabilization of the liquid sheet.

Both regimes can be found in the study of Fernandez et al [14], in the case of a planar liquid sheet. Research is in progress to detect the edge of the liquid sheet and study the decrease of the break up length during air velocity increase. To study the transition between both regimes, Fernandez et al [14] and before them Lorenzo et al [15] have introduced the MFR parameter (for Momentum Flux Ratio) (4), to compare the momentum flux ratio between the gas and the liquid.

$\operatorname{MFR}=\left(\rho_{G} \cdot U_{G}{ }^{2}\right) /\left(\rho_{L} \cdot U_{L}{ }^{2}\right)$

The regime transition is observed for MFR 1; it's rather close to the value obtained by Fernandez et al [14] (MFR 0.5). The difference could result from the air flow which shears only a side of the liquid film, contrary to the study of Fernandez and al.

As the film and the spray are observed together, it is possible to make the link between the structure of the liquid film and the regimes of atomization.

When the liquid film is in "initial regime", primary atomization is dominated by the cellular breakup mode. If the end of the film is in the "disrupted regime", the predefined atomisation regime is in the mode "stretched ligament breakup".

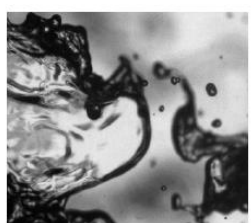

(a) $t=0 m s$

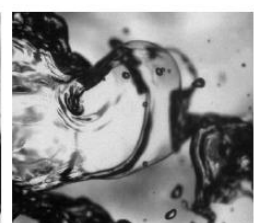

(b) $t=4 \mathrm{~ms}$

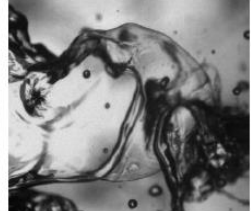

(c) $t=7 \mathrm{~ms}$

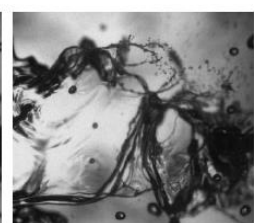

(d) $t=9 \mathrm{~ms}$

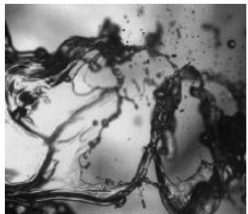

(e) $t=12 m s$

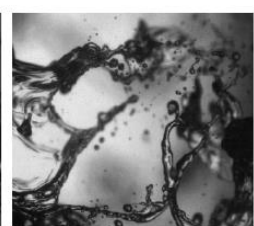

(f) $t=15 \mathrm{~ms}$

Figure 9 Zoom of a bag break structure.

But as the atomization regime depends on the air velocity, it is difficult to know if the liquid film regime changes the primary break up mode.

To make the link between the liquid film and the primary atomization, we compared the structure of the liquid film to the injector exit with the initial structure of the liquid sheet.

For this, the correlation coefficient (Corr) defined previously can be used with some modifications. Now three windows called $W_{1}, W_{2}, W_{3}(1 \mathrm{~mm} \times 20.8 \mathrm{~mm})$ are needed (figure 10a): $W_{1}$ and $W_{2}$ are located in the prefilming zone at $x_{1}=43.9 \mathrm{~mm}$ and at $x_{2}=46.6 \mathrm{~mm}$ respectively. $W_{3}$ is located after the injector exit $\left(x_{3}=49.3 \mathrm{~mm}\right)$ at the beginning of the liquid sheet. The distance between each window is the same $(2.7 \mathrm{~mm})$.

Figures 10.b and 10.c show zooms $(1 \mathrm{~mm} \times 5 \mathrm{~mm})$ of the same wave in each three windows. An image processing was made on $W_{1}$ and $W_{2}$ to correct the effects of curvature due to the glass cylinder.

From the three windows, two correlation coefficients can now be calculated: Corr ${ }_{1-2}$ and Corr $2-3$ corresponding to the maximum value of the spatial correlations between the pairs $\left[W_{1}, W_{2}\right]$ and $\left[W_{2}, W_{3}\right]$ respectively $(10000$ images are used).

Figure 11, shows the evolution of both correlation coefficients (Corr $1-2$ and Corr $_{2-3}$ ) for different air velocities.

Corr $_{1-2}$ provides the level of spatial correlation for a displacement of the liquid film of $1.7 \mathrm{~mm}$ and can be used as a "reference" inside the liquid film. Corr $2-3$ characterizes the loss due to the rough change in the conditions of the liquid flow at the injector exit.

Corr $_{1-2}$ decreases for the air velocity range $\left[27 \mathrm{~m} . \mathrm{s}^{-1}-33 \mathrm{~m} . \mathrm{s}^{-1}\right]$ and becomes stable around 0.4 for higher air velocities. Corr ${ }_{1-2}$ is calculated at location $\mathrm{x}=45.2 \mathrm{~mm}$, and this value $(0.4)$ is coherent with the last measures in This work is licensed under a Creative Commons 4.0 International License (CC BY-NC-ND 4.0). 
figure $7(x=40.7 \mathrm{~mm})$. The coefficient Corr $1-2$ and Corr $_{2-3}$ follows the same trend, but the values of Corr $2-3$ are $20 \%$ lower. A first analysis of the instantaneous images (figure 10) indicates that the structure of the film near the injector exit remains still present beyond this zone (i.e. in the first millimeters of the liquid sheet).

a) Schema (this model is not to size)

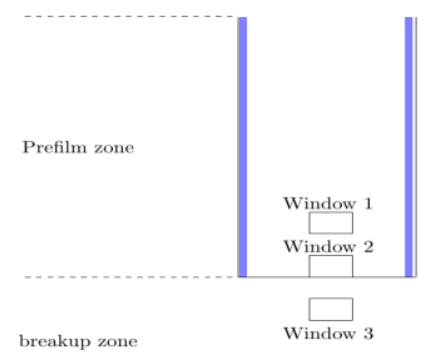

b) $U_{G}=27 m \cdot s^{-1}$

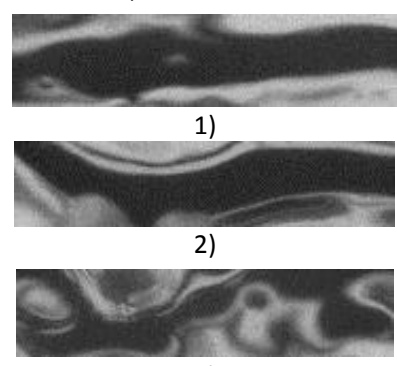

3) c) $U_{G}=48 m \cdot s^{-1}$

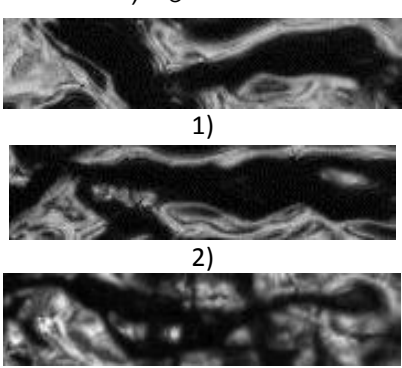

3)

Figure 10 a) Schema of each window's position. Zoom of a wave for an air velocity of $27 \mathrm{~m} \cdot \mathrm{s}^{-1}$ (b) and $48 \mathrm{~m} \cdot \mathrm{s}^{-1}$ (c). Each pitures are separated by $2.7 \mathrm{~mm}$. Pictures 1,2 are inside the prefilming zone and 3 are outside (Window size: $1 \mathrm{~mm} \times 5 \mathrm{~mm}$ )

Nevertheless it is normal that Corr $_{2-3}$ is smaller because the liquid flow is not forced anymore to adhere to the wall of the tube. 3D displacements can occur in the liquid sheet.

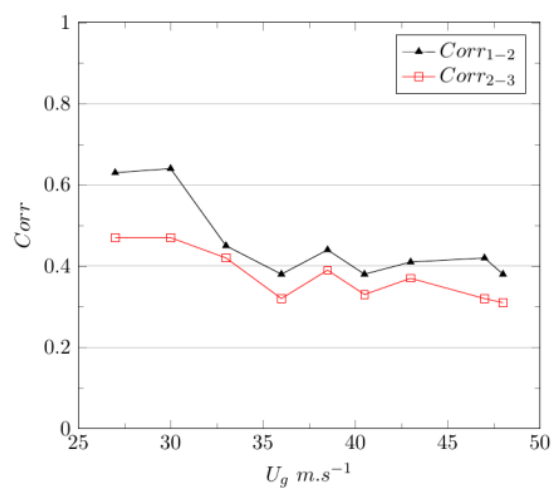

Figure 11 Evolution of the correlation coefficient $\left(\operatorname{Corr}_{12}, \operatorname{Corr}_{23}\right)$ in function of air velocity, black line with triangle is for the correlation inside the prefilmer, and the red line is between the prefilmer and the liquid sheet.

Another possible explanation is given by Koch et al [16]: a liquid accumulation at the end of the glass tube could modify the structure of the film before the liquid sheet. In spite of this effect, liquid waves are preserved after the injector exit (figure 10) and will play a role in the primary atomisation process.

\section{Conclusion}

Experiments were performed on a prefilming airblast atomizer designed at IMFT to visualize the liquid film and the primary atomization together using high-speed imaging.

Concerning the liquid film different regimes of wave structure can be observed. The first one called "initial", close to the liquid injection, reveals wide and quasi-periodic 2D waves. The second regime called "disrupted" near the injector exit, develops without specific spatial frequency and waves are in 3D shape.

An image processing, based on spectra analysis, was developed to study both the frequencies and the velocity of the waves. In the "initial zone" a well-pronounced peak appears around $F_{\max }=600 \mathrm{~Hz}$ and $F_{\max }$ increases quasi linearly with $U_{G}$

Whatever the air velocity, the wave velocity increases with the axial distance as the main air flow accelerates the liquid film continuously from the liquid inlet up to the injector exit.

To quantify the progressive disintegration of the waves, a specific image processing based on spatial correlations (coefficient Corr) was developed. For low air velocity, stable waves are detected in the initial zone and the value of $\operatorname{Corr}(\mathrm{x})$ is high. A small increase in air velocity strongly modifies the structure of the liquid film and the coefficient Corr $(x)$ drops sharply. The "initial zone" becomes shorter and the "transition zone" and the "disrupted zone" are moved upstream.

Concerning the spray different breakup regimes have been observed: cellular breakup with membranes and spanwise ligaments (low air velocity) and stretched ligament breakup with bag-like structure and longitudinal ligaments (high air velocity).

To make the link between the liquid film and the primary atomization, the analysis based on the coefficient of correlation (Corr) was extended to the zone of primary breakup. 
The results clearly show that the structure of the liquid film at the outlet of the injector remains still very present in the primary atomization zone in terms of local and instant thickness of the liquid sheet.

The work will be continued by improved post-treatment to reduce the atomization in the background to obtain quantitative atomization characteristics.

\section{Acknowledgements}

Authors want to acknowledge the federation FERMAT (Fluides, Energie, Réacteurs, Matériaux et Transferts), for the optical diagnostic material.

\section{Nomenclature}

$x \quad$ axial direction

y transvers direction

D injector's diameter [m]

$\delta \quad$ height of liquid film [m]

$U_{L} \quad$ liquid velocity $\left[\mathrm{m}_{\mathrm{L}} \mathrm{s}^{-1}\right]$

$U_{G} \quad$ gas velocity $\left[\mathrm{m} . \mathrm{s}^{-1}\right]$

$\sigma \quad$ surface tension [N.m $\left.{ }^{-1}\right]$

$\mu \quad$ viscosity [Pa.s]

$U_{\text {wave }} \quad$ wave velocity $\left[\mathrm{m} . \mathrm{s}^{-1}\right]$

$\mathrm{Re}_{\delta} \quad$ liquid Reynold number

$\begin{array}{ll}\operatorname{Re}_{\mathrm{D}} & \text { air Reynold number } \\ \mathrm{F}_{\max } & \text { frequency maximal }\left[\mathrm{s}^{-1}\right] \\ \Delta \mathrm{t} & \text { time step[s] } \\ \Delta \mathrm{X}_{\text {corr }} & \text { spatial step[m] } \\ \text { Corr } & \text { correlation coefficient } \\ \text { MFR } & \text { Momentum flux ratio } \\ \text { We } & \text { Weber number } \\ \text { Oh } & \text { Orhnesorge number } \\ \text { St } & \text { Strouhal number }\end{array}$

\section{References}

[1] Lefebvre A. H., 1989, "Atomization and Sprays."

[2] Déjean, B., Berthoumieu, P., Gajan, P., 2016, International Journal of Multiphase Flow, 79, pp. $214-224$.

[3] Warncke, K., Gepperth, S., Sauer, B., Sadiki, A., Janicka, J., Kock, R., Bauer, H.-J., 2017, International Journal of Multiphase Flow, DOI: 10.1016/j.ijmultiphaseflow.2016.12.010.

[4] Gepperth, S., Bärow, E., Koch, R., Bauer, H.-J. Bauer Sep 2014, $26^{\text {th }}$ Annual Conference on Liquid Atomization and Spray Systems.

[5] Alekseenko, S. V., Cherdanstev A. V., Cherdantsev, M. V., Isaenkov, S. V., Markovich, D. M., 2015, International Journal of Multiphase Flow , 77, pp.65-75.

[6] Han, Y.M., Seol, W. S., Lee, D. S., Yagokin, V. I., Jeung I.S., 2001, Journal of Engineering for Gas Turbines and Power, 123(1), pp. 33-40.

[7] Matas, J., Hong, M., Cartellier, A., 2014, Physics of Fluids, 26(4), 042108.

[8] Agbaglah, G., Chiodi, R., Desjardins, O., 2017, Journal of Fluid Mechancis, 812, pp1024-1038.

[9] Carvalho,I., Heitoyr, L.V., Santos, 2002, International Journal of Multiphase Flow,28 (5), pp773-789.

[10] Dumouchel, C., 2008, Experiments in fluids, 45 (3), pp 371-422.

[11] Gepperth, S., Müller, A., Koch, R., Bauer, H.-J. Bauer Sep 2012, $12^{\text {th }}$ Triennial Conference on Liquid Atomization and Spray Systems.

[12] Stapper, B., Sowa, W. Samuelsen, G.S., 1992, Journal of Engineering for Gas Turbines and Power, 114(1), pp39-45.

[13] Park, J., Huh, K., Li, X., Renksizbulut, M. 2004, Physics of Fluids, 16(3), pp625-632.

[14] Fernandez, V.,G., Berthoumie, P., Lavergne, G., 2009, Comptes Rendus Mecanique, 337(6-7),pp 481-491.

[15] Lozano, A., Barreras, F. 2001, Experiments in Fluids, 31(4), pp367-376.

[16] Koch, R., Braum, S., Wieth, L., Chaussonnet, G., Dauch, T., Bauer, H.,J., 2016, International Symposium on Transport Phenomena and Dynamics of Rotating Machinery. 


\title{
Influence of droplet clustering in sprays on liquid deposition rate on spherical targets
}

\author{
Paul Andrade*, Georgios Charalampous, Yannis Hardalupas \\ Mechanical Engineering Department, Imperial College London, SW7 2AZ, UK \\ *Corresponding author: p.andrade14@imperial.ac.uk
}

\begin{abstract}
The origin of temporal fluctuations of liquid mass deposition rates, obtained from a spray of droplets impinging on a solid spherical target, was investigated by correlation with droplet clusters in the spray. The droplet clusters were quantified using a Voronoi analysis on instantaneous images of the droplets, to obtain the number of droplet clusters, the area of the clusters and the number of droplets in each cluster. It was found that the normalised area of the droplet clusters had a distribution with a peak around $10^{-1}$ and a right tail which followed a power law of exponent -1.8. As the number density of the droplets inside the clusters increased, the temporal fluctuations of the liquid mass deposition rates increased, as a greater variation of droplet sizes impinged the target. However, as the standard deviation of the distribution of the normalised droplet cluster areas was increased, the temporal fluctuations in the liquid mass deposition rates reduced, as variations to the droplet number density and droplet sizes inside the clusters were averaged out.
\end{abstract}

\section{Keywords}

Clustering, Spray Impingement, Liquid Deposition.

\section{Introduction}

Multi-phase flows occur in a wide range of natural and industrial applications, such as rain formation, atmospheric emission, spray drying and combustion. In many of these applications, the surrounding fluid might be turbulent and this may influence how the particles disperse in such systems. For instance, during spray drying, an industrial process which produces powder from a liquid slurry feed, the change in dispersion caused by the surrounding hot turbulent air may alter the rate of collisions which occur between droplets of the atomized slurry, as well the outcome of the collisions. This may then change the morphology of the produced powder [1], and so it is important therefore to gain physical insight into how these physical processes behave. Whilst a unified theory which describes completely the dispersion of particles in a turbulent flow is lacking, two non-dimensional numbers, the Stokes number and the Gravitational settling parameter, have been used to characterize the dispersion.

The Gravitational settling parameter, $\phi$, characterizes the influence of gravity to the dispersion of the particle and is defined in Equation (1) as the ratio of the terminal velocity of a particle, $\mathrm{V}_{\mathrm{T}}$, to a characteristic velocity scale of a turbulent flow, $V_{F}$, typically the Kolmogorov scale [2]. Particles with a high settling parameter may have little lateral dispersion as they fall. This is because these particles have a very high terminal velocity and therefore spend little time in local turbulent flow eddies, so their response to these eddies occurs over a reduced period of time [3]. Alternatively, particles with a very low settling parameter will spend more time in the local eddies and therefore may have greater ability to disperse.

$$
\phi=\mathrm{V}_{\mathrm{T}} / \mathrm{V}_{\mathrm{F}} .
$$

The Stokes number, St, characterizes the influence of the particle inertia to its ability to disperse in the fluid flow. It is typically defined as the ratio of two time scales, $\tau_{\mathrm{P}}$ and $\tau_{\mathrm{F}}$, as shown in Equation (2) [4]. ' $\tau_{\mathrm{P}}$ ' is the particle response time and is the time required for a particle to respond to the local turbulent velocity fluctuations. ' $\tau_{\mathrm{F}}$ ' is a characteristic time scale of the turbulent flow and, for small particles, the Kolmogorov time scale is typically selected as the characteristic time [5].

$$
\mathrm{St}=\tau_{\mathrm{P}} / \tau_{\mathrm{F}} .
$$

If particles have a very low Stokes number, i.e. St $<<1$, the particles offer little resistance to turbulent velocity fluctuations and only slightly deviate from the instantaneous streamlines of the flow. If St $>>1$, particles have a high inertia and therefore follow trajectories defined by their initial boundary conditions, ignoring the majority of the fluctuations imparted on them by the flow. If however, St $\sim 1$, the particles only partially respond to the local fluctuations. This leads to particles aggregating closer together, creating regions of the flow field where a large number of particles accumulate, forming 'clusters' and regions of the flow field where there are few particles, forming 'voids' [6]. Since clusters have a high particle density, they can increase the probability of collisions [7,8].

A wide range of Stokes numbers and Gravitational settling parameters may exist in multi-phase flows. One such example, is during operation of an industrial spray dryer. At the beginning of the process, an atomizer creates a polydispersed spray of droplets containing liquid, fine solids and air. As the droplets are dried by the surrounding hot turbulent air, the remaining particles become 'sticky', after their surface reaches a critical viscosity [9]. The turbulent 

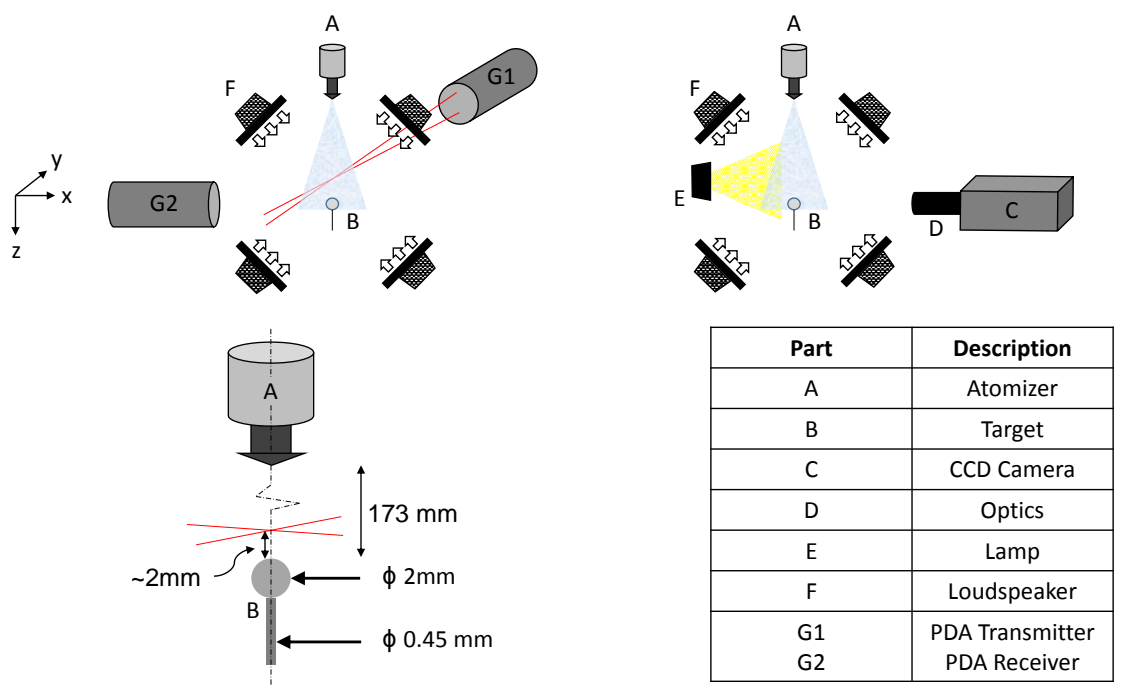

Figure 1. Details of the experimental set-up.

air flow promotes collisions between the different particles, with the outcome of the collisions dependent on the geometric and kinematic properties of particles, their trajectories and the surrounding air [10-13]. This can lead to formation of larger particles due to agglomeration, where a drying droplet or a sticky particle deposits on another sticky particle [9]. It can also produce much smaller particles due to the formation of 'secondary droplets' during droplet splashes on a sticky particle, causing a part of the droplet to re-atomise during impact [11]. The secondary droplets are then dried by the hot air to form 'fines'. These different physical processes lead to the formation of a wide range of droplet and particle sizes with variable composition inside the spray drier. Since the particle response time and terminal velocity, used to calculate St and $\phi$, depend on the droplet/particle size and composition, there must also be a wide range of Stokes numbers and settling parameters. It is therefore very difficult to assess how the particles disperse in the turbulent air flow inside the spray drier, even if the distribution of Stokes numbers and settling parameters is known. This makes the production of powders with desired characteristics quite inefficient.

To better understand the influence of the surrounding turbulent air on droplet/particle dispersion and particle-droplet collisions, imaging-based experiments of binary collisions involving a droplet impinging on a spherical solid target were carried out [13], inside a facility called the 'Box of Turbulence' [14]. This facility produces homogeneous isotropic turbulence without mean flow, allowing for the study of turbulent fluctuations on droplet dispersion. The surrounding turbulent air flow caused large lateral dispersion of the impinging droplets and changed the collision geometry, which influenced the outcome of the collisions. In more recent work [15], a spray, produced by an ultrasonic atomizer, impinged on the target, and imaging techniques were used to quantify the rate of accumulation of liquid on the target. The experiment was implemented inside the box of turbulence, which allowed the influence of the surrounding air turbulence on the rate of liquid depositing on the target to be investigated. As the intensity of the turbulent velocity fluctuations increased, the rate of liquid accumulation reduced. This is because fewer droplets from the spray impinged on the target, as they dispersed laterally due to the flow turbulence. Additionally, the collision outcomes may change due to the variation of the relevant parameters, as demonstrated by previous work on binary collisions.

It was noted that, for a fixed set of spray operating conditions, the temporal fluctuations of the accumulated liquid on the target increased with time. These temporal fluctuations propagated on the measurements of the liquid deposition rates. The aim of this paper is to investigate the origin of these fluctuations by correlating the estimated liquid deposition rates to characteristics of instantaneous clusters of droplets, which may occur in the spray. The paper is organized as follows. The next section describes the experimental set-up. This includes details of the characteristics of the droplets in the spray and the estimated liquid deposition rates, both of which were obtained previously but are relevant to the current paper. Also included in the methodology is a description of how recorded instantaneous images of droplets in the spray were processed using Voronoi analysis, which allowed droplet clusters to be identified. The following section presents the findings in terms of the droplet cluster characteristics, quantified from the Voronoi analysis, and correlates them to the deposition rate information on the solid target and discusses the droplet clustering implications on the time-dependent liquid deposition rates. The paper ends with a summary of the main findings.

\section{Methodology}

An experiment was set up inside the 'box of turbulence' facility, where a spray, produced by an ultrasonic atomizer, (Sono-Tek Ultrasonic $48 \mathrm{KHz}$ ) impinged on a spherical target (Figure 1). The target material was Stainless Steel 
316 , with a diameter of $2 \mathrm{~mm}$ and a maximum surface roughness of $0.125 \mu \mathrm{m} \mathrm{R}_{\mathrm{a}}$. The target was supported by a hypodermic needle (BD Microlance $326 \mathrm{G}$ ), of diameter $0.45 \mathrm{~mm}$. The box of turbulence uses eight loudspeakers mounted on a cubic frame to create synthetic jets. Each loudspeaker is directed towards the centre of the frame and homogeneous isotropic turbulence, without mean flow, is generated at the centre, as shown in Refs [14, 16]. The target was placed at the centre of the cubic frame, and the atomizer placed approximately $17.3 \mathrm{~cm}$ directly above the target, positioned just above the loudspeakers to avoid interference with the flow field. The flow field was characterized using the turbulent Reynolds number, $\mathrm{Re}_{\lambda}$, obtained from Particle Image Velocimetry (PIV) measurements of the flow field using a commercial system (LaVision). Three different Reynolds numbers were investigated, namely $\operatorname{Re}_{\lambda}=92,110$ and 136 .

The characteristics of the droplets in the spray were measured using Phase Doppler Anemometry (PDA), using a commercial system (Dantec Dynamics). The probe volume was placed just a few millimetres above the target. The droplet velocity and size were measured, from which the number density was also estimated. Depending on the intensity of the turbulent air and the atomization conditions, the droplet velocities were found to be around $1 \mathrm{~m} / \mathrm{s}$, the Sauter mean diameter of the droplets was approximately $40 \mu \mathrm{m}$ and the number density of the droplets were of the order of $10^{5}$ droplets per cubic centimetre. Since the atomizer imparted some momentum to the droplets, the instantaneous droplet velocity and turbulent flow velocity of the large eddies were used as characteristic velocities for the settling parameter. The settling parameter varied between approximately 13 and 55, whilst the Stokes numbers varied between approximately 0 to 1.4 .

A CCD camera then recorded images of the spray impinging on the target at a frame rate of $5 \mathrm{~Hz}$, with an exposure time of $1000 \mu \mathrm{s}$ and a magnification of approximately $6 \mu \mathrm{m} / \mathrm{px}$. The droplets deposited on the target, creating an initially thin liquid film around its surface. With further droplet impingements, the liquid film grew with time and drained underneath the target, before detaching due to gravity [15]. After detachment, the process was then repeated. The accumulation of liquid on the target was estimated using image processing (Matlab). First, Canny edge detection was used to detect the edges of the liquid film and the target. The edges were filled in, providing an estimate of the combined area of the target and the liquid film. The target area was deducted using a baseline image of a dry target. By assuming the liquid film developed symmetrically around the target, the liquid volume could be calculated. The mass of liquid, which accumulated around the target, was then plotted as a function of time. A fitting procedure was used to obtain the gradient of the line of best fit for the data, which corresponded to the liquid mass deposition rate. For a given experimental condition, since the process was repeated several times, several estimates of the liquid mass deposition rate were obtained.

In a separate experiment, for each experimental condition, 500 instantaneous droplet images were obtained in the spray, at a frame rate of $2.5 \mathrm{~Hz}$ and a magnification of $90 \mu \mathrm{m} / \mathrm{px}$. For the current work, a Voronoi analysis was applied to the droplet images of the spray (Matlab), as described by Ref [17], and quantified the number of droplet clusters present at each image, the number of droplets in each cluster and the area of each cluster. First, droplets below a given intensity threshold were removed from the image. This is because the images were obtained through illumination by a laser sheet and droplets out of the plane of the laser sheet, corresponding to low light scattering intensity droplets, could contribute adversely to the analysis. Next, for the remaining droplets, the Voronoi cells were computed. The boundary of each Voronoi cell defined a region of points on the image, which were closest to a given droplet. In other words, if a location on the image was chosen at random, the nearest droplet to that location is immediately known by virtue of the Voronoi cell and therefore, by definition, each Voronoi cell must contain only one droplet. If the droplets on the image were located close together, the Voronoi cells around these droplets were small. If droplets on the image were spaced far apart, their Voronoi cells would be larger. By using the area of the Voronoi cell as a measure of its size, clusters of droplets were identified based on connected Voronoi cells whose area fell below a given threshold. The threshold used is based on the intersection of the distribution of Voronoi cells in the image with a Poisson distribution of Voronoi cells of the same particles [17]. The latter distribution represents a random spatial distribution of droplets in the image domain and is obtained using the $2 \mathrm{D}$ analytical result by Ref [18].

\section{Results \& Discussion}

The results are presented in two sections. In the first section, the characteristics of the droplet clusters in the spray are quantified, when the surrounding gas is quiescent or turbulent. This then provides statistics which can be used to correlate to the liquid deposition rates in the second section.

\section{Quantification of droplet cluster characteristics in the spray}

The probability density function (PDF) for the detected area of the droplet clusters in the spray from all images for a given experimental condition was computed using a histogram consisting of 1000 bins (Figure 2). The area of each cluster, denoted $A_{C}$, was normalized with the average cluster area, $\overline{A_{C}}$. The distribution of the area of the droplet clusters displays a peak at around $10^{-1}$ for all Reynolds number. The results for liquid atomization by the ultrasonic atomiser driven by powers of $2.7 \mathrm{~W}$ and $3.2 \mathrm{~W}$ at the same liquid flow rate are qualitatively similar. Some authors have observed power law distributions with exponents of $-5 / 3$ to -2 for the probability of the droplet cluster areas [17, 19, $20]$, or of the qualitatively equivalent voids [17, 20-22]. However, a power-law distribution has only a straight line on log-log axes, but some of the aforementioned authors also observed peaks in their droplet cluster/void distributions $[17,19,20]$. A power law, with an exponent of -1.8 , is also shown in Figure 2 for comparison and appears to match 


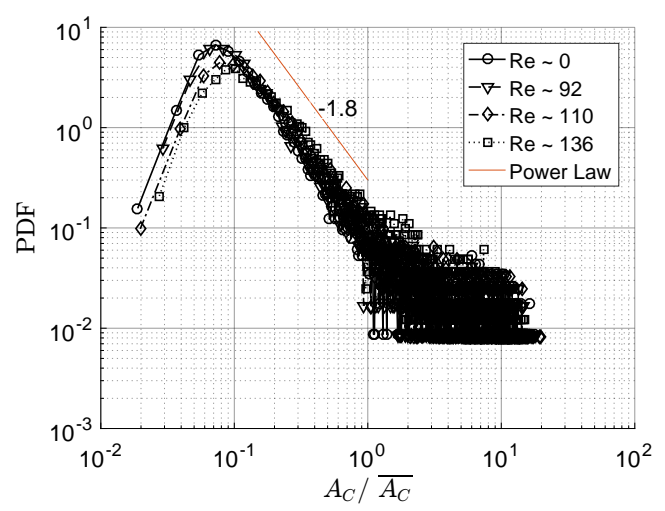

Figure 2. PDF of area of droplet clusters for operation of the ultrasonic atomiser with power and liquid flow rate of $2.2 \mathrm{~W}$ and 50 $\mathrm{cm}^{3} /$ min respectively.

the data only on the right tail of the distribution. A power law probability distribution of the droplet cluster area would suggest that droplet clusters are self-similar, implying that the clustering effect is due to a range of turbulent flow eddy sizes and not wholly dependant on the smallest eddies quantified by the corresponding Kolmogorov scales only [21].

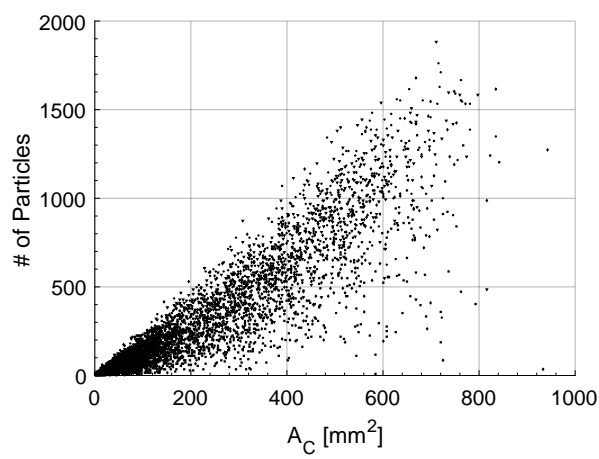

(a) Linear axes showing magnitude of variation

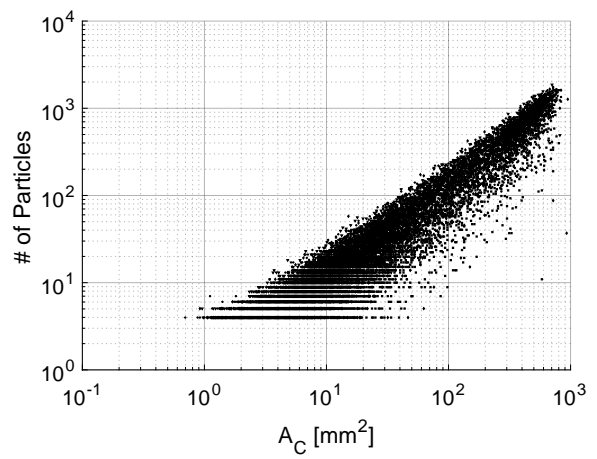

(b) Log-Log axes showing relative variation

Figure 3. Number of droplets detected in each cluster, for all Reynolds number, for the spray from the ultrasonic atomiser operating with power of $2.2 \mathrm{~W}$ and a liquid flow rate of $50 \mathrm{~cm}^{3} / \mathrm{min}$.

Next, the number of droplets inside each cluster was evaluated. Generally, the number of detected particles per cluster increased, as the area of the identified cluster increased (Figure 3). Whilst, for clusters with a large area, there was a greater magnitude of variation in the number of detected droplets (Figure 3a), the change in the number of detected droplets was actually more significant for clusters with a smaller area (Figure $3 \mathrm{~b}$ ).

Figure 3 may be explained physically by the response and dispersion of droplets to the local flow fluctuations. The number of droplets inside a cluster will depend on first, how many droplets existed in the neighbourhood of a cluster, at a short time interval, $\delta \tau$, before the cluster was formed (Figure 4). The response of the droplets to the local flow velocity fluctuations then determines which droplets will aggregate to form a cluster and which droplets do not. Since there is a wide distribution of Stokes numbers and settling parameters in the spray, there is a wide variation of response times to flow velocity fluctuations, influencing how many of those droplets, in a neighbourhood of the spray, aggregate to form the cluster.

If a cluster has a small area, it only covers a small region of the flow field. Prior to the formation of the small cluster, any droplets in its local neighbourhood may have only been exposed to a small range of flow velocity fluctuations (Figure 4a and Figure 4b). In Figure 4a, two droplets do not aggregate to form the cluster whereas in Figure 4b, four droplets do not aggregate to form the cluster. Whilst the change in the number of droplets aggregating is small (Figure $3 a$ ), the relative variation, i.e. the change in the number of droplets relative to the size of the cluster, is significant (Figure 3b). Alternatively, if a cluster has a large area, it covers a large region of the flow field. The droplets inside the large cluster may, therefore, have been exposed to a much wider range of flow velocity fluctuations before they aggregated into the cluster (Figure 4c). This in turn means that there may have been significant variations to how the different droplet sizes responded to different intensity of velocity fluctuations, leading to a large magnitude of variation in the number of droplets that formed the cluster, as shown in Figure 3a. However, since the cluster is so large, even if several droplets do not aggregate to form the cluster, its local number density is still high and, as a result, its relative variation is low (Figure $3 b$ ). 


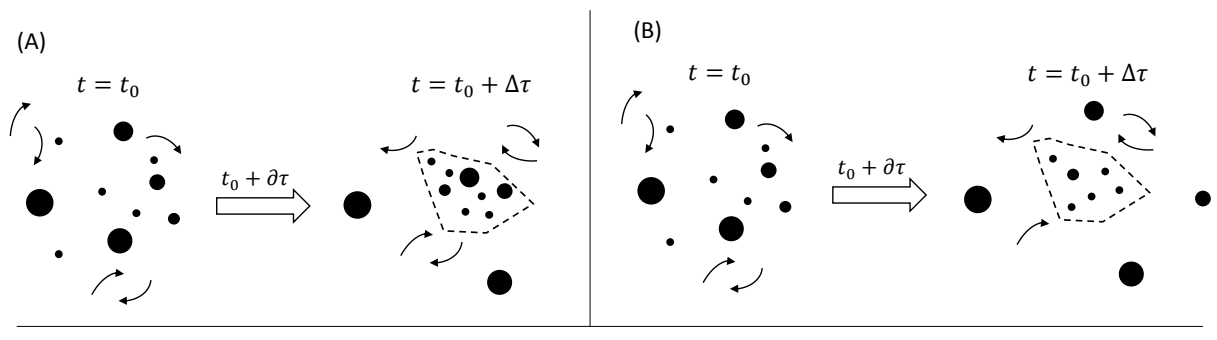

(C)
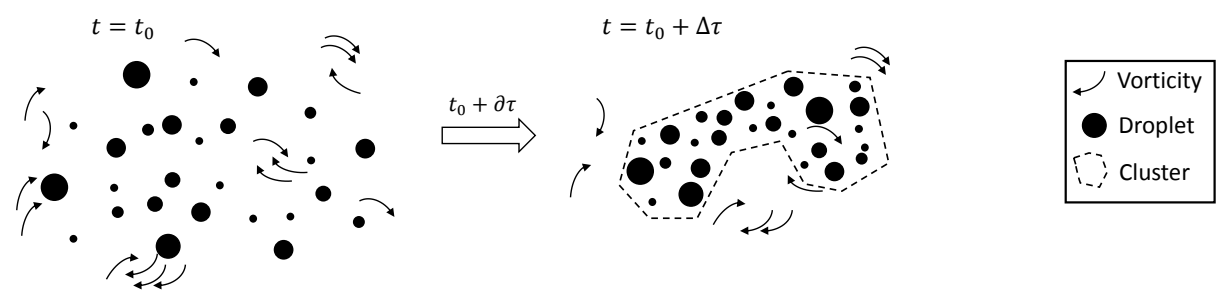

Figure 4. Schematic of cluster formation in polydispersed spray where ' $\mathrm{t}$ ' is the time, $t_{0}$ is the time as the cluster begins to form and $\delta \tau$ is a short interval for the cluster to form. (A) A group of droplets forms a small cluster. Two droplets do not aggregate into the cluster. (B) A group of droplets forms a small cluster, but several droplets do not aggregate into the cluster. (C) A group of droplets form a large cluster. The larger cluster may be formed from a larger range of local flow velocity fluctuation intensities.

Table 1. Coefficient of Determination values, $R^{2}$, for linear regression fit of number of droplets against area of cluster

\begin{tabular}{c|cccc}
\hline & $\operatorname{Re} \sim 0$ & $\operatorname{Re} \sim 92$ & $\operatorname{Re} \sim 110$ & $\operatorname{Re} \sim 136$ \\
\hline $2.2 \mathrm{~W}$ & 0.94 & 0.95 & 0.89 & 0.81 \\
$2.7 \mathrm{~W}$ & 0.90 & 0.90 & 0.88 & 0.79 \\
$3.2 \mathrm{~W}$ & 0.85 & 0.87 & 0.83 & 0.69
\end{tabular}

A linear regression fit was attempted to obtain the gradient of the number of droplets against the area of the cluster from Figure 3. The Coefficient of Determination values for the regression lines, $R^{2}$, are shown in Table 1 and the gradients of the regression line, denoted $\mathrm{D}_{\mathrm{ND}}$, are shown in Table 2. As the Reynolds number of the surrounding gas is increased, $R^{2}$ is reduced, suggesting the linear fit becomes less appropriate. This is true, regardless of the conditions used to generate the spray. As the Reynolds number is increased, the droplets have a greater lateral dispersion. If the atomization conditions are kept constant, the greater dispersion may lead to a greater variation of distances between droplets. This results in droplet clusters with a wider spread in the number of droplets they contain, which corresponds to a lower $\mathrm{R}^{2}$ value. The greater lateral dispersion means fewer droplets may also be located in the vicinity of the target and, therefore, there may be, for a cluster with a given size, fewer droplets inside the cluster, as reflected by $D_{N D}$ reducing with increasing Reynolds number in Table 2.

The frequency at which various sized clusters impinged on the target was also investigated. Since different cluster areas contain different number of droplets (Figure 3), the local droplet number density in each cluster may differ. The number density for each cluster was estimated by first, calculating the total number of droplets inside the cluster, $\sum N_{P}$ and then, dividing this by the cluster area, $A_{C}$. The clusters in each image of a given experimental condition were then 'binned' according to their area. The number density in these binned clusters was calculated and their frequency of occurrence was plotted (Figure 5). The liquid flow rate and the power supplied to the atomizer were kept fixed $\left(50 \mathrm{~cm}^{3} / \mathrm{min}\right.$ and $2.2 \mathrm{~W}$ respectively). It appears that very small clusters are more frequent than very large clusters (c.f. Figure 5a and Figure 5d). For a given Reynolds number, the droplet number density of the clusters is consistent regardless of the size of the cluster though as before (Figure $3 b$ ), there is greater relative variation in smaller cluster areas.

Table 2. Gradients, $D_{\mathrm{ND}}$, from the linear regression fit of number of droplets against area of cluster

\begin{tabular}{c|cccc}
\hline & $\mathrm{Re} \sim 0$ & $\mathrm{Re} \sim 92$ & $\mathrm{Re} \sim 110$ & $\mathrm{Re} \sim 136$ \\
\hline $2.2 \mathrm{~W}$ & 1.81 & 1.79 & 1.43 & 1.05 \\
$2.7 \mathrm{~W}$ & 1.35 & 1.34 & 1.20 & 0.89 \\
$3.2 \mathrm{~W}$ & 1.21 & 1.15 & 1.01 & 0.72
\end{tabular}




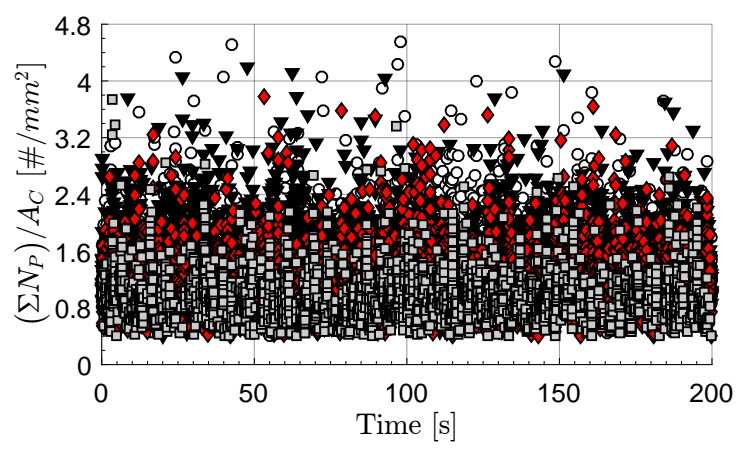

$\circ \operatorname{Re} \sim 0 \nabla \operatorname{Re} \sim 92 \diamond \operatorname{Re} \sim 110 \quad \square \operatorname{Re} \sim 136$

(a) $0 \mathrm{~mm}^{2} \leqslant A_{C}<10 \mathrm{~mm}^{2}$

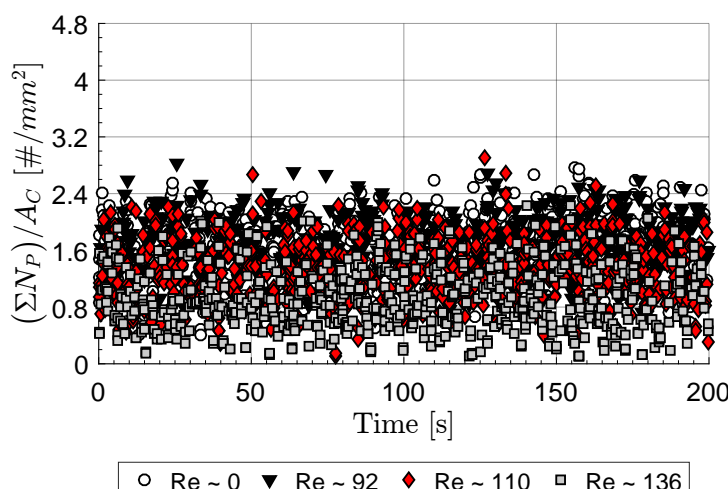

(c) $100 \mathrm{~mm}^{2} \leqslant \mathrm{~A}_{\mathrm{C}}<500 \mathrm{~mm}^{2}$

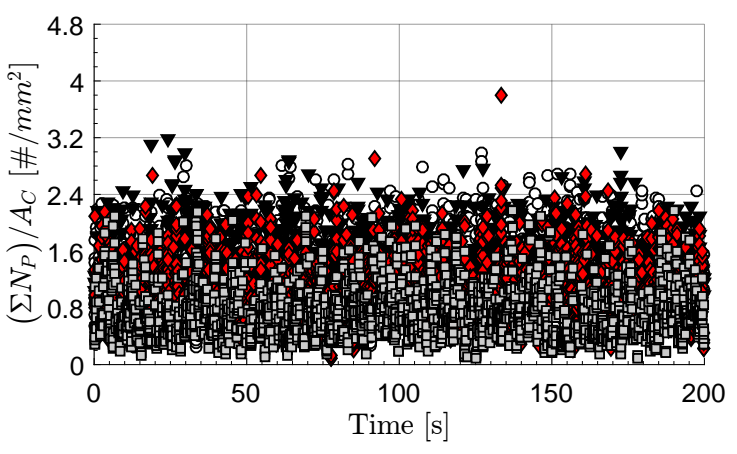

$\circ \operatorname{Re} \sim 0 \quad \boldsymbol{R e} \sim 92 \diamond \operatorname{Re} \sim 110 \quad$ a $\operatorname{Re} \sim 136$

(b) $10 \mathrm{~mm}^{2} \leqslant \mathrm{~A}_{\mathrm{C}}<100 \mathrm{~mm}^{2}$

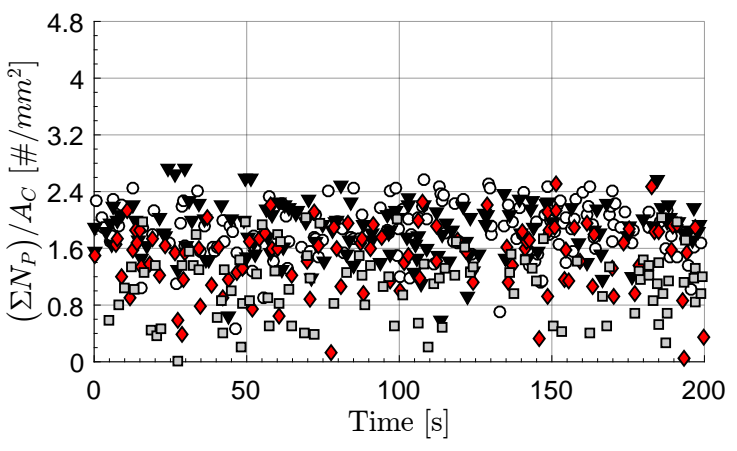

$\circ \operatorname{Re} \sim 0 \quad \boldsymbol{R e} \sim 92 \diamond \operatorname{Re} \sim 110 \quad \square \quad \operatorname{Re} \sim 136$

Figure 5. The relationship between the estimated number of droplets contained in each cluster, $\left(\sum N_{P}\right) / A_{C}$, and their probability of occurrence during $\sim 200$ seconds (500 images) of measurements.

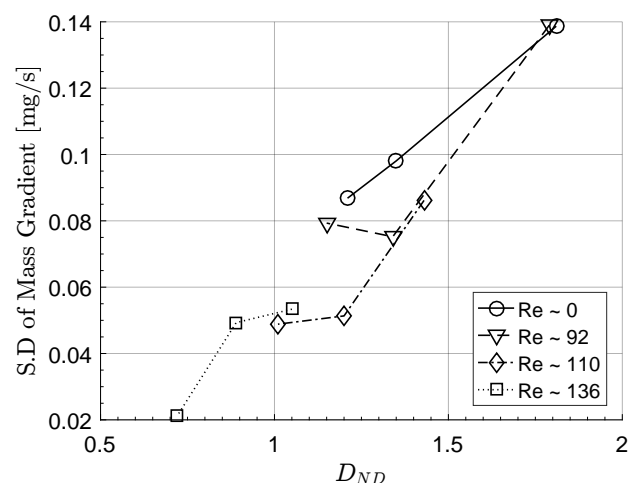

(a) Variation of liquid mass temporal gradients with gradients from Table 2.

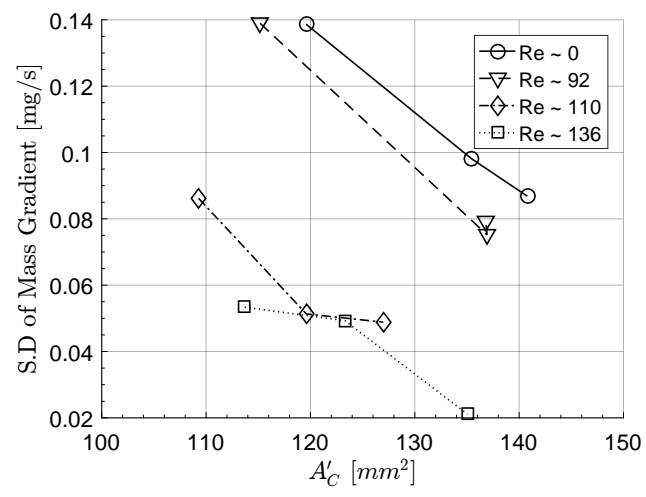

(b) Variation of liquid mass temporal gradients with standard deviation of cluster area, $A_{C}^{\prime}$.

Figure 6. Variation of standard deviation (S.D) of liquid mass temporal gradients with statistics from the clusters at different atomizer operating powers and at a fixed liquid flow rate of $50 \mathrm{~cm}^{3} / \mathrm{min}$ supplied to the atomizer. 


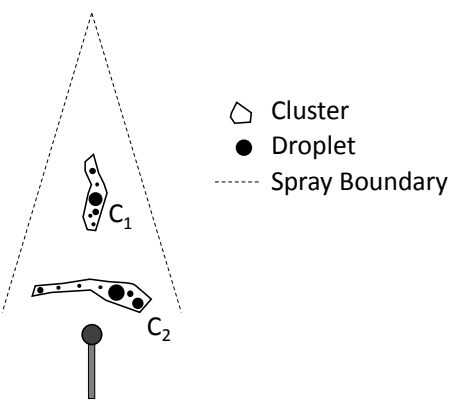

Figure 7. Droplet clusters may have different orientations relative to the target. This may influence the liquid deposition rate. Cluster $\mathrm{C}_{1}$ may deposit more droplets than $\mathrm{C}_{2}$, provided its properties do not change prior to impinging on the target.

\title{
Relationship between droplet clusters and liquid deposition rate
}

The influence of the droplet clusters to the estimated liquid deposition rates was then assessed and presented in Figure 6. Figure $6 a$ shows the standard deviation of the liquid mass temporal gradients increases as $D_{\mathrm{ND}}$ increases. $D_{N D}$ represents the average droplet number density of the clusters, for a given experimental condition. As the number density increases, there may be greater variation to the size of droplets inside the cluster. This may result in greater variation to the amount of liquid, which deposits on the target, corresponding to an increase to the spread of the liquid mass temporal gradients. Figure $6 \mathrm{~b}$ shows the standard deviation of the liquid mass temporal gradients decreases as the standard deviation of the area of the clusters, denoted by $A_{C}^{\prime}$, is increased. ' $A_{C}^{\prime}$ ' represents the spread of the distribution of the area of the clusters. If the spread is increased, a greater range of droplet cluster areas impinges on the target. This means any variations to the droplet number density (and the size of the droplets) inside the cluster are averaged out, leading to less variation of the liquid mass temporal gradients.

The shape and orientation of the cluster, relative to the target, as well as the size of the droplets inside the cluster must also be considered. If all the droplets were of similar size inside a cluster and all impinged on the target, Figure 5 may suggest that the intermittent clusters lead to temporal variations in how the liquid accumulates on the target. However, as well as there actually being a wide rage of droplet sizes in the spray, the shape and orientation of each cluster, relative to the target, can also vary substantially, and may be gravity dependant [23]. This may result in only part of a droplet cluster impinging on the target as shown in Figure 7. Provided that the clusters in the figure do not vary before they impinge on the target, most of the droplets contained in cluster $\mathrm{C}_{1}$ will deposit on the target, whereas only part of the droplets contained in $\mathrm{C}_{2}$ will impinge on the target.

\section{Conclusions}

The temporal fluctuations of the rate of liquid mass accumulation on a spherical target during spray impingement were studied by assessing the influence of characteristics of instantaneous droplet clusters formed in the spray. The main findings are summarized below:

- For a given Reynolds number, both large and small clusters appear to have similar local droplet number densities. Large clusters are much less frequent than small clusters. This may cause some of the variations to the rate of liquid deposition on the target.

- The greater the number density of droplets inside a cluster, the greater the fluctuations of the rate of liquid accumulation on a target.

\section{Acknowledgements}

We would like to acknowledge financial support from Procter and Gamble through an EPSRC industrial case studentship and EPSRC grant EP/K019732/1.

\author{
Nomenclature \\ $A_{C} \quad$ Area of Cluster $\left[\mathrm{mm}^{2}\right]$ \\ $A_{P} \quad$ Area of Particle $\left[\mathrm{mm}^{2}\right]$ \\ $\overline{A_{C}} \quad$ Mean Cluster Area $\left[\mathrm{mm}^{2}\right]$ \\ $\widetilde{A_{C}} \quad$ Standard Deviation of Cluster Area $\left[\mathrm{mm}^{2}\right]$ \\ Re Turbulent Reynolds Number \\ St Stokes Number
}

\section{References}

[1] D. F. Fletcher et al. "What is important in the simulation of spray dryer performance and how do current CFD models perform?" In: Applied Mathematical Modelling 30.11 (2006), pp. 1281-1292. ISSN: 0307-904x. DOI: DOI10.1016/j .apm. 2006.03.006. 
[2] A. Aliseda et al. "Effect of preferential concentration on the settling velocity of heavy particles in homogeneous isotropic turbulence". In: Journal of Fluid Mechanics 468 (2002), pp. 77-105. ISSN: 0022-1120. DOI: Doi10. 1017/S0022112002001593.

[3] M. R. Wells and D. E. Stock. "The effects of crossing trajectories on the dispersion of particles in a turbulent flow". In: Journal of Fluid Mechanics 136 (1983), pp. 31-62. ISSN: 1469-7645. DOI: doi : 10 . 1017 / S0022112083002049.

[4] C. T. Crowe, R. A. Gore, and T. R. Troutt. "Particle Dispersion by Coherent Structures in Free Shear Flows". In: Particulate Science and Technology 3.3-4 (1985), pp. 149-158. ISSN: 0272-6351. DOI: 10. 1080/02726358508906434.

[5] A. M. Wood, W. Hwang, and J. K. Eaton. "Preferential concentration of particles in homogeneous and isotropic turbulence". In: International Journal of Multiphase Flow 31.10-11 (2005), pp. 1220-1230. ISSN: 0301-9322. DOI: DOI10.1016/j.ijmultiphaseflow .2005.07.001.

[6] R. Monchaux, M. Bourgoin, and A. Cartellier. "Analyzing preferential concentration and clustering of inertial particles in turbulence”. In: International Journal of Multiphase Flow 40 (2012), pp. 1-18. ISSN: 0301-9322. DOI: DOI10.1016/j.ijmultiphaseflow .2011.12.001.

[7] S. Sundaram and L. R. Collins. "Collision statistics in an isotropic particle-laden turbulent suspension .1. Direct numerical simulations”. In: Journal of Fluid Mechanics 335 (1997), pp. 75-109. ISSN: 0022-1120. DOI: Doi10. 1017/S0022112096004454. URL: \%3CGo\%20to\%20ISI\%3E : //WOS : A1997WW06300004.

[8] W. C. Reade and L. R. Collins. "Effect of preferential concentration on turbulent collision rates". In: Physics of Fluids 12.10 (2000), pp. 2530-2540. ISSN: 1070-6631. DOI: Doi10.1063/1.1288515. URL: \%3CGo\%20to\% 20ISI\%3E : //WOS : 000089171000016.

[9] R. E. M. Verdurmen et al. "Agglomeration in Spray Drying Installations (The EDECAD Project): Stickiness Measurements and Simulation Results". In: Drying Technology 24.6 (2006), pp. 721-726. ISSN: 0737-3937. DOI: $10.1080 / 07373930600684973$.

[10] J. Qian and C. K. Law. "Regimes of coalescence and separation in droplet collision". In: Journal of Fluid Mechanics 331 (1997), pp. 59-80. ISSN: 1469-7645. DOI: doi : 10.1017/S0022112096003722.

[11] Y. Hardalupas, A. M. K. P. Taylor, and J. H. Wilkins. "Experimental investigation of sub-millimetre droplet impingement on to spherical surfaces". In: International Journal of Heat and Fluid Flow 20.5 (1999), pp. 477485. ISSN: 0142-727X. DOI: http://dx.doi .org/10.1016/S0142-727X (99) 00045-4.

[12] A. L. Yarin. "Drop impact dynamics: Splashing, spreading, receding, bouncing..." In: Annual Review of Fluid Mechanics 38 (2006), pp. 159-192. ISSN: 0066-4189. DOI: DOI10.1146/annurev .fluid.38.050304.092144.

[13] G. Charalampous and Y. Hardalupas. Droplet-Particle Collisions and Breakup in Homogenous and Isotropic Turbulence. 27th European Conference on Liquid Atomization and Spray Systems. Sept. 2016.

[14] G. Charalampous and Y. Hardalupas. Preferential concentration of water droplets in a volume of homogeneous and isotropic turbulence. 49th AIAA Aerospace Sciences Meeting including the New Horizons Forum and Aerospace Exposition. Jan. 2011.

[15] P. Andrade, G. Charalampous, and Y. Hardalupas. Spray impingement on spherical targets in homogenous isotropic turbulence. 27th European Conference on Liquid Atomization and Spray Systems. Sept. 2016.

[16] H. Lian, G. Charalampous, and Y. Hardalupas. "Preferential concentration of poly-dispersed droplets in stationary isotropic turbulence". In: Experiments in Fluids 54.5 (2013). ISSN: 0723-4864. DOI: Artn1525Doi10. 1007/S00348-013-1525-3.

[17] R. Monchaux, M. Bourgoin, and A. Cartellier. "Preferential concentration of heavy particles: A Voronoi analysis". In: Physics of Fluids 22.10 (2010). ISSN: 1070-6631. DOI: Doi10.1063/1.3489987.

[18] F. Jarai-Szabo and Z. Neda. "On the size-distribution of Poisson Voronoi cells". In: eprint arXiv:cond-mat/0406116 (June 2004). eprint: cond-mat/0406116.

[19] M. Obligado et al. "Preferential concentration of heavy particles in turbulence". In: Journal of Turbulence 15.5 (2014), pp. 293-310. ISSN: 1468-5248. DOI: Doi10.1080/14685248.2014.897710.

[20] Sholpan Sumbekova et al. "Preferential concentration of inertial sub-Kolmogorov particles: The roles of mass loading of particles, Stokes numbers, and Reynolds numbers". In: Physical Review Fluids 2.2 (2017), p. 024302. DOI: 10.1103/PhysRevFluids.2.024302.

[21] S. Goto and J. C. Vassilicos. "Self-similar clustering of inertial particles and zero-acceleration points in fully developed two-dimensional turbulence". In: Physics of Fluids 18.11 (2006). ISSN: 1070-6631. DOI: Artn115103Doi10. 1063/1.2364263.

[22] G. Boffetta, F. De Lillo, and A. Gamba. "Large scale inhomogeneity of inertial particles in turbulent flows". In: Physics of Fluids 16.4 (2004), pp. L20-L23. ISSN: 1070-6631. DOI: Doi10.1063/1.1667807.

[23] A. Dejoan and R. Monchaux. "Preferential concentration and settling of heavy particles in homogeneous turbulence". In: Physics of Fluids 25.1 (2013). ISSN: 1070-6631. DOI: Doi10.1063/1.4774339. 


\title{
Viability of coaxial atomization for disintegration of cell solutions in cell spray applications
}

\author{
Malte Bieber*, Sarah Menzel ${ }^{2}$, Anja Lena Thiebes ${ }^{2}$, Christian Gabriel Cornelissen ${ }^{2}$, Stefan \\ Jockenhoevel $^{2}$, Reinhold Kneer ${ }^{1}$, Manuel Armin Reddemann ${ }^{1}$ \\ ${ }^{1}$ Institute of Heat and Mass Transfer, RWTH Aachen University, Germany \\ ${ }^{2}$ Department of Biohybrid \& Medical Textiles (BioTex) at AME-Helmholtz Institute for \\ Biomedical Engineering, ITA-Institut für Textiltechnik, RWTH Aachen University, Germany and \\ at AMIBM-Masstricht University, The Netherlands \\ *Corresponding author: bieber@wsa.rwth-aachen.de
}

\begin{abstract}
Treating Leukemia with intravenous stem cell transplantation represents a well-established therapy technique. For applications, that require high local cell concentrations, transplantation by conventional intravenous injection is less potent, due to cell distribution with blood circulation. Instead, spraying them directly onto the injured or diseased area shows promising results in various applications, e.g. superficial treatment of topographically challenging wounds, in situ seeding of cells on implants, deposition of cells in tubular organs for stem cell therapy.

The present work aims for a basic knowledge about viability boundaries for coaxial cell-spray atomization and the reciprocal influence between cells in solution and primary breakup mechanics. A generic modular nozzle is developed, to ensures reproducible boundary conditions. Investigations are conducted regarding primary breakup and relations between resulting droplet size distribution and cell survival. Measurements are performed, utilizing microscopic high-speed visualization with suitable image post processing. Cell viability is analyzed using phase contrast microscopy prior and after atomization. A relation between Rayleigh-Taylor instability wavelength and droplet size distributions by means of Sauter mean diameter (SMD) and cell survival rate (CSR) is suggested. A power law is presented, exclusively dependent on dimensionless measures $\left(\lambda_{\perp} \sim \operatorname{Re}^{-1 / 2} \mathrm{We}^{-1 / 3}\right)$ which is found to be proportional to SMD and CSR.
\end{abstract}

\section{Keywords}

cell spray, coaxial atomization, cell survival

\section{Introduction}

Stem cell therapy is a research field in regenerative medicine with increasing importance due to the ability of cells to support healing of afflicted tissue. A well-established example represents the treatment of Leukemia by means of intravenous stem cell transplantation, where cells distribute by blood circulation within the organism after injection. To utilize the full potential of stem cells for applications that require high local cell concentration, alternative approaches for transplantation would be desirable, that enable local treatment rather than homogeneous distribution. An example for the requirement of high concentrations is the treatment of Acute Lung Injury (ALI) or Acute Respiratory Distress Syndrome (ARDS), both resulting from inhalation of biological toxins or toxic chemicals [1]. Despite extensive research, the mortality rate remains high (40\%) for both diagnoses [2]. As stated in [3], the anti-inflammatory properties of intrapulmonary instilled MSCs decrease the severity of endotoxin-induced ALI and improves survival in animal studies, though the increased liquid feed may further damage the lung tissue. Minimization of liquid feed into the lung could be achieved by using droplets as a carrier medium. A well-established technique for drug delivery in the lung using droplets, is the nebulization of liquids into a fine mist, that is directly inhaled. However, nebulization of cell solutions leads to high cell mortality rates [4]. In addition, direct inhalation is characterized by high deposition at the throat and at the first bifurcation, while in case of ALI/ARDS, the area of treatment is located at the alveolar regions. Therefore, the development of a suitable technique is required, that ensures high cell survival and reliable deposition at the area of treatment.

In this context, spraying of stem cells represents a promising approach. Suitability of cell spraying was already shown for external/superficial applications e.g. treatment of burn injuries by [5], treatment of topographically challenging wounds by [6] and most recently in [7]. However, application of cell-sprays within the human body was not realized so far. A flexible endoscopic spraying device was introduced by [8], enabling the atomization of therapeutic liquids and thus reliable local deposition of liquids in hollow organs. Utilized components are i) cell-thrombinsuspension and ii) fibrinogen, each dissolved in a tris-buffered-saline solution (TBS). Both solutions are injected through an endoscopic nozzle and are atomized by a coaxial air stream. After application, the fibrinogen polymerizes to fibrin gel, gluing the cells onto the area of treatment.

Cells in buffered solution are spherical and move with the surrounding fluid. However, they are prone to external forces, that possibly lead to destruction of cell membrane. Three destruction mechanisms for cell membranes have been investigated for syringe needle flow: cell deformation by pressure, linear shear flow and extensional flow origin from inlet flow conditions [9]. Pressure and shearing took minor roles in cell destruction, since spheres are generally pressure resistant and linear shearing causes them to spin within the surrounding fluid. Spinning reduces the stress applied on the cell membrane, due to circumferential distribution of the force application point [10]. However, while 
not being the main cause of cell damage, shearing influences the function of cells after application, as it influences cell differentiation [11]. Contrary, extensional flow imposes a local force onto the cell, causes deformation and potentially destroys the membrane as shown for synthetic capsules [12]. Hence, extensional flow is assumed to be the main cause of membrane damage. Yet, in coaxial air assisted endoscopic atomization, all three destruction mechanisms are presumably relevant, as cells are first injected into the working channel, continue to flow through catheter and nozzle exit and until they are finally atomized via coaxial airflow. In addition, the atomization process itself is characterized by a combination of high shearing and extensional flows. The liquid phase ejects through the nozzle, where it is sheared and accelerated by the coaxial air stream. Transverse instabilities of the liquid jet grow in amplitude and eventually form ligaments [13]. Further acceleration of these ligaments results in extensional flow and possibly in cell damage. Nonetheless, viability of endoscopic cell spray has been shown with high survival rates of $>90 \%$ [14] and a profound investigation of endoscopic cell spray has revealed no influence of the injection system on cell mortality [15].

In order to reach alveolar regions, directly inhaled particles need to be smaller than $10 \mu \mathrm{m}$ in diameter [16]. Droplets generated with an endoscopic nozzle can be deposited directly into the lung airflow and bypass first bifurcations. Nevertheless, based on the findings of Heyder et al. [16] a high atomization level is necessary with droplet sizes in the range of cell diameter $(\sim 15 \mu \mathrm{m})$.

In this work, an overview is presented regarding the effect of high atomization on cell viability. In addition, the primary breakup at nozzle exit is investigated with respect to reciprocal influence between cells in solution and breakup mechanics.

\section{Material and methods}

Potential influence of cells in tris-buffered solution (TBS) on the atomization process and the resulting spray character is investigated in a series of spray experiments with cell suspension and pure TBS for reference. Therein, the mass gas-liquid-ratio (GLR) is varied and optical measurement techniques are utilized for data collection. The cell suspension is injected into the nozzle, atomized, recollected and analyzed with respect to cell survival rate. The analysis of the spray character in combination with cell survival measurements might lead to findings regarding a link between cell survival and droplet size distributions or atomization mechanics.

\section{Injected substances:}

Reference measurement without cells are performed using pure tris-buffered saline (TBS), prepared by dissolving $4.36 \mathrm{~g}$ Tris/HCL, $0.64 \mathrm{~g}$ Tris base, $8 \mathrm{~g} \mathrm{NaCl}$ and $0.2 \mathrm{~g} \mathrm{KCl}$ diluted to $1000 \mathrm{ml}$ with $\mathrm{H} 2 \mathrm{O}$ with $\mathrm{pH}$ value adjustment to 7.4 with $\mathrm{HCl}$. For experiments including cells, TBS solution is enriched with living cells of A549 human cell line until a concentration of $1.6 \cdot 10^{6} \mathrm{cells} / \mathrm{ml}$ is achieved. Note, that cells in TBS are spherical with an average cell diameter of $15 \mu \mathrm{m}$, hence in the same order as mesenchymal stem cells, utilized in stem cell therapy [18]. Additionally, the selected cell concentration does not influence the fluid properties and is low enough for the fluid to be considered newtonian [17]. All substances are stored and injected at ambient conditions of $T=293.15 \mathrm{~K}$ and $p=100 \mathrm{kPa}$, thus fluid temperature matches ambient temperature with corresponding material properties $\left(\rho=1000 \mathrm{~kg} / \mathrm{m}^{3}\right.$ and $\nu=$ $\left.1.06 \cdot 10^{-6} \mathrm{~m}^{2} / \mathrm{s}[15]\right)$.

Test setup for spray generation and analysis:

The general test setup is presented in Figure 1 (left). The spray, generated by coaxial atomization, is injected into a flow chamber with side windows for optical access. Visualization of the propagating spray is provided by a combination of high-speed shadowgraphy using incoherent pulsed light. The liquid mass flow can be adjusted through a linear translation stage, that is utilized to actuate the syringe. The gas volume flow rate is adjusted and monitored by a combination of valve and rotameter within a range of $0.5 \mathrm{~m}^{3} / \mathrm{h} \leq 6 \mathrm{~m}^{3} / \mathrm{h}$. A schematic of the generic coaxial nozzle is shown in Fig: 1 (right). It's geometry is based on previous works, in which endoscopic catheter nozzles have been utilized. Thus, the generic nozzle mimics catheter nozzle flow conditions but enables generic reproducible boundary conditions. Core of the nozzle is a straight tube with a connection possibility for sterile syringes at the top and small wall thickness $(200 \mu \mathrm{m})$, to ensure a minimum gap between liquid and gas phase. This way, the interface boundary layer matches the gas boundary layer at nozzle exit, since the gap between liquid and gas phase is negligible. The tube is surrounded by an annular flow channel for coaxial atomization. The outer diameter of the annular flow channel is chosen to $D=3 \mathrm{~mm}$ and thus matches the working channel diameter of bronchoscopes.

The tube is exclusively attached to the upper body at the liquid inlet. The upper body is loosely connected to the lower body via three screws and tightened with 3 compression springs. This way, the upper body including the tube can be tilted relative to the lower body, which enables a precise coaxial and axial alignment of the tube relative to the annular air flow channel. The gas inlet is oriented perpendicular to the air flow channel, forcing the gas stream to turn directly into a flow straightener. From there on, the air flow enters an exchangeable nozzle tip, until it ejects parallel with the liquid flow.

Spray visualization:

To analyse the propagating spray, high-speed imaging (Fastcam SA-X, Photron Limited, 1024x1024) is utilized at $12,500 \mathrm{fps}$ in combination with suitable image post processing. The qualitative spray character is determined via macroscopic imaging with a constant Xenon light source and Zeiss makro planar t* $2 / 100 \mathrm{~mm}$ zf camera lens, to monitor the general spray character and to define the ideal position for microscopic imaging. Microscopic imaging is performed to capture the primary breakup and droplet propagation, by using a monochromatic and incoherent 


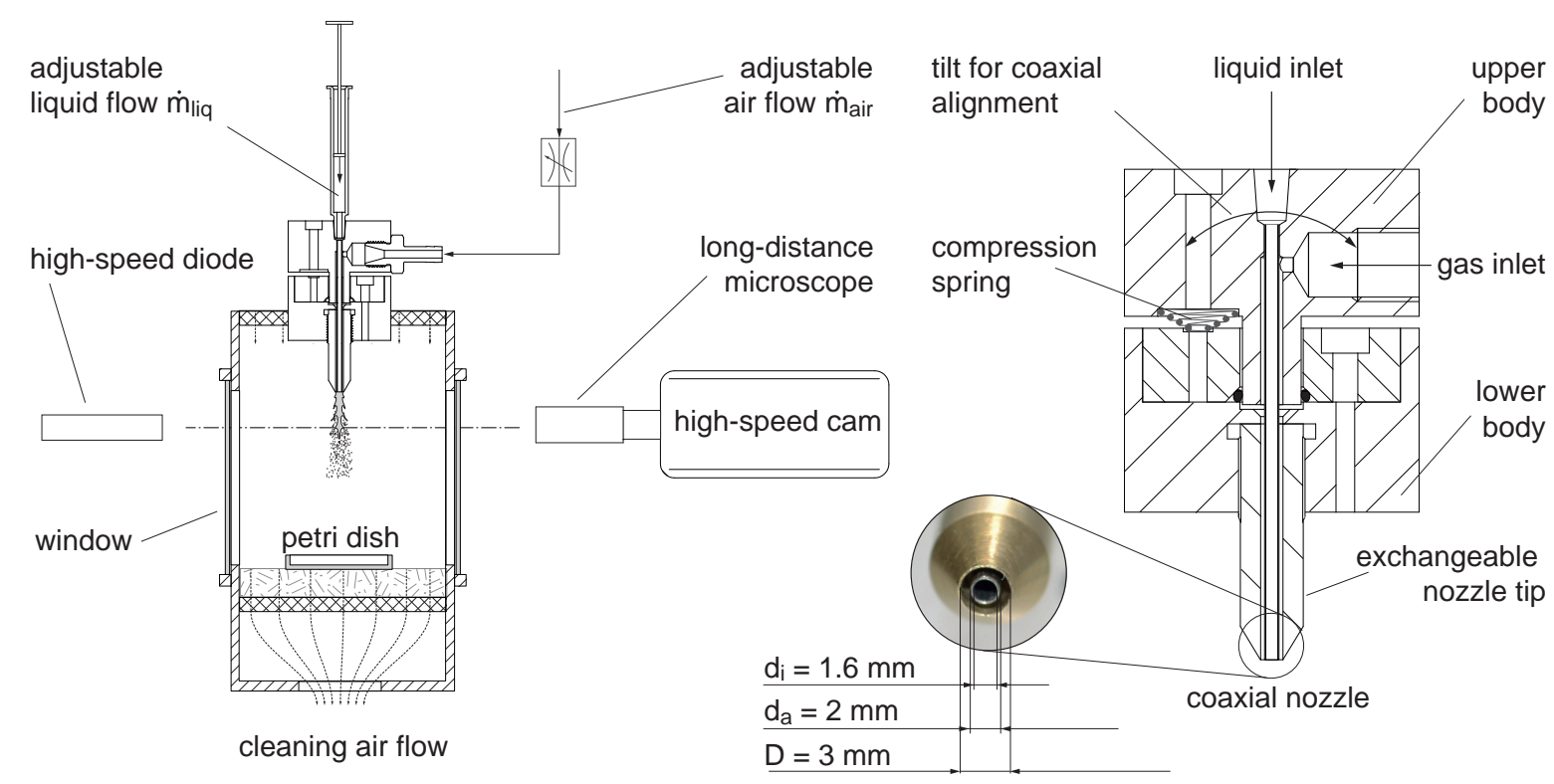

Figure 1. Left, experimental setup for spray investigation of coaxial gas stream driven atomization. Characterization of dispersed phase is done using macroscopic backlight visualization and long-distance transmitted light microscopy. Survival rates are determined before injection and after collection of droplets. Right, detailed presentation of modular coaxial nozzle design.

pulsed light source (Cavilux Smart, $640 \mathrm{~nm}$ wavelength, $10 \mathrm{~ns}$ pulse duration) and a Navitar Mag Zoom Lens with $12: 1$ zoom ratio set to $4.66 \mu \mathrm{m} / \mathrm{pixel}$, resulting in a $4.8 \mathrm{~mm} \times 4.8 \mathrm{~mm}$ frame size. Determination of resulting droplet diameters requires microscopic capturing sufficiently far from nozzle exit to ensure a fully atomized spray. Therefore, the investigated window ranges from $12 \mathrm{~mm}$ to $16.8 \mathrm{~mm}$ below nozzle exit. The typical spray cone angle for coaxial air assisted atomization is $20^{\circ}[21]$. To capture the entire spray, it is scanned horizontally in several highspeed videos, that are recombined afterwards in conducted image post processing. To investigate primary breakup, additional microscopic images are taken directly at nozzle exit with identical frame size.

\section{Image post processing:}

The image post-processing divides each high-speed video into individual images with a constant interframing time of $0.4 \mathrm{~ms}$. This way, over-weighting of slow droplets (with minimal velocity of $12.5 \mathrm{~m} / \mathrm{s}$ ) by multiple detection in one frame is avoided. For each considered frame a binarized image is generated, suitable for detection and evaluation of coherent areas, representing droplets. Pixels of coherent areas are counted and the corresponding eccentricity, as well as the equivalent circle diameter evaluated, which is convertible into metric units. In two dimensional pictures overlapping droplets are treated as single droplets with high eccentricity. Droplets with high eccentricity have high potential for secondary droplet breakup and overlapping or high aspect ratio droplets are potential error sources for calculation of Sauter mean diameter (SMD). Droplets with eccentricity e $>0.7$ are therefore not taken into account resulting in a maximum error potential caused by eccentricity of $5.77 \%$.

To evaluate whether identified droplets are off focus or not, two distinct filter sets (with different gamma value, contrast and grey threshold) are applied. The sensitivity regarding intensity gradients differs for each filter leading to differing detected droplet sizes for out of focus droplets. This can be used for droplet selection, in order to consider exclusively in focus droplets. For more information see $[15,17]$.

Test parameter:

The hydrodynamic entrance length is a function of characteristic length and Reynolds number. For laminar pipe

Table 1. Investigated parameter set for the gas flow with resulting mass gas-liquid-ratio (GLR), vorticity thickness $\delta$ and Weber number constructed on vorticity thickness

\begin{tabular}{|c|c|c|c|c|c|}
\hline$\dot{\mathrm{V}}_{\text {gas }}$ & $\dot{\mathrm{m}}_{\mathrm{gas}}$ & $\mathrm{Re}_{\text {gas }}$ & GLR & $\delta$ & $\mathrm{We}_{\delta}$ \\
\hline $\mathrm{m}^{3} / \mathrm{h}$ & $10^{-3} \cdot \mathrm{kg} / \mathrm{s}$ & - & - & $10^{-5} \cdot \mathrm{m}$ & - \\
\hline 1.39 & 1.17 & 6848 & 2.95 & 7.52 & 15.58 \\
\hline 1.81 & 1.52 & 8879 & 3.82 & 6.77 & 23.01 \\
\hline 2.28 & 1.92 & 11232 & 4.84 & 5.94 & 32.74 \\
\hline 2.86 & 2.40 & 14036 & 6.04 & 5.28 & 45.74 \\
\hline 3.53 & 2.97 & 17352 & 7.47 & 4.73 & 62.87 \\
\hline 4.41 & 3.71 & 21687 & 9.34 & 3.8 & 87.84 \\
\hline 6.07 & 5.11 & 29833 & 12.84 & 3.24 & 141.73 \\
\hline
\end{tabular}


flow, it can be determined according to $L_{h} / d_{i}=0.06 \cdot R e$ [20]. With a tube aspect ratio of $L / d_{i}=37.5$ and liquid Reynolds number $\mathrm{Re}_{\text {liq }} \leq 625$, a fully developed laminar velocity profile can be assumed at nozzle exit for every combination of parameter. The liquid Reynolds number for tube flow is calculated according to

$$
\operatorname{Re}_{\text {liq }}=\frac{4 \cdot \dot{m}_{\text {liq }}}{d_{\mathrm{i}} \cdot \pi \cdot \eta_{\text {liq }}}
$$

The corresponding mass flow rate is determined by the syringe actuation velocity, density and the syringe diameter and adjusted to a resulting liquid Reynolds number $R e_{\text {liq }}=300$. This way, the hydrodynamic entry length $\left(L_{h} / d_{i}=18\right)$ is reasonably smaller than the tube aspect ratio presented above.

The gas flow channel is characterized as an annular tube with a corresponding air Reynolds number

$$
\mathrm{Re}_{\mathrm{air}}=\frac{u_{\text {exit }} \cdot 2 \cdot s}{\nu_{\text {gas }}}
$$

where $s=\left(D-d_{\mathrm{a}}\right) / 2$ (see Figure 1) represents the gap size between tube and coaxial air flow channel. Air flow monitoring is performed through pressure corrected ${ }^{\star}$ rotameter measurement. In Tab. 1, the investigated parameter settings for the air flow are presented. The minimum gas volume flow rate is set, to always ensure a fully atomized jet. The massflow rate $\dot{m}_{\text {gas }}$ is calculated through the ideal gas law and velocity $u_{\text {exit,gas }}$ through continuity. The GLR is calculated according to $\mathrm{GLR}=\dot{\mathrm{m}}_{\text {gas }} / \dot{\mathrm{m}}_{\text {liq }}$.

Determination of cell survival rates:

For determination of cell survival rates, the spray is collected in a Petri dish, recollected by pipetting, dyed with Trypan blue and finally analyzed using an Axiovert $40 \mathrm{C}$ phase-contrast microscope with a Zeiss AxioCam ERc $5 \mathrm{~s}$. Thereby, the amount of living cells within a control area is determined and compared with the cell concentration of untreated cell suspension. The resulting sample size of cells varies around 300 detected cells in the control area. Two "positive controls" are evaluated: First, the cell survival rate in the original cell suspension pre injection and secondly the cell survival rate after surpassing the entire injection system including target impact but without air flow and thus without atomization. Comparing the cell survival rates before and after injection without airflow leads to conclusions about the influence of injection system as well as target impact on the cell survival rate. With knowledge of the original cell survival rate as well as the injection influence, exclusively the influence of the atomization process on the cells can be investigated through subtraction of critical side effects, e.g. extensional syringe flow, tube flow or target impact.

\section{Primary breakup theory of coaxial atomization}

The following brief description of theoretical background is based on the findings of Marmottant and Villermaux [13]. When the liquid jet emerges into a stationary atmosphere, capillary Plateau-Rayleigh instabilities are evolve. An additional axisymmetric destabilization occurs, when a coaxial gas flow is added, with an exit velocity $u_{\text {exit,gas }}$ significantly larger than the liquid exit velocity $u_{\text {exit,liq }}$ (see Figure 2 ). The acceleration of the interface in radial direction is oscillating, i.e. the local direction of acceleration alternates towards gas phase and liquid phase respectively. Pulsation of the surface waves can be expressed as $\omega_{\mathrm{L}}=2 \pi \cdot\left(\mathrm{u}_{\mathrm{u}}\right.$ $\left.\mathrm{u}_{\text {liq }}\right) / \lambda \sim\left(\rho_{\text {gas }} / \rho_{\text {liq }}\right)^{1 / 2} \cdot \mathrm{u}_{\text {gas }} / \lambda$ with $\lambda \sim \delta\left(\rho_{\text {liq }} / \rho_{\text {gas }}\right)^{1 / 2}$ representing the wavelength of the axial perturbation. A perpendicular acceleration $\left(g=a \cdot \omega_{\mathrm{L}}^{2} \cdot \sin (\omega \mathrm{t})\right)$ can be determined including the wave amplitude a. While directing towards the liquid phase, this acceleration is unstable, causing transverse Rayleigh-Taylor instabilities, that are oriented in azimuthal direction (see Figure 2). As a consequence, instabilities cause indentations of the rim, ligament formation and finally disintegration into droplets.

The azimuthal perturbation wavelength $\left(\lambda_{\perp}\right)$ depends on surface tension and is proportional to the undulation velocity $u_{u}$. For a plane surface, the temporal Rayleigh-Taylor growth rate is given as

$$
\omega_{\mathrm{i}, \mathrm{RT}} \sim\left(\frac{\rho_{\text {liq }} \cdot g^{3}}{\sigma}\right)^{\frac{1}{4}} \quad \text { and } \quad \lambda_{\mathrm{RT}} \sim\left(\frac{\sigma}{\rho_{\text {liq }} \cdot g}\right)^{\frac{1}{2}} .
$$

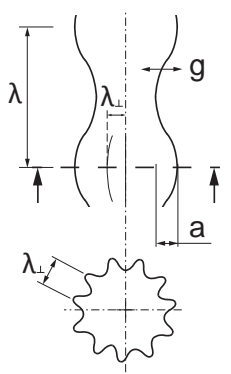

Figure 2. Schematic of transvese and azimuthal instabilities

${ }^{*}$ The rotameter, calibrated for ambient pressure $\left(p_{\text {amb }}\right)$ and monitoring the air flow, is located prior the coaxial nozzle. Thus, the static pressure inside the rotameter increases, due to pressure losses inside the nozzle and the measurement requires correction, regarding the resulting change of gas density. The measured flow-rate is based on force equilibrium between the weight force of the float body (index s) and aerodynamic force imposed by the gas (index g). Using continuity and with the assumption, that the density of the solid float body is significantly higher than the gas density, a proportionality relation of the flow-rate can be developed.

$$
\dot{V} \sim \sqrt{\frac{\left(\rho_{\mathrm{s}}-\rho_{\mathrm{g}}\right) \cdot g \cdot V_{\mathrm{s}}}{\rho_{\mathrm{g}}}} \sim \sqrt{\frac{\rho_{\mathrm{s}}}{\rho_{\mathrm{g}}}}
$$

With knowledge of the increased pressure the measured rotameter scale value can be corrected according to

$$
\frac{\dot{V}_{\text {meas }}}{\dot{V}_{\text {corr }}}=\frac{\sqrt{\rho_{\mathrm{s}} / \rho_{\mathrm{g}, \mathrm{cal}}}}{\sqrt{\rho_{\mathrm{s}} / \rho_{\mathrm{g}, \text { real }}}}=\sqrt{1+\frac{p_{\text {amb }}}{p_{\text {amb }}+\Delta p}}
$$

The obtained volume flow rate is assigned to the increased pressure inside the rotameter. To evaluate the volume flow rate at the nozzle exit, further adjustment is necessary, that includes the expansion towards nozzle exit by utilizing the ideal gas law.

$$
\dot{V}_{\text {exit,nozzle }}=\dot{V}_{\text {meas }} \cdot \sqrt{1+\frac{p_{\text {amb }}}{p_{\text {amb }}+\Delta p}} \cdot\left(1+\frac{p_{\text {amb }}}{p_{\text {amb }}+\Delta p}\right)=\dot{V}_{\text {meas }}\left(1+\frac{p_{\text {amb }}}{p_{\text {amb }}+\Delta p}\right)^{\frac{3}{2}}
$$


A critical condition for significant azimuthal instability development exists, when the inverse of the Rayleigh-Taylor growth rate is in the order of the transit time $\lambda / u_{\mathrm{u}}$. In this case, a correlation between maximum acceleration of the interface $\left(g_{\max }\right)$ and selected wavelength $(\lambda)$ and Weber number $\left(\mathrm{We}_{\lambda}\right)$ can be defined.

$$
g_{\max } \sim\left(\frac{\sigma}{\lambda \cdot \mathrm{We}_{\lambda}^{-\frac{1}{3}}}\right)^{2} \quad \text { with } \quad \mathrm{We}_{\lambda}=\frac{\rho_{\text {gas }}^{2} \cdot u_{\mathrm{gas}}^{2}}{\sigma / \lambda}
$$

With $\lambda \sim\left(\rho_{\text {liq }} / \rho_{\text {gas }}\right)^{(1 / 2)} \delta$ and a constant density ratio, the ligament spacing $\lambda_{\perp}$ can be expressed as a function of the vorticity thickness $\delta$.

$$
\lambda_{\perp} \sim\left(\frac{\sigma}{g_{\max }}\right)^{\frac{1}{2}} \sim \delta \cdot \mathrm{We}_{\delta}^{-\frac{1}{3}}\left(\frac{\rho_{\mathrm{gas}}}{\rho_{\text {liq }}}\right)^{-\frac{1}{3}} \quad \text { with } \quad \delta \simeq 5.6 \cdot h \cdot \mathrm{Re}_{\text {gas,exit }}^{-\frac{1}{2}} \quad \Rightarrow \lambda_{\perp} \sim \mathrm{Re}_{\text {gas,exit }}^{-\frac{1}{2}} \cdot \mathrm{We}_{\delta}^{-\frac{1}{3}}
$$

Ligament spacing is assumed to be directly proportional to resulting droplet size distributions, due to mutual correlation between ligament spacing, ligament diameter and resulting droplet sizes after ligament breakup. Ligament formation and potential extensional flows caused by ligament acceleration and thinning are assumed to be the main mechanic for cell damage [15]. Hence, maximum interface acceleration $g_{\max }$ is a potential measure for correlation between primary breakup and CSR and ligament spacing for resulting droplet size distributions of the spray.

\section{Results and discussion}

Investigation of mutual interaction between cells in solution and atomization is performed through variation of mass gas-liquid-ratio (GLR) in air assisted atomization of pure tris-buffered saline (TBS) and TBS enriched with living human cells. An overview is presented regarding jet breakup in air assisted atomization, followed by a brief discussion of resulting droplet size distributions, cell survival rates (CSR) and break up phenomena.

\section{Primary breakup analysis}

In Figure 3 four breakup regimes are presented. Left, a laminar jet exits the nozzle, that is perturbed in axial di-
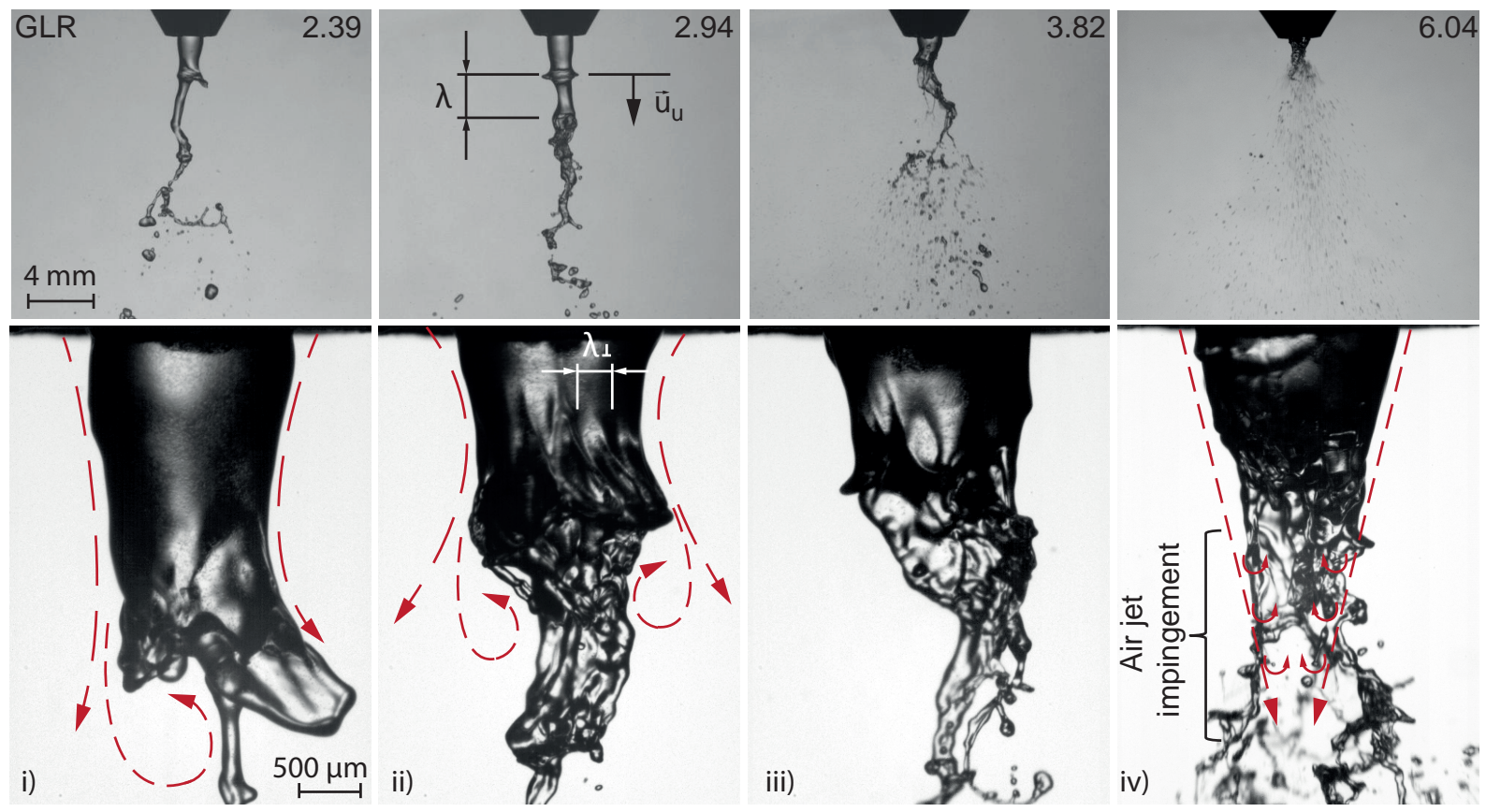

Figure 3. Macroscopic (top half) and microscopic (bottom half) images of the primary breakup of cell suspension are presented in dependency of the mass gas-liquid-ratio (GLR). Four different atomization regimes are shown; i) flapping: Axial modulation and high reciprocal flow interaction between gas and liquid face, ii) pulsing: Axial modulations superimposed by transverse Rayleigh-Taylor modulations and decreasing gas vortex sizes and liquid instability wavelengths, iii) transition: further decreasing flow characteristics, iv) disintegration: direct jet disintegration in the gas impingement region, liquid structures follow the gas flow.

rection $(\lambda)$. Disintegration of the jet occurs exclusively at the tip. Interaction between liquid and gas phase causes the jet tip to flap, which eventually leads to jet breakup. The second case $(G L R=2.94)$ is characterized by axial pulsation of the jet, superimposed by transverse Rayleigh-Taylor instabilities $\left(\lambda_{\perp}\right)$, causing ligament formation and jet breakup. This case is followed by a transition regime of decreasing perturbation wavelength $\lambda_{\perp}$. With further increasing GLR, the jet directly disintegrates in the air jet impingement region. Macroscopic perturbations are no longer visible and the liquid is directly dragged from a stationary liquid bulk, that is stabilized by the passing air flow. Primary breakup phenomena of the generic coaxial nozzle correspond well with the findings of Marmottant and Villermaux [13]. Hence, the gap thickness between gas and liquid phase is small enough to be negligible and 
the nozzle enables reliable boundary conditions for experimental data comparison with the theoretical findings described above.

Droplet size distribution and cell survival

In flexible endoscopic cell spray applications, droplet size distributions, expressed by Sauter mean diameter (SMD), as well as CSR decrease exponentially with increasing GLR. Main difference between atomization of pure buffered solution (TBS) and atomization of cell suspensions (TBS+cells) have been found in the resulting SMDs for identical test settings [15]. Until today, there is no profound explanation for these results, since the material properties of cell suspension are identical to TBS and show strict newtonian behavior [17]. Even though, the correlation between increasing airflow (i.e. increasing hydrodynamic forces) and resulting cell damage are comprehensibly, but gathered without reproducible boundary conditions. In this work, a generic nozzle is utilized to guarantee reliable boundary conditions and test data. In Figure 4 droplet size distribution by means of SMDs are presented in dependency on Reynolds and Weber number, as well as corresponding CSRs. Reynolds and Weber power laws utilized on the
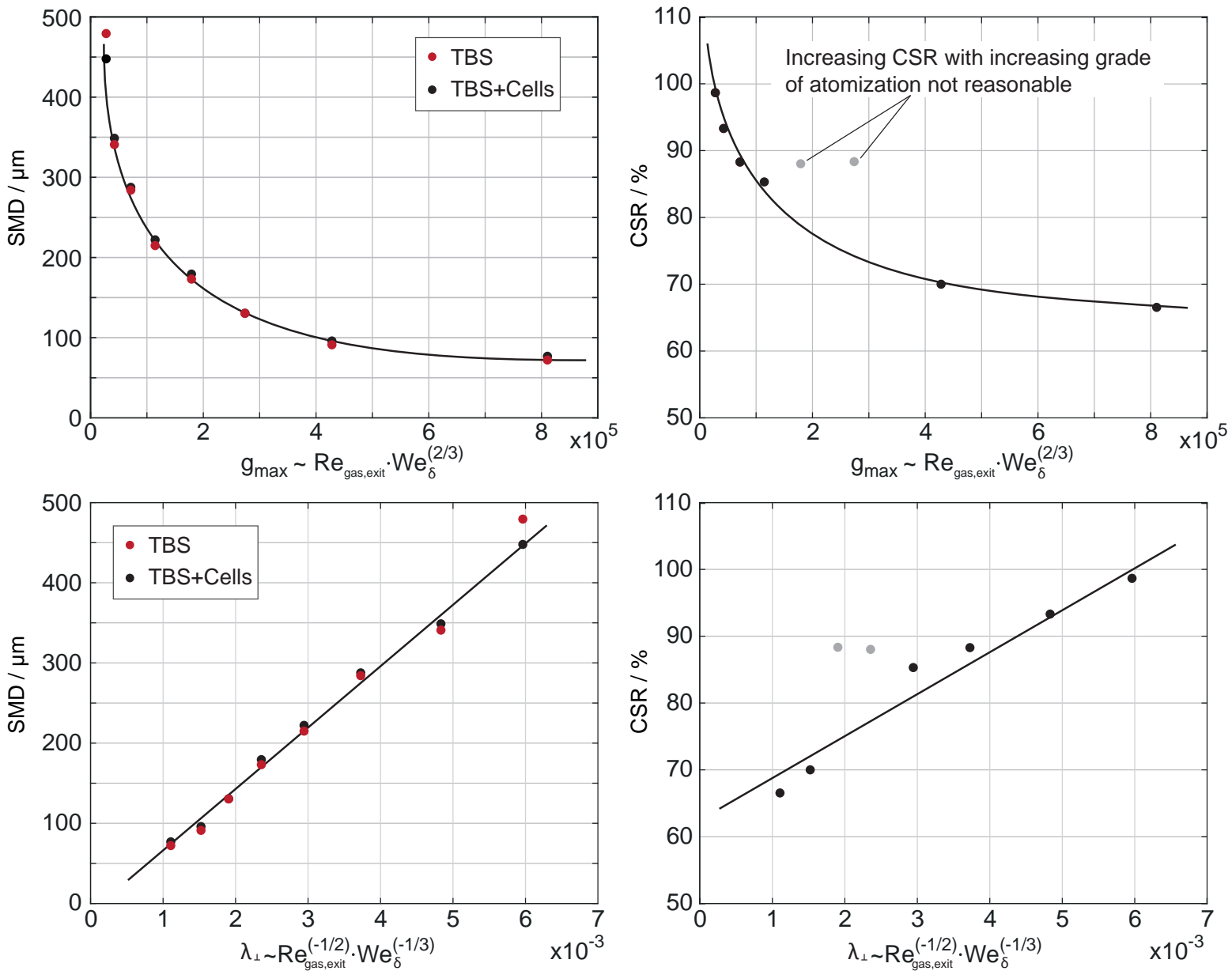

Figure 4. Left, droplet size distribution by means of Sauter mean diameter (SMD) is presented in dependency on Reynolds and Weber number. The Reynolds and Weber power law origins from Rayleigh-Taylor instability theory and is proportional to the maximum interface acceleration $g_{\max }$ (top) and transverse instability wavelength $\lambda_{\perp}$ (bottom). Right, the corresponding cell survival rates (CSR) are shown in dependency on equivalent dimensionless measures.

$\mathrm{X}$-axis are developed from Rayleigh-Taylor instability theory and are proportional to the maximum interface acceleration $g_{\max }$ and transverse instability wavelength $\lambda_{\perp}$ (i.e. ligament spacing). The droplet size distribution decreases exponentially with increasing Reynolds and Weber number, hence with increasing air flow and interface acceleration. The cell survival rate reveals a similar behavior with a lowest CSR of $64.64 \%$. Note, that grey values in cell survival are not taken into account, as increasing CSRs assigned to higher grades of atomization contradicts earlier findings and presumably origin from statistical errors in CSR determination. The observation of increasing SMDs with cells added to the solution $[15,17]$ could not be reproduced and presumably origin in unreliable boundary conditions. This assumption is further supported by the finding, that the present cell concentration neither influences material properties nor newtonian behavior [17].

From (7) a proportionality between maximum interface velocity and ligament spacing can be determined.

$$
\lambda_{\perp} \sim\left(\frac{1}{g_{\max }}\right)^{\left(\frac{1}{2}\right)}
$$


By adjusting the scaling of the abscissa accordingly, a linear dependency for SMD and CSR is found (see Figure 4 , bottom half) exclusively dependent on dimensionless measures, that can be further utilized for development of a Reynolds and Weber power law and for prediction of SMD and CSR. Additionally, it represents a basis for future test series aiming for a deeper understanding of the main mechanics causing cell damage. Unlike assumed earlier, CSR is thus proportional to ligament spacing instead of maximum interface acceleration and should be in focus for future investigations regarding hydrodynamics and cell damage.

Particles in primary breakup

Microscopic investigation of primary breakup for $\mathrm{GLR} \leq 3.82$ is conducted regarding main breakup phenomena. Thereby, ligament and bag breakup of liquid sheets are found to be the dominant. In Figure 5, an exemplary propagating bag breakup and ligament formation of cell suspension is presented for $G L R=3.82$, captured at $20 \mathrm{k}$ frames per second. A liquid sheet evolves from the coherent jet, that is captured and thinned out by the coaxial air stream until it collapses. Part of the sheet disintegrates into droplets, while the rest recombines into a coherent ligament, driven by surface tension. The result is a circular coherent ligament, that further disintegrates into droplets by ligament breakup. At the beginning of sheet formation, black dots can be detected, that represent cells in the
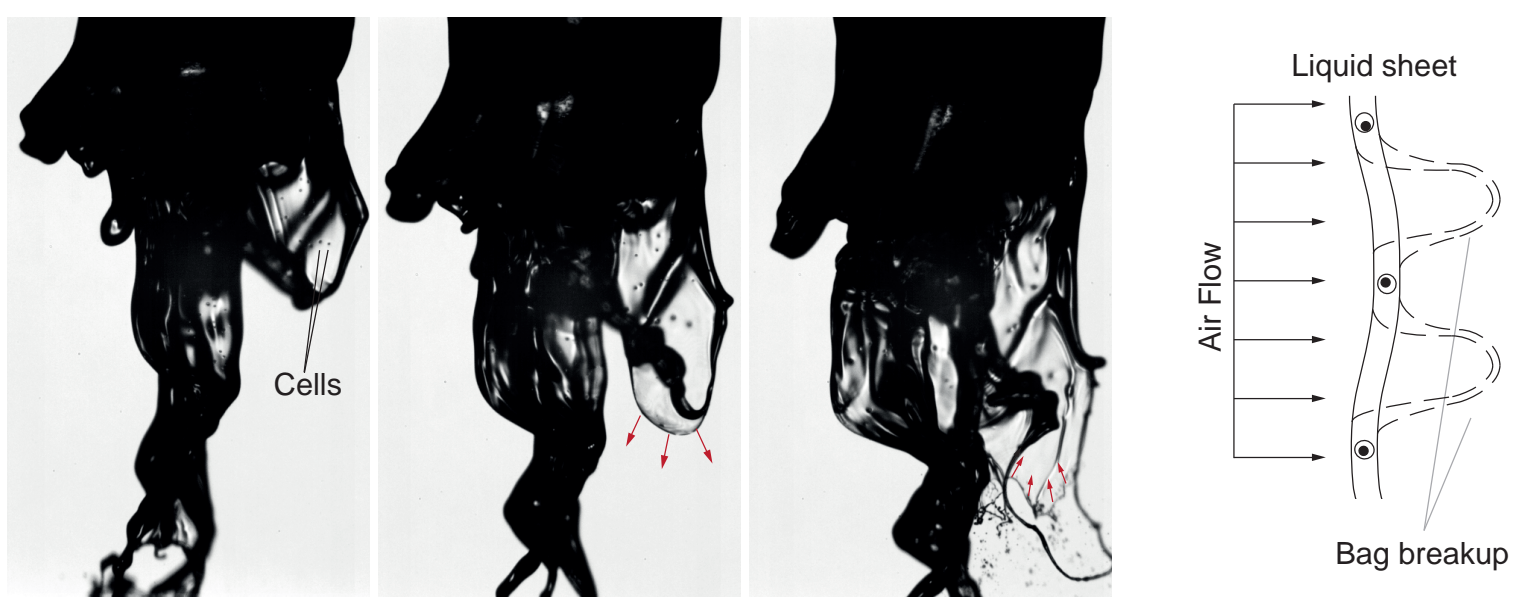

Figure 5. From left to right, propagating bag breakup and ligament formation of cell suspension is presented for $G L R=3.82$, captured at 20k frames per second. Black spots, visible exclusively in thicker parts of liquid sheets and ligaments, represent cells, that are surrounded by buffered solution. The lack of cells in thinning liquid sheets indicates, that the cells presumably act as a

flow barrier/stabilizer. This way, the propagation of bag breakup happens favorably in regions with low cell concentration.

disintegrating jet. Note, that the exemplary images are representative for all bag breakups captured, and cells are exclusively located in thicker regions of the liquid sheet, rims or ligaments but rarely in further thinning regions, that are typical for bag breakup. The cells presumably act as a stabilizer and since the flow will always chose the path of least resistance, bag breakup is favorably located in areas with low cell concentration (see Figure 5, right). After breakup, a small mass fraction of the bag disintegrates into droplets, while the main fraction collapses and gathers into a coherent ring shapes ligament, including the cells. During atomization, cells are therefore most likely located in ligaments and not in liquid sheets. This phenomena supports the findings mentioned above, in which ligament formation and sizing directly correlates with cell survival rather then other breakup phenomena. Note, that this hypothesis requires further investigation with generic test setups and high particle concentration.

\section{Conclusions}

The present work represents a systematic investigation of a spray generated through coaxial air assisted atomization. The data is evaluated regarding primary breakup, droplet size distribution and the influence of atomization on cell survival. A series of spray experiments is conducted with varying mass gas-liquid-ratios (GLR) of air flow and cell suspension.

General phenomena of primary breakup are discussed in dependency of GLR and assigned to the context of Rayleigh-Taylor instability driven ligament formation. A relation between ligament spacing and droplet size distributions by means of Sauter mean diameter (SMD) and cell survival rate (CSR) is suggested, including a corresponding power law. The relation is exclusively dependent on dimensionless measures $\left(\lambda_{\perp} \sim \mathrm{Re}^{-1 / 2} \mathrm{We}^{-1 / 3}\right)$ and found to be proportional to SMD and CSR.

Primary breakup in coaxial air assisted atomization is characterized by ligament and liquid sheet formation and breakup. Analysis of bag breakup reveals, that even though cells can be found in liquid sheets, evolving and thereby thinning areas of the sheet leading to bag breakup tend to lack presence of cells. The cells rather stay in thicker regions of the sheet, that are less influenced by the coaxial airflow. Therefore, the cells might act as a flow barrier and thereby stabilizing specific areas of high cell concentration. In this case airflow and liquid would always chose the path of least resistance and bypass the cells. The cells remain in ligaments, thus ligament formation and breakup are presumably the main phenomena influencing cell survival, which corresponds well to the proportional dependency of cell survival on ligament spacing. 


\section{Acknowledgements}

We gratefully acknowledge financial support from the Excellence Initiative of the German federal state governments (Exploratory Research Space, RWTH Aachen University).

\section{References}

[1] Angelini, D., Dorsey, R., Willis, K., Hong, C., Moyer, R., Oyler, J., Jensen, N., Salem, H., 2013, Inhalation Toxicology, 25(1), pp. 37-62.

[2] Lee, J., Gupta, N., Serikov, V., Matthay, M., 2009, Expert opinion on biological therapy, 9(10), pp. 1259-1270.

[3] Gupta, N., Su, X., Popov, B., Lee, J., Serikov, V., Matthay, M., 2007, The Journal of Immunology, 179, pp. 1855-1863.

[4] Sosnowski, T., Kurowska, A., Butruk, B., Jablczynska, K., 2013, AIDIC Conference Series, 11, pp. 371-380.

[5] Duncan, C., Shelton, R., Navsaria, H., Balderson, D., Papini, R., Barralet, J., 2005, Journal of Biomedical Materials Research Part B: Applied Biomaterials, 73(2), pp. 221-228.

[6] Gerlach, J. C., Johnen, C., McCoy, E., Bräautigam, K., Plettig, J., Corcos, A., 2011, Burns, 37(4), pp. e19-e23.

[7] Esteban-Vives, R., Choi, M., Young, M., Over, P., Ziembicki, J., Corcos, A., Gerlach, J., 2016, Burns, 42, pp. e99-e106.

[8] Thiebes, A. L., Reddemann, M. A., Palmer, J., Kneer, R., Jockenhoevel, S., Cornelissen, C. G., 2016, Tissue Engineering Part C, 22(4), pp. 322-331.

[9] Aguado, B., Mulyasasmita, W., Su, J., Lampe, K., Heilshorn, S., 2012, Tissue Engineering Part A, 18(7-8), pp. 806-815.

[10] Bluemink, J., Lohse, D., Prosperetti, A., and Wijngaarden, L., 2008, Journal of Fluid Mechanics, 600, pp. 201-233.

[11] Luo,W., Xiong,W., Zhou, J., Fang, Z., Chen,W., Fan, Y., Li, F.. 2011, Acta biochimica at biophysica Sinica, 43(3), pp. 210-216.

[12] Chang, K. and Olbricht, W. (1993). Journal of Fluid Mechanics, 250, pp. 587-608.

[13] Marmottant, P. and Villermaux, E. (2004). Journal of fluid mechanics, 498, pp. 73-111.

[14] Thiebes, A. L., Albers, S., Klopsch, C., Jockenhoevel, S.,Cornelissen, C. G., 2015, BioResearch open access, 4(1), pp. 278-287.

[15] Bieber, M., Thiebes, L.,Cornelissen, C.G., Jockenhoevel, S., Kneer, R., Reddemann, M.A., Sep. 5.-7. 2016, 27th European Conference on Liquid Atomization and Spray Systems, Brighton, UK.

[16] Heyder, J., Gebhart, J. Rudolf, G. Schiller, C.F. Stahlhofen, W., 1985, Journal of Aerosol Science,5, pp. 811-825.

[17] Bieber, M., Thiebes, L.,Cornelissen, C.G., Jockenhoevel, S., Kneer, R., Reddemann, M.A., 2017, Atomization and Sprays, submitted

[18] Ge, J., Guo, L., Wang, S., Zhang, Y., Cai, T., Zhao, R. C., Wu, Y., 2014, Stem Cell Reviews and Reports, 10(2), pp. 295-303.

[19] Gage, F. H., 1998, Nature, 392(6679), pp. 18-24.

[20] Kuemmel, W., 2007, "Technische Strömungsmechanik". B.G. Teubner Verlag.

[21] Wozniak, G., 2013, "Zerstäbungstechnik". Springer Verlag Berlin Heidelberg. 


\title{
Numerical Simulation of the Dispersion and Deposition of a Spray Carried by a Pulsating Airflow in a Patient-Specific Human Nasal Cavity
}

\author{
Ali Farnoud ${ }^{1 *}$, Xinguang $\mathrm{Cui}^{2}$, Ingo Baumann ${ }^{3}$, Eva Gutheil ${ }^{1}$ \\ ${ }^{1}$ Interdisciplinary Center for Scientific Computing, Heidelberg University, Germany \\ ${ }^{2}$ Lawrence Berkeley National Laboratory, Berkeley, CA \\ ${ }^{3}$ Department of Otorhinolaryngology, Head and Neck Surgery, Medical Center of Heidelberg \\ University, Heidelberg, Germany \\ ${ }^{*}$ Corresponding author: ali.farnoud@iwr.uni-heidelberg.de
}

\begin{abstract}
The present numerical study concerns the dispersion and deposition of a nasal spray in a patient-specific human nose. The realistic three-dimensional geometry of the nasal cavity is reconstructed from computer tomography (CT) scans. Identification of the region of interest, removal of artifacts, segmentation, generation of the .STL file and the triangulated surface grid are performed using the software packages ImageJ, meshLab, and NeuRA2. An unstructured computational volume grid with approximately 15 million tetrahedral grid cells is generated using the software Ansys ICEM-CFD 11.0. An unsteady Eulerian-Lagrangian formulation is used to describe the airflow and the spray dispersion and deposition in the realistic human nasal airway using two-way coupling. A new solver called pimpleParcelFoam is developed, which combines the lagrangianParcel libraries with the pimpleFoam solver within the software package OpenFOAM 3.0.0. A large eddy simulation (LES) with the dynamic sub-grid scale (SGS) model is performed to study the spray in both a steady and a pulsating airflow with an inflow rate of $7.5 \mathrm{~L} / \mathrm{min}$ (or maximum value in case of the pulsating spray) and a frequency of $45 \mathrm{~Hz}$ for pulsation as used in commercial inhalation devices. 10,000 mono-disperse particles with the diameters of $2.4 \mu \mathrm{m}$ and $10 \mu \mathrm{m}$ are uniformly injected at the nostrils. In order to fulfil the stability conditions for the numerical solution, a constant time-step of $10^{-5} \mathrm{~s}$ is implemented. The simulations are performed for a real process time of $1 \mathrm{~s}$, since after the first second of the process, all particles have escaped through the pharynx or they are deposited at the surface of the nasal cavity. The numerical computations are performed on the high-performance computer bwForCluster MLS\&WISO Production using 256 processors, which take around 32 and 75 hours for steady and pulsating flow simulation, respectively. The study shows that the airflow velocity reaches its maximum values in the nasal valve, in parts of the septum and in the nasopharynx. A complex airflow is observed in the vestibule and in the nasopharynx region, which may directly affect the dispersion and deposition pattern of the spray. The results reveal that the spray tends to deposit in the nasal valve, the septum and in the nasopharynx due to the change in the direction of the airflow in these regions. Moreover, it is found that due to the pulsating airflow, the aerosols are more dispersed and penetrate deeper into the posterior regions and the meatuses where the connections to the sinuses reside.
\end{abstract}

\section{Keywords}

Particle deposition, nasal cavity, pulsating airflow, nebulized aerosol.

\section{Introduction}

The human nasal cavity is part of the upper respiratory system and it is connected to the face by the nostrils, which are the only visible parts of the nasal airway [1]. The nasal cavity is divided into right and left parts by the nasal septum and it is lined by a densely vascularized mucus [2]. The nasal passage consists of different regions which are illustrated in Figure 1: the nasal valve, inferior, middle and superior meatuses, the olfactory region and the nasopharynx. These complex regions inside the nasal cavity are related to the physiological functions of the nasal airway. For instance, the olfactory region which is lined by olfactory nerves, is responsible for the sense of smell. The complex system of the nasal cavity is responsible for filtering the inhaled particles available in air and cleaning the mucus layer by the mucociliary clearance process [1]. During inhalation, the airflow and the particles enter the nasal cavity at the nostrils, pass through a 90 degree bend

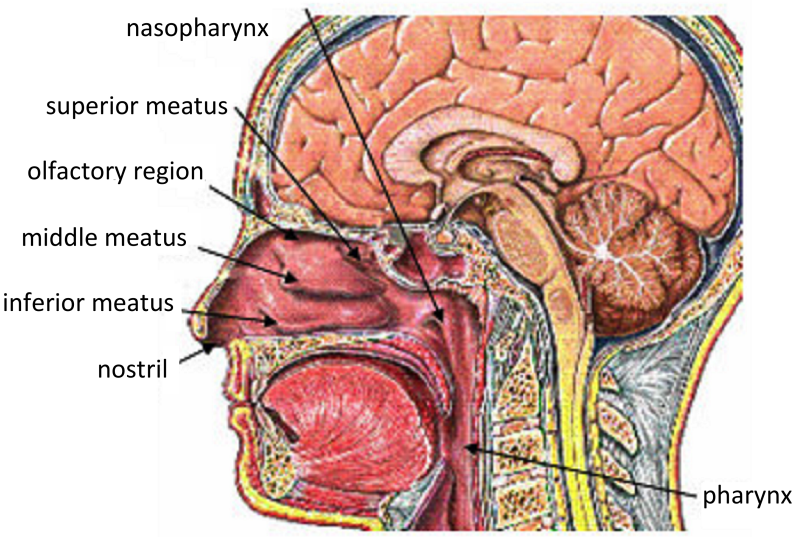

Figure 1. The anatomy of the nasal cavity [4] and a constricted area (nasal valve). Afterwards, the air and the particles enter the main nasal cavity, where they pass through the three airways of inferior, middle, and superior meatuses. The streaming air from the meatuses 
finally unifies in the nasopharynx and experiences another 90 degree bend which connects the nasal cavity with the pharynx [1]. The complex geometry of the meatuses and two 90 degree bends at the nasal valve and the nasopharynx help the respiratory system to trap the bacteria and potentially toxic particles on the sticky mucus. The particles that are trapped on the mucus layer finally dissolve in the mucus layer and absorb into the blood [2].

The nasal cavity is surrounded by paranasal sinuses which are air-filled cavities. There are four pairs of the sinuses which are connected to the main nasal airway: the maxillary, the ethmoid, the frontal and the sphenoid sinuses. The paranasal sinuses have various functions such as reducing the weight of the skull and resonating the speech [1]. These normal functions of the nose may be disturbed by various serious health conditions.

Chronic rhinosinusitis (CRS) is a common upper respiratory disease, which is defined as the inflammation of the nasal and paranasal cavity for the duration of more than 12 weeks [5]. CRS could occur due to the bacterial, fungal or viral infections, allergies, exposure to the inhaled irritants, septum deviation, or nasal polyps. The symptoms of CRS are mucosal swelling, mucus secretion into the nasopharynx, loss of cilia, airway obstruction, and no sinus drainage [1]. The prevalence of CRS disorder in Europe and USA is $10.9 \%$ [6] and 9-14\%, respectively [7]. The quality of life (QOL) of CRS patients is significantly effected by this disorder. Functional Endoscopic Sinus Surgery (FESS) is one of the main solutions for the patients suffering from CRS which has a success rate of 70-90\% [8,9]. It is found that after FESS there is a remarkable improvement in the QOL of the CRS patients [10].

The nasal airway is a potential path for drug delivery for CRS patients due its large surface area and its vascular surface [3]. However, conventional nasal drug delivery devices could not efficiently transport the drug to the desired target. Since CRS occurs in sinuses and connections of the sinuses which are not ventilated properly, the use of a conventional nasal sprays leads to an inefficient delivery of the medication. By using a nasal pump sprays, the particles tend to deposit in the anterior parts of the nasal airway [11] and medication cannot penetrate into the posterior regions of the nasal cavity, the ostia and the sinuses. This is due to the high inertial impaction caused by large particles with diameters of around $60 \mu \mathrm{m}$ [11]. Recent nebulizers are producing smaller particles in the range of $1-10 \mu \mathrm{m}$ [11], which could lead to an even distribution of the particles and and improvement of the penetration of the particles to posterior parts of the nasal cavity. Accordingly, it is important to study the airflow pattern and the particle dispersion and deposition in different patient-specific nasal cavities. Generating a pulsating airflow may improve the penetration of the airflow into the meatuses which are the connections to the sinuses [11]. Conclusively, the accessibility of the medication into the meatuses and sinuses is improved. Möller et al. [11] experimentally showed that the implementation of a pulsating airflow improves the delivery of the aerosols into the posterior regions of the nasal cavity and the sinuses. They experimentally compared a nasal pump spray and a pulsating aerosol delivery device. They concluded that the utilization of the nasal pump spray led to deposition of the most of the aerosols in the nasal valve region. Hilton et al. [12] experimentally studied the effect of the diameter of the particles on the deposition in the paranasal sinuses. They found that the particles with a diameter smaller than $5 \mu \mathrm{m}$ led to a higher deposition efficiency compared to the particles with diameters larger than $5 \mu \mathrm{m}$.

In order to gain a better understanding on the parameters affecting the airflow pattern and the aerosol dispersion and deposition in the nasal cavity, several in-vivo $[13,14,15]$ and in-vitro $[16,17,18]$ studies have been performed. Since performing experimental measurements in several human nasal airway models are time consuming, and technical difficulties with respect to the precision of in-vivo measurement due to complex geometry of the nose as well as unwanted side effects caused by the probes exist that may affect the gas flow [19], computational fluid dynamic (CFD) studies of the nasal airflow are an affordable alternative. In addition, CFD allows for parametric studies of variables which are difficult to change in the experiment. The advancement of CFD as well as the digital image processing sciences in the recent decades allows for a precise simulation of the nasal airflow and the particle dispersion and deposition pattern in the patient-specific nasal cavity. The first numerical studies of the airflow pattern in the nasal airway were accomplished by Keyhani et al. [20] and Subramaniam et al. [21]. Recently, particle dispersion and deposition have been studied in realistic nasal airways [22, 23, 24]. These simulations were performed with the commercial software packages CFX [22] and Fluent [23, 24]. Most of the research in this area considers a steady inlet airflow [25] or an unsteady flow representing inhalation-exhalation cycles [26]. Frank-Ito et al. [27] studied the effect of the volume grid cells on the deposition efficiency of the particles while modeling the realistic airflow and particle transport in a realistic human cavity including the paranasal sinuses. They found that by increasing the number of the tetrahedral elements and specially the density of the volume grid cells at the wall, the particle deposition fraction decreased. They concluded that a coarse mesh could lead to overestimation of the deposition efficiency of the particles.

In the present study, a patient-specific geometry of the nasal airway (without sinuses) is constructed from CT images, and the effect of the pulsating airflow on the dispersion and deposition of the particles in the nasal airway is studied using a large eddy simulation. Moreover, the effect of the diameter of the particles on the deposition efficiency in the nasal cavity is investigated.

\section{Methods}

The airflow and particle dispersion and deposition during steady and pulsating inlet airflow conditions is studied in a CFD simulation. The steps involved are the identification of the region of interest in the CT scan and the generation of a numerical surface and volume grids for the simulation. After determination of appropriate governing equations, a large eddy simulation (LES) is performed with appropriate boundary and inlet conditions. 


\section{Geometry}

A realistic three-dimensional geometry of the nasal cavity is constructed from the CT images of a patient [28]. The images with slice thickness of $1 \mathrm{~mm}$ are obtained in .DICOM format. Subsequently, identification of the region of interest, artifacts removal, segmentation, extraction of the .STL file and generation of the triangulated surface are preformed by using the software packages ImageJ, meshLab, NeuRA2 [28]. Due to complexity of the geometry including sharp curves and edges, a volume grid with approximately 15 million unstructured tetrahedral cells is generated using the software package ICEM-CFD Ansys 11.0. Figure 2 illustrates the sagittal view of the CT images (left) and the constructed geometry (right). The airflow enters from the inlet and leaves the nasal passage from the outlet.
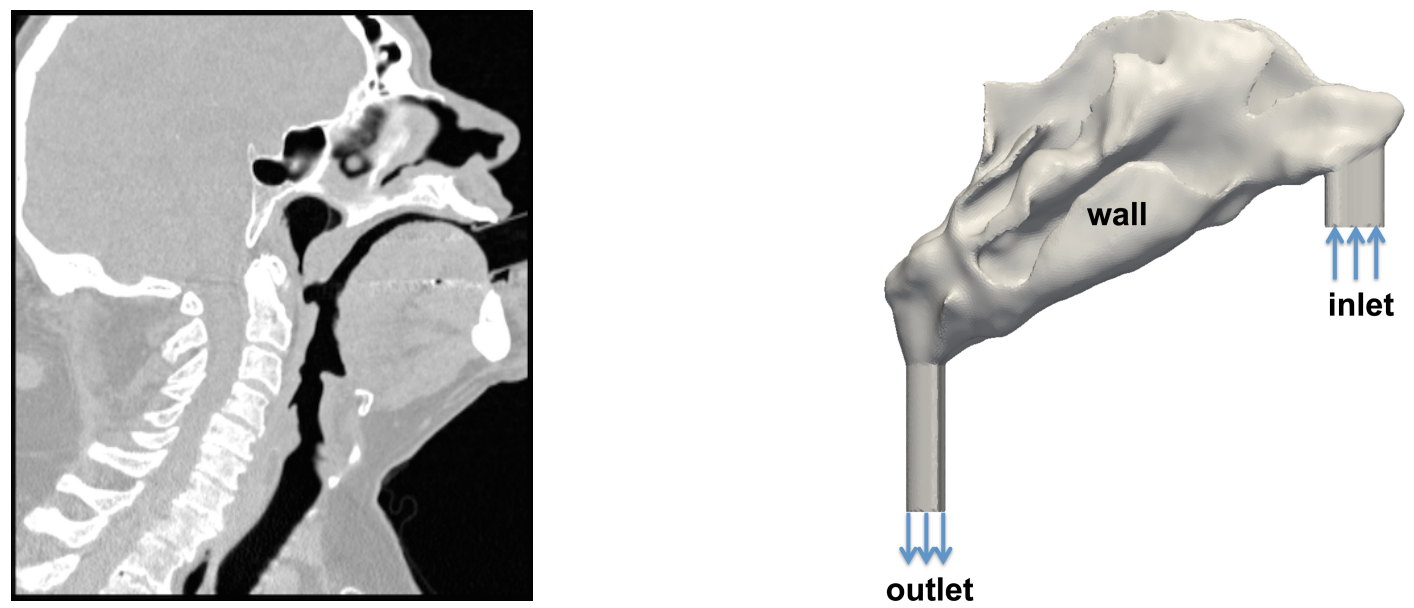

Figure 2. The CT image of the patient (left) and the constructed three dimensional nasal cavity (right)

\section{Governing Equations}

A Eulerian-Lagrangian description of the gas and liquid phases is employed where a large eddy simulation with the dynamic sub-grid scale model [32] is used to describe the laminar-transitional-turbulent gas flow. Two-way coupling [28] of the gas-phase equations with the particles is used.

In the Smagorinsky SGS model, the grid filter $\bar{G}$

$$
\bar{f}(\mathbf{x})=\int_{D} f\left(\mathbf{x}^{\prime}\right) \bar{G}\left(\mathbf{x}, \mathbf{x}^{\prime}\right) \mathrm{d} \mathbf{x}^{\prime}
$$

with grid-dependent filter width $\bar{\Delta}$ is applied to both the continuity and the momentum equations. The sub-grid stress tensor $\tau_{i j}^{\mathrm{r}}$ appearing in the filtered equations [31] is closed using an eddy viscosity hypothesis $\tau_{i j}^{\mathrm{r}}-\left(\delta_{i j} / 3\right) \tau_{k k}^{\mathrm{r}} \approx$ $-2 v_{\mathrm{r}} \bar{S}_{i j}$, where the residual stress is related to the filtered rate of strain, $\bar{S}_{i j}$. The eddy viscosity then is closed using the Smagorinsky coefficient $C_{\mathrm{S}}, v_{\mathrm{r}}=\left(C_{\mathrm{S}} \bar{\Delta}\right)^{2}|\bar{S}|$ with $\bar{S}_{i j}=1 / 2\left(\partial \bar{U}_{i} / \partial x_{j}+\partial \bar{U}_{j} / \partial x_{i}\right)$ and $|\bar{S}|=\left(2 \bar{S}_{i j} \bar{S}_{i j}\right)^{1 / 2}$ [31] to yield

$$
\tau_{i j}^{\mathrm{r}}-\left(\delta_{i j} / 3\right) \tau_{k k}^{\mathrm{r}} \approx-2 C_{\mathrm{s}} \bar{\Delta}^{2}|\bar{S}| \bar{S}_{i j} .
$$

The assumption of a constant Smagorinsky coefficient is relaxed in the dynamic SGS model [29], where a so-called test filter $\widetilde{G}$ with filter width $\widetilde{\Delta}$

$$
\widetilde{f}(\mathbf{x})=\int_{D} f\left(\mathbf{x}^{\prime}\right) \widetilde{G}\left(\mathbf{x}, \mathbf{x}^{\prime}\right) \mathrm{d} \mathbf{x}^{\prime}
$$

is performed after the filtering given by Eq. (1). Then the continuity and momentum equations for an unsteady, incompressible and isothermal airflow field yield [29]

$$
\begin{aligned}
& \frac{\partial \widetilde{\bar{U}}_{j}}{\partial x_{j}}=0 \\
& \frac{\partial \widetilde{\bar{U}}_{i}}{\partial t}=-\frac{\partial\left(\widetilde{\bar{U}}_{i} \widetilde{\bar{U}}_{j}\right)}{\partial x_{j}}-\frac{\partial \widetilde{\bar{p}}}{\partial x_{i}}-\frac{\partial T_{i j}}{\partial x_{j}}+\frac{1}{\operatorname{Re}} \frac{\partial^{2} \widetilde{\bar{U}}_{i}}{\partial x_{j} \partial x_{j}}+\widetilde{\bar{S}}_{\mathrm{p}, i}
\end{aligned}
$$

In the equations above, $i, j=1, \ldots, 3$, and the Einstein summation convention is used. Note that in the momentum equation (5), the filtered source term $\widetilde{\bar{S}}_{\mathrm{p}, i}$

$$
\widetilde{\bar{S}}_{\mathrm{p}, i}=\sum_{i=1}^{n}\left[\frac{m_{\mathrm{p}, i}}{\Delta V} \frac{\left(\mathbf{U}_{\mathrm{p}, i}\right)_{t_{\mathrm{out}}}-\left(\mathbf{U}_{\mathrm{p}, i}\right)_{t_{\mathrm{in}}}}{t_{\mathrm{out}}-t_{\mathrm{in}}}\right]
$$


appears that accounts for the interaction of the particles and gas phase, i.e. two-way coupling is used [28]. For one-way coupling, the effect of particle motion on the gas phase is neglected, i.e. the source term in Eq. (5), $S_{\mathrm{p}, i}$ is set to zero. $T_{i j}$ is the dynamic Smagorinsky stress tensor, which replaces the residual stress tensor $\tau_{i j}{ }^{r}$ used for the constant Smagorinsky-coefficient approach above. The sub-grid stress tensor then yields [29] $T_{i j}={\widetilde{U_{i} U_{j}}}_{-} \widetilde{\bar{U}}_{i} \widetilde{\bar{U}}_{j}$, which is related to the resolved turbulent stress $L_{i j}=\widetilde{\bar{U}}_{i} \bar{U}_{j}-\widetilde{\bar{U}}_{i} \widetilde{\bar{U}}_{j}$ through the Germano identity [29]

$$
L_{i j}=T_{i j}-\tilde{\tau}_{i j}^{\mathrm{r}}
$$

In analogy to the Smagorinsky stress tensor $\tau_{i j}^{\mathrm{r}}$, the dynamic Smagorinsky stress tensor is described through the viscosity hypothesis as [29]

$$
T_{i j}-\left(\delta_{i j} / 3\right) T_{k k} \approx-2 \widetilde{\bar{\Delta}}^{2} \mid \widetilde{S}^{\mid} \widetilde{\bar{S}}_{i j}
$$

After substitution of the test-filtered Eq. (2) and Eq. (8) in the Eq. (7) and multiplying both sides of the equation by $\bar{S}_{i j}$, and finally averaging both sides of the equations, the Smagorinsky constant $C_{\mathrm{s}}$ is derived as [29]

$$
C_{\mathrm{s}}=-\frac{1}{2} \frac{\left\langle L_{k l} \bar{S}_{k l}\right\rangle}{\widetilde{\bar{\Delta}}^{2}\left\langle\mid \tilde{\bar{S}}_{\bar{S}} \tilde{\bar{S}}_{m n} \bar{S}_{m n}\right\rangle-\bar{\Delta}^{2}\left\langle\widetilde{\tilde{S}}_{\mid \bar{S}} \bar{S}_{p q}\right\rangle}
$$

The Smagorinsky coefficient $C_{\mathrm{s}}$ is a function of time and space and is locally calculated in every time step, leading to a more appropriate calculation of $C_{\mathrm{s}}$ for laminar-transitional-turbulent flows [29].

The mono-disperse particles are uniformly injected through both nostrils, and the initial particle velocity are taken equal to the airflow velocity at the nostrils. Lagrangian particle tracking is used to calculate the location $\mathbf{x}_{\mathrm{p}}$ and the velocity $\mathbf{U}_{\mathrm{p}}$ of the particles

$$
\begin{aligned}
& \frac{\mathrm{d} \mathbf{x}_{\mathrm{p}}}{\mathrm{d} t}=\mathbf{U}_{\mathrm{p}} \\
& \frac{\mathrm{d} \mathbf{U}_{\mathrm{p}}}{\mathrm{d} t}=\mathbf{F}_{\mathrm{D}}+\mathbf{g}=\frac{18 \mu}{\rho_{\mathrm{p}} d_{\mathrm{p}}^{2}} \frac{C_{\mathrm{D}} \operatorname{Re}_{\mathrm{p}}}{24}\left(\widetilde{\mathbf{U}}-\mathbf{U}_{\mathrm{p}}\right)+\mathbf{g},
\end{aligned}
$$

where drag $\mathbf{F}_{\mathrm{D}}$ and gravitational forces $\mathbf{g}$ are exerted on the particles, and the drag coefficient $C_{\mathrm{D}}$ is [30]

$$
C_{\mathrm{D}}= \begin{cases}\frac{24}{\operatorname{Re}_{\mathrm{p}}} & \text { if } \mathrm{Re}_{\mathrm{p}} \leq 10^{3} \\ \frac{24}{\operatorname{Re}_{\mathrm{p}}}\left(1+0.15 \mathrm{Re}_{\mathrm{p}}^{0.678}\right) & \text { if } \operatorname{Re}_{\mathrm{p}}>10^{3} .\end{cases}
$$

In Eq. (11), $\mu$ is the dynamic gas viscosity, $\operatorname{Re}_{\mathrm{p}}$ denotes the particle Reynolds number, and $\rho_{\mathrm{p}}$ is the particle density.

\section{Physical properties and boundary conditions}

The physical properties of air are considered for the gas phase, and for the particles, the properties of water are assigned since most of the drugs are aqueous solutions with the approximate density of water [32]. Due to the stickiness of the mucus layer on the inner surface of the nasal airway, it is assumed that the particles are trapped on the mucus once they hit the wall. The particles which are trapped on the surface of the mucus move towards the pharynx via the mucociliary clearance function of the nasal cavity. During the mucociliary clearance process, the movement of the cillia directs the particles towards the pharynx. However, since the velocity of the particles during the mucociliary clearance process is very slow in comparison to both the velocity of the air and the particle motion in the nasal cavity, it is reasonable to consider the nasal wall as stationary (no-slip) [33]. In the present study it is assumed that the wall is smooth and stationary. In order to control the inlet and outlet effects, the inlet and the outlet of the nasal cavity are

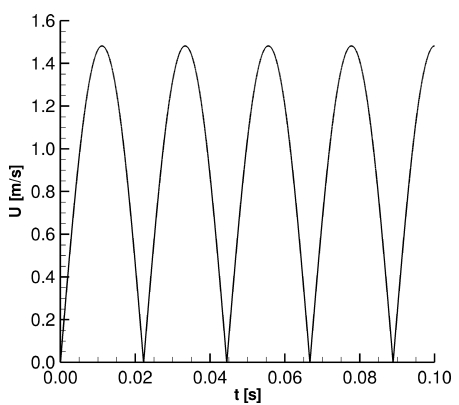

Figure 3. Profile of the pulsating air inflow extended by pipes [34] which are shown in the right part of Figure 2.

The present study compares steady and pulsating airflow at the inlet of the nostrils. For the steady inhalation, a constant airflow rate of $7.5 \mathrm{~L} / \mathrm{min}$ is considered. In case of the pulsating airflow, a sinusoidal shape with a frequency of $45 \mathrm{~Hz}$ and an maximum value of $7.5 \mathrm{~L} / \mathrm{min}$ is used, cf. Figure 3. This condition corresponds to $63.7 \%$ of the present steady inhalation rate. The pulsating flow is described by

$$
U=U_{0}\left|\sin \left(2 \pi \omega^{*} t\right)\right|
$$

with $\omega^{*}=\omega / 2$ and $\omega=45 \mathrm{~Hz}$. A total of 10,000 mono-disperse particles are injected at the nostrils with the corresponding initial gas velocity of $1.48 \mathrm{~m} / \mathrm{s}$ over the first time step of $10^{-5} \mathrm{~s}$ [35]. In order to study the effect of the particle diameter on the deposition efficiency of the particles in the nasal airway, two different particle diameters of $2.4 \mu \mathrm{m}$ and $10 \mu \mathrm{m}$ at fixed particle number density are studied. The particle diameter of $2.4 \mu \mathrm{m}$ is selected since it represents the Sauter mean diameter of the aerosols generated by the PARI SINUS nebulizers [11]. 


\section{Numerical method}

The governing equations of the gas flow and those for particle motion are solved by a hybrid Eulerian-Lagrangian solver. The LES simulations with the dynamic SGS model are performed using the solver pimpleFoam in the software package OpenFOAM 3.0.0, which includes the PIMPLE (PISO combined with SIMPLE) algorithm to couple the gas phase pressure and the gas velocity. This solver is coupled to the lagraniganParcel library, and a new solver called pimpleParcelFoam is developed, which enables the two-way coupled Eulerian-Lagrangian solution for the airflow and the particles. This numerical model is suitable to solve a three-dimensional, transient, incompressible, isothermal and Newtonian gas phase flow with particle motion. The simulations are carried out for a real process time of $1 \mathrm{~s}$, since after the first second more than $99 \%$ of the particle are attached to the surface of the wall or have escaped from the outlet. The computations are performed on the bwForCluster MLS\&WISO using 256 processors with a constant time step of $10^{-5} \mathrm{~s}$ for both the gas and particle phases. For the simulations with steady and pulsating airflow, the clock times are approximately 32 and 75 hours, respectively.

\section{Results and discussion}

First, the gas flow is analyzed for both the steady and pulsating flows before the particle dispersion and deposition is discussed for the different conditions under consideration.

\section{Gas phase}

Figure 4 shows the contour plot of the gas velocity at different coronal cross-sections with equal intervals of $9.22 \mathrm{~mm}$ between the nasal valve (right) and nasopharynx (right) for steady inhalation. The airflow velocity attains its maximum values in the nasal valve region, the nasopharynx and the inferior parts of the septum. The olfactory region and the tips of the meatuses are not ventilated in accordance with results by Chung and Kim [36]. Figure 5 illustrates the streamlines of the gas velocity for steady inhalation. The steady airflow uniformly enters the nostrils

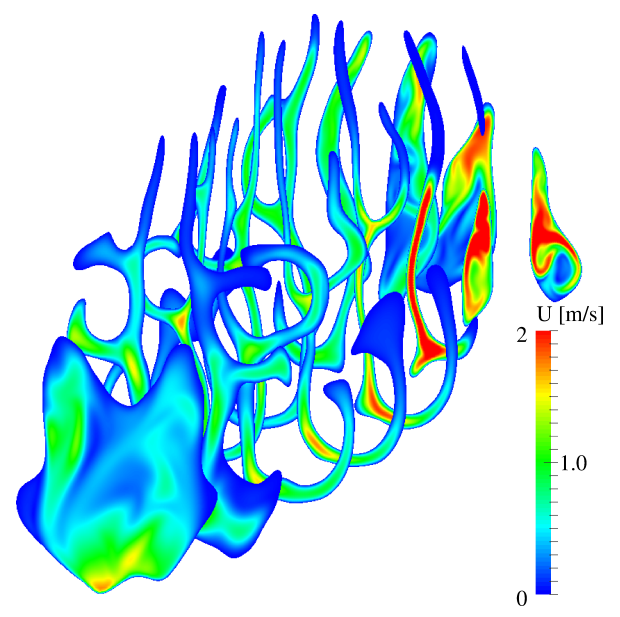

Figure 4. Gas velocity for steady inhalation at different coronal planes between the nasal valve on the right, and the nasopharynx on the left, cf. Figure 1, right part

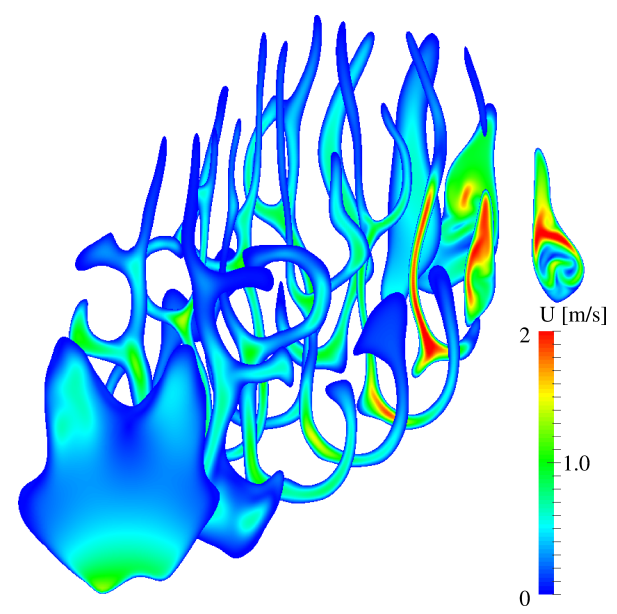

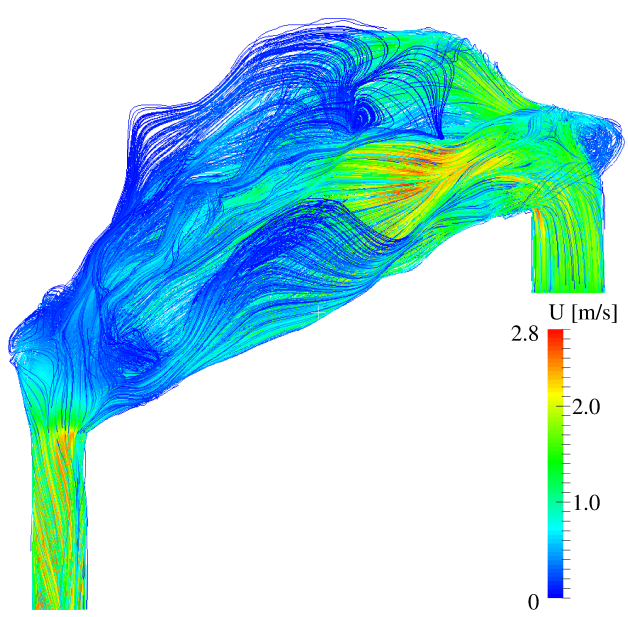

Figure 5. Streamlines of the gas velocity for steady inhalation

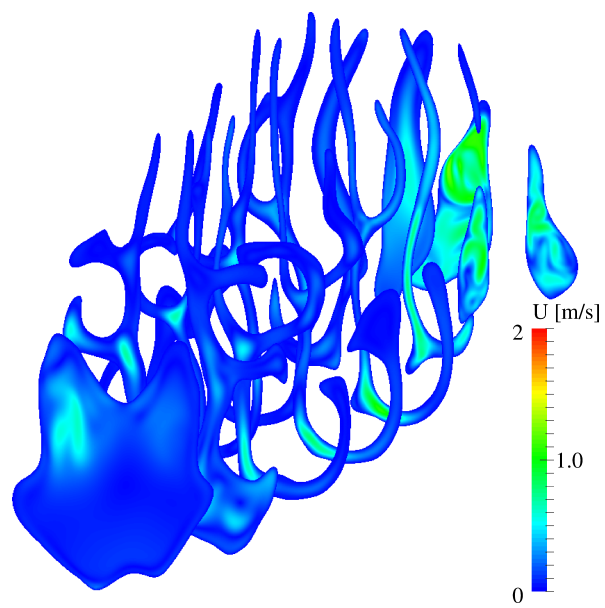

Figure 6. Pulsating airflow: gas velocity at different cross-sections for the second pulse left: at $(t=0.0333 \mathrm{~s})$ and right: at $(t=0.0444 \mathrm{~s})$, cf. Figure 3 
and passes through a $90^{\circ}$ bend at the vestibule, inducing vortices and a complex aflow pattern. Depending on the patient-specific anatomy of the nasal cavity, the location and shape of the vortices in the airway may change [21]. In the present study, the complex flow and vortices in the nasal valve region and immediately after the nasal valve are similar to experimental results of Doorly et al. [19]. The airflow field in the nasal cavity plays a quite important role, since the best treatment method for each patient may be decided based on the flow conditions in nasal passage [36]. The airflow from the upper, middle and lower meatuses combine and unify in the nasopharynx region. The combination of the flows from the three meatuses and a $90^{\circ}$ bend in the nasopharynx causes complex vortices and a swirling flow in the nasopharynx region.

Figure 6 shows the corresponding contour plots of the gas velocity for the pulsating flow during the second pulse, cf. Figure 3 , at the peak at $t=0.0333 \mathrm{~s}$ (left) and at the end of the second pulse at $t=0.0444 \mathrm{~s}$ (right). A comparison of the steady and pulsating inhalation shows not much difference at the peak of the second pulse except for that the absolute values are somewhat reduced for the pulsating flow. This is due to the fact that the steady inflow occurs at a constant flow rate of $7.5 \mathrm{~L} / \mathrm{min}$ whereas this value is only reached at the peak of the pulse during pulsating inflow, leading to an overall (integral) average value of $4.78 \mathrm{~L} / \mathrm{min}$. At the end of the second pulse, cf. Figure 6 , the velocity of the gas phase in the nasal airway is dramatically reduced except for in the upper parts of the nasal valve. A future simulation of an average pulsed air inflow rate of $7.5 \mathrm{~L} / \mathrm{min}$ may help to a better evaluation of the differences in results of steady versus pulsating airflow.

\section{Particle dispersion and deposition}

This section concerns the study of the influence of the steady and pulsating particle injection as well as the particle size on the particle dispersion and deposition efficiency. Two different particle diameters of $2.4 \mu \mathrm{m}$ and $10 \mu \mathrm{m}$ are studied for a fixed value of 10,000 mono-disperse particles that are injected uniformly at the nostrils.

Figure 7 depicts the evolution of the particles with a diameter of $2.4 \mu \mathrm{m}$, carried by a pulsating airflow at different times, cf. Figure 3. At $t=0.00555 \mathrm{~s}$, which is at half of the ascending part of the first pulse, the particles reside in the extended inlet pipe. At $t=0.0222 \mathrm{~s}$, i.e. at the end of the first pulse, the particles pass through the nasal valve and enter into the main nasal cavity. At the peak of the third pulse at $t=0.0555 \mathrm{~s}$, the particles enter the nasopharynx, the inferior and the middle meatuses. At the end of the fifteenth pulse at $t=0.333 \mathrm{~s}$, some particles have moved out of the domain towards the pharynx, some are attached to the wall, and others are still moving inside the nasal airway. In general, the particles tend to deposit in the nasal valve, nasopharynx and some parts of the septum. In particular, particles penetrate into the tips of the meatuses or into the upper and posterior parts of the nasal cavity. Figure 8 illustrates the corresponding particle evolution of the same particle size carried by the steady airflow. Even though the principal pattern of particle evolution and deposition is similar for steady airflow, the aerosols penetrate deeper into the posterior regions and the meatuses for the pulsating airflow, where the connections to the sinuses reside, see Figure 7.

It is observed that the particle evolution occurs faster for steady inhalation compared to the current pulsating flow condition which, however, corresponds to only $63.72 \%$ of the steady inhalation rate. A better evaluation of the different conditions with respect to the particle evolution rate may be achieved if the present maximum value of the
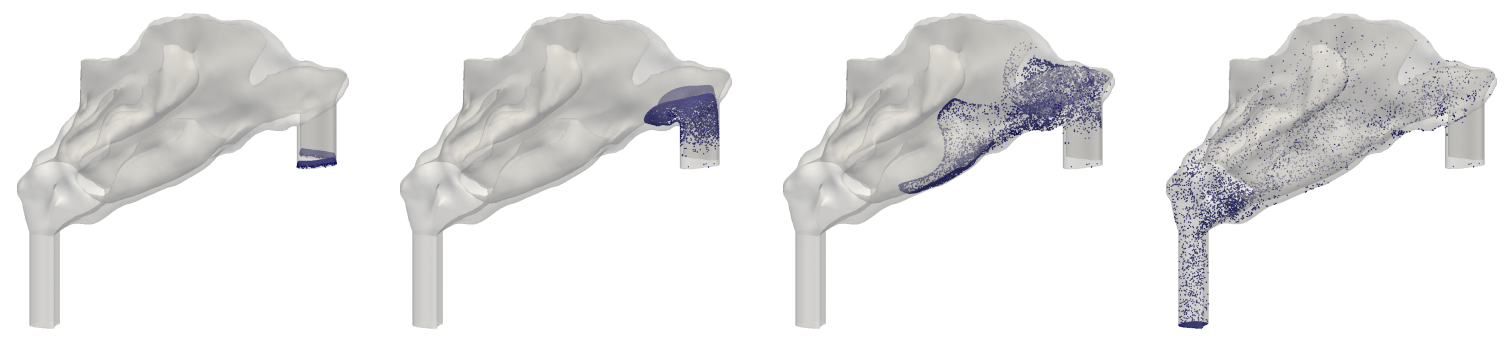

Figure 7. Temporal evolution of the particles of diameter $2.4 \mu \mathrm{m}$ for the pulsating airflow from left to right at $t=0.00555 \mathrm{~s}$, $t=0.0222 \mathrm{~s}, t=0.0555 \mathrm{~s}$, and $t=0.333 \mathrm{~s}$
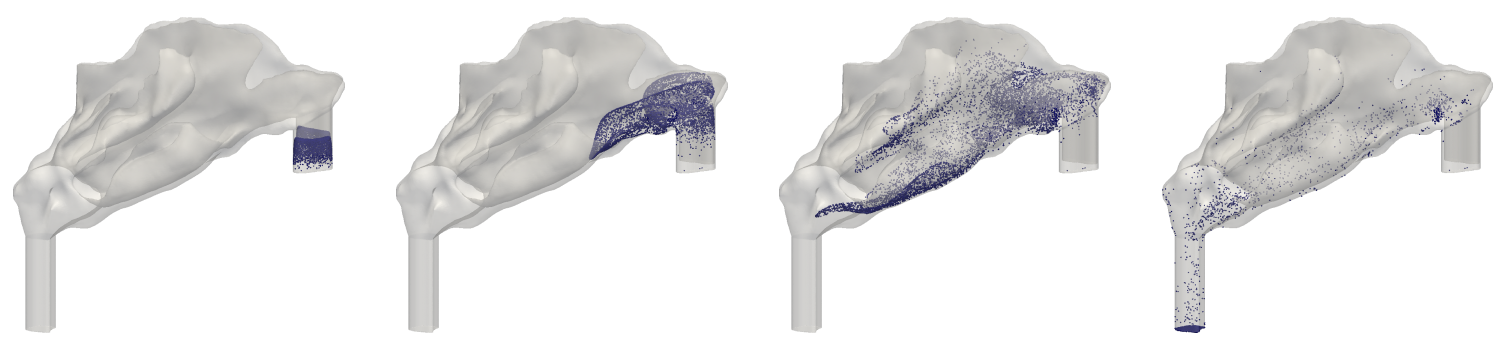

Figure 8. Temporal evolution of the particles of diameter $2.4 \mu \mathrm{m}$ for the steady inhalation from left to right at $t=0.00555 \mathrm{~s}$, $t=0.0222 \mathrm{~s}, t=0.0555 \mathrm{~s}$, and $t=0.333 \mathrm{~s}$ 


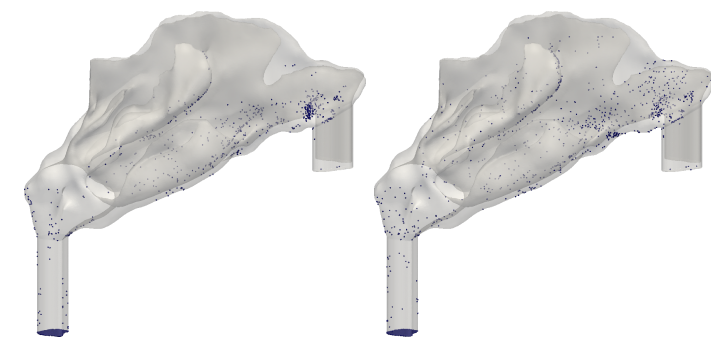

Figure 9. Particle deposition pattern, $d_{0}=2.4 \mu \mathrm{m}$ after $1 \mathrm{~s}$ for left: steady and right: pulsating airflow

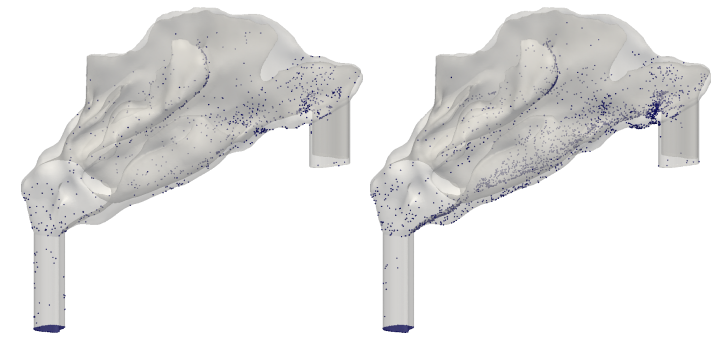

Figure 10. Particle deposition pattern, $d_{0}=2.4 \mu \mathrm{m}$ (left) and $d_{0}=10.0 \mu \mathrm{m}$ (right) after $1 \mathrm{~s}$ for the pulsating airflow

pulsating flow should be enhanced to 1.56 times of the initial velocity of the steady inhalation, resulting in the same integral inhalation rate for both situations.

Figure 9 illustrates the deposition pattern of the particles with diameter of $2.4 \mu \mathrm{m}$ after $1 \mathrm{~s}$ for steady (left) and pulsating (right) airflow. Steady airflow leads to accumulation of particle deposition in some regions of the nasal airway, in particular in the nasal valve and the nasal pharynx. The pulsating airflow leads to improved ventilation of the olfactory region, the superior meatuses and the tips of the middle meatuses, which is associated with particles deposition in theses regions. However, for the simulation of the steady airflow, no deposition is observed in the olfactory region, superior meatuses and tips of the middle meatuses.

The variation of initial particle diameter for steady inhalation shows that an increase of the particle diameter from $2.4 \mu \mathrm{m}$ to $10 \mu \mathrm{m}$ results in an enhancement of the deposition efficiency (DE) in the nasal cavity from $7.4 \%$ to $24.8 \%$ (not shown). The larger particles are trapped on the surface of the nasal cavity due to higher inertial impaction [11]. However, smaller particles penetrate deeper into the nasal airway and they enter the pharynx. For this reason, nasal drug delivery devices which are designed to target the drug into the lower respiratory system, are to generate finer particles. A similar behavior is observed for the pulsating airflow with particle diameters of $2.4 \mu \mathrm{m}$ and $10 \mu \mathrm{m}$ shown in Figure 10. A variation of the particle diameters of $2.4 \mu \mathrm{m}$ and $10 \mu \mathrm{m}$ results in deposition efficiencies of $8.3 \%$ and $24.8 \%$, respectively. The DE for the steady and pulsating flow carrying particles with a diameter of $10 \mu \mathrm{m}$ lead to the same results with a different deposition pattern showing that the pulsating spray shows less particle accumulation and is favored for the particles to reach into the meatuses and deeper posterior regions. However, those deposition efficiencies are likely to be better for the pulsating airflow if the same integral airflow rate was used in the pulsating case compared to the steady case. Future studies should take this into account.

Moreover, the influence of one-way versus two-way coupling is studied. For the simulations with a steady inhalation of $7.5 \mathrm{~L} / \mathrm{min}$ and a particle diameter of $10 \mu \mathrm{m}$, the simulations with one-way and two-way coupling between two phases result in deposition efficiencies of $18.3 \%$ and $21.2 \%$, respectively. The higher DE is attributable to the higher gas velocity in case of two-way coupling since the drag forces causes a flow acceleration, leading to deeper penetration into the nasal cavity and thus, to a higher DE. Furthermore, the simple and the dynamic SGS using twoway coupling are compared for the steady inhalation with a particle diameter of $10 \mu \mathrm{m}$. The simulated values of DE with the simple and the dynamic Smagorinsky SGS are $21.2 \%$ and $24.8 \%$, respectively. The evaluation of these results is difficult due to a lack of experimental data for the deposition efficiency. However, due to the transitional laminar-turbulent character of the flow and its small length scales, it is likely that the dynamic Smagorinsky model leads to better results.

\section{Conclusions}

A study of the airflow and the dispersion and deposition of mono-disperse particles in a realistic model of a patient is performed using a large eddy simulation with dynamic sub-grid scale model and Lagrangian particle tracking. Both steady and pulsating airflows at the inlet are studied for different particle sizes. The results depict that the injection of larger particles leads to a higher deposition efficiency due to higher inertial impaction. Furthermore, it is observed that the DE is lower if one-way coupling between the gas phase and the particles is used compared to two-way coupling, where the influence of the particle motion on the gas is considered. Similarly, the simple Smagorinsky SGS model predicts a lower DE in comparison to the dynamic Smagorinsky SGS model.

The steady inhalation leads to particle deposition in the nasal valve, nasopharynx and parts of the septum, where the flow experiences a change in flow direction. For the pulsating airflow, a better ventilation of the meatuses and the olfactory regions is achieved compared with the steady inhalation. Even though the total DE is the same for the steady and the pulsating airflow, the perspective of the pulsating spray is better because of its improved ventilation of regions that are of particular interest for the medical treatment of critical diseases such as CRS. The deposition efficiency for the pulsating flow is likely to be enhanced if not the maximum value of the pulse is equal to the steady airflow velocity but the integral value, which would increase the current initial value of the pulsating flow by about a factor of 1.56 compared to the present value. Thus, the pulsating aerosol delivery is a potential drug delivery method for the CRS patients. Future studies concern the parametric dependence of the pulsation frequency and its amplitude on the DE of the drug delivery during pulsating drug delivery. 


\section{Acknowledgements}

The authors thank Dr. H. Mentzel from PARI Respiratory Equipment, Inc. for intense discussions about the pulsating spray initial conditions. The current study is part of a project supported by the German Research Foundation (DFG) through a research fellowship of the HGS MathComp. Furthermore, high-performance computing time at the bwForCluster MLS\&WISO Production which is funded by the state of Baden-Württemberg through bwHPC and DFG through grant INST 35/1134-1 FUGG.

\section{References}

[1] Tu, J., Inthavong, K., Ahmadi, G., 2013, "Computational Fluid and Particle Dynamics in the Human Respiratory System". Springer.

[2] Kimbell, J., Shroeter, D., Asgharian, B., Wong, B. A., Segal, R. A., Dickens, C. J., Southall, J. P. and Millerk F. J. 2004 Respiratory Drug Delivery, 9, pp. 233-238.

[3] Suman, J. D, 2013 Drug Delivery and Translational Research, 3, pp. 4-15.

[4] Shi, H., Kleinsteuer, C., Zhang, Z., 2007 Journal of Aerosol Science, 38, pp. 398-419.

[5] Fokkens, W. J., Lund, V. J., Mullol, J. Bachert, C., Alobid, I., Baroody, F., Cohen, N.,Cervin, A., Douglas, R.,Cevaert, P., Georgalas, C., Goossens, H., Harvey, R., Hellings, P., Hopkins, C.,Jones, N., Joos, G., Kalogjera, L., Kern, B., Kowalski, M., Price, D., Riechelmann, H., Schlosser, R., Senior, B., Thomas, M., Toskala, E., Voegels, R., Deyun, W., Wormald, P. J., 2012 Rhinology, Suppl. 23, pp. 1-299.

[6] Hastan, D., Fokkens, W. J., Bachert, C., Newson, R. B., Bislimovska, A., Bousquet, P. J., Brozek, G.,Dahlen, S. E., Forsberg, B., Gunnbjornsdottir, M.,Kasper, L., Krämer, U., Kowalski, M. L., Lange, B., Lundbäack, B., Salagean, E.,Todo-Bom, A.,Tomassen, P., Toskala, E.,van Drunen, C. M., Bousquet, J., Zuberbier, T., Jarvis, D., Burney, P., 2011 Allergy, 66, pp. 1216-1223.

[7] Blackwell, D. L., Lucas, J. W., and Clarke, T. C., 2014 National Center of Health Statistics, Vital Health Stat, 10(260).

[8] Iro, H., Mayr, S., Wällisch, C., Wigand, M. E., 2004 Rhinology, 42, pp. 200-206.

[9] Stammberger, H., Posawetz, G., 1990 European Archives of Oto-Rhino-Laryngology, 247, pp. 63-76.

[10] Baumann, I., Blumenstock, G., Praetorius, M., Sittel, C., Piccirillo, J. F., Plinkert, P. K., 2006 HNO, 54, pp. 544549.

[11] Möller, W., Schuschnig, U., Bartenstein, P., Meyer, G., Häussinger, K., Schmid, O., Becker, S., 2014 Journal of Aerosol Medicine and Pulmonary Drug Delivery, 27, pp. 255-263.

[12] Hilton, C., Wiedmann, T., Martin, M. S., Humphrey, B., Schleiffarth, R., Rimell, F., 2008 American Journal of Rhinology, 22, pp. 395-398.

[13] Proetz, A. W., 1951 Annals of Otology, Rhinology and Laryngology, 60, pp. 439-467.

[14] Elad, D., Liebenthal, R., Wenig, B. L., Einav, S., 1993 Med. Biol. Eng. Comput., 31, pp. 585-592.

[15] Swift, D. L., Storng, J. C., 1996 Journal of Aerosol Science, 27, pp. 1125-1996.

[16] Hornung, D. E., Leopold, D. A., Youngentob, S. L., Gagne, G. M., Thomas, F. D, 1987 Archives of Otolaryngology Head and Neck Surgery, 113, pp. 169-172.

[17] Hahn, I., Schere, P. W., Mozell, M. M., 1993 Annals of Otology, Rhinology and Laryngology, 75, pp. $2273-2287$.

[18] Kim, S. K., Chung, S. K., 2004 Measurement Science and Technology, 14, pp. 1090-1096.

[19] Doorly, D. J., Taylor, D. J., Schroter, R. C., 2008 Respiratory Physiology \& Neurobiology, 163, pp. 100-110.

[20] Keyhani, K., Scherer, P. W., Mozell, M. M., 1995 Journal of Biomedical Engineering, 117, pp. 429-441.

[21] Subramaniam, R. P., Richardson, R. B., Morgan, K. T., Kimbell, J. S., 1998 Inhalation Toxicology, 10, pp. 91-120.

[22] Liu, Y., Matida, E. A., Gu, J., Johnson, M. R., 2007 Journal of Aerosol Science, 38, pp. 683-700.

[23] Inthavong, K., Ge., Q., Se., C. M. K., Yang, W., Tu, J. Y., 2011 Journal of Aerosol Science, 42, pp. 100-113.

[24] Ghahramani, E., Abouali, O., Emdad, H., Ahmadi, G., 2014 Journal of Aerosol Science, 67, pp. 188-206.

[25] Moghadas, H., Abouali, O., Faramarzi, A., Ahmadi, G., 2011 Respiratory Physiology \& Neurobiology, 177, pp. 9-18.

[26] Zhu, J. H., Lim, K. M., Thong, K. T. M., Wang, D. Y., Lee, H. P., 2014 Respiratory Physiology \& Neurobiology, 194, pp. 29-36.

[27] Frank-Ito, D. O., Wofford, M., Schroeter, J. D., Kimbell, J. S., 2015 Journal of Aerosol Medicine and Pulmonary Drug Delivery, 28, pp. 1-11.

[28] Cui, X. G., 2012, "CFD Study of the Flow Field and Particle Dispersion and Deposition in the Upper Human Respiratory System". PhD Dissertation, Heidelberg University, Heidelberg, Germany.

[29] Germano, M., Piomelli, U., Moin, P., Cabot, W. H., 1991 Phys. Fluids, 3(7), pp. 1760-1765.

[30] Schiller, L., Naumann, A. Z., 1933 VDI Zeitschrift, 77, pp. 318-320.

[31] Pope, S.B., Turbulent Flows, first ed., Chambridge University Press, 2000.

[32] Edwards, D. A., Hanes, J., Caponetti, G., Hrkach, J., Ben-Jebria, A., Eskew, M. L., Mintzes, J., Deaver, D., Lotan, N. and Langer, R., 1997 Science, 276, pp. 1868-1872.

[33] Xi, J., Longest, P. W., 2009 Aerosol Science and Technology, 43, pp. 808-827.

[34] Shi, H., Kleinstreuer, C., Zhang, Z., 2006 Journal of Aerosol Science, 38, pp. 398-419.

[35] Farnoud, A., Cui, X. G., Baumann, I., Gutheil, E., May 22-27, 2016, 9th International Conference on Multiphase Flow, Florence, Italy.

[36] Chung, S. K., and Kim, S. K, 2008 Respiratory Physiology \& Neurobiology, 163, pp. 111-120. 


\section{B}

Automotive 6 


\title{
Analysis of PDA measurements on a double injection GDI spray
}

\author{
Lucio Araneo ${ }^{\star 1,2}$, Roberto Dondè ${ }^{2}$, Lucio Postrioti ${ }^{3}$, Andrea Cavicchi ${ }^{3}$ \\ ${ }^{1}$ Politecnico di Milano, Energy Department, via Lambruschini 4A, Milano, Italy \\ ${ }^{2}$ CNR-ICMATE, via Cozzi 53, Milano, Italy \\ ${ }^{3}$ Università di Perugia, Dipartimento di Ingegneria, via Duranti 67, Perugia, Italy \\ ${ }^{*}$ Corresponding author: lucio.araneo@polimi.it
}

\begin{abstract}
A N-heptane spray from a GDI multi-hole injector operated in ambient air at fixed conditions and with double injection commands is studied with different experimental techniques to better understand the spray behaviors, focusing the analysis on the effect of different dwell times between the two pulses. Results from spray photographic analysis, fuel injected quantity, droplet velocity and sizing by Phase Doppler Anemometry are presented and compared. The peculiarities and usefulness of a complementary application of the different techniques is illustrated. The two spray pulses have the same time length, so that the first spray evolves in a nearly quiescent and clean ambient, while the second, nominally identical to the first one, evolves in its trailing edge. The direct comparison allows an immediate perception of the differences among the two sprays, at the different dwell times, where the shorter tested, 160 microseconds, was chosen as the one that shows the first appreciable effect with at least one of the used techniques; the differences are clearly evident in the PDA results, sufficiently visible from the injection rate, not appreciable in the imaging at short distance. The effect of the longer dwell times becomes more evident and is illustrated.
\end{abstract}

\section{Keywords}

GDI, Spray, Double Injection, PDA, droplet sizing

\section{Introduction}

The process of fuel injection is crucial for the mixture preparation in automotive engines, and the use of fuel direct injection is considered also in gasoline engine an efficient way to attain better performances in terms of energy efficiency and pollution reduction. The experimental effort to characterize the GDI engine with its component has been huge in the last decades. The multi-hole injectors geometry and the electronic control allow to use a large number of parameters to attain the desired results, among which injector hole sizes and spray pattern, fuel pressure, injection timing and duration, where also split or multiple injections can be used [3, 8]. Different experimental techniques are continuously improved and used to characterize GDI fuel sprays, both with the aim of understanding the effect of the controlling parameters, and to provide accurate data for modeling and CFD tune-up and calibration. Being split injections and GDI spray droplet sizing [5] widely studied since years, the present work accurately reports a parametric study focused on the effect of the dwell time on the spray droplet velocity and size and generally on the second spray behavior.

\section{Experimental set-up}

The used injector is a standard production injector, (part number VW 03C 906 036N produced by Magneti Marelli) with static flow rate of $13 \mathrm{~mm} / \mathrm{ms}$ (N-heptane at 100bar), used in GDI engines with relatively small cylinder capacity. Its spray pattern presents five plumes, one of which is nearly axially oriented, and at the same time widely accessible from its sides, thus resulting favorable for the PDA measurements nearly like with a single hole injector. The same injector sample was used for all the tests, although different samples were available. Nheptane was chosen as model fuel, widely used for better result reproducibility, although it is not really representative of real gasoline behavior due to its higher boiling point [2]. The experiment were conducted in two different laboratories with different experimental set-ups; slight differences exist among the two fuel pressurizing systems, in the geometries of the rail volumes, and in the injector power stage, with current timing and intensity that may differ slightly, with a difference in the rise-up of the current peak evaluated as 20-30 microseconds.

Injection Rate set-up

The tested injector was characterized in terms of injection rate profile by means of the proprietary instrument 'UniPg Injection Analyzer'. The fuel injected into the closed measuring chamber causes a pressure increment. By time-deriving the chamber pressure signal, the injection rate time-history can be obtained according to the Zeuch method [7]. After each actuation cycle, a fast electro-valve is opened, discharging the fuel and restoring the original base pressure before the next injection cycle starts. As the fluid bulk modulus is significantly influenced by 
both temperature and pressure, the instrument is able to continuously calibrate by measuring the mean injected mass and the fuel density by means of a Coriolis mass flow meter (Siemens Sitrans CF 2100) placed downstream the measuring chamber. After a proper thermal stabilization in each operating condition, the acquisition procedure is repeated for some hundreds of consecutive injection cycles in order to allow a statistically significant analysis of the process mean characteristics and of its shot-to-shot dispersion. Further details about this instrument can be found in [4].

\section{Photographic set-up}

The spray is directed with the injector axis $\left(Z_{I_{N J}}\right)$ oriented vertically downward, with the spray pattern symmetry plane corresponding to the electric connector, marked as $X_{I N J}$ axis and used as rotation reference. The injector is mounted in a rotating flange on the top of the closed bomb detailed in [2], kept with open windows (90 mm diameter) to have a confinement but not a pressurization, and a continuous air renewal. A schlieren system operated without the knife edge is used to obtain back-light photographies without any perspective distortion. A PCO Sensicam Fast shutter is used to capture single images of the spray in the $X Z_{\text {INJ }}$ and $Y Z_{\text {INJ }}$ planes. The image resolution is set at $10 \mathrm{pixel} / \mathrm{mm}$, the exposure about 1 microsecond.

PDA set-up

A Dantec BSA P80 Phase Doppler Anemometer is used, equipped with a $310 \mathrm{~mm}$ focal length lens on the transmitter, and a $500 \mathrm{~mm}$ lens on the receiver oriented at $70^{\circ}$ side scattering angle. Axial velocity is set in the range -10 to $130 \mathrm{~m} / \mathrm{s}$, with the positive direction directed downward in the laboratory, the droplet maximum diameter is set at 80 microns.

\section{Experimental conditions}

The experimental conditions are fixed, with only the command pulse shape used as a parameter, with single pulse and double pulse injections with variable dwell time as reported in the following Figure 1.

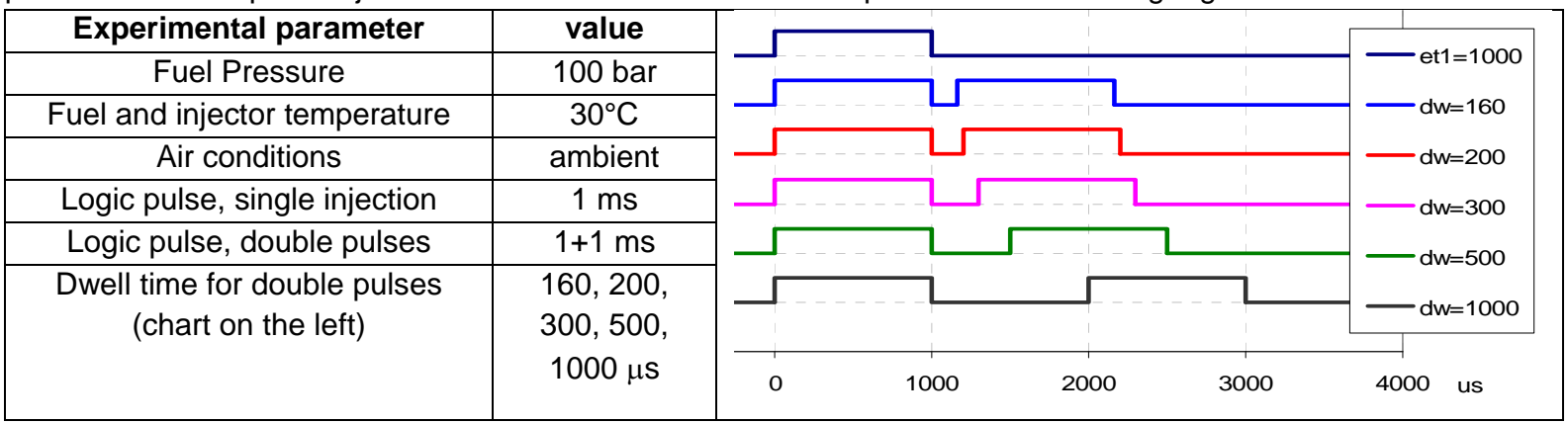

Figure 1. Experimental parameter values and pulse time diagram for the different dwell times

\section{Injection rate results}

The injection rate, reported in figure 2, reflects quite directly the injection command. The initial negative injection rate values before the injection hydraulic start may be speculated to reflect the inward needle opening, that results as a minimum suction of fuel from the instrument measurement volume as discussed in [6].

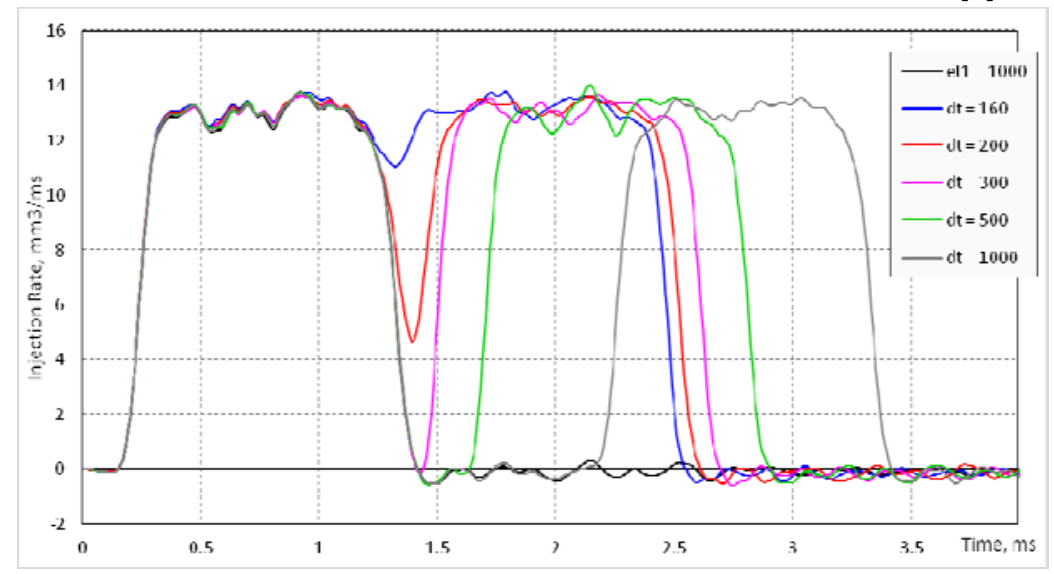

Figure 2. Injection rate results $(\mathrm{mg} / \mathrm{ms})$, for the different dwell times $(\mu \mathrm{s})$

The first increase quantifies the injector opening delay, in the same way the decrease after the closing command reflects the closing delay. Both delays depend on the constructive and operative parameters of the injector; in the 
present case the closing delay is longer than the opening one thus causing the fuel injection period being longer than the logic pulse. The duration of both opening and closing slope is in the order of $100 \div 150 \mu \mathrm{s}$. The quasi steady injection rate value is about $13 \mathrm{~mm}^{3} / \mathrm{ms}$, with slight oscillations due to typical hydraulic wave effects.

In this set-up the minimum dwell time tested, $160 \mu \mathrm{s}$, was the one that showed a visible decrease of the injection rate, about $10 \%$, sufficient to be distinguished from the hydraulic pulsations. It should be mentioned again that the injection rate set-up uses a different electronic power stage, and a possible difference in the order of few microseconds compared to the optic bench may be reasonable. For shorter dwell times the injector inertias, electrical and mechanical, merges the two logical pulses in a unique continuous injection event.

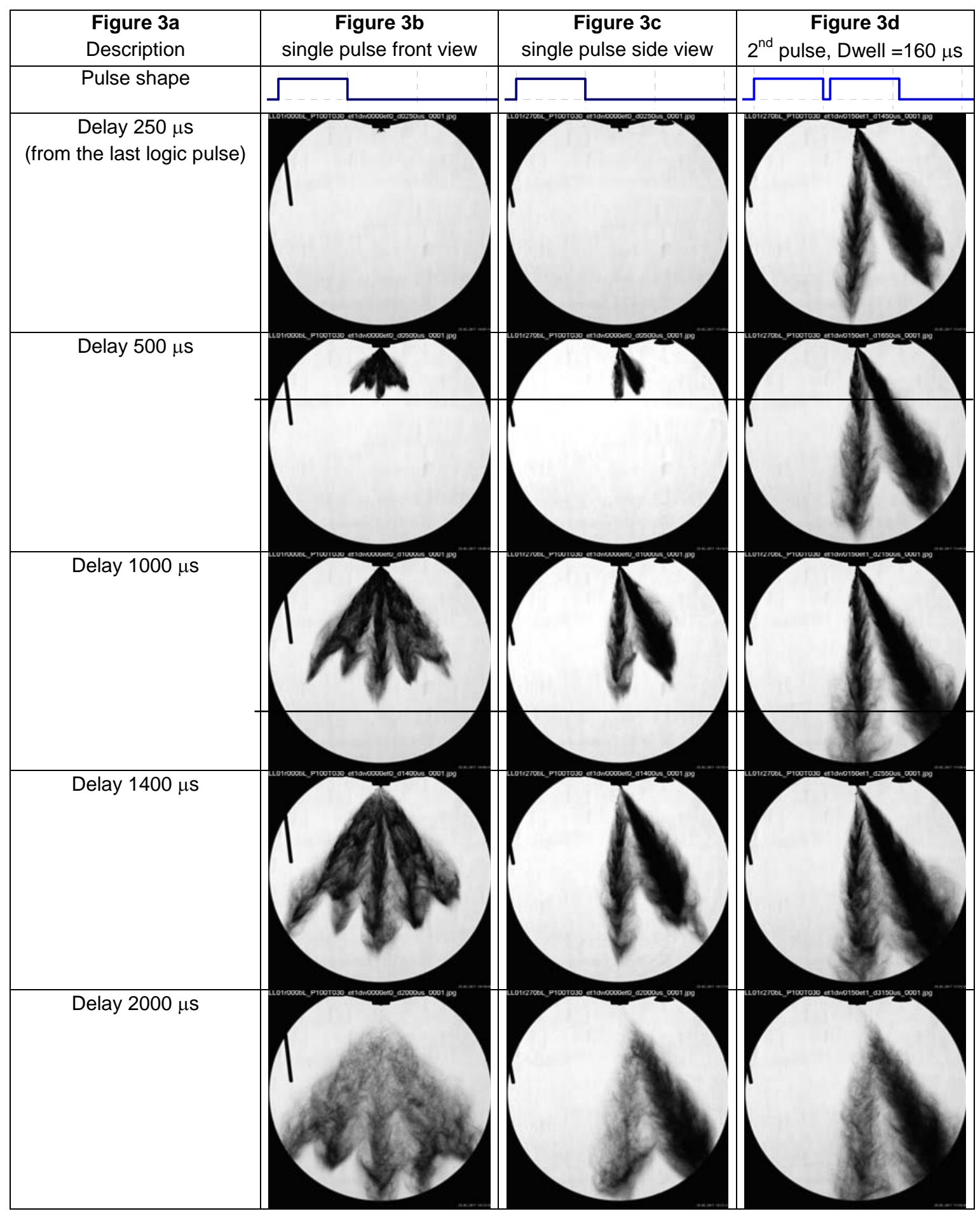

Figure 3. Evolution of the spray with the different pulse shapes 
With dwell time of $200 \mu$ s the injection rate is reduced by $30 \%$ before increasing again due to the second pulse command. With dwell time of $300 \mu$ s the closing is complete before the second pulse, whose trace reproduces quite similarly the trace of the first one. Longer dwell times confirm that, for what concerns the injected quantity, the two pulses look mostly independent; this coherence in terms of injection rate between the first and the second pulses is due to both the relatively long dwell time (pre-magnetization effects are negligible) and the rather long energizing time that ensures the complete needle lify displacement (no ballistic behavior).

\section{Photographic results}

Figure 3 presents a short extract of the photographic analysis showing a short history of the spray evolution.

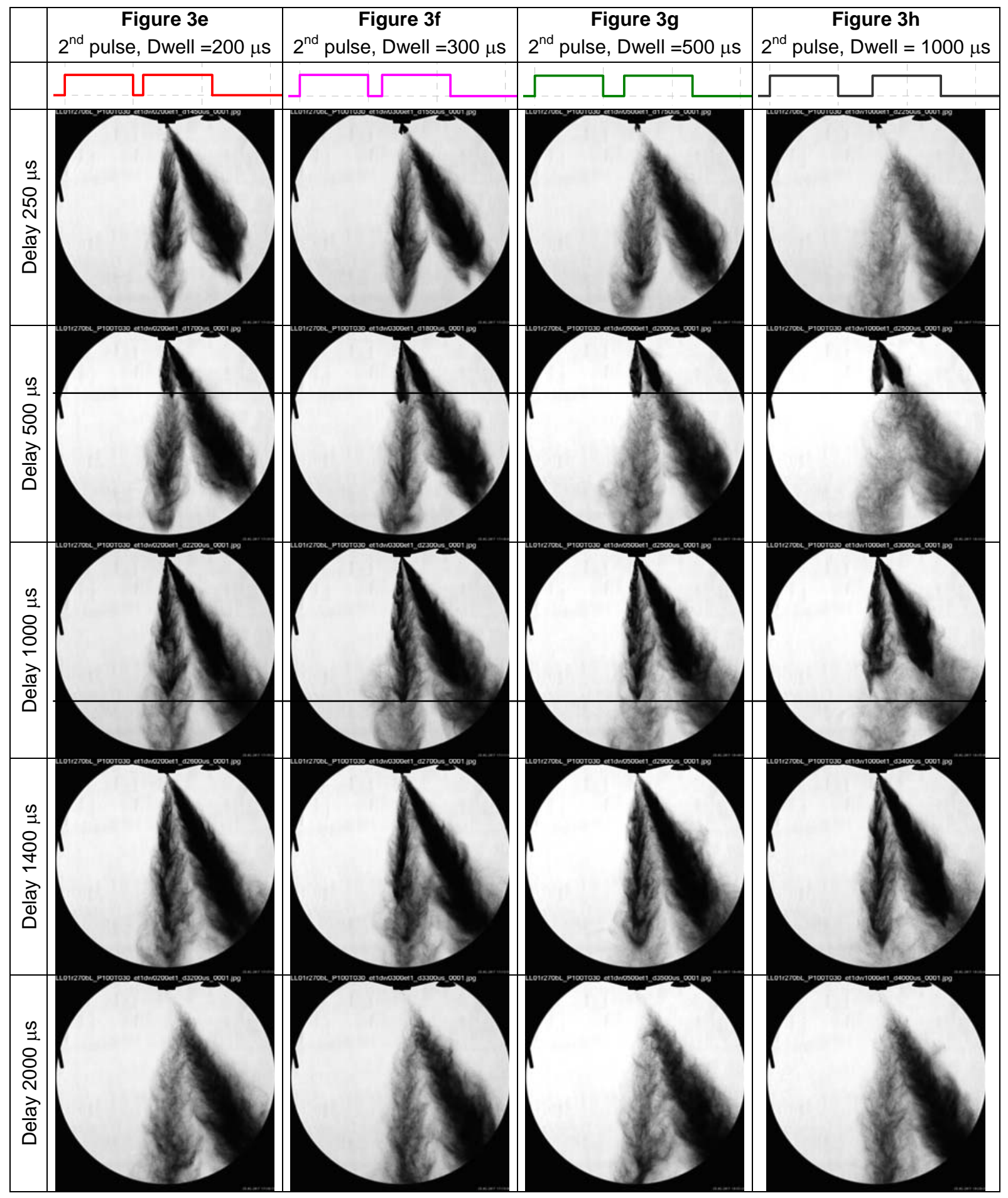

Figure 3. Continuation

This work is licensed under a Creative Commons 4.0 International License (CC BY-NC-ND 4.0).

EDITORIAL UNIVERSITAT POLITÈCNICA DE VALÈNCIA 
The delay of each image is indicated starting from the beginning of the last logic injection pulse, so that the differences can be more easily perceived.

With a single pulse spray, that is the same as the first of a double pulse injection, the spray evolves in undisturbed air, starts to be visible $250 \mu \mathrm{s}$ after the trigger due to the opening delay, and the needle closure is clearly visible at $1400 \mu$ s, i.e. $400 \mu \mathrm{s}$ after the logic command end. A shorter closing delay can be supposed, but not clearly observed with photography.

For the dwell time of $160 \mu \mathrm{s}$, the shortest tested, in the images of figure $3 \mathrm{~d}$ it is difficult to perceive a clear distinction between the two pulses, that becomes more and more visible as the dwell time becomes longer. $\mathrm{A}$ reference line is added over the Figure 3 to help in locating the spray tip at 500 and $1000 \mu$ s delays.

\section{PDA centering}

The PDA traversing system is installed with its origin and $Z_{P D A}$ axis coinciding with the injector ones, used as rotation axis to optimize the injector position for easier experimental configuration and access to the investigated plume. The PDA transmitting optical axis is directed as the YPDA traverse axis of Figure 4, where different colors are used to easily identify the different PDA and Spray reference systems. The two systems are centered with accuracy better than $0.2 \times 0.4 \times 0.2 \mathrm{~mm}$, along the $(X, Y, Z)_{P D A}$ reference directions illustrated in Figure 4a.

For the PDA measurements, after the correct centering of the two reference systems, the PDA measurement volume is centered on the selected spray plume at the axial position $Z=45$. Measurements are performed along a cross pattern composed by $9+9$ positions spaced by $1.5 \mathrm{~mm}$ along the injector $X Y_{\mathbb{I N J}}$ reference system. For this purpose a longer injection is used, $2 \mathrm{~ms}$, to measure the average velocity during the quasi-steady injection period, and 200 injections are recorded at each position for good statistics. The results reported in figure 4 show the good centering of the plume, that is symmetric along its $Y_{\mathbb{I N J}}$ axis, and slightly asymmetric in its $X_{\mathbb{I N J}}$ direction due to the suction effect of the other four plumes. Such effect was clearly visible in the photos, with the central plum attracted by the other ones after the needle closure. These results are quickly processed on, so are not bias corrected and may differ from the following results.

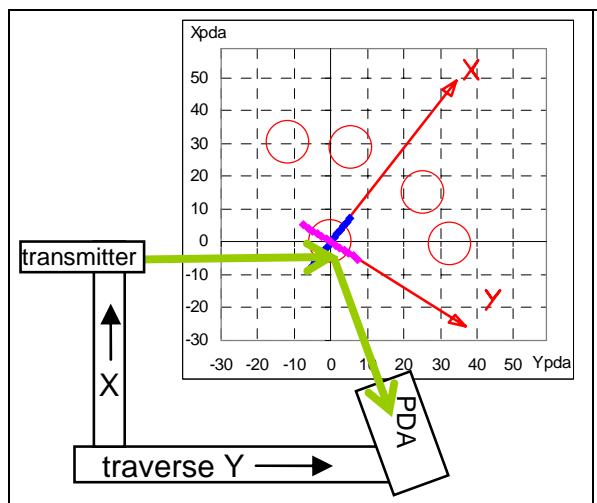

a) The spray $X Y$ axes, the nominal pattern at $Z=45 \mathrm{~mm}$, and the investigated plume with the PDA optics position, the

$X Y_{P D A}$ axes and the measurement positions pattern

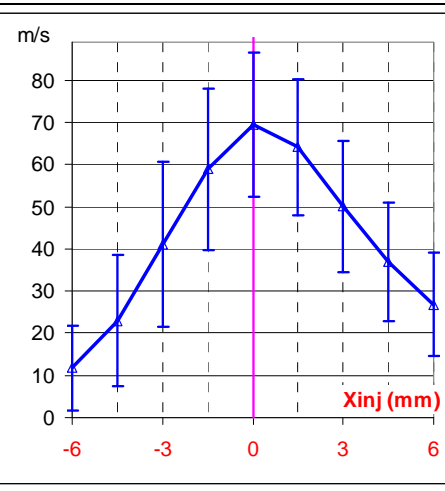

b) The quasi-steady axial velocity profile (average $\pm \sigma$ ) along the $X_{I N J}$ axis, at $Z_{P D A}=Z_{I N J}=45 \mathrm{~mm}$. Note the slight asymmetry.

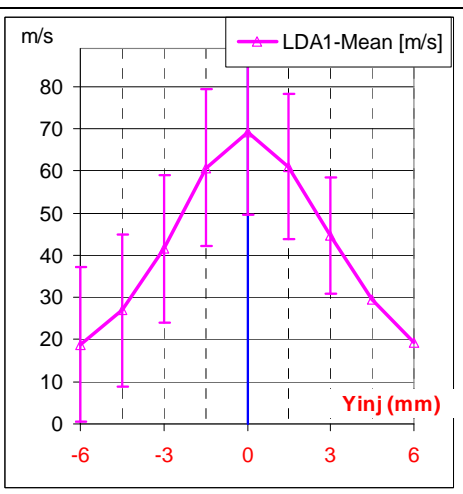

c) The quasi-steady axial velocity profile (average $\pm \sigma$ ) along the $Y_{I N J}$ axis at $Z_{P D A}=Z_{I N J}=45 \mathrm{~mm}$

Figure 4. PDA cantering on the spray plume. Colours are used to ease the identification of the different axes. These averages are not Bias.

\section{PDA results}

To study the effect of the dwell time, PDA data are recorded on the central position of the plume found in figure 4. For each condition, 1000 injections are recorded to improve the statistics. Results are averaged in time windows of $100 \mu \mathrm{s}$ duration, centered around their nominal value, and reported in figure 5. For each time window, the typical average results for droplet population are computed: average velocity, diameters $D_{10} D_{20} D_{30} D_{32}$, and the number of counted droplets, that normally is more than 10000 in each time window. The PDA bias is corrected for by weighting the droplet data in function of their diameter with an empirical function. Together with the count plots, in Figure $5 \mathrm{c}$ is reported the real timing of the pulse logic command. In the column $4 \mathrm{~b}$, the pulse is displayed shifted in time until the first droplets are measured, to ease the understanding of the spray time evolution and direct comparison of results.

\section{The single injection}


The single pulse injection, that is the same as the first of a sequence, allows a comparison also to the second pulse also after the needle closure, so that the two trailing edges can be compared.

Average velocity and diameters are compared using as reference the quasi steady values. Different phases can be distinguished

First fast single droplets

The graphs show quite often few large and fast droplets at the beginning of the injection, with velocity $10 \%$ higher and diameters $50 \%$ higher than in the quasi steady period. It should be noted that the graphs are the accumulation of 1000 injections, while those fast droplets are only few tens, meaning that in cycle resolved results, they would appear only randomly once every many tens of injections, while the following time windows collect many thousands of droplets.

The faster droplets presence can be supposed as the effect of an aerodynamic selection at the spray front, and in the average results they may show up or not, hidden by the more numerous slower droplets, depending also on the chosen time window positions. Their relative weight is limited: even with $50 \%$ larger diameter, that is 4 times the individual volume and mass, they represent less than $0.5 \%$ in number, so $2 \%$ of the mass of one of the following windows, but in a graph the two groups are represented each by one symbol, being perceived at first sight as having the same relative importance.

Initial slow droplets

The following two time windows show droplets that, compared to the quasi steady ones, are initially $15 \%$ slower ( 50 vs. $58 \mathrm{~m} / \mathrm{s}$ ), and $40 \%$ larger $\left(D_{30} 14\right.$ vs. $10 \mu \mathrm{m}$ ), and then converging to the steady values. The number of detected droplets quickly reaches the maximum value, 5 to 10 or more thousands per window.

Quasi steady period

Velocity, size and number of detected droplets per time window keep quite stable, with values around 55-57 m/s, diameters ranging from $D_{10}=9 \mu \mathrm{m}$ to $D_{30}=15 \mu \mathrm{m}$, and 10 to 15 thousands counts per window. Some small oscillation are repetitive in all the tested cases.

Trailing edge

The needle closing is followed by a fast but not sharp velocity decrease, with smaller dimensions, averaged diameters are roughly halved, while the number of detected droplets that decreases as the velocity, typical of the PDA technique that relies on the single counting of particles (when their volume concentration in the flow is constant, the detection rate is proportional to the flow velocity).

Far trail

For times after $3 \mathrm{~ms}$, the velocity falls down below few meters per second, and only few and slightly larger droplets are left suspended in the air.

\section{The second injection}

The second injection is quite similar to the first one, except in its initial phase, where its tip merges the trailing edge previous pulse.

The first fast single droplets never show up, may be because statistically masked by the largest number of very slow droplets present in the ambient.

Initial slow droplets

In this phase the second injection shows quite different results. The rise up is much slower and requires more time, nearly $1 \mathrm{~ms}$ to, reach the same steady value of the first pulse. This behavior is a consequence of the merging and partial mixing with the slow trailing edge of the previous pulse, as visible in Figure 6, where the two population may still be distinguished The slow droplets suspended in the air, are only partially accelerated, while at the same time there is a lack of fastest droplets. The average result is a decrease of the mean velocity. The effect is less visible for very short dwell time, where the trailing edge has no time to slow down. The measured diameter do not show the initial large droplet, but it can not be argued if they do not exist, or if their presence is also masked in the average values by large quantity of suspended small droplets of the trailing edge.

Quasi steady period

It shows the same average values as the first pulse, it keeps just shorter in the present experiments, It should be noted that in the case of a short second pulse as often used in a real engine, the steady period would not be reached, as only the longer transient part would appear in the measurements.

Trailing edge

No clear evidence of any difference can be observed in this part. 


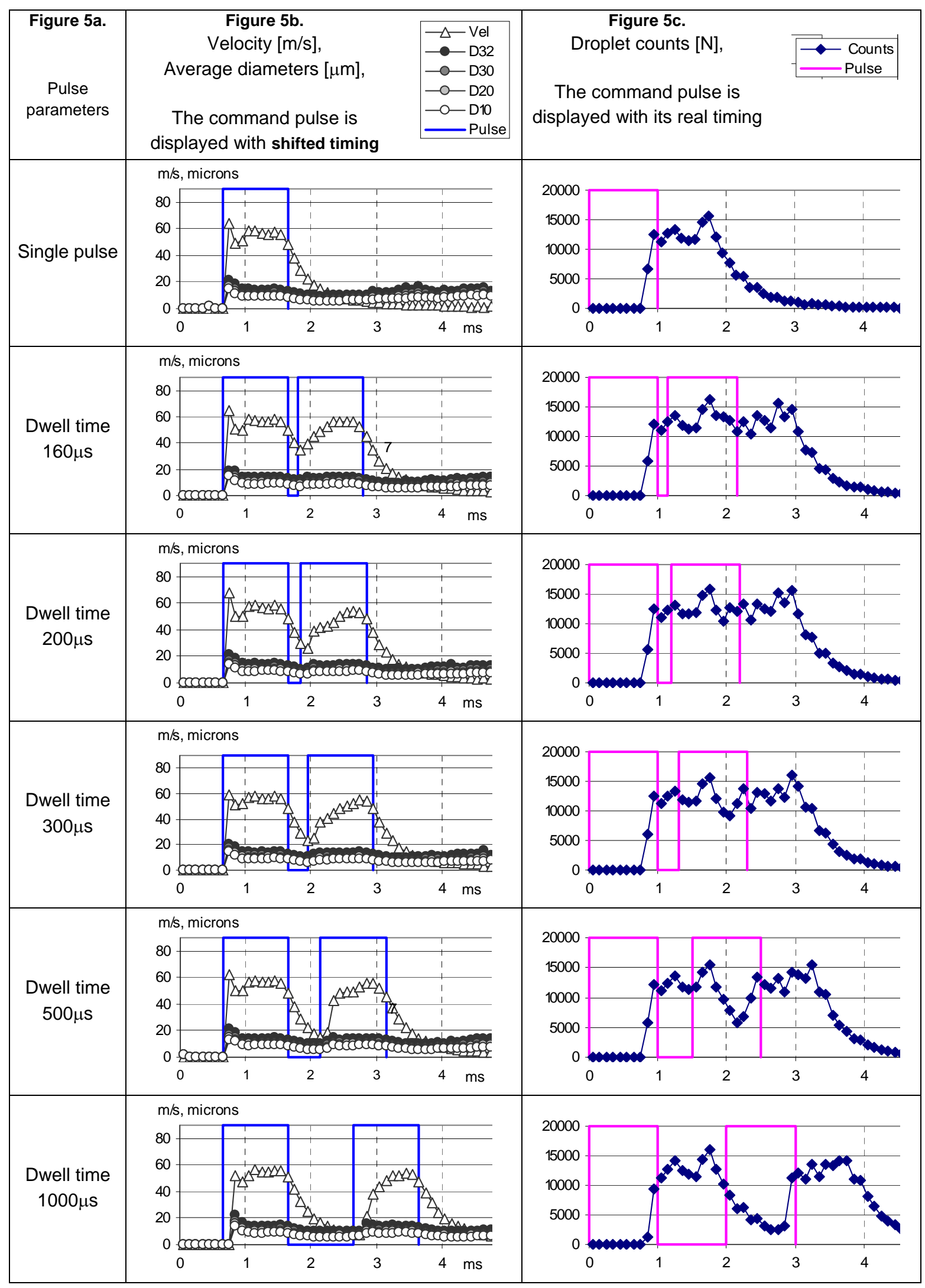

Figure 5. PDA average results

This work is licensed under a Creative Commons 4.0 International License (CC BY-NC-ND 4.0).

EDITORIAL UNIVERSITAT POLITÈCNICA DE VALÈNCIA 


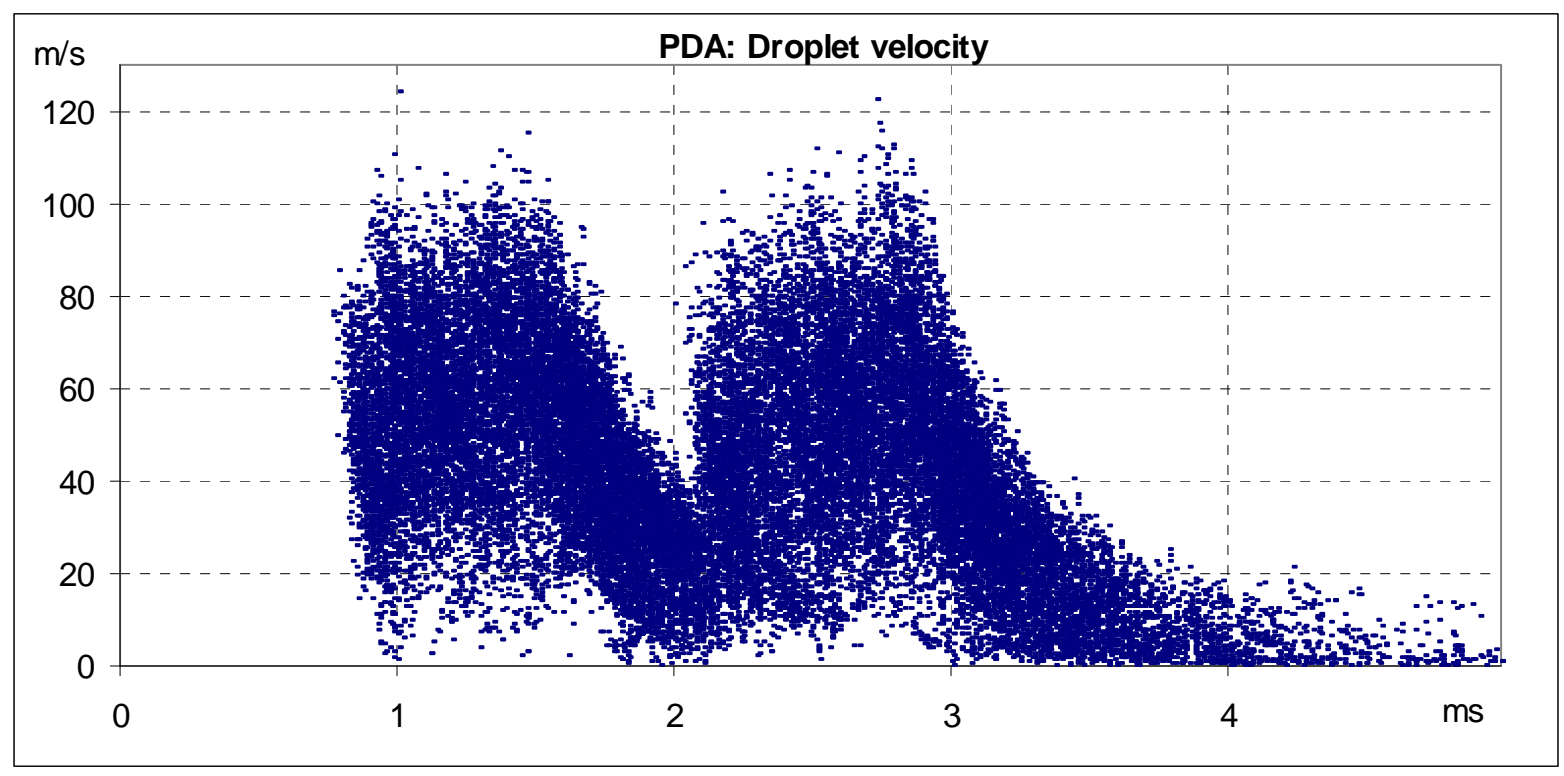

Figure 6. PDA results: droplet velocity for dwell time $=300$ microseconds

\section{Conclusions and perspective}

The comparison of the three techniques offers complementary results.

The fuel rate results can detect in the fuel flow, minimal variations that are not shown by photos, but the information is limited the nozzle exit position, and can not give any further information on what may happen more downstream in the spray. Further, the hydraulic analysis is relevant to all the jets emerging from the nozzle. Photographic results offer a global view of the spray, thus also of the interaction and merging of the two pulses, but are blind in the most dense regions of the inside spray or in its denser wake, so for example can give precise estimate of the opening delay, but not of the closing delay or of the events for short dwell times. Laser Doppler results can gives many complete and time resolved information but in a single position; different positions may be investigated, but the process is extremely time consuming. The PDA can detect some effect for very short dwell times, but may become blind in regions that are optically very dense. The use of the three techniques is useful to build up a more detailed and comprehensive understanding of the spray evolution

\section{Nomenclature}

LDV Laser Doppler Velocimetry

PDA Phase Doppler anemometry

GDI Gasoline Direct Injection

\section{References}

[1] Albrecht, Borys, Damaschke and Tropea, 2001, "Laser Doppler and Phase Doppler Measurement Techniques" Springer.

[2] Araneo L., and Dondè, R., 2017, Fuel 191, pp 500-510

[3] Benajes J., Molina S., García A., Monsalve-Serranoa J., and Durrett R., Applied Energy 134 (2014): 90-101.

[4] Postrioti, L., Cavicchi, A., Paolino, D., Guido, C., Parotto, M., and Di Gioia, R. 2016. Fuel, 173, 8-28.647-655,

[5] Kawahara N, Tomita E, Nakasuji H, and Sumida M. 20067, ICLASS06-186

[6] Postrioti, L., Bosi, M., Cavicchi, A., AbuZahra, F. Di Gioia, R. and Bonandrini, G.., 2015 SAE Technical Paper 2015-24-2480,

[7] Zeuch, W...1961 Motortech. Z, 22(9), 344-349.

[8] Zheng, Z., Tian, X., and Zhang, X. 2015, Applied Thermal Engineering, 84, 237-245. 


\title{
Imaging of Gasoline-like Sprays with Planar Laser-induced Exciplex Fluorescence using a Stereoscopic Imaging System
}

\author{
Mats Andersson*1, Akichika Yamaguchi², Hua Wang ${ }^{3}$ \\ ${ }^{1}$ Department of Applied Mechanics, Chalmers University of Technology, Göteborg, Sweden \\ ${ }^{2}$ DENSO Corporation, Showa-cho, Kariya-shi, Aichi-ken, Japan \\ ${ }^{3}$ Dantec Dynamics A/S, Skovlunde, Denmark \\ *Corresponding author: f3cma@chalmers.se
}

\begin{abstract}
The role of the fuel injection systems in direct injected gasoline engines is to achieve a suitable fuel vapor distribution, homogeneous or with some degree of stratification, while avoiding unwanted effects such as wall wetting. Planar laser-induced exciplex fluorescence (PLIEF) is a method suitable for the characterization of such sprays since it enables separate imaging of both vapor and liquid phase of fuel simultaneously. In this study a hollow-cone spray generated with an outwards-opening piezo-actuated injector is investigated, with the injector mounted in a constant volume, constant pressure spray chamber with quartz windows, providing a controlled steady test environment. $\mathrm{N}$-hexane is used as surrogate fuel of gasoline, together with exciplex-forming fluorescence tracers - fluorobenzene and diethylmethylamine. Fluorescence excitation is carried out with a parallel laser sheet from the fourth harmonic light of a Nd:YAG-laser $(266 \mathrm{~nm})$ running at $10 \mathrm{~Hz}$. Exciplex fluorescence images from liquid phase and monomer fluorescence spray images from vapor phase can be acquired by a single UV-sensitive CMOS camera equipped with a stereoscope having filters selectively transmitting monomer fluorescence at 295 $\mathrm{nm}$ and exciplex fluorescence at $355 \mathrm{~nm}$. Since the fluorescence is strongly quenched by oxygen, most of the experiments were carried out in a nitrogen atmosphere.

Images were recorded during the injection and at various time steps after the end of the injection, and typical spray development for this type of injector was observed, i.e. the fuel forms an expanding cone, the sheet breaks up to form a vortex structure and the vortices continue to expand after the end of the injection. Fuel vapor is firstly observed at the same locations as the liquid drops, and is then accumulated into the center of the vortices. In addition, penetration of liquid phase and vapor phase are found to be very similar. Various injection pressures have been tested, which shows that increasing the fuel pressure from 10 to $20 \mathrm{MPa}$ results in a larger vortex structure. The fuel evaporation can be followed by studying the evolution of the monomer and exciplex fluorescence as a function of time. At room temperature the vaporization is found to be very slow, but above $40^{\circ} \mathrm{C}$ there is a noticeable presence of vapor at the end of the injection, and at higher temperatures, the vaporization goes even faster.
\end{abstract}

\section{Keywords}

Hollow-cone spray, Exciplex LIF, Phase separation, GDI, LIEF

\section{Introduction}

The role of the fuel injection systems in direct injected gasoline engines is to achieve a suitable fuel vapor distribution, homogeneous or with some degree of stratification, while avoiding unwanted effects such as wall wetting. Therefore it is desirable to characterize the fuel injection systems and how the fuel vaporizes and becomes dispersed in the combustion chamber, in order to investigate and improve the combustion process.

As an extension technique of planar laser-induced fluorescence (LIF), which is proved to be a powerful imaging technique to visualize distributions of fuel, air or even combustion species, planar laser-induced exciplex fluorescence (PLIEF) provides spectrally separated fluorescence from the liquid and vapor phases and therefore is a novel and significant advance for imaging and quantifying vaporizing sprays [1,2]. In this method, the fuel is doped with two different tracers. The two tracers are selected so that one, or both, of the tracers are excited by the incoming laser light and upon excitation forming an excited state complex, an exciplex, with the other tracer molecule. The fluorescence of this exciplex is red-shifted compared to the fluorescence of the individual molecules, the monomers. The exciplex formation is efficient only in liquid phase where the tracers are in closer proximity and the solvent can act stabilizing for the complex. One of the advantages with LIEF as compared with LIF is that it enables imaging of the vapor phase without interference from the liquid phase.

This technique has been used for visualization $[3,4]$ and quantitative measurements with both diesel-like $[5,6]$ and gasoline-like tracer-fuel mixers [7,8]. For most of the studies in the literature, the fluorescence from the spray was 
imaged using two cameras, equipped with filters transmitting wavelengths corresponding to monomer and exciplex emission, respectively. These two images ideally correspond to the fuel distribution in vapor and liquid phase.

In this study, a stereoscopic imaging system is used to image both monomer and exciplex fluorescence from a gasoline-like spray, which generates a hollow-cone spray. The imaging system includes a stereoscope and therefore only one camera is needed. Spray development of the vapor and liquid phase with time after start of injection (asoi) is well captured by this imaging system. In addition, influences from injection pressure and ambient conditions have also been observed and analyzed to investigate how these factors influence the location and relative abundance of liquid and vapor phase fuel.

\section{Experiment setup}

Spray development was investigated in a constant volume/constant pressure spray chamber, shown in Figure 1. The chamber can provide a constant air flow, in order to ventilate the injected fuel; however, the air velocity is significantly lower than the spray velocity and therefore it has negligible influence on the spray development. The chamber is equipped with three quartz windows during the study, providing good optical access for both laser light sheet and fluorescence signals.
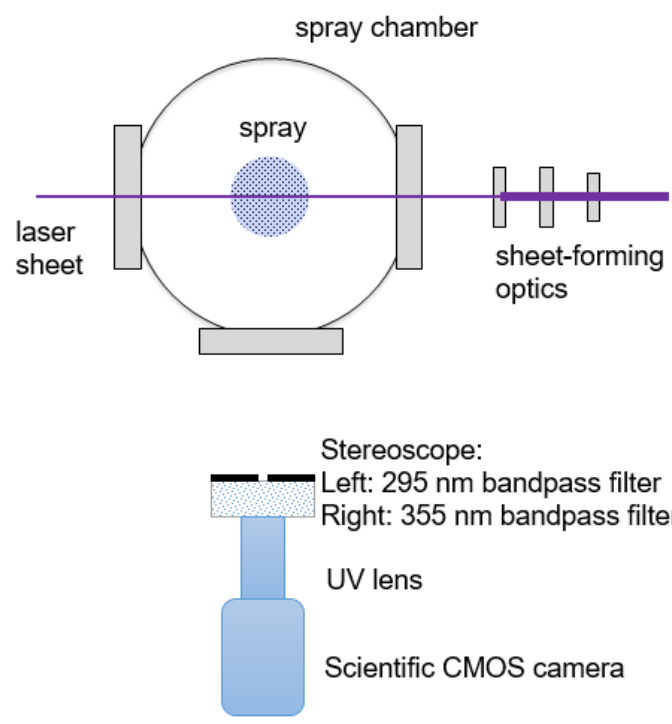

Figure 1. Schematic of the experimental setup as seen from above.

An outward opening piezo actuated GDI injector (Bosch type) was used during the experiments and mounted at the top of the spray chamber. The outward opening needle creates a hollow cone spray shape with vorticies [9-13]. The injector driver supplies a certain voltage over the piezo stack, which expands rapidly and acts on the needle. The needle lift will increase with increased voltage. Due to the fast opening and closing of the needle, the injector can be opened and closed multiple times during one engine cycle and this in addition to the possibility to use varying needle lifts provide many degrees of freedom for spray control but is also a challenge for engine calibration. The injector type is used up to 200 bar fuel injection pressure and provides great atomisation, and limited vertical penetration.

The fuel temperature was kept constant by regular fuel injections. The chamber gas temperature was constantly monitored by thermocouples during all measurements. The fuel pressure is well controlled by a pressurized air driven Haskel pump connected to a pressure accumulator. The nominal electrical control pulse was $0.4 \mathrm{~ms}$, but due to the ramping of the control voltage for opening and closing, the total time during which fuel was injected was observed to be $\sim 0.8 \mathrm{~ms}$.

$\mathrm{N}$-hexane was used as surrogate fuel of gasoline, together with exciplex fluorescence-forming tracers fluorobenzene (FBZ) and diethylmethylamine (DEMA). This is a well-characterized tracer combination [14] with minimal spectral cross-talk and good co-evaporation properties, and has been applied in several studies [15-18]. The concentrations of tracers used in this work was $3 \% \mathrm{FBZ}$ and $7 \%$ DEMA by volume.

Fluorescence excitation was carried out with a parallel laser sheet from the fourth harmonic light of a Nd:YAG-laser $(266 \mathrm{~nm}$ ) running at $10 \mathrm{~Hz}$, and the energy of each pulse was around $20 \mathrm{~mJ}$. The laser light was formed into a 50 
$\mathrm{mm}$ high sheet passing just under the injector. Exciplex fluorescence image from liquid phase and monomer fluorescence spray image from vapor phase was acquired by a single UV-sensitive scientific CMOS camera equipped with a stereoscope having filters selectively transmitting monomer fluorescence at $295 \mathrm{~nm}$ and exciplex fluorescence at $355 \mathrm{~nm}$, a simpler system than the 4 way-mirror arrangement reported for fuel and temperature imaging [19]. The image recording rate was the same as the fuel injection rate, $1 \mathrm{~Hz}$. Since the fluorescence is strongly quenched by oxygen, most of the experiments were carried out in a nitrogen atmosphere.

The influence from variant injection pressure (100 or 200 bar fuel pressure) and ambient temperature (21, 39, 56 or $68^{\circ} \mathrm{C}$ ) has been investigated in this study. For all tested conditions, images were recorded at various time steps during and after the end of the injection and consequently the spray development of both vapor and liquid phase can be observed and analyzed.

\section{Results and discussion}

Images of the spray have been captured with the spray chamber pressurized with air or with nitrogen to assess the influence of quenching by oxygen molecules on the fluorescence yield. Figure 2 shows exciplex-monomer image pairs, recorded at the same conditions except the spray chamber being fed by air or nitrogen. In addition to the images, horizontal intensity profiles calculated at the centre of the two images are shown. The two exciplex images are very similar with vortices having a low drop density at the middle, the main difference being a lower signal intensity of about a factor of 3 in air. The monomer image recorded with a nitrogen atmosphere shows as expected a vapor fuel distribution significantly different from that of the liquid drops (exciplex), whereas the monomer image recorded in air has a very low signal intensity, more than 50 times lower than in nitrogen. The character of the monomer images is also slightly different, likely due to the strong quenching of the vapor monomer signal resulting in monomer fluorescence from the liquid phase contributing to the detected monomer fluorescence. A monomer fluorescence image in air with a good signal intensity could be obtained using an image intensifier in front of the camera. However, for this study of spray properties analysing liquid and vapour fuel distribution all further imaging was carried out in nitrogen atmosphere since this provided good quality monomer and exciplex images of comparable intensity using the stereoscopic imaging system without image intensifier.
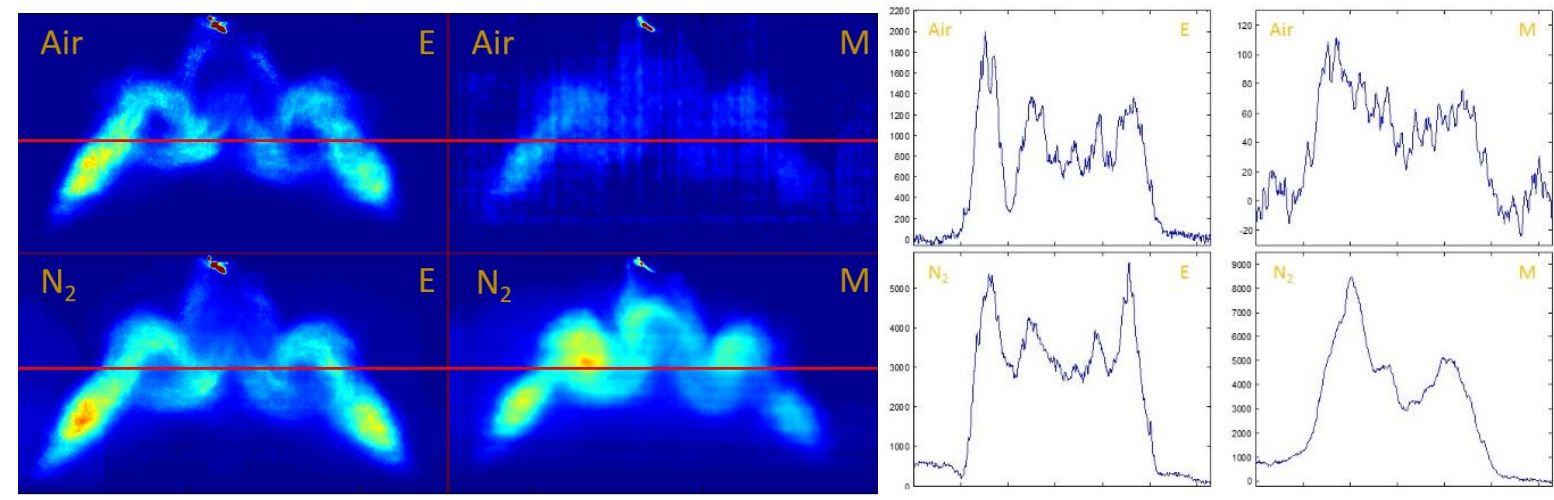

Figure 2. Left: averaged exciplex (left column) and monomer (right column) images recorded in air (upper row) and nitrogen (lower row) at 4.2 bar pressure and $56^{\circ} \mathrm{C}$ gas temperature, $3 \mathrm{~ms}$ asoi with a fuel pressure of 100 bar. Image size $95 \times 53 \mathrm{~mm}$. Right: Intensity profiles taken through the middle of each image of Left side (labeld as red line).

In each measurement point (pressure, temperature and time asoi) 20 images were recorded to obtain reasonable statistics, and from the 20 images average images were calculated. In Figure 3 averaged images at some selected measurement points are shown together with two of the individual image pairs. In the further description of the results averaged images will be displayed since general trends are more easily identified in these and the arbitrariness in the selection of one individual image can be avoided. On the other hand there are important features, which only can be seen in the individual images that will be commented on in the further analysis. Naturally the structures in the averaged images appear more smeared out, whereas the liquid structures, especially at later times appear as drops concentrated to streaks in the individual images. The vapour clouds are more homogeneous, although with internal concentration differences, and have sharp boundaries. 


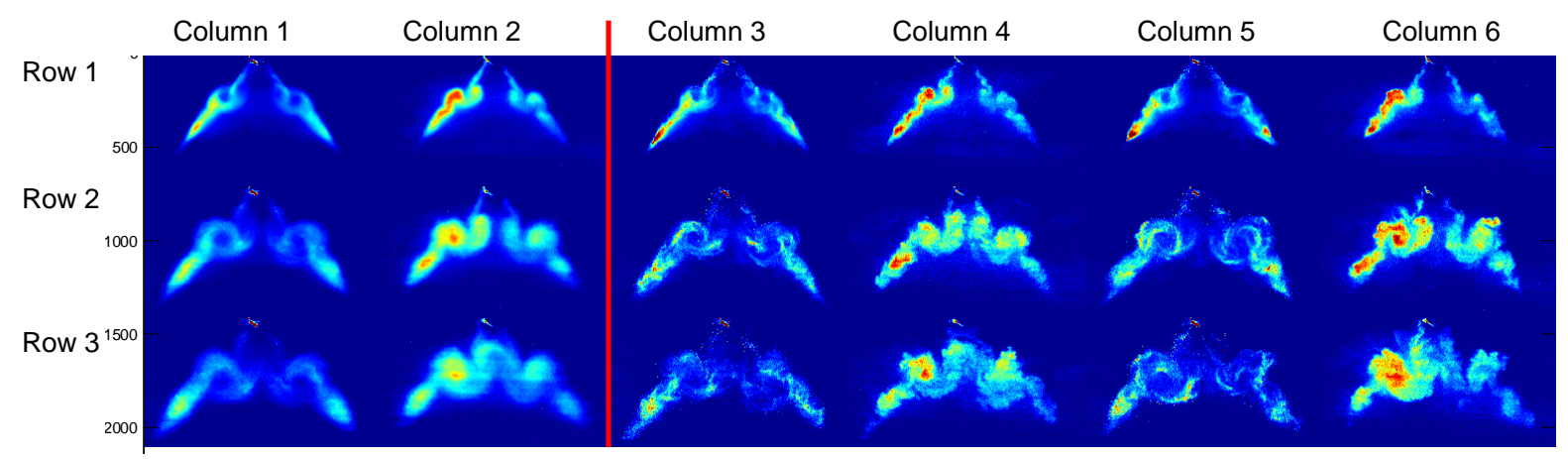

Figure 3. Averaged (Column 1 \& 2) and individual (Columen 3-6) image pairs, where left image in each pair (columns 1, 3 and

5 ) is an exciplex image and the right image (columns 2, 4 and 6 ) is a monomer image. The images are recorded at 4.2 bar nitrogen pressure and $56^{\circ} \mathrm{C}$ temperature, a fuel pressure of 100 bar and 1 (row 1 ) 2 (row 2) and 3 (row 3 ) ms asoi. Image size $95 \times 53 \mathrm{~mm}$

An overview of the spray development with time can be followed by a series of exciplex images displayed in Figure 4. The images, in particular the ones recorded at the earlier time steps, show a non-symmetric intensity where the left part of the spray is much more intense. The reason for this is that the laser light enters from the left and due to the high drop density the light is strongly attenuated and only a fraction of the incident light continues to the right part of the spray. Another effect of the high drop density is multiple light scattering resulting in light being scattered by drops outside of the laser sheet, and some intensity appears to be generated inside the cone.

Initially the cone develops rapidly during the injection, and soon there is a perturbation about $20-25 \mathrm{~mm}$ from the injector. This perturbation leads to the formation of the vortex structure, with two counter-rotating vortices, a larger outer vortex and a smaller inner one. The vortices continue to grow also long after the end of injection. At the bottom of the spray, i.e. downstream of the point where the vortices are formed, the cone structure is preserved without large movements the fuel. Close to the injector traces of the cone can still be observed, along with scattered larger drops (seen in individual images).

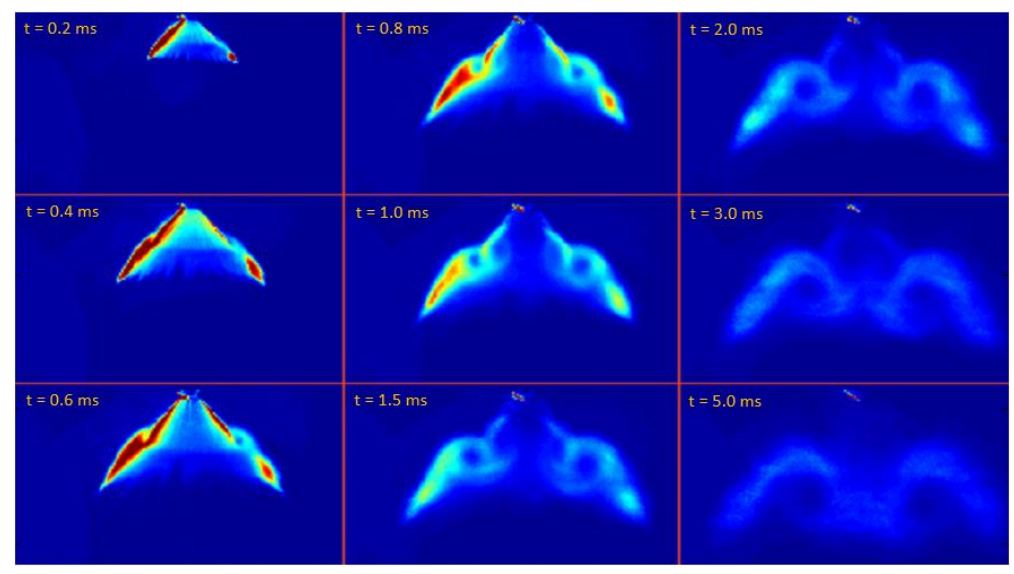

Figure 4. A series of exciplex fluorescence images recorded at 4.2 bar nitrogen pressure and $56^{\circ} \mathrm{C}$ temperature, a fuel pressure of 100 bar. The images are recorded at 0.2, 0.4, 0.6 (left column), 0.8, 1.0, 1.5 (middle column), 2.0, 3.0, 5.0 ms asoi (right column).

To analyse the fuel evaporation, both monomer fluorescence and exciplex fluorescence images at different time after start of injection are shown in the Figure 5, under four different ambient temperatures. At all the temperatures tested, fuel vapor is firstly observed at the same locations as the liquid drops, and then it is accumulated into the center of the vortices, which can be well observed in all exciplex images from shortly after start of injection (1ms). In the exciplex images there is a local minimum in the center of the votices, whereas there is a local maximum at the same position, which is in agreement with earlier observations using a combination of planar LIF and Mie scattering [20]. In addition, penetration of liquid phase and vapor phase are found to be very similar.

The fuel evaporation can be followed by studying the evolution of the monomer and exciplex fluorescence as a function of time. At room temperature $\left(21^{\circ} \mathrm{C}\right)$ the vaporization is found to be very slow, and consequently the 
exciplex signal (corresponding to the liquid phase) is much stronger than the monomer signal (corresponding to the vapor phase). When the ambient temperature is increased to around $40^{\circ} \mathrm{C}$, vaporization of fuel is more observed, particularly after end of injection. Correspondingly, the intensity value of exciplex fluorescence images is slightly reduced after end of injection, proving more fuel was vaporized. It can also be noted the smaller inner vortex, which is barely seen in the exciplex images, appears more clearly in the monomer images and this fuel vapor moves towards the center of the cone to fill the volume about $20 \mathrm{~mm}$ below the injector.

When in-chamber temperature is above $40^{\circ} \mathrm{C}$ there is a noticeable presence of vapor at the end of the injection. At this temperature, monomer fluorescence images generally show a higher intensity value in the spray region, compared with exciplex fluorescence images. At higher temperatures, the vaporization goes even faster. Nonetheless, there are still some big drops remaining in the spray a relatively long time after end of injection at the higher temperatures. The influence of temperature on the appearance of vortices seems to be small, but their size is smaller at later times at higher temperature, likely due to a loss of momentum as the drops vaporize. Increasing of temperature leads to more vapor and less liquid in the vortices region.
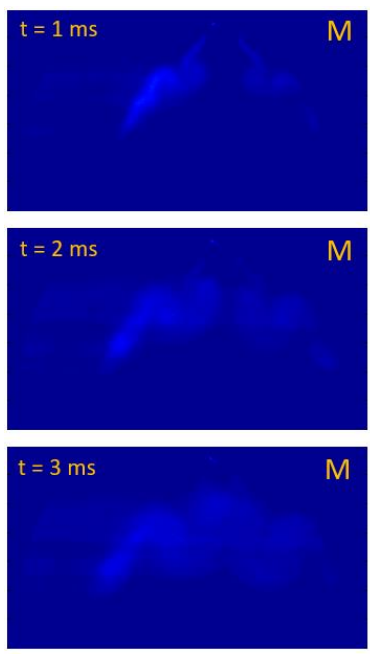

(a) Ambient temperature $21^{\circ} \mathrm{C}$
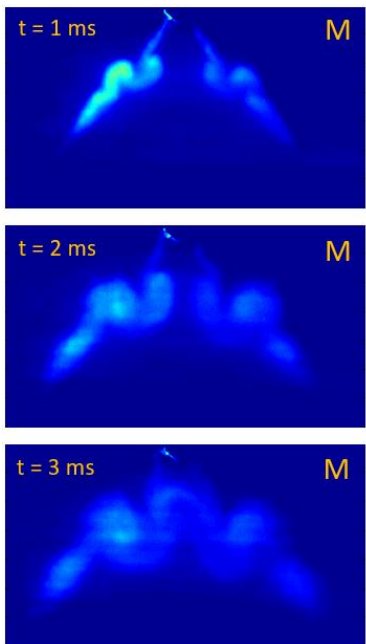

(c) Ambient temperature at $56^{\circ} \mathrm{C}$
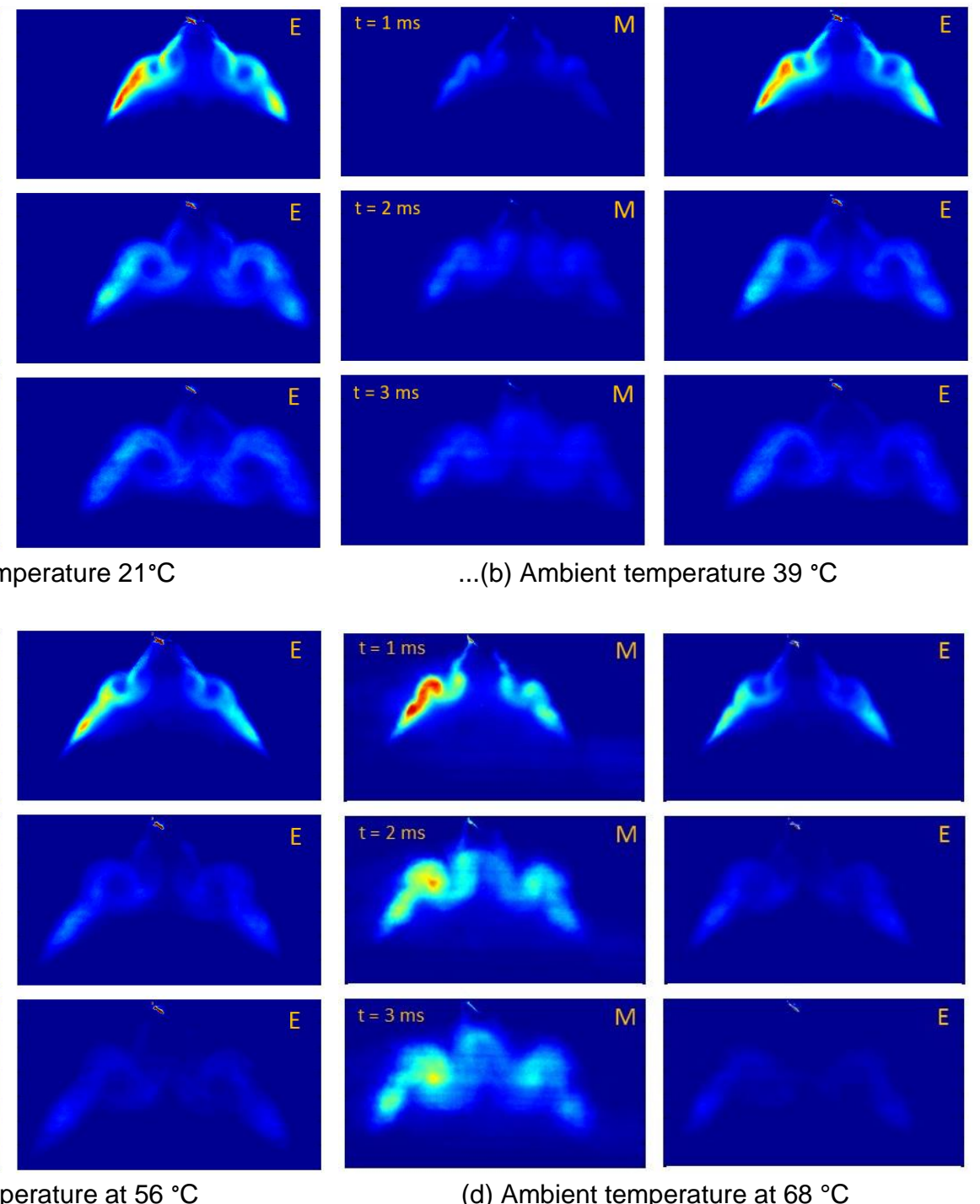

(d) Ambient temperature at $68^{\circ} \mathrm{C}$

Figure 5. Monomer fluorescence $(\mathrm{M})$ and exciplex fluorescence $(\mathrm{E})$ images at different time after start of injection under different ambient temperature, 4.2 bar nitrogen pressure, 100 bar injection pressure

The influence of fuel pressure was investigated by performing measurements at 100 and 200 bar. Figure 6 shows a two sets of images (temperature 39 and $56^{\circ} \mathrm{C}$ ) recorded with a fuel pressure of 200 bar, but otherwise the same conditions as in the corresponding images shown in Figure 5. It is clear that the higher fuel pressure leads to a much stronger vortex formation, with larger vortices at each of the time steps. It should in particular be noted that 
much of the fuel remaining at the bottom of the cone at 100 bar, is dragged into the vortices at a fuel pressure of 200 bar. Furthermore, it can be noted that the intensity ratio between monomer and exciplex fluorescence is higher at 200 bar compared to 100 bar, which shows that vaporization is faster at the higher fuel pressure.

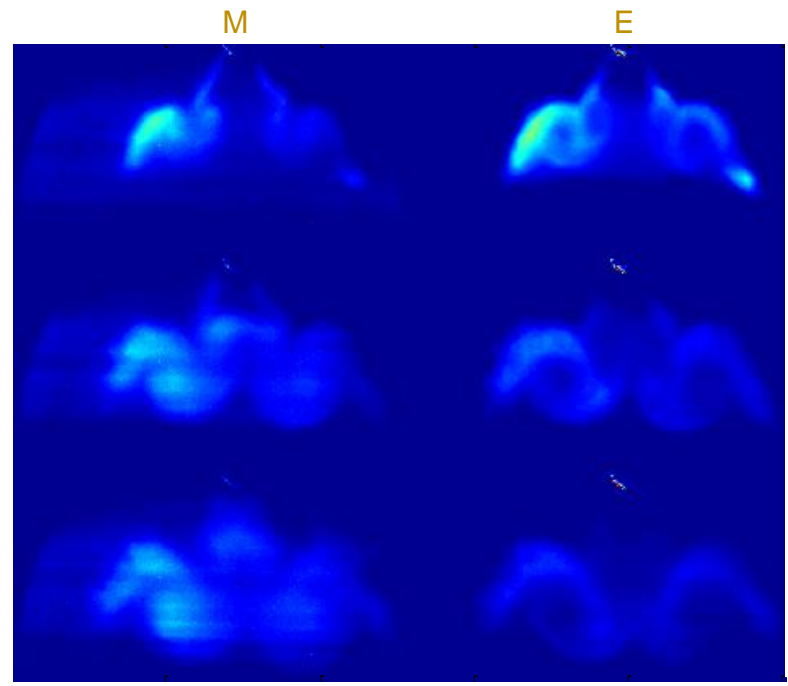

Left panel: $\mathrm{T}=39^{\circ} \mathrm{C}$

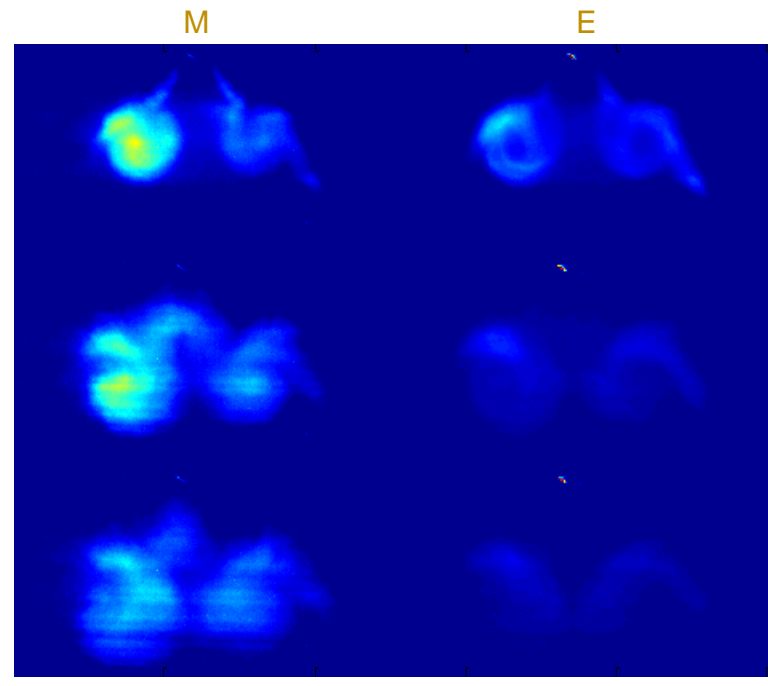

Right panel: $\mathrm{T}=56^{\circ} \mathrm{C}$

Figure 6. Averaged monomer fluorescence (left) and exciplex fluorescence (right) image pairs recorded at chamber temperature $39^{\circ} \mathrm{C}$ (left panel) and $56^{\circ} \mathrm{C}$ (right panel), pressure 4.2 bar and fuel pressure 200 bar, at various times asoi:

$1 \mathrm{~ms}$ top row, $2 \mathrm{~ms}$ middle row and $3 \mathrm{~ms}$ bottom row.

The variations in fuel distribution from injection to injection was analyzed by calculating images representing the standard deviation and coefficient of variation (standard deviation divided by the average). Figure 7 shows a representative example of standard deviation and coefficient of variation images recorded at $T=56^{\circ} \mathrm{C}$ and $\mathrm{P}_{\text {fuel }}=$ 100 bar together with the averaged image. In the standard deviation images it can be seen that the largest values are obtained where there is a high fuel concentration. This shows that even if the major part of the fuel is concentrated to certain areas, there are still significant fluctuations between injections of the actual distribution. This is also what can be seen in individual images as the ones shown in Figure 3. The strongest relative variations in fuel concentrations as seen in the coefficient of variation images are found along the borders of the spray and particularly on the outside of the cone. This is an effect of a high-concentration fuel cloud with a sharp edge, but the edge appearing at slightly different locations in each injection. In the coefficient of variation images there are dots appearing in the area close to the nozzle. This is due to larger fuel drops, likely generated during the injector closing event, still remaining in the area around the injector.

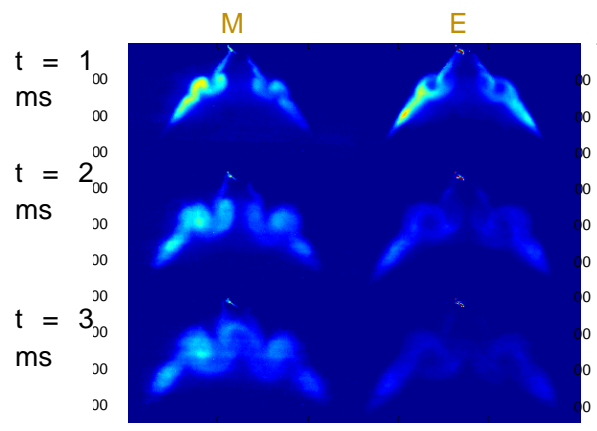

averaged images

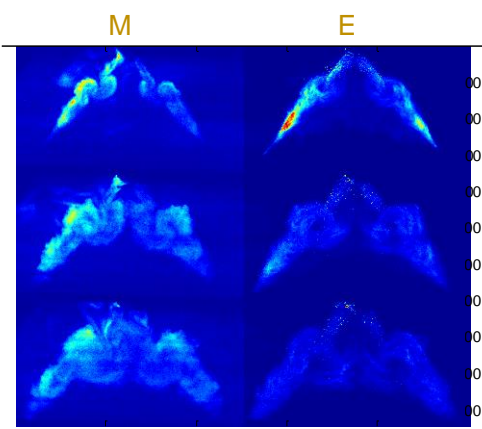

standard-deviation images

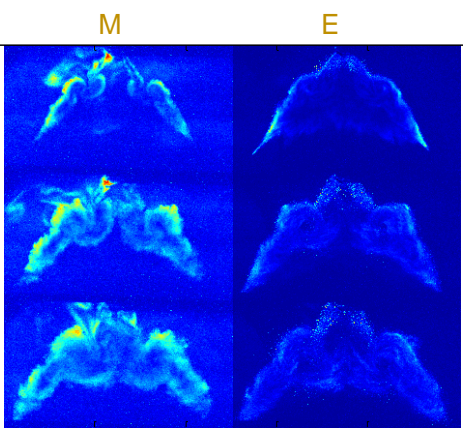

coefficient-of-variation images

Figure 7. Averaged images (left), standard-deviation images (middle) and coefficient-of-variation images (right). In each panel monomer image is in the left column and the exciplex image in the right column. The images are recorded at 4.2 bar nitrogen pressure and $56^{\circ} \mathrm{C}$ temperature, a fuel pressure of 100 bar and 1 (top row) 2 (middle row) and 3 (bottom row) ms asoi. Image size $95 \times 53 \mathrm{~mm}$. 


\section{Conclusions}

Fuel sprays from a piezo-actuated outwards-opening gasoline injector were investigated using the planar laserinduced exciplex fluorescence technique. In this application a single CMOS camera without intensifier was used with a stereoscope. With appropriate filters, images of monomer and exciplex fluorescence with good signal strengths could be obtained. The distribution of fuel in liquid and vapor phase was imaged at various times after start of injection, at different gas temperatures in the spray chamber and with different fuel injection pressures. It was found that the double vortex structure, characteristic for this type of injector was found, and that the drops predominantly remain at the outer part of the vortices, whereas the fuel vapor moves towards the center of the vortices, but also towards the center of the cone. With the $n$-hexane-based fuel, there is a significant difference in the observed fractions of fuel in liquid and vapor phase when temperature is varied in the $20-70^{\circ} \mathrm{C}$ range. When the fuel injection pressure is increased from 100 to 200 bar, the vortices become significantly larger and also the evaporation becomes faster.

\section{Acknowledgements}

Financial support was obtained from the Combustion Engine Research Centre (CERC) at Chalmers, and the Swedish Energy Agency. The imaging system was provided by Dantec Dynamics. Stimulating discussions with Petter Dahlander are gratefully acknowledged.

\section{References}

[1] Melton, L.A., 1983, Applied Optics, 22, 2224-2226.

[2] Fansler, T. and Parrish, S., 2015, Measurement Science and Technology, 26, 012002(34pp).

[3] Ghandhi, J. B., Felton, P. G., Gajdeczko, B. F., and Bracco, F. V., 1994, SAE Paper 940394.

[4] Fujimoto, H., Choi, D., Shima, Y., and Senda, J., 2002, Measurement Science and Technology, 13, pp. 391

[5] Payri, F., Pastor, J.V., Pastor, J.M. and Juliá, J.E., 2006, International Journal of Engine Research, 7, pp. 7789

[6] Rogler, P., Grzeszik, R., Arndt, S. and Aigner, M., 2007, SAE Paper 2007-01-1827

[7] Chen, H., Xu, M., Zhang, G., Zhang, M. and Zhang, Y., 2010, ASME Conf. Proc. 2010 (49446) 391-403

[8] Froba, A., P., Rabenstein, F., Munch, K., U., and Leipertz, A., 1998, Combust. Flame, 112, pp. $199-209$.

[9] Skogsberg, M., Dahlander, P., Denbratt, I., 2007, SAE Technical Paper, 2007-01-1409.

[10] Baecker, H., Kaufmann, A., Tichy, M., 2007, SAE Technical Paper, 2007-01-1407.

[11] Hemdal, S., Denbratt, I., Dahlander, P., Wärnberg, J., 2009, SAE Technical Paper, 2009-01-1496.

[12] Zigan, L., Schmitz, I., Flügel, A., Wensing, M., Leipertz, A., 2011, Fuel, 90, 348-363.

[13] Schmid, A., Schneider, B., Boulouchos, K., Wigley, G., 2014, Atomization and Sprays, 24, 859-873.

[14] Düwel, I., Koban, W., Zimmermann, F.P., Dreier, T., Schulz, C., 2009, Applied Physics B, 97, pp 909-918.

[15] Fansler, T.D., Drake, M.C., Gajdeczko, B. Düwel, I., Koban, W., Zimmermann, F.P., Schulz, C., 2009, Measurement Science and Technology, 20, 125401.

[16] Zhang, G.M., Xu, M., Zhang, Y.Y., Zhang, M., Cleary, D.J., 2012, Atomization and Sprays, 22, pp 861-878.

[17] Zeng, W., Xu, M., Zhang, Y., Wang, Z., 2013, Proceedings of the Combustion Institute, 34, pp 1677-1685.

[18] Shim, Y.S., Choi, G.M., Kim, D.J., 2009, International Journal of Multiphase Flow, 35, pp 885-895.

[19] Wieske,P., Wissel, S., Grünefeld, G., Pischinger, S., 2006, Applied Physics B, 97, pp 323-329.

[20] Andersson, M., Wärnberg, J., Hemdal, S., Dahlander, P., Denbratt, I., SAE Technical Paper 2011-01-1889. 


\title{
Transcritical mixing of sprays for multi-component fuel mixtures
}

\author{
Julien Manin*1,2, Cyril Crua ${ }^{3}$, Lyle M. Pickett ${ }^{1}$ \\ ${ }^{1}$ Combustion Research Facility, Sandia National Laboratories, Livermore CA, USA \\ ${ }^{2}$ Artium Technologies, 470 Lakeside Dr., Sunnyvale CA, USA \\ ${ }^{3}$ Advanced Engineering Centre, University of Brighton, Brighton BN2 4GJ, United Kingdom \\ ${ }^{*}$ Corresponding author: imanin@artium.com
}

\begin{abstract}
The mixing of fuels with oxidizer has been an increasingly interesting area of research with new engine technologies and the need to reduce emissions, while leveraging efficiency. High-efficiency combustion systems such as diesel engines rely on elevated chamber pressures to maximize power density, producing higher output. In such systems, the fuel is injected under liquid state in a chamber filled with pressurized air at high temperatures. Theoretical calculations on the thermodynamics of fuel mixing processes under these conditions suggest that the injected liquid can undergo a transcritical change of state. Our previous experimental efforts in that regard showed through highspeed imaging that spray droplets transition to fluid parcels mixing without notable surface tension forces, supporting a transcritical process. Only mono-component fuels were used in these studies to provide full control over boundary conditions, which prevented extrapolation of the findings to real systems in which multi-component fuels are injected. Multi-component fuels add another layer of complexity, especially when detailed experiments serve model development, requiring the fuels to be well characterized. In this work, we performed high-speed microscopy in the near-field of high-pressure sprays injected into elevated temperature and pressure environments. A reference diesel fuel and several multi-component surrogates were studied and compared to single component fuels. The results support that a transition occurs under certain thermodynamic conditions for all fuels. As anticipated, the transition from classical evaporation to diffusive mixing is affected by ambient conditions, fuel properties, droplet size and velocity, as well as time scales. Analogous to previous observations made with the normal alkane sprays, the behavior of the multi-component fuels correlate well with their bulk critical properties.
\end{abstract}

\section{Keywords}

Diesel sprays, Transcritical mixing, Multi-component fuels.

\section{Introduction}

Despite the growth of alternative technologies for power generation in transportations systems, these technologies only represent a fraction of the worldwide vehicle fleet. Forecasts predict that thermal engines are still going to be highly dominant in decades to come [1]. The same predictions expect a $75 \%$ increase in fuel demand between air, marine and rail transportation, while heavy-duty alone would account for $40 \%$ of the total transportation energy demand. Even with the substantial improvements in gasoline direct-injection and the development of promising new combustion strategies (e.g., homogeneous-charge compression-ignition, spray-guided spark ignition or gasoline compression-ignition), heavy-duty, marine and rail transportation are expected to rely on diesel combustion for years to come. The mixing process between the injected fuel and the oxidizer in diesel engines has been long identified as a key parameter to efficient and clean combustion. Experiments have been devoted to understanding mixing under such conditions, but gaps remain regarding the vaporization process at these extreme thermodynamic conditions. Further, the high velocities and small flow scales produced by the elevated injection pressures limit our ability to fully study and understand the complex processes at play in the confined environment of an engine's combustion chamber.

A substantial part of the research over the last decade has been dedicated to build computational fluid dynamic models to understand these processes. One major limitation is encountered when the fundamental knowledge about the physics of the process to be numerically predicted is inaccurate or incomplete. Such an issue concerns the multi-phase mixing process under elevated pressures and temperatures. Recent theoretical studies aiming at resolving the interface between the liquid and the ambient gas under diesel-like conditions, as well as the state of the mixture, suggest that the high pressures and temperatures the injected fluid is subjected to may broaden this interface [2,3]. As a result, the surface tension between the two fluids may decrease dramatically to the point that the interface enters the continuum regime. Under such molecular state, the integrity of the interface may easily be challenged by external forces such as aerodynamic or turbulence, potentially leading to a single-phase type of mixing. The conditions found in diesel engines are favorable to such process, analogous to the transcritical mixing observed in cryogenic liquid injection applied to liquid rockets $[4,5]$. 
Beyond studies carried out by the rocket community at very different conditions from those of diesel engines, detailed experiments convincingly showing the transcritical mixing process of spray droplets were inexistent until the recent experiments performed by the authors [6]. Other works [7, 8] and past efforts by our group [9] suggested the different mixing behavior based on macroscopic observations or optically-degraded microscopic features. But they failed at capturing the mixing process at the droplet scale, thus leaving room for controversy. The authors' most recent work [6] succeeded in visualizing the transition from classical evaporation to diffusive mixing at the droplet level. The analysis of the high-speed visualizations revealed that the mixing processes could be classified into three regimes. It was confirmed that the classical perspective on evaporation and mixing occurred well beyond the critical point of the injected fluid, with spherical droplets presenting a surface-based vaporization process. Two new regimes were identified, where vaporization and mixing were accelerated due to fluid structure deformation and stretching or a behavior resembling a single-phase mixing process. Both these regimes seem to be affected by a severe drop in intermolecular forces. These two regimes were classified into transitional mixing and diffusive mixing, respectively. Transitional mixing was defined when the fluid structures were seen to deform and oscillate, contributing to an accelerated vaporization. Diffusive mixing corresponds to a regime where the fluid features eventually stretch and deform to a point where intermolecular forces can no longer hold the structure together, leading to a rapid single-phase-like mixing process. One criticism from this work has been that the experiments were performed on pure components, i.e., normal alkanes from n-heptane to n-hexadecane, and that the complex distillation properties of multi-component fuels may radically affect the conclusions. The lack of knowledge on multicomponent mixtures at the fundamental level limit the usefulness of the aforementioned theoretical calculations to extract interfacial properties under the thermodynamic conditions relevant to diesel spray mixing.

The nature of multi-component fuels is expected to affect the evaporation and mixing processes depending on the thermodynamic conditions inside the chamber at the time of injection. The Coordinating Research Council (CRC) has developed advanced surrogates for diesel combustion research purposes. They produced several multicomponent surrogates with the objective of determining which surrogates best capture the physical and chemical properties of the target diesel fuel and why $[10,11]$. The target fuel, as well as the different surrogate fuels created by the CRC have undergone detailed characterization. These fuels represent the ideal candidates to study the mixing processes of multi-component fuel mixtures under high pressures and temperatures at the microscopic level. In a way similar to our previous campaign on alkane fuels, we applied long-distance microscopy at high speed to resolve in space and track in time the spray-generated droplets under relevant diesel conditions. The ambient conditions were varied to map out the thermodynamic space under which the different mixing regimes were observed, from classical evaporation to diffusive mixing. Time-resolved sequences of the spray processes are presented, and the information extracted from the high-speed visualizations is summarized in pressure and temperature charts highlighting the relationships between mixing behavior and critical properties.

Following the present introduction, the equipment used in this study is described, starting with the high-pressure and high-temperature chamber, followed by the high-speed microscope imaging system, and finishing with the injection system and the different fuels, along with their properties. The results first present an example of the visualization capabilities to resolve and track spray-generated droplets under extreme conditions with alkane fuels. Side-by-side sequences of the classical and diffusive evaporation and mixing processes are shown for the different fuels tested in this study. The results of the observations are then presented on thermodynamic diagrams for the different fuels, showing slight but noticeable variations with respect to mixing behavior.

\section{Experimental apparatus}

The different multi-component fuels and the equipment used to carry out the experimental work are presented in the following paragraphs. The CRC Project AVFL-18a panel $[10,11]$ described the methodology to obtain multicomponent surrogate fuels that match the physico-chemical properties of a reference diesel fuel, as well as the combustion characteristics. The following paragraphs regarding the multi-component fuels briefly describe the methods, but more importantly summarize the information relevant to the present study. As explained in the introduction, previous studies on transcritical mixing from our group used single component fuels, namely normal alkanes. These fuels were used in this study to provide examples as well as baseline to the comparisons with the multi-component fuels detailed thereafter. The CRC formulated a set of four ultralow sulfur diesel surrogate fuels to enable detailed numerical and experimental studies in research engines or dedicated combustion vessels. The different surrogates were designed to match the characteristics of a reference fuel, referred to as CFA target, which corresponds to a sample of the batch A grade no. 2-D S15 diesel emission-certification fuel from Chevron-Philips Chemical Co. The surrogates were formulated with various chemical compounds in order to capture the composition of the target fuel from a hydrocarbon structural standpoint. The objective behind the different surrogate formulations regarding the number and types of components was to identify the appropriate requirements in terms of 
compositional accuracy to effectively match the performance characteristics of the target fuel under different engine operating conditions. The whole process to formulate, blend and characterize the different surrogates was described in great detail in Refs. [10, 11].

Two-dimensional gas chromatography with flame ionization detection performed as part of the target fuel characterization undertaken in Refs. $[10,11]$ revealed that the target fuel contained on the order of 5000 individual components or isomers. The surrogate formulation method relied on a predefined palette of components, as mentioned above. The palette consists of 13 pure compounds, extracted from the normal, cyclo- and branched alkane families, as well as naphtha-aromatic and aromatic species. A regression model was applied to determine the composition that best matches the following parameters: Mole fraction of the different carbon types, derived cetane number, distillation properties and fuel density. The surrogates created through this process, called V0a, V0b, V1 and V2, are made of four, five, eight and nine components, in that order. In this study, we decided to test the CFA target fuel, as well as three of the four surrogates: V0a, V0b and V2. We refer to Ref. [11] for the detailed compositions of the different surrogates. The relevant properties of the target fuel and surrogates are listed in Table 1. Also listed in Table 1 for comparison purposes, are the fluid properties for the normal-alkane fuels used in the present work and in previous studies by the authors [6]: n-heptane, n-dodecane and n-hexadecane.

Table 1. Physico-chemical properties of the various fuels used in this study.

\begin{tabular}{c|ccccccc}
\hline \hline Properties & $\mathrm{n}_{-} \mathrm{C}_{16} \mathrm{H}_{34}$ & $\mathrm{n}-\mathrm{C}_{12} \mathrm{H}_{26}$ & $\mathrm{n}-\mathrm{C}_{7} \mathrm{H}_{16}$ & CFA & V0a & V0b & V2 \\
\hline \hline Number of components & 1 & 1 & 1 & $\sim 5000$ & 4 & 5 & 9 \\
Density $^{1}\left[\mathrm{~kg} / \mathrm{m}^{3}\right]$ & 770 & 750 & 684 & 848 & 818 & 838 & 853 \\
Viscosity $^{2}[\mathrm{mPa} . \mathrm{s}]$ & 2.92 & 1.36 & 0.39 & 2.28 & 2.45 & 2.30 & 2.38 \\
Surface tension $^{3}[\mathrm{mN} / \mathrm{m}]$ & 27.5 & 25.4 & 20.1 & 28.2 & 26.4 & 27.4 & 27.7 \\
Boiling point $^{4,5}[\mathrm{~K}]$ & 554 & 489 & 372 & 345 & 287 & 317 & 344 \\
Critical temperature $^{6}[\mathrm{~K}]$ & 722 & 658 & 540 & - & 440 & 446 & 451 \\
Critical pressure $^{6}[\mathrm{MPa}]$ & 1.43 & 1.82 & 2.73 & - & 1.94 & 2.21 & 2.29
\end{tabular}

${ }^{1}$ Density was measured according to the ASTM D4052 standard method.

2 Kinematic viscosity was measured according to the ASTM D445 standard method.

${ }^{3}$ Surface tension for the multi-component fuels was measured via the plate-method [11]

${ }^{4}$ Boiling point for the surrogate fuels corresponds to the heaviest component property.

${ }^{5}$ Boiling point for the target fuel was measured according to the ASTM D 86 standard method and corresponds to $100 \%$ distillation.

${ }^{6}$ Critical temperature and pressure for the surrogate fuels was estimated from their mole-weighted critical properties.

The fuel sprays were injected in an optically-accessible constant-volume pre-burn vessel with high temperature and pressure capabilities (up to $35 \mathrm{MPa}$ and $1400 \mathrm{~K}$ ). A solenoid-actuated common-rail injector equipped with an axiallydrilled single-hole research nozzle of conical ( $\mathrm{k}$-factor $=1.5), 0.180-\mathrm{mm}$ diameter orifice was used to generate the sprays. The vaporizing and mixing sprays were qualitatively analyzed thanks to a high-speed microscopic system specifically developed for this application. The system consists of a commercial high-speed camera, a long-distance microscope objective and an ultrafast illumination source. The illumination was provided by an LED system designed and developed in our laboratories. A state-of-the-art high-speed CMOS camera was employed to acquire full frame resolution images at 40,000 frames per second (fps). The high-speed camera was equipped with a custom-assembled long-distance microscope objective, producing an effective optical magnification of approximately $8 x$ (digital scale factor $=3.5 \mu \mathrm{m} / \mathrm{pix}$ ). The experimental arrangement, as well as a detailed analysis of the optical performance of the system, paramount in this case, are provided in Ref. [9].

\section{Results and discussion}

As noted earlier, the analysis of the high-speed movies is mostly qualitative. Some efforts have been made to quantify certain parameters that the time-resolved nature of the experiment can extract, such as droplet size or shape as function of time. Size-related information under these conditions is important as it may provide guidance regarding the rate of evaporation, which is key to predict liquid penetration and mixing field in spray and engine simulations. The extracted evaporation rate can also be used to estimate ambient temperature or relative velocity (the difference in velocity between the droplet and the surrounding gas), but such an exercise is complicated in practice because of the many parameters affecting evaporation. Furthermore, accurate species properties are necessary for these results to leverage modelling capabilities, and even the well-characterized multi-component fuels injected in this work are beyond the current numerical capabilities. 
To present the spray system from a global standpoint, we first show a sequence of photographs capturing a relatively large field of view. In this case, the imaging system was set up with an overall magnification of $2 x$, as opposed to the $8 x$ magnification used in the rest of the experiments. Such imaging setup provides a scale factor slightly over $10 \mu \mathrm{m} /$ pixel (compared to the $3.5 \mu \mathrm{m} /$ pix achieved by the system described above), which is still enough to resolve large droplets, especially those generated by the lower injection velocities at the end of injection. The high-speed movies of the image sequences shown in Fig. 1 were acquired at $10 \mathrm{kfps}$. In these sequences, the injector is on the left of the images, and the spray exits the axially-drilled nozzle orifice penetrating horizontally from left to right. A simple intensity normalization has been performed on the image to keep the intensities and contrasts at similar levels across the different sequences. The two time-sequences of Figure 1 show sprays of $n$-hexadecane fuel injected into environments corresponding to different thermodynamic conditions in modern diesel engines. The temperature and pressure for the left panel were $900 \mathrm{~K}$ and $6.0 \mathrm{MPa}$, respectively; the sequence on the right corresponds to an ambient held at $1200 \mathrm{~K}$ temperature and 10.7 MPa pressure. The timing shown at the top left corner is given relative to the end of injection and is synchronized for both sequences. The scale of the images is indicated at the bottom left corner of the top photograph.

The image sequences of Fig. 1 show the injection event at different timings from the middle of the injection to 2.4 $\mathrm{ms}$ after the end of injection, and oriented from left to right for two conditions. The corresponding high-speed movies present a clear perspective over the processes, which contrasts with the somewhat limited effect of the still images presented therein. The left photographs present the spray during the injection event, with tiny droplets on the periphery of the spray on the top sequence. It is much more difficult to identify droplets on the bottom images, even though they appear to be comparable to the top sequence in terms of sharpness and clarity. Both sprays present a dark core, highlighting the optical thickness of high pressure sprays. The following images present the droplet field after the end of injection. It appears that the field of droplets decrease in number substantially with time, across the last two images of the sequence.

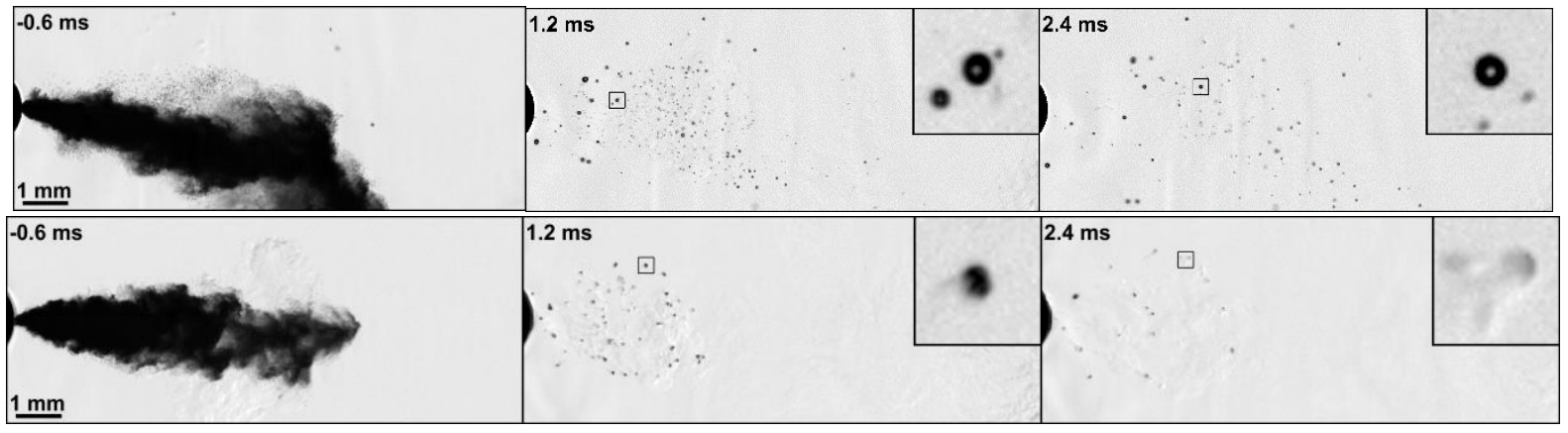

Figure 1: Time-sequence photographs showing the end of injection sequence of $\mathrm{n}$-hexadecane fuel injected under liquid state (363 K, $50 \mathrm{MPa}$ ) into highly heated and pressurized inert environments. Top: Environment at $900 \mathrm{~K}, 6.0 \mathrm{MPa}$ the classical view of liquid breakup and vaporization. Bottom: Environment at $1200 \mathrm{~K}, 10.7 \mathrm{MPa}$ highlighting the effect of high pressure and temperature on mixing.

A magnified droplet, representative of the field, is shown at the top right corner on both sequences to assist with the observations. Both droplets are initially about $50 \mu \mathrm{m}$ in diameter and travel at similar speeds of only a few meters per second. The droplet speed must be compared to the high injection velocities of several hundred meters per second during injection, responsible for droplet size distributions centered around a few micrometers, typical of diesel sprays [12]. The droplet fields produced at the end of injection highlight the differences between the conditions. The droplets of the left panel remain spherical after a brief oscillating period following their formation, and until complete evaporation. The droplets spherical shape reinforces the influence of surface tension, while they decrease in size, until they are no longer successfully identified from the background, corresponding the droplet diameters a little above $10 \mu \mathrm{m}$. Even though it is not readily apparent in the magnified droplet, the evaporation process and associated decrease in droplet diameter is visible through the reduction in droplet number throughout the last two photographs of the sequence. This case exemplifies the classical viewpoint on droplet evaporation, with a mass transfer from liquid to vapor happening on the surface or surrounding of the fluid feature. On the other hand, the droplets produced by the end of injection on the bottom sequence do not remain spherical throughout the images, but deform and mix with the ambient without apparent influence of surface tension. This perspective is clearly shown in the magnified inset, where the photograph taken $1.2 \mathrm{~ms}$ after the end of injection shows a quasispherical droplet that just started vaporizing and deforming, while the following image of the sequence shows the remains of a deformed fluid structure. The optical thickness of the middle photograph contrasts with the relatively clear shadow observed in the last image. The lack of sharpness denotes that the interfacial gradient may not be as 
steep as in the top sequence. These observations are compatible with the broadening of the interface proposed by Dahms and coworkers [2], and described in the introduction. The disruption of the fluid feature and ostensible mass transfer to the surrounding continue in the subsequent images, with only faint disconnected structures remaining in the last image of this sequence. The mass transfer from the fluid structure to the surrounding can be interpreted as a mixing process controlled by aerodynamic forces and molecular diffusion, rather than by the strong intermolecular forces exhibited by surface tension. As such, this process was called diffusive mixing. It can be noted that a number of publications have been referring to this process as supercritical evaporation $[13,14]$, but diffusive mixing appears to be more descriptive of such process.

The two sequences of Fig. 1 also aid as example to detail the criteria employed to qualitatively analyze the various high-speed spray visualizations. Following the descriptions given above for the top sequence, the droplets keep their spherical shape and integrity thanks to surface tension. Most droplets refract light, as shown by the bright spot in their center, denoting that the interface between the droplet and the ambient is sharp, and that the droplet coincides with the imaging system's focal position. Such droplets are tracked to observe whether the optical sharpness of the interface and refraction spot remain, indicating classical evaporation, or degrade, suggesting diffusive mixing. As commented in the previous paragraph, the disappearance of the refraction spot and the observed degradation of the droplet boundaries can be associated with interface broadening and surface tension drop, as suggested by the theoretical calculations carried out by Dahms et al. [2]. Such modifications in the interface state result in lower intermolecular forces, driving the feature to rapidly lose cohesion and disappear in the ambient when subjected to local turbulence and other aerodynamic forces. These observations classify the event as diffusive mixing. Not featured in Fig. 1, transitional mixing was identified when the fluid structures were seen to deform and oscillate, contributing to an accelerated vaporization while the structure remained connected.

Figures 2 to 5 present image sequences for the different fuels under conditions highlighting the two processes observed on Fig. 1 and described above. Similar to Fig. 1, the top sequences of the following figures present the classical evaporating spray viewpoint, showing the droplet formation at the end of injection and the subsequent droplet size reduction until complete evaporation for most of the droplet field. The bottom image panels offer examples of diffusive mixing, where atomization and droplet formation can initially be observed, followed by surface evaporation, until the intermolecular forces can no longer hold the spherical shape of the fluid structures, resulting in deformation and rapid disintegration of the structure to mix with the ambient. The injector is also located on the left side of the images, and the sprays penetrate from left to right. These sprays were injected at pressures between 20 and $25 \mathrm{MPa}$, to keep pressure difference above $10 \mathrm{MPa}$. Unlike Fig. 1, these images were acquired at a higher optical magnification, with a digital resolution on the order of $3.5 \mu \mathrm{m}$ per pixel, allowing better visualization of the atomization process and the generated fluid structures. The main limitation of this optical arrangement is the smaller field of view of approximately $4 \mathrm{~mm}$ in the axial direction (scale displayed at bottom left corner of top image), allowing most droplets to leave the visualized region before being fully vaporized or mixed with the ambient. The acquisition frequency was also increased by a factor of four compared to that of Fig. 1, even though the still images shown thereafter do not highlight the improved temporal resolution. The image panels of Figs. 2 to 5 all present the same timings. All timings reported at the top left corner referred to the end of injection. It should be noted that the ambient conditions shown in Figs. 2 to 5 are provided as examples and should not be used to draw conclusions about the relationship between mixing processes and thermodynamic space for the different fuels. It is important to acknowledge that the image sequences presented therein are limited to describe the processes, and that most of the observations and descriptions surrounding these images were extracted from the original or processed highspeed movies.

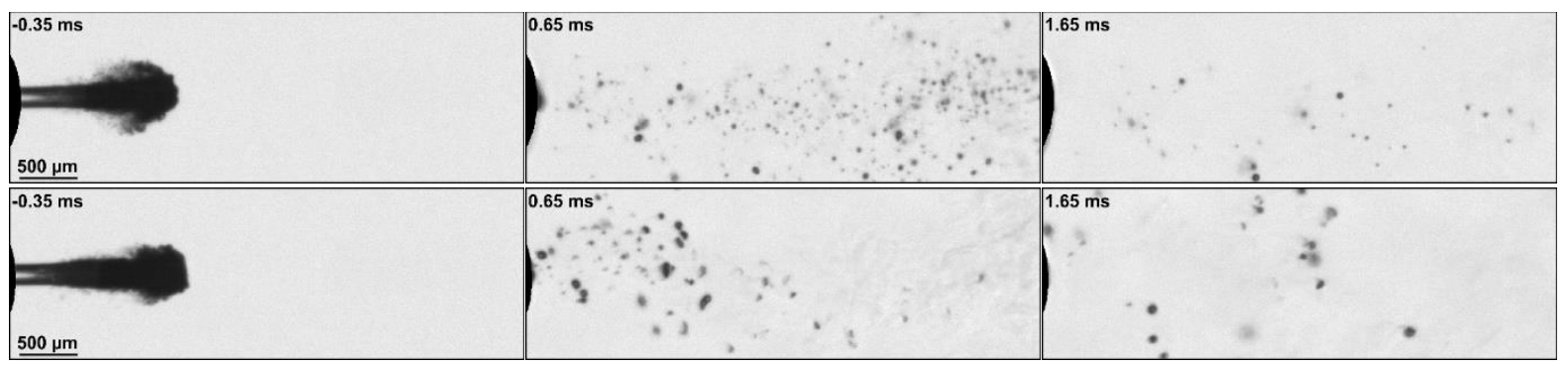

Figure 2: Time-sequence showing the injection, evaporation and mixing processes of sprays of the CFA target fuel into environments at $1000 \mathrm{~K}, 9.2 \mathrm{MPa}$ (left) and 1300, $11.9 \mathrm{MPa}$ (right). The sequence on the left features the classical evaporation, while the right sequence shows signs of diffusive mixing. 


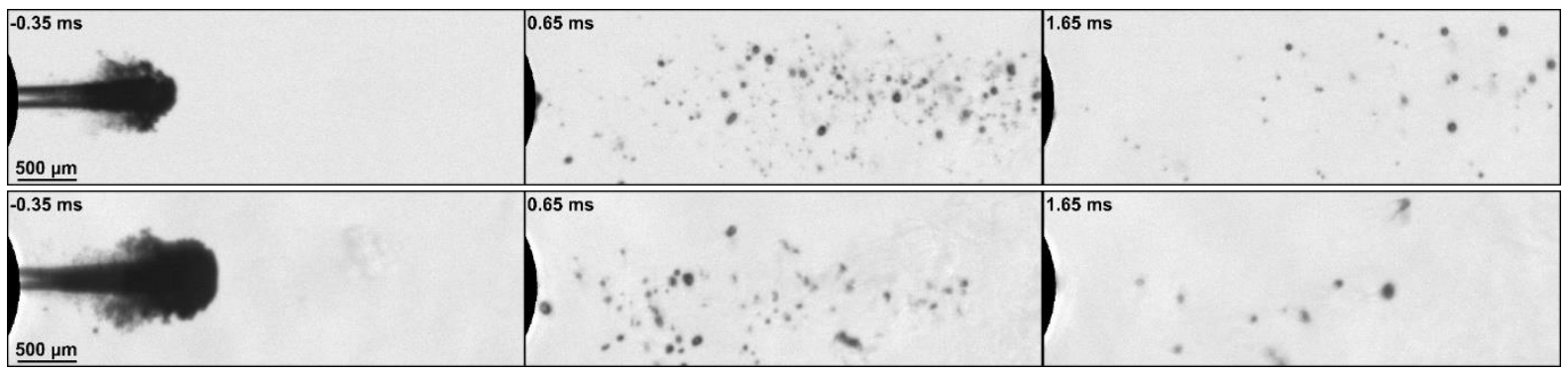

Figure 3: Time-sequence showing the injection, evaporation and mixing processes of V0a surrogate fuel sprays into environments at $900 \mathrm{~K}, 8.2 \mathrm{MPa}$ (left) and 1300, 11.9 MPa (right). The sequence on the left features the classical evaporation, while the right sequence shows signs of diffusive mixing.

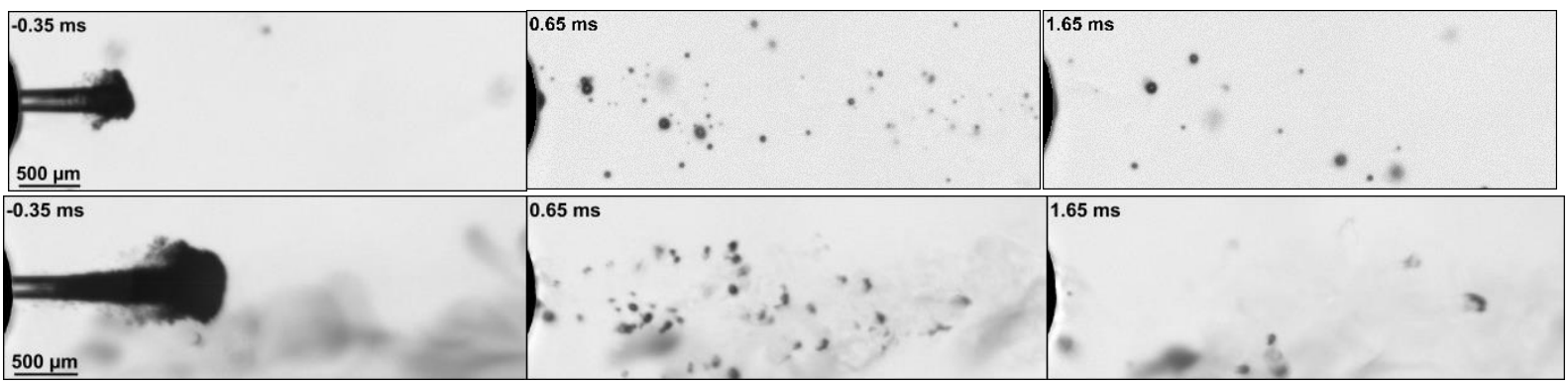

Figure 4: Time-sequence showing the injection, evaporation and mixing processes of V0b surrogate fuel sprays into environments at $900 \mathrm{~K}, 12.2 \mathrm{MPa}$ (left) and 1400, 12.8 MPa (right). The sequence on the left features the classical evaporation, while the right sequence shows signs of diffusive mixing.

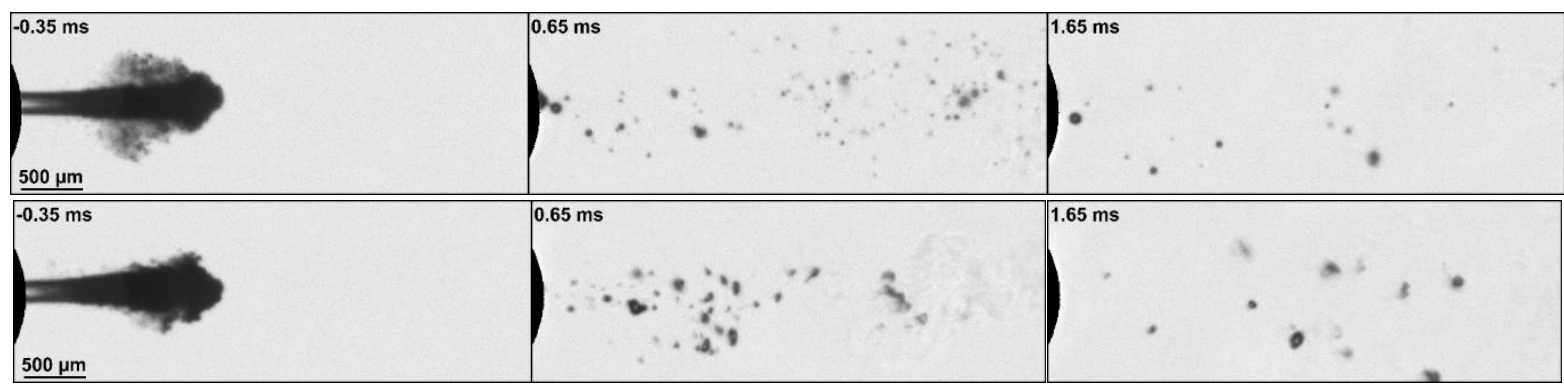

Figure 5: Time-sequence showing the injection, evaporation and mixing processes of $\mathrm{V} 2$ surrogate fuel sprays into environments at $900 \mathrm{~K}, 12.2 \mathrm{MPa}$ (left) and 1400, $12.8 \mathrm{MPa}$ (right). The sequence on the left features the classical evaporation, while the right sequence shows signs of diffusive mixing.

As mentioned earlier for Fig. 1, the sequences presented in Figs. 2 to 5 are synchronized together with respect to the end of injection. The first image of the sequence shows the developing spray, acquired shortly after the start of injection, corresponding to approximately $350 \mu$ s before the end of injection in these examples. Paying attention to the periphery of the plume, small fluid structures can be observed on both first images of most sequences. It is however difficult to assess whether these features are spherical or deformed due to their small dimensions, and possible motion blurring. Fluid features are in general visible on the spray periphery throughout the injection event, but the short life-times and small sizes render the analysis complicated, as detailed earlier. The spray rapidly dissipates in the next images of the various sequences, with the faster moving features exiting the visualized domain, while the slower structures are left behind. The remaining images in the sequences highlight the differences between the mixing processes previously described alongside Fig. 1, with spherical droplets decreasing in size in the top sequences, and deforming fluid features eventually mixing with the ambient via diffusion on the bottom images. It should be noted that the ambient conditions of the lower panels are somewhat above the transition limit between classical/transitional mixing and diffusive mixing. These elevated temperature and pressure conditions are necessary to ensure that the fluid structures are deforming and mixing with the ambient with substantially reduced intermolecular forces within the 4-mm long field of view.

In order to summarize the experiments and compare the different fuels in the scheme of the diffusive mixing process, the tests have been classified on pressure - temperature diagrams. Figure 6 shows these diagrams for the four 
multi-component fuels tested in this study. All diagrams are set to the same limits to facilitate comparisons across the different fuels. White symbols represent conditions where only classical evaporation and mixing was observed, while the black symbols correspond to conditions where diffusive mixing was identified. The transitional mixing regime is identified by grey markers.
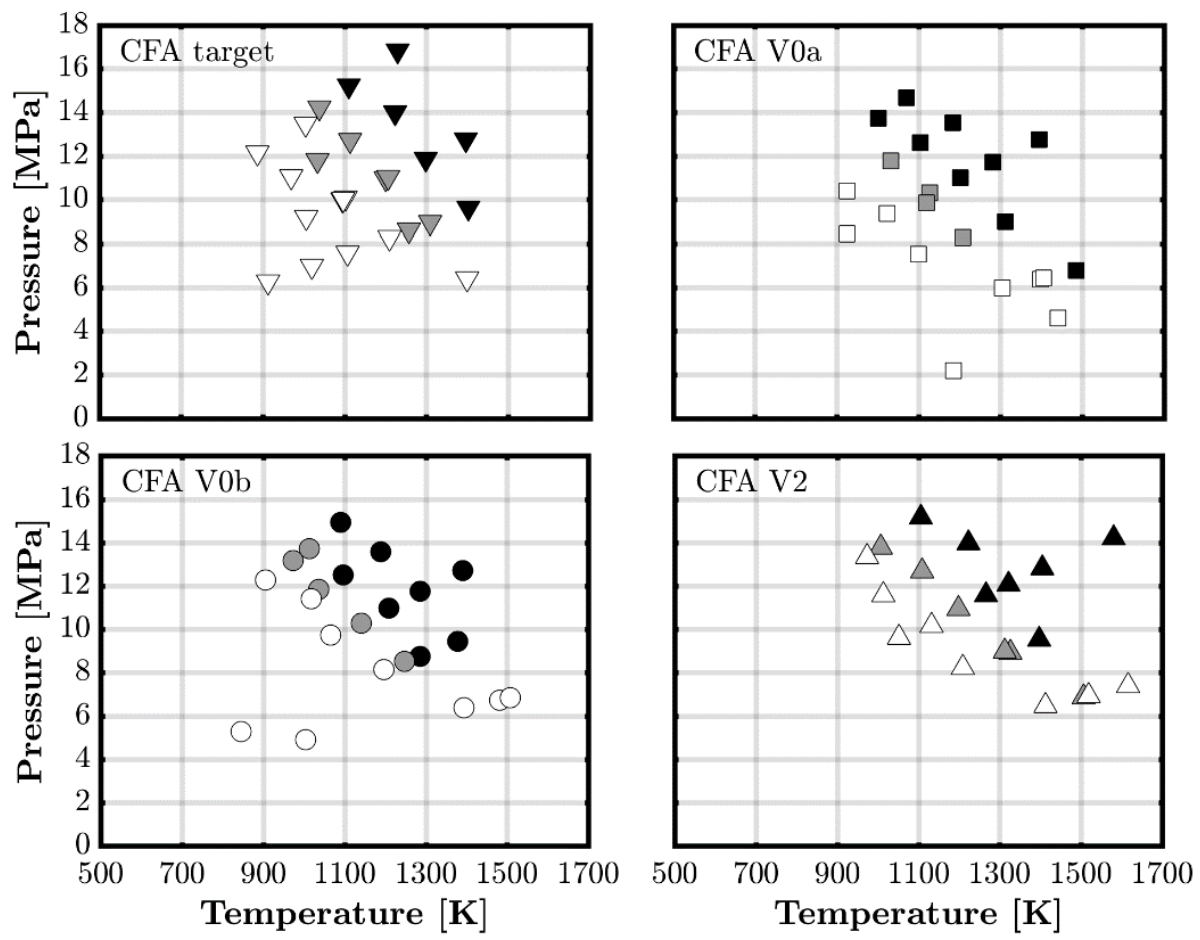

Figure 6: Pressure-temperature diagrams showing the test points for each of the four multi-component fuels. The white symbols represent test conditions where only classical evaporation is observed; the gray markers represent conditions showing transitional mixing; the black symbols represent test conditions where the fluid exhibits diffusive mixing.

The relatively large thermodynamic space of the diagrams in Fig. 6 does not highlight the differences between the fuels. At the same time, it is not surprising that the fuels show similarities because the surrogates were produced to match certain characteristics of the CFA target fuel. It should be noted though, that matching the critical properties was not part of the objectives of the formulation process. Despite these apparent similarities, a closer look at the black symbols shows that CFA target fuel is best matched by V2, while V0a and V0b present relatively similar behavior on the pressure - temperature space. As noted after Table 2, the closest surrogate to CFA target in terms of physico-chemical properties in this study is the nine-component V2 surrogate fuel. Characteristics such as density and boiling point are matched within $1 \%$ between these two fuels. These two fuels present transcritical mixing behavior at higher temperature and pressure regions in the thermodynamic charts of Fig. 6 compared to the other two fuels. It should be noted that the ambient pressures and temperatures where diffusive mixing has been observed are typical conditions near or past top-dead-center for modern diesel engines. These tests therefore confirm our previous findings about mixing properties under realistic thermodynamic conditions on singlecomponent fuels.

\section{Conclusions}

Microscopic observations of diesel sprays injected in high pressure and temperature environments have been carried out to understand the vaporization and mixing processes of multi-component fuels above the critical point. Three multi-component diesel surrogate fuels were used, in addition to a well-characterized commercial diesel target fuel. The surrogate fuels were compared to the target fuel by means of detailed high-speed visualizations of spray processes in the near-nozzle region.

As previously observed for the alkane sprays, the four multi-component fuel sprays present three mixing processes: classical evaporation, transitional mixing and diffusive mixing. These experiments demonstrate that diesel fuels are also subject to transcritical mixing, where the effects of surface tension are significantly reduced to allow the fluid structures to rapidly mix with the surrounding. Differences between the multi-component fuels have been observed when plotting the regime classification on pressure - temperature diagrams. The thermodynamic spaces where the different mixing regimes were identified shift from one fuel to another, and agree with the particular fuel composition 
and physico-chemical properties. Interestingly, the most complex surrogate formulation proved to be the best match for the target fuel in these experiments, despite the fact that critical properties were not accounted for during the formulation. Future efforts will focus on determining the fluid structure lifetime under the different regimes, to establish a relationship between thermodynamic properties and vaporization/mixing rate for single and multicomponent fuels.

\section{Acknowledgements}

This work was supported by the UK's Engineering and Physical Science Research Council [grant number EP/K020528/1]. The authors gratefully acknowledge Coordinating Research Council Project AVFL-18a for formulating, characterizing, and providing the target and surrogate fuels used in this study. This study was performed at the Combustion Research Facility, Sandia National Laboratories is a multi-mission laboratory managed and operated by National Technology and Engineering Solutions of Sandia, LLC., a wholly owned subsidiary of Honeywell International, Inc., for the U.S. Department of Energy's National Nuclear Security Administration under contract DE-NA0003525.

\section{References}

[1] ExxonMobil. The outlook for energy: A view to 2040. Outlook Series, 2015.

[2] R. N. Dahms, J. Manin, L. M. Pickett, and J. C. Oefelein. Understanding high-pressure gas-liquid interface phenomena in diesel engines. Proceedings of the Combustion Institute, 34(1):1667-1675, 2013.

[3] R. N. Dahms. Gradient theory simulations of pure fluid interfaces using a generalized expression for influence parameters and a Helmholtz energy equation of state for fundamentally consistent two-phase calculations. Journal of colloid and interface science, 445:48-59, 2015.

[4] N. Zong and V. Yang. Cryogenic fluid jets and mixing layers in transcritical and supercritical environments. Combustion science and technology, 178(1-3):193-227, 2006.

[5] B. Chehroudi. Recent experimental efforts on high-pressure supercritical injection for liquid rockets and their implications. International Journal of Aerospace Engineering, 2012, 2012.

[6] C. Crua, J. Manin, and L. M. Pickett. On the transcritical mixing of fuels at diesel engine conditions. Submitted to Fuel, 2017.

[7] Z. Falgout, M. Rahm, Z. Wang, and M. Linne. Evidence for supercritical mixing layers in the ECN Spray A. Proc. Combust. Inst., 35(2):1579-1586, 2015.

[8] M. Wensing, T. Vogel, and G. Gotz. Transition of diesel spray to a supercritical state under engine conditions. International Journal of Engine Research, 17(1):108-119, 2016.

[9] J. Manin, M. Bardi, L. M. Pickett, R. N. Dahms, and J. C. Oefelein. Microscopic investigation of the atomization and mixing processes of diesel sprays injected into high pressure and temperature environments. Fuel, 134:531-543, 2014.

[10] C. J. Mueller, W. J. Cannella, T. J. Bruno, B. Bunting, H. D. Dettman, J. A. Franz, M. L. Huber, M. Natarajan, W. J. Pitz, M. A. Ratcliff, and Others. Methodology for formulating diesel surrogate fuels with accurate compositional, ignition-quality, and volatility characteristics. Energy \& Fuels, 26(6):3284-3303, 2012.

[11] C. J. Mueller, W. J. Cannella, J. T. Bays, T. J. Bruno, K. DeFabio, H. D. Dettman, R. M. Gieleciak, M. L. Huber, C.-B. Kweon, S. S. McConnell, W. J. Pitz, and M. A. Ratcliff. Diesel surrogate fuels for engine testing and chemical-kinetic modeling: Compositions and properties. Energy \& Fuels, 30(2):1445-1461, 2016.

[12] C. Crua, G. de Sercey, M. R. Heikal, and Gold M. Dropsizing of near-nozzle Diesel and RME sprays by microscopic imaging. ICLASS 2012, Heidelberg, Germany, 2012.

[13] S. D. Givler and J. Abraham. Supercritical droplet vaporization and combustion studies. Prog. Energ. Combust., 22(1):1-28, 1996.

[14] G. S. Zhu and S. K. Aggarwal. Transient supercritical droplet evaporation with emphasis on the effects of equation of state. International Journal of Heat and Mass Transfer, 43(7):1157-1171, 2000. 


\title{
Influence of the Fuel Properties on the Injection Process and Spray Development in a Large Ship Diesel Engine
}

\author{
I. Najar ${ }^{* 1}$ B. Buchholz ${ }^{1}$, B. Stengel ${ }^{1}$, C. Fink ${ }^{1}$, E. Hassel ${ }^{2}$ \\ ${ }^{1}$ Institute of Piston Machines and Internal Combustion Engines, Rostock University, Germany \\ ${ }^{2}$ Department of Technical Thermodynamics, Rostock University, Germany \\ *Corresponding author: ibrahim.najar2@uni-rostock.de
}

\begin{abstract}
The present paper deals with the influence of fuel properties on the spray behaviour. This influence was studied experimentally using a common rail injection system from a medium speed diesel engine. The experiments have been performed with diesel fuel (EN-590) and heavy fuel oil (RMG 180) on a constant volume chamber at room temperature. Comparison of the spray characteristics shows that the heavy fuel oil penetrates deeper in the chamber. However, the diesel spray has a bigger cone angle. These results formed the basis for a further development of the 1D-model [1] to predict the spray penetration by considering the fuel properties and temperature.
\end{abstract}

\section{Keywords}

Fuel properties, Diesel, Heavy Fuel Oil, Injection, 1D-Spray Model.

\section{Introduction}

The dependency of mixture formation on fuel properties is known and investigated by several researchers e.g. [25]. In [2] different biodiesel blends $\left(B_{5}, B_{10}, B_{20}\right.$ and $\left.B_{50}\right)$ in diesel fuel, pure biodiesel $\left(B_{100}\right)$ and diesel fuel were experimentally examined in a cold chamber (room temperature) and on a single-cylinder diesel engine. The study shows that the physical properties of biodiesel and its blends influence the spray breakup as well as the emissions output. It was also noticed that the bigger the proportion of the biodiesel the longer the spray tip penetration. This result agrees with those in [3]-[5] and [14]. In [14] leads an increase in the fuel density to increase the spray length and decrease in the spray cone angle in the fully developed zone. A similar influence has the fuel viscosity in the same zone. Besides the influence on the spray development, the fuel physical properties affect the hydraulic behaviour of the injector and thus on the injection rate and injection duration. Increasing the fuel density leads to a higher injection rate [12], [14-15], which can be theoretically concluded from Bernoulli's equation. Viscosity influence on the hydraulic behaviour of the injector is more complicated which causes a discrepancy in literature. For too small injection quantities the dynamic of the injector needle is affected by fuel viscosity which changes the injection rate shape and timing [16]. If the injector needle reaches its highest position than the close curve depends on the viscosity [17]. This phenomenon could not be noticed in [15] and [18].

The introduction of the IMO-TIER-III emission legislation demands a drastic reduction of $\mathrm{NO}_{\mathrm{x}}$-emission within the $\mathrm{NO}_{x}$ Emission Control Areas (NECAs). The demanded reduction can only be made by developing and introducing novel technologies for ship diesel engines. The discussed strategies for $\mathrm{NO}_{\mathrm{x}}$-reduction is based on different technologies from automotive sector which usually can be realized only by the use of distillate fuels. Outside these control areas, IMO-TIER-II limits are applied and in force. This legislation can be achieved by utilizing engine internal measures such as miller cycle combined with high pressure intake air, heavy fuel oil and common rail injection system. HFO and the distillate fuels have different physical properties which affect the spray structure, and thus, the combustion and the emission formation as well. Therefore, the relationship between the physical properties of the fuel and the development of spray structure has been experimentally investigated using HFO (RMG 180) and diesel fuel (EN-590). Table 1 presents the corresponding physical properties of the HFO (RMG 180) and the diesel fuel.

\section{Experimental setup}

Injection experiments were carried out using a large engine solenoid valve CR-injector with integrated fuel reservoir and a nozzle containing eight cylindrical holes. The injector can be operated with both distillate fuel as well as heavy fuel oil (HFO). Injection rate measurements were performed using an injection rate analyser [7] (based on $\mathrm{BOSCH}$ principle [8]), Figure 1. Fuel spray characteristics were quantified using a high-pressure/high-temperature chamber [9] with optical access, Figure 1. The chamber can be operated at a constant flow of nitrogen at temperatures and pressures up to $900 \mathrm{~K}$ and 40 bar. For optical accessibility the chamber is equipped with three windows while the injector was mounted with a customized flange to achieve a full frame of one injection jet for analysis with a maximum length of $130 \mathrm{~mm}$. The other injection jets were hidden using a deflection cap that was mounted on the injector tip. 
The injector was operated with diesel fuel (DF) according to DIN EN-590 specification and with heavy fuel oil (RMG 180), Table 1. In order to assure a typical injection viscosity during heavy fuel oil operation the fuel system was heated properly using electrical heating. Furthermore, the injector temperature was controlled using heated oil in the area of the nozzle.

For quantification of the spray characteristics in terms of spray penetration and spray cone angle image sequences were taken at cold chamber conditions (room temperature) using a Phantom V7.2 high-speed camera. The images have a resolution of $800 \times 300 \mathrm{px}$ and were recorded at a frame frequency of up to $12820 \mathrm{kHz}$ and shutter times $\leq$ 5 s. For proper lightning of the injection jet two xenon flashes were mounted orthogonally to the camera, Figure 2. Injection and camera were controlled via a main test bench computer. To avoid shot-to-shot deviations results of at least 10 injections were averaged. Jet volume was calculated through a numeric rotation of the jet profile defined by the measured spray penetration and spray cone angle. The operating pints used in the current study to validate and modify the spray model are shown in Table 2.

Table 1. Test fuel properties during injection tests. *values from DIN EN 590 fuel specification

\begin{tabular}{l|ll}
\hline \hline & DF $($ EN 590) & HFO (RMG 180) \\
\hline \hline Density $\left[\mathrm{kg} / \mathrm{m}^{3}\right]$ & $820-845\left(15^{\circ} \mathrm{C}\right)^{\star}$ & $995\left(50^{\circ} \mathrm{C}\right)$ \\
Viscosity $[\mathrm{cSt}]$ & $<4,5\left(40{ }^{\circ} \mathrm{C}\right)^{*}$ & $12\left(120^{\circ} \mathrm{C}\right)$ \\
Operation temperature $\left[{ }^{\circ} \mathrm{C}\right]$ & 40 & 120
\end{tabular}

\begin{tabular}{ccc}
\multicolumn{3}{c}{ Table 2. Test Matrix } \\
\hline \hline PInj in bar & $\rho_{g}$ in $\mathrm{kg} / \mathrm{m}^{3}$ & $T_{g}$ in $\mathrm{K}$ \\
\hline \hline 800 & 22 and 33 & 293 \\
1600 & 22 and 33 & 293 \\
1800 & 22 and 33 & 293
\end{tabular}

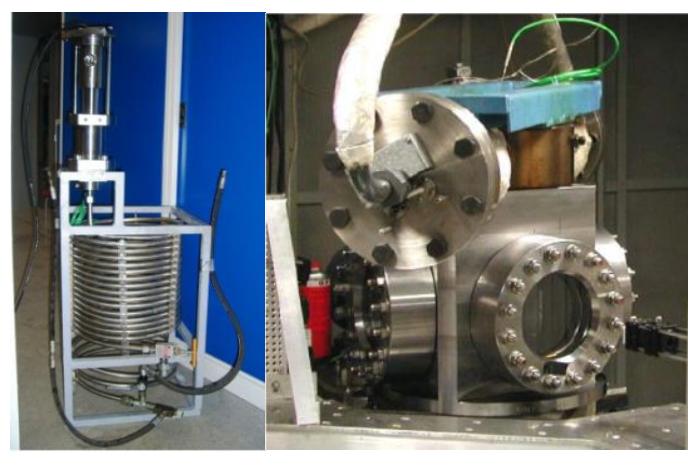

Figure 1. (Left) Injection rate analyser, (Right) high-pressure/high-temperature injection chamber
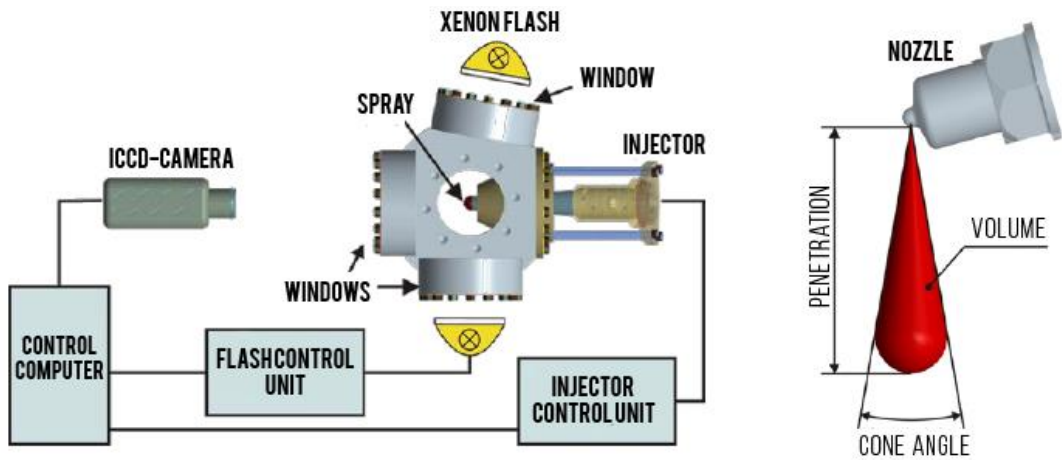

Figure 2. (Left) Schematic layout of scatter light measurements, (Right) definition of jet parameters

\section{D-modell to predict the spray tip penetration}

The empiric model in [1] bases on two phenomena, which were noticed in a framework of an experimentally analysis of the mixture formation at medium speed diesel engines [6]. First, it has been noticed that the spray penetration from a nozzle hole depends only on the gas density and the cumulated injected mass, Figure 3. Furthermore, the 
correlation between the spray penetration and the cumulated injected mass proves to be valid regardless of the injection pressure, the nozzle holder and the nozzle geometry [1]. The left diagram in Figure 3 reveals the known influence of the injection pressure on spray length. Rising the injection pressure leads to a faster penetration. If the penetration was plotted over the cumulated injected mass per nozzle hole, then the injection pressure has no influence on the spray penetration, which can be recognized in the right diagram in Figure 3.
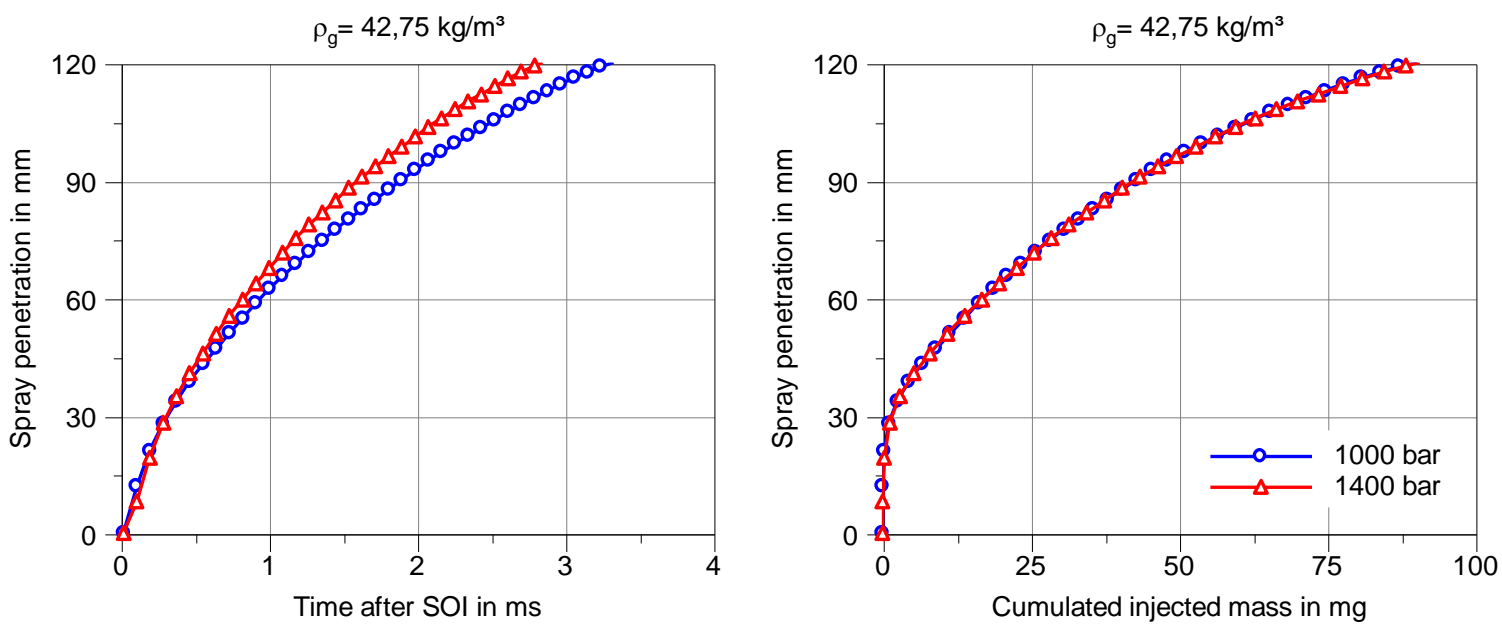

Figure 3. Plot of Spray penetration at 1000 bar and 1400 bar rail pressure against time (Left) and against cumulated injection mass per nozzle hole (Right)
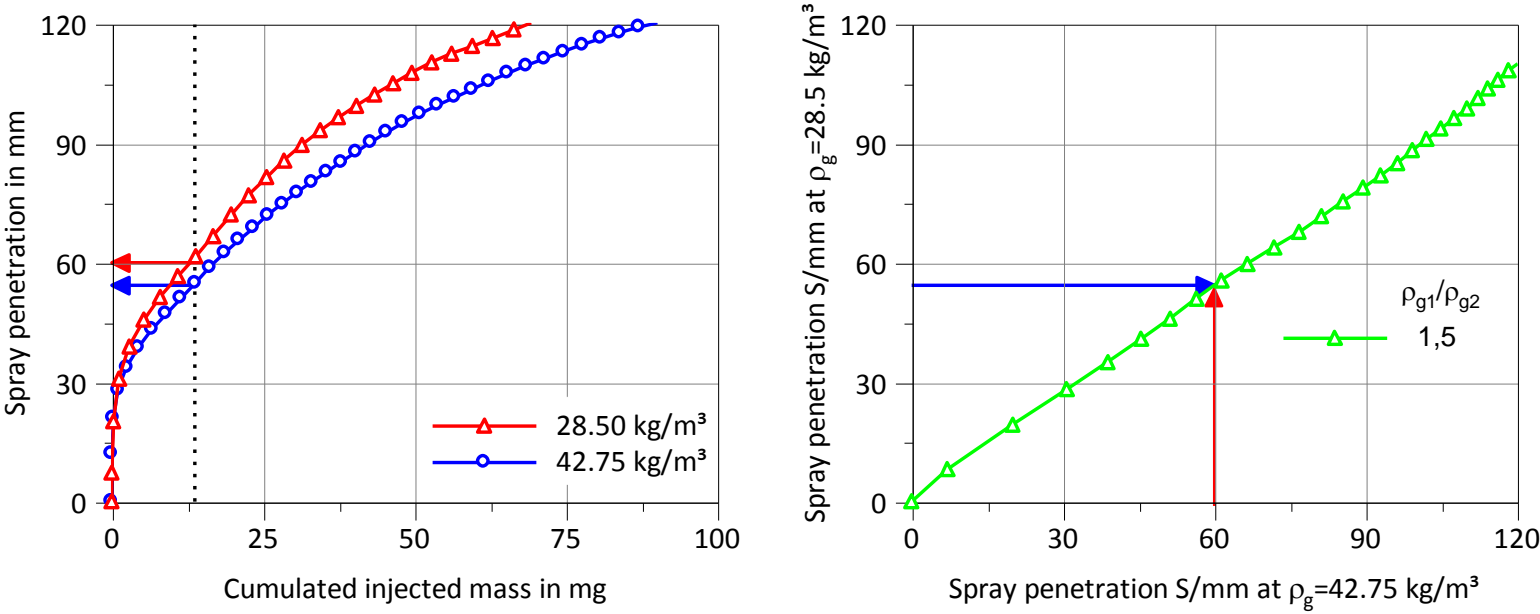

Figure 4. Spray penetration at 28.5 and $42.75 \mathrm{~kg} / \mathrm{m}^{3}$ against the cumulated injected mass (Left), and against each other (Right)

The second phenomenon includes the influence of the gas density on the spray tip penetration. To investigate this influence spray penetration at gas density $\rho_{2}$ (As an example $28.5 \mathrm{~kg} / \mathrm{m}^{3}$ in Figure 4) has been plotted over another penetration at different gas density $\rho_{1}\left(42.75 \mathrm{~kg} / \mathrm{m}^{3}\right.$ in the same figure) at the same cumulated injected mass (notice the red and the blue arrow). The relationship between these two penetrations seems to be linear, as can be seen from the previous figure (Right). The linear curve slope changes with the gas densities and depends only on the density ratio, see Figure 5 .

At the end the spray penetration can be calculated as a function of the cumulated injected mass, the hydraulic flow rate and gas density, equation 1 . The hydraulic flow rate as well as the cumulated mass are taken for one hole not the whole nozzle. This means, that the used cumulated injected mass should be equal to the cumulated injected mass of the whole nozzle divided by number of the nozzle holes. The model coefficients from equation 1 were determined to be $a=265.7, b=0.445$ and $c=-0.387$ with fuel temperature of $40^{\circ} \mathrm{C}$. It can be shown that the coefficients are the same for all analyzed nozzles and injectors. More details about the deviation of equation 1 and its validation can be found in [1].

$$
S_{\rho_{g}}=a \cdot\left(\frac{m_{f}}{\sqrt{Q_{h y d}}}\right)^{b} \cdot \rho_{g}^{c}
$$


Under the assumption that the injection rate is constant and using for example the model from Naber and Siebers [19], which is based on momentum conservation, a similar equation can be derived, equation 2 , which validates this model.

$$
S_{\rho_{g}} \propto\left(\frac{m_{f}}{\sqrt{Q_{h y d}}}\right)^{b 1} \cdot\left(\rho_{f} \cdot \rho_{g}\right)^{c 1}
$$

The coefficients $b_{1}$ and $c_{1}$ are 0.5 and -0.25 respectively, and as can be noticed they are similar to empirical values in equation 1 . Although that the fluid density $\rho_{f}$ shows up explicitly in equation 2 , it is expected a better agreement between the measured and using equation 1 calculated penetrations. The reason for this lies in considering the transient behaviour of the injection rate during the opening and closing phase and thus the hydraulic of the injector. Furthermore, equation 1 considers implicitly not only the fuel density but also its surface tension by the coefficient $a$. This dependency will be explained later, see equation 3. Moreover the coefficient $a$ has the same value for all nozzles and remains the same for different injector types and designs [1], which is not the case in equation 2.

Equation 1 was developed and validated for diesel fuel at fuel temperature of $40^{\circ} \mathrm{C}$. To use this model with other fuels and/or at different fluid temperature the model coefficients should be modified.

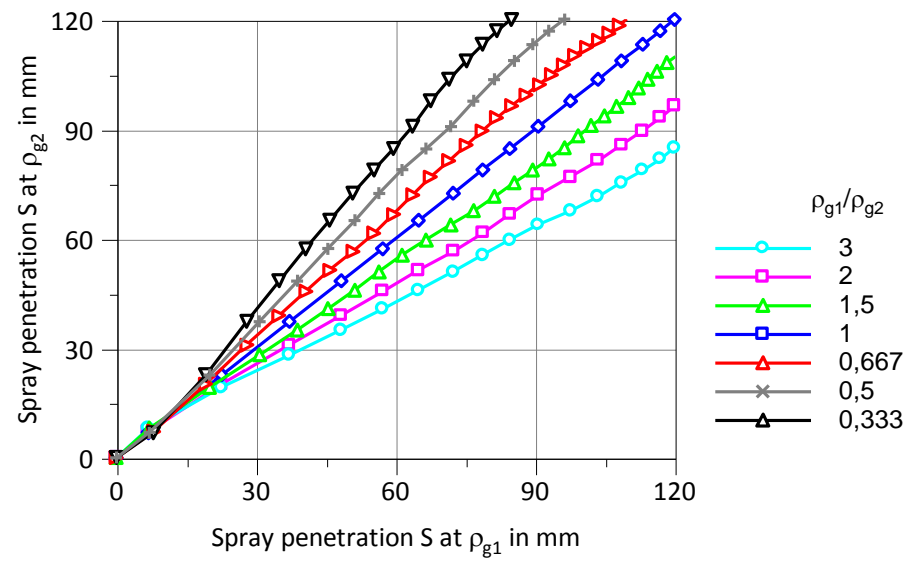

Figure 5. Correlation of spray penetrations at different gas densities at times of equal cumulated mass
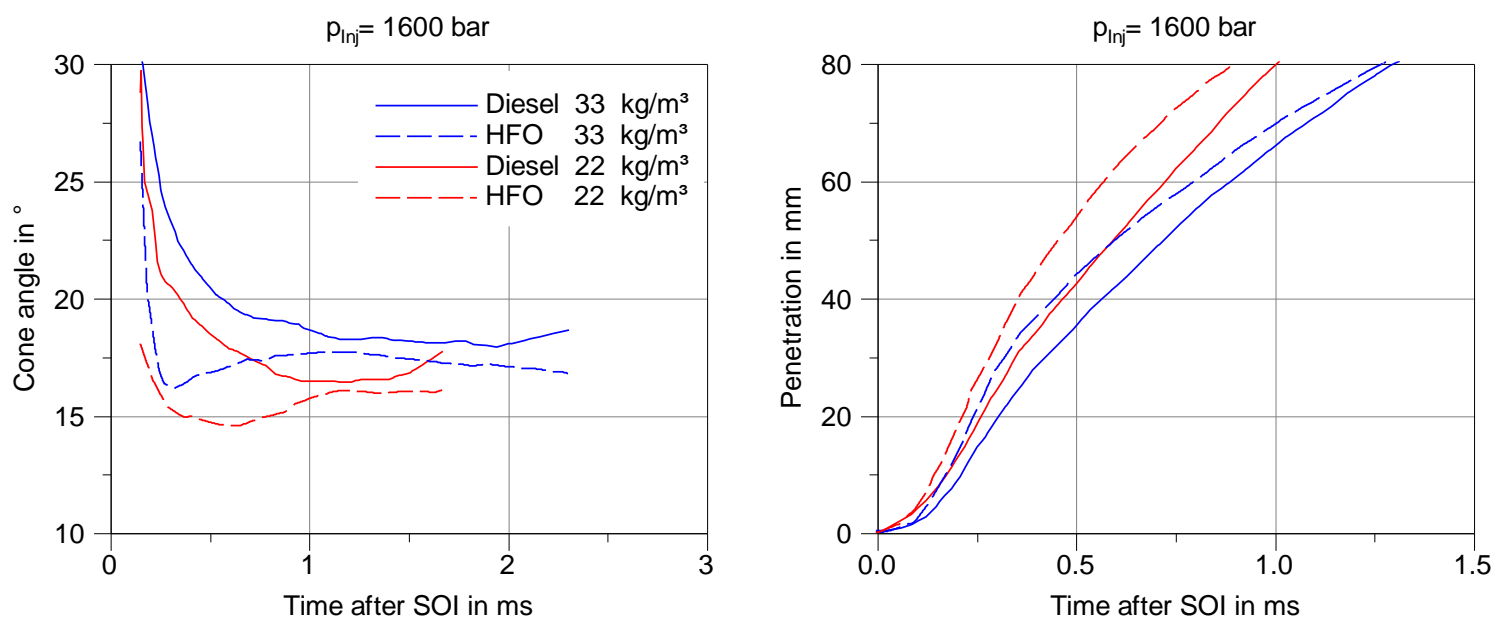

Figure 6. Cone angle and spray penetrations of the two fuels at varying gas densities and 1600 bar injection pressure

\section{Experimental and numerical results}

Figure 6 presents the development of the measured spray tip penetration and cone angle of diesel fuel and HFO at 1600 bar and two gas densities 22 and $33 \mathrm{~kg} / \mathrm{m}^{3}$. The standard deviation of the measurement is lower than $2 \mathrm{~mm}$ and $1.5^{\circ}$ for spray penetration and cone angle respectively. Due to the high surface tension i.e. low We-number of HFO its primary break up results in bigger droplets and ligaments with more inertia than by diesel fuel. This leads to a longer spray penetration. However, the diesel obtains a higher cone angle. To explain this the nozzle flow has been simulated using the commercial CFD-code AVL-FIRE. 
To calculate the needle lift curve, which is needed for the nozzle flow simulation, a 1D-hydraulic-model of the injector was developed using the program AVL BOOST HYDSIM and subsequently calibrated with utilizing the measurements on the injection rate analyzer. Figure 7 presents a comparison of a calculated and measured injection rate and also a comparison between calculated and measured injected mass at different injector actuation time. As can be seen, the data agrees very well. The deviation of the cumulated injection mass is less than $5 \%$ which lies in the range of the measurement accuracy.

The CFD-simulation of the nozzle flow shows that turbulent kinetic energy at the nozzle outlet in diesel case is much larger than in HFO-case, Figure 8. The high viscosity of the heavy fuel oil suppresses the formation of cavitation. In diesel case the fuel vapour reaches the hole outlet, Figure 8. Collapsing of the cavitation bubbles in diesel case enhances the turbulence and the radial components of the velocity which increase the initial angle of the spray. Furthermore the low value of turbulent kinetic energy and the high viscosity of the heavy fuel lead to a compact spray core with a lower air entrainment in the spray and thus to a low cone angle value.
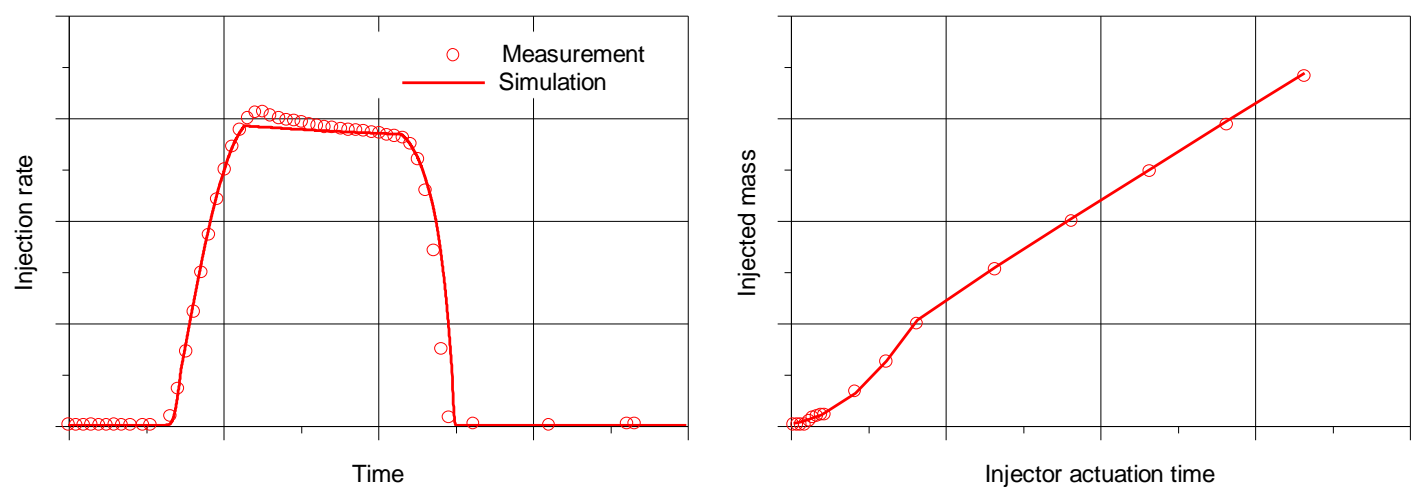

Figure 7. Comparison of the measured and calculated injection rate (Left) and the injected mass at different actuation time
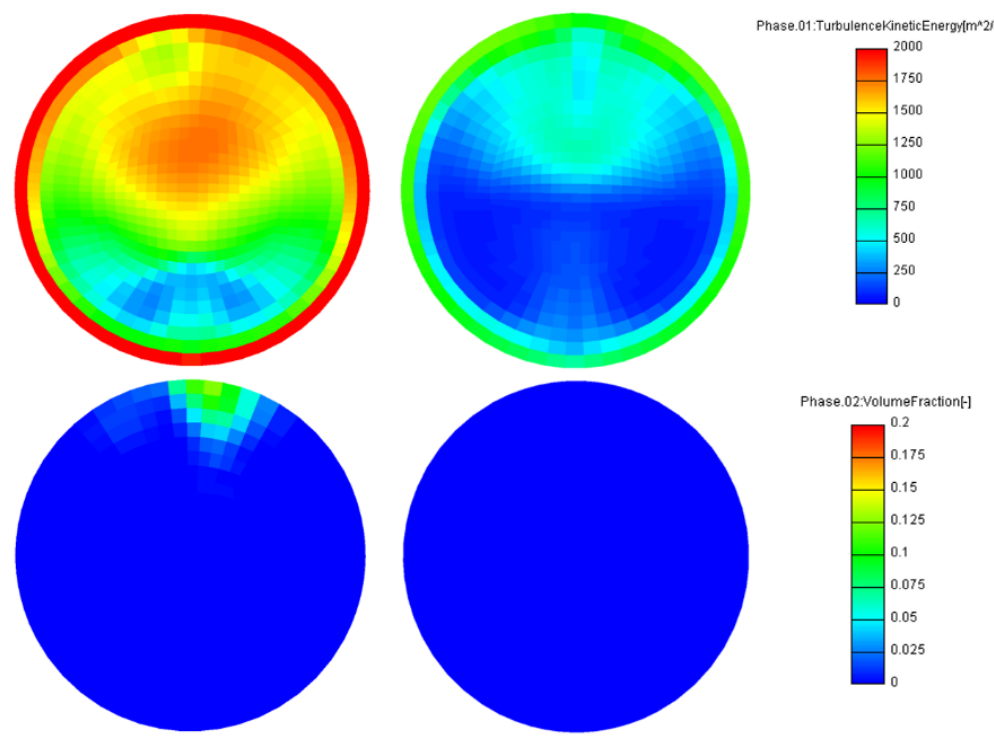

Figure 8. Turbulent kinetic energy and vapour volume fraction at nozzle outlet for diesel case (Left) and for HFO (Right)

\section{Modification of the 1D-modell to predict the HFO-spray tip penetration}

Figure 9 presents the measured spray tip penetration of the HFO by 1600 and 1800 bar injection pressure and $33 \mathrm{~kg} / \mathrm{m}^{3}$ gas density and of diesel at the same density. The results are plotted in the mass domain. As can be seen from this figure, the HFO curves are nearly identical, which confirms that the first phenomenon of the 1D-Model is also valid for HFO. From the same figure is to be noticed that the HFO-curves differ from the diesel one. This means that the parameter $(a)$ and $(b)$ in equation 1 should be modified for HFO case. The new values for $(a)$ and $(b)$ are 275 and 0.37 respectively.

To calculate the influence of the gas density on the spray penetration in HFO-case a spray penetration at gas density $\rho_{1}$ has been plotted over another one at different gas density $\rho_{2}$ but at the same cumulated injected mass 
(similar to Figure 4). The correlation between the sprays is linear and has the same slope like in diesel case, which means that the influence of the gas density on the spray tip penetration is the same for both fuels and do not depend on the fuel properties, Figure 10.

Figure 11 contains a representative selection of spray penetrations at different operating points, calculated from the measured injection rates using modified parameters for HFO and measured directly within the high-pressure injection chamber. As the comparison shows, the calculated and measured data agree very well.

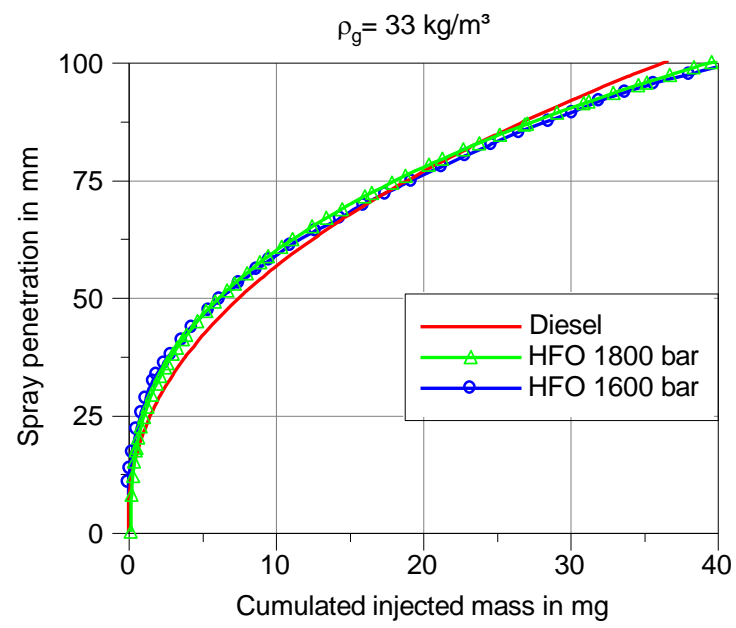

Figure 9. Spray tip penetration against the cumulated injected mass for diesel and HFO

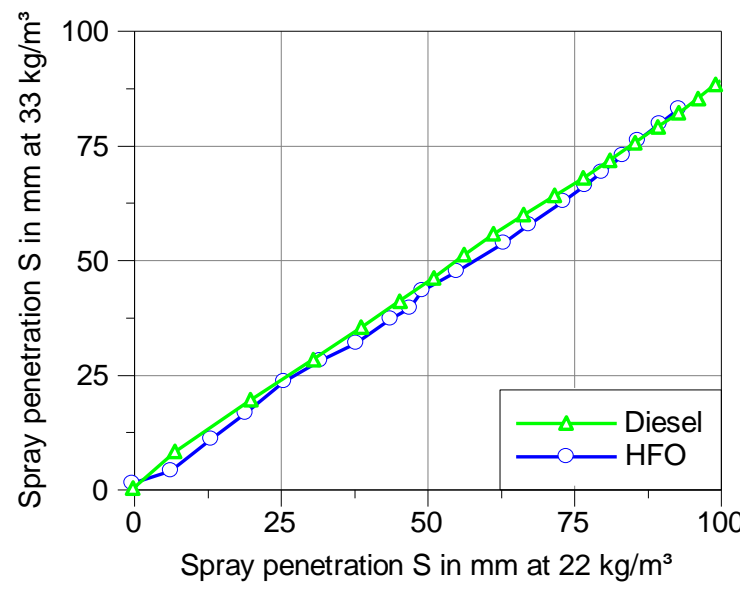

Figure 10. Correlation of spray penetrations at different gas densities at times of equal cumulated mass for diesel and HFO
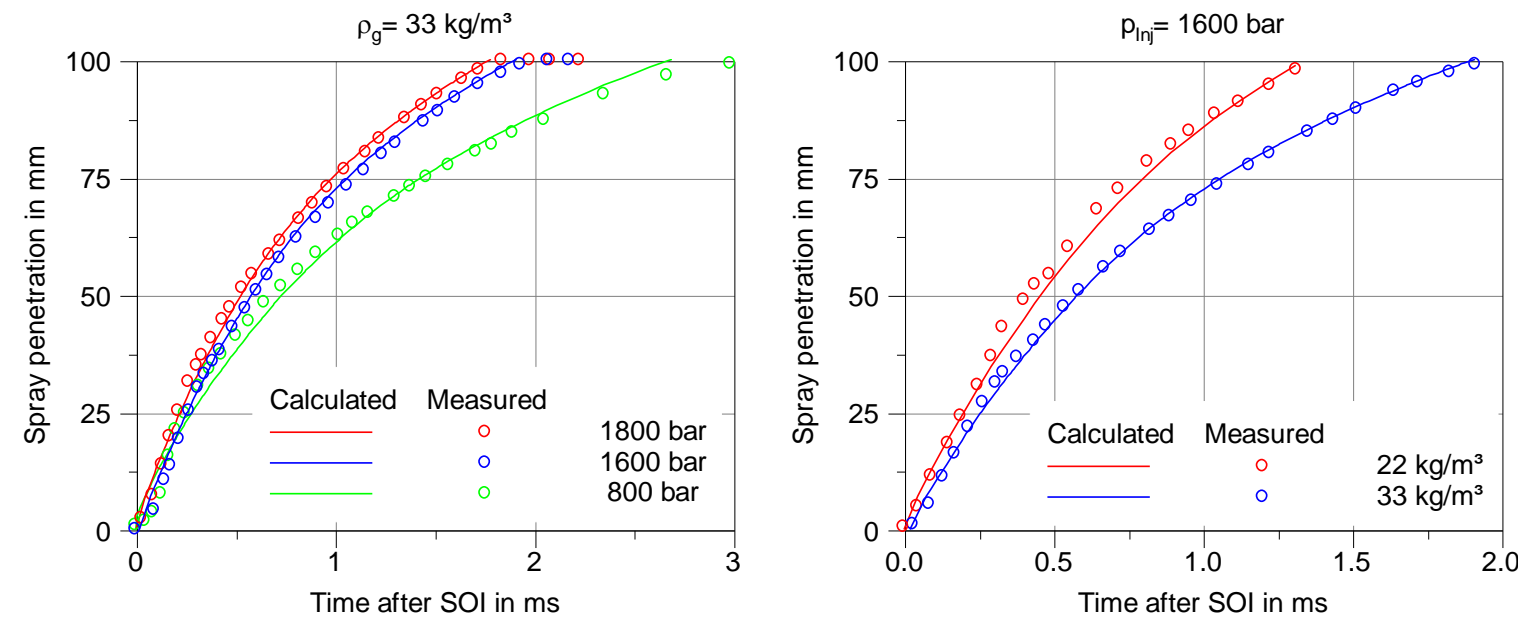

Figure 11. A comparison between the calculated and measured HFO-spray penetration for different injection pressure at 33 $\mathrm{kg} / \mathrm{m}^{3}$ gas density (Left) and for two gas densities at 1600 bar injection pressure (Right)

This work is licensed under a Creative Commons 4.0 International License(CC BY-NC-ND 4.0). 


\section{Prediction of spray tip penetration at fuel with different fuel temperature}

Temperature dependency of diesel properties is known and investigated by several researchers e.g. [10] and [11]. As the temperature increases both the fluid density and viscosity decrease. This leads to an enhanced break up because of the high value of Weber number [12]. In [12] the authors studied numerically the influence of the fuel temperature on the spray length. To calculate the tip penetration under different fuel temperatures the authors in [12] assumed that the parameters $(b)$ and $(c)$ are temperature independent and suggested a simple correlation to predict the parameter $\left(a_{T}\right)$ at fuel temperature $\mathrm{T}$ as a function of $(a)$ and Weber number at a reference temperature $T_{0}=313 \mathrm{~K}$, equation 3 .

$$
a_{T}=a_{T_{0}} \cdot \sqrt{\frac{W e_{T_{0}}}{W e_{T}}}=a_{T_{0}} \cdot \sqrt{\frac{\rho_{T_{0}}}{\sigma_{T_{0}}} \cdot \frac{\sigma_{T}}{\rho_{T}}}
$$

To validate this equation for diesel fuel experimental data from Engine Combustion Network (ECN) are used. The measured data are located on [13]. On the home page a numerous experimental data with different parameter like fuel type, nozzle hole diameter, gas density and temperature are available. Figure 12 presents a comparison between the data from ECN at fluid temperature of $450 \mathrm{~K}$ and using equation 1 and 3 calculated spray length. Using equation 3 the calculated value of $\left(a_{T}\right)$ is 225. The left diagram in Figure 12 shows the good agreement between ECN and calculated curves for different nozzles. This high ability to calculate the penetration for different nozzles is explained by considering the injection rate and thus the injector dynamic within the opening phase. Further comparisons, carried out at 1400 bar injection pressure, $0.33 \mathrm{~mm}$ outlet diameter of the injection hole and different gas densities confirm the high accuracy of the model, see the right diagram in Figure 12.
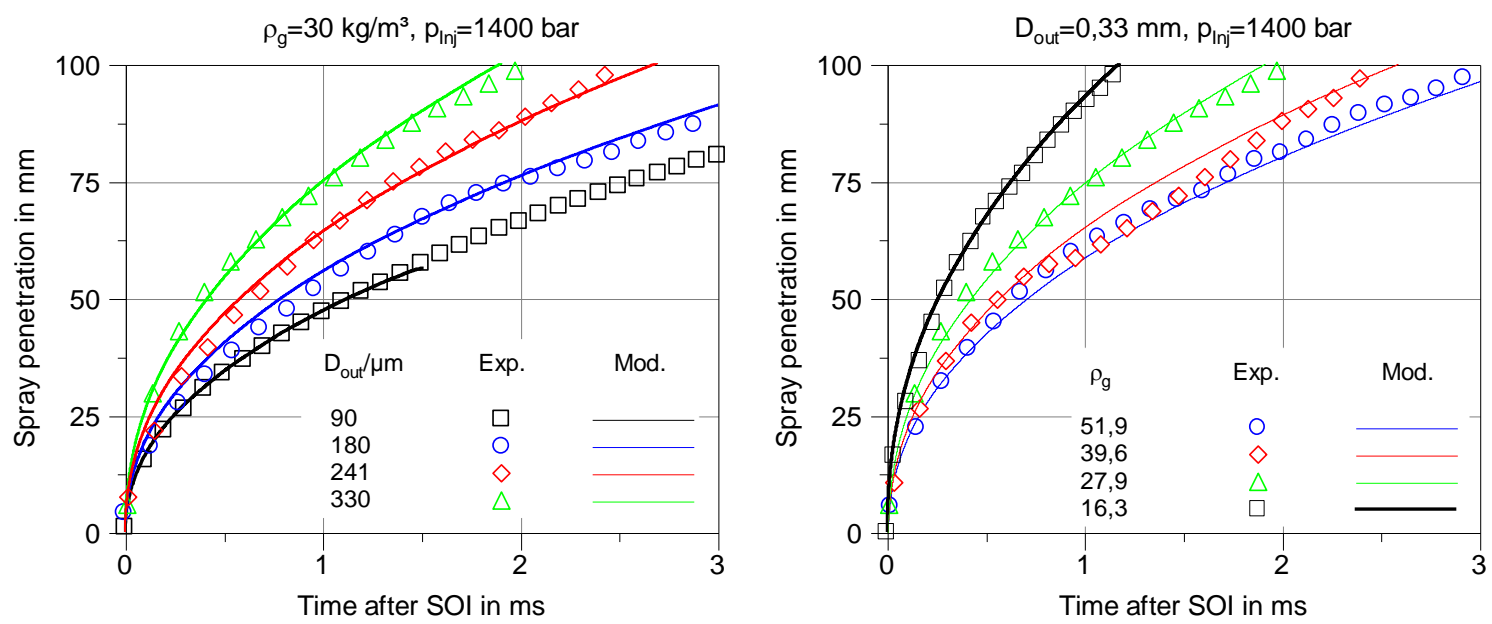

Figure 12. Measured and calculated spray penetration for different nozzles (Left) and various gas densities (Right)

\section{Conclusions}

In the course of the development of an engine, combustion engineers have great interest in a simple mean to predict the spray penetration within the cylinder. A flow rate analyser has been used to understand the hydraulic behaviour of the injector under different fuels. The influence of fuel on the mixture formation under none evaporating conditions has been studied using a high-pressure/high-temperature chamber of the Institute of Piston Machines and Internal Combustion Engines at Rostock University. The measurements emphasize that the fuel properties influence the spray break up and furthermore the spray length and cone angle.

To calculate the spray length with HFO an existing model has been modified and used. This model is originally developed for diesel sprays from large injectors and is based on two phenomena which include in particular the behaviour of the spray in mass domain and the correlation between two sprays at different gas densities. At the beginning these two phenomena have been verified for the HFO. The results show that the influence of the gas density on the spray length is independent from the fuel type. On the other hand the two other parameters which describe the influence of the fuel properties on the break-up had to be modified. Using the new values of these parameters sprays at various rail pressures and gas densities could be calculated for HFO. The results agree very well with the measurements. Because of the strong influence of the fluid temperature on the fuel properties and therefore on the spray penetration a correlation to calculate the first parameter in the model $(a)$ is investigated. The results has been validated utilizing experimental data from ECN. The comparison shows the high prediction accuracy of the model for a wide range of conditions. 
Furthermore the influence of the fuel type on the spray cone angle is experimentally investigated. To explain the results a 1D-model of the measured injector was coupled with a 3D-CFD-simulation of the nozzle internal hole. Because of the low values of the turbulent kinetic energy and Weber number HFO-spray-core will be much more compact than by diesel, which leads to poor air entrainment in the spray and to a smaller spray cone angle.

\section{Acknowledgements}

The presented results are part of the research project "Multiple Injection Strategies for Optimizing Mixture Formation and Combustion in Large Diesel Engines to meet lowest $\mathrm{CO}_{2}$ and Pollutant Emissions using Maritime Fuels". We would like to thank the German Research Fondation (DFG) for funding this project. We would also like to thank Bosch company for their assistance.

\section{Nomenclature}

\begin{tabular}{|c|c|c|c|}
\hline$a, b$ and $c$ & model coefficients & $T$ & fuel temperature $[\mathrm{K}]$ \\
\hline$a_{T}$ & model coefficient $(a)$ at fuel temperature $\mathrm{T}$ & $T_{g}$ & gas temperature $[\mathrm{K}]$ \\
\hline$D_{\text {out }}$ & hole outlet diameter & $\rho_{f}$ & fuel density [kg/m³] \\
\hline$S$ & spray tip penetration [mm] & $\rho_{g}$ & gas density $\left[\mathrm{kg} / \mathrm{m}^{3}\right]$ \\
\hline$S_{\rho_{g}}$ & $\begin{array}{l}\text { spray tip penetration at gas density } \rho_{g} \\
{[\mathrm{~mm}]}\end{array}$ & $m_{f}$ & $\begin{array}{l}\text { cumulated injected mass from one nozzle } \\
\text { hole }[\mathrm{mg}]\end{array}$ \\
\hline$Q_{\text {hyd }}$ & $\begin{array}{l}\text { hydraulic flow rate from one nozzle hole } \\
{\left[\mathrm{cm}^{3} / 30 \mathrm{~s} @ 100 \text { bar }\right]}\end{array}$ & $p_{\text {Inj }}$ & injection pressure [bar] \\
\hline $\mathrm{CO}_{2}$ & Carbon dioxide & $W e$ & Weber number [-] \\
\hline$N O_{x}$ & Nitrogen oxide & $\sigma$ & surface tension $[\mathrm{N} / \mathrm{m}]$ \\
\hline NECAs & $\mathrm{NO}_{x}$ Emission Control Areas & $D K$ & diesel fuel \\
\hline$E C N$ & Engine Combustion Network & HFO & heavy fuel oil \\
\hline SOI & Start of Injection & & \\
\hline
\end{tabular}

\section{References}

[1] Najar, I., Fink, C., Pinkert, F., Harndorf, H., 2014, October 2014, 26th European Conference on Liquid Atomization and Spray Systems

[2] Gostic, I., Senghaas, C., Willmann, M., Wensing, M., September 2010, 23th European Conference on Liquid Atomization and Spray Systems.

[3] Hüttl, C., Leidenberger, U., Brüggemann, D., September 2010, 23th European Conference on Liquid Atomization and Spray Systems.

[4] Heilig, A., Kaiser, M., Dinkelacker, F., Sep. 5.-7. 2011, 24th European Conference on Liquid Atomization and Spray Systems.

[5] Grimaldi, C., Postrioti, L., SAE Technical Paper 2000-01-1252, 2000, doi: 10.4271/2000-01-1252.

[6] Rostock University, BMWi-Project, Fuel and Air Management for Emission reduction, Final report

[7] Fink, C., Rabe, J. R., Drescher, M., Harndorf, H., Sep. , 2012, $2^{\text {nd }}$ Rostocker Großmotorentagung

[8] Bosch, W., 1964, , MTZ-MotortechnischeZeitschrift, 25(7)

[9] Fink, C., Pinkert, F., Harndorf, H., Sep. 5.-7. 2011, 24th European Conference on Liquid Atomization and Spray Systems.

[10] Payri, R., Salvador,F.J., Gimeno, J., Bracho, G., Fuel 90 (2011) 1172-1180

[11] Safarov, J., Hassel, U., Shahverdiyev, 27-29 June 2017, 11th International Colloquium Fuels

[12] Najar I., Fink, C., Stengel, B., Buchholz, B., Drescher, M., Harndorf, H., Hassel, E., March. 16.-17. 2017. $13^{\text {th }}$ Engine combustion processes

[13] Engine Combustion Network, https://ecn.sandia.gov/ecn-data-search/?nam=1

[14] Dernotte, J., Hespel, C., Foucher, F., Houillé, S., Mounaïm-Rousselle, C., Sep. 5.-7. 2011, 24th European Conference on Liquid Atomization and Spray Systems.

[15] Desantes, J., Arrègle, J., Pastor, J., Delage, A., SAE Technical Paper 980802, 1998, doi:10.4271/980802

[16] Desantes, J., Payri, R., Garcia, A., Manin, J., Energy \& Fuels, 23 (6) (2009) 3227\{3235, ISSN 0887-0624, doi:nbibinfofdoigf10.1021/ef801102wg.

[17] Payri, R., Salvador,F.J., Carreres, M., De la Morena, J., Energy Conversion and Management 114 (2016) 376\{391, ISSN 01968904, doi:nbibinfofdoigf10.1016/j.enconman.2016.02.043g.

[18] Chang, C., Farrell, P., SAE Technical Paper 970353, 1997, doi:10.4271/970353.

[19] Naber, J. and Siebers, D., SAE Technical Paper 960034, 1996, doi:10.4271/960034. 


\title{
Characteristics of Control Piston Motion and Pressure Inside of a Common Rail Diesel Injector
}

\author{
Sebastian Schuckert*1, Georg Wachtmeister ${ }^{1}$ \\ ${ }^{1}$ Chair of Internal Combustion Engines, Technical University of Munich, Germany \\ ${ }^{*}$ Corresponding author: schuckert@Ivk.mw.tum.de, ORCID ID: 0000-0002-8668-7733
}

\begin{abstract}
In this paper the experimental setup of a commercial third generation common rail solenoid injector with advanced measurement is discussed. The motion of the control piston is measured while performing injection rate investigations using a purpose-built injection rate analyzer of the Bosch type. At the same time fuel pressure in the feed line of the nozzle is gauged and contrasted to fuel pressure before the inlet connector.

In contrast to the steady rise observed in a similar study, the motion of the control piston in this case is characterized by a changing gradient in the upward movement. The magnitude of the negative displacement of the upper part of the control piston due to the fuel pressure in the control volume corresponds to simulation results of the elastic deformation.

Pressure before the inlet connector and pressure in the feed line exhibit a similar course with a difference in magnitude that is rising with higher rail pressures. Precisely with the end of injection the pressure in the feed line surpasses the pressure before the inlet connector for a short moment. The measurement results of control piston motion and pressure inside the injector are of particular interest because these parameters are to serve as indicators for changes in the injection rate caused by phenomena like wear and coking amongst others.
\end{abstract}

\section{Keywords}

common rail injector, injection rate measurement, eddy current sensor, control piston motion, feed line pressure

\section{Introduction}

The act of injecting diesel not only supplies the fuel for the subsequent combustion, but at the same time also determines the start of combustion with the diesel combustion process. This is unlike the gasoline combustion process, where injection and ignition are separated. Thus, the injection process is a major influence factor to consider in order complying with the increasingly severe emission legislation for compression ignition engines.

Furthermore, alterations affecting the injection parameters of common rail diesel injectors that emerge during engine operation have been identified. Research efforts focused on brittle external nozzle deposits mostly consisting of carbon referred to as coking. In recent years, a type of sticky, the so-called internal diesel injector deposits (IDID) have appeared in production engines and caused needles to stick in common rail diesel injectors. It has been observed that the addition of certain additives (polyisobutylene succinimide; PIBSI) to diesel fuel, which are to inhibit the formation of coking deposits, have the side effect of contributing to the formation of IDID [1][2]. These PIBSI react with acids that emerge from fatty acid methyl ester (FAME), a biodiesel supplement to common diesel in the European Union, to form IDID. Other major factors that influence the occurrence of IDID are high fuel temperatures and the content of aromatics and oxygen in the diesel fuel [2].

Deposits on the nozzle tip and inside the nozzle holes due to coking cause numerous adverse effects. It is proven that this type of deposits exerts a great influence on the injection rate of an injector, impairs the break-up of the fuel spray and leads to an inferior mixing of fuel and air in the combustion chamber [3]. In addition, coked nozzles exhibit larger cyclic variations of cylinder peak pressure than clean nozzles, even though the visible spray pattern appears unchanged [4]. Ultimately, external deposits lead to a deterioration of combustion efficiency, a decrease in rated power and cause a rise in brake specific fuel consumption [5]. The formation of coking deposits is driven by higher temperatures at the nozzle and in its spray holes, while cavitation inside the nozzle serves as an inhibitor to their built-up [6][7]. Cavitation inside the nozzle induced by a lower discharge coefficient proved to be beneficial against coking. A positive effect also applies to high spray hole conicity due to a built-up of deposits which is predominant in the inlet region of the hole [6]. In summary, coking deposits will be a key influencing parameter for future nozzle designs [8].

Legislative constraints together with the problem of internal and external injector deposits have led to the development of systems that are to maintain optimal fuel consumption and emissions optimal over the entire engine lifetime [9][10][11][12]. These closed-loop control strategies have been presented for both light [9][12] and heavy duty engines [11]. All these principles have in common that the fuel pressure inside the injector is measured and 
evaluated applying pressure transducers to the injectors [9][11] or utilizing the piezo-actuator of the injector itself to calculate the inside pressure [12]. Algorithms are utilized to determine key injection parameters like start and end of injection as well as the maximum injection rate and quantity [10]. These injection controls enable a very high injection quantity accuracy and close timings between pilot and main injection that are favorable for reducing nitrogen oxide emissions [9].

It is postulated that these closed-loop control systems allow to compensate for detrimental effects like wear or coking by maintaining injection rate and quantities over engine lifetime [10][11]. The German Research Foundation has funded a project at Technical University of Munich to investigate the influence of these alterations like wear and coking onto critical parameters as the injection rate and eventually the engine-out emissions. The key goal is to find parameters related to the deterioration of the injection rate and to develop an open-loop control that enables to restore the desired injection parameters. The model approach for the identification of these aging effects is presented in [13].

\section{Experimental Setup}

The measurement setup consists of a third generation common rail diesel injector that is equipped with multiple sensors. A solenoid valve in the upper part regulates the start and end of injection by opening and closing the discharge throttle of the control volume. When the solenoid is energized, diesel flows out of the control volume through the discharge throttle and less fuel is flowing in through the inlet throttle, which possesses a smaller diameter. Hence, fluid pressure inside the control volume decreases. The upward force generated by the rail pressure on the annular upper part of the nozzle needle exceeds the downward force of the control volume pressure, which is acting on the top area of the control piston. This difference in forces leads to the lift of the needle, which in turn drives the control piston. When the energizing of the solenoid ends, the downward force of the control volume pressure together with the nozzle spring force surpasses the nozzle sac pressure force. The nozzle subsequently closes and injection ends.

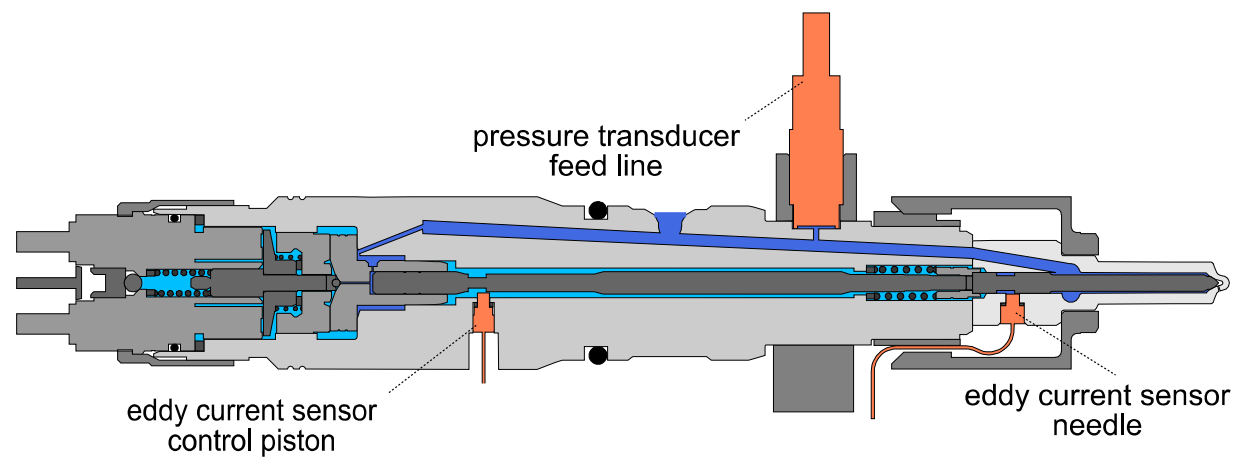

Figure 1. Schematic sensor setup of the modified solenoid injector (note: some sensors and lines are projected to the section plane for clarity)

For the measurement of needle or control piston motion measurement principles like opto-electronical sensors have been presented [14]. Furthermore, a solution to detect needle opening and closing by employing an electric circuit, which is closed by the needle in its extreme positions, has been introduced [15]. Nevertheless, as they prove high robustness and a high signal-to-noise ratio, two eddy current sensors are utilized to measure the motions of control piston and needle, respectively. A piezo-resistive pressure transducer gauges the fuel pressure inside the feed line of the injector (see Figure 1). The sensors are mounted in different parts of the injector for measuring independent parameters. These parameters in turn are the basis for the development of an open-loop control compensating for alterations of injection behavior caused by aging effects.

The linear measurement range of the eddy current sensors of $0.5 \mathrm{~mm}$ is not significantly larger than the lift of both control piston and needle with $0.35 \mathrm{~mm}$ and previous measurements showed an overlay of control piston motion and its deformation caused by fluid pressure. To clarify the extent of this deformation those two components are simulated using the finite element method.

The simulation model uses a force (D) to represent the diesel fluid acting downward on top of the control piston with rail pressure. The upper part of the piston that is guided in the control volume unit is represented by a frictionless support (B). All inside faces of the nozzle are pressurized with rail pressure $(E)$ while the ring-shaped outside face serves as a fixed support (A). The needle is guided frictionless inside the nozzle (F) and the needle spring force (C) acts on top of the needle (see Figure 2). 
Simulation results show that outfitting both control piston and needle with circular grooves does not have a significant effect on their deformation compared to their unaltered counterparts in the production injector (see Figure 2). These grooves are necessary for the measuring principle of the eddy current sensor. The simulation proves that the stiffness properties of the two moving parts remain unchanged, thus the injection process is left uninfluenced. As the static deformation is barely greater than the difference between linear measurement range of the eddy current sensor and the operational lift of the control piston (for the needle it is considerably smaller), the eddy current sensors are able to measure both deformation and lift of control piston and needle in the linear measurement range.
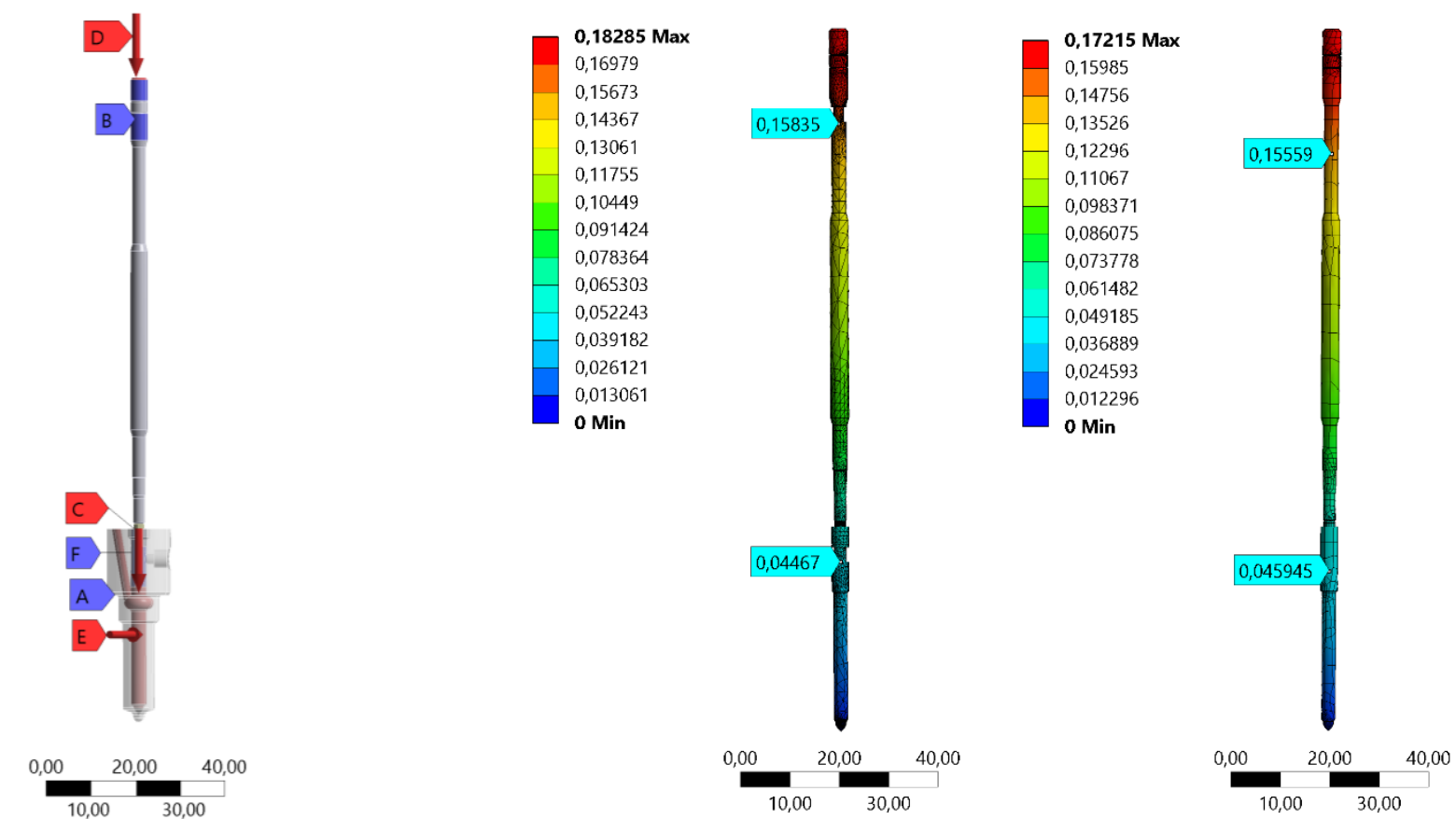

Figure 2. Comparison of needle $\boldsymbol{x}_{N}$ and control piston $\boldsymbol{x}_{C p}$ deformations due to rail pressure $\boldsymbol{p}_{\text {Railset }}=180 \mathrm{MPa}$ (left: boundary conditions of finite element simulation; middle: needle and control piston for lift measurements; right: unaltered needle and control piston)

A custom software is used for the data acquisition and the processing of measurement data. The analysis steps are shown in Table 1. The injection rate $\dot{m}$ is derived from measuring the dynamic pressure in the fuel-filled coiled tube using a piezoelectric pressure transducer. Because of this measurement principle, the signal undergoes a drift compensation, a filtering process and a subsequent conversion to a mass-flow using the cross-section of the tube, the speed of sound and the density of diesel (compare [16]).

Table 1. Data processing steps applied to measurement parameters

\begin{tabular}{|c|c|c|c|c|}
\hline Data processing & 1. & 2. & 3. & 4. \\
\hline Parameter & $\begin{array}{l}\text { Drift } \\
\text { compensation }\end{array}$ & Filtering & Conversion & Averaging \\
\hline Injection rate $\dot{m}$ & $\bullet$ & $\bullet$ & $\mathrm{V}$ to $\mathrm{mg} / \mathrm{ms}$ & $\bullet$ \\
\hline Injector inside pressure $p_{I n j}$ & - & $\bullet$ & $\mathrm{V}$ to $\mathrm{MPa}$ & $\bullet$ \\
\hline Measured rail pressure $p_{\text {Rail,mea }}$ & - & $\bullet$ & $\mathrm{V}$ to $\mathrm{MPa}$ & $\bullet$ \\
\hline Control piston lift $x_{C p}$ & - & $\bullet$ & $V$ to $\mathrm{mm}$ & $\bullet$ \\
\hline Needle lift $x_{N}$ & - & $\bullet$ & $V$ to $\mathrm{mm}$ & $\bullet$ \\
\hline
\end{tabular}

All measurements in this paper are performed while measuring the injection rate utilizing a purpose-built injection rate analyzer applying the Bosch working principle [16]. The fuel supply system for the common rail injector consists of a commercial high-pressure system with low-pressure pump, high-pressure pump with suction valve and common rail. Figure 3 outlines the experimental setup and the most import measurement parameters. 
All other signals are filtered likewise with a second order Butterworth filter to reduce high-frequent noise and are converted from current or voltage signal into their physically correct dimension. Generally every signal is averaged using multiple (here: five) single measurements in order to avoid statistically distributed deviating signals.

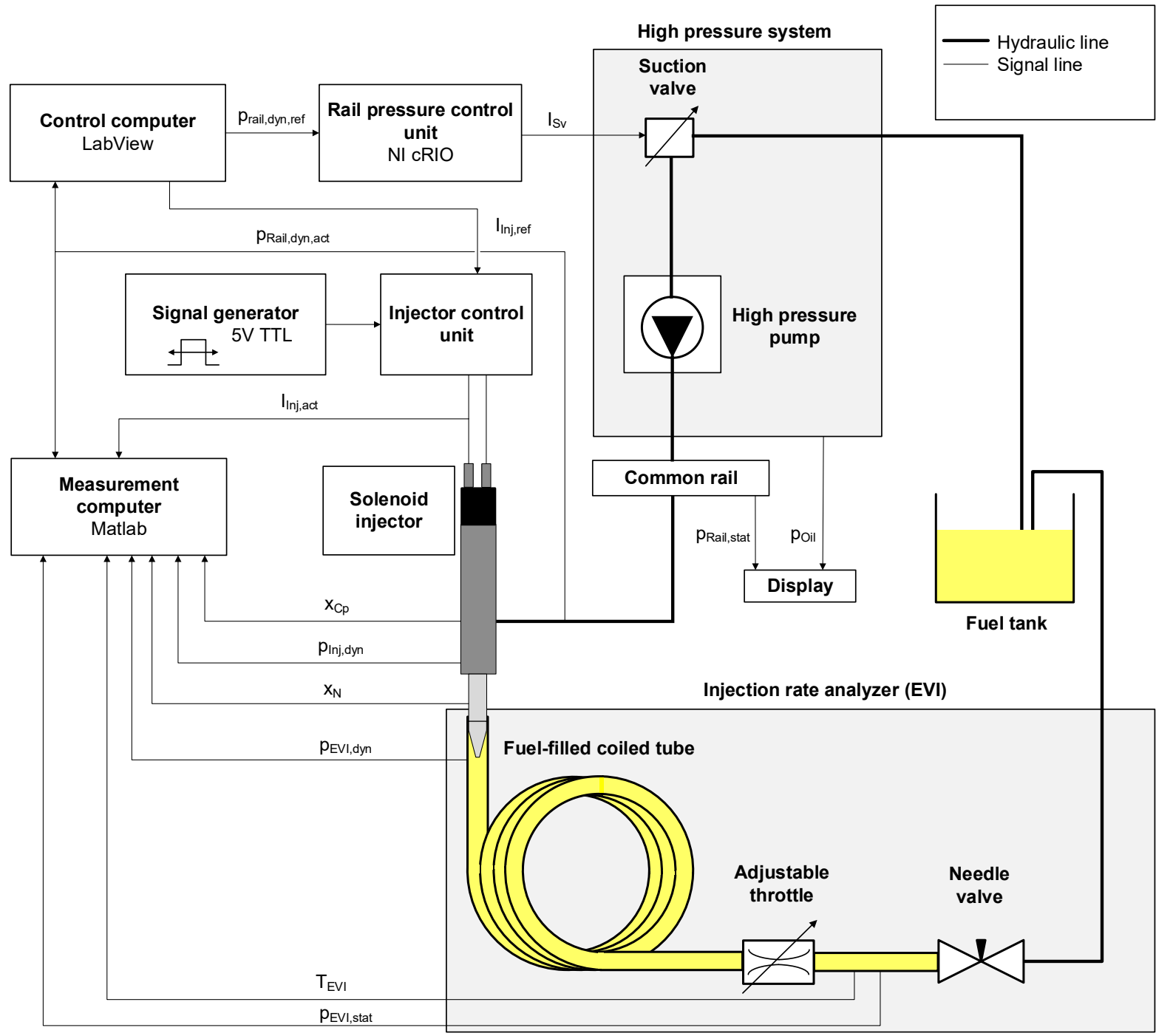

Figure 3. Schematic overview of measurement setup and main parameters

\section{Measurement Results}

Although the motion of the nozzle needle regulates the fluid flow from feed line to nozzle sac, it is neglected in this study. The implementation of the eddy current sensor for measuring control piston motion leaves the external geometry of the injector body unaltered. The nozzle with the applied eddy current sensor changes the external geometry of the injector as it necessitates a different union nut. This union nut is to make room for the minimum bending radius of the wire of the eddy current sensor. In the future, engine investigations will be performed with the developed injector and different nozzles. Under the premise of using the existing cylinder head, no major change in the external geometry of the injector is admissible. Thus, the main aim of the presented investigations is to clarify correlations of control piston motion and fuel pressure at two distinct positions to key injection parameters.

Figure 4 illustrates the measured injection rates for four different set rail pressures. Higher rail pressures cause a steeper increase and an earlier decrease of injection rate while at the same time a rise in maximum injection rate is observed.

The reason for this behavior is the increase in nozzle sac pressure that exerts a higher upward force on the needle. This causes a faster acceleration of the needle and a faster increase of the hydraulic flow area between needle seat and nozzle tip. Ultimately, a higher injection rate results for higher rail pressures at the same instant of time in 
the opening phase of the nozzle needle. The same but inverse causal relationship applies to the closing phase of the needle.

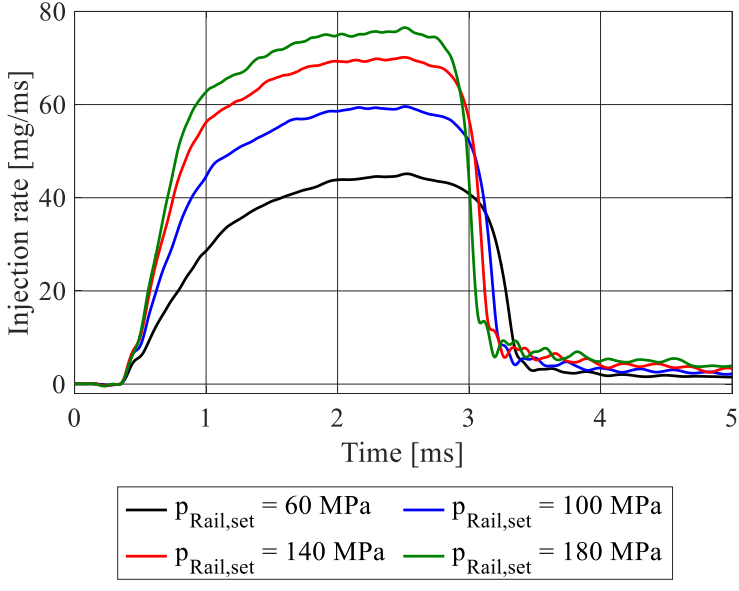

Figure 4. Injection rate profiles of the modified common rail injector utilized for control piston and needle lift measurements $\left(t_{E T}=2.2 \mathrm{~ms}\right)$

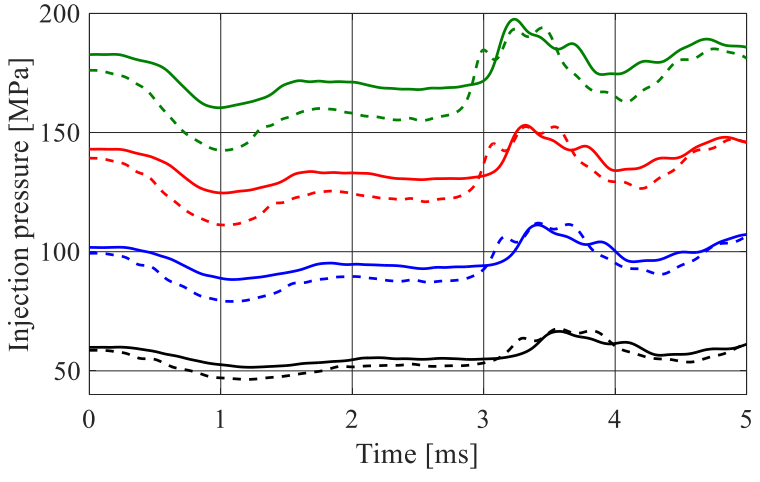

$$
\begin{aligned}
& -\mathrm{p}_{\text {Rail,set }}=60 \mathrm{MPa}-\mathrm{p}_{\text {Rail,set }}=100 \mathrm{MPa} \\
& -\mathrm{p}_{\text {Rail,set }}=140 \mathrm{MPa}-\mathrm{p}_{\text {Rail,set }}=180 \mathrm{MPa}
\end{aligned}
$$

Figure 5. Pressure inside feed line $p_{\text {Inj }}$ (dashed lines) and before inlet connector $p_{\text {Rail,act }}$ (solid lines) $\left(t_{E T}=2.2 \mathrm{~ms}\right.$ )

The maximum injection rate is a function of the difference of common rail pressure and backpressure inside of the injection rate analyzer (see Equation (1) according to [17]). Therefore, it increases with higher common rail pressures as the backpressure is kept constant for all investigations.

$$
\dot{m}=C_{d} \cdot A_{h} \cdot \rho_{f} \cdot \sqrt{\frac{2 \cdot\left(p_{\text {Rail,mea }}-p_{E V I, \text { dyn }}\right)}{\rho_{f}}}
$$

Various studies have demonstrated the feasibility of detecting injection events by analyzing the rail pressure trace [9]. It has also been proven that by subjecting the rail pressure signal to a short-time Fourier analysis, it is possible to discriminate between pilot and main injection as well as to detect injector malfunction [18]. Therefore, both pressure before inlet connector and pressure inside the feed line are evaluated.

The comparison of pressure before the inlet connector and in the feed-line of the injector shows an overlap of both pressures between $3.2 \mathrm{~ms}$ and $3.6 \mathrm{~ms}$. Here the pressure in the feed line reaches the level of the pressure before the inlet connector or even slightly surpasses its level (see Figure 5). The temporal concurrence of the peaks of both measured pressures demonstrates a correlation to the end of injection.

At the end of injection a pressure wave is generated by the closing needle [19]. This pressure wave propagates from the needle seat upstream through feed line and inlet connector. Thus, a local maximum is detectable first in the signal of the pressure transducer in the feed line and only afterwards at the sensor before the inlet connector. When evaluating the temporal difference between these first local maxima on feed line pressure and pressure before inlet connector, a distance of around $400 \mathrm{~mm}$ between the two pressure transducers is calculated (see Table 5 lists the data for the calculation of the distance between the two pressure sensors.

Table 5 in Annex). This is in good agreement with the actual distance between the sensors and supports the thesis of the wave propagation as the cause for the local pressure peaks on both signals.

The difference between the first minimum of the injection rate (used here as end of injection) and the coinciding pressure peaks is in the magnitude of under $100 \mu \mathrm{s}$, while the temporal deviation of the concurrence of pressures peak with regard to the end of injection is under $3 \%$ for rail pressures between 60 and $180 \mathrm{MPa}$ (see Table 2). Higher set rail pressures show a smaller discrepancy between end of injection and concurrence of pressures peak. This temporal accuracy of detecting the end of injection by indirect means is remarkably higher than the one of a different approach, which determines the end of injection by detecting and evaluating the sound-borne noise in engine parts utilizing knock sensors [20].

Furthermore, a method to detect start and end of injection by assessing the derivative of the common rail pressures signal has been presented [19]. The here presented method by regarding the concurrence of the pressure peaks of the two sensors as a detection of end of injection is more precise than that method. Table 2 presents the difference between the maximum derivative of the pressure before the inlet connector, which is only more accurate for a set rail pressure of $60 \mathrm{MPa}$. 
Table 2. Assessment of correlation between injection rate and pressure inside feed line $\left(\boldsymbol{t}_{\boldsymbol{E} T}=2.2 \mathrm{~ms}\right)$

\begin{tabular}{|c|c|c|c|c|c|c|c|}
\hline $\begin{array}{l}\text { Set rail } \\
\text { pressure } \\
p_{\text {Rail,set }} \\
{[\mathrm{MPa}]}\end{array}$ & $\begin{array}{l}\text { 1. Minimum } \\
\text { of control } \\
\text { piston lift } \\
t_{M c p} \\
{[\mathrm{~ms}]}\end{array}$ & $\begin{array}{l}\text { 1. Minimum } \\
\text { of injection } \\
\text { rate } t_{\text {Mir }} \\
\text { [ms] }\end{array}$ & $\begin{array}{l}\text { Concur- } \\
\text { rence of } \\
\text { pressure } \\
\text { peaks } t_{C p p} \\
{[\mathrm{~ms}]}\end{array}$ & $\begin{array}{l}\text { Difference } \\
t_{D i f 1}= \\
t_{C p p}-t_{M i r} \\
{[\mathrm{~ms}]}\end{array}$ & $\begin{array}{l}\text { Deviation } \\
t_{D e v}= \\
t_{D i f} / t_{D i} \\
{[\%]}\end{array}$ & $\begin{array}{l}\text { Max. derivative } \\
\text { of pressure } \\
\text { before inlet } \\
\text { connector } \\
t_{M d p} \\
{[\%]}\end{array}$ & $\begin{array}{l}\text { Difference } \\
t_{D i f 2}= \\
t_{M d p}-t_{M i r} \\
{[\mathrm{~ms}]}\end{array}$ \\
\hline 60 & 3.448 & 3.485 & 3.568 & 0.083 & 2.64 & 3.432 & -0.053 \\
\hline 100 & 3.323 & 3.350 & 3.415 & 0.065 & 2.17 & 3.285 & -0.065 \\
\hline 140 & 3.245 & 3.273 & 3.323 & 0.050 & 1.71 & 3.192 & -0.081 \\
\hline 180 & 3.175 & 3.195 & 3.240 & 0.045 & 1.58 & 3.112 & -0.083 \\
\hline
\end{tabular}

Figure 6 shows that the gradient in control piston upward motion is considerably steeper for higher set rail pressures. Thus, the maximum lift is attained earlier and maintained for a longer period of time. The comparison of control piston motions with another investigation [21], in which an eddy current sensor is utilized, reveals a remarkable difference as in this study there is no change in gradient in the upward motion (see Figure 7).

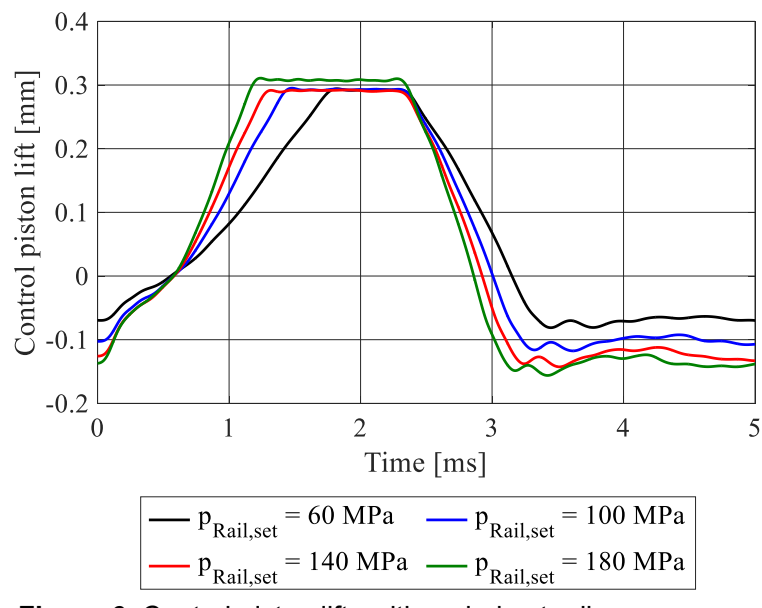

Figure 6. Control piston lifts with varied set rail pressures $p_{\text {Rail }, \text { set }}\left(t_{E T}=2.2 \mathrm{~ms}\right)$

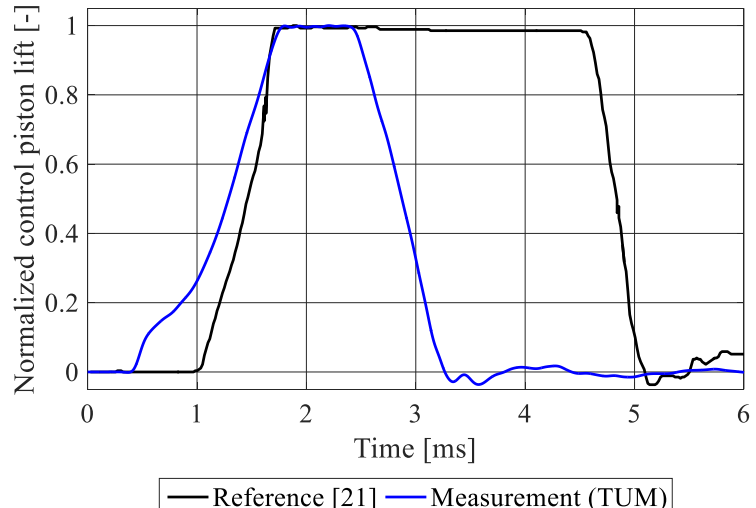

Figure 7. Comparison of control piston lift of reference [21] and measured control piston lift $\left(p_{\text {Rail,set }}=140 \mathrm{MPa}, t_{E T, T U M}\right.$ $\left.=2.2 \mathrm{~ms}, t_{E T,[21]}=2.0 \mathrm{~ms}\right)$

The temporal starting point for the descent of the control piston is identical for every set rail pressure while the gradient of the downward motion is again depending on the set rail pressure. The changing gradient in the rising motion is noteworthy, as it points to the elastic behavior of the steel control piston. After energizing the solenoid valve the pressure in the control volume decreases what leads to a lower downward force on top of the control piston and a reduction of the elastic deformation. The elongation of the control piston overlays with the upward motion of the piston in the ballistic stage of the injection process and causes the change of gradient. This observation is in accordance with control piston behavior in one-dimensional hydraulic injector simulations.

Table 3. Comparison of simulated and measured elastic deformation of the control piston due to applied rail pressure and closed solenoid valve

\begin{tabular}{|c|c|c|c|c|}
\hline $\begin{array}{l}\text { Set rail pressure } \\
p_{\text {rail,set }} \\
\text { [MPa] }\end{array}$ & $\begin{array}{l}\text { Simulated control } \\
\text { piston lift } x_{c p, s}[\mathrm{~mm}]\end{array}$ & $\begin{array}{l}\text { Measured control } \\
\text { piston lift } x_{c p, m}[\mathrm{~mm}]\end{array}$ & $\begin{array}{l}\text { Difference } D_{c p a}= \\
x_{c p, m}-x_{c p, s} \\
{[\mathrm{~mm}]}\end{array}$ & $\begin{array}{l}\text { Deviation } D_{c p r}= \\
D_{c p a} I x_{c p, m} \\
{[\%]}\end{array}$ \\
\hline 60 & -0.0533 & -0.0696 & -0.0163 & 23.4 \\
\hline 100 & -0.0882 & -0.1026 & -0.0144 & 14.0 \\
\hline 140 & -0.1235 & -0.1258 & -0.0023 & 1.8 \\
\hline 180 & -0.1585 & -0.1371 & -0.0214 & 15.6 \\
\hline
\end{tabular}


The comparison of measured (lift at $t_{E T}=0 \mathrm{~ms}$ in Figure 6) and simulated elastic deformation (see paragraph Experimental Setup) illustrates a strong congruence in terms of magnitude (see Table 3). One possible reason for the deviation of measured control piston deformation is the non-linearity of the controller of the eddy current sensor at the end of its linear measuring range. As the nozzle needle is significantly shorter than the control piston of the investigated injector, elastic deformation exerts a minor influence on its motion and the findings of the control piston motion are not transferrable to needle motion.

\section{Conclusions}

A common rail injector was equipped with two eddy current sensors and a piezoelectric pressure transducer. This novel setup allowed to measure control piston and needle motion along with injector inside pressure simultaneously for the first time.

Finite elements simulations proved that the geometrical alterations of control piston and needle did not affect their mechanical behavior. Moreover, these simulations clarified the magnitude of their deformation due to fluid pressure in the control volume and confirmed the measured initial deformation of these two parts.

A concurrence of local maxima of pressure before the inlet connector and pressure in the feed line of the injector occurred in close temporal vicinity to the end of injection. The measurement results showed that the gradient of control piston upward motion changes as the control piston is lifted due to the decrease of its elastic deformation. This non-linear motion of the control piston was different to the linear motion in a similar study, but was supported by a one-dimensional hydraulic simulation of the injector.

In general measuring the control piston motion of a common rail diesel injector with an eddy current sensor exhibited a low level of noise on the raw signal and proved a very high repeatability (see Figure 8 and Figure 9 in Annex). Additionally no detrimental electromagnetic influence of the energization of the solenoid valve on the raw signal was detectable as asserted in a previous study [14].

In summary, the presented sensor arrangement exhibited a high temporal accuracy and an ability to detect small differences in the measured parameters. It is suited for detecting aging effects if these alterations affect the control piston motion and the injector inside pressure. Therefore, these two parameters are an appropriate basis for the development of an open-loop control strategy countering diesel injector aging. Furthermore, the measured control piston lifts are a validation basis for hydraulic injector simulations and the concurrence of local pressure maxima is useful as indirect means to detect the end of injection in future investigations.

\section{Acknowledgements}

The authors thank the German Research Foundation DFG for the funding of the project (reference number WA 2468/4-1) in which the here presented results originated. Furthermore, the authors thank Martin Niedermeier for the development the injector and Mario Meinhardt for the development of the data acquisition.

\section{Nomenclature}

$\begin{array}{lll}\text { Symbol } & \text { Unit } & \text { Measured parameter } \\ A_{h} & \mathrm{~m}^{2} & \text { Total cross-section of nozzle holes } \\ C_{d} & - & \text { Discharge coefficient of nozzle hole } \\ I_{\text {Inj,ref }} & \mathrm{A} & \text { Reference injector current } \\ I_{\text {Inj,mea }} & \mathrm{A} & \text { Measured injector current } \\ I_{S v} & \mathrm{~A} & \text { Suction valve current } \\ k & - & \text { Conicity factor } \\ \dot{m} & \mathrm{mg} / \mathrm{ms} & \text { Injection rate } \\ p_{E V I, \text { dyn }} & \mathrm{MPa} & \text { Dynamic pressure injection rate analyzer } \\ p_{E V I, \text { stat }} & \mathrm{MPa} & \text { Static pressure injection rate analyzer } \\ p_{\text {Inj }} & \mathrm{MPa} & \text { Pressure inside feed line of injector } \\ p_{\text {Oil }} & \mathrm{MPa} & \text { Oil pressure inside gear box } \\ p_{\text {Rail,mea }} & \mathrm{MPa} & \text { Measured rail pressure } \\ p_{\text {Rail,set }} & \mathrm{MPa} & \text { Set rail pressure } \\ p_{\text {Rail,stat }} & \mathrm{MPa} & \text { Static rail pressure } \\ t_{\text {Dif } 1} & \mathrm{~ms} & \text { Difference between } t_{C p p} \text { and } t_{\text {Mir }} \\ t_{\text {Dif } 2} & \mathrm{~ms} & \text { Difference between } t_{M d p} \text { and } t_{M i r} \\ t_{E T} & \mathrm{~ms} & \text { Energizing time } \\ t_{C p p} & \mathrm{~ms} & \text { Concurrence of pressure peaks } \\ t_{M c p} & \mathrm{~ms} & \text { First minimum of control piston lift } \\ & & \end{array}$




$\begin{array}{lll}t_{M d p} & \mathrm{~ms} & \text { Maximum derivative of pressure before inlet connector } \\ t_{M i r} & \mathrm{~ms} & \text { First minimum of injection rate } \\ T_{E V I} & \mathrm{~K} & \text { Fuel temperature injection rate analyzer } \\ x_{C p} & \mathrm{~mm} & \text { Control piston lift } \\ x_{N} & \mathrm{~mm} & \text { Needle lift } \\ \rho_{f} & \mathrm{~ms} & \text { Fuel density }\end{array}$

\section{References}

[1] Galante-Fox, J., and Bennett, J., Diesel injector internal deposits in High Pressure Common Rail diesel engines, Conference Fuel Systems for IC Engines, London, 2012, doi:10.1533/9780857096043.5.157

[2] Fink, C., Crusius, S., Junk, R., Schümann, U. et al., Fuel-related Deposits in Common-Rail Injectors, $24^{\text {th }}$ Aachen Colloquium Automobile and Engine Technology, 2015

[3] d'Ambrosio, S., and Ferrari, A., Diesel Injector Coking: Optical-Chemical Analysis of Deposits and Influence on Injected Flow-Rate, Fuel Spray and Engine Performance, Journal of Engineering for Gas Turbines and Power 134(6): 062801, 2012, doi:10.1115/1.4005991

[4] Winterbone, D., Clough, E., Rao, K., Richards, P. et al., The Effect of DI Nozzle Fouling on Fuel Spray Characteristics, SAE Technical Paper 922232, 1992, doi:10.4271/922232

[5] Williams, R., Smith, A., and Buttery, I., Formation and Removal of Injector Nozzle Deposits in Modern Diesel Cars, SAE International Journal of Fuels and Lubricants 6(1):230-240, 2013, doi:10.4271/2013-01-1684

[6] Ikemoto, M., Omae, K., Nakai, K., Ueda, R. et al., Injection Nozzle Coking Mechanism in Common-rail Diesel Engine, SAE Technical Paper 2011-01-1818, 2011, doi:10.4271/2011-01-1818

[7] Tang, J., Pischinger, S., Lamping, M., Körfer, M. et al., Coking Phenomena in Nozzle Orifices of DI-Diesel Engines, SAE Technical Paper 2009-01-0837, 2009

[8] Ullrich, J., Steininger, R., Semisch, R., and Reke, M., New Methodology for Early Injector Qualification in Real Engine, $10^{\text {th }}$ Conference Diesel and Gasoline Direct Injection, Berlin, 2016

[9] Miyaki, M., Takeuchi, K., Ishizuka K., and Sasaki, S., The Breakthrough of Common Rail System: Closed-loop Control Strategy Using Injector with Built-in Pressure Sensor, 30. Internationales Wiener Motorensymposium, Vienna, 2009

[10] Miyaura, T., Morikawa, A., Ito, Y., Ishizuka, K. et al., Development of Diesel Engine using New Fuel Injection System - Direct Monitoring of Fuel Injection Pressure using Injector with Built-in Sensor, and its Applications, SAE Technical Paper 2013-01-1739, 2013

[11] Marker, J., Willmann, M., Mlicki, F., Mehr, A. et al., Potential of INSITU closed-loop control of fuel injection in large LFO engines, $15^{\text {th }}$ Conference The Working Process of the Internal Combustion Engine, Graz, 2015

[12] Schöppe, D., Stahl, C., Krüger, G., and Dian, V., Servogetriebene Piezo-Common-Rail-Dieseleinspritzung, Motortechnische Zeitschrift 73(3):208-213, 2012, doi:10.1365/s35146-012-0266-9

[13] Hofmann, O., Strauß, P., Schuckert, S., Huber, B. et al., Identification of Aging Effects in Common Rail Diesel Injectors Using Geometric Classifiers and Neural Networks, SAE Technical Paper 2016-01-0813, 2016, doi:10.4271/2016-01-0813

[14] Coppo, M., Dongiovanni, C., and Negri, C., A Linear optical sensor for measuring needle displacement in common-rail diesel injectors, Sensors and Actuators A 134(2):366-373, 2007, doi:10.1016/j.sna.2006.05.038

[15] Voigt, P., Schiffgens, H.-F., Béduneau, J.-L., Cochet, T. et al., Delphi Injector Closed Loop Control Strategy Using the "Switch" Technology for Diesel Passenger Cars, 25th Aachen Colloquium Automobile and Engine Technology, Aachen, 2016

[16] Bosch, W., Der Einspritzgesetz-Indikator, ein neues Meßgerät zur direkten Bestimmung des Einspritzgesetzes von Einzeleinspritzungen, Motortechnische Zeitschrift 25(7):268-282, 1964

[17] Payri, R., Gimeno, J., Novella, R., and Bracho, G., On the rate of injection modeling applied to direct injection compression engines, International Journal of Engine Research 17(10):1015-1030, 2016, doi:10.1177/1468087416636281

[18] Payri, R., Lujan, J. M., Guardiola, C., and Rizzoni, G., Injection diagnosis through common-rail pressure measurement, Proceedings of the Institution of Mechanical Engineers, Part D: Journal of Automobile Engineering 220(3):347-357, 2006, doi:10.1243/09544070JAUTO34

[19] Delphi International Operations Luxembourg S.À.R.L., Novel Fuel Rail for Injection System, Patent Application WO 2017/025348 A1, 2017

[20] Christ, K., Kalibrierung von Magnet-Injektoren für Benzin-Direkteinspritzsysteme mittels Körperschall, Ph.D. thesis, Karlsruhe Institute of Technology, 2011

[21] Seykens, X.L.J., Somers, L.M.T., and Baert, R.S.G., Detailed Modeling of Common Rail Fuel Injection Process, Journal of Middle European Construction and Design of Cars 3(2-3):30-39, 2005 


\section{Annex}

In Figure 8 and Figure 9 five consecutive control piston lift are depicted. A low level of noise on the lift curves is clearly visible.

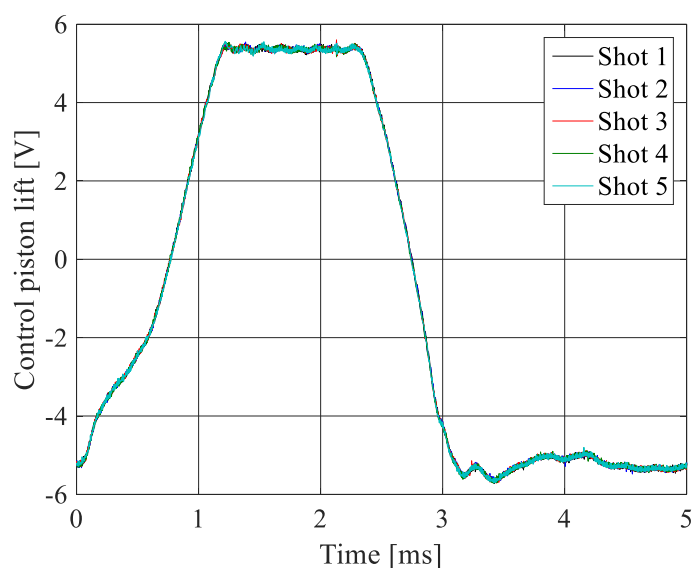

Figure 8. Raw data of five single control piston motion measurements $\left(p_{\text {Rail,set }}=180 \mathrm{MPa}, t_{E T}=2.2 \mathrm{~ms}\right.$ )

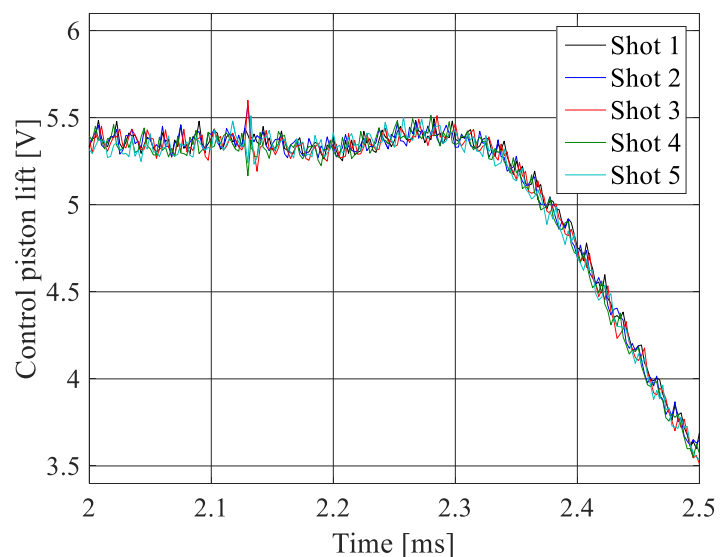

Figure 9. Illustration of noise level of raw signal of control piston motion (magnification of Figure 8)

Table 4 summarizes the key properties of the injector and of its production nozzle that are utilized for the here presented investigations.

Table 4. Parameters of nozzle and injector

\begin{tabular}{|l|l|}
\hline \multicolumn{2}{|l|}{ Injector } \\
\hline Type & $\begin{array}{l}\text { Commercial third generation } \\
\text { common rail }\end{array}$ \\
\hline Rail pressure & $180 \mathrm{MPa}$ \\
\hline Nozzle & Sac \\
\hline Type & 1.5 \\
\hline Conicity factor $k$ & $0.177 \mathrm{~mm}$ \\
\hline Spray hole diameter & 0.87 \\
\hline Discharge coefficient $C_{d}$ &
\end{tabular}

Table 5 lists the data for the calculation of the distance between the two pressure sensors.

Table 5. Evaluation of pressure data

\begin{tabular}{|l|l|l|l|l|l|l|}
\hline $\begin{array}{l}\text { 1. Maximum } \\
\text { pressure feed line } \\
{[\mathbf{m s}]}\end{array}$ & $\begin{array}{l}\text { 1. Maximum } \\
\text { pressure before inlet } \\
\text { connector [ms] }\end{array}$ & Difference [ms] & $\begin{array}{l}\text { Bulk modulus } \\
{\left[\mathbf{N} / \mathbf{m m}^{2}\right]}\end{array}$ & $\begin{array}{l}\text { Density } \\
{\left[\mathbf{k g}^{3} \mathbf{m}^{3}\right]}\end{array}$ & $\begin{array}{l}\text { Speed of } \\
\text { sound }[\mathrm{m} / \mathbf{s}]\end{array}$ & $\begin{array}{l}\text { Length } \\
{[\mathrm{m}]}\end{array}$ \\
\hline 3,305 & 3,567 & 0,262 & 1738 & 840 & 1438 & 0,377 \\
\hline 3,152 & 3,395 & 0,243 & 2155 & 857 & 1585 & 0,385 \\
\hline 3,070 & 3,315 & 0,245 & 2567 & 871 & 1716 & 0,421 \\
\hline 3,000 & 3,225 & 0,225 & 2961 & 884 & 1830 & 0,412 \\
\hline
\end{tabular}




\title{
LIF/Mie Droplet Sizing of Water Sprays from SCR System Injector Using Structured Illumination
}

\author{
Łukasz Jan Kapusta \\ Warsaw University of Technology, Faculty of Power and Aeronautical Engineering, Institute \\ of Heat Engineering, Warsaw, Poland \\ Lukasz.Kapusta@itc.pw.edu.pl
}

\begin{abstract}
Recent trends in SCR (Selective Catalytic Reduction) systems development increase requirements for UWS (Urea Water Solution) injection. Close-coupled SCR system designs decrease the distance available for water evaporation and urea decomposition. Due to that, much effort is put into static mixing elements design improvement and injection process enhancement. So far, most experimental studies on UWS spray formation were based on Mie scattering visualization using global illumination and shadowgraphy imaging. High speed imaging of Mie signal with global illumination allows to determine global spray parameters such as penetration and angle but does not give information on droplet sizes. Droplet size determination, due to relatively large droplets generated by SCR injectors, can be done with Mie scattering or backlight imaging methods. Then the visualized area becomes narrowed since high magnification is required. Determination of droplet size distribution across whole spray in such arrangement requires number of measurements. LIF/Mie (Laser Induced Fluorescence/Mie scattering) technique provides an attractive alternative for rapid determination of droplet size distribution across the whole spray. This method however suffers from multiple scattering effects which might affect droplet size distribution results even in relatively dilute sprays.

In this study, LIF/Mie ratio distribution across sprays from commercial automotive injector for SCR systems was determined by simultaneous LIF and Mie detection using structured illumination. Moreover, the results were compared with conventional LIF/Mie imaging. Nd:YAG pulse laser was used as a light source. Second harmonic beam of $532 \mathrm{~nm}$ was used to illuminate the sprays. Instead of UWS pure water doped with Eosin $Y$ was used. The results showed that conventional images exhibited much stronger background signal. Moreover, the conventional imaging was sensitive to reflections from experimental setup elements, specifically reflections from LIF camera filter. These two observations prove the importance of using SLIPI for LIF/Mie droplets sizing in sprays for SCR systems. At the same time the obtained results showed that under certain conditions (no accidental reflections in the background) conventional imaging provides similar LIF/Mie ratio as structured illumination. The results showed that the LIF/Mie ratio remains unchanged over the spray cloud. This suggests that SMD remains unchanged as well. The slight increase of LIF/Mie ratio far from the injector outlet could be caused by absence of small droplets due to lower momentum and thus lower penetration distance. This assumption however should be verified with PIV measurement.
\end{abstract}

\section{Keywords}

SLIPI, LIF/Mie, droplet sizing, SCR, spray.

\section{Introduction}

The SCR aftertreatment concept for NOx removal from exhaust gases has over 10-year history in automotive sector. At the beginning, the SCR aftertreatment systems were implemented into heavy-duty vehicles [1]. Recently introduced NOx limitations [2] forced their application into passenger cars. As shown by Jaworski et al. [3] and Zheng et al. [4] previous aftertreatment system designs based on in-line arrangement of separate components such as oxidation catalyst, SCR catalyst, particulate filter, and ammonia trap allowed to place the injector relatively far upstream from the inlet of the SCR catalyst. The injector position could be easily optimized. The static mixer could be placed at distance of several duct diameters upstream the SCR catalyst as well. This allowed to provide sufficient distance for water evaporation and urea decomposition. Newly designed SCR systems are supposed to provide higher NOx reduction efficiency than in-line systems since the real driving conditions emissions measurement is supposed to be introduced in near future [5]. The stricter requirements for NOx emissions are the reason for close-coupled to the engine SCR systems designs. Such designs, due to higher exhaust gas temperature, offer huge potential of NOx reduction [6] but meet serious constraints in terms of packaging [7] and cause challenges for UWS injection. Integration of oxidation catalyst, SCR catalyst and particulate filter in one unit result in dramatically decreased space for water evaporation and urea decomposition. Therefore, each close-coupled SCR unit requires 
specific design for certain engine and vehicle. In many cases only one injector location is possible, which can be only slightly modified. Then, the injection process becomes of high importance since the spray pattern and initial droplet size distribution generated by the injector are the only factors which can be used to optimize spatial liquid particles distribution in the SCR system in terms of efficient liquid-gas mixing process. The spray pattern and droplet size distribution need to be adjusted to specific SCR unit design. Thus, fast and reliable method for spray characterization is very important for proper injector selection and spray pattern optimization.

Injectors for UWS injection which are used in the newest SCR systems provide relatively dilute sprays with high SMD (Sauter Mean Diameter), especially when compared to Diesel sprays. SMD in modern SCR system injector is typically around $100 \mu \mathrm{m}$ (measured at distance of $50 \mathrm{~mm}$ from the injector outlet). Thus, broad variety of measurement techniques can be used for droplet size determination starting from Mie scattering [8], backlight imaging [8,9], ending up with PDA (Phase Doppler Anemometry) [9] and LIF/Mie. Grout et al. [8] used Mie scattering to determine the size of individual droplets and calculate the evaporation rate according to D-square evaporation law. The droplets taken into account for diameter calculation covered at least several pixels what made possible to directly determine their diameters basing on Mie scattering images. This approach requires high magnification, what in turn limits the visualised area. Droplet size determination by means of backlight illumination is also based on individual droplets visualisation, and the same conditions need to be met (visualised droplet needs to cover at least several camera pixels). Therefore, in this case the visualised area becomes limited as well. In order to visualize individual droplets, Postrioti et al. [9] limited field of view of $2048 \times 2048$ pixel camera to $15 \times 15 \mathrm{~mm}$. This gave the scale factor of $7.3 \mu \mathrm{m} /$ pixel. In order to visualize the whole spray, they used backlight method. Then the field of view was of $100 \times 100 \mathrm{~mm}$. In that case however, the droplet size determination could not be made, and the results were used only to conclude on liquid mass distribution over the visualized area. Postrioti et al [9] used also PDA to determine droplet diameters. Due to the fact that PDA is a point measurement method, the spatial distribution required number of measurements. In their study the SMD was determined at 16 locations at distance of $90 \mathrm{~mm}$ and $140 \mathrm{~mm}$ from the injector outlet. They made 5 additional measurements for methods comparison which showed good accordance between the results obtained with PDA and backlight imaging. The 21 measurement locations done with PDA methods gave information only on part of the spray. In order to build the SMD distribution basing on PDA measurements the number of measurement points needs to be much higher. LIF/Mie technique can be an attractive alternative to discussed methods as it allows a rapid determination of droplet size distribution across the spray at the same time providing information on global spray parameters, specifically the spray angle. It has been successfully used for port fuel injection studies [10] where injection pressure is very similar to the one in SCR systems. The advantage of this method in reduced measuring times comes along with various challenging issues, such as accuracy limited by multiple-scattering, absorption effects and calibration procedures [10]. The calibration procedure is especially difficult for 90 deg detection angle since increase of the scattered light intensity with the droplet diameter is irregular [11]. Therefore, LIF/Mie ratio results shown in this study shall treated as qualitative indicator, not as direct SMD result. The multiple scattering effects are especially strong in case of optically dense sprays like non-evaporating diesel spray, where more than $65 \%$ of multiple light scattering is usually detected [12]. However, even in fairly dilute spray, where single scattering events are in majority, the conventional LIF/Mie technique still remains largely affected by errors introduced by multiple light scattering [13]. Structured Laser Illumination Planar Imaging (SLIPI) technique is an illumination and processing method which can remove signal coming from scattering at the droplets located outside illuminated area and therefore limits the errors caused by multiple scattering. It was developed for sprays by Berrocal et al. [14] and Kristensson et al. [15], and commercially implemented by LaVision [16]. The SLIPI concept is based on recording several modulated images where the modulation is vertically shifted between each recording [16]. Due to necessity of recording three images where sprays are illuminated with different modulation shift and the transient nature of spray, the image averaging is required. In high pressure conditions this causes setup complication since the flow conditions are required to scavenge the test chamber during the time between two consecutive injections [17]. Spray visualisation in ambient conditions doesn't pose any challenge since the recording can be usually repeated many times, and the averaging for each modulation phase can be done for high number of images. In this study, the injection process was observed in ambient conditions, therefore LIF/Mie ratio was determined using structured illumination. SLIPI LIF/Mie images were compared with conventional LIF/Mie images in order to determine if using structured illumination and SLIPI processing brings any benefit in such dilute sprays as SCR sprays.

The LIF/Mie experiments were supported by primary breakup visualization using shadowgraphy with long distance microscopy in order to determine relevant area for SMD calculation. Moreover, before main experiments the initial liquid jet velocity was measured using high speed imaging. The sprays were generated by a commercial automotive three-hole injector used in SCR systems. 


\section{Experimental setup}

The spray cloud created by three liquid jets emerging from the injector was illuminated using structured light sheet. In order to create modulated light sheet, the SLIPI optics delivered by LaVison was used. In the setup used in the study the three modulated images were used to create SLIPI image. The modulation shift was done by electric motor integrated into the SLIPI optics. In order to visualise whole spray cloud, the modulated light sheet in the area of interest was expanding in vertical direction. The schematic setup of the SLIPI optics is shown in Figure 1.

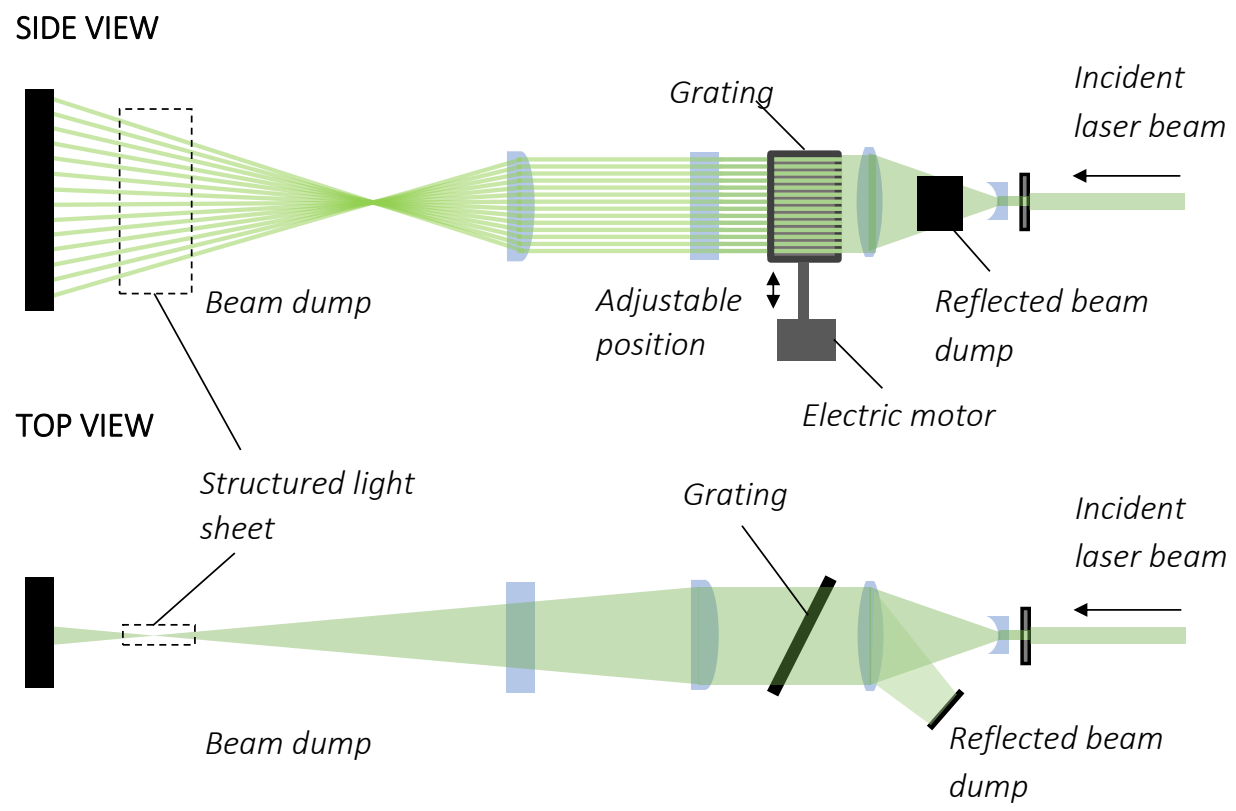

Figure 1. Schematic diagram of complete SLIPI optics.

Structured light sheet was created from second harmonic beam generated by Spectra-Physics Quanta-Ray Pro$230 \mathrm{Nd}$ :YAG $10 \mathrm{~Hz}$ pulse laser. The images were recorded by LaVision sCMOS cameras equipped with different filters. For Mie detection $532 \mathrm{~nm}$ bandpass filter was used, while for LIF $532 \mathrm{~nm}$ notch filter was applied. The shutter duration was adjusted to $15 \mu \mathrm{s}$ in both cameras. The schematic diagram of experimental setup is shown in Figure 2.

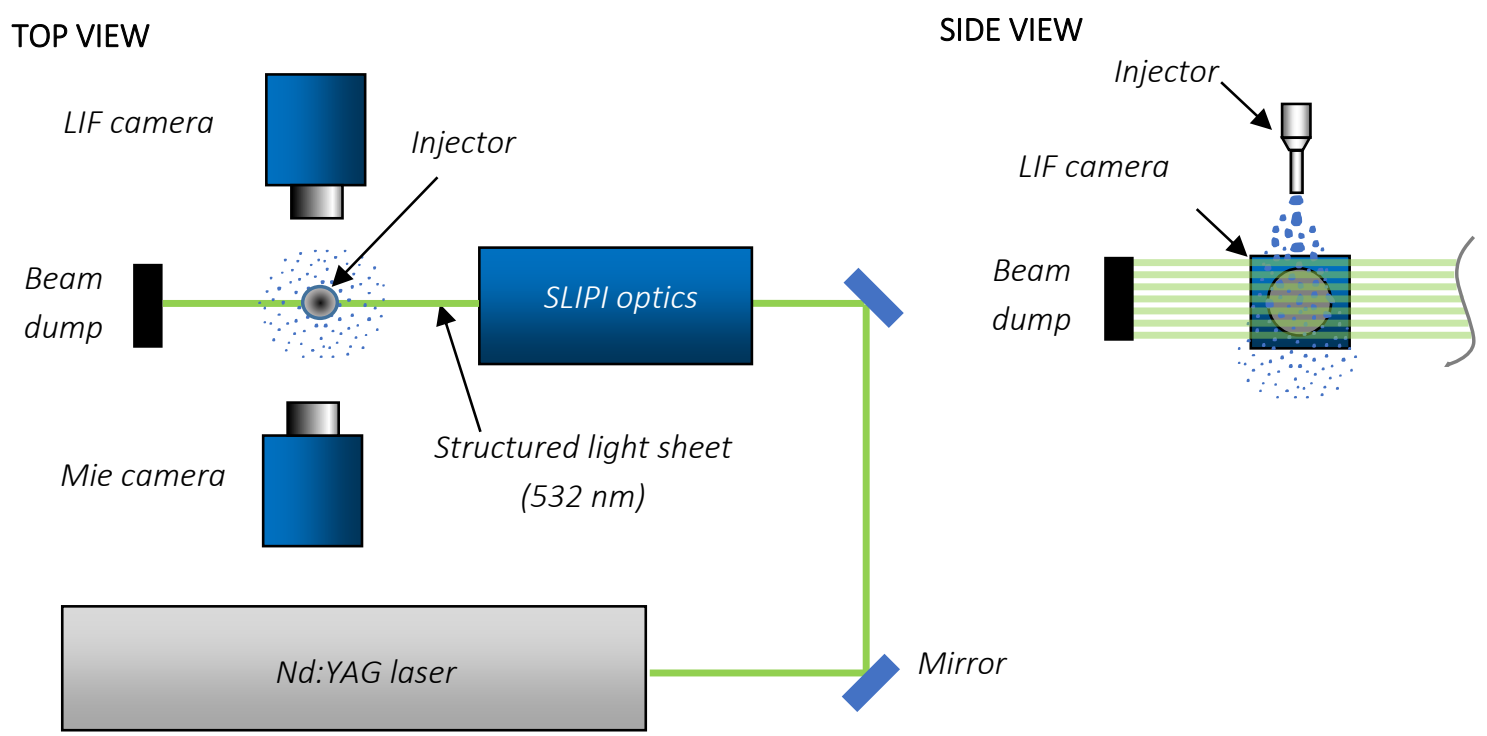

Figure 2. Schematic diagram of complete experimental setup. 
Because the modulated light sheet was expanding the period of modulation was dependent on the distance from the SLIPI optics. Therefore, the modulation period for calibration purposes was determined for the most relevant area - at distance of injector axis. The beam modulation period was calibrated using quartz cuvette filled with water doped with Eosin Y. The structured light sheet in the cuvette visualised with LIF and Mie cameras together with intensity profiles are shown in Figure 3 . Note that laser light sheet enters the visualisation area with $\mathrm{x}$ axis.
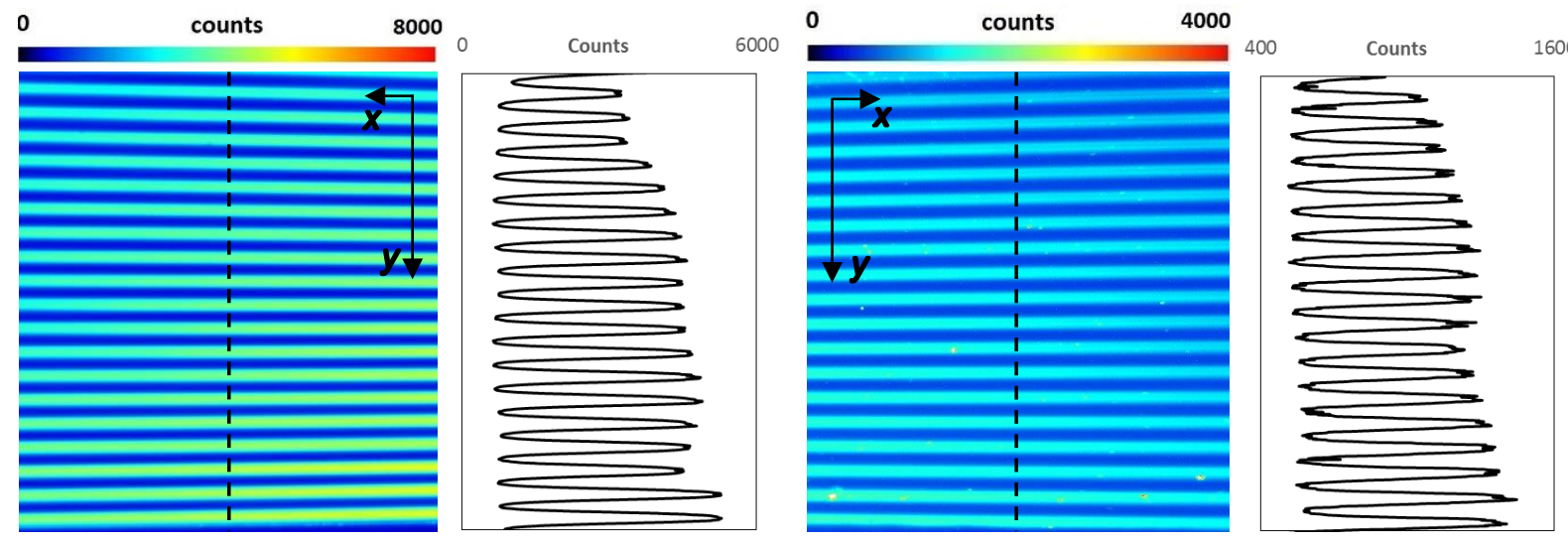

Figure 3. Structured light sheet used for calibration and the intensity profiles, LIF and Mie respectively from left.

The optics used in each camera was different, therefore the visible area in each camera was different as well. The system was calibrated in order to account for that difference and make possible to calculate LIF/Mie ratio. For this purpose, transparent plate with evenly distributed black dots was used.

Sprays were generated by a commercial 3-hole Bosch injector for SCR systems (no. 0280158 720). Instead of UWS pure water doped with Eosin $Y$ was used. Injection pressure was of 5 bar and the injection duration was of $15 \mathrm{~ms}$. The initial velocity of the liquid jet emerging the injector nozzle measured by high speed imaging at these conditions was of $25 \mathrm{~m} / \mathrm{s}$. During LIF and Mie recording the sprays were illuminated $10 \mathrm{~ms}$ after SOI (Start of Injection), when the sprays were fully developed. One LIF and one Mie image per injection was captured (for the same laser pulse). In order to specify the relevant area for LIF/Mie-based SMD calculation, the experiments were supported by shadowgraphy-based long distance microscopy measurements. Based on the microscopy results, the distance from injector outlet where no ligaments and strongly deformed droplets were present was determined. The setup for long distance microscopy is shown in [18].

\section{Results and discussion}

The image acquisition process for SLIPI visualisation consisted of capturing three series of images, each for different modulation phase, which could be then averaged. Berrocal et al. [12] recorded 100 images per each phase for diesel spray. According to the authors it was sufficient number for proper averaging. In this study 200 images were recorded for each phase. Capturing 200 images required 200 separate injections since only one image was captured per injection. The total number of recorded images for three modulation phases of the light sheet was of 600. One of two hundred instantaneous images and the averaged image for each modulation phase are shown in Figures 4 (LIF) and 5 (Mie). At each image the visualised area is of $90 \times 50 \mathrm{~mm}$. Both, instantaneous and averaged images are raw images without any processing and background subtraction. The injector axis is parallel to y axis.
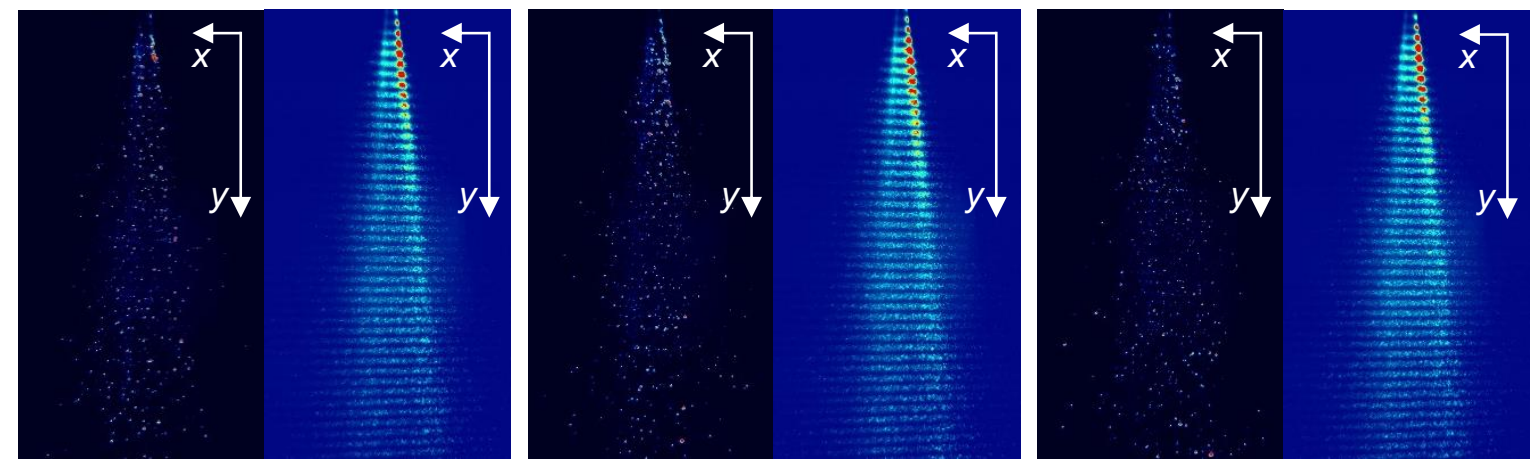

Figure 4. Instantaneous (left) and averaged (right) LIF images for each modulation phase; colour scale range 0-16000 counts (instantaneous images) and 0-8000 counts (averaged); the field of view at single image is of $90 \times 50 \mathrm{~mm}$.

This work is licensed under a Creative Commons 4.0 International License (CC BY-NC-ND 4.0). 

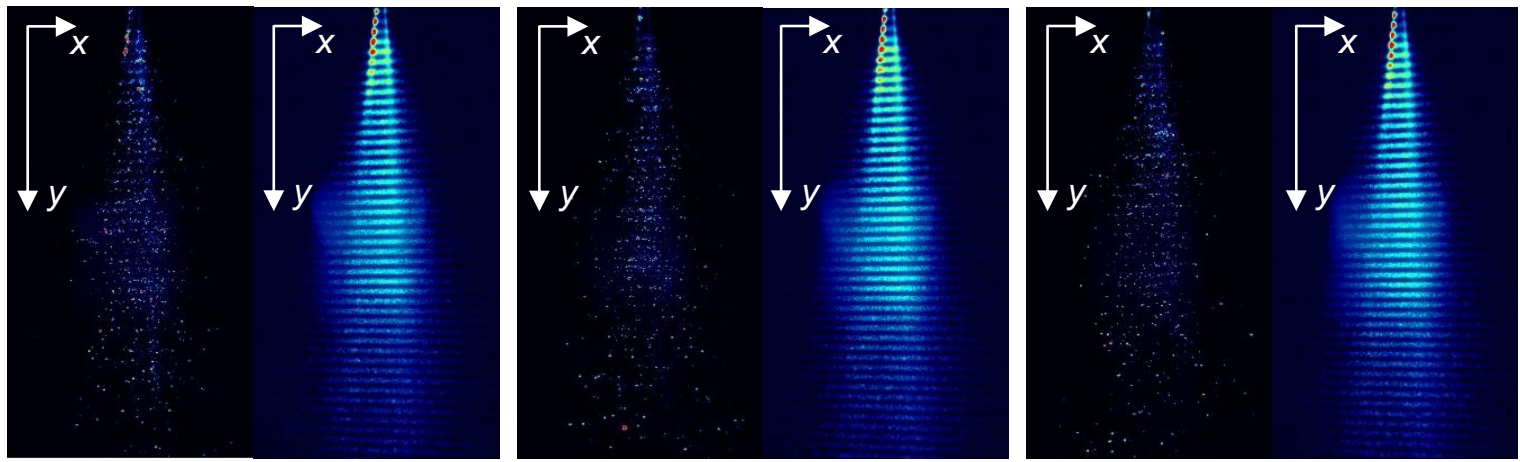

Figure 5. Instantaneous (left) and averaged (right) Mie images for each modulation phase; colour scale range $0-64000$ counts (instantaneous images) and 0-16000 counts (averaged); the field of view at single image is of $90 \times 50 \mathrm{~mm}$.

Averaged LIF images exhibit relatively high background signal when compared to averaged Mie signal. Note that colour scale range is different for LIF and Mie images. Moreover, in case of LIF the background signal is uniform, while in case of Mie there is a clearly distinguishable area where it is strongly increased. This signal most probably comes from $532 \mathrm{~nm}$ notch filter placed at LIF camera, which reflects Mie signal scattered at droplets. That suggests that the arrangement based on dichroic mirror and location of both cameras at one side of the spray would be better solution in terms of background noise at raw images. This would be important when using conventional LIF/Mie but should not pose any challenge for SLIPI processing. The observed phase results shown in Figures 4 and 5 exhibit much stronger signal on one side of the spray, right and left respectively (note that the images are mirrored due to opposite locations of the cameras). This signal enhancement is not the effect of laser signal extinction as observed in single nozzle sprays $[12,17,19]$ but results from the fact that the spray cloud observed here is formed by three jets. The light sheet crosses one jet emerging from the injector (right side of the LIF image, and left side of Mie image) while going between two other jets.

Basing on three averaged modulated images (shown in Figures 4 and 5) conventional image was reconstructed (see Figure 6a). Strong background signal in the centre of Mie image was still present. The conventional images were processed to subtract the background signal (see Figure 6b). The background image was taken with laser pulse but without the spray. Although the overall background signal was substantially decreased, the signal in the centre of Mie image was still present. Therefore, additionally the measurement of Mie signal was done with masked LIF filter on the opposite camera. The average Mie image for masked LIF filter after background subtraction is shown in Figure 6c. After masking the filter the brighter region was not present, what confirms the assumption that the laser light was first scattered at the spray cloud and then reflected from the opposite camera filter.
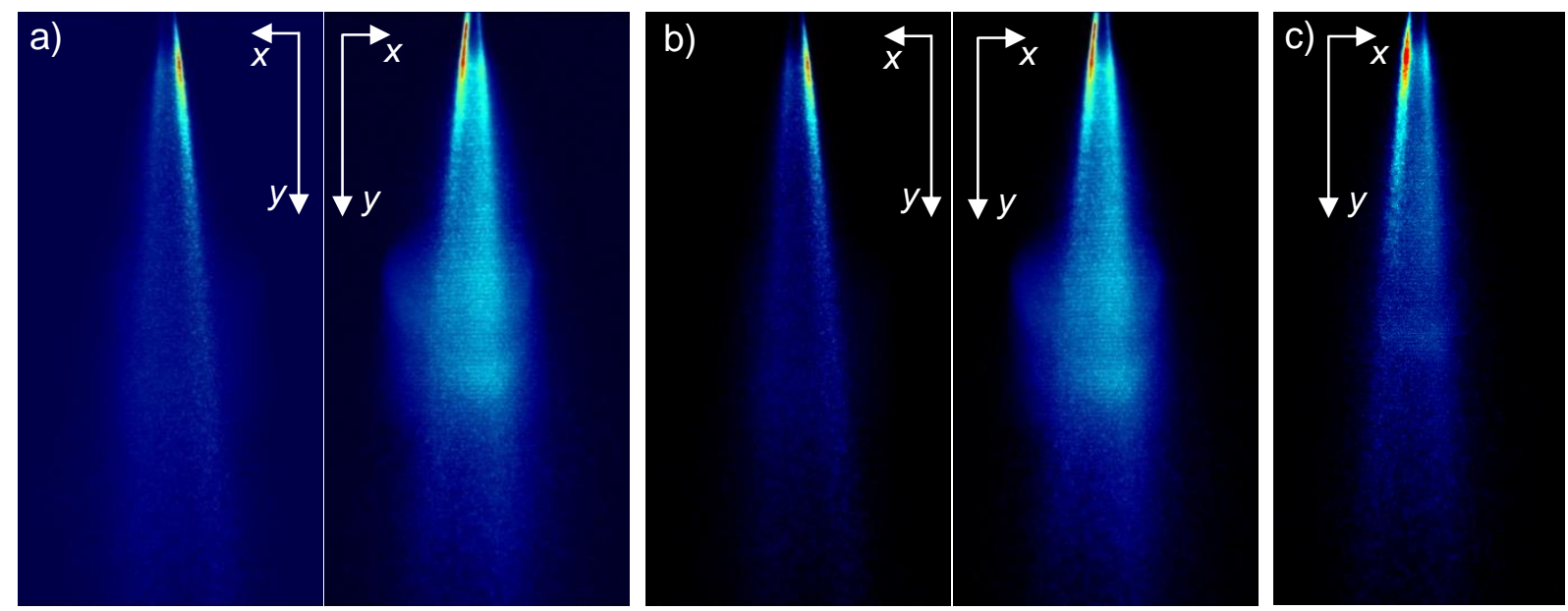

Figure 6. a) and b) Conventional LIF (left) and Mie (right) images reconstructed from three modulated averaged images; a) without background subtraction, b) with background subtraction c) Conventional Mie image obtained with masked filter on opposite camera (with background subtraction); colour scale range 0-8000 counts (LIF images) and 0-16000 counts (Mie images); the field of view at single image is of $90 \times 50 \mathrm{~mm}$.

The average phase images shown in Figures 4 and 5 were used to construct SLIPI image (shown in Figure 7a). Although Mie images shown in Figure 5 were affected by reflection from the filter they were selected for further analysis due to two reasons. The main reason was to construct LIF/Mie ratio basing on LIF and Mie signals from 
the same droplets. The other reason was to verify how SLIPI technique performs in removing this kind of reflections. Both, LIF and Mie SLIPI images were characterized by low background signal when compared to conventional image. The Mie SLIPI image appeared to be insensitive to recorded reflections from the camera filter, which were present at images used to build SLIPI image (averaged images shown in Figures 4 and 5).

SLIPI and conventional images were then used to calculate LIF/Mie ratio. The conventional images after background subtraction were taken for this analysis. The results are shown in Figure 7b. Note that LIF/Mie ratio was multiplied by 100 . LIF/Mie ratio distribution in case of conventional imaging was affected by discussed above camera reflections while SLIPI image was unaffected.
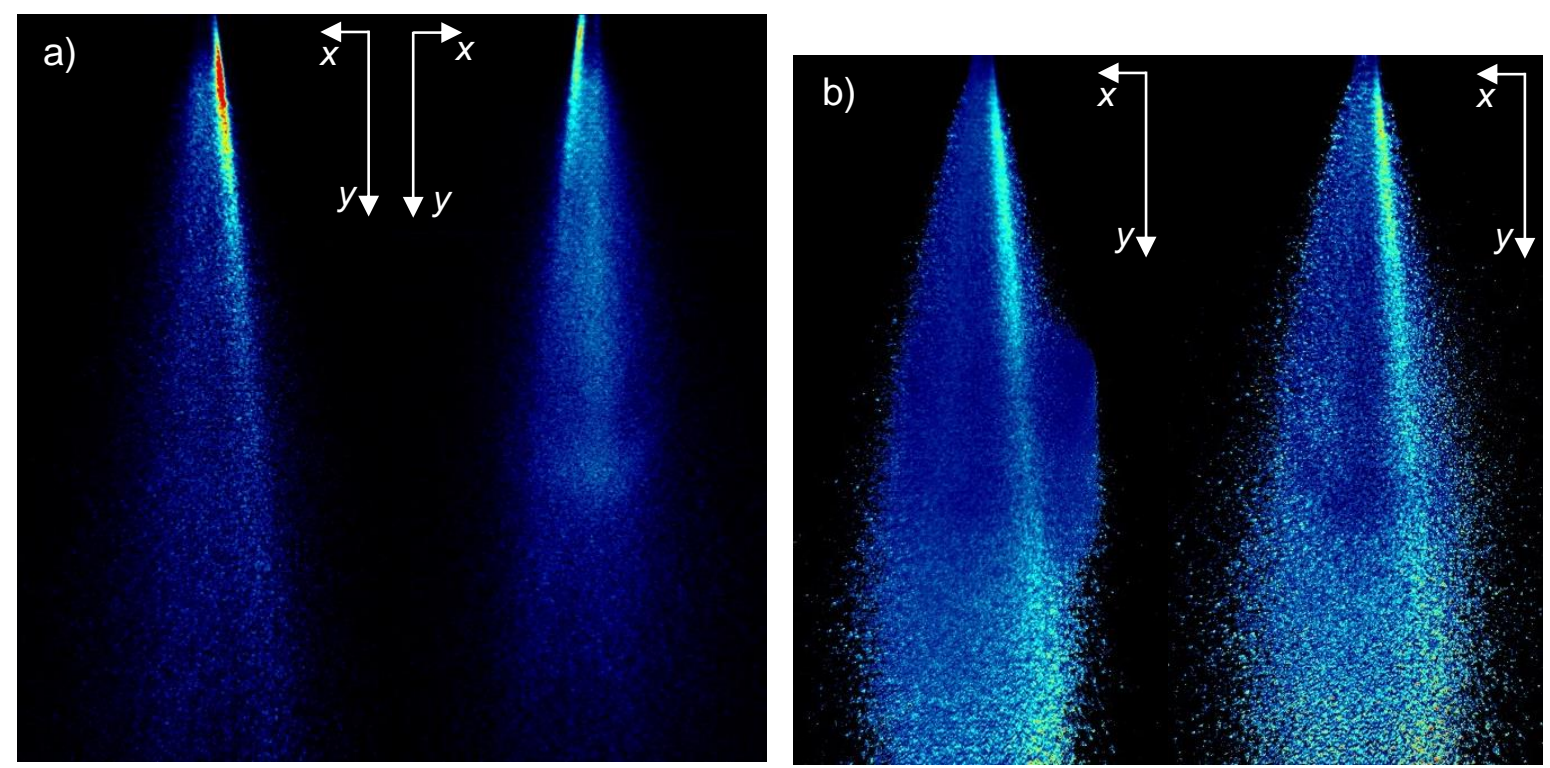

Figure 7. a) SLIPI LIF (left) and Mie (right) images, the field of view at single image is of $90 \times 50 \mathrm{~mm}$, colour scale range $0-4000$ counts (LIF) and 0-16000 counts (Mie). b) LIF/Mie ratio calculated from conventional images (after background subtraction) and from SLIPI images, respectively from left, the field of view at single image is of $85 \times 50 \mathrm{~mm}$, colour scale range $0-120$ counts.

In general, LIF/Mie ratio results are very similar for both type of imaging what indicates low multiple scattering effects. Nevertheless, the results obtained by SLIPI imaging seem to be more accurate. In case of conventional imaging LIF/Mie ratio decreases in the middle of the image. This observation suggests that the diameters of the droplets decrease in the middle of the visualised area to increase again at the end of the spray. This is questionable especially in case of the nozzle which was illuminated with the light sheet directly in the middle. In this case, it was expected to see either constant or gradually decreasing SMD along the nozzle hole axis. It needs to be considered that the LIF/Mie ratio could be affected by the non-spherical shape of droplets. Therefore, in order to determine how the LIF/Mie results could be affected by ligaments and strongly deformed droplets, the near-nozzle region was visualised with shadowgraphy-based long distance microscopy. The primary breakup visualisation showed that the unbroken liquid length oscillates between 5.2 and $6.4 \mathrm{~mm}$. Detached ligaments and strongly deformed droplets, however, were still observed further downstream up to around $11 \mathrm{~mm}$ from the injector outlet. The primary breakup and droplet formation is shown on two instantaneous images presented in Figure 8.

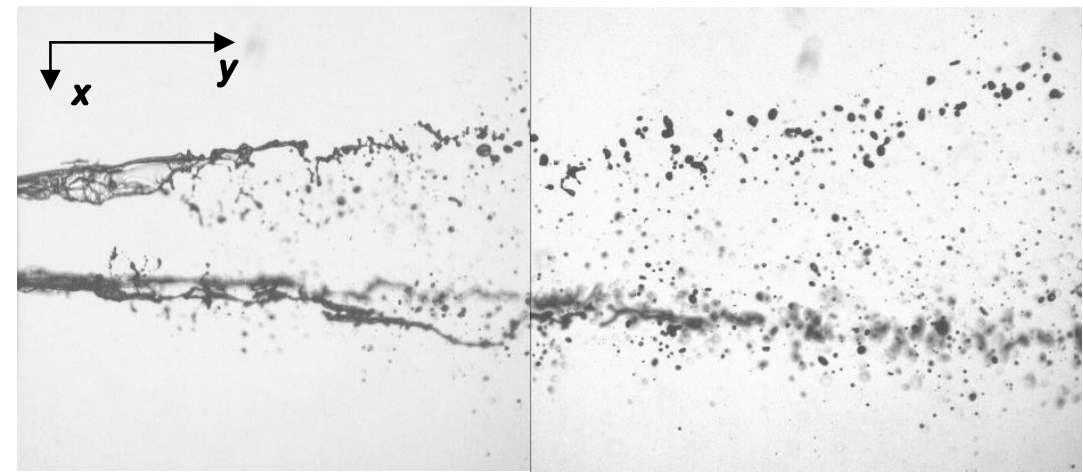

Figure 8. Long distance microscopy images - field of view $16.5 \times 7 \mathrm{~mm}$ (two images together); note that the images were taken during different injections. 
The injector position during shadowgraphy measurements was the same as during SLIPI recording. The images shown in Figure 8 were rotated by $90 \mathrm{deg}$ in order to connect them properly. Note that these images were taken during different injection events. The primary breakup experiments indicated that the area between the injector outlet and the distance of $11 \mathrm{~mm}$ from injector outlet is not relevant for LIF/Mie based SMD conclusions. In the area where ligaments were observed, typically high value of LIF/Mie ratio suggests high SMD. This area should be excluded from the analysis. Taking this into account one may notice that the LIF/Mie ratio does not change much with the distance from the injector. This is clearly visible in LIF/Mie ratio calculated from SLIPI images (shown in right image in Figure 7b). Constant LIF/Mie ratio suggests constant SMD. This in turn indicates that the secondary breakup does not play important role in the spray formation. This is in accordance to statement made by Birkhold et al. [20].

In both images shown in Figure $7 \mathrm{~b}$ LIF/Mie ratio is slightly increased at higher distance $(60-90 \mathrm{~mm})$. This can be related to the fact that bigger droplets are more prone to penetrate further, while the smaller ones due to lower momentum are decelerated easily by the aerodynamic forces. Lack of small droplets and the same size of large ones in that area causes the increase of SMD. This assumption however should be verified with PIV measurement. As stated above, the LIF/Mie-based droplet sizing is attractive since it can provide also information on global spray parameters, specifically the spray angle. Therefore, the conventional and SLIPI results obtained here were also used to determine the visualization angle of the entire spray cloud. For this purpose, only LIF images were used since conventional Mie images were affected by the reflections in background. Again, for this purpose the conventional images after background subtraction were used. The visualisation angle was determined assuming spray border as $90 \%$ of the recorded intensity. The visualisation angle determined from SLIPI image was of 18.06 deg while for conventional image was of 13.49 deg. $25 \%$ lower spray angle in case of conventional image suggests that conventional imaging performs relatively poor in the areas where signal is low. Then it is hardly distinguishable from the background.

\section{Conclusions}

Sprays emerging from the commercial automotive injector for SCR system were characterized by means of LIF/Mie method using structured illumination. SLIPI images were compared with conventional LIF/Mie images reconstructed from three modulated images. Additionally to LIF/Mie experiments near-nozzle area was visualized by means of shadowgraphy-based long distance microscopy in order to determine the area relevant for LIF/Mie-based conclusions on SMD. Ligaments and strongly deformed droplets were observed at distance of up to around $11 \mathrm{~mm}$ from the injector outlet indicating that the conclusions on SMD should not concern this area.

In general, LIF/Mie ratio results were very similar for both types of imaging, conventional and SLIPI. This indicates low multiple scattering effects. Moreover, the LIF/Mie ratio didn't change much with the distance from the injector. This suggests that the secondary breakup events are very rare. This is in accordance to statement made by Birkhold et al. [20].

Higher value of LIF/Mie ratio was observed at higher distance from the injector outlet $(60-90 \mathrm{~mm})$. This can be related to the fact that bigger droplets penetrate further while the smaller ones due to lower momentum are easily decelerated by the aerodynamic forces. Lack of small droplets and the same size of large ones (due to nonevaporating conditions) causes the increase of SMD. This assumption however should be verified with PIV measurement.

It needs to be noted that the conventional LIF/Mie images were affected by the incident reflections, coming from the camera filter located on the other side of the spray. This reflection was especially strong in Mie image.

Locating LIF and Mie cameras at one side of the laser light sheet and splitting LIF and Mie signals on dichroic mirror could be the solution for that. However, in certain cases it is not possible to get rid of background reflections. In case of SLIPI imaging the background signal didn't pose any challenge.

As far the experiments in the geometries resembling engine exhaust system are concerned this might be an important feature. Then the background reflections are expected to be present.

In the setup used in this study combination of these two approaches, conventional and structured illumination for LIF/Mie droplet sizing, could allow to conclude on the background signal origin and at the same time gave information on instantaneous droplet size distribution over the whole spray. In the setup used here the instantaneous LIF/Mie ratio is available only for conventional illumination since SLIPI used here requires three images obtained for different modulation phases of the structured light sheet. Interesting option for instantaneous imaging is two-pulse SLIPI based only on two phases as discussed by Payri et al. [21] and Kristensson et al. [22]. This approach however requires a dual cavity laser. This is planned to be done in the future.

In general, one can conclude that both methods, conventional and SLIPI, can be used for LIF/Mie ratio determination in such dilute sprays, provided that the sprays are visualised on non-reflecting background. SLIPI however should be used if a test rig generates any reflections. 


\section{Acknowledgements}

Current work was supported by the European Smart Growth Operational Programme 2014-2020 through the project "Development of mixing and urea-water solution conversion unit in SCR systems in order to start production of exhaust system for compression ignition engine that meets the Euro 7 emission standards", grant number: POIR.04.01.04-00-0060/15-02.

\section{References}

[1] Hirata, K., Masaki, N., Ueno, H., and Akagawa, H., 2005, "Development of Urea-SCR System for HeavyDuty Commercial Vehicles," SAE Tech. Pap. 2005-01-1860.

[2] European Comission, 2012, "Amendment No 459/2012 as Regards Emissions from Light Passenger and Commercial Vehicles (Euro 6)," Off. J. Eur. Union, 2012(459/2012), pp. 16-24.

[3] Jaworski, P., Kapusta, Ł. J., Jarosiński, S., Ziółkowski, A., Capetillo, A. C., and Grzywnowicz, R., 2015, "SCR Systems for NOx Reduction in Heavy Duty Vehicles," J. KONES, 22(4), pp. 139-146.

[4] Zheng, G., Fila, A., Kotrba, A., and Floyd, R., 2010, "Investigation of Urea Deposits in Urea SCR Systems for Medium and Heavy Duty Trucks," SAE Technical Paper, SAE International.

[5] European Commission, "Real-Driving Emissions in the EURO 6 Regulation on Emissions from Light Passenger and Commercial Vehicles (RDE3) - DRAFT REGULATION" [Online]. Available: https://ec.europa.eu/info/law/better-regulation/initiatives/ares-2016-6339064_en. [Accessed: 11-Jan2017].

[6] Kojima, H., Fischer, M., Haga, H., Ohya, N., Nishi, K., Mito, T., and Fukushi, N., 2015, "Next Generation All in One Close-Coupled Urea-SCR System," SAE Technical Paper, SAE International.

[7] Michelin, J., Nappez, P., Guilbaud, F., Hinterberger, C., Ottaviani, E., Gauthier, C., Maire, P., and Couturier, T., 2015, "Advanced Close Coupled SCR Compact Mixer Architecture," SAE Technical Paper, SAE International.

[8] Grout, S., Blaisot, J.-B., Pajot, K., and Osbat, G., 2013, "Experimental Investigation on the Injection of an Urea-water Solution in Hot Air Stream for the SCR Application: Evaporation and Spray/wall Interaction," Fuel, 106(x), pp. 166-177.

[9] Postrioti, L., Brizi, G., Ungaro, C., Mosser, M., and Bianconi, F., 2015, "A Methodology to Investigate the Behaviour of Urea-Water Sprays in High Temperature Air Flow for SCR," Fuel, 150(x), pp. 548-557.

[10] Bareiss, S., Fuhrmann, N., Dreizler, A., Bacher, H., Höffner, J., Weishäupl, R., and Kügler, D., 2013, "Planar Droplet Sizing for Characterization of Automotive Sprays in Port Fuel Injection Applications Using Commercial Fuel," Flow and Combustion in Advanced Gas Turbine Combustors, J. Janicka, A. Sadiki, M. Schäfer, and C. Heeger, eds., Springer Netherlands, Dordrecht, pp. 445-461.

[11] Charalampous, G., and Hardalupas, Y., 2011, "Numerical Evaluation of Droplet Sizing Based on the Ratio of Fluorescent and Scattered Light Intensities (LIF/Mie Technique).," Appl. Opt., 50(9), pp. 1197-1209.

[12] Berrocal, E., Kristensson, E., Hottenbach, P., Aldén, M., and Grünefeld, G., 2012, "Quantitative Imaging of a Non-Combusting Diesel Spray Using Structured Laser Illumination Planar Imaging," Appl. Phys. B, 109(4), pp. 683-694.

[13] Mishra, Y. N., Kristensson, E., and Berrocal, E., 2014, "Reliable LIF/Mie Droplet Sizing in Sprays Using Structured Laser Illumination Planar Imaging," Opt. Express, 22(4), p. 4480.

[14] Berrocal, E., Kristensson, E., Richter, M., Linne, M., and Aldén, M., 2008, "Application of Structured Illumination for Multiple Scattering Suppression in Planar Laser Imaging of Dense Sprays," Opt. Lett., 16(22), pp. 17870-17881.

[15] Kristensson, E., Berrocal, E., Richter, M., Pettersson, S., and Aldén, M., 2008, "High-Speed Structured Planar Laser Illumination for Contrast Improvement of Two-Phase Flow Images," Opt. Lett., 33(23), pp. 2752-2754.

[16] Leick, P., Grzeszik, R., Arndt, S., and Wissel, S., 2011, "Suppression of Multiple Scattering Using Structured Light Sheets - A First Assessment for Diesel and Gasoline Spray Visualization," Proceedings of 24th European Conference on Liquid Atomization and Spray System, Estoril, Portugal, pp. 1-10.

[17] Pastor, J., and Benajes, J., 2002, "Planar Laser-Induced Fluorescence Fuel Concentration Measurements in Isothermal Diesel Sprays," Opt. Express, 10(7), pp. 309-23.

[18] Kapusta, Ł. J., Jaworski, P., Teodorczyk, A., and Kowalski, J., 2015, "Laser Based Diagnostic System for Spray Measurements," J. KONES. Powertrain Transp., 22(3), pp. 91-98.

[19] Pastor, J. V, Payri, R., Salavert, J. M., and Manin, J., 2012, "Evaluation of Natural and Tracer Fluorescent Emission Methods for Droplet Size Measurements in a Diesel Spray," Int. J. Automot. Technol., 13(5), pp. 713-724.

[20] Birkhold, F., Meingast, U., Wassermann, P., and Deutschmann, O., 2006, "Analysis of the Injection of UreaWater-Solution for Automotive SCR DeNOx-Systems: Modeling of Two-Phase Flow and Spray/WallInteraction," SAE Technical Paper, SAE International.

[21] Payri, R., Gimeno, J., Martí, P., and Manin, J., 2012, "Fuel Concentration in Isothermal Diesel Sprays through Structured Planar Laser Imaging Measurements," Int. J. Heat Fluid Flow, 34, pp. 98-106.

[22] Kristensson, E., Berrocal, E., and Aldén, M., 2014, "Two-Pulse Structured Illumination Imaging.," Opt. Lett., 39(9), pp. 2584-7. 


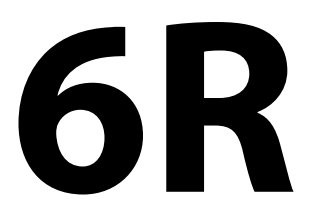

Atomizers 6 


\title{
Numerical simulation of superheated jets using an Eulerian method
}

\author{
Konstantinos Lyras ${ }^{1}$, Siaka Dembele ${ }^{1}$, C. Madhav Rao Vendra ${ }^{2}$, Jennifer Wen ${ }^{2 *}$ \\ ${ }^{1}$ Department of Mechanical and Automotive engineering, Kingston University London, United \\ Kingdom \\ ${ }^{2}$ School of Engineering, University of Warwick, Coventry, United Kingdom \\ *Corresponding author: jennifer.wen@warwick.ac.uk
}

\begin{abstract}
Flash boiling is the rapid phase change of a pressurised fluid that emerges in ambient conditions below its vapour pressure. Flashing can occur either inside or outside the nozzle depending on the local pressure and geometry and the bubble formation leads to interfacial interactions that eventually influence the emerging spray. Lagrangian methods which exist in literature to simulate the flash atomisation and inter-phase heat transfer employ many simplifying assumptions. Typically, sub-models used for the break-up, collisions and evaporation introduce an extensive empiricism that might result in unrealistic predictions for cases like flashing. In this study, a fully Eulerian approach is selected employing the $\Sigma-Y$ model proposed by Vallet and Borghi. The model tracks liquid structures of any shape and computes the spray characteristics comprising a modified version for the transport equation of the surface density. The main goal of this study is to investigate the performance of this model in flash boiling liquids using the Homogeneous Relaxation Model (HRM) developed by Downar-Zapolski, a model capable of capturing the heat transfer under sudden depressurisation conditions accounting for the non-equilibrium vapour generation. The model in this present study considers that the instantaneous quality would relax to the equilibrium value over a given timescale which is calculated using the flow field values. A segregated approach linking the HRM and $\Sigma-Y$ is implemented in a compressible formulation in an attempt to quantify the effects of flash boiling in the spray dynamics. The developed model is naturally implemented in RANS in a dedicated solver HRMSonicELSAFoam. Results from simulations of two-phase jets of different subcooled fluids through sharp-edged orifices show that the proposed approach can accurately simulate the primary atomisation and give reliable predictions for the droplet sizes and distribution. Strong effects of the flashing and turbulent mixing on the jet are demonstrated. The model is tested for turbulent flows within small nozzles and was developed within the open source code OpenFOAM.
\end{abstract}

\section{Keywords}

Flash-boiling, atomisation, ELSA model.

\section{Introduction}

Flashing jets occur when a high-pressure liquid flowing through a nozzle or an orifice is suddenly exposed to a low-pressure environment, becoming superheated if it is not already so. Flashing is characterised by a rapid phase change along the jet and bubble nucleation within the liquid core that influences the spray formation [1, 2]. Flashing is very important in safety studies in cases of accidental releases of a liquefied flammable gas through a small crack in the pipeline system. In the aerosol industry it can be used to control nucleation having the advantage of producing sprays with very fine droplets within small domains [3]. In its dense part, the two-phase jet might appear in different forms like bubbly, slug or annular and the nucleation is possible to start upstream of the orifice (a process generally termed internal flashing) or at some distance downstream of the orifice (external flashing). The geometry and the nozzle length-to-diameter ratio, the degree of superheat or subcooling, the storage and ambient conditions play a crucial role in the atomisation process. Flashing occurs either if a liquid follows an isothermal depressurisation or an isobaric heating. In both cases, the fluid fails to adjust to the local changes in pressure and temperature admitting a metastable state which makes the process more challenging to understand.

Lagrangian modelling approaches usually rely on submodels for flash vaporisation (such as in [4]) and incorporate them in the concept of liquid parcels. The idea of parcels splits the modelling of the rapid boiling and aerodynamic fragmentation of the jet employing single-droplets models. Despite the simplifications it suffers from, it is a logical approach to the problem since after the primary atomisation and the consequent break-up, the liquid structures become small enough to resemble spherical droplets. Experimental studies for water [5-7] and various cryogenic fluids like liquid nitrogen in [8] indicate that two-phase jets emerging through a nozzle might begin flashing with a severe impact on the spray. Numerical works of Lee et al. in [9] and Schmidt et al. in [10] also agree with this change in the disintegration regime. In spite of its significance, detailed numerical studies of flash boiling atomisation are scarce. Here, a novel approach is proposed to simulate the nozzle flow and the primary atomisation in an Eulerian framework. The method tracks the interface of the spray in a compressible conservative manner using the volume of fluid (VOF) method and is combined with the HRM implementation of Schmidt et al. [10]. The method switches to a Lagrangian behaviour for the smaller liquid structures employing the $\Sigma-Y$ model of Vallet and Borghi [11]. The simulations considered here concern R134a jets which is an example a cryogenic liquid with strong vaporisation effects when released in the atmosphere. For this reason, a modified formulation is suggested for the $\Sigma-Y$ model, adjusting the surface density equation to accommodate simulations of superheated jets. 


\section{Mathematical formulations and methods}

The Eulerian-Lagrangian Spray Atomisation (ELSA) model was established after the first formulation of $\Sigma-Y$ model in [11-12]. In ELSA, the two-phase flow consists of the liquid and gas, which vary in density and the flow is studied as one single phase, solving one equation for mass and momentum which are omitted here for the sake of brevity. The same stands for the VOF method. The liquid mass fraction $Y_{l}$ is used as a marker function to track the liquid. The liquid mass fraction is calculated by the following equation,

$$
\frac{\partial \bar{\rho} \tilde{Y}_{l}}{\partial t}+\frac{\partial \bar{\rho} \tilde{Y}_{l} \tilde{u}_{j}}{\partial x_{j}}=-\frac{\partial \bar{\rho} R_{j l}}{\partial x_{j}}
$$

where $R_{j l}$ is the turbulent liquid flux given by,

$$
R_{j l}=-\frac{\nu_{t}}{S c_{t}} \frac{\partial \tilde{Y}_{l}}{\partial x_{j}}
$$

where $u_{j}, \rho, \nu_{t}, S c_{t}$ are the common velocity of the liquid and gas in the $j$ direction, the mixture density, the kinematic turbulent viscosity and the turbulent Schmidt number respectively. Here only RANS approach is treated and $\tilde{\phi}$ denotes the mass weighted Favre average of a variable $\phi$ decomposed into a mean part $\bar{\phi}$ and a fluctuating part $\phi^{\prime \prime}$. The classical Navier-Stokes equations are solved in a fully Eulerian context using VOF and the flow variables such as density, viscosity are averaged using the vapour mass fraction $x$, which is calculated with the following transport equation,

$$
\frac{\partial \bar{\rho} \tilde{x}}{\partial t}+\frac{\partial \bar{\rho} \tilde{u_{j}} \tilde{x}}{\partial x_{j}}=\Gamma
$$

Here $\Gamma$ is the source term that stands for the interfacial mass transfer and is related to the vapour mass production rate. This term is calculated using the HRM developed by Downar Zapolski in [13] and is written as a first order approximation as,

$$
\Gamma=\rho \frac{D x}{D t}=\rho \frac{x^{*}-x}{\Theta}
$$

Here $x^{*}$ is the equilibrium value of $x$ and $\Theta$ is a timescale in which $x$ would relax to $x^{*}$. The equilibrium value $x^{*}$ is calculated here assuming isenthalpic conditions as,

$$
x^{*}=\frac{h-h_{l, s a t}}{h_{v, s a t}-h_{l, s a t}}
$$

In this formulation, $h_{l, s a t}, h_{v, s a t}$ are the saturated enthalpies of liquid and vapour state. The timescale for the model is calculated as,

$$
\Theta=\Theta_{0} \alpha^{-0.257} \psi^{-2.24}
$$

and is validated for flashing releases of water at pressures up to ten bar. The non-dimensional pressure $\psi$ is equal $\left(p_{\text {sat }}-p\right) / p_{\text {sat }}$. The timescale $\Theta$ is a function of the constant $\Theta_{0}=6.51 \times 10^{-4}[s]$ and the void fraction given by,

$$
\alpha=\frac{\rho_{l}-\rho}{\rho_{l}-\rho_{v}}
$$

where $\rho_{l}$ and $\rho_{v}$ are the liquid and vapour densities. The ideal gas law is used for $\rho_{v}$ and $\rho_{l}$ is calculated using compressibility and a reference state as,

$$
\rho_{l}=\rho_{r e f}+\frac{\partial \rho}{\partial p}\left(p-p_{r e f}\right)
$$

\section{The Eulerian-Lagrangian Spray Atomisation (ELSA) model}

The ELSA model is used to model the atomisation process and has been extensively tested for spray injection in the automotive industry. It is used here to predict the characteristics of the liquid structures in the dense and dilute parts of the spray. The innovative idea that is introduced in ELSA, is the mean liquid/gas interface density $\Sigma$ for describing the spray. This definition is not limited to the assumption of spherical droplets which is a standard in many Lagrangian methods in commercial and open-source software. The interface density can be considered as the amount of spatial surface of liquid per unit volume at a given position, hence $\Sigma$ has units of inverse length, $m^{-1}$. In finite volume methods, the volume is the control volume as shown in figure 1. 


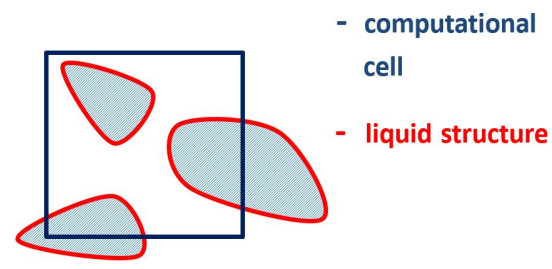

Figure 1. Surface density at a control volume. Bold red colour inside the computational cell indicates the liquid surface and the bold blue line is the control volume in a 2D projection.

The ELSA model has been originally developed and validated in RANS and is proven to accurately model the turbulent mixing $[15,20]$. It has been modified since its first appearance. Here we use the formulation proposed by Menard et al. [14]. The model was modified by Lebas et al. [15] and the $\Sigma$ equation can be written as,

$$
\frac{\partial \bar{\Sigma}}{\partial t}+\frac{\partial \tilde{u}_{j} \bar{\Sigma}}{\partial x_{j}}=\frac{\partial}{\partial x_{j}}\left(\frac{\nu_{t}}{S c_{t}} \frac{\partial \bar{\Sigma}}{\partial x_{j}}\right)+\Psi\left(S_{\text {init }}+S_{\text {turb }}\right)+(1-\Psi)\left(S_{\text {coll }}+S_{2 n d B U}\right)+S_{v a p}
$$

The model consists of several source terms on the RHS, which are associated to different processes that might have impact on the atomisation. The source terms can be calculated via different approaches. Here they are split for the dense and dilute part of the spray using an indicator function $\Psi$ which is equal to one if the liquid mass fraction, $\tilde{Y}_{l}$ is between 1 and 0.5 and is zero for cells with liquid mass fraction less than 0.1 . The indicator function can be written as a function of the liquid volume fraction, $\phi_{l}$, where $\phi_{l}=\bar{\rho} \tilde{Y}_{l} / \bar{\rho}_{l}$ as,

$$
\Psi\left(\phi_{l}\right)=H\left(\phi_{l}-0.1\right) H\left(\phi_{l}-0.5\right)+\left(H\left(\phi_{l}-0.1\right)-H\left(\phi_{l}-0.5\right)\right)\left(2.5 \phi_{l}-0.25\right)
$$

where $H()$ is the Heaviside step function. The term $S_{\text {init }}$ corresponds to the minimum liquid/gas surface produced in the atomisation process and is larger where the gradient of liquid mass fraction is higher according to,

$$
S_{\text {init }}=2 \frac{\mu_{t}}{S c_{t}} \frac{6 \bar{\rho}}{\rho_{l} \rho_{g} l_{t}} \frac{\partial \tilde{Y}_{l}}{\partial x_{i}} \frac{\partial \tilde{Y}_{l}}{\partial x_{i}}
$$

This expression is used in cases where $\tilde{Y}_{l}\left(1-\tilde{Y}_{l}\right)<0.001$. Otherwise the second fraction in equation 11 is equal to $\frac{\bar{\Sigma}}{\tilde{Y}_{l}\left(1-\tilde{Y}_{l}\right)}$. In the previous equation, $\rho_{g}$ is the density of ambient gas (still air in the present study) and $l_{t}$ is the turbulent length scale. Since the $S S T k-\omega$ model is used here, it is written as,

$$
l_{t}=C_{\mu}^{3 / 4} \frac{k^{1 / 2}}{\omega}
$$

where $C_{\mu}$ is a constant equal to 0.09. $S_{\text {turb }}$ is the term responsible for the production or destruction of the interface density due to stretching caused by turbulence and collisions/coalescences in the dense part of the spray. It is assumed that interface will be created or destroyed due to turbulence until $\Sigma$ reaches an equilibrium value, $\Sigma_{\text {turb }}^{*}$. The turbulent time-scale $\tau_{t}$ for this process is calculated from the turbulence model as,

$$
\tau_{t}=\frac{1}{\omega}
$$

Considering a Weber number equal to one at the equilibrium, the source term is then calculated as,

$$
S_{\text {turb }}=\frac{\bar{\Sigma}}{\tau_{t}}\left(1-\frac{\bar{\Sigma}}{\Sigma_{\text {turb }}^{*}}\right)
$$

At the dilute part of the spray, the liquid structures are treated in a Lagrangian manner. The source term for the surface creation/destruction due to collisions in the dilute areas is written in a similar way as in equation 14 . The collision time-scale $\tau_{\text {coll }}$ is calculated as,

$$
\tau_{\text {coll }}=\frac{1}{\bar{\Sigma} \sqrt{\frac{2 \tilde{k}}{3}}}
$$

where $\tilde{k}$ is the turbulent kinetic energy. Hence, the collisions source term is equal to,

$$
S_{\text {coll }}=\frac{\bar{\Sigma}}{\tau_{\text {coll }}}\left(1-\frac{\bar{\Sigma}}{\Sigma_{\text {coll }}^{*}}\right)
$$


The additional equilibrium term $\Sigma_{\text {coll }}^{*}$ is calculated via an equilibrium Weber number,

$$
W e_{\text {coll }}^{*}=\frac{\rho_{l} u_{\text {coll }}^{2} D_{32}}{\sigma_{l}}
$$

Where $\sigma_{l}$ is the surface tension of liquid. The characteristic collision velocity $u_{\text {coll }}$ is calculated via,

$$
u_{\text {coll }}=\sqrt{\frac{2}{3} \tilde{k}}
$$

The diameter $D_{32}$ is the characteristic size of the droplet, equivalent to the Sauter mean diameter and equals to,

$$
D_{32}=\frac{6 \bar{\rho} \tilde{Y}_{l}}{\rho_{l} \bar{\Sigma}}
$$

The equilibrium Weber $W e_{\text {coll }}^{*}$ is taken equal to 12 and the $\Sigma_{\text {coll }}^{*}$ is now calculated for equation 16 . The next term to model in equation 9 is $S_{2 n d B U}$, which comprises the surface production due to secondary break-up and is updated using an expression like equation 16. The time-scale $\tau_{2 n d B U}$ of the break-up is calculated using the experimental work of Pilch and Erdman [16]. This is a characteristic time where the liquid droplet will break-up due to RayleighTaylor or Kelvin-Helmholtz instabilities and is given by,

$$
\tau_{2 n d B U}=T \frac{D_{32}}{u_{r e l}} \sqrt{\frac{\rho_{l}}{\rho_{g}}}
$$

where the relative velocity between the two phases, $u_{r e l}$ can be computed using equation 2 as,

$$
u_{r e l}=\frac{R_{j l}}{\tilde{Y}_{l}\left(1-\tilde{Y}_{l}\right)}
$$

$T$ is the total dimensionless time defined as the time when the drop and its fragments no longer break-up and is taken from [16]. The equilibrium Weber number for the secondary break-up is equal to,

$$
W e_{2 n d B U}^{*}=12\left(1+1.077 O h^{1.6}\right)
$$

and assuming the effect of viscosity negligible, an equilibrium $\Sigma_{2 n d B U}^{*}$ is obtained and $S_{2 n d B U}$ is updated. Finally, the last term in equation 9 is $S_{v a p}$, which is responsible for the change in interface density due to evaporation. This term is usually omitted in the literature since there is no available model valid for all the spray regions. In superheated liquids such as Liquefied Natural Gas (LNG) or in many refrigerants, evaporation could be important in the equation for $\Sigma$. A simple correlation is introduced here,

$$
S_{\text {vap }}=-\frac{\bar{\Sigma}}{\Theta}\left(\frac{x^{*} \rho^{*}}{\rho}-x\right)
$$

where the "*" symbol stands for the thermodynamic equilibrium of each illustrated variable. The equations presented are solved in a segregated approach using the finite volume method. The method in this study was developed using OpenFOAM [17]. All the variables are stored in the centre of the control volumes and the interpolated fluxes are stored at the centre of the cell faces. The PIMPLE algorithm is used for the pressure velocity coupling. The algorithm proposed by Schmidt et al. in [10] is used inserting the contribution of phase change into the pressure equation. The PIMPLE algorithm is coupled with HRM in a way that considers the non-equilibrium vapour generation in the pressure-velocity coupling which is absent in standard OpenFOAM. Additionally, the available models for modelling the droplet evaporation were found to give less accurate predictions in the vapour mass fraction and velocity, especially in the dense spray region, something that is not observed with HRM.

\section{Results and discussion}

Numerical simulations were performed using the series of experiments from the FLIE project [18-19] investigating flashing releases of R134A (1,1,1,2 - Tetrafluoroethane: CF3-CH2F). The experimental domain consists of a high pressure region where R134A is stored at a pressure above its vapour pressure at ambient conditions (663 $k P a$ at $293.15 \mathrm{~K}$ ). The liquid passes through a nozzle of diameter $D$ and length $L$ and is released into a low-pressure region which is equal to the atmospheric pressure. The computational domain and its discretisation consisting of 2.0 million hexahedral cells are shown in figure 2. The curvature at the walls in the nozzle exit is not considered for the present simulations. The liquid core is more dense around the centreline of the jet, which indicates that finer mesh is needed there. The physical parameters for the simulations are shown in Table 1. The cases tested here concern the geometry in which $L=4 \mathrm{~mm}$ and $D=2 \mathrm{~mm}$. Keeping in mind the importance of the flash boiling or the geometry impact on the spray, the flow is simulated inside the nozzle avoiding arbitrary assumptions for the jet inlet in the lower pressure region. Figure 3 illustrates the velocity distribution. The jet is emerging in the atmosphere with a velocity of approximately $32 \mathrm{~m} / \mathrm{s}$, which is in good agreement with the experimental observations. The liquid core that is observed in the vicinity of the centreline of the jet, has the highest velocity. 
Table 1. Physical properties for simulations.

\begin{tabular}{c|c}
\hline & Physical parameters for simulations \\
\hline Inlet pressure & $800 \mathrm{kPa}$ \\
Inlet temperature & $293.35 \mathrm{~K}$ \\
Outlet pressure & $100 \mathrm{kPa}$ \\
Outlet temperature & $298 \mathrm{~K}$ \\
$L / D$ & 2 \\
Superheat degree & $47 \mathrm{~K}$
\end{tabular}

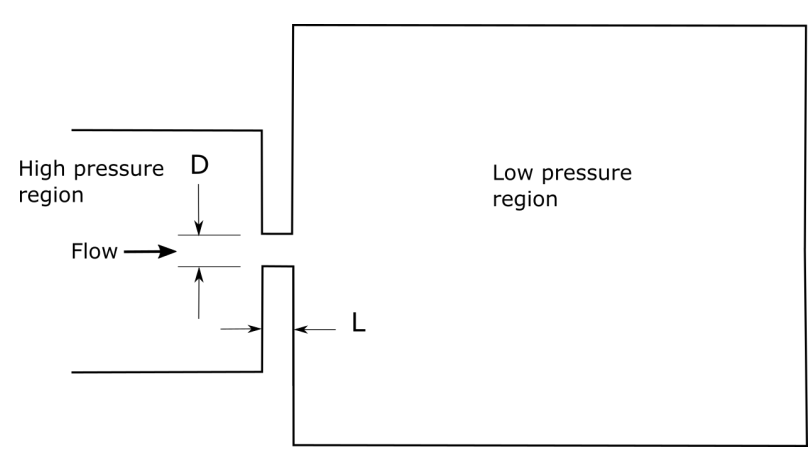

(a) Simplified sketch of the domain for the experiments in [19].

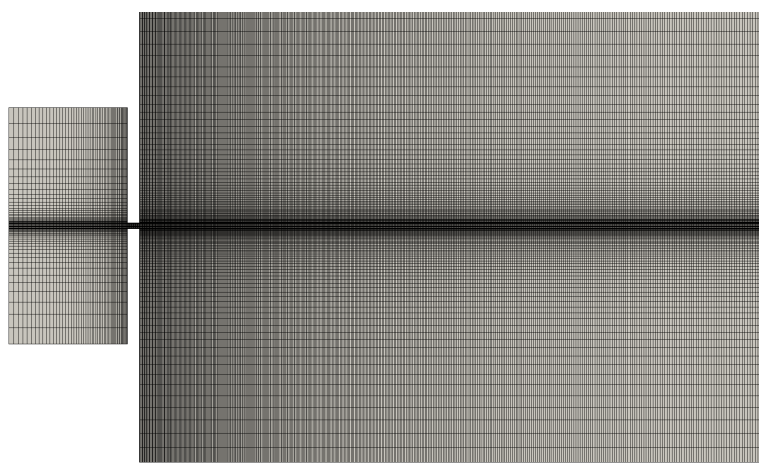

(b) Discretisation of the domain.

Figure 2. Representation of the domain

This is obvious in both inside and outside the nozzle. As long as we move along the centreline the jet morphology changes with an enhanced evaporation on its surface. The change in the jet regime at the beginning of the release is clearer in figure 4.

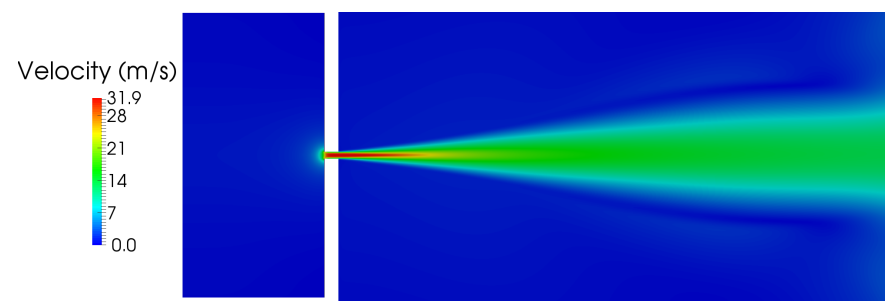

(a) Velocity profile projection for the selected case.

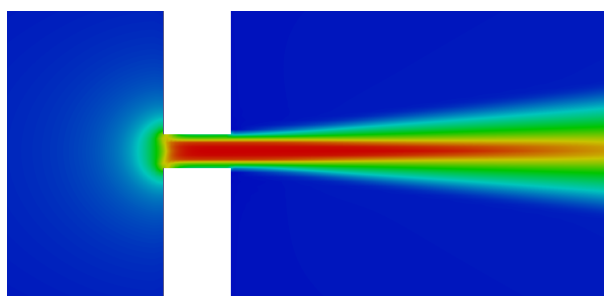

(b) A closer caption of the velocity.

Figure 3. Converged jet velocity.

The liquid core is dense with a high number of liquid ligaments close to the nozzle and the liquid volume fraction has its highest value, close to one. A few diameters away from the nozzle exit, the liquid volume fraction decreases until it reaches the lowest limit of zero. For a distance up to $200 \mathrm{D}$, two regions of this decrease can be identified which correspond to different slopes in the graph in figure 4-b. The same trends are observed in other numerical works (see in [20]). The bandwidth of these regions is affected by the thermophysical properties and is expected to differ for cryogenic releases and non-evaporating jets. Previous studies in flashing jets indicate a rapid phase change as soon as the local pressure drops lower than the saturation pressure. The initiation of this phase change is more likely to happen within the nozzle for longer nozzles but is also possible for short nozzles with $L / D$ less than seven like here, even in low superheat degrees [8]. The jet vaporises within the nozzle, and the flow separation at the sharp inlet corners results in a pressure drop and consequently in phase change (for more details see in [21]). Because of this, the flow becomes two-phase and the liquid volume fraction decreases close to the nozzle walls as shown in figure 4-a. The same behaviour is observed in the density. Hence, the jet at the nozzle exit, cannot be considered as a pure liquid. Additionally, the method presented in the previous chapter, can predict the amount of the vaporised liquid giving a realistic prediction for the jet morphology downstream.

Another important parameter in the atomisation modelling is the mean interface density and its evolution. $\Sigma$ is calculated via equation 9 comprising all the possible processes that could cause an increase or decrease in the liquid/gas interface. For this reason a second order of accuracy bounded scheme for the convective terms in the $\Sigma$-equation is used. A typical distribution of $\Sigma$ is illustrated in figure 5 . It is expected to be higher closer to the nozzle exit where the initiation term Sinit dominates. The source term $S_{\text {init }}$ dominates the $\Sigma$ generation initially and together with $S_{\text {turb }}$ are the most important source terms in the primary atomisation region. On the contrary the 


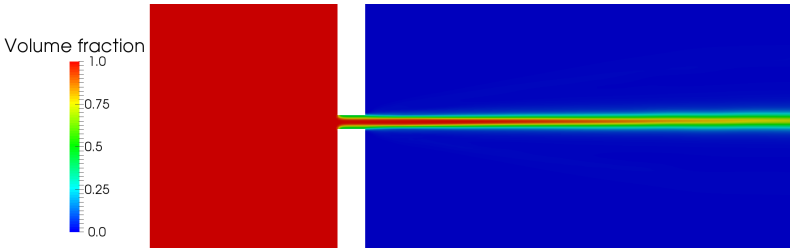

(a) In the whole domain.

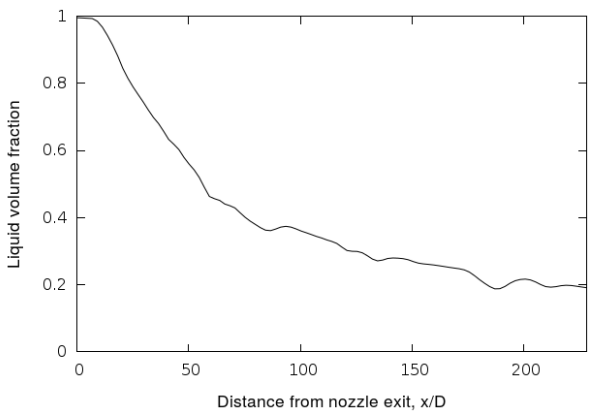

(b) In the centreline of the jet.

Figure 4. Liquid volume fraction for the properties of Table 1.

terms $S_{\text {coll }}, S_{2 n d B U}$ are less important in the vicinity of the nozzle exit hence $\Psi$ is equal to 1 in this case. The terms $S_{\text {coll }}, S_{2 n d B U}$ are dominant in the secondary break-up region where droplets are generated due to aerodynamic break-up and collisions. These terms prevail in the $\Sigma$-equation and $\Psi$ tends to zero for the diluted regions. The terms $S_{\text {init }}$ and $S_{\text {turb }}$ are expected to be of minor importance in this case. The source term $S_{\text {vap }}$ is more important in the primary atomisation and two/three dimensional numerical investigations showed that usually is smaller than the other source terms in equation 9. Definitely, further examination of equation 23 must be conducted. From equation 11, the initial interface density is a function of the liquid mass fraction gradient which is greater close to the injection region. After the injection, the jet starts to fragment and interface is created. Consequently, $\Sigma$ is increasing. During the primary atomisation, large blobs and ligaments form, which is also reported in [19] in the experiments. After reaching a peak value, $\Sigma$ starts to decrease rapidly. Some diameters after the peak value, the interface density reduces to $\mathcal{O}(10)$. In this region, apart from the evaporation process, the secondary break-up takes place and causes the large ligaments to burst into smaller droplets which leads to an increase in the interface density. That is why the source term $S_{2 n d B U}$ is positive in equation 9 . The contribution of the source term due to droplet collisions, $S_{\text {coll }}$ is also included in this part of the spray. The droplet size is defined in the regions where the liquid mass fraction and interface density are non-zero. It is calculated via equation 19 and some two-dimensional results are shown in figure 5 for the Sauter mean diameter $\left(D_{32}\right)$ along the jet centreline. The $D_{32}$ is higher close to the nozzle exit where the jet is almost pure liquid at the centreline. After almost 5 diameters, the $D_{32}$ order is of some hundreds of microns. As mentioned before, flashing is a process very efficient in the aerosol industry for producing fine sprays. Hence, the resulting $D_{32}$ values are reasonable and in relatively good agreement with the experimental data from [19]. Three-dimensional simulations performed showed that along the radial axis, $D_{32}$ is maximum at the jet centreline and decreases along the radial axis.

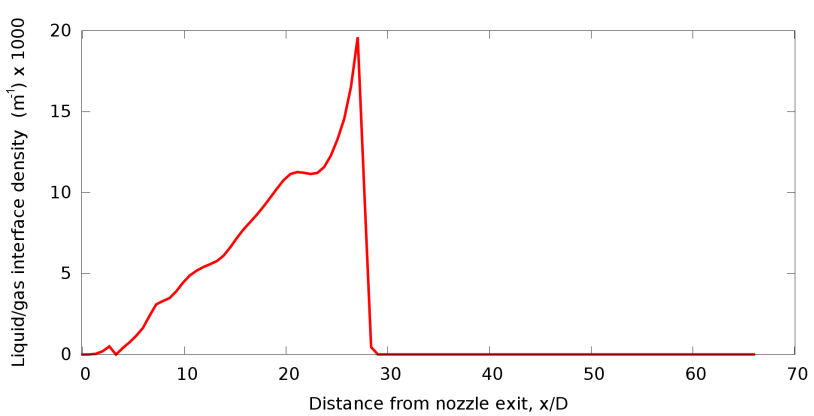

(a) Axial interface density distribution.

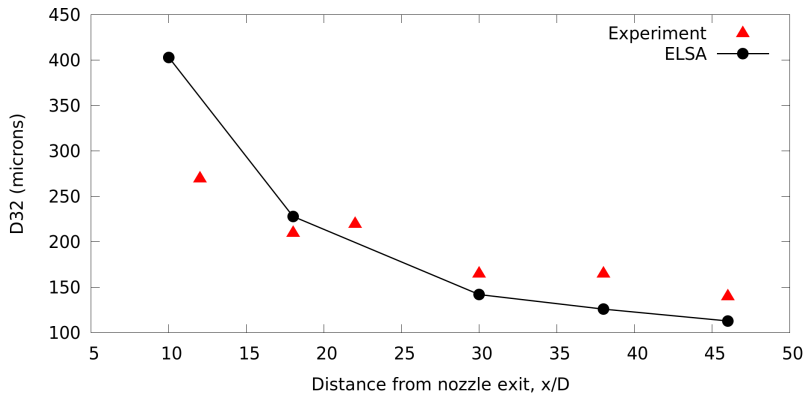

(b) Sauter mean diameter in the centreline of the jet.

Figure 5. ELSA model simulations.

The axial velocity obtained from ELSA at the primary atomisation region is shown in figure 6 compared to the PIV and PDA experimental results. The velocity ranged in the simulations between $32 \mathrm{~m} / \mathrm{s}$ in the dense region, and $1 \mathrm{~m} / \mathrm{s}$ in the diluted regions. In the simulations, the peak velocity is observed a few diameters after the jet exits to the atmosphere whereas in the experiments the peak velocity is reported in the region between $10-18 D$ as illustrated in figure 6 . The velocity obtained from ELSA model is high and almost constant within this range, but decreases with further increasing the distance from the nozzle exit. For example it gets half at $x / D=110$ [19].

\section{Conclusions}

An Eulerian-Lagrangian Atomisation Spray method using RANS, implemented within OpenFOAM, is presented. The model follows the original implementation of $\Sigma-Y$ model, solving two equations, one for the liquid mass fraction, and another one for the interface density. Source terms for accounting for the creation/destruction of the interface due to mechanical and thermodynamic effects are used in the modelling. A novel expression for the change in sur- 


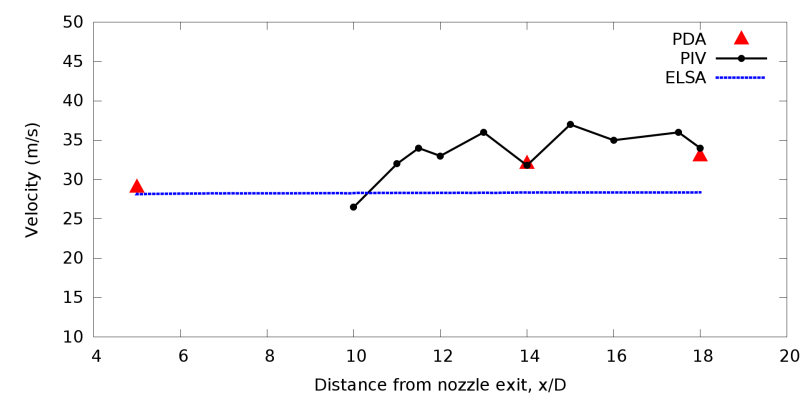

Figure 6. Velocity along the jet centreline.

face density in cryogenic jets is used here and is inserted in the $\Sigma$-equation. The applications of the method include fuel atomisers, accidental releases and other situations of a sudden pressure drop of a superheated/sub-cooled liquids in a low pressure environment.

The ELSA approach, combines the advantages of Eulerian and Lagrangian frameworks, and uses explicit formulations for modelling the droplet characteristics like the Sauter mean diameter. Preliminary results show that the method can properly describe the droplet sizes along the jet centreline. Further research should be made for the implicit/explicit treatment of the source terms in the $\Sigma-Y$ model and the impact of the turbulence modelling approach on the primary atomisation.

\section{Acknowledgements}

The authors gratefully thank the financial support by the Marie Curie Action of the 7th framework Programme of the European Commission. The current work is part of the SafeLNG project (project ID: 606754) on the numerical characterisation and simulation of the complex physics underpinning the safe handling of liquefied natural gas (2014-2018).

\section{Nomenclature}

$C_{\mu} \quad$ constant of turbulence model [-]

$D \quad$ diameter $[m]$

$D_{32} \quad$ Sauter mean diameter, SMD $[m]$

$h \quad$ enthalpy $[\mathrm{J} / \mathrm{kg}]$

$H \quad$ Heaviside step function [-]

$k \quad$ kinetic energy $\left[\mathrm{m}^{2} \mathrm{~s}^{-2}\right]$

$L \quad$ length of nozzle $[\mathrm{m}]$

$l_{t} \quad$ turbulent length scale $[m]$

Oh Ohnesorge number, $\mu_{l} / \rho_{l} D_{32} \sigma_{l}[-]$

$p \quad$ pressure $[k P a]$

$S_{\text {init }} \quad$ minimum liquid/gas interface production $\left[\mathrm{m}^{-1} \mathrm{~s}^{-1}\right]$

$S_{\text {turb }} \quad$ source term for interface production/destruction due to turbulence $\left[\mathrm{m}^{-1} \mathrm{~s}^{-1}\right]$

$S_{\text {coll }} \quad$ interface density source term due to collisions $\left[\mathrm{m}^{-1} \mathrm{~s}^{-1}\right]$

$S_{2 n d B U}$ interface density source term due to secondary break-up $\left[m^{-1} s^{-1}\right]$

$S_{v a p} \quad$ interface density source term due to evaporation $\left[\mathrm{m}^{-1} \mathrm{~s}^{-1}\right]$

$S c_{t} \quad$ turbulent Schmidt number, $\mu / \rho D_{m}[-]$, where $D_{m}=$ mass diffusivity $\left[m^{2} s^{-1}\right]$

$t \quad$ time $[s]$

$u_{j} \quad$ velocity $[\mathrm{m} / \mathrm{s}]$

$u_{\text {coll }} \quad$ characteristic collision velocity $[\mathrm{m} / \mathrm{s}]$

$W e_{\text {coll }}^{*}$ equilibrium Weber number characteristic for droplets collisions $[\mathrm{m} / \mathrm{s}]$

$W e_{2 n d B L}^{*}$ equilibrium Weber number characteristic for secondary break-up $[\mathrm{m} / \mathrm{s}]$

$x \quad$ vapour mass fraction [-]

$x^{*} \quad$ equilibrium vapour mass fraction [-]

$\phi_{l}, \tilde{Y} \quad$ liquid volume fraction, mass weighted Favre averaged mass fraction [-]

$\alpha \quad$ void fraction [-]

$\Gamma \quad$ interfacial mass transfer in the HRM $\left[\mathrm{kgm}^{-3} \mathrm{~s}^{-1}\right]$

$\Theta \quad$ relaxation time scale $[s]$

$\Theta_{0} \quad$ constant in the HRM $[s]$

$\mu, \mu_{t} \quad$ dynamic viscosity, dynamic turbulent viscosity $\left[\mathrm{kgm}^{-1} \mathrm{~s}^{-1}\right]$

$\nu_{t} \quad$ kinematic eddy viscosity $\left[\mathrm{m}^{2} / \mathrm{s}\right]$

$\rho \quad$ density $\left[\mathrm{kgm}^{-3}\right]$

$\sigma \quad$ surface tension $\left[\mathrm{kgs}^{-2}\right]$ 


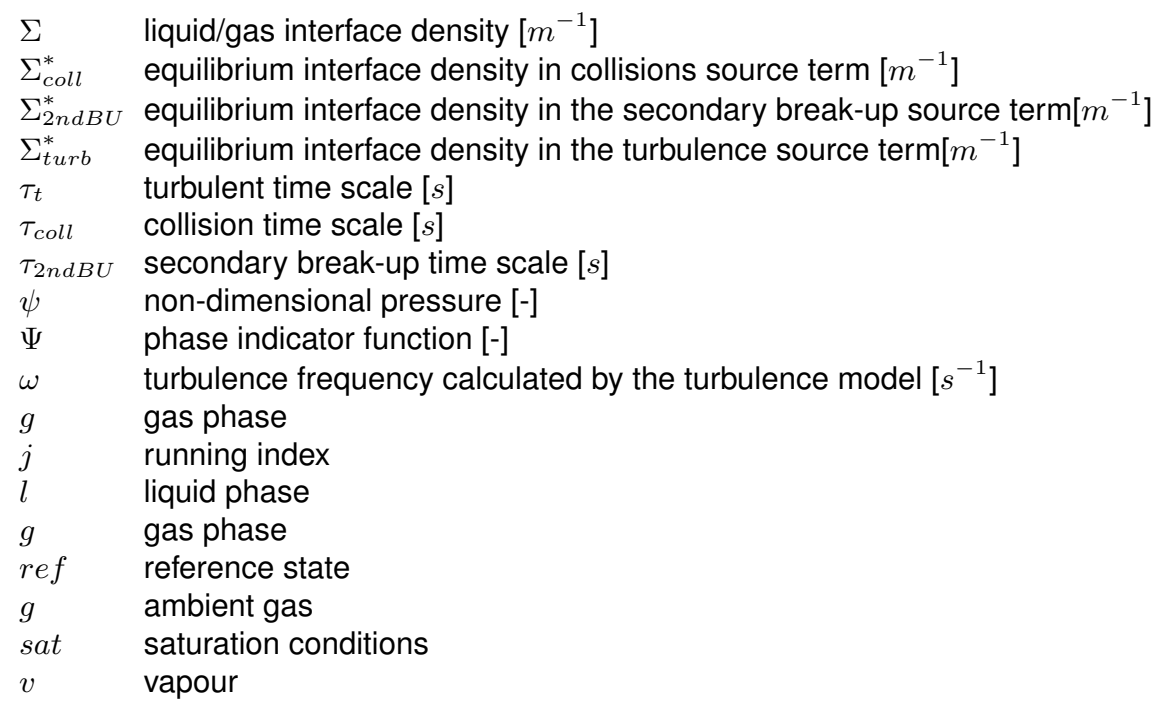

\section{References}

[1] Oza, R.D.,Sinnamon, J.F., 1983, "An experimental and analytical study of flash boiling fuel injection”, SAE paper, 830590.

[2] Witlox, H., Bowen, P., 2002, "Flashing liquid jets and two-phase dispersion. A review". Contract research report 403/2002, HSE.

[3] Sher, E.,Bar-Kohany, T., Rashkovan A. 2008, "Flash boiling atomization", Progress in Energy and Combustion Science, 34 (4), pp 417-439.

[4] Zuo B., Gomes A.M., Rutland C.J., 2000, "Modelling Superheated Fuel Spray Vaporization”, Int. Journal of Engine Research, (1), pp. 321-326.

[5] Reitz R., 1990, "A Photographic Study of Flash-Boiling Atomization", Aerosol Science and Technology, (12) 3, pp. 561-569.

[6] Park C.K., Park J. W., Chung M. K., Chun M. K., 1997, "An empirical correlation for Critical Flow Rates of Subcooled Water Through Short Pipes with Small diameters", Journal of the Korean Nuclear society, (29) 1, pp. 35-44.

[7] Xu J., Chin T., Yang L., 1995, "Two-Phase Critical Discharge of Initially Saturated or Subcooled Water Flowing in Sharp-Edged Tubes at High Pressure”, Journal of Thermal Science, (4) 3, pp. 193-199.

[8] Simoneau R.,1975, "Maximum two-phase flow rates of subcooled Nitrogen through a sharp-edged orifice", NASA TM X-71760.

[9] Lee J., Madabhushi R., Fotache C., Gopalakrishnan S., Schmidt D., 2009, "Flashing flow of superheated jet fuel", Proceedings of the Combustion Institute, (31), pp. 3215-3222.

[10] Schmidt D.P., Gopalakrishnan S., Jasak H., 2010, "Multi-dimensional simulation of thermal non-equilibrium channel flow", International Journal of Multiphase Flow, (36), pp. 284-292.

[11] Vallet A., Borghi R., 1999, C. R. Acad. Sci., Paris, Sér. II b, (327), pp. 1015-1020.

[12] Vallet A., Burluka A., Borghi R., 2001, "Development of an eulerian model for the "atomization" of a liquid jet", Atomisation and Sprays, (31), pp. 619-642.

[13] Downar-Zapolski, P., Bilicki, Z., Bolle, L., Franco, J., 1996, "The non-equilibrium relaxation model for onedimensional flashing liquid flow", International Journal of Multiphase Flow, (22), pp. 473-483.

[14] Menard T., Beau P. A., Tanguy S., Demoulin F. X., Berlemont A., 2005, "Primary break-up: DNS of liquid jet to improve atomization modelling", Computational Methods in Multiphase Flow III, (50), pp. 343-352.

[15] Lebas, R., Menard, T., Beau, P., Berlemont, A., Demoulin, F., 2009, "Numerical simulation of primary break-up and atomization: DNS and modelling study", International Journal of Multiphase Flow, (35), pp. 247-260.

[16] Pilch, M., Erdman, C.A., 1987, "Use of breakup time data and velocity history data to predict the maximum size of stable fragments for acceleration-induced break-up of a liquid drop", International Journal of Multiphase Flow, 13 (6), pp. 741-757.

[17] Weller H. G., Tabor G., Jasak H., Fureby C., 1998, "A tensorial approach to computational continuum mechanics using object-oriented techniques”, Comput. Phys., 12, pp. 620-631.

[18] Yildiz D., Van Beeck J., Rambaud P., Buchlin J. M., 17-20 July 2006, Proceedings of FEDSM2006, Joint U.S.European Fluids Engineering Summer Meeting.

[19] Yildiz D., 2005, Experimental investigation of superheated liquid jet atomization due to flashing phenomena, $\mathrm{PhD}$ thesis Université Libre de Bruxelles/von Karman Institute. ISBN: 978-2-930389- 49-4.

[20] Navarro-Martinez S., 2014, "Large eddy simulation of spray atomization with a probability density function method", International Journal of Multiphase Flow, 63, pp. 11-22.

[21] Schmidt, David P., 1997, Cavitation in Diesel Fuel Injector Nozzles. PhD thesis, Univ. of Wisconsin Madison. 


\title{
Supercritical and transcritical real-fluid mixing using the PC-SAFT EOS
}

\author{
C. Rodriguez ${ }^{\star 1}$, A. Vidal ${ }^{1}$, P. Koukouvinis ${ }^{1}$, M. Gavaises ${ }^{1}$ \\ ${ }^{1}$ School of Mathematics, Computer Science and Engineering, City, University of London, UK \\ *Corresponding author: Carlos.Rodriguez@city.ac.uk
}

\begin{abstract}
A numerical framework has been developed to simulate the mixing of supercritical and transcritical fluids using an equation of state based on statistical associating fluid theory. In a Diesel engine the liquid fuel is injected into supercritical air. After the injection, the Diesel is heated over its critical temperature reaching a supercritical state. Modelling real-fluid effects is critical in order to properly characterize the air/fuel mixing in the combustion chamber. By using the PC-SAFT EoS (Perturbed Chain Statistical Association Fluid Theory Equation of State) real fluids effects can be taken into account in a CFD simulation. The PC-SAFT EoS shows best results than cubic EoS computing liquid density, compressibility, speed of sound, vapor pressures and density derivatives. Unlike cubic EoS, this model accounts for the shape and size of the molecules. Gas, liquid, supercritical and vapor-liquid equilibrium states can be simulated. PT FLASH (Isothermal Multiphase Flash Calculation) is applied to compute the phase diagram used by the code. Shock tube problems were conducted in a wide range of pressures and densities using $n$-dodecane to show the capability of the developed algorithm. The results were compared with the solution of an exact Riemann solver which has the PC-SAFT EoS implemented showing a high degree of agreement. In addition, a two-dimensional simulation of supercritical nitrogen jet mixing was carried out to check the multidimensional capability of the code.
\end{abstract}

\section{Keywords}

Supercritical, transcritical, PC-SAFT EoS

\section{Introduction}

Small soot particles generated in Diesel engines are linked to important health issues such as heart attacks, premature death, bronchitis or asthma among children. Recent studies [1] has shown that a supercritical Diesel combustion can reduce the emissions of particulate matter and nitrogen oxides. The significant reduction (or even almost disappearance) of the fuel vaporization time has been linked with reduced harmful emissions [2, 3] while at the same time, it may improve engine thermodynamic efficiency due to engine operation at higher compression ratios.

In Diesel engines the liquid fuel is injected into air that has pressure and temperature conditions higher than the critical point of the fuel. The liquid injection temperature is lower than the fuel critical temperature but as the liquid is heated up, it may reach supercritical temperature before its full vaporisation. This process is known as transcritical injection. A single fluid or a mixture reaches a supercritical state when the pressure and the temperature exceed its critical values. Under such conditions, the repulsive atomic forces overcome the surface tension, resulting in the existence of a single phase. A supercritical phase shows properties of both gases and liquids (e.g., gas-like diffusivity and liquid-like density).

Simulating supercritical flows makes necessary the implementation of a real-gas equation of state (EoS), which takes into account the intermolecular repulsive forces in the relationship among pressure, density and temperature. Nowadays, cubic EoS are standard to compute supercritical and transcritical thermodynamics in CFD simulations. Soave-Redlich-Kwong and Peng-Robinson EoS are widely utilised. By using the Péneloux volume correction [4], the low values of liquid density provided by the original version of these models can be fixed, but the calculation of gas compressibility factors and pressure derivatives are still inaccurate [5].

In the last few decades more advanced models have been developed. The SAFT EoS is an advanced molecular model which can precisely compute the thermodynamic properties of complex mixtures. It is based on the perturbation theory extensively studied by Wertheim [6-9]. Chapman et al. [10,11] developed this EoS by applying Wertheim's theory and extending it to mixtures. 
In the SAFT model the reference fluid is formed by spherical segments of equal size. Then attractive forces are added between molecules. After this, chains are forms with hard-sphere segments and finally associative forces are added between molecules. Figure 1 shows a physical representation of the different contributions.
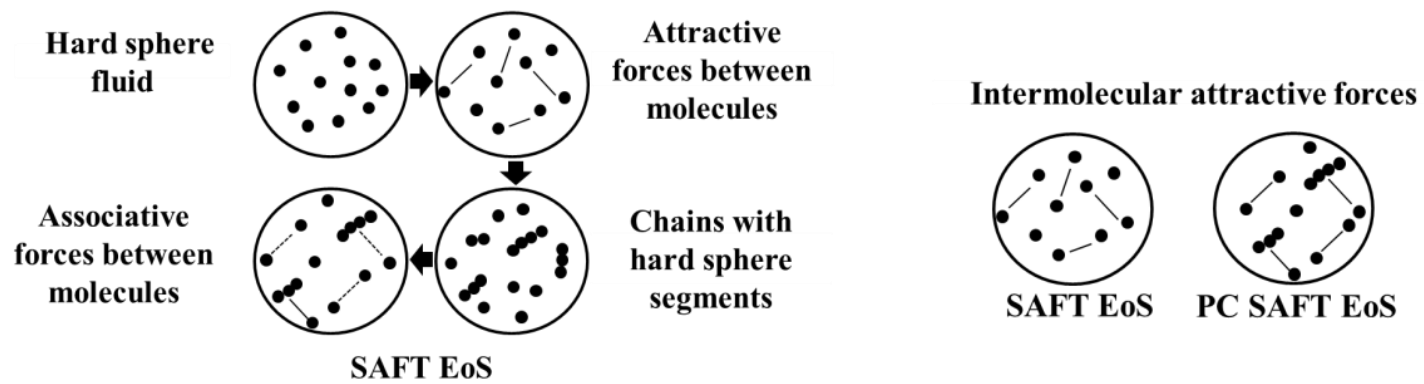

Figure 1. Physical description of the attractive and repulsive contributions of the SAFT EoS and the PC-SAFT EoS [12]

Many variants of the SAFT model exist. Among them, the PC-SAFT EoS (Perturbed Chain Statistical Association Fluid Theory EoS) is the one implemented here. In this variant, hard chains are used as the reference fluid instead of hard spheres. The SAFT EoS compute the attractive interaction between individual molecules and chain segments while in the PC-SAFT EoS the interactions between entire molecules are considered. This improves the thermodynamic description of chain-like fluid mixtures [12].

Although this EoS is based on advance molecular theory, only three parameters of each component have to be specified to compute the thermodynamic properties of the mixtures. The performance of the PC-SAFT model is superior to cubic EoS computing heat capacities, speed of sound, pressure-volume derivative and pressuretemperature derivative [13]. The results for gas Z-factors and compressibility are more accurate as well. It shows a good prediction of liquid densities and pure component vapor pressures. Additionally, it accounts for the shape and size of the molecules as it is based on statistical mechanics.

\section{Mathematical formulation}

The Navier-Stokes equations for a non-reacting multi-component mixture containing $\mathrm{N}$ species in a $2 \mathrm{D}$ flow are given by:

$\frac{\partial \mathbf{U}}{\partial \mathrm{t}}+\frac{\partial \mathbf{F}}{\partial \mathrm{x}}+\frac{\partial \mathbf{G}}{\partial \mathrm{y}}=\frac{\partial \mathbf{F}_{\mathrm{v}}}{\partial \mathrm{x}}+\frac{\partial \mathbf{G}_{\mathrm{v}}}{\partial \mathrm{y}}$

The vectors of eq. 1 are:

$\mathbf{U}=\left[\begin{array}{c}\rho \mathrm{Y}_{1} \\ \vdots \\ \rho \mathrm{Y}_{\mathrm{N}} \\ \rho u \\ \rho \mathbf{v} \\ \rho E\end{array}\right\rfloor, \mathbf{F}=\left[\begin{array}{c}\rho u Y_{l} \\ \vdots \\ \rho u Y_{N} \\ \rho u^{2}+p \\ \rho u v \\ (\rho E+p) u\end{array}\right\rfloor, \mathbf{G}=\left[\begin{array}{c}\rho u Y_{l} \\ \vdots \\ \rho u Y_{N} \\ \rho u v \\ \rho \mathrm{v}^{2}+p \\ (\rho E+p) v\end{array}\right\rfloor, \mathbf{F}_{\mathrm{v}}=\left[\begin{array}{c}-\mathrm{J}_{\mathrm{x}, 1} \\ \vdots \\ -\mathrm{J}_{\mathrm{x}, \mathrm{N}} \\ \sigma_{\mathrm{xx}} \\ \sigma_{\mathrm{xy}} \\ \mathrm{u} \sigma_{\mathrm{xx}}+\mathrm{v} \sigma_{\mathrm{xy}}-\mathrm{q}_{\mathrm{x}}\end{array}\right\rfloor$

$\mathbf{G}_{\mathbf{v}}=\left[\begin{array}{c}-\mathbf{J}_{\mathrm{y}, 1} \\ \vdots \\ -\mathbf{J}_{\mathrm{y}, \mathrm{N}} \\ \sigma_{\mathrm{yx}} \\ \sigma_{\mathrm{yy}} \\ \mathrm{u} \sigma_{\mathrm{yx}}+\mathrm{v} \sigma_{\mathrm{yy}}-\mathrm{q}_{\mathrm{y}}\end{array}\right]$ 
where $\rho$ is the fluid density, $\mathrm{u}$ and $\mathrm{V}$ are the velocity components, $\mathrm{p}$ is the pressure, $\mathrm{E}$ is the total energy, $\mathrm{J}_{\mathrm{i}}$ is the mass diffusion flux of species $\mathrm{i}, \sigma$ is the deviatoric stress tensor and $\mathrm{q}$ is the diffusion heat flux vector.

A finite volume scheme is employed in this work. The PC-SAFT EoS is implemented in order to simulate supercritical and transcritical states. Operator splitting is adopted to divide the physical processes into hyperbolic and parabolic sub-steps. The global time step is computed using the CFL criterion of the hyperbolic operator.

\subsection{Hyperbolic sub-step}

The HLLC solver is used to solve the Riemann problem. The conservative variables are interpolated onto the cell faces using a fifth-order WENO (Weighted Essentially Non-Oscillatory) scheme due to its high order accuracy and non-oscillatory behaviour. Time integration is performed by using a third order TVD RK (Total Variation Diminishing Runge-Kutta) method.

\subsection{Parabolic sub-step}

The method developed by Chung et al. [14] is used to calculate the values of the dynamic viscosity and thermal conductivity of the mixture. In order to compute the diffusion coefficient the model of Riazi et al. [15] is utilised. A TVD scheme of third order is used to perform the time integration of this sub-step. The diffusion fluxes are computed conservatively using a second order method.

\subsection{PC-SAFT EOS}

In perturbation theory, the potential energy of the molecular fluid is described as the sum of the potential energy of the reference fluid and a perturbation. The PC-SAFT EOS (based on perturbation theory) is expressed as the sum of all the residual Helmholtz free energy contributions. These contributions correspond to the different types of molecular interactions. The Helmholtz free energy was computed using eq.3 [16]

$\tilde{a}^{r e s}=\tilde{a}^{h c}+\tilde{a}^{\text {disp }}$

$\tilde{a}^{h c}=\bar{m} \tilde{a}^{h s}-\sum_{i} x_{i}\left(m_{i}-1\right) \ln g_{i i}^{h s}\left(\sigma_{d i i}\right)$

$\tilde{a}^{h s}=\frac{1}{\varsigma_{0}}\left|\frac{3 \varsigma_{1} \varsigma_{2}}{\left(1-\varsigma_{3}\right)}+\frac{\varsigma_{2}^{3}}{\varsigma_{3}\left(1-\varsigma_{3}\right)^{2}}+\left(\frac{\varsigma_{2}^{3}}{\varsigma_{3}^{2}}-\varsigma_{0}\right) \ln \left(1-\varsigma_{3}\right)\right|$

$\tilde{a}^{\text {disp }}=-2 \pi \rho_{m} I_{1}(\eta, \bar{m}) \overline{m^{2} \varepsilon \sigma_{d}^{3}}-\pi \rho_{m} \bar{m} C_{1} I_{2}(\eta, \bar{m}) \overline{m^{2} \varepsilon^{2} \sigma_{d}^{3}}$

$$
\varsigma_{n}=\frac{\pi}{6} \rho_{m} \sum_{i} x_{1} m_{i} d_{i}^{n} \quad n \in\{0,1,2,3\}
$$

The thermodynamic variables computed by the PC-SAFT model are the temperature, pressure, sonic fluid velocity, enthalpy, entropy, fugacity and Gibbs free energy. The algorithm inputs are the density, internal energy and three pure component parameters (number of segments per chain, energy parameter of each component and segment diameter). The density and the internal energy are obtained from the conservative variables of the CFD code. The pure component parameters are specified in the initialization of the simulation.

A Newton Raphson method is employed to compute the temperature. The temperature is required to calculate the value of all other thermodynamic variables. The temperature dependent function used in the iterative method is the internal energy. When this converges to the value provided by the conservative variables, the iterative process is terminated.

\subsubsection{Iteration process}

a) Guessing an initial temperature value

The value of the temperature from the previous time RK sub-step or the previous time step is used to initialize the iteration process. In most cells this value will be close to the solution (assuming a small time step). 
b) Computing the compressibility factor

It is calculated as the sum of the ideal gas contribution (considered to be 1), the dispersion contribution and the residual hard-chain contribution [16].

$Z=1+Z^{h c}+Z^{\text {disp }}$

$Z^{h s}=\frac{\varsigma_{3}}{\left(1-\varsigma_{3}\right)}+\frac{3 \varsigma_{1} \varsigma_{2}}{\varsigma_{0}\left(1-\varsigma_{3}\right)^{2}}+\frac{3 \varsigma_{2}^{3}-\varsigma_{3} \varsigma_{2}^{3}}{\varsigma_{0}\left(1-\varsigma_{3}\right)^{3}}$

$Z^{h c}=\bar{m} Z^{h s}-\sum_{i} x_{i}\left(m_{i}-1\right)\left(g_{i i}^{h s}\right)^{-1} \rho_{m} \frac{\partial g_{i i}^{h s}}{\partial \rho_{m}}$

$Z^{d i s p}=-2 \pi \rho_{m} \frac{\partial\left(\eta I_{1}\right)}{\partial \eta} \overline{m^{2} \varepsilon \sigma_{d}^{3}}-\pi \rho_{m} \bar{m}\left\lfloor C_{1} \frac{\partial\left(\eta I_{2}\right)}{\partial \eta}+C_{2} \eta I_{2}\right\rfloor \overline{m^{2} \varepsilon^{2} \sigma_{d}^{3}}$

Where $\mathrm{C}_{1}, \mathrm{C}_{2}$ are abbreviations [16].

c) Computing the pressure

The pressure can be calculated using eq.12 once the compressibility factor is known [16].

$P=Z k T \rho_{m}\left(10^{10}\right)^{3}$

d) Computing the internal energy

It is the sum of the ideal internal energy and the residual internal energy. The residual internal energy is calculated by using eq.13 [17].

$\frac{U^{r e s}}{R T}=-T\left(\frac{\partial \tilde{a}^{r e s}}{\partial T}\right)_{\rho, x_{i}}$

If the computed total internal energy is not close enough to the value obtained from the conservative variables the Newton Raphson method is applied to calculate a new value of the temperature and repeat steps b-d.

\subsubsection{Other thermodynamic properties}

Once the temperature value is known the following properties are computed.

Heat capacities: They are computed as the sum of the ideal contribution (Poling et al. [18]) and the correction done by the PC-SAFT EoS (eq.15) [17].

$$
C_{v}^{r e s}=-R T\left[2\left(\frac{\partial \tilde{a}^{r e s}}{\partial T}\right)_{\rho, x_{i}}+T\left(\frac{\partial^{2} \tilde{a}^{r e s}}{\partial T^{2}}\right)_{\rho, x_{i}}\right], C_{p}^{r e s}=C_{v}^{r e s}+R \frac{\left[\rho_{m} T\left(\frac{\partial^{2} \tilde{a}^{r e s}}{\partial \rho_{m} \partial T}\right)_{x_{i}}+\rho_{m}\left(\frac{\partial \tilde{a}^{r e s}}{\partial \rho_{m}}\right)_{T, x_{i}}+1\right]^{2}}{\left[\rho_{m}^{2}\left(\frac{\partial^{2} \tilde{a}^{r e s}}{\partial \rho_{m}{ }^{2}}\right)_{T, x_{i}}+2 \rho_{m}\left(\frac{\partial \tilde{a}^{r e s}}{\partial \rho_{m}}\right)_{T, x_{i}}+1\right]}
$$

Total enthalpy: It is used to compute the thermal diffusion vector in the parabolic sub-step. The total enthalpy is the sum of the ideal contribution (obtained by integrating the ideal heat capacity at constant pressure with respect to the temperature) and the residual enthalpy (eq.14) [16].

$\frac{\hat{h}^{r e s}}{R T}=-T\left(\frac{\partial \tilde{a}^{r e s}}{\partial T}\right)_{\rho, x_{i}}+(Z-1)$ 
Total entropy: Likewise the enthalpy, it is the sum of the ideal and the residual contributions. The ideal contribution is computed using the formula of the entropy for ideal gases and the residual contribution is computed using eq. 16 [16].

$$
\frac{\hat{s}^{r e s}(P, T)}{R}=-T\left\lfloor\left(\frac{\partial \tilde{a}^{r e s}}{\partial T}\right)_{\rho, x_{i}}+\frac{\tilde{a}^{r e s}}{T}\right\rfloor+\ln (Z)
$$

Sonic fluid velocity: The equation applied by Diamantonis et al. (eq.17) [19] was used.

$$
\omega=\sqrt{\frac{C_{p}}{C_{v}}\left(\frac{\partial P}{\partial \rho_{m}}\right)_{T}}
$$

Fugacity: It is an input of the PT FLASH model used to perform phase equilibrium calculations. Eq.18 [16] is applied to compute the fugacity.

$$
\ln \varphi_{k}=\frac{\mu_{k}^{r e s}(T, v)}{k T}-\ln Z
$$

\subsubsection{Calculation of the phase diagram}

The calculation of the number of phases present in a mixture in a certain condition is a recognized problem in the utilization of any EoS. In some cases, the number of phases is assumed a priori and then the composition in every phase is calculated by imposing the conditions of equilibrium. However, this technique often leads to divergence in the iterative methods used to achieve these. In our case, this is solved by an isothermal flash calculation after a stability analysis using the Tangent Plane Criterion Method proposed by Michelsen [20] and applied to the PCSAFT EoS by Justo-Garcia et al. [21]. Nevertheless, for a case in which we have a single component, this problem gets simplified vastly and the rudimentary method of assuming a fixed number of phases can be applied. First, we calculate the saturation curve for the single component by imposing the equilibrium conditions:

$$
\begin{aligned}
& T_{\text {SATURATED_VAPOR }}=T_{\text {SATURATED_LIQUID }} \\
& p_{\text {SATURATED_VAPOR }}=p_{\text {SATURATED_LIQUID }} \\
& \mu_{\text {SATURATED_VAPOR }}=\mu_{\text {SATURATED_LIQUID }}
\end{aligned}
$$

where $\mathrm{T}, \mathrm{p}$ and $\mu$ are the temperature, pressure and chemical potential of the component. Then for the inputs, we compare with the calculated saturation curve, knowing then if the conditions for the component are inside or outside of it. In case of being outside, the EoS is applied directly. On the other hand, if it is inside, the saturation values are weighted by the amount of vapor-liquid calculated. The phase diagram is computed in the initialization of the program in order to reduce the computational time of the simulation.

\section{Results and discussion}

In this section, the hyperbolic part of the code is validated against the exact solution of the Riemann problem. In addition, a two-dimensional simulation of a cryogenic nitrogen jet was performed to demonstrate the twodimensional capability of the numerical framework.

\subsection{Shock tube problem}

A shock tube problem is a one-dimensional Riemann problem frequently applied to validate computational fluid codes. The Euler equations are solved in this validation so a direct comparison with the exact solver can be done. The component used to perform the simulation was $n$-dodecane. The exact Riemann solver uses an unstructured table which contains the thermodynamic properties derived from the PC-SAFT EoS. This table is generated before performing the simulations. The domain used was $\mathrm{x} \in[-2,2]$, the initial conditions of the left state are $\rho_{L}=751.167 \mathrm{~kg} / \mathrm{m}^{3}, \quad p_{L}=40.2 \mathrm{kPa}, U_{L}=0 \mathrm{~m} / \mathrm{s}$ and the initial conditions of the right state are $\rho_{R}=719.249 \mathrm{~kg} / \mathrm{m}^{3}$, $p_{R}=170 \mathrm{bar}, u_{R}=0 \mathrm{~m} / \mathrm{s}$. 800 equally spaced cells were used with a fifth order spatial accuracy. Wave transmissive boundary conditions are implemented in the left and right sides. Figure 3 shows a high degree of agreement between the exact solution and the computed results. 

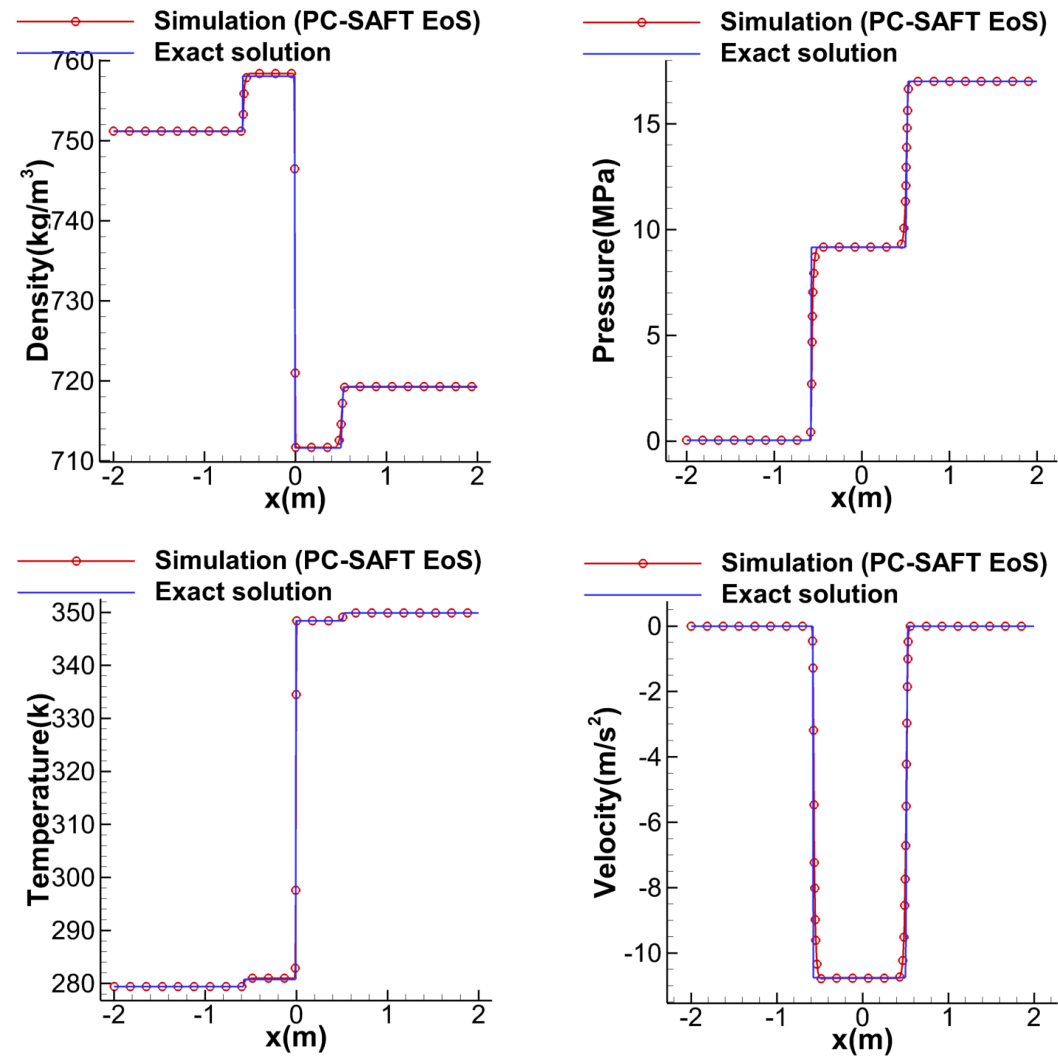

Figure 2. Validation of the hyperbolic sub-step in the Riemann problem. Comparison between the exact and the numerical solution of the density (upper left), pressure (upper right), temperature (lower left) and $\mathrm{x}$-velocity (lower right) at $\mathrm{t}=5 \times 10^{-4} \mathrm{~S}$.

\subsection{Two-dimensional test case}

A two-dimensional supercritical nitrogen jet mixing case was simulated using the developed numerical framework. Cryogenic liquid nitrogen is injected into a chamber filled with high temperature nitrogen. This simulation was previously done by Ma et al. [22] employing similar conditions to the case 3 of Mayer et al. [23]. The ambient pressure in the combustion chamber is $4 \mathrm{MPa}$, the density of the jet is $440 \mathrm{~kg} / \mathrm{m}^{3}$ and the density of the nitrogen in the combustion chamber is $40 \mathrm{~kg} / \mathrm{m}^{3}$. The velocity of the jet is $50 \mathrm{~m} / \mathrm{s}$ and the diameter of the exit nozzle is $1.0 \mathrm{~mm}$. The domain used is $30 \mathrm{~mm} \times 15 \mathrm{~mm}$. A structured mesh is applied with a uniform cell distribution. The cell size is $0.06 \mathrm{~mm} \times 0.06 \mathrm{~mm}$. A flat velocity profile is imposed at the inlet. Transmissive boundary conditions are used in all boundaries with the exception of the inlet. It was not utilised a sub-grid scale model. The parabolic sub-step is included into the simulation.

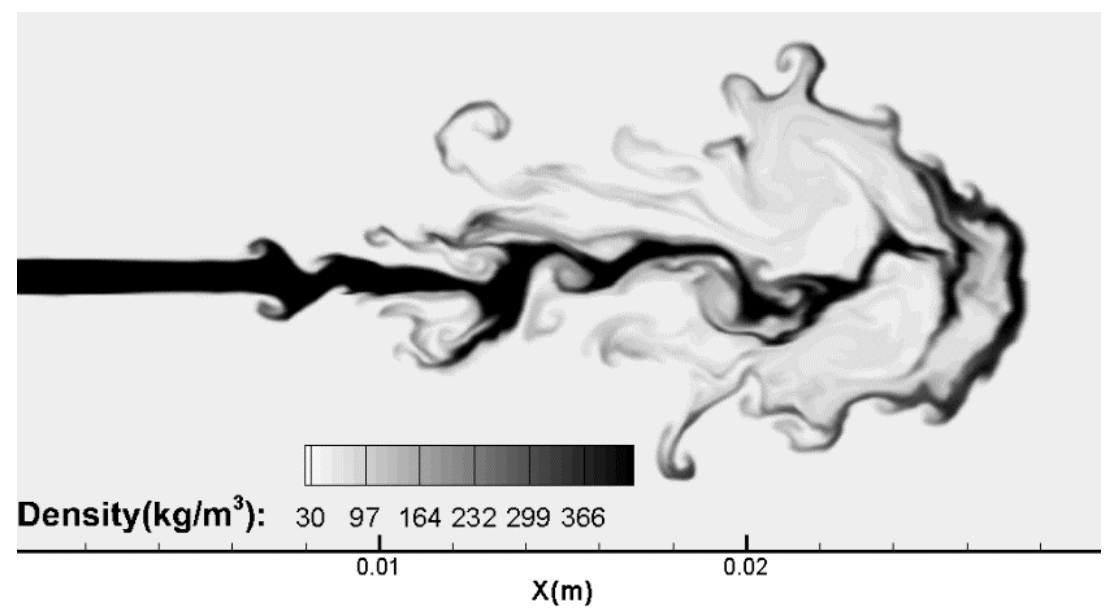

Figure 3. Density results of the simulation of the planar cryogenic nitrogen jet at $t=7.90 \times 10^{-4} \mathrm{~s}$ 
At high pressures the intermolecular interactions in the gas-liquid interphase are symmetrical so the atomization phenomena is suppress. In Figure 3 it can be appreciated that the mixing process depends on the turbulence where the large density gradients play an important role. When the jet is introduced in the high temperature environment of the chamber the velocity gradients at the jet surface generate a rollup which finally breakup into ligament-shaped structures. The Kelvin Helmholtz instability can be observed in the shear layer.

The mixing layer is similar to a gas/gas turbulent mixing case. This feature was observed by the experiments performed by Chehroudi el al. [24]. No droplets are formed at this conditions. Figure 4 shows how the jet is quickly heated to a gas-like supercritical state after the injection takes place.

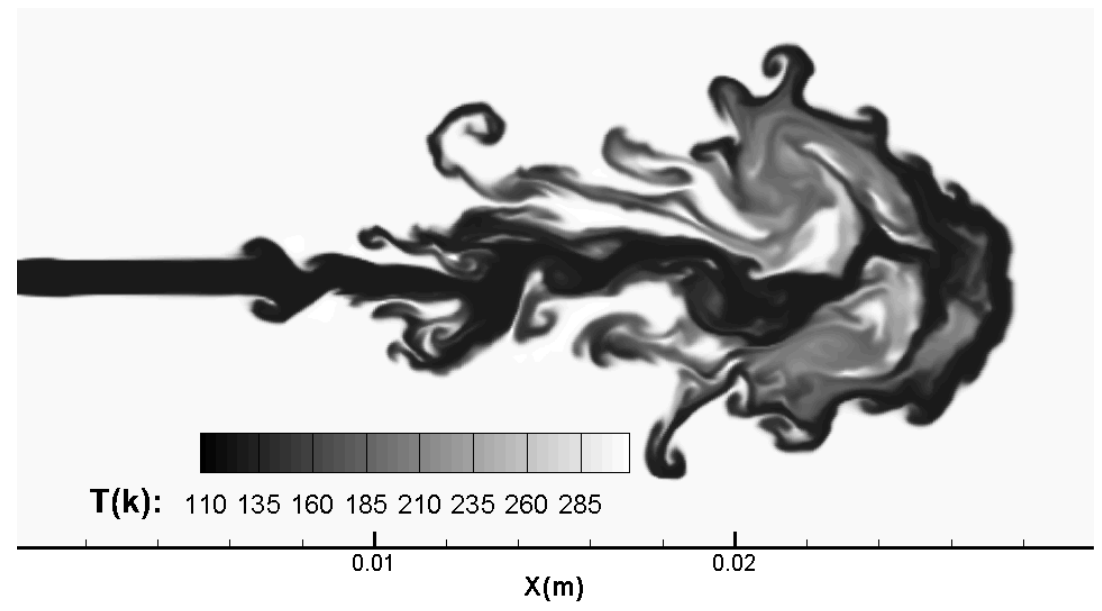

Figure 4. Temperature results of the simulation of the planar cryogenic nitrogen jet at $t=7.90 \times 10^{-4} \mathrm{~S}$

By setting the CFL number at 1.0 and using a second order RK method the number of time steps needed to simulate $7.9 \mathrm{E}-4 \mathrm{~s}$ is approximately 50000 . The average CPU time for a time step is $14.2 \mathrm{~s}$. It has to be taken into account that this is a serial code used to validate the numerical framework and it is not optimized. If the operator splitting is not applied the CPU time can be reduced to 8.6s. Unlike the fifth order WENO scheme, the evaluated first and second order schemes are not able to properly capture the large density gradients. Third and fourth order reconstruction schemes have not been evaluated.

\section{Conclusions}

A numerical framework was developed to simulate the mixing of supercritical and transcritical flows using the PCSAFT EoS. By using this advance molecular model, real fluid effects are taken into account in the simulations. Apart from supercritical state, the developed code is able to simulate gas, liquid and vapor-liquid phase equilibrium. The algorithm applied to couple the PC-SAFT EoS and the Navier-Stokes equations allows a quick computation of the thermodynamic properties of the flow. The hyperbolic part of the code was validated against the exact solution of the Riemann problem solved for the PC-SAFT EoS. Furthermore, the simulation of the two-dimensional supercritical nitrogen jet mixing case shows the multidimensional capability of the numerical framework. Future work will include the simulation of transcritical and supercritical mixing cases with a composition more similar to Diesel in temperature and pressure conditions closer to those of a diesel engine.

\section{Acknowledgements}

This project has received funding from the European Union Horizon 2020 Research and Innovation programme. Grant Agreement No 675528

\section{Nomenclature}

$$
\begin{aligned}
& \text { List of Symbols } \\
& \qquad \begin{array}{cl}
\tilde{a}^{\text {res }} & =\text { reduced Helmholtz free energy } \\
d & =\text { temperature-dependent segment diameter }[\AA] \\
g & =\text { radial distribution function } \\
I & =\text { integrals of the perturbation theory }
\end{array}
\end{aligned}
$$

$$
\begin{aligned}
& \text { Greek } \text { Letters } \\
& \begin{array}{ll}
\varepsilon & =\text { depth of pair potential }[\mathrm{J}] \\
\eta & =\text { packing fraction } \\
\rho & =\text { density }\left[\mathrm{kg} / \mathrm{m}^{3}\right] \\
\rho_{m} & =\text { total number density of molecules }\left[1 / \AA^{3}\right]
\end{array}
\end{aligned}
$$




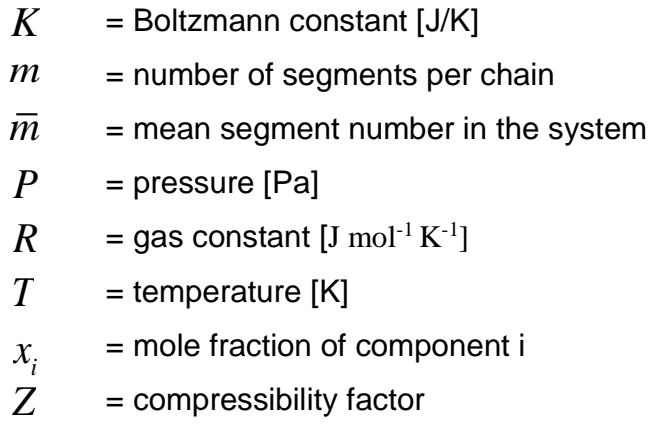

$\sigma_{d} \quad=$ segment diameter $\left[\AA^{3}\right]$

Superscripts

disp = contribution due to dispersive attraction

$h c=$ residual contribution of hard-chain system

$h s \quad=$ residual contribution of hard-sphere system

id = ideal gas contribution

\section{References}

[1] Lin, R. and Tavlarides, L.L., 2012. Thermophysical properties needed for the development of the supercritical diesel combustion technology: Evaluation of diesel fuel surrogate models. The Journal of Supercritical Fluids, 71, pp.136-146.

[2] Tavlarides, L.L. and Antiescu, G., Syracuse University, 2009. Supercritical diesel fuel composition, combustion process and fuel system. U.S. Patent 7,488,357.

[3] Vogel T. et. al, (2013). Transition of Fuel Components into Supercritical State under Diesel Process Conditions. In ILASS - Europe. Chania.

[4] Péneloux, A., Rauzy, E. and Fréze, R., 1982. A consistent correction for Redlich-Kwong-Soave volumes. Fluid Phase Equilibria, 8(1), pp.7-23.

[5] Schou Pedersen, K. and Hasdbjerg, C., 2007, January. PC-SAFT equation of state applied to petroleum reservoir fluids. In SPE Annual Technical Conference and Exhibition. Society of Petroleum Engineers.

[6] Wertheim, M.S., 1984. Fluids with highly directional attractive forces. I. Statistical thermodynamics. Journal of statistical physics, 35(1), pp.19-34.

[7] Wertheim, M.S., 1984. Fluids with highly directional attractive forces. II. Thermodynamic perturbation theory and integral equations. Journal of statistical physics, 35(1-2), pp.35-47.

[8] Wertheim, M.S., 1986. Fluids with highly directional attractive forces. III. Multiple attraction sites. Journal of statistical physics, 42(3), pp.459-476.

[9] Wertheim, M.S., 1986. Fluids with highly directional attractive forces. IV. Equilibrium polymerization. Journal of statistical physics, 42(3), pp.477-492.

[10] Chapman, W.G., Gubbins, K.E., Jackson, G. and Radosz, M., 1989. SAFT: equation-of-state solution model for associating fluids. Fluid Phase Equilibria, 52, pp.31-38.

[11] Chapman, W.G., Jackson, G. and Gubbins, K.E., 1988. Phase equilibria of associating fluids: chain molecules with multiple bonding sites. Molecular Physics, 65(5), pp.1057-1079.

[12] Khare, N.P., 2003. Predictive modeling of metal-catalyzed polyolefin processes.

[13] De Villiers, A.J., Schwarz, C.E., Burger, A.J. and Kontogeorgis, G.M., 2013. Evaluation of the PC-SAFT, SAFT and CPA equations of state in predicting derivative properties of selected non-polar and hydrogen-bonding compounds. Fluid Phase Equilibria, 338, pp.1-15.

[14] Chung, T.H., Ajlan, M., Lee, L.L. and Starling, K.E., 1988. Generalized multiparameter correlation for nonpolar and polar fluid transport properties. Ind. Eng. Chem. Res, 27(4), pp.671-679.

[15] Riazi, M.R. and Whitson, C.H., 1993. Estimating diffusion coefficients of dense fluids. Industrial and Engineering Chemistry Research, 32(12).

[16] Gross, J. and Sadowski, G., 2001. Perturbed-chain SAFT: An equation of state based on a perturbation theory for chain molecules. Industrial \& engineering chemistry research, 40(4), pp.1244-1260.

[17] Gord, M.F., Roozbahani, M., Rahbari, H.R. and Hosseini, S.J.H., 2013. Modeling thermodynamic properties of natural gas mixtures using perturbed-chain statistical associating fluid theory. Russian Journal of Applied Chemistry, 86(6), pp.867-878.

[18] Poling, B.E., Prausnitz, J.M. and O'connell, J.P., 2001. The properties of gases and liquids (Vol. 5). New York: Mcgraw-hill. Appendix A.

[19] Diamantonis, N.I. and Economou, I.G., 2011. Evaluation of SAFT and PC-SAFT EoS for the calculation of thermodynamic derivative properties of fluids related to carbon capture and sequestration.

[20] Michelsen, M.L., 1982. The isothermal flash problem. Part I. Stability. Fluid phase equilibria, 9(1), pp.1-19.

[21] Justo-García, D.N., García-Sánchez, F. and Romero-Martínez, A., 2008, March. Isothermal Multiphase Flash Calculations with the PC-SAFT Equation of State. In E. Díaz-Herrera and E. Juaristi eds.,, AIP Conference Proceedings (Vol. 979, No. 1, pp. 195-214). AIP.

[22] Ma, P.C., Bravo, L. and Ihme, M., 2015. Supercritical and transcritical real-fluid mixing in diesel engine applications (No. ARL-RP-0551). ARMY RESEARCH LAB ABERDEEN PROVING GROUND MD VEHICLE TECHNOLOGY DIRECTORATE.

[23] Mayer, W., Telaar, J., Branam, R., Schneider, G. and Hussong, J., 2003. Raman measurements of cryogenic injection at supercritical pressure. Heat and Mass Transfer, 39(8-9), pp.709-719.

[24] Chehroudi, B., Talley, D. and Coy, E., 2002. Visual characteristics and initial growth rates of round cryogenic jets at subcritical and supercritical pressures. Physics of Fluids, 14(2), pp.850-861. 


\title{
Where does the drop size distribution come from?
}

\author{
Romain Canu ${ }^{* 1}$, Christophe Dumouchel ${ }^{1}$, Benjamin Duret ${ }^{1}$, Mohamed Essadki², Marc \\ Massot $^{2}$, Thibaut Ménard ${ }^{1}$, Stefano Puggelli ${ }^{3}$, Julien Réveillon ${ }^{1}$, François-Xavier Demoulin ${ }^{1}$ \\ 1UMR 6614-CORIA, Technopôle du Madrillet, B.P. 12, Avenue de I'Université, 76801 Saint \\ Etienne du Rouvray Cedex, France \\ ²Laboratoire EM2C - UPR CNRS 288, Ecole Centrale Paris, Grande Voie des Vignes, 92295 \\ Chatenay-Malabry Cedex, France \\ ${ }^{3}$ Dipartimento di Ingegneria Industriale, Università degli Studi di Firenze, Via S. Marta 3, \\ 50139 Firenze, Italia \\ *Corresponding author: romain.canu@coria.fr
}

\begin{abstract}
This study employs DNS of two-phase flows to enhance primary atomization understanding and modelling to be used in numerical simulation in RANS or LES framework. In particular, the work has been aimed at improving the information on the liquid-gas interface evolution available inside the Eulerian-Lagrangian Spray Atomization (ELSA) framework. Even though this approach has been successful to describe the complete liquid atomization process from the primary region to the dilute spray, major improvements are expected on the establishment of the drop size distribution (DSD). Indeed, the DSD is easily defined once the spray is formed, but its appearance and even the mathematical framework to describe its creation during the initial breakup of the continuous liquid phase in a set of individual liquid parcels is missing. This is the main aim of the present work to review proposals to achieve a continuous description of the DSD formation during the atomization process.

The attention is here focused on the extraction from DNS data of the behaviour of geometrical variable of the liquidgas interface, such as the mean and Gauss surface curvatures. A DNS database on curvature evolution has been generated. A Rayleigh-Plateau instability along a column of liquid is considered to analyse and to verify the capabilities of the code in correctly predicting the curvature distribution. A statistical analysis on the curvatures data, in terms of probability density function, was performed in order to determine the physical parameters that control the curvatures on this test case. Two different methods are presented to compute the curvature distribution and in addition, the probability to be at a given distance of the interface is studied. This approach finally links the new tools proposed to follow the formation of the spray with the pioneering work done on scale distribution analysis.
\end{abstract}

\section{Birth of drop size distribution}

A standard output expected from any atomization model or theory is the drop size distribution (DSD). There are several definitions of this function. Most generally, it is $F_{N}(D)$ in such way that $F_{N}\left(D^{\prime}\right) d D$ is the number of droplet per unit of volume with a diameter $\in\left[D^{\prime}, D^{\prime}+d D[\right.$. In this case, it is the number diameter distribution (NDD) and it may be also normalized to define a probability density function $P_{N}(D)$.

The function $F_{N}(D)$ requires the possibility to count the number of droplets. Thus, it is necessary to separate the liquid phase in a set of discrete elements. Usual atomization starts with a continuous liquid flow (for instance a liquid jet) and during the atomization process the splitting of the continuous liquid phase occurs. This phase of the atomization process can be associated to the so-called primary breakup. Once it is created, for fixed external conditions and generally considering the whole spray, the NDD may evolve towards an asymptotic state, for which numerous theoretical and experimental works are reported in the literature [1]. To address more complex situations or to determine its function in space and time, a transport equation for the DSD is required and in the case of the $F_{N}(D)$ this is the Williams-Boltzmann equation (WBE) [2].

Although the flow inside the injector as well as surface instabilities and the way to solve the WBE have been the subject of important researches, few works deal with the droplet generation step to estimate the initial DSD. One reason is the lack of data to justify any proposal and the other reason is the non-applicability of the NDD definition during the primary breakup. Indeed, considering a liquid jet before the detachment of a liquid parcel, the notion of a diameter cannot be reasonably introduced. However, considering an initial instability over a liquid surface, such unsteadiness will evolve in time and create wrinkles at a certain length scale that should be in some way related on a theoretical point of view to the diameter of the droplet that will be then generated. It is clear that a link between these two subsequent steps is still missing. Regarding the data in this transition zone during primary breakup, there have been great progresses both in experimental techniques and in numerical simulations. 
The main goal of the present study is therefore the proposition of an innovative extended definition of the drop size distribution to be used all along the atomization process, leading to the so called Surface Curvature Distribution (SDF). The definition of the SDF will be first introduced and related to some characteristic geometrical properties of the liquid/gas interface, such as the mean and Gauss curvatures. Then, numerical simulations will be then used to extract its evolution on a fundamental breakup test case. The well-known Archer code [3], [4], where a combined VOF-Level set approach is used to capture the interface and a ghost-fluid method is applied to represent accurately the jump of variable across the liquid-gas interface, has been used to this end.

The paper is structured as follows: the next section is devoted to the description of the SDF and to the introduction of a relation between the SDF and the NDF for a spherical droplet based on surface curvatures. In the second part of the paper, a Rayleigh-Plateau test case has been considered to determine the evolution of the two curvatures to show how the breakup process from a liquid jet to the final droplet can be directly described employing such surface geometrical properties.

\section{An extended definition of the drop size distribution}

The purpose of this section is to propose an extended definition of the drop size distribution that may applicable all along the atomization process including the primary breakup zone. Before looking at the DSD definition, it should be pointed out that the same problem appears to define a mean diameter: it is a moment of the NDD and therefore it can be defined only once the continuous liquid jet has been broken in a set of liquid droplets. From the pioneering work of Vallet and Borghi [5], [6] it is known that the definition of the mean diameter can be replace beneficially by the more general mean surface density $\Sigma$, that is the surface area per unit of volume. This quantity can be defined everywhere whatever is the liquid phase topology and, combined with the liquid volume fraction $\alpha$, it gives the mean Sauter diameter once the spray is formed: $D_{32}=\frac{6 \alpha}{\Sigma}$. Recently, Essadki et al [7] used high order fractional moments of the DSD for disperse phase, where the size is given by the surface area of droplet, to recover some interface geometrical quantities already used in describing the gas-liquid interface in [9]. These quantities are the volume fraction, the mean surface density and the two averaged Gauss $G=\kappa_{1} * \kappa_{2}$ and mean $H=\frac{\kappa_{1}+\kappa_{2}}{2}$ curvatures, where $\kappa_{1}$ and $\kappa_{2}$ are the two principal curvatures of the surface.

To overcome the limits of the DSD in modelling a gas-liquid interface, some surface characteristics can be used since they should carry the information of the DSD which is hidden during the wrinkling process.

The proposal here is to look at the curvature $(\kappa)$ distribution along the liquid-gas surface. In this case the distribution of curvature is $F_{S}(\kappa)$ and the $F_{S}\left(\kappa^{\prime}\right) d \kappa$ is the area of surface with a curvature $\kappa \in\left[\kappa^{\prime}, \kappa^{\prime}+d \kappa[\right.$. This leads to the definition of a surface curvature distribution (SCD). However, an accurate description of the interface cannot be restricted to one geometrical variable as it is often used in the DSD for disperse phase. Therefore, we use the Gauss and mean curvatures as phase-space variable, leading to a bi-variate $\operatorname{SCD}: F_{S}(H, G)$. Once the spray is formed, the DSD is a particular case of $F_{S}(H, G)$ since for spherical droplets $\kappa_{1}=\kappa_{2}=2 / D$.

Indeed, for a disperse phase of spherical droplets, the Gauss curvature can be simply expressed as $G=4 \pi / S$, where $S=\pi D^{2}$ is the droplet surface area. Therefore, the two functions $4 \pi / G F_{S}(H=\sqrt{G}, G)$ and $F_{D}(D)$ are equivalents, since they count the probable number of droplets per volume and per radius or Gauss curvature. The link between the two distributions has been already generalized in [8] for non-spherical droplets by using some interesting topological properties of Gauss curvature.

Therefore, considering that during primary atomization the liquid-gas interface undergoes different instabilities and deformation, the interface state can be described geometrically by the local curvatures. When the curvatures reach high values, the liquid breakup mechanism is activated and lead to generate droplets of diameter related to the curvatures of the previous interface state. Hence, the first proposal has been to carry the curvature distribution by the surface. However, any spray may be characterised by a volumetric distribution. For a spray, the DSD becomes $F_{V}(D)$ in such way that $F_{V}\left(D^{\prime}\right) d D$ is the liquid volume fraction of droplets with a diameter $\in\left[D^{\prime}, D^{\prime}+d D[\right.$. This volumetric distribution can be also extended to any liquid topology, leading to the volumetric distance function distribution $(\mathrm{V} \varphi \mathrm{D})$, that can be a second proposal. It is defined by $F_{V}(\varphi)$ in such way that $F_{V}\left(\varphi^{\prime}\right) d \varphi$ is the volume fraction of flow that is at a distance $\varphi$ from the interface, with $\varphi \in\left[\varphi^{\prime}, \varphi^{\prime}+d \varphi[, \varphi\right.$ is positive in the liquid phase and negative in the gas phase. With this definition the following relation stands:

The gas volume fraction is $\alpha_{g}=\int_{-\infty}^{0} F_{V}(\varphi) d \varphi$, the liquid volume fraction is $\alpha_{l}=\int_{0}^{+\infty} F_{V}(\varphi) d \varphi$, and the mean surface density $\Sigma=F_{V}(0)$.

The $\mathrm{V} \varphi \mathrm{D}$ gives the distribution of the flow with respect to the distance to the interface. The link with the DSD is not straightforward. However, this distribution can be clearly identified on a given geometric object and in particular for a spherical droplet. Indeed, this idea has been explored by Dumouchel and co-workers [12,13] with the concept of scale distribution, $E_{3}(d)$. The definition of this function for a given object (for instance a droplet) is based on the total object volume $V_{0}$ and the volume $V(d)$ defined by all points at a distance $d$ or greater to the object surface. 
The scale distribution is given by $E_{3}(d)=\frac{\left(V_{0}-V(d)\right)}{V_{0}}$. The link between the scale distribution and the $\mathrm{V} \varphi \mathrm{D}$ can be express readily by $E_{3}(d)=\frac{F_{V}(\varphi=d)}{\alpha_{l}}$. Dumouchel et al. have worked on experimental data in 2D [12] and more recently they have used 3D simulation (ICM + DNS) to extract 3D results [13]. A link must exist between the scale distribution or the volume fraction distribution with the curvature or at least an average curvature of the surface. This point is still under investigation inside our research group.

\section{First application of the definitions}

As a first step a very simple configuration corresponding to a Rayleigh-Plateau instability, where a set of droplet is created from an initial column of liquid, is here used to show how the surface curvatures and $\mathrm{V} \varphi \mathrm{D}$ can be used to describe a breakup event. This is a standard test case for the code Archer that is known to give good results on this configuration. Two methods have been used to extract the curvature distribution. The first one is based on the distance function that is part of the numerical procedure in the resolution of the two-phase flow in Archer code. The normal to the surface $\vec{n}=-\frac{\vec{\nabla} \varphi}{|\vec{\nabla} \varphi|}$ is first calculated. Then, the matrix $P=I-\vec{n} \vec{n}^{T}$ with $I$ the identity matrix, and the Hessian matrix $H m=\frac{\partial^{2} \varphi}{\partial x_{i} \partial x_{j}}$ are calculated in order to have the matrix $G m=-\frac{P H m P}{|\vec{\nabla} \varphi|}$. Finally, the two principal curvatures $\kappa_{1}$ and $\kappa_{2}$ are obtained by :

$\kappa_{1}=\frac{T+\sqrt{2 F^{2}-T^{2}}}{2}$ and $\kappa_{2}=\frac{T-\sqrt{2 F^{2}-T^{2}}}{2}$

with $T$, the trace of the matrix $G m$ and $F$, the Frobenius norm of the matrix $G m$. For a more detailed explanation of the method, see [11]. In Archer, these curvatures are calculated on the center of the mesh cells, so in order to have the right curvatures on the interface, the curvature radius is corrected by the distance function which gives the distance from the center of the cell to the interface.

The second one has been developed in [8], where the gas-liquid interface is discretized with a 2D triangulated mesh using the Marching Cube algorithm. The curvature is computed at each vertex of the created mesh, as spatial averages around this vertex, based on some results of discrete differential geometry [10]. Then, in order to eliminate the noise of the estimated curvatures, the authors of [8] used an adequate spatial-averaging process, which preserves some topological feature of the gas-liquid interface such as computing the droplet number from the bivariate SCD: $F_{S}(H, G)$, while filtering the noise.

Finally, the first result on the $\mathrm{V} \varphi \mathrm{D}$ have been obtained through the distance function. All these concepts have been explored in a simple test case described in the next section before addressing more complex test cases up to the final atomization.

\section{Numerical Results}

The validation case used here is the Rayleigh-Plateau instability, which consists on the formation of droplets from an initial liquid jet due to surface tension with the surrounding gas. We computed a quarter of a cylinder and used symmetric boundary conditions in a computational box of $1,5.10^{-4} \times 1,0.10^{-4} \times 1,0.10^{-4} \mathrm{~m}$ with a $96 \times 64 \times 64$ mesh. The liquid and gas properties are the following ones:

$\rho_{l}=1000 \mathrm{~kg} \cdot \mathrm{m}^{-3} \quad \rho_{g}=1 \mathrm{~kg} \cdot \mathrm{m}^{-3}$

$\mu_{l}=1,0 \cdot 10^{-3} \mathrm{~kg} \cdot \mathrm{m}^{-1} \cdot \mathrm{s}^{-1} \quad \mu_{g}=1,879 \cdot 10^{-5} \mathrm{~kg} \cdot \mathrm{m}^{-1} \cdot \mathrm{s}^{-1}$

$\sigma=0,072 \mathrm{~kg} \cdot \mathrm{s}^{-2}$

The cylinder has a radius $R=3,34 \cdot 10^{-5} \mathrm{~m}$ and the initial perturbation, essential to observe the instability, has an amplitude of $10 \%$ of the radius of the cylinder and a wavelength of $3.10^{-4} \mathrm{~m}$ (twice the length of the box). The initial conditions have been chosen in order to have a wave number satisfying $k R=0,7$ which correspond to the fastest growth rate. The two previous methods to extract the curvature distribution were tested (Figure 1) but no significant differences were observed, which shows that this case is well resolved with a high mesh resolution.

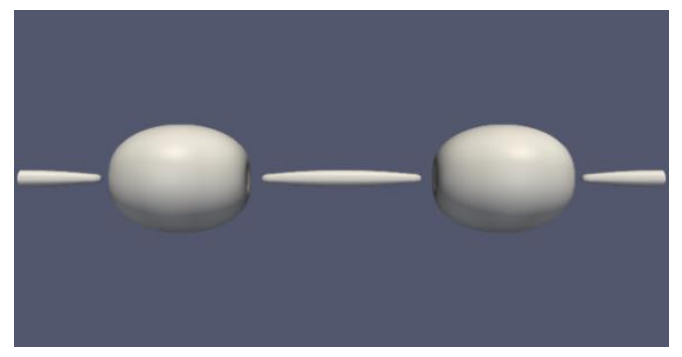



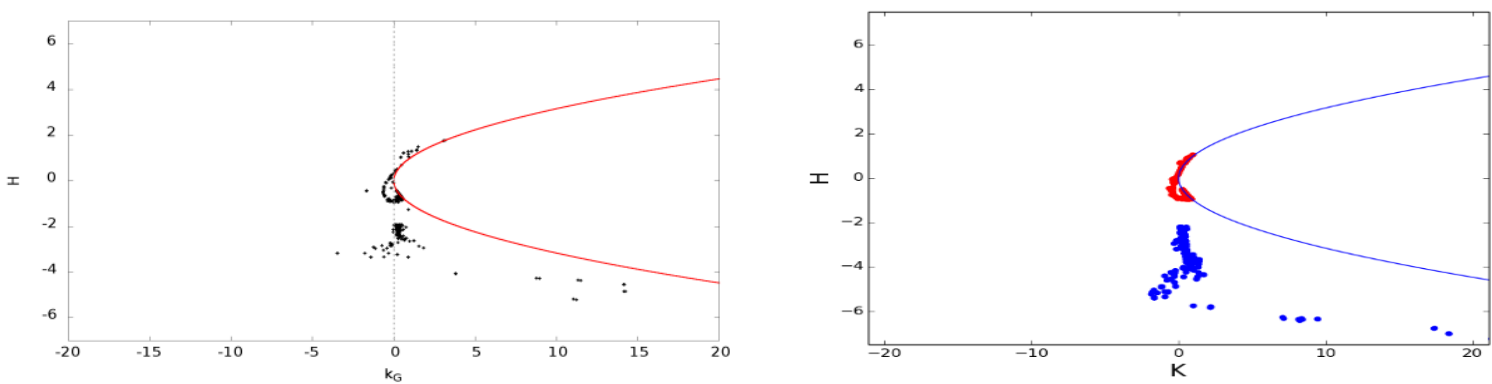

Figure 1. Top left : Rayleigh Plateau instability at $t=1,88.10^{-4} \mathrm{~s}$; Bottom left : Mean curvature in function of Gauss curvature with the first method (red curve represents $\kappa_{1}=\kappa_{2}$ and the dotted line represents $G=0$ ) ; Bottom right : Mean curvature in function of Gauss curvature with the second method (blue curve represents $\kappa_{1}=\kappa_{2}$, red points represents the right part of the simulation and blue points, the left part)

In the following figures, the mean and total curvatures are provided as dimensionless using the radius of the initial cylinder as reference length, whereas for the Gauss curvature the radius squared has been used to this end. The mean curvature of a sphere with a radius of $d x$ is 21,4 and its Gauss curvature is 457,5 .

On Figures 6 and 7, the distance function $\varphi$ is reported as dimensionless using $d x$ as reference and the curves are shown between $-l_{x}$ and $l_{x}$. The same validation case is used in these figures but with a $36 \times 24 \times 24$ mesh sizing. The surface evolution of the Rayleigh-Plateau instability in different four time steps is shown in Figure 2, where it is possible to see the initial configuration ( $t a$ ), the deformation of the cylinder $(t b)$, the moment of the break-up (tc) and the final droplet's formation (td). It is now interesting to analyse the evolution of surface curvatures during this breakup process.

It should be pointed out that when the cylinder is weakly deformed (Figure 6), in terms of PDF profile we are very close to the theoretical profile of a cylinder with a linear curve inside the liquid part. For the total curvature (Figure 5 left) and Gauss curvature (Figure 4 left) cumulative, a step profile at values corresponding to this cylinder is obtained. Furthermore, on the graph showing mean curvature in function of Gauss curvature (Figure 3 top left), all the points are around the vertical line representing the cylinder.

Then, when such cylinder is deformed (tb), we observe negative values for Gauss curvature (Figure 4 right) corresponding to all the saddle points in the middle of the cylinder. This portion of cylinder is visible on the mean and Gauss curvatures graph (Figure 3 top right) and it is the arc formed by the points. Besides, on this graph, the points, which represent the big part of the cylinder, get closer to the red curve corresponding to a sphere.

When the break-up happens ( $t c$ ), the previous arc breaks up and two sets of points appear (Figure 3 bottom left), one of which is located on the red curve. These points correspond to the big droplet. Indeed, the mean curvature is approximately 0.5 and the Gauss curvature about 0.25 which correspond to a radius twice as big as the initial jet radius. The other points, located below the red curve, correspond to the small ellipsoid. Here, the mean curvature is quite high because of the small thickness and the Gauss curvature is weaker because the curvature is close to zero along the length. The big droplet is characterised by the step on the total curvature cumulative (Figure 5 right) and the small ellipsoid by all the high negative values. It should be pointed out that such break-up process is mainly evident on the curvature plot since small variations on the interface geometry can lead strong changes in the curvature values.

Finally, when the two droplets are formed (td), for the mean and Gauss curvatures graph (Figure 3 bottom right) most of the points are on the red curve, which represents the spheres. The points corresponding to the big droplet are located approximately at the same place than the previous time. The points corresponding to the small droplet are those having an higher curvature because of, obviously, its small radius. On Figure 7, PDF profile becomes parabolic in the liquid part, as expected for spheres, with higher values of the distance function because the biggest droplet is bigger than the initial cylinder. 

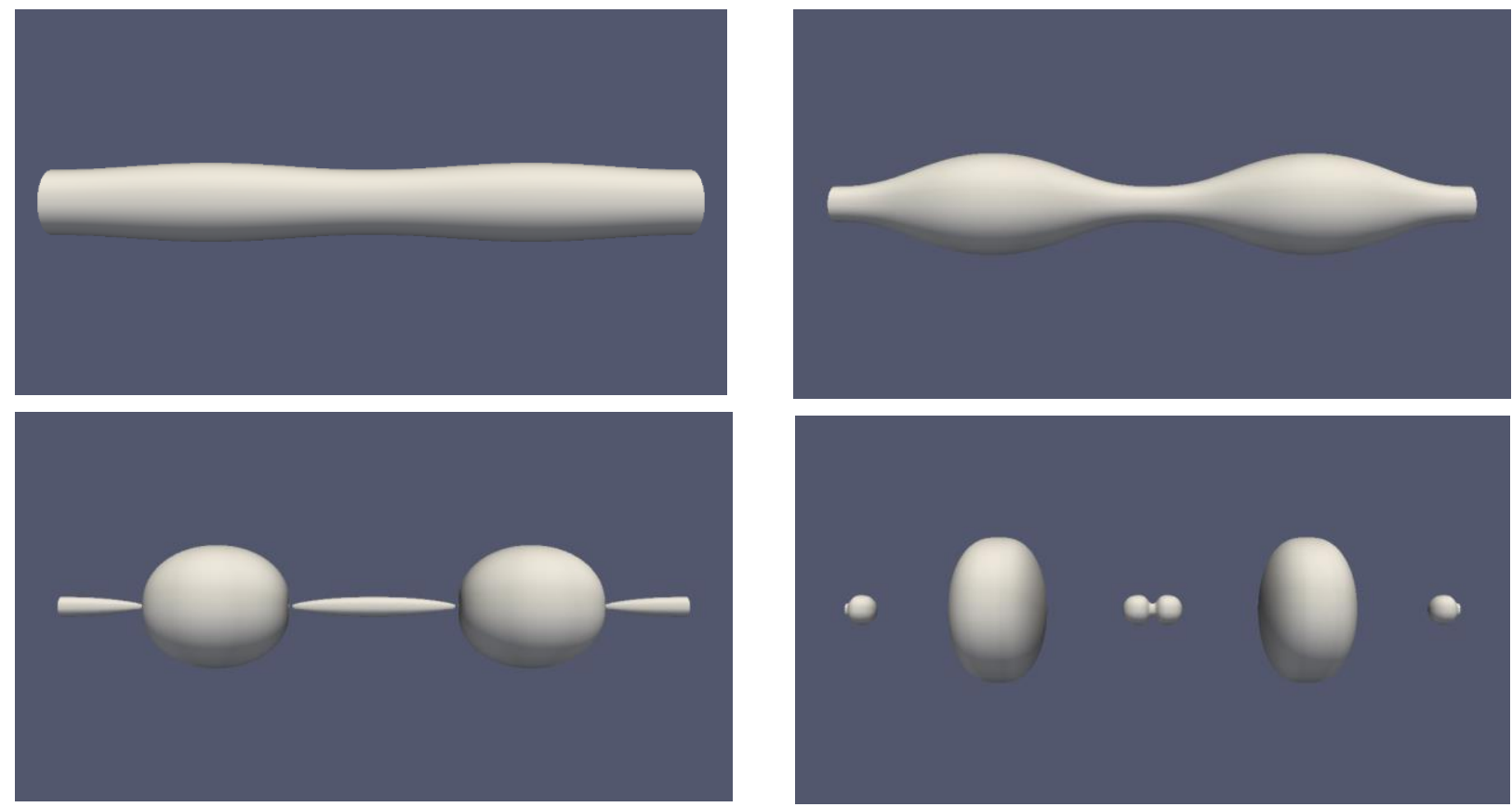

Figure 2. Top left : Rayleigh Plateau instability at $t_{a}=3,92.10^{-7} \mathrm{~s}$; Top right : Rayleigh Plateau instability at $t_{b}=1,49.10^{-4} s$; Bottom left : Rayleigh Plateau instability at $t_{c}=1,86.10^{-4} \mathrm{~s}$; Bottom right : Rayleigh Plateau instability at $t_{d}=2,03.10^{-4} \mathrm{~s}$
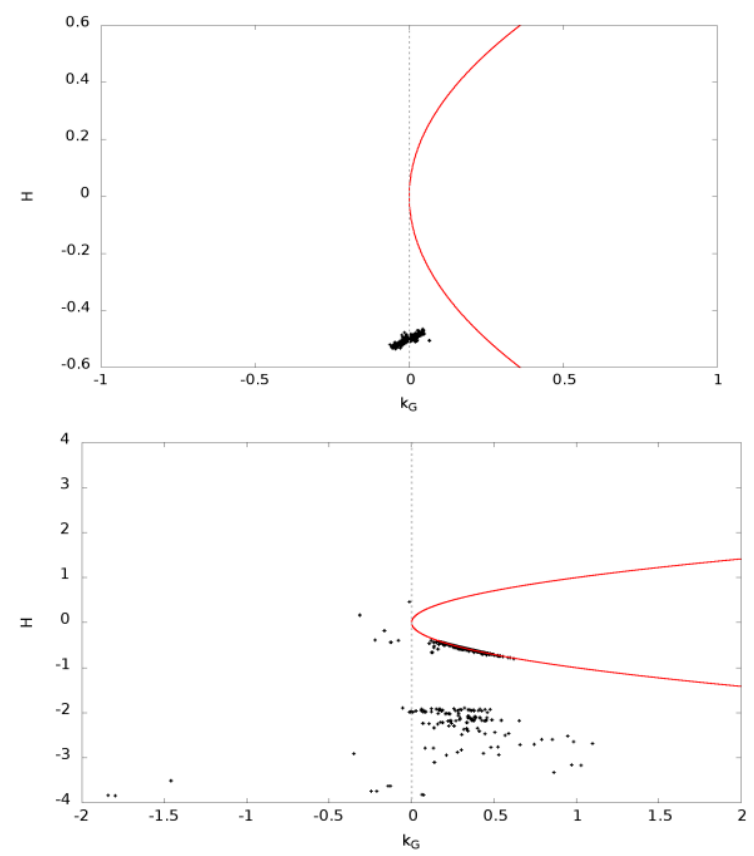
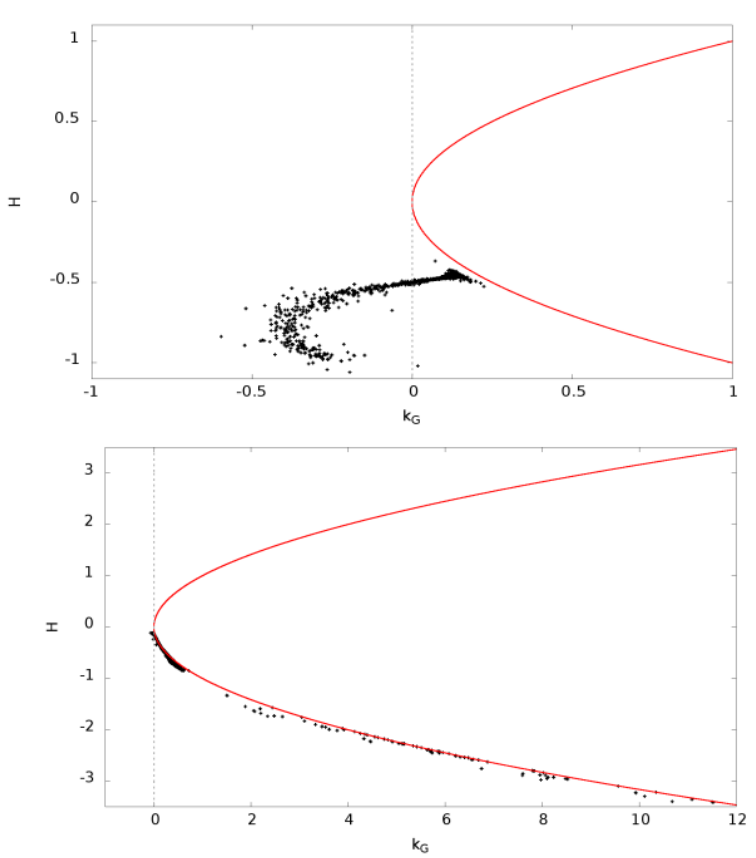

Figure 3. Mean curvature in function of Gauss curvature (Top left : at $t_{a}$; Top right : at $t_{b}$; Bottom left : at $t_{c}$; Bottom right : at $t_{d}$ (red curve represents $\kappa_{1}=\kappa_{2}$ so a sphere and the dotted line represents $G=0$ so a cylinder) 

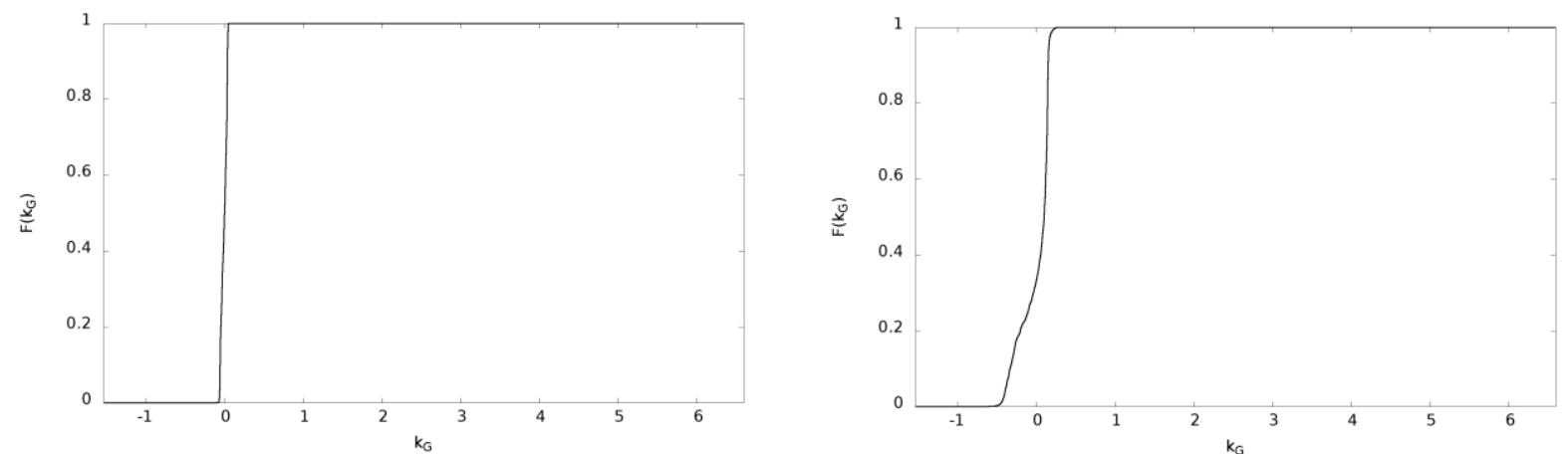

Figure 4. Cumulative of the Gauss curvature (Left : at $t_{a} ;$ Right : at $t_{b}$ )
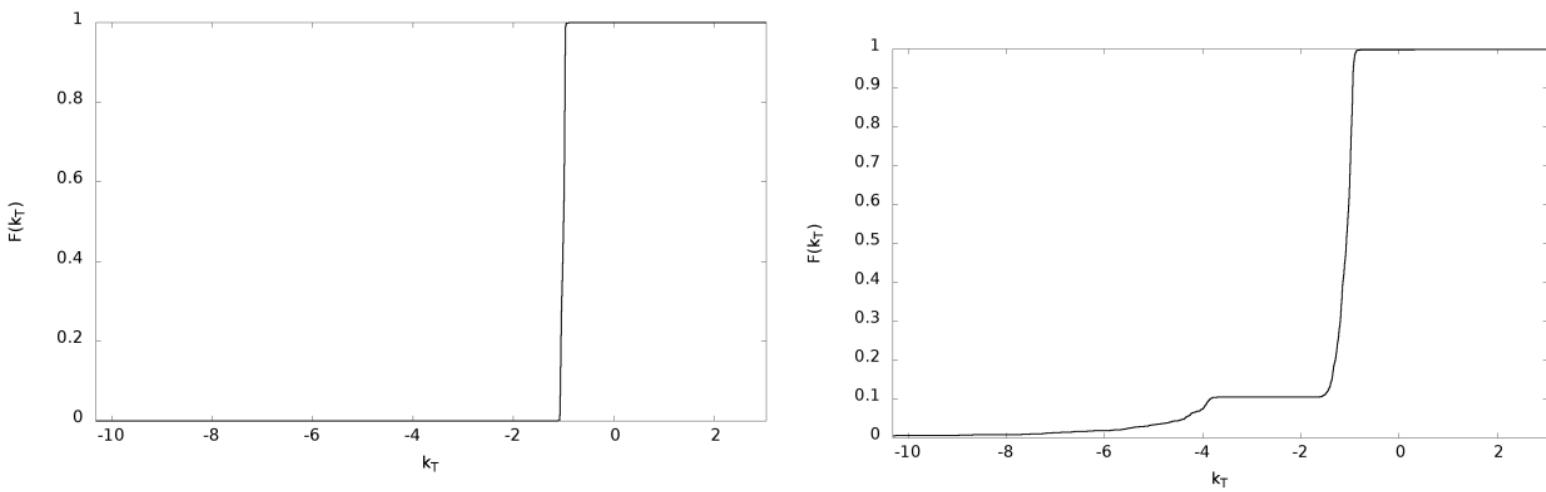

Figure 5. Cumulative of the total curvature (Left : at $t_{a}$; Right: at $t_{c}$ )
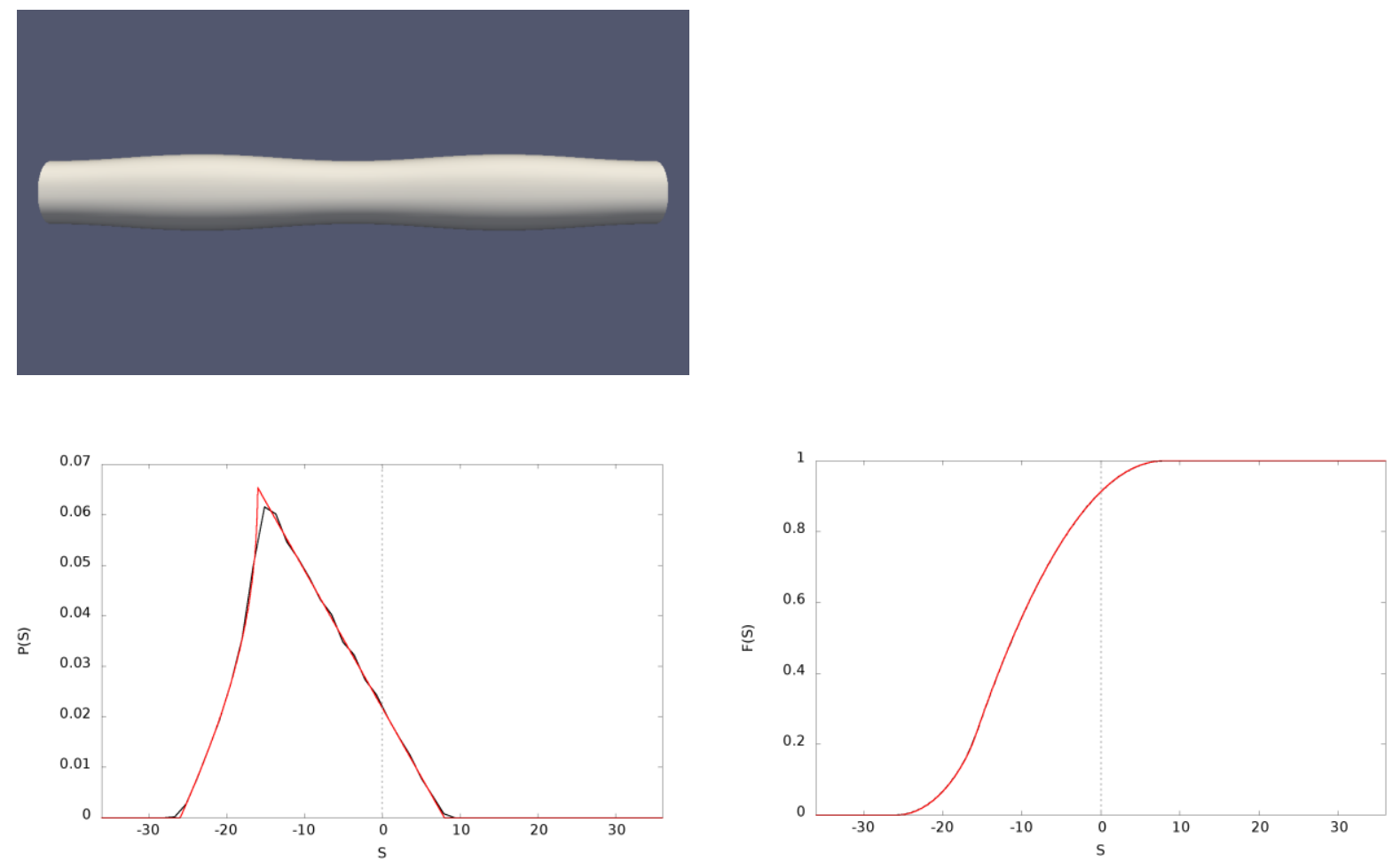

Figure 6. Top left : Rayleigh Plateau instability at $t_{a}$; Bottom left : pdf of the distance function (red curve represents the initial pdf profile for a cylinder) ; Bottom right : cumulative of the distance function (red curve represents the initial cumulative profile for a cylinder) ; the dotted line separates liquid (positive values) and gas (negative values) 

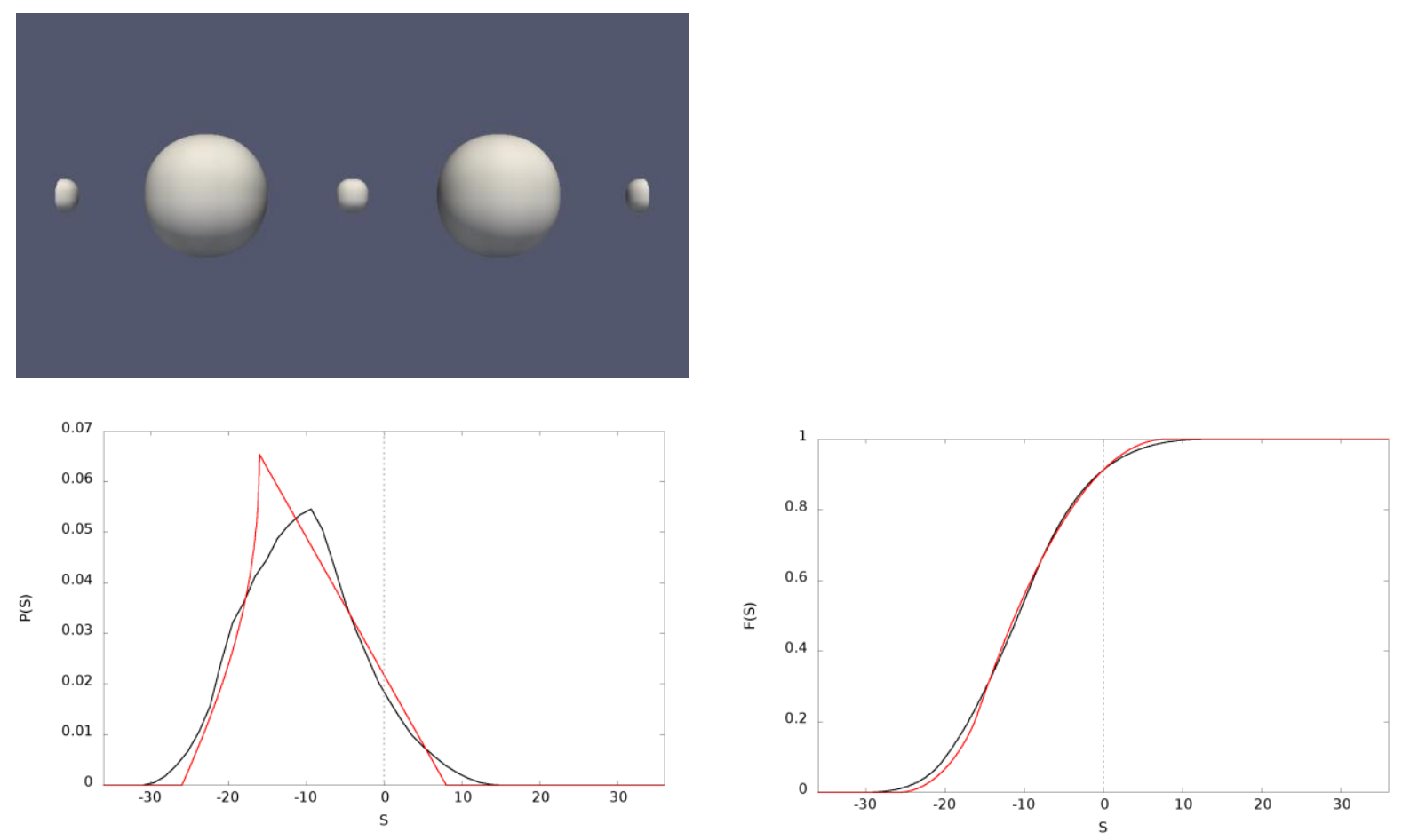

Figure 7. Top left : Rayleigh Plateau instability at $t_{d}$; Bottom left : pdf of the distance function (red curve represents the initial pdf profile for a cylinder) ; Bottom right : cumulative of the distance function (red curve represents the initial cumulative profile for a cylinder) ; the dotted line separates liquid (positive values) and gas (negative values)

\section{Conclusions}

This study shows how DNS of two-phase flows can be employed to improve understanding and modelling of primary atomization to be then used in RANS or LES framework. In particular, the study is aimed at enhancing the information available in ELSA framework through the introduction of liquid/gas interface curvatures. Firstly, on a mathematical point of view, an extended definition of the drop size distribution to be used all along the atomization process is proposed, leading to the so called surface curvature distribution. Then, the extraction of surface curvatures is shown for a simple Rayleigh-Plateau test case before to go on with more complex and computationally costly tests. Two different methods to extract the curvature distributions are tested, even if they do not here determine appreciable differences. The evolution of Gauss and mean curvatures is analysed in detail in order to show how the breakup process from a liquid jet to the final droplet can be easily described in terms of such surface geometrical properties.

\section{Nomenclature}

$\begin{array}{ll}\text { D } & \text { Droplet diameter [m] } \\ \text { G } & \text { Gauss curvature }[\mathrm{m}-2] \\ \text { H } & \text { Mean curvature }[\mathrm{m}-1] \\ \text { R } & \text { Cylinder radius }[\mathrm{m}]\end{array}$

\section{Greek}

$\begin{array}{ll}\alpha & \text { Liquid volume fraction [-] } \\ \Sigma & \text { Mean surface density [m-1] } \\ \kappa & \text { Principal curvature [m-1] } \\ \varphi & \text { Distance function from the interface [m] } \\ \rho & \text { Density [kgm-3] }\end{array}$


$\begin{array}{ll}\mu & \text { Viscosity [kgm-1 s-1] } \\ \sigma & \text { Surface tension [kgs-2] }\end{array}$

\section{Subscripts}

l Liquid

g Gas

\section{Acronyms}

DNS Direct Numerical Simulation

DSD Drop Size Distribution

ICM Interface Capturing Method

LES Large Eddy Simulation

NDD Number Diameter Distribution

RANS Reynolds Average Navier Stokes

SCD Surface Curvature Distribution

$\mathrm{V} \varphi \mathrm{D} \quad$ Volumetric distance function Distribution

\section{References}

[1] E. Babinsky and P. E. Sojka, "Modeling drop size distributions," Prog. Energy Combust. Sci., vol. 28, no. 4, pp. 303-329, 2002.

[2] F. Williams, "Spray Combustion and Atomization," Phys. Fluids, vol. 1, no. 6, pp. 541-545, 1958.

[3] S. Tanguy and A. Berlemont, "Application of a level set method for simulation of droplet collisions," Int. J. Multiph. Flow, vol. 31, no. 9, pp. 1015-1035, 2005.

[4] T. Menard, S. Tanguy, and A. Berlemont, "Coupling level set/VOF/ghost fluid methods: Validation and application to 3D simulation of the primary break-up of a liquid jet," Int. J. Multiph. Flow, vol. 33, no. 5, pp. 510-524, 2007.

[5] A. Vallet and R. Borghi, "Modélisation Eulerienne de L'atomisation d'un Jet Liquide," C R Acad Sci Paris Sér II $B$, vol. 327, pp. 1015-1020, 1999.

[6] A. Vallet, A. A. Burluka, and R. Borghi, "Development of a Eulerian model for the 'Atomization' of a liquid jet," At. Sprays, vol. 11, no. 6, pp. 619-642, 2001.

[7] M. Essadki, S. de Chaisemartin, F. Laurent, and M. Massot, "High Order Moment Model for Polydisperse Evaporating Sprays Towards Interfacial Geometry," submitted to SIAM J. Appl. Math, (2016). Available on HAL, https://arxiv.org/pdf/1608.07148

[8] M. Essadki, F. Drui, S. de Chaisemartin, A. Larat, T. Ménard and M. Massot, "Statistical modeling of the gasliquid interface: Toward a unified description of the disperse and separated phases flows," submitted to IJMF, (2017) pp1--25. Available on HAL

[9] M. Essadki, S. de Chaisemartin, M. Massot, F. Laurent, A. Larat, S. Jay, "Adaptive mesh refinement and high order geometrical moment method for the simulation of polydisperse evaporating sprays," Oil \& Gas Science and Technology, vol. 71, no. 5, (2016), 1-25

[10] M. Meyer, M. Desbrun, P. Schröder, A. H. Barr, "Discrete differential-geometry operators for triangulated 2manifolds." Visualization and Mathematics III pp 35-57 (2001) 1-26.

[11] G. Kindlmann, R. Whitaker, T. Tasdizen, T. Möller, "Curvature-based transfer functions for direct volume rendering : Methods and applications" In Proc. of IEEE Visualization 2003 (2003), pp. 513-520.

[12] C. Dumouchel, T. Ménard, W. Aniszewski, "Towards an Interpretation of the scale Diffusivity in Liquid Atomization Process : An Experimental Approach," Physica A, vol. 438, 612-624, 2015

[13] C. Dumouchel, W. Aniszewski, T. T. Vu, T. Ménard, (2017) International Journal of Multiphase Flow, DOI : 10.1016/j.jimultiphaseflow.2017.03.012 


\title{
Weakly nonlinear instability of a viscous liquid jet
}

\author{
Marie-Charlotte Renoult ${ }^{1}$, Günter Brenn ${ }^{2, *}$, Innocent Mutabazi ${ }^{3}$ \\ ${ }^{1}$ CORIA, Université de Rouen, France \\ ${ }^{2}$ ISW, Graz University of Technology, Austria \\ ${ }^{3}$ LOMC, Université du Havre, France \\ ${ }^{*}$ Corresponding author: guenter.brenn@tugraz.at
}

\begin{abstract}
A weakly nonlinear stability analysis of an axisymmetric viscous liquid jet is performed. The calculation is based on a small-amplitude perturbation method and restricted to second order. Contrary to the inviscid jet and the planar viscous sheet cases studied by Yuen in 1968 [1] and Yang et al. in 2013 [2], respectively, a part of the solution results from a polynomial approximation of Bessel functions. Results on interface shapes for a small wave number and initial perturbation amplitude, four different Ohnesorge numbers, taking into account the approximate part or not, are used to predict the influence of liquid viscosity on satellite drop formation and evaluate the influence of the approximation. It is observed that the liquid viscosity has a retarding effect on satellite drop formation, in agreement with previous experimental and numerical work. In addition, it is found that the approximate terms can be reasonably ignored, providing a simpler viscous weakly nonlinear model for the description of the first nonlinearity growth in liquid jets. The present work replaces the ILASS 2016 paper [3] by the authors on the same subject.
\end{abstract}

\section{Keywords}

Viscous liquid jet, nonlinear capillary instability, satellite drop formation.

\section{Introduction}

A liquid jet breaks up forming main and satellite drops. The present work is concerned with the influence of both liquid viscosity and nonlinearities on satellite drop formation.

The first linear stability analysis of the capillary instability of a liquid jet in an ambient medium was conducted by Rayleigh [4, 5], more than a century ago. In this reference work, the liquid is assumed inviscid and the ambient medium is the vacuum. His analysis shows that, in order to destabilize the jet, the wavelength $\lambda$ of a varicose surface disturbance must be greater than the circumference of the undeformed circular jet cross section, as observed initially by Savart and Plateau [6, 7]. Two associated amplitude growth rates correspond to such an unstable perturbation wavelength, one being the opposite of the other, with the magnitude given by the well-known Rayleigh's linear dispersion relation for the inviscid jet in a vacuum.

The effect of liquid viscosity was then investigated by Weber [8], about fifty years later. The generalization of the previous dispersion relation introduces a growth rate and viscosity dependent modified wave number making the dispersion relation transcendental, yet numerically solvable, which is turned into a closed-form expression in the long-wave approximation. Contrary to the inviscid case, there are two different real growth rates with opposite signs for each unstable wavelength, with distinct absolute values below the corresponding inviscid one. This difference leads to dispersion relation curves always lower than the inviscid ones, as expected by the classical damping effect inferred from liquid viscosity.

Due to the linearity of the previous analysis, the interaction of disturbances with different wavelengths is not accounted for, and the drops produced by the jet breakup are predicted to be monodisperse in size. In particular, with no imposed perturbation, the most probable wavelength, i.e. the wavelength with maximum growth rate, is often used to get a good approximation for the main drop size in liquid jet breakup.

The effect of nonlinearities was first studied by Yuen [1]. In his weakly nonlinear analysis of an inviscid jet in a vacuum he described the jet interface shape up to order three at small initial deformation amplitude. Plotting jet surface profiles for various wave numbers and a fixed small initial amplitude perturbation, he observed the appearance of an undulation between the primary crests of the initial perturbation in the low wave number case. This observation is considered today as the first prediction of the formation of satellite drops. Good agreement of the predictions of his model with experimental results was found for the deviation of the jet surface shape from the single sinusoidal one [9], the satellite drop size $[10,11]$ and the growth rate of the two first nonlinear harmonics for wave numbers less than the fastest growing mode [12]. For larger wave numbers (more precisely, greater than the fastest growing mode), Yuen's model does not predict the formation of satellite drops, even though they are still observed in the experiment [10]. This stands as one limitation of the weakly nonlinear stability analysis.

Based on Yuen's model, the effect of liquid viscosity on the formation of drops was deduced. For inviscid jets, satellite drops are predicted to become smaller when the wave number converges to the fastest growing mode. With increasing viscosity, the fastest growing mode is shifted to lower wave numbers, and consequently the amplitude of the undulation is reduced, while its wavelength is increased, as pointed out by Goedde \& Yuen [13]. This qualitative description is in agreement with experimental and numerical results showing that satellite drops can be completely suppressed for sufficiently high Ohnesorge numbers [14].

Recently, the effect of both liquid viscosity and nonlinearities was combined for the study of a liquid sheet, the planar counterpart of the axisymmetric jet, revealing a complicated influence of liquid viscosity on the nonlinear 


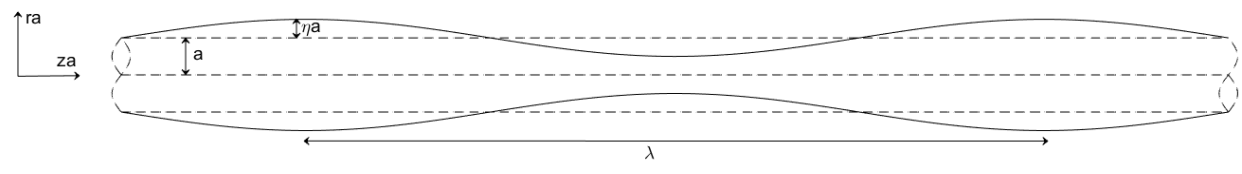

Figure 1. Sketch of the geometry of a capillary jet under varicose deformation.

sheet stability [2]. In a 2016 ILASS paper [3], the jet geometry was considered by the present authors, introducing the need for a polynomial approximation of one part of the viscous contribution, that could not be fully solved analytically, due to the presence of Bessel function products with different arguments. However, in this weakly nonlinear analysis, the two values of growth rates were incorrectly taken, as real numbers of the same absolute value but opposite signs, like in the inviscid case.

Here, we revisit our weakly nonlinear model of the temporal instability of a viscous jet in a vacuum to gain insight into the role of the jet liquid viscosity on satellite drop formation. The expansion of the jet interface shape is restricted to second order terms in the small initial perturbation amplitude, the second-order solution being sufficient to predict satellite drop formation [3]. In the following section we derive the equations of motion, as well as their boundary and initial conditions. Thereafter we solve the equations derived in the sequence of the order and present our method of approximation of one part of the viscous contribution containing products of Bessel functions with different arguments. Results on surface shapes are then presented and the effect of both the liquid viscosity and the level of approximation discussed by comparison to the inviscid solution of Yuen [1]. The paper ends with the conclusions.

\section{Formulation of the problem}

We study the weakly nonlinear temporal instability of a viscous liquid jet as sketched in Figure 1. The jet is assumed to be axisymmetric around the $z$ axis of the cylindrical coordinate system. The liquid is treated as incompressible and Newtonian. The dynamic influence from the ambient air is neglected, i.e. we treat the ambience as a vacuum. Body forces are not accounted for, since Froude numbers are large. The problem is formulated in cylindrical coordinates to account for its geometry.

The flow variables and equations are adimensionalized with the undeformed jet radius $a$, the capillary time scale $\left(\rho a^{3} / \sigma\right)^{1 / 2}$ and the capillary pressure $\sigma / a$ for length, time and pressure, respectively. Here, $\rho$ is the liquid density and $\sigma$ the air-liquid interfacial tension. The jet surface is described as a place where $r_{s}(t, z)=1+\eta(t, z)$, where $\eta$ is the non-dimensional deformation against the undisturbed cylindrical shape (cf. Figure 1).

For the problem at hand, the equation of continuity and the two components of the momentum equation in the radial $(r)$ and axial $(z)$ directions read

$$
\begin{aligned}
\frac{1}{r} \frac{\partial}{\partial r}\left(r u_{r}\right)+\frac{\partial u_{z}}{\partial z} & =0 \\
\frac{\partial u_{r}}{\partial t}+u_{r} \frac{\partial u_{r}}{\partial r}+u_{z} \frac{\partial u_{r}}{\partial z} & =-\frac{\partial p}{\partial r}+O h\left[\frac{\partial}{\partial r}\left(\frac{1}{r} \frac{\partial}{\partial r}\left(r u_{r}\right)\right)+\frac{\partial^{2} u_{r}}{\partial z^{2}}\right] \\
\frac{\partial u_{z}}{\partial t}+u_{r} \frac{\partial u_{z}}{\partial r}+u_{z} \frac{\partial u_{z}}{\partial z} & =-\frac{\partial p}{\partial z}+O h\left[\frac{1}{r} \frac{\partial}{\partial r}\left(r \frac{\partial u_{z}}{\partial r}\right)+\frac{\partial^{2} u_{z}}{\partial z^{2}}\right]
\end{aligned}
$$

where $O h=\mu /(\sigma a \rho)^{1 / 2}$ is the Ohnesorge number, the characteristic dimensionless parameter distinguishing the viscous from the inviscid case, with the liquid dynamic viscosity $\mu$. The closed set of the above equations must be solved subject to initial and boundary conditions. The kinematic boundary condition states that the material rate of deformation of the jet surface equals the radial velocity component at the place of the deformed surface. The kinematic boundary condition therefore reads

$$
u_{r}=\frac{D \eta}{D t}=\frac{\partial \eta}{\partial t}+u_{z} \frac{\partial \eta}{\partial z} \text { at } r=1+\eta
$$

The first dynamic boundary condition states that the shear stress parallel to the jet surface is zero, since the ambient gas phase dynamic viscosity is very small, so that momentum cannot be transferred across the jet boundary at an appreciable rate. The second dynamic boundary condition states that the stress normal to the jet surface, composed from the flow-induced pressure and a viscous contribution, differs across the interface by the contribution due to the surface tension. The zero-shear stress boundary condition reads

$$
(\vec{n} \cdot \tau) \times \vec{n}=\overrightarrow{0} \quad \text { at } r=1+\eta
$$

where the outward unit normal vector $\vec{n}$ is given as $\vec{n}=\left(\vec{e}_{r}-\frac{\partial \eta}{\partial z} \vec{e}_{z}\right) / \sqrt{1+\left(\frac{\partial \eta}{\partial z}\right)^{2}}$ and the viscous extra stress tensor $\tau$ in (5) is the one for the incompressible Newtonian fluid. The corresponding normal stress boundary condition reads

$$
-p+O h(\vec{n} \cdot \tau) \cdot \vec{n}+(\vec{\nabla} \cdot \vec{n})=0 \quad \text { at } r=1+\eta
$$


We obtain the divergence of the normal unit vector in this equation as

$$
(\vec{\nabla} \cdot \vec{n})=(1+\eta)^{-1}\left[1+\left(\frac{\partial \eta}{\partial z}\right)^{2}\right]^{-1 / 2}-\frac{\partial^{2} \eta}{\partial z^{2}}\left[1+\left(\frac{\partial \eta}{\partial z}\right)^{2}\right]^{-3 / 2} \text { at } r=1+\eta
$$

The initial surface disturbance is assumed to be purely sinusoidal with the amplitude $\eta_{0}$ and wave number $k=$ $2 \pi a / \lambda$. With this assumption, volume conservation leads to the following expression for the initial non-dimensional jet shape [1]:

$$
r_{s}(0, z)=1+\eta(0, z)=\eta_{0} \cos k z+\left(1-\eta_{0}^{2} / 2\right)^{1 / 2}=1+\eta_{0} \cos k z-\frac{1}{4} \eta_{0}^{2}-\frac{1}{32} \eta_{0}^{4}-\ldots
$$

As usual in weakly nonlinear analysis, the initial deformation amplitude is assumed to be small, i.e. $\eta_{0} \ll 1$. For analyzing these equations in a weakly nonlinear form, the two velocity components and the pressure in the flow field, as well as the deformed interface shape, are expanded into power series with respect to the parameter $\eta_{0}$. This means that we formulate the dependencies as for, e.g., the radial velocity, pressure and jet shape:

$$
u_{r}=u_{r 1} \eta_{0}+u_{r 2} \eta_{0}^{2}+\ldots ; \quad p=p_{1} \eta_{0}+p_{2} \eta_{0}^{2}+\ldots ; \quad r_{s}=1+\eta_{1} \eta_{0}+\eta_{2} \eta_{0}^{2}+\ldots
$$

Furthermore, one important difference between the linear analysis and the present weakly nonlinear one is that the boundary conditions are satisfied on the deformed jet surface, not on the undeformed, circular cylindrical shape. For doing this, but still allowing for the functions in the boundary conditions to be evaluated on the undeformed jet surface, their values on the deformed shape are represented by Taylor expansions, such as

$$
\left.u_{r}\right|_{r=1+\eta}=\left.u_{r}\right|_{r=1}+\left.\frac{\partial u_{r}}{\partial r}\right|_{r=1} \eta+\ldots ;\left.\quad p\right|_{r=1+\eta}=\left.p\right|_{r=1}+\left.\frac{\partial p}{\partial r}\right|_{r=1} \eta+\ldots
$$

Substituting these approaches into the flow equations (1) - (3) and into the boundary conditions (4), (5) and (6), and representing the flow properties and their derivatives as given in (9), we obtain sets of first and second order equations of motion with the boundary conditions consisting of all the terms with the deformation parameter $\eta_{0}$ to the first and second powers, respectively.

\section{First-order equations}

To obtain the first-order equations we collect all the terms in the above expansions with the parameter $\eta_{0}$ to the first power. The first-order continuity and momentum equations read

$$
\begin{aligned}
\frac{1}{r} \frac{\partial}{\partial r}\left(r u_{r 1}\right)+\frac{\partial u_{z 1}}{\partial z} & =0 \\
\frac{\partial u_{r 1}}{\partial t} & =-\frac{\partial p_{1}}{\partial r}+O h\left[\frac{\partial}{\partial r}\left(\frac{1}{r} \frac{\partial}{\partial r}\left(r u_{r 1}\right)\right)+\frac{\partial^{2} u_{r 1}}{\partial z^{2}}\right] \\
\frac{\partial u_{z 1}}{\partial t} & =-\frac{\partial p_{1}}{\partial z}+O h\left[\frac{1}{r} \frac{\partial}{\partial r}\left(r \frac{\partial u_{z 1}}{\partial r}\right)+\frac{\partial^{2} u_{z 1}}{\partial z^{2}}\right]
\end{aligned}
$$

For the boundary conditions of first order we obtain

$$
\begin{aligned}
u_{r 1} & =\frac{\partial \eta_{1}}{\partial t} \text { at } r=1 \text { kinematic } \\
\frac{\partial u_{z 1}}{\partial r}+\frac{\partial u_{r 1}}{\partial z} & =0 \text { at } r=1 \text { zero shear stress } \\
-p_{1}+2 O h \frac{\partial u_{r 1}}{\partial r}-\left(\eta_{1}+\frac{\partial^{2} \eta_{1}}{\partial z^{2}}\right) & =0 \text { at } r=1 \text { zero normal stress }
\end{aligned}
$$

Furthermore, the initial conditions of first order are

$$
\eta_{1}(0, z)=\cos k z \quad \text { and } \quad \frac{\partial \eta_{1}}{\partial t}(0, z)=0
$$

\section{Second-order equations}

To obtain the second-order equations we collect all the terms in the above expansions with the parameter $\eta_{0}$ to the second power. The second-order continuity and momentum equations read

$$
\begin{aligned}
\frac{1}{r} \frac{\partial}{\partial r}\left(r u_{r 2}\right)+\frac{\partial u_{z 2}}{\partial z} & =0 \\
\frac{\partial u_{r 2}}{\partial t}+u_{r 1} \frac{\partial u_{r 1}}{\partial r}+u_{z 1} \frac{\partial u_{r 1}}{\partial z} & =-\frac{\partial p_{2}}{\partial r}+O h\left[\frac{\partial}{\partial r}\left(\frac{1}{r} \frac{\partial}{\partial r}\left(r u_{r 2}\right)\right)+\frac{\partial^{2} u_{r 2}}{\partial z^{2}}\right] \\
\frac{\partial u_{z 2}}{\partial t}+u_{r 1} \frac{\partial u_{z 1}}{\partial r}+u_{z 1} \frac{\partial u_{z 1}}{\partial z} & =-\frac{\partial p_{2}}{\partial z}+O h\left[\frac{1}{r} \frac{\partial}{\partial r}\left(r \frac{\partial u_{z 2}}{\partial r}\right)+\frac{\partial^{2} u_{z 2}}{\partial z^{2}}\right]
\end{aligned}
$$


The boundary conditions of second order at $r=1$ are

$$
\begin{aligned}
u_{r 2} & =\frac{\partial \eta_{2}}{\partial t}+u_{z 1} \frac{\partial \eta_{1}}{\partial z}-\eta_{1} \frac{\partial u_{r 1}}{\partial r} \quad \text { kinematic } \\
\frac{\partial u_{z 2}}{\partial r} & +\frac{\partial u_{r 2}}{\partial z}+\eta_{1} \frac{\partial}{\partial r}\left(\frac{\partial u_{z 1}}{\partial r}+\frac{\partial u_{r 1}}{\partial z}\right)+ \\
& +2\left(\frac{\partial u_{r 1}}{\partial r}-\frac{\partial u_{z 1}}{\partial z}\right) \frac{\partial \eta_{1}}{\partial z}=0 \quad \text { zero shear stress } \\
-p_{2} & -\eta_{1} \frac{\partial p_{1}}{\partial r}+2 O h\left[\eta_{1} \frac{\partial^{2} u_{r 1}}{\partial r^{2}}+\frac{\partial u_{r 2}}{\partial r}-\frac{\partial \eta_{1}}{\partial z}\left(\frac{\partial u_{z 1}}{\partial r}+\frac{\partial u_{r 1}}{\partial z}\right)\right]- \\
& -\left[\eta_{2}+\frac{\partial^{2} \eta_{2}}{\partial z^{2}}+\frac{1}{2}\left(\left(\frac{\partial \eta_{1}}{\partial z}\right)^{2}-2 \eta_{1}^{2}\right)\right]=0 \quad \text { zero normal stress }
\end{aligned}
$$

Furthermore, the initial conditions of second order are

$$
\eta_{2}(0, z)=-1 / 4 \text { and } \frac{\partial \eta_{2}}{\partial t}(0, z)=0
$$

Solving these sets of equations will reveal the weakly nonlinear role of the viscous stresses in the jet liquid on the capillary instability of a Newtonian viscous liquid jet in a vacuum.

\section{Solutions of the governing equations First-order solutions}

The first-order equations describe the linear problem. They exhibit well known solutions expected to be recovered by our equations. In particular, we expect to recover the special form of the dispersion relation of the viscous jet first presented by Weber [8] without the ambient gas influence.

Since we are looking at two-dimensional flow fields, for determining the first-order velocity and pressure fields we apply the method of the Stokesian stream function. The stream function $\psi$ represents the liquid motion due to the disturbance from the cylindrical form of the jet. The stream function is defined by its relations to the two velocity components $u_{r 1}$ and $u_{z 1}$ as per [15]

$$
u_{r 1}=-\frac{1}{r} \frac{\partial \psi}{\partial z} \quad, \quad u_{z 1}=\frac{1}{r} \frac{\partial \psi}{\partial r}
$$

Using this definition of the velocity components as derivatives of the stream function, the resulting first-order velocity field satisfies the continuity equation identically.

The first-order interface deformation is assumed to remain sinusoidal. $\eta_{1}$ is thus searched under the form

$$
\eta_{1}=\hat{\eta}_{1} \exp \left(i k z-\alpha_{1} t\right)
$$

with $\alpha_{1}$ the first-order angular frequency of the jet problem.

Taking the curl of the momentum equation in a vector form based on (12) and (13), we obtain the equation

$$
\left(\frac{1}{O h} \frac{\partial}{\partial t}-E^{2}\right)\left(E^{2} \psi\right)=0, \text { where } \quad E^{2}=r \frac{\partial}{\partial r}\left(\frac{1}{r} \frac{\partial}{\partial r}\right)+\frac{\partial^{2}}{\partial z^{2}}
$$

for the stream function. The solution of (27) for a flow field containing the position $r=0$ reads [16]

$$
\psi(r, z, t)=\left[C_{1} r I_{1}(k r)+C_{3} r I_{1}(l r)\right] \exp \left(i k z-\alpha_{1} t\right)
$$

where $l^{2}=k^{2}-\alpha_{1} / O h$. The two constants $C_{1}$ and $C_{3}$ are determined by the kinematic and the dynamic zero tangential stress boundary conditions (14) and (15) and read

$$
C_{1}=-\frac{i \alpha_{1} \hat{\eta}_{1}}{k I_{1}(k)} \frac{l^{2}+k^{2}}{l^{2}-k^{2}}=\frac{i \hat{\eta}_{1}}{k I_{1}(k)}\left(2 k^{2} O h-\alpha_{1}\right), \quad C_{3}=\frac{i 2 \alpha_{1} \hat{\eta}_{1}}{I_{1}(l)} \frac{k}{l^{2}-k^{2}}=-\frac{i 2 \hat{\eta}_{1} O h k}{I_{1}(l)}
$$

With these constants, the stream function of the disturbance is known, but the angular frequency $\alpha_{1}$ remains to be determined. From the stream function we may calculate the velocity field in the jet due to the disturbance as

$$
\begin{array}{rrr}
u_{r 1} & = & -i k\left[C_{1} I_{1}(k r)+C_{3} I_{1}(l r)\right] \exp \left(i k z-\alpha_{1} t\right) \\
u_{z 1} & = & {\left[C_{1} k I_{0}(k r)+C_{3} l I_{0}(l r)\right] \exp \left(i k z-\alpha_{1} t\right)}
\end{array}
$$

The pressure due to the disturbance in the liquid field is obtained by integrating one component of the momentum equation. For this, the $z$ component is the right choice since it offers an easy integration with respect to the $z$ coordinate. The result is

$$
p_{1}=-i \alpha_{1} C_{1} I_{0}(k r) \exp \left(i k z-\alpha_{1} t\right)+f(t, r)
$$


where $f(t, r)$ must be equal to zero to satisfy the boundary condition (16). The dispersion relation of the jet is now found by introducing the velocity field and the pressure in the jet into the dynamic zero normal stress boundary condition (16). The result is the well-known relation

$$
\alpha_{1}^{2}-2 \alpha_{1} k^{2} O h\left[1-\frac{1}{k} \frac{I_{1}(k)}{I_{0}(k)}-\frac{2 k l}{l^{2}+k^{2}} \frac{I_{1}(k)}{I_{0}(k)}\left(\frac{I_{0}(l)}{I_{1}(l)}-\frac{1}{l}\right)\right]=k\left(1-k^{2}\right) \frac{I_{1}(k)}{I_{0}(k)} \frac{l^{2}-k^{2}}{l^{2}+k^{2}}
$$

which was first presented by Weber [8]. For zero liquid viscosity $(O h \rightarrow 0)$, this relation reduces to the result of Rayleigh [4] for the inviscid jet in a vacuum.

For disturbance wave numbers $0 \leq k \leq 1$, the relation (33) has two real solutions $\alpha_{1}^{+}$and $\alpha_{1}^{-}$, one positive and one negative, where, due to the formulation of the time dependency by the exponential function, the unstable behaviour of the jet is associated with the negative one. Note that this is the main point of difference from our previous version of the analysis [3], where the two solutions were incorrectly assumed to be of same absolute value like in the inviscid case. For wave numbers $k>1$, the relation has two conjugate complex roots with a positive real part. The two values of $\alpha_{1}$ represent two waves on the jet surface travelling in different directions and with different phase velocity. Accounting for both these waves, we formulate the first-order jet surface shape as

$$
\eta_{1}(z, t)=\hat{\eta}_{1}^{+} \exp \left(i k z-\alpha_{1}^{+} t\right)+\hat{\eta}_{1}^{-} \exp \left(i k z-\alpha_{1}^{-} t\right)
$$

The first-order initial conditions (17) require that initially the jet surface is governed by the function $\cos k z$ and is at rest. For the amplitudes $\hat{\eta}_{1}^{+}$and $\hat{\eta}_{1}^{-}$these conditions reveal the equations

$$
\hat{\eta}_{1}^{+}=-\frac{\alpha_{1}^{-}}{\alpha_{1}^{+}-\alpha_{1}^{-}} \quad \text { and } \quad \hat{\eta}_{1}^{-}=\frac{\alpha_{1}^{+}}{\alpha_{1}^{+}-\alpha_{1}^{-}}
$$

so that the amplitudes are known. The first-order stream function, velocity components and pressure are then obtained. As an example, the radial velocity component reads

$$
\begin{aligned}
u_{r 1}(r, z, t) & =\hat{\eta}_{1}^{+}\left[\left(2 k^{2} O h-\alpha_{1}^{+}\right) \frac{I_{1}(k r)}{I_{1}(k)}-2 k^{2} O h \frac{I_{1}\left(l^{+} r\right)}{I_{1}\left(l^{+}\right)}\right] \exp \left(i k z-\alpha_{1}^{+} t\right)+ \\
& +\quad \hat{\eta}_{1}^{-}\left[\left(2 k^{2} O h-\alpha_{1}^{-}\right) \frac{I_{1}(k r)}{I_{1}(k)}-2 k^{2} O h \frac{I_{1}\left(l^{-} r\right)}{I_{1}\left(l^{-}\right)}\right] \exp \left(i k z-\alpha_{1}^{-} t\right) \\
=: & \hat{\eta}_{1}^{+} f_{r}^{+}(r) \exp \left(i k z-\alpha_{1}^{+} t\right)+\hat{\eta}_{1}^{-} f_{r}^{-}(r) \exp \left(i k z-\alpha_{1}^{-} t\right)
\end{aligned}
$$

where the modified wave number $l$ appearing in the coefficients $C_{1}$ and $C_{3}$ was formulated with the two different values of $\alpha_{1}$ and denoted with the superscripts corresponding to their signs. And analogously, the axial velocity component reads:

$$
u_{z 1}(r, z, t)=: \quad i \hat{\eta}_{1}^{+} f_{z}^{+}(r) \exp \left(i k z-\alpha_{1}^{+} t\right)+i \hat{\eta}_{1}^{-} f_{z}^{-}(r) \exp \left(i k z-\alpha_{1}^{-} t\right)
$$

For zero liquid viscosity, as already stressed, the two solutions of $\alpha_{1}$ exhibit the same absolute value, but have different signs. Furthermore the two amplitudes of the first-order jet surface shape in (35) assume the same value of $1 / 2$. The two velocity components, pressure and jet surface shape therefore reduce to the inviscid solutions by Yuen [1]. E.g., the inviscid first-order radial velocity component and pressure read

$$
u_{r 1,0}(r, z, t)=\alpha_{1} \frac{I_{1}(k r)}{I_{1}(k)} \exp (i k z) \sinh \alpha_{1} t ; \quad p_{1,0}(r, z, t)=-\alpha_{1}^{2} \frac{I_{0}(k r)}{k I_{1}(k)} \exp (i k z) \cosh \alpha_{1} t
$$

This serves as a validation of the first-order viscous solution. The respective real solutions arise by reducing the exponential functions $\exp (i k z)$ to their real parts.

\section{Second-order solutions}

We now proceed to the second-order equations. e.g., the second-order solutions for the radial velocity component and the jet surface shape are sought under the forms

$$
\begin{aligned}
u_{r 2}(r, z, t) & =u_{r 21}^{+}(r) e^{2 i k z-2 \alpha_{1}^{+} t}+u_{r 21}^{-}(r) e^{2 i k z-2 \alpha_{1}^{-} t}+u_{r 21}^{ \pm}(r) e^{2 i k z-\left(\alpha_{1}^{+}+\alpha_{1}^{-}\right) t}+ \\
& +u_{r 22}^{p}(r) e^{2 i k z-\alpha_{2}^{p} t}+u_{r 22}^{m}(r) e^{2 i k z-\alpha_{2}^{m} t} \\
\eta_{2}(z, t) & =\hat{F}_{21}^{+} e^{2 i k z-2 \alpha_{1}^{+} t}+\hat{G}_{21}^{+} e^{-2 \alpha_{1}^{+} t}+\hat{F}_{21}^{-} e^{2 i k z-2 \alpha_{1}^{-} t}+\hat{G}_{21}^{-} e^{-2 \alpha_{1}^{-} t}+ \\
& +\hat{F}_{21}^{ \pm} e^{2 i k z-\left(\alpha_{1}^{+}+\alpha_{1}^{-}\right) t}+\hat{G}_{21}^{ \pm} e^{-\left(\alpha_{1}^{+}+\alpha_{1}^{-}\right) t}+\hat{\eta}_{22}^{p} e^{2 i k z-\alpha_{2}^{p} t}+\hat{\eta}_{22}^{m} e^{2 i k z-\alpha_{2}^{m} t}
\end{aligned}
$$

These forms result from the mathematical structures of the second-order equations of motion (18) - (20), with the nonlinear terms of first order involved, and the boundary conditions (21) - (23). We look at wave numbers $k<1$ yielding linear instability.

The various contributions to the second-order solutions are now determined. Looking at the second-order equations of motion (18) - (20) we see that it is convenient to eliminate the second-order velocities from the momentum 
equations using the continuity equation (18) to obtain a differential equation for the second-order pressure $p_{21}$. The result is the equation

$$
\Delta p_{21}=-\operatorname{div}\left[\left(\vec{v}_{1} \cdot \vec{\nabla}\right) \vec{v}_{1}\right]
$$

In this equation $\vec{v}_{1}$ represents the first-order velocity field. Using the Lamé identity for the convective derivative of $\vec{v}_{1}$, (41) becomes

$$
\Delta\left[p_{21}+\vec{v}_{1}^{2} / 2\right]=+\operatorname{div}\left[\vec{v}_{1} \times\left(\vec{\nabla} \times \vec{v}_{1}\right)\right]
$$

This is a Poisson equation for the total pressure $\mathcal{P}_{21}:=p_{21}+\vec{v}_{1}^{2} / 2$. The structure of the solution in terms of its dependency on the axial coordinate and on time is determined by $\vec{v}_{1}^{2} / 2$ and by the terms on the right of (42). Both groups of terms contain exponential functions of twice the two rates of growth or decay, $-2 \alpha_{1}^{+}$and $-2 \alpha_{1}^{-}$, and the sum of the two rates, $-\left(\alpha_{1}^{+}+\alpha_{1}^{-}\right)$, each multiplied by time. In view of the limited space, we present the terms depending on $\exp \left(-2 \alpha_{1}^{+} t\right)$ only. The others are derived analogously. Using the representation of the stream function by modified Bessel functions, we re-write (42) and obtain the form

$$
\begin{aligned}
\Delta\left[\mathcal{P}_{21}^{+}\right] & =\frac{l^{+^{2}}-k^{2}}{r^{2}}\left\{C_{3}^{+^{2}}\left[\left(l^{+^{2}}-2 k^{2}\right) r^{2} I_{1}^{2}\left(l^{+} r\right)+l^{+^{2}} r^{2} I_{0}^{2}\left(l^{+} r\right)\right]+\right. \\
& \left.+C_{1}^{+} C_{3}^{+}\left[k r l^{+} r I_{0}(k r) I_{0}\left(l^{+} r\right)-k^{2} r^{2} I_{1}(k r) I_{1}\left(l^{+} r\right)\right]\right\} e^{2 i k z-2 \alpha_{1}^{+} t}
\end{aligned}
$$

for the dependency of time represented here. The solutions may be composed from the general solutions of the homogeneous equations and the particular solutions of the inhomogeneous equations. The final form of the solution, formulated for the pressure contribution $p_{21}^{+}$, reads

$p_{21}^{+}(r, z, t)=\left\{\left[-\frac{1}{4} \hat{\eta}_{1}^{+2}\left({f_{r}^{+}}^{2}-{f_{z}^{+}}^{2}\right)+C_{21}^{+} I_{0}(2 k r)+\sum_{i=1}^{I} \delta_{2 i}^{+} r^{2 i}\right] e^{2 i k z}+\left[\hat{P}_{21}^{+}+\frac{1}{4} \hat{\eta}_{1}^{+2}\left({f_{r}^{+}}^{2}+f_{z}^{+2}\right)\right]\right\} e^{-2 \alpha_{1}^{+} t}$ where the coefficients $\delta_{2 i}^{+}\left(k, l^{+}, C_{1}^{+}, C_{3}^{+}, q_{2 i}\right)$ arise from a polynomial approximation at order $I$ of the Bessel functions in the right-hand side of equation (43) according to $I_{0, I}(x)=\sum_{i=1}^{I} q_{2(i-1)} x^{2(i-1)}$ and $I_{1, I}(x)=I_{0, I}^{\prime}(x)$. From the second-order pressure field and the equations of motion (18) and (19), the corresponding second-order contributions to the two velocity components are determined. The differential equation (19) for $u_{r 2}$ yields the solution

$$
u_{r 21}^{+}=\left\{D_{21}^{+} I_{1}\left(2 m^{+} r\right)+\frac{k C_{21}^{+}}{\alpha_{1}^{+}} I_{1}(2 k r)+\sum_{i=1}^{I-1} \zeta_{2 i+1}^{+} r^{2 i+1}\right\} e^{2 i k z-2 \alpha_{1}^{+} t}
$$

where $m^{+^{2}}=k^{2}-\alpha_{1}^{+} /(2 O h)$ and the coefficients $\zeta_{2 i}^{+}\left(k, l^{+}, m^{+}, C_{1}^{+}, C_{3}^{+}, O h, q_{2 i}\right)$ again arise from approximations of the Bessel functions in the right-hand side of the radial velocity component differential equation (not reported here). From the velocity $u_{r 21}$ and the continuity equation (18), the contributions to $u_{z 21}$ are determined easily. The part depending on $\exp \left(-2 \alpha_{1}^{+} t\right)$ reads

$$
u_{z 21}^{+}=\frac{i}{2 k}\left\{D_{21}^{+} 2 m^{+} I_{0}\left(2 m^{+} r\right)+\frac{2 k C_{21}^{+}}{\alpha_{1}^{+}} I_{0}(2 k r)+\sum_{i=1}^{I-1} 2(i+1) \zeta_{2 i+1}^{+} r^{2 i}\right\} e^{2 i k z-2 \alpha_{1}^{+} t}
$$

The coefficients $C_{21}^{+}$and $D_{21}^{+}$, as well as the amplitudes $\hat{P}_{21}^{+}, \hat{F}_{21}^{+}$and $\hat{G}_{21}^{+}$(see equation (40)) are unknown constants, which are determined using the three boundary conditions of the second-order problem. The terms depending on $\exp \left(-2 \alpha_{1}^{-} t\right)$ and $\exp \left(-\left(\alpha_{1}^{-}+\alpha_{1}^{+}\right) t\right.$, and thus the amplitudes $\hat{P}_{21}^{-}, \hat{F}_{21}^{-}, \hat{G}_{21}^{-}, \hat{P}_{21}^{ \pm}, \hat{F}_{21}^{ \pm}, \hat{G}_{21}^{ \pm}$are obtained following the same method.

The second parts of the second-order solutions, with subscripts 22 , which depend on time through the frequency $\alpha_{2}$ of the second-order problem, are directly deduced from the equations for the linear problem, since they are of the same structure as for first order, with the wave number $k$ replaced by $2 k$, the angular frequency $\alpha_{1}$ by $\alpha_{2}$, and the deformation amplitude $\hat{\eta}_{1}$ by $\hat{\eta}_{22}$. The frequency $\alpha_{2}$ is therefore obtained as a solution of a dispersion relation which is formally equal to the first order relation (33), but formulated with double the wave number. The only unknown remaining is therefore the amplitude $\hat{\eta}_{22}$. e.g., the equation for the radial velocity component with subscripts 22 read

$$
\begin{aligned}
u_{r 22}(r, z, t) & =\hat{\eta}_{22}^{p}\left[\left(8 k^{2} O h-\alpha_{2}^{p}\right) \frac{I_{1}(2 k r)}{I_{1}(2 k)}-8 k^{2} O h \frac{I_{1}\left(m_{2}^{p} r\right)}{I_{1}\left(m_{2}^{p}\right)}\right] \exp \left(2 i k z-\alpha_{2}^{p} t\right)+ \\
& +\quad \hat{\eta}_{22}^{m}\left[\left(8 k^{2} O h-\alpha_{2}^{m}\right) \frac{I_{1}(2 k r)}{I_{1}(2 k)}-8 k^{2} O h \frac{I_{1}\left(m_{2}^{m} r\right)}{I_{1}\left(m_{2}^{m}\right)}\right] \exp \left(2 i k z-\alpha_{2}^{m} t\right)
\end{aligned}
$$

where we have defined $m_{2}^{2}=4 k^{2}-\alpha_{2} / O h$ and denote the two solutions of the dispersion relation for $\alpha_{2}$ by superscripts $p$ and $m$, since they may be either real or (conjugate) complex, depending on the wave number $\mathrm{k}$. In the case of the real solutions, the superscripts denote the positive and negative values, and for the complex solutions they denote the positive and negative imaginary parts. The real part is positive for all the wave numbers 


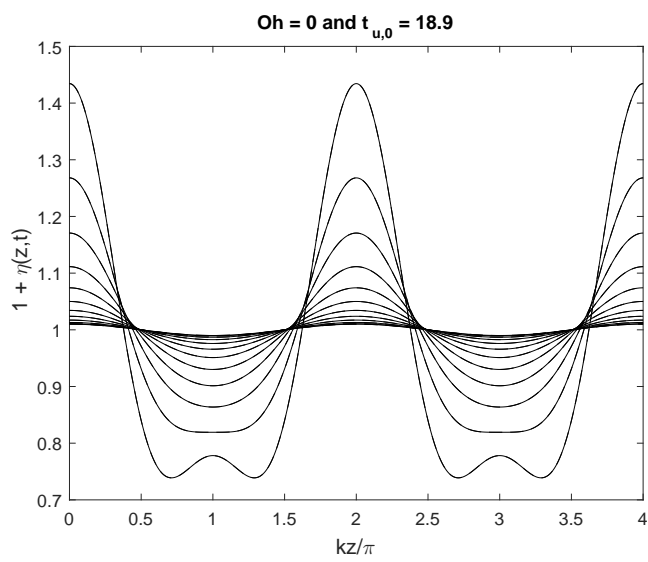

a)

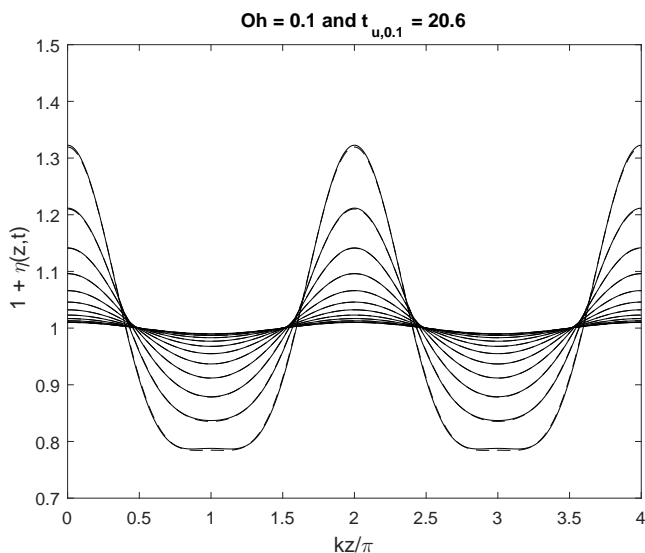

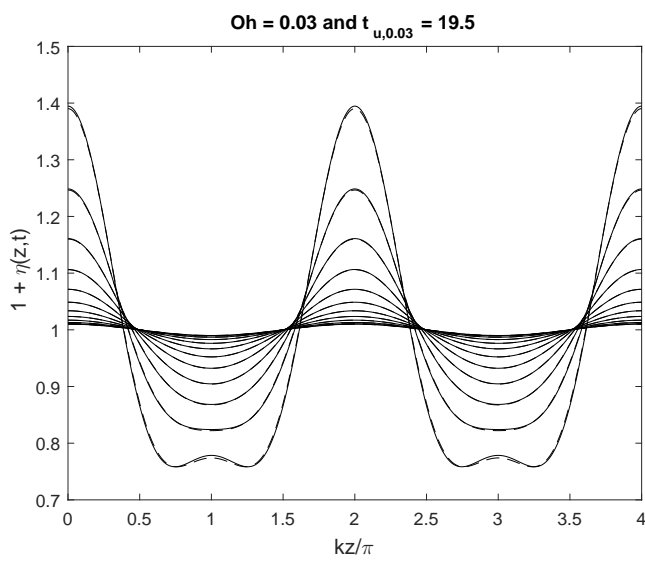

b)

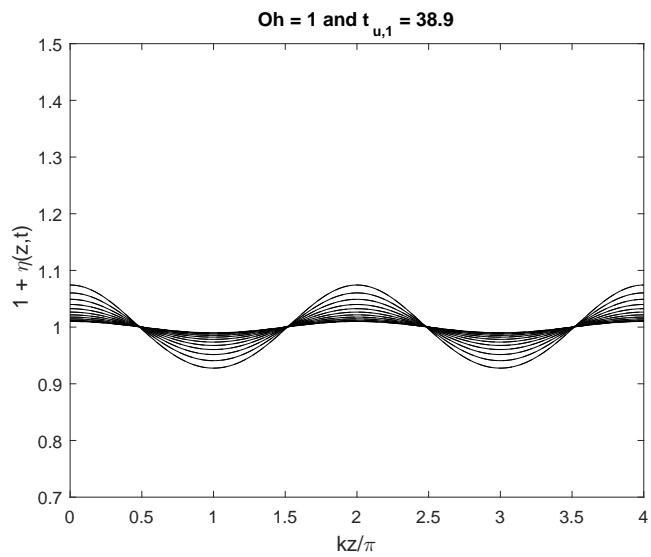

Figure 2. Surface shapes for various Ohnesorge numbers: a) $O h=0$ (inviscid case), b) $O h=0.03$, c) $O h=0.1, \mathrm{~d}$ ) $O h=1$ for discrete times $t=\left[0, \Delta t, 2 \Delta t, \ldots, t_{u}, t_{u}+\Delta t\right]$ with $\Delta t=t_{u} / 10$. The value of $t_{u}$ is indicated in each figure. Disturbance wave number $k=0.3$ and initial deformation amplitude $\eta_{0}=0.01$. Lines are drawn as solid for surface shapes taking into account the approximate viscous contribution with $I=6$ and as dashed for neglecting it.

$1 \leq 2 k \leq 2$. The values of $\hat{\eta}_{22}^{p}$ and $\hat{\eta}_{22}^{m}$ are deduced from the two initial conditions. From the first-order and secondorder contributions we finally construct the description of the velocity components, the pressure, and the deformed jet surface to second order as defined in Eq. (9). For example, the deformed jet surface to second order reads:

$$
r_{s}(z, t)=1+\eta(z, t)=1+\eta_{0} \eta_{1}(z, t)+\eta_{0}^{2} \eta_{2}(z, t)
$$

where

$$
\eta_{1}(z, t)=\left(\hat{\eta}_{1}^{+} e^{-\alpha_{1}^{+} t}+\hat{\eta}_{1}^{-} e^{-\alpha_{1}^{-} t}\right) \cos k z
$$

and

$$
\begin{aligned}
\eta_{2}(z, t) & =\frac{1}{2}\left(\hat{\eta}_{22}^{p} e^{-\alpha_{2}^{p} t}+\hat{\eta}_{22}^{m} e^{-\alpha_{2}^{m} t}+\hat{F}_{21}^{+} e^{-2 \alpha_{1}^{+} t}+\hat{F}_{21}^{-} e^{-2 \alpha_{1}^{-} t}+\hat{F}_{21}^{ \pm} e^{-\left(\alpha_{1}^{-}+\alpha_{1}^{+}\right) t}\right) \cos 2 k z+ \\
& -\frac{1}{4} e^{-2\left(\alpha_{1}^{-}+\alpha_{1}^{+}\right) t}\left(\hat{\eta}_{1}^{-} e^{\alpha_{1}^{+} t}+\hat{\eta}_{1}^{+} e^{\alpha_{1}^{-} t}\right)^{2}
\end{aligned}
$$

The problem of second-order weakly nonlinear viscous jet instability is thus fully solved. From our above equations, the inviscid solution of Yuen [1] is retrieved for zero liquid viscosity $(O h \rightarrow 0)$.

\section{Results and discussion}

With the presently developed viscous weakly nonlinear model, the influence of viscosity on the phenomenon of satellite drop formation is investigated. Satellite drop formation is here identified by the appearance of an undulation, i.e., a local maximum between two consecutive crests, prior to jet breakup $(\eta=-1)$. Based on the second-order representation of the jet surface in (47), the undulation occurs as soon as $4 \eta_{2} \eta_{0} / \eta_{1}=1$. We denote the time for which the previous relation is satisfied as $t_{u}$. Like in the inviscid case [1], the undulation is not predicted for wave numbers greater than the fastest growing mode. Since the latter decreases with increasing liquid viscosity, a relatively small value of $k$ is selected to allow for comparison between different values of the Ohnesorge number. The initial deformation amplitude is imposed too, since its influence on the jet surface wave does not depend on the liquid viscosity and has already been studied by Yuen [1]. Jet surface shapes are thus calculated for a fixed wave number $k=0.3$ and initial deformation amplitude $\eta_{0}=0.01$. 
In Figure 2, jet surface shapes are drawn at different times for four selected Ohnesorge numbers: 0 (inviscid case), $0.03,0.1$ and 1 . For all these values, the non-dimensional fastest growing mode, given as $[2(1+3 O h)]^{-1 / 2}$ by the long-wave approximation [8], remains greater than 0.3 , which means that the formation of an undulation is expected by the viscous model. For each case, the time increment between two consecutive shapes is $\Delta t=t_{u} / 10$, the first time is 0 and the last time is $t_{u}+\Delta t$. Solid lines represent the viscous solutions for which the approximate terms are considered up to order $I=6$, whereas dashed lines correspond to the solutions for which the approximate terms are omitted.

The comparison between jet surfaces drawn for different Ohnesorge numbers reveals a retarding effect of the jet liquid viscosity on the apparition of the undulation. This is in agreement with experimental [13] and numerical [14] results, where it is found that satellite droplets are fewer with jets of more viscous liquids. Moreover, the comparison between solutions of different levels of approximation shows that the solution neglecting the viscous term on the right-hand side of equation (42) is enough to represent the jet surface shape for the wave number investigated. This result allows us to simplify the viscous model by ignoring the approximate terms.

These first results will be completed in the next future by considering the effect of the surrounding ambient medium and liquid viscoelasticity.

\section{Conclusions}

A weakly nonlinear stability analysis of a viscous Newtonian liquid jet in a vacuum was performed. By including the viscous stresses in the liquid, the model complements the results of Yuen [1] for the inviscid case and Yang and co-authors [2] for a plane viscous liquid sheet. In a weakly nonlinear analysis, velocity, pressure and jet surface shape are expanded in series with respect to a small deformation parameter, here the initial amplitude of the jet deformation, yielding a set of equations with different powers of the parameter. Our analysis is restricted to second order for the sake of simplicity, in a first attempt to solve the viscous jet problem. The first-order solution corresponds to the linear one first derived by Weber [8] which converges to Rayleigh's solution for the inviscid case [4]. The second-order solution represents the nonlinear influence from the first-order fields and is obtained by solving a Poisson equation for the second-order pressure field. A polynomial approximation was used to take into account the viscous terms on the right hand side of this equation, leading to a viscous model with an approximation representing a part of the viscous influence. At vanishing Ohnesorge number of the jet, our equations reduce to Yuen's inviscid results [1]. Varying the jet Ohnesorge number between 0.03 and 1 reveals a retarding effect of the viscosity on the formation of the undulation, thus on satellite drop formation in liquid jet breakup. This is in agreement with the experiment $[13,17]$. The influence of the level of approximation was also explored by comparing the solutions with and without the approximate part. The results suggest that the approximate part can be ignored, thus yielding a simpler viscous weakly nonlinear model of capillary liquid jet instability. The analysis will be carried further to include the influence from a gaseous ambient medium and viscoelastic liquid behaviour.

\section{Acknowledgements}

G.B. is indebted to I.M. and his team at the LOMC of the CNRS in Le Havre, and to Christophe Dumouchel at CORIA in Rouen, for their hospitality during three sabbatical stays in September 2014, September 2015, and December 2016 and acknowledges the inspiring atmospheres at the two laboratories. MC.R. was supported by the LABEX $E M C^{3}$ under the project TUVECO.

\section{References}

[1] Yuen, M.C., 1968, J. Fluid Mech., 33, pp. 151-163.

[2] Yang, L. J., Wang, C., Fu, Q. F., Du, M. L., Tong, M. X., 2013, J. Fluid Mech., 735, pp. 249-287.

[3] Renoult, M. C., Brenn, G., Mutabazi, I., 2016, ILASS 2016.

[4] Rayleigh, J.W.S. Lord, 1878, Proc. London Mathematical Society, 10, pp. 4-13.

[5] Rayleigh, J.W.S. Lord, 1879, Proc. Royal Society London A, 29, pp. 71-97.

[6] Savart, F., 1833, Annales de Chimie et Physique, 53, pp. 337-386.

[7] Plateau, J., 1873, Gauthiers-Villars, Paris, pp. 450-495.

[8] Weber, C., 1931, Zeitschrift für Angewandte Mathematik und Mechanik, 11, pp. 136-154.

[9] Rutland, D.F., Jameson, G.J., 1971, J. Fluid Mech., 46, pp. 267-271.

[10] Rutland, D.F., Jameson, G.J., 1970, Chemical Engineering Science, 25, pp. 1689-1698.

[11] Lafrance, P., 1975, Physics of Fluids, 18, pp. 428-432.

[12] Taub, H.H., 1976, Physics of Fluids, 19, pp. 1124-1129.

[13] Goedde, E.F., Yuen, M.C., 1970, J. Fluid Mech., 40, pp. 495-511.

[14] Ashgriz, N., Mashayek, F., 1995, J. Fluid Mech., 291, pp. 163-190.

[15] Bird, R.B., Stewart, W.E., Lightfoot, E.N., 1960, "Transport Phenomena". J. Wiley and Sons, New York.

[16] Brenn, G., 2017, "Analytical solutions for transport processes". Springer, Heidelberg, New York.

[17] Brenn, G., Frohn, A., 1993, Experiments in Fluids, 15, pp. 85-90. 


\title{
Flexible Piezoelectric Drop-On-Demand Droplet Generation
}

\author{
Norbert Riefler*1, Thomas Wriedt ${ }^{2}$, Udo Fritsching ${ }^{1}$ \\ ${ }^{1}$ Institute of Material Science (IWT), Bremen, Germany \\ ${ }^{2}$ Particle and Process Engineering, University Bremen, Germany \\ *Corresponding author: riefler@iwt.uni-bremen.de
}

\begin{abstract}
The size of droplets generated by piezoelectric drop-on-demand (DOD) droplet generators can be varied to a certain degree within one order of magnitude. This variation means that the droplet size is not solely determined by the nozzle diameter, and the droplet generation process is not restricted to drops extruded through a nozzle in conventional operation. By varying the electronic driving pulse, different droplet sizes can be obtained. To investigate the interaction of piezoelectric pulse excitation and the finally produced droplets, different approaches are applied. A comparison of a modal analysis of a pure piezo based on mechanical admittance calculations proofs the usability of electrical impedance measurements. This kind of measurements are then compared to finite-element simulations of a coupled piezo system - one as actuator, the other as pressure sensor - to extend the usable methods with the result that the fluid is of minor influence on the modal frequencies. Last, two phase fluid flow simulations with consequent pressure wave evaluations of the fluid show different pressure wave frequency specta than the modal analysis.
\end{abstract}

\section{Keywords}

Piezoelectic droplet-on-demand droplet generator, modal analysis, compressible two-phase flow

\section{Introduction}

Piezoelectric droplet generators are used commercially in inkjet printers as well as scientific instruments to, e.g., investigate single droplet phenomena like combustion [1] or drop collisions [2]. For controlled production of monodisperse droplets, drop-on-demand offer the possibility of generating droplets of different size solely by applying appropriate electrical signals to the piezoelectric actuator in 'w'-waveform [3] or in the form of staircase voltages [4]. Temple [5] suppossed that droplets can be generated with a much smaller diameter than the nozzle orifice and this is related to a 'Faraday Resonance', i.e. a resonance phenomenon that occurs in vertical oscillation of a column of liquid with a free surface. These surface wave resonances are a consequence of a pressure rise near the nozzle and lead to a transfer of energy and momentum from the acoustic field to the droplet.

The generation of droplets smaller than the nozzle orifice is of interest, e.g., to produce small droplets of a suspension. In this case, typical DOD droplet generators with a nozzle diamater well below $70 \mu \mathrm{m}$ tend to clogg with the consequence of a laborious cleaning process. Therefore, a dismountable droplet generator is developed to enable simple cleaning. Additionally, the nozzle diameter is increased to $200-300 \mu \mathrm{m}$ which is unusual for common fluids like water [6]. The generation parameters (i.e. pulse voltage and pulse time) for fluids with different viscosities and surface tension is experimentally tested. The transfer of momentum of a piezo actuator to the tube structure in the way of a modal analysis is also investigated and delivers preferred excitation frequencies of the solid tube part to the fluid. Last, the response of a compressible fluid to a pressure pulse is analyzed and interpreted as pressure waves by Fourier transformation.

\section{DOD generation experiments}

A principal sketch of a piezoelectric droplet generator can be seen in figure 1. It consists of a titanium tube with screw threads on each end, see figure 2, top, shown in upward directed operation in the bottom of this figure. In an experimental series, fluids with varying viscosities $\eta$, densities $\rho$ and surface tensions $\sigma$ given in table 1 are used.

Table 1. Fluid properties and generated droplet diameters

\begin{tabular}{lcccc}
\hline & $\eta[\mathrm{mPa} \cdot \mathrm{s}]$ & $\rho\left[\mathrm{kg} / \mathrm{m}^{3}\right]$ & $\sigma[\mathrm{N} / \mathrm{m}]$ & $d_{\text {drop }}[\mu \mathrm{m}]$ \\
\hline DI water & 1 & 1000 & 0.0725 & $24 \ldots 170$ \\
\hline water-glycerol & 2 & 1054.35 & 0.0715 & $135 \ldots 180$ \\
\hline ethanol-glycerol & 3 & 867.67 & 0.0225 & $113 \ldots 130$ \\
\hline
\end{tabular}




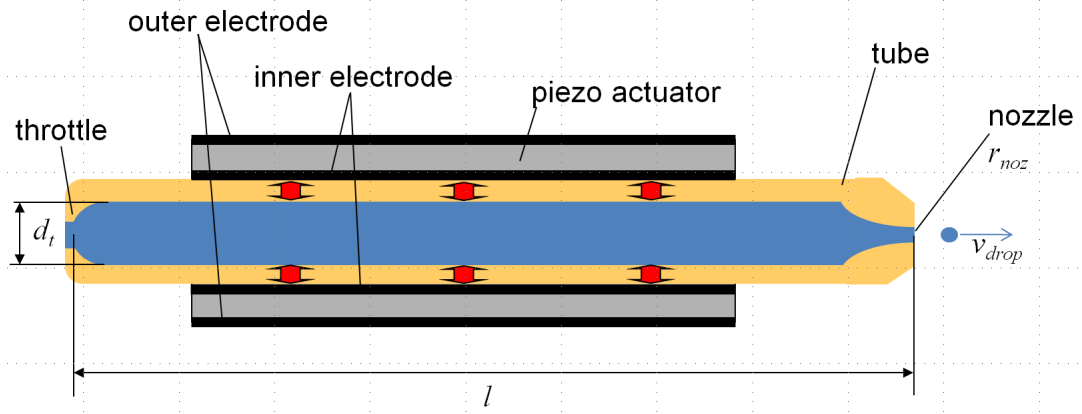

Figure 1. Sketch of a piezelectric droplet generator; the actuator moves the tube in radial direction (displacement $\leq 100 \mathrm{~nm}$ ).

The generated monodisperse and - at least one hour - stable droplets show different diameters and velocites. The dimensionless Reynolds and Ohnesorge numbers:

$$
R e=\frac{\rho d v_{d r o p}}{\eta}, \quad O h=\frac{\sqrt{W e}}{R e}=\frac{\eta}{\sqrt{\sigma \rho d}}
$$

are calculated based on these data to characterize the operating regime [7] of the droplet generator. The findings are shown in figure 3 together with the principal limits. The droplet diameters vary with driving signal in particular with DI water almost one order of magnitude and emphasize the flexibility of piezoelectric droplet generators.

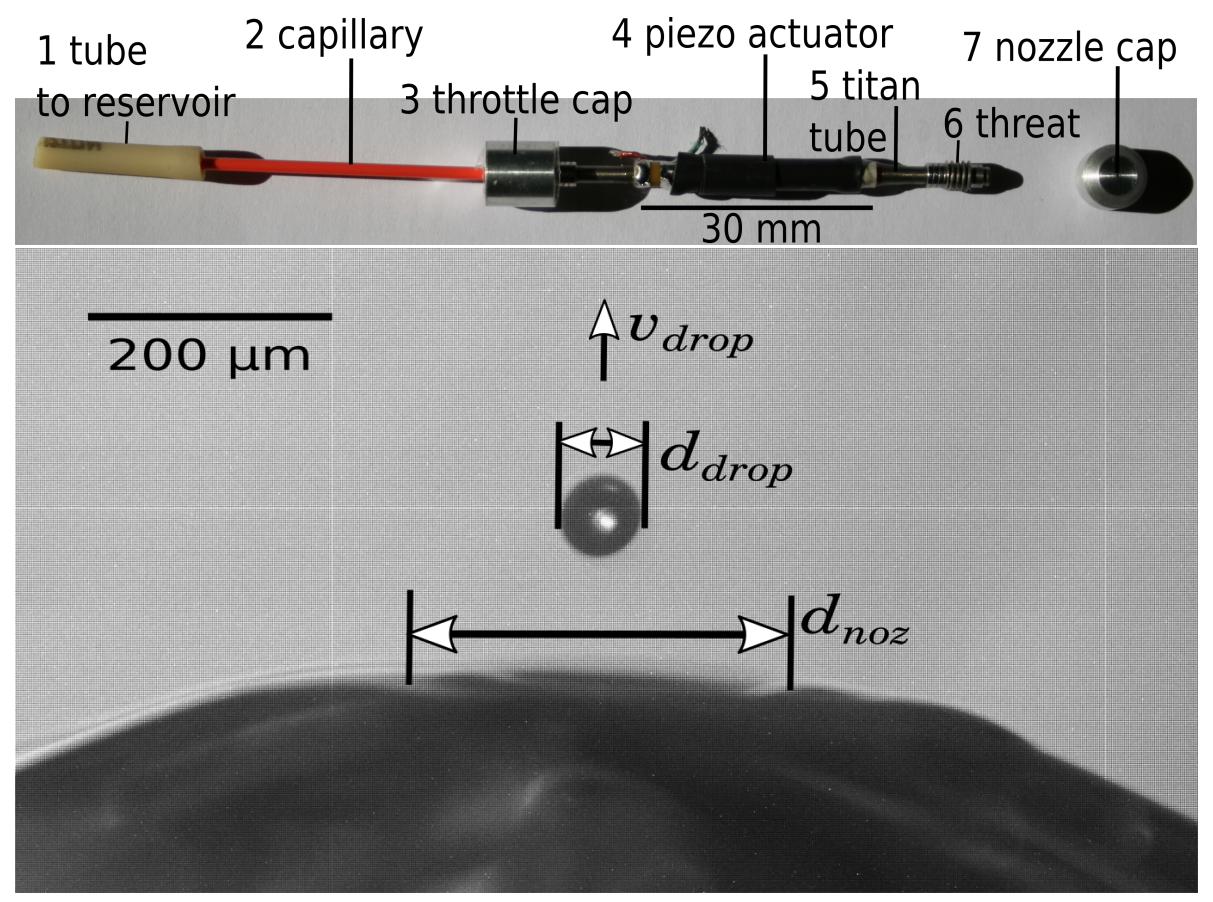

Figure 2. Droplet generator with the screwed off nozzle (7, top right) and piezo actuator in the middle (4); the red PEEK capillary

(2) on the left can be moved into the titan tube (5); image of a droplet with diameter of $d_{d r o p}=70 \mu \mathrm{m}$ from a nozzle with diamater of $d_{n o z}=300 \mu \mathrm{m}$ (below).

The restriction to a few stable operating points of the droplet generator for the ethanol-glycerol mixture is due to the low contact angle of this fluid with many solid materials. This results in a strong tendency to nozzle wetting which leads to a unstable generation process. The reason of the low number of operating points in case of deionized water is mainly due to a restricted maximum voltage of the used power supply of $\Delta V=200 \mathrm{~V}$. 


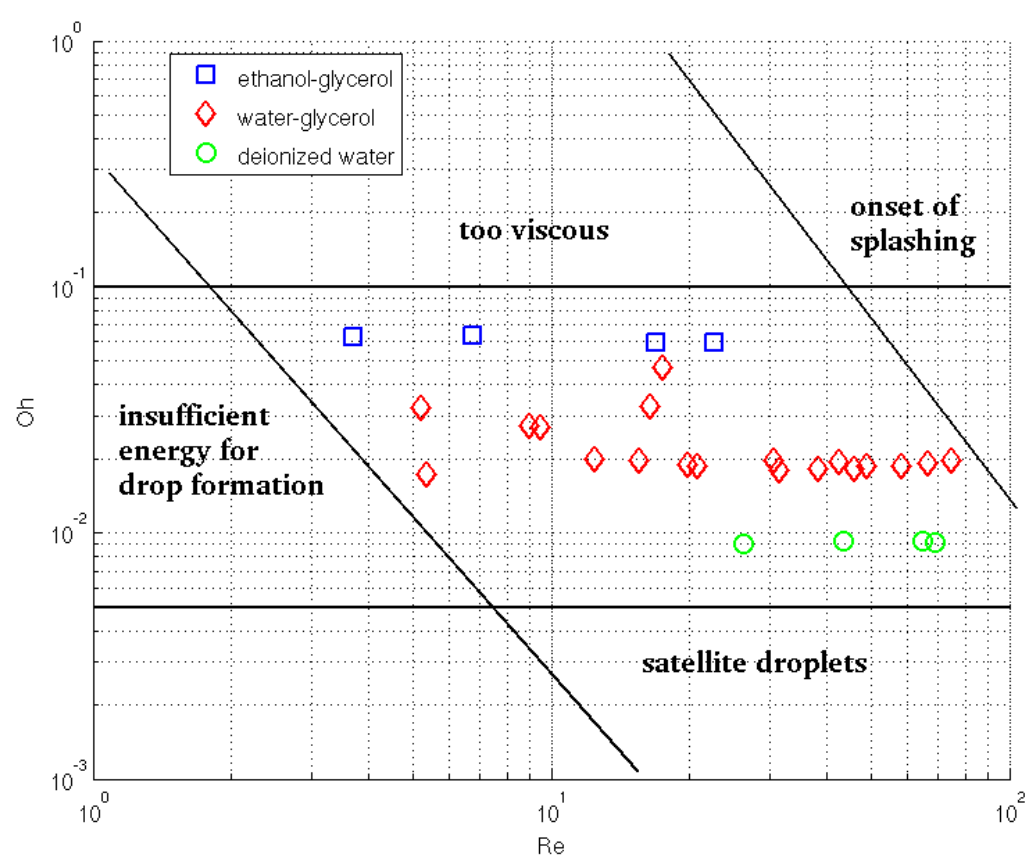

Figure 3. Stable droplet regime of the used DOD device.

\section{Modal analysis \\ One piezo system}

The piezoelectric actuator is characterized using a vector network analyzer (VNA) in reflection mode to investigate the electrical response of the system to different frequencies of the electrical drive signal. A low voltage sine signal with frequency range from about $10 \mathrm{kHz}$ to $1 \mathrm{MHz}$ is applied first to the piezo alone and in a second measurement to the piezo bonded to the titan tube. These measurements correspond to the excitation with a Dirac delta pulse and are equivalent to the impulse response function. At resonance frequencies strong responses can be observed, and these eigenfrequencies are the modes of the acousto-mechanical system [8]. From analytical considerations of a finite cylindrical beam with the properties given in table 2 the mechanical admittance can be expressed by modal summation [8]:

$$
Y(\omega)=i \omega \sum_{n=1}^{\infty} \frac{\phi_{n}(x)^{2}}{M\left(\omega_{n}^{2}\left(1+i \eta_{\text {loss }}\right)-\omega^{2}\right.}
$$

with the angular frequency $\omega=2 \pi f$, the modal mass $M=m l$ with the mass per unit area $m=\rho 2 \pi a h$, the mean radius of the shell $a=\left(d_{o}+d_{i}\right) / 2$, the shell wall thickness $h=\left(d_{o}-d_{i}\right) / 2$, the second moment of area (areal moment of intertia) $I=\pi a^{3} h$, the damping coefficient $\eta_{l o s s}$, and the modal function of $n$-th order:

$$
\phi_{n}(x)=\sqrt{2} \sin \left(\frac{n \pi x}{l}\right)
$$

for any position $x$ along the beam and $n=1,2, \ldots$. The natural frequencies are given by:

$$
\omega_{n}=\frac{n^{2} \pi^{2}}{l^{2}} \sqrt{\frac{E I}{m}}
$$

and they agree with the modal frequencies of a cylindrical beam. However, due to mechanical constraints, not all modal frequencies can be excited. In our case, a voltage applied to the piezoelectric actuator excites the coupled tube-piezo system mainly in radial direction, so it is expected that not every mode is experimentally observable.

The measured electrical impedances $Z$ are compared to the mechanical admittances $Y$ calculated by eqn. (2) because the admittance of a lumped mass-spring system corresponds to the impedance of a corresponding electrical equivalent, see Firestone [9]. However, not every acousto-mechanical mode contributes to the admittance spectrum. Only by using modes number $n=1,2,6$ and 21 the admittance spectrum shown in figure 4 with an estimated damping factor of $\eta=0.02$; lower damping inflates the calculated peaks while larger values overdamp the admittance curve. 
Table 2. Geometrical and material properties of the used lead circonate titanate piezoelectric material PIC151 of length $l$, inner diameter $d_{i}$, outer diameter $d_{o}$, Youngs modulus $E$ and density $\rho$

\begin{tabular}{ccccc}
\hline$l[\mathrm{~mm}]$ & $d_{i}[\mathrm{~mm}]$ & $d_{o}[\mathrm{~mm}]$ & $E[\mathrm{GPa}]$ & $\rho\left[\mathrm{kg} / \mathrm{m}^{3}\right]$ \\
\hline 30 & 2.2 & 3.2 & 10 & 7800 \\
\hline
\end{tabular}

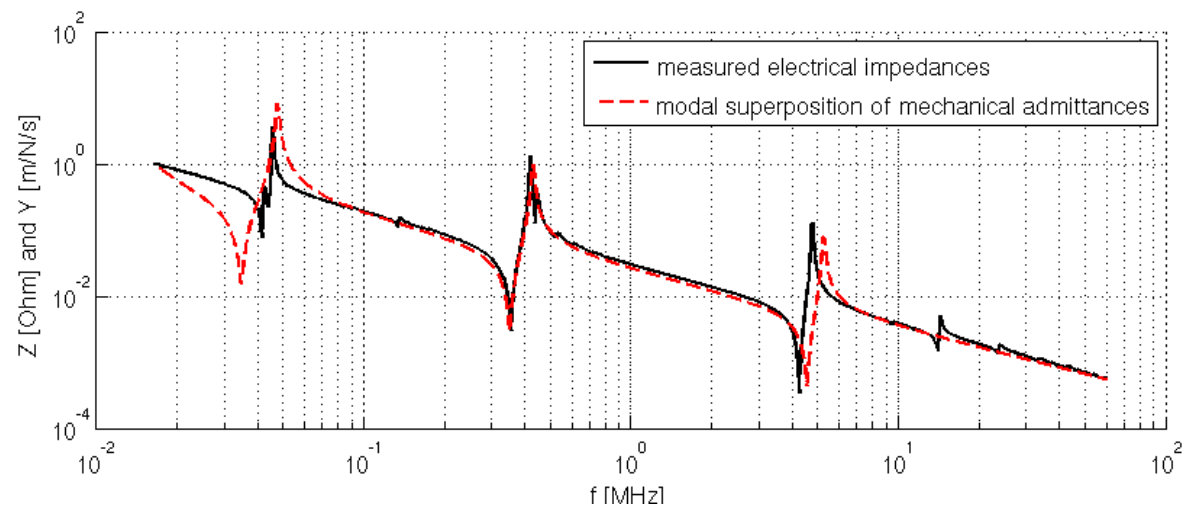

Figure 4. Comparison of measured impedance $Z$ with calculated admittance spectra $Y$.

This modal analysis considers the piezo tube only. As far as the piezo is adhesively bonded to the titan tube, the electrical impedance spectra changes as shown in figure 5.

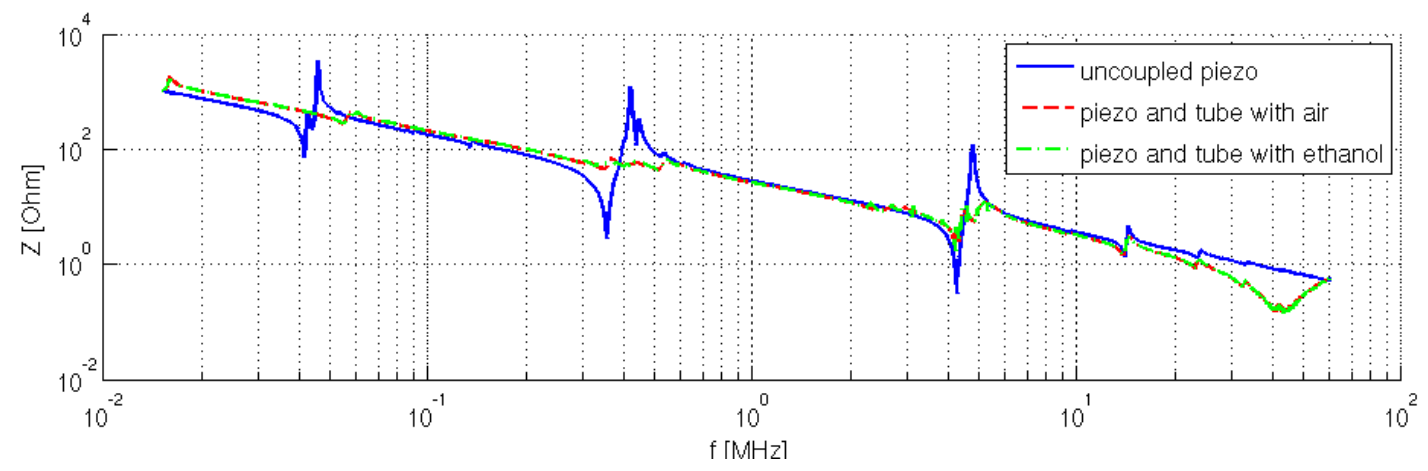

Figure 5. Measured impedance spectra $Z$ for the umcoupled piezo (blue, straight line) and the coupled system with air (red, dashed line) and filled with ethanol (green, dash-dotted line).

The tube strongly damps the pronounced modes of the uncoupled piezo (see the dashed and dash-dotted lines). However, the resonance frequencies changes only in case of the first resonance from about $42 \mathrm{kHz}$ to $55 \mathrm{kHz}$, and a new resonance at $\approx 15 \mathrm{kHz}$ (leftmost) occurs. The kind of fluid within the tube seems to be of minor importance to the system as can be seen from the curves with air and ethanol as fluid, that are only slightly different.

\section{Two coupled piezos}

The effect of the fluid - either air or ethanol - of the modal analysis on the droplet generator is small because the response is dominated by the solid materials titan and the PZT piezo ceramic. However, the influence of the mechanical vibrations on the fluid is crucial for a descrption of the fluidal droplet generation process. Therefore, it is intended to perform a fully coupled fluid-structure interaction simulation based on the finite element method (FEM). This will replace the analytical approach, and the reliability of the FEM simulations is therefore proved using a specical double piezo system, where the mechanical response function can be compared to an electrical stimulated excitation by a piezo actuator on the titan tube. I.e., the recording of the response on a second piezo, also bonded to the same titan tube, serves as a mechanical sensor.

The mesh of the FEM system, generated by the 'DesignModel' of ANSYS ${ }^{\odot}$ Academic Research, Release 17.2 within the Workbench framework, is shown in figure 6. A modal analysis is performed and compared to the resonance spectrum of the electrical driven system, where one piezo is fed with a sine wave of $U=5 \mathrm{~V}$ and the second piezo is connected to an oscilloscope for visualization and measurement of voltage. The resonance frequencies are determined at the local maximum of the piezo sensor signal in the frequency domain. 


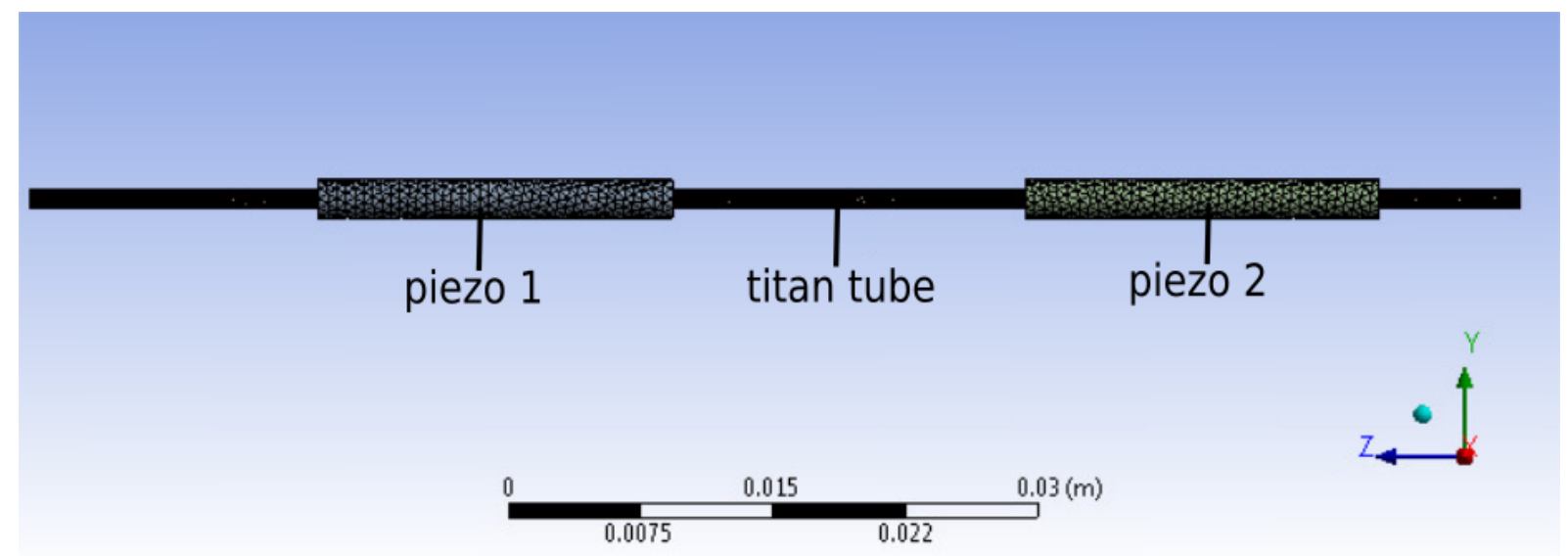

Figure 6. FEM model of a coupled system with two piezo actuators.

The modal frequencies from the FEM modal analysis and the frequencies at the resonance peaks show a strong similarity, although some values in the sweep test are missing. This can be explained by the large outputs of the piezo sensor in the frequency range starting from about $50 \mathrm{kHz}$ up to about $110 \mathrm{kHz}$. However, the results indicate that the FEM modal analysis is sufficiently precise and can be used for system characterizations.

Table 3. Comparison of modal frequencies from FEM analysis $\left(f_{F E M}\right)$ and measured frequencies of peak signals $\left(f_{\text {sweep }}\right)$

\begin{tabular}{ccccccccccc}
\multicolumn{6}{c}{ during frequency sweep; empty fields mean that there is mean no maximum detectable. } \\
\hline$f_{F E M}$ & 10.5 & 12.1 & 14.4 & 16.2 & 23.1 & 25.6 & 33.0 & 33.1 & 41.9 & $\mathrm{kHz}$ \\
$f_{\text {sweep }}$ & 8.1 & 12.7 & 13.2 & 17.5 & 23.5 & 28.5 & 32.3 & 33.5 & & $\mathrm{kHz}$ \\
\hline$f_{F E M}$ & 45.2 & 53.9 & 59.7 & 65.9 & 66.0 & 66.6 & 68.2 & 72.7 & 75.2 & $\mathrm{kHz}$ \\
$f_{\text {sweep }}$ & 44.3 & - & - & 63.0 & 63.2 & 66.6 & 67.2 & - & - & $\mathrm{kHz}$ \\
\hline$f_{F E M}$ & 78.4 & 85.6 & 88.1 & 92.7 & 92.8 & 101.5 & 113.2 & 117.3 & & $\mathrm{kHz}$ \\
$f_{\text {sweep }}$ & - & - & - & - & - & - & 115.3 & - & & $\mathrm{kHz}$ \\
\hline
\end{tabular}

\section{Compressible two phase flow}

The fluid cell of the droplet generator employed above is mapped as a 2D simulation case with cylinder symmetry in OpenFOAM, see figure 7. The transient simulation is performed with a compressible solver applying a volume of fluid method for the two phases water and air ('compressiblelnterFoam'). A pressure inlet in place of the piezo actuator (indicated by the black line in figure 7) is charged with a trapezoidal pressure pulse. The symmetry line is on the bottom and except the right inlet-outlet atmosphere patch, all other boundaries are walls.

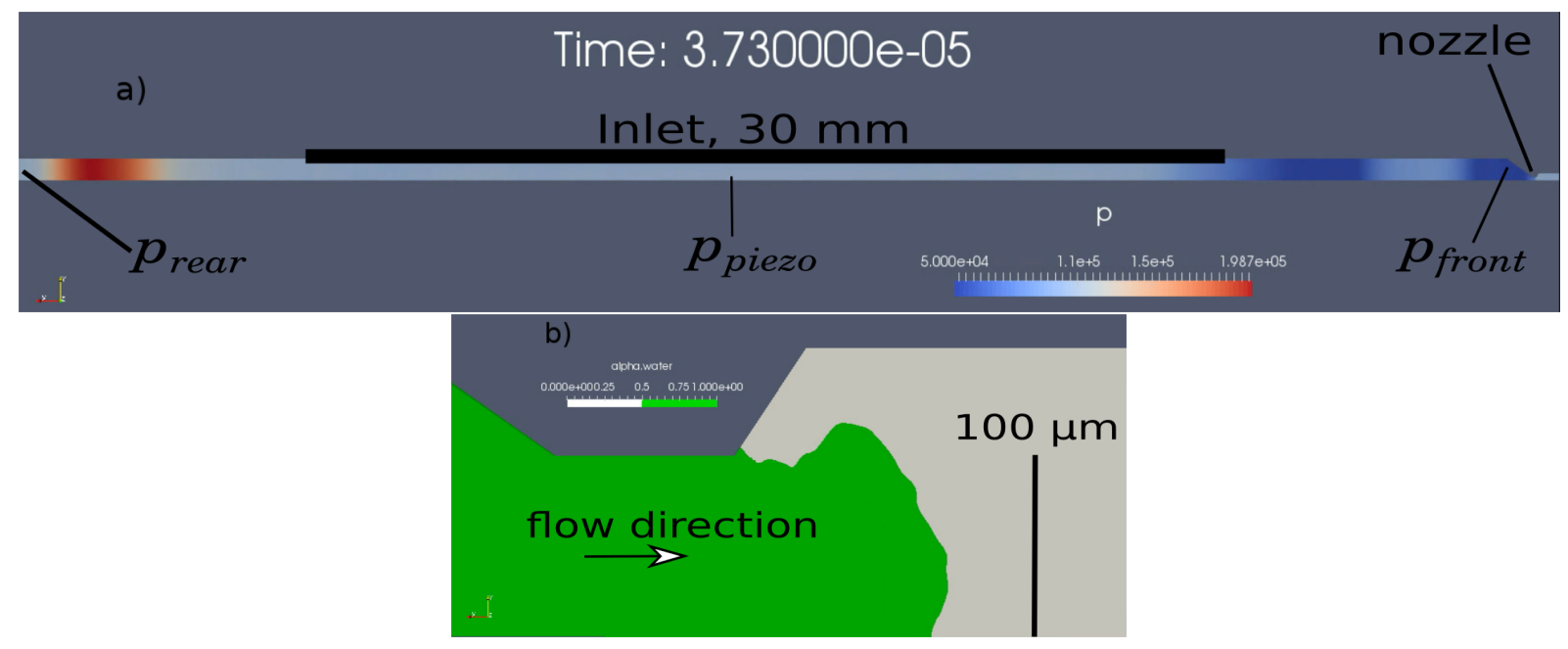

Figure 7. Two phase flow simulation with a compressible solver; a) pressure distribution along the fluid cell at $t=37.3 \mu$ s after start of the pressure pulse of length $t_{\text {pulse }}=20 \mu \mathrm{s}$ at the inlet (indicated with the black line); b) extruded fluid (green) with waves on the surface; orifice, i.e. nozzle radius $r_{n o z}=100 \mu \mathrm{m}$.

A pressure pulse of length $t_{p u l s e}=20 \mu \mathrm{s}$ and pressure increase of $\Delta p=1$ bar (additionally to the $1013 \mathrm{mbar}$ ambient pressure) excites the fluid in the chamber of figure 7 to oscillate at several frequencies. There is no 
response on the water-air interface on the nozzle until about $t=30 \mu \mathrm{s}$, but then water starts to extrude and a large droplet is generated. The free surface shows slight oscillations, however no Faraday resonances - proposed by Temple [5] - are observable due to the 2D mesh geometry of the simulations. Furthermore, the extruded fluid volume yields a droplet greater than those from experimental findings given in table 1.

To investigate the pressure values within the fluid cell in time, three pressure recording probes - positioned in the middle close to the piezo $\left(p_{\text {piezo }}\right)$, in the front cone close to the nozzle $\left(p_{\text {front }}\right)$ and in the rear $\left(p_{\text {rear }}\right)-$ are represented in figure 8 . The applied pressure pulse at the inlet can be clearly seen together with some overshoots (blue, straight line). At about $12 \mu$ s the pressure wave arrives simultaneously at the front cone and the rear. However, due to the conical constriction close to the nozzle on the right, the pressure wave form differ from the rectangluar rear side (left) with the result of different pressure curves.
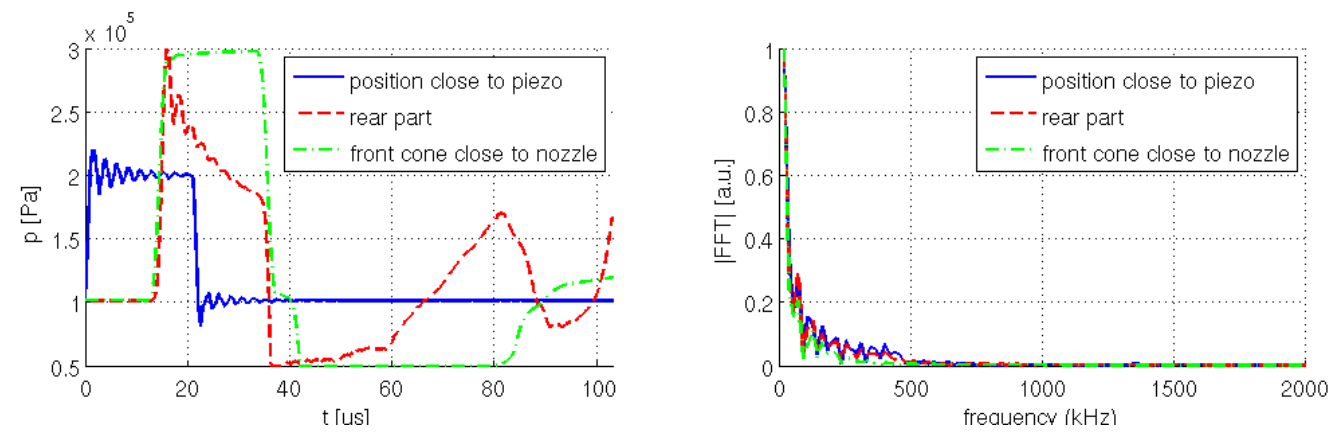

Figure 8. Pressure values at three probes located along the fluid cell after a pulse excitation length of $t_{\text {pulse }}=20 \mu \mathrm{s}$ (left); FFT's of the pressure waves (right).

If the pressure pulse is shorten to a triangular pulse, where the pressure rises up to $\Delta p=1$ bar at $\Delta t=1 \mu \mathrm{s}$, and falls afterwards back to ambient pressure at $\Delta t=1 \mu \mathrm{s}$ again, the response at the same probe locations delivers different curves, see figure 9.
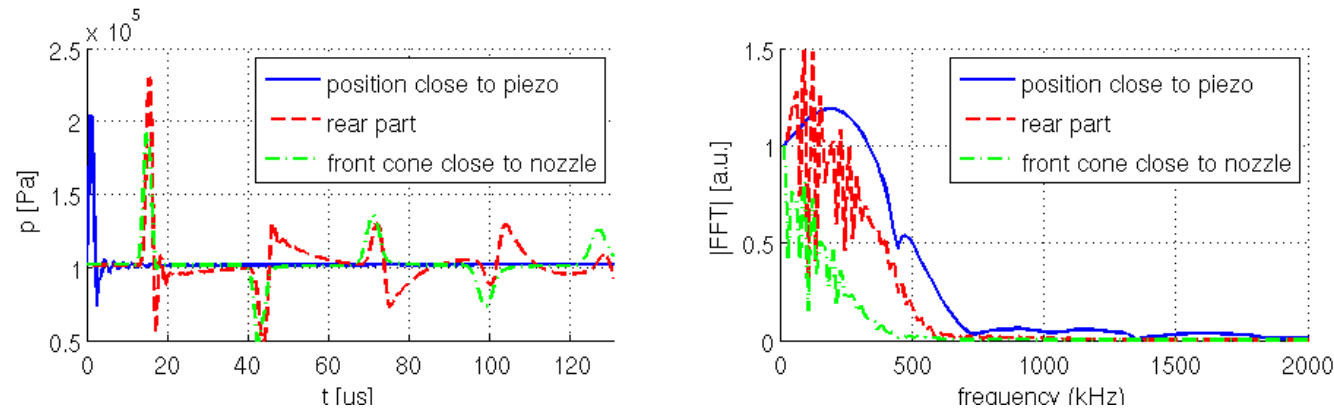

Figure 9. Same as in figure 8 but $t_{p u l s e}=2 \mu \mathrm{s}$.

Each pressure curve given in the left parts of figures 8 and 9 are Fourier transformed and represented in the right part. Therefore, the short pulse $\left(t_{\text {pulse }}=2 \mu \mathrm{s}\right)$ triggers a much larger frequency spectrum of pressure waves than the longer pulse. A thorough evaluation of the pressure response in time at about 1000 different probe positions distributed within the fluid cell serves as data basis for an evaluation of the frequency response of the fluid cell, where the Fourier transformed pressure function of each probe is stored. Some of these resonance frequencies appear many times and their occurence are counted.

To clarify if the the pressure wave frequencies and their number of counts can be reflected by the resonances in the mechanical impedance spectra of figure 5 , both results are represented in figure 10 . Every frequency is projected to the measured impedance value, and the number of counts, i.e. how often this frequency occurs in the FFTs of the pressure waves, are represented by the size of the dots. It can be seen that the longer excitation pulse $\left(t_{\text {pulse }}=\right.$ $20 \mu \mathrm{s}$, see figure 8) excites a lower frequency spectra, however with more pronounced single resonances, while the shorter pulse $\left(t_{\text {pulse }}=1 \mu \mathrm{s}\right.$, see figure 9$)$ excites a much broader spectra. However, the mechanical vibrations, i.e. the eigenmodes of the mechanical (solid) coupled piezo-tube system, are not represented by the fluid flow simulations. This means that the pressure waves within the fluid cell are not related to the mechanical piezo-tube vibrational system, a result found already by the impedance spectra, see figure 5 .

\section{Conclusions}

Analytic and FEM simulations are done to infer the eigenmodes of the solid material of the investigated droplet generators showing good agreement of the peak resonances of the piezo. Further comparisons of FEM simulations with electrical measured resonances on a system with two piezos coupled via a titan tube show also good agree- 
ment. These results permit the usage of the different methods to investigate the behaviour of the droplet generator which is essentially dominated by the coupling of the piezo and the tube and to a minor degree the coupling with the liquid in the tube chamber.

Additionally performed compressible and multiphase fluid flow simulations show rich spectra of pressure wave frequencies, however they cannot be directly attributed to the mechanical modes. Therefore, a complete fluidstructure-interaction model is actually developed to see the influence of the mechanical vibrations on the fluid and the generation of droplets in DOD generator.
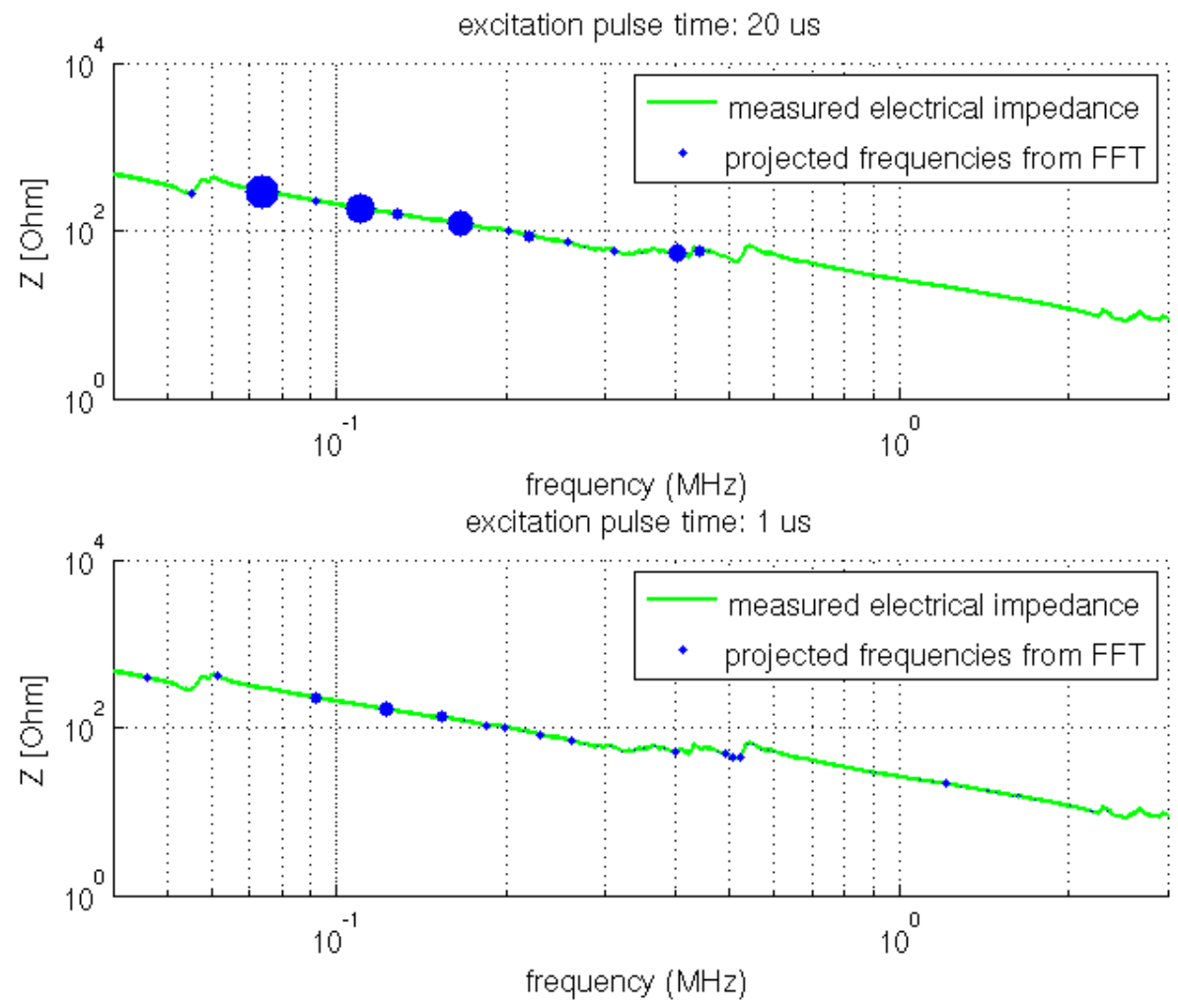

Figure 10. Comparison of the pressure wave frequencies after an excitation of $t_{\text {pulse }}=20 \mu \mathrm{s}$ (top) with those after $t_{\text {pulse }}=1$ $\mu \mathrm{s}$; the dots are the frequencies from the FFT, and their size corresponds to their number how often they occur.

\section{Acknowledgements}

We would like to thank the German Research Foundation (DFG) for financial support of this project.

\section{References}

[1] C. D. Rosebrock, N. Riefler, T. Wriedt, L. Mädler, and S. D. Tse. Disruptive burning of precursor/solvent droplets in flame-spray synthesis of nanoparticles. AlChE J., 59:4553-4566, 2013.

[2] O. Kurt, U. Fritsching, and G. Schulte. Secondary droplet formation during binary suspension droplet collisions. Atom. Spray, 19:457-472, 2009.

[3] O. A. Basaran, H. Gao, and P. P. Bhat. Nonstandard inkjets. Annu. Rev. Fluid Mech., 45:85-113, 2013.

[4] N. Riefler and T. Wriedt. Generation of monodisperse micron-size droplets using free adjustable signals. Part. Part. Syst. Charact., 25:176-182, 2008.

[5] S. Temple. Small fast inkdrop emission from a nozzle. Technical report, 43rd European Study Group with Industry, Lancaster University, 2-5th April 2002, 2002.

[6] H. Ulmke, T. Wriedt, and K. Bauckhage. Piezoelectric droplet generator for the calibration of particle-sizing instruments. Chem. Eng. Technol., 24:265-268, 2001.

[7] I. M. Hutchings and G. D. Martin, editors. Inkjet Technology for Digital Fabrication. Wiley, Chichester, 2012.

[8] F. Fahy and P. Gardonio. Sound and Structural Vibration. Elsevier, Academic Press, Amsterdam, 2006.

[9] F. A. Firestone. A new analogy between mechanical and electrical systems. J. Acoust. Soc. Am., 4:249-267, 1933. 


\title{
A Comprehensive Predictive Model to Anticipate Droplets Size Distribution in Pressure Swirl Atomizer
}

\author{
Sajad jafari ${ }^{1}$, Azadeh Kebriaee*1, Shahin sohrabi ${ }^{2}$ \\ ${ }^{1}$ Department of Aerospace engineering, Sharif University of Technology, Iran \\ ${ }^{2}$ Department of Mechanical engineering Universitat Politècnica de Catalunya, Spain \\ *Corresponding author: kebriaee@sharif.ir
}

\begin{abstract}
A predictive model is proposed to determine the droplet size and velocity distribution at the beginning of the secondary breakup. Liquid bulk is subjected to three stages of atomization including flow inside the injector, primary atomization, and secondary atomization to change a spray. The flow inside pressure swirl injector consisting an axial air core is a strongly complex two phase flow studied by highly precision coupled level set and volume of fluid method (CLSVOF). Subsequently, breakup lengths, interface deformation, as well as the most unstable frequency mode are calculated by nonlinear stability theory applied to emanating liquid sheet. Application of maximum entropy principle (MEP) is the final stage to predict size and velocity distribution of spray droplets. Based on liquid sheet breakup and conservative equations involving continuity, momentum, and energy, Newton-Raphson method is used to solve MEP equations. The proposed model has no dependency on empirical data in comparison with the usual past researches. The predicted results are shown to be in good agreement with the experimental findings related to performance characteristics and droplets size.
\end{abstract}

Keywords

Atomization, CLSVOF, Nonlinear Planar Liquid Sheet Stability, MEP, SMD.

\section{Introduction}

SPRAY modelling is a fundamental tool in the development of combustion chamber study. Fuel injectors have a key role on the combustion instability in gas turbine engines, internal combustion engines, and industrial furnaces [1]. Different correlations used to predict droplets size have no sufficient credit to apply in any arbitrary conditions. An objective procedure considering effective parameters, therefore, strongly helps designer in the parametric study. In the proposed method, representation of a comprehensive model for spray simulation leads to elicit the plenary information of injector performance with regarding the whole stages of atomization. Liquid bulk is subjected to different phases including flow inside the injector, primary atomization, and secondary atomization to change the spray droplets. Regardless of simple geometry of pressure swirl atomizer, the transition from a bulk of flow to the micron droplets is extremely complex. The atomization especially pressure swirl injector involves a transition twophase flow with recirculation region inside the injector and a complex unstable flow after issuing from injector [2]. The studies on injector are usually restricted to simulation and modelling a specific stage of atomization [3]. The numerical or experimental study on the flow inside the injector is the first step to determine macroscopic parameters of injector $[4,5]$. Primary Atomization attributed to liquid sheet breakup is the second stage of atomization. The transition from liquid bulk to ligaments and subsequently parent droplets is a strongly complex phenomenon. Two traditional methods are instability theory and breakup visualization to understand breakup mechanism $[6,7,8]$. The last stage in atomization is attributed to the secondary atomization. Final Sauter mean diameter, mean velocity, and uniformity of spray are the most important microscopic parameters usually studied experimentally [9, 10]. In the present work, we introduce a comprehensive model to predict the droplet size distribution. The two phase flow of axial air core inside the injector is simulated by coupled level set and volume of fluid method CLSVOF [11]. CLSVOF is a remedy to resolve the problems of level set and VOF methods corresponding to mass conservation and interface construction, respectively. Since extending the flow domain to outside of injector is a costly solution to identify the primary atomization of liquid sheet, a more inexpensive method should be introduced to determine the breakup procedure. The nonlinear hydrodynamic instability theory is applied to calculate the breakup length as well as interface deformation induced by the most unstable frequency [12]. The requirements of nonlinear instability theory are thickness of liquid sheet and velocity magnitude of film derived by numerical simulation of flow inside the injector. The calculation of size and velocity distribution of droplets is the last stage to determine injector performance. Maximum entropy principle (MEP) is a method to directly compute probability density function of droplets size and velocity $[13,14]$. What calculated is the spray information in the secondary atomization threshold and a designer can apply this straight forward procedure to predict injector behaviour. 


\section{Mathematical models and equations}

\subsection{Flow Simulation inside the Injector}

The tangential slots in pressure swirl injector causes to form an air core in the axial zone of the atomizer. One of the most current methods to simulate multi-phase flow is volume of fluid (VOF) [15]. The low storage requirement makes VOF a popular method to apply in numerical two phase flow simulations. In VOF, zero value is attributed to empty cell of fluid and subsequently a cell included the interface is evaluated by a value in a range of zero to one [16]. Besides wide applications of VOF in two phase flow simulations, the disadvantages of VOF involve the difficulty of interface reconstruction, the lack of adequate precision in the location of interface, and high dependency of solution accuracy to domain grid. So, Level Set (LS) method was developed as the VOF alternative by Osher [17]. The LS function is a signed function defined to specify the cell distance from the interface [18]. Based on LS function definition, the point on the interface is set to zero and the positive value is attributed to one fluid and negative to another. Unlike VOF, the interface location and its characteristics can be predicted accurately in LS method. In contrast, the continuity equation is not satisfied due to non-conservative inherent of LS function. A remedy to eliminate of disadvantages of these two-phase flow simulation methods is to introduce a hybrid solution. In the method of VOF and LS coupling, two methods are simultaneously solved to cover the problems [18]. In our first attempt, results were validated with experimental finding of Horvay and Leuckel [19]. The injector evaluates our simulation as figure. 1 .

\subsection{Instability in Planar Liquid Sheet Emanating from the Nozzle}

The first theoretical study on non-viscous liquid sheet instability was reported by Squire [20]. Following Squire, various studies on instability of round jet and sheet were performed with more accurate considerations either about assumptions on liquid and gas flow or methods of solution. For example, nonlinear instability analyses on sheet and jet were performed to understand more details about breakup mechanism. Following, two-dimensional inviscid and incompressible liquid sheet with $2 a^{*}$ thickness is schematically shown in Fig.2, where the liquid sheet with axial uniform velocity of $U_{l}$ moves through the gaseous medium [12]. When the basic non-rotational flow is perturbed by small disturbances, the liquid-gas interfaces are shifted to the new location with $y^{*}=(-1)^{j+1}+\eta_{j}$ coordinates. Where $j=1$ indicates the top and $j=2$ the bottom interface. Based on none-dimensional velocity potentials, the continuity equations of liquid and gas phases are defined as equations $(1,2)$.

$$
\begin{array}{llc}
\phi_{l, x x}+\phi_{l, y \mathrm{y}}=0 & \text { for } & -1+\eta_{2}<y<1+\eta_{1} \\
\phi_{\mathrm{g}, x x}+\phi_{\mathrm{g}, \mathrm{yy}}=0 & \text { for } & 1+\eta_{1}<y<\infty
\end{array}
$$
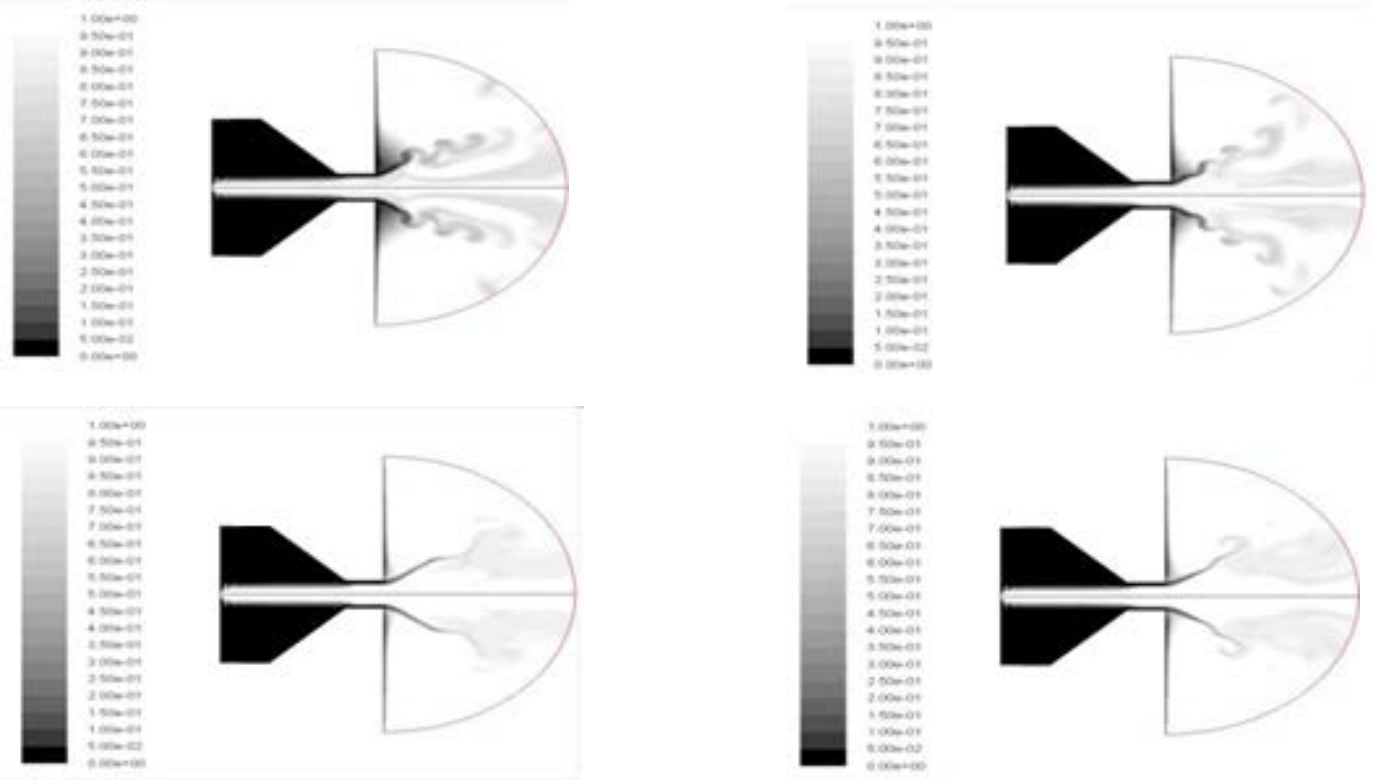

Fig 1. Numerical simulation by CLSVOF code for capturing of the interfaces for Horvay \& Leuckel's injector 
Where length, time, and density are normalized by half thickness of $a^{*}$, convection time and liquid density, respectively. In addition, the interfacial boundary condition is derived as,

$$
v_{i}=\frac{D \eta_{i}}{D t}=\frac{d \eta_{i}}{d t}+U_{i} \frac{d \eta_{i}}{d x}
$$

Where $U_{i}=\frac{d \phi}{d x}$ and $v_{i}=\frac{d \phi}{d y}$ are velocity components and the kinematic boundary conditions are simplified as,

$$
\begin{aligned}
& \phi_{l, y}-\eta_{j, t}-\phi_{l, \mathrm{x}} \eta_{j, \mathrm{x}}=0 \\
& \phi_{\mathrm{g}, y}-\eta_{j, t}-\phi_{\mathrm{g}, \mathrm{x}} \eta_{j, \mathrm{x}}=0
\end{aligned}
$$

The force balance at the interface leads to the dynamic boundary conditions as,

$$
\frac{1}{2}-\frac{1}{2} \rho U^{2}+\rho \phi_{g, t}-\phi_{l, t}+\frac{1}{2} \rho\left(\phi_{g, x}^{2}+\phi_{g, y}^{2}\right)-\frac{1}{2}\left(\phi_{l, x}^{2}+\phi_{l, y}^{2}\right)=\frac{(-1)^{j} \eta_{j, x x}}{W e\left(1+\eta_{j, x}{ }^{2}\right)^{\frac{3}{2}}}
$$

Applying regular perturbations method to expand the interface displacement, the following forms can be assumed to specify the first and second order surface deformations, respectively,

$$
\begin{aligned}
& \eta_{j, 1}(x, t)=A_{1, s} \cosh \left(\omega_{1, s} t\right) \cos \left(\alpha_{1, s} t+k x\right) \\
& \eta_{j, 2}(x, t)=p_{2, s}(t) \cos (2 k x)+Q_{2, s}(t) \sin (2 k x)
\end{aligned}
$$

Where $\omega_{1, s}$ and $\alpha_{1, s}$ are the growth rate and angular frequency, respectively, and $A_{1, s}$ is the amplitude of disturbed deformation. After sorting governing equations as well as kinematic and dynamic boundary conditions in terms of first order and second order in terms of $\eta_{0}$, we have the equations of (10-20). By simplifying the calculations based on the coefficient of sinus and cosine for the first and second order of surface deformations, respectfully the equations are solved. After tedious mathematical manipulations, the growth rate of surface deformation can be determined in the eigenvalue equation as equation (15). The variations of growth rate vs. wave number are shown in Fig 3 for the case of $\rho=0.001, U=4, W e=40$. As seen, $\omega_{1, s}$ has a peak in terms of wave number, corresponding to the most unstable mode of liquid sheet breakup. According to Fig 3 , the most unstable frequency is related to $k=0.181$ wave number for the first mode of instability discussed in [12]. In Fig.4, the growths of interface deformation in different times are depicted for $\rho=0.001, U=4, W e=40$, and $k=0.181$. Fig 4 prescribes the temporal interface disturbance growth and consequently sheet breakup occurs about $t \approx 145$. Based on Gaster's transformation [21], Fig 5 represents the locus of interfaces deformation for the case of $\rho=0.001$, $U=4, W e=40$.

$$
\begin{aligned}
& \eta_{j}(\mathrm{x}, \mathrm{t})=\sum_{n=1}^{\infty} \eta_{0}^{n} \eta_{j, n}(\mathrm{x}, \mathrm{t}) \\
& \phi_{l 1, x x}+\phi_{11, \mathrm{yy}}=0 \text { for }-1<y<1 \\
& \phi_{\mathrm{g} 1, x x}+\phi_{\mathrm{g} 1, \mathrm{yy}}=0 \text { for }+1<y<+\infty,-\infty<y<-1 \\
& \phi_{l 1, y}-\eta_{j, 1, t}-\eta_{j, 1, \mathrm{x}}=0 \\
& \phi_{l 1, y}-\eta_{j, 1, t}-\eta_{j, 1, \mathrm{x}}=0 \\
& \rho \phi_{g 1, x}+\phi_{11, \mathrm{t}}+\rho U \phi_{g 1, x}-\phi_{11, x}-\frac{(-1)^{j}}{w e} \eta_{j, 1, x x}=0 \\
& \omega_{1, s}=\frac{\tanh _{\tan (k) \rho(U-1)^{2}-k \frac{\rho+\tanh (k)}{W e}}^{\rho+\tanh (k)}}{}
\end{aligned}
$$

\section{First order of $\eta_{0}$}




$$
\begin{aligned}
& \phi_{12, x x}+\phi_{12, y \mathrm{y}}=0 \text { for }-1<y<1 \\
& \phi_{12, y}-\eta_{j, 2, \mathrm{t}}-\eta_{j, 2, \mathrm{x}}=\eta_{j, 1, \mathrm{x}} \phi_{11, x}-\eta_{j, 1} \phi_{11, \mathrm{yy}} \\
& \phi_{12, y}-\eta_{j, 2, \mathrm{t}}-\eta_{j, 2, \mathrm{x}}=\eta_{j, 1, \mathrm{x}} \phi_{11, x}-\eta_{j, 1} \phi \\
& \phi_{g 2, y}-\eta_{j, 2, \mathrm{t}}-U \eta_{j, 2, \mathrm{x}}=\eta_{j, 1, \mathrm{x}} \phi_{g 1, x}-\eta_{j, 1} \phi_{g 1, \mathrm{yy}} \\
& \phi_{g 2, \mathrm{t}}-\phi_{12, \mathrm{t}}+\rho U \phi_{g 2, x}-\phi_{12, x}-(-1)^{j} \frac{\eta_{j, 2, x x}}{w e}=-\rho\left[\eta_{j, 1, \mathrm{t}} \phi_{g 1, y}+\eta_{j, 1} \phi_{g 1, y t}\right] \\
& +\left[\eta_{j, 1, \mathrm{t}} \phi_{11, y}+\eta_{j, 1} \phi_{11, y t}\right]-\frac{1}{2} \rho\left[\phi_{g 1, x}^{2}+\phi_{g 1, y}^{2}+2 \mathrm{U}\left(\eta_{j, 1, \mathrm{x}} \phi_{g 1, y}+\eta_{j, 1} \phi_{g 1, y x}\right)\right]+\frac{1}{2}\left[\phi_{11, x}^{2}+\phi_{1, y}^{2}+2\left(\eta_{j, 1, x} \phi_{11, y}+\eta_{j, 1} \phi_{11, y x}\right)\right]
\end{aligned}
$$

\subsection{Velocity and Droplet Size Distribution in Spray}

The physics beyond the nozzle flow and sheet breakup are deterministic concepts [2], while spray atomization is a perfectly stochastic process with different size and velocity droplets [22,23] . As known, the probability density function (PDF) of droplets size and velocity is introduced to define spray characteristics. A solution to confront with difficulty of spray study is to use the Shannon maximum entropy principle (MEP), independent of experimental primary data [23]. This method was introduced by [13] and later developed by Li and Takin [24]. In addition, recent investigations attributed to Movahednejad have improved the modified momentum equations in MEP [25]. Shannon stated the form of entropy as, [23,25]

$$
s=-\kappa \sum f \ln f
$$

Where $\mathbf{s}$ is the statement of statistical thermodynamic entropy $\kappa$ is the Boltzmann's constant and $\mathbf{f}$ is the probability density function. Besides to establish the maximum condition of thermodynamic entropy, the conservation equations including mass, momentum, and energy should be satisfied in sheet breakup. Supposed spherical droplets, the conservation equations are introduced based on PDF as equations (22-26)

$$
\begin{aligned}
& \int_{\bar{D}_{\min }}^{\bar{D}_{\max }} \int_{\bar{u}_{\min }}^{\bar{u}_{\max }} f \bar{D}^{3} d \bar{D} d \bar{u}=1+\bar{s}_{m} \\
& \int_{\bar{D}_{\max }}^{\bar{D}_{\max }} \int_{\bar{u}_{\min }}^{\bar{u}_{\min }} f \bar{D}^{3} \bar{u} d \bar{D} d \bar{u}=1+\bar{s}_{m u} \\
& \int_{\bar{D}_{\min }}^{\bar{D}_{\max }} \int_{\bar{u}_{\min }} f\left(\frac{\bar{D}^{3} \bar{u}^{2}}{H}+\frac{B \bar{D}^{2}}{H}\right) d \bar{D} d \bar{u}=1+\bar{s}_{e}
\end{aligned}
$$

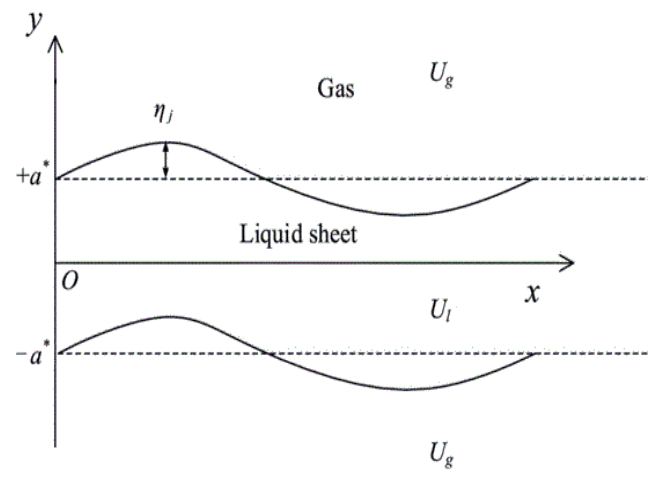

Figure 2. Planar liquid sheet [1]

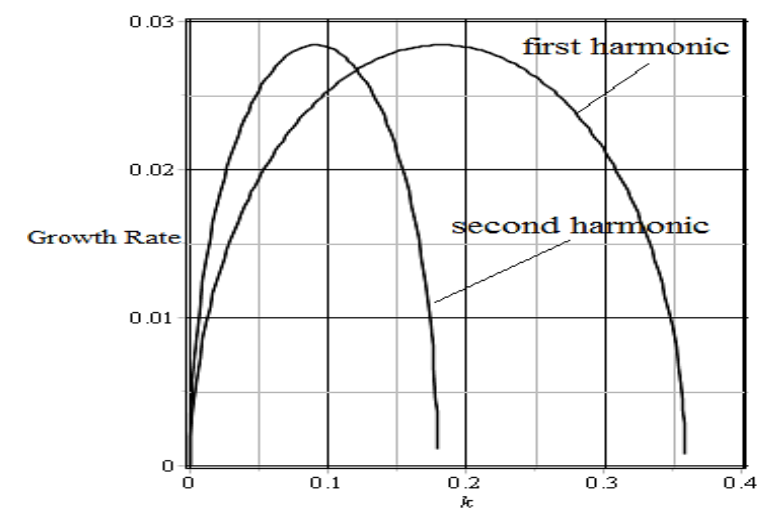

Figure 3. Instability growth rate vs. wave number for $\rho=0.001$,

$$
U=4, W e=40
$$



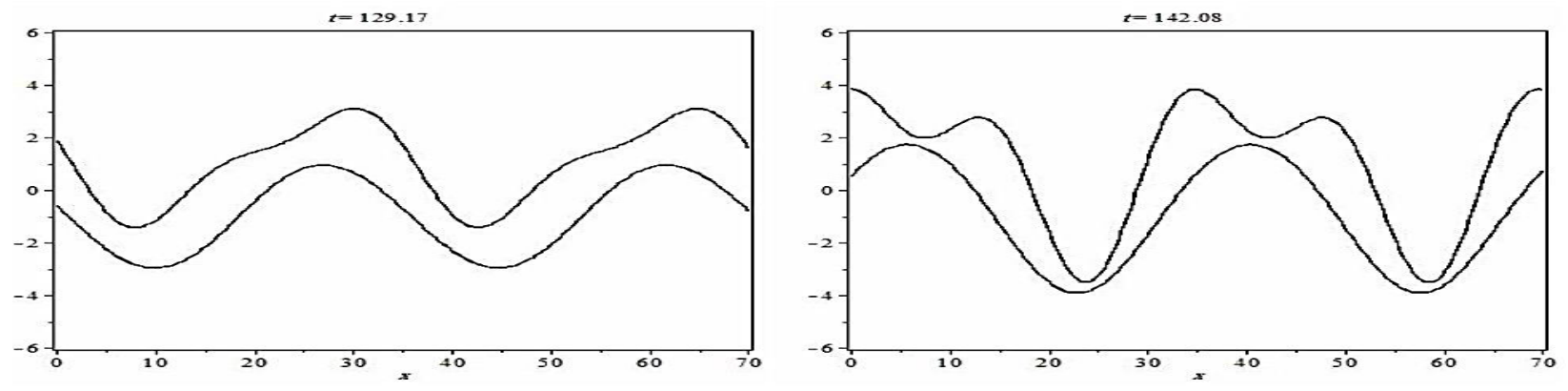

Fig 4. Temporal surface deformation evolution for $\rho=0.001, U=4$, We $=40$, and $k=0.181$

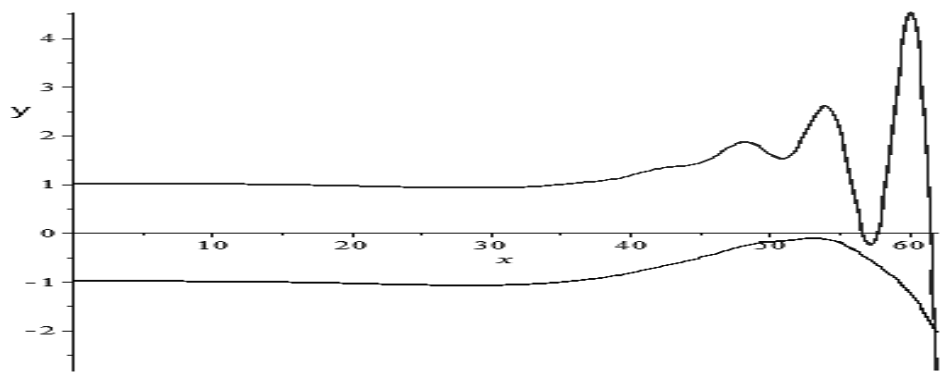

Fig 5. How breakup of the liquid film after issuing from the nozzle

$$
B=\frac{12}{W e}, W e=\frac{\rho_{l} U_{l}^{2} D_{30}}{\sigma}
$$

According PDF concept, the following equation is also introduced,

$$
\int_{\bar{D}_{\min }}^{\bar{D}_{\max }} \int_{\bar{u}_{\min }}^{\bar{u}_{\max }} f \bar{D}^{3} d \bar{D} d \bar{u}=1
$$

It is worthy to note that Eqs. (22-24) and (26) are non-dimensionlaized by $D_{30}$ and $U_{l}$ known as mean diameter and the potential velocity of liquid sheet. Also, the probability density function using Lagrange multipliers method can be obtained as equation (27).

$$
f=f_{0} \exp \left[-\lambda_{0}-\lambda_{1} \bar{D}^{3}-\lambda_{2} \bar{D}^{3} \bar{u}-\frac{\lambda_{3}}{H}\left(\bar{D}^{3} \bar{u}^{2}+B \bar{D}^{2}\right)\right]
$$

If the velocity profile at the injector outlet is assumed uniform, the shape factor of velocity profile $H$ will be unity. But if the outlet flow from the injector is assumed to be fully developed and turbulent, this factor will be equal to [25, 26]. The accuracy of this formulation is confirmed with comparing with experimental work of Li [27].

\section{RESULTS \& DISCUSION}

As mentioned, following different stages of atomization will be conducted to determine the velocity and droplet size distribution at the secondary atomization threshold. The prediction of droplet size and velocity distribution is categorized in three stages including flow inside the injector, sheet instability near the nozzle, and primary atomization. The interaction of air core and highly swirling flow inside the injector is simulated by CLSVOF Capturing accurate location and geometry of air core. Two key findings of flow simulation inside the injector are liquid film thickness and liquid velocity used to calculate the sheet breakup length in nonlinear instability theory. Extending instability theory to nonlinear terms causes directly to predict deformation topology of sheet in the breakup of moment. At the last stage, the droplets velocity and size distribution are calculated by solving MEP equations. The probability density function of size and velocity is a comprehensive map to derive all of spray characteristics included mean diameter, Sauter mean diameter, mean velocity as well as the parameters of RosinRammler function to specify droplet size distribution [2]. 

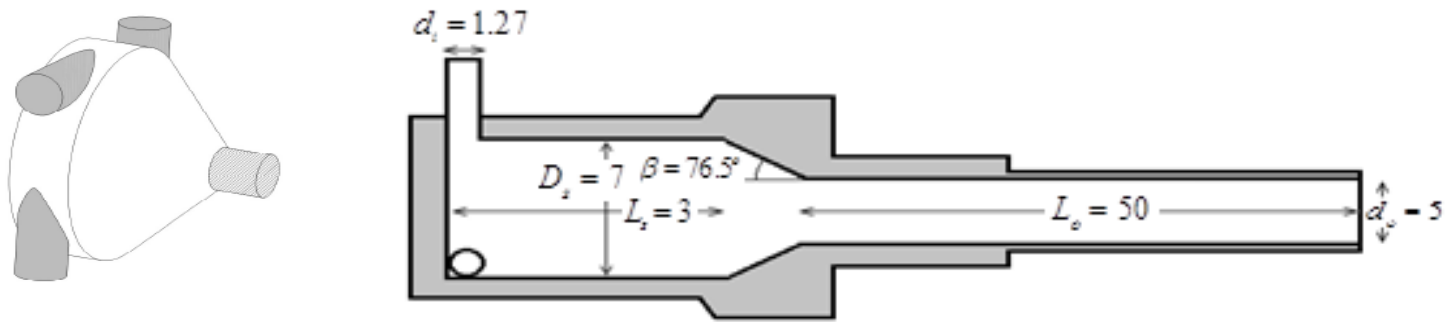

Fig 6. Geometrical schematic of injector [28]

In this paper, the results of Eberhart's experimental results were used to evaluate the proposed comprehensive model. Eberhat examined a pressure swirl injector to study spray angle, liquid film thickness, and droplets size by PDPA method, at different flow rates [28]. The geometrical schematic of injector has been illustrated in Fig 6 and injector dimensions are numerically shown in the figure in millimetre [28]. The case with mass flow rate $2.809 \times 10^{-3} \mathrm{~kg} / \mathrm{s}$ was chosen to simulate. As mentioned, CLSVOF was used to model two phase flow field of injector by 110000 structural grid cells after validating mesh dependency [4]. The method of RANS equations solution has been completely demonstrated in and the validation of solution was confirmed based on experimental results of [19]. Fig 7 illustrates how the air core is formed in the axial zone by representation of volume of water inside the injector. Attenuation of liquid sheet is seen due to accelerating axial velocity in orifice part by decreasing cross section of flow. The recirculating flow near the nozzle represents positive pressure gradient caused by free vortex flow inside the injector. The results in Fig 8 confirm the last claim about pressure distribution. As seen, free vortex flow leads to increase of swirl velocity near the axis and subsequently decrease of pressure in the whole length of injector. The numerical results of injector performance characteristics included injector discharge coefficient, liquid film thickness, and spray half angle are quantitatively compared with experimental results. Results describe the maximum error in the numerical simulation of injector is less than $13 \%$ to determine performance characteristics inside the injector. The most unstable wave number of liquid sheet is $k=1.158$ based on Eq. (10), corresponding to $\rho=0.001, U=0$, and $W e=1913$.

Where the magnitude velocity of flow at the exit of injector $U_{l}=28$ and half thickness of liquid sheet $2 a^{*}=0.41 \mathrm{~mm}$ are applied to non-dimensionalize the nonlinear instability equations. It is worthy to note that nonlinear instability analysis (NLISA) is an alternative of LISA (Linear Instability Analysis) for more accurate location of issuing the primary droplets in the computational flow field. In Fig 9, the shape of liquid sheet is depicted for $\eta_{0}=0.01$, and consequently the length of breakup is predicted about $23 \mathrm{~mm}$ in NLISA. The experimental breakup length equals $26 \mathrm{~mm}$ has been compared with NLISA. The more precise prediction of breakup in NLISA is obviously seen by quantitative calculation.

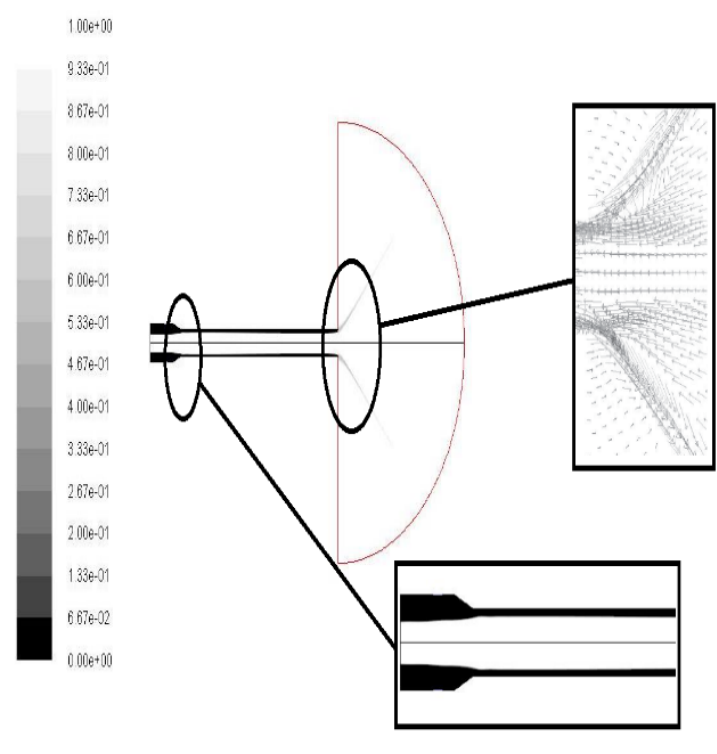

Fig 7. Contour of water volume in pressure swirl injector

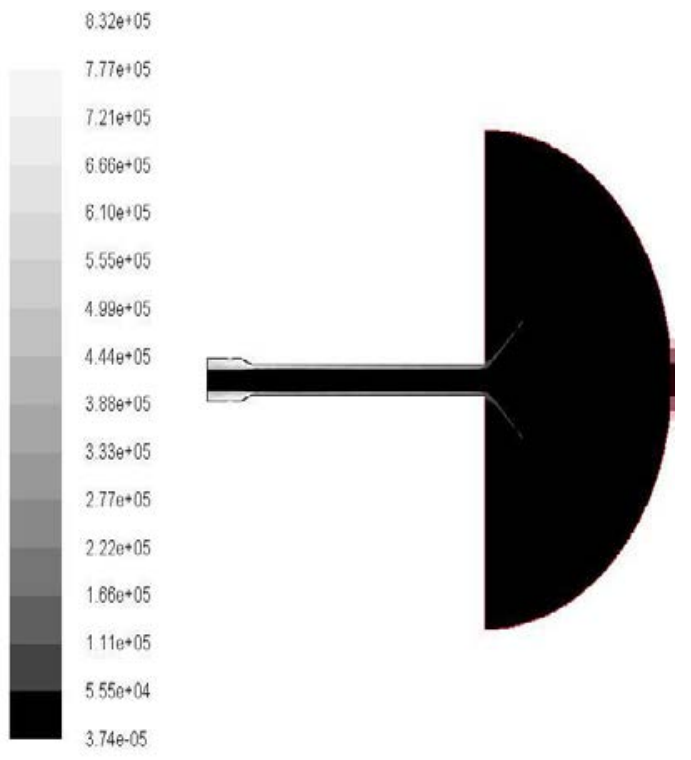

Fig 8. Contour of pressure in the flow domain 


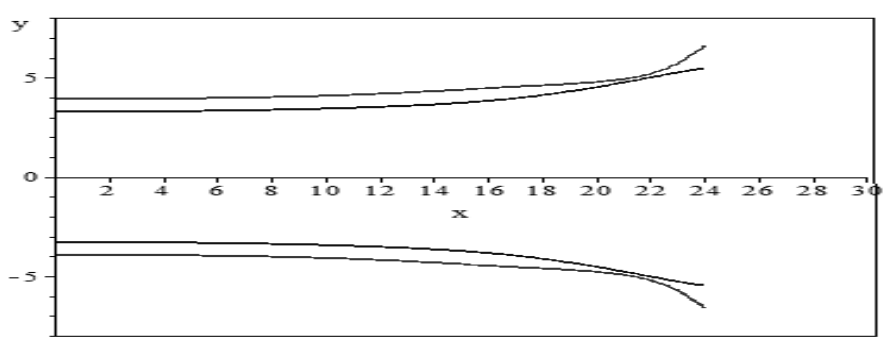

Fig 9. Liquid interfaces simulation by nonlinear instability theory

The droplets velocity and size distribution for current flow are achieved by solving MEP equations. After calculating Lagrange's multipliers, PDF of droplets size $(d)$ and velocity $(u)$ are represented in Fig 10. PDF includes the whole information of spray characteristics. For example, the Sauter mean diameter (SMD) is numerically integrated by following equation.

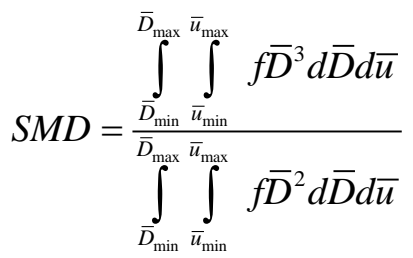

Based on PDF calculated by MEP equations, Sauter mean diameter is calculated about $80 \mu \mathrm{m}$, reported about $100 \mu \mathrm{m}$ in experiment, [28]. Although the results depict an error about $20 \%$ in this calculation, it should be noted this is an accumulated error in different simulation stages. It should be noted that Sauter mean diameter is not a unique parameter to validate the solution and it may be some other parameters not reported in literature is completely near the simulation results like mean diameter or average velocity of droplets.

\section{Conclusion}

A comprehensive model is a unique solution to predict the injector performance in spray production. The tangential slot embedded on the injector body leads to a free vortex flow inside the injector causing air core in the axial zone. The complex two phase flow in the small size of the injector necessitates application of CLSVOF to determine thickness of liquid film, tangential and axial velocity in the injector nozzle. The output of flow simulation inside the injector is used in calculation of planar hydrodynamic instability to determine the manner of breakup process. The knowledge about the most unstable frequency calculated in this part helps to predict the size and velocity distribution function in spray. Probability density function of size and velocity in spray is computed by maximum entropy principle method. In MEP method, the maximum entropy is applied as a supplementary constraint to introduce a unique function of size and velocity distribution of droplets. Newton-Raphson is used to solve conservative governing equations. After calculating Lagrange's multipliers, PDF is determined, and subsequently Sauter mean diameter is calculated. The acceptable results compared with experiments guaranty the idea of this model to predict macroscopic as well as microscopic parameters of injector.

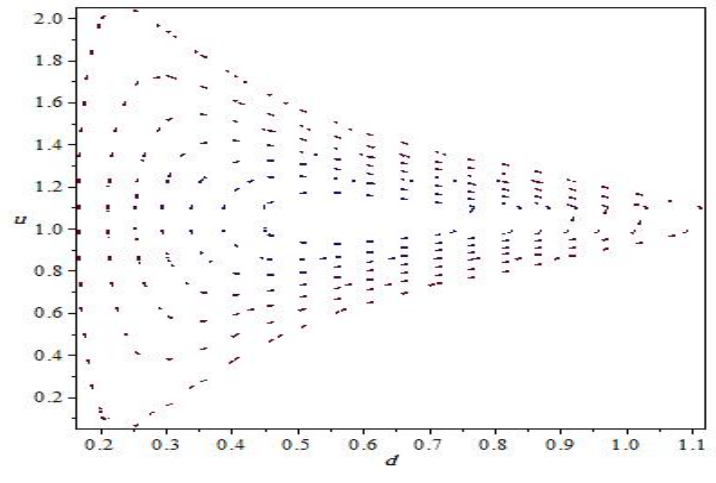

A) Two-dimensional contour

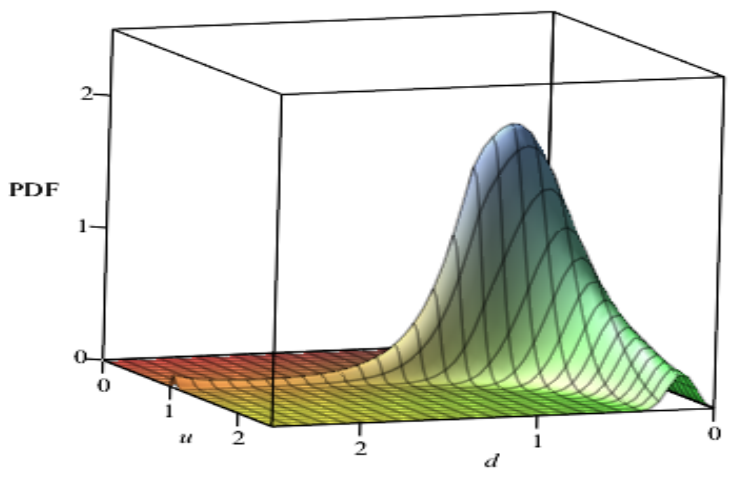

B) Three-dimensional contour

Fig 10. PDF of droplets size and velocity 


\section{REFERENCES}

[1] Ashgriz N. ed. (2011), "In Handbook of Atomization and Sprays", Springer, 339-358.

[2] Lefebvre A. H. (1989), "Atomization and Sprays, Hemisphere", Bristol Pa, U.S.A.

[3] Tharakan, T., Mukhopadhyay, A., Datta, A., and Jog, M. (2013), "Trends in Comprehensive Modeling of Spray Formation," International Journal of Spray and Combustion Dynamics, vol. 5, no. 2, pp. 123-180.

[4] Nouri-Borujerdi, A., and Kebriaee, A. (2012). "Numerical Simulation of Laminar and Turbulent Two-Phase Flow in Pressure-Swirl Atomizers", AIAA Journal, Vol. 50, No. 10, pp. 2091-2101.

[5] Shafaee, M., Banitabaei, S. A., Esfahanian, V., Ashjaee, M.(2012), "An investigation on effect of geometrical parameters on spray cone angle and droplet size distribution of a two-fluid atomizer", Journal of Mechanical Science and Technology, vol. 25, No. 12, pp. 3047-3052

[6] Ibrahim, A. A., Jog, M. A.(2003), "Effect of liquid and air swirl strength and relative rotational direction on the instability of an annular liquid sheet ",Acta Mechanica, vol. 186, no. 1, pp. 113-133.

[7] Lad, V. N., Murthy, Z. V. P.(2016)," Effects of the geometric orientations of the nozzle exit on the breakup of free liquid jet", Journal of Mechanical Science and Technology, vol. 30, no. 4, pp. 1625-1630.

[8] Inamura, T., Tamura, H. \& Sakamoto, H.(2003), "Characteristics of Liquid Film and Spray Injected from Swirl Coaxial Injector," Journal of Propulsion and Power, vol. 19, no. 4, pp. 632-639.

[9] T. Marchione, C. Allouis, A. Amoresano, and Federico Beretta(2007), "Experimental Investigation of a Pressure Swirl Atomizer Spray", Journal of Propulsion and Power, Vol. 23, No. 5, pp. 1096-1101.

[10] Shim, Y. S., Choi, G. M., Kim, D. J. (2008), "Numerical and Experimental Study on Hollow-Cone Fuel Spray of High Pressure Swirl Injector under High Ambient Pressure Condition", Journal of Mechanical Science and Technology, vol. 22, no. 2, pp. 320-329.

[11] Sussman, M., Puckett E. G. (2000), "A Coupled Level Set and Volume-of-Fluid Method for Computing 3D and Axisymmetric Incompressible Two-Phase Flows", Journal of Computational Physics, vol. 162, No. 2, pp. $301-337$.

[12] Mitra, S.K. (2001), "Breakup Process of Plane Liquid Sheets and Prediction of Initial Droplet Size and Velocity Distribution in Sprays," Ph. D Thesis, University of Waterloo.

[13] Sellens R.W., Brzustowski T. A.(1985), "A Prediction of Drop Size Distribution in a Spray from Principle", Atomization and Spray Technology, 1, 89-102.

[14] Sellens R.W., Brzustowski T.A. (1986), "A Simplified Prediction of Droplet Velocity Distribution in a Spray", Combustion and Flame, 65, 273-279.

[15] Xue, J. (2004), "Computational Simulation of Flow inside Pressure Swirl Atomizers", Ph. D. Thesis, University of Cincinnati.

[16] Yeh, Ch. L. (2007), "Numerical Simulation of a Turbulent Liquid Jet Emanating from a Plain-Orifice Atomizer and a Pressure-Swirl Atomizer", Numerical Heat Transfer Applications, vol. 51, no. 12, pp. 1187-1212.

[17] Osher, S. and Sethian, J.A. (1988), "Fronts propagating with curvature-dependent speed: Algorithms based on Hamilton-Jacobi formulations," Journal of Computational Physics, vol. 79, no. 1, pp. 12-49.

[18] Sussman M., Fatemi E., Smereka P., Osher S.(1998), "An improved level set method for incompressible two-phase flows," Computers \& Fluids, vol. 27, no. 5-6, pp. 663-680.

[19] Horvay M., Leuckel W.(1986), "Experimental and Theoretical Investigation of Swirl Nozzles for Pressure-Jet Atomization", German Chemical Engineering, 5, 276-283.

[20] Squire, H.B. (1953), "Investigation of the instability of a moving liquid film", British Journal of Applied Physics, vol. 4, no. 6, pp. 167-169.

[21] Gaster M. (1962),"A note on the relation between temporally-increasing and spatially-increasing disturbances in hydrodynamic stability," Journal of Fluid Mechanics, vol. 14, no. 02, p. 222

[22] Dumouchel C. (2006), "A New Formulation of the Maximum Entropy Formalism to Model Liquid Spray Drop-Size Distribution", Particle \& Particle Systems Characterization, vol. 23, no. 6, pp. 468-479.

[23] Dumouchel C. (2009), "The Maximum Entropy Formalism and the Prediction of Liquid Spray Drop-Size Distribution", Journal of Entropy, 11, 713-747.

[24] Li X., Tankin R.S. (1987), "Droplet size distribution: A Deviation of a Nukiyama-Tanasawa Type Distribution Function", Combustion Science and Tech., 56, 65-76.

[25] Movahednejad E., Ommi F., Hosseinalipour S. M., Chen .C. P., Mahdavi S. A.(2011), "Application of maximum entropy method for droplet size distribution prediction using instability analysis of liquid sheet," Heat Mass Transfer, vol. 47, no. 12, pp. 1591-1600.

[26] Mahdavi S.A., Movahednejad E., Ommi F., Hosseinalipour S.M.(2013), "Estimation of the Breakup Length for the Annular and the Round Liquid Jet Using Linear Instability Analysis", IJST, Transactions of Mechanical Engineering, 37, No. M2, 217-232.

[27] Li, X.; Chin, L.P., Tankin, R.S.; Jackson, T.; Stutrud, J., Switzer, G.(1991)“Comparison between experiments and predictions based on maximum entropy for sprays from a pressure atomizer," Combustion and Flame, vol. 86, no. 1-2, pp. 73-89.

[28] Eberhart, C. J, Lineberry, D. M. and Moser, M. D. (2009),"Experimental Cold Flow Characterization of a Swirl Coaxial Injector Element," 45th AIAA/ASME/SAE/ASEE Joint Propulsion Conference \&amp; Exhibit. 


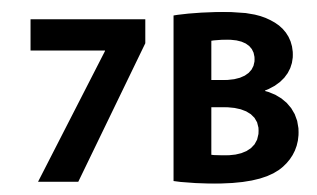

Experimental techniques 1 


\title{
Determination of Heat and Flow Characteristics in Spray Cooling with Rectangular Finned Heat Sink
}

\author{
Altug Karabey*1, Kenan Yakut ${ }^{2}$ \\ ${ }^{1}$ Ercis Vocational High School, Yuzuncu Yil University, Turkey \\ ${ }^{2}$ Faculty of Engineering, Ataturk University, Turkey \\ ${ }^{*}$ Corresponding author: akarabey@yyu.edu.tr
}

\begin{abstract}
Spray cooling process has many parameters such as extended surface, angle of inclination, effect of gravity, diameter of nozzle, angle of spray, mass flux, geometry of cooled surface, thermal performance and critical heat flux of spray etc. Many effective parameters to carry out the experiments with conventional test methods are both expensive and time consuming. As a solution in these circumstances, Taguchi method, which is one of the modern experimental design and optimization methods and very effective in solving such problems, was used in this study. Taguchi method, as well as being in effective to improve the quality of products, also gives the opportunity to achieve better results with much less experiment. Using Taguchi method, as well as to reach the target value exactly, the sensitivity of the design against uncontrollable factors is reduced to a minimum. Thus, the optimum tolerance range in cost and quality factors is determined. When compared to conventional experimental design methods, Taguchi method has many advantages.

In these experiments with using rectangular pin fin heat sinks, the effects of the longitudinal and lateral distances of the consecutively arranged nozzle or diffuser-like fin pairs, widths of the fins, angle of fins, heights of fins, spraying time, air flow rate, liquid flow rate (ALR, the ratio of air-liquid flow rate) and the ratio of the nozzle-heat sink distance to the nozzle diameter $(\mathrm{h} / \mathrm{d})$ on heat and flow characteristics have been investigated by using Taguchi experimental design method. For this reason, characteristics of flow and heat transfer are considered separately. Nusselt number considered as performance statistic, $L_{27}\left(3^{11}\right)$ orthogonal array has been selected as an experimental design plan for the eleven parameters mentioned above. The Nusselt number was calculated by taking into account the characteristic length of heat sink and the optimized results were found to be fin width of $45 \mathrm{~mm}$, fin angle of $45^{\circ}$, fin height of $15 \mathrm{~mm}$, $x$ direction distance between fins of $20 \mathrm{~mm}$, y direction distance between fins of $20 \mathrm{~mm}$, $x$ direction distance between slices of $15 \mathrm{~mm}$, y direction distances between slices of $20 \mathrm{~mm}$, air flow rate of $10^{-3} \mathrm{~m}^{3} / \mathrm{s}$, liquid flow rate of $5,83^{*} 10^{-6} \mathrm{~m}^{3} / \mathrm{s}$, spraying time of $5 \mathrm{~s}$ and the ratio of the nozzle-heat sink distance to the nozzle diameter $(\mathrm{h} / \mathrm{d})$ of 667 .
\end{abstract}

\section{Keywords}

Spray cooling, Taguchi Methods, heat sink.

\section{Introduction}

Atomization is used to transfer energy from wide surface areas in low temperatures throughout cooling applications. From the outlet of an atomizer, an unsteady liquid mass performs a fragmentation process respectively in the order of layer formation, ligament formation, and droplet formation. The velocity and diameter rates of the droplets to compose spray are important. When the droplets with a certain speed and diameter range are grouped, the droplet speed and diameter range occur, and these two ranges are fundamental to characterizing the spray.

Effective energy transfer is ensured with spray cooling, a technology characterized by high heat transfer, heat convection homogeneity, and a low droplet impact speed, which are explored for engineering applications today. Having gained momentum especially in the last 20 years, studies on the atomization of liquids have been used widely in industry. The cooling of metal surfaces and electronic components, drying operations, humidification, washing, watering and fire extinguishing operations, pulverized fuel injection systems, surface coating and spray painting operations, agricultural disinfection, drug manufacturing, and spray medicine mechanisms taken orally and through the nose can be cited as applications of atomization. Because atomization has an extensive area of use, empirical studies are required so spray parameters can be analyzed qualitatively and quantitatively [10]. Analyzing the impacts of all determined parameters on the performances of thermal systems and system elements is almost impossible in terms of both time and testing costs when using the strategy of making tests by changing a parameter at any time. By not changing a parameter at every turn, it is possible to ensure a significant decline in the number of experiments by using only those selected carefully from full factorial designs. With this testing method, randomization is used to eliminate the impact of heterogeneity on testing methods. Thanks to such a course of 
action, it is possible to reduce the adverse effects of unexpected changes (i.e., ambient temperature, humidity, pressure, etc.) on uncontrolled factors [11].

With the method first developed by Japanese engineer Dr. Taguchi, it is possible to minimize the variations in product and process by selecting the most appropriate levels of controllable factors against the factors that create these variations and that are uncontrolled. In his testing strategy, Taguchi uses high fractional experiments, including orthogonal sequences, to specify optimum values of controllable factors. With the analysis of the data obtained after the experiments, whether the anticipated result would be achieved is controlled for by conducting confirmation experiments in optimum conditions determined thereof. After the performance values and signal-tonoise ratio-obtained following the experiments-are analyzed and this classification is made, variation is reduced with the help of control factors, and average targeted values are achieved with the help of correction factors. Furthermore, the most suitable and economic values of inefficient factors are selected.

In Taguchi's testing strategy, high fractional experiments, including orthogonal sequences, are used to specify the optimum values of controllable factors. With the analysis of the data obtained after the experiments, whether the anticipated result would be achieved is controlled for by conducting confirmation experiments in optimum conditions determined thereof [11]. Phadke [5], states Taguchi developed 18 different orthogonal sequences. Taguchi also developed linear graphics and triple charts to be based on to ensure the placement of factors and the combined effects in these sequences. In many problems, one of the standard orthogonal sequences may be used as an experimental design directly. By making use of linear graphics, triple charts, and similar instruments in some cases, an experimental design suitable for the problem may be developed with partial arrangements in standard orthogonal sequences. In other words, simplicity or perfect flexibility is ensured in planning multi-factor or multi-level experiments.

After the performance values and signal-to-noise ratio-obtained following the experiments—are analyzed and this classification is made, variation is reduced with the help of control factors, and average targeted values are achieved with the help of correction factors. Furthermore, the most suitable and economic values of inefficient factors are selected [1].

In this study, the impact of 11 parameters with three levels on the optimum operation conditions of a heat sink was determined by means of Taguchi's experimental design method, which was applied to spray cooling. Positive and negative aspects of the method were discussed in studies regarding the increase in the heat transfer in heat exchangers.

\section{Material and methods}

Experimental parameters and plan

In this study, the optimum parameters of heat sink in Figure 1, which are sequenced in the form of a longitudinal and lateral distances were determined in terms of heat transfer using Taguchi's experimental design technique.

Parameters having an impact on heat transfer were selected as the fin width, fin angle, fin height, horizontal distance between fins, vertical distance between fins, horizontal distance between slices, vertical distance between slices, rate of nozzle-heat sink distance to nozzle diameter, air flow, water flow, and spraying time. As well, the $L_{27}\left(3^{11}\right)$ orthogonal array was selected as the experimental design. Experiments were performed in the spray cooling testing apparatus indicated in Figure 2. This study used a heat sink $300 \times 300 \mathrm{~mm}$ in size with a testing pin fin on the heat sink and $\mathrm{h} / \mathrm{d}$ distance, which is adjustable, on which an air-supported atomizer $1.2 \mathrm{~mm}$ in diameter can be mounted, along with a flow meter and manometer for air-water fluids. Air and water were preferred as fluids in the system. A 15-liter water/oil bath that operates in a closed system was placed near the testing area so experiments could be performed at constant temperatures. With the experimental setup set (Figure 2), such parameters as nozzle outlet speed of spray, droplet diameter, distribution of spray droplets in the testing area, and amount of surface contact were obtained with the help of a charge coupled device (CDD) camera and stroboscope, and the temperature distributions were obtained via thermal cameras.

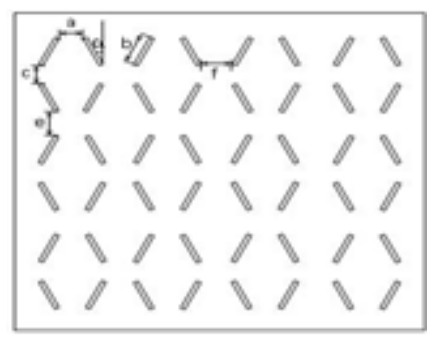

Figure 1. Geometrical definitions of the tested models. 


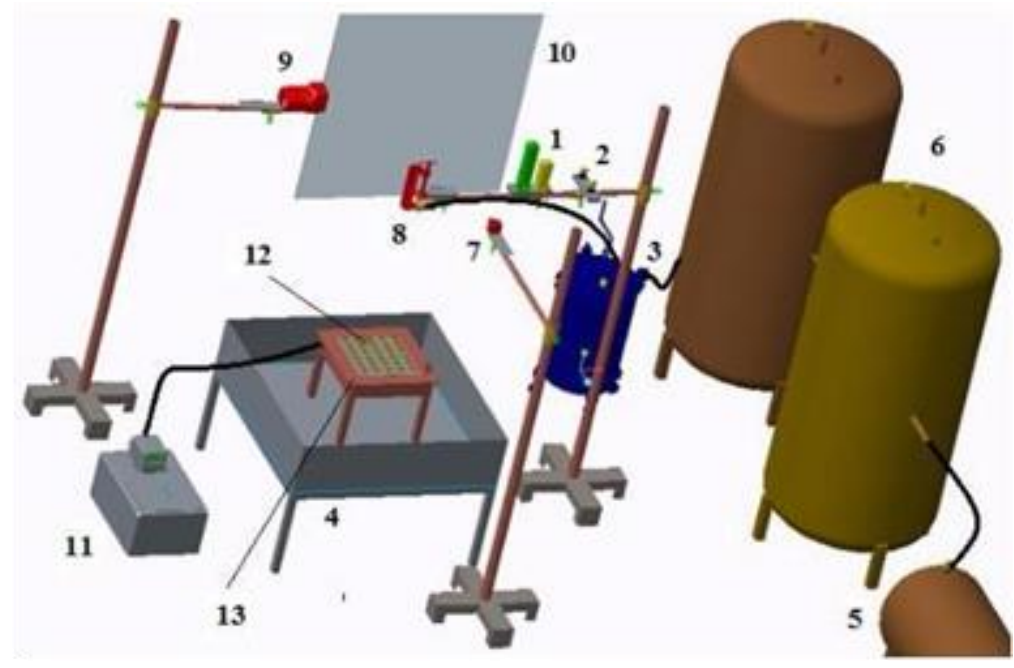

Figure 2. A schematic display of the experimental setup:

(1) Flow meter, (2) manometer, (3) pressurized water tank, (4) reservoir, (5) compressor, (6) air tank, (7) CCD camera, (8) nozzle, (9) stroboscope, (10) background, (11) water/oil bath, (12) test-section, (13) reactor.

Table 1 shows the controllable parameters selected in consideration of the fact that they are likely to affect the characteristics of heat transfer and the values of these parameters and that were analyzed in the tests.

Table 1. Parameters and their values studied in the experiments.

\begin{tabular}{|c|c|c|c|c|}
\hline & & \multicolumn{3}{|l|}{ Level } \\
\hline \multicolumn{2}{|c|}{ Parameter } & 1 & 2 & 3 \\
\hline $\mathrm{A}$ & $\mathrm{h} / \mathrm{d}$ ratio & 166.6 & 333.3 & 666.6 \\
\hline B & Fin width, b [mm] & 15 & 30 & 45 \\
\hline C & Angle of fin, $\alpha$ [degree] & 15 & 30 & 45 \\
\hline D & Fin height, $\mathrm{H}[\mathrm{mm}]$ & 15 & 25 & 35 \\
\hline$E$ & Horizontal distance between fins, a [mm] & 10 & 15 & 20 \\
\hline $\mathrm{F}$ & Vertical distance between fins, $\mathrm{c}[\mathrm{mm}]$ & 10 & 15 & 20 \\
\hline G & Vertical distance between slices, e [mm] & 10 & 15 & 20 \\
\hline $\mathrm{H}$ & Horizontal distance between slices, $f$ [mm] & 10 & 15 & 20 \\
\hline I & Air flow, $Q_{a}\left[\mathrm{~m}^{3} / \mathrm{h}\right]$ & 2.1 & 2.9 & 3.6 \\
\hline $\mathrm{J}$ & Water flow, Q। $\left[\mathrm{m}^{3} / \mathrm{h}\right]$ & 0.012 & 0.021 & 0.03 \\
\hline $\mathrm{K}$ & Spraying time, $\mathrm{T}[\mathrm{s}]$ & 5 & 10 & 15 \\
\hline
\end{tabular}

Table 1 shows that all parameters were selected to have three levels, and the $L_{27}\left(3^{11}\right)$ orthogonal array $(O A)$ experimental design method was selected accordingly as the experimental plan [5].

Experimental plan shows that only 54 experiments were conducted with 27 heat sink unit using the $\operatorname{L}_{27}\left(3^{11}\right)$ experimental design and Taguchi's experimental design instead of conducting $3^{11}=177,147$ experiments with a full factorial experimental design from the classical testing design methods. Each experiment was repeated twice at different times so the effects of deforming and the random factors in the experiments could be observed. "Performance statistics" was selected as the optimization criteria. Performance values in terms of the Nusselt number were calculated to be able to observe the impacts of the parameters on the optimization criteria. The Nusselt number was calculated based on nozzle diameter in consideration of the convection heat transfer coefficient, which is calculated based on the total area of the heat transition surface, and a general optimum was found. The "the bigger the better" performance statistic was used for Nusselt numbers.

$$
Z_{B}=-10 \log \left(\frac{1}{n} \sum_{i=1}^{n} \frac{1}{Y_{i}^{2}}\right)
$$

Kackar [6], states that many (more than 60) performance statistics likely to be used based on the analysed problem were developed. The following performance statistic, was developed for the "the bigger the better" status and is one of the alternatives that could be selected for the optimization criteria, where $Z_{B}$ and $Z_{K}$ indicate performance 
statistics, $n$ indicates the number of repetitions made in an experimental combination, and $Y_{i}$ shows the performance value of the test.

In the Taguchi method, the experiment corresponding to the optimum working conditions might not have been undertaken during the whole period of experimentation. In such cases, the performance value corresponding to optimum working conditions can be predicted by utilizing the balanced characteristic of the OA. For this aim, the additive model may be used [7]

$Y_{i}=\mu+X_{i}+e_{i}$

where indicates the general average performance value, $X_{i}$ indicates the fixed impact of the parameter-level combination in the test, and $e_{i}$ indicates the random fault in the experiment. As the equation is a point estimation, calculated using experimental data, a confidence interval must be calculated to determine whether this value is significant. A confidence interval at the selected error level may be calculated with the help of the following equation [8],

$Y_{i} \pm \sqrt{F_{\alpha ; 1, D F_{M S e}} * M S e *\left(\frac{1+m}{N}+\frac{1}{n_{r}}\right)}$

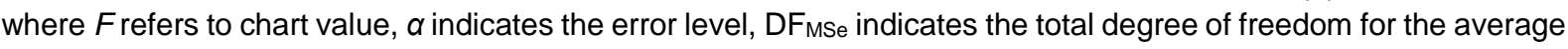
of the error squares, $m$ refers to the total degree of freedom for the parameters used in estimating optimum working conditions, $N$ refers to the total number of tests/experiments, and $n_{i}$ indicates the number of repetitions in the confirmation experiment. If the experimental results are in terms of percentage (\%), an omega conversion of percentage values is performed with the help of the following equation before calculating Equations (3) and (4). After, the values of concern are determined by applying a reverse-conversion with the help of the same equation [9],

$\Omega(d b)=-10 \log \left(\frac{1}{P}-1\right)$

where $\Omega(d b)$ indicates the decibel value, which was found with the omega conversion of the percentage value, and $p$ indicates the percentage value of the experimentally obtained product.

Calculating the heat transfer coefficient

For this study, the Bi number was found to be significantly low for the aluminum heat sinks with rectangular fins at a constant surface temperature in the non-boiling regime, and it was decided to use a total mass approach. Accordingly, the amount of heat generated on the aluminum surface was calculated with the following equations,

$Q_{\text {total }}=Q_{\text {konv }}+Q_{\text {rad }}+Q_{\text {vap }}$

and

$Q=m_{A l} C_{p} \Delta T$

Where $\Delta T$ refers to average surface temperatures of the surface before and after spray.

Tahat et al. [2] reported that radiative heat-transfer rate from a pin-fin depends on: (i) the temperature of its radiating surface; (ii) the temperature distribution of its surroundings; (iii) the emissivities of the fin, base and environment; and (iv) the shielding effect of adjacent fins. The radiating surface temperature and the shielding effect determine the grey-body shape-factor $F$.

The total steady-state rate of radiative heat-transfer can be evaluated from

$Q_{\text {rad }}=F A_{S}\left(T_{S}^{4}-T_{a}^{4}\right)$

In similar studies Tahat et al. [2], Tahat et al. [3], El-sayed et al. [4], etc., reported that the total radiative heat-losses from a similar test surface would be about $0.5 \%$ of the total heat-input. Also the base plate and fins were of aluminum; their surfaces being highly matted using black spray painting and so of low emissivity. Therefore, the radiative heat loss could be neglected. Using these findings, together with the fact that the test section was well insulated and the readings of the thermojunction placed at the outer surface of the heating section was nearly equal to the ambient temperature, then one could assume with some confidence that the last two terms of Eq. (6) may be ignored. Then, the Eq. (6) reduces to 
$Q_{\text {total }}=Q_{\text {Conv }}$

As for the heat transfer coefficient for the spray, sent via the air-supported nozzle, it was identified as follows;

$h_{s p}=\frac{\left(Q-Q_{r a d}-Q_{b u h .}\right)}{A_{y}\left(T_{y}-T_{s p}\right)}$

When the studies in the literature are reviewed, both the projection area and total heat transfer area are used as the heat transfer surface area. In this study, the total heat transfer area was used. This area refers to the total heat transfer surface area created by the fins and base plate, and it can be identified as follows:

$$
A_{s}=W L+N\left[\left(2 b h_{k}\right)+\left(2 t h_{k}\right)\right]
$$

The Nusselt number was considered a performance statistic; it was calculated with the following equation based on the inner diameter of the nozzle while calculating the Nusselt number:

$N u=\frac{h_{s p} D_{h}}{k}$

In addition, the heat thrown due to vaporization was calculated for several spraying times, according to the change in the relative humidity on the surface. This value was determined to be $14.72 \%, 16.92 \%$, and $19.31 \%$, respectively, for $5 \mathrm{~s}, 10 \mathrm{~s}$, and $15 \mathrm{~s}$ of spraying. The forces playing a role in the fragmentation of unsteady liquid masses may be identified as inertia forces, surface tension, and viscous forces. This is why pure numbers to characterize these kinds of flows consist of different combinations of these three different forces. The first that comes to mind among the pure numbers used to characterize any forced flow problem is the Reynolds number, which is known as the rate of inertia forces to viscous forces:

$\operatorname{Re}=\frac{D_{h} U_{o r t}}{v}$

In the equation, thermo-physical specifications and the average speed Uort of the liquid in the nozzle cross-section were determined in consideration of the segment of the fluid in the nozzle outlet.

\section{Results and discussion}

Following the tests and measurements, the collected data were analyzed using the ANOVA ${ }^{\mathrm{TM}}$ packet program to determine the impact of each parameter on the performance statistic. The average surface temperatures were calculated for all experiments with a thermal camera before and after the spraying operation. An example, performed for Experiment-4, is included in Figure 3 below.

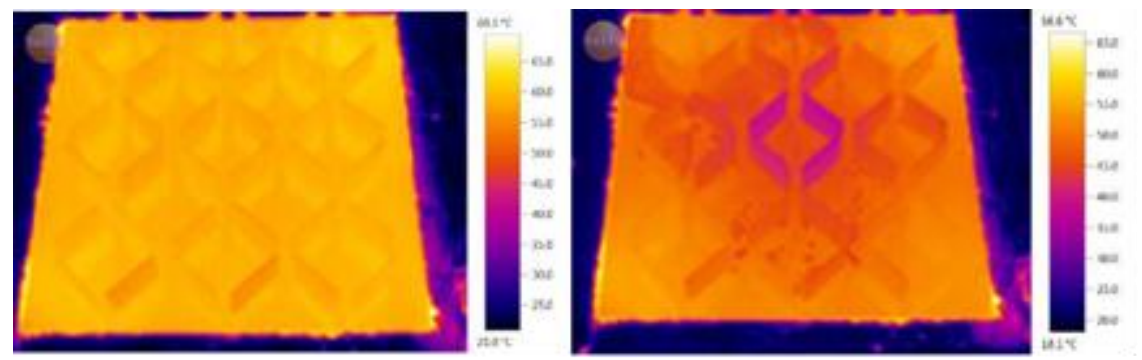

Figure 3. Thermal camera images.

The impact of each parameter on optimization was calculated and results are presented in Figure 4 . The order of graphics was determined in accordance with the degree of the impact of the parameters on the performance statistic. In the graphics, the numerical value of the maximum point indicates the best value of the respective parameter, and the numerical value of the minimum point indicates the worst value of the respective parameter. In the effect graphic to the Nusselt number (Figure 5), the maximum points for all parameters indicate the optimum levels of the parameter selected in the experimental study conducted thereof. 


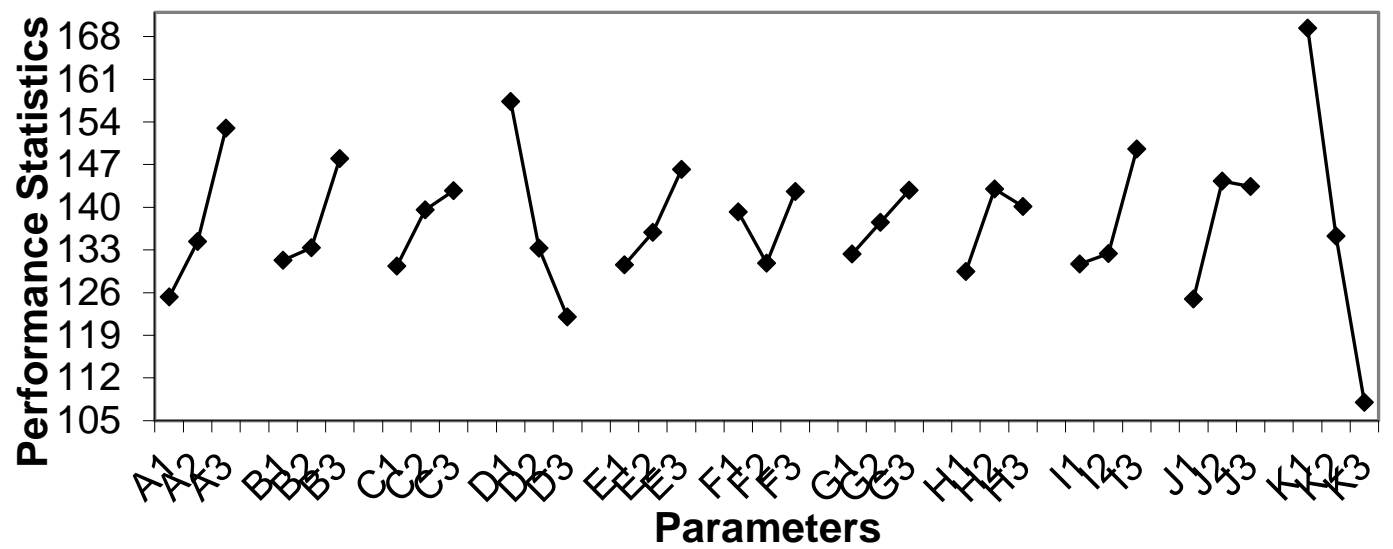

Figure 4. The effect of each parameter on the Nusselt number.

It seems difficult and complex to understand experimental results from the graphics at first glance. If the procedure is reviewed with an example, the fin width and variation in the performance statistic B are indicated in Table 1. The fin width is $15 \mathrm{~mm}$ at the first point, and this corresponds to the first level of the parameter (see Table 1). Experiments corresponding to Level 1 can be found in experimental plan. Test numbers from experimental plan are 1, 2, 3, 10, $11,12,19,20,21$. The performance statistic value is the average of the data obtained from these tests. In Figure 4 , the experimental conditions for the second data point in th plan and the average of the experiments are indicated with 2 in experimental plan (i.e., 4, 5, 6, 13, 14, 15, 22, 23, and 24). The numerical value of the maximum point in each graphic gives the best value of the respective parameter. These values are included in Table 3 for each parameter, referring to the optimum value of each parameter under experimental conditions. Besides, performance values of the combinations corresponding to optimum conditions, estimated with the help of Equation (2) in Table 3 and the confidence interval at a 5\% error level of these estimations, were calculated with the help of Equation (4), and they are included in Table 3. Moreover, confirmation experiments were conducted under the determined optimum conditions to be able to test the accuracy of these estimations, and the results are presented in the "Real" line. Because the performance values obtained in confirmation experiments are within the calculated confidence interval, it can be stated that experimental results are acceptable at a $5 \%$ error level.

Table 3. Optimum conditions and performance values for tested models.

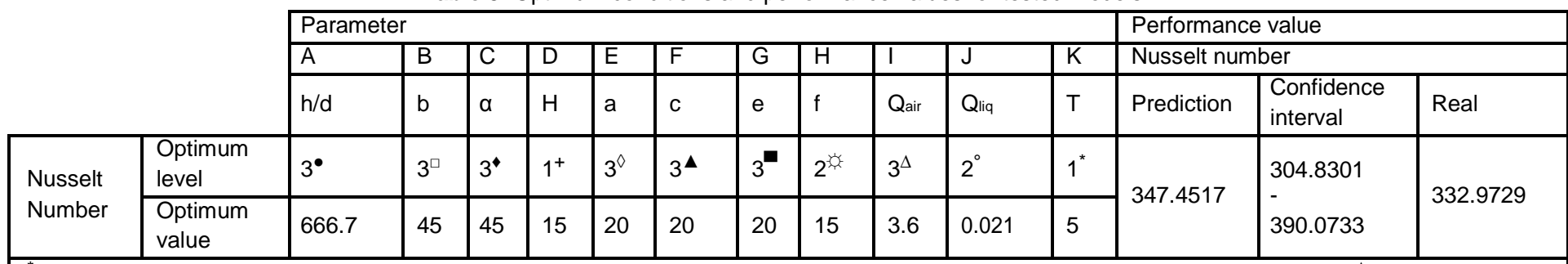

*: The first effective parameter ${ }^{+}$: The second effective parameter $\bullet:$ The third effective parameter ${ }^{\circ}:$ The fourth effective parameter ${ }^{\Delta}:$ The fifth effective parameter ${ }^{\square}$ : The sixth effective parameter ${ }^{\diamond}$ : The seventh effective parameter ${ }^{\star}$ : The eighth effective parameter $\downarrow$ : The ninth effective parameter

$\boldsymbol{\Delta}:$ The tenth effective parameter

Contribution percentages of the impacts of each parameter on the selected performance characteristic are indicated in Figures 5. Contribution percentage indicates the impact of the respective parameter on the performance statistic, and it is calculated as follows:

$\mathrm{KY}=([$ Squares total-(Degree of freedom*Total errors) $]) /($ Total of squares $)$ 


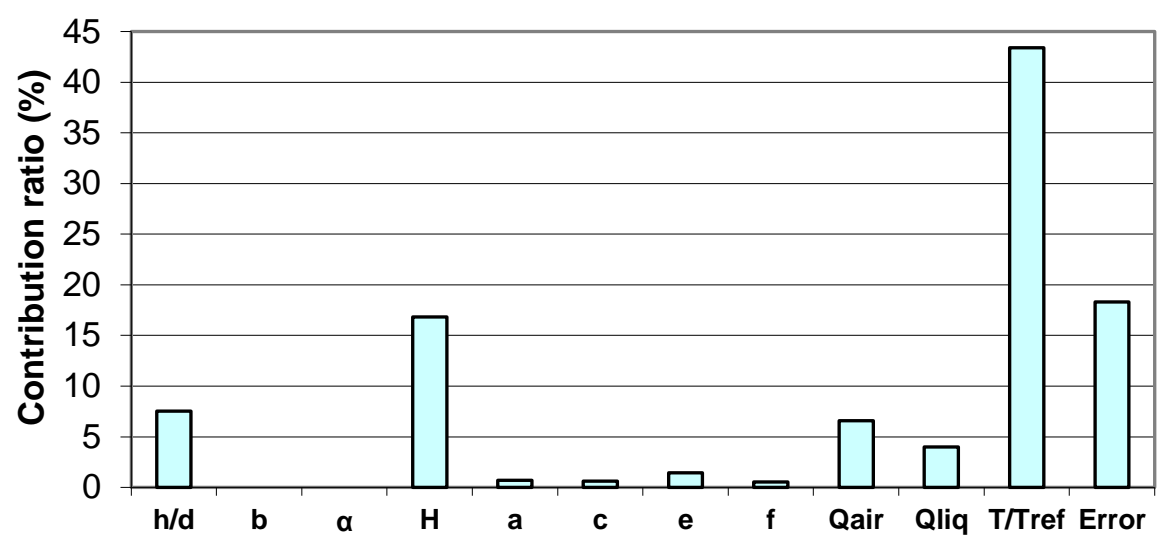

Figure 5. Contribution ratio of each factor to the Nusselt number.

The parameters having the greatest impact on the Nusselt number are, respectively, spraying time $(T)$, fin height $(\mathrm{H})$, the rate of nozzle-heat sink distance to nozzle diameter (h/d), water flow (Qliq), air flow (Qair), fin width (b), horizontal distance between fins (a), horizontal distance between slices (f), fin angle $(\alpha)$, vertical distance between fins (c), and vertical distance between slices (e). It was determined that the parameter having the greatest effect on the Nusselt number is spraying time. Based on the surface geometry, the highest Nusselt number was calculated as 234.4 when the spraying time was $5 \mathrm{~s}$. This indicates there is no additional spraying operation to achieve an optimum cooling load. It was determined that fin height is the parameter having the second-greatest effect on the Nusselt number. In cases where the fin height is $15 \mathrm{~mm}$, the highest Nusselt number calculated was 234.4 based on surface geometry. While the Nusselt numbers were being calculated, the average heat convection coefficient was determined according to the total heat transition area. In the equation, the heat transition surface area from the fins was also considered, and the heat convection coefficient was reduced, as the heat transition area would increase in the event that fin height increases.

The parameter having the third-greatest effect on the Nusselt number was the rate of the nozzle-heat sink distance to nozzle diameter $(\mathrm{h} / \mathrm{d})$. The highest Nusselt number calculated was 234.4 based on the surface geometry at the point where the $\mathrm{h} / \mathrm{d}$ ratio is a maximum 666.7 .

\section{Conclusions}

This experimental study was conducted to determine the heat transfer and flow characteristics of a spray cooling application for a heat sink consisting of rectangular fins with a longitudinal and lateral geometry. Performance criteria regarding the Nusselt number were calculated to be able to observe the impacts of parameters on the optimization criteria by making use of the Taguchi experimental design method and the $L_{27}\left(3^{11}\right)$ experimental design, and the optimum heat sink geometry was obtained. Results for a general optimum heat sink, obtained based on the Nusselt number, were determined to include a $15-\mathrm{mm}$ fin height, $45-\mathrm{mm}$ fin width, $45^{\circ}$ fin angle, $20-\mathrm{mm}$ distance between fins, 20-mm vertical distance between fins, 20-mm horizontal distance between slices, 15-mm vertical distance between slices, 666.7 rate of nozzle-heat sink distance to nozzle diameter, $3.6-\mathrm{m}^{3} / \mathrm{h}$ air flow, $0.021-\mathrm{m}^{3} / \mathrm{h}$ water flow, and 5-s spraying time. The parameters having the greatest impact on the Nusselt number are, respectively, spraying time $(T)$, fin height $(H)$, the ratio of nozzle-heat sink distance to nozzle diameter $(\mathrm{h} / \mathrm{d})$, water flow (Qliq), air flow (Qair), fin width (b), horizontal distance between fins (a), horizontal distance between slices (f), fin angle ( $\alpha$ ), vertical distance between fins, (c) and vertical distance between slices (e). It was determined that spraying time is the parameter having the greatest effect on the Nusselt number. Studies to determine spray flow characteristics are rather complex. Therefore, it is essential to measure via a laser that enables a detailed analysis of the flow structure (PIV). Florinert liquids, such as FC-72 and FC-87, can be used in spray cooling as an operation liquid. The heat transfer process must be reviewed by adding surface-active agents. This study was performed below the boiling point, but studies hereafter may be conducted above the boiling point or at the Leidenfrost temperature. It is essential to research air-supported nozzles, which are also used in this study to design different nozzle geometries. Heat transfer and the flow process can be analyzed mathematically using such software as ANSYS-FLUENT.

\section{Acknowledgements}

This research was supported as project (BAP-2013/103) of the Research Fund of Ataturk University. 


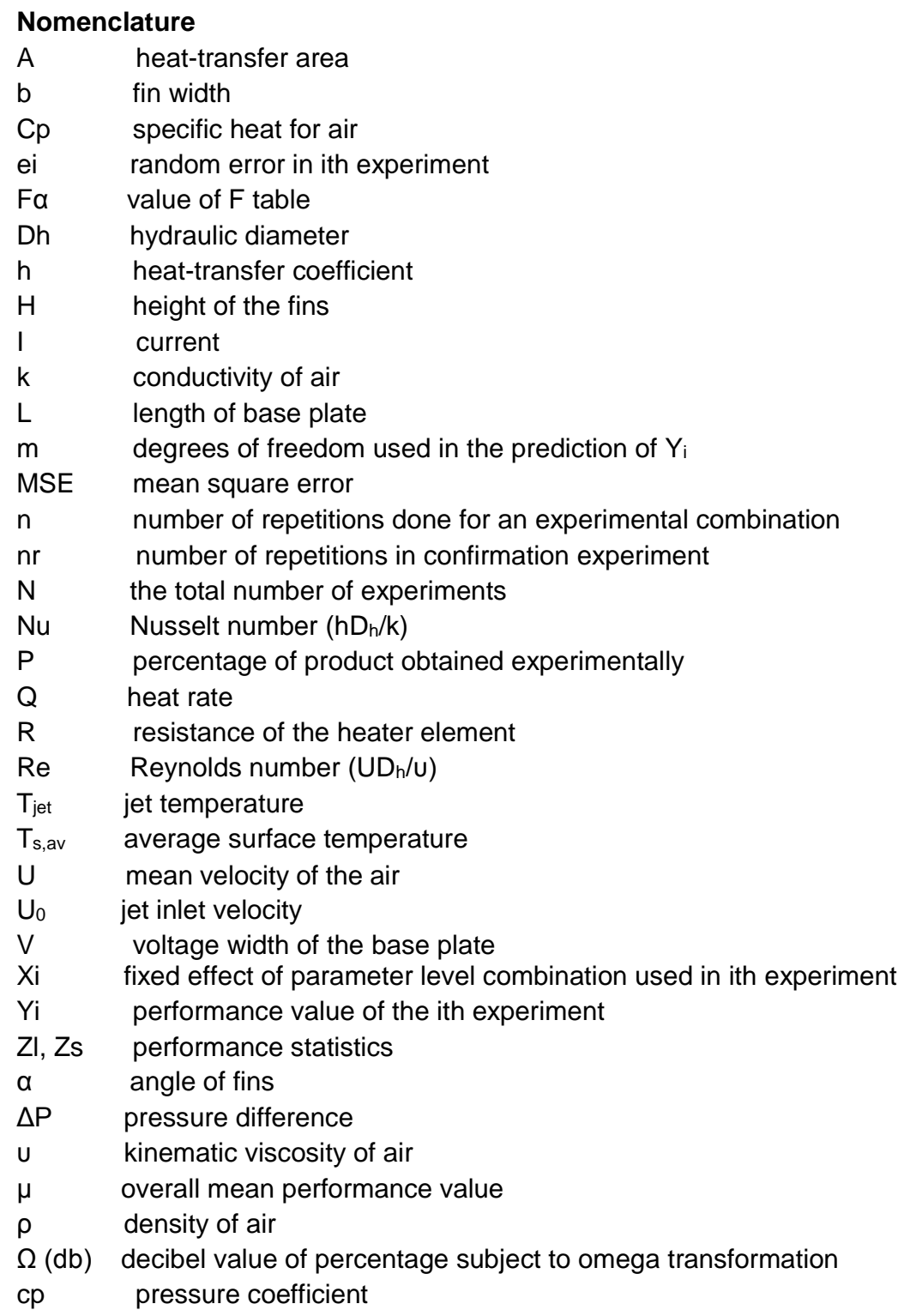

\section{References}

[1] Sahin B., Yakut K., Kotcioglu I., Celik C. Optimum design parameters of a heat exchanger. Applied Energy 2005; 82:90-106.

[2] Tahat MA, Babus_Haq RF, Probert SD. Forced steady-state convection from pin-fin arrays. Appl Energy 1994;48:335-51.

[3] Tahat M, Kodah ZH, Jarrah BA, Probert SD. Heat transfers from pin-fin arrays experiencing forced convection. Appl Energy 2000;67/4:419-42.

[4] El-Sayed SA, Mohamed MS, Abdel-latif AM, Abouda AE. Investigation of turbulent heat transfer and fluid flow in longitudinal rectangular-fin arrays of different geometries and shrouded fin array. Exp Therm Fluid Sci 2002;26:879900.

[5] Phadke MS. Quality engineering using robust design. Englewood Cliffs (NJ): Prentice-Hall; 1989.

[6] Kackar RN. Off-line quality control, parameter design and Taguchi methods. J Qual Technol 1985;17:176-209.

[7] Phadke MS, Kackar RN, Speeney DV, Grieco MJ. Off-line quality control in integrated fabrication using an experimental design. Bell Sys Tech J 1983;62/5:1273-309.

[8] Ross PJ. Taguchi techniques for quality engineering. Singapore: McGraw-Hill; 1989.

[9] Taguchi G. System of experimental design, quality resources. New York: International Publications; 1987.

[10] Karabey A. Determination of spray characteristics of impingement jet for heat exchanger. PhD Thesis [In Turkish], Ataturk University; 2016.

[11] Celik, C. (1996). Guided experimental techniques in design. Industrial Engineering, 7(6), 14-20. 


\title{
Liquid-liquid secondary fragmentation with solidification
}

\author{
M. Hadj-Achour, N. Rimbert*, M. Gradeck \\ Laboratoire d'Énergétique et de Mécanique Théorique et Appliquée (LEMTA), Lorraine \\ University-CNRS, France \\ ${ }^{*}$ Corresponding author: nicolas.rimbert@univ-lorraine.fr
}

\begin{abstract}
In the event of a hypothetical core disruptive accident in nuclear power plants, the molten core may flow out the reactor vessel and interact with the cold water. The evolution of the accident is strongly affected by the fragmentation of the jet of molten metallic fuel due to its interaction with the water (i.e. this situation is known as fuel coolant interaction, $\mathrm{FCl}$ ). In order to evaluate and predict the various consequences of a $\mathrm{FCl}$, many researches are conducted with either corium or high melting temperature molten metal, where premixing stage evolves with an important production of steam. This steam production that is unavoidable because the high temperature of corium leads to difficulties for using optical diagnostics. Hence, in our case, we use a eutectic alloy (Field's metal) with a low melting point $\left(62^{\circ} \mathrm{C}\right)$ in order to be able to visualize correctly the droplet fragmentation processes.
\end{abstract}

The present work focuses on the fragmentation of a single Field's metal liquid droplet with mass equals to $0.27 \mathrm{~g}$ $( \pm 0.01 \mathrm{~g})$. The liquid droplet interacts with a water pool whose temperature range between $20^{\circ} \mathrm{C}$ to $60^{\circ} \mathrm{C}$. According to its Weber number, it fragments in different ways. For each experiment, a single droplet has been visualized using a high-speed camera (at $8000 \mathrm{fps}$ ). All measurements (drop size, velocity, impact parameter and geometrical properties of the drops after the penetration) into the pool are evaluated using an open source image processing. Solidified fragments can then be sieved and the size PDF determined. Focus of the present work is put on the evolution of the Sauter Mean Diameter with increasing Weber number and varying bath pool temperature. It is shown that using a simple crust model during solidification and defining an effective Weber number which include the crust elasticity all the curves collapse on the same master curve for all the water bath temperature considered.

\section{Keywords}

Liquid Metal Spray, Nuclear Safety, Powder production, Fragmentation, Solidification

\section{Introduction}

One of the worst sequels of nuclear accident is nuclear core meltdown. In the event of primary containment vessel failure, the melted core (or corium) can pour outside and interact with the surrounding water (presence of surrounding water is uncertain and is related to the handling of the accident). The size and the distribution of the resulting fragments is still a scientific open problem. Yet it may be related to atomization and spray studies and to the field of metallic powder production as the interplay between fragmentation and solidification is a common mechanism. Present work focuses on what is traditionally called secondary atomization or more exactly to the fragmentation mechanism of a single droplet in a surrounding flow field. However, the problem will be simplified as vapour production (which may result in a vapour explosion in the hottest cases and prevent using simple optical diagnostics) will be prevented. Therefore present work will be quite close to previous studies by Hsiang and Faeth [1], Landeau et al. [2] or Ranger and Nicholls [3] for instance. However these works focus on either very high or very low density ratio, whereas for the intermediate density ratio (around ten) results are scarcer (see Gelfand [4], Kim et al. [5] for instance). Present work will therefore focus on the fragmentation and solidification mechanisms of a mildly hot single liquid metal droplet in water with a density ratio between phases close to 8 .

Fragmentation mechanisms are strongly impacted by value of the Weber, Ohnesorge and Reynolds numbers and fragmentation regimes are determined as a function of these numbers:

$$
W e=\frac{\rho_{A} D_{0} U_{L}^{2}}{\sigma}, O h=\frac{\mu_{L}}{\sqrt{\sigma \rho_{L} D_{0}}}, \operatorname{Re}=\frac{\rho_{A} D_{0} U_{L}}{\mu_{L}} .
$$


However, in order to take into account solidification during fragmentation, an apparent Weber number is proposed, following Haraldsson [6]. In this model surface tension is increased during solidification to take into account the elasticity of the solidified crust. Thought initially proposed to modify Kelvin-Helmholtz during solidification, it will be shown that it can also be used to propose a correlation for the Sauter Mean Diameter of the solidified fragments as a function of this new Weber number.

As present work is dominantly experimental, the experimental setup will be detailed and results discussed but a comparison with recent results obtained using Direct Numerical Simulation with the Open Source Code Basilisk (cf. Popinet [7]) will illustrate the similarity between experiments and simulation.

Last section will present how introducing a modified Weber number leads to an efficient data reduction.

\section{Material and methods}

Experimental setup is detailed on figure 1, a droplet generator is located above a 500 L liquid water pool that can be maintained at a given temperature thanks to a plunging heating element. Measurements are made using shadowgraphy thanks to a LED projector and a high speed camera. Solidified fragments are then collected (after several hundredth droplet impingements) and sieved to obtain the fragments size distribution. The liquid metal used in present experiments is Field's metal whose properties are given in Table 1. It is a eutectic alloy of Tin, Bismuth and Indium whose melting temperature is $62^{\circ} \mathrm{C}$. Therefore dropping $85^{\circ} \mathrm{C}$ liquid metal droplets into water does not produce any vapour.

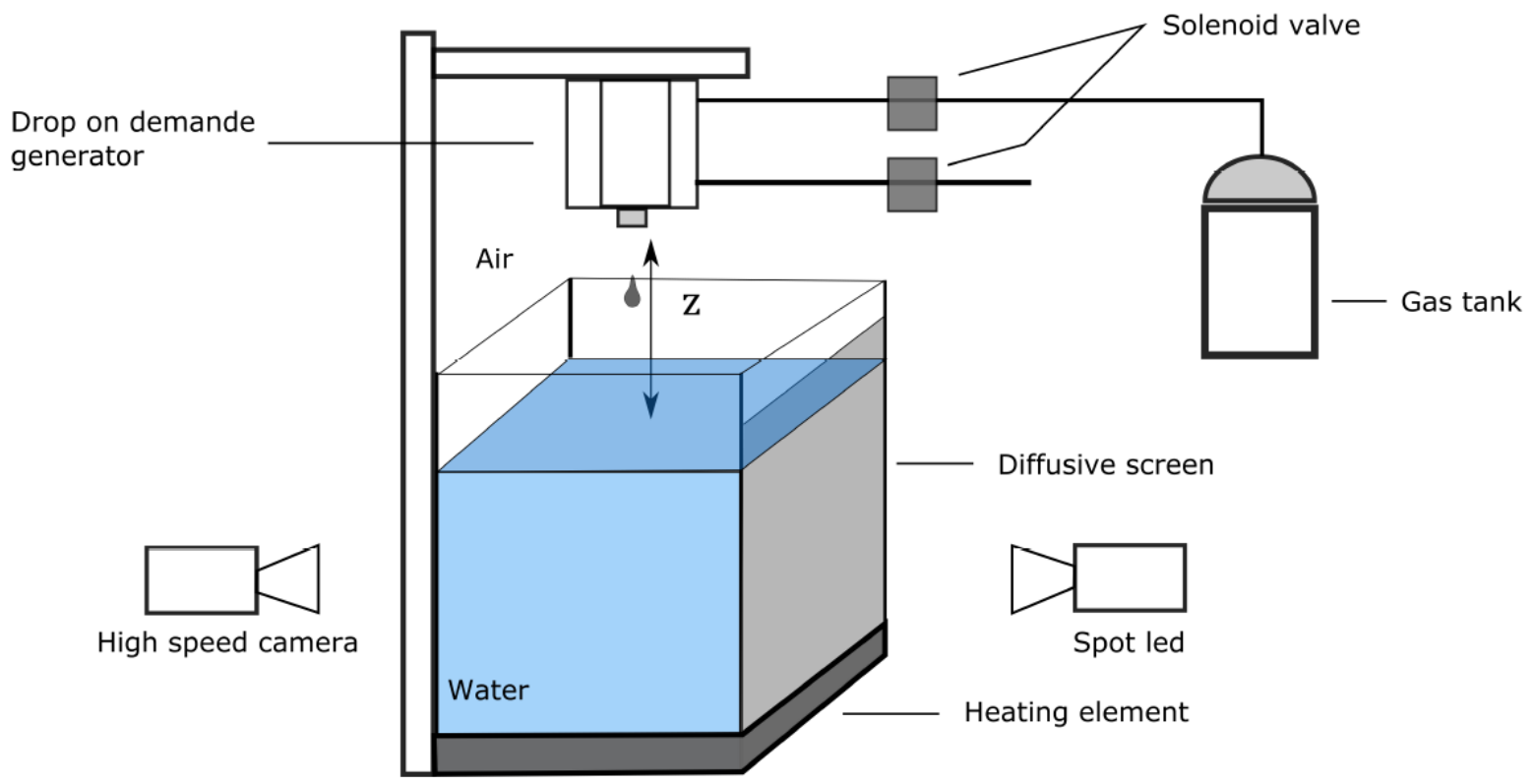

Figure 1. Picture of the experimental setup.

Liquid metals have commonly a very high surface tension, $\sigma=0.4 \mathrm{~N} / \mathrm{m}$, in the present case. However they are very sensitive to oxidation, cf. Xu et al. [8]. A thin oxide layer can appear that gives them yield stress rheological behavior. Therefore their initial shapes are close to yield stress fluid droplets (cf. figure 2). However value of this yield stress threshold is low enough to have no effects on the ulterior behavior of the droplet. Yet the initial shape of the droplet is close to a prolate spheroid whose length to width ratio equals 2 (up to $4 \%$ deviation).

Table 1. Liquid properties.

\begin{tabular}{cccccc}
\hline & $\square$ & $k$ & $\mu$ & $C_{p}$ & $H_{f}$ \\
\cline { 2 - 6 } & $\mathrm{kg} \cdot \mathrm{m}^{-3}$ & $\mathrm{~W} /(\mathbf{m} . \mathbf{k})$ & $\mathrm{Pa.s}$ & $\mathrm{J} /(\mathrm{Kg} \cdot \mathrm{K})$ & $\mathrm{J} / \mathrm{Kg}$ \\
\cline { 2 - 6 } $\begin{array}{c}\text { Field Metal } \\
\text { Water }\end{array}$ & 7994 & 5.44 & 0.01 & 300 & 26415 \\
\cline { 2 - 6 } & 997 & 0,6071 & 0.001 & 4185 & 334000 \\
\hline
\end{tabular}



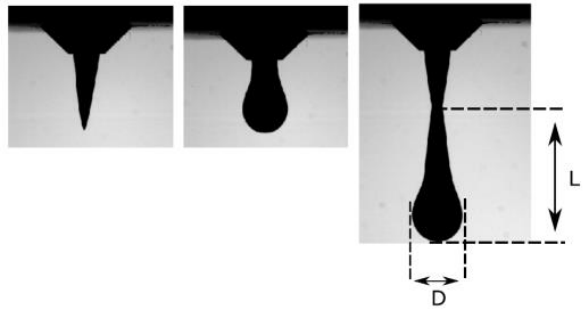

Figure 2. Liquid Metal Droplet Pinch-Off.

Experiments range over six different Weber number and 3 bath temperatures $\left(28^{\circ} \mathrm{C}, 40^{\circ} \mathrm{C}\right.$ and $\left.50^{\circ} \mathrm{C}\right) . A$ fter $50^{\circ} \mathrm{C}$, fragments remains liquid long enough to merge in a pool and cannot be collected anymore. Initial temperature and mass of the droplets are kept constant at, respectively, $85^{\circ} \mathrm{C}$ and $0.27 \mathrm{~g}\left(\mathrm{D}_{0}=4 \mathrm{~mm}\right)$. Initial velocity of the droplet range from $0.5 \mathrm{~m} / \mathrm{s}$ à $3.5 \mathrm{~m} / \mathrm{s}$ according to their initial height above the bath (cf. table 2). As drag effects are negligible over such small heights, their impact velocity can be estimated to be

$$
U=\sqrt{2 g z}
$$

Table 2. Different Weber numbers tested.

\begin{tabular}{llll}
\hline We & $z(\mathrm{~cm})$ & $U(\mathrm{~m} / \mathrm{s})$ & $R e$ \\
\hline 118 & 60 & 3.43 & 13809 \\
\hline 99 & 50 & 3.13 & 12606 \\
\hline 79 & 40 & 2.80 & 11275 \\
\hline 59 & 30 & 2.42 & 9765 \\
\hline 39 & 20 & 1.98 & 7973 \\
\hline 20 & 10 & 1.40 & 5637
\end{tabular}

Time will be non dimensionalized with the Ranger and Nicholls [3] characteristic atomization time given by

$$
\begin{aligned}
& t_{R N}=\frac{U_{0}\left(\frac{\rho_{L}}{\rho_{A}}\right)^{0.5}}{D_{0}} . \\
& t^{*}=\frac{t}{t_{R N}}
\end{aligned}
$$

\section{Results}

Figure 3 shows the impact of the droplet on the water surface. On such small height (i.e. less than 60 $\mathrm{cm})$, neither large water deformation nor air entrainment is observed.

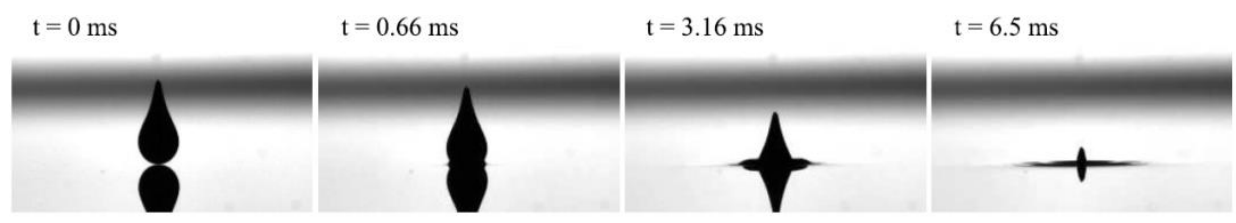

Figure 3. Liquid Metal Droplet Impact on the water surface

Figure 4 depicts the ulterior behaviour of the droplet. According to the Weber number the droplet can either oscillate or deform into a spherical bag (whose concavity is opposite to the gas-liquid case). Fragmentation mechanism is therefore very similar to classical bag breakup in gas liquid case except the reversed concavity of the bag. This may be related to the production of an intense vortex ring in the wake of the droplet (CastrillonEscobar [9]). Rayleigh-Taylor waves can be observed but the breakup of the bag is closer to the bursting of soap bubble as can be seen on figure 4 . This produces very small droplets while larger ligaments eventually destabilize to produce larger droplets leading to a multi-peaked distribution. 

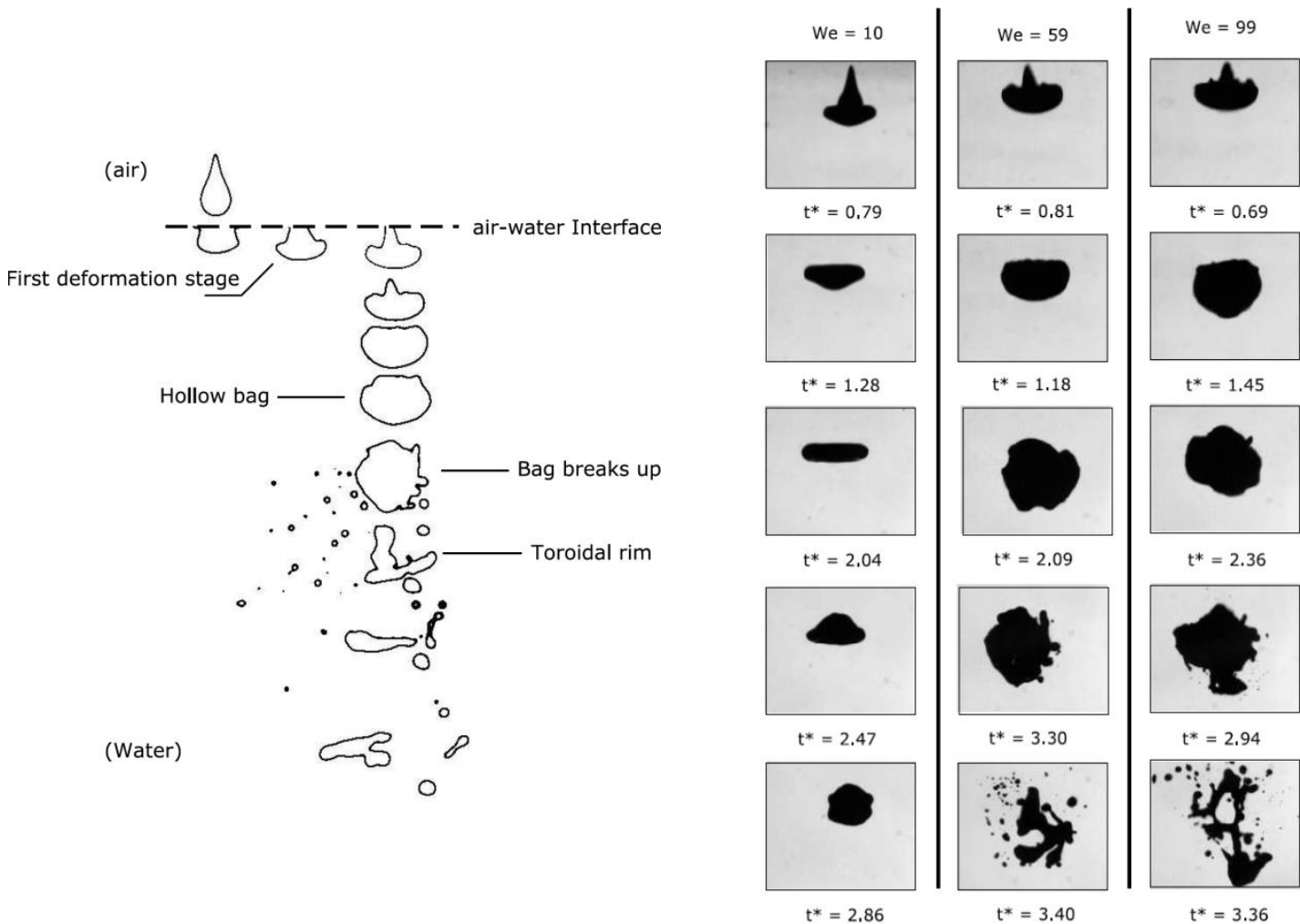

Figure 4. Deformation and fragmentation of the droplet without solidification. $T_{L}=85^{\circ} \mathrm{C} \cdot T_{A}=50^{\circ} \mathrm{C}$.

According to the Weber number, different behaviors are observed. For We $<12$, droplet oscillates between an oblate and a prolate shape while for $12<\mathrm{We}<20$ this oscillation may produce fragmentation when oscillating in the prolate shape. After We $>20$ and up to the maximum tested value of 120 , the droplet deforms into a spherical hollow cap which can eventually be solidified when the water temperature is cold enough (cf. figure 5). This bubble can then burst leading to a population of fine droplet and large ligaments threaded into cobweb like structures.
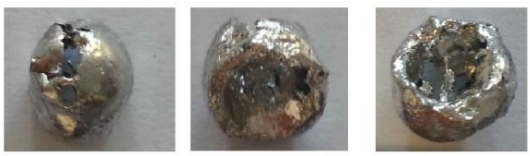

Figure 5. Liquid Metal Droplet Frozen during the reverse bag formation. $T_{A}=20^{\circ} \mathrm{C}$

Thought not detailed in present paper, figure 6 shows a comparison between a DNS obtained using the Basilisk solver and present experiments. Lambda 2 criterion is used to visualize the vortex ring that appear in the wake of the droplet (cf. Castrillon Escobar [9]). That criterion looks for the eigenvalue of the tensor $\mathrm{S}^{2}+\Omega^{2}$ which can be considered as a good approximation of the pressure Hessian. Zones where the second eigenvalue is negative $\left(\lambda_{2}<0\right)$ are likely to be low pressure region owing to the presence of a vortex. As the density ratio between droplet and surrounding liquid is lower than in the gas liquid case, the inertia created by this rotary motion can strongly affect the droplet shape. This seems in agreement with early 2D DNS results obtained by (Han and Tryggvasson, [10]) who however did not look for these vortices. 


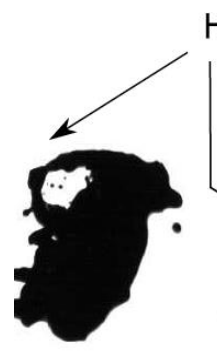

$0 \mathrm{~ms}$
Hole growth

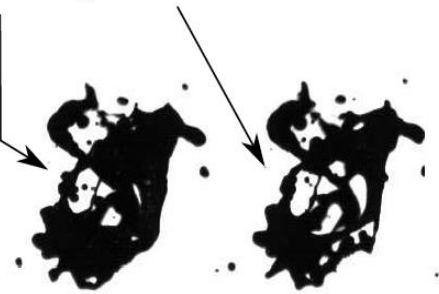

$2 \mathrm{~ms}$

$2.5 \mathrm{~ms}$ $t^{*}=0$

$t^{*}=0.63$

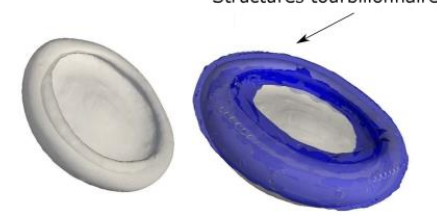

$\mathrm{t}^{*}=0.95$

Ligaments Small fragments

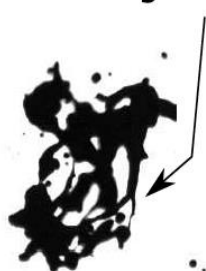

$3 \mathrm{~ms}$

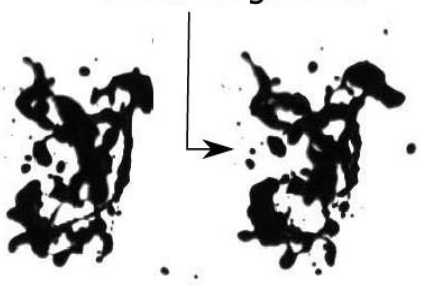

$4 \mathrm{~ms}$

$5 \mathrm{~ms}$

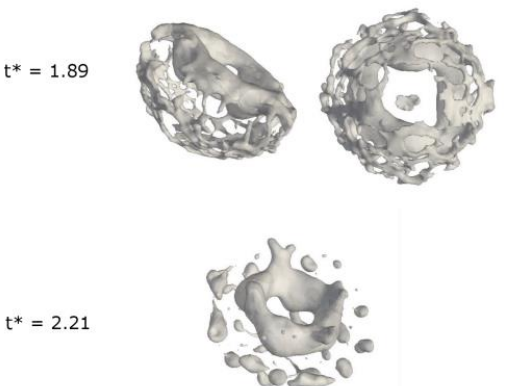

Flow $\uparrow \uparrow \uparrow \uparrow$

Figure 6. Comparison between experimental results and numerical simulations with Basilisk.

Figure 7 shows the values of measured Sauter Mean Diameter (SMD) and Mass Mean Diameter (MMD) after sieving and weighting the solidified fragments (the sieve sizes are 20,50, 100, 500,1000 and $2000 \mu \mathrm{m}$ ). For Weber number 20, the droplet fragments during its prolate elongation generating two main daughter droplets of roughly equal shape, therefore $S M D / D_{0}$ ratio equals $1 / 2$. Ratio between $S M D$ and MMD range from 1 to 1,5 in agreement with DNS results of Jalaal and Mehravaran [11]. As Weber number increases these average size of fragments decreases and it can also be seen that, as expected, the colder the water, the larger the fragments. A possible interpretation to these graphs is that, as surrounding temperature decreases, a larger solidified crust can form at the interface between liquid metal and water. This crust will then increase the apparent surface tension of the droplet. Therefore the real Weber number should be lower owing to this increased surface tension and the corrected curve should shift to the left. This will be the focus of next section.
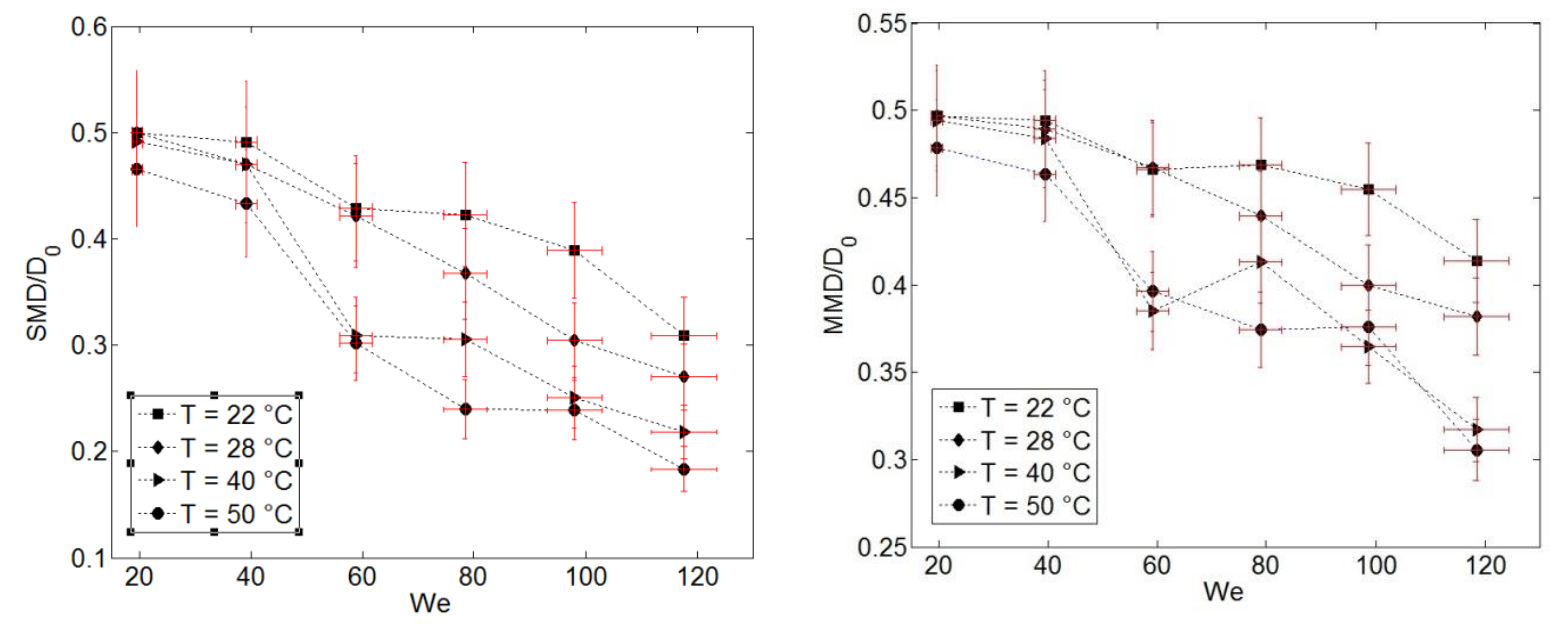

Figure 7. Sauter Mean Diameter and Mass Mean Diameter of the solidified fragments as a function of Weber number. 


\section{Discussion}

Owing to the previous results, it seems to be interesting to find a model that can account for the shift to the right of the curve as the bath temperature is decreased. Haraldsson et al. [6] gave us a hint as they devised a simple model for the crust growth and used it to see how it could affect the growth rate of Kelvin-Helmholtz instability. However this crust does grow over time, therefore a specific time instant must be selected to create a new Weber number. In the present work, it has been decided to use the fragmentation inception time given by eq. 7.

$$
t_{\text {frag }}=8(W e-12)^{-0.25}\left(1+2.2 O h^{1.6}\right) t_{R N}
$$

In the following the droplet is assumed to be still spherical and the crust thickness $\delta$ is assumed to be thin. The solidification time is supposed to be the sum of the time needed to remove the sensible heat from the droplet and the time needed for the crust to grow.

$$
t_{\text {sol }}=t_{\text {sup }}+t_{\text {fus }}, \quad t_{\text {sup }}=\frac{\rho_{L} C p_{L} \Delta T_{\text {sup }} \delta}{q^{\prime \prime}}, t_{\text {fus }}=\frac{\rho_{L} H f_{L} \delta}{q^{\prime \prime}}
$$

The heat flux $q$ " is obtained from a classical correlation for a sphere:

$$
q^{\prime \prime}=\frac{k_{A}}{D_{0}}\left(2+0.6 \operatorname{Re}^{0.5} \operatorname{Pr}^{1 / 3}\right)\left(T_{L}-T_{A}\right)
$$

Therefore crust thickness at time $t_{\text {frag }}$ is given by

$$
\delta\left(t_{\text {frag }}\right)=\frac{t_{\text {frag }} q^{\prime \prime}}{\rho_{L}\left(C p_{L} \Delta T_{\text {sup }}+H f\right)} .
$$

This thin solidified shell is elastic and possesses a Young modulus $E$ of $9.25 \mathrm{GPa}$ (cf. Shan et al. [12]). Following Haraldsson et al. [6], it is possible to convert the elastic energy of deformation into a surface energy thanks to

$$
\sigma_{\text {elas }}=\frac{E \delta^{3}}{12\left(1-v^{2}\right)^{3} D_{0}^{3}}
$$

where $v$ is Poisson ratio (taken to be $1 / 3$ ). Lastly, the new Weber number is

$$
W e_{a p p}=\frac{\rho_{A} D_{0} U_{L}^{2}}{\sigma_{a p p}}
$$

where apparent surface tension is the sum of the liquid surface tension and of this elastic surface tension

$$
\sigma_{\text {app }}=\sigma+\sigma_{\text {elas }}
$$

Figure 7 shows the evolution of the ratio $S M D / D_{0}$ as a function of this new Weber number. As expected all curves are shifted to the left and seem to collapse on a common master curve.

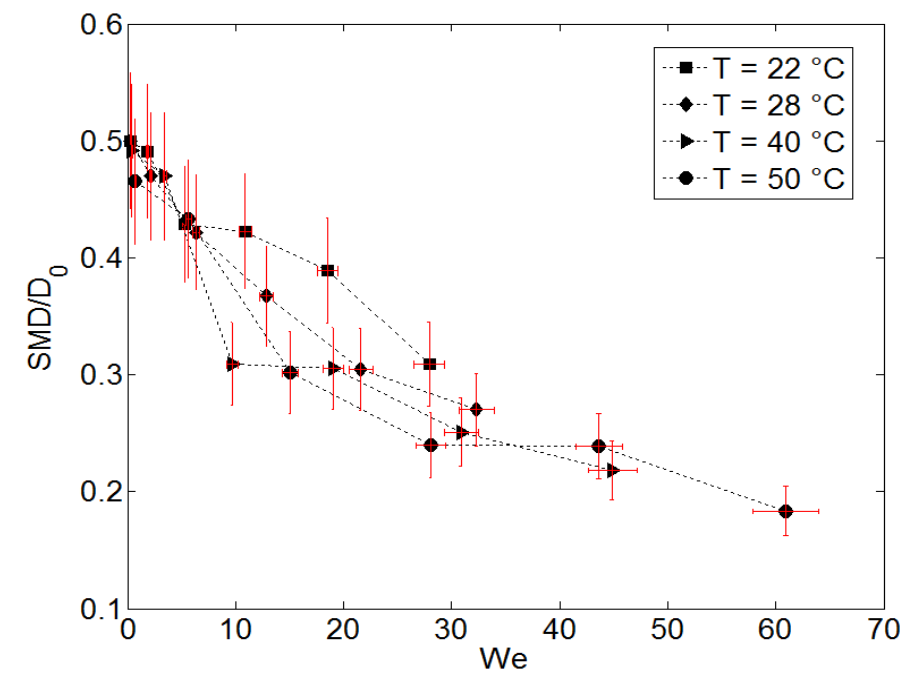

Figure 8. Sauter Mean Diameter of the solidified fragments as a function of the modified Weber number. Initial droplet temperature is $85^{\circ} \mathrm{C}$. 


\section{Conclusions}

In this work, a single heated liquid metal droplet impact into cold water has been studied thanks to a specifically designed experimental setup. The temperature of the water bath has been increased from $20^{\circ} \mathrm{C}$ to $50^{\circ} \mathrm{C}$. The initial temperature of the droplet has been kept constant $85^{\circ} \mathrm{C}$ way above the liquid metal solidification point $62^{\circ} \mathrm{C}$ ). For the lowest value of the bath temperature $\left(20^{\circ} \mathrm{C}\right)$ and for high enough value of the Weber number (We>20), the droplet can be frozen before its breakup during its initial deformation into a hollow shell showing that this experiment is well designed to study the interplay between solidification and fragmentation. For the higher value of the water temperature, different fragmentation regimes have been put into evidence: below $\mathrm{We}<12$, the droplet does not fragment and oscillates. Between $12<\mathrm{We}<20$, the droplet oscillates and fragments during the prolate part of its oscillation. It mainly produces two daughter droplets of equal size. Bag breakup happens when We $>20$, but the concavity of the bag is opposite to the liquid-gas case owing to the strong influence of the vortex ring that is produced in the wake of the droplet by flow separation. The liquid metal bubble then bursts and produces a mixture of fine droplets and a cobweb of ligaments which then fragments into bigger droplets. As the temperature of the bath is lowered, the size of the fragments increases. This can be related to a stronger influence of solidification on the fragmentation mechanism. A simple way to account for this is to create a new Weber number which sums up the surface tension and the elastic energy of the solidified crust at the inception of the droplet fragmentation. This allows the different curves (SMD and MMD as a function of the droplet Weber number for the different bath temperature) to collapse on a common master curve.

\section{Acknowledgements}

This work has been funded by French Government through "programme d'investissements d'avenir" AnR RSNR ICE under the grant $n^{\circ}$ ANR-10-RSNR-01. The authors would also like to mention support from EU-COST Action MP1305 "Flowing Matter".

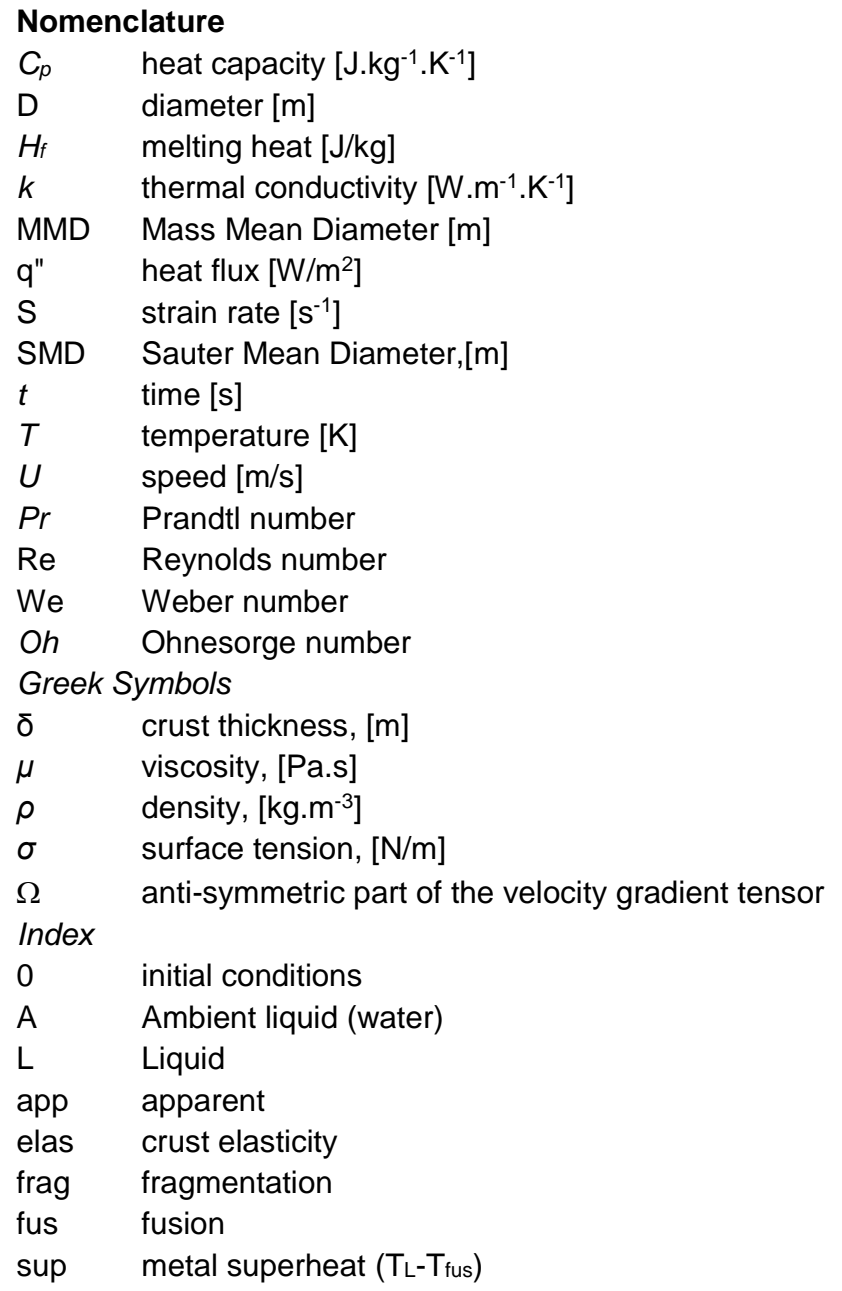




\section{References}

[1] Hsiang and Faeth, (1995) Int. J. Multiph. Flow 21, 545.

[2] Landeau et al., (2014) J. Fluid Mech. 749, 478.

[3] Ranger and Nicholls (1969), AIAA J. 7, 285.

[4] Gelfand (1996), Prog. Energy Combust. Sci. 22, 201.

[5] Kim et al., (1983) in Proc. Int. Meet. Light Water Sev. Accid. Eval., edited by American Nuclear Society (Stone \& Webster Engineering Corp,), p. 4.1-4.7.

[6] Haraldsson et al., (2001) Heat Mass Transf. 37, 417.

[7] Popinet, (2003) J. Comput. Phys. 190, 572.

[8] Xu et al., (2012) Phys. Fluids 24, 63101.

[9] Castrillon Escobar (2016), Ph.D. Thesis, Univ. Lorraine.

[10] Han and Tryggvason, (2001), Phys. Fluids 13(6), 1554

[11] Jalaal and Mehravaran (2012), Int. J. Multiph. Flow 47, 115.

[12] Shan et al. (2013), Smart Mater. Struct. 22, 85005. 


\title{
Planar Droplet Sizing: Application to a spray of Jet A-1 kerosene
}

\author{
P. Doublet ${ }^{\star 1,2}$, C. Lempereur ${ }^{2}$, V. Bodoc ${ }^{2}$, M. Orain ${ }^{2}$, P. Gajan ${ }^{2}$ \\ ${ }^{1}$ Safran Aircraft Engines, France \\ ${ }^{2}$ ONERA The French Aerospace Lab, France \\ ${ }^{*}$ Corresponding author: pierre.doublet@onera.fr
}

\begin{abstract}
Optical techniques are widely employed for their non-intrusive behavior and are applied to two-phase flow investigations. Until now, the most commonly used technique to determine the droplet size is the Phase Doppler Anemogranulometry, although it is time consuming for an overall injector characterization. An imaging technique called Planar Droplet Sizing has been used to offer an alternative and provide a spatially-resolved 2D map of the Sauter Mean Diameter (SMD). The measurement is based on the ratio between laser-induced fluorescence and scattered light intensities which are assumed to be proportional respectively to the droplet volume and droplet surface area. However, previous studies revealed that the dependence of fluorescence intensity on the droplet volume can be altered by the absorption of light in the liquid. The scattered light intensity depends on the scattering angle and intensity variations within the field of view must be avoided.

The aim of this study is to make the PDS technique operational for a Jet A-1 kerosene spray. A strong absorption of liquid kerosene appears under UV excitation at $266 \mathrm{~nm}$ making the technique unsuitable. Under visible excitation at $532 \mathrm{~nm}$, a fluorescent tracer (Pyrromethene 597) must be added to the kerosene to enhance the fluorescence signal. To prevent scattered light intensity variations within the field of view, an optimal scattering angle close to $115^{\circ}$ is required. An image processing algorithm is proposed in order to reduce the effects of multiple scattering.
\end{abstract}

\section{Keywords}

Planar Droplet Sizing, kerosene, spray, Sauter Mean Diameter

\section{Introduction}

Context

Sprays are used in many industries where, depending on the application, there are particular demands on the atomization of the liquid. In aeronautical gas turbines, the injection of liquid fuel into the highly turbulent gas flow of a combustion chamber gives rise to many complex physical phenomena: the liquid stream is stretched into ligaments and atomized into droplets that are then transported further downstream, while evaporating and enabling the combustion process. Fine atomization is required to promote vaporization and combustion. In this context, knowledge of the particle size and velocity distribution of both phases is a key factor for the design of an injection system.

The application of CFD codes to such unsteady and dense sprays is still challenging; if numerical parameters need to be tuned to fit experimental results, these data must in turn be reliable. In this respect, experiments yield essential information for the development and evaluation of atomization and spray flow models.

In order to fulfill the accuracy requirement, laser-based optical diagnoses are in steady progress. These techniques are preferred to physical probes because they do not disturb the spray and can be applied in a harsh environment where a physical probe would not survive. Although a classical pointwise technique, Phase Doppler Anemogranulometry, has been widely investigated over the last decades, whole-field measurement techniques are a promising means to reduce the test duration and cost.

\section{Planar Droplet Sizing (PDS)}

The Planar Droplet Sizing method also called LIF/Mie firstly appeared in 1993 for the characterization of a transient fuel spray [1]. This technique consists in illuminating a spray with a laser sheet. Contrary to the classical techniques as PDA (Phase Doppler Anemogranulometry) or ILIDS (Interferometric Laser Imaging for Droplet Sizing), PDS does not yield the individual particle size, but a statistical value at each point, namely the SMD, which is widely used to characterize injection devices. The pros and cons of PDS and traditional techniques are summarized in the table 1 . This is an important quantity for mass transfer processes: a small SMD indicates fine evaporation, weak penetration, efficient mixture and combustion processes. This technique is based on Mie 
scattering and fluorescence emission from droplets. The fluorescence and scattered light intensities $I_{F}$ and $I_{M}$ are assumed to be proportional respectively to the volume and the surface of the illuminated droplet [1]. A ratio of both images is therefore proportional to the SMD, also called $d_{32}$, of the size distribution of the $\mathrm{N}$ measured droplets. Thanks to this technique, a two-dimensional map of $d_{32}$ can be obtain in a few minutes.

$$
\frac{I_{F}}{I_{M}}=\frac{k_{F} \sum_{i}^{N} n_{i} d_{i}^{3}}{k_{M} \sum_{i}^{N} n_{i} d_{i}^{2}}=k \cdot d_{32}
$$

where $k_{F}, k_{M}$ and $k$ are coefficients determined experimentally. A numerical and experimental study allowed Domann and Hardalupas to analyze the accuracy of this technique [2]. More recently, Lempereur and al. [3] followed this approach in order to characterize a real aeronautical engine spray under atmospheric conditions. This work showed that the scattering angle (Figure 1.c), the droplet size and the refractive index modify the proportionality between the scattering intensity and the droplet surface. In parallel, the influence of the concentration of fluorescent dye on the proportionality between the fluorescence intensity and the droplet volume [4] was demonstrated. Optimum values of the scattering angle and the fluorescent dye concentration were obtained to improve the sizing measurement accuracy $[5,6]$ and recent studies compared the PDS measurements with the PDA ones $[3,7]$. Nevertheless in that study, ethanol was used as a surrogate fluid.

Table 1. Pros and cons of main technique used for measuring droplet diameters.

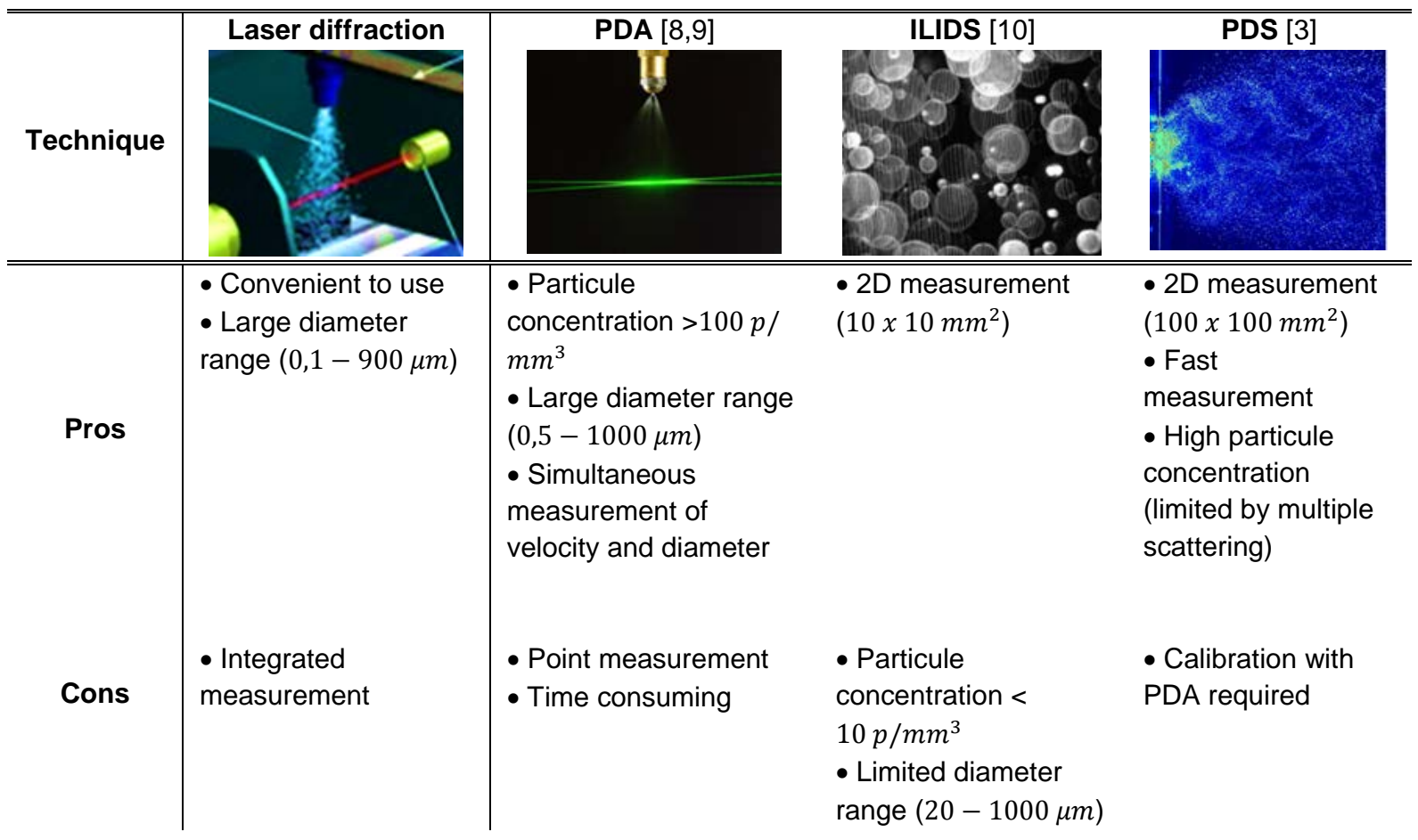

Focus of the study

The aim of this paper is to extend the Planar Droplet Sizing technique to a spray of Jet A-1. The first part of this article highlights a review of the implementation of this technique on a swirled spray using ethanol and Rhodamine $6 \mathrm{G}[3,6]$. The extension to the Jet A-1 requires knowledge of its optical properties in order to accurately define the experimental setup. These issues will be discussed in the second part. The PDS technique implementation and first results will be the topic of the last part.

\section{PDS implementation on an aeronautical swirled spray}

Recent investigations at ONERA focused on the implementation of this technique to characterize the spatial droplet size distribution in the spray of an aeronautical swirled injection system used in actual combustion chambers [3, 6]. Tangential air intakes create a swirl around the injection axis in order to promote flame stabilization and flow mixture. Experiments are conducted at ambient temperature on the unsteady spray generated downstream of the injector. The liquid used for the simulation is ethanol, because its surface tension is close to that of kerosene $\left(\sigma_{e t h}=22 \mathrm{mN} / \mathrm{m}\right.$ and $\left.\sigma_{k e r}=23,3 \mathrm{mN} / \mathrm{m}\right)$. Since ethanol is not naturally fluorescent, it was doped with a dye. Rhodamine $6 \mathrm{G}$ was chosen because of its optimal excitation wavelength (529 $\mathrm{nm})$ and its 
high quantum yield (0.95) in these conditions. An intensified camera was used with filters for fluorescence and scattered light images. The study focuses on the parameters which alter respectively fluorescence and scattered light as the particle number concentration, the scattering angle or the dye concentration. The main parameter that affects the relation between fluorescence intensity and particle volume is the dye concentration $\mathrm{c}$. The relation can be rewritten as follows:

$$
I_{F}=k_{F} \sum n_{i} d_{i}^{F(c)}
$$

On one hand, fluorescence intensity is generally low and the dye concentration must be large enough in order to yield a sufficient signal. On the other hand, the light absorption increases with the dye concentration. A concentration of Rhodamine $6 \mathrm{G}$ of $1 \mathrm{mg} / \mathrm{L}$ was sufficient to give an exploitable fluorescent signal on the intensified camera. [4].

The droplet size and the scattering angle $\alpha$ alter the relation between the scattered light intensity and the droplet surface area. The relation can be expressed as follows:

$$
I_{S}=k_{S} \sum n_{i} d_{i}^{M\left(d_{i}, \alpha\right)}
$$

Investigations on mean scattered light images enabled to determine the parameters that affect the measurement accuracy. As can be seen in Figure 1, contrary to the fluorescence image, the scattering image was not symmetric on both sides of the nozzle axis and an attenuation of more than $20 \%$ between the amplitudes of the two peaks $A$ and $B$ can be measured. This attenuation was due to the variation of the scattering angle within the field of view (scattering angle centered at $90^{\circ}$ ). To limit this effect, two corrections were proposed. First, the angle of view was reduced by increasing the focal length and observation distance. Then, a favourable mean scattering angle was determined (close to $100^{\circ}$ ) to lessen the intensity variation within the field of view. In this angular range, the exponent $\mathrm{M}$ only depends on the droplet diameter. A theoretical approach enables to estimate the relation between the scattered intensity and the surface area. In the range of interest $(1-100 \mu \mathrm{m})$, the $\mathrm{d}^{2}$ law is invalidated: the $\mathrm{M}$ exponent steadily increases from 1 to 2 with the diameter. The best power law fit for diameters between 5 and $100 \mu \mathrm{m}$ is obtained for $M=1,7$.
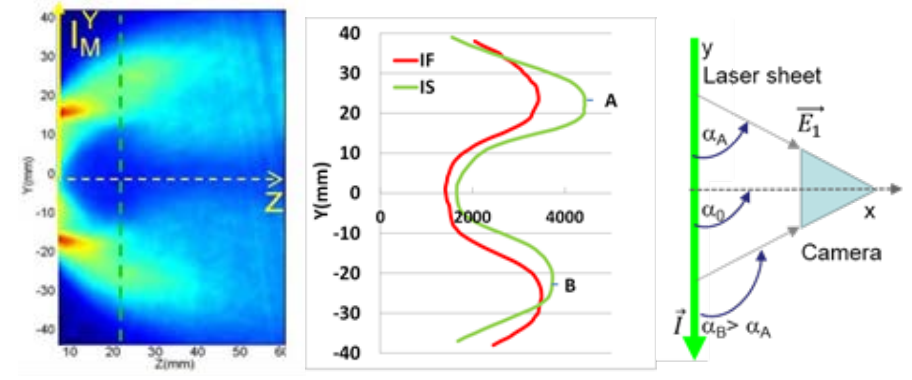

Figure 1. (a) Mean Mie scattered light image (b) vertical Y-profiles on Mie and LIF images at $Z=20 \mathrm{~mm}$ from the injection point (c) definition of the local scattering angle for $\alpha_{0}=90^{\circ}$.

PDS and PDA measurements were compared in order to validate the PDS results. The knowledge of exponents $\mathrm{M}$ and $\mathrm{F}$ helped understanding the differences between both techniques: the diameter $d_{F M}$ calculated with the PDS technique has the unit of a diameter at a power roughly equal to 1,3 for $F=3$ and $M=1,7$.

$$
\left(d_{F M}\right)^{F-M}=\frac{\sum d^{F}}{\sum d^{M}}
$$

Since the PDS ratio was not found to be proportional to $d_{32}$, a calibration is required for the whole diameter range. The method consists in creating a correspondence between values of Sauter Mean Diameter measured with PDA and ratios obtained by PDS image processing at the same points. A power law fit was obtained and can be used to build a Look Up Table (LUT) to convert PDS images into $d_{32}$ maps. This calibration has been validated on several diameters beyond a distance of $Z=20 \mathrm{~mm}$ from the injection point. Closer to the injection, some discrepancies occurred between PDS and PDA measurements due to the presence of large, non-spherical liquid structures. Moreover, a higher density of particles may induce multiple scattering. As a result, none of those techniques is reliable close to the injector where the density of particles becomes high. 
For more applied studies involving on the influence of fluid properties variation over the atomisation process, it is necessary to transpose these results obtained with ethanol to the Jet A-1.

\section{PDS extension to Jet A-1}

Characterization of jet A-1 optical properties

Before implementing the PDS technique on a Jet A-1 spray, some investigations of its optical properties have to be carried out in order to define precisely the experimental setup (excitation wavelength, camera orientation, ...) and to estimate the exponents $\mathrm{M}$ and $\mathrm{F}$. The idea is to quantify the absorption coefficient $\mathrm{k}$ and to insure that the kerosene fluorescence is high enough to obtain reliable fluorescence images.

The first attempt consisted in exciting in the UV (at $266 \mathrm{~nm}$ ) because of the well-known fluorescence of the aromatics contained in kerosene vapours [11]. In order to extend these results to liquid kerosene, experiments are realized with a $10 \mathrm{~mm}$-side cubic quartz test cell containing liquid kerosene. As shown in Figure 2.a, a spectrometer and an intensified camera are placed on opposite sides of the cell to enable simultaneous fluorescence and absorption measurements. The laser is a Quantel Brilliant one, the spectrometer is a HR2000+ from Ocean Optics and the intensified camera is a Princeton PIMAX II.

The normalized fluorescence spectrum of liquid kerosene, plotted in Figure 2.b, exhibits the same shape as the vapour spectrum one obtained in previous studies [11]. The first fluorescent band is due to the mono-aromatic molecules whereas the di-aromatics ones are responsible for the second band [11]. However, a shift of the emission spectrum of liquid kerosene to higher wavelengths is observed, probably due to the differences in energy levels between liquid and vapour phases.

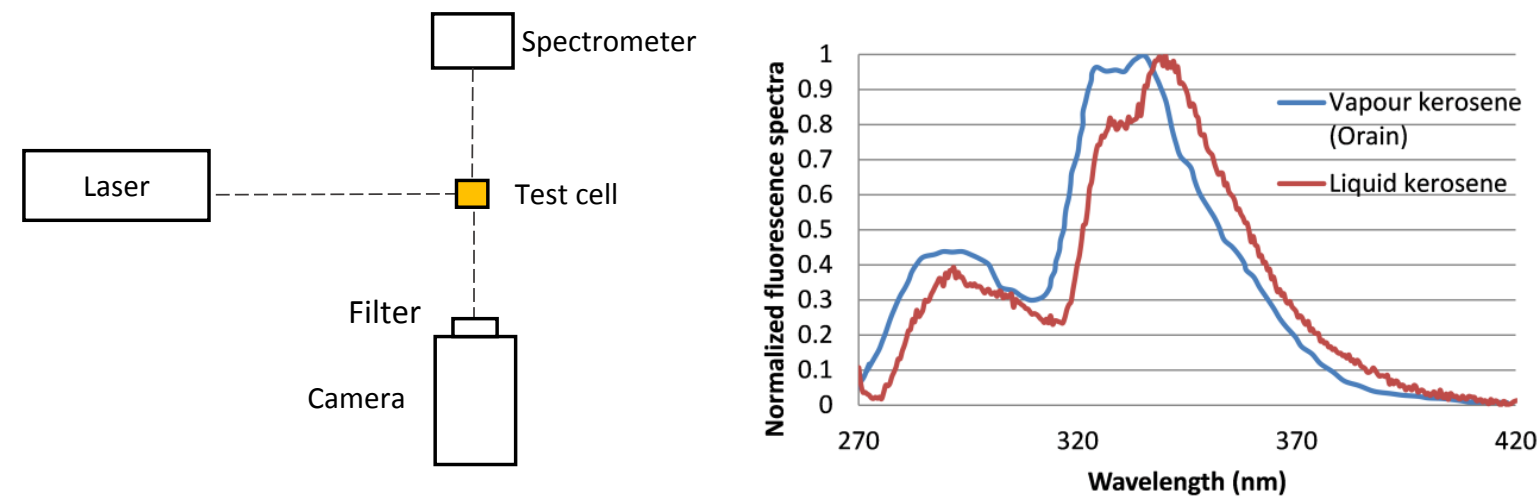

Figure 2. (a) Experimental set-up for the fluorescent and absorption measurements (b) Normalized fluorescence spectra of liquid and vapour Jet A1.

The images point out a strong absorption of the liquid kerosene at this excitation wavelength: laser beam extinction appeared a few millimetres downstream from the entrance window. In order to quantify this absorption, the parameter of interest is the extinction coefficient $k$ which is the imaginary complex part of the refractive index. This parameter is involved in the Beer-Lambert law:

$$
I=I_{0} \exp \left(-\frac{4 \pi k}{\lambda} x\right)
$$

where $I_{0}$ is the laser intensity at the entrance window of the test cell, $x$ the optical path in the medium and $I$ is the laser intensity at the probe volume. The factor $\mathrm{k}$ is obtained from the processing of the images of the laser beam propagation in the cell. For a $266 \mathrm{~nm}$ excitation wavelength, the value of the extinction coefficient $k$ is high, close to $1,3 \cdot 10^{-2}$. The exponent $F$ involved in the relation between fluorescence intensity and droplet volume can be estimated thanks to simulations of internal laser field excitation [12]. It is not equal to 3 but close to 2: as a result, the fluorescence intensity is not proportional to the volume of the droplet but rather to its surface area. Therefore, the PDS technique using this excitation wavelength seems to be unsuitable because the ratio of fluorescence to scattered light intensities is not proportional to the Sauter Mean Diameter but is roughly constant. To solve this problem, the excitation wavelength is changed from $266 \mathrm{~nm}$ to $532 \mathrm{~nm}$. At this wavelength, a low absorption is measured $\left(k=7,9.10^{-9}\right)$ and the coefficient $\mathrm{F}$ is much closer to $3(F=2,91)$. Nevertheless, considering an excitation wavelength of $532 \mathrm{~nm}$, the fluorescence signal is very low and will be a limiting factor. The use of a fluorescent dye is thus required as in the ethanol case. Rhodamine 6G was used with ethanol but is not miscible with kerosene; Pyrromethene 597-8C9 is selected: its wavelength absorption peak is centered on $525 \mathrm{~nm}$ corresponding to a fluorescent emission between $540 \mathrm{~nm}$ and $700 \mathrm{~nm}$. Several concentrations are tested in order 
to determine the best compromise: a concentration of $1 \mathrm{mg} / \mathrm{L}$ leads to an extinction coefficient of $3.10^{-7}$ and a coefficient $F$ of 2,90 . Table 2 summarizes the values of the absorption coefficient and coefficient $F$ according to the mixture types and excitation wavelengths.

Table 2. Extinction and $\mathrm{F}$ coefficients for the different mixtures and excitation wavelengths.

\begin{tabular}{c|ccc}
\hline \hline Mixture & Excitation wavelength $(\mathrm{nm})$ & $\begin{array}{c}\text { Extinction } \\
\text { coefficient } \mathrm{k}\end{array}$ & Coefficient $\mathrm{F}$ \\
\hline \hline Kerosene & 266 & $1,3.10^{-2}$ & 1,99 \\
Kerosene & 532 & $7,9.10^{-9}$ & 2,91 \\
Kerosene + Pyrromethene $(1 \mathrm{mg} / \mathrm{L})$ & 532 & $3.10^{-7}$ & 2,90
\end{tabular}

\section{PDS implementation and measurements}

Experimental set-up

A hollow-cone Jet A-1-spray, generated from a Delavan nozzle, is investigated at ambient temperature, atmospheric pressure and with a liquid injection pressure set to 5 bars which corresponds to a liquid flow rate of $8 \mathrm{~L} / \mathrm{h}$. In this case, the properties of liquid kerosene are the following: $\sigma=23,30 \mathrm{mN} / \mathrm{m}, \rho=798,78 \mathrm{~kg} / \mathrm{m}^{-3}$ and $v=1.6405 .10^{-6} \mathrm{~m}^{2} / \mathrm{s}$. In the present study, visualisations in longitudinal sections of the spray are considered. As shown in Figure 3, the spray core is illuminated at $532 \mathrm{~nm}$ with a Quantel Twins BSL Nd:Yag laser. A Princeton PIMAX IV camera with a GenIII intensifier is chosen for these experiments. The quantum efficiency (QE) of the camera is close to $50 \%$ in the dye emission spectral range $(>532 \mathrm{~nm})$. For the fluorescence signal, a high pass filter with a cut-off wavelength at $540 \mathrm{~nm}$ is used (the optical density is equal to 7 in the blocking range), whereas, for the Mie signal, a band pass filter centered at $532 \mathrm{~nm}$ (FWHM 10nm) is used (the optical density is equal to 6 in the blocking range). The optical settings are facilitated thanks to sequential acquisitions. Both LIF and Mie signals come from the same camera and viewing point, which suppresses potential discrepancies originating from the sensors, the dewarping or from different optical paths. Moreover, to ensure reproducible measurements, the pressurized tank is equipped with an expansion valve which limits the pressure fluctuations under 0,01 bar. The dynamic range of the camera is, for the LIF signal, a critical parameter to visualize the largest droplets without saturation while enabling the detection of the smallest ones, keeping in mind that the intensity is proportional to the volume of the particle. The best figures are obtained when operating the camera at the lowest pixel readout rate, in order to generate the lowest readout noise. Thus, the frame rate is kept at its minimum, namely 5 images/s in these experiments. The LIF and Mie averaged images should be obtained from several thousands of images to ensure a good statistical convergence. The top of the fluorescence and scattered images corresponds to the nozzle exit. In order to prevent saturations the spray formation region is not illuminated by the laser sheet. All of the images are dewarped in the measurement plane and presented in millimeters.

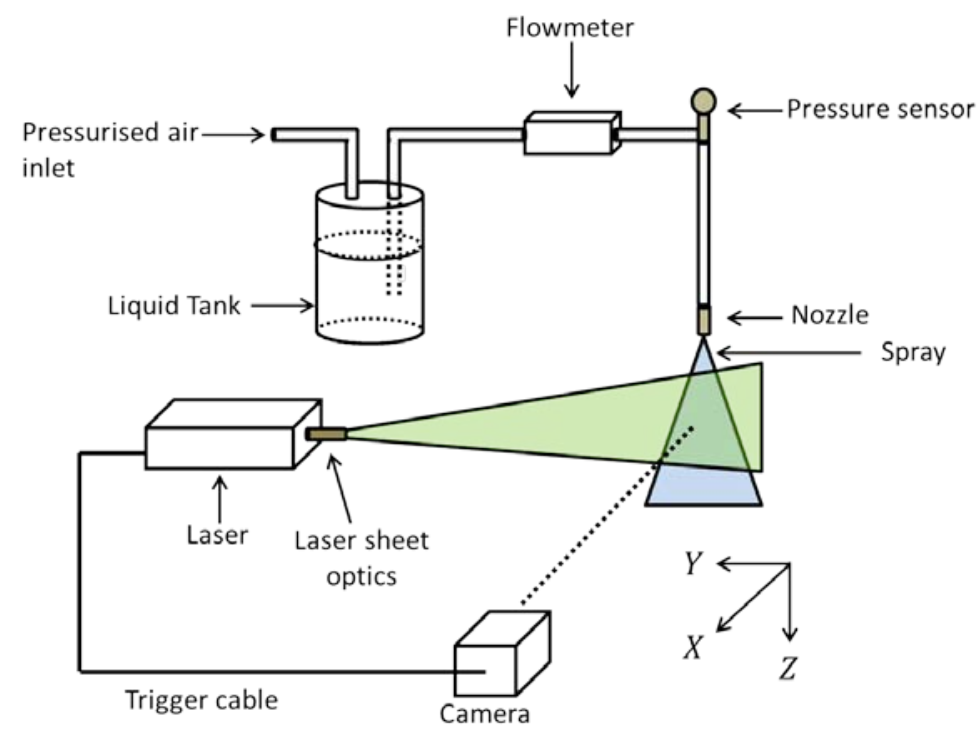

Figure 3. Experimental set-up for the LIF/MIE technique implementation. 


\section{Scattered light images}

Since the variation of the scattering angle within the field of view may be responsible for strong intensity variations, it is interesting to look for an optimal configuration where this fluctuation is minimized. The theoretical evolution of the scattered light intensity versus $\alpha$ is calculated for $\alpha$ ranging from $60^{\circ}$ to $120^{\circ}$ and for various droplet diameter distributions. As shown in Figure 4, the slope of $I(\alpha)$ is important in forward scattering and tends towards zero in the $110-120^{\circ}$ range. For this PDS implementation on kerosene, a mean scattering angle close to $115^{\circ}$ is chosen. To study the effect of the scattering angle on the scattered light intensity, two critical values are considered: the camera is placed at $70^{\circ}$ and $115^{\circ}$. With a $105 \mathrm{~mm}$ focal length and a $13 \mathrm{~mm} \times 13 \mathrm{~mm} \mathrm{CCD}$ sensor, an angle of view of $\pm 2,5^{\circ}$ is achieved. At a distance of $840 \mathrm{~mm}$, a $90 \mathrm{~mm}$-side field can be visualized.

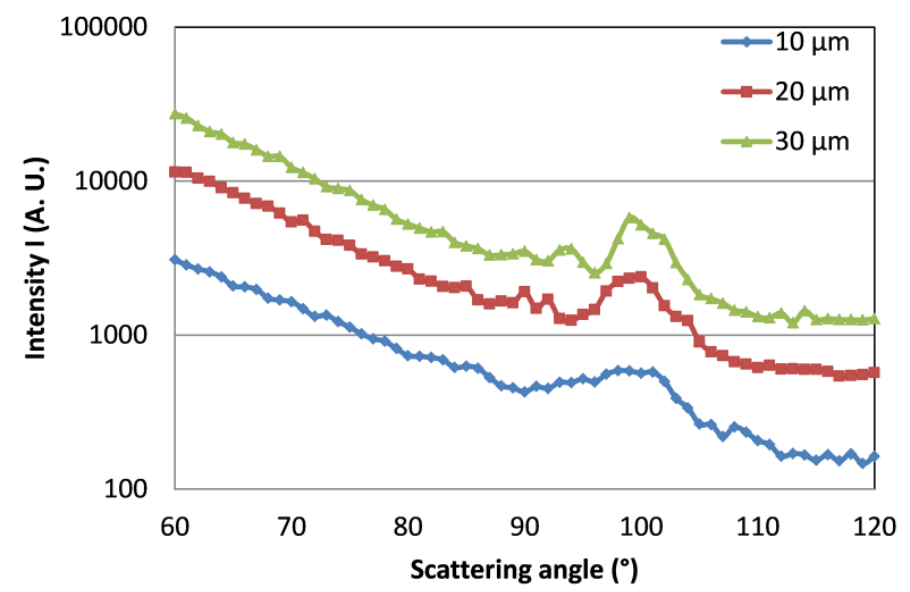

Figure 4. Variation of the scattered light intensity versus $\alpha$ for several log-normal droplet distributions (radius mean values equal to $10,20,30 \mu \mathrm{m}$ and standard deviation equal to $10 \%$ ). The calculations have been computed with an laser excitation wavelength of $532 \mu \mathrm{m}$ and a refractive index of 1,45.

As can be seen on the averaged images (Figure 5), for a scattering angle of $70^{\circ}$, a strong dissymmetry appears on the Mie image only. In each case $\left(70^{\circ}\right.$ and $\left.115^{\circ}\right)$, the laser sheet is coming from the left side. The left side of this image exhibits higher levels than the right one due to the angular sensitivity described in Figure 4 . At a scattering angle of $115^{\circ}$, the scattered image is roughly symmetrical about the injection axis. The choice of this angle results from a theoretical study to limit the angular effect. As expected, the fluorescence signal does not depend on the scattering angle because fluorescence is supposed to be an isotropic phenomenon. For the following experiments and investigations, a scattering angle of $115^{\circ}$ is chosen.

Scattering angle

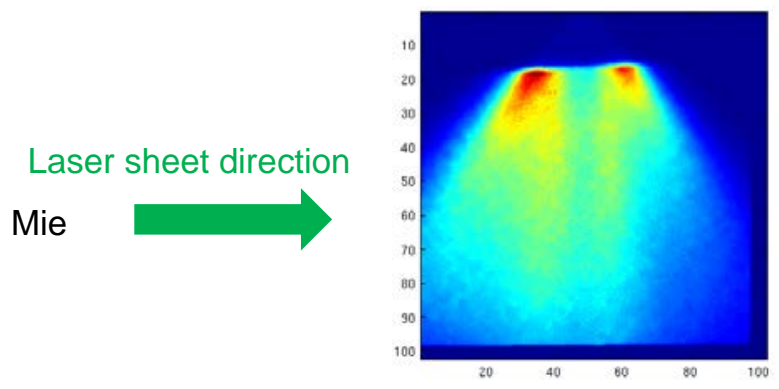

LIF
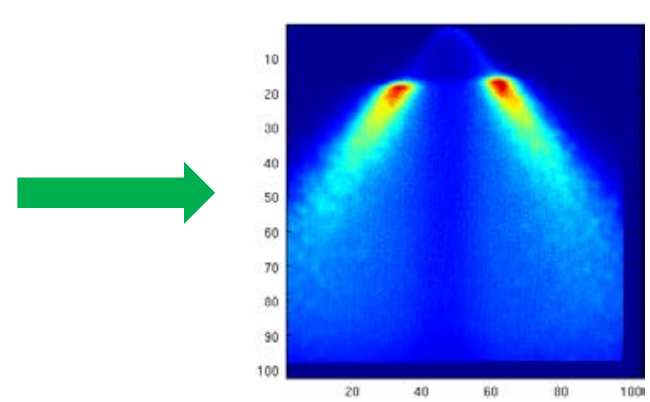

$115^{\circ}$
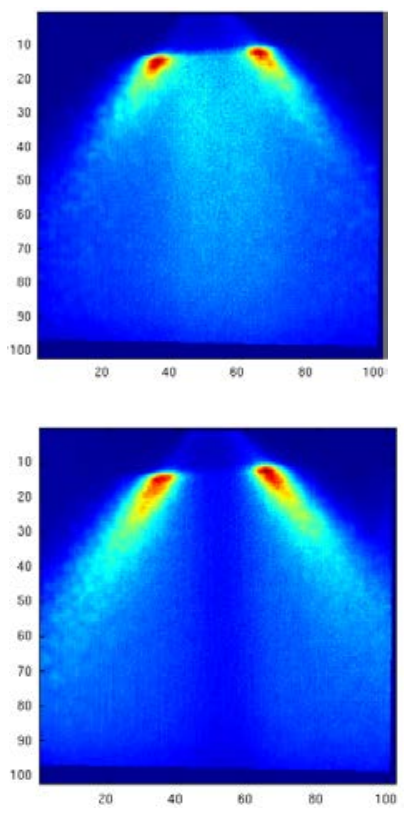

Figure 5. LIF/Mie averaged images for $\alpha=70^{\circ}$ and $115^{\circ}$. 
In both configurations, the intensity level of the Mie images seems overestimated inside the spray. Signal is detected where no droplets are present because the light scattered by a droplet illuminated by the laser may in turn be scattered by droplets that are not contained in the laser sheet. This multiple scattering effect is here mainly visible on the Mie images. Actually, when the density of droplets becomes high, a method based on spatial intensity modulation of the laser sheet, SLIPI [13] has proved effective to differentiate between the contributions arising from direct and multiple scattered light. The approach enables the removal of undesired diffuse light coming from the spray itself or from reflections in the background. This technique will be implemented in a near future on this spray.

Meanwhile, in order to reduce the contribution of multiple scattering in the signal, an image processing algorithm based on top hat filtering is applied to the images. The idea consists in filtering out the low frequency image information which corresponds to the surrounding background (from multiple scattering) while preserving the high frequencies. Since the spray is not too dense, individual droplets are visible in each image and can be extracted by this procedure which removes the contribution of the local background as illustrated in Figure 6 . The result is still noisy here and should be obtained from a longer sequence of images - at least a few thousand - in order to achieve a better description of the spray. The idea of high pass filtering in spray images was firstly shown by [14]. Some precautions have to be taken because this approach can also remove some relevant information. Anyhow, this method seems promising and requires further investigation and comparison with the SLIPI solution.
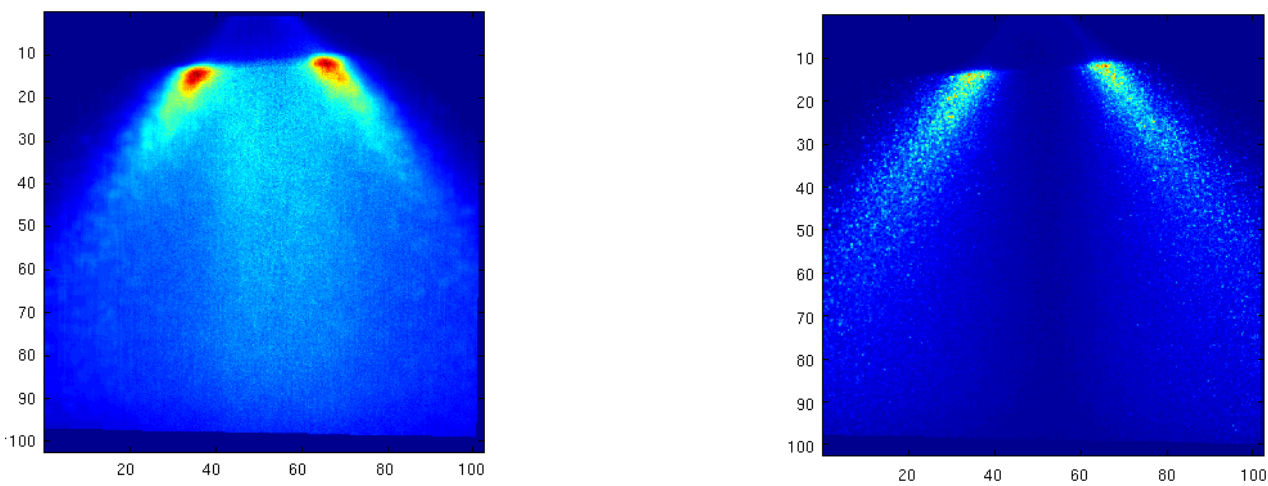

Figure 6. (a) Averaged scattered image (b) Averaged image from top hat filtered images of the sequence.

\section{Sauter Mean Diameter mapping}

The LIF/Mie ratio calculated from averaged images is presented in Figure 7. Although the Mie image needs to be improved, the result is qualitatively satisfactory: the map is roughly symmetrical about the injection axis, the minimum value of the ratio obtained in the spray core is three to four times smaller than the one at the edges, which is consistent with the SMD measured by PDA. Complementary PDA measurements will enable to achieve absolute PDS mapping.
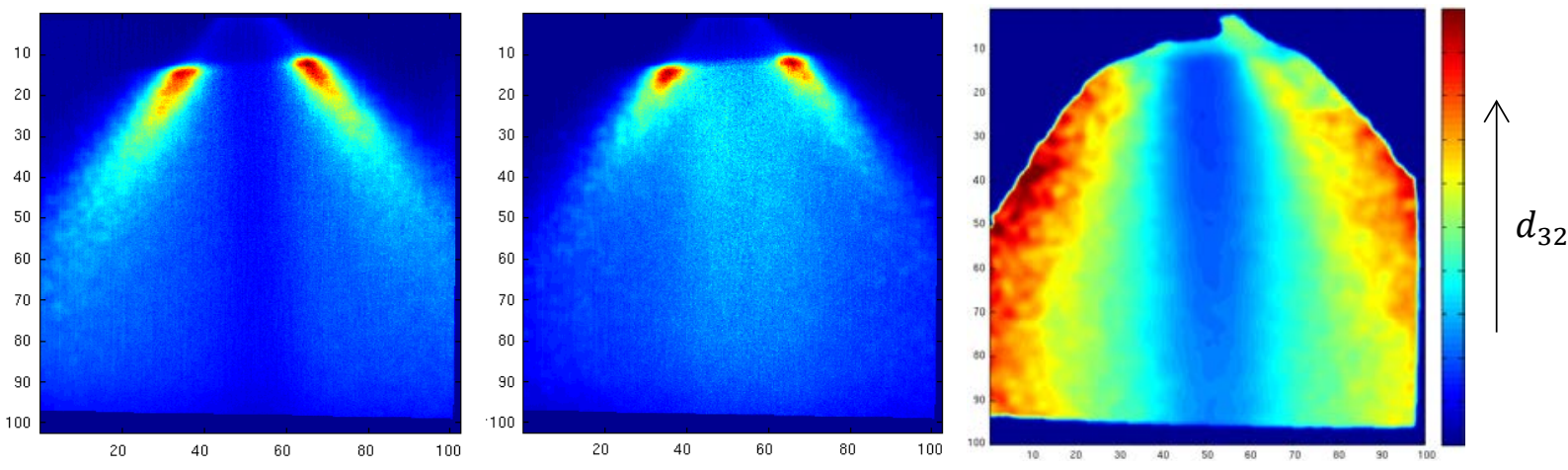

Figure 7. LIF and MIE images and their ratio proportional to the droplet SMD. 


\section{Conclusions and perspectives}

Planar Droplet Sizing is an imaging technique providing a spatially-resolved 2D map of the Sauter Mean Diameter in a few minutes contrary to point measurement techniques. A preliminary study enabled to adapt it to an ethanol spray by investigating the influence of optical and geometrical parameters on the measurement accuracy. The present investigation highlights its extension to Jet A-1 spray. The optical properties of liquid kerosene result in a strong absorption under UV excitation which makes the PDS technique unsuitable. The use of fluorescent dye appears necessary in order to increase the fluorescence signal of liquid kerosene under visible excitation at 532 $\mathrm{nm}$. The effect of the scattering angle is also investigated with theoretical and experimental approaches. A scattering angle close to $115^{\circ}$ seems to be adapted to prevent scattered light intensity variation within the field of view. Under these conditions, the LIF/Mie ratio exhibits the symmetry of the spray around the injection axis and provides a relevant order of magnitude of the SMD variations within the spray. Since scattered light images are altered by multiple scattering, an image processing algorithm is proposed to reduce this effect, prior to the implementation of the SLIPI technique.

\section{Acknowledgements}

This work is conducted in cooperation between ONERA and SAFRAN AIRCRAFT ENGINES (Safran Group). The authors gratefully acknowledge the ANRT (Association Nationale de la Recherche et de la Technologie) which supports part of the PhD.

\section{Nomenclature \\ $\sigma_{e t h}$ Ethanol surface tension $\left[\mathrm{kg} / \mathrm{s}^{2}\right]$. \\ $\sigma$ Kerosene surface tension $\left[\mathrm{kg} / \mathrm{s}^{2}\right]$. \\ $\rho$ Kerosene density $\left[\mathrm{kg} / \mathrm{m}^{3}\right]$. \\ $v$ Kerosene kinematic viscosity $\left[\mathrm{m}^{2} / \mathrm{s}\right]$.}

\section{References}

[1] Kamimoto, T., Yokota, H., and Kobayashi, H., 1989, SAE Transactions Journal of Engines, 98, paper\#890316.

[2] Domann, R., Hardalupas, Y., 2001, Particle \& Particle Systems Characterization, 18, pp. 3-11.

[3] Lempereur, C., Bodoc, V., Brettar, J. and Gajan, P., Sep. 2016, $15^{\text {th }}$ CFTL.

[4] Charalampous, G. and Hardalupas, Y., 2011, Applied Optics, 50 (9), pp.1197-1209.

[5] Charalampous, G. and Hardalupas, Y., 2011, Applied Optics, 50 (20), pp. 3622-3637.

[6] Brettar, J., Thesis, 2015, ONERA

[7] Mishra, Y., Kristensson, E. and Berrocal, E., 2014, Optics Express, 22 (4), pp. 4480-4492.

[8] Sankar, S. V., Inenaga, A. S. and Bachalo, W. D., July 20.-23. 1992, $6^{\text {th }}$ International Symposium on Applications of Laser Anemometry to Fluid Mechanics.

[9] Kawahara, N., Tomita, E. and Okano, H., 2012, ICLASS.

[10] Hardalupas, Y., Sahu, S., Taylor, A. MKP and Zarogoulidis, K., 2010, Experiments in fluids, 49 (2), pp. $417-$ 434.

[11] Orain, M., Baranger, P., Ledier, C., Apeloig, J. and Grisch, F., 2014, Applied Physics B, 116 (3), pp. $729-745$.

[12] Mees, L., Grehan, G. and Gouesbet, 2001, Optics Communications, 194, pp. 59-65.

[13] Berrocal, E., Kristensson, E., Hottenbach, P., Grünefeld, G. and Aldén M., May 15. - 18. 2011, 23rd Annual Conference on Liquid Atomization and Spray Systems.

[14] Smallwood, G. J, Guelder, O. L and Snelling, D. R,1994, $25^{\text {th }}$ Symposium on Combustion/ The Combustion Institute, pp. 371-379. 


\title{
Development steps of 2-color laser-induced fluorescence with MDR-enhanced energy transfer for instantaneous planar temperature measurement of micro-droplets and sprays
}

\author{
Johannes Palmer*, Manuel A. Reddemann, Valeri Kirsch, Reinhold Kneer \\ ${ }^{1}$ Institute of Heat and Mass Transfer, RWTH Aachen University, Germany \\ *Corresponding author: palmer@wsa.rwth-aachen.de
}

\begin{abstract}
A new method for instantaneous measurement of temperature, size and velocity of micro-droplets has been developed. The method is based on the well-known 2-color laser-induced fluorescence (2cLIF) technique, but uses a pulsed laser for 2-dimensional imaging without motion blur and an adjusted dye mixture for suppression of LIFMDRs by utilizing the MDR-enhanced energy transfer effect. This work presents the development steps that are necessary to verify feasibility of pulsed $2 D$-2cLIF-EET for micro droplet and hollow-cone spray applications.
\end{abstract}

\section{Keywords}

droplets, temperature, 2cLIF, morphology dependent resonances, Pyrromethene 597-8C9, Oil Blue N

\section{Introduction}

Temperature measurements of micro droplets are an important tool to understand various physical processes, e.g. mixture formation of fuel and air in an engine's cylinder. Most contact-free temperature measurement approaches for micro droplets are based on the so called 2-color laser-induced fluorescence (2cLIF) thermometry method developed at LEMTA [1]. The technique uses a fluorescence dye dissolved in a liquid of interest. After excitation by a continuous wave $(\mathrm{CW})$ laser, the dye re-emits absorbed laser light as spontaneous fluorescence with temperature dependent intensity ${ }^{1}$ (Fig 1, A). Fluorescence intensity is evaluated in two wavelength bands (two colors): one wavelength band with a strong and one with weak temperature dependence. The ratio of both signals remains temperature sensitive, but is ideally independent of local and temporal fluctuations in laser light excitation, droplet size and dye concentration.[1] Fluorescence from two separate wavelength bands may be recorded by different types of detector-configurations [2]:

- OD-2cLIF uses two photomultiplier tubes (PMT) for point-wise fluorescence detection (first shown by [1]).

- 1D-2cLIF is based on a spectrograph coupled to an intensified CCD. The detector's entrance slit allows one dimensional fluorescence detection. Fluorescence bands can be flexibly selected during post-processing [2, 3]. As a CW laser is used for excitation, recorded images are affected by motion blur.

- 2D-2CLIF uses two CCDs for two dimensional (planar) fluorescence detection. Motion blur becomes a major issue due to long CCD exposure duration and processes can only be investigated on average: e.g. images of a micro droplet chain appear jet-like shaped with the width of the droplets [4] or in case of spray investigations, turbulent effects such as vortices are lost [5].

To explore more specific types of problems, basic 2cLIF has been continuously extended or modified: e.g. different types of fluorophores for various liquids $[6,7]$ or a third PMT for an additional wavelength band that accounts for self-absorption effects of a single dye [8]. More interestingly, latest 2cLIF publications consider a pulsed laser for fluorescence excitation [2, 9, 10]. Pulsed lasers solve the issue of motion blur. In case of a planar detectorconfiguration, instantaneous measurements of temperature and size are possible. However, high excitation power of a pulsed laser inevitably leads to so called morphology dependent resonances (MDRs) inside micro droplets $[3,11]$. MDRs occur regardless of the presence of any fluorescent dye. If a fluorescent dye is present, its emission spectrum is changed by the MDRs and reliable 2cLIF thermometry is prevented [2, 12, 13]. The term MDR describes light being trapped inside a droplet due to almost total internal reflection at the droplet's inner surface and going into resonances. The droplet acts as micro-cavity and causes constructive interference of light waves with an increased photon density of state [11]. A higher density of state increases the probability of nonlinear optical processes. In fact, fluorescence starts to resonate during MDR occurrence as well. This phenomenon is called MDR-lasing (or simply lasing) and is caused by the increased density of state. The dye's cross-section becomes increased and stimulated fluorescence emission starts as soon as the cavity round-trip gain succeeds cavity losses due to leakage and absorption [14]. This is always the case for high excitation power provided by a pulsed laser. The process of lasing is represented in images by ring-like bright structures at the droplet surface with two additional opposing bright spots in case of plane wave illumination (Fig. 1, C). The bright spots are a consequence of the super-position of light circling in standing waves around the droplet surface.[14] Modes of light resonances become visible at specific frequencies in the dye's emission spectrum (Fig 1, B), depending on droplet size [15], physical properties [16] and excitation power $[15,17]$.

${ }^{1}$ 2cLIF-theory is specifically formulated for spontaneous fluorescence emission of a single dye only. 
A spontaneous emission of droplets $\lambda_{11} \lambda_{12} \lambda_{21} \lambda_{22}$

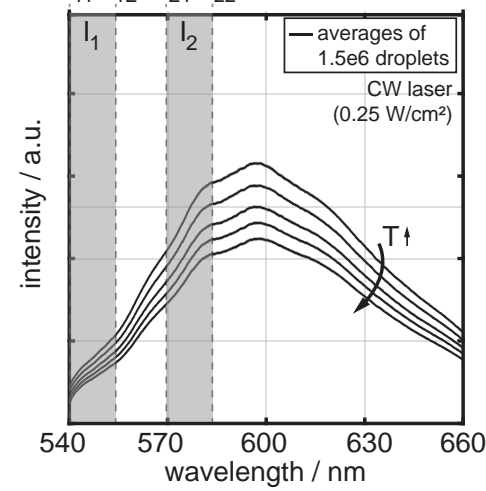

B MDRs-lasing of droplets

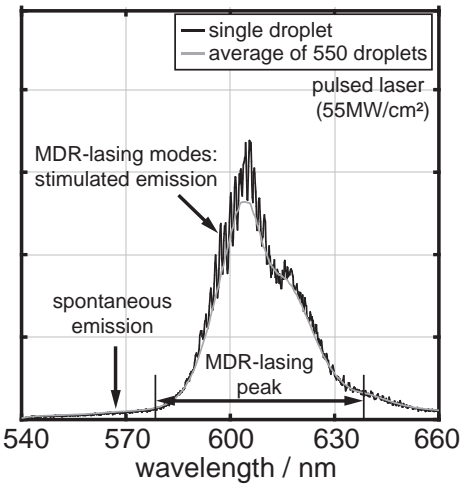

C droplet image with MDRs

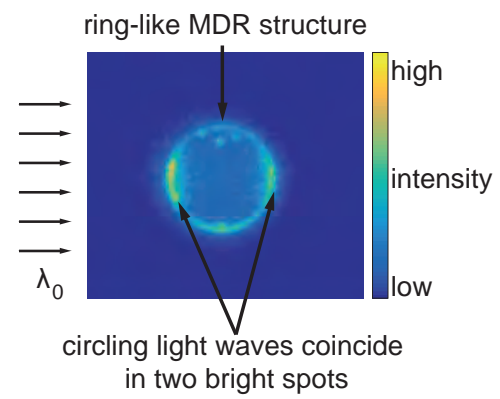

Figure 1. Fluorescence spectra and images of $70 \mu \mathrm{m}$ droplets consisting of PM597 dissolved in Ethanol (10 $\mu \mathrm{M})$. Details about the measurement setup are listed at a later section. Diagram A: Illumination by a CW laser causes spontaneous emission. Wavelength regions $\left(I_{1}, I_{2}\right)$ for 2cLIF thermometry are highlighted. Diagram B: Pulsed excitation causes MDRs. Fluorescence spectra become a super-position of spontaneous and stimulated emission. A single droplet spectrum allows identification of MDR modes. Image C: MDR-lasing appears as ring-like structure with two additional bright spots.

MDR-lasing makes 2cLIF thermometry unfeasible, especially for planar detection, where bright intensity peaks limit the usability of intensified CCDs. In order to clear the emission spectrum of pyrromethene 597-8C9 (PM597) from MDR-lasing, Perrin et al. suggest using an additional dye called Oil Blue N (OBN) [13]. The two-dye combination allows MDR-enhanced energy transfer (EET) from one dye to another. Energy that would originally be spend on PM597-lasing is transferred to OBN instead. In turn, lasing occurs for OBN at higher wavelengths. Spectroscopic pulsed 1D-2cLIF measurements approve this theory and also show that pulsed 2cLIF thermometry is feasible.[2]. Until now, pulsed 2D-2cLIF with MDR-enhanced energy transfer has not been tested on droplets or sprays. However, due to the importance of temperature determination of sprays, more simple 2cLIF setups have been tested: Occurrence of MDRs has only been considered in a single publication [12], where liquid spray temperature is determined point-wise using CW-OD-thermometry. The same type of setup has been used in numerous approaches afterwards, but without consideration of MDR-lasing (e.g. [18]). The average temperature field of a hollow cone spray has been measured by Mishra et al. [5] using CW-2D-2cLIF in combination with SLIPI ${ }^{2}$. MDR-lasing has not been discussed in that work. Only a single pulsed 2D-2cLIF approach has been found in commonly available literature [9]. The pulsed laser that is used in the approach definitively causes MDR-lasing of the fluorophor, but the phenomenon has not been considered at all.

In the following, basic 2cLIF theory is explained and experimental setups are described. Afterwards, three development steps are presented, describing how pulsed 2D-2cLIF with MDR-enhanced energy transfer (pulsed 2D2cLIF-EET) is designed: i) Dyes need to be selected for targeted MDR-enhanced energy transfer and tested in well defined droplet chain experiments using 1D-spectroscopy. ii) The 2cLIF setup is upgraded with a planar detectorconfiguration (pulsed 2D-2cLIF-EET). The setup is tested in well-defined droplet experiments. iii) The new setup is put to the test in with a complex hollow cone-spray under engine-like conditions.

\section{Theory of 2CLIF thermometry for spontaneous emission of a single dye}

$2 \mathrm{cLIF}$ thermometry requires a fluorescent dye with wavelength dependent temperature sensitivity. Fig. 1(A) shows fluorescence emission of pyrromethene 597-8C9 for a set of temperatures measured in bulk. The dye's spontaneous emission spectrum $I(\lambda)\left[\mathrm{W} / \mathrm{m}^{2}\right]$ can be described by eq. 1 . The equation contains an Arrhenius approach to model temperature dependence of the absorption cross-section and quenching effects [1]:

$$
I(\lambda)=K_{\mathrm{opt}}(\lambda) K_{\mathrm{spec}}(\lambda) V I_{0} C \exp \left[\frac{\beta(\lambda)}{T}\right]
$$

The equation contains optical and spectral constants $K_{\mathrm{opt}}(\lambda)$ [-] and $K_{\mathrm{spec}}(\lambda)$ [1/mol], the illuminated measurement volume in the focal plane $V\left[\mathrm{~m}^{3}\right]$, excitation intensity $I_{0}\left[\mathrm{~W} / \mathrm{m}^{2}\right]$, temperature $T[\mathrm{~K}]$ and the dye's molecular concentration $C\left[\mathrm{~mol} / \mathrm{m}^{3}\right.$ ] and temperature sensitivity $\beta(\lambda)[\mathrm{K}][6]$. For a wavelength range from $\lambda_{i 1}$ to $\lambda_{i 2}$, fluorescence $I_{i}$ is described by the integral $[1,6]$

$$
I_{i}=\int_{\lambda_{i 1}}^{\lambda_{i 2}} K_{\mathrm{opt}}(\lambda) K_{\mathrm{spec}}(\lambda) V I_{0} C \exp \left[\frac{\beta(\lambda)}{T}\right] \mathrm{d} \lambda=K_{\mathrm{opt}, i} K_{\mathrm{spec}, i} V I_{0} C \exp \left[\frac{a_{i}}{T^{2}}+\frac{b_{i}}{T}\right]
$$

where $\beta(\lambda)$ is approximated by a second order polynomial with coefficients $a_{i}$ and $b_{i}$. The 2cLIF thermometry approach uses the ratio $R(T)$ [-] of two different wavelength regions $\left(I_{1}, I_{2}\right)$, to cancel out fluctuating values such as excitation intensity $I_{0}$, measurement volume $V$ and dye concentration $C$ [1]:

$$
R(T)=\frac{I_{2}}{I_{1}}=\frac{K_{\mathrm{opt}, 2} K_{\text {spec }, 2}}{K_{\mathrm{opt}, 1} K_{\mathrm{spec}, 1}} \exp \left[\frac{a_{2}-a_{1}}{T^{2}}+\frac{b_{2}-b_{1}}{T}\right]=\exp \left[\frac{a_{21}}{T^{2}}+\frac{b_{21}}{T}+c\right]
$$

\footnotetext{
${ }^{2}$ Structured Laser Illumination Planar Imaging
} 
This simplified equation contains only three unknown parameters: temperature sensitivities $a_{21}\left[\mathrm{~K}^{2}\right], b_{21}[\mathrm{~K}]$ and setup constant $c[-]$. All parameters are determined by calibration at known temperatures. Wavelength bands are selected by evaluating the measured temperature sensitivity $\beta(\lambda)$ of a dye (see [6, 2] for details). One wavelength band is selected as temperature independent reference $\left(I_{1}\right.$ in Fig. $\left.1(\mathrm{~A})\right)$, the other wavelength band must be strongly temperature dependent $\left(I_{2}\right)$.

The theory above is derived for spontaneous fluorescence emission of a single dye only. This boundary condition is not necessarily given for pulsed laser excitation of dye-seeded micro droplets due to occurrence of MDR-lasing. Nevertheless, our latest publication has proven validity of this theory for pulsed excitation combined with a two dye MDR-enhanced energy transfer [2]. Therefore, validity of 2cLIF theory is assumed to be given for this works pulsed $2 D-2 c L I F-E E T$ setup as well.

\section{Experimental setups}

Four slightly different experimental setups are used for the pulsed planar 2cLIF development: i) \& ii) CW \& pulsed $1 \mathrm{D}$ spectroscopy of droplets iii) pulsed 2D-2cLIF of droplets and iv) pulsed 2D-2cLIF of hollow-cone sprays.
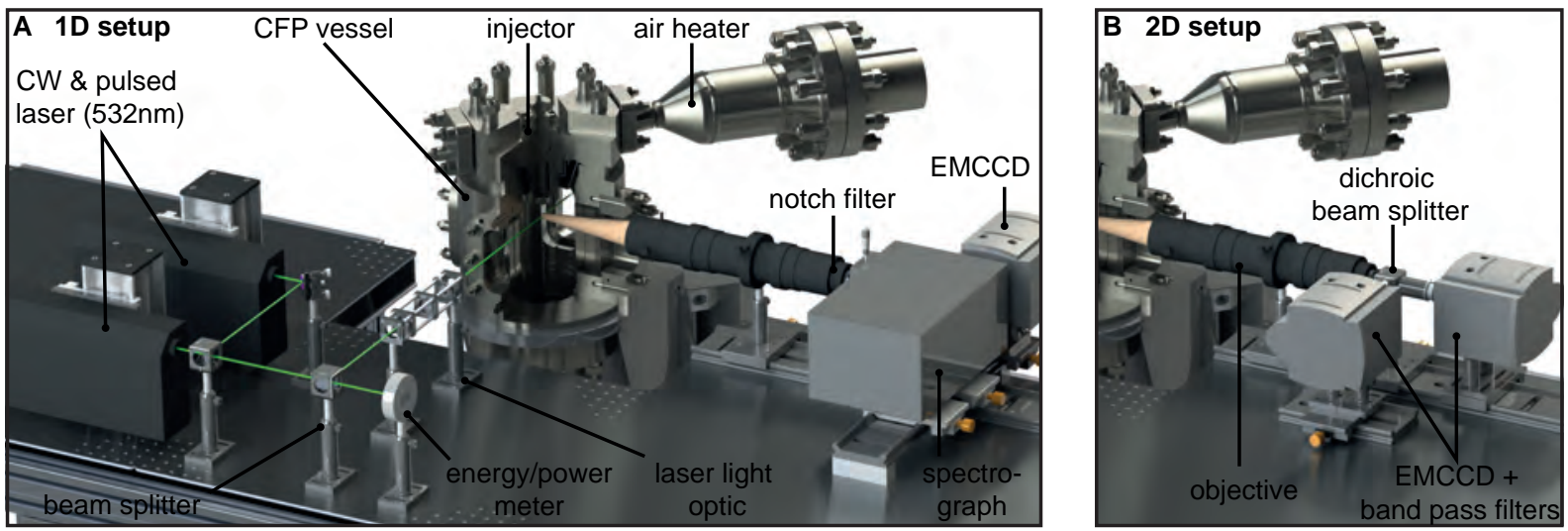

Figure 2. Experimental setup contains either a pulsed or CW laser for fluorescence excitation. Before the beam reaches the droplets, its cross section is expanded to about the size of the detector. After passing a high pass filter, a fluorescence image is magnified with a far-field microscope. The detector is a spectrograph that is connected to an electron multiplied CCD.

Micro droplets are produced by a vibrating orifice generator with an outlet diameter of $35 \mu \mathrm{m}$. Droplets with a size of approximately $70 \mu \mathrm{m}$ consist of Ethanol and are created with a constant frequency of $77 \mathrm{kHz}$. Droplets are injected into quiescent air with a pressure of 2.5 bar, corresponding to a measured droplet velocity of about $10.5 \mathrm{~m} / \mathrm{s}$. Liquid temperature is controlled by a heating cartridge with an integrated thermocouple. Cooling is provided by a surrounding water circuit. The droplet generator is placed in the central position of the head of a heatable constant pressure-flow (CPF) vessel (Fig. 2, A). The CPF chamber is only required for spray experiments. Ambient conditions remain atmospheric during the droplet experiments (293 K, 1 bar). The droplet measurement position lies $6 \mathrm{~mm}$ under the generator's outlet. This proximity guarantees isothermal conditions of liquid inside the injector and droplets. During spray experiments, a piezo-driven hollow-cone injector replaces the droplet generator. Ethanol as fuel is injected with a pressure of 150 bar and an injection duration of $2 \mathrm{~ms}$. The injector is temperature controlled at $305 \mathrm{~K}$ by a water circuit. The true injector outlet temperature is assumed to be higher due to heated air flowing $(<0.1 \mathrm{~m} / \mathrm{s})$ through the chamber. Experiments are conducted with an ambient temperatures of $400 \mathrm{~K}$ and an ambient pressure of 10 bar.

Table 1. Optical setting of the experimental setups.

\begin{tabular}{c|cccc}
\hline & CW 1D-spec. & pulsed 1D-spec. & pulsed 2D-2cLIF & pulsed 2D-2cLIF \\
droplets & droplets & droplets & spray \\
\hline illumination spot & circular beam & circular beam & circular beam & light sheet \\
illumination dimension & $\varnothing 7 \mathrm{~mm}$ & $\varnothing 2.7 \mathrm{~mm}$ & $\varnothing 2.7 \mathrm{~mm}$ & $0.3 \mathrm{~mm} \times 50 \mathrm{~mm}$ \\
$I_{0}$ & $0.25 \mathrm{~W} / \mathrm{cm}^{2}$ & $55 \mathrm{MW} / \mathrm{cm}^{2}$ & $55 \mathrm{MW} / \mathrm{cm}^{2}$ & $55 \mathrm{MW} / \mathrm{cm}^{2}$ \\
exposure time & $1 \mathrm{~s}$ & $10 \mu \mathrm{s}$ & $10 \mu \mathrm{s}$ & $10 \mu \mathrm{s}$ \\
image height & $2.15 \mathrm{~mm}$ & $2.15 \mathrm{~mm}$ & $2.15 \mathrm{~mm}$ & $47.89 \mathrm{~mm}$ \\
image width & - & - & $2.15 \mathrm{~mm}$ & $47.89 \mathrm{~mm}$ \\
magnification & 6.2 & 6.2 & 6.2 & 0.61 \\
image resolution & $0.477 \mathrm{px} / \mu \mathrm{m}$ & $0.477 \mathrm{px} / \mu \mathrm{m}$ & $0.477 \mathrm{px} / \mu \mathrm{m}$ & $0.0467 \mathrm{px} / \mu \mathrm{m}$ \\
wavelength scale & $7.24 \mathrm{px} / \mathrm{nm}$ & $7.24 \mathrm{px} / \mathrm{nm}$ & - & - \\
images per condition & 20 & 50 & 200 & 30 (injections) \\
\hline
\end{tabular}

A continuous wave (CW) and a pulsed Nd:YAG laser (pulse duration: $4 \mathrm{~ns}$ ) are available for fluorescence excitation, 
both operating at a wavelength of $532 \mathrm{~nm}$ and identical polarization (Fig. 2, A). The CW laser is applied for spectroscopic reference experiments of micro droplet chains only, where spontaneous fluorescence emission must be guaranteed. The pulsed laser is operated differently for micro droplet chain and spray investigations. Micro droplets are excited with a laser beam (flat-top intensity profile) and a pulse repetition rate of $2.5 \mathrm{~Hz}$. The laser beam is reshaped to a light sheet for the spray experiments and synchronized to the injection events. The light sheet allows to illuminate a thin cut-out section of the spray's hollow-cone. The specific laser excitation intensity of the pulsed laser is kept constant during droplet chain and spray experiments. Changes of the specific excitation intensity due to a different illumination spot size (circular beam for droplet and light sheet for spray) are compensated by alteration of the laser output energy. Laser output power is monitored continuously using a beam splitter that separates $15 \%$ of the laser output intensity (laser stability deviation $<3 \%$ ). See table 1 for details of the laser configuration.

Fluorescence of micro droplets is collected by a far-field microscope (Infinity optics K2/SC) (Fig. 2, A). A Notch filter (Thorlabs NF533-17) removes scattered laser light from the spectrum. The spectroscopic setup that is used for droplet investigations only, uses a spectrograph (Princeton Instruments, Acton SP 2300i) attached to an electron multiplied CCD (EMCCD, Andor iXon3 DU-888) for fluorescence evaluation. The spectrograph's entrance slit is opened far enough to collect light from entire droplets $(1 \mathrm{~mm})^{3}$. The 2D-2cLIF imaging setup uses two identical EMCCDs (model as before) for fluorescence detection (Fig. 2, B). After passing the Notch filter, fluoresce is separated at $573 \mathrm{~nm}$ wavelength using a dichroic beam splitter (Chroma ZT561rdc). Each light path is further band pass filtered before reaching the EMCCDs (Semrock 549/15 nm BL, Semrock, 586/15 nm BL). Band pass filters (wavelength bands) are selected in accordance with our previous investigations of PM597 [2]. EMCCDs amplify detected light with a gain of $200 \mathrm{x}$ and are cooled to $178 \mathrm{~K}$ for an optimum signal to noise ratio. Spray investigations require a larger field of view and thus a switching of the microscope for a macro objective (Zeiss Makro-Planar T 100mm ZF.2). Depending on the particular setup, EMCCDs record a different number of images to justify reproducibility. Image numbers and details of the optical setup are listed in table 1.

\section{Development steps of pulsed 2D-2cLIF with MDR-enhanced energy transfer}

Step I: Dye selection and 1D-spectroscopic verification of MDR-enhanced energy transfer for droplet chains Pyrromethene 597-8C9 (PM597) is selected as temperature sensitive fluorescent dye for all experiments. The dye is dissolved in Ethanol with a molar concentration of $10 \mu \mathrm{M}^{4}$. PM597 is known for its low probability of self-absorption [6], sufficient solubility and an invariable emission spectrum for many liquids [7]. Fig. 1(A) illustrates the emission behavior of the dye for spontaneous emission at different temperatures and the spectrum change due to MDR-lasing (B). In order to clear the temperature dependent wavelength region of PM597 from lasing, MDR-enhanced energy transfer is enabled by adding Oil Blue $\mathrm{N}(\mathrm{OBN})$ with a concentration of also $10 \mu \mathrm{M}$ [2].

A single dye absorbance (cuvette)

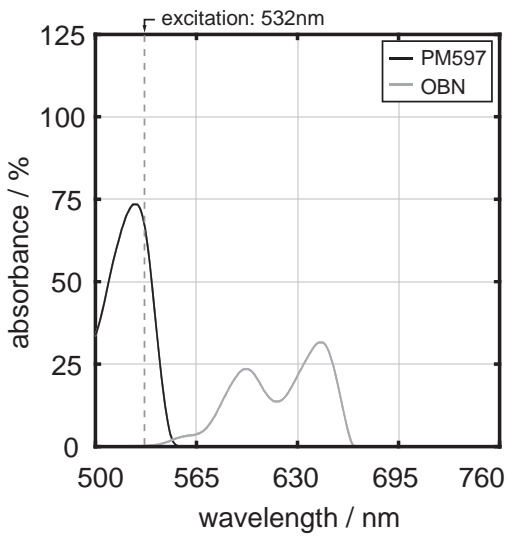

B single dye emission (cuvette)

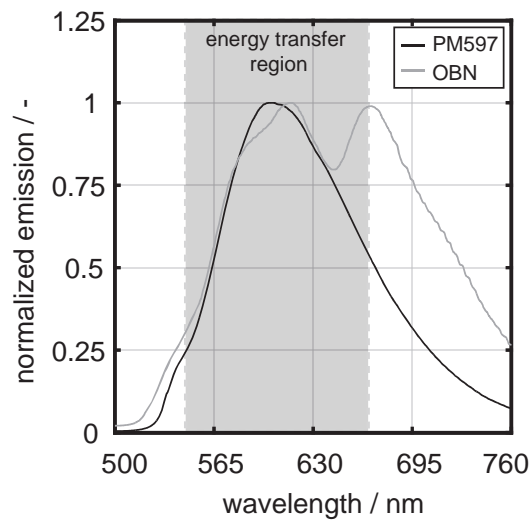

C average droplet chain emission

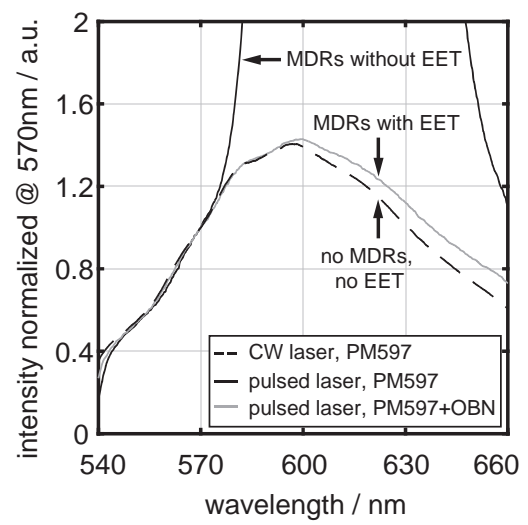

Figure 3. In bulk absorbance (A) and emission (B) spectra of pyrromethene 597-8C9 and Oil Blue N dissolved in Ethanol (each $10 \mu \mathrm{M}$. Diagram C: droplet chain fluorescence emission of an PM597-OBN mixture with and without MDR-EET in comparison with an MDR-free PM597 reference spectrum (operating conditions are listed in the previous chapter). Droplet results are averaged.

OBN is hardly excited by laser light (Fig. 3, A), such that energy absorption occurs almost exclusively by energy transfer from PM597 [2]. However, the shown emission spectrum of OBN (Fig. 3, B) is based on an excitation wavelength of $532 \mathrm{~nm}^{5}$. An energy transfer of both dyes takes place in the overlap region of the emission spectrum of PM597 and the absorption spectrum of $\mathrm{OBN}^{6}$ (compare Fig. $3 \mathrm{~A} \& \mathrm{~B}$ ). The major part of transferred energy is subsequently quenched or self-absorbed and only a minor part of energy is re-emitted. OBN emission based on energy transfer produces a much more red-shifted spectrum compared to the graph shown in Fig. 3(B), because dye excitation is not monochromatic and occurs at wavelengths much higher than $532 \mathrm{~nm}$. An energy transfer occurring

\footnotetext{
${ }^{3}$ The relatively large slit-width causes spectral averaging that needs to be remembered during result interpretation.

${ }^{4}$ The unit $\mu \mathrm{M}$ is a common abbreviation for $\mu \mathrm{mol} / \mathrm{L}$.

${ }^{5}$ Showing normalized emission removes the fact that absolute emission of OBN is almost negligible compared to PM597.

${ }^{6}$ Energy transfer happens only by radiation not by Förster resonant energy transfer, since molecule distances are large (low concentrations).
} 
in a droplet would cause an emission of OBN that includes a high amount of energy originally responsible for PM597lasing (compare Fig. 1, B), but becomes transferred to OBN before visible emission. As a consequence, PM597lasing is suppressed and OBN starts lasing instead, whereas lasing wavelengths are significantly red-shifted. One important condition for complete suppression of PM597-lasing is a targeted matching of both dye concentrations [2]. The selected concentrations of $10 \mu \mathrm{M}$ per dye, or rather their ratio of one, secure similar absorption crosssections and thus comparable probabilities of absorption. Stimulated emission of PM597 becomes interrupted and no cavity-round trip is completed.

Fig. 3(C) shows a comparison of fluorescence emission of droplets with and without energy transfer ${ }^{7}$. The emission spectrum with MDR-EET (pulsed laser, PM597+OBN) proves complete suppression of PM597-lasing. Due to the energy transfer, emission is a super-position of both dyes. Up to wavelengths of $600 \mathrm{~nm}$, the normalized emission spectrum is identical to spontaneous emission of PM597 that is based on CW excitation. Negligible OBN emission is caused by three facts: i) comparably weak emission of PM597, ii) weak absorption of OBN and iii) a strong self-absorption tendency of OBN. For wavelengths higher than $600 \mathrm{~nm}$, energy transfer conditions are different. Emission of OBN becomes visible in addition to PM597's fluorescence. Lasing that occurs for OBN now, is not visible in the given diagram, but definitively takes place for wavelengths higher than $660 \mathrm{~nm}^{8}$.

Validity of 2cLIF theory for the chosen dye concentrations and subsequent energy transfer has been proven in our previous investigations [2]: although EET causes saturation of spontaneous fluorescence emission in the 2cLIF wavelength bands, self-similarity of the normalized emission spectrum remains and thus 2cLIF theory yields. This conclusion is valid regardless of the chosen excitation intensity and droplet size. [2]

\section{Step II: Applicability of pulsed 2D-2cLIF with MDR-enhanced energy transfer for micro droplets}

Spectroscopic investigations of the chosen dye combination (PM597 and OBN, both seeded with $10 \mu \mathrm{M}$ in Ethanol) proof successful shifting of MDR-lasing from PM597 to OBN and validity of 2cLIF theory. In this development step, the spectrograph is replaced by two EMCCDs to enable imaging of temperature fields (Fig. 2, B). Stereo camera vision requires calibration to secure an identical field of view. Images of a reference target are matched using bi-linear interpolation. Calibration results are applied to every set of recorded images. The calibration is highly sensitive to miss-alignments, such that mistakes on the sub-pixel scale result in severe measurement errors (see [4] for details). After successful camera calibration, images of both devices are divided pixel by pixel. Camera calibration is followed by a temperature calibration that requires measurements of average droplet intensity ratios for a set of known temperatures under isothermal conditions. Average droplet ratios are determined as the follows: i) Droplets of each ratio-image are isolated and saved as images one after another in an array, whereas droplet centers are aligned. ii) The array is averaged to receive a locally resolved average droplet ratio-image. A following occurrence-refining ${ }^{9}$ improves the image quality at the droplet surface. iii) Further horizontal and vertical image averaging allows determining an overall droplet-ratio for each temperature (Fig. 4, B).

A average images of isothermal droplets

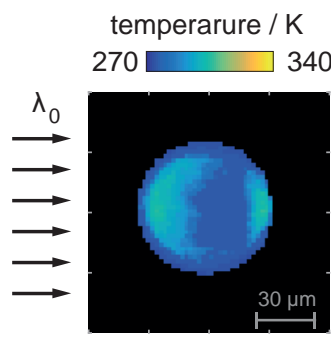

temperature: $285 \mathrm{~K}$

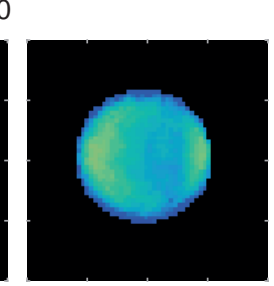

297 K

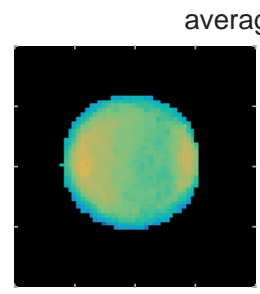

$309 \mathrm{~K}$ averages of 2700 droplets

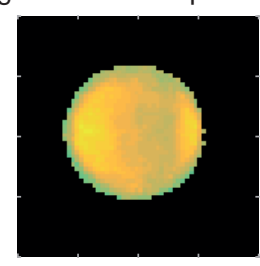

$321 \mathrm{~K}$
B overall temperature calibration

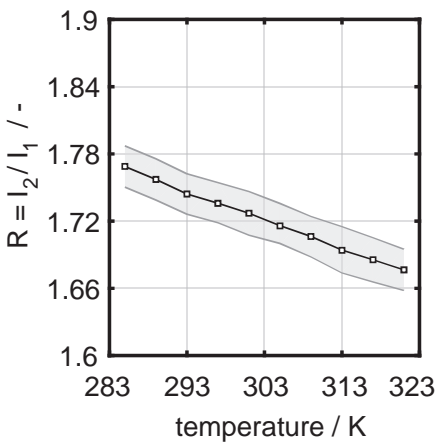

Figure 4. Temperature calibration results of the pulsed 2D-2cLIF-EET droplet investigation. Images (A): Average droplet temperature images under isothermal condition are affected by a nonphysical, but systematic temperature fluctuation across the droplet. The cause of this effect is still unknown. Diagram (B): The corresponding temperature calibration that is used to convert ratio into temperature images. Uses experimental operating conditions are listed in the section Experimerntal setups.

The measured temperature-ratio function is used to determine polynomial constants in eq. 3 . Eq. 3 is then applied pixel-wise to ratio-images to convert them into temperature fields. Fig. 4(A) shows average temperature-images of isothermal droplets. Although different temperatures can clearly be distinguished, an unexpected inhomogeneity pattern is evident for each measured temperature. The inhomogeneity explains the standard deviation of the calibration that corresponds to about 3 to $4 \mathrm{~K}$. Locally increased temperatures are found to be nonphysical, because the laser pulse itself causes only a negligible temperature change of $\Delta T=0.07 \mathrm{~K}$ for the given experimental configura$\operatorname{tion}^{10}$. The cause of the emerged inhomogeneity pattern is more likely caused by a locally changed laser excitation intensity that affects the fluorescence spectrum of one detection wavelength band stronger than the other. Excitation

\footnotetext{
${ }^{7}$ How to evaluate CW and pulsed 1D-spectroscopic images can be found at [2].

${ }^{8}$ This fact will be shown in a future work.

${ }^{9}$ Averaged pixel values that are based on information of less than half of the total number of evaluated droplets are sorted out.

${ }^{10}$ This value can be determined by a simple approximation.
} 
intensity may be reduced due to strong light absorption by the dyes on the light entering side of the droplet (concentration effect). On the opposing side, laser intensity may be increased due to focusing effects as the droplet acts as lens (hypothesis according to [19]). Nevertheless, the cause for changes in the fluorescence emission spectrum due to fluctuations in laser intensity remains unknown and requires further research.

In total, pulsed 2D-2cLIF-EET of micro droplets shows convincing results. Although droplet temperature-images show evidence of an unexplained physical mechanism, average droplet temperature can be reliably measured. Applying pulsed 2D-2cLIF-EET for a macroscopic case, i.e. a spray, makes influences of inhomogeneities inside droplets negligible. Reduced optical magnification makes droplets appear as objects smaller than a single pixel with a single average intensity value. Moreover, the amount of droplets and the depth of field contribute to a further averaging. Applying pulsed 2D-2cLIF-EET for a complex spray seems therefore feasible.

\section{Step III: Applicability of pulsed 2D-2cLIF with MDR-enhanced energy transfer for hollow-cone sprays}

In the third development step, pulsed 2D-2cLIF-EET is applied to a complex macroscopic droplet phenomenon: a poly-disperse, evaporating hollow-cone spray. Dye seeded Ethanol is injected into heated and pressurized air (400 K, 10 bar). EMCCDs record single-shot images for four different moments of the injection (from $0.5 \mathrm{~ms}$ to $2.0 \mathrm{~ms}$ in steps of $0.5 \mathrm{~ms}$ ). For each of the four moments, 30 consecutive fuel injections are recorded with a time separation of five seconds. After stereo-camera calibration, temperature calibration is conducted by placing a closed cuvette filled with fuel in the focal measurement plane. Step-wise increased ambient air temperature heats up the liquid to isothermal and known temperatures (up to the normal boiling point of Ethanol, $351 \mathrm{~K}$ ). Measurements lead to a linear calibration (similar to Fig. 4, B), but with low standard deviation. An additional calibration is performed for varying ambient pressure, showing no intensity-ratio change for the tested range up to 15 bar.

A

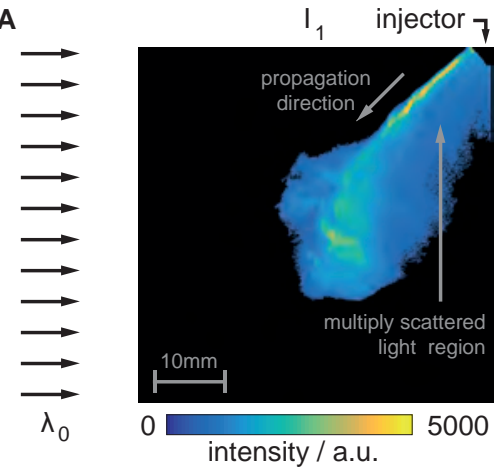

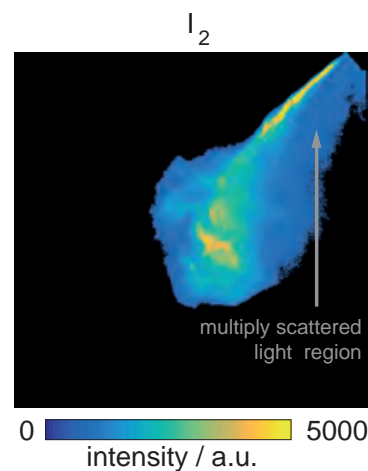

intensity / a.u.
B

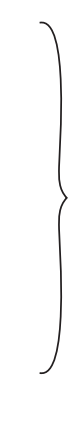

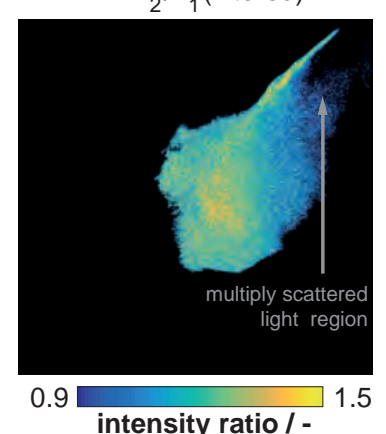

Figure 5. A shows both single-shot intensity images of the hollow-cone spray 2 ms after start of injector energizing. Dense spray regions are identified by high intensity values. Disruptive multiply scattered light causes severe "noise". B shows the corresponding single-shot ratio image. Ratio values below 0.9 are nonphysical and thus sorted out. Operating conditions: 150 bar injection with a duration of $2 \mathrm{~ms}$; atmosphere with 10 bar and $400 \mathrm{~K}$.

Recorded images are evaluated with regard to temperature only. The post-processing is simplified compared to the droplet evaluation, because no droplets need to be isolated. Fig. 5(A) shows both single-shot intensity images of the spray and Fig. 5(B) their corresponding ratio. Since rotational symmetry of the spray is assumed, only half the illuminated cut-out section is recorded ${ }^{11}$. Both intensity images are affected by multiply scattered light, detected as "noisy" fluorescence on the inside of the hollow-cone. Multiply scattered light is a common phenomenon of hollowcone sprays and can only be suppressed by applying SLIPI ${ }^{12}$. The ratio of both intensity images is affected by multiply scattered light as well. Division of two "noisy" intensity values results in a ratio of in average one. According to the temperature calibration, a ratio value of one corresponds to a fluid temperature of about $350 \mathrm{~K}$ and thus a valid temperature $^{13}$. Therefore, regions of multiply scattered light cannot be clearly distinguished from the actual spray. In order to minimize influences from multiply scattered light, ratio images are filtered. Pixels with ratio values lower than 0.9 are sorted out. This value has been chosen for two reasons: i) Corresponding temperature values of $365 \mathrm{~K}$ and above are too high to occur in the spray and thus, are nonphysical. This is obvious, when the temperature field of the multiply scattered light region is compared with the temperature values of the actual spray. ii) It is commonly known, how liquid spray formation has to look like in the near nozzle region without multiply scattered light. Using the ratio-threshold of 0.9 allows to cut-out regions where liquid is not supposed to be (compare fig. $5 \mathrm{~A}$ and $\mathrm{B}$ ).

Fig. 6 shows four average temperature-images for differernt moments of the fuel injection. Although images are refined by the ratio-threshold filter, regions of multiply scattered light are still visible as zones of nonphysical high temperatures. Temperature images allow identification of consequences of flow phenomena such as a temperature change due to a toroidal vortex (Fig. 6, C).

In the following, measured temperature fields are judged with regard to credibility. During mixture formation of the spray, droplets are expected to increase their temperature continuously ${ }^{14}$. However, spray images reveal the

\footnotetext{
${ }^{11}$ The injector is therefore located in the top right corner of the images.

${ }^{12}$ Structured Laser Illumination Planar Imaging, see [5] for CW-2D-2cLIF thermometry combined with SLIPI.

${ }^{13} \mathrm{Ambient}$ pressure of 10 bar causes an increased boiling point of Ethanol of $424 \mathrm{~K}$.

${ }^{14}$ The initial droplet temperature is assumed to be below adiabatic droplet temperature.
} 

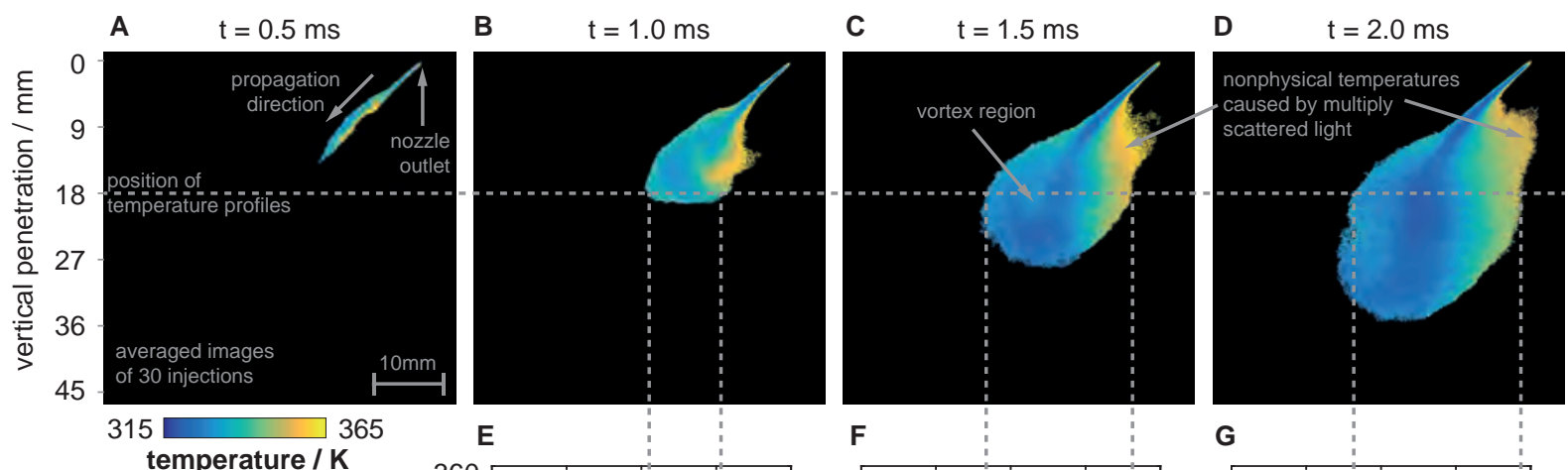

temperature / K

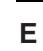

F
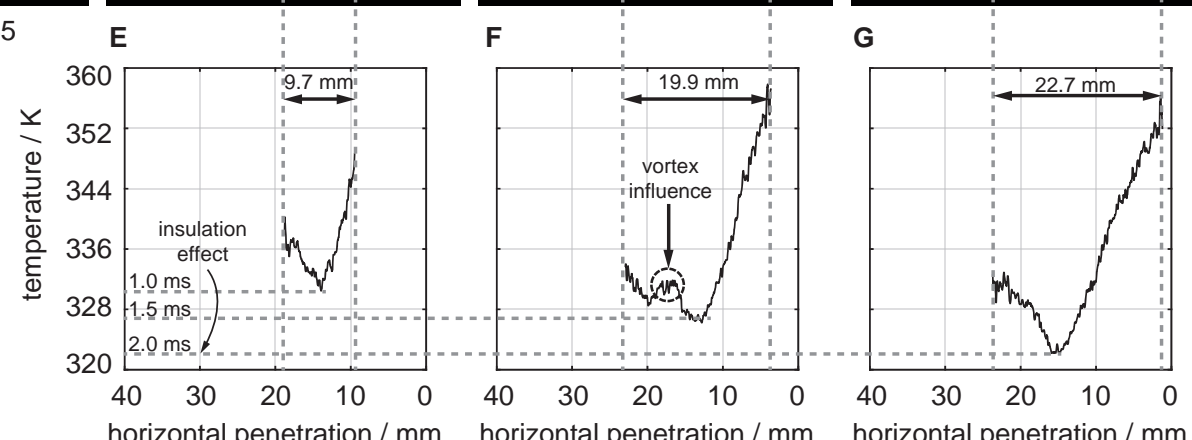

Figure 6. Average temperature images of the spray propagation in four time steps after start of injector energizing (A-D). All images show signs of multiply scattered light on the cone's inside. Comparison of different time steps needs consideration of the fact that not droplets, but positions are followed over time. As the spray propagates, the spray-sheet-core becomes more and more insulated by liquid and vapor. As a consequence droplet heating is slowed down. Diagrams (E) to (G) quantify the spray's diffusion-limited mixture formation by comparing temperature profiles $18 \mathrm{~mm}$ below injector outlet. Operating conditions: 150 bar injection with a duration of $2 \mathrm{~ms}$; atmosphere with 10 bar and $400 \mathrm{~K}$.

opposite: a more or less isothermal spray-sheet-core that cools down with an advancing time after start of injection (Fig. $6 \mathrm{~A}$ to D). To resolve this contradiction, content of temperature-images must be interpreted: Comparing images of different times steps need consideration of the fact that only fixed positions are compared and not droplets over their lifetime. A thought experiment helps to understand this statement: Only one fixed position below the injector outlet shell be evaluated. Fuel droplets that arrive at this position are pushing away previous droplets, which become an insulating layer of liquid and vapor for the new droplets. Due to the insulation layer, no hot unsaturated air reaches the new droplets. Heating at the fixed position is therefore decelerated. Basically, this progress describes a diffusion-limited mixture formation, where evaporation is much faster than heating and air-vapor mixing is hindered. Occurrence of this mechanism depends on the type of nozzle and injection and ambient conditions.

Diagrams $E$ to $G$ of fig. 6 quantify the insulation-effect for the recorded spray temperature fields of this work. The diagrams show temperature profiles of the spray, evaluated $18 \mathrm{~mm}$ vertically below the injector outlet for three different time steps of the injection. All graphs show the coldest spray region in the center of the spray-sheet. Horizontal positions further distant from the sheet-core have higher temperatures, because the insulation layer is thinner. For a later moment of the injection ( $E$ to $F$ ), the spray-sheet becomes wider. A wider spray-sheet has a thicker insulation layer and thus a lower sheet-core temperature. The entire process is superimposed by horizontal movement of the spray-cloud and occurrence of a toroidal vortex that is positioned at the outer side of the hollowcone. Diagram $6(F)$ indicates the influence of the vortex as local temperature increase. The vortex transports hot unsaturated air inside the spray-sheet-core. A higher heat flux is assumed to increase droplet heating.

In total, temporal changes of the spray temperature seem to be reasonable. The overall temperature level is credible as well: Coldest spray temperatures are always above injection temperature (305 K). Highest spray temperatures (excluding regions of multiply scattered light) are below the boiling point of Ethanol ( $424 \mathrm{~K}$ at 10 bar) and more importantly below adiabatic droplet temperature of the selected ambient condition $(340 \mathrm{~K})^{15}$.

\section{Conclusions}

This work presents the development of a new temperature measurement method named pulsed planar two color laser-induced fluorescence thermometry with MDR-enhanced energy transfer or in short: pulsed 2D-2cLIF-EET. The method is based on a previous 1D-spectroscopic version that is specifically designed for droplet applications, where morphology dependent resonances (MDRs) are suppressed to enable a reasonable temperature measurement. Novelty of this work is the planar detection setup. The second detection dimension extends the number of possible research-applications significantly - fast and turbulent processes, e.g. engine-like sprays, can now be measured at once in size and temperature. The method design of this work is presented step-wise from initial dye selection that is required for MDR-shifting, over explanation to the required equipment, to two application cases that led to new findings and open questions:

\footnotetext{
${ }^{15}$ The value is determined by applying an analytic one dimensional single droplet evaporation model.
} 
- Droplet application: Temperature imaging of micro droplets shows overall reliable results. However, locally resolved average droplet images reveal a systematic temperature fluctuation across isothermal droplets that is nonphysical and thus must be caused by the measurement technique. The cause of this phenomenon is presumably based on local changes of fluorescence excitation intensity. Further research would allow to optimize the droplet application and also answer open research questions of other publications.

- Macroscopic spray application: Spray investigations show reasonable temperature results as well. Temporal temperature changes of the hollow-cone spray reveal a diffusion-limited mixture formation process. Main challenge of the spray application are issues due to multiply scattered light that causes "noisy" fluorescence. Corresponding spray regions on the spray-cone's inside appear as zones with nonphysical high temperatures. Only a SLIPI extension of the setup may solve the problem.

\section{Acknowledgement}

This work was performed as part of the Cluster of Excellence "Tailor-Made Fuels from Biomass", which is funded by the Excellence Initiative of the German federal and state governments to promote science and research at German universities.

\section{References}

[1] Lemoine, F., Antoine, Y., Wolff, M., and Lebouche, M., 1999. "Simultaneous temperature and 2d velocity measurements in a turbulent heated jet using combined laser-induced fluorescence and Ida". Experiments in Fluids, 26(4), pp. 315-323.

[2] Palmer, J., Reddemann, M. A., Kirsch, V., and Kneer, R., 2016. "Temperature measurements of micro-droplets using pulsed 2-color laser-induced fluorescence with mdr-enhanced energy transfer". Experiments in Fluids, 57(12).

[3] Chang, R. K., and Campillo, A. J., eds., 1996. Optical Processes in Microcavities. Advanced Series in Applied Physics. WORLD SCIENTIFIC.

[4] Dunand, P., Castanet, G., and Lemoine, F., 2012. "A two-color planar lif technique to map the temperature of droplets impinging onto a heated wall". Experiments in Fluids, 52(4), pp. 843-856.

[5] Mishra, Y. N., Abou Nada, F., Polster, S., Kristensson, E., and Berrocal, E., 2016. "Thermometry in aqueous solutions and sprays using two-color lif and structured illumination". Optics Express, 24(5), p. 4949.

[6] Deprédurand, V., Miron, P., Labergue, A., Wolff, M., Castanet, G., and Lemoine, F., 2008. "A temperaturesensitive tracer suitable for two-colour laser-induced fluorescence thermometry applied to evaporating fuel droplets". Measurement Science and Technology, 19(10), p. 105403.

[7] Palmer, J., Mathieu, F., Reddemann, M., and Kneer, R., 08.- 10. Spetember 2014. "Temperature measurements of evaporating biofuel droplets". ILASS - Europe, 26th Annual Conference on Liquid Atomization and Spray Systems, Bremen - Germany.

[8] Lavieille, P., Delconte, A., Blondel, D., Lebouché, M., and Lemoine, F., 2004. "Non-intrusive temperature measurements using three-color laser-induced fluorescence". Experiments in Fluids, 36(5), pp. 706-716.

[9] Zhang, G., Hung, D. L. S., and Xu, M., 2014. "Experimental study of flash boiling spray vaporization through quantitative vapor concentration and liquid temperature measurements". Experiments in Fluids, 55(8).

[10] Chaze, W., Caballina, O., Castanet, G., and Lemoine, F., 2016. "The saturation of the fluorescence and its consequences for laser-induced fluorescence thermometry in liquid flows". Experiments in Fluids, 57(4).

[11] Tzeng, H.-M., Wall, K. F., Long, M. B., and Chang, R. K., 1984. "Evaporation and condensation rates of liquid droplets deduced from structure resonances in the fluorescence spectra". Optics Letters, 9(7), p. 273.

[12] Wolff, M., Delconte, A., Schmidt, F., Gucher, P., and Lemoine, F., 2007. "High-pressure diesel spray temperature measurements using two-colour laser-induced fluorescence". Measurement Science and Technology, 18(3), pp. 697-706.

[13] Perrin, L., Castanet, G., and Lemoine, F., 2015. "Characterization of the evaporation of interacting droplets using combined optical techniques". Experiments in Fluids, 56(2).

[14] Serpenguzel, A., Kucuksenel, S., and Chang, R., 2002. "Microdroplet identification and size measurement in sprays with lasing images". Optics Express, 10(20), p. 1118.

[15] Anand, M., Dharmadhikari, A., Dharmadhikari, J., Mishra, A., Mathur, D., and Krishnamurthy, M., 2003. "Twophoton pumped lasing from methanol micro-droplets doped by a weakly fluorescent dye". Chemical Physics Letters, 372(1-2), pp. 263-268.

[16] Chen, G., Mohiuddin Mazumder, M., Chang, R. K., Christian Swindal, J., and Acker, W. P., 1996. "Laser diagnostics for droplet characterization: Application of morphology dependent resonances". Progress in Energy and Combustion Science, 22(2), pp. 163-188.

[17] Tzeng, H.-M., Wall, K. F., Long, M. B., and Chang, R. K., 1984. "Laser emission from individual droplets at wavelengths corresponding to morphology-dependent resonances”. Optics Letters, 9(11), p. 499.

[18] Labergue, A., Pena-Carillo, J.-D., Gradeck, M., and Lemoine, F., 2017. "Combined three-color lif-pda measurements and infrared thermography applied to the study of the spray impingement on a heated surface above the leidenfrost regime". International Journal of Heat and Mass Transfer, 104, pp. 1008-1021.

[19] Frackowiak, B., and Tropea, C., 2010. "Numerical analysis of diameter influence on droplet fluorescence". Applied optics, 49(12), pp. 2363-2370. 


\title{
High Speed X-Ray Imaging for Nozzle Exit Velocity and Density Distribution Measurements of GDI Nozzles
}

\author{
Raphael Distler ${ }^{1}$, Dr. Christoph Hamann ${ }^{1}$, Dr. Martin Krämer ${ }^{1}$, Dr. Eberhard Kull ${ }^{1}$, Prof. Dr. \\ Michael Wensing ${ }^{2}$, Dr. Zhilong $\mathrm{Li}^{3}, \mathrm{Ya} \mathrm{Gao}^{3}$, Dr. Jin Wang ${ }^{3}$ \\ ${ }^{1}$ Continental Automotive $\mathrm{GmbH}$, Regensburg, Germany \\ ${ }^{2}$ Lehrstuhl für technische Thermodynamik, Friedrich-Alexander-Universität \\ Erlangen-Nürnberg, Germany \\ ${ }^{3}$ Advanced Photon Source, Argonne National Laboratory, United States of America \\ *Corresponding author: raphael.distler-ext@continental-corporation.com
}

\begin{abstract}
Investigation of the primary breakup region of gasoline sprays is important for future nozzle development. It improves the principal understanding of inner nozzle flow and spray breakup. It also allows validating and developing CFD models. Due to the high optical density common measurement techniques like Phase Doppler Anemometry reach their limit in optical dense sprays as in the primary breakup region. High Speed X-Ray Imaging is capable to measure 2D velocity distributions directly at the spray hole exit. For generating the intense X-Ray beam the synchrotron Advanced Photon Source at Argonne National Laboratory is used. Passing through the spray the X-Ray beam is changed by two different physical principles: absorption and phase contrast. Absorption can be applied to measure the density of the spray. Phase contrast is used to visualize the borders of droplets and ligaments with high contrast. The accelerated electron bunches inside the synchrotron have a constant period length to each other. This leads to an accurate pulsed X-Ray beam (periodicity: $68 \mathrm{~ns}$ ). The use of multi exposure with very short X-Ray pulses (17 ns) shows the traveled distance of the spray droplets and ligaments. The spray speed $(150-250 \mathrm{~m} / \mathrm{s})$ is calculated by dividing these distances with the time gap between two X-Ray pulses. The X-Ray measured density distributions and velocity distributions are combined to calculate the spray force rate. The so gained force rate is validated with a spray force measurement performed at the Spray Momentum Test Bench (SMTB) at Continental Automotive $\mathrm{GmbH}$. The study is focusing on the measurement setup of High Speed X-Ray Imaging at Argonne National Laboratory and the evaluation algorithms.
\end{abstract}

\section{Keywords}

High Speed X-Ray, Gasoline Direct Injection, Gasoline Spray, Primary Breakup, Spray Density, Spray Velocity

\section{Introduction}

The spray performance of a GDI injector is highly influencing the efficiency and pollutant emissions of modern gasoline combustion engines. To optimize the whole process from injection over mixture formation and the final combustion, the behavior of a single spray plume has to be understood. To find a direct link between nozzle geometry and generated spray, the primary breakup region has to be analyzed. The primary spray velocity and density distribution are the key factors for further spray breakup. Measurement techniques like Phase Doppler Anemometry and Laser Induced Fluorescence reach their limit in optical dense sprays as in the primary breakup region. Therefore no information about the velocity and density distribution at the nozzle exit is available for comparison of nozzles and as input for CFD simulations. High Speed X-Ray Imaging is able to capture both. Out of the density and velocity distributions, the spray momentum can be calculated and for verification compared with a mechanically measured spray momentum distribution. $[1,2,3,4]$

\section{Hydraulic Injector and Spray Parameters}

The correlation between massflow, flow velocity and resulting momentum can be theoretically calculated by the law of Bernoulli. Equation 1 gives the theoretically massflow $\dot{m}_{t h}$ depending on the flow cross section $A$ the fuel density $\rho$ and the relative fuel pressure $\Delta p$. Equation 2 shows the correlation between the flow speed $v_{t h}$, the relative fuel pressure $\Delta p$ and the fuel density $\rho$. [5]

$$
\begin{aligned}
& \dot{m}_{t h}=A v \rho=A \sqrt{2 \rho \Delta p} \\
& v_{t h}=\sqrt{\frac{2 \Delta p}{\rho}}
\end{aligned}
$$

Due to phenomena like turbulence and cavitation, the flow velocity and massflow in real nozzles is smaller. For this reason different types of discharge coefficients are defined. $C_{D}$ is giving the ratio of the effective massflow 
$\dot{m}_{\text {eff }}$ to the theoretically calculated massflow $\dot{m}_{t h}$. (Equation 3) The $C_{A}$ is defining the ratio of the effective used hydraulic flow cross section $A_{\text {eff }}$ related to the theoretical flow cross section $A_{0}$. (Equation 4) This equation is directly linked to the model of the vena contracta. The ratio of the theoretical flow velocity to the real flow velocity is given by Equation 5. The product of $C_{V}$ and $C_{A}$ delivers the $C_{D}$. (Equation 6) [5]

$$
\begin{aligned}
& C_{D}=\frac{\dot{m}_{e f f}}{\dot{m}_{t h}} \\
& C_{A}=\frac{A_{\text {eff }}}{A_{0}} \\
& C_{v}=\frac{v_{e f f}}{v_{t h}} \\
& C_{D}=C_{A} \cdot C_{v}
\end{aligned}
$$

By adding these discharge coefficients to the Bernoulli equations the following correlations are developed. (Equation 7, Equation 8) [5]

$$
\begin{aligned}
& m_{\text {eff }}=C_{D} A_{0} \sqrt{2 \rho \Delta p} \\
& v_{e f f}=C_{v} \sqrt{\frac{2 \Delta p}{\rho}}=C_{D} \frac{A_{0}}{A_{e f f}} \sqrt{\frac{2 \Delta p}{\rho}}
\end{aligned}
$$

By multiplication of the massflow and the velocity the momentum flow $\dot{p}_{e f f}$ is calculated. (Equation 9) [5]

$$
\dot{p}_{e f f}=C_{D}^{2} \frac{A_{0}^{2}}{A_{e f f}} 2 p
$$

\section{Basics on X-Ray Radiation and its Interaction with Fuel}

Hard X-Ray radiation describes light with a wavelength from $<5-250 \mathrm{pm}$ which corresponds to a photon energy of $5-250 \mathrm{keV}$. For the measurements at ANL X-Ray radiation with a photon energy of up to $9 \mathrm{keV}$ is used. X-Ray radiation can be produced in different ways: [6]

- highly energetic transients in electron shells of atoms or molecules: In X-Ray tubes a cathode is producing free electrons. These electrons are accelerated by an electromagnetic field towards a target. If a free electron has enough energy and pushes out an orbital electron of an atom in the target, by hitting the atom, the vacancy of the left electron has to be filled up with an higher energetic electron. While filling up the vacancy, the higher energetic electron has to emit a photon to reach the correct energy level. This photon is the produced X-Ray photon and has a material characteristic wavelength.

- deceleration of charged particles like electrons and positive ions: Particles which are getting deflected by an electrical field send out so called Bremsstrahlung: This effect delivers X-Ray radiation with a continuous spectrum.

- synchrotron radiation is a special type of Bremsstrahlung: In a synchrotron charged particles are bent at every edge of the ring. This bent equals a deceleration of the particle in one direction while acceleration in another direction. A consequence of this is Bremsstrahlung. Undulators and wigglers are used for adding oscillations on the electron bunches and to produce synchrotron radiation with a broad spectrum. As the electron bunches in a synchrotron are highly collimated, a very intensive and collimated beam can be produced. The radiation is linearly or circularly polarized.

The used synchrotron, the Advanced Photon Source (APS) at the Argonne National Laboratory, is providing a special running mode: the hybrid-singled mode. Figure 1 illustrates the used measurement setup. In the APS electron storage ring with its perimeter of $1104 \mathrm{~m}$, nine bunches of electrons are circulating in a ring with nearly the velocity of light. This results in a running frequency of $274 \mathrm{kHz}$. Eight of the nine bunches build up a so called bunch train. The ninth bunch with a current of $16 \mathrm{~mA}$ is brighter than the single bunches in the bunch train with a current of $11 \mathrm{~mA}$ each. The periodicity of the bunches of the bunch train is $68 \mathrm{~ns}$. The overall bunch train duration is $500 \mathrm{~ns}$. The duration of each bunch is $17 \mathrm{~ns}$. [7] The time gap between the super bunch and the bunch train is in each direction $1.569 \mu \mathrm{s}$. When one bunch is passing the undulator of the used beamline, X-Ray radiation is decoupled into the laboratory. The produced X-Ray radiation has a continuous spectrum and is characteristically collimated. [7] A setup of different shutters is used to cut off the not needed X-Ray pulses. The injector is mounted tip down into a spray chamber. The X-Ray pulses are passing and interacting with the spray. The X-Ray images 


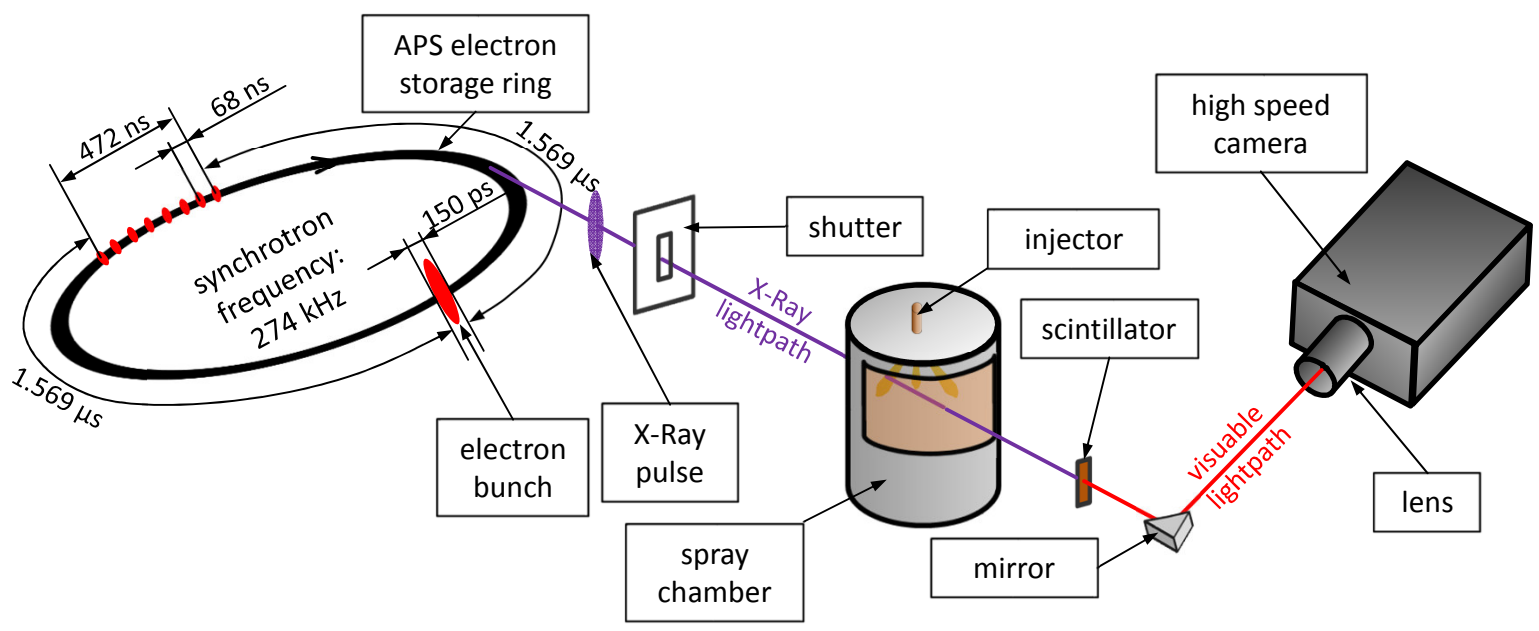

Figure 1. Measurement setup for High Speed X-Ray Imaging at the Advanced Photon Source of the Argonne National Laboratory based on [7]

are converted to visible light images by a fast scintillator. The visible light is reflected into a high speed camera by a mirror. [7]

The interaction of X-Ray radiation with matter can be separated into two different phenomena: absorption and phase contrast. The phenomena of absorption is described by the law of Lambert-Beer-Bonguer. Light looses a constant fraction of its intensity by passing a constant material thickness. This results in a exponentially relationship between the light intensity $(I(x))$ and the material thickness the light passed through. $I(x)$ is calculated by its material specific and wavelength depended absorption coefficient $\alpha$ and the passing distance of the light $x$. (Equation 10) [8]

$$
I(x)=I_{0} e^{-\alpha x}
$$

The second phenomena, the phase contrast, is caused by refraction of a coherent X-Ray beam on phase borders where a gradient in X-Ray refraction indices is occurring. The reflection angle is depended on the X-Ray spectrum's wavelenghts and the difference of the refraction indices. Phase contrast imaging in common is used to separate small structures in material compounds where both materials have very similar absorption coefficients. The refraction leads to high contrast on the image. Its visibility on the captured image is mainly influenced by the distance between the measurement object and the detector, or in the here used setup of the scintillator. Detailed information about phase contrast imaging can be found in [9].

\section{Basics of the Used Measurement Process and Evaluation Algorithms}

For obtaining the velocity of the spray in the near field a multiexposure of the injection is captured by using three $X$ Ray pulses of the described bunch train. By knowing the time interval between the single bunches and by measuring the distance a structure traveled during the exposures, its' velocity can be calculated. To enhance the phase contrast intensity and therefore to visualize the structure edges clearly, a longer distance between spray and scintillator is used. For obtaining the density distribution of the fuel in the spray the effect of phase contrast is minimized by shortening the distance between spray and scintillator and by using an X-Ray mirror inside the beam path to select only low-energy photons at $7 \mathrm{keV}$.

\section{Algorithm for the Spray Velocity Evaluation}

For evaluating the spray velocity a novel algorithm is developed to enhance the spatial and temporal resolution of the velocity result. Like common Particle Image Velocimetry algorithms, it is based on auto correlation of the image. Figure 2 gives an exemplaric overview of the single evaluation steps. The raw image (1) gets background corrected by subtracting an image without any spray. (2) Furthermore the contrast of the image gets enhanced. In (3) the image is divided into 26 by 25 squares and the resolution of the image is enlarged by calculating subpixels. The square size defines the spatial velocity resolution in the final evaluation. A parameter study with synthetic generated velocity images was done to find a suitable velocity resolution without a systematic result distortion. The viable resolution is mainly influenced by the average spray velocity, the pixelsize and the exposure gap. The single squares are getting autocorrelated (4). To identify the realistic peaks from the autocorrelation the image gets rotated that the flow is roughly from upside down. (5) The rotation angle is defined at $30^{\circ}$ (= height angle of the single spray jet). In (6) the autocorrelated image is cropped to the relevant part of the image for the velocity information. As the main peak of an autocorrelation image is always in the middle of the image it is cut to a region which is able to show all 5 peaks of a multiexposed structure with a maximum velocity of $300 \mathrm{~m} / \mathrm{s}$ in axial $(\mathrm{z})$ and $50 \mathrm{~m} / \mathrm{s}(\mathrm{y})$ in radial direction. The experiments shown in this paper are done with $\mathrm{n}$-heptane at 200 bar injection pressure which results in a Bernoulli velocity of $240 \mathrm{~m} / \mathrm{s}$. In a next step the positions of the peaks are getting located. Plotting the row/column position of the maxima against their number and applying straight fits delivers the average slopes, 
which are the corresponding average moved distances the spray passed during two exposures in axial and radial distance. With this information the velocities are calculated. A reversing of the before done image rotation (5) by using basic trigonometry is delivering the correct axial and radial spray velocities. The theorem of Pythagoras is used to calculate the absolute spray velocity.

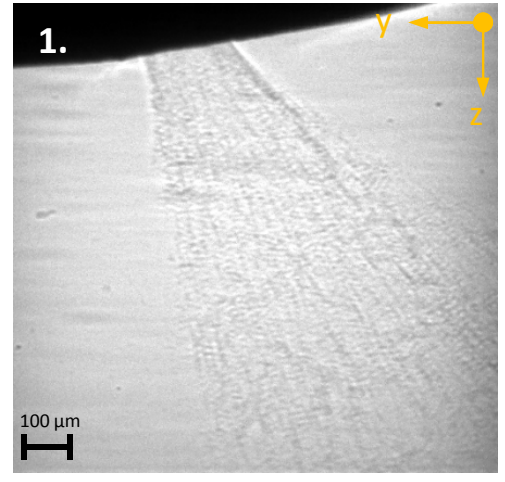

raw image (multi exposure)

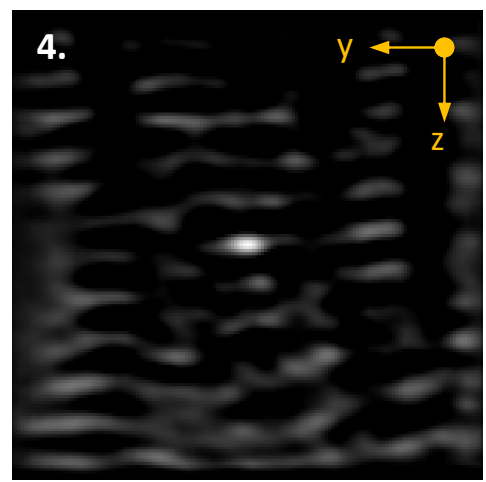

auto correlated image

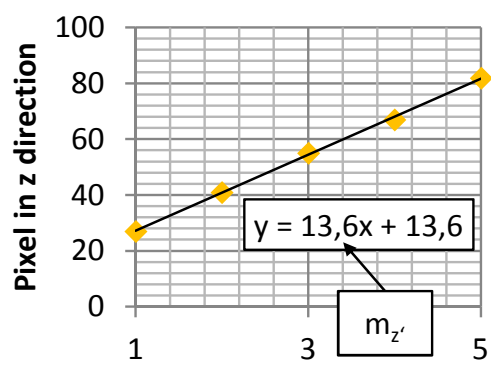

no. of maximum

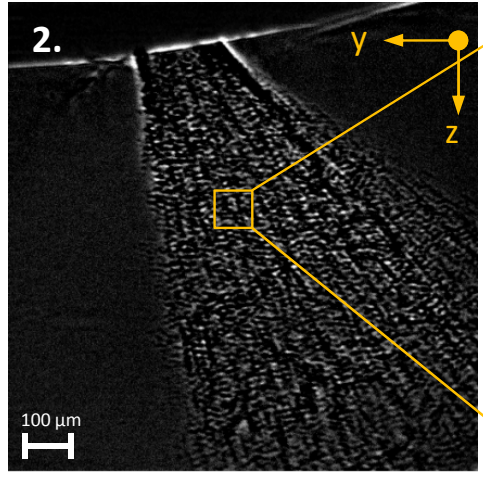

background corrected

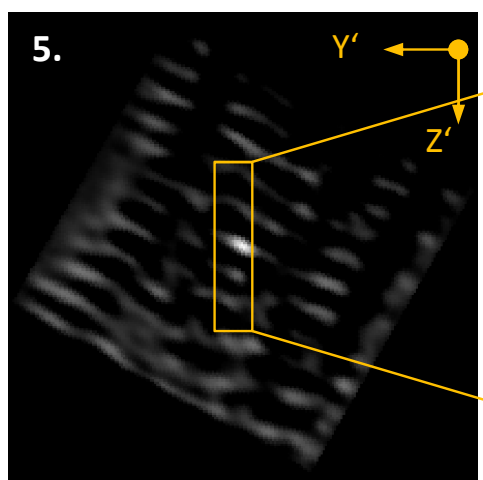

roughly rotated auto correlated image

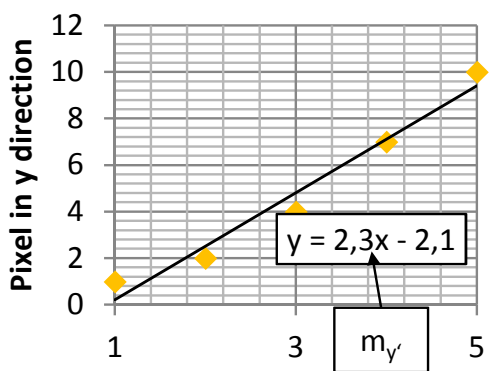

no. of maximum

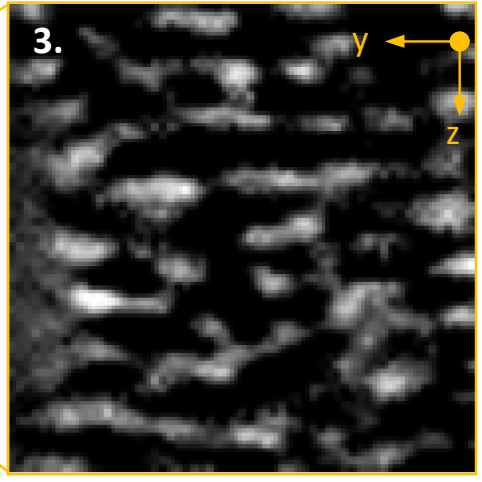

cutting of the image into pieces

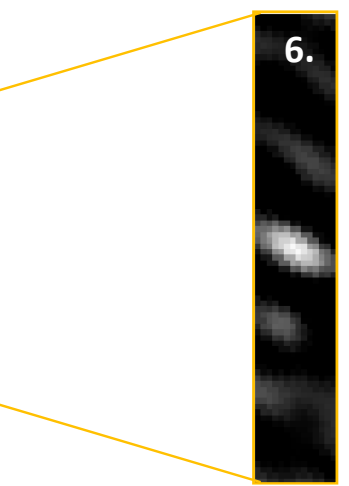

cropped to the relevant image region

$$
\begin{gathered}
v_{z^{\prime}}=m_{z^{\prime}} * \frac{\text { pixelsize }}{\text { exposuregap }} \\
v_{y^{\prime}}=m_{y^{\prime}} * \frac{\text { pixelsize }}{\text { exposuregap }} \\
v_{\text {res }}=\sqrt{v_{z^{\prime}}^{2}+v_{y}^{2}}
\end{gathered}
$$

Figure 2. Basic process of the velocity evaluation algorithm

\section{Algorithm for the Spray Density Evaluation}

Although the effect of phase contrast is minimized by shortening the distance between spray and scintillator, the phase contrast signal still is by order of magnitudes higher than the absorption signal in the image. For this reason the developed density evaluation algorithm has to filter the phase contrast. One basic idea of the spray density evaluation is that the effect of phase contrast is not producing any light, it just leads to a local redistribution of the intensities. Therefore the integrated intensity of the X-Ray is not influenced by the effect of phase contrast. Figure 3 shows the two steps which are used for the density evaluation. The raw image gets background corrected by subtracting an image without any spray. For the filtering of the background corrected image different types of filters and filter settings are analyzed on synthetic density images which are distorted by phase contrast. As a result a median filter is chosen. Also in literature the recommended filter for phase contrast is a median filter. [10]

The filter error has to be corrected by adjusting the overall grey value sum of the image to the grey value sum of the background corrected image.

For calibrating the measurement setup, capillaries of different diameters filled with $n$-heptane are used. By knowing the density of the n-heptane and the size of the capillaries the absorption coefficient of the fuel for the used setup and evaluation calibration can be calculated. Figure 4 shows the X-Ray transmission against thickness of a n-heptane pillar. By applying the law of Lambert Beer-Bonquer the transmission coefficient $\mu$ can be calculated. Applying this 


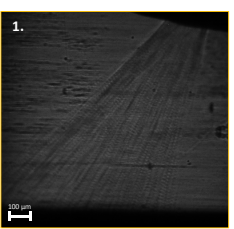

raw image

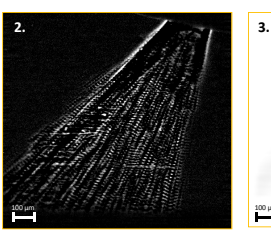

background corrected

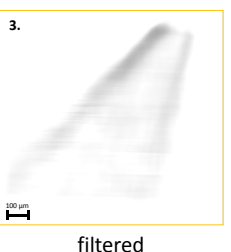

filtered

Figure 3. Basic process of the density evaluation algorithm

coefficient to the image the density distribution inside the spray is delivered. (e.g. Figure 5) The scale can be given in fluid path length and projected spray density. The fluid path length describes how thick a liquid fuel pillar has to be to deliver the same absorption. The projected spray density gives the density distribution integrated in the line of sight.

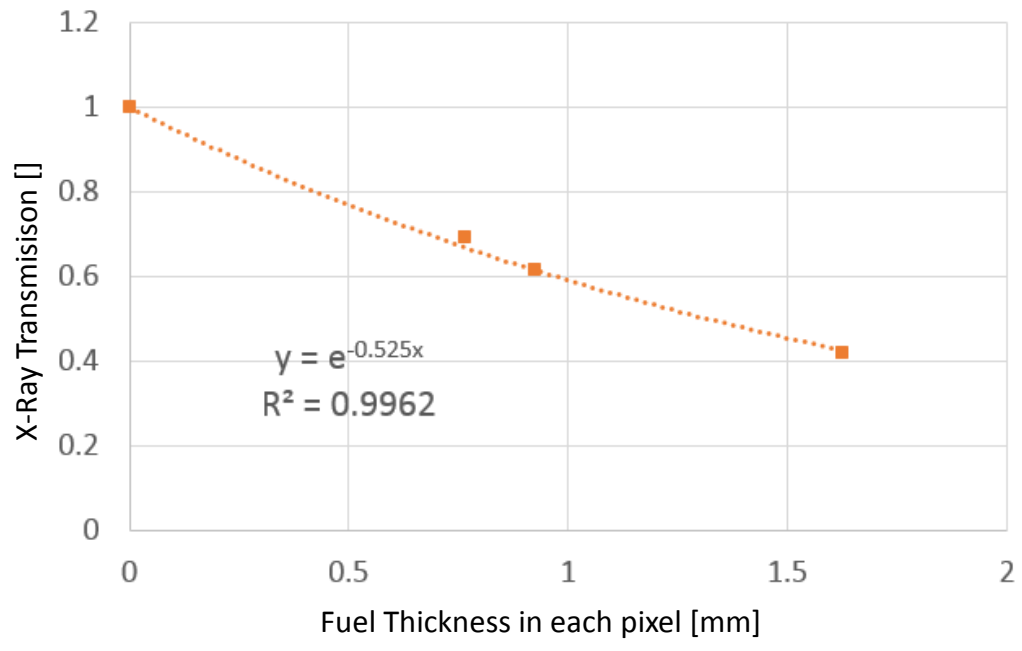

Figure 4. Calibration result of the used measurement setup for different capillaries filled with $\mathrm{n}$-heptane
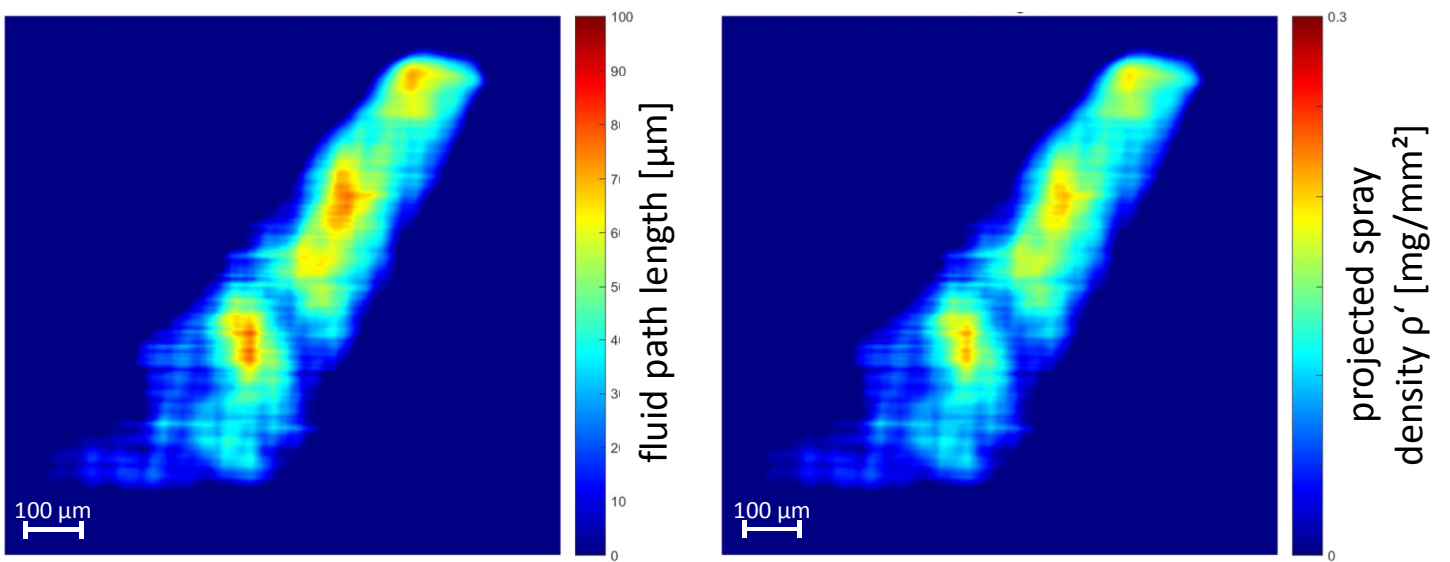

Figure 5. Example for the spray density evaluation, left = fluid path length the X-Ray is passing; right = projected spray density

\section{Calculation of the Momentum out of X-Ray Measurements}

Figure 6 illustrates the information the X-Ray measurement is providing. The velocities and densities are depended on the two spatial coordinates $z$ and $y$ and the point of time $t$. For calculating the spray momentum, spray force and massflow rate, a cut through the spray rectangular to the flow direction is done. For each pixel on this cutting line the spray parameters are getting calculated.

The massflow in $z$ direction is calculated in Equation 11 by the product of the density $\rho^{\prime}(y, z, t)$, the velocity $v_{a b s}(y, z, t)$ and the pixelsize.

$$
\dot{m}_{a b s}(y, t)=\rho^{\prime} \cdot v_{a b s} \cdot l_{\text {pixel }}
$$




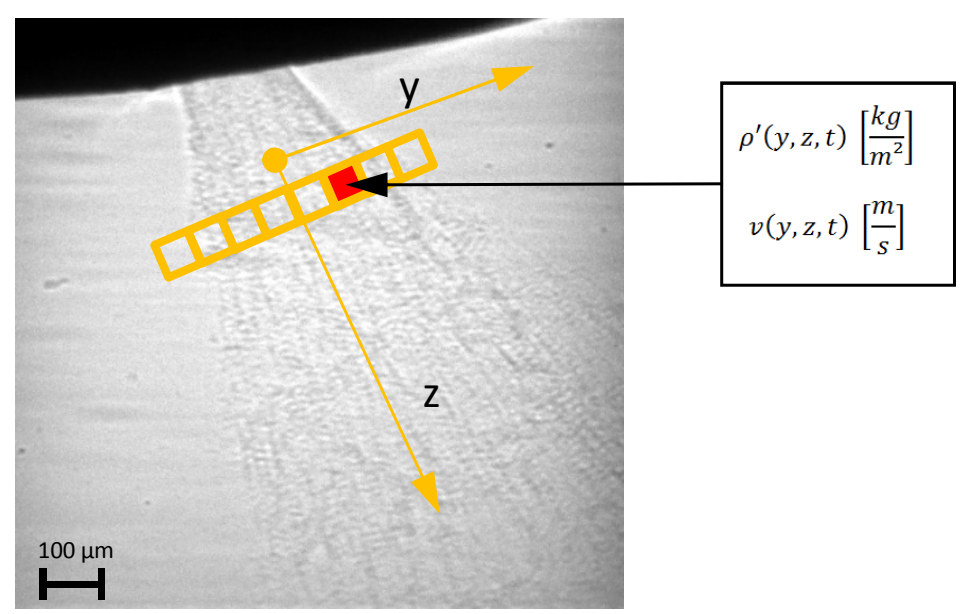

Figure 6. Information provided by the X-Ray measurement

The spray force is calculated by the product of the massflow $\dot{m}_{a b s}(y, t)$ and the velocity $v_{a b s}(y, t)$. (Equation 12)

$$
F_{a b s}(y, t)=\dot{m}_{a b s}(y, t) \cdot v_{a b s}(y, t)
$$

By integrating the spray forces $F_{a b s}(y, t)$ in $y$ direction, the overall spray force rate is calculated. Integrating this force rate over time delivers the spray momentum. (Equation 13)

$$
p_{a b s}=\iint F_{a b s}(y, t) d y d t
$$

\section{Basics of Spray Momentum Measurements}

The measurement principle used on the SMTB is illustrated in Figure 7. A target pin is mounted on a piezoelectric force sensor and can be freely positioned in a spherical coordinate system around the injector tip. As the target pin in the used setup has a diameter of $1 \mathrm{~mm}$ a shielding cap is used to ensure the force sensor only measures the force which is induced on the pin and not the force of spray which is hitting the sensor itself. The target pin surface is always faced to the origin of the spherical coordinate system. Therefore only the axial force of the spray can be measured. Force on the piezoelectric sensor is producing electric charge. This charge gets amplified and converted into a time depended voltage signal which corresponds to a force rate. The force rate has to get filtered at the sensor's critical frequency. By integration of the force rate, the momentum is calculated. As a small pin is used which is not able to capture the whole spray, the momentum of a plume has to be calculated out of several measurement points. A basic theory about the mathematical principle can be found in [5].

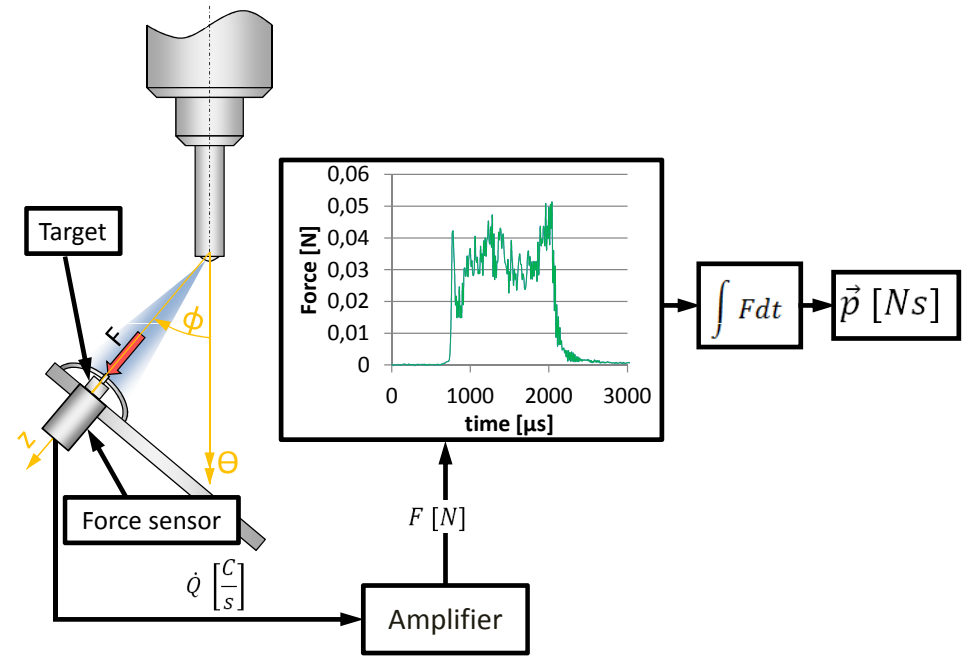

Figure 7. Measurement principle of the SMTB 


\section{Results}

Figure 8 shows an exemplaric mass and velocity rate for a gasoline spray an the distance of $400 \mu m$ to the sprayhole exit. By averaging the rates the discharge coefficients are calculated by using Equation $3\left(C_{D}\right)$, Equation $5\left(C_{V}\right)$ and Equation $6\left(C_{A}\right)$.

$$
\begin{aligned}
& \text { - } C_{v}=0,85 \\
& \text { - } C_{A}=0,65 \\
& \text { - } C_{D}=0,55
\end{aligned}
$$
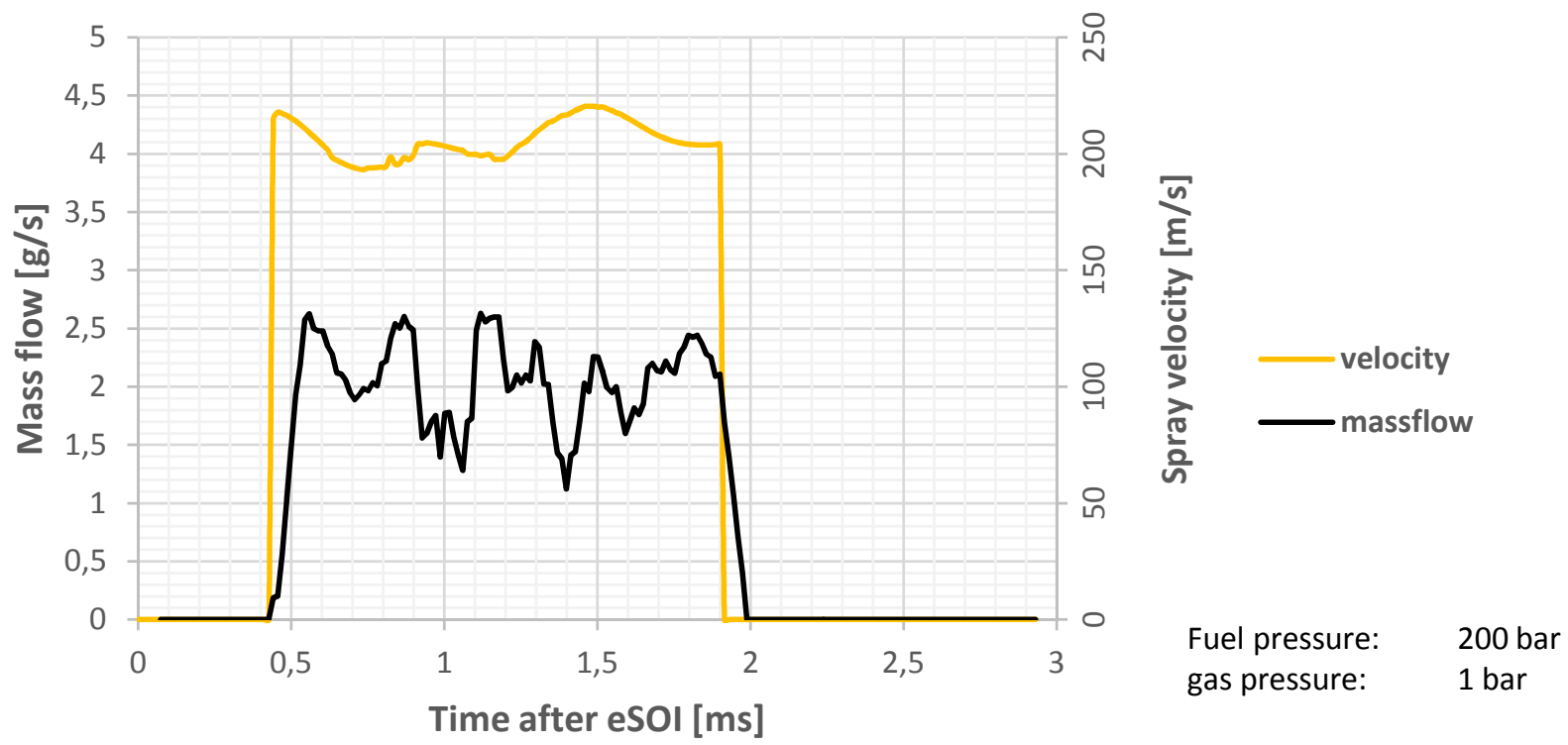

Figure 8. Exemplaric mass and velocity rate measured with High Speed X-Ray Imaging for a Gasoline Spray at 200 bar

For the comparison of the resulting spray force rates and the spray momentum the mechanically captured force rate has to be aligned. The SMTB runs with Exxsol D40. The High Speed X-Ray measurements are performed with $\mathrm{n}$-heptane. For this reason the spray momentum force rate is corrected by the density ratio of $n$-heptane to Exxsol D40. Also the measurements are done at different positions due to the limitations of the measurement techniques. The X-Ray evaluation cut is at $400 \mu \mathrm{m}$ distance to the nozzle hole exit. The local spray momentum measurement is done on a sphere with a radius of $10 \mathrm{~mm}$ distance. The calculated force rate out of the local spray momentum measurement and the calculated force rate out of the X-ray measurement are given in Figure 9. Additionally the integrated spray momentum curves are displayed.

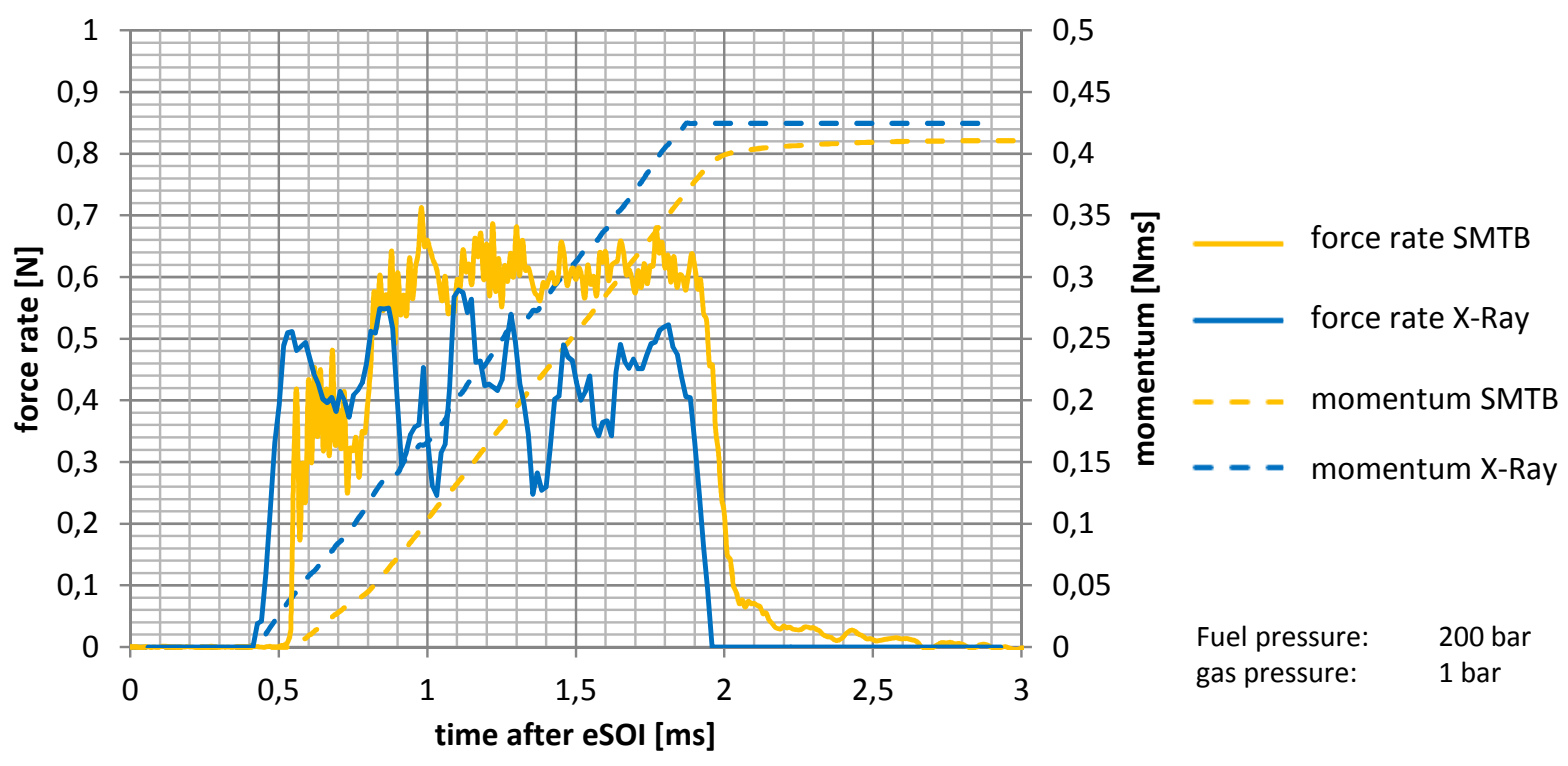

Figure 9. Comparison of the calculated spray momentum of High Speed X-Ray Imaging and the SMTB

The spray force rate of the SMTB is shifted to later points in time as the spray has to pass the distance of $10 \mathrm{~mm}$ before reaching the measurement pin. Also the filtering of the raw spray force signal will result in a jitter. It can be 
seen that both measurement techniques deliver spray momentum values in the same range. The SMTB delivers a slightly smaller spray momentum due to dissipation while traveling the distance to the measurement pin.

\section{Conclusions}

Within this study methods for evaluating velocity and density measurements captured with High Speed X-Ray Imaging are developed. The algorithms are applied exemplary. The results are compared and validated with measurements of a mechanical SMTB. High Speed X-Ray Imaging turns out to be a powerful tool to measure velocity and density distributions inside a GDI spray in the nearfield. The measurement of the density and velocity distributions inside a gasoline spray allow to split up the mechanically easily measurable spray momentum distribution to find the main drivers for momentum differences in different gasoline jets. It will reach its limits in far away distances as the structural velocities and therefore the traveled distances of the structures between two exposures are too small for the very high pulse frequency of the X-ray source.

\section{Acknowledgements}

This work is supported by the US Department of Energy, Basic Energy Sciences, Office of Science, under Contract No. 852 W5.

\section{References}

[1] M. Krämer, E. Kull, Markus Heldmann, M. Wensing. Investigations on gasoline spray porpagation behaviour characteristics for multihole injectors: Sae technical paper 2014-01-2732. 2014.

[2] R. Distler, M. Krämer, E. Kull, M. Wensing. Spray characterization of multihole gdi injectors with spray momentum measurements. Motorische Verbrennung Aktuelle Probleme und moderne Lösungsansätze (XIII. Tagung), 2017.

[3] M. Krämer. Einfluss motorischer Randbedingungen auf die Spraycharakteristik bei direkteinspritzenden Ottoverfahren, volume 2016,2 of Berichte zur Thermodynamik und Verfahrenstechnik. 1. auflage edition, 2016.

[4] M. Krämer, E. Kull, M. Wensing. Flashboiling - induced targeting changes in gdi sprays, 2015.

[5] J. Ernst. Experimentelle und numerische Analyse des Impulses von aufbereitetem Kraftstoffspray im düsennahen Bereich der Dieseleinspritzung. Wissenschftliche Reihe Fahrzeugtechnik Universität Stuttgart. Wiesbaden, 2014.

[6] C. Gerthsen. Gerthsen Physik: Mit 89 Tabellen und 105 durchgerechneten Beispielen und 1065 Aufgaben mit vollständigen Lösungswegen. Springer-Lehrbuch. Springer, Berlin and Heidelberg and New York and Barcelona and Hongkong and London and Mailand and Paris and Singapur and Tokio, 20., aktualisierte aufl. edition, 1999.

[7] Yujie Wang, Xin Liu, Kyoung-Su Im, Wah-Keat Lee, Jin Wang, Kamel Fezzaa, David L.S. Hung, James R. Winkelman. Ultrafast $\mathrm{x}$-ray study of dense-liquid-jet flow dynamics using structure-tracking velocimetry. nature physics, 2008.

[8] A. Kastengren, C. F. Powell. Synchrotron x-ray techniques for fluid dynamics. Exp Fluids, (55:1686), 2014.

[9] P. Cloetens. Contribution to Physe Contrast Imaging, Reconstruction and Tomography with Hard Synchrotron Radiation. 1999.

[10] D. Mukherjee, Sanjoy Das, B.K. Shah. Evaluation of coating layer thickness for simulated triso coated fuel particle by micro focus radiography. Proceedings of the National Seminar \& Exhibition of Non-Destructive Evaluation, 2011. 


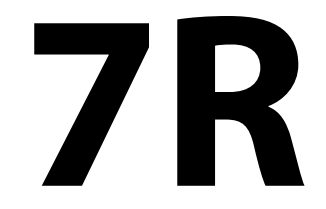

Combustion 1 


\title{
Influence of the chemical mechanism in the frame of diesel-like CFD reacting spray simulations using a presumed PDF flamelet-based combustion model
}

\author{
F. Payri, J. M. García-Oliver, R. Novella, E. J. Pérez-Sánchez* \\ CMT Motores Térmicos, Universitat Politècnica de València, Spain \\ ${ }^{*}$ Corresponding author: edpresnc@mot.upv.es
}

\begin{abstract}
The ability of a computational fluid dynamics (CFD) simulation to reproduce the diesel-like reacting spray ignition process and its corresponding flame structure strongly depends on both the fidelity of the chemical mechanism for reproducing the oxidation of the fuel and also on how the turbulence-chemistry interaction (TCl) is modeled. Therefore, investigating the performance of different chemical mechanisms not only in perfect stirred reactors but directly in the diesel-like spray itself is of great interest in order to evaluate their suitability for being further applied to CFD engine simulations.

This research work focuses on applying a presumed probability density function (PDF) unsteady flamelet combustion model to the well-known spray A from the Engine Combustion Network (ECN), using three chemical mechanisms widely accepted by the scientific community as a way to figure out the influence of chemistry in the key characteristics of the combustion process in the frame of diesel-like spray simulations. Results confirm that in spite of providing all of them correct trends for ignition delays (ID) and lift-off lengths (LOL), when comparing with experimental results, the structure of the flame presents noticeable differences, especially in the low and intermediate temperatures and high equivalence ratio regions. Consequently, the selection of the chemical mechanism has an impact on the zones of influence of key species as observed in both spatial coordinates and also in the equivalence ratio-temperature maps. These differences are expected to be relevant considering the implications when coupling pollutant emissions models. The analysis of temperature and oxygen concentration parametric studies evidences how the observed differences are consistent and moderately dependent on the ambient conditions.
\end{abstract}

\section{Keywords}

Combustion modeling, Chemical mechanism, Spray A, Flamelet

\section{Introduction}

The improvement of the combustion technologies in industrial devices, such as diesel engines, in terms of efficiency and pollutant emissions, evidence that a comprehensive knowledge of the processes involved is mandatory. Between the different processes that concur in the energetic transformation, turbulent non-premixed combustion appears as one of the most relevant. Nevertheless, its modeling is a complex issue due to the different length and time scales of the turbulence, the fuel oxidation and the interaction between them [1].

These considerations point out that for a proper modeling of the turbulent non-premixed combustion two main aspects are essential. The first one is the chemical mechanism, that determines the fuel oxidation, while the second one is the turbulence-chemistry interaction (TCl).

Promoted by the need of gaining a very detailed knowledge in these issues in diesel spray conditions, the Engine Combustion Network (ECN) has proposed a set of experiments in controlled conditions that shed light for these concerns by means of experiments and numerical simulations.

More particularly, the well-known spray A models a diesel-like spray with n-dodecane as a surrogate diesel fuel. The boundary conditions encompass low temperatures and moderate EGR in correspondence with boundary conditions of interest for modern diesel engines.

On the one hand, with regard to experimental measurements, different institutions have carried out experiments with different facilities that are classified in constant-volume pre-burn (CVP) combustion vessels and constant-pressure flow (CPF) rigs [3]. With such facilities, measurements of global combustion parameters, such as ignition delay (ID) and lift-off length (LOL), together with more detailed information, such as species spatial evolution by means of planar laser-induced fluorescence (PLIF), can be captured. This valuable data permits to validate the combustion models and figure out the main aspects of the turbulent combustion.

On the other hand, with regard to the combustion modeling, it is desirable that the model reproduces the combustion structure as well as the $\mathrm{TCl}$ with low computational cost while managing complex chemical mechanisms for the sake of a chemically accurate evolution. These aspects are covered by flamelet models used together with tabulated chemistry and, more particularly, by the Approximated Diffusion Flamelet (ADF) approach [4]. This model, used in the present work, has been demonstrated to offer powerful capabilities and provides satisfactory results in the frame of diesel sprays and diesel engines simulations $[5,6]$.

Nevertheless, the fuel oxidation process, given by the chemical mechanism, arises as one of the cornerstones in the combustion numerical simulation and is one of the inputs to the problem which causes a greater uncertainty. These reasons justify the effort invested by the scientific community for properly describing the chemistry evolution that is patent in the great variety of chemical mechanisms available for a given fuel. Encouraged by this situation, 
a comparison between different chemical schemes is carried out not only in homogeneous conditions, as in homogeneous reactors (HRs) calculations, but on the diesel spray itself in order to figure out the final implications of the selection of the chemical mechanism.

The aim of this work is to simulate the spray A by means of the presumed PDF ADF unsteady flamelet model with different chemical mechanisms of interest in order to clear up the implication on the choice of each of them. Experimental results were measured in CPF experimental facility available at CMT-Motores Térmicos [7, 8].

This work starts with a brief description of the model and the spray A parametric studies included. The validation is sequentially performed, first, by means of HRs and flamelet solutions and, second, by the comparison of the spray evolutions. In this case, a first analysis is given by the ID and LOL parameters, later a more detailed insight is accomplished by the species fields in spatial coordinates and equivalence ratio-temperature maps. Finally, a temporal evolution of the production of species of interest in the whole domain closes the comparison. The work ends with the main conclusions extracted from the analysis.

\section{Methodology \\ Model description}

Spray A has been modeled and computed in the open tool-box OpenFoam environment [9]. Cylindrical symmetry is supposed and, consequently, calculation is performed on a meridian cut, with dimensions $54 \times 108 \mathrm{~mm}$. The mesh is structured with constant cell size of $0.25 \times 0.5 \mathrm{~mm}$ as in previous works $[6,10]$.

A RANS (Reynolds Averaged Navier-Stokes) approach is used for simulating the turbulence. More particularly, a standard $k-\varepsilon$ model with $C_{\varepsilon 1}=1.52$ [11] is applied to calculations (the rest of constants with standard values). The liquid phase is modeled with a DDM (discrete droplet method) based on the Kelvin-Helmholtz and Rayleigh-Taylor instabilities for describing the atomization and breakup processes in conjunction with the Ranz-Marshall model for droplet evaporation. A detailed description of the model constants used for these simulations can be found in a previous work of the authors [6].

The combustion model is based on the flamelet concept which describes the turbulent combustion as an ensemble of laminar flames, called flamelets, embedded in the turbulent flame. The flamelet equations lead to the Diffusion Flamelet (DF) model. This flame description has been successfully applied for diesel engine and gas turbines simulations [12].

The DF model solves eq. (1) for all the species $(N)$ appearing in the chemical mechanism [1].

$$
\frac{\partial Y_{k}}{\partial t}=\frac{\chi}{2} \frac{\partial^{2} Y_{k}}{\partial Z^{2}}+\dot{\omega}_{k} \quad k=1, \ldots, N
$$

where $Y_{k}$ is the mass fraction for species $k, Z$ is the mixture fraction, $\chi$ is the scalar dissipation rate and the chemical source term is represented by $\dot{\omega}_{k}$, which is obtained from the chemical ODE system.

The initial mixture fraction-temperature profile for the laminar flamelet calculations is obtained assuming an adiabatic mixing process between the air and the fuel streams, which accounts for the enthalpy exchange due to the fuel evaporation. Thus, the initial temperature for each mixture fraction decreases compared to the non-evaporative adiabatic mixing process ([13]). As the fuel is injected in liquid phase and $Z$ only accounts for the contribution of the gas phase, $Z$ varies between 0 and the saturation mixture fraction $Z_{s a t}$, defined as the maximum mixture fraction for which the fuel does not condensate.

The value of $\chi$ is given by $\chi=2 D|\nabla Z|^{2}$, with $D$ the molecular mass diffusion. If a steady profile is imposed, it follows the formula [1]

$$
\chi(a, Z)=\frac{a}{\pi} Z_{\text {sat }}^{2} \exp \left[-2\left(\operatorname{erfc}^{-1}\left(2 Z / Z_{\text {sat }}\right)\right)^{2}\right]
$$

where $a$ is the strain rate.

When complex mechanisms are used for diesel engine simulations, where boundary conditions vary significantly, the number of flamelets to be calculated is in the order of several hundred or thousand and solving the ODE system given by the chemical mechanism together with the PDE system given by eq. (1) is unaffordable [14]. This situation appeals for the introduction of new hypotheses that reduce the computational cost while still managing complex chemical mechanisms.

With this purpose, the ADF (Approximated Diffusion Flamelet) model was suggested several years ago in order to perform diesel engine simulations while keeping the combustion flamelet structure and using complex chemical schemes. Based on the fact that eq. (1) shows that the flamelet evolution corresponds to a homogeneous reactor $(\mathrm{HR})$ when the strain rate tends to zero, the ADF model decouples the chemistry from diffusion taking the chemical source term from an equivalent homogeneous reactor. Equation for the ADF model reads

$$
\frac{\partial Y_{c}}{\partial t}=\frac{\chi}{2} \frac{\partial^{2} Y_{c}}{\partial Z^{2}}+\dot{\omega}_{c}^{H R}\left(Z, Y_{c}\right)
$$

In eq. (3), $Y_{c}$ is the progress variable, defined as a mass species fractions linear combination, which describes the chemical evolution. Consequently, the ADF model establishes two hypotheses, namely, to solve only the flamelet equation for the progress variable and to decouple the chemical source term of the diffusion processes. Although, 
at first glance, it may seem very restrictive constrains this model has been successfully applied to diesel-like sprays [6], diesel engines and furnaces $[5,14,15]$. For this work, the progress variable is defined as $Y_{c}=Y_{\mathrm{CO}}+Y_{\mathrm{CO}_{2}}$ [4]. Once the flamelet solutions are available, that depend on $\left(Z, Y_{c}, a\right)$ for both DF and ADF models, the TCl is accounted for by means of presumed probability density functions (PDFs). In order to obtain the turbulent flame manifold, a beta PDF is used for mixture fraction fluctuations while a log-normal PDF is used for the variability in the scalar dissipation rate direction [1]. No fluctuations are accounted for the progress variable dimension. This TCl description leads to

$$
\widetilde{\psi}\left(\tau, \widetilde{Z}, S, \overline{\chi_{s t}}, \sigma\right)=\int_{0}^{\infty} \int_{0}^{Z_{s a t}} \psi\left(\tau, Z, \chi_{s t}\right) P_{Z}(Z, \widetilde{Z}, S) P_{\chi_{s t}}\left(\chi_{s t}, \overline{\chi_{s t}}, \sigma\right) \mathrm{d} Z \mathrm{~d} \chi_{s t}
$$

where $\psi$ represents any variable and $\tau$ corresponds to the flamelet time. Mixture fraction PDF is defined by the mean mixture fraction, $\widetilde{Z}$, and its variance, $\widetilde{Z^{\prime \prime 2}}$, parametrized with the segregation factor $S$ determined by $S=$ $\widetilde{Z^{\prime \prime 2}} /\left(\left(Z_{\text {sat }}-\widetilde{Z}\right) \widetilde{Z}\right)$. The variance of $\ln (\chi), \sigma^{2}$, is assumed to be constant and equal to 2 [16].

The same procedure is applied to the scalar dissipation rate profile which gives $\tilde{\chi}$. The ratio $\tilde{\chi}$ and $\widetilde{Z^{\prime \prime 2}}$ is usually related to the inverse of a turbulent mixing time by a constant $\left(C_{\chi}\right)$ which leads to

$$
\tilde{\chi}=C_{\chi} \frac{\varepsilon}{k} \widetilde{Z^{\prime \prime 2}}
$$

where $k$ and $\varepsilon$ are the turbulent kinetic energy and the rate of dissipation of turbulent kinetic energy, respectively. In the CFD calculation, transport equations for $\widetilde{Z}, \widetilde{Z^{\prime \prime 2}}$ and for species mass fractions are solved together with the continuity, Navier-Stokes and enthalpy equations. The species chemical source terms are retrieved from the turbulent flame manifold, obtained from the integration by means of presumed PDFs of the flamelet database, following the formula:

$$
\dot{\omega}_{k}=\frac{\widetilde{Y}_{k}^{t a b}\left(\widetilde{Z}, S, \overline{\chi_{s t}}, \widetilde{Y}_{c}(t+\delta \tau)\right)-\widetilde{Y_{k}}(t)}{\delta \tau}
$$

where $\widetilde{Z}, S, \overline{\chi_{s t}}$ and $\widetilde{Y_{k}}(t)$ are values related to the CFD cell and ${\widetilde{Y_{k}}}^{t a b}$ is tabulated in the turbulent flame manifold. $\delta \tau$ is the time step for advancing in this manifold. The value $\widetilde{Y}_{c}(t+\delta \tau)$ is obtained from

$$
\widetilde{Y}_{c}(t+\delta \tau)=\widetilde{Y}_{c}(t)+\frac{\partial \widetilde{Y}_{c}}{\partial t}\left(\widetilde{Z}, S, \overline{\chi_{s t}}, \widetilde{Y}_{c}(t)\right) \delta \tau
$$

For this work, the flamelet manifolds have been solved with around 160 points in the mixture fraction direction (it depends on the $Z_{\text {sat }}$ value) and a mesh of 504 points has been imposed for the progress variable with the aim of reducing uncertainties. For the turbulent combustion manifold around 32 values for $\widetilde{Z}$ have been kept, 17 for $S$ ranging between 0 and 0.3 and around 35 values in the $\overline{\chi_{s t}}$ direction are retained. The mesh imposed for the progress variable direction has 51 values following a parabolic distribution. The CFD time step is fixed at $10^{-7} \mathrm{~s}$, which is considered enough small for describing the auto-ignition process and then, $\delta \tau$ is chosen equal to the CFD time step.

Finally, this work pretends to shed light on the influence of the chemical mechanism in turbulent spray simulations. For this purpose, three well-known mechanisms for the n-dodecane fuel, which is a diesel surrogate, available in the literature, have been used to carry out this study. The largest of them is the Narayanaswamy et al. with 255 species and 2289 reactions [17]. The Yao et al. mechanism, which has been specially addressed to the spray $A$, is an skeletal mechanism that consists of 54 species and 269 reactions [18]. Finally, the Wang et al. mechanism with 100 species and 432 reactions is the third and last mechanism that has been analyzed in the present work [19].

\section{Parametric study}

Experimental results correspond with those measured in the CPF facility available at CMT - Motores Térmicos [7, 8]. Two representative parametric sweeps have been chosen to accomplish this work. Boundary conditions are varied around the nominal conditions that is defined by an ambient temperature of $T_{a m b}=900 \mathrm{~K}$, an oxygen concentration of $X_{\mathrm{O}_{2}}=0.15$ and a density of $\rho=22.8 \mathrm{~kg} / \mathrm{m}^{3}$. The first sweep is a parametric temperature variation with $X_{\mathrm{O}_{2}}=$ 0.15 and $\rho=22.8 \mathrm{~kg} / \mathrm{m}^{3}$. The second one varies in the oxygen concentration direction while keeping the ambient temperature at $T_{a m b}=900 \mathrm{~K}$ and the density at $\rho=22.8 \mathrm{~kg} / \mathrm{m}^{3}$. The injection pressure is $150 \mathrm{MPa}$ and the fuel temperature is $363 \mathrm{~K}$ for all the cases. The parametric cases description is gathered in table 1.

The injector has a nominal diameter of $90 \mu \mathrm{m}$, with nozzle reference 210675 [2]. The injection rate lasts for more than $4 \mathrm{~ms}$ in order to describe the quasi-steady fields. More details about the injection rate can be found in $[6,20]$. 
Table 1. Definition of the spray A parametric studies.

\begin{tabular}{ccccccc}
\hline$X_{\mathrm{O}_{2}}$ & $T_{a m b}(\mathrm{~K})$ & $\rho_{a m b}\left(\mathrm{~kg} / \mathrm{m}^{3}\right)$ & $p_{a m b}(\mathrm{MPa})$ & $p_{\text {inj }}(\mathrm{MPa})$ & $Z_{\text {st }}$ & $Z_{\text {sat }}$ \\
\hline \hline 0.13 & 900 & 22.8 & 5.98 & 150 & 0.040 & 0.326 \\
\hline 0.15 & 750 & 22.8 & 4.97 & 150 & 0.046 & 0.251 \\
\hline 0.15 & 800 & 22.8 & 5.3 & 150 & 0.046 & 0.278 \\
\hline 0.15 & 850 & 22.8 & 5.63 & 150 & 0.046 & 0.303 \\
\hline 0.15 & 900 & 22.8 & 5.96 & 150 & 0.046 & 0.326 \\
\hline 0.21 & 900 & 22.8 & 5.91 & 150 & 0.063 & 0.325 \\
\hline
\end{tabular}

\section{Results and discussion}

The set-up of the model is adjusted from the experimental inert spray A solution. For the sake of brevity this comparison is here omitted and the interested reader is referred to [6] whose set-up configuration has been directly taken for the present work.

Given that a flamelet model is used which in turn is based on the HRs solutions, the reactive comparison starts by the analysis of the ignition delay for the different chemical mechanisms provided by the HRs and the flamelet solutions. In this way, the behavior of the mechanisms is analyzed at different levels of complexity and the discrepancies between them can be tracked from the very beginning of the process until the final stage, that is, the turbulent spray. Figure 1 shows the ignition delay for the HRs and the flamelet with strain rate $a=100 \mathrm{1} / \mathrm{s}$, belonging to the representative strain rates of the flamelets related to the high temperature region of the turbulent flow [21], for the nominal case. The ignition delay is defined as the time of maximum rate of rise of the temperature for each mixture fraction, adopting a similar definition to that suggested by the ECN [2].
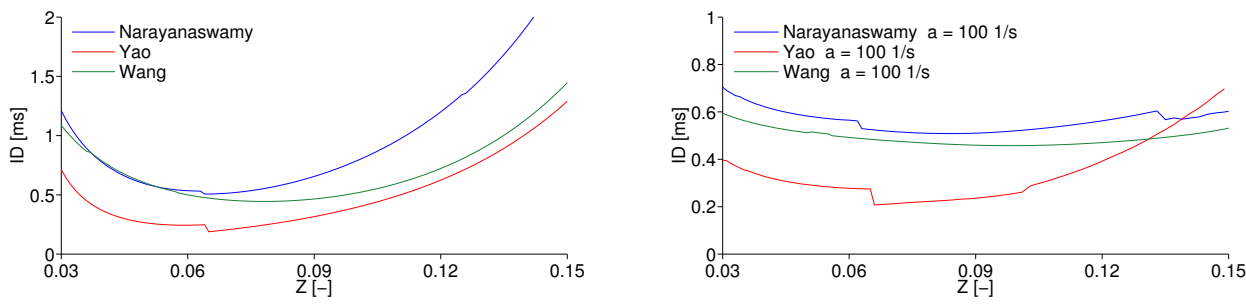

Figure 1. Ignition delay curves as a function of the mixture fraction for the HRs (left) and the flamelet with strain rate $a=1001 / \mathrm{s}$ (right) ignition for the nominal case.

As it can be deduced from figure 1, the Narayanaswamy and Wang mechanisms provide similar ignition delay profiles, in lean and slightly rich mixtures for the case of the ignition of the HRs and in the whole range of mixture fraction for the case of the flamelet, while the Yao mechanism exhibits the fastest ignition, with a minimum value around $200 \mu \mathrm{s}$, as it has been reported in the frame of the ECN [25].

The previous comparison is followed by the global parameters of the turbulent reactive spray, namely, the ignition delay and lift-off length. The ECN ignition delay criterion, namely, the time spent from start of injection (SOI) until the maximum rise of maximum Favre-averaged temperature takes place [2], is here applied. Although different definitions can be found for the LOL in the literature [2, 22], in this work, the criterion based on the minimum axial distance to the nozzle where $14 \%$ of the maximum value of $\widehat{Y_{\mathrm{OH}}}$ in the domain is reached is adopted, given the satisfactory results that provides [6]. Figures 2 and 3 show the ID and LOL results for both parametric studies for the different chemical mechanisms. Additionally, experimental results are included with the nominal value and the uncertainty, given by error bars. In the temperature parametric variation none of the LOL values stabilized during the injection time ( $>4 \mathrm{~ms}$ ) for the $750 \mathrm{~K}$ case and, hence, no value has been assigned.
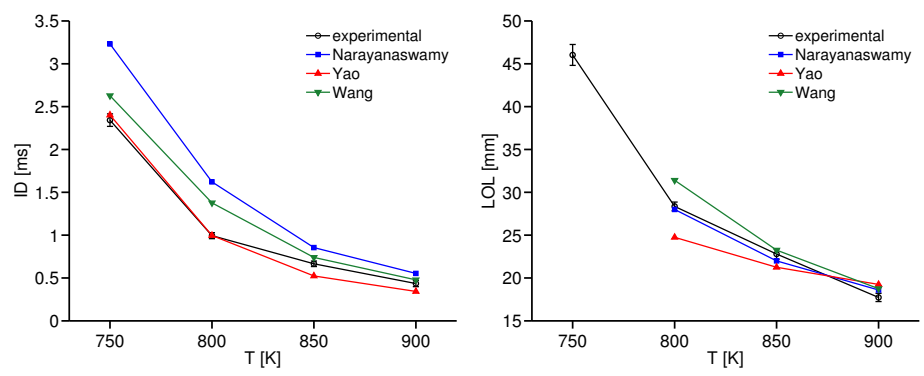

Figure 2. ID (left) and LOL (right) parameters for the temperature parametric variation $\left(X_{\mathrm{O}_{2}}=0.15, \rho=22.8 \mathrm{~kg} / \mathrm{m}^{3}\right)$.

Attending to figure 2 it is clear that the Yao mechanism provides excellent results for ID even though, as it was mentioned before, it is addressed for spray A calculations [18]. Both Narayanaswamy and Wang mechanisms 

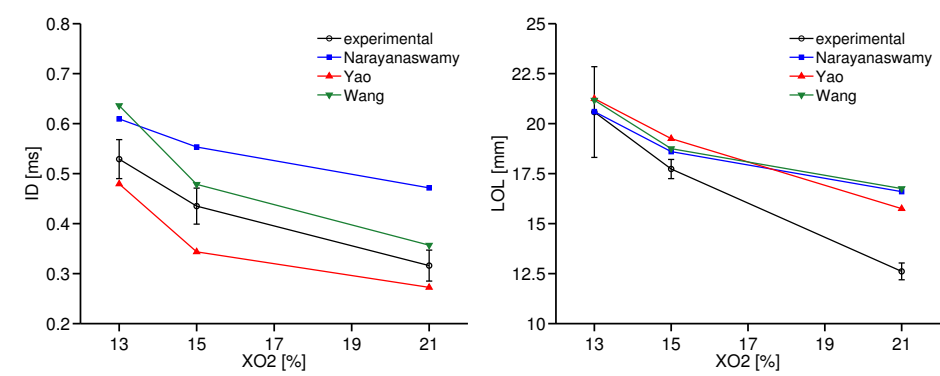

Figure 3. ID (left) and LOL (right) parameters for the oxygen parametric variation $\left(T_{a m b}=900 \mathrm{~K}, \rho=22.8 \mathrm{~kg} / \mathrm{m}^{3}\right)$.

overestimate the ID and the values provided by the Narayanaswamy are greater than those corresponding to the Wang mechanism [23]. Nevertheless, the experimental trends are correctly reproduced. In terms of LOL, the Narayanaswamy mechanism is the one which better fits the LOL although the others give reasonable results.

With regard to oxygen sweep the Yao and Wang mechanisms follow a similar trend for the ID although the first one underestimates it while the second one overestimates it. The Narayanaswamy overestimates ID measurements [23] and is the mechanism which shows less sensitivity with the oxygen variation. For the LOL parameter, all three mechanisms provide very close values between them that are far from the experimental result for $X_{\mathrm{O}_{2}}=0.21$ and show low sensitivity when changing the oxygen concentration compared to the experiment.

In conclusion, in qualitative terms, the three mechanisms show acceptable trends for ID and LOL. In quantitative terms, the Yao mechanism seems that is the one that best fits the ID, especially for the temperature parametric variation [18]. As it is observed, in general, the Yao mechanism shows the fastest ignition followed by the Wang mechanism and, finally, by the Narayanaswamy mechanism, in line with the trends given by the ignition for the HRs and the flamelet shown in figure 1 for the nominal case.

Finally, it is important to note that the previous results show that when the ID is decreased the LOL is reduced too [26], pointing out to the auto-ignition phenomenon as an stabilization flame mechanism and, hence, to the relevance of the chemical kinetics involved in the combustion process for diesel engine conditions [1].

Apart from the global parameters trends, a deeper insight is provided by analyzing the temperature and species spatial fields for the different chemical mechanisms. For that purpose, figure 4 shows the solutions for the nominal case for the temperature and three representative species, namely, formaldehyde $\left(\mathrm{CH}_{2} \mathrm{O}\right)$, a tracer of the low and intermediate temperature reactions, hydroxide $(\mathrm{OH})$, a tracer of the high temperature reactions and, finally, acetylene $\left(\mathrm{C}_{2} \mathrm{H}_{2}\right)$, a soot precursor. The experimental and modeled LOL values are included together with the stoichiometric level curve. The fields correspond to very advanced time instants $(3000 \mu \mathrm{s})$ when a considerable section of the spray has reached quasi-steady regime.
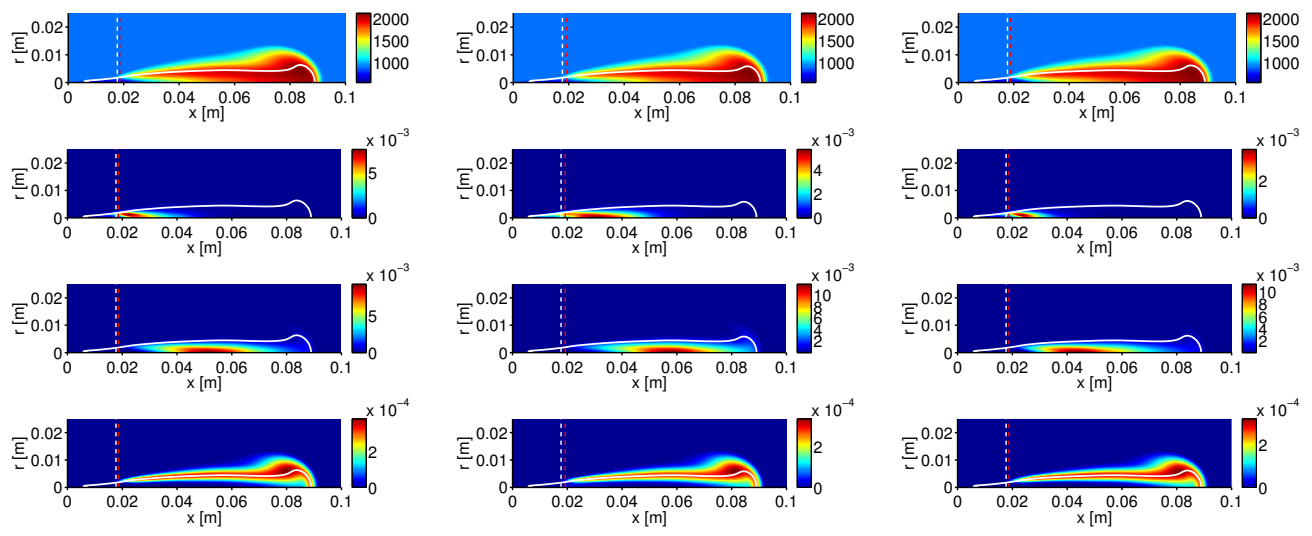

Figure 4. Temperature (top) and species mass fractions fields of $\mathrm{CH}_{2} \mathrm{O}$ (middle top), $\mathrm{C}_{2} \mathrm{H}_{2}$ (middle bottom) and $\mathrm{OH}$ (bottom) for the nominal case $\left(T_{a m b}=900 \mathrm{~K}, X_{\mathrm{O}_{2}}=0.15\right.$ and $\left.\rho=22.8 \mathrm{~kg} / \mathrm{m}^{3}\right)$ for the Narayanaswamy (left), Yao (center) and Wang (right) chemical mechanisms. LOL values are included with dashed lines: experimental (white) and $14 \% \widetilde{Y_{\mathrm{OH}}} \max$ (red). Additionally, the stoichiometric line is shown (solid white).

Attending to the results gathered in figure 4, it is clear how the three mechanisms provide similar results for the high temperature regions and, consequently, the spatial fields and the maximum values for the temperature and $\mathrm{OH}$ fields do not show noticeable discrepancies. Nevertheless, the low temperature regions are the zones where differences are more stringent as can be deduced from the $\mathrm{CH}_{2} \mathrm{O}$ field and, in a less accused way, from the $\mathrm{C}_{2} \mathrm{H}_{2}$ field. The $\mathrm{CH}_{2} \mathrm{O}$ field considerably changes among mechanisms as well as its maximum value reached in the domain.

In general, the fields provided by the Narayanaswamy and the Wang mechanisms are similar [23] and the Yao mech- 
anism is the one that shows greater differences, especially for the $\mathrm{CH}_{2} \mathrm{O}$ that extends in a wider region compared to the other mechanisms.

A clearer description of the combustion process is evidenced when the species are represented in the equivalence ratio-temperature or $\phi$-T maps. Species $\mathrm{CH}_{2} \mathrm{O}, \mathrm{C}_{2} \mathrm{H}_{2}$ and $\mathrm{OH}$ are drawn, only including the points of the domain that verify the condition $Y_{i}>0.3 \cdot Y_{i}^{\max }$ where $i$ represents the species $\mathrm{CH}_{2} \mathrm{O}, \mathrm{C}_{2} \mathrm{H}_{2}$ or $\mathrm{OH}$ and the superscript max refers to maximum value in the domain. Additionally, the curve of maximum temperature as a function of $\phi$ during the combustion onset is included. Figures 5 and 6 show these $\phi$-T maps for the parametric variation in temperature and oxygen concentration. Results correspond to very advanced instants ( $>3500 \mu \mathrm{s})$.
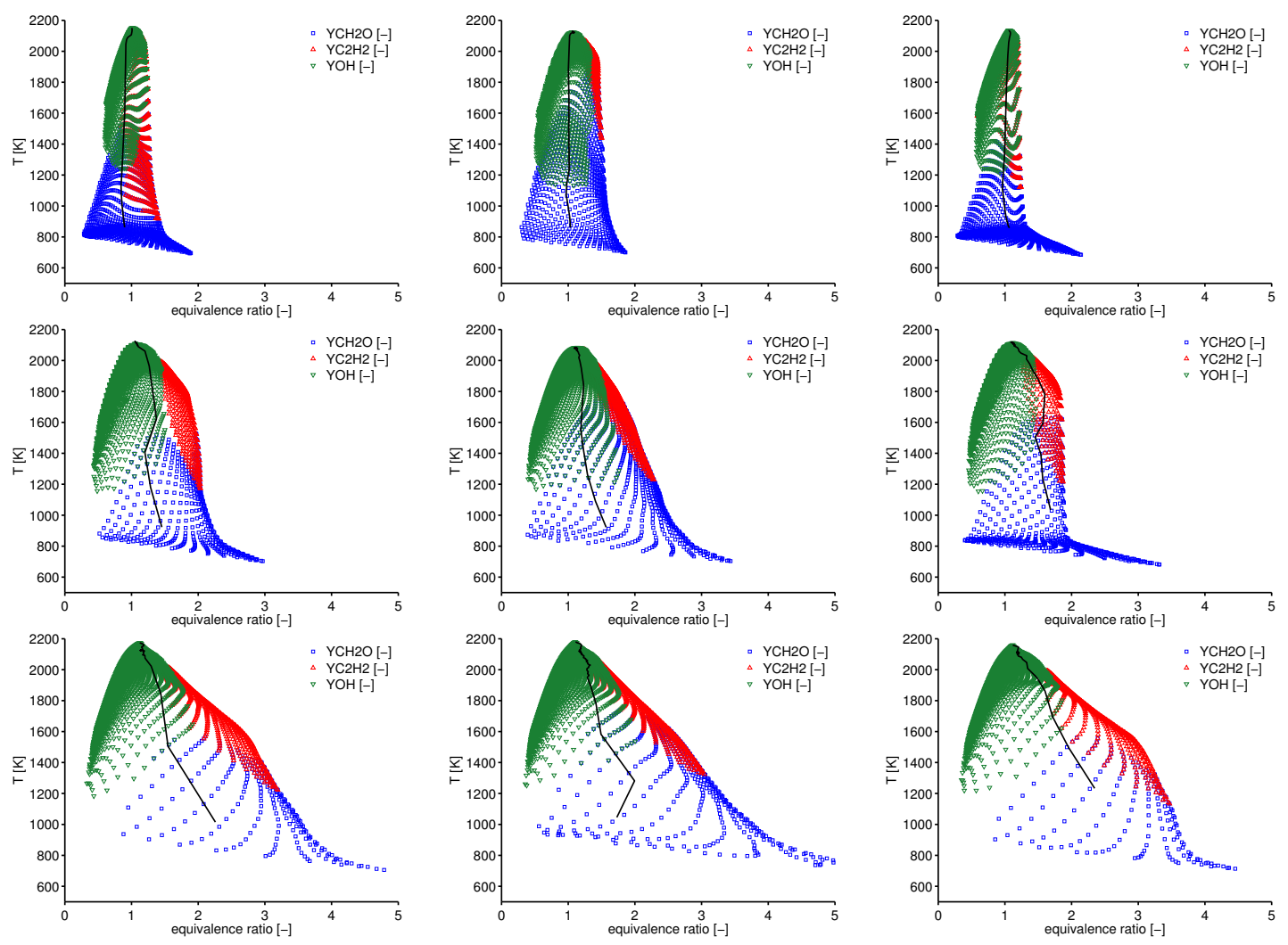

Figure 5. $\phi$-T maps for the temperature parametric variation $\left(X_{\mathrm{O}_{2}}=0.15, \rho=22.8 \mathrm{~kg} / \mathrm{m}^{3}\right)$ for $750 \mathrm{~K}$ (top), $800 \mathrm{~K}$ (middle) and $900 \mathrm{~K}$ (bottom) for the Narayanaswamy (left), Yao (center) and Wang (right) chemical mechanisms. The onset of the combustion in terms of maximum temperature as a function of $\phi$ is included in solid black line. Scales are common for all cases.

Figures 5 and 6 show that the low and intermediate temperatures and rich mixtures $\left(\mathrm{CH}_{2} \mathrm{O}\right.$ and $\left.\mathrm{C}_{2} \mathrm{H}_{2}\right)$ are the zones more affected by the selection of the chemical mechanism while the high temperature region $(\mathrm{OH})$ is very similar for all the mechanisms, as was pointed out in figure 4. Consequently, the formaldehyde is especially affected by the chemistry as well as the contour of the map, which delimits the region of reactive mixtures, that is clearly different for rich mixtures between mechanisms. The zone where the reactive mixtures extend in the $\phi$-T map is important because it determines the prediction of pollutant emissions by the model [24]. It is observed that the Wang mechanism shows the most accused slopes in the contour of the map for rich mixtures and, on the contrary, the softest slopes at these mixtures correspond to the Yao mechanism.

The differences between chemical mechanisms are accentuated when decreasing (by the boundary conditions) the reactivity of the mixture either by the reduction of the ambient temperature or the oxygen concentration.

With regard to the high temperature combustion onset, represented by the transient evolution of the maximum temperature as a function of the equivalence ratio, the Wang mechanism shows that the ignition kernels appear at $\phi>2$ or even richer, the Narayanaswamy provides a similar evolution although at leaner mixtures while, following the Yao mechanism, this onset occurs at slightly rich mixtures. The maximum temperature reached in the domain does not depend on the chemical kinetics.

In terms of species, the Narayanaswamy and Wang mechanisms show similar behavior while the Yao mechanism is the one that exhibits more stringent discrepancies denoted by the wide region where formaldehyde appears and the greater reactivity of the rich mixture fractions.

In conclusion, it is deduced that the low temperature region, where the LOL establishes and close to the region where soot is nucleated, is the most affected zone by the chemical mechanism while no relevant differences arise for the high temperature chemistry. 

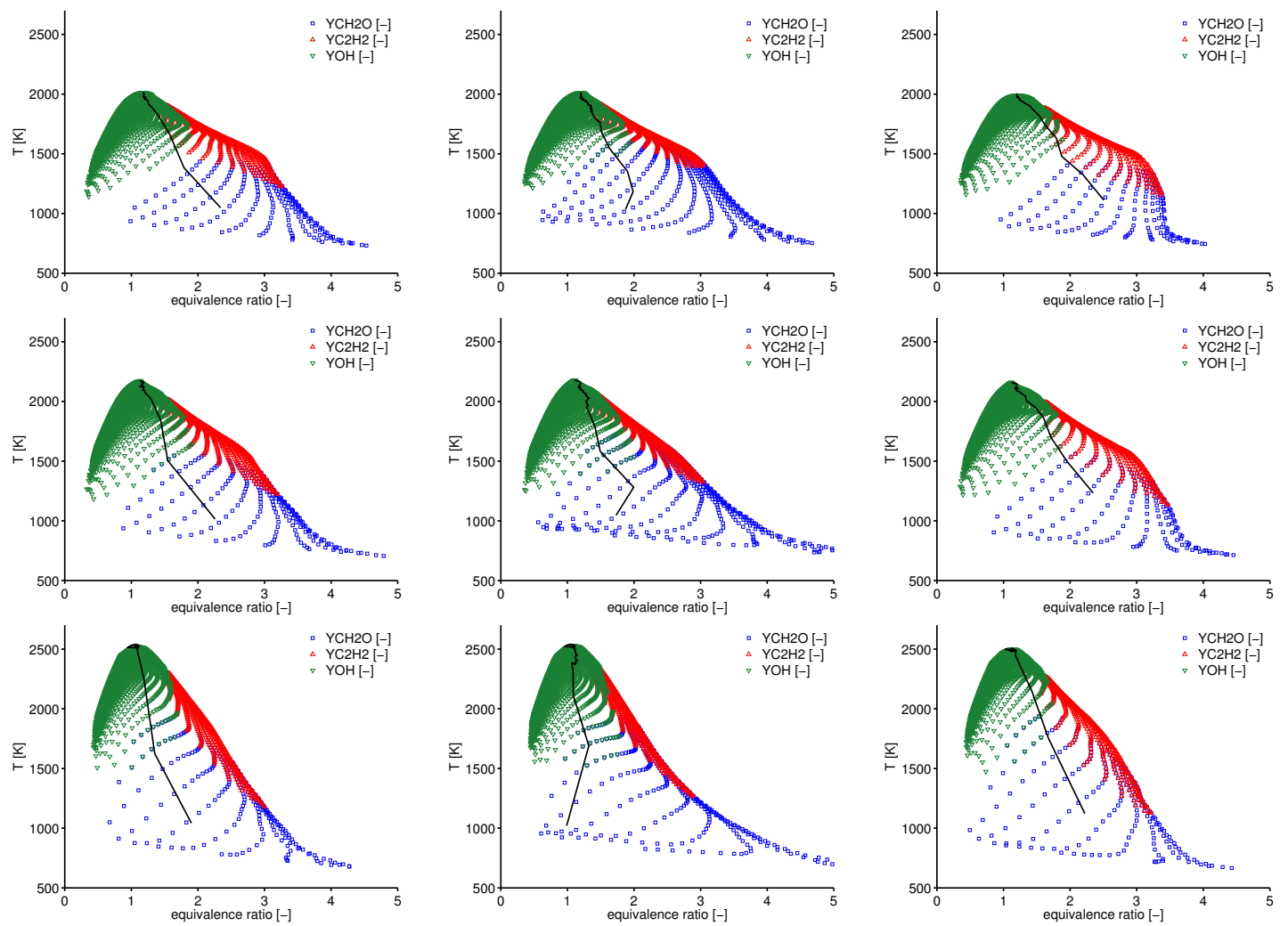

Figure 6. $\phi$-T maps for the oxygen parametric variation $\left(T_{a m b}=900 \mathrm{~K}, \rho=22.8 \mathrm{~kg} / \mathrm{m}^{3}\right.$ ) for $X_{\mathrm{O}_{2}}=0.13$ (top), 0.15 (middle) and 0.21 (bottom) for the Narayanaswamy (left), Yao (center) and Wang (right) chemical mechanisms. The onset of the combustion in terms of maximum temperature as a function of $\phi$ is included in solid black line. Scales are common for all cases.

Finally, to end up this comparison, the temporal evolution of the total mass fraction of some relevant species appearing in the combustion $\left(\mathrm{CH}_{2} \mathrm{O}, \mathrm{C}_{2} \mathrm{H}_{2}, \mathrm{CO}, \mathrm{CO}_{2}\right.$ and $\left.\mathrm{H}_{2} \mathrm{O}\right)$ are included in figures 7 for the nominal case.

It is observed in figure 7 that the three mechanisms give an excellent agreement for the temporal evolution of the masses related to final species, such as $\mathrm{CO}_{2}$ and $\mathrm{H}_{2} \mathrm{O}$ and intermediate species such as CO. Nevertheless, intermediate species that appear in the region of rich mixtures and intermediate/high temperatures, such as $\mathrm{CH}_{2} \mathrm{O}$ and $\mathrm{C}_{2} \mathrm{H}_{2}$, show important discrepancies between mechanisms. Hence, it is concluded that the selection of the chemical scheme is relevant for describing the structure of the combustion as well as for predicting soot formation but not for the calculation of thermochemical parameters related to the high temperature chemistry and/or equilibrium flame conditions.

As a final remark, figure 7 evidences that the $\mathrm{C}_{2} \mathrm{H}_{2}$, a soot precursor, is notably greater for the Yao mechanism with regard to the other schemes and, hence, it is expected that the Yao mechanism predicts greater soot formation.

\section{Conclusions}

One of the major concerns when simulating combustion devices is related to the description of the chemistry provided by the chemical mechanism. Hence, it is considered of scientific interest to give some insight about the influence of the mechanism not only in homogeneous conditions but in the turbulent spray itself.

For that purpose, in this work, the spray A, which is representative of the modern diesel engine combustion, has been modeled by means of the presumed PDF ADF unsteady flamelet model. Three chemical mechanisms for the $\mathrm{n}$-dodecane, widely extended in the scientific community, have been analyzed, namely, Narayanaswamy, Yao and Wang mechanisms.

The three schemes well reproduce the trends of the parametric sweeps in terms of global parameters (ID and LOL) although the Yao mechanism systematically provides faster ignition.

In general terms, the Narayanaswamy and Wang mechanisms show similar behavior while the Yao mechanism departs from the other two.

According to the $\phi$-T maps it is observed that the region of high temperatures is hardly affected by the selection of the chemical mechanism but the zone of low and intermediate temperatures and rich mixtures, in the vicinity where the LOL is established and the soot is nucleated, is the region where the mechanism shows more clear influence. Finally, the mass temporal evolution of relevant species appearing during the combustion confirms that the chemical kinetics scheme has a considerable impact for tracking the evolution of the intermediate species, particularly, for soot predictions, as well as for the combustion flame structure. Nevertheless, the selection of the mechanism has 

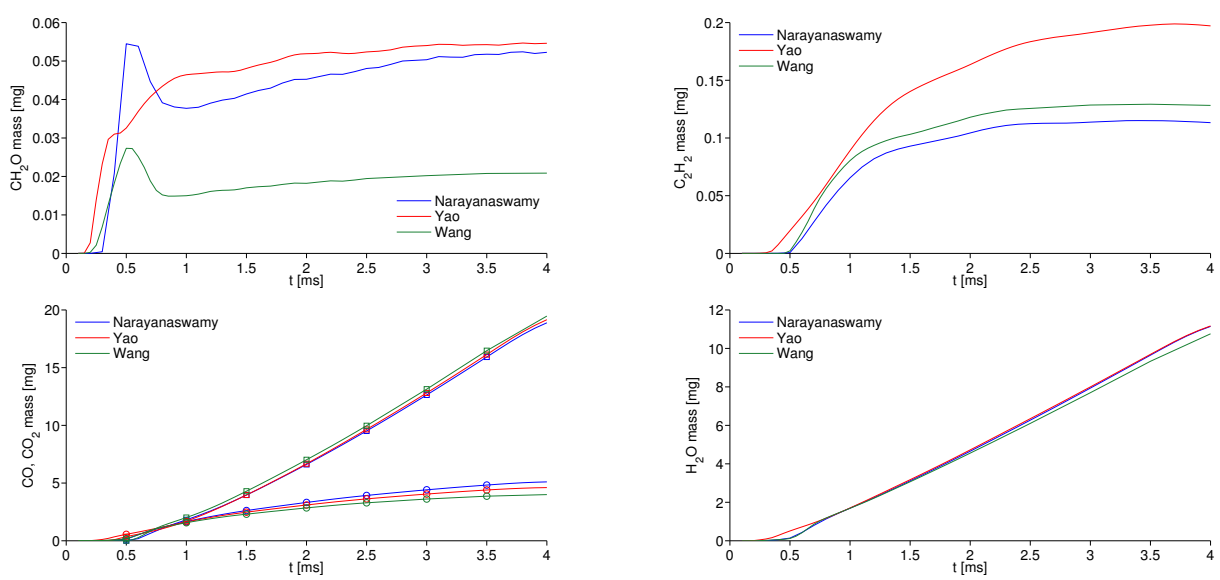

Figure 7. Mass temporal evolution of $\mathrm{CH}_{2} \mathrm{O}$ (top left), $\mathrm{C}_{2} \mathrm{H}_{2}$ (top right), $\mathrm{CO}$ (circles) and $\mathrm{CO}_{2}$ (squares) (bottom left) and $\mathrm{H}_{2} \mathrm{O}$ (bottom right) species for the nominal case and the different mechanisms.

less influence for final species and thermochemical parameters related to the high temperature combustion.

\section{Acknowledgements}

Authors acknowledge that this work was possible thanks to the Ayuda para la Formación de Profesorado Universitario (FPU 14/03278) belonging to the Subprogramas de Formación y de Movilidad del Ministerio de Educación, Cultura y Deporte from Spain. Also this study was partially funded by the Ministerio de Economía y Competitividad from Spain in the frame of the COMEFF (TRA2014-59483-R) national project.

\section{References}

[1] Poinsot, T. and Veynante, D., 2005, "Theoretical and numerical combustion". RT Edwards Inc.

[2] Engine combustion network, https: //ecn. sandia.gov/ ([cit. 2017-03-09]).

[3] Bardi, M., Payri, R., Malbec, L. M., Bruneaux, G., Pickett, L. M., Manin, J., Bazyn, T., Genzale, C. L., 2012, Atomization and Sprays, 22 (10).

[4] Michel, J. B., Colin, O., Veynante, D., 2008, Combustion and Flame, 152 (1), pp. 80-99.

[5] Tillou, J., Michel, J. B., Angelberger, C., Veynante, D., 2014, Combustion and Flame, 161 (2), pp. 525-540.

[6] Desantes, J. M., García-Oliver, J. M., Novella, R., Pérez-Sánchez, E. J., 2017, Applied Thermal Engineering, 117, pp. 50-64.

[7] Benajes, J., Payri, R., Bardi, M., Martí-Aldaraví, P., 2013, Applied Thermal Engineering, 58 (1), pp. 554-563.

[8] Payri, R., García-Oliver, J. M., Xuan, T., Bardi, M., 2015, Applied Thermal Engineering, 90, pp. 619-629.

[9] OpenFOAM http://www .openfoam.com/ ([cit. 2017-03-09]).

[10] Desantes, J. M., Garcia-Oliver, J. M., Pastor, J. M., Pandal, A., 2016, Atomization and Sprays, 26 (7).

[11] Pope, S. B., 1978, AIAA journal, 16 (3), pp.279-281.

[12] Barths, H., Hasse, C., Bikas, G., Peters, N., 2000, Proceedings of the Combustion Institute.

[13] Akkurt, B., Akargun, H. Y., Somers, L. M. T., Deen, N. G., Novella, R., Pérez-Sánchez, E. J., 2017, SAE Technical Paper

[14] Michel, J. B., Colin, O., 2013, International Journal of Engine Research, 15 (3), 346-369.

[15] Colin, O., Michel, J. B., 2016, Flow, Turbulence and Combustion, 97 (2), 631-662.

[16] Naud, B., Novella, R., Pastor, J. M., Winklinger, J. F., 2015, Combustion and Flame, 162 (4), pp. 893-906.

[17] Narayanaswamy, K., Pepiot, P., Pitsch, H., 2014, Combustion and Flame, 161 (4), pp. 866-884.

[18] Yao, T., Pei, Y., Zhong, B. J., Som, S., Lu, T., Luo, K. H., 2017, Fuel, 191, pp. 339-349.

[19] Wang, H., Ra, Y., Jia, M., Reitz, R. D., 2014, Fuel, 136, pp. 25-36.

[20] CMT - Motores Térmicos, Universitat Politècnica de València, Spain, http://www.cmt.upv.es/ECN03.aspx ([cit. 2017-03-09]).

[21] Bekdemir, C., Somers, L. M. T., de Goey, L. P. H., 2011, Proceedings of the Combustion Institute.

[22] Bajaj, C., Ameen, M., Abraham, J., 2013, Combustion Science and Technology, 185 (3), pp. 454-472.

[23] Frassoldati, A., D’Errico, G., Lucchini, T., Stagni, A., Cuoci, A., Faravelli, T., Onorati, A., Ranzi, E., 2015, Combustion and Flame, 162 (10), pp. 3991-4007

[24] Kamimoto, T., Bae, M., 1988, SAE Technical Paper.

[25] Hawkes, E., Sep. 6 2015, Fourth Workshop of the Engine Combustion Network, https://ecn.sandia.gov/ ([cit. 2017-03-09]).

[26] Pickett, L. M., Siebers, D. L., Idicheria, C. A., 2005, SAE Technical Paper. 


\title{
Analytical / Computational Approach to Liquid Spray Heating and Vaporization at Supercritical Pressures
}

\author{
Albert Jordà Juanós ${ }^{1}$ and William A. Sirignano*,1 \\ ${ }^{1}$ Department of Mechanical and Aerospase Engineering, University of California, Irvine, CA \\ 92697-3975, U.S.A. \\ ${ }^{*}$ Corresponding author: sirignan@uci.edu
}

\section{Abstract}

Our findings from two areas of background research will define an approach to the study of liquid spray heating and vaporization in gases at supercritical pressure: (i) vaporizing droplets at supercritical pressure and (ii) supercritical combustion in simple configurations, e.g., counterflow. The a priori conclusion that only one phase exists at supercritical pressure is based on false "lore" and not physical law. The question about the phases must be left open until the analysis reaches a conclusion; a proper approach will be defined. Proper equations of state for density and enthalpy and the determination of phase equilibrium, liquid composition due to dissolved gas, energy of vaporization, surface tension, and transport properties for high pressures will be discussed. The case of an isolated droplet will be reviewed and origin of the transcritical concept will be explained. A counterflow spray configuration at pressures above the liquid critical pressure will be analyzed. The concept of shifting phase equilibrium will be applied as the droplets in the spray heat. Hydrocarbon liquids and oxidizing gaseous environments will be studied. Differences between real fluids and ideal fluids at high pressures will be emphasized. Proper rules for gaseous mixtures and liquid solutions will be discussed.
\end{abstract}

\section{Keywords}

High pressure, supercritical, phase equilibrium.

\section{Introduction}

Combustion of hydrocarbons at high pressure is becoming more important in a broad range of engineering applications, such as diesel engines, gas turbines, and rocket engines, where fuels are usually in liquid phase. There are several issues related to these situations that are not mastered in the combustion literature and must be addressed. (1) The critical pressure of a mixture is commonly substantially higher than the critical pressure of any component. Thus, two phases can easily exist at so-called "supercritical" conditions. (2) Since critical pressure varies with composition, a volume (even at uniform pressure and temperature) can have subcritical portions and supercritical portions in space and/ or time. (3) At subcritical mixture conditions, large amounts of ambient gas will dissolve in the fluid and diffuse away from the liquid-gas interface into the liquid interior. Thus, an injected liquid cannot be assumed to have its original composition or any uniform composition; its density, surface tension, specific heat, and transport properties can change with time and space. (4) Consequently, because of density gradients on both sides of the interface, an experimental image showing a fuzzy interface cannot be sufficient proof of single-phase behavior. (5) Even at true supercritical conditions, there can be a pseudo-two-phase behavior. A narrow domain can exist in temperature-pressure space across which sharp changes in density and can occur. Although the changes are not discontinuous like the true two-phase case, there can be similar consequences. (6) Transport rates decrease and chemical rates increase with increasing pressure. Therefore, the rate-controlling mechanism can change. In the presentation, most of these issues will be discussed. They are not all addressed herein.

In real application combustors, the fuel often enters the combustion chamber at sub-critical temperatures but supercritical pressures, resulting in a "trans-critical" situation [1]. While droplets can be immersed in a gas, the situation is not simply traditional non-premixed combustion. The ambient hot fluid is supercritical but the cold liquid is not supercritical although existing at supercritical pressure. The gaseous mixture in the film neighboring can have a higher critical pressure than any component of the mixture. So, the mixture critical pressure can vary significantly both spatially and temporally. For the same nearly uniform pressure and at the same instant, there can be fluid regions at sub-critical conditions and other regions that are supercritical.

Liquid-vapor phase equilibrium calculations have been used in the past for applications primarily related to the oil and refinery industries. Less attention has been given to situations where liquid-fuel injection is used. Analysis of length- and time-scales associated to the a liquid jet indicates that diffusion of species from the surrounding fluid into the liquid jet occurs much faster than the break-up process of the liquid, and the diffusion layer is much greater than the molecular spacing, even at very high pressures. Thus, continuum theory must be applied in such region with the proper evaluation of thermodynamic properties for a gaseous mixture and liquid solution. The first approach in the analysis of such situations is the study of vapor-liquid equilibrium at high pressure. Results are presented in the next section both for binary mixtures and for mixtures with more than two components. The following section presents the model that accounts for variations in the system composition and temperature due to heat addition. 


\section{High-Pressure Phase Equilibrium}

The formulation for high-pressure phase equilibrium is well known and can be found in several thermodynamic and chemical engineering books (i.e. [2, 3]). Qualitative behavior of mixture critical pressure and temperature was also described in these and other references. The main novelty in our results is in the evaluation of the SRK EOS for particular hydrocarbon-oxygen mixtures as well as the methane-water mixture. The formulation is reviewed below, and results of interest for current combustion mixtures are included.

Thermal, mechanical and chemical potential must be balanced between the liquid and the gas phases for a multicomponent mixture to be in equilibrium:

$$
T^{\ell}=T^{\vartheta} \quad ; \quad P^{\ell}=P^{q} \quad ; \quad \mu^{\ell}=\mu^{\ell}
$$

The relationship between chemical potential and fugacity is:

$$
\mu_{i}^{\diamond}-\mu_{i}=R_{u} T \ln \frac{f_{i}}{f_{i}^{\diamond}} \quad \text { and } \quad \lim _{P \rightarrow 0}\left(\frac{f_{i}}{P_{i}}\right) \rightarrow 1
$$

The chemical potential equality may be expressed in terms of the fugacity as $f_{i}^{\ell}=f_{i}^{q}$, whereby, bringing in the fugacity coefficient gives

$$
\chi_{i}^{\ell} \phi_{i}^{\ell}=\chi_{i}^{\mathcal{Q}} \phi_{i}^{\text {, }} \quad \text { where } \quad \sum_{i=1}^{N} X_{i}^{\ell}=1, \sum_{i=1}^{N} X_{i}^{\mathcal{g}}=1
$$

For systems with only two species, Equations 3 are used to solve for the mole fractions of each species in both liquid and gas phases. A dedicated code has been written to obtain solutions. Iterations are performed using the "fsolve" Matlab function until convergence is achieved.

The Soave-Redlich-Kwong Equation of State (SRK EoS) is selected because of its reasonable accuracy for a wide range of fluid states [4]. It is presented below both in its original form and in its cubic form in terms of compressibility factor $Z$. In the following, $p, T, R_{u}$, and $\bar{v}$ stand for pressure, temperature, universal gas constant, and molar specific volume, respectively.

$$
p=\frac{R_{u} T}{\bar{v}-b}-\frac{a}{\bar{v}(\bar{v}+b)} ; \quad Z^{3}-Z^{2}+\left(A-B-B^{2}\right) Z-A B=0 ; \quad Z \equiv \frac{p \bar{v}}{R_{u} T} ; \quad A \equiv \frac{a p}{\left(R_{u} T\right)^{2}} ; \quad B \equiv \frac{b p}{R_{u} T}
$$

This empirical equation has two parameters $a$ and $b$, which are constants for single-component fluids, but become composition, pressure, and temperature dependent in the multicomponent version. The parameter mixing rules of the Soave-Redlich-Kwong EoS are employed [5]:

$$
a=\sum_{i=1}^{K} \sum_{j=1}^{K} X_{i} X_{j}\left(a_{i} a_{j}\right)^{0.5}\left(1-k_{i j}\right) \quad ; \quad b=\sum_{i=1}^{K} X_{i} b_{i}
$$

The pure species attractive and repulsive parameters $a_{i}$ and $b_{i}$ may be obtained from the species critical points as

$$
\begin{aligned}
& a_{i}=a_{c i} \alpha_{i} ; a_{c i}=0.42748 \frac{\left(R_{u} T_{c i}\right)^{2}}{P_{c i}} ; \alpha_{i}^{0.5}=1+S_{i}\left(1-T_{r i}^{0.5}\right) \\
& S_{i}=0.48508+1.5517 \omega_{i}-0.15613 \omega_{i}^{2} ; b_{i}=0.08664 \frac{R_{u} T_{c i}}{P_{c i}}
\end{aligned}
$$

where $T_{c i}$ and $P_{c i}$ are the critical temperature and critical pressure of mixture component $i, k_{i j}$ is the characteristic binary interaction constant and $\omega_{i}$ are the acentric factors. These values are taken from the literature [6]. Sub-index $r$ stands for "reduced" and equals the property temperature or pressure divided by its critical value.

Note that the mixing rules are associated to each equation of state. Binary interaction coefficients $k_{i} j$ are also dependent on the chosen equation of state, and they can be found in the literature, at least for the major species. From basic thermodynamic relations, the fugacity coefficient can be expressed in terms of temperature and volume (or temperature and pressure). For the SRK EoS case, the fugacity coefficient of species $i$ is

$$
\ln \left[\Phi_{i}\right]=\frac{b_{i}}{b}(Z-1)-\ln [Z-B]-\frac{A}{B}\left(2\left(\frac{a_{i}}{a}\right)^{0.5}-\frac{b_{i}}{b}\right) \ln \left[1+\frac{B}{Z}\right]
$$

The specific enthalpy departure function for the SRK EoS is given by [7]:

$$
h-h^{*}=\frac{1}{\bar{W}}\left[R_{u} T(Z-1)+\frac{T \frac{d a}{d T}-a}{b} \ln \frac{Z+B}{Z}\right]
$$

where $h^{*}$ is the enthalpy for an ideal gas at the given temperature.

At high pressure values and a given temperature, the energy required to vaporize one mole of component $i$ from the liquid mixture to the gaseous mixture is substantially different from the latent heat of vaporization. The former is the enthalpy of vaporization of component $i$ while the latter is defined as the energy required to vaporize one mole 


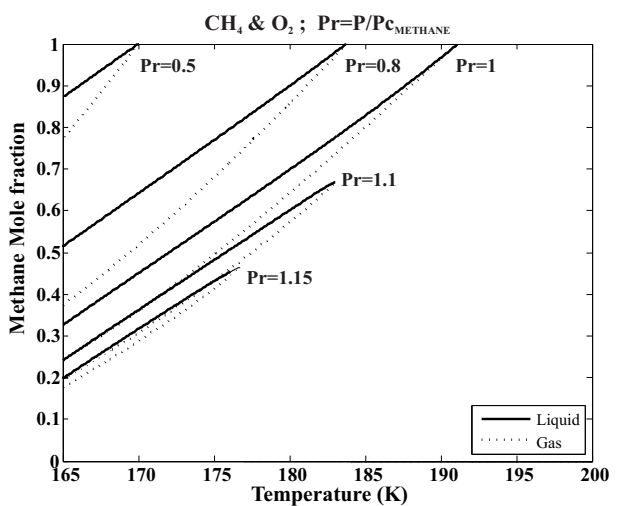

(a) Methane

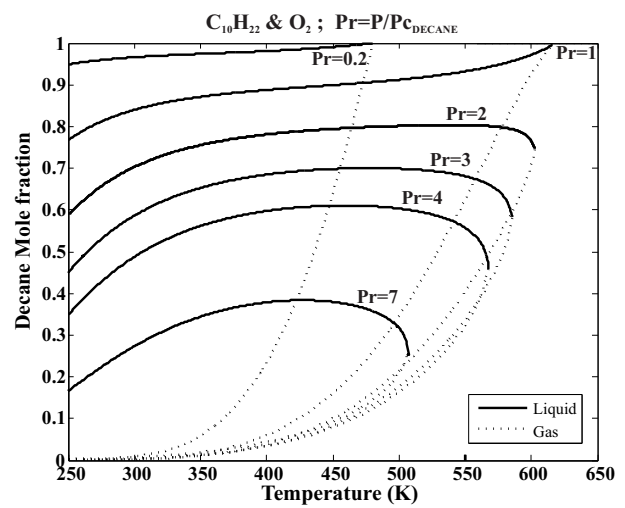

(b) Decane

Figure 1. Binary Systems of (a) methane and oxygen, (b) decane and oxygen, in phase equilibrium (normalizing pressure corresponds to fuel).

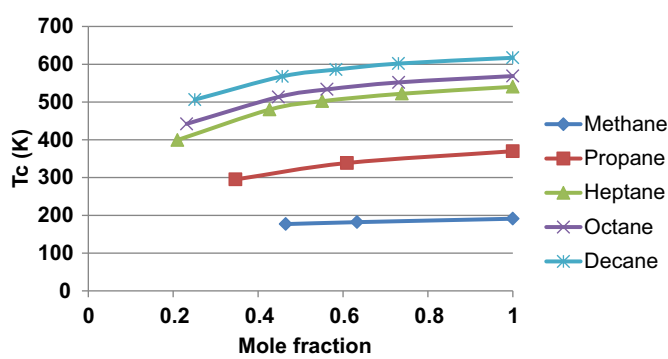

(a) Critical Temperature

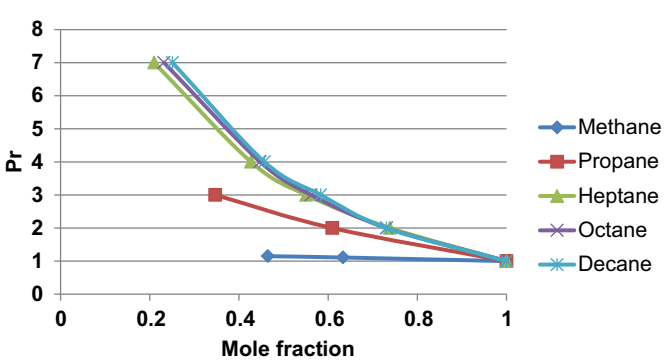

(b) Reduced Pressure

Figure 2. Critical properties of hydrocarbon/oxygen mixtures vs. mole fraction.

of pure liquid $i$ in its own vapor at a given temperature and the corresponding saturation pressure. The enthalpy of vaporization as a function of the fugacity coefficient is:

$$
\Delta \bar{h}_{v, i}=\bar{h}_{i}^{g}-\bar{h}_{i}^{\ell}=R_{u} T^{2} \frac{\partial}{\partial T}\left[\ln \frac{\phi_{i}^{q}}{\phi_{i}^{\ell}}\right]
$$

The surface tension coefficient of the liquid $\sigma_{i}$ is evaluated using the Parachor parameter for each species $P_{i}$, which is taken from the literature [8]:

$$
\sigma_{i}=\left(\frac{P_{i}}{\bar{v}}\right)^{4}
$$

where $\bar{v}$ is the molar specific volume given by the EoS.

\section{Two Species}

Contrary to most of the hydrocarbons, methane has critical pressure and temperature that are much closer to the critical values for oxygen. Thus, the mixture of these two components are described by curves that are sharper, and the range of pressures for which two phases are obtained is narrower. This effect can be seen in Figure 1a. For a prescribed temperature, increasing the pressure reduces the liquid mole fraction of methane, implying that there is more oxygen being dissolved into the liquid mixture. The oxygen mole fraction in the liquid decreases when the temperature is increased at a given pressure. As heavier hydrocarbons are computed, the differences between the critical properties of the hydrocarbon and the oxygen become greater and the mole fractions of liquid and gas phases are more unequal. This effect may be seen in Figure 1b. For pressures greater than the critical pressure of the hydrocarbon, increasing temperature at a fixed pressure raises the mole fraction of the hydrocarbon in the liquid. However, as the critical pressure is approached, the mole fraction slightly drops again.

The mixture critical temperature values vary monotonically between the maximum and the minimum critical temperature of the two pure species (see Figure 2a). The critical pressure, however, rises higher than the critical pressure of any component species and there can still be two phases (or sub-critical conditions; see Figure 2b). In this last plot, the normalizing pressure for each hydrocarbon is the critical pressure of the pure hydrocarbon. At temperatures below $550 \mathrm{~K}$ the methane gaseous phase and the water liquid phase are dominant for all pressures equal or above the critical pressure of water. For temperatures above $550 \mathrm{~K}$, gaseous-water mole fraction increases above $50 \%$. Liquid-water mole fraction is still much greater than liquid methane, with more methane dissolving into the liquid. 


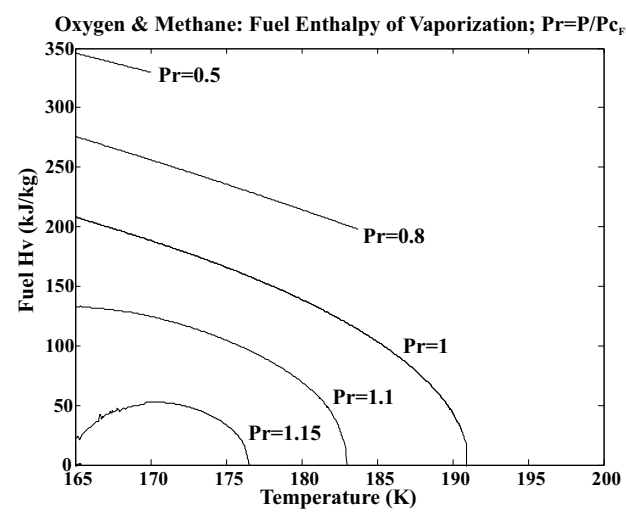

(a) Methane

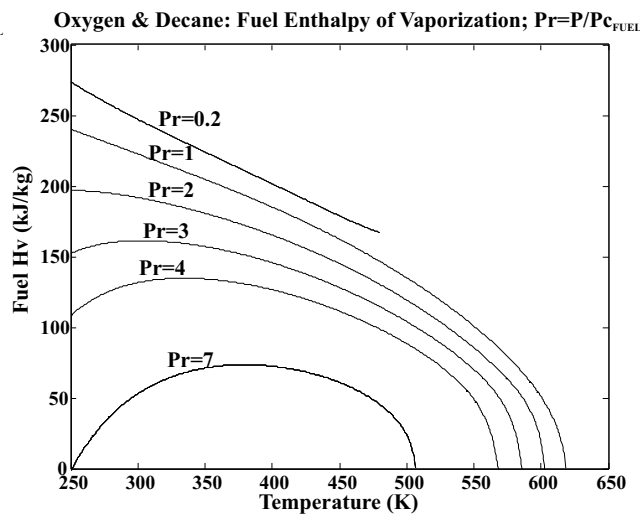

(b) Decane

Figure 3. Hydrocarbon Fuel and Oxygen Enthalpy of Vaporization (Normalizing pressure corresponds to Fuel).

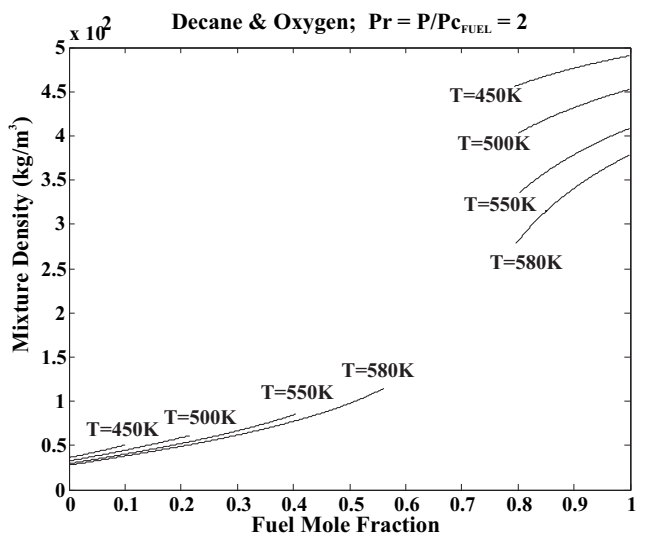

(a) Density vs. Composition

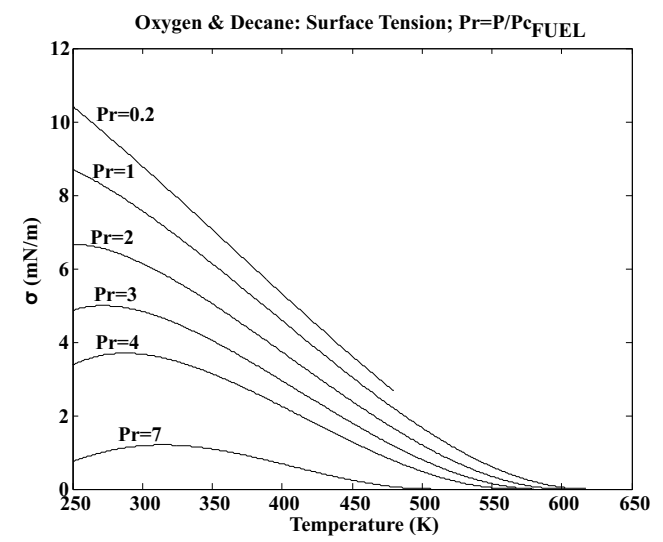

(b) Surface Tension

Figure 4. Decane and Oxygen: Density and Surface Tension.

The variation of the enthalpy of vaporization with temperature and pressure is given in Figure 3 for methane, or decane, with oxygen. The enthalpy of vaporization decreases with pressure at constant temperature. At constant pressure, however, the enthalpy of vaporization may increase at low temperatures and then decrease monotonically until it reaches 0 at the critical point.

Figure 4 shows density and surface tension variations for decane with oxygen. In Figure 4a the composition is varied from the liquid/gas phase equilibrium values at prescribed pressure and temperatures. Density increases with increasing decane fraction both for the liquid and for the gas phases. Density increases faster in the liquid than in the gas with increasing fuel fraction. Figure $4 \mathrm{~b}$ shows how surface tension tends to decrease with temperature at constant pressure until it reaches 0 at the critical point. However, surface tension increases with temperature at low temperature ranges and high pressures. At constant temperature, surface tension decreases with increasing pressure.

\section{Four Species}

Analysis of phase-equilibrium for a mixture composed of reactants and products is of interest for applications such as Exhaust Gas Recirculation (EGR). A single-step stoichiometric reaction between decane and oxygen results in mixtures with four major species: $\mathrm{C}_{10} \mathrm{H}_{22}, \mathrm{O}_{2}, \mathrm{H}_{2} \mathrm{O}$, and $\mathrm{CO}_{2}$. Two mole fraction ratios must be constrained to conduct the phase equilibrium calculation. We will consider $\mathrm{CO}_{2}$ and $\mathrm{H}_{2} \mathrm{O}$ predominantly in the gas phase and always in the product stoichiometric molar proportion of $11 / 10$. The other ratio is selected to be between the oxygen and the products, and will be varied as a parameter. See Figure 5, which applies at twice the critical pressure of the decane, and every curve belongs to one of the oxygen-to-products ratio. As expected, for the higher proportions of oxygen vs. products, concentrations of the reactants are greater in the liquid phase while concentration of products is lower. As temperature is increased, more decane is dissolved into the liquid and less of the other species. For a pure concentration of decane, we get its critical temperature. For low temperatures, more water is in the liquid phase. As temperature is increased, the fraction of water in either phase decreases. The same occurs for oxygen and carbon dioxide, although these two are predominantly in the gas phase. For pressures greater than the critical pressure of decane, altering the ratio between oxygen and products changes the critical temperature of the mixture. Ratios with more oxygen weight result in higher critical temperatures. As it happened for the decane-oxygen binary system, decane mole fraction decreases for a pressure increment. Liquid oxygen mole fraction, however, increases. The fraction of oxygen that dissolves into the liquid increases with increasing pressure and also with increasing 


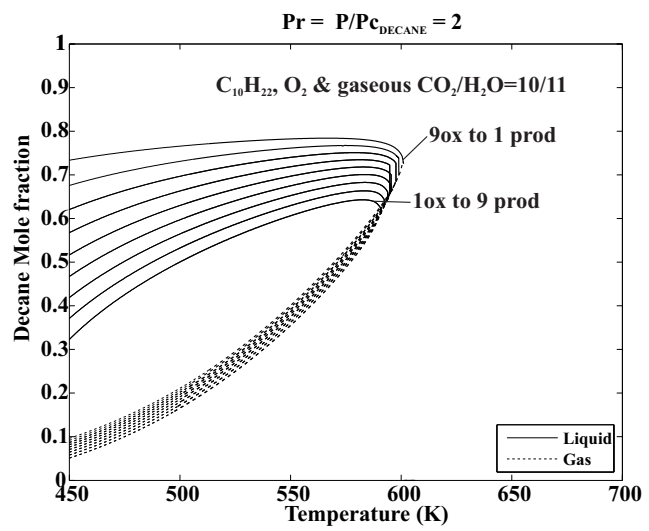

(a) Decane Mole Fraction

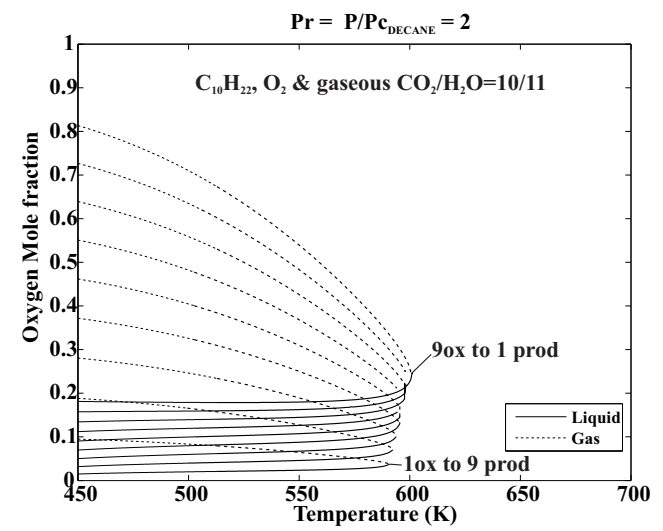

(b) Oxygen Mole Fraction

Figure 5. Decane, Oxygen and Products Phase Equilibrium $\left(P_{r}=P / P_{c_{D E C A N E}}=2\right)$.

oxygen-to-product ratio. The same effect occurs with $\mathrm{CO}_{2}$.

\section{Two-Phase Shifting Equilibrium}

High-pressure phase equilibrium was described in the previous section and solutions for different binary and multicomponent mixtures were reported. Here, we analyze the case where a shift in the species mass fractions and density is caused by a temperature change. The latter may be due to heat transferred from combustion in the gas phase occurring in the vicinity of the phase-change location.

With only phase change and no chemical change, $N_{i}$ (the number of moles of species $i$ ) remains fixed at its initial value $N_{i 0}$; that is $N_{g i}+N_{l i}=N_{i}=N_{i 0}=$ constant. Defining the mole number of the gas mixture as $N_{g}=\sum_{i=1}^{K} N_{g i}$ and the mole number of the liquid mixture as $N_{l}=\sum_{i=1}^{K} N_{l i}$, where $K$ is the total number of species, we also have $N_{g}+N_{l}=N=N_{0}=$ constant. With this, it is convenient to express the mole fractions normalized over both phases. Let $\xi_{g} \equiv N_{g} / N_{0} ; \xi_{l} \equiv N_{l} / N_{0} ; \xi_{g i} \equiv N_{g i} / N_{0}$; and $\xi_{l i} \equiv N_{l i} / N_{0}$. Their relation with the common mole fractions are

$$
\xi_{g i}=X_{g i} N_{g} / N_{0}=X_{g i} \xi_{g} ; \xi_{l i}=X_{l i} N_{l} / N_{0}=X_{l i} \xi_{l} ; X_{g i}=\xi_{g i} / \xi_{g} ; X_{l i}=\xi_{l i} / \xi_{l} ; i=1, \ldots, K-1
$$

Consequently,

$$
\xi_{g i}+\xi_{l i}=\frac{N_{i 0}}{N_{0}}=\xi_{i}=\xi_{i 0} ; i=1, \ldots, K
$$

The relations for phase equilibrium were presented in the previous section (see Equation 3). There are $K$ species and $K$ phase-equilibrium relations. Taking changes in Equation 3 due to pressure and temperature, we obtain

$$
\begin{aligned}
& {\left[\Phi_{g i}+X_{g i} \frac{\partial \Phi_{g i}}{\partial X_{g i}}\right] d X_{g i}-\left[\Phi_{l i}+X_{l i} \frac{\partial \Phi_{l i}}{\partial X_{l i}}\right] d X_{l i}+X_{g i}\left(\sum_{j \neq i} \frac{\partial \Phi_{g i}}{\partial X_{g j}}\right) d X_{g j}-X_{l i}\left(\sum_{j \neq i} \frac{\partial \Phi_{l i}}{\partial X_{l j}}\right) d X_{l j}} \\
& =\left[X_{l i} \frac{\partial \Phi_{l i}}{\partial p}-X_{g i} \frac{\partial \Phi_{g i}}{\partial p}\right] d p+\left[X_{l i} \frac{\partial \Phi_{l i}}{\partial T}-X_{g i} \frac{\partial \Phi_{g i}}{\partial T}\right] d T ; i=1, \ldots, K
\end{aligned}
$$

In a phase change without chemical reaction, we have $\Delta N=0 ; d N_{l}=-d N_{g} ; d \xi_{l}=-d \xi_{g} ; d N_{l i}=-d N_{g i}$; and $d \xi_{l i}=-d \xi_{g i}$. It follows that, for small changes in composition due to vaporization or condensation,

$$
d X_{l i}=\frac{1}{\xi_{l}} d \xi_{l i}-\frac{\xi_{l i}}{\xi_{l}^{2}} d \xi_{l}=-\frac{1}{\xi_{l}} d \xi_{g i}+\frac{\xi_{l i}}{\xi_{l}^{2}} d \xi_{g} \quad ; \quad d X_{g i}=\frac{1}{\xi_{g}} d \xi_{g i}-\frac{\xi_{g i}}{\xi_{g}^{2}} d \xi_{g} ; i=1, \ldots, K
$$

Substitution of Equation 14 into Equation 13 yields

$$
\beta_{i i} d \xi_{g i}+\sum_{k \neq i} \beta_{i k} d \xi_{g k}=\delta_{i} d p+\epsilon_{i} d T ; i=1, \ldots, K
$$

where the following definitions are made:

$$
\begin{aligned}
\beta_{i i} & \equiv \frac{\Phi_{g i}}{\xi_{g}}+\frac{\Phi_{l i}}{\xi_{l}}+\frac{X_{g i}}{\xi_{g}} \frac{\partial \Phi_{g i}}{\partial X_{g i}}+\frac{X_{l i}}{\xi_{l}} \frac{\partial \Phi_{l i}}{\partial X_{l i}}-\frac{\Phi_{g i} X_{g i}}{\xi_{g}}-\frac{\Phi_{l i} X_{l i}}{\xi_{l}}-\frac{X_{g i}}{\xi_{g}}\left(\sum_{j=1}^{K} X_{g j} \frac{\partial \Phi_{g i}}{\partial X_{g i}}\right)-\frac{X_{l i}}{\xi_{l}}\left(\sum_{j=1}^{K} X_{l j} \frac{\partial \Phi_{l i}}{\partial X_{l i}}\right) ; \\
\beta_{i k} & \equiv \frac{X_{g i}}{\xi_{g}} \frac{\partial \Phi_{g i}}{\partial X_{g i}}+\frac{X_{l i}}{\xi_{l}} \frac{\partial \Phi_{l i}}{\partial X_{l i}}-\gamma_{i}, k \neq i ; \\
\gamma_{i} & \equiv \frac{\Phi_{g i} X_{g i}}{\xi_{g}}+\frac{\Phi_{l i} X_{l i}}{\xi_{l}}+\frac{X_{g i}}{\xi_{g}}\left(\sum_{j=1}^{K} X_{g j} \frac{\partial \Phi_{g i}}{\partial X_{g i}}\right)+\frac{X_{l i}}{\xi_{l}}\left(\sum_{j=1}^{K} X_{l j} \frac{\partial \Phi_{l i}}{\partial X_{l i}}\right) ; \\
\delta_{i} & \equiv X_{l i} \frac{\partial \Phi_{l i}}{\partial p}-X_{g i} \frac{\partial \Phi_{g i}}{\partial p} ; \epsilon_{i} \equiv X_{l i} \frac{\partial \Phi_{l i}}{\partial T}-X_{g i} \frac{\partial \Phi_{g i}}{\partial T} ; i=1, \ldots, K ; k=1, \ldots, i-1, i+1, \ldots, K
\end{aligned}
$$


Equation 15 presents a linear system of $K$ equations for the $K$ values of $d \xi_{g}$. It can be solved to yield the solutions. We define $A_{i} \equiv\left[\beta_{i k}\right]^{-1}\left[\delta_{k}\right]$ and $B_{i} \equiv\left[\beta_{i k}\right]^{-1}\left[\epsilon_{k}\right]$ :

$$
\begin{aligned}
& d \xi_{g i}=-d \xi_{l i}=A_{i} d p+B_{i} d T ; \\
& d X_{l i}=\left[\frac{X_{l i} \sum_{j=1}^{K} A_{j}-A_{i}}{\xi_{l}}\right] d p+\left[\frac{X_{l i} \sum_{j=1}^{K} B_{j}-B_{i}}{\xi_{l}}\right] d T ; \\
& d X_{g i}=\left[\frac{A_{i}-X_{g i} \sum_{j=1}^{K} A_{j}}{\xi_{g}}\right] d p+\left[\frac{B_{i}-X_{g i} \sum_{j=1}^{K} B_{j}}{\xi_{g}}\right] d T ; \quad i=1, \ldots, K
\end{aligned}
$$

Assume a small amount of heat per unit mole $\delta q$ is added to the mixture at constant pressure. As equilibrium shifts, we have

$$
d \psi_{g}=-d \psi_{l} ; \quad d \psi_{g i}=-d \psi_{l i}=\frac{W_{i}}{\sum_{j=1}^{K} \xi_{j} W_{j}} d \xi_{g i}=\frac{W_{i} A_{i}}{\sum_{j=1}^{K} \xi_{j} W_{j}} d p+\frac{W_{i} B_{i}}{\sum_{j=1}^{K} \xi_{j} W_{j}} d T
$$

When a differential amount of heat per unit mass $\delta q$ is added to the mixture at constant pressure,

$$
d h=\xi_{g}\left(d h_{g}-d h_{l}\right)+d h_{l}+\left(h_{g}-h_{l}\right) d \xi_{g}=\delta q
$$

The differential of enthalpy per unit mole for a multi-component gas or liquid is given as

$$
d h=\left(\left.\frac{\partial\left(h-h^{*}\right)}{\partial T}\right|_{p, X_{i}}+\left.\frac{\partial h^{*}}{\partial T}\right|_{X_{i}}\right) d T+\sum_{i=1}^{K}\left(\left.\frac{\partial\left(h-h^{*}\right)}{\partial X_{i}}\right|_{p, T, X_{j}, j \neq i}+\left.\frac{\partial h^{*}}{\partial X_{i}}\right|_{T, X_{j}, j \neq i}\right) d X_{i}
$$

Through Equations 19 and 20, the added heat $\delta q$ causes a change $d T$ in the temperature. The change in the temperature causes a shift in $X_{g i}, X_{l i}, \xi_{g i}$, and $\xi_{l i}$ through Equations 17.

These equations can be used to consider a flow where methane and water flow together with two phases at the same temperature. One phase is a gas with methane and water vapor. The other phase has liquid water with dissolved methane. The above equations can be recast to give moles per unit volume (or mass per unit volume) and coupled with the energy equation. Results are expected over the next several months.

\section{Zero-dimensional Model}

In this model, composition and temperature vary with time but remain uniform in space. Pressure is constant and volume is allowed to change accordingly. Initial conditions must be provided. It is necessary that phase equilibrium is enforced in the initial state. Heat must be provided to drive the change of phase; combustion can occur only at higher temperatures where vaporization is completed. For this model, oxygen and fuel must be present in the initial state; they may enter through either phase or both phases.

From Equations 17, 18, 19, and 20, we may construct a system of ordinary differential equations governing the two-phase behavior as it proceeds through the shifting equilibrium change at constant pressure.

$$
\frac{d \xi_{g i}}{d t}=-\frac{d \xi_{l i}}{d t}=B_{i} \frac{d T}{d t} ; \quad \frac{d X_{l i}}{d t}=\left[\frac{X_{l i} \sum_{j=1}^{K} B_{j}-B_{i}}{\xi_{l}}\right] \frac{d T}{d t} ; \quad \frac{d X_{g i}}{d t}=\left[\frac{B_{i}-X_{g i} \sum_{j=1}^{K} B_{j}}{\xi_{g}}\right] \frac{d T}{d t} ; i=1, \ldots, K
$$

The differentials for the fractional change $\dot{r}_{i}$ in gas mass of species $i$ and for the fractional change $\dot{r}$ in total gas mass are given by

$$
\dot{r}_{i} \equiv \frac{1}{\psi_{g}} \frac{d \psi_{g i}}{d t}=\frac{W_{i} B_{i}}{\sum_{j=1}^{K} \xi_{g j} W_{j}} \frac{d T}{d t} ; \dot{r} \equiv \sum_{i=1}^{K}=\frac{1}{\psi_{g}} \frac{d \psi_{g}}{d t}=\frac{\sum_{i=1}^{K} W_{i} B_{i}}{\sum_{j=1}^{K} \xi_{g j} W_{j}} \frac{d T}{d t} ; i=1, \ldots, K
$$

Now, the rate of enthalpy change can be related to the rate of heat addition (or subtraction) also to the time derivatives of temperature and mole fractions.

$$
\frac{d h}{d t}=\psi_{g}\left(\frac{d h_{g}}{d t}-\frac{d h_{l}}{d t}\right)+\frac{d h_{l}}{d t}+\left(h_{g}-h_{l}\right) \frac{d \psi_{g}}{d t}=\frac{\delta q}{d t}=\dot{q}
$$

The following relation can be applied for both the gas enthalpy $h_{g}$ and the liquid enthalpy $h_{l}$.

$$
\frac{d h}{d t}=\left(\left.\frac{\partial\left(h-h^{*}\right)}{\partial T}\right|_{p, X_{i}}+\left.\frac{\partial h^{*}}{\partial T}\right|_{X_{i}}\right) \frac{d T}{d t}+\sum_{i=1}^{K}\left(\left.\frac{\partial\left(h-h^{*}\right)}{\partial X_{i}}\right|_{p, T, X_{j \neq i}}+\left.\frac{\partial h^{*}}{\partial X_{i}}\right|_{T, X_{j \neq i}}\right) \frac{d X_{i}}{d t}
$$

Substitution of Equation 24 into Equation 23 for the rates $d h_{g} / d t$ and $d h_{l} / d t$ yields a governing equation for the temperature derivative $d T / d t$. These first order ODEs are sufficient together with initial conditions to determine the $6 K+6$ variables $X_{g i}, X_{l i}, \xi_{g i}, \xi_{l i}, \psi_{g i}, \psi_{l i}, \psi_{g}, \psi_{l}, h_{g}, h_{l}, h, T$ as functions of time during the heating and phase change at constant pressure.

Once the vaporization is completed or the mixture critical temperature has been exceeded, most of these equations have lost their usefulness; only Equation 24 would still be applicable for $h_{g}$. Since the heats of formation are included in $h_{g}$, that equation would still apply when gas-phase chemistry begins. It has been assumed however that 


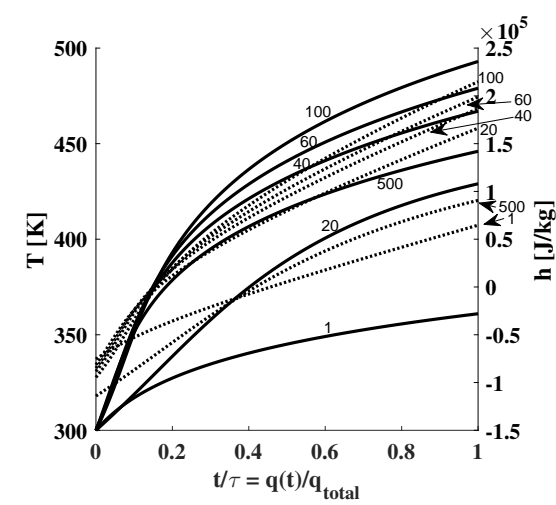

(a) Temperature and specific enthalpy

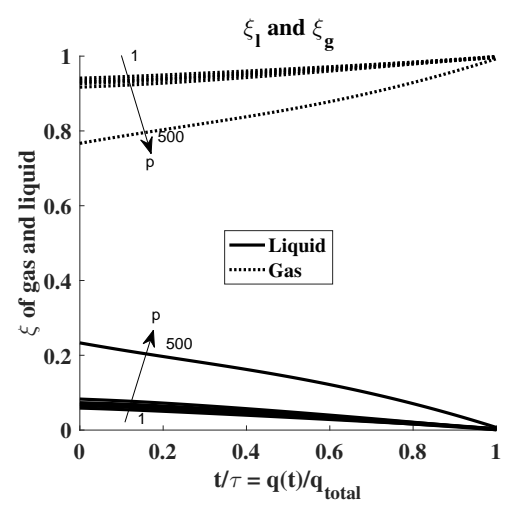

(b) Mole fraction of liquid and gas

Figure 6. (a) Temperature and specific enthalpy, (b) Mole fraction of liquid and gas, $p=[1-500]$ atm

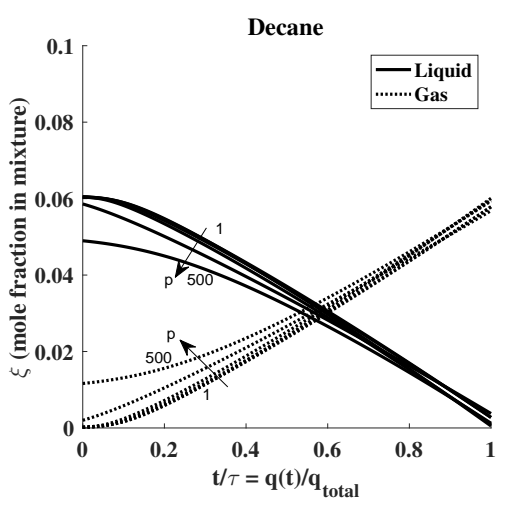

(a) Fraction of decane in mixture

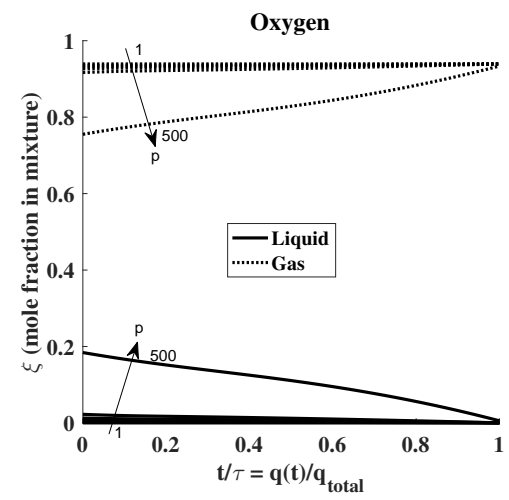

(b) Fraction of oxygen

Figure 7. Mole fraction of species in mixture, $p=[1-500]$ atm

gas-phase chemistry begins after vaporization is completed or after the mixture critical temperature is exceeded. Once vaporization is completed, $h=h_{g}=$ constant and Equation 24 provides a relation between temperature change and composition change. Equations 21 and 22 no longer are relevant in the post-vaporization period; rather, changes in $X_{g i}, \xi_{g i}$, and $\psi_{g i}$ will be driven by chemical kinetic laws.

Below are results for a binary mixture of decane and oxygen, which will be interesting to study both ignition and laminar flame problems. Results for a mixture of water and methane are not included for brevity, but they will also be discussed during the presentation. This second case relates directly to the methane-hydrate diffusion flames.

Let us consider 1 mole of decane and 15.5 moles of oxygen for a stoichiometric mixture between these two components. The mole fractions of each species in each phase are given from phase equilibrium at a given temperature and pressure.

The total number of moles in the liquid and in the gas are computed first. Then, $\xi_{g}$ and $\xi_{l}$ are obtained. In the following expression, subscript 1 stands for decane.

$$
N_{l}=\frac{\left(\xi_{1}-X_{g 1}\right)}{\left(X_{l 1}-X_{g 1}\right)} N_{0} \quad ; \quad N_{g}=N_{0}-N_{l} \quad ; \quad \xi_{l}=\frac{N_{l}}{N_{0}} \quad ; \quad \xi_{g}=\frac{N_{g}}{N_{0}}
$$

Finally, $x i_{g i}$ and $x i_{l i}$ are calculated using Equation 11.

The horizontal axis in the following plots corresponds to the time $t$ normalized by the heating time $\tau=q_{\text {total }} /(\delta q / d t)$, considering that heat is added at a constant rate. The values marked next to each curve are pressure magnitudes in [atm].

Figure 6a shows the evolution of temperature and mixture specific enthalpy from the beginning of the process (always at $300 \mathrm{~K}$ ) until the liquid solution is completely vaporized, for pressures ranging from 1 to $500 \mathrm{~atm}$. The difference between initial and final enthalpy represents the total heat added to vaporize all the liquid. This quantity increases with pressure from 1 to $100 \mathrm{~atm}$, but the trend is inverted from 100 to $500 \mathrm{~atm}$. Figure $6 \mathrm{~b}$ shows the fractions of matter that belong to each phase as a function of the normalized time, for the various studied pressures. Because the considered mixture is composed predominantly of oxygen, the gas phase dominates initially. The liquid fraction is reduced almost linearly as heat is added and vaporization occurs. The fraction of liquid is greater for greater pressures, at any given instant throughout the process. The information in Figure $6 \mathrm{~b}$ is dissected for the two species in Figures $7 \mathrm{a}$ and $7 \mathrm{~b}$. Liquid decane fraction decreases slower initially, and approximately after $25 \%$ of the process, it continues decreasing linearly. There is less liquid decane at higher pressures and more gaseous decane. The opposite effect occurs with oxygen, where more of it disolves into the liquid phase at higher 


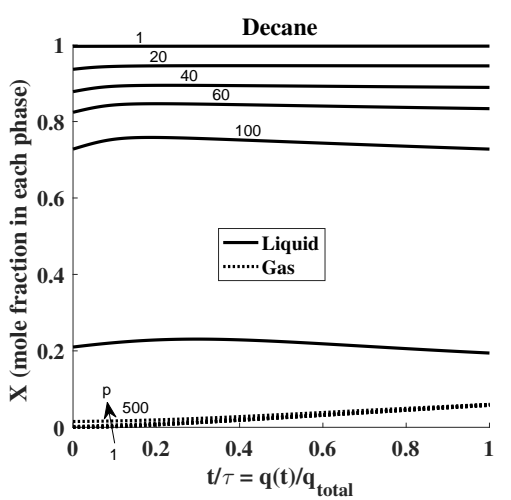

(a) Mole fraction of decane

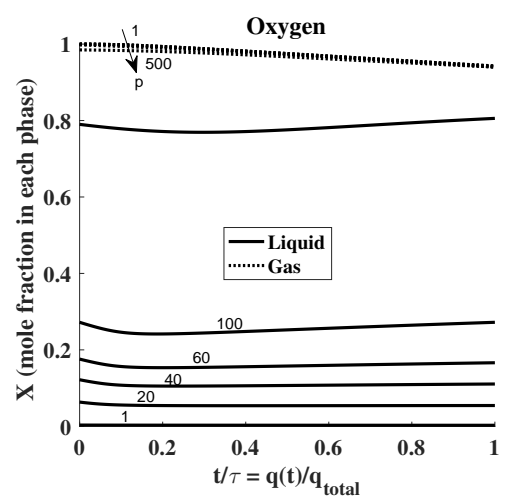

(b) Mole fraction of oxygen

Figure 8. Mole fraction of species, $p=[1-500]$ atm

pressures. Figures $8 \mathrm{a}$ and $8 \mathrm{~b}$ show the mole fraction of each species in each phase throughout the whole process. The proportions of each species in the liquid phase are almost constant with time, except of slight variations at the beginning. For the gas phase, we see a slight monotonic increase in decane mole fraction while oxygen mole fraction is reduced.

\section{Conclusions}

Two-phase multicomponent mixtures are studied analytically and numerically using a cubic equation of state with appropriate high-density relations for enthalpies and potential functions at phase equilibrium. Results for vapor-liquid phase equilibrium are presented for binary mixtures of several hydrocarbons with oxygen and methane with water. These mixtures are relevant to practical situations in which a liquid is injected into a gas that is at supercritical conditions and for methane-hydrate combustion. Given this scenario, researchers usually assume single-phase behavior, while we show that two-phases exist at pressures that are several-fold the critical pressure of any single component within the mixture. Calculations are extended to mixtures with more than two components, where mole fraction ratios must be prescribed to close the system of equations.

A shifting equilibrium is considered as heat is added. Based on the shifting equilibrium assumption, a model for zero-dimensional, unsteady heating, vaporization, and exothermic reaction is presented. The one-dimensional, steady flow model is not presented for brevity. Nevertheless, together with the presented zero-dimensional model, it provides a template by which formulation can readily be made for a steady multidimensional model or unsteady models in one, two, or three dimensions. This would allow for the study of diffusion flames among other cases. The $x$-derivatives in the one-dimensional analysis can be replaced by gradient vector and divergence operators. Of course, boundary conditions will differ for diffusion flames but they are described in classical literature for singlephase behavior. The shifting equilibrium problem with decane and oxygen could be coupled with ignition delay or diffusion flame problems in future work. The case with a mixture of methane and water is interesting for methanehydrate diffusion flames as well as for other applications where water is injected while being premixed with the fuel.

\section{Acknowledgements}

This research was supported by the NSF under Grant CBET-1333605 and AFOSR under Grant FA9550-15-1-0033. The first author appreciates the Balsells Fellowship support.

\section{References}

[1] J. P. Delplanque and W. A. Sirignano, "Transcritical vaporization and combustion of lox droplet arrays in a convective environment," Combust. Sci. Technol., vol. 105, no. 4-6, pp. 327-344, 1995.

[2] S. M. Wallas, Equilibria in Chemical Engineering. Butterworth Pub., 1985.

[3] J. P. O,Connell and J. M. Haile, Thermodynamics Fundamentals for Applications. Cambridge University Press, 2005.

[4] G. Soave, "Equilibrium constants from a modified redlich-kwong equation of state," Chem. Eng. Sci., vol. 27, pp. 1197-1203, 1972.

[5] G. Soave, S. Gamba, and L. A. Pellegrini, "Srk equation of state: Predicting binary interaction parameters of hydrocarbons and related compounds," Fluid Phase Equilib., vol. 299, pp. 285-293, 2010.

[6] J. M. Prausnitz, R. N. Lichtenthaler, and E. G. de Azevedo, Molecular Thermodynamics of Fluid-Phase Equilibria. Englewood Cliffs, N.J.: Prentice Hall PTR, 1999.

[7] Y. V. C. Rao, Chemical Engineering Thermodynamics. Universities Press, 1997.

[8] B. E. Poling, J. M. Prausnitz, and J. P. O,Connell, The Properties of Gases and Liquids, 5th Ed. McGraw-Hill, 2001. 


\title{
Theory of Stagnation-Point Spray Flame Ignition
}

\author{
G. Kats ${ }^{1}$, J.B. Greenberg ${ }^{* 1}$ \\ ${ }^{1}$ Aerospace Engineering, Technion, Israel Institute of Technology, Haifa, Israel \\ *Corresponding author: aer9801@technion.ac.il
}

\begin{abstract}
A theory of stagnation-point spray flame ignition by an isothermal hot surface is presented for the first time. A mixture of fuel droplets and air flowing against an isothermal hot surface (such as a hot ignition probe) is considered. The spray of droplets is modelled using the sectional approach and a mono-sectional case is adopted for simplicity. A single global chemical reaction is assumed for the case when ignition occurs. The mathematical analysis makes use of a small parameter that is exploited for an asymptotic approach. The analysis produces a criterion for ignition that includes effects of the flow field, the reactants and the fuel spray-related parameters. Numerical computations reveal the way in which the latter impact on whether ignition will occur or not.
\end{abstract}

\section{Keywords}

spray flame; ignition; stagnation-point flow

\section{Introduction}

The theory of spray flame ignition has received rather sparse attention in the literature. The ignition process is generally classified by two possibilities: (a) auto-ignition - which is caused by chain branching or thermal feedback in homogeneous/heterogeneous mixtures without exposure to an external heat source, or (b) forced ignition which involves an external source of energy, e.g. an electrical spark, a heated surface, a shock wave, a pilot flame etc. The most common type of forced ignition is a spark generated by a breakdown voltage between two electrodes. The reason for spark ignition popularity in aerospace systems is the aircraft platform requirement to place the ignition system in a constant location and to ignite the cold mixture within a specific time [1]. In the current work we consider forced ignition of a spray of liquid fuel droplets in an oxidising environment.

The problem of forced ignition in gaseous or two phase (gas-liquid) mixtures can be analysed using two quite different mathematical approaches. For analysis of single droplet ignition it was pointed out [2] that either a quasisteady state analysis can be adopted, in which conditions for the existence of a steady state solution are sought as being indicative of the occurrence of ignition, or a full time-dependent description of the physics can be employed to determine if evolution to a successful or failed state is achieved. These two different approaches were previously used in the context of laminar gaseous diffusion flames for examining conditions for flame extinction [3-5]. Interestingly, it was demonstrated in [3] that the so-called static condition for flame extinction did not necessarily coincide with the dynamic condition. Nevertheless, the static or quasi-steady state approach does provide useful information concerning those factors that play a role in extinction/ignition.

For spray ignition the dynamic approach generally requires numerical simulations [6-8]. Recently, a combination of analytical/numerical tools was utilized to tackle the time-dependent approach to spray flame ignition $[9,10]$.

In $[1,11]$ a full three-dimensional DNS study of turbulent mono-size spray flame spark ignition was carried out using a detailed chemical kinetic scheme. This enabled the different scenarios resulting from use of either dispersed or dense sprays to be studied comprehensively. Such DNS studies provide valuable information but are computationally intensive and, as such, have limitations.

In the current paper we adopt a static/steady-state approach for studying the problem of laminar spray flame ignition at the stagnation point of an isothermal hot surface. This configuration, for gas flames, was investigated by Law [12], Chen et al. [13] and Hsu and Lin [14]. It is a useful paradigm, from both fundamental and practical perspectives. In terms of the theory, a similarity solution is admitted which enables the two-dimensional configuration to be reduced to a one-dimensional form thereby facilitating insight into the basic physics/chemistry. In practical terms, such configurations appear in chemically reacting flows such as the hot tip of a projectile or a heated ignition probe. Furthermore, the influence of stretch can be readily controlled and studied in such a set-up $[13,14]$.

Here we consider for the first time the ignition of a fuel spray-air mixture in stagnation-point flow. We derive a new analytical condition for ignition of the mixture and examine the role of the spray in determining whether ignition is achieved or not. 


\section{Mathematical Model}

The configuration we consider is shown in Fig. 1. A steady laminar flow of a constant density fluid, comprised of fuel vapor, fuel droplets, oxygen and a diluent, approaches the stagnation point $\eta=0$, where $\eta$ is a similarity variable to be described later. A hot spot is generated at the stagnation point due to a prescribed temperature $T_{W}$. Under appropriate conditions the consequent thermal field will enable the formation of steady state premixed spray flame. At the current stage we assume a global, irreversible, one step reaction described by Arrhenius's law can be employed: fuel + oxidant $\rightarrow$ products + heat. In the context of flame ignition a more detailed chemical mechanism is probably more accurate but the one-step mechanism we use provides a reasonable starting point if the role of the spray on ignition is the main focus of attention.

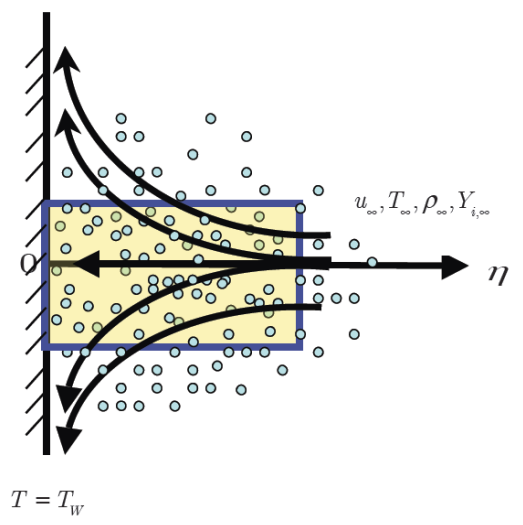

Figure 1: Configuration for stagnation-point ignition by an isothermal hot surface of a premixed fuel spray oxidant mixture.

In Cartesian coordinates the governing equations are the conventional boundary-layer-type conservation equations [15], supplemented by a conservation equation relating to the liquid fuel in the spray.

Continuity:

$\frac{\partial(\rho u)}{\partial x}+\frac{\partial(\rho v)}{\partial y}=0$

Momentum:

$\rho u \frac{\partial u}{\partial x}+\rho v \frac{\partial u}{\partial y}-\frac{\partial}{\partial y}\left(\mu \frac{\partial u}{\partial y}\right)=\rho_{\infty} u_{\infty} \frac{d u_{\infty}}{d x}$

Energy:

$\rho u \frac{\partial T}{\partial x}+\rho v \frac{\partial T}{\partial y}-\frac{\partial}{\partial y}\left(\frac{\lambda}{c_{p}} \frac{\partial T}{\partial y}\right)=\omega-L S_{v}$

Fuel vapor:

$\rho u \frac{\partial Y_{F}}{\partial x}+\rho v \frac{\partial Y_{F}}{\partial y}-\frac{\partial}{\partial y}\left(\rho D_{F} \frac{\partial Y_{F}}{\partial y}\right)=-\omega+S_{v}$

Oxygen:

$\rho u \frac{\partial Y_{O}}{\partial x}+\rho v \frac{\partial Y_{O}}{\partial y}-\frac{\partial}{\partial y}\left(\rho D_{O} \frac{\partial Y_{O}}{\partial y}\right)=-\omega$

Liquid Fuel:

$$
\rho u \frac{\partial Y_{d}}{\partial x}+\rho v \frac{\partial Y_{d}}{\partial y}=-S_{v}
$$

where the chemical source term is

$\omega=\rho \frac{A}{a} Y_{F} Y_{O} \exp \left(-\frac{E}{R T}\right)$

and the vaporization term is

$S_{v}=\rho C Y_{d} H\left(T-T_{v}\right)$ 
In these equations $u, v$ are the velocity components in the $x, y$ directions, respectively, $\rho$ is the density, $T$ is the temperature, $\lambda$ the thermal conductivity, $c_{p}$ the specific heat, $L$ the latent heat of vaporization of the liquid fuel, $Y_{F}, Y_{O}, Y_{d}$ are the mass fractions of the fuel vapor, oxygen and liquid fuel, respectively, $a$ is the strain rate, $A$ the pre-exponential constant, $E$ the activation energy, $R$ the universal gas constant, $C$ the vaporization coefficient and $T_{v}$ is the prespecified temperature at which droplet evaporation is initiated (such as the liquid fuel's boiling temperature).

The governing equations are to be solved subject to the following boundary equations:

$$
\begin{aligned}
& u(x, 0)=v(x, 0)=0, u(x, \infty)=u_{\infty} \\
& T(x, 0)=T_{W}, T(x, \infty)=T_{\infty} \\
& \frac{\partial Y_{F}(x, 0)}{\partial x}=0, Y_{F}(x, \infty)=m_{F u} \\
& Y_{d}(x, \infty)=m_{d u}
\end{aligned}
$$

where the subscript "u" refers to conditions in the unburned mixture.

Eq. (1) is the continuity equation for the mixture. Its form follows from the implicit assumption that the liquid fuel volume fraction is sufficiently small. Therefore, the transport properties will be supposed to be determined primarily by the properties of the gaseous species. It is further assumed that the various transport coefficients such as thermal conductivity, diffusion coefficients, specific heat at constant temperature, latent heat of vaporization of the liquid droplets, etc., can be satisfactorily specified by representative constant values. It is assumed that the velocity of propagation of the flame is much less than the velocity of sound so that dynamic compressibility effects in the mixture can be neglected. Thus, the density becomes only a function of the temperature through the gas law. The droplets are taken to be in dynamic equilibrium with their host surroundings so that no momentum equations need to be solved for the droplets, and the appropriate drag-related term in the gas phase momentum Eq. (2) need not be considered. Eq. (3) is the energy conservation equation written in terms of temperature. Note the source/sink terms on the right hand side corresponding to (a) heat release by chemical reaction (second term) and (b) heat loss due to absorption of heat by droplets for vaporization (third term). Eq. (4) is the mass fraction conservation equation for fuel vapor with the last term on the right hand side the source term for the production of fuel vapor by evaporating droplets. In order to model the spray of liquid fuel droplets the sectional approach was exploited and a mono-sectional description is given here for simplicity at the current stage [16]. As mentioned, the droplets are viewed from a far-field vantage point, i.e. their average velocity is equal to that of their host environment. In addition, the temperature of the droplets is taken as equal to that of the surroundings; essentially, the droplets heat-up time is small compared with the characteristic time associated with their motion. Here, use of these latter two assumptions is made mainly for mathematical tractability but also in order to reduce some of the physical clutter to allow us to focus on the main mechanisms at play. Eq. (5) is the mass fraction conservation equation for the oxidiser. The use of the Heaviside function, $H$, in Eq. (8) implies that appreciable evaporation of the fuel droplets only commences when the local temperature reaches a prescribed value, $T_{v}$, say the liquid fuel's boiling temperature. The conditions under which the aforementioned spray-related assumptions (based on relevant scaling) are applicable are discussed at length in [17].

By applying appropriate transformations [15], assuming a unity Lewis number and then normalizing (see APPENDIX) this set of equations can be written in terms of a similarity variable, $\eta$, as follows:

$$
\begin{aligned}
& f^{\prime \prime \prime}+f f^{\prime \prime}+1-\left(f^{\prime}\right)^{2}=0 \\
& \theta^{\prime \prime}+f \theta^{\prime}=-\alpha \omega+\alpha L^{\prime} S_{v} \\
& y_{F}^{\prime \prime}+f y_{F}^{\prime}=\alpha \omega / \vartheta_{F}-\alpha S_{v} \\
& y_{O}^{\prime \prime}+f y_{O}^{\prime}=\alpha \omega / \vartheta_{O} \\
& f y_{d}^{\prime}=\alpha S_{v}
\end{aligned}
$$

where $f$ is related to the stream function which satisfies the continuity equation identically (for details see [12] and [15]), and

$$
\begin{aligned}
& \omega=\rho \alpha A m_{O u}\left(m_{F u}+m_{d u}\right) y_{F} y_{O} \exp \left(-\theta / \theta_{a}\right) \\
& S_{v}=\rho C y_{d} H\left(\eta_{v}-\eta\right) \\
& \alpha=(1 / a \rho)
\end{aligned}
$$


with $\eta_{v}$ being the location where the temperature at which the onset of evaporation occurs.

The boundary conditions become:

$\left\{\begin{array}{l}f(0)=f^{\prime}(0)=0, f^{\prime}(\infty)=1 \\ \theta(0)=\theta_{W}, \theta(\infty)=\theta_{\infty} \\ y_{f}^{\prime}(0)=0, y_{f}(\infty)=1-\delta \\ y_{O}^{\prime}(0)=0, y_{O}(\infty)=1 \\ y_{d}(\infty)=\delta\end{array}\right.$

which must be supplemented with matching conditions of continuity on $\theta$ and $y_{f}$ and their first derivatives at $\eta_{v}$ where evaporation begins.

\section{Solution}

The solution for the stream function (Eq. (9)) is found numerically, and used when solving for the thermal and species fields. As far as the latter are concerned, in the limit of infinitely large activation energy chemical reaction will be negligible, but for large yet finite values chemical activity will be initiated close to the wall. The structure of the solution we seek should therefore be comprised of an outer "frozen" solution of a convective-diffusive nature merging with an inner solution near the wall which is dominated by a reactive-diffusive nature. We therefore determine the solutions in the outer region first taking the nonlinear chemical kinetic terms to be negligible. For ease, use is made of a new coordinate

$$
\xi=N(\eta) / N(\infty)
$$

with

$$
N(\eta) \equiv \int_{0}^{\eta} \exp \left(-\int_{0}^{\eta^{\prime}} f\left(\eta^{\prime \prime}\right) d \eta^{\prime \prime}\right) d \eta^{\prime}
$$

and the thermal field is found to be

$$
\theta_{\text {frozen }}(\xi)= \begin{cases}\frac{\left(\theta_{v}-\theta_{\infty}\right) \xi-\theta_{v}+\theta_{\infty} \xi_{v}}{\xi_{v}-1} & , 1 \geq \xi \geq \xi_{v} \\ \theta_{\text {Wall }}+\left[\theta_{v}-\theta_{\text {Wall }}-L^{\prime} \Delta \delta m\left(\xi_{v}\right)\right] \frac{\xi}{\xi_{v}}+L^{\prime} \Delta \delta m(\xi) & , \xi_{v} \geq \xi \geq 0\end{cases}
$$

The fuel vapor profile is given by

$$
y_{F, \text { frozen }}(\xi)=\left\{\begin{array}{lr}
1-\delta-\Delta \delta(\xi-1) m^{\prime}\left(\xi_{v}\right) & , 1 \geq \xi \geq \xi_{v} \\
1-\delta-\Delta \delta\left[\left(\xi_{v}-1\right) m^{\prime}\left(\xi_{v}\right)+m(\xi)-m\left(\xi_{v}\right)\right], & \xi_{v} \geq \xi \geq 0
\end{array}\right.
$$

The oxygen profile is simply

$$
y_{O, \text { frozen }}(\xi) \equiv 1
$$

and in Eqs. (19) and (20) use was made of the liquid fuel mass fraction profile

$y_{d}=\delta, \quad \eta \geq \eta_{v}$

$y_{d}=\delta \exp \left[\Delta\left(\Lambda(\eta)-\Lambda\left(\eta_{v}\right)\right)\right], \quad \eta_{v} \geq \eta \geq 0$

with

$$
\Lambda(\eta) \equiv \int \frac{d \eta}{f(\eta)}
$$

and the spray related evaporation function $m(\xi)$ which is the $\xi$-coordinate version of the following function written in terms of the similarity variable

$r(\eta)=\int_{0}^{\eta}\left[\exp \left(-\int_{0}^{\eta^{\prime}} f\left(\eta^{\prime \prime}\right) d \eta^{\prime \prime}\right)\left\{\int_{0}^{\eta^{\prime}} \exp \left[\Delta\left(\Lambda\left(\eta^{\prime \prime}\right)-\Lambda\left(\eta_{v}\right)\right)+\int_{0}^{\eta^{\prime \prime}} f\left(\eta^{\prime \prime}\right) d \eta^{\prime \prime}\right]\right\} d \eta^{\prime}\right.$

The location of the onset of evaporation is found from the implicit equation

$$
\frac{\left(\theta_{v}-\theta_{\infty}\right) N^{\prime}\left(\eta_{v}\right)}{N\left(\eta_{v}\right)-N(\infty)}=\frac{\left(\theta_{v}-\theta_{W}-L^{\prime} \Delta \delta r\left(\eta_{v}\right)\right) N^{\prime}\left(\eta_{v}\right)}{N\left(\eta_{v}\right)}+L^{\prime} \Delta \delta r^{\prime}\left(\eta_{v}\right)
$$


Armed with the outer frozen solution, we are in a position to develop the inner solution close to the stagnation point at which chemical reaction will occur. Asymptotic methods are tailor-made for this purpose once a small parameter has been identified. Here we choose to use

$\varepsilon=\frac{\theta_{W}^{2}}{\theta_{a}} ; \theta_{a}=\frac{E c_{p}}{R q} ; \theta_{W}=\frac{T_{W} c_{p}}{q} ; \beta=\theta_{W}-\theta_{v}$

The temperature distribution in the outer region has the following form

$\theta_{\text {outer }}=\theta_{v}+A_{0}\left(\xi_{v}-\xi\right)+\varepsilon A_{1}\left(\xi_{v}-\xi\right)+O\left(\varepsilon^{2}\right)$

where the constants $A_{0}, A_{1}$ will be found by matching the inner and outer solutions.

In order to continue the analysis we introduce a stretched inner coordinate

$\chi=\beta \xi / \varepsilon$

Now, the solution in the inner region will be the frozen outer solution with the addition of a perturbation due to chemical reaction, viz.

$\theta_{\text {inner }}(\chi)=\theta_{\text {frozen }}(\chi)+\varepsilon \phi(\chi)$

After much algebraic manipulation it can be shown that the function $\phi$ must satisfy

$\frac{d^{2} \phi}{d \chi^{2}}=-\frac{\sigma}{2} \exp \left(\phi-\frac{\chi}{\xi_{v}}\left(1+\frac{L^{\prime} \Delta \delta m\left(\xi_{v}\right)}{\beta}\right)-\frac{\theta_{a} L^{\prime} \Delta \delta m\left(\frac{\varepsilon \chi}{\beta}\right)}{\theta_{W}^{2}}\right)$

where

$\sigma \equiv \frac{2 \mu \varepsilon N(\infty)^{2}}{\beta^{2}} \cdot\left(1-\left.\frac{\xi_{v}}{\vartheta_{O}} \beta \frac{d \phi}{d \chi}\right|_{0}\right)\left(\begin{array}{l}1-\delta-\Delta \delta\left[\left(\xi_{v}-1\right) m^{\prime}\left(\xi_{v}\right)-m\left(\xi_{v}\right)\right] \\ -\left.\frac{\xi_{v}}{\vartheta_{F}} \beta \frac{d \phi}{d \chi}\right|_{0}\end{array}\right) \exp \left(-\frac{\theta_{a}}{\theta_{W}}\right)$

with $\sigma$ being a suitably defined Damkohler number. When $\sigma \rightarrow 0$ chemical reaction will be negligible, whereas when $\sigma \rightarrow \infty$ it is very fast. Eq. (30) is subject to the boundary condition $\phi(\chi=0)=0$ which stems from the $O(1)$ nature of the temperature at the stagnation point. The second boundary condition is obtained by matching the inner and outer solutions and is given by:

$\left.\frac{\partial \phi}{\partial \chi}\right|_{\chi \rightarrow \infty}=\frac{L^{\prime} \Delta \delta m\left(\xi_{v}\right)}{\xi_{v} \beta}$

The solution of Eq.(30) is

$\phi^{*}(\tau)=\tau+\ln \left[c_{1}\left(1-\tanh ^{2}\left(\frac{\sqrt{c_{1}\left(c_{2}+\tau\right)^{2}}}{2}\right)\right) / \sigma^{*}\right]$

where

$\tau=\frac{\chi}{\xi_{v}}\left(1+\frac{L^{\prime} \Delta \delta m\left(\xi_{v}\right)}{\beta}\right)$

$\phi^{*}=\phi-\frac{\theta_{a} L^{\prime} \Delta \delta m\left(\frac{\varepsilon \chi}{\beta}\right)}{\theta_{W}^{2}}$

$\sigma^{*}=\frac{\sigma}{\left[\frac{1}{\xi_{v}}\left(1+\frac{L^{\prime} \Delta \delta m\left(\xi_{v}\right)}{\beta}\right)\right]^{2}}$

and where $c_{1}, c_{2}$ are constants to be found from the boundary conditions. The condition at the wall leads to:

$\phi^{*}(0)=\ln \left(\frac{c_{1}}{\sigma^{*}}\right)+\ln \left[1-\tanh ^{2}\left(\frac{\sqrt{c_{1} c_{2}^{2}}}{2}\right)\right]=0$ 
whereas the outer condition yields

$$
c_{1}=\left(1-\frac{L^{\prime} \Delta \delta m\left(\xi_{v}\right)}{\beta+L^{\prime} \Delta \delta m\left(\xi_{v}\right)}\right)^{2}
$$

By substituting back into Eq.(38), after some algebra it is found that

$$
\left|c_{2}\right|=\ln \left[\frac{1 \pm \sqrt{1-\frac{\sigma^{*}}{c_{1}}}}{1 \sqrt{1-\frac{\sigma^{*}}{c_{1}}}}\right] / \sqrt{c_{1}}
$$

from which it can deduced that in order to guarantee a solution the condition $\sigma^{*} \leq c_{1}$ must be satisfied. This implies that $\sigma^{*}=c_{1}$ is a transition point between a burning and non-burning solution or, putting it another way, between a situation in which ignition occurs or does not. In full, the condition reads

$\sigma=1 / \xi_{v}^{2}$

where $\sigma$ is defined in Eq. (31). The role that the spray plays in this ignition criterion is clearly represented by the heat loss terms resulting from the heat absorbed by the droplets for evaporation, by the production of fuel vapor that exhibits itself via the second bracketed term on the RHS of Eq. (31) and by the location of the onset of evaporation $\xi_{v}$. It is of interest to note that if no spray is present, i.e. $\delta=0$, a single phase gas situation is under consideration, the criterion Eq. (40) collapses to that of [12].

\section{Results and discussion}

Use was made of the analytical solution in the previous section to examine the effect of heat loss and fuel spray parameters on conditions for spray flame propagation and extinction. The data used for the calculations was as follows (unless otherwise specified): $Q=1.279 \times 10^{7} \mathrm{~J} / \mathrm{kg}, L=0.04 Q, \lambda=0.02512 \mathrm{Wm} / \mathrm{K}, \mathrm{A}=10^{10} \mathrm{~s}^{-1}$, $E=2 \times 10^{8} \mathrm{~J} / \mathrm{kmol}, \quad c_{p}=1255.92 \mathrm{~J} / \mathrm{kgK}, T_{u}=300 \mathrm{~K}, T_{v}=400 \mathrm{~K}, \quad a=1000 \mathrm{sec}^{-1}$. The chemical kinetic scheme employed concerns the burning of $n$-decane and relevant thermochemical data was taken from [18] and [19]. By specifying the initial fraction of liquid fuel in the total fuel (vapor +liquid) in the fresh mixture, $\delta$, it can be shown that the mass fractions in the fresh mixture are given by the following expressions:

$\left(m_{O u}, m_{d u}, m_{F u}\right)=\left(s\left\{1-m_{d u}-m_{F u}\right\}, \frac{s \delta}{\left(1+\alpha_{O F} / \phi\right)}, m_{d u} \frac{(1-\delta)}{\delta}\right)$

unless $\delta=0$ for which

$m_{F u}=\frac{s}{\left(s+\alpha_{O F} / \phi\right)}$

where $s$ is the mole fraction of oxygen in the fresh mixture, $\alpha_{O F}$ is the stoichiometric coefficient and $\phi$ is the equivalence ratio. Here $\phi$ is taken as $5, \alpha_{O F}=3.5$ and $s=1$.

It is clear that, given a set of data relating to the gas and liquid phases, condition (40) and Eq. (31) provide a relationship from which the critical value of the stagnation point temperature can be extracted. To examine the influence of the spray-related parameters on this temperature these parameters were varied whereas the gasrelated parameters were held fixed. Fig. 2 is an example in which contours of selected critical temperatures are drawn in the $C-\delta$ plane, where it will be recalled that $C$ is the evaporation coefficient and $\delta$ is the fraction of liquid fuel in the initial vapor+liquid fuel mass fraction. Consider, for example, the contour of $T_{W}=1570 \mathrm{~K}$. Points (i.e. liquid phase conditions to the right of this contour) will not permit ignition, whereas points to the left will support the existence of a flame in the vicinity of the stagnation point. Moving to the right of any contour for a fixed value of $C$ implies that the initial liquid load is greater, thereby effectively increasing the heat loss to the system required to evaporate the droplets. Similarly, for a fixed value of $\delta$ increasing the evaporation coefficient produces more focusing of the heat loss borne by the system. In both cases a greater value of $T_{W}$ would therefore be required to initiate chemical reaction.

Another way to view this behaviour is illustrated in Fig. 3 where the actual critical temperature for ignition is plotted as a function of the evaporation coefficient for different initial liquid loads. Also, appearing is the horizontal curve for a pure gaseous fuel, for the sake of comparison. The Influence of both the spray parameters is rather clear. Take, for example, $C=600$. The presence of only liquid fuel instead of purely gaseous fuel produces a $4 \%$ increase in the critical temperature for ignition. 


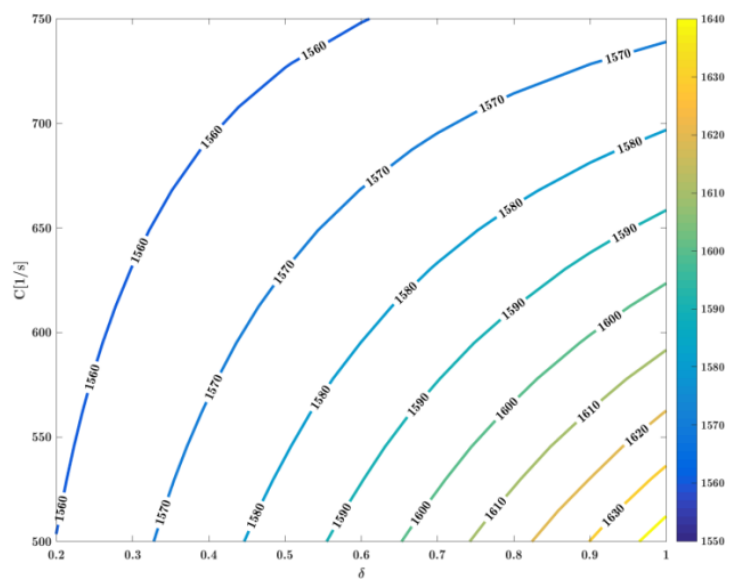

Figure 2: Influence of vaporization coefficient and total initial liquid load on conditions for ignition in stagnation point flow; points to the left of a given temperature contour permit ignition.

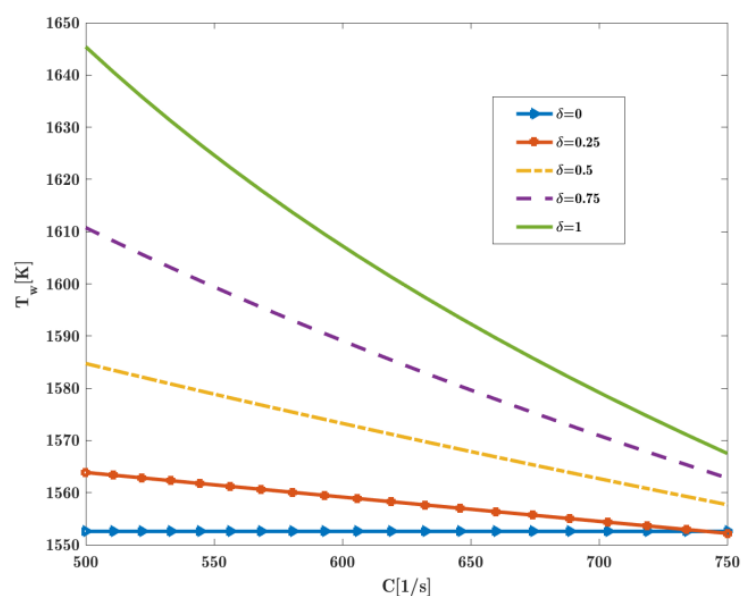

Figure 3: Influence of vaporization coefficient on critical stagnation-point temperature for ignition, for various total initial liquid loads, in stagnation point flow.

Finally, in Fig. 4 we show the influence of the vaporization coefficient on the mass fraction of fuel vapor at the stagnation point. This term is the second bracketed term on the right hand side of Eq. (31) before the exponential term, and represents the role of the fuel vapor produced by droplet evaporation in the chemical reaction.

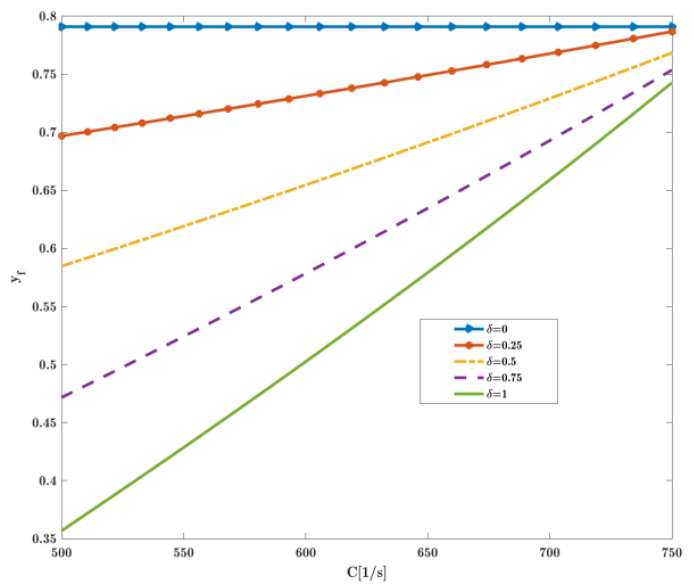

Figure 4: Influence of vaporization coefficient on stagnation-point mass fraction of fuel vapor at ignition, for various total initial liquid loads, in stagnation point flow. 
It can be observed that the higher the initial liquid load the lower the value of fuel vapor mass fraction. As a consequence, to compensate a higher temperature must be supplied at the stagnation point for ignition to occur. This is readily seen to be the case by referring to Fig. 3 .

\section{Conclusions}

A new steady-state/static analysis of conditions for ignition of an air-fuel spray mixture in stagnation point flow impinging on an isothermal wall was presented. The analysis leads to a criterion for ignition that includes effects of the flow field, the reactants and the fuel spray-related parameters. Numerical calculations using the formula mapped the way in which the latter impact on whether ignition will occur or not.

The analysis is predicated on a number of simplifying assumptions which, nevertheless, enable a first insight into the complexities of spray ignition to be uncovered. Further research into relaxing some of these assumptions without having to resort to a complete CFD code to obtain a solution are currently ongoing and will be reported in the future. Experimental evidence to validate or suggest modification of the theory will be welcomed by the authors.

\section{Acknowledgements}

JBG thanks the Lady Davis Chair in Aerospace Engineering for partial support of this research.

\section{Appendix}

In this Appendix the normalized quantities appearing in the governing equations are listed:

$$
\left\{\begin{array}{l}
\theta=\frac{c_{p} T}{q} ; \theta_{a}=\frac{E c_{p}}{R q} ; L^{\prime}=\frac{L\left(m_{d u}+m_{f u}\right)}{q} ; y_{f}=\frac{Y_{f}}{m_{d u}+m_{f u}} ; y_{d}=\frac{Y_{d}}{m_{d u}+m_{f u}} ; y_{O}=\frac{Y_{O}}{m_{O u}} ; \delta=\frac{m_{d u}}{m_{d u}+m_{f u}} ; \\
\Delta=\frac{C}{a} ; \vartheta_{O}=m_{O u} ; \vartheta_{F}=m_{d u}+m_{f u} ; \mu=\frac{A m_{O u}\left(m_{F u}+m_{d u}\right)}{a} ; \\
s=\int_{0}^{x} \rho_{0}\left(x^{\prime}\right) \mu_{0}\left(x^{\prime}\right) u_{0}\left(x^{\prime}\right) d x^{\prime} ; \eta=\frac{u_{0}}{\sqrt{2 s}} \int_{0}^{y} \rho\left(x, y^{\prime}\right) d y^{\prime}
\end{array}\right.
$$

In these definitions $q$ is the heat of reaction.

\section{References}

[1] Neophytou, A., "Spark ignition and flame propagation”, PhD dissertation, University of Cambridge, 2010.

[2] Aggarwal, S.K., 2014, Progress in Energy and Combustion Science, 45, pp. 79-107.

[3] Kikby, L.L. and Schmitz, R.A., 1966, Combustion and Flame, 10, pp. 205-219.

[4] Linan, A., 1974, Acta Astronautica, 1, pp. 1007-1039.

[5] Matalon, M. and Ludford, G.S.S., 1979, Acta Astronautica, 6, pp. 1377-1386.

[6] Aggarwal, S.K. and Sirignano, W.A., 1984, Proceedings of the Combustion Institute, 20, pp. 1773-1780.

[7] Aggarwal, S.K. and Sirignano, W.A., 1986, Combustion Science and Technology, 46(3-6), pp. 289-300.

[8] Aggarwal, S.K. and Sirignano, W.A., 1988, AIAA Journal of Propulsion, 4(1), pp. 14-21.

[9] Kats, G. and Greenberg, J.B., Scitech 2016, AIAA Paper 2016-1029, San Diego, USA, 4-8 January 2016.

[10] Kats, G. and Greenberg, J.B., ILASS - Europe 2016, 27th Annual Conference on Liquid Atomization and Spray Systems, 4-7 September 2016, Brighton, UK.

[11] Neophytou, A., Mastorakos, E. and Cant, R.S., 2011, Proceedings of the Combustion Institute, 33, pp. 21352142.

[12] Law, C.K., 1978, International Journal of Heat and Mass Transfer, 21, pp. 1363-1368.

[13] Chen, Z.H., Lin, T.H. and Sohrab, S.H., 1988, Combustion Science and Technology, 60(1-3), pp. 63-77.

[14] Hsu, A-K. and Lin, T.H., 2003, Combustion Science and Technology, 175(12), pp. 2141-2159.

[15] Chung, P.M., Chemically reacting non-equilibrium boundary layers, in Advances in Heat Transfer, Academic press, NY, 1965.

[16] Greenberg, J.B., Silverman, I. and Tambour, Y., 1993, Combustion and Flame, 93, pp. 90-96.

[17] Sanchez, A.L, Urzay, J. and Linan, A., 2015, Proceedings of the Combustion Institute, 35, pp. 1549-1577.

[18] Stull, D.R. and Prophet, H., JANAF Thermochemcial Tables (2nd ed.), NSRDS-NBS 37., Washington, DC 20234, 1971.

[19] Vargaftik, N.B., Tables on the Thermophysical Properties of Liquids and Gases (2nd ed.), John Wiley and Sons, 1975 


\title{
Polydisperse Spray Flames in Vortex Flows
}

\author{
Yuval Dagan ${ }^{1, *}$, David Katoshevski², J. Barry Greenberg ${ }^{3}$ \\ ${ }^{1}$ Department of Mechanical Engineering, Massachusetts Institute of Technology, \\ Cambridge, MA 02139, USA \\ ${ }^{2}$ Environmental Engineering Unit, Ben-Gurion University of the Negev, Beer-Sheva, Israel \\ ${ }^{3}$ Faculty of Aerospace Engineering, Technion - Israel Institute of Technology, Haifa, Israel \\ ${ }^{*}$ Corresponding author: yuvalda@mit.edu
}

\begin{abstract}
A new mathematical analysis of the dynamics of laminar spray diffusion flames in the vicinity of a vortex flow field is presented. The governing equations for a spray evaporating in an unsteady vortex are studied. New similarity solutions are found for the dynamics of the spray and the flame it supports. Analytical solutions for the spray flames are derived using Schvab-Zeldovich parameters, through which the radial evolution of the flames is found. The results based on the solution reveal the significant influence the droplets size has on the diffusion flame dynamics in the vicinity of vortical flows.
\end{abstract}

\section{Introduction}

The dynamics of evaporating sprays play an important role in many practical combustion applications such as gasturbines and swirl combustion chambers. The interactions between a gaseous diffusion flame and a vortex flow-field were extensively studied [1, 2, 3]. In a previous study of Dagan et al. [4], unsteady turbulent spray-flame instability in a concentric jet combustion chamber was studied using large eddy simulations (LES). In their study, droplet grouping and ligament structures were identified in the vicinity of vortices in large recirculation zones.

Using direct numerical simulations (DNS) of Diesel spray combustion in the vicinity of a recirculation zone, Shinjo et al. [5] showed that when droplets are larger than the Kolmogorov microscale, mixing is strongly enhanced by the presence of droplets and fuel vapor clusters are likely to form quickly when the droplet number density is high. They suggested that external group combustion is likely to occur near the recirculation zone. The effects of droplet clustering on evaporation were thoroughly discussed by Sirignano [6] and Harstad et al. [7]. Clusters of droplets are formed in vortical flows, as they accelerate towards the outer region of the vortex[8]. The dynamics of dropletvortex interactions and their influence on the structure of an evaporating spray was numerically studied by [9]. Droplet-vortex interaction in the Karman-vortex street was studied by Burger et al. [10], using DNS and a theoretical approach imposing a harmonically oscillating flow field. Recently, Franzelli et al. [11] numerically characterized the regimes of spray flame-vortex interactions. They used a two-dimensional Oseen type vortex in their study. However, their study relates to a vortex moving perpendicularly through an opposed flow spray sheet. These studies emphasize the need for a more fundamental understanding of the influence of droplet dynamics on combustion in vortical environments and recirculating flows, which appear in turbulent, as well as laminar environments.

The objective of the present work is to analytically study the influence of a vortex flow-field has on the evaporation and combustion of polydisperse sprays. A new mathematical formulation for the dynamics of polydisperse sprays in the vicinity of a vortex flow field is presented. The governing equations for a polydisperse spray evaporating in an unsteady vortex and the diffusion flame they support are studied and new similarity solutions are found for the dynamics of the spray flames, using the sectional approach (following Tambour, Greenberg and Katoshevski [14, $15,16,17])$. Finally, preliminary results are shown for the influence of polydispersity on the reacting diffusion sprayflames in the vicinity of vortical flows.

\section{Governing equations \\ Gas phase}

The equations for a polydisperse spray evaporating in a two-dimensional unsteady axisymmetric vortex flow are presented. A polar coordinate system $(r, \theta)$ is employed in the following derivation of equations. As a result of our assumption of an axisymmetric flow, all derivatives with respect to the angular coordinate $\theta$ are assumed zero.

A constant density for the host gas is assumed. The constant density assumption is frequently adopted in diffusion flame studies under conditions in which the fuel and the oxidant are heavily diluted so that the heat released by chemical reaction is small in comparison with the thermal energy of the mixture and gas expansion is negligible. Under these assumptions, the governing gas-phase equations are

Gas-phase momentum:

$$
\begin{aligned}
& \frac{\partial v_{r}}{\partial t}+v_{r} \frac{\partial v_{r}}{\partial r}-\frac{v_{\theta}^{2}}{r}=-\frac{1}{\rho} \frac{\partial p}{\partial r}+\nu \frac{\partial}{\partial r}\left(\frac{1}{r} \frac{\partial}{\partial r}\left(r v_{r}\right)\right)-\sum_{j=1}^{N_{s}} \tilde{Q}_{j} F_{r, j}-\dot{S}_{e v, r} \\
& \frac{\partial v_{\theta}}{\partial t}+v_{r} \frac{\partial v_{\theta}}{\partial r}+\frac{v_{r} v_{\theta}}{r}=\nu \frac{\partial}{\partial r}\left(\frac{1}{r} \frac{\partial}{\partial r}\left(r v_{\theta}\right)\right)-\sum_{j=1}^{N_{s}} \tilde{Q}_{j} F_{\theta, j}-\dot{S}_{e v, \theta}
\end{aligned}
$$


where $\rho$ is the gas-phase density, $v_{r}$ is the gas radial velocity, $v_{\theta}$ is the tangential velocity of the host gas, $\nu$ is the kinematic viscosity and $p$ is the pressure. $\tilde{Q}_{j}$ denotes mass fraction of the liquid fuel in size section $j$ and $N_{s}$ is the total number of sections. $\dot{S}_{e v}$ accounts for the momentum transferred to the host gas by the vapors. $F_{r, j}$ and $F_{\theta, j}$ describe the interaction between the gas phase and the droplets of size section $j$ in the radial and tangential directions, respectively, being proportional to the relative velocity between the droplets and the gas

$$
F_{r, j}=\tau_{j}^{-1}\left(v_{r}-u_{r, j}\right) ; \quad F_{\theta, j}=\tau_{j}^{-1}\left(v_{\theta}-u_{\theta, j}\right)
$$

where $u_{r, j}$ and $u_{\theta, j}$ are the radial and tangential velocities of the droplets of section $j$, respectively. $\tau_{j}$ is the sectional droplet relaxation time-scale.

The conservation equations for the vapour mass fraction of the fuel, $m_{f}$ and the oxidizer, $m_{o}$, and energy equation are given by

$$
\begin{aligned}
& \frac{\partial m_{f}}{\partial t}+\frac{1}{r} \frac{\partial}{\partial r}\left(r v_{r} m_{f}\right)=D_{f} \frac{1}{r} \frac{\partial}{\partial r}\left(r \frac{\partial m_{f}}{\partial r}\right)+C Q-\frac{1}{\rho} \tilde{S}_{R, f} \\
& \frac{\partial m_{o}}{\partial t}+\frac{1}{r} \frac{\partial}{\partial r}\left(r v_{r} m_{o}\right)=D_{o} \frac{1}{r} \frac{\partial}{\partial r}\left(r \frac{\partial m_{o}}{\partial r}\right)-\frac{1}{\rho} \tilde{S}_{R, o} \\
& \frac{\partial T}{\partial t}+\frac{1}{r} \frac{\partial}{\partial r}\left(r v_{r} T\right)=\frac{k}{\rho C_{p}} \frac{1}{r} \frac{\partial}{\partial r}\left(r \frac{\partial T}{\partial r}\right)-\frac{h_{v}}{C_{p}} C Q+\frac{1}{\rho C_{p}} \tilde{S}_{R, T}
\end{aligned}
$$

following Katoshevski et al. [16]. $T$ is the temperature, $k$ and $C_{p}$ are the gas conduction coefficient and specific heat at constant pressure, respectively. $h_{v}$ accounts for the latent heat of vaporization, $D_{f}$ and $D_{o}$ are the diffusion coefficients, and we assume all species have the same $C_{P}$ value. The $S_{R}$ terms are the reaction source terms for each equation, based on the assumption of a global reaction of the form Fuel $+\zeta_{s}$ Oxidant $\rightarrow$ Products, where $\zeta_{s}$ is the stoichiometric coefficient. However, as will be showed in the following sections, Schvab-Zeldovich type variables will be formulated in the similarity coordinate. Hence, an explicit treatment of the reaction terms will not be required.

\section{Liquid spray phase}

In the present study the equations for the reacting flow and their solutions are obtained only for a mono-sectional case. However, the sectional equations for the dynamics of sprays are presented here in a polydisperse formulation for the sake of generality. The multi-size droplet population is represented by a set of $N_{s}$ sectional conservation equations of the form

$$
\frac{\partial \tilde{Q}_{j}}{\partial t}+\frac{1}{r} \frac{\partial}{\partial r}\left(r u_{r, j} \tilde{Q}_{j}\right)=-C_{j} \tilde{Q}_{j}+B_{j, j+1} \tilde{Q}_{j+1} ; \quad j=1,2, \ldots, N_{s}
$$

where the coefficient $B_{j, j+l}$ accounts for droplets from section $(j+1)$ which are added to section $j$ during their evaporation, whereas $C_{j}$ accounts for evaporation of droplets within section $j$ and for droplets that move from section $j$ to section $(j-1)$.

In reality, the evaporation coefficient is a complicated function of the temperature differential between the droplets and the surrounding gas, the diffusivity and other properties of the fuel and its surroundings. Here the $d^{2}-$ law underlies the definition of the evaporation coefficient. Reasonably accurate estimates of droplet size and vaporization time do provide some evidence of the validity of this law even under transient temperature conditions [15, 21, 22]. In addition, Labowsky [19] showed that the $d^{2}-l a w$ provides a reasonable prediction of the actual vaporization history of an interacting droplet, especially in the initial period of combustion. Considerable progress in going beyond the limitations of Labowsky's model and its findings [19] can be found in the work of [20]. In principle, any more sophisticated model could be used as a basis for constructing the sectional evaporation coefficients. Inclusion of such details is likely to affect our results in a quantitative rather than a qualitative way [24]. In a similar manner, no separate energy conservation equation is solved for the spray as the current model assumes instantaneous thermal adjustment of the gas-liquid mixture to a common temperature; although this may not always be the case in transient situations [23]. The present analysis is therefore limited to fairly volatile fuels and small droplets for which the $d^{2}-$ law is valid.

Under these assumptions, the spray sectional momentum equations are

$$
\begin{aligned}
& \frac{\partial u_{r, j}}{\partial t}+u r, j \frac{\partial u_{r, j}}{\partial r}-\frac{u_{\theta, j}^{2}}{r}=F_{r, j}+\frac{1}{\tilde{Q}_{j}}\left(S_{j, r}^{L, M}-u_{r, j} S_{j}\right) \\
& \frac{\partial u_{\theta, j}}{\partial t}+u_{r, j} \frac{\partial u_{\theta, j}}{\partial r}+\frac{u_{r, j} u_{\theta, j}}{r}=F_{\theta, j}+\frac{1}{\tilde{Q}_{j}}\left(S_{j, \theta}^{L, M}-u_{\theta, j} S_{j}\right)
\end{aligned}
$$

The last term in the brackets on the RHS of equations 8 and 9 represents loss of linear momentum $\left(u_{j} S_{j}\right)$ due to evaporation of droplets in section $j$, and the linear momentum $\left(S_{j}^{L M}\right)$ added to section $j$ due to droplets from higher sections that are added to section $j$ during their evaporation (see [16]).

Here,

$$
S_{j}=-C_{j} \tilde{Q}_{j}+B_{j, j+1} \tilde{Q}_{j+1}
$$

and

$$
\begin{aligned}
& S_{j, r}^{L, M}=-C_{j} \tilde{Q}_{j} u_{r, j}+B_{j, j+1} \tilde{Q}_{j+1} u_{r, j+1} \\
& S_{j, \theta}^{L, M}=-C_{j} \tilde{Q}_{j} u_{\theta, j}+B_{j, j+1} \tilde{Q}_{j+1} u_{\theta, j+1}
\end{aligned}
$$




\section{Similarity transformation of the gas and spray equations}

We shall now use a similarity variable, $\eta=r^{2} / 4 \nu t$ and rewrite the host gas velocities as a function of $\eta$ :

$$
\bar{v}_{r}(\eta)=\frac{r v_{r}}{\nu} ; \quad \bar{v}_{\theta}(\eta)=\frac{r v_{\theta}}{\nu} .
$$

Next, the droplet velocities are defined as

$$
\bar{u}_{r, j}(\eta)=\frac{r u_{r, j}}{\nu} ; \quad \bar{u}_{\theta, j}(\eta)=\frac{r u_{\theta, j}}{\nu} .
$$

Substituting the above definitions into the droplet momentum equations one obtains

$$
\begin{aligned}
& \bar{u}_{r, j}^{\prime}-\frac{1}{2 \eta} \bar{u}_{r, j}^{\prime} \bar{u}_{r, j}+\frac{1}{4 \eta^{2}} \bar{u}_{r, j}^{2}+\frac{1}{4 \eta^{2}} \bar{u}_{\theta, j}^{2}=-\frac{1}{4 \eta^{2}} \frac{1}{\nu^{2}} r^{3} F_{r, j}-\frac{1}{\eta} \frac{1}{Q_{j}} \bar{B}_{j, j+1} Q_{j+1}\left(\bar{u}_{r, j+1}-\bar{u}_{r, j}\right) \\
& \bar{u}_{\theta, j}^{\prime}-\frac{1}{2 \eta} \bar{u}_{\theta, j}^{\prime} \bar{u}_{r, j}=-\frac{1}{4 \eta^{2}} \frac{1}{\nu^{2}} r^{3} F_{\theta, j}-\frac{1}{\eta} \frac{1}{Q_{j}} \bar{B}_{j, j+1} Q_{j+1}\left(\bar{u}_{\theta, j+1}-\bar{u}_{\theta, j}\right)
\end{aligned}
$$

where the primes denote differentiation with respect to $\eta$. The drag terms are written explicitly as

$$
\frac{1}{\nu^{2}} r^{3} F_{r, j}=A_{j}\left(\bar{v}_{r}-\bar{u}_{r, j}\right) ; \quad \frac{1}{\nu^{2}} r^{3} F_{\theta, j}=A_{j}\left(\bar{v}_{\theta}-\bar{u}_{\theta, j}\right)
$$

where $A_{j}=\tau^{-1} r^{2} / \nu$. The characteristic time of the droplets is taken as $\tau=\frac{\rho_{d} d^{2}}{18 \mu_{g}}$, where $d, \rho_{d}$ and $\mu_{g}$ are the averaged droplet diameter in section $j$, liquid fuel density and the host gas viscosity, respectively. It can be demonstrated that for the range of radii involved $A_{j}$ does not change much. In order to obtain the spray equations in terms of $\eta$ only, the sectional evaporation coefficients are defined as

$C_{j}=\bar{C}_{j} / t, \quad B_{j, j+1}=\bar{B}_{j, j+1} / t$. The use of an inverse time dependence of the sectional evaporation coefficients is reasonable in a general sense as evaporation will indeed peter out after sufficient droplet life time has elapsed. Thus, in similarity coordinates, the spray equations take the form

$$
\left(1-\frac{1}{2 \eta} \bar{u}_{r, j}\right) Q_{j}^{\prime}-\frac{1}{2 \eta} \bar{u}_{r, j}^{\prime} Q_{j}=-\frac{1}{\eta}\left(-\bar{C}_{j} Q_{j}+\bar{B}_{j, j+1} Q_{j+1}\right)
$$

\section{Shvab-Zeldovich formulation of diffusion spray-flames in a vortex}

In the mono-sectional framework, the following set of equations are derived in the similarity coordinate, $\eta$, for the fuel mass fraction, the oxidizer mass fraction and temperature, respectively:

$$
\begin{aligned}
& \left(1-\frac{1}{2 \eta} \bar{u}_{r}\right) Q^{\prime}-\frac{1}{2 \eta} \bar{u}_{r}^{\prime} Q=\frac{1}{\eta} C Q \\
& \frac{m_{f}^{\prime \prime}}{S c_{f}}+\left(1+\frac{1}{\eta S c_{f}}-\frac{\bar{v}_{r}}{2 \eta}\right) m_{f}^{\prime}-\frac{\bar{v}_{r}^{\prime}}{2 \eta} m_{f}=-\frac{1}{\eta} C Q+\zeta_{f} M_{f} \frac{1}{\eta} S_{R} \\
& \frac{m_{o}^{\prime \prime}}{S c_{o}}+\left(1+\frac{1}{\eta S c_{f}}-\frac{\bar{v}_{r}}{2 \eta}\right) m_{o}^{\prime}-\frac{\bar{v}_{r}^{\prime}}{2 \eta} m_{o}=\zeta_{o} M_{o} \frac{1}{\eta} S_{R} \\
& \frac{T^{\prime \prime}}{S c_{o}}+\left(1+\frac{1}{\eta P r}-\frac{\bar{v}_{r}}{2 \eta}\right) T^{\prime}-\frac{\bar{v}_{r}^{\prime}}{2 \eta} T=\frac{h_{v}}{C_{P} T_{C}} C Q-\sum_{i} \zeta_{i} M_{i} h_{i} \frac{1}{\eta} S_{R}
\end{aligned}
$$

Next, we assume a unity Lewis number, $S c_{f}=S c_{o}=\operatorname{Pr}=S c$ and use the following notation:

$$
\hat{\zeta}=\frac{\zeta_{o} M_{o}}{\zeta_{f} M_{f}} ; \quad \hat{h}=\frac{h_{v}}{C_{P} T_{C}} ; \quad \zeta^{*}=\frac{\zeta_{o} M_{o}}{-1 / \hat{h}} \sum_{i} \zeta_{i} M_{i} h_{i}+\zeta_{f} M_{f}
$$

Finally, defining two Schvab-Zeldovich variables

$$
\beta=\frac{1}{S c}\left(m_{f}-\frac{m_{o}}{\hat{\zeta}}\right), \quad \gamma=\frac{T}{\hat{h}}+m_{f}-\frac{m_{o}}{\zeta^{*}}
$$

one obtains the following equations

$$
\begin{aligned}
& \beta^{\prime \prime}+S c\left(1+\frac{1}{\eta S c}-\frac{\bar{v}_{r}}{2 \eta}\right) \beta^{\prime}-\frac{\bar{v}_{r}^{\prime}}{2 \eta} \beta=\frac{1}{\eta} C Q \\
& \gamma^{\prime \prime}+S c\left(1+\frac{1}{\eta S c}-\frac{\bar{v}_{r}}{2 \eta}\right) \gamma^{\prime}-\frac{\bar{v}_{r}^{\prime}}{2 \eta} \gamma=0
\end{aligned}
$$

The boundary conditions for the above functions are

$$
\begin{aligned}
& \beta(\infty)=\frac{1}{S c} m_{f, \infty} ; \quad \beta(0)=-\frac{1}{S c} \frac{m_{o, 0}}{\hat{\zeta}} \\
& \gamma(\infty)=\frac{T_{\infty}}{\hat{h}}+m_{f, \infty} ; \quad \gamma(0)=\frac{T_{f}}{\hat{h}}-\frac{m_{o, 0}}{\zeta^{*}}
\end{aligned}
$$




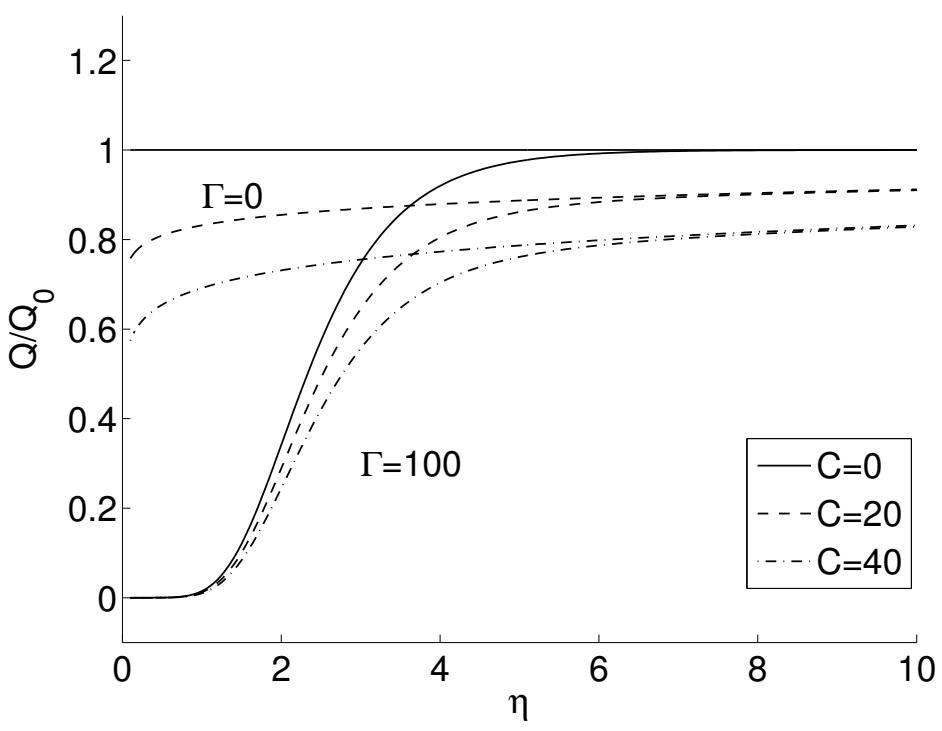

Figure 1. Liquid fuel mass fraction divided by the initial mass fraction $Q_{0}$ as a function of the similarity coordinate for different values of vortex intensity $\Gamma$ and evaporation coefficients $\bar{C}$.

It should be mentioned that for the actual solution we use the similarity variable boundaries from some small time and radius so that we avoid any singularity. The flame location is where $\beta=0$. This is the same as where the leading order solution (in terms of the usual Zeldovich number) locates the flame front.

In order to solve the equations for vortex spray dynamics analytically we shall first derive an expression for the droplet velocities $u_{r}$ and $u_{\theta}$ as follows. Appropriate manipulation of Eq. 15,16 leads to

$$
\bar{u}_{r}^{2} \bar{u}_{\theta}^{\prime}+\bar{u}_{\theta}^{2} \bar{u}_{\theta}^{\prime}=-A\left[\left(\bar{v}_{r}-\bar{u}_{r}\right) \bar{u}_{\theta}^{\prime}-\left(\bar{v}_{\theta}-\bar{u}_{\theta}\right) \bar{u}_{r}^{\prime}\right] .
$$

For the host velocity field, a two-dimensional Oseen-vortex will be used,

$$
\bar{v}_{\theta}=\bar{\Gamma}\left(1-e^{-\eta}\right) ; \quad \bar{v}_{r}=0 .
$$

which is also an exact solution of Eq. 2 for $\eta>>1$. Here, $\bar{\Gamma}=\Gamma / 2 \pi \nu$, where $\Gamma$ is the angular velocity. For the fine sprays under consideration, we assume that the droplets closely track the vortex flow-field in the transverse coordinate $\theta$. On the other hand, we expect the presence of the vortex to significantly increase the droplets radial velocity $u_{r}$, especially at lower values of $\eta$. Hence, droplets will not track the gas radial velocity which is initially assumed zero (Eq. 30). Thus, $\bar{u}_{\theta}=\bar{v}_{\theta}$ and equation 29 takes the following simple form

$$
\bar{u}_{r}^{2}+\bar{u}_{\theta}^{2}-A \bar{u}_{r}=0,
$$

from which $\bar{u}_{r}$ can be extracted.

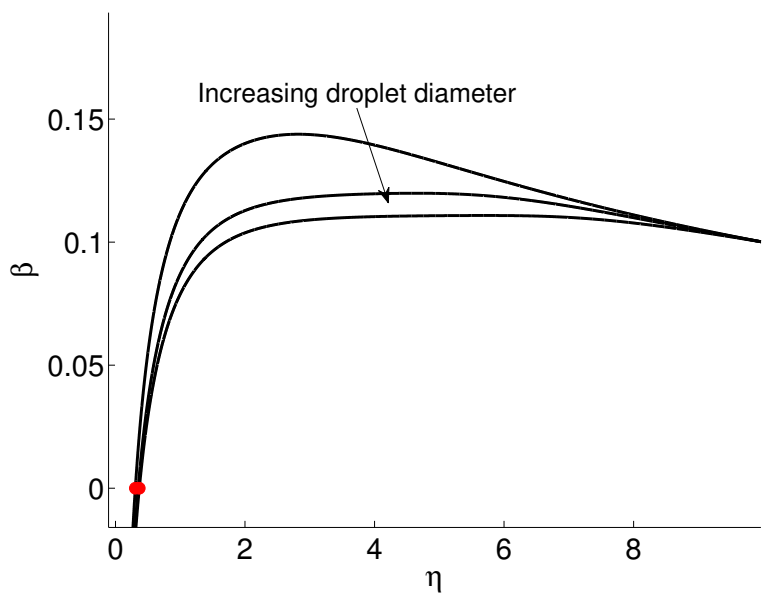

(a)

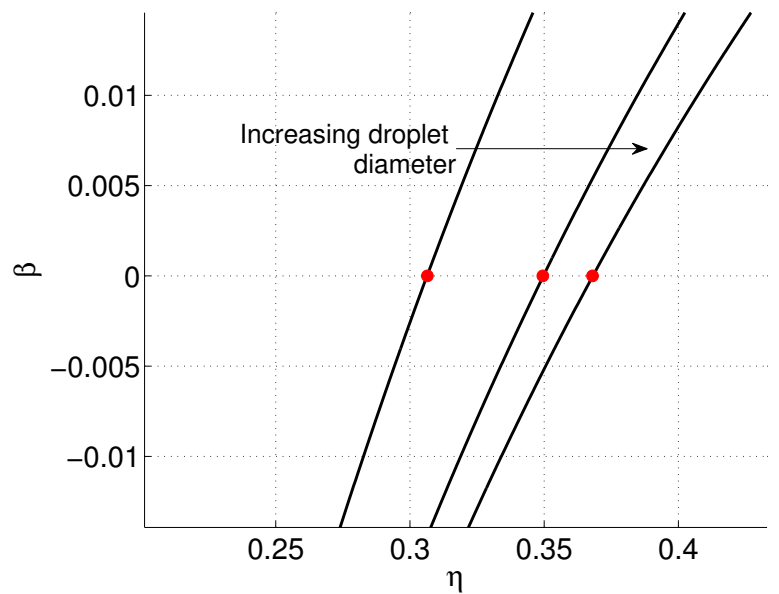

(b)

Figure 2. Schvab-Zeldovich function $\beta$ as a function of the similarity coordinate $\eta$ for three different initial droplet diameters: $d_{0}=10,50,100 \mu \mathrm{m}$. The flame is located where $\beta=0$ (shown in solid circles). (a) Whole region of the analysis (b) a closeup on the flame region at lower values of $\eta$. 


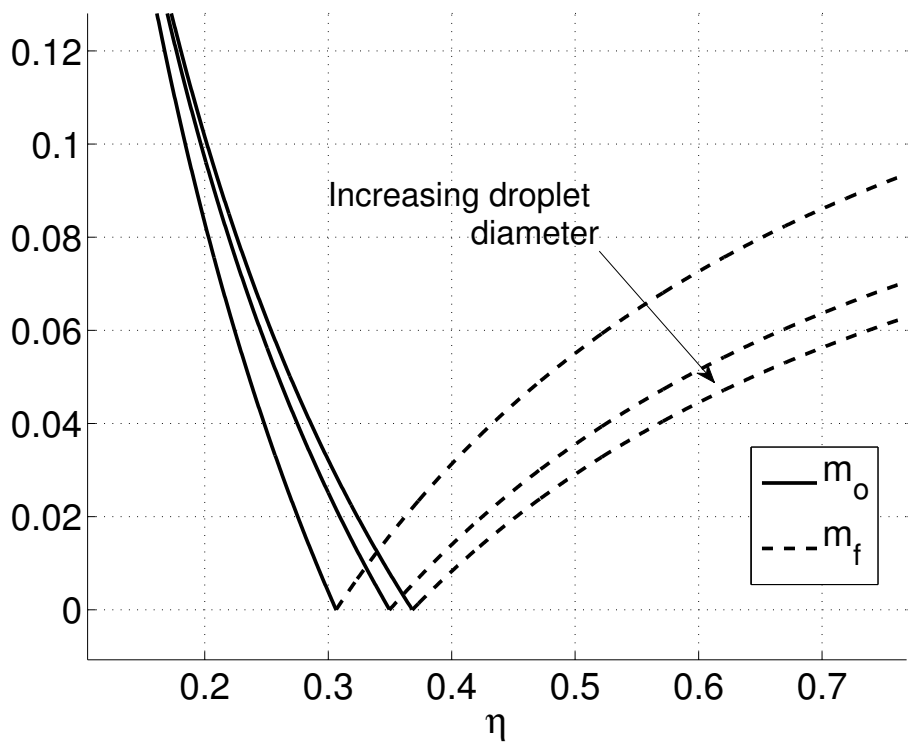

Figure 3. Profiles of fuel mass fraction $\left(m_{f}\right)$ and the oxidizer mass fraction $\left(m_{o}\right)$ in the similarity framework for three different initial droplet diameters: $d_{0}=10,50,100 \mu \mathrm{m}$.

Next, the equation for the liquid fuel mass fraction $Q$ (Eq. 19) is solved by separation of variables:

$$
Q^{\prime} / Q=\left(2 \bar{C}+\bar{u}_{r}^{\prime}\right) /\left(2 \eta-\bar{u}_{r}\right),
$$

from which $Q(\eta)$ can be solved by integration. Assuming $\bar{v}_{r}=\bar{v}_{r}^{\prime}=0$, we may also evaluate $\beta(\eta)$ numerically. The solution for the second Schvab-Zeldovich function, $\gamma$ is

$$
\gamma(\eta)=c_{3} E i(-S c \eta)+c_{4}
$$

where $E i$ is the exponential integral function, and $c_{1}, c_{2}, c_{3}$ and $c_{4}$ are integration constants found using the boundary conditions (Eq. 27, 28). In the present study equation 33 is also evaluated numerically.

\section{Results and discussion}

The liquid fuel mass fraction divided by the initial mass fraction $Q_{0}$ is presented in Figure. 1 as a function of the similarity coordinate for different values of vortex values of $\Gamma$ and evaporation coefficients $\bar{C}$. For non-evaporating droplets, $\bar{C}=0$, and zero vortex intensity, $\Gamma=0$, the liquid mass fraction does not change, as expected. On the other hand, for evaporating droplets and $\Gamma=0$ the liquid mass fraction decreases with decreasing values of $\eta$, which corresponds to increasing time values for a given radial location. The vortex intensity has a substantial effect, especially at lower values of $\eta$, in effect pushing the fuel droplets from the center outwards as time goes by. No droplets are found below $\eta \approx 1$. The calculation of liquid mass fraction was carried out between $0.1<\eta<100$. Only a a partial view is presented in the figure for clarity. Hence, for $\eta \rightarrow 100, Q / Q_{0} \rightarrow 1$.

\section{Polydispersity effect}

In our previous study [18] it was shown that in the vicinity of a vortical flow, spray dynamics is highly affected by the droplets' size, but also by the spray initial distribution, even when the same Sauter mean diameter is considered. It will therefore be interesting to capture the influence of initial droplet size on the propagation of the diffusion flame.

The equations for the dynamics of the polydisperse spray were derived here in the previous sections. In this section, as a first attempt to assess the influence polydispersity will have on the dynamics of the diffusion flame in the vicinity of vortical flows, the flame equations were solved analytically for different initial droplet sizes. It should be noted that the solution is essentially mono-sectional, and that the coupling effect between each section (e.g the intersection transfer of momentum) is out of the scope for this study and will not be considered here.

The Schvab-Zeldovich function $\beta$ is presented in Fig. 2a as a function of the similarity coordinate $\eta$ for three different initial droplet diameters: $d_{0}=10,50,100 \mu \mathrm{m}$. The droplet size effect on the flame location (at $\beta=0$ ) is significant, pushing the flame away from the vortex core (which s located at $\eta=0$ ). This is demonstrated in a closeup view in Fig. 2b. Although small droplets acceleration due to the presence of a vortex is higher than that of larger droplets, it can be shown by solving equation 15 that the maximum radial velocity reached is higher for the larger droplets. This affects the flame, as the vortex tends to push the larger droplet farther away and thus reducing the available fuel vapor required for the flame.

Knowing the functional behaviour of $\beta(\eta)$, and assuming that the species are totally consumed at the flame zone, one can easily extract the fuel vapour and oxidizer mass fraction as a function of $\eta$. This is shown in Fig. 3 for lower values of $\eta$, where the flame is located. Results for three different initial droplet diameters are presented, showing a significant droplet size effect; for a given value of $\eta$ (i.e at a given time and radial location in the physical space) lower fuel mass fraction is observed for larger droplets. The flame is located at the point where all the fuel and oxidizer are consumed $\left(m_{o}=m_{f}=0\right)$.

Finally, solutions of the temperature distribution in the similarity framework for the same vortex intensities and evaporation coefficients are presented in Fig. 4. For larger droplets that are pushed farther than smaller droplets, the temperature curve is pushed to higher values of $\eta$, which means that for a given time the flame will be pushed farther away from the vortex core. Increased heat 


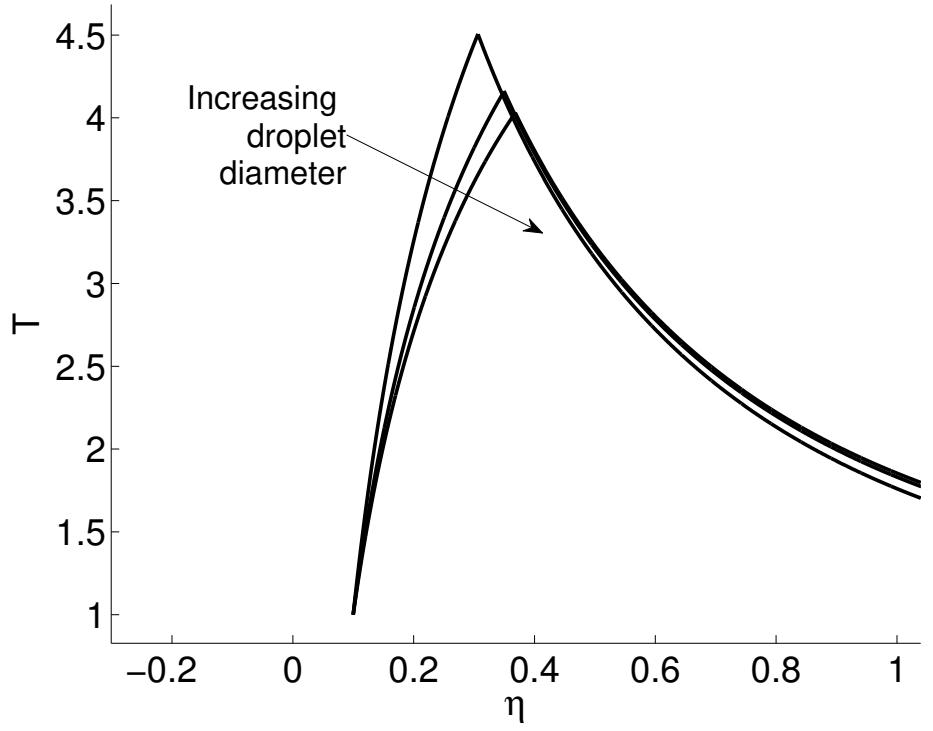

Figure 4. Solutions of the temperature distribution in the similarity framework for three different initial droplet diameters:

$$
d_{0}=10,50,100 \mu m \text {. }
$$

loss due to evaporation also plays an important role in lowering the temperature, which in some cases might lead to extinction. However, this is beyond the scope of the present study. Further work to elucidate the relative importance of the different factors controlling the spray-flame in vortex flows will be reported in the future.

\section{Conclusions}

A new mathematical analysis of the dynamics of laminar spray diffusion flames in the vicinity of a viscous vortex flow field was presented. The governing equations for sprays evaporating in an unsteady vortex were derived and new similarity solutions were found for the dynamics of the spray in a mono-sectional framework. New analytical solutions for the spray flames were obtained using Schvab-Zeldovich parameters, through which the radial evolution of the flames was found. The vortex flow field was shown to have a significant effect on the location and extent of the flame, pushing the droplets outwards and therefore shifting the flame location to larger radii for a given instant of time.

Spray flame with larger initial diameter droplets shows different flame dynamics; they are pushed away farther than spray flame with smaller droplets and exhibit lower temperatures. Certainly, future experimental data would be helpful in validating the theory presented in the current study.

\section{Nomenclature}

$A_{j} \quad$ dimensionless sectional drag coefficient

$B_{j, j+1}$ dimensionless sectional vaporization coefficient

$C_{j} \quad$ dimensionless sectional vaporization coefficient

$C_{P} \quad$ specific heat capacity at constant pressure $[\mathrm{J} / \mathrm{kgK}]$

$D \quad$ diffusion coefficient $\left[\mathrm{m}^{2} / \mathrm{s}\right]$

$d \quad$ droplet diameter $[m]$

$F \quad$ function of $\eta$

$h \quad$ specific enthalpy $[\mathrm{J} / \mathrm{kg}]$

$k \quad$ thermal conductivity $[\mathrm{W} / \mathrm{mK}]$

$M \quad$ molecular weight $[\mathrm{g} / \mathrm{mol}]$

$m$ mass fraction

$N_{s} \quad$ number of sections

$\operatorname{Pr} \quad$ Prandtl number

$p \quad$ pressure $[\mathrm{Pa}]$

$Q_{j} \quad$ sectional liquid mass-fraction

$r$ radial coordinate $[\mathrm{m}]$

$S \quad$ source term

$S c \quad$ Schmidt number

$T$ temperature $[K]$

$t \quad$ time $[s]$

$u \quad$ droplet velocity $[\mathrm{m} / \mathrm{s}]$

$v \quad$ host gas velocity $[\mathrm{m} / \mathrm{s}]$

\section{Greek symbols}

$\beta \quad$ Shvab-Zel'dovich function

$\Gamma \quad$ angular velocity $[\mathrm{rad} / \mathrm{s}]$ 


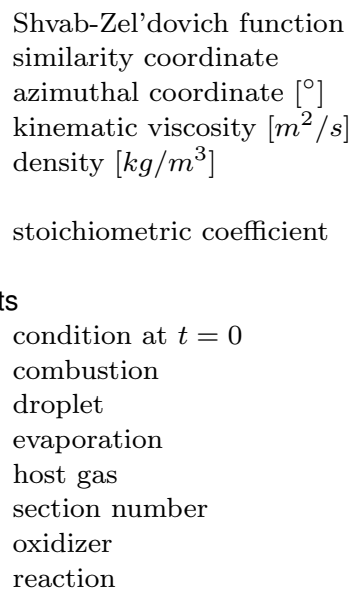

\section{References}

[1] Marble, F., 1985, Recent advances in the aerospace sciences, Springer, 395-413.

[2] Alain, M. and Candel, S., 1988, Combustion science and technology 60:79-96.

[3] Renard, P.H., Thevenin, D., Rolon, J.C. and Candel S., 2000, Progress in energy and combustion science 26: 225-282.

[4] Dagan, Y., Arad, E. and Tambour, Y., 2015, Proc. Combust. Inst. 35:1657-1665.

[5] Shinjo, J. and Umemura, A., 2013, Proc. Combust. Inst. 34:1553-1560.

[6] Sirignano, W.A., 1999, "Fluid Dynamics and Transport of Droplets and Sprays", Cambridge University press.

[7] Harstad, K. and Bellan, J., 2001, Combust. Flame 121:1861-1879.

[8] Bellan, J. and Harstad, K., 1991, Symposium (International) on Combustion 23:1375-1281.

[9] Park, T.W., Aggarwal, S.K. and Katta, V.R., 1996, Intl J. Heat Mass Transfer, 39:2205-2219.

[10] Burger, M., Schmehl, R., Koch, R., Wittig, S. and Bauer, H-J., 2006, Int. J. Heat Fluid FI. 27:181-191.

[11] Franzelli, B., Vié, A. and Ihme, M., 2014, Center for Turbulence Research Annual Research Briefs.

[12] Shiah, S. and Sichel, M., 1993, AIAA paper, 93-0901.

[13] Santoro, V., Kyritsis, D. and Gomez A., 2000, Proc. Combust. Inst. 28:1023-1030.

[14] Tambour, Y., 1985, Combust. Flame 60:15-28.

[15] Greenberg, J.B., Silverman, I. and Tambour, Y., 1993, Combust. Flame 93:90-96.

[16] Katoshevski, D. and Tambour, Y., 1993, Phys. Fluids, A 5:3085-3098.

[17] Greenberg, J.B., 2007, Combust. Flame, 148:187-197.

[18] Dagan, Y., Katoshevski, D. and Greenberg, J.B., 2016, ILASS - Europe, 27th Annual Conference on Liquid Atomization and Spray System, At Brighton, UK.

[19] Labowsky M., 1980, Combust. Sci. Technol., 22(5-6):217-26.

[20] Imaoka, R T and Sirignano, WA, 2005, Proc. Combust. Inst., 30(2):1981-1989.

[21] Law, CK, 1976, Combust. Flame, 26:17-22

[22] Law, CK,1977, Combust. Sci. Technol., 15(1-2):65-74.

[23] Abramzon, B and Sirignano, WA, 1989, 32(9):1605-1618.

[24] Greenberg, J. B. and Katoshevski, D., 2016, Combust. Theor. Model, 20(2):349-372. 


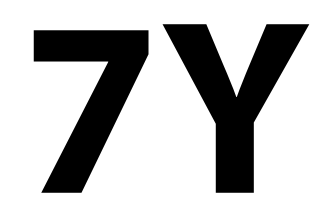

Droplet 4 


\title{
Experimental Investigation of Size Effects in Colliding Droplets
}

\author{
Lars Pasternak ${ }^{* 1}$, Martin Sommerfeld ${ }^{2}$ \\ ${ }^{1}$ Otto-von-Guericke-Universität Magdeburg, Institut für Verfahrenstechnik, Germany \\ ${ }^{2}$ Otto-von-Guericke-Universität Magdeburg, Institut für Verfahrenstechnik, Germany \\ *Corresponding author: lars.pasternak@ovgu.de
}

\begin{abstract}
The present work focuses on the size effect of binary collisions of PVP droplets (viscosity $5.5 \mathrm{mPas}$ ). The aim was the identification of the boundaries between bouncing, coalescence, stretching and reflexive separation.

These boundaries are necessary for numerical simulations of droplet collisions in spray drying processes. Therefore, droplet chains were generated by droplet generators with oscillating membrane and directed towards each other at different angles for producing binary collisions. In the experiments two droplet properties (i.e. droplet size and size ratio) were varied. Two synchronised high-speed cameras were used to observe the collision process and outcome perpendicularly and parallelly to the collision plane. The variation of the impact parameter $B$ was performed by a frequency offset for one droplet generator. The relative velocity (i. e. 0.5 to $4.7 \mathrm{~m} / \mathrm{s}$ ) was set by changing the collision angle.
\end{abstract}

\section{Keywords}

droplet collision, droplet size ratio, modelling separation

\section{Introduction}

Spray drying processes constitute a very complex multiphase system due to the different elementary processes involved, i.e. atomization, drying, collision of droplets and formation of agglomerates. After atomization, droplet collisions with the different possible collision outcomes result in a modification on the product particle size distribution. Typical results of a collision of two droplets may be bouncing, coalescence and separation and were plotted in the well-known collision maps (see Figure 1). In these maps the non-dimensional impact parameter $B$ (see Eq. 1) is plotted against collision Weber number (see Eq. 2). However, the collision map depends on droplet parameters, i.e. liquid and size properties of the droplets. For Euler/Lagrange calculation of sprays it is necessary to know the pattern of the collision map without running experiments.

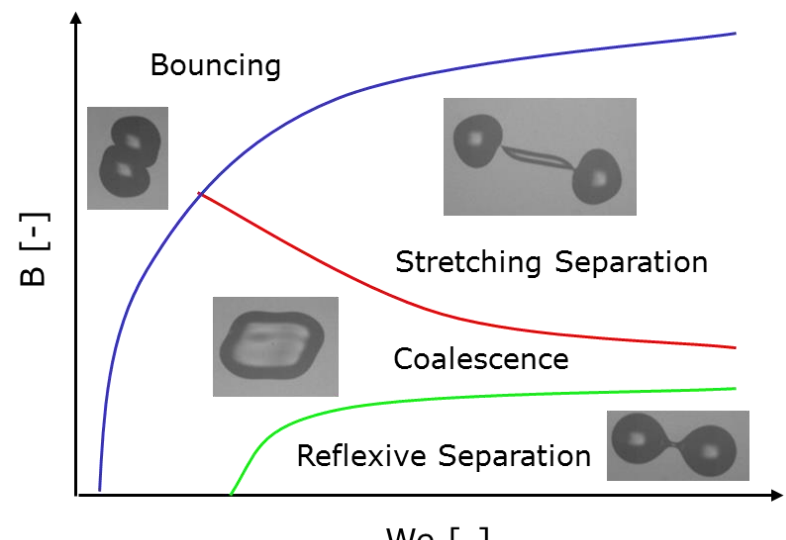

We $[-]$

Figure 1. Typical droplet collision map, with samples of collsion outcomes

During the last decades a number of models were developed to predict the collision outcome. The boundary between bouncing and coalescence/stretching separation can be identified by means of the experimental data based model by Estrade et al. [1]. The models by Ashgriz and Poo [2], for water droplets, are determining the lower boundaries for stretching separation and coalescence. The momentum theory based model by Jiang et al. [3] observes the viscosity effect for the lower boundary of the stretching separation. Based on the Jiang [3] model, Gotaas et al. [4] used experimental data from glycols to specify values of $C_{a}$ and $C_{b}$. This model was improved by 
Sommerfeld [5] by finding a dependency between $C_{a}$ and the Ohnesorge number (see Eq. 3) and setting the other involved constant $C_{b}=1$. The data from the experiments by Kuschel and Sommerfeld [6] were obtained only from $\sim 380 \mu \mathrm{m}$ mono-sized droplets. To validate the 3rd order polynomial fit of $\mathrm{C}_{\mathrm{a}}$ and Ohnesorge number, it was necessary to run experiments with variation of the droplet size properties. Furthermore, this study is devoted to describe the triple point, where all regimes are in coincidence, as well as the reflexive separation.

\section{Material and methods}

The experiments were carried out using two oscillating membrane droplet generators (producer: Encap BioSystems, model: IE-0010H-P). The Polyvinylpyrrolidone K17 solution (see Table 1) was supplied by a pressure vessel (see Figure 2). Droplet chains were created by means of excitation of the liquid inside the droplet generator and pressed throw 200, 300 and $400 \mu \mathrm{m}$ nozzles. The excitation frequency for the break-up was nozzle size dependant and in the range between 1800 and $4690 \mathrm{~Hz}$. The amplitude of the excitation signal was set by an amplifier (Thomann TA1050). A rotation stage was mounted on a three axis translation stage system in order to control the position of each nozzle. In combination with one of the two synchronised high-speed cameras (perpendicular to the collision plane) and the translation stage, off centre collisions could be avoid. The relative velocity (i. e. 0.5 to $4.7 \mathrm{~m} / \mathrm{s}$ ) was set by changing the droplet chain angle by the use of the rotation stages of each droplet generator.

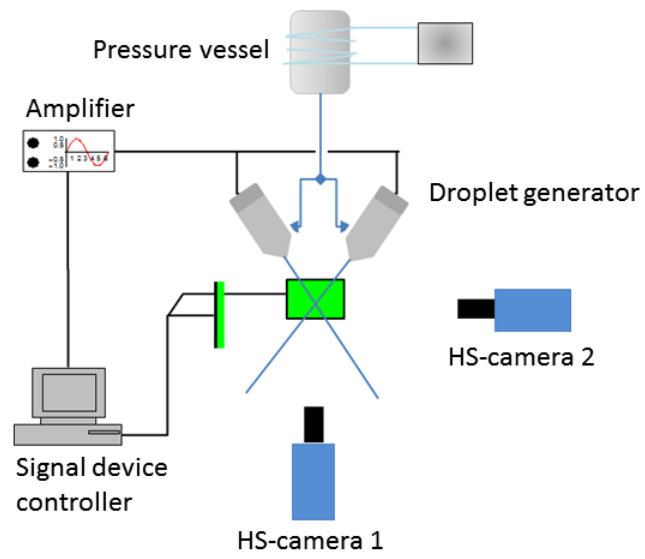

Figure 2. Sketch of the experimental setup [6]

Table 1. Liquid properties the PVP - Water solution at $22^{\circ} \mathrm{C}$

\begin{tabular}{c|cccc}
\hline \hline Specie & $\begin{array}{c}\text { Mass } \\
\text { fraction [\%] }\end{array}$ & $\begin{array}{c}\mathrm{M} \\
{[\mathrm{mPas}]}\end{array}$ & $\begin{array}{c}\sigma \\
{[\mathrm{mN} / \mathrm{m}]}\end{array}$ & $\begin{array}{c}\rho \\
{\left[\mathrm{kg} / \mathrm{m}^{3}\right]}\end{array}$ \\
\hline \hline PVP (BASF) & 20 & 5.5 & 62.2 & 1043.2
\end{tabular}

The illumination of the collision process was done by two $8 \times 10 \mathrm{~cm}^{2}$ backlight LED arrays. The observation of the droplet collision event was based on two Photron SA4 high speed cameras operating up to 10,000 frames per second. The synchronisation of the cameras was controlled by a signal generator. The cameras were equipped

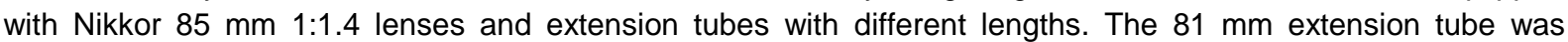
mounted on both cameras. For Case 1 (see Table 2) the tube on camera 1 was extended to $110 \mathrm{~mm}$. In the combination of lens and extension tube the resolution was 13.2 (only camera 1 in Case 1 ) and $17.2 \mu \mathrm{m} /$ pixel.

Table 2. Nozzle configuration for PVP droplets (see Table 1)

\begin{tabular}{c|c|ccccccc}
\hline \hline & $\begin{array}{c}\text { Nozzle } \\
\text { Combination } \\
{[\mu \mathrm{m}]}\end{array}$ & $\begin{array}{c}\text { Size } \\
\text { drop 1 } \\
{[\mu \mathrm{m}]}\end{array}$ & $\begin{array}{c}\text { Size } \\
\text { drop 2 } \\
{[\mu \mathrm{m}]}\end{array}$ & $\begin{array}{c}\text { Size } \\
\text { ratio } \\
\Delta[-]\end{array}$ & $\begin{array}{c}\text { Oh } \\
\text { drop 1 [-] }\end{array}$ & $\begin{array}{c}\text { Oh } \\
\text { drop 2 [-] }\end{array}$ & $\begin{array}{c}\text { max. Re } \\
\text { drop 1 [-] }\end{array}$ & $\begin{array}{c}\text { max. Re } \\
\text { drop 1[-] }\end{array}$ \\
\hline Case 1 & $200-200$ & 349 & 353 & 0.99 & 0.0364 & 0.0362 & 256 & 260 \\
Case 2 & $300-300$ & 560 & 557 & 0.99 & 0.0286 & 0.0289 & 502 & 490 \\
Case 3 & $300-400$ & 580 & 679 & 0.85 & 0.0282 & 0.0261 & 320 & 378 \\
Case 4 & $200-300$ & 411 & 563 & 0.73 & 0.0335 & 0.0286 & 234 & 310 \\
Case 5 & $200-400$ & 440 & 673 & 0.65 & 0.0324 & 0.0262 & 277 & 391
\end{tabular}


Due to the droplet velocity up to $4 \mathrm{~m} / \mathrm{s}$, the high resolution and the camera frame rate, the droplet displacement between two images was up to $400 \mu \mathrm{m}$. To avoid issues with the particle tracking it was necessary to develop a new approach for the detection of the droplet position. In the first image of both collision partners an eccentric box (see Figure 3 left, middle) is set around both droplets. The eccentric shape was necessary as a result of the different droplet velocities of both chains. This box ensures that only droplets inside are detected and only two collision partners are existing. In the next image the box is shifted by a default offset, by determining the droplet pixel velocities and droplet sizes the real offset is calculated for the next image. The positions and velocities from the droplets are based on their centre of mass. In the following images the offset is always recalculated, the collision possibility is verified and the size and shape from the box readjusted. The droplet detection ends in the last image before contact. Inside the box the droplets are identified by Laplacian of Gaussian operator, which limited the accuracy of the droplet position and size results. The expected error by the edge detection is less than one pixel. Due to the fluctuation of the instantaneous velocities, each collision sequence consists of at least six images. Out of the instantaneous data the mean droplet velocities are calculated and the droplet contact point is estimated by the velocities and the last droplet position (see Figure 3 right).
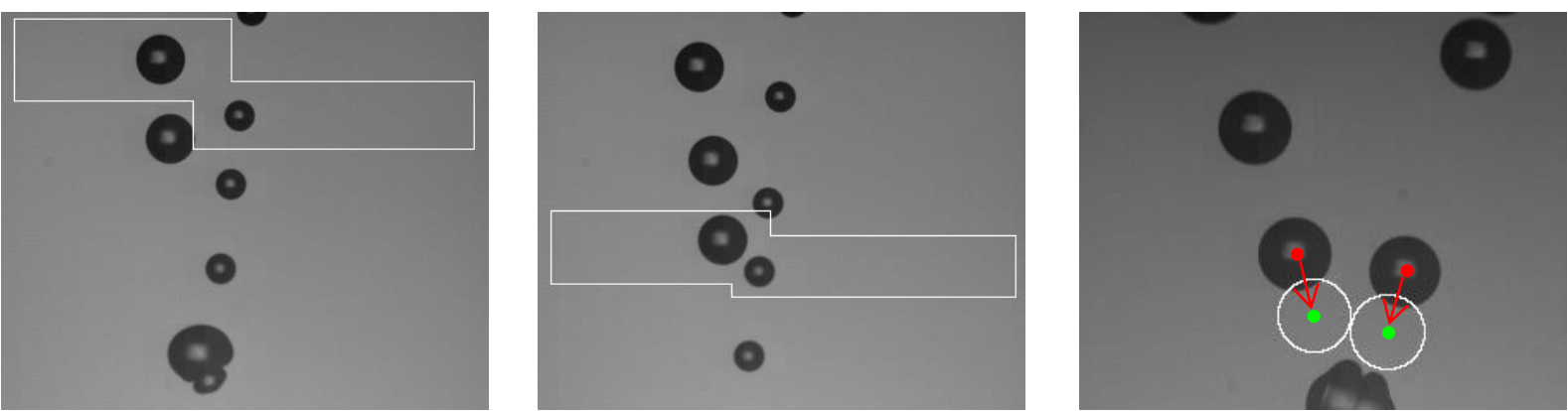

Figure 3. Variable box for droplet detection, left: first image of collision pair with large eccentricity, middle: last image before collision, right: estimated droplet contact point

The contact point position is used to calculate the non-dimensional impact parameter $\mathrm{B}$, which describes only the geometry of the collision without any liquid properties. The impact parameter is the sinus of the enclosed angle between the relative velocity and the position vector $\mathrm{P} 1,2$ of the droplets centre points (see Figure 4).

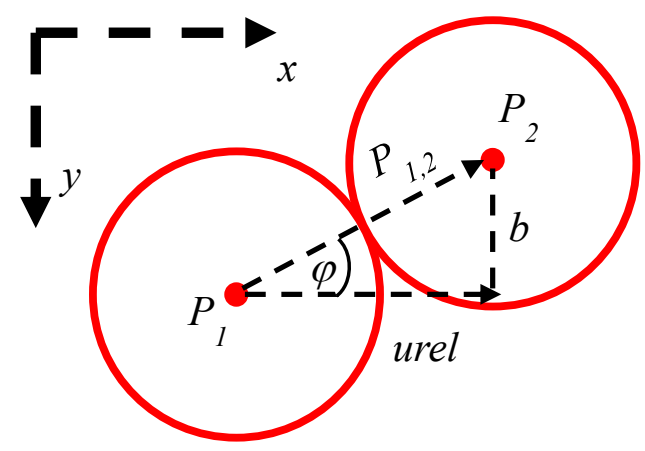

Figure 4. Droplet collision geometry [7]

The mean droplet relative velocity and diameter are used for the other non-dimensional numbers: Weber number We, size ratio (see Eq. 4) and the droplet Reynolds number Re (see Eq. 5). The effect of the viscosity is included in the Ohnesorge number Oh.

$$
\begin{aligned}
& B=\frac{2 b}{d_{1}+d_{2}}=\sin \varphi \\
& W e=\frac{\rho d_{1} u_{r e l}^{2}}{\sigma}
\end{aligned}
$$




$$
\begin{aligned}
& O h=\frac{\mu}{\sqrt{\rho \sigma d_{1}}} \\
& \Delta=\frac{d_{1}}{d_{2}} \\
& R e=\frac{\sqrt{W e}}{O h}=\frac{\rho d_{1} u_{r e l}}{\mu}
\end{aligned}
$$

For the collision outcome prediction the authors focus in this study only on the boundary line between stretching separation and coalescences. The Ashgriz and Poo [2] approach (see Eq. 6) for water droplets takes into consideration the size ratio, but not on the liquid properties. For higher viscos liquids the liquid properties had to be included. Based on this, Sommerfeld [5] improved the Gotaas et al. model (see Eq. 7) by implementing a dependency of Oh and the constant $C_{a}$ (see Eq. 8), and setting $C_{b}=1$.

$$
\begin{aligned}
& W e=\frac{4\left(1+\Delta^{3}\right)^{2}\left[3(1+\Delta)(1-B)\left(\Delta^{3} \phi_{S}-\phi_{l}\right)\right]^{0.5}}{\Delta^{2}\left[\left(1+\Delta^{3}\right)-\left(1-B^{2}\right)\left(\phi_{S}+\Delta^{3} \phi_{l}\right)\right]} \\
& B=\frac{C_{a}}{\sqrt{W e}}\left[1+C_{b} \frac{\mu}{\sigma}\left(\frac{\rho d_{1}}{\sigma}\right)^{0.5}\right] \\
& C_{a}=2.63-7.2 \cdot O h+7.86 \cdot O h^{2}+1.4 \cdot O h^{3}
\end{aligned}
$$

\section{Results and discussion}

The collision maps B over We are approved to describe the collision outcome of spray processes. The following collision maps are representing the complete outcome of 20 Ma\% PVP solution with constant material properties (see Table 1) and variation of droplet sizes and size ratios (see Table 2). The experiments were conducted up to We $=100$, with a special resolution of $\mathrm{We}<10$ and including the whole range of the impact parameter $\mathrm{B}<1$. Beside the experimental collision outcome, the fit of Ashgriz and Poo [2] and Sommerfeld [5] are plotted in the collision maps. The Gotaas et al. model is ignored because of the two variable constants $\mathrm{C}_{\mathrm{a}}$ and $\mathrm{C}_{\mathrm{b}}$.

For a better understanding of the collision outcome, droplets with $\Delta=1$ collided in Case 1 and 2 (see Table 2). In the collision maps the identification of triple point plays an imported role, because for higher We-number the stretching separation fraction increases. In Case 1 the triple point is located at We $\approx 26$ (see Fig. 5 left). By increasing the droplet size to $560 \mu \mathrm{m}$, in Case 2 the triple point is also located at We $\approx 26$ (see Fig. 5 right). The critical We- number (beginning of reflexive separation) occur out of head-on collision [2] with small B. The critical We-number in Case 2 is shifted from $W e \approx 46$ in Case 1 to $W e \approx 37$, as result of the higher kinetic energy and the lower influence of the surface tension. For We $<16$ bouncing is the only collision outcome in Case 2 , in contrast to coalescence detected from the beginning in Case 1 (min. We-number). In Case 1 and 2 (see Fig. 5) the curve by Eq. 7 and 8 match with the lower boundary of the stretching separation, while the Ashgriz and Poo model [2] underpredict the boundary in Case 1 and fit in Case 2.
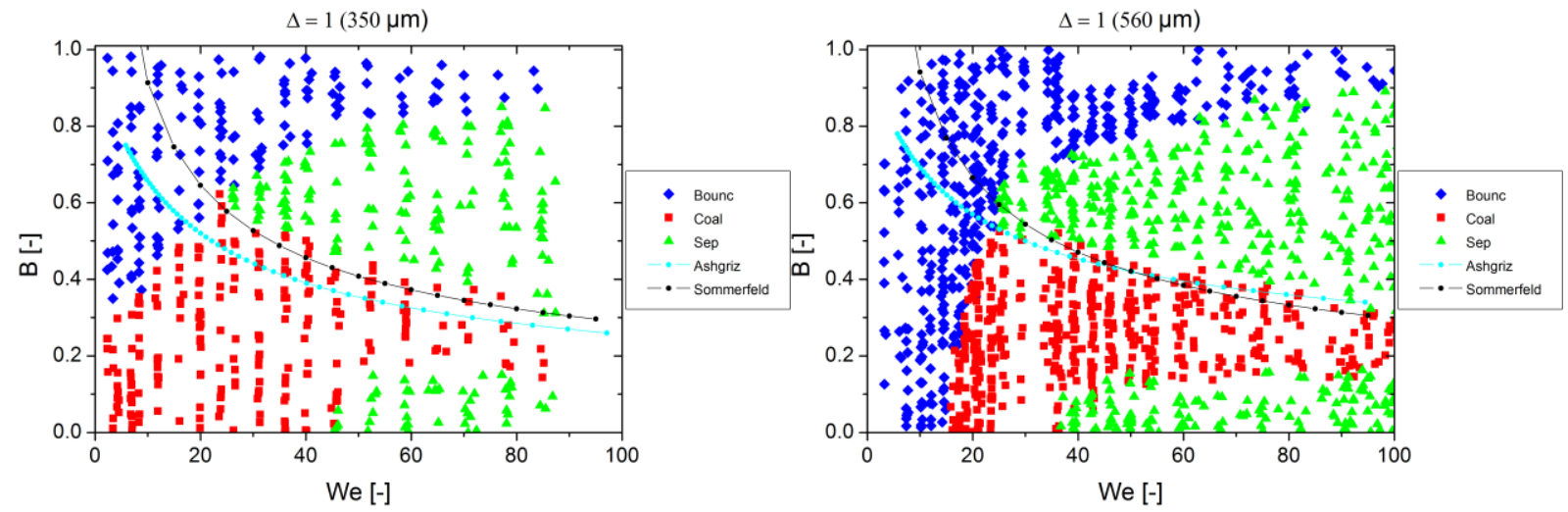

Figure 5. Collision maps of $5.5 \mathrm{mPas} \mathrm{PVP}$; left: Case $1, \Delta=1$, mean droplet size $=350 \mu \mathrm{m}$; right: Case $2, \Delta=1$, mean droplet size $=560 \mu \mathrm{m}$ 
According to the triple point results in Case 1 and 2, the point in Cases 3 to 5 (see Fig. 6) is located closed to We $\approx 26$. The critical We-number increases for smaller size ratio. A lack of data in Case 4 (see Fig. 6 top right), in the area in front of the reflexive separation and $B \approx 0.1$, suggests that the critical We-number is lower than We $\approx 52$. In opposite to the critical We-number the beginning of coalescences decreases for smaller size ratio. A lack of data in Case 3 (see Fig. 6 top left), in the area in front of the coalescence and $B \approx 0.45$, suggests that the beginning of coalescence is lower than $W e \approx 20$. In comparison to the Ashgriz and Poo model [2], which underpredict the lower boundary of the stretching separation, the Sommerfeld [5] curve (see Fig. 6 centre) shows a deviation from the experiments only in Case 5.

For small We-numbers (depending on the liquid properties) early coalescence could be observed. This outcome was detected for one collision in Case 3 (see Fig. 6 top left).
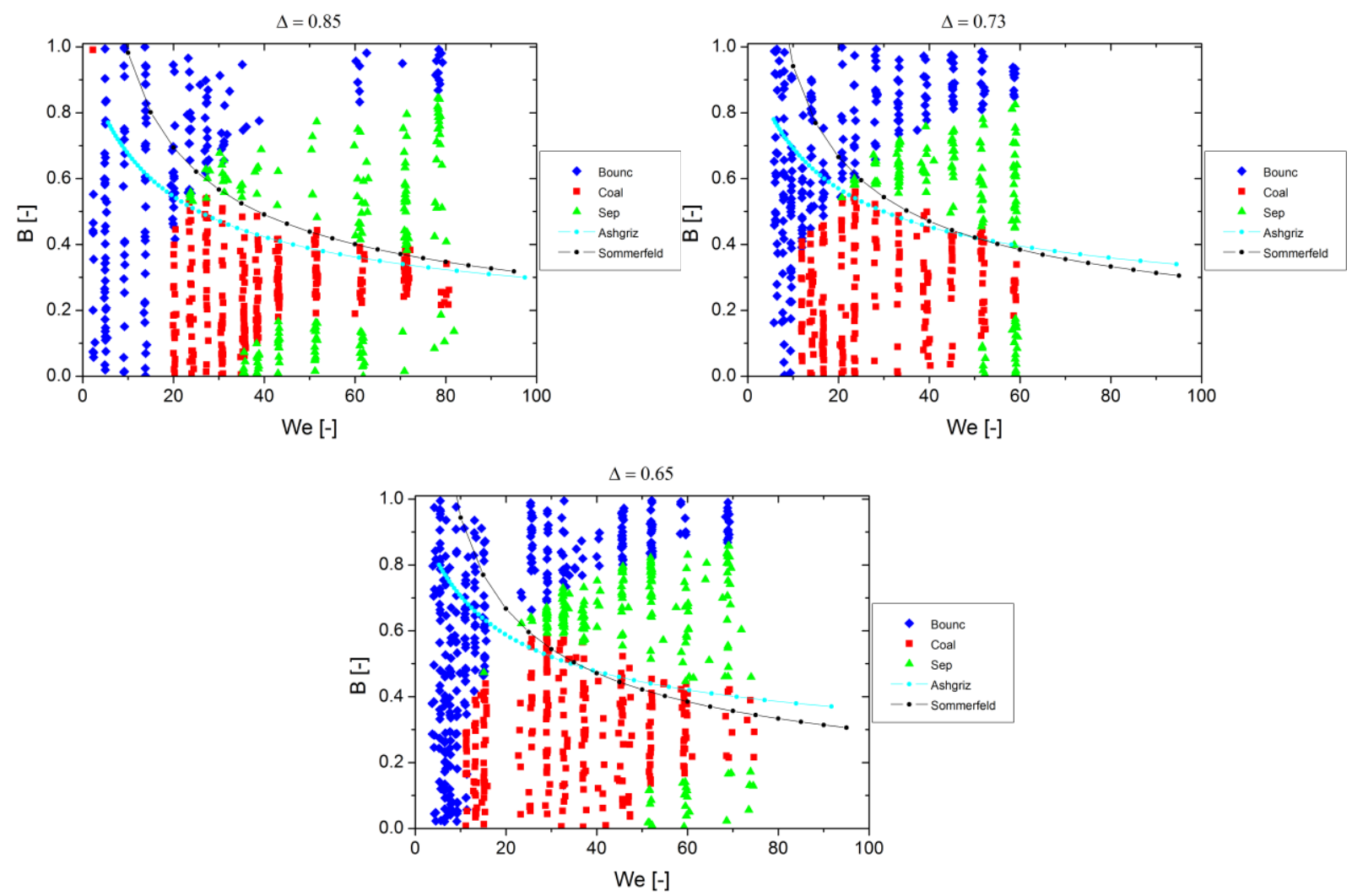

Figure 6. Collision maps of $5.5 \mathrm{mPas} \mathrm{PVP}$; top left: Case $3, \Delta=0.85$; top right: Case $4, \Delta=0.73$; centre: Case $5, \Delta=0.65$

The relevant We-numbers, related to the collision maps of Case 1- 5 (see Fig. 5, 6), and the corresponding Ohnumbers are used to predict the dependency between Oh and We (see Fig. 7) [7].

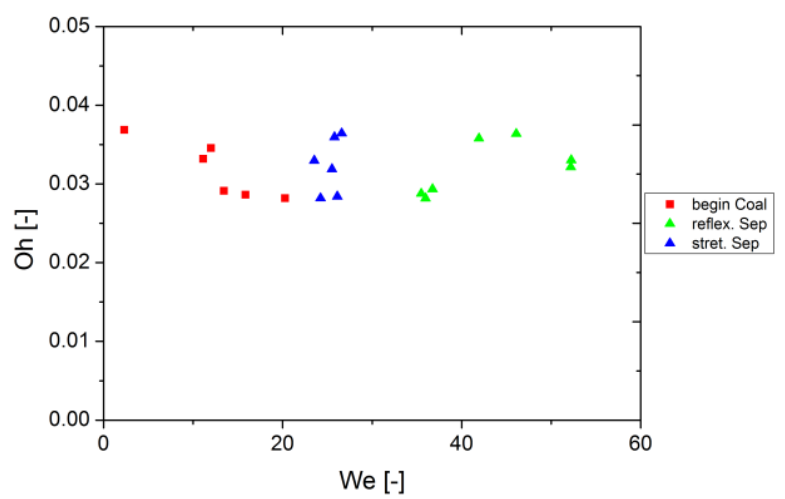

Figure 7. Onset of coalescence, reflexive and stretching separation; for Cases 1 - 5 and two additional experiments with PVP (see Table 1), Case 6: $\Delta=1$, mean droplet size $=360 \mu \mathrm{m}$; Case 7: $\Delta=1$, mean droplet size $=570 \mu \mathrm{m}$ 


\section{Conclusions}

The influence of droplet size properties was identified by the use of a new droplet detection method. For different droplet size properties of the same liquid the important We-numbers: beginning of coalescence, beginning stretching separation and beginning of reflexive separation were presented. The results for the coalescence and reflexive separation indicate a dependency between Oh-number and We-number. Smaller size ratio resulting in smaller We-number for the beginning of coalescence and larger We-number for the beginning of reflexive separation. The collision maps of the five Cases are showing that triple point is not influenced by the droplet size properties. The Sommerfeld [5] approach is demonstrating that the lower boundary of stretching separation can be described only by liquid properties and droplet size.

\section{Nomenclature}

b Lateral displacement of the droplet centres upon collision [m]

B Non-dimensional impact parameter [-]

$\mathrm{C}_{\mathrm{a}}, \mathrm{C}_{\mathrm{b}} \quad$ Parameter of Jiang et al. [3] model

$\mathrm{d}_{1} \quad$ Small droplet diameter [m]

$\mathrm{d}_{2} \quad$ Large droplet diameter [m]

Oh Ohnesorge number [-]

$\mathrm{Re} \quad$ Droplet Reynolds number [m]

urel Relative velocity

We Weber number

$\Delta \quad$ Size ratio [-]

$\mu \quad$ Dynamic viscosity of the fluid [Pa s]

$\Phi_{\mathrm{S}}, \Phi_{\mathrm{l}} \quad$ Parameter for small and large droplets in the Ashgriz and Poo [2] model [-]

$\rho \quad$ Density of the liquid $\left[\mathrm{kg} / \mathrm{m}^{3}\right]$

$\sigma \quad$ Surface tension of the liquid $[\mathrm{N} / \mathrm{m}]$

$\varphi \quad$ Enclosed angle between the relative velocity and the position vector $\mathrm{P} 1,2$ of the droplets centre points $\left[^{\circ}\right]$

\section{References}

[1] Estrade, J.-P., Carentz, H., Lavergne, G., Biscos, Y. "Experimental investigation of dynamic binary collision of ethanol droplets - a model for droplet coalescence and bouncing", 1999, International Journal of Heat and Fluid Flow 20, pp. 486-491.

[2] Ashgriz, N.; Poo, J.Y.:"Coalescence and separation in binary collisions of liquid drops", 1990, Journal of Fluid Mechanics 221, pp. 183-204.

[3] Jiang, Y. J., Umemura, A. and Law, C.K.: "An experimental investigation on the collision behavior of hydrocarbon droplets", 1992, Journal of Fluid Mechanics 234, pp. 171-190.

[4] Gotass , C., Havelka, P. Jakobson, H. A. and Svendsen H.F, Hase, M., Roth, N., and Weigand, B. : "Effect of viscosity on droplet droplet collision outcome Experimental study and numerical comparison", 2007, Physics of Fluids 19, 102106

[5] Sommerfeld, M., Sep. 4.-7. 2011, 27th European Conference on Liquid Atomization and Spray Systems.

[6] Kuschel, M., Sommerfeld, M., Investigation of droplet collisions for solutions with different solids content, 2013, Exp Fluids 54:1440.

[7] Sommerfeld, M, Kuschel, M., "Modelling droplet collision outcomes for different substances and viscosities", 2016, Exp Fluids 57:187. 


\title{
Splashing of a very viscous liquid drop impacting onto a solid wall wetted by another liquid
}

\author{
Hannah M. Kittel*, Ilia V. Roisman, Cameron Tropea \\ Institute for Fluid Mechanics and Aerodynamic, Technische Universität Darmstadt, Germany \\ *Corresponding author: kittel@sla.tu-darmstadt.de
}

\begin{abstract}
In this experimental work the main focus is on the impact of a single drop of a very viscous liquid onto a thin, horizontal wall film of different liquid. Splashing resulting from drop impact onto a wetted wall occurs in many natural and engineering applications like in internal combustion engines and spray cooling. While the splashing threshold for low viscosity liquid drops has been extensively examined, impact of a very viscous drop is much less studied. The viscosities of drop and wall film liquids are varied up to kinematic viscosities of $100,000 \mathrm{~mm}^{2} / \mathrm{s}$. The liquids used in the experiments are miscible.

The impact outcome is determined by the impact parameters and fluid properties. The effect of very viscous liquids used as drop fluid and as wall film liquid on the kinematic of the corona expansion is investigated in the experiments. The results of drop impact onto solid walls are compared to obtain the limiting asymptotic values for the splashing threshold.

Finally, a semi-empirical model for the splashing threshold, for the maximum spreading radius $D_{\max }$ and for the maximum spreading times $t_{\max }$ are developed for extremely viscous liquids.
\end{abstract}

\section{Keywords}

Drop impact, high viscous liquids, wetted wall

\section{Introduction}

Splashing as a result of a drop impact onto a thin liquid film [1-3] or onto a dry substrate [4-6] occurs in many natural and engineering applications like spray coating, spray cooling, paint spraying, ink-jet printing and in internal combustion engines. Impact of a supercooled drop onto a surface of an aircraft can lead to ice accretion, while collision with a heated substrate can lead to drop evaporation or intensive boiling [7]. The drop/wall interaction is affected by the fact that the drop and the liquid film are different liquids and may exhibit different degrees of miscibility and viscosity. A comprehensive review on the phenomena, modeling and application of drop impact can be found in [8]. Drop impact onto a wetted wall can lead to various different outcomes. In the case of splashing
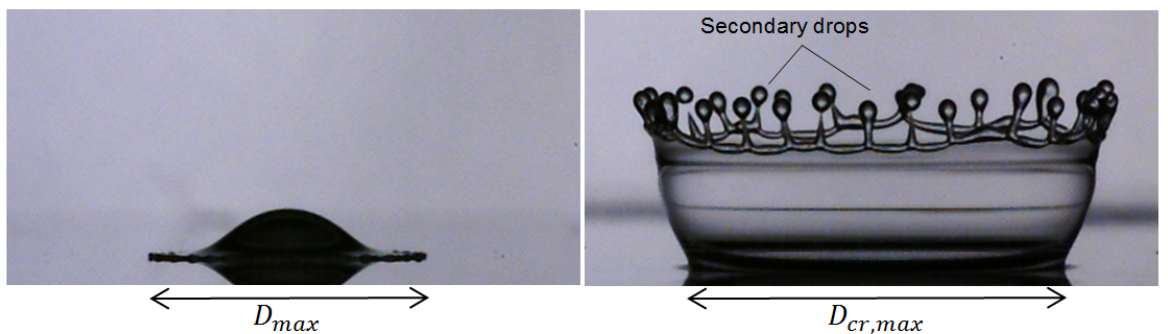

Figure 1. Different impact outcomes, on the left deposition, on the right corona splash.

a radial expanding flow in the lamella is formed after the drop impact. Due to the interaction with the outer liquid wall film an uprising liquid sheet is generated which is bounded by a Taylor rim [10-12]. If the rim gets unstable, finger-like jets are formed which lead to the generation of secondary drops [14]. In this case the inertial effects in the spreading lamella dominate over the surface tension and viscosity effects, and if the crown jets are unsteady, secondary droplets are formed. It is well-known that the splashing threshold of a drop impacting onto a wetted wall is determined by the inertia, viscous and capillary forces. If no instabilities occur or the instabilities do not lead to secondary drops, the phenomena is called corona. If the drop spreads on the impact surface, it is called deposition. In this case the spreading diameter is determined by the flow in the lamella and the propagation of the rim [9]. The dimensionless Weber and Reynolds number as well as the substrate wettability determine furthermore the maximum spreading diameter. If the drop rebounds completely or partially, it is called rebound or partial rebound. The impact outcome is highly determined by the impact parameters, like the impact velocity $u_{0}$ and the initial drop diameter $d_{0}$, and in the case of different liquids by the fluid properties, like the kinematic viscosity $\nu$ and the surface tension $\sigma$. To describe these phenomena the relative film thickness $\delta=h / d_{0}$ and the viscosity ratio $\nu_{0}=\nu_{f} / \nu_{d}$ are 
introduced. In order to define a splashing threshold, whether splashing occurs or not, the dimensionless $\mathrm{K}$ number is introduced [13]. A critical value is determined that defines the splashing threshold. The splashing threshold is dependent on the impact velocity as well as on the viscosity of both liquids of drop and wall film. While the splashing threshold of low viscosity liquid drops has been extensively examined in the past [13], impact of a very viscous drop is much less studied, despite its relevance to such intensively developing fields like additive manufacturing.

The main subject of this study is the experimental investigation of the effect of very high viscous liquids on the outcome of drop impact. The maximum spreading diameter $D_{\max }$ in the case of deposition and the maximum corona diameter $D_{c r, \max }$ in the case of corona/corona splash as well as the maximum spreading time $t_{\max }$ are determined for various fluid combinations. The splashing/deposition limit is determined using the modified $\mathrm{K}^{*}$ number as a function of the viscosity ratio $\nu_{0}$.

\section{Material and methods}

The experimental setup is shown schematically in Fig. 2 and consists of three main parts, the droplet generating unit, the impact substrate and the observation system. In order to generate a single droplet, the micro pump transports the liquid from a tank to the cannula. At the end of the tip the droplet is collected until it reaches a critical mass and drips off. Since the droplet dripping is driven by gravity the impact velocity is determined by the distance of the tip of the cannula and the impact substrate. In this study the impact velocity is set to $u=3.2 \mathrm{~m} / \mathrm{s}$. In order to vary the initial droplet diameter $d_{0}$ different cannula diameter are used. As impact substrate a horizontal glass plate is used. In order to generate a wall film the glass plate is sandblasted up to a recess. A ring with a diameter of $60 \mathrm{~mm}$ is glued on this sandblasted area. The unsealed area is used to create the wall film. To measure the film thickness a micrometer screw is used. The tip of the screw is first lowered till it touches the glass substrate to determine the reference value. After the liquid is filled in the screw is lowered till it reaches the liquid surface. The difference of both values determines the film thickness.

The impact substrate is located between the observation system. The observation system consists of a highspeed camera (Photron FASTCAM SA-X2) and a light-emitting diode (LED, Veritas Constellation 120E) with 12,000 Lumen. A diffusing plate is placed in front of the LED in order to receive a consistent light. In this study a frame rate of $30,000 \mathrm{fps}$ is used with a resolution of $896 \times 480$ pixel. The shutter time is set to $12.5 \mu \mathrm{s}$. The physical properties of the liquids which are used in this study can be found in Table 1. The viscosities of drop and wall film liquids are varied up to kinematic viscosities of $100,000 \mathrm{~mm}^{2} / \mathrm{s}$. The liquids used in these experiments are miscible.

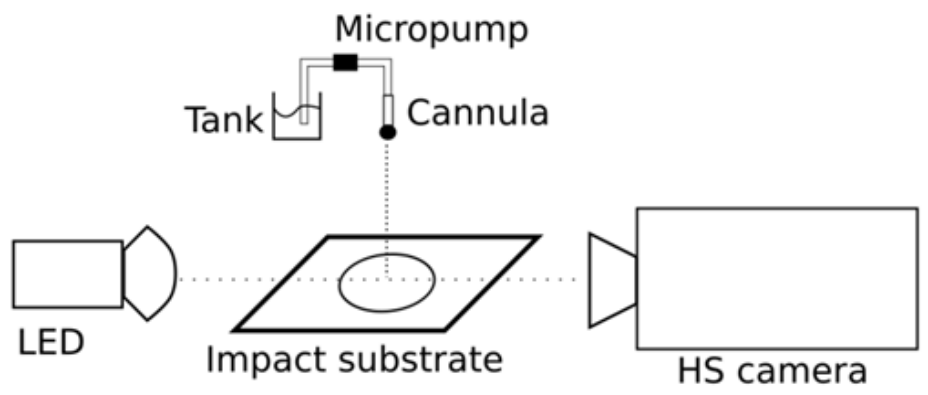

Figure 2. Schematic illustration of the experimental setup

Table 1. Physical properties of the liquids used in this study.

\begin{tabular}{c|ccc}
\hline & Kinematic viscosity $\left[\mathrm{mm}^{2} / \mathrm{s}\right]$ & Surface tension $[\mathrm{mN} / \mathrm{m}]$ & Density $\left[\mathrm{kg} / \mathrm{m}^{3}\right]$ \\
\hline H2O & 1.004 & 72.24 & 997 \\
Hexadecane & 4.11 & 27.61 & 769.15 \\
S5 & 5 & 17.72 & 910 \\
S10 & 10 & 18.19 & 920 \\
S20 & 20 & 18.2 & 925 \\
S65 & 65 & 18.2 & 950 \\
S350 & 350 & 18.56 & 972 \\
S500 & 500 & 18.83 & 972 \\
S750 & 750 & 18.6 & 972 \\
S1000 & 1,000 & 18.59 & 972 \\
S10000 & 10,000 & 18.81 & 972 \\
S30000 & 30,000 & 18.81 & 972 \\
S100000 & 100,000 & 18.81 & 972
\end{tabular}




\section{Results and discussion}

In order to see if there is a connection between the outcome of drop impact onto a solid wall and onto a high viscous liquid film the viscosity of the wall film is varied up to $100,000 \mathrm{~mm}^{2} / \mathrm{s}$. A solid surface can be considered as a substrate with infinite viscosity.

The impact of a drop leads to different outcomes dependent on the impact substrate. If the impact substrate is covered by a very high viscosity liquid or is a solid surface the impact results in deposition, as it can be seen in Fig. 3. The maximum spreading diameter $D_{\max }$ as well as the maximum spreading time $t_{\max }$ are mainly determined by the viscosity of the drop and the impact substrate. In this case the viscosity of the wall film determines the phenomena itself, deposition, but the viscosity of the drop determines the geometrical and temporal parameters like $D_{\max }$ and $t_{\max }$. Comparing the outcomes of the same drop impacting onto different wall film liquids, as it can be seen in Fig. 3, the outcome does not differ much from each other, whereby comparing the outcomes of the same wall film liquid but different drop liquids the outcomes differ significantly from each other. The impact substrate determines the motion of the drop after the impact, whether the drop can build a corona or the fluid of the drop is stopped and end in deposition. The higher viscous the film gets the less motion of the drop is enabled due to the viscous forces. In the case of deposition the higher viscous the drop gets the less spreading occurs also due to the viscous forces. The motion of the drop is stopped due to the inaction of the drop. Directly after the drop impact a very high viscous drop only sits on the impact substrates, but does not spread what is caused by the high viscosity and its loss of ambition to change its shape. In the first phase of a couple milli seconds a very high viscous drop gets only compressed breadth wise, then the motion of the drop is stopped. In the second phase which lasts much longer, several seconds, the contact angle decreases slowly. The viscosity of the liquids highly influences the impact outcome due to its characteristic of motion depending on its magnitude.

If the liquid of the impact substrate is less viscous the drop impact leads to the evolution of a corona. Furthermore
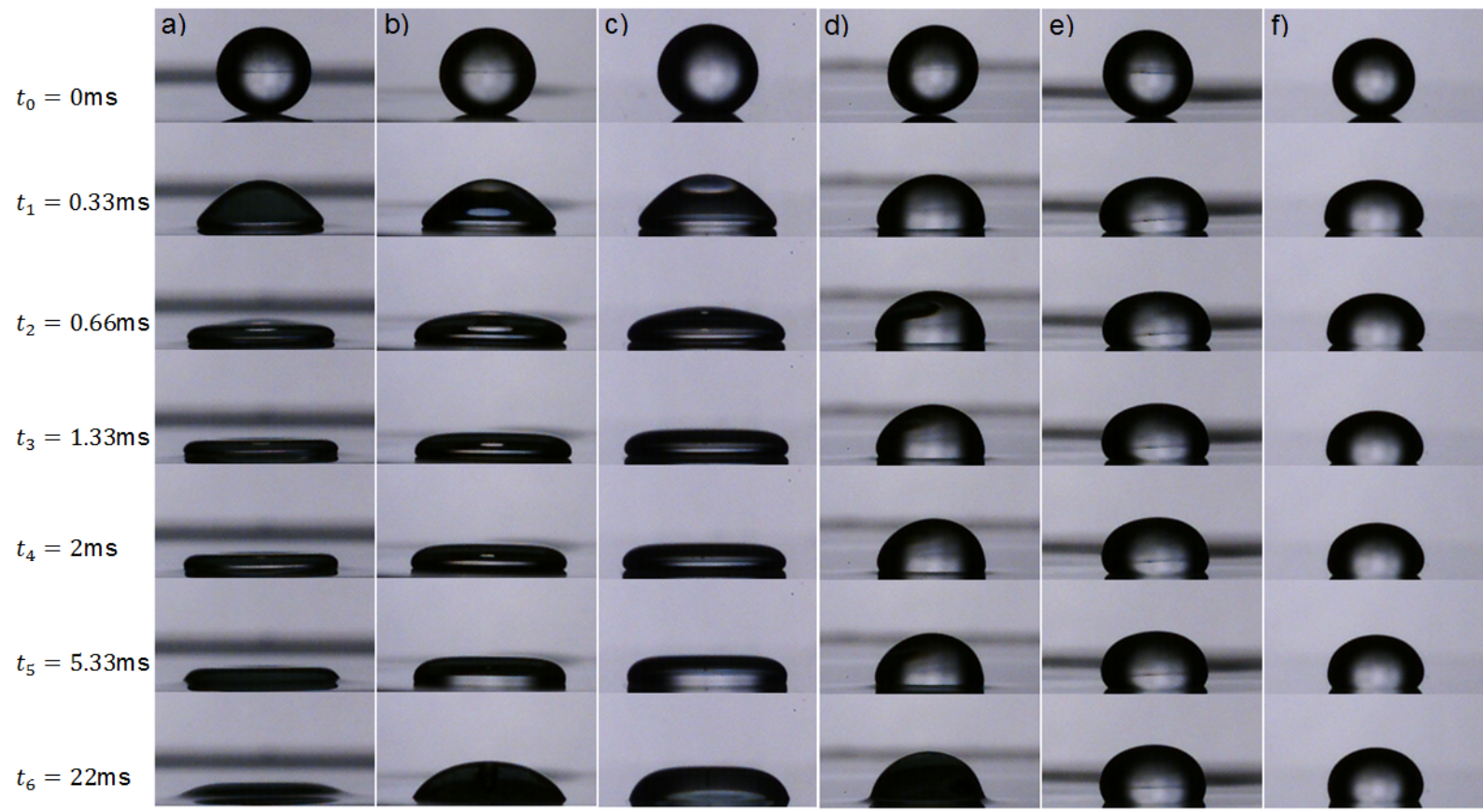

Figure 3. Temporal development of the drop spreading of a $S 350$ drop (a)-c)) and a $S 10000$ drop (d)-f)) impacting onto different impact substrates, wall film of $S 1000(a), d)$ ) and of $S 100000(b), e)$ ) with the same relative film thickness $\delta=0.227$ and glass (c), f)).

it can also lead to corona splash depending on the impact substrate. The impact outcome itself is influenced by the viscosity of the wall film whereby the maximum spreading diameter $D_{\max }$ and maximum corona diameter $D_{c r, \max }$ are highly influenced by the viscosity of the drop as it can be also observed in the case of deposition. It can be observed that with increasing viscosity of the drop $D_{c r, \max }$ decreases whereas the increase of the viscosity of the wall film does not significantly influence $D_{c r, \max }$ as shown in Fig. 4.

The fluid of the drop seems to predominate the impact outcome. In the case of splashing though it can be observed that the fluid of the wall film determines the splashing threshold as it can be seen in Fig. 4. In the case of a highly viscous drop the splashing threshold is already reached at a wall film viscosity of $\nu_{f}=20 \mathrm{~mm}^{2} / \mathrm{s}$.

The impact substrate also determines the impact outcome. For a high viscous wall film the drop cannot evolve to a corona since the resistance of the wall film is too high and does not admit the evolution of a corona. The drop can only slightly penetrate the wall film. Therefore the drop spreads on the high viscous wall film alike on a solid surface, like glass. If the wall film is less viscous the drop impact leads to corona or even to corona splash. In this case the drop is able to penetrate into the wall film, the liquid of the wall film is pushed aside and a corona is built. In both 


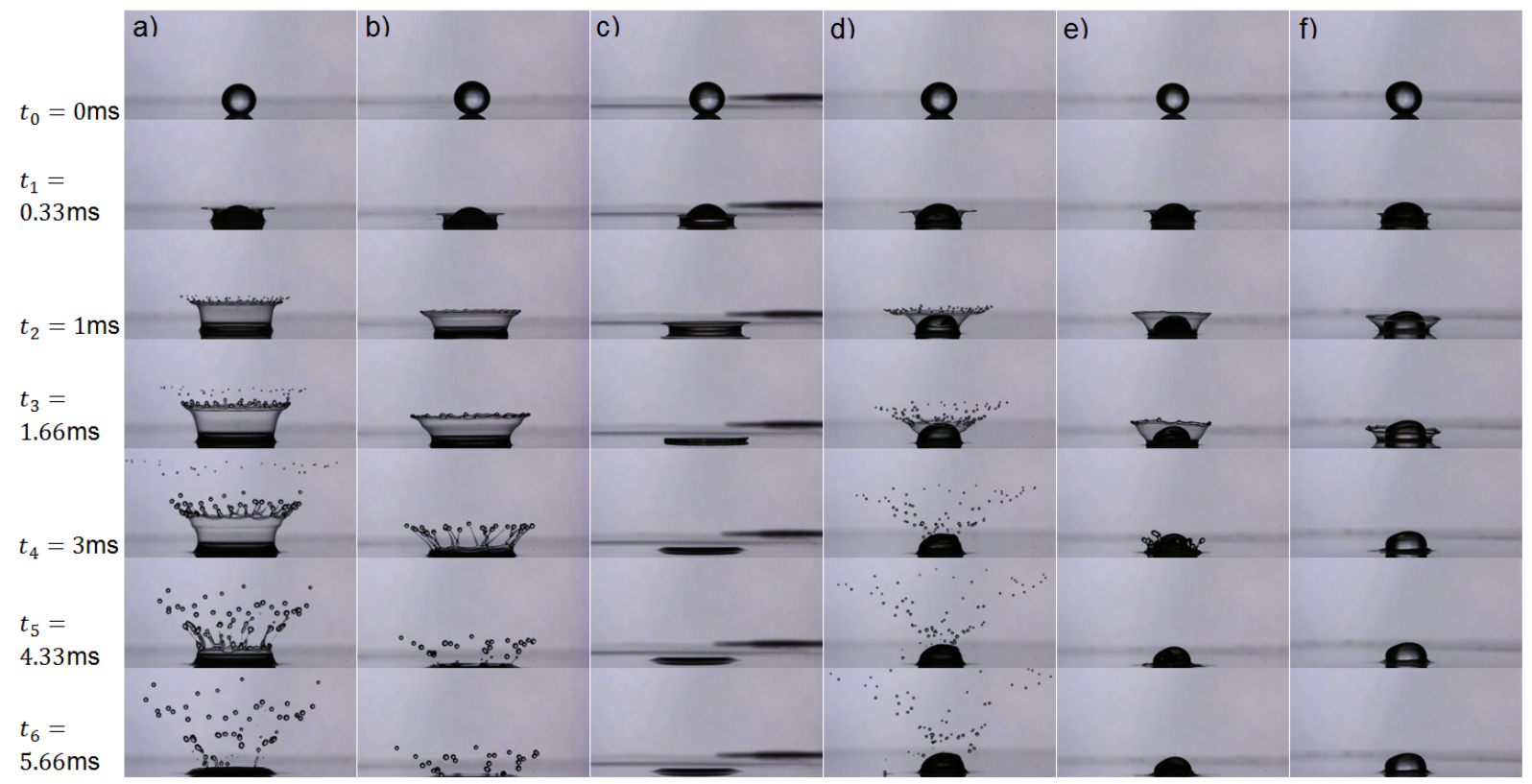

Figure 4. Temporal development of the corona evolution of a $S 350$ drop (a)-c)) and a $S 10000$ drop (d)-f)) impacting onto different impact substrates, wall film of S5 (a), d)), S10 (b), e)) and S20 (c), f)) for the same relative film thickness $\delta=0.045$.

cases the maximum spreading diameter and the maximum corona diameter vary dependent on the viscosity of the drop. Similar to a high viscous wall film a high viscous drop does not change its shape too much. In contrast a less viscous drop extends into the wall film.

In order to determine the effect of a high viscosity liquid on the process of spreading the dimensionless maximum spreading diameter $D_{\max } / D_{0}$ and the dimensionless maximum spreading time $\tau$ are evaluated. The dimensionless maximum spreading time $\tau$ is defined below in Eq.1,

$$
\tau=t_{\max } \frac{u_{0}}{d_{0}} R e_{d}^{-1 / 5}
$$

with the impact velocity $u_{0}$, the initial drop diameter $d_{0}$ and the Reynolds number of the drop

$$
R e_{d}=\frac{u_{0} d_{0}}{\nu_{d}}
$$

The indices $d$ and $f$ represent $d r o p$ and $f i l m$, respectively. $\frac{u_{0}}{d_{0}} R e^{-1 / 5}$ is the typical time of expansion of the boundary in the drop [9].

In Fig. 5a) the dimensionless maximum spreading time $\tau$ is shown as a function of the relative film thickness $\delta$ and the viscosity ratio $\nu_{0}$. For a range of viscosities the value of $\tau$ is near to unity. Therefore the time of drop deformation is determined by the expansion of the boundary layer. It can be seen that with increasing viscosity of the drop impacting drop onto different high viscous liquids the dimensionless time $\tau$ decreases respectively its dependence on $\delta \nu_{0}^{1 / 6}$. However, the dependency of $\tau$ on $\delta$ is higher than the dependency on $\nu_{0}$ what is expressed by $\delta \nu_{0}^{1 / 6}$. The dependency of the dimensionless time $t_{\max } u_{0} / d_{0}$ as a function of the Reynolds number of the drop on the viscosity of the drop in the case of glass as impact substrate is shown in Fig. 5b). In the transition region of low and high viscous liquids expressed by the Reynolds number a jump can be seen. For $R e_{d}<20$ the dimensionless spreading time is not dependent on $R e_{d}$ anymore. The gap of the transition region seems to occur due to the characteristic of the low viscous and high viscous liquids to move very fast and accordingly to stop its motion very fast what leads to comparable dimensionless spreading times in the case of glass as impact substrate.

The effect of the viscosity of the wall film on the dimensionless maximum spreading diameter is shown in Fig. 6a). A clear correlation between $D_{\max } / d_{0}$ and $R e_{d}$ can be seen for the different impact substrates. Furthermore all results are rather in line with each other. This shows that the drop impact onto a high viscous wall film can be compared to the drop impact onto a glass substrate. The experimental results are compared with a model of the maximum spreading diameter by Roisman [9] and is valid for high Reynolds numbers.

$$
D_{\text {max }} \approx 0.87 R e^{1 / 5}-0.4 R e^{2 / 5} W e^{1 / 2} .
$$

The theoretical $D_{\max }$ matches the experimental data rather well. For $R e \geq 0$ the theoretical $D_{\max }$ and the experimental $D_{\max } / d_{0}$ differ from each other.

If the fluid of the wall film is less viscous, but the fluid of the drop is high viscous, a corona is evolved. The maximum corona diameter $D_{c r, \max }$ is in this connection highly dependent on the viscosity of the drop as it can be seen in Fig. 6b). Since all results are rather in line with each other the viscosity of the wall film does not seem to influence 


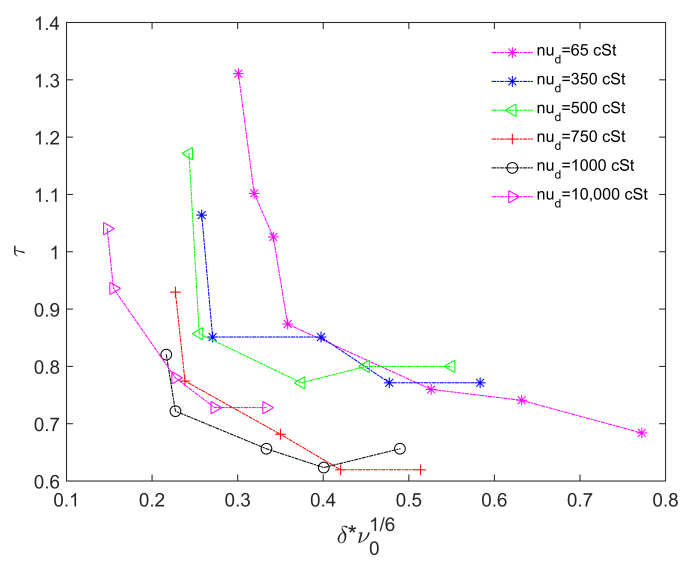

(a)

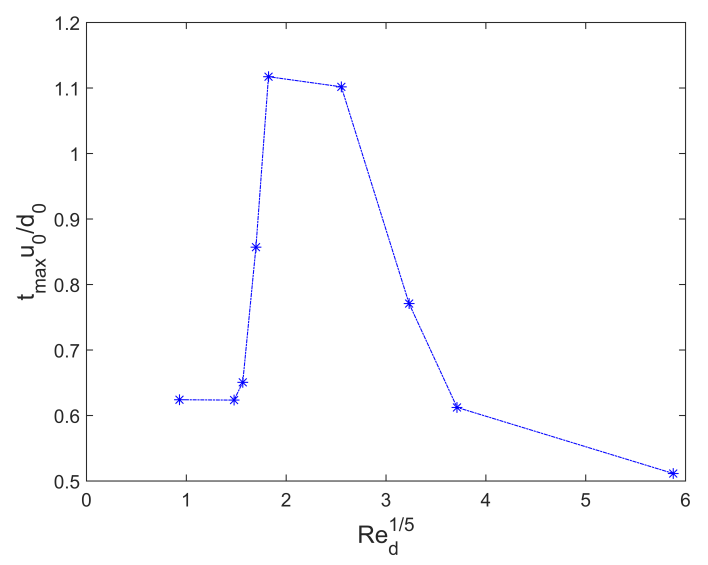

(b)

Figure 5. a) $\tau$ defined in Eq. (1) as a function of the relative film thickness $\delta$ and the viscosity ratio $\nu_{0}$ and of b) the dimensionless spreading time $t_{\max } u_{0} / d_{0}$ as a function of the Reynolds number of the drop $R e_{d}$ for a drop impacting onto glass.

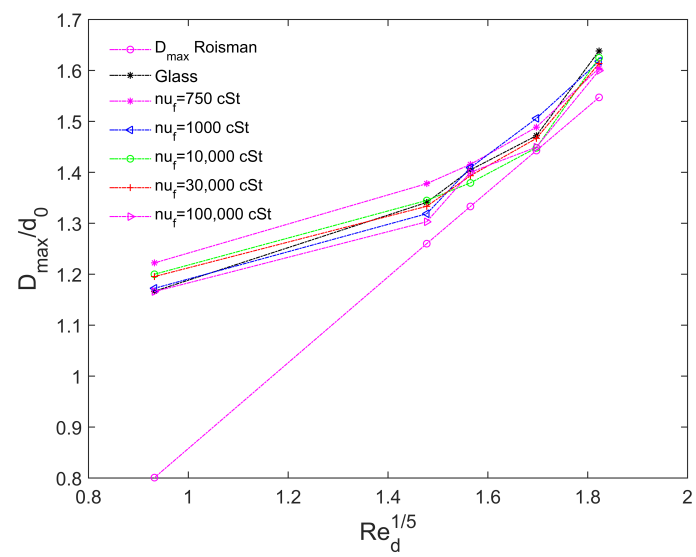

(a)

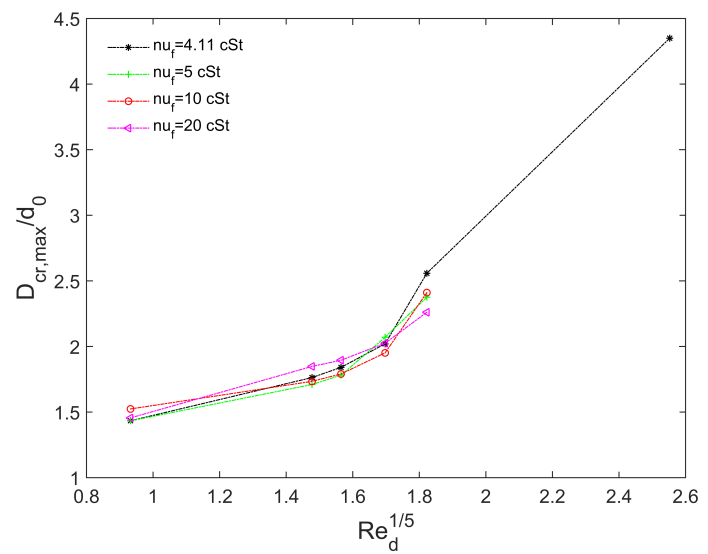

(b)

Figure 6. a) Dimensionless maximum spreading diameter as a function of the Reynolds number of the drop $R e_{d}$ and b) Dimensionless maximum corona diameter as a function of the Reynolds number of the drop $R e_{d}$ for the relative film thickness $\delta=0.045 \mathrm{~mm}$.

$D_{c r, \max }$ significantly. Solely the impact outcome itself, corona or corona splash, is influenced by the viscosity of the wall film as shown in Fig. 4. A high viscous drop does not tend to spread after the drop impact what can already be seen in Fig. 3 in the case of deposition. Due to its contour accuracy which comes from the high viscosity the drop only pushes the liquid of the wall film aside but does not extend itself. Due to the kinetic energy resulting from the impact the shape of the corona is determined by the film liquid which is pushed aside while the drop nearly keeps its initial form. Therefore the maximum corona diameter decreases with decreasing Reynolds number.

If splashing occurs, it is desirable to define a splashing threshold. The dimensionless $\mathrm{K}$ number has been introduced in order to define a critical value to determine the splashing threshold in the case of a one component system [13] as

$$
K=W e R e^{1 / 2}
$$

with the Weber number

$$
W e=\frac{u_{0}^{2} d_{0} \rho}{\sigma}
$$

with the density $\rho$ and the surface tension $\sigma$ and the Reynolds number as described in Eq.2.

If the liquids of droplet and wall film are different, the modified $\mathrm{K}^{*}$ number is used as introduced in [15] since both fluid properties influence the impact outcome. The modified $\mathrm{K}^{*}$ number is defined as

$$
K^{*}=\frac{d^{3 / 2} \rho u^{5 / 2}}{\sqrt{\bar{\nu}} \sigma}
$$


with

$$
\begin{aligned}
& \bar{\nu}=\frac{\nu_{f}^{3 / 2}+\nu_{d}^{3 / 2}}{\nu_{f}^{1 / 2}+\nu_{d}^{1 / 2}}, \\
& \bar{\sigma}=\frac{\sigma_{f}+\sigma_{d}}{2}
\end{aligned}
$$

and

$$
\bar{\rho}=\frac{\rho_{f}+\rho_{d}}{2} .
$$

The effective viscosity is estimated on the assumption that the average film thickness in the corona is scaled by the

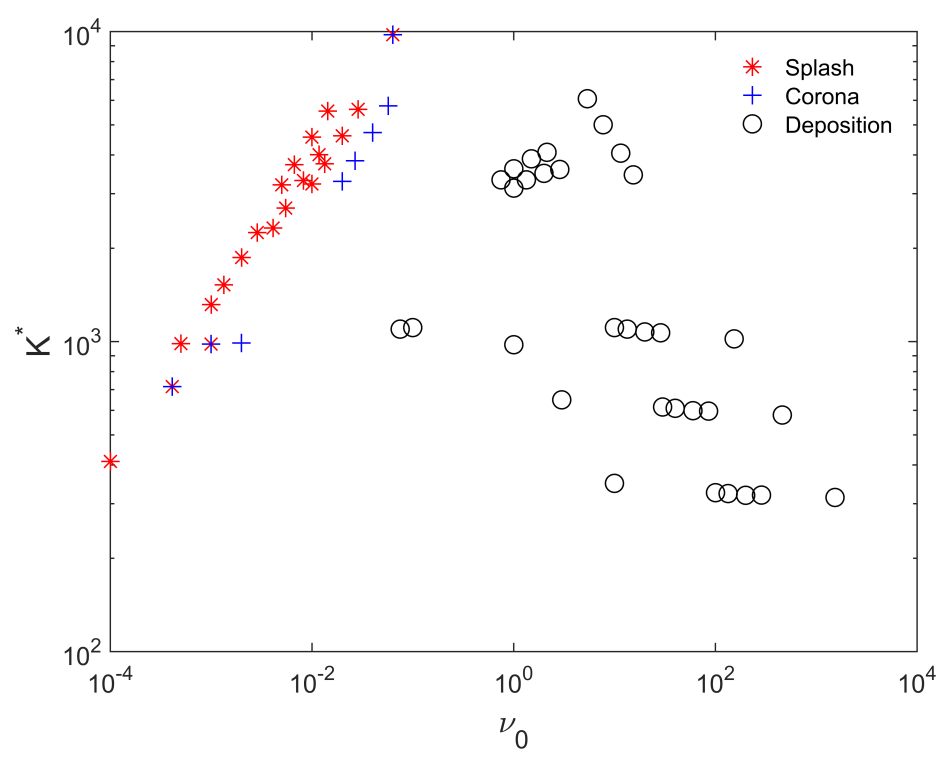

Figure 7. Impact outcome illustrated by the $\mathrm{K}^{*}$ number as function of the viscosity ratio $\nu_{0}$.

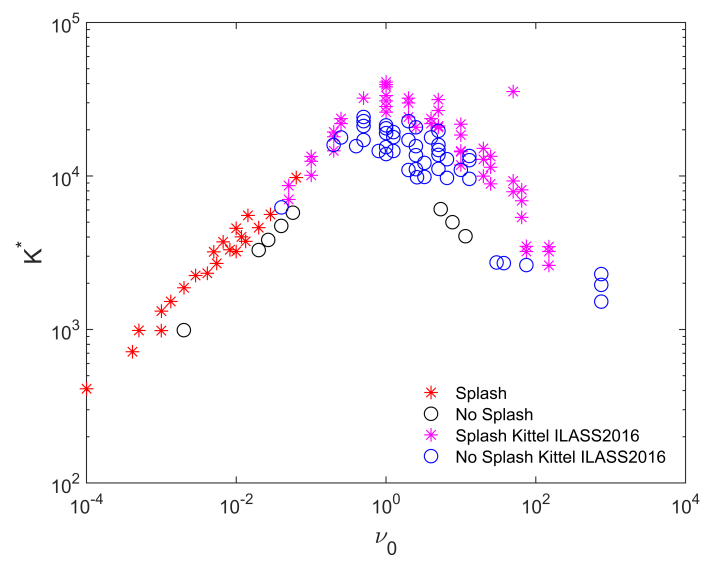

(a)

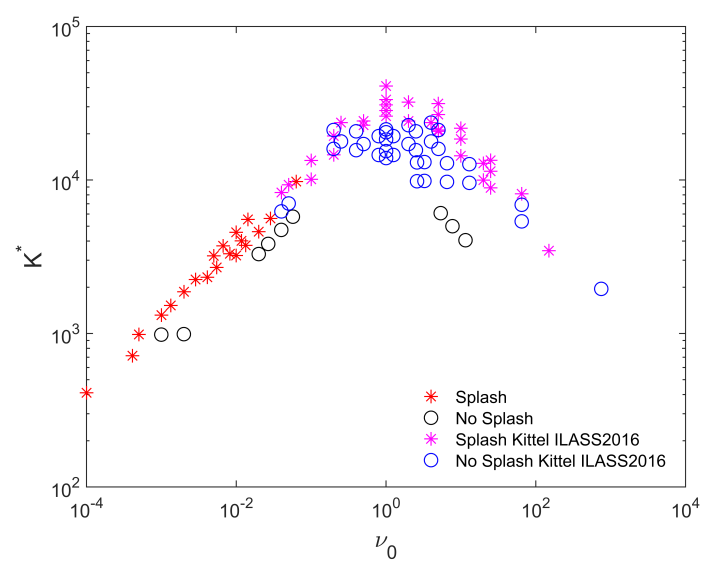

(b)

Figure 8. The modified $\mathrm{K}^{*}$ number as a function of the viscosity ratio $\nu_{0}$ compared to the results from Kittel [15] for the film thickness a) $h=0.1 \mathrm{~mm}$ and b) $h=0.25 \mathrm{~mm}$.

thickness of the viscous boundary layer $\sqrt{\nu t}$. The average viscosity in the composed film of both liquids is estimated as the film thickness average.

In Fig. 7 the $\mathrm{K}^{*}$ number is shown as a function of the viscosity ratio $\nu_{0}$ for the film thicknesses used in this study. A clear splashing/corona limit can be seen. More experimental data is needed to sharpen the corona/deposition limit, a fist tendency can be seen so far. A transition of splashing to corona and finally to deposition can be seen. The in 
this study observed phenomena,deposition, corona and corona splash, can be differentiated using the modified $\mathrm{K}^{*}$ number as a first approximation. It has to be noted that more experimental data is needed.

In Fig. 8 the results relating to the film thickness $h=0.1 \mathrm{~mm}$ in a) and $h=0.25 \mathrm{~mm}$ in b) are compared to the results of Kittel et al. [15]. The results of this study for both film thicknesses fit the data of Kittel et al. [15] very well. The correlation between the modified $\mathrm{K}^{*}$ number and the viscosity ratio $\nu_{0}$ seems to work for numerous liquid combinations, like low viscous - low viscous or low viscous - high viscous.

\section{Conclusions}

In this study the effect of a high viscous drop impacting onto a wetted wall on the impact outcome is investigated. The viscosity of the drop and wall film are varied up to $100,000 \mathrm{~mm}^{2} / \mathrm{s}$ as well as the relative film thickness. The dependency of the maximum spreading diameter in the case of deposition and maximum corona diameter in the case of corona on the Reynolds number is shown. The viscosity of the wall film only influence the impact outcome itself, but the viscosity of the drop determine maximum spreading diameter and maximum corona diameter. In the case of a high viscous drop impacting onto a high viscous wall film the outcome is comparable to the impact on glass except for its final resting shape. The dimensionless maximum spreading time $\tau$ has been introduced to determine the effect of the viscosity of the drop. In the case of a high viscous drop impacting onto a less viscous wall film the maximum corona diameter decreases with increasing drop viscosity. To determine the splashing threshold the modified $K^{*}$ number as a function of the viscosity ratio shows a good correlation.

\section{Acknowledgements}

This research was supported by the the German Scientific Foundation (Deutsche Forschungsgemeinschaft) in the framework of the SFB-TRR 150 collaborative research center, subproject A02.

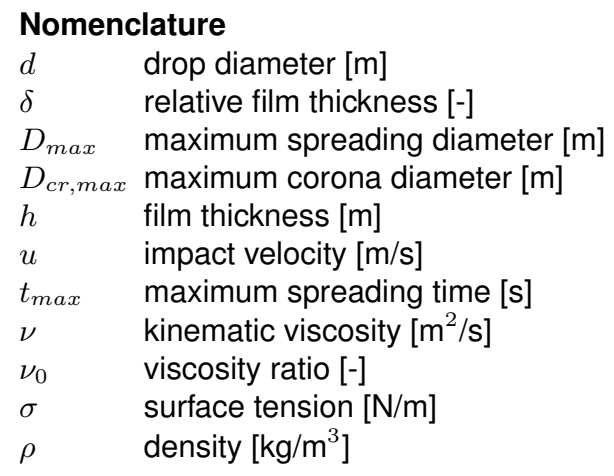

\section{References}

[1] Rein, M., 1993, Fluid Dynamics Research, 12, pp. 61-93.

[2] Yarin, A.L., 2006, Annual Review of Fluid Mechanics, 38, pp. 159-192.

[3] Thoroddsen, S. T., Etoh, T. G., Takehara, K., 2008, Annual Review of Fluid Mechanics, 40, pp. 257-285.

[4] Marengo, M., Antonini, C., Roisman, I. V., Tropea, C., 2011, Current Opinion in Colloid \& Interface Science, 16, pp. 292-302.

[5] Josserand, C. and Thoroddsen, S. T., 2016, Annual Review of Fluid Mechanics, 48, pp. 365-391.

[6] Liang, G., Mudawar, I., 2017, International Journal of Heat and Mass Transfer, 106, pp. 103-126.

[7] Quéré, D., 2013, Annual Review of Fluid Mechanics, 45, pp. 197-215.

[8] Yarin, A. L., Roisman I. V., Tropea, C., 2017, Collision Phenomena in Liquids and Solids, Cambridge University Press.

[9] Roisman, I. V., 2009, Physics of Fluids, 21(5), pp.052104.

[10] Taylor, G., 1959, Proceedings of the Royal Society, 253(1274), pp. 313-321.

[11] Yarin, A.L., Weiss, D.A., 1995, Journal of Fluid Mechanics, 283, pp. 141-173.

[12] Roisman, I.V., Tropea, C., 2006, Journal of Fluid Mechanics, 472, pp. 373-397.

[13] Cossali, G.E., Coghe, A., Marengo, M., 1997, Experimental of Fluids, 22 (6), pp. 463-472.

[14] Roisman, I.V., Horvat, K., Tropea, C., 2006, Physics of Fluids, 18(10), pp. 102-104.

[15] Kittel, H.M., Roisman, I.V. and Tropea, C., Sep. 4.-7. 2016, $27^{\text {th }}$ European Conference on Liquid Atomization and Spray Systems. 


\title{
Drop Stream - Immiscible Jet Collisions: Regimes and Fragmentation Mechanisms
}

\author{
Carole Planchette*, Hannes Hinterbichler, Günter Brenn \\ Institute of Fluid Mechanics and Heat Transfer, Graz University of Technology, Austria \\ *Corresponding author: carole.planchette@tugraz.at
}

\begin{abstract}
We investigate the collision of a continuous liquid jet with a regular stream of immiscible droplets. The immiscible liquids, namely silicon oil for the continuous jet and an aqueous glycerol solution for the drop stream, are selected to enable the total wetting of the drops by the jet liquid. Four different regimes are experimentally identified: drops in jet, encapsulation without satellites, encapsulation with satellites from the jet liquid and mixed fragmentation. The drops in jet regime, potentially of great interest for new applications, corresponds to a regular stream of drops embedded in a continuous jet and is described and reported for the first time. Using well known aspects of drop collision and jet stability, we propose to model the transition between the drops in jet regime and the others. Two dimensionless parameters are derived from this analysis which are thus used to produce a simple regime map where the drops in jet regime can be well distinguished from the other outcomes.
\end{abstract}

\section{Keywords}

drops, jet, breakup/fragmentation, collisions, capillary flows

\section{Introduction}

Interactions and stability of fluid systems with free surfaces have been of scientific interest since more than a century due to their importance for processes in nature and industry. Collisions between drops have been widely studied, involving, for example, two identical drops [1], two drops of immiscible liquids [2], or even three drops that come simultaneously into contact [3]. Liquid jets and ligaments, which may among others originate from drop collisions and impacts, have also been deeply investigated $[4,5]$. Their fragmentation, as well as the collisions between two jets [6], have been in focus since they may result in the production of drops which must be occasionally avoided or enhanced and controlled for processes such as fiber production, atomization, printing, etc.

In the present study, the collisions of a drop stream with a continuous jet consisting of different immiscible liquids are experimentally investigated. Special interest is given to the possibility to incorporate a regular drop stream into a continuous liquid jet without causing its fragmentation. Such a structure may lead to promising applications, especially in the field of encapsulation. We refer to it as the drops in jet regime and focus on its limit of stability.

In the following section we present our materials and methods, including the experimental setup. Our results are presented thereafter. The observed regimes are described, and we propose an analysis to model the transition between the drops in jet regime and the others. The paper ends with the conclusions.

\section{Materials and methods}

The jet and the drops (as well as the associated liquids) are designated by the subscripts $j$ and $d$, respectively. The full description of the collisions requires knowing the fluid properties of the immiscible liquids, as well as some geometric and kinetic parameters.

\section{Immiscible liquids}

We use an aqueous glycerol solution (50\% glycerol by weight) for the drop stream and a silicon oil of low viscosity for the jet (silicon oil M5, from Carl Roth, Austria). The aqueous glycerol solution is colored with food dye to distinguish it from the silicon oil on the images.

The relevant physical properties of these liquids, density $\rho$, dynamic viscosity $\mu$ and the surface tension $\sigma$ are listed in table 1. The interfacial tension between them, denoted $\sigma_{d j}$, is equal to $35 \mathrm{mN} / \mathrm{m}$. Note that, due to the relative values of the surface and interfacial tensions, the total wetting of the drops by the jet liquid is ensured.

Table 1. Physical properties of the used liquids at $23 \pm 2{ }^{\circ} \mathrm{C}$. The glycerol content is given in mass percent.

\begin{tabular}{lccc}
\hline Liquid & $\begin{array}{c}\text { Density } \\
\rho\left[\mathrm{kg} / \mathrm{m}^{3}\right]\end{array}$ & $\begin{array}{c}\text { Dynamic visocity } \\
\mu[\mathrm{mPa} \mathrm{s}]\end{array}$ & $\begin{array}{c}\text { Surface tension } \\
\sigma[\mathrm{mN} / \mathrm{m}]\end{array}$ \\
\hline Glycerol 50\% (G50) & 1131.3 & 5.24 & 66.53 \\
Silicon oil m5 (SO M5) & 913.4 & 4.57 & 19.50 \\
\hline
\end{tabular}




\section{Experimental setup}

To produce controlled collisions between the continuous jet and the monodisperse droplet stream, the experimental setup of figure 1 is used. It consists of two independent pressurized tanks which supply the immiscible liquids (G50 and SO M5) allowing for independent flow rate adjustment. Two nozzles with variable orifice diameter produce liquid jets that can be disturbed at an adjustable frequency via a piezo electric crystal to make use of the Plateau-Rayleigh instability and produce regular droplet streams [7]. Thus, in this work, one nozzle is connected to a signal generator to provide a regular droplet stream, while the other one is used unconnected to supply a continuous liquid jet.

The typical frequency of drop formation $f$ is in the order of $10 \mathrm{kHz}$. The jet and drop diameters are not necessarily identical and range between $100 \mu \mathrm{m}$ and $300 \mu \mathrm{m}$. Both nozzles are mounted on micro-stages to allow fine translational and rotational adjustment of the liquid trajectories. The collisions are illuminated by stroboscopic lighting with the help of an LED lamp. Pictures are taken with a PCO Sensicam video camera. The typical resolution of our imaging system is in the order of $2 \mu \mathrm{m} / \mathrm{px}$.

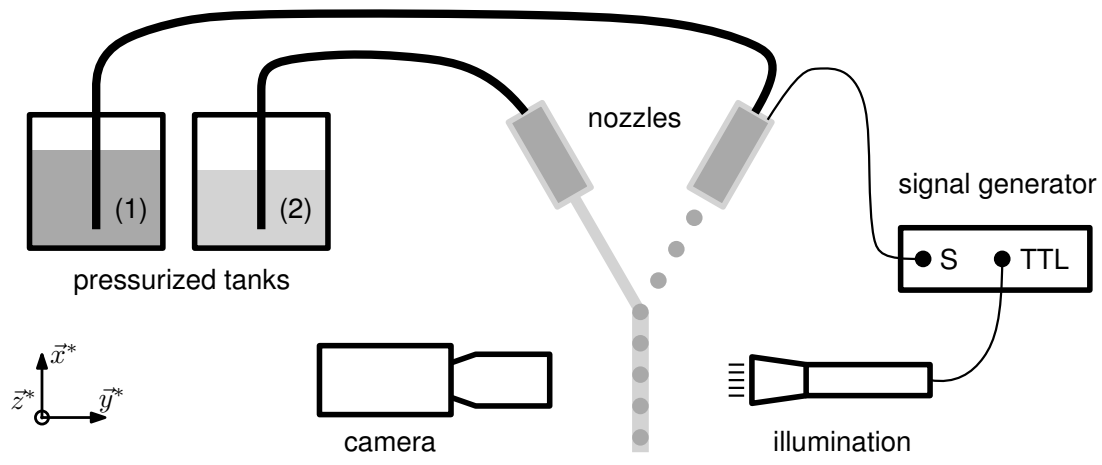

Figure 1. Experimental setup for collisions of a droplet stream with a continuous and immiscible liquid jet.

\section{Kinetic and geometric parameters}

The geometric and kinetic parameters of the studied collisions are illustrated in figure 2. In the laboratory reference frame $\left(\vec{x}^{*}, \vec{y}^{*}, \vec{z}^{*}\right)$, the jet (diameter $D_{j}$ ) and the drops (diameter $D_{d}$ ) have the velocities $\vec{u}_{j}$ and $\vec{u}_{d}$, respectively. In the reference frame moving with the jet $(\vec{x}, \vec{y}, \vec{z})$, the velocities $\vec{u}_{j}$ and $\vec{u}_{d}$ can be replaced by the relative velocity $\vec{U}$, which is collinear with $\vec{y} . l_{j}$ and $l_{d}$ are the spatial periods, where $l_{d}$ can be tuned varying the flow rate of the stream and the frequency of drop production $f$. The spatial period of the jet can then by calculated by

$$
l_{j}=l_{d} \frac{u_{j}}{u_{d}} .
$$

The impact parameter $p$, see the right hand side of figure 2, is defined as the distance separating the droplet center of mass and the one of the corresponding volume for the jet (length $l_{j}$ ) perpendicular to their relative direction of movement. For the present study, only head-on collisions $(p=0)$ are considered. Practically, the position $p=0$ can be found by observing the evolution of the collision outcome during the translation of one of the nozzles along $\vec{z}^{*}$. Detecting the position where this evolution reverses accurately provides $p=0$. Furthermore, the collisions are symmetric with respect to $\vec{x}^{*}$ ( $\alpha$ is bisected by $\vec{x}^{*}$ ).

$D_{j}, D_{d}, l_{d}$ and all the relevant angles (see figure 2) are obtained by image analysis using the public domain software ImageJ [8]. The velocity of the drops is given by $u_{d}=l_{d} f$. The velocity of the jet $u_{j}$ is deduced from continuity,
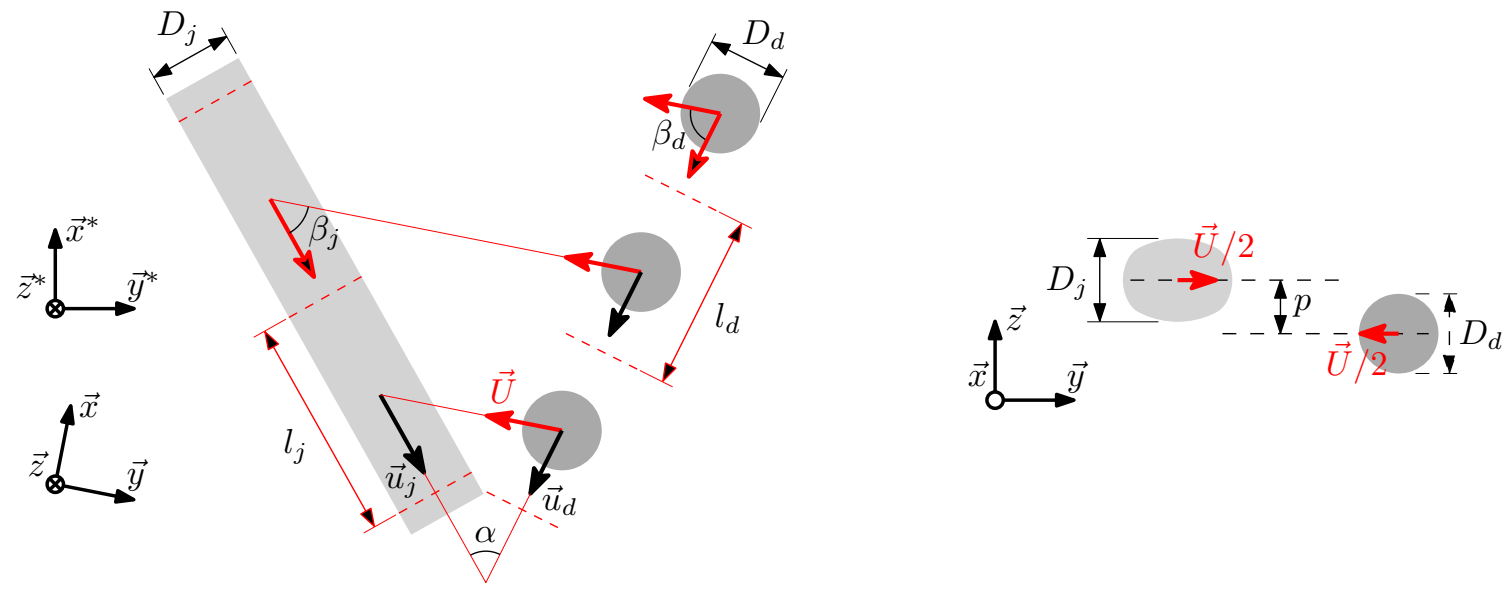

Figure 2. Geometric and kinetic parameters of the collision. $\left(\vec{x}^{*}, \vec{y}^{*}, \vec{z}^{*}\right)$ represents the laboratory reference frame and $(\vec{x}, \vec{y}, \vec{z})$ the reference frame moving with the jet, where $\vec{y}$ is collinear with the relative velocity $\vec{U}$. 
measuring the flow rate of the jet and its diameter. The relative velocity $\vec{U}$ yields

$$
\vec{U}=\vec{u}_{j}-\vec{u}_{d}
$$

and can be modified by varying $u_{j}, u_{d}$ or $\alpha$.

\section{Results and Discussion Regimes}

We identified four different outcomes for the collision of a droplet stream with an immiscible continuous jet. These regimes are described below with the help of collision photographs. In all photographs (figures 3-6), the droplets and the jet move from left to right. An (almost) horizontal white breaking line in a picture indicates that separate pictures were needed to capture the full process.

\section{- Drops in jet}

The drops in jet regime (see figure 3) exhibits a regular stream of droplets embedded in an immiscible liquid jet which does not fragment. In figure 3a, the jet and drops have the same diameter of approximately $200 \mu \mathrm{m}$. The two velocities $\left(u_{d}=6.48 \mathrm{~m} / \mathrm{s}, u_{j}=3.43 \mathrm{~m} / \mathrm{s}\right)$ represent similar momentum fluxes in the laboratory reference frame, as it can be seen downstream from the direction of movement of the resulting stream. In figure $3 \mathrm{~b}$, the jet momentum flux (measured in the laboratory reference frame) is greater than the one of the droplet stream. As a result, the direction of the resulting drops in jet structure is almost similar to the one of the jet alone before the collision. In both cases, the regularity of the drop spacing is preserved, although its actual value may be modified by the collision. This effect originates from the relative orientation of the jet and drop velocities, and a geometric analysis leads to a drop spacing after encapsulation equal to $l_{j}=l_{d} \sin \left(\beta_{d}\right) / \sin \left(\beta_{j}\right)=l_{d} u_{j} / u_{d}$.

(a)

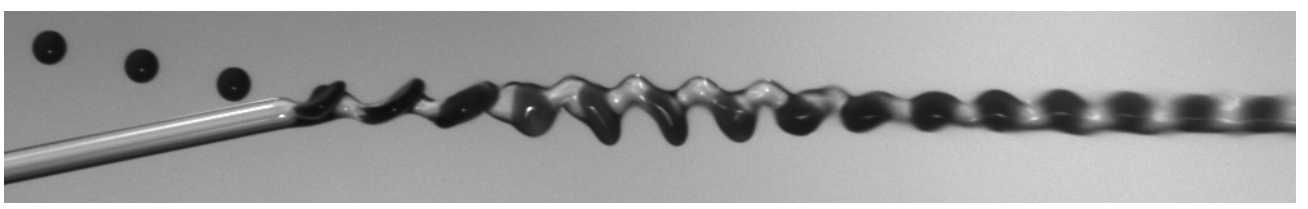

(b)

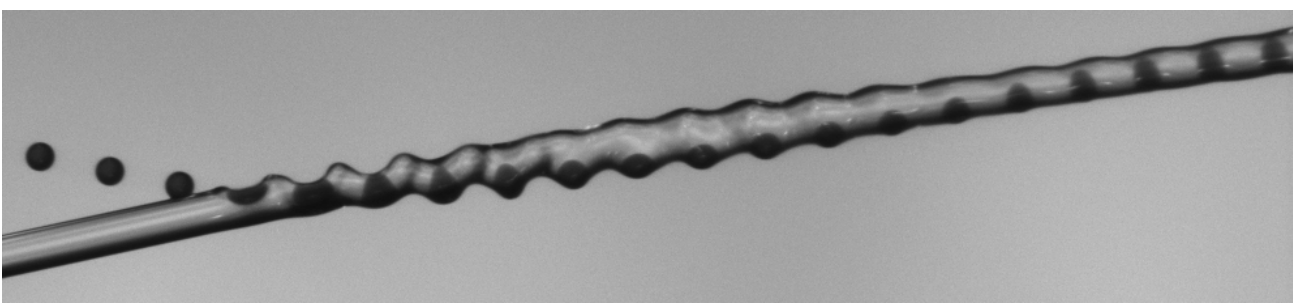

Figure 3. Regime drops in jet: (a), $D_{d}=202 \mu \mathrm{m}, D_{j}=200 \mu \mathrm{m}, u_{d}=6.48 \mathrm{~m} / \mathrm{s}, u_{j}=3.43 \mathrm{~m} / \mathrm{s}, U=3.56 \mathrm{~m} / \mathrm{s} ;$ (b), $D_{d}=192 \mu \mathrm{m}$, $D_{j}=300 \mu \mathrm{m}, u_{d}=5.55 \mathrm{~m} / \mathrm{s}, u_{j}=4.92 \mathrm{~m} / \mathrm{s}, U=2.30 \mathrm{~m} / \mathrm{s}$.

- Encapsulation without satellites

In figure 4, two examples of encapsulation without satellites can be seen. The drops of the stream are embedded by an immiscible shell made of the liquid from the fragmented jet. The resulting structure is a stream of individual spherical capsules in the surrounding air. No satellites are formed, and each drop initially present in the drop stream gives rise to a unique capsule. Thus, the shell volume corresponds to the volume of a cylinder of diameter $D_{j}$ and length $l_{j}$. The resulting stream of capsules is very regular, showing a spacing potentially different from the initial drop spacing. Here again, the spacing modification originates from the

(a)

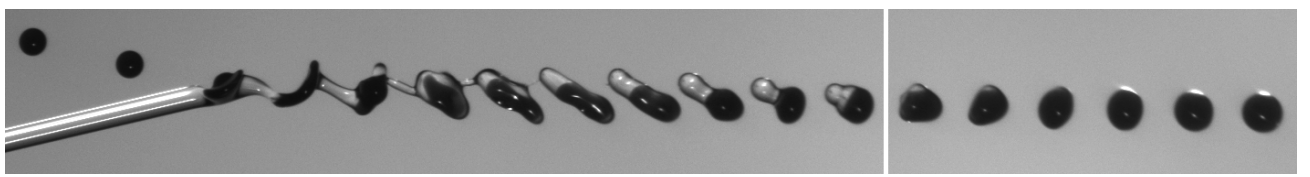

(b)

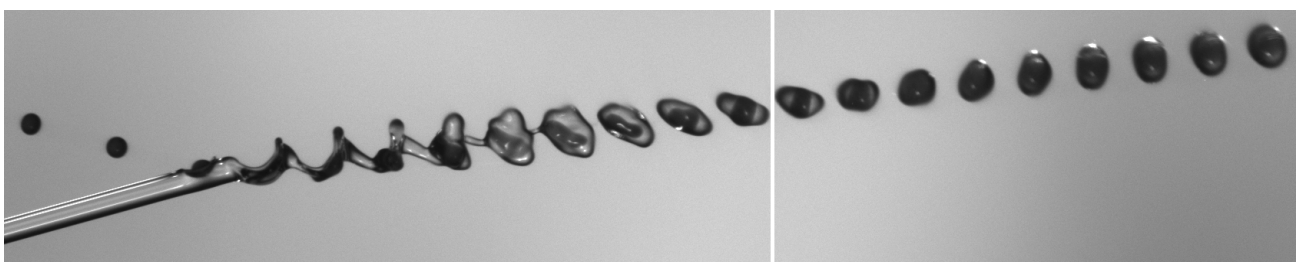

Figure 4. Regime encapsulation without satellites: (a), $D_{d}=227 \mu \mathrm{m}, D_{j}=200 \mu \mathrm{m}, u_{d}=6.56 \mathrm{~m} / \mathrm{s}, u_{j}=3.43 \mathrm{~m} / \mathrm{s}$, $U=3.80 \mathrm{~m} / \mathrm{s} ;(\mathrm{b}), D_{d}=238 \mu \mathrm{m}, D_{j}=300 \mu \mathrm{m}, u_{d}=8.09 \mathrm{~m} / \mathrm{s}, u_{j}=4.93 \mathrm{~m} / \mathrm{s}, U=4.60 \mathrm{~m} / \mathrm{s}$. 
relative orientation of the jet and drop velocities. As for the drops in jet regime, the relative importance of the initial jet and initial drop stream momentum fluxes is visible in the orientation of the final structure, balanced for picture (a) and dominated by the jet for picture (b).

- Encapsulation with pure oil satellites

Two illustrations of the encapsulation with pure oil satellites regime are reproduced in figure 5 . The drops cross over the jet which fragments. Its liquid is distributed between the shell of spherical capsules and satellite droplets. The spherical capsules form a regular stream. The core of each capsule has the volume of one drop initially present in the drop stream, while its immiscible shell has a volume corresponding to a portion of the volume of a cylinder of diameter $D_{j}$ and length $l_{j}$. The excess of jet liquid forms a regular stream of satellite drops.

(a)

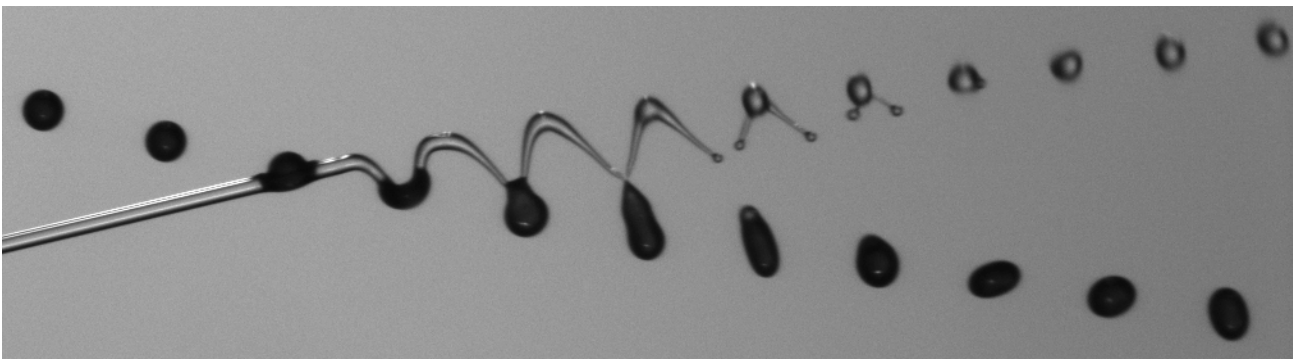

(b)

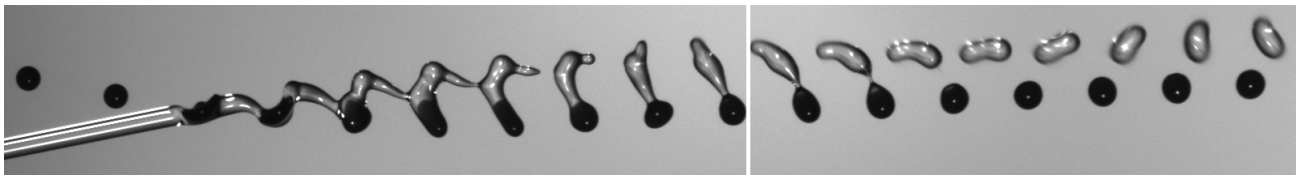

Figure 5. Regime encapsulation with pure oil satellites: (a), $D_{d}=210 \mu \mathrm{m}, D_{j}=100 \mu \mathrm{m}, u_{d}=7.60 \mathrm{~m} / \mathrm{s}, u_{j}=6.03 \mathrm{~m} / \mathrm{s}$, $U=3.58 \mathrm{~m} / \mathrm{s} ;(\mathrm{b}), D_{d}=229 \mu \mathrm{m}, D_{j}=200 \mu \mathrm{m}, u_{d}=6.75 \mathrm{~m} / \mathrm{s}, u_{j}=4.98 \mathrm{~m} / \mathrm{s}, U=2.89 \mathrm{~m} / \mathrm{s}$.

\section{- Multiple or mixed fragmentation}

In figure 6, the last identified regime, multiple or mixed fragmentation, can be observed. This regime typically occurs at large relative velocities, which can be seen by the large deformations of the jet and drops upon impact. In contrast to all the other regimes, not only the jet fragments, but also the drops initially present in the

(a)

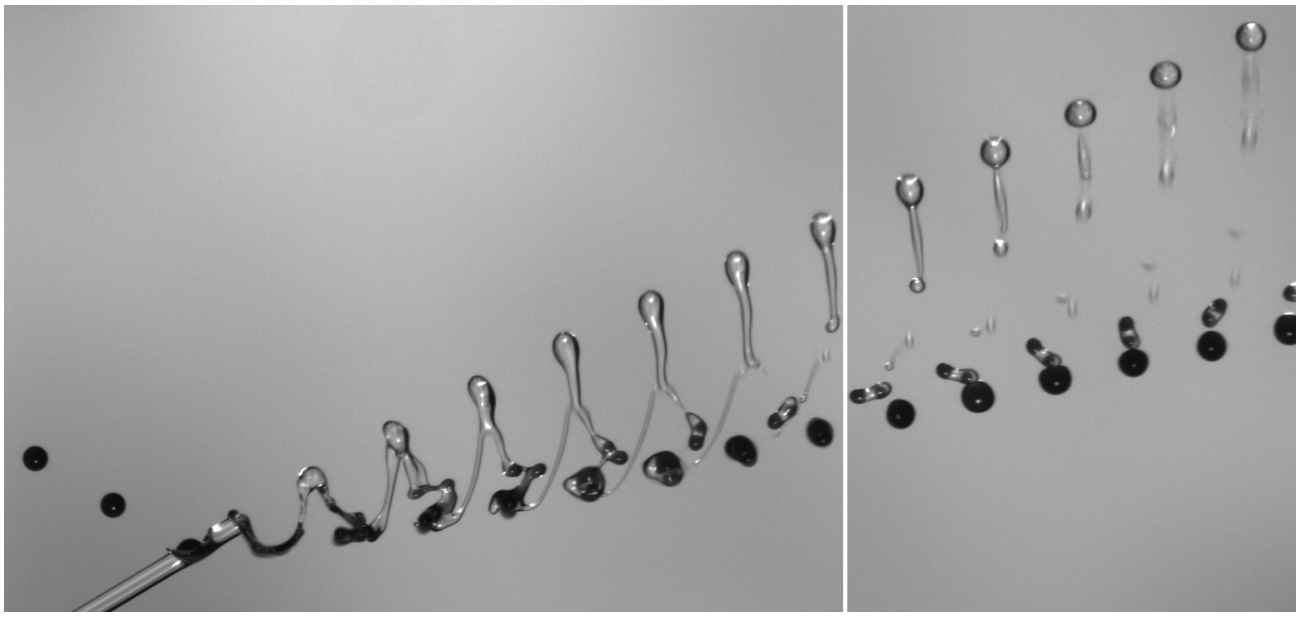

(b)

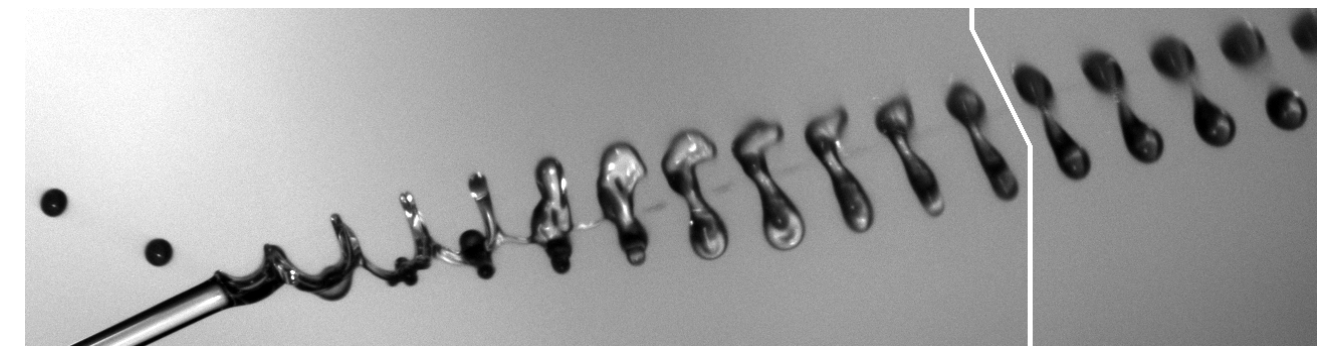

Figure 6. Regime multiple or mixed fragmentation: (a), $D_{d}=223 \mu \mathrm{m}, D_{j}=200 \mu \mathrm{m}, u_{d}=6.60 \mathrm{~m} / \mathrm{s}, u_{j}=7.22 \mathrm{~m} / \mathrm{s}$, $U=7.12 \mathrm{~m} / \mathrm{s} ;$ (b), $D_{d}=236 \mu \mathrm{m}, D_{j}=300 \mu \mathrm{m}, u_{d}=8.08 \mathrm{~m} / \mathrm{s}, u_{j}=4.93 \mathrm{~m} / \mathrm{s}, U=6.23 \mathrm{~m} / \mathrm{s}$. 
stream. As a result, a structure is formed exhibiting several droplets containing both liquids. Oil satellites may also be formed (see figure 6a), but not necessarily (see figure $6 \mathrm{~b}$ ).

\section{Regime map and boundaries of the drops in jet regime}

In view of using the drops in jet regime for new applications such as the production of advanced fibers, it is required to describe the conditions of its occurrence. Since the full description of such collisions involves 13 independent parameters, we aim to focus on the most critical contributions and propose an analysis based on our experimental observations, studies of immiscible drop collisions, and knowledge about jet stability. Thus, two different fragmentation mechanisms of the continuous jet can be identified.

First, the jet fragmentation can be driven by inertia, as also observed for immiscible drop collisions. More precisely, this may occur if the kinetic energy of the impacting drops is too high by comparison to surface energy and viscous losses opposing jet distortion. In this case, it is expected that the drops simply cross over the jet - similarly to the crossing separation observed for immiscible drop collisions [2] - and fragment it. Based on this picture, and neglecting for simplicity the viscous losses, a first dimensionless parameter can be proposed that should help distinguishing the drops in jet regime from the others. The impacting drop kinetic energy can be estimated by $\rho_{d} D_{d}{ }^{3} U^{2}$, while the surface energy opposing the crossing of the jet scales as $\sigma_{j} D_{j} l_{j}$. The ratio of these two quantities provides a modified Weber number defined by $W e^{*}=\rho_{d}\left(D_{d}{ }^{3} / D_{j} l_{j}\right) U^{2} / \sigma_{j}$. Using this parameter as the abscissa of the two-dimensional regime map of figure 7 , we observe a good representation of the experimental results. The threshold value of $W e^{*}$ is found to be approximately 240 . Above this value, no drops in jet can be observed any more. Instead, mixed fragmentation becomes dominating in agreement with an inertial regime.

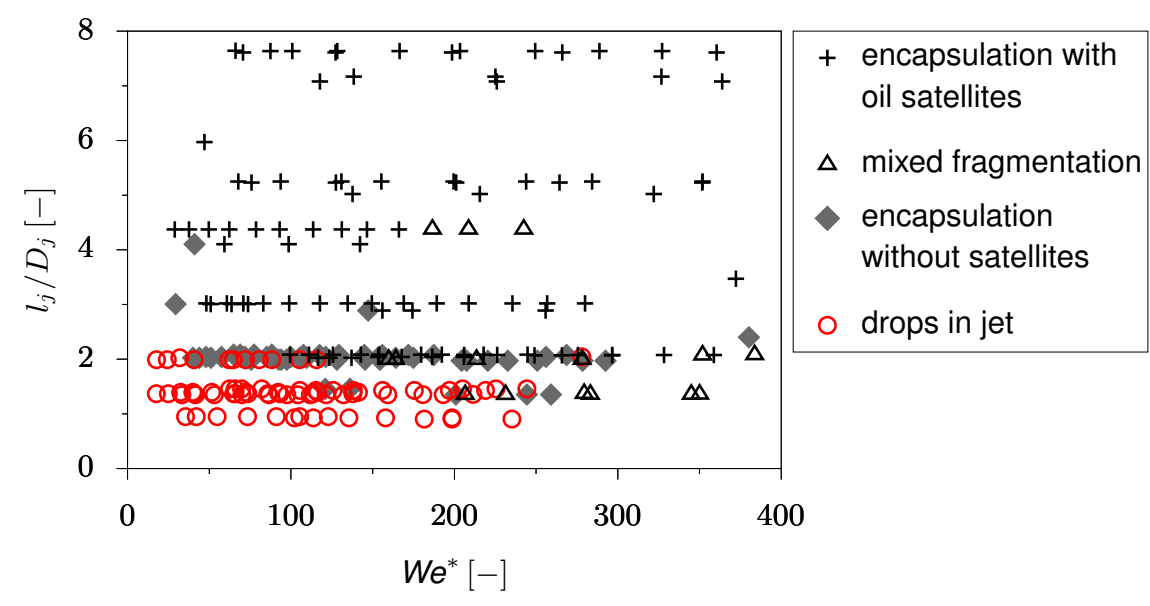

Figure 7. Two-dimensional regime map. Using $l_{j} / D_{j}$ and $W e^{*}$ allows to describe the boundaries of the drops in jet regime.

A second mechanism of fragmentation can be observed which corresponds to a Plateau-Rayleigh like instability where the continuous jet is destabilized by the periodic disturbances caused by the impacting drops. For jets of one liquid only, it is well known that the disturbances are unstable as soon as their wavelength $\lambda$ reaches the critical value of $\pi D_{j}$ [9]. The Rayleigh criterion is often formulated as $\lambda / D_{j} \geq \pi$. By extension, it appears relevant for the studied collisions to consider the ratio between the drop spacing after contact with the jet and the jet diameter. By definition, the drop spacing after contact with the jet corresponds to $l_{j}$, the spacial period of the jet. It yields $l_{j} / D_{j} \geq \pi$.

The use of $l_{j} / D_{j}$ as the ordinate variable of the two-dimensional regime map of figure 7 confirms that $l_{j} / D_{j}$ is a critical parameter for the occurrence of drops in jet. Yet, the threshold value is found to be approximately 2, $50 \%$ below the expected value of $\pi$, and seems to slightly decrease with the modified Weber number. This discrepancy can be explained by observing the typical pictures of figure 8 . The pictures of figure 8 correspond to low We* and have been chosen to illustrate collisions where $l_{j} / D_{j}<2 ; 2<l_{j} / D_{j}<\pi$ and $l_{j} / D_{j}>\pi$. While theoretically both collisions with $l_{j} / D_{j}<\pi$ should lead to drops in jet, only the one with $l_{j} / D_{j}<2$ does. We attribute this shift to the distortion of the jet subjected to the drop impacts. More precisely, the jet portion located between two impacting drops tends to be elongated by the relative movements of the drops and the jet whose relative momentum, normal to the final drops in jet trajectory, is not yet fully dissipated. From $W e^{*}$ definition, we expect that the greater $W e^{*}$ is, the longer and thinner this cylindrical portion becomes. Thus, the underestimation of its aspect ratio when using $l_{j} / D_{j}$, e.g. the undistorted dimensions, increases with increasing $W e^{*}$ 
(a)

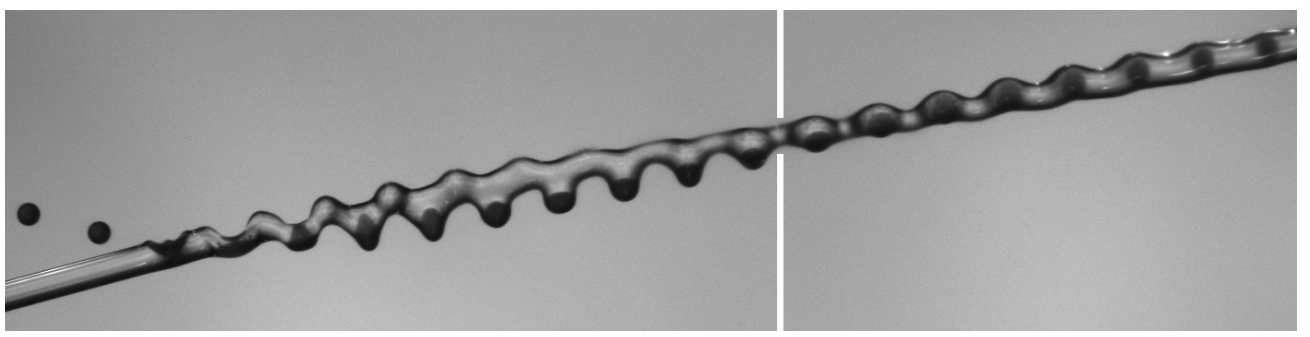

(b)

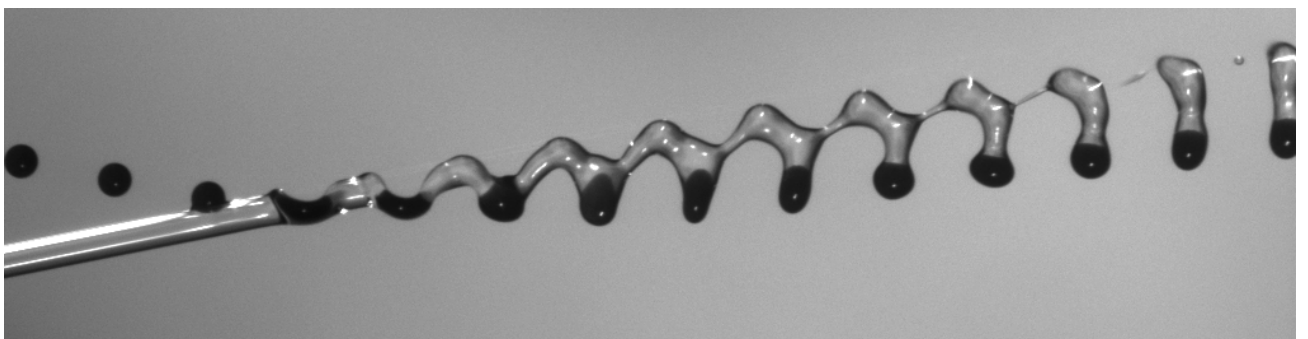

(c)

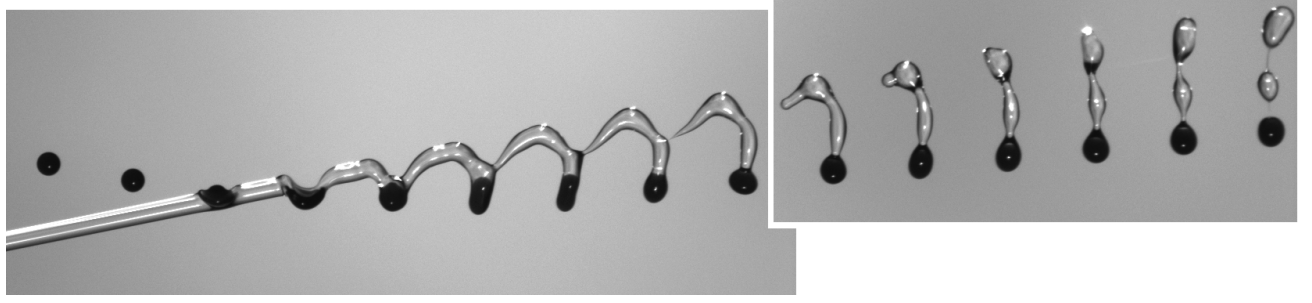

Figure 8. (a) $D_{d}=216 \mu \mathrm{m}, D_{j}=300 \mu \mathrm{m}, u_{d}=5.55 \mathrm{~m} / \mathrm{s}, u_{j}=4.92 \mathrm{~m} / \mathrm{s}, U=2.73 \mathrm{~m} / \mathrm{s}$ yielding $l_{j} / D_{j}=1.99$ and $W e^{*}=24$; (b) $D_{d}=202 \mu \mathrm{m}, D_{j}=200 \mu \mathrm{m}, u_{d}=6.73 \mathrm{~m} / \mathrm{s}, u_{j}=4.90 \mathrm{~m} / \mathrm{s}, U=3.15 \mathrm{~m} / \mathrm{s}$ yielding $l_{j} / D_{j}=2.80$ and $W e^{*}=34$ and (c) $D_{d}=225 \mu \mathrm{m}, D_{j}=200 \mu \mathrm{m}, u_{d}=6.67 \mathrm{~m} / \mathrm{s}, u_{j}=7.21 \mathrm{~m} / \mathrm{s}, U=2.77 \mathrm{~m} / \mathrm{s}$ yielding $l_{j} / D_{j}=4.04$ and $W e^{*}=29$.

\section{Conclusions}

By colliding a drop stream with a continuous immiscible liquid jet we generate different kinds of liquid structures. These structures have been classified in 4 distinct regimes: drops in jet for which both initial structures combine without any fragmentation; two regimes of encapsulation, both leading to the coating of the initial drops by a liquid shell originating from the jet. The regimes can be distinguished by the formation (or not) of satellites and referred to as encapsulation with and without oil satellites. Finally, mixed fragmentation occurs, where both the drops and the jet fragment into several droplets of various compositions. To our knowledge, it is the first time that the formation of the very regular drops in jet structure is reported. Two critical dimensionless parameters, corresponding to two distinct fragmentation mechanisms, have been proposed to describe the transitions between this regime and others. On the one hand, we identified a Plateau-Rayleigh like instability that results in the disappearance of the drops in jet structure if $l_{j} / D_{j}$ reaches a critical value of approximately 2 . On the other hand, the fragmentation of the jet may also originate from an excess of kinetic energy. In this case, capillary forces are insufficient to keep the drops in the jet, and the drops fragment it by crossing over it. We derived a modified Weber number We* and verified that above a threshold value of approximately 240 , no drops in jet structure can be obtained.

\section{Nomenclature}

$D \quad$ diameter $[\mathrm{m}]$

$l \quad$ spatial period length [m]

$f \quad$ frequency of drop formation $[\mathrm{Hz}]$

impact parameter [m]

$\vec{u}, u \quad$ velocity $[\mathrm{m} / \mathrm{s}]$

$\vec{U}, U$ relative velocity $[\mathrm{m} / \mathrm{s}]$

We* modified Weber number

$\vec{x}, \vec{y}, \vec{z} \quad$ frame of reference [m]

$\alpha \quad$ angle between jet and drop velocity vector [rad]

$\beta \quad$ angle between relative velocity vector and velocity vector [rad]

$\lambda \quad$ wavelength [m]

$\mu \quad$ dynamic viscosity [Pa s]

$\rho \quad$ density $\left[\mathrm{kg} / \mathrm{m}^{3}\right]$

$\sigma \quad$ surface or interfacial tension $[\mathrm{N} / \mathrm{m}]$ 


\section{Subscripts \\ d drop \\ $j \quad$ jet}

\section{References}

[1] Jiang, Y. J., Umemura, A. and Law, C. K., 1992, Journal of Fluid Mechanics, 234 , pp. 172-190.

[2] Planchette, C., Lorenceau, E. and Brenn, G., 2012, Journal of Fluid Mechanics, 702, pp. 5-25.

[3] Hinterbichler, H., Planchette, C., and Brenn, G., 2015, Experiments in Fluids, 56, 190.

[4] Villermaux, E., 2007, Annual Review of Fluid Mechanics, 39, pp. 419-446.

[5] Eggers, J. and Villermaux, E., 2008, Reports on Progress in Physics, 71, pp. 1-79.

[6] Bremond, N. and Villermaux, E., 2006, Journal of Fluid Mechanics, 549, pp. 273-306.

[7] Brenn, G., Durst, F., and Tropea, C., 1996, Particle \& Particle Systems Characterization, 13, pp. 179-185.

[8] ImageJ - Image processing and analysis in Java, https://imagej .nih.gov/ij/ ([cit. 2017-03-06]).

[9] Rayleigh, J. W. S., 1878, Proceedings of the London Mathematical Society, 10, pp. 4-13. 


\title{
Generalized analysis of the deposition/splashing limit for one- and two-component droplet impacts upon thin films
}

\author{
Ronan Bernard ${ }^{* 1}$, Patrick Foltyn ${ }^{1}$, Anne Geppert ${ }^{1}$, Grazia Lamanna ${ }^{1}$, Bernhard Weigand ${ }^{1}$ \\ ${ }^{1}$ Institut für Thermodynamik der Luft- und Raumfahrt, Universität Stuttgart, Germany \\ *Corresponding author: ronan.bernard@itlr.uni-stuttgart.de
}

\begin{abstract}
Single drop impacts on thin liquid layers are of particular interest because of the ejection of secondary droplets, the so-called splashing. Only a few studies handle the deposition/splashing limit for two-component interaction, where the liquid properties of the impacting drop and wall film differ significantly.

This study aims at identifying a unified approach for one- and two-component interactions to determine the deposition/splashing limit. Therefore, a large database of both interactions is considered, which includes data from literature for one-component interactions plus the following binary combinations: hyspin-hexadecane, diesel-hexadecane and diesel-motor oil. Furthermore, a systematic study of two-component interactions with several silicon oils and hexadecane is performed. To map the outcomes, the Ohnesorge number $O h$ and the Reynolds number Re calculated with arithmetically averaged fluid properties between droplet and wall film fluid are chosen. The dimensionsless film thickness $\delta$ is added to form a $3 D$ plot, where one- and two-component experiments are combined.

Existing correlations from the literature are revised regarding both interactions and their consistency is checked. The investigated range of high viscosity fluids allow us to propose an improvement of the correlation for high $O h$. Our results show that the arithmetically averaged fluid properties lead to a good repartition of both one- and twocomponents interactions toward the deposition/splashing limit. They also corroborate the previous findings that an increase of $\delta$ inhibits splashing but its influence is decreasing with increasing $O h$.
\end{abstract}

\section{Keywords}

droplet impact, thin film, two-components interaction, splashing treshold.

\section{Introduction}

Single drop impact on a thin liquid layer (i.e. wall film) covers a wide range of industrial applications like coating, printing, cooling or combustion. They are of particular interest since they lead to the ejection of secondary droplets, the so-called splashing. The investigations concerning the droplet impact on wetted walls show that for a given Reynolds number $(R e)$, Ohnesorge number $(O h)$ and dimensionsless wall film thickness ( $\delta$, film thickness $h$ over droplet diameter $D$ ), the outcome of one-component droplet impact can be determined. Roughness of the wall can be neglected in the case of $0.1 \leq \delta \leq 1$ [9], when it is completely embedded in the liquid wall film. However, drop-film interactions are affected by the use of different fluids for the droplet and the liquid film. Very little is known for this two-component interaction: the available database is still sparse and therefore the validity of proposed correlations for the deposition/splashing limit cannot be yet generalized to any type of fluid combinations.

In this study, we consider a droplet impact of Newtonian fluids onto a wetted, cold, horizontal, solid surface. In the following, the denomination fluid1/fluid2 means that a fluid1 droplet impacts onto a fluid2 wall film and fluid1-fluid2 that both permutations were performed.

This study focuses on the deposition/splashing limit by incorporating both one- and two-component interactions into a single correlation. For this purpose, different existing correlations will be considered.

Many studies were performed for the deposition/splashing limit of one-component interaction such as Yarin and Weiss [7], Cossali et al. [2], Coghe et al. [8], Marengo and Tropea [9], Wang and Chen [11], Rioboo et al. [10], Vander Wal et al. [1] just to name a few.

The latter [1] encompasses a study on one-component interaction of twelve different fluids (ranging from alkanes to alcohols and including mixtures of glycerol-water). The dimensionsless parameters chosen to map the impacts outcome were $\mathrm{Oh}$ and $\mathrm{Re}$. The ranges of investigation were approximately [0.0026;0.017] and [800;14,000] for $\mathrm{Oh}$ and $R e$, respectively. The dimensionsless film thickness was kept constant and equal to 0.1 (obtained from the data $D=2 \mathrm{~mm}$ and $h=0.2 \mathrm{~mm}$ ). This restricts the validity of this correlation to $\delta=0.1$. The best fit of the data was found to be:

$$
\text { Oh } R e^{1.17}=63 \quad \text { (only valid for } \delta=0.1 \text { ). }
$$

Another well-known correlation is the one of Cossali et al. [2] that emphasizes the influence of the dimensionsless film thickness $\delta$ and of the dimensionsless roughness $R_{n d}=R_{a} / D$ on the onset of splashing. This correlation was obtained from experimental results with a mixture of water and glycerol, offering a quite wide predictive range. The traditional non-dimensionless numbers (based on single fluid properties) were $W e \in[200 ; 1600], O h \epsilon$ $[0.0022 ; 0.141]$ and $\delta \in[0.08 ; 1.2]$. The empirical correlation is based on the so-called impact factor $K=W e O h^{-0.4}$ inspired from a model on dry surfaces from Stow and Stainer [6]. In the case of thin film $\left(\delta \gg R_{n d}\right.$, to distinguish from very thin film), the influence of roughness can be neglected and the correlation for one-component interaction 

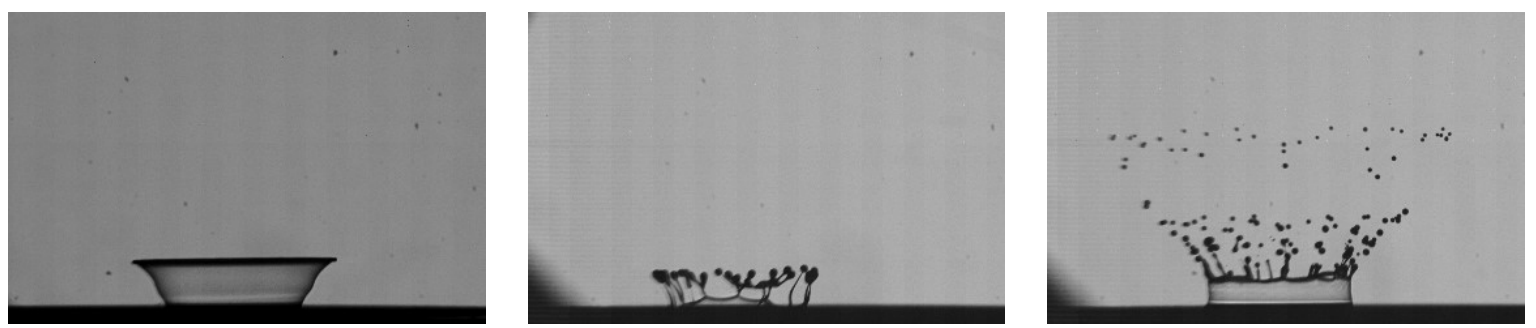

Figure 1. Classification of droplet impact outcomes: deposition (left), transition (middle) and splashing (right).

was found to be:

$$
W e O h^{-0.4}=2100+5880 \delta^{1.44}
$$

In [2], the splashing was defined as the formation of secondary droplets which has to be distinguished from deposition without secondary droplets. Cossali et al. [2] observed different outcomes for the same impact conditions near the deposition/splashing limit and so defined an upper and lower value of the critical $K$. Recently, Geppert et al. [3] introduced a regime, where a mixed impact behavior occurs. The latter could neither be distinctly assigned to splashing nor deposition, and was therefore classified as transition. In this study, we will also consider this transition state as a distinct outcome. The mapping is split into three regions, whose characteristic outcomes are depicted in Figure 1. On the right side, the splashing with the formation of liquid fingers along the upper lamella rim and the ejection of secondary droplets is depicted. On the left side, the deposition, where the crown originates from the wall film and then descends back to it without generating secondary droplets is shown. In between, the transition zone can be seen, where the crown is formed, secondary droplets are formed but not ejected and coalesce with the wall film.

Geppert et al. [3] also worked on a unified approach of the splashing/deposition limit for one- and two-component interaction. Their empirical correlation was based on the impact factor $K$ and $\delta$, similarly to Cossali et al. [2]. In [4], the authors noticed a major influence of the wall film fluid properties on the impact outcomes for two-component interaction (permutations of hyspin and hexadecane), that led to a different definition of the traditional dimensionsless parameters. They calculated the Ohnesorge number $O h_{v}$ with arithmetical averaged viscosities. The Weber number instead, was based on droplet properties since the kinetic energy is provided by the impacting drop and the surface tension of the two fluids did not differ significantly from each other. The resulting correlations for one- and two-component interactions were given as follow:

$$
\begin{aligned}
& W e^{0.625} O h_{v}^{-0.25}=114+145 \delta^{1.2} \\
& W e^{0.625} O h_{v}^{-0.25}=114+163 \delta^{1.2}
\end{aligned}
$$

The equations (3) and (4) are based on the hexadecane/hexadecane and hyspin-hexadecane interactions, respectively. This correlations are close to each other, and also close to the previous correlation of Cossali et al. [2]. Another study using even more averaged properties for two-component interaction was performed by Kittel et al. [5]. Based on experiments with silicon oils and water, they found that the conventional impact factor $K$ is not accurate for two-component interaction, because the wall film properties are not taken into account. They proposed a modified impact factor $K^{*}=R e_{v-B L}^{0.5} W e_{\sigma}$, where $v-B L$ and $\sigma$ indicate averaged viscosities based on the boundary layer theory and arithmetical averaged surface tensions, respectively. Plotting $K^{*}$ as a function of viscosity ratio between wall film and droplet fluids, Kittel et al. [5] reported that a tendency of splashing treshold can be seen, unlike the conventional impact factor $K$. This shows that both properties of the droplet and the wall film have to be taken into account. In this study, all properties (viscosities, surface tensions and densities) will be arithmetically averaged, leading to the following dimensionsless parameters:

$$
W e_{\text {ave }}=\frac{\left(\rho_{f}+\rho_{d}\right) V^{2} D}{\sigma_{f}+\sigma_{d}}, \quad O h_{\text {ave }}=\frac{\nu_{f}+\nu_{d}}{2} \sqrt{\frac{\rho_{f}+\rho_{d}}{D\left(\sigma_{f}+\sigma_{d}\right)}}, \quad R e_{\text {ave }}=\frac{2 V \cdot D}{\nu_{f}+\nu_{d}}
$$

with the density $\rho$, the kinematic viscosity $\nu$, the surface tension $\sigma$, the impact velocity $V$ and the droplet diameter $D$. The subscripts $f$ and $d$ denote film and droplet. The arithmetical average allows to take both, droplet and film fluids, into account for two-component interactions, and has no influence in case of one-component interaction. Therefore, it allows the use of the previous correlations from the literature.

The purpose of this study is to propose an unified approach for the correlation of the deposition/splashing limit for one- as well as two-component interactions. This can be attained with the use of arithmetically averaged parameters in the conventional dimensionsless numbers. The governing parameters will be put together to offer an overall view of their influence. The existing correlations are put into perspective and their consistency will be checked. To achieve these goals, a large database is needed. Therefore, a systematic study of two-component interactions 
was performed by including variations in viscosities, surface tensions and densities. Specifically, we permutated several silicon oils and hexadecane for the droplet and wall film liquids. Furthermore, a large database of one- and two-component interactions from [1, 2, 3] is combined with the performed experimental results.

\section{Material and methods \\ Material}

The experimental setup used in this work is the same as the one used by Geppert et al. [4]. It consists of three main systems: the dropper, the impingement area and a two-perspectives shadowgraphy imaging system.

The droplets are generated with a pump, which delivers the fluid from the reservoir to a needle having a fixed diameter of $0.8 \mathrm{~mm}$. The flow rate is low enough to make the droplet drip off by gravity and then take advantage of the gravitational acceleration. The impact velocity is adjusted with the height of the needle.

During its fall, the droplet is passing through a laser light barrier that triggers the high-speed camera (Photron Fastcam SA1.1). The imaging system allows the simultaneous recording of a frontal and lateral view making the observation of non-axisymmetric features during the impact possible. The target zone is constantly back-lighted by two photodiodes. The images are then redirected by a set of mirrors and both light paths are focused by a prime lens on the camera sensor $(896 \times 320$ pixels $)$. The frame rate of the camera and the shutter speed were set to $20,000 \mathrm{fps}$ and 1/92,000 s, respectively. The magnification of the optical set is $1: 4$ to allow the observation of the entire impact area.

The impact area consists of a smooth sapphire glass plate on which a thin metallic ring (diameter $60 \mathrm{~mm}$, height $0.6 \mathrm{~mm}$ ) is glued. The inner region of the ring is filled with the wall film fluid till the desired thickness is reached. The measurement of the wall film thickness is realized with a commercial device (Micro-Epsilon - Model, IFC2451 controller and IFS2405-3 sensor) using the confocal chromatic imaging (CCl) technique, a non-intrusive distance measurement method. This method uses the difference in refractive index between the wall film fluid and the plate analyzing the spectral distribution of the reflected light. More details about the experimental setup can be found in [3].

\section{Method}

A systematic study of two-component interaction is carried out where the properties of the test liquids was varied. Specifically, we interchanged silicon oils with different viscosities and hexadecane for the droplet and wall film liquid, respectively. Fluid properties are listed in table 1 . The viscosity is doubled each time for $B 5, B 10$ and $B 20$.

Table 1. Fluid properties at ambient temperature and pressure.

\begin{tabular}{c|ccc}
\hline & density $\left[\mathrm{kg} \cdot \mathrm{m}^{-3}\right]$ & surface tension $\left[10^{-3} \mathrm{~N} \cdot \mathrm{m}^{-1}\right]$ & viscosity $\left[10^{-6} \mathrm{~m}^{2} \cdot \mathrm{s}^{-1}\right]$ \\
\hline Hexadecane & 773 & 27.5 & 4.46 \\
B3 & 900 & 18.0 & 3.0 \\
B5 & 920 & 19.2 & 5.0 \\
B10 & 945 & 20.2 & 10.0 \\
B20 & 955 & 20.6 & 20.0
\end{tabular}

The wall film thickness was adjusted to systematically achieve a dimensionsless film thickness of approximately $\delta=0.11$, depending on the droplet diameter. The droplet diameter is related to the constant needle diameter and the fluid properties. The averaged droplet diameters are roughly $1.9 \mathrm{~mm}$ for silicon oils and $2.4 \mathrm{~mm}$ for hexadecane. The free fall height was equal to $1.28 \mathrm{~m}$ and $0.87 \mathrm{~m}$, resulting in an impact velocity of approximately $4.3 \mathrm{~m} / \mathrm{s}$ and $3.6 \mathrm{~m} / \mathrm{s}$, respectively. For each test condition, a set of at least three experiments was performed, ensuring reproducibility and reducing measurement errors. The experimental procedure was carried out as follow: the dropper was installed for a specific droplet fluid. A series of experiments was performed with another fluid as wall film. Before each experiment, the pool was cleaned with ethanol and refilled. The wall film fluid was progressively added to the pool depending on the measured wall film thickness. The thickness was measured after a specific delay time to ensure the film liquid at rest and averaged over 10,000 values.

After all fluids were tested as wall film, the entire dropper (pipes and needle) was replaced for the next droplet fluid. The experimental uncertainties for this experimental setup are detailed in [3]. The measurement of droplet diameter and impact velocities are performed with an in house MATLAB routine from consecutive images just before impact. The measurement accuracy for the droplet diameter and the impact velocity is in the range of $2,1 \%$ and $2 \%$, respectively. The error related to the measurement of the film thickness is less than $1 \%$ (which is also reported in Geppert et al. [3]). The classification of the outcomes (splashing, transition or deposition) was judged visually for each experiment.

To enlarge the database, other experimental results from Geppert et al. [4, 3] were added, performed on the same experimental setup for the interactions hexadecane/hexadecane and hyspin-hexadecane. In addition, previous unpublished experimental results were also added for the interactions hyspin/hyspin, diesel/diesel, diesel/Castrol 5W-30 and diesel/hexadecane. Fluid properties are listed in Table 2. The investigations with these additional combinations were performed for a wide range of film thicknesses and Weber number.

Overall, the entire database from this experimental setup was given by: $\operatorname{Re}_{\text {ave }} \in[98 ; 3654] ; O h_{\text {ave }} \in[0.012 ; 0.283]$; $\delta \in[0.03 ; 0.55]$ and contains more than 2000 experiments. 
Table 2. Additional fluid properties at ambient temperature and pressure.

\begin{tabular}{c|ccc}
\hline & density $\left[\mathrm{kg} \cdot \mathrm{m}^{-3}\right]$ & surface tension $\left[10^{-3} \mathrm{~N} \cdot \mathrm{m}^{-1}\right]$ & viscosity $\left[10^{-6} \mathrm{~m}^{2} \cdot \mathrm{s}^{-1}\right]$ \\
\hline Hyspin & 878 & 28.7 & 18.0 \\
Diesel & 827 & 28.0 & 4.0 \\
Castrol 5W-30 & 846 & 31.0 & 156.2
\end{tabular}

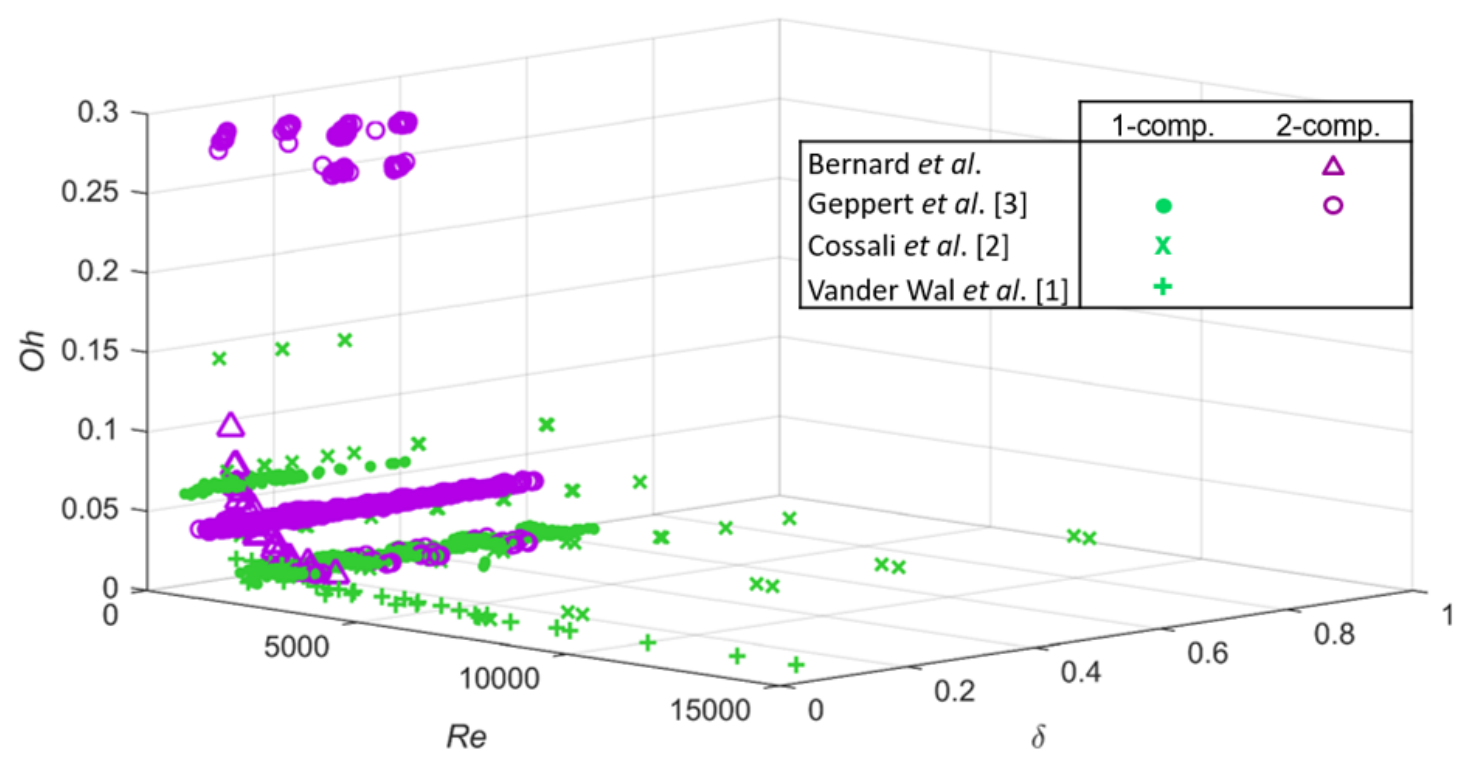

Figure 2. Common mapping of the investigated range of one- and two-components interactions (green and purple symbols, respectively) in $\left(O h_{\text {ave }}, R e_{\text {ave }}, \delta\right)$ from the referenced sources.

\section{Results and discussion}

To map the different outcomes, we follow the approach of Vander Wal et al. and use arithmetically averaged properties, meaning that $O h_{\text {ave }}$ and $R e_{\text {ave }}$ are chosen as defined in equation 5. Using the third governing parameter $\delta$, a $3 D$ plot containing the entire database is created and shown in Figure 2.

Each point represents one experiment from the considered authors, putting the investigated ranges for one- and two-component interactions into perspective (green and purples symbols, respectively). The dots and circles are the experiments from [3], where each array corresponds to a detailed investigation for a given combination. Similarly, the data from Cossali et al. [2] are plotted with green crosses. The data from Vander Wal et al. [1] varying the fluid properties are distributed along $\delta=0.1$ in green plus signs. Finally, the sets of two-component experiments of this work are drawn in purple triangles, completing the investigated range of [1] and filling the gaps between the steps experiments of [3].

Several slices in Figure 2 will be considered to enhance the influence of each parameter separatly and compare correlations. In the following figures, each symbol represents a particular fluid combination, as summarized in Table 3. The red symbols belong to the splashing state, the blue ones to deposition and the green ones were classified to be in the transition state, as defined in the introduction.

The chosen reference correlations are the ones of Vander Wal et al. [1] and Cossali et al. [2], expressed in equations (1) and (2), respectively. Given that $O h, R e$ and $W e$ are linked by $O h=\sqrt{W e} / R e$, these correlations are equivalent and can be compared.

First, we focus on the slice $\delta=0.1$ where the Vander Wal correlation [1] is valid. We consider the range $\delta \in[0.1 ; 0.12]$, which contains the series of two-component experiments of silicon oils and hexadecane. The slice is shown in Figure 3 with the legend reference given in Table 3.

The yellow dashed line represents the correlation of Vander Wal et al. [1]. It gives a good trend for the deposition/splashing limit, but seems to lie below what could be seen as the best fit. This is particularly the case for Re smaller than 1000, which also corresponds to the lower limit of the investigated range by Vander Wal et al. [1] (roughly $R e \in[800 ; 14,000]$ ). In fact, the correlation fits well for higher Re. The purple dashed line corresponds to the correlation of Cossali et al. [2] adapted to the formalism of Vander Wal et al. [1]. Both correlations show high consistency with each other. Similarly, the correlation of Geppert et al. [3] based on the interaction hyspin/hexadecane - equation (4) with averaged viscosities only - is drawn as dashed cyan line in Figure 3. Both are very close together, as shown in [3], highlighting the similarity of their approaches.

All these correlations show the same asymptotical behaviour at high $R e$ but differ for $R e<1000$. In fact, the different exponents on $R e$ in the correlations change the curvature for a similar asymptotical behaviour. A higher exponent (e.g. Cossali vs Vander Wal) leads to an increase of the deposition/splashing limit. 
Table 3. Legend of fluid combinations and droplet impact outcomes for Figures 3, 4, 6.

\begin{tabular}{c|c}
\hline & Symbol \\
\hline Hyspin-Hexadecane & $\circ$ \\
Hexadecane/Hexadecane & $\times$ \\
Hyspin/Hyspin & $\star$ \\
Diesel/Castrol 5W30 & $\cdot$ \\
Diesel/Diesel & $\square$ \\
Diesel/Hexadecane & $\diamond$ \\
Oil-Oil & $\triangle$ \\
Oil-Hexadecane & $\nabla$ \\
Cossali et al. experiments & + \\
Vander Wal et al. experiments & \multirow{2}{}{} \\
blue & deposition \\
green & transition \\
red & splashing
\end{tabular}

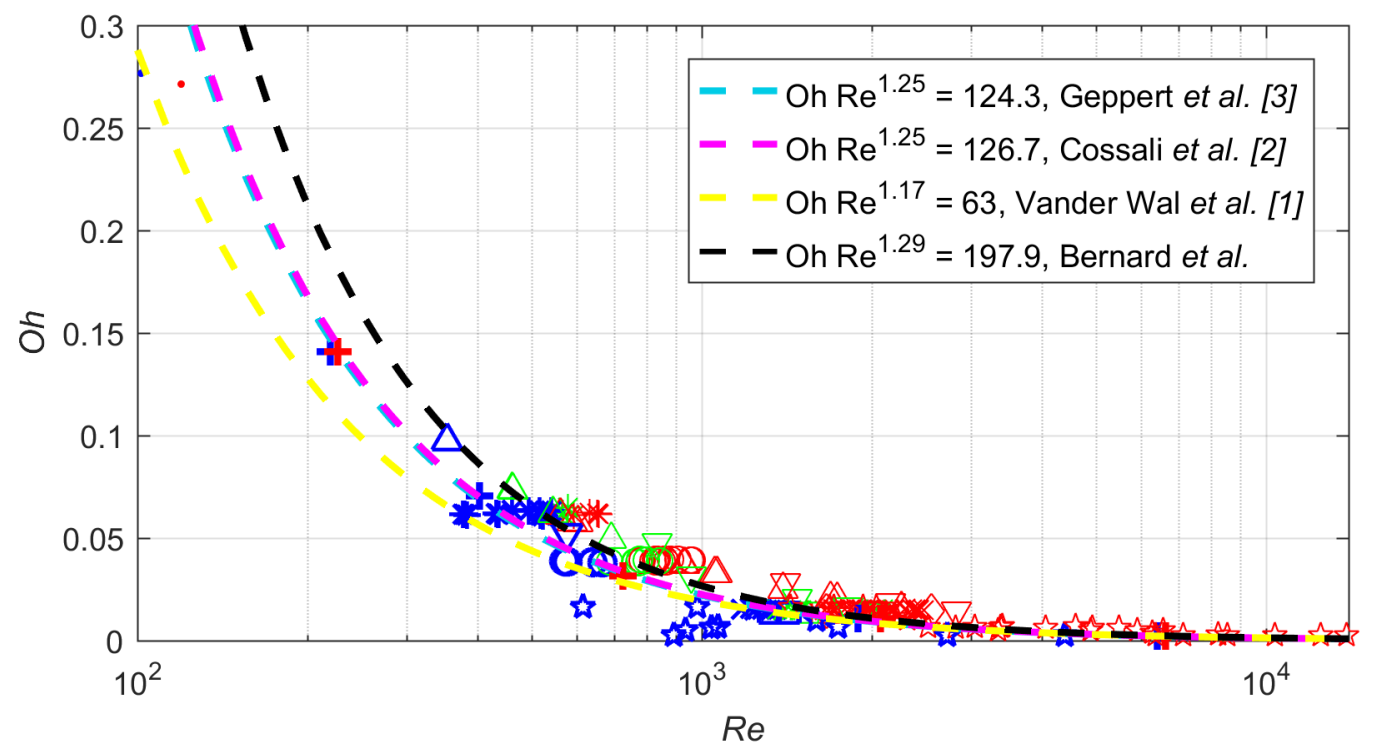

Figure 3. Mapping of droplet impact outcomes for $\delta=0.1$ on a logarithmic scale. Comparison of the correlations of Vander Wal et al. [1] (yellow dashed line), Cossali et al. [2] (purple dashed line), Geppert et al. [3] (cyan dashed line) and from equation (7) (black dashed line). Legend referenced in Table 3.

The experiments performed with high $\mathrm{Oh}$ apparently show an even higher deposition/splashing limit in the range of $R e<1000$. To better fit the transition zone in this range, the following correlation, drawn as a black dashed line in Figure 3, can be used:

$$
\text { Oh } R e^{1.29}=197.9 \quad \text { (only valid for } \delta=0.1 \text { ). }
$$

Note that the two-component interactions (Hyspin-Hexadecane $\circ$, Diesel/Hexadecane $\diamond$, Oil-Oil $\triangle$, Oil-Hexadecane $\nabla$ ) fit the correlation (6), as well as the one-component interactions (Hexadecane/Hexadecane $\times$, Hyspin/Hyspin $\star$, Diesel/Diesel $\square$ ), despite significant differences in viscosity, surface tension and density. This supports the use of averaged properties to take into account both droplet and wall film fluids. The correlation (6), expressed for $\delta=0.1$ offers the same behaviour as the previous correlations from literature for high $R e$. For low $R e$, the deposition/splashing limit was adjusted to be higher.

The influence of the wall film thickness will be now considered in the top-view of the Figure 2, presenting $R e$ as function of $\delta$ for several values of $O h$ in Figure 4. To distinguish between the several steps of Ohnesorge numbers, vertical dashed lines are drawn. The arrow indicates increasing $O h$. The domain on the right side contains mostly points from the slice $O h=0.015$, the middle one from $O h=0.04$ and the left one from all points where $O h$ is higher than 0.14 (correlations are drawn with $O h=0.141$ and $O h=0.25$ ). The interactions hyspin/hyspin $(\star)$, oil-oil $(\triangle)$, oil-hexadecane $(\nabla)$ as well as the data from Cossali et al. [2] and Vander Wal et al. [1] were removed to avoid overlapping (except for $O h=0.141)$. 


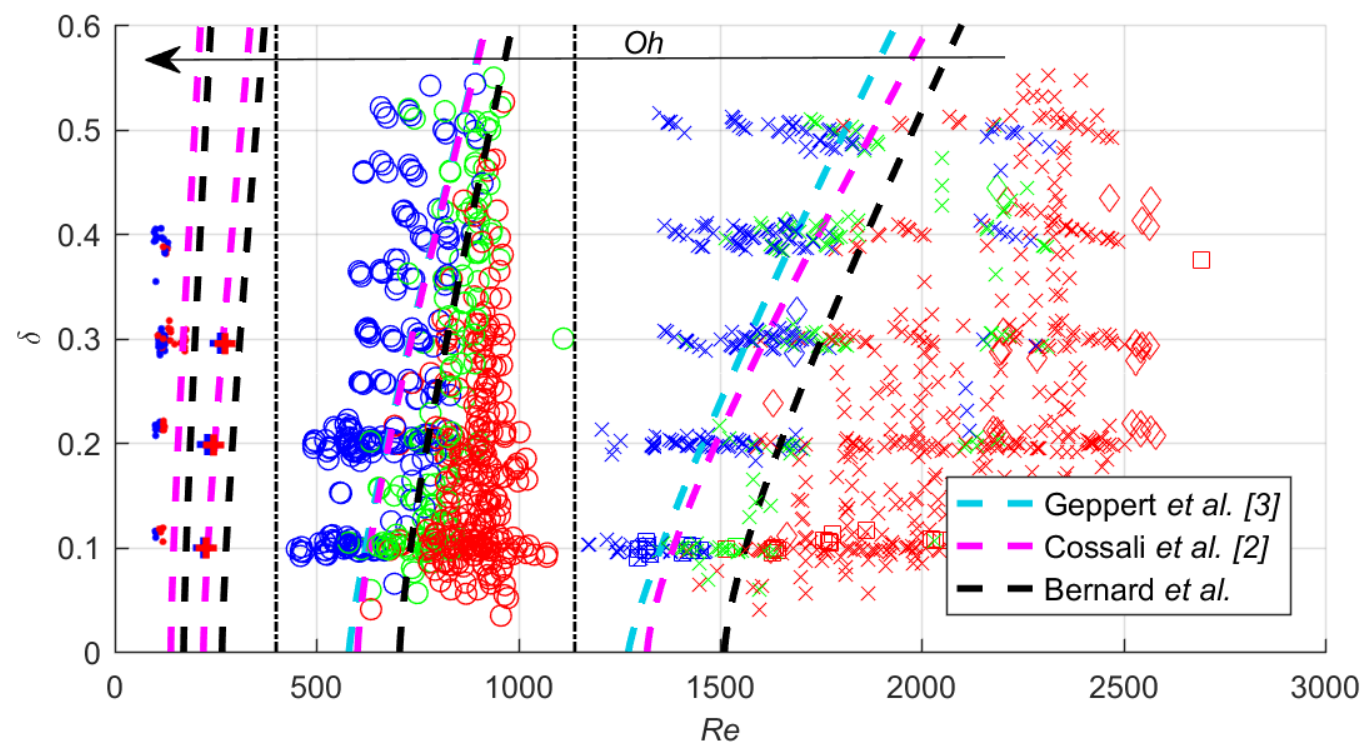

Figure 4. Influence of $\delta$ on the deposition/splashing limits for $O h=0.015$ (right), $O h=0.04$ (middle), $O h=0.141$ and $O h=0.25$ (left). Legend referenced in Table 3.
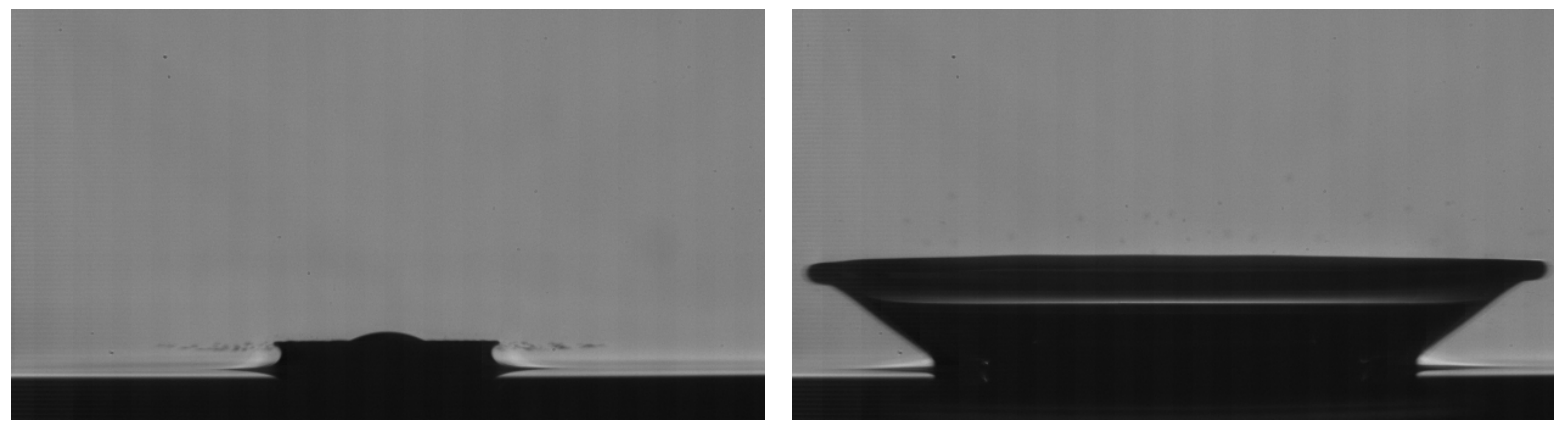

Figure 5. Impact of a diesel droplet onto a Castrol 5W-30 wall film. Left: ejection of tiny droplets at the beginning of the impact. Right: typical deposition morphology of the crown just before receding

For each domain, the correlation of Cossali et al. [2] is drawn as dashed purple line. The adjusted correlations of Geppert et al. [3] for hexadecane/hexadecane $(O h=0.015)$, equation (3), and hyspin-hexadecane $(O h=0.04)$, equation (4), are also plotted in their validity range as cyan dashed lines. In each domain, these correlations are very close together, both in the transition zone and comprised in the experimental uncertainties, see [3]. The dark dashed correlations in Figure 4 corresponds to the one expressed previously for $\delta=0.1$ in equation (6), with a dependency on $\delta$ adapted from the correlation of Cossali et al. [2]. The best fit, consistent with equation (6), was found to be:

$$
\text { Oh } \operatorname{Re}^{1.29}=\left(4400+8900 \delta^{1.44}\right)^{\frac{1}{1.6}}
$$

Once again, it is located close to the other correlations, in the transition zone. However, one can observe that for high $O h$ (left domain), the splashing/deposition limit seems to be much higher than the real one, where red dots (splashing of Diesel/Castrol 5W30) can be seen on the left side of the correlation. Considering the very high viscosity of the employed wall film, and also the high viscosity ratio between droplet and film, the classification of this outcomes as splashing can be discussed: the ejection of tiny droplets at the very beginning of the impact can be observed (Figure 5, left) similar to prompt splashing, explaining the classification. But considering now the crown wall that follows (Figure 5, right) no droplets are formed and this feature is closer to deposition. This explains also that no transition state is observed in this domain. Further investigations have to be performed to draw a conclusion in this domain of high viscosity fluids.

Considering the influence of $\delta$ on the deposition/splashing limit, one can easily see in Figure 4 that the required $R e$ to onset splashing are increasing with increasing $\delta$. In other words, an increase of $\delta$ inhibits splashing, as reported in literature [2, 3]. However, the deposition/splashing limits are becoming straighter with increasing $O h$, as one can see in the middle and left domain in Figure 4, meaning that the influence of $\delta$ on the deposition/splashing limit is decreasing. 
All these effects are now summarized by defining a deposition/splashing limit surface, depicted in Figure 6 . The equation of this surface, expressed in equation (7), is made of the correlation deducted in slice $\delta=0.1$ for the left side and of the adapted dependency of $\delta$ inspired from Cossali et al. on the right side. This surface, plotted in Figure 6, splits the $3 D$ domain in two parts: above the surface (top view, left) are the splashing outcomes -red symbols- and below the surface (bottom view, right) are the deposition outcomes -blue symbols-. The transition outcomes mostly overlap the surface limit.
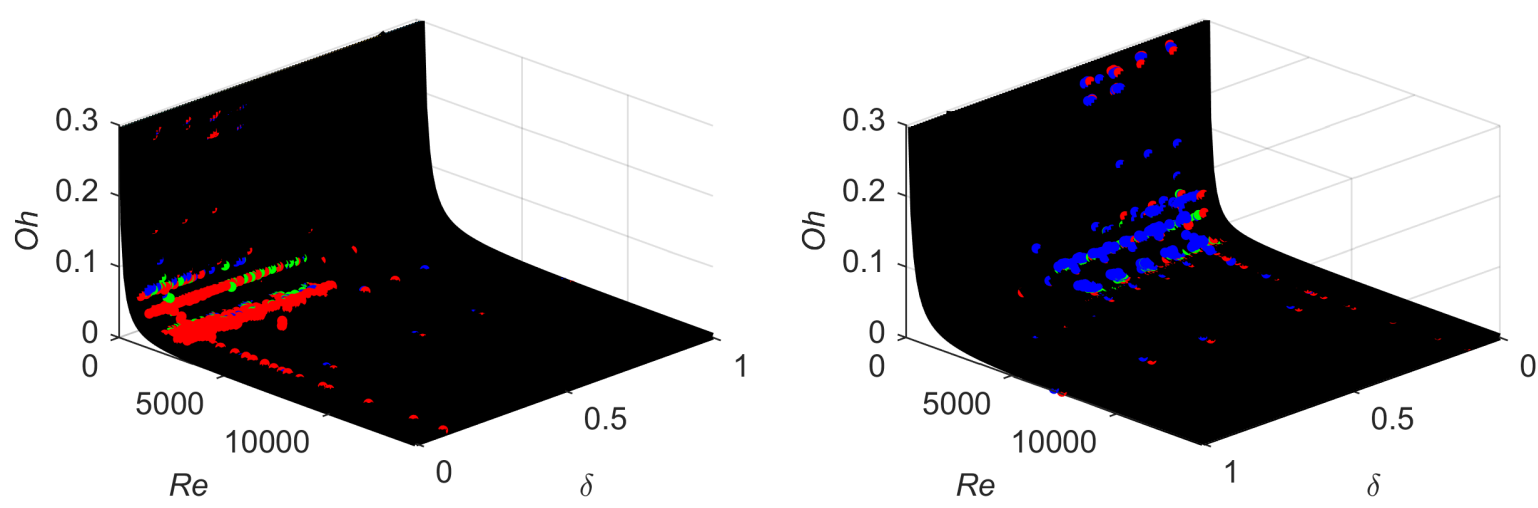

Figure 6. Mapping of the outcomes split by the deposition/splashing surface limit defined in equation (7), view from the top -splashing- (left) and from the bottom -deposition- (right). Legend referenced in Table 3.

Considering the available database, 1660 outcomes over 2197 experiments are on the correct side of the deposition/splashing surface limit, which represents a accuracy of $75.6 \%$. Transition outcomes can be in both deposition and splashing regions. For comparison, the surface based on the correlation of Cossali et al. [2] would bring an accuracy of $72.7 \%$. This improvement is due to the domain for $O h$ larger than 0.025 , where the curvature of the limit seemed to be underestimated. However, more experiments in this range have to be performed. For lower $O h$, the surface limit stays consistent with the previous correlations from literature $[1,2,3]$. Furthermore, the arithmetically averaged properties lead to a good repartition toward the limit, which makes this correlation valid also for two-component interaction.

\section{Conclusion}

The deposition/splashing limit is of particular interest for single drop impacts upon thin liquid layers due to the ejection of secondary droplets. Many studies investigated this limit for one-component interactions, but only a few focused on two-component splashing, where the two liquids differ significantly.

In this study, an unified approach of the deposition/splashing limit for both one- and two-component interactions is investigated. A large database is used: for one-component, the results from Cossali et al. [2] and Vander Wal et al. [1] are combined. Furthermore, experiments from Geppert et al. [3, 4] for one- and two-component interactions of hyspin and hexadecane, are added, plus the combinations of diesel-hexadecane and diesel-motor oil. Lastly, a systematic study of two-component interactions with several silicon oils and hexadecane is performed.

To map the outcomes, the Ohnesorge number $O h$ and the Reynolds number Re are chosen. These are calculated with arithmetically averaged properties between droplet and wall film fluid. Besides, the dimensionsless film thickness $\delta$ is added to form a 3D plot where one- and two-component experiments are combined.

An analyse of the mapping shows that the arithmetically averaged properties lead to a good repartition of the one- and two-component outcomes toward the deposition/splashing limit, despite significant differences in the fluid properties of the combinations.

This study enhances also that all previous reported correlations (Vander Wal et al. [1], Cossali et al. [2] and Geppert et al. [3]) show a high consistency among each other, within the experimental uncertainty.

Since the database includes high viscous fluids, it was possible to propose an improved correlation for the deposition/splashing limit in the range of low $\operatorname{Re}(\operatorname{Re}<1000)$.

\section{Acknowledgements}

The authors kindly acknowledge the financial support of this work by the Deutsche Forschungsgemeinschaft (DFG) in the frame of the International Research Training Group "Droplet Interaction Technologies" (DROPIT).

\section{Nomenclature}

Greek symbols

$\rho \quad$ density $\left[\mathrm{kgm}^{-3}\right]$

$\nu \quad$ kinematic viscosity $\left[m^{2} s^{-1}\right]$ 


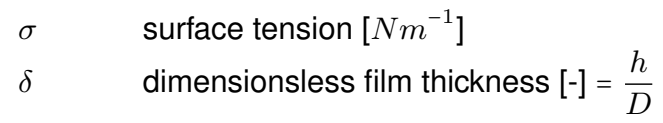

Latin symbols

$V \quad$ impact velocity $\left[\mathrm{m} \mathrm{s}^{-1}\right]$

$D \quad$ droplet diameter [m]

$h \quad$ film thickness [m]

Re Reynolds number [-] $=\frac{V D}{\nu}$

Oh Ohnesorge number [-] $=\nu \sqrt{\frac{\rho}{\sigma D}}$

$W e \quad$ Weber number [-] $=\frac{\rho V^{2} D}{\sigma}$

$K \quad$ impact factor [-] $=$ We $\stackrel{\sigma}{O} h^{-0.4}$

$K^{*} \quad$ impact factor from [5] with averaged viscosities and surface tensions [-]

$R_{a} \quad$ arithmetic averaged roughness

Subscripts

$d \quad$ droplet

$f \quad$ wall film

$v \quad$ averaged viscosities

$v-B L$ averaged viscosities based on boundary layer theory

$\sigma \quad$ averaged surface tensions

ave all properties averaged

\section{References}

[1] Vander Wal, R. L., Berger, G. M., \& Mozes, S. D. (2006). The splash/non-splash boundary upon a dry surface and thin fluid film. Experiments in fluids, 40(1), 53-59.

[2] Cossali, G. E., Coghe, A., \& Marengo, M. (1997). The impact of a single drop on a wetted solid surface. Experiments in Fluids, 22(6), 463-472.

[3] Geppert, A., Chatzianagnostou, D., Meister, C., Gomaa, H., Lamanna, G., \& Weigand, B. (2016). Classification of Impact Morphology and Splashing/Deposition Limit for n-Hexadecane. Atomization and Sprays, 26(10), 9831007.

[4] Geppert, A., Gomaa, H., Meister, C., Lamanna, G., \& Weigand, B. (2014). Droplet wall-film interaction: impact morphology and splashing/deposition boundary of hyspin/n-hexadecane two-component systems. In Proceedings of the 26th Annual Conference on Liquid Atomization and Spray Systems, ILASS-Americas.

[5] Kittel, H.M., Roisman, I.V., Tropea, C. (2016). Outcome of Drop Impact onto a Liquid Film of Different Viscosities. In Proceedings of the 27th Annual Conference on Liquid Atomization and Spray Systems, ILASS-Europe.

[6] Stow, C. D., \& Stainer, R. D. (1977). The physical products of a splashing water drop. Meteorological Society of Japan Journal, 55, 518-532.

[7] Yarin, A. L., \& Weiss, D. A. (1995). Impact of drops on solid surfaces: self-similar capillary waves, and splashing as a new type of kinematic discontinuity. Journal of Fluid Mechanics, 283, 141-173.

[8] Coghe, A., Brunello, G., Cossali, G. E., Marengo, M., \& TeMPE-CNR, M. I. (1999). Single drop splash on thin film: measurements of crown characteristics. In ILASS Europe.

[9] Tropea, C., \& Marengo, M. (1999). The impact of drops on walls and films. Multiphase Science and Technology, 11(1), 19-36.

[10] Rioboo, R., Bauthier, C., Conti, J., Voue, M., \& De Coninck, J. (2003). Experimental investigation of splash and crown formation during single drop impact on wetted surfaces. Experiments in fluids, 35(6), 648-652.

[11] Wang, A. B., \& Chen, C. C. (2000). Splashing impact of a single drop onto very thin liquid films. Physics of fluids, 12(9), 2155-2158. 


\title{
Experimental study of liquid-vapor mass transfer in non-reacting and reacting droplet chains
}

\author{
Michael Stöhr*1, Stefanie Werner ${ }^{1}$, Wolfgang Meier ${ }^{1}$ \\ ${ }^{1}$ German Aerospace Center (DLR), Institute of Combustion Technology, \\ Pfaffenwaldring 38-40, 70569 Stuttgart, Germany \\ *Corresponding author: michael.stoehr@dlr.de
}

\begin{abstract}
The dynamics of liquid-vapor mass transfer largely determines the performance of internal and gas turbine spray combustors. The key mechanisms however typically take place on small spatial scales of less than $100 \mu \mathrm{m}$ which have been difficult to measure. The present work thus aims at the development and application of an experimental technique for the characterization of droplet evaporation with high spatial resolution. Single chains of monodisperse acetone droplets with diameters of 125 and $225 \mu \mathrm{m}$ are injected into a channel with a cross-section of $60 \times 60 \mathrm{~mm}^{2}$ and quartz glass side walls for optical access. The droplet chains are surrounded by a laminar air flow with velocity and temperature of about $0.1 \mathrm{~m} / \mathrm{s}$ and $300 \mathrm{~K}$, respectively. The distribution of acetone vapor around the droplets is measured using planar laser-induced fluorescence (PLIF) excited by the 4th harmonic of a $\mathrm{Nd}$ :YAG laser at $266 \mathrm{~nm}$. The measurements are performed in thin transversal sections between the droplets in order to avoid signal corruption by halation effects that occur when the laser directly hits the droplets as reported in previous studies. In addition, the spatial resolution of the PLIF setup was enhanced by using proper sheetforming and imaging optics. The resulting in-plane resolution and out-plane-resolution (i.e. thickness of the laser sheet) are both determined to about $20 \mu \mathrm{m}$, which thus allows an accurate characterization of the small-scale vapor distribution near the droplets. Using a separate calibration measurement, quantitative acetone concentrations are obtained for non-reacting conditions. As a complementary technique, the droplet evaporation is measured using shadowgraphy droplet sizing. Both non-reacting and reacting droplet chains are studied. The results for the non-reacting cases show that the droplet chains are surrounded by a column of nearly-saturated acetone vapor with a concentration maximum at the centerline. For increasing radial distances, the vapor concentration decays quickly with a half width of $0.5 \mathrm{~mm}$ and reaches almost zero for $r>1 \mathrm{~mm}$. It is further seen that the width of the vapor column increases with streamwise distance. For the experiment with a reacting droplet chain, which is continuously ignited by a heating wire at the channel inlet, a cylindrical reaction zone around the chain with a radius of about $1.5 \mathrm{~mm}$ is observed. The shadowgraphy measurements show that the rate of droplet evaporation is significantly enhanced for the reacting conditions. This is attributed to the high rate of heat transfer from the flame to the droplets and the resulting enhanced acetone mass transfer to the sink at the reaction zone.
\end{abstract}

\section{Keywords}

Droplet evaporation, Laser-induced fluorescence, Monodisperse droplet chain, Liquid-vapor mass transfer

\section{Introduction}

Several important energy conversion devices such as internal combustion engines and gas turbines are driven by the combustion of liquid fuels. The liquid fuels are typically first atomized into a spray of small droplets, which are then dispersed in a turbulent flow, and subsequently evaporate, and then mix and react with air. In many cases the evaporation is the slowest, i.e., rate-controlling process. The detailed experimental characterization of droplet evaporation, especially for an increasing number of alternative fuels, is therefore an important basis for an enhanced understanding of the physical mechanisms and the advancement of numerical models needed for the design of improved combustors.

A classical canonical configuration for the investigation of droplet evaporation and combustion is the linear monodisperse droplet chain. Several quantities have been characterized experimentally for this configuration, such as, e.g., gas phase temperature using coherent anti-stokes Raman spectroscopy (CARS) [1-3], droplet temperature using laser-induced fluorescence (LIF) [4-5] and rainbow thermometry (RT) [6-8], vapor concentration using planar LIF (PLIF) [9, 10], and droplet size using RT, interferometric laser imaging for droplet sizing (ILIDS) [10,11] and droplet imaging $[12,13]$ (mentioning only a part of the numerous works).

The dynamics of the liquid-vapor mass transfer, however, is governed by strong gradients of concentration and temperature in the close vicinity of the gas-liquid interface. Due to the typically small size of droplets, the key mechanisms therefore take place on small spatial scales of significantly less than $100 \mu \mathrm{m}$, which have been 
difficult to measure. The spatial resolution of methods for measurement of vapor concentration near droplets, which are usually performed using PLIF of a fluorescent species such as acetone [9, 10], e.g., was limited by the thickness of the laser sheet of at least $150 \mu \mathrm{m}$, and therefore an improvement is desirable.

The measurement of vapor concentration in the gas phase near the droplet interface is further complicated by socalled halation effects. These effects are caused by interaction of laser light with the fluorescent liquid and lead to corruption of the PLIF signal within a distance of about one droplet diameter from the interface as reported in Refs. $[9,10]$.

The present work thus aims at the development and application of a PLIF method with enhanced spatial resolution that allows the accurate characterization of liquid-vapor mass transfer close to droplet boundaries. For this purpose, the halation effects, which occurred in previous studies using an axial PLIF configuration (Figure 1a) are avoided by a transverse PLIF setup where the horizontal laser sheet is located between two adjacent droplets (Figure 1b). In addition, a significantly enhanced spatial resolution of the PLIF setup was achieved by usage of selected sheet-forming and imaging optics. Using a separate calibration measurement, quantitative acetone concentrations are obtained for non-reacting conditions.

The measuring technique is applied to monodisperse droplet chains operated in a laminar flow channel with welldefined boundary conditions. Both non-reacting and reacting droplet chains are studied and then compared, and the benefits and limitations of the measuring technique are discussed.
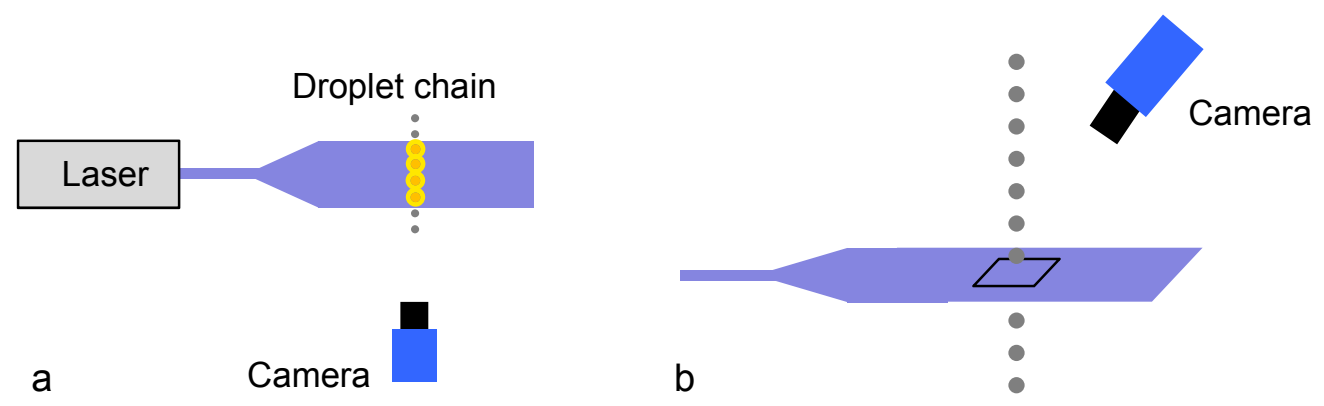

Figure 1: Configurations for (a) axial and (b) transverse vapor PLIF imaging around droplet chains.

\section{Experimental setup}

Measurements are performed in a flow channel (shown in Figure 2) with a quadratic cross-section of $60 \times 60 \mathrm{~mm}^{2}$ and a height of $200 \mathrm{~mm}$. All four side walls consist of glass windows (thickness $8 \mathrm{~mm}$ ) held by corner posts in order to provide optical access. Air is introduced into a plenum at the channel base and then passes through a porous sintered bronze plate into the channel. Air preheating is possible, but not used in the present work. Monodisperse chains of acetone droplets are produced by Rayleigh decay of a laminar liquid jet using a piezoelectric droplet generator (FMP Technology). Orifice plates with diameters of $50 \mu \mathrm{m}$ and $100 \mu \mathrm{m}$ are used, leading to droplet diameters of $125 \mu \mathrm{m}$ and $225 \mu \mathrm{m}$ as described further below. In all cases a laminar air flow with a temperature of about $\mathrm{T}=300 \mathrm{~K}$ and an average velocity of about $0.1 \mathrm{~m} / \mathrm{s}$ is applied. The droplet chains can be operated under both non-reacting and reacting conditions. In the latter case, a Kanthal heating wire wrapped onto a ceramic pipe surrounding the droplet chain at the channel inlet provides a continuous ignition source. The outlet of the ceramic pipe is defined as the origin $y=0 \mathrm{~mm}$ of the axial distance $\mathrm{y}$.

The acetone vapor concentration in the gas phase near the droplets is measured using the vapor fluorescence induced by laser light with a wavelength of $\lambda=266 \mathrm{~nm}$. The output of a frequency-quadrupled Nd:YAG laser (Innolas Spitlight 600, pulse duration ca. $7 \mathrm{~ns}$, operated at a repetition rate of $8 \mathrm{~Hz}$ and a pulse energy of $6 \mathrm{~mJ}$ at $266 \mathrm{~nm}$ ) is formed to a laser sheet using a cylindrical lens of $f=150 \mathrm{~mm}$ focusing on the droplet chain. The fluorescence emission in the range of $350-550 \mathrm{~nm}$ is recorded by two cameras: The first (Camera 1 in Figure 2a, Lavision Imager Intense equipped with a $\mathrm{f}=\mathbf{2 0 0} \mathrm{mm}$ Nikon F/4 macro lens) is located at the same height as the laser sheet, and thus records a 1D profile of the PLIF signal integrated across the laser sheet. The second camera (Camera 3 in Figure 2a, Lavision Imager SCMOS equipped with the same macro lens and mounted on a Scheimpflug adapter) is located laterally above the laser sheet and thus records the 2D PLIF distribution in the plane of the laser sheet. Perspective distorsions due to the diagonal view are corrected in the post-processing 
using an image transformation based on a geometrical calibration. The fields of view (FOV) of the two cameras are specified in Figure 2a.

Droplet sizes are measured using shadowgraphy. The $\lambda=532 \mathrm{~nm}$ output of the PLIF laser is transformed into a diverging beam using a $\mathrm{f}=-20 \mathrm{~mm}$ concave spherical lens, such that the beam illuminates a fluorescent screen with a diameter of about $100 \mathrm{~mm}$. The incoherent red fluorescence of the screen is recorded by a CCD camera (Camera 2 in Figure 2a, Lavision Imager Intense) equipped with a long-distance microscope (Questar QM 100) with a working distance of about $250 \mathrm{~mm}$ and a field of view of $0.97 \times 0.73 \mathrm{~mm}^{2}$.

Both the PLIF and shadowgraphy measurements are synchronized to the droplet formation by using the signal that drives the piezo-electric droplet generator as an external trigger for laser and cameras.
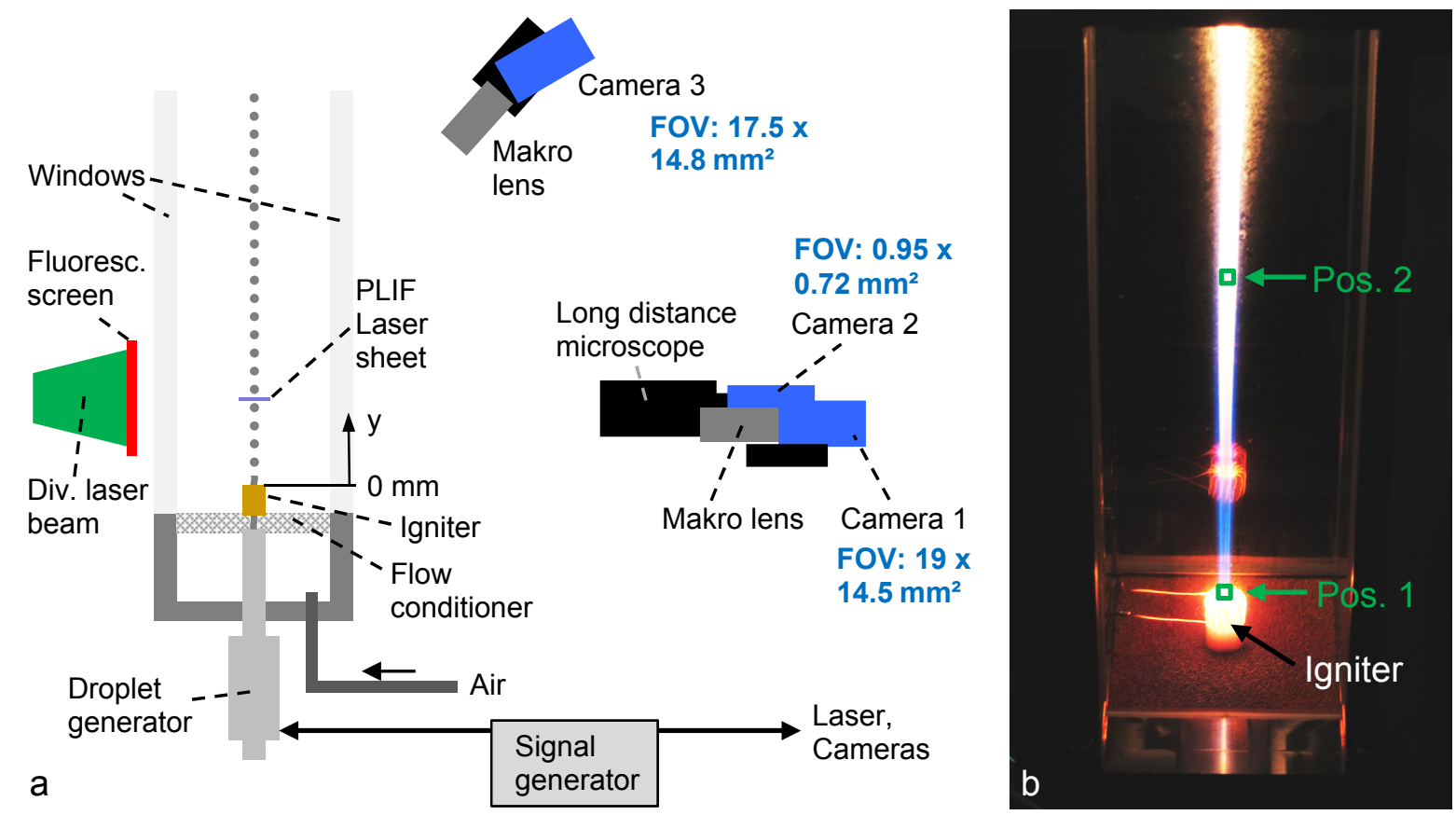

Figure 2: (a) Sketch of experimental setup and (b) photograph of reacting droplet chain in the flow channel.

\section{Characterization of spatial resolution}

In order to measure vapor distributions in the gas phase between droplets with diameters in the range of 100$250 \mu \mathrm{m}$, a spatial resolution of significantly less than $100 \mu \mathrm{m}$ is desirable. For planar measurement techniques such as PLIF, spatial resolution generally has two aspects: The 2D in-plane resolution that is governed by the imaging optics, and the out-of-plane resolution that is determined by the thickness of the laser sheet. The 2D inplane resolution was measured by imaging the line pattern of a USAF 1951 target as shown in Figure 3 . With the standard windows of $8 \mathrm{~mm}$ thickness it was found that the astigmatism due to the diagonal view through the glass limits the resolution to about $150 \mu \mathrm{m}$. As a consequence, the side window towards the PLIF cameras was replaced by a $1 \mathrm{~mm}$ glass plate mounted onto a metal frame. The central part of the resulting target image shown in Figure $3 b$ shows that the resolution is then significantly enhanced such that lines of at least $20 \mu \mathrm{m}$ can be resolved.

The thickness of the laser sheet in the vicinity of the droplet chain is characterized using a beamprofiler (Gentec Beamage $4 \mathrm{M}$ ) that is traversed across the focus of the sheet-forming cylindrical lens as shown in Figure 4a. The results plotted in Figure $4 \mathrm{~b}$ show that the thickness is in the range 15-20 $\mu \mathrm{m}$ within a distance of $\pm 0.5 \mathrm{~mm}$ around the focus. Since the acetone vapor is mostly confined to a radius of also $0.5 \mathrm{~mm}$ around the droplet chain as shown below, an out-of-plane resolution of $\leq 20 \mu \mathrm{m}$ can be assumed. This fits well to the similar value of $20 \mu \mathrm{m}$ for the in-plane resolution, resulting in an overall 3D resolution of about $20 \mu \mathrm{m}$.

The resolution of the shadowgraphy imaging system using the long-distance microscope is about $2 \mu \mathrm{m}$. 
a
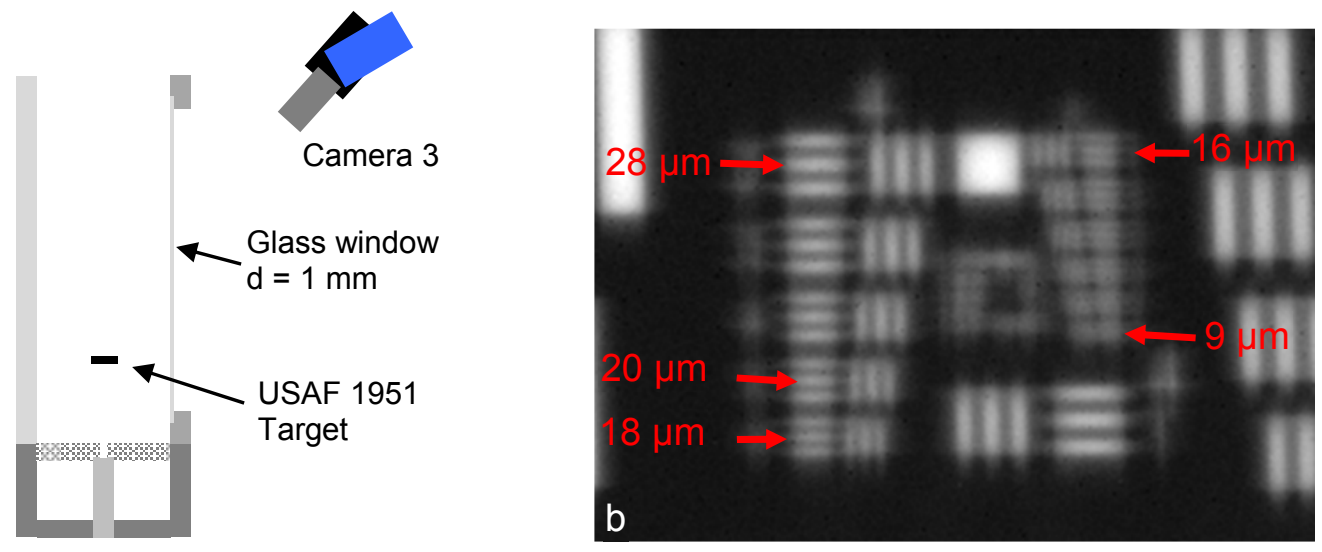

Figure 3: (a) Setup für determination of in-plane spatial resolution, (b) image of USAF 1951 target.

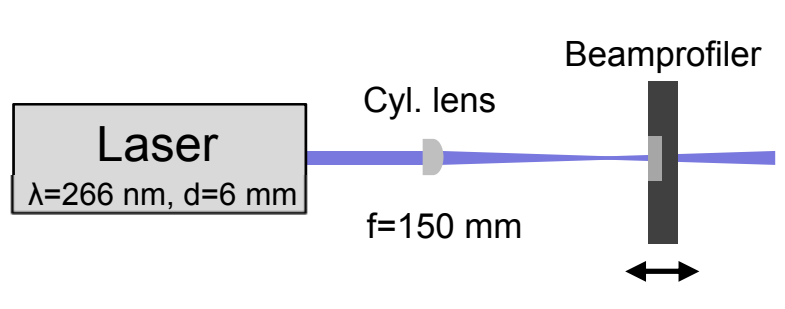

a

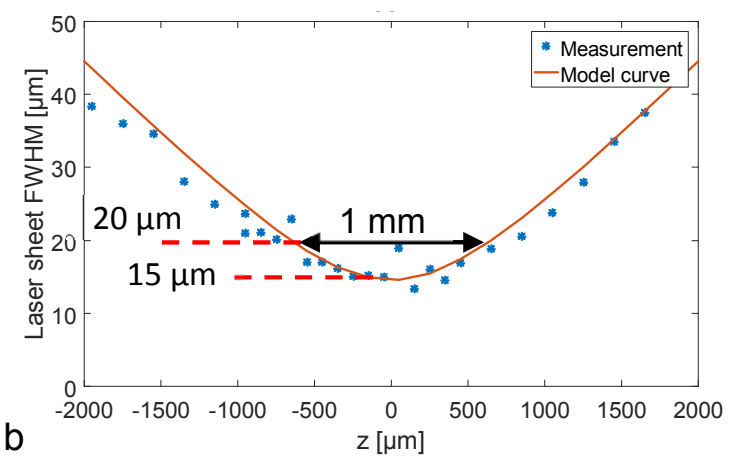

Figure 4: (a) Setup for measurement of laser sheet thickness, (b) sheet thickness near focus $(z=0 \mu m)$.

\section{Calibration of PLIF signal}

In order to determine quantitative acetone vapor concentrations from the $2 \mathrm{D}$ acetone PLIF signals measured with camera 3, a separate calibration measurement is performed. A stream of saturated acetone at a temperature of $300 \mathrm{~K}$ is introduced into the channel directly below the PLIF laser sheet through a small pipe as shown in Figure 5. For this air/vapor mixture, which has a known acetone concentration of $0.013 \mathrm{~mol} / /$, the PLIF signal is then recorded with same laser and camera settings as in the droplet chain measurements.

The resulting PLIF image is shown in the right part of Figure 5. Intensity variations in the vertical direction are caused by inhomogeneities of the laser beam. In the horizontal direction, an intensity minimum is observed in the central part where the focus is located. This intensity reduction is caused by saturation of the fluorescence due to excessive fluence of the laser pulse, which is highest at the focus. A further increase of laser pulse energy therefore would not lead to an increased PLIF signal.

The droplet PLIF measurements are then corrected for the effects from laser beam inhomogeneity and the fluorescence saturation through a division by the calibration measurement. The resulting data then provides a $2 \mathrm{D}$ distribution of volumetric acetone concentration in $\mathrm{mol} / \mathrm{l}$.

It is noted that the acetone fluorescence quantum yield generally depends on temperature [14]. For an excitation at $\lambda=266 \mathrm{~nm}$, however, the variation of fluorescence per molecule is less than $5 \%$ per $100 \mathrm{~K}$ for temperatures around $300 \mathrm{~K}$ that are relevant for the present non-reacting conditions [14], and therefore uncertainties due to the variation of fluorescence quantum yield are very low. It is further noted that effects from collisional quenching on acetone fluorescence are negligible [15]. 


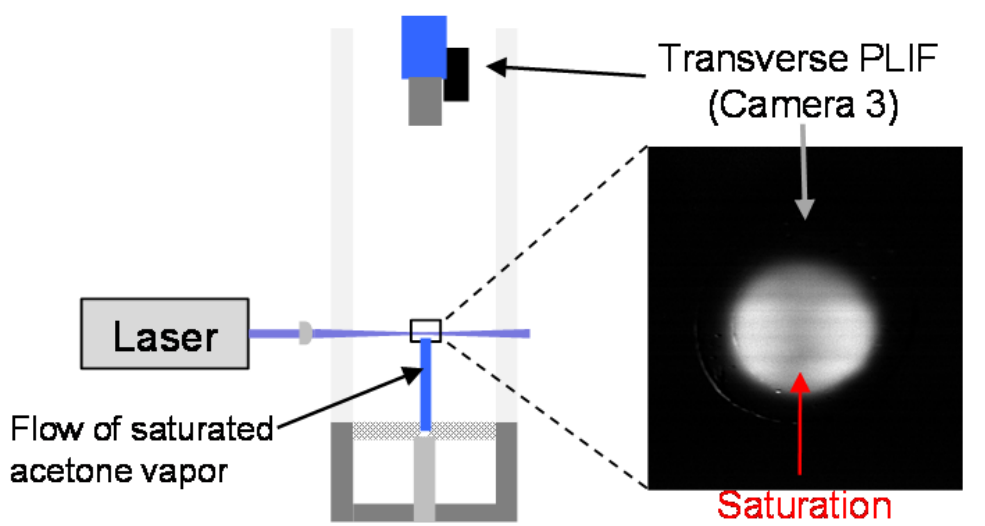

Figure 5: Setup for PLIF signal calibration.

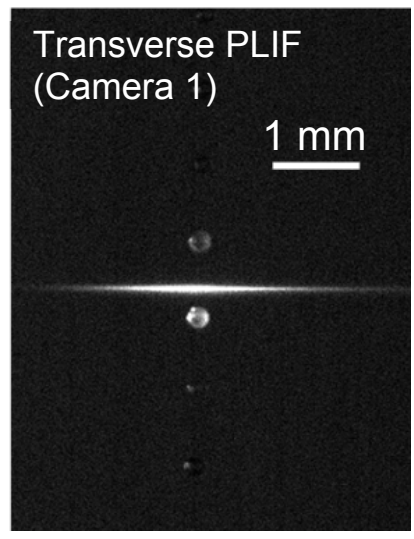

Figure 6: Transverse 1D PLIF distribution for case $A$ at $y=50 \mathrm{~mm}$.

\section{Results for non-reacting condition}

In the following sections, measurements for two droplet chain parameters are presented. In the first case $A$, an orifice plate diameter $d_{0}=100 \mu \mathrm{m}$, droplet diameter $d_{d} \approx 225 \mu \mathrm{m}$, droplet spacing $C \approx 3.9 d_{d}$, and droplet velocity of $v \approx 15 \mathrm{~m} / \mathrm{s}$ were chosen. In the second case $B$, the parameters were $d_{o}=50 \mu \mathrm{m}, d_{d} \approx 125 \mu \mathrm{m}, C \approx 4.1 d_{d}$ and $v \approx 19$ $\mathrm{m} / \mathrm{s}$. For case $B$, measurements were also performed under reacting condition, and are discussed in the next section.

In Figs. 6 and 7, a PLIF measurement for case $A$ at a height of $y=50 \mathrm{~mm}$ in the channel is shown. The 1D horizontal, line-of-sight integrated profile obtained with camera 1 (Figure 6) clearly shows that the laser sheet passes through droplet chain axis without impinging on a droplet. Some droplets near the laser sheet are visible due to laser light reflected at the channel window. Although no quantitative concentrations can be obtained from the line-of-sight integrated 1D profile, the measurement reveals the position of the laser sheet (in this case, about $230 \mu \mathrm{m}$ above the upper border of the droplet below), and indicates that the droplet chain is surrounded by a column of acetone vapor with a radius of roughly $1 \mathrm{~mm}$.

The corresponding 2D PLIF distribution obtained with camera 3 is shown in Figure 7a. Here, 100 instantaneous measurements with the same position relative to the droplets were averaged. The PLIF image shows a continuous distribution of acetone vapor with up to 20 counts as well as superimposed signals from the droplets below and above the laser sheet that are illuminated by reflected laser light as mentioned above. In the region with superimposed droplets, apparently no vapor concentrations can be estimated. The resulting quantitative distribution of vapor concentration, obtained with the calibration described in the previous section, is shown in Figure $7 \mathrm{~b}$. From the 2D distribution, a $1 \mathrm{D}$ profile through the centerline is plotted in Figure 7c. The profile shows that the vapor concentration reaches a maximum at the centerline of about $60 \%$ of the saturation concentration at $\mathrm{T}=300 \mathrm{~K}$, i.e. about $7.810^{-3} \mathrm{~mol} / \mathrm{l}$, and has a half width of about $0.5 \mathrm{~mm}$. The concentration distribution roughly resembles a Gaussian, which would be consistent with a classical diffusion-like radial mass transport.

From the measurements for case B, horizontal line-of-sight integrated PLIF profiles and shadowgraphy images obtained at heights of $y=3 \mathrm{~mm}$ and $\mathrm{y}=93 \mathrm{~mm}$ (labeled as Pos.1 and 2, respectively, in Figure 2b) are shown in Figure 8. While at $y=3 \mathrm{~mm}$ the column of acetone vapor around the droplets has a width of about $2 \mathrm{~mm}$, further downstream at $y=93 \mathrm{~mm}$ the width is increased to about $4 \mathrm{~mm}$. This demonstrates the radial transport of acetone vapor in the laminar channel flow. A similar growth of vapor column width for increasing distance from the injector has also been observed in a recent numerical simulation of a monodisperse droplet chain [16]. From the corresponding shadowgraphy images, the droplet size is estimated as $\approx 125 \mu \mathrm{m}$ for both positions, i.e. no significant decrease of droplet size appears. This is expectable since the droplets move through a near-saturated acetone vapor, and the heat transfer from the ambient air at $\mathrm{T} \approx 300 \mathrm{~K}$ is low. 


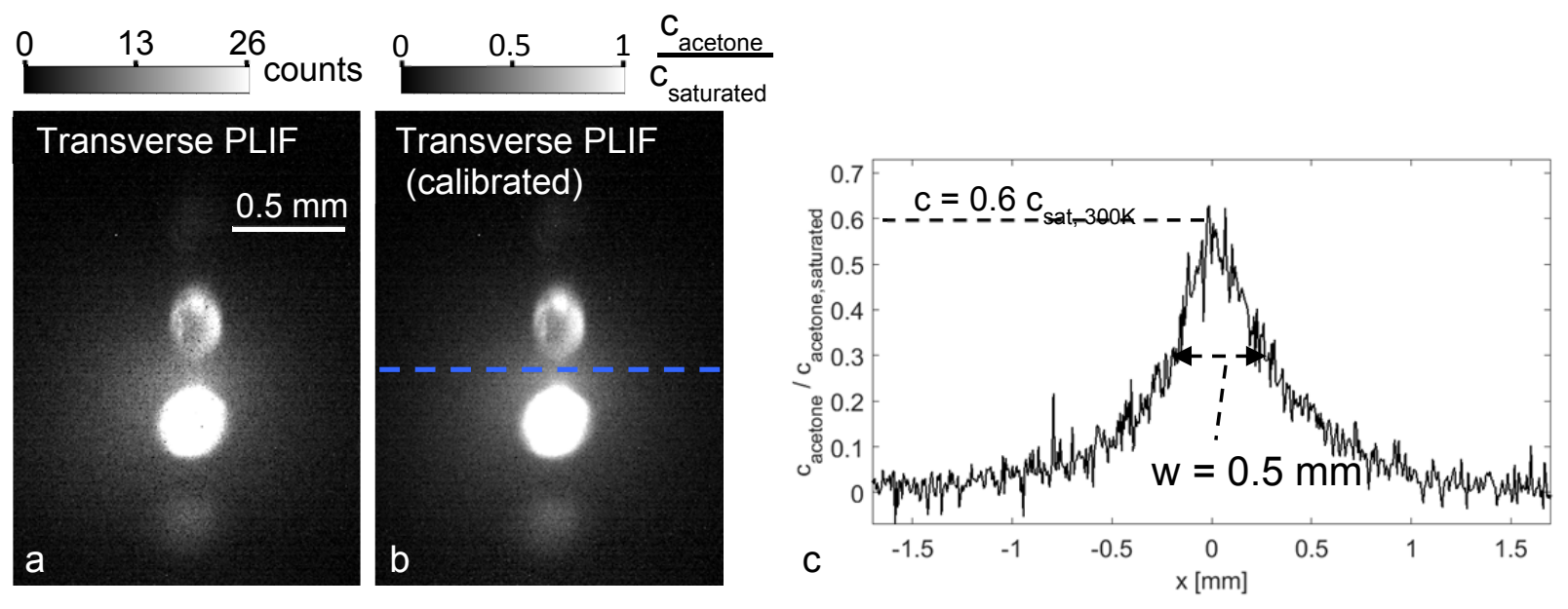

Figure 7: (a) Raw and (b) calibrated transverse distributions of PLIF signal for case A at $y=50 \mathrm{~mm}$, (c) concentration profile along a line (marked blue in (b)) across the droplet chain axis.

\section{Results for reacting condition}

The droplet evaporation and liquid-vapor mass transfer for the reacting case B differ significantly from those of the non-reacting cases discussed above. When the fuel vapor is continuously ignited by the heating wire at the channel base, a cylindrical flame encloses the droplet chain as shown in Figure $2 \mathrm{~b}$. Chemiluminescence images (not shown) indicate that the flame front is located at a radius of about $r=1.5 \mathrm{~mm}$. It is further observed that for heights of $y>50 \mathrm{~mm}$, significant levels of soot are formed in the flame.

The horizontal PLIF profiles at $y=3 \mathrm{~mm}$ displayed in Figure 8 show that the PLIF intensities are significantly lower for the reacting condition than for the corresponding non-reacting case. This is expected for two reasons: First, CARS measurements for reacting monodisperse droplet chains showed that gas temperatures on the centerline are typically in the range of 1200-2000 K [1-3], which implies a strongly reduced gas density and thereby a reduced volumetric vapor concentration. Secondly, the fluorescence quantum yield of acetone for excitation at $\lambda=266 \mathrm{~nm}$ is significantly reduced for temperatures larger than, say, $600 \mathrm{~K}$ [14]. Since the gas temperatures for the present experiment are unknown, these two effects cannot be corrected and therefore no quantitative vapor concentrations can be obtained for the reacting case.

At the height $\mathrm{y}=93 \mathrm{~mm}$, by contrast, a much stronger PLIF signal appears for the reacting condition compared to the non-reacting case. The photograph in Figure $2 \mathrm{~b}$ indicates that intense soot formation takes place in this part of the flame. The region of soot formation can further be identified as a gray vertical column caused by soot luminosity in the PLIF image for $y=93 \mathrm{~mm}$ in Figure 8. The high PLIF signal is therefore attributed to PAH molecules, which are considered as soot precursors and strongly fluoresce when excited at $\lambda=266 \mathrm{~nm}$ [17]. While the PAH fluorescence further inhibits the quantification of acetone vapor in the sooting regions of the reacting droplet chain, it may enable insights into the onset of soot formation.

The corresponding shadowgraphy images show that the droplet diameters have reduced considerably from 125 $\mu \mathrm{m}$ at the channel base to $112 \mu \mathrm{m}$ at $\mathrm{y}=93 \mathrm{~mm}$. This is apparently caused by the intense heat transfer from the flame front at $r=1.5 \mathrm{~mm}$ to the droplets at $r=0 \mathrm{~mm}$. As a rough estimate, the CARS measurements for a reacting chain of monodisperse ethanol droplets by Virepinte et al. [3] showed that the gas temperature reaches a maximum of $2000 \mathrm{~K}$ at the flame front at $\mathrm{r}=1.5 \mathrm{~mm}$ and reduces to $1300-1500 \mathrm{~K}$ at the centerline, which corresponds to a substantial temperature gradient of $330-470 \mathrm{~K}$ per $\mathrm{mm}$ driving the fuel vaporization. 


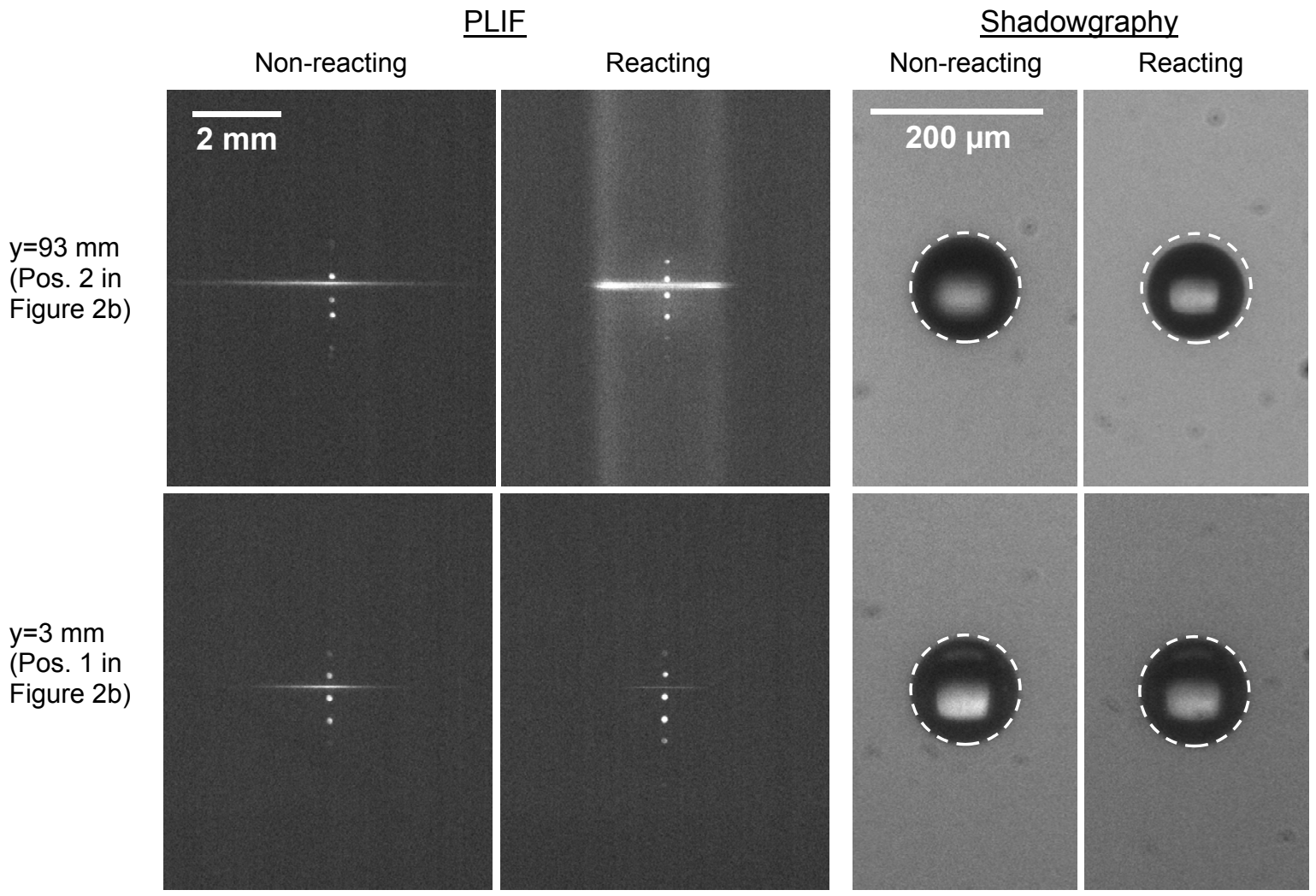

Figure 8: Transverse vapor PLIF (left) and droplet shadowgraphy (right) for non-reacting and reacting conditions for case $B$ at heights $y=3 \mathrm{~mm}$ and $y=93 \mathrm{~mm}$. The dashed circles represent a diameter of $125 \mu \mathrm{m}$.

\section{Conclusions}

The present work demonstrated the development and application of a PLIF technique for the characterization of the fuel vapor distribution around monodisperse chains of acetone droplets in a laminar flow channel. The technique features a high spatial resolution of around $20 \mu \mathrm{m}$, which is significantly improved compared to prior approaches. Furthermore the signal corruption by halation effects due to interaction of laser light and droplets, which was reported in previous studies, could be avoided by measurements in thin transversal sections between the droplets. Together with a calibration using the signal from air with known acetone vapor concentration, quantitative acetone concentrations could be obtained for the first time, to our knowledge, in the close vicinity of droplets.

Measurements were performed for two cases denoted as A and B with different droplet diameters and spacings. The latter case $B$ was also studied under reacting conditions using a heating wire as a continuous ignition source. The results for the non-reacting case A revealed a cylindrical column of acetone vapor around the droplet chain with a Gaussian-like concentration distribution that reaches a maximum value of $7.810^{-3} \mathrm{~mol} / \mathrm{l}$ near the centerline at a height of $y=50 \mathrm{~mm}$. The results for the non-reacting case $B$ showed that the radius of the column of nearsaturated acetone vapor increases in streamwise direction from $r \approx 1 \mathrm{~mm}$ at the channel base to $r \approx 2 \mathrm{~mm}$ at a height of $y=93 \mathrm{~mm}$. The evaporation rate of the non-reacting droplets determined using shadowgraphy imaging was found to be very low, as expected from the low heat transfer and the nearly-saturated vapor in the vicinity of the droplets.

For the corresponding reacting case $\mathrm{B}$, the droplet chain is enclosed by a cylindrical flame front with a radius of about $r=1.5 \mathrm{~mm}$. The acetone PLIF signal around the droplets is significantly reduced compared to the nonreacting case as expected from the reduced gas density and reduced fluorescence yield at higher gas temperature. For heights of $y>50 \mathrm{~mm}$, significant levels of soot are formed in the flame. Due to the elevated, yet not precisely known temperatures, and additional interference with PAH fluorescence in the soot-forming regions, acetone vapor concentrations could not be quantified for the reacting case. Shadowgraphy measurements of droplet size, on the other hand, indicated a significantly enhanced rate of vaporization for the reacting case, which is consistent with the strong heat transfer from the flame front. 


\section{Acknowledgements}

The contribution of Tobias Biesner to the design and setup of the experiment is appreciated. Financial support from the DLR project R2F is gratefully acknowledged.

\section{References}

[1] Zhu, J., Dunn-Rankin, D., Applied Optics, 1991, 30, pp. 2672-2674.

[2] Zhu, J., Dunn-Rankin, D., 1992, Symposium (International) on Combustion, 24, pp. 1473-1481.

[3] J.F. Virepinte, Y. Biscos, G. Lavergne, P. Magre, G. Collin, 2000, Combustion Science and Technology, 150, pp. 143-159.

[4] Castanet, G., Lemoine, F., 2007, Proceedings of the Combustion Institute, 31, pp. 2141-2148.

[5] Lemoine, F., Castanet, G., 2013, Experiments in Fluids, 54, 1572.

[6] Roth, N., Anders, K., Frohn, A., 1991, Applied Optics, 30, pp. 4960-4965.

[7] Castanet, G., Lavielle, P., Lemoine, F., Lebouche, M., Atthasit, A., Biscos, Y, Lavergne, G., 2002, International Journal of Heat and Mass Transfer, 45, pp. 5053-5067.

[8] Saengkaew, S., Godard, G., Blaisot, J. B., Grehan, G., 2009, Experiments in Fluids, 47, pp. 839-848.

[9] Orain, M., Mercier, X., Grisch, F., 2006, Combustion Science and Technology 177, pp. 249-278.

[10] Sahu, S., Hardalupas, Y., Taylor, A. M. K. P., 2014, Experiments in Fluids, 55, 1673.

[11] Glover, A. R., Skippon, S. M., Boyle R. D., 1995, Applied Optics, 34, pp. 8409-8421.

[12] Silverman, M. A., Dunn-Rankin, D., 1994, Combustion Science and Technology, 100, pp. 57-73.

[13] Perrin, L., Castanet, G., Lemoine, F., 2015, Experiments in Fluids, 56, 29.

[14] Thurber, M. C., Grisch, F., Kirby, B. J., Votsmeier, M., Hanson, R.K., 1998, Applied Optics, 37, pp. 49634978.

[15] Lozano, A., Yip, B., Hanson, R. K., 1992, Experiments in Fluids, 13, pp. 369-376.

[16] Castanet, G., Perrin, L., Caballina, O., Lemoine, F., 2016, International Journal of Heat and Mass Transfer, 93, pp. 788-802.

[17] Sun, R., Zobel, N., Neubauer, Y., Chavez, C. C., Behrendt, F., 2010, Optics and Lasers in Engineering, 48, pp. 1231-1237. 


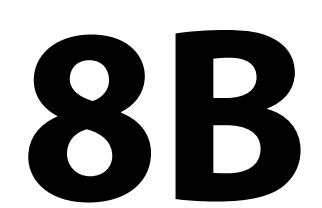

Experimental techniques 2 


\title{
Vapor phase penetration measurements with both single and double-pass Schlieren for the same injection event
}

\author{
Raul Payri*, F. J. Salvador, Gabriela Bracho, Alberto Viera \\ CMT Motores Termicos, Universitat Politècnica de València, Spain \\ *Corresponding author: rpayri@mot.upv.es
}

\begin{abstract}
Schlieren imaging has been adopted as a standard optical technique for the analysis of diesel sprays under engine like conditions. A single-pass Schlieren arrangement is typically used for the study of single-orifice nozzles, as vessels with multiple optical accesses regularly allow line of sight visualization. Contrarily, for multi-spray nozzles, measurements are commonly performed through a single optical access, in which case a double-pass arrangement is employed. As a consequence, the light beams pass through the test section twice, increasing the optical sensitivity of the Schlieren setup. However, the impact this has on the macroscopic spray characteristics is still unclear. The scope of this study is to analyze the differences in vapor phase penetration for the same injection event, through high-speed imaging, for both single and double-pass Schlieren configurations. Experiments were carried out with a three hole nozzle with a nominal orifice diameter of $90 \mu \mathrm{m}$, named Spray B from the Engine Combustion Network, using commercially available diesel fuel and in non-reactive conditions. The impact of different injection pressures and chamber densities on the spray captured by each setup was assessed. On the results, vapor phase penetration followed the expected trend found in the literature, as it increases with increasing injection pressure and decreasing chamber density. Comparing the optical setups, vapor phase penetration obtained with the double-pass arrangement was marginally higher. The deviation was observed throughout all tested conditions. Although the discrepancy was approximately constant for different injection pressures and chamber temperature, it increased with increasing density. These results highlight the importance of a proper understanding regarding the limitations of optical diagnostics, in particular for results used in calibration of computational models.
\end{abstract}

\section{Keywords}

Diesel injection, Schlieren, vapor phase penetration

\section{Introduction}

In recent years, the implementation of Schlieren imaging in the experimental field has expanded to numerous applications that include military, industrial and scientific research. As Settles [1] stated, it allows seeing optical inhomogeneities in transparent media, like air. In small scale applications, these optical inhomogeneities can relate to density gradients in the medium through which light beams propagate. More specifically, in the field of diesel injection, a Schlieren setup, coupled with high-speed cameras and a proper image processing methodology, has become an essential tool for the study of vaporizing diesel sprays.

Researchers have used Schlieren imaging to analyze the transient vapor phase of the diesel sprays in both nonreactive and reactive conditions. With this technique it is possible to measure simple macroscopic parameters, like spray tip penetration and spreading angle [2,3], to more complex variables, like ignition delay [4-7] or even lift-off length [8]. It is not only fundamental knowledge to understand how boundary conditions affect these variables, but the experimental data is necessary to validate computational tools, such as 1-D models or computational fluid dynamics (CFD).

The two most common Schlieren configurations are single-pass (SP) [2, 5-9] and double-pass (DP) [3, 10-13]. Typically, the first setup is used for axially drilled single-orifice nozzles, where vessels with multiple optical accesses allow line of sight visualization. Contrarily, the double-pass setup is commonly used for multi-hole nozzles, where the sprays tend to be visualized through a single optical access. Consequently, the light crosses the test section twice, and theoretically the Schlieren sensitivity increases by a factor of two [1]. Subsequently, as each region of the spray contour is determined by the amount of refraction the optical configuration can capture, the boundary observed by each system could differ, because is determined by its sensitivity.

The objective of the present study is to compare two different Schlieren imaging setups and their capabilities in measuring vapor phase penetration. Experiments were carried out using a Spray B injector from the Engine Combustion Network (ECN) dataset (http://www.sandia.gov/ecn/). This is a multi-orifice nozzle in which the three holes are not equally spaced. In consequence, one spray is optically isolated from the others, often referred as the spray of interest. Therefore, it can be visualized both as a single and multi-orifice nozzle for the same injection event. Parametric variations of injection pressure, chamber temperature and density were carried out.

This report is divided into four sections. Followed by this introduction, the experimental facility is briefly mentioned, along with a description of the Schlieren principle, optical setups, and image processing methodology. Then, the results regarding background structures and vapor phase penetration are presented, with a short discussion for each case. In the last section, the main conclusions are drawn. 


\section{Materials and methods \\ Testing facility}

A high pressure and temperature vessel, with three optical accesses, was used. The facility can reach nearly quiescent and steady thermodynamic conditions, relevant to the diesel engine. The test chamber presents constant pressure and flow throughout its section, while a group of compressors, high-pressure reservoirs, and heaters provide the necessary conditions for testing purposes. The facility is explained in more detail in the work of other authors $[3,5]$. The fuel delivery system is made up of commercially available components. A Bosch CP3 pump, powered by an electric engine, supplies high-pressure fuel to a common rail with a pressure regulator driven by a PID controller.

The Spray B nozzle (reference 211200) is thoroughly described in the ECN website (http://www.sandia.gov/ ecn/). It consists of a three orifice nozzle, where only the spray of interest is studied, which is located at $180^{\circ}$ relative to the fuel inlet port. The nozzle outlet diameter is $93.2 \mu \mathrm{m}$, with a $k$-factor of 1.5 , and a nominal inclination angle of $17.5^{\circ}$.

\section{Schlieren principle}

Light rays propagate uniformly through homogeneous media, but they are refracted proportional to the refractive index of the medium they enter [1]. Gladstone and Dale [14] found that there is a linear relationship between the refractive index and gas density, presented in Eqn. 1. Where $n$ is the refractive index, $\rho$ is the gas density, and $k_{G D}$ is the Gladstone-Dale coefficient. Additionally, the angular deflection $\varepsilon$ of a ray in the perpendicular plane $x-y$, of a light beam that is traveling in a direction $z$ can be obtained with the expression in Eqn. 2 [1], where $L$ represents the optical path length, and $n_{0}$ the refractive index of the surroundings.

$$
\begin{aligned}
& n-1=\rho \cdot k_{G D} \\
& \varepsilon_{x}=\frac{L}{n_{0}} \frac{\partial n}{\partial x} \quad, \quad \varepsilon_{y}=\frac{L}{n_{0}} \frac{\partial n}{\partial y}
\end{aligned}
$$

From equations 1 and 2, there is a clear relationship between the changes in density and angular deflection of light rays, that can be visualized through Schlieren imaging with a simple optical setup. In Figure 1.a, where a basic single-pass arrangement is presented, beams from a point light source are parallelized by a lens. Any rays deflected by density gradients in the test section (trajectory $i$ in the figure) are blocked by a slit located at the focal distance of the second lens (or cut-off plane). On the contrary, non-deflected beams ( $i i$ in the figure) can pass through the slit and reach the screen. In theory, this produces a black and white image, because the light source is infinitesimal and located exactly at the focal distance of the first lens. Thus each point in the test section is illuminated by a single light ray. In real practice, sources of illumination are finite. Thus the diagram of the setup is more similar to the one presented in Figure 1.b. Here, the Schlieren object in the test section is illuminated by multiple infinitesimal $\partial j$ light sources. In consequence, the image assembled by the second lens is a composition of sub-images, formed by each bundle of rays from each $\partial j$ source [1]. More importantly, this gives the Schlieren setup a continuous dynamic range, in the form of gray-scale intensities (combination of trajectories $i$ and $i i i$ ), compared to the black and white resolution from the first example.

To resemble a double-pass setup, we introduce a mirror where the Lens* is, in Figure 1.b, thus light rays would travel twice through the Schlieren object. In consequence, they are diverted once again by the density gradients, boosting the deflection angle [1]. Additionally, the optical sensitivity can be controlled by the size of the slit. Reducing the aperture is directly related to a decline in minimum deflection angle not blocked by the cutoff [9].

\section{Optical setup for vapor phase visualization}

Following the principles explained in the previous section, both single and double-pass Schlieren imaging were used to study the vapor phase penetration of a diesel spray. Table 1 presents a summary of the optical setup, and Figure

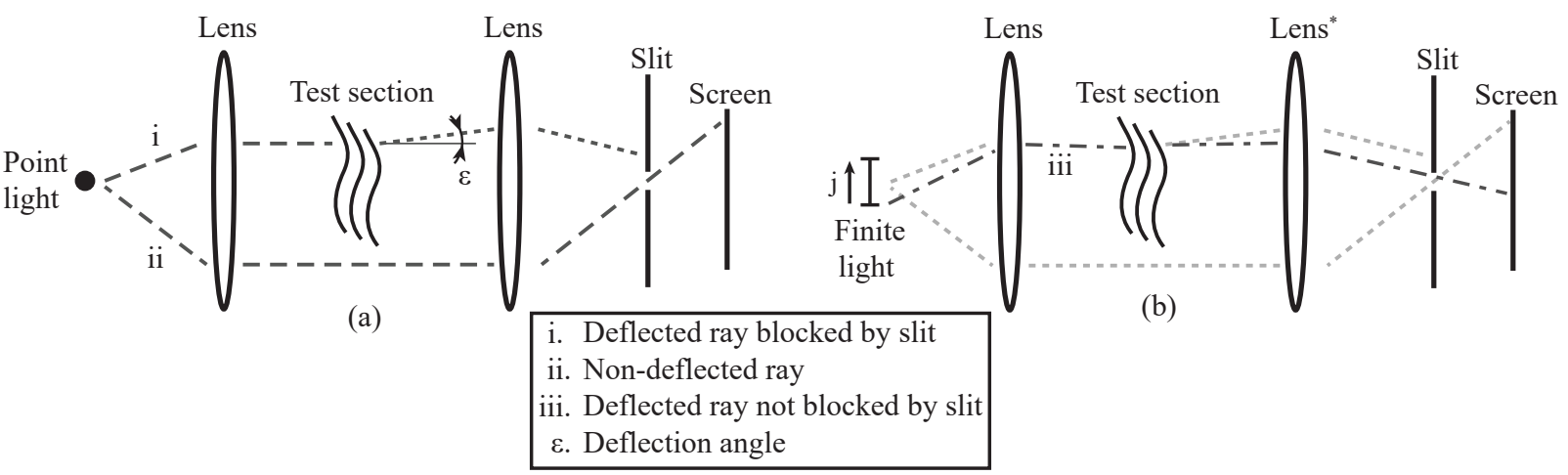

Figure 1. Point (a) and finite (b) light source in Schlieren setup. 
2 the assembly of this configuration for visualization.

For the single-pass arrangement (light trajectory in green), the illumination source was located at the focal distance of a parabolic mirror instead of a regular lens, in contrast to the previous diagram. The angle between the light source and the optical axis of the SP setup was minimized as a result of two factors. Firstly, the long focal distance of the mirror. Secondly, because only one plume is visualized, the source was positioned just above the optical axis, with the minimum angle that allowed the visualization of the spray of interest. As a result, coma aberration was minimized, and the uniformity of the background illumination was improved [1].

In the double-pass configuration (light trajectory in orange), because the spray is visualized through one optical access, a high-temperature mirror is used. Therefore, a beam splitter (50:50) is needed to maintain the optical axis aligned with the other components of the DP setup. As a consequence, only $25 \%$ of the original light reached the cut-off plane. However, the power output of the illumination source was not a constraint, so both setups were calibrated with the same background intensity before measurement.

Additionally, the top left corner of Figure 2 presents the optical perspective inside the test chamber, where the spray of interest is colored in orange. Note that, due to the fact that the high-temperature mirror did not fully enclose the nozzle of the injector, the minimum penetration measurable by the DP setups was $6 \mathrm{~mm}$. Dashed and continuous lines represent the rays before and after passing through the spray, respectively.

The diameter for both diaphragms was set to $4 \mathrm{~mm}$, to have the same sensitivity at the cut-off plane. Previous authors $[3,5,9]$ proved that this diaphragm size provides a proper balance for spray and background segmentation for the Schlieren setups in the facility. Both cameras were set to the same speed, resolution, exposure, and equipped with the same lens.

Table 1. Optical configuration.

\begin{tabular}{ll}
\hline Camera & Photron SA5 \\
\hline Camera lens & Zeiss 100mm \\
\hline Frame rate & $50 \mathrm{kfps}$ \\
\hline Exposure & $10 \mu \mathrm{s}$ \\
\hline Light source & Continous xeon-arc \\
\hline Pixel-mm & $11.4 \mathrm{px} / \mathrm{mm}$ \\
\hline Cut-off & $4 \mathrm{~mm}$ \\
\hline
\end{tabular}
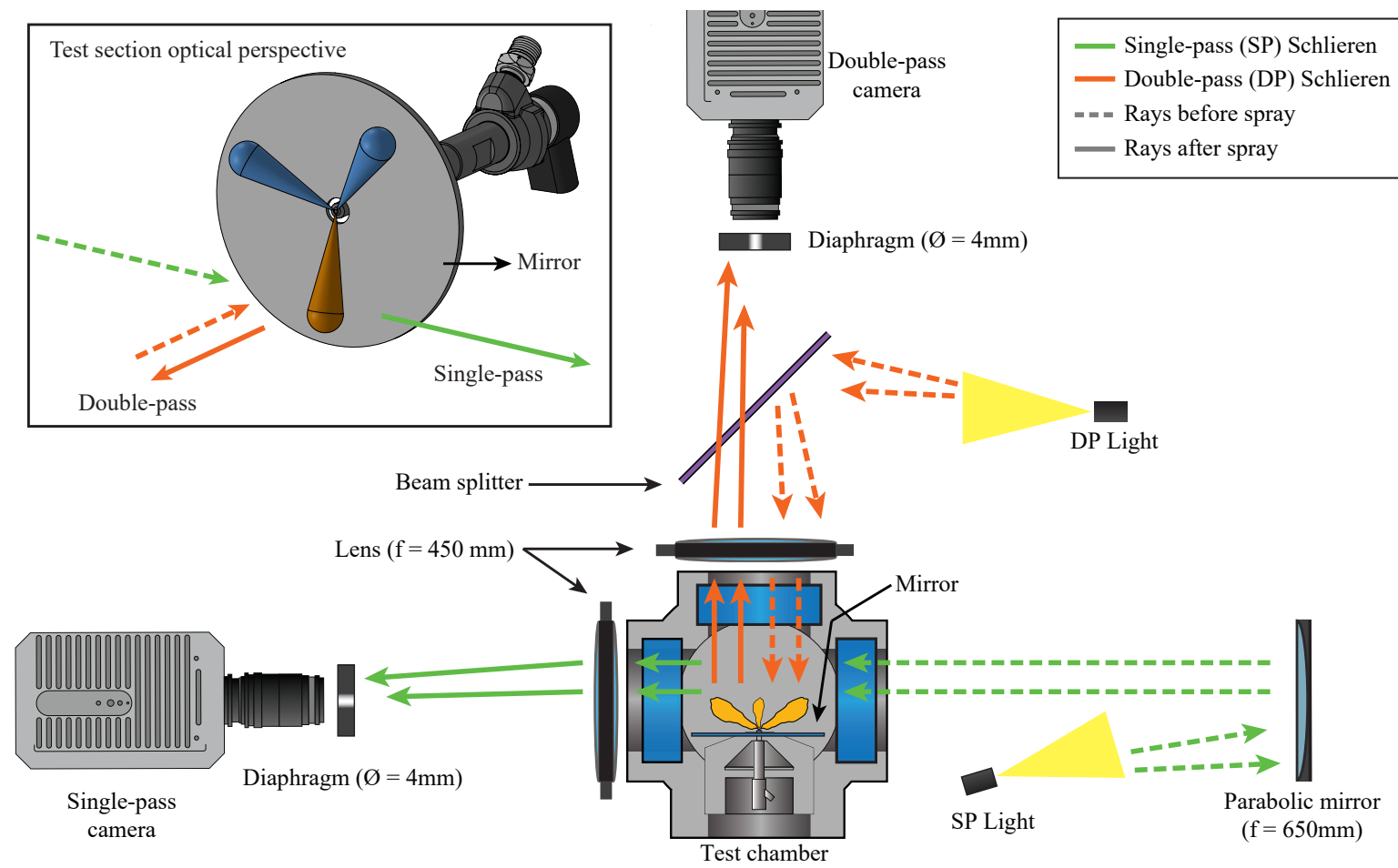

Figure 2. Optical setup used for vapor phase visualization with both single and double-pass Schlieren arrangements. The top right corner presents the optical perspective of the test section, with the spray of interest highlighted in orange. For a better color interpretation of this figure, the reader is referred to the web version of this article. 


\title{
Image processing methodology
}

The procedure used to depict the spray contour is a composition of two extensively used approaches. The core of the segmentation algorithm is based in a fixed threshold intensity-sensitive method $[3,5,10,15]$. But to improve the contour detection capabilities, an image-temporal-derivative approach $[9,16,17]$ was added. Each produces gray-scale images, which are then combined with an adjustable weighted average. This way, the two approaches complement each other. A detailed description of the combined methodology is found in the work of Payri et al. [17]

\section{Definition of the macroscopic spray variables}

With the contour of the spray defined, the next step is to calculate the macroscopic variable for comparison. Regularly, the spray tip penetration is defined as the furthest pixel from the orifice exit, measured in polar coordinates. But, as shown in Figure 2, the spray is observed through different perspectives. The single-pass setup visualizes the real development of the spray, because its axis is parallel to the plane of the camera sensor. Contrarily, the double-pass arrangement sees a projection of the spray evolution, so it is necessary to correct the tip penetration with the inclination angle. Because of the different perspectives, and with the schematics presented in Figure 3 , the following considerations are done:

- The furthest pixel might not be located at the same position for each setup. In consequence, tip penetration (S) could be measured in different geometrical positions of the spray. But the low value of the nozzle inclination angle, and the plume-shaped spray, minimizes the chances of this happening, and its impact on the results.

- The spray tip penetration, measured through the double-pass setup $\left(S_{D P}\right)$, needs to be corrected with the inclination angle $\psi$. But, as mentioned by Payri et al. [18], this angle fluctuates during the injection event.

- The furthest pixel is not contained in the spray axis. Consequently, the correction has to account also the off-axis angle $\delta$.

- Considering the previous points, the variable proposed for comparison is the furthest axial penetration. Since it is parallel to the spray axis, it is always corrected with a constant inclination angle, thus reducing uncertainties due to the spray axis and off-axis angle corrections for the DP spray tip penetration.

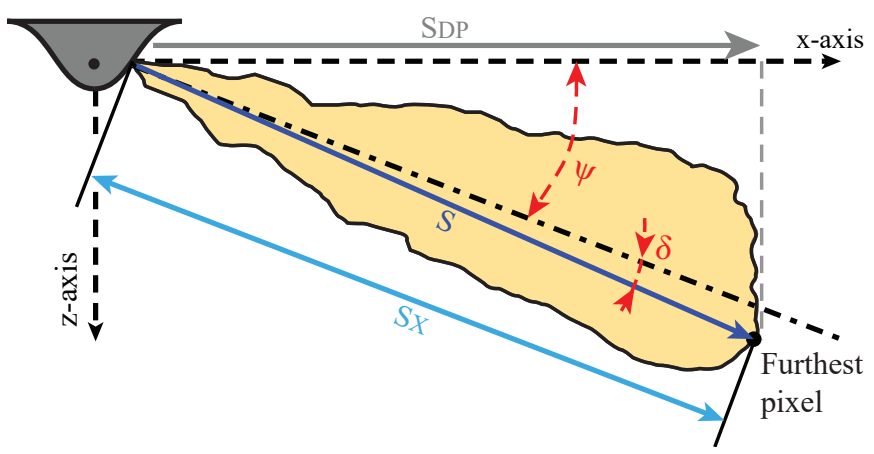

\author{
-.- $\quad$ Spray axis \\ -S Furthest pixel penetration \\ -Sx Furthest pixel axial penetration \\ -SDP Double pass projected penetration \\ $\psi \quad$ Inclination angle \\ $\delta \quad$ Off-axis angle
}

Figure 3. Definition of the spray tip penetration used for comparing single and double-pass Schlieren setups

\section{Data averaging and start of injection}

With the procedures explained in the previous section, the raw results, gathered through high-speed imaging, produce a significant amount of data repetition-wise. Therefore, a moving average technique, described in detail by Payri et al. [19], is used to calculate a single time-dependent curve for the spray tip penetration. For a time instant $t_{i}$, the correspondent penetration value $S\left(t_{i}\right)$ is computed with the linear regression $S=k t+b$. The fitting constants $k$ and $b$ are calculated by solving with $t$ and $S$ consisting of all the data contained in the time interval $t_{i} \pm \Delta t$, with $\Delta t=150 \mu \mathrm{s}$. Subsequently, the average value $\bar{S}$ is estimated by evaluating $t_{i}$ in the regression. This process is repeated for each time-step up to the end of injection. The SOI is calculated by extrapolating the penetration curve to zero, that is $t(S=0)$, with a quadratic fit [10,17-19]. Since the high-temperature mirror in the double-pass setup makes it not possible to observe the first few millimeters of penetration, the SOI was calculated only with the SP data, and then copied to the double-pass data.

\section{Test conditions}

Experimental conditions are listed in Table 2. Values include a parametric sweep in injection pressure, chamber temperature, and density, following ECN recommendations. Experiments were carried out in a non-reactive environment with commercially available diesel fuel. 
Table 2. Test conditions

\begin{tabular}{lll}
\hline Parameter & Value & Units \\
\hline Fuel & Diesel & - \\
\hline Energizing time & 2.5 & $\mathrm{~ms}$ \\
\hline Injection pressure $\left(P_{i n j}\right)$ & $50-100-150$ & $\mathrm{MPa}$ \\
\hline Chamber temperature $(T)$ & $800-900$ & $\mathrm{~K}$ \\
\hline Chamber density $(\rho)$ & $7.6-15.2-22.8$ & $\mathrm{~kg} / \mathrm{m}^{3}$ \\
\hline Gas & Nitrogen & - \\
\hline Repetitions & 10 & - \\
\hline
\end{tabular}

\section{Results and discussion}

\section{Background distribution and spray contrast}

The background of images captured with a Schlieren setup displays a unique structure, because it contains the density gradient information of all the optical path. Not only there is a difference in the distance and trajectory traveled by the light rays inside the test section, but as previously commented, the double-pass arrangement has, theoretically, twice the sensitivity [1]. Figure 4.a-d presents the backgrounds before injection for different chamber temperature and densities. Each column represents images from the SP (left) and DP (right) setups. From this perspective, the hot gas from the heater enters the test section from the right side of the frames.

Focusing in Figure 4.a, the mirror mounted for the DP setup created a stream of flow cooler than the surroundings, marked with a dashed red arrow, and observed throughout the experiments in the SP arrangement. In contrast, this was not visible in the double-pass images, because of the difference in length (thickness) of the optical path that this region represents in each perspective.

The influence of chamber density in the background for each optical setup is presented in Figure 4.a-c. For both systems, increasing the density increases the disturbances captured by the Schlieren, as observed by Pickett et al. [20]. On the contrary, comparing Figures 4.a and 4.d, suggests that chamber temperature produces fewer disturbances in the background, at least for the conditions tested. The marked impact of chamber density in the background is because of its linear scaling with gradients of refractive index [1,20]. On the other side, the effect of temperature depends mainly on its distribution along the test section. Additionally, the double-pass setup presents more complex background structures, with more details in local regions of the frame than the SP configuration. More interestingly, in Figure 4.d, the DP system captures with more detail the hot gases entering the test section. The difference between optical setups on background structures, observed in Figures 4.a-d, are a consequence of at least two factors. On one side, the flow distribution of the hot gases is not strictly equal in each optical plane. On the other, and more importantly, the increased sensitivity for the DP system. Moreover, the optical path length $L$ of the Schlieren object, in this case the test section, is also bigger for the double-pass setup. That is $\approx 250 \mathrm{~mm}$ compared to $\approx 200 \mathrm{~mm}$ for the SP, which is the distance traveled by the light rays inside the test section for each configuration.

The last comparison, Figure 4.e, presents a random time step in the latter part of the injection event for fixed boundary conditions. The clear difference of contrast between spray and background grants more evidence of the higher sensitivity of the double-pass configuration, which provides images with sharper contours and a darker core to the image processing methodology. In consequence, the raw data gathered through the DP setup is less sensitive to differences in thresholding, and the tip region is very well differentiate from the background throughout the injection event, even when local equivalence ratios are low and the density in the region tends to values similar to the surroundings.

\section{Spray tip penetration}

Following the definition from Figure 3, spray tip penetration results, for both Schlieren setups, at different injection pressures are presented in the left part of Figure 5, for fixed conditions of chamber temperature and density. The dashed and continuous line represent both single and double-pass arrangements, respectively. The same line style format is maintained throughout the section. From that figure, both Schlieren setups captured the effect of injection pressure in the tip penetration $[2,17,18]$ equally, as it increases in about the same ratio with increasing rail pressure. More interestingly, the DP configuration depicts higher spray penetration values, being more notably in the later part of the injection event. No effect of the rail pressure in the difference is observed.

The right side of Figure 5, depicts the effect of chamber density on the spray tip penetration. As observed in the previous case, both Schlieren arrangements capture the effect of density in the spray development in agreement with the literature $[10,17]$. However, even though the double-pass setup still depicts higher penetration values, the difference between the optical arrangements is reduced when decreasing the density, and reports almost identical values at the lowest level in the conditions tested.

The effect of the density on the difference between optical setups can be understood by analyzing the sensitivity of each configuration. The deflection caused by the diluted regions of the fuel injected in the test section, given 


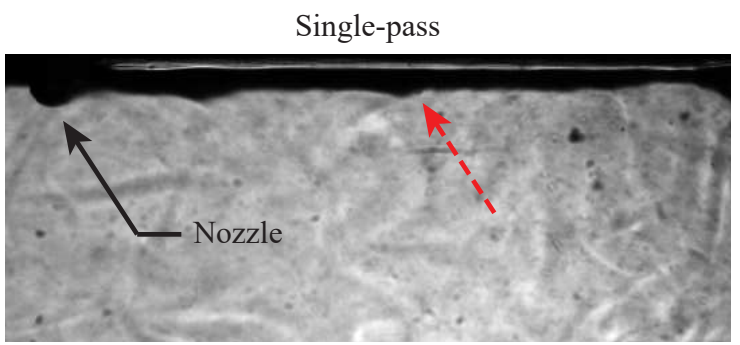

Double-pass

(a) Chamber temperature $=800 \mathrm{~K}$, density $=7.6 \mathrm{~kg} / \mathrm{m}^{3}$
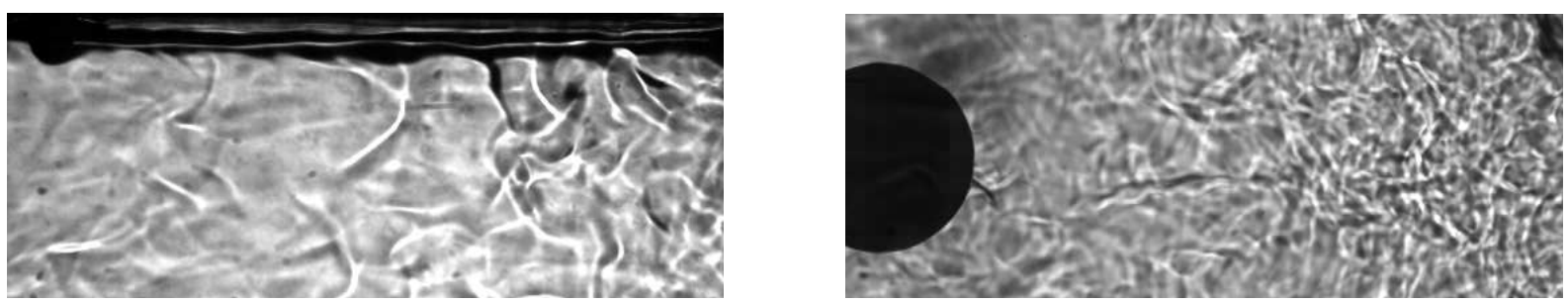

(b) Chamber temperature $=800 \mathrm{~K}$, density $=15.2 \mathrm{~kg} / \mathrm{m}^{3}$.
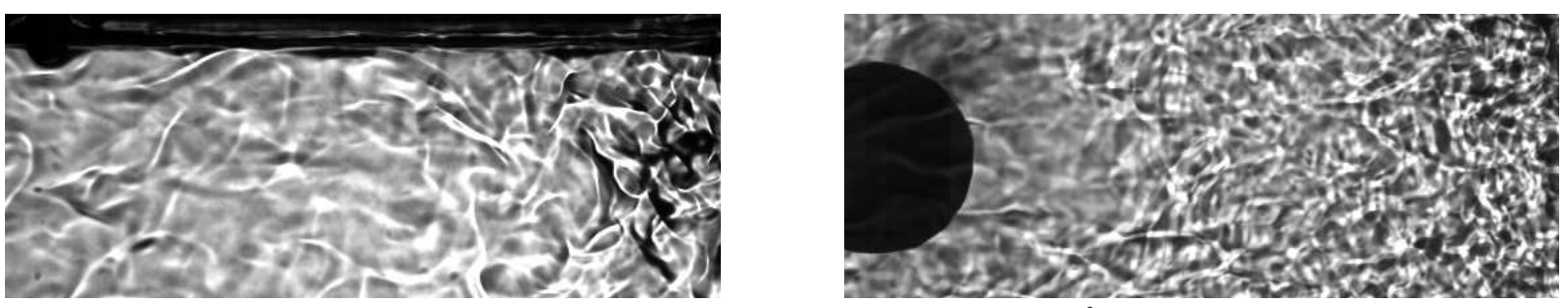

(c) Chamber temperature $=800 \mathrm{~K}$, density $=22.8 \mathrm{~kg} / \mathrm{m}^{3}$.
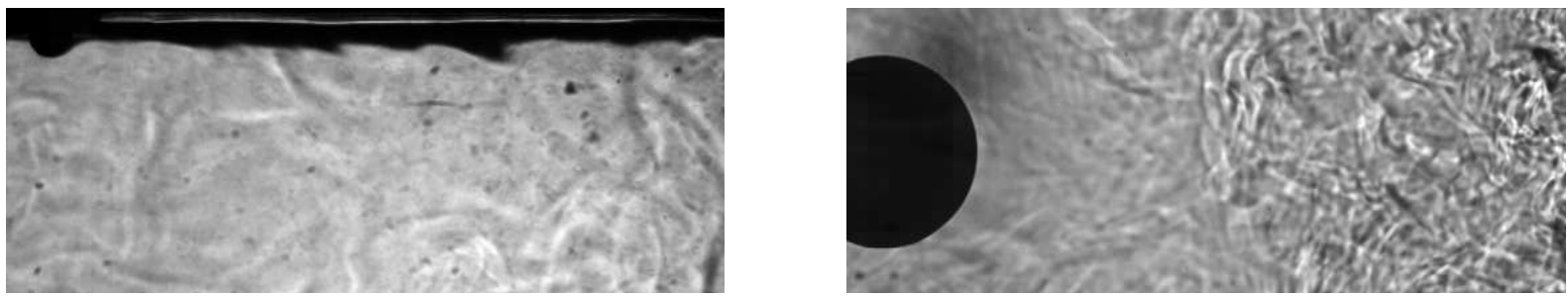

(d) Chamber temperature $=900 \mathrm{~K}$, density $=7.6 \mathrm{~kg} / \mathrm{m}^{3}$.
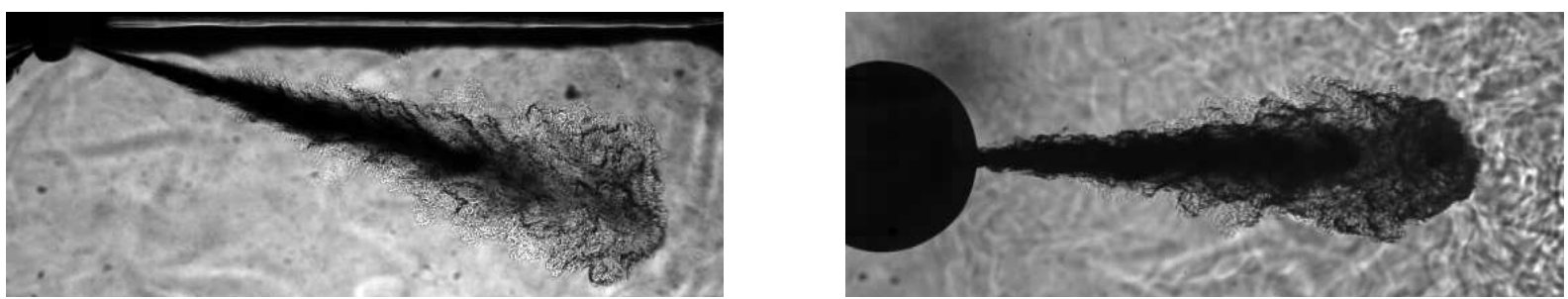

(e) Difference in spray contrast, chamber temperature $=800 \mathrm{~K}$, density $=7.6 \mathrm{~kg} / \mathrm{m}^{3}$.

Figure 4. Differences in background structures and spray contrast for the single (left) and double-pass (right) Schlieren

in Eqn. 2, depends on the refractive index of the surroundings, thus its density (Eqn. 1). Therefore, with the test section at $22.8 \mathrm{~kg} / \mathrm{m}^{3}$, the resultant deflection of a light ray passing through the spray is less than at $7.6 \mathrm{~kg} / \mathrm{m}^{3}$. Furthermore, the density gradients between these diluted regions of the sprays (contours or tip region at a latter part of the injection event) and the surroundings are lower, also producing smaller deflection angle in these areas. Consequently, due to the increased sensitivity of the DP configuration [1], the setup captures "more" spray in the same time step than the SP setup, even though they are visualizing the same injection event.

\section{Further analysis of the two Schlieren configurations}

From the results analyzed in the previous sections, the overall trend found was that the double-pass Schlieren system depicts higher spray tip penetration for all conditions tested. Figure 4.e showed a big difference in contrast and contour sharpness between optical configurations, suggesting that the higher sensitivity in the DP caused this difference in the macroscopic description of the spray. But still, other factors need to be accounted for.

Regarding the optical setup and experimental measurements, both diaphragms were set to $4 \mathrm{~mm}$, resulting in an equal sensitivity at the cut-off plane. Background illumination intensity was calibrated to achieve the same levels before starting the experiments campaign. Additionally, reflections, or double-imaging, were filtered in the cut-off 

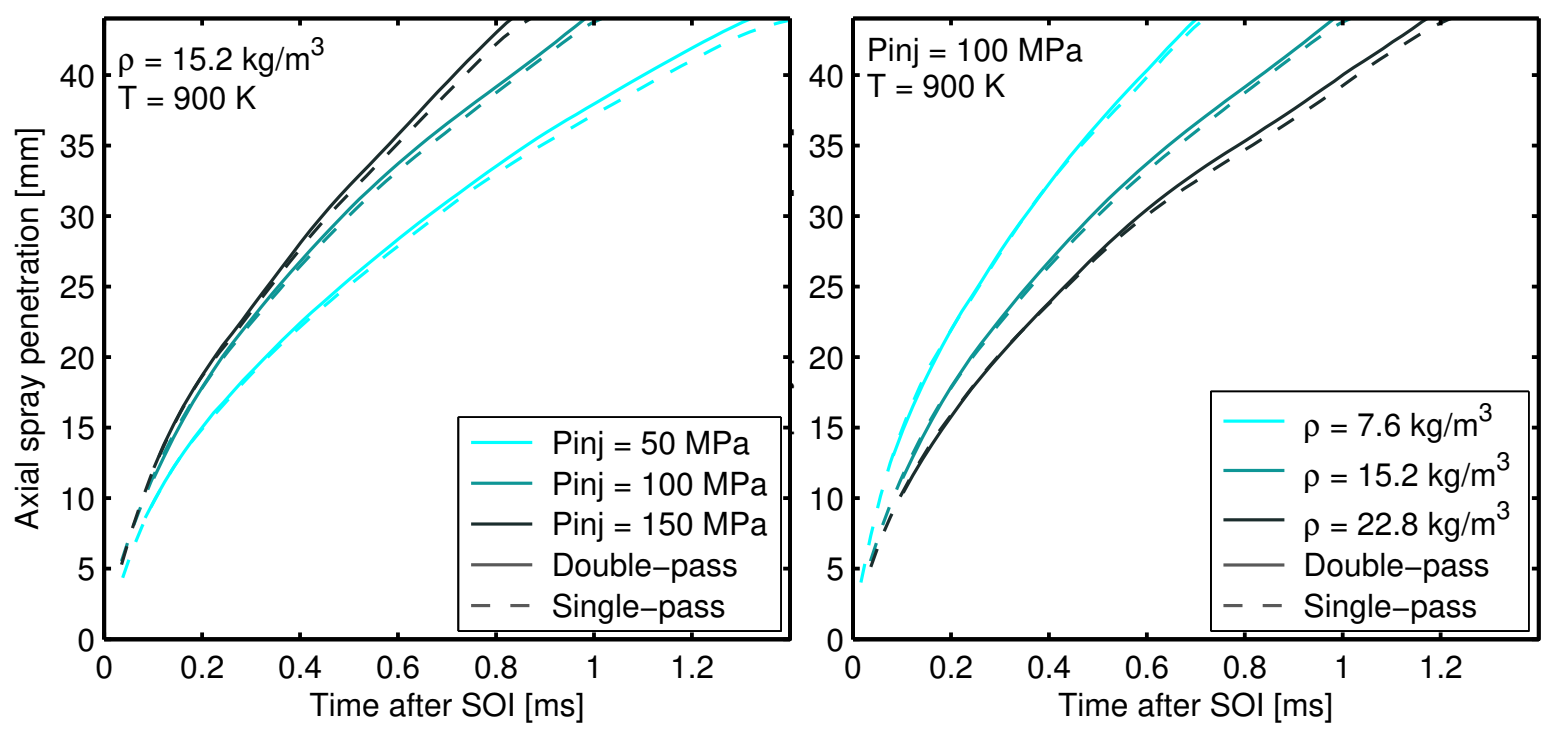

Figure 5. Spray tip penetration for both Schlieren setups at different injection pressures (left) and chamber densities (right).

plane, due to a similar phenomenon as reported by Pastor et al. [11]. Optical accesses typically present small misalignments, caused by differences in the stress of the sealing gaskets, facility assembly, among others, which turn difficult to control. But, curiously, such loss of parallelism becomes an advantage, because these reflections, caused by the optical elements, can be filtered out in the cut-off plane, preventing them from reaching the camera. Regarding the data processing, the same configuration was set for the image processing methodology and moving average technique. But, as stated before, the images provided by the DP system presented more contrast between the spray and the background, that results in easier contour segmentation and less dependence in the thresholding value. In addition, with the spray tip penetration definition used (presented in Figure 3 and previously discussed), the influence of the variable observed for each optical perspective on the final results was diminished.

The differences between setups observed in Figures 4.e and 5, provides some evidence that, because the light rays are passing twice through the test section, boosting its deflection angle, the DP configuration depicts a longer spray. Particularly in regions where the difference between the local density of the spray and its surroundings is low, and the deflection angle is small. Furthermore, the added contrast and sharper contours resulted in images easier to segment with the processing methodology.

\section{Conclusions}

In this research, vapor phase visualization of a diesel spray was performed with both single and double-pass Schlieren imaging setups. Both optical configurations were set up identically in terms of background illumination intensity, camera speed, exposure, pixel to millimeter ratio and diaphragm diameter at the cut-off plane. A three orifice nozzle from the ECN dataset, known as Spray B, was used. Experiments were carried out in a high pressure and temperature vessel, with commercially available diesel, and in a non-reactive environment. A parametric sweep of injection pressure, chamber temperature, and density was performed. The differences between the optical configurations in the background structures and spray development were analyzed for the conditions tested.

The high-temperature mirror used for the double-pass configuration created a stream of flow, cooler than the surroundings, which affected measurements of the single-pass setup near the nozzle (less than 3-4 mm of penetration). On the contrary, this was not visualized by the DP system, due to the differences in the optical path length (thickness) for each perspective. However, because the double-pass mirror did not fully enclose the nozzle, the first 6 $\mathrm{mm}$ of tip penetration were not captured.

The disturbances of background structures, in the frames before injection, notably increase with increasing chamber density, with less influence for increasing chamber temperature, for the range of conditions tested. The marked impact of density on the background distribution was expected because of its linear scaling with gradients of refractive index. The effect of temperature depends on its distribution along the vessel, as the biggest disturbances appear near the inlet of the hot gases to the test section. The double-pass configuration captures more disruptions (gradients) in the background, compared to the single-pass setup.

For all conditions tested, the spray tip penetration results follow the trend expected from other works in the literature. It increases with increasing injection pressure and decreasing chamber density.

Comparing the optical setups, the double-pass system depicts higher spray tip penetration in the latter part of the injection event. No effect from the injection pressure in the difference was observed. On the contrary, decreasing the density reduces the discrepancy between optical configurations, as they present almost identical values at the lowest density tested.

The double-pass setup produces images with a sharper spray contour and more contrast, that are easier to segment from the background. Additionally, due to its higher sensitivity (as bigger deflection angles), the DP system captures 
vaporized fuel in regions where the difference between the local density of the spray and its surroundings is low, and thus the deflection angle is small. Decreasing the chamber density reduces the influence of the sensitivity because the dissimilarities between densities of these local regions are increased.

\section{Acknowledgments}

This research has been partially funded by FEDER and Spanish Ministerio de Economía y Competitividad through project TRA2015-67679-c2-1-R. Additionally, Alberto Viera is supported through the FPI contract 2016-S2-1361 of "Programa de Apoyo para la Investigación y Desarrollo (PAID)" of Universitat Poltècnica de València.

\section{References}

[1] G. S. Settles, Schlieren and Shadowgraph Techniques, Springer Berlin Heidelberg, Berlin, Heidelberg, 2001.

[2] J. D. Naber, D. L. Siebers, Effects of Gas Density and Vaporization on Penetration and Dispersion of Diesel Sprays, SAE Paper 960034 (1996).

[3] R. Payri, F. J. Salvador, J. Gimeno, A. Viera, in: H. Tschöke, R. Marohn (Eds.), 10. Tagung Diesel- und Benzindirekteinspritzung 2016, 1, Springer Vieweg, 2017, pp. 133-152.

[4] R. Payri, J. P. Viera, Y. Pei, S. Som, Experimental and numerical study of lift-off length and ignition delay of a two-component diesel surrogate, Fuel 158 (2015) 957-967.

[5] R. Payri, F. J. Salvador, J. Manin, A. Viera, Diesel ignition delay and lift-off length through different methodologies using a multi-hole injector, Applied Energy 162 (2016) 541-550.

[6] R. Payri, J. P. Viera, V. Gopalakrishnan, P. Szymowitz, The effect of nozzle geometry over ignition delay and flame lift-off of reacting direct-injection sprays for three different fuels, Fuel (2017).

[7] P. M. Lillo, L. M. Pickett, H. Persson, O. Andersson, S. Kook, Diesel Spray Ignition Detection and Spatial/Temporal Correction, SAE Paper 2012-01-1239 (2012) 1-21.

[8] J. V. Pastor, R. Payri, J. M. Garcia-Oliver, F. J. Briceño, Schlieren Methodology for the Analysis of Transient Diesel Flame Evolution, SAE International Journal of Engines 6 (2013) 1661-1676.

[9] J. V. Pastor, R. Payri, J. M. Garcia-Oliver, J.-g. Nerva, Schlieren Measurements of the ECN-Spray A Penetration under Inert and Reacting Conditions, SAE Technical Paper 2012-01-0456 (2012).

[10] R. Payri, J. Gimeno, J. P. Viera, A. H. Plazas, Needle lift profile influence on the vapor phase penetration for a prototype diesel direct acting piezoelectric injector, Fuel 113 (2013) 257-265.

[11] J. V. Pastor, J. M. Garcia-Oliver, J. M. Pastor, L. D. Zapata, Evaporating Diesel Spray Visualization using a Double-pass Shadowgraphy / Schlieren Imaging, SAE technical Paper 2007-24-0026 (2007).

[12] R. Payri, F. J. Salvador, A. Garcia, A. Gil, Combination of visualization techniques for the analysis of evaporating diesel sprays, Energy \& Fuels 26 (2012) 5481-5490.

[13] J. V. Pastor, R. Payri, J. M. Garcia-Oliver, F. J. Briceño, Analysis of Transient Liquid and Vapor Phase Penetration for Diesel Sprays Under Variable Injection Conditions, Atomization and Sprays 21 (2011) 503-520.

[14] J. H. Gladstone, T. P. Dale, Researches on the Refraction, Dispersion, and Sensitiveness of Liquids, Philosophical Transactions of the Royal Society of London 153 (1863) 317-343.

[15] R. Payri, F. J. Salvador, P. Martí-Aldaraví, D. Vaquerizo, ECN Spray G external spray visualization and spray collapse description through penetration and morphology analysis, Applied Thermal Engineering 112 (2017) 304-316.

[16] M. Bardi, R. Payri, L.-M. Malbec, G. Bruneaux, L. M. Pickett, J. Manin, T. Bazyn, C. L. Genzale, Engine Combustion Network: Comparison of Spray Development, Vaporization, and Combustion in Different Combustion Vessels, Atomization and Sprays 22 (2012) 807-842.

[17] R. Payri, J. P. Viera, V. Gopalakrishnan, P. G. Szymkowicz, The effect of nozzle geometry over the evaporative spray formation for three different fuels, Fuel 188 (2017) 645-660.

[18] R. Payri, G. Bracho, P. Marti-Aldaravi, A. Viera, Near field visualization of diesel spray for different nozzle inclination angles in non-vaporizing conditions., Atomization and Sprays 27 (2017) 1-17.

[19] R. Payri, J. Gimeno, M. Bardi, A. H. Plazas, Study liquid length penetration results obtained with a direct acting piezo electric injector, Applied Energy 106 (2013) 152-162.

[20] L. M. Pickett, J. Manin, C. L. Genzale, D. L. Siebers, M. P. B. Musculus, C. A. Idicheria, Relationship Between Diesel Fuel Spray Vapor Penetration/Dispersion and Local Fuel Mixture Fraction, SAE International Journal of Engines 4 (2011) 764-799. 


\title{
X-ray diagnostics of dodecane jet in spray A conditions using the new one shot engine (NOSE)
}

\author{
Ibrahim Chiboub", Hugo Ajrouche*2, Ob Nilaphai ${ }^{2}$, Sébastien Dozias ${ }^{1}$, Bruno Moreau², \\ Camille Hespel $^{2}$, Fabrice Foucher ${ }^{2}$, Christine Mounaïm-Rousselle², Jean-Michel Pouvesle ${ }^{1}$, \\ Eric Robert ${ }^{1}$ \\ 1'GREMI, CNRS-Université d'Orléans, Orléans, France \\ ${ }^{2}$ PRISME, Université d'Orléans, Orléans, France \\ *Corresponding author: hassan.ajrouche@univ-orleans.fr
}

\begin{abstract}
Quantifying liquid mass distribution data in the dense near nozzle area to develop and optimize diesel spray by optical diagnostic is challenging. Optical methods, while providing valuable information, have intrinsic limitations due to the strong scattering of visible light at gas-liquid boundaries. Because of the high density of the droplets near the nozzle, most optical methods are ineffective in this area and prevent the acquisition of reliable quantitative data. $\mathrm{X}$-ray diagnostics offer a solution to this issue, since the main interaction between the fuel and the X-rays is absorption, rather than scattering, thus $X$-ray technique offers an appealing alternative to optical techniques for studying fuel sprays. Over the last decade, $x$-ray radiography experiments have demonstrated the ability to perform quantitative measurements in complex sprays. In the present work, an X-ray technique based on X-ray absorption has been conducted to perform measurements in dodecane fuel spray injected from a single-hole nozzle at high injection pressure and high temperature. The working fluid has been doped with DPX 9 containing a Cerium additive, which acts as a contrast agent. The first step of this work was to address the effect of this dopant, which increases the sensitivity of X-ray diagnostics due its strong photon absorption, on the behavior and the physical characteristics of $n$-dodecane spray. Comparisons of the diffused back illumination images acquired from $n$ dodecane spray with and without DPX 9 under similar operating conditions show several significant differences. The current data show clearly that the liquid penetration length is different when DPX 9 is mixed with dodecane. To address this problem, the dodecane was doped with a several quantities of DPX containing $25 \% \pm 0.5$ of Cerium. Experiments show that $1.25 \%$ of Ce doesn't affect the behaviour of spray. Radiography and density measurements at ambient pressure and 60 bars are presented. Spray cone angle around $5^{\circ}$ is obtained. The obtained data shows that the result is a compromise between the concentration of dopant for which the physical characteristics of the spray do not change and the visualization of the jet by X-ray for this concentration.
\end{abstract}

\section{Keywords}

Diesel Spray, Engine, X-ray radiography, high pressure high temperature chamber, dodecane, Cerium, spray diagnostics

\section{Introduction}

New One Shot Engine (NOSE) has been designed to simulate the thermodynamic conditions at High PressureHigh Temperature (HPHT) like an actual common-rail diesel engine to study the diesel spray and combustion. The first objective was to share the experimental results required by Engine Combustion Network (https://ecn.sandia.gov). In a first step, the penetration lengths for the vapor and liquid spray at the non-reactive standard Spray-A condition ( $900 \mathrm{~K}, 60 \mathrm{bar}$, and $22.8 \mathrm{~kg} / \mathrm{m}^{3}$ with pure nitrogen) were achieved from focused shadowgraphy and diffused-back illumination (DBI) [1]. A detailed understanding of the mixing between fuel and air can lead to more efficient combustion. This mixing process is controlled by injection and spray propagations. For this reason, several work has been done to further characterizing the impact of different injection parameters on spray development [2-3] and to create correlations between near-nozzle flow behavior and diesel engine performance which increase the ability of designing more efficient diesel engines. Detailed experimental data not only lead to further understanding of the spray behavior from the injector, but also provide valuable knowledge to enhance computational modelling capabilities.

Over the past few years, different optical diagnostics have been widely developed to characterize and optimize diesel spray [4] (such as Laser induced fluorescence [5], Spontaneous Raman scattering [6-7], Mie scattering [8], PIV [9], DBI, etc...). However, due to the visible light scattering, which is strongly refracted from droplets in the dense near nozzle area, the analysis of the spray dense core by these techniques has been limited. Since 2000, the absorption of X-ray, generated by Argonne's Advanced Photon Source has been developed to understand the 
near-nozzle spray better [9-12]. The X-ray radiography technique is perhaps one of few methods able to measure the liquid mass distributions in otherwise complex sprays [7-9] permitting analyses that cannot be performed using optical spray diagnostics. This greatly simplifies the analysis as the attenuation signal can be related to the liquid mass density in the path of the X-rays with minimal sensitivity to the droplet size. Recently, Xray micro-source has been used for the estimation of the temporal-averaged vapour volume fraction within high-speed cavitating flow orifices [17].

In general, three common anode elements iron, copper and tungsten, can be used to produce X-ray photons for spray diagnostics. The higher $\mathrm{X}$-ray fluxes are obtained with tungsten anodes but the X-ray spectrum is composed of several characteristic $L$ lines which is not a very favorable situation for spray density calculation, ideally achievable with a monochromatic radiation. Iron electrodes exhibit the lower energy photons in the $\mathrm{K}$ lines around $6.4 \mathrm{keV}$ but, in this case, the X-ray flux is much lower and the sputtering of the anode during electron bombardment is to high impeding to operate this material in repetitive experiments over few hundreds of shots. View that the $\mathrm{X}$ ray propagation path from the $\mathrm{X}$-ray source to the CCD camera, the $\mathrm{X}$-ray source used in this work is a table top micro focus X-ray tube equipped with a copper anode [14] to get the best compromise between the X-ray flux and the contrast between the jet and the chamber absorption with high dynamic. The purpose of this paper is to present the benefits and limitations of using the X-ray source and to suggest some recommendations for improving performance.

The technique was applied in the case of Cerium (Ce) doped dodecane sprays through micrometric nozzles (hole diameter $90 \mu \mathrm{m}$ ) in NOSE at HPHT. Cerium is used for its strong impact on the X-ray diagnostics due to the strong photon absorption of $\mathrm{Ce}$ at relatively low X-ray energy and this additive is used in engine technology for the regeneration of diesel filters issues. This additive has been used in synchrotron based diagnostics which have been performed with energy beams around $8 \mathrm{keV}$ [15-17]. The experiments were conducted for different pressures and different concentrations of $\mathrm{Ce}$ in order to visualize the jet of dodecane with the polychromatic X-ray micro source with $\mathrm{Cu}$ anode. Experimental radiography of dodecane spray expansion, cone angle and density measurements will be performed by several shots X-ray measurement and also compared with the average measurement over a large number of shots which enhance the quality of the signal and allow a more detailed analysis. The spray cone angle is determined from the width of the spray at several axial sections. Absorption and density measurement obtained from $5 \mathrm{~ms}$ injection of $20 \%$ Ce doped n-dodecane spray are presented. Finally, the vertical binning being key point of $\mathrm{X}$-ray measurement to achieve a good value of signal to noise ratio is analysed.

\section{Material and methods}

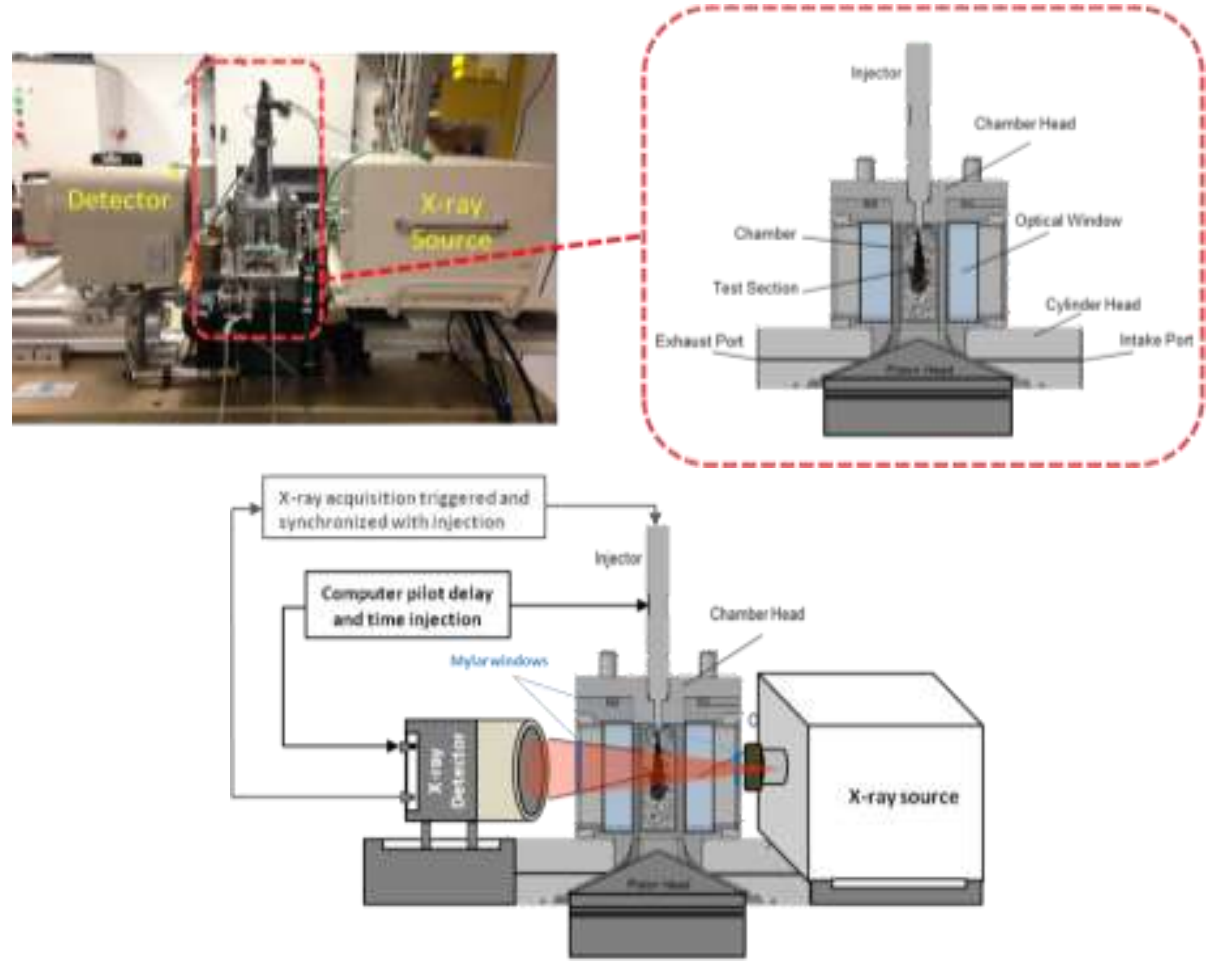

Figure 1: Photo shows the X-ray micro-source and detector and that on the right details the black spray chamber. At the bottom, a schematic of the experimental setup is shown thus the radiography device associated and synchronized with the injection system of spray chamber. 
The layout of the experimental set-up is shown in Figure 1. It composed from four main subassemblies: a high pressure and high temperature chamber, micro X-ray source, an X-ray CCD camera and a pilot for the triggering and synchronization of the X-ray source shots and the needle injector control. The experimental set-up about the new combustion chamber based on concept of rapid cycling machine called "New One Shot Engine (NOSE) developed to study the diesel spray has been described in detail elsewhere [1] and only a short summary will be given here. NOSE is based on the use of one-cylinder low-speed diesel engine of 18 horse power HP at $750 \mathrm{rpm}$. This cylinder has $155 \mathrm{~mm}$ bore diameter and a $177.8 \mathrm{~mm}$ stroke. The original cylinder head was replaced by a dedicated chamber, designed by extending the combustion chamber for supporting optical technique measurements.

For X-ray diagnostic, the transmission through the window depends on its thickness and the material it is made of. The X-ray beam passes through a pressurized spray chamber with $\mathrm{X}$-ray rectangular windows $(25 \mathrm{~mm}$ wide, $80 \mathrm{~mm}$ high) where rectangular slits equipped with $125 \mu \mathrm{m}$ thick Mylar films, with high X-ray transmission, have been used as entrance and exit windows along the $\mathrm{X}$-ray propagation path from the $\mathrm{X}$-ray source to the $\mathrm{X}$-ray detector. The layer of Mylar will slightly decrease (a few percent) the transmission of the X-rays. Other advantages of Mylar is its resistance to high pressure and it can also support more than hundreds of cycles. After the beam passes through the spray chamber, the X-ray radiographies are acquired using X-ray CCD camera. This camera offers approximately high quantum efficiency with 12 bits of dynamic range. The detector is optimized for low energy $X-$ rays $(20 \mathrm{KeV})$ using high contrast $X$-ray image intensifier (4 inch). X-rays are converted in visible light through an efficient scintillator and this emission is then detected with $1920 \times 1440$ pixels CMOS image sensor and $0.5 \mathrm{~mm}$ of Beryllium as input window. The camera is placed $25 \mathrm{~cm}$ from the high pressure chamber giving a magnification equal to 1. The X-ray diagnostic is triggered and synchronized with zero delay between time of acquisition and time of injection. The X-ray diagnostics of liquid jet depends on strong absorption of the X-ray beam by the liquid fuel. This is essential at high ambient density levels, since the ambient gas in the chamber can absorb a significant amount of the x-ray intensity. To enhance the x-ray absorption coefficient of dodecane, a cerium fuel additive has been added. The properties of the fuel are listed in Table 1.

Table 1. Fuel Physical properties

\begin{tabular}{c|cc}
\hline \hline Properties & n-dodecane & DPX 9 \\
\hline \hline Density $\left(\mathrm{kg} \cdot \mathrm{m}^{-3}\right)$ & 753 & 1107 \\
Dynamic viscosity $(\mathrm{mPa}$.s $)$ & 0.97954 & 4.1786 \\
kinematic viscosity $\left(\mathrm{mm}^{2} . \mathrm{s}^{-1}\right)$ & 1.3335 & 3.7756
\end{tabular}

The average energy measurement of the micro X-ray source used in this work was determined. This quantity is relevant for the spray density calculation. For this purpose, X-ray transmission of increasing number of Kapton® $\left(\mathrm{C}_{12} \mathrm{H}_{22} \mathrm{O}_{2} \mathrm{~N}_{2}\right)$ foils each having a thickness of $50 \mu \mathrm{m}$ with a $1.42 \mathrm{g.cm}{ }^{-3}$ density are performed for calibration, knowing that the $\mathrm{x}$-ray absorption of this material is well known. The measurement of these test objects transmission was realized in the same configuration of that used later for spray diagnostics with rigorous Signal-to-Noise ratio study.

Table 2. Description of ECN spray $A$ boundary conditions and X-ray radiography test conditions

\begin{tabular}{c|c}
\hline \hline Parameter & Dodecane \\
\hline Injector body type & Bosch CR 2.16 \\
Nozzle type & Single hole, axially oriented \\
Nozzle outlet diameter $(\mu \mathrm{m})$ & 90 \\
Fill gas & Nitrogen $\left(\mathrm{N}_{2}\right)$ \\
Ambient gas temperature $(\mathrm{K})$ & 900 \\
Ambient gas pressure $(\mathrm{MPa})$ & 6.0 \\
Ambient gas density $\left(\mathrm{kg} \cdot \mathrm{m}^{-3}\right)$ & 22.8 \\
Fuel & n-dodecane \\
Fuel injection pressure $(\mathrm{MPa})$ & 150 \\
Fuel temperature at nozzle $(\mathrm{K})$ & 363 \\
Injection duration $(\mathrm{ms})$ & 5
\end{tabular}

The radiography measurements fundamentally measure the path-length-integrated amount of liquid in the $x$-ray beam. At each measurement location, the projected mass per unit area along the beam pass $\Delta M$ is related to the measured transmission by the Lambert-Beer law:

$$
\Delta M=\int \rho d z=\frac{-1}{\mu} \log \left(\frac{I_{(t)}}{I_{0}}\right)
$$


In this equation, $I_{(t)}$ and $l_{0}$ are the transmitted and incident beam intensities (i.e. during and before the injection), $\mu$ is the absorption coefficient (per mass/area) and $\rho$ the local fuel density in the spray, which will generally be lower than the density of undisturbed liquid.

\section{Results and discussion}

\section{Example of typical Data}

Data were obtained for two levels of pressure (1bar and 60 bars). The impact of the added amount of doping agent (Eolys Rhodia DPX 9) on the physical characteristics of the spray is illustrated in Figure 2. Comparisons between the diffused back illumination images acquired from n-dodecane spray without DPX 9 containing Ce (Figure 2 a) and these obtained from n-dodecane spray with $20 \%$ of Ce (Figure 2 b) showing clearly that liquid penetration length is higher when DPX is present. The current data also show that for $20 \%$ of DPX mixed with n-dodecane, the physical characteristics of $n$-dodecane spray and its behavior show several significant differences from the $n$ dodecane spray without DPX (i.e. viscosity, density). Moreover, the data presents different rate of evaporation and it is clearly observed that there is no possible determination of liquid length of the spray obtained with $20 \%$ of DPX, as seen by the shadow and the larger dark area in Figure $2 \mathrm{~b}$ showing the unevaporated part of the fuel. Thus measurements with lower concentrations of dopant under similar boundary and injection conditions were performed in order to determine the lowest concentration of dopant making it possible to keep similar physical properties of the spray while enabling visualization of the jet by X-rays. As illustrated in Figure 2 when the percentage of Ce decreases, the DPX 9 starts to evaporate efficiently. For $2.5 \%$ of $\mathrm{Ce}$, appearance of two regions where we can observe that the darker region decreases to $10.5 \mathrm{~mm}$ and for $0.25 \%$, an important evaporation of DPX is obtained. Liquid length around $10.5 \mathrm{~mm}$ is achieved from the Figure 2 (f).
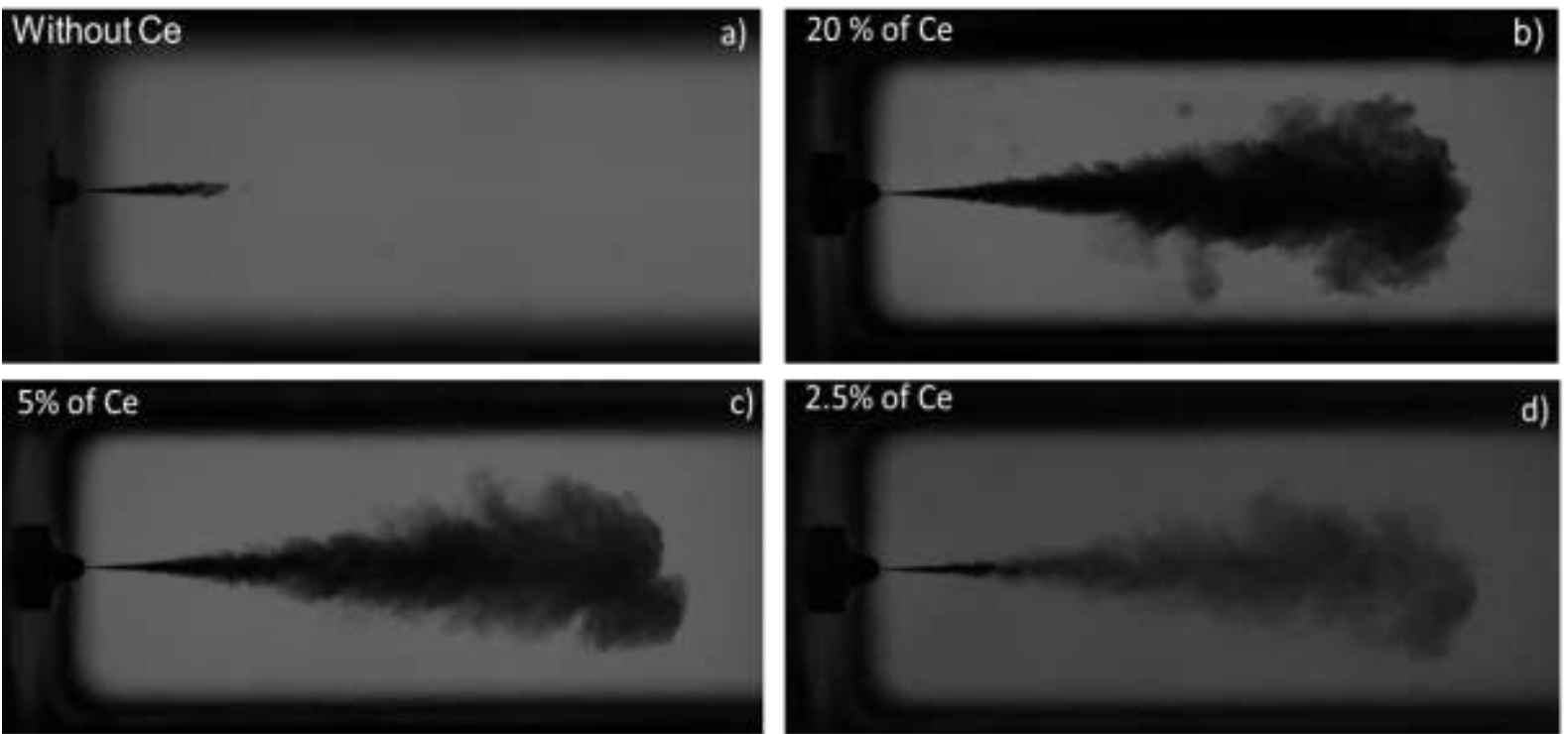

\section{$1.25 \%$ of $\mathrm{Ce}$}

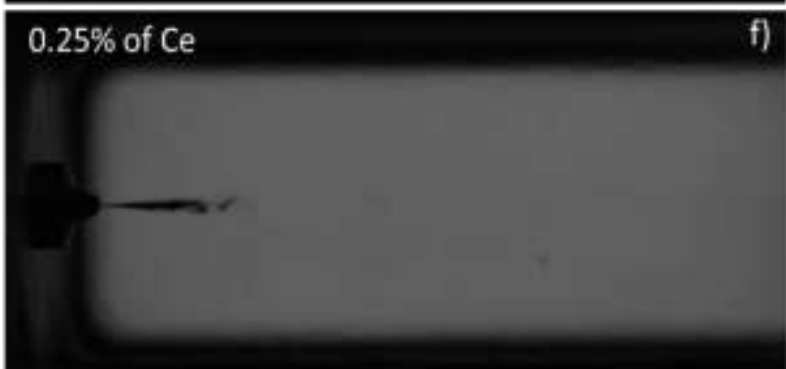

Figure 2: Examples of Diffused Back Illumination instantaneous image of the spray at 60 bar $900 \mathrm{~K}$ (a) dodecane without DPX (b) dodecane doped with $20 \%$ of $\mathrm{Ce}$ (c) dodecane doped with $5 \%$ of $\mathrm{Ce}$ (d) dodecane doped with $2.5 \%$ of $\mathrm{Ce}$ (e) dodecane doped with $1.25 \%$ of $\mathrm{Ce}(\mathrm{f})$ dodecane doped with $0.25 \%$ of $\mathrm{Ce}$. 


\section{Spatial resolution}

The resolution of the full X-ray radiography setup was first calculated from the analysis of the grey level profiles of $1 \mathrm{~mm}$ thick lead and air line pairs with a number of line pairs per $\mathrm{mm}$ extracted from the radiograph illustrated in Figure $3 \mathrm{a}$ ). The calculation of the modulation transfer function involving the ratio of the peak-to-valley grey level amplitude to the maximum grey level value amplitude, for the different spatial frequencies, associated with the number of line pairs per $\mathrm{mm}$, together with the calculation of the signal to noise ratio.

Figure 3 presents the grey level profiles obtained in the geometrical configuration of this experiment averaging over 100 shots and showing that significant contrasts are obtained for patterns as small as $100 \mu \mathrm{m}$ which is appropriate for this work. The estimated spatial resolution of the $\mathrm{X}$ ray setup is around $120 \mu \mathrm{m}$.
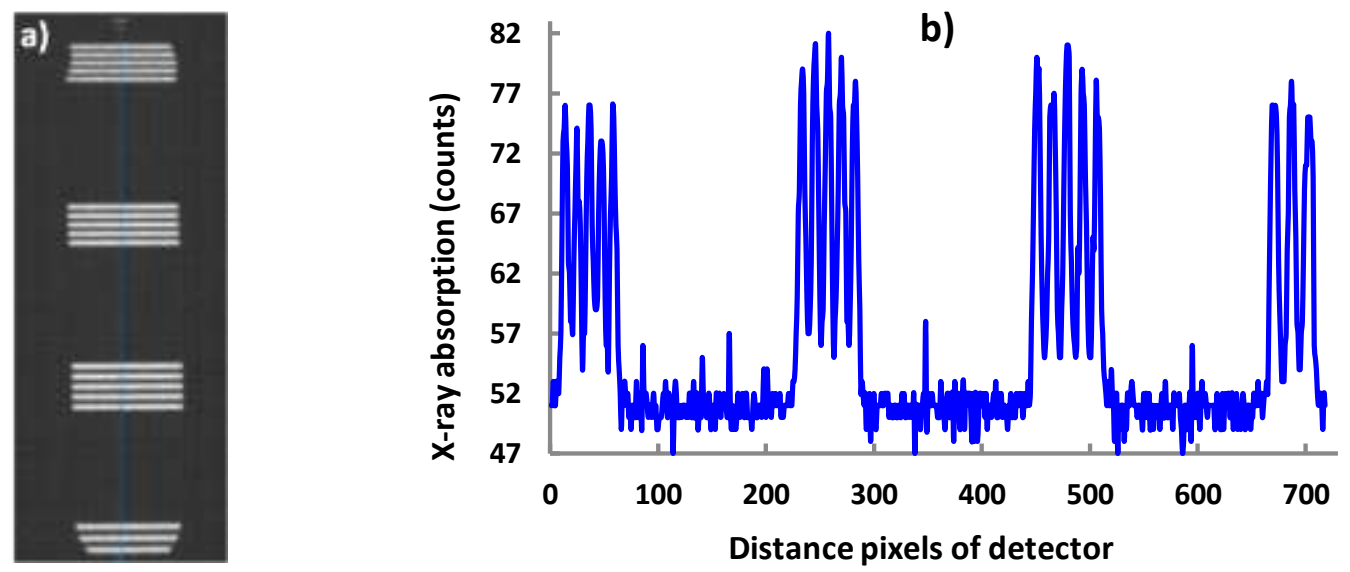

Figure 3: a) Radiograph of calibrated line pair pattern with detector at $25 \mathrm{~cm}$ from injector. b) Grey level profiles extracted from radiograph (a). From left to right, the pattern consists in $4.55,4,3.15$ and 3.55 , line pairs per $\mathrm{mm}$.
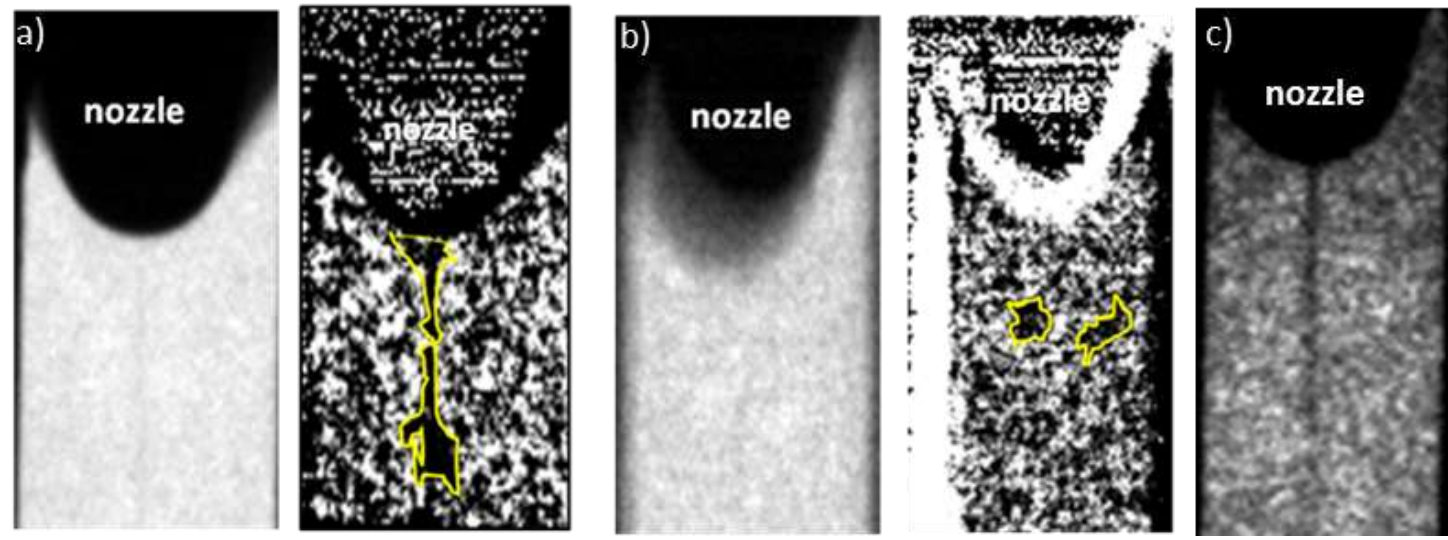

Figure 4: $20 \%$ Ce doped $\mathrm{n}$-dodecane at $900 \mathrm{~K}$ sprays radiographs, (a), (b) radiograph at ambient pressure and 60 bars, respectively, left: transmitted beam and right: absorption, (c) $40 \% \mathrm{Ce}$ doped $\mathrm{n}$-dodecane at $850 \mathrm{~K}$ and 20 bars sprays radiographs

Radiography data was obtained from averaging 10 shots from the X-ray measurements. A slight asymmetry in the flow is evident, even though the nozzle geometry is normally axisymmetric. The measurements were realized over several averaged X-ray shots in the same configuration of that used later for diagnostic of $20 \%$ Ce doped $n$ dodecane spray (894 Kg.m ${ }^{-3}$ at $300 \mathrm{~K}$ and ambient conditions). The X-ray CCD camera was set $25 \mathrm{~cm}$ from the spray chamber integrate the filtering of ambient air, high pressure chamber and Mylar window. In these experimental conditions the mean X-ray energy was determined to be of $8 \mathrm{KeV} \pm 0.3$. The injection duration and X-ray camera duration were both $5 \mathrm{~ms}$ close to the emission of the characteristic Ka copper line of $\mathrm{X}$-ray micro source. As shown in Figure 4, radiography collected at 1 bar and 60 bar chamber pressure shows the absorption by the jet near to the nozzle. Notable observation from the radiographs (a) and (c), that spray present a cone angle focused near of nozzle and the measurements from radiography can be based on the behavior of the dense core of the spray, which contains most of the spray mass. Examples of Ce doped dodecane sprays line profile and their radiographies at different pressure are shown in Figure 5. 

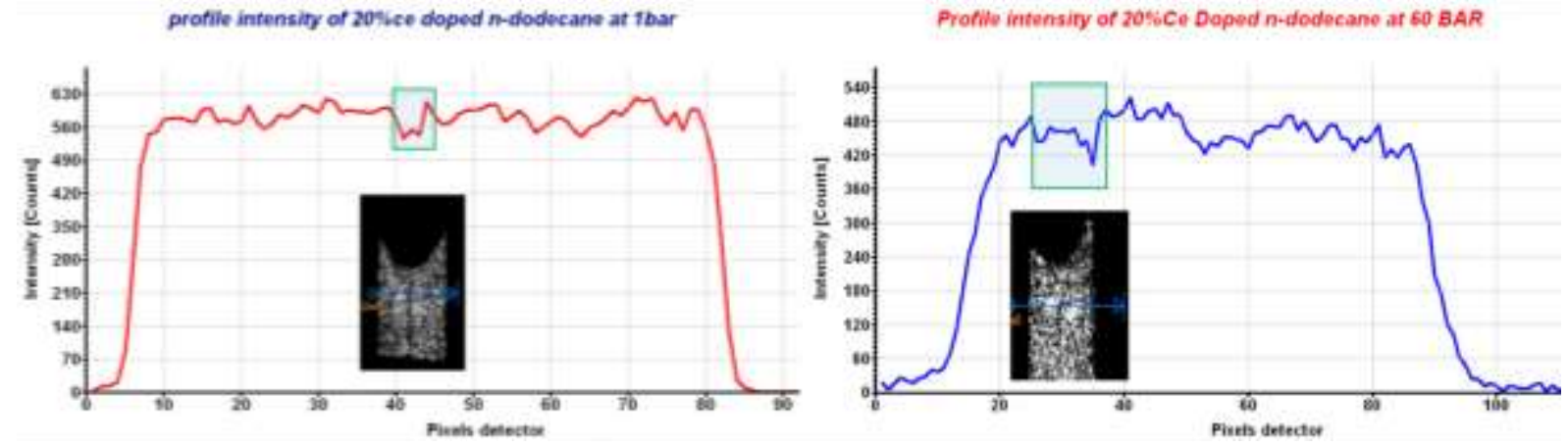

Figure 5: Ce doped dodecane sprays line profile and their radiographies (right) radiographies at 1 bar of pressure (left) radiographies at pressure of 60 bars.

Determining the spray cone angle is to define a width of the spray at several axial sections. In this study, the fullwidth, half-maximum (FWHM) is determined by fitting a transmitted or absorption signal with a Gaussian function. Figure 6 indicates that the FWHM values near the nozzle are quite similar for both operating conditions and the FWHM increases significantly with axial distance for the nozzle.

From the limited quality radiograph with this percentage of $\mathrm{Ce}$, the spray propagation occurs in a very stable approach from one injection event to another due to similar result obtained along the line where the continuous jet is obtained over a straight vertical propagation. The full angle of aperture deduced from the measurement of the ratio of the radial extension to the downstream propagation length has a constant value of about $5^{\circ}$ with no significant evidence for the presence of dodecane out of this cone. The maximum density inferred from the ratio grey level value measured in and out of the jet and accounting for an average X-ray energy of $8.0 \pm 0.3 \mathrm{keV}$ and the dodecane $X$ ray absorption cross section for this $X$-ray energy, is of about $0.6 \mathrm{~g} . \mathrm{cm}^{-3}$.
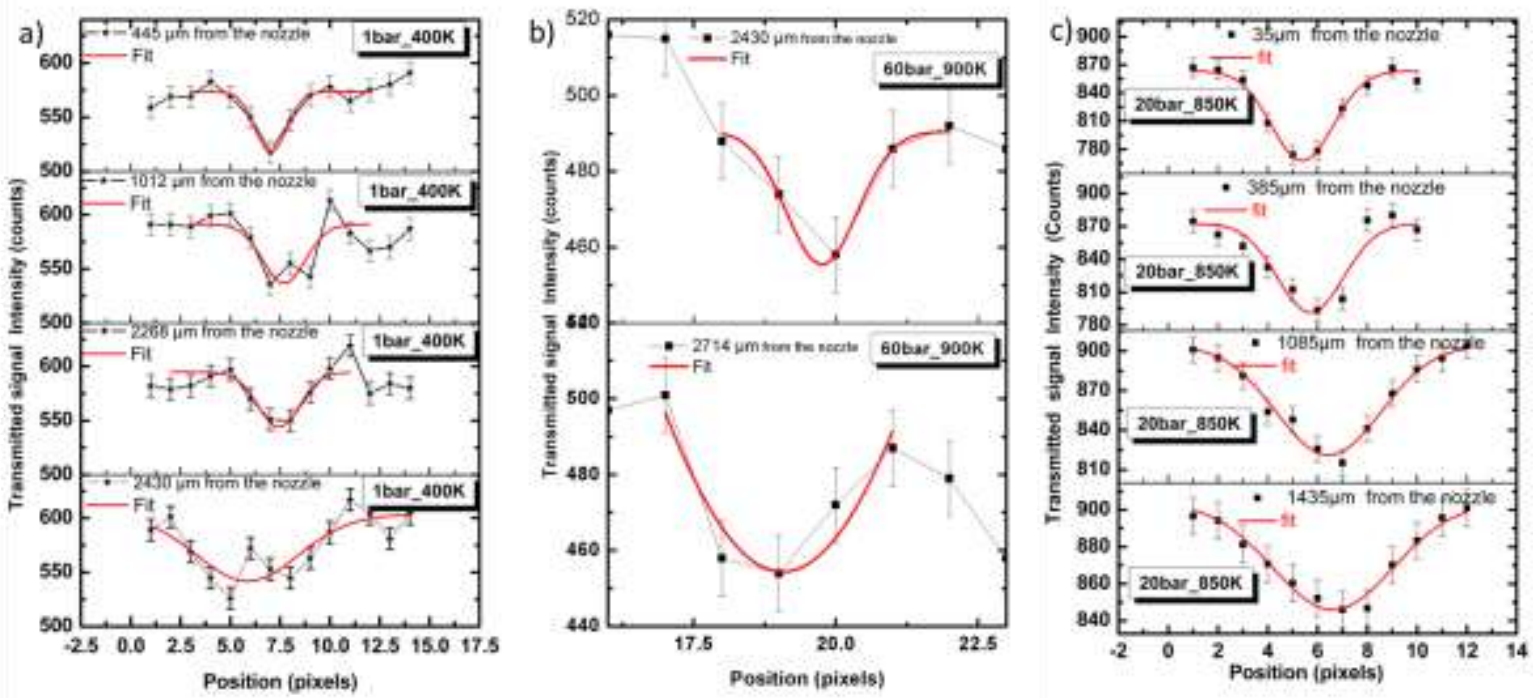

Figure 6: Fits and lines profiles of transmitted signals of $20 \%$ Ce doped dodecane sprays at several axial positions from the nozzle. (a) 1 bar of pressure, (b) radiograph at pressure of 60 bars, (c) radiograph at pressure of 20 bars.

Table 3 shows the density measurement obtained from 5 ms injection of Ce doped n-dodecane spray calculated from radiographs at different position of spray from the nozzle. Reminding that the density of the mixing of $20 \% \mathrm{Ce}$ doped $\mathrm{n}$-dodecane spray is $894 \mathrm{Kg} \cdot \mathrm{m}^{-3}$ at $300 \mathrm{~K}$ and ambient conditions. During the propagation of the spray, the density decreases due to the air entrainment. A considerable increasing of the density is obtained at high pressure (60 bar) and high temperature $(900 \mathrm{k}$ ). Under this condition, the fuel evaporates. During the vaporization of dodecane the cerium level increases causing the increasing of the density. The uncertainty in the density determination is calculated by determining the uncertainty in measuring the absorption coefficient and the photon shot noise, which follows a Poisson distribution. By propagating the error through the logarithm of equation 1 , the resulting uncertainty in density is less than $8 \%$. 
Table 3. Parameters of $5 \mathrm{~ms}$ of injection duration of $20 \%$ and $40 \%$ of Ce doped $\mathrm{n}$-dodecane spray calculated from radiographs (presented in Figure 4) at different position of spray from the nozzle.

\begin{tabular}{c|ccc}
\hline \hline $\begin{array}{c}\text { Pressure in the } \\
\text { chamber }\end{array}$ & $\begin{array}{c}\text { Position from the } \\
\text { nozzle }(\mu \mathrm{m})\end{array}$ & FWHM & Density $\left(\mathrm{kg} / \mathrm{m}^{3}\right)$ \\
\hline \hline & 445 & $2.103 \pm 9.5 \%$ & $875 \pm 1.1 \%$ \\
1 bar & 1012 & $2.477 \pm 6 \%$ & $870 \pm 1.1 \%$ \\
$(20 \%$ of Ce) & 2268 & $2.536 \pm 8.2 \%$ & $868 \pm 1.2 \%$ \\
& 2430 & $6.047 \pm 5.8 \%$ & $852 \pm 3.5 \%$ \\
& 35 & $3.124 \pm 6.7 \%$ & $960 \pm 2 \%$ \\
20 bar & 385 & $3.398 \pm 23.8 \%$ & $958 \pm 4.8 \%$ \\
$(40 \%$ of $\mathrm{Ce})$ & 1085 & $4.968 \pm 9 \%$ & $957 \pm 4.2 \%$ \\
& 1435 & $6.094 \pm 13.9 \%$ & $952 \pm 2.1 \%$ \\
60 bar & 2430 & $1.463 \pm 34.1 \%$ & $950 \pm 6.2 \%$ \\
$(20 \%$ of $\mathrm{Ce})$ & 2714 & $4.432 \pm 7.8 \%$ & $964 \pm 7.1 \%$
\end{tabular}

\section{Signal to Noise Ratio (SNR)}

To quantify the capacity of the actual setup with micro X-ray source to detect the cerium doped dodecane sprays through micrometric nozzles in a HPHT injection chamber, SNR analysis was also performed on single-shot $X$ ray measurement where we make binning along one, and several line profiles, respectively. Each line profile of the detector correspond to a vertical resolution of $30 \mu \mathrm{m}$. So that considering the spatial resolution of the diagnostics in the present experimental setup, pixel binning to enhance signal to noise ratio is not a severe limitation.

The SNR values obtained from the X-ray measurement are defined as the ratio of mean peak intensity of the minima subtracted from the mean environment intensity to the root-mean-square ( $\mathrm{rms}$ ) fluctuations of the pixel signal in the image. The radiograph images from the $X$ ray source were dark-current subtracted.

$S N R=\frac{\mid I_{\text {hole }}-\text { Mean of environment Intensity } \mid}{\sigma_{\text {fluctuation of pixels signal }}}$
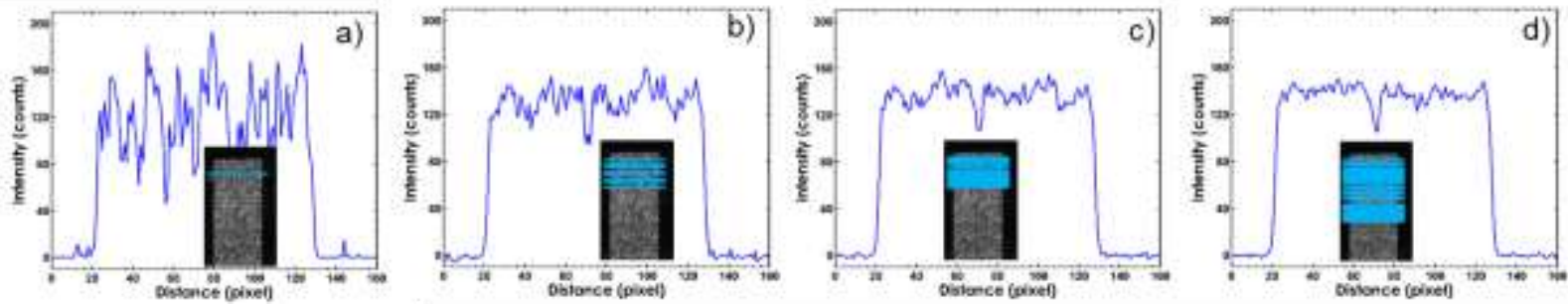

Figure 7: Single-shot X-ray Ce doped dodecane sprays profile and their radiographies at 1 bar and 300K by binning over (a) 1 line (b) 10 lines (c) 50 lines (d) 100 lines.

Table 4. SNR obtained from radiographies at 1 bar by binning over different number of lines.

\begin{tabular}{c|cccc}
\hline \hline Number of lines & 1 & 10 & 50 & 100 \\
\hline \hline SNR & 0.8 & 2.3 & 3.7 & 4.2
\end{tabular}

Due to the low absorption of a few percent of the dodecane sprays, injection events require to be averaged over a few hundred of X-ray shots to get sufficient SNR from radiographs. If we increase the number of shots the SNR for $20 \%$ dopant is improved. In our experiment conditions, good compromise to achieve a reasonable SNR is 10 shots for 1 bar and 100 shots for 60 bars. However, as we can see in the single shot $X$ ray measurement applied at ambient condition to Ce doped dodecane (Figure 7), signal of the jet is completely drowned inside the backgroundnoise. When binning over large number of line increases, a considerable reduction of RMS is obtained leading to increase the SNR and then the absorption of jet is clearly seen. This analysis suggests to reduce the percentage of dopant and to make vertical binning on X-ray radiography. An improvement in SNR is clearly observed when vertical binning is performed and the presence of the jet of dodecane can be clearly detected.

\section{Conclusions}

To the best of our knowledge, this is the first time that X-ray radiography of a dodecane jet at high pressure and high temperature have been obtained in spray A conditions using new one shot engine. X-ray radiography has demonstrated its potential as a useful quantitative diagnostic for studying dense fuel sprays. From the various 
studies carried out to study the effect of DPX dopant on the physical characteristics of dodecane spray, it has been shown that the behavior of the spray is completely different when high concentration of dopant is mixed with the dodecane. Liquid penetration length was determined when decreasing the Ce dopant concentration in dodecane till $1.25 \%$. Further analysis suggested to reduce the percentage of dopant and to make vertical binning on $\mathrm{X}$-ray radiography, for which improvement in signal to noise-ratio was clearly observed and the presence of the jet of dodecane can be detected. The full angle of aperture deduced from the measurement is around $5^{\circ}$. Density and absorption measurement obtained from $5 \mathrm{~ms}$ injection of $20 \%$ Ce doped n-dodecane spray at $900 \mathrm{~K}$ are around $950 \mathrm{~kg} / \mathrm{m}^{3}$. First experiments show that measurement output is a compromise between, on the one hand, the percentage of dopant for which the physical characteristics and the behavior of the spray do not change, and on the other hand the visualization of the jet by X-ray.

\section{Acknowledgements}

This work is supported by ANR Research National Agency (ECN-France project). I.C. is supported by ANR PLANEX ANR-11-EQPX-0036-01. The authors would like to thank Thierry Seguelong for DPX9 supply and Gilles Bruneaux for scientific discussions.

\section{References}

[1] O. Nilaphai et al., "New high pressure and high temperature chamber for Diesel spray characterization," in ILASS - Europe 2016, 27th Annual Conference on Liquid Atomization and Spray Systems, 4-7 September, 2016, pp. 1-10.

[2] E. Giannadakis, M. Gavaises, and C. Arcoumanis, "Modelling of cavitation in diesel injector nozzles," J. Fluid Mech., vol. 616, no. September, p. 153, 2008.

[3] S. Tonini, M. Gavaises, C. Arcoumanis, and a. Theodorakakos, "Prediction of Liquid and Vapor Penetration of High Pressure Diesel Sprays," SAE Tech. Pap., vol. 2006, no. 724, 2006.

[4] D. L. Siebers, "Liquid-Phase Fuel Penetration in Diesel Sprays." SAE International , 1998.

[5] M. Wolff, a Delconte, F. Schmidt, P. Gucher, and F. Lemoine, "High-pressure Diesel spray temperature measurements using two-colour laser-induced fluorescence," Meas. Sci. Technol., vol. 18, no. 3, pp. 697706, 2007.

[6] H. Ajrouche, A. Lo, P. Vervisch, and A. Cessou, "Detector assessment for 1D single-shot spontaneous Raman scattering for temperature and multi-species measurements in flames," in 18th International Symposium on Applications of Laser Techniques to Fluid Mechanics, 04-07 July, 2016.

[7] H. Ajrouche, A. Lo, P. Vervisch, and A. Cessou, "Assessment of a fast electro-optical shutter for 1D Spontaneous Raman Scattering in flames," Meas. Sci. Technol., vol. 26, no. 7, p. 075501 (9pp), 2015.

[8] A. Adam, P. Leick, G. Bittlinger, and C. Schulz, "Visualization of the evaporation of a diesel spray using combined Mie and Rayleigh scattering techniques," Exp. Fluids, vol. 47, no. 3, pp. 439-449, 2009.

[9] Z.-M. Cao, K. Nishino, S. Mizuno, and K. Torii, "PIV measurement of internal structure of diesel fuel spray," Exp. Fluids, vol. 29, no. 7, pp. S211-S219, 2000.

[10] B. R. Halls, T. J. Heindel, A. L. Kastengren, and T. R. Meyer, "Evaluation of X-ray sources for quantitative two- and three-dimensional imaging of liquid mass distribution in atomizing sprays," Int. J. Multiph. Flow, vol. 59, pp. 113-120, 2014.

[11] P. Leick, T. Riedel, G. Bittlinger, C. F. Powell, A. L. Kastengren, and J. Wang, "X-ray measurements of the mass distribution in the dense primary break-up region of the spray from a standard multi-hole common-rail diesel injection system," Proc. ILASS - Eur. Meet., pp. 1-6, 2007.

[12] D. J. Duke, A. B. Swantek, A. L. Kastengren, and C. F. Powell, "X-ray Diagnostics for Cavitating Nozzle Flow," J. Phys. Conf. Ser., vol. 656, p. 12110, 2015.

[13] D. J. Duke et al., "X-ray radiography of cavitation in a beryllium alloy nozzle," Int. J. Engine Res., pp. 113, 2016

[14] E. Robert, S. Dozias, C. Cachoncinlle, and J. M. Pouvesle, "Flash X-ray diagnostics of dodecane injection through micrometric nozzle."

[15] a. I. Ramírez et al., "Quantitative X-ray measurements of high-pressure fuel sprays from a production heavy duty diesel injector," Exp. Fluids, vol. 47, no. 1, pp. 119-134, 2009.

[16] A. L. Kastengren, C. F. Powell, Y. Wang, K.-S. Im, and J. Wang, "X-Ray Radiography Measurements of Diesel Spray Structure At Engine-Like Ambient Density," At. Sprays, vol. 19, no. 11, pp. 1031-1044, 2009.

[17] M. Lorenzi, N. Mitroglou, M. Santini, and M. Gavaises, "Novel experimental technique for 3D investigation of high-speed cavitating diesel fuel flows by X-ray micro computed tomography," Rev. Sci. Instrum., vol. 88, p. 33706, 2017.

[18] A. L. Kastengren et al., "Axial Development of Diesel Sprays at Varying Ambient Density Argonne National Laboratory Advanced Photon Source Argonne National Laboratory," in ILASS Americas, 22nd Annual Conference on Liquid Atomization and Spray Systems, Cincinnati, OH, 2010, no. May.

[19] A. L. Kastengren, C. F. Powell, K.-S. Im, Y.-J. Wang, and J. Wang, "Measurement of Biodiesel Blend and Conventional Diesel Spray Structure Using X-Ray Radiography," J. Eng. Gas Turbines Power, vol. 131, no. 6, pp. 62802-62807, Jul. 2009. 


\title{
A quantitative analysis of nozzle surface-bound fuel for diesel injectors
}

\author{
Jack Turner ${ }^{1}$, Dan Sykes ${ }^{1}$, Guillaume de Sercey ${ }^{1}$, Viacheslav Stetsyuk ${ }^{1}$, Martin Gold², \\ Richard Pearson ${ }^{2}$, Cyril Crua ${ }^{1}$ \\ ${ }^{1}$ Advanced Engineering Centre, University of Brighton, Brighton, UK \\ ${ }^{2}$ BP Formulated Products Technology, Pangbourne, UK \\ *Corresponding author: j.turner3@brighton.ac.uk
}

\begin{abstract}
In a fuel injector at the end of the injection, the needle descent and the rapid pressure drop in the nozzle leads to discharge of large, slow-moving liquid structures. This unwanted discharge is often referred as fuel 'dribble' and results in near-nozzle surface wetting, creating fuel-rich regions that are believed to contribute to unburnt hydrocarbon emissions. Subsequent fluid overspill occurs during the pressure drop in the expansion stroke when residual fluid inside the nozzle is displaced by the expansion of trapped gases as the pressure through the orifices is equalised, leading to further surface wetting. There have been several recent advancements in the characterisation of these near nozzle fluid processes, yet there is a lack of quantitative data relating the operating conditions and hardware parameters to the quantity of overspill and surface-bound fuel. In this study, methods for quantifying nozzle tip wetting after the end of injection were developed, to gain a better understanding of the underlying processes and to study the influence of engine operating conditions. A high-speed camera with a longdistance microscope was used to visualise fluid behaviour at the microscopic scale during, and after, the end of injection. In order to measure the nozzle tip temperature, a production injector was used which was instrumented with a type $\mathrm{K}$ thermocouple near one of the orifices. Image post-processing techniques were developed to track both the initial fuel coverage area on the nozzle surface, as well as the temporal evolution and spreading rate of surface-bound fluid. The conclusion presents an analysis of the area of fuel coverage and the rate of spreading and how these depend on injection pressure, in-cylinder pressure and in-cylinder temperature. It was observed that for this VCO injector, the rate of spreading correlates with the initial area of fuel coverage measured after the end of injection, suggesting that the main mechanism for nozzle wetting is through the impingement of dribble onto the nozzle. However, occasional observations of the expansion of orifice-trapped gas were made that lead to a significant increase in nozzle wetting.
\end{abstract}

\section{Keywords}

Diesel; end of injection; dribble; surface wetting; image processing

\section{Introduction}

Increasingly strict emissions standards place considerable pressure on the automotive industry to increase the efficiency of, and reduce the emissions of the engine. This is primarily done by optimising the fuel-air mixing processes that occur inside the combustion chamber. Since the fuel air mixing is affected by the fuel injection equipment (FIE), much of the research efforts have been in improving the injector design and spray characteristics. This has resulted in the numbers of holes in the injector tip increasing with subsequent designs, as well as increases in the operating pressure, with typical systems operating with common rail pressures of over $200 \mathrm{MPa}$. These increasingly harsh conditions are believed to accelerate the formation and growth of deposits in and around the injector tip.

The accumulation of deposits in and around injector tips is associated with reduced engine performance and lifetime. This reduction in performance is known to manifest in a variety of ways including increased acoustic and pollutant emissions [1-3]. The deposits can also reduce the hydraulic diameter of the nozzle hole, resulting in a reduction in the quantity of injected fuel and reduced quality and consistency of injection [3-5]; all of which cause a reduction in engine power [6]. The deposits can also increase cavitation, which can then lead to further coking of the nozzle [7]. Injector needle sticking can occur and eventually injector failure [8, 9]. The issue of deposit formation is further compounded by both the strict tolerances of critical parts of the FIE 
Recent efforts have been directed at optimisation of the injection event itself. Shifts towards piezo-actuated injectors mean that dynamic response times are improved, reducing the time spent in the transient injection phase. This has been shown to give superior fuel air mixing when compared with older systems using solenoid actuated injectors $[5,6]$. A more recent technique in injection optimisation utilises split injection strategies. This gives increased control of the rate of injection. Split injections have been shown to reduce unburnt hydrocarbon (UHC) emissions. There are, however, diminishing returns, suggestive of a persistent source of UHC emissions [10].

Transient injection phases, in particular the end of injection, are believed to be related to this persistent source of emissions [11], as well as the continuing issues around injector deposits. During the end of injection, as the needle descends, the complex fluid flow inside the nozzle and orifices results in the discharge of large, slow and deformed liquid structures during the collapse of the spray. This leads to two problems. Firstly, the liquid often lands on the near-nozzle surface where it is then exposed to the high pressures and temperatures inside the cylinder. Since this creates locally rich regions in an overall lean environment, incomplete combustion, cracking and degradation are likely to occur. This is particularly true if the combustion flame front contacts the surface-bound fluid. It is likely that this process would impact the initial formation of carbonaceous deposits in and around the injector tip. Secondly, the large, slow-moving droplets that were ejected may not be atomized sufficiently to undergo complete evaporation, thus producing unwanted combustion by-products. There is also potential for these droplets to be drawn directly through the exhaust valve where they contribute directly to the UHC emissions. Increased use of split injection strategies increases the time spent by the spray within the transient regime. This increases the occurrence of liquid structures associated with the end of injection and the related problems with emission and deposits. As such, the end of injection has recently attracted considerable efforts to characterise and explain the observed phenomena [12-15].

There have been numerous simulations and numerical studies that have predicted the complex fluid behaviour inside nozzle during the closing of the needle [16-19] these studies predict the breaking up of the jet into ligaments as well as rapid pressure fluctuations during this time. There is a point in which the experimental observation and numerical simulations are in very good agreement. There does, however, exist a lack of quantitative analysis relating to the volume of fluid on the surface of the near nozzle region after the end of injection. There have been simulations on predicting mass outflux [18], but this includes fluid that is discharged into the cylinder as well as onto the surface.

Whilst there are interesting fluid dynamic processes occurring at the orifices throughout the engine cycle [15, 20], this investigation is focused on nozzle wetting processes that occur within a few milliseconds from the end of injection. Development of quantitative methods to measure both the spreading area of surface-bound fluid in the near-nozzle region after the end of injection, as well as the spreading rate, allows elucidation of the underlying mechanism of the initial fuel spreading. In order to decouple the effects of pressure and momentum on the initial coverage area, injections were carried out at significantly different timing during the engine cycle, resulting in greatly different in-cylinder pressure and temperature. This allowed the effect of capillary action and spreading to be investigated since the physical properties of the fuel are functions of pressure and temperature and the spreading dynamics are a function of both liquid physical properties and ambient conditions. In fact, at higher pressures and temperatures nozzle surface wetting is expected to increase. This is due to the pressure effect on viscosity and the temperature effect on viscosity and surface tension. Increasing both pressure and temperature relaxes the contact angle and increases surface wetting [21]. Based on this it can be hypothesised that the surface wetting will be more pronounced when injecting at top-dead-centre (TDC). Given the timescales involved, it is likely that pressure would be the dominant effect, since the nozzle tip will experience a reduced temperature variation due to thermal inertia.

\section{Experimental setup}

The principal apparatus used in this research was a single cylinder reciprocating rapid compression machine. The fuel was delivered by a Delphi common-rail system, comprising a DFP-3 high-pressure pump rated at $200 \mathrm{MPa}$, and a six-hole Siemens instrumented injector with a VCO type nozzle. The setup allows observation of all six orifices simultaneously as they all sit within the plane of focus. The injector contained two integrated type $\mathrm{K}$ thermocouples accurate to $\pm 1 \mathrm{~K}$. These thermocouples record temperature at a point just below the tip of the nozzle surface and another inside the body of the injector to measure the fuel temperature around the needle prior to discharge. The injector was mounted orthogonal to the incident light to allow visualisation of all orifices simultaneously. 
Table 1. Test conditions and hardware characteristics

\begin{tabular}{c|c}
\hline \hline Parameter & Value \\
\hline Nozzle type & Siemens DW10, 6-hole VCO \\
Orifice diameter & $145 \mu \mathrm{m}$ \\
Peak motored pressure & $60 \mathrm{bar}$ \\
Peak motored temperature & $640 \mathrm{~K}$ \\
Injection pressures & $500 ; 1500 \mathrm{bar}$ \\
Injection timing & $-30 ; 0 ; 30 \mathrm{CA}$ aTDC \\
Injection pulse duration & $200 ; 400 ; 600 \mu \mathrm{s}$ \\
Nozzle tip temperature & $405 \mathrm{~K}+/-3 \mathrm{~K}$
\end{tabular}

The light source consisted of two synchronised high-speed lasers illuminating the opposite sides of a 6-hole Siemens DW10 instrumented injector. Laser 1 is a CAVITAR Cavilux solid state diode laser emitting at $690 \mathrm{~nm}$ and Laser 2 is a copper vapour laser emitting at 510 and $578.2 \mathrm{~nm}$ with the beam being delivered via optical fibre. Laser 1 is controlled via a software interface and can be used to trigger a signal generator which, in turn, emits a pulse to drive Laser 2. The frame exposure of the high-speed camera was set at $1 \mu \mathrm{s}$, although the exposure was controlled by the shorter laser pulse durations. More details can be found in the specification table below.

Table 2. Imaging equipment specification. Frame rates shown are at minimum and maximum resolution and then a representative resolution from the data presented in this project.

\begin{tabular}{c|c}
\hline \hline Parameter & Value \\
\hline \hline Camera & Phantom V12.1 \\
Pixel size & $20 \mu \mathrm{m}$ \\
Bit depth & 12 -bit \\
Frame exposure time & $1 \mu \mathrm{s}$ \\
Frame rate (704 $\times 704$ pixels) & $10,000 \mathrm{fps}$ \\
Image resolution & $6.58 \mu \mathrm{m}$ per pixel \\
Field of view & $4.63 \mathrm{~mm} \times 4.63 \mathrm{~mm}$
\end{tabular}

With this setup, video data was acquired showing all six orifices for one entire engine revolution. The orifice were numbered clockwise as seen in Figure 1.

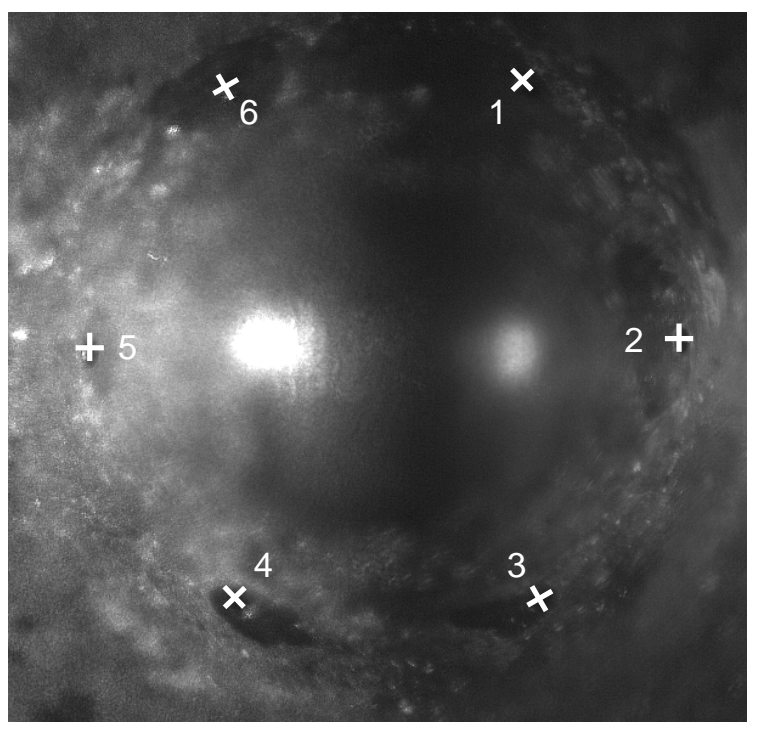

Figure 1. Video frame showing the entire injector tip and all orifices in focus. Note how each orifice is illuminated differently, in particularly how the equatorial orifices 2 and 5 seem to afford little contrast as they lie directly in the path of the incident laser light. The remaining orifices $1,3,4,6$ offer better contrast as light from the liquid layers is scattered away from the imaging system. The surface-bound fluid appears darker, whereas this effect is reduced for orifices 2 and 5. 


\section{Quantification of coverage area and spreading rate}

In order to obtain quantitative data to explore the key hypothesis that the surface wetting will be more pronounced when injecting at TDC, image processing algorithms were developed to semi-automatically extract the coverage area of liquid fuel on the nozzle tip. The key stages of the image processing algorithm are listed and described below:

1. Normalisation of pixel intensities: the intensity histogram was stretched in relation to the top and bottom $10 \%$ pixel intensities across the frame to account for laser pulse to pulse variation.

2. Flat fielding: the illumination across the frame was not homogeneous due to the curvature of the nozzle tip surface. The intensities across the frame were homogenised using a non-linear gradient filter.

3. Removal of high frequency noise: high frequency noise was removed, and small regions with similar pixel intensity distributions were merged using morphological closing with a non-flat, inverted ball-shaped structuring element, followed by morphological opening.

4. Removal of reflections from the orifice edge: an elliptical mask layered over the orifice with constant pixel intensities, equal to the mean of those surrounding it, was applied to remove high intensity reflections from the orifice's edge.

5. Semi-automatic binarisation: a function using the mean and spread of pixel intensities across the frame, in conjunction with a user input coefficient, was used for the binarisation threshold. The automatic thresholding performed well, but a user adjustment was required in $33 \%$ of the videos to faithfully track the coverage area. These tended to be for higher injection pressure conditions.

6. Smoothing of the binarised image: morphological operators were applied to remove small-scale features.

Binarised regions that did not overlap with the orifice were removed, therefore videos showing no coverage did not show any binarised regions. The fuel coverage area was converted from pixels to $\mathrm{mm}^{2}$ via scaling of the final binarised region, accounting for the $30^{\circ}$ inclination of the nozzle tip surface with respect to the optical axis. A visual inspection of the image processing output was performed for all frames to ensure that the automated measurements accurately represented the coverage area. Videos which could not be processed were discarded, and some videos were re-processed with a custom binarisation threshold.

This approach gave consistent results for orifice 3 due to its orientation relative to the illumination and the imaging system, therefore the image processing was only applied in the area surrounding orifice 3 . Visual inspection of the videos confirmed that this particular orifice was representative of the nozzle's behaviour. An example of the user interface for the image processing code is shown in Figure 2.
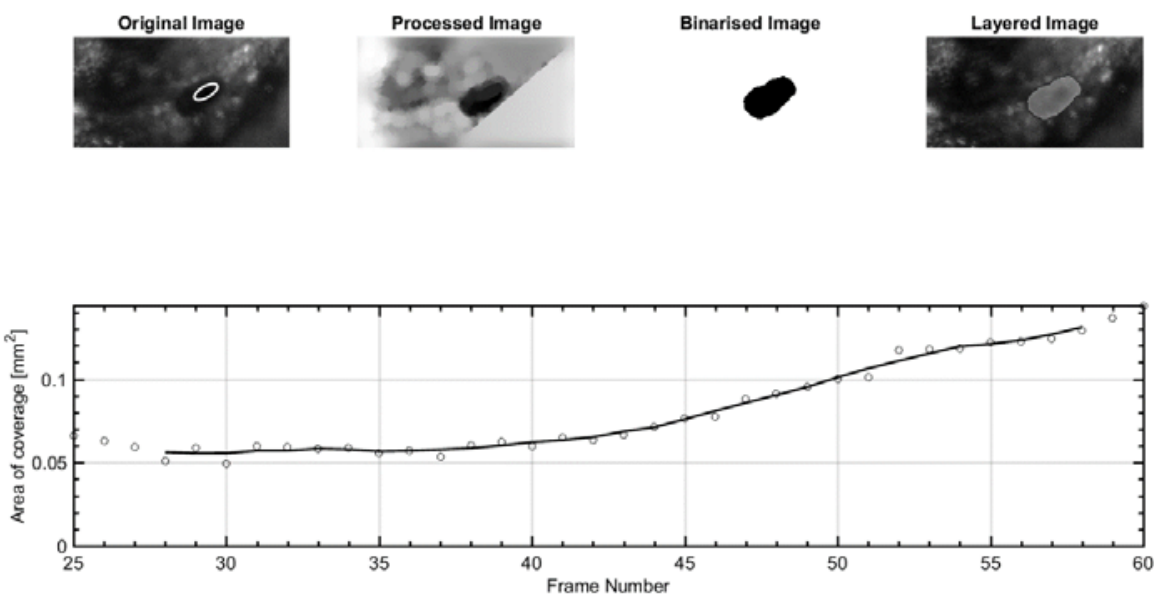

Figure 2. User interface showing the results of image analysis, with individual steps displayed so that the performance of the algorithm can be assessed in real time.

A mean value was extracted for data points between 3 and $3.5 \mathrm{~ms}$ ASOI. This was able to yield an approximation for the initial quantitative coverage of the fluid, shown in Figure 3. The individual data points on that chart represent the mean values from all injections at matching injection pressures and injection timings in which coverage occurred. 


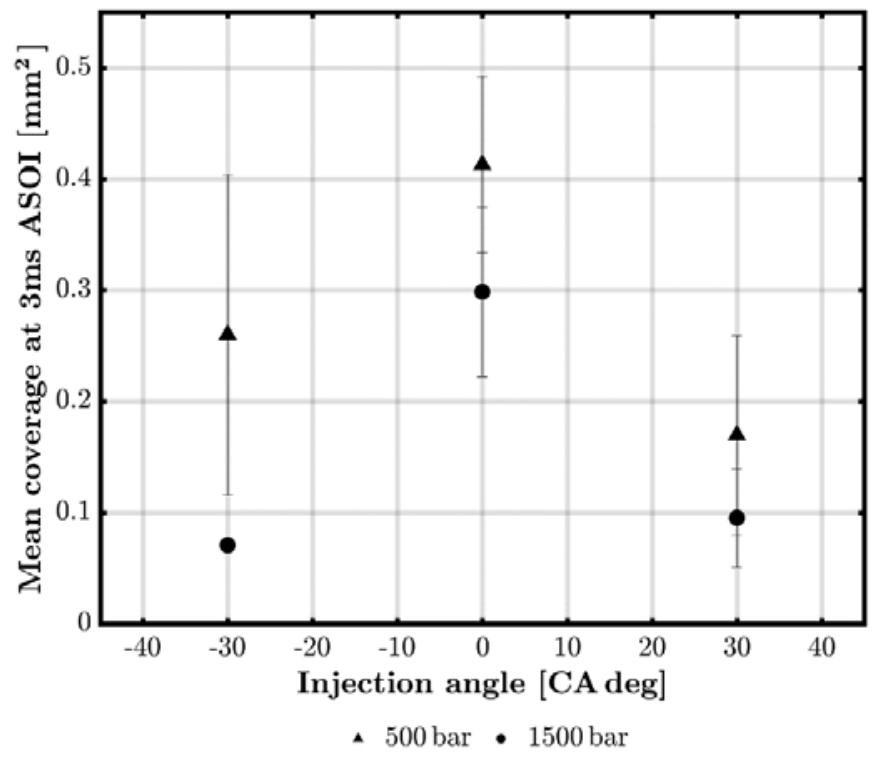

Figure 3. Mean initial coverage area measured $3 \mathrm{~ms}$ after the end of injection trigger. This shows that increasing injection pressure reduces the amount of initial fuel coverage once the spray has collapsed. Each data point represents the mean of 3 to

15 individual injections, and the error bars represent \pm 1 standard deviation. The orifice area has been subtracted.

\section{Results and discussion}

A key hypothesis that was tested in the present work is whether the presence of gas trapped inside the nozzle orifices could influence the wetting of the nozzle tip during the expansion stroke. As the in-cylinder pressure drops rapidly during expansion, gas trapped within the orifices should expand and displace liquid fuel onto the external surface of the nozzle tip. In order to test this hypothesis 3 injection timings were investigated:

1. During the compression stroke, when the gas pressure rise should inhibit the expansion of trapped gas

2. At top-dead-centre when the gas pressure is nearly constant

3. During the expansion stroke when the rapid gas pressure drop should promote the expansion of trapped gas.

The mean coverage area was found to depend heavily on the crank angle at which the injection pulse was sent. Injection at TDC is where the temperature and pressure are the greatest, higher pressure and temperatures relax contact angles and are conducive of more surface wetting. The lower in-cylinder pressures found when injecting away from TDC lead to greater fluid momentum at the instant when spray collapse occurs. This means there is less chance of splashback or ligament retraction towards the nozzle tip. It is interesting to note that the initial coverage areas measured when injecting during the compression (-30 CA) and expansion (+30 CA) strokes are almost identical. This confirms that the expansion of trapped gas does not significantly contribute to the initial wetting of this nozzle's surface.

The data recorded in Figure 3 shows a clear trend where increasing injection pressure leads to a reduction of the initial fuel coverage area. This can be explained by an increase of the fluid momentum at higher injection pressures, which in turn reduces the likelihood of ligament impingement or splashback onto the injector surface. The rate of liquid spreading was measured (Figure 4) by analysing the nozzle wetting shortly after the end of injection, using a linear regression where the gradient of the line represents the temporal evolution of the wetted surface area (Figure 5). The same as those described for the initial coverage trend was observed for both injection timing and injection pressure. This suggests that the spreading rate highly correlates with the initial coverage area. This correlation indicates that the temporal increase in wetted surface area is due to the spreading of the initial liquid deposition, rather than the emergence of additional liquid from the orifice. This is further evidence that the expansion of orificetrapped gas bubbles is not the main contributor to liquid spreading across the near-nozzle surface after the end of injection. However, some exceptions were occasionally recorded, with the emergence of a bubble being observed and followed by an abrupt increase of the wetted area (Figure 5). Although such bubble expansions were not 
common, when they did occur, the coverage area could rapidly double; hence, the importance of this phenomenon may not be negligible.

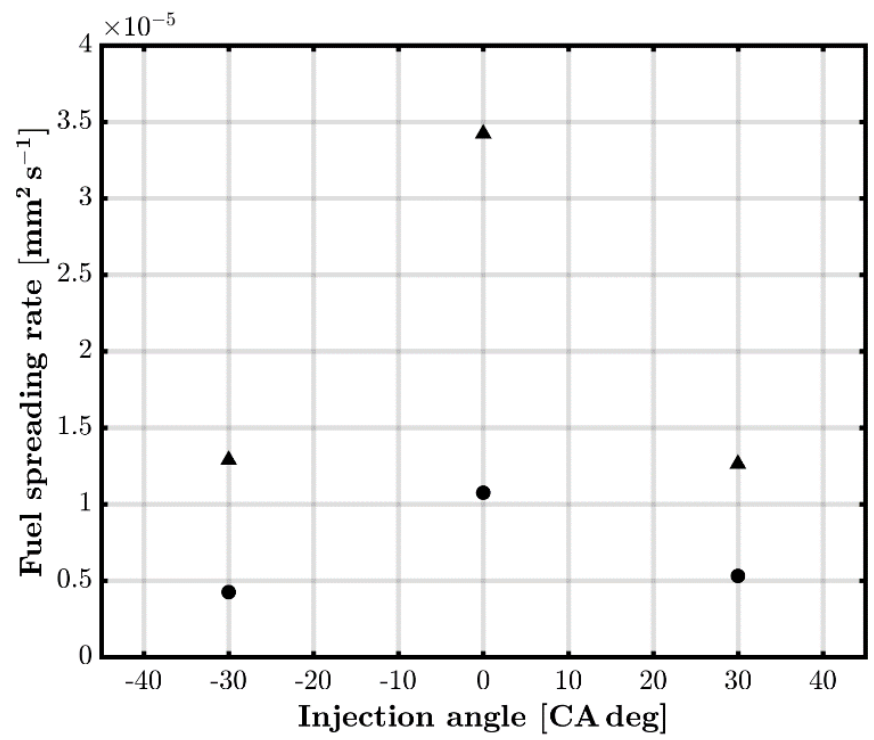

- 500 bar • 1500 bar

Figure 4. Illustration of how the rate of liquid spreading correlates with both crank angle and injection pressure. The rate of liquid spreading was calculated using linear regression analysis where the gradient of the line of best fit denotes the spreading rate in $\mathrm{mm}^{2} \mathrm{~s}^{-1}$ 


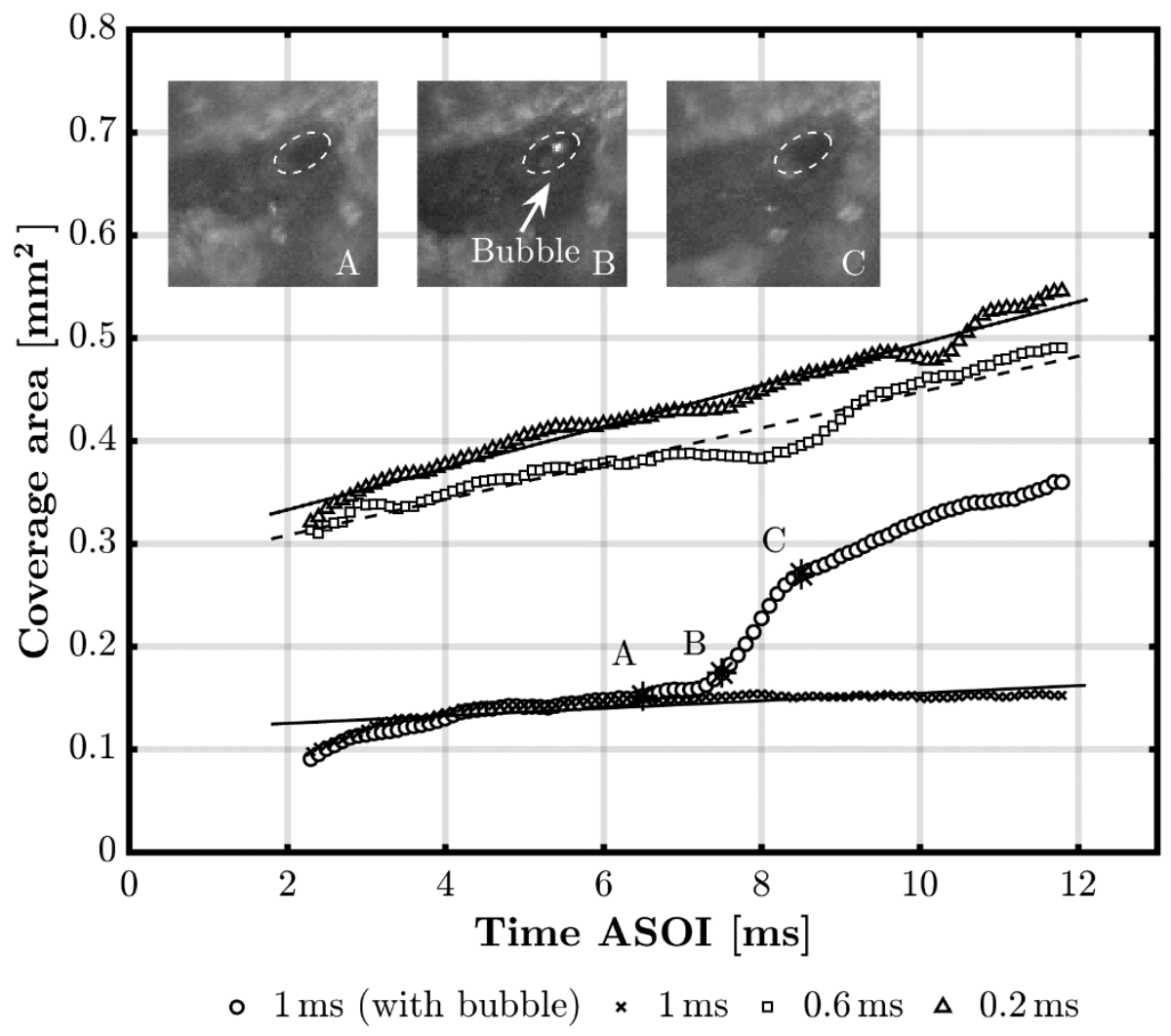

Figure 5. Temporal evolution of liquid coverage area as a function of time after start of injection trigger. In the majority of cases, the spreading rate observed was constant, as indicated by the linear trend lines. Occasionally, the emergence of a bubble was observed and followed by an abrupt increase of the detected coverage (white circles), however, this phenomena only occurred outside the image processing period. The dashed ellipses highlight the orifice location and the white arrow in frame B points to the distinctive scattering caused by the emergence of a liquid/gas interface within the orifice. Although such bubble expansions were not common, when they did occur, the coverage area rapidly increased.

\section{Conclusions}

In this study, a method for quantifying nozzle tip wetting after the end of injection was developed to gain a better understanding of the underlying processes and to study the influence of engine operating conditions. A high-speed camera with a long-distance microscope was used to visualise fluid behaviour at the microscopic scale during, and after, the end of injection. In order to measure the nozzle tip temperature a production injector was instrumented with a type $\mathrm{K}$ thermocouple near one of the orifices. Post-processing techniques were developed to track both the initial fuel coverage area on the nozzle surface, as well as the temporal evolution and spreading rate of surfacebound fluid.

Increasing the injection pressure from 500 bar to 1500 bar reduced the initial nozzle tip wetting. This is believed to be the result of increased momentum of the end of injection dribble, which reduces the likelihood of fluid contacting the surface of the near nozzle region, either by retraction of surface-bound ligaments or spray splashback. It was observed that the rate of spreading correlated with the initial area of fuel coverage measured after the end of injection, suggesting that the main mechanism for the wetting of a VCO nozzle is through the impingement of dribble onto the nozzle tip. 
For this nozzle the expansion of orifice-trapped gas bubbles was not found to be the main contributor to liquid spreading across the near-nozzle surface after the end of injection. However, on some occasions the emergence of a bubble was observed and followed by an abrupt increase of wetted surface area as liquid fuel inside the orifice was displaced onto the nozzle tip. When this occurred the coverage area could rapidly double, suggesting that this phenomenon may not be negligible.

\section{Acknowledgements}

This work was supported by BP Formulated Products Technology and the UK's Engineering and Physical Science Research Council [EPSRC grant EP/K020528/1 and EP/N509607/1].

\section{References}

1. H. Sandquist, I. Denbratt, F. Owrang, J. Olsson, Influence of fuel parameters on deposit formation and emissions in a direct injection stratified charge SI engine, in: SAE International Spring Fuels \& Lubricants Meeting, Orlando, Florida, USA, May 7-9, 2001. doi:10.4271/2001-01-2028.

2. R. E. Olsen, M. C. Ingham, G. M. Parsons, A fuel additive concentrate for removal of injector deposits in light-duty diesels, in: SAE International Fall Fuels \& Lubricants Meeting and Exhibition, Fairborn, Ohio, USA, October 20-23, 1984. doi:10.4271/841349.

3. R. Caprotti, W. J. Fowler, G. Lepperhoff, M. Houben, Diesel additive technology effects on injector hole erosion/corrosion, injector fouling and particulate traps, in: SAE International Fuels \& Lubricants Meeting \& Exposition, Philadelphia, Pennsylvania, USA, October 18-21, 1993. doi:10.4271/932739.

4. G. Lepperhoff, M. Houben, Mechanisms of deposit formation in internal combustion engines and heat exchangers, in: SAE International Congress \& Exposition, Detroit, Michigan, USA, October 17-20, 1993. doi:10.4271,931032.

5. A. Birgel, N. Ladommatos, P. Aleiferis, S. Zülch, N. Milovanovic, V. Lafon, A. Orlovic, P. Lacey, P. Richards, Deposit formation in the holes of diesel injector nozzles: A critical review, in: SAE Powertrains, Fuels \& Lubricants Meeting, Rosemont, Illinois, USA, October 6-9, 2008. doi:10.4271/2008-01-2383.

6. R. Caprotti, A. Breakspear, O. Graupner, T. Klaua, Detergency requirements of future diesel injection systems, in: SAE Powertrain \& Fluid Systems Conference \& Exhibition, San Antonio, Texas, USA, October 24-27, 2005. doi:10.4271/2005-01-3901.

7. B. Argueyrolles, S. Dehoux, P. Gastaldi, L. Grosjean, F. Levy, A. Michel, D. Passerel, Influence of injector nozzle design and cavitation on coking phenomenon, in: SAE International Fuels \& Lubricants Meeting, Kyoto, Japan, July 23-27, 2007. doi:10.4271/2007-01-1896.

8. J. Barker, C. Snape, D. Scurr, Information on the aromatic structure of internal diesel injector deposits from time of flight secondary ion mass spectrometry (ToF-SIMS), in: SAE 2014 World Congress \& Exhibition, Detroit, Michigan, USA, April 8-10, 2014. doi:10.4271/2014-01-1387.

9. J. Reid, S. Cook, J. Barker, Internal injector deposits from sodium sources, SAE International J. Fuels Lubricants 7 (2014) 436-44. doi:10.4271/2014-01-1388.

10. C. P. Koci, Y. Ra, R. Krieger, M. Andrie, D. E. Foster, R. M. Siewert, R. P. Durrett, Multiple-event fuel injection investigations in a highly-dilute diesel low temperature combustion regime, SAE International J. Engines 2 (2009) 837-57. doi:10.4271/2009-01-0925.

11. C. Koci, A. Dempsey, J. Nudd, B. Knier, Understanding hydrocarbon emissions in heavy duty diesel engines combining experimental and computational methods, SAE International J. Engines 10 (2017). doi:10.4271,2017-01-0703.

12. A. Swantek, D. Duke, N. Sovis, End of injection, mass expulsion behaviors in single hole diesel fuel injectors, in: Proceedings of ILASS Americas, Portland, Oregon, USA, September 18-21, 2014.

13. A. B. Swantek, A. L. Kastengren, D. Duke, F. Z. Tilocco, N. Sovis, C. F. Powell, A further examination of fuel dribble from single hole diesel nozzles, in: ILASS-Europe 26th Annual Conference, Bremen, Germany, September 8-10, 2014.

14. S. Moon, W. Huang, Z. Li, J. Wang, End-of-injection fuel dribble of multi-hole diesel injector: Comprehensive investigation of phenomenon and discussion on control strategy, Applied Energy 179 (2016) 7-16. doi:10.1016/j.apenergy.2016.06.116.

15. W. E. Eagle, Cinema-stereo imaging of fuel dribble after the end of injection in an optical heavy-duty diesel engine, in: THIESEL, Valencia, Spain, September 9-12, 2014.

16. N. Papadopoulos, P. Aleiferis, Numerical modelling of the in-nozzle flow of a diesel injector with moving needle during and after the end of a full injection event, 12th International Conference on Engines \& Vehicles 8 (2015) 2285-302. doi:10.4271/2015-24-2472. 
17. M. Battistoni, A. L. Kastengren, C. F. Powell, S. Som, Fluid dynamics modeling of end-of-injection process, in: ILASS Americas 26th Annual Conference, Portland, Oregon, USA, May 5-8, 2014.

18. M. Battistoni, C. Poggiani, S. Som, Prediction of the nozzle flow and jet characteristics at start and end of injection: Transient behaviors, SAE International J. Engines 91 (2015) 84-97. doi:10.4271/2015-011850.

19. M. Battistoni, Q. Xue, S. Som, Large-eddy simulation (LES) of spray transients: Start and end of injection phenomena, Oil \& Gas Science Technology-Revue d'IFP Energies nouvelles 71 (2015) 1-4. doi:10.2516/ogst/2015024.

20. J. E. Turner, V. Stetsyuk, C. Crua, R. J. Pearson, M. R. Gold, The effect of operating conditions on postinjection fuel discharge in an optical engine, in: ICLASS 13th Triennial International Conference, Tainan, Taiwan, August 23-27, 2015.

21. D. R. Caudwell, J. P. M. Trusler, V. Vesovic, W. A. Wakeham, The viscosity and density of n-dodecane and n-octadecane at pressures up to $200 \mathrm{MPa}$ and temperatures up to $473 \mathrm{~K}$, International Journal of Thermophysics 25 (2004) 1339-52. doi:10.1007/s10765-004-5742-0. 


\title{
Design and experiments to investigate spray and impingement characteristics of a common rail type lubrication system
}

\author{
Matthias Stark*1, Arturo de Risi², Marcello Giangreco², Simon Diggelmann ${ }^{1}$ \\ ${ }^{1}$ WinGD, Winterthur Gas \& Diesel Ltd, Switzerland \\ ${ }^{2}$ University of Salento, Department of Engineering for innovation, Lecce, Italy \\ *Corresponding author: matthias.stark@wingd.com
}

\begin{abstract}
This paper reports on the establishment of a testing facility to investigate spray and impingement characteristics of a recently developed, fully adaptive lubrication system for large two stroke marine diesel engines. The reported part of this research relates to development steps towards the new lubrication system as well as the implementation of a dedicated testing facility and the establishment of validation approaches in order to corroborate technology developments in this context.
\end{abstract}

The major objective of this development yields an optimization of lubricant utilization and at the same time a significant reduction of lubricant fraction in the exhaust gas. A requisite to obtain the desired level of flexibility calls for a revised approach in injecting the lubricant. The desired flexibility of the new lubrication approach foresees a cycle based adjustment of the injected amount of lubricant as well as the injection pressure in order to provide the possibility to shape the injection spray pattern. Concept studies nominate the application of a common rail system with integrated needle lift type injectors and adjustable injection pressure as compliant with the requisite to inject the lubricant with a high level of flexibility.

This paper hence encompasses design and development aspects of the new lubrication system but most of all highlights steps in developing a validation concept in order to compare common lubrication systems with the new type of lubrication system.

Initial results of the test cell provide an insight regarding relevant information on the injection spray characteristics over the full engine load range as well as the possibility to compare common lubrication system performance with the new common rail lubrication system.

A comparison between measurement and simulation results provide the possibility to implement experimentally gained data in a neuronal network in order to enhance the predictive quality of the simulation tool.

\section{Keywords}

Injection system development and validation, computational injection spray simulation, experimental spray investigation

\section{Introduction}

Detailed investigations related to lubricant flow optimizations and transportation mechanisms clearly demonstrate the importance of an appropriate lubricant injection. [1-4] A characterization of relevant contributors to the total lubricant balance of a large two stroke marine diesel engine was performed to address the optimization potential of single components of the tribosystem. Optimizations of piston ring pack geometries showed superior functionality in terms of considerably reducing the amount of lubricant in the exhaust gas. Another, yet equal important aspect which must be taken into consideration when looking at further reducing lubricant consumption of such an engine type, relates to a detailed investigation regarding the application of the lubricant by means of a properly designed lubrication system. Design aspects of such a recent development focus on controlling lubricant spray characteristics over the complete engine load range and related variations of boundary conditions. Thus, investigations in the context of this paper, focus on developing an evaluation approach to support lubricant spray simulation tool developments as well as an experimental quantification of the lubrication system performance under close to real engine like boundary conditions.

One of the differences of a large two stroke diesel marine engine, compared to automotive engines, is found in the application of such a lubrication system. Electronically controlled lubrication systems are designed to meet 
appropriately defined lube oil feed rates in order to optimize cylinder lube oil consumption without compromising life time performance of a tribosystem. Mentioned lubrication systems deliver accurately metered, main engine load and fuel quality dependent quantities of lubricant to the cylinder liner with a very precise timing. This circumstance provides the possibility of adjusting lubrication system parameters on basis of operational requirements.

Fig. 1 shows a schematic of a "Pulse Jet" two stroke lubrication system, a typical setup of a lubrication system applied on WinGD's large two stroke marine diesel engines. It consists of a lubricant tank, a filter system, lube oil dosage pumps and non-return valve based lube oil injectors. The cylinder lube oil is applied onto the running surface of a cylinder liner by means of appropriately designed lube oil injector nozzle tips.

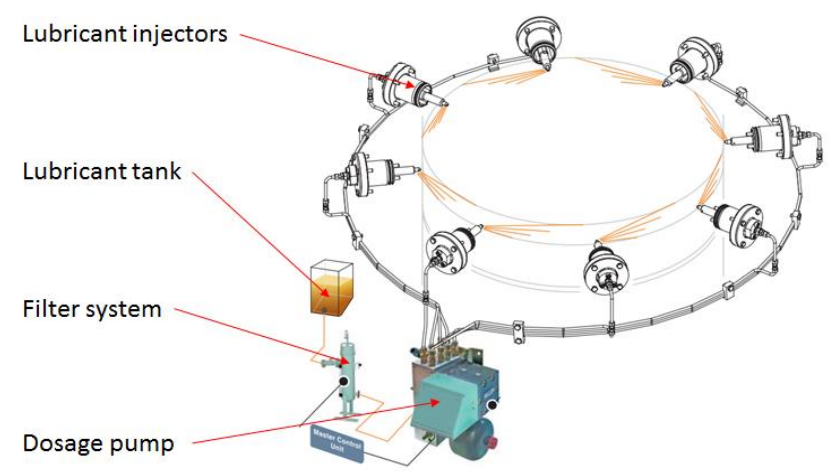

Fig.1: Typical arrangement of a large two stroke diesel engine lubrication system [2,3]

The lubricant is delivered to the injectors by a dosage pump which is powered by pressurized servo oil drawn from the engines system oil circuit. The lube oil feed rate and timing are electronically controlled by a solenoid valve of the dosage pump providing full flexibility in setting the injection timing over the full load range of the engine. A short coming of such a system is found in significant variations of the lubricant spray pattern following well investigated effects of a strong temperature dependency of the dynamic viscosity and related spray behaviour. A necessity to control the spray characteristics in order to optimize lubricant utilization thus leads to the development of a needle lift type in injection system in conjunction with a common rail approach.

\section{Lubrication system development}

Complying with the requirements of a fully adaptive lubrication system and the requisite to actively control lubricant spray characteristics over the entire engine load range, calls for a review of existing lubrication approaches. A significant part of this investigation hence focuses on the development of such a system to optimize lubricant utilization and to guarantee optimal life time performance. Fig.2 shows a schematic of the common rail type lubrication system incorporating needle lift type lubricant injectors.

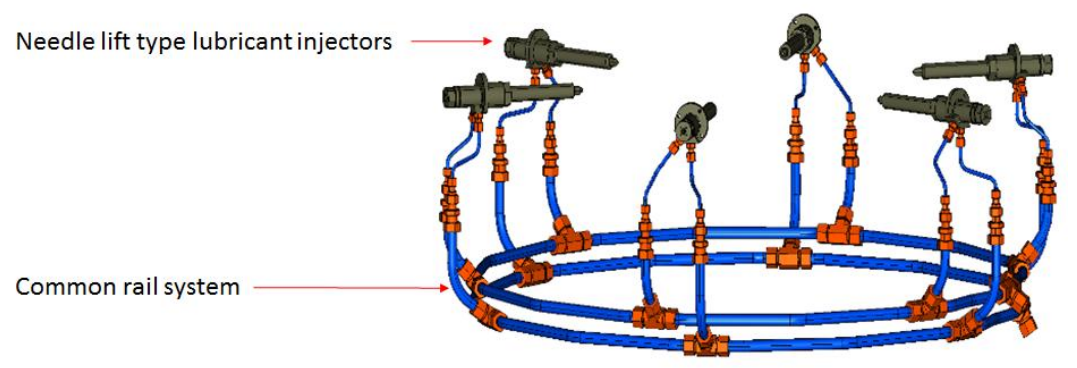

Fig.2: Schematic of a new common rail type lubrication system

Such an approach provides the possibility to adjust the injection pressure and timing over the complete engine load range and related temperature condition variations. Flexibility in shaping the lube oil spray pattern can thus achieved by applying main engine load dependent injection parameters. 
A typical development approach in this respect encompasses computational simulation support to optimize geometrical aspects and to predict lubrication system performance. A 1D fortran code based on concentrated volume method is applied to simulate the new lubricant injectors, taking friction effects on dynamics of moving components of the injector into consideration. Basic equations of the simulation tool are mass and momentum equations, whereof the integration of momentum equations on a solid body allows to model the moving parts, such as valves and needles. Forces due to the pressure on the surfaces, to the spring load and preload, to the friction forces were taken into account as well as the collision forces calculated by the elastic collision theory. An integral approach on a generic volume for the equation of mass is convenient when the main phenomenon is the pressure variation due to the flow rate and volume variation, as in the several chambers of the injection system. The discharge coefficient was evaluated according to Schmitt's formulation [14]; furthermore, the leakage between different chambers was evaluated considering laminar flow in the gap. Mass and momentum equations were employed to simulate the fluid dynamic phenomena inside the pipes and connections between different chambers of the system. Using a further relation between pressure and density, also provides the possibility to solve the equations in onedimensional form using the method of characteristics. $[9,11]$ This relation is based on the bulk modulus of the fluid, which is considered linear according to the pressure, interpolating the data obtained from measurements, the presence of air in the oil could also be considered. Nikuradse's theory was used to calculate the friction forces while the boundary conditions were calculated according to Matsuoka. All the equations were solved using an explicit first order Runge-Kutta scheme. A schematic of the simulated component is shown in Fig.3. a) and b) as explained by de Risi. [5]

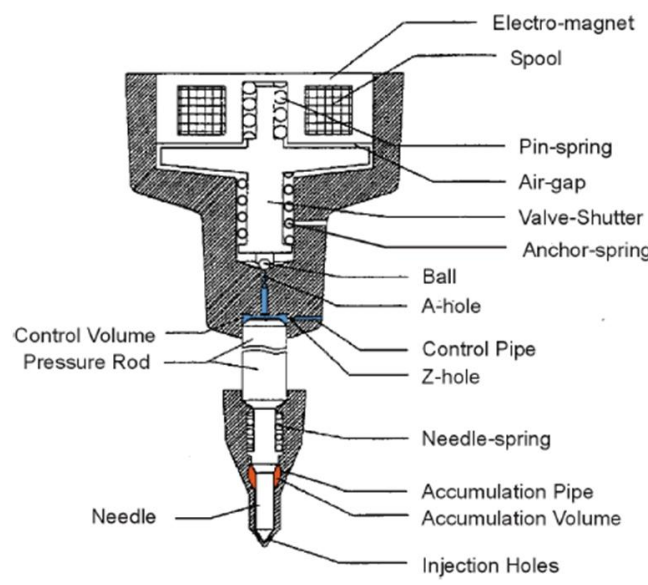

a)

Fig.3: a) Schematic of the simulated components in the LubeFluid 1D code; b) detailed view of the simulated scheme for the control zone, displaying the open position of a needle lift type injector. [5]

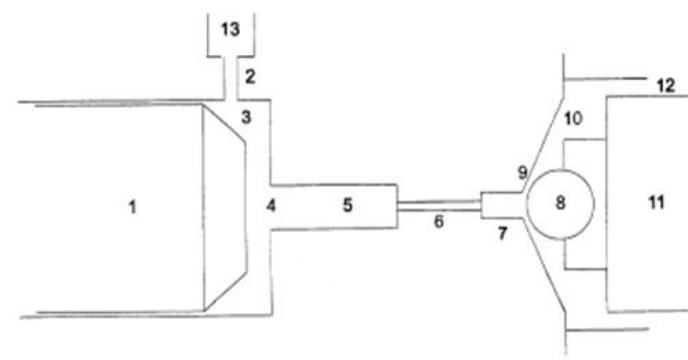

1) pressure rod; 2) Z-hole; 3) control volume; 4) A-hole entrance; 5) A-hole pre-chamber; 6) A-hole; 7) prehole; 8) ball; 9) valve seat; 10) ball-chamber; 11) valve actuator "pin"; 12) backflow pipes; 13) Zhole pre-chamber.

b)

A brief description of the new type of lubricant injector is described hereafter. In the upper part of the injector there is a magnetic circuit which is controlled by an Electronic Control Unit. When a voltage signal arrives from the ECU, the magnetic circuit attracts the valve-shutter and opens the valve. The control volume and the accumulation volume are high pressure chambers fed by the rail via adequate pipes. These chambers limit upwards and downwards the pressure rod. At rest (no voltage signal) both the control volume and the accumulation volume are at the same pressure and the pressure rod due to the different cross section at the bottom and at the top, keeps the needle in its seat. When the magnetic circuit open the valve, the pressure in the control volume gets lower since the fluid moves from this zone to the upper region through the open valve. As a consequence, the pressure rod is moved upwards by the pressure gradient, the needle is raised from its seat and the injection starts. When the electrical signal stops, the valve is closed and the injection ceases. The injection profile resulting from this process depends on the dynamic characteristic of the injector. In particular, the raising of the needle from its seat is governed by the fluid dynamics of both the control zone and the accumulation volume. The geometric features of these zones will be used as parameters in the optimization with genetic algorithms that will be carried out on basis of a first draft of the injector. [5]

Assuming a dependence of density on pressure only, it should be possible to introduce the fuel bulk modulus as defined by Parson [15]; moreover, the elasticity of the pipe walls has been calculated by reducing the bulk modulus of the fluid volume contained between them. The equations of flow inside a pipe were solved writing continuity and 
momentum equations considering one-dimensional isothermal flow. Taking into account those hypotheses, the equation system is:

$$
\begin{aligned}
& \left(\frac{\partial p}{\partial t}\right)+\left(\frac{u \partial p}{\partial x}\right)+\frac{\left(\rho c^{2} u \delta\right)}{A}+\rho c^{2}\left(\frac{\partial u}{\partial x}\right)=0 \\
& \left(\frac{\partial u}{\partial t}\right)+u\left(\frac{\partial u}{\partial x}\right)+\frac{\left(\frac{\partial p}{\partial x}\right)}{\rho}+f=0
\end{aligned}
$$

where $\delta=\left(\frac{d A}{d x}\right), u$ and $c$ are the oil and sound velocity, respectively, whereas $\rho$ is the fluid bulk density and $f$ is the friction factor.

Supposing that the density of both phases is only function of the pressure and does not vary, as the section pressure is constant and equal to the vapor pressure if there is the presence of a vapor phase, the continuity equation can be written as:

$$
\left(\rho_{v}-\rho_{l}\right) \frac{\partial \alpha}{\partial t}+u\left(\rho_{v}-\rho_{l}\right) \frac{\partial \alpha}{\partial x}+\rho \frac{\partial u}{\partial x}=0
$$

The pressure drop due to the friction in two-phase flow cannot be treated as mono-phase, since the vapor completely modifies the fluid dynamic conditions in the pipe; therefore, its evaluation was computed through the Lockhart's and Martinelli's empirical correlation. [13] A typical simulation result, obtained using the 1D LubeFluid code, is shown in Fig.4, which compares different injection timing effects on injection velocity profiles at 900 bar injection pressure.

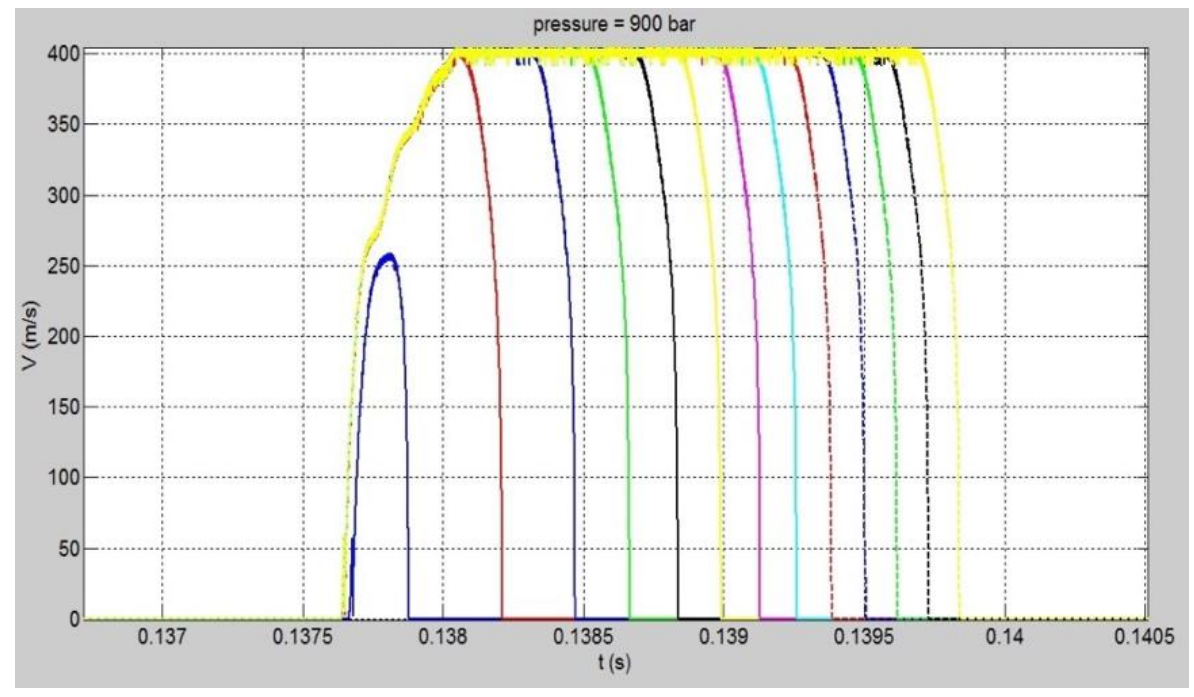

Fig.4: Injection velocity profile as function of excitation time. The first blue curve is representative of an injection in the ballistic regime. [5]

Initial simulation results highlight, that the time response of the injector significantly is affected by stiffness and preloading of its three springs: the pin spring, the anchor spring and the needle spring. These dynamic parameters hence are key parameters in the final optimization process to be performed in the next step of the ongoing project.

\section{Experimental setup}

Investigating mentioned injection characteristics calls for the establishment of a sound testing environment. Therefore a system was designed to provide testing conditions similar to real engine application boundary conditions in order to investigate thermo - physical properties and related effects on the lubricant spray. The test cell hence provides the possibility to address parameter variations such as pressure, temperature, injection pressure, nozzle geometry and lubricant property variations over the complete load range of a large two stroke marine diesel engine. 
The test cell design features a cylinder liner segment between two lubrication quill positions in order to simulate the lube oil spray under engine like conditions. Fig. 5 shows the segment which is covered and provided with an optical access in order to investigate on different effects on the lube oil spray pattern and the impingement characteristic on the cylinder liner wall.

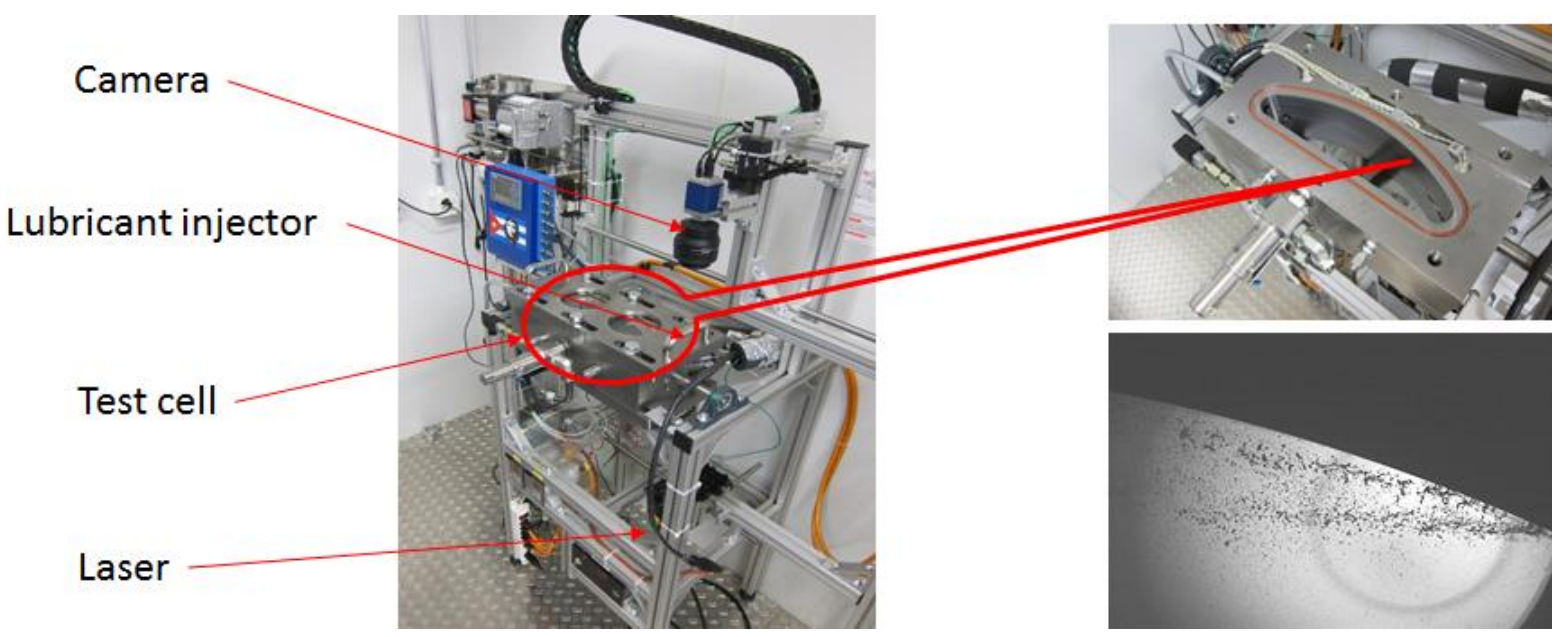

Fig.5: Schematic of the lube oil injection test cell and first impressions of the testing environment

In order to comply with real engine like conditions, a pre-study was performed to determine relevant system boundary parameters. The system is designed to provide pressure and temperature characteristics comparable to the full engine load range. Efforts in developing a fully operative test cell focus on the application of measurement technologies and instrumentation. A variety of Software and Hardware adaptions lead to the desired final test cell design. Adapting a standard Pulse Jet lubrication system and a high speed camera provides first impressions of a lube oil spray and gives a feedback on how the test cell performs.

Further adaptions of system components lead to preliminary information regarding the injection spray characteristics over the full engine load range. Fig. 6 shows a comparison between $25 \%$ and $100 \%$ engine load equivalent system boundary conditions and related lubricant spray patterns of a single-hole nozzle based on a typical diffused back-illumination measurement approach.
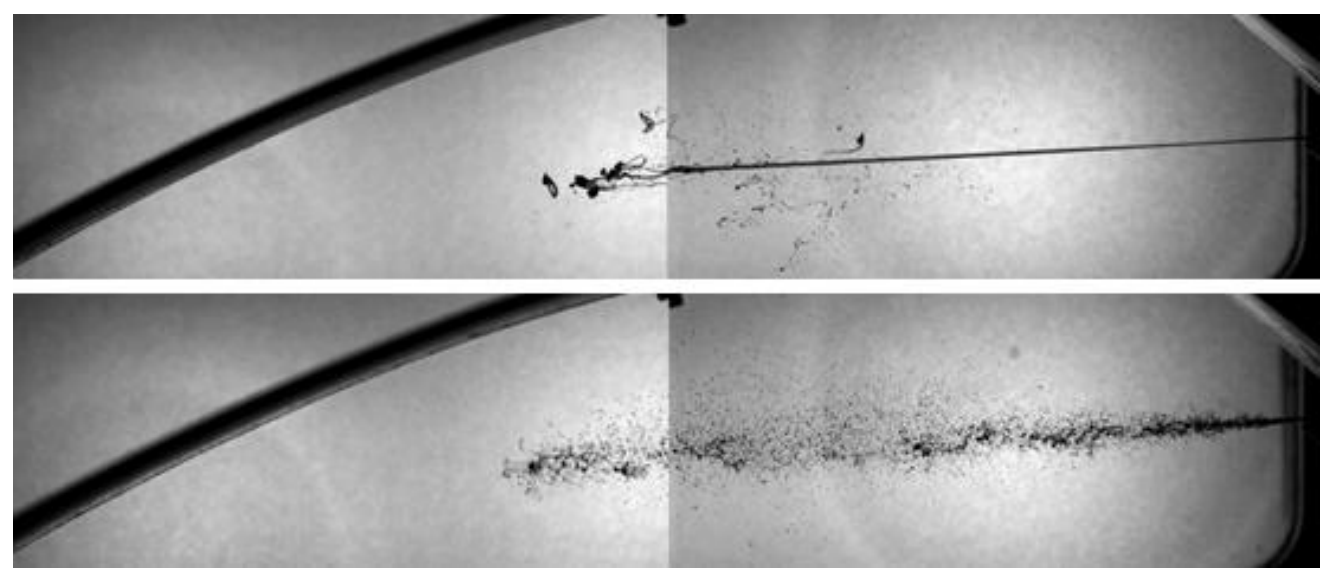

Fig.6: Diffused back-illumination approach measurement results of lube oil injection spray characteristics for 25\%engine load (1,6 bar of absolute cell pressure and $421 \mathrm{~K}$ lube oil temperature) and $100 \%$ engine load equivalent system boundary conditions (4.8 bar of absolute cell pressure and $476 \mathrm{~K}$ lube oil temperature)

Fig.6 also demonstrates the strong dependence between lube oil temperature and injection pressure. In fact, considering a lube oil temperature of $421 \mathrm{~K}$, an injection pressure of 1.6 bar absolute and a nozzle diameter of 0.75 $\mathrm{mm}$, the behaviour of the liquid phase is simply a liquid column. Instead, with an oil temperature of $476 \mathrm{~K}$ and an injection pressure of 4,8 bar absolute, a break-up in the liquid phase is visible, with the formation of a certain number of droplets. This break-up depends on the Ohnesorge number, which is the ratio between the Weber number square 
root and the Reynolds number. Thus, the decrease of the viscosity results in an increasing of the Reynolds number and a decay of the Ohnesorge number. The tested oil is characterized by an SAE 50 viscosity index with a kinematic viscosity of $19,2 \mathrm{~mm} / \mathrm{s}^{\wedge} 2 @ 100^{\circ} \mathrm{C}$ and for this reason the spray condition can shift from a no-break-up regime to a first wind induced break-up.

The acquired images are used to study spray characteristics. A Labview code is currently being developed to standardize measurement results for all testing conditions. Definitions of regions of interest (ROI) are utilized to characterize the development of the spray. Fig.7 shows a schematic of such an attempt in characterizing distinct injection characteristics. Two ROls are related to the upper and lower part of the spray (red and green rectangle) whereas a third one follows the spray tip along the distance from the nozzle towards the simulated cylinder wall. (yellow rectangle)

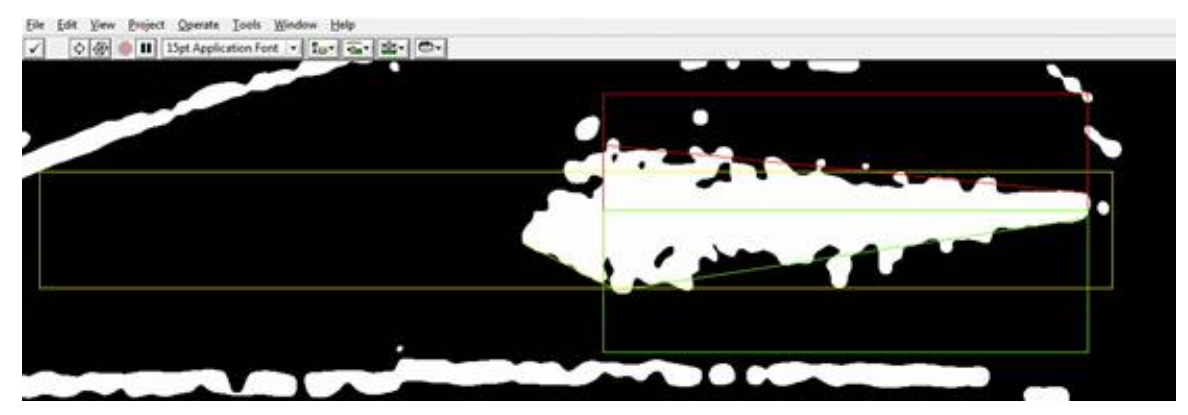

Fig.7: Schematic of a typical spray characterization approach

Next steps in this development relate to further optimizations of the system and the implementation of algorithms to quantify lubricant spray and impingement characteristics.

\section{Simulation tool development}

This section briefly discusses the numerical approach to develop a code which is capable of simulating lubricant sprays. A 1D CFD and a neural network approach is used with the objective to create a fast running application to compute spray characteristics variations and to quickly visualize the effect on the cylinder wall. A particular approach of predicting the lubricant spray characteristics has been applied by making use of a CFD numerical approach. Literature review and experimental data reveal the most suitable analytical spray models which best describe the phenomenology of lubricant sprays. [4]

The first step of the numerical code development relates to the computation of the jet velocity $v_{i}$ at the nozzle hole exit. This has been made using the relation derived from Bernoulli:

$$
v_{i}=k_{v} \sqrt{\frac{2 \Delta p}{\rho_{l}}}
$$

Where $k_{v}$ is the discharge coefficient from Hiroyasu et al, $\rho_{l}$ is the liquid phase density and $\Delta p$ is the pressure difference across the nozzle. Subsequently, the volume of the injected liquid for every time-step has been computed using a typical injection profile loaded from the acquired data, which is similar to the one in figure, to calculate the pressure-drop at every time-step (dt). Thus, the expression for the instant volume of oil is the following:

$$
V_{f}=A_{n z} v_{i} d t
$$

At this point it is necessary to compute the Sauter mean diameter (SMD) of the droplets so that it is possible to apply resulting drag forces. For this reason, a series of SMD prediction models has been implemented providing the possibility to change the model in the software graphic unit interface according to literature information related to the specific spray measurement results. The implemented models are derived from Hiroyasu-Kadota [6], Elkotb et al. [7], Hiroyasu-Arai [8], Hiroyasu-Arai-Tabata [9] respectively:

$$
S M D=23.9 \Delta p^{-0.135} \rho_{g}^{0.121} V_{f}^{0.131}
$$




$$
\begin{gathered}
S M D=3.08 \Delta p^{-0.54} \rho_{g}{ }^{0.06}\left(\sigma \rho_{l}\right)^{0.737} v_{l}^{0.385} \\
S M D=0.38 d_{n z} R e_{l}^{0.25} W e_{l}^{-0.32}\left(\frac{\mu_{l}}{\mu_{g}}\right)^{0.37}\left(\frac{\rho_{l}}{\rho_{g}}\right)^{-0.47} \\
S M D=8.7 d_{n z}\left(R e_{l} W e_{l}\right)^{-0.28}
\end{gathered}
$$

Equations (6) and (7) are related to the injection pressure rather than inlet velocity derived by injected droplet measurement into stagnant air. The equation by Hiroyasu and Kadota considers the volumetric flow rate of the liquid, but does not consider any liquid properties. Thus the equation is not strictly valid for liquids with physical characteristics like the lubricant. Equation (7), instead, considers liquid density, surface tension, kinematic viscosity, air density and injection pressure, which is the most suitable approach for the modelled lubricant spray. Equation (8) is suitable for incomplete spray, in fact, the atomization process depends mainly on the injection velocity in the nozzle hole. The spray cannot be formed correctly (incomplete spray) for low injection velocities, causing an insufficient atomisation, with a long transformation process from liquid column to droplets. This is the case that can occur in the lube oil spray, which can be characterized from low velocity and laminar flow due to the high viscosity of the liquid phase. However, to describe the complete spray, equation (9) appears to be the most suitable one. [4]

At this stage, a computation of the injected mass, $m_{i n j}$, for each time-step is performed with equation

$$
m_{i n j}=k_{v} A_{n z} \sqrt{2 \Delta p \cdot \rho_{l}} \mathrm{dt}
$$

Using the computed SMD provides the possibility to estimate the Reynolds field of the mean droplet as a function of time. Thus, according to the studies of Desantes et al [8] [9], who presented a description of the drag coefficient $C_{d}$ for sprays, equation (11) has been used to calculate the drag force:

$$
\begin{cases}C_{d}=\frac{24}{R e}\left(1+0.15 R e^{0.687}\right) & \text { if } R e \leq 10^{3} \\ C_{d}=0.44 & \text { if } \operatorname{Re}>10^{3}\end{cases}
$$

Finally, It is possible to calculate the mean force acting on the jet, as a weighted arithmetic mean to the injected masses of the j-th drag forces.

So far, the dynamic characteristics of the spray has been considered. Now, it is necessary to make an estimation of the spray angle which will be applied to the definition of the $3 \mathrm{~d}$ behaviour. The approach followed was to use a literature expression of the spray cone angle and to correct it introducing the dependencies from the viscosity to make a best fit to the value measured during the tests. Considering the equation for the spray angle provided by [10], corrected with a nozzle length-diameter ratio provided by [11], a best fit relation (12) for the cone spray angle has been found:

$$
\vartheta=457 \cdot d_{n z}^{0.512} v_{l}^{-1.53} \operatorname{atan}\left[\frac{1}{C_{n z}} 4 \pi \frac{\sqrt{3}}{6}\left(\frac{\rho_{g}}{\rho_{l}}\right)^{0.5}\right]
$$

With

$$
C_{n z}=3+0.28 \frac{L_{n z}}{d_{n z}}
$$

The source code of this numerical simulation tool is based on Labview for a full integration with the previously exhibited neural network code and with the designed graphical user interface (GUI). Main details and most important features of the simulation tool are shown in Fig. 8 


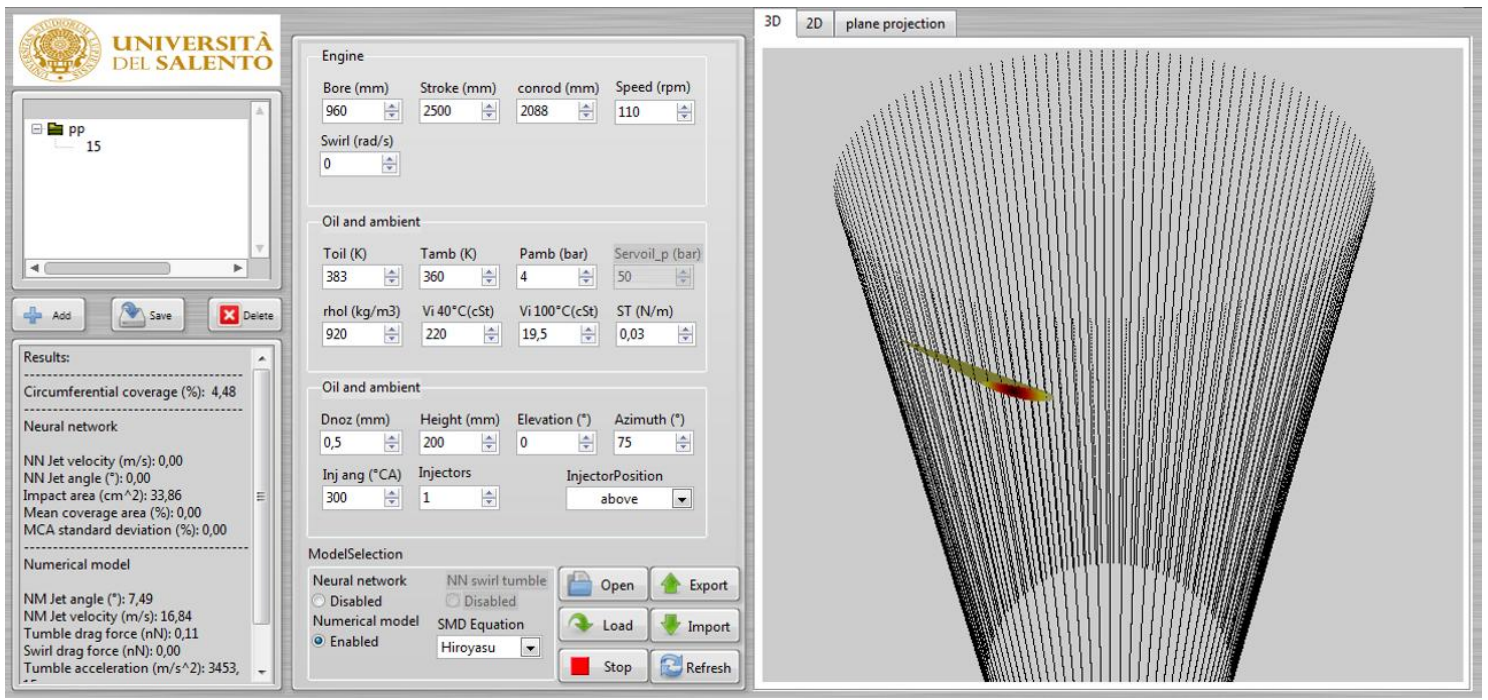

Fig.8: Main screen of the Lube oil simulation tool [4]

The main screen of the application is split into three main parts:

- Tree of configuration and calculation results

- Input parameters and computation model selection. In this mask, it is possible to insert the geometrical engine specifications (bore diameter, stroke, etc...), lubricant properties and injection system properties

- The graphical section shows a series of three tabs for the visualization in 3d render and on a 2D plane of the performed computation.

The developed application is capable of predicting the performance of a complete lubrication system, composed by a number of up to eight lubricant injectors with a maximum of five holes per injector. [4]

Another feature of the new simulation tool relates to the prediction of the shape of the lube oil spray. Fig. 9 compares the predicted spray characteristics with the measured ones of the above investigated ambient conditions at $25 \%$ and $100 \%$ engine load equivalent system conditions.

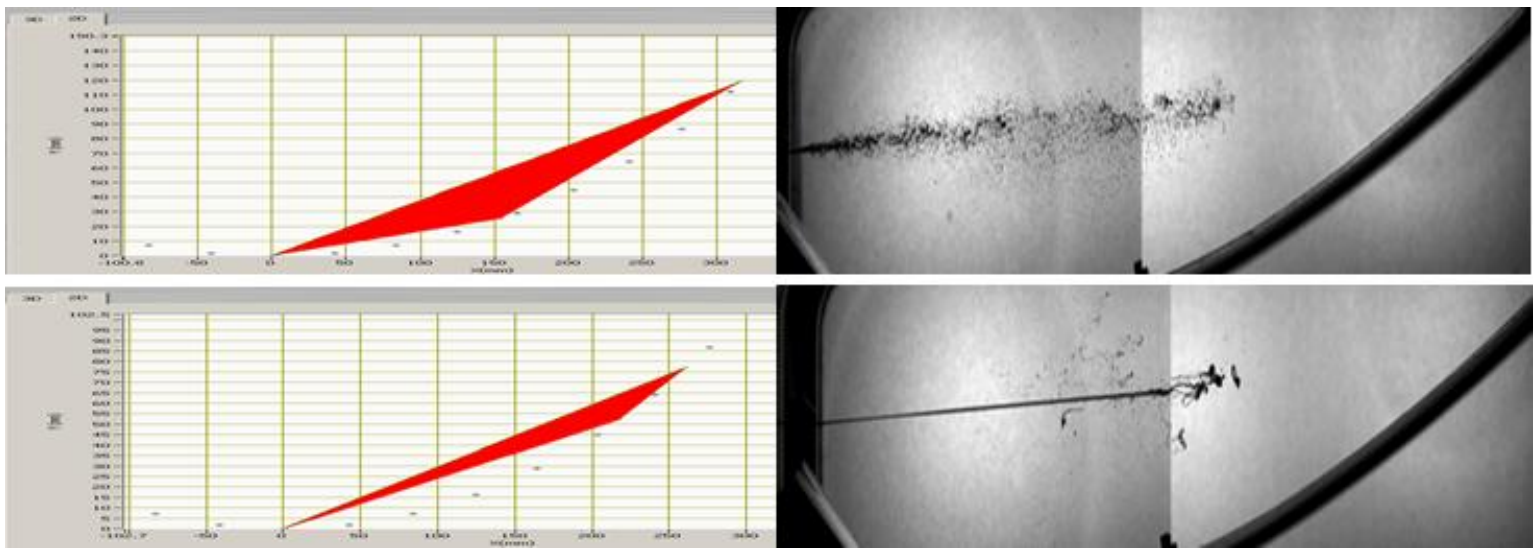

Fig.9: Comparison between predicted and measured lube oil injection spray characteristics for $25 \%$ engine load equivalent system conditions (1,6 bar of absolute cell pressure and $421 \mathrm{~K}$ lube oil temperature) and 100\% engine load equivalent system conditions (4.8 bar of absolute cell pressure and $476 \mathrm{~K}$ lube oil temperature)

Next steps of this investigation yield the implementation of experimentally gained data in a neuronal network of the simulation tool in order to enhance predictive accuracy of the model and to support design optimization of the new lubrication system development. 


\section{Conclusions}

The analysis presented here highlights key steps towards the development of a new, common rail based lubrication system comprising the application of needle lift type lubricant injectors as well as the consequent application of a dedicated testing facility and furthermore focuses on relevant simulation tool development activities in order to provide all the required tools for a successful lubrication system development. The simulation of relevant injector kinetics allows to predict lubricant flow characteristics in the injector and highlights the optimization potential of such an approach. A distinct testing facility supports design variation investigations of relevant lubrication system components under close to real engine boundary conditions and the same time provides the possibility to validate a new lubricant spray and impingement simulation tool. The combination of all activities represents a sound basis for further developments and optimization cycles in order to obtain the desired lubrication performance.

\section{Acknowledgements}

This project has received funding from the European Union's Horizon 2020 research and innovation programme under grant agreement No 634135

$\begin{array}{ll}\text { Nomenclature } & \\ \Delta p: & \text { pressure drop across the nozzle hole } \\ \rho_{g} \text { and } \rho_{l}: & \text { gas and liquid density respectively } \\ V_{f}: & \text { injected volume of liquid } \\ \sigma: & \text { liquid/air surface tension } \\ v_{l}: & \text { kinematic viscosity of the liquid } \\ d_{n z}: & \text { nozzle diameter } \\ L_{n z}: & \text { nozzle length } \\ R e_{l}: & \text { Reynolds number of the liquid } \\ W e_{l}: & \text { Weber number of the liquid } \\ \mu_{l}: & \text { dynamic viscosity of the liquid } \\ \mu_{g}: & \text { dynamic viscosity of the gas phase } \\ d_{n z}: & \text { nozzle diameter [mm]; } \\ v_{l}: & \text { lubricant viscosity [cSt]; } \\ \rho_{g} \text { and } \rho_{l}: & \text { the gas and liquid density respectively } \\ C_{n z}: & \text { nozzle geometry coefficient }\end{array}$

\section{References}

[1] P. Harper, M. Stark, Measurement of Piston Ring lubricant Film Thickness in Fired Engine using Ultrasonic Reflectometry, Cimac Paper, 2013

[2] M. Stark, R. Mittler, Lubricant Flow Optimization of Large Two Stroke Marine Diesel Engine Piston Ring Packs, ICEF2016-9326, Proceedings of the ASME 2016 INternal Combustion Fall Technical Conference

[3] M. Stark, R. Mittler, Optimization of Tribodynamic Effects to improve the Reduction Potential of Particulate Matter Concentrations in the Exhaust Gas of Large Two Stroke Marine Diesel Engines, 14FFI-0396, SAE2014

[4] M. Giangreco, Numerical and experimental study of two stroke marine engine lubrication systems, pHD thesis, University of Salento, 2013"

[5] A. de Risi, "Evaluation of Piston Ring Pack Phenomena," University of Salento - Internal report

[6] N. Ashgriz, "Handbook of atomization and sprays-Theory and applications," Springer, 2011, p. 101

[7] J. B. Heywood, "Internal combustion engine fundamentals," McGraw-Hill, 1988

[8] R. D. Reitz and F. V. Bracco, "On the Dependence of Spray Angle and Other Spray Parameters on Nozzle Design and Operating Conditions," SAE paper, no. 790494, 1979

[9] H. Hiroyasu and T. Katoda, "Fuel Droplet Size Distribution in a Diesel Combustion Chamber," SAE Trans, no. Paper 74017, 1974

[10] M. M. Elkotb, "Fuel Atomization for Spray Modeling," Prog. Energy Combust. Sci., vol. 8, no. 1, pp. 61-91, 1982

[11] H. Hiroyasu, M. Arai and M. Tabata, "Empirical Equations for the Sauter Mean Diameter of a Diesel Spray," SAE Technical Paper, no. 890464, 1989

[12] J. M. Desantes, X. Margot, J. M. Pastor, M. Chavez and A. Pinzello, " CFD-Phenomenological diesel spray analysis under evaporative conditions," Energy Fuels, vol. 23, p. 3919-3929, 2009

[13] Lockhart, R.W., Martinelli, R.C.; Chem. Eng. Prog., Vol. 45. 1949, pp. 39-48

[14] Josef Kunes: Dimensionless Physical Quantities in Science and Engineering. Elsevier, 2012, ISBN 0-12391458-2, S. 263

[15] I. Parson (ed.), Fieldspars and Their Reactions, 449 - 499, 1994 Kluwer Academic Publishers 


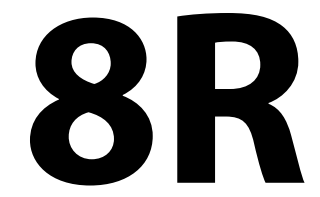

Combustion 2 


\title{
Multi-scale Eulerian-Lagrangian simulation of a liquid jet in cross-flow under acoustic perturbations
}

\author{
Swann Thuillet"1 ${ }^{*}$ Davide Zuzio ${ }^{1}$, Olivier Rouzaud ${ }^{1}$, Pierre Gajan ${ }^{1}$ \\ ${ }^{1}$ ONERA - The French Aerospace Lab \\ *Corresponding author: Swann.Thuillet@onera.fr
}

\begin{abstract}
The design of modern aeronautical propulsion systems is constantly optimized to reduce pollutant emissions while increasing fuel combustion efficiency. In order to get a proper mixing of fuel and air, Liquid Jets Injected in gaseous Crossflows (LJICF) are found in numerous injection devices. However, should combustion instabilities appear in the combustion chamber, the response of the liquid jet and its primary atomization is still largely unknown. Coupling between an unstable combustion and the fuel injection process has not been well understood and can result from multiple basic interactions.

The aim of this work is to predict by numerical simulation the effect of an acoustic perturbation of the shearing air flow on the primary breakup of a liquid jet. Being the DNS approach too expensive for the simulation of complex injector geometries, this paper proposes a numerical simulation of a LJICF based on a multiscale approach which can be easily integrated in industrial LES of combustion chambers. This approach results in coupling of two models: a two-fluid model, based on the Navier-Stokes equations for compressible fluids, able to capture the largest scales of the jet atomization and the breakup process of the liquid column; and a dispersed phase approach, used for describing the cloud of droplets created by the atomization of the liquid jet. The coupling of these two approaches is provided by an atomization and re-impact models, which ensure liquid transfer between the two-fluid model and the spray model. The resulting numerical method is meant to capture the main jet body characteristics, the generation of the liquid spray and the formation of a liquid film whenever the spray impacts a solid wall.

Three main features of the LJICF can be used to describe, in a steady state flow as well as under the effect of the acoustic perturbation, the jet atomization behavior: the jet trajectory, the jet breakup length and droplets size and distribution.

The steady state simulations provide good agreement with ONERA experiments conducted under the same conditions, characterized by a high Weber number (We>150). The multiscale computation gives the good trajectory of the liquid column and a good estimation of the column breakup location, for different liquid to air momentum flux ratios. The analysis of the droplet distribution in space is currently undergoing. A preliminary unsteady simulation was able to capture the oscillation of the jet trajectory, and the unsteady droplets generation responding to the acoustic perturbation.
\end{abstract}

\section{Keywords}

multiscale numerical simulation, liquid jet in gaseous crossflow, acoustic perturbation, Euler-Lagrange coupling

\section{Introduction}

The liquid jet in crossflow (LJICF) configuration covers several applications in engineering systems, such as combustion, chemical or even agriculture fields. Particularly in aircraft combustion chambers, systems where the fuel jet is injected normally into an air crossflow are commonly used. Compared with a free jet into a quiescent flow, this configuration enables a better mixing of fuel and air and fuel evaporation before delivery to the combustor. Therefore LJICF are found in numerous injection devices as the "Lean Preximed Prevaporizer" (LPP) device. However, this device operates on lean premixed combustion, a regime that easily leads to the apparition of combustion instabilities. Unstable combustion is the consequence of a thermo-acoustical coupling between instationary heat release from combustion and acoustic pressure oscillations in the combustion chamber. These instabilities lead to excessive engine vibration and possible irreversible damage of the propulsion system. Among the mechanisms that can be responsible for the variation of heat release from combustion, Apeloig [1] has shown the effect of : acoustic perturbations in the incoming airflow that creates fluctuations of the acoustic speed and pressure, state of the liquid phase injected in the combustion chamber (atomization, spray, filming), acoustic boundary conditions (including multiperfored liners [2]). The complete understanding of coupling mechanisms between unstable combustion and fuel injection process remains essential. The aim of our work is to perform a numerical simulation describing the behavior of a liquid jet in a subsonic gaseous crossflow under acoustic perturbation, thus improving the knowledge on the interaction between atomization and flow fluctuations.

Many studies have focused on steady-state LJICF. Mashayek et al. [2] gather the important parameters for the study of LJICF : the liquid and gaseous related physical parameters (density, viscosity, surface tension) and the parameters linked to the configuration, such as diameter of the jet and injector geometry. Pai et al. [3] identify four adimensional numbers for the jet behavior and atomization : the jet-to-crossflow momentum flux ratio $q$, the aerodynamic Weber number $W e_{g}$, the liquid Reynolds number $R e_{l}$ and the Ohnesorge number $O h_{l}$. Wu et al. [4] studied experimentally the influence of these parameters on the behavior of the jet. They observed similarities 


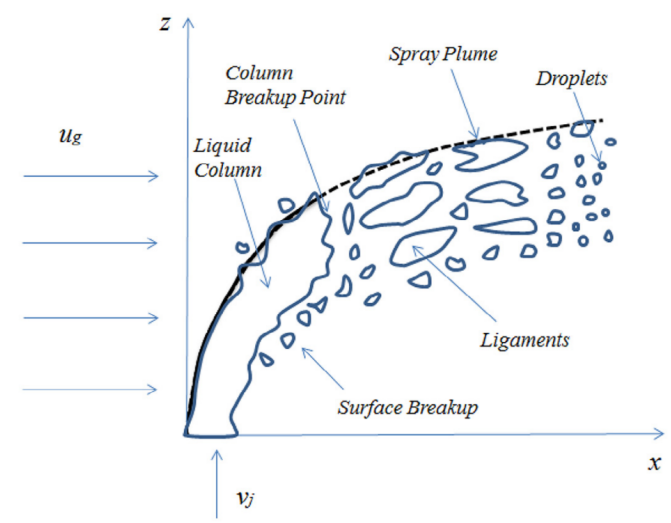

Figure 1. Liquid jet in crossflow configuration, from Broumand et al. [5]

between liquid jet primary breakup regimes and those reported by Hsiang and Faeth [6] for secondary breakup of droplets. Hence, they proposed a $q-W e_{g}$ map to classify the jet primary breakup. These map shows different breakup regimes, such as the column breakup, the multimode breakup or shear breakup.

The liquid jet breakup generates different liquid structures : ligaments, droplets and blobs (Fig. 1). Experimentally, the jet breakup is usually characterized by four features [5] : liquid jet primary breakup regimes, liquid jet trajectory, liquid jet breakup length and spray characteristics. The first experimental studies [4] focused on large-scale features of the liquid jet breakup, such as trajectory of the jet and column breakup location. In a review paper, No [7] makes an inventory of the empirical correlations for trajectory of the jet. They showed a huge discrepancy between the different correlations obtained from experimental studies. Indeed, there are many factors that impacts the jet trajectory, and a restricted number of dimensionless numbers cannot characterize totally the jet trajectory over several regimes [7].

A few experimental studies on the effect of acoustic perturbations on LJICF have been performed. Carpentier et al. [8] investigated the comportment of a liquid jet in the presence of acoustic waves generated by a loudspeaker upstream a Kundt tube. The position of the jet orifice to the acoustic velocity nodes influences directly the jet destabilization, up to the jet atomization. Song et al. [9] studied the effect of a modulated crossflow on the spray formed by the liquid jet injection. They found out that an oscillating gaseous crossflow faintly changes the trajectory of the jet whereas it affects its atomization, resulting in smaller and more numerous droplets than in the steady case. They lately showed a periodic behavior regarding mean drop sizes and mean drop velocity. Unlike Song, Anderson et al. [10] observed a cyclical oscillation of the jet penetration when they increase the modulation level rate $\tau=\frac{\mathcal{V}^{\prime}}{\bar{V}}$. They came to the following conclusion : for a small $\tau$ only atomization process is impacted, when $\tau$ becomes stronger, the jet trajectory will also be modified by the modulated crossflow.

This study focuses on a high aerodynamic Weber number $\left(W e_{g}>110\right)$, which is typical for shear breakup. In this type of primary breakup, the liquid jet is slightly deformed by the crossflow. Instabilities appears on the windward side of the liquid jet due to the strong shearing of the crossflow. These instabilities keep growing along the liquid jet and cause the breakup of the liquid column as a whole. Other instabilities are observed, creating ligaments then droplets stripped from the jet sides. These ligaments sizes have been widely studied by Sallam et al. [11], finding out that both the sizes of the ligaments and droplets increase with the distance from the orifice. Mazallon et al. [12] concluded that the length of the ligament increases progressively with the Ohnesorge number. This paper presents a multi-scale simulation of a LJICF in both stationary and perturbed gas flow up to the formation of the spray in the form of a dispersed phase.

\section{Numerical Approaches}

Phenomenological models have been developed to represent the mechanisms of primary breakup of the LJICF. Wang et al. [13] uses linear stability to describe the instability (especially Rayleigh-Taylor waves) of a round liquid jet in crossflow. They determined that three main terms contribute to the instability of the jet : jet velocity, surface tension and aerodynamic force. The analogy between jet atomization and "blobs" breaking up under Kelvin-Helmoltz instability enabled the development of two breakup models : the Taylor Analogy Breakup (TAB) [14] and the Wave breakup models [15]. These models can be easily integrated in Reynolds Averaged Navier-Stokes (RANS) computations.

Many numerical studies have been led with higher grid resolution, such as large eddy simulation (LES) [16]. This type of simulations represents large-scales features of the jet, but needs the development of sub-grid models to represent the drop generation process.

Direct numerical simulation (DNS) describes the whole atomization process from the larger scales to the smaller 
ones. They are generally based on sharp interface computed by Level-Set method [17], Volume-of-Fluid [18], and derived methods such as Refined Level-Set [19] and Coupled Level-Set and Volume-of-Fluid [20]-[21]. These approaches require a large computational effort and can be combined with AMR method [22] to reduce the number of cells in the numerical domain or Euler-Lagrangian coupling (in a discrete element sense)[23].

The aim of this paper is to assess a new numerical approach based on a multi-scale methodology for the breakup of a liquid jet in cross-flow. The multi-scale methodology is described in the following section. While the twofluid model captures the larger scale phenomena of the liquid jet breakup, the spray evolution is described by a well established dispersed phase model resolved in a Lagrangian framework. The Eulerian-Lagrangian coupling enables the simultaneous resolution of the two previous models. Being the DNS approach too expensive for the simulation of complex injector geometries, the multiscale approach provides a less expensive alternative that can be easily integrated in industrial LES of combustion chambers.

This multi-scale method is validated in the case of a liquid jet in steady gaseous crossflow. A preliminary unsteady simulation shows the effect of an oscillating crossflow on the jet behavior.

\section{Numerical method}

The multi-scale approach has been proposed by Blanchard [24] as a tool to be integrated in a LES framework. This approach has been already validated on the primary atomization of a sheared liquid sheet. This approach is based upon three numerical models :

- a two-fluid model based on finite volume discretization of conservation equation for both liquid and gas,

- a dispersed phase model with a Lagrangian solver which describes the motion of the droplets stripped from the jet,

- a sub-scale atomization model that detects zones where the droplets are produced. This model enables the exchanges between the two previous models.

The methodology has been implanted in to the ONERA legacy CEDRE code [25].

\section{Two-fluid model}

The two-fluid model (usually gas/liquid) considers two fluids simultaneously present in any point of the domain. The hypothesis of local mechanical equilibrium imposes that the two fluids have the same velocity and pressure within the given cell. The mass and momentum balance equation resolved by the model are :

$$
\begin{aligned}
& \frac{\partial \tilde{\boldsymbol{\rho}}}{\partial t}+\operatorname{div}(\tilde{\boldsymbol{\rho}} \otimes \boldsymbol{v})=0 \\
& \frac{\partial \rho \boldsymbol{v}}{\partial t}+\operatorname{div}(\rho \boldsymbol{v} \otimes \boldsymbol{v}+p \mathbf{I})=\operatorname{div}\left(\boldsymbol{\tau}_{\boldsymbol{v}}+\boldsymbol{\tau}_{\boldsymbol{c}}\right)+\rho \boldsymbol{g}+\boldsymbol{s}_{p}
\end{aligned}
$$

with $\tilde{\boldsymbol{\rho}}=\left[\alpha_{l} \rho_{l}, \alpha_{g} \rho_{g}\right]^{T}=\left[\tilde{\rho}_{l}, \tilde{\rho_{g}}\right]^{T}$ the fluid bulk densities, $\alpha_{l, g}$ the volume fractions of the liquid and the gas, $\rho_{l, g}$ the densities of the liquid and the gas, $\tau_{c}$ and $\tau_{v}$ respectively the capillary and viscous stress tensor, $g$ the gravity acceleration and $s_{p}$ the two-way coupling source term. The mixture density is $\rho=\alpha_{l} \rho_{l}+\alpha_{g} \rho_{g}$. As the configuration studied in this paper is iso-thermal, we do not consider the energy balance equation.

The numerical resolution of the system is done by finite volume scheme on unstructured 3D meshes. The time discretization is based on an explicit RK2 scheme. A second-order MUSCL scheme is used to achieve robust second order space accuracy, while a special attention has been paid to the multislope limitation procedure [26] in order to limit the diffusion of the interface.

Thermodynamic closure laws are considered for each phase : perfect gas and weakly compressible liquid [27].

\section{Dispersed phase model}

In a typical propulsion system, the number of droplets created from the fuel atomization can be huge, thus it is impossible to simulate each droplet individually. A statistical approach is chosen, based on the resolution of a Boltzmann equation [28] where a scalar function represents the average liquid droplets density. The resolution is based on a Lagrangian method, called particulate method. The dispersed phase model assumptions are :

- the droplets are rigid spheres,

- the spray is sufficiently diluted that the volume occupied by the droplet can be neglected,

- the evaporation, the secondary breakup and the collisions between droplets are not taken into account in the present simulations. Nevertheless, these features can be activated in the CEDRE code.

The concept of numerical particles is introduced, each particle being characterized by a numeric weight carrying the information of many physical droplets. These numerical particles obey transport equations. The spray is coupled to the carrier phase in a full two-way coupling. The numerical integration of this model is fully described in [24]. 


\section{Atomization model}

The atomization model couples the the two-fluid model and the dispersed phase model. This model must determine the location where the liquid is transferred from one model to another. The conservation of liquid mass and momentum is imposed between the two models.

The activation of the atomization model in a given cell is based on several criteria :

- the atomization should occur when the two-fluid solution is too diffuse,

- the model should not affect multifluid resolved liquid structures.

These criteria are rewritten with threshold values imposed by the user on the liquid volume fraction $\alpha_{l}$ and its gradient $\nabla \alpha_{l}$ calculated in the two-fluid model. The threshold on the volume fraction is set to $\alpha_{l}^{\text {atom }}=0.1$, this value is a good compromise to trigger the atomisation where the liquid phase is diluted enough. The value of $\left\|\nabla \alpha_{l}\right\|^{\text {atom }}$ is directly linked to the interface thickness :

$$
\delta_{\text {int }} \approx \frac{1}{\left\|\nabla \alpha_{l}\right\|^{\text {atom }}}
$$

Thus, the threshold value must indicate a zone where the interface is diffuse enough, that is why we choose $\left\|\nabla \alpha_{l}\right\|^{\text {atom }}=C . \Delta x$ with $C=0.5$ and $\Delta x$ the local cell dimension.

Once the atomization is activated, the particles are injected in each cell $\Omega_{i}$ by respecting : the number of injected particles, the initial velocity of each droplet, the initial position randomly taken inside $\Omega_{i}$, the physical properties of the droplets (same as the liquid), and diameter of the droplets. The diameter of the droplet is still a user-defined parameter at the present time. In this work, the diameter is chosen with the use of experimental correlations, thus representing the final diameter after primary and secondary atomization.

\section{Results and discussion \\ Experimental set-up}

Because of the few experimental studies that show the liquid jet in presence of an oscillationg crossflow, ONERA carried out an experimental investigation [29]. The purpose of this study is to understand the behavior of the spray generated from the breakup of a liquid jet in gaseous crossflow in the presence of combustion instabilities, and particularly the formation of liquid films when the droplets impact the duct walls.

The experimental configuration consists of a duct with rectangular cross-section where the liquid is injected by an circular orifice located on the bottom wall. Upstream, a loudspeaker delivers an imposed periodic acoustic perturbation. The figure 2 shows the experimental disposition, the highlighted rectangular area corresponds to the domain calculated by the numerical simulations. This domain has the following dimensions : $0.23 \mathrm{~m}$ in length, $0.05 \mathrm{~m}$ in width and $0.05 \mathrm{~m}$ in height. A convergent section is placed just before the nozzle to reduce the duct height to $0.02 \mathrm{~m}$, and thus stabilize the mean flow : the gas flow is considered as non-turbulent. The airflow velocities given in this paper refers to the velocity at the channel outlet. Several measurement methods provide us a significant database for the primary breakup of the liquid jet with a gaseous crossflow. The comparison between the numerical and experimental results on the same configuration allow to minimize the discrepancies due to the different geometrical and physical parameters (such as confinement or high-temperature effects).

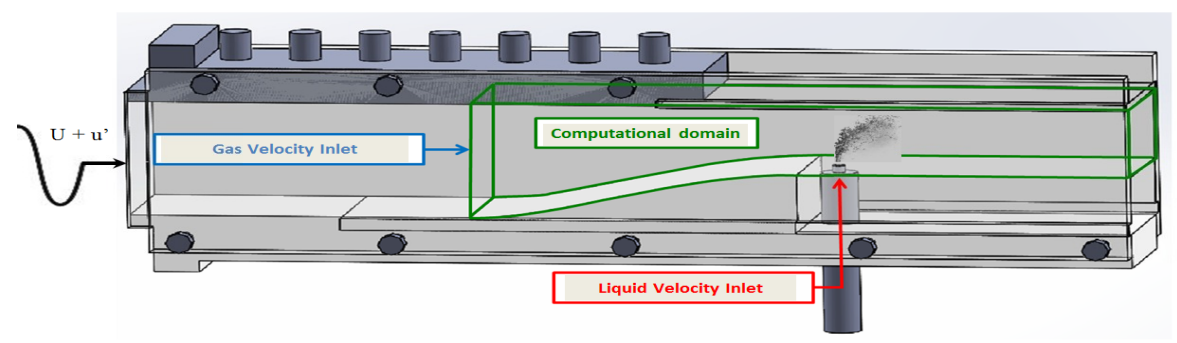

Figure 2. Experimental configuration set up at ONERA [29], the computational domain is highlighted in green

\section{Steady flow simulations}

First, we focus on a liquid jet injected in a steady gaseous crossflow. This configuration is chosen as representative as possible as a multipoint fuel injection device. The flow description is :

- the two fluids are respectively water for the liquid jet and air for the crossflow,

- the gaseous crossflow is laminar, the liquid jet is supposed laminar,

- the study is conducted at standard temperature and pressure (STP). 
Table 1. Parameters of the simulation of the liquid jet in steady crossflow

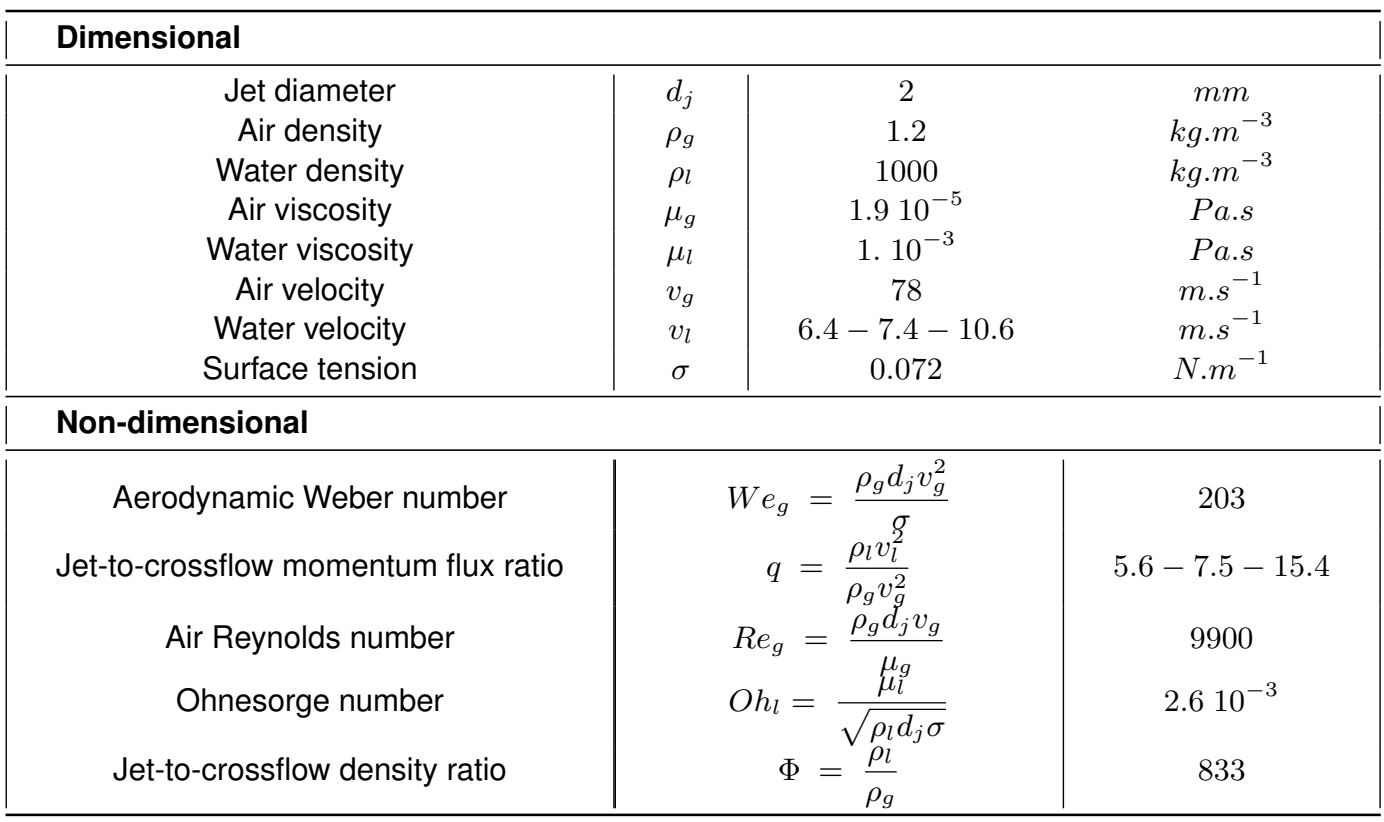

The computational domain is the same as the experimental setting presented in the previous subsection. The mesh consists of about 3.8 M elements, the most refined zone is along the jet inlet surface where the smallest cell size is $\Delta x=80 \mu \mathrm{m}$. There are 30 cells in the section of the jet. The mesh becomes coarser downstream the injection point, where the dispersed phase model entirely describes the liquid phase. At the moment, no subgrid turbulence model has been activated as we are mainly interested in largest scales liquid/gas interactions. Turbulent effects will be taken in account in the future simulations. We choose an explicit time discretization, with a time step $\Delta t=5.10^{-8} \mathrm{~s}$. An effective parallelization on 480 processors enables to get a steady regime in approximately 10 hours CPU time.

The inlet gas velocity has a constant value of $v_{g}=78 \mathrm{~m} . \mathrm{s}^{-1}$. The other physical parameters are summarized in table 2. As the liquid considered is water, the Ohnesorge number is $O h_{l}=2.6410^{-3}$. As long as this number is smaller than 0.3 , Sallam at al. [11] states that the viscous effects are small enough that the Ohnesorge number do not play any role to classify the jet primary breakup. The present Weber number is representative of the shear breakup regime.

Based on the correlations from Sallam's experimental observations [11], the droplet diameter has been fixed at $d_{l}=100 \mu \mathrm{m}$. This diameter represents the droplets at the channel exit, after secondary breakup.

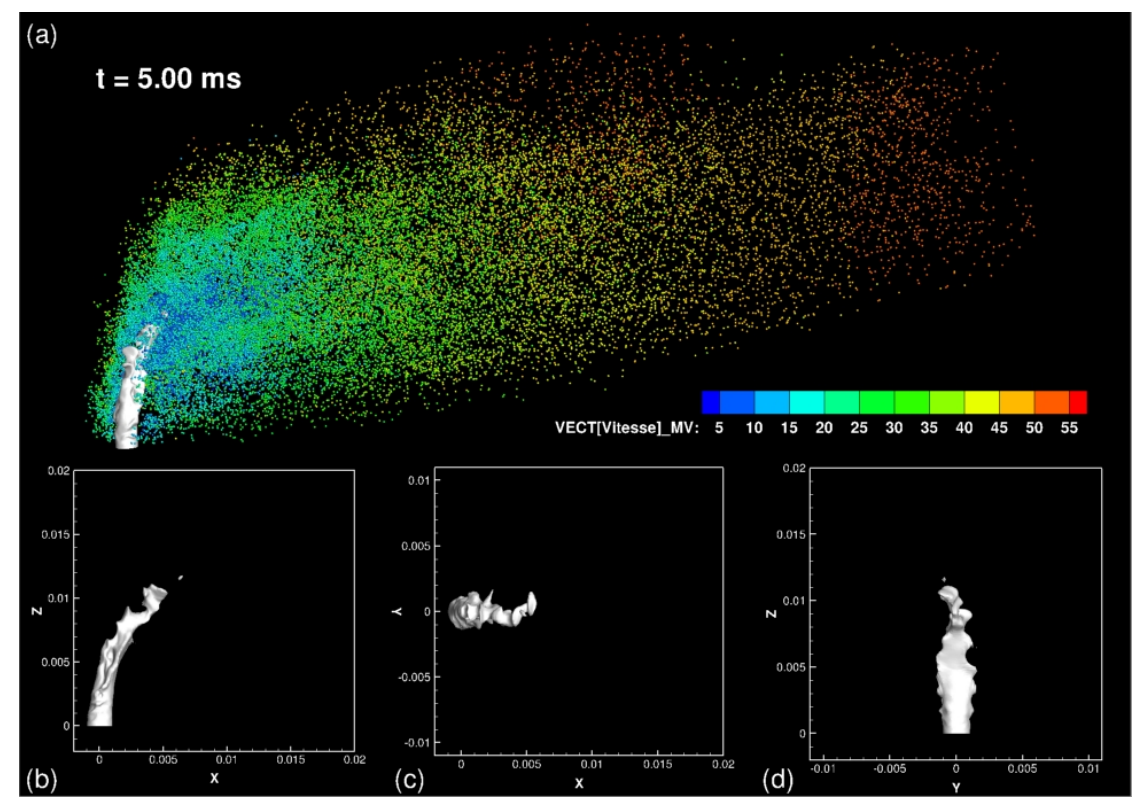

Figure 3. Simulation of the liquid jet in a gaseous steady crossflow 
We firstly focus on the simulation with $q=7.5$. The figure 3-(a) shows a snapshot of the simulated liquid jet in cross-flow, $5 \mathrm{~ms}$ after the beginning of the liquid injection. The white shape represents the liquid core of the jet, depicted by the iso-contour $\alpha_{l}=0.5$. This value of the liquid volume fraction $\alpha_{l}$ is chosen to define as precisely as possible the envelope which encloses most of the liquid. The particles are the Lagrangian droplets created by the atomization model.

The first step is to analyze the large scale features of the jet. We observe the development of the liquid jet bent by the crossflow. Waves propagate on the windward part of the jet, and these instabilities keep growing as they move along the jet up to provoke the breakup of the liquid column. A first estimation shows that the wavelength of these waves are slightly under the diameter jet (Fig. 3-(b)). Sallam et al. [11] give a correlation based on his experimental results, that leads to a wavelength of $\lambda_{s}=0.3$. A protocol is actually developed to determine precisely the wavelength and the frequency of these waves.

As experimentally observed, the section of the jet is deformed and tends to a "kidney shape" (Fig. 3-(c)).

The analysis of the side view pictures of the jet enables to define jet trajectories for three differents value of $q$. These results have been compared to the experimental data [29] on the Figure 4.

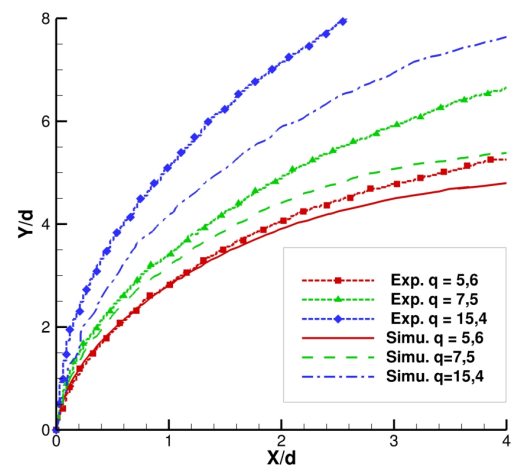

Figure 4. Effect of the $q$ parameter on the jet trajectory

The numerical simulation results shows a further penetration of the liquid jet as the parameter $q$ increases, in accordance with the experimental results [7]. Near the injection orifice $\left(\frac{x}{d_{j}}=0.5\right)$, the trajectories from numerical simulations are in a good agreement with the experimental observations. Beyond this point, the numerical curves tend to underestimate the jet trajectory, especially with increasing values of $q$. This may be explained by the liquid inlet conditions : laminar or turbulent profiles [16]. In our case the laminar profile seems to leads to a larger area exposed to the air flow, involving a stronger drag; this force increasing the jet bending.

With the side view of the liquid jet 3-(b), we can also estimate the mean column breakup location. The liquid jet breakup length corresponds to the streamwise distance reached by the liquid jet before its complete breakup. Even if the determination of the breakup length is not very accurate for several reasons [5], we will compare our results to experimental and numerical studies. The three numerical cases provides a constant normalized length $\frac{x_{b}}{d_{j}}$, around 5.2. Such a tendancy has also been observed in previous experimental studies [4]. Indeed, when the inlet liquid velocity increases, the penetration of the jet will tends to be more important while the breakup characteristic time is shorter. We compared our value of 5.2 with the data provided in the paper of Sallam et al. [11] and the theoretical value given by Wu et al. [4] of 8.6. Although our value appears far from Wu's value, the comparison with the experimental results from Sallam gives a good agreement, especially when the experimental conditions are closer to the considered configuration. Other numerical studies, such as Li and Soteriou [20] or Xiao et al. [16] also obtained similar values for the normalized breakup length.

Concerning the column breakup height $y_{b}$, the simulations give a proportionality $y_{b} \propto q^{0.47}$. Several authors [5] integrates other non-dimensional parameters in their correlation, such as $R e_{g}$ or $W e_{l}$. Here again, there is a large degree of discrepancies in literature for the prediction of the column breakup height, nevertheless the relation found in that study is in the range of the correlations given by Wu et al. [4]..

On the figure 3-(a), the particles are colored by their velocity magnitude. The initial velocity of the particles when they are generated is close to the liquid jet velocity magnitude. The particles velocities near the domain outlet are still mush smaller that the airflow velocity. Considering a characteristic length $l_{0}=0.1 \mathrm{~m}$ equal to the distance between the jet orifice and the outlet to and $v_{g}$ the gas flow velocity, the characteristic time of a particle and the Stokes number are :

$$
t_{0}=\frac{\rho_{p} d_{p}^{2}}{18 \mu_{g}}=0.029 \mathrm{~s}, \quad S t=\frac{t_{0} v_{g}}{l_{0}}=21.8
$$


As the Stokes number is greater than 1 , the particles are mostly driven by inertia and their relaxation to the air flow velocity is weak. The transit time of a particle in the domain is calculated with the length of the channel $l_{0}$ and a gas velocity $v_{0} \approx 50 \mathrm{~m} . \mathrm{s}^{-1}$ slightly lower than the airflow velocity because of the jet wake:

$$
t_{p}=\frac{l_{0}}{v_{0}} \approx 0.002 s<<t_{0}
$$

This explains that the particles velocity does not reach the airflow velocity before the channel exit.

A further analysis of the liquid jet and spray is currently in progress and will provide more details about the instabilities on the surface of the jet and the droplets distribution. These results will be compared to the data from ONERA experiments.

\section{Unsteady flow simulation}

The aim of this simulation is to show the capability of the multi-scale simulation to handle the propagation of an acoustic wave and its effect on the liquid jet behavior.

Table 2. Parameters of the simulation of the liquid jet in unsteady crossflow

\begin{tabular}{|c|c|c|c|}
\hline \multicolumn{4}{|l|}{ Dimensional } \\
\hline $\begin{array}{c}\text { Air velocity } \\
\text { Water velocity }\end{array}$ & $\begin{array}{l}v_{g} \\
v_{l}\end{array}$ & $\begin{array}{c}39 \rightarrow 91 \\
6.2\end{array}$ & $\begin{array}{l}m . s^{-1} \\
m . s^{-1}\end{array}$ \\
\hline \multicolumn{4}{|l|}{ Non-dimensional } \\
\hline $\begin{array}{l}\text { Aerodynamic Weber number } \\
\text { Jet-to-crossflow momentum flux ratio } \\
\text { Air Reynolds number }\end{array}$ & $\begin{array}{c}W e_{g} \\
q \\
R e_{g}\end{array}$ & $\begin{array}{r}51 \\
3.9 \\
4900\end{array}$ & $\begin{array}{l}6 \\
1 \\
500\end{array}$ \\
\hline
\end{tabular}

The parameters of the simulation are given in the table 2. Starting from a steady state regime has been reached, then a periodic oscillation on the inlet acoustic velocity is imposed. The frequency of the oscillation is set to its experimental value $177 \mathrm{~Hz}$. This frequency ensures the presence of a pressure node near the injection point, thus maximizing the acoustic velocity variations at this location. The modulation rate is $\tau=40 \%$.

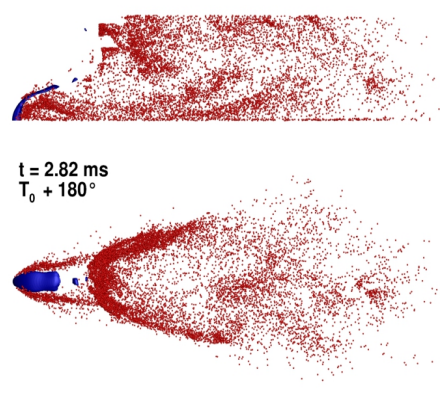

(a)
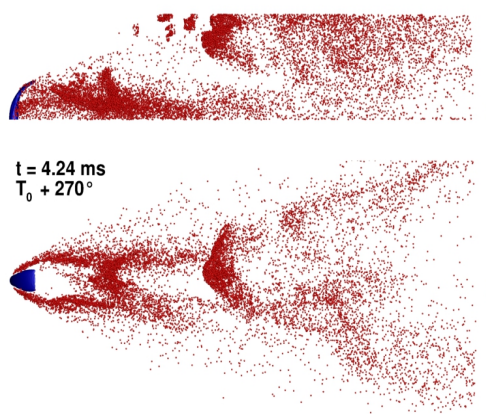

(b)
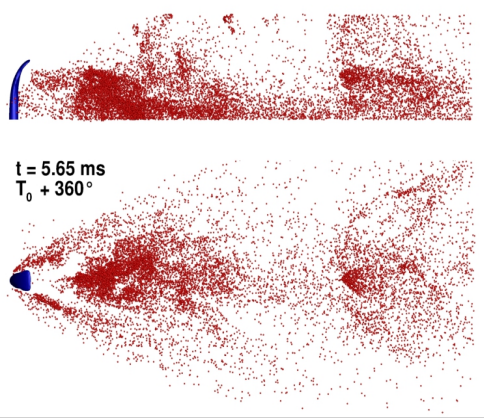

(c)

Figure 5. Simulation of the liquid jet in unsteady crossflow

Figure 5 shows snapshots of the jet at three different times after the beginning of the oscillation. The temporal phase is given in relation to the velocity oscillation imposed at the inlet. The blue shape is the liquid core of the jet, the Lagrangian particles are colored in red. Note that the atomization model has been activated at the beginning of the unsteady state, which can explain that the number of droplets is lower than the steady simulations presented in the previous part. The side views show that the liquid jet shape and penetration changes with the inlet velocity oscillation. Image processing of the numerical side views provides a frequency of the jet flapping around $177 \mathrm{~Hz}$, consistent with the frequency of the upstream velocity oscillation.

A qualitative analysis of the pictures shows that the spray distribution depends on the time. For instance, a dense cloud of droplets reaches the upper wall at $t=T_{0}+180^{\circ}$ (Fig. 5-(a)) whereas the most dense region is located near the bottom wall at $t=T_{0}+270^{\circ}$ (Fig. 5-(b)). For all the three images, droplets are mainly clearly on the sides of the jet, as expected in such a flow regime. Besides, the unsteady spray distribution is qualitatively different from its steady counterpart (Fig. 3).

The simulation demonstrates the unsteady and heterogeneous characters of the spray distribution. When coupled with an evaporation model, it is expected to observe strong local variations of the mixture stoichiometry. Such a feature is consistent with the experimental observations of Apeloig [1].

This simulation is still in progress and additional analysis will provide more information about the liquid jet oscillation and the spray distribution. The numerical results will be compared to the experimental data from ONERA [29]. 


\section{Conclusions}

The simulation of a liquid jet in both steady and unsteady gaseous crossflow have been presented in this paper. The steady computation gives the good trajectory of the liquid column and a good estimation of the column breakup location, for different liquid to air momentum flux ratios. The unsteady simulations has shown the oscillation of the jet trajectory and the unsteady droplets generation responding to the acoustic perturbation. A quantitative study of the spray properties (distribution, velocities ...) is currently undergoing. The numerical data will be compared to the experiments conducted in ONERA.

The numerical approach is based on a multi-scale methodology. The results prove that the multi-scale methodology is able to capture the large scales of the LJICF and to reproduce the droplet generation from its breakup.

In a next step, evaporation processes will be taken into account [25]. Since there are still uncertainties on the data setting, the impact of the flow regime (laminar, turbulent ...) for both the liquid and gaseous phases will be also investigated.

One of the most important constraint to this method is the determination of the droplet radius. A predicting diameter model, such as an ELSA method, is considered to improve the proposal approach. This model will determine the local droplet size according to the actual atomization mechanisms. The model will be validated against the experimental observations.

\section{References}

[1] Apeloig, J., 2013, "Étude expérimentale du rôle de la phase liquide dans les phénomènes d'instabilités thermo-acoustiques agissant au sein de turbomachines diphasiques", Ph.D. thesis, ONERA

[2] Mashayek, A., and Ashgriz, N. , 2011, "Atomization of a Liquid Jet in a Crossflow", Handbook of Atomization and Sprays, pp. 657-683.

[3] Pai, M., Pitsch, H., and Desjardins, O., 2009, "Detailed numerical simulations of primary atomization of liquid jets in crossflow", 47th AIAA Aerospace Sciences Meeting, p. 373

[4] Wu, P. K., Kirkendall, K. A., Fuller, R. P., and Nejad, A. S., 1997, "Breakup processes of liquid jets in subsonic crossflows", Journal of Propulsion and Power, 13(1), pp. 64-73.

[5] Broumand, M., and Birouk, M., 2016, "Liquid jet in a subsonic gaseous crossflow: Recent progress and remaining challenges", Progress in Energy and Combustion Science, 57, 1-29.

[6] Hsiang, L. P., and Faeth, G. M., 1995," Drop deformation and breakup due to shock wave and steady disturbances", International Journal of Multiphase Flow, 21(4), pp. 545-560.

[7] No, S. Y., 2015, "A review on empirical correlations for jet/spray trajectory of liquid jet in uniform cross flow", International Journal of Spray and Combustion Dynamics, 7(4), pp. 283-313

[8] Carpentier, J. B., Baillot, F., Blaisot, J. B., and Dumouchel, C., 2009, "Behavior of cylindrical liquid jets evolving in a transverse acoustic field", Physics of Fluids, 21(2)

[9] Song, J., Ramasubramanian, C., and Lee, J. G., 2014, "Response of Liquid Jet to Modulated Crossflow", Atomization and Sprays, 24(2).

[10] Anderson, T. J., Proscia, W., and Cohen, J. M., 2001, "Modulation of a liquid-fuel jet in an unsteady cross-flow", ASME Turbo Expo 2001 : Power for Land, Sea, and Air, American Society of Mechanical Engineers.

[11] Sallam, K. A., Aalburg, C., and Faeth, G. M., 2004, "Breakup of round nonturbulent liquid jets in gaseous crossflow", AIAA journal, 42(12), pp. 2529-2540.

[12] Mazallon, J., Dai, Z., and Faeth, G. M., 1999, "Primary breakup of nonturbulent round liquid jets in gas crossflows", Atomization and Sprays, $9(3)$.

[13] Wang, S., Huang, Y., and Liu, Z. L., 2014, Theoretical analysis of surface waves on a round liquid jet in a gaseous crossflow, Atomization and Sprays, 24(1)

[14] O'Rourke, P. J., and Amsden, A. A., 1987, "The TAB method for numerical calculation of spray droplet breakup", Los Alamos National Lab., NM (USA).

[15] Reitz, R. D., and Bracco, F. V., 1982, "Mechanism of atomization of a liquid jet", The Physics of Fluids, 25(10)

[16] Xiao, F., Dianat, M., and McGuirk, J. J., 2013, "Large eddy simulation of liquid-jet primary breakup in air crossflow", AIAA journal, 51(12), pp. 2878-2893.

[17] Desjardins, O., and Pitsch, H., 2010, "Detailed numerical investigation of turbulent atomization of liquid jets", Atomization and Sprays, 20(4).

[18] Tomar, G., Fuster, D., Zaleski, S., and Popinet, S., 2010, "Multiscale simulations of primary atomization", Computers \& Fluids, 39(10), pp. 1864-1874.

[19] Herrmann, M., 2008, "A balanced force refined level set grid method for two-phase flows on unstructured flow solver grids", Journal of Computational Physics, 227(4), pp. 2674-2706.

[20] Li, X., and Soteriou, M. C., 2014, "High-fidelity simulation of high density-ratio liquid jet atomization in crossflow with experimental validation", 26th Annual Conference on Liquid Atomization and Spray Systems, ILASS Americas (pp. 18-21).

[21] Ménard, T., Tanguy, S., and Berlemont, A., 2007, "Coupling level set/VOF/ghost fluid methods: Validation and application to 3D simulation of the primary break-up of a liquid jet", International Journal of Multiphase Flow, 33(5), pp. 510-524.

[22] Zuzio, D., and Estivalezes, J. L., 2011, "An efficient block parallel AMR method for two phase interfacial flow simulations", Computers \& Fluids, 44(1), pp. 339-357.

[23] Herrmann, M., 2010, "A parallel Eulerian interface tracking/Lagrangian point particle multi-scale coupling procedure", Journal of Computational Physics, 229(3), pp. 745-759

[24] Blanchard, G., 2014, "Modélisation et simulation multi-échelles de l'atomisation d'une nappe liquide cisaillée", Ph.D. thesis, ONERA.

[25] Refloch, A., Courbet, B., Murrone, A., Villedieu, P., Laurent, C., Gilbank, P., Troyes, J., Tessé, L., Chaineray, G., Dargaud, J., Quémerais, E., Vuillot, F., 2011, "CEDRE software", AerospaceLab, (2), p-1.

[26] Le Touze, C., Murrone, A., and Guillard, H., 2015, "Multislope MUSCL method for general unstructured meshes", Journal of Computational Physics, 284, pp. 389-418.

[27] Dutoya, D., and Matuszewski, L.,2011, "Thermodynamics in CEDRE", AerospaceLab, (2), p-1.

[28] Pai, M. G., and Subramaniam, S., 2009, A comprehensive probability density function formalism for multiphase flows, Journal of Fluid Mechanics, 628, p. 181

[29] Desclaux A., 2016, "Étude de l'influence d'une excitation acoustique sur le comportement d'un jet liquide débouchant transversalement dans un écoulement d'air", Masters thesis, ONERA. 


\title{
Investigation of the Influence of Microexplosions on a Laminar Premixed Water-in-Fuel Emulsion Spray Flame
}

\author{
N. Yokev ${ }^{1}$, J.B. Greenberg ${ }^{* 1}$ \\ ${ }^{1}$ Aerospace Engineering, Technion, Israel Institute of Technology, Haifa, Israel \\ *Corresponding author: aer9801@technion.ac.il
}

\begin{abstract}
A model is presented for a one-dimensional laminar premixed flame, propagating into a rich, off-stoichiometric, fresh homogenous mixture of water-in-fuel emulsion spray, with air and inert gas. The main purpose is to investigate the steady-state burning velocity and burnt temperature as functions of parameters such as initial water content in the emulsified droplet and total liquid droplet loading. In particular the influence of microexplosion of the spray's droplets on the flame's characteristics will be highlighted for the first time.
\end{abstract}

\section{Keywords}

water-in-fuel droplets; spray; microexplosions; laminar flame.

\section{Introduction}

It is common knowledge that tremendous effort is currently being invested in the search for viable biofuel blends for combustion engineering in an effort to reduce harmful NOx and PM emissions to the atmosphere. This effort has been spurred on by increasingly stringent international protocols governing the restricted emission of harmful pollutants to the atmosphere (see, for example, [1]). In practical terms, biofuel blends must be created to be fit for utilization in existing combustors (such as jet engines, for example) thereby circumventing the need to perform expensive modifications to current combustors or, more critically, to designing new suitable combustors.

Of the many ideas being considered to attempt to meet the strict standards dictated by protocols is the use of water-in-fuel emulsions. Consideration of water-in-fuel emulsion (hereinafter referred to as WIFE) droplets for combustion involves understanding a number of aspects of the physical behavior of these droplets as they evaporate, in terms of processes occurring within the droplet and their impact on the immediate vicinity outside the droplet. As will be described it is these processes that are responsible for the benefits that the droplets potentially possess, which may be advantageously harnessed under appropriate operating conditions.

Kadota and Yamasaki [2] appear to be the first authors to present a review dedicated to theoretical and experimental research on water fuel emulsion combustion until the year 2002. Subsequently, in 2014, Khan et al. [3] reviewed current trends in water-in-diesel emulsion as a fuel. Their paper focuses exclusively on experimental studies that were carried out to investigate the physics of both NOx and particulate reduction and the phenomenon of micro-explosion that may occur under appropriate operating conditions in WIFE (see later). In addition, there is a discussion of research into the actual influence of water-in-diesel emulsions on engine performance for a wide range of engine types and loading conditions. It is of interest to note that, due to the variety of engine set-ups and experimental methodologies employed, results reported in the literature were not consistent. However, generally, a water content of between $5-40 \%$ by volume was successfully utilized. Although there was a consensus concerning the ability of the water-in-fuel emulsions to reduce NOx and particulate production the reported extent of the reduction was far from uniform.

The first models of emulsified water/oil droplets appeared in 1977 [4, 5]. Jacques [4] solved numerically a heat transfer model containing essential elements of the underlying behavior of the transient heating of a single emulsified fuel droplet. His main concern was to try to quantify the primary effect of the water in the droplet. Attention was particularly focused on attempting to replicate the experimentally observed reduction of particulate formation by the water's presence based on the premise suggested by Shyu et al. [6] that, for waterless fuel droplets, this occurs during latter stages of heavy fuel oil combustion as a result of cracking of liquid phase hydrocarbons within the droplet at temperatures above $700 \mathrm{~K}$. The addition of water was shown to act as an extra source for heat loss thereby lowering the temperature within the droplet so that the extent of cracking reactions (and, therefore, subsequent formation of particulates) was reduced.

Micro-explosions seem to have been first reported in 1965 [7]. Experimental studies showed that combustion of compound droplets with different volatile fluids may be accompanied by violent explosion of the droplets. As the temperature increases, the higher volatile fluid, which is strategically located at the core, will evaporate sooner in the form of a bubble. The fast expansion of the gas at the core will result in partial or full disintegration of the 
parent droplet and it is this behavior that is called a micro-explosion. A similar occurrence called puffing happens when the nucleation bubble ruptures the parent droplet along with fine steams of tiny droplets. This happens when the bubble expands very rapidly and tears through the host droplet at a specific location. These phenomena are very important to combustion because they provide a secondary mechanism of atomization by breaking down large droplets into a smaller, faster evaporating form. Since the fuel combusts in gaseous form only, microexplosions help more fuel to change phase from liquid to gas, thereby improving the efficiency of fuel consumption .

One of the most important factors promoting the occurrences of micro-explosions is the superheat limit. Superheated liquid is defined as a fluid at extremely high temperatures - well above the boiling point, and thus occurs mainly when the working fluid undergoes a significant increase in pressure (Fu et al [8]). Since pressure is the main mechanism for controlling the boiling point of the fluid, micro-explosions could have major application in diesel engines, where the pressure is already designed to be extremely high (Law $[5,9,10])$. This phenomenon has a greater effect on heavier components (Fu et al [8]), such as fuel versus water, which means that a fuel shell will have higher tolerance to evaporation than water and it will allow more pressure to build up inside the emulsion droplet, promoting stronger micro-explosions. In [10], Law and Wang experimented with micro-explosions under different pressures, and showed an agreement with their previous mathematical theory. They theorized that increasing the pressure will enhance the occurrences of micro-explosions, and demonstrated this it up to 5 atmospheres.

Shinjo et al [11] described the detailed physics behind micro-explosions and puffing using numerical simulations to solve several problems such as the dynamics of the surfaces between emulsion droplet's fluids. The process of puffing begins with the growth of a single bubble between the water and fuel surfaces until it bursts. The outcome of the puffing depends on the following three mechanisms: (1) oscillation of the emulsified water droplet, (2) thrust created by the jet of vapor when the water boils, and (3) inertia of the bubble when it bursts - or in other words, the smaller the water content within the droplet is, the more effective the burst will be at detaching the water from the oil shell. On the other hand, if the water sub-droplet is large, the force applied by water vapor ejected from the emulsion droplet will push the water sub-droplet further within the oil shell - causing an increase in the effectiveness of the secondary atomization, but also the breakup time period. Since effective micro-explosions can improve fuel efficiency, a discussion of the increased time period is warranted. Tarlet et al [12] experimentally found that increasing either the water content or the droplet radius would promote longer micro-explosion delays. This concurs with the theoretical work of Shinjo et al [11], with respect to the addition of water content. Tarlet et al [12] also found that shorter micro-explosions delays occur when the ambient temperature and the relative velocity are higher, as well as an increase in the liquid content. He validated these conclusions with 27 different experiments under different conditions that would either promote or suppress the onset of micro-explosions. Finally, we mention a very recent theoretical study by Girin [13] which provides a solid analytical/theoretical foundation to the understanding of micro-explosions, as well as predicting the resulting number and size of the smaller fuel droplets that are formed.

Due to the complexity involved in modeling WIFE spray combustion under conditions in which micro-explosions take place, all theoretical studies we are aware of have only been concerned with the sequential evaporation of the fuel outer "shell" followed by the evaporation of the water inner "core". Thus, in a series of papers Hsuan et al [14], Hsuan and Lin [15,16] and Hou et al [17] considered WIFE spray flames. The mathematical analysis presented by these authors related to both fuel rich and fuel lean flames and took account of the fact that the major effect of the water evaporation was as a heat sink due to the heat loss incurred as the water absorbed heat for evaporation. Actually, such heat loss is also present when the outer fuel layer evaporates but the latent heat of evaporation of water is typically much larger than that of hydrocarbon fuels. For example, for n-octane it is of the order of $300 \mathrm{~kJ} / \mathrm{kg}$ in comparison to $2254 \mathrm{~kJ} / \mathrm{kg}$ for water. This discrepancy can have a strong effect on the laminar flame speed, depending on the relative amount of water in the droplets and the location of onset of pure water evaporation. With the respect to the latter both possible scenarios were examined (i.e. pre- and post- flame front water evaporation) combined with the nature of the fuel evaporation (completely before the flame front or only partially so). The methodology used was based on Lin et al's [18] analysis of fuel spray combustion which made use of asymptotic methods for finding the flame structure and an expression for the burning velocity. Critical conditions for flame extinction and propagation were determined as a function of the initial water content in the droplets.

An allied study, motivated by the behavior of emulsion explosives made up of a mixture of a fuel and an ammonium nitrate-water solution, was conducted by Hughes et al. [19]. A one-dimensional theoretical model was solved numerically to examine combustion waves driven by competing exothermic and endothermic reactions in the presence of water evaporation. A rich variety of influences of the water content on the flame propagation and its stability were noted, mainly because of its impact on the temperature and thereby on the fraction of ammonium 
nitrate consumed by the competing exothermic and endothermic reactions. Interestingly, the water evaporation was treated as a first-order chemical reaction with a specially constructed rate coefficient. However, once again, micro-explosions were not accounted for.

In the current work we describe a preliminary model of the behavior of a laminar off-stoichiometric water-in-fuel emulsion spray flame. We present for the first time a simplified theory for the steady state propagation of such flames and incorporate the overall effect of micro-explosions in the model. The governing equations are solved analytically and a formula for the burning velocity of the flame is derived which highlights the influence of the relative amount of water to liquid fuel in the sprays droplets and the impact of droplet micro-explosions.

\section{The model and governing equations}

\section{Assumptions}

We consider a laminar one-dimensional premixed flame propagating into a fuel rich off-stoichiometric fresh homogeneous mixture of fuel vapor, water-in-fuel emulsion droplets, oxygen and an inert gas. The flame is taken to propagate from right to left. The droplets are viewed from a far-field vantage point, i.e. their average velocity is equal to that of their host environment. For qualitative purposes this approach has been demonstrated to be quite valid [20]. The temperature of the droplets is taken to be that of the surroundings until the fuel's boiling temperature is reached; essentially the droplets heat-up time is small compared to the characteristic time associated with their motion. The initial ratio of the amount of water to the amount of liquid fuel in the droplets is assumed to be constant irrespective of the size of the droplets. Droplet evaporation is assumed negligible until a prescribed reference temperature (such as the boiling temperature of the liquid fuel) is attained at which point the fuel begins to evaporate at a finite rate. In the meantime, there is a buildup of water vapor within the compound droplets as the liquid water evaporates until conditions are attained for which the water vapor erupts through the outer layer of evaporating fuel thereby shattering the liquid fuel into smaller fuel-only droplets which, of course, continue to evaporate.

The stoichiometry of the gas mixture that the flame front meets is taken to be fuel rich, so that the limiting reactant consumed by chemical reaction is oxygen. It is assumed that the various transport coefficients, such as thermal conductivity, diffusion coefficient, specific heat at constant pressure, latent heat of vaporization of the droplets etc., can be satisfactorily specified by representative constant values. An overall reaction of the form $v_{F}$ fuel $+v_{O}$ oxidant $\rightarrow$ products is taken to describe the chemistry. However, in this way the water vapor does not participate in the chemical reaction. Nevertheless, neutralizing the role of chemical effects associated with water vapor by utilizing a one-step chemical reaction is not unreasonable at a first attempt, as it was found [21] that evaporated water vapor has less than a $10 \%$ effect on flame extinction conditions whereas thermal effects, that are included in our model, influence them significantly

As the velocity of propagation of the flame is much less than the velocity of sound, dynamic compressibility effects in the mixture can be neglected. Thus, the density becomes only a function of the temperature through the gas law.

A realistic polydisperse spray can be described using the sectional method [22] in which the droplet sizedistribution is divided into sections (or bins) and conservation equations are derived for the liquid fuel in each section allowing for droplet evaporation from a given section, say $\mathrm{j}$, and addition to that section as droplets evaporate in the next section up and become eligible for membership in j. For simplicity at the current stage we assume a mono-sectional description of the spray's droplets.

Droplet evaporation is assumed negligible until a prescribed reference temperature $T_{v}$ (such as the boiling temperature of the liquid fuel) is attained. The pre-vaporization region will be denoted: $R_{1}=\left\{\xi:-\infty<\xi \leq-\xi_{v f}\right\}$ where $\xi=-\xi_{v f}$ is the point at which the ambient temperature equals $T_{v}$. At this point the liquid fuel in the droplets begins to evaporate. Micro-explosion is supposed to occur instantaneously at a predefined temperature $T_{e}$, producing smaller droplets which will evaporate more rapidly than the parent droplets from which they were created. This region will be denoted by $R_{2}=\left\{\xi:-\xi_{v f} \leq \xi \leq-\xi_{e}\right\}$. The stoichiometry of the gas mixture that the flame front meets will be determined by the particular conditions under consideration. We restrict our analysis to those circumstances in which a homogeneous flame front actually exists. If the flame front is located at $\xi=0$ in the moving coordinate system we adopt, the pre- and post-flame zones can be denoted by $R_{3}=\left\{\xi:-\xi_{e} \leq \xi \leq 0\right\}$ and $R_{4}=\{\xi: 0 \leq \xi<\infty\}$, respectively.

\section{Governing equations}

Under the aforementioned assumptions the governing equations for the gas-phase describe energy and oxygen mass fraction conservation: 


$$
\begin{aligned}
& T^{\prime}=T^{\prime \prime}+D a \cdot m_{O} \exp \left[\theta\left(1-T^{-1}\right)\right]-L_{W} S_{v W}-L_{f} S_{v f} \\
& m_{O}^{\prime}=m_{O}^{\prime \prime}-D a \cdot m_{O} \exp \left[\theta\left(1-T^{-1}\right)\right] \\
& m_{d f}^{\prime}=-S_{v f} \\
& m_{d W}^{\prime}=-S_{v W}
\end{aligned}
$$

where ( $)^{\prime}$ denotes the derivative with respect to $\xi$, the normalized mass fractions of oxygen, liquid fuel and water are defined by

$m_{O}=\frac{m_{O}^{*}}{m_{O u}^{*}}, m_{d f}=\frac{m_{d f}^{*}}{m_{f u}^{*}+\left(m_{d f u}^{*}+m_{d W u}^{*}\right)}, m_{d W}=\frac{m_{d W}^{*}}{m_{f u}^{*}+\left(m_{d f u}^{*}+m_{d W u}^{*}\right)}$

with the upper asterisk denoting actual mass fraction and the suffixes $O, u, f, d$ and $W$ denoting oxygen, unburned value, fuel, droplets and water, respectively. $T$ is the non-dimensional temperature, $D a$ is the usual chemical Damkohler number, $\theta$ is the dimensionless activation energy, $L_{W}, L_{f}$ are the latent heats of vaporization of the water and fuel, respectively, and where the evaporation source terms for fuel and water are

$$
S_{v f}=S_{f}\left(H\left(\xi+\xi_{v f}\right)\left(1-H\left(\xi_{e}+\xi\right)\right)\right)+f S_{f} H\left(\xi+\xi_{e}\right), \quad S_{v W}=S_{W} H\left(\xi+\xi_{e}\right)
$$

in which, following the sectional approach to spray modelling [22]:

$$
S_{f}=C_{f} m_{d f}, S_{W}=C_{W} m_{d W}
$$

where $C_{f}$ and $C_{W}$ are evaporation Damkohler numbers. In Eqs.(6a) and (6b) use is made of the Heaviside function $H$ to define the regions in which the expressions it multiplies apply. Thus, in Eq.(6a) the first term on the right hand side is applicable once the fuel starts to evaporate through to the point $\xi=-\xi_{e}$ where the microexplosion occurs. From that point onwards the rate of evaporation of the fuel is increased by a factor of $f$ due to the shattering of the droplets by the micro-explosion. Incorporating a result of Girin's micro-explosion theory [13] within the framework of the sectional approach to spray modelling, an expression for this factor can be shown to be

$$
f \approx\left(0.27\left(1-\alpha^{1 / 3}\right)\right)^{-2}
$$

where $\alpha$ is the initial ratio of water to liquid fuel in the spray's droplets. As mentioned previously this ratio is assumed to be the same for all the droplets initially in the spray. In what may be considered as the current first order model the sudden micro-explosion at $\xi=-\xi_{e}$ simply results in the same amount of remaining fuel instantaneously changing its rate of evaporation. Other features of the occurrence of a micro-explosion, such as complete droplet rupturing puffing [11] and the spatial location of the new sized droplets, are not accounted for at the current stage. The evaporation of the water is assumed to occur within the original compound droplets and the micro-explosion releases all water vapor instantaneously at $\xi=-\xi_{e}$ so that $C_{W} \rightarrow \infty$. This fact will express itself via the matching conditions between regions $R_{2}$ and $R_{3}$.

To close this mathematical formulation boundary and matching conditions are required. These are readily shown to be

$$
\begin{aligned}
& \xi \rightarrow-\infty \quad T=T_{u}, m_{O}=1, m_{d f}=m_{d f u}, m_{d W}=m_{d W u} \\
& \xi \rightarrow \infty \quad T=T_{b}, m_{O}=0 \\
& \xi=-\xi_{v f} \quad T=T_{v},\left[m_{O}\right]=\left[m_{O}^{\prime}\right]=\left[T^{\prime}\right]=0, m_{d f}=m_{d f u} \\
& \xi=-\xi_{e} \quad T=T_{e},\left[m_{o}\right]=\left[m_{o}^{\prime}\right]=[T]=\left[m_{d f}\right]=0,\left[T^{\prime}\right]=L_{W} m_{d W u} \\
& \xi=0 \quad[T]=\left[m_{O}\right]=\left[m_{d f u}\right]=0,\left[T^{\prime}\right]=-Q,\left[m_{O}^{\prime}\right]=Q
\end{aligned}
$$

where the subscript $u$ denotes unburned conditions.

\section{Solution}

The solution to the afore-defined problem is readily developed using known asymptotic methods, and is found to be

In $R_{l}$ :

$T(\xi)=\left[1-L_{w} \alpha \tilde{\delta} e^{\xi_{e}}-\frac{C_{f} f L_{f}[(1-\alpha) \widetilde{\delta}] e^{-C_{f}\left(-\xi_{e}+\xi_{v f}\right)+\xi_{e}}}{C_{f} f+1}-\frac{C_{f} L_{f}[(1-\alpha) \widetilde{\delta}] e^{\xi_{v f}}}{C_{f}+1}+\frac{C_{f} L_{f}[(1-\alpha) \widetilde{\delta}] e^{-C_{f}\left(-\xi_{e}+\xi_{v f}\right)+\xi_{e}}}{C_{f}+1}\right] e^{\xi}$

$m_{0}=1-e^{L e \xi}$ 
$m_{d f}=(1-\alpha) \tilde{\delta}$

In $R_{2}$ :

$T(\xi)=$

$-L_{f}[(1-\alpha) \tilde{\delta}]+\left(1-L_{w} \alpha \tilde{\delta} e^{\xi_{e}}-\frac{C_{f} f L_{f}[(1-\alpha) \tilde{\delta}] e^{-C_{f}\left(-\xi_{e}+\xi_{v f}\right)+\xi_{e}}}{C_{f} f+1}+\frac{C_{f} L_{f}[(1-\alpha) \tilde{\delta}] e^{-C_{f}\left(-\xi_{e}+\xi_{v f}\right)+\xi_{e}}}{C_{f}+1}\right) e^{\xi}+\frac{L_{f}[(1-\alpha) \tilde{\delta}] e^{-C_{f}\left(\xi+\xi_{v f f}\right)}}{C_{f}+1}(15 a)$

$m_{0}=1-e^{L e \xi}$

$m_{d f}=(1-\alpha) \tilde{\delta} e^{-C_{f}\left(\xi+\xi_{v f}\right)}$

In $R_{3}$ :

$T(\xi)=-L_{w} \alpha \tilde{\delta}-L_{f}[(1-\alpha) \tilde{\delta}]+e^{\xi}+\frac{L_{f}[(1-\alpha) \widetilde{\delta}] e^{-C_{f}\left[\left(-\xi_{e}+\xi_{v f}\right)+f\left(\xi+\xi_{e}\right)\right]}}{C_{f} f+1}$

$m_{0}=1-e^{L e \xi}$

$m_{d f}=(1-\alpha) \tilde{\delta} e^{-C_{f}\left[\left(-\xi_{e}+\xi_{r f}\right)+f\left(\xi+\xi_{e}\right)\right]}$

In $R_{4}$ :

$T(\xi)=1-L_{w} \alpha \tilde{\delta}-L_{f}[(1-\alpha) \tilde{\delta}]+\frac{L_{f}[(1-\alpha) \tilde{\delta}] e^{-C_{f}\left[\left(-\xi_{e}+\xi_{v f}\right)+f\left(\xi+\xi_{e}\right)\right]}}{C_{f} f+1}$

$m_{O}=0$

$m_{d f}=(1-\alpha) \tilde{\delta} e^{-C_{f}\left[\left(-\xi_{e}+\xi_{v f}\right)+f\left(\xi+\xi_{e}\right)\right]}$

The locations of the onset of evaporation and the micro-explosion are determined according to predefined temperatures and can be readily found to be:

$$
\begin{aligned}
& -\xi_{v_{f}}=\ln \left\{\frac{T_{v f}+\frac{C_{f} L_{f}[(1-\alpha) \tilde{\delta}]}{C_{f}+1}}{1-L_{w} \alpha \widetilde{\delta} \xi_{e}-\frac{C_{f} f L_{f}[(1-\alpha) \tilde{\delta}] e^{-C_{f}\left(-\xi_{e}+\xi_{v f}\right)+\xi_{e}}}{C_{f} f+1}+\frac{C_{f} L_{f}[(1-\alpha) \tilde{\delta}] e^{-C_{f}\left(-\xi_{e}+\xi_{v f}\right)+\xi_{e}}}{C_{f}+1}}\right\} \\
& -\xi_{e}=\ln \left\{T_{e}+L_{f}[(1-\alpha) \tilde{\delta}]+L_{w} \alpha \tilde{\delta}-\frac{L_{f}[(1-\alpha) \tilde{\delta}] e^{-C_{f}\left(-\xi_{e}+\xi_{v f}\right)}}{C_{f} f+1}\right\}
\end{aligned}
$$

The burning velocity is determined to be:

$U=\frac{R T_{a} \sqrt{2 L e \lambda A}}{E} * \exp \left\{\frac{-E}{2 R T_{a}^{*}}-\frac{L_{f^{\prime}}[(1-\alpha) \widetilde{\delta}]}{2}\left[1-\frac{U^{2} e^{-\frac{C_{f}^{*} \lambda\left[\left(-\xi_{e}+\xi_{v f}\right)+f \xi_{e}\right]}{U^{2}}}}{C_{f}{ }^{*} \lambda \cdot f+U^{2}}\right]-\frac{L_{w^{\prime} \alpha \widetilde{\delta}}}{2}\right\}$

in which $T_{a}$ is the adiabatic flame temperature, $\lambda$ is the thermal conductivity of the gas mixture, $R$ is the gas constant, $E, A$ are the activation energy and pre-exponential coefficient of the global chemical reaction and $\left(L_{f}^{\prime}, L_{W}^{\prime}\right)=\theta\left(L_{f}, L_{W}\right)$ are related to the latent heats of evaporation of the fuel and water, respectively.

Note that since $C_{f}$ is in non-dimensional form according to $C_{f}=C_{f}^{*} \lambda / U^{2}$ Eq.(20) becomes an implicit equation for the burning velocity. In the aforementioned solutions the total initial fraction of liquid in the total fuel and water (vapor +liquid) in the fresh mixture, $\tilde{\delta}$, appears. It can be shown that if there are no droplets present initially (i.e. $\tilde{\delta}=0)$ and the premixture is purely gaseous the expression for the flame propagation velocity collapses to that for a gaseous flame (see, e.g. Buckmaster and Ludford [23]). If there is no water present in the droplets (i.e. $\alpha=0$ ) the formula reduces to that of Greenberg et al. [24] in the limit of infinite and finite vaporization rate. The second and third terms in the exponential represent the contributions to reducing the burning velocity (relative to that of a purely gaseous flame) due to evaporation of the liquid fuel and the water, respectively. 


\section{Results and discussion}

By specifying the initial fraction of liquid in the total fuel and water (vapor +liquid) in the fresh mixture, $\tilde{\delta}$ and the initial ratio of water to liquid in the droplets, $\alpha$, it can be shown that the mass fractions in the fresh mixture are given by the following expressions:

$$
\begin{aligned}
& m_{O u}^{*}=s\left\{1-m_{d f u}^{*}-m_{f u}^{*}-m_{d W u}^{*}\right\} \\
& m_{d f u}^{*}=\frac{\frac{\delta \delta \varphi}{\hat{\alpha}_{o f}}}{\left[\left(1+\frac{s \varphi}{(1-\alpha) \hat{\alpha}_{o f}}\right)+\frac{\alpha(1-\tilde{\delta})}{(1-\alpha)}\right]} \\
& m_{d W u}^{*}=\frac{\alpha m_{d f u}^{*}}{(1-\alpha)} \\
& m_{f u}^{*}=1-\frac{m_{o u}^{*}}{s}-m_{d f u}^{*}-m_{d w u}^{*}
\end{aligned}
$$

where $S$ is the mole fraction of oxygen in the fresh mixture, $\alpha_{O F}$ is the stoichiometric coefficient and $\varphi$ is the equivalence ratio. Here, $\varphi$ is taken as 2 and $\alpha_{O F}=3.5$.

Other data used was as follows (unless otherwise specified):

$q=1.279 \cdot 10^{7}\left[\frac{\mathrm{J}}{\mathrm{kg}}\right], \rho=1\left[\frac{\mathrm{kg}}{\mathrm{m}^{3}}\right], \kappa=0.02512\left[\frac{\mathrm{W}}{\mathrm{mK}}\right], C_{p}=1255.92 \frac{\mathrm{J}}{\mathrm{kg}}, A=10^{10}\left[\frac{1}{\mathrm{sec}}\right], E=2 \cdot 10^{8}\left[\frac{\mathrm{J}}{\mathrm{kmol}}\right], T_{u}^{*}=$ $300[K], T_{v f}^{*}=400[K], T_{e}^{*}=600 K, L_{f} / q=0.04, L_{W} / q=0.12$.

In Figure 1 we illustrate the major effect the micro-explosion has on the burning velocity. The burning velocity is plotted against the total initial liquid mass fraction (i.e. water+liquid fuel). Three different initial water content fractions are considered. Curves are drawn accounting for the occurrence of micro-explosions (the solid lines) and for the cases when no micro-explosion is accounted for (the broken lines) for comparison. The impact of the microexplosion is very pronounced, reducing the burning velocity by a factor of almost $43 \%$ at the most, when no fuel vapor is initially present in the fresh mixture. This factor decreases as less liquid is replaced by pre-vaporized fuel. In addition, it is seen that the more water initially present in the spray's droplets the greater the impact in reducing the burning velocity. This is evidently due to the greater heat absorbed by the water for the sudden evaporation at the point of the micro-explosion which, in turn, reduces the temperature and thereby the propagation velocity. This effect is likely play a critical role in the stability (and extinction of these flames). The combined effect of volumetric heat loss and liquid fuel evaporation heat loss on flame extinction was addressed in [25] and compared to the case of volumetric heat loss alone [26]. However, an analysis of these features in the current context is beyond the scope of the current paper.

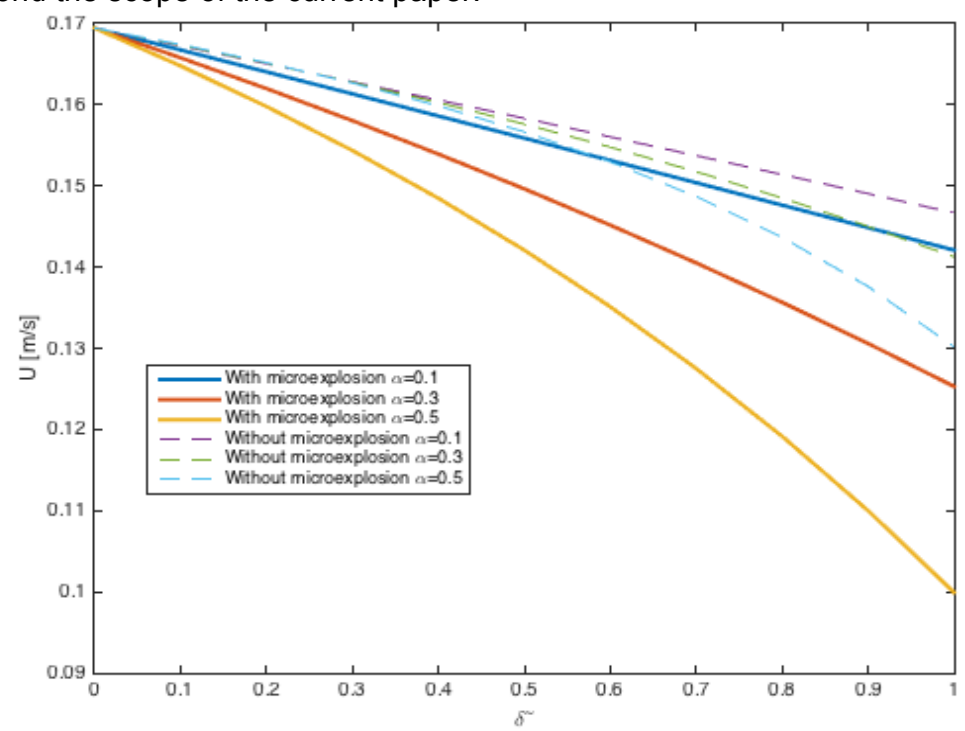

Figure 1: Influence of droplets micro-explosion on the burning velocity of water-in-fuel laminar spray flames as a function of total initial liquid mass fraction.

In Figure 2 the influence of the micro-explosion parameter $f$ on the flame temperature is illustrated for different initial liquid mass fractions. Recall that the factor $f$ depends on the initial fraction of water in the droplets (Eq.(8)). It is important to note that the flame temperature depends amongst others on both droplet loading and the water content. Thus, when there are no droplets present the top portion of the graph is unchanged with no heat loss accrued. As liquid droplets are introduced the effect that the water content $\alpha$ has on the temperature comes to the 
fore. Since $f$ is a function of alpha, the more water in the droplets the larger $f$ becomes with the consequent microexplosion producing smaller droplets which evaporate more rapidly thereby focusing heat loss due to the absorption of heat by the rapidly evaporating fuel droplets. This yields lower temperatures.

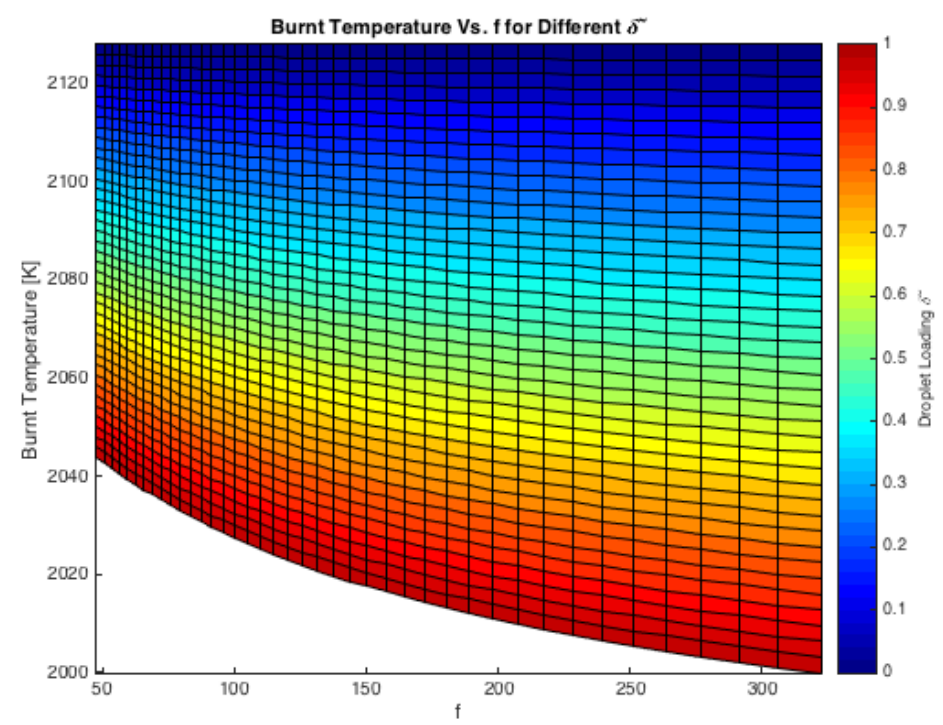

Figure 2: Influence of micro-explosion factor $f$ on flame temperature in water-in-fuel spray flame propagation.

In Figure 3 the effect of the micro-explosions on the behavior of the liquid fuel mass fraction is drawn. The upper curve is for the case when no water is present in the sprays droplets so that they are comprised of liquid fuel only. For the data considered here this spray evaporates fairly slowly and droplets actually traverse the flame front, which is located at $\xi=0$. The lower curve illustrates the behavior when micro-explosions occur at about $\xi=-2.2$

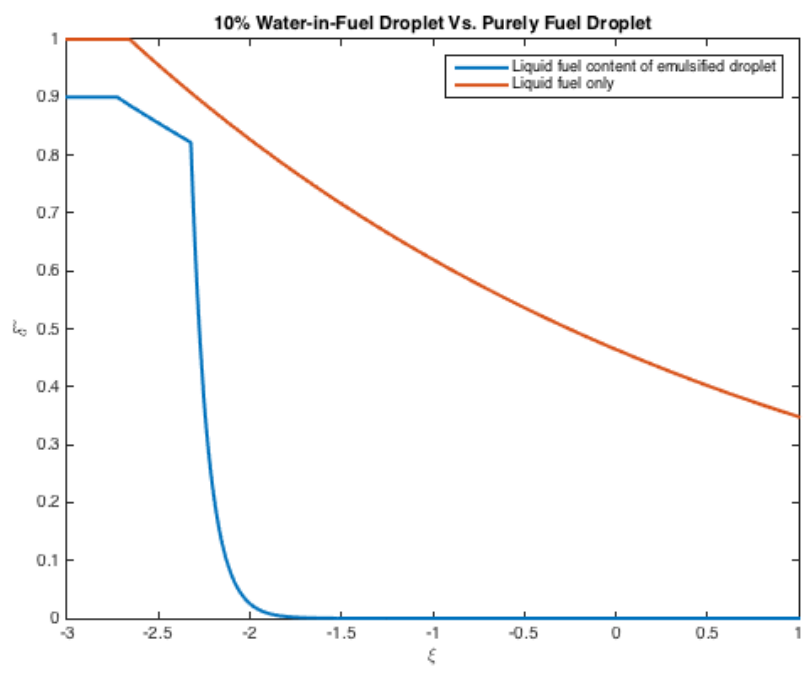

Figure 3: Comparison between liquid fuel spatial profiles with and without accounting for droplet micro-explosions.

The influence of the micro-explosions is dramatic, with the smaller droplets produced evaporating very quickly. In contrast to the purely liquid fuel spray, the water-in-fuel spray considered here are completely evaporated upstream of the flame front. Nevertheless, as shown above, the price paid for the premature fuel evaporation is a drastic loss in the flame temperature which may, of course, be detrimental in terms of combustion efficiency. It would seem that micro-explosions are likely to be more efficient in near stoichiometric or fuel lean mixtures for which the prematurely produced fuel vapor is actively involved in the combustion at the flame front. Such a 
situation is precluded here as the mixture is off-stoichiometric and fuel rich so that the limiting reactant accounted for in the global chemical reaction assumed is the oxidant.

\section{Conclusions}

A simple model of water-in-fuel laminar spray flame propagation was presented for the first time, including the influence of micro-explosions. The analytical solutions provide insight into the essential processes competing in such combustion. For the fuel-rich off-stoichiometric flames considered it was found that the micro-explosions are effective in enhancing the rapid production of fuel vapour but are rather disadvantageous in terms of lowering the flame temperature and burning velocity. The latter effects are likely to impinge directly on the stability and/or extinction of such flames - an issue that is the subject of ongoing research. The primary advantage of microexplosions is likely to be prominent in lean and near-stoichiometric mixtures. Work in this direction will be reported on in the future.

\section{Acknowledgements}

JBG thanks the Lady Davis Chair in Aerospace Engineering for partial support of this research.

\section{References}

[1] The Kyoto Protocol Mechanisms, International Emissions Trading Clean Development Mechanism Joint Implementation, United Nations Framework Convention on Climate Change, 2007.

[2] Kadota, T. and Yamasaki, H. , 2002, Progress in Energy and Combustion Science, 28, pp.385-404.

[3]. Khan, M.Y., Karim, Z.A.A, Hagos, F.Y., Aziz, A.R.A and Tan, I.M., 2014, The Scientific World Journal, Article ID 527472.

[4] Jacques, M.T., 1977, Combustion and Flame, 29, pp. 77-85.

[5] Law, C.K.,1977, Combustion Science and Technology,17, pp. 29-38.

[6] Shyu, R.R., Chen, C.S., Goudie, G.O. and El-Wakil, M.M., 1972, Fuel, 51, pp. 135-145.

[7] Ivanov, V.M and Nefdov, P.I., 1965, NASA TT F-258.

[8] Fu, W.B., Hou, L.Y., Ma, F.H. and Wang, L., 2002, Fuel Processing Technology, 79, pp. 107-119.

[9] Law, C.K., Lee, C.H. and Srinivasan, N., 1980, Combustion and Flame, 37, pp. 125-143.

[10] Law, C.K and Wang, C.H., 1985, Combustion and Flame, 59, pp. 53-62.

[11] Shinjo, J., Xia, J., Ganippa, L.C. and Megaritis, A., 2014, Physics of Fluids, 26, pp. 103302, 1-22.

[12] Tarlet, D., Bellettre, J.,Tazerout, M. and Rahmouni, C., 2009, International Journal of Thermal Science, 48, pp. 449-460.

[13] Girin, O.G., 2016, ILASS-Europe 2016, 27th Annual Conference on Liquid Atomization and Spray Systems, 4-7 September, Brighton, UK.

[14] Hsuan, C.-Y., Hou, S.-S and Lin, T.-H., 2014,, Mathematical Problems in Engineering, Article ID 584926.

[15] Hsuan, C.-Y. and Lin, T.-H, 2012, Combustion and Flame,159, pp. 1652-1662.

[16] Hsuan, C.-Y. and Lin, T.-H, 2012, Combustion Theory and Modeling,16(3), pp.483-505.

[17] Hou, S.-S., Lin, J.-C. and Hsuan, C.-Y., 2014, International Journal of Heat and Mass Transfer, 71, pp. 503514.

[18] Lin, T.H., Law, C.K. and Chung, S.H., 1988, International Journal of Heat and Mass Transfer, 31(5), pp. 1023-1034.

[19] Hughes, K.J., Brindley, J. and McIntosh, A.C., 2013, Proceedings of the Royal Society A, 469: 20130506, http://dx.doi.org/10.1098/rspa.2013.0506.

[20] Greenberg, J.B. and. Kalma, A., 1998, "Computational Aspects of the Sectional Modeling Method for Predicting Spray Combustion", HTD-Vol. 361-2, Proceedings of the ASME Heat Transfer Division, pp. 3-10, ASME International Mechanical Engineering Congress, Anaheim, Ca.

[21] Lentati, A.M. and Chelliah, H.K., 1998, Proceedings of the Combust Institute,.27, pp. 2839-46.

[22] Greenberg, J.B., Silverman, I. and. Tambour, Y., 1993, Combustion and. Flame,. 93, pp. 90-963.

[23] Buckmaster, J.D. and Ludford, G.S.S., Theory of Laminar Flames, Cambridge University Press, Cambridge, UK, 1982.

[24] Greenberg,J.B.,McIntosh, A.C. and Brindley, J.,2001, Proceedings of the Royal Society of London, Series A: Mathematical, Physical and Engineering Sciences, 457, No.2005, pp.1-31.

[25] Kats, G. and Greenberg, J.B., 2016, Journal of Combustion, Article ID 1069873, Vol. 2016, http://dx.doi.org/10.1155/2016/1069873.

[26] Joulin, G. and Clavin, P., 1979, Combustion and Flame, 35, pp. 139-153. 


\title{
Spray and Combustion Characterizations of Acetone-Butanol- Ethanol (ABE) blend in High-Pressure and High-Temperature Conditions
}

\author{
Ob Nilaphai ${ }^{* 1}$, Hugo Ajrouche ${ }^{1}$, Camille Hespel ${ }^{1}$, Bruno Moreau ${ }^{1}$, Somchai Chanchaona ${ }^{2}$, \\ Fabrice Foucher ${ }^{1}$, Christine Mounaim-Rousselle ${ }^{1}$ \\ 'PRISME, Université d'Orléans, France \\ ${ }^{2}$ CERL, King Mongkut's University of Technology Thonburi, Thailand \\ *Corresponding author: ob.nilaphai@etu.univ-orleans.fr
}

\begin{abstract}
The intermediate fermentation mixture of butanol production, Acetone, Butanol and Ethanol (ABE), is increasingly considered as a new alternative fuel in $\mathrm{Cl}$ engines due to its physical and chemical properties, which are similar to those of butanol, and its advantages of no additional cost or energy consumption due to butanol separation. In a previous study, the High-Pressure and High-Temperature (HPHT) chamber, called 'New One Shot Engine" (NOSE), was used to investigate macroscopic spray-combustion parameters by validating Spray-A conditions of the Engine Combustion Network. The present study concerns the spray-combustion characteristics of the ABE mixture (volume ratio $3: 6: 1$ ), blended with $n$-dodecane at a volumetric ratio of $20 \%$ (ABE20), compared to n-dodecane as reference fuel. The macroscopic spray and combustion parameters were investigated, for non-reactive conditions, in pure Nitrogen and for reactive conditions, in $15 \%$ oxygen, at ambient pressure (60 bar), ambient density $\left(22.8 \mathrm{~kg} / \mathrm{m}^{3}\right)$ and different ambient temperatures $(800 \mathrm{~K}, 850 \mathrm{~K}$ and $900 \mathrm{~K})$. The liquid and vapor spray penetrations were investigated by the Diffused Back Illumination (DBI) and Schlieren techniques in non-reactive conditions. In reactive conditions, the lift-off length was measured by $\mathrm{OH}^{*}$ chemiluminescence images at $310 \mathrm{~nm}$. The Schlieren technique was also used to verify the choice of detection criterion. The ignition delay results of the two fuels were compared. It was found that the behavior of the two fuels as a function of temperature was similar even if the liquid length of ABE20 was shorter than that of $n$-dodecane at all ambient temperatures. On the other hand, no real difference in vapor spray penetration between the two fuels was observed. The vaporization properties and the lower autoignition ability of ABE20 led to longer ignition delays and lift-off length.
\end{abstract}

\section{Keywords}

Spray and Combustion Characterizations, Acetone-Butanol-Ethanol (ABE), High-Pressure and High-Temperature Conditions.

\section{Introduction}

Due to the increase in energy demand and the depletion of oil resources during the last few decades, butanol has become an alternative fuel, considered in the transportation sector as a sustainable energy and also a means to reduce greenhouse gases compared to conventional fuel [1], as it can be produced from renewable bioresources in the form of agricultural biomass and waste [2]. Moreover, butanol induces lower fuel consumption as its energy content is higher than ethanol, up to $30 \%$, and its lower water solubility decreases the tendency to microbial-induced corrosion in fuel storage and pipelines during transportation [1]. The higher cetane number $(\mathrm{CN})$ of butanol $(\mathrm{CN}=$ 25) compared to ethanol $(\mathrm{CN}=8)$ leads to easier ignition in compression ignition $(\mathrm{Cl})$ engines [3]. It is suitable for diesel injection systems thanks to its high level of viscosity like diesel fuel and no water content, unlike ethanol. Finally, researchers have considered butanol as one means to reduce $\mathrm{CO}, \mathrm{HC}$, NOx, and Soot emissions [4] and also to improve combustion efficiency especially in advanced combustion modes [5].

Bio-butanol can be produced from agricultural crops and lignocellulosic biomass by using Clostridium bacteria, Clostridium beijerinckii or Clostridium acetobutylicum, to ferment lignocellulosic hydrolysate sugars to produce a mixture of Acetone, Butanol and Ethanol $(A B E)$ in a volume ratio of 3:6:1 [6], [7], after which it is distilled to separate butanol from the ABE mixture. However, the intermediate fermentation product, $A B E$, can also be considered as a potential new alternative fuel itself for $\mathrm{Cl}$ engines because its physical and chemical properties are similar to those of butanol [3], [7], [8]. In addition, if the ABE mixture can be used as fuel, the cost and energy consumption of the separation process of butanol from ABE would be eliminated. 
The Engine Combustion Network (ECN), initiated by Sandia National Lab, is an international network for the experimental and simulation analysis of combustion phenomena for diesel and gasoline engines to provide new accurate data about diesel spray and combustion processes and thereby reach a better understanding of the physical processes involved and also of the modelling concepts and models themselves [9], [10]. To participate in this network, PRISME Laboratory University of Orleans developed a new chamber to reach the High Pressure-High Temperature (HPHT) thermodynamic conditions of current common-rail diesel engines, called 'New One Shot Engine" (NOSE), designed based on the concept of the Rapid Cycling Machine (RCYM).

In a previous study, NOSE was successfully used to investigate the macroscopic spray parameters of n-dodecane by using standard conditions and standard measurement methods in the Spray-A conditions of the Engine Combustion Network (ECN) [10]. The present study focuses on the spray - combustion parameters in the case of an $\mathrm{ABE}$ mixture, blended with $\mathrm{n}$-dodecane in a volume ratio of $20 \%$ (ABE20) in comparison to the reference fuel, $\mathrm{n}$-dodecane. The macroscopic spray parameters and combustion parameters were investigated in non-reactive conditions (pure Nitrogen) and reactive conditions (15\% oxygen) at ambient pressure (near 60 bar), ambient density $\left(22.8 \mathrm{~kg} / \mathrm{m}^{3}\right)$ and different ambient temperatures $(800 \mathrm{~K}, 850 \mathrm{~K}$, and $900 \mathrm{~K})$. The injection conditions were controlled at 1,500 bar of injection pressure, $90 \stackrel{\circ}{\circ}$ of injection fuel temperature, and $1.5 \mathrm{~ms}$ of injection duration. The results of macroscopic spray, the liquid length (LL) and vapor spray penetration (S) were investigated by the Diffused Back Illumination (DBI) and Schlieren techniques for non-reactive conditions. In reactive conditions, the Lift-off Length (LOL) was measured by $\mathrm{OH}^{*}$ chemiluminescence images at $310 \mathrm{~nm}$. In the last section, the results of ignition delay (ID) for the two fuels are discussed and compared. The Schlieren technique was used to verify the detection criterion to determine the ignition delay by $\mathrm{OH}^{*}$ chemiluminescence.

\section{Experimental set-up and operating conditions}

NOSE is a High Pressure-High Temperature (HPHT) combustion chamber which operates based on the concept of the Rapid Cycling Machine (RCYM). It was developed to study diesel spray and combustion with the objective of sharing experimental conditions defined by the ECN [10]. The NOSE design is based on the use of a single-cylinder low-speed diesel engine with a $155 \mathrm{~mm}$ bore diameter and $177.8 \mathrm{~mm}$ stroke. The original cylinder head was replaced by a dedicated chamber, designed by extending the combustion chamber to support optical measurements. The compression ratio was set at 15:1 by modifying the shape of the piston head. There are 4 quartz windows, $25 \mathrm{~mm}$ thick, $25 \mathrm{~mm}$ wide, and $80 \mathrm{~mm}$ long to support several kinds of optical measurement. Further details about the chamber design, thermal characterization chamber, specification of the injection system, and data acquisition system were presented in our previous study [10].

To achieve target conditions in an adiabatic compression process, the initial conditions such as initial pressure, temperature, and injection temperature must be defined before starting operation. For example, in the non-reactive condition of $900 \mathrm{~K}$, the piston was first positioned at BDC with a cooling temperature fixed at $83^{\circ} \mathrm{C}$. The chamber was heated up to $100.5^{\circ} \mathrm{C}$ to achieve $90^{\circ} \mathrm{C}$ inside the injector 'sac'. The chamber was filled with Pure Nitrogen $\left(\mathrm{N}_{2}\right)$ until 1.8 bar of initial pressure was reached. The electric motor drives the piston from BDC to TDC to compress gas inside the chamber. Around TDC, the control system generates the TTL signal to drive fuel injection during 1.5 $\mathrm{ms}$ and to trigger image recording. The main testing conditions are summarized in Table 1.

Table 1. The main conditions of test fuels at the Start Of Injection (SOI)

\begin{tabular}{l|l}
\hline \hline \multicolumn{1}{c|}{ Parameters } & \multicolumn{1}{c}{ Value } \\
\hline \hline Ambient gas temperature & $800,850,900 \mathrm{~K}$ \\
Ambient gas pressure & near $60 \mathrm{bar}$ \\
Ambient gas density & $22.8 \mathrm{~kg} / \mathrm{m}^{3}$ \\
Ambient gas oxygen & $0 \% \mathrm{O}_{2}$ for non-reactive \\
& $15 \% \mathrm{O}_{2}$ for reactive \\
Ambient gas velocity & $\mathrm{Near}-q$ quiescent, less than $1 \mathrm{~m} / \mathrm{s}$ \\
Fuel injection pressure & $150 \mathrm{MPa}(1,500$ bar $)$ \\
Fuels & $\mathrm{n}$-dodecane, and ABE20 \\
Fuel temperature at nozzle & $363 \mathrm{~K}\left(90^{\circ} \mathrm{C}\right)$ \\
Injection duration & $1.5 \mathrm{~ms}$
\end{tabular}

In this study, the ABE used was based on the typical volume ratio 3:6:1 [7] (30\% of acetone, $60 \%$ of butanol, and $10 \%$ of ethanol with acetone $(98.5 \%)$, butanol $(99.5 \%)$, and ethanol $(99.5 \%)$ ). The ABE mixture was blended with $\mathrm{n}$-dodecane, at a volumetric ratio of $20 \%$ (ABE20), as n-dodecane is a conventional surrogate of diesel fuel. The physical properties of the fuels are given in Table 2 . The density and viscosity were measured by a viscosity meter (Anton Paar SVM300) and the other properties were taken from the literature [11]-[13], if available. 
Table 2. Fuel properties

\begin{tabular}{l|c|c|c|c|c}
\hline \multicolumn{1}{c|}{ Properties } & n-Dodecane & Acetone & Butanol & Ethanol & ABE20 \\
\hline \hline Molecular formula & $\mathrm{C}_{12} \mathrm{H}_{26}$ & $\mathrm{C}_{3} \mathrm{H}_{6} \mathrm{O}$ & $\mathrm{C}_{4} \mathrm{H}_{9} \mathrm{OH}$ & $\mathrm{C}_{2} \mathrm{H}_{5} \mathrm{OH}$ & - \\
Density at $15^{\circ} \mathrm{C}\left(\mathrm{kg} / \mathrm{m}^{3}\right)$ & 752.8 & 789 & 813.3 & 794.1 & 760.7 \\
Viscosity at $15{ }^{\circ} \mathrm{C}\left(\mathrm{mm}^{2} / \mathrm{s}\right)$ & 2.032 & 0.149 & 4.119 & 1.489 & 1.642 \\
Cetane number [11] & 74 & - & 17 & 8 & - \\
Boiling point $(\mathrm{K})[12]$ & 489.3 & 329.1 & 390.6 & 351.3 & - \\
Vapor pressure at 298 K (kPa) [12] & 0.018 & 30.80 & 0.58 & 7.91 & - \\
Latent heat of Vaporization at 298 K & 362 & 518 & 582 & 904 & - \\
$(\mathrm{kJ} / \mathrm{kg})[12],[13]$ & & & & &
\end{tabular}

\section{Optical techniques}

In this part the details of the different optical techniques and post-processing tools are explained. Table 3 summarizes the optical set-ups, for the 4 different techniques needed to characterize macroscopic spray of nonreactive and combustion parameters, following the $\mathrm{ECN}$ recommendations to ensure accurate experimental results.

Table 3. Summary of the optical set-ups

\begin{tabular}{|c|c|c|c|c|c|}
\hline & \multicolumn{2}{|c|}{ Non-Reactive } & \multicolumn{3}{|c|}{ Reactive } \\
\hline $\begin{array}{l}\text { Optical Technique } \\
\text { Parameters }\end{array}$ & $\begin{array}{l}\text { DBI } \\
\text { Liquid } \\
\text { Penetration } \\
\text { Length (LL) }\end{array}$ & $\begin{array}{l}\text { Schlieren } \\
\text { Vapor Spray } \\
\text { Penetration } \\
\text { (S) }\end{array}$ & $\begin{array}{l}\mathrm{OH}^{*} \text { Chemilu. } \\
\text { Lift-Off Length } \\
\text { (LOL) }\end{array}$ & $\begin{array}{l}\text { Schlieren } \\
\text { Ignition Delay } \\
\text { (ID) }\end{array}$ & $\begin{array}{l}\mathrm{OH}^{*} \text { Chemilu. } \\
\text { Ignition Delay } \\
\text { (ID) }\end{array}$ \\
\hline Camera / Detector & $\begin{array}{l}\text { Phantom- } \\
\text { V1611 }\end{array}$ & $\begin{array}{l}\text { Phantom- } \\
\text { V1611 }\end{array}$ & $\begin{array}{l}\text { Photron - } \\
\text { APX-12 }\end{array}$ & $\begin{array}{l}\text { Phantom- } \\
\text { V1611 }\end{array}$ & $\begin{array}{l}\text { Newport } \\
\text { Photomultiplier } \\
\text { tube } 70680\end{array}$ \\
\hline Sensor type & CMOS & CMOS & ICCD & CMOS & - \\
\hline Light source & $\begin{array}{l}\text { LED plate } \\
\text { (white) } \\
100 \times 100 \mathrm{~mm}\end{array}$ & $\begin{array}{l}\text { LED (white) } \\
\text { with } 1 \mathrm{~mm} \\
\text { pinhole }\end{array}$ & - & $\begin{array}{l}\text { LED (white) } \\
\text { with } 1 \mathrm{~mm} \\
\text { pinhole }\end{array}$ & - \\
\hline Lens & $60 \mathrm{~mm} \mathrm{f} / 2.8$ & - & $\begin{array}{l}\text { UV } 60 \mathrm{~mm} \\
\mathrm{f} / 3.5\end{array}$ & - & - \\
\hline Mirrors & - & $\begin{array}{l}2 \text { Parabolic } \\
\text { Mirrors } 34 \text { inch }\end{array}$ & - & $\begin{array}{l}2 \text { Parabolic } \\
\text { Mirrors } 34 \text { inch }\end{array}$ & - \\
\hline Pin hole & - & $6 \mathrm{~mm}$ & - & $6 \mathrm{~mm}$ & - \\
\hline Filter & - & - & $\begin{array}{l}\text { BPF } 310 \mathrm{~nm} \\
\text { FWHM } 10 \mathrm{~nm}\end{array}$ & LPF $\leq 550 \mathrm{~nm}$ & $\begin{array}{l}\text { BPF } 307 \mathrm{~nm} \\
\text { FWHM } 10 \mathrm{~nm}\end{array}$ \\
\hline Frame speed & $49 \mathrm{kHz}$ & $39 \mathrm{kHz}$ & $2 \mathrm{kHz}$ & $39 \mathrm{kHz}$ & - \\
\hline Exposure time & $3 \mu \mathrm{s}$ & $5 \mu \mathrm{s}$ & $499 \mu \mathrm{s}$ & $5 \mu \mathrm{s}$ & - \\
\hline Image size & $512 \times 384 \mathrm{pi}^{2}$ & 1024 x $400 \mathrm{pi}^{2}$ & $512 \times 1024 \mathrm{pi}^{2}$ & $1024 \times 400 \mathrm{pi}^{2}$ & - \\
\hline Magnification & $12 \mathrm{pix} / \mathrm{mm}$ & 12.3 pix/mm & $18.2 \mathrm{pix} / \mathrm{mm}$ & 12.3 pix/mm & - \\
\hline Repetitions & 12 tests & 12 tests & 12 tests & 12 tests & 12 tests \\
\hline
\end{tabular}

Diffused Back Illumination (DBI)

The liquid penetration length (LL) is an important parameter when designing and optimizing the operation of modern diesel engines. The length of liquid-phase fuel needs to be optimized to avoid fuel impinging on and collecting at the piston bowl or cylinder wall, which causes higher emissions. The liquid penetration length is defined as the maximum distance of liquid-phase from the injector tip to liquid phase fuel penetration [14], [15]. To avoid uncertainties due to the light source, the calibration and the post-processing, DBI is recommended by the ECN as the standard technique to measure LL [9], [16]. Moreover, DBI is easy to implement as it consists of only two main components: the light source and the camera, as shown in Figure 1 (left).

A white LED light plate measuring $100 \times 100 \mathrm{~mm}^{2}$ was used to illuminate the liquid phase of spray. The light intensity through the optical window and images from the test section were collected by the high speed camera (Phantom V1611), as shown in Figure 1 (left). The flame rate, exposure time, and image resolution were set at suitable values to capture high quality images $\left(512 \times 512 \mathrm{pi}^{2}\right)$ at a high frequency $(49 \mathrm{kHz})$, as shown in Table 3. 
For post-processing, the light intensity of background images $\left(I_{b g}\right)$ was processed from the average of images before start of injection (SOI). The average light intensity $\left(I_{\text {avg }}\right)$ was normalized by $I_{b g}$. The images during steadystate of injection, i.e. 1.1 to $1.5 \mathrm{~ms}$ after SOI, were averaged to avoid the transient phases due to the start and end of injection. Then the light extinction factor, $\tau$, along the spray core was calculated from equation (1). The light extinction image is shown in Figure 1 (right-top). To avoid the effect of the beam steering phenomenon due to variation in the refraction index on the medium at the liquid spray tip, the distance where the linear fit line (red line) crosses the X-axis was determined as the liquid length (LL) of spray penetration, as shown in Figure 1 (rightbottom).

$$
\tau(x, y)=-\log \left(\frac{I_{a v g}(x, y)}{I_{b g}(x, y)}\right)
$$
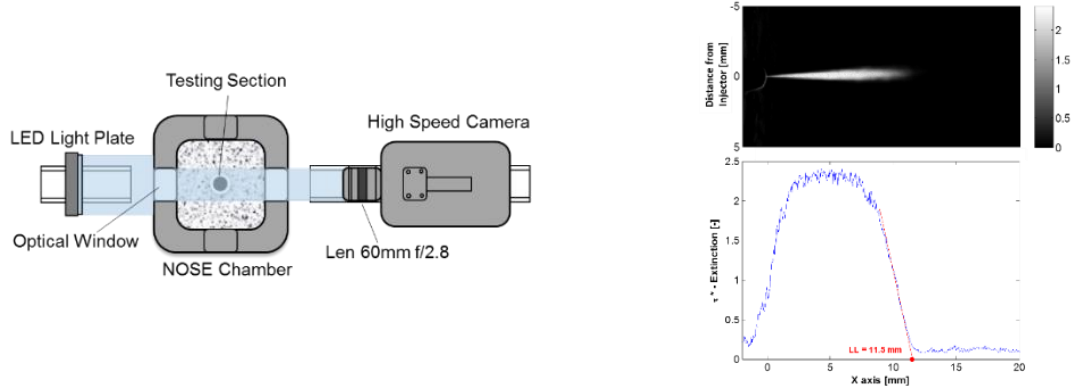

Figure 1. Scheme of DBI set-up (left), the light extinction image (right-top), and LL determination criterion (right-bottom)

\section{Schlieren visualization}

To detect the vapor phase of the spray, the high-sensitivity Schlieren technique was used, based on the measurement of the bending of the light source though the test section, which is sensitive enough to capture the spray boundary. The pinhole or aperture Schlieren stop was used to give a bright field and dark background. The image processing and determination of $S$ were done by using the standardized post-processing code of Sandia to reduce uncertainties and to compare and validate the values obtained. Figure 2 (left) shows an example of Schlieren images and an example of the spray boundary (red line). The vapor spray penetration length is defined at the spray front that crosses the spray axis (vertical blue line). The Schlieren set-up, presented in Figure 2 (right), is composed of a continuous white LED with a $1 \mathrm{~mm}$ pinhole, mirrors and collimating mirrors to reflect light through the test section in the NOSE chamber. A $6 \mathrm{~mm}$ pinhole was set up to record Schlieren light by the high speed camera (Phantom V1611). The image resolution was $1024 \times 400$ pixels, and the highest flame rate was set at $39 \mathrm{kHz}$, with an exposure time of $5 \mu \mathrm{s}$.
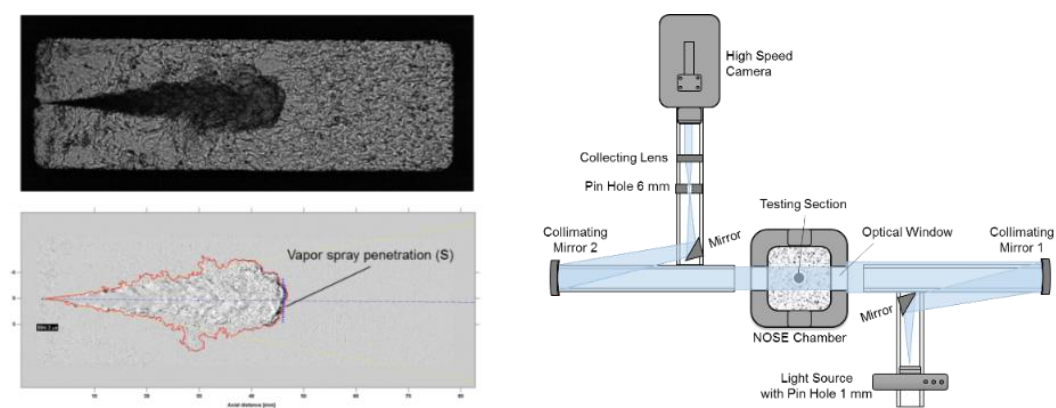

Figure 2. An example of Schlieren images (left-top) and an example of the spray boundary (left-bottom). Experimental scheme of Schlieren set-up. (right)

In reactive conditions three optical techniques were set up to measure the combustion parameters at the same time, namely a lift-off length by $\mathrm{OH}^{*}$ chemiluminescence images at $310 \mathrm{~nm}$, an ignition delay from high speed Schlieren images, and the $\mathrm{OH}^{\star}$ chemiluminescence temporal signal recorded by the photomultiplier as shown in Figure 3. 

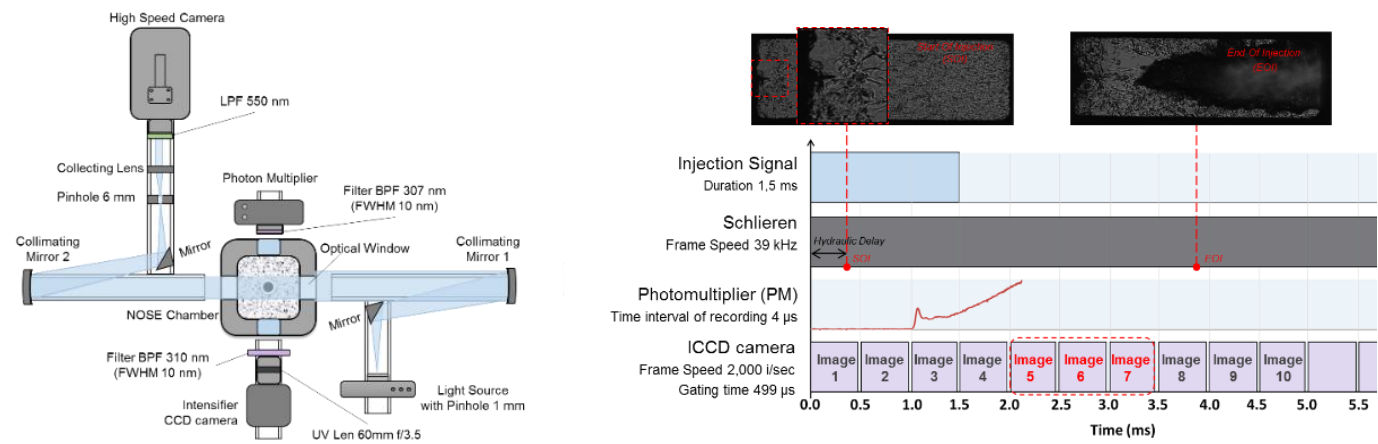

Figure 3. The optical set-ups for reactive condition (left) and synchronization signals diagram (right). The images 5, 6 and 7 (2.0 ms after SOI) of ICCD camera were used to calculate the LOL.

\section{Lift-Off Length}

Generally, Lift-Off Length is defined as the distance between the injector tip and the stabilized flame of the high temperature reaction zone [17], [18]. As is well known, at high temperature the chemiluminescence spectrum of $\mathrm{OH}^{*}$ is centered at $310 \mathrm{~nm}$, so OH${ }^{*}$ is a good indicator to measure the LOL. As shown in Figure 3, an ICCD Photron Fastcam APX 12 was used with a $60 \mathrm{~mm}$ f/3.5 UV lens and equipped with a $310 \mathrm{~nm}$ (FWHM10) band-pass filter (BFP). A long constant gating time of $449 \mu$ s was used to allow turbulent fluctuation in the LOL to reach an average so as to provide an image of the quasi-steady mean LOL after start of injection (SOI) [17]. 10 images were recorded and the image taken during quasi-steady state, as well as images 5, 6 and 7 (2.0 ms after SOI) were used to calculate the LOL as shown in Figure 3 (left). Figure 4 (left) shows the post-processing criterion; the intensity along the upper (red) and lower (blue) profile of the spray centerline is considered to define LOL. In this study LOL was defined as the average distance between the injector tip and the first value above $50 \%$ of the maximum intensity of each profile, following the ECN method [9]. Figure 4 (right) shows an example of the LOL of $n$-dodecane at $850 \mathrm{~K}$.
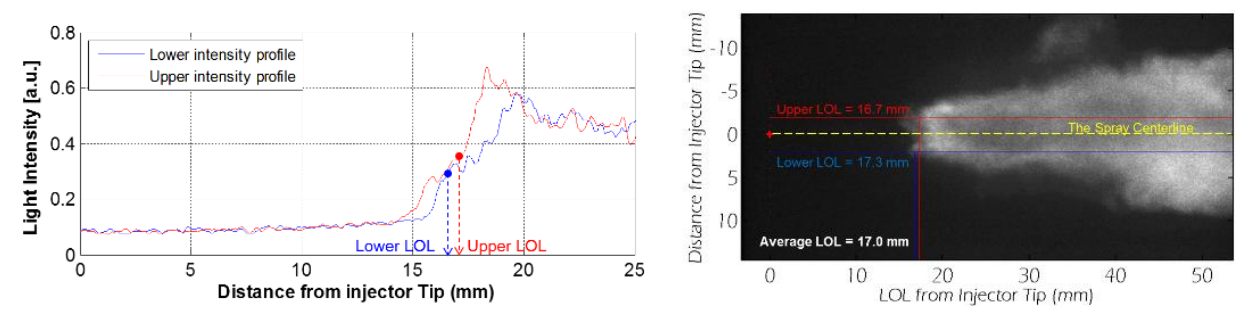

Figure 4. The light intensity along the upper and lower profile of the spray centreline and the dots represent the $50 \%$ of the maximum intensity of each profile (left) and an example of LOL result at $850 \mathrm{~K}$ of $\mathrm{n}$-dodecane (right)

Ignition Delay

To determine the ability of fuels to auto-ignite, the ignition delays of $n$-dodecane and ABE20 were characterized and compared. During the combustion process, there are two states of ignition: the first state of ignition or cool flame, and the second state of ignition or hot flame [15]. The cool flame is the moment at which the first light is emitted by natural luminosity, when the parent fuel molecules are broken down and raise the temperature slightly. However, during this stage it cannot be detected by an increase in cylinder pressure but by the emission of $\mathrm{CH}$ radicals [15], [19]. After that the hot flame occurs, which can be characterized by OH radicals chemiluminescence in UV at $310 \mathrm{~nm}$ [9], [15], [18]. In this paper the ignition delay was defined as the time between the start of injection and the second stage of ignition or hot flame ignition delay. The start of injection (SOI) is an important parameter that should be clearly defined before calculating the ignition delay. It was defined here as the time between the start of injection current and the first injection image of the spray obtained by Schlieren visualization, usually called "hydraulic delay". To use the same criterion to define ignition delay with both techniques, the results shown in this work were corrected by the hydraulic delay.

The hot flame ignition delay was detected by the Schlieren technique, which was set as for the reactive condition, but a $550 \mathrm{~nm}$ low-pass filter was added to limit emissions due to soot radiation. Figure 5 (left) shows the Schlieren images during cool flame and hot flame events. As mentioned before, the hot flame ignition delay appears after the cool flame. The cool flame can be detected at the extinction region in the reactive Schlieren images. This can be explained by the fact that during this period the slight increase in temperature changes the refractive index of the spray to match the refractive index of the ambient gas, leading to more transparent images than the original Schlieren images [20]. Figure 5 (left) shows the series of cool flame images and post-processing images. The vapor 
spray length in transparent images is shorter after post-processing when compared with non-reactive events, as shown in Figure 5 (right) from $312.9 \mu$ s to $373.1 \mu \mathrm{s}$. After that, the head of spray images reappear at $398.7 \mu \mathrm{s}$ and the curve again becomes similar to that of the non-reactive events. In the next images the head of spray smoothly expands in the radial and axial directions. The red point in Figure 5 (right) marks the onset of combustion, which corresponds to the second image after the return to the normal trend. This enables the hot flame ignition delay of the different fuels to be compared.

To verify the criterion defined for Schlieren images, $\mathrm{OH}^{*}$ chemiluminescence was also used to detect the hot flame ignition delay. As can be seen in Table 3, a Newport Oriel Photomultiplier tube side-on with a PMT 70705 high voltage power supply and a band-pass filter of $307 \mathrm{~nm}$ (FWHM 10) was used to follow $\mathrm{OH}^{*}$ chemiluminescence temporally. It was set up close to the NOSE window and positioned on the opposite side to the ICCD camera, as shown in Figure 3 (left). The low light intensity signal from the combustion event was multiplied and recorded in an in-house Labview data acquisition system (DAQ) with a $4 \mu$ s recording interval. The time at the start of combustion of the hot flame is defined at the maximum of the first peak. Figure 5 (right) shows that the two techniques used to identify ignition delays are in good agreement.
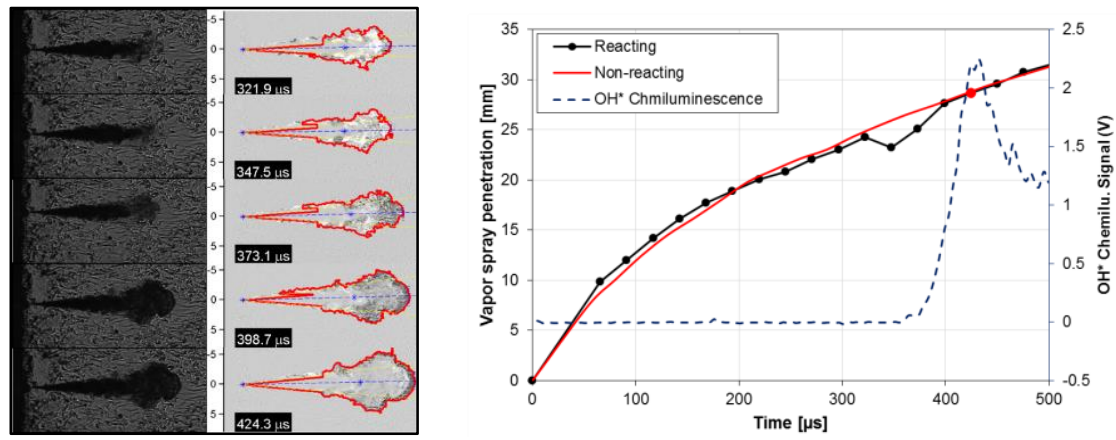

Figure 5. The series of Schlieren images and post-processed images during cool flame and hot flame events (left) and validation of the ignition delay criterion on Schlieren images by the $\mathrm{OH}^{*}$ chemiluminescence collected by a Photomultiplier at $900 \mathrm{~K}$ on n-dodecane (right).

\section{Result and discussion}

This section presents the results of spray and combustion parameters at different ambient conditions for both fuels, $\mathrm{n}$-dodecane and ABE20. All the results are averages of 12 tests; standard deviation bars are indicated.

\section{Liquid penetration length}

Figure 6 shows the results of LL for both fuels at different ambient temperatures. As expected, LL is longer at lower ambient temperature than at high temperatures, there is more energy entrainment on the liquid fuel, leading to an increase in the evaporation rate and also as the surface tension and viscosity of the fuel are lower, the atomization process is enhanced [7], [14]. The LL of ABE20 are shorter than those of $n$-dodecane for all ambient conditions, mainly due to the higher volatility of solution fuels in ABE20. From the physical properties shown in Table 2, the boiling point of the fuel components of ABE20 is $32.7 \%, 20.2 \%$, and $28.2 \%$ lower than that of $n$-dodecane for acetone, butanol, and ethanol, respectively. Moreover, the vapor pressure of acetone, butanol, and ethanol is 30.8 $\mathrm{kPa}, 0.58 \mathrm{kPa}$, and $7.9 \mathrm{kPa}$, which is much higher than that of $\mathrm{n}$-dodecane $(0.018 \mathrm{kPa})$. This leads to a faster evaporation rate for ABE20.

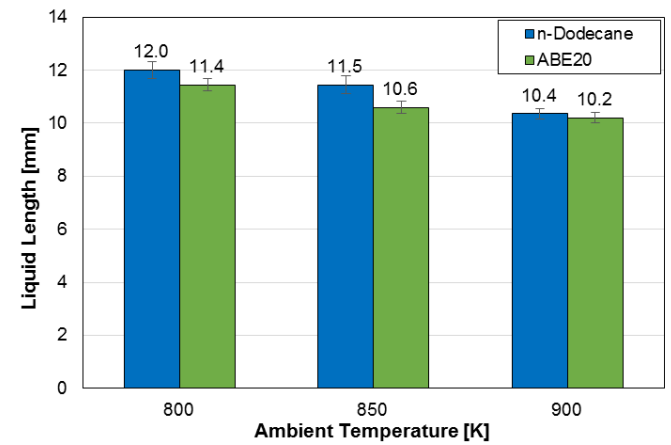

Figure 6. Liquid penetration length (LL) results for different tests. 
Vapour spray penetration length

Figure 7 (left) shows the vapor spray penetration of ABE20 for different ambient temperatures. It can be observed that there is no significant difference ( $1 \%$ of variation in average) and this trend is also the same in $n$-dodecane. The vapor spray penetration depends mainly on the spray momentum which is controlled by the injection pressure (1500 bar), the fuel density and the gas density, set at $22.8 \mathrm{~kg} / \mathrm{m}^{3}$ whatever the ambient temperature. The effect of fuel on vapor length is presented in Figure 7 (right). The blue line represents the average $S$ (from 3 ambient temperatures) of ABE20 and the red line the average $S$ of $n$-dodecane. There is no significant difference in the trends of the two fuels ( $2 \%$ of variation in average).
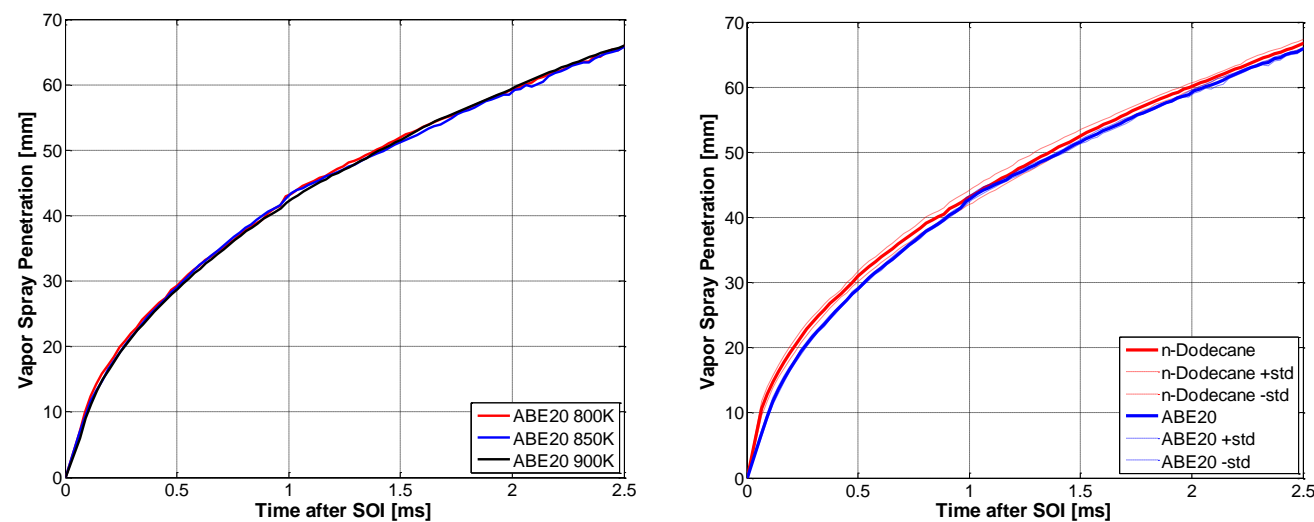

Figure 7 Vapor spray penetration length profile averaged on 12 repetitive tests.

\section{Lift-Off Length (LOL)}

The LOL is determined by the region where the flame stabilizes, and where the spray velocity and flame front velocity are balanced. A longer LOL will provide more time and space for fuel droplets to evaporate and mix with the ambient air [3]. From Figure 8 (left), it can be seen that both fuels show shorter LOL at higher ambient temperature as also found by [7], [18], because the increase in ambient temperature improves evaporation and mixing processes. LOL for ABE20 are longer than for $n$-dodecane for all temperatures. As mentioned previously, even though ABE20 has a faster evaporation rate, it is a blend of fuels with higher latent heat than n-dodecane, up to $250 \%$ for ethanol. This induces a cooling effect in the chamber and an increase in the vaporization time for ABE20, and therefore a longer LOL and ID are expected.
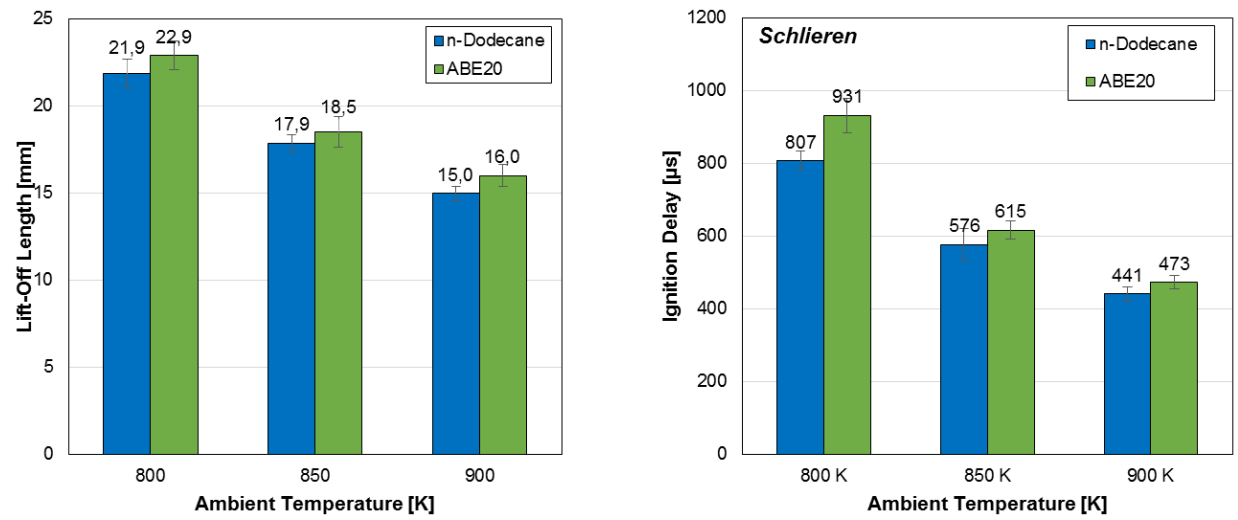

Figure 8 Lift-Off Length (left) and Ignition Delay (right) of n-dodecane and ABE20 at different ambient gas temperatures.

Ignition Delay (ID)

This part presents the results of ignition delay (ID) of the Schlieren technique, identified as the hot flame ignition delay, as shown in Figure 8 (right). As mentioned earlier, there is a relationship between LOL and ID. Both ID and LOL increase with the decrease in ambient temperature. This is due to the fact that the fuel atomization and evaporation rate at lower ambient temperature are lower than at high temperature, lowering the chemical reaction rate [7]. As expected, the higher resistance to auto-ignition of ABE20 induces a higher ID for all testing conditions, 
because the ABE20 was blended with lower cetane fuels than $n$-dodecane $(\mathrm{CN}=74)$, such as ethanol $(\mathrm{CN}=8)$ and butanol $(\mathrm{CN}=17)[11]$.

\section{Conclusions}

To characterize spray and combustion parameters, the standard optical set-ups and image post-processing defined by the ECN were installed on the NOSE, a high-pressure high-temperature combustion chamber. From the experimental results presented, focusing on a comparison between ABE20 fuel and n-dodecane for 3 different ambient temperatures, it can be concluded that:

- $\quad$ The LL of both fuels decreases with an increase in ambient temperature due to more energy entrainment on the liquid fuel which increases the evaporation rate and reduces the surface tension and viscosity of the fuel.

- The increase in ambient temperature improves the evaporation and mixing process because of shorter liftoff length and ignition delay.

- $\quad$ The LL of ABE20 is shorter than that of $n$-dodecane due to its being blended with high volatility fuels that evaporate more easily, but ABE20 exhibits a similar trend of $S$ compared to $n$-dodecane.

- The higher latent heat of vaporization in ABE20 decreases the saturation temperature, increasing the liftoff length.

- $\quad$ The lower auto-ignition ability of ABE20 is indicated by the lower cetane number of blended fuel in ABE20.

- The ignition delay results of the two techniques are in good agreement.

\section{Acknowledgements}

The authors acknowledge the National Research Agency (contract ANR-14-CE22-0015-01) for financial support to the ECN-France project and Region Centre Val de Loire (CPER 2007-2013 Energies du Futur) and FEDER for financial support to build the experimental set-up. We also thank G.Bruneaux and M.Bardi from IFPen for interesting discussions that helped us to refine the analysis.

\section{References}

[1] Wu, M., Wang, M., Liu, J., and Huo, H., 2008, Biotechnology Progress, pp. 1204-1214.

[2] Nigam, P. S., and Singh, A., 2011, Progress in Energy and Combustion Science, 37 (1), pp. 52-68.

[3] Wu, H., Nithyanandan, K., Zhou, N., Lee, T. H., Lee, C. F. F., and Zhang, C., 2015, Fuel, 142, pp. 109-116.

[4] M. Tuner, 2016, SAE Technical Paper, 2016-01-0882.

[5] No, S. Y., 2016, Fuel, 183, pp. 641-658.

[6] Karimi, K., Tabatabaei, M., Horváth, I. S., and Kumar, R., 2015, Biofuel Research Journal, 8, pp. 301-308.

[7] Wu, H., Nithyanandan, K., Zhang, J., Lin, Y., Lee, T. H., and Lee, C. F., 2015, Applied Energy, 149, pp. 367378.

[8] Chang, Y. C., Lee, W. J., Lin, S. L., and Wang, L. C., 2013, Applied Energy, 109, pp. 182-191.

[9] Engine Combustion Network, https://ecn.sandia.gov ([cit. 14-03-2017]).

[10] Nilaphai, O., Hespel, C., Moreau, B., Contino, F., Bourgeois, N., Chanchaona, S., Foucher, F., and MounaïmRousselle, C., Sep. 4.-7. 2016, 27th Annual Conference on Liquid Atomization and Spray Systems.

[11] National Renewable Energy Laboratory, http://www.nrel.gov/docs/fy14osti/61693.pdf ([cit. 14-03-2017]).

[12] National Center for Biotechnology Information, https://www.nih.gov ([cit.14-03-2017]).

[13] Couch, H. T., Kozicki, W., Sage, B. H., 1963, Journal of Chemical and engineering data, 8 (3), pp. 346-349.

[14] Siebers, D. L., 1998, SAE Technical Paper, 107 (724), pp. 1205-1227.

[15] Meijer, M., Galle, J., Somers, L. M. T., Griensven, J. G., and Verhelst, S., 2013, SAE International Journal of Engines, 6 (2), pp. 1238-1248.

[16] Bardi, M., Bruneaux, G., and Malbec, L., 2016, SAE Technical Paper, 2016-01-0845.

[17] Siebers, D. L., and Higgins, B., SAE Technical Paper, 2001-01-0530.

[18] Payri, R., Salvador, F. J., Manin, J., and Viera, A., 2016, Applied Energy, 162, pp. 541-550.

[19] Dec, J. E., and Espey, C., 1998, SAE Technical Paper, 982685.

[20] Pickett, L. M., Kook, S., and Williams, T. C., 2009, SAE International Journal of Engines, 2009-01-0658. 


\title{
Computational Prediction of Primary Breakup in Fuel Spray Nozzles for Aero-Engine Combustors
}

\author{
Thilo F. Dauch*, Samuel Braun ${ }^{2}$, Lars Wieth ${ }^{3}$, Geoffroy Chaussonnet ${ }^{4}$, Marc C. Keller ${ }^{5}$, \\ Rainer Koch ${ }^{6}$, Hans-Jörg Bauer ${ }^{7}$ \\ Institut für Thermische Strömungsmaschinen (ITS), \\ Karlsruhe Institute of Technology (KIT), Germany \\ *Corresponding author: thilo.dauch@kit.edu
}

\begin{abstract}
Primary breakup of liquid fuel in the vicinity of fuel spray nozzles as utilized in aero-engine combustors is numerically investigated. As grid based methods exhibit a variety of disadvantages when it comes to the prediction of multiphase flows, the "Smoothed Particle Hydrodynamics" (SPH)-method is employed. The eligibility of the method to analyze breakup of fuel has been demonstrated in recent publications by Braun et al, Dauch et al and Koch et al $[1,2,3,4]$. In the current paper a methodology for the investigation of the two-phase flow in the vicinity of fuel spray nozzles at typical operating conditions is proposed. Due to lower costs in terms of computing time, 2D predictions are desired. However, atomization of fluids is inherently three dimensional. Hence, differences between 2D and 3D predictions are to be expected. In course of this study, predictions in 2D and based on a 3D sector are presented. Differences in terms of gaseous flow, ligament shape and mixing are assessed.
\end{abstract}

\section{Keywords}

Multiphase Flows - Fuel Atomization - Smoothed Particle Hydrodynamics - Aero-Engine - Combustor

\section{Introduction}

Aiming at a reduction of pollutant emissions of air-traffic, academia and industry both invest in research to investigate processes causing the formation of pollutants of aero-engine combustors. One aspect influencing the formation of pollutants is the quality of the injected fuel spray and its placement within the gaseous flow field of the combustion chamber.

Because of limited optical access and challenging thermodynamic conditions, experimental studies are costly and cannot provide detailed information about breakup of the fuel in the close vicinity of fuel injectors. Hence, numerical investigations analyzing the local two-phase flow are desired.

At the "Institut für Thermische Strömungsmaschinen" (ITS) a numerical code based on the Lagrangian "Smoothed Particle Hydrodynamics" (SPH)-method has been developed by Koch, Hoefler and Braun [5, 4]. The objective is to predict the liquid fuel breakup in the close vicinity of the fuel spray nozzle. Conventional grid based methods exhibit a variety of inherent shortcomings, which can be overcome by a fully Lagrangian approach. In recent publications the potential of the code in terms of two-phase flow predictions has been demonstrated successfully by Braun et al, Dauch et al and Keller et al [1, 3, 6].

Current state-of-the art methods for combustor design do not take into account the details of primary breakup. Correlations based on empirical studies are employed to impose droplet initial conditions for subsequent EulerLagrangian CFD predictions. These correlations must be tuned to each individual setup and need to be calibrated. Most of the correlations are valid only for low pressures and temperatures. A detailed simulation of primary breakup can overcome these shortcomings. It might provide transient droplet initial conditions, which can be used as input for subsequent Euler-Lagrangian predictions. Even if SPH computations are too costly for every day design studies, they can help deriving simplified primary breakup models such as presented by Chausonnet et al [7], that are efficient and accurate enough to be integrated in conventional CFD-codes.

The current study demonstrates, how SPH predictions can be used to investigate the details of primary breakup at fuel spray nozzles typically utilized in aero-engines at realistic operating conditions. As fuel spray atomization is inherently three dimensional, predictions in 3D are desired. Because of the lower costs for 2D predictions, design studies in 2D are preferred if possible. However, deviations between 2D and 3D predictions are to be expected. To identify the deviations this study will address the comparison between $2 \mathrm{D}$ and $3 \mathrm{D}$ results.

\section{Numerical Method}

The original purpose of the "Smoothed Particle Hydrodynamics"(SPH)-method is the analysis of phenomena occurring in astrophysics as presented by Gingold et al and Lucy [8, 9]. Nowadays, SPH is employed for the analysis of different problems in science and engineering as demonstrated by Chaussonnet et al, Dauch et al and Keller et al $[10,3,6]$. In contrast to conventional grid based CFD methods, SPH is a fully Lagrangian method and does not require a computational mesh. The flow domain is discretized by means of moving discretization points, referred to as "particles".

In grid based methods the location of the phase interface depends on the definition of the limited volume fraction resulting in reduced accuracy. The phase interface needs to be reconstructed, which is computationally expensive and prone to interface diffusion. 
Due to the Lagrangian character of the SPH method, phase interfaces of multi-phase flows are inherently advected and do not have to be reconstructed. Once particles are initialized as liquid, they remain liquid. Thus, the contour of the ligaments is uniquely defined by the location of the liquid particles. Hence, interface reconstruction is avoided. As primary breakup is entirely resolved, no breakup criterion is required. Additionally, particle methods can be parallelized more efficiently and therefore are less computationally expensive.

One drawback inherent to the method is its instability referred to as "Tensile Instability" as presented by Swegle et al [11]. Even though a variety of mitigations has been developed, which are summarized by Liu et al [12], the problem is still not solved in general. Another problem of SPH is the consistency issue, as described by Liu et al and Quinlan et al $[12,13]$. Both aspects are subject of ongoing research as described by Chaussonnet et al and Litvinov et al $[10,14]$. However, despite these known issues, SPH has been demonstrated to capture primary atomization with high accuracy as presented by Koch et al [4].

The fluid flow is modeled by means of the Navier-Stokes equations and corresponding boundary conditions. In the present work the flow is considered to be isothermal. Hence, the energy equation is not considered here. The substantial derivatives of density and velocity are defined by continuity and momentum equation:

$$
\begin{aligned}
\frac{D \rho}{D t} & =-\rho(\vec{\nabla} \cdot \vec{v}), \\
\rho \frac{D \vec{v}}{D t} & =-\vec{\nabla} p+\vec{\nabla} \cdot \tau+\rho \vec{f} .
\end{aligned}
$$

In order to illustrate the formalism of the SPH-method, the formulation of any function $f(\vec{r})$ in terms of the convolution integral is utilized:

$$
f(\vec{r})=\int f\left(\overrightarrow{r^{\prime}}\right) \delta\left(\vec{r}-\overrightarrow{r^{\prime}}\right) d \overrightarrow{r^{\prime}}
$$

Replacing the Dirac function by an approximation called kernel function $W\left(\vec{r}_{a}-\vec{r}_{b}, h\right)$ yields an approximated convolution integral. A variety of kernel functions exists as pointed out and discussed by Dehnen in [15]. In the present study a quintic kernel function is used. The typical shape of a kernel function is depicted in Fig. 1a. The magnitude $h$ is called the smoothing length. Depending on $h$, the so called radius of influence, exemplified in Fig. $1 \mathrm{~b}$, is varied.

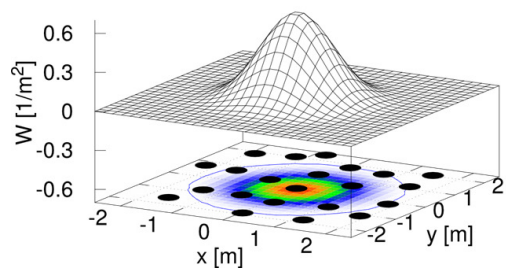

(a) Kernel Function [16]

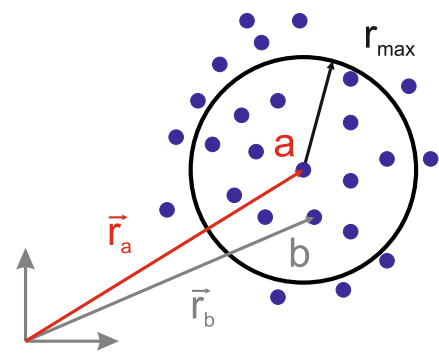

(b) Radius of Influence

Figure 1. SPH Methodology

Approximating the integral by a finite sum leads to a quadrature. Consequently, a scheme for the computation of derivatives can be determined as presented by Liu et al [12]:

$$
\begin{aligned}
\langle f\rangle_{a} & =\sum_{b} f\left(\vec{r}_{b}\right) W\left(\vec{r}_{a}-\vec{r}_{b}, h\right) V_{b}, \\
\langle\nabla \cdot f\rangle_{a} & =-\sum_{b} f\left(\vec{r}_{b}\right) \nabla W\left(\vec{r}_{a}-\vec{r}_{b}, h\right) V_{b} .
\end{aligned}
$$

The brackets \langle\rangle represent the SPH approximation. The index $a$ denominates the central particle and index $b$ any particle inside the radius of influence. The variable $V_{b}$ indicates the volume of each individual particle $b$. The resulting quadratures are the basic SPH schemes. Equation 4 demonstrates that any magnitude $\langle f\rangle_{a}$ at the position of particle $a$ can be determined in terms of a weighted sum $f$ over the function $f\left(r_{b}\right)$ of all other particles $b$ located inside the radius of influence.

By means of the quadratures, the terms on the right hand side of the Navier-Stokes equations as presented in Eq. 1 and 2 can be approximated. The substantial derivatives are computed and integrated in time by means of a predictor-corrector time integration scheme. In the present study, the density is not computed by means of the discretized continuity equation, but by the direct SPH scheme according to Colagrossi et al [17]:

$$
\langle\rho\rangle_{a}=m_{a} \sum_{b} W\left(\vec{r}_{a}-\vec{r}_{b}, h\right)
$$


The pressure gradient is computed as proposed by Monaghan in [18]:

$$
\left\langle-\frac{\vec{\nabla} p}{\rho}\right\rangle_{a}=-\frac{1}{\rho_{a}} \sum_{b} \frac{m_{b}}{\rho_{b}}\left(p_{a}+p_{b}\right) \vec{\nabla} W\left(\vec{r}_{a}-\vec{r}_{b}, h\right) .
$$

Viscosity is modeled by means of a viscosity model presented by Szewc [19]. These approximations conserve momentum and ensure stability in case of large density ratios. As equation of state the Tait-Volume-Equation according to Batchelor [20] is used:

$$
p=\frac{\rho_{\text {nom }} c^{2}}{\kappa} \cdot\left[\left(\frac{V_{\text {nom }}}{V}\right)^{\kappa}-1\right]+p_{\text {back }} .
$$

where $V$ is the volume and $p$ the pressure of each particle. The quantitiy $c$ is the speed of sound and $\kappa$ the polytropic exponent. Index nom designates the reference value of the corresponding quantity. The background pressure $p_{\text {back }}$ is used in weakly compressible SPH to mitigate the inherent tensile instability of the SPH method. As to the assumptions of the weakly compressible approach, pressure depends only on density or volume, respectively. Hence, the equation of state is applied after the volume of each particle has been computed by means of Eq. 6 . Following Wieth et al [21], a modified version of the surface tension model by Adami et al [22] is employed. Static and dynamic contact angles are accurately predicted based on the prescribed surface tension coefficients. According to Young's equation, static contact angles of $60^{\circ}$ are recovered for the present case. In dynamic flows contact angles deviate from the static values. The current implementation takes this effect into account as a result of the balance of forces. The prediction of static and dynamic contact angles has been demonstrated and validated by Wieth et al [21].

As pointed out by Violeau and Rogers [23] three different types of wall treatment exist: Fictitious particles, repulsive functions and boundary integrals. The objective of all wall boundary treatments is to provide kernel support within the radius of influence of all fluid particles across the solid wall boundary. In the current implementation, prescribed dummy particles of at least three layers are used as solid wall boundary representation. Additionally, a repulsive function resembling the Lennard-Jones potential is employed as presented by Wieth et al [21] to avoid particle intrusion. By means of these wall treatments the no-slip condition at the wall is imposed.

In 3D simulations, the circumferential velocity component can easily be imposed the inlet. Centrifugal forces are inherently imposed by the swirling flow. In order to mimic the centrifugal forces in the 2D setup, a model aiming at a representation of centrifugal forces is incorporated. For each SPH particle entering the domain, the radius and the circumferential velocity is stored. In other words, the angular momentum of the particle entering the domain is recovered. When assuming the conservation of the angular momentum, radial equilibrium as well as incompressible fluids, the variation of the circumferential velocity of a particle can be determined as function of its actual radius. Then the corresponding centrifugal forces can be assigned. The model is presented previously by the author in [3].

\section{Fuel Spray Nozzle}

The fuel spray nozzle investigated in the present study consists of three annular, coaxial air ducts referred to as inner air, outer air and dome air according to the setup presented by Mansour et al [24]. All air flows exhibit a swirl component because of the arrangement of swirl generators in each duct as depicted in Fig. 2a. Because of the high angular momentum of all air flows, high centrifugal forces occur forcing the flow in radial direction. As the ratio of angular and axial momentum is large, it comes to vortex breakdown. A stagnation point (SP) is formed, in the vicinity of the middle axis as indicated in Fig. $2 b$.

The main objective of the dome air flow is to shape the recirculation zone inside the combustor. In between the inner and the outer air duct, fuel is supplied through an annular, coaxial slot across the whole perimeter. The fuel is released into the shear zone between inner and outer air. The shear stress imposed by the air will act against the surface tension and finally the fuel will be disintegrated.

Local recirculation zones are to be expected downstream the bluff edges of each shroud as indicated in Fig. 2b. The flows of inner and outer air form a stream tube. Per definition, no mass transfer takes place across the boundaries of the tubes. These boundaries are indicated as dashed lines in Fig. $2 b$.

\section{Computational Setup}

Main objective of this study is the analysis of the multi-phase flow in the vicinity of the prefilming edge in 2D and 3D. The focus is on the interaction of the inner air flow with the fuel in the fuel supply duct, the formation of ligaments and their disintegration. Dispersion of the fuel droplets, their placement inside the combustor and secondary breakup are not the objective of the present study.

The numerical domain is a three dimensional sector based on the axisymmetric cross-section depicted in Fig. $3 a$. There are two inlet boundaries for air and one inlet boundary for fuel. The velocity profiles for both air flows are extracted from grid-based CFD predictions and imposed as boundary conditions. In Fig. 4a and 4b the shape of the velocity profiles imposed at each inlet is presented. The axial velocity profile of the fuel flow at the inlet is based on a correlation for laminar flows. At the fuel inlet, the circumferential velocity recovers a free vortex with sharp velocity gradients at the sides. This profile is used in order to mimic an established annular flow with no slip condition at 


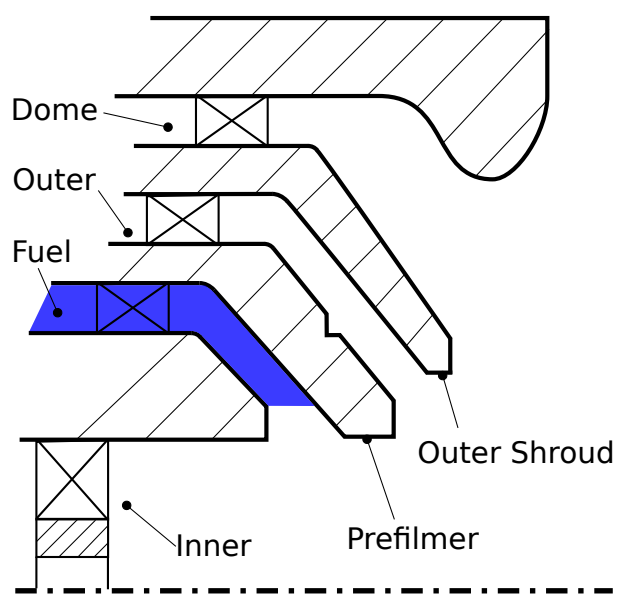

(a) Geometry

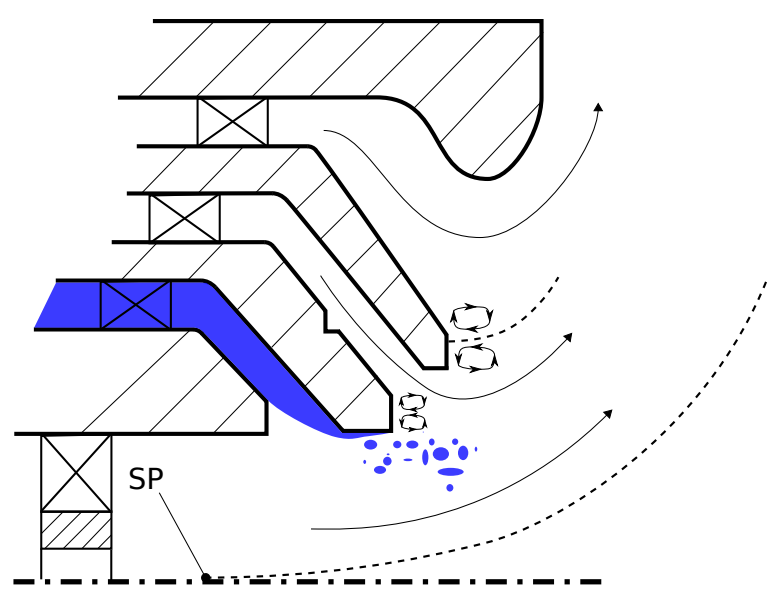

(b) Local Air Flow

Figure 2. Fuel Spray Nozzle

Table 1. Thermodynamic Conditions and Fluid Properties

\begin{tabular}{cccc} 
& & Air & Fuel \\
\hline $\mathrm{T}$ & $\mathrm{K}$ & 649 & 293.15 \\
$\rho$ & $\mathrm{kg} \mathrm{m}^{-3}$ & 6.51 & 770 \\
$\mu$ & $10^{-3} \mathrm{Pas}$ & 0.03255 & 1.56 \\
\hline
\end{tabular}

the walls. The pressure gradient is set to zero at all inlet boundaries. At the outlet cross-section of the domain, the pressure is set constant and the velocity gradient in normal direction is imposed to be zero.

In order to capture the influence of vortex breakdown, the boundaries of the stream tube containing the inner and outer air are extracted from grid based one-phase flow predictions and imposed as slip walls in the SPH setup as depicted in Fig. $3 a$.

The thermodynamic conditions of the underlying operating point are presented in Tab. 1. Surface tension coefficients are set for the liquid to recover static contact angles of $60^{\circ}$. The pressure is $1.213 \mathrm{MPa}$ and the surface tension $27.510^{-3} \mathrm{~N} \mathrm{~m}^{-1}$.

As exemplified in Fig. 3b, the cross-section of the fuel duct at the inlet constitutes an annulus. Further downstream, the duct is deflected in radial direction and forced to smaller radii. Whenever the main direction of the flow exhibits a radial component, the cross-section constitutes a frustoconical surface. The area of the frustoconical surface scales with the radius. The same effect applies to the outer air duct.

The presented setup enables the computation of the local multi-phase flow in the vicinity of the atomizer at a high spatial resolution of $10 \mu \mathrm{m}$. The 3D sector covers 5 degrees. We are aware of the fact, that in order to resolve details of the flow in circumferential direction, the size of the segment is too small. However, swirl effects can inherently be captured. Furthermore, the sector recovers the inherently three dimensional shape of the flow cross-section along the flow path as illustrated in Fig. 3b.

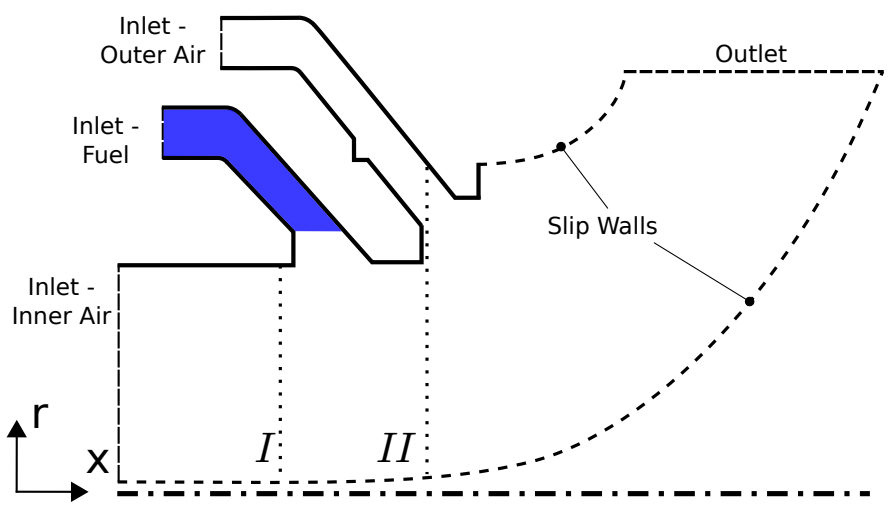

(a) Boundary Conditions

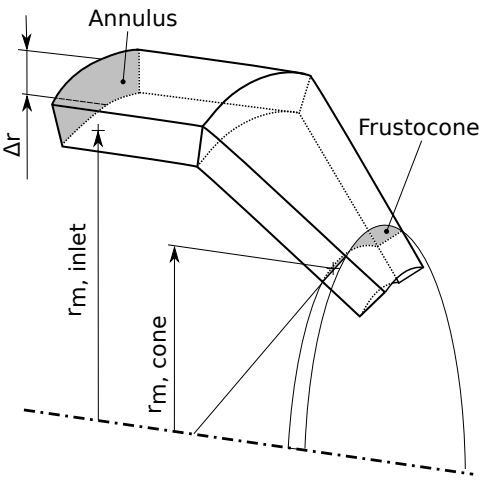

(b) Cross-Sections in Fuel Duct

Figure 3. Computational Domain

Aiming at lower computational costs, predictions based on two-dimensional domains are desired. However, 2D 


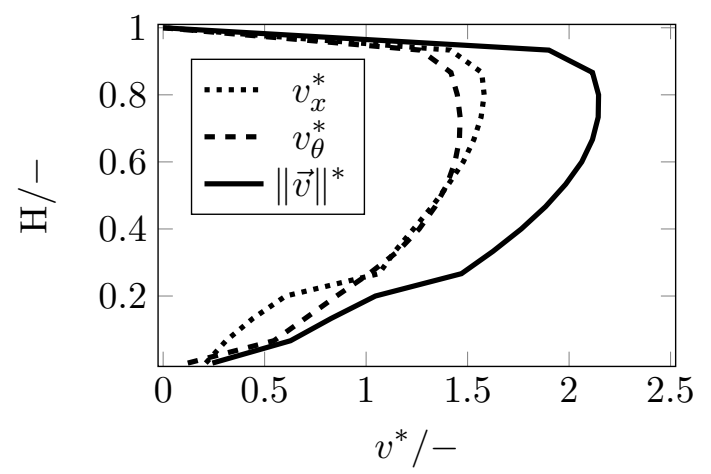

(a) Inner Air Duct

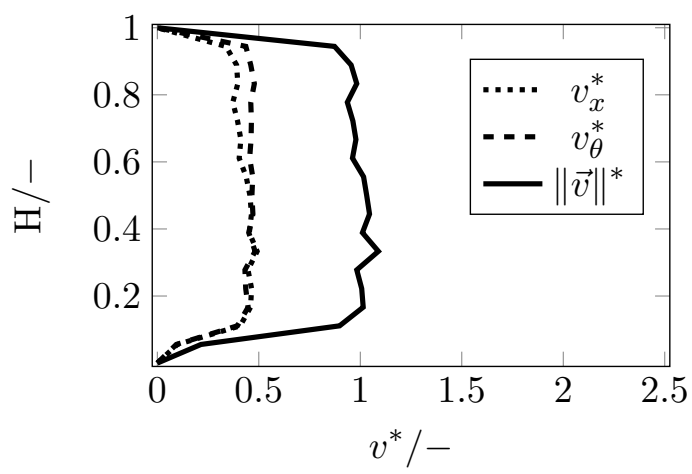

(b) Outer Air Duct

Figure 4. Inlet Boundary Conditions

predictions violate the inherently three dimensional character of fuel atomization. They furthermore imply a planar extension in lateral direction, also violating the frustoconical shape of the flow cross-sections as presented before. In order to identify and quantify differences between 2D and 3D predictions, 2D computations are also performed. In $2 \mathrm{D}$ the circumferential velocity at the inlet of each duct is used as input to the 2D swirl model. All the other boundary conditions are kept constant. A discretization study in 2D up to a resolution of $2.5 \mu \mathrm{m}$ has been conducted, demonstrating that a spatial resolution of at least $10 \mu \mathrm{m}$ is required.

\section{Results and Discussion}

\section{Gaseous Flow - Velocity Profiles}

In Fig. 5a the non-dimensional velocity profiles of the axial velocity in $2 \mathrm{D}$ and $3 \mathrm{D}$ are depicted at the axial position I. The co-ordinate $\mathrm{H}$ is the non-dimensional channel height. The non-dimensional channel height is 1 at the radius of the prefilmer lip. As to be expected, the shape of the profiles is similar to the shape of the axial velocity imposed at the inlet boundary. Obviously, the maximum of the axial velocity component in 2D is located at a larger radius than in the inherently three dimensional case. As the flow is not developed at position I, it comes to a radial velocity component. Comparing 2D and 3D, larger values of the radial velocity can be stated in case of the 2D prediction. Both, the shift of the maximum of the axial velocity and the larger value of the radial velocity indicate that the virtual centrifugal forces seem to be slightly stronger than the inherent centrifugal forces in 3D. The 2D swirl model is based on the assumption of conserved angular momentum. Contrary, in 3D angular momentum is not conserved because of friction losses. Hence, lower centrifugal forces in 3D are to be expected.

In Fig. $5 \mathrm{c}$ the resulting velocity magnitudes in $2 \mathrm{D}$ and $3 \mathrm{D}$ are depicted. In $2 \mathrm{D}$ only a representative centrifugal force is modeled, but the circumferential velocity component is not present. Therefore, there is no contribution to the magnitude of the absolute velocity $\|v\|^{*}$. Hence, the magnitude of the velocity is smaller in $2 \mathrm{D}$ compared to 3D. The velocity distribution of air at position I acts on the liquid fuel flow exiting the fuel gallery. The centrifugal forces of the inner air flow lead to a force in radial direction and, hence, to an interaction with the fuel flow inside the exit of the fuel duct.

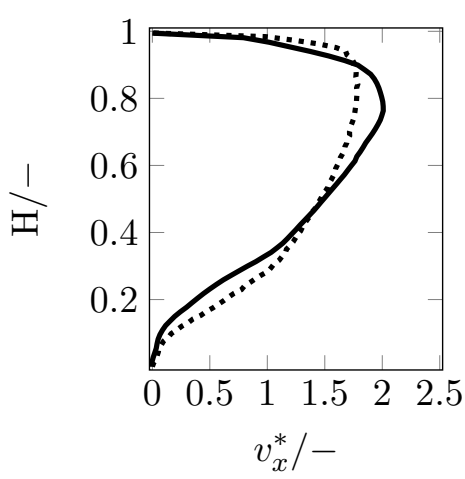

(a) Axial Velocity

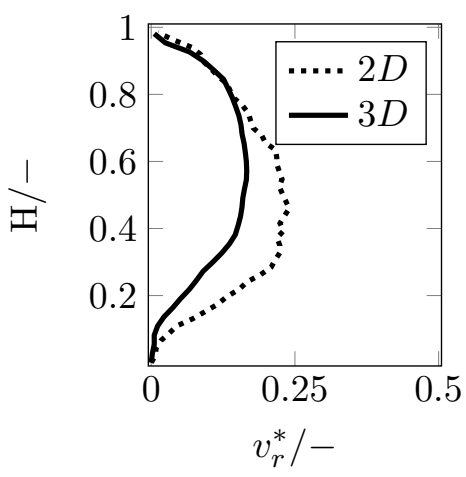

(b) Radial Velocity

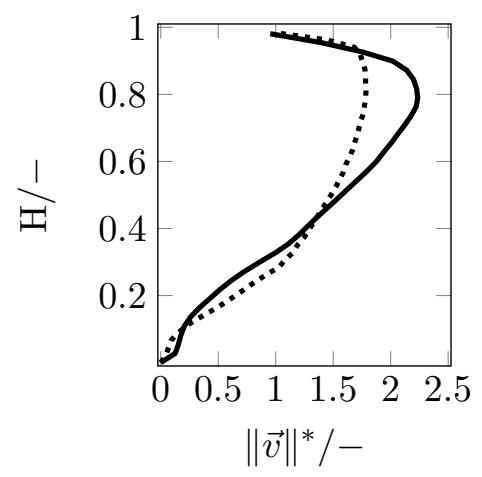

(c) Velocity Magnitude

Figure 5. Comparison of Velocity Profiles at Position I - 2D vs. 3D

In Fig. 6 the velocity profiles at position II obtained from 2D and 3D predictions are presented. In contrast to the inner air flow, the differences of the velocity magnitude in the outer air flow are significantly larger. Again in 2D there is no contribution of the circumferential velocity to the magnitude. Furthermore, the flow is accelerated according to the continuity equation because of the decrease of the flow cross-section as illustrated in Fig. 3b. In 2D this effect is 
omitted. Hence, only a deflection of the flow, but no acceleration takes place resulting in a much smaller magnitude of the velocity.

One can conclude from the velocity profiles, that deviations of the gaseous flow between 2D and 3D occur. The differences inside the inner air duct are comparably small. The largest differences are to be expected downstream the prefilming surface in the vicinity of the shear zone between inner and outer air.

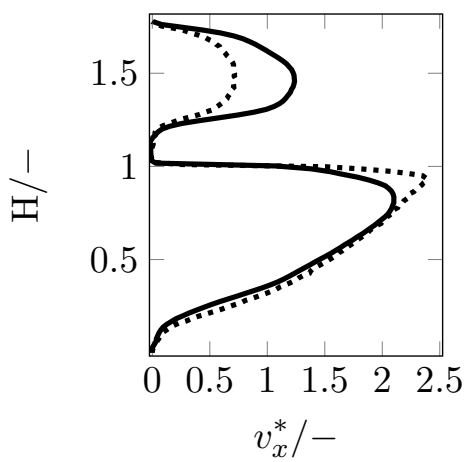

(a) Axial Velocity

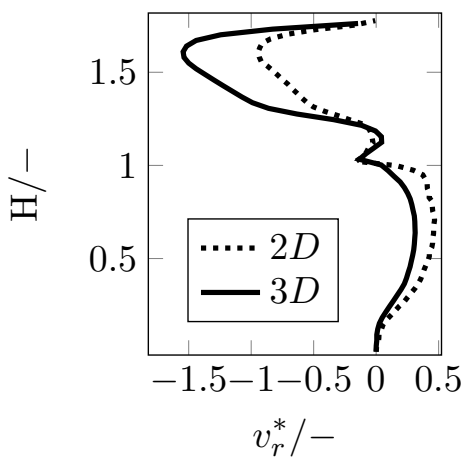

(b) Radial Velocity

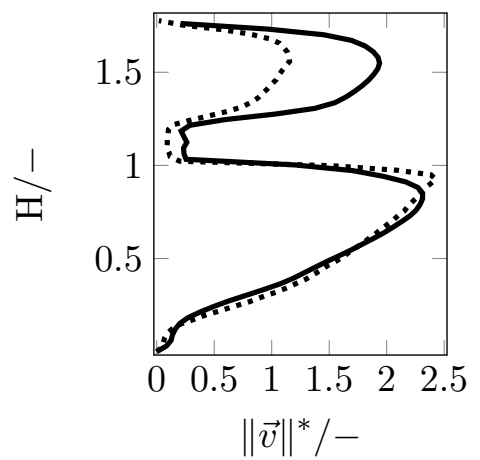

(c) Velocity Magnitude

Figure 6. Comparison of Velocity Profiles - 2D vs. 3D - Position II

\section{Gaseous Flow - Flow Instabilities}

Due to the Lagrangian nature of the SPH method, a color coding can easily be applied, which serves as a tool to distinguish between inner and outer air flow. Fuel is colored in blue and air originating from the outer air inlet is indicated as orange. Air that entered the domain through the inner air inlet is not visible. By means of the color coding the Kelvin-Helmholtz instability between inner and outer air flow can be visualized. Two instantaneous snapshots are presented in Fig. 7. The snapshots are recorded at the beginning of the simulation, when there is no fuel emerging from the supply duct. Despite the differences with regard to the velocity field in the outer air duct, the Kelvin-Helmholtz instability is qualitatively captured in 2D and 3D. Because of the higher velocity in the outer air duct in 3D, the shear zone is located at a smaller radius than in 2D. In each case, two vortices occur in the snapshots. The fuel exiting the fuel gallery will be released into the corresponding shear zone and exposed to the local flow instabilities of the gaseous flow. The Kelvin-Helmholtz instability presented here is one example of a transient flow instability that might occur in the vicinity of the nozzle. Instabilities in circumferential direction might also occur, but just a subset of wavelengths is captured in the present numerical setup due to the small circumferential extension. Later in time, as soon as fuel emerges from the supply duct, the distinct vortices due to the Kelvin-Helmholtz instability will vanish. However, instabilities of the gaseous flow in radial direction still occur and interact with the liquid.
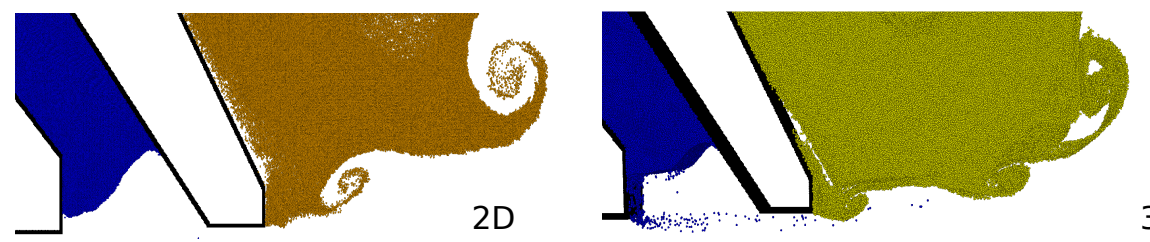

Figure 7. Comparison of Kelvin-Helmholtz Instability

\section{Liquid Flow}

The design of the air flow with fuel emerging from the fuel gallery, leads to a periodic filling and discharge effect at the exit region of the fuel duct. In Fig. 8 three snapshots presenting the different phases of the periodic discharge are depicted. In the beginning fuel is accumulated in the exit region of the fuel duct. Once the cavity is filled up with fuel, a ligament is formed reaching out into the inner air duct. The momentum of the inner air flow pushes the ligament against the prefilming surface. The ligament is released into the shear zone and breaks up into smaller ligaments. Once the ligament is extracted, the fill level of the cavity reaches its minimum. Then for a period of time fuel is again accumulated in the cavity, before the formation of a new ligament starts again. In 3D and 2D the same periodic effect can be observed.

In Fig. 9 the time evolution of liquid volume flow rate across the circular cross-section of the inner air duct at axial position II is depicted. The periodic discharge effect is recovered in terms of periodic peaks of the liquid volume flow rate. Facing comparably large computational effort in 3D, only a few discharge events are captured. Comparing $2 \mathrm{D}$ and $3 \mathrm{D}$, one can conclude from the time histories that the peaks do not coincide in time. The reason for the 


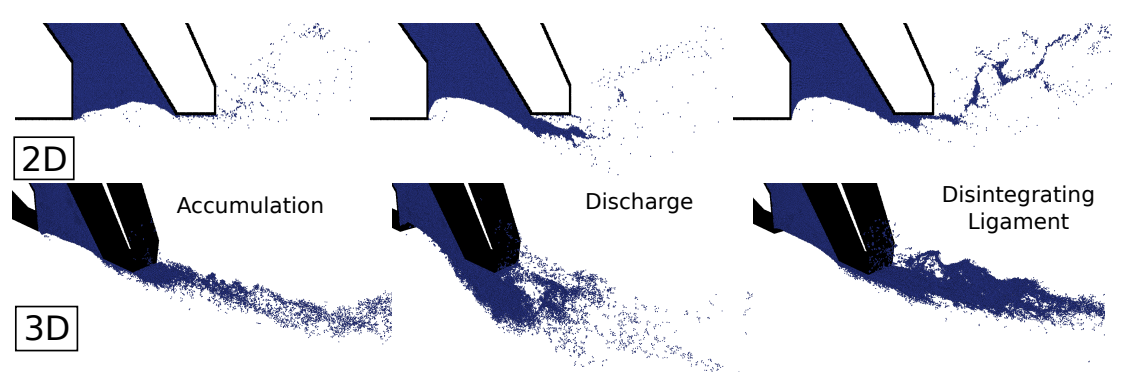

Figure 8. Periodic Filling and Discharge

difference is the higher velocity of fuel at the outlet of the fuel gallery in 3D due to the reduced frustoconical crosssection compared to 2D. Based on the few available events, the frequency of the periodic discharge is determined to be approximately $1.6 \mathrm{kHz}$. However, the number of events is far below statistical significance.

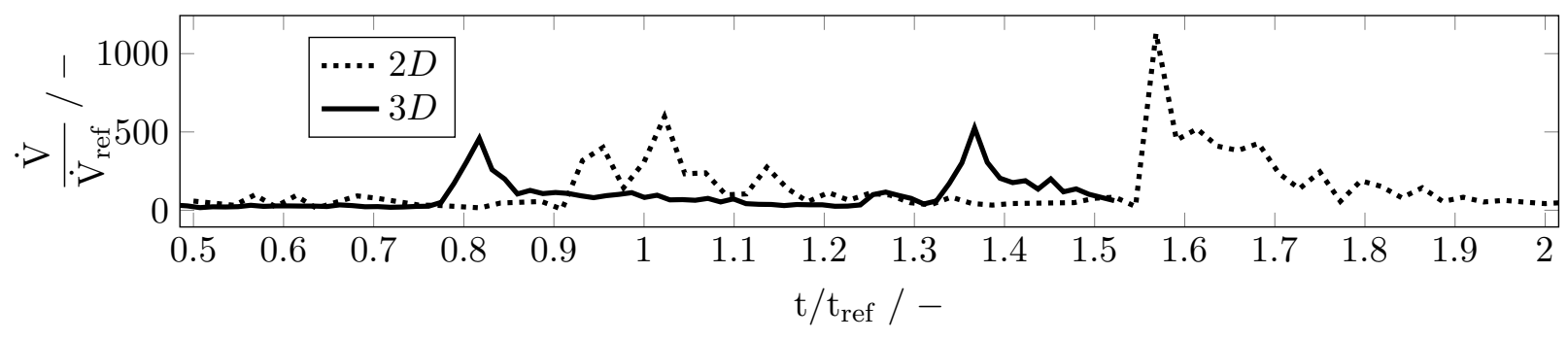

Figure 9. Evolution of the Non-Dimensional Volume Flow Rate over Time

\section{Conclusions}

A methodology for the numerical analysis of primary breakup by means of SPH in the vicinity of fuel spray nozzles at typical engine conditions is presented. A typical airblast-nozzle for aero-engines with three air flow ducts is analyzed. Predictions based on a 3D sector are performed and compared to predictions based on simplified two dimensional setups. To the knowledge of the authors this is the first analysis of primary breakup in a fuel spray nozzle at engine conditions in 3D by means of SPH. The following conclusions can be drawn:

1. SPH is suitable for the analysis of primary breakup phenomena in realistic fuel spray nozzle configurations.

2. Unsteady effects in the gaseous and the liquid flow field are identified.

3. Temporal fluctuations and flow instabilities can be studied in 3D, which resemble those observed in experimental data.

4. Large quantitative discrepancies between 2D and 3D simulations occur.

5. However, 2D simulations are capable of giving qualitative insights into local, unsteady flow phenomena.

\section{Acknowledgements}

This work was performed on the computational resource ForHLR Phase II funded by the Ministry of Science, Research and Arts Baden-Würrtemberg and DFG ("Deutsche Forschungsgemeinschaft"). In addition the authors would like to thank Rolls-Royce Deutschland Ltd \& Co KG for the outstanding cooperation. The authors also are grateful for many lively and fruitful discussions with Simon Holz.

\section{Nomenclature}

\section{Abbreviations}

ITS Institut für Thermische Strömungsmaschinen

PDF Probability Density Function

SMD Sauter Mean Diameter

SPH Smoothed Particle Hydrodynamics

SP Stagnation Point

VoF Volume of Fluid

\section{Latin Symbols}

$c \quad$ Speed of Sound $\left[\mathrm{m} \mathrm{s}^{-1}\right]$

$d \quad$ Diameter $[\mathrm{m}]$

$h \quad$ Smoothing Length [m]

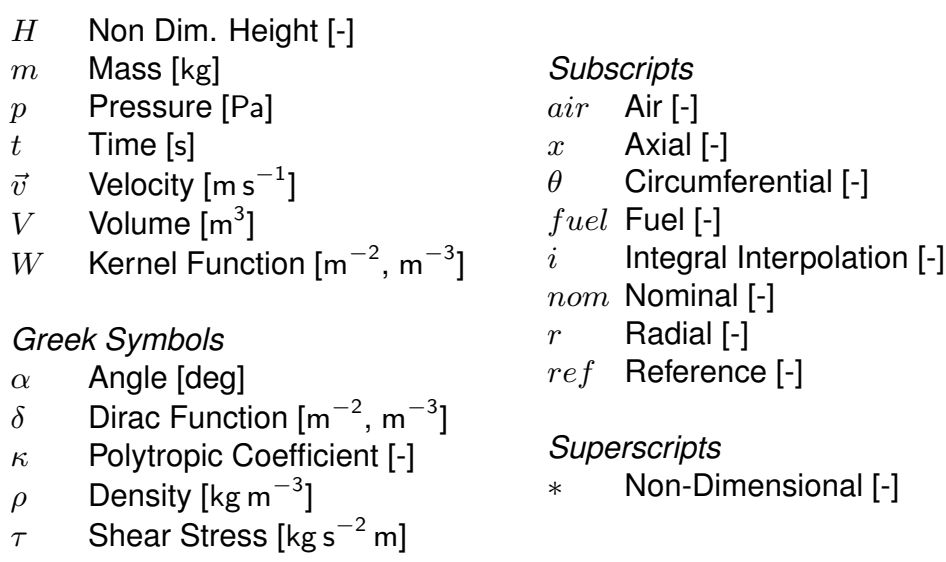




\section{References}

[1] S. Braun, M. Krug, L. Wieth, C. Höfler, R. Koch, and H.-J. Bauer, "Simulation of primary atomization: Assessment of the smoothed particle hydrodynamics (sph) method," in 13th Triennial International Conference on Liquid Atomization and Spray Systems (ICLASS), Tainan, Taiwan, 2015.

[2] S. Braun, L. Wieth, R. Koch, and H.-J. Bauer, "Influence of trailing edge height on primary atomization: Numerical studies applying the smoothed particle hydrodynamics (sph) method," in 13th Triennial International Conference on Liquid Atomization and Spray Systems, Tainan. Taiwan., 2015.

[3] T. Dauch, S. Braun, W. L., G. Chaussonnet, M. Keller, R. Koch, and B. H.-J., "Computation of liquid fuel atomization and mixing by means of the SPH method: application to a jet engine fuel nozzle," ASME 2016 Turbo Expo: Turbine Technical Conference and Exposition, Seoul, 2016.

[4] R. Koch, S. Braun, L. Wieth, G. Chaussonnet, T. Dauch, and H.-J. Bauer, "Prediction of primary atomization using smoothed particle hydrodynamics," European Journal of Mechanics - B/Fluids, vol. 61, Part 2, pp. 271 278, 2017. Rotating Flows.

[5] C. Höfler, S. Braun, R. Koch, and H.-J. Bauer, "Modeling spray formation in gas turbines-a new meshless approach," Journal of Engineering for Gas Turbines and Power, vol. 135, pp. 011503-011503, Nov. 2012.

[6] M. C. Keller, S. Braun, L. Wieth, G. Chaussonnet, T. Dauch, R. Koch, C. Höfler, and H.-J. Bauer, "Numerical modeling of oil-jet lubrication for spur gears using smoothed particle hydrodynamics," in Proceedings of the 11th International SPHERIC Workshop, pp. 69-76, 2016.

[7] G. Chaussonnet, O. Vermorel, E. Riber, and B. Cuenot, "A new phenomenological model to predict drop size distribution in large-eddy simulations of airblast atomizers," International Journal of Multiphase Flow, vol. 80, pp. 29-42, Apr 2016.

[8] R. Gingold and J. Monaghan, "Smoothed particle hydrodynamics-theory and application to non-spherical stars," Monthly notices of the royal astronomical society, vol. 181, pp. 375-289, 1977.

[9] L. Lucy, "A numerical approach to the testing of the fission hypothesis," The astronomical journal, vol. 82, pp. 1013-1024, 1977.

[10] G. Chaussonnet, S. Braun, L. Wieth, R. Koch, and H.-J. Bauer, "Influence of particle disorder and smoothing length on sph operator accuracy," in 10th International SPHERIC Workshop, Tainan, Taiwan, 2015.

[11] J. Swegle, D. Hicks, and S. Attaway, "Smoothed particle hydrodynamics stability analysis," Journal of Computational Physics, vol. 116, no. 1, pp. 123 - 134, 1995.

[12] M. Liu and G. Liu, "Smoothed particle hydrodynamics (sph): an overview and recent developments," Archives of Computational Methods in Engineering, vol. 17, no. 1, pp. 25-76, 2010.

[13] N. Quinlan, M. Basa, and M. Lastiwka, "Truncation error in mesh-free particle methods," International Journal for Numerical Methods in Engineering, vol. 66, p. 2064-2085, 2006.

[14] S. Litvinov, X. Hu, and N. Adams, "Towards consistence and convergence of conservative sph approximations," Journal of Computational Physics, vol. 301, pp. 394-401, Nov. 2015.

[15] W. Dehnen and H. Aly, "Improving convergence in smoothed particle hydrodynamics simulations without pairing instability," Monthly Notices of the Royal Astronomical Society, vol. 425, no. 2, p. 1068, 2012.

[16] L. Wieth, C. Lieber, W. Kurz, S. Braun, R. Koch, and B. H.-J., "Numerical modeling of an aero-engine bearing chamber using the meshless smoothed particle hydrodynamics method," in Proceedings of ASME Turbo Expo 2015: Turbine Technical Conference and Exposition, Montréal, Canada, GT2015-42316, vol. 2B, 2015.

[17] A. Colagrossi and M. Landrini, "Numerical simulation of interfacial flows by smoothed particle hydrodynamics," Journal of Computational Physics, vol. 191, no. 2, pp. 448 - 475, 2003.

[18] J. J. Monaghan, "Smoothed particle hydrodynamics," Annual Review of Astronomy and Astrophysics, vol. 30, no. 1, pp. 543-574, 1992.

[19] K. Szewc, J. Pozorski, and J.-P. Minier, "Analysis of the incompressibility constraint in the smoothed particle hydrodynamics method," International Journal for Numerical Methods in Engineering, vol. 92, no. 4, pp. 343369, 2012.

[20] G. Batchelor, An introduction to fluid dynamics. Cambridge University Press, 2000.

[21] L. Wieth, S. Braun, R. Koch, and H.-J. Bauer, "Modeling of liquid-wall interaction using the meshless smoothed particle hydrodynamics (sph) method," in ILASS Europe, 26th Annual Conference on Liquid Atomization and Spray Systems, Bremen, Germany, 2014.

[22] S. Adami, X. Hu, and N. Adams, "A new surface-tension formulation for multi-phase $\{\mathrm{SPH}\}$ using a reproducing divergence approximation," Journal of Computational Physics, vol. 229, no. 13, pp. 5011 - 5021, 2010.

[23] D. Violeau and B. D. Rogers, "Smoothed particle hydrodynamics (sph) for free-surface flows: past, present and future," Journal of Hydraulic Research, vol. 54, no. 1, pp. 1-26, 2016.

[24] A. Mansour and M. Benjamin, "Pure airblast nozzle," 2003. 


\section{$8 Y$ \\ Droplet 5}




\title{
Experimental and computational investigation of binary drop collisions under elevated ambient pressure
}

\author{
Louis Reitter ${ }^{1}$, Muyuan Liư ${ }^{2}$, Jan Breitenbach ${ }^{1 *}$, Kuan-Ling Huang ${ }^{3}$, Dieter Bothe ${ }^{2}$, \\ Günter Brenn ${ }^{4}$, Kuo-Long Pan ${ }^{3}$, llia V. Roisman ${ }^{1}$, Cameron Tropea ${ }^{1}$ \\ ${ }^{1}$ Fluid Mechanics and Aerodynamics, TU Darmstadt, Germany \\ ${ }^{2}$ Mathematical Modeling and Analysis, TU Darmstadt, Germany \\ ${ }^{3}$ Mechanical Engineering, National Taiwan University, Taiwan \\ ${ }^{4}$ Fluid Mechanics and Heat Transfer, TU Graz, Austria \\ ${ }^{*}$ Corresponding author: jbreitenbach@sla.tu-darmstadt.de
}

\begin{abstract}
Spray systems often operate under extreme ambient conditions like high pressure, which can have a significant influence on important spray phenomena. One of these phenomena is binary drop collisions. Such collisions, depending on the relative velocity and the impact parameter (eccentricity of the collision), can lead to drop bouncing, coalescence or breakup. This experimental and computational study is focused on the description of the phenomenon of drop bouncing, which is caused by a thin gas layer preventing the drops coalescence. To identify the main influencing parameters of this phenomenon, experiments on binary drop collisions are performed in a pressure chamber. This experimental system allows us to investigate the effect of an ambient pressure (namely the density and viscosity of the surrounding gas) on the bouncing/coalescence threshold.
\end{abstract}

\section{Keywords}

drop collision, breakup/coalescence, multiphase flow

\section{Introduction}

Understanding the collisions of drops is of interest for many fields of application. They play an important role in raindrop formation [1, 2] and in the modeling of fuel combustion [3, 4]. Systematic experimental studies visualizing binary drop collisions provide information allowing to construct regime maps of the collision outcomes $[5,6,7]$. The mechanisms of bouncing, coalescence, stretching separation, reflexive separation, and shattering are observed. These mechanisms are determined by the kinetic and geometrical parameters of the collision, as well as the properties of the drop liquids and the surrounding gas. The main kinetic and geometrical parameters are defined in Figure 1, where $D_{1}$ and $D_{2}$ are the drop diameters and $V$ is the relative drop velocity. $B$ is the distance of closest approach of the drop centers, measured orthogonal to $V$ at the instance of the collision. Dimensional analysis reveals that the collision Weber number We $=V^{2} D_{1} \rho / \sigma$, the Ohnesorge number $\mathrm{Oh}=\mu / \sqrt{\sigma D_{1} \rho}$, the impact parameter $X=2 B /\left(D_{1}+D_{2}\right)$ and the drop size ratio $\Delta D=D_{1} / D_{2}$ determine the outcome of the collision, if the medium ambient to the colliding drops is not varied.

The present investigation concentrates on the mechanism of bouncing. Outcomes from drop collsion with a solid substrates shown, that small droplets are able to bounce on solid substrate, irrespectivly of their wettability [8]. The bouncing is caussed by a thin squeezing air film beetween the droplet and the substrate leading to squeeze forces. For the binary drop collsion, Rayleigh [9] pointed out that the drop collision mechanism may be influenced by electrical charge on the drops. The occurrence of bouncing or coalescence of electrified drops may depend on their charge density. Electrically charged drops formed from vertically upward pointing electrified liquid jets may either coalesce (at moderate charge density) or rebound (at high charge density).

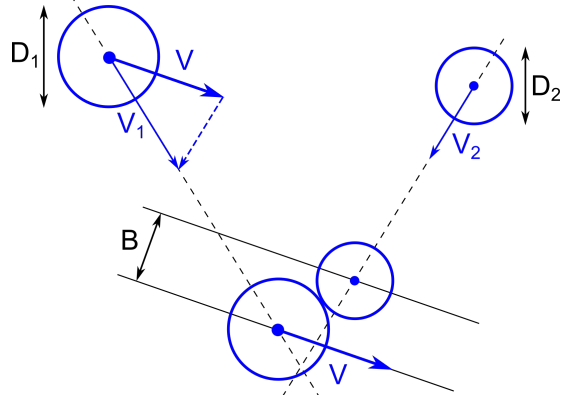

Figure 1. Definition of collision geometry This behaviour determines the appearance of the jet, which may be either fairly coherent or strongly scattered, respectively. Qian and Law [7] carried out experiments in atmospheres of different gases at different pressures, showing that the extension of the bouncing regime in the collision charts varies with the ambient gas pressure. At higher gas pressure, the bouncing regime widens. The experimental study [10] demonstrates that the bouncing regime relies on the presence of the ambient gas. The regime disappears if the drops collide in a vacuum. This important result clearly indicates the role of the medium ambient to the colliding drops in the occurrence of bouncing. Consequently, the study [11] shows that bouncing is enhanced with drops colliding in another viscous fluid.

From the literature we know several models for binary drop collision outcomes applied in the framework of CFD codes, such as the KIVA-II code. The first model for bouncing was presented in [12]. It assumes that, upon impact, 
the drops deform into semi-spherical shapes. If the deformation produces an increase in surface energy larger than the initial kinetic energy of the pair, the drops will bounce. The resulting criterion is a threshold of the impact Weber number as a function of the impact parameter and drop size ratio. The model is empirical, built on collision charts, and does not represent the flow details in the space between the approaching drops [13]. This model is used by the group of Reitz to predict drop collision outcomes, accounting for all the four mechanisms at moderate Weber numbers, except shattering [14]. The conclusion is that, in the present literature, we do not see any detailed investigation of the flow in and around colliding drops for the bouncing mechanism.

The effect of the ambient pressure on the thresholds between the regimes is not yet completely understood. The main subject of this study is the experimental and computational investigation of binary drop collisions under elevated ambient pressure with emphasis on bouncing. The goal is to better understand this mechanism caused by the viscous air flow in a very thin gap between the colliding drops, and to model the transition conditions between coalescence and bouncing. The regime maps allow the threshold Weber number between bouncing and coalescence to be determined as a function of the impact parameter. One important result of this study is that only a minor dependence of the drop coalescence on the elevated ambient pressure is observed. It should be noted that, for high pressures, the value of the kinematic viscosity of air is almost constant.

\section{Experimental and Computational Methods \\ Experimental setup}

The experimental setup consists of two drop generators mounted in a pressure chamber, a high-speed video system and a data acquisition system. The scheme of the setup is shown in Figure 2. The pressure chamber can be operated in the range of absolute pressure from 1 bar to 20 bar. It has four glass windows to provide optical access to the studied phenomena.

Two drop generators are used to generate a chain of drops and can be controlled independently. They are connected to a pressurized fluid supply tank and a signal generator. The electrical signal powers the drop generators' piezo actuators, which are used to impose disturbances onto the continuous fluid jets. If the disturbance frequency is properly set, the jet undergoes a controlled Rayleigh-type breakup and a stream of monodisperse drops is produced. The drop diameter can be varied by the disturbance frequency and by changing the orifice diameter of the drop generator.

Two drop generators are positioned in front of one chamber window. For precise positioning, one drop generator is mounted on a manual micromanipulator and the other drop generator is mounted on a highprecision traversing system. The piezo motor of the traversing system can adjust one drop generator position in $5 \mu \mathrm{m}$ steps. This fine adjustment of the drop generator position allows to ensure equal distances between each drop generator and the camera objective. The high-speed camera is operated with a frame rate of 22000 frames per second. Continuous illumination using an LED light source is used in the experiments. A data acquisition and control system is used to control the fluid flow rate through the drop generators and to measure the experimental properties. These are the air pressure, temperature and relative humidity in the pressure chamber, the supply fluid temperature and the pressure in the fluid reservoir. The relative velocity of the colliding drops, their diameters and impact parameter are calculated via image processing.

In the experiments $42 \mathrm{wt} . \%$ glycerol-water solution at a

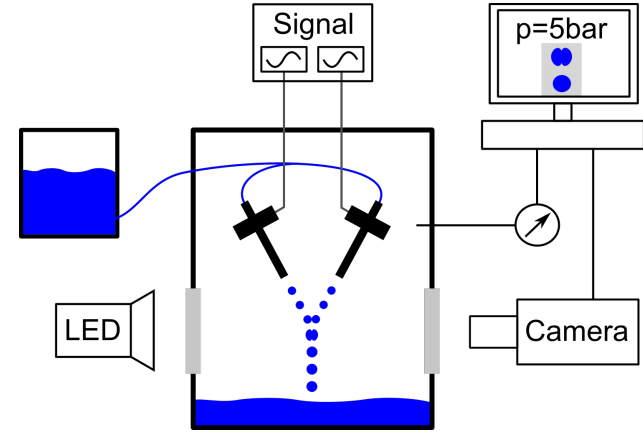

Figure 2. Scheme of the experimental setup

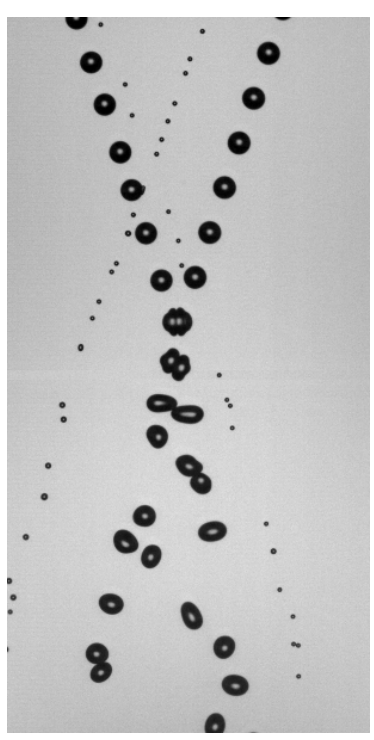

(a) bouncing

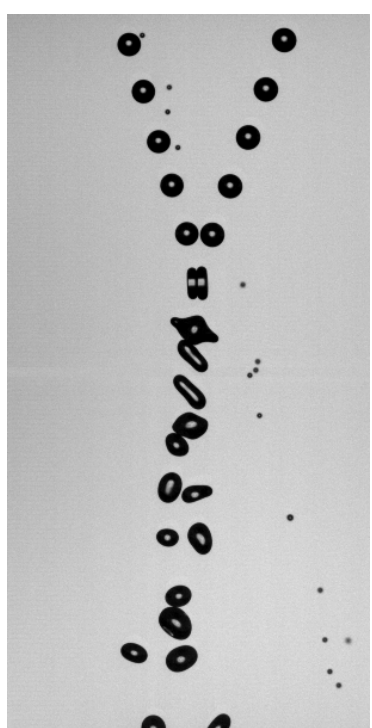

(b) coalescence
Figure 3. Exemplary observations of binary drop collisions leading to bouncing or coalescence. temperature of $T_{f}=28^{\circ} \mathrm{C}$ is used for the drops, whose diameter ranges from $D_{1}=335-400 \mu \mathrm{m}$ with a constant drop size ratio $\Delta D \approx 1$. The impact velocity varied from $V_{1 / 2}=1.5-3.3 \mathrm{~m} / \mathrm{s}$. The dynamic viscosity, surface tension coefficient and density of the liquid are $\mu_{L}=3.25 \times 10^{-3}$ Pa.S, $\sigma=68.44 \times 10^{-3} \mathrm{~N} / \mathrm{m}$ and $\rho_{L}=1058 \mathrm{~kg} / \mathrm{m}^{3}$, respectively. The corresponding Ohnesorge number $\mathrm{Oh} \approx 0.02$ is almost constant in all the experiments. The air temperature in the pressure chamber was $T_{0}=28^{\circ} \mathrm{C}$ and the relative humidity $\phi=78 \%$.

Exemplary observations of drop collisions leading to different outcomes, bouncing or coalescence, are shown in Figure 3. These images are then processed to measure the drops sizes and the impact velocity and to identify the 


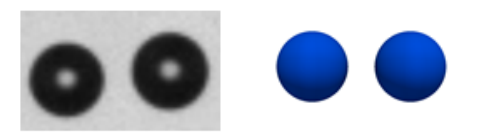

$t=0 \mathrm{~ms}\left(t^{*}=-0.379\right)$
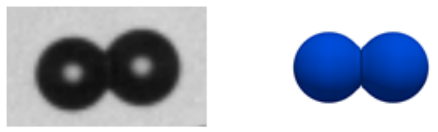

$0.091 \mathrm{~ms}(0.102)$
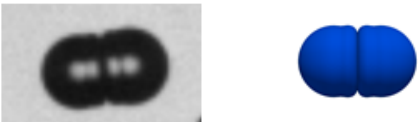

$0.136 \mathrm{~ms}(0.343)$
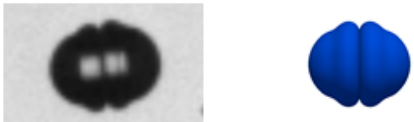

$0.182 \mathrm{~ms}(0.584)$
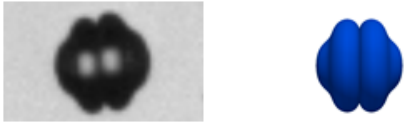

$0.227 \mathrm{~ms}(0.824)$
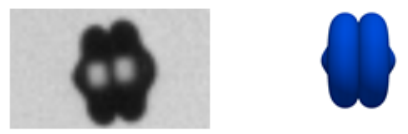

$0.273 \mathrm{~ms}(1.065)$

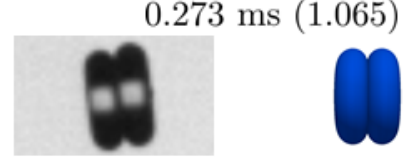

$0.318 \mathrm{~ms}(1.305)$
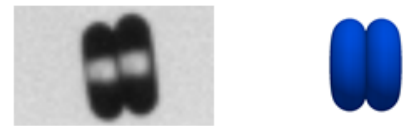

$0.364 \mathrm{~ms}$ (1.546)
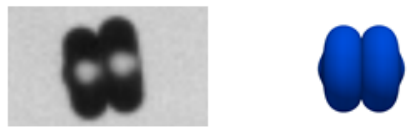

$0.409 \mathrm{~ms}(1.787)$
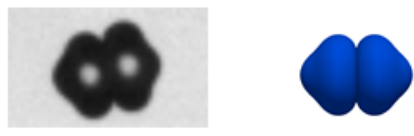

$0.455 \mathrm{~ms}(2.027)$
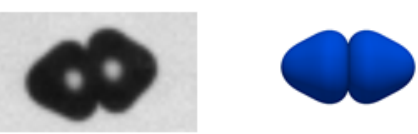

$0.500 \mathrm{~ms}(2.268)$
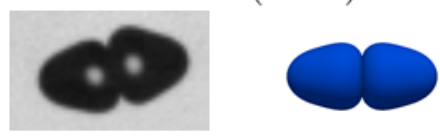

$0.546 \mathrm{~ms}(2.508)$
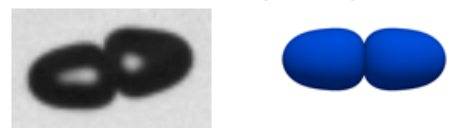

$0.591 \mathrm{~ms}(2.749)$
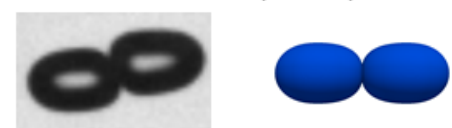

$0.636 \mathrm{~ms}(2.990)$
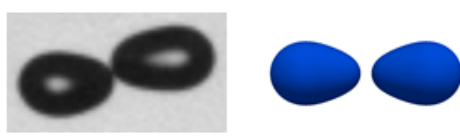

$0.773 \mathrm{~ms}(3.471)$

Figure 4. Drop bouncing near the coalescence threshold. Comparison of the computed drop shapes after axisymmetric drop collision with the experimental observations. The parameters are: $\mathrm{Re}=204.92$, We $=17.76, X=0.0135$, the pressure is $p=7$ bar. The three columns compare experiments (left) with numerical simulations (right).

main collision regimes. The two drop chains in the background are produced in the jet breakup region. Due to the high ambient pressure small satellite drops bounce off the main drop chain. These pre-collision droplets do not affect the binary collision.

\section{Computational method}

In order to better understand the mechanisms of binary drop collisions, this phenomenon is simulated numerically, solving the full Navier-Stokes equations in the liquid and gas phases and accounting for surface tension effects. The numerical simulations are performed with the in-house code Free Surface 3D (FS3D), which was originally developed at the ITLR (University of Stuttgart) [15] and has been extended both at the ITLR and in the Mathematical Modeling and Analysis (MMA) group of the Technische Universität of Darmstadt. FS3D is based on the Volume-ofFluid (VOF) method and solves the incompressible transient two-phase flow in 3D on a staggered Cartesian mesh. A recent overview of FS3D is given in [16]. FS3D has been validated in $[15,17,18,19]$ for the simulation of binary droplet collisions.

In FS3D, for integrating the surface tension force, the balanced-CSF [20] and CSS models [21] are implemented. The balanced-CSF model has the advantage of much less parasitic currents. However, it is not able to deal with the topology change when merging of two interfaces occurs. The CSS model can deal with the topology change in a natural manner; however, it suffers from parasitic currents. Based on their advantages and disadvantages, the balance-CSF model is used before possible coalescence in the simulations, while at the instant of coalescence, the computation of the surface tension force is computed according to the CSS model.

In experiments, prior to possible coalescence, there is a gas layer between the colliding droplets with a thickness generally of the order of a few $10 \mathrm{~nm}$ [22]. The gas layer cannot be resolved due to current restrictions in computational effort. This results in a numerical coalescence of droplets in standard VOF simulations, since the computation of the surface tension force of one droplet is affected by its counterpart. In order to simulate bouncing, specifically in case of a symmetric collision, we temporarily remove the symmetric counterpart of one droplet while computing the surface tension force. This treatment is also valid for the case of coalescence before the merging occurs. At the instant of possible coalescence, this treatment is discarded so that coalescence then occurs. The time instant of coalescence is defined as the averaged value of the instants that correspond to the last cuspy contour and the first smooth contour between the two droplets [23], which are obtained from the experiment.

In the simulations, one droplet with diameter $D_{1}$ is initialized, colliding towards its symmetric counterpart with velocity $V$. A rectangular box with the dimensions of $\left(2 D_{1}\right)^{3}$ is discretized with $256^{3}$ equidistant Cartesian cells. Three symmetry planes are employed for reducing the computational effort. Slip conditions are prescribed on the symmetry planes while homogeneous Neumann boundary conditions for the velocity and zero pressure are imposed on the other parts of the boundary. 


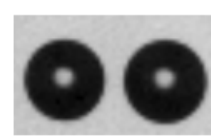

$t=0 \mathrm{~ms}\left(t^{*}=-0.0002\right)$

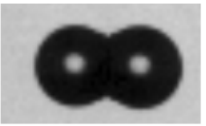

$0.091 \mathrm{~ms}(0.434)$

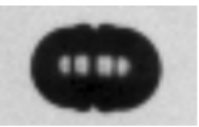

$0.136 \mathrm{~ms}(0.651)$
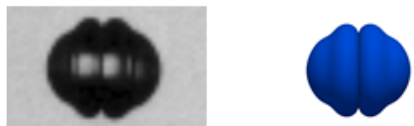

$0.182 \mathrm{~ms}(0.869)$

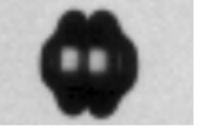

$0.227 \mathrm{~ms}(1.086)$

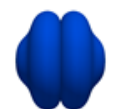

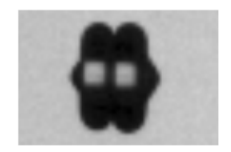

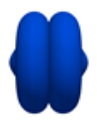

$0.273 \mathrm{~ms}(1.303)$
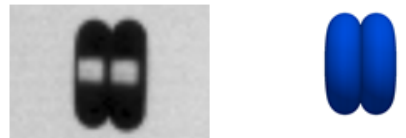

$0.318 \mathrm{~ms}(1.520)$
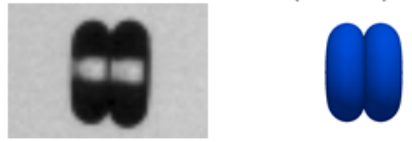

$0.364 \mathrm{~ms}(1.737)$
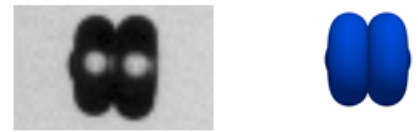

$0.409 \mathrm{~ms}(1.955)$
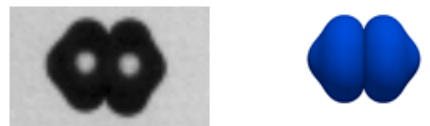

$0.455 \mathrm{~ms}(2.172)$
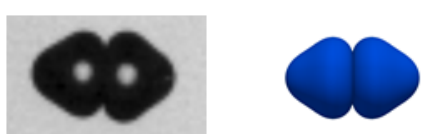

$0.500 \mathrm{~ms}(2.389)$
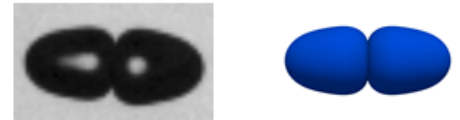

$0.590 \mathrm{~ms}(2.824)$
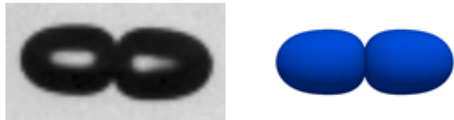

$0.636 \mathrm{~ms}(3.041)$
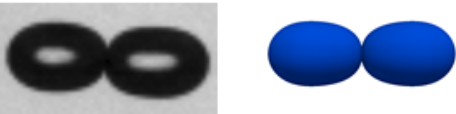

$0.682 \mathrm{~ms}(3.258)$
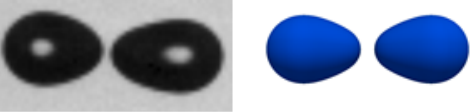

$0.773 \mathrm{~ms}(3.692)$

Figure 5. Drop bouncing. Comparison of the computed drop shapes after axisymmetric drops collision with the experimental observations. The parameters are: $\operatorname{Re}=208.10$, We $=17.27, X=0.005$, the pressure is $p=9$ bar.
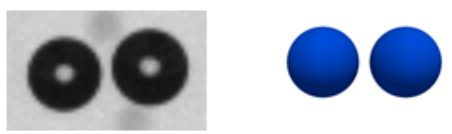

$t=0 \mathrm{~ms}\left(t^{*}=-0.0002\right)$
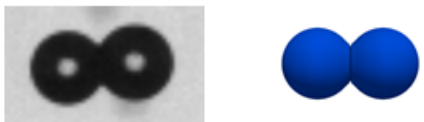

$0.045 \mathrm{~ms}(0.242)$
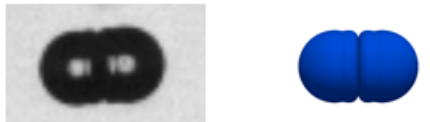

$0.091 \mathrm{~ms}(0.484)$
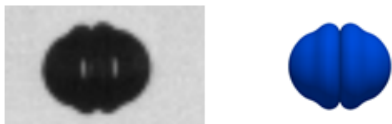

$0.136 \mathrm{~ms}(0.726)$
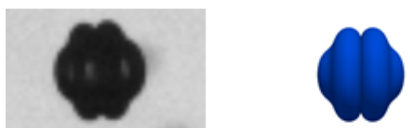

$0.182 \mathrm{~ms}(0.968)$
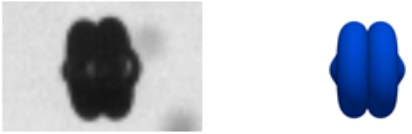

$0.227 \mathrm{~ms}(1.209)$
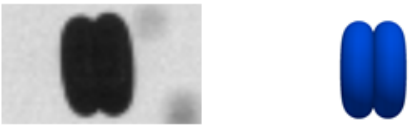

$0.272 \mathrm{~ms}(1.451)$
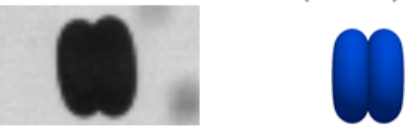

$0.318 \mathrm{~ms}(1.693)$
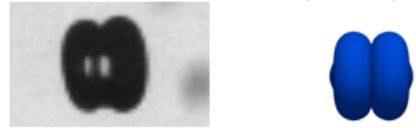

$0.364 \mathrm{~ms}(1.935)$

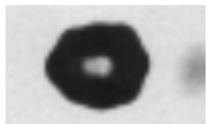

$0.409 \mathrm{~ms}(2.177)$
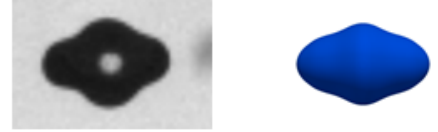

$0.455 \mathrm{~ms}$ (2.419)
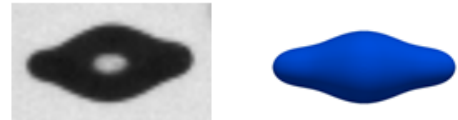

$0.591 \mathrm{~ms}(3.145)$
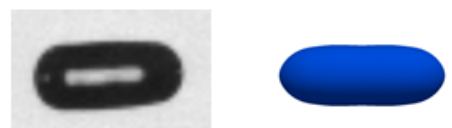

$0.727 \mathrm{~ms}(3.871)$
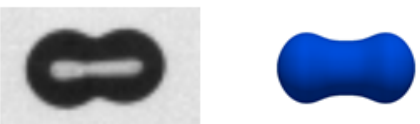

$0.864 \mathrm{~ms}(4.596)$

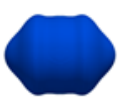

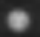

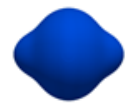

$1.136 \mathrm{~ms}$ (6.048)

Figure 6. Drop coalescence. Comparison of the computed drop shapes after axisymmetric drops collision with the experimental observations. The parameters are: $\operatorname{Re}=210.01, \mathrm{We}=18.48, X=0$, the pressure is $p=7$ bar. The coalescence is imposed in the computations. 


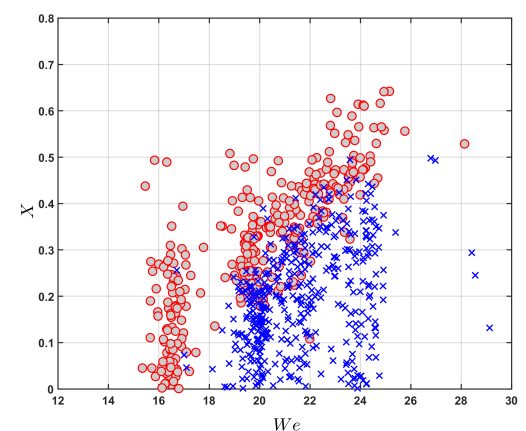

(a)

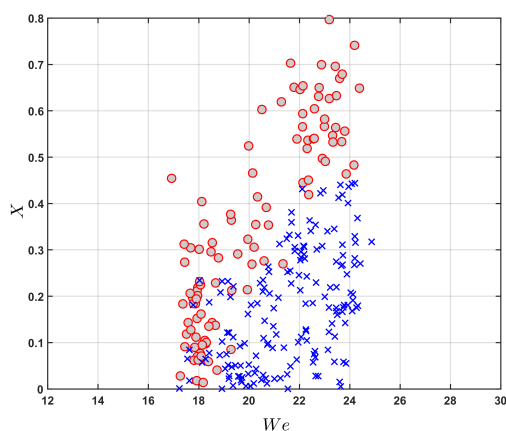

(b)

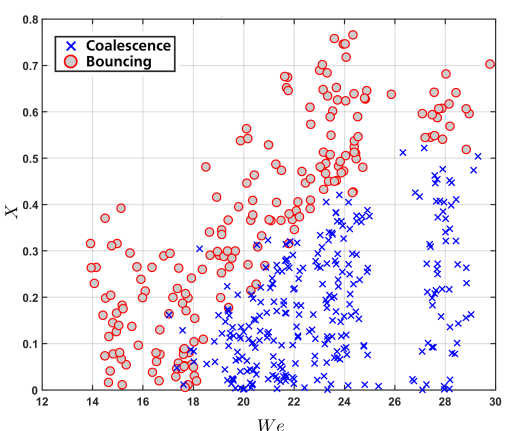

(c)

Figure 7. Bouncing-coalescence diagrams of the experimental outcome for various Webern numbers We and impact parameters $X$ at different ambient pressures: (a) $p=5.3$ bar, (b) $p=7.0$ bar, and (c) $p=9.0$ bar.

\section{Results and discussion}

The theoretically predicted shapes of the drops during collision are compared with the experimental data in Figures 4 - 6. The agreement is rather good for both bouncing cases (Figs. 4 and 5) and the coalescence case (shown in Figure 6). It should be noted, that since the coalescence still cannot be described numerically, the instant of coalescence during drop deformation was externally imposed. Some deviation between the predictions and experimental data at the later stages of drop coalescence can be explained by the sensitivity of the phenomena to the instant of coalescence (which is not known a priori).

The outcome from the binary drops collision is determined by many factors, like gas density, the impact Weber number and the impact parameter, which determine the duration of contact. The duration $t_{c}$ of the binary collision in the bouncing regime is governed by inertia and surface tension. It has been shown [10] that for high Weber numbers this time is scaled very well by half of the Rayleigh period of oscillation,

$$
t_{c}=A \sqrt{\frac{\pi^{2} \rho_{L} D_{1}^{3}}{8 \sigma}}
$$

where $A \approx \mathrm{We} \mathrm{e}^{0.043}$ is obtained by fitting to the experimental data reported in [10], $\rho_{L}$ and $\sigma$ are the liquid density and surface tension. It should be noted that in the case of drop rebound the contact time will be different, since the rim experiences an additional force associated with two liquid interfaces bounding the air gap. Therefore, the estimated corrected contact time is

$$
t_{c} \approx \mathrm{We}^{0.043} \sqrt{\frac{\pi^{2} \rho_{L} D_{1}^{3}}{16 \sigma}}
$$

for rebound. For the set of parameters corresponding to the experiments shown in Figures 4 and 5 , the contact time predicted by (2) is $t_{c}=0.708 \mathrm{~ms}$ and $t_{c}=0.772 \mathrm{~ms}$, respectively, which agrees rather well with the observed contact times. In any case, since the Weber number enters equation (2) only with a small power, we can assume

$$
t_{c} \sim \sqrt{\frac{\rho_{L} D_{1}^{3}}{\sigma}}=\frac{D_{1}}{V_{n}} \mathrm{We}^{1 / 2}
$$

for a certain range of Weber numbers.

The bouncing-coalescence diagrams for different ambient pressures, showing the types of collision outcome for various impact parameters $X$ and Weber numbers We, are shown in Figure 7. It is interesting that these diagrams for elevated pressures are very similar. This counter-intuitive result is explained by the fact that the influence of the pressure on the dynamic viscosity $\mu_{A}$ of the air in the gap is only minor. It indicates also that the viscosity effects in the gap between the drops are dominant. It is therefore obvious that the Weber number is not a relevant dimensionless group for description of the bouncing/coalescence threshold.

To develop a reliable expression for this threshold an accurate analysis of the air flow in the gap between the drop and further its stability analysis are necessary. In this study only rough estimations of the relevant parameters are performed. The typical air gap thickness $h_{\text {air }}$ between the drops can be roughly estimated using the lubrication approximation in the description of the gas flow and using the expression for the pressure $p$, produced by drop impact [24], i.e.

$$
p \sim \rho_{L} V_{n}^{2} \exp \left[-b \frac{t V_{n}}{D_{1}}\right], \quad h_{a i r} \sim\left[\frac{\mu_{A} D_{1}}{\rho_{L} V_{n}}\right]^{1 / 2}=D_{1} \operatorname{Re}_{A}^{-1 / 2}
$$

where $V_{n} \equiv V \sqrt{1-X^{2}}$ is the normal component of the relative impact velocity, $\mu_{A}$ is the air dynamic viscosity, $b$ is a dimensionless constant ( $b=3.1$ found from the computations in [24]), $\operatorname{Re}_{A}$ is the Reynolds numbers based on the air density and normal velocity component $V_{n}$. 


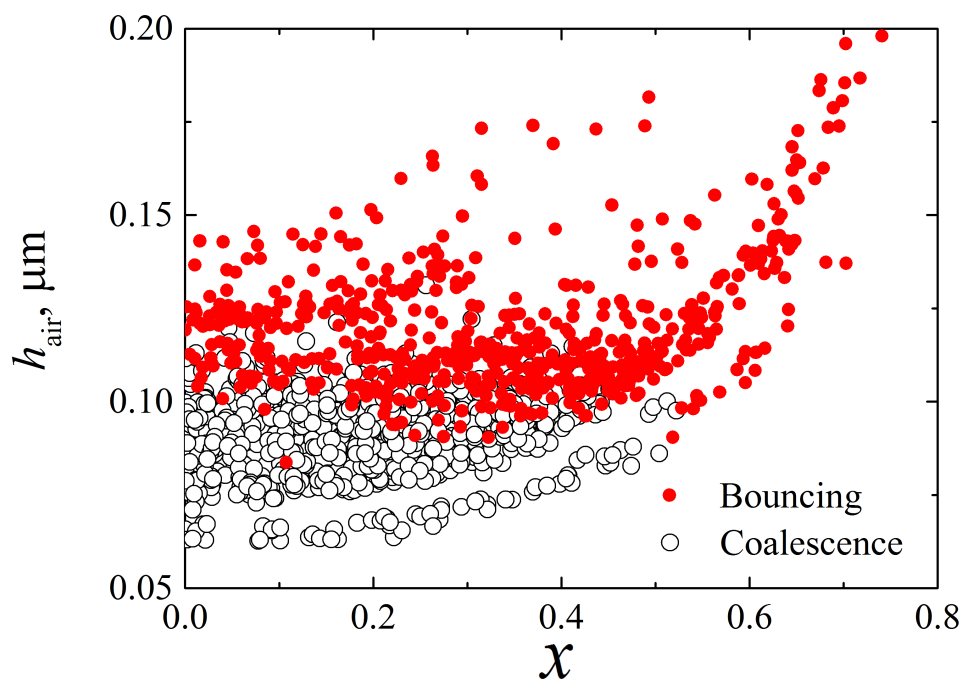

Figure 8. The predicted thickness of the air layer for bouncing and coalescence, roughly estimated in (5), as a function of the impact parameter.

For a long time after impact the thickness of the gap is governed by the flow in the lamellae. Such flow has been analyzed in [24, 25], leading to the evolution of the film thickness in the form $h \sim t^{-2}$. This analysis has been confirmed in the experiments with drop impact onto convex surfaces [26]. Finally, we estimate the thickness of the air layer during drop collision according to

$$
h_{a i r} \sim\left[\frac{\mu_{A} D_{1}}{\rho_{L} V_{n}}\right]^{1 / 2} \frac{D_{1}^{2}}{t_{c}^{2} V_{n}^{2}}=\frac{\mu_{A}^{1 / 2} \sigma}{\rho_{L}^{3 / 2} D_{1}^{1 / 2} / V_{n}^{5 / 2}}=D_{1} \operatorname{Re}_{A}^{-1 / 2} \mathrm{We}_{n}^{-1},
$$

where $\mathrm{We}_{n}$ is the Weber number based on the normal component of the impact velocity.

The dependence of the predicted values of $h_{\text {air }}$ on the dimensionless impact parameter $X$ for the cases of drop bouncing and coalescence is shown in Fig. 8. For small values of the impact parameter, $X<0.5$, the estimated minimum air gap thickness $h_{\text {air }}$ is approximately $100 \mathrm{~nm}$. Locally, the minimum gap thickness can be much smaller, since the gap thickness is strongly non-uniform and its value reduces near the rim area. Therefore, the physics of coalescence can depend on the intermolecular forces. Nevertheless, the value of $h_{\text {air }}$ is probably a relevant scale determining the coalescence threshold. Further analysis is still required to describe the mechanism of coalescence.

\section{Conclusions}

In this study, the bouncing and coalescence of two Newtonian drops in air under elevated pressure conditions have been observed using a high-speed camera. Additionally, the kinematics of drop deformation has been described numerically using a computational code based on the volume-of-fluid method. The typical times of contact are estimated and compared to existing expressions.

The thickness of the air gap between the drops is roughly estimated. It is shown that the predicted smallest gap thickness of the air gap is in our case approximately $100 \mathrm{~nm}$. This value can be used as a scale for the bouncing/coalescence threshold. However, more data are required for validation of this assumption and for development of a more precise model.

\section{Acknowledgements}

This research was supported by the the German Scientific Foundation (Deutsche Forschungsgemeinschaft) in the framework of the SFB TRR 75 Collaborative Research Center, subprojects C04 and A07. The author Louis Reitter has contributed to the present manuscript in the framework of the course "Sprays and Atomization".

\section{References}

[1] Brazier-Smith, P., Jennings, S. and Latham, J., Proc. R. Soc. Lond. A. 326(1566):393-408 (1972).

[2] Park, R., Ph.d. thesis, University of Wisconsin (1970).

[3] Faeth, G., Prog. Energy Combust. Sci. 3(4):191-224 (1977).

[4] O'Rourke, P. J. and Bracco, F. V., Proc. Inst. Mech. Eng. 9:101-106 (1980).

[5] Ashgriz, N. and Poo, J., J. Fluid Mech. 221:183-204 (1990).

[6] Jiang, Y., Umemura, A. and Law, C., J. Fluid Mech. 234:171-190 (1992).

[7] Qian, J. and Law, C., J. Fluid Mech. 331:59-80 (1997).

[8] de Ruiter, J., Lagraauw, R., Mugele, F. and van den Ende, D., J. Fluid Mech. 776:531-567 (2015). 
[9] Rayleigh, J., Proc. R. Soc. London 28:405-409 (1878).

[10] Willis, K. and Orme, M., Exp. Fluids 34(1):28-41 (2003).

[11] Kim, J. and Longmire, E. K., Exp. Fluids 47(2):263-278 (2009).

[12] Estrade, J., Carentz, H., Lavergne, G. and Biscos, Y., Int. J. Heat Fluid Flow 20:486-491 (1999).

[13] Post, S. and Abraham, J., Int. J. Multiphase Flow 28:997-1019 (2002).

[14] Munnannur, A. and Reitz, R., Int. J. Multiphase Flow 33:873-896 (2007).

[15] Rieber, M., Numerische Modellierung der Dynamik freier Grenzflächen in Zweiphasenströmungen, FortschrittBerichte VDI: Reihe 7, Strömungstechnik, VDI-Verlag, Düsseldorf (2004), PhD Thesis, Institut für Thermodynamik der Luft- und Raumfahrt (ITLR), Universität Stuttgart.

[16] Eisenschmidt, K., Ertl, M., Gomaa, H., Kieffer-Roth, C., Meister, C., Rauschenberger, P., Reitzle, M., Schlottke, K. and Weigand, B., Appl. Math. Comput. 272:508-517 (2016).

[17] Focke, C., Kuschel, M., Sommerfeld, M. and Bothe, D., Int. J. Multiph. Flow 56:81-92 (2013).

[18] Liu, M. and Bothe, D., J. Fluid Mech. 789:785-805 (2016).

[19] Planchette, C., Hinterbichler, H., Liu, M., Bothe, D. and Brenn, G., J. Fluid Mech. 814:277-300 (2017).

[20] Popinet, S., J. Comput. Phys. 228(16):5838-5866 (2009).

[21] Lafaurie, B., Nardone, C., Scardovelli, R., Zaleski, S. and Zanetti, G., J. Comput. Phys. 113(1):134-147 (1994).

[22] Zhang, P. and Law, C. K., Phys. Fluids 23(4):042102 (2011).

[23] Pan, K.-L., Law, C. K. and Zhou, B., Journal of Applied Physics 103(6):064901 (2008).

[24] Roisman, I. V., Berberović, E. and Tropea, C., Phys. Fluids 21(5):052103 (2009).

[25] Yarin, A. and Weiss, D., J. Fluid Mech. 283:141-173 (1995).

[26] Bakshi, S., Roisman, I. V. and Tropea, C., Phys. Fluids 19(3):032102 (2007). 


\title{
Experimental investigation on puffing and micro-explosion occurrence of water in rapeseed oil emulsions droplets. Effect of the surfactant concentration.
}

\author{
Eliezer Ahmed Melo-Espinosa*1, Jérôme Bellettre ${ }^{2}$, Dominique Tarlet², Ramón Piloto- \\ Rodríguez ${ }^{1}$ and Sebastian Verhelst ${ }^{3}$
}

${ }^{1}$ Center for the Study of Renewable Energy Technologies, Faculty of Mechanical Engineering, Instituto Superior Politécnico “José Antonio Echeverría”(CUJAE), Marianao, 19390, Habana (Cuba)

2 Laboratoire de Thermique et Energie de Nantes, LTEN UMR CNRS 6607, BP 50609, 1 rue Christian Pauc, 44306 Nantes cedex 3, France

${ }^{3}$ Department of Flow, Hot and Combustion Mechanics, Faculty of Engineering and Architecture, Ghent University, Sint-Pietersnieuwstraat 41, B-9000, Ghent (Belgium)

\author{
*Corresponding author: emelo@ceter.cujae.edu.cu and \\ eliezer.ahmed.melo.espinosa@gmail.com
}

\begin{abstract}
In this paper, the assessment of puffing and micro-explosion occurrence in emulsion drops with different water percentage is studied. The emulsified fuels are formulated using micro channel emulsifier, rapeseed oil and diesel fuel as continuous phase, as well as water and Sorbitan Sesquioleate as surfactant. The formulated dispersed systems are covered under different experimental factors such as water ratio and surfactant percentage. The puffing occurrence is reported in all emulsified fuels tested (i.e. with and without surfactant). A sudden puffing and highest number of occurrence is noted when the water amount increases in all emulsified fuels. The micro-explosion phenomenon is only noted in emulsified fuel formulated without surfactant.
\end{abstract}

Keywords

Micro-explosion, puffing, Leidenfrost effect, rapeseed oil, diesel fuel

\section{Introduction}

The direct use of vegetable oils in diesel engines causes several problems such as a less efficient combustion process due to the poor atomization process and changes in the ignition delay. For these reasons and seeking for a more engine-friendly fuel, it is necessary to change the feedstock properties applying different methods such as: preheating, blending with diesel fuel, transesterification, cracking / pyrolysis or emulsification. The advantage and drawbacks of each method have been pointed out in different researches [1, 2].

Among these methods, the emulsification has an additional advantage linked to its capacity to decrease diesel engine exhaust emissions such as nitric oxides, solid and carbonaceous residues [3]. It is a cheaper method because modifications of the original engine design, special and sophisticated equipments are not necessary. The preparation of an emulsion involves no complex chemical reactions [4]. It also produces no by-products unlike transesterification [4].

According to Tran et al. [5] the combustion of emulsified fuel droplets is largely characterized by this difference between water and fuel volatility. The droplet is heated by convective and radiative heat transfer and its temperature reaches the superheat limit. Inside the droplet, this is followed by a rapid bubble nucleation, and then, internal formation of vapor bubbles [5]. The vaporization of water then blows up the oil layer and thereby forms smaller oil droplets, which increases the oil droplet's surface to volume ratio [6]. This phenomenon is called "micro-explosion" [6, 7].

The micro-explosion causes the secondary atomization which forms the bulk of much finer droplets [5]. Such secondary drops evaporate very quickly and are dispersed over a large volume, improving fuel/air 
mixing and the overall combustion efficiency [8]. This mechanism is fundamental in reducing particulate emission in the combustion of medium and heavy oils [8]. In addition, the presence of water influences the physics and chemical kinetics of combustion and has a beneficial effect on the rate-of-heat release and the reduction of pollutant emissions [9].

On the other hand, during combustion, the vaporized water reduces the flame temperature, changes the chemical composition of the reactants, resulting in higher $\mathrm{OH}$ radical concentration controlling the $\mathrm{NO}$ formation rate and soot oxidation, and dilutes the rich zones in the combustion chamber [9]. In addition, due to the micro-explosion phenomenon as a consequence of the dispersed water into emulsified fuel, it is also possible to improve the atomization process and recover the combustion efficiency when it is used as diesel engine fuel. However, it is important to point out that the physicochemical properties of emulsified fuel play a decisive role.

Nevertheless, a large scientific effort has been devoted to the experimental evaluation of the microexplosion phenomenon [10-26]. However, the micro-explosion phenomenon has been also questioned [27]. Califano et al. [8] pointed out that the micro-explosion does not always occur, and that its occurrence depends on a number of parameters. An important factor affecting the micro-explosion could be the coalescence or phase separation of the dispersed water droplets into the continuous phase $[8,10]$.

On the other hand, several studies [11,14-16, 28-31] reported the occurrence of a particular phenomenon (i.e. puffing or bubbling) prior to micro-explosion phenomenon. The occurrence of puffing is also linked to the thermal effect of dispersed phase into continuous phase under intense heat flux. This phenomenon is not always observable, consists in the formation of irregular bubble of steam that perturbs the free surface of the droplet $[16,32]$.

In spite of the fact that puffing and micro-explosion phenomenon are well-known, discovered in 1965 by Ivanov and Nefedov [29] and defined separately by Watanabe et al. [32], their assessment involving emulsified fuels have not been studied thoroughly. For this reason, the scope of this investigation is to analyze the puffing and micro-explosion occurrence in emulsified fuels obtained through a micro-channel emulsifier.

\section{Experimental set-up and procedures}

The experimental investigation of puffing and micro-explosion occurrence is conducted in emulsified fuel drops on a hot surface. The emulsion fuel drops are placed on a heated plate leading to the Leidenfrost effect. Recently, several researches have been conducted in this topic using Leidenfrost burning [11, 1416, 22].

A pipettor VWR with tip ejector (volume: $2-20 \mu \mathrm{L}$, precision: $\leq 1.5-0.3 \%$ and accuracy of $\pm 1.0-0.6 \%$ ) is used as a fuel droplet generator. Additional equipment such as a high-speed camera model $675 \mathrm{~K}-\mathrm{M} 1$ (HighSpeedStar series) provided by LaVision, a hot plate controlled by a power station Weller and a Type$\mathrm{K}$ thermocouple coupled to a K-Thermocouple thermometer Hanna are used for the visualization and control of the studied phenomena. The schematic diagram of the experimental setup used is shown in Figure 1.

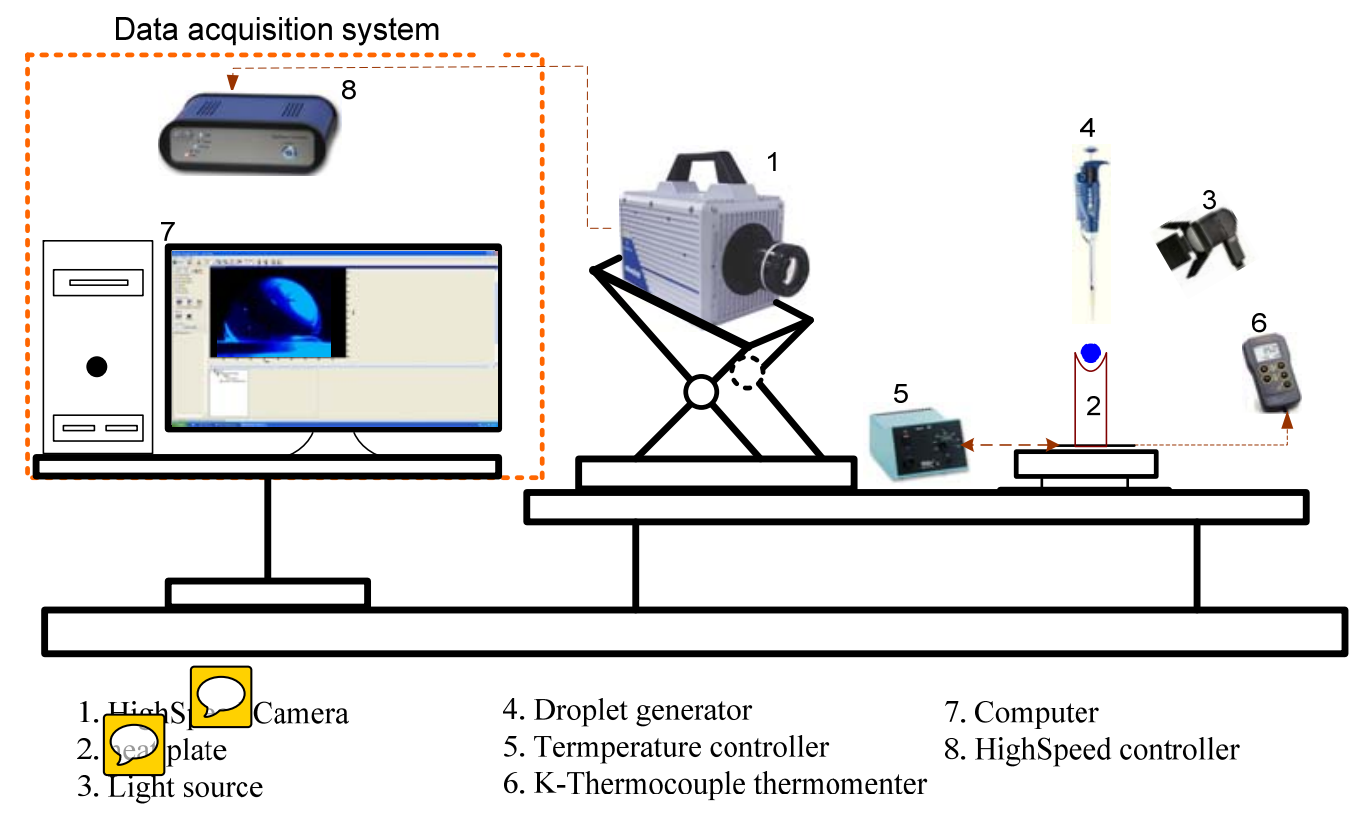

Figure 1. Schematic diagram of the micro-explosion setup 
The assessment of the puffing and micro-explosion occurrence is conducted under selected test conditions keeping fixed the hot plate temperature at $365^{\circ} \mathrm{C}$ and atmospheric pressure. This temperature was found as a proper value to ensure the Leidenfrost effect occurrence (emulsified droplet lifted) avoiding the quick warm-ups and vaporization, in agreement with previous experiments developed by Mura et al. [33] and Tarlet et al. [13, 15]. The test conditions are shown in Table 1.

Table 1. Puffing and micro-explosion test conditions

\begin{tabular}{c|ccc}
\hline $\begin{array}{c}\text { Hot plate } \\
\text { temperature } \\
\left({ }^{\circ} \mathrm{C}\right)\end{array}$ & $\begin{array}{c}\text { Droplet } \\
\text { volume }(\mu \mathrm{L})\end{array}$ & $\begin{array}{c}\text { Droplet } \\
\text { diameter } \\
(\mathrm{mm})\end{array}$ & $\begin{array}{c}\text { Acquisition } \\
\text { frequency } \\
(\mathrm{Hz})\end{array}$ \\
\hline 365 & 12 & 2.84 & 750
\end{tabular}

In this study several emulsified fuels are prepared based on previous results reported in [35]. The materials used are rapeseed oil and diesel fuel as continuous phase, water as dispersed phase and Sorbitan Sesquioleate as surfactant. The emulsified fuels studied here are ERO7, ERO8, ERO9, ERO10, ERO11 and ERO12, which are prepared through a micro-channel emulsifier. The continuous phase and surfactant were previously blended and stirred during 15 minutes (at $1000 \mathrm{rpm}$ ) as a first step before the use of micro-channel emulsifier. The experimental facilities used for the emulsification process were three piston displacement pumps (ARMEN-AP-TRIX-500-200) feeding a micro-channel with an optimized geometry, which has been recently presented in details by Belkadi et al. [35].

The emulsions' composition and properties are shown in Table 2. Moreover, additional emulsified fuels are formulated avoiding the use of any surfactant and keeping fixed the water ratio $(10 \%, 20 \%$ and $30 \%)$. The aim of this proposal is to analyze the effect of surfactant and dispersed phase concentration on puffing and micro-explosion occurrence. That is why the most stable and the less stable emulsions are going to be compared.

Table 2. Composition and properties of the emulsified fuels - ERO: Emulsified rapeseed oil, ${ }^{*}$ RO-Diesel: Blend of $20 \%$ of rapeseed oil-in $80 \%$ of diesel fuel

\begin{tabular}{c|ccccc}
\hline \hline $\begin{array}{c}\text { Emulsified } \\
\text { fuel }\end{array}$ & \multicolumn{3}{|c}{ Composition } & \multicolumn{2}{c}{ Properties } \\
\hline & $\begin{array}{c}\text { RO-Diesel } \\
(\% \text { Vol. })\end{array}$ & $\begin{array}{c}\text { Surfactant }(\% \\
\text { Vol. })\end{array}$ & $\begin{array}{c}\text { Water } \\
(\% \text { Vol. })\end{array}$ & $\begin{array}{c}\text { Dynamic } \\
\text { viscosity } \\
(\mathrm{mPa} . \mathrm{s})\end{array}$ & $\begin{array}{c}\text { Average } \\
\text { droplet } \\
\text { size }(\mu \mathrm{m})\end{array}$ \\
\hline \hline ERO7 & 88 & 2 & 10 & 4.6 & 4.97 \\
ERO8 & 78 & 2 & 20 & 16.1 & 8.12 \\
ERO9 & 68 & 2 & 30 & 20.4 & 9.2 \\
ERO10 & 86 & 4 & 10 & 5 & 4.88 \\
ERO11 & 76 & 4 & 20 & 16.5 & 7.96 \\
ERO12 & 66 & 4 & 30 & 21 & 8.89
\end{tabular}

\section{Experimental results}

The emulsified droplets undergo a Leidenfrost effect as a result of the heat flux on the dispersed water within emulsions. Under this effect, a continuous process of vapor bubbles formation within emulsified fuel matrix is expected. For this reason, this section focuses on effect of dispersed water quantity into emulsified droplets exploring vapor bubble growth itself. The vapor bubble growth in the emulsified droplets was studied in a data set of captured images prior the first puffing or micro-explosion occurs. The time lapse in the vapor bubble formation and growth are shown in Fig 2. In general, an increase of surfactant percentage in the emulsions leads to a disruptive effect on vapor bubble formation and its further development.

The time lapse between generating droplet on the hot surface and vapor bubble formation varies significantly from an emulsion to another depending of their composition. For each set, bigger vapor bubbles are observed in a shorter period of time for emulsions without surfactant.

In this regard, comparable results have been attained for other emulsified fuels [10-12], in spite of the effect of additional factors such as the surfactant percentage, the analyzed size of the emulsion droplet and characteristics of continuous phase (e.g. volatility, viscosity, carbon-chain length, etc.) have not been studied thoroughly. The vapor bubbles formations are linked to several phenomena such as nucleation, 
agglomeration, coalescence and bubbling which might lead to a further occurrence of puffing and microexplosion. Nevertheless, an analysis about the effect of surfactant on puffing and micro-explosion occurrence will be discussed in the next section.

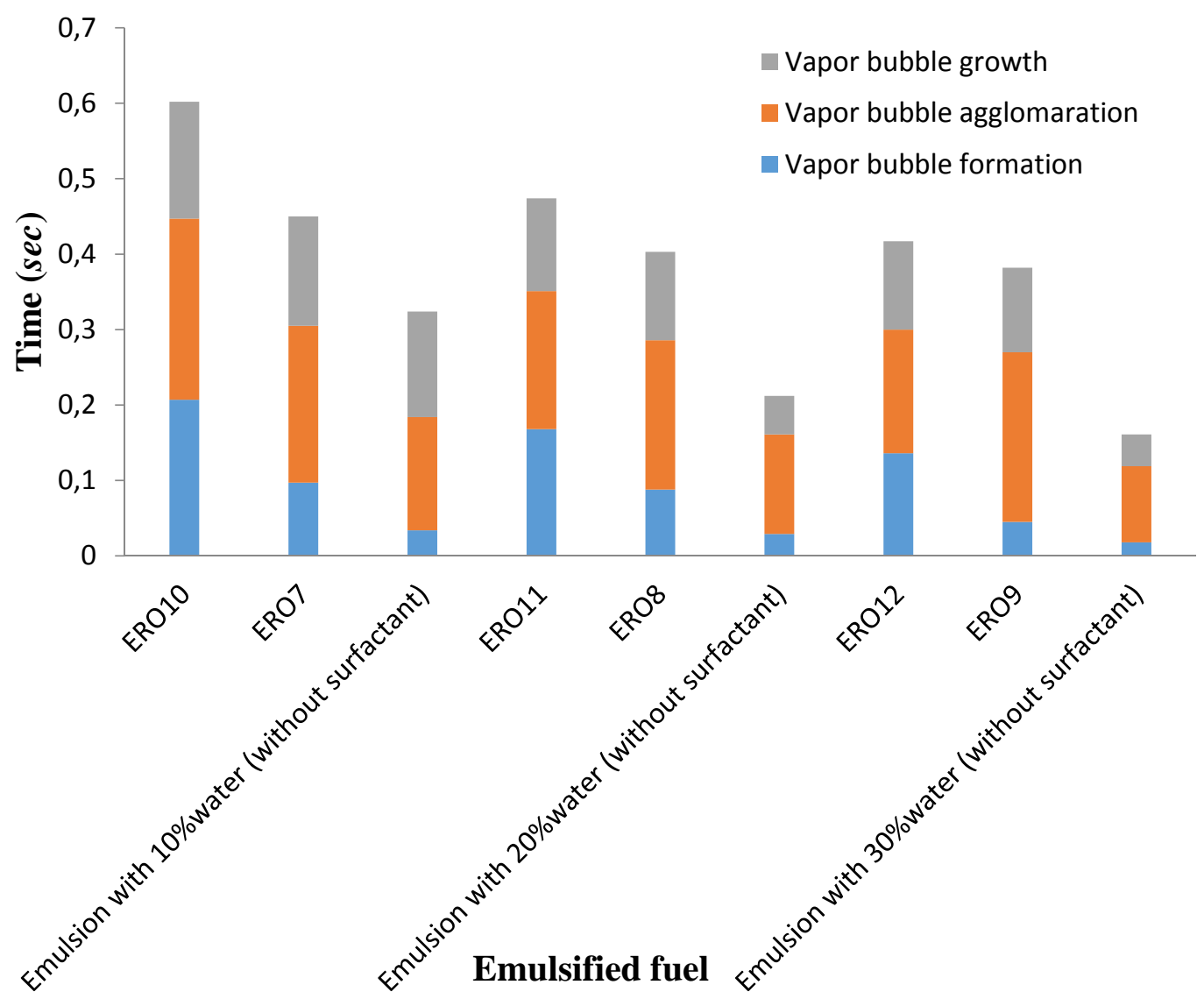

Figure 2. Time lapse between generating droplet, vapor bubble formation and growth

The experimental results of puffing and micro-explosion occurrence in the formulated emulsions with/without surfactant are shown in Fig 3 and Fig. 4. For all cases, the light luminous areas around the droplet surface represent the surrounding vaporized fuel.

As can be noted, an increase of water percentage promotes a sudden occurrence of the puffing phenomenon. The number of puffing occurrence per set of emulsions also increases with the water ratio, but contrary when the surfactant percentage is increased. A delay of puffing process with the increase of surfactant percentage was also attained. This behavior is a consequence of the surfactant activity within the interphase boundary inside the emulsified fuels.

On the other hand, the micro-explosion phenomenon only occurs in emulsified fuels formulated without surfactant (see Fig 3, Fig 4). This fact might be correlated with the sudden agglomeration and coalescence of the dispersed water in the bottom of the emulsified droplet [12], which is associated to differences between dispersed and continuous phase density. Moreover, a strong puffing process like weak microexplosion is noted in the emulsified fuel prepared with $2 \%$ of surfactant and higher water amount (i.e. ERO12, not shown here du to lake of place).

An average water droplet size of $5 \mu \mathrm{m}$ is seen as an adequate size for a good micro-explosion as shown by Mura et al. [33] and Tarlet et al. [13, 15]. However, micro-explosion phenomenon might occur also for smaller or bigger droplets size, although it may be more or less strong according to Tarlet et al. [15]. In our study, only two emulsified fuel reach this value (i.e. ERO7 and ERO10) but they do not micro-explode. This behavior might be associated with different factors such as the composition of both sets of dispersed systems (e.g. water and surfactant percentage) and physicochemical properties of continuous phase (e.g. volatility and boiling temperature). 


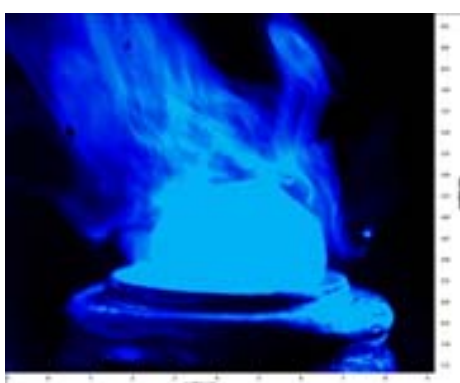

$\mathrm{t}=0 \mathrm{sec}$

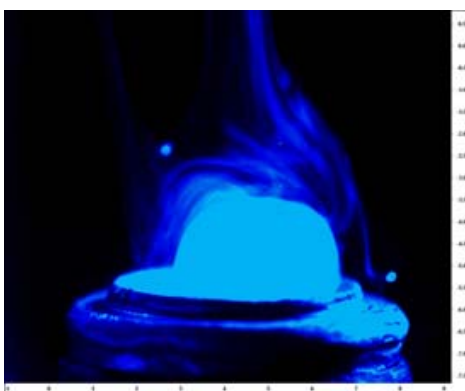

$\mathrm{t}=0$ sec

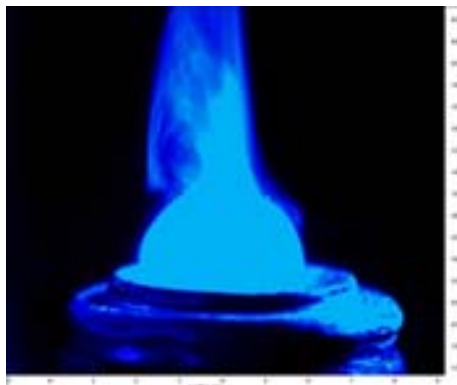

$\mathrm{t}=0 \mathrm{sec}$

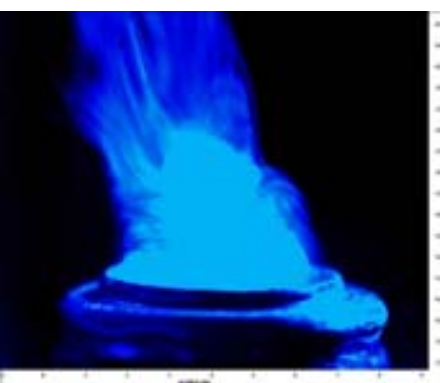

$\mathrm{t}=0.736 \mathrm{sec}$

(a)

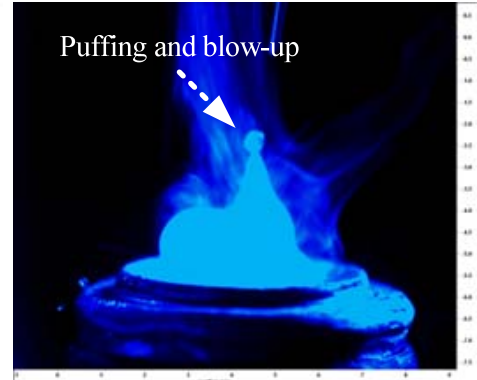

$\mathrm{t}=0.569 \mathrm{sec}$

(b)

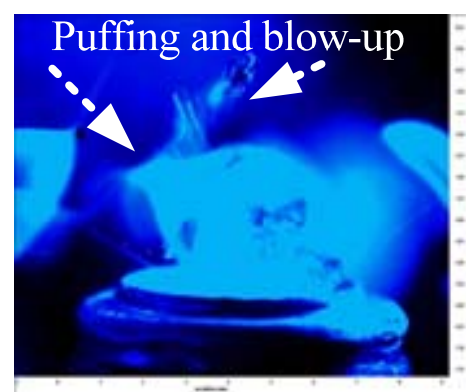

$\mathrm{t}=0.416 \mathrm{sec}$

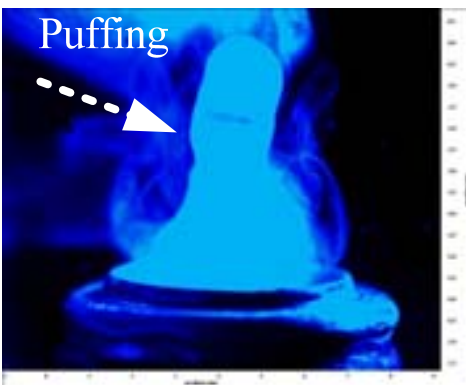

$\mathrm{t}=1.035 \mathrm{sec}$

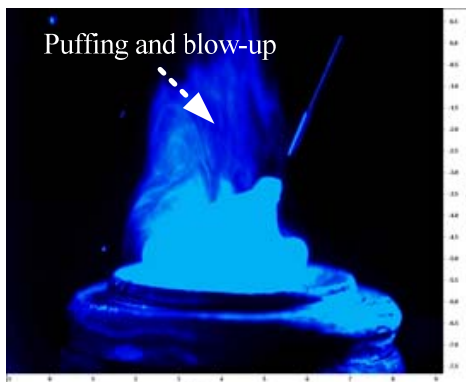

$\mathrm{t}=0.745 \mathrm{sec}$

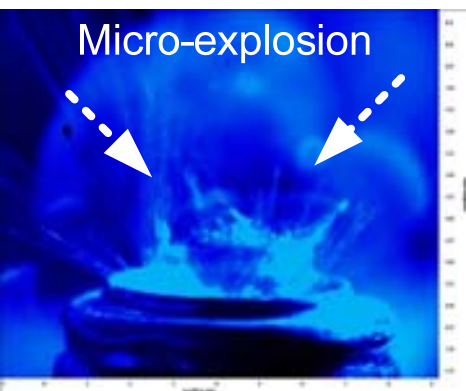

$\mathrm{t}=0.68 \mathrm{sec}$

(c)

Figure 3. Sequence of puffing and micro-explosion occurrence: a) ERO10, b) ERO7, c)Emulsified fuel prepared with90\% of diesel-rapeseed oil blend and $10 \%$ of water (without surfactant)

A summary of emulsified fuels burning droplets plotted in a ternary diagram is shown in Fig 5 . The black zone represents a zone where the puffing and micro-explosion phenomena are often reported in the literature [10-16, $18-20,22-26,32-34]$ using different continuous phases with increasing water emulsification up to $30 \%$. In our study, both phenomena took place in all emulsified fuels prepared without surfactant (see section 3.2 and Fig 5a). In addition, a set of emulsions formulated without surfactant but involving pure diesel fuel and different water ratio also reported the occurrence of puffing and micro-explosion phenomenon (i.e. see red-blue points in Fig 5a).

As it was previously mentioned, emulsified fuels prepared with surfactant reported puffing and vaporized fuel process (see yellow-blue points in Fig 5b). An additional set of emulsified fuels using surfactant were analyzed in order to broaden the investigate zone. In concordance with previous results, a puffing and vaporization process was also attained.

For each case of emulsions formulated with a blend of rapeseed oil-in diesel, an increase of the rapeseed oil ratio in the blend conducted to highest surrounding vaporized fuel. This assessment is characterized by differences between rapeseed oil and diesel fuel volatility, mainly. 


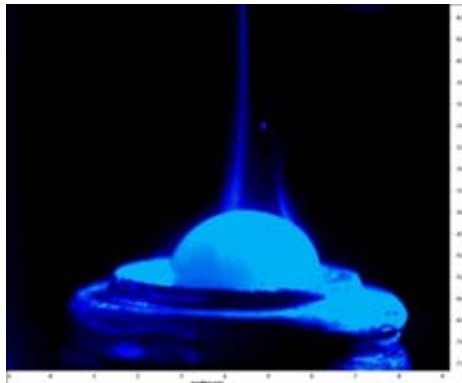

$\mathrm{t}=0 \mathrm{sec}$

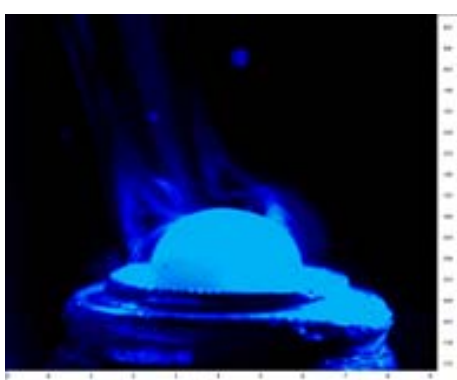

$\mathrm{t}=0 \mathrm{sec}$

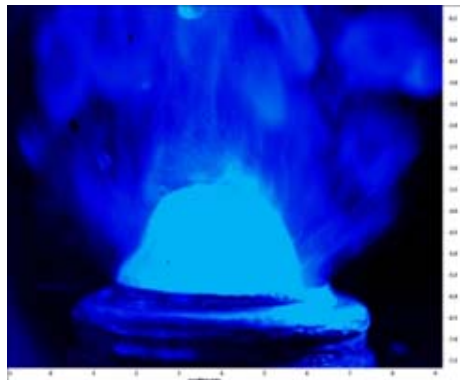

$\mathrm{t}=0 \mathrm{sec}$

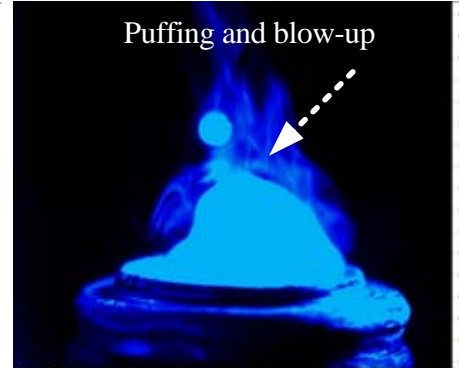

$\mathrm{t}=0.584 \mathrm{sec}$

(a)

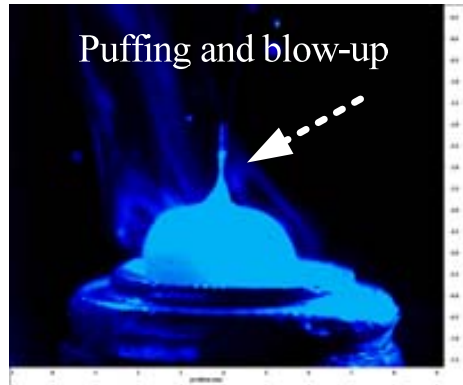

$\mathrm{t}=0.512 \mathrm{sec}$

(b)

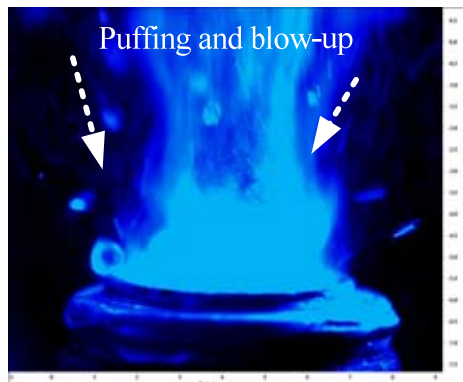

$\mathrm{t}=0.254 \mathrm{sec}$

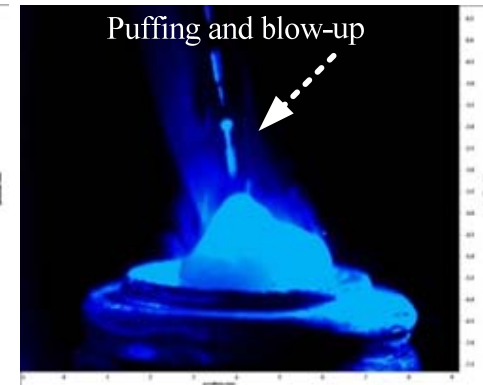

$\mathrm{t}=0.699 \mathrm{sec}$

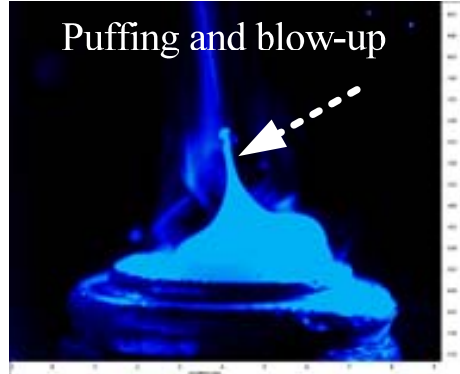

$\mathrm{t}=0.604 \mathrm{sec}$

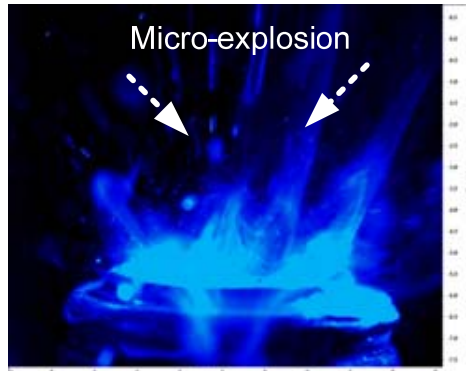

$\mathrm{t}=0.540 \mathrm{sec}$

(c)

Figure 4. Sequence of puffing and micro-explosion occurrence: a) ERO11, b) ERO8, c) Emulsified fuel prepared with80\% of diesel-rapeseed oil blend and $20 \%$ of water (without surfactant)

\section{Conclusions}

The results of several experiments varying the amount of water, surfactant and continuous phase depicted the occurrence of puffing and/or micro-explosion phenomena, even for lower water amounts. A summary of emulsified fuels burning droplets was plotted in a ternary diagram denoting an interesting zone where puffing and micro-explosion occur. A sudden puffing and highest number of occurrence is noted when the water amount was increased, but contrary when the surfactant percentage is increased. This fact is also correlated with a disruptive effect achieved on vapor bubble growth. On the other hand, the micro-explosion phenomenon only took part in emulsified fuel formulated without surfactant. All this denote that the occurrence of puffing and micro-explosion involves a multifactorial analyze based on several factors such as the dispersed water size, water amount, surfactant usage and physicochemical properties of continuous phase (e.g. volatility, boiling temperature, viscosity, carbon-chain length). 


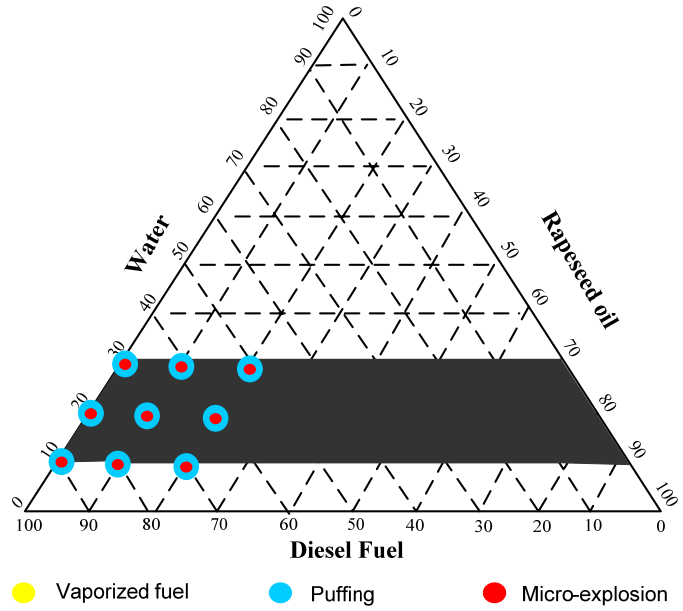

a)

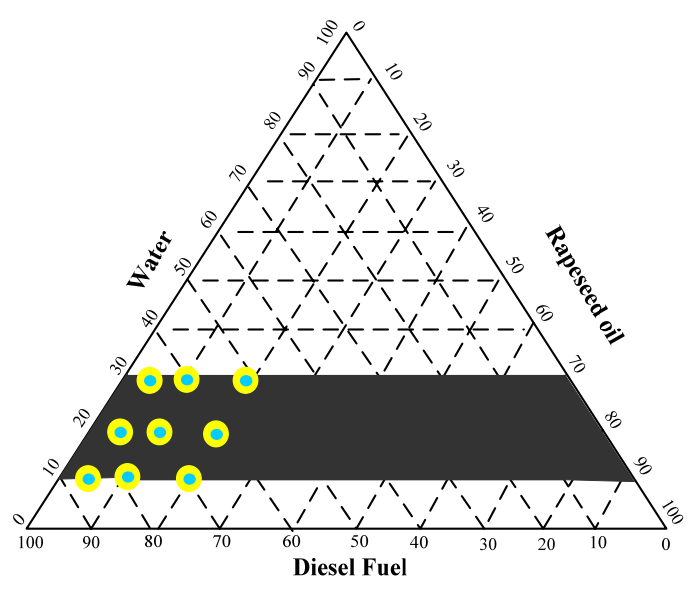

Vaporized fuel
Puffing

Micro-explosion

b)

Figure 5. Summary of emulsified fuel burning droplet plotted in a ternary diagram: a) Emulsified fuel prepared without surfactant, b) Emulsified fuel prepared with surfactant

\section{Acknowledgements}

The authors wish to express their acknowledgement to the Centre National de la Recherche Scientifique (CNRSFrance) and Flemish Interuniversity Council's (VLIR) University Development Cooperation, funding a South Initiatives Program entitled "Emulsified systems for biofuels. Assessment of their performance in diesel engines", because of their support to this research.

\section{References}

[1] M. Senthil Kumar, A. Kerihuel, J. Bellettre, and M. Tazerout, "A comparative study of different methods of using animal fat as a fuel in a compression ignition engine," Journal of Engineering for Gas Turbines and Power, vol. 128, pp. 907-914, 2006.

[2] E. Melo, R. Piloto, L. Goyos, R. Sierens, and S. Verhelst, "Emulsification of animal fats and vegetable oils for their use as a diesel engine fuel: An overview," Renewable and Sustainable Energy Reviews, vol. 47, pp. 623633, 2015.

[3] R. Ocampo-Barrera, R. Villasenora, and A. Diego-Marin, "An experimental study of the water content on combustion of heavy fuel oil/water emulsion droplets," Combustion Flame, vol. 126, pp. 1845-1855, 2001.

[4] M. Mubarak and M. Senthil Kumar, "An experimental study on waste cooking oil and its emulsions as diesel engine fuel " in Advances in Engineering, Science and Management (ICAESM), Nagapattinam, Tamil Nadu 2012, pp. $61-66$.

[5] X. Tran and I. Ghojel, "Impact of Introducing Water into the Combustion Chamber of Diesel Engines on Emissions - an Overview," in 5th Asia-Pacific Conference on Combustion,, The University of Adelaide, Adelaide, Australia, 2005, pp. 233-236.

[6] B. Debnath, N. Sahoo, and U. Saha, "Adjusting the operating characteristics to improve the performance of an emulsified palm oil methyl ester run diesel engine," Energy Conversion and Management, vol. 69, pp. 191198, 2013.

[7] R. Crookes, et al., "Systematic assessment of combustion characteristics of biofuels and emulsions with water for use as diesel engine fuels," Energy Conversion and Management, vol. 38 pp. 1785-1795, 1997.

[8] V. Califano, R. Calabria, and P. Massoli, "Experimental evaluation of the effect of emulsion stability on microexplosion phenomena for water-in-oil emulsions," Fuel vol. 117, 2014.

[9] N. Samec, B. Kegl, and R. Dibble, "Numerical and experimental study of water/oil emulsified fuel combustion in a diesel engine," Fuel, vol. 81, pp. 2035-2044, 2002.

[10] E. Mura, P. Massoli, C. Josset, K. Loubar, and J. Bellettre, "Study of the micro-explosion temperature of water in oil emulsion droplets during the Leidenfrost effect," Experimental Thermal and Fluid Science, vol. 43 pp. 63-70, 2012

[11] M. Yahaya, Z. Abdul, A. Abd, M. Heikal, and C. Crua, "Puffing and microexplosion behavior of water in pure diesel emulsion droplets during Leidenfrost effect " Combustion Science and Technology, 2016. 
[12] D. Tarlet, C. Josset, and J. Bellettre, "Comparison between unique and coalesced water drops in microexplosions scanned by differential calorimetry," International Journal of Heat and Mass Transfer vol. 95, pp. 689692, 2016

[13] D. Tarlet, C. Allouis, and J. Bellettre, "The balance between surface and kinematic energies within an optimal micro-explosion," International Journal of Thermal Sciences, vol. 107, pp. 179-184, 2016.

[14] M. Avulapati, L. Ganippa, J. Xia, and A. Megaritis, "Puffing and micro-explosion of diesel-biodiesel-ethanol blends," Fuel, vol. Fuel pp. 59-66, 2016.

[15] D. Tarlet, E. Mura, C. Josset, J. Bellettre, C. Allouis, and P. Massoli, "Distribution of thermal energy of childdroplets issued from an optimal micro-explosion," International Journal of Heat and Mass Transfer vol. $77 \mathrm{pp}$. 1043-1054, 2014.

[16] E. Mura, R. Calabria, V. Califano, P. Massoli, and J. Bellettre, "Emulsion droplet micro-explosion: Analysis of two experimental approaches," Experimental Thermal and Fluid Science vol. 56, pp. 69-74, 2014.

[17] S. Hou, F. Rizal, T. Lin, T. Yang, and H. Wan, "Microexplosion and ignition of droplets of fuel oil/bio-oil (derived from lauan wood) blends," Fuel vol. 113, pp. 31-42, 2013.

[18] H. Watanabe, Y. Suzuki, T. Harada, Y. Matsushita, H. Aoki, and T. Miura, "An experimental investigation of the breakup characteristics of secondary atomization of emulsified fuel droplet," Energy vol. 35, pp. 806-813, 2010.

[19] H. Watanabe, Y. Matsushita, H. Aoki, and T. Miura, "Numerical simulation of emulsified fuel spray combustion with puffing and micro-explosion," Combustion and Flame, vol. 157, pp. 839-852, 2010.

[20] Y. Morozumi and Y. Saito, "Effect of physical properties on microexplosion occurrence in water-in-oil emulsion droplets," Energy Fuels vol. 24, pp. 1854-1859, 2010.

[21] D. Tarlet, J. Bellettre, M. Tazerout, and C. Rahmouni, "Prediction of micro-explosion delay of emulsified fuel droplets," International Journal of Thermal Sciences vol. 48, pp. 449-460, 2009.

[22] T. Kadota, H. Tanaka, D. Segawa, S. Nakaya, and H. Yamasaki, "Microexplosion of an emulsion droplet during Leidenfrost burning," Proceedings of the Combustion Institute, vol. 31, pp. 2125-2131, 2007.

[23] S. Chung and K. Kim, "An experiment on vaporization and microexplosion of emulsion fuel droplets on a hot surface," 23 Symposium International on Combustion, vol. 23, pp. 1431-1435, 1991.

[24] K. Tsao and C. Wang, "Puffing and microexplosion phenomena of water emulsion fuels," SAE Technical Paper, 1986.

[25] C. Wang and C. Law, "Microexplosion of fuel droplets under high pressure," Combustion and Flame, vol. 59, pp. 53-62, 1985.

[26] C. Law, C. Lee, and N. Srinivasan, "Combustion characteristics of water-in-oil emulsion droplets," Combustion and Flame, vol. 37, pp. 125-143, 1980.

[27] W. Fu, et al., "There is no micro-explosion in the diesel engines fueled with emulsified fuel," Chinese Science Bulletin, vol. 51, pp. 1261-1265, 2006.

[28] D. Ogunkoya, S. Li, O. Rojas, and T. Fang, "Performance, combustion, and emissions in a diesel engine operated with fuel-in-water emulsions based on lignin," Applied Energy, vol. 154, pp. 851-861, 2015.

[29] A. Muhsin, H. Noge, H. Abdul, and W. Jazair, "An overview of utilizing water-in-diesel emulsion fuel in diesel engine and its potential research study," Journal of the Energy Institute vol. 87, pp. 1-16, 2014.

[30] Y. Li, S. Hou, and W. Sheu, "Investigation on boiler efficiency and pollutant emissions of water/heavy oil emulsions using edge-tone resonant homogenizer," Fuel vol. 119, pp. 240-251, 2014.

[31] M. Huo, S. Lin, H. Liu, and C. Lee, "Study on the spray and combustion characteristics of water-emulsified diesel," Fuel vol. 123, pp. 218-229, 2014.

[32] H. Watanabe, T. Harada, Y. Matsushita, H. Aoki, and T. Miura, "The characteristics of puffing of the carbonated emulsified fuel," International Journal of Heat and Mass Transfer vol. 52 pp. 3676-3684, 2009.

[33] E. Mura, C. Josset, K. Loubar, G. Huchet, and J. Bellettre, "Effect of dispersed water droplets in microexplosion phenomenon for water in oil emulsion," Atomization and sprays, vol. 20, pp. 791-799, 2010.

[34] J. Gong and W. Fu, "A study on the effect of more volatile fuel on evaporation and ignition for emulsified oil," Fuel vol. 80, pp. 437-445, 2001.

[35] A. Belkadi, A. Montillet, and J. Bellettre, "Biofuel emulsifier using high velocity impinging flows and singularities in micro-channels," presented at the ASME 2016 Internal Combustion Fall Technical Conference (ICEF2016), Greenville, USA, 2016. 


\title{
Experimental Investigation of Droplet Injections in the Vicinity of the Critical Point: A comparison of different model approaches
}

\author{
Christoph Steinhausen*1, Grazia Lamanna ${ }^{1}$, Bernhard Weigand ${ }^{1}$, \\ Rolf Stierle ${ }^{2}$, Joachim Gross ${ }^{2}$, \\ Andreas Preusche ${ }^{3}$, Andreas Dreizler ${ }^{3}$ \\ ${ }^{1}$ Institute of Aerospace Thermodynamics, University of Stuttgart, Germany \\ ${ }^{2}$ Institute for Thermodynamics and Thermal Process Engineering, University of Stuttgart, \\ Germany \\ ${ }^{3}$ Institute of Reactive Flows and Diagnostics, Technical University of Darmstadt, Germany \\ ${ }^{*}$ Corresponding author: christoph.steinhausen@itlr.uni-stuttgart.de
}

\begin{abstract}
The disintegration process of liquid fuel within combustion chambers is one of the most important parameters for efficient and stable combustion. Especially for high pressures exceeding the critical value of the injected fluids the mixing processes are not fully understood yet. Recently, different theoretical macroscopic models have been introduced to understand breakdown of the classical two phase regime and predict the transition from evaporation to a diffuse-mixing process. In order to gain deeper insight into the physical processes of this transition, a parametric study of free-falling $n$-pentane droplets in an inert nitrogen atmosphere is presented. Atmospheric conditions varied systematically from sub- to supercritical values with respect to the fluid properties. An overlay of a diffuse lighted image with a shadowgram directly in the optical setup (front lighted shadowgraphy) was applied to simultaneously detect the presence of a material surface of the droplet as well as changes in density gradients in the surrounding atmosphere. The experimental investigation illustrates, that the presence of a material surface cannot be shown by a direct shadowgram. However, reflections and refractions caused by diffuse ambient illumination are able to indicate the presence of a material surface. In case of the supercritical droplet injections in this study, front lighted shadowgraphy clearly revealed the presence of a material surface, even when the pre-heated droplets are released into a supercritical atmosphere. This detection of the droplet interface indicates, that the droplet remains subcritical in the region of interest, even though it is injected into a supercritical atmosphere. Based on the adiabatic mixing assumption recent Raman-scattering results in the wake of the droplet are re-evaluated to compute the temperature distribution. Presented experimental findings as well as the re-evaluation of recent Raman scattering results are compared to thermodynamic models to predict the onset of diffuse-mixing and supercritical disintegration of the droplet. Additionally, a one dimensional evaporation model is used to evaluate the validity of the adiabatic mixing assumption in the estimation of the droplet temperature. The presented findings contribute to the understanding of recent theoretical models for prediction of spray and droplet disintegration and the onset of diffuse-mixing processes.
\end{abstract}

\section{Keywords}

Front lighted Shadowgraphy, Raman-scattering, Supercritical fluid injection

\begin{abstract}
Introduction
The disintegration process of liquid droplets in a high pressure and high temperature environment is one of the most important parameters for stable and efficient combustion. Therefore, the behaviour of droplets in such an environment has long been a matter of analytical, numerical and experimental investigation. Especially the transition process from a two phase behaviour of the droplet or jet to a diffusion dominated mixture is not yet well understood. Recently, three different models predicting the onset of this diffuse-mixing have been introduced. The first model by Banuti and Hannemann [1] exploits the supercritical transition at the Widom-line of the fluid and introduces a new breakup model called pseudo-boiling. Another, second criterion, is proposed by Dahms and Oefelein [2] and later extended by Dahms [3]. Dahm's criterion compares the thickness of the interface layer with the characteristic length scale of heat transfer to calculate the probability of a supercritical breakup. Qiu and Reitz [4] proposed a third model, which extends classical thermodynamic conditions for a single component fluid to binary mixtures. The transition from sub- to supercritical fluid behaviour occurs, when the local temperature and pressure exceed the critical values of the mixture. Whereas the pressure of the mixture at the droplets interface is controlled in the experimental setup the temperature has to be estimated. Qiu and Reitz [4] suggested a adiabatic mixing assumption, which takes phase stability and phase separation into account, to calculate the local temperature based on the local concentration. In the following, we will focus on a discussion of the Qiu and Reitz [4] model using two different experimental techniques, namely front lighted shadowgraphy and Raman-scattering. Complementary, the evaporation model of Abramzon and Sirignano [5] is used to evaluate the validity of the adiabatic mixing assumption.
\end{abstract}




\section{Experimental Setup}

The experimental investigations described in this paper were done in two nearly identical heatable pressure chambers. The chambers are both designed for investigation of free falling droplets in near critical environments. In case of phenomenological investigations the droplets are generated in a closed chamber, detached and measured. For statistical investigations the chamber is used as a continuous flow chamber. The gas inside the chamber is a pressurized pure nitrogen atmosphere controlled by either solenoid valves mounted in the fluid supply and exhaust system or with a pneumatic valve at the system exhaust (Badger Meter). The pressure chamber can be operated at pressures up to 60 bar and temperatures up to $553 \mathrm{~K}$. Pressure inside the chamber is measured at the fluid exhaust with a temperature compensated pressure transducer (Keller $35 \mathrm{X}-\mathrm{HTC}$ ) with an uncertainty of \pm 300 mbar. Several T-type thermocouples with an uncertainty rated at $\pm 1 \mathrm{~K}$ are installed in the chamber wall and the core to monitor the steel temperature as well as the temperature of the nitrogen atmosphere, respectively.

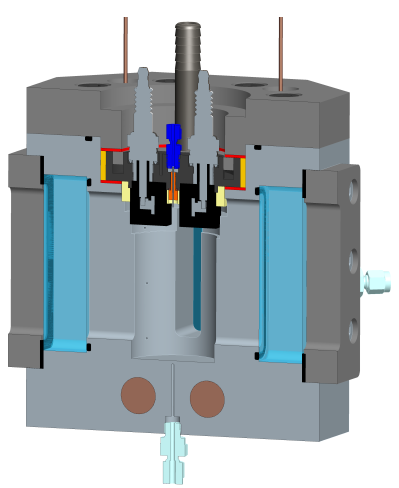

(a) $3 \mathrm{D}$ cross section of the chamber

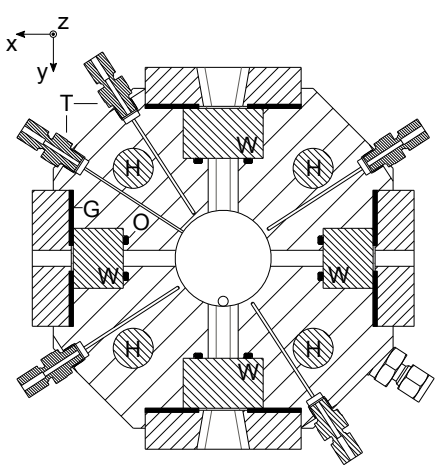

(b) Horizontal cross-section through chamber. G: Graphite gaskets; H: Heating cartridges; O: FFKM-O-Rings T: Type-T thermocouples; W: quartz windows.

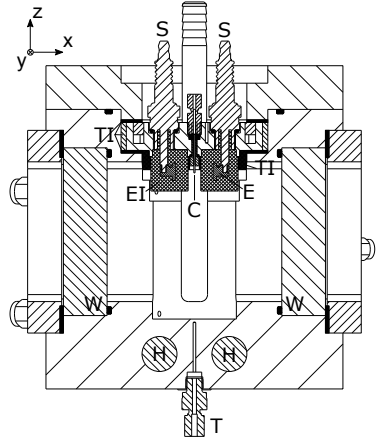

(c) Vertical cross-section through chamber and droplet generator. C: Capillary; $\mathrm{El}$ : Electric insulation; $\mathrm{H}$ : Heating cartridges; $\mathrm{S}$ : Spark plugs; T: Type-T thermocouples; TI: Thermal insulation; W: quartz windows.

Figure 1. Horizontal and vertical cut of the pressure chamber.

The pressure chamber is made out of temperature resistant stainless steel (EN-1.4571). The cylindrical core has a diameter of $48 \mathrm{~mm}$ and a height of $85 \mathrm{~mm}$. Optical accessibility is granted through $4 \mathrm{UV}$-transparent quartz windows which are arranged in a $90^{\circ}$ angle to each other. Six heating cartridges, four mounted vertically and two mounted horizontally, are inserted in the chamber body. The cartridges are controlled with type J-thermocouples in the heater cartridges as well as type T-thermocouples in the chamber. In figure 1 vertical and horizontal cross-sections of the chamber are depicted. Droplets are generated by an electric detachment system. The system utilizes the interactions between strong, pulsed electric fields and the fluid of the droplet. A detailed description of the droplet generator can be found in Weckenmann et al. [6] and Oldenhof et al. [7]. A analytical and numerical framework of the detachment process was done by Ouedraogo et al. [8]. For further information about the experimental setup the reader is referred to Bork et al. [9], Weckenmann et al. [6] and Oldenhof et al. [7].

\section{Raman Scattering}

The objective of the Stokes-shifted Raman scattering layout is the quantitative investigation of species concentrations in the wake of a free-falling droplet. A detailed description of the Raman scattering setup as well as the calibration and data evaluation can be found in Bork et al. [9]. Based on the concentration measurements a temperature estimation is performed to gain a deeper insight into the thermodynamic state of the fluid-mixture in the droplet wake. As a first approximation, the adiabatic mixing model is used for estimation of the temperature. Dahms and Oefelein [2], and later Dahms [3] used this assumption for their simulation study of supercritical jets. Qiu and Reitz [4] used an extended adiabatic mixing assumption, which includes phase instabilities and phase separation for their study on high pressure fuel injection.

\section{Front Lighted Shadowgraphy}

Front lighted shadowgraphy is an optical technique to indicate the thermodynamic state of the droplet and simultaneously visualize the changes in density gradients in its vicinity. It applies a direct overlay of a diffusive or scattered lighted image with a common shadowgram in the optical setup. Due to surface tension of the droplet, a strong density gradient at the interface occurs. Using this steep density gradient, the material surface of the droplet is detectable by refractions and reflections of the incoming light on the droplet surface. Changes in density gradients in the vicinity of the droplet are displayed by direct shadowgraphy in parallel light due to the Laplacian of the refractive index $\left(\partial^{2} n / \partial x^{2}\right)[10]$. 


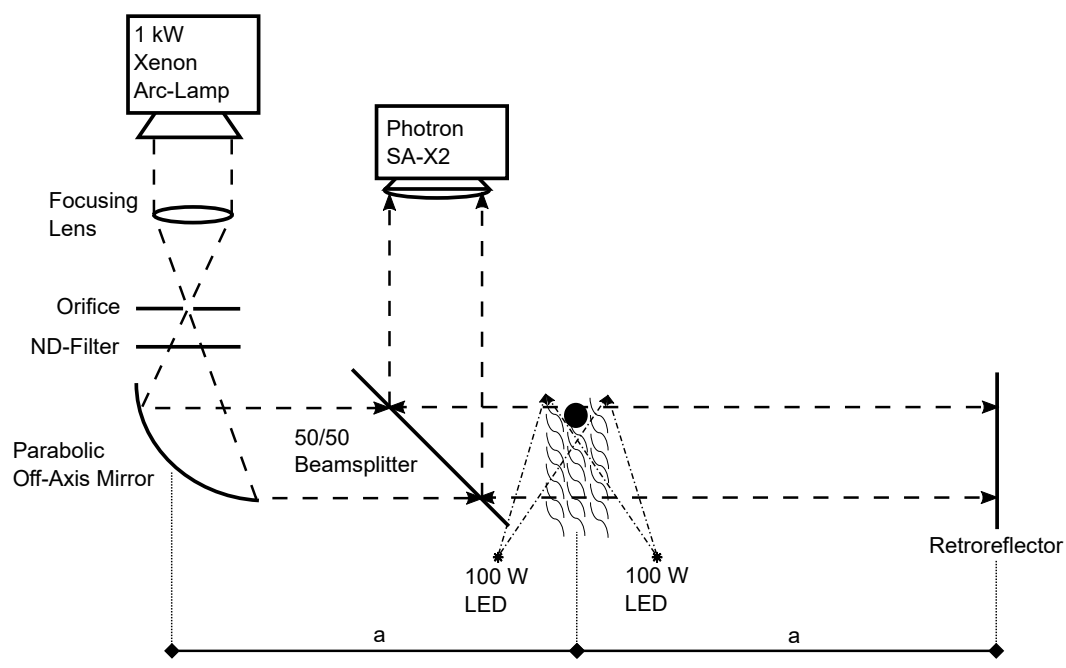

Figure 2. Front lighted shadowgraphy setup.

Figure 2 depicts the front lighted shadowgraphy setup. The retroreflector, which reflects the incoming light with the same incidence angle, on the right side of the chamber overlays a frontal illumination of the droplet with a backlighted shadowgraph. Both images are focused on the capillary and are aligned as well as overlayed by a $50 / 50$ beam splitter. Note, that optical distortions caused by the reflected shadowgram are not visible. This is due to the fact, that the focal depth of the used long distance microscope is at least one magnitude smaller than the distance between the chamber and the retroreflector. To increase reflection and refraction on the material surface of the droplet two LED arrays are located at the bottom of the pressure chamber illuminating the droplet detachment system. By removing the neutral density filter (ND-filter), located behind the orifice, the intensity of the frontal and back illumination can be adjusted. Without the ND-filter the intensity of the arc lamp overpowers the LED illumination leading to a common shadowgraph.

\section{Experimental results: Characterization of the thermodynamic state during droplet evolution}

A parametric study of free falling $n$-pentane droplets in a sub- to supercritical nitrogen atmosphere has been conducted using front lighted shadowgraphy. Experimental test conditions reach from subcritical chamber conditions to supercritical conditions. The injection temperature of injected fluid remains subcritical to anchor the droplet on the capillary. The reduced chamber temperature and pressure are referred to as $T_{r, c h}=T_{c h} / T_{c}$ and $p_{r, c h}=p_{c h} / p_{c}$, respectively. The reduced fluid injection temperature is $T_{r, f}=T_{f} / T_{c}$. Reduced values are normalized by the critical values of $\mathrm{n}$-pentane $\left(p_{c}=33.7\right.$ bar, $\left.T_{c}=469.7 \mathrm{~K}\right)$ [11].

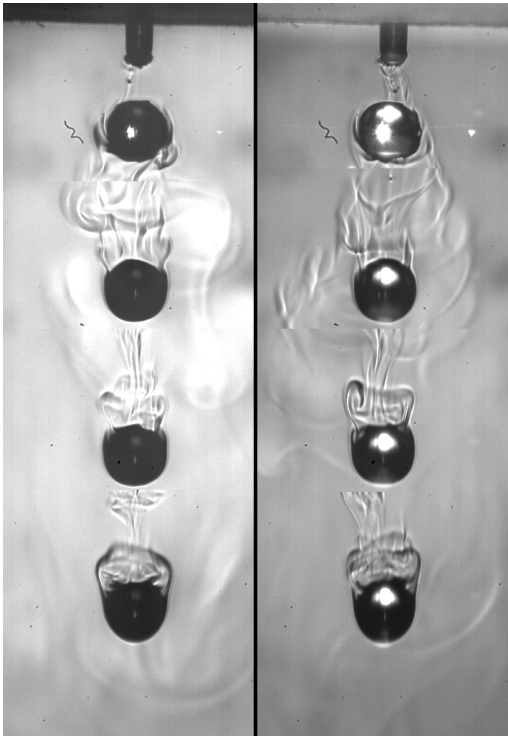

(a) $T_{r, c h}=0.81, T_{r, f}=0.81$

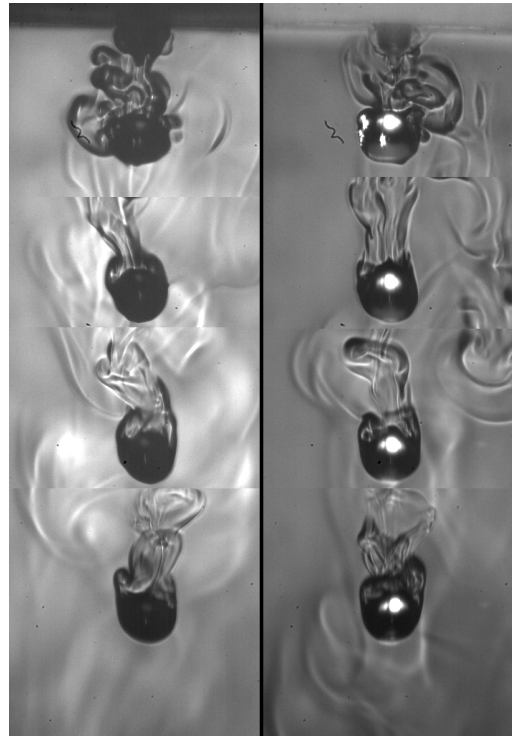

(b) $T_{r, c h}=0.95, T_{r, f}=0.95$

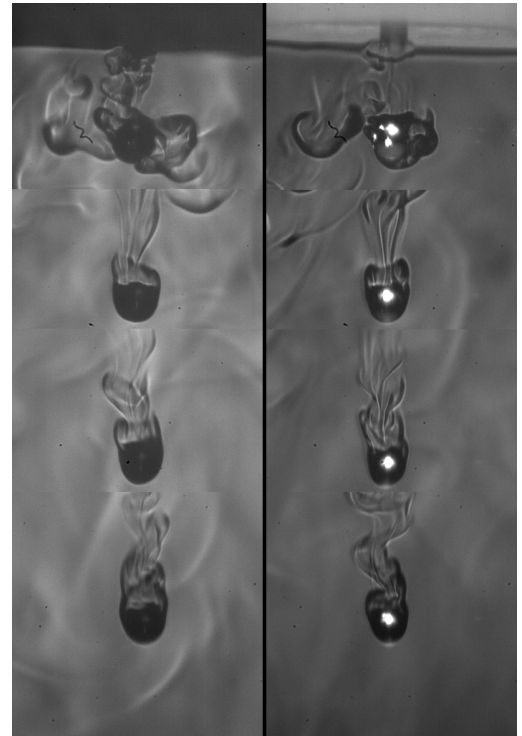

(c) $T_{r, c h}=1.11, T_{r, f}=0.95$

Figure 3. Front lighted shadowgraphs at four different positions for supercritical chamber pressures $\left(p_{r, c h}=1.78\right)$ and sub- to supercritical temperatures. Left: shadowgraph without ND-filter; Right: front lighted shadowgraph with ND-filter. 
The temporal evolution of the droplet under sub- to supercritical ambient temperature and supercritical chamber pressure $\left(p_{r, c h}=1.78\right)$ is depicted in figure 3 . The right side of each image displays the front lighted shadowgram, whereas on the left hand side the common shadowgraph is depicted. Every image is a compilation of snapshots recorded during the same experiment at four different locations. The shadowgrams and front lighted shadowgrams were recorded directly after each other and care was taken to insure similar chamber conditions during the experiment. As can be seen from figure 3, the mounting of the ND-filter increases the reflections and refractions on the droplet surface by reducing the intensity of the arc lamp. The bright spot at the top of the droplet in the upmost location is a result of reflections caused by the illuminated droplet detachment system, whereas the glare point in the centre of the droplet is a result of the refraction of the laser beam used for triggering. Without ND-filtering the reflection caused by the illuminated droplet detachment system is just slightly visible. This shows the overpowering of the LED arrays by the arc lamp. Changes in density gradients in the vicinity of the droplet are visible in both images.

A visual comparison of reflections and refractions in the front lighted shadowgrams depicted in figure 3 reveals similar behaviour for all conditions. Changes in density gradients are displayed by both common and front lighted shadowgraphy. The material interface of the droplet, however, can only by revealed by reflections and refraction caused by the diffuse illumination in the front lighted shadowgrams. Additionally, presence of a droplet surface is clearly indicated at all conditions, even when injected into supercritical atmospheres. At the transition from sub- to supercritical fluid states the phase boundary of the droplet should be vanishing, due to vanishing surface tension. Weckenmann et al. [12] illustrated, that a diffuse lighted image is sensitive to phase boundaries. Reflections and refractions on the droplet surface would concordantly vanish or show a different optical behaviour. A supercritical droplet, to be precise, a supercritical gas mixture, will therefore show no or different signs of reflective spots or refractive glare points compared to subcritical droplets. By the virtue of that fact, detection of the material surface and similar optical behaviour compared to subcritical temperatures indicates, that the droplet remains subcritical in the field of view although it is injected into a supercritical atmosphere. One possible explanation for this fluid behaviour are cooling effects due to evaporation of the droplet, which mitigate the heat fluxes from the supercritical atmosphere into the droplet. A more detailed explanation will be given in the next section by both experimental and analytical investigations of the droplet wake and the evaporation of the droplet, respectively.

\section{Assessment of thermodynamic models}

In this section experimental investigations regarding the thermodynamic state of the droplet are affiliated with thermodynamic analysis of the interfacial temperature as well as the thermodynamic state of the droplet. Our thermodynamic model is based on the true adiabatic mixing temperature calculation by Qiu and Reitz [4], which takes phase stability into account and uses the PC-SAFT equation of state (EOS). The PC-SAFT EOS is formulated as a fundamental equation in the Helmholtz energy utilizing results from statistical physics [13]. PC-SAFT uses chains of tangentially bonded segments of hard spheres as molecular model coarse graining the real molecular structure considering hard-sphere repulsion, chain formation, van der Waals attraction association and polar interactions. One-fluid treatment allows easy transfer from pure components to mixtures. Consideration of the molecular structure allows for good qualitative and quantitative representation of thermodynamic properties with a minimum number of parameters. Since Qiu and Reitz [4] used the Peng-Robinson EOS, figure 4 shows a direct comparison of the theoretical adiabatic mixing temperature calculated by Qiu and Reitz and the PC-SAFT EOS. The fluid system consist of a dodecane-nitrogen mixture at 60 bar and $900 \mathrm{~K}$ ambient pressure and temperature, respectively. The fluid temperature is $363 \mathrm{~K}$. As can been seen in figure 4 both calculation coincide very well.

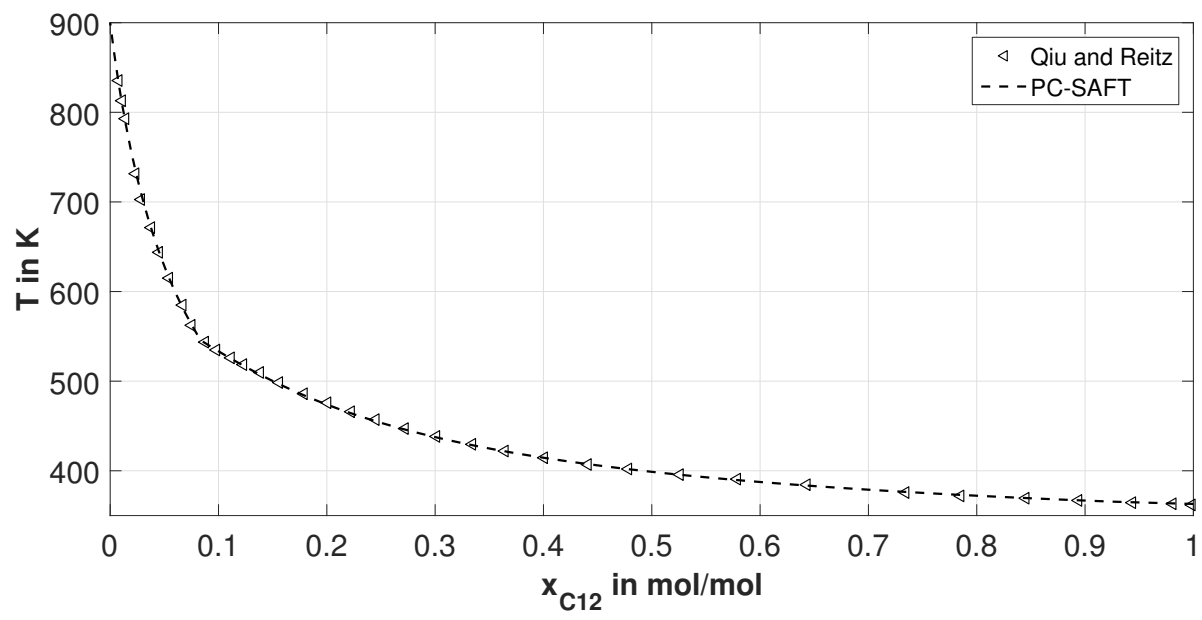

Figure 4. Comparison of the theoretical adiabatic mixing temperature calculations for a n-dodecane-nitrogen $\left(\mathrm{C} 12-\mathrm{N}_{2}\right)$ mixture. 


\section{Characterization of the droplet wake}

A comprehensive Raman test campaign was conducted to analyse the concentration field in the droplet wake for a variety of test fluids injected into a nitrogen atmosphere. In this paper a re-evaluation of the results for the $n$ heptane/nitrogen binary mixture done by Bork et. al [9] is discussed. A similar re-evaluation of the experimental results using the thermodynamic model of Dahms [3] is currently in development but not yet published. In this paper we will focus on the thermodynamic model presented by Qiu and Reitz [4]. The measured concentration field in the droplet wake is complemented with an estimation of the temperature field to provide insight into the role of evaporation on the droplet's surface temperature evolution. The temperature field is estimated using the concentration data applying the theoretical adiabatic mixing assumption by Qiu and Reitz [4]. The data is afterwards fitted by an iteration of the initial droplet temperature using the adiabatic mixing assumption at constant chamber conditions. Whilst the adiabatic mixing assumption has not been validated by independent measurements of temperature and concentration, we will apply this assumption to the experimental results to enable a direct comparison with the predictions from the theoretical models of Qiu and Reitz [4].
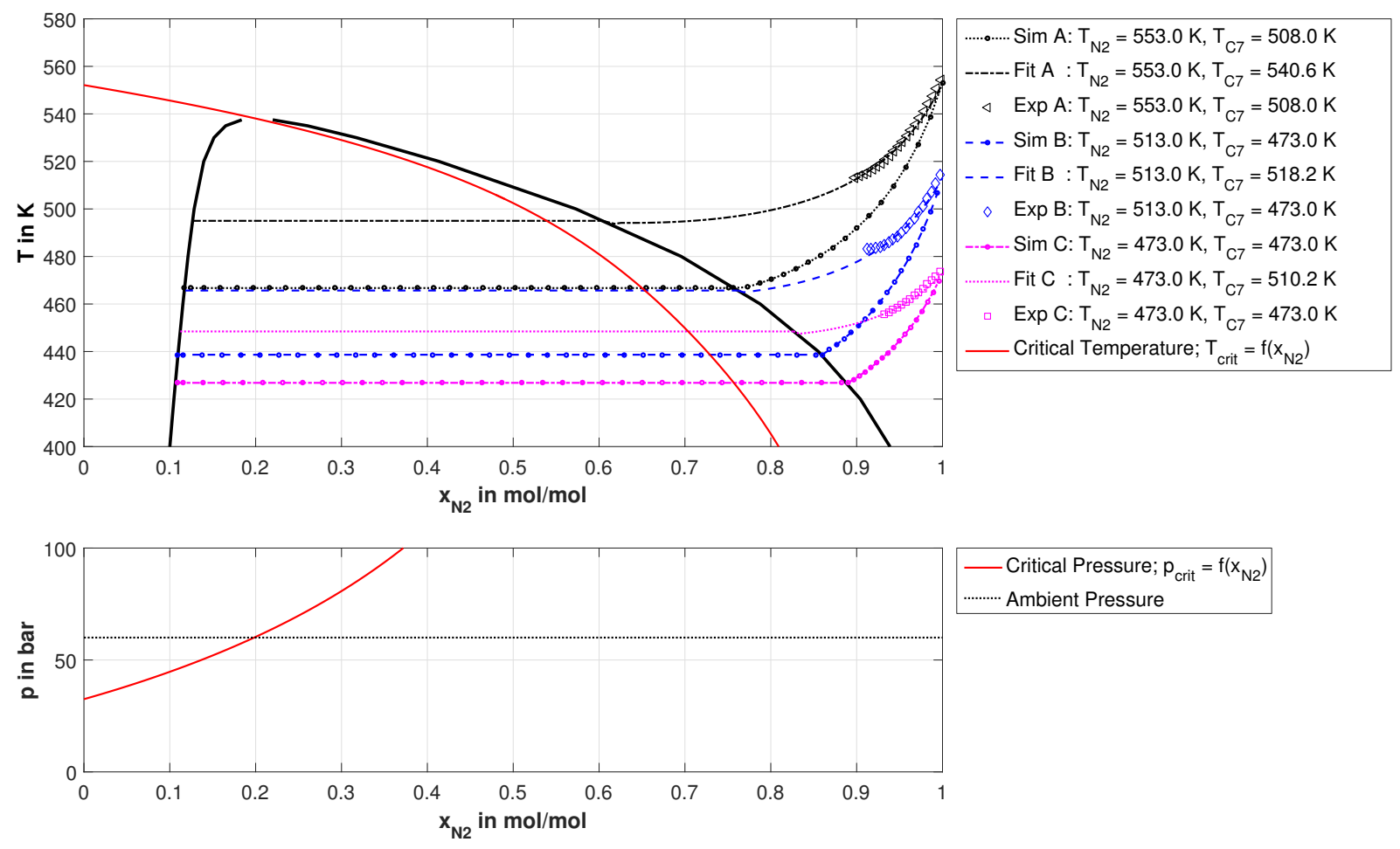

Figure 5. Correlation between measured mole fraction in the droplet wake and mixing temperature plotted with the vapour liquid equilibrium for a n-heptane-nitrogen $\left(\mathrm{C}^{-}-\mathrm{N}_{2}\right)$ mixture at 60 bar and the critical locus. The temperature in the vapour phase is estimated assuming adiabatic mixing using the PC-SAFT EOS.

Figure 5 shows adiabatic mixing lines fitted onto the experimental data (curves without markers), plotted together with the adiabatic mixing lines, derived according to the Qiu and Reitz [4] model (curves with markers). The latter are calculated for the experimental test conditions of the Raman scattering investigations. In contradiction to Qiu and Reitz [4], we assume a homogeneous droplet temperature, which is equal to the estimated surface temperature. Hence, the temperature inside the two phase region is constant. The different colours distinguish between the experimental cases $\mathrm{A}, \mathrm{B}$ and $\mathrm{C}$ of the Raman scattering investigations. The critical temperature as well as the critical pressure of the mixture are plotted as well. Note, that critical temperature and pressure are solely depending on the composition of the mixture. Comparing the theoretical adiabatic mixing lines derived from the Qiu and Reitz [4] model (curves with marker) with the fitted experimental data (curves without markers) the results deviate systematically from each other. Especially the resulting interfacial temperature of the theoretical adiabatic mixing line, which is the intersection of the mixing line with the dew point line, is significantly lower. Additionally the necessary initial droplet temperature to fit the experimental data is higher for all cases. The same discrepancies can be observed using the adiabatic mixing assumption from Dahms [3]. Note, that this similar behaviour is to be expected, since adiabatic mixing by Dahms [3], and Qiu and Reitz [4] is similar as long as the phase stability limit is not reached. The observed discrepancy can be explained by an evaluation of the inherent assumptions of the two models. Both models consider the system in stable thermodynamic equilibrium for the initial conditions and couple the temperature in the vapour phase with the mole fraction assuming adiabatic mixing. However, the injection time and dwell time of the droplet or jet is in the range of seconds or milliseconds, respectively. Hence, it is highly questionable that thermal equilibrium is reached within this short residence time. The next section will focus on this by applying the evaporation model of Abramzon and Sirignano [5] to the discussed experimental cases. 
Besides the mixing lines, figure 5 shows critical temperature and critical pressure of the mixture. In compliance with the transition criterion predicting the onset of diffuse-mixing by Qiu and Reitz [4], the droplet remains subcritical when the droplet's temperature and pressure remain subcritical. Hence, they are below the critical values of the mixture. In order to reveal the thermodynamic state of the droplet, the mole fraction in the liquid phase has to be taken into account. The latter can be extracted from the bubble point line assuming a homogeneous droplet temperature. The corresponding temperature is therefore the estimated interface temperature. Following this criterion $\mathrm{n}$-heptane droplets remain subcritical for all our experimental conditions, since the droplet temperature is below the critical temperature of the liquid compositions. In the vapour phase in the vicinity of the droplet the critical pressure of the mixture exceeds the ambient pressure leading again to a subcritical thermodynamic state. In figure 6, the n-pentane-nitrogen $\left(\mathrm{C}_{5}-\mathrm{N}_{2}\right)$ fluid system of the front lighted shadowgraphy investigations are depicted. Applying the transition criterion by Qiu and Reitz [4] to the n-pentane cases, a subcritical droplet as well as a subcritical vapour phase at the boundary layer of the droplet is observed. Hence, for both fluid systems our experimental observations agree with the transition criterion proposed by Qiu and Reitz [4].
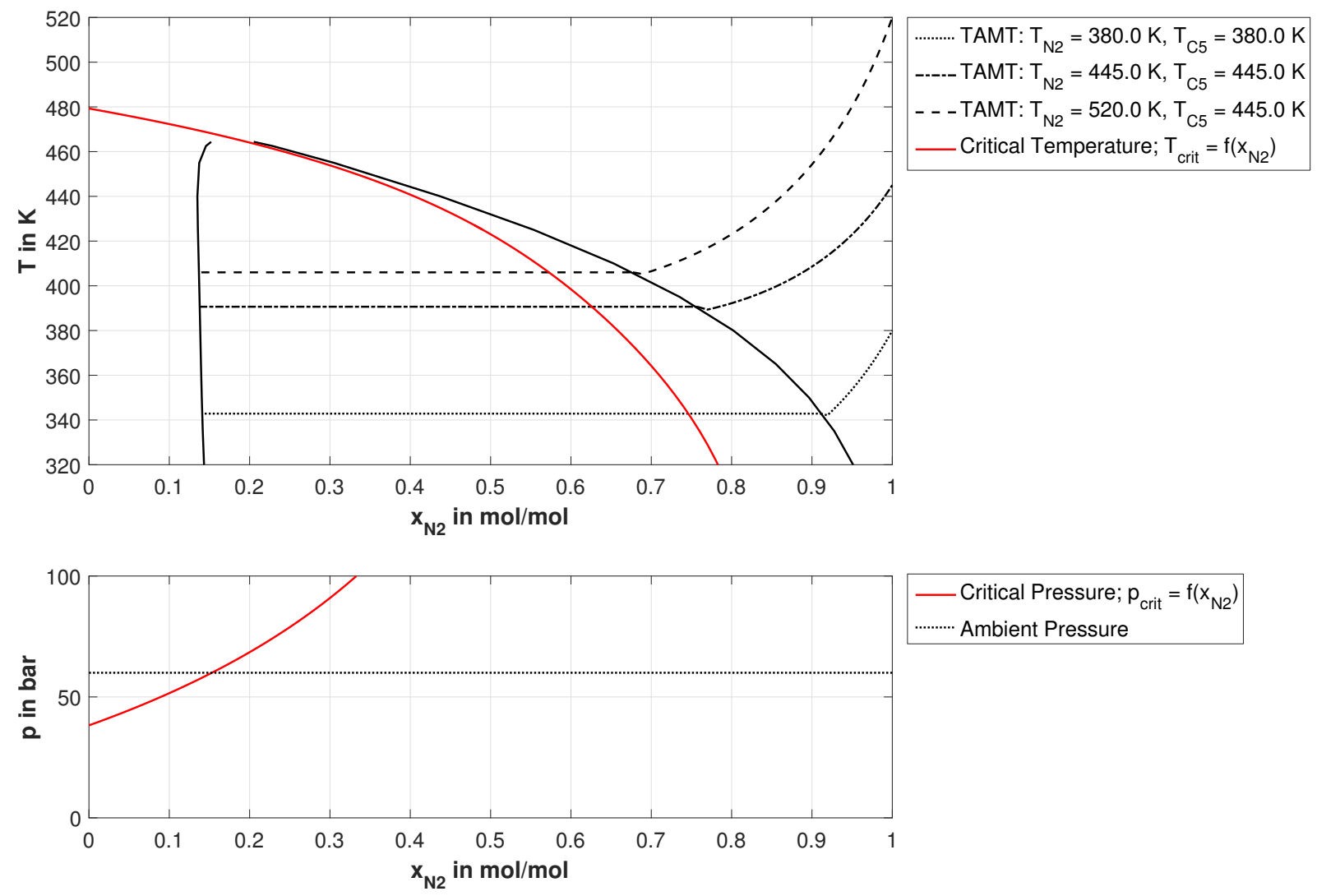

Figure 6. Theoretical adiabatic mixing temperature over mole fraction plotted with the vapour liquid equilibrium for a $\mathrm{n}$-pentane-nitrogen $\left(\mathrm{C} 5-\mathrm{N}_{2}\right)$ mixture at 60 bar and the critical locus. The temperature in the vapour phase is estimated using the PC-SAFT EOS.

\section{Characterization of the droplet surface temperature}

The observations in the previous section reveal, that investigations of the surface temperature of the droplet are highly important to confirm the thermodynamic state of the droplet and account for evaporative cooling of the droplet. Since experimental investigations regarding the droplets surface temperature in the discussed setup have not been done yet, we will focus on an analytical approach to estimate the temperature history of the droplet. As a first strategy the equilibrium evaporation model of Abramzon and Sirignano [5] is used. Thermodynamic properties of the pure fluids are extracted from the NIST Database [11], whereas properties of the mixture are calculated according to Amagats law for gas mixtures. Diffusion coefficients are calculated using the method of Wilke and Lee [14] with Lennard Jones parameter extracted from Hirschfelder et al. [15] for n-pentane and nitrogen. For nheptane the Lennard Jones parameter are listed in Chea and Violi [16]. Furthermore, the saturation pressure of the liquid in the vapour phase is corrected by the binary mixture composition in equilibrium using an enhancement factor as explained by Luijten [17]. The temporal evolution of the droplet temperature is shown in figure 7 for both $\mathrm{n}$-pentane and $\mathrm{n}$-heptane droplets. The initial droplet diameter is $D_{0}=1 \mathrm{~mm}$ and the atmospheric pressure is $p_{a m b}=60$ bar. 


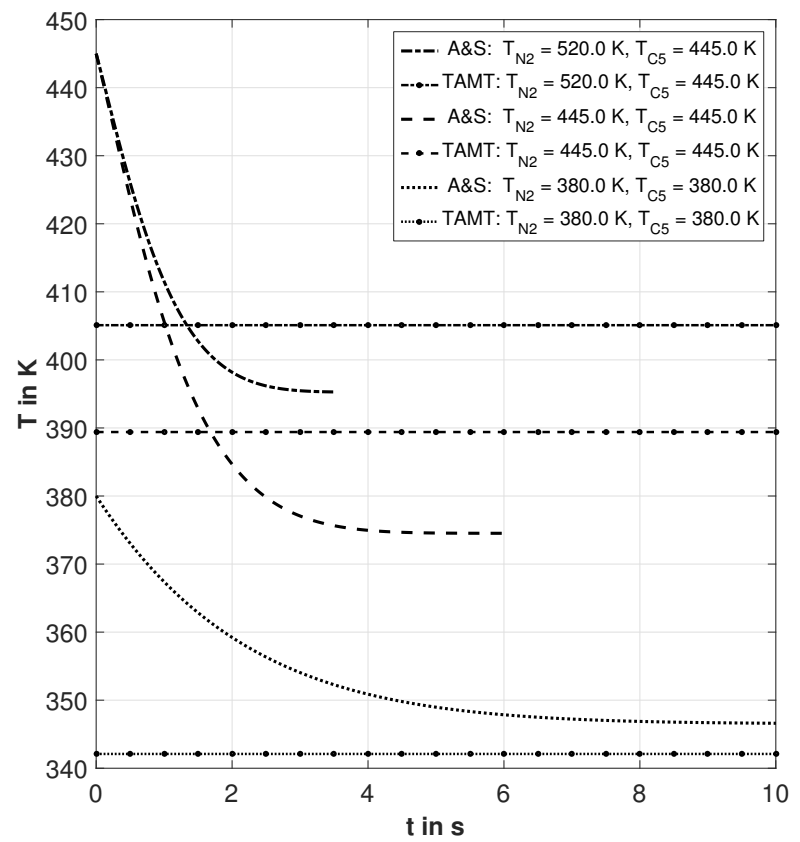

(a) n-pentane droplet in nitrogen atmosphere.

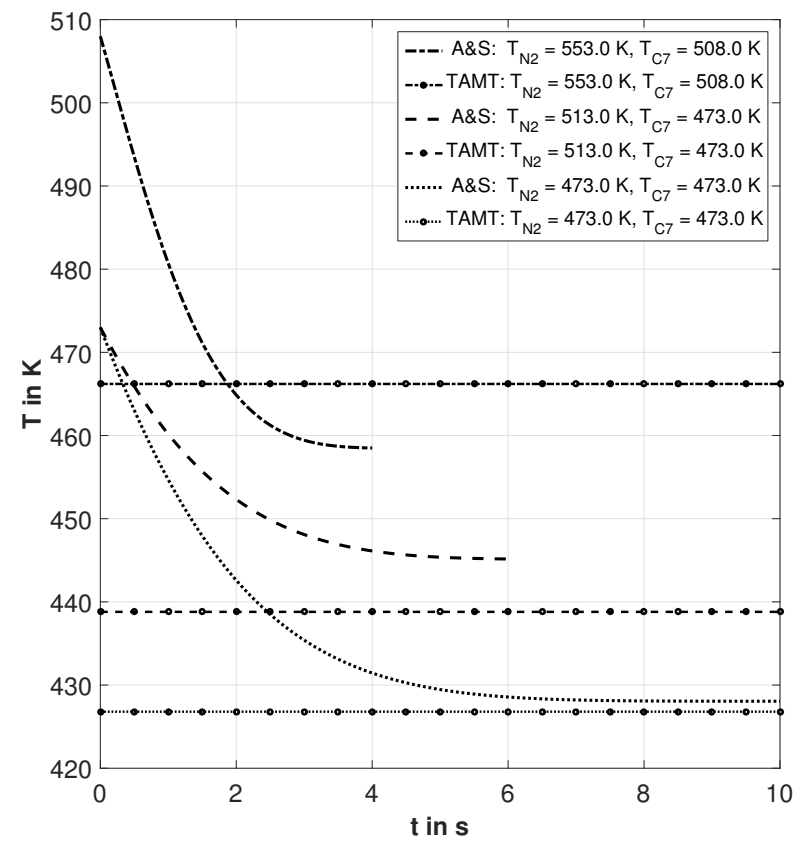

(b) n-heptane droplet in nitrogen atmosphere.

Figure 7. Droplet surface temperature evolution over time compared to the theoretical adiabatic mixing temperature (TAMT) at the surface of the droplet. The latter is the intersection of the TAMT with the dew point line at vapour liquid equilibrium.

In figure 7, a comparison between the estimated droplet surface temperature with the theoretical value TAMT (true adiabatic mixing temperature) at the droplet's interface is depicted. The latter is the intersection between the theoretical adiabatic mixing line calculated as described by Qiu and Reitz [4] and the dew point line of the binary mixture. The fluid systems and experimental conditions are the same for figure 5 and 6 . The comparison of the surface temperature shows, that for low initial droplet temperatures the TAMT at the interface sightly underestimates the wet bulb temperature. However, the agreement at high dwell times is good, due to the attainment of local thermodynamic equilibrium. Indeed, for lower initial droplet temperatures the equilibrium vapour mole fraction at the drop interface is rather low as shown in figure 5 and 6 . Consequently the wet bulb temperature coincides with the TAMT. For higher initial droplet temperatures the equilibrium vapour mole fraction increases significantly. Hence, the vapour boundary layer at the droplet interface remains undersaturated, leading to a sustained evaporation rate. Concordantly, the surface temperature drops below the TAMT values. This discrepancy increases with decreasing latent heat as can be seen by comparing n-pentane (low latent heat) and n-heptane (high latent heat) in figure 7. This leads to the following conclusion: The TAMT can be applied to estimate the wet bulb temperature only for low initial droplet temperatures. However, one must consider that the latter is reached after a characteristic dwell time, which depends on the initial droplet size. In the described experiments the dwell time is approximately $0.5 \mathrm{~s}$ for the $n$-heptane cases and circa $2 \mathrm{~s}$ for $n$-pentane. Hence, using the TAMT to calculate the surface temperature would lead to an underestimation of up to $40 \mathrm{~K}$ of the droplet temperature and therefore seems, as discussed in the previous section, not applicable to calculate the temperature of the droplet surface. For high temperature cases models based on the assumption of global thermodynamic equilibrium between the droplet and the gaseous phase are not suited to characterise the droplet temperature at any time.

\section{Conclusions}

An experimental and analytical study of drop dynamics at high pressure and temperature conditions has been conducted. We employed front lighted shadowgraphy to investigate the thermodynamic state of the droplet by detecting the material surface of the droplet as well as Raman scattering to investigate the concentration field in the droplet wake. Both investigations were carried out on falling alkane droplets in a nitrogen atmosphere. These experiments were complemented with an analysis of a transitional criterion to predict the onset of diffuse-mixing. Additionally, an evaporation model was applied to study the evolution of the droplet temperature compared to the adiabatic mixing assumption. Our main conclusions are the following:

- The front lighted shadowgraphy setup was able to reveal the presence of a material surface by means of reflections and refractions on the material surface of the droplet. In all experimental cases the presence of a material surface was observed, even when the droplet was injected into a supercritical atmosphere. This observation can be explained by the evaporative cooling effects. Evaporation prevents the droplet from gasification by mitigating the heating of the droplet interface.

- The temperature field in the droplet wake, calculated from the concentration field assuming adiabatic mixing, further confirm the role of evaporation for the evolution of the droplet. 
- From the presented experimental studies the Qiu and Reitz model [4] predicting the onset of diffuse-mixing shows good agreement. Hence, the critical locus of the mixture seems to be a suitable criterion to determine the thermodynamic state of a fluid injection. However, since the critical locus has not been reached yet, further experimental investigations at higher temperature levels are necessary.

- The comparison of the evaporation model of Abramzon and Sirignano [5] with the adiabatic mixing assumption of Qiu and Reitz [4] or Dahms [3], corroborates the role of evaporative cooling in the droplet evolution. Furthermore, the time scales of the fluid injection and dwell time have to be taken into account when calculating the temperature distribution in high pressure injection process.

- By applying the critical locus as the onset criterion of diffuse-mixing a global thermodynamic equilibrium is assumed. Evaporative cooling effects, however, lead to strong departure from global thermodynamic equilibrium and therefore may deduce erroneous results. This is especially true, for fast evaporation processes and short injection times. Hence, a detailed evaluation of the prediction of the onset of diffuse-mixing requires further investigations in the vicinity of the critical locus and eminently above the critical line.

\section{Acknowledgements}

The authors gratefully acknowledge the German Research Foundation (DFG) for the financial support through the collaborative research centre SFB/Transregio 75.

\section{References}

[1] Banuti, D. and Hannemann, K., "Supercritical Pseudo-Boiling and its Relevance for Transcritical Injection," 50th AIAA/ASME/SAE/ASEE Joint Propulsion Conference, No. 3571 in Conference Proceedings online, American Institute of Aeronautics and Astronautics, 2014, pp. 1-16.

[2] Dahms, R. N. and Oefelein, J. C., "On the transition between two-phase and single-phase interface dynamics in multicomponent fluids at supercritical pressures," Physics of Fluids, Vol. 25, 2013, pp. 092103.

[3] Dahms, R. N., "Understanding the breakdown of classic two-phase theory and spray atomization at enginerelevant conditions," Physics of Fluids, Vol. 28, No. 4, 2016, pp. 042108.

[4] Qiu, L. and Reitz, R. D., "An investigation of thermodynamic states during high-pressure fuel injection using equilibrium thermodynamics," International Journal of Multiphase Flow, Vol. 72, 2015, pp. 24 - 38.

[5] Abramzon, B. and Sirignano, W., "Droplet vaporization model for spray combustion calculations," Aerospace Sciences Meetings, American Institute of Aeronautics and Astronautics, Jan. 1988, pp. -.

[6] Weckenmann, F., Bork, B., Oldenhof, E., Lamanna, G., Weigand, B., Böhm, B., and Dreizler, A., "Single acetone droplets at supercritical pressure: droplet generation and characterization of PLIFP," Zeitschrift für Physikalische Chemie, Vol. 225, No. 11-12, 2011, pp. 1417-1431.

[7] Oldenhof, E., Weckenmann, F., Lamanna, G., Weigand, B., Bork, B., and Dreizler, A., "Experimental investigation of isolated acetone droplets at ambient and near-critical conditions, injected in a nitrogen atmosphere," Progress in Propulsion Physics, Vol. 4, EDP Sciences, 2013, pp. 257-270.

[8] Ouedraogo, Y., Gjonaj, E., Weiland, T., Gersem, H. D., Steinhausen, C., Lamanna, G., Weigand, B., Preusche, A., Dreizler, A., and Schremb, M., "Electrohydrodynamic simulation of electrically controlled droplet generation," International Journal of Heat and Fluid Flow, Vol. 64, 2017, pp. 120 - 128.

[9] Bork, B., Preusche, A., Weckenmann, F., Lamanna, G., and Dreizler, A., "Measurement of species concentration and estimation of temperature in the wake of evaporating n-heptane droplets at trans-critical conditions," Proceedings of the Combustion Institute, Vol. 36, No. 2, 2017, pp. $2433-2440$.

[10] Settles, G. S., Schlieren and shadowgraph techniques: Visualizing Phenomena in Transparent Media, Springer, 2001.

[11] Lemmon, E. W., Huber, M. L., McLinden, M. O., et al., "NIST Standard Reference Database 23," NIST Reference Fluid Thermodynamic and Transport Properties, Vol. 9, 2010, pp. 55.

[12] Weckermann, F., Bork, B., Lamanna, G., Dreizler, A., and Weigand, B., "Experimental investigation of droplet injections in the vicinity of the critical point." Marseille, France, 2014.

[13] Gross, J. and Sadowski, G., "Perturbed-Chain SAFT: An Equation of State Based on a Perturbation Theory for Chain Molecules," Industrial \& Engineering Chemistry Research, Vol. 40, No. 4, 2001, pp. 1244-1260.

[14] Wilke, C. R. and Lee, C. Y., "Estimation of Diffusion Coefficients for Gases and Vapors," Industrial \& Engineering Chemistry, Vol. 47, No. 6, 1955, pp. 1253-1257.

[15] Hirschfelder, J. O., Curtiss, C. F., and Bird., R. B., Molecular theory of gases and liquids, Wiley, New York, corr. printing with notes added, 3. printing ed., 1966.

[16] Chae, K. and Violi, A., "Mutual diffusion coefficients of heptane isomers in nitrogen: A molecular dynamics study," The Journal of Chemical Physics, Vol. 134, No. 4, 2011, pp. 044537.

[17] Luijten, C. C. M., Nucleation and droplet growth at high pressure, Ph.D. thesis, TU Eindhoven, 1998. 


\title{
Breakup Length of Urea Water Solution Jet in a Hot Cross Flow
}

\author{
Senthil Kumar. $\mathrm{P}^{1}$, Shamit Bakshi ${ }^{1}$, Anand T.N.C *1 \\ ${ }^{1}$ Department of Mechanical Engineering and National Centre for Combustion Research and \\ Development, Indian Institute of Technology Madras, India \\ *Corresponding author: anand@iitm.ac.in
}

\begin{abstract}
Selective Catalytic Reduction (SCR) using Urea-Water Solution (UWS) as an ammonia precursor is considered as one of the best choices to meet the current stringent emission norms for reduction of $\mathrm{NO} x$ in diesel engines. UWS sprayed in the engine exhaust line forms ammonia, and this ammonia reduces $\mathrm{NO}_{\mathrm{x}}$ into nitrogen. The $\mathrm{NO}_{\mathrm{x}}$ reduction efficiency depends on the mixing and evaporation behavior of the UWS spray in the hot exhaust gas. Spray characteristics decide the evaporation rate and hence the $\mathrm{NO}_{x}$ reduction efficiency. The spray structure is closely related to the breakup point and breakup mode of the jet. Hence, in this study, breakup length and breakup mode were investigated by injecting UWS (32.5\% by weight) through a nozzle in a hot air cross flow. A CCD camera and pulsed $\mathrm{Nd}$ :Yag laser were used for capturing the images. Experiments were conducted with varying nozzle size $\left(150,250\right.$ and 400 micron), injection pressure $\left(0.5\right.$ to 3 bar), temperature $\left(32{ }^{\circ} \mathrm{C}, 150{ }^{\circ} \mathrm{C}\right.$ and $200^{\circ} \mathrm{C}$ ) and air flow rate. The effect of operating parameters (nozzle size, injection pressure, air temperature and velocity) in terms of dimensionless numbers (Weber number and momentum flux ratio) on jet breakup mode and jet breakup length was studied. It was observed that the breakup length for UWS was close to that of water. The jet breakup length increases with momentum flux ratio since a jet having a higher momentum is able to penetrate a larger distance in the cross flow. Increasing the air temperature increases the velocity of the cross flow and hence reduces the breakup length. A correlation for jet breakup length was developed. The effect of inclusion of Weber number in the breakup length correlation, in addition to the momentum flux ratio, was studied. Visual observation shows that droplet sizes obtained from the plain orifice injector without preheating is large. Preheating the UWS before injection is recommended to reduce the droplet size.
\end{abstract}

\section{Keywords}

UWS, cross flow, breakup length and laser diagnostics.

\section{Introduction}

Diesel engines deliver a high thermal efficiency, high torque density and good fuel economy. However, emissions produced by diesel engines, especially oxides of nitrogen (NOx), pose a major problem to the environment and to human health. In-cylinder treatment methods like retarding injection timing, Exhaust Gas Recirculation (EGR) and exhaust after treatment methods like three-way catalysts and lean $\mathrm{NO}$ traps are not suitable to meet future US and European emission norms. Urea-SCR after treatment method has the ability to meet these norms [1].

In this method, ammonia reacts with $\mathrm{NO}_{\mathrm{x}}$ in hot exhaust gas and reduces it to Nitrogen in the presence of a catalyst which accelerates the reduction reaction. Liquefied ammonia poses issues relating to storage, handling and transportation. Gaseous ammonia storage leads to problems of toxicity and possible explosions. Hence, the direct use of ammonia is avoided. Urea is used as a precursor for ammonia. Requirement of uniform distribution of ammonia in exhaust gas and the hygroscopic nature of urea prevent the use of solid urea. Hence, urea (32.5\% by weight) is mixed with water. The eutectic point of this solution is at $-11^{\circ} \mathrm{C}$. This provides the lowest crystallization point and ensures a constant concentration even when the solution is partially frozen [1]. UreaWater Solution namely UWS is injected into hot exhaust gas. Thermal decomposition of UWS consists of three successive stages namely evaporation, thermolysis and hydrolysis. First, water evaporates from the solution. Then, urea decomposes into ammonia and isocyanic acid in the thermolysis process. Finally, isocyanic acid reacts readily with water vapor and produces ammonia and carbon dioxide in the hydrolysis process.

The efficiency of the $\mathrm{NO}_{x}$ reduction reaction is affected by the availability and uniformity of ammonia present before the catalyst. However, ammonia formation is influenced by mixing and evaporation of UWS which are affected by the spray quality. All the above processes: evaporation of urea, ammonia formation, ammonia mixing with exhaust gas and reduction reaction need to be completed within a short period of time or distance. This is the major challenge in mobile engines, especially at low exhaust gas temperatures, since only a short distance is available for mixing, evaporation and reaction. Typical cross flow spray characteristics are shown in Figure1. The 
actual spray structure starts from the jet breaking point. This will decide the other parameters of the spray such as the width, trajectory, droplet size and distribution. This motivates the study of the breakup length of UWS jet in hot cross flow. The objective of this study is to find a correlation for breakup length of UWS jet in the hot cross flow by conducting experiments with different nozzle sizes, injection pressures, air velocities and temperatures. Here, breakup length refers to the vertical distance between the nozzle exit and the point where the jet breaks and forms ligaments. This is also referred to as the transverse breakup length, jet transverse penetration or column fracture height [2].

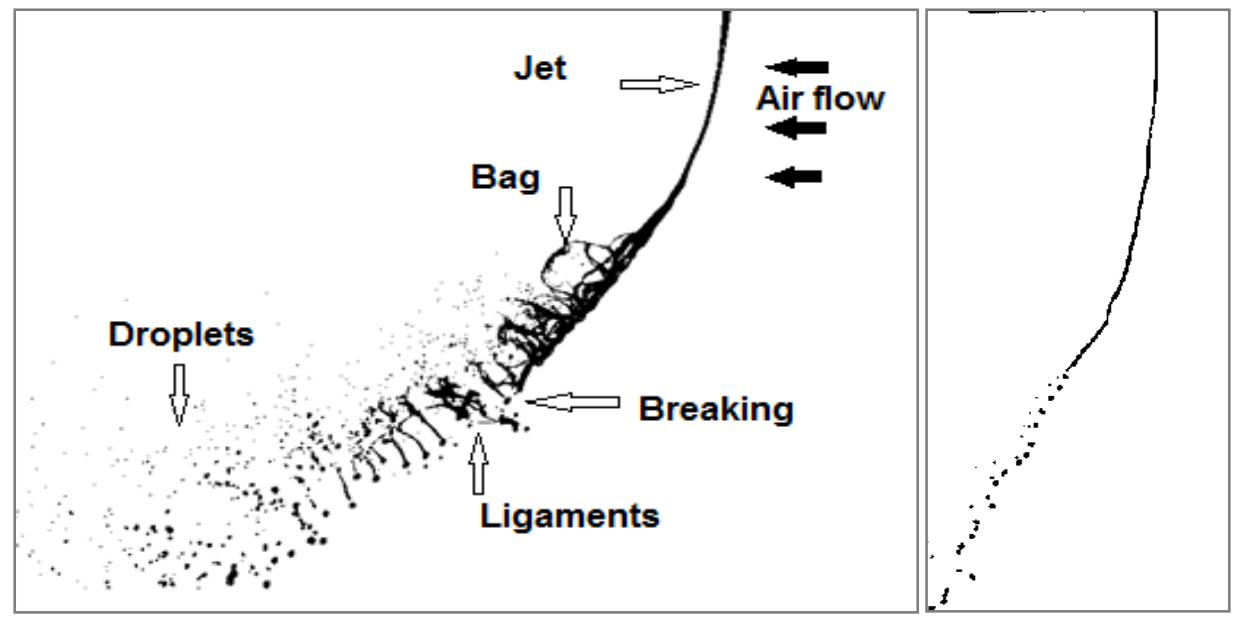

Figure 1. Typical jet in cross flow showing bag breakup and column breakup regimes (not to scale)

In previous studies, researchers have developed several correlations for breakup length in different forms. Table 1 shows the correlations for breakup length from different studies. In studies [3] to [7], correlations were developed for breakup length in terms of the momentum flux ratio. In addition to the momentum flux ratio, Ohnesorge number was included when liquid viscosity exceeds $0.019 \mathrm{~Pa}$.s in [4]. Two separate correlations were proposed based on Weber number $(\leq 8$ and $\geq 12)$ in [7]. Aerodynamic Weber number and Reynolds number were added along with momentum flux ratio in [8] and [9] respectively.

Table 1. Correlations for breakup length from literature

\begin{tabular}{|l|l|l|}
\hline Author & Correlation & Equation number \\
\hline Wu et al. [3], 1997 & $\frac{y_{b}}{d}=3.07 q^{0.53}$ & $(1)$ \\
\hline Birouk et al. [4], 2003 & $\frac{y_{b}}{d}=3.13 q^{0.53 \quad \mu_{l}<0.019 \quad \text { Pa.s }}$ & $(2 \mathrm{a})$ \\
& $\frac{y_{b}}{d}=8.60 q^{0.87} \mathrm{Oh}^{2} \quad \mu_{l}>0.019 \quad$ Pa.s & $(2 \mathrm{~b})$ \\
\hline Costa et al. [5], 2006 & $\frac{y_{b}}{d}=8.05 q^{0.5}$ & $(3)$ \\
\hline Wang et al. [6], 2011 & $\frac{y_{b}}{d}=2.50 q^{0.53}$ & $(4)$ \\
\hline Y.Zheng et al. [7], 2011 & $\frac{y_{b}}{d}=10.7 q^{0.40}$ for We(air) $\leq 8$ & $(5 \mathrm{a})$ \\
\hline Ragucci et al. [8], 2007 & $\frac{y_{b}}{d}=4.46 q^{0.40}$ for We(air) $\geq 12$ & $(5 \mathrm{~b})$ \\
\hline Bellofiore et al. [9], 2007 & $\frac{y_{b}}{d}=4.355 q^{0.416} \mathrm{We}_{\mathrm{aero}}^{0.135}$ & $(6)$ \\
\hline
\end{tabular}


Correlations (1), (2a), (3) and (4) appear close to each other in terms of the constants and exponents, even though different liquids (water, ethyl alcohol, lubrication oil, Jet $A$ fuel) were used for the experiment. Experiments were conducted in different air flow conditions: normal temperature and pressure in [3] to [7], elevated pressure (10 bar) in [8], and elevated temperature $\left(327^{\circ} \mathrm{C}\right)$ and pressure (10-20 bar) in [9]. Higher Weber number and lower length to diameter of the nozzle $(\mathrm{I} / \mathrm{d})$ were used in [3], where as lower Weber numbers and higher length to diameter ratio of the nozzle were used in [7]. Studies [3] and [6] gave a single equation for a wide range of momentum flux ratio. Liquid was injected at an angle of $15^{\circ}, 30^{\circ}$ and $45^{\circ}$ with the air flow direction in [5].

Table 2. Experiment Input conditions for the cases 1 and 2

\begin{tabular}{|c|c|c|c|c|c|c|c|}
\hline Parameters & \multicolumn{3}{|c|}{ Case1 } & Case2 & Parameters & Case1 & Case2 \\
\hline Weber number & \multicolumn{3}{|l|}{$7-16$} & $2.4-5$ & $\begin{array}{l}\text { Liquid Injection } \\
\text { pressure (bar) }\end{array}$ & $0.5-3$ & $0.5-3$ \\
\hline Momentum flux ratio & \multicolumn{3}{|c|}{$22-149$} & $36-436$ & $\begin{array}{l}\text { Liquid Injection } \\
\text { Velocity }(\mathrm{m} / \mathrm{s})\end{array}$ & $8-20$ & $8-20$ \\
\hline Liquid & \multicolumn{3}{|c|}{ Water, UWS } & UWS & Air Temperature $\left({ }^{\circ} \mathrm{C}\right)$ & 150,200 & $32,150,200$ \\
\hline \multirow{2}{*}{$\begin{array}{l}\text { Nozzle diameter (micron) } \\
\text { Length/diameter ratio }\end{array}$} & 152 & 254 & 406 & \multirow[t]{2}{*}{152,254} & \multirow[t]{2}{*}{ Air Velocity (m/s) } & \multirow[t]{2}{*}{$45-55$} & \multirow[t]{2}{*}{$35-50$} \\
\hline & 0.62 & 1 & 1.67 & & & & \\
\hline
\end{tabular}

In the present study, the experiments were conducted by injecting UWS into the cross flow of air at elevated temperature. The input conditions used in this experiment are given in Table 2. Surface tension and density of UWS are taken as $0.065 \mathrm{~N} / \mathrm{m}$ and $1090 \mathrm{~kg} / \mathrm{m}^{3}$, respectively [10]. Maximum uncertainty values calculated for Weber number and momentum flux ratios are $8.8 \%$ and $9.4 \%$ respectively.

\section{Experimental set up and procedure}

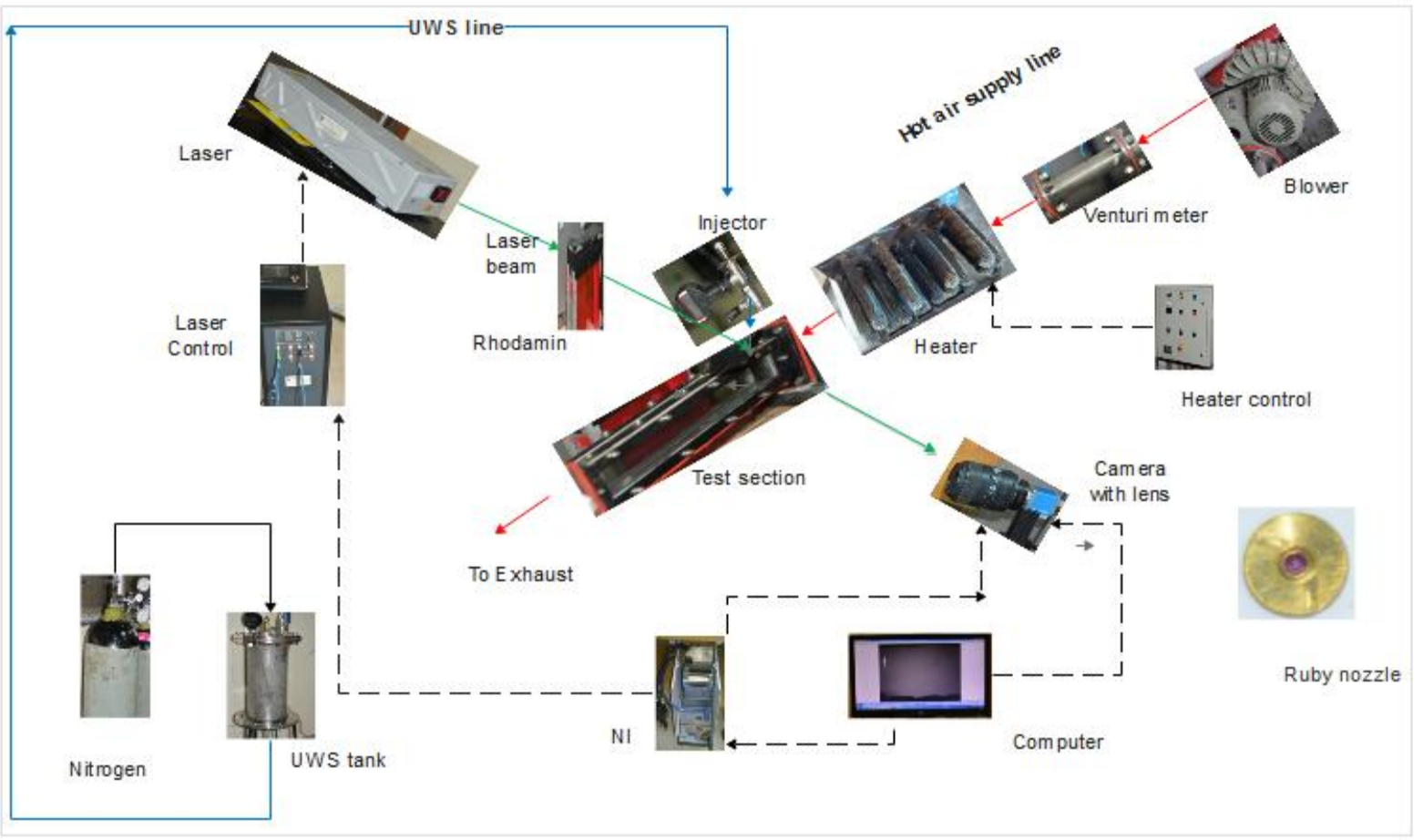

Figure 2a. Schematic of the experimental set up. 
A schematic view of the experimental set up is shown in Figure $2 a$. The experimental setup includes three main subsystems: hot air supply line, UWS injection line and optical components for imaging. The air supply line consists of a blower to draw air, a venturi meter to measure the volume flow rate of air, a heater ( $30 \mathrm{~kW})$ to heat the air, a test section and an exhaust arrangement. The test section has a length of $250 \mathrm{~mm}$ and a cross section of $40 \mathrm{~mm}$ (height) $\times 25 \mathrm{~mm}$ (width). The walls of the test section are made of toughened glass to visualize the spray. The urea injection line consists of a $\mathrm{N}_{2}$ cylinder, pressure vessel to store the UWS and an injector with a ruby nozzle. Nitrogen pressurizes the vessel and the liquid was injected through the injector into the test section. A cylindrical, straight hole ruby nozzle having diameter of 152, 254 or 406 micron size with the corresponding length/diameter ratio of $0.62,1$ and 1.67 respectively was used. The dimension, shape and assembly of the ruby nozzle with brass plate fixed in the injector are shown in Figure $2 \mathrm{~b}$. The plain orifice injector has been selected for this experiment due to the following reasons: it is simple in design, allows for easy cleaning and can be easily mounted or removed from the test section for change of nozzle size. The jet coming from the hole does not get disturbed by the jet coming from the other holes which would be present in a multi hole injector and this helps to understand the spray structure and make it suitable for image processing.
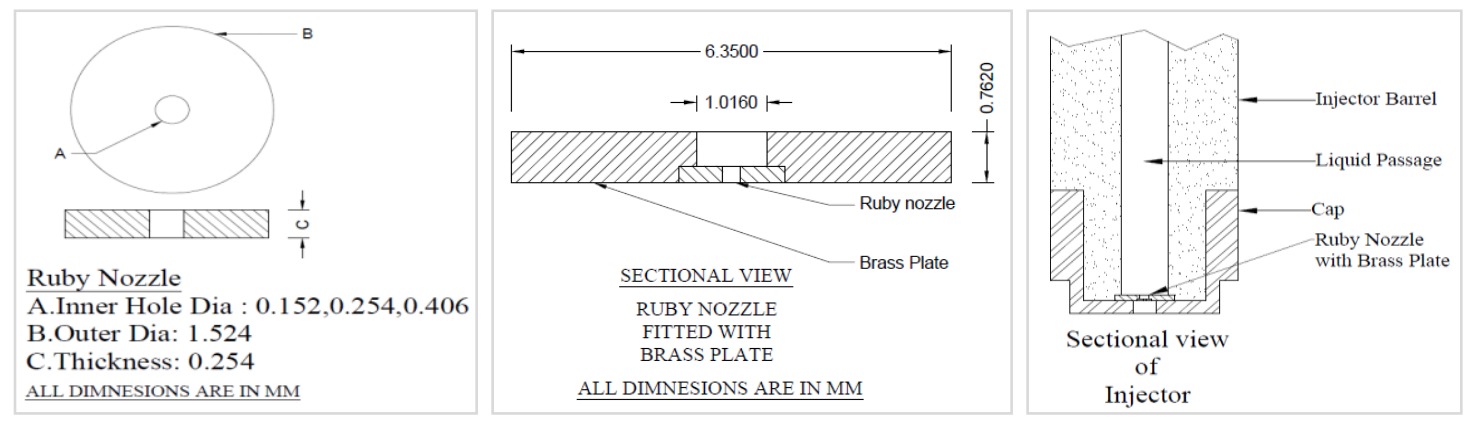

Figure 2b. Ruby Nozzle, Ruby nozzle fitted with brass plate and the Injector

The optical sub system contains an Nd:Yag laser $(532 \mathrm{~nm})$ for illumination, a CCD camera fitted with a lens (105 $\mathrm{mm}$ ) to capture the spray image and an National Instruments device to trigger and synchronize the laser and the camera at $10 \mathrm{fps}$. Rhodamine-B solution based diffuser is placed in between the laser and the test section to reduce the speckle in the images [11]. The hot air from the heater was passed through the test section and the UWS in room temperature was injected perpendicular to this hot air stream. The bottom of the injector (cap) was exposed to the hot air. The liquid temperature inside the injector, near the nozzle was measured using a thermocouple while conducting some of the trials. During continuous injection of the liquid, the temperature of the liquid was observed to be slightly $\left(5^{\circ} \mathrm{C}\right)$ above the room temperature $\left(30^{\circ} \mathrm{C}\right)$. In the calculation it was taken as room temperature. Backlight imaging technique was employed to capture spray images by using the pulsed laser and camera. Finally, the images were processed using MATLAB [12].

\section{Image processing}

Two thousand images of the spray structure were captured for each test condition. Each gray scale image was subtracted from the reference (background) gray scale image. Then, the subtracted image was converted into a binary (black and white) image using a suitable threshold (cut-off intensity) value, which is shown in Figure 3. From this binary image, the distance between the jet origin and the jet breakup point in transverse direction was calculated in terms of pixels. Breakup length was obtained by pixel-to-millimeter conversion using a scale image, taken during the experiment. Typically, a $50.7 \mathrm{~mm} \times 37.9 \mathrm{~mm}$ and $55.5 \mathrm{~mm} \times 41.5 \mathrm{~mm}$ field of view with a pixel resolution of 36 and $40 \mu \mathrm{m} /$ pixel was imaged.
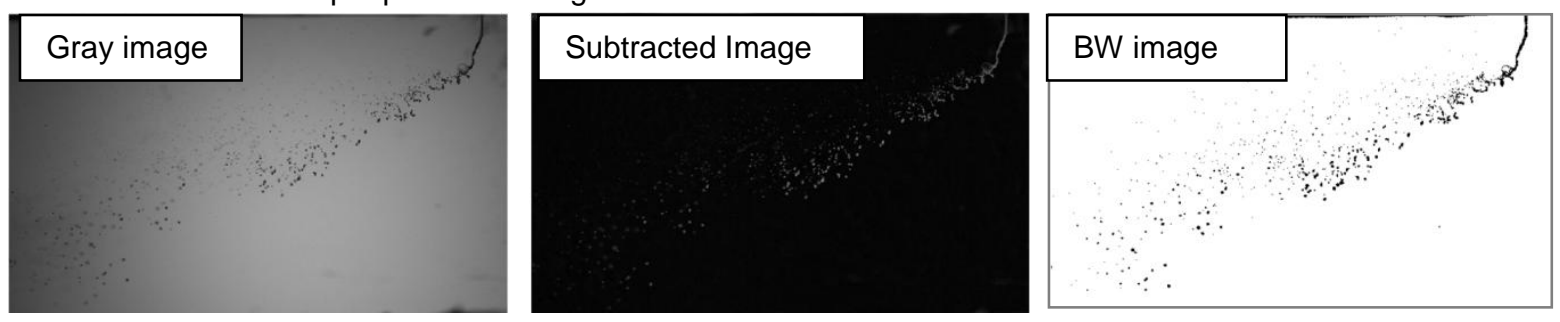

Figure 3. Stages of image processing employed in the present study. 


\section{Results and discussion}

\section{Breakup mode}

Bag breakup mode was observed in the Weber number range of 7-16 (case 1) and column breakup mode was observed in the Weber number range of 2.4-5 (case 2) as shown in Figure 1. A large number of droplets are formed during bag breakup and only a few are formed during column breakup.

\section{Breakup length}

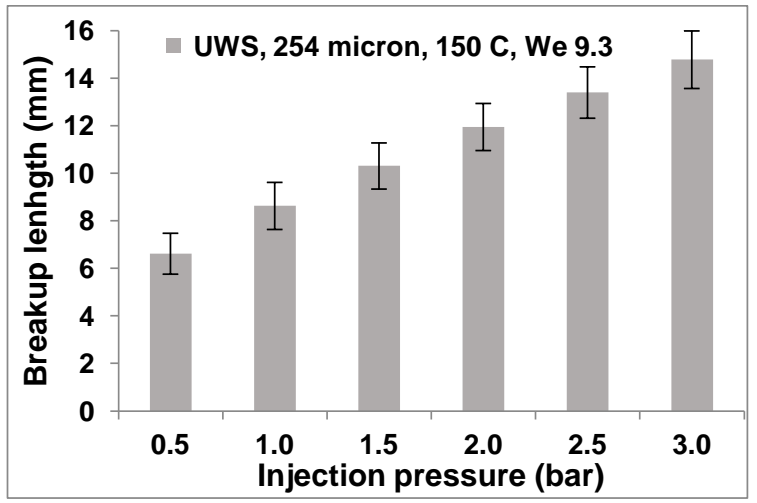

Figure 4a. Effect of injection pressure on the breakup length

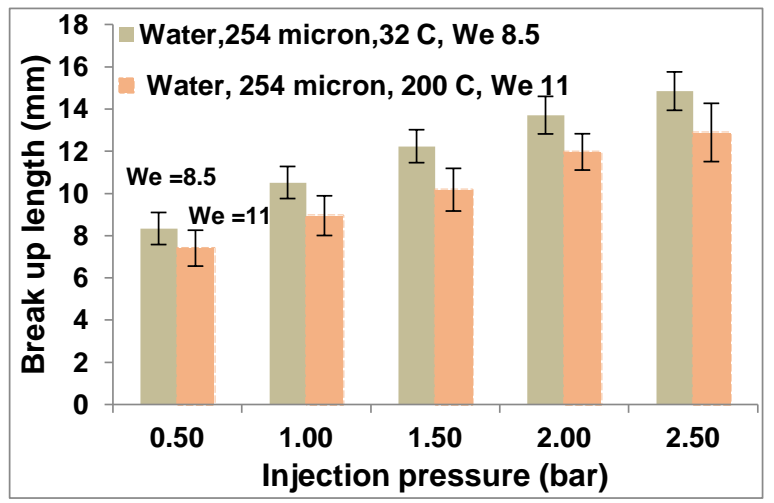

Figure 4b. Effect of temperature on the breakup length

Figure 4a shows the breakup length results for two experimental conditions: liquid: UWS, nozzle diameter: 254 micron, temperature: $150^{\circ} \mathrm{C}$, We: 9.3. Breakup length increases with an increase in the momentum flux ratio. Momentum flux ratio was increased by increasing the injection pressure, and hence the injection velocity.

Figure $4 \mathrm{~b}$ shows the breakup length results for two experimental conditions (liquid: water, nozzle diameter: 254 micron, temperature: $32{ }^{\circ} \mathrm{C}$, We: 8.5 and liquid: water, nozzle diameter: 254 micron, temperature: $150{ }^{\circ} \mathrm{C}$, We: 11). An increase in air temperature $\left(32^{\circ} \mathrm{C}\right.$ to $\left.200{ }^{\circ} \mathrm{C}\right)$ increases air velocity and hence the Weber number, but reduces the momentum flux ratio. A decrease in the breakup length was observed with an increase in air temperature for the same liquid injection velocity. There is not much difference between the breakup length for water and UWS as the properties (surface tension, viscosity) of both liquids are close to each other.

\section{Breakup length Correlation}

Correlations for breakup length were obtained for the entire Weber number range (2-16) and momentum flux ratio (22-436) in terms of momentum flux ratio, without Weber number and with Weber number in equations (8) and (9) respectively as:

$$
\begin{aligned}
& Y_{b}=d * 2.32 * q^{0.687} \\
& Y_{b}=d * 8.06 * q^{0.516} W e^{-0.227}
\end{aligned}
$$

Figure (5a) shows the plot between momentum flux ratio and breakup length at a Weber number of 16 (nozzle size: 406 micron, air temperature: $208^{\circ} \mathrm{C}$ ). Including Weber number in equation (9) reduces the average deviation between the predicted breakup length values with the experimental values (from $13.3 \%$ to $10.3 \%$ ), in comparison with equation (8). This is observed for all the experimental conditions.

Figure (5b) shows the plot between momentum flux ratio and breakup length at a Weber number of 5 (Nozzle size: 152 micron, Air temperature: $156^{\circ} \mathrm{C}$ ). Similar to the previous case, inclusion of Weber number in the correlation brings the predicted breakup length values close to the experimental values.

Separate correlations were obtained for the Weber numbers in the range 7-16 (bag breakup) and Weber numbers in the range 2.4-5 (column beak up) as shown in equations (9a) and (9b) respectively. These correlations further reduce the average deviation between the predicted and the experimental values $(9.2 \%$ for $9 \mathrm{a}$ and $9.5 \%$ for $9 \mathrm{~b})$ and bring the result much closer to the experimental values, as shown in Figure $5 \mathrm{a}$ and $5 \mathrm{~b}$. 


$$
\begin{aligned}
& Y_{b}=d * 4.69 * q^{0.55} W e^{-0.073} \\
& Y_{b}=d * 9.41 * q^{0.483} W e^{-0.204}
\end{aligned}
$$

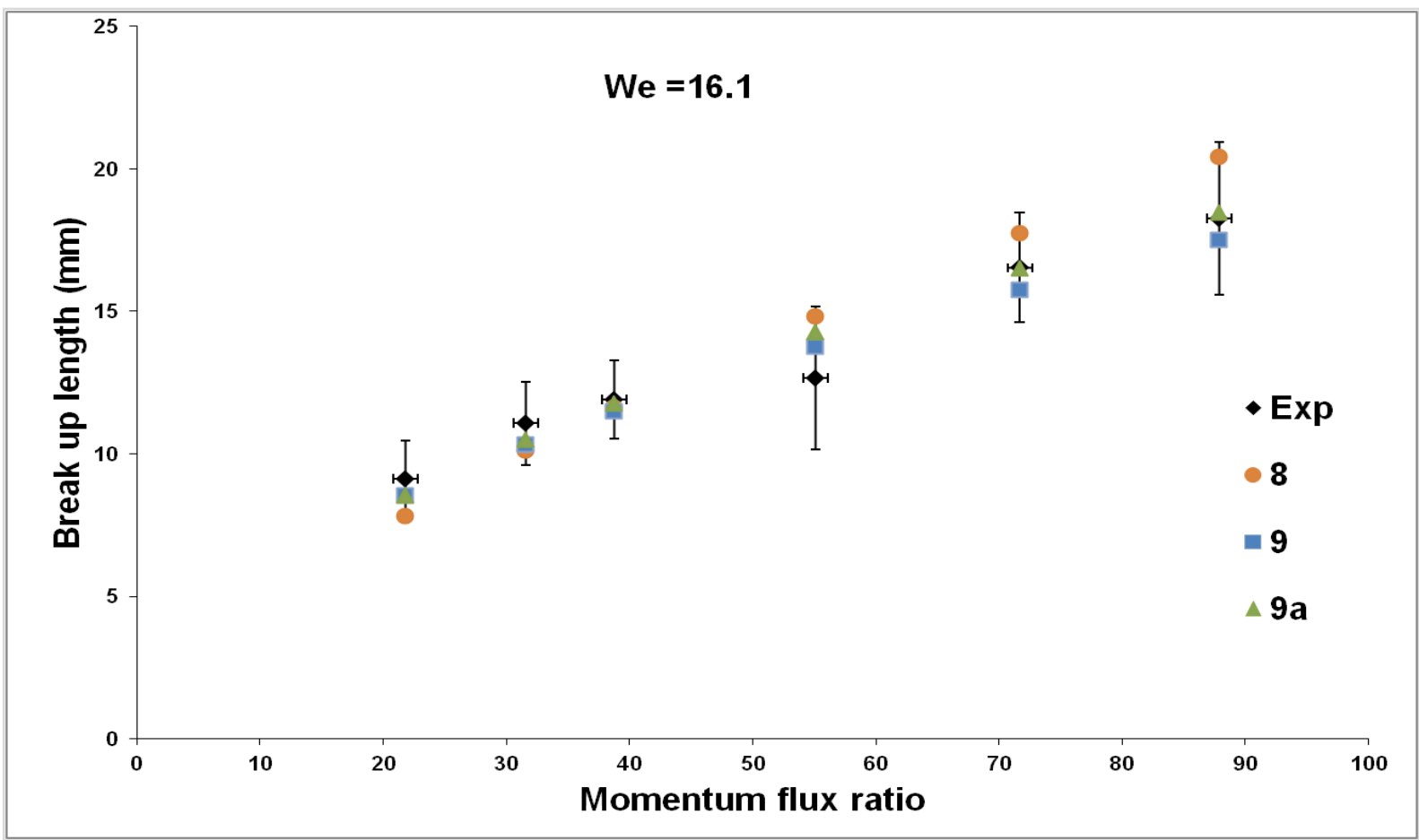

Figure 5a. Effect of momentum flux ratio on the breakup length $(\mathrm{We}=16)$. Experimental values are plotted along with the values predicted by equations (8), equation (9) and equation (9a)

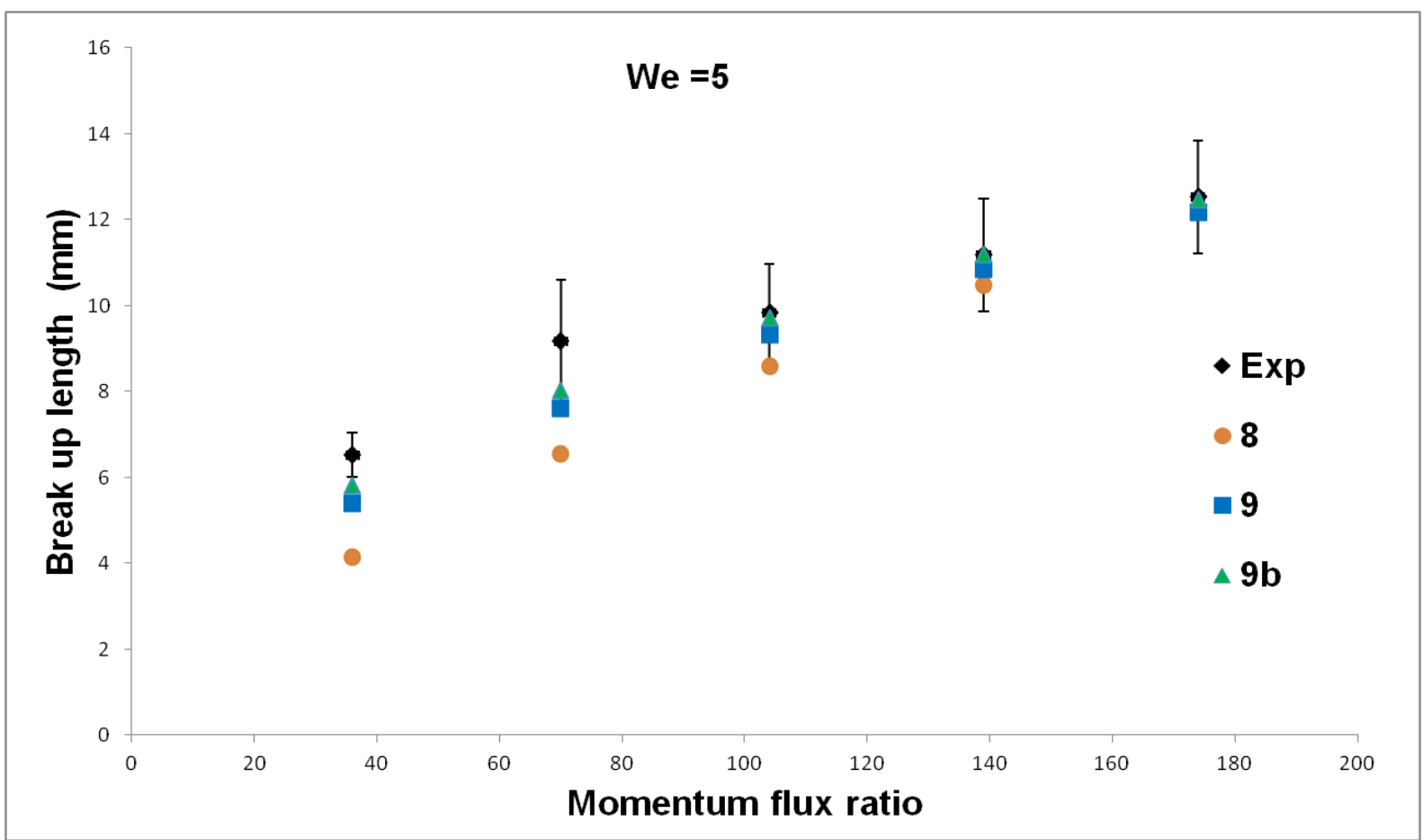

Figure 5b. Effect of momentum flux ratio on the breakup length $(\mathrm{We}=5)$. Experimental values are plotted along with the values predicted by equations (8), equation (9) and equation (9b) 


\section{Droplet}

Visual observation shows that the maximum size of the droplet is nearly equivalent to the nozzle size. Droplets of this size are not small enough to evaporate before they reach the catalyst in the exhaust pipe. Injecting the liquid through the plain orifice injector without preheating the liquid is thus not suitable for injecting UWS. Preheating the UWS before the injection is could help to reduce the droplet size and obtain more efficient evaporation.

\section{Conclusion}

An attempt was made to study UWS jet in a hot cross flow in Weber number range 2.4-16 and momentum flux ratio range 22-436. Bag breakup mode was observed for Weber numbers in the range 7-16 and column breakup mode for Weber number range 2-5 for the jet. The breakup length increases with an increase in the momentum flux ratio. The effect of temperature is incorporated in the Weber number and momentum flux ratio. Including the Weber number along with the momentum flux ratio in the breakup length correlation, reduces the deviation from the experimental values. Individual correlations for particular breakup modes (Weber number range) predict the breakup length values closer to the experimental values. This preliminary experimental investigation using plain orifice injector shows that the size of the droplet is large particularly at low air velocities. The droplet may not evaporate within the limited exhaust pipe length and time. Preheating the UWS before the injection is required to reduce the droplet size and obtain more efficient evaporation.

\section{Acknowledgement}

The authors gratefully acknowledge the financial support from Caterpillar India which made this work possible and Dr. Kameswara Rao Anupindi, IIT Madras, for proof-reading this article.

\section{Nomenclature}

Dimensionless numbers

$$
\begin{array}{ll} 
& W e=\frac{\rho_{a} v_{a}^{2} d}{\sigma_{l}} \\
& q=\frac{\rho_{l} v_{l}^{2}}{\rho_{a} v_{a}^{2}} \\
& \\
\text { We }(\text { aero }) & =\frac{\rho_{a} v_{l}^{2} d}{\sigma_{l}} \\
& \\
& R e_{a}=\frac{\rho_{a} v_{a} d}{\mu_{a}} \\
& \text { Oh }=\frac{\mu_{l}}{\sqrt{\rho_{l} d \sigma_{l}}} \\
\mathrm{~d} & \text { Diameter of nozzle [m] } \\
\mathrm{v} & \text { Velocity [m/s] } \\
\text { Oh } & \text { Ohnesorge number } \\
\mathrm{q} & \text { Momentum flux ratio } \\
\text { We } & \text { Weber number } \\
\mathrm{Y}_{\mathrm{b}} & \text { Breakup length [mm] } \\
R e_{a} & \text { Reynolds's number } \\
\mathrm{l} & \text { Liquid } \\
\mathrm{a} & \text { Air } \\
\rho & \text { Density [kg/m } \left.{ }^{3}\right] \\
\mu & \text { Dynamic viscosity }\left[\mathrm{Ns} / \mathrm{m}^{2}\right] \\
\sigma & \text { Surface tension }[\mathrm{N} / \mathrm{m}]
\end{array}
$$

\section{References}

[1] M. Koebel., M. Elsener., and M. Kleemann. , 2000, Catalysis Today, 335-345.

[2] Soo-Young No., Sep. 2.-6. 2012, ICLASS 2012, $12^{\text {th }}$ Triennial International Conference on Liquid Atomization and Spray Systems, Heidelberg, Germany.

[3] Wu, P-K., Kirkendall, K.A., Fuller, R.P.,1997, Journal of Propulsion and Power, 13(1):64-73.

[4] Birouk, M., Stabler,T., Azzopardi, B.J., 2003, Particle and Particle Systems Characterization, 20:39-46. 
[5] Costa, M., Melo, M.J., Sousa, J.M.M., Levy, Y., 2006, AIAA Journal, 44(3): 646-653.

[6] Wang, Q., Mondragon, U.M., Brown, C.T., McDonell, V.G., 2011, Atomization and Sprays, 21(3): $203-219$.

[7] Yinghui Zheng., Andr'eW. Marshall., 2011, Atomization and Sprays, 21 (7): 575-589.

[8] Ragucci, R., Bellofiore, A., Cavaliere, A., 2007, Atomization and Sprays 17:47-70.

[9] Bellofiore, A., Cavaliere, A., Ragucci,R., 2007, Combustion Science and Technology,179:319-342.

[10] Sauli Halonen., Teija Kangas ., Mauri Haataja., Ulla Lassi ., Emiss. Control Sci. Technol, 10.1007/ s40825016-0051- 1

[11] Saransh Jain., S Somasundaram., and T N C Anand., 2016, Measurement Science and Technology, 27(2016) 025406 (7pp)

[12] MATLAB and Statistics Toolbox Release 2012a, The MathWorks, Inc., Natick, Massachusetts, United States, http://www.Mathwork.com. 


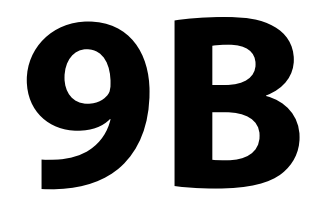

Combustion 3 


\title{
Spray Ignition and Local Flow Properties in a Swirled Confined Spray-Jet Burner: Experimental Analysis
}

\author{
J. Marrero-Santiago ${ }^{\star 1}$, A. Verdier ${ }^{1}$, A. Vandel ${ }^{1}$, G. Godard ${ }^{1}$, G. Cabot ${ }^{1}$, B. Renou ${ }^{1}$ \\ ${ }^{1}$ Normandie Univ, INSA Rouen, UNIROUEN, CNRS, CORIA, 76000 Rouen, France \\ ${ }^{*}$ Corresponding author: marreroj@coria.fr
}

\begin{abstract}
Laser ignition was investigated in the swirled, confined CORIA Rouen Spray Burner under ultra-lean conditions $(\phi=0.61)$ with $n$-heptane as the liquid fuel. Ignition probability was calculated for different spark locations and compared to the non-ignited local flow properties. Mean velocity components of the carrier flow were measured by PDA under spray presence and without spray, and are compared to mean values from PIV. PIV measurements provide information on the instantaneous airflow and the total strain rate. Fuel droplet size-velocity data was measured by PDA. Toluene-PLIF images were acquired to provide information on the local equivalence ratio and the flammability factor. Results show that the outer recirculation zone (ORZ) has a flammability factor close to 1 and the highest ignition probability $(\sim 80 \%)$. These results have a high correlation with the air velocity field and turbulent kinetic energy. Instantaneous equivalence ratio images and shear rate-velocity fields give important information on local segregation of the flow properties that help to understand the ignition process. The present work provides a useful database for numerical simulations and industry, plus new insight on spray ignition.
\end{abstract}

\section{Keywords}

Spray ignition, PDA, PIV, Toluene PLIF, Ignition probability, Local flow properties.

\section{Introduction}

The ignition process in gas turbines involves a wide range of parameters which makes it a multi-physical complex problem. New aeronautical burner designs demand a better knowledge of the mechanisms here involved to assure, for instance, re-ignition in high altitude of lean-combustion engines. Real combustors work with two-phase flows with strongly varying local properties. Indeed, turbulence, flow velocity, droplet size and velocity, and fuel vapour repartition are parameters governing the ignition mechanisms. Several studies on spray ignition [1-3] have been carried out. Studies on spray ignition in industrial configurations are still scarce although some pioneer experiments and simulations in linear [4,5] and annular combustors [6, 7] have been done. Investigations on the influence of parameters such as turbulence $[8,9]$ also exist. The knowledge is still not sufficient to identify and understand the real mechanisms of ignition. More experiments are also needed to validate numerical simulations.

Ignition in aeronautical engines can be divided into four steps: (i) energy deposition through a spark and its evolution into a flame kernel; (ii) kernel propagation; (iii) flame stabilisation on one injector; (iv) injector-to-injector propagation of the flame. Sometimes one of these phases is not successful and leads to a missed ignition. Even with a sufficient energy supply at phase (i), phase (ii) appears to be a critical step, namely in lean conditions. During its propagation in the chamber, the flame kernel will be exposed to varying local properties of the flow that may be adverse for its survival. This depends on both, the initial location of the kernel (spark location) and on its subsequent trajectory. Ignition probability is, therefore, very important for designers and for numerical simulations.

The present investigation addresses the ignition of a mono-injector swirled confined jet-spray burner. An ignition probability map is obtained through a probabilistic approach varying the spark location inside the chamber. The gaseous flow velocity field is characterised by phase Doppler anemometry (PDA), as well as the fuel ( $n$-heptane) droplet distribution, size and velocity. High-speed particle image velocimetry (PIV) provides the gaseous instantaneous velocity fields. In order to complete the study of the local properties, the fuel vapour distribution is also measured by planar laser induced fluorescence (PLIF) on a tracer (toluene). This technique enables the simultaneous visualisation of the fuel droplets, the air issuing jet and the fuel vapour concentration in the gaseous phase, providing the local fuel-to-air equivalence ratio along all regions in the burner.

\section{Material and methods}

Facility

Experiments are carried out in a swirled version of the CORIA Rouen Spray Burner [10, 11], confined with quartz windows in order to allow full optical access (Fig.1). The system is composed of a simplex pressure atomizer (Danfoss, $1.46 \mathrm{kgh}^{-1}, 80^{\circ}$ hollow cone) and an external annular swirling air co-flow with an inner and outer diameter 
of 10 and $20 \mathrm{~mm}$ respectively. The radial swirler is composed of 18 rectangular $(6 \mathrm{~mm} \times 8 \mathrm{~mm})$ channels inclined at $45^{\circ}$ with a corresponding swirl number of 0.76 [12]. Air and liquid fuel ( $n$-heptane) mass flow rates are controlled by thermal and Coriolis mass flow controllers. The airflow leaves the annular pipe to enter the combustion chamber as a turbulent jet with a strong swirling motion establishing low pressures and a consequent recirculation region at the centre of the burner. The air and fuel inlet conditions are $8.2 \mathrm{gs}^{-1}\left(\mathrm{~T}=416 \pm 2 \mathrm{~K}\right.$ ) and $0.33 \mathrm{gs}^{-1}$ ( $\left.\mathrm{T} 350 \mathrm{~K}\right)$ respectively, leading to a global equivalence ratio $(\phi)$ of 0.61 representing ultra-lean conditions. The four windows form a chamber of $10 \mathrm{~cm} \times 10 \mathrm{~cm}$ base. Under these conditions, the system reaches a stable internal window temperature of $387 \mathrm{~K}$, which is constantly measured by a thermocouple to assure the repeatability of experiments. For the ignition experiment, the mixture is ignited with a laser induced spark, by focusing a $532 \mathrm{~nm}$ laser beam in a selected location inside the combustion chamber. Ignition details are provided at the end of the following section.
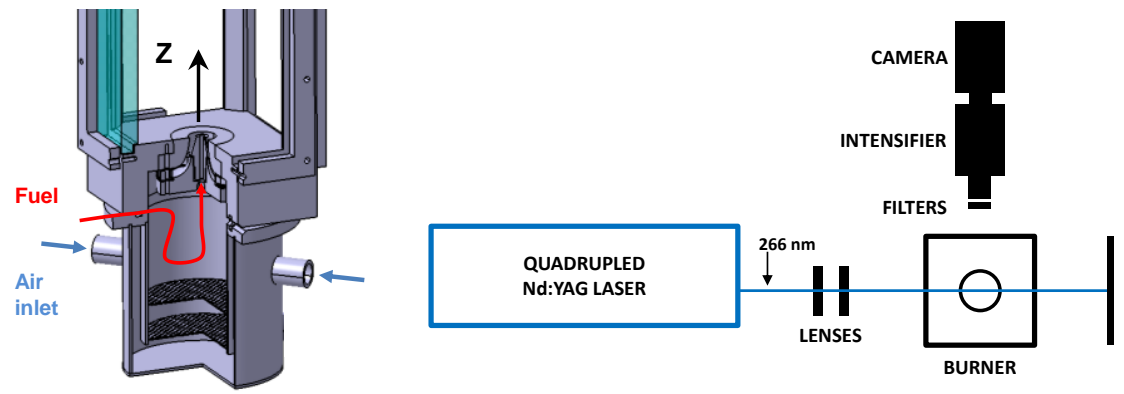

Figure 1. Cut of the burner with detail of the injection system and experimental setup for toluene-PLIF.

\section{Diagnostics}

All diagnostics were applied to the non-reactive flow in order to describe the local flow properties present in the burner at the moment of ignition.

- Phase Doppler anemometry (PDA):

Droplet size and velocity were measured by a commercial PDA system (DANTEC) operating in FIBER mode. An argon laser provided green $(514.5 \mathrm{~nm})$ and blue $(488 \mathrm{~nm})$ beams. Beam spacing was $50 \mathrm{~mm}$; transmitting and receiving lenses focal lengths were $350 \mathrm{~mm}$ and $310 \mathrm{~mm}$, respectively. The off-axis angle of the receiving optics was $50^{\circ}$ (in front scattering position), which is close to the Brewster angle. Laser energy before beam separation was $2 \mathrm{~W}$. The used aperture mask allows a detection diameter range of $139 \mu \mathrm{m}$. The measurement volume can be approximated by a cylinder of $120 \mu \mathrm{m}$ in diameter and $200 \mu \mathrm{m}$ in length. Data sampling was limited to 40,000 droplets or to $30 \mathrm{~s}$ of measuring time, allowing converged statistics of size-classified data. Due to the spray structure and particle concentration distribution, the measurements were not possible below $z=10 \mathrm{~mm}$. The gain and voltage in the photomultipliers (PMs) were adjusted so as not to saturate the anode currents in order to correctly detect the dispersed phase. The carrier phase velocity measurements were performed by seeding the air with $2 \mu \mathrm{m}$ olive oil droplets and increasing the gain and voltage in the PMs. Provided that the PDA system was 2D, only two velocity components (in the perpendicular direction to the interference fringes) could be measured simultaneously. PDA data are presented here through statistical estimators and the spray properties are assumed to be of rotational symmetry. Therefore, one radial profile reveals the axial and radial components of the velocity field and a second radial profile (perpendicular to the first one) reveals the axial (again) and azimuthal components.

- High-speed particle image velocimetry (HS-PIV):

It is also important to analyse the instantaneous airflow velocity fields given that the processes that are analysed in this paper are of a transitory and stochastic nature. For this purpose, the carrier phase was seeded far upstream with $<2 \mu \mathrm{m}$ zirconium oxide $\left(\mathrm{ZrO}_{2}\right)$ particles. Particle image velocimetry was applied on the airflow without spray presence at a $5 \mathrm{kHz}$ repetition rate on the $\mathrm{RZ}$ plane (vertical cut) with a magnification factor of $0.05 \mathrm{~mm} / \mathrm{px}$. A double cavity $527 \mathrm{~nm}$ Nd:YLF laser (Darwin Dual Quantronix) and a Phantom V2512 high-speed camera were used. The time between laser pulses was set to $7 \mu$ s enabling to correctly capture the wide range of velocities present in the flow and preventing the seeding particles from leaving the interrogation plane between pulses due to perpendicular velocity components (azimuthal). The confinement windows increase the complexity of the experiments enabling a short acquisition time before becoming covered by dust. 5000 raw single images were selected to calculate 2500 instantaneous vector fields. Raw images were normalised by their corresponding mean images to reduce any spatial energy variations and background scattering. The $1280 \times 800 \mathrm{px}^{2}$ images were processed with Dynamic Studio from DANTEC using the Adaptive PIV calculation, with initial and final interrogation windows of $64 \times 64$ and $16 \times 16 \mathrm{px}^{2}, 50 \%$ of overlap and a maximum iteration number of 10, yielding 159x99 vector fields. The signal-tonoise ratio acceptance was set over 1.5 and a $5 \times 5 \mathrm{px}^{2}$ median validation was applied. Post-processing with Matlab 
enabled to further analyse the data. The mean velocity field and the total strain rate field (instantaneous and mean) were calculated.

- Planar laser induced fluorescence (PLIF) on toluene:

Local equivalence ratio is a very important parameter in the ignition process. Its characterisation is, indeed, vital in order to understand the mechanisms that govern ignition. At these lean-mixture configurations slight equivalence ratio variations imply very big variations of the minimum ignition energy (MIE) [13]. PLIF on a tracer enabled to measure the molar fraction of fuel vapour and calculating the local gaseous equivalence ratio in the chamber. Toluene was selected as a tracer of the fuel vapour as it presents a boiling point $(383.75 \mathrm{~K})$ close to that of $n$ heptane $(371.57 \mathrm{~K})$. Figure 3 presents the experimental setup. Toluene was excited with a laser sheet at $266 \mathrm{~nm}$ by a quadrupled Nd-YAG laser at a repetition rate of $10 \mathrm{~Hz}$. The fluorescence light was collected by an intensified camera (EMICCD PIMAX 4) equipped with two $275 \mathrm{~nm}$ high-pass filters (ZUL275 x $1 \mathrm{~mm}$ ) and a $280 \mathrm{~nm}$ high-pass filter (WG280 x $3 \mathrm{~mm}$ ), so as to reduce the signal from droplets. The tracer was mixed at $2 \%$ in volume in the $n$ heptane reservoir.

Toluene is very sensitive to $\mathrm{O}_{2}$-quenching and its fluorescence signal $\left(\mathrm{S}_{\mathrm{f}}\right)$ varies with temperature. For this reason, a preliminary study of the dependence of $S_{f}$ on temperature variations (dT) was performed by varying the amount of $\mathrm{O}_{2}$ injected in the chamber. It was found that, for the nominal gas temperature $(\mathrm{T}=416 \pm 2 \mathrm{~K})$, a configuration with $1 \% \mathrm{O}_{2}$ and $99 \% \mathrm{~N}_{2}$ in mass (in the co-flow) nullifies the dependence of $\mathrm{S}_{\mathrm{f}}$ on $\mathrm{dT}$ for $\mathrm{dT}<15 \mathrm{~K}$. This agrees with the analysis performed in [14]. Therefore, the air inlet conditions for this experiment were modified to $0.4 \mathrm{gs}^{-1}$ of air and $7.8 \mathrm{gs}^{-1}$ of $\mathrm{N}_{2}$. In this way, $\mathrm{S}_{f}$ only depends on the molar concentration of toluene and, making the hypothesis that the fuel evaporates at the same rate under the new configuration, it provides useful information on the fuel vapour repartition. Raw images were normalized by a laser sheet mean image, recorded at the same flow conditions at the top part of the burner where droplets are no longer present. The mean noise image was subtracted from the raw images. 500 noise images were recorded at both burner locations; 1000 laser sheet images were acquired at the top part and 3000 raw images of the spray region (bottom) were acquired. This was performed on a $43 \times 43 \mathrm{~mm}$ field comprising one half zoom of the chamber. The laser sheet mean image is a reference of the molar concentration of toluene present in the fully evaporated homogeneous fuel-gas mixture. This allows performing the calculation of the fuel mixture fraction and of the equivalence ratio in the corrected images.

- Ignition probability exploration:

Ignition in the burner was addressed using a probabilistic approach. The mixture was ignited by a laser-induced spark in a selected location inside the burner using a focused $532 \mathrm{~nm}$ laser beam. A convergent lens of $200 \mathrm{~mm}$ focal length is used. The experimental setup is similar to the one used in [8]. The energy of the laser pulse was controlled and adjusted through the use of a coated variable dielectric attenuator (Laser Optik, Ref. 1Q2) without modifying the Q-switch delay in order to keep the physical beam properties constant. A chopper was used to operate in a single-shot mode. The deposited laser energy was around $405 \mathrm{~mJ}$. This high value is necessary to ensure a full dynamic range of ignition probability within the domain, due to very lean operating conditions $(\phi=0.61)$. The energy deposited in the spark was obtained from the measurements of the reference and transmitted laser pulse energies with two precision laser pyroelectric energy meters (Ophir: PE-25). The spark position was varied through a mesh of 132 interrogation points. For each spark position, 30 independent ignition tests were performed and ignition probability in each point was calculated dividing the number of successful events by the total test number. To make the experiment repeatable, it was crucial to keep the wall temperature constant. For this purpose, a thermocouple constantly measured the inner wall temperature ( $T=385 \mathrm{~K}$ in stable conditions with non-reacting flow). A Labview program permitted precise a control of the parameters and automatization of the process, triggering a spark only when the nominal conditions were reached. Time between sparks was $~ 20$ s.

\section{Results and discussion}

\section{Air velocity}

PDA data was post-processed to extract the mean components of the gaseous velocity field, the liquid Sauter mean diameter and size-classified velocity field. In this section, the mean gaseous velocity field is presented and compared to mean data from PIV measurements. The PIV instantaneous velocity fields are presented in second place.

- Mean velocity profiles (PDA vs PIV):

Figure 2 shows the axial (left), radial (middle) and azimuthal (right) mean velocity components of the airflow. Black dots represent the airflow velocity without spray presence (by PDA) and green triangles represent the airflow velocity with spray presence (by PDA). The PIV mean velocities (axial and radial) are stressed by the orange line. The global observation of the figure reveals that there is a very good agreement between all plots. This means that the spray induces minor modifications in the flow motion. A closer look at the axial velocities shows two peaks at $\mathrm{r}=10 \mathrm{~mm}\left(\sim 45 \mathrm{~ms}^{-1}\right)$ and central negative velocities $\left(\sim-23 \mathrm{~ms}^{-1}\right)$ due to the inner recirculation zone (IRZ), typical 
from swirling flows. Maximum velocities are observed at low axial stations $(\mathrm{z}=10 \mathrm{~mm})$ and relax downstream with a widening of the air jet. At $z=45 \mathrm{~mm}$, axial velocity ranges from -7 to $16 \mathrm{~ms}^{-1}$. Radial velocities present much lower values with maximum peaks of $\sim 10 \mathrm{~ms}^{-1}$ at $\mathrm{z}=10, \mathrm{r}=10 \mathrm{~mm}$, describing the lateral opening of the jet. Values show the same type of downstream relaxation. The outer recirculation zone (ORZ) is captured at $z=10 \mathrm{~mm}$ for $r>15 \mathrm{~mm}$ with small centripetal values. Azimuthal velocities show a narrow peak of $33 \mathrm{~ms}^{-1}$ at $z=10, r=8 \mathrm{~mm}$ that decreases to $10 \mathrm{~ms}^{-1}$ at $z=45, r=20 \mathrm{~mm}$. PIV measurements show a good agreement with PDA data.
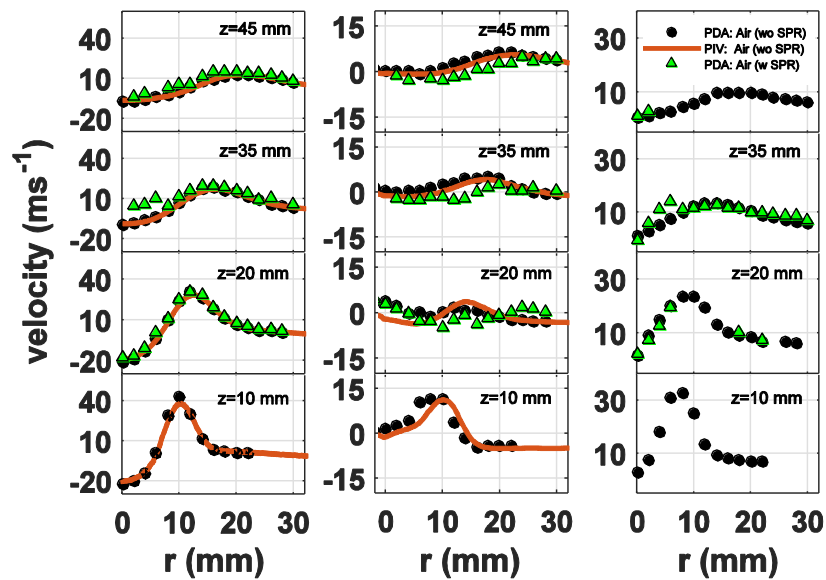

Figure 2. Mean components of air velocity. Left: axial; middle: radial; right: azimuthal. Black and green markers: PDA data without and with spray presence, respectively. Orange lines: PIV data without spray presence.

- Instantaneous velocity fields (PIV):

Instantaneous snapshots of the velocity field reveal more information of the gaseous behaviour. Figure 3 shows the vectors of three temporally independent instantaneous velocity fields. The colour map represents the total instantaneous strain rate $(\sigma)$ of the flow calculated from the 2D strain rate tensor, as shown in Fig.3. The real strain rate would take into account the third velocity component, but 2D data already adds some valuable information. Shearing structures are mostly generated in the highly turbulent region at the exit of the air duct, at low axial stations, where high velocities meet with movements in different directions (IRZ and ORZ). Some of these structures are transported by a global flow motion downstream and can be found at different points in the entire domain. The ORZ is generally free of high strain rates. Velocity fluctuations from one instant to another result in variations in the size of the IRZ and ORZ (i.e.: A vs C). Changes in velocity magnitude of the main jet structure can be also observed (i.e.: A vs B). These variations introduce significant differences in the behaviour of passive scalars present in the flow and in ignition mechanisms.

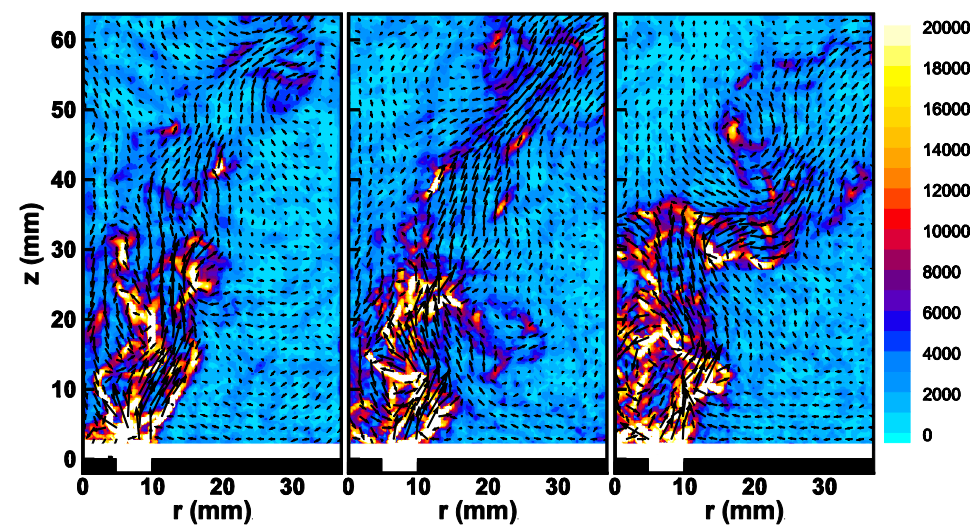

$$
\begin{gathered}
E_{i j}=\frac{1}{2}\left(\partial_{j} v_{i}+\partial_{i} v_{j}\right) \\
\sigma=\left(\sum E_{i j}^{2}\right)^{1 / 2}
\end{gathered}
$$

Figure 3. Instantaneous air velocity fields from PIV. Colours represent the total strain rate $\left(\mathrm{s}^{-1}\right)$.

\section{Fuel droplet size and velocity}

\section{- Sauter mean diameter and droplet distribution:}

The fuel spray presents a complex heterogeneous distribution, with droplet sizes ranging from 2 to $80 \mu \mathrm{m}$. The Sauter mean diameter (SMD) (Fig.4 (left)) takes values around $30 \mu \mathrm{m}$ and shows higher values at the borders of the spray $(\sim 35 \mu \mathrm{m})$ and lower values at the centre $(\sim 20 \mu \mathrm{m})$. This tendency is reproduced for all axial stations. The 
droplets are expelled from the atomizer with a strong radial component, forming a hollow cone of $80^{\circ}$ opening. Big droplets have ballistic trajectories whereas small droplets follow better the carrier flow motion, entering in the recirculation zones (IRZ and ORZ). This explains the lower SMD observed at the centre and at $Z=10 \mathrm{~mm}, \mathrm{r}>15 \mathrm{~mm}$. Figure 4 (right) presents the presence of droplets in the domain by the number of droplets detected per second by the PDA. A void can be observed at the centre, confirming the hollow nature of the cone. All size classes are distributed in the same way, meaning that a heterogeneous size mixture is present everywhere. The predominant size-classes are [10-20] and [20-30] $\mu \mathrm{m}$.
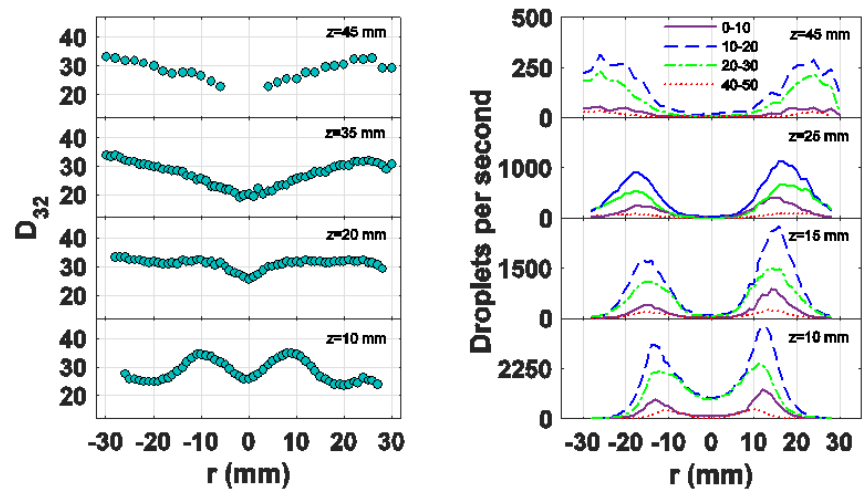

Figure 4. Left: Sauter mean diameter of the spray droplets. Right: droplet detection frequency by size classes.

\section{- Size-classified velocity:}

The three mean velocity components are shown in Figure 5 for three different size classes: [0-10], [20-30] and [4050] $\mu \mathrm{m}$. Values for the last group are not shown in the central region above $z=15 \mathrm{~mm}$ because less than 100 droplets were detected during the measurements. When comparing Figure 5 to Figure 2, it can be noticed that [0-10] $\mu \mathrm{m}$ droplets (named small droplets hereafter) follow more closely the airflow velocity field than [40-50] $\mu$ m droplets (named big droplets hereafter). The main difference with the air velocity is observed for the mean radial velocity profiles. Spray droplets are ejected from the nozzle with strong radial velocities. At the first axial station, the three groups have important radial velocities $\left(\sim 30 \mathrm{~ms}^{-1}\right)$ but farther downstream small droplets adapt their velocity to the airflow. Big droplets follow more ballistic trajectories and continue to present important radial centrifugal velocities until $z=35 \mathrm{~mm}$, where they nearly join the other groups. When overlapped to the air stream, droplet axial and azimuthal mean velocities are smaller for the spray than for the air, so small droplets are strongly accelerated by the co-flow while big droplets continue flying at smaller velocities until farther downstream. When fuel droplets exit the high air velocity region, the opposite occurs: they find a quiescent area where they still conserve a high axial velocity. This is more remarkable at low axial stations and for big droplets, and it is also observed for the azimuthal component to a lesser extent. Differences of more than $20 \mathrm{~ms}^{-1}$ are observed between the [0-10] group and the [4050] group.
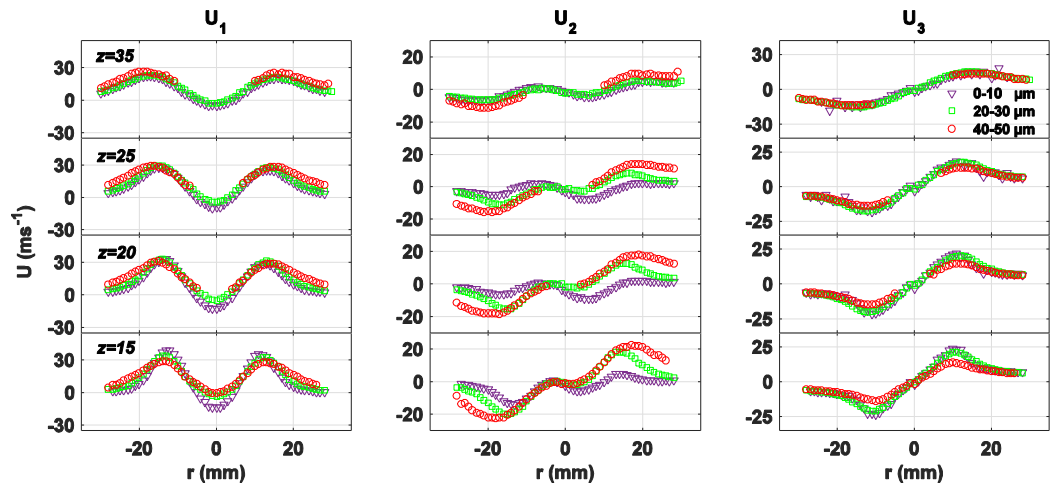

Figure 5. Mean components of fuel droplet velocity separated into three size classes. Left: axial; middle: radial; right: azimuthal.

The other size-classes present the same trends, but they are not presented here so as to facilitate the comprehension of the figure. The behaviour of [50-60] and [60-70] groups is very close to the [40-50] group, but, as they contain less droplets, they are not presented. During their trajectories, droplets experience important slip velocities, which certainly control the evaporation process and, therefore, vapour production and local air-to-fuel 
ratio. Slip velocities correspond to the relative velocities between droplets and the airflow. They are a key parameter in two-phase evaporation and combustion.

\section{Local gaseous equivalence ratio}

Toluene-PLIF corrected images represent the local equivalence ratio in the burner. A non-linear diffusion filter presented in [15] was applied on them so as to delete incoherent fluctuations at high spatial frequency coming from the camera noise. Figure 6 (left) shows an instantaneous filtered equivalence ratio image. Fuel droplets can be observed on the image. Indeed, there is a much higher tracer concentration in the liquid phase than in the gaseous phase. It was verified that $S_{f}$ has a linear response for gaseous equivalence ratios of $\phi<4.3$ and, through a calibration curve, this enabled to determine a threshold on top of which pixel values are rejected. These pixels and their neighbours are coloured in black on the image and are not taken into account for the further calculations, provided that the objective is to characterize the gaseous equivalence ratio. Some interesting observations can be extracted from Fig.6 (left). The droplet jet is coloured in red and big droplets in black. Droplets are expelled away from the nozzle at an angle to exit the image at the top right corner. Fuel droplets and high equivalence ratio zones near droplets are organized in the flow following a curvy pattern and around rotational structures in 1, 2 and 3, recalling, indeed, the airflow patterns observed in Fig.3. This type of preferential segregation was investigated in [16]. The core of the eddies in 1, 2 and 3 reveals low equivalence ratio values (below flammability limits $(\phi \sim 0.555$ [17])). Next to the dense spray, the airflow entrance can be observed in black and dark blue $(\phi \sim 0)$. The ORZ presents a homogeneous equivalence ratio close to the global equivalence ratio $(\phi \sim 0.61)$ whereas the IRZ shows lower values (below flammability limits sometimes). Figure 6 (middle) shows the mean equivalence ratio calculated from 3000 instantaneous images and reveals a similar $\phi$ distribution with a stoichiometric iso-line surrounding the mean spray branch. Points named A to $F$ represent single-pixel probes used to extract $\phi$ PDFs in the 3000 instantaneous images. Histograms in Fig.6 (right) show the equivalence ratio PDFs at the probe positions. The IRZ shows an increase in equivalence ratio from $A$ to $B$. The majority of the PDS at point $A$ is beyond the flammability limits. Both points in the ORZ reveal constant values of $\phi$ inside the flammability limits. In contrast to these narrow histograms, PDFs in the spray branch range from $\phi \sim 0.5$ to $\phi \sim 4$ due to the alternation of rich and lean structures. As the probe moves towards the spray core ( $F$ to E), PDFs become wider and richer because the fuel is more concentrated.
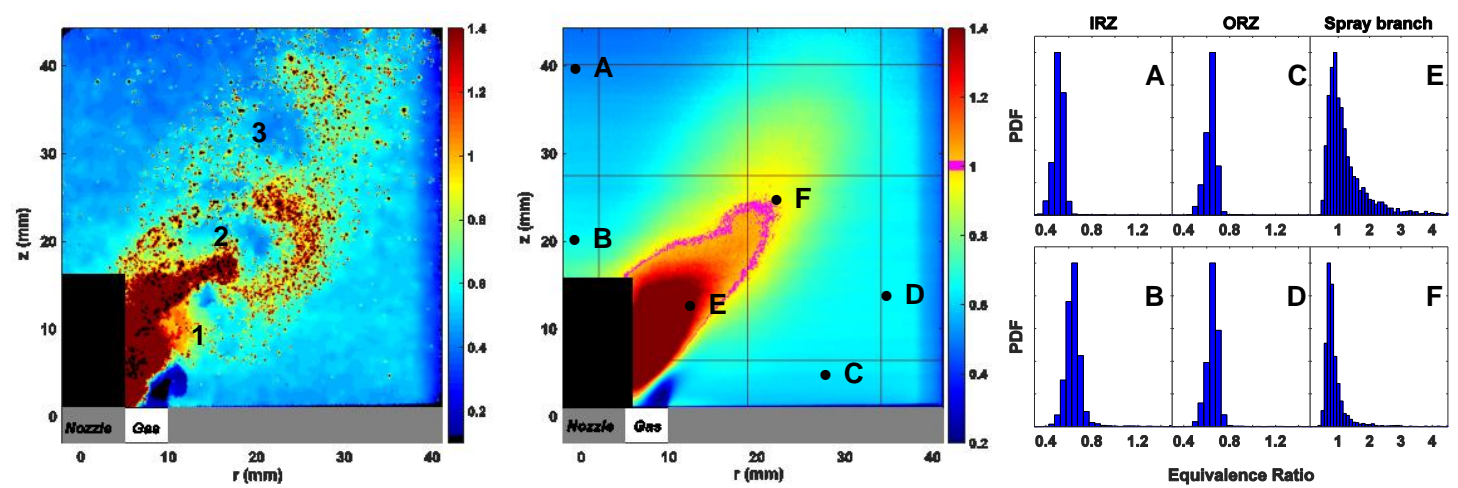

Figure 6. Left: instantaneous equivalence ratio image. Middle: mean equivalence ratio image. Right: Equivalence ratio PDFs at different probe positions ( $\mathrm{A}$ to $\mathrm{F}$ marked on the mean image).

The flammability factor $(F)$ is defined as the probability of finding a mixture between the low and high flammability limits $(0.555<\phi<2.008$ for $n$-heptane, calculated from [17]). Indeed, it corresponds to the area of the PDFs between these values. The reader can refer to the review done in [18]. The flammability factor was calculated for each pixel and it is presented in Fig.7 (left). It is here where the ORZ clearly stands out as the best ignitable zone, with $F$ values very close to 1 , meaning that the mixture is always ignitable here. The IRZ shows much lower values except near the spray $(z<20 \mathrm{~mm})$. The dense spray core and the air inlet present very low or zero $F$ and the mean spray branch can be identified as a region of lower $F$ than the ORZ.

Ignition probability

Ignition probability was evaluated in function of the spark location through the laser-induced ignition experiment. Figure 7 (right) indicates by black-magenta iso-contour lines the ignition probability map of the combustion chamber. Colour maps represent the turbulent kinetic energy $k$ and the velocity magnitude $|\mathrm{V}|$ of the airflow locally. The ignition probability map reveals that ignition becomes an impossible task in the centre of the burner (globally for $\mathrm{r}<20 \mathrm{~mm}$ ). There is a steep growth in probability from $r \sim 20$ to $r \sim 30 \mathrm{~mm}$ to reach a plateau of of $\sim 70$ and $\sim 80 \%$ in the ORZ close to the walls. Lean conditions ( $\phi_{\text {global }} \sim 0.61$ ) make ignition a very complicated task because the initial flame kernel is very vulnerable to any variations of the local flow properties and, depending on its initial location, it 
will be generated under very different flow conditions. The detailed description of the flow in the chamber reveals a very strong variation of local properties. This directly determines the trajectory of the flame kernel and whether it will survive or not. An analysis of the influence of the local flow properties is now presented.
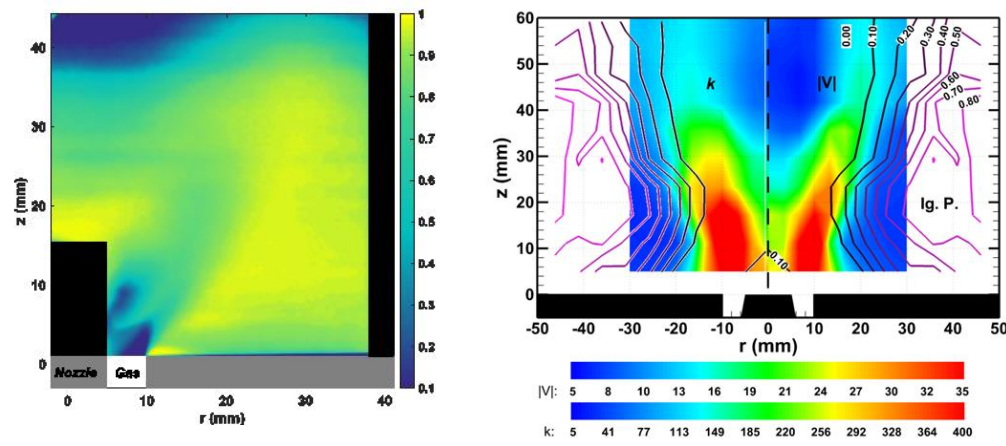

Figure 7. Left: Flammability factor. Right: Ignition probability map (lines); airflow turbulent kinetic energy " $k$ " $\left(m^{2} s^{-2}\right)$ and airflow velocity magnitude $|\mathrm{V}|\left(\mathrm{ms}^{-1}\right)$.

\section{- Comparison to local flow properties:}

During the kernel's initial life, its surrounding environment will quickly determine its properties (size, shape, wrinkling, reaction rate, temperature, growth rate, movement, etc.) and strongly condition the probability of establishing a stable flame on the injector. The trajectory of the flame kernel also has an important impact on ignition probability since it forces the kernel to change location, hence, flow properties. The cartography of ignition probability shown in Fig.7 (right) presents a very high correlation with the velocity magnitude $|\mathrm{V}|$ and the turbulent kinetic energy $k$, being the shape of the probability iso-lines the same as those of $|\mathrm{V}|$ and $k$. Ignition probability appears to be zero where velocity magnitude reaches $\sim 20 \mathrm{~ms}^{-1}$ and where $k$ reaches $\sim 250 \mathrm{~m}^{2} \mathrm{~s}^{-2}$, and it is very high when $|\mathrm{V}|$ and $k$ are very low. This is true for the $O R Z$ whereas the IRZ is not ignitable. Moreover, black-magenta iso-lines describe a typical ORZ shape. The flammability factor map explains why the IRZ has zero ignition probability: $F$ values are too low or reach acceptable values (at low axial stations) where $k$ starts to grow. The stoichiometric line in the mean equivalence ratio image (Fig.6 (middle)) shows no correlation with ignition probability because histograms in E and F (Fig.6 (right)) and the instantaneous $\phi$ (Fig.6 (left)) reflect the importance of the instantaneous mixture distribution. The latter demonstrates the stratified nature of the present lean mixture. When sparking in the spray branch region, local $\phi$ can be $\sim 0.5$ once and $\sim 4$ in the following instant of time, while the $O R Z$ always presents constant $\phi$ values. Figure 4 (right) shows that very few fuel droplets are still present beyond $r \sim 30$ $\mathrm{mm}$, where ignition probability grows. In fact, fuel droplets have antagonist effects on the kernel. On the one hand, they intensely supply fuel vapour elevating the equivalence ratio near the flame, thus, elevating laminar flame speeds, heat release and flame temperature (which is positive in a globally lean mixture). In contrast, fuel droplets are below their boiling temperature $(371.53 \mathrm{~K}$ for $n$-heptane) and evaporation implies a cooling of the fuel. This has a negative impact on the system, lowering the flame temperature by boosting heat dissipation from the flame front and from the burnt gases. In this sense, small, pre-evaporated droplets would perturb the flame to a lesser extent. The flame kernel is very vulnerable to strain too. Shear and vorticity affect the flame stretch and wrinkling. When the kernel enters a region with too much shear stress during its propagation, it is ripped and divided into smaller pieces. This can lead to misfire if the kernel is still too small due to an increase in surface and heat losses. Figure 3 reveals high strain rates in regions with low ignition probability. The ORZ is generally free of shearing structures, which are distributed along the main air jet trajectory in the surroundings of the ORZ. For $z>30$ and $r>20 \mathrm{~mm}$, high strain rates alternate with lower strain rates along the main jet. If the spark is triggered near a high strain rate location, the initial kernel may be suddenly teared until extinction or into several pieces. A second spark in the same location an instant later will possibly not find this shearing structure and ignition will be possibly successful. This explains that in regions with alternating properties ignition probability presents high variability. When a flame kernel is initiated in the ORZ, free from shearing flow movements, it will have the time and opportunity to develop and grow robust enough to support future strains and local extinctions.

\section{Conclusions}

Spray ignition was investigated under ultra-lean conditions through experiments to determine ignition probability against spark location (laser-induced) inside the swirled, confined CORIA Rouen Spray Burner. Local flow properties were characterised by advanced laser techniques. Phase Doppler anemometry (PDA) measurements informed on fuel droplet size, distribution and velocity and on airflow velocity. High-speed particle image velocimetry 
(PIV) enabled the description of the instantaneous gaseous velocity field and comparison to the mean data from PDA. Planar laser induced fluorescence (PLIF) on toluene as a tracer of $n$-heptane was used to characterise the local gaseous equivalence ratio throughout the burner.

The flammability factor $(F)$, extracted from the equivalence ratio images, reveals that the ORZ presents good ignitable properties, being $F \sim 1$ here. The ignition probability map, in accordance to $F$, shows that the ORZ is the best region to start a flame kernel in the present configuration, with $\sim 80 \%$ of success. The IRZ, on the opposite, is not appropriate to start ignition. Probability iso-lines reveal a steep increase at the interphase between the strong air jet and the start of the ORZ. A very high correlation is observed between the ignition probability and the air velocity and turbulent kinetic energy, finding high probabilities for low velocities and low turbulent kinetic energies. Equivalence ratio images describe the stratification of the mixture and explain why the IRZ is non-ignitable. Instantaneous PIV air velocity fields enable the calculation of local strain rates that also explain the survival or extinction of flame kernels, being the ORZ globally free of high shearing structures. Fuel droplets are mainly present in the spray branch and are rare in the IRZ and ORZ. The present work provides new insight on spray ignition and a detailed description of the local flow properties in a swirled confined jet-spray burner in non-reactive conditions. It is a useful database for industry and for the initialisation and validation of numerical simulations.

\section{Acknowledgements}

The authors acknowledge financial support from ANR under the project TIMBER ANR-14-CE23-0009.

\section{References}

[1] Marchione, T., Ahmed, S. F., and Mastorakos, E., 2009, "Ignition of turbulent swirling n-heptane spray flames using single and multiple sparks," Combustion and Flame, 156(1), pp. 166-180.

[2] Moesl, K. G., Vollmer, K. G., Sattelmayer, T., Eckstein, J., and Kopecek, H., 2009, "Experimental Study on Laser-Induced Ignition of Swirl-Stabilized Kerosene Flames," Journal of Engineering for Gas Turbines and Power, 131(2), pp. 021501-021508.

[3] Wandel, A. P., 2014, "Influence of scalar dissipation on flame success in turbulent sprays with spark ignition," Combustion and Flame, 161(10), pp. 2579-2600.

[4] Kao, Y.-H., Denton, M., Wang, X., Jeng, S.-M., and Lai, M.-C., 2015, "Experimental Spray Structure and Combustion of a Linearly-Arranged 5-Swirler Array," (56680), p. V04AT04A038.

[5] Cordier, M., Vandel, A., Renou, B., Cabot, G., Boukhalfa, A. M., Esclapez, L., Barré, D., Cuenot, B., and Gicquel, L., "Experimental and numerical analysis of an ignition sequence in a multiple-injectors burner, GT 2013 - 94681," Proc. Proceeding of the the ASME Turbo Expo.

[6] Prieur, K., Durox, D., Beaunier, J., Schuller, T., and Candel, S., 2017, "Ignition dynamics in an annular combustor for liquid spray and premixed gaseous injection," Proceedings of the Combustion Institute, 36(3), pp. 3717-3724.

[7] Machover, E., and Mastorakos, E., 2017, "Experimental investigation on spark ignition of annular premixed combustors," Combustion and Flame, 178, pp. 148-157.

[8] Cardin, C., Renou, B., Cabot, G., and Boukhalfa, A. M., 2013, "Experimental analysis of laser-induced spark ignition of lean turbulent premixed flames: New insight into ignition transition," Combustion and Flame, 160(8), pp. 1414-1427.

[9] Ahmed, S. F., Balachandran, R., Marchione, T., and Mastorakos, E., 2007, "Spark ignition of turbulent nonpremixed bluff-body flames," Combustion and Flame, 151(1-2), pp. 366-385.

[10] Shum-Kivan, F., Marrero Santiago, J., Verdier, A., Riber, E., Renou, B., Cabot, G., and Cuenot, B., 2017, "Experimental and numerical analysis of a turbulent spray flame structure," Proceedings of the Combustion Institute, 36(2), pp. 2567-2575.

[11] Verdier, A., Marrero Santiago, J., Vandel, A., Saengkaew, S., Cabot, G., Grehan, G., and Renou, B., 2017, "Experimental study of local flame structures and fuel droplet properties of a spray jet flame," Proceedings of the Combustion Institute, 36(2), pp. 2595-2602.

[12] Cordier, M., 2013, "PhD Thesis, Allumage et propagation de flamme dans les écoulements fortement swirlés : études expérimentales et numériques," CORIA, INSA de Rouen, France.

[13] Beduneau, J.-L., Kim, B., Zimmer, L., and Ikeda, Y., 2003, "Measurements of minimum ignition energy in premixed laminar methane/air flow by using laser induced spark," Combustion and Flame, 132(4), pp. 653-665.

[14] Rossow, B., 2011, "PhD Thesis, Processus photophysiques de molécules organiques fluorescentes et du kérosène applications aux foyers de combustion : applications aux foyers de combustion," Paris 11 , France.

[15] Xavier, P., Vandel, A., Godard, G., Renou, B., Grisch, F., Cabot, G., Boukhalfa, M. A., and Cazalens, M., 2016, "Investigation of combustion dynamics in a cavity-based combustor with high-speed laser diagnostics," Experiments in Fluids, 57(4), p. 50.

[16] Reveillon, J., and Demoulin, F.-X., 2007, "Effects of the preferential segregation of droplets on evaporation and turbulent mixing," Journal of Fluid Mechanics, 583, pp. 273-302.

[17] Neophytou, A., 2010, "PhD Thesis, Spark Ignition and Flame Propagation in Sprays," University of Cambridge, England.

[18] Mastorakos, E., 2009, "Ignition of turbulent non-premixed flames," Progress in Energy and Combustion Science, 35(1), pp. 57-97. 


\title{
Visualization of pilot flame of an optically-accessible coaxially- staged aero-engine lean-burn fuel injector
}

\author{
Kazuaki Matsuura*1, Shunya Uesaka², Tomoyuki Iwasaki², Yoji Kurosawa1, Hideshi Yamada', \\ Takeshi Yamamoto ${ }^{1}$ and Shigeru Hayashi ${ }^{2}$ \\ ${ }^{1}$ Institute of Aeronautical Technology, Japan Aerospace Exploration Agency, Japan \\ ${ }^{2}$ Department of Mechanical Engineering, Hosei University, Japan \\ ${ }^{*}$ Corresponding author: matsuura.kazuaki@jaxa.jp
}

\begin{abstract}
The visualization of the pilot flame of a coaxially-staged aero-engine lean-burn fuel injector, not only downstream but also inside of the pilot nozzle, was successfully performed at realistic aero-engine conditions. Optical access toward the inside of the nozzle was achieved through the inner and outer shrouds, both of which were made of transparent quartz. The image distortion caused by complex contours of the two shrouds was corrected by a method based on optical ray tracing, which realized precise determination of spatial intensity distribution of optical signals. Line-of-sight $\mathrm{OH}$ chemiluminescence, cross-sectional OH-LIF, kerosene LIF and kerosene Mie scattering were employed as diagnostic tools. The effects of pilot local air-to-fuel ratio on spray flame structure were revealed, both inside and downstream of the pilot nozzle under stable combustions. As the pilot mixture got rich, the main reaction zone moved from inside of the pilot nozzle to the region near the injector lip downstream of the injector exit. The OH-LIF signal was detected near the central axis surrounded by the fuel spray. It was also observed near the back-step of the pilot nozzle for the rich cases. The experiments under combustion oscillation were also conducted and the correlation of phenomena inside and downstream of the pilot nozzle was captured. It was clarified that the reaction enhancement in the outer part of the lip vortex region was caused by the convection of rich mixture, which appeared near the pilot atomizer lip at 150 210deg earlier oscillation phase angle.
\end{abstract}

\section{Keywords}

Aero-engine combustor, Optical burner/injector, Staged lean-burn injector, Laser induced fluorescence, Ray tracing, Optical refraction correction, Elevated temperature and pressure

\section{Introduction}

For reduction of NOx from aircrafts, coaxial-staging lean burn fuel injection systems have been studied intensively these days [1-15] and some of them have been already in service. Typically, they consist of a non-premixedmode pilot nozzle at the center for flame stability and a lean premixed-mode main nozzle surrounding the pilot for drastic NOx reductions. For such an injector, both fuel injection and fuel/air mixing start a few centimeter upstream from the injector exit (i.e. entrance of the combustion chamber). Moreover, this is also true for its pilot flame. Therefore, many important phenomena take place in regions enclosed by the metal walls of the injector, where the application of optical diagnostics is not straightforward compared with a conventional combustor.

In our previous work, visualization of fuel spray/vapor behavior and fuel/air mixing inside the main nozzle (i.e. the premixing duct) was performed by high speed visualization and/or LIF techniques $[10,11,15]$. Through a fullycontoured quartz-made transparent outer-shroud, dynamic motion of fuel sprays under combustion oscillations at an elevated pressure and temperature was clearly captured and discussed in relation to the flame dynamics downstream [15]. However, visualization of phenomena in the pilot nozzle has not been attempted yet.

In this work, the inner shroud of the staged injector, which was an annular metal block radially separating the pilot nozzle form the main, was also made of quartz. Therefore, the investigation of the pilot flame of the coaxial staged injector was made possible through both the inner and outer shrouds at realistic aero-engine conditions, which had been hardly found in published literatures to the authors' best knowledge. The present paper provides discussions on structures of spray flames both inside and downstream of the pilot nozzle at stable combustion conditions. In addition, the results on the correlation of the phenomena between these two regions under combustion oscillation are presented.

\section{Experiments}

Schematic drawings of the fuel injector are presented in Fig.1. Detailed descriptions of the coaxial-staging fuel injector can be found in Refs. [8,9]. The design of the pilot nozzle is briefly mentioned here for convenience of the discussions below. Two types of counter-rotating double-swirl airblast atomizers (for example, see Ref. [17]) were 
used, designated as U4D4 and U8. As for U4D4 type, liquid fuel (kerosene) is injected through four injection holes upstream and an annular exit at its atomizer lip. The liquid injected through the latter fuel circuit was metered by four grooves upstream of the exit to make annular liquid film as uniform as possible. The axes of the holes and grooves are inclined so that the liquid fuel is injected with swirling motions. As for U8, only upstream injection was employed through eight injection grooves and no injection at its atomizer lip. The U4D4 type is the standard design in our research project, whereas the U8 type was one of the prototype during the development. As mentioned later, the latter was used for study of combustion oscillation.
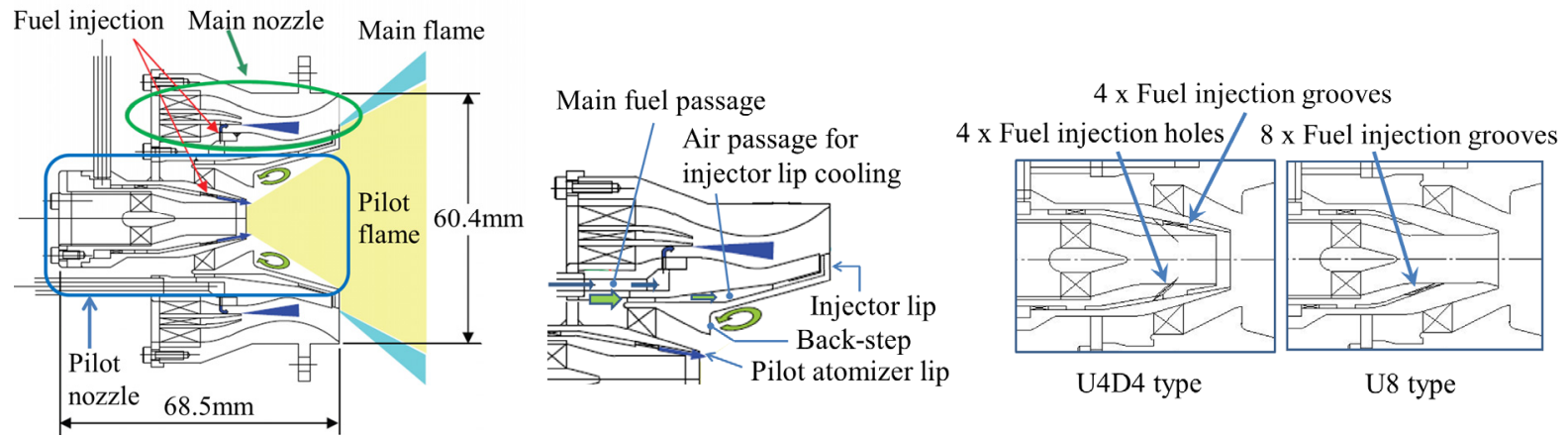

Figure 1. Staged fuel injector (Note the figure is for metal version with injector lip cooling).

The experimental setup is presented in Fig.2, which is similar to that used in our previous experiments $[11,15]$. The coordinate system used in this study is also shown in the figure, whose origin corresponds to the center of the exit of the fuel injector. For optical measurements, three optical accesses from top and sides were provided through quartz windows installed on the walls of the combustor liner and outer casing. The optical combustor has a square cross-section $(85 \times 85 \mathrm{~mm})$. For dynamic pressure measurements, a pressure transducer (Vibrometer CP211) was mounted on the sensor port on the lower wall of the combustor with a $28 \mathrm{~mm}$ recess. This enables introduction of cooling air for the sensor surface and also avoids its direct exposure to the flame.

To realize optical access toward the inside of the pilot nozzle, some modification was made from the original injector design. In addition to its outer shroud [15], its inner shroud was also partially made of transparent quartz. An air passage for the injector lip cooling and the main fuel passage in Fig. 1 were eliminated for simplification. Though this optical injector was originally designed to visualize the region of $z=-20.8 \sim-6.5 \mathrm{~mm}$, it was actually possible to observe phenomena close to the atomizer lip $(z \sim-22.8 \mathrm{~mm})$, owing to refraction effects. The region of $-6.5<z<1.5 \mathrm{~mm}$ is not visible because of the metallic heat shield.

The setup for visualization is shown in Fig.3, similar to Refs. [10-12,15] where its detailed descriptions are found. The laser sheet was parallel to the $y z$ plane and introduced from the top. Its thickness at the test section was approximately $0.5 \mathrm{~mm}$. In this study, the location of the laser sheet was fixed at the central plane $(x=0)$. The imaging optics was also similar to those in the references, except the CCD for Imaging optics A (PCO pixelfly

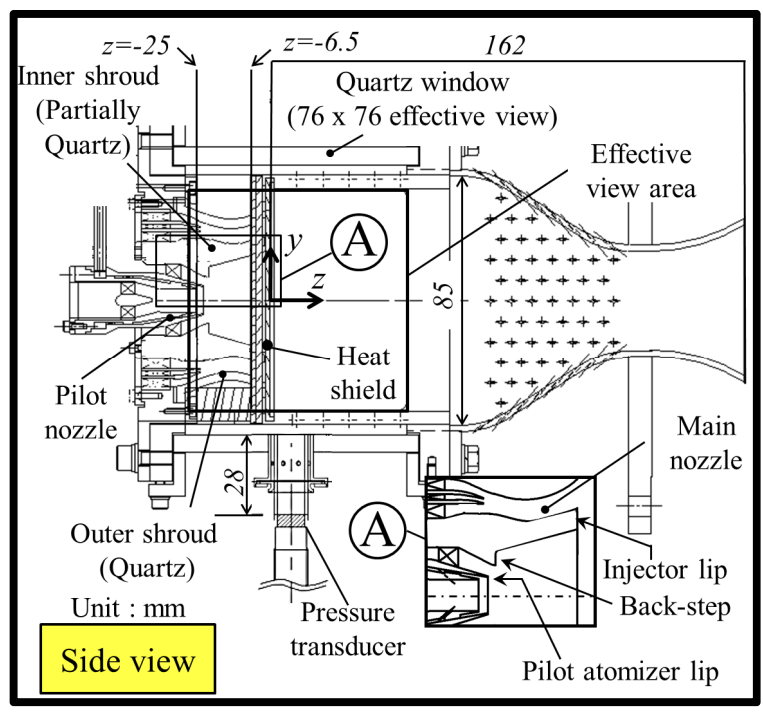

a) Optical combustor with optical injector.

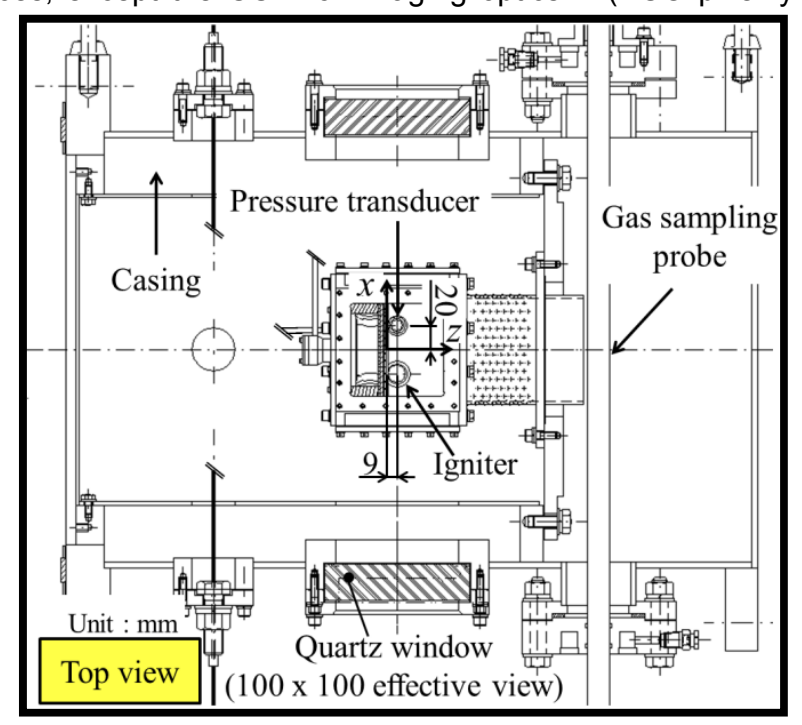

b) Combustor installed in test rig.

Figure 2. Schematic drawing of experimental setup. 
USB, 14bit). Imaging optics A could take images at higher acquisition rate $(10 \mathrm{~Hz})$ than imaging optics $\mathrm{B}(0.83 \mathrm{~Hz})$, but its image quality was not as good as that of the latter because the matching between relay-lens optics and the CCD was not optimized. They were originally used for simultaneous imaging, but not for the present experiments. Instead, only either of them was used depending on whether the image quality or acquisition rate was important. Images of $\mathrm{OH}$ chemiluminescence $\left(\mathrm{OH}^{*}\right.$, qualitative marker of heat release, location of reaction zones), OH-LIF (reaction zones and following burnt gas), kerosene-LIF (distribution of fuel in both liquid and gas phase, here tracing naphthalene-like molecules with two fused benzene rings,) and kerosene-Mie scattering (liquid phase fuel distribution) were taken by using appropriate optical band-pass filters. Those for $\mathrm{OH}^{*}, \mathrm{OH}-\mathrm{LIF}$, kerosene-LIF and kerosene-Mie scattering were CVI F10-307.1-3-2.00 (307.1nm, FWHM 10nm), Asahi Spectra MZ0310 (310nm, FWHM 10nm), Edmund \#84-108 (340nm, FWHM 29nm) and Asahi-Spectra MZ0280 (280nm, FWHM 10nm), respectively. The images of $\mathrm{OH}^{*}$ were those of line-of-sight, whereas the rests were those on the cross-sectional plane $(x=0)$. The signals for the laser trigger and camera acquisition timing were stored with the pressure oscillation signal by a multi-channel data recorder (TEAC, GX-1) at $100 \mathrm{kHz}$ sampling rate.

Experiments were conducted for two cases, Case A and B, as described in Table 1. Only pilot fuel was injected in all of the present experiments. Case $\mathrm{A}$ was to investigate the effects of pilot (local) air-to-fuel ratio $\left(A F R_{p}\right)$ on flame structures at stable combustion conditions, and Case B was to study dynamic behavior of the spray flame under combustion oscillation. For case $\mathrm{A}$, the variations of $A F R_{p}$ was carried out by changing the pilot fuel flow rate $\left(m_{f, p}\right)$ at a fixed inlet air condition. Here, $A F R_{p}$ was calculated using the ratio of effective opening areas between the pilot and the combustor in total, 110 and $760 \mathrm{~mm}^{2}$, respectively. A $10 \mathrm{~mm}^{2}$ reduction of the effective opening area of the combustor from the value in Ref. [15] was due to the elimination of the injector lip cooling. The inlet pressure, temperature, and combustor pressure loss for Case A were simulating a cruise condition of a target engine for small aircrafts $[8,9]$. Those of case B were close to its idle condition, but the inlet temperature was set about $40 \mathrm{~K}$ higher than the target value. This condition was a "trade-off" choice: The higher inlet temperature was, the better the image quality was, due to the less soot deposition to the transparent inner shroud, but the smaller amplitude of oscillation pressure it resulted in.

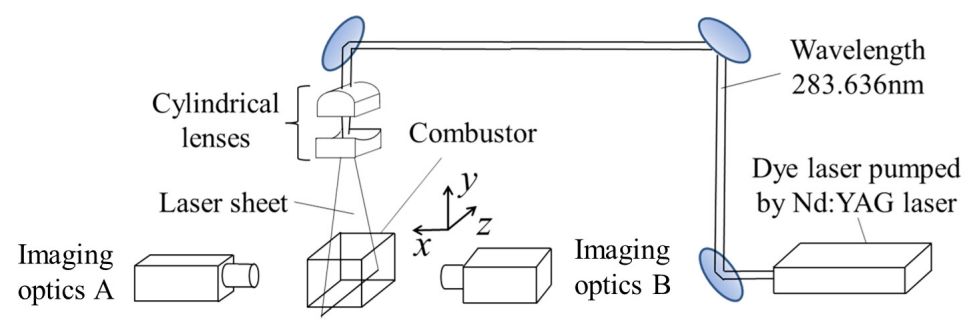

Figure 3. Optical setup.

Table 1 Test conditions.

\begin{tabular}{l|cc}
\hline \hline & Case A (Stable combustion) & Case B (Combustion oscillation) \\
\hline \hline Pilot nozzle type & U4D4 & U8 \\
Inlet pressure, $\mathrm{kPa}$ & 700 & 487 \\
Inlet temperature, $\mathrm{K}$ & 760 & 545 \\
Combustor pressure loss & $4 \%$ & $4 \%$ \\
Airflow rate in total $, m_{a}, \mathrm{~g} / \mathrm{s}$ & 316 & 259 \\
Pilot fuel flow rate, $m_{f, p}, \mathrm{~g} / \mathrm{s}$ & $1.52-5.44$ & 1.58 \\
Pilot air-to-fuel ratio, $A F R_{p}$ & $30.1-8.4$ & 23.8 \\
Imaging optics & B & $\mathrm{A}$
\end{tabular}

\section{Results and discussions}

A typical photograph of the pilot flame of the present staged-injector is presented in Fig. 4. The structure of the upstream part of the pilot flame is successfully revealed by means of the "optical staged-injector" developed for this study. The flame shows a conical structure, since the airflow is detached from the back-step of the pilot nozzle as illustrated in Fig. 1.

Image distortion is one of the serious problems on the visualization of phenomena inside the pilot nozzle. The complex contours of the inner and outer shrouds result in refractions of optical rays. To compensate this effect, a method was developed for optical refraction correction based on optical ray tracing. A pin-hole model was used to 


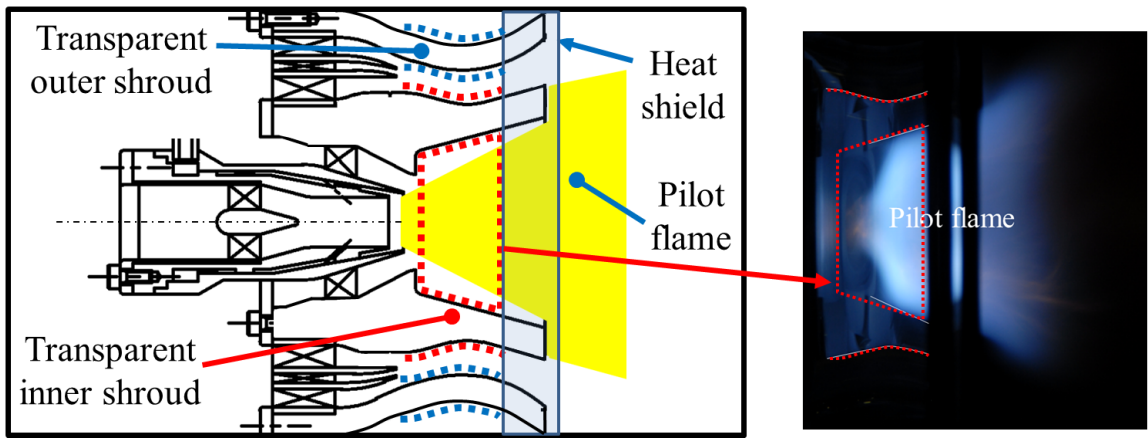

Figure 4. Visualization of flame in pilot nozzle and combustion chamber (Case A, $A F R_{p}=30.1$, Exposure time; 20ms).

model the imaging optics. The correction method is partially described in Ref. [16] with an example of its application to the visualization of fuel distribution inside the premixing duct (i.e. main nozzle) of the same injector. In this study, the same method was applied to the imaging of the pilot zone. The calculated degree of distortion in terms of displacements $(\Delta y, \Delta z)$ when imaging phenomena on a laser sheet at the central plane $(x=0)$ is presented in Fig. 5-a. Here, $\Delta y, \Delta z$ are defined so that the refraction correction was achieved by the transform of intensity distribution $I(y, z)$ to $I(y+\Delta y, z+\Delta z)$. The displacements were not calculated for regions without interests of this study, as masked by grey color. The importance of the correction is clear especially close to the pilot atomizer lip where the displacements are significantly large $(\Delta z>3.5 \mathrm{~mm})$. An original image for kerosene-LIF and that corrected by the present method are shown in Fig. 5-b. After the correction, the intensity distribution becomes realistic; for example, the upstream part of the fuel distribution attaches the pilot atomizer lip. The example shows that precise determination of spatial distribution of LIF signals can be realized by means of the present method.

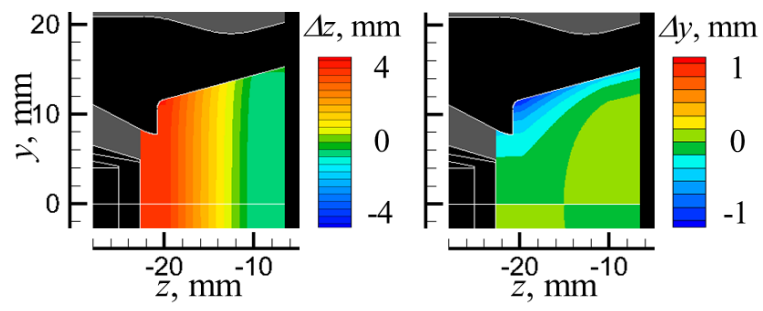

(a) Displacement caused by refraction
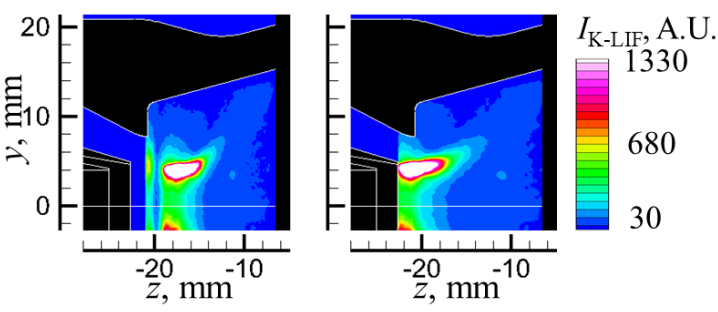

(b) kerosene-LIF intensity before(left) and after(right) correction

Figure 5. Optical refraction correction.

As mentioned in the following discussions, the interpretation of the results obtained from the present optical measurements is not always straightforward. Some items which should be taken into account for correct data interpretation are summarized in advance, in Table 2.

Spatial distribution of time-averaged intensity of line-of-sight $\mathrm{OH}$ chemiluminescence $\left(\mathrm{OH}^{*}\right.$-LoS), cross-sectional $\mathrm{OH}$-LIF, kerosene LIF and kerosene Mie scattering are presented in Fig.6 for Case A. Results for roughly upper half of the combustor $(y>0)$ are presented because the phenomena were nearly symmetric with respect to $z$ axis in a qualitative sense. The regions where the cautions are needed for data interpretation are indicated with the item numbers in Table 2. The structures of spray combustion field for two cases, a lean and a slightly rich ones $\left(A F R_{p}\right.$ $=25.1$ and 12.6) are summarized in schematic illustrations in Fig. 7. For convenience of explanation, reaction zones are separately numbered, (i) near the central axis and the atomizer lip close to the liquid spray, (ii) from middle and downstream part of the pilot nozzle extending to the injector lip region, (iii) close to the injector lip, (iv) from the injector lip extending to central region in the combustion chamber reflecting the inner recirculation zone (IRZ) flow structure, and (v) near the back-step.

For the leaner cases, the reactions in zones (i) (iii) are dominant, and they are in connection as seen in Fig. 6 $\left(\mathrm{OH}^{*}\right.$ and $\left.\mathrm{OH}-\mathrm{LIF}\right)$. OH-LIF signal is observed near the central axis surrounded by the fuel spray (designated as "A"). As $A F R_{p}$ decreases, zones (i) and (ii) look separated evidently ("B"), meaning the reaction is suppressed between the two. In this region, rich mixtures after evaporation exist, where the kerosene LIF signal is observed but the kerosene Mie is not. Thus, the separation is due to quick evaporation causing local temperature decrease due to its latent heat and too rich mixture, and both cause local suppression of reactions. On the other hand, reactions in the downstream part of zone (ii), and in zone (iii) are enhanced and still continue into zone (iv), as favorable mixture for combustion is established more downstream for low $A F R_{p}$ cases. For $A F R_{p}=12.6$, the 
Table 2. Items to be considered for data interpretation.

\begin{tabular}{|c|c|c|c|}
\hline Item No & Description & Location & Type of image \\
\hline 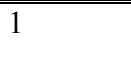 & Noises due to reflection and stray light & Close to walls and back-step & $\begin{array}{l}\text { All, especially } \\
\text { kerosene LIF }\end{array}$ \\
\hline 2 & $\begin{array}{l}\text { Noises due to reflection and stray light: } \\
\text { Correlated with signal intensity in combustion } \\
\text { chamber close to injector lip }\end{array}$ & $\begin{array}{c}\text { Downstream part of pilot } \\
\text { nozzle }\end{array}$ & OH LIF \\
\hline 3 & Insufficient cut-off by optical band-pass filter & $\begin{array}{l}\text { Near pilot atomizer lip where } \\
\text { kerosene is in liquid phase }\end{array}$ & $\mathrm{OH}$ LIF \\
\hline 4 & Transparency loss due to soot deposition & $\begin{array}{l}\text { Inside pilot nozzle, possibly } \\
\text { low } A F R_{p} \text { for Case } \mathrm{A} \text {, and } \\
\text { Case } \mathrm{B}^{* 1}\end{array}$ & All \\
\hline 5 & $\begin{array}{l}\text { Validity of correction of } \mathrm{OH} \text { chemiluminescence } \\
\text { line-of-sight image by applying displacement } \\
\text { values at the central plane }{ }^{\star 2}\end{array}$ & Inside pilot nozzle & $\begin{array}{l}\text { OH chemi- } \\
\text { luminescence }\end{array}$ \\
\hline
\end{tabular}

${ }^{*}$ For Case A, no soot deposition was observed for $A F R_{p}>18$ by visual inspection during the experiments. For $A F R_{p}<18$, direct observation was not reliable because of too bright background due to luminous flames so it was difficult to tell if it affects the signal quality. It should be noted that, at least, at $A F R_{p}=12.6$ for kerosene LIF experiment, its effect on the image is hardly observed. For Case $B$, the effect was apparent and must be considered for all the images.

${ }^{*}$ Present correction method should not be applied to line-of-sight images theoretically, but was still applied as the corrected images gave better qualitative information than the original images in practice.

kerosene vapor signal is detected even downstream of the injector exit ("C"). The $\mathrm{OH}^{*}$ signal overlaps it and the strong OH-LIF signal is observed its outside, behind the injector lip. This structure is caused by the lip vortex and some of the burned gas is trapped in this region. The existence of the lip recirculation zone (LRZ, [2]) was confirmed by our previous PIV measurements [13,14]. For further low $A F R_{p}$ cases (not shown), the location with OH-LIF signals in zone (iii) further moves towards outside with decreasing its intensity, as the mixture downstream of the injector becomes too rich. As for zone (v), the OH-LIF signal begins to be evident for $A F R_{p}=12.6$. This is mainly from that of burned gas. For rich cases such as for $A F R_{p}=8.4, \mathrm{OH}^{*}$ as a sign of heat release is detected ("D"), though its intensity is not strong.

The results for Case B are discussed in the followings. A typical pressure oscillation signal and its frequency spectrum after an adequate band-pass frequency filtering $(200-2500 \mathrm{~Hz})$ are shown in Fig. 8 . The number of FFT points was 8192 . The filtering was performed to remove low frequency noises mainly from the power supply line of the electric heater and its harmonics (especially 50 and $150 \mathrm{~Hz}$ ), and high frequency noises due to the acoustic resonance inside the pressure sensor mount port $(2800 \sim 4200 \mathrm{~Hz})$. The characteristic frequency of pressure oscillation is $390 \mathrm{~Hz}( \pm 8 \%)$.

The phase-averaged kerosene/OH LIF intensity distributions on $x=0$ plane are shown in Fig. 9 by line-contours and color plots, respectively. Here, the phase angle $\varphi$ is defined assuming that the pressure oscillation signals are in a sinusoidal shape, i.e., $p ; \sin (\varphi)$. The regions where the cautions are needed are again indicated with the item numbers. Even after the trade off choice of the test condition, the soot deposition (item 4 in Table 2) was still inevitable. Nevertheless, qualitative and semi-quantitative assessments were possible, providing information such as the correlation of phenomena inside and downstream of the pilot nozzle as described below: The phasedependent variations of the sum of LIF signals in some specified regions are presented in Fig. 10 as percentages of their average values. As shown in Figs. 9 and 10, the kerosene LIF intensity just downstream of the pilot atomizer lip (R1) shows its maximal value at $\varphi$ 330deg. This high concentration fuel parcel convects towards downstream at local flow speeds. Eventually, when assuming near-sinusoidal behaviour of fuel concentration variations and neglecting ripple-like structure of the curves in Fig. 10, the highest fuel concentration takes place at $\varphi \sim 0,60$, and $90 \mathrm{deg}$ for regions R2,R3 and R4 (z -13, -8 and 3mm), respectively. After the arrival of the high concentration fuel parcel, a reaction enhancement in the lip vortex region takes place. The rich mixture extends around the the lip vortex flow and encounters a flesh airflow from the main nozzle, which probably causes the reaction enhancement and apparent flash-back-like motion of the flame (designated as "A"). As a result, the $\mathrm{OH}$ LIF in the outer part of the lip vortex region (R5) shows its high values for $\varphi=120 \sim 180 \mathrm{deg}$, which is about 150 210deg later than the occurrence of the maximal fuel concentration in region R1. 

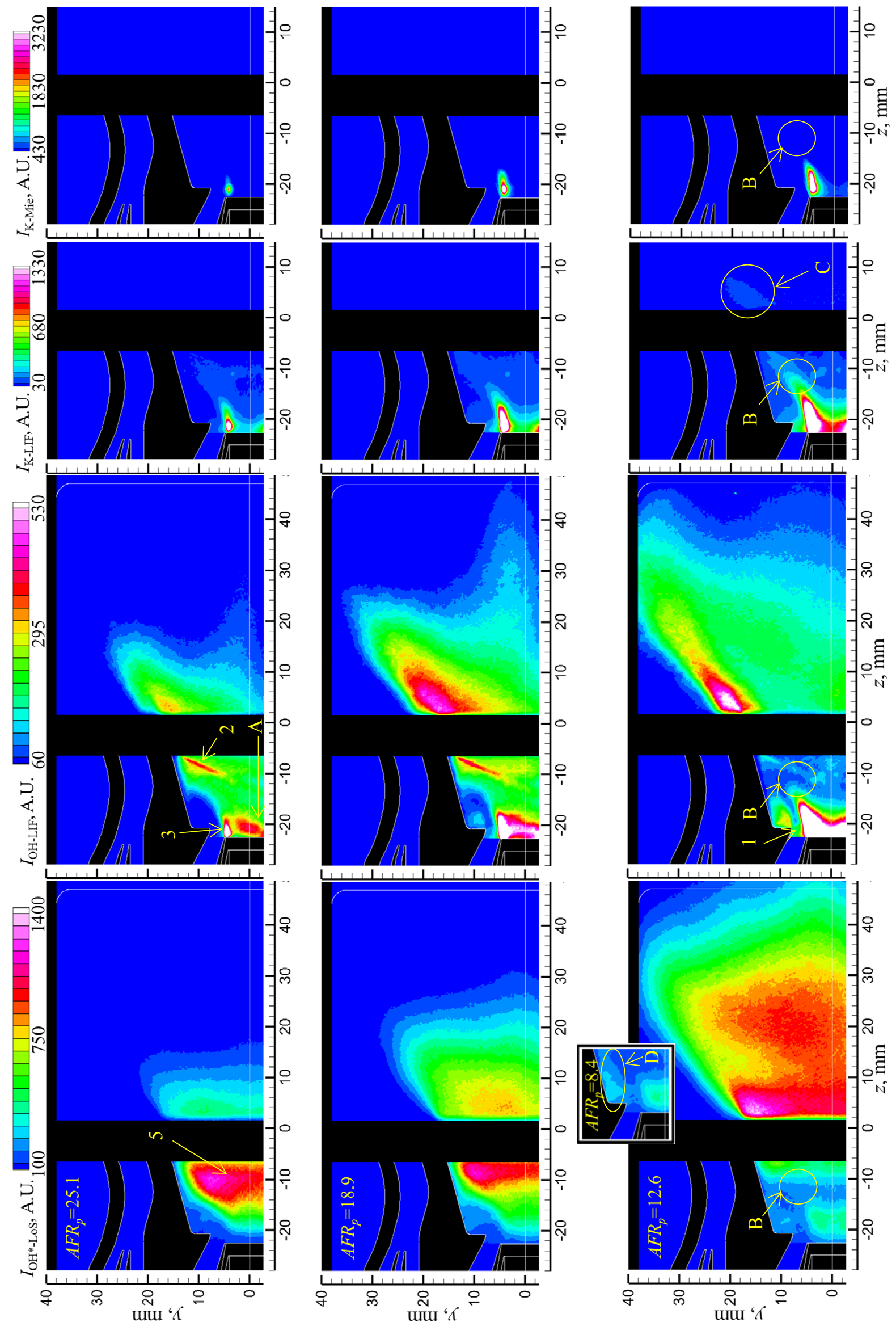

Figure 6. Spatial distribution of time-averaged intensity of line-of-sight $\mathrm{OH}$ chemiluminescence, cross-sectional OH-LIF, kerosene- LIF and kerosene Mie scattering on $x=0$ plane for Case $\mathrm{A}$ (note the figure is rotated).

This work is licensed under a Creative Commons 4.0 International License (CC BY-NC-ND 4.0).

EDITORIAL UNIVERSITAT POLITĖCNICA DE VALĖNCIA 


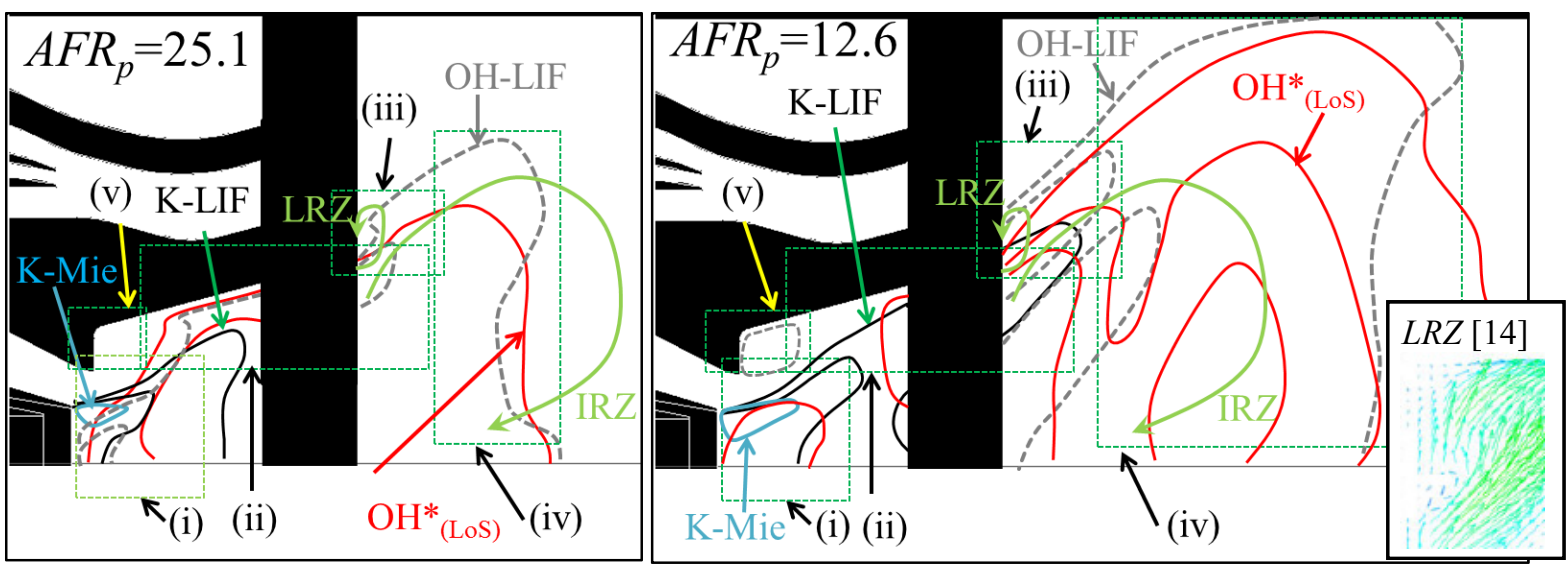

Figure 7. Schematic illustrations of pilot flame structure.
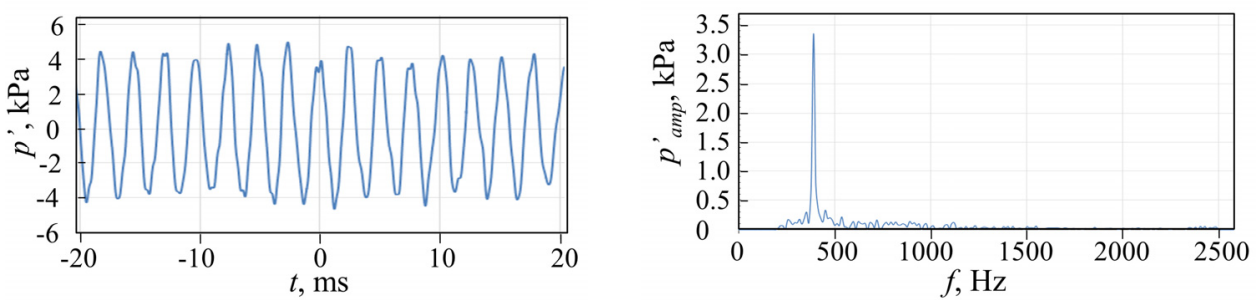

Figure 8. Examples of pressure oscillation signal and its frequency spectrum.

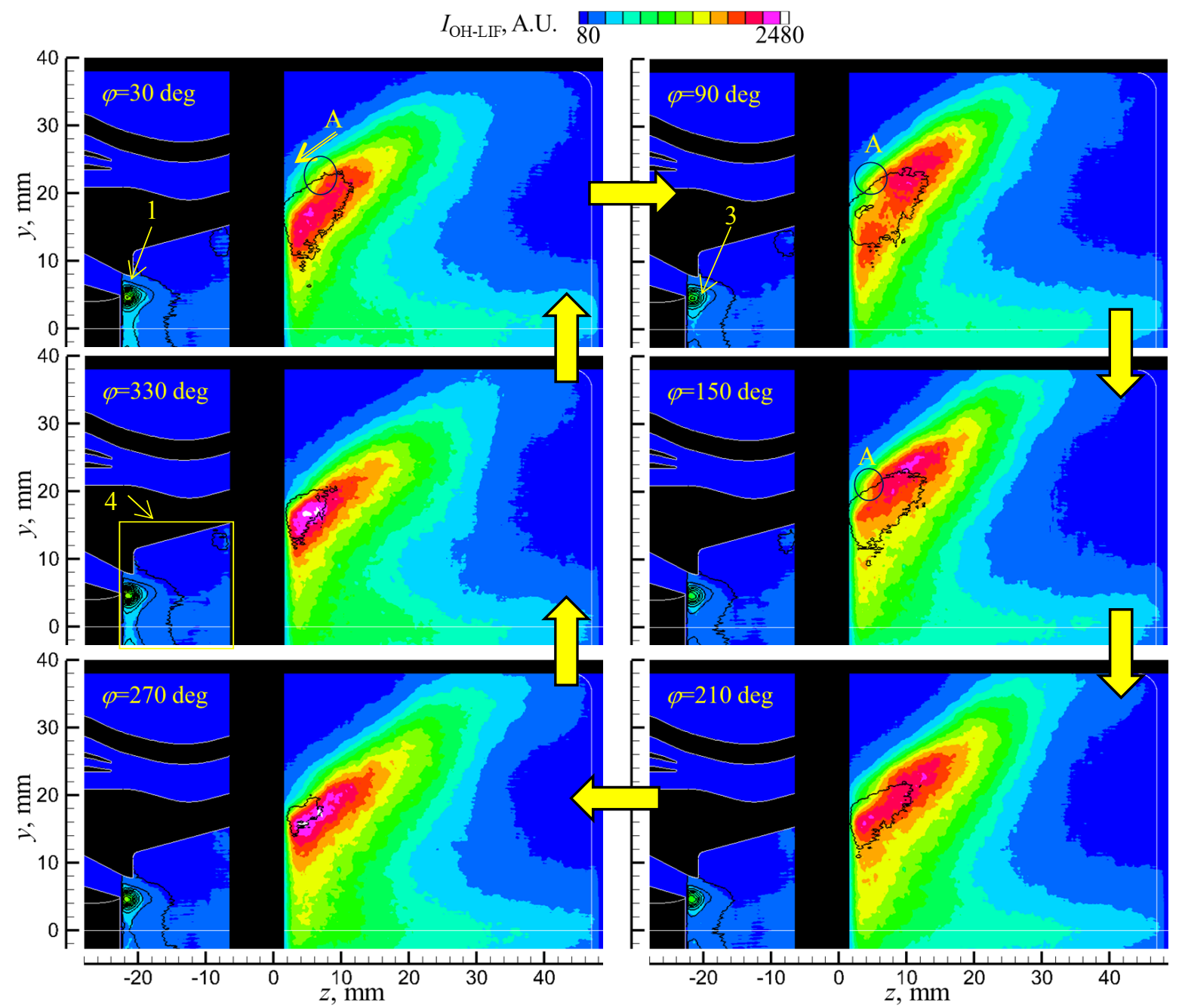

Figure 9. Phase-averaged kerosene/OH-LIF intensity distribution on $x=0$ plane for Case $\mathrm{B}$.

This work is licensed under a Creative Commons 4.0 International License (CC BY-NC-ND 4.0).

EDITORIAL UNIVERSITAT POLITÈCNICA DE VALÈNCIA 

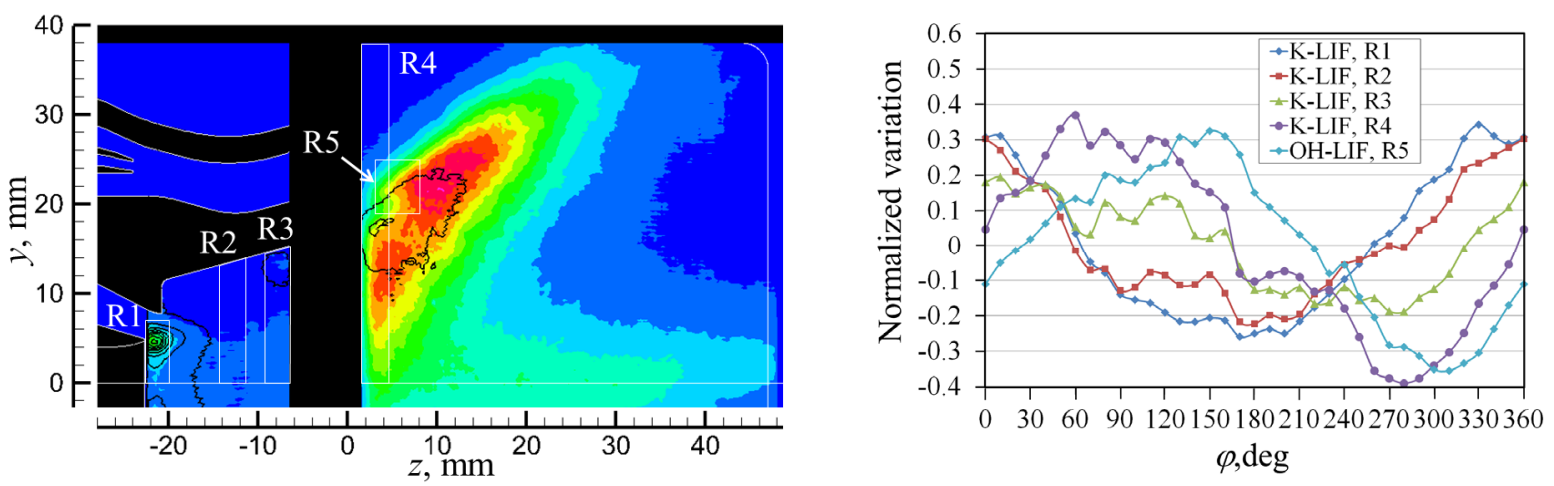

Figure 10. phase-dependent variation of the sum of LIF signals in specified regions.

\section{Conclusions}

The visualization of the pilot flame of the coaxially-staged aero-engine lean-burn fuel injector, not only downstream but also inside of the pilot nozzle, was successfully performed at the realistic aero-engine conditions. The optical access toward the inside of the nozzle was achieved through the inner and outer shrouds, both of which were made of transparent quartz. The image distortion caused by the complex contours of the two shrouds was corrected by the method based on the optical ray tracing. The line-of-sight $\mathrm{OH}$ chemiluminescence, crosssectional OH-LIF, kerosene LIF and kerosene Mie scattering were employed as diagnostic tools. The effects of the pilot local air-to-fuel ratio on the spray flame structure were revealed. As the pilot mixture got rich, the main reaction zone moved from inside of the pilot nozzle to the region near the injector lip downstream of the injector exit. The OH-LIF signal was detected near the central axis surrounded by the fuel spray. It was also observed near the back-step for the rich cases. The experiments under combustion oscillation were also conducted. The reaction enhancement in the outer part of the lip vortex region was observed as a result of the convection of rich mixture, which appeared near the pilot atomizer lip at 150 210deg earlier oscillation phase angle.

\section{References}

[1] Mongia, H.C., 2003, AIAA/ICAS International Air and Space Symposium and Exposition, AIAA 2003-2657. [2] Dhanuka, S. K., Temme, J. E., Driscoll, J. F., Mongia, H. C., 2009, Proc. Combustion Institute, 32, pp. 29012908.

[3] Lazik, W., Doerr, Th., Bake, S., v. d. Bank, R., Rackwiz, L., 2008, Proc. ASME Turbo Expo, GT2008-51115.

[4] Apeloig, J. M., d'Herbigny, F.X., Simon, F., Gajan, P., Orain, M., Roux, S., 2015. J. Prop. Power, 31(1), pp. 309-319.

[5] Fujiwara, H., Matsuura, K., Shimodaira, K., Hayashi, S., Kobayashi, M., Oda, T., Horikawa, A., Matsuyama, R., Ogata, H., Kinoshita, Y., 2011, Proc. ASME Turbo Expo, GT2011-46256.

[6] Matsuyama, R., Kobayashi, M., Ogata, H., Horikawa, A., Kinoshita, Y., Proc. ASME Turbo Expo, GT201268272.

[7] Yamamoto, T., Shimodaira, K., Kurosawa, Y., Matsuura, K., and Yoshida, S., 2009, Proc. ASME Turbo Expo, GT2009-59852.

[8] Yamamoto, T., Shimodaira, K., Yoshida, S., Kurosawa, Y., 2013, J. Eng. Gas Turbines Power, 135 (3), pp. 031502-031509.

[9] Yamamoto, T., Shimodaira, K., Kurosawa, Y., and Yoshida, S., 2013, Proc. ASME Turbo Expo, GT201395496.

[10] Matsuura, K., Ohori. S., Yoshiura, Y., Kurosawa, Y., Yamada, H., Shimodaira, K., and Hayashi, S., 2012, Poster presentation, 12th International Conference on Liquid Atomization and Spray Systems.

[11] Ohori. S., Eguchi, T., Matsuura, K., Yamada, H., Kurosawa, Y., Yamamoto, T., Shimodaira, K., Hayashi, S., 2013, Proc. 22th Japanese Conference on Liquid Atomization and Spray Systems, pp. 406-409 (in Japanese).

[12] Tachibana, S., Saito, K., Yamamoto, T., Makida, M., Kitano, T., Kurose, R., 2015, Combust. Flame, 162, pp. 2621-2637.

[13] Yoshiura, Y., Matsuura, K., Kurosawa, Y., Yamada, H., Shimodaira, K., Yamamoto, T., Hayashi, S., 2012, Proc. Gas Turbine Society of Japan, 40, pp. 159-164 (in Japanese).

[14] Matsuura, K., Ide, K., Yoshiura, Y., Ohori, S., Kurosawa, Y., Yamada, H., Shimodaira, K., Yamamoto, T., Ohota, Y., Hayashi, S., 2013, Proc. 25th European Conference on Liquid Atomization and Spray Systems, 2013.

[15] Matsuura, K., Eguchi, T., Oide, S., Yamada, H., Kurosawa,Y., Yamamoto, T., Hayashi, S., 2015, 51st AIAA/SAE/ASEE Joint Propulsion Conference, AIAA 2015-4085.

[16] Uesaka, S., Eguchi, T., Oide, S., Matsuura, K., Hayashi, S. 2016, Proc. 25th Japanese Conference on Liquid Atomization and Spray Systems, pp. 58-61 (in Japanese).

[17] Zheng, Q. P., Jasuja, A. K. Lefebvre, A. H., 1997, J. Eng. Gas Turbines Power, 119, pp. 512-518. 


\title{
Spray Combustion Chamber: History and Future of a Unique Test Facility
}

\author{
Andreas Schmid ${ }^{\star 1}$, Naoki Yamada ${ }^{1}$ \\ ${ }^{1}$ Future Technologies, Winterthur Gas \& Diesel Ltd., Switzerland \\ *Corresponding author: andreas.schmid@wingd.com
}

\begin{abstract}
Large marine two-stroke diesel engines still represent the major propulsion system for merchant shipping. With steadily increasing transport demands, rising operational costs and stricter environmental legislations, the global marine shipping industry finds itself facing the challenge to future-proof its fleet. In order to comply with international maritime organizations emission standards (TIER II and TIER III), highly sophisticated and flexible combustion systems are demanded. With the help of spray and combustion research such systems can be developed and continuously improved. A highly valuable tool to investigate sprays of large marine diesel injectors under engine relevant conditions is the Spray Combustion Chamber (SCC). This paper reviews the history of the SCC, shows todays possibilities and looks into the near future of research involving large marine two-stroke engines. The SCC was built during the first Hercules project (I.P.-HERCULES, WP5, [1]). The initial setup focused on fundamental investigations comprising the application of highly flexible thermodynamic conditions. During follow-up projects (Hercules beta [2] and Hercules C [3]) the SCC was continuously developed, and a variety of influences on spray and combustion were experimentally assessed. The initial SCC design focused on maximum optical access as well as the applicability of a wide span of optical techniques. Single-hole nozzles were utilized to generate reference data to optimize existing spray and combustion simulation models. Different fuel types and fuel qualities were investigated and effects of the in-nozzle flow on spray morphology was identified. A sound set of results was achieved and published in several (internal and public) reports. Over the years, spray research at Winterthur Gas \& Diesel has turned its focus from basic spray investigations to more detailed cavitation and in-nozzle flow examinations [4], [5]. Future research on the SCC will focus on investigations of more engine related topics, as, for example, the application of a fuel flexible injection system as is currently developed in the HERCULES-2 project [6]. Significant design modifications of the initial setup were necessary, as the injector positions and therefore exposure of the spray relative to the swirl were not fully congruent with real engine conditions. As a consequence, the new setup includes some minor drawbacks, e.g. the optical access of the nozzle tip is only visible from one side of the chamber. This means that line-of-sight methods are currently only possible at selected positions in the centre of the chamber. Therefore, a new setup was installed to illuminate the spray, consisting of a high speed, high energy laser $(100 \mathrm{kHz}, 100 \mathrm{~W})$ and special optics. In order to obtain enhanced optical access, tangential windows were rearranged, now pointing directly at the nozzle. With this setup, a first set of images was realized, showing a real spray as it occurs in large marine two-stroke diesel engines.
\end{abstract}

\section{Keywords}

Large marine diesel engines, spray, combustion, high pressure high temperature conditions, heavy fuel oil, residual fuel

\section{Introduction}

Due to their high thermal efficiency and reliability, Diesel engines are still relied upon extensively for seaborne freight transport. With steadily increasing transport demands, rising operational costs and increasing environmental restrictions, the global marine shipping industry finds itself facing the challenge to future-proof its fleet. Ship owners and operators are looking for alternative fuels in order to remain economically competitive while complying with strict environmental regulations. Today's injection systems already are capable of handling a variety of fuel qualities, ranging from light fuel oils to residual fuels of very high viscosity and density. Nevertheless, in order to provide even higher fuel flexibility, injection systems are designed to facilitate the injection of fuels with for example very poor self-ignition properties or poor lubricity.

To develop such an injection system, highly sophisticated tools are required: From state of the art injection- and component test rigs up to full scale test engines (e.g. RTX-6, located in Winterthur, Switzerland). 


\section{The past}

Before the SCC was built, experimental data, regarding spray and combustion of large marine diesel engines was scarce. Especially for common rail type engines - also for large marine diesel engines a cornerstone - the injection behaviour could only be estimated based on up-scaled models from (much!) smaller experiments. This lack of data lead to a great partnership between several stake holders: In close collaboration with ETH Zürich and PSI Villigen, a setup was designed, developed and established which still represents state-of-the-art.

The SCC was built during the first Hercules project, IP-HERCULES (WP5, [1]). The setup allowed for fundamental spray and combustion investigations (fundamental in the context of industrial research) with highly flexible thermodynamic conditions and impeccable optical access. Unlike a specific test engine, pressure, temperature and swirl can be adjusted almost independently, while on a (large marine two-stroke) test engine variations of pressure and temperature can only be achieved by changing the compression ratio or the scavenge air temperature. This gives the possibility to investigate combustion under conditions which are out of the scope of the classic compression curve of an engine. Figure 1 shows the schematic of the initial SCC design.

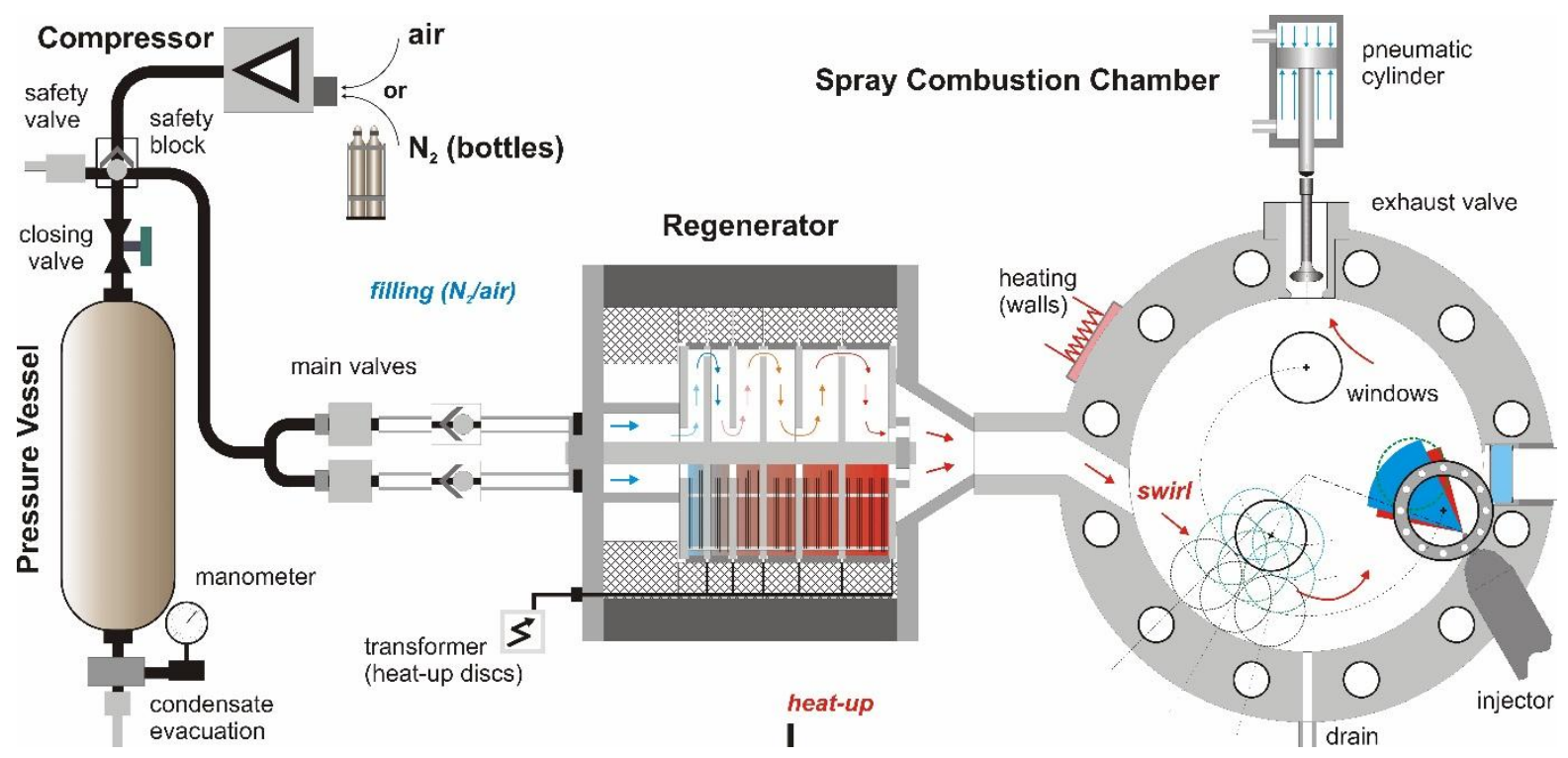

Figure 1: Schematic drawing of the entire test facility setup, also indicating operation phases such as heat up of the process gas or swirl production as well as functional aspects (window position, exhaust valve, etc.) [7]

The field of operation of the SCC is much larger compared to any constant volume chamber. The principle consists of three main parts:

a) Pressure vessels

These can be filled with nitrogen, air or a mixture of inert gases and oxygen in order to simulate EGR conditions for example. Fast acting valves control the pressure in the SCC: starting from a minimum filling time of about $150 \mathrm{~ms}$ up to $1000 \mathrm{~ms}$ to reach 140 bar chamber pressure. From the pressure bottles, the inert gas flows through the

b) Regenerator

The so-called regenerator is actually a large surface which heats the process gas and allows for process gas temperature between $300 \mathrm{~K}$ up to $940 \mathrm{~K}$, depending on the pressure and swirl conditions aimed for. The pressurised and/or hot gas then feeds the main chamber, the

c) SCC main body

This disc shaped volume represents the volume and geometric dimensions of a medium sized twostroke marine diesel engine. The process gas is introduced via tilted inlet ports into the chamber producing the characteristic swirl. The swirl velocity is a function of time. By setting a different timing of the injection event, different swirl velocities can be accomplished. The main body itself consist of three pieces:

i. A cover, representing the piston

ii. The ring section, representing the liner

iii. A second cover (mirrored), representing the engines cylinder cover 
The three pieces are held together by $50 \mathrm{~mm}$ bolts. The two covers are independently rotatable by steps of $15^{\circ}$.

The optical access is granted via three sapphire windows featuring an optical width of $100 \mathrm{~mm}$. The three windows are mounted on individual radial positions on the cover. In combination with the rotational freedom, the complete area of the SCC can be observed. Additionally, three smaller windows in the ring section - pointing to the centre of the combustion chamber - allow for optical access from the side. The left image in Figure 2 shows the setup before the installation of the control system. Well visible in the front is the SCC main body. The right side of the image shows the common rail system and the Injection Control Unit (ICU) mounted on top of the fuel rail (yellow). This is a standard injection system as found on a serial engine.
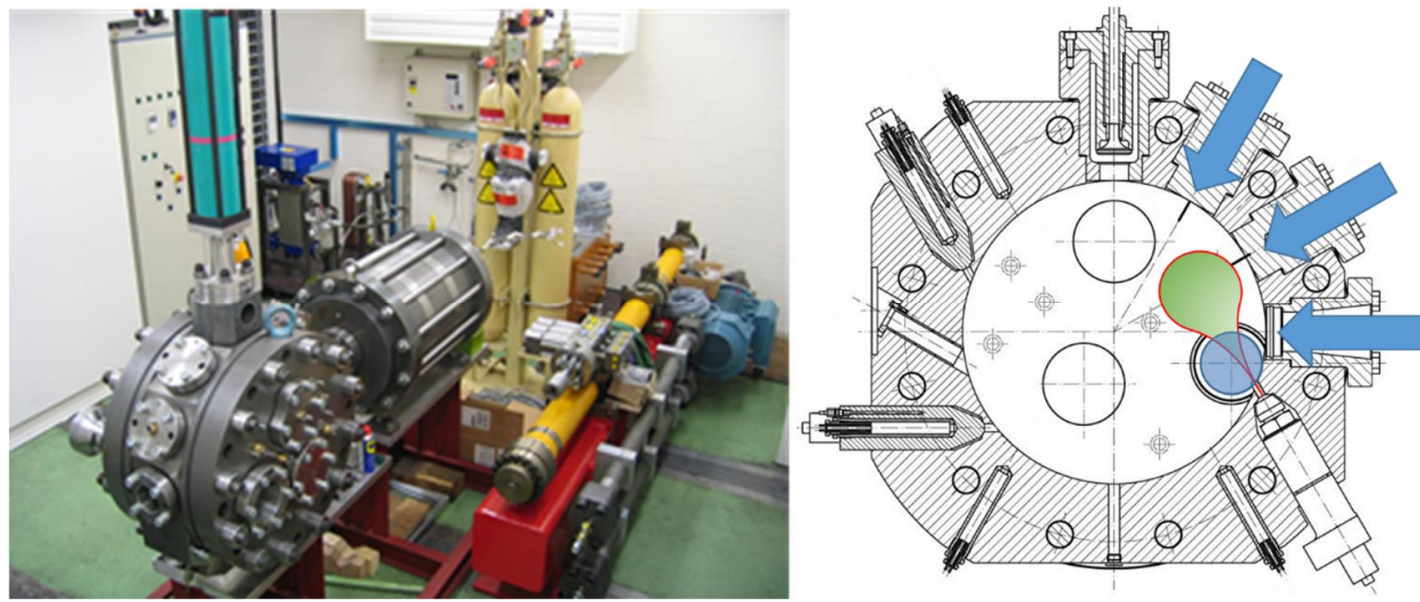

Figure 2: Installation in the laboratory, short before finalization (left), sectional view with optical accesses from the side indicated with arrows (right)

The fuel valve is a standard injector, instrumented with a needle lift sensor, as well as a pressure sensor, measuring the pressure before the nozzle. The injector is mounted on the SCC main body at an angle of $30^{\circ}$ from the vertical axis. The nozzle tip, in most cases, is a single-hole nozzle with co-axial layout as indicated on Figure 2 , right side.
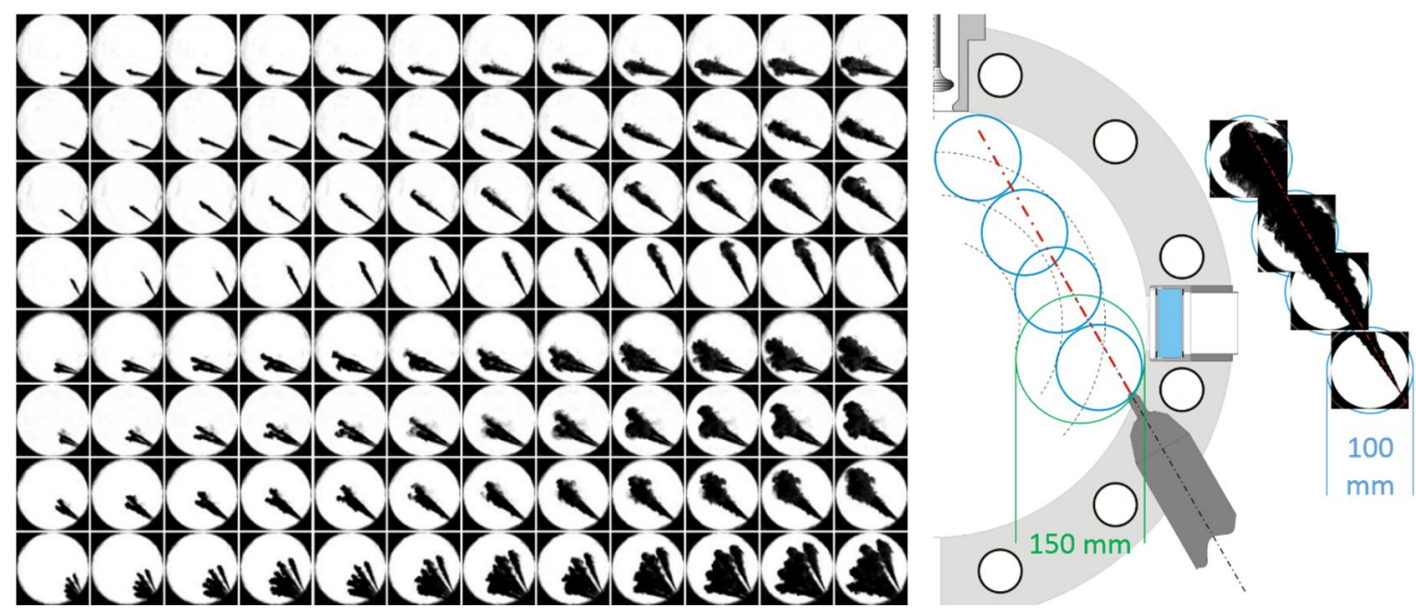

Figure 3: Extract of the different spray investigations [8] (left side) and an example of different window positions, necessary to follow the spray [9]

The setup was extensively used to produce data of sprays at different conditions, as can be seen in Figure 3 (left side). Due to the size of the spray compared to the optical access of the chamber, investigations of the complete spray are very time consuming: Including the time needed to heat up the system, pump up the pressure vessels and cleaning the windows, a single experiment can take up to 20 minutes. For each spray, several window positions were required to record the complete spray, as can be seen at the right side of Figure 3 . In the first version of the SCC the covers had to be rotated to change the window position. The rotation of the $500 \mathrm{~kg}$ (each) disks took about 
half a day as the setup had to be taken apart, rotated and reassembled again. The interested reader can estimate the effort to make spray investigations with the first design: It was tremendous.

In the following projects (HERCULES-B [2], HERCULES-C [3]) the SCC was continuously improved and extended, while various influences on spray and combustion have been experimentally assessed, as several internal and external publications proof [7], [8][10],[14][18]. Windows with increased optical width - eccentrically arranged in a large window-holder - allowed for wider spray areas to be observed and much quicker change of the window position. The green circle in Figure 3 (right side) illustrates the increased diameter of the windows, compared to the blue circles of the initial design.

The larger windows provide maximum optical access and support the application of a wide span of optical techniques such as: Shadow imaging, Mie scattering, Phase Doppler Anemometry or $\mathrm{OH}^{*}$-chemiluminescence. However, harsh combustion conditions and the size of the experimental setup called for ... adaptions of measurement techniques to properly investigate typical effects on spray and ignition. For example, during combustion, soot incandescence is so strong that it easily outshines the background illumination applied for shadow imaging. The solution was an "improved shadow imaging" setup, consisting of a laser emitting at $640 \mathrm{~nm}$ in combination with a narrow band pass filter before the camera. The result can be seen in Figure 4: The image, acquired with the classic setup, is dominated by the light from soot incandescence, whereas the spray on the right side - for the same thermodynamic conditions, at the same time after SOI -is clearly visible.

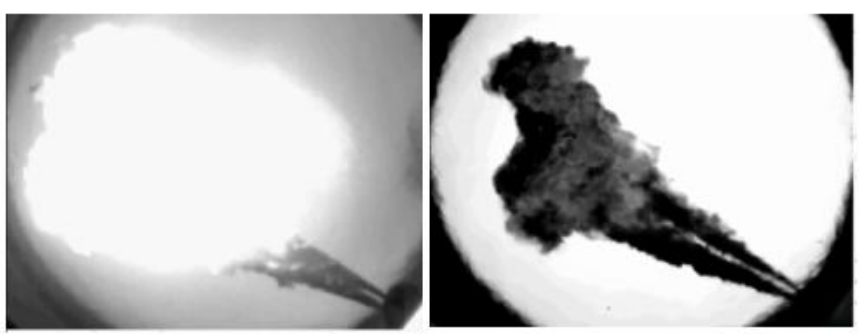

Figure 4: Classic shadow imaging (left) compared to the improved shadow imaging (right)

The same problem occurred for $\mathrm{OH}^{*}$ chemiluminescence; As presented in Ref. [10] radiation from soot fully hides $\mathrm{OH}^{*}$ chemiluminescence shortly after ignition. In order to distinguish between UV light from the $\mathrm{OH}$ radical and light from soot radiation, both signals were captured and $\mathrm{OH}^{*}$ could be detected in the beginning of the combustion. Droplet size measurement by means of Phase Doppler Anemometry (PDA) under these conditions is even more complicated, especially investigating an opaque fluid. Therefore, a study was made to design the ideal setup to do so: The result is a tailor-made PDA system as described in Ref.[11], allowing to measure droplet sizes with PDA in the harsh environment of the SCC, even with heavy fuel oil. With the focus of in-nozzle flow special illumination techniques have been compared to assess the spray very close to the nozzle [12].

With single hole nozzles, reference data for the optimisation of spray and combustion simulations has been generated [8], [9], [13],[14],[15],[16],[17]. Different fuel types and fuel qualities have been investigated on their spray behaviour, ignition characteristics and combustion [18]. With special nozzles effects of the in-nozzle flow on spray morphology have been identified [19], [20]. Between 2010 and 2015 a detailed and comprehensive analysis of spray and combustion of large marine diesel sprays under the presence of swirl flow [21] was performed.

\section{The present}

Today the SCC comes in a complete new dress, with a main chamber design representing the actual combustion chamber geometry of an engine. To investigate nozzle tips with the same orifice orientation as on the engine, the cylinder cover follows real engine specifications. With the RTX-6 test engine next door to the SCC, its cylinder cover design was taken to guarantee best agreement with engine measurements. By doing so, the SCC can be considered as an optical access to the combustion space of the RTX-6 test engine - except for some limitations like the $100 \%$ load case (which is not fully applicable due to the high peak pressure). Nevertheless, the orientation and position of the injectors is exactly the same as on the engine. Figure 5 shows the sketch of the new main body with all injector positions occupied with standard injectors (left). The right side shows the actual installation. 

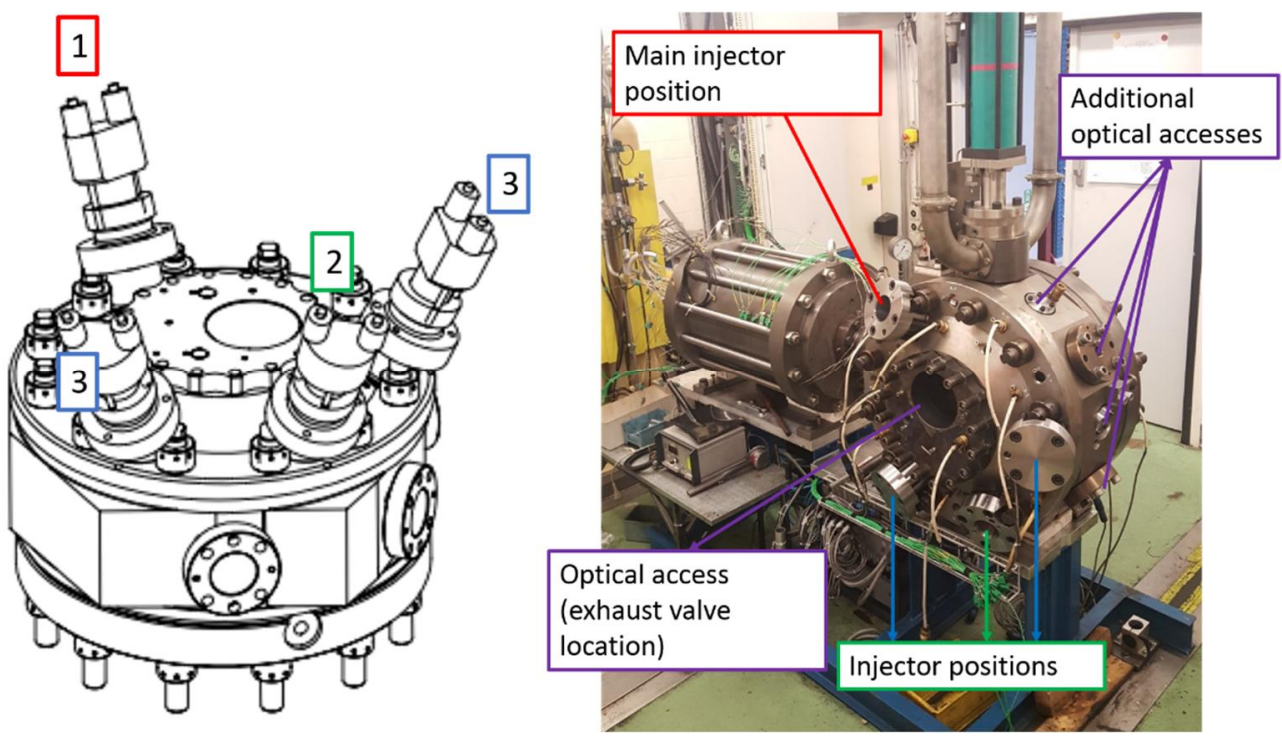

Figure 5: New SCC main body with cover of the test engine (RTX-6)

Well visible are the adapters on the injector positions " 3 ". All the adapters were made larger than the actual injector, so that also future injector designs can fit todays body: Individual adapters fit any actual (and most probably future) injector to be tested on the SCC, without re-machining the main body. The injector positions without injector are closed with so called dummies. The four possible injector positions are divided in:

1) The main injector

2) A secondary injector for the two-injector setup (setup on smaller two-stroke engines)

3) Two additional injectors for the three-injector setup (setup on large and very large two-stroke engines).

Where in the real engine the exhaust valve would be located, the SCC has an eccentric window holder with a sapphire window of $150 \mathrm{~mm}$ optical width. This allows the observation of the centre of the combustion chamber. The back side of the SCC - the piston side - is closed with one of the existing covers, allowing to observe the complete combustion chamber.

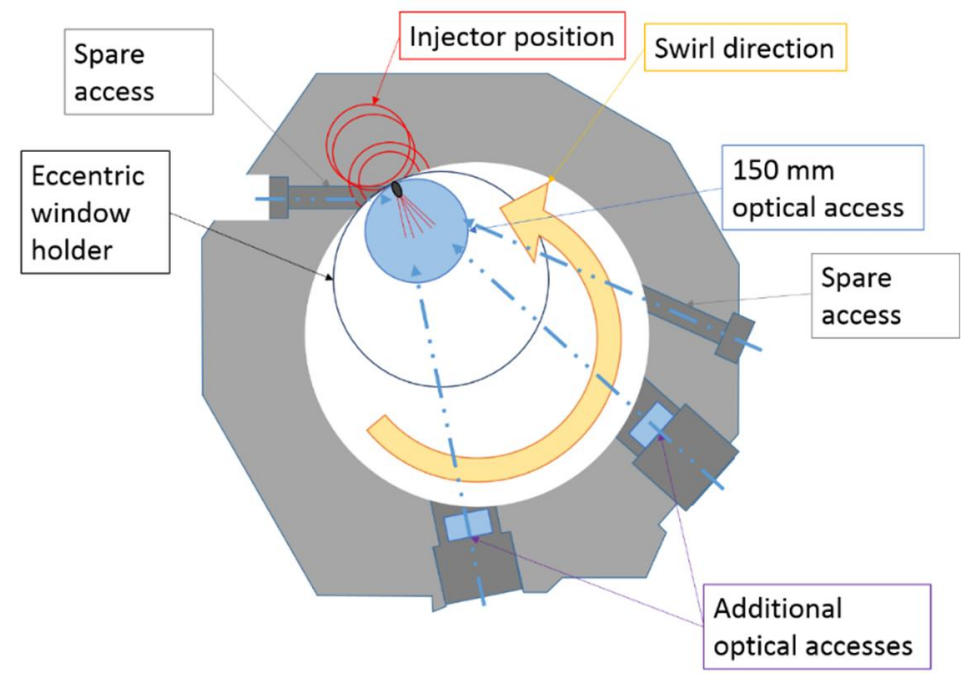

Figure 6: Sectional view with optical accesses from the side

Another distinct modification compared to the initial arrangement is the orientation of additional optical accesspossibilities. As visible in Figure 2, the windows located in the ring section of the SCC are all pointing to the centre of the main body. This restricts the application of measurement techniques which are based on laser sheets to illuminate the spray. Laser Induced Fluorescent measurement methods were therefore only applicable in a very limited way. The new setup has the additional windows not pointing to the centre of the chamber anymore, as can 
be seen in Figure 6: To illuminate the spray with laser light, the additional optical accesses are pointing to the tip of the injector. This allows the observation of the spray origin from the side and/or enables the admission of a laser (sheet) for the application of Mie scattering techniques to illuminate the liquid phase of the spray or even LIF-based methods. The smaller holes from the side of the chamber are for the so called spare accesses. These holes are intended for additional measurement techniques like fibre optics or can be used to additionally illuminate the spray (additional light from the side or an additional laser wavelength for excitation) which might be implemented in the future.

A very first spray investigation has been conducted with the new setup: As a reference case the injection with a standard fuel nozzle has been chosen, under conditions as they are found at about $25 \%$ engine load. Figure 7 shows a series of spray images, from SOI until the fully developed flame is visible.
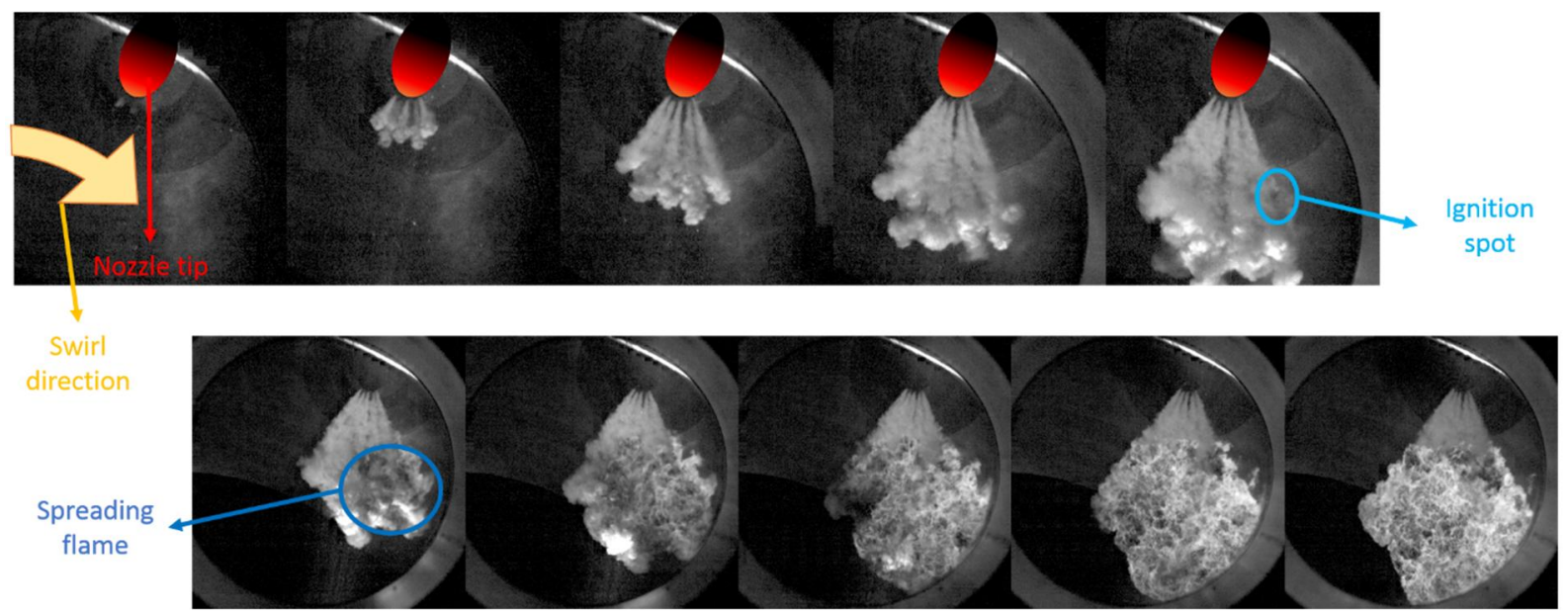

Figure 7: Example of a real sized spray nozzle under engine like conditions

Unlike in smaller engines, the spray pattern is not symmetric, as already discussed in earlier investigations looking at eccentric sprays ([19], [20]). To allow for maximum scavenging efficiency, the exhaust valve is located in a centred position of the cylinder. Therefore, the fuel injectors are protruding from the side into the combustion chamber. The swirl, coming from the left side, pushes the leading fuel portions to the side. This effect is more evident when looking at the flame: Instead of an even distribution of the flame, it is pushed out of the visible area into the lower right corner. On the lower left side, no flame can therefore be seen. Compared to the investigations from the single-hole nozzles another interesting fact can be observed: The rather narrow arrangement of the fuel orifices produces 5 individual sprays, very early during the injection. But after a certain progress of the spray, these single sprays seem to merge to a single spray front, acting as one large spray/flame volume.

\section{The future}

Even though the SCC has been adapted to apply engine relevant injection systems, basic spray and combustion research still represents a fundamental requirement: $A$ detailed investigation has started to understand the interrelation between in-nozzle flow, cavitation, spray breakup - by means of Ballistic Imaging - spray morphology, ignition and combustion. This research will partly take place in the laboratory under non-evaporating, no-swirl conditions and later transfer this knowledge into the SCC, where the spray morphology, ignition and combustion will be assessed.

For the HERCULES-2 project [6] research on the SCC will focus on more engine relevant problematics, as for example the application of a fuel flexible injection system. Future injection systems are designed to provide full flexibility with regards to fuel qualities and the operation of classic fuels like HFO and MDO as well as on fuels with very low viscosity or ignitability like ethanol or methanol. Such systems could not have been tested on the initial setup, as the injector position was not optimised for such investigations and the adaption of the main body would have been too costly.

The new main body on the SCC also included the installation of a new illumination setup, consisting of a high speed, high energy laser $(100 \mathrm{kHz}, 100 \mathrm{~W})$ and special optics. The very short illumination pulses of 2 ns allow not only the pin sharp visualisation of the spray within the SCC, but should also visualize the fast exiting spray close to the orifice as suggested in earlier works [12]. 


\title{
Conclusions
}

Ten years ago, a worldwide unique experimental setup, allowing for the investigation of large scale marine diesel sprays under engine relevant conditions, has been taken into service. Since then the installation has been improved with respect to optical access, fuel flexibility and reduction of service time. In parallel its sphere of action has been continuously increased, from the investigation of spray morphology and combustion to a device which allows for the examination of small quantities of alternative fuels, new injection systems or new injection strategies with a variety of measurement techniques. Over the years also several measurement methods have therefore been adapted and improved in order to be applied under the demanding conditions of large marine diesel engines.

Now the next chapter in the life of the SCC has been opened, the application of real engine hardware to establish the direct link between irreplaceable measurements on test engines and the possibility to access the combustion with highly sophisticated methods and measurement techniques.

A very first series of experiments showed the successful application of the new setup and already helped to better understand combustion under these demanding and unique conditions.

In the future, this new setup will directly help to improve combustion of different fuels, support the development of combustion systems and methods and therefore do one's bit in the reduction of exhaust emissions, increase of fuel flexibility and efficiency.

\section{Acknowledgements}

The installation of such a complex setup would not have been possible without the support of several partners over the past years. The design, setup and continuous improvement of the SCC has been conducted within the framework of different projects:

- $\quad$ I.P. HERCULES project within EC's 6th Framework Program: Contract TIP3-CT-2003-506676

- $\quad$ HERCULES- $\beta$ project within EC's 7th Framework Program: Contract SCP7-GA-2008-217878

- $\quad$ HERCULES-C project within EC's 7th Framework Program: Contract SCP1-GA-2011-284354

- HERCULES-2 project within European Union's Horizon 2020 research and innovation programme under grant agreement No 634135

The additional financial support by the Swiss Federal Government during several projects:

- Contract 154269, Project 103241,

- SFOE Contract SI/500940, TP Nr 8100075, Project name "FlexFuel Combustion"

- SFOE Contract SI/501299-01, TP Nr 8100075, Project name "INFLOSCOM"

is gratefully acknowledged.

Over the past years, a fantastic collaboration has been established with and among the different partners. The sincere thank therefore goes to the experts and people who took part in the long journey and are still invaluable allies:

- $\quad$ ETH Zürich

- PSI Villigen

- $\quad$ EMPA Dübendorf

- $\quad$ FHNW Brugg-Windisch

- IFPEN Paris

- NTUA Athens

- Chalmers University of Technology Gothenburg

\author{
Abbrevations \\ EGR Exhaust Gas Recirculation \\ EMPA Eidgenössische Materialprüfanstalt \\ FHNW Fachhochschule Nordwestschweiz \\ ETH Eidgenössische Technische Hochschule \\ HFO Heavy Fuel Oil \\ ICU Injection Control Unit \\ IFPEN Institut Français du Pétrole, Energie Nouvelle \\ LIF Laser Induced Fluorescence \\ MDO Marine Diesel Oil \\ NTUA National Technical University of Athens \\ PSI Paul Scherrer Institute \\ SCC Spray Combustion Chamber \\ SOI Start Of Injection \\ WinGD Winterthur Gas \& Diesel Ltd.
}




\section{References}

[1] IP-HERCULES, www.ip-HERCULES.com

[2] HERCULES-B, www.HERCULES-B.com

[3] HERCULES-C, www.HERCULES-C.com

[4] C. Habchi, J. Bohbot, A. Schmid, K. Herrmann, A comprehensive Two-Fluid Model for Cavitation and Primary Atomization Modelling of liquid jets - Application to a large marine Diesel injector, CAV2015, 9th International Symposium on Cavitation, Lausanne, Switzerland, 6-10 December 2015.

[5] Balz R., "In-nozzle flow and spray morphology investigations of marine diesel injectors", Poster, ECCOMATE 1st Conference on Combustion Processes in Marine and Automotive Engines, 07. to 08.06.2016, Lund, Sweden

[6] HERCULES-2, http://www.hercules-2.com/work-package-1

[7] Herrmann K., Schulz R., Weisser G., „Development of a reference experiment for large diesel engine combustion system optimization", Paper No. 98, CIMAC Congress 2007, Vienna

[8] Herrmann K., von Rotz B., Schulz R., Weisser G., Boulouchos K., Schneider B., „Reference Data Generation of Spray Characteristics in Relation to Large 2-Stroke Marine Diesel Engines Using a Novel Spray Combustion Chamber Concept", ILASS - Europe 2010, Brno, Czech Republic

[9] von Rotz B., Herrmann K., Weisser G., Cattin M., Bolla M., Boulouchos K., „Impact of Evaporation, Swirl and Fuel Quality on the Characteristics of Sprays typical of Large 2-Stroke Marine Diesel Engine Combustion Systems", ILASS - Europe 2011, Estoril, Portugal

[10] Schmid A., von Rotz B., Bombach R., Weisser G., Herrmann K., Boulouchos K., „Investigation of Marine Diesel Ignition and Combustion at Engine-Like Conditions by means of $\mathrm{OH}^{*}$ Chemiluminescence and Soot Incandescence", Paper No. Cl-8, COMODIA 2012, Fukuoka, Japan

[11] von Rotz B., Kammermann T., Schneider B., Schmid A., Herrmann K., Weisser G., Boulouchos K., „Evaluation of PDA Applicability in Regard to Heavy Fuel Oil Spray Investigations”, $17^{\text {th }}$ International Symposium on Applications of Laser Techniques to Fluid Mechanics, Lisbon, Portugal, 07-10 July, 2014

[12] Schürch C., "Evaluation of Spray Visualization Techniques for the Investigation close to the Orifice", Master thesis, ETH Zürich 2014

[13] Schulz R., Herrmann K., von Rotz B., Hensel S., Seling F., Weisser G., Wright Y.M., Bolla M., Boulouchos K., „Assessing the Performance of Spray and Combustion Simulation Tools against Reference Data Obtained in a Spray Combustion Chamber Representative of Large Two-Stroke Diesel Engine Combustion Systems“, Paper No. 247, CIMAC Congress 2010, Bergen

[14] Herrmann K., "Entwicklung, Validierung und erste Anwendung eines neuartigen Brennkammer-konzepts für die Generierung von Referenzdaten hinsichtlich der Charakterisierung des Einspritz-vorganges in Bezug auf grosse 2-Takt Schiffsdieselmotoren“, 1. GMT Rostock, Deutschland

[15] Herrmann K., von Rotz B., Schulz R., Weisser G., Schneider B., Boulouchos K., „A "Spray Combustion Chamber" facility for investigations in relation to large 2-stroke marine diesel engine combustion system optimization", Abstract of the International Symposium on Marine Engineering (ISME), 2011, Kobe, Japan

[16] Bolla M., Cattin M.A., Wright Y.M., Boulouchos K., Schulz R., „3D-CFD Lagrangian Spray Simulations for Large Two Stroke Marine Diesel Engines compared with Experimental Data of a Spray Combustion Chamber", ASME ICES2012-81016, Torino, Italy

[17] Nagy, I. G., Schmid, A., et al., Computational analysis of spray primary breakup in 2-stroke marine diesel engines with different nozzle layouts, ICLASS 2015, 13th Triennial International Conference on Liquid Atomization and Spray Systems, Tainan, Taiwan, August 23-27, 2015, Tainan, Taiwan

[18] Schmid A., von Rotz B., et al. Ignition behaviour of marine diesel fuels under engine like conditions, SAE 2014 International Powertrain, Fuels \& Lubricants Meeting, October 2014, Birmingham, UK

[19] Schmid, A., von Rotz, B., Schulz, R., et al., Influence of nozzle hole eccentricity on spray morphology, ILASS - Europe 2013, 25th European Conference on Liquid Atomization and Spray Systems, 1-3 Sep. 2013, Chania, Greece

[20] Schmid, A., Von Rotz B., et al. Influence of in-nozzle flow on spray morphology, ILASS - Europe 2014, 26th Annual Conference on Liquid Atomization and Spray Systems, 8-10 Sep. 2014, Bremen, Germany

[21] von Rotz, B., Experimental Investigation of Spray Characteristics and Ignition Processes at Conditions representative of Large Two-Stroke Marine Diesel Engines. Dissertation, ETH Zürich, 2015, No. 22968 (2015). http://dx.doi.org/10.3929/ethz-a-010836986 


\title{
Study of the soot quantification for two-stage injection of diesel in a constant-volume combustion chamber
}

\author{
Jiawei Cao' ${ }^{1}$, Zhixia He ${ }^{* 2}$, Bei $\mathrm{Li}^{1}$, Wenjun Zhong ${ }^{1}$, Mei Wang ${ }^{1}$ \\ ${ }^{1}$ School of Energy Power Engineering, Jiangsu University, Zhenjiang, China \\ 2 Institute of Energy Research, Jiangsu University, Zhenjiang, China \\ *Corresponding author: zxhe@ujs.edu.cn
}

\begin{abstract}
The effect of two-stage injection strategies on the soot formation of $0 \#$ fossil diesel were investigated experimentally using a constant volume combustion chamber. The ambient conditions was kept constant as injection pressure 150 $\mathrm{MPa}$, ambient gas temperature $900 \mathrm{~K}$, ambient gas pressure 45 bar. A high-speed diffused back-illumination extinction imaging technique was employed to make quantitative measurement on temporal soot evolution and reacting spray liquid length and a direct high-speed camera was used to measure the ignition delay. Two-stage injection strategies were varied with different pilot and main injection time, including a sweep of dwell time in pilotmain injection and pilot injection duration. The results show that the ignition delay decreases with the increasing dwell time. It may result from the entrained surrounding gas enhance the spray combustion process. In the reacting condition, the liquid-phase penetration is slightly longer with the shorter dwell time. However, the pilot injection duration shows slighter impact. The longer dwell time contributes to more total soot mass while the different pilot injection duration barely affect the total soot mass of the main injection.
\end{abstract}

\section{Keywords}

Diesel; two-stage injection; Soot; Combustion chamber

\section{INTRODUCTION}

Diesel engines have been employed extensively due to its outstanding efficiency and power output. However, enormous soot emission from the conventional diesel cannot meet the strict emission regulation. Multiple injection technology has been a practical method to reduce the carbon-monoxide (CO), unburned hydrocarbons (UHC), NOx and soot emissions without the penalty of fuel consumption [1][2][3]. Interactions between the individual injections in a multiple injection process are the key to comprehend the soot generation mechanism which could reduce soot emission [1][3]. In the previous literatures, multiple injections have shown potent for simultaneous reduction of NOx and soot emission in the diesel engine [4][5][6][7].

In non-reacting conditions, the spray of the main injection penetrates faster within a shorter dwell time investigated by Mie-scatter and CFD models [8]. When the two-stage injection duration are same, the second injection vapor phase penetration is longer and faster [9][10][11][12]. For combustion characteristics, ignition delay of main injection is much shorter than that of the pilot injection and it may be shorter or longer with the dwell time increasing due to the different entrainment of surrounding gas [8]. In the multiple injection strategy investigation, the dwell time between injections and the pilot injection duration were seen as the two main factors. Seong-Young Lee et al. [13] found that it exists a slight reduction of the soot mass at the 0.3/0.65/1.2 ms condition. Fang et al. [14]measured the soot production of the biomass to liquid fuel using multiband flame measurement and two-color pyrometry. They found that the pilot injection results in a lower soot level compared with single injection with the same injection quality, while the pilot injection shown little impact on No. 2 diesel soot generation. However, in the study of [15], more fuel quantity during the first injection resulted in less soot emission with no increase in NOx emission when the pilot injection quantity is relative small to the main injection. Coupling the two factors, Carlucci et al. [16] found that both injection timing and duration show similar effect on the soot formation and oxidation. From the discussion before, in conslusion the soot formation in the multiple injection strategy isn't still precisely understanding.

In this study, the ignition delay, reacting spray liquid length and total soot mass evolution of 0\# fossil diesel were measured in a high-temperature high-pressure constant volume combustion chamber, the objective is to explore the effects on soot formation of $0 \#$ diesel under different dwell time and pilot injection duration in the constant volume chamber. In the next research, the dwell time will be extended. Schlieren and $\mathrm{OH}$ chemiluminescence imaging are going to be used for further analysis.

The rest of this article is structured as follows: the material and methods section present the test rig, test matrix and optical setup. Results are composed of three sections: ignition delay, the reacting spray liquid length, the spatial soot concentration distribution and total soot mass evolution under dwell time and pilot injection duration. 


\section{Material and methods \\ Test Rig}

The experiments were carried out in a high-temperature high-pressure constant volume combustion chamber. The cross section of the chamber is depicted in Fig. 1. Optical access is provided by four quartz windows at each side, $100 \mathrm{~mm}$ in diameter and $70 \mathrm{~mm}$ thick. The volume of the chamber is 12 liters, including the volume of the heating cartridges which are installed at the bottom inside the chamber. A single-hole injector with $0.18 \mathrm{~mm}$ diameter nozzle was mounted at the top of the chamber. The gas inside the chamber is injected by the high-pressure air holders and controlled by the inlet and outlet valves mounted at the bottom of the chamber. More details on the experimental equipment can be found in Ref. [17].

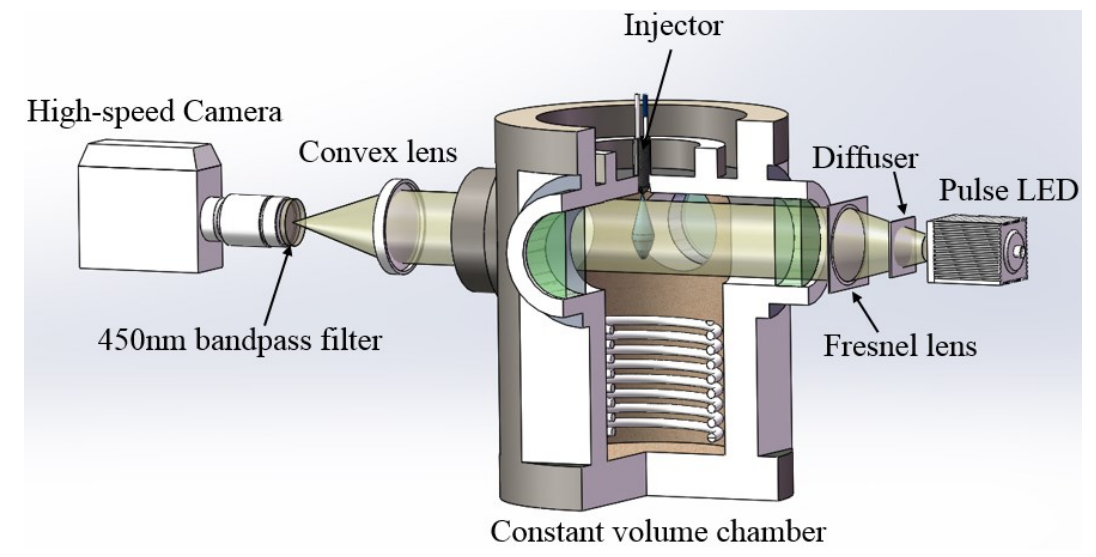

Fig. 1. Schematic of experimental setup

\section{Test matrix}

The operating condition of ignition delay and soot mass tests was maintained with ambient temperature $900 \mathrm{~K}$, ambient pressure 45 bar and injection pressure $150 \mathrm{MPa}$. The gas inside the chamber was pure air. Point 1 was employed for ignition delay and all the points was used for the soot evolution and reacting spray liquid length tests. In the study, all the set time were energizing timing. A wide range of dwell time (DT=400 $\mu \mathrm{s}, 600 \mu \mathrm{s}, 800 \mu \mathrm{s})$ was tested at a fixed pilot injection and the actual dwell time defined as the interval time of the beginning timing of the two-stage injection is $667 \mu \mathrm{s}, 1066 \mu \mathrm{s}$ and $1366 \mu \mathrm{s}$, respectively. The pilot injection energizing time (PIE) sweeps from $532 \mu \mathrm{s}$ to $709 \mu \mathrm{s}$ and the main injection(MI) is constant at $1773 \mu$ s energizing time. The single injection was seen as the reference. During the measurement, each condition was set as 10 times, and the interval $10 \mathrm{~s}$ to decline the uncertainty of the slight fluctuation of ambient temperature and pressure between two injections. The detailed test matrix is given in Table 1.

Table 1. Test matrix

\begin{tabular}{c|ccc}
\hline \hline Points & PIE $[\mu \mathrm{s}]$ & MI $[\mu \mathrm{s}]$ & DT $[\mu \mathrm{s}]$ \\
\hline \hline 1 & 532 & 1773 & $400,600,800$ \\
2 & 621 & 1773 & 800 \\
3 & 709 & 1773 & 800 \\
4 & 0 & 1773 & 0
\end{tabular}

\section{Optical setup}

Several optical diagnostics were employed in the measurements. Small aperture direct high-speed imaging was used for ignition delay and high-speed diffused back-illumination extinction imaging (DBIEI) was employed for soot mass evolution and liquid length in oxydic conditions.

\section{Diffused background illumination extinction imaging}

Fig. 1 shows the schematic of optical setup for DBIEI measurement. The setup implemented in this work consists of a pulse LED illumination system and a high-speed camera on opposing side of the spray in a line-of-sight arrangement. The pulse LED $(140 \mathrm{~W})$ triggered by the high-speed camera provides the successively stroboscopic light. The Fresnel lens $(\mathrm{d}=100 \mathrm{~mm}, \mathrm{f}=72 \mathrm{~mm})$ mounted close to the access window was used to magnify the illumination intensity and background light area. The engineer diffuser $\left(a=203 \mathrm{~mm}, \alpha=20^{\circ}\right)$ directs a bundle of 
diffused rays that will reduce beam steering caused by gas density gradients with minimal light intensity loss in hightemperature conditions.

The transmitted light of the LED and flame luminosity was collected by high-speed CMOS camera running at 60 $\mathrm{kHz}$ (Photron SA-Z). The camera lens used was a 105-mm f/2.8 Nikkor lens. The filter used was a $450 \mathrm{~nm}$ bandpass filter (FWHM $10 \mathrm{~nm}$ ). The convex lens $(\mathrm{d}=150 \mathrm{~mm}, \mathrm{f}=400 \mathrm{~mm})$ was employed dwindle the images size that focused on the lens of the camera. The measurement mechanism of DBIEI technology is based on Beer-Lambert Law [18]:

$$
\frac{I-I_{\text {flame }}}{I_{0}}=\exp (-K L)
$$

In Eq. $1, I$ is the sum of the transmitted LED intensity and the flame natural luminosity, $I_{\text {flame }}$ is the flame intensity corrected between every two successive LED pulses, $I_{0}$ is the incident intensity acquired at the time of injection, $K$ is the dimensional extinction coefficient and $L$ is the path length through the soot cloud. Soot volume fraction is based on small particle Mie theory [18][19]:

$$
f_{v}=\frac{\lambda}{k_{e}} \cdot k
$$

Where $f_{v}$ is soot volume fraction, $\lambda$ is the wavelength of the captured light $450 \mathrm{~nm}, k_{e}$ is the dimensionless optical extinction coefficient. $k_{e}$ can be obtained from the Rayleigh-Debye-Gans (RDG) theory as $k_{e}=7.61$. The details of the RDG theory and all the parameters used for calculating the $k_{e}$ are referenced from [19][21]. As a consequence, the sum of soot mass $\left(\mathrm{m}_{\text {soot }}\right)$ along the line-of-sight at each pixel was derived from Eq. 3 using an assumed density of $1.8 \mathrm{~g} / \mathrm{cm}^{3}$ for soot $(\rho)[22]$.

$$
m_{\text {soot }}=\rho K L \frac{\lambda}{\kappa_{e}} \times \text { pixel area }
$$

\section{Ignition delay and liquid-phase penetration}

For the ignition delay measurement, the transient combustion process images were captured by the same high speed camera with DBIEl at 60000 frames per second with a resolution of $896 \times 368$ pixels and an exposure time of $0.248 \mu \mathrm{s}$.

As mentioned before, the DBIEI is based on the light extinction by the soot particles. Obviously, the liquid phase can reduce the light intensity and the sign can be shown in the window. Moreover, the spray penetrates from the left to the right in the access. The liquid length (LL) was also quantified from DBIEl images following the approach recommended from Engine Combustion Network (ECN) [23]. The only difference is that the temporal liquid length evolution here was obtained from images averaged from 10 repetitions at each time position rather than just a one time-averaged value. The details for this process can be found in [24].

\section{Results and discussion \\ Flame luminosity and ignition delay}

Fig. 2 shows the time-sequence flame luminosity images after the start of the main injection (AMI) for the Point 1. For all the dwell time, main combustion started while the pilot combustion was still active. It means that the dwell time should be extended for further investigation. As the DT became longer, main combustion started when the pilot combustion was extinguished, as shown in the cases DT=600 $\mu \mathrm{s}, 800 \mu \mathrm{s}$. Under all dwell time, the flame of the main injection tends to be stable after $1 \mathrm{~ms} \mathrm{AMI}$.

Fig. 3 shows the ignition delay of pilot injection, main injection and the lag time between them. It can be found that the ignition delay of all DT cases was shorter than the single injection, when ignition delay was observed to be approximately $0.73 \mathrm{~ms}$. One thing should be pointed that the ignition delay was elapsed time from the start of the main injection. Short dwell time in two-stage injection would reduce the premixed combustion phase and the slightly increased surrounding gas temperature enhance main injection combustion process. For the case DT=400 $\mu \mathrm{s}$, the fresh air was consumed by the pilot injection and the main injection phase was ignited in a high-concentration exhaust lately due to the fresh air consumption in the pilot combustion. With the dwell time increasing, the ID of the main injection is shorter because of the increased space for a better combustion mixture, which can be seen at 0.5 ms AMI in DT $=800 \mu$ s case. 

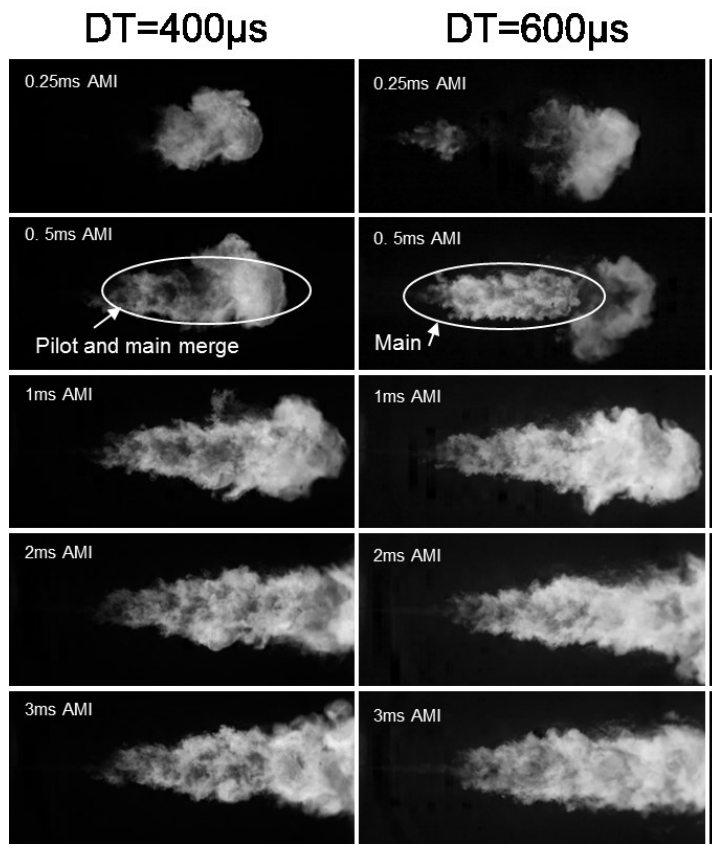

\section{DT $=800 \mu s$}
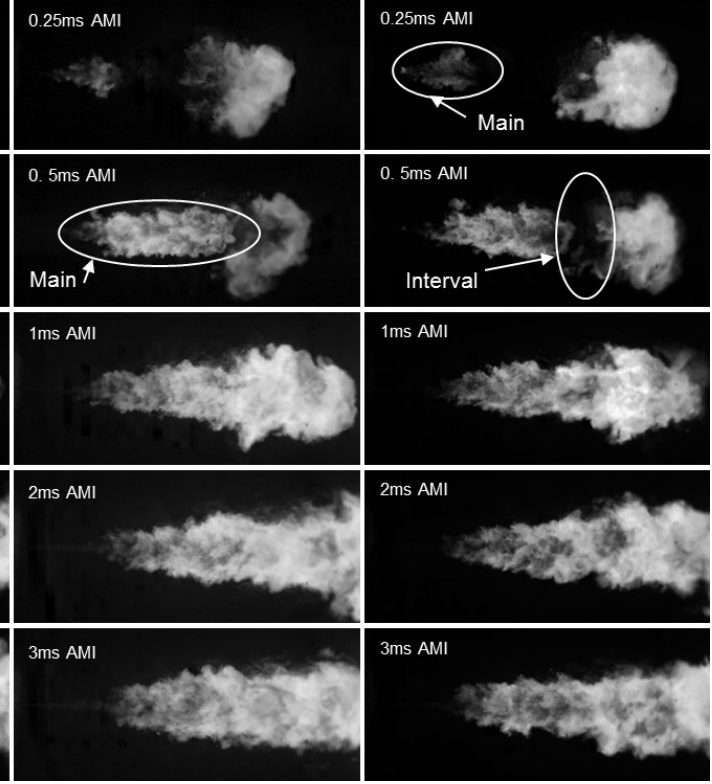

Fig. 2. Pilot and main flame luminosity after main injection (AMI) for DT $=400 \mu$ s (left column), DT=600 $\mu$ s (middle column), and $\mathrm{DT}=800 \mu \mathrm{s}$ (right column)

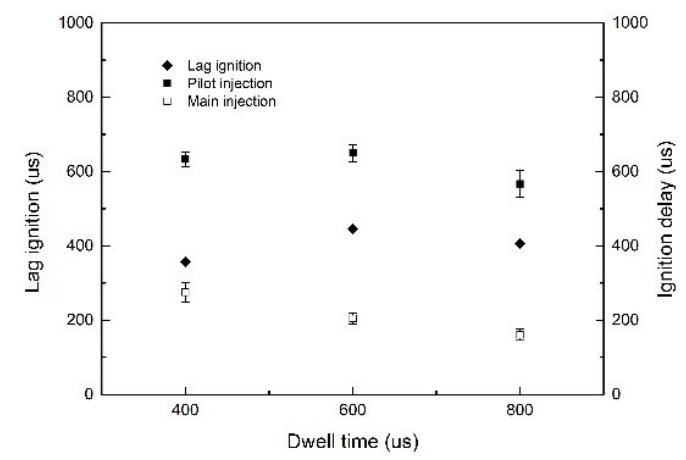

Fig. 3. Ignition delay with different dwell time (DT=400 $\mu \mathrm{s}, 600 \mu \mathrm{s}, 800 \mu \mathrm{s})$

\section{Liquid length}

Previous research shows that the LL has a great influence on the spray combustion and emission characteristics. Proper LL contributes to a good fuel/air mixing, while long liquid phase impinges on the piston bowl wall leading to a larger soot emission and reduced engine efficiency [25]. In-house image processing using Matlab from experimental shadowgraph images provided measurements of vapor penetration shown in Fig. 4.

Shown in the Fig. 4, it can be found that the reacting LL has been stabilized in all cases after $500 \mu$ s AMI. From Fig. 4 (a), it can be seen that the LL of DT=400 $\mu$ s is slightly higher than that of the other cases during the time from 500

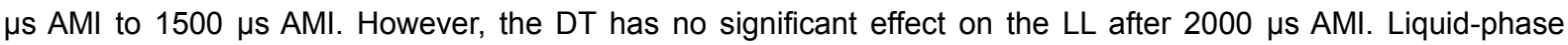
penetration is more associated with the ambient gas density and fuel density and viscosity. This trend is different with the spray-vapor penetration under two-stage injection. Coupled with Fig. 3, the spray in MI needs more time to ignite the spray due to low fresh air concentration. In Fig. 4 (b), it is shown that the LL overlap in the main evolution in all pilot injection cases. The results were found to be identical with the research in [8]. 


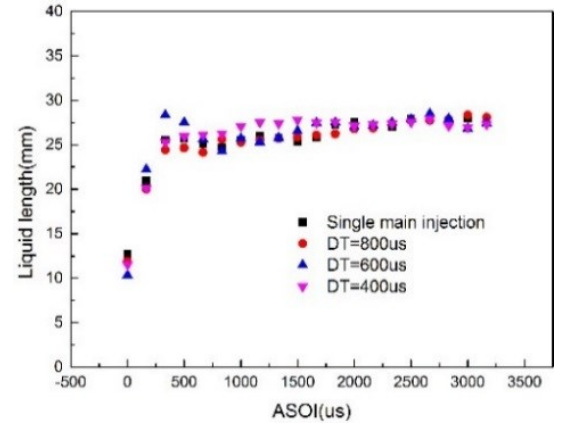

(a) Vapor penetvolumen length for DT

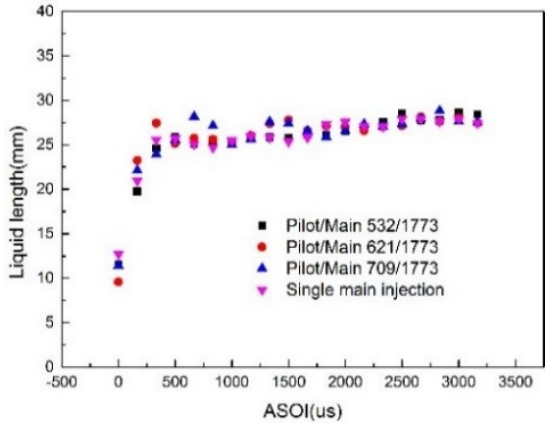

(b) Vapor penetration length for pilot

Fig. 4. Vapor penetration length for $D T=400 \mu \mathrm{s}, 600 \mu \mathrm{s}, 800 \mu \mathrm{s}$, (a) and PIE $=532 \mu \mathrm{s}, 621 \mu \mathrm{s}, 709 \mu \mathrm{s}$, (b)

\section{Soot analysis}

Taking into consideration Fig. 3, the stabilized time for comparison which was chosen for the study is kept constant at $2.5 \mathrm{~ms}$ AMI. Fig. 5 shows the typical combustion process of all PIE energizing timings and the single injection (without pilot injection) as the reference. The time chosen is $0.2 \mathrm{~ms}, 0.5 \mathrm{~ms}, 1 \mathrm{~ms}$ and $2.5 \mathrm{~ms}$ AMl. The yellow vertical line marks the illumination limitation. As mentioned before, the extinction in the left part inside the picture is the spray liquid.

Shown in Fig. 5, soot intensity can be found near the tip of the spray at $0.25 \mathrm{~ms}$ AMl in the case DT=800 $\mu$ s. However, the spray is not obvious in the case DT=400 $\mu \mathrm{s}$. It may be caused by the dissatisfaction with the injector needle electromechanical delay due to the such short dwell time. It can also be found that the highest soot concentration located in the middle section of the flame at $0.5 \mathrm{~ms}$ AMl that is consistent with the results in [8]. Generally, the high temperature and high $\mathrm{C} / \mathrm{O}$ location exists near the flame in the diffused flame combustion. In addition, the highest soot concentration appears in the tip of the flame. However, the soot in the flame tip is much lower than that in the middle of the flame. Similar to the results of ignition delay in dwell time, the longer dwell time accelerates the soot formation in main injection due to the wide space between two flames for entrainment. Lack of the interactive effect of multiple injection, it needs more time for the reference case to reach the soot formation environment. In addition, the soot concentration of main injection is lowest in the case DT=400 $\mu$ s. At the time of $2.5 \mathrm{~ms} \mathrm{AMl}$, it can also be found that the soot formation increases with the longer dwell time. Especially, the soot concentration of the main injection in DT=400 $\mu$ s is slightly lower than that in single injection. shown in the soot distribution evolution, the soot formation is obviously affected by the surrounding emission caused by the pilot injection. The main injection spray was ignited in the burning environment caused by the pilot injection combustion. Fig. 6 gives the total soot mass of all DT cases. The first soot peak level also appears in all cases which is also observed in [26]. It can be seen that the soot peak level and the total soot mass are higher than others when the dwell time became longer.

Fig. 7 shows the soot distribution of all PIE cases, the 2-D soot concentration distribution was shown in the Fig. 7(a) and the total soot mass evolution was shown in the Fig. 7(b). From the Fig. 7(a), it can be seen that the soot formation of the main under PIE cases is slightly higher than that of the reference. However, the pilot injection duration has no significant effect on the soot formation. Fig. 7(b) shows the soot mass evolution of pilot injection duration. It exists overlap among three pilot injection duration in the time between $2.5 \mathrm{~ms}$ to $3.5 \mathrm{~ms}$ after the first energizing timing. In the whole evolution, the main injection soot mass has no difference except the lower in the case $\mathrm{PIE}=621 \mu \mathrm{s}$. 


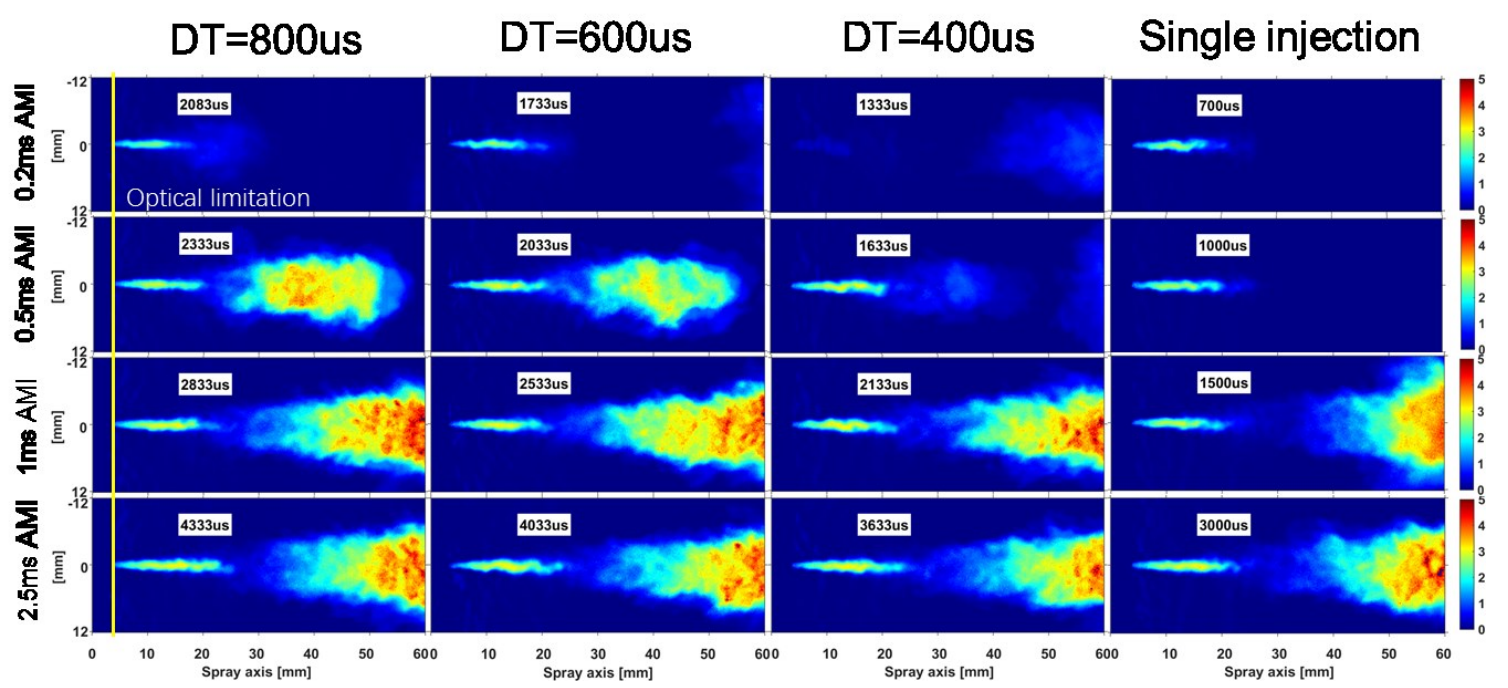

Fig. 5. Soot evolution of DT cases

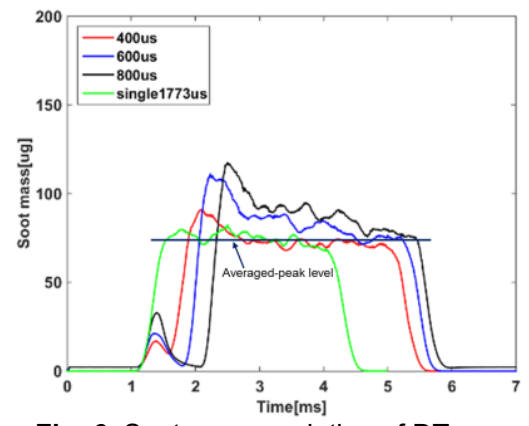

Fig. 6. Soot mass evolution of DT cases

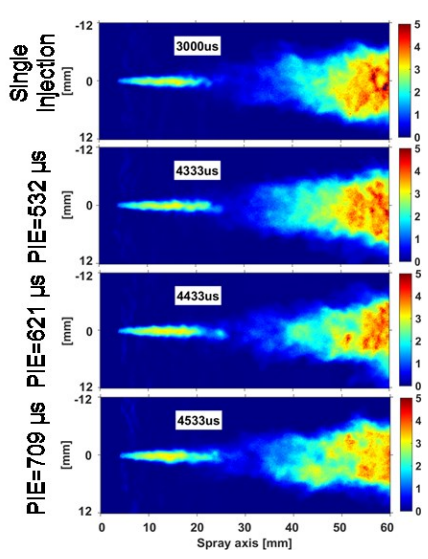

(a) 2-D soot concentration

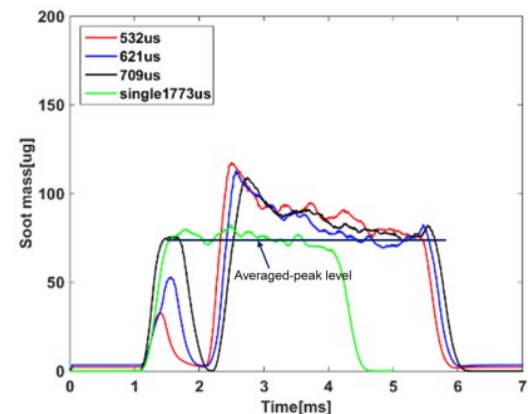

(b) Total soot mass evolution

Fig. 7. Soot distribution of PIE cases

\section{Conclusions}

In the paper, BDIEI technique was employed to detect liquid length and total soot mass, ignition delay was investigated by direct high-speed camera. The test matrix contains different DT (400 $\mu \mathrm{s}, 600 \mu \mathrm{s}, 800 \mu \mathrm{s})$ and PIE $(532 \mu \mathrm{s}, 621 \mu \mathrm{s}, 709 \mu \mathrm{s})$. The constant ambient condition inside the chamber is ambient temperature $900 \mathrm{~K}$, injection pressure $150 \mathrm{MPa}$ and ambient gas pressure $45 \mathrm{bar}$. All the points were measured in a constant volume combustion chamber equipped with a single-hole injector. The fuel used is $0 \#$ diesel. Conclusions are summarized as follows:

- In a such short dwell time, ignition delay decreases with the increasing dwell time, and the flame boundary of two injection is blurry in the case $D T=400 \mu \mathrm{s}$. DT $=400 \mu \mathrm{s}$ is dissatisfied with the injector needle delay

- For the liquid length, shorter dwell time leads to slightly longer liquid-phase penetration. However, pilot/main ratio shows slight impact, all the curves are overlapped. 
- It can be seen that the max soot concentration is located in the center of the soot distribution. The soot formation of the reference is lower than that of the DT cases, except the DT=400 $\mu$ s is similar. In the total soot mass evolution, the first peak level is found in all cases except the reference. The comparison shows that the total soot mass increases with the increase of dwell time while the pilot injection duration shows no effect. In all the comparison, the soot production of the main injection in the two-stage injections are higher than that of the reference.

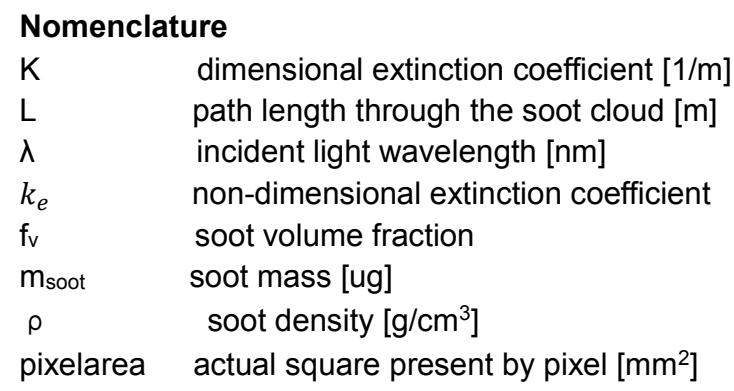

\section{Acknowledgements}

This research was supported by the National Natural Science Foundation of China (No. 51176066). Practice Innovation Project of Jiangsu Province (SJLX15 0491) and Natural Science Foundation of Jiangsu Province (BK20161349).

\section{References}

[1] Y. Imaoka, K. Shouji, T. Inoue, T. Noda. A study of a multistage injection mechanism for improving the combustion of direct-injection gasoline engines, SAE Int. J. Engines 8 (2015) 1080-1087.

[2] D. Martin, J. Stratmann, P. Pischke, R. Kneer, M.-C. Lai, Experimental investigation of the interaction of multiplegdi injections using laser diagnostics, SAE Int. J. Engines 3 (2010) 372-388

[3] J. O'Connor, M. Musculus. In-cylinder mechanisms of soot reduction by close-coupled post-injections as revealed by imaging of soot luminosity and planar laser-induced soot incandescence in a heavy-duty diesel engine, SAE Int. J. Engines 7 (2014) 673-693.

[4] Chen SK. Simultaneous reduction of NOx and particulate emissions by using multiple injections in a small diesel engine. SAE paper. 2000:01-3084

[5] Mohammad Reza Herfatmanesh, Pin Lu, Hua Zhao et al. Experimental investigation into the effects of twostage injection on fuel injection quantity, combustion and emissions in a high-speed optical common rail diesel engine[J]. Fuel, 109 (2013) 137-147

[6] Kim H, Lee J, Kim K, Lee K. Effect of the Atkinson cycle combined with calibration factors on a two-stage injection-type premixed charge compression ignition engine[J]. Energy Fuel 23 (2009) 4908-4916

[7] Kim YJ, Kim KB, Lee KH. Effect of a 2-stage injection strategy on the combustion and flame characteristics in a PCCl engine. Int J Autom Technol, 2011;12:639-44

[8] Khanh Cung, Seong-Yong Lee, Abdul Moiz et al. Spray-combustion interaction mechanism of multipleinjection under diesel engine conditions[J]. Proceedings of the Combustion Institute, 35(2015) 3061-3068.

[9] L.M. Pickett, S. Kook, T.C. Williams. Transient liquid penetration of early-injection diesel sprays, SAE Int. J. Engines 2 (2009) 785-804.

[10] S. Skeen, J. Manin, L.M. Pickett, Visualization of Ignition Processes in high-pressure sprays with multiple injections of n-dodecane, SAE Int. J. Engines 8 (2015) 696-715.

[11] Scott Skeen, Julien Manin, Lyle M Pickett. Visualization of Ignition Processed in High-pressure Sprays with Multiple Injections of n-Dodecane[C]. SAE Paper, 2015-01-0799

[12] Ahmed Abdul Moiz, Khanh D. Cung, Seong-Young Lee. Simultaneous Schlieren-PLIF Studies for Ignition and Soot Luminosity Visualization with Close-Coupled High-Pressure Double Injections of n-Dodecane[J]. Journal of Energy Resources Technology, 2017/ 012207-1

[13] Ahmed Abdul Moiz, Muhsin M Ameen, Seong-Young Lee et al. Study of soot production for double injections of $\mathrm{n}$-dodecane in $\mathrm{Cl}$ engine-like conditions[J]. Combustion and Flame, 173(2016) 123-131.

[14] Wei Jing, Zengyang Wu, Tiegang Fang. Spray combustion of biomass-based renewable diesel fuel using multiple injection strategy in a constant volume combustion chamber[J]. Fuel, 181(2016) 718-728.

[15] Shayler et al. SAE Paper, 2005-01-0375

[16] Carlucci P, Ficarella A, Laforgia D. Effects of pilot injection parameters on combustion for common rail diesel engines. SAE 2003-01-0700; 2003

[17] Da li, Zhixia He, Tiemin Xuan et al. Simultaneous capture of liquid length of spray and flame lift-off length for second-generation biodiesel/diesel blended fuel in a constant volume combustion chamber[J].Fuel,2017(189):260-269

[18] H. Zhao, N. Ladommatos. Optical diagnostics for soot and temperature measurement in diesel engine[J]. Prog. Energy combust.1998(24):221-255 
[19] Liang Zheng, Xiao Ma, Zhi Wang et al. An optical study on liquid-phase penetration, flame liftoff location and soot volume fraction distribution of gasoline-diesel blends in a constant volume vessel[J]. Fuel,139(2015),365373

[20] Julien Manin, Lyle M. Pickett, Scott A. Skeen et al. Two-color diffused back-illumination imaging as diagnostics for time-resolved soot measurements in reacting sprays[C]. SAE paper,2013

[21] Koylu U. O. and Faeth G. M. Optical properties of overfire soot in buoyant turbulent diffusion flames at long residence times. Journal of heat transfer, 116(1):152-159, 1994.

[22] Scott A. Skeen, Julien Manin, Kristine Dalen et al. Extinction-based imaging of soot processes over a range of diesel operating conditions[C]. 8th U. S. National Combustion Meeting,2013

[23] Engine Combustion Network webpage: http://www.sandia.gov/ecn (online)

[24] Manin, J., Bardi, M., and Pickett, L.M., "Evaluation of the liquid length via diffused back-illumination imaging in vaporizing diesel sprays," International Symposium COMODIA 2012 SP2-4, 2012

[25] Lequien Guillaume, Berrocal Edouard, Gallo Yann, et al. Effect of jet-jet interactions on the liquid fuel penetration in an optical heavy-duty DI diesel engine, SAE 2013-01-1615.

[26] Yuri Martin Wright, Pickett, L.M et al. Onset and progression of soot in high-pressure n-docecane sprays under diesel engine conditions[J]. International Journal of Engine Research. 


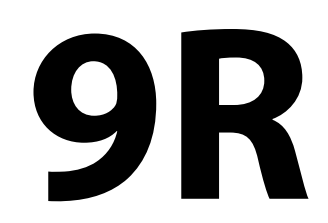

Internal flow 1 


\title{
Towards design optimization of high-pressure gasoline injectors using Genetic Algorithm coupled with Computational Fluid Dynamics (CFD)
}

\author{
Robin Hellmann*, Paul Jochmann ${ }^{1}$, Karl Georg Stapf ${ }^{1}$, Erik Schuenemann ${ }^{1}$, László Daróczy² \\ Dominique Thévenin ${ }^{2}$ \\ ${ }^{1}$ Robert Bosch GmbH, Schwieberdingen, Germany \\ ${ }^{2}$ The Laboratory of Fluid Dynamics and Technical Flows, Otto-von-Guericke-University, \\ Magdeburg, Germany \\ *Corresponding author: robin.hellmann@de.bosch.com
}

\begin{abstract}
The spray pattern of high-pressure multi-hole injectors as well as the atomization process are of uttermost importance regarding efficiency and emissions in gasoline combustion engines. Ensuring optimal homogenization while meeting the engine individual specifications regarding spray targeting and massflow is a crucial development goal. High effort is put on the layout of the nozzle seat to meet the engine requirements. Success is only possible with a deep knowledge of the influencing quantities, considering that many design parameters affect the inner nozzle flow. Based on this knowledge improvement in spray penetration length and atomization can be achieved.

In the current investigation a segment model of the injector is considered. A fully automated, highly parallelized workflow enables a systematic examination of the constrained design space with acceptable computational time. The CFD workflow is implemented in the OPtimization Algorithm Library++ (OPAL++) developed at the "Otto von Guericke" University of Magdeburg.

First, inner nozzle flow 3D-CFD calculations of two selected nozzle geometries are validated by comparison with shadowgraphy and Long-Distance-Microscope (LDM) measurements. Using these simulations, correlations between nozzle flow parameters and the key spray characteristics, serving as optimization objectives, are analyzed. Second, a Design-of-Experiment (DoE) is created to understand the interdependency between design variables and objectives. Based on the DoE, metamodels are constructed, validated, compared with each other and used for optimization. Afterwards, a direct 3D CFD-optimization is carried out for the nozzle geometry. It relies on a Genetic Algorithm in OPAL++ to identify the Pareto front of the multi-objective problem. Finally, the Pareto front is analyzed and conclusions are drawn for future research.
\end{abstract}

\section{Keywords}

Nozzle flow, High Pressure Injector, CFD, Metamodeling, Genetic Algorithm, Optimization

\section{Introduction}

In the recent years the demand for high power output and high efficiency combined with stricter emission regulations has led to a broad use of Gasoline Direct Injection (GDI) engines. To fulfill the engine individual requirements as well as the legislation emission regulations high development effort was put towards the injection system. Different fields of investigations cover system pressure, type and position of injector, injection timing as well as geometric studies of the key valve seat parameter, e.g. [17,22]. The latter are principally responsible for the primary spray characteristics like spray plume trajectory, spray plume cone angle, atomization and penetration. Further engine-specific demands, e.g., cylinder geometry, charge motion, mass flow rate, etc., require a flexible design. The designs of nozzle seat and spray hole are crucial for the development of current combustion systems [11,21].

Most published studies consider only a few designs obtained by changing a single parameter $[14,15,20]$. For multihole high pressure gasoline injectors the manufacturing process has reached a high degree of design flexibility, e.g., due to laser-drilled spray holes. A systematic exploration of this design space with manufactured injectors would be very expensive and time consuming. However, rising computational power together with more efficient simulation models opens the field for extensive design parameter studies; it is then possible to use genetic algorithms (GA) for design optimization. Optimization based on Computational Fluid Dynamics (abbreviated CFD-O) with the help of GA has been already successfully applied to various engineering problems, e.g., [7,8]. In the present paper the goal of the optimization is to achieve the best possible atomization and to lower the penetration length of a single plume by maximizing the Turbulent Kinetic Energy (TKE) and the spray opening angle $\beta$ at the spray hole outlet plane. For this purpose, four design variables are selected: spray hole length $s h_{l}$, spray hole conicity $s h_{c o n}$, counterbore diameter $c b_{d}$ and the wall thickness $w t$ of the valve seat.

Current state-of-the-art computational fluid dynamic (CFD) methods are capable to predict the massflow and spray characteristics with sufficient accuracy. In the present study idealized conditions (1 bar cylinder pressure with an incompressible and isothermal flow at $20^{\circ} \mathrm{C}$ ) are applied to the Reynolds-averaged Navier-Stokes (RANS) simulation method using an Eulerian multiphase treatment. The setup is first validated with Long Distance Microscope (LDM) and shadowgraphy measurements of a generic two-hole injector. Correlations between the two chosen objectives and the nozzle flow data from the simulations are identified. 
As 3D-CFD simulations of the inner nozzle flow are still computationally time-consuming, only a segment of the injector with a smaller computational domain is considered. A fully automatic workflow is set up consisting of geometry creation, meshing, definition and solving of the problem, postprocessing as well as coupling with the GA. Using OPAL++, approximation models can be considered, so that response surface models (RSM) are built on a design of experiments (DoE), like suggested in $[16,18,19]$. For each objective a RSM is built by a regression analysis and used to identify interrelationships between design variables and objectives. Furthermore, the RSM models are deployed to a multi-objective optimization process, which later is compared with the results of a pure CFD-based optimization.

\section{Geometry and Numerical Method}

Figure 1 shows the high pressure gasoline injector (left), the valve seat including the pintle (middle) and the simulation domain of a segment model (right). To better understand the interdependency between inner nozzle flow simulations and spray characteristics, measurements were performed with a purpose-built two-hole injector, each hole arranged at the same radius from the injector axis at $180^{\circ}$ circumferential spacing. The nozzle inclination angles $\alpha$, skew angle between vertical injector axis and spray hole axis, are $10^{\circ}$ - hereinafter referred to as "SH1" - and $50^{\circ}$ - hereinafter referred to as "SH2" - leading to two different sharp bends at the transition from the nozzle seat to the spray hole inlet. This region of transition influences the highly transient, non-homogeneous and turbulent inner nozzle flow. Therefore it is indispensable that the numerical model should reflect the flow characteristics of both holes. The remaining geometric parameter of the two holes are identical. The length-to-diameter ratio $s h_{l} / s h_{d}$ of the slightly diverging hole with conicity angle $s h_{c o n}$ is approximately 1 with a nozzle length $s h_{l}$ of order $200 \mu \mathrm{m}$. A cylindrical "counterbore" with length-to-diameter ratio $c b_{l} / c b_{d}$ of approximately 0.75 is attached to the nozzle. The wall thickness $w t$ of the valve seat is hence the sum of spray hole and counterbore length. Figure 2 depicts the parameter being investigated in the present study. Compared to production type multi-hole injectors the nozzle flow and spray plume interaction for the two-hole injector is, due to the distance, negligible [10]. The assumption of spray hole independency permits the analysis of a segment model representing one single hole from a five-hole injector. The simulation domain covers the smallest gap between pintle and valve seat, the spray hole with downstream counterbore and the cylinder volume, partly represented by an ambient domain ending after an axial expansion of $0.5 \mathrm{~mm}$, like depicted in Figure 1. All subsequent investigations do not include pintle motion as this phase is only about ten percent of the whole injection duration. Thus this impact on the penetration length is negligible for a typical homogeneous gasoline combustion process. Additionally limited computational effort required for CFD-O can be ensured. Hence, the transient simulations are performed for a static geometry with a pintle displacement at full lift.
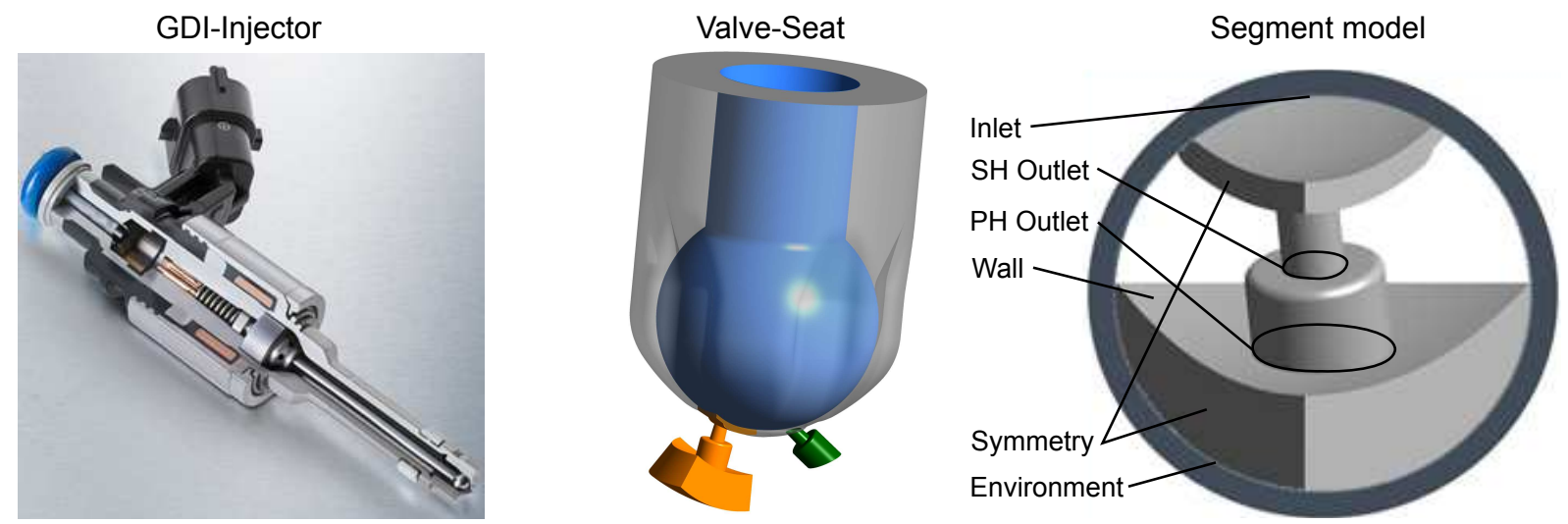

Figure 1. Simulation domain of segment model [23]

ANSYS CFX, a commercial CFD tool, is employed to simulate the two-component and two-phase flow (n-heptane, $\mathrm{n}$-heptane vapor, air) of the high-pressure injector. The three-fluid system is represented with a homogeneous model, an Eulerian-Eulerian method where all phases share a single velocity field [24]. Mass transfer between fuel and fuel vapor is considered with the use of the Rayleigh-Plesset cavitation model. Turbulence is treated with the RANS approach where flow variables are decomposed into mean and fluctuation terms. Stochastic effects of turbulence is represented through the two additional transport equations of the $k$ - $\omega$-SST turbulence model, used to close the system of partial differential RANS equations for mass and momentum conservation. Furthermore, the flow is considered as incompressible and isothermal at $293.15 \mathrm{~K}$. A total pressure of 200 bar serves as inlet boundary condition and an opening with a reference pressure of 1 bar is applied to the boundaries of the ambient domain. The sidewalls are treated as symmetry planes.

The simulation setup for validation covers a physical time of $0.125 \mathrm{~ms}$ with a timestep of $10^{-7}$ seconds. As the flow field has to develop from rest the analysis starts after $0.025 \mathrm{~ms}$. ANSYS CFX uses the Finite Volume Method for spatial discretization so that a hexahedral mesh is generated with ANSYS Meshing. Mesh refinements in the regions of high pressure and velocity gradients, i.e., close to the walls, in the spray hole and counterbore, ensure an adequate solution of the complex 3D-flow. Results from a preliminary mesh study lead to the mesh consisting of roughly 3 million nodes with average axial cell size in the spray hole of $3.5 \mu \mathrm{m}$. 


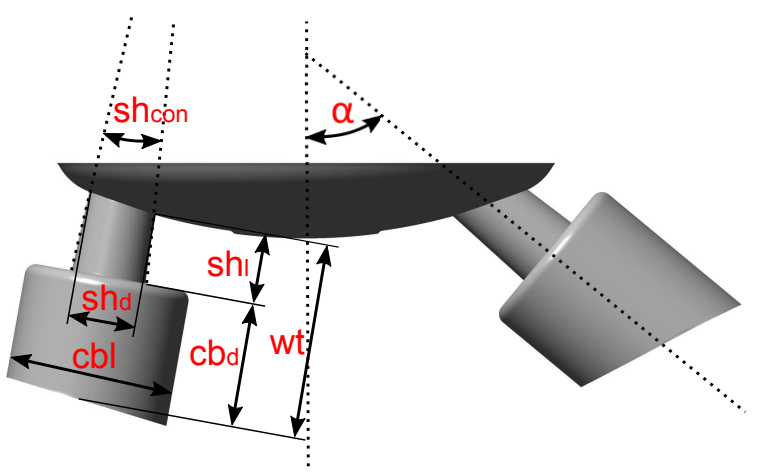

Figure 2. Geometry parameter

\section{Validation and Correlations}

To validate the simulation setup, the outflow and the spray in the nearfield of the injector are recorded with a LongDistance-Microscope (LDM) at $100 \mathrm{kHz}$ in a spray chamber. Additionally, shadowgraphy measurements at $25 \mathrm{kHz}$ visualize the penetration for a large image section of $124 \mathrm{~mm} \times 100 \mathrm{~mm}$.

Table 1. Simulation setup and measurement conditions

\begin{tabular}{r|ccccc}
\hline & $\begin{array}{c}\text { Pressure } \\
\text { difference }\end{array}$ & Fuel & Temperature & $\begin{array}{c}\text { Chamber } \\
\text { fluid }\end{array}$ & Duration \\
\hline Measurement & $200 \mathrm{bar}$ & n-heptane & $293.15 \mathrm{~K}$ & $\mathrm{~N}_{2}$ & $1.5 \mathrm{~ms}$ \\
Simulation & $200 \mathrm{bar}$ & n-heptane & $293.15 \mathrm{~K}$ & Air & $0.125 \mathrm{~ms}$
\end{tabular}

The conditions of the spray chamber compared to the simulation setup are given in Table 1. For safety reason the spray chamber is purged with $\mathrm{N}_{2}$ instead of air, as used in the simulation setup. For the spray chamber tests a realistic injection duration of $1.5 \mathrm{~ms}$ is chosen to ensure a static massflow in the middle of the injection sequence. First, shadowgraphy measurements (Figure 3) visualize the spray plume penetration with a frequency of $200 \mu \mathrm{s}$. The pintle motion at the beginning of injection results in a lower penetration for $\mathrm{SH} 2$, however, this effect levels out so that after $1000 \mu$ s the penetration length of both holes is approximately equal.

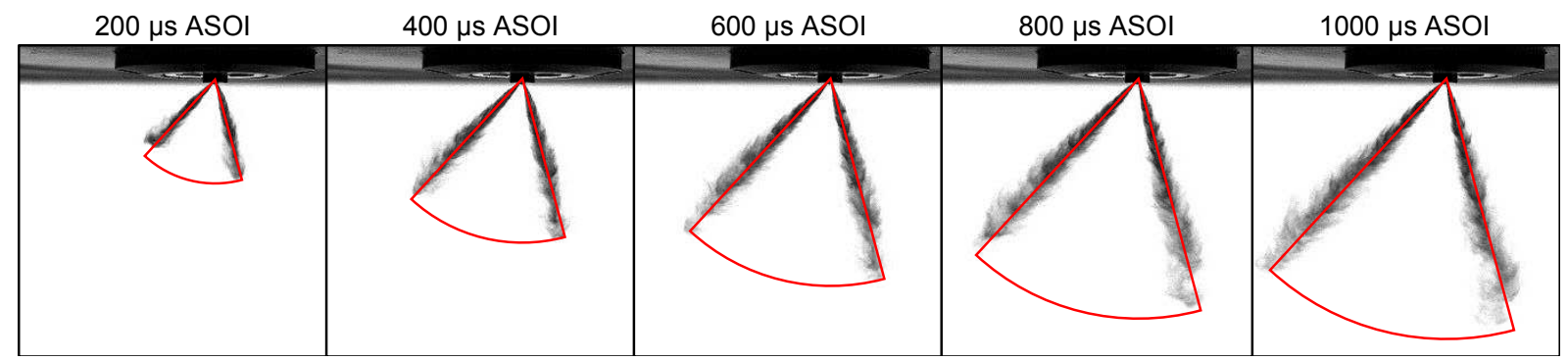

Figure 3. Shadowgraphy at five timesteps

Then, the simulation results of the segment model are compared with LDM measurements of the two single plumes. Figure 4 shows the spray in the injector nearfield after an injection duration of approximately $1 \mathrm{~ms}$. The fuel is represented by isosurfaces with a constant volume fraction of 0.1 colored with the corresponding velocity. The red lines represents the plume opening angle $\beta$ closest to the counterbore exit plane. In Table 2 the penetration after $600 \mu \mathrm{s}$ is linked to the spray opening angle, confirming the expected relationship of a declining penetration with increasing opening angle. Furthermore, the direction of the simulated spray plumes match the measured ones, see Figure 4. It is worth noting that the RANS simulation shows a starting breakup, so that also the onset the atomization process is approximately covered. Anyhow, the focus of this paper will be limited to the spray opening angle $\beta$.

Figure 5 shows contours of fuel volume fraction as well as velocity vectors to compare the spray direction and spray opening angle $\beta$. With help of the nozzle flow data the measured and simulated spray opening angles $\beta$ can be analyzed, see Table 2. The simulation is able to predict the opening angle for both holes at the counterbore exit planes in close agreement with the measurement. The simulated $\beta$-angles are for both holes approximately two degree too low, which can also be slightly seen in Figure 4. However, it is crucial to note that SH2 has, compared to $\mathrm{SH} 1$, an almost two degree higher opening angle in the simulation as well as in the measurements. Hence, the 


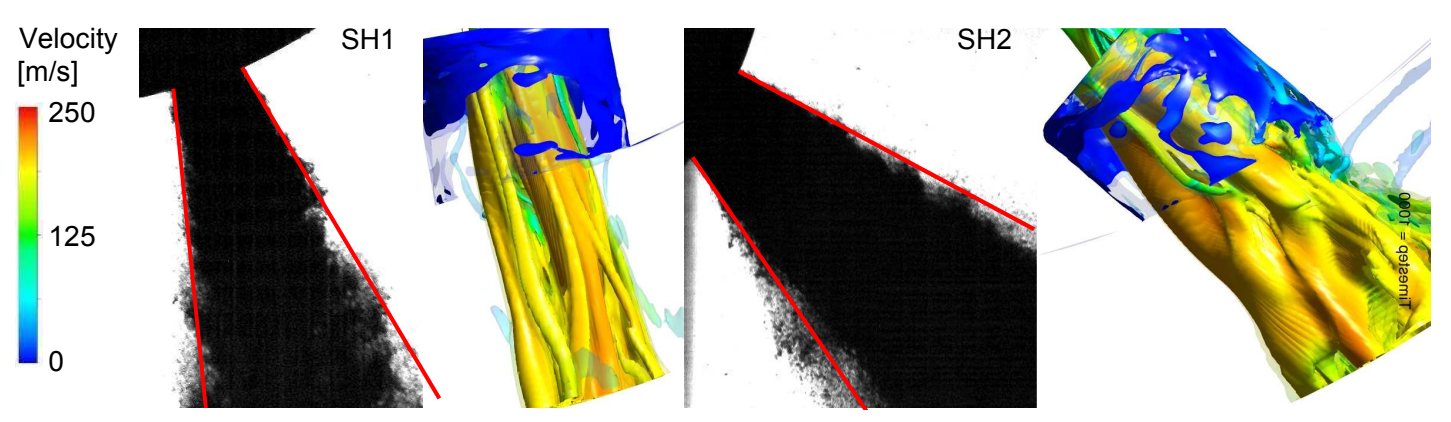

Figure 4. LDM vs. simulated isosurfaces

correlation between spray opening angle and penetration length is found identically in simulation and experiment. To limit computational effort, the spray opening angle at the spray hole outlet will be used as an objective for the optimization tasks. At this plane the simulated values are higher, as the spray guidance of the counterbore walls is missing, see Table 2. Hence, the described tendency is even more intense at this plane, leading to a high $\beta$-angle of $42.94^{\circ}$ for $\mathrm{SH} 2$ compared to $32.10^{\circ}$ for $\mathrm{SH} 1$.

Table 2. Correlations between measurements, objective $\beta$ and inner nozzle flow data

\begin{tabular}{lc|cc|cc}
\hline \multicolumn{2}{c|}{ Geometry } & \multicolumn{2}{c|}{ Measured } & \multicolumn{2}{c}{ Simulation at Outlet of } \\
referred as & $\alpha$ & Penetration $(600 \mu s)$ & $\beta$ & $\beta$ CB & $\beta$ SH \\
\hline SH1 & $10^{\circ}$ & $58.13 \mathrm{~mm}$ & $24.55^{\circ}$ & $22.82^{\circ}$ & $32.10^{\circ}$ \\
SH2 & $50^{\circ}$ & $56.40 \mathrm{~mm}$ & $26.68^{\circ}$ & $24.96^{\circ}$ & $42.94^{\circ}$
\end{tabular}

\section{Contour: \\ 1.0 \\ 0.5 \\ 0.0}

Fuel Volume

Fraction [-]
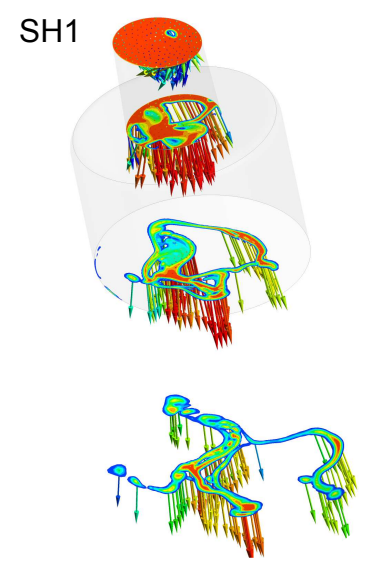

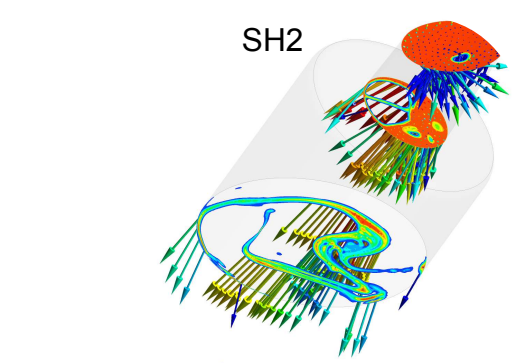

Vector: Velocity $[\mathrm{m} / \mathrm{s}]$

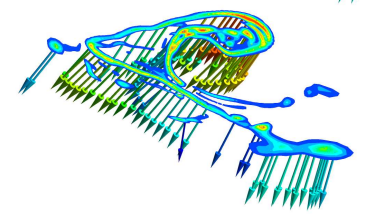

Figure 5. Distribution of fuel volume fraction and velocity vectors

Finally the validation shows that the simulation setup is able to capture the nozzle flow characteristics. A correlation between the objective (low spray penetration) and the spray opening angle $\beta$ can be clearly seen. The values of the predicted spray opening angle $\beta$ at counterbore outlet are in close agreement to the measured angles. At spray hole exit, much higher values are found, but the proper tendency can be clearly identified. Thus, the $\beta$ angle at spray hole exit can be used for optimization.

\section{Metamodeling and Optimization}

The aim of a multi-objective problem is to improve simultaneously the values of all, at least two, objectives, without violating any predefined constraint. A minimization problem can, without any loss of generality, be defined as in equation 1 ,

$$
\begin{aligned}
& \min _{\mathbf{x} \in \mathbb{R}^{n}} y(\mathbf{x}), \text { with } y(\mathbf{x})=\left(f_{1}(\mathbf{x}), f_{2}(\mathbf{x}), \ldots, f_{m}(\mathbf{x})\right), m=1,2, \ldots, M \\
& \text { subject to } g_{k}(\mathbf{x}) \leq 0, k=1,2, \ldots, K \\
& h_{l}(\mathbf{x})=0, l=1,2, \ldots, L \\
& x_{i}^{l} \leq x_{i} \leq x_{i}^{u}, i=1,2, \ldots, N
\end{aligned}
$$

where $\mathbf{x}$ is the $N$-dimensional vector of design variables, $f_{m}(\mathbf{x})$ are the $M$ objective functions, $g_{k}(\mathbf{x})$ are the $K$ inequality constraints and $h_{l}(\mathbf{x})$ are the $L$ equality constraints. In the present study only inequality constraints are 
used $(L=0)$ so that the feasible design space $\mathbb{X}$ must satisfy the $K$ inequality constraints, lay in between the lower $x_{i}^{l}$ and upper $x_{i}^{u}$ parameter boundaries and be part of $\mathbb{R}^{n}\left(\mathbf{x}^{n} \in \mathbb{X}^{n} \in \mathbb{R}^{n}\right)$. The definition of the multi-objective minimization problem is given in equation 2 .

$$
\text { (I) }: y(\mathbf{x}) \stackrel{\mathbf{x}}{\rightarrow} \min , \text { with } \mathbf{x}^{n} \in \mathbb{X}^{n}
$$

As the selected objectives might be incompatible with each other, a global optimum often does not exist for a multi-objective problem. Hence, comparing two designs and deciding which one is better can become difficult or even impossible. Pareto-dominance is a sorting method that allows identification of the best design from a set of solutions. This method delivers an a posteriori analysis and is defined as follows:

$$
\hat{\mathbf{x}} \prec \mathbf{x} \Leftrightarrow\left\{\begin{array}{l}
\forall i \in[1 \ldots M]: f_{i}(\hat{\mathbf{x}}) \leq f_{i}(\mathbf{x}) \\
\exists j \in[1 \ldots M]: f_{j}(\hat{\mathbf{x}})<f_{j}(\mathbf{x})
\end{array}\right.
$$

Pareto-dominance in equation 3 states that design variable $\hat{\mathbf{x}}$ dominates design variable $\mathbf{x}$ if $f(\hat{\mathbf{x}})$ is not worse for each objective $i$ of $f(\mathbf{x})$ and is at least better in one objective $f_{j}(\mathbf{x})$. A dominant design $\hat{\mathbf{x}}$ is always the preferred solution. With this analysis, the optimization process delivers the Pareto front, i.e. a set of non-dominated solutions, defined as:

$$
P(\mathbb{X}):=\{\mathbf{x} \in \mathbb{X} \mid \nexists \hat{\mathbf{x}} \in \mathbb{X}: \hat{\mathbf{x}} \prec \mathbf{x}\}
$$

Equation 4 states that all Pareto-optimal solutions are of the same quality. The final goal is to find as many as possible Pareto-optimal solutions in one single run [5]. Except for some simple analytical cases the exact Pareto front cannot be reached, so that iterative methods are required to approximate the front $[3,13]$. Genetic Algorithms are easy to use, widely applicable, and deliver a global optimum. They are therefore broadly used as search and optimization tools [4]. GA use the principle of natural selection including semi-stochastic processes. There are many common GA available [9]. NSGA-II is one of the most well-known approach for multi objective optimization problems [5]. The detailed implementation of the NSGA-II algorithm in OPAL++, e.g. dominance, constraint handling and validity, can be found in [2].

To initialize the population various DoE approaches exist $[1,12]$. Based on the first generation a new set of individuals, the offspring, are generated using the genetic operators selection, crossover and mutation. During the iterative optimization process the GA prefer the individuals with high performance for further genetic operations. Therefore, the new offspring will drift iteratively towards the Pareto front.

Table 3. Optimization settings

\begin{tabular}{c|cccc|cc}
\hline & \multicolumn{4}{|c|}{ Design variable } & \multicolumn{2}{c}{ Objectives at SH Outlet } \\
& $c b_{d}$ & $s h_{\text {con }}$ & $s h_{l}$ & $w t$ & $\beta$ & TKE \\
\hline Lower bound & $300 \mu \mathrm{m}$ & $0^{\circ}$ & $125 \mu \mathrm{m}$ & $300 \mu \mathrm{m}$ & - & - \\
Upper bound & $550 \mu \mathrm{m}$ & $25^{\circ}$ & $250 \mu \mathrm{m}$ & $600 \mu \mathrm{m}$ & $\max$ & $\max$
\end{tabular}

Table 3 represents the four input parameters with their ranges and the two objectives: maximize TKE and $\beta$ at the spray hole outlet. Furthermore, a minimum counterbore length is set with an inequality constraint $g_{1}(\mathbf{x})$. To construct a polynomial approximation for the described problem a DoE of size 142 is first generated using Sobol sampling, a quasi-random sequence delivering a uniform distribution. This method is recommended for limited sample sizes, as here [1]. Based on the DoE an Iterative Reweighted Least Square (IR_LSQ), a global polynomial regression method of total order three is applied as a Response Surface having 35 coefficients $c_{m}$. The estimated value $s$ of the IR_LSQ is given by:

$$
\begin{array}{r}
s(\mathbf{x})=p^{T}(\mathbf{x}) c_{m} \\
\text { with } p^{T}(\mathbf{x})=\left[p_{1}(\mathbf{x}), p_{2}(\mathbf{x}), \ldots p_{m}(\mathbf{x})\right]
\end{array}
$$

The unknown coefficients $c_{m}$ are iteratively determined by minimizing the weighted least square errors between predicted values $s$ and the simulated values $Y$ at the $N$ sample points:

$$
\sum_{i=1}^{N}\left[p^{T}\left(\mathbf{x}_{i}\right) c_{m}-Y\right]^{2} \stackrel{c}{\rightarrow} \min
$$

Due to software failures, poor mesh quality or constraint violations 37 design have been found to be invalid, so that in total 105 designs have been evaluated on a High Performance Computing (HPC) cluster. In Figure 6 a poor fit for the objective TKE is seen with a R-square value of only 0.34 . This result could probably be improved by adding more coefficients to the IR_LSQ model [6], which is here impossible due to the high computational effort of each single evaluation. Especially the prediction of the individual with a simulated TKE of approx. $300 \mathrm{~m}^{2} / \mathrm{s}^{2}$ is poor, showing that the metamodel does not properly predict the region of interest (high TKE values). Fortunately, the predicted values for the spray opening angle $\beta$ show over the whole range an excellent agreement with the simulated ones, see Figure 7. 
In Figure 8 the 3D-response for TKE and in Figure 9 the response for $\beta$ over the spray hole conicity $s h_{\text {con }}$ and wall thickness $w t$ of the nozzle seat are shown. The two normalized 3D-responses are only valid for selected values of counterbore diameter and spray hole length $\left(c b_{d}=400 \mu \mathrm{m}, s h_{l}=200 \mu \mathrm{m}\right)$. For this configuration the spray opening angle $\beta$ is dominated by the spray hole conicity $s h_{c o n}$, leading to a higher spray opening angle $\beta$ when decreasing spray hole conicity $s h_{c o n}$.
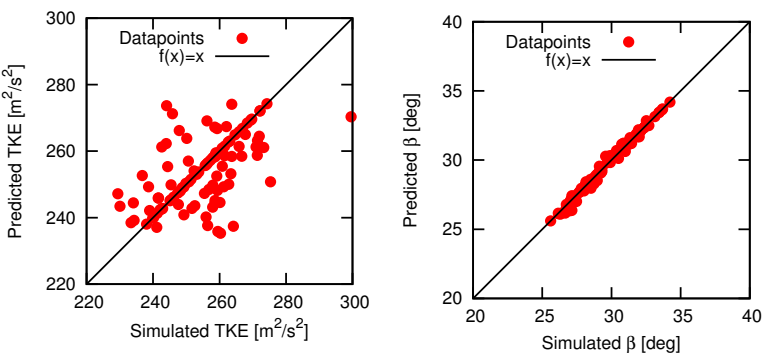

Figure 6. Metamodeling TKE
Figure 7. Metamodeling for $\beta$

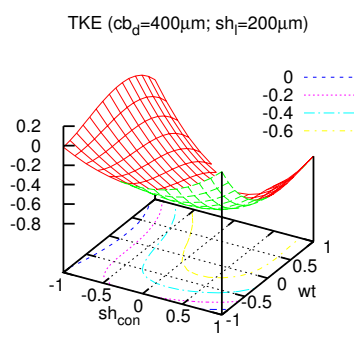

Figure 8. Reponse for TKE

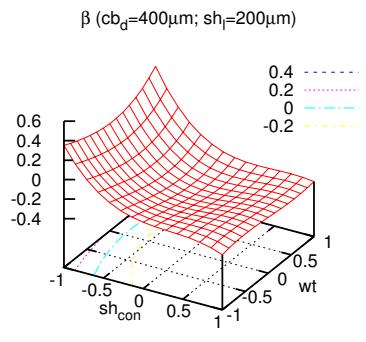

Figure 9. Response for $\beta$

The two IR_LSQ models are then used in a metamodel-driven optimization, which evaluates a parameter set by using two algebraic equations. This fast evaluation allows a NSGA-II multi-objective optimization with a large population size of 200 for 50 generations. Figure 10 shows the distribution of the DoE, the Pareto front of the first and of the last $\left(50^{\text {th }}\right)$, generation. Additionally, three design points (ID1 - ID3), at the most meaningful positions of the Pareto front, are selected for validation; the corresponding CFD results are shown with colored triangles. In agreement with Figure 7, the prediction of $\beta$ obtained by CFD agrees with the metamodel; the triangles stay roughly at the same height in the diagram; however, CFD leads to a much different value of TKE, so that the triangles are widely shifted to the left side.

Figure 11 links the objectives of the three selected Pareto-optimal solutions with the design variables. The TKE at spray hole outlet plane as well as velocity vectors are also shown. All configurations correspond to the lowest possible counterbore diameter and spray hole length. The high difference in spray opening angle $\beta$ between ID1 and the rest of the Pareto-optimal solutions is due to the small spray hole conicity and the highest possible wall thickness. ID2 and ID3 correspond to small geometry changes in wall thickness and spray hole conicity. Therefore, the simulated results from ID2 and ID3 only differ slightly from each other. It appears that TKE metamodel shows excessive sensitivity, leading to poor prediction accuracy, especially in the region of high TKE values.

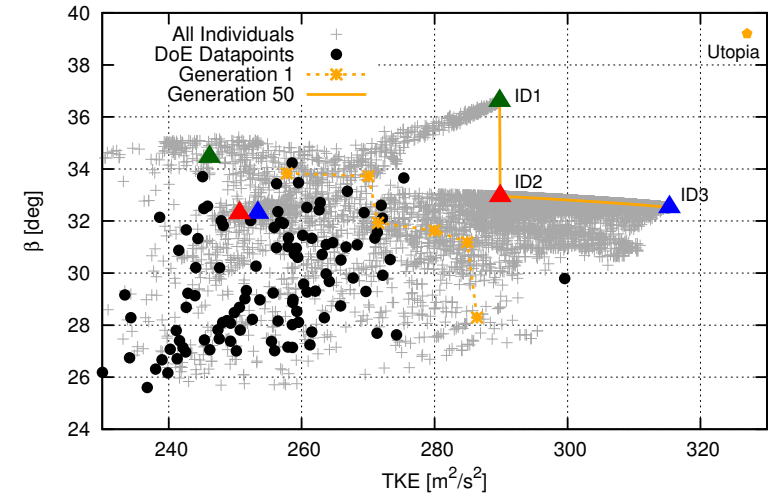

Figure 10. Pareto front for the metamodel-based NSGA II
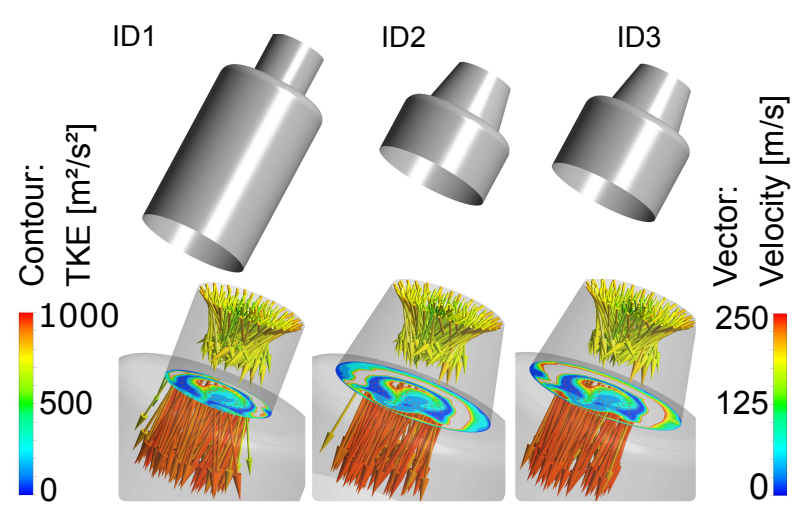

Figure 11. Geometry and flow of Pareto-optimal designs

In order to check how the true Pareto front looks like, a direct multi objective optimization with the NSGA-II algorithm has been carried out. Unfortunately, due to computational cost, it involves only a population size of 48 . Figure 12 depicts the DoE and the Pareto front of the first and last $\left(10^{\text {th }}\right)$ generation. A design improvement can clearly be seen as the whole Pareto front moves towards the top-right corner. Again, three meaningful optimal individuals have been selected for a detailed analysis, illustrated in Figure 13.

A low spray hole conicity shows a positive effect on TKE and on $\beta$, so that all three designs correspond to minimal conicity. A broader range for this parameter could deliver even better designs. The remaining three design variables show large differences within the Pareto-optimal solutions. For the spray hole length two contradictory trends can be observed for the objectives: The higher the spray hole length, the higher the TKE; the lower the spray hole length, the higher the spray opening angle $\beta$. ID1 has the maximum TKE with an intermediate counterbore diameter, a maximum spray hole length and a low wall thickness resulting in a very short counterbore length. On the opposite, ID5 shows the highest simulated value in $\beta$, but a low TKE value.

Like in the validation section, the flow is investigated with the help of contours representing the fuel volume fraction 


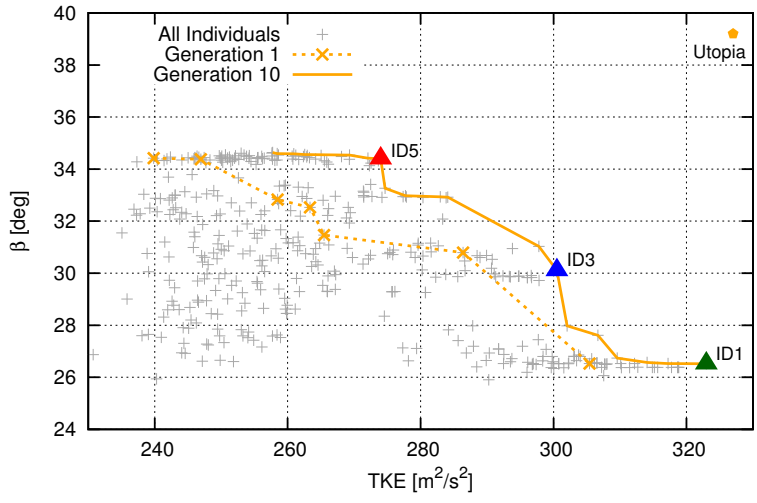

Figure 12. Pareto front for the direct NSGA II

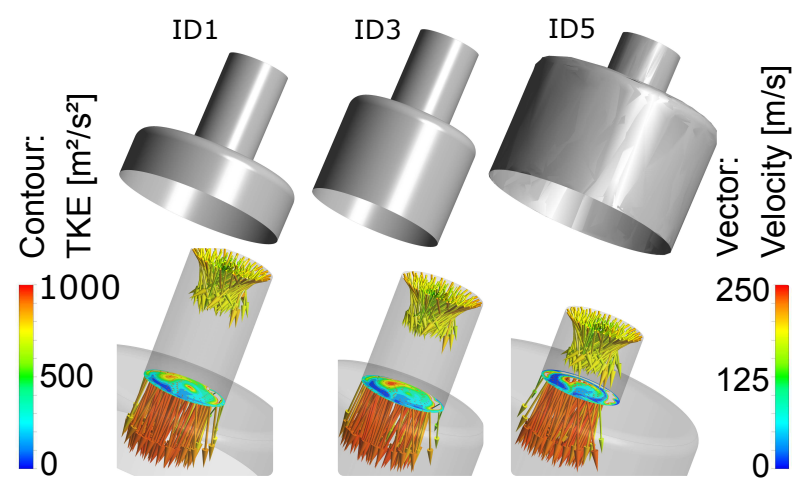

Figure 13. Geometry and flow of Pareto-optimal designs

and velocity vectors (Figure 14). The volume fractions at the bottom show the distributions at the opening boundary of the segment model. Looking at the velocity vectors it is confirmed that a longer spray hole leads to a lower $\beta$ angle. Therefore, ID5 shows the widest spread of the spray plume. The trends detected by the direct optimization can also be seen further downstream, e.g. at the counterbore outlet and at the boundary condition of the simulation domain.

\section{Contour: \\ Fuel Volume \\ Fraction [-]}

1.0
0.5
0.0

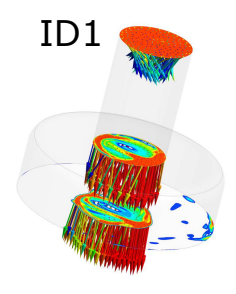

ID1

(n)

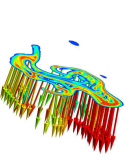

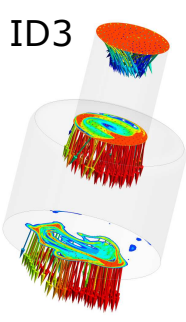

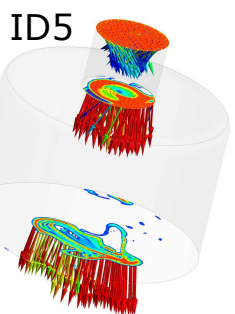

Vector:

Velocity $[\mathrm{m} / \mathrm{s}]$

175

Figure 14. Distribution of Fuel Volume Fraction and velocity vectors

\section{Conclusions}

The RANS simulation setup of a segment model has the capability to reveal the main flow characteristics for a GDI inner nozzle flow. A comparison with LDM and shadowgraphy measurements show correct trends concerning spray direction, spray opening angle and distribution of the plume at counterbore outlet. First correlations between inner nozzle flow and penetration length have been derived and used as objective functions for a multi-objective optimization. Reaching a low penetration while conserving a high turbulent breakup should be obtained thanks to a high TKE and a high spray opening angle at the spray hole outlet.

An optimization setup with four design variables has been presented. Response surfaces (IR_LSQ of order three) for a Sobol-sampled DoE containing 105 valid design points have been analyzed leading to following conclusions:

- The metamodel for the spray opening angle $\beta$ shows promising results and is suitable for optimization.

- The TKE-metamodel is not accurate enough to capture the influence of geometry changes.

Hence, selected Pareto-optimal solutions of the metamodel-based optimization show high deviations in TKE when comparing with CFD. For comparison with this fast metamodel optimization, a computationally challenging direct optimization was carried out using NSGA2 within approximately 250,000 CPU hours. After 10 generations with a population size of 48 , considerably improved designs can be obtained. The two most noticeable results affecting nozzle design are:

1. A low spray hole conicity has a positive effect for both objectives. Therefore, all the optimal solutions nearly share the lowest possible spray hole conicity.

2. The longer the spray hole length, the lower the spray opening angle, but the higher the TKE. 
The use of GA for nozzle seat optimization will be pursued. The parameter range for the investigated variables will be increased towards manufacturing constraints. The influence of turbulence modelling on complex nozzle flows will be further investigated by comparing the results of RANS with LES (Large-Eddy-Simulations). Using a second validation case, the prediction reliability of the simulation model regarding spray plume penetration will be verified, increasing confidence in the presented approach.

\section{Acknowledgements}

The authors would like to thank Dipl.-Ing. M. Lausch for his support regarding the simulation domain and setup. Furthermore we would like to thank Dr. P. Leick for providing the shadowgraphy and LDM measurements.

\section{Nomenclature}

$\begin{array}{ll}\text { DoE } & \text { Design-of-Experience } \\ \text { LDM } & \text { Long-Distance-Microscope } \\ \text { GDI } & \text { Gasoline-Direct-Injection } \\ \text { CFD } & \text { Computational Fluid Dynamics } \\ \text { CFD-O } & \text { CFD-Optimization } \\ \text { GA } & \text { Genetic Algorithm } \\ \text { RSM } & \text { Response Surface Method } \\ \text { RANS } & \text { Reynolds Averaged Navier Stokes } \\ \text { LES } & \text { Large-Eddy-Simulation } \\ \text { IR_LSQ } & \text { Iterative Reweighted Least Square }\end{array}$

$\begin{array}{ll}\text { HPC } & \text { High Performance Computing } \\ \text { NSGA } & \text { Non-Sorting Genetic Algorithm } \\ \text { TKE } & \text { Turbulent Kinetic Energy }\left[\mathrm{m}^{2} \mathrm{~s}^{-2}\right] \\ c b_{d} & \text { counterbore diameter }[\mu \mathrm{m}] \\ s h_{d} & \text { spray hole diameter }[\mu \mathrm{m}] \\ s h_{\text {con }} & \text { spray hole conicity [deg] } \\ s h_{l} & \text { spray hole length }[\mu \mathrm{m}] \\ w t & \text { wall thickness }[\mu \mathrm{m}] \\ \alpha & \text { inclination angle of spray hole }[\mathrm{deg}] \\ \beta & \text { spray plume opening angle [deg] }\end{array}$

\section{References}

[1] Burhenne, S., Jacob, D., Henze, G., Nov. 14.-16. 2011, 12th Conference of International Building Performance Simulation Association.

[2] Daróczy, L., 2016, "Practical issues in the optimization of CFD based engineering problems". PhD thesis, University Magdeburg.

[3] Daróczy, L., Janiga, G., Thévenin, D., 2014, Energy, 65, pp. 364-373.

[4] Deb, K., 1999, Sadhana, 24 (4-5), pp. 293-315.

[5] Deb, K., Pratap, A., Agarwal, S., Meyarivan, T., 2002, IEEE Transactions on Evolutionary Computation, 6 (2), pp. 182-197.

[6] Ender, T., Balestrini-Robinson, S., 2015, "Modeling and Simulation in the Systems Engineering Life Cycle". Springer

[7] Gen, M., Cheng, R., 2000, "Genetic Algorithms and Engineering Optimization". John Wiley and Sons.

[8] Janiga, G., Thévenin, D., 2008, "Optimization and Computational Fluid Dynamics". Springer.

[9] Konak, A., Coit, D., Smith, A. E., 2006, Reliability Engineering \& System Safety, 91 (9), pp. 992-1007.

[10] Köpple, F., Jochmann, P., Kufferath, A., Bargende, M., 2013, SAE International Journal of Engines, 6, pp. 911-925.

[11] Kufferath, A., Wiese, W., Samenfink, W., Dageförde, H., Knorsch, T., Jochmann, P., Mar. 10.-11. 2015, Fuel Systems for IC Engines.

[12] Macdonald, I. A., July 27.-30. 2009, Eleventh International IBPSA Conference.

[13] Marler, T. R., Arora, J. S., 2004, Structural and Multidisciplinary Optimization, 26 (6), pp. 369-395.

[14] Matsumoto, A., Xie, X., Zheng, Yi., Lai, M., Moore, W., May 16.-19. 2010, 22nd Annual Conference on Liquid Atomization and Spray Systems.

[15] Mitroglou, N., Nouri, J. M., Gavaises, M., Arcoumanis, C., 2006, International Journal of Engine Research, 7 (3), pp. 255-270.

[16] Myer, R. H., Montgomery, D. C., 2002, "Response Surface Methodology: Process and Product Optimization Using Designed Experiments". John Wiley and Sons.

[17] Payri, R., Viera, J., Gopalakrishnan, V., Szymkowicz, P. G., 2016, Fuel, 183, pp. 20-33.

[18] Sacks, J., Welch, W. J., Mitchell, T. J., Wynn, H. P., 1989, Statistical science, 4 (4), pp. 409-423.

[19] Simpson, T., Mistree, F., Korte, J., Mauery, T., 1998, 7th AIAA/USAF/NASA/ISSMO Symposium on Multidisciplinary Analysis and Optimization.

[20] Tu, P. W., Xu, H., Srivastava, D. K., Dean, K., Jing, D., Cao, L., Weall, A., Venus, J. K., Sep. 1. 2015, JSAE/SAE 2015 International Powertrains, Fuels \& Lubricants Meeting.

[21] Wiese, W., Kufferath, A., Storch, A., Rogler, P., Feb. 24.-25. 2015, 2. Internationaler Motorenkongress.

[22] Zhao, F.-Q., Yoo, J.-H, Liu, Y., Lai, M.-C., Oct. 1. 1996, 1996 SAE International Fall Fuels and Lubricants Meeting and Exhibition.

[23] Robert Bosch GmbH, http://www.bosch-mediaspace.de/ ([cit. 2017-02-22]).

[24] ANSYS Inc., CFX Solver Theory Guide, http://www . ansys .com/ ([cit. 2017-02-28]). 


\title{
Investigation of the effects of domain representation and boundary condition selection in numerical simulations of micro scale flows with phase change
}

\author{
Daniel Pearce ${ }^{* 1}$, Konstantina Vogiatzaki ${ }^{2}$,A.M.K.P Taylor ${ }^{3}$, Y. Hardalupas ${ }^{3}$ \\ ${ }^{1}$ Delphi Diesel Systems Ltd, Imperial College, London,UK \\ ${ }^{2}$ Advanced Engineering Center, University of Brighton, UK \\ ${ }^{3}$ Mechanical Engineering Department, Imperial College, London, UK \\ *Corresponding author: Daniel.Pearce@delphi.com
}

\begin{abstract}
Cavitation is a phenomenon affected considerably by the underlying pressure waves that occur on similar time and length scales as the bubble dynamics. Thus appropriate representation of wave dynamics within numerical frameworks is of paramount importance for the prediction of the phase change process in the nozzle as well as the subsequent spray formation. In this paper we focus on investigating the sensitivity of the wave dynamics within a compressible Large Eddy Simulation framework with regards to downstream geometry and boundary representation. Diesel was used as working fluid and was injected at various pressures through a micro-channel. Results in terms of vapour fraction, velocity and pressure are compared with the experimental data of Winklhofer [30,31]. The downstream domain length and reflectivity properties are shown to exert a significant effect on in-nozzle processes.
\end{abstract}

\section{Keywords}

cavitation, LES, pressure waves, OpenFOAM

\section{Introduction}

The term cavitation is used to describe the phenomenon of vapour production within a homogeneous liquid due to the pressure reduction below the vapour pressure of the liquid. Dynamically occurring cavitation arises when the local flow conditions are such that the fluid detaches from a wall (sometimes at a "salient point" such as a corner) and if the associated streamline curvature is sufficiently small, low pressure regions are formed on the surface as in propellers, hydrofoils etc. A different mechanism can arise in orifices and nozzles, where the high speed flow through the throat causes - essentially according to Bernoulli's theorem - so that this local low pressure arises far from walls and cavitation is generated within the stream. An example of the latter cavitation is Craya-Curtet coflowing, confined jets where the entrainment rate may be strong enough to generate pressures below the vapour pressure and hence cavitate [3]. A more comprehensive review of the various forms of cavitation may be found in Franc [12], Bergant [4], Dumont [6] and Arndt [2,3].

In the present study, we examine cavitation as it occurs within micro channels, particularly those used as a simplified approximation for a nozzle. Nozzles are fundamental to a wide range of industrial processes such as spray painting, jet cutting and fuel injection systems. This application was chosen for a number of reasons: the geometry is simple and experiments for which measurements are available such as Winklhofer et al. [31] or Mauger et al. [18], the problem is of commercial interest (e.g. to support emissions regulations) and there exists a wide body of previous studies upon which to build. The specific difficulties around the conditions found in typical injection systems may also be seen as an extreme case with which a numerical modelling scheme would need to cope. This flow has also, therefore, a wider field of application to the more general problem of numerical simulation of hydrodynamic cavitating flows.

In this paper we asses the predictive capabilities of a numerical framework within the Large Eddy Simulation context when Diesel fuel is injected at various downstream pressures through a micro-channel. In particular, the aim was to improve model behaviour in the occurrence of critical cavitation and beyond. Critical cavitation is defined as the point at which the flow ceases to be dependent on the driving pressure difference and has reached a maximum mass flow rate (known as 'choked' conditions). If the pressure difference is greater than that required for choked conditions, the cavitation region may cover the full spanwise extent of the channel and be 'fully choked'. This flow regime between choked and fully choked is currently poorly reproduced by simulation in the literature. To address this poor understanding, the transient nozzle exit pressure was identified as the focus for investigations. Thus, in order to correctly model the processes within the nozzle, the nozzle exit pressure and its relationship to the farfield pressure were explored. Since it is difficult experimentally to measure the pressure at the nozzle exit, typically it is the mean value of the farfield pressure that is reported instead. The novelty of this paper is to investigate this discrepancy between experimentally measured downstream pressure and its correct representation in terms of model parameters. For a model to be validated against such experimental pressure data therefore, the downstream 
domain must contain the relevant features that affect this pressure. From this, the downstream length and boundary condition were varied parametrically. The second feature of importance is the presence of pressure waves in the domain which can substantially change the nozzle exit pressure in a time dependent way. For these reasons, the experimental data for pressure at a measured downstream station were compared to different pressure values of both fixed and non-reflective (wave transmitting) domain terminations in order to attempt to replicate the correct conditions at the nozzle exit.

The structure is as follows: We first present our numerical framework as well as the experimental data source. We explain in detail the important sub-models used (phase change, turbulence and compressibility) as well as the numerical parameters that affect the results (physical properties, grid and boundary conditions). A sensitivity analysis for all the aforementioned models and parameters is not possible here due to space limitations. Thus, in the result section, we chose to present the sensitivity of the predictions to the selection of pressure boundary conditions that are not well represented in the literature up to date. We present the numerical results in terms of void fraction, velocity and pressure and we compare these with the corresponding experimental data [31].

\section{Experimental Setup}

The simulations in this work are compared to experiments described in [31] and the model nozzle is shown in Fig. 1. Diesel fuel was pumped through a micro channel eroded into a $0.3 \mathrm{~mm}$ thick steel sheet which was sandwiched between two sapphire windows, allowing the visualisation of the channel and forming a nozzle-like structure. The geometry analysed here corresponds to the throttle geometry called "U" in [31], which is a rectangular shaped channel of $100 \times 301 \times 284 \mathrm{~mm}$ with an inlet radius of $20 \mu \mathrm{m}$. The channel extended $10 \mathrm{~mm}$ upstream and downstream of the nozzle to minimise the effect of the boundary conditions within the nozzle. In all of the experimental cases, the upstream pressure was 100bar, and the outlet pressure was varied.

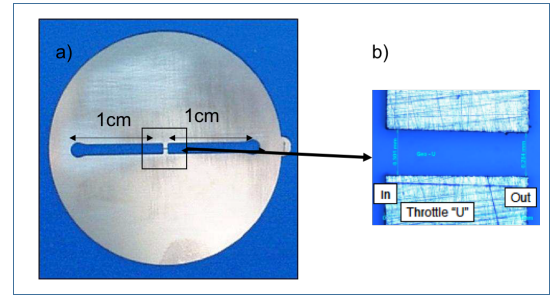

Figure 1. (a) View of the experimental nozzle (b) Zoom-in in throttle geometry U. [31]

\section{Numerical Parameters}

In this work, OpenFOAM [11] was chosen as the code base due to its extensible nature with access to the source code in order to create and modify the governing numerics. A brief description of the governing equations, phase change, turbulence and compressibility sub-models as well as grid specifics are presented in the following section. Special focus is given to the choice of the boundary conditions, that is the main topic of this work.

\section{Multiphase modelling including compressibility}

For the simulation of the two phases present (fuel vapour and liquid fuel) in the domain we are using the so called "one-fluid approach" within a compressible framework. Instead of having an individual set of transport equations for each phase, in every computational cell liquid and vapour are treated as a single continuum. The compressibilities of both phases are taken into account and the temperature is assumed to be uniform. The one fluid approach is suitable for problems where the second phase may be considered to be fully dispersed within the first such as 'cloud cavitation' or strongly turbulent cavitation of numerous small, discrete bubbles. It is less well suited to slug flow or stratified flow situations where the two phases might reasonably be expected to have differing velocities. In our case, and given the high pressure differences for the geometry under consideration, this approach can be considered as a reasonable assumption and has been validated previously by authors such as Salvador et al. [25]. The starting point of forming the numerical framework used is the following equation of continuity

$$
\frac{D \bar{\rho}}{D t}=\psi \frac{D \bar{\rho}}{D t}
$$

which is based on a barotropic equation of state. The term $\psi$ is the compressibility of the mixture which is defined as $\psi=1 / c^{2}$ (where $\mathrm{c}$ is the speed of sound) and needs to be determined. Equation 1 can be used either directly in the continuity equation to formulate a pressure equation, or integrated to obtain the pressure as a function of the density as suggested by Schmidt et al. [26]. In our case we use the former approach, following [14]. We use the notation with bars over the quantities since the framework is formulated within the LES context. The equation of state for the mixture should then be consistent with the liquid and the vapour equations of state, both at the limits 
when there is pure liquid or pure vapour, and also at the intermediate states when there is a mixture. The densities of the two phases are defined as:

$$
\bar{\rho}_{v}=\psi_{v} \bar{p} \quad \text { and } \quad \bar{\rho}_{l}=\bar{\rho}_{l}^{0}+\psi_{l} \bar{p}
$$

The terms $\psi_{v}$ and $\psi_{l}$ are the compressibility factors of vapour and liquid respectively. In order to separate the amount of volume that each phase occupies in this "mixture" fluid a phase indicator function known as the vapour fraction, $\alpha$, is used. $\alpha$ is defined as

$$
\bar{\alpha}=\frac{\bar{\rho}-\bar{\rho}_{\text {lsat }}}{\bar{\rho}_{\text {vsat }}-\bar{\rho}_{\text {lsat }}} \quad \text { where } \quad \bar{\rho}_{v s a t}=\psi_{v} \bar{p}_{\text {sat }}
$$

It can be seen that if there is no cavitation within a cell then $\bar{\rho}=\bar{\rho}_{\text {lsat }}$ resulting in $\alpha=0$ while for the case that a cell is full of vapour $\bar{\rho}=\bar{\rho}_{\text {vsat }}$ and $\alpha=1$. The equilibrium model as described above has as its main advantage its simplicity. There is no need to solve an additional transport equation for the vapour volume fraction, since the fluid above the vapour pressure is defined as a liquid, the fluid below the vapour pressure as vapour and the change of density with pressure is defined by an equation of state. Moreover, since it is based on local equilibrium assumption it ignores the effect of the interfacial forces between different phases and thus the surface tension term in the momentum equation is considered negligible. Models of this category are more appropriate for high speed nozzle flows in that the effect of surface tension is minimised resulting in an homogeneous mixture of bubbles and liquid in each cell such as in diesel fuel injectors [7]. The model has been compared with alternative models accounting for interfacial forces in our previous work [27] for various cavitation numbers but for a larger scale geometry (lower velocity) and results indicated a similar behaviour of the two models as the cavitation number was increasing. The choice of an appropriate closure relation was studied by Gonclaves and Patella [8] using a one fluid model applied to a venturi geometry. This combined a stiffened gas model with the barotropic model, described above, using a compressible RANS solver. They used a sinusoidal form of the gas closure relation which gave a smooth transition between pure liquid and pure vapour speed of sound (and hence compressibility) but this may be unphysical since it is known that the speed of sound in a mixture can be significantly less than that in either of the two phases individually.

Combining Eqs. 2,3 we form the mixture's equilibrium equation of state.

$$
\bar{\rho}=(1-\alpha) \bar{\rho}_{l}^{0}+\left[\alpha \psi_{v}+(1-\alpha) \psi_{l}\right] p_{\text {sat }}+\psi\left(p-p_{\text {sat }}\right)
$$

The equations of motion are then closed with the constitutive relations for the density, $\rho$, the dynamic viscosity (using a linear model), $\mu$ and the compressibility $\psi$.

\section{Compressibility and Thermophysical properties}

A linear model for compressibility was used since it is considered more consistent with the volume of fluid method, where viscosity and mass fraction are similarly described by linear equations [14].

$$
\bar{\psi}=\alpha \bar{\psi}_{v}+(1-\alpha) \bar{\psi}_{l}
$$

When Eq.5 is used, then the equation of state for the liquid can be reduced to a linear equation as well with $\bar{\rho}_{l, \text { vap }}=\rho_{l, \text { sat }}+\psi_{p}$. Alternative models are the Chung [5] and the Wallis model [28]. Literature [13] suggests that the Wallis model represents the lower speed of sound (compressibility) in a bubbly mixture in a more physical way, but it is rather unstable when used for high speed flows.

In terms of the choice of the individual compressibility factors $\psi_{l}$ and $\psi_{v}$ the physical properties of the diesel fuel have to be considered. The difficulty in the use of Diesel in numerical simulations is that the fuel typically varies seasonally as well as geographically so its properties are generally defined in terms of a range rather than a specific value. For example in Europe, the specification of common pump diesel must conform to EN590 [1] (which is more concerned with sulfur content and biofuel components due to their effect on emissions) so that the stated density must be between 820 and $845 \mathrm{~kg} / \mathrm{m}^{3}$. Koukouvinis et al. [15] chose to use a value well outside this range, presumably in order to tune their model and reduce the propensity of the code to develop significant negative pressures. Habchi et al. [9] also chose a value outside the EN590 range from some of their earlier work on lumped parameter modelling [10] which was focused on vaporisation rates and simulating spray conditions at $400 \mathrm{~K}$. The selection of subcomponents and use of a gas derived equation of state may explain this lower than usual density value. All other authors reviewed chose values close to the EN590 range but it is generally unclear whether this is for convenience or whether it served to help drive model outputs. For the current simulations the thermodynamic properties in Table 1 have been adopted. 


\section{Turbulence and the Sub - Grid scales}

Turbulence in the context of small scale, high speed flow is difficult to interpret within the realm of multiphase flows since not only are the mechanisms poorly understood [23] but the transition to fully developed turbulent flow, which might be expected as the Reynolds number increases, can be delayed significantly. Established vapour regions near the wall can reduce the effective viscosity and associated frictional losses such that the central jet can persist in a turbulent transition regime for a wide range of pressure differences. In this study, therefore, a transient methodology is required (LES) even though the problem is quasi-steady state. It should be noticed that one of the difficulties in performing LES in the context of cavitation is that it is hard to determine the length scale spectrum and the resolution requirements. Although in single phase flows the smallest scale is defined through the Kolmogorov scale, in two phase flows the definition of an equivalent scale is difficult. In the current work we model the sub-grid tensor term with an algebraic eddy viscosity models in which the stress tensor $\tau_{i j}^{s g s}$ is related to the resolved strain rate tensor $\widetilde{S}_{i j}$ by means of a scalar eddy viscosity given by an algebraic equation. It is based on the Boussinesq hypothesis associating $\tau_{i j}^{\text {sgs }}$ with a sub-grid scale (sgs) turbulent viscosity, $\mu_{T}$. The idea is that the momentum transfer caused by turbulent eddies can be modelled with an eddy viscosity in the same way the momentum transfer caused by the molecular motion in a gas can be described by a molecular viscosity. The model is known as the Wall-Adapting Local Eddy-viscosity model (WALE) [19]. The sgs viscosity is dynamically computed with the square of the velocity gradient tensor rather than the resolved strain rate used in Smagorinsky-type models. This velocity tensor can not only account for the effects of both strain and rotation rate of the smallest resolved turbulence fluctuations, but also recovers the proper near-wall scaling for the eddy viscosity without requiring dynamic procedures. Moreover, as the WALE model is invariant to any coordinate translation or rotation and no test-filtering operation is needed, it is therefore well suited for LES in complex geometries [19].

\section{Boundary conditions}

In the course of reviewing existing literature on the simulation of cavitating channels, it was found that there exists a dearth of information on the downstream length requirements of placing the exit boundary condition in order to correctly satisfy a pressure governed boundary condition. Previous authors have assumed (implicitly) that the pressure at the nozzle exit is the same as that found downstream and thus experimental values are often applied at unphysical domain locations (e.g an experimental value from a sensor distant from the nozzle is applied at the nozzle exit). This is done to reduce the computational domain and hence computational cost. In the experiments of Winklhofer, fuel pressure levels are measured $35 \mathrm{~mm}$ upstream and downstream of the observation element. This will be referred for the rest of the paper as " $P_{\text {out }}$ " (the real physical outlet) while the pressure used in the numerical simulations as " $P_{\text {boundary" }}$ ". These two pressures do not necessarily coincide even if the same case is under consideration since "Pboundary" is dependent on the size of the simulated geometry that imposes a specific pressure drop in between the exit of the nozzle and the experimentally measured boundary condition of the domain. It may also allow different levels of pressure wave reflection. It should also be noted that, in the experiment, the gauge used may be damped in order to observe a smooth value for monitoring purposes. Even with a high frequency pressure sensor, the reported values have lost the richness of the full signal content which would have been an indicator of the frequency and amplitude of any transient fluctuations. " $P_{\text {out }}$ " should then be considered to be time averaged with the possible existence of an unknown fluctuating component. Examples of zero downstream length include [29], [20] and [17] while those classified as 'short' -between 1-5 diameters include [15] while [22] utilise larger exit domains with extra geometrical features. In this study, we consider the effects of the downstream length to the exit boundary of the computational domain by varying the distance from the outlet of the channel section to the boundary by 5,9 and $12 \mathrm{~mm}$ (keeping in mind that the channel length is $1 \mathrm{~mm}$ while the length of the geometry before the nozzle is also simulated as $1 \mathrm{~mm}$ ). Thus, the $12 \mathrm{~mm}$ long case essentially represents the size of the downstream experimental geometry closely. One additional case with $3 \mathrm{~mm}$ length was tested: however it is not reported here since the close proximity of the exit boundary to the nozzle outlet led to considerable numerical instabilities.

The boundary conditions were treated as a total pressure condition at the inlet to the domain with a small component of turbulent kinetic energy and negligible velocity. For the the outlet boundary condition, two conditions were tested. The first configuration is typical for this type of problem and uses a fixed value condition (FP) imposed as a pressure constraint to ensure that the equations are well bounded. This type of problem is the classic 'pressure driven flow' scenario in which the velocity at the domain exit is considered to be a dependant variable. The second configuration tested was a novel variant of the pressure wave transmissive (PWT) condition that was modified to be compatible for multiphase problems. The PWT condition attempts to transmit all incident pressure waves outside the domain and maintain a given far field value of pressure. This is done by attempting to keep the instantaneous value 'close' to the given far field value. Here, 'close' is defined by a separate parameter, the far field distance. A smaller far field distance implies that a smaller deviation is allowed from its prescribed value whilst a larger far field distance allows greater deviation. The core scheme for this is derived from Poinsot and Lele [21] and is accomplishing by assuming

Table 1. Thermodynamic properties of Diesel

\begin{tabular}{ccccc}
\hline \hline$p_{\text {sat }}(P a)$ & $\rho_{l, s a t}\left(\mathrm{~kg} / \mathrm{m}^{3}\right)$ & $\rho_{v, \text { sat }}\left(\mathrm{kg} / \mathrm{m}^{3}\right)$ & $\psi_{l}$ & $\psi_{v}$ \\
\hline \hline 5400 & 832 & 0.1361 & 0.0000005 & 0.0000025 \\
\hline
\end{tabular}


that, for high Reynolds flows, the hyperbolic part of the Navier Stokes equations represent the wave component of the local pressure field.

\section{Grid}

The cell size in the nozzle, that is the area of interest, is $0.02 \mathrm{~mm}$ while outside the nozzle it is $0.06 \mathrm{~mm}$. The Reynolds numbers considered for all three outlet pressure cases (15bar, 30bar, 50bar) is of the order of $10^{6}$ while the Taylor scale is of the order of $0.001 \mathrm{~mm}$. The total number of cells was varied depending the size of the geometry in order to maintain a constant resolution. The near wall region was resolved to typical values of $y^{+}$of the order 1-5 such that the LES terms are considered to be implicit. To ensure a stable solution, transient calculations with a step of $250 \mu s$ were performed. The convergence criteria used for the calculations are based on the values of the residuals (which have to reach a value of $10^{-7}$, at least, for all equations) and the mass flow rate (which has to be stabilized as much as possible). The time step is limited by both the Courant number (taken as 0.2 ) and the acoustic Courant number (taken as 12).

\section{Results}

Figure 2 shows the vapour fraction, pressure and velocity instantaneous fields for the $5 \mathrm{~mm}$ domain geometry at time $\mathrm{t}=0.075 \mathrm{~ms}$ using FP boundary conditions. Three different outlet pressure values are presented. Looking at the $\alpha$ profiles it can be seen that as the outlet pressure is increased, the amount of vapour within the nozzle reduces and this is comparable with the experimental observations [31]. Also, although a structure resembling a cavitation cloud is evident after the nozzle exit for $P_{\text {boundary }}=15 \mathrm{bar}$, this disappears for higher pressures. Looking at the velocity and pressure plots (especially at the area downstream of the nozzle), it can be seen that using a FP boundary condition leads to 'pulses' in the jet velocity with alternating fast and slow regions with similar vector directions, implying that the rotation is low and there is no significant reverse flow. This spatial oscillation of the velocity magnitude has several implications for the overall flow structures that may be observed. We suggest that these velocity fluctuations are a result of the pressure waves trapped in the domain reflecting and interacting at nodal points so local high and low pressure regions act to retard or accelerate the jet as it moves away from the nozzle. The challenge that arises in numerical simulations using FP boundary conditions is that there is no straight forward way to separate pressure waves associated with the pressure outlet conditions and pressure waves that occur "naturally" because of the nozzle operating conditions. By considering a PWT boundary condition as we suggest in this work, we have the potential to better control of the waves that are allowed to reflect within the domain and thus help us separate which phenomena are associated with naturally occurring waves and which ones are related to numerical artefacts One implication relevant to pressure prediction that is evident from the plots of Fig. 2 is that local pressure differences in streamwise sections of the downstream domain drive velocity profile differences in the shear layer which in turn affect local low pressure regions in which cavitation is likely. It is interesting to note that an examination of the vorticity associated with this flow shows very little correlation and no clear vortical structures could be observed. The pressure and velocity plots in Fig. 2 have additional black contours added to highlight those areas where the pressure is less than the vapour pressure. Cavitation is not observed for the 55bar outlet pressure case and only observed in the region of the vena contracta for the 30bar case. The 30bar velocity plot shows how the jet shear layer undergoes velocity differences along the jet centreline while the 15 bar velocity plot shows how greater pressure differences can lead to vapour formation in the shear layer. It is evident that correct prediction of the pressure waves in the domain is an important factor in the overall solution.

Another implication is that the variable pressure difference across the nozzle due to these wave effects can lead to instabilities in the cavitation which is generated in the usual vena contracta regions and along the nozzle walls. Supercavitation is partially suppressed and the vapour along the nozzle walls may not always extend fully to the exit with small backflow into the channel as the local pressure at the exit corner is higher than the vapour pressure. The momentum of the jet inside the nozzle is high enough that the boundary between the fast moving fluid and the near static vapour is essentially fixed after the initial jet acceleration.

An additional important remark from these plots is relevant to the cavitation evolution in the nozzle for the $30 \mathrm{bar}$ exit pressure that is identified in the experiments as the critical cavitation case (see Fig 3). The experimental data indicates that cavitation and the subsequent 'bubbly' mixture could extend over the entire spanwise section of the channel as critical cavitation pressure differentials are exceeded. For the $U$ geometry, this evolution occurs rapidly after the exit pressure is reduced below 30bar, as even the 29bar exit pressure condition has substantially increased cavitation probability. This could explain why that the simulated 30bar FP case (seen in Fig. 2), which is particularly close to a high sensitivity region, shows a low cavitation region within the nozzle.

In order to better understand the effect of pressure waves within the domain, the wave transmissive boundary condition is applied at the outlet. This configuration was tested at 20bar far field pressure with 5,9 and $18 \mathrm{~mm}$ far field distance values. Although with this approach waves are able to exit the domain as expected, (in theory limiting the effect of artificial pressure oscillations), some pressure variations are still present because of the high Reynolds number of the flow. These instabilities interact with the vapour fraction leading to vapour collapse events in the downstream area. These collapse events trigger pressure waves which are then able to travel upstream towards the nozzle in a similar way as that which occurs in the experiment. We first zoom into the nozzle cavitation development for the 15bar FP case and 20bar PWT (see Fig. 4). The experimental evidence [31] is that under these conditions the cavitation should extend to the nozzle centreline leading to fully choked conditions (as in Fig 3b). In our simulations we can also see some indication of the cavitation regions beginning to close on the centreline for the FP conditions with instabilities growing in the phase transition region, something that is largely missing from other 


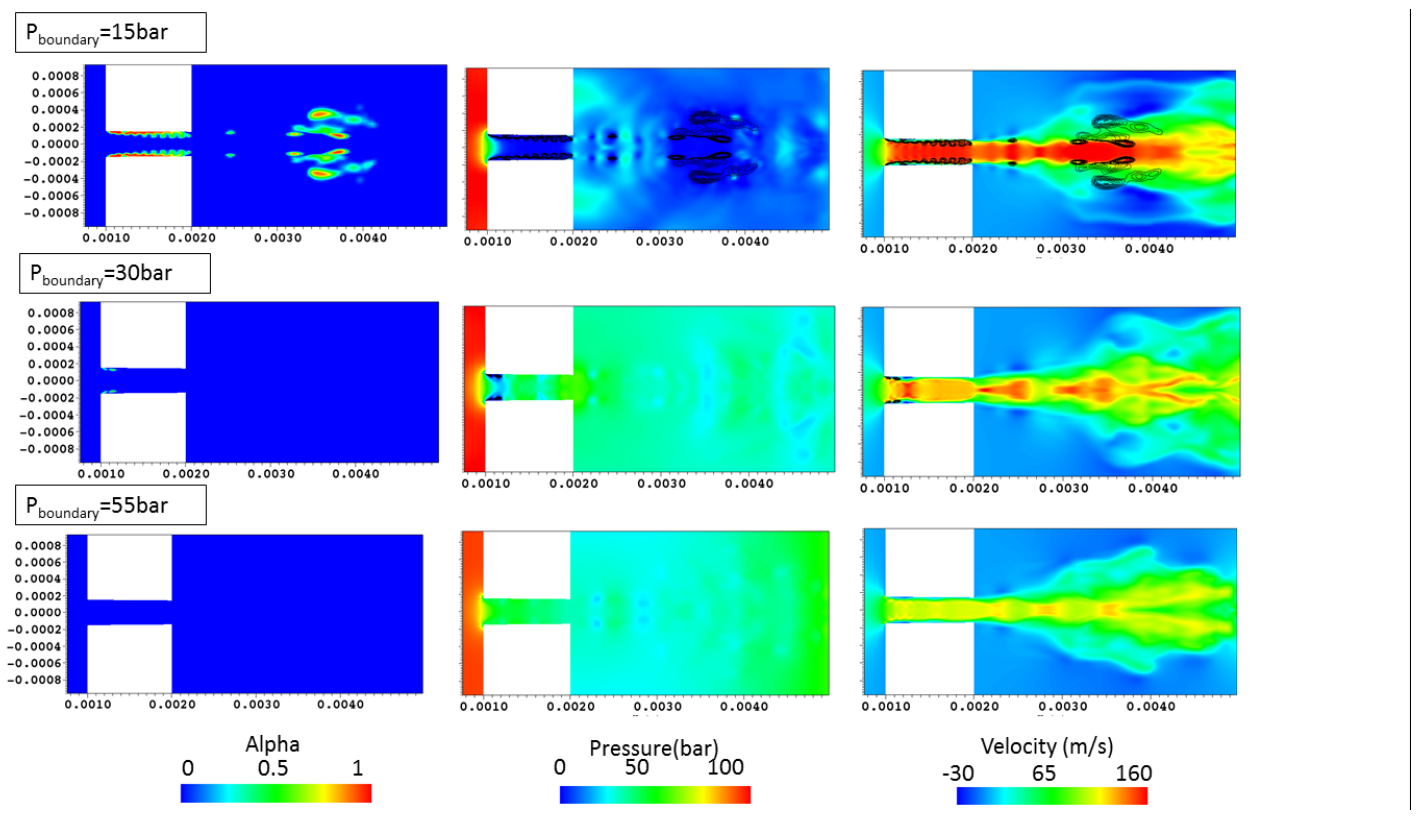

Figure 2. Instantaneous contour plots of vapour fraction, pressure and axial velocity at $t=.075 \mathrm{~s}$. Boundary condition is considered as fixed pressure. Axis scale is $m$.

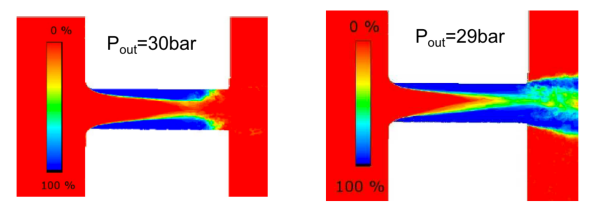

Figure 3. Experimental cavitation probability at critical cavitation point and beyond [31].

simulations of similar geometries. In contrast it can be noted that as the pressure waves are allowed to escape the domain, the cavitation in the PWT case lacks any turbulent features and more closely resembles lower pressure cases. This highlights the impact that reflections from the boundary (either physical or artificial) have on the nozzle cavitation regime. Previous work such as [16] and [24], for example, have examined the same geometry but have not addressed this issue, i.e that fully choked flow conditions are met. In contrast in Fig. 4 we can see that when waves are allowed to travel back to the domain then the framework is better capable of representing some of the important features. Of course further understanding is needed since the waves in the case of FP are more relevant to the length of the domain rather than the actual present in the domain because of the injection conditions. In previous work of [14], in order to increase the amount of vapour within the nozzle the upper and lower walls were considered with a slip condition for the velocity, similar to that used in supersonic compressible flow. This was done under the assumption that a no-slip boundary condition would lead to overestimates of the thickness of the boundary layer, resulting in strong vortices in the centre of the channel and incorrect modelling of the flow's cavitation. The assumption of a slip condition seems rather unphysical and according to our observations, a re-examination of the boundary conditions might be more appropriate.

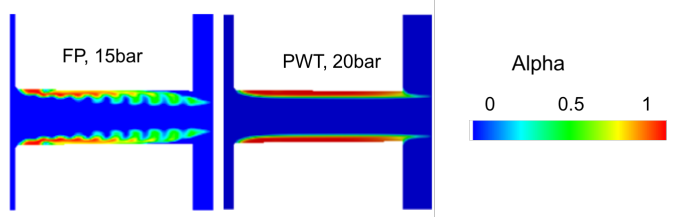

Figure 4. Vapour fraction in nozzle region, Left: $15 \mathrm{bar}$ FP, $5 \mathrm{~mm}$ downstream length. Right 20bar PWT, far field $5 \mathrm{~mm}$

Figure 5 shows a comparison of the pressure profiles along the centreline of the nozzle for a selection of the tested configurations. The experimental profile inside the nozzle is characterised by an initial pressure drop because of the flow turn at the inlet as well as a sudden pressure rise at around $0.5 \mathrm{~mm}$ downstream. It can be seen that for the $15 \mathrm{bar}$ cases where FP conditions are used, only the case of $5 \mathrm{~mm}$ captures the correct behaviour. This does not necessarily mean that this boundary condition is accurate. It indicates only that since we have set the boundary $5 \mathrm{~mm}$ downstream the exit of the nozzle and this specific boundary imposes reflection of pressure waves, these 
waves travelled back upstream inside the nozzle recovering some of the behaviour present in experiments. For the other two cases $(9 \mathrm{~mm}$ and $12 \mathrm{~mm}$ ), that in reality are closer to the real experimental geometry, the pressure drop is over-predicted inside the nozzle. The pressure only partially recovers because of the pressure increase at the exit. These results are similar to previous studies in the literature [13]. Looking at the poor agreement of the prediction of the $9 \mathrm{~mm}$ and the $12 \mathrm{~mm}$ case even downstream of the nozzle exit (at $1.5 \mathrm{~mm}$ ), one can argue that since we impose 15bar pressure at $9 \mathrm{~mm}$ and $12 \mathrm{~mm}$ boundary we cannot really predict accurately the pressure difference measured by the experiment at $35 \mathrm{~mm}$ downstream of the nozzle. In an effort to better tune our results, we include the results of a $9 \mathrm{~mm}$ geometry with $P_{\text {boundary }}=20 \mathrm{bar}$. We can see that with this re-adjustment although the results match better at $1.5 \mathrm{~mm}$, the predictions inside the nozzle are still poor. Changing the boundary to wave transmissive conditions gives two important advantages. One is that for both the $5 \mathrm{~mm}$ far field and the $18 \mathrm{~mm}$ far field values, part of the in nozzle pressure profile is correctly recovered. Moreover, with this method, the size of the simulated geometry is not changed (this is always $5 \mathrm{~mm}$ ): instead the far field parameter is controlled and hence so is the amount of waves allowed within the domain. This considerably reduces the computational cost in comparison to FP conditions in which the geometry was varied in order to control the boundary response.

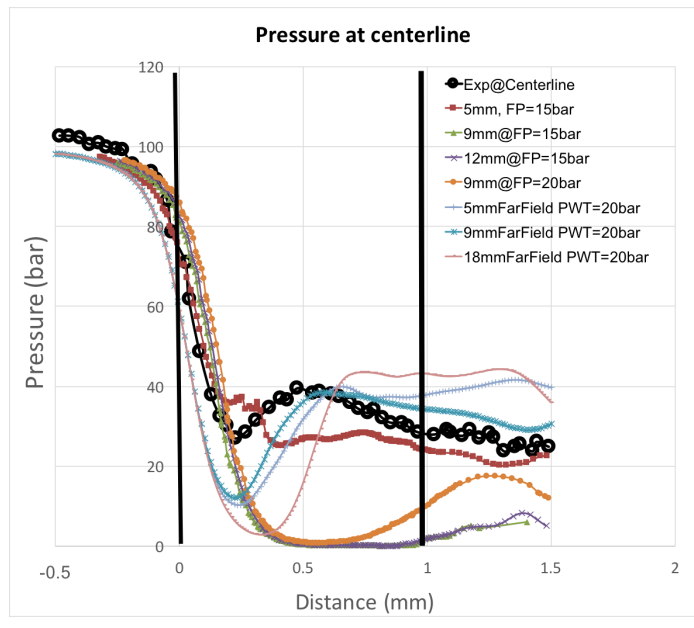

Figure 5. Pressure profiles along nozzle centreline comparing experimental data at 30bar to various downstream pressure values

Figure 6 shows the spanwise velocity profiles for the $15 \mathrm{bar}$ experimental condition at $\mathrm{x}=0.17 \mathrm{~mm}$. Five numerical cases are considered (three with FP boundaries and two with PWT). The experimental data shows a peak in velocity towards the nozzle upper and lower boundaries which is indicative of the lower viscosity and hence frictional losses in this area because of cavitation. It also shows a recovery of the velocity in the nozzle core although it does not achieve a maximum. The $15 \mathrm{bar}, 5 \mathrm{~mm}$ downstream FP case is best able to reproduce this result although it does not show the higher central area. The PWT condition shows a flatter profile which indicates that the shape of the FP conditions is being influenced by wave reflections present in the downstream area. The wave transmissive cases also demonstrate the differences seen across the various far field values that were used. Figure 6 has far field values of $5 \mathrm{~mm}$ and $9 \mathrm{~mm}$, all at the same $P_{\text {boundary }}$ of $20 \mathrm{bar}$. A linear trend in the maximum velocity as the far field values increase can be seen. Note that the $9 \mathrm{~mm}$ case with FP and the $9 \mathrm{~mm}$ PWT both at 20 bar predict similar velocity magnitude at the centerline but they differ in the prediction at the layer closer to the wall. This can be considered as an indication that capturing the pressure waves better within the domain is not expected to change substantially the main jet behaviour but it is expected to change the cavitation predictions. Further variations of the wave transmissive case are currently running in order to better explore this relationship although they were not available at the time of writing.

\section{Conclusions}

The downstream boundary conditions of a micro-channel geometry with in nozzle cavitation at critical cavitation and fully choked flow conditions were examined through numerical experiment and their impact assessed. The domain bounds and definition have been shown to be an area poorly understood with regards to their effect on in nozzle results. Using rigid exit conditions mean that pressure waves generated either within the nozzle or the downstream domain are reflected and continue to influence the local flow conditions. Downstream domain length and choice of boundary condition are therefore important in order to accurately model the pressure field and cavitation within the nozzle. The outlet from the nozzle section is prone to external cavitation in the shear layer (for choked flow conditions) which can also impact the nozzle exit pressure, particularly when attempting to validate against experimental results where the downstream pressure may be measured at a location some distance from the nozzle itself. It was found that vorticity (especially for cavitation developed outside the nozzle) did not play a large part in external cavitation formation, rather the velocity difference in the shear zone interacting with pressure waves had the greatest influence. A novel wave transmissive boundary condition for multiphase flow was tested and shown to be promising in replicating in-nozzle pressure profiles compared to more traditional fixed boundary conditions. However it as proved less accurate in replicating the velocity profile close to the wall. Further work needs to be done 


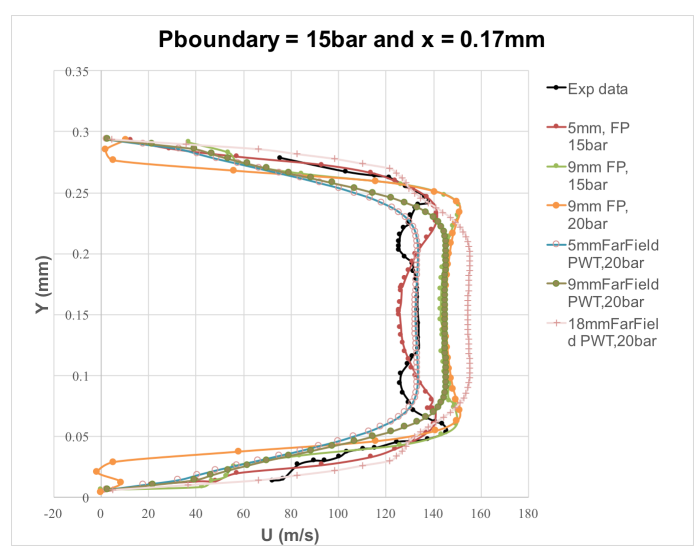

Figure 6. Spanwise Velocity plots at $\mathrm{x}=.17 \mathrm{~mm}$ comparing experimental data at $15 \mathrm{bar}$ to various downstream pressure values

in order to confirm the stability of the wave transmissive boundary with regards to vapour formation in the far field.

\section{References}

[1] Anon. BS EN590:2013, 2013.

[2] R. Arndt. Annual Review of Fluid Mechanics, pages 273-328, 1981.

[3] R. Arndt. Annual Review of Fluid Mechanics, page 175, 2002.

[4] A. Bergant, R. Simpson, and S. Tijsseling. Journal of Fluids and Structures, 22(2):135-171, feb 2006.

[5] M. S. Chung, S. B. Park, and H. K. Lee. Journal of Sound and Vibration, 276(1-2):13-26, 2004.

[6] N. Dumont, O. Simonin, and C. Habchi. 8th International conference on Liquid Atomisation and Spray systems, 2000.

[7] C.P. Egerer, S. Hickel, S. J. Schmidt, and N. Adams. Physics of Fluids, 26(8), 2014.

[8] Eric Goncalves and Regiane Fortes Patella. Computers and Fluids, 38(9):1682-1696, 2009.

[9] C. Habchi, N. Dumont, and O. Simonin. Atomization and Sprays, 18(2):129-162, 2008.

[10] C Habchi, FA Lafossas, P Beard, and D Broseta. SAE paper 2004-01-1996, 2004.

[11] C.W Hirt and B.D Nichols. Journal of Computational Physics, 39(1):201 - 225, 1981.

[12] Jean-Pierre Franc and Jean-Marie Michel. Fundamentals of Cavitation. Kluwer Academic Publishers, Dordrecht, 2004.

[13] F.P. Kärrholm. Numerical modelling of diesel spray injection, turbulence interaction and combustion. PhD thesis, Mech Eng, Goteborg, 2008.

[14] F.P. Kärrholm, H. Weller, and N. Nordin. 5th Joint ASME/JSME Fluids Engineering Conference, pages 465474, 2007.

[15] P. Koukouvinis, M. Gavaises, J. Li, and L. Wang. Journal of Physics: Conference Series 9th International Symposium on Cavitation, 656:012088, 2015.

[16] X. Margot, S. Hoyas, A. Gil, and S. Patouna. Engineering Applications of Computational Fluid Mechanics, 6(1):15-24, 2012.

[17] Sergey Martynov. Analysis, pages 1-226, 2005.

[18] Cyril Mauger, Loic Mees, Marc Michard, Alexandre Azouzi, and Stephane Valette. Experiments in Fluids, 53(6):1895-1913, 2012.

[19] F. Nicoud and F. Ducros. Flow, Turbulence and Combustion, 62(3):183-200, 1999.

[20] Stavroula Patouna. A CFD STUDY OF CAVITATION IN REAL SIZE DIESEL INJECTORS. PhD thesis, University Polytechnica Valencia, 2012.

[21] T.J. Poinsot and S.K Lele. Journal of Computational Physics, 99(2):352, apr 1992.

[22] Tao Qiu, Xin Song, Yan Lei, Xinghua Liu, Xiaodong An, and Mingchia Lai. Applied Thermal Engineering, 109:364-372, 2016.

[23] F. Ruiz and L. He. Atomization and Sprays, 9:419-429, 1999.

[24] Kaushik Saha, Ehab Abu-Ramadan, and Xianguo Li. Journal of Engineering for Gas Turbines and Power, 135(6):062801, 2013.

[25] F. J. Salvador, J. V. Romero, M. D. Roselló, and J. Martínez-López. Mathematical and Computer Modelling, 52(7-8):1123-1132, 2010.

[26] David P. Schmidt, Christopher J. Rutland, and Michael L. Corradini. Atomization and Sprays, 9(3):255-276, 1999.

[27] K. Vogiatzaki, P. Koukouvinis, and J. Carlton. ILASS 2016, pages 1-8, 2016.

[28] G. B. Wallis. One-dimensional two-phase flow. McGraw-Hill, 1969.

[29] Xiang Wang and WanHua Su. Chinese Science Bulletin, 54(10):1655-1662, may 2009.

[30] E. Winklhofer, E. Kelz, and A. Morozov. Proceedings of the ILASS-Europe Conference, Sorrento, 2003.

[31] E. Winklhofer, E. Kull, E. Kelz, and A. Morozov. Proceedings of the ILASS-Europe Conference, Zurich, page 574 ? 579, 2001. 


\title{
Numerical simulation of compressible cavitating two-phase flows with a pressure-based solver
}

\author{
Marco Cristofaro ${ }^{1,2, *}$, Wilfried Edelbauer ${ }^{1}$, Manolis Gavaises ${ }^{2}$, Phoevos Koukouvinis ${ }^{2}$ \\ ${ }^{1}$ AVL List GmbH, Hans List Platz 1, 8020 Graz, AT \\ ${ }^{2}$ City University London, Northampton Square EC1V OHB, UK \\ ${ }^{*}$ Corresponding author: Marco.Cristofaro@avl.com
}

\begin{abstract}
This work intends to study the effect of compressibility on throttle flow simulations with a pressure-based solver. The simple micro throttle geometry allows easier access for obtaining experimental data compared to a real injector, but still maintaining the main flow features. For this reasons it represents a meaningful and well reported benchmark for validation of numerical methods developed for cavitating injector flows.

An implicit pressure-based compressible solver is used on the filtered Navier-Stokes equations. Thus, no stability limitation is applied on the time step. A common pressure field is computed for all phases, but different velocity fields are solved for each phase, following the multi-fluid approach. The liquid evaporation rate is evaluated with a Rayleigh-Plesset equation based cavitation model and the Coherent Structure Model is adopted as closure for the sub-grid scales in the momentum equation.

The aim of this study is to show the capabilities of the pressure-based solver to deal with both vapor and liquid phases considered compressible. A comparison between experimental results and compressible simulations is presented. Time-averaged vapor distribution and velocity profiles are reported and discussed. The distribution of pressure maxima on the surface and the results from a semi-empirical erosion model are in good agreement with the erosion locations observed in the experiments. This test case aims to represent a benchmark for further application of the methodology to industrial relevant cases.
\end{abstract}

\section{Keywords}

cavitation erosion, compressible pressure-based, multi-fluid LES

\section{Introduction}

Diesel injectors can suffer from cavitation erosion. Recent regulations in term of emissions forced engine manufacturers to increase the injection pressure to values above 2000 [bar]. The consequent higher velocities in the nozzle lead to the formation of a more diffused spray and, thus, better combustion. This causes higher engine efficiency and less emissions, but, at the same time, higher risk of cavitation. If the pressure reaches locally values below saturation pressure due to the high velocity, the liquid evaporates. Under certain conditions, the repetitive collapse of the generated vapor that is convected further downstream, where pressure is above the saturation pressure, may be an aggressive phenomenon. Fatigue damages can then appear on the nearby surfaces. Many works have been published in the last decades to predict cavitation erosion. Experimental campaigns are of crucial importance to gain a better understanding of the physical phenomena involved and to provide usable data for the validation of new models. Numerical approaches based on multi-phase Computational Fluid Dynamics (CFD) are able to model cavitating flow fields. Useful information can then be obtained to assess the probability of cavitation erosion appearance.

A wide range of flow aggressiveness and erosion risk indices were developed in the recent years. A review of some selected cavitation erosion models, together with a description of the physical mechanism leading to cavitation erosion, was provided by Van Terwisga et al. in [1]. The model of Bark et al. presented in [2, 3] is based on the analysis of collapsing vapor cavities dynamics and it well correlates the erosion risk with the underlying physical phenomena. On the other hand it may be difficult to use it when the erosive cavities are small. The model described in [4] by Fortes-Patella et al. is instead based on two efficiencies in which the energy is transferred from the collapsing cloud to the wall. The theoretical basis fit with the energy cascade phenomena and it could be easily applicable, but it still needs detailed studies to assess the efficiency values. In [5], Koukouvinis et al. defined a cavitation aggressiveness index based on the Lagrangian derivative of pressure and the time scales of a single bubble and of the whole vapor cavity. Bergeles et al. used instead the acoustic pressure of a single bubble collapse to develop an erosion aggressiveness index in [6]. The authors validated it on a real eroded injector geometry, that was also used by Koukouvinis et al. in [7] to test the cavitation aggressiveness index.

Multi-phase compressible CFD simulations were able to directly reproduce pressure waves generated by collapsing vapor clouds and impacting on the nearby surfaces. A 2-D inviscid density-based solver was used by Skoda et al. in [8] for a micro-channel flow and proved able to detect these pressure waves. In [9] Egerer et al. detected similar pressure peaks on the same geometry, but using a 3-D density-based solver with the single-fluid Large Eddy Simulation (LES) approach. A similar solver was also used by Mihatsch et al. in [10]. A grid dependency study of pressure waves intensity was performed and a scaling law was defined to fit the the pressure peaks rate to the one recorded during the experiments. Koukouvinis et al. presented in [11] the recorded pressure peaks obtained with a pressure-based solver with a single-fluid LES approach. Results are presented for a micro-channel flow, as 
well as for a real injector case. Another cavitating injector simulation was presented by Örley et al. in [12], where a density-based solver with explicit time integration and the single-fluid approach were applied. Pressure peak intensities in the order of 1000 [bar] were recorded in both works.

The present work exploits the pressure-based commercial solver AVL FIRETM resolving a fully compressible multiphase cavitating flow. Implicit time integration is adopted to avoid stability constraints on the simulation time step. The multi-fluid modeling approach avoids the need of mixture properties definition, instead required for the singlefluid approach. Both vapor and liquid compressibility are considered with separate equations of state. Furthermore the slip velocity between the phases is modeled and analyzed. Pressure peaks recorded on the surface are then presented and compared with the semi-empirical erosion model presented by Greif et al. in [13]. Experimental visualization of cavitation erosion is also included for comparison.

\section{Numerical model}

The set of Navier-Stokes equations describing the iso-thermal compressible 2-phase cavitating flow is hereby presented. The equations are iteratively solved with a pressure-based solver. In the applied methodology a single pressure is solved for all phases, but different velocity fields are obtained for each of them. This is known as Eulerian-Eulerian modeling approach, or multi-fluid model [14]. The dispersed phase (vapor) is then treated as a second continuous phase interpenetrating the continuous phase (liquid). The mass conservation equations are first solved independently to compute the volume fraction of each phase, $\alpha_{k}{ }^{*}$, and then combined to obtain the common pressure, $p$. Once the phase densities, $\rho_{k}$, are computed using appropriate equations of state (see Sec. Geometrical model and simulation set-up), two separate momentum conservation equations are solved to find the phase velocity fields, $\bar{v}_{k}$. The interaction between phases must be included in the mass and momentum exchange terms. In the present methodology they are modeled considering a mono-dispersed bubbly flow model [15].

The mass conservation of each phase is represented by a transport equation with a source term modeling the inter-phase mass transfer, $\Gamma_{k}$. Since the total mass must be conserved, the mass lost by one phase is absorbed by the other and then: $\sum_{k} \Gamma_{k}=0$. Equation 1 shows the mass conservation equation in the differential conservative formulation for the generic phase $k$.

$$
\frac{\partial \alpha_{k} \rho_{k}}{\partial t}+\nabla \cdot \alpha_{k} \rho_{k} \bar{v}_{k}=\Gamma_{k}
$$

Equation 1 is used to compute the volume fraction of both the liquid and the vapor phase, assuming densities and velocities as known. Since the values of volume fraction do not satisfy a priori the condition of exactly filling the available volume, a compatibility condition is also imposed $\sum_{k} \alpha_{k}=1$.

The inter-phase mass transfer is modeled with the Original FIRE linear cavitation model [16]. The model considers the mass transfer contribution from each vapor bubble dynamic, as presented in Eq. 2.

$$
\Gamma_{d}=-\Gamma_{c}=N \rho_{d} 4 \pi R^{2} \dot{R}=\rho_{d}\left(3 \alpha_{d}\right)^{2 / 3}(4 \pi N)^{1 / 3} \dot{R}
$$

The vapor bubble number density is indicated with $N$, the average bubble radius with $R$ and its rate of change $\dot{R}$. Positive values of $\Gamma_{d}$ mean a growth of the bubble size, thus evaporation of the liquid into vapor. The bubble radius rate of change is extracted from the simplified Rayleigh-Plesset equation for a single bubble [17, 18]. No thermal, gas content, viscosity and surface tension effects are considered in the equation, and the second order time derivative term is neglected. The final formulation for $\dot{R}$ is then given in Eq. 3 .

$$
\dot{R}= \pm \sqrt{\frac{2}{3} \frac{\left|p_{\text {sat }}-p\right|}{\rho_{c}}} \text { with } \begin{cases}+ & \text { if } p \leq p_{\text {sat }} \\ - & \text { if } p>p_{\text {sat }}\end{cases}
$$

The saturation pressure of the liquid is indicated with $p_{\text {sat }}$. The number density is instead calculated according to an empirical function, formed by a constant part and a diminishing linear ramp, see Eq. 4 .

$$
N= \begin{cases}N_{0} & \text { if } \alpha_{d} \leq 0.5 \\ \max \left[2\left(N_{0}-1\right)\left(1-\alpha_{d}\right)+1, N_{\text {min }}\right] & \text { if } \alpha_{d}>0.5\end{cases}
$$

This is a rather heuristic model used to model coalescence effects: as soon as the vapor volume fraction is greater than 0.5 the bubbles start to merge. The initial number density, $N_{0}$, strongly depends on the characteristics of the liquid phase and it is the main cavitation model parameter. A minimum value, $N_{m i n}$, is also imposed.

$A$ joined mass conservation equation is then obtained by combining all the mass transport equations. Considering $\alpha_{k}$ and $\bar{v}_{k}$ as known, Eq. 5 is solved to compute the pressure shared among all phases. It is important to notice that the term $\Gamma_{k}$ is not elided but it is a volumetric flux when phase change occurs.

$$
\sum_{k} \frac{1}{\rho_{k}}\left\{\frac{\partial \alpha_{k} \rho_{k}}{\partial t}+\nabla \cdot \alpha_{k} \rho_{k} \bar{v}_{k}-\Gamma_{k}\right\}=0
$$

\footnotetext{
* The subscript $k$ is used to indicate a quantity related to a generic phase. The letter $c$ is instead used for the continuous liquid phase and $d$ for the dispersed vapor phase.
} 
The density time derivative of the first term of Eq. 5 is elaborated considering the compressibility in Eq. 6 . The isentropic phase speed of sound is defined as $c_{k}^{2}=\left(\partial p / \partial \rho_{k}\right)_{s}$. The final formulation shows how the presented pressure equation is in agreement with Wood's mixture speed of sound $1 / \rho_{m} c_{m}^{2}=\sum_{k} \alpha_{k} / \rho_{k} c_{k}^{2}$, [19] (being $\rho_{m}=\sum_{k} \alpha_{k} \rho_{k}$ ).

$$
\sum_{k} \frac{\alpha_{k}}{\rho_{k}} \frac{\partial \rho_{k}}{\partial t}=\sum_{k} \frac{\alpha_{k}}{\rho_{k}} \frac{\partial \rho_{k}}{\partial p} \frac{\partial p}{\partial t}=\left\{\sum_{k} \frac{\alpha_{k}}{\rho_{k}} \frac{1}{c_{k}^{2}}\right\} \frac{\partial p}{\partial t}=\frac{1}{\rho_{m} c_{m}^{2}} \frac{\partial p}{\partial t}
$$

One momentum conservation equation is then solved for each phase separately as presented in Eq. 7 . The momentum transfer and turbulence models provide the closure terms.

$$
\frac{\partial \alpha_{k} \rho_{k} \bar{v}_{k}}{\partial t}+\nabla \cdot \alpha_{k} \rho_{k} \bar{v}_{k} \bar{v}_{k}^{T}=-\alpha_{k} \nabla p+\nabla \cdot \alpha_{k}\left(\boldsymbol{T}_{k}+\boldsymbol{T}_{k}^{s g s}\right)+\bar{M}_{k}+\bar{v}_{i} \Gamma_{k}
$$

The vector $\bar{M}_{k}$ is the inter-phase momentum transfer and $\bar{v}_{i}$ is the interface velocity. The superscript $T$ indicates the transpose of a vector or a matrix, so that $\bar{v}_{k}^{T}$ is an horizontal vector, being $\bar{v}_{k}$ considered vertical. The term $\bar{v}_{i} \Gamma_{k}$ represents the momentum carried by the mass transferred between phases. The $3 \times 3$ matrix $\boldsymbol{T}_{k}$ is the shear stress tensor, while $\boldsymbol{T}_{k}^{\text {sgs }}$ encloses the effect of the turbulence on the momentum conservation equation. In the present work the Large Eddy Simulation Coherent Structure Model (LES-CSM) is adopted as closure model [20].

The interfacial momentum exchange is modeled with the drag force acting on each bubble. The bubbles move into the liquid with a certain relative velocity: $\bar{v}_{r}=\bar{v}_{d}-\bar{v}_{c}$. This relative velocity causes a pressure distribution on the bubble surface, whose integral is a force opponent to the relative motion. Equation 8 presents the sum of drag forces in the mono-dispersed case.

$$
\left(\bar{M}_{c}\right)_{D}=N 4 \pi R^{2} \frac{1}{2} \rho_{c}\left|\bar{v}_{r}\right| \bar{v}_{r} C_{D}=(36 \pi N)^{1 / 3} \alpha_{d}^{2 / 3} \frac{1}{2} \rho_{c}\left|\bar{v}_{r}\right| \bar{v}_{r} C_{D}
$$

The number density is taken consistently from the cavitation model, see Eq. 4, and the drag coefficient, $C_{D}$, is modeled as presented in [15, 21].

The presented equations are solved on the 3-D domain with the finite volume discretization method and the convergence of the system of equations is obtained with a Semi-Implicit Method for Pressure-Linked Equations (SIMPLE) algorithm, for further details see [15]. One benefit of pressure-based solvers is that the same numerical procedure can be used for both compressible and incompressible simulations. Originally pressure-based solvers were developed to solve incompressible flow fields. Thanks to further developments, their applicability was extended also to compressible flows. Consequently, a direct comparison between compressible and incompressible flow fields simulations can be obtained, with no major modifications in the numerical algorithm. The opportunity to use wellconsolidated solution procedure, along with existing cavitation models, makes pressure-based solvers of great interest for both scientific and industrial communities.

\section{Experimental setup}

The experimental data used as reference in this work are already published in [22]. The setup was designed to study cavitation erosion for a micro-throttle flow, representing a simplified Diesel injector nozzle. A sharp-edge geometry is considered, also known as I-channel geometry. The main components of the hydraulic system were a tank, an high pressure pump, a metal throttle and a cooling system. The throttle is enclosed between two sapphire glasses that allow optical access to the flow. The channel is $993[\mu \mathrm{m}]$ long, $295[\mu \mathrm{m}]$ high and $300[\mu \mathrm{m}]$ depth. The working fluid was commercially available Diesel fuel and it was demonstrated that small variations of the properties (e.g. due to a different manufacturer) did not influence the results [22]. Figure 1 shows an exploded view of the test chamber with the flow path (left), the steel plate with the throttle (middle) and a schematic visualization of the throttle with dimensions in microns (right). An extensive description of the hydraulic system, fluid dynamic field, measurements techniques and data elaboration can be found in [8, 23, 22, 24, 25, 26].

In the present work the case with an inlet pressure of 300 [bar] and outlet pressure of 120 [bar] is considered. At this condition the pressure waves intensity induced by collapsing vapor clouds are maximum [8]. This working condition is also known as cavitation critical point (CCP) [22]. It separates the states for which by increasing the pressure difference between inlet and outlet, the mass flow increases (following the Bernoulli curve) or remains constant (choked flow). The CCP can change with inlet and outlet pressure, temperature and fluid quality (e.g. nuclei presence and dissolved gas).

\section{Geometrical model and simulation set-up}

Since cavitation is an inertial driven phenomenon, thermal effects are here neglected, assuming an iso-thermal flow field. The fluid properties of Diesel described in [22] have been used. The liquid continuous phase density is modeled with a linearized equation of state as described in Eq. 9.

$$
\rho_{c}(p)=\rho_{r e f}+\left(\frac{\partial \rho}{\partial p}\right)_{r e f}\left(p-p_{r e f}\right)=\rho_{r e f}+\frac{p-p_{r e f}}{c^{2}}
$$



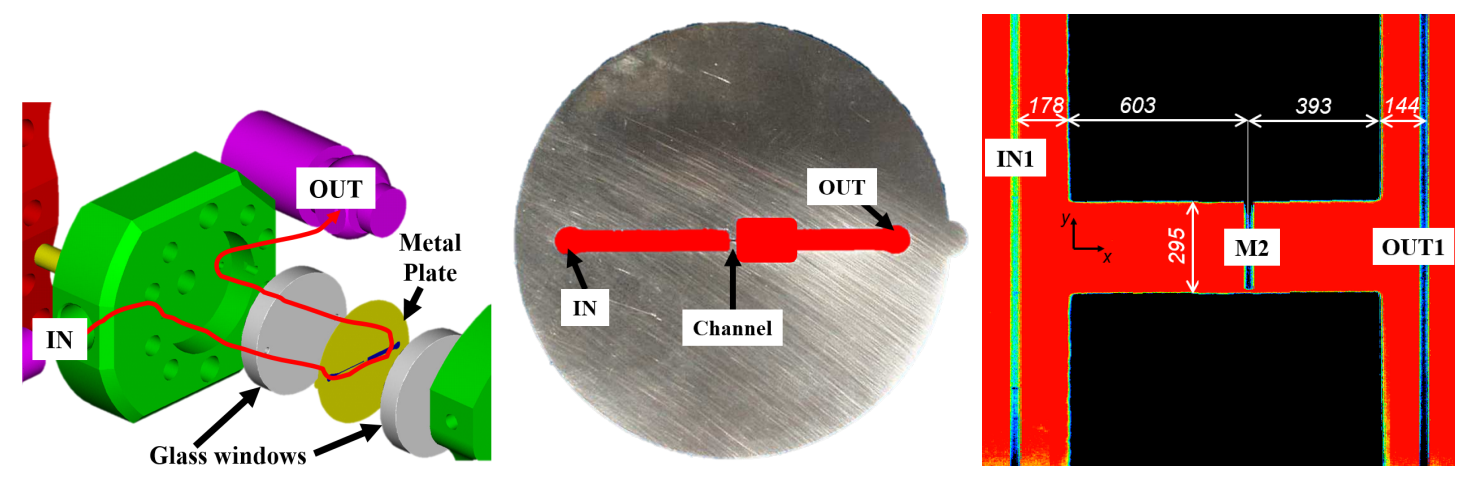

Figure 1. Micro throttle of the Prevero test case: flow path (left), steel plate (middle) and geometry with dimensions in microns of the throttle (right). Reproduced from [22]

The reference density, $\rho_{\text {ref }}$, was set to $820\left[\mathrm{~kg} / \mathrm{m}^{3}\right]$, reference pressure, $p_{\text {ref }}$, to 1 [bar] and the iso-thermal liquid speed of sound, $c$, to $1320[\mathrm{~m} / \mathrm{s}]$. The saturation pressure, $p_{\text {sat }}$, was of $4500[\mathrm{~Pa}]$ and a dynamic viscosity of 0.0021 [Pas] was used accordingly to [23]. The Diesel vapor density was instead modeled with the ideal gas law, see Eq. 10.

$$
\rho_{d}(p)=\frac{p}{R_{g} T}
$$

The specific gas constant, $R_{g}$, was of $48[\mathrm{~J} / \mathrm{kg} \mathrm{K}]$ and a constant temperature, $T$, of $321.15[\mathrm{~K}]$ was used. The vapor phase dynamic viscosity was set to $10^{-5}$ [Pas].

The used computational mesh was presented in [23] for the LES simulations. It is a block structured volume mesh and it is composed of 17 million cells. The smallest cell dimension is of $8.210^{-7}$ [m] close to the wall, while the characteristic cell size inside the channel is of $2.910^{-6}[\mathrm{~m}]$. The characteristic length scales of the liquid flow can be estimated from preliminary Reynolds-Averaged Navier-Stokes (RANS) simulations. The Kolmogorov length scale is computed as $\eta=\left(\nu^{3} / \mathcal{E}\right)^{1 / 4} \simeq 210^{-7}[\mathrm{~m}]$ and the Taylor length scale is estimated as $\lambda=\sqrt{10} \operatorname{Re}^{-1 / 2} L \simeq 510^{-6}[\mathrm{~m}]$. The used values are: turbulence dissipation rate $\mathcal{E} \simeq 10^{10}\left[\mathrm{~m}^{2} / \mathrm{s}^{3}\right]$, kinematic viscosity $\nu \simeq 2.510^{-6}\left[\mathrm{~m}^{2} / \mathrm{s}\right]$, velocity $|\bar{v}|=270[\mathrm{~m} / \mathrm{s}]$ and length $L=310^{-4}[\mathrm{~m}]$. The characteristic cell size inside the channel is then circa fifteen times the Kolmogorov length scale and half of the Taylor length scale. From the preliminary RANS results it is possible to compute the first cell $y^{+}$value as $y^{+}=y(1) / \ell_{\tau} \simeq 1.4$ (being $\ell_{\tau} \simeq 510^{-7}[\mathrm{~m}]$ ).

Static pressure was imposed at the open boundaries: 300 [bar] was applied at the inlet and 120 [bar] at the outlet. The central differencing scheme was applied to the momentum equation, while the MINMOD scheme was used for pressure and volume fraction equations, [27]. A small blending with the upwind scheme was added to the momentum equation to damp spurious numerical oscillations. A constant time step of $10^{-8}[\mathrm{~s}]$ was adopted, corresponding to a maximum value of convective CFL number of 3 . The time marching is done with an unconditionally stable second order accurate three time level implicit scheme. Although the maximum value of the acoustic CFL number based on the liquid speed of sound is 16 , the compressibility of the mixture corresponds to a speed of sound lower than the one of the pure liquid phase [19], leading to acoustic CFL numbers in liquid-vapor mixture regions smaller than one. The following numerical procedure was adopted to decrease the computational effort of the simulation. Firstly a RANS simulation was run for $210^{-4}$ [s] on a coarse grid with no mass transfer allowed from vapor to liquid. A quasi-steady solution was then obtained, with pure liquid in the whole domain. The obtained flow field was used to initialize the LES on the finer grid. The simulation run then for further $510^{-5}$ [s] for the evaluation of the results. This allowed to strongly reduce the overall computational cost compared to start the simulation directly with LES on the fine mesh.

\section{Results and discussion}

Results from the compressible numerical simulation are presented in this section. Both time-averaged and instantaneous values are included in the analysis. The integral mass flow and the vapor cavity distribution from simulations are compared with the experimental data reported in [22]. Mid-cut flow fields are then presented, together with the corresponding velocity profiles. Two cavitation erosion prediction approaches are finally compared with the experiments.

Table 1 presents the time-averaged mass flow values obtained in the experiment and in the simulation. For the simulation, a time average of the integral mass flow over the cross section M2 (see Fig. 1) was computed. The simulation shows a $4.25 \%$ smaller average mass flow compared to the experiments. The reason for this discrepancy is attributed to the different vapor distribution obtained from the simulation compared to the experiment. A bigger area occupied by the vapor in the channel cross section leads to sligthly smaller mass flow rate values. A further reason for the difference is the very small averaging time obtained from the simulation compared to the experiments, due to the high computational cost. However, considering the standard deviation of the simulation, the experiment result lays in the statistical range of the values from the simulation. 
Table 1. Average mass flow comparison. Experimental value from [22] against the simulation average integral mass flow at $M 2$ with the corresponding standard deviation.

\begin{tabular}{ccc}
\hline Experimental & Simulation & Relative difference \\
\hline $12.7[\mathrm{~g} / \mathrm{s}]$ & $12.16 \pm 0.55[\mathrm{~g} / \mathrm{s}]$ & $-4.25 \%$
\end{tabular}

In Fig. 2 the vapor distribution obtained from 50 transmission images (left) is compared with the maximum value of time-averaged vapor volume fraction along the channel depth (right). The flow moves from the left to the right. The maximum value along the $z$ direction (being $z$ the coordinate starting from the bottom glass window, perpendicular to it and orientated into the channel) was adopted to mimic the physics of the experiments, for which the light is blocked by the presence of vapor at any location along its path.

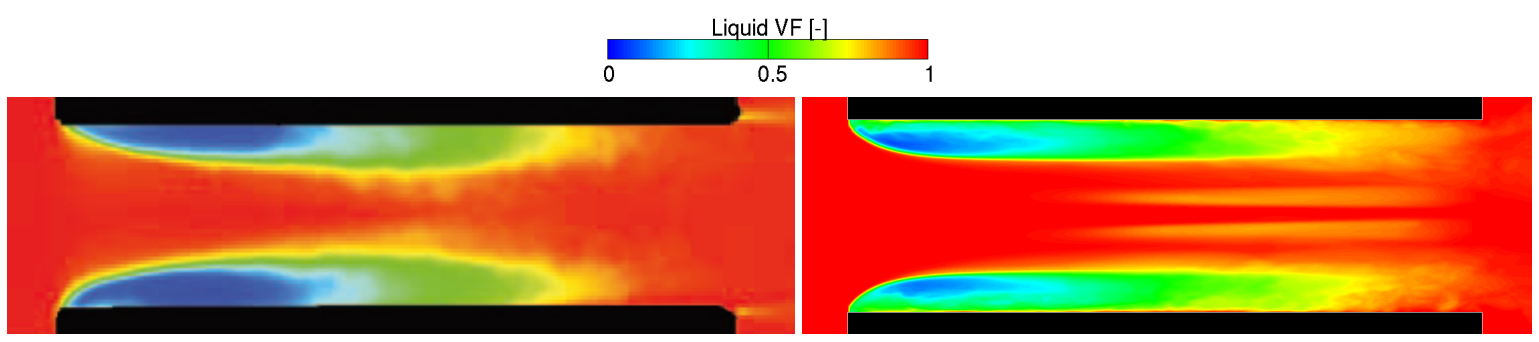

Figure 2. Time-averaged vapor cavity distribution (red liquid, blue vapor). Experimental transmission images reproduced from [23] (left) and minimum value along depth from simulation results (right).

The average flow field obtained from the simulation is further described in Fig. 3. A cut at the half of the domain depth is presented for the liquid velocity and the average pressure fluctuations fields. A vapor cavity along the entire channel length close to the walls can be observed in both the vapor (Fig. 2) and the velocity field. The cavity closes at the right end of the channel, where the highest values of time-averaged pressure fluctuations appear.

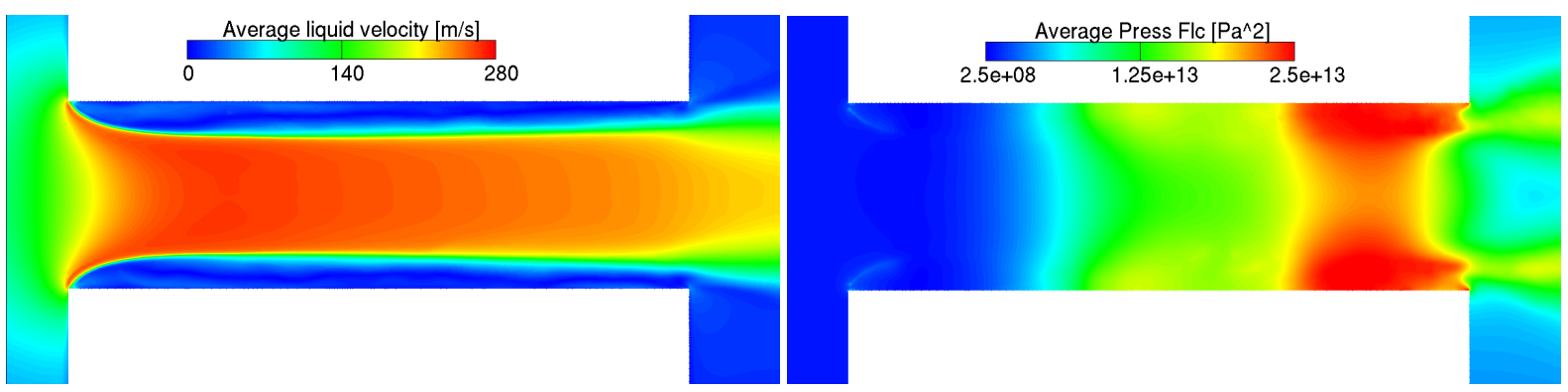

Figure 3. Time-averaged liquid velocity magnitude (left) and pressure fluctuations (right). Mid-cut of simulation results.

Figure 4 shows a sample of an instantaneous velocity and a volume fraction field. The laminar to turbulent transition of the shear layer can be detected in the velocity field at around half of the channel length. The dynamics of the vapor phase is instead shown in the volume fraction field: unstable vapor clouds detach from the main cavity and are convected downstream. They are then subjected to further break-up, collapse and rebounding. A strong dynamic interaction is detected between collapsing vapor clouds and pressure waves. This is recorded by the strong impact pressure on the nearby surfaces, usually related with possible cavitation erosion, presented later in this work.

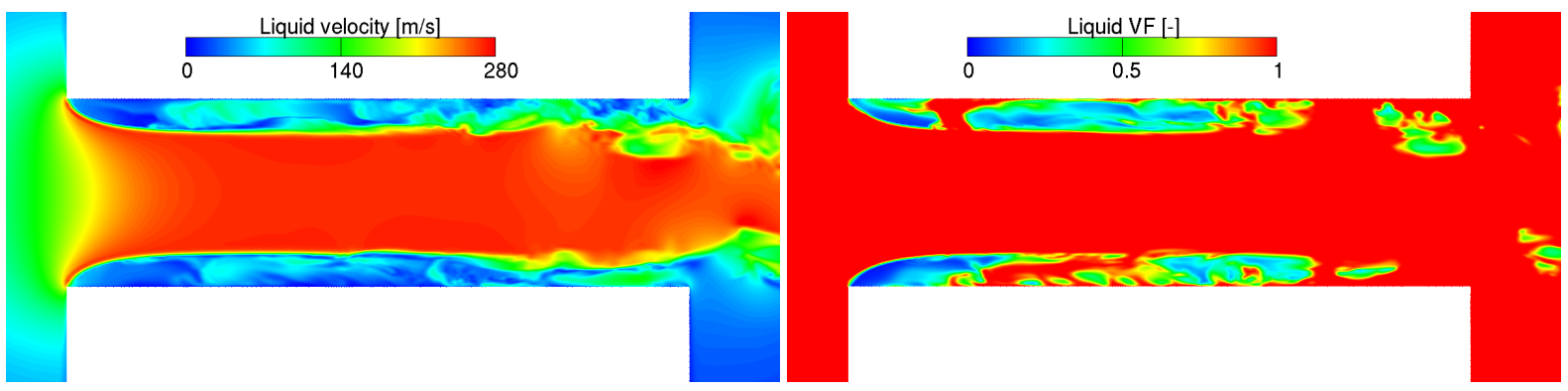

Figure 4. Instantaneous velocity magnitude (left) and liquid volume fraction (right). Mid-cut of simulation results at $t=3.510^{-5}[\mathrm{~s}]$. 
The numerical model used in the current work is based on the solution of two separate momentum equations. This leads to the computation of a separate velocity field for each phase. The slip velocity can then be evaluated as the difference between the two velocity fields. Figure 5 presents an example of instantaneous liquid velocity and relative slip velocity (defined as the velocity difference non-dimensionalised with the liquid velocity). The values are taken from three measurements planes IN1, M2 and OUT1 according to Fig. 1 (right) and then extracted at half of the channel depth $(z=150[\mu \mathrm{m}])$. The three different locations are presented on the same graphs. Slip velocities of the order of $15 \%$ of the liquid velocity were detected. It is important to notice that the highest values appear for M2 and OUT1 sections in liquid-vapor mixture regions.
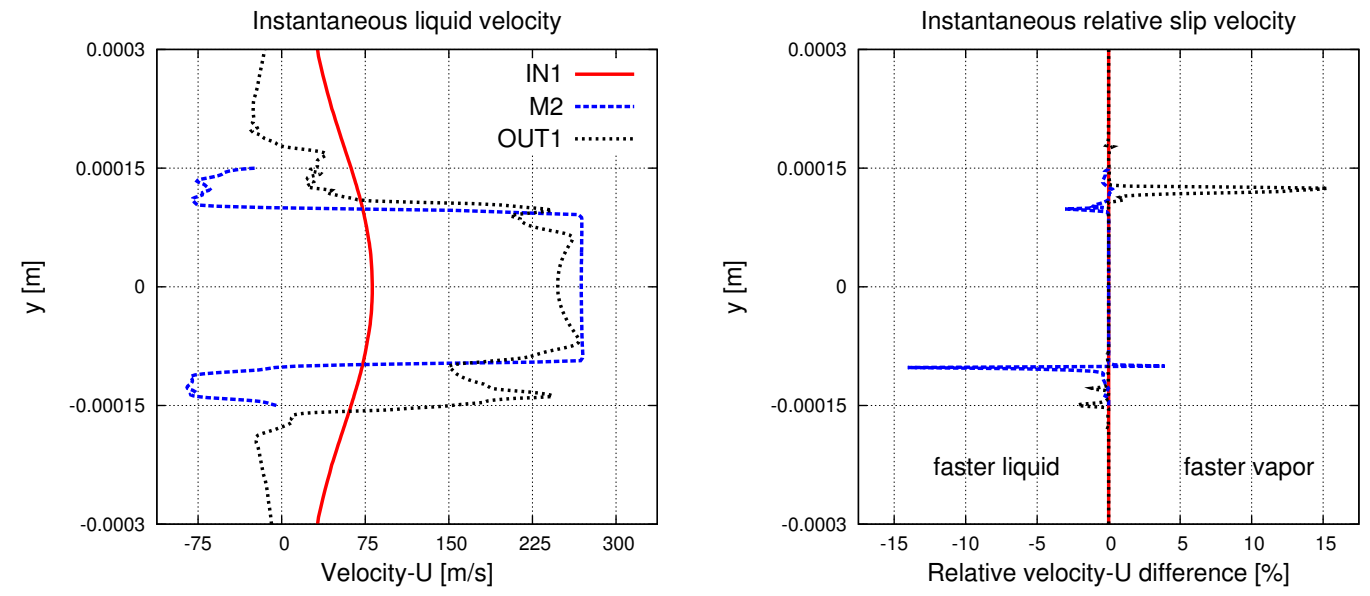

Figure 5. Instantaneous liquid velocity profiles (left) and relative slip velocity (right). Local values from a mid-cut of the simulation results at $t=3.510^{-5}[\mathrm{~s}]$.

In Fig. 6 the same analyses as in Fig. 5 is presented but for time-averaged velocities. The maximum magnitude of the average slip velocity is of the order of $1.5 \%$ of the liquid velocity. This is 10 times lower than the instantaneous value, but it still appears in the liquid-vapor mixture regions along M2 and OUT1 sections.
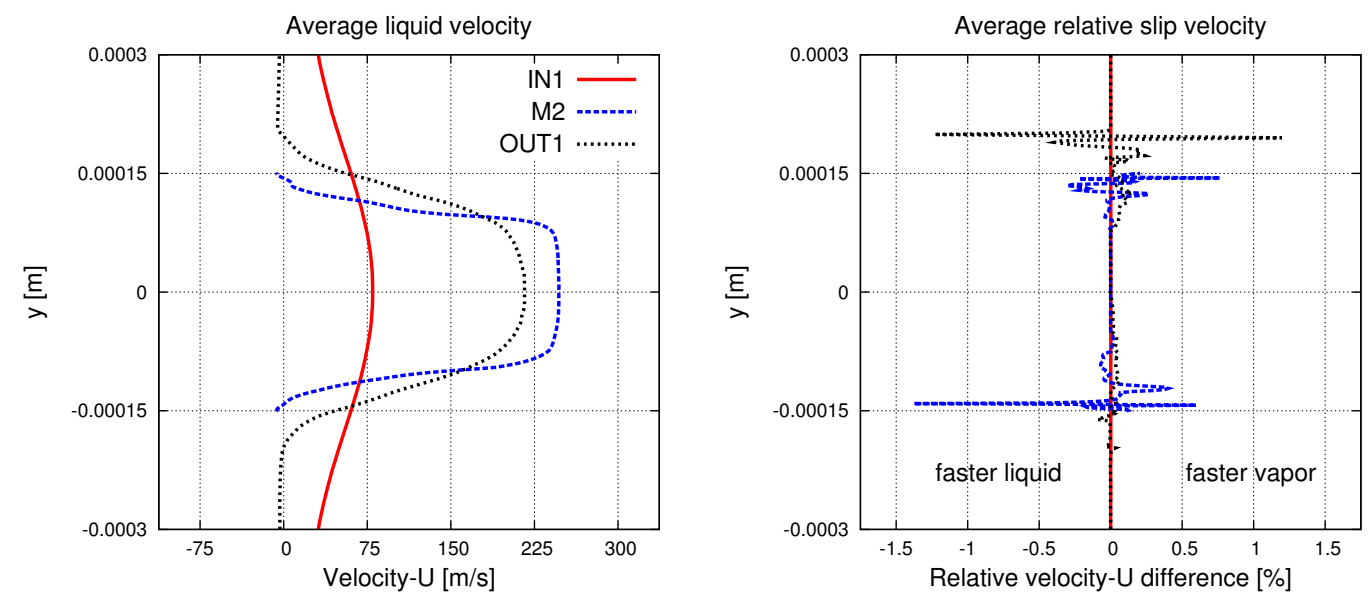

Figure 6. Average liquid velocity profiles (left) and relative slip velocity (right). Local values from a mid-cut of the simulation results.

The results of two different cavitation erosion modeling approaches are then presented and compared with experiments. The cavitation erosion prediction model described in [15] and already applied in [13, 23], is compared with the pressure peaks recorded on the channel surface during the simulation. The compressibility of both liquid and vapor phases was essential to resolve pressure waves starting from collapsing vapor clouds.

The maximum recorded pressure on the surface was 1500 [bar], however it is important to mention that this value is mesh and time step dependent [10]. In the present work the distribution of recorded pressure peaks is only used to obtain a qualitatively distribution of possible erosion locations. Figure 7 presents the qualitative results of erosion risk prediction from the modeling of the Mean Depth of Penetration Rate (MDPR) (left above) and the recorded pressure peaks (right above), together with experimental visualization of the erosion time evolution from [8] (below). A black cut at half of the depth of the channel is included for the simulation results, showing the average vapor presence in gray-scale. The same geometry was used for both the experiment and the simulation. The experimental data were obtained with 125 [bar] at the outlet and a temperature of $60\left[{ }^{\circ} \mathrm{C}\right]$. The identical operational point was not simulated due to the lack of flow experimental analyses for this exact condition. The red arrows show 
an area that both MDPR model and pressure peaks detect as regions with high cavitation erosion risk. A similar cavitation erosion location was also found in the experiments. This demonstrates the capability to detect correct cavitation erosion locations with both modeling approaches. The evolution of experimental erosion patterns after the first material removal cannot be directly compared with simulations due to the modifications in the geometry.
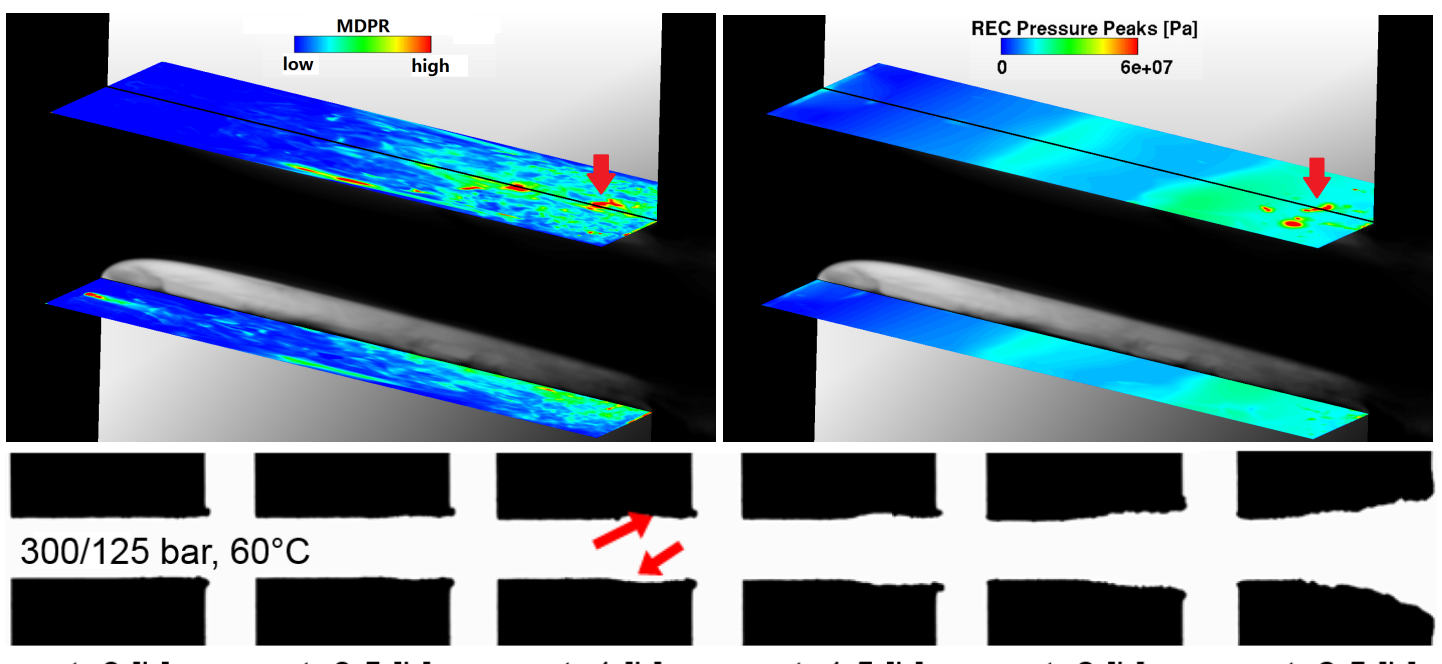

$t=1.5[h]$

$\mathrm{t}=2[\mathrm{~h}]$

$\mathrm{t}=2.5[\mathrm{~h}]$

Figure 7. Cavitation erosion risk predictions comparison. Averaged modeled MDPR (left), maximum recorded pressure peaks (right) and experimental cavitation erosion time evolution reproduced from [8] (below).

\section{Conclusions}

A numerical approach to solve compressible multi-phase flow fields is presented and results for a micro-throttle cavitating flow are discussed. A pressure-based solver is used to resolve iso-thermal multi-phase flow fields, with all phases considered compressible. Separate velocity fields are also computed for each phase. The computational methodology is presented, including mass and momentum transfer models for cavitating flows. The capability of the pressure-based solver to resolve compressible multi-phase cavitating flows is then assessed.

The I-channel test case is then used for verification. Average mass flow and vapor distribution are showed in good agreement with experimental data. The average and instantaneous flow fields resulting from the simulation are then illustrated, including an analysis of the two velocity profiles differences. It is demonstrated that the slip velocity modeled with the multi-fluid approach is significant for local and instantaneous values, but it is reduced on time-averaged velocity fields.

The capabilities of different cavitation erosion prediction models are finally analyzed, with reference to experimental visualizations. Surface pressure peaks above the driving pressure could be detected with the presented compressible solver. A good agreement exists between the erosion locations of the semi-empirical erosion model and the recorded surface pressure peaks with the experimental erosion patterns.

\section{Acknowledgements}

Financial support from the MSCA-ITN-ETN of the European Union's H2020 programme, under REA grant agreement n. 642536 is acknowledged.

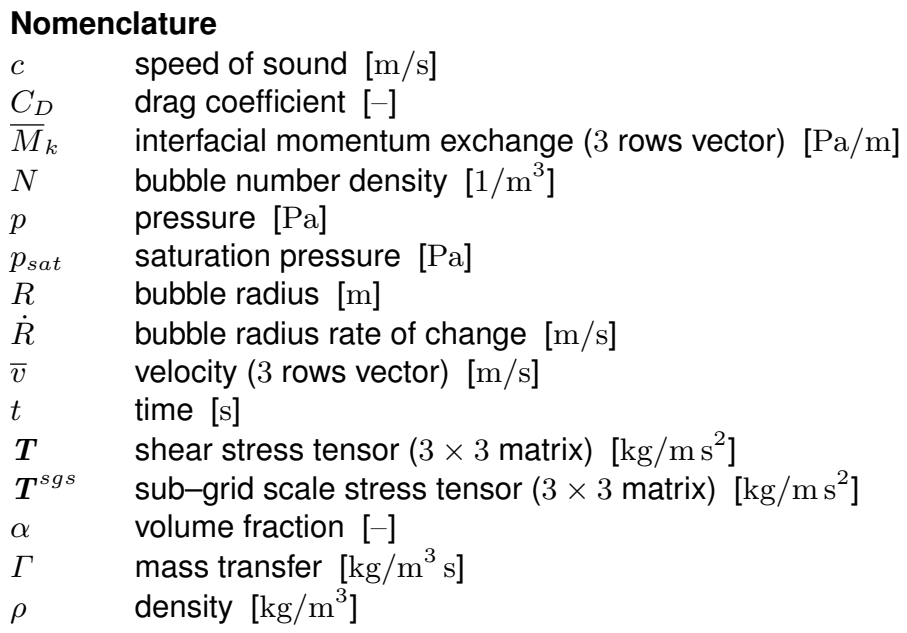




\section{References}

[1] Van Terwisga, T. J. C., Fitzsimmons, P. A., Ziru, L., and Foeth, E. J., 2009. "Cavitation erosion - a review of physical mechanisms and erosion risk models". In 7th International Symposium on Cavitation.

[2] Bark, G., Berchiche, N., and Grekula, M., 2004. Application of principles for observation and analysis of eroding cavitation - the EROCAV observation handbook. Edition 3.1.

[3] Grekula, M., and Bark, G., 2009. "Analysis of video data for assessment of the risk of cavitation erosion". In 1st International Conference on Advanced Model Measurement Technology for the EU Maritime Industry.

[4] Fortes-Patella, R., Reboud, J. L., and Briancon-Marjollet, L., 2006. "A phenomenological and numerical model for scaling the flow agressiveness in cavitation erosion". In EROCAV Workshop.

[5] Koukouvinis, P., Bergeles, G., and Gavaises, M., 2015. "A cavitation aggressiveness index within the Reynolds averaged Navier Stokes methodology for cavitating flows". Journal of Hydrodynamics, 27(4), pp. 579-586. http://dx.doi.org/10.1016/S1001-6058(15)60519-4.

[6] Bergeles, G., Li, J., Wang, L., Koukouvinis, F., and Gavaises, M., 2015. "An erosion aggressiveness index (EAI) based on pressure load estimation due to bubble collapse in cavitating flows within the RANS solvers". SAE Int. J. Engines, 8(5). http://dx.doi.org/10.4271/2015-24-2465.

[7] Koukouvinis, P., Bergeles, G., Li, J. Z., Wang, L., Theodorakakos, A., and Gavaises, M., 2015. "Simulation of cavitation inside diesel injectors, including erosion modelling". In Fuel Systems for IC Engines, IMECHE, pp. 125-135.

[8] Skoda, R., Iben, U., Mozorov, A., Mihatsch, M., Schmidt, S. J., and Adams, N. A., 2011. "Numerical simulation of collapse induced shock dynamics for the prediction of the geometry, pressure and temperature impact on the cavitation erosion in micro channels". In WIMRC, 3rd International Cavitation Forum.

[9] Egerer, C. P., Hickel, S., Schmidt, S. J., and Adams, N. A., 2014. "Large-eddy simulation of turbulent cavitating flow in a micro channel”. Physics of Fluids, 26(085102), pp. 190-200. http://dx.doi.org/10.1063/1.4891325.

[10] Mihatsch, M. S., Schmidt, S. J., and Adams, N. A., 2015. "Cavitation erosion prediction based on analysis of flow dynamics and impact load spectra". Physics of Fluids, 27(103302). http://dx.doi.org/10.1063/1.4932175.

[11] Koukouvinis, P., Gavaises, M., Li, J. Z., and Wang, L., 2016. "Large eddy simulation of diesel injector including cavitation effects and correlation to erosion damage". Fuel, 175, February, pp. 26-39. http://dx.doi.org/10.1016/j.fuel.2016.02.037.

[12] Örley, F., Hickel, S., Schmidt, S. J., and Adams, N. A., 2016. "Large-eddy simulation of turbulent, cavitating fuel flow inside a 9-hole diesel injector including needle movement". International Journal of Engine Research, April, p. 1468087416643901. https://doi.org/10.1177/1468087416643901.

[13] Greif, D., and Wang, D. M., 2006. "Aspects of modeling cavitation effects within injection equipment using advanced two-fluid techniques". Turbulence, Heat and Mass Transfer, 5, pp. 769-772. http://dx.doi.org/10.1615/ICHMT.2006.TurbulHeatMassTransf.1680.

[14] Brennen, C. E., 2005. Fundamentals of Multiphase Flows. Cambridge University Press. ISBN 0521848040.

[15] AVL FIRE TM , 2017. CFD User Manual, v2017 ed.

[16] Grogger, H. A., and Alajbegović, A., 1998. "Calculation of the cavitating flow in Venturi geometries using two fluid mode". In ASME Fluids Engineering Division Summer Meeting, A. FEDSM, ed.

[17] Lord Rayleigh, J. W. S., 1917. "On the pressure developed in a liquid during the collapse of a spherical cavity". Philosophical Magazine Series 6, 34(200), pp. 94-98. http://dx.doi.org/10.1080/14786440808635681.

[18] Plesset, M. S., 1949. "The dynamics of cavitation bubbles". ANSME Journal of Applied Mechanics, 16, pp. 228-231.

[19] Wood, A. B., 1930. A textbook of sound: Being an account of the physics of vibrations with special reference to recent theoretical and technical developments. Macmillan.

[20] Kobayashi, H., 2005. "The subgrid-scale models based on coherent structures for rotating homogeneous turbulence and turbulent channel flow". Physics of Fluids, 17(4), p. 045104.

[21] Ishii, M., and Mishima, K., 1984. "Two-fluid model and hydrodynamic constitutive relations". Nuclear Engineering \& Design, 8(107).

[22] Morozov, A., and Iben, U., 2008. "Experimental analysis and simulation of cavitating throttle flow". In HEFAT, 6th International Conference on Heat Transfer, Fluid Mechanics and Thermodynamics.

[23] Edelbauer, W., Strucl, J., and Morozov, A., 2014. "Large eddy simulation of cavitating throttle flow". In SimHydro: Modelling of rapid transitory flows.

[24] Winklhofer, E., Kull, E., Kelz, E., and Morozov, A., 2001. "Comprehensive hydraulic and flow field documentation in model throttle experiments under cavitation conditions". In ILASS-Europe.

[25] Iben, U., Mozorov, A., Winklhofer, E., and Skoda, R., 2011. "Optical investigations of cavitating flow phenomena in micro channels using a nano second resolution". In WIMRC, 3rd International Cavitation Forum.

[26] Iben, U., Mozorov, A., Winklhofer, E., and Wolf, F., 2011. "Laser-pulse interferometry applied to high-pressure fluid flow in micro channels". Exp Fluids(50), pp. 597-611. http://dx.doi.org/10.1007/s00348-010-0950-9.

[27] Sweby, P. K., 1984. "High resolution schemes using flux limiters for hyperbolic conservation laws". SIAM journal on numerical analysis, 21(5), pp. 995-1011. 


\title{
Role of heat transfer in bubble dynamics neglecting phase change. A numerical study
}

\author{
Stavros Fostiropoulos ${ }^{* 1,2,}$, Ilias Malgarinos ${ }^{1,2}$, George Strotos ${ }^{3}$, Nikolaos Nikolopoulos ${ }^{1}$, \\ Emmanouil Kakaras ${ }^{1}$, Phoevos Koukouvinis ${ }^{2}$ and Manolis Gavaises ${ }^{2}$ \\ ${ }^{1}$ Centre for Research and Technology Hellas/Chemical Process and Energy Resources \\ Institute (CERTH/CPERI), Egialeias 52, Marousi, Greece \\ ${ }^{2}$ City University London, School of Engineering and Mathematical Sciences, Northampton \\ Square, EC1V OHB London, UK \\ ${ }^{3}$ Piraeus University of Applied Sciences, Mechanical Engineering Department, 250 Thivon \\ and P. Ralli str., Aegaleo 12244, Greece \\ "Corresponding author: fostiropoulos@certh.gr
}

\begin{abstract}
Bubble dynamics is generally described by the well-known Rayleigh-Plesset (R-P) equation in which the bubble pressure (or equivalently the bubble density) is predefined by assuming a polytropic gas equation of state with common assumptions to include either isothermal or adiabatic bubble behaviour. The present study examines the applicability of this assumption by assuming that the bubble density obeys the ideal gas equation of state, while the heat exchange with the surrounding liquid is estimated as part of the numerical solution. The numerical model employed includes the solution of the Navier-Stokes equations along with the energy equation, while the liquidgas interface is tracked using the Volume of Fluid (VOF) methodology; phase-change mechanism is assumed to be insignificant compared to bubble heat transfer mechanism. To assess the effect of heat transfer and gas equation of state on bubble behaviour, simulations are also performed for the same initial conditions by using a polytropic equation of state for the bubble phase without solving the energy equation. The accuracy of computations is enhanced by using a dynamic local grid refinement technique which reduces the computational cost and allows for the accurate representation of the interface for the whole duration of the phenomenon in which the bubble size changes significantly. A parametric study performed for various initial bubble sizes and ambient conditions reveals the cases for which the bubble behaviour resembles that of an isothermal or the adiabatic one. Additional to the CFD simulations, a $0-D$ model is proposed to predict the bubble dynamics. This combines the solution of a modified R-P equation assuming ideal gas bubble content along with an equation for the bubble temperature based on the $1^{\text {st }}$ law of thermodynamics; a correction factor is used to represent accurately the heat transfer between the two phases.
\end{abstract}

\section{Keywords}

Bubble dynamics, heat transfer, CFD-VOF model, 0-D model.

\section{Introduction}

The need for the inclusion of thermal effects in bubble dynamics was first addressed in [1] among others; it was shown that the polytropic gas assumption may provide inaccurate predictions of the bubble behaviour when thermal processes are taken into consideration. Since then, the effect of heat and mass transfer on bubble dynamics were examined in a large number of studies, either by CFD numerical models that are capable of solving the complex equations that characterize the physical processes of the bubble motion, or by reduced order models which include various assumptions but are computationally more efficient. In the framework of the CFD studies, the effect of heat transfer by solving the equation of gas-vapour bubble including variation of liquid temperature and assuming liquid incompressibility was examined in [2]. The main assumption in this study, was that the temperature distribution inside the bubble to be uniform, which allowed the authors to integrate analytically the continuity and momentum equations inside the bubble. In [3], the motion of a single bubble during a mild collapse was simulated, by solving the mass, momentum and energy equations when the ambient pressure increases stepwise. The authors highlighted the effect of heat and mass transfer on the bubble dynamics, while their main conclusion was that the mean bubble temperature and the decaying time of the bubble motion depend on the initial bubble radius. Regarding strong bubble collapses, where liquid compressibility has to be considered; the motion of a collapsing bubble subjected to a strong pressure field was investigated in [4] by using the Keller equation [5]. High pressure and temperature values at the collapse instant which are associated with the sonoluminescence, were observed

Regarding the reduced order models, in [6] thermal effects in bubble behaviour were investigated by using perturbation analysis method in the nearly adiabatic and isothermal regimes; the results are accurate only for very low Peclet numbers (isothermal limit). In high Peclet numbers, the model results in a convolution integral which is computationally expensive to be solved. In the context of sonoluminescence, a reduced order model has been proposed in [7]. Finally, a reduced order model has been developed in [8] by applying proper orthogonal 
decomposition to the full set of equations for a spherical gas-vapour bubble, including heat and mass diffusion. A transfer coefficient, for heat and mass diffusion which directly depends on the Peclet number, was derived.

In the current study, the effect of heat transfer on bubble motion is examined by CFD, solving the energy equation and assuming that the bubble density follows the ideal gas equation of state. The detailed physical phenomena are analyzed based primarily on a CFD numerical model, the latter however used as well to propose an easily manageable in terms of computational cost 0-D model, following a sensitivity analysis among the 0-D and the CFD results.

\section{Mathematical models}

\section{CFD numerical model}

The bubble dynamics, for conditions that phase change is not considered, are investigated with a CFD simulation model which solves the Navier-Stokes equations in a 2-D axisymmetric domain along with the VOF methodology [9] to track the liquid-gas interface. For the simulations where heat exchange between the gas bubble (pure air bubble) and the surrounding liquid is considered, the energy equation is also solved and the bubble density is assumed to obey the compressible ideal gas equation of state. Additionally, for the evaluation of the heat transfer effects but also for validation purposes, CFD simulations were performed for the same initial conditions without solving the energy equation. For the latter cases, the bubble density obeys a polytropic gas equation of state $\left(p_{g}=\kappa \rho^{n}\right)$, where the constant parameter $\kappa$ is set according to a reference state for gas pressure $p_{g}$ and density. The thermal VOF model has been extensively used in a number of studies from the authors' group in deforming droplet simulations such as in [10-12] and in [13-15], but also in cases with polytropic bubble dynamics as in [16, 17]. The model equations have been presented in detail in the aforementioned works and thus they are not repeated here. The present work is not considering any phase change effects and ignores any vapour presence (thus a pure air bubble is considered), any compressibility effects in the liquid phase, while the surface tension has been ignored since its effect is significant only for very small bubble sizes $(\mathrm{nm})$. Additionally, the gas properties are kept constant (except of the density), without considering any dependence on temperature. Regarding the assumption of no phase change, this is proved to be true for the conditions examined (water at atmospheric conditions) since the thermodynamic parameter $\Sigma$ proposed in [18] is small and bubble dynamics can be considered "inertially controlled". In any case, the aim of the present work is to isolate the effect of heat transfer on bubble dynamics and to propose a first basis for a 0-D model, while resulting to the fact that the results presented here cannot be directly related to the actual multi-phase actual phenomena appearing in bubble dynamics.

The 2-D axisymmetric computational domain is semi-circular and extends to a distance of $100 \mathrm{R}_{0}$ to minimize any influence of the boundary conditions on the solution. The computational cells are squares at the bubble region $\left(1.5 \mathrm{R}_{0}\right)$ and quadrilateral at the rest of the domain; this is shown in Figure 1. To enhance the accuracy of computations and achieve a low computational cost, an adaptive local grid refinement technique [19] is used. The grid resolution expressed as cells per Radius $(\mathrm{cpR})$ range between $400 \mathrm{cpR}$ for the maximum bubble radius and $8 \mathrm{cpR}$ for the minimum one. Far from the bubble, boundary conditions of constant pressure and temperature are applied, while only the half of the bubble is simulated by using symmetry boundary condition. The CFD simulations are performed with the commercial CFD tool ANSYS FLUENT v16 [20], along with various user defined functions (UDFs) for the implementation of the adaptive local grid refinement method in Malgarinos et al. [19] and the adaptive time-step for the implicit VOF solver.

\section{0 -D model}

Alongside with the CDF model, a 0-D model, which is capable of predicting the bubble size evolution under both pressure and thermal effects, is presented in this subsection. The model solves a modified Rayleigh-Plesset (R-P) equation along with an equation for the mean bubble temperature derived from an energy balance in the bubble. Apart from the aforementioned assumptions, the model further assumes uniform temperature and pressure inside the bubble, which are widely used in the works mentioned in the introduction. The present model is similar to the reduced order model in [8], which was derived by reducing the full set of equations using the proper orthogonal decomposition method (POD); nevertheless, the cases presented here correspond to different physical conditions, while Diesel liquid is also examined.
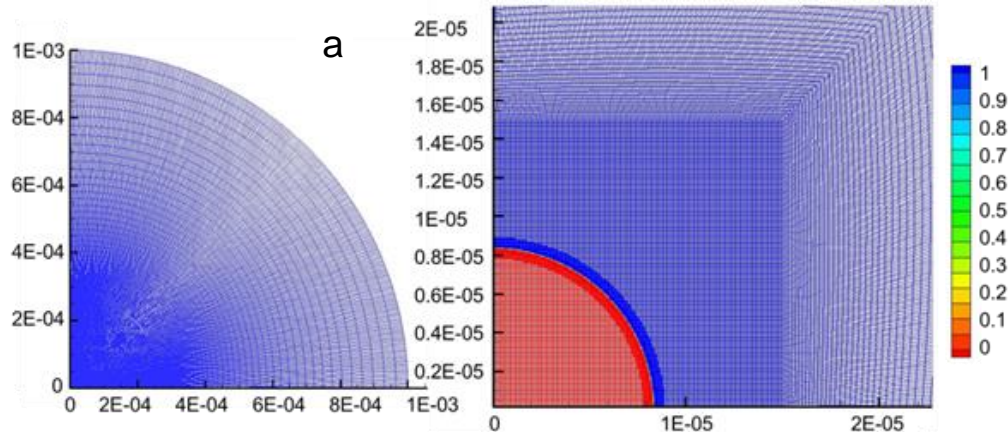

b

Figure 1. (a) Grid detail at the bubble region and (b) whole computational domain. Blue (red) colour indicates the ID of the gas (liquid) phase. 
The classical Rayleigh-Plesset equation, which is an effective tool for the prediction of the bubble dynamic behaviour, is used as a starting point for the development of the 0-D model. In this study Rayleigh-Plesset equation is written as

$\ddot{R}=\frac{1}{R}\left(-\frac{3 \dot{R}^{2}}{2}+\frac{p_{g}-p_{\infty}}{\rho_{l}}-\frac{2 \sigma}{\rho_{l} R}-4 \mu_{l} \frac{\dot{R}}{\rho_{l} R}\right), \quad p_{g}=p_{g, 0}\left(\frac{R_{0}}{R}\right)^{3} \frac{T_{B, m}}{T_{B, m, 0}}$

Recall that the present work assumes a pure air bubble, thus the vapour pressure $p_{v}$ is not appearing in eq. 1 . In order to obtain an equation for $T_{B, m}$, the first law of thermodynamics for the bubble with ideal gas content is employed in the following form:

$\dot{Q}-\dot{W}=m \cdot\left(c_{v} \frac{d T_{B, m}}{d t}+\frac{3}{5} \dot{R} \ddot{R}\right)$

The first and second terms on the left hand side express the heat entering the bubble and the work done by the bubble, respectively. On the right hand side, $m$ remains constant in the absence of phase change. The first and second terms inside the parentheses stand for the change of the mean internal energy and the change of the average bubble kinetic energy respectively; nevertheless, the second term can be ignored since it has been found to play a minor role conducting series of numerical tests. The formulation for the work done by the bubble is:

$\dot{W}=p_{B} A_{\text {surf }} \dot{\mathrm{R}}, \quad A_{\text {surf }}=4 \pi R^{2}$

Here, $p_{B}$ is equal to $p_{g}$ for the present cases. Finally, the most sensitive part of the present model is $\dot{Q}$. This is assumed to be equal to the product of $\dot{Q}_{\text {ref }}$ with a correction factor $f_{\text {heat }}$ :

$\dot{Q}=f_{\text {heat }} \dot{Q}_{\text {ref }}, \quad \dot{Q}_{\text {ref }}=k_{g} \frac{T_{\infty}-T_{B, m}}{R} A_{\text {surf }}$

More details on the heat flux will be further discussed in the next section. After substituting Eq. 3,4 into Eq. 2, the resulting model consists of a system of coupled O.D.E's (Eq. 1,2) which predicts the bubble dynamics when thermal effects are taken into account. The set of equation is integrated in time with a fourth order Runge-Kutta scheme.

\section{Results and Discussion}

I. Model results for an ideal gas bubble

In the first part of this section, results of the CFD ideal gas simulations are presented in Figure 2, along with those obtained from the commonly used assumption of polytropic gas bubble (either isothermal or adiabatic). In the ideal gas ones, the heat transfer between the bubble and the liquid is not predefined (adiabatic or isothermal), but becomes part of the solution. In Figure $2 \mathrm{a}$, the case of bubble collapse $\left(p_{\infty}>p_{g}\right)$ is considered and the conditions examined are identical to those examined in [16] $\left(p_{\infty}=10^{5} \mathrm{~Pa}, p_{g, 0}=6900 \mathrm{~Pa}\right)$; for the ideal gas case, both the liquid and the gas have an initial temperature of $300 \mathrm{~K}$. In Figure $2 \mathrm{~b}$, the case of bubble growth is considered $\left(p_{\infty}=10^{5} \mathrm{~Pa}, p_{g, 0}=30 \cdot 10^{5} \mathrm{~Pa}\right)$; with an initial bubble temperature of $2500 \mathrm{~K}$, higher than the initial liquid temperature. A first glimpse shows that the effect of heat transfer is quite important for the bubble expansion case compared to the bubble collapse one, while the ideal gas curve is not between the limiting conditions of isothermal and adiabatic behaviour as expected. Regarding the collapsing case (Figure 2a), the effect of heat transfer becomes more important after the first collapse and it is getting intensified as time evolves. These results are in agreement with previous studies in [6] and in [21] which state that not only pressure differences but also heat exchange between liquid and gas could affect bubble's behavior. Additional to the bubble radius behaviour, the temporal evolution of the mean bubble temperature is also of importance. The latter is shown in Figure 2c for the case of bubble collapse, where the differences at the bubble temperature profiles are more intense compared to those of the bubble radius. In the adiabatic regime, where there is no heat exchange between the gas and the liquid, there is a smooth variation of the mass averaged temperature in time and a high peak of $1800 \mathrm{~K}$ at the collapse instant is predicted from the 0-D model which for the moment neglects any heat transfer mechanism; in the isothermal regime the bubble is in thermal equilibrium with the surrounding liquid.

For the ideal gas case, the bubble temperature profile is located between the adiabatic and the isothermal regimes. For most of the time, the average bubble temperature is nearly constant and near the collapse there is a steep change of the mean temperature which reaches values up to $1120 \mathrm{~K}$; this behaviour pertains to a rather isothermal bubble. 

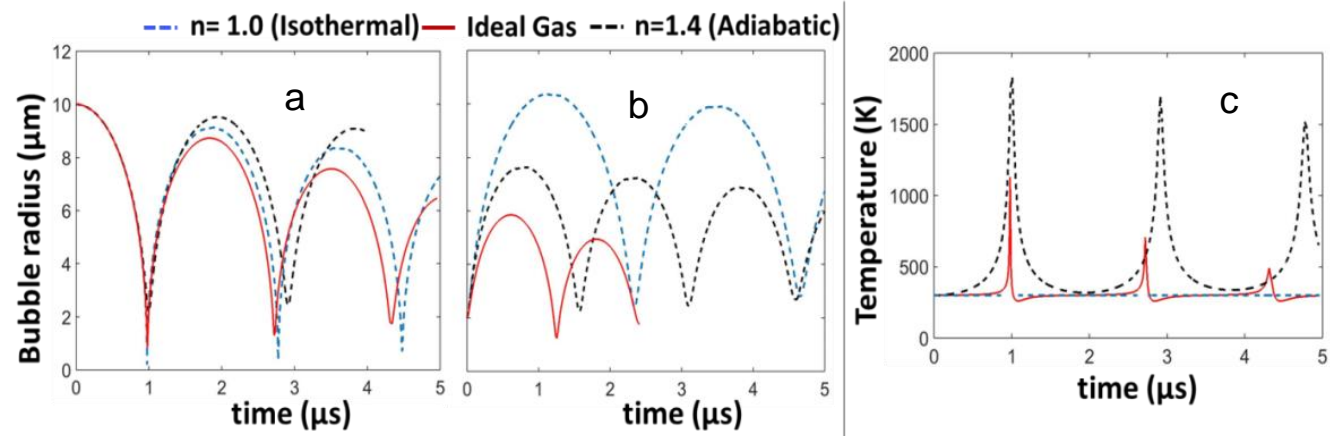

Figure 2. Results from CFD simulations: Bubble radius for collapse $\left(\boldsymbol{p}_{\infty}=10^{5} \mathrm{~Pa}, \boldsymbol{p}_{\boldsymbol{g}, \mathbf{0}}=6900 \mathrm{~Pa}\right)(\mathrm{a})$ and growth $\left(\boldsymbol{p}_{\infty}=10^{5}\right.$ $\mathrm{Pa}, \boldsymbol{p}_{g, 0}=3 \cdot 10^{5} \mathrm{~Pa}$ ) (b) for the Ideal gas case, adiabatic case and isothermal case; $\mathrm{c}$ ) temperature profile for the case of bubble collapse.

An interesting characteristic here is the cooling of the bubble below the liquid temperature, which is observed in the rebound right after the collapse. This occurs due to a time lag between the heat entering and the work received by the bubble [3]. This is a characteristic phenomenon that is captured also by the 0-D model.

The heat exchange between the gas and liquid can be quantified based on the CFD simulations by using Eq. 2, and solving for $\dot{Q}$. This is shown in Figure 3, which illustrates the temporal evolution of the heat flux at the bubble, which is smoothed by using a moving average filter, and the work done by the bubble normalized with the maximum absolute work value; the corresponding temporal evolution of non-dimensional bubble radius is also presented. During the collapse period, the work that the bubble receives from the liquid phase and the heat directed from the bubble to the liquid phase, increase in terms of absolute values, while upon collapse the work decreases abruptly. Later during the expansion phase, the bubble supplies work to the liquid and heat changes direction and flows towards the bubble. During the whole phenomenon, heat and work are in phase between them, while the ratio of their maximum absolute was computed equal to $\dot{Q}_{\max } / \dot{W}_{\max } \sim 0.2$; the latter manifests the significance of heat transfer to the evolution of the phenomenon.

The analysis that follows in the rest of the paper concerns only cases of bubble collapse, while the reference operating conditions in [16] are used, as in Figure 2a. In order to relate the temporal evolution of $\dot{Q}$ provided by the CFD simulations (considered to be the actual one) with that of $\dot{Q}_{r e f}$ (Eq. 4), a graph between the two is given in Figure 4a. Each collapse-expansion cycle is characterized by a circle-like trajectory which diminishes with time. The ratio of the aforementioned quantities provides $f_{\text {heat }}$, which works as a tuning parameter between the actual heat $\dot{Q}_{C F D}$, and the assumed expression for heat transfer $(\dot{Q})$ that has been used in the 0-D model (Eq. 4). In [8], it was found that the corresponding optimal correction factor is equal to 5 for a nearly isothermal behaviour; this parameter was kept constant during the simulation without making any distinction between the collapsing or the expanding phase. In this study the optimal $f_{\text {heat }}$ value, which was found to be constant as well, is determined from the deviation between the CFD values and those predicted by the 0-D model for the bubble radius and the mean bubble temperature at the first collapse.

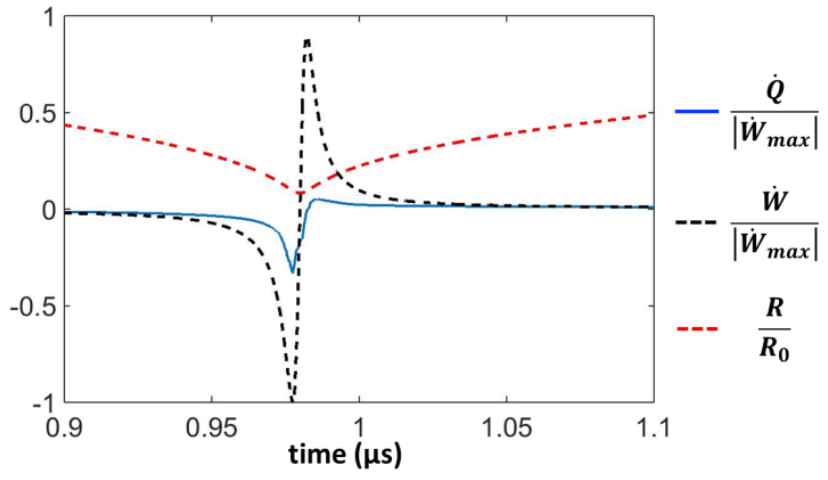

Figure 3. Evolution of the normalized heat (blue line), normalized work (black line) and bubble radius (red line) with time; results are demonstrated only for a time frame close to the bubble collapse.

The predicted deviation for a range of $f_{\text {heat }}$ values is shown in Figure $4 \mathrm{~b}$. As seen, there is no value of the parameter $f_{\text {heat }}$ resulting in a simultaneously optimum 0-D model performance for both the bubble radius and the temperature. The value chosen for the simulation is the one corresponding to the optimum performance in predicting the bubble radius, i.e. $f_{\text {heat }}=10$. Additionally and judging from the error values, one could see in Figure $4 \mathrm{~b}$ that the model performs well for a wide range of values of $f_{\text {heat }}$ between 5 and 20 . Next, the settings used in the CFD ideal gas case, are now implemented in the 0-D model. Figure 5 shows results of the 0-D model indicated by black solid line with $f_{\text {heat }}=10$, against the results of the CFD ideal gas case. Note that there is a 
deviation on the prediction of the maximum temperature value by the $0-D$ model compared to that by the CFD model equal to $218 \mathrm{~K}$, however regarding the radius profile there is an identical match between $0-\mathrm{D}$ model and CFD.
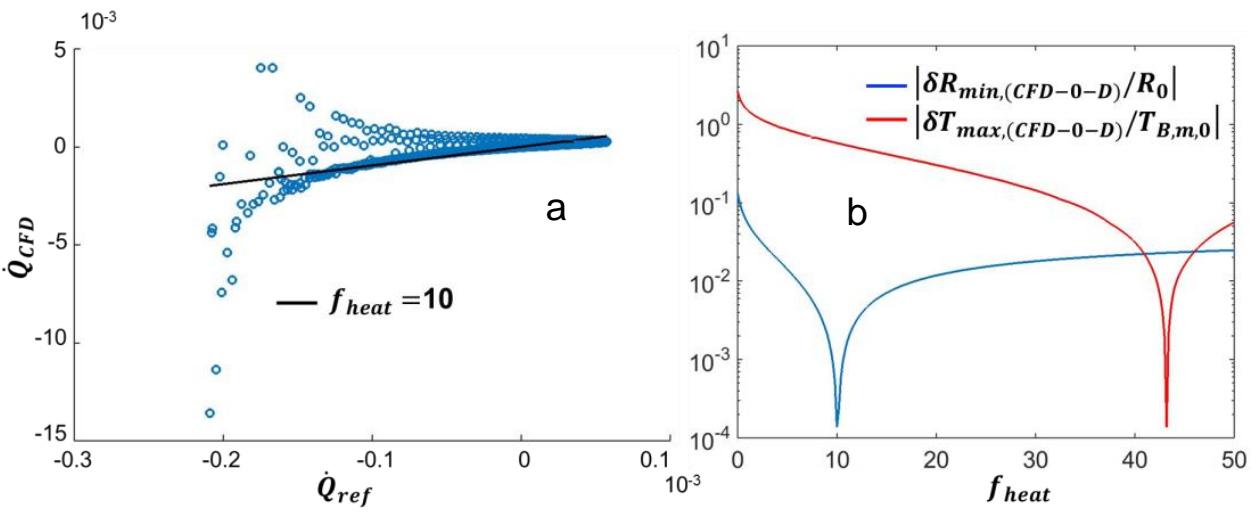

Figure 4. a) $\dot{Q}_{C F D}$ provided by CFD against $\dot{Q}_{\text {ref }}$ which is the assumed heat in the 0-D (blue scattered spots); constant correction factor $f_{\text {heat }}=10$ (black solid line) .b) Deviation between the CFD values and those predicted with the 0-D model plotted in logarithmic scale for bubble radius (blue solid line) and temperature (red solid line) for various $f_{\text {heat }}$ values.

II. Parametric study

In order to assess the effect of heat transfer on the bubble dynamics, a number of parametric cases with different properties and physical parameters, is performed. These include the effect of different initial bubble size and the effect of different ambient conditions with varying initial pressure difference, temperature and conductivity in both the liquid and the gas phase. The aforementioned parameters were varied with respect to the reference settings with the corresponding results to be always compared against the results of the reference case shown in Figure 5 . It has to be mentioned that a limited amount of the examined cases will be presented in the context of this paper, for reasons of space limits. Figure 6 quantifies the bubble dynamics for various initial bubble radius (a) and initial liquid pressure (b). The results of the 0-D model show very good agreement with CFD for the parametric initial radius cases (a). The results of the cases with various initial pressure show rather satisfactory agreement (right panels).
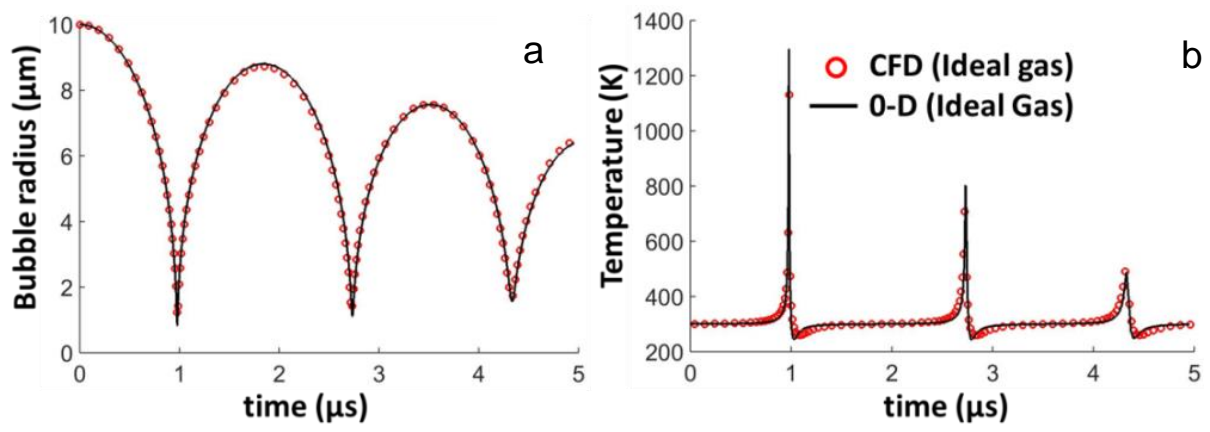

Figure 5. Radius (a) and temperature (b) profile of the CFD (red scatter) and 0-D model (black solid line).

Here, the examined case with increased liquid pressure $\left(2 \cdot p_{\infty}\right)$ exhibits discrepancies, probably due to the fact that, for large pressure differences, the assumption of a uniform pressure is violated [22]. Regarding the examined temperature profiles which are not presented here, the 0-D model is not capable of capturing accurately the temporal evolution of bubble temperature. Particularly, in the examined case with increased liquid pressure, the deviation between the temperature peak predicted from the CFD and the 0-D model is quite high. Moreover, parametric runs with various liquid and gas conductivities were conducted. The corresponding results which are not presented in this study, reveal that the model used is able to capture all the cases examined and that the liquid conductivity is playing a minor role relative to the gas conductivity. Finally, the performance of the $0-D$ model is examined for liquid Diesel with properties computed from Kolev [23] at atmospheric conditions. Specifically, the properties assumed are the following; liquid density $\left(\rho_{l}=826 \mathrm{~kg} \mathrm{~m}^{-3}\right)$, heat capacity $\left(c_{p}=\right.$ $\left.1993.7 \mathrm{~J} \mathrm{~kg}^{-1} \mathrm{~K}^{-1}\right)$, liquid thermal conductivity $\left(k_{l}=0.12 \mathrm{~W} \mathrm{~m}^{-1} \mathrm{~K}^{-1}\right)$ and liquid dynamic viscosity $\left(\mu_{l}=0.003 \mathrm{~Pa} \mathrm{~s}\right)$. Results show that the $0-\mathrm{D}$ model with the same correction factor $\left(f_{\text {heat }}=10\right)$, is capable of capturing the behaviour of Diesel liquid (Figure 7), which exhibits lower peak temperature values and the bubble energy dissipates faster compared to water liquid due to its larger viscosity. 


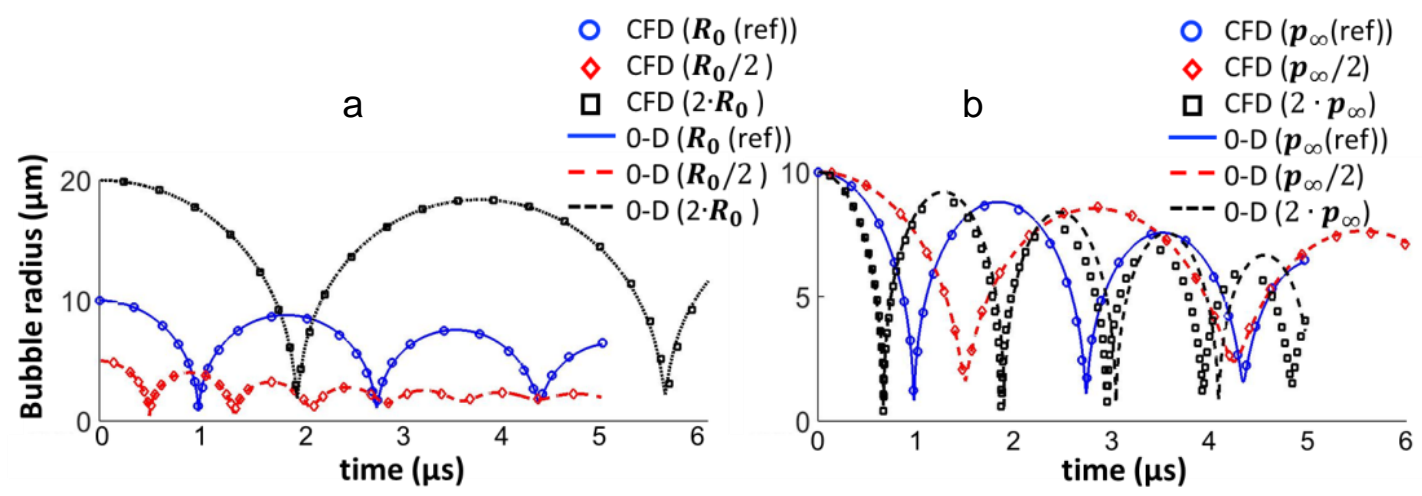

Figure 6. Bubble radius and temperature profiles for 0-D model (solid and dashed lines) against CFD (scatter spots) for parametric initial bubble radius (a) and initial liquid pressure (b).

In the last part of this subsection, quantification of the overall effect of heat transfer is carried out. For each parametric case the following factor is determined based on the minimum bubble radius only for the first collapse.

$F_{R}=\frac{R_{\text {min,thermal }}-R_{\min , n=1}}{R_{\min , n=1.4}-R_{\min , n=1}}$

, where $R_{\min , n=1.4}$ and $R_{\min , n=1}$ are the minimum bubble radius at the adiabatic and the isothermal regime, respectively. The aforementioned factors range between 0 and 1 . When factor values are closer to 0 the bubble motion is close to that of an isothermal bubble, while when they approach 1 the bubble tends to behave adiabatically.
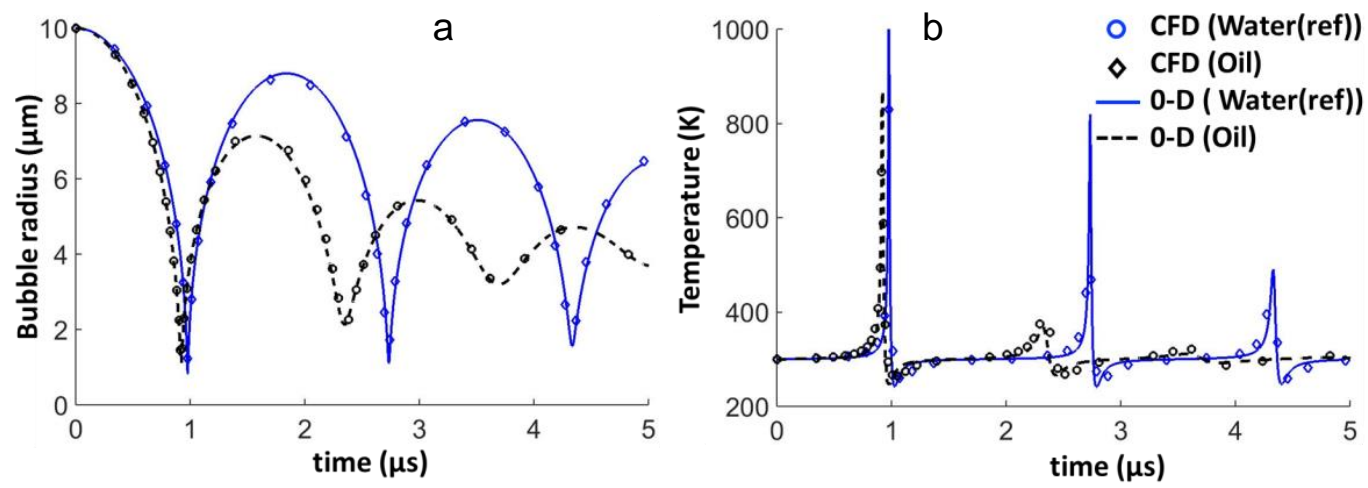

Figure 7. Bubble radius (a) and temperature (b) profile for the 0-D model (solid and dashed lines) against CFD (scatter spots).

The isothermal-adiabatic behaviour is evaluated against the gas phase Peclet number, which reads:

$P e_{g, r e f}=\frac{R_{0} u_{r e f}}{a_{g, 0}}, u_{\text {ref }}=\sqrt{3 p_{\infty} / \rho_{l}}$

Here, $a_{g, 0}$ is based on a reference velocity $u_{r e f}$ which is derived from a bubble natural frequency used in [8]. Figure 8 shows the factors computed from both CFD and 0-D models (denoted as $F_{R_{C} \text { CFD }}$ and $F_{R_{0} \text { OD }}$ respectively) plotted against the $P e_{g, r e f}$ number. Regarding the bubble radius, it is clearly demonstrated that for the low Peclet number limit the bubble tends to perform isothermally, while for higher Peclet numbers the bubble motion approaches the behaviour of an adiabatic bubble. Moreover, it is demonstrated that in the range of Peclet number with values between $10^{-2}$ and 1 , there is small deviation between the $F_{R_{-} C F D}$ and the $F_{R_{-} 0-D}$. On the other hand, for Peclet number larger than 1, it seems that the aforementioned deviations become more significant. Consequently, the proposed 0-D model with $f_{\text {heat }}=10$, can provide reliable results for $P e_{g, r e f}<1$. 


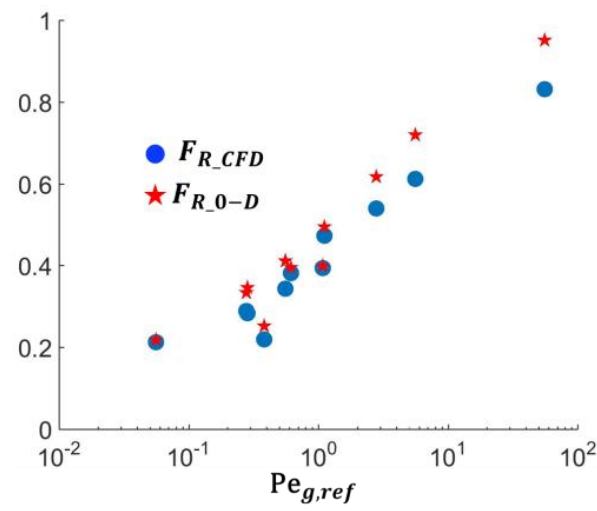

Figure 8. Computed factor from CFD and 0-D model for both minimum bubble radius.

\section{Conclusions}

In this study, the individual effect of heat transfer on bubble dynamics was examined by solving the energy equation and assuming that the bubble density follows the ideal gas equation of state, always targeting conditions where phase change can be neglected as in the considered cases where liquid temperature is not higher than $300 \mathrm{~K}$. The detailed physical phenomena were investigated with a 2-D axisymmetric CFD numerical model coupled with the VOF methodology, while an adaptive local grid refinement technique enhanced the accuracy of computations with a low computational cost. Alongside with the CFD numerical model, a 0-D model that predicts bubble motion, is proposed. The latter combines a modified Rayleigh-Plesset equation along with an equation for the mean bubble temperature based on the first thermodynamic law. The model also requires the estimation of the heat flux towards the bubble. This was determined by processing the CFD data and it was modeled with a simplified expression using a constant tuning factor; nevertheless, this was found adequate to capture the basic physics and simulate with acceptable accuracy a wide range of cases. These include various bubble sizes, various bubble-liquid pressure differences and liquid/gas properties. Specifically, in the low Peclet number limit with values between $10^{-2}$ and 1 , where bubble performs rather isothermally, the $0-D$ model is capable to provide reliable results due to the identical matching of the latter with the corresponding results from the CFD model. On the other hand, for Peclet numbers larger than 1, the 0-D model with the specific constant correction factor does not provide very accurate results, though more cases need to be examined. Finally, a future plan, related to the current study, is to examine the same phenomenon under evaporating conditions. For that reason further development of the 0-D model, with the inclusion of mass diffusion terms is needed.

\section{Acknowledgements}

Financial support from the MSCA-ITN-ETN of the European Union's H2020 programme, under REA grant agreement n. 675676 is acknowledged.

\section{Nomenclture Roman symbols}

$p$
$T$
$\rho$
$R$
$m$
$A_{\text {surf }}$
$\dot{W}$
$\dot{Q}$
$k$
$c_{p}$
$c_{v}$
$P e$
$n$
$F_{R}$
$f_{h e a t}$
Greek symbols
$\sigma$
$a$
$\mu$
$u$
$\kappa$

Subscripts
$\infty$
0
$\mathrm{~g}$
$\mathrm{l}$
$\mathrm{V}$
$\mathrm{B}$
$\mathrm{m}$
ref
CFD
0-D
Dotted symbols
$\dot{\varphi}$
$\ddot{\varphi}$

far-field quantity initial value

gas

liquid

vapour

bubble

mass-averaged

reference value values provided by CFD values provided by $0-D$

first time derivative second time derivative 


\section{References}

1. Prosperetti, A., L.A. Crum, and K.W. Commander, Nonlinear bubble dynamics. The Journal of the Acoustical Society of America, 1988. 83(2): p. 502-514.

2. Nigmatulin, R., N. Khabeev, and F. Nagiev, Dynamics, heat and mass transfer of vapour-gas bubbles in a liquid. International Journal of Heat and Mass Transfer, 1981. 24(6): p. 1033-1044.

3. Matsumoto, Y. and F. Takemura, Influence of Internal Phenomena on Gas Bubble Motion. Effects of Thermal Diffusion, Phase Change on the Gas-Liquid Interface and Mass Diffusion between Vapor and Noncondensable Gas in the Collapsing Phase. JSME International Journal Series B, 1994. 37(2): p. 288296.

4. Vuong, V.Q. and A.J. Szeri, Sonoluminescence and diffusive transport. Physics of Fluids, 1996. 8(9): p. 2354-2364.

5. Keller, J.B. and M. Miksis, Bubble oscillations of large amplitude. The Journal of the Acoustical Society of America, 1980. 68(2): p. 628-633.

6. Prosperetti, A., The thermal behaviour of oscillating gas bubbles. Journal of Fluid Mechanics, 1991. 222: p. 587-616.

7. Kreider, W., et al., A reduced-order, single-bubble cavitation model with applications to therapeutic ultrasound. The Journal of the Acoustical Society of America, 2011. 130(5): p. 3511-3530.

8. Preston, A., T. Colonius, and C. Brennen, A reduced-order model of diffusive effects on the dynamics of bubbles. Physics of Fluids, 2007. 19(12): p. 123302.

9. Hirt, C.W. and B.D. Nichols, Volume of fluid (VOF) method for the dynamics of free boundaries. Journal of Computational Physics, 1981. 39(1): p. 201-225.

10. Strotos, G., et al., Predicting the evaporation rate of stationary droplets with the VOF methodology for a wide range of ambient temperature conditions. International Journal of Thermal Sciences, 2016. 109: $p$. 253-262.

11. Strotos, G., et al., Numerical investigation of aerodynamic droplet breakup in a high temperature gas environment. Fuel, 2016. 181: p. 450-462.

12. Strotos, G., et al., Aerodynamic breakup of an n-decane droplet in a high temperature gas environment. Fuel, 2016. 185: p. 370-380.

13. Malgarinos, I., N. Nikolopoulos, and M. Gavaises, A numerical study on droplet-particle collision dynamics. International Journal of Heat and Fluid Flow, 2016. 61: p. 499-509.

14. Malgarinos, I., N. Nikolopoulos, and M. Gavaises, Numerical investigation of heavy fuel droplet-particle collisions in the injection zone of a Fluid Catalytic Cracking reactor, Part I: Numerical model and 2D simulations. Fuel Processing Technology, 2017. 156: p. 317-330.

15. Malgarinos, I., N. Nikolopoulos, and M. Gavaises, Numerical investigation of heavy fuel droplet-particle collisions in the injection zone of a Fluid Catalytic Cracking reactor, part II: 3D simulations. Fuel Processing Technology, 2017. 156: p. 43-53.

16. Koukouvinis, P., et al., Numerical simulation of a collapsing bubble subject to gravity. Physics of Fluids, 2016. 28(3): p. 032110.

17. Koukouvinis, P., et al., Simulation of bubble expansion and collapse in the vicinity of a free surface. Physics of Fluids, 2016. 28(5): p. 052103.

18. Brennen, C.E., Cavitation and bubble dynamics. 2013: Cambridge University Press.

19. Malgarinos, I., N. Nikolopoulos, and M. Gavaises, Coupling a local adaptive grid refinement technique with an interface sharpening scheme for the simulation of two-phase flow and free-surface flows using VOF methodology. Journal of Computational Physics, 2015. 300: p. 732-753.

20. Fluent, A., Release 16.0, ANSYS. 2014, Inc.

21. Nigmatulin, R. and N. Khabeev, Heat exchange between a gas bubble and a liquid. Fluid Dynamics, 1974. 9(5): p. 759-764.

22. Storey, B.D., H. Lin, and A.J. Szeri, Physically realistic models of catastrophic bubble collapses. http://resolver. caltech. edu/cav2001: sessionB6. 001, 2001.

23. Kolev, N.I., Thermodynamic and transport properties of diesel fuel, in Multiphase Flow Dynamics 4. 2011, Springer. p. 293-327. 


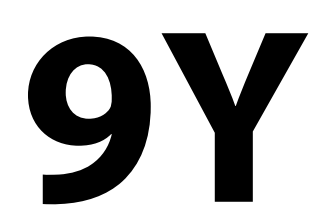

Droplet 6 


\title{
Evaluation of the combustion characteristics of isolated droplets of Jet $A$ blended with ethanol and butanol
}

\author{
Álvaro Muelas, Pilar Remacha, Javier Ballester* \\ Laboratory for Research on Fluid Dynamics and Combustion Technologies (LIFTEC) \\ University of Zaragoza / CSIC, Zaragoza, Spain \\ *Corresponding author: ballester@unizar.es
}

\begin{abstract}
In light of the potential of ethanol and butanol as alternative fuels for blending with conventional kerosene in gas turbine engines, experimental data regarding the burning characteristics of these blends are required in order to better understand their combustion process. In this study, free-falling droplets of Jet A, ethanol, butanol and their mixtures $(20 \%$ alcohol in Jet $A$ by volume) were examined in a combustion chamber which provides representative conditions of real flames, both in terms of temperature and oxygen availability. Results show that the evolution of droplet diameter for Jet $A$ and its blends with both alcohols are very similar, regardless of the obvious compositional differences. On the other hand, sooting behaviors are found to be quite different, with a clear reduction in the sooting propensity of the Jet A/alcohol mixtures when compared to neat kerosene. These results are consistent with previous studies in gas turbines, suggesting that such blends are viable alternative fuels with similar combustion characteristics to Jet $A$, but with much less propensity to produce soot. Moreover, this study provides new results on the combustion properties of Jet $A /$ ethanol and Jet $A / b u t a n o l$ mixtures, for which very scarce data exist in the open literature.
\end{abstract}

\section{Keywords}

Droplet combustion, Jet A, Butanol, Ethanol.

\section{Introduction}

Renewable fuels derived from biofeedstocks (i.e. biofuels) are recently gaining importance due to environmental and sustainability reasons. Biofuels are reported to have negligible sulphur content and to inhibit $\mathrm{PAH}$ and soot formation [1]. Furthermore, the biomass used to produce biofuels is renewable, carbon-neutral and domestically available. Although fossil fuels are likely to remain dominating transportation and energy systems in the near future [2], the use of biofuels as additives can significantly reduce their consumption, and therefore their environmental footprint. The most widely used biofuel today is by far bio-ethanol [1,2], which is commonly added to gasoline for its use in internal combustion engines. Ethanol production via fermentation of plants and starches was among the very first developments in biofuels because of the already well-established alcohol industry [3]. Although ethanol production from edible crops poses serious concerns, such as competition with the food industry or indirect land-use change, it remains nowadays as the major source for bio-ethanol feedstock [2]. These concerns have motivated efforts to find more environmentally-friendly processes for ethanol production, such as lignocellulosic biomass or residual waste conversion (second generation bio-ethanol).

Even though ethanol is the most used biofuel nowadays, some of its properties entail significant limitations. A research on potential new fuel molecules has been carried out in order to overcome these drawbacks. One of the most promising candidates is butanol, which has several well-known advantages when compared with ethanol [28]:

- $\quad$ Higher energy density.

- $\quad$ Lower propensity for water absorption.

- Higher miscibility with hydrocarbons.

- $\quad$ Boiling point closer to the gasoline/diesel fuel range: lesser impact on the fuel distillation curve.

- Better material compatibility: corrosion towards ferrous metals and elastomers swelling are reported to be much less severe than in the case of ethanol.

- $\quad$ Lower vapor pressure: safer manipulation and less volatile organic compounds emission.

- $\quad$ Lower heat of vaporization, which facilitates ignition.

- Current engines can run on more enriched butanol blends without any modification.

Bio-butanol can be both produced by fermentation of edible crops (first generation biofuel) or through more advanced technologies, using lignocellulosic biomass or algae as feedstock (second generation biofuel) [3-5]. 
Although there is currently much less butanol production capacity compared to ethanol, several companies are developing technologies for bio-butanol synthesis, and equipping ethanol plants with butanol production capabilities through cost-effective retrofitting $[4,7]$.

A comparatively large number of studies have analyzed the performance of ethanol and butanol in sparkignition engines, both as pure fuels or blended with gasoline (e.g. [9, 10]). Much less work has been done regarding its possible use in gas turbine engines, even though some recent studies point to the feasibility of kerosene-alcohol mixtures for their use in stationary gas turbines. Mendez et al. investigated Jet A/butanol [7] and Jet A/ethanol [11] mixtures in a $30 \mathrm{~kW}$ experimental gas turbine. Results suggest that blending Jet $A$ with both alcohols is a viable alternative for achieving $\mathrm{CO}$ and NOx emissions reduction while maintaining similar performance to that of pure kerosene. Patra et al. studied the combustion characteristics of kerosene / ethanol mixtures in an experimental axi-symmetric combustor [12], concluding that ethanol addition significantly decreases the flame luminosity due to soot reduction, while also decreasing $\mathrm{CO}$ emissionsi.

Even though engine studies provide very useful information regarding fuel performance under realistic conditions, results can be dependent on several non-controllable variables (e.g. fuel spray patterns, temperature profiles inside the combustion chamber, etc.), and therefore results can depend to some extent on the experimental facility used. On the contrary, in the much more simplified single droplet configuration all the parameters affecting the results are completely known and controllable, and therefore the combustion characteristics observed are intrinsically attributable to the fuel. Recent studies have examined the combustion characteristics of pure ethanol (e.g. [13, 14]) and butanol (e.g. [8, 15]) droplets, but to the authors' knowledge, none has addressed the droplet combustion characteristics of these alcohols blended with conventional kerosene. The main objective of this work is therefore to provide new experimental data on the combustion and sooting characteristics of unsupported, free-falling droplets of ethanol/Jet $A$ and butanol/Jet $A$ blends.

\section{Experimental}

Experiments were conducted in the Droplet Combustion Facility (DCF) developed at LIFTEC and displayed in Figure 1. Details of the facility can be found in a previous work [16], and therefore only the most important features of the experimental method will be explained here.

\section{Droplet combustion facility}

Fuel droplets were generated in the DCF by means of a piezoelectric device with initial diameters $\left(D_{0}\right)$ of 150 $\pm 1.7 \mu \mathrm{m}$ for all the cases studied. This size was chosen because it is considered to be a good compromise between actual sizes in practical applications and a good accuracy of the experimental results. Droplet spacing always exceeded 120 droplet diameters, so that interactions between droplets can be considered to be negligible. As droplet generation stability is perhaps the most critical variable in the experiment, initial droplet diameter was thoroughly checked by means of repeated size measurements at a given location ( $2 \mathrm{~mm}$ after injection), at least at the beginning and end of each test.
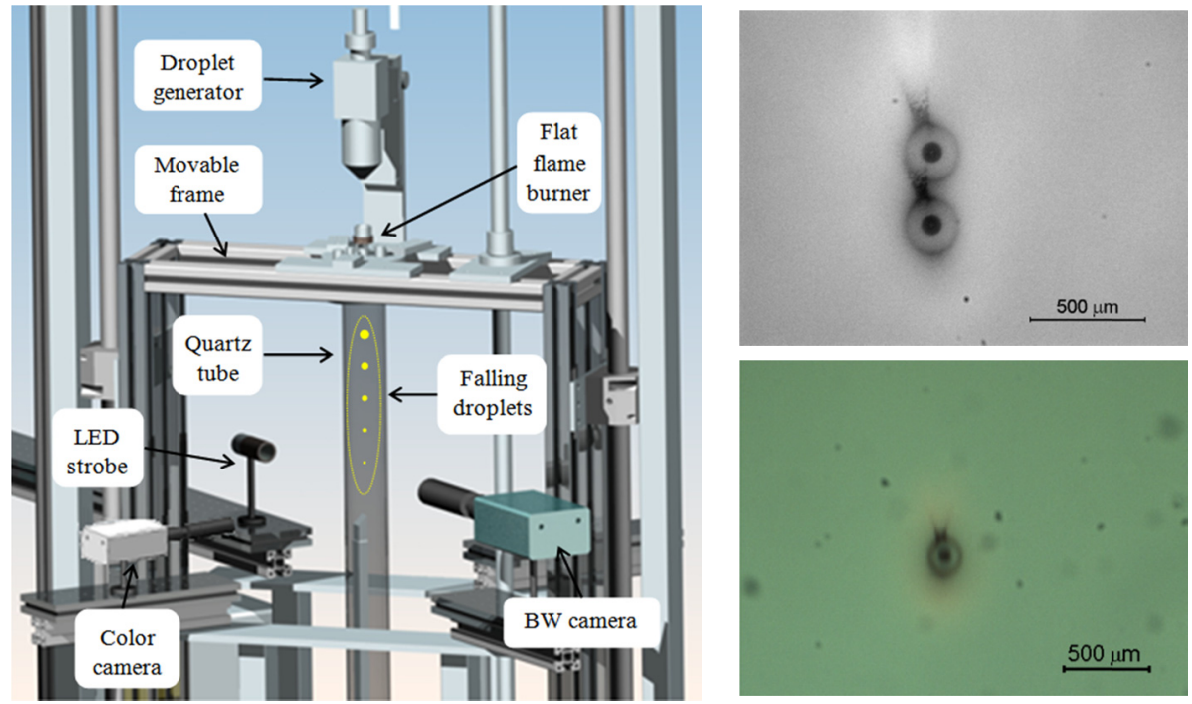

Figure 1. Schematic of the droplet combustion facility (left) and images taken by both cameras for a same droplet (right). 
The monosized droplets were injected along the axis of a quartz tube, coaxially with the combustion products generated by a flat-flame premixed methane-air burner (McKenna). This coflow provided a controllable and realistic environment to study the evolution of fuel droplets under representative conditions of real flames, both in terms of oxygen availability and temperature. As in real flames a droplet might be subjected to different oxygen conditions, the droplet combustion process was studied both at 3 and $10 \%$ of oxygen (by volume, dry basis) in the coflow.

Different imaging methods were used to record visual information describing the various aspects of droplet combustion. Size and velocity evolution of droplets were recorded through a black and white (BW) high sensitivity CCD camera (QImaging Retiga SRV, 12-bit Mono) fitted with a long distance microscope and backlighted via LED strobe. This optical system was programmed to record two sequential shots of a droplet in the same frame with a time lapse of $500 \mu$ s between them. As it can be seen in Figure 1, this imaging method not only gives information about the droplet size and motion, but also about the existence of a soot shell for certain conditions. A color camera (Teledyne DALSA Genie HC1024, 8-bit) perpendicularly placed to the BW camera and faintly backlighted was used to record the flame images. The weak backlight was provided in order to make the droplet distinguishable from the self-illuminated flame, capturing in the same image the free-falling droplet surrounded by its flame, as also shown in Figure 1.

Pictures taken with both imaging methods were post-processed in order to extract the relevant droplet combustion features in the most precise and repeatable way. However, for the fuels and conditions examined in this study, the flames registered in the color images were so weak that the involved uncertainties are considered too high to extract reliable quantitative data.

\section{Fuels investigated}

Commercial Jet A obtained from a local airport, ethanol (99.8\% purity) and 1-butanol (99.4\% purity) were used for the tests. Additionally, two mixtures were prepared with $80 \%$ Jet A and $20 \%$ alcohol by volume, denoted as B20 and E20 for blends of Jet A with butanol and ethanol, respectively. The main properties for the pure fuels are shown in Table 1. Ethanol and butanol properties are extracted from the literature, whereas a specific analysis of the Jet A sample was performed at the Instituto de Carboquímica (ICB-CSIC) in order to obtain its composition and most significant properties.

Table 1. Selective properties of the fuels investigated.

\begin{tabular}{|c|c|c|c|c|c|c|c|c|c|}
\hline & $\begin{array}{l}\text { Molecular } \\
\text { formula }\end{array}$ & $\begin{array}{c}\mathrm{MW} \\
(\mathrm{g} / \mathrm{mol})\end{array}$ & $\begin{array}{c}\% \mathrm{C} \\
\text { (mass) }\end{array}$ & $\begin{array}{c}\% \mathrm{H} \\
\text { (mass) }\end{array}$ & $\begin{array}{c}\% 0 \\
\text { (mass) }\end{array}$ & $\begin{array}{c}\text { Boiling point } \\
\left({ }^{\circ} \mathrm{C}\right)\end{array}$ & $\begin{array}{l}\text { Lower Heating } \\
\text { Value }(\mathrm{MJ} / \mathrm{kg})\end{array}$ & $\begin{array}{c}\text { Density at } \\
20^{\circ} \mathrm{C}\left(\mathrm{kg} / \mathrm{m}^{3}\right)\end{array}$ & $\begin{array}{l}\text { Vaporization } \\
\text { Enthalpy }(\mathrm{kJ} / \mathrm{kg})\end{array}$ \\
\hline Jet $A$ & $\mathrm{C}_{10.6} \mathrm{H}_{19.6}$ & 146.8 & 86.6 & 13.4 & - & $99-286$ & 42.60 & 791 & $330^{a}$ \\
\hline 1-Butanol ${ }^{\mathrm{b}}$ & $\mathrm{C}_{4} \mathrm{H}_{10} \mathrm{O}$ & 74.1 & 64.9 & 13.5 & 21.6 & 117 & 33.10 & 810 & 584 \\
\hline Ethanol ${ }^{b}$ & $\mathrm{C}_{2} \mathrm{H}_{6} \mathrm{O}$ & 46.0 & 52.2 & 13.0 & 34.8 & 78 & 26.95 & 789 & 838 \\
\hline \multicolumn{10}{|c|}{${ }^{\mathrm{a}}$ From ref. [7] } \\
\hline \multicolumn{10}{|c|}{${ }^{\mathrm{b}}$ From ref. [17] } \\
\hline
\end{tabular}

\section{Results and discussion}

Droplet size and burning rate evolution

As stated above, tests were performed for Jet A, B20, E20, butanol and ethanol for two different oxygen conditions: 3 and $10 \% \mathrm{O}_{2}$. The normalized droplet size evolutions for these two conditions are shown in Figures $2 \mathrm{a}$ and $2 \mathrm{~b}$ respectively. Based on the quasi-steady theory of droplet burning, measurements are displayed as normalized square diameter versus normalized time. Normalization is performed with the droplet diameter value at injection $\left(D_{0}\right)$. 


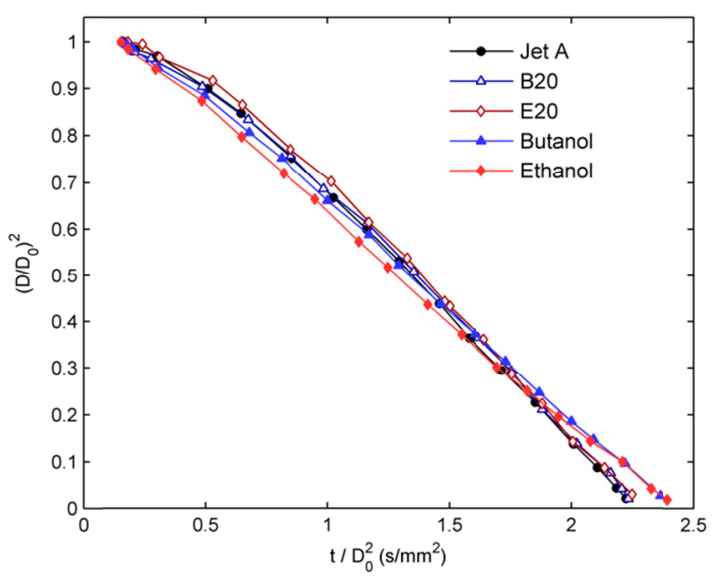

a) $3 \% \mathrm{O}_{2}$

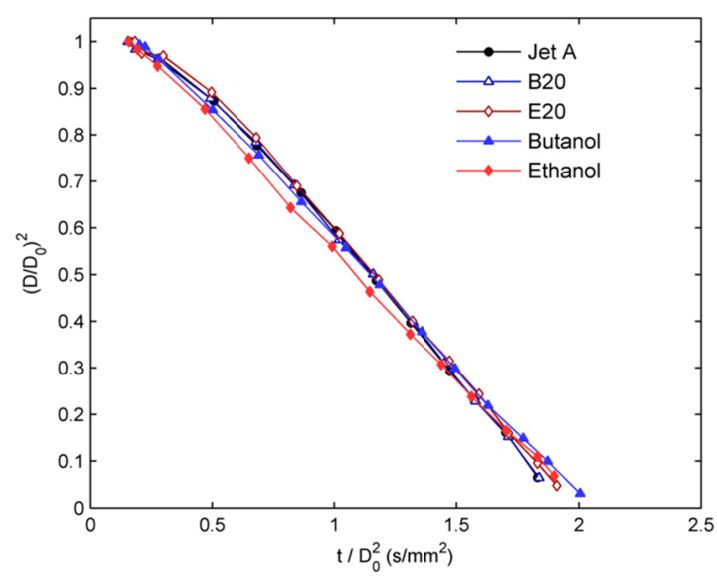

b) $10 \% \mathrm{O}_{2}$

Figure 2. Evolution of normalized droplet size for Jet A, B20, E20, butanol and ethanol for both oxygen conditions studied.

Results displayed in Figure 2 are in good agreement with the ' $\mathrm{d}^{2}$ law', and after a short heat-up period, the normalized square diameter decreases linearly with time with a nearly constant slope (the burning rate $K$ ) for all the cases studied. For each oxygen condition, the observed behaviors are found to be quite similar between fuels, as all of them display close burning rates and droplet consumption times, particularly among Jet A, B20 and E20. Pure alcohols exhibit the most distinctive behaviors, with shorter heat-up periods and smaller burning rates. The lower boiling points of both alcohols compared to most of the Jet A constituents can be responsible for the shorter heat-up periods, whereas their higher latent heats of vaporization could account for the smaller burning rates. Comparing ethanol and butanol, the former appears to show a slightly faster heating period and a lower burning rate, results in accordance with the properties listed in Table 1. On the other hand, E20 and particularly B20 display almost indistinguishable behaviors from pure Jet $A$ throughout all the vaporization process. As both alcohols' boiling points are located at the beginning of the Jet A distillation curve, and the burning rates of the three pure fuels are relatively close, this almost coincident behavior can either imply that there are no preferential vaporizations, or that the similarities between the pure fuels' behavior are masking them. For a certain fuel, it is clear that as oxygen concentration in the coflow increases, so does the burning rate, accelerating substantially the droplet evaporation process. This is ascribed to the higher temperature in the shell flame around the droplet, leading to an enhanced heat transfer to the droplet.

In order to gain insight into the slight differences found between fuels, their burning rates were quantified as the instantaneous slopes of the curves displayed in Figure 2: $K=-d\left(D^{2}\right) / d t$. Since differentiation greatly amplifies any small experimental uncertainty in $D^{2}$ curves, a five-point centred moving average was performed for smoothing purposes. The resulting curves are displayed in Figure 3, where the evolution of $K$ with normalized time is shown. Even though the slopes in Figure 2 seemed practically constant, the calculated burning rates plotted in Figure 3 display clearly unsteady behaviors. The burning rates extracted for Jet A, B20 and E20 continuously increase with time, whereas those of butanol and ethanol show an initial growth followed by a decrease near the final stages of droplet combustion. The initial growth is possibly caused by the droplet heating transient, whilst the final decay could be ascribed to different factors such as flame extinction or the absorption of water vapour by the alcohol droplets. Ambient water vapour absorption was proven to decrease the burning rate of pure ethanol droplets in a recent study [18], mainly in the final stages of droplet combustion. As the droplet environment is rich in water vapour generated in the flat flame burner, this could be indeed a possible reason for the burning rate decay found for ethanol and, to a certain extent, also for butanol (water solubility in 1-butanol is $20 \% \mathrm{w} / \mathrm{w}$ at $20^{\circ} \mathrm{C}$ [17]). As Jet $\mathrm{A}$ is a highly multicomponent fuel, its burning rate variations can be also dependent on fuel composition evolution, complicating therefore its analysis. 


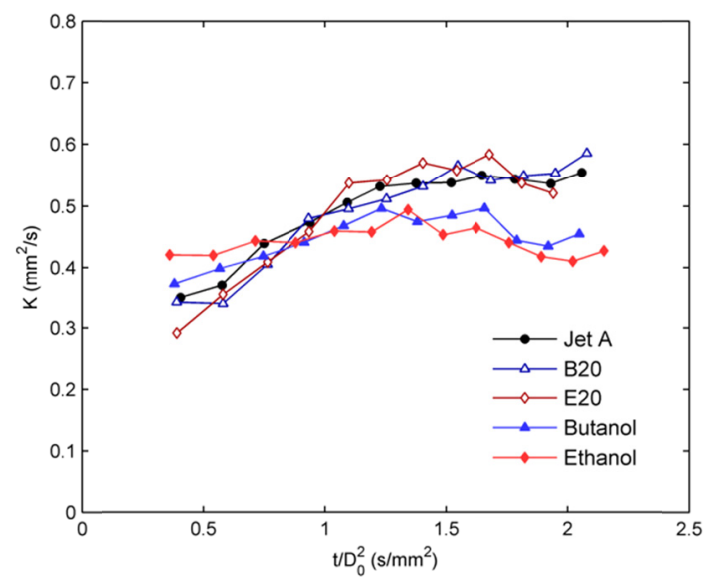

a) $3 \% \mathrm{O}_{2}$

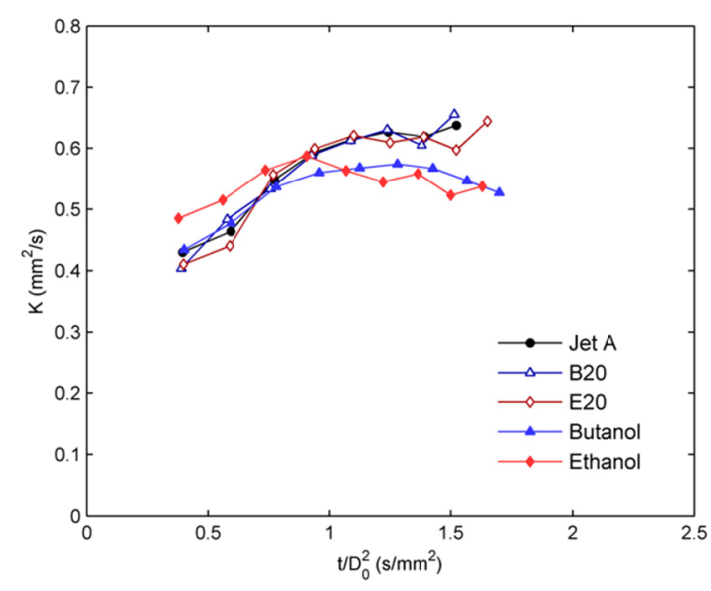

b) $10 \% \mathrm{O}_{2}$

Figure 3. Evolution of burning rate $K\left(\mathrm{~mm}^{2} / \mathrm{s}\right)$ with normalized time for Jet A, B20, E20, butanol and ethanol for both oxygen conditions studied. Five-point centered moving average was performed for smoothing purposes.

\section{$\underline{\text { Soot shell evolution }}$}

Although the droplet size evolutions presented in Figure 2 are found to be quite similar for all the fuels studied, the sooting behaviors differ considerably, as it can be clearly seen in Figure 4, which shows the evolution of soot shells for the $10 \% \mathrm{O}_{2}$ condition. Similar soot shells are usually achieved in totally converction-free environments obtained through microgravity such as [8]. In these configurations, the soot aggregates formed during combustion remain trapped at the radial location where thermophoresis and Stefan flux drag forces balance. Soot shells occurrence is much rarer for free-falling droplets, as the relative velocity between the droplet and the surrounding gases would sweep the soot away. The experimental conditions used in this work provide very low relative velocities between the droplets and the coflow, allowing the formation of an almost perfectly spherical soot shell surrounding the droplets for certain conditions. Even so, as it can be seen in Figure 4, some soot aggregates drifted away from the soot shell due to a small droplet slip velocity, perhaps also with some contribution of buoyancy-induced convection. Conclusions on the evolution and the amount of soot visible around droplets should be therefore taken with caution. However, as droplet velocities are found to be very close among fuels, the authors consider that a qualitative comparison between fuels' sooting propensity can be drawn from their soot shell evolution.

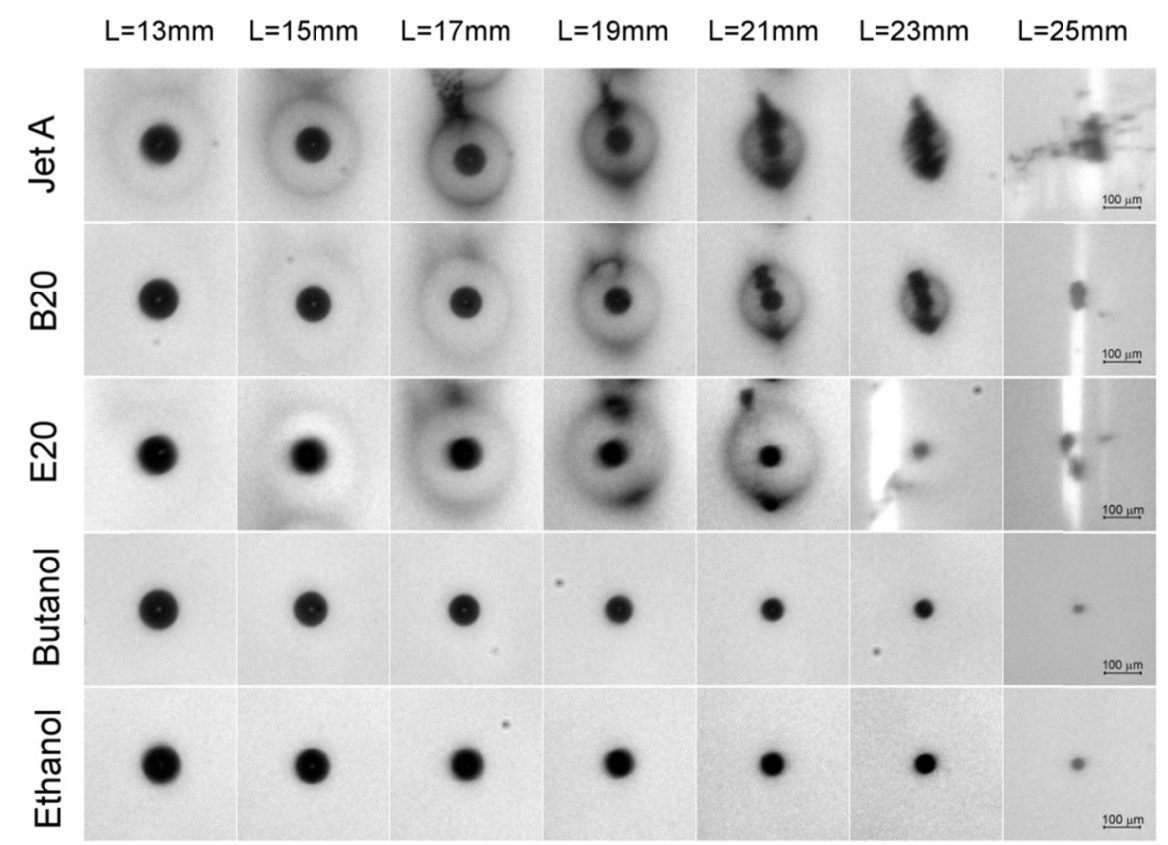

Figure 4. Evolution of droplet and soot shell for the examined fuels (cropped double-exposure BW images).

For a better comparison, pictures are arranged according to their approximate length after injection $(L)$. 
Figure 4 reveals qualitative differences between the soot shells formed for the various fuels, all of them subjected to exactly the same experimental conditions. As expected, no soot shell was found for ethanol and butanol, as no soot was formed. Jet A droplets display perfectly spherical soot shells which become thicker as the droplet combustion proceeds. Near the end of droplet burning, the soot particles agglomerate into a selfsupported crust which completely encloses the droplet, hindering droplet identification (for this reason, some curves in Figure $2 b$ lack a few points at the end of the droplets lifetimes). Droplet depletion occurs with a weak microexplosion, macroscopically visible as a tiny spark at the end of the flame trace. This feature has been reported in other free-falling droplet combustion works (e.g. [13]). B20 and E20 show much thinner soot shells compared to those of pure Jet $\mathrm{A}$, with a significant delay in the soot shell formation and no microexplosion during droplet depletion. These results point to a considerable soot reduction for alcohol blends. B20 displays a more steady behavior, with the soot shell progressively enclosing the droplet, as happened for neat Jet A. On the other hand, E20 produces a soot shell located at a larger radius and with much more unsteady characteristics: the spherical shape eventually breaks, with uneven soot distributions changing continuouslly with time. When the droplet becomes small enough, the wide soot shell quickly oxidizes and breaks down when reaching contact with the receding flame (as happens in the $L=23 \mathrm{~mm}$ picture). After the breakdown, the remaining soot agglomerates continue their burning with the coflow oxygen, their combustion giving a luminous trace.

\section{Visual appearance and analysis of the flame traces}

As the droplet stream falls along the quartz tube, its combustion generates a macroscopically visible flame streak. This streak can provide valuable information regarding the amount of soot present in the flame region, as incandescent soot gives a characteristic yellow luminosity. Therefore, long exposure images of the flame traces were taken with a DSLR camera. Figure 5 shows a comparison of the flame traces captured for all the studied fuels for the $3 \% \mathrm{O}_{2}$ condition.

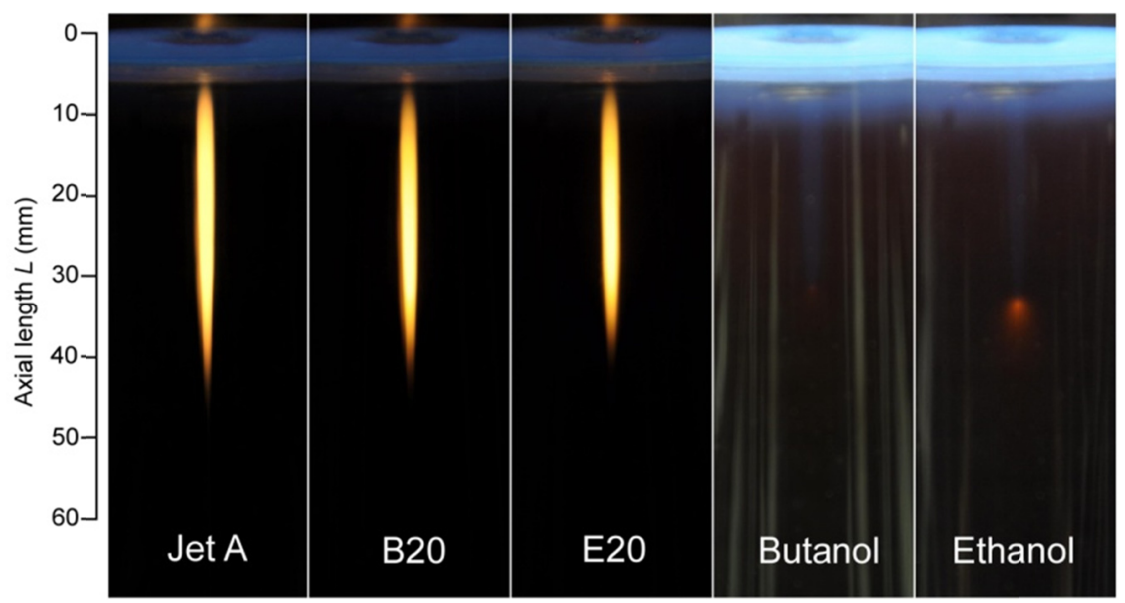

Figure 5. Long exposure photographs for $150 \mu \mathrm{m}$ droplets of different fuels at $3 \% \mathrm{O}_{2}$. The exposure time used for the Jet A, B20 and E20 pictures is $1 / 5 \mathrm{~s}$ ( 5 injected droplets), whereas for both pure alcohols $4 \mathrm{~s}$ (100 injected droplets) were required.

As it can be clearly seen in Figure 5, Jet A, B20 and E20 share the same camera settings, whereas butanol and ethanol required a considerable higher exposure for their bluish traces to appear visible. This is due to the much lower intensity of the chemiluminescence luminosity compared to the black-boidy emission from soot particles. Comparing the flame streaks of Jet A, B20 and E20, it is noted that alcohol addition appears to slightly decrease the flame luminosity within the first millimetres of the trace, where the sooty emission looks somewhat more orangish and less intense. It is noteworthy that these flame traces reach longer axial distances than the droplets themselves (the droplet depletion lengths for the $3 \% \mathrm{O}_{2}$ condition lie between $31 \pm 2 \mathrm{~mm}$ after injection). This is due to the relatively low oxygen availability in the coflow, which lowers the combustion rate of the already formed soot particles. For both alcohols, it is interesting the occurrence of a tiny reddish spot following droplet depletion. This red spot looks much more intense in the ethanol trace, and its origin is unknown, being the emission of external compounds (either present in the original alcohol or absorbed as a contamination) a possible cause. To the authors' knowledge, this feature has not been observed so far in ethanol droplet combustion works, and therefore further work is needed in order to ascertain its cause. 
Since the flame traces for Jet A, B20 and E20 were captured with exactly the same camera settings, a direct comparison of flame brightness can provide a soot propensity indicator for each fuel and condition. Figure 6 shows an analysis of the flame traces displayed in Figure 5. The axial profiles of luminasity were calculated by adding the values at all pixels across a given cross section of the flame trace. Although the quantitative value of this result is unclear (e.g., due to eventual differences in soot temperature), the luminosity curves can be interpreted in terms of the differences in the amount of soot for the various fuels at fixed experimental conditions.

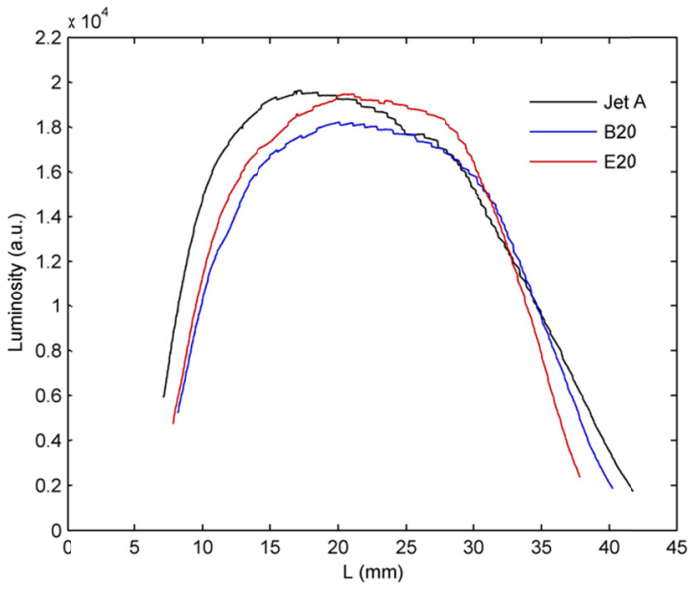

Figure 6. Axial variation of the flame traces' luminosity for $150 \mu \mathrm{m}$ droplets off Jet $\mathrm{A}, \mathrm{B} 20$ and $\mathrm{E} 20$ for the $3 \% \mathrm{O}_{2}$ condition in the coflow.

Figure 6 illustrates in more quantitative terms the differences in the visual aspect of flame traces noted above. Within the first millimetres, B20 and E20 traces are less luminous than that of Jet A, as the luminosity profiles of these two mixtures are slightly shifted to the right. This could imply a preferential vaporization of the more volatile alcohols in the first region, although this result was not observed in the droplet size evolutions presented in Figure 2 (maybe as a consequence of the similar behaviors in the droplet evaporation process among fuels). While the B20 luminosity profile remains below that of pure Jet $A$ for the whole trace length, the E20 trace surpasses the Jet A luminosity for axial distances comprised between $L \sim 20$ and $L \sim 31 \mathrm{~mm}$ (which is approx. the droplet consumption length). This could be ascribed to the unstable behavior of the E20 mixture in this last stage, as it was stated above for the $10 \% \mathrm{O}_{2}$ condition. In this region, the E20 flame trace becomes slightlly wider than the rest, analogously to the wider soot shells found in Figure 4. This greater soot dispersion for the E20 trace could enhance soot consumption within this region, justifying the higher luminosity prior to droplet depletion and the shorter flame trace recorded afterwards. The integration along the axial coordinate of the whole luminosity profiles displayed in Figure 6 yields a reduction of $10.8 \%$ in luminosity for the B20 and a $9.1 \%$ reduction for the E20 when compared to the pure Jet $\mathrm{A}$ trace.

\section{Conclusions}

The droplet combustion characteristics of Jet A, ethanol, butanol, E20 and B20 were studied under the same experimental conditions, which are thought to be representative of temperature and oxygen availability in real flames. In addition, relative velocities between droplets and the surrounding gases were minimized, so that droplets burnt in a practically 1-D configuration, as proven by the formation of almost perfectly spherical soot shells. As a consequence, the obtained experimental results may be compared with theoretical one-dimensional droplet combustion models. All the droplet combustion curves are in good agreement with the $d^{2}$ law after the initial heat up period. The B20 and E20 mixtures show very close burning rates to pure Jet A throughout all the combustion process, whereas pure alcohols display a slightly different behavior, with a noticeable burning rate reduction for the latter stages of droplet combustion. This could be ascribed to vapour water absorption by the hydrophilic alcohol droplets, although further work would be needed to corroborate or discard this hypothesis. The most significant combustion characteristic of Jet A/alcohol mixtures was found to be their lower soot formation tendency. This was consistently demonstrated through the close-up images of the soot shells and the lower luminosity of the visible flame traces. The strong similarities in combustion rates between pure Jet $A$ and its mixtures with butanol and ethanol, in addition to the lower propensity to form soot of the latter support the potential of both alcohols as attractive additives to Jet A. 


\section{Acknowledgements}

This work was supported by the Spanish Ministry of Education through the pre-doctoral grant FPU15/01866 and the Ministry of Science and Innovation through the research projects CSD2010-00011 and ENE2016-76436-R. The analysis of the Jet A sample was kindly provided by Drs. R. Murillo and M. Callen, from the Instituto de Carboquímica (ICB-CSIC). The authors are also grateful to Dr. Álvaro Sobrino for his assistance with data capture and analysis, and to Luis Ojeda for his support in the experimental tasks. The work of Mario Angeloni in the development of the Droplet Combustion Facility, without which this study would not have been possible, is also gratefully acknowledged.

\section{References}

[1] Saxena, R., Adhikari, D., and Goyal, H., 2009, Renewable and Sustainable Energy Reviews, 13(1), pp. 167178.

[2] Sarathy, S. M., Oßwald, P., Hansen, N., and Kohse-Höinghaus, K., 2014, Progress in Energy and Combustion Science, 44, pp. 40-102.

[3] Bergthorson, J. M., and Thomson, M. J., 2015, Renewable and Sustainable Energy Reviews, 42, pp. 13931417.

[4] Jin, C., Yao, M., Liu, H., Chia-fon, F. L., and Ji, J., 2011, Renewable and Sustainable Energy Reviews, 15(8), pp. 4080-4106.

[5] Bruno, T. J., Wolk, A., and Naydich, A., 2009, Energy \& Fuels, 23(4), pp. 2295-2306.

[6] Hoxie, A., Schoo, R., and Braden, J., 2014, Fuel, 120, pp. 22-29.

[7] Mendez, C., Parthasarathy, R., and Gollahalli, S., 2014, Applied Energy, 118, pp. 135-140.

[8] Xu, Y., and Avedisian, C. T., 2015, Energy \& Fuels, 29(5), pp. 3467-3475.

[9] Galloni, E., Fontana, G., Staccone, S., and Scala, F., 2016, Energy Conversion and Management, 110, pp. 319-326.

[10] Zhang, Z., et al., 2014, Fuel, 130, pp. 177-188.

[11] Mendez, C., Parthasarathy, R., and Gollahalli, S., 2012, 50th AIAA Aerospace Sciences Meeting including the New Horizons Forum and Aerospace Exposition, pp. 522.

[12] Patra, J., et al., 2015, Fuel, 144, pp. 205-213.

[13] Botero, M., Huang, Y., Zhu, D., Molina, A., and Law, C., 2012, Fuel, 94, pp. 342-347.

[14] Nakaya, S., Segawa, D., Kadota, T., Nagashima, Y., and Furuta, T., 2011, Proceedings of the Combustion Institute, 33(2), pp. 2031-2038.

[15] Alam, F. E., Liu, Y., Avedisian, C., Dryer, F., and Farouk, T., 2015, Proceedings of the Combustion Institute, 35(2), pp. 1693-1700.

[16] Angeloni, M., Remacha, P., Martínez, A., and Ballester, J., 2016, Fuel, 184, pp. 889-895.

[17] National Institute of Standards and Technology, http://webbook.nist.gov/. ([cit. 2017-03-08]).

[18] Saharin, S., et al., 2011, ASME 2011 Turbo Expo: Turbine Technical Conference and Exposition, pp. 669676. 


\title{
Experimental and Numerical Investigation of Dispersed and Continuous Liquid Film under Boiling conditions
}

\author{
C. Habchi' ${ }^{* 1,2}$, N. Lamarque ${ }^{3}$, J. Helie ${ }^{3}$ and S. Jay ${ }^{1,2}$ \\ ${ }^{1}$ IFP Energies nouvelles, 1 et 4 avenue de Bois-Préau, 92852 Rueil-Malmaison, France \\ ${ }^{2}$ Institut Carnot IFPEN Transports Energie \\ ${ }^{3}$ Continental Automotive SAS, 1 avenue Paul Ourliac BP 1149, 31036 Toulouse, France \\ ${ }^{*}$ Corresponding author: $\underline{\text { Chaouki.Habchi@ifpen.fr }}$
}

\begin{abstract}
In this work, both experimental and numerical investigations have been carried out in order to improve the modelling of the vaporization of wall liquid-deposits in internal combustion engines. A comprehensive model is suggested for the vaporization of liquid films in the different boiling regimes, including nucleate boiling regime, the Leidenfrost boiling regime, as well as the transition boiling regime occurring between the two latter. This work extends the validity of the Liquid Film Boiling model (Habchi, Oil \& Gas Science and Technology - Rev. IFP, Vol. 65 , No. 2, 2010) for dispersed liquid films that may be formed when a dilute spray impinges a wall. A sub-grid liquid film is indeed considered when the wetted-area is smaller than the wall cell-face area. A sessile droplet model is used to estimate the wall area wetted by the liquid film and whether it is resolved by the grid or located in the sub-grid scale (SGS). In addition, a novel Leidenfrost vaporization model is proposed for spray droplets located near a hot wall. The above vaporisation/boiling models has been implemented in the Large-Eddy simulation (LES) AVBP code. The validation has been carried out using two different experiments. First, the experimental lifetime curve of a sessile droplet (Stanglmaier et al., SAE paper 2002-01-0838) has been used for a quantitative validation in the different boiling regimes. Second, the wall impingement of a heptane spray from a typical gasoline injector from Continental Automotive, has been simulated. The numerical results obtained under boiling conditions, are compared to the liquid film footprints and lifetime provided by the Refractive Index Matching (RIM) experiment which is described in this article.
\end{abstract}

\section{Keywords}

Liquid film, Boiling, Leidenfrost, LES, RIM

\section{Introduction}

In Gasoline Direct Injection (GDI) engines, the impingement of the spray on the walls cannot be avoided in all operating conditions. Therefore, a fine tuning of the operating points involving impact of the spray droplets on the inner walls of the engine must be performed. Computational Fluid Dynamics (CFD) codes are often used for this purpose. However, the modelling of all the spray-wall interactions, including evaporation and the different boiling regimes, remains challenging. In this work, both an experimental and a numerical investigation have been carried out in order to improve the understanding and the modelling of the vaporization of liquid deposits in the boiling regimes. The liquid film sub-models previously developed by the authors [1-5] using a RANS approach have been improved and implemented in the Large-Eddy Simulation (LES) code AVBP [6]. In particular, this work extends the validity of the Liquid Film Boiling (LFB) model [5] for dispersed liquid films or tiny deposits that may be formed when a dilute spray impinges a wall. In addition, experimental measurements have been carried out using the Refractive Index Matching (RIM) method described in Section 1. Next, the different physical sub-models and improvements suggested in the present article are detailed in Section 2. Several sessile droplet test cases have been used in Section 3 for the numerical and physical validations of the sub-models proposed for the different liquid film boiling regimes. Then, a typical GDI spray/wall impact configuration has been used for the validation of the liquid film numerical results (footprints, lifetime ...) under boiling regime.

\section{Experimental setup}

The purpose of the Refractive Index Matching (RIM) experimental set-up is to observe the liquid film generated by one plume of a GDI injector on a transparent plate. This methodology has been introduced by Drake et al. [7] and used by Maligne and Bruneaux [8] to study wall film thickness in an optical Direct injection engine. The principles are depicted in Figure 1. The impingement plate is in quartz and has a known average roughness $R_{a}=15 \mu \mathrm{m}$, which order of magnitude is typical of a piston roughness. Its refractive index, with its roughened surface, is around 1.46 [8], which is close to liquid hydrocarbons studied here (1.385 for n-heptane). When liquid, with a refractive index which closely matches the quartz wall index, is on the window, light scattering is modified. The RIM then easily provides the liquid film footprint and a sufficiently accurate total lifetime. Figure 2 gives a picture of the global set-up. Images are captured by a high-speed CMOS video camera. Exposure time is fixed and equal to $100 \mu \mathrm{s}$ and the frame rate is up to $10^{4} \mathrm{fps}$. The objective on the camera has a fixed focal length of $60 \mathrm{~mm}$ and for 
this study; the $\mathrm{f}$-number is $\mathrm{f} / 8$. Resolution is then $83.3 \mu \mathrm{m} /$ pixel. The quartz plate $(50 \mathrm{~mm}$ diameter) is put on an aluminium hollow cylinder (to allow visualization from below, with a flat mirror), which is heated with an electrical collar, driven by a PID controller, that ensures the quartz window has a known and constant temperature. Temperature is measured with a thermocouple located on the quartz plate, as depicted on Figure 2. The quartz window is enlightened with one light guide (diameter: $1 \mathrm{inch}$ ) connected to a $150 \mathrm{~W}$ continuous light illuminator (halogen lamp). Fuel n-heptane is injected with a GDI 3-holes nozzle.

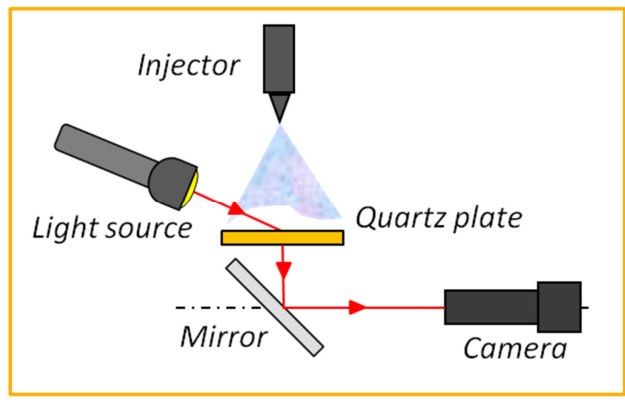

Figure 1. Sketch of the RIM experimental set-up.

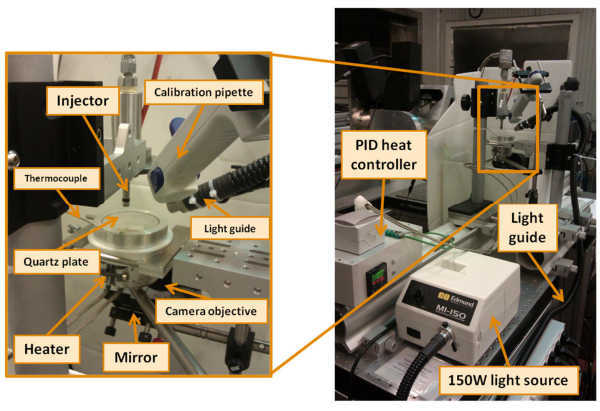

Figure 2. Pictures of the experimental set-up.

As explained in [9], despite the fine calibration, the liquid film thickness is very difficult to be accurately measured. Indeed, it is most often underestimated. Many factors are modifying local and global light scattering, as bubbles (entrapped air) and waves on the film surface, as well as undesired late and slow droplets which may remain several milliseconds levitating above the film. Therefore, only the footprints of the impinging spray and the estimation of the total lifetime of the liquid film are used for the validation in section 3.3. However, many additional useful observations have been made available by our RIM experiments. For instance, it is important to note the existence of a lot of isolated liquid spots all around the continuous liquid film, particularly visible at cold conditions. As they are thin, the associated mass is small in comparison with the central liquid film. Moreover, as their contact line length/surface ratio is high, they then vaporize very fast. The above remark highlights the huge difficulties of the modelling of liquid films (i.e. its formation, evaporation or boiling) at the sub-grid scale (SGS), especially during the impingement of dilute sprays. The main features of the modified SGS LFB model are described in Section 2.2. The validation of such modification is discussed in Section 3. In addition, Figure 3 shows that air can be entrapped during the spray-wall impact in our experiments. Very small bubbles are entrapped in the wall (quartz) troughs as depicted in the zoom of Figure $3(b)$. Those bubbles can survive dozens of milliseconds after end of injection. They can act as first nucleus in boiling conditions.

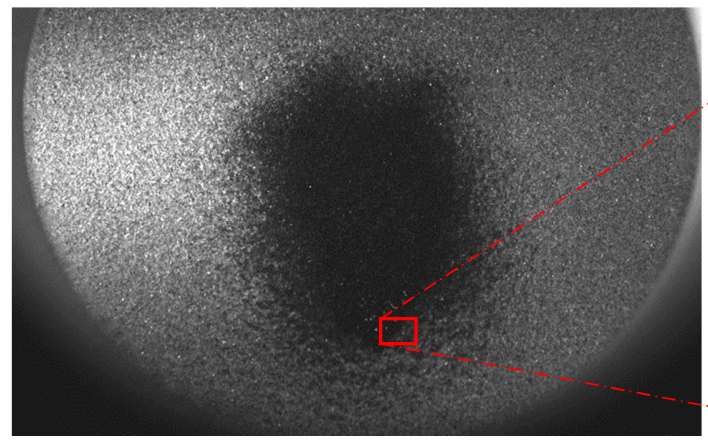

Figure 3(a) Liquid film for : Injection pressure $=100 \mathrm{bar}$ Average roughness $\mathrm{Ra}=15 \mu \mathrm{m}-\mathrm{Ti}=1500 \mu \mathrm{s}-\mathrm{Tw}=30^{\circ} \mathrm{C}$. Image size $(608,376)$ pixel, $83.3 \mu \mathrm{m} /$ pixel.

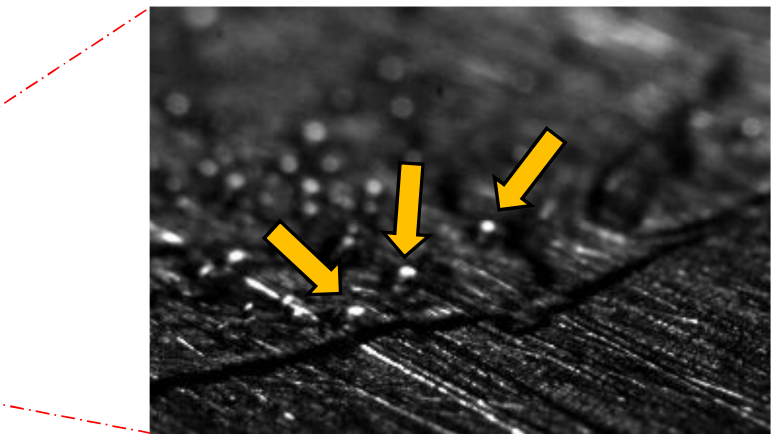

Figure 3(b) Highly zoomed visualisation of a wall film generated by a GDI injector. Image extracted from a highspeed sequence. Some bubbles are shown by orange arrows

\section{Fundamental of boiling of thin films and models description}

For the modelling of a liquid film formed on the surface of a combustion chamber, heat transfer and phase changes are of primary importance. One may distinguish four regimes of vaporization in the literature [10,11] which can be classified according to the superheat degree $\Delta T_{w, s a t}=\left(T_{w}-T_{\text {sat }}\right)$ and uses as limits for these regimes, the saturation temperature $T_{\text {sat }}$, the Nukiyama temperature $T_{N}$ and the Leidenfrost temperature $T_{L}$. $T_{N}$ corresponds to the point of maximum heat flux ( $\mathrm{CHF}$ in boiling curves) or to the minimum point of the lifetime curve (Figure 4); and $T_{L}$ corresponds to local minimum of the curve of the heat flux or to the maximum of the 
lifetime curve separating the Leidenfrost regime IV and boiling regime III, depicted in Figure 4 . These critical temperatures depend on the properties of the wall (roughness, presence of impurity, wettability, thermal conductivity, specific heat, etc.) as well as on gravity, gas pressure, liquid flow rate, etc. (see [5] for a complementary bibliographical review. These critical temperatures may also be determined experimentally from the boiling or lifetime curve of a droplet as depicted in Figure 4 obtained by measuring the total time that it takes to a droplet to completely evaporate after it has been gently deposited on a hot wall [12] :

Regime I, complete wetting regime $\left(T_{w}<T_{\text {sat }}\right)$

The sprays, impinging a wall having a $T_{w}$ smaller than $T_{\text {sat }}$, always form a liquid film which evaporates slowly. In this regime, the evaporation rate strongly depends on the turbulence level in the ambient gas $[3,4,13,14]$.

Regime II, nucleate boiling regime $\left(T_{\text {sat }}<T_{w}<T_{N}\right)$

In this regime, the liquid very close to the wall (e.g. in the thermal boundary layer) is overheated. This leads to the formation of vapour cavities which are born starting from germs (or sites) often hidden in the wall roughness (heterogeneous nucleation) and from the small bubbles are entrapped in the wall troughs as shown in Figure 3(b). While growing, these bubbles may be detached from the wall, especially in case of pool boiling or a raltively thick liquid film. This process of phase change at subcritical conditions prevents the temperature of the liquid $T_{1}$ from exceeding to much its saturation (or spinodal) temperature by consuming most of the heat flux $Q_{w, I}$ transferred to the liquid mainly by the wall. In contrary to pool boiling, the growing vapour cavities cannot be detached from the wall in the case of thin liquid film of interest in this work. Indeed, it has been shown experimentally in [15] that while growing, the vapour cavity diameter increases. After vapour cavities bursting the thin liquid film, tiny droplets are formed as observed in [16] and a dry-spot may appear. In addition, by increasing the superheat degree $\Delta T_{w, s a t}$ in this regime, the number of dry-spots increases. This process leads to a rapid increase of the dry area and the contact line length.

Regime III, transition boiling regime $\left(T_{N}<T_{W}<T_{L}\right)$

This is the transition regime between the nucleate boiling regime and the subsequent Leidenfrost regime. The small vapor cavities and the dry-spots on the wall coalesce progressively and form vapor columns and/or a larger vapor cavity. Indeed, several authors $[11,17,18]$ explained the decrease of the heat flux provided by the wall and the increase of the droplet lifetime, by the coalescence of the vapor cavities formed on the wall. As a consequence, in this transition boiling regime, the heat flux passes more and more through a vapor cushion between the wall and the liquid, and therefore strongly increases the droplet lifetime (Figure 4) with increasing $T_{w}$.

Regime IV, Leidenfrost or "film boiling" regime $\left(T_{w}>T_{L}\right)$

In this regime, there is no liquid film formation on the wall. The impingement always results in the rebound/splashing of the impinging spray droplets. This behavior has been explained by an intense heating of the droplets front, which leads to the formation of a vapor cushion and prevents the contact of the liquid with the wall. In this condition, the wall heats the vapor cushion which in its turn heats the droplets generated by the thermal instabilities during the transition boiling regime. Therefore, the heat flux between the wall and the spray droplets through the vapor cushion must be accounted for in the energy balance in droplets evaporation models. Obviously, this additional heat flux vanishes when the droplet goes far from walls. This additional heat flux will be shown to be of primary importance for the correct prediction of the vaporization lifetime of a sessile droplet in Section 3.1 .

\subsection{Modelling scope}

The modelling scope of this work is the implementation in AVBP [6] of a comprehensive set of models for liquid film boiling. The convective liquid film evaporation, prevailing in regime I, is described by Desoutter et al. [3,4]. The LFB model proposed in [5] is summarized, along with the following improvements:

a) A sub-model for a more accurate sub-grid-scale (SGS) modelling of liquid film boiling.

b) A modified spray evaporation model for droplets near hot walls, particularly for those under the Leidenfrost regime vaporization.

In addition, the wall film droplets formed near the end of the boiling transition regime are released and become free/spray droplets as soon as $T_{L}$ becomes smaller than $T_{w}$ (see the schematics illustrating the boiling states near the limits of the boiling regimes in Figure 4 and 5). It is also worth noting that the spray/wall interaction models such as the rebound or splashing model will not be discussed in this article since they are available elsewhere [19]. In addition, the reader may refer for instance to [20] for the estimation of $T_{N}$ and $T_{L}$.

\subsection{Thin liquid film boiling models}

Il well known that evaporation takes place mostly at the meniscus of a liquid film. According to the LFB model [5], the boiling phenomenon may therefore be modelled using the following two main parameters:

- $\quad$ The dry fraction of liquid film area due to boiling (i.e. vapour cavities and dry-spots), denoted $\boldsymbol{a}_{\boldsymbol{d r y}}$; 
- The solid-liquid-vapour contact line length density (index clld) of the liquid film, denoted $\boldsymbol{k}_{\text {clld }}$.

According to each boiling regime main characteristics, the behaviour of the above two parameters have been correlated to the wall temperature as depicted in Figure 5. $a_{\text {dry }}$ varies inevitably between 0 and 1 when the wall temperature increases from $T_{\text {sat }}$ to $T_{L}$ (depicted in red color in Figure 5 for the case of iso-octane), respectively. Moreover, the experimental observations mentioned above showed that $a_{d r y}$ increases quickly during the nucleate boiling regime. It reaches a value of about 0.7 to 0.9 when $T_{w} \approx T_{N}$. This behaviour of the function $a_{d r y}\left(T_{w}\right)$ has been expressed by the following correlation:

$$
\boldsymbol{a}_{\text {dry }}=\left[\frac{T_{w}-T_{\text {sat }}}{T_{L}-T_{\text {sat }}}\right]^{\frac{1}{4}}
$$

As for the Solid-liquid-vapour contact lines length density $\boldsymbol{k}_{\text {clld }}\left(T_{w}\right)$ of the liquid film, it is defined as an increasing function from 0 to 1 in the nucleate regime of boiling. Then, it decreases in the transition regime of boiling up to a minimal value, denoted $k_{c l l d}^{\text {min }}$. This behaviour is specified by the following correlations:

$$
\begin{gathered}
k_{\text {clld }}=\frac{\boldsymbol{a}_{\text {dry }}}{\boldsymbol{a}_{\text {dry }}\left[T_{w}=T_{N}\right]} \\
k_{\text {clld }}=\left(1-k_{\text {cld }}^{\text {min }}\right)\left[\frac{T_{L}-T_{w}}{T_{L}-T_{\text {sat }}}\right]^{4}+k_{\text {clld }}^{\text {min }}
\end{gathered}
$$

$$
\begin{aligned}
& \text { when } T_{w} \leq T_{N} \\
& \text { when } T_{N}<T_{w} \leq T_{L}
\end{aligned}
$$

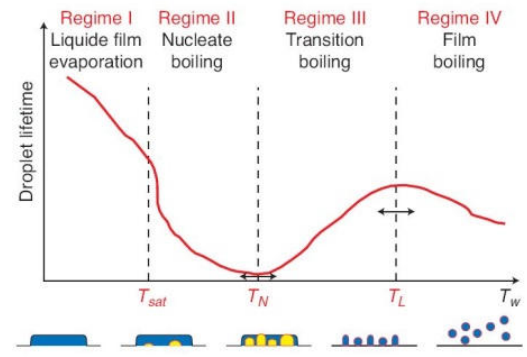

Figure 4. Droplet lifetime curve and schematics illustrating the boiling states near the limits of the boiling regimes (reproduced from [5])

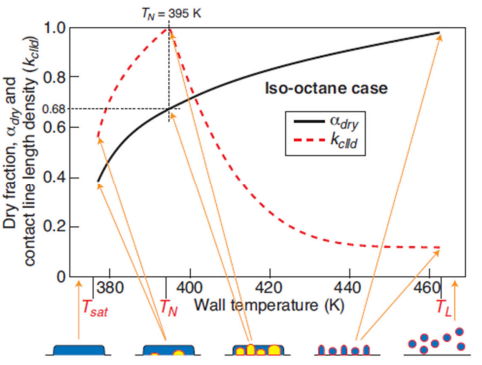

Figure 5. Dry fraction $a_{d r y}$ function and Contact Line Length Density $k_{\text {clld }}$ function drawn for the iso-octane case at atmospheric pressure. The schematics illustrate the boiling states near the limits of the boiling regimes (reproduced from [5]).

For the boiling phenomenon, instantaneous thermodynamic equilibrium is assumed (i.e. no metastable state is permitted). This assumption makes sense for liquid film already including bubbles even in cold conditions, as depicted in Figure 6 . Therefore, the vaporized mass $\dot{M}_{v}$ may be calculated by the following energy balance:

$$
\dot{M}_{v}=\frac{\left[\beta_{1}\left(1-\boldsymbol{a}_{\text {dry }}\right) Q_{w, l}+\beta_{2} \boldsymbol{a}_{\text {dry }} Q_{w, v, l}\right] S_{f i l m}}{L_{\text {sat }}}
$$

where $L_{s a t}$ is the liquid latent heat of vaporization at $T_{s a t .} Q_{w, l}$ is the heat flux per unit area between the wall and the liquid in direct contact. It is calculated by Equation (4) where $\lambda_{l, s a t}$ is the thermal conductivity of the liquid at $T_{s a t}$ and $\delta_{t h}$ has been estimated in [5] of the order of few microns. $Q_{w, v, l}$ is the heat flux per unit area between the wall and the liquid through the vapour cushion. It is the driving heat flux compared to the former in transition boiling regime III, particularly when $T_{w}$ tends towards $T_{L}$. The wall heats the vapour cushion which in its turn heats the liquid. It is calculated by Equation (5) where $\lambda_{v, s a t}$ is the thermal conductivity of the vapour at $T_{\text {sat. }}$ The area $S_{\text {film }}$ is obviously equal to the wall cell-face for a resolved liquid film. But, it is unknown for the tiny liquid film, such as the one depicted around the main footprint of the continuous liquid film in Figure 6(a). In this case, the model of Nagaoka [21] is used for the estimation of the SGS liquid film area, $S_{f i l m}$. In addition, this model has proved sufficiently accurate for the estimation of the average film thickness, $h_{\text {film }}$ for the SGS modelling of non-resolved liquid film transport and vaporisation. Equation (3) also includes two additional coefficients: $\beta_{1}$ represents the fraction of the wetted area $\left(1-\boldsymbol{a}_{d r y}\right)$ where most liquid vaporization is produced (i.e. area of the meniscus); whereas $\beta_{2}$ represents the fraction of $\boldsymbol{a}_{\boldsymbol{d r y}}$ where the liquid film is separated from the wall by a vapour cushion. In our previous work [5], the thickness of the vapour cushion has been correlated to the ambient pressure in accordance with the experimental observations of Temple-Pediani [22]. 


$$
\begin{gathered}
Q_{w, l}=\lambda_{l, s a t} \frac{\left(T_{w}-T_{s a t}\right)}{\delta_{t h}} \\
Q_{w, v, l}=\lambda_{v, s a t} \frac{\left(T_{w}-T_{s a t}\right)}{\delta_{v}} \quad \text { with } \quad \delta_{v}=10^{-4}\left(\frac{P}{P_{0}}\right)^{2}
\end{gathered}
$$

\subsection{Droplets vaporisation near hot walls}

In the AVBP code [6], a typical spray evaporation model, named SIGLE is used. It is a modified Abramzon and Sirignano model [23]. In this work, another modification of this model is carried out in order to account for an additional heat flux coming from hot walls. Indeed, this complementary heat flux is necessary when the spray is close to hot walls, especially in the Leidenfrost regime. Such heat flux is suggested here under the following form:

$$
Q_{w}=\lambda_{g} \frac{\left(T_{w}-T_{d}\right)}{\delta_{g}} S_{d}
$$

Where $T_{d}$ is the droplet temperature and $\delta_{g}$ (respectively $\lambda_{g}$ ) is the thickness (respectively thermal conductivity) of the gas cushion between the wall and the droplet. $\delta_{g}$ is computed as the normal distance to the wall of the droplet centre, minus its radius. Note that this distance cannot be smaller than $\delta_{v}$ given by Equation (5). $\lambda_{g}$ is estimated at a temperature equal to $T_{d}+\left(T_{g}-T_{d}\right) / 3$. Finally, since spray droplets in internal combustion engine are small, the area $S_{d}$ is computed simply as the half of the droplet surface facing the wall. The flux given by Eq. (6) is added to the heat flux coming from the gas phase in the SIGLE droplets evaporation model.

\section{Models validation}

The different models suggested in this paper have been implemented in the LES code AVBP [6]. The validation is carried out using two different experiments. First, the experimental lifetime curve of a sessile droplet from [12] is used for a quantitative validation in the different boiling regimes at resolved and SGS numerical conditions. Second, the wall impingement of a heptane spray from a prototype GDI injector from Continental Automotive, has been simulated. The numerical results obtained under boiling conditions, are compared to the liquid film footprints and lifetime provided by the RIM experiments described in section 1.

\subsection{Sessile drop test cases : Numerical validations}

In the experiments of Stanglmaier et al. [12], a rather big droplet $\left(V_{0}=5 \mu l\right)$ is deposited on an aluminium heated plate. Once deposited gently on the wall, this droplet spreads and forms a liquid film. In this work, we assumed the droplet after impact takes the form of a cylinder with radius $r_{f}$ and height $h_{f}$. In order to compute $r_{f}$ and $h_{f}$, Nagaoka et al. [21] model is used as described in [5]. A sub-grid liquid film is indeed considered when the obtained area $S_{\text {film }}=\pi r_{f}^{2}$ is smaller than the area $S_{w}$ of the wall-cell face where the liquid film is located. In this case, the SGS liquid film height is also taken equal to $h_{f}$ as this value is more accurate than the one usually computed in most CFD codes as $h_{f}=V_{0} / S_{w}$, which value is often underestimated. The initial conditions given by the Nagaoka et al. [21] model and the physical properties are summarized in Table 1 and Table 2, respectively.

Figure 6 shows the initial liquid film deposited on the bottom wall for the SGS case (a) and resolved case (b). The grid used for case (b) is refined five time more than in case (a). The grid size in case (b) is specified in such a way that the wall-area covered by the liquid film, $S_{f i l m}$ is equal to the sum of the 25 central-cell areas. In addition, 25 droplets have been distributed at the centre of each of these 25 wall-cell faces (Figure 6(b)). The initial droplets size is specified in such a way that the same initial volume is used for both cases (a) and (b). For these two cases, two wall temperatures $T_{\text {wall }}$ equal to $388 \mathrm{~K}$ and $423 \mathrm{~K}$ have been simulated. The first wall temperature is chosen in the middle of the nucleate boiling regime and the second one in the transition boiling regime (see $T_{N}$ and $T_{L}$ in Table 2). The numerical results are depicted in Figure 7. First, the resolved and SGS cases are compared in Figure 7(a) in terms of liquid film volume. The liquid film temporal evolutions are somewhat different. But, their total lifetimes are similar, around $0.6 \mathrm{~s}$ and $4.5 \mathrm{~s}$ for $T_{\text {wall }}$ equal to $388 \mathrm{~K}$ and $423 \mathrm{~K}$, respectively. Second, Figure $7(\mathrm{~b})$ shows that even if the liquid film is initially resolved by the grid in case (b), vaporization/boiling reduces the wetted area relatively quickly and makes its calculation required in the SGS.

Table 1 : Initial conditions for sessile drop test cases using iso-octane

\begin{tabular}{|c|c|c|c|c|c|c|c|}
\hline$V_{0}(\mu l)$ & $r_{0}(\mathrm{~mm})$ & $r_{f} / r_{0}$ & $h_{f}(\mu \mathrm{m})$ & $S_{\text {film }}\left(\mathrm{mm}^{2}\right)$ & $P_{\text {amb }}(\mathrm{Pa})$ & $T_{a m b}(\mathrm{~K})$ & $T_{d}$ \\
\hline 5 & 1.061 & 4.32 & 86.9 & 57.6 & $1.01 \times 10^{5}$ & $T_{\text {wall }}$ & $T_{\text {sat }}$ \\
\hline
\end{tabular}




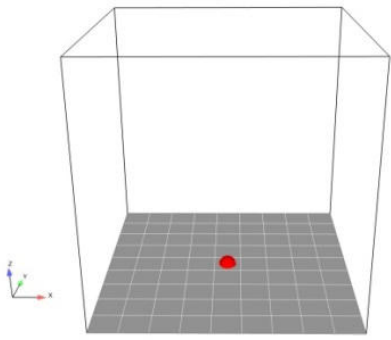

Case (a): Un-resolved liquid film SGS case defined using a single droplet with $r_{0}=1.061 \mathrm{~mm}$, on one wall-cell face.

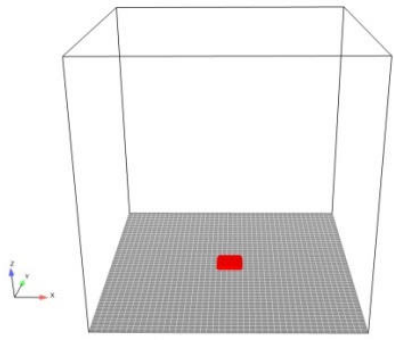

Case (b): Resolved liquid film case defined using 25 droplets with $r_{o}=0.3628 \mathrm{~mm}$, on 25 wall-cell faces.

Figure 6. Initial conditions for SGS and resolved liquid film. The same initial volume is used for both cases (a) and (b).

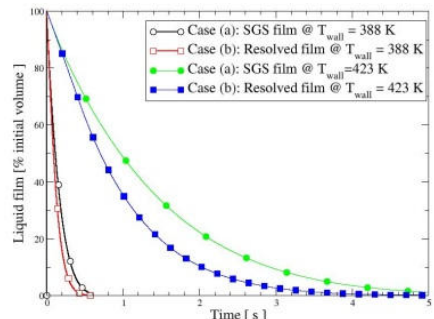

(a) Liquid film volume temporal evolutions.

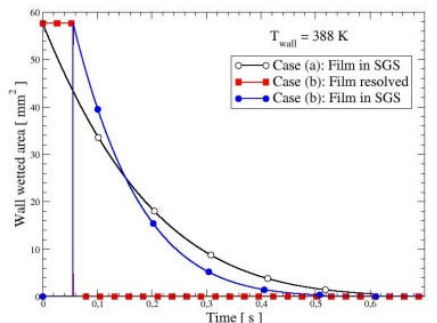

(b) Liquid film areas. SGS vs. resolved liquid film.

Figure 7. SGS vs. resolved liquid film. The same initial volume is specified for both cases (a) and (b).

\subsection{Sessile drop test cases : Experimental validations}

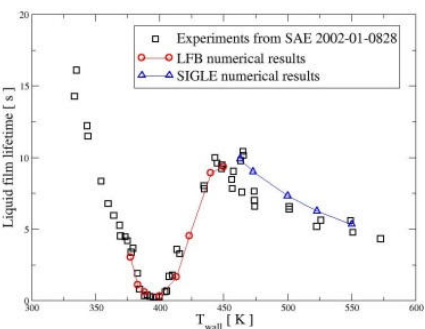

Figure 8. Iso-octane liquid film total lifetime. LFB model is used in nucleate and transition boiling regimes. SIGLE is the droplets vaporisation model used in the Leidenfrost regime.

In this subsection, the suggested models in the different boiling regimes are evaluated. Several simulations similar to the above case (a) have been carried out. For the simulations under the Leidenfrost regime, the gas temperature is assumed initially equal to the wall temperature. In this case, the sessile droplet is deposited at the wall, at $h_{\text {d,init }}=1 \mathrm{~mm}$ with a zero initial velocity. In addition, the sessile droplet initial temperature is assumed equal to $T_{s a t}$. This is true at least on the sessile droplet surface faced to the wall. Note that the gravity acceleration acting towards the wall is switched on. In these conditions, the rebound regime is invoked each time the droplet hits the wall. The numerical results in terms of liquid volume temporal evolutions are depicted in Figure 9. One may see in this Figure the lifetime decrease (increase) in the nucleate and Leidenfrost regimes (in the transition boiling regime) as wall temperature is increasing (decreasing), respectively. A good agreement of these numerical results with the experiments of Stanglmaier et al. [12] in terms of total lifetimes, is shown in Figure 8. Each symbol ( $\circ$ or $\mathbf{\Delta}$ ) in this Figure is the result of a single AVBP simulation. Although the LFB model (see Section 2.2) is obviously very different from the SIGLE vaporization model (see Section 2.3) proposed in this work, the numerical results show a smooth transition between them.

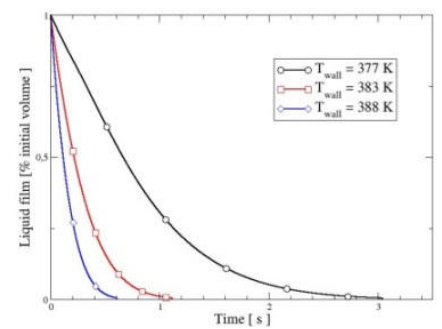

(a) Nucleate boiling regime

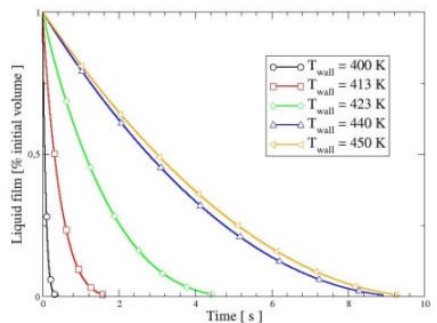

(b) Transition boiling regime

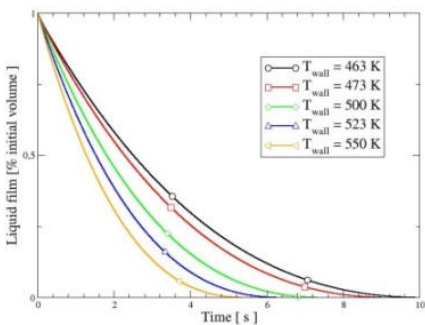

(c) Leidenfrost regime

Figure 9. Liquid volume temporal evolutions from sessile droplets simulations in SGS for 13 different wall temperatures.

\subsection{The GDI spray/wall interaction configuration}

In this section we evaluate the different models suggested in this work for a typical GDI spray impinging a wall. The RIM experimental liquid film results generated by one plume of a GDI injector on a transparent plate are used for comparisons (see section 1). The impingement plate is in quartz and has a known average roughness 
$R_{a}=15 \mu \mathrm{m}$. It is located $40 \mathrm{~mm}$ from the nozzle. The computational domain hexahedral grid is shown in Figure 10(a). It consists of six million nodes. A refined zone has been provided to suitably describe the coupling of the gas with the spray and the liquid film. The cell size in the refined region is $25 \mu \mathrm{m}$. Outlet boundary conditions are specified for the four lateral faces of the computational domain. The bottom face is the heated wall at $T_{\text {wall }}=423 \mathrm{~K}$ for which an isothermal wall law is specified. However, the top wall is assumed at ambient temperature, $T_{a m b}=323 \mathrm{~K}$ for which a free slip isothermal wall is specified. In addition, Figure $10(\mathrm{~b}-\mathrm{c})$ shows the impinging $\mathrm{n}$ heptane spray configuration. Relatively to the vertical direction, the spray axis have an angle of $31^{\circ}$ in the $(x, z)$ plane. The rest of the parameters is given in Table 3. Of course, the spray injection model and the spray-wall interaction and the liquid film formation models including the rebound/splashing phenomena are very important for this configuration. Here, the DVI injection model [24] with an intact liquid core length equal to $2 \mathrm{~mm}$ is used for the numerical simulation of the injection process. The liquid is injected using a given trapezoidal injection rate assuming opening and closing periods equal to $0.3 \mathrm{~ms}$. A velocity coefficient equal to 0.7 is also used in order to take into account the momentum lost inside the nozzle. Moreover, one hundred-thousand parcels have been used for the discretized injected mass $\left(m_{i n j}=3.25 \mathrm{mg}\right)$. With the DVI model, a Rosin-Rammler distribution is specified with a Sauter Mean Diameter, $S M D=12 \mu m$ and a parameter, $q=2.2$. In addition, the $S A B$ secondary breakup model [25] is used, while assuming no-collision between the droplets in the present LES. Spray droplets evaporation is performed using the modified SIGLE model (see Section 2.3). For the spray-wall interaction and liquid film formation models, in-house IFPEN models [19] have been used. Figure 11 shows a fairly good comparison of the LES footprints results relative to the experimental RIM images. In addition, the evaporation rate in the transition boiling regime of $n$-heptane (since $T_{N}<T_{\text {wall }}<T_{L}$ ) is well predicted as the total lifetime of the liquid film is very close to the experimental value, $70 \mathrm{~ms}$.

Table 3 : Injection and physical parameters

\begin{tabular}{|c|c|c|c|c|c|c|}
\hline$P_{a m b}(\mathrm{~Pa})$ & $T_{a m b}(\mathrm{~K})$ & $\rho_{\text {heptane }}\left(\mathrm{kg} / \mathrm{m}^{3}\right)$ & $T_{\text {wall }}(\mathrm{K})$ & $T_{\text {sat }}(\mathrm{K})$ & $T_{N}(\mathrm{~K})$ & $T_{L}(\mathrm{~K})$ \\
\hline $1.01325 \times 10^{5}$ & 323 & 688 & 423 & 368 & 390 & 456 \\
\hline \hline$P_{\text {inj }}(\mathrm{bar})$ & $m_{\text {inj }}\left(10^{-6} \mathrm{~kg}\right)$ & $t_{\text {inj }}(\mathrm{ms})$ & $d_{\text {nozzle }}(\mu \mathrm{m})$ & $S M D_{\text {inj }}(\mu \mathrm{m})$ & Cone angle $(9)$ & $T_{\text {inj }}(\mathrm{K})$ \\
\hline 200 & 3.25 & 1.36 & 200 & 12 & 12.5 & 300 \\
\hline
\end{tabular}

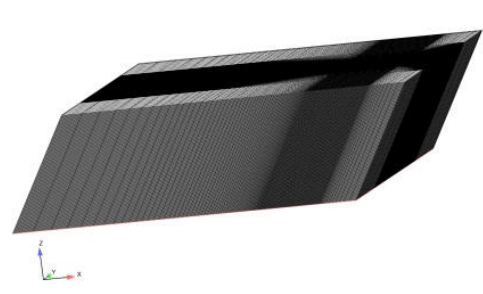

$$
\text { Time }=0.0007 \mathrm{~s}
$$

$$
\text { Time }=0.0014 \mathrm{~s}
$$

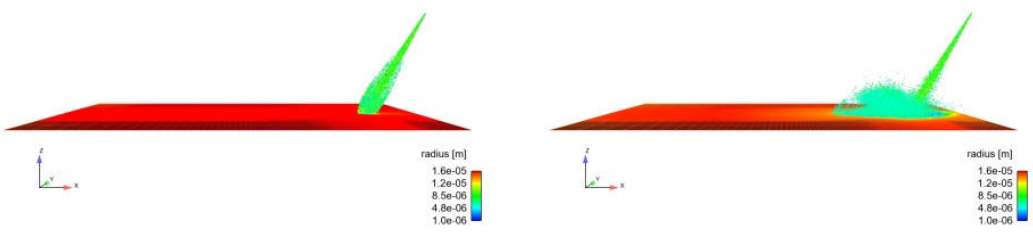

Figure 10. (Left) Mesh (6Mi nodes) and spray orientation (centre) before (right) after impingement.
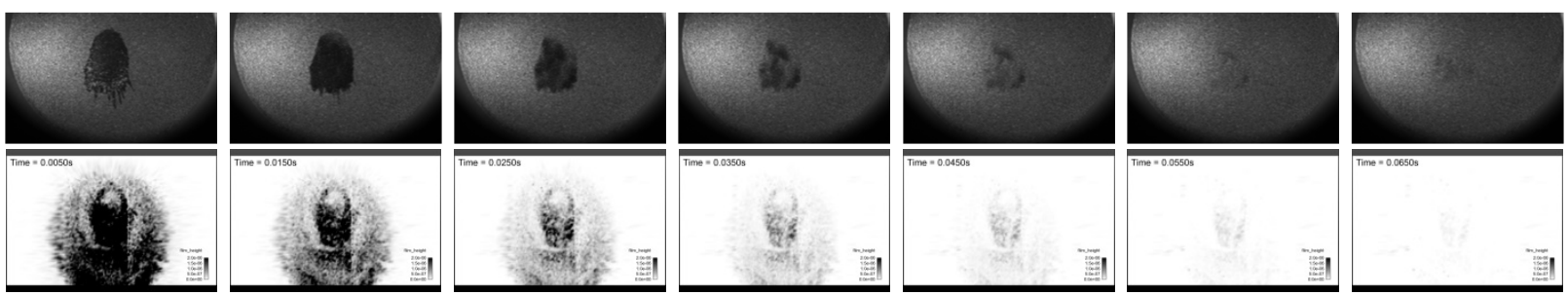

Figure 11. Experimental (top) and LES (bottom) liquid film footprints every $10 \mathrm{~ms}$ starting from $5 \mathrm{~ms}$.

\section{Conclusions}

Two fundamental improvements for the liquid film boiling modelling have been suggested in this article. First, a model allowing the simulation of resolved and sub-grid liquid film boiling is proposed. Second, a modified droplets evaporation model is proposed and applied to the vaporization of droplets located near hot walls, especially for the Leidenfrost boiling regime. These models have been implemented in AVBP and applied to the simulation of the vaporization of an iso-octane sessile droplet in the nucleate boiling regime, the transition boiling regime as well as in the Leidenfrost boiling regime. In addition, RIM experiments have been performed and used for the overall validation the suggested models. The obtained LES results have proved to compare well with our experiments which are of a big interest in several devices like in GDI engines. 


\section{Acknowledgements}

This work has been supported by the French ANR agency in the framework of the project ASTRIDE (reference ANR-12-VPTT-07). It was granted access to the High Performance Computing (HPC) resources of CCRT under the allocations x20162b6139 made by GENCI (Grand Equipement National de Calcul Intensif).

\section{References}

[1] Foucart H., Habchi C., Le Coz J.-F., and Baritaud T., 1998,"Development of a Three Dimensional Model of Wall Fuel Liquid Film for Internal Combustion Engines," SAE paper N`980133.

[2] Habchi C., and Foucart H., 2000,"Multidimensional modelling of gasoline spray impingement and liquid film heat transfer and boiling on heated surfaces," ICLASS Conference Pasadena Proceedings.

[3] Desoutter G., Habchi C., Cuenot B., and Poinsot T., Single component liquid film evaporation model development and validation using direct numerical simulations, 10th ICLASS Conference, Kyoto, Japan, 28/08 - 01/09/2006.

[4] G. Desoutter, C. Habchi, B. Cuenot, and T. Poinsot, 2009,"DNS and modeling of the turbulent boundary layer over an evaporating liquid film," International Journal of Heat and Mass Transfer, 52(25-26), pp. 6028-6041.

[5] Habchi C., 2010,"A Comprehensive Model for Liquid Film Boiling in Internal Combustion Engines," Oil \& Gas Science and Technology - Rev. IFP Energies nouvelles, 65(2), pp. 331-343.

[6] IFPEN-CERFACS LES code,"The AVBP HandBook: http://www.cerfacs.fr/avbp7x/handbook.php (2017),"

[7] Drake M. C., Fansler T. D., Solomon A. S., and Szekely G. A., 2003,"Piston Fuel Films as a Source of Smoke and Hydrocarbon Emissions from a Wall-Controlled Spark-Ignited Direct-Injection Engine," SAE Technical Paper 2003-01-0547.

[8] Maligne D., and Bruneaux G., 2011,"Time-Resolved Fuel Film Thickness Measurement for Direct Injection SI Engines Using Refractive Index Matching," SAE Technical Paper 2011-01-1215.

[9] N. Lamarque, C. Schmit, and J. Hélie, Eds., 2016. Refractive Index Matching Method to Study Gasoline Spray-Wall Impingement:: 9th ICMF, Florence, Italy, 22 - 27 May 2016, Florence, Italy.

[10] Rein M., Ed., 2002. Drop-surface interactions, Springer, Wien, New York.

[11] Dhir V. K., 1998,“Boiling Heat Transfer,” Annu. Rev. Fluid Mech., 30(1), pp. 365-401.

[12] Stanglmaier R. H., Roberts C. E., and Moses C. A., 2002,"Vaporization of Individual Fuel Drops on a Heated Surface: A Study of Fuel-Wall Interactions Within Direct-Injected Gasoline (DIG) Engines," SAE Technical Paper 2002-01-0838.

[13] O'Rourke P. J., and Amsden A. A., 2000,"A Spray / Wall Interaction Submodel for the KIVA-3 Wall Film Model," SAE Technical Paper 2000-01-0271.

[14] G. Desoutter, B. Cuenot, C. Habchi, and T. Poinsot, 2005,"Interaction of a premixed flame with a liquid fuel film on a wall," Proc. of the Combust. Inst., 30(1), pp. 259-266.

[15] Gong S., Ma W., and Gu H., 2014,"An experimental investigation on bubble dynamics and boiling crisis in liquid films," International Journal of Heat and Mass Transfer, 79, pp. 694-703.

[16] Bertola V., and Sefiane K., 2005,"Controlling secondary atomization during drop impact on hot surfaces by polymer additives," Phys. Fluids, 17(10), p. 108104.

[17] NISHIO S., and TANAKA H., 2002,"Advanced Fluid Information. Simplified Model Predicting Contact-LineLength Density at Critical Heat Flux Based on Direct Observation of Boiling Structure," JSME Int. J., Ser. B, 45(1), pp. 72-78.

[18] Nigmatulin B. I., Vasiliev N. I., and Guguchkin V. V., 1993,"Interaction between liquid droplets and heated surface," Wärme- und Stoffübertragung, 28(6), pp. 313-319.

[19] C. Habchi, 2005,"Modélisation de l'interaction spray-film liquide par une approche lagrangienne incluant splashing et évaporation," report №58472, IFPEN.

[20] C. Habchi, New Correlations for Leidenfrost and Nukiyama Temperatures with Gas Pressure Application to Liquid Film Boiling Simulation: 23rd ILASS - Europe, Brno, Czech Rep, 6-9 Sept 2010.

[21] Nagaoka M., Kawazoe H., and Nomura N., Eds., 1994. Modeling Fuel Spray Impingement on a Hot Wall for Gasoline Engines: SAE Technical paper 940525.

[22] R. W. Temple-Pediani, 1969, "Fuel Drop Vaporization under Pressure on a Hot Surface," Proceedings of the Institution of Mechanical Engineers, 184(1), pp. 677-696.

[23] B. Abramzon, and W.A. Sirignano, 1989,"Droplet vaporization model for spray combustion calculations," International Journal of Heat and Mass Transfer, 32(9), pp. 1605-1618.

[24] C. Habchi, and G. Bruneaux, Eds., 2012. LES and Experimental investigation of Diesel sprays: ICLASS, Heidelberg, Germany, 2-6 sept, 2012.

[25] Habchi C., 2012, "The energy Spectrum Analogy Breakup (SAB ) model for the numerical simulation of sprays," ATOMIZATION AND SPRAYS, 21(12), pp. 1033-1057. 


\title{
Development of an evaporation model for the dense spray region in Eulerian-Eulerian multiphase flow simulations
}

\author{
Stefano Puggelli, ${ }^{*}$, Lorenzo Palanti ${ }^{1}$, Antonio Andreini ${ }^{1}$, François-Xavier Demoulin ${ }^{2}$ \\ ${ }^{1}$ Department of Industrial Engineering (DIEF), University of Florence, Italy \\ ${ }^{2}$ CNRS CORIA UMR 6614, University of Rouen, France \\ ${ }^{*}$ Corresponding author: stefano.puggelli@htc.de.unifi.it
}

\begin{abstract}
In the present study, a novel implicit numerical model to describe evaporation phenomena in the dense spray region is proposed. The main aim is to go beyond the limits of standard vaporization models, which are normally based on a dilute spray assumption, to deal with high liquid volume fractions. The proposed method is based on an a priori computation of steady state equilibrium conditions reached by a system composed by liquid, vapour and air at constant pressure combined with a modelled characteristic time of evaporation. Such equilibrium composition and temperature is then used inside numerical calculations to compute evaporation source terms. The new formulation allows to simulate evaporation process in the dense zone of the spray, where, due to the extremely low thermal relaxation time, classical explicit method can lead to unphysical results. Such innovative approach has been implemented in a multiphase solver in the framework of the CFD suite OpenFOAM. An Eulerian-Eulerian solver, derived from the Eulerian Lagrangian Spray Atomization (ELSA) model, has been used, in order to correctly describe the liquid-gas flow without assumptions on the topology of the liquid phase. Evaporation source terms have been modelled as function of the amount of surface available for mass and heat transfer. An analysis of the solver has been carried out in RANS framework in order to highlight the capabilities of the approach in dealing with high liquid volume fraction regions with a physically consistent representation of evaporation phenomena.
\end{abstract}

\section{Keywords}

Spray modelling, ELSA, Eulerian-Eulerian methods, Evaporation modelling.

\section{Introduction}

The future standards on pollutants emissions expected by ICAO-CAEP [1] for the next generation of civil aeroengines have pushed the attention towards the introduction of lean burn technology in aeronautical framework. Here, a drastic reduction of NOx levels can be achieved working on a narrow range of temperature and equivalence ratio. Therefore, all the issues related to liquid fuel atomization and air-fuel mixing have to be carefully investigated and Computational Fluid Dynamics (CFD) has been gaining strong attention for the design process.

In this framework, the numerical method chosen for the modelling of the liquid phase can have a strong impact on both simulation accuracy and computational effort. Standard Eulerian-Lagrangian (E-L) approaches, which are based on tracking single liquid discrete entities (i.e. parcels), are characterized by a straightforward introduction of the main interactions between the gas and the liquid phase, even if they are not theoretically suitable in the near injection region where the spray is really dense. An extensive use of experimental correlations in order to introduce the effects of primary breakup inside numerical calculations is therefore required. However, this strategy is not general since a strong spreading between the huge number of available experimental correlations can be determined for the same configuration and operating conditions.

Besides, Eulerian-Eulerian (E-E) methods are very attractive since several ways, associated to different computational costs, can be here used to solve the kinetic Boltzmann-Williams equation [2]. This has determined a copious research on Eulerian methods starting from approaches based on a discretization of the spray distribution along the diameter space (i.e. sectional approaches [3]) to other built on the calculation of some moments of the spray probability density function (such as Quadrature Method Of Moments [4] or Direct Quadrature Method Of Moments [5]. Other approaches, such as entropy maximization [6] or moments with interpolative closure [7], can be also found in the literature but their description goes beyond the scope of the present work. Recent contributions in this area have shown the suitability of these methods in describing the most important features of liquid sprays [8]. Nevertheless, since all these approaches are theoretically based on the Boltzmann-Williams equation, they can be used only when the spray is composed by individual droplets with well-defined features as position or diameter. Therefore, in order to consider also the dense zone, diffuse interface models have been considered in the present work. In these models, the interface is considered as a mixing zone where both liquid and gas phases coexist at the same macroscopic position with an occupied portion of volume defined by the liquid volume fraction $\left(\alpha_{l}\right)$. In this context, several family of models have been developed in technical literature and in the present analysis the Eulerian-Lagrangian Spray Atomization (ELSA) model has been considered $[9,10]$. In ELSA framework, an Eulerian mixture model is employed to describe the near-nozzle dense spray region, whereas a lagrangian population is initialized when the spray becomes dilute. Therefore, a full atomization process from pure liquid to dispersed droplets can be considered. Such approach has been already validated on different spray configurations $[9,10]$ and its capabilities extended to consider the strong interactions between liquid and gas phase that characterize aero-engine applications [11]. 


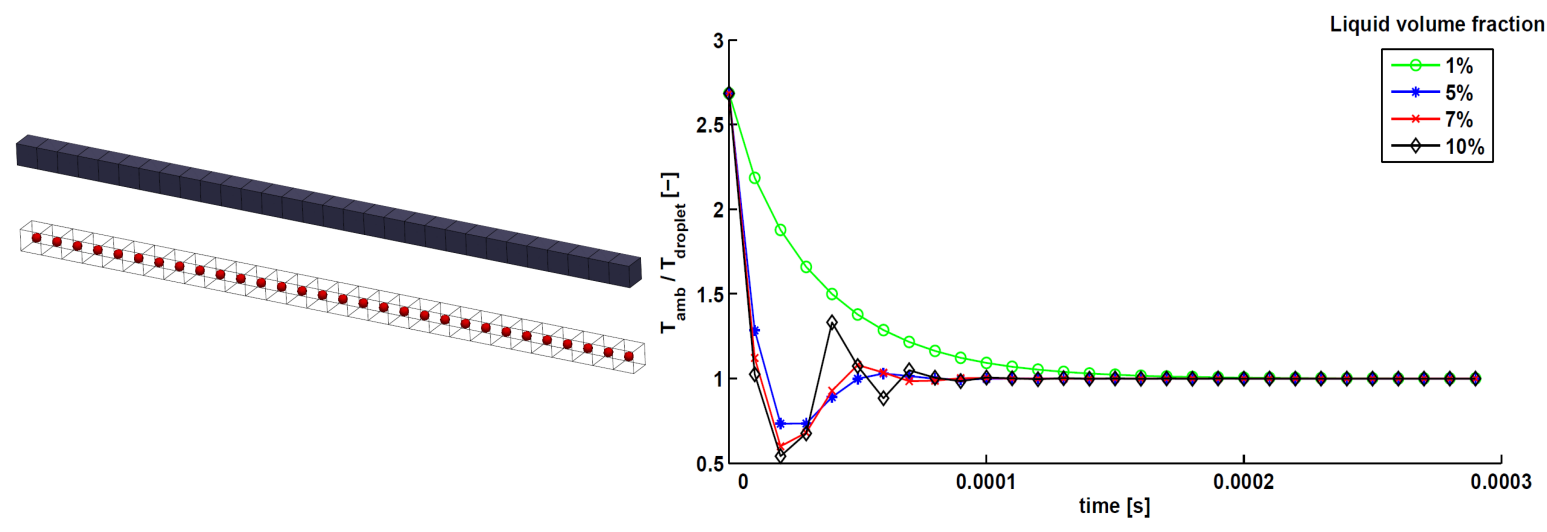

Figure 1. Ratio of ambient and droplet temperature with the respect of time for several liquid volume fraction using an explicit formulation for evaporation modelling.

However, large part of such contributions have been performed in iso-thermal test conditions or using modelling strategies for the evaporation process directly derived from a lagrangian context. For instance, in [9] evaporation effects in the dense zone are neglected, whereas in [12] an explicit formulation is employed for the Eulerian region of ELSA and the vaporization rate is calculated from the local temperature considering the fuel vapour as saturated on the liquid surface. However, such explicit formulation can lead to strong temperature undershootings on the gas side in regions where $\alpha_{l}$ is really high (i.e. near injector regions). Considering for instance the 1-D test case shown in Figure 1 where a cloud of droplets (highlighted in red) is introduced inside a hot stagnant environment, it is possible to calculate the ratio between the gas phase and liquid temperature with respect to time for several values of cell liquid volume fraction. The source/sink terms due to evaporation are here implemented through an explicit formulation and in particular the sink term employed for the gas phase energy equation can be written as:

$$
\frac{d h}{d t}=H A_{d r o p}\left(T_{l}-T_{g}\right)-\frac{d m}{d t} L
$$

where $h$ stands for gas phase enthalpy, $d h / d t$ for its own variation in time, $H$ and $A_{d r o p}$ for heat transfer coefficient and droplet surface area respectively. Liquid temperature and gas phase temperature are mentioned as $T_{l}$ and $T_{g}$ while $d m / d t$ and $L$ are evaporation rate and latent heat of vaporization. It should be pointed out that increasing the liquid volume fraction, this explicit formulation can possibly lead to unbounded values of gas phase temperature. In fact, as soon as $d m / d t$ has been estimated and temperatures have been fixed, depending on $d t$ a certain value of $d h$ can be computed. If $d t$ it is not sufficiently short, $d h$ may be large enough to determine a sharp decrease of gas phase temperature. Then, dealing with a very small gas phase fraction it is possible to obtain temperature undershoots as reported on the right side of Figure 1. Clearly, such issue can be overcome by reducing the simulation time step but with a strong increase of the computational cost considering that as the liquid volume fraction tends to 1.0 the time step should go to zero in order to avoid such instabilities.

Therefore, an implicit formulation can be used to robustly include evaporation inside numerical methods and to avoid this unphysical behaviour in the dense spray region. Implicit approaches are based on a priori calculation of a local equilibrium state established between the volume of liquid and gas that coexist in the same control volume. Such thermodynamic equilibrium condition is then used to compute the evaporation rate as will be explained in detail in the next section. Such implicit approaches are normally based on the assumption that locally the liquid is completely evaporated at equilibrium state [13]. This is not generally true, since for non diluted cases some amount of liquid remains even at equilibrium state. This is the case of the near injector region where the liquid core may be slightly affected by evaporation, but can heavily determine the overall atomization. In [14, 15] an original implementation of evaporation modelling based on local adiabatic saturation conditions is shown, but it is assumed that the characteristic time scale of vaporization is the calculation time step $(d \tau)$. It is essentially based on the hypothesis that the vaporization is so quick that it is completed within the simulation time-step. However, this is not generally true since the particle relaxation time can vary inside the domain based on the local flow-field. For instance in [16], a security factor is introduced between the simulation and particle time step in order to correctly catch the dynamic and thermal evolution of the liquid phase.

To overcome such limitations and to extend the ELSA capabilities in dealing with evaporation in the dense spray region with a more physical characteristic time scale, a novel implicit evaporation modelling strategy has been developed in the framework of the open source code OpenFOAM. Considering that the attention here is mainly focused on the dense zone, only the Eulerian-Eulerian solver derived from ELSA has been considered for the present study. The next section is therefore devoted to the description of such E-E solver and to the characterization of the modelling strategy employed to include evaporation. In the second part of the paper, the available experimental data from the Engine Combustion Network (ECN) database for a diesel jet in evaporating test conditions are used to assess the proposed model on a realistic configuration. 


\section{Numerical methods Compressible ELSA model}

In the E-E model derived from ELSA model, the two phase flow is analysed as a single phase flow composed of two species with highly variable density. Therefore, beyond the equation for the mixture momentum $(U)$, the solver essentially consists of an equation for the liquid volume fraction to predict the liquid evolution and of an equation for liquid/gas interface per unit of volume $(\Sigma)$ to model the breakup process and to consider a polydisperse spray distribution. Locally the spray Sauter Mean Diameter (SMD) can be directly calculated from $\alpha_{l}$ and $\Sigma$ as $S M D=6 \alpha_{l} / \Sigma$. The reader interested in a detailed description of the ELSA formalism and its possible extensions to include the effects of a slip velocity between liquid and gas (i.e. Quasi Multiphase Eulerian approach) is addressed to the specific literature $[9,10,11]$

Starting from such E-E approach, a fully compressible solver has been firstly developed for evaporative test condition. In fact, the energy transfer from gas to liquid plays a key role and, due to the high temperature variation, the density of the gas phase can not be clearly considered constant. A transport equation for vapour volume fraction has been first of all introduced alongside the one for $\alpha_{l}$. Both of them are now solved in a compressible fashion and source terms due to evaporation have been added. The equation are written in RANS context, thus all variables are considered as mean Reynolds variables. A standard gradient closure is applied for turbulent fluxes but it is not here discussed for the sake of brevity and only the vaporisation term is explained in detail.

$$
\begin{aligned}
& \frac{\partial \rho_{l} \alpha_{l}}{\partial t}+\frac{\partial \rho_{l} \alpha_{l} U_{i}}{\partial x_{i}}-\frac{\partial}{\partial x_{i}}\left[\left(\rho_{l} D_{l}+\frac{\mu_{t}}{S c_{t l}}\right) \frac{\partial \alpha_{l}}{\partial x_{i}}\right]=-\dot{\alpha}_{e v} \\
& \frac{\partial \rho_{v} \alpha_{v}}{\partial t}+\frac{\partial \rho_{v} \alpha_{v} U_{i}}{\partial x_{i}}-\frac{\partial}{\partial x_{i}}\left[\left(\rho_{v} D_{v}+\frac{\mu_{t}}{S c_{t v}}\right) \frac{\partial \alpha_{v}}{\partial x_{i}}\right]=\dot{\alpha}_{e v}
\end{aligned}
$$

where $\rho$ and $D$ represents the bulk density and diffusivity for liquid and vapour, $S c_{t}$ is the turbulent Schmidt number, $\mu_{t}$ is the turbulent viscosity and $\dot{\alpha}_{e v}$ is the contribution of evaporation. The subscripts $l$ and $v$ stand for liquid and vapour respectively. Clearly, the air volume fraction is directly calculated from $\alpha_{l}+\alpha_{v}+\alpha_{a}=1$, where the subscript $a$ refers to air.

Two energy equations, one for liquid and one for gas phase, written in terms of temperatures have been also introduced and the contribution of evaporation appears as additional source term $\left(\dot{T}_{e v}\right)$. Below, the energy equation for the liquid phase is shown.

$$
\frac{\partial \rho_{l} \alpha_{l} c_{p_{l}} T_{l}}{\partial t}+\frac{\partial \rho_{l} \alpha_{l} c_{p_{l}} T_{l} U_{i}}{\partial x_{i}}-\frac{\partial}{\partial x_{i}}\left(\alpha_{l} \frac{\mu_{t}}{P r_{t l}} \frac{\partial T_{l}}{\partial x_{i}}\right)=\dot{T_{e v}}
$$

where $c_{p}$ is the specific heat and $P r_{t}$ is the turbulent Prandtl number. A sink term due to evaporation should be introduced also for the $\Sigma$ equation, but it has been neglected in the present study since it should have a minor impact in the dense region that is dominated by the breakup process because of the high value of the Weber number. In the further diluted part, a model similar to the one used in [9] may be applied.

Clearly, special attention has been devoted to the dynamic expression of the evaporation source terms in the previous system of equations, exploiting the following implicit formulation:

$$
\begin{aligned}
& \dot{\alpha}_{e v}=\rho_{l}\left(\frac{\alpha_{l e q}-\alpha_{l}}{\tau_{m}}\right) \\
& \dot{T}_{e v}=\rho_{l} \alpha_{l} c p_{l}\left(\frac{T_{l e q}-T_{l}}{\tau_{T}}\right)
\end{aligned}
$$

where $\alpha_{l e q}$ and $T_{l e q}$ represent the equilibrium state that locally the liquid/gas system reaches in terms of remaining liquid volume and temperature with two rates defined by $\tau_{m}$ and $\tau_{T}$. Similar formulations are employed also for the gas phase and they have not been reported here for the sake of brevity. Equations 5 and 6 lead to an unconditionally stable system on a mathematical point of view, even if a proper calculation of equilibrium state and evaporation rates has to be provided and it will be detailed in the next sub-section.

\section{Equilibrium calculation and evaporation rates}

Firstly, it is important to recall that two phases of a pure substance are in an equilibrium state when both phases share the same value of the specific Gibbs function. Mass transfer plays a key role in equilibrium composition for a vaporizing system and it is of primary importance in calculating the equilibrium temperature since if some liquid evaporates (or some vapour condensates) a variation in total enthalpy occurs due to the latent heat of vaporization. Therefore, considering the evaporation process in a two-phase, two-component system inside an isolated volume at constant pressure, where mass transfer takes place from liquid to vapour phase, it is theoretically possible to calculate the equilibrium temperature of the system as:

$$
T_{e q}=\frac{m_{a} c_{p_{a}} T_{g}+m_{l} c_{p_{l}} T_{l}+m_{v} c_{p_{v}} T_{g}-\left(m_{v e q}-m_{v}\right) L}{m_{a} c_{p_{a}}+m_{l e q} c_{p_{l}}+m_{v e q} c_{p_{v}}}
$$




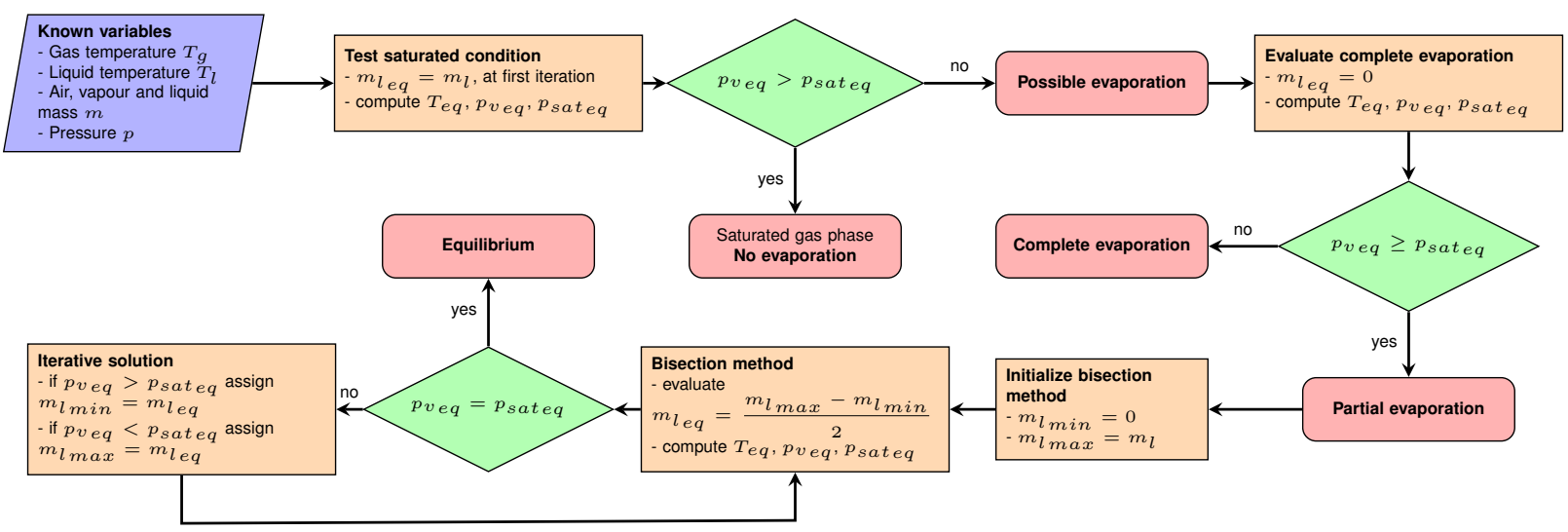

Figure 2. Method implemented for computation of equilibrium composition and temperature

Another relationship is now necessary to estimate the composition at equilibrium. In the present study, it has been chosen to directly calculate the mass of liquid at equilibrium $\left(m_{l e q}\right)$ and consequently the vapour mass as $m_{v e q}=m_{v}-\left(m_{l e q}-m_{l}\right)$, where $m_{l}$ and $m_{v}$ are the local mass of liquid and vapour at time $t$. In addition, it has been considered that phase equilibrium for a two phase system can be defined also when the vapour pressure in the air is equal to the saturation pressure of liquid at the liquid temperature. It means that:

$$
p_{v}=p_{\text {sat }}\left(T_{e q}\right)
$$

where partial vapour pressure $\left(p_{v}\right)$ can be easily calculated through the number of moles of air and vapour in the fraction of volume available for the gas phase. Therefore, using Equations 7 and 8, an iterative cycle can be set to determine the equilibrium state varying $m_{v e q}$ until Equation 8 is satisfied. The loop is briefly summarized in Figure 2. At the beginning, the saturated condition is evaluated by placing $m_{l e q}=m_{l} . T_{e q}$ is therefore calculated by equation $7, p_{v_{e q}}$ by equation 8 and $p_{s a t}$ by the standard Antoine equation using $T_{e q}$. If the obtained vapour partial pressure is higher than the saturation pressure, no evaporation will be allowed since the gas phase is already saturated by vapour. In this case, only an heat transfer is introduced between phases and a proper $T_{e q}$ is calculated.

On the contrary, if some evaporation is possible, the case of complete evaporation is evaluated and two possible situations are therefore considered:

1. if $p_{v_{e q}}<p_{\text {sat }}$, the whole liquid will be evaporated and the final equilibrium temperature for the liquid is set equal to the wet bulb value, whereas the one for the gas is computed thanks to Equation 7 .

2. if $p_{v_{e q}} \geq p_{s a t}$, only partial evaporation will take place. This situation leads to an iterative cycle based on Equation 8 and in the present study a simple bisection method has been employed because of his stability and boundedness. Further developments are surely required on this point to decrease the overall computational effort.

Finally, the obtained $m_{l e q}$ and $T_{e q}$ are used to compute source terms for transport equation through Equations 5 and 6.

The remaining parameters that needs now to be defined are $\tau_{m}$ and $\tau_{T}$. There is not modelling proposal for the rate of vaporisation to be applied for any liquid volume fraction yet. To overcome this difficulty a first proposal is based on the Abramzon-Sirignano model [17]. The advantage is to recover on more diluted part the correct vaporisation rate, but the characteristic vaporisation time scale is underestimated in the dense part. However, it is worth mentioning that this error should be partially compensated by the implicit method. In fact, the equilibrium value $m_{l e q}$ should be correctly computed through the equilibrium cycle and the modelling approximation is only given by the $\tau$ computation. In addition to that, in the dense zones, where $\alpha_{l}$ approaches to one, the volume left for gas phase is generally small and it will be rapidly cooled and saturated by vapour. Therefore, the mass of liquid allowed to evaporate will be small as well as the characteristic $\tau$. This suggests that the time required by the system to achieve the equilibrium is not so relevant and far more attention must be paid to the estimation of the correct equilibrium state at least for the near injector region. Further developments are required on this point, introducing an increasing number of geometrical properties of the liquid/gas interface: a model for the curvature of the liquid surface is now under investigation to this end. However, using previous assumption, it is possible to obtain the expressions of $\tau_{m}$ and $\tau_{T}$ shown below:

$$
\begin{aligned}
\tau_{m} & =\frac{m_{l}-m_{l e q}}{\dot{m}}=\frac{m_{l}-m_{l e q}}{\pi d n_{d} S h^{*} D_{g} \rho_{g} \ln \left(1+B_{M}\right)} \\
\tau_{T} & =\frac{T_{l_{e q}}-T_{l}}{\frac{\dot{Q}-\dot{m} L}{c_{p_{l}} m_{l}}}
\end{aligned}
$$


Table 1. Operating conditions of the ECN test cases under investigation.

\begin{tabular}{c|ccc}
\hline & Test case 1 & Test case 2 & Units \\
\hline Orifice nominal diameter & 0.084 & 0.084 & $\mathrm{~mm}$ \\
Mean injection pressure & 150 & 50 & $\mathrm{MPa}$ \\
Mean injection velocity & $\sim 600$ & $\sim 300$ & $\mathrm{~m} / \mathrm{s}$ \\
Fuel density & 713 & 713 & $\mathrm{~kg} / \mathrm{m}^{3}$ \\
Fuel temperature & 363 & 363 & $\mathrm{~K}$ \\
Ambient temperature & 900 & 900 & $\mathrm{~K}$ \\
Ambient pressure & 6.05 & 6.05 & $\mathrm{MPa}$ \\
Area contraction coefficient & 0.98 & 0.98 & -
\end{tabular}

where $d$ and $n_{d}$ can be directly calculated from $\alpha_{l}$ and $\Sigma$ as shown below:

$$
\begin{aligned}
& d=\frac{6 \alpha_{l}}{\Sigma} \\
& n_{d}=\frac{V_{\text {liquid }}}{V_{\text {droplet }}}=\frac{V_{\text {liquid }}}{V_{\text {cell }}} \frac{V_{\text {cell }}}{V_{\text {droplet }}}=\alpha_{l} \frac{6}{\pi d^{3}} V_{\text {cell }}
\end{aligned}
$$

Moreover, in Equation 10, $\dot{Q}$, which is the heat transfer between phases, appears and it can be calculated as:

$$
\dot{Q}=\pi d n_{d} N u^{*} k_{g} \frac{\ln \left(1+B_{T}{ }^{\prime}\right)}{B_{T}{ }^{\prime}}\left(T_{g}-T_{l}\right)
$$

where $k_{g}$ is the gas thermal conductivity, $N u^{*}$ is the corrected Nusselt number to account for the effects of Stefan flow and $B_{T}{ }^{\prime}$ is the thermal Spalding number. Due to the lack of slip velocity between phases, Reynolds number employed in the definition of $N u^{*}$ and $S h^{*}$ is calculated using the fluctuating component of velocity [18]. Such approximation will be avoided employing QME [11] in a future implementation of the solver. For the sake of brevity, the complete expression of these terms is not here detailed but it is possible to recast a formulation of each one in terms of the characteristics variables of ELSA (i.e. $\alpha_{l}$ and $\Sigma$ ). It should be pointed out that all these quantities are valid both for the dilute and dense spray region since they are based only on geometrical properties of the droplet-gas interface, which are defined in all the domain.

\section{Validation of ELSA under evaporating test conditions Description of the test case}

The experimental test case investigated in the present work has been studied at Sandia National Laboratories and it is composed by a common rail injection system, used to supply fuel to a solenoid-actuated diesel injector [19]. The nominal diameter of the injector here analysed is $0.084 \mathrm{~mm}$ and the experimental apparatus is described in detail in [19].

Several experimental data are available and, for the evaporating non-reacting test conditions here studied, the specific rail pressure ranges from 50 to $150 \mathrm{MPa}$ and the spray is injected into an ambient density of $22.8 \mathrm{~kg} / \mathrm{m}^{3}$, corresponding to an ambient pressure of approximately $6 \mathrm{MPa}$ at $900 \mathrm{~K}$. A single component $\mathrm{n}$-dodecane fuel is used to allow a complete knowledge of the physical and chemical properties. The injected fuel, thanks to the injection velocity and to the high temperature of the combustion chamber, rapidly breakups and evaporates, even if no combustion happens, because of the non-reactive environment. Injection duration is different for the various measurements, but it always overcome $4 \mathrm{~ms}$ in order to achieve the quasi-steady period of spray development.

Liquid length is measured through high-speed Mie scatter images using a $3 \%$ threshold of maximum intensity. For steady liquid penetration the period between 0.5 and $1.4 \mathrm{~ms}$ is considered [19]. Furthermore, Rayleigh scatter imaging is used to obtain several contour data for vapour fraction in order to determine both its axial and radial distributions. Data for vapour are available starting from $17.85 \mathrm{~mm}$ after the injection point until $50 \mathrm{~mm}$ downstream. In Table I the main operating conditions of the analysed test cases are summarized.

It should be pointed out that in terms of evaporation modelling the test case is really challenging since a large region where $\alpha_{l}$ tends to 1.0 can be found. On a theoretical point of view, this would constrain E-E solvers based on an explicit strategy to strongly reduce the time step size to avoid an unphysical behaviour of the vaporization source terms. Such issue should not appear using the modelling strategy here proposed, since the employment of an implicit formulation leads to a stable treatment of vaporization and to a time step definition just related to the local Courant-Friedrichs-Lewy (CFL) condition, determined by the flow-field velocity and mesh size.

\section{Numerical setup}

Calculations here reported have been realized using OpenFOAM v. 3.0.1. Simulations have been carried out on the axi-symmetric computational domain shown in Figure 3 where a zoom of the mesh in the near injection region is also shown. The domain is a 5 degrees sector of the whole domain with 1 element in the azimuthal direction. The axial and radial extensions of such domain are smaller than the experimental chamber (i.e. $108 \mathrm{~mm} \times 108 \mathrm{~mm}$ 
in experiments against $100 \mathrm{~mm} \times 20 \mathrm{~mm}$ in present calculations), but it has been shown $[15,20]$ that it should not impact the jet evolution and the atomization process. Furthermore, considering that the focus of the present work is on evaporation modelling, the injector duct has not been included and the diameter has been reduced based on the area contraction coefficient experimentally measured (see Table I).
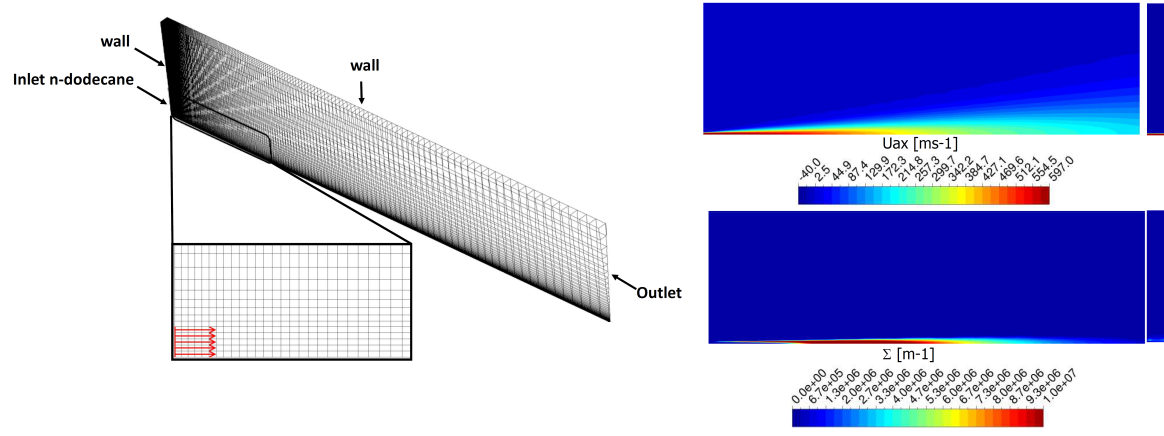

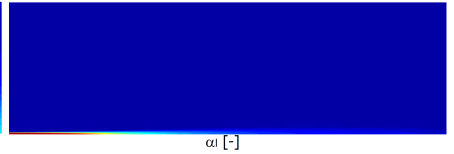

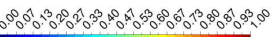

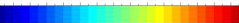

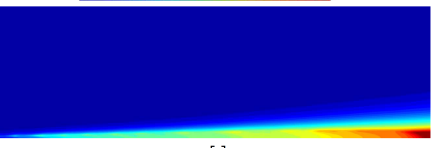

$\alpha v[-]$

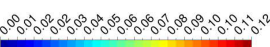

Figure 3. Computational domain (left) and evolution of ELSA variables for Test case 1 (right).

Such assumption allows us to neglect the cavitation inside the nozzle that would affect the real velocity profile and to concentrate the study just on the downstream region. As shown in [15, 20], where ELSA formulation is used to model the liquid/gas interface for the same test case, a mesh sizing with 10 elements along the injector diameter is able to properly reproduce the main features of the flow-field together with the liquid-air mixing. As suggested in [20], the aspect ratio of the cells comprised in the inlet patch is kept close to 1.0, while they are stretched in radial and axial direction. Therefore, a structured mesh counting 12500 cells with a size of $0.008 \mathrm{~mm}$ at the injector exit was generated.

Mass flow rate is imposed at the injector inlet following the available experimental data, whereas a static pressure is prescribed at the outlet. All the walls are treated as smooth, non-slip and adiabatic, whereas cyclic conditions have been applied on the two lateral patches.

The used time step has been chosen in order to ensure a control of the Courant number lower than one inside the computational domain. Therefore, all calculations were performed with $\mathrm{d} \tau=1.5 \mathrm{e}-8 \mathrm{~s}$ for Test case 1 considering the high velocity of the liquid jet, whereas it has been increased to $\mathrm{d} \tau=2.5 \mathrm{e}-8 \mathrm{~s}$ for Test case 2 .

With regard to turbulence modelling, a standard $\kappa-\epsilon$ model has been employed. Following results shown in [15], a sensitivity study on the value of the characteristic constant $C_{\epsilon 1}$, in order to better predict the evolution of the jet, has been also performed. The investigated values are 1.44, 1.52 and 1.60 but for the sake of brevity, such analysis is not reported here. In fact, it has been pointed out that standard values of $\kappa$ - $\epsilon$ constants (i.e. $C_{\epsilon 1}=1.44$ ) are able to reproduce vapour jet spreading with an overall good agreement, while the modified ones are not able to catch the lateral turbulent dispersion of the vapour.

The solver follows a classical segregated PISO method to solve the pressure-velocity coupling. Both convective and diffusive fluxes have been discretized following second order schemes whereas first order Euler scheme has been employed for time advancement.

\section{Results}

Figure 3 shows velocity and liquid-gas interface density evolutions together with liquid and vapour fraction contours obtained with ELSA on Test case 1 on a window of $10 \mathrm{~mm} \times 3 \mathrm{~mm}$ after the injector exit. The liquid jet, due to its high Weber and Reynolds numbers, enters the chamber and undergoes a quick atomization process, which is pointed out by the zone where the production of $\Sigma$ is really high. Such violent atomization is related to the growth of instabilities on the liquid surface due to the turbulent interactions with the gas phase, but it is also strongly affected by the heat-up and evaporation of liquid that take place immediately in the near injection region. A liquid core is therefore generated and the spray tends progressively to evaporate producing a region with a non-negligible volume of $n$-dodecane vapour. It should be pointed out that at the end of the selected window, the vapour volume fraction exceeds already the $10 \%$ and this can have an important effect in the stabilization mechanism if reacting test conditions are considered. Furthermore, even in regions where the liquid volume fraction is really high (i.e. $\alpha_{l} \simeq 0.8-0.9$ ) the code is able to robustly determine a non zero evaporation rate with a consequent production of fuel vapour. In these regions it is likely that an explicit method shows strong numerical instabilities and undershootings in gas phase temperature.

In Figure 4, a comparison of the obtained mixture fraction contours (in the same experimental window, i.e. 33.71 $\mathrm{mm} \times 14.081 \mathrm{~mm}$ from $17.85 \mathrm{~mm}$ after injector exit [19]) together with its axial and radial distributions at two axial distances are shown for Test case 1. Clearly, considering that non reacting test conditions are here considered, the mixture fraction has been directly calculated using locally the vapour and air mass fractions. An overall good agreement has been obtained both in terms of axial and radial distributions. Considering the contour plots, vapour concentration seems to be slightly overpredicted mainly in the near axis zone and the difference with experiments tends to decrease going further downstream. This is also confirmed by radial profiles: the jet spreading is underpredicted with a negative impact on the vapour concentration in centerline proximity especially on the first experimental 

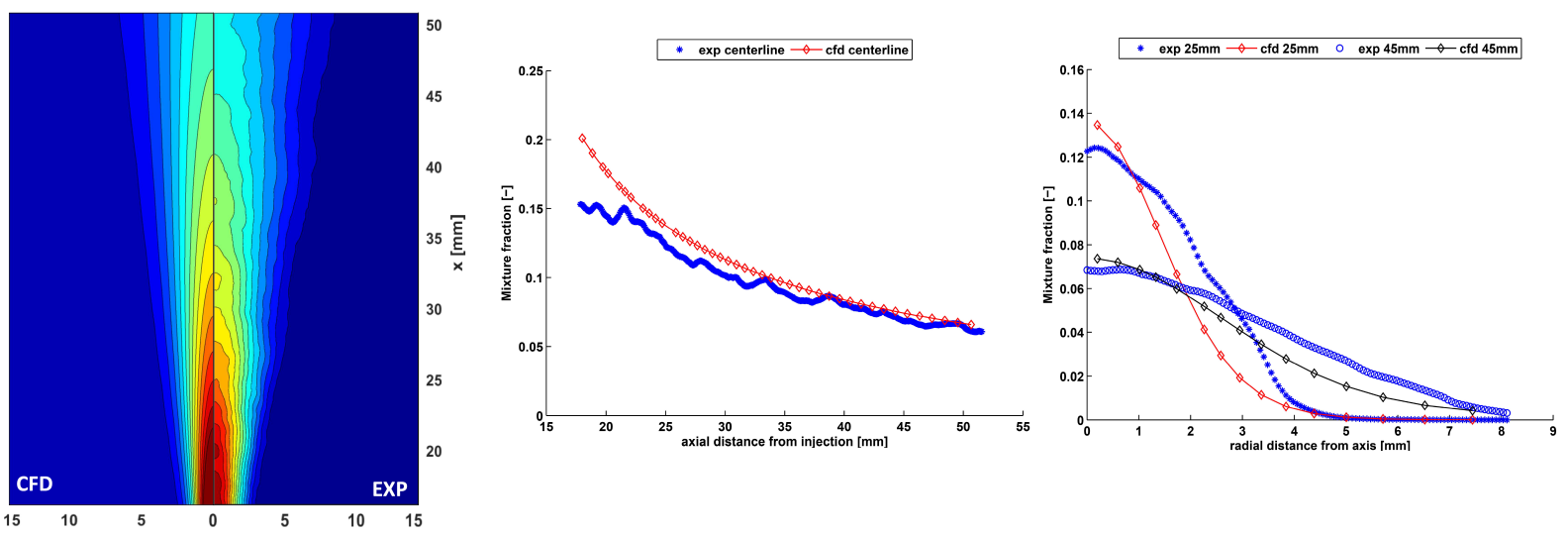

Figure 4. Contours of mixture fraction (left) and axial (center) and radial (right) mixture fraction distributions for Test case 1.
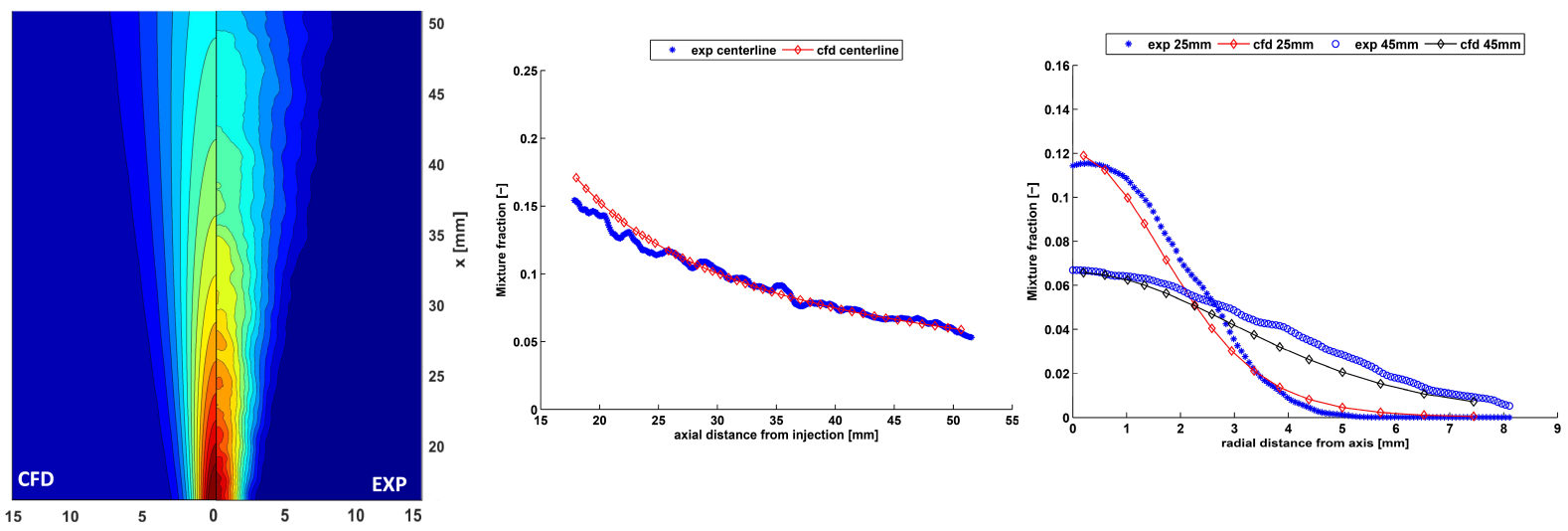

Figure 5. Contour of mixture fraction (left) and axial (center) and radial (right) mixture fraction distributions for Test case 2.

plane. At higher axial distances, the effect of turbulence modelling is less pronounced and numerical results properly reproduce the vapour concentration both in terms of axial and radial distribution. Therefore, based on a physically consistent representation of the gas and turbulence flow-field, the developed code is able to properly predict the local equilibrium state and the final vapour concentration. On the other hand, reducing the axial distance, the effect of turbulence modelling on the solution is more and more important leading, for instance, to an overestimation of mixture fraction at the beginning of the experimental window. Further developments are surely required on this point exploiting scale resolving techniques, such as Large Eddy Simulation, for better catching the liquid/gas interactions. Moreover, the liquid jet penetration was also available on an experimental point of view. Strong attention should be paid on the definition of phase penetration. In technical literature about numerical simulations of the ECN data, several and different definitions on this topic can be found and in the present study, following the work of [20] and [21], the liquid penetration has been evaluated using iso-contour of $\alpha_{l}=0.1 \%$. In this way it has been possible to evaluate a liquid jet penetration of $10.2 \mathrm{~mm}$ which nearly corresponds to the experimental one (i.e. $10.4 \mathrm{~mm}$ ).

However, to reduce the impact of turbulence modelling and to focus the attention just on evaporation modelling, Test case 2, which is characterized by a lower injection pressure and liquid velocity, has been also considered. Numerical results in terms of mixture fraction contour as well as axial and radial distributions are shown in Figure 5. It should be pointed out that a really good agreement has been obtained for this test case. Thanks to a more physically consistent representation of the flow-field and of the interactions between gas and liquid phase, obtained mixture fraction profiles mimic well the experimental evolution both in terms of axial profile and radial spreading. The pressure and velocity fields together with liquid volume fraction, even in RANS framework, are now correctly reproduced and this leads to properly calculate both the equilibrium state as well as the global evaporation rate.

Finally, it is interesting to point out that on an experimental point of view also the time-dependent penetration of vapour was available for these two test cases. As for the liquid phase, the instantaneous penetration of vapour has been evaluated using the iso-contour of $\alpha_{v}=0.1 \%$ and obtained results are shown in Figure 6 . With a higher injection pressure, the vapour penetration grows faster and this is due to the higher velocity at the exit of the injector. The instantaneous vapour penetration is directly linked to the mass flow rate of the injector but it is also the result of two conflicting phenomena: the increase of velocity makes the liquid penetration growing faster and the turbulence mixing increases the vaporization rate which reduces the liquid penetration. All these phenomena are observed experimentally and recovered by the numerical model. A slight underprediction of penetration can be anyway pointed out mainly considering Test case 2 and it is probably related to a low momentum exchange between vapour and the 

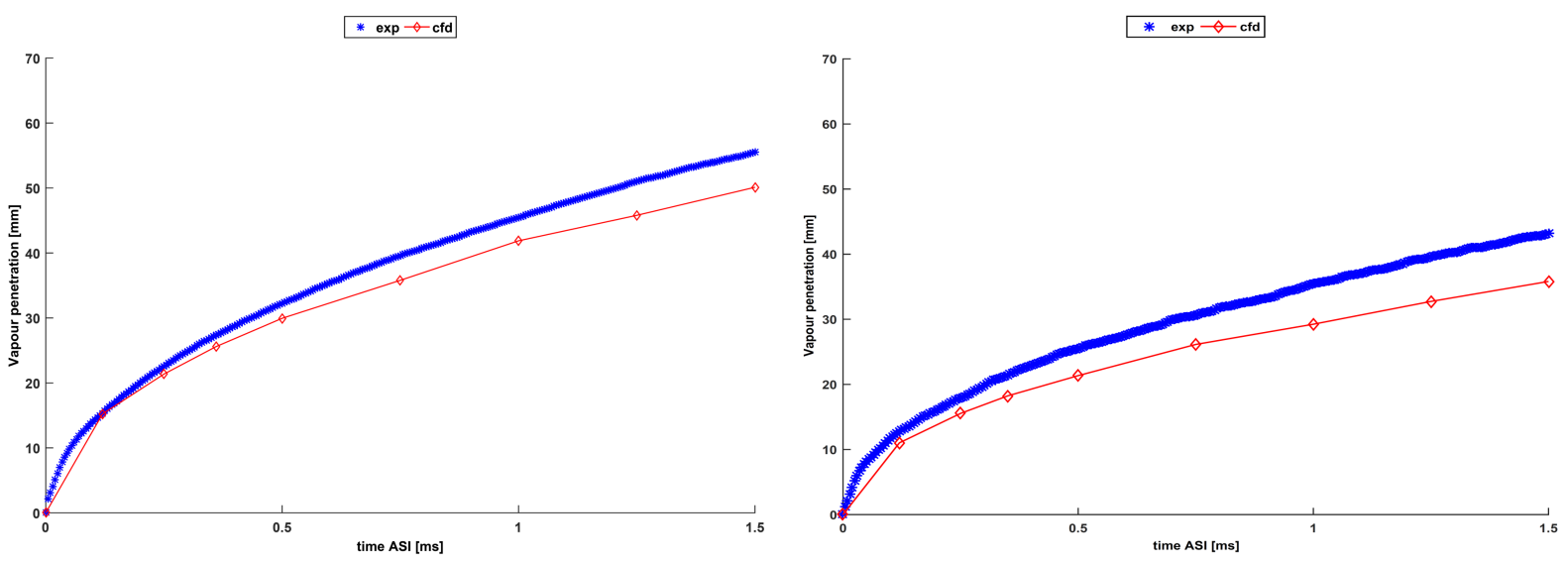

Figure 6. Time-dependent vapour penetrations for Test case 1 (left) and 2 (right).

surrounding air, which can be again related to the performances of the turbulence model. Probably, it is underpredicting the jet spreading and also the interactions between vapour and air at the periphery of the jet, leading to a slower vapour volume fraction mainly concentrated in the near axis region and therefore to a reduced penetration. As already said, scale resolving techniques would help in reducing this gap thanks to a realistic representation of the turbulent flow-field.

\title{
Conclustions
}

This study shows an innovative strategy in modelling spray atomization and in particular in the description of the evaporation in dense spray region where primary break-up takes place. The near injector region is really difficult to characterize experimentally and here the ELSA model has been chosen since it ensures a reliable characterization of the whole atomization process from purely liquid to the dispersed phase. An implicit vaporization model, based on local equilibrium state computation, has been introduced and the entire approach has been successfully tested on an experimental test case representative of diesel injection for two different values of injection pressure. The comparison with experiments shows that the whole approach is able to correctly reproduce the atomization process and to properly catch how evaporation affects the liquid distribution. For both test cases an overall good agreement has been obtained and in particular for Test case 2, where the impact of turbulence modelling should be more reduced, the mixture fraction evolution is well reproduced by numerical computation. In particular, reducing the injection pressure, the model is able to catch the time-dependent reduction of vapour penetration and remaining discrepancies have been justified considering the employed turbulence model. A preliminary validation of the proposed setup is therefore presented and further developments are under investigation for the extension of the whole approach in LES framework.

\section{Nomenclature}

Bt Thermal Spalding number

cp Specific heat $\left[\mathrm{m}^{2} \mathrm{~s}^{-2} \mathrm{~K}^{-1}\right]$

$D \quad$ Laminar Diffusivity $\left[\mathrm{m}^{2} \mathrm{~s}^{-1}\right]$

$k \quad$ Thermal conductivity [ $\left.\mathrm{kgms}^{-3} \mathrm{~K}\right]$

$m \quad$ Mass [kg]

$p \quad$ Pressure $\left[\mathrm{kgm}^{-1} \mathrm{~s}^{-2}\right]$

$\mathrm{Nu} \quad$ Nusselt number

$S c \quad$ Schmidt number

$S M D \quad$ Sauter Mean Diameter [m]

$\operatorname{Pr} \quad$ Prandtl number

$T \quad$ Temperature [K]

$U \quad$ Mixture Velocity $\left[\mathrm{ms}^{-1}\right]$

\author{
Greek \\ $\alpha_{l} \quad$ Liquid Volume Fraction [-] \\ $\Sigma \quad$ Liquid-gas interface density $\left[\mathrm{m}^{-1}\right]$ \\ $\rho \quad$ Density $\left[\mathrm{kgm}^{-3}\right]$ \\ $\mu \quad$ Viscosity $\left[\mathrm{kgm}^{-1} \mathrm{~s}^{-1}\right]$ \\ $\tau \quad$ Evaporation rate $[\mathrm{s}]$
}




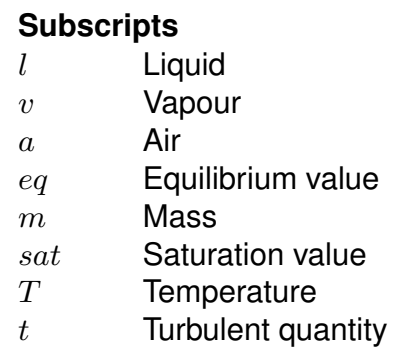

\section{Acronyms}

$C F D \quad$ Computational Fluid Dynamics

ELSA Eulerian Lagrangian Spray Atomization

$E C N \quad$ European Combustion Network

LES Large Eddy Simulation

QME Quasi Multiphase Eulerian

\section{References}

[1] ICAO. Enviromental report, aviation and climate change, 2010.

[2] Williams, F. A. Spray combustion and atomization. Physics of Fluids, 1(6):541-545, 1958.

[3] F., Laurent and M., Massot. Multi-fluid modelling of laminar polydisperse spray flames: origin, assumptions and comparison of sectional and sampling methods. Combustion Theory and Modelling, 5(4):537-572, 2001.

[4] Marchisio, D. L., Pikturna, J. T., Fox, R. O., Vigil, R. D., and Barresi, A. A. Quadrature method of moments for population-balance equations. AIChE Journal, 49(5):1266-1276, 2003. ISSN 1547-5905. .

[5] Fox, R.O., Laurent, F., and Massot, M. Numerical simulation of spray coalescence in an eulerian framework: Direct quadrature method of moments and multi-fluid method. Journal of Computational Physics, 227(6):3058 - 3088, 2008. ISSN 0021-9991. .

[6] Kah, D., Laurent, F., Massot, M., and Jay, S. A high order moment method simulating evaporation and advection of a polydisperse liquid spray. Journal of Computational Physics, 231(2):394 - 422, 2012. ISSN 0021-9991.

[7] F., Michael. Method of moments with interpolative closure. Chemical Engineering Science, 57(12):2229 2239, 2002. ISSN 0009-2509. Population balance modelling of particulate systems.

[8] Massot, M. Eulerian Multi-Fluid Models for Polydisperse Evaporating Sprays, pages 79-123. Springer Vienna, Vienna, 2007. ISBN 978-3-211-72464-4.

[9] Lebas, R., Menard, T., Beau, P.A., Berlemont, A., and Demoulin, F.X. Numerical simulation of primary break-up and atomization: DNS and modelling study. International Journal of Multiphase Flow, 35(3):247 - 260, 2009. ISSN 0301-9322.

[10] Vallet, A. and Borghi, R. Modélisation eulerienne de l'atomisation d'un jet liquide. Comptes Rendus de l'Académie des Sciences - Series IIB - Mechanics-Physics-Astronomy, 327(10):1015 - 1020, 1999. ISSN 1287-4620.

[11] Andreini, A., Bianchini, C., Puggelli, S., and Demoulin, F.X. Development of a turbulent liquid flux model for eulerian-eulerian multiphase flow simulations. International Journal of Multiphase Flow, 81:88 - 103, 2016. ISSN 0301-9322. .

[12] Ning, W., Reitz, R. D., Lippert, A. M., and Diwakar, R. Development of a next-generation spray and atomization model using an eulerian-lagrangian methodology. In 17th International Multidimensional Engine Modeling User's Group Meeting, Detroit, MI, April 2007, 2007.

[13] Kösters, A. and Karlsson, A. A comprehensive numerical study of diesel fuel spray formation with openfoam. Technical report, SAE Technical Paper, 2011.

[14] Garcia-Oliver, J. M, Pastor, J. M, Pandal, A., Trask, N., Baldwin, E., and Schmidt, D. P. Diesel spray cfd simulations based on the $\sigma$-y eulerian atomization model. Atomization and Sprays, 23(1), 2013.

[15] Desantes, J. M., Garcia-Oliver, J. M., Pastor, J. M., and Pandal, A. A comparison of diesel sprays cfd modeling approaches: Ddm versus $\sigma$-y eulerian atomization model. Atomization and Sprays, 26(7):713-737, 2016. ISSN 1044-5110. 
[16] Chrigui, M., Gounder, J., Sadiki, A., Masri, A. R., and Janicka, J. Partially premixed reacting acetone spray using LES and FGM tabulated chemistry. Combustion and Flame, 159(8):2718-2741, 2012. ISSN 0010-2180. Special Issue on Turbulent Combustion.

[17] Abramzon, B. and Sirignano, W.A. Droplet vaporization model for spray combustion calculations. International Journal of Heat and Mass Transfer, 32(9):1605 - 1618, 1989. ISSN 0017-9310.

[18] Chin, J.S. and Lefebvre, A.H. The Role of the Heat-up Period in Fuel Drop Evaporation. International Journal of Turbo and Jet Engines, 1985. .

[19] ECN. Engine combustion network, 2012. URL https://ecn.sandia.gov/.

[20] Khuong, A. D. The Eulerian-Lagrangian Spray Atomization (ELSA) model of the jet atomization in CFD simulations: evaluation and validation. PhD thesis, Universitat Politécnica de Valéncia, 2012.

[21] Kralj, C. Numerical simulation of Diesel spray processes. PhD thesis, University of London, 1996. 


\title{
Drop Impact onto a Metallic Porous Layer: Effect of Liquid Viscosity and Air Entrapment
}

\author{
Cristina Boscariol ${ }^{1}$, Dipak Sarker ${ }^{2}$, Boseon Kang ${ }^{1,3}$, Cyril Crua ${ }^{1}$, Marco Marengo ${ }^{1}$
}

\author{
${ }^{1}$ School of Computing Engineering and Mathematics, University of Brighton, United Kingdom \\ ${ }^{2}$ School of Pharmacy and Biomolecular Science, University of Brighton, United Kingdom \\ ${ }^{3}$ Chonnam National University, Gwangju, Korea \\ ${ }^{*}$ Corresponding author: c.boscariol@brighton.ac.uk
}

\begin{abstract}
The drop impact onto porous surfaces has important applications in many fields, such as painting, paper coating, drug delivery and cosmetic sprays. In most of these applications, the optimisation of the deposition process is carried out empirically, without a proper understanding of the physics and a theoretical modelling of the spreading and the imbibition phenomena. The purpose of this study is to analyse droplet impacts on metallic meshes to define a general modelling strategy of the impact regimen on particular 2D regular porous surfaces. The application of this structure is relevant in process like filtration but also in the medical field, considering for example reconstructive surgery. By analysing the impact of droplets of water, acetone and a mixture of glycerol and water, having a diameter and an impact velocity in a range of $1.5-3 \mathrm{~mm}$ and $2-4 \mathrm{~m} / \mathrm{s}$, respectively, on meshes with a pore size ranging between 25 and $400 \mu \mathrm{m}$, a regime map was built considering 6 different impact outcomes. The outcomes were characterised by a deposition of the droplet on the substrate, or a partial imbibition, or a total imbibition. By increasing the impact velocity, a splash region was defined, which is still characterised by a final deposition, a partial imbibition and a total imbibition. It is found that the most influencing parameters are closely linked to the liquid properties and the impact velocity, more specifically liquid surface tension plays a major role in defining the impact outcome. In the case of Acetone, the lower surface tension brings to an almost instantaneous total imbibition whereas the experiments conducted using water and glycerol solution, showed a major distribution of the deposition regimes with respect to the other outcomes, due to the effect of a higher viscosity. It was found that the geometrical characteristics of the mesh such as pore size and wire diameter, play an important role as well in defining the total imbibition outcome. Finally, the defined transition maps, shows that for a certain combination of physical properties and initial condition, the outcome of the droplet impact is predictable.
\end{abstract}

\section{Keywords}

Drop impact, porous surface, metallic mesh, impact regimes, imbibition

\section{Introduction}

The phenomenon linked to droplet impact on porous surfaces has important applications in many fields. Considering the sheer number of practical applications that involve surfaces of this level of complexity, it is important to remark the fact that the number of parameters that can affect the impact outcome is vast. For this reason, a range of numerical and experimental investigations is still required, for example, to quantify the imbibition due to porosity and identify the outcome of the impacts [1] [6]. The understanding of the parameters that play the most important role in the evolution of the droplet inside the pore is still an open question. Depending on its size, the droplet spreading will be affected by porosity and its evolution might be modified by pore distribution. For example, in the distribution of agrochemicals, droplets are distributed as aqueous solution and sprayed on plants using pumps [2]. Focusing on environmental applications, it is possible to refer to the infiltration of rain and surface water into soil and the migration of oil in permeable porous media [3]. Porous surfaces find an application even in internal combustion engines. In fact, by using a layer with a specific porosity in cylinder-process, it is possible to obtain homogeneous and low emission combustion by enhancing fuel vaporization and distribution in space [4]. Another application is given by the deposition of dyes on papers in the ink-jet printing process [5]. In comparing the impact of droplets on rough and porous surfaces, Roisman et al. [6] developed a model describing the different regimes of splashing thresholds. In their model, it is shown that in the case of porous surfaces, a deposition outcome, without splash, is more probable considering the partial penetration of the liquid in the pore. They proposed an experimental map showing that two most significant parameters influencing the prompt splash-deposition are the Weber number and the ratio given by two geometrical characteristics linked to roughness. Neyval et al. [7], presented a numerical model, based on the finite volume method, to analyse the dynamics of the impact absorption of a liquid droplet impinging on a porous medium. To model the dynamics of the fluid flow, they enhanced the effects of surface 
tension and capillary forces. They compared their results with experimental data resulting in good agreement. Sahu et al. [8] analysed the impact of nanoparticle suspension into porous filter membranes focusing on penetration given by the hydrodynamic effect. This phenomenon is caused by the kinetic energy brought by a drop, which impacts on the porous media having a very small pore size with respect to drop size. They compared this aspect with the effects given by dynamic and capillary pressures and concluded that penetration into porous medium is possible when the dynamic pressure is higher than the capillary pressure, but also when hydrodynamic focusing, that occurs when the drop diameter is much larger than pore diameter, is observed. Kumar et al. [9] pointed out that the overall imbibition is influenced both by the material of the porous media and by capillary and showed that increasing drop size brought to slower imbibition. Karepetsas et al. [10] investigated droplet interaction, considering both smooth and structured surfaces. Specifically, they focused on the droplet sliding on an inclined surface. The model they performed, treats the liquid-gas and liquid-solid interfaces in a unified content and defines the dynamic contact angle by combining the action of disjoining and capillary pressure and viscous stresses without applying a boundary condition. They pointed out how dynamic hysteresis can be linked to the topography of the substrate and in the case of a structured surface, they predicted the effect of static hysteresis by observing that the droplet slides only beyond a certain critical inclination angle. Moquaddam et al. [11], focused on the regime of bouncing on macro-textured superhydrophobic surfaces that are characterised by a reduction of the contact time. They based the study of these effects using the entropic lattice Boltzman model for multiphase flows, investigating numerically a liquid droplet impacting a surface with tapered posts. They could focus on pancake bouncing phenomenon in complete detail. In this way, it was possible to accurately estimate the transformation of kinetic energy in surface tension and viceversa. This research aims at defining a general modelling strategy of the impact regimes given by experiments conducted on metal porous meshes, for different combinations of pore dimension, impact velocity, drop radius, liquid surface tension and viscosity.

\section{Material and methods}

The experimental analysis was conducted using three liquids: water, acetone and a solution composed by water and glycerol, to analyse the effect of viscosity and surface tension of liquid. The target surfaces were selected from a set of stainless steel metal meshes mainly used for filtration applications, with a range of pore size between 25 and $400 \mu \mathrm{m}$, purchased from (Plastock Plastok® Meshes and Filtration Ltd.). The characteristics of the meshes and liquid properties are listed in Table 1 and 2.

Table 1 Mesh characteristics.

\begin{tabular}{ccc}
\hline Sample Number & Pore Diameter $(\mu \mathrm{m})$ & Wire Diameter $(\mathrm{mm})$ \\
\hline 1 & 25 & 0.025 \\
2 & 50 & 0.036 \\
3 & 80 & 0.05 \\
4 & 100 & 0.065 \\
5 & 125 & 0.1 \\
6 & 150 & 0.1 \\
7 & 200 & 0.125 \\
8 & 250 & 0.1 \\
9 & 400 & 0.22 \\
\hline
\end{tabular}

Table 2 Liquid Properties

\begin{tabular}{cccc}
\hline Liquid & $\begin{array}{c}\text { Density } \\
\left(\mathrm{kg} / \mathrm{m}^{3}\right)\end{array}$ & $\begin{array}{c}\text { Viscosity } \\
(\mathrm{mPa} \mathrm{s})\end{array}$ & $\begin{array}{c}\text { Surface } \\
\text { Tension }(\mathrm{N} / \mathrm{m})\end{array}$ \\
\hline Water & 996 & 1 & 0.073 \\
Acetone & 793 & $0.30-0.543$ & 0.023 \\
Water \& Glycerol & 1118.6 & 10 & 0.067 \\
\hline
\end{tabular}

The experiments were organised in different groups. In order to obtain a range of impact velocity between $2 \mathrm{~m} / \mathrm{s}$ and $4 \mathrm{~m} / \mathrm{s}$, the height of release was varied between $20 \mathrm{~cm}$ and $80 \mathrm{~cm}$. Two different needle sizes were used to analyse the effect of drop diameter on the porous surface. 
The optical setup included a Photron Fastcam SA4 high speed camera (with a resolution of $1024 \times 800$ pixels), angled at $60^{\circ}$ with respect to the horizontal plane. The test area was illuminated using a custom-built high-speed LED light source, synchronised to the high-speed camera. The image analysis was conducted using a MATLAB code for image processing, capable to identify the dimension of the spreading, the droplet initial diameter, and the impact velocity by tracing its centre of mass. Table 4 and 5 report the error analysis for each measurement.

Table 4 Error Analysis of Impact Velocity for Water, Acetone and Water \& Glycerol

\begin{tabular}{ccccccc}
\hline $\begin{array}{c}\text { Height of } \\
\text { Release } \\
(\mathrm{cm})\end{array}$ & $\begin{array}{c}\text { Needle } \\
\text { Gauge No. }\end{array}$ & Liquid & $\begin{array}{c}\text { Mean } \\
\text { Velocity } \\
(\mathrm{m} / \mathrm{s})\end{array}$ & $\begin{array}{c}\text { Standard } \\
\text { Deviation } \\
(\mathrm{m} / \mathrm{s})\end{array}$ & $\begin{array}{c}\text { Maximum } \\
\text { Value }(\mathrm{m} / \mathrm{s})\end{array}$ & $\begin{array}{c}\text { Minimum } \\
\text { Value }(\mathrm{m} / \mathrm{s})\end{array}$ \\
\hline 20.3 & 21 & Water & 1.8 & 0.17 & 2.2 & 1.6 \\
44.3 & 21 & Water & 2.9 & 0.11 & 3.1 & 2.7 \\
80.3 & 21 & Water & 3.9 & 0.16 & 4.2 & 3.5 \\
20.3 & $26 \mathrm{~s}$ & Water & 1.9 & 0.11 & 2.1 & 1.7 \\
44.3 & $26 \mathrm{~s}$ & Water & 2.9 & 0.14 & 3.1 & 2.7 \\
80.3 & $26 \mathrm{~s}$ & Water & 3.9 & 0.16 & 4.6 & 3.6 \\
20.3 & 21 & Acetone & 1.9 & 1.9 & 2.1 & 1.7 \\
44.3 & 21 & Acetone & 2.9 & 2.9 & 3.2 & 2.7 \\
80.3 & 21 & Acetone & 3.9 & 3.9 & 4.1 & 3.7 \\
20.3 & $26 \mathrm{~s}$ & Acetone & 1.9 & 0.13 & 2.2 & 1.7 \\
44.3 & $26 \mathrm{~s}$ & Acetone & 2.9 & 0.17 & 3.2 & 2.7 \\
80.3 & $26 \mathrm{~s}$ & Acetone & 3.7 & 0.20 & 4.3 & 3.5 \\
20.3 & 21 & Water \& Glycerol & 1.8 & 0.07 & 2.1 & 1.7 \\
44.3 & 21 & Water \& Glycerol & 2.7 & 0.10 & 3.1 & 2.6 \\
80.3 & 21 & Water \& Glycerol & 3.7 & 0.07 & 3.9 & 3.5 \\
20.3 & $26 \mathrm{~s}$ & Water \& Glycerol & 1.8 & 0.12 & 2.0 & 1.6 \\
44.3 & $26 \mathrm{~s}$ & Water \& Glycerol & 2.7 & 0.15 & 3.1 & 2.5 \\
80.3 & $26 \mathrm{~s}$ & Water \& Glycerol & 3.8 & 0.11 & 3.9 & 3.6 \\
\hline
\end{tabular}

Table 5 Error Analysis of Initial Diameter for Water, Acetone and Water \& Glycerol

\begin{tabular}{cccccc}
\hline $\begin{array}{c}\text { Needle } \\
\text { Gauge No. }\end{array}$ & Liquid & $\begin{array}{c}\text { Mean } \\
\text { Diameter } \\
(\mathrm{mm})\end{array}$ & $\begin{array}{c}\text { Standard } \\
\text { Deviation } \\
(\mathrm{mm})\end{array}$ & $\begin{array}{c}\text { Maximum } \\
\text { Value }(\mathrm{mm})\end{array}$ & $\begin{array}{c}\text { Minimum } \\
\text { Value }(\mathrm{mm})\end{array}$ \\
\hline 21 & Water & 3.0 & 0.12 & 3.3 & 2.6 \\
$26 \mathrm{~s}$ & Water & 1.9 & 0.09 & 2.1 & 1.7 \\
21 & Acetone & 2.0 & 0.11 & 2.2 & 1.7 \\
$26 \mathrm{~s}$ & Acetone & 1.7 & 0.10 & 2.1 & 1.6 \\
21 & Water \& Glycerol & 2.9 & 0.06 & 3.1 & 2.8 \\
$26 \mathrm{~s}$ & Water \& Glycerol & 1.5 & 0.18 & 1.8 & 1.00 \\
\hline
\end{tabular}

\section{Results and discussion}

By observing the result of the experiments, it is possible to identify 6 different outcomes. For a lower velocity impact, these outcomes are a deposition, a partial imbibition and a total imbibition. For a higher velocity impact, it is possible to observe a transition to a splash region, which is still characterised by a final deposition, a partial imbibition and a total imbibition. The deposition outcome is characterised by the fact that, in a range of 15-30ms after the drop impacts on the substrate, and after the spreading and the recoiling, it is still possible to observe a liquid pancake on the surface without a proper imbibition, and the droplet recoils in an asymmetrical shape. On the opposite, for the imbibition, the liquid penetrates completely through the mesh pores after the impact. The partial imbibition can be considered as a transition outcome in which during the recoiling process after the impact, part of the liquid penetrates under the surface and part of the liquid is deposited on the substrate in a time range of 4-6ms. To distinguish the different outcome regimes, a first attempt was made, considering a dimensionless parameter given by the ratio between pore size and droplet size, $\boldsymbol{\beta}=\frac{\boldsymbol{D}_{p}}{\boldsymbol{d}}$. The results are shown in Figures 1 to 3 . 


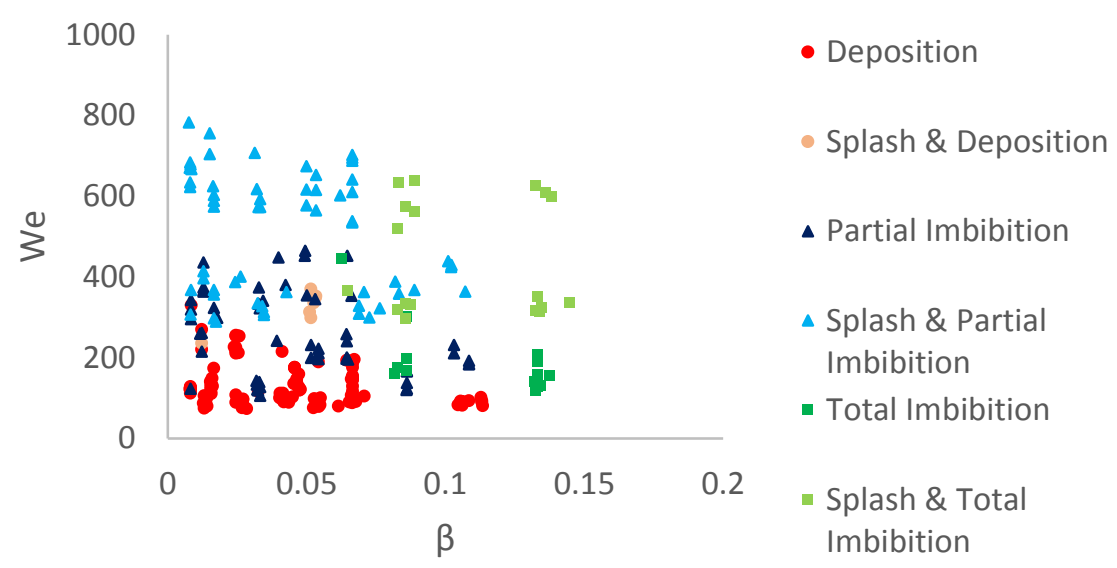

Figure 1 Regime distribution for water as a function of $\beta$.

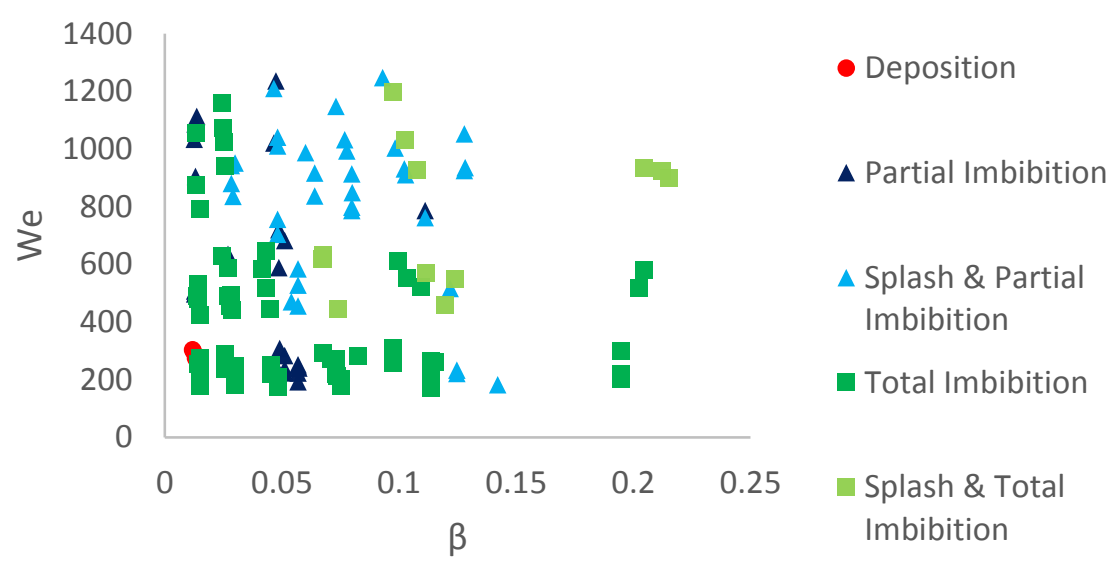

Figure 2 Regime distribution for acetone as a function of $\beta$

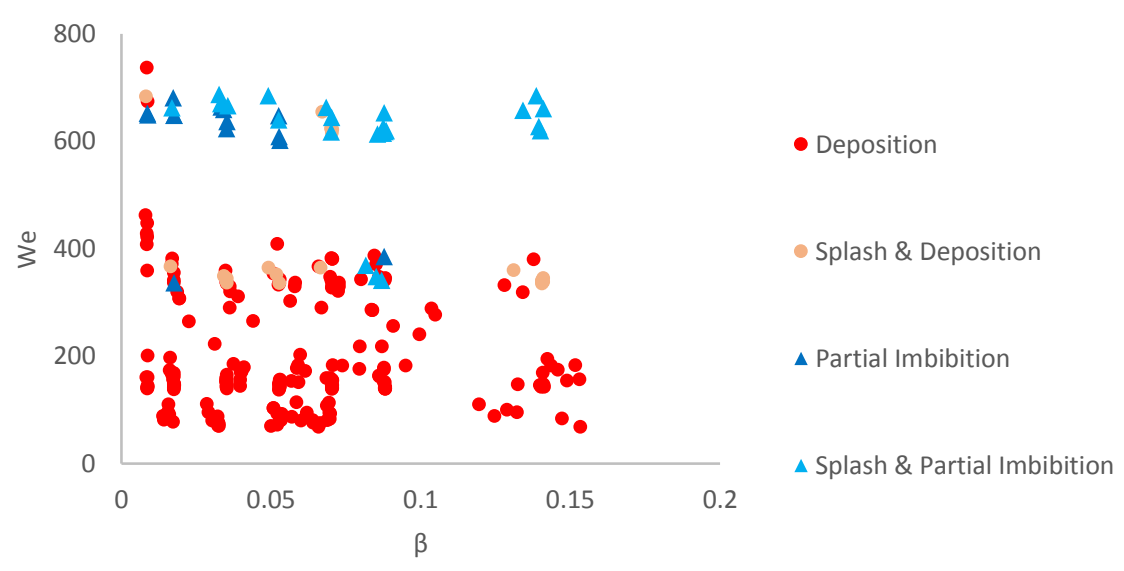

Figure 3 Regime distribution for water and glycerol as a function of $\beta$ 
From the figures above, it is possible to observe that in the case of water, an almost equal distribution of deposition, partial imbibition and total imbibition is achievable. On the contrary for the acetone, due to the lower surface tension of the liquid, the dominant outcome is given by a total imbibition. For the solution composed of water and glycerol, due to the higher viscosity of the liquid, the dominant outcome is given by the deposition. It can be seen that the separation of the outcomes is not clearly defined and, consequently, $\beta$ is not the best parameter to define the different outcome regions. A second attempt was made, introducing a new geometrical parameter given by the ratio of the empty area over the full area of the mesh pore, $\gamma=\left(1+2 \cdot \frac{D_{w}}{D_{p}}\right)^{2}$ where $D_{w}$ is the mesh wire diameter. This number was obtained considering that the ratio of the 2 areas can be written as $\frac{A_{\text {full }}}{A_{\text {empty }}}=\frac{\left(D_{p}+2 D_{w}\right)^{2}}{D_{P}^{2}}=D_{p}^{2} \cdot \frac{\left(1+\frac{2 D_{w}}{D_{p}}\right)^{2}}{D_{p}^{2}}=\left(1+\frac{2 D_{w}^{2}}{D_{p}^{2}}\right)$

Figure 4 shows schematically the interaction of the droplet with the mesh geometry.

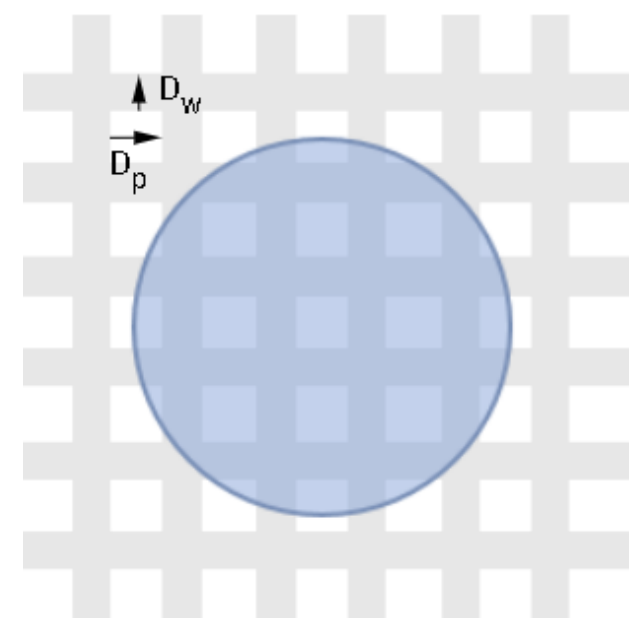

Figure 4 Impact and spreading of the droplet on the porous surface.

The results are shown in Figures 5.

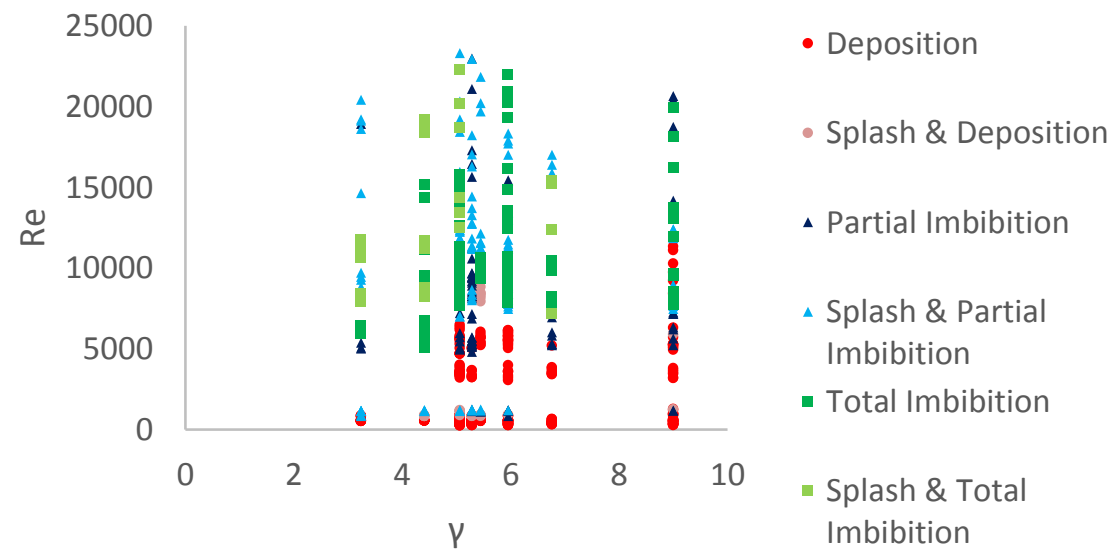

Figure 5 Regime distribution for all the liquids as a function of $\gamma$. It is shown that a clear distribution of the regions is still not achievable. 
By using $\gamma$, a clearer distribution of the outcomes is achieved in the case of water and a solution of water and glycerol but the case of acetone is still not clarified. Moreover, considering Figure 5, in which all the liquids data are reported, it is still not possible to clearly define the regions of deposition, partial imbibition and total imbibition having liquids with different characteristics of physical properties. Therefore, it is necessary to modify the dimensionless numbers used to define the regime map, and find a better combination capable to take in account of all the effects that can influence the impact outcome. Considering the previous results, it is possible to remark that $\gamma$, even if still not satisfactory to achieve the regime separations, plays an important role, under certain conditions, especially in the case in which the value of surface tension does not go under a certain value as for acetone. Consequently, it is possible to assume that the surface tension must be taken in consideration as well to define the regime distribution. Conversely, the splash region is mainly influenced by a higher impact velocity and a lower viscosity. It was chosen to represent the data introducing new dimensionless numbers. The dimensionless parameter on $\mathrm{x}$-axis is given by $\xi=p_{c}^{\star} \cdot \gamma$. Here, $p_{c}^{\star}$ is the capillary pressure $p_{c}=\frac{\sigma}{D_{p}}$ scaled by a critical value which still has to be empirically defined. In this way, the effect of geometry is coupled with the effects given by surface tension, in terms of imbibition effectiveness. The parameter on y-axis is given by $M=R e^{2} O h^{-1}$. In fact, considering the previous graph, it is clear that Reynolds number alone is not sufficient to obtain a satisfactory description of outcome distribution. For such reason it has been coupled with Ohnesorge number, which includes the surface tension effects. Even if this combination of dimensionless numbers brings to a satisfactory result (see Figure 6), it is still necessary to clarify which is the best value of the exponents attributed to Re and Oh to obtain the clearest separation of the different regimes.

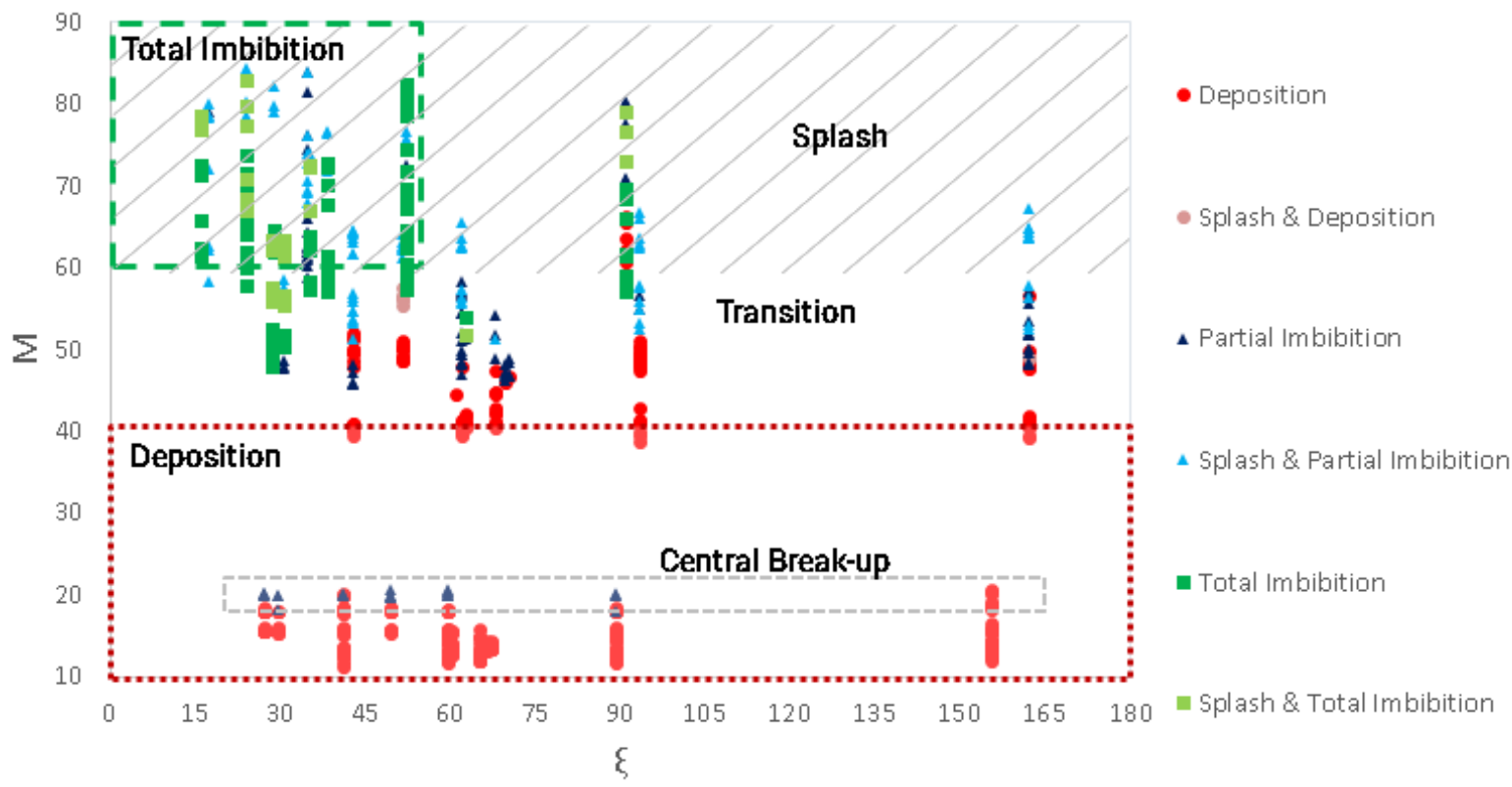

Figure 6 Regime distribution for all the liquids as a function of $\xi$ and $M$

It is now possible to observe that, the regime characterised by deposition, is localized in the lower part of the figure. In the middle part, a transition regime occurs, in which both deposition and partial imbibition are probable. In the upper part of the figure, a splash regime is identified, which is mainly characterised by a total imbibition for a value of $\xi<50$. In a small area in the deposition region, an outcome was detected characterised by a central break-up, mainly due to the experimental data collected for the solution composed by water and glycerol. Table 5 reports the region classification in terms of $\mathrm{M} / \xi$ space coordinates.

Table 5 Definition of regions in $M-\xi$ coordinates

\begin{tabular}{ccc}
\hline $\begin{array}{c}\text { Region } \\
\text { Definition }\end{array}$ & $M$ & $\xi$ \\
\hline Deposition & $0-45$ & $0-180$ \\
Transition & $45-55$ & $0-180$ \\
$\quad$ Total & $55-90$ & $0-50$ \\
Imbibition & $55-90$ & $0-180$ \\
Splash & 5
\end{tabular}

This work is licensed under a Creative Commons 4.0 International License (CC BY-NC-ND 4.0). 


\section{Conclusions}

This study is focused on the investigation of droplet impact on metallic meshes with a wide range of pore sizes. It was found that the attempt to represent the different outcome regimes excluding a geometrical parameter referred to mesh wire diameter was not satisfactory to obtain a proper identification of regimes. In addition, to achieve a clear distinction of the impact regimes, it is fundamental to refer to a dimensionless number that also takes account the liquid properties, specifically, the viscosity and the surface tension. To reflect the above points, new dimensionless parameters, $\boldsymbol{M}$ and $\boldsymbol{\xi}$, are here introduced, and the outcomes of the impact are therefore predicted for the given range of experimental parameters. In considering this numbers, comparing the results to the literature, it was seen that conversely to what Roisman et al. pointed out in their study [6], our result show that the two most important parameters to describe the impact of droplet on a porous material may not be the We number and a ratio given by considering roughness geometrical parameter. The present results show a good agreement with Neyval et al. [7], and Sahu et al. [8], considering respectively the major role given to the surface tension, and the fact that an imbibition outcome is mainly observable for droplet with a larger diameter.

\begin{tabular}{ll}
\multicolumn{2}{l}{ Nomenclature } \\
$p_{c} \quad$ Capillary pressure $[\mathrm{Pa}]$ \\
$\sigma$ & Surface tension $[\mathrm{N} / \mathrm{m}]$ \\
$\rho$ & Liquid density $\left[\mathrm{kg} / \mathrm{m}^{3}\right]$ \\
$D_{p}$ & Pore diameter $[\mathrm{m}]$ \\
$D_{w}$ & Wire diameter $[\mathrm{m}]$ \\
$d$ & Droplet diameter $[\mathrm{m}]$ \\
$v_{i}$ & Impact velocity $[\mathrm{m} / \mathrm{s}]$ \\
$W e$ & Weber number \\
$\operatorname{Re}$ & Reynolds number \\
$\mu$ & Viscosity $[\mathrm{Pa} \mathrm{s}]$ \\
$\xi$ & $p_{c}^{\star} \cdot \gamma$ \\
$\mathrm{M}$ & $R e^{2} O h^{-1}$ \\
$\beta$ & $\frac{D_{\text {pore }}}{d}$ \\
$\gamma$ & $\left(1+2 \cdot \frac{D_{w}}{D_{\text {pore }}}\right)$
\end{tabular}

\section{References}

[1] Marengo, M., Antonini, C., Roisman, I. V., Tropea, C., (2011), Drop collision with simple and complex surfaces, Current Opinion in Colloid \& Interface Science, 16, pp. 292-302.

[2] Bertola, V., (2008), Some applications of controlled drop deposition on solid surfaces, Recent Patents on Mechanical Engineering, 1, pp. 167-174.

[3] Yarin, A.L., (2006), Drop impact dynamics: Splashing, Spreading, Receding, Bouncing, Annual Review of Fluid Mechanics, 38, pp. 159-192.

[4] Weclas, M., (2010), Potential of porous media combustion technology as applied to internal combustion engines, Journal of Thermodynamics, VOL 201039 pp.

[5] Woo Shik Kim, Sang Yong Lee, (2014), Behaviour of a drop impinging on heated porous surfaces, Experimental Thermal and Fluid Science, 55, pp. 72-70.

[6] Roisman, Ilia V., Lembach, A.Tropea, C., (2015). Drop splashing induced by target roughness and porosity: The size plays no role, Advances in Colloid and Interface Science ,VOL 222 p. 615-621.

[7] Neyval, C. R. Jr., Griffiths, R.F., Santos, J. M., (2004). Numerical simulation of the impact of liquid droplets on porous surfaces, Journal of Computational Physics,198, pp. 747-770.

[8]Sahu, R.P., Sett, S., Yarin, A.L., Pourdeyhimi, B., (2015), Impact of aqueous suspension drops onto nonwettable porous membranes: Hydrodynamic focusing and penetration of nanoparticles, Colloids and Surfaces A: Physicochem. Eng. Aspects, 467, pp. 31-45.

[9] Kumar, S., Deshpande, A.P.,(2006), Dynamic of drop srepading on fibrous porous media, Colloids and Surfaces A: Physicochem. Eng. Aspects, 277, pp. 157-163.

[10] Karapetsas, G., Chamakos, N. T., Papathanasiou, A.G., (2016), Efficient modelling of droplet dynamics on complex surfaces, Condens. Matter, 28, 16 pp.

[11] Mazloomi Moquaddam, A., Chikatamarla, Shyam S., Karlinm I., 2016, Drop bouncing off macro-textured superhydrophobic surfaces, Langmuir 


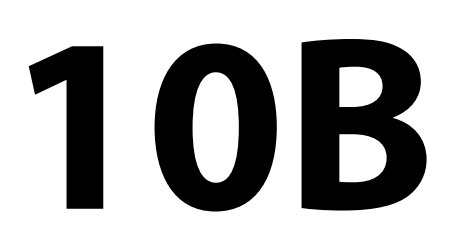

Experimental techniques 3 


\title{
Visualisation and quantitative analysis of the near nozzle formation and structure of a high pressure water jet in air and in water
}

\author{
Sarah Jasper*1, Jeanette Hussong ${ }^{2}$, Ralph Lindken ${ }^{1}$ \\ ${ }^{1}$ Institute of Thermodynamics and Fluid Mechanics, Bochum University of Applied Sciences, \\ Lennershofstr. 140, 44801 Bochum \\ ${ }^{2}$ Chair of Hydraulic Fluid Machinery, Ruhr-University Bochum, Universitätsstr. 150, \\ 44801 Bochum \\ ${ }^{*}$ Corresponding author: sarah.jasper@hs-bochum.de
}

\begin{abstract}
High pressure water jets (HPWJ) with Reynolds numbers in the scale of $10^{4}$ are visualised by high speed photography in air and water. Moreover, suitable measurement techniques are tested and verified by quantitative analysis of the emerging jet to identify the influence of the surrounding fluid on the HPWJ.

The HPWJ process known from industrial applications can be adapted to the field of rock drilling. In this specific case, the HPWJ is used to cut and destroy rock in deep geothermal reservoirs. The process is known as jet drilling. Although there have been research activities in this field, the process itself is not well understood so far and practical applications are rare. Therefore, the aim of our work is the visualisation of the process to increase the knowledge of waterjet and rock interactions.

High speed photography in terms of shadowgraph experiments is used for visualisation. Moreover, an estimation of the fluid velocity on the boundary of the HPWJ in air is performed. For this, the shadowgraph images are evaluated with the double-frame technique well known with particle image velocimetry (PIV). Analysis of both the structure and the velocity distribution of the HPWJ in water is done by combined PIV and laser induced fluorescence (LIF) analysis with fluorescent dye.
\end{abstract}

\section{Keywords}

PIV, LIF, velocity distribution, jet flow, spray analysis

\section{Introduction}

The use of geothermal energy for heat and power exploitation as a renewable energy resource can contribute to a more sustainable energy supply. Enhanced geothermal systems (EGS) enable the recovery of geothermal energy even in surroundings that do not have a naturally hydrothermal system [1,2]. Based on an injection well drilled into hard crystalline rock in up to $3000 \mathrm{~m}$ depth, several laterals are drilled into the rock formation to create an EGS.

The creation of these laterals can be realized by the use of a HPWJ, a process known as radial jet drilling. Jet drilling provides major advantages over conventional mechanical drilling techniques, namely higher drilling speed, less tool wear and reduced environmental risk. However, jet drilling shows drawbacks when drilling into hard crystalline rocks, which represents a challenge to the utilization for EGS. To overcome this drawback, the jetting process has to be optimized towards the implementation for hard crystalline rock drilling. Although there has been research on jet drilling since the 1960s, the process of water jetting of rocks itself is barely understood in terms of physics and fluid mechanics [3-5].

Therefore, the objective of our research project is to enhance this process knowledge with a focus on the interaction between the HPWJ and the rock surface under realistic conditions. The interaction of the HPWJ and the rock surface can be accessed by determination of the jet energy impact on the rock surface. The energy impact is characterized by geometrical information about the jet as well as the corresponding velocity distribution. For the purpose of quantitative analysis of the geometrical information, the HPWJ can be considered to be a highly dense spray. Thus, relevant spray parameters can be evaluated with common spray analysis techniques to quantify the different influences on the formation of the HPWJ. PIV analysis and the appendant correlation algorithm give information about velocities of the HPWJ. Combining these data, the energy impact of the HPWJ for different operating parameters on the rock surface can be estimated. The determination of the energy impact also contributes to the understanding of the rock destroying mechanism of a HPWJ in different stand-off distances to the rock surface. 
In this work, visualisation and optical measurement techniques are chosen to investigate the process. As there is a lack of optical access in a genuine bore hole in high depth, this visualisation is done gradually starting with the development of a suitable optical measurement technique and progresses towards more realistic designs and conditions. The optical measurement technique is further developed for the analysis of a highly dense, high Reynolds number (currently up to $12.3 \times 10^{4}$ ) jet flow in different fluids. For this purpose, two different methods are tested and successfully applied to the jet flow:

- The application of the shadow method with double-pulsed, non-coherent back illumination in combination with PIV evaluation based on a cross-correlation algorithm and

- A combined PIV and LIF technique with double-pulsed laser light sheet illumination under a $90^{\circ}$ angle relative to the observation direction.

The potential application is verified by a parameter study of the operation parameter's influence as well as the surrounding fluid's influence on the formation of the HPWJ.

The visualisation of a flat nozzle jet at high Reynolds numbers has been performed under atmospheric conditions in air by Zelenak et al. [6]. They demonstrated the applicability of the shadow method with combined PIV correlation algorithm on this less dense, high Reynolds number jet to visualise the jet structure and analyse velocity fields. However, this method shows disadvantages in the near-nozzle region where the jet has a high density. For high Reynolds number water jets, visualisation has been frequently done by shadow method (e.g. $[7,8])$. In addition to that, analysis of the velocity fields of both low and high Reynolds number water jets is commonly done by PIV (e.g. $[9,10]$ ). By combining both the PIV and LIF technique, it is possible to access the jet structure as well as velocity fields at the same time. Fukushima et al. [11] and Webster et al. [12] applied this combination to turbulent jets with Reynolds numbers of 2,000 and 3,000, respectively, with one camera for PIV and one camera for LIF, respectively.

For our research aim, a suitable measurement technique for high Reynolds number, highly dense water jets in a limited space and in different fluids is required. Concerning this, possible limitations are the high density of the jet for the shadow analysis in air, whereas the combination of the high Reynolds number and the small spatial expansions are possible limitations to the PIV and LIF analysis in water. Therefore, the shadow method with PIV correlation algorithm for the jet in air and the combined PIV and LIF technique with one camera for the jet in water are tested and verified to obtain simultaneous information about the jet structure and the velocity.

\section{Experimental setup}

In this contribution, the applicability of established optical measurement techniques for investigation of the HPWJ cutting process are tested and validated. Challenges to the application of these optical measurement techniques are the high velocities, the very limited spatial expansions and the dense liquid jet. Therefore, experiments with a broad parameter variety are performed to establish a reliable and reproducible measurement technique. A successful establishment of the measurement technique can be used for further experiments concerning the interaction between the HPWJ, the surrounding fluid and the rock surface.

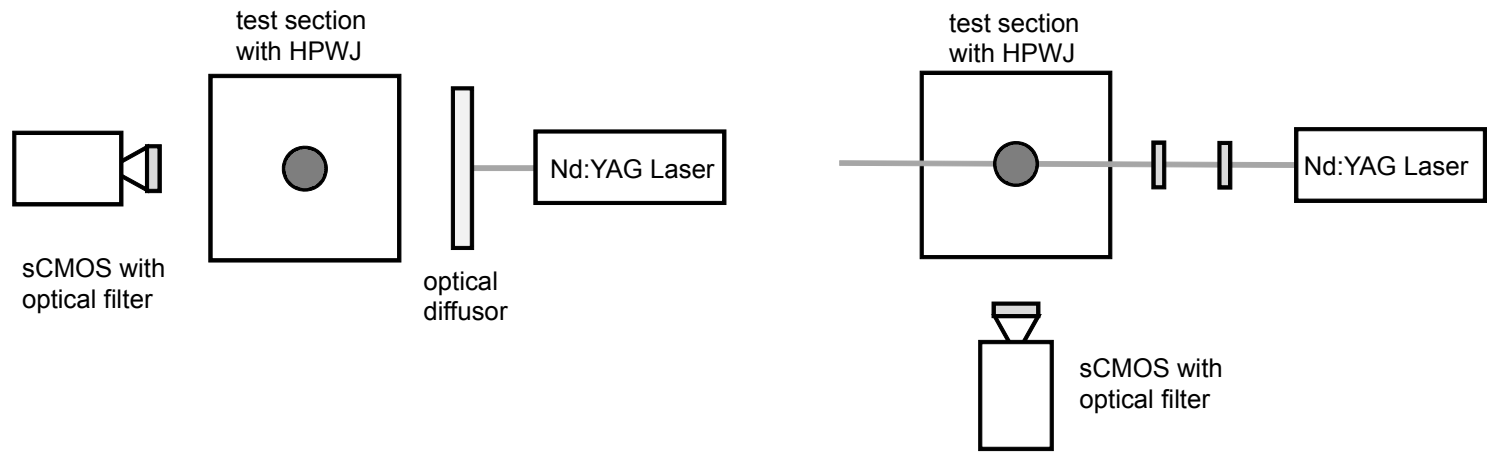

Figure 1. Schematic view of shadowgraph setup for experiments in air (left) and the PIV setup for experiments in water (right) from the top

A high pressure pump (delivering up to $15 \mathrm{dm}^{3} \mathrm{~min}^{-1}$ at a maximum operating pressure of $15 \mathrm{MPa}$ ) and a pressure atomizer nozzle are used to generate the water jet. Experiments are performed for three different nozzle diameters $d_{N}(1.8 \mathrm{~mm}, 2.0 \mathrm{~mm}, 2.3 \mathrm{~mm})$, allowing a parameter range for the nozzle output pressure $p_{N}$ of up to 
$10 \mathrm{MPa}$ and maximum flow rates $\mathrm{Q}_{\mathrm{N}}$ of up to $14 \mathrm{dm}^{3} \mathrm{~min}^{-1}$. The flow rate is measured by electromagnetic inductive flow metering (Optiflux 1300, Krohne). The output pressure is determined by a pressure sensor (BSP00JP, Balluff) $30 \mathrm{~mm}$ upstream of the nozzle. For the two different experiments, the HPWJ is introduced in air or water, respectively. A schematic assembly of the experimental set up for both the shadowgraph system and the PIV system is illustrated in figure 1. The shadowgraph setup is used for experiments in air, whereas the PIV setup is used for experiments in water. A summary of the experimental conditions is given in table 1.

Table 1. Summary of experimental conditions.

\begin{tabular}{ll|c|c}
\hline \hline \multicolumn{2}{c|}{ Parameter } & Unit & Value \\
\hline \hline nozzle diameter & $\mathrm{d}_{\mathrm{N}}$ & $\mathrm{mm}$ & $1.8,2.0,2.3$ \\
nozzle pressure & $\mathrm{p}_{\mathrm{N}}$ & $\mathrm{MPa}$ & $0.1-10$ \\
flow rate & $\mathrm{Q}$ & $\mathrm{dm}^{3} \mathrm{~min}^{-1}$ & $2.0-14.0$ \\
jet velocity & $\mathrm{u}_{\mathrm{J}}$ & $\mathrm{m} \mathrm{s}^{-1}$ & $8.0-130.0$ \\
Reynolds number & $\mathrm{Re}_{\mathrm{N}}$ & - & $1.2 \times 10^{4}-12.3 \times 10^{4}$
\end{tabular}

\section{Visualisation and velocity measurements in air}

High speed photography with a shadowgraph system is used for visualisation of the HPWJ in air and estimation of the velocity distribution. Due to the high velocities of up to $130 \mathrm{~m} \mathrm{~s}^{-1}$ and the small size of the field of view $(20 \mathrm{~mm} \times 20 \mathrm{~mm})$, very short light pulses are needed to capture images of the flow structures without motion blur. Therefore, a shadowgraph system with a light pulse of $20 \mathrm{~ns}$ is selected. This shadowgraph system consists of a 16 bit CMOS camera (sCMOS, LaVision) with a Zeiss Yashica macro lens and a Nd:YAG double-pulse laser (repetition rate $15 \mathrm{~Hz}$, wavelength $532 \mathrm{~nm}$, pulse length $5 \mathrm{~ns}$, maximum energy output per pulse $200 \mathrm{~mJ}$, Quantel Evergreen200) and a laser guiding arm with an optical diffuser, transforming the laser pulse in 20 ns non-coherent pulse. The laser is equipped with a Metrolux laser attenuator that allows stable laser operation at high laser output power and use of an optical diffuser with a maximum laser power input of $50 \mathrm{~mJ}$. The optical diffuser itself consists of laser beam shaping optics, a fluorescently labelled plastic plate and a diffuse screen. The $5 \mathrm{~ns}$ laser light pulse enters the diffuser, is widened by the optics and excites the florescence on the plastic plate positioned under a $45^{\circ}$ angle. A frequency-shifted, non-coherent $20 \mathrm{~ns}$ light pulse is emitted from the plastic plate and the light is guided to the diffuse screen. Applying this setup, it is possible to acquire images with a motion blur of $1-$ 2 pixels under the given experimental conditions. The camera and the laser are synchronized by a timing unit. Using the double-frame mode of the camera and the dual pulse laser, it is also possible to evaluate velocity information from the shadowgraph images. This is especially applied for the HPWJ in air as the reflecting phase boundary makes PIV measurements difficult.

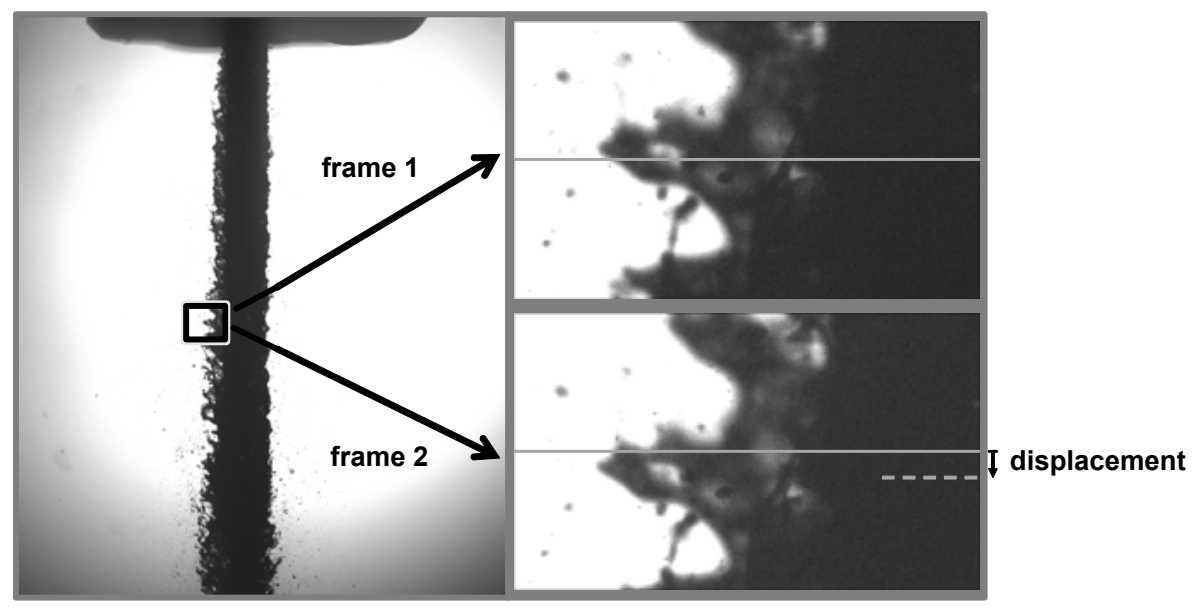

Figure 2. Illustration of the cross-correlation evaluation on the interface structures of the HPWJ in air $\left(\mathrm{d}_{\mathrm{N}}=1.8 \mathrm{~mm}, \mathrm{p}_{\mathrm{N}}=6 \mathrm{MPa}\right.$, $\left.\mathrm{Q}=11 \mathrm{dm}^{3} \mathrm{~min}^{-1}, \operatorname{Re}_{\mathrm{N}}=9.9 \times 10^{4}\right)$. The detailed images on the right show one frame of the double-frame image with dimensions of $1 \times 2 \mathrm{~mm}$, respectively, also including a reference line and the resulting displacement. The structures show a pixel shift of 10 pixels in average from frame 1 to frame 2 .

For every shadow experiment a series of 1000 double-frame images is taken. For spray parameter analysis, image pre-processing is needed. In a first step, the images are inverted, afterwards an appropriate intensity threshold for the determination of the jet boundary is set. The set of 1000 double-frame images are analysed and the mean value of each spray parameter for a single set is taken. Evaluation of the velocity information from the shadowgraph images is done by correlation on interface structures on the boundary of the HPWJ. Therefore, the 
double-frame images are masked before correlation. The principle of correlation on interface structures is illustrated in figure 2.

\section{Visualisation and velocity measurements in water}

In addition to the shadowgraph images, combined PIV and LIF analysis is performed for visualisation of the HPWJ in water and measurement of the velocity distribution. Due to the high density of the spray, fluorescent tracers are used to obtain information of the velocity distribution in the HPWJ. The camera is equipped with a high pass filter with a cut-on at $540 \mathrm{~nm}$ to ensure that no undesired reflections from the laser light but only the light emitted by the fluorescent dye is recorded. Water soluble vinyl paint with rhodamine B (emission peak at $565 \mathrm{~nm}$ ) as fluorophore is used both as fluorescent dye and tracer, as the vinyl paint does also contain fluorescent, solid particles. The use of this fluorescent paint permits simultaneous PIV and LIF analysis. The evaluation of this simultaneous measurement technique has been introduced by Lindken and Merzkirch [13] and has also been successfully adapted to other applications $[14,15]$. The technique is based on the separation of different grey level values. In this work, the grey levels are obtained from the background, the image of the dissolved dye and the fluorescent particles in increasing order, illustrated in figure 3. Consequently, simultaneous measurement of the HPWJ interface as well as velocity distribution can be applied by appropriate thresholding.

Similar to the shadow experiments, a series of 1000 double-frame images is also taken for every measurement point. PIV analysis is done by cross-correlation of the double-frame images. To obtain the shape of the HPWJ, image post-processing with an appropriate threshold is needed. Subsequently, the images can also be used to analyse spray parameters.
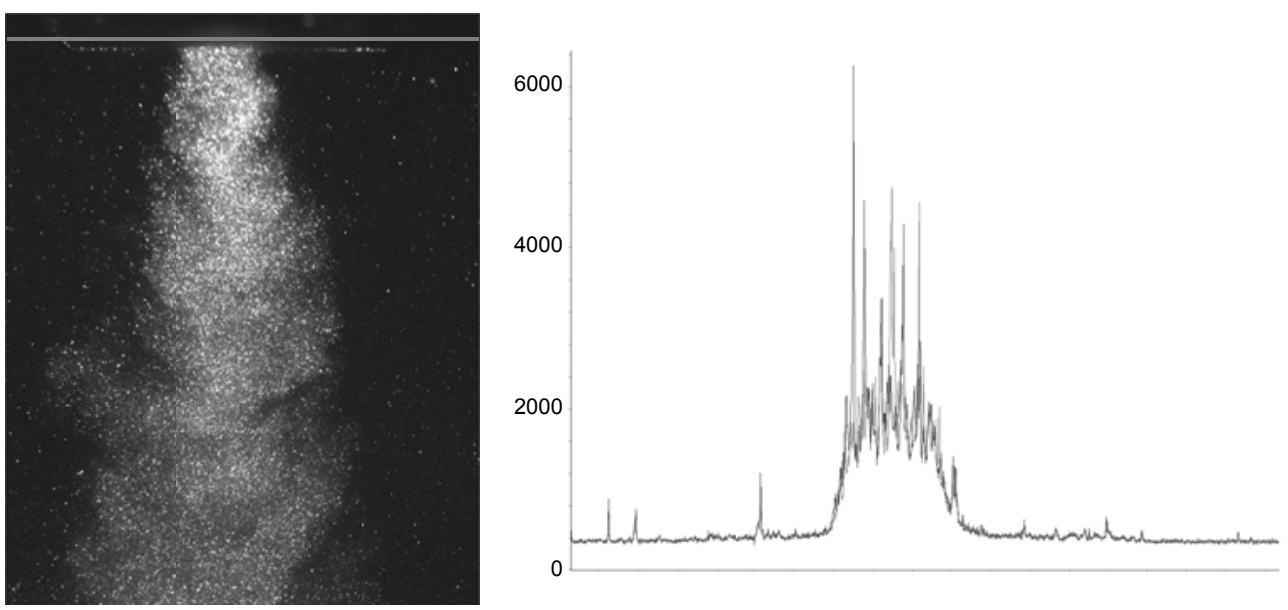

Figure 3. Example of a combined instantaneous PIV and LIF image with different grey levels of the HPWJ in water $\left(\mathrm{d}_{\mathrm{N}}=1.8 \mathrm{~mm}, \mathrm{p}_{\mathrm{N}}=0.1 \mathrm{MPa}, \mathrm{Q}=2.3 \mathrm{dm}^{3} \mathrm{~min}^{-1}, \mathrm{Re}_{\mathrm{N}}=1.5 \times 10^{4}\right)$. On the right, the grey value distribution along the grey line in the left image is shown. The fluorescent dye seeded jet creates grey values about 1500 counts, while the unseeded surrounding water is at 300 counts. Fluorescent tracer particles appear as peaks at around 4000 counts.

\section{Velocity distribution of the HPWJ}

Applying the cross-correlation algorithm on the interface structures of the HPWJ in air, the velocity distribution at the boundary of the HPWJ can be determined. To prevent uncertainties in the correlation algorithm, the core of the HPWJ is masked out [6]. For verification of the correlation results, the jet velocity $u_{J}$ can be estimated by the continuity equation, taking into account the measured flow rate $Q$ and the physical nozzle cross-sectional area $A_{N}$. Figure 4 contrasts the obtained velocity values. Differently than expected, the velocity distribution on the boundary of the HPWJ by correlation of the shadow images is higher than the one obtained by the above mentioned calculation. This discrepancy is due to the assumption of single phase, liquid flow. In air, the visualisation does not show the existence of a second, gaseous phase. That is why the first velocity estimation is done with the assumption of single phase, liquid flow. In contrast to that, visualisation in water shows the formation of gas bubbles right at the nozzle outlet. 

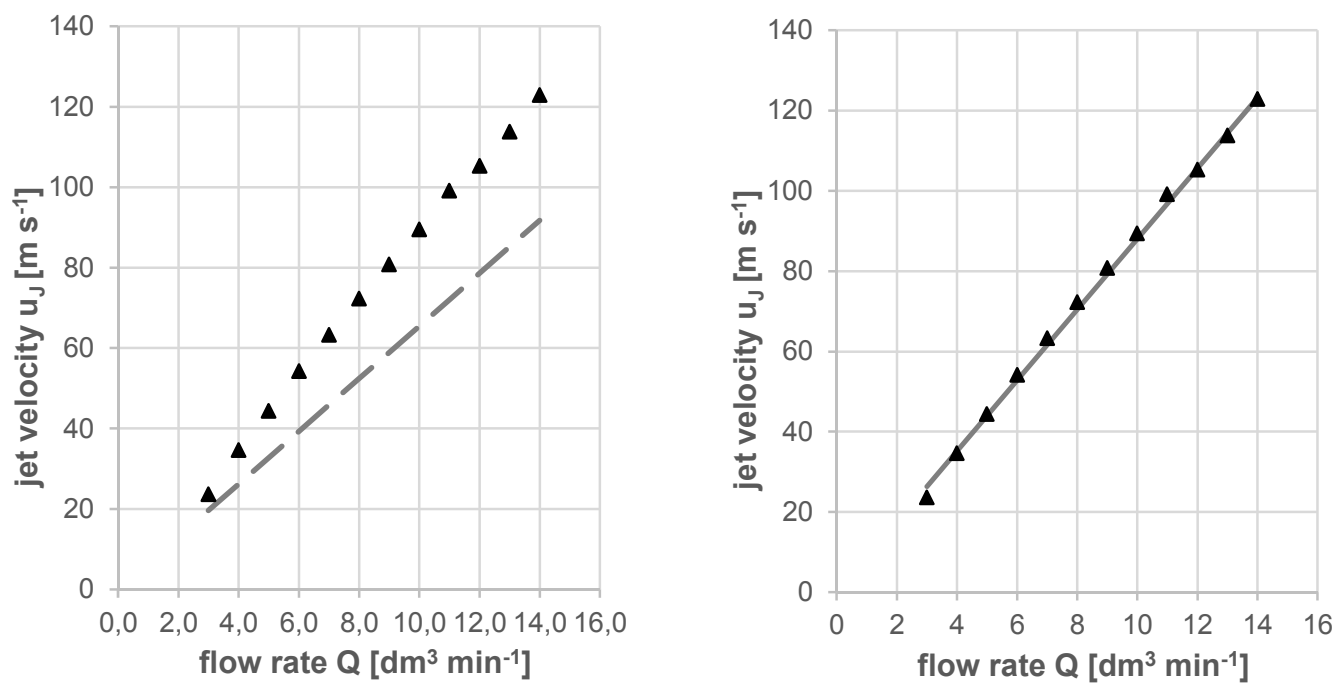

Figure 4. Velocity at the interface of the HPWJ in air, $d_{N}=1.8 \mathrm{~mm}$. Values determined by eq. (1) with assumption of single phase flow (left, grey dashed line) and two phase flow (right, grey line). Values determined by PIV cross-correlation are given in both diagrams (black triangles).

Possible reasons for the formation of a gaseous phase are already dissolved gas in the water or the occurrence of cavitation, which, in addition, can be favoured by outgassing of the dissolved gas. The existence of a gaseous phase can block a part of the nozzle outlet cross-sectional area. This blocking reduces the effective outlet crosssectional area $A_{\text {eff, }}$ resulting in higher outlet velocities [15]. Therefore, the correlated velocities are higher due to a smaller effective outlet cross-sectional area in comparison to the physical cross-sectional area used for velocity estimation.

Due to the occurrence of the gaseous phase, the application of the combined PIV and LIF analysis for the HPWJ in water is limited. The gas bubbles cause scattering both of the laser light and the light emitted by the fluorescent dye. This is a major drawback because an analysis of the velocity distribution in the core of the jet close to the nozzle outlet is not feasible with this experimental setup. Nevertheless, from a distance of about 6-7 nozzle diameters downwards, no gaseous phase is present and a correlation of the double-frame images is possible. Thus, comparing the velocity at the boundary of the HPWJ both in air and water is plausible in this region. As expected, the correlation results indicate that the velocity of the HPWJ in water is smaller than in air [e.g. 17].

\section{Estimation of the gas proportion from velocity data}

Due to the presence of a gaseous phase, the effective nozzle outlet cross-sectional area is reduced. However, the resulting effective nozzle outlet cross-sectional area $A_{\text {eff }}$ can be determined by the correlated velocities in air $\mathrm{u}_{\mathrm{J}^{*}}$ and the measured flow rate $\mathrm{Q}$ in the nozzle feeding pipe before cavitation occurs, eq. (1).

$$
A_{\text {eff }}=Q / u_{J^{*}}
$$

This leads to an effective outlet cross-sectional area which is up to one fourth smaller than the physical outlet cross-section. According to the relationship between the effective outlet cross-sectional area $A_{\text {eff }}$ and the gas proportion $\mathrm{x}$, the latter can be estimated at the nozzle outlet, eq. (2).

$$
x=\left(A_{N}-A_{\text {eff }}\right) / A_{\text {eff. }}
$$

Consequently, the void fraction can be quantified to be $20-26 \%$, increasing with decreasing physical nozzle outlet cross-sectional area or diameter. For example, the estimated velocity values by continuity equation with a void fraction of $26 \%$ for the smallest nozzle outlet diameter $d_{N}=1.8 \mathrm{~mm}$ are shown in figure 4 . Although the gas bubbles are a limitation to the optical access of the near nozzle jet, the existence of those gas bubbles can be favourable in the rock destroying mechanism [18].

\section{Structure of the HPWJ}

The pressure atomizer nozzles used for the experiments are designed for applications in the open air. Under these conditions, they have a very small spray angle to generate a full jet with a high energy impact. The spray 
cone angle of the HPWJ is a relevant spray parameter to analyse the influence of the surrounding fluid. An increase in the spray angle indicates an increase in the interaction of the HPWJ and the surrounding fluid.

For the HPWJ in air, the spray angle for all examined nozzles is smaller than $4^{\circ}$, showing a moderate increase with increasing flow rate, pressure and nozzle outlet diameter, respectively. In contrast to that but similar to literature, the HPWJ in water shows a significantly wider spray angle at the same experimental conditions. The increase in the spray angle is caused by higher shear forces at the jet interface [e.g. 17,19]. As an example, figure 5 shows the comparison of the time averaged HPWJ structure in air and water respectively.

In addition to that, the structure on the boundary of the HPWJ changes from a smooth to a rougher interface, showing clearly visible interface structures. Comparing instantaneous images, it is also visible that, in the field of view, the HPWJ does not break up in air, whereas in water the HPWJ does break up. This is also in accordance with literature [e.g. 17,19]. Two examples of instantaneous images are shown in figure 6 . The break up in water is due to the higher viscosity of water in contrast to air. The resulting shear forces are higher, causing the HPWJ to break up or atomize.
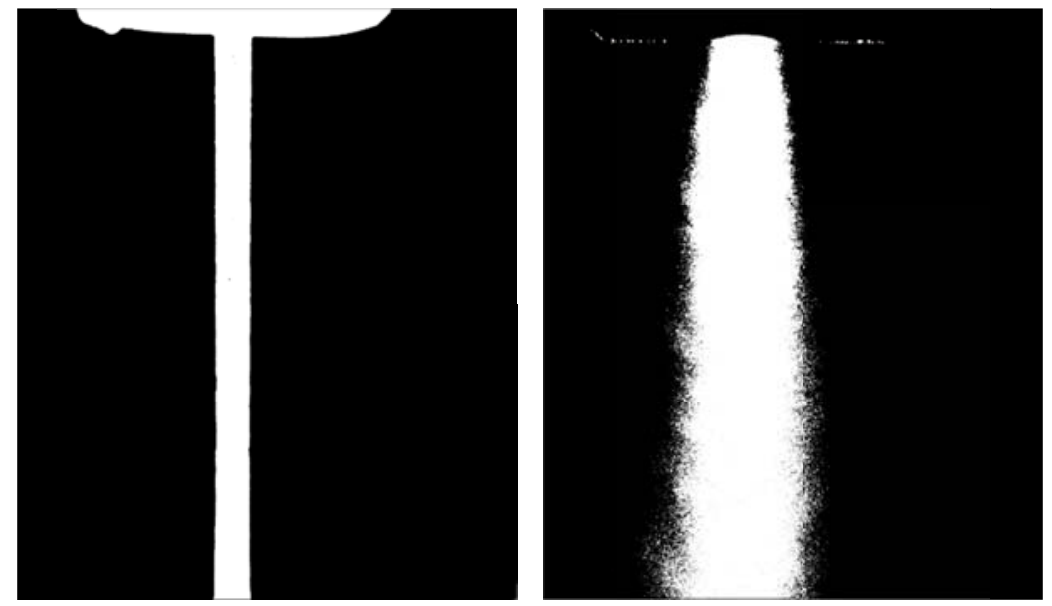

Figure 5. Comparison of the time averaged structure of the HPWJ in air (left) and water (right), $\mathrm{d}_{\mathrm{N}}=1.8 \mathrm{~mm}, \mathrm{p}_{\mathrm{N}}=0.1 \mathrm{MPa}$, $\mathrm{Q}=2.3 \mathrm{dm}^{3} \mathrm{~min}^{-1}, \operatorname{Re}_{\mathrm{N}}=1.5 \times 10^{4}$
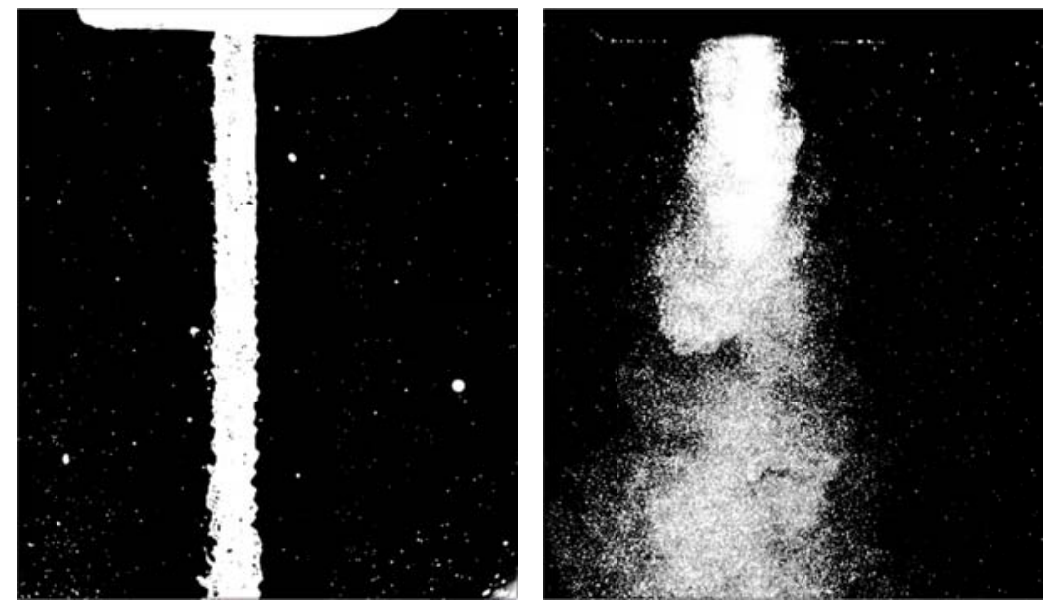

Figure 6. Comparison of the instantaneous structure of the HPWJ in air (left) and water (right), $\mathrm{d}_{\mathrm{N}}=1.8 \mathrm{~mm}, \mathrm{p}_{\mathrm{N}}=0.1 \mathrm{MPa}$, $Q=2.3 \mathrm{dm}^{3} \min ^{-1}, \operatorname{Re}_{\mathrm{N}}=1.5 \times 10^{4}$

\section{Conclusions}

Both high speed photography in terms of shadowgraph images and the combined PIV and LIF technique are successfully adopted to the present experimental setup and conditions. Both techniques can be applied to simultaneously visualise and analyse the HPWJ with high Reynolds numbers of currently up to $12.3 \times 10^{4}$ for drilling applications. To ensure the applicability to the given circumstances, further experiments with absence of a gaseous phase will be performed. Nevertheless, the experiments presented in this contribution give first hints about the influence of the surrounding fluid on a HPWJ which are in accordance with literature data. 
The here proven measurement techniques can be used to obtain a model for the energy impact on the rock surface. Both the structural and velocity information indicate that the energy impact under realistic borehole conditions, which means in a fluid other than air, is less. Therefore, it is key to expand the knowledge of the generation and development of the HPWJ in different fluids to determine the optimal set up for rock destruction and a successful technology transfer of the HPWJ process to drilling applications. To achieve this objective, the proven experimental techniques for water can also be applied for measurements in slurry and drilling mud in future work.

\section{Acknowledgements}

This work is being funded through the "FH-Struktur2016" venue for universities of applied sciences by the ministry for innovation, science and research of the state of Nordrhein-Westfalen, Germany (AZ: 322-8.03.04.02).

\section{Nomenclature}

$A_{\text {eff }} \quad$ effective nozzle cross-sectional area [mm]

$\mathrm{A}_{\mathrm{N}} \quad$ nozzle cross-sectional area [mm]

$\mathrm{d}_{\mathrm{N}} \quad$ nozzle diameter $[\mathrm{mm}]$

$\dot{m} \quad$ mass flow $\left[\mathrm{kg} \mathrm{s}^{-1}\right]$

$\mathrm{p}_{\mathrm{N}} \quad$ nozzle pressure [MPa]

$Q \quad$ flow rate $\left[\mathrm{dm}^{3} \mathrm{~min}^{-1}\right]$

$\mathrm{Re}_{\mathrm{N}} \quad$ Reynolds number at nozzle exit [-]

$\mathrm{u}_{\mathrm{J}} \quad$ jet velocity $\left[\mathrm{m} \mathrm{s}^{-1}\right.$ ]

$\mathrm{u}_{\mathrm{J}^{*}} \quad$ correlated jet velocity $\left[\mathrm{m} \mathrm{s}^{-1}\right]$

$\rho_{\mathrm{L}} \quad$ liquid density $\left[\mathrm{kg} \mathrm{m}^{-3}\right]$

\section{References}

[1] Tester, J.W., et al., 2006, "The Future of Geothermal Energy - Impact of Enhanced Geothermal Systems (EGS) on the United States in the 21st Century".

[2] Finsterle, S., Zhang, Y., Pan, L., Dobson, P, Oglesby, K., 2013, Geothermics, 47, pp. 104-115.

[3] Farmer, I. and Attewell, P., 1965, International Journal of Rock Mechanics and Mining Sciences \& Geomechanics Abstracts, 2 (2), pp. 135 - 153.

[4] Brook, N., Summers, D. A., 1969, International Journal of Rock Mechanics and Mining Sciences \& Geomechanics Abstracts, 6 (3), pp. 249-258.

[5] Hood, M., Nordlund, R., Thimons, E., 1990, International Journal of Rock Mechanics and Mining Sciences \& Geomechanics Abstracts, 27 (2), pp. 77-86.

[6] Zelenak, M., Foldyna, J., Scucka, J., Hloch, S., Riha, Z., 2015, Measurement, 72, pp. 1-8.

[7] Donald, M.B., Singer, H., 1959, Transactions of the Institution of Chemical Engineers, 37, pp. 255-267.

[8] Soyama, H., Yanauchi, Y., Sato, K., Ikohagi, T., Oba, R., Oshima, R., 1996, Experimental Thermal and Fluid Science, 12 (4), pp. 411-416.

[9] Or, C. M., Lam, K. M., Liu, P., 2011, Journal of Hydro-environment Research, 5 (2), pp. 81-91.

[10] Lacagnina, G., Romano, G.P., 2015, Experiments in Fluids, 56 (1), pp. 480-490.

[11] Fukushima C., Aanen L., Westerweel J., Laser Techniques for Fluid Mechanics, pp. 339-356.

[12] Webster, D.R., Roberts, P.J.W., Ra'ad, L., 2001, Experiments in Fluids, 30 (1), pp. 65-72.

[13] Lindken, R., Merzkirch, W., 2002, Experiments in Fluids, 33 (6), pp. 814-825.

[14] Nogueira, S., Soursa, R.G., Pinto, A.M.F.R., Riethmuller, M.L., Campos, J.B.L.M., 2003, Experiments in Fluids, 35 (6), pp. 598-609.

[15] Westerweel, J., Petracci, A., Delfos, R., Hunt, J. C., 2011, Philosophical transactions. Series A, Mathematical, physical, and engineering sciences, 369 (1937), pp. 723-737.

[16] Payri, F., Bermúdez, V., Payri, R., Salvador, F.J., 2004, Fuel, 83 (4-5), pp. 419-431.

[17] Abramomovich, G.N., 1963, "The theory of turbulent jets".

[18] Field, J.E., Camus, J.J., Tinguely, M., Obreschkow, D., Farhat, M., 2012, Wear, 290-291, pp. 154-160.

[19] Hussein, J.H., Capp, S.P., George, W., 1994, Journal of Fluid Mechanics, 258 (1), pp. 31-75. 


\title{
Experimental investigation of Ethane and Propane injection under sub- and super-critical conditions
}

\author{
N. Vallée, G. Ribert*, J.-B. Blaisot, D. Lisiecki \\ Normandie Univ., INSA Rouen Normandie, UNIROUEN, CNRS, CORIA \\ 76000 Rouen, France \\ *Corresponding author: ribert@coria.fr
}

\begin{abstract}
Studying a fluid flow under high-pressure conditions through reliable experiments is still nowadays a challenge. When the chamber pressure exceeds the critical pressure of working fluids the supercritical state of matter is reached and the distinction between gas and liquid becomes blurred. For such special conditions, experimental data are scarce and need to be consolidated. Indeed, the modification of the local refractive index induced by density gradient needs to be analyzed with appropriate image-based technique.

In the present study, the REFINE test bench (Real-gas Effect on Fluid Injection: a Numerical and Experimental study) has been designed at CORIA Lab to study the non-reactive injection of Ethane and Propane into Nitrogen under sub- and supercritical conditions. The ambient gas pressure can be raised up to $6 \mathrm{MPa}$ and warmed up to $573 \mathrm{~K}$ to scan sub- and trans-critical injections. The chamber is equipped with two perpendicular optical axes allowing simultaneously different optical diagnostics. Experimental data are collected from shadowgraph and diffused backlight illumination techniques. Quantitative measurements of jet spreading angle and breakup length are compared to results coming from literature exhibiting multiple flows topologies.
\end{abstract}

\section{Keywords}

Supercritical, Experimental, Injection.

\section{Introduction}

In propulsion applications like in rocket engines, gas turbines or diesel engines when late injections are performed, injection often occurs at pressures that are above the critical point of the incoming fluids, i.e. at supercritical pressures [1, 2]. At such high-pressure conditions, injection is quite different from classical known behaviors observed at low pressure, and studying these new phenomena is challenging. Very few experiments dealing with non-reacting supercritical injection exists in the world, but a substantial experimental data set has been developed over the last few decades at DLR and AFRL laboratories [3]. The main issue is the ambient high pressure that locally affects the refractive index gradient making the delivery of experimental data highly challenging. Therefore this lack of reliable data is damaging for the development of models for turbulence and combustion $[4,5,6]$.

The objectives of the research program REFINE (Real-gas Effects on Fluid Injection: a Numerical and Experimental study) are to bridge the gap between experimental and numerical observations in providing reliable experimental data and new strategies of modeling. The experimental aspect is presently addressed through the development of a new test-bench (next section) and for which a first qualitative analysis is presented. Indeed, many combustion devices involve thermodynamic phase transition from subcritical to supercritical state as in high-pressure combustion chambers of rocket or diesel engines. When the pressure exceeds the critical pressure $p_{c}$ of any substance, the distinction between gas and liquid vanishes and the injection and combustion are quite different from classical known behaviors observed at low pressure. $p_{c}$ (resp. $T_{c}$ ) is the highest pressure (resp. temperature) for which distinct liquid and gas phases can be observed [7]. Above these values spray atomization is changed [3, 8, 9]: drops are no longer observed on Shadowgraph or Schlieren images due to the reduced surface tension and heat of vaporization [10,11]. Nevertheless a dense and dark core is still visible at the injector exit on shadowgraph [12]. The analysis of the spreading angle shows that supercritical jet has a behavior closer to a gaseous jet than to a liquid one [13, 14]. Only a few studies are dealing with experimental investigations of high-pressure injection and mixing process. Moreover, existing test benches often include additional effects that add a degree of complexity to a process already complex in nature. Indeed, particular conditions such as reactive flows, assisted-jet, cryogenic injection or acoustic interactions are often considered.

This paper introduces a new test bench and brings the first quantitative results that are compared to the literature. Injection of Propane was first studied but more investigations were explored for Ethane jets with in particular, the influence of the injection velocity, the temperature of the surrounding fluid, and the pressure. A first set of data on breakup length and spreading angle is also provided.

\section{Experimental setup}

The experiment has been designed to study injection and atomization of a non-assisted jet under high pressures conditions. REFINE is equipped with a steel chamber able to withstand pressures up to $10 \mathrm{MPa}$ and moderate temperatures $(573 \mathrm{~K})$. Two optical axes through circular silica windows of $\varnothing 120 \mathrm{~mm}$ are available for simultaneous measurements (see Fig. 1). The chosen working fluids are Ethane $\left(\mathrm{C}_{2} \mathrm{H}_{6}, T_{c}=305.3 \mathrm{~K}\right.$ and $\left.p_{c}=4.87 \mathrm{MPa}\right)$ and Propane $\left(\mathrm{C}_{3} \mathrm{H}_{8}, T_{c}=369.8 \mathrm{~K}\right.$ and $\left.p_{c}=4.25 \mathrm{MPa}\right)$. Their moderate critical pressure and temperature are within 
the range of operating conditions achievable in the chamber. A one-liter pump supplies the working fluid at the liquid-gas equilibrium pressure. Before injection, the pump is filled up, and then the fluid contained inside the vessel of the pump is compressed to the target pressure plus $0.05 \mathrm{MPa}$ to prevent the ambient fluid in the main chamber from entering the pump and creating an effervescent jet.

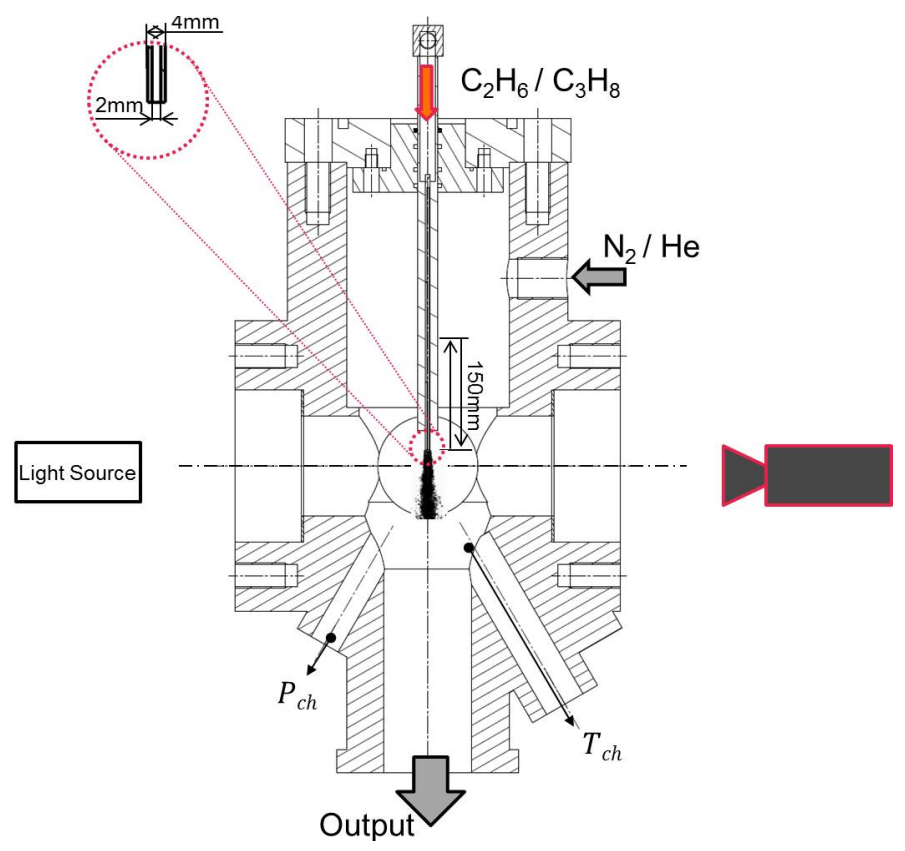

Figure 1. Section of the REFINE injection chamber.

The injection is performed with a sharp-edged stainless steel tube of $210 \mathrm{~mm}$ long, with a $4 \mathrm{~mm}$ outer diameter and a $2 \mathrm{~mm}$ inner diameter. The injector can be moved vertically over a $150 \mathrm{~mm}$ stroke by a motorized system located on the top of the chamber, to expand the field of view. The rig is fully instrumented with thermocouples, pressure gauges and mass flow meters as indicated in Fig. 2. The chamber is filled with Nitrogen and the pressure is controlled with a pressure regulator having a precision of $\pm 0.1 \mathrm{MPa}$. To warm up the ambient fluid, two MICA heating plates are placed at the bottom of the chamber and a heater (Fig. 2) is inserted just before the feeding of $\mathrm{N}_{2}$.

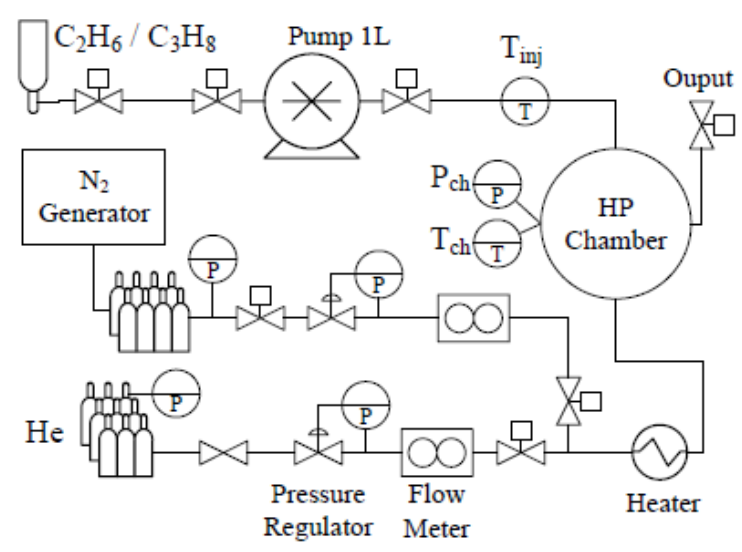

Figure 2. Flowchart of REFINE test bench.

Two optical diagnostics, diffused back-light illumination (DBI) and shadowgraphy, are used to quantify the injection and atomization of the working fluids. The main difference between these two techniques lies in the light source arrangement. For shadowgraphy, a collimated laser diode (Cavitar) is used whereas a stroboscope flashlamp (MVS2601) with a glass diffuser is used for DBI. Depending on the situation and on the desired information, different camera configurations were used, as indicated in Tab. 1. The DBI allows detecting the largest density gradients, which are essentially located at the liquid-gas interface contrary to shadowgraphy that detects smoother density gradient.

All the cases studied hereafter are summarized in Tab. 2, which provides the values of pressure $(P)$, temperature in the chamber $\left(T_{c h}\right)$ or upstream the injector $\left(T_{i n j}\right)$ and velocity $(V)$ calculated as the average of measurements made during the injection process. The \pm number indicates the variability of the measured quantity during this injection. The dimensionless numbers (Reynolds, Weber and Ohnesorge) are also provided. 
Table 1. Table of the different configurations of camera and light source with the associate designation code used in this manuscript.

\begin{tabular}{c|c|c|c|c}
\hline Code & Camera & Resolution $(\mathrm{px} / \mathrm{mm})$ & Field $(\mathrm{mm})$ & Light \\
\hline BG1 & BlueCougar-x24 & 27.0 & $59.3 \times 44.5$ & $\mathrm{DBI}$ \\
BG2-1 & BlueCougar-x25a & 53.5 & $45.8 \times 38.1$ & $\mathrm{DBI}$ \\
BG2-2 & BlueCougar-x25a & 130.5 & $18.8 \times 15.7$ & Shadowgraphy \\
PCO-1 & PCO Edge 5.5 & 58.0 & $44.1 \times 37.2$ & $\mathrm{DBI}$ \\
PCO-2 & PCO Edge 5.5 & 121.5 & $294.3 \times 17.8$ & $\mathrm{DBI}$
\end{tabular}

Table 2. Tests case conditions with dimensionless numbers: Reynolds $(R e)$, liquid and gaseous $\mathrm{Weber}_{(\mathrm{We}}$, We $\left.\mathrm{We}_{g}\right)$ and Ohnesorge $(O h)$.

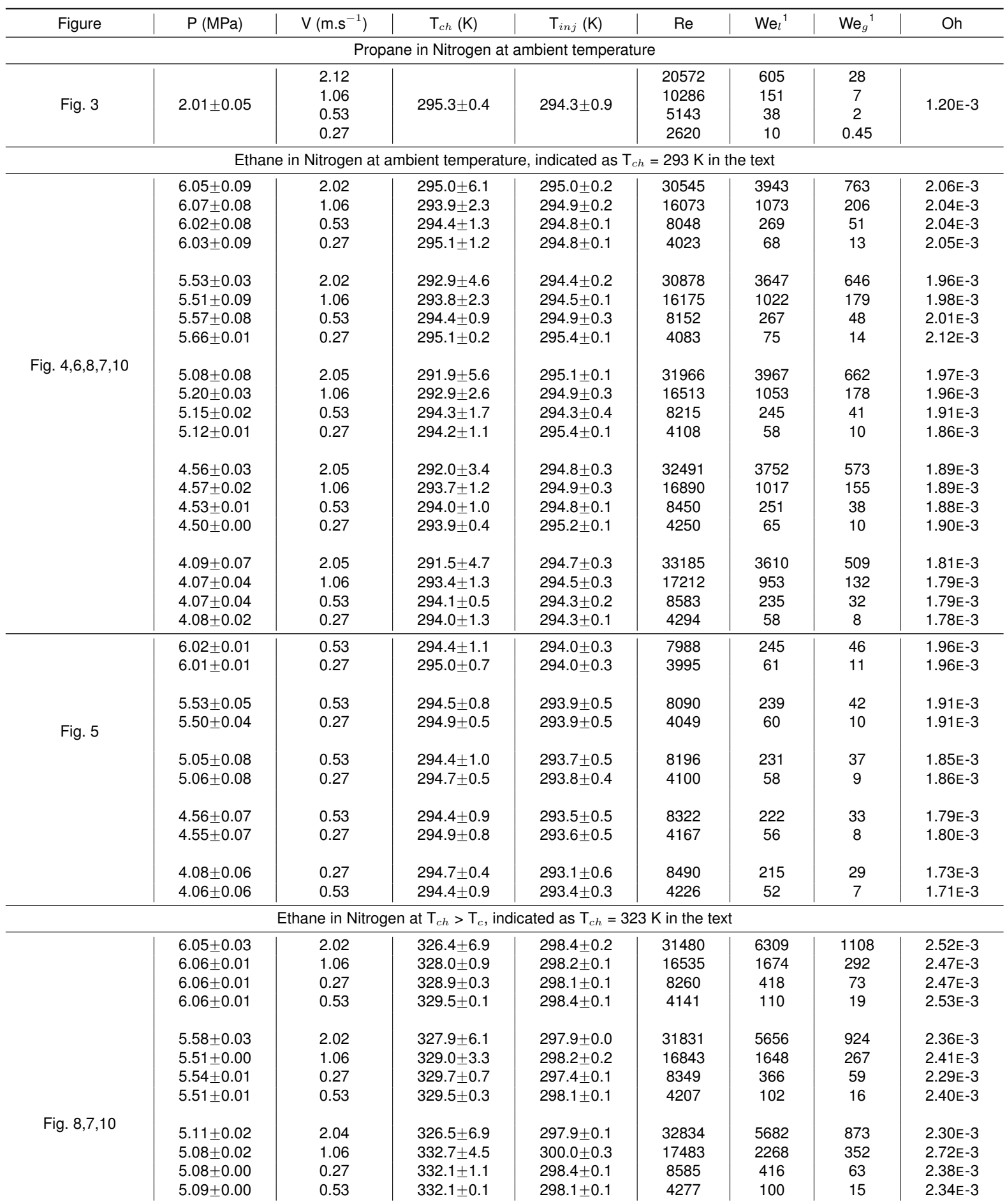

\footnotetext{
${ }^{1}$ Considering pure Ethane at $\mathrm{T}_{i n j}$ position and taken a surface tension from NIST (http://webbook.nist.gov/chemistry/) in liquid phase on the evaporation line at the $\mathrm{T}_{i n j}$
} 


\section{Results and discussion}

The behavior of a cylindrical jet discharging into a quiescent atmosphere is commonly characterized [15] by the jet stability curve as shown in Fig. 3. This curve shows the variation of the jet breakup length $\left(L_{b u}\right)$ as a function of the discharge velocity. The breakup length is defined as the distance between the injection orifice and the first discontinuity in the liquid jet. Five flow regimes are identified. They are respectively named the dripping regime $(a \rightarrow b)$, Rayleigh $(b \rightarrow c)$, first wind-induced $(c \rightarrow d)$, second wind-induced $(d \rightarrow e)$ and atomization regimes $(>e)$. The dripping regime is not of interest here.

Images of a Propane jet discharging into an atmosphere of nitrogen at $293 \mathrm{~K}$ and $2 \mathrm{MPa}$ are presented along the stability curve in Fig. 3. Refraction of light near the liquid-gas interface yields to steep grey level gradient making easier the localization of the interface. Brighter areas in the center are also due to the refraction of light which is less deviated when going through the jet axis. The more the surface is corrugated, the more deviated is the light making the jet darker. For $V=0.25 \mathrm{~m} . \mathrm{s}^{-1}$, the jet morphology is characteristic of the Rayleigh regime. Increasing the velocity up to $0.5 \mathrm{~m} . \mathrm{s}^{-1}$, the breakup length becomes greater than the field of view. The discharge velocity then approaches the critical velocity corresponding to point $c$. For $V=1 \mathrm{~m} \cdot \mathrm{s}^{-1}$ the breakup length becomes shorter, confirming that the critical velocity has been exceeded. The surface of the jet becomes corrugated and the first wind-induced regimes is reached. For $V=2 \mathrm{~m} . \mathrm{s}^{-1}$, disturbances on the jet surface are getting stronger and $L_{b u}$ is no longer observable within this field of view, i.e. the point $d$ is passed and the jet is in the second wind-induced regime.

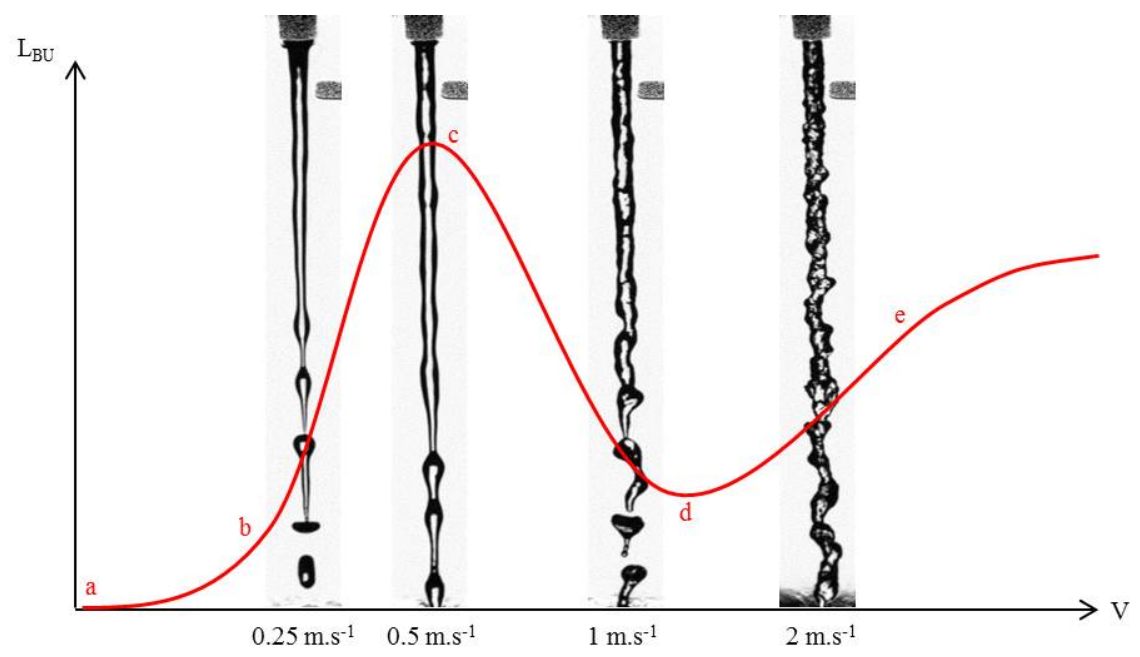

Figure 3. Injection of Propane at 2.0 MPa and ambient temperature along the stability curve [15](BG1).

Images of a jet of Ethane into Nitrogen at $293 \mathrm{~K}$ and 6.0 MPa are shown in Fig. 4. The breakup length of Ethane jet has been investigated for $V=0.25 \mathrm{~m} . \mathrm{s}^{-1}$ and $V=0.5 \mathrm{~m} . \mathrm{s}^{-1}$. For $V \geqslant 1 \mathrm{~m} . \mathrm{s}^{-1}$, the atomization starts only a few diameters downstream the injection orifice and the cloud of drops around the jet makes difficult the estimation of $L_{b u}$. The intact core length is thus commonly used in these conditions to characterize the jet atomization [16].

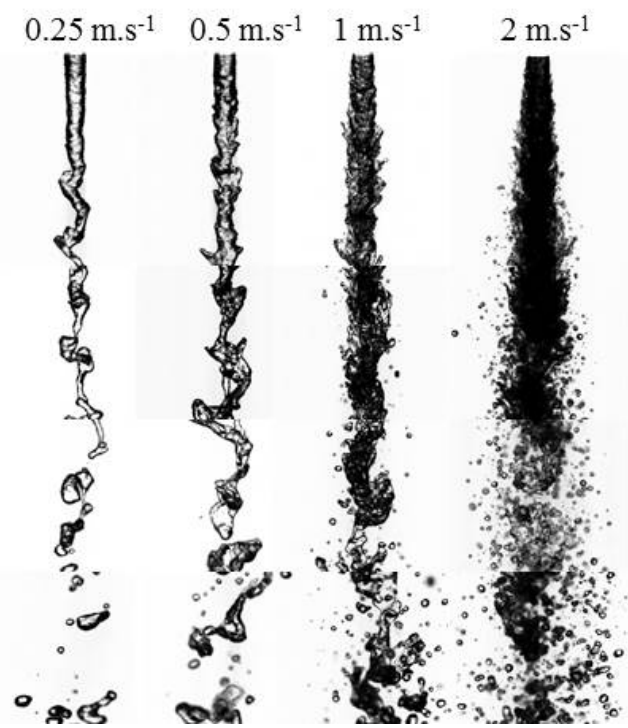

Figure 4. Injection of Ethane at 6.0 MPa and ambient temperature (PCO-2). 
Statistics on $L_{b u}$ of the Ethane jet was estimated for the two lowest discharge velocities. Since the jet deviates from its central axis, both perpendicular visual axes were used (BG2-1 \& PCO-1) to make sure that no drop or fluid deformations hide the first discontinuity of liquid. 600 two-level images were taken and analyzed for each case to compute the probability density functions (pdf) for $L_{b u}$ (see Fig. 5). For all the studied ambient pressures, the slowest velocity gives the shortest breakup length, meaning that $L_{b u}$ increases with the discharge velocity. Since the shape of the jet (see Fig. 4) has nothing in common with a Rayleigh jet, those jets can be classified in the second wind-induced regime. It can also be observed that the pdf of $L_{b u}$ are wider for a larger velocity and for $V=0.25 \mathrm{~m} \mathrm{s.}^{-1}$ the maximum neak is hetter defined. No conclusion could be drawn on the influence of nressure on thi
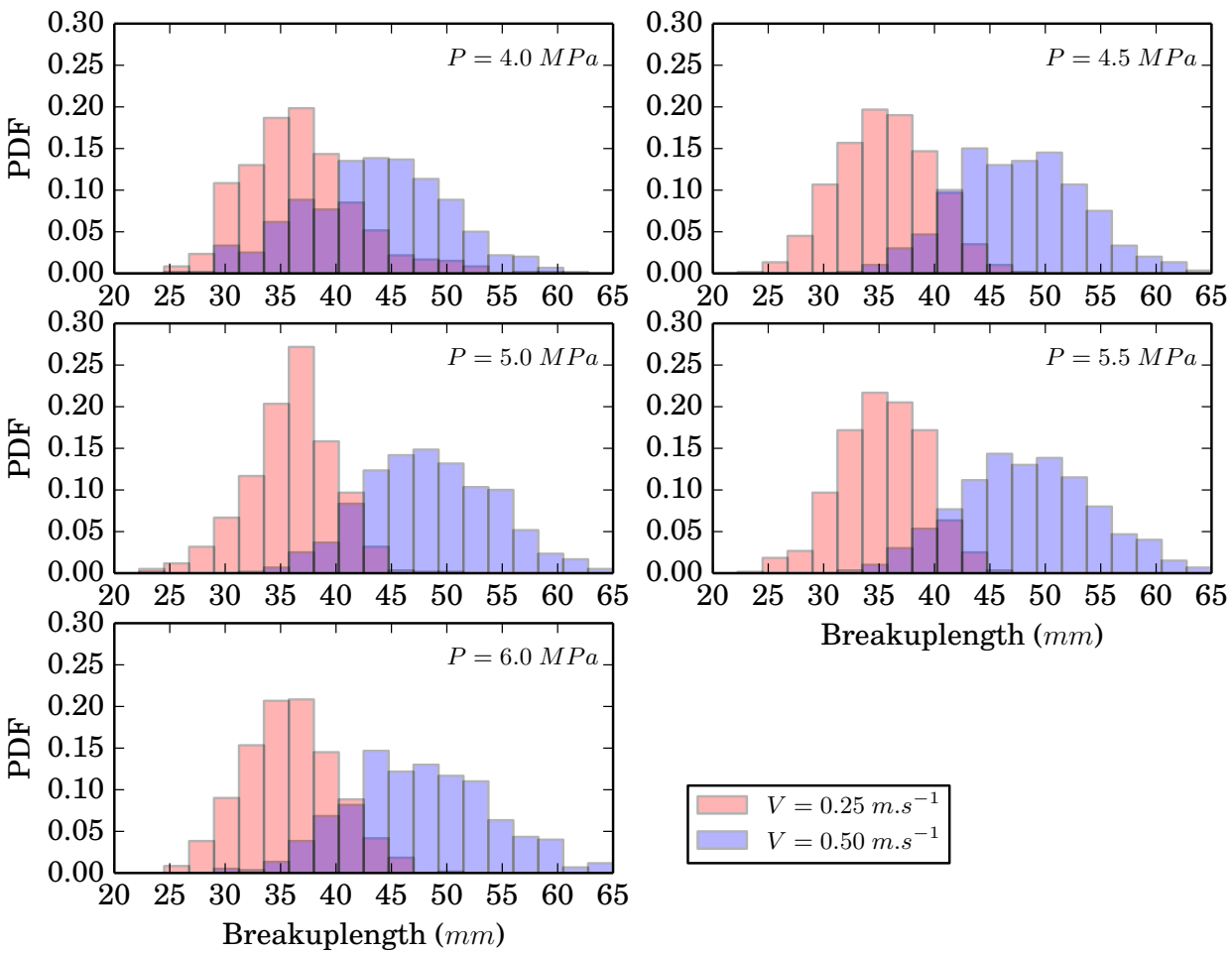

Figure 5. PDF of 600 breakup lengths of Ethane jet at $293 \mathrm{~K}$. Pressure from $4.0 \mathrm{MPa}$ to $6.0 \mathrm{MPa}$

The impact of the chamber pressure on jet disintegration is illustrated in Fig. 6 for Ethane injected into $\mathrm{N}_{2}$ at $293 \mathrm{~K}$ with a fixed discharge velocity of $1 \mathrm{~m} . \mathrm{s}^{-1}$. The pressure ranges from 4.0 MPa to 6.0 MPa, i.e. from subcritical to supercritical pressure if referring to Ethane critical pressure. Increasing the pressure leads to more perturbed jets with smaller drops, similarly to the observations done by Oschwald [17] for an injection of $\mathrm{N}_{2}$ at $5 \mathrm{~m} . \mathrm{s}^{-1}$ into $\mathrm{N}_{2}$ at rest.

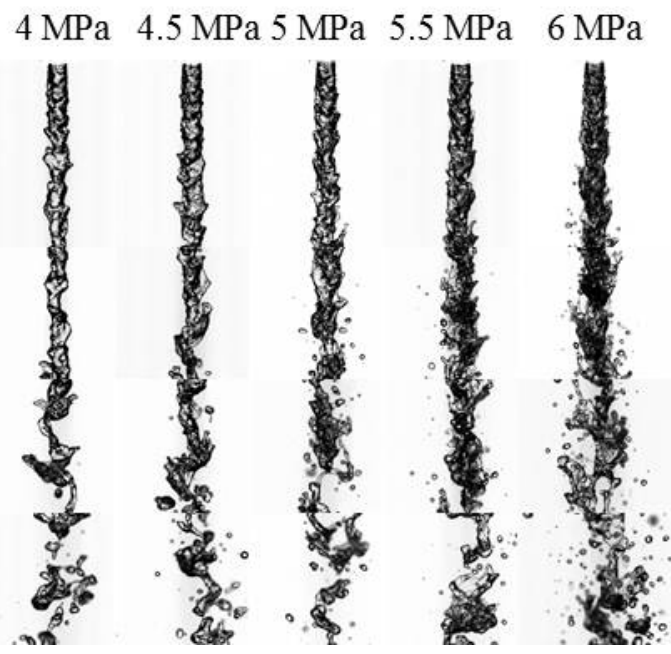

Figure 6. Injection of Ethane into Nitrogen at $V=1 \mathrm{~m} . \mathrm{s}^{-1}$ for different levels of ambient pressure with $T=293 \mathrm{~K} .4$ images are used to create a field of visualization of $66.1 \mathrm{~mm}$ length (PCO-2). 
Table 3. Measured temperatures $T_{c h}, T_{i n j}$ and $T_{j e t}$ in the case of an injection of Ethane into $\mathrm{N}_{2}$ for different velocities.

\begin{tabular}{c|c|c|c|c|c}
\hline$P(\mathrm{MPa})$ & $V\left({\left.\mathrm{~m} . \mathrm{s}^{-1}\right)}\right.$ & $T_{c h}(\mathrm{~K})$ & $T_{i n j}(\mathrm{~K})$ & $T_{j e t}(\mathrm{~K})$ at $6 \mathrm{~mm}$ & State $^{2}$ \\
\hline 6.0 & 0.25 & 327 & 298 & 308 & Supercritical \\
6.0 & 0.50 & 325 & 297 & 303 & Liquid \\
6.0 & 1.00 & 323 & 298 & 300 & Liquid \\
6.0 & 2.00 & 328 & 298 & 298 & Liquid
\end{tabular}

To explore the effect of the ambient temperature, various configurations of Ethane jets into Nitrogen for four discharge velocities and a temperature of $\mathrm{N}_{2}$ set to $T=293 \mathrm{~K}<T_{c}$ or $T=323 \mathrm{~K}>T_{c}$ are shown in Fig. 7. Pressure is fixed to $6.0 \mathrm{MPa}$, i.e. above the critical pressure of Ethane. The temperature of the jet $\left(T_{j e t}\right)$ measured in the fluid at the nozzle exit with an exposed junction thermocouple placed $6 \mathrm{~mm}$ downstream from the nozzle exit are summarized in Tab. 3. These temperature measurements were done on different experiments, i.e. not simultaneously to image acquisition. This temperature, $T_{j e t}$, decreases with the increase of the velocity. The discharge velocity must be considered as a key-point parameter since it induces modification in the ratio between characteristic convection and thermal times

For the lowest injection velocities $\left(V=0.25 \mathrm{~m} . \mathrm{s}^{-1}\right.$ and $\left.V=0.5 \mathrm{~m} . \mathrm{s}^{-1}\right)$, the temperature has a clear impact on jet disintegration since $T_{j e t}$ is close to the critical temperature of Ethane ( $T_{c}=305 \mathrm{~K}$ ). For example, a diffuse jet with dense pockets appears for $T_{c h}=323 \mathrm{~K}$ and $V \leqslant 0.5 \mathrm{~m} . \mathrm{s}^{-1}$ whereas a liquid-gas interface is clearly visible for $T_{c h}=293 \mathrm{~K}$.

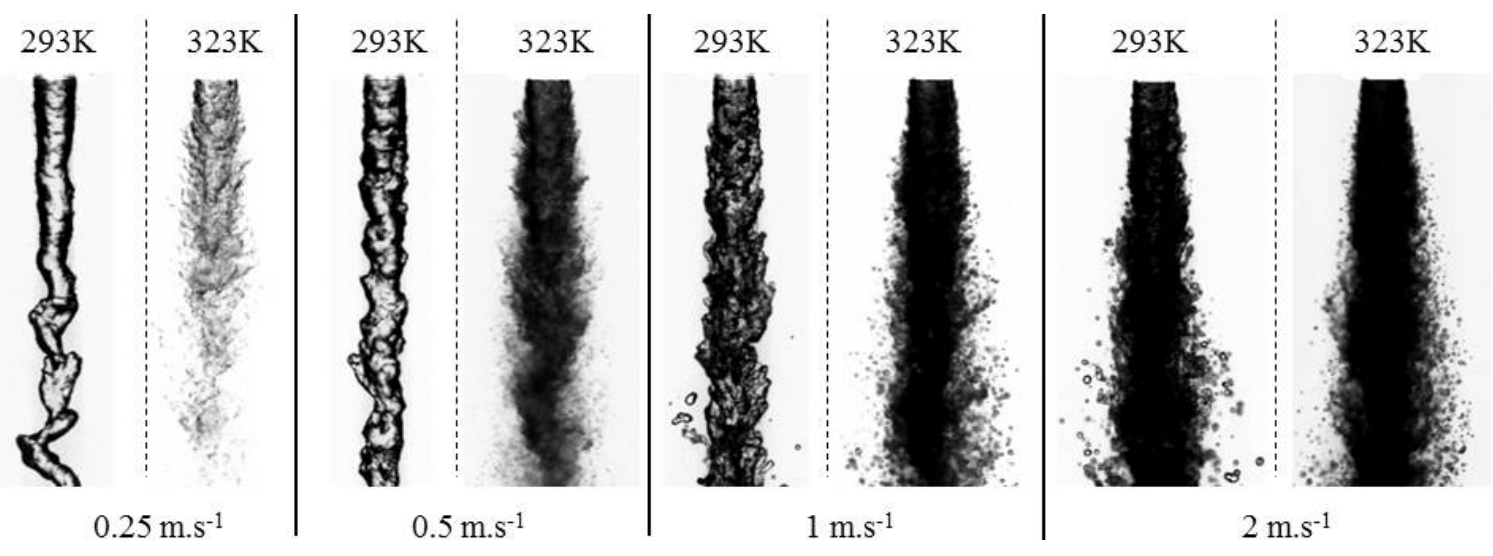

Figure 7. Jets of Ethane in Nitrogen at $6 \mathrm{MPa}(\mathrm{PCO} 2)$.

Increasing the velocity to $V=1 \mathrm{~m} . \mathrm{s}^{-1}$, small fluid elements identified as drops appear whatever $T_{c h}$. However, there are more droplets identified for $T=323 \mathrm{~K}$. The same observation is done for $V=2 \mathrm{~m} . \mathrm{s}^{-1}$. Hence, having a temperature of the ambient fluid above the critical temperature of the injected fluid strongly modifies the jet behavior. The interaction between the injected fluid and the ambient one is greater for low injection velocities where a diffusion process is observed, but modifies also the atomization process at higher injection velocity as stated in $[3,18]$.

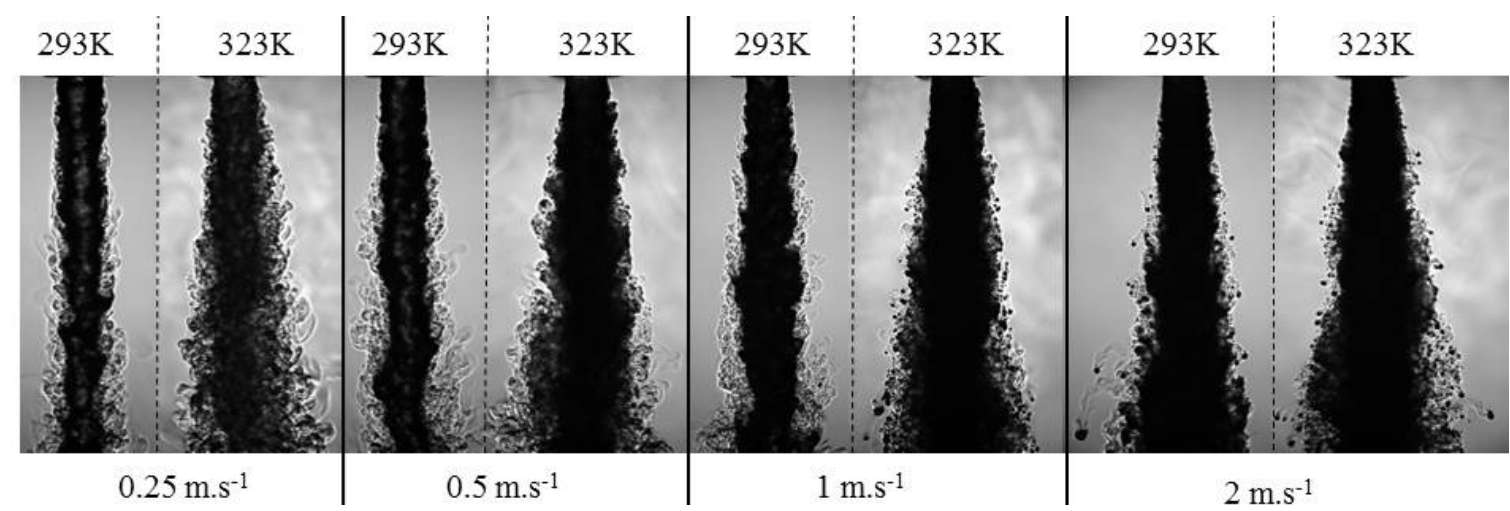

Figure 8. Jets of Ethane in Nitrogen at $6 \mathrm{MPa}$ (BG2-2).

With DBI visualizations some finger-like structures are observed at the jet interface. In order to analyze those structures, shawdowgraph visualizations were achieved to explore the density gradient around the liquid jet (or around the dense core). Accordingly, shadowgraph images (Fig. 8) have been taken simultaneously to DBI images ( Fig. 7) on the perpendicular axis. A grey level gradient clearly appears around the liquid. It consists of a $\mathrm{C}_{2} \mathrm{H}_{6}-\mathrm{N}_{2}$

\footnotetext{
${ }^{2}$ Considering pure Ethane at the $T_{\text {jet }}$ position.
} 
mixing layer varying radially from pure Ethane in the center of the jet to pure Nitrogen far from the jet.

Two topologic parameters are then defined from shadowgraph images: the dark-core angle and the spreading angle also named growth rate [19]. From the normalized images of raw shadowgraphs, a two-level image is created. The black and white delimitation gives an interface between dense and light fluid. The calculation of the standard deviation of 120 two-level shadowgraph images yields the probability of presence of this interface on a grey-level scale. Only the values above $90 \%$ of the maximum of this probability are retained to define the area delimited by the dark-core angle $\theta_{d}$ (inside boundary in red on Fig. 9) and the spreading angle $\theta_{s}$ (outside boundary in green). The spreading angle for different pressures, velocities and chamber temperatures of an Ethane jet are plotted in Fig. 10.
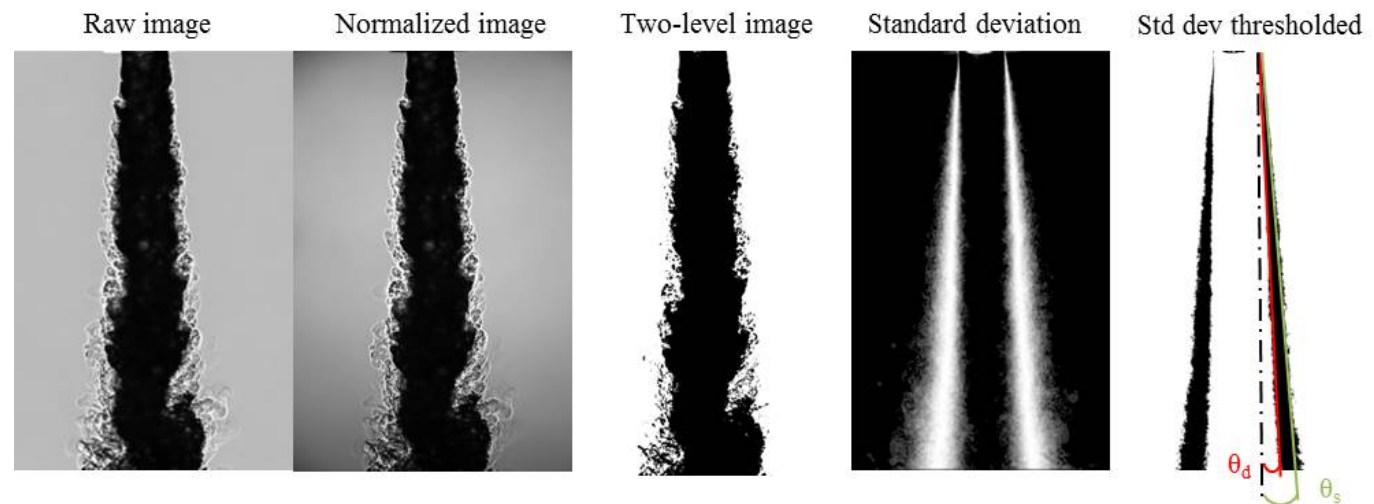

Figure 9. Image processing from raw image to the threshold of the standard deviation. On the last image: the dark-core angle $\theta_{d}$

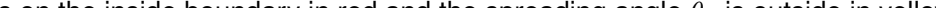

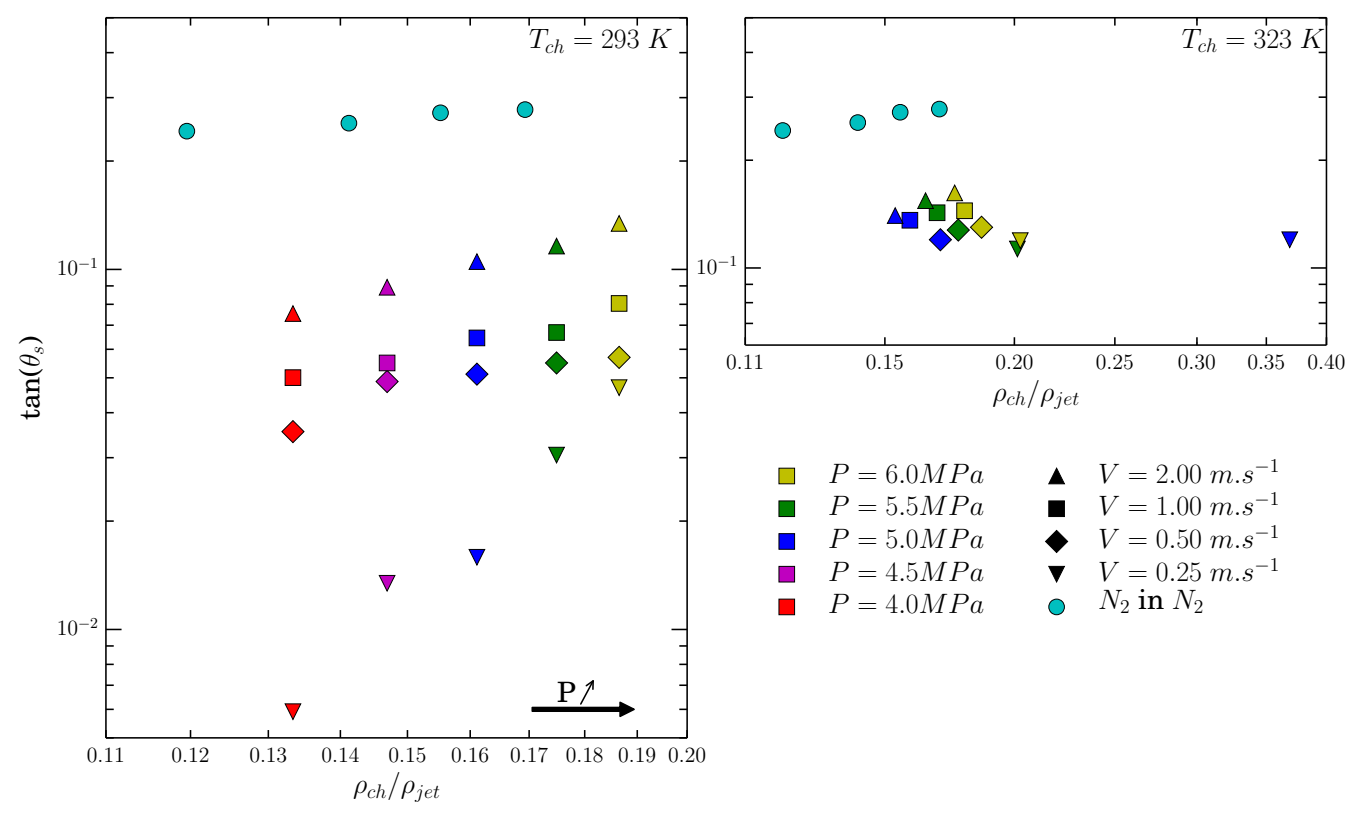

Figure 10. Spreading angle versus the chamber to injectant density ratio. For comparison, spreading angle of liquid $\mathrm{N}_{2}$ injection in $\mathrm{N}_{2}$ at ambient temperature from Fig. 8 of Chehroudi's paper [12].

To be compared to the results summarized by Chehroudi in [12], the spreading angle is plotted against the ambient over injectant density ratio. The density of pure fluids is based on the measured temperature (Tab. 3) and estimated from NIST tables. For example, if $T_{c h}=323 \mathrm{~K}$, then the temperature of Ethane considered for NIST tables is $T_{j e t}$. For each velocity, the growth rate increases with the pressure (also with the density ratio), which is in agreement with the other studies. At a given ambient pressure, the spreading angle increases with the velocity. Ethane spreading angles are smaller to the AFRL data for liquid $N_{2}$ in $N_{2}$ [12] having similar density ratios. This difference can be due to the velocity range in our experiments, which is one of the lowest in the literature but no indication of velocity is given on the original plot of Chehroudi (Fig. 8 in [12]). Increasing the temperature makes a significant change of flow topology at low velocities. In Fig. 10 (right), the injection of Ethane at $V=0.25 \mathrm{~m} . \mathrm{s}^{-1}$ under a pressure of $5.0 \mathrm{MPa}$ exhibits a density ratio of $\rho_{c h} / \rho_{\text {jet }}=0.35$ contrary to configurations at higher pressure $\left(\rho_{c h} / \rho_{\text {jet }} \approx 0.20\right)$. Indeed decreasing the pressure makes the working conditions closer to the critical point. The limit of plotting the spreading angle against the density ratio is that when the chamber is heated, the jet temperature varies along the jet. There is even a temperature gradient in Nitrogen evidenced by the non-uniform grey level in shadowgraph images far from the jet at $323 \mathrm{~K}$ in Fig. 8. 


\section{Conclusions}

A new fully instrumented experimental test-bench has been developed at CORIA lab to study the injection of inert fluids under supercritical conditions. In this study, Ethane and Propane are injected into a cold or warm environment of Nitrogen. The knowledge of the behavior of those fluids has been detailed. Results for the breakup length and the spreading angle are in agreement with the literature. It has been shown that the temperature of the ambient fluid played a major role in jet disintegration when pressure exceeds the critical pressure of the injected fluid. In case of an ambient temperature higher than the critical temperature of injected fluids a diffuse mixing process occurs for low discharge velocities. For a higher injection velocities, a process of atomization appears but with a larger spatial distribution of drops. These results were obtained by shadowgraphy and diffused backlight illumination that gave complementary information. Other optical diagnostics as schlieren and CBOS will be soon implemented to complement these first results. A X-ray analysis is currently under progress to deliver a density gradient measurement and to give additional characterization of the jet behaviors.

\section{Acknowledgements}

This work was funded by the ANR (Agence Nationale de la Recherche) research project REFINE (Real-gas Effects on Fluid Injection: a Numerical and Experimental study), Grant No. ANR-13-BS09-0007.

\section{References}

[1] Dahms, R. N., and Oefelein, J. C., 2013. "On the transition between two-phase and single-phase interface dynamics in multicomponent fluids at supercritical pressures". Physics of Fluids (1994-present), 25(9), p. 092103.

[2] Dahms, R. N., and Oefelein, J. C., 2015. "Liquid jet breakup regimes at supercritical pressures". Combustion and Flame, 162(10), pp. 3648-3657.

[3] Oschwald, M., Smith, J. J., Branam, R., Hussong, J., and Schik, A., 2006. "Injection of fluids into supercritical environments". Combust. Sci. and Tech., 178, pp. 49-100.

[4] Petit, X., Ribert, G., Lartigue, G., and Domingo, P., 2013. "Large-eddy simulation of supercritical fluid injection". The Journal of Supercritical Fluids, 84, pp. 61-73.

[5] Petit, X., Ribert, G., and Domingo, P., 2015. "Framework for real-gas compressible reacting flows with tabulated thermochemistry". The Journal of Supercritical Fluids, 101, pp. 1-16.

[6] Ribert, G., Petit, X., and Domingo, P., 2016. "High-pressure methane-oxygen flames. analysis of sub-grid scale contributions in filtered equations of state". The Journal of Supercritical Fluids, 121, pp. 78-88.

[7] Turns, S. R., 2006. Thermodynamics: concepts and applications. Cambridge University Press.

[8] Manin, J., Bardi, M., Pickett, L., Dahms, R., and Oefelein, J., 2014. "Microscopic investigation of the atomization and mixing processes of diesel sprays injected into high pressure and temperature environments". Fuel, 134, pp. 531-543.

[9] Segal, C., and Polikhov, S., 2008. "Subcritical to supercritical mixing". Phys. Fluids, 20, p. 052101.

[10] Mayer, W., Schik, A. H., Vielle, B., Chauveau, C., Gškalp, I., Talley, D. G., and Woodward, R. D., 1998. "Atomization and breakup of cryogenic propellants under high-pressure subcritical and supercritical conditions". Journal of Propulsion and Power, 14(5), pp. 835-842.

[11] Chehroudi, B., Talley, D., and Cohn, R., 2002. "Visual characteristics and initial growth rates of round cryogenic jets at subcritical and supercritical pressures". Physics of Fluids, 14-2, pp. 850-861.

[12] Chehroudi, B., Talley, D., Mayer, W., Branam, R., and Smith, J., 2003. Understanding injection into high pressure supercritical environments. Tech. rep., DTIC Document.

[13] Mayer, W., Telaar, J., Branam, R., Schneider, G., and Hussong, J., 2003. "Raman measurements of cryogenic injection at supercritical pressure". Heat and Mass Transfer, 39, pp. 709-719.

[14] Manin, J., Bardi, M., Pickett, L., and Payri, R., 2016. "Boundary condition and fuel composition effects on injection processes of high-pressure sprays at the microscopic level". International Journal of Multiphase Flow, 83, pp. 267-278.

[15] Dumouchel, C., 2008. "On the experimental investigation on primary atomization of liquid streams". Exp. Fluids, 45, pp. 371-422.

[16] Charalampous, G., Hadjiyiannis, C., and Hardalupas, Y., 2016. "Comparative measurement of the breakup length of liquid jets in airblast atomisers using optical connectivity, electrical connectivity and shadowgraphy". Measurement, 89, pp. 288-299.

[17] Oschwald, M., Schneider, G., and Clauss, W., 2005. "Application of visualization techniques and quantitative optical diagnostics for the investigation of supercritical jet atomization". In The 8th International Symposium on Fluid Control, Measurement and Visualization.

[18] Chehroudi, B., 2012. "Recent experimental efforts on high-pressure supercritical injection for liquid rockets and their implications". International Journal of Aerospace Engineering, 2012.

[19] Chehroudi, B., Cohn, R., and Talley, D., 2002. "Cryogenic shear layers: experiments and phenomenological modeling of the initial growth rate under subcritical and supercritical conditions". Int. J. of Heat Fluid Flow, 23, pp. 554-563. 


\title{
An Improved Image Processing Method for Particle Characterization by Shadowgraphy
}

\author{
Hua Wang ${ }^{* 1}$, Francisco Felis ${ }^{2,3}$, Severine Tomas ${ }^{2}$, Fabien Anselmet ${ }^{3}$ and Muriel Amielh ${ }^{3}$ \\ ${ }^{1}$ Dantec Dynamics A/S, Skovlund, Denmark \\ ${ }^{2}$ Institut national de recherche en sciences et technologies pour l'environnement et \\ l'agriculture (IRSTEA), UMR G-EAU France \\ ${ }^{3}$ Institut de Recherche sur les Phenomenes Hors Equilibre (IRPHE), France \\ ${ }^{*}$ Corresponding author: hwg@dantecdynamics.com
}

\begin{abstract}
Shadowgraphy is one of the most popular imaging techniques to characterize moving particles by their size, geometry as well as velocity, due to its simplicity. However, it requires advanced image processing to handle various image defects such as non-uniform illumination, overlapped particles, etc., which are normally only solved for individual applications. This study proposes a robust image processing method for particle shadowgraphy, aiming to process imperfect particle shadow images. The proposed method first detects qualified particles from particle shadow images, and then processes detected particles individually. Therefore different defects from different particles can be handled separately and locally. An overlapped particles detection and separation algorithm is also implemented to improve the accuracy of size and geometry characterization.

The proposed method is first proved by synthetic generated particle shadow images, followed by a proof test with shadow images from a transparent dot pattern target. Finally this method is successfully applied to a shadow image acquired from a water spray and proved to be able to handle various issues of shadowgraphy.
\end{abstract}

\section{Keywords}

Shadowgraphy, image processing, particle characterization

\section{Introduction}

Over past few years, there has been a growing interest and also increasing availability of commercial image analysis software for atomization characterization by means of shadowgraphy for spherical as well as irregular shape particles. Compared with point measurement techniques such as phase Doppler anemometry, it is easy to setup and the results are more straightforward to understand. Additionally, it can be applied for particles with any shape [1, 2] and it is not affected by multi-scattering effects [3, 4].

However, most of imaging process methods for shadowgraphy are made for individual applications and often demand high image quality [5], which cannot be achieved in many cases due to the complexity of the spray or the limitation from optical access. In general, these image processing methods are facing various challenges like too many droplets with various shapes in the field of view - which results in overlapped particles [6] or out-of-focus particles, non-uniform illumination, etc.

In this study, an advanced shadowgraphy image processing method is proposed to handle shadow images from complex sprays. The principle of this method will be firstly introduced, followed by verification from synthetic images, dot target images with known dot sizes, and then a validation with shadow images from a real spray.

\section{Principle of shadow image processing}

This method firstly detects particles based on a global threshold determined by the users - which depends on individual measurement. Optionally, a 2D Mexican hat filter can be applied to convolute the raw image and consequently a map of intensity gradient can be obtained, which indicates the edge of each particle. This procedure is proved to be more effective to detect particles when the back illumination is not uniform and the particles are out of focus [7]. Then each particle is segmented from the global image (gradient image in the case of optional filtering) with a bounding box which is slightly larger than itself for further process. This process is shown in Figure 1. Then, the particle segmentation is performed from this gradient image. 


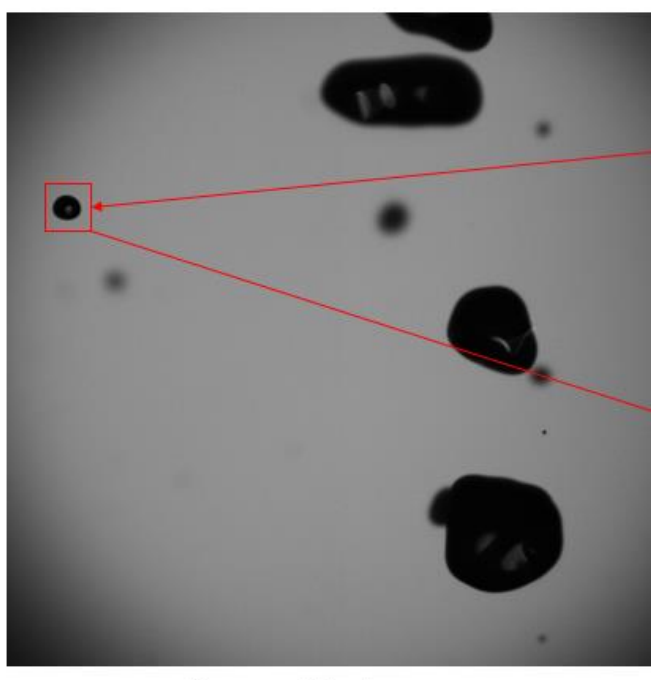

Raw particle image

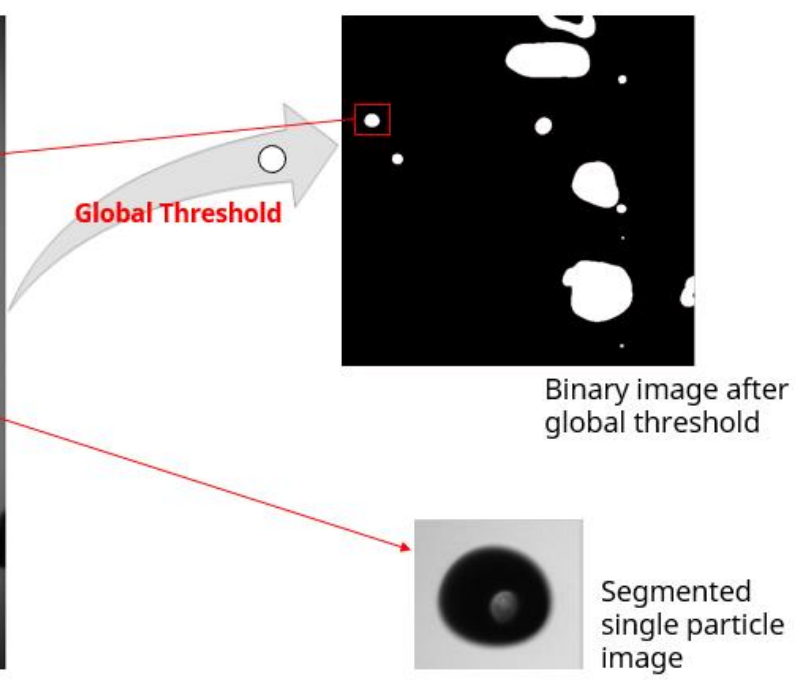

(a)
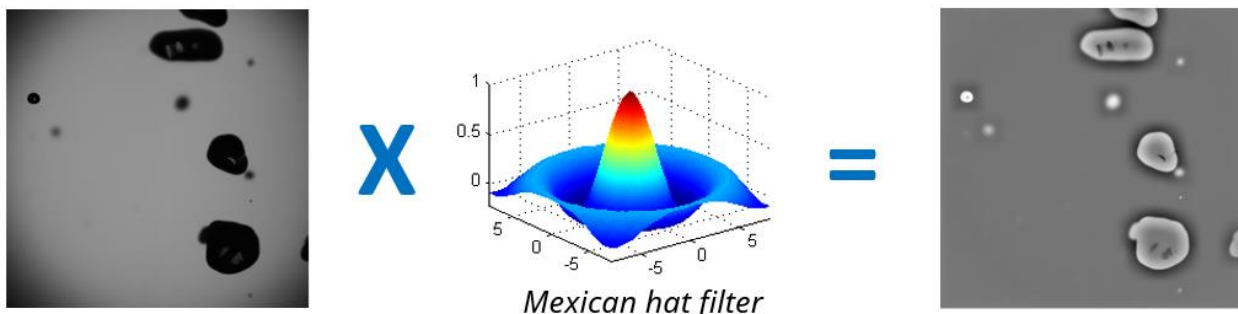

(b)

Figure 1. Particle detection and segmentation: (a) general routine, (b) Optional Mexican hat filter to be used instead of global threshold

After the particle is segmented from raw image, a local threshold will be applied to the bounding box to extract the particle. The local threshold is calculated by:

Threshold $_{\text {local }}=i_{\text {min }}+\left(i_{\text {max }}-i_{\text {min }}\right) * 0.61$

Where $i_{\min }$ and $i_{\max }$ are the minimal and maximum gray values in the segmented single particle image. The factor of 0.61 is proposed by [8]. This value is the one which allows obtaining, based on point spread function, a polynomial relationship between contrast of particle image and measured particle diameter. Consequently, there is a potential to correct the measured size of those out-of-focus particles or to define a depth-of-field thickness where all particles are focused. None of these solutions is presently implemented in our algorithm.

Segmented single particle image uses this threshold to be binarized and particle will be extracted for further geometry characterization. By using a local threshold for each detected particle, the issues coming from nonuniform back illumination can be minimized. In addition, using a fixed factor to determine local threshold for particle diameter evaluation can also avoid subjectivity introduced from different users. More specifically, global threshold selected by the user would only affect the identification of particles - number of particles identified; once the particle is identified, its size only depends on the local threshold calculated from formula (1).

After the particle extraction, its diameter and other geometry information will be calculated by standard geometry characterization functions from OpenCV.

After geometry characterization, three validation methods can be applied if necessary:

a) Particle area (range).

b) Particle eccentricity, which is defined as the ratio between the long axis and short axis.

c) Particle roundness, also called as inertia moment, which measures the spread of points around the centre of mass. Its definition is given by the formula below:

inertia $=n u_{20}+n u_{02}$ 
$n u_{j i}=\frac{m u_{j i}}{m_{00}^{\frac{i+j}{2}+1}}$

$m u_{j i}=\sum_{x, y}(x-\bar{x})^{j} \cdot(y-\bar{y})^{i}$

Where $\mathrm{x}$ and $\mathrm{y}$ are coordinates of pixels within the particle, $\bar{x}$ and $\bar{y}$ are the centroid coordinates of the particle.

All the three validations above are aiming to reject noise caused by dirt in the imaging system, which are often very small in the field of view and have different shapes from expected particles.

In addition, a method to detect and separate overlapped particles is also implemented here. This method assumes that overlapped particles (seen in Figure 2) can be separated into two (or more) smaller particles by shrinking from its boundary, which represents the kernels of those overlapped particles. If two (or more) smaller particles can be identified after shrinking, these particles will be identified as overlapped particles, and a reexpand process will be applied to those kernels to calculate the size of each separated particle. The amount of reexpand is identical as the amount of shrink. The process is described in Figure 2.

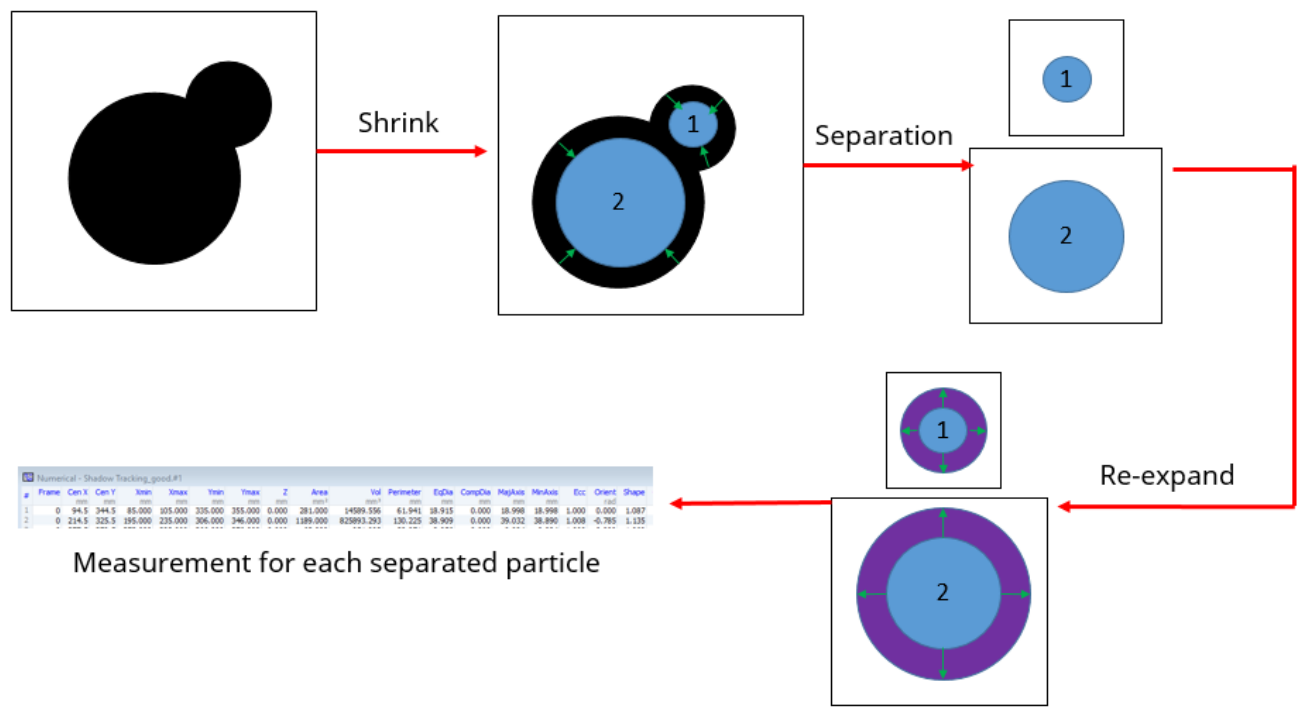

Figure 2. Method to detect and correct overlapped particles

This overlapped particles detection and correction is based on the assumption mentioned above. In some applications, particles shape can be highly irregular and therefore they are identified as overlapped particles. In this case, the proposed method is no longer suitable.

\section{Results and discussion}

The proposed shadow image processing method has firstly been evaluated by synthetic particle image, which is shown in Figure 3, together with the contour provided by shadow image processing. The synthetic particle image is a binary image made from Microsoft Paint program, with particles having diameters from 10 to 80 pixels. The diameter results provided by shadow image processing are given in Table 1.

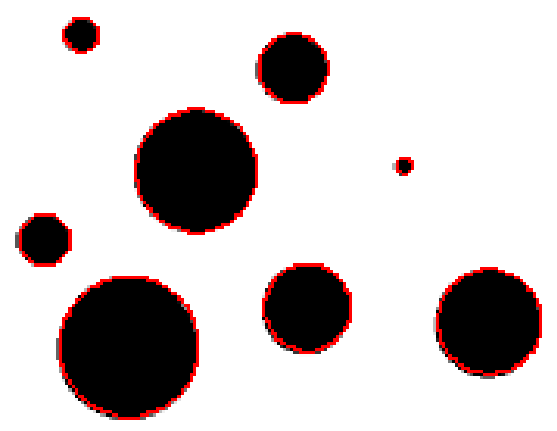

Figure 3. Synthetic particles image and contours (red) determined by shadow processing 
Table 1. Comparison of the ground truth and diameter measured by shadow processing on the synthetic particles image

\begin{tabular}{c|c}
\hline \hline Ground truth (pix) & Diameter measured by shadow processing (pix) \\
\hline \hline 10 & 9.933 \\
20 & 19.93 \\
30 & 29.821 \\
40 & 39.960 \\
50 & 49.861 \\
60 & 59.869 \\
70 & 69.901 \\
80 & 79.869
\end{tabular}

The comparison between measured diameter from shadow processing and ground truth shows a very good agreement between each other. This proves the accuracy of particle edge detection and diameter evaluation.

The overlapped particles detection and separation is also firstly tested by synthetic particles image. The left side of Figure 4 shows a group of synthetic particles with slight overlapping and the right side of the same figure shows the particle contour given by shadow processing. Overlapped particles have been well detected and recovered by this method. This method has been also tested by synthetic particles images with severe overlapping but it failed to identify the overlapping.

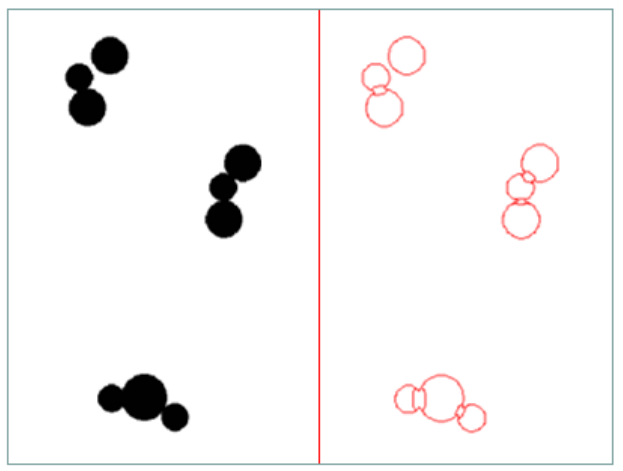

Figure 4. Left, synthetic overlapped particles; Right, contour of each particle detected by shadow image processing

The next test has been carried out with a shadow image from a transparent target with dots pattern (Thorlabs R3L3S4P2), which is shown in Figure 5. The dots on this target have five different size groups but only three of them are shown in the field of view: $62.5 \mu \mathrm{m}, 125 \mu \mathrm{m}$ and $250 \mu \mathrm{m}$. The back illumination is slightly non-uniform to increase the challenges for the shadow processing. The scale in the image is $7.2 \mu \mathrm{m}$ per pixel.

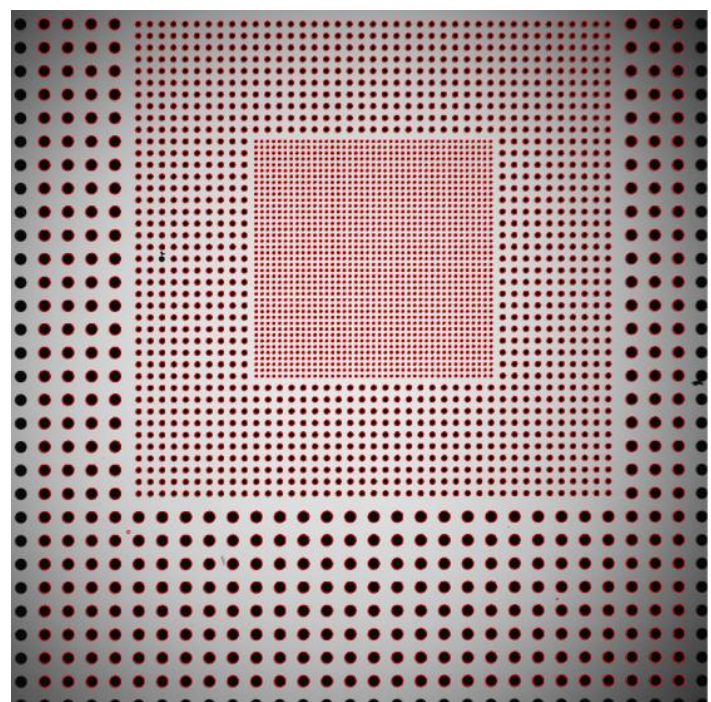

(a)

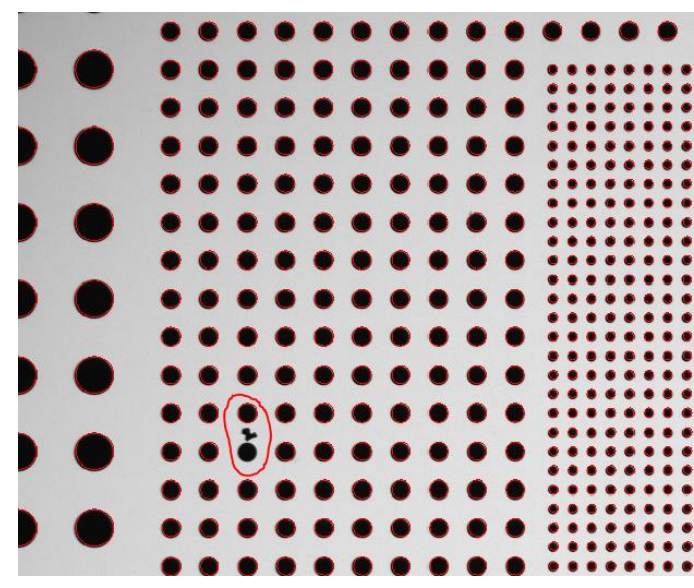

(b)

Figure 5. (a) Shadow image of a transparent dot target, with three groups of dots (62.5 $\mu \mathrm{m}, 125 \mu \mathrm{m}$ and $250 \mu \mathrm{m})$. Contour of each dot detected by shadow image processing are shown as red. (b) Zoom: missed dot due to dirt on the target

This work is licensed under a Creative Commons 4.0 International License (CC BY-NC-ND 4.0). 


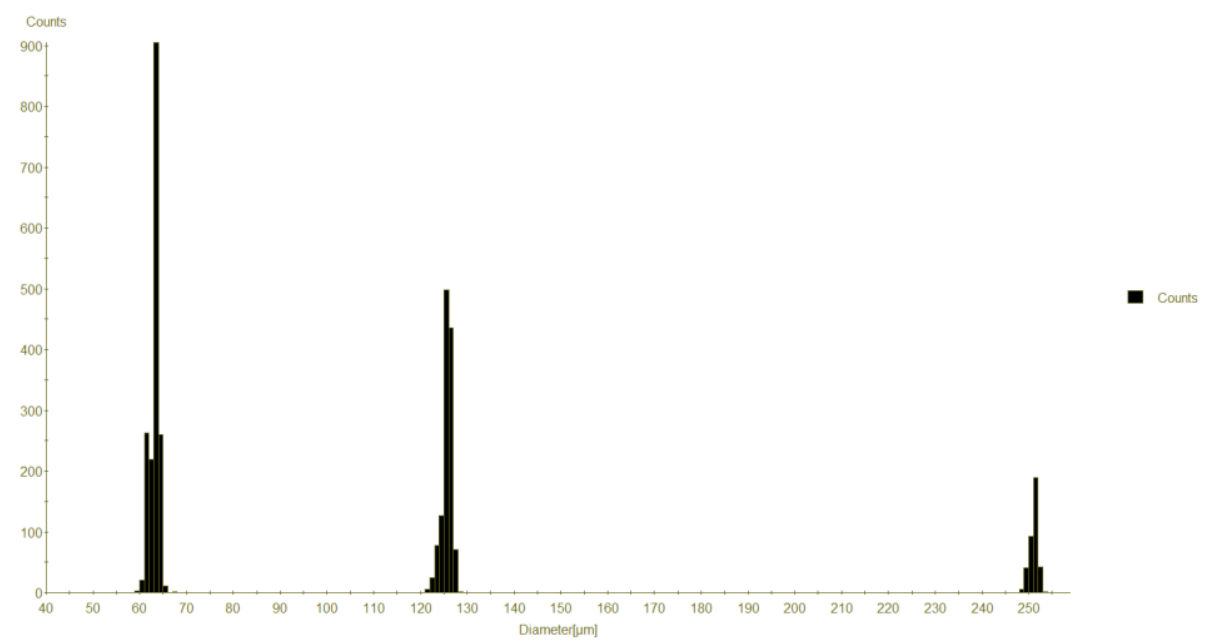

Figure 6. Diameter histogram of transparent dot target shadow image provided by shadow image processing

Table 2. Summary of measurement results from shadow imaging processing on the transparent dot target shadow image

\begin{tabular}{l|cccc}
\hline \hline Dot Size $(\mu \mathrm{m})$ & Number of Dots & Dots detected & $\begin{array}{c}\text { Average size measured } \\
\text { by shadow processing } \\
(\mu \mathrm{m})\end{array}$ & $\begin{array}{c}\text { Standard deviation of size } \\
\text { measured by shadow } \\
\text { processing }(\mu \mathrm{m})\end{array}$ \\
\hline \hline 62.5 & 1681 & 1681 & 63.98 & 0.92 \\
125 & 1240 & 1239 & 127.75 & 1.08 \\
250 & 400 & 371 & 255.73 & 0.90
\end{tabular}

Figure 6 shows the diameter histogram from shadow image processing, which indicates a very narrow distribution of each size group. Table 2 shows the comparison of target specifications and the results measured from shadow processing, which also shows a very good agreement. All small dots and middle dots are detected except only one middle dot which is missed because it is connected to a dirt on the target (shown in Figure 5.b). The missed big dots from shadow processing are due to the fact that they are very close to the frame border and are therefore rejected.

Different global thresholds have been also tested to study the influence of this parameter. Tests with various global thresholds show very similar result (variance $<1 \%$ ). The slight difference is introduced by different numbers of particles identified with different global thresholds and therefore the statistical results are changed.

Finally the proposed method has been tested with a shadow image acquired from a water spray, which is shown in Figure 7, together with the contour determined by shadow image processing. Typical challenges of shadow measurements on water spray can be all identified here, such as non-spherical particles, out-of-focus particles, overlapped particles, non-uniform back illumination, and so on.

Most of the particles can be detected and segmented correctly from this image with the proposed method. Particularly, that in the right bottom side (on top of a big out-of-focus particle) has been correctly detected. In addition, the two overlapped particles next to this big out-of-focus particle have also been detected and separated correctly. There are several sharp particles which are not identified, but this is because they are close to the image boundary and therefore are rejected. 


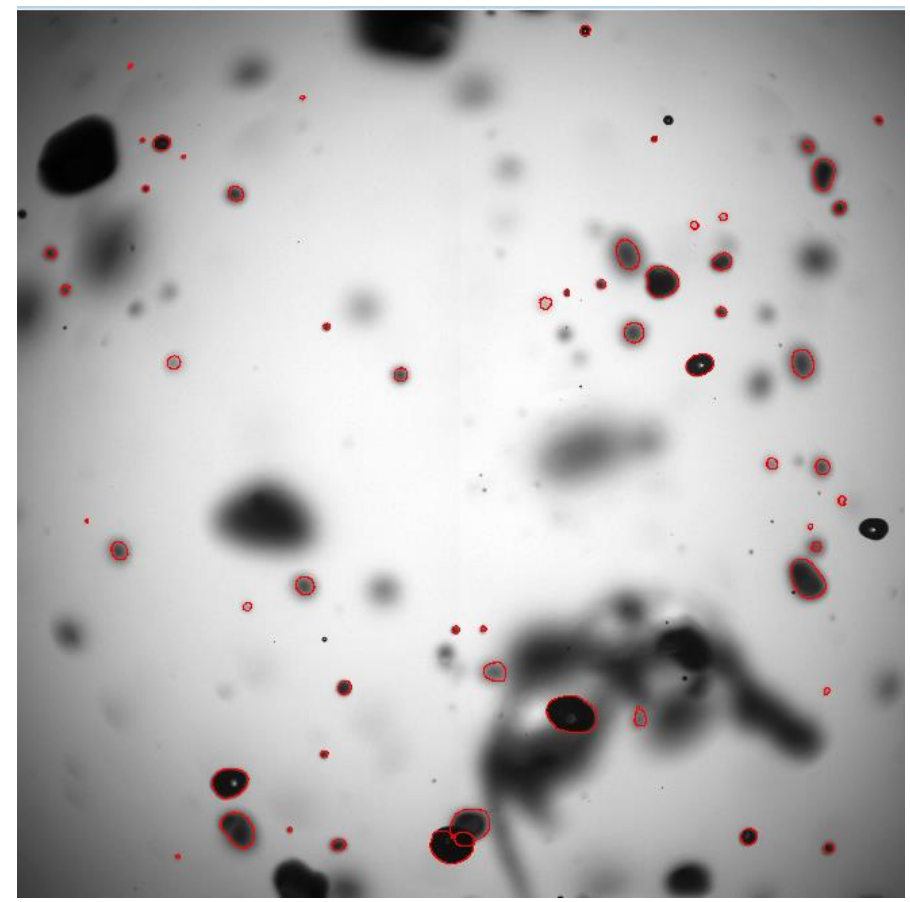

Figure 7. Shadow images acquired from a water spray, together with red contour of each detected particle given by shadow image processing

Several out-of-focus particles are also detected, which can cause a bias on the size result. Correction to these out-of-focus particles is not included in this study. Fdida and Blaisot proposed a method to correct the out-of-focus particles or to define a depth-of-field thickness where all particles are focused, based on the point spread function [8]. These methods will be implemented and investigated in the next stage of this project.

\section{Conclusions}

A new shadow processing method is proposed with advanced particle detection and segmentation algorithm, together with an overlapping particles detection and separation method. This method is firstly qualified by synthetic particle images and then further evaluated with shadow images of a transparent target with dot patterns. Later the new method is applied to shadow images from a water spray.

All test results show that this new shadow processing method can handle various issues of shadowgraphy measurement on water sprays. Particularly, this method is robust and less sensitive to parameters compared with existing shadow processing methods. In addition, this method can handle overlapped particles in the shadow image and provide more accurate results.

\section{Acknowledgements}

This work is supported by a collaboration project among IRSTEA, IRPHE and Dantec Dynamics A/S, aiming to improve characterization of moving irregular particles by shadowgraphy measurements.

\section{References}

[1] Bachalo, W. and Sankar. S., 2003, Spray diagnostics. In: Mercer, C.R. (Ed.). Optical Metrology for Fluids, Chap 5, Combustion and Solids. Springer-Verlag.

[2] Tropea, C., 2011, Annual Review Fluids Mechanics, 43, pp. 399-426.

[3] Lecuona, A., Rodriguez, P., Sosa, P., and Zequeira, R., 2000, Measurement Science and Technology, 11 (8), pp. 1152-1161.

[4] Ghaemia, S., Rahimib, P. and Nobesc, D., 2010, International Journal of Spray and Combustion Dynamics, 2 (2), pp. 103-124.

[5] Stevenin C., Tomas, S., Vallet, A., Amielh, M. and Anselmet, F., 2016, International Journal of Multiphase Flow, 84, pp. 264-278.

[6] Blaisot, J. and Yon, J., 2005, Experemental Fluids, 39 (6), pp. 977-994.

[7] Yon, J., 2003, Doctoral thesis, University of Rouen.

[8] Fdida, N. and Blaisot, J., 2009, Measurement Science and Technology, 21(2), pp. 255-269. 


\title{
Measurement of polymeric time scales from linear drop oscillations
}

\author{
Gregor Plohl*, Günter Brenn \\ Institute of Fluid Mechanics and Heat Transfer, Graz University of Technology, Austria \\ *Corresponding author: gregor.plohl@tugraz.at
}

\begin{abstract}
The oscillating drop method allows material properties of liquids to be measured from damped drop oscillations. The literature discusses, e.g., the measurement of the liquid dynamic viscosity and the surface tension against the ambient medium, predominantly for Newtonian liquids. We use this method for measuring pairs of material properties of polymeric liquids. Pairs of properties may be measured, since the quantity measured is a complex frequency with a real and an imaginary part. For the measurements, individual drops are levitated in air by an ultrasonic levitator and imaged with a high-speed camera. Amplitude modulation of the ultrasound drives shape oscillations of the levitated drop. When the modulation is switched off, with the levitating force maintained, the drop performs free oscillations which are damped due to the liquid viscosity. The data acquired from the images recorded are the angular frequency and the damping rate which are used as an input into the characteristic equation of the oscillating drop. Our measurements intend to yield either two viscoelastic time scales with the zero-shear viscosity known, or one time scale and the zero-shear viscosity, with the other time scale known. The two time scales are the stress relaxation and the deformation retardation times. The latter is difficult to get for polymer solutions.

The present contribution presents results from a large set of measurements of the deformation retardation time. Liquids studied are aqueous solutions of poly(acryl-amides) at varying concentration. The corresponding values of the zero-shear viscosity agree well with the values from shear rheometry. Values of the deformation retardation time differ substantially from the values commonly used in viscoelastic flow simulations. Furthermore, the measured values disagree with the predictions from the viscous-elastic stress splitting approach in linear viscoelasticity. With our study we will provide a consistent set of material properties for the Oldroyd-B model in linear viscoelasticity. This will be important for material modelling in viscoelastic spray simulations.
\end{abstract}

\section{Keywords}

Linear drop shape oscillations, damped oscillations, polymeric liquid, ultrasonic levitation.

\section{Introduction}

The deformations of a drop surface due to shape oscillations may influence transport processes across the liquid/gas interface, such as the evaporation of the drop or the absorption of gases from the environment. For their relevance for transport processes, and for scientific interest, oscillations of liquid drops have been under investigation since the time of Lord Rayleigh, who derived the angular frequency $\alpha_{m, 0}=\sqrt{m(m-1)(m+2)} \sqrt{\sigma / \rho a^{3}}$ of linear oscillations of mode $m$ for an inviscid drop with density $\rho$, radius $a$, and surface tension $\sigma$ against the ambient vacuum [1]. Rayleigh's work was extended by Lamb [2], who included the influence of viscosity of the drop liquid and obtained the oscillation frequency and the rate of decay of the oscillations in the limits of very high and very low drop viscosity.

The idea to measure material properties of liquids from damped drop shape oscillations has brought about the oscillating drop method $[3,4]$. To date, the existing literature discusses the measurement of material parameters, such as the dynamic viscosity of the liquid and its surface tension against the ambient medium, predominantly for Newtonian liquids [5-8].

For viscoelastic systems, the oscillating drop method was used for investigating the surface rheology [9, 10]. The materials were surfactant solutions, and the drops were levitated due to the microgravity conditions of the experiment. In these studies, complementary effects of the bulk and the surface viscosities were found [9] and quantified [10]. Most recently, the oscillating drop method was proposed and developed for measuring polymeric time scales [11-13]. The basis of the method is the characteristic equation for the complex frequency of the drop. The experiments were carried out with aqueous solutions of the two different poly(acryl-amides) Praestol 2500 and Praestol 2540 with different degree of hydrolysis and hence different mechanical flexibilities of the macromolecules. The drops were levitated using an ultrasonic levitator. The aim of the first study [11] was to quantify the influences of the two polymeric time scales on the oscillation behavior of the drop, and to propose an experimental method to determine the deformation retardation time by measuring damped oscillations of the drop. A proof-of-concept experiment was 
presented to show the potential and limitations of the method. In [12], the characteristic equation was further analyzed, and a numerical method for determining a pair of liquid properties from the characteristic equation was presented. The method was tested for three different aqueous polymer solutions ( 0.3 and 0.8 wt.\% Praestol 2500 and 0.05 wt. \% Praestol 2540). The values of the deformation retardation time $\lambda_{2}$ obtained by this method deviate strongly from the values typically used in viscoelastic flow simulations. The sensitivity and uncertainty analysis have shown the values of $\lambda_{2}$ depend weakly on the uncertainty of the experimental data.

In the present study, the oscillating drop method [11-13] was used to study the deformation retardation time dependency on the polymer concentration. We have studied aqueous solutions of Praestol 2500 with polymer mass fractions between 0.1 and $1.0 \mathrm{wt}$ \%. Our paper is organized as follows: first the theoretical foundations of drop shape oscillations are presented. We then outline the experiment for measuring the complex oscillation frequency. Further, we present and discuss the experimental results and draw the conclusions.

\section{Theoretical Foundations}

The equations governing linear viscoelastic drop shape oscillations are the equation of continuity $\boldsymbol{\nabla} \cdot \boldsymbol{v}=0$ and the linearized equation of motion

$$
\rho \frac{\partial v}{\partial t}=-\nabla \cdot(p \boldsymbol{\delta}-\boldsymbol{\tau})
$$

The extra stress tensor $\boldsymbol{\tau}$ is related to the flow field through the appropriate linear viscoelastic material model. Material models in fluids relate rates of deformation to the extra stresses, which appear on top of some isotropic stress, such as pressure. Material models follow either from micro-rheological or from phenomenological approaches. The former consists in a transport equation for the dyadic of the end-to-end position vector of the macromolecules with itself, which yields information about the state of deformation of the molecules in the solution and, thereby, about the stress. The phenomenological approach models extra stresses and their changes by a transport equation. The rheological equations of state representing the material model must satisfy invariance criteria in coordinate transformations and must be independent of the motion of the material as a whole [14].

The most general form of a phenomenological material model for a viscoelastic fluid is the Oldroyd eight-constant model [15]. It allows non-linear material behavior to be modelled and relies on the eight constant parameters of the model, which all have the dimension of time, except the dynamic viscosity involved. Simplifying this model to the linear case, i.e., treating a liquid as linear viscoelastic, the extra stress in the liquid at small rates of deformation may be described by the differential equation

$$
\tau+\lambda_{1} \frac{d \tau}{d t}=-\eta_{0}\left(\dot{\gamma}+\lambda_{2} \frac{d \dot{\gamma}}{d t}\right)
$$

where $\lambda_{1}$ and $\lambda_{2}$ are the stress relaxation and deformation retardation times, $\eta_{0}$ is the zero shear viscosity and $\dot{\gamma}$ is the rate of deformation tensor. This equation is also known as the Jeffreys model. The relaxation time characterizes the time scale on which the stress relaxes after removal of strain, and the retardation time describes the strain relaxation after removal of stress. The case $\lambda_{1}=\lambda_{2}=0$ describes the purely viscous Newtonian fluid, and the case $\lambda_{2}=0$, $\lambda_{1}>0$ describes the purely elastic fluid. With the time dependency of motion given by an exponential function $\exp \left(-\alpha_{m} t\right)$, where $\alpha_{m}$ is the complex angular frequency of mode $m$, the stress tensor $\tau$ satisfying Eq.(2) reads

$$
\boldsymbol{\tau}=\eta_{0} \frac{1-\alpha_{m} \lambda_{2}}{1-\alpha_{m} \lambda_{1}} \dot{\gamma}=\eta\left(\alpha_{m}\right) \dot{\gamma}
$$

This equation indicates that the linear viscoelastic extra stress may be represented formally in the same way as for a Newtonian fluid, with the difference that the dynamic viscosity $\eta\left(\alpha_{m}\right)$ depends on the complex frequency $\alpha_{m}$ (correspondence principle). In our discussions on the linear stability behaviour of viscoelastic liquid systems below we will assume material behaviour according to this model. Shear thinning, as a non-linear phenomenon, is therefore not accounted for, which is strictly correct for Boger fluids, but neglects some potentially important phenomenon in shear thinning liquids. In the latter case, however, linear liquid behaviour on the first Newtonian plateau of the flow curve may still be represented correctly by the linear approach [16].

In polymer science and in computational rheology, it is customary to decompose the stress into its Newtonian and non-Newtonian components (referred to as the viscous-elastic stress splitting) or, more generally, to write the extra stress $\boldsymbol{\tau}=\boldsymbol{\tau}_{s}+\boldsymbol{\tau}_{p}$ and the dynamic viscosity $\eta_{0}=\eta_{s}+\eta_{p}$ as sums of contributions from the Newtonian solvent and the polymer (solvent-polymer stress splitting) [17, 18]. A similar approach can be applied also for the Giesekus model [19]. This approach yields the Jeffreys model (Oldroyd B) if the deformation retardation time is defined as 


$$
\lambda_{2}=\lambda_{1} \frac{\eta_{s}}{\eta_{s}+\eta_{p}}=\lambda_{1} \frac{\eta_{s}}{\eta_{0}}=: \lambda_{2 G}
$$

The damped oscillatory motion of the drop is analyzed in detail in [11,12]. The fundamental outcome of the analysis is the characteristic equation for the complex frequency of the drop

$$
\left(\frac{\alpha_{m, 0}}{\alpha_{m}}\right)^{2}=\frac{2\left(m^{2}-1\right)}{q^{2} a^{2}-2 q a j_{m+1} / j_{m}}-1+\frac{2 m(m-1)}{q^{2} a^{2}}\left[1+\frac{2(m+1) j_{m+1} / j_{m}}{2 q a j_{m+1} / j_{m}-q a}\right]
$$

where $q=\sqrt{\alpha_{m} / v\left(\alpha_{m}\right)}$ and $j_{m}$ and $j_{m+1}$ are spherical Bessel functions of the first kind at the value $q a$ of their arguments. The equation is formally identical to the results of Lamb [2] obtained for Newtonian liquids. In the present case of a viscoelastic liquid, however, the kinematic viscosity involved in the equation is a function of the complex oscillation frequency $\alpha_{\mathrm{m}}$. The equation is transcendental in qa and must therefore be solved numerically to determine, e.g., the zero-shear dynamic viscosity $\eta_{0}$ and the deformation retardation time $\lambda_{2}$ involved in the dynamic viscosity $\eta\left(\alpha_{m}\right)=v\left(\alpha_{m}\right) \rho=\eta_{0}\left(1-\alpha_{m} \lambda_{2}\right) /\left(1-\alpha_{m} \lambda_{1}\right)$. Knowing the real and imaginary parts of the complex oscillation frequency $\alpha_{\mathrm{m}}$ from the experiment, we get two pieces of information from the characteristic equation. In the following section we present an experiment suitable for measuring the complex oscillation frequency $\alpha_{2}$ of the drop.

\section{Experimental method}

The experiment bases on the levitation of an individual drop in a standing ultrasound wave [20]. The acoustic resonator consists of a transducer and a curved reflector allowing for the stable placement of a drop with a diameter $\leq$ $3 \mathrm{~mm}$ (Fig. 1). Liquids studied are aqueous solutions of poly(acryl-amides) at varying concentration. The drops are produced and placed into the acoustic field with an insulin syringe (Fig. 2 left, center). Oscillation frequencies excited by ultrasound modulation are $\mathrm{O}(120 \mathrm{~Hz})$, close to the resonance frequency of the base-mode $\mathrm{m}=2$. After switch-off of the modulation, the drop returns to its equilibrium shape by damped oscillations (Fig. 2 right).

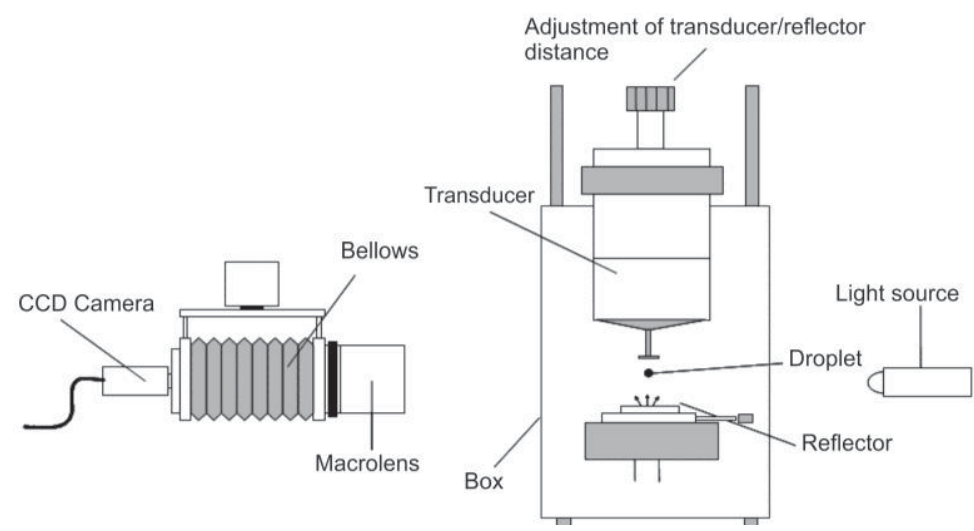

Figure 1. Experimental setup with acoustic levitator for drop shape oscillation studies.
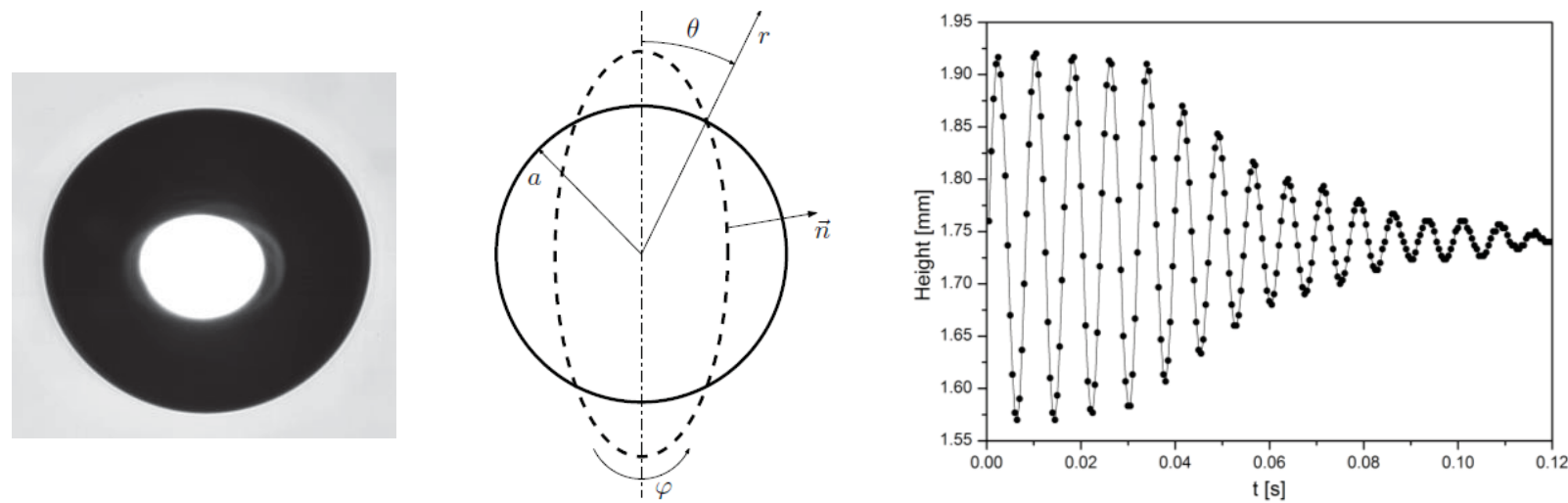

Figure 2. (Left) Shape of a levitated $1.7 \mathrm{~mm}$ drop, (center) equilibrium and deformed drop shapes in the spherical coordinate system. The deformed shape (dashed line) is represented by $r_{s}(\theta, t)=a+\epsilon_{0} P_{m}(\cos \theta) \exp \left(-\alpha_{m} t\right)$ [11], (right) distance between the north and south poles in a damped drop oscillation as a function of time. 
The data acquired from the high-speed camera recording of the damped oscillations are the angular frequency $\alpha_{m, i}$ and the damping rate $\alpha_{m, r}$. For determining the pair of liquid properties $\left(\eta_{0}, \lambda_{2}\right)$, the two values $\alpha_{m, i}$ and $\alpha_{m, r}$ are used as an input into the characteristic equation (5) of the drop, so that the left-hand side of the equation is known.

Our experiments were carried out with aqueous solutions of the poly(acryl-amide) Praestol 2500 from Solenis Technologies (Germany). This is a non-ionic polymer with a degree of hydrolysis of $3-4 \%$ and molecular weight about $15-20 \cdot 10^{6} \mathrm{~kg} / \mathrm{kmol}$. The aqueous solutions were prepared in demineralized water, producing a master solution with a solute mass fraction of 10000 ppm by mass, which was then diluted to achieve the various mass fractions. The shear viscosity of the liquids was measured as a function of shear rate with a rotational rheometer Anton Paar MCR 300. The values of the zero-shear viscosity $\eta_{0}^{*}$ (first Newtonian plateau) were determined from measured flow curves approximated by the empirical Carreau-Yasuda model. The temperature was kept constant at the value of $22^{\circ} \mathrm{C} \pm 1{ }^{\circ} \mathrm{C}$ in the laboratory where the drop oscillation experiments were carried out. The densities of the liquids were measured with an oscillating U-tube device with an accuracy of $\pm 0.1 \mathrm{~kg} / \mathrm{m}^{3}$. They are all in the order of $10^{3} \mathrm{~kg} / \mathrm{m}^{3}$. The surface tension of the liquids against the ambient air was measured with a drop volume tensiometer. The stress relaxation time $\lambda_{1}$ of the liquids was measured with a filament stretching elongational rheometer yielding mean values with standard deviations between 6 and 15\% [20]. The properties of the aqueous solutions of the poly(acryl-amide) Praestol 2500 investigated in the present study are listed in Table 1 and presented in Fig. 3.

Table 1. Properties of the aqueous Praestol 2500 polymer solutions at $22^{\circ} \mathrm{C}$

\begin{tabular}{ccccc}
\hline \hline $\begin{array}{c}\text { Solute mass } \\
\text { fraction } w \\
{[w t . \%]}\end{array}$ & $\begin{array}{c}\text { Density } \\
\rho\end{array}$ & $\begin{array}{c}\text { Surface } \\
\text { tension } \sigma \\
{\left[\mathrm{kg} \mathrm{m}^{-3}\right]}\end{array}$ & $\begin{array}{c}\text { Zero-shear } \\
\text { viscosity } \eta_{0}{ }^{*} \\
{[\mathrm{~Pa} \mathrm{~s}]}\end{array}$ & $\begin{array}{c}\text { Stress relaxation } \\
\text { time } \lambda_{1} \\
{[\mathrm{~s}]}\end{array}$ \\
\hline \hline 0.1 & 1000 & 0.0722 & $0.013 \pm 10 \%$ & $0.025 \pm 10 \%$ \\
0.2 & 1000 & 0.0727 & $0.033 \pm 10 \%$ & $0.066 \pm 10 \%$ \\
0.3 & 1000 & 0.0732 & $0.08 \pm 10 \%$ & $0.11 \pm 10 \%$ \\
0.4 & 1000 & 0.0737 & $0.12 \pm 10 \%$ & $0.12 \pm 10 \%$ \\
0.5 & 1000 & 0.0743 & $0.39 \pm 10 \%$ & $0.16 \pm 10 \%$ \\
0.6 & 1000 & 0.0748 & $0.56 \pm 10 \%$ & $0.18 \pm 10 \%$ \\
0.7 & 1000 & 0.0753 & $0.9 \pm 10 \%$ & $0.21 \pm 10 \%$ \\
0.8 & 1000 & 0.0758 & $1.6 \pm 10 \%$ & $0.23 \pm 10 \%$ \\
0.9 & 1000 & 0.0763 & $2.3 \pm 10 \%$ & $0.25 \pm 10 \%$ \\
1.0 & 1000 & 0.0769 & $3.9 \pm 10 \%$ & $0.285 \pm 10 \%$
\end{tabular}
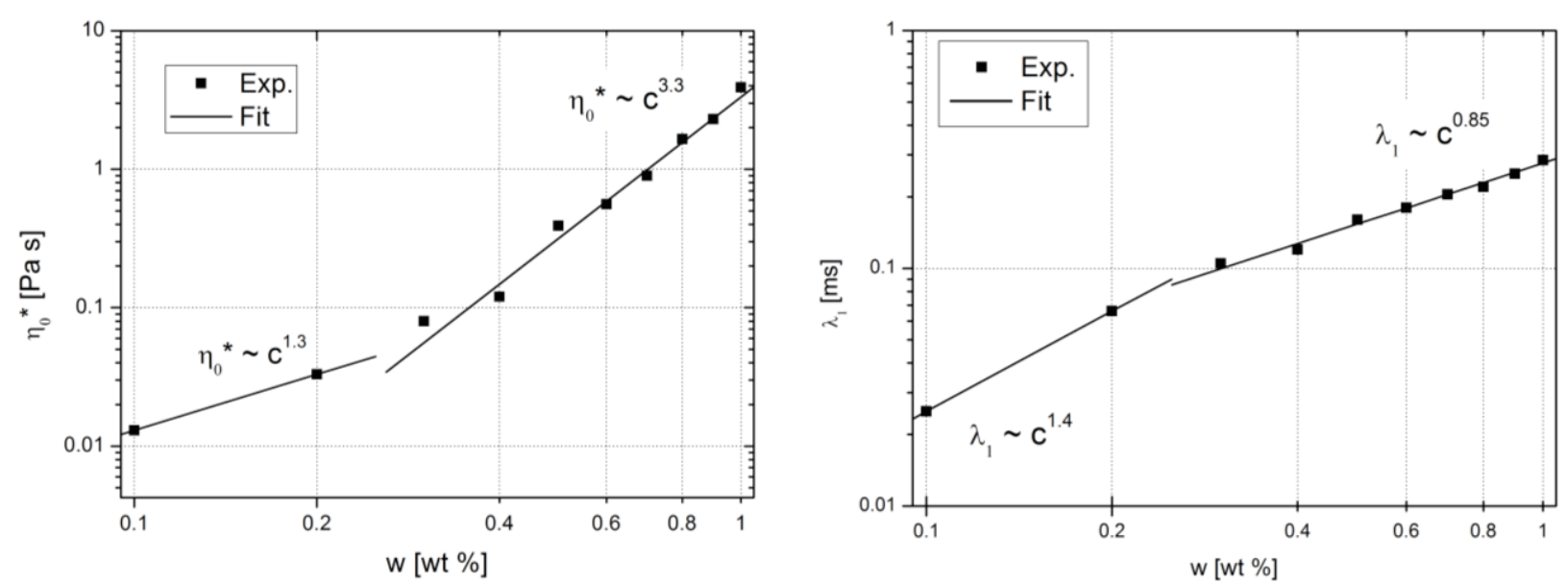

Figure 3. Praestol 2500 aqueous solutions. (Left) Zero-shear viscosity and (right) relaxation time against polymer mass fraction.

Figure 3 (left) shows the zero-shear viscosity against polymer mass fraction. The measured data are approximated by the scaling law

$\eta_{0} \propto w^{S}$

where $S \approx 1.3$ at low polymer mass fractions $w$, and $S \approx 3.3$ for high polymer mass fraction. The transition between these two regimes lies between $w=0.2$ and 0.3 wt.\% (Fig. 3 left). Figure 3 (right) shows the dependence of the 
relaxation time on the polymer mass fraction $w$. The measured values are approximated using the similar scaling law $\lambda_{1} \propto \mathrm{w}^{T}$, where $\mathrm{T} \approx 1.4$ for low concentrations and $\mathrm{T} \approx 0.85$ for higher concentrations. The transition between these two regimes lies again between $w=0.2$ and $0.3 \mathrm{wt} . \%$. The region between 0.3 and $1.0 \mathrm{wt} . \%$ can be seen as a semidilute regime, and the region between 0.1 and $0.2 \mathrm{wt} . \%$ as the transition from the dilute to the semi-dilute regime. Images of the levitated drop are recorded by a high-speed camera at a framing rate of $2 \mathrm{kHz}$ under backlight illumination. An uncertainty in the length measurement of $\pm 2 \mathrm{Px} / \mathrm{s}$ with the resolution of $300 \mathrm{Px} / \mathrm{s} / \mathrm{mm}$ results in a sizing uncertainty of $\pm 6.7 \mu \mathrm{m}$, which is equivalent to $\pm 0.3 \%$ for a $2 \mathrm{~mm}$ drop. Within at most $10 \mathrm{~s}$ after the drop has been placed in the acoustic levitator, several pictures of the drop are taken in order to have its initial shape and volume. This initial state, where the evaporation of the solvent has had no influence on the solution concentration yet, allows the concentration of the drop liquid at all later times to be deduced from the volume. The equilibrium radius $a$ is calculated from the recorded instantaneous images. The zero-shear viscosity obtained from the experiment is the value corresponding to the concentration of the solution present during the related experiment.

The experiment for determining the polymeric deformation retardation time from damped drop oscillations is subject to influences from the experimental method of acoustic levitation and the non-Newtonian behavior of the polymeric liquid, which is shear-thinning in many cases. In order to fulfill the limitations set by the linear theory underlying the characteristic equation of the drop, and in order to avoid influences from the shear thinning of the liquid, we measure the drop oscillation frequency and damping rate in the late stages of the damped oscillation.

The oscillation shown in Figure 2 (right) was recorded for a 0.3 wt. \% Praestol 2500 solution drop with the equilibrium diameter of $1.83 \mathrm{~mm}$. The drop was driven at $130 \mathrm{~Hz}$ before the modulation was switched off. From these data, the frequency and damping rate in the last part of the motion were extracted, so that both the linear oscillation behavior was ensured and the shear-thinning of the polymer solution did not have any influence on the oscillation. The frequency and damping rate are determined using the least-squares method to achieve the best fit of a prescribed function to the damped oscillations measurement data. The fitting procedure was done of both oscillating directions, as shown in Figure 4 (left) and the average value for the frequency and the damping rate were calculated. The volume-equivalent spherical radius was also calculated (Figure 4 right) and compared to initial radius in order to determine the correct polymer concentration.

The angular frequency and the damping rate of the drop depicted in Fig. 2, determined by this procedure, are $\alpha_{2, i}=(2 \pi) \cdot 134.2 \mathrm{~Hz}$ and $\alpha_{2, r}=36.2 \mathrm{~s}^{-1}$. The real and imaginary parts of the complex angular frequency $\alpha_{2}=\alpha_{2, r}+$ $i \alpha_{2, i}$ are therefore known.
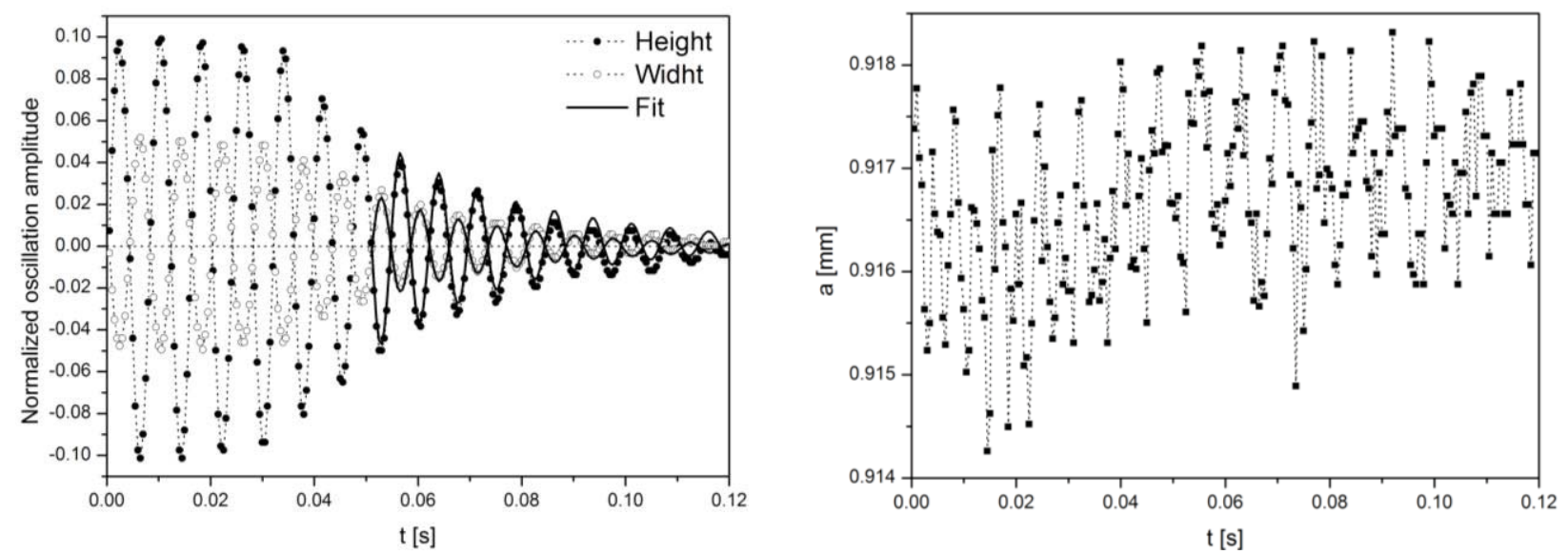

Figure 4. Damped oscillations of a levitated $1.72 \mathrm{~mm} 0.3 \mathrm{wt} \%$ aqueous Praestol 2500 solution drop as a function of time. (Left) Normalized oscillation amplitude and the fitting curve (solid line) in the last part of the motion. (Right) Volume-equivalent spherical radius of the drop as a function of time, varying by no more than $0.3 \%$.

\section{Solutions of the characteristic equation}

The characteristic equation (5) is transcendental in the argument of the spherical Bessel functions involved and must therefore be solved numerically. The method for determining $\eta_{0}$ and $\lambda_{2}$ by solving this equation and the detailed analysis of the characteristic equation are presented in [12]. For the numerical analysis we use the computer algebra software MATHEMATICA. As a prerequisite, the complex frequency $\alpha_{m}$ must be accurately measured in the experiment, and the radius of the drop as well as the density, surface tension and stress relaxation time of the liquid in contact with the ambient air must be known. Due to the influence from the spherical Bessel functions, the equation 
exhibits a complicated set of solutions. Identification of the right solution among the calculated pairs of $\eta_{0}$ and $\lambda_{2}$ follows from comparison of the value of $\eta_{0}$ with the result $\eta_{0}^{*}$ from the measurements with a rotational viscosimeter. The solutions $q a$ of the characteristic equation and corresponding calculated values of zero shear viscosity $\eta_{0}$ and deformation retardation time $\lambda_{2}$ for a 0.3 wt\% aqueous Praestol 2500 solution drop are listed in Table 2.

Table 2. Positive roots qa of the characteristic equation and corresponding calculated values of zero shear viscosity $\eta_{0}$ and deformation retardation time $\lambda_{2}$ for the $0.3 \mathrm{wt} \%$ Praestol 2500 solution drop in Fig. 3 . The correct solution is highlighted.

\begin{tabular}{c|c|c|c|c|c}
\hline $\begin{array}{c}\text { Radius } \\
{[\mathrm{mm}]}\end{array}$ & $\begin{array}{c}\text { Frequency } \\
{[\mathrm{Hz}]}\end{array}$ & $\begin{array}{c}\text { Damping } \\
\text { rate }\left[\mathrm{s}^{-1}\right]\end{array}$ & $q a$ & $\begin{array}{c}\eta_{0} \\
{[P a \cdot s]}\end{array}$ & $\begin{array}{c}\lambda_{2} \\
{[s]}\end{array}$ \\
\hline \hline 0.917 & 134.2 & 36.2 & $4.385+7.8312 i$ & 0.0002 & $2130 \cdot 10^{-4}$ \\
\pm 0.006 & \pm 0.4 & \pm 2.5 & $4.974+0.0791 i$ & 2.656 & $1.267 \cdot 10^{-4}$ \\
& & & $8.767+0.0426 i$ & 0.856 & $1.005 \cdot 10^{-4}$ \\
& & $12.116+0.0263 i$ & 0.448 & $0.941 \cdot 10^{-4}$ \\
& & $15.363+0.0164 i$ & 0.279 & $0.915 \cdot 10^{-4}$ \\
& & $18.569+0.0106 i$ & 0.191 & $0.903 \cdot 10^{-4}$ \\
& & $21.755+0.0071 i$ & 0.139 & $0.897 \cdot 10^{-4}$ \\
& & $24.928+0.0049 i$ & 0.106 & $0.894 \cdot 10^{-4}$ \\
& & $28.093+0.0036 i$ & $\mathbf{0 . 0 8 3}$ & $\mathbf{0 . 8 9 3 \cdot 1 0 ^ { - 4 }}$ \\
& & $31.254+0.0027 i$ & 0.067 & $0.892 \cdot 10^{-4}$ \\
& & $34.411+0.0020 i$ & 0.056 & $0.891 \cdot 10^{-4}$ \\
& & $37.564+0.0016 i$ & 0.047 & $0.891 \cdot 10^{-4}$
\end{tabular}

From the error analysis [12] follows that $\lambda_{2}$ can be accurately determined even if the right solution qa cannot unambiguously be identified. This is due to the weak dependency of $\lambda_{2}$ on the solution qa of the characteristic equation. This weak dependency is demonstrated in Table 2. On the other hand, in order to accurately determine $\eta_{0}$, very accurate measurements of all the input parameters are required.

\section{Results and discussion}

With each of the ten polymer solutions we performed a set of about 35 measurements in order to get statistically reliable results. From each polymer solution, five drops with different radius were levitated and with each of these drops, we performed 6 to 8 oscillation measurements. The results are presented in Table 3 and in Fig. 5. Table 3 shows the set of viscosities and deformation retardation times of the aqueous polymer solutions (Table 1), where values obtained as solutions of the characteristic equation are compared to values of the deformation retardation time from the viscous-elastic stress splitting approach $\lambda_{2 G}=\lambda_{1} \eta_{s} / \eta_{0}^{*}$. The value of the solvent viscosity $\eta_{s}$ used was $1 \mathrm{mPa}$ $\mathrm{s}$ for water. The uncertainty of the values listed in Table 3 is of the order of $10 \%$.

Table 3. The viscosities $\eta_{0}$ and deformation retardation times $\lambda_{2}$ obtained from oscillating drop measurements. For comparison the zero shear viscosity $\eta_{0}^{*}$ and the deformation retardation $\lambda_{2 \mathrm{G}}$ from the viscous-elastic stress splitting approach are also listed.

\begin{tabular}{c|c|c|c|c}
\hline \hline $\begin{array}{c}\text { Solute mass } \\
\text { fraction w } \\
{[w t \%]}\end{array}$ & $\begin{array}{c}\text { Viscosity } \eta_{0} \\
{[P a s]}\end{array}$ & $\begin{array}{c}\text { Zero-shear } \\
\text { viscosity } \eta_{0}^{*} \\
{[P a s]}\end{array}$ & $\begin{array}{c}\text { Retardation } \\
\text { time } \lambda_{2} \\
{\left[10^{-4} s\right]}\end{array}$ & $\begin{array}{c}\text { Retardation } \\
\text { time } \lambda_{2 \mathrm{G}} \text { with } \eta_{0}^{*} \\
{\left[10^{-4} s\right]}\end{array}$ \\
\hline \hline 0.1 & 0.013 & 0.013 & 0.35 & 19.23 \\
0.2 & 0.037 & 0.033 & 0.47 & 20.00 \\
0.3 & 0.08 & 0.08 & 0.9 & 13.75 \\
0.4 & 0.127 & 0.12 & 2 & 10.00 \\
0.5 & 0.43 & 0.39 & 2.1 & 4.10 \\
0.6 & 0.51 & 0.56 & 2.5 & 3.21 \\
0.7 & 0.82 & 0.9 & 2.7 & 2.33 \\
0.8 & 1.65 & 1.6 & 2.9 & 1.38 \\
0.9 & 2 & 2.3 & 3.1 & 1.09 \\
1.0 & 6 & 3.9 & 3.5 & 0.73
\end{tabular}



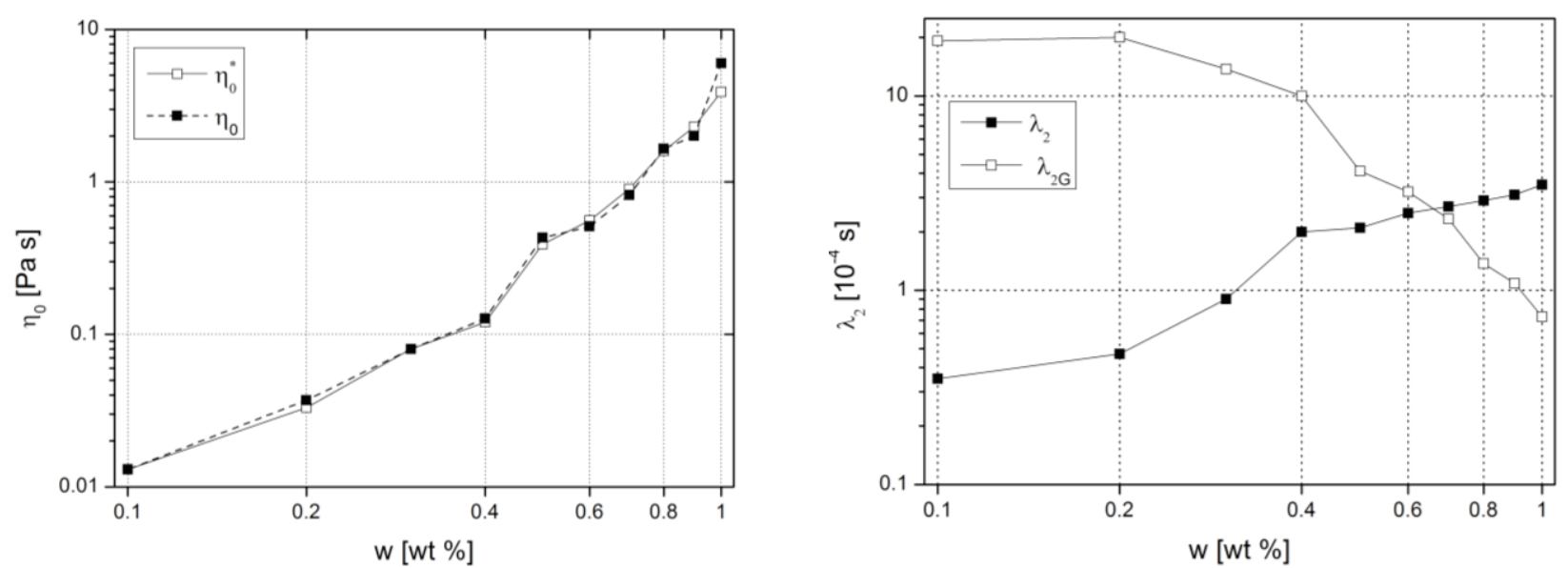

Figure 5. (Left) Calculated $\eta_{0}$ and measured $\eta_{0}^{*}$ and (right) $\lambda_{2}$ and $\lambda_{2 \mathrm{G}}$ against Praestol 2500 polymer mass fraction $w$.

\section{Zero shear viscosity}

The values of the zero-shear dynamic viscosity agree with the values from the shear viscosimetry to within $\pm 10 \%$, except for the $1.0 \mathrm{wt}$ \% polymer solution. This disagreement could be due to experimental error, i.e. the measurement error of the input parameters is too large. A second reason for the disagreement could be that this case may be close to the limit of applicability of our method regarding the polymer concentration, since we must keep in mind that our experimental method relies on the ability of the drop to perform damped periodic oscillations. Once the drop Ohnesorge number threshold to aperiodic behavior is exceeded, the deformed drop returns to its equilibrium state in an aperiodic manner, so that our method cannot be applied any more. This will be the subject of further studies.

\section{Deformation retardation time}

The results show that the measured deformation retardation time $\lambda_{2}$ increases monotonically with the polymer mass fraction $w$. For the concentrations studied, values of $\lambda_{2}$ between 35 and $350 \mu$ s are obtained. On the other hand, $\lambda_{2 G}$ as defined by the viscous-elastic stress splitting approach decreases with increasing polymer mass fraction in the range of polymer mass fractions above $w=0.2 \mathrm{wt} \%$. The two trends are equal only in the range of polymer mass fractions below $0.2 \mathrm{wt} . \%$. The trend of $\lambda_{2 G}$ to vary with the polymer concentration is quantified as $\left(\partial \lambda_{2 G} / \partial c\right) / \lambda_{2 G}=$ $\left(\partial \lambda_{1} / \partial c\right) / \lambda_{1}-\left(\partial \eta_{0} / \partial c\right) / \eta_{0}$. With the dependencies of $\lambda_{1}$ and $\eta_{0}$ on the polymer concentration given above, we arrive at the condition that $\left(\partial \lambda_{2 G} / \partial c\right) / \lambda_{2 G}$ is positive only when $\mathrm{T}>\mathrm{S}$, which is the case in the sufficiently dilute regime below $w=0.2$ wt.\% only. We conclude from this that the calculation of $\lambda_{2}$ with the viscous-elastic stress splitting approach reproduces the trend from the experiment correctly only in this sufficiently dilute regime, which corresponds to different polymer mass fraction regimes for different polymers and solvents. The absolute values of $\lambda_{2 G}$ obtained, however, still differ substantially from the result of the measurement. In the case of the present polymer solution, the measured values of $\lambda_{2}$ agree well with the calculated $\lambda_{2 G}$ for polymer mass fractions between 0.5 and 0.8 wt. $\%$ only, and this rather by coincidence. For the most dilute solutions studied, the measured and calculated values strongly deviate from each other, which finding remains to be explained once more polymer solutions were investigated. As one reason for these differences due to the different concentrations of the solutions we may see the fact that the more dilute solutions may be better described by the viscous-elastic stress splitting approach than the more concentrated solutions. This could indicate a limitation of the validity of the equation for $\lambda_{2 G}$ for concentrated systems. The absolute values of this time scale, however, still remain quite different even in the dilute solutions.

\section{Conclusions}

In this study we use linear damped shape oscillations of drops for measuring the zero-shear viscosity and the deformation retardation time of viscoelastic polymeric drop liquids. The solution of the linearized equations of change governing the drop shape oscillations yields the characteristic equation for the complex oscillation frequency which is used for determining the material properties. For a given drop, the oscillation frequency and the damping rate are measured in an experiment using acoustic levitation. Liquid material properties relevant for the oscillations, such as liquid density, surface tension and stress relaxation time, are measured by appropriate standard methods. The zeroshear viscosity and the deformation retardation time of the liquid are obtained as solutions of the characteristic equation of the oscillating drop. Values of the liquid dynamic viscosity are close to those from shear rheometry. They 
allow the correct solution of the characteristic equation to be identified from a manifold and support the correctness of the deformation retardation times determined.

The values of $\lambda_{2}$ are found to deviate strongly from values often used in simulations of viscoelastic liquid flow. The values of $\lambda_{2}$ agree with $\lambda_{2 G}$ only for small region of polymer mass fractions (between 0.5 and 0.8 wt. \%). Further work will be devoted to investigating the deformation retardation behaviour of various polymers at different concentrations in solvents of different quality.

\section{Nomenclature}

a equilibrium drop radius [m]

$\mathrm{j}_{\mathrm{m}}, \mathrm{j}_{\mathrm{m}+1} \quad$ spherical Bessel functions [1]

m mode number [1]

$\mathrm{p} \quad$ pressure $[\mathrm{Pa}]$

$\mathrm{P}_{\mathrm{m}} \quad$ Legendre polynomials

$q=q_{r}+i q_{i}$ complex inverse oscillatory length scale $\left[\mathrm{m}^{-1}\right]$

$r \quad$ radial coordinate

$\mathrm{S}, \mathrm{T} \quad$ scaling exponents [1]

$\mathrm{t} \quad$ time $[\mathrm{s}]$

$v \quad$ velocity $\left[\mathrm{m} \mathrm{s}^{-1}\right]$

$w \quad$ polymer mass fraction [wt.\%]

$\alpha_{\mathrm{m}}=\alpha_{\mathrm{m}, \mathrm{r}}+i \alpha_{\mathrm{m}, \mathrm{i}}$
$\alpha_{\mathrm{m}, 0}$
$\varepsilon_{0}$
$\dot{\gamma}$
$\eta$
$\eta_{0}$
$\lambda_{1}, \lambda_{2}, \lambda_{2 \mathrm{G}}$
$v$
$\rho$
$\sigma$
$\tau$

\author{
complex angular frequency $\left[\mathrm{s}^{-1}\right]$ \\ inviscid angular frequency $\left[\mathrm{s}^{-1}\right]$ \\ oscillation amplitude [m] \\ rate of deformation tensor $\left[\mathrm{s}^{-1}\right]$ \\ dynamic viscosity [Pa s] \\ zero-shear dynamic viscosity [Pa s] \\ polymeric time scales [s] \\ kinematic viscosity $\left[\mathrm{m}^{2} \mathrm{~s}^{-1}\right]$ \\ density $\left[\mathrm{kg} \mathrm{m}^{-3}\right]$ \\ surface tension $\left[\mathrm{N} \mathrm{m}^{-1}\right]$ \\ extra stress tensor $[\mathrm{Pa}]$
}

\section{References}

[1] Rayleigh, Lord, J.W.S., 1879, Proc. R. Soc. London A, 29, pp. 71-97.

[2] Lamb, H., 1881, Proc. London Math. Soc., 13, pp. 51-66.

[3] Kovalchuk, V., Krägel, J., Aksenenko, E., Loglio, G., Liggieri, L., 2001, in Möbius, D. \& Miller, R. (Eds.): Novel methods to study interfacial layers, Elsevier Sci. B.V., pp. 485-516.

[4] Ravera, F., Loglio, G., Kovalchuk, V., 2010, Curr. Opin. Colloid Interface Sci., 15, pp. 217-228.

[5] Perez, M., Salvo, L. Suéry, M., Bréchet, Y., Papoular, M., 2000, Phys. Rev. E, 61, pp. 2669-2675.

[6] Egry, I., Lohöfer, G., Seyhan, I., Schneider, S., Feuerbacher, B., 1998, Appl. Phys. Lett., 73, pp. 462-463.

[7] Hiller, W.J., Kowalewski, T.A., 1989, Physico-Chem. Hydrodyn., 11, pp. 103-112.

[8] Hsu, C.J., Apfel, R.E., 1985, J. Colloid Interface Sci., 107, pp. 467-476.

[9] Tian, Y.R., Holt, R.G., Apfel, R.E., 1995, Phys. Fluids, 7, pp. 2938-2949.

[10] Apfel, R.E., Tian, Y.R., Jankovsky, J., Shi, T., Chen, X., Holt, R.G., Trinh, E., Croonquist, A., Thornton, K.C., Sacco, A. Jr., Coleman, C., Leslie, F.W., Matthiesen, D.H., 1997, Phys. Rev. Lett, 78, pp. 1912-1915.

[11] Brenn, G., Teichtmeister, S., 2013, J. Fluid Mech., 733, pp. 504-527.

[12] Brenn, G., Plohl, G., 2015, J. Non-Newton. Fluid Mech., 223, pp. 88-97.

[13] Yang, L., Kazmierski, B.K., Hoath, S.D., Jung, S., Hsiao, W.K., Wang, Y., Berson, A., Harlen, O., Kapur, N., Bain, C.D., 2014, Phys. Fluids, 26, 113103.

[14] Oldroyd, J., 1950, Proc. R. Soc. London A, 200, pp.523-541.

[15] Oldroyd, J., 1958, Proc. R. Soc. London A, 245, pp.278-297.

[16] Brenn, G., Plohl, G., 2017, Atomization Sprays, 27.

[17] Bird, R.B., Armstrong, R.C., Hassager, O., 1987, Dynamics of polymeric liquids. John Wiley, New York.

[18] Amoreira, L.J., Oliveira, P.J., 2010, Adv. Appl. Math. Mech., 2, pp. 483-502.

[19] Giesekus, H.W., 1994, Phänomenologische Rheologie - Eine Einführung (Phenomenlogical Rheology - An Introduction, in German), Springer, Berlin, Heidelberg, New York.

[20] Yarin, A.L., Brenn, G., Kastner, O., Rensink, D., Tropea, C., 1999, J. Fluid Mech., 399, pp. 151-204.

[21] Stelter, M., Brenn, G., Yarin, A.L., Singh, R.P., Durst, F., 2000, J. Rheology, 44, pp. 595-616. 


\title{
Visualization of water droplet deformation and breakup in a continuously accelerated flow field.
}

\author{
A. García-Magariño*1, S. Sor ${ }^{2}$, A. Velazquez ${ }^{3}$ \\ ${ }^{1}$ Engineering Systems for Spanish Defense (ISDEFE) Madrid, Spain \\ ${ }^{2}$ National Institute of Aerospace Technology Madrid, Spain \\ ${ }^{3}$ Universidad Politécnica de Madrid, Madrid, Spain \\ *Corresponding author: agarcia-magarino@isdefe.es
}

\begin{abstract}
A new specific direct illumination technique is proposed for studying the deformation and breakup process of droplets that are exposed to a continuously increasing flow field. This type of flow field is in contrast to the traditional shock-tube experiments where droplets are suddenly exposed to a constant high airstream. In order to generate a continuously accelerated flow field the rotating arm facility at INTA is used. Droplets are allowed to fall in the path of an incoming airfoil mounted at the end of a rotating arm. Under certain conditions, these droplet deform and breakup before impinging on the airfoil. The incoming airfoil is a key element in the illumination technique. Droplets of $0.8 \mathrm{~mm}$ of diameter immersed in the flow field generated by an airfoil model of chord $0.690 \mathrm{~m}$ approaching at $80 \mathrm{~m} / \mathrm{s}$ were chosen for this study and were found to undergo a breakup process similar to the bag and stamen breakup found in the literature. Each of the stages at this type of breakup process was investigated by means of this new direct illumination technique and a high resolution camera. These images were compared to those images obtained using shadowgraph illumination technique and a high speed camera under the same conditions. Five different stages were studied and characterized. The final objective in this study was to visualize and identify fluid structures that occur during the deformation and breakup process in a continuously accelerated flow field in order to develop and fine tune physical-mathematical models.
\end{abstract}

\section{Keywords}

Droplet Breakup, Visualization, Accelerating Flow.

\section{Introduction}

Droplet aerobreakup has been studied mainly in shock tube [1,2] or wind tunnels facilities [3,4,5], where the droplet suddenly experiences a high constant air speed. Two comprehensive reviews on this field are those of Guildenbecher et al. [6] and Theofanous [7]. In the first one, attention was paid to the breakup morphology and a similar classification of breakup mode as the one found in Pilch and Erdman [8] was established: Vibrational breakup, Bag Breakup, Multimode (Bag and Stamen), Sheet Stripping (Sheet Thinning), and Catastrophic Breakup. The second review made a good discussion on the two main mechanisms leading to breakup: RayleighTaylor piercing and shear-induced entrainment. However, these two reviews [6,7] cover data obtained in facilities where droplets are suddenly exposed to a high constant velocity air stream. This is in contrast with the problem studied in this paper, where droplet are initially in a quiescent flow and then the air velocity starts to increase gradually with an acceleration that also increases, until the acceleration and the velocity reach values that allow for droplet breakup. The problem is a non-stationary problem and transient effects need to be considered. Accelerating and decelerated non-uniform flow fields have only been studied for non-deformable spheres [9,10], or droplets that are small enough to neglect deformation [11] and it has proved to be a very complex problem since there still exist contratdicting results. Therefore, one of the novelties of this work is to study the aerobreakup of droplets in a non-stationary flow.

In order to visualize and identify fluid structures that occur during the water droplet deformation and breakup process, different techniques of image acquisition can be employed: shadowgraph images [3,4,12,13,14], interpherometry [1,15] and direct illumination images [16]. Due to the difficulty associated to using direct illumination in transparent liquids, the shadowgraph technique has mainly been used in the past, providing only the distorted droplet contour. Previous studies by the authors were based on data obtained by shadowgraph illumination technique [17]. However, direct illumination allows for obtaining more information of the structure, which can provide a better understanding of the details of the process. This article compares visualization of the breakup of droplets in a non-stationary accelerating flow field using direct illumination technique to previous 
shadowgraph images. Only the bag and stamen breakup type is addressed. First the specific direct illumination technique is described, then the results are discussed and finally the main conclusions are summarized.

\section{Material and methods}

In order to achieve a continuously accelerated flow field the rotating arm facility at INTA was employed. The rotating arm facility had already been used to study water droplet deformation and breakup in a continuously accelerated flow field using the shadowgraph technique [17]. A photograph of the experimental setup can be observed in Figure 1. An airfoil model was attached to the end of a rotating arm whose length was $2.2 \mathrm{~m}$. At the same time, droplets of $0.8 \mathrm{~mm}$ of diameter were allowed to fall in the path of the incoming airfoil. These droplets were generated by means of a TSI MDG-100 droplet generator functioning on the bases of the Rayleigh theory. As the airfoil model approaches the droplets, the air velocity, as seen by the droplets, increases continuously. The flow field generated by the airfoil was characterized in advance using the Particle Image Velocimeter (PIV) technique. A complete description of this characterization can be found in [17]. This characterization allows us to correlate the conditions at which each breakup stage take place. The velocity flow field measurements, as seen from the airfoil reference system, can be observed in Figure 2. The airfoil model profile shape used is plotted in Figure 3. The airfoil model chord was $690 \mathrm{~mm}$, its leading edge radius was $103 \mathrm{~mm}$ and its span length was 200 $\mathrm{mm}$.

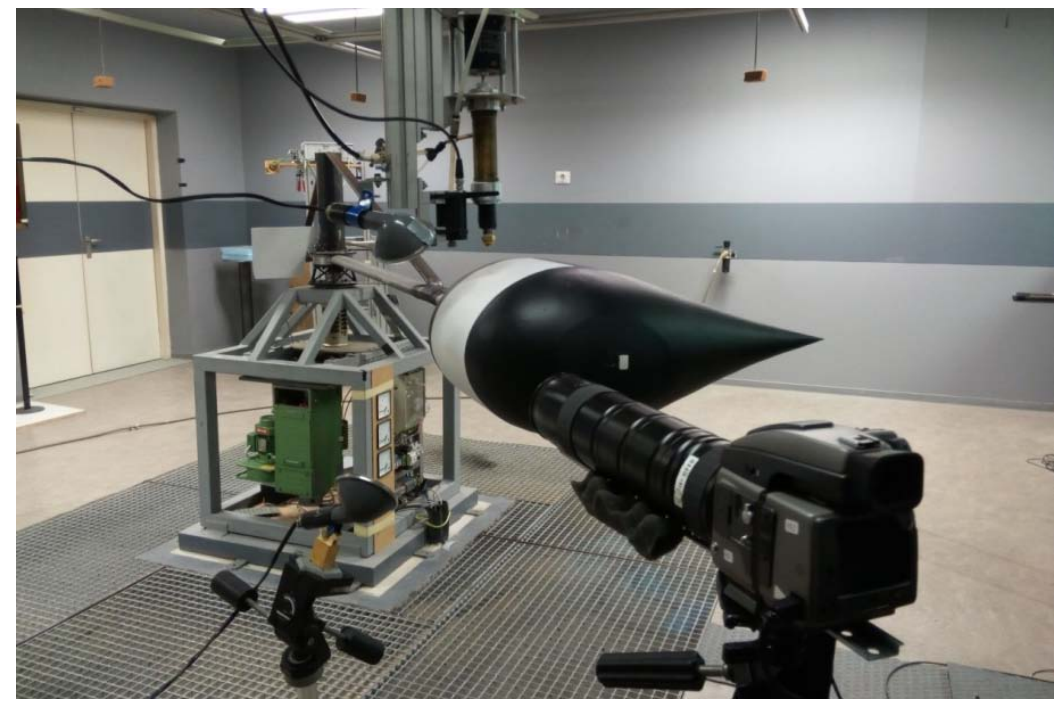

Figure 1. Photograph of the experimental setup.

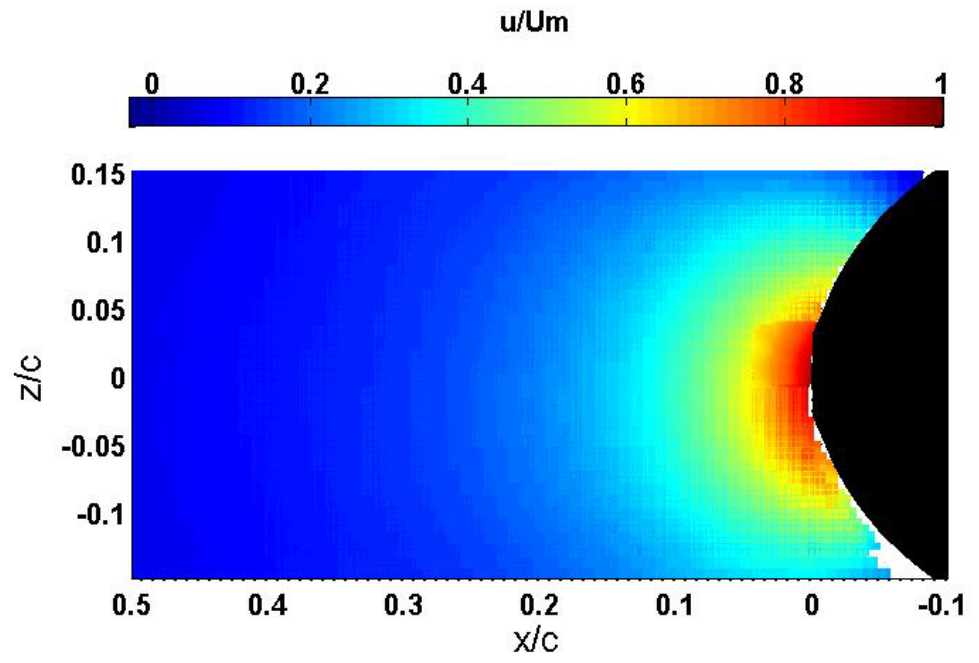

Figure 2. PIV measurements of the 2D vector magnitude of the velocity flow field as seen from the airfoil reference system. 


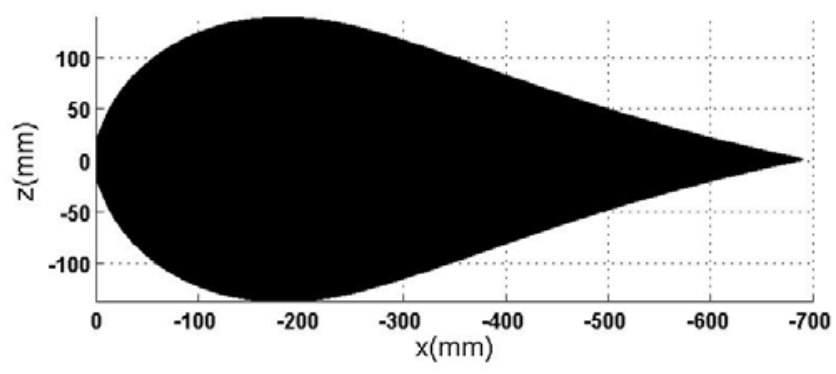

Figure 3. Airfoil model profile.

\section{Direct illumination technique}

The key element in the acquisition data technique presented in this paper is this illumination technique. The front part of the model was painted white to reflect the light and to increase the droplet illumination. The advantage of using the model as a light reflector is that the model is very close to the droplets when they breakup providing the maximum amount of light. Two flashes lamps, the 1538-A Strobotac Electronic Stroboscopic and the1539-A Stroboslave, illuminate the droplets and the airfoil model when it approaches the droplets (See Figure 4). Each flash lasted on the order of $3 \mu \mathrm{s}$. A diode photodetector trigger was installed at a certain position of the model path. The flashes are delayed a certain time from the trigger signal using a Programmable Timing Control Hub IDT. By choosing the delay time, the distance of the model to the droplets is varied. This allows for taking images at different stages of the deformation and breakup process. Photographs are taken by the high resolution camera Hasselblad H3DII-39, which has a resolution of 7216 pixels (width) $\times 5412$ pixels (height). The magnification was $145 \mathrm{pix} / \mathrm{mm}$. The lens used were: HC 4/200 mm, extensions H52 mm, H26 mm, H13 mm and a converter H1.7x. The camera setup was ISO-200 and f/6.8.

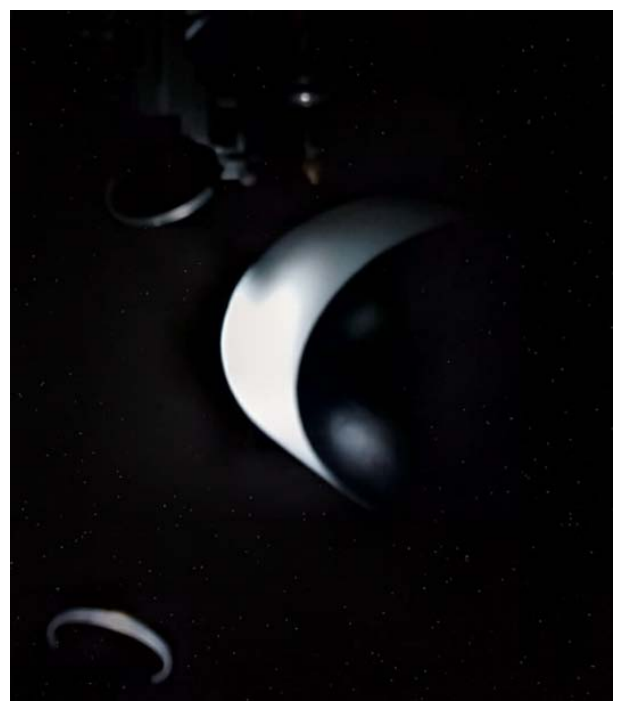

Figure 4. New illumination technique

An example of the images recorded by this illumination technique can be observed in Figure 5. The airfoil model was moving at $80 \mathrm{~m} / \mathrm{s}$ and the initial droplets diameter was $0.8 \mathrm{~mm}$. 


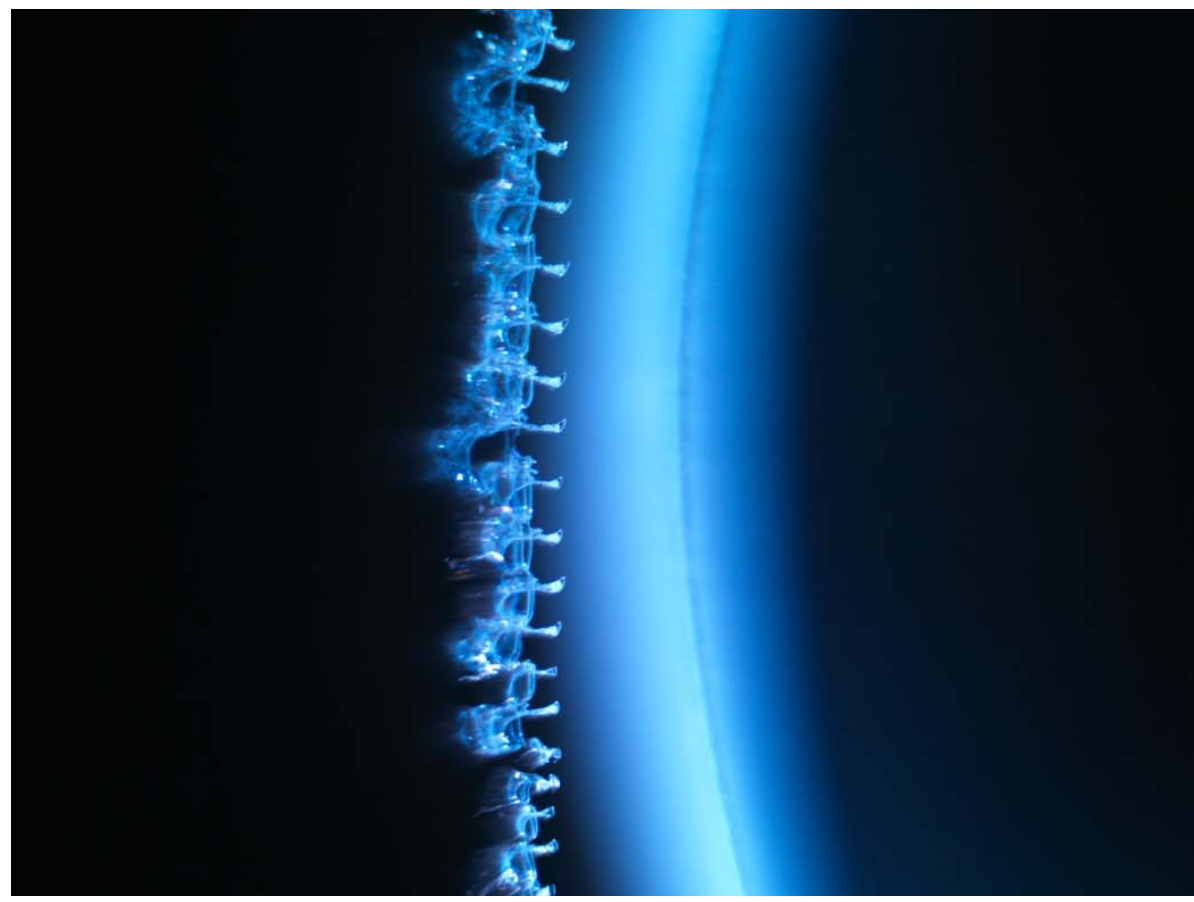

Figure 5. An example of the images taken by the direct illumination technique.

\section{Shadowgraph illumination technique}

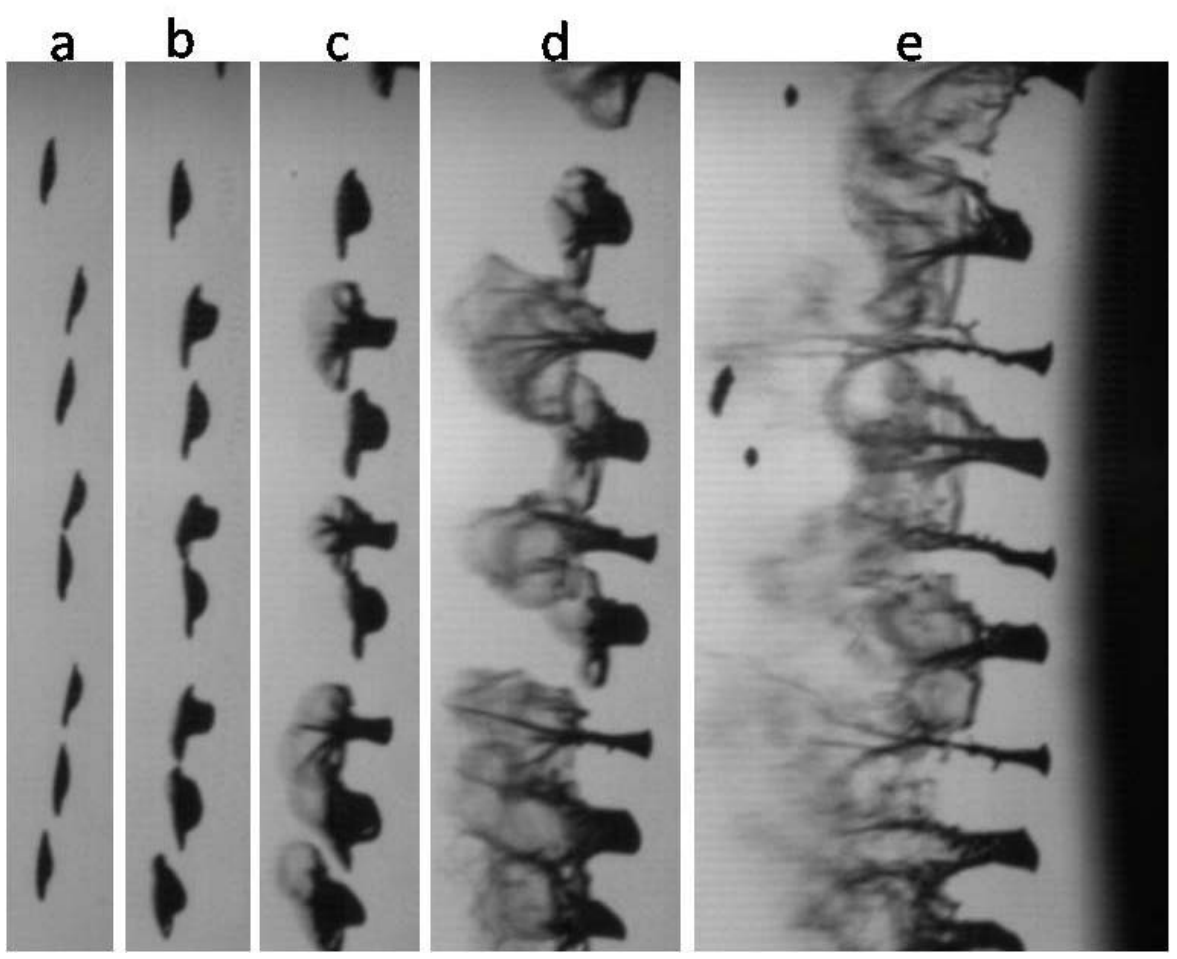

Figure 6. Five shadowgraph images that corresponds to the five stages found: a) Oblate spheroid shape (distance to the model $22 \mathrm{~mm}$, air velocity $59 \mathrm{~m} / \mathrm{s}$ ); b) Hat-like shape (distance to the model $16 \mathrm{~mm}$, air velocity $64 \mathrm{~m} / \mathrm{s}$ ); c) Mushroom-like shape (distance to the model $10 \mathrm{~mm}$, air velocity $69 \mathrm{~m} / \mathrm{s}$ ); d) Parachute-like shape (distance to the model $6 \mathrm{~mm}$, air velocity $74 \mathrm{~m} / \mathrm{s}$ ) and e) Fountain-like shape (air velocity $79 \mathrm{~m} / \mathrm{s}$ ).

In order to compare the photographs taken using direct illumination technique to the traditional shadowgraph technique, additional tests using shadowgraph illumination were also employed. Instead of a high resolution 
camera, a high speed camera at $75000 \mathrm{fps}$ was used with a resolution of $192 \times 312$ pixels. A xenon lamp of 2000 W illuminated back from the droplet background. The magnification was $14.26 \mathrm{pix} / \mathrm{mm}$. The advantage of using a high speed video camera was that a single droplet can be tracked, while the higher resolution camera was only able to take a single shot (photo camera). Either of the two ways may be helpful in faster understanding. An image of the evolution of deformation and breakup process can be observed in Figure 6 .

\section{Results and discussion}

A sequence of the images as the droplets approach the model using both direct illumination technique and shadowgraph illumination are shown in Figures 6 and 7 respectively. It can be observed that, despite of the differences in the incoming flow field topology, droplets undergo a similar process to that found in the literature known as "bag and stamen" or "multimode" breakup mode. The breakup process has been divided into five stages summarized in Table 1, where characteristic distances from the leading edge of the droplet to the leading edge of the model at the centerline and their corresponding air velocities are given. Additionally Reynolds numbers and Weber numbers based on the droplet diameter and the instantaneous air velocity are also provided in Table 1.

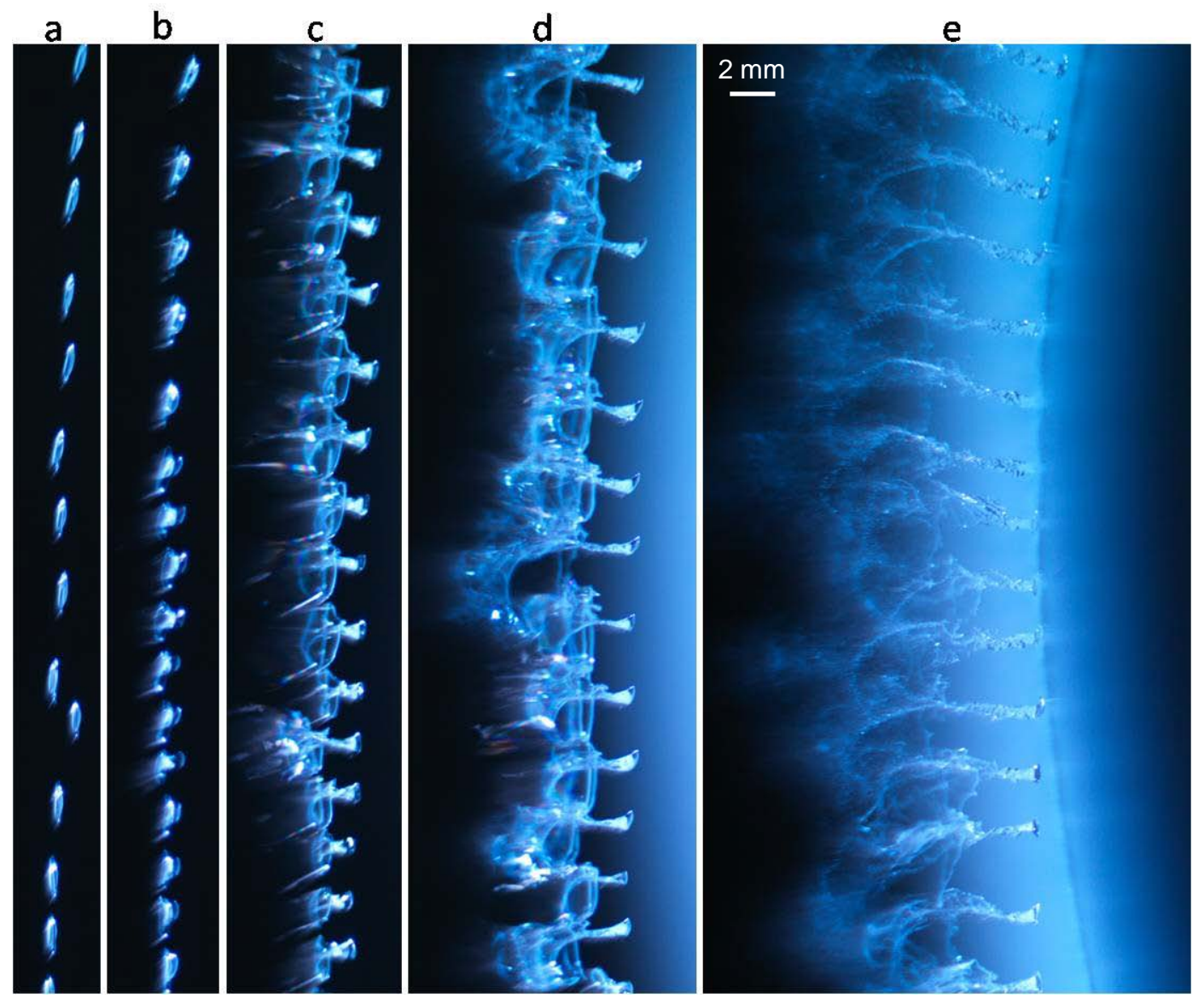

Figure 7. Five cropped images that correspond to the five stages found: a) Oblate spheroid shape (distance to the model 22 $\mathrm{mm}$ ); b) Hat-like shape (distance to the model $16 \mathrm{~mm}$ ); c) Mushroom-like shape (distance to the model $10 \mathrm{~mm}$ ); d) Parachutelike shape (distance to the model $5 \mathrm{~mm}$ ) and e) Fountain-like shape.

Table 1. Breakup stages found.

\begin{tabular}{c|c|c|c|c|c}
\hline \hline $\begin{array}{c}\text { Breakup } \\
\text { Stage }\end{array}$ & $\begin{array}{c}\text { Droplet Contour Shape } \\
\text { (observed in shadowgraph image) }\end{array}$ & $\begin{array}{c}\text { Distance to the } \\
\text { model }(\mathrm{mm})\end{array}$ & $\begin{array}{c}\text { Air velocity } \\
(\mathrm{m} / \mathrm{s})\end{array}$ & $\mathrm{Re}$ & We \\
\hline \hline a & Oblate Spheroid & 20 & 60 & 3230 & 48 \\
b & Hat-like shape & 15 & 65 & 3450 & 55 \\
c & Mushroom-like shape & 10 & 69 & 3700 & 63 \\
d & Parachute-like shape & 5 & 74 & 3970 & 73 \\
e & Fountain-like shape & 0 & 80 & 4270 & 84 \\
\hline \hline
\end{tabular}


Droplets that are initially spherical undergo a deformation process where they come to resemble an oblate spheroid due to the higher pressures found in the front and rear sides of the droplet and the lower pressures zone in the droplet rim. During this process, the maximum diameter of this oblate spheroid increases while the minimum diameter decreases. Then, there is an instant where the minimum diameter no longer decreases, but starts to increase. Theofanous et al [7] argued that this is due possibly to the Rayleigh-Taylor piercing at the forward side of the droplet. Figure 8 shows an image at this point. It can be observed the onset of a bulge starting on the right side, while an incipient recess in the left side is also evidenced thanks to the direct illumination technique. The incipient recess in the left side was not considered previously in the theoretical modelling [18]. Additionally the ratio of the bulge height to the droplet maximum diameter observed is greater than the typical multimode or bag and stamen morphology [19]. Then this bulge evolves towards to a hat cup that increases in length. A possible physical explanation for the increase in length of the hat cup is that the mass of the bulge is greater than the mass of the brim, which leads to a greater inertia.

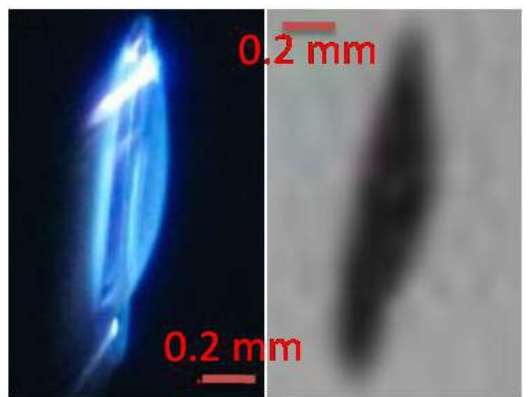

Figure 8. Oblate Spheroid comparison. Distance from the leading edge of the droplet to the model of $20 \mathrm{~mm}$.

Figure 9 shows the next stage, where a hat-like shape is evidenced. It can be observed the brim of the hat, where a concavity seems to be discerned.

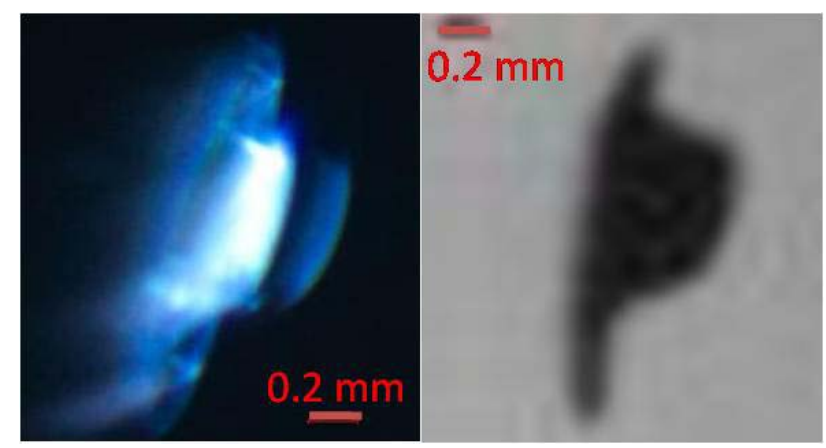

Figure 9. Hat-like shape comparison. Distance from the leading edge of the droplet to the model of $15 \mathrm{~mm}$.

In the next image, in Figure 10, the initial concavity in the brim of the hat has grown considerably by the time the traditionally mushroom-like shape is observed in the shadowgraph image. It can be observed that the rear side of the droplet (the left-side in the picture), has a hole in the middle whose length could even be guessed by the different colour observed in the image. The diameter of the brim has also increased being now of the order of 2.2 $\mathrm{mm}$. The depth of the concavity of the brim was measured to be of the order of $0.75 \mathrm{~mm}$. This direct illumination technique provides new information on that very feature at this point. From the shadowgraph image, it could be concluded that the rear part of the droplet was a bag; however from the new images it is evident the appearance of the central hole. The same is inferred from the parachute stage in Figure 10 also: although it could be inferred that there exists a half spherical big bag acting as a parachute, a toroidal bag with a central rear hole is evidenced actually in the new figure. Further studies are needed to explain from a physical point of view the new features found. 

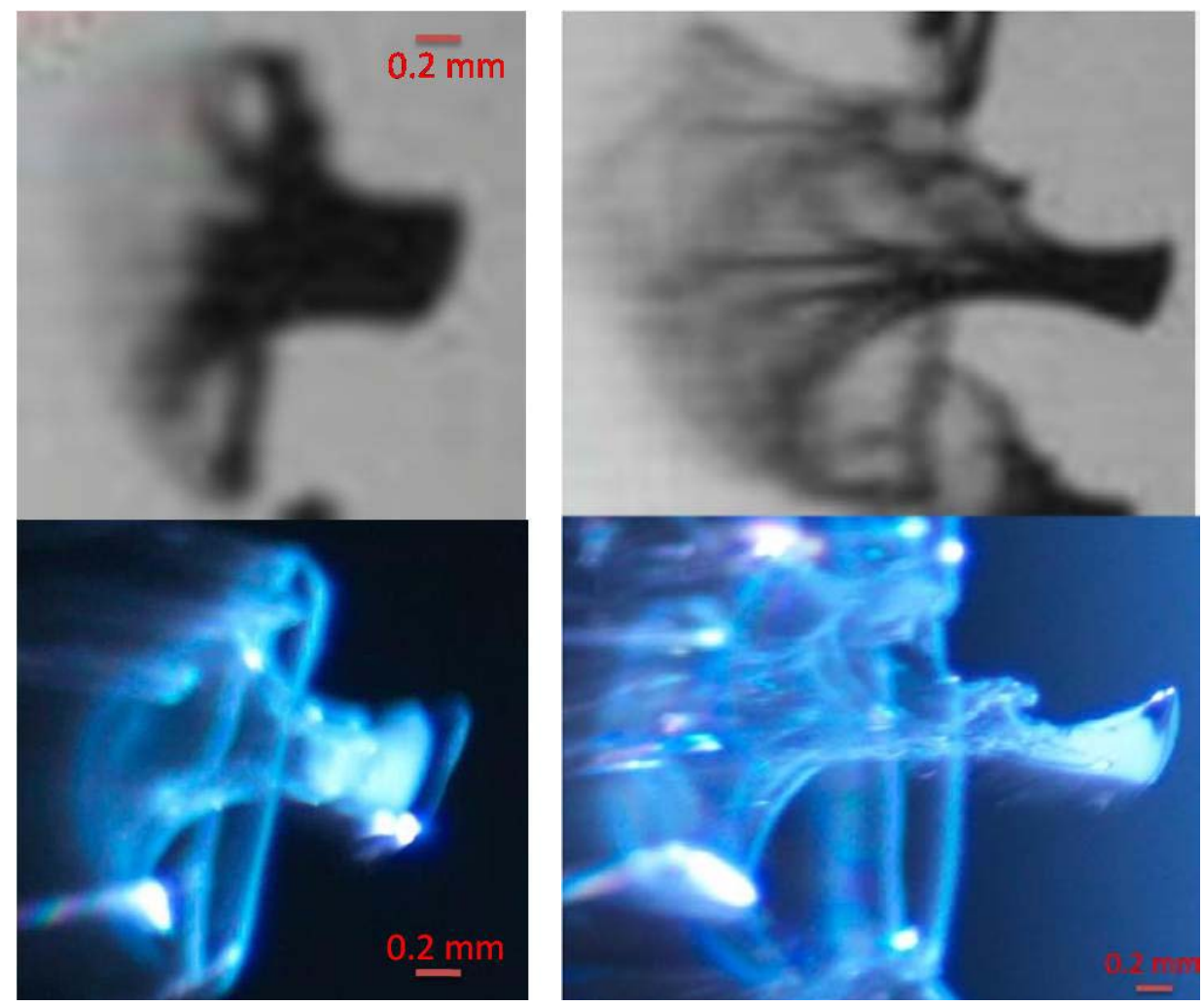

Figure 10. On the left: Mushroom-like shape comparison; distance from the leading edge of the droplet to the model of $10 \mathrm{~mm}$. the right: Parachute-like shape comparison; distance from the leading edge of the droplet to the model of $5 \mathrm{~mm}$.

Finally, the toroidal bag breaks and a fountain-like shape appear. Then the stamen, which was the tube that connected the toroidal bag to the rest of the droplet starts to break up as well. Figure 11 shows this last stage. It seems that most of the remaining droplet mass is located in the right hand side of the stamen.

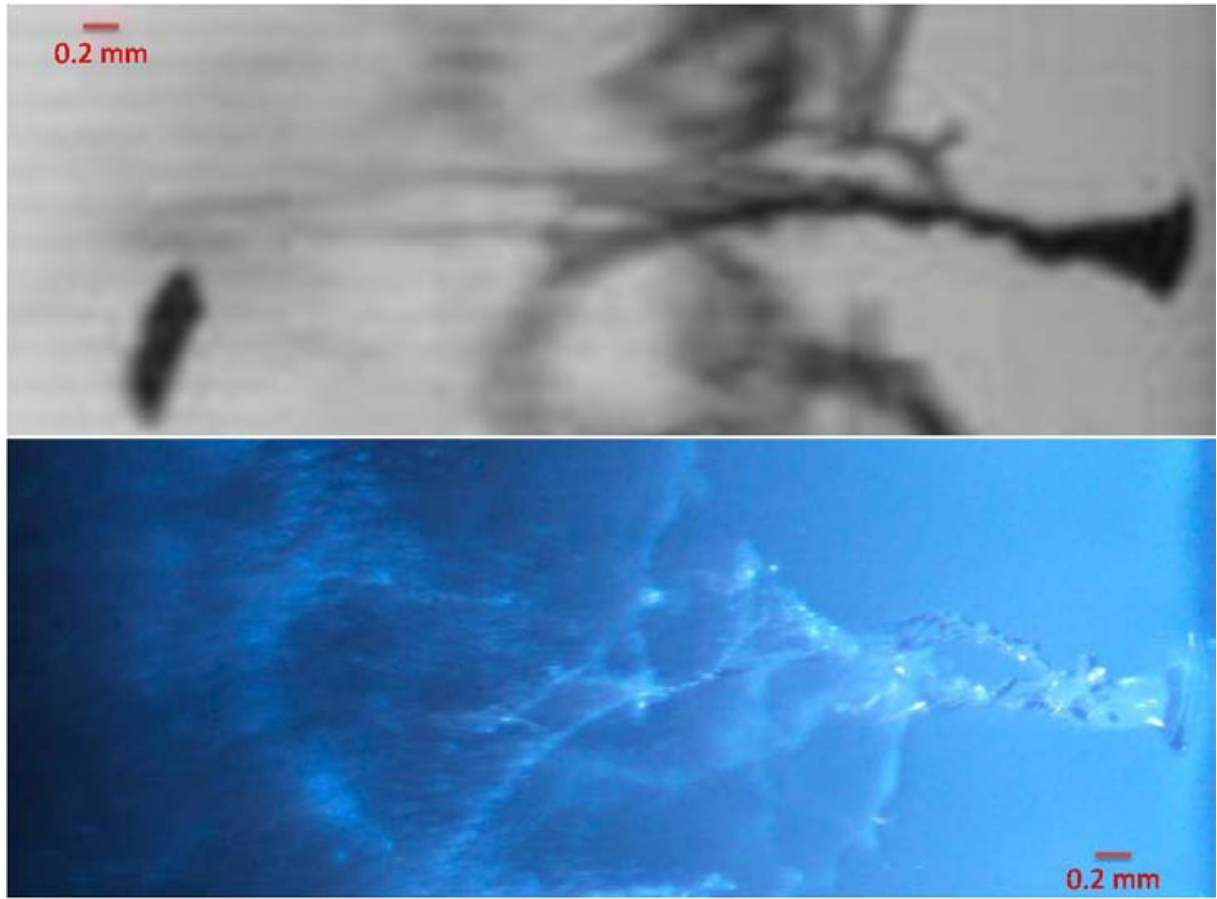

Figure 11. Fountain-like shape comparison.

This work is licensed under a Creative Commons 4.0 International License(CC BY-NC-ND 4.0). 


\section{Conclusions}

The deformation and breakup process of $0.8 \mathrm{~mm}$ diameter droplets subjected to a continously accelerating flow field has been studied by means of a new direct illuminaiton technique. The topology of the breakup was the type of "bag and stamen" or "multimode" breakup. Five different stages in the process were identified and characterized: a) Oblate spheroid shape, b) Hat-like shape, c) Mushroom-like shape, d) Parachute-like shape and e) Fountain-like shape. Direct illumination images obtained provide additional information regarding fluid structures that occur during the deformation and breakup process. Out from the beginning of the breakup process, a hole appears in the rear part of the droplet that was not inferred from previous observations. This hole is increasing in size as the stamen increases until the stamen finally breaks up. Further studies are needed to explain from a physical point of view the new features found.

Though the direct illumination technique has proved to be a good approach in this case, some problems have also been encountered. Very high light intensity was needed mainly due to two reasons: the short duration times of the phenomena (which led to the necessity of short flash duration) and the low reflection of the droplet due to its transparency. In order to obtain more light intensity, the reflector (the incoming airfoil model painted in white) could be placed very close to the droplet, thereby allowing for a larger amount of light. Due the direct illumination used, glints also appear in the photographs.

\section{Acknowledgements}

A. Velazquez was funded by the Spanish Ministry of Economy and Competitivenes (Ministerio de Economia y Competitividad) under research contract DPI2016-75296-P and gratefully acknowledges this support. A. GarcíaMagariño was funded by Spanish Ministry of Defense under the INTA program "Termofluidodinámica".

\section{Nomenclature}

C chord [mm]

u velocity $\left[\mathrm{m} \mathrm{s}^{-1}\right]$

$\mathrm{U}_{\mathrm{m}} \quad$ model velocity $\left[\mathrm{m} \mathrm{s}^{-1}\right]$

$\mathrm{x} \quad$ horizontal distance $[\mathrm{mm}]$

z vertical distance $[\mathrm{mm}]$

\section{References}

[1] Wierzba, A., Takayama, K., 1988, AIAA Journal, 26(11), pp. 1329-1331

[2] Hsiang, L.P., and Faeth, G. M., 1992, International Journal of Multiphase Flow, 18(5), pp. 635-652.

[3] Luxford, G., Hammond, W., and Ivey, P., January 2005, 43rd Aerospace Sciences Meeting and Exhibit.

[4] Wierzba, A., 1990, Experiments in Fluids, 9, pp. 59-64.

[5] Theofanous, T. G., and Li, G.J., 2008, Physics of Fluids, 20, 052103 pp:1-14

[6] Guildenbecher, D. R., Lopez-Rivera, C., and Sojka, P. E., 2009, Experiments in Fluids, 46(3), pp. 371-402.

[7] Theofanous, T. G., 2011, Annual Review of Fluid Mechanics, 43, pp. 661-690

[8] Pilch, M., and Erdman, C. A., 1987, International Journal of Multiphase Flow, 13(6), pp. 741-757.

[9] Journal G., Houas L., Igra, O., Estivalez, J.-L., Devals, C., Meshkow, E. E., 2007, Proceeding of Real Society A, 463, pp. 3323-3345.

[10] Igra, O., Takayama, K., 1993, Proceeding of Real Society A, 442, pp. 231-247.

[11] Temkin, S., Metha, H. K., 1982, Journal of Fluid Mechanics, 116, pp. 297-312.

[12] Theofanous, T. G., Li, G.J., and Dinh, T. N., 2004, Transactions of the ASME, 126, pp: 516-527.

[13] Kwon, K.-S., Kim, H.-S., and Choi, M., 2016, Review of Scientificic Instruments, 87, 035101 pp: 1-10.

[14] Gobyzov, O., Lozhkin, Y., Ryabov, M. and Markovich, D., 2016, EPJ Web of Conferences, 114, 02031 pp: 16.

[15] Pavlov, I. N., Rinkevichyus, B. S., and Tolkachev, A. V., June 25-28, 2012, $15^{\text {th }}$ International Symposium on Flow Visualization, 114, $02031 \mathrm{pp}:$ 1-6.

[16] Illias, S., Ishak, M. S. A., Hussain, S. and Ismail, K. A., 2016, International Journal of Applied Engineering Research, 11(22), pp: 10832-10837.

[17] Garcia-Magariño, A., Sor, S., and Velazquez, A. 2015, Aerospace Science and Technology, 45, pp. $190-500$.

[18] Wang, C., Chang, S., Wu, H., Ding, L., and Thompson, J. M. 2015, Atomization and Sprays, 25(10), pp. 857869.

[19] Theofanous, T. G., Li, G. J., and Dihn, T. N. 2004, Transactions of the ASME, 126, pp. 516-527. 


\title{
Analysis of Heat and Flow Characteristics on Spray Cooling Heat Transfer with an Optimized Hexagonal Finned Heat Sink
}

\author{
Faruk Yesildal ${ }^{* 1}$ and Kenan Yakut ${ }^{2}$ \\ ${ }^{1}$ Mechanical Engineering Department, Agri Ibrahim Cecen University, Agri, Turkey \\ ${ }^{2}$ Mechanical Engineering Department, Atatürk University, Erzurum, Turkey \\ ${ }^{*}$ Corresponding author: fayesildal@agri.edu.tr
}

\begin{abstract}
An experimental study was carried out with a hexagonal finned heat sink to obtain valuable information about the thermal and flow characteristics on spray cooling. Water was used as the refrigerant fluid and was atomized by an air-assisted atomizer and some of the variables that affect spray cooling process, such as breakup length and Sauter Mean Diameter (SMD) were obtained using appropriate correlations. The parameters most influential on the Nusselt number were determined and analyzed. Experiments were conducted for optimized conditions. The jet diameter and spray angle were determined via image processing using macroscopic aspects. Nusselt were demonstrated of hexagonal finned heat sink which optimized according to the Taguchi optimization method. The results of the spray analysis showed that SMD decreases with an increase in either air-liquid mass ratio (ALR) or operating pressure resulting in a more uniform spray. As the liquid flow rate increases in all ALR values, the heat transfer rate also increases markedly. As a results of the experiments, Nusselt number, jet width and spray angle correlations were developed. The relationship of ALR-Nu was demonstrated according to fin height and spraying time.
\end{abstract}

Keywords:

Spray cooling, Spray parameters, Taguchi, Non-boiling regime

\section{Introduction}

Spray can be used for large amounts of energy transfer in low temperatures during cooling, and heat can be removed from the surface more easily than pool boiling. Thus, the heat transfer rate is higher, and the drops can be affected by mass, energy, and momentum interchanges between them. Air-assisted spray cooling is utilized more often in the industry, compared to forced air jets and pressured nozzles. Air-assist spray is currently used during some applications in which rapid cooling is required, such as cooling of plates that have a normal thickness, cooling of hot-rolled steel, tempering glass in the automotive industry, and cooling of electronic chips. Additionally, air-assisted nozzles are widely used in the food processing industry. Spray cooling is also used in advanced technology applications, such as thermal control of space stations, safety of nuclear reactors, and cooling of turbine blades [1]. The studies related to spray flow, heat transfer, and image processing in the literature are summarized below.

Researchers in [2] investigated the effects of spray on a square heated test surface was investigated to ascertain the cooling characteristics of PF-5052. Full-cone spray nozzles were used in the experiments to compare FC-72, FC-87, and PF-5052 liquids in terms of spray orientation and cooling performance. Sauter Mean Diameter (SMD) and its cooling effects also investigated. The obtained database was used to develop a general correlation between single-phase heat transfer, nucleate boiling, and CHF. The authors offered a single correlation related to nucleate boiling data for different fluids based on density ratio, We and Ja number. They also showed that the orientation was not a measurable effect on spray cooling regimes.

Researchers [3] compared continuous and intermittent spray cooling for low temperature fluxes in a single-phase regime and for high temperatures in a two-phase regime. They obtained the required cooling rate on a target surface by controlling flow rate and frequency. The efficiency of intermittent spray was higher than continuous spray, and it was also found that intermittent spray increased spray efficiency in low flow rates. Additionally, the spray angle was obtained using image processing.

Liu et al. [4] investigated the heat transfer performance on augmented surfaces during cooling by water-spraying in non-boiling regime. Surface augmentation was carried out by straight fins. The surfaces were placed on copper blocks with cross sectional areas of $0.01 \mathrm{~m} \times 0.01 \mathrm{~m}$. They showed that the heat transfer was enhanced on straight fin surfaces much more than on flat surfaces. However, they also investigated that increasing rate decreases as fin height increases.

A comprehensive review of flow-visualization techniques for analysing spray characteristics was conducted and experiments of different lighting techniques performed. Researchers [5] introduced the spray photos of airblast and swirl atomizers by using rear, slit, incident and scattered light methods. The authors showed that the photos taken 
using these methods are useful for jet breakup, internal details, and external appearances of spray. They also reached and the conclusion that combining different techniques may be necessary for achieving suitable specifications.

Charalampous et al. [6] used three techniques to measure the breakup length in airblast atomizers. First, a single high-resolution photo was taken using a high-speed shadowgraphy method to determine the breakup length and duration. Second, using an electrical conductivity method, conductivity was vertically measured in the jet. Third, the optical connectivity method was used to take photos by lighting the jet with a laser. The authors compared the advantages and constraints of these three methods.

Karwa et al. [7] cooled a copper surface with a diameter of $0.02 \mathrm{~m}$ using water spray from a pressured atomizer in the non-boiling regime. The surface was isolated and heated using electricity. The experiments were conducted in the following conditions: $35-85 \mathrm{~W} / \mathrm{cm}^{2}$ heat flux, $95^{\circ} \mathrm{C}$ surface temperature, and $2.6-9.9 \mathrm{~kg} / \mathrm{m}^{2}$ mass flow. The heat transfer coefficient was calculated between the ranges of 9,000-24,000 W/m² $\mathrm{K}$, and heat transfer increased with the increase of mass flow. The authors developed an empirical correlation related to average Nusselt and Reynolds numbers. The correlation was found to be $N u=20.344(R e)^{0.659}$. The distribution of target surface temperature was calculated by extrapolation in accordance with the method given by [2]. The results showed that the heat transfer coefficient was dramatically larger than the forced convection under non-boiling conditions.

In [8], heat transfer was investigated using deionized water in a non-boiling spray cooling system. The experiments were conducted using two full cone spray nozzles in an open-loop test system. The effects of liquid volumetric flow rate, distance between nozzle to surface and liquid inlet temperature were also investigated. The authors observed that the heat transfer coefficient increased dramatically with the increase of volumetric flow rate and decrease of liquid inlet temperature. They also pointed out that adding a surfactant to the liquid increased the heat transfer.

There are various studies in the literature on spray cooling, image processing, droplet size distribution, atomization performance [9], drop morphology [10], characterization of spray [11], injection parameters [12], and drop impact [13].

In this study, the optimum spray parameters in hexagonal finned heat sinks in the conditions of constant surface temperature and a non-boiling regime are determined using Taguchi experimental design method. In the first step, the parameters that have impacts on heat transfer, such as the distance between nozzle to surface, fin height, fin width, distance between the fins in directions $x$ and $y$, liquid and air flow rates and spraying time, were determined. The temperature of the surface was lower than the boiling temperature of the refrigerant in the non-boiling regime. The droplets accumulated on the heated surface as a moving liquid film during spraying. The heat transfer mechanism in the non-boiling regime was composed of forced convection and liquid film vaporization. As the surface temperature increased to the point of boiling in the cooler, phase transition gives the expected increase in heat flux. Spray cooling provides a controlled cooling process particularly during electronic cooling in a non-boiling regime. Spray cooling is the most effective method for metal cooling due to increasing contact between droplets and the surface. Air-assisted spray also provides advantages to this process [14].

Biot number $(\mathrm{Bi})$ should always be enumerated at the outset to identify transient conduction problems which may be treated simply as lumped parameter problems, for which $\mathrm{Bi}<0.1$ and for which it is seldom necessary to solve the conduction equation. Convection is the dominant controlling process [15]. If $\mathrm{Bi}<0.1$ it is generally accepted that lumped system analysis [16]. In this study, the Biot number was calculated to be very low for hexagonal finned aluminium heat sinks with constant surface temperature in a non-boiling regime; thus, the total mass approach was used. In this case, convection was the dominant heat transfer mechanism and rate-controlling process. The heat removed from the aluminium surface was calculated as;

$$
Q=m_{A l} C_{p} \Delta T
$$

$\Delta T$ is the difference of average surface temperatures before and after spraying. The heat transfer coefficient is defined below as a spray heat transfer coefficient $\left(h_{s p}\right)$;

$$
h_{s p}=\frac{\left(Q-Q_{r a d}-Q_{e v p}\right)}{A_{y}\left(T_{s}-T_{s p}\right)},
$$

where the Nu number defined as

$$
N u=\frac{h_{s p} D}{k}
$$

The effect of heat transfer by radiation was calculated according to the average temperature of the surface during spraying. The heat removed by evaporation was calculated for different spraying times based on the change in relative humidity on the surface. The humidity was measured before and after spraying. Measurements were taken over the finned surface $(1 \mathrm{~cm})$ with different stations by humidistat. The average evaporation rates are obtained as $16 \%, 17 \%$ and $22 \%$ for spraying time of 5 s, 10 s, and 15 s, respectively.

\section{Material and methods}

Spray cooling is an effective heat convection method and is widely used in engineering applications. Most experimental data and explicative models have been developed over years for all stages. These stages are drop forming, diameter-velocity distribution, drop-air interaction, impact and dispersion mechanisms, and drop-surface 
heat transfer. Surface tension, outlet velocity of liquid drop, and flow rate affect the liquid breakup mechanisms. Spray drops are characterized by diameter, velocity, and three-dimensional distributions.

In the hexagonal finned heat sinks, the advantages of air-assisted spray cooling over air jet and pressured water spray cooling were investigated in terms of optimized geometrical and operational spray parameters. Using an experimental system (Figure 1), the outlet velocity of spray from the nozzle, droplet diameter, SMD, and jet diameter parameters were determined by image processing and relevant correlations. The images were processed using Matlab GUI to investigate the spray flow parameters. SMD values were calculated using the correlations offered by Shanawany [17] (Eq. 4) and Feras [18] (Eq. 5).

$$
\frac{\mathrm{D}_{32}}{\mathrm{D}_{\mathrm{h}}}=\left(1+\frac{\dot{\mathrm{m}}_{\mathrm{L}}}{\dot{\mathrm{m}}_{\mathrm{A}}}\right)\left[0.33\left(\frac{\sigma}{\rho_{\mathrm{A}} \mathrm{U}_{\mathrm{A}} \mathrm{D}_{\mathrm{p}}}\right)^{0.6}\left(\frac{\rho_{\mathrm{A}}}{\rho_{\mathrm{L}}}\right)^{0.1}+0.068\left(\frac{\mu_{\mathrm{L}}^{2}}{\sigma \rho_{\mathrm{L}} \mathrm{D}_{\mathrm{p}}}\right)^{0.5}\right]
$$

In this dimensionless correlation, the hydraulic diameter $\left(D_{h}\right)$ was calculated as $D_{h}=2 d_{0}$. $D_{p}$ is the characteristic diameter for the atomizer [19].

$$
D_{32}=0.95 \frac{\left(\sigma \mu_{L}\right)^{0.33}}{\rho_{L}^{0.37} \rho_{A}^{0.3} U_{A}}\left[1+\frac{\dot{m}_{L}}{\dot{m}_{A}}\right]^{1.7}+0.13\left(\frac{\mu_{L}^{2} d_{0}}{\sigma \rho_{L}}\right)^{0.5}\left(1+\frac{\dot{m}_{L}}{\dot{m}_{A}}\right)^{1.7}
$$

The instruments used for measurements were installed in accordance with the system guidelines. Surface heat dissipation was measured and processed using a TESTO 875-2 thermal camera. The thermal camera in steadystate conditions was used to evaluate the average surface temperature before and immediately after spraying. The changes in Nusselt-ALR were investigated for different spraying times (Fig. 5) and fin heights (Fig. 6). For the heat sink to be heated at a constant temperature, water/oil bath with a circulator was established and the reactor was designed and manufactured. The top of the reactor was heated by the water/oil bath according to the heat sink's dimensions. The base plates were made of aluminum and were $0.3 \mathrm{~m}$ long, $0.3 \mathrm{~m}$ wide, and $0.008 \mathrm{~mm}$ height. It was surrounded by heat insulation.

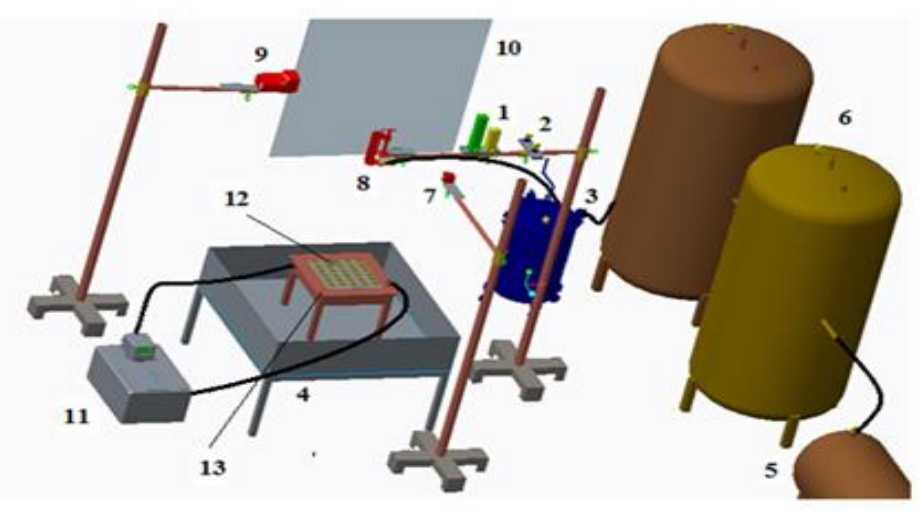

Figure 1. Experimental apparatus (1-Flowmeters, 2-Manometers, 3-Pressurized water tank, 4-Reservoir, 5-Compressor, 6-Air tank, 7- CCD camera, 8-Nozzle, 9-Stroboscope, 10-Black background, 11-Water/oil Bath, 12-Hexagonal finned heat sinks, 13Reactor)

Previously, experiments were conducted to determine the effects of parameters and dispersion on heat transfer during air-assisted spray cooling. Variables examine were the parameters affecting flow and heat transfer processes, arrangement of fins, air and liquid flow rates, fin heights, and spray duration in hexagonal heat sinks to determine the specified optimum geometric and flow parameters for hexagonal finned heat sinks. Experiments were conducted at a constant surface temperature. A Taguchi $L_{18}\left(2^{1 *} 3^{7}\right)$ standard orthogonal array was chosen as the experimental layout. Based on the results of the Taguchi experiments, we performed the experiments under optimum conditions. Experiments were conducted under optimized conditions using manufactured optimum heat sinks. (Fig. 2). 

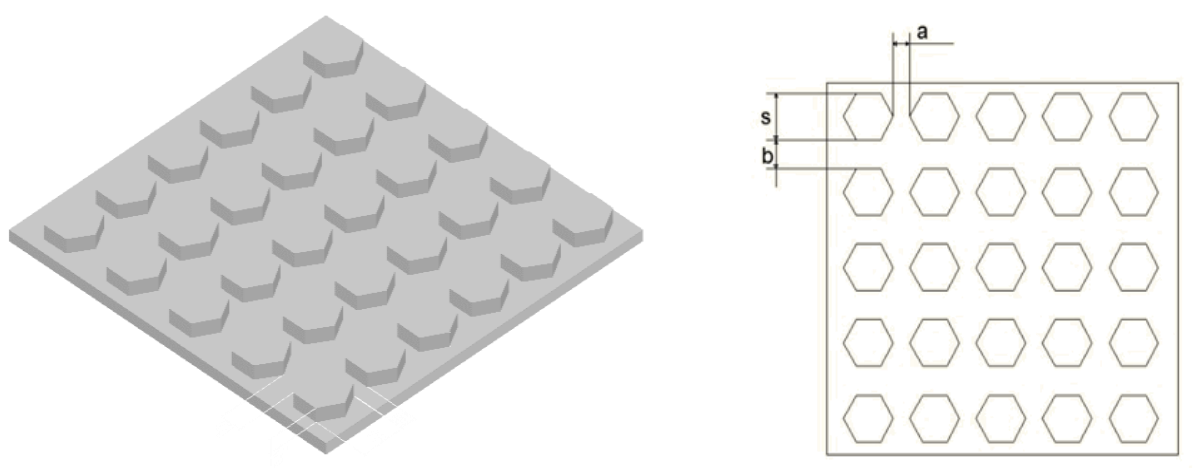

Figure 2. Optimal geometry of the hexagonal finned heat sink

Optimum geometric parameters investigated in this study are shown in Figure 2. All optimized parameters are classified in Table 1.

Table 1. Optimized Parameters

\begin{tabular}{c|c}
\hline \hline Symbol & Main parameters \\
\hline \hline$H / d$ & $\mathrm{H} / \mathrm{d}$ rate \\
$h_{k}$ & Fin height [mm] \\
$s$ & Fin width [mm] \\
$a$ & Distance to $\mathrm{x}$ direction between fins $[\mathrm{mm}]$ \\
$b$ & Distance to y direction between fins $[\mathrm{mm}]$ \\
$Q_{a}$ & Air flow rate $\left[\mathrm{m}^{3} / \mathrm{h}\right]$ \\
$Q_{l}$ & Liquid flow rate $\left[\mathrm{m}^{3} / \mathrm{h}\right]$ \\
$t$ & Spraying time $[\mathrm{s}]$
\end{tabular}

\section{Results and discussion}

Examined parameters and their values are given in Table 2. Eight parameters were examined: one at two levels $(\mathrm{H} / \mathrm{d})$ and seven at three levels. The optimized results were found to be a nozzle-surface distance of $400 \mathrm{~mm}$, fin height of $10 \mathrm{~mm}$, fin width of $36 \mathrm{~mm}$, distance to $x$ direction between fins of $15 \mathrm{~mm}$, distance to y direction between fins of $10 \mathrm{~mm}$, air-flow rate of $3.6 \mathrm{~m}^{3} / \mathrm{h}$, water flow rate of $0.03 \mathrm{~m}^{3} / \mathrm{h}$ and spraying time of 5 seconds. Later, detailed graphs were drawn according to experiments with these optimum values. In this study, the variations of the two most influential parameters are presented related to Nusselt number and ALR (Figure 5-6).

Table 2. Investigated flow parameters and their levels

\begin{tabular}{c|ccc}
\hline \hline Factor & Level 1 & Level 2 & Level 3 \\
\hline \hline$H / d$ & $800 / 1.2$ & $400 / 1.2$ & --- \\
$h_{k}$ & 10 & 15 & 20 \\
$s$ & 14 & 26 & 36 \\
$a$ & 10 & 15 & 20 \\
$b$ & 10 & 15 & 20 \\
$Q_{a}$ & 2.1 & 2.9 & 3.6 \\
$Q_{l}$ & 0.012 & 0.021 & 0.03 \\
$t$ & 5 & 10 & 15
\end{tabular}

Using the estimation method proposed by Kline and McClintock [20], the maximum uncertainties of the investigated parameters were calculated (Table 3). Uncertainty analysis was carried out with respect to the Nusselt number, Weber number, Reynolds number, and ALR. 
Table 3. Uncertainties in the values of variables

\begin{tabular}{l|c}
\hline \hline \multicolumn{1}{c|}{ Variable } & Relative Uncertainty $\left(\mathbf{W}_{\mathbf{R}}\right)$ \\
\hline \hline Mass of aluminium, $m$ & 0.09 \\
Surface temperature, $T_{s}$ & 0.09 \\
Fluid temperature, $T_{s p}$ & 0.09 \\
Water flow-rate, $Q_{l}$ & 0.03 \\
Air flow-rate, $Q_{a}$ & 0.05 \\
Nozzle diameter, $d_{0}$ & 0.025 \\
Surface tension, $\sigma$ & 0.001
\end{tabular}

The changes of ALR-SMD for water and air-flow rates presented in Figure 3 were drawn with the correlations of Feras and Shanawany. The value of SMD decreased as ALR increased.

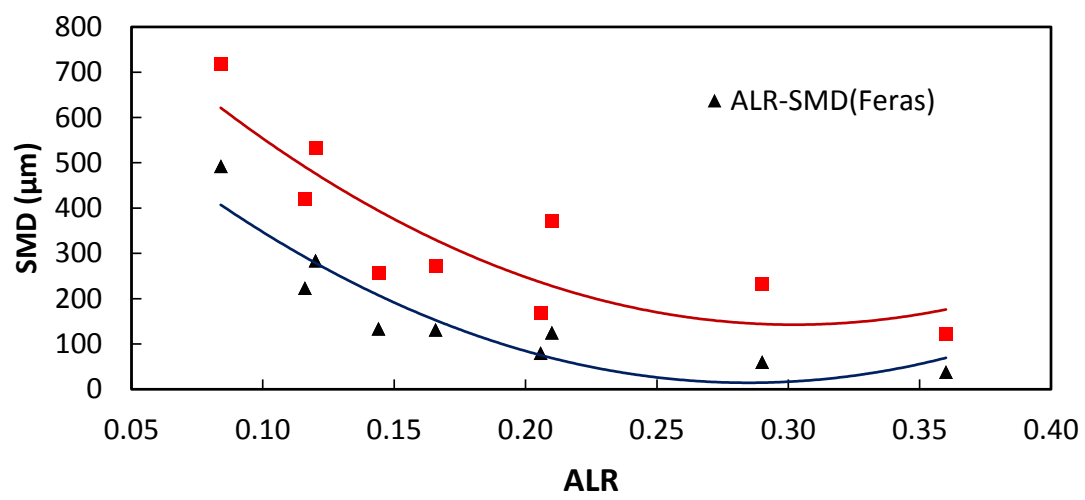

Figure 3. The relationship of ALR-SMD with the correlations of Feras and Shanawany.

In Figure 3, SMD is seen to decrease as ALR increases in accordance with the literature. These correlations were chosen to show the decline. There are many SMD correlations in the literature for various operational and geometric conditions [19], [21].

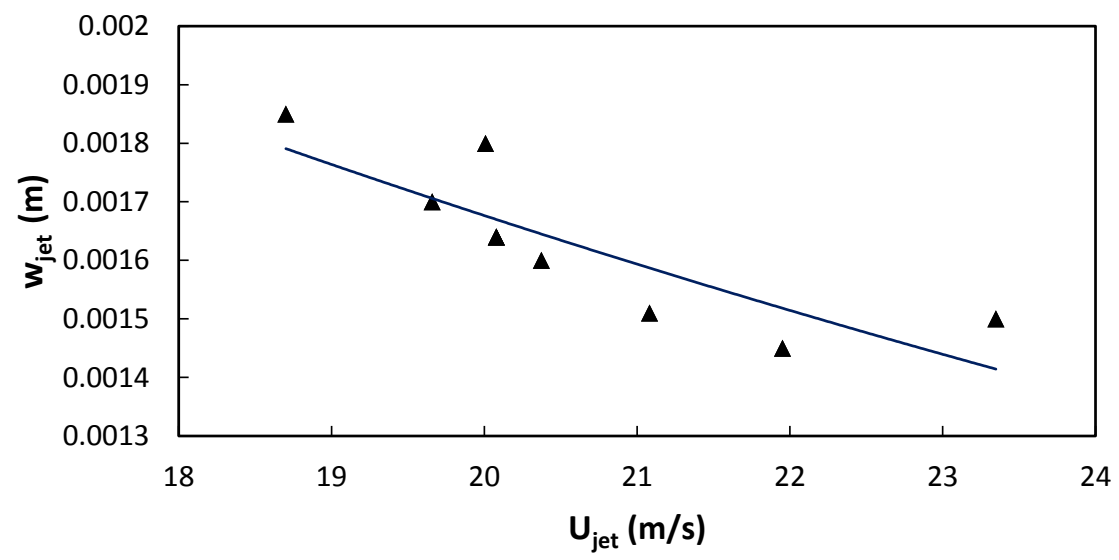

Figure 4. The relationship of jet width to jet velocity

It can be seen in Figure 4 that jet velocity decreased as jet width increased. The parameters that affect Nusselt number are spraying time $(t)$, fin height $\left(h_{k}\right)$, air-flow rate $\left(Q_{a}\right)$, fin width $(s)$, water flow rate $\left(Q_{l}\right)$, the distance between fins in the directions of $y(b)$ and $x(a)$, and the distance between nozzle and surface $(H / d)$. The parameters with the greatest impact on the Nusselt number is spraying time. When the spraying time was $5 \mathrm{~s}$, the Nusselt number reached the maximum value. No investigation has been done for spraying times below this value. According to the experiments designed using the Taguchi method, the changes in ALR-Nu are based on spraying time $(t)$ and fin height $\left(h_{k}\right)$. 


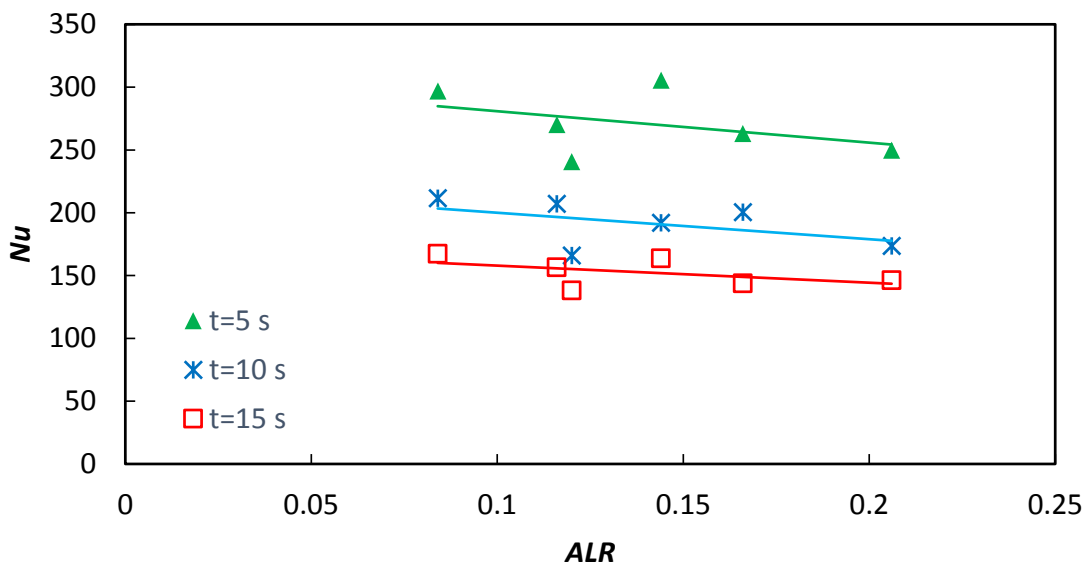

Figure 5. Nusselt vs. ALR as a function of spraying time

The changes in ALR-Nu related to spraying time are presented in Figure 5. The maximum Nusselt number was obtained for $5 \mathrm{~s}$ spraying time. Nusselt number decreased as the spraying time decreased.

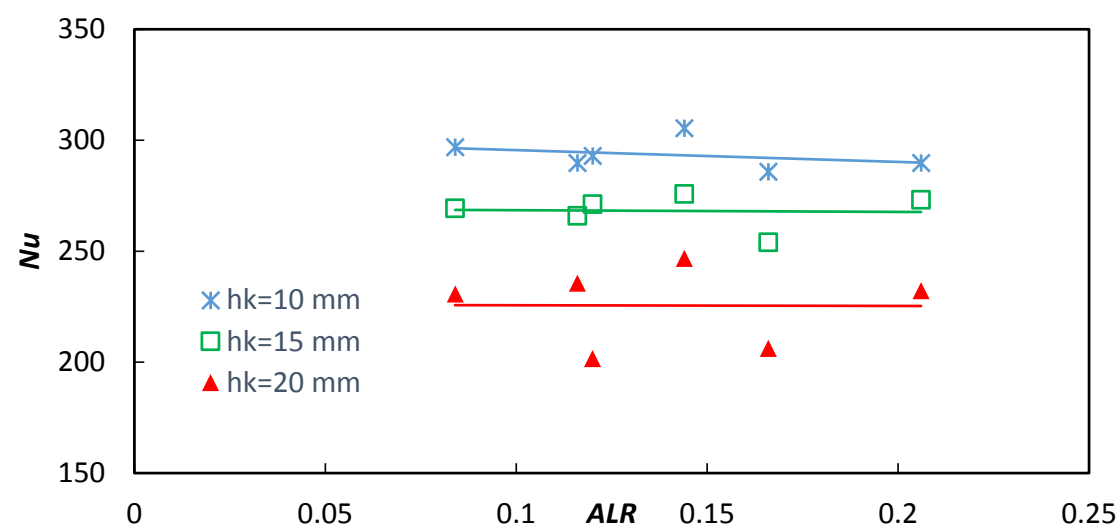

Figure 6. Nusselt vs. ALR as a function of fin height

The change in ALR-Nu correlate to fin height in Figure 6. The maximum Nu value was obtained when the fin height was $10 \mathrm{~mm}$. A significant increase was observed in Nu number with the decrease of $h_{k}$. There is an inverse relationship fin height and Nu number: As the fin height increases, the Nusselt number decreases. The Nusselt number does not change significantly as ALR increases. The jet diameter and spray angle were determined via image processing with Matlab GUI using macroscopic aspects (Fig. 7). There are various ways to define the spray cone angle [22], but in this study it is defined as the angle between the maximum left and right positions at a halflength of the spray tip penetration from the nozzle tip [23].

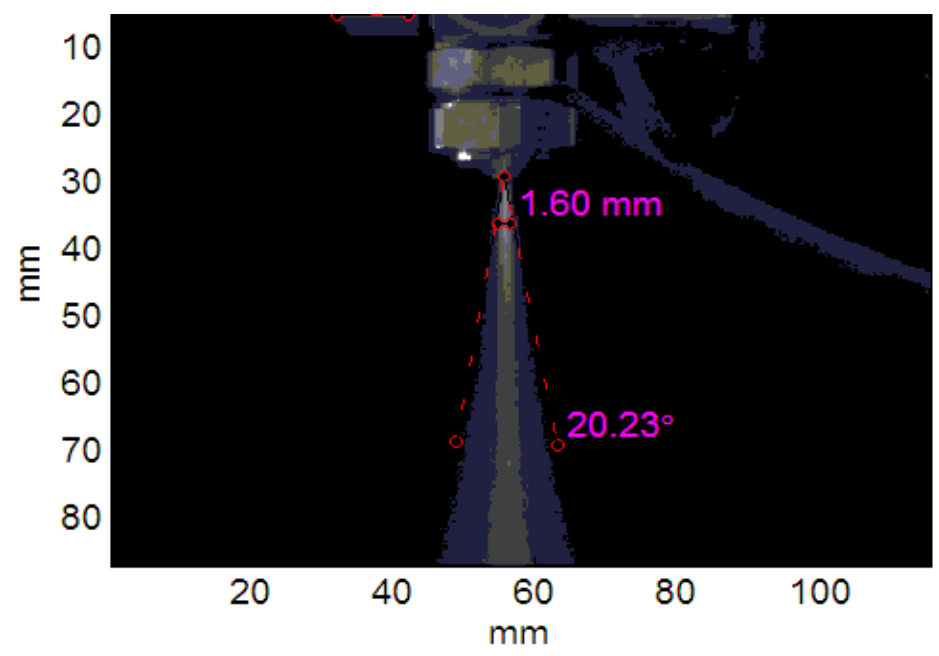

Figure 7. Image-processing analysis with MATLAB GUI for spray angle and jet width 
Nu number, spray-angle and jet-diameter correlations were obtained as follows in the experiments performed on the optimized heat sink geometry. These correlations were obtained as a result of 54 experiments. Experimental findings obtained at various ALR ratios in the DXD-HS1 air-assisted nozzle and correlations with the "Custom Loss" function in the STATISTICA program for Nu number, spray angle and jet width were obtained. Correlations were obtained according to a least square procedure.

$$
\begin{array}{ll}
N u=0.7797 A L R^{-0.1} t^{-0.58} h_{k}^{-0.18} s^{34.37} a^{76.13} b^{-38.43}\left(\frac{H}{d}\right)^{-40.25} & (\mathrm{R}=0.90) \\
\theta=1.16347 * A L R^{-0.18} W e^{-0.05}\left(P_{a} / P_{l}\right)^{0.17} \mathrm{Pr}^{1.56} & (\mathrm{R}=0.98) \\
w_{j e t}=0.06782 * A L R^{0.65} W e^{0.1}\left({ }^{P} / P_{l}\right)^{-0.14} \mathrm{Pr}^{-1.65} & (\mathrm{R}=0.84)
\end{array}
$$

\section{Conclusions}

An experimental study was carried out to determine heat transfer and flow characteristics of spray cooling for aluminum hexagonal finned heat sinks. The optimized geometric and flow parameters of water spray cooling were analyzed under a non-boiling regime. Experiments were conducted for optimum conditions, and based on these, the following observations were drawn. The changes in Nu-ALR for different spraying time, fin heights, and fin width parameters significantly affected the Nusselt number. The maximum Nusselt number was obtained for a $5 \mathrm{~s}$ spraying time. It was observed that the Nusselt number increased as the spraying time decreased. But did not increase significantly with $A L R$ for all fin heights. There is an inverse relationship between fin height and Nu number. Convective heat transfer increased with the liquid flow rate in all ALR values, while heat flux increased with water flow because of the increase in heat transfer related to increasing droplet intensity. Nu number, spray angle and jet width correlations were obtained in the experiments performed on the optimized heat sinks.

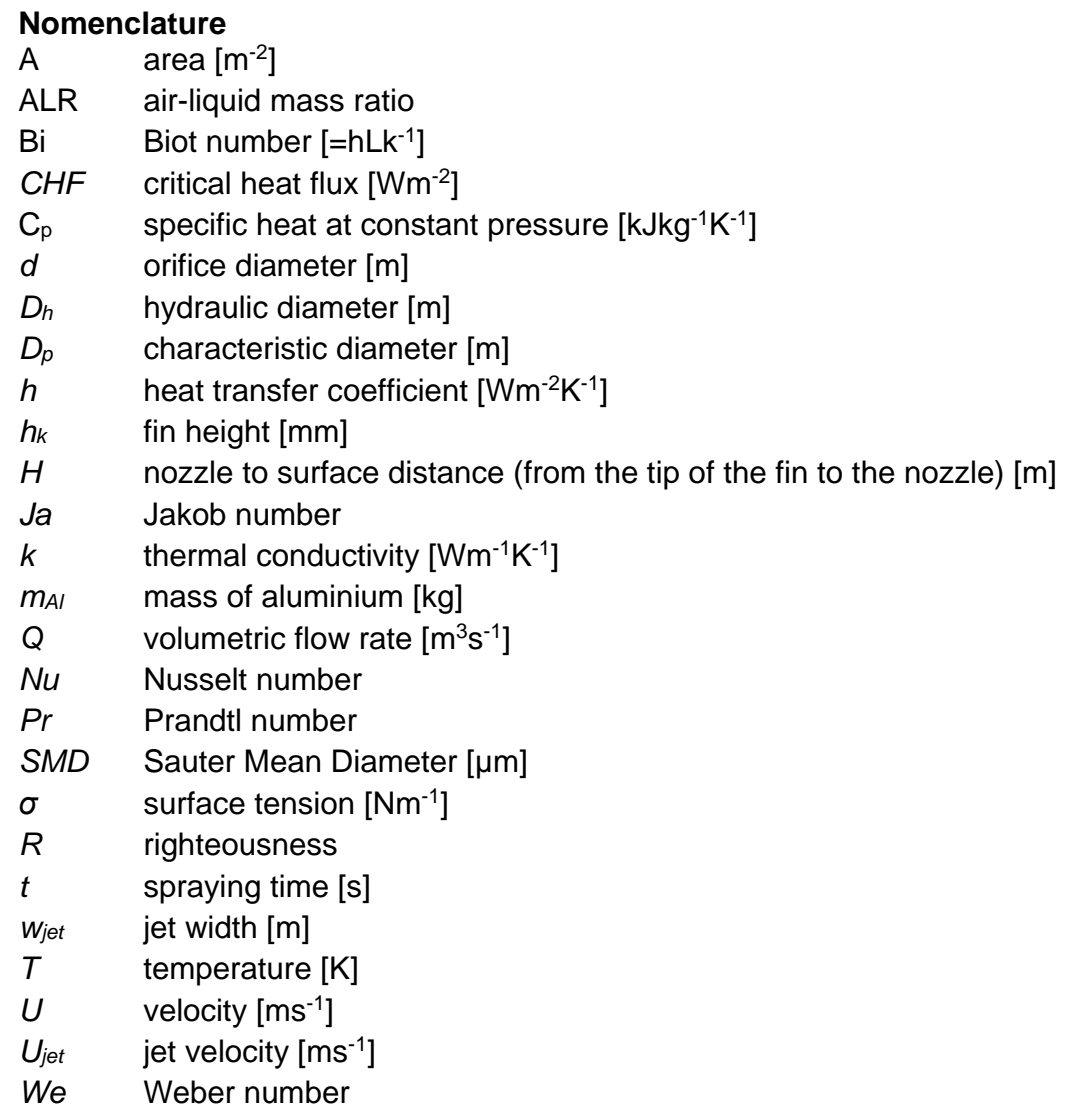




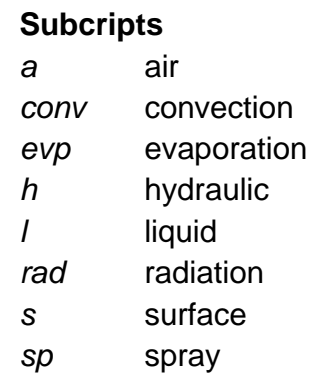

\section{References}

[1] Wang, X., Lu, G., Peng, X., and Wang, B., "Evaporation and nucleate boiling of an individual droplet on surfaces," Proc. ASME 2005 Summer Heat Transfer Conference collocated with the ASME 2005 Pacific Rim Technical Conference and Exhibition on Integration and Packaging of MEMS, NEMS, and Electronic Systems, American Society of Mechanical Engineers, pp. 703-710.

[2] Rybicki, J. R., and Mudawar, I., 2006, "Single-phase and two-phase cooling characteristics of upward-facing and downward-facing sprays," International Journal of Heat and Mass Transfer, 49(1), pp. 5-16.

[3] Somasundaram, S., and Tay, A., 2013, "Comparative study of intermittent spray cooling in single and two phase regimes," International Journal of Thermal Sciences, 74, pp. 174-182.

[4] Liu, M., Wang, Y., Liu, D., Xu, K., and Chen, Y., 2011, "Experimental study of the effects of structured surface geometry on water spray cooling performance in non-boiling regime," Frontiers in Energy, 5(1), pp. 75-82.

[5] Sridhara, S., and Raghunandan, B., 2010, "Photographic investigations of jet disintegration in airblast sprays," Journal of applied fluid mechanics, 3(2), pp. 111-123.

[6] Charalampous, G., Hadjiyiannis, C., Hardalupas, Y., and Taylor, A., "Measurement of continuous liquid jet length in atomizers with optical connectivity, electrical conductivity and high-speed photography techniques," Proc. 23rd Annual Conference on Liquid Atomization and Spray Systems, ILASS-Europe.

[7] Karwa, N., Kale, S. R., and Subbarao, P., 2007, "Experimental study of non-boiling heat transfer from a horizontal surface by water sprays," Experimental Thermal and Fluid Science, 32(2), pp. 571-579.

[8] Tao, Y., Huai, X., Wang, L., and Guo, Z., 2011, "Experimental characterization of heat transfer in non-boiling spray cooling with two nozzles," Applied Thermal Engineering, 31(10), pp. 1790-1797.

[9] Sharief, R. A., Jeong, J. R., and James, D. D., 2000, "The performance characteristics of solid-cone-spray pressure-swirl atomizers," 10(6), p. 20.

[10] Nikolopoulos, N., Strotos, G., Nikas, K.-S. P., Gavaises, M., Theodorakakos, A. P., Marengo, M., and Cossali, G. E., 2010, "Experimental investigation of a single droplet impact onto a sessile drop," 20(10), pp. 909-922.

[11] Batarseh, F. Z., Roisman, I. V., and Tropea, C., 2010, "Characterization of a spray generated by an airblast atomizerwith prefilmer," 20(10), pp. 887-903.

[12] Desantes, J. M., Payri, R., Pastor, J. M., and Gimeno, J., 2005, "Experimental characterization of internal nozzle flow and diesel spray behavior. part i: nonevaporative conditions," 15(5), pp. 489-516.

[13] Marengo, M., Antonini, C., Roisman, I. V., and Tropea, C., 2011, "Drop collisions with simple and complex surfaces," Current Opinion in Colloid \& Interface Science, 16(4), pp. 292-302.

[14] Issa, R. J., 2009, Multiphase spray cooling technology in industry, INTECH Open Access Publisher.

[15] Davies, T., 2011, "Biot number."

[16] Çengel, Y. A., and Ghajar, A. J., 2015, Heat and mass transfer : Fundamentals \& Applications.

[17] Batarseh, F. Z. M., 2009, "Spray generated by an airblast atomizer: atomization, propagation and aerodynamic instability," Technische Universität.

[18] El-Shanawany, M., and Lefebvre, A., 1980, "Airblast atomization: effect of linear scale on mean drop size," Journal of Energy, 4(4), pp. 184-189.

[19] Lefebvre, A., 1988, Atomization and sprays, CRC press.

[20] Kline, S., and McClintock, F., 1953, "Analysis of uncertainty in single-sample experiments," Mechanical Engineering, 75.

[21] Ashgriz, N., 2011, Handbook of atomization and sprays: theory and applications, Springer Science \& Business Media.

[22] Payri, R., Salvador, F., Gimeno, J., and De la Morena, J., 2008, "Macroscopic behavior of diesel sprays in the near-nozzle field," SAE International Journal of Engines, 1(2008-01-0929), pp. 528-536.

[23] Kim, H. J., Park, S. H., and Lee, C. S., 2010, "A study on the macroscopic spray behavior and atomization characteristics of biodiesel and dimethyl ether sprays under increased ambient pressure," Fuel processing technology, 91(3), pp. 354-363. 


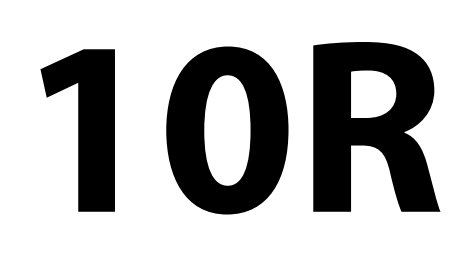

Internal flow 2 


\title{
Statistical Methods of Images Analysis as an Essential Tool in the Assessment Process of Computer Methods Intended for Numerical Simulations of Cavitating Flows
}

\author{
Agnieszka Niedźwiedzka*1, Seweryn Lipiński² \\ ${ }^{1}$ Department of Mechanics and Basics of Machine Design, Faculty of Technical Sciences, \\ University of Warmia and Mazury in Olsztyn, Poland \\ 2Department of Electric and Power Engineering, Electronics and Automatics, Faculty of \\ Technical Sciences, University of Warmia and Mazury in Olsztyn, Poland \\ *corresponding author: agnieszka.niedzwiedzka@uwm.edu.pl
}

\begin{abstract}
Cavitation, because of its negative effects, like e.g. erosion, noise or vibration, is usually an undesirable phenomenon. However, in devices where spraying and atomization are expected, cavitation is required. This group of devices includes e.g. diesel injectors. Appearance of vapour bubbles results in increase of the maximum flow velocity. It is possible for the following reasons. Firstly, bubbles start to form in the throat, so its diameter reduces. Secondly, appearance of vapour bubbles along the wall results in a slip boundary condition. Moreover, cavitation has a positive influence on a spray cone angle. However, regardless of the place of occurrence, research on cavitation bases primarily on numerical simulations. The area of numerical methods intended for cavitating flows includes many solutions which differ not only in the basic assumptions, i.e. considering flow either as a multiphase mixture with the average density or just as two independent liquids, fluid and vapour, with a distinct boundary between them, but also in many methods applied in particular approaches. Currently, to choose the best way of the prediction of cavitation phenomenon for the undertaken issue, many aspects should be considered. The most important factor is the assessment level between the results of numerical simulation and experimental data. Secondary are computing time, requirements for the hardware, price of software and additional costs connected with the selected software. The final decision about the chosen way of the cavitation prediction results from all the above-considered elements. The main aim of the work is to present the methods of image analysis, which can be very helpful in this process. The main advantage of these methods is the quantitative answer about the correlation degree between analysed images. It eliminates subjective decisions based solely on a raw imaging material. The image material used in the work was obtained via numerical simulations performed in ANSYS Fluent. Presented methodology bases on their statistical analysis that considers the shape and intensity of cavitating area, as well as on basic methods of image processing and analysis. The conclusion is that the obtained results demonstrate the usefulness of the proposed methods in the aspect of a reliable comparison of images obtained in the numerical studies of the cavitation phenomenon.
\end{abstract}

\section{Keywords}

Cavitating flow, numerical simulations, assessment process, statistical analysis, image analysis.

\section{Introduction}

Cavitation is one of the most investigated topics in the area of fluid flow research. This phenomenon itself consists in growth of vapour bubbles in a fluid under the influence of drop in pressure below the saturated liquid pressure and it is usually considered as an undesirable phenomenon, because of its negative effects, which are inter alia erosion, noise, vibration and loss of efficiency [1], [7], [10]. However, cavitation can be useful; moreover, there are situations where it can be desired. Cavitation accompanies and determines such phenomena as spraying and atomization that are required in many devices. The example of such device is diesel injector [10], [1]. Through the appearance of vapour bubbles, the maximum flow velocity increases; it is possible for the following two reasons. Firstly, bubbles start to form in the throat, so its diameter reduces. Secondly, appearance of vapour bubbles along the wall results in a slip boundary condition. Cavitation has also a positive influence on a spray cone angle [19]. Collapse of the cavitation bubbles enhances fuel atomization. The collapse results in decrease of droplets size what is beneficial for vaporization. A faster vaporization helps to mix fuel and air and to reduce ignition delay [24]. The biggest difficulty for experimental and numerical investigations of engine diesel injectors are small dimensions (the inner diameter is about $200 \mu \mathrm{m}$ ) and a very fast flow velocity, even about $500 \mathrm{~m} / \mathrm{s}$. For that reasons, to extract data for validation of the results of numerical simulations special tools should be applied [20]. 
Regardless of the place of occurrence, research on cavitation bases primarily on numerical simulations [13], [21]. The area of numerical methods intended for cavitating flows includes many solutions. Primarily they differ in the basic assumptions, i.e. considering flow as a multiphase mixture either with the average density or just as two independent liquids, fluid and vapour, with a distinct boundary between them. Other differences stems from the use of many methods applied in particular approaches. All methods base on the governing equations of mass, momentum and energy [23]:

$$
\begin{aligned}
& \frac{\partial \rho}{\partial t}+\operatorname{div}(\rho \vec{u})=0 \\
& \frac{\partial}{\partial t}(\rho \vec{u})+\operatorname{div}(\rho \vec{u} \otimes \vec{u})=\operatorname{div}\left(-p \vec{I}+\vec{\tau}^{m}+\vec{\tau}^{R}\right)+\rho s_{b} \\
& \frac{\partial}{\partial t}(\rho e)+\operatorname{div}(\rho e \vec{u}+\rho \vec{u})=\operatorname{div}\left[\left(\vec{\tau}^{m}+\vec{\tau}^{R}\right) \vec{u}+\vec{q}^{m}+\vec{q}^{R}\right]+\rho s_{e}
\end{aligned}
$$

This set of equations can be solved independent or jointly for each of the phases, which are liquid and vapour. The first solution (based on independent solutions), because of longer computing time and bigger system requirements, is rarely preferred. In the second, more economical option, two ways are considered: solving an additional transport or a barotropic equation. The additional transport equation describes conditions of change of physical state from liquid to vapour and vice versa and it is expressed in a form of source terms. The most solutions of source terms base on the Rayleigh equation [17]:

$$
R \frac{d^{2} R}{d t^{2}}+\frac{3}{2}\left(\frac{d R}{d t}\right)^{2}=\frac{p_{s a t}-p}{\rho_{l}}
$$

In order to choose the best way of the prediction of cavitation phenomenon for the undertaken issue many aspects should be considered. The most important factor is the assessment level between the results of numerical simulation and experimental data. Secondary are computing time, requirements for the hardware, price of software and additional costs connected with the selected software. The final decision on the chosen way of the cavitation prediction should be a result taking into account all the above-mentioned elements. The raw image material, showing contours of vapour volume fraction, is a vexed basis for the key question concerning the assessment level of the results. The qualitative answer is the best possible solution. It eliminates a subjective view and gives unequivocal and irrefutable proofs of the made choice. The literature concerning the statistical methods of image analysis aiming to define correlation coefficients is very poor and the proposed methods have not gained popularity in the research area of cavitating flows. The image analysis was used e.g. for calculating the Volumetric Index necessary for determination of the fouling degree of the injectors [25] or to determine the mean error between vapour penetration measurements and simulations [4]. The statistical methods dominate either in validation of quantitative data, such as mass flow rate or temperature [16]. Many reasons can be listed for this state of affair, beginning from a small interest in such comparisons methods and ending in lack of any strictly specified requirements in evaluation of the achieved numerical results. Despite the fact that in the area of numerical simulations of the flow in engine diesel injectors a progress is visible each year, validation of the achieved results stays usually at the same level and the traditional raw image analyses still dominate [2], [3], [5], [8], [18]. Paying more attention to the statistical image analysis could be a real and desired support for the rapidly growing area of numerical calculations, not only for devices like diesel injectors. This work is an attempt to popularize the statistical methods of image comparisons as an excellent, unequivocal and simple solution that help to make the best choice without any doubts about the influence of subjective perspective.

\section{Aim}

The main aim of the work is to present the methods of statistical image analysis of cavitating area. Using basic methods of image processing two correlation coefficients are proposed. The first correlation coefficient bases on the intensity of the cavitation cloud while the second on the changes in the shape of the cavitation cloud. Both proposed coefficients can be helpful in the process of the assessment of results obtained in numerical simulations. The original achievement are also the results of the numerical simulations of cavitating flow in form of the contours of vapour volume fraction that are used in the further statistical image analysis.

It should be emphasized, that proposed coefficients can successfully be used for the purpose of comparing results of simulations with experiment as well; as it is described in the "Conclusions" section. 


\section{Material and methods}

The analysed axisymmetric and two-dimensional geometry is an orifice which dimensions are shown in Figure 1. The total length of the orifice is equal to $48 \mathrm{~mm}$, the length of the inlet part is equal to $16 \mathrm{~mm}$, the inlet diameter is equal to $23.04 \mathrm{~mm}$ and the outlet diameter equals to $8 \mathrm{~mm}$. The inspiration for the geometry is the "Cavitation modeling" tutorial [29]. In accordance with the guide, the inlet pressure is equal to 5 bar and the outlet pressure to 9.5 bar. The numerical calculations are performed for steady state.

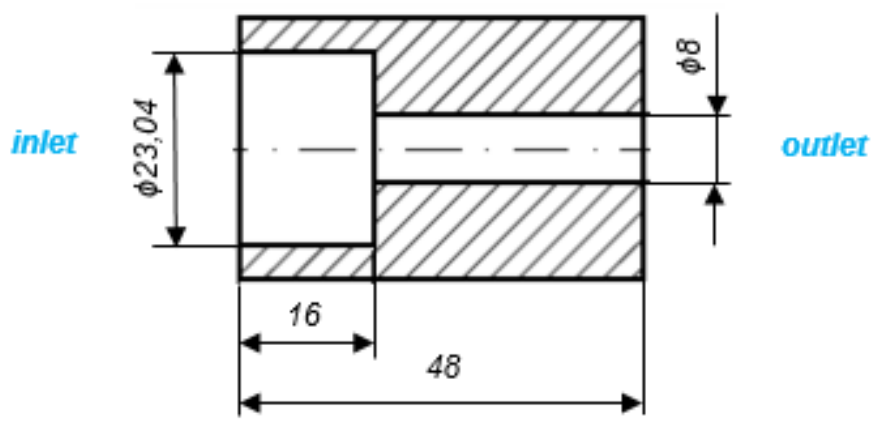

Figure 1. Dimensions of the analysed orifice.

The material for the analysis of the validity of the application of statistical methods of image processing in the assessment of the compliance degree of achieved results are the contours of the vapour volume fraction achieved in numerical simulations of cavitating flow in the orifice. Seven homogeneous cavitation models are considered: Iben [9], Kunz et al. [11], Merkle et al. (1998) [12], Merkle et al. (2006) [13], Schnerr and Sauer [21], Singhal et al. [22] and Zwart et al. [26]. The source terms of the chosen models are presented in Table 1. The numerical simulation are performed using Fluent software, a part of the Ansys 14.5 package. All the models are implemented using UDFs (User Defined Functions) with the built-in macro Define Cavitation Rate [27]. Verification of the used UDFs is presented in the work by Niedźwiedzka et al [15].

Table 1. Source terms of the analysed cavitation models.

\begin{tabular}{|c|c|c|}
\hline Authors & Source terms for condensation & Source terms for evaporation \\
\hline Iben [9] & $\dot{m}^{+}=C_{p} \rho_{v} \frac{6 \alpha_{v}}{2 R} \sqrt{\frac{2}{3} \frac{\left(p-p_{s a t}\right)}{\rho_{l}}}$ & $\dot{m}^{-}=-\rho_{l} \frac{6 \alpha_{v}}{2 R} \sqrt{\frac{2}{3} \frac{\left(p_{s a t}-p\right)}{\rho_{l}}}$ \\
\hline $\begin{array}{l}\text { Kunz et al. } \\
\quad[11]\end{array}$ & $\dot{m}^{+}=\frac{C_{p} \rho_{v} \alpha_{l}^{2}\left(1-\alpha_{l}\right)}{t_{\infty}}$ & $\dot{m}^{-}=-\frac{C_{d} \rho_{v} \alpha_{l}\left(p_{s a t}-p\right)}{\left(0.5 \rho_{l} U_{\infty}^{2}\right) t_{\infty}}$ \\
\hline $\begin{array}{l}\text { Merkle et } \\
\text { al. (1998) } \\
\quad[12]\end{array}$ & $\dot{m}^{+}=\frac{C_{p} \rho_{v}\left(1-\alpha_{l}\right)\left(p-p_{s a t}\right)}{\left(0.5 \rho_{l} U_{\infty}^{2}\right) t_{\infty}}$ & $\dot{m}^{-}=-\frac{C_{d} \rho_{l} \alpha_{l}\left(p_{s a t}-p\right)}{\left(0.5 \rho_{l} U_{\infty}^{2}\right) t_{\infty}}$ \\
\hline $\begin{array}{l}\text { Merkle et } \\
\text { al. (2006) } \\
\quad[13]\end{array}$ & $\dot{m}^{+}=k_{l} \frac{\left(\rho_{v} \alpha_{l}\right)}{t_{\infty}}\left(\frac{\left(p-p_{s a t}\right)}{k_{p} p_{v}}\right)$ & $\dot{m}^{-}=-k_{v} \frac{\rho_{v} \alpha_{l}}{t_{\infty}}\left(\frac{\left(p_{s a t}-p\right)}{k_{p} p_{v}}\right)$ \\
\hline $\begin{array}{l}\text { Schnerr } \\
\text { and Sauer } \\
\text { [21] }\end{array}$ & $\dot{m}^{+}=\frac{\rho_{v} \rho_{l}}{\rho_{m}} \alpha_{v}\left(1-\alpha_{v}\right) \frac{3}{R} \sqrt{\frac{2}{3} \frac{\left(p-p_{s a t}\right)}{\rho_{l}}}$ & $\dot{m}^{-}=-\frac{\rho_{v} \rho_{l}}{\rho_{m}} \alpha_{v}\left(1-\alpha_{v}\right) \frac{3}{R} \sqrt{\frac{2}{3} \frac{\left(p-p_{s a t}\right)}{\rho_{l}}}$ \\
\hline $\begin{array}{l}\text { Singhal et } \\
\text { al. [22] }\end{array}$ & $\dot{m}^{+}=C_{p} \frac{\sqrt{k}}{\sigma} \rho_{l} \rho_{l} \sqrt{\frac{2}{3} \frac{\left(p-p_{s a t}\right)}{\rho_{l}}} f_{v}$ & $\dot{m}^{-}=-C_{d} \frac{\sqrt{k}}{\sigma} \rho_{l} \rho_{v} \sqrt{\frac{2}{3} \frac{\left(p_{s a t}-p\right)}{\rho_{l}}}\left(1-f_{v}-f_{g}\right)$ \\
\hline $\begin{array}{l}\text { Zwart et } \\
\text { al. [26] }\end{array}$ & $\dot{m}^{+}=C_{p} \frac{3 \alpha_{v} \rho_{v}}{R} \sqrt{\frac{2}{3} \frac{\left(p-p_{s a t}\right)}{\rho_{l}}}$ & $\dot{m}^{-}=-C_{d} \frac{3 \rho_{v}\left(1-\alpha_{v}\right) \alpha_{n u c}}{R} \sqrt{\frac{2}{3} \frac{\left(p_{s a t}-p\right)}{\rho_{l}}}$ \\
\hline
\end{tabular}


The images obtained in the simulations are shown in Figure 2. It can be seen that the contours of the vapour volume fraction are very similar for the each considered solution. The length of the cavitating area is close to the outlet of the orifice or finishes at the outlet edge. The intensity of the cavitating cloud is also variable for each of the analysed variant what is the most visible at the edges of the part with the smaller diameter. The biggest intensity of cavitation is located at place where the diameter rapidly changes. For each of the contours the same scale was used where the maximum value of the vapour volume fraction is 0.99 .

Images shown in Figure 2 were used to achieve the main aim of the work, which was to present the methods of statistical image analysis, which can help in the process of the assessment of results obtained in numerical simulations of cavitation phenomenon.

a)

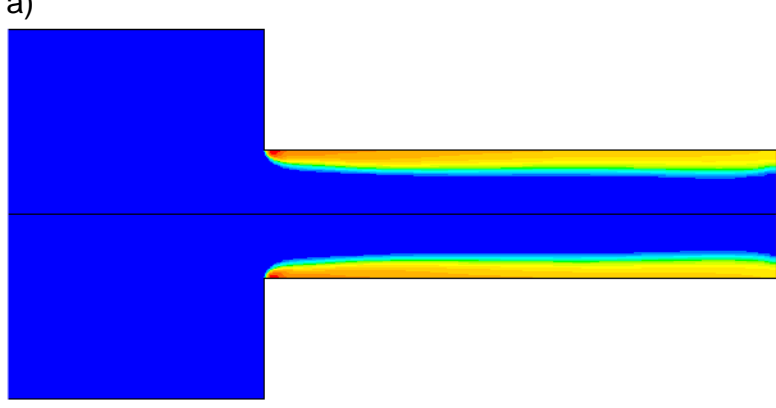

c)

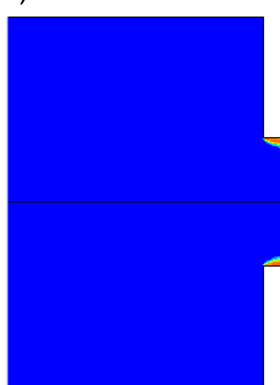

e)

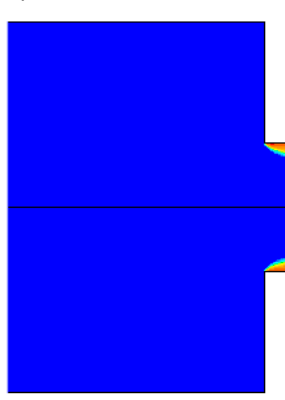

g)

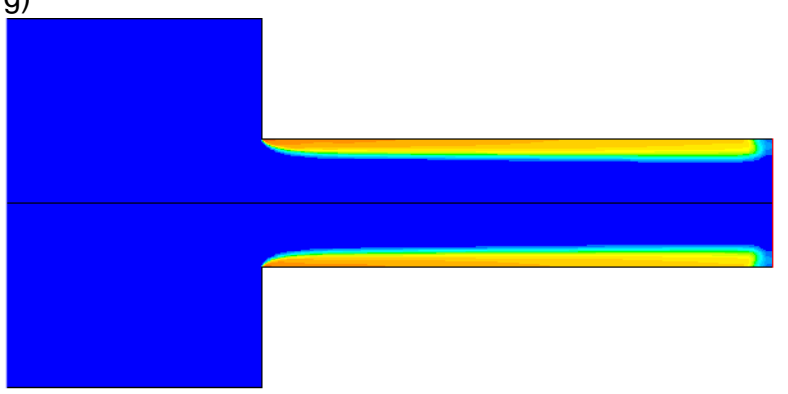

b)

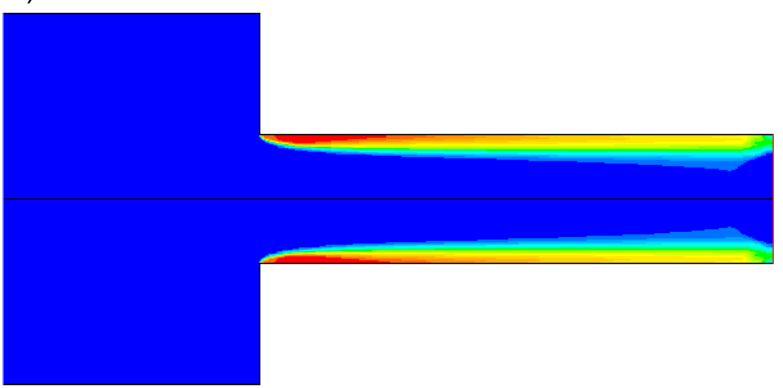

d)

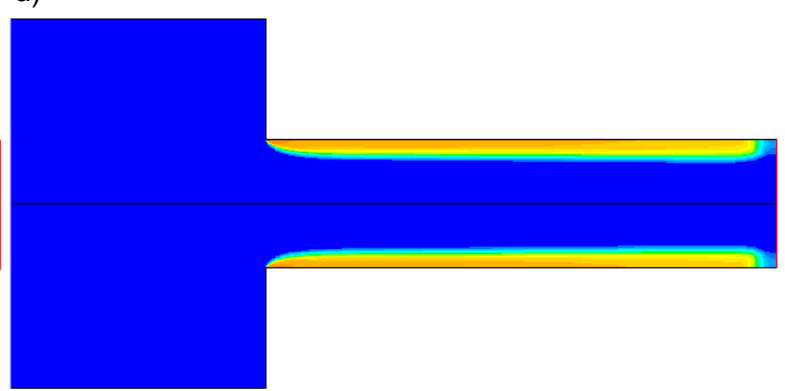

f)
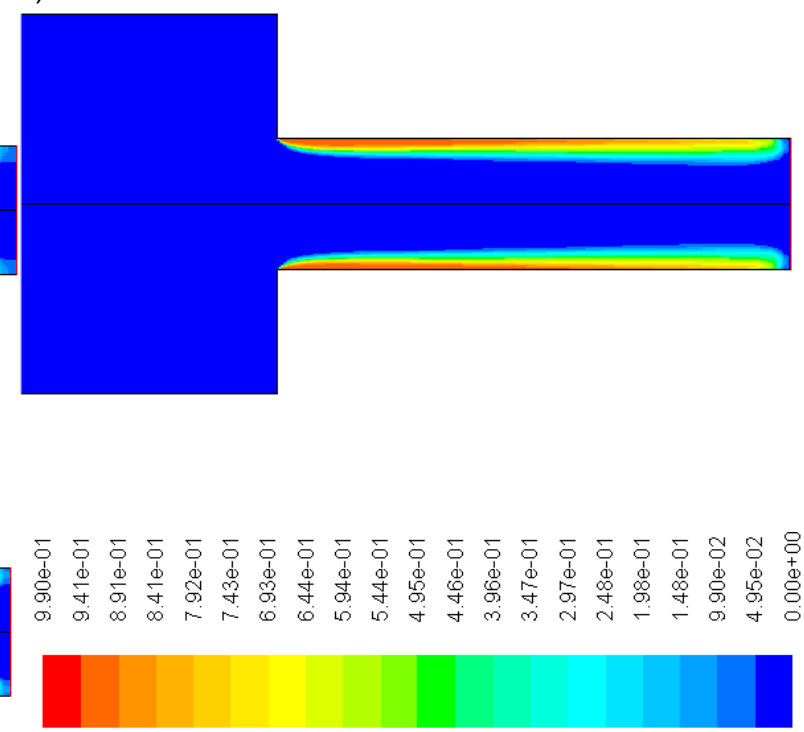

Figure 2. Images of cavitating flow obtained via numerical simulations in the described orifice: Iben model (a), Kunz et al. model (b), Merkle et al. model 1998 (c), Merkle et al. model 2006 (d), Schnerr and Sauer model (e), Singhal et al. model (f) and Zwart et al. model (g). 
Presented methodology bases on their statistical analysis that considers the shape and intensity of cavitating area, as well as on basic methods of image processing and analysis. In order to evaluate obtained results in an objective manner, we propose to introduce two correlation coefficients. Each of them has a different purpose. The first coefficient ( $r_{1}$ - intensity correlation coefficient) bases on the intensity of cavitation; it is a 2-D equivalent for a Pearson product-moment correlation coefficient and is defined as follows:

$$
r_{I}=\frac{\sum_{x=1}^{X} \sum_{y=1}^{Y}\left(A_{x y}-\bar{A}\right)\left(B_{x y}-\bar{B}\right)}{\sqrt{\left(\sum_{x=1}^{X} \sum_{y=1}^{Y}\left(A_{x y}-\bar{A}\right)^{2}\right)\left(\sum_{x=1}^{X} \sum_{y=1}^{Y}\left(B_{x y}-\bar{B}\right)^{2}\right)}} .
$$

This coefficient gives information on how correlated are intensities of cavitation in images being compared. Typical values of this coefficients are in range from -1 to 1 , where 1 is the total positive correlation, 0 is no correlation, and -1 is total negative correlation.

The second correlation coefficient ( $r_{s}$ - shape correlation coefficient) is supposed to show similarity in changes of the shape of cavitation cloud. It is calculated using formula (5) as well, but in that case, the operation is performed on $I_{D}$ images, which are created in order to show changes in cloud intensities (not intensities per se). Individual pixels (with $x$ and $y$ coordinates) of $I_{D}$ images are obtained as:

$$
I_{D_{x y}}=I_{(x+1)(y+1)}-I_{x y}, x=1,2, \ldots, X-1, y=1,2, \ldots, Y-1 .
$$

Obtained values of this coefficient should be interpreted just as values of the rıcoefficient.

\section{Results and discussion}

Table 2 shows obtained values of the $r$ c coefficient for each combination (pair) of considered models.

\begin{tabular}{|c|c|c|c|c|c|c|c|}
\hline & Iben & Kunz et al. & $\begin{array}{c}\text { Merkle et al. } \\
(1998)\end{array}$ & $\begin{array}{c}\text { Merkle et al. } \\
(2006)\end{array}$ & $\begin{array}{c}\text { Schnerr and } \\
\text { Sauer }\end{array}$ & $\begin{array}{c}\text { Singhal et } \\
\text { al. }\end{array}$ & $\begin{array}{c}\text { Zwart et } \\
\text { al. }\end{array}$ \\
\hline Iben & 1 & 0.93 & 0.92 & 0.92 & 0.92 & 0.92 & 0.93 \\
\hline Kunz et al. & 0.93 & 1 & 0.96 & 0.93 & 0.94 & 0.95 & 0.94 \\
\hline $\begin{array}{c}\text { Merkle et al. } \\
\text { (1998) }\end{array}$ & 0.92 & 0.96 & 1 & 0.93 & 0.94 & 0.97 & 0.94 \\
\hline $\begin{array}{c}\text { Merkle et al. } \\
\text { (2006) }\end{array}$ & 0.92 & 0.93 & 0.93 & 1 & 0.99 & 0.96 & 0.99 \\
\hline Schnerr and Sauer & 0.92 & 0.94 & 0.94 & 0.99 & 1 & 0.97 & 0.99 \\
\hline Singhal et al. & 0.92 & 0.95 & 0.97 & 0.96 & 0.97 & 1 & 0.97 \\
\hline Zwart et al. & 0.93 & 0.94 & 0.94 & 0.99 & 0.99 & 0.97 & 1 \\
\hline
\end{tabular}

Table 2. Values of the $r_{i}$ correlation coefficients for each combination of considered models.

Table 3 shows obtained values of the $r_{s}$ coefficient for each combination (pair) of considered models.

Table 3. Values of the $r_{S}$ correlation coefficients for each combination of considered models.

\begin{tabular}{c|cccccccc}
\hline \hline & Iben & Kunz et al. & $\begin{array}{c}\text { Merkle et } \\
\text { al. (1998) }\end{array}$ & $\begin{array}{c}\text { Merkle et al. } \\
(2006)\end{array}$ & $\begin{array}{c}\text { Schnerr and } \\
\text { Sauer }\end{array}$ & $\begin{array}{c}\text { Singhal et } \\
\text { al. }\end{array}$ & $\begin{array}{c}\text { Zwart et } \\
\text { al. }\end{array}$ \\
\hline \hline Iben & 1 & 0.21 & 0.17 & 0.15 & 0.16 & 0.15 & 0.16 \\
Kunz et al. & 0.21 & 1 & 0.39 & 0.44 & 0.42 & 0.35 & 0.43 \\
$\begin{array}{c}\text { Merkle et al. } \\
\text { (1998) }\end{array}$ & 0.17 & 0.39 & 1 & 0.29 & 0.34 & 0.47 & 0.34 \\
$\begin{array}{c}\text { Merkle et al. } \\
\text { (2006) }\end{array}$ & 0.15 & 0.44 & 0.29 & 1 & 0.77 & 0.38 & 0.85 \\
$\begin{array}{c}\text { Schnerr and Sauer } \\
\text { Singhal et al. }\end{array}$ & 0.16 & 0.42 & 0.34 & 0.77 & 1 & 0.45 & 0.85 \\
Zwart et al. & 0.15 & 0.35 & 0.47 & 0.38 & 0.45 & 1 & 0.43 \\
\end{tabular}


Figures 3 and 4 shows the values from the Tables 2 and 3 in a graphical form.

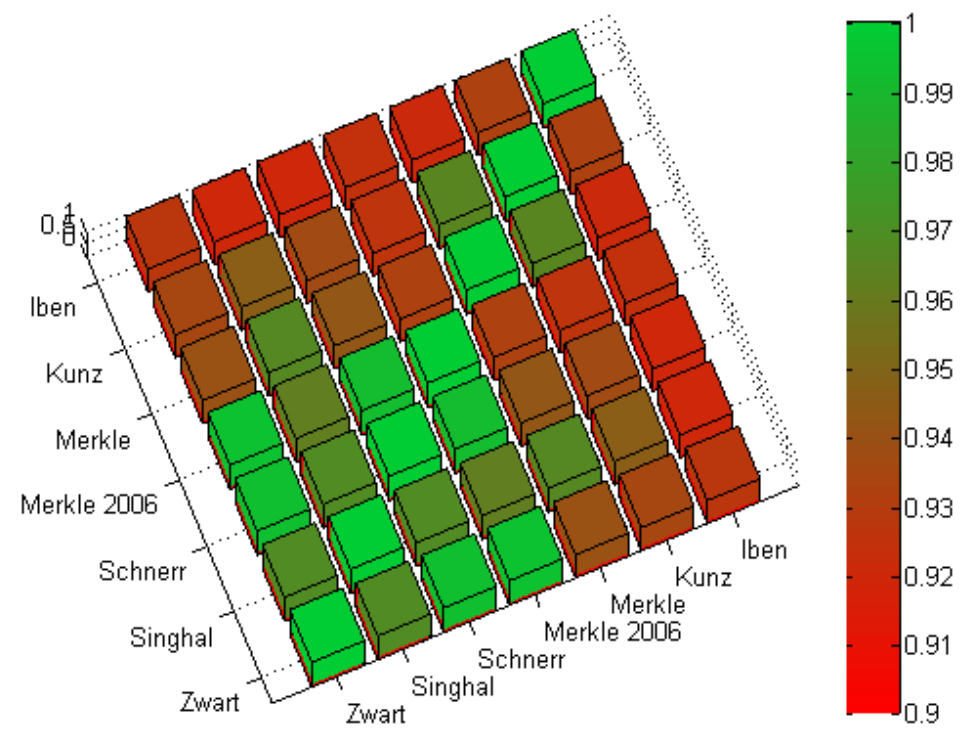

Figure 3. Values of the $r_{i}$ correlation coefficients for each combination of considered models.

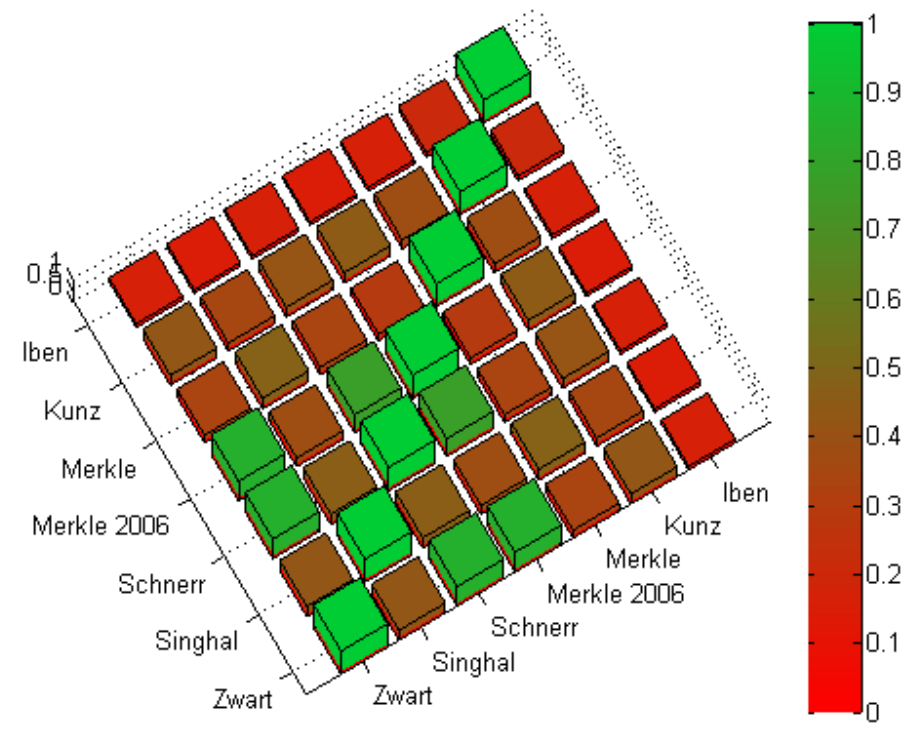

Figure 4. Values of the $r_{s}$ correlation coefficients for each combination of considered models.

The analysis of Table 2 and Figure 3 shows that considering the intensity of the cavitation cloud the best correlated models are three pairs: the Merkle et al. model (2006) and the Schnerr and Sauer model, the Merkle et al. model (2006) and the Zwart et al. model and the Schnerr and Sauer model and the Zwart et al. model. Ale the mentioned pairs achieved an excellent value of correlation, namely $99 \%$. The next group of the best correlated models constitutes of six pairs: the Singhal et al. model and the Merkle et al. model (1998), the Singhal et al. model and the Schnerr and Sauer model, the Singhal et al. model and the Zwart et al. model, the Singhal et al. model and the Merkle et al. model (2006), the Merkle et al. model (1998) and the Kunz et al. model and the Singhal et al. model and the Kunz et al. model. The values of the first correlation coefficients are as follows: $97 \%, 97 \%, 97 \%, 96 \%, 96 \%$ and $95 \%$. The last twelve pairs, i.e. showing the worst values of the used correlation coefficient, correlate with others on the level of $94 \%, 93 \%$ and $92 \%$. The worst correlated model is the lben model. It achieves the correlation value of $92 \%$ with four models: the Merkle et al. model (1998), the Merkle et al. model (1998), the Schnerr and Sauer model and the Singhal et al. model. 
The analysis of Table 3 and Figure 4 shows significantly lower values of the second correlation coefficient than these from Table 2. Two pairs are best-correlated: the Merkle et al. model (2006) and the Zwart al. model and the Merkle et al. model (2006) and the Schnerr and Sauer model. The values of the correlation coefficient are $85 \%$ and $77 \%$, respectively. The next well correlated group has six pairs: the Merkle et al. model and the Singhal et al. model, the Schnerr and Sauer model and the Singhal et al. model, the Merkle et al. model (2006) and the Kunz et al. model, the Zwart et al. model and the Kunz et al. model, the Zwart et al. model and the Singhal et al. model, the Schnerr and Sauer model and the Kunz et al. model. The values of the first correlation coefficients are as follows: $47 \%$, $45 \%, 44 \%, 43 \%, 43 \%$ and $42 \%$. The worst correlated is once again the lben model. The values of the correlation coefficient are even 15\% for the Merkle et al. model (2006) and the Singhal et al. model.

From the both analysis results unambiguously that the best-correlated pair is the Merkle et al. model and the Zwart et al. model. The second correlation coefficient turned out very useful even though it initially seemed to be superfluous. Thanks to this coefficient, we managed to extract the best-correlated pair from the five pairs with the same correlation coefficient considering the intensity of the cavitation cloud.

\section{Conclusions}

One of the major challenges during analyses of images obtained in numerical analysis of cavitating flows is their objective comparison. In other words, the goal here is not to just state that different models produce different results, but to get an objective measure of the stated difference. Our work proposes the solution for that problem.

The presented methods allow obtaining the quantitative answer about the correlation degree between analysed images in terms of shape of cavitation cloud and intensity of cavitation. The main advantage here is the elimination of subjective decisions based solely on a raw imaging material.

Obtained results demonstrate the usefulness of the proposed methods as they allow comparing images obtained in the numerical studies of the cavitation phenomenon in a reliable and objective way. One of the conditions to make such a claim are the differences between obtained correlation coefficients of shape and intensity.

It should be noted that proposed coefficients can successfully be as well used for the purpose of comparing results of simulations with experiment. For that purpose, it is only necessary to ensure the coherence of the geometrical dimensions of the image obtained in the experiment with the dimensions of the image obtained in the numerical simulations, and to normalize the intensity of the images being compared. Depending on the experimental method, it may also be necessary to properly pre-process the image. The volume and scope of this work does not allow for such comparison; it will be done in our future work.

\section{Nomenclature}

\begin{tabular}{|c|c|}
\hline$A, B$ & compared images \\
\hline$C_{d}$ & evaporation model constant [-] \\
\hline$C_{p}$ & condensation model constant $[-]$ \\
\hline$e$ & energy [J] \\
\hline$f_{g}$ & mass fraction of noncondensible gases [-] \\
\hline$f_{y}$ & vapour mass fraction [-] \\
\hline$I_{D}$ & differential image \\
\hline$k$ & turbulence kinetic energy $\left[\mathrm{m}^{2} / \mathrm{s}^{2}\right]$ \\
\hline$k_{p}$ & scaling constant $[-]$ \\
\hline$k_{y}$ & scaling constant [-] \\
\hline$\dot{m}^{+}$ & mass source for condensation $\left[\mathrm{kg} /\left(\mathrm{m}^{3} \cdot \mathrm{s}\right)\right]$ \\
\hline $\begin{array}{l}\dot{m}^{-} \\
p\end{array}$ & $\begin{array}{l}\text { mass source for evaporation }\left[\mathrm{kg} /\left(\mathrm{m}^{3} \cdot \mathrm{s}\right)\right] \\
\text { local fluid pressure }[\mathrm{Pa}]\end{array}$ \\
\hline$p_{\text {sat }}$ & saturated vapour pressure $[\mathrm{Pa}]$ \\
\hline$p \vec{I}$ & spherical stress tensor [Pa] \\
\hline$\vec{q}^{m}$ & molecular heat flux $\left[\mathrm{kg} / \mathrm{s}^{3}\right]$ \\
\hline$\overleftrightarrow{q}^{R}$ & turbulent heat flux $\left[\mathrm{kg} / \mathrm{s}^{3}\right]$ \\
\hline$R$ & bubble radius [m] \\
\hline$r_{I}$ & intensity correlation coefficient [-] \\
\hline
\end{tabular}




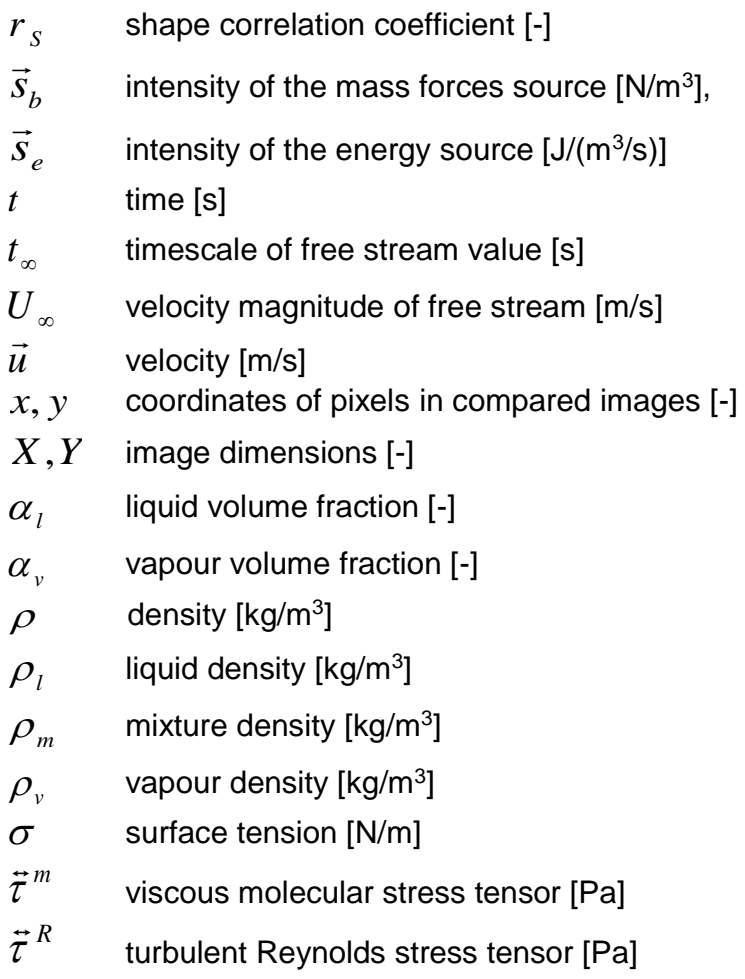

\section{References}

[1] Andriotis, A., Gavaises, M., Arcoumanis, C., 2008, Journal of Fluid Mechanics, 610, pp. 195-215.

[2] Bunnell, R. A., Heister, S. D., Yen, C., Collicott, S. H., 1999, Atomization and Sprays, 9 (5), pp. 445-465.

[3] Chen, Y., Heister, S. D., 1996, Atomization and Sprays, 6 (6).

[4] Fitzgerald, R. P., Gehrke, C., Svensson, K., Martin, G., 2017, SAE Technical Paper, No. 2017-01-0826).

[5] Giannadakis, E., Gavaises, M., Arcoumanis, C., 2008, Journal of Fluid Mechanics, 616, pp. 153-193.

[6] Gohil, P. P., Saini, R. P., 2014, Renewable and Sustainable Energy Reviews, 33, pp. 280-289.

[7] Gupta, M. K., Sharma, D. S., Lakhera, V. J., 2016, Applied Mechanics Reviews, 68 (3), p. 030801.

[8] Habchi, C., Dumont, N., Simonin, O., 2008, Atomization and Sprays 18 (2).

[9] Iben, U., 2002, Systems Analysis Modeling Simulations, 42, pp. 1283-1307.

[10] Kim, K. H., Chahine, G., Franc, J. P., Karimi, A., 2014, "Advanced experimental and numerical techniques for cavitation erosion prediction".

[11] Kunz, R. F., Boger, D. A., Stinebring, D. R., Chyczewski, T. S., Lindau, J. W., Gibeling, H. J., Venkateswaran, S., Govindan, T. R., 2000, Computers and Fluids, 29 (8), pp. 849-875.

[12] Merkle, C. L., Feng, J., Buelow, P. E. O., April 7. - 10. 1998, 3rd International Symposium on Cavitation.

[13] Merkle, C. L., Li, D., Venkateswaran, S., Jul. 9. - 12. 2006, 42nd AIAA/ASME/SAE/ASEE Joint Propulsion Conference.

[14] Mirshahi, M., Yan, Y., Nouri, J. M., 2015, Journal of Physics: Conference Series, 656 (1), p. 012093.

[15] Niedźwiedzka, A., Lipiński, S., Kornet, S., 2017, Journal of Hydroinformatics, DOI: 10.2166/hydro.2017.004.

[16] Payri, R., Salvador, F. J., Carreres, M., De la Morena, J., 2016, Energy Conversion and Management, 114, pp. 376-391.

[17] Rayleigh, L., 1917, Philosophical Magazine, 34, pp. 94-98.

[18] Salemi, R., Koukouvinis, P., Strotos, G., McDavid, R., Wang, L., Li, J., Marengo, M., Gavaises, M., 2015, Journal of Physics: Conference Series, 656 (1), p. 012083.

[19] Salvador, F. J., Romero, J. V., Roselló, M. D., Martínez-López, J., 2010, Mathematical and Computer Modelling, 52 (7), pp. 1123-1132.

[20] Schmidt, D. P., Rutland, C. J., Corradini, M. L., 1999, Atomization and sprays, 9 (3), pp. 255-276.

[21] Schnerr, G. H., Sauer, J., May 27. - Jun. 1. 2001, 4th International Conference on Multiphase Flow.

[22] Singhal, A. K., Athavale, M. M., Li, H., Jiang Y., 2002, Journal of Fluids Engineering, 124, pp. 617-624.

[23] Sobieski, W., 2011, Technical Sciences, 14, 299-313.

[24] Whitelaw, J. H., Payri, F., Desantes, J. M., 2002. "Thermo-and Fluid-dynamic Processes in Diesel Engines: Selected papers from the THIESEL 2000 conference held in Valencia, Spain, September 13-15, 2000".

[25] Zampilli, M., Bartocci, P., Bidini, G., Fantozzi, F., 2016, Energy Procedia, 101, pp. 693-700.

[26] Zwart, P. J., Gerber, G., Belamri, T., May 30. - Jun. 4. 2004, 5th International Conference on Multiphase Flow.

[27] ANSYS Fluent, http://www.ansys.com/Products/Fluids/ANSYS-Fluent ([cit. 2017-02-08])

[28] Ansys Fluent Tutorial Guide, http://www.ansys.fem.ir/ansys fluent tutorial.pdf ([cit. 2017-02-08]). 


\title{
Effect of geometry of real-size transparent nozzles on cavitation and on the atomizing jet in the near field
}

\author{
Y. Cao ${ }^{1}$, S. Idlahcen ${ }^{1}$, J.-B. Blaisot*1, C. Rozé ${ }^{1}$, L. Méès ${ }^{2}$, D. Maligne ${ }^{3}$ \\ ${ }^{1}$ CORIA UMR-6614, Université de Rouen, 76801 Saint-Etienne-du-Rouvray, France \\ ${ }^{2}$ LMFA UMR-5509, CNRS, École Centrale de Lyon, 69134 Écully, France \\ ${ }^{3}$ DELPHI France, 41000 Blois, France \\ ${ }^{*}$ Corresponding author: blaisot@coria.fr
}

\begin{abstract}
Cavitation inception is very sensitive to the nozzle geometry. Two diesel-like transparent nozzles with sharp and round inlet edge were investigated under moderate injection pressure. By means of backlight imaging technique, images were recorded in two regions near the orifice: within ten orifice diameter downstream the nozzle and inside the nozzle, for different times after start of the injection. A cross-correlation technique was applied to images downstream the orifice to estimate the average velocity of the spray. The development of the spray was studied at the transient and full-opened stage, related to the needle lift motion. To analyse the flow inside the nozzle, the relative size of the vapour pockets was measured on the image and the relative frequency of the occurrence of cavitation was determined over image series. This approach permits to examine the time variation of the cavitation occurrence from the inlet to the outlet of the orifice. The results highlight the sensitivity of the cavitating flow to the shape of the inlet edge. Moreover, the trends of the variation over time of the velocity and of the cavitation frequency are very similar to each other but different for each geometry, indicating that the characteristics of the diesel spray in the near field is closely affected by the way cavitation is developing in the nozzle.
\end{abstract}

\section{Keywords}

diesel injection, index matching, backlight, liquid interface tracking, frequency of cavitation

\section{Introduction}

Fuel consumption and engine emission are important issues concerning transportation industries. Engine optimization still needs to be improved, not only to balance the global reduction of fossil fuel reserves, but also to reduce their environmental impact. There is a pressing need to address clean combustion objectives, by developing a better understanding and control of every process steps of internal combustion engines. Atomization of fuel is a key element as it impacts the whole combustion process by affecting the initial conditions of this process.

In modern diesel engines, atomization efficiency was improved by considering high injection pressure in combination with small orifice diameter of only about a hundred micrometres or less. Large pressure variations of the fuel flow occurring inside injector nozzles promote cavitation occurrence. Indeed, cavitation occurs when the relative pressure drops locally below the vapour pressure of the fuel or when local stress is very high, resulting in the formation of vapour pockets or cavities in the nozzle orifice [1].

Nurick [2] proposed the first theoretical model to predict nozzle flow under cavitation, based on two-dimensional transparent single-orifice nozzles. Since then, similar nozzle designs were used to analyze the development of the cavitating flow and improve the model, by means of experiments $[3,4,5,6,7]$ and simulations $[8,9]$. The flows in the nozzle were compared by changing either the $L / D$ ratio or the inlet geometry of the orifice. Cavitation inception was found to be very sensitive to the nozzle geometry. Prasetya et al. [6] claimed that the cavitation thickness and the resulting contraction coefficient depend on the inlet edge radius.

For a real-size nozzle, the inlet geometry affects the spray development as well. By comparing cylindrical and conical nozzles, Payri et al. $[10,11,12]$ pointed out that the conicity of the orifice, representing the difference between inlet and outlet diameter, had an impact on the spray formation, specially in the near-field downstream the orifice. Furthermore, in terms of the manufacturing process, the machining precision of the rounding operation at the inlet edge is facing the high sensitivity of cavitation to this geometry. Indeed, a recent analysis of the internal structure of two similar diesel nozzles with a synchrotron source was reported by Wu et al. [13]. A significant difference in inlet edge radius was found even if the design dimensions were the same.

Non-intrusive optical diagnostics are able to provide the velocity information both for the flow inside the nozzle $[4,14,15,16]$ and for the downstream spray $[17,18]$. In their work, Moon et al. [18] argued that the sharp inlet edge induced a higher velocity gradient than the round inlet edge in the transverse direction. In this paper, we use the velocity measurement developed at CORIA $[19,20]$ to investigate the velocity field in the near field of the orifice. This work mainly focuses on the effects of the nozzle geometry, by studying cavitation inception in diesel-like orifices of different shapes, and the effect cavitation on the velocity of a diesel spray in the near nozzle region.

\section{Injector and transparent nozzle}

Injection tests have been conducted with a prototype injector based on a modified DELPHI solenoid Diesel injector. To visualize the flow inside the discharging orifice, transparent nozzles (PMMA) are coupled to the end of the 
stainless nozzle part of the injector body. Two different transparent nozzles have been used in the experiments whose geometry includes a sac and the discharging orifice of diameter $D=0.35 \mathrm{~mm}$ as shown in figure 1 . The main geometry is identically asymmetrical for the two nozzles, with the orifice axis shifted relatively to the sac axis in the side view. As shown after, the asymmetry induces non symmetry in the development of the cavitation inside the orifice, that makes it easier to characterize. In the perpendicular direction (front view), the nozzles are symmetric. The difference between the geometries lies in the connection between the sac and the orifice (see detail in 1), one being sharp and referred to as Sharp edge and the other one being smooth and referred to as Round edge. To visualize cavitation in the transparent nozzles, a refractive index matching method was used by adding high refractive index liquid to calibration oil (ISO 4113) [21]. At ambient temperature $\left(T=20^{\circ} \mathrm{C}\right)$, the vapor pressure of the mixed liquid was found to be around $P_{v}=5 \mathrm{~Pa}$ in a previous study [22].

The current activation signal delivered to the injector by an injector driving system IPOD from EFS company is shown in figure 2. The injection duration is fixed to $T_{i n j}=4 \mathrm{~ms}$ and the injection pressure to $P_{i n j}=30 \mathrm{MPa}$ for both nozzles. The back pressure is kept at ambient value, i.e. $P_{b}=0.1 \mathrm{MPa}$.

The dynamic pressure $P_{\text {tube }}$ is monitored by a pressure sensor mounted on a straight stainless steel tube upstream the fuel inlet of the injector (red curves in figure 2). To characterize the cavitation regime, the cavitation number is defined as $\sigma=\frac{P_{l}-P_{v}}{P_{l}-P_{b}}$ in [2,10], whereas $P_{l}$ is the fuel pressure. To highlight the difference in sac geometry, the pressure in the sac need to be considered in this computation: $P_{l}=P_{s a c}$. However, this measurement is not available in this experiment. Thus, by considering $P_{l}=P_{i n j}$, it leads to an identical cavitation number for both geometries: $\sigma($ Sharp $)=\sigma($ Round $)=1.003$, indicating that cavitation is likely to occur [10, 23]

The injector is equipped with a needle lift sensor (micro-epsilon LS04(04)). The needle lift signal (blue curves in figure 2) presents three parts as usual, i.e. an increase of the needle lift from closed position, a maximum that can have a plateau and a decrease at needle closing. The plateau is observed when the fully opened stage is reached. Here, the plateau is observed at a delay after start of activation ( $A S O A$ ) about $2.2 \mathrm{~ms}$ with the Sharp inlet edge and about $3 \mathrm{~ms}$ with the Round inlet edge. This means that under the same pressure conditions, the Round inlet edge leads to a lower dynamics of the injector needle lift as the time to reach the fully opened stage is increased by almost $50 \%$. It is thus reasonable to assume that the pressure in the sac is significantly different for the two nozzle shapes as this pressure directly controls the needle lift motion speed.

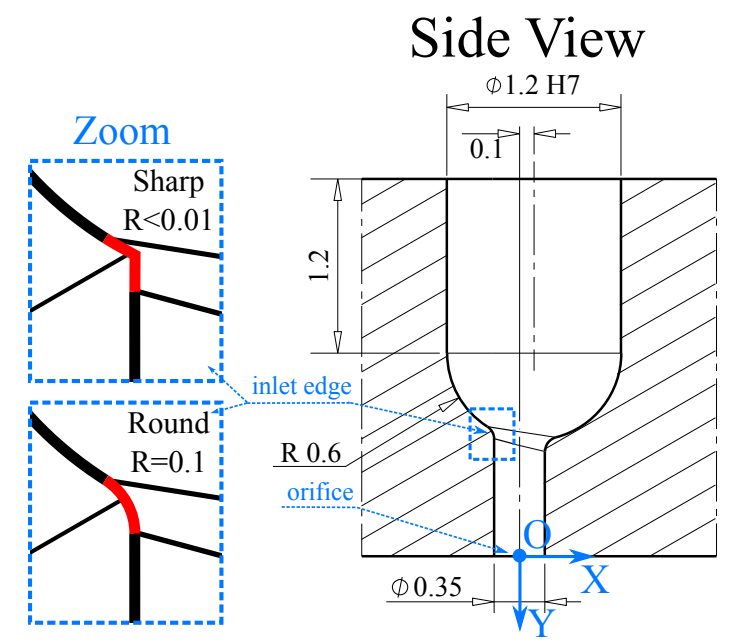

Figure 1. Geometric difference between two nozzle designs. The unit of length is millimetre.

\begin{abstract}
Velocity measurements at fully opened stage
A backlight imaging technique is used to visualize the dynamics of the flow in the nozzle and in the spray, within a distance of about ten nozzle diameters downstream the nozzle orifice. The velocity fields for the Sharp inlet edge were obtained in a previous work [21]. A similar optical setup has been used in the present work for the Round inlet edge. The pulsed light source is a laser diode (Cavitar) with a pulse duration of 10ns (in place of a double cavity femtosecond laser source in [21]). A double frame camera is set to record image-pairs separated by a delay of about $d t=200 \mathrm{~ns}$. The image size is $2048 \mathrm{pixel} \times 2048 \mathrm{pixel}$ and the spatial resolution is about $800 \mathrm{pixel} / \mathrm{mm}$ corresponding to a field of view of $2.58 \mathrm{~mm} \times 2.58 \mathrm{~mm}$ for both views. One should notice that the field of view are different for the Sharp inlet edge, which are $3.47 \mathrm{~mm} \times 3.47 \mathrm{~mm}$ and $3.00 \mathrm{~mm} \times 3.55 \mathrm{~mm}$ for the front and side view respectively. Considering the highest velocity measured in the spray of the order of $200 \mathrm{~m} / \mathrm{s}$, the blurring effect remains about 1 pixel with this optical setup, which has a marginal impact on the image quality [24].

The system operates at low frequency: one injection occurs every second and one image-pair is acquired during one injection cycle. In the reference frame used to give the positions in the spray, the origin $O$ is fixed at the geometric centre of the orifice (see Figure 1), $Y$ represents the axial injection direction and $X$ represents the transverse direction. Image-pairs are used to compute velocity fields in the spray from the displacement of the liquid structures and ligaments in the images. The instantaneous velocities are denoted as $U_{n}$ and $V_{n}$ along $X$ and $Y$ directions,
\end{abstract}




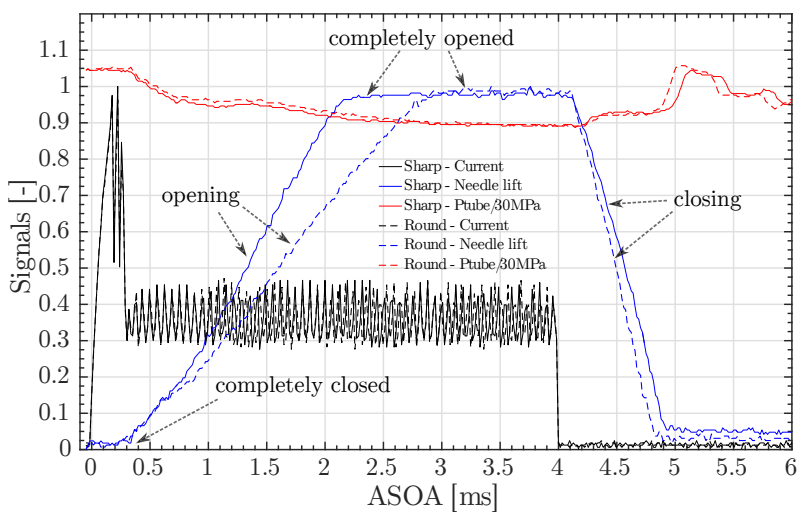

Figure 2. Analog signals vs delay After Start of Activation (ASOA). The current and needle lift signals are rescaled. The pressure signal upstream the injector $\left(P_{\text {tube }}\right)$ is rescaled by the value of $30 \mathrm{MPa}$.

respectively.

Displacements are estimated by liquid interface tracking algorithm. By using the in-house code, the cross-correlation technique is applied to liquid elements (ligaments and droplets) identified in the image. For the sake of brevity, the method is not described here and details can be found in $[19,20]$. Over 250 velocity fields are considered in a statistical analysis to estimate the average and fluctuating velocity in the spray at a given delay $A S O A$.

The spray dynamics is investigated at fully opened stage. The average spray image is shown in figure 3 in gray scale in the background. Liquid elements appear as black over white (for the surrounding air) as commonly obtained with backlight techniques. The mean spray images seem to be quite similar in both cases for the front view, with a narrower spray span for the Round case. More precisely, at a distance of 5D from the orifice, the width is about $30 \%$ less.

The average velocity along $X$ direction over $N$ samples is computed as $\bar{U}=\int_{n} U_{n} / N$. Reynolds decomposition gives the fluctuation term: $u^{\prime}=U-\bar{U}$ and then the variance is computed as $\frac{n}{u^{\prime} u^{\prime}}$, which is the square of standard deviation (std). The average velocity maps in color scale overlap the average spray images in figure 3 . The statistical convergence for Round edge is investigated at two locations in the side view: on the left side (1) and on the right side (2). These locations are represented in figure 3 (lower left) and their coordinates are given in Table 1.

The curves in Figure $4 \mathrm{a}$ indicates the variations of the statistical quantities in both directions when increasing the number of samples. At any location, values are normalised by the value computed with all available samples $(N=250)$, in order to highlight the trends of the curves. Here, filled symbols indicate the values within an interval of $\pm 5 \%$. It is clear that average velocities converge quickly as about 130 samples are sufficient in both directions to reach the final value. For the variances, 200 samples are necessary to obtain the convergence. The statistics for the entire sample set are given in Table 1. The standard deviation is of the same order of magnitude for each side: about $22 \mathrm{~m} / \mathrm{s}$ in the spray direction and $6.5 \mathrm{~m} / \mathrm{s}$ in the transverse direction. Finally, we estimate the precision of the velocity measurement by computing the confidence interval. For uncorrelated samples where $\mathrm{N}>30$, the true mean falls within $95 \%$ of the confidence interval given by $\bar{U} \pm 1.96 \sqrt{\overline{\overline{u^{\prime} u^{\prime}}}} / \sqrt{N}$ [25]. With the values in Table 1, confidence intervals are about $\pm 3 \mathrm{~m} / \mathrm{s}$ and $\pm 0.8 \mathrm{~m} / \mathrm{s}$ for $\bar{U}$ and $\bar{V}$ in $\mathrm{x}$ and y directions respectively. So the estimation of average velocity is accurate within less than $3 \%$ by using this measurement method.

For the Round case, the order of magnitude of the maximum velocity is about $150 \mathrm{~m} / \mathrm{s}$, which is about $10 \%$ less than for the Sharp case. The velocity map on the front view seems to be symmetric for the Sharp edge whereas it is not for the Round edge. Also, velocity values on the side view are significantly reduced on the right side of the spray. It is obvious that the change of radius of curvature at the inlet edge has a huge impact on the spray dynamics from both views. It is most likely a direct consequence of the modification of the flow upstream the orifice, cavitation inception being sensitive to the sac geometry. To better understand the leading reason at the origin of such a difference in the velocity fields for the two nozzle geometries, we focused our attention to the transient phase, i.e., during the lifting of the needle, in the following sections.

\section{Transient phases of injection}

Image-pairs were recorded for different delays $A S O A$, giving average velocity maps along injection duration. The time evolution of the average velocity is considered hereafter (see Figure 5). Let's first consider the front view. The average velocity is computed as follows. Two rectangular regions are considered for computing mean velocities $(5 \mathrm{x}$ 5 pixels) (see gray rectangles in Figure 3). They are located within $X=1.25 D$ on either side of the orifice axis and at a distance of $Y=5 D$ downstream the orifice. According to the needle dynamics for the two cases, the injection duration is about $2.5 \mathrm{~ms}$ and $3.5 \mathrm{~ms}$ for the Sharp and Round cases respectively.

For the Sharp edge a steep increase of the velocity is observed at the early stage of the injection (up to a delay $A S O A \simeq 1 \mathrm{~ms}$ ). The curves have similar shapes and values for both sides, as a result of the symmetry of the spray in this case. A short plateau of $\simeq 0.5 \mathrm{~ms}$ in duration followed by a decrease is observed on the left side of the spray whereas the plateau remains up to the end of the curve on the right side. 

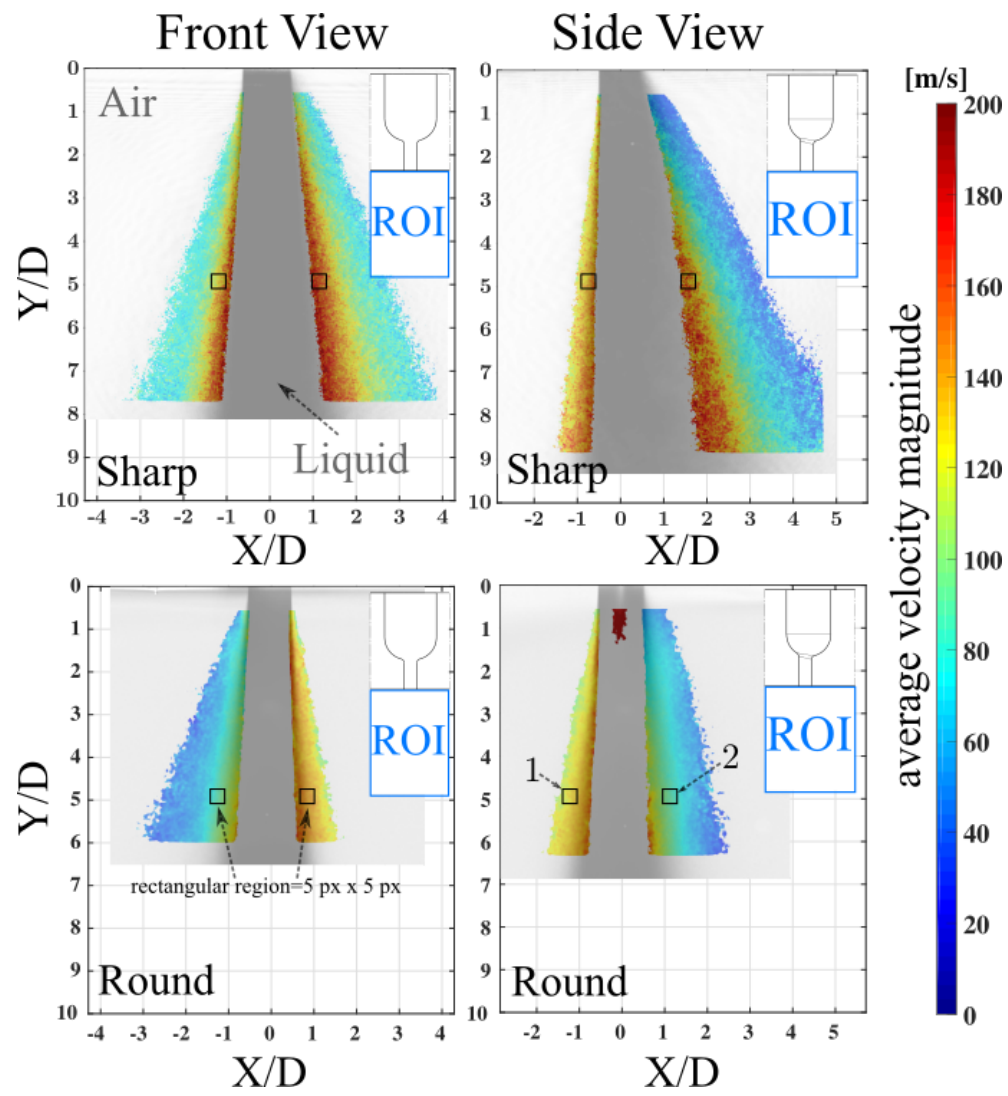

Figure 3. Average velocity map at fully opened stage. The average velocity (in color scale) and the average spray image (in the background) is overlapped. Two upper images, obtained in [21], concern the Sharp inlet edge at $A S O A=2.2$ ms with a spatial resolution of $590 \mathrm{pixel} / \mathrm{mm}$. And the image-pairs are separated by $d t=314 \mathrm{~ns}$; Two lower images concern the Round inlet edge at $A S O A=3 \mathrm{~ms}$ with a spatial resolution of $759 \mathrm{pixel} / \mathrm{mm}$ and $d t=200 \mathrm{~ns}$.

This leads to a non-negligible difference of about $40 \mathrm{~m} / \mathrm{s}$ between the two sides of the spray. A pressure oscillation in the sac induced by a needle rebound when the needle reaches complete lift position could be the reason for this asymmetry. This pressure oscillation could also be induced by pressure wave reflections in the high pressure line, even if the delay for such reflections is sought to be longer in practice (see for example $P_{\text {tube }}$ signals in Figure 2).

\begin{tabular}{rcc}
\hline Location & 1 & 2 \\
\hline$(x / D, y / D)$ & $(-1.25 ; 5)$ & $(1.25 ; 5)$ \\
$\bar{U}[\mathrm{~m} / \mathrm{s}]$ & 126,6 & 89,0 \\
$\sqrt{\overline{u^{\prime} u^{\prime}}}[\mathrm{m} / \mathrm{s}]$ & 21.3 & 22.1 \\
$\bar{V}[\mathrm{~m} / \mathrm{s}]$ & -21.6 & 13 \\
$\sqrt{\overline{v^{\prime} v^{\prime}}}[\mathrm{m} / \mathrm{s}]$ & 6.5 & 6.5 \\
\hline
\end{tabular}

Table 1. Statistics of velocity in the Round case.

Despite this unexpected outcome, average velocity and needle lift curves have common trends for the Sharp case. For the Round edge, the velocity increase at the beginning of injection is slower than for the Sharp edge. This is expected since the relatively slow needle lift motion for the Round edge indicates a slower pressure increase in the sac in this case. The average velocity curves have similar shapes but reach different values on each side of the spray for the Round edge, with a difference that increases up to $30 \mathrm{~m} / \mathrm{s}$ (starting from a delay $A S O A=1 \mathrm{~ms}$ ). Whereas nozzle geometries are supposed to be symmetrical for this view, it is guessed that a very little asymmetry can subsists in the nozzle geometry for this view. In particular, a slight turn of the PMMA plate could happened during the machining of transparent nozzles. Thus, as cavitation development is very sensitive to the geometry, such a small non visible asymmetry can be the cause for this non expected asymmetrical development of the spray. The right figure in 5 shows the time evolution of velocity in the side view. For the Sharp geometry, it should be mentioned that the rectangular regions are changed to new locations: $X=-0.8 D$ and $X=1.7 D$ on each side of the spray with the same distance to the orifice (5D), due to the asymmetric spreading of the spray. The shape of curves are not much different for each side, neither from those in the front view whereas the values differs during the transient phase. For Round shape, it should be noticed that the locations of rectangular regions are unchanged. For both sides, the velocities are of the same order as the Sharp edge at the early stage. The same shape is found for each side of the jet, but with a difference about $40 \mathrm{~m} / \mathrm{s}$ between left and right velocities when the plateau is 

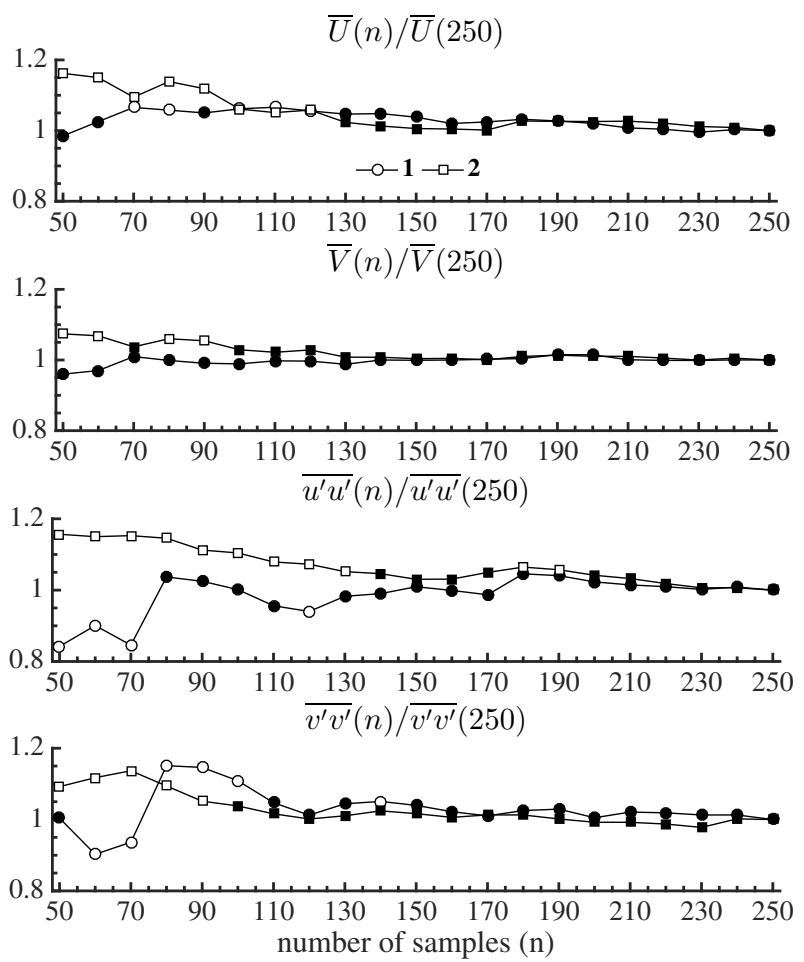

Figure 4. Statistical convergence in the Round case. Filled symbols indicate the values within $[0.95 ; 1.05]$ interval.

reached. This difference is expected in this case.

It is interesting to notice that the difference between each side appears around $A O S A=1 \mathrm{~ms}$. And this seems to be amplified during the transient phase and ends at a value of about $40 \mathrm{~m} / \mathrm{s}$. To develop a better understanding of this results, three instantaneous images of the flow inside the Round edge nozzle for $A S O A=1 \mathrm{~ms}$ are shown in Figure 5). We recall that the aim of asymmetrical configuration is to obtain an asymmetrical development of cavitation allowing a better visualization of the cavities and liquid-vapour interfaces. On these images, the liquid phase or the transparent nozzle appear in light gray, and the vapour cavities induced by cavitation appears in black. It is obvious that cavitation occurs at nozzle entrance on the right side in all images but not always down to the nozzle orifice. We can remark also that large vapour pockets reach the nozzle orifice on the left side of the image. As the presence of cavitation reduce the hydraulic section, the liquid velocity is locally increased in region where there is no cavitation pockets, i.e., mainly on the right side of the flow. In consequence, it is not surprising to obtain larger velocity on the right side than the left side when cavitation is sufficiently developed for the vapour pockets to reach the orifice. Fluctuation levels (std) estimated for the Round edge to be larger than $10 \mathrm{~m} / \mathrm{s}$ are though to be linked to the statistical variation of the properties of the vapour pockets. Indeed, the nozzle orifice is filled with vapour cavities: partially on left and right sides in image No 31; almost completely in image No 32; and mainly on the left side in image No 33. Again, during the development of the spray, the fluctuation level is doubled: almost $25 \mathrm{~m} / \mathrm{s}$ of fluctuation is found at fully opened stage. The fluctuation of the vapour cavities reaching the orifice might be one of the main reasons of this high fluctuation level of the velocity in the near field of the spray.

In the next section, attention is put on the generation of cavitation in the nozzle along the injection duration.

\section{Cavitation analysis}

To investigate the cavitation occurring in the nozzle, backlight images of the flow in the nozzle are recorded (for the side view only) with a similar optical set-up. It is necessary to be pointed out that as for any imaging method, no data can be provided for the size and shape of the vapour pockets in the dimension perpendicular to the image plane due to the 2D projection proceeded by image formation. Thus, a low gray level pixel in the image indicates that vapour phase is present in the nozzle at the corresponding $(X, Y)$ position, but without any indication on the position in the third direction nor on the depth of the local the vapour phase pocket. These images thus need to be interpreted with caution. Whereas the volume of cavitation pockets cannot be determined, the relative frequency of the occurrence of cavitation was estimated by measuring the relative size of the projection of these pockets on the images.

The method to estimate the relative frequency of cavitation is demonstrated through an example in the figure 6 . The gray level raw image is first transformed in a two-level (black and white, bw) image by applying a threshold value determined by the Ostu's threshold method [26] on an average image at fully opened stage. The bw image is then inverted, in order to have white pixels (coded with value 1) corresponding to the presence of the vapour phase. Finally, the average image is computed over 25 samples at the same delay $A S O A$. This average image has a null gray level value, i.e. null relative frequency in region where cavitation never occurred and a gray level of 1 , i.e. a 


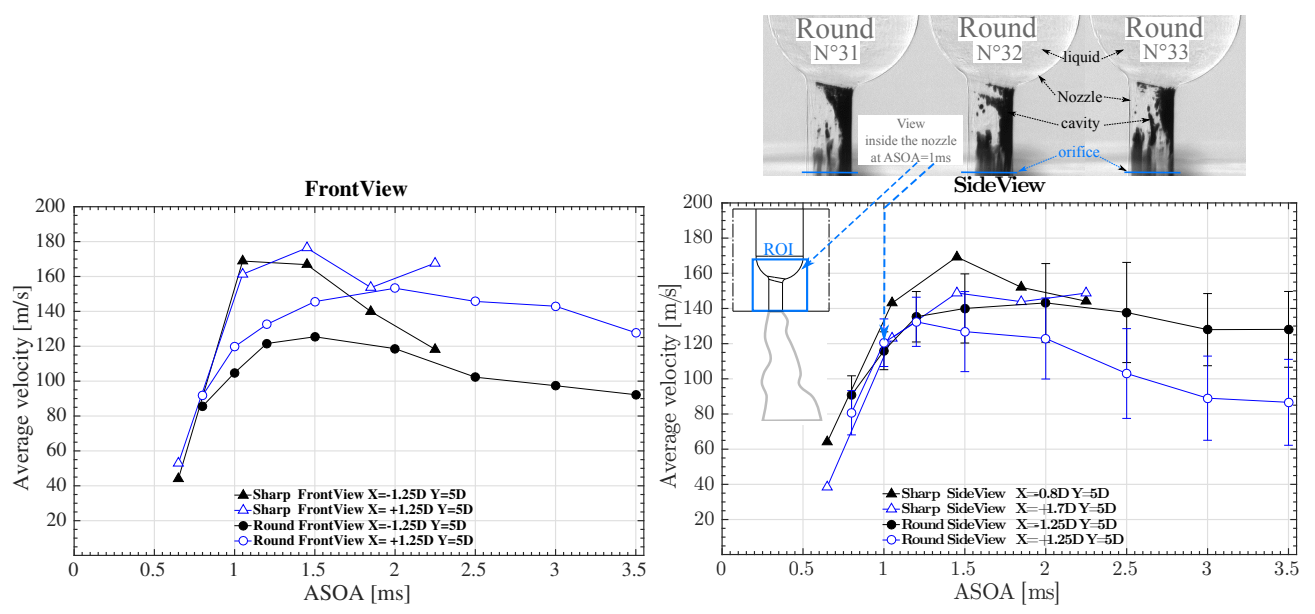

Figure 5. Time evolution of the average velocity from the front (left) and side (right) view.
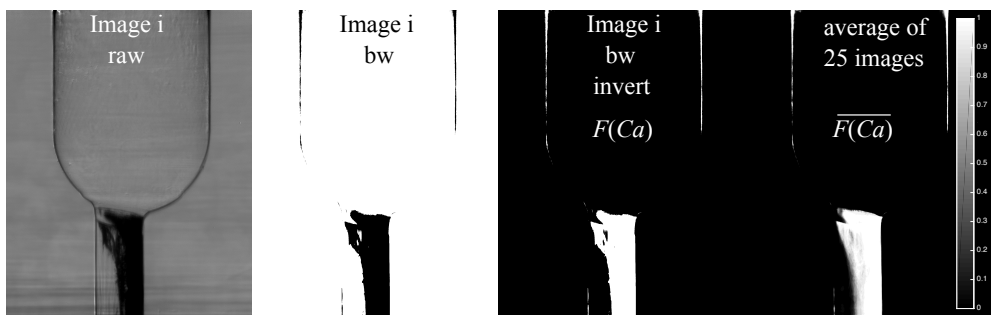

Figure 6. Computation of the average relative frequency of cavitation.

relative frequency of 1 , where cavitation is always detected in the image series.

The average images giving the cavitation frequency $\overline{F(C a)}$ at fully opened stage for both geometries are shown on the right side of the figure 7 . Despite the similar shape of the light gray area in the region of interest, the gray level is clearly different for the different geometries. The variation of the frequency along the nozzle axis was determined by averaging the gray level values over each row in the image. The reference position of these row are given in distance over $Y$ axis from the nozzle orifice, i.e., with negative values.

The evolution of the frequency $\overline{F(C a)}$ at the entrance and exit of the orifice channel is first examined. At the entrance $(Y=-0.75 \mathrm{~mm})$, the increase of the frequency $\overline{F(C a)}$ is fast for the Sharp inlet edge, and the plateau is reached at a very early stage, before $1 \mathrm{~ms}$. The increase is also very steep for Round edge but cavitation occurrence starts with a delay of $\simeq 0.1 \mathrm{~ms}$ and followed later on by a plateau. This means that cavitation is initiated in a very short time at orifice entrance whatever the velocity of the needle lift motion. Moreover, for the Sharp edge, the plateau value over 0.9 indicates that for any injection event, cavitation is mostly present at the entrance of the orifice.

At the orifice outlet $(Y=-0.05 \mathrm{~mm})$, the cavitation frequency starts increasing up to a delay $A S O A \simeq 1 \mathrm{~ms}$ and then decreases slowly. When the needle is lifted completely, we find that the width of the white region is approximately half the orifice diameter (see image in Figure 7), which is consistent with the value of $\overline{F(C a)} \simeq 0.5$. It can be noticed that the curves for the frequency and for the average velocity look very similar for the Sharp edge (see Figure 5). Indeed, the fast increase of the near-field velocity occurs at the same time (for $A S O A<1 \mathrm{~ms}$ ) and the same way as for the start of the cavitation frequency.

We can also notice that for the Round edge cavitation starts nearly simultaneously at the entrance and at the exit of the orifice, with a delay of about $0.2 \mathrm{~ms}$ compared to the Sharp edge. At the entrance, the frequency continue to increase up to a plateau of about 0.7 , which is $30 \%$ lower than for the Sharp edge, showing that cavitation is less prompt to occur for a smooth shaped orifice inlet. At the nozzle exit, the frequency curves are very similar for both shapes, i.e., it decreases to a value of $\overline{F(C a)} \simeq 0.5$ meaning that cavitation occurred here about half of the time. In addition, a slow transition along with fluctuations are both encountered in the time variation of velocity and cavitation frequency curves. It is reasonable to suggest that the development of cavitation at nozzle exit is very sensitive to the inlet edge geometry, and the spray atomization in the near-field is related to the way cavitation is developing up to the nozzle exit.

The variation of cavitation frequency along the orifice at any time after the start of injection is shown in the figure 8 . For the Sharp edge, the profiles of $\overline{F(C a)}$ are quite similar during the injection and present a quasi-continuous decrease of cavitation frequency from inlet to outlet. For the Round edge, the variation of cavitation present a minimum at mid distance from $0.7 \mathrm{~ms}$ to $2.0 \mathrm{~ms}$. Cavitation frequency becomes continuously decreasing only for a delay $A S O A \geq 2.5 \mathrm{~ms}$.

At fully opened stage, cavitation frequency reaches similar values for both nozzles, which is not consistent with the difference observed previously on the velocity maps (see Figure 3). As mentioned earlier, images do not contain 


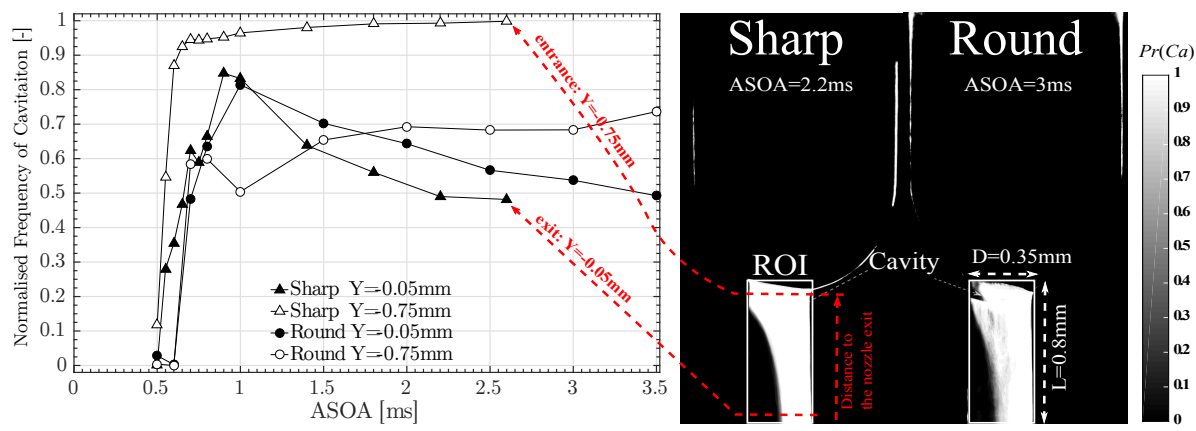

Figure 7. Average relative frequency of cavitation $\overline{F(C a)}$ at the entrance $(Y=-0.75 \mathrm{~mm})$ and the exit $(Y=-0.05 \mathrm{~mm})$ of the nozzle orifice.
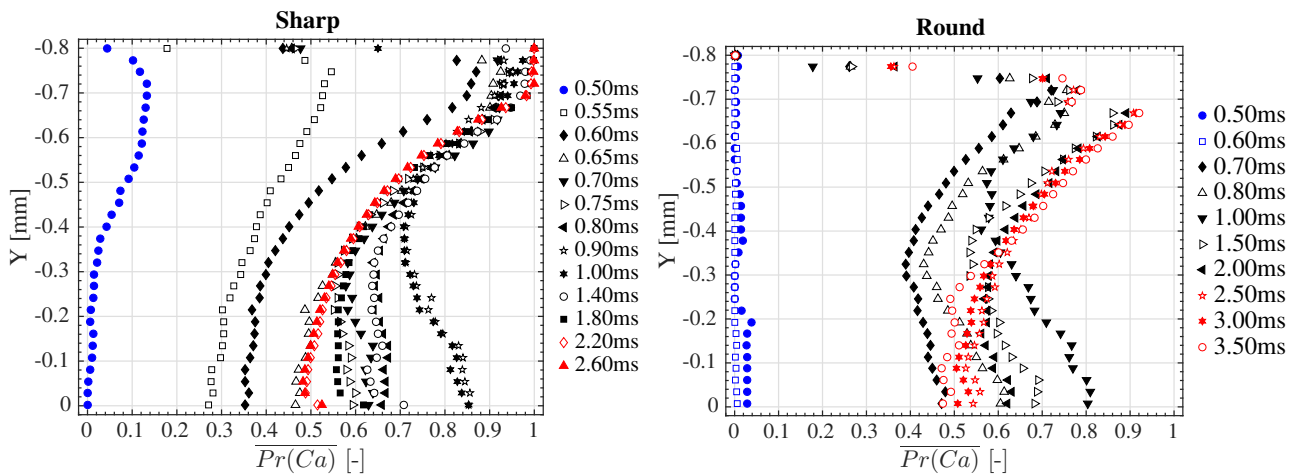

Figure 8. Average relative frequency of cavitation $\overline{F(C a)}$ profiles for the Sharp case (left) and Round case (right).

any information on the third dimension. Two vapour pockets having the same projection area in image can have totally different depths in the third dimension. These pockets leads to similar cavitation frequency with the above mentioned analysis, but the spray dynamics will probably be different. Thus, the missing information in the third dimension is believed to be a key element to well understand the effect of cavitation on atomization.

\section{Conclusions}

This experimental work investigates the flow through a diesel-like transparent nozzle. Two nozzles with different inlet edge shapes were compared. A backlight technique was used to visualize the flow downstream the orifice within ten orifice diameters and the flow in the nozzle.

Velocities of the spray estimated by a cross-correlation method were statistically averaged. During the transient phase, the velocity variations were compared to report the high sensitivity of the flow pattern to the orifice shape. This yields to different velocity maps at fully opened stage. The relative frequency of cavitation in the nozzle were computed by measuring the relative size of the vapour pockets on the image. For the Sharp edge, cavitation occurs immediately and a continuous decrease of the frequency of the cavitation is observed after $A S O A=1 \mathrm{~ms}$ and from inlet to outlet. For the Round edge, cavitation occurs with a short delay compared to the Sharp edge, and the cavitation frequency varies gradually after $A S O A=1 \mathrm{~ms}$ and present a minimum value at mid nozzle distance. The spatial distribution of cavitation frequency from inlet to outlet of the orifice is thus found to be different between the two nozzles. The results indicate that the velocity in the spray and the frequency of cavitation in the nozzle have similar trends for both nozzle designs.

Perspectives for this work include improvement of the determination of the properties of vapour pockets in the nozzle. This would imply a measure of the depth of the vapour pockets in the third dimension. Due to the complex 3D geometry of the small-size nozzle, this will constitute a real challenge to overcome, mostly from an optical point of view. In a more accessible way, the temporal correlation of the flow inside the nozzle and downstream the orifice will have to be deeply explored by simultaneous visualization of inside and outside flows.

\section{Acknowledgements}

This work was supported by CANNEx program (ANR-13-TDMO-03), funded from French National Research Agency (ANR). 


\section{References}

[1] Lefebvre, A., 1988. Atomization and sprays, Vol. 1040. CRC press.

[2] Nurick, W., 1976. "Orifice cavitation and its effect on spray mixing". ASME, Transactions, Series I-Journal of Fluids Engineering, 98, pp. 681-687.

[3] Winklhofer, A. H. A. M. E., and Phillip, H., 2000. "Cavitation and spray formations in diesel flow situations". In ILASS-Europe, pp. 11-13.

[4] Winklhofer, E., Kull, E., Kelz, E., and Morozov, A., 2001. "Comprehensive hydraulic and flow field documentation in model throttle experiments under cavitation conditions". In ILASS-Europe, pp. 2-6.

[5] Sou, A., Hosokawa, S., and Tomiyama, A., 2007. "Effects of cavitation in a nozzle on liquid jet atomization". International journal of heat and mass transfer, 50(17), pp. 3575-3582.

[6] Prasetya, R., Mashida, M., Yamada, Y., and Sou, A., 2015. "Effect of nozzle inlet roundness and nozzle length on cavitation and liquid jet". In ILASS-Europe.

[7] Pratama, R. H., Sou, A., Wada, Y., and Yohohata, H., 2015. "Cavitation in mini-sac nozzle and injected liquid jet". In ILASS-Europe.

[8] Dabiri, S., Sirignano, W., and Joseph, D., 2007. "Cavitation in an orifice flow". Physics of Fluids, 19(7), p. 072112.

[9] Bastawissi, H. A.-E., Elkelawy, M., et al., 2014. "Investigation of the flow pattern inside a diesel engine injection nozzle to determine the relationship between various flow parameters and the occurrence of cavitation". Engineering, 6(13), p. 923.

[10] Payri, F., Bermudez, V., Payri, R., and Salvador, F., 2004. "The influence of cavitation on the internal flow and the spray characteristics in diesel injection nozzles". Fuel, 83(4), pp. 419-431.

[11] Payri, R., García, J., Salvador, F., and Gimeno, J., 2005. "Using spray momentum flux measurements to understand the influence of diesel nozzle geometry on spray characteristics". Fuel, 84(5), pp. $551-561$.

[12] Payri, R., Salvador, F. J., Gimeno, J., and Viera, J. P., 2015. "Experimental analysis on the influence of nozzle geometry over the dispersion of liquid n-dodecane sprays". Frontiers in Mechanical Engineering, 1, p. 13.

[13] Wu, Z., Gao, Y., Gong, H., and Li, L., 2016. "Evaluation and optimization of the structural parameter of diesel nozzle basing on synchrotron radiation imaging techniques". Journal of Instrumentation, 11(04), p. C04004.

[14] Sou, A., Tomiyama, A., Hosokawa, S., Nigorikawa, S., and Maeda, T., 2006. "Cavitation in a two-dimensional nozzle and liquid jet atomization". JSME International Journal Series B Fluids and Thermal Engineering, 49(4), pp. 1253-1259.

[15] Mauger, C., Méès, L., Michard, M., Azouzi, A., and Valette, S., 2012. "Shadowgraph, schlieren and interferometry in a $2 \mathrm{~d}$ cavitating channel flow". Exp. Fluids.

[16] Hult, J., Simmank, P., Matlok, S., Mayer, S., Falgout, Z., and Linne, M., 2016. "Interior flow and near-nozzle spray development in a marine-engine diesel fuel injector". Experiments in Fluids, 57(4), pp. 1-19.

[17] Suh, H. K., and Lee, C. S., 2008. "Effect of cavitation in nozzle orifice on the diesel fuel atomization characteristics". International journal of heat and fluid flow, 29(4), pp. 1001-1009.

[18] Moon, S., Gao, Y., Wang, J., Fezzaa, K., and Tsujimura, T., 2014. "Near-field dynamics of high-speed diesel sprays: Effects of orifice inlet geometry and injection pressure". Fuel, 133, pp. 299-309.

[19] Sedarsky, D., Idlahcen, S., Rozé, C., and Blaisot, J.-B., 2013. "Velocity measurements in the near field of a diesel fuel injector by ultrafast imagery". Experiments in fluids, 54(2), pp. 1-12.

[20] Berlemont, A., Blaisot, J.-B., Bouali, Z., Cousin, J., Desjonqueres, P., Doring, M., Dumouchel, C., Idlahcen, S., Leboucher, N., Lounnaci, K., et al., 2013. "Numerical simulation of primary atomization: Interaction with experimental analysis". Atomization Sprays, 23(12), pp. 1103-1138.

[21] Purwar, H., Lounnaci, K., Idlahcen, S., Roze, C., Blaisot, J.-B., Méès, L., and Michard, M., Aug. 23.-27. 2015. "Effect of cavitation on velocity in the near-field of a diesel nozzle". In 13th Triennial International Conference on Liquid Atomization and Spray Systems.

[22] Chorążewski, M., Dergal, F., Sawaya, T., Mokbel, I., Grolier, J.-P. E., and Jose, J., 2013. "Thermophysical properties of normafluid (iso 4113) over wide pressure and temperature ranges". Fuel, 105, pp. 440-450.

[23] Desantes, J., Payri, R., Salvador, F., and De la Morena, J., 2010. "Influence of cavitation phenomenon on primary break-up and spray behavior at stationary conditions". Fuel, 89(10), pp. 3033-3041.

[24] Purwar, H., Wang, H., Tang, M., Idlahcen, S., Rozé, C., Blaisot, J.-B., Godin, T., and Hideur, A., 2015. "Ultrafast high-repetition imaging of fuel sprays using picosecond fiber laser". Optics express, 23(26), pp. 33396-33407.

[25] Benedict, L., and Gould, R., 1996. "Towards better uncertainty estimates for turbulence statistics". Experiments in fluids, 22(2), pp. 129-136.

[26] Otsu, N., 1979. "A threshold selection method from gray-level histograms". IEEE transactions on systems, man, and cybernetics, 9(1), pp. 62-66. 


\title{
VOF Simulation of The Cavitating Flow in High Pressure GDI Injectors
}

\author{
F. Giussani ${ }^{1 *}$, A. Montorfano ${ }^{1}$, F. Piscaglia ${ }^{1}$, A. Onorati ${ }^{1}$, J. Hélie $^{2}$ \\ ${ }^{1}$ Dip. di Energia, Politecnico di Milano, via Lambruschini 4, I-20156 Milan (Italy) \\ ${ }^{2}$ Continental Automotive SAS, 1 av. Paul Ourliac BP 1149, 31036 Toulouse Cedex 1 (France) \\ *Corresponding author: filippo.giussani@polimi.it
}

\begin{abstract}
The paper describes the development in the OpenFOAM ${ }^{\circledR}$ technology of a dynamic multiphase Volume-of-Fluid (VoF) solver, supporting mesh handling with topological changes, that has been used for the study of the physics of the primary jet breakup and of the flow disturbance induced by the nozzle geometry during the injector opening event in high-pressure Gasoline Direct Injection (GDI) engines. Turbulence modeling based on a scale-resolving approach has been applied, while phase change of fuel is accounted by means of a cavitation model that has been coupled with the VOF solver. Simulations have been carried out on a 6-hole prototype injector, especially developed for investigations in the framework of the collaborative project FUI MAGIE and provided by Continental Automotive SAS. Special attention has been paid to the domain decomposition strategy and to the code development of the solver, to ensure good load balancing and to minimize inter-processor communication, to achieve good performance and also high scalability on large computing clusters.
\end{abstract}

\section{Keywords}

Volume-of-fluid, GDI injectors, topologically changing mesh, hybrid RANS/LES, cavitation, OpenFOAM ${ }^{\circledR}$

\section{Introduction}

Turbulence certainly has a direct impact on thermodynamic efficiency, brake power and emissions of the engine, since its influence extends from volumetric efficiency to air/fuel mixing, combustion and heat transfer. In a gasoline engine, there are two strategies for injecting the fuel: Port Fuel Injection (PFI), a well known technology to favor air/fuel mixing, and Gasoline Direct Injection (GDI), which consists in injecting the fuel directly into the cylinder. GDI is becoming a common solution in automotive industry since it allows for a great flexibility in the air/fuel ratio, leading to low fuel consumption and emissions at light loads (lean or stratified mode) and high power output during rapid accelerations and heavy loads (power mode) [1,2]. These different conditions are not only related to the amount of fuel injected into the combustion chamber, but also to the way the injection is performed: level of atomization, penetration and diffusion of fuel. Experimental campaigns showed that spray formation is mainly influenced by the geometry of the injector itself (L/D ratio, number of holes, internal geometry, etc.) [3, 4], the operating pressure and the needle lift curve [5]. Fuel is characterized by high velocities (order of hundreds of meters per second) and reduced timescales, so that time-transient phenomena play an important role. Moreover, because of the strong acceleration of the liquid phase inside the injector nozzle, pressure may drop below the saturation value causing the onset of cavitation, which strongly modifies the internal flow field due to the presence of bubbles. Hence, a better comprehension of spray characteristics could help to improve engine performance and injector design. Simulating the injector opening event and the spray primary breakup are still on the frontier of modern modeling science. Since primary breakup is strictly related to turbulence inside the injector, a correct way to describe turbulence is crucial for a successful simulation. In this sense, a time-resolved approach like seems the most appropriate to model turbulence in injectors and also a straightforward way to overcome the intrinsic lack of time resolution of the Unsteady Reynolds-Averaged Navier-Stokes (URANS) equations [6]. Disperse sprays are usually simulated by a Lagrangian/Eulerian approach [7]: particles reside in the Eulerian grid cells and introduce source terms in the Eulerian conservation laws to account for interactions between the two phases. To simulate fuel injection, the continuous liquid core in the near-nozzle region is modeled by discrete parcels which typically are assumed to have the same characteristic size as the nozzle exit diameter and phenomenological models are used to account for breakup [8], as well as collision and coalescence of liquid droplets [9]. The above mentioned method requires a precursor nozzle flow simulation of the Eulerian liquid phase to predict the flow conditions at the nozzle exit [10]. The coupling between the internal and the external nozzle simulation is inherently weak, due to potential inconsistencies of the two-phase models in the two separate simulations [10]. On the other hand, engine sprays, and sprays in general, could be better described using a continuum for both the liquid and the gas phases, where conservation laws are solved under Eulerian flow assumptions and grid is refined until its resolution allows for solving droplets or bubbles without introducing any conceptual particles. In such a case, a transport equation for an indicator function is used to track the liquid-gas interface $[11,12,13]$. In this work the Volume-of-fluid (VOF) approach is adopted: governing equations are solved for a fluid whose thermophysical properties are a weighted average of the liquid and the vapor phases according to the liquid fraction. Additional terms are inserted in the equations to account for the presence of a physical interface. See Sec. for more details. Besides, since pressure inside the injector nozzle may drop below saturation pressure, a source term for cavitation in the momentum and continuity equations has been included. 
Finally, a mesh motion strategy based on topological changes $[14,15,16]$ has been coupled with the VOF solver and applied to simulate the needle movement: the aim was to study the early stage of the cavitating flow in the internal nozzle at the injector opening, where the continuum hypothesis is assumed to be valid. Some simplifying assumptions have been introduced in the present version of the solver, which represents an initial step towards a more comprehensive framework for injectors simulation: the fluid behavior can be regarded as incompressible and all gaseous phases (air + fuel vapor) can be represented by only one component (i.e., the fuel vapor). The developed code in the OpenFOAM technology has been tested on large supercomputers at the Argonne National Laboratory. New algorithms to perform load-balanced constrained decomposition, compatible with topological changes, have been developed.

\section{Test case}

The selected test case chosen for validation and testing of the dynamic VOF solver is a 6-hole prototype injector prototype provided by Continental (Fig. 1, left), especially developed for investigations in the framework of the collaborative project FUI MAGIE. Each nozzle is cylindrical with diameter $d=182 \mu \mathrm{m}$ and length $L=210 \mu \mathrm{m}$. Needle lift measurements (Fig. 1, right) were made available by Dr. Jin Wang, at Argonne National Laboratory, through X-ray propagation-based phase-enhanced micro-imaging [17].
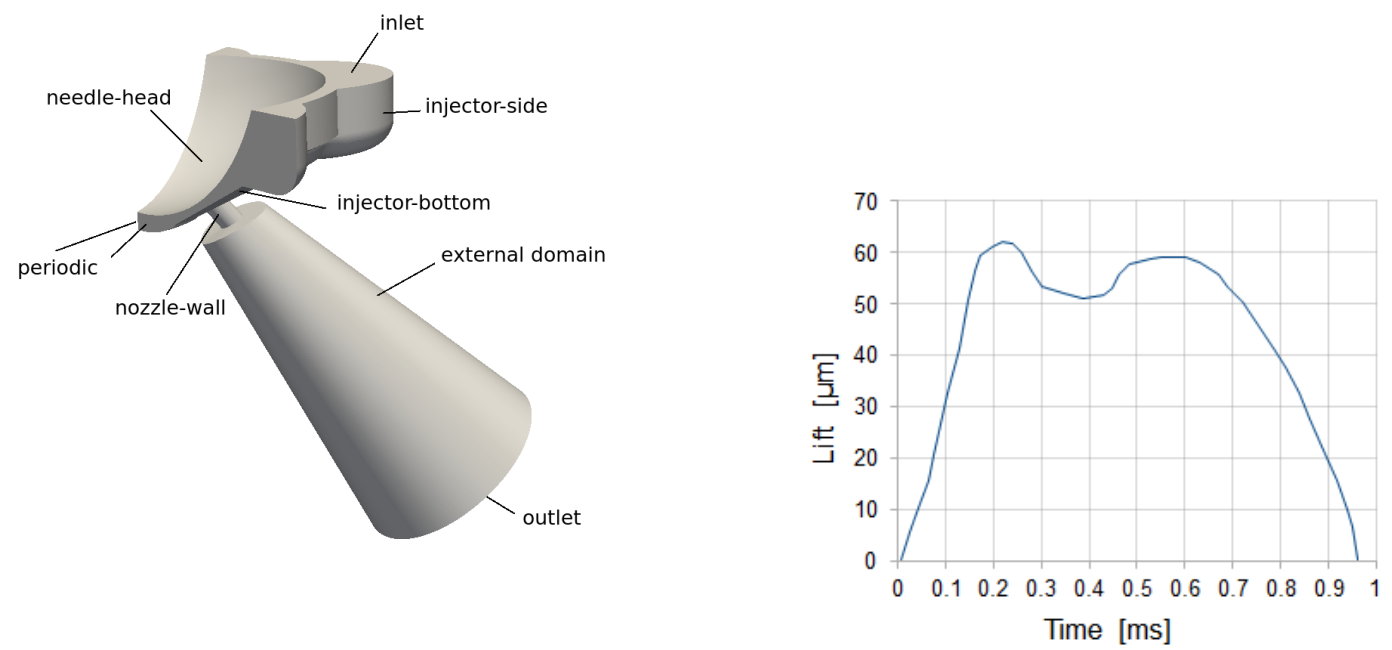

Figure 1. 6-hole prototype injector; only $1 / 6$ of the real component has been simulated (left). Injector lift curve (right).

In the experiments, conditions of liquid gasoline were $T=293 \mathrm{~K}$ and $p=30$ bar. Fuel was injected at ambient temperature in a large transparent chamber filled with air at ambient pressure and temperature. When the static pressure inside the nozzle drops below the vaporization pressure of the fuel, the liquid starts to cavitate and turns into the gaseous state of the fuel. Under these conditions, three phases of the fluid are observed: air, fuel vapor and liquid.

\section{Two-phase VOF solver and cavitation model}

A two-phase dynamic (isothermal and immiscible) VOF incompressible solver, including a cavitation model, has been used to simulate the injector opening event; in the simulations the liquid is assumed to be injected into a plenum chamber. The dynamic VOF solver includes a transport equation for the liquid fraction $\alpha_{l}$ (Eq. (1)), that is defined as the ratio of the liquid volume contained in a cell to the total liquid volume. The conservation equation for $\alpha_{l}$ is:

$$
\frac{\partial \alpha_{l}}{\partial t}+\nabla \cdot\left(\alpha_{l}\left(\mathbf{U}-\mathbf{U}_{b}\right)\right)+\nabla \cdot\left[\alpha_{l}\left(1-\alpha_{l}\right) \mathbf{U}_{c}\right]=\frac{\dot{m}}{\rho_{l}}
$$

where $\dot{m}$ is the mass source due to cavitation and $\left(\mathbf{U}-\mathbf{U}_{b}\right)$ is the relative velocity field between the fluid and the moving boundary. From the definition of $\alpha$, it results that $\alpha_{l}+\alpha_{v}=1$ where subscripts $l$ and $v$ stand for liquid and vapor respectively. The volume fraction is used to scale the physical properties of the mixture as:

$$
\rho=\rho_{l} \alpha_{l}+\left(1-\alpha_{l}\right) \rho_{v} \text { and } \mu=\mu_{l} \alpha_{l}+\left(1-\alpha_{l}\right) \mu_{v}
$$

The weighted physical properties of mixture are then used in the momentum equation:

$$
\frac{\partial(\rho \mathbf{U})}{\partial t}+\nabla \cdot\left(\rho\left(\mathbf{U}-\mathbf{U}_{b}\right) \mathbf{U}\right)-\nabla \cdot \mu \nabla \mathbf{U}-\rho \mathbf{g}=-\nabla p-\mathbf{F}_{s}
$$


On the right-hand side, $\mathbf{F}_{s}$ is surface force defined as $\sigma \kappa(x) \mathbf{n}$ with curvature $\kappa(x)=\nabla \cdot \mathbf{n}$, interface normal face unit vector $\mathbf{n}=\nabla \alpha_{l} /\left|\nabla \alpha_{l}\right|$, and surface tension $\sigma$. The last term on the left-hand side of Eq. 1 is known as the artificial compression term and it is non-zero only at the interface due to $\alpha_{l}\left(1-\alpha_{l}\right)$. The compression term has the role of shrinking the phase-interface towards a sharper one [18]. The compression term does not bias the solution in any way and it only corrects the flux of $\alpha_{l}$ in the interface-normal direction. In order to enforce this procedure, Weller [19] suggested the compression velocity to be calculated as:

$$
\mathbf{U}_{c}=C_{\alpha}|\mathbf{U}| \mathbf{n}
$$

The intensity of the interface compression is controlled by a constant $C_{\alpha}$, which yields no compression when zero, a conservative compression for $C_{\alpha}=1$ and high compression for $C_{\alpha}>1$ [18]. On the right-hand side, the mass source for cavitation is modeled according to the theory by Schnerr and Sauer [20]. According to it, there are several vapor bubbles (or nuclei) inside the liquid, that act as the initial sources of the phase change; cavitation starts from their locations and due to their presence. The mass transfer rate between liquid and vapor phases can be defined as follows:

$$
\dot{m}=\alpha_{l} \dot{m}_{\alpha v}+\left(1-\alpha_{l}\right) \dot{m}_{\alpha c}
$$

where:

$$
\begin{aligned}
& \dot{m}_{\alpha c}=C_{c} \alpha_{l} \frac{3 \rho_{v} \rho_{l}}{R_{b} \rho} \sqrt{\frac{2}{3 \rho_{l}\left|p-p_{s a t}\right|}} \cdot \max \left(p-p_{s a t}, 0\right) \\
& \dot{m}_{\alpha v}=C_{v}\left(1+\alpha_{n}-\alpha_{l}\right) \frac{3 \rho_{v} \rho_{l}}{R_{b} \rho} \cdot \sqrt{\frac{2}{3 \rho_{l}\left|p-p_{s a t}\right|}} \min \left(p-p_{s a t}, 0\right)
\end{aligned}
$$

with $C_{c}$ and $C_{v}$ as, respectively, the condensation and vaporization rate coefficients chosen by the user. These coefficients represent the relaxation time that either phase needs to be transferred into the other one. According to previous works [21] $C_{v}$ and $C_{c}$ are chosen equal to 1, that equals to consider the same behavior for condensation and vaporization rate. Moreover the reciprocal bubble radius is defined as:

$$
\frac{1}{R_{b}}=\left[\frac{4}{3} \frac{\pi n \alpha_{l}}{\left(1+\alpha_{n}-\alpha_{l}\right)}\right]^{1 / 3}
$$

$\alpha_{n}=V_{n} / 1+V_{n}$ is the nuclei volume fraction; the latter is computed as:

$$
V_{n}=\frac{n \pi d_{n}{ }^{3}}{6}
$$

with $n$ as the number of nuclei per unit volume and $d_{n}$ their diameter. Since to authors' knowledge there are no experiments about nucleation characteristics of liquid fuels, size and distribution of nuclei have been taken from experiments carried out with water. In this work, it is assumed a uniform nuclei distribution of $n=1.6 \cdot 10^{-8} \mathrm{~m}^{-3}$ with a diameter $d_{n}=2 \mathrm{~nm}$. Moreover, depending on the local properties of the flow, the extra diagonal terms in the linear system matrix of Eq. (1) can become very large (due to very high phase change rate), thus impairing the convergence rate. In order to improve the solution stability, the source term needs to be rewritten, so that Eq. 1 is formulated by decomposing the volume source $\dot{V}$ in an implicit and an explicit part:

$$
\dot{V}=\left[\frac{1}{\rho_{l}}-\alpha_{l}\left(\frac{1}{\rho_{l}}-\frac{1}{\rho_{v}}\right)\right] \dot{m}=A_{\alpha} \dot{m}=A_{\alpha}\left(\dot{m}_{\alpha v}-\dot{m}_{\alpha c}\right) \alpha_{l}+A_{\alpha} \dot{m}_{\alpha c}=\left(\dot{V}_{v}-\dot{V}_{c}\right) \alpha_{l}+\dot{V}_{c}
$$

the former being $\left(\dot{V}_{v}-\dot{V}_{c}\right)$, which enters the system matrix as a diagonal coefficient; on the other hand, the explicit part of the source term $\dot{V}_{c}$ will be written on the right-hand side of the linear system. The final form of the conservation equation of $\alpha_{l}$ (Eq. (11)) therefore reads:

$$
\frac{\partial \alpha_{l}}{\partial t}+\nabla \cdot\left(\alpha_{l}\left(\mathbf{U}-\mathbf{U}_{b}\right)\right)+\nabla \cdot\left[\alpha_{l}\left(1-\alpha_{l}\right) \mathbf{U}_{c}\right]=\left(\dot{V}_{v}-\dot{V}_{c}\right) \alpha_{l}+\dot{V}_{c}
$$

Turbulence has been accounted for by means of a Hybrid RANS/LES approach called Dynamic Length-Scale Resolution Model (DLRM) developed by the authors [22]. The underlying principle of DLRM is that the turbulent viscosity computed by a RANS model (the $k-\omega$ SST in this case) can be scaled down to Implicit LES when time and mesh resolution locally allow to solve turbulent structures. The momentum equation (3) is then complemented by the insertion of a turbulent stress tensor $\mathbf{B}=\mu_{t}\left(\nabla \mathbf{U}+\nabla^{T} \mathbf{U}\right)$ :

$$
\frac{\partial(\rho \mathbf{U})}{\partial t}+\nabla \cdot\left(\rho\left(\mathbf{U}-\mathbf{U}_{b}\right) \mathbf{U}\right)-\nabla \cdot \mu \nabla \mathbf{U}-\rho \mathbf{g}-\nabla \cdot \mathbf{B}=-\nabla p-\mathbf{F}_{s}
$$

whereas all other variables (including velocity) have now the meaning of partially-averaged quantities. A more detailed discussion on the physical significance of "partial average" can be found in e.g. [23]. 


\section{Dynamic mesh handling and parallel optimization}

The moving mesh functionality used in this work is described in $[14,15]$ and it has been applied as described in [16]. The injector opening event is simulated by dynamically attaching and detaching the conformal interface represented by the set of faces detachFaces in Fig. 2a, while a prescribed vertical motion is set for the boundary needleHead and for the corresponding moving cell set. Initially the first layer cell attached to bottomFaces and topfaces are respectively stretched and shrunk. Due to the very small gap between the injector needle and the injector body at closure $(O(\delta)=10 \mu \mathrm{m})$, the mesh handling could look particularly difficult: cell sizes change by orders of magnitude and this can impair the mesh smoothness in the near-nozzle region. To overcome this issue, when cell layers reach the $\mathrm{max} / \mathrm{min}$ layer thickness specified by the user, automatic addition/removal is triggered, ensuring constant cell quality during the whole simulation. In the needle region, the maximum thickness (triggering addition) was set to $6 \mu \mathrm{m}$, whereas the minimum threshold (triggering removal) was $0.35 \mu \mathrm{m}$.

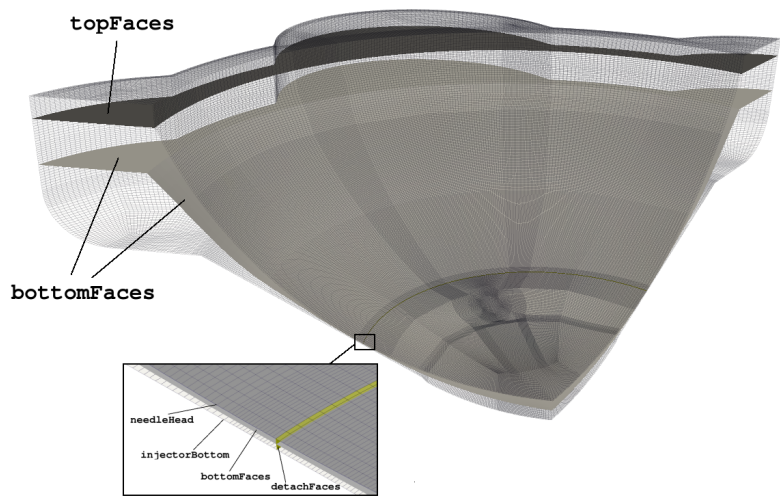

(a)

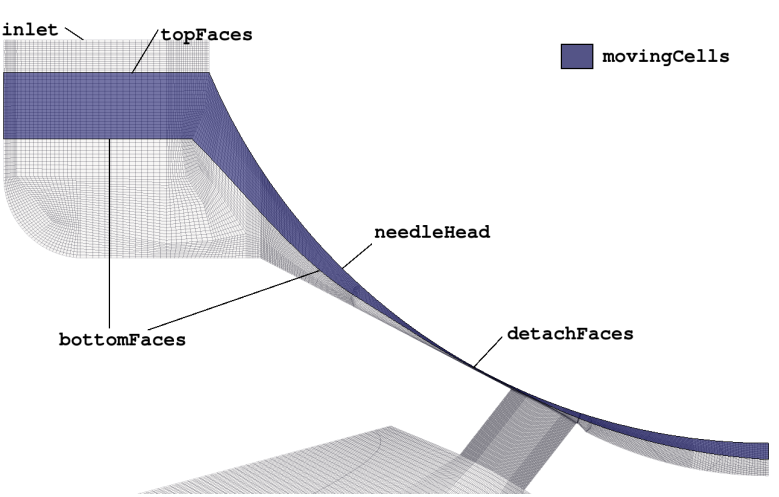

(b)

Figure 2. (a) Detail of face sets used by the solver: topFaces and bottomfaces are where layer A/R occurs, detachFaces are used to dynamically attach/detach the mesh; needleHead is the moving patch. (b) Cell sets used for decomposition

To run parallel simulations, the Finite Volume (FV) mesh has to be decomposed into a set of sub-domains, each to be assigned to a single core for processing. In simulations involving mesh motion with topological changes, new constraints in domain decomposition arise. In OpenFOAM ${ }^{\circledR}$, as well as in most of the CFD codes, topological changes cannot occur across inter-processor patches between neighboring sub-domains and the decomposition algorithm must be constrained in this sense. In other words, in regions where layer Addition/Removal (A/R) occurs, inter-processor faces cannot be parallel to layer A/R surface as shown in Fig. $2 b$. Nevertheless, it is important for the decomposition to remain balanced despite the aforementioned restrictions; in addition, the algorithm should require minimal user intervention, allowing the automation of case setup for large simulation campaigns. A new strategy for mesh decomposition, which considers all the aforementioned aspects, has been developed and applied to the GDI injector. The decomposition algorithm proceeds as follows:

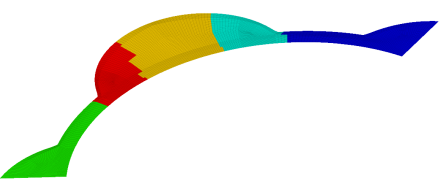

(a)

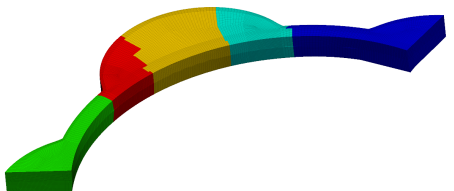

(b)

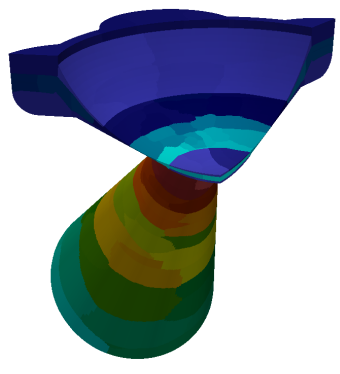

(c)

Figure 3. Overview of decomposition strategy: (a) Decomposition of face set topfaces using Scotch method; (b) Propagation of face set decomposition tho whole cell Set; (c) Complete decomposition

1. The grid is divided into several regions, in accordance with the decomposition constraints (layer $A / R$ zones, attach/detach zones, static parts Fig. 2b). Each mesh region is in turn decomposed into a number of subdomains, depending on the size of the region;

2. All face sets where dynamic layering is triggered (e.g. bottomFaces) are decomposed and subsets of faces are distributed among processors. For specified cell sets, cell decomposition is propagated perpendicularly 
to the face set using a cell-face walk algorithm, over the entire mesh region where layer addition/removal can be triggered, as shown in Figs. 3a-3b;

3. The remaining cells of the mesh are distributed among processors using non-constrained decomposition Fig. $3 c$

The decomposition strategy outlined above is very flexible, allowing for an almost perfect cell distribution (load balancing) over the processors with complex geometries and topological changes. The domain decomposition and overall balancing of the mesh used in this work is shown in Fig. 4. For similar geometries, the steps for domain decomposition can be easily automated, thus enabling automatic case setup in optimization/validation campaigns. Scalability tests were performed with the same solver on a simplified test case. Results are shown in Fig. 5. Three meshes with progressive refinement were tested, i.e., with $1 \mathrm{M}, 8 \mathrm{M}$, and $64 \mathrm{M}$ cells. Speedup is linear at least for a certain number of sub-domains per each mesh. The reason for the degradation of parallel performance over a certain threshold is still under investigation. However, since the present case has a number of cells that is very similar to one mesh used in the test (respectively $11 \mathrm{M}$ cells vs. 8M cells), a decomposition over 256 sub-domains has been chosen. Moreover, the same tests [24, 25] proved that using Preconditioned Conjugate Gradient (PCG) method instead of Geometric-Algebraic Multi-Grid (GAMG) to solve Poisson equation for pressure, can reduce the overall wall clock time but does not affect the scalability behavior, especially when a large amount of cores are used. On the other hand, there is also a chance that the performance decay is caused by a different convergence behavior of some sub-domains rather than an increased communication overhead. In case the interface-tracking resolution is delegated only to a few processors out of many, the consequent increased difficulty in solving the Poisson Equation (because of sharp pressure and density changes across the liquid/gas interface) on those few sub-domains can act as a bottleneck on the overall clock time. As a consequence, the parallel performance of the run might be impaired.

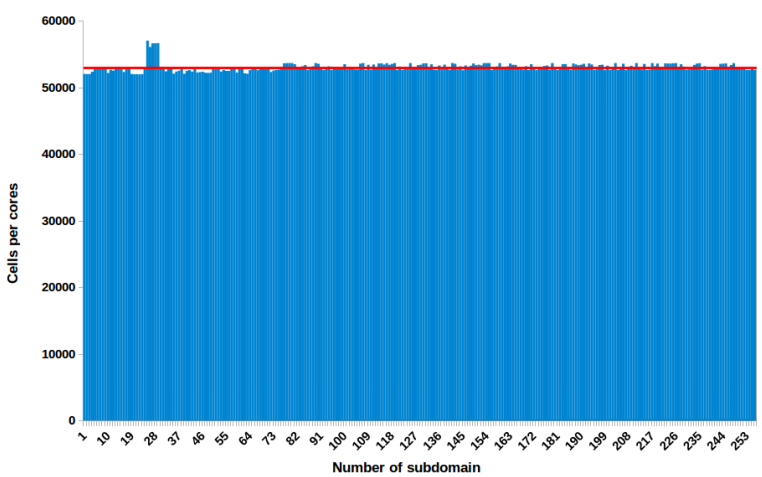

Figure 4. Histogram of number of cells per core. Red line represents mean value

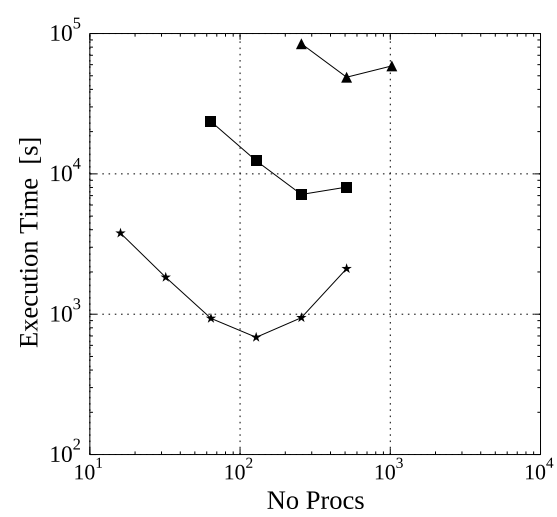

Figure 5. Left: Wall-clock time vs number of sub-domains. Right: Wall-clock time vs cells per processor. $-\star-1 \mathrm{M}$ mesh - $-8 \mathrm{M}$ mesh , $-\mathbf{\Delta}-64 \mathrm{M}$ mesh. Simulation time is $0.05 \mathrm{~s}$ for $1 \mathrm{M}$ and $8 \mathrm{M}$ cases, $0.025 \mathrm{~s}$ for $64 \mathrm{M}$ case.

\section{Case Setup \\ Geometry}

The computational mesh is of the order $11 \mathrm{M}$ cells ( $3 \mathrm{M}$ cells within the injector domain and $8 \mathrm{M}$ cells within the ambient) that allows a spatial resolution in the range $0.3-5 \mu \mathrm{m}$ (within the injector seat-nozzle) to $20 \mu \mathrm{m}$ (within the ambient domain). The mesh presents a maximum non-orthogonality of 76 degrees in the near-needle region, that forces the adoption of a limited scheme for laplacian discretization, together with at least 2 non-orthogonal correctors in the Poisson equation. Skewness maximum value it is around 1.35 which fulfills the good quality requirements. The injection chamber has been approximated by a conical volume (about $135 \mathrm{~mm}^{3}$ ), to limit the overall number of cells while keeping the lateral outlet patches sufficiently far not to interfere with the internal flow. Its length, along nozzle axis direction, is about 14 nozzle diameters, and its outlet diameter is about 10 nozzle diameters. From the previous work of Lu [21] it has been seen that such geometry approximation coupled with non-reflecting outlet condition [26] does not influence break-up phenomena. The block-structured, fully hexahedral FV mesh has 11M cells at injector closure. The approach chosen for turbulence modeling allows for the use of an increased cell size in the external region, where velocities are lower and turbulence dynamics are less critical for the global solution, limiting the overall number of cells. On the other hand, where cavitation occurs and a large amount of turbulence is generated, highly-resolved meshes are required to accurately capture the physics. Because of the CourantFriedrichs-Lewy (CFL) criterion, this greatly limits the size of time steps. n-Heptane has been chosen to simulate the fuel since its properties approximate the behavior of gasoline quite well [27]. 


\section{Numerical setup}

Time derivatives have been approximated by a second-order backward Euler scheme. Extensive development was done to preserve second order temporal accuracy with dynamic addition of cell layers in OpenFOAM [16, 28]. Pressure-velocity coupling is achieved by means of a transient-SIMPLE algorithm. The phase-fraction equation (Eq. (1)) is solved explicitly at the beginning of each time iteration by the Multi Universal Limiter for Explicit Solution (MULES) [29], a Flux Corrected Transport method developed by Weller to ensure boundedness of the $\alpha$ flux on all cell faces, which requires CFL to be lower than 1. In addition, special Total Variation Diminishing (TVD) methods are used for the compression velocity term $\nabla \cdot\left[\alpha_{l}\left(1-\alpha_{l}\right) \mathbf{U}_{c}\right]$ and $\nabla \cdot\left(\mathbf{U} \alpha_{l}\right)$. The former has been discretized with interface Compression scheme [30], while the latter with van Leer, as suggested by Weller [19]. For the convective term $\nabla \cdot(\phi \mathbf{U})$ a Linear Upwind with Stabilized Transport (LUST) scheme has been selected [31], while pure second order spatial discretization has been used for transport term of turbulent variables. A total-pressure boundary condition has been applied on the inlet section, and normal velocity is indirectly obtained from the pressure gradient on the boundary. Moreover, the sac domain has been initialized as containing only liquid fuel, for purging of fuel at considered pressure is not significant. Turbulence is mainly generated by the fluid/wall interaction in the needle region, and by the shear layer at the nozzle outlet, thus no synthetic turbulence generation has been deemed as required at the inlet. This allowed a reduction in the computational cost of about $10 \%$.
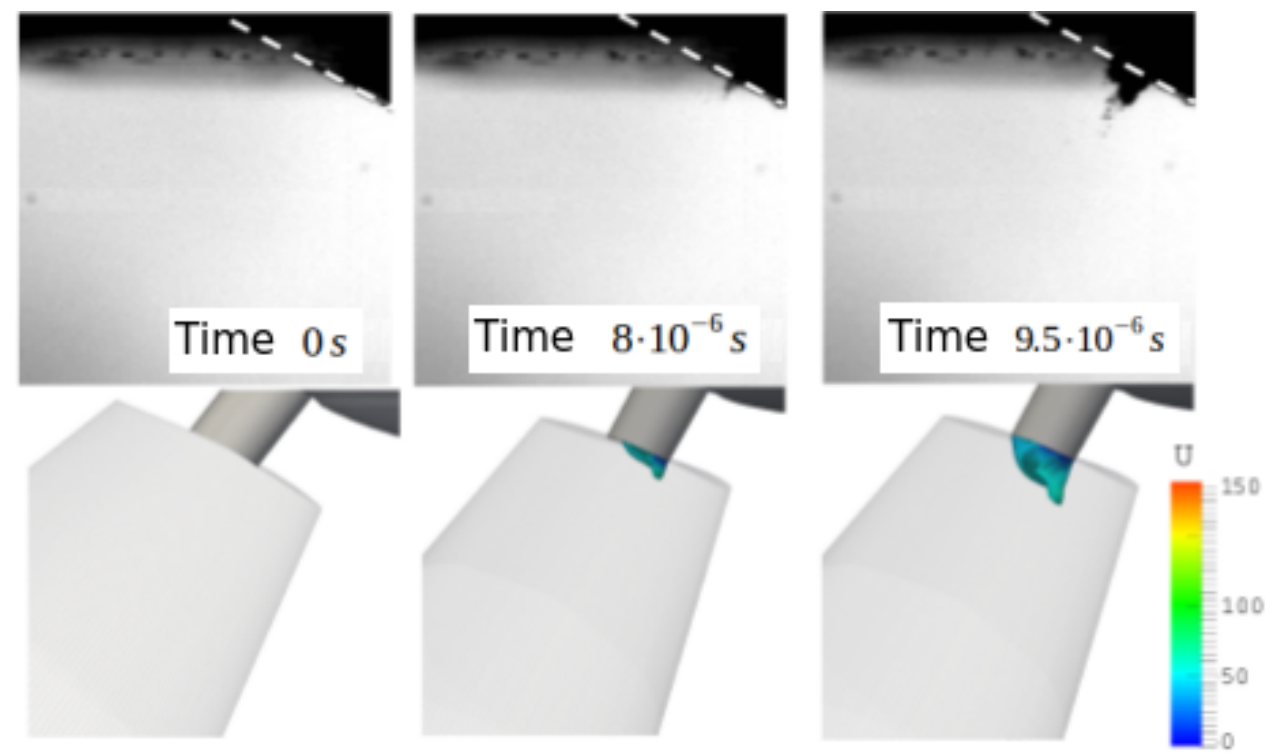

Figure 6. Comparison of experiments (top) and simulation (bottom) at early stage of injection.

\section{Injector opening: preliminary results}

Since the simulation is very demanding in terms of computational resources, only partial results are available at the time this paper is written. Fig. 6 shows the evolution of $n$-Heptane spray inside the combustion chamber up to $0.095 \mathrm{~ms}$ After Start Of Injection (ASOI). Simulation results (isosurface of liquid fraction at $\alpha=1$ ) is compared with high-speed shadowgraphs. The agreements in terms of shape and size of the jet is very good. As the needle starts to open, the nozzle is filled progressively with a non-uniform distribution of liquid due to the high level of turbulence; therefore, the tip of the spray jet does not have the typical mushroom shape described by other authors [21, 32], who analyzed the primary breakup at fixed needle position (maximum lift). This mechanism can be seen in Fig. 7 where the nozzle injector has been cut to show the internal flow. By looking at the constant pressure line at $p=p_{\text {sat }}$ in Fig. 7, the main cavitation region can be detected in correspondence of the vena contracta between the needle sac and the nozzle entry. A recirculation vortex is formed in this point, where pressure drops below the saturation value. The pocket of vapor is then transported towards the jet core and it is disrupted by the turbulent structures that reside there. A clearer idea of the turbulent structures that are formed in the nozzle can be observed in Fig. 8. Similarly to [21], a pair of counter-rotating vortices, roughly aligned with the nozzle axis, is formed in the jet core together with finer turbulent structures responsible of the instability of the jet (Fig. 8). The pressure drop occurring at the vortex cores causes again the fuel to cavitate, and elongated vapor regions are formed along the nozzle axis. This phenomenon is clearly visible in Fig. 9 where an iso-line of pressure at $p_{\text {sat }}$ is superposed to a contour plot of liquid phase fraction. The aforementioned vortex pair can be also the cause of the symmetric shape of the jet tip observed in Figs. 6 and 7. Finally, hints of primary breakup can be observed in Fig. 8: the development of some unstable structures like instability waves on the upper side of the jet and wrinkling of the liquid front as it enters the injection chamber are clearly visible. 


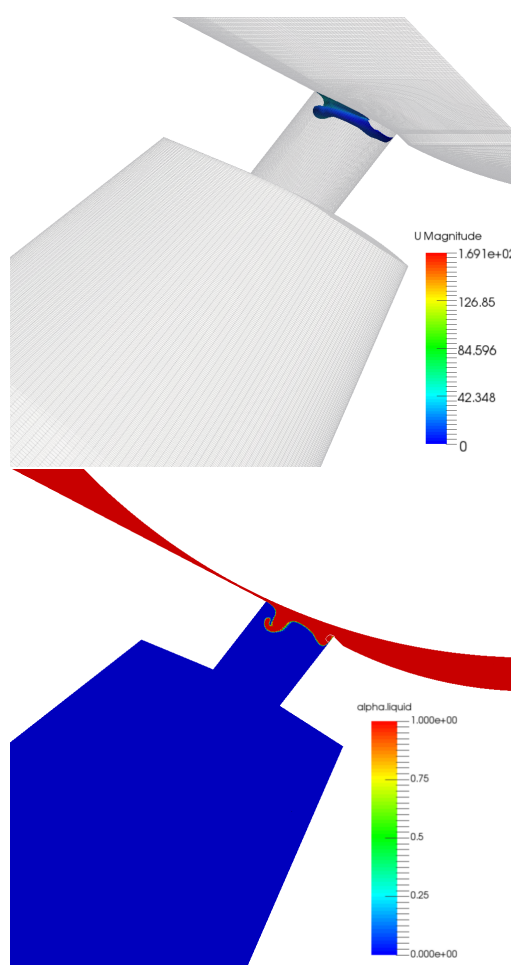

(a) $t=0.001 \mathrm{~ms}$

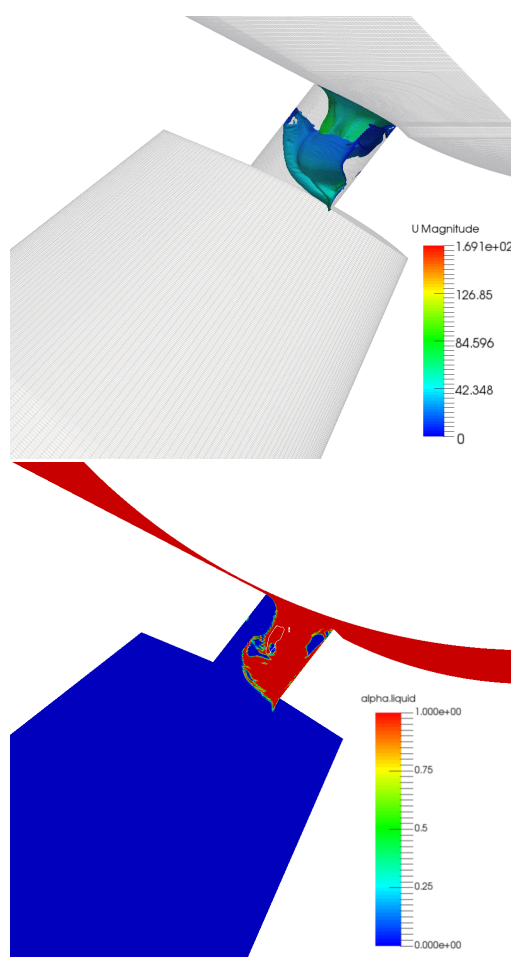

(d) $t=0.0065 \mathrm{~ms}$

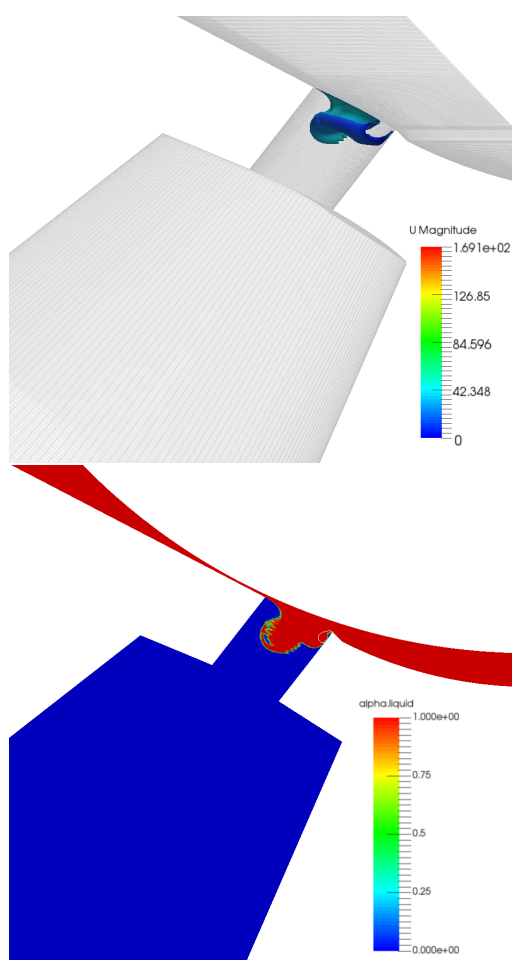

(b) $t=0.0025 \mathrm{~ms}$

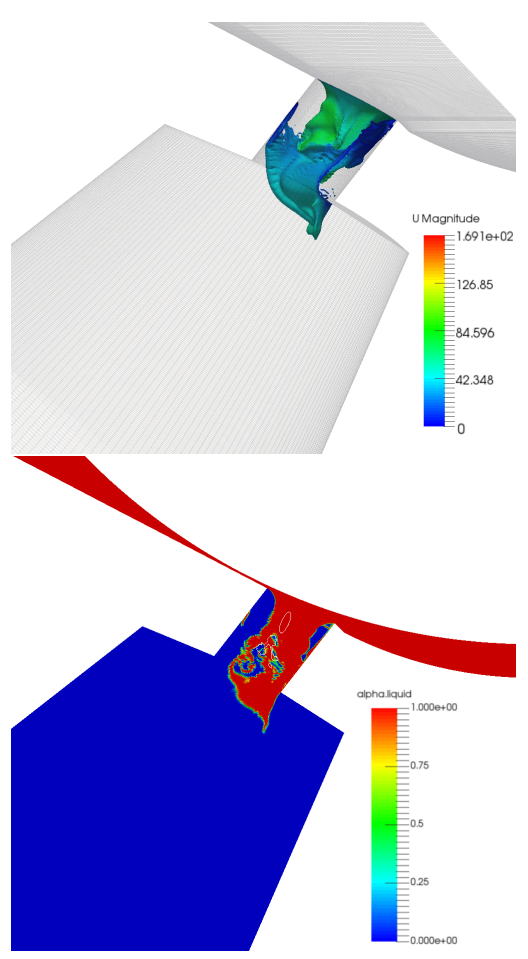

(e) $t=0.008 \mathrm{~ms}$

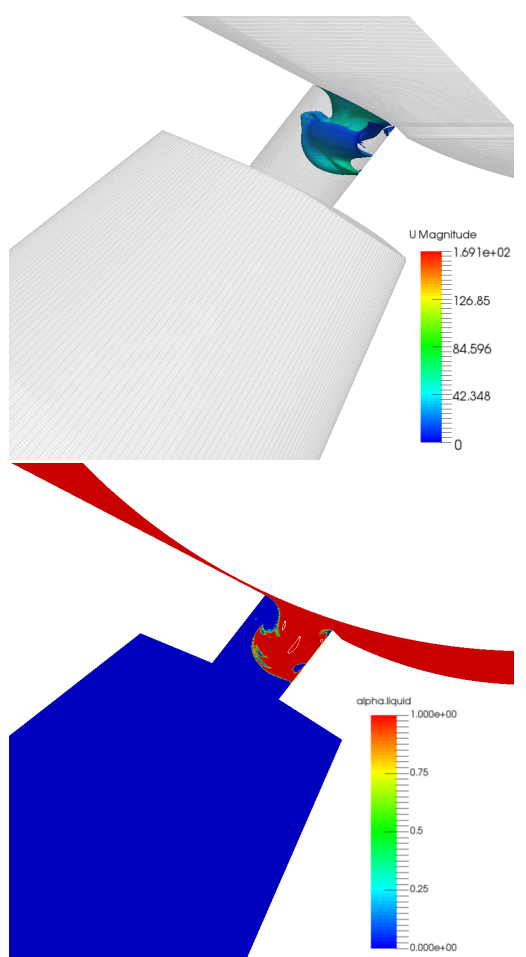

(c) $t=0.005 \mathrm{~ms}$
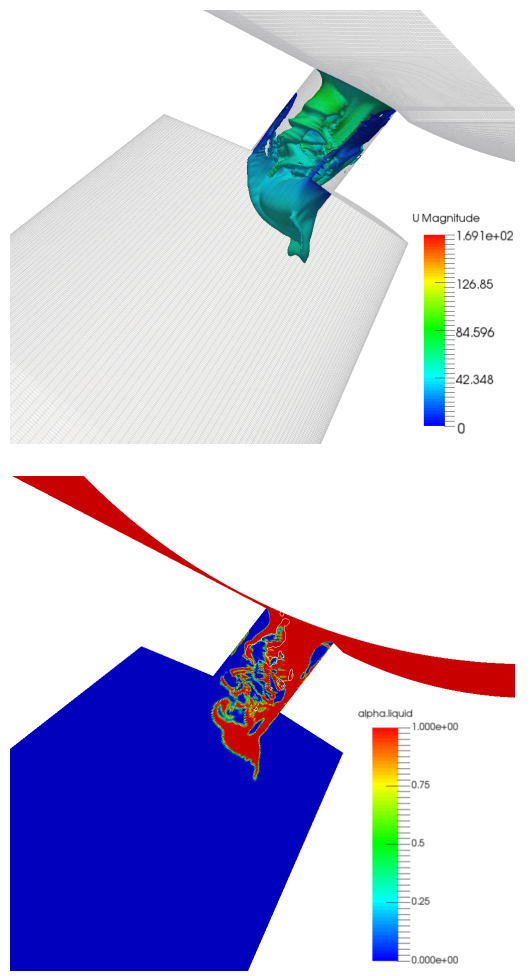

(f) $t=0.01 \mathrm{~ms}$

Figure 7. Sequence of snapshots of the spray exiting the nozzle following SOI. Top row: isosurface with $\alpha_{l}=0.5$, bottom row: sliced view of the above, with constant pressure line at $p=p_{\text {sat }}$. 

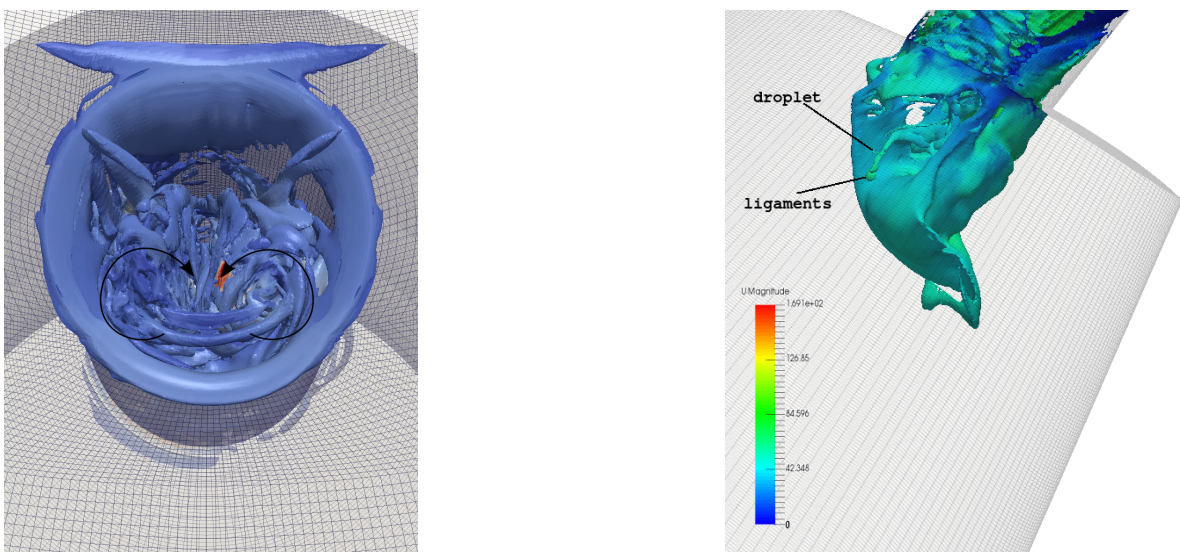

Figure 8. Left: vortical structures (isosurface of $Q=10^{4}$ ) in the nozzle at $t=9.5 \cdot 10^{-6} \mathrm{~s}$; right: liquid-vapor interface
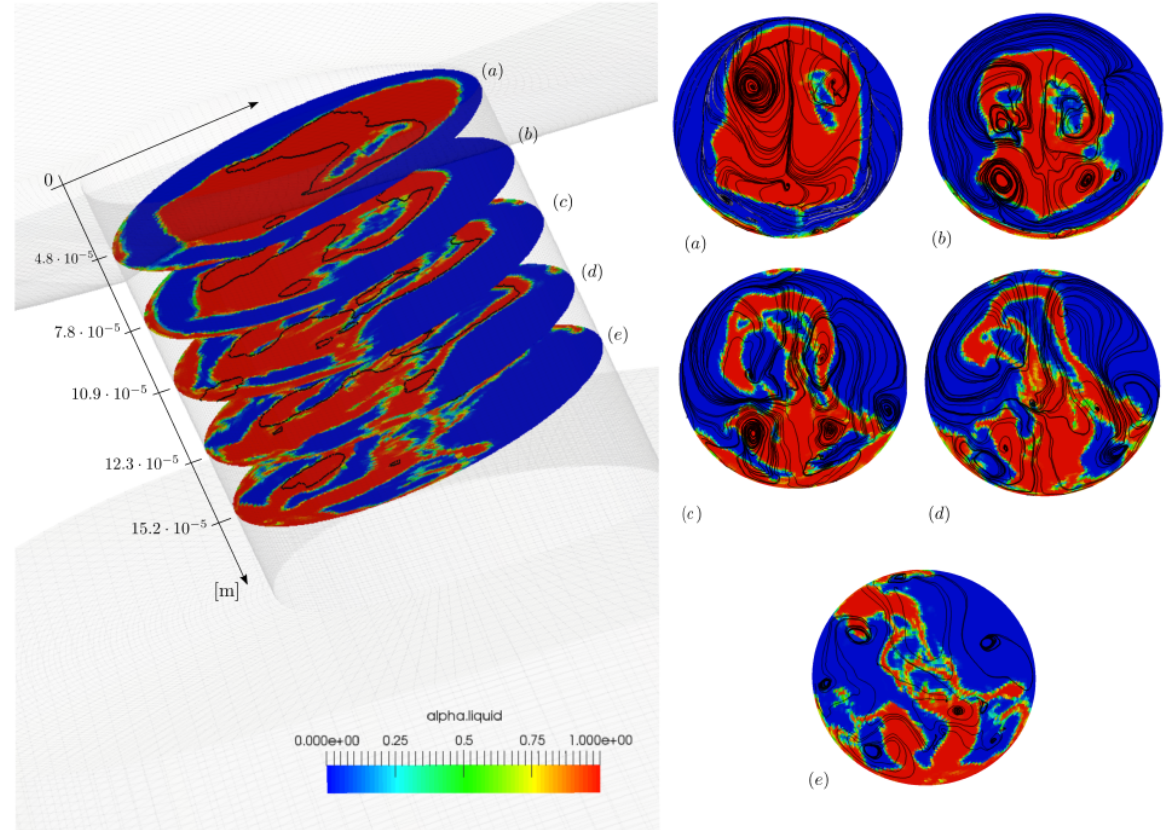

(c)

(d)

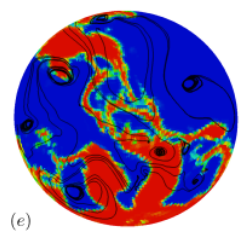

Figure 9. Horizontal cuts inside the nozzle at different height from nozzle inlet at time $9.5 \cdot 10^{-6} \mathrm{~s}$. The thin black line represents iso-contour of $p$ at $p_{\text {sat }}=4720 \mathrm{~Pa}$

\section{Conclusions}

The paper shows current developments done on dynamic simulation of multi-phase flows of GDI injectors, with particular focus on the coupling of an incompressible two-phase VOF solver with the cavitation model in a framework where the dynamic mesh based on topological changes is automatically handled in parallel. The solver has been applied to the simulation of a gasoline injector specifically designed for GDI engines and provided by Continental Automotive SAS. The case has been set up to simulate the early stages of the gasoline injection, using topological changes to implement mesh motion during injector opening. Details of the flow structures during the primary breakup are detected, which are in accordance with the findings of [21]. In this sense, the approach can be considered as promising and worthy of further investigation to assess its applicability during the design stage of injectors, with a particular focus on cavitation behavior. In particular, the initial assumptions about the incompressible nature of the flow have to be investigated, along with any effect arising from the consideration of only two gaseous phases. The solver also proved to be very stable: boundedness of the liquid fraction $\alpha_{l}$ was always preserved during the simulations even in presence of topological changes, so the method can be assumed to be fully conservative. Also, second-order accuracy was preserved both in space and time, which is not trivial in presence of dynamic topologically changing grids. Finally, a new decomposition method enabled good scalability of the code on large supercomputers. The developed code presented in this paper uses the most recent versions of OpenFOAM released by the OpenFOAM ${ }^{\circledR}$ Foundation and by OpenCFD ${ }^{\circledR}$ as development base frameworks. 


\section{Acknowledgments}

Authors would like to kindly thank the LCRC (Laboratory Computing Resource Center), Argonne National Lab, for making available the computing resources through the HPC cluster Blues within the PETSC-FOAM project.

\section{Nomenclature}

$T \quad$ temperature $[\mathrm{K}]$

$P \quad$ pressure [bar]

$\alpha_{l} \quad$ liquid volume fraction [-]

$t \quad$ time [s]

$\mathbf{U} \quad$ velocity [ $\mathrm{m} \mathrm{s}^{-1}$ ]

$\mathbf{U}_{b} \quad$ boundary velocity [ $\mathrm{m} \mathrm{s}^{-1}$ ]

$\mathbf{U}_{c} \quad$ compression velocity [ $\mathrm{m} \mathrm{s}^{-1}$ ]

$\alpha_{v} \quad$ vapor volume fraction [-]

$\rho_{l} \quad$ liquid density $\left[\mathrm{kg} \mathrm{m}^{-3}\right]$

$\rho \quad$ mixture density $\left[\mathrm{kg} \mathrm{m}^{-3}\right]$

$\rho_{V} \quad$ vapor density $\left[\mathrm{kg} \mathrm{m}^{-3}\right]$

$\mu \quad$ dynamic viscosity of mixture [Pa s]

$\mu_{l} \quad$ liquid dynamic viscosity [Pa s]

$\mu_{v} \quad$ vapor dynamic viscosity [Pa s ]

g gravity acceleration $\left[\mathrm{m} \mathrm{s}^{-2}\right]$

$\mathbf{F}_{s} \quad$ surface forces $\left[\mathrm{N} \mathrm{m}^{-2}\right]$

$\sigma \quad$ surface tension forces $\left[\mathrm{Nm}^{-1}\right]$

\begin{tabular}{|c|c|}
\hline$\kappa(x)$ & interface curvature $\left[\mathrm{m}^{-1}\right]$ \\
\hline n & normal vector [-] \\
\hline$C_{\alpha}$ & compression velocity coefficient \\
\hline$C_{c}$ & condensation coefficient [-] \\
\hline$R_{b}$ & bubble radius $\left[\mathrm{m}^{-1}\right]$ \\
\hline$p_{\text {sat }}$ & saturation pressure [Pa] \\
\hline & vaporization coefficient [-] \\
\hline & nuclei concentration $\left[\mathrm{m}^{-3}\right]$ \\
\hline & nuclei volume fraction [-] \\
\hline & total volume of nuclei $\left[\mathrm{m}^{-3}\right]$ \\
\hline & nuclei diameter $[\mathrm{m}]$ \\
\hline & alpha equation source term $\left[\mathrm{s}^{-1}\right]$ \\
\hline & vaporization source term $\left[\mathrm{s}^{-1}\right]$ \\
\hline & condensation source term $\left[\mathrm{s}^{-1}\right]$ \\
\hline & turbulent stress tensor $\left[\mathrm{N} \mathrm{m}^{-2}\right]$ \\
\hline & turbulent dynamic viscosity $[\mathrm{Pa}$ \\
\hline
\end{tabular}

\section{References}

[1] F. Zhao, D. Harringotn, and M. Lai, Automotive Gasoline Direct-Injection Engines, 2002. http: //books.sae.org/r-315/

[2] F.-Q. Zhao, M.-C. Lai, and D. L. Harrington, "A review of mixture preparation and combustion control strategies for spark-ignited direct-injection gasoline engines," in SAE Technical Paper. SAE International, 021997. http://dx.doi.org/10.4271/970627

[3] W.-M. Ren and H. Sayar, "Influence of nozzle geometry on spray atomization and shape for port fuel injector," in SAE Technical Paper. SAE International, 03 2001. http://dx.doi.org/10.4271/2001-01-0608

[4] M. Skogsberg, P. Dahlander, R. Lindgren, and I. Denbratt, "Effects of injector parameters on mixture formation for multi-hole nozzles in a spray-guided gasoline di engine," in SAE Technical Paper. SAE International, 04 2005. http://dx.doi.org/10.4271/2005-01-0097

[5] X. Zhang, L. J., and J. Wang, "Effect of fuel and nozzle geometry on the off-axis oscillation of needle in diesel injectors using high-speed x-ray phase contrast imaging," Journal of Instrumentation, vol. 11, no. 05, p. C05015, 2016. http://stacks.iop.org/1748-0221/11/i=05/a=C05015

[6] R. Payri, B. Tormos, J. Gimeno, and G. Bracho, "The potential of large eddy simulation (les) code for the modeling of flow in diesel injectors," Mathematical and Computer Modelling, vol. 52, no. 7-8, pp. 1151 - 1160, 2010, mathematical Models in Medicine, Business \& amp; Engineering 2009. http://www.sciencedirect.com/science/article/pii/S089571771000097X

[7] K. John and Dukowicz, "A particle-fluid numerical model for liquid sprays," Journal of Computational Physics, vol. 35, no. 2, pp. 229-253, 1980. http://www.sciencedirect.com/science/article/pii/002199918090087X

[8] Reitz and D. Rolf, "Modeling atomization processes in high-pressure vaporizing sprays," Atomization and Spray technology, vol. 3, no. 4, pp. 309-337, 1987.

[9] A. Amsden, "A block-structured program for engines with vertical and canted valves," 1997.

[10] E. Giannadakis, M. Gavaises, and C. Arcoumanis, "Modeling of cavitation in Diesel injector nozzles," International Journal of Fluid Mechanics, vol. 616, pp. 153-193, 2008.

[11] T. Menard, S. Tanguy, and A. Berlemont, "Coupling level set/VOF/ghost fluid methods: Validation and application to 3D simulation of the primary break-up of a liquid jet," International Journal of Multiphase Flow, vol. 33, no. 5, pp. $510-524$, 2007. http://www.sciencedirect.com/science/article/pii/S0301932206001832

[12] H. Pitsch and O. Desjardins, "Deatailed umerical investigation of turbulent atomization of liquid jets," Atomization and Sprays, vol. 20, no. 4, pp. 311-336, 2010.

[13] S. S. Deshpande, M. F. Trujillo, X. Wu, and G. Chahine, "Computational and experimental characterization of a liquid jet plunging into a quiescent pool at shallow inclination," International Journal of Heat and Fluid Flow, vol. 34, no. 0, pp. 1 - 14, 2012. http://www.sciencedirect.com/science/article/pii/S0142727X12000197

[14] F. Piscaglia, A. Montorfano, and A. Onorati, "An extension of the dynamic mesh handling with topological changes for LES of ICE in OpenFOAM," SAE paper 2015-01-0384, 2015, SAE World Congress Exhibition, Detroit, Michigan (USA), 2015, http://dx.doi.org/10.4271/2015-01-0384. 
[15] F. Piscaglia, A. Montorfano, and A. Onorati, "Development of fully-automatic parallel algorithms for mesh handling in the OpenFOAM-2.2.x technology," SAE Technical Paper 2013-24-0027, 2013, http://dx.doi.org/10.4271/ 2013-24-0027.

[16] F. Piscaglia, A. Montorfano et al., "Hybrid RANS/LES of Moving Boundary Problems: Application to Cavitating Sprays and In-Cylinder Flows," in International Multidimensional Engine Modeling User's Group Meeting At the SAE Congress, April 11th 2016, https://imem.cray.com/agenda.html.

[17] W.-K. Lee, K. Fezzaa, and J. Wang, "Metrology of steel micronozzles using x-ray propagationbased phase-enhanced microimaging," Applied Physics Letters, vol. 87, no. 8, p. 084105, 2005. http://dx.doi.org/10.1063/1.2034099

[18] E. Berberovic, "Investigation of free-surface flow associated with drop impact: Numerical simulations and theoretical modeling," Ph.D. dissertation, Technische Universität, Darmstadt, November 2010. http://tuprints.ulb.tu-darmstadt.de/2319/

[19] H. Weller, "A new approach to vof-based interface capturing methods for incompressible and compressible flow," Technical Report TR/HGW/04,OpenCFD Ltd., Technical Report TR/HGW/04,OpenCFD Ltd., 2008.

[20] G. Schnerr and J. Sauer, "Unsteady cavitating flow - a new cavitation model based on a modified front capturing method and bubble dynamics." ASME Fluids Engineering Summer Meeting, June 2011-15, Boston (USA), 2000.

[21] N. Lu, F.-X. Demoulin, J. Reveillon, and J. Chesnel, "Large eddy simulation of cavitation and atomization in injector flows using openfoam," ILASS - Europe 2014, 26th Annual Conference on Liquid Atomization and Spray Systems, Bremen, Germany, 2014.

[22] F. Piscaglia, A. Montorfano, and A. Onorati, "A Scale Adaptive Filtering Technique for Turbulence Modeling of Unsteady Flows in IC Engines," SAE Int. J. Engines, Paper n. 2015-01-0395, 2015, http://dx.doi.org/10.4271/ 2015-01-0395.

[23] P. Sagaut, Large-Eddy Simulation for Incompressible Flows: an Introduction, ser. Scientific computation. Springer-Verlag, 2006.

[24] J. Hélie, J. Chesnel, and N. LU, "Parallel performance of OpenFOAM in industrial applications," PRACE project pa1507, Tech. Rep., 2015.

[25] A. Montorfano, F. Giussani, F. Piscaglia, and J. Hélie, "Parallel performance of OpenFOAM in industrial applications," PRACE project pa3053, Tech. Rep., 2016.

[26] F. Piscaglia, A. Montorfano, and A. Onorati, "Development of a non-reflecting boundary condition for multidimensional nonlinear duct acoustic computatio," Journal of Sound and Vibration, vol. 332, no. 4, pp. 922-935, 2013.

[27] D. L. Hung, D. L. Harrington, A. H. Gandhi, L. E. Markle, S. E. Parrish, J. S. Shakal, H. Sayar, S. D. Cummings, and J. L. Kramer, "Gasoline fuel injector spray measurement and characterization - a new sae j2715 recommended practice," SAE International Journal of Fuels and Lubricants, vol. 1, 042008. http://gen.lib.rus.ec/scimag/index.php?s=10.4271/2008-01-1068

[28] F. Piscaglia, "Ongoing and future perspective developments in OpenFOAM," Keynote invited talk at the 4th Annual ESI-OpenCFD OpenFOAM User Conference 2016, Cologne (Germany).

[29] The OpenFOAM Foundation. Free open-source CFD. http://www.openfoam.org.

[30] O. Ubbink, "Numerical prediction of two fluid systems with sharp interfaces," Ph.D. dissertation, Imperial College, University of London, 1997.

[31] H. Weller, "Controlling the computational modes of the arbitrarily structured C grid," Monthly Weather Review, vol. 140, pp. 3220-3234, 2012. http://journals.ametsoc.org/doi/pdf/10.1175/MWR-D-11-00221.1

[32] G. Stiesch, Modeling Engine Spray and Combustion Processes, ser. Heat and Mass Transfer. Springer Berlin Heidelberg, 2003. https://books.google.co.uk/books?id=4A35ABlw1wgC 


\title{
High-speed X-Ray Phase-Contrast Imaging of String Cavitation in an Enlarged Diesel-Injector Orifice Replica
}

\author{
I.K. Karathanassis ${ }^{*}$, P. Koukouvinis ${ }^{1}$, M. Lorenzi ${ }^{1}$, E. Kontolatis ${ }^{1}$, Z. Li ${ }^{2}$, J. Wang ${ }^{2}$, \\ N. Mitroglou ${ }^{1}$ and M. Gavaises ${ }^{1}$ \\ ${ }^{1}$ School of Mathematics, Computer Science and Engineering, City, University of London, UK \\ ${ }^{2}$ Advanced Photon Source, Argonne National Laboratory, Lemont, IL 60439, USA \\ *Corresponding author: Ioannis.Karathanassis@city.ac.uk
}

\section{Abstract}

The present investigation illustrates the temporally-resolved, phase-contrast visualization of the cavitating flow within an enlarged injector replica conducted at the ANL Advanced Photon Source. The flow was captured through side-view, x-ray radiographies at 67890 frames per second with an exposure time of $347 \mathrm{~ns}$. The orifice employed for the experiments has an internal diameter of $1.5 \mathrm{~mm}$ and length equal to $5 \mathrm{~mm}$. A parametric investigation was conducted considering various combinations of the Reynolds and cavitation numbers, which designate the extent of in-nozzle cavitation. Proper post-processing of the obtained radiographies enabled the extraction of information regarding the shape and dynamical behaviour of cavitating strings. The average string extent along with its standard deviation was calculated for the entire range of conditions examined $(\mathrm{Re}=18000-36000, \mathrm{CN}=1.6-7.7)$. Furthermore, the effect of the prevailing flow conditions on quantities indicative of the string dynamic behaviour such as the breakup frequency and lifetime was characterized and the local velocity field in the string region was obtained.
\end{abstract}

\section{Keywords}

Fuel injection, synchrotron radiation, nozzle flow, high-speed radiography, velocimetry

\section{Introduction}

Modern fuel injectors operate at extreme pressures of the order of 3000 bars, as it has been demonstrated that this degree of pressurization leads to increased engine performance and reduced pollutant emissions [1]. The turbulent nature of injector flows gives rise to highly-transient cavitation phenomena emanating due to the geometrical layout and/or the emerging secondary flow pattern [2]. The onset of vortical (string) cavitation within the injector holes has been demonstrated to lead to atomization enhancement and spray cone angle increase [3]. In contrast, as demonstrated in [4], the collapse of unstable vapour clouds appears to be more aggressive compared to attached cavities in terms of cavitation-induced erosion.

Referring to two-phase flow visualization, optical techniques, e.g. shadowgraphy, Schlieren or LIF/LIEF imaging, have been traditionally employed and thus a transparent, durable material such as Perspex or sapphire must be utilized for the manufacturing of the nozzles. Several experimental investigations dealing with the characterization of the cavitating flow arising within fuel injectors have been performed on enlarged nozzle replicas [5], since the lower pressure of injection along with the larger geometrical length scale compared to real-size injectors facilitate the attainment of higher temporal and spatial resolution and, thus, allow a more thorough elucidation of the flow phenomena setting in. Concurrent studies having been conducted on real-size injectors with transparent tips have verified the main conclusions established by the investigations on replicas with regard to the flow topology and main features [6]. Flow similarity between the different examinations performed on varying length scales is ensured by the adjustment of the non-dimensional quantities that designate the flow conditions, i.e. the cavitation (CN) and Reynolds $(\mathrm{Re})$ numbers.

$\mathrm{X}$-ray imaging is currently gaining momentum as a technique suitable for the visualization of two-phase flows with relevance to fuel injectors. Both x-ray phase-contrast and attenuation imaging have been performed so as to illustrate the spray structure and local flow dynamics in the near-nozzle region [7-8]. The work of [7] visualized the spray expelled by a single and a three-hole injector configuration using X-ray Phase Contrast Imaging (XPCI) and provided quantitative data on the axial velocity and turbulence intensity up to a location of approximately $50 \mathrm{~mm}$ downstream the nozzle outlet. Besides, in [8], by making use of x-ray attenuation imaging, provided quantitative data for the spray density in a region up to $10 \mathrm{~mm}$ downstream the outlet of a single-orifice injector. Referring to innozzle flows, [9] quantified the vapour extent that emerged within an enlarged orifice, while they also managed to obtain time-resolved measurements, yet for a very small area of interest of the order of $\mu m^{2}$. More recently, the group of the authors [10] relied on micro-computed tomography to obtain the average three-dimensional structure and vapour volume fraction distribution of the cloud cavitation that arose in a simplified injector layout with an orifice 
diameter of $3 \mathrm{~mm}$. In this study, an enlarged orifice similar to that employed in [10] is examined and focus is given on elucidating the topological and dynamical features of the highly-fluctuating vortical structures, conventionally termed as string cavitation. For this reason, high-speed XPCI has been selected for the flow visualization, which allows the acquisition of high-speed, high-resolution radiographies with a relatively wide field of view.

\section{Experimental Setup}

The custom-made nozzle replica used in this investigation along with the upstream and downstream flow manifolds are shown in Fig. 1a, along with an indicative shadowgraphy image of the emerging two-phase flow. The prototype nozzle, the layout and main dimensions of which are shown in the detail-view of the figure, was manufactured from carbon fibre (TORAYCA TFO0S), which, firstly, causes low radiation attenuation compared to metals and, furthermore, is able to withstand injection pressures up to 150 bar and outlet pressures up to 50 bar without deformation of its shape. A metallic needle with a hemispherical tip is inserted into the flow chamber (sac) upstream the injector hole and adjusted to a fixed position. A manifold layout precedes the examined nozzle configuration to ensure that the incoming flow is parallel and unperturbed, whereas the mechanism responsible for the adjustment of the needle is also seated on the flow device realizing the manifold, as can be seen on the top part of Fig. 1a.The hydraulic flow loop that was developed for the present investigation and is depicted on Fig. 1b, was operated at steady-state flow-rate conditions, while the fuel temperature was monitored through type-K thermocouples inserted in taps properly placed at the storage tank feeding the pump and the inlet manifold and adjusted to a specified setpoint with the use of a heat exchanger and a PID controller. Control valves (flow regulators) placed at the outlet of the feed pump were employed for imposing the liquid volumetric flow rate, which was monitored through an axialturbine flow meter. Liquid pressure at the inlet and outlet of the test bench was monitored through pressure transducers inserted in taps.

a

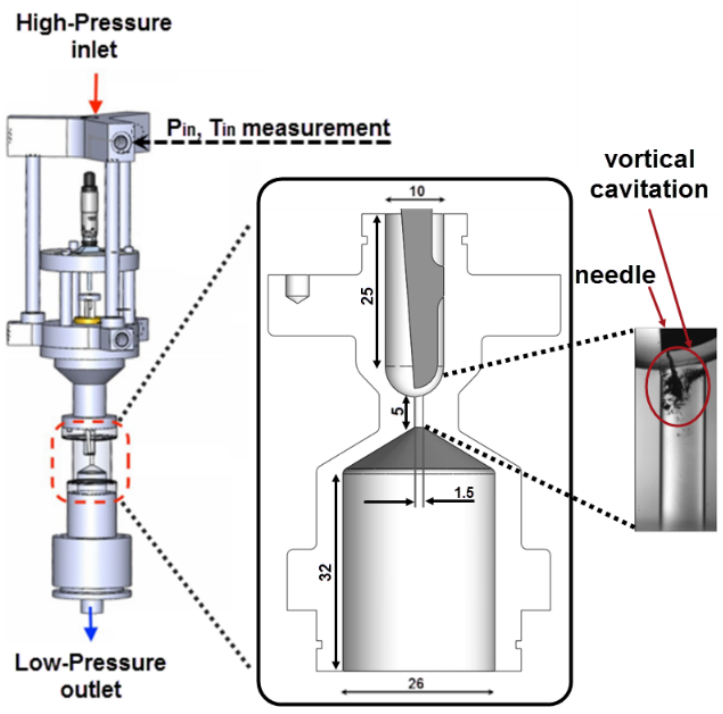

b

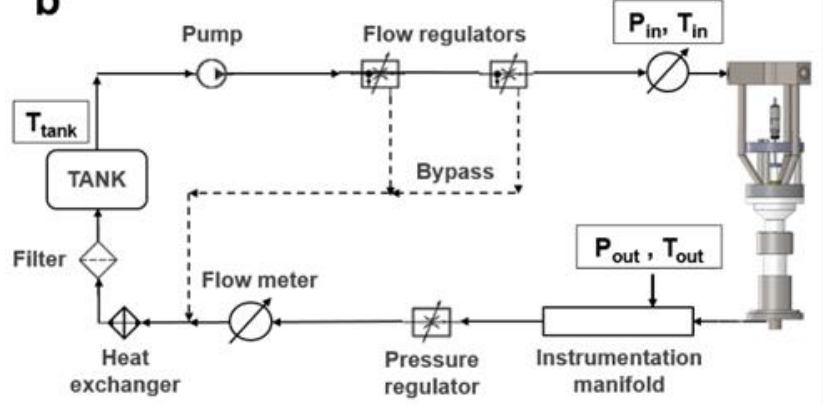

Figure 1. Schematic layout of (a) the test-bench and (b) the hydraulic flow loop.

The XPCI measurements were performed at the 7ID beamline, which has access to a white $x$-ray beam generated by an undulator in the APS electron storage ring. Referring to the beam pulsation mode, the so called "hybrid mode" allowing for time-resolved measurements, was selected. According to the specific operating mode, an x-ray pulse having a duration of $150 \mathrm{ps}$ and carrying $16 \mathrm{~mA}$ of current is followed, after a gap of $1.569 \mu \mathrm{s}$, by eight short pulses, each one carrying $11 \mathrm{~mA}$, separated by time intervals of $51 \mathrm{~ns}$. The operating cycle is completed by an additional gap of $1.569 \mu \mathrm{s}$ and hence its entire duration is equal to $3.682 \mu \mathrm{s}$. The attenuated x-ray beam, after its interaction with the injector orifice, impinges on the scintillator crystal, which converts the $x$-ray radiation to visible light (432 $\mathrm{nm}$ ). The image is then reflected by a 450 mirror and captured by a CCD camera. In the specific investigation, only the bunch of eight pulses was utilized, which allowed a shutter exposure time of 347 ns to be achieved and hence the captured flow can be considered as frozen. Side-view radiographies at 67890 frames per second were obtained with a field of view of $2.56 \mathrm{~mm} \times 2.56 \mathrm{~mm}$ discretized by $512 \times 512$ pixels, thus producing a spatial resolution of $5 \mu \mathrm{m} /$ pixel. However, it must be noted that the $x$-ray beam is collimated to a circular cross-section and therefore the 
overall orifice length was segmented into five positions, which were radiated in a consecutive manner with overlapping regions between the locations.

The different test cases considered in the present evaluation are summarized in Table 1. As can be seen, four cases have been included in this study, corresponding to different values of the cavitation and Reynolds numbers. The position of the needle was adjusted to $0.5 \mathrm{~mm}$ upstream of its seat, since preliminary tests verified that strings with clearly discernible interfaces set in for the specific needle-lift value. The (nominal) Reynolds number characterizing the flow was defined on basis of the mean flow velocity $u_{\text {mean }}=4 \dot{V} / \pi D^{2}$ and the nozzle diameter $(D=1.5 \mathrm{~mm})$ and lies in the range of 18000-35500; thus flow is expected to exhibit highly transient features within short time scales. Besides, the cavitation number was defined as follows:

$C N=\frac{p_{\text {inj }}-p_{\text {back }}}{p_{\text {back }}-p_{\text {sat }}}$

where $p_{i n j}, p_{b a c k}$ and $p_{\text {sat }}$ stand for the injection (upstream), back and saturation pressures, respectively. Based on the error propagation [11] due to the accuracy in the measurement of pressure, velocity and temperature, the uncertainty in the values of $\mathrm{Re}$ and $\mathrm{CN}$ are associated with global uncertainties of 3.5 and $5 \%$, respectively. The working fluid employed for the investigation was commercial Diesel fuel provided by Lubrizol Corp., while the thermophysical properties required for the determination of Re and $\mathrm{CN}$ were calculated according to the data provided by [12]. The Diesel fuel density in the temperature range $312-315 \mathrm{~K}$ is of the order of $810 \mathrm{~kg} / \mathrm{m}^{3}$.

Table 1. Experimental test-cases investigated.

\begin{tabular}{c|cccccc}
\hline \hline No. & lift [mm] & $\mathbf{R e}$ & $\mathbf{C N}$ & $\mathbf{P}_{\text {inj }}[$ bar] & $\mathbf{P}_{\text {back }}[\mathbf{b a r}]$ & $\mathbf{T}_{\text {in }}\left[{ }^{\circ} \mathbf{C}\right.$ ] \\
\hline \hline 1 & & 18000 & 1.6 & 14.56 & 5.64 & 40.7 \\
2 & \multirow{2}{*}{0.5} & 35500 & 1.6 & 39.3 & 15.3 & 40.0 \\
3 & & 35500 & 2.0 & 36.4 & 12.2 & 42.3 \\
4 & & 35500 & 7.7 & 31.8 & 3.8 & 39.2
\end{tabular}

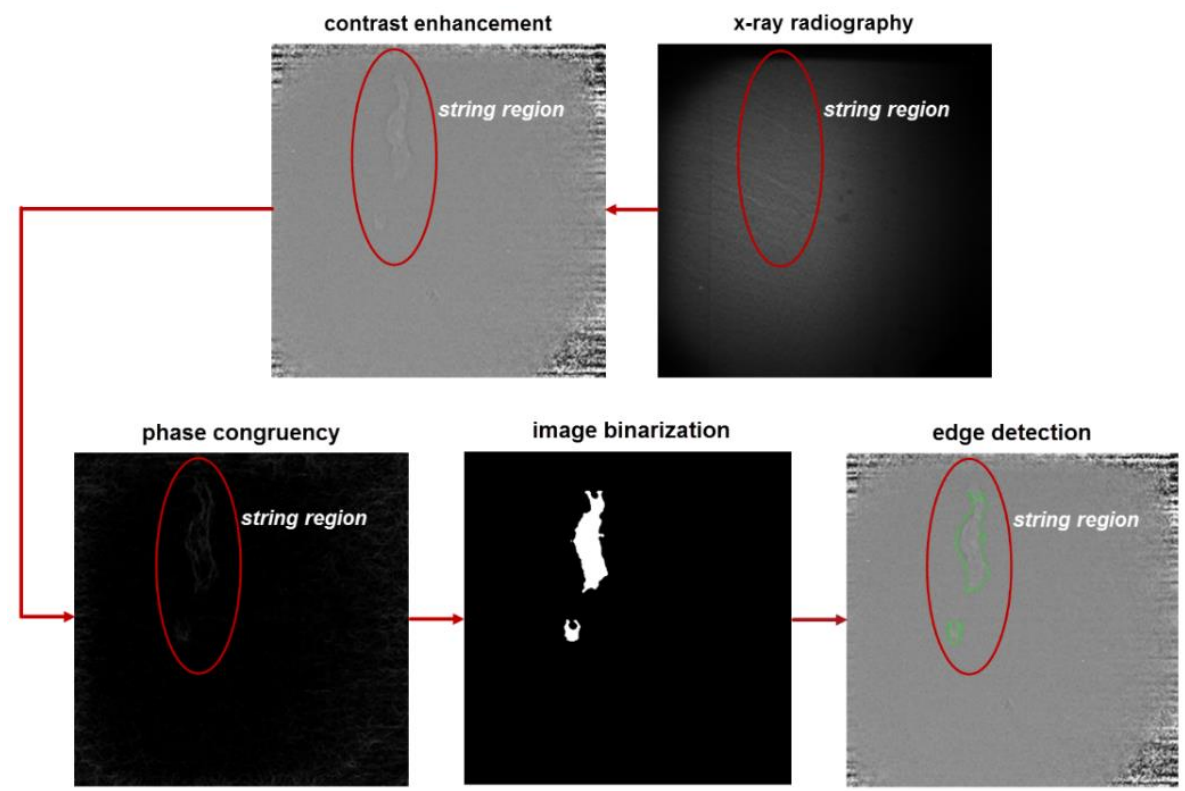

Figure 2. Sequence of the post-processing techniques employed.

\section{Post-processing methodology}

The camera used allows for the acquisition of 12-bit grayscale images, where a change in contrast signifies the presence of cavitation. A typical, "raw" x-ray radiography can be seen in the top-right part of Fig. 2, where the shadow of the needle is also visible at the top part of the figure. It can be easily perceived that the contrast fluctuation in the region of cavitation is low and therefore, in order to enhance contrast, the flow radiographies were divided by background images and the pixel brightness of each resulting image was adjusted to a specified range of values. All image-manipulation techniques were performed using the Image Processing toolbox in Matlab. A phase congruency method, as described in [13], was implemented to recognise the features of interest in the enhanced- 
contrast images (bottom left part of Fig. 2). Subsequently, the image was binarized by applying a mean filter and proper thresholding [6], since after phase congruency the "string" edges appeared much brighter compared to the rest of the image. In subsequent step, the Canny's algorithm was employed to detect the edges of the identified structure. Finally, an additional ad-hoc algorithm was imposed to fill the gaps possibly arising in the detected edges and, thus, to produce a closed line corresponding to the string interphase (green line on right bottom part of Fig. 2).

\section{Results}

The actual string topology arising in the injector region adjacent to the needle tip $(X=0)$ for $R e=35500$ is illustrated in Fig. 3. Fig 3a corresponds to a moderately cavitating flow $(\mathrm{CN}=1.6)$ and, as can be seen, a slender string sets in, which transforms to almost separated structures of irregular shape as the phenomenon progresses in time. It must be noted that post processing of the entire set of radiographies obtained for the specific location revealed a variability in the forms of the cavitating structures, comprising elongated strings, separated ligaments and clusters of bubbles. Increase of the cavitation number to 7.7 leads to the emergence of string that occupies a significant part of the injector hole cross-section having a relatively invariant core and, in general, a more stable topology compared to the respective for $\mathrm{CN}=1.6$. The fact that the string attaches to the needle tip constitutes proof that the underlying cause is a longitudinal vortex, as according to the second Helmholtz theorem, a vortex filament must extend to a boundary or form a closed loop (see [14] for the flow field arising in a similar orifice layout). The strong centrifugal forces prevailing at the vortex core cause the liquid to cavitate. In the case of cloud cavitation, the cavity should be attached to the nozzle wall, as it arises due to the flow separation downstream the constriction. The temporally resolved images reveal significant interfacial fluctuations especially for the low value of the cavitation number due to the conflicting surface tension and viscous effects. It is a well-known fact that string cavitation exhibits a highly transient behaviour (e.g. refer to [10]). The string topology has been resolved in much more detail compared to conventional optical imaging and a far more complex topology has been revealed compared to typical shadowgraphy images (e.g. see Fig. 1). The interaction of the longitudinal vortices with the inherent flow turbulence causes the fluctuating appearance behaviour of the string cavities for low $\mathrm{CN}$. In essence, vortices of smaller scale temporarily disrupt the coherence of the prevailing vortical motion thus causing the string to collapse.

a

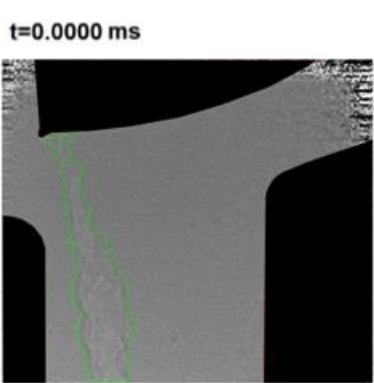

b

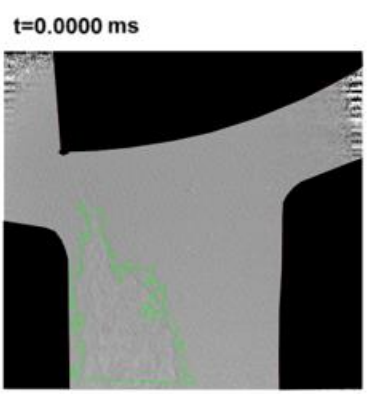

$\mathrm{t}=0.0147 \mathrm{~ms}$

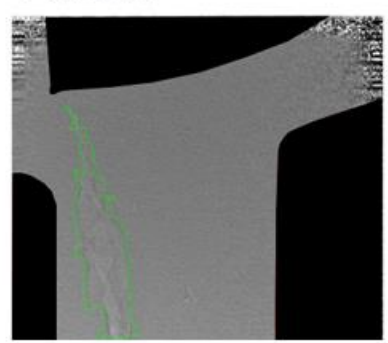

$\mathrm{t}=0.0147 \mathrm{~ms}$

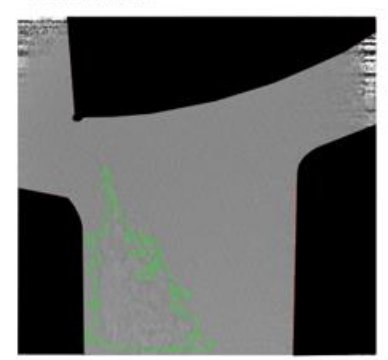

$\mathrm{t}=0.0294 \mathrm{~ms}$

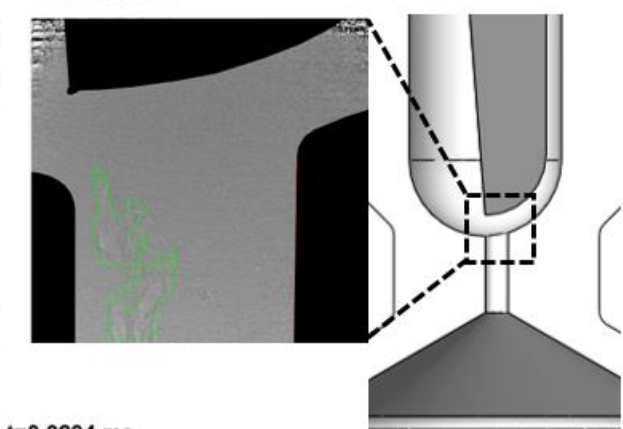

$\mathrm{t}=0.0294 \mathrm{~ms}$

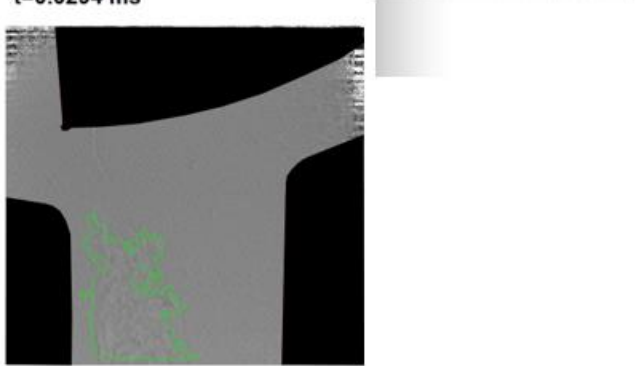

Figure 3. Topology of the cavitation cloud at the region adjacent to the needle tip for $\mathrm{Re}=35500$ : (a) $\mathrm{CN}=1.6$ and (b) $\mathrm{CN}=7.7$.

The spatial probability of vapour occurrence per pixel of the visualized region is depicted in Fig. 4a, as calculated by the averaging of 16000 radiographies for each region along the nozzle length also shown on the top part of Fig. 4a. Vapour occurrence probability is relatively low for $\mathrm{CN}=1.6$ and reaches values up to 0.8 indicating that the formation of cavitating structures is a highly dynamical flow process. On the contrary for $\mathrm{CN}=7.7$, the string core obtains a probability value of 1 , signifying that a temporally-invariant, in the sense that its core does not exhibit significant fluctuations, string establishes with a well-defined interface to the surrounding liquid. It can be therefore 
deduced that the string topology corresponds to a structure occupied by pure vapour. It is important to point out that the nozzle layout with the rounded inlet vertices (see nozzle wall at $\mathrm{X}=0$ ) prevents geometrical cavitation to emerge. Hence, the onset of string cavitation is attributed solely to the secondary flow pattern, i.e., strong longitudinal vortices set in at the nozzle core due to the flow inherent instabilities and the effect of the geometrical constriction. The low pressure prevailing at the vortex cores causes the liquid to vaporize and the string to form. Furthermore, Fig. $4 \mathrm{~b}$ depicts the image standard deviation, i.e. variability in pixel brightness compared to the mean image, which is indicative the magnitude of the shape variability that the vortical structures exhibit. Referring to $\mathrm{CN}=1.6$, the highly fluctuating behaviour of the cavitating structures is clearly demonstrated, since the standard deviation obtains high values up to 0.5 in all the visualized positions. It can be deduced considering Figs. $3 a$ and $4 a-b$ regarding $\mathrm{CN}=1.6$, that, despite the temporal variations in shape, a string-shaped structure is the prevailing cavitation form even for low cavitation numbers. For $\mathrm{CN}=7.7$, the standard-deviation plot makes evident that the largest part of the string core remains invariant in time. Only the string part located close to the needle tip exhibits considerable temporal variation, while further downstream, high standard-deviation values occur at the outer edges.

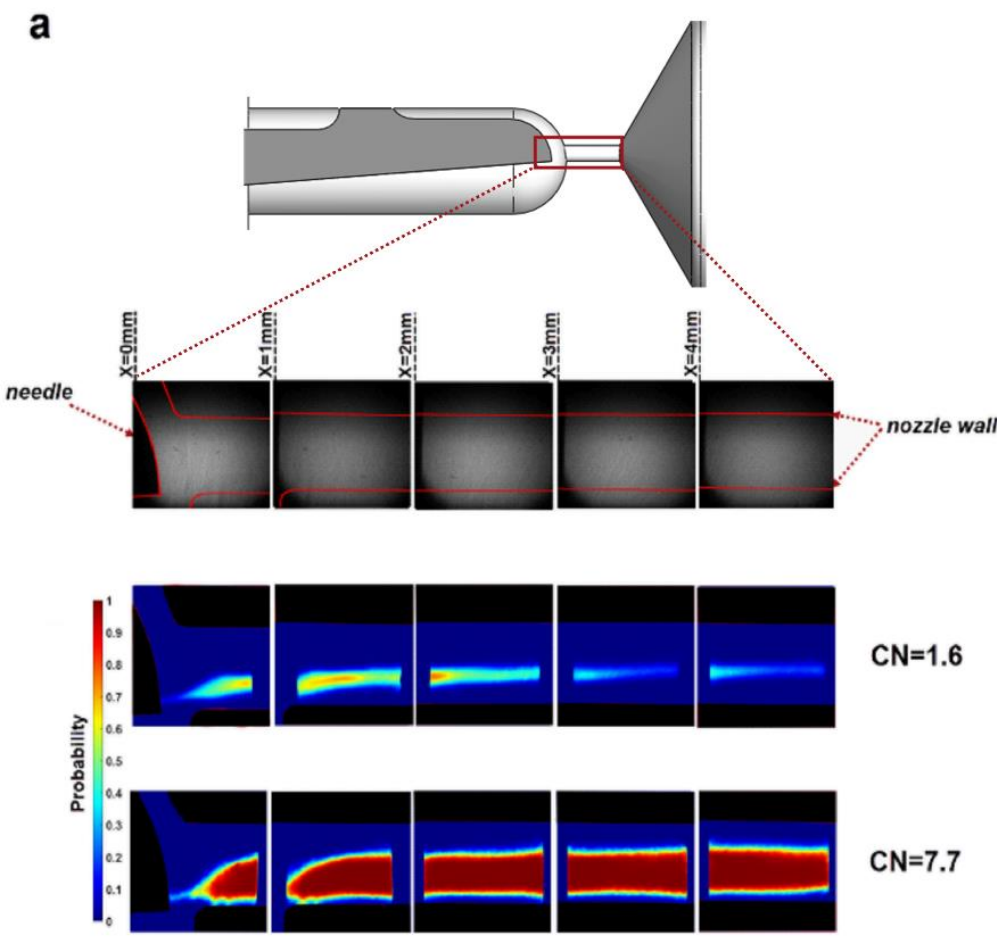

b

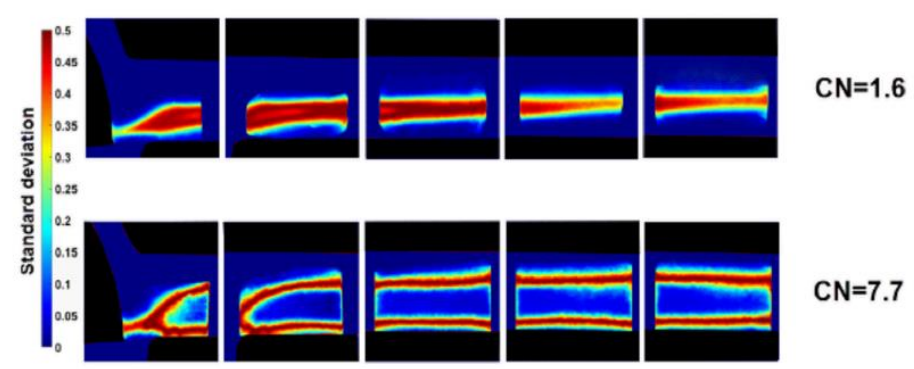

Figure 4. (a) Mean image and (b) standard deviation of the cavitating string for $R e=35000$.

As it has already been pointed out, string cavities for low cavitation numbers constitute dynamical flow features with highly varying morphology. In order to allow a straightforward comparison between the different cases investigated, the occurrence frequency of a repetitive, distinct flow process is presented in the dimensionless form of the Strouhal number $(S t=f L / u)$, with an associated uncertainty of the order of $3.5 \%$. For instance, Fig. 5, illustrates the break-up behaviour of single strings into additional cavitating structures. The region immediately downstream the needle tip was selected due to the highly fluctuating string topology there, as verified by Fig. $4 \mathrm{a}$. As depicted on Fig. 5a, string break-up is declared by examining frames (the control window is shown at the inset of Fig. 5b) corresponding to successive time instances and verifying that the detected edge has transformed from a single to two (or more) 
closed loops. As made evident by Fig. 5b, depicting the break-up probability at a specific frequency, the string becomes more stable, i.e the break-up frequency decreases, as the Reynolds and cavitation numbers increase, while the repetitive pattern of the phenomenon is enhanced, as higher occurrence probabilities are obtained for the dominant frequencies. For instance, the cumulative probability the string break-up to occur at frequencies corresponding to $\mathrm{St} \leq 3$ for $\mathrm{Re}=18000$ and $\mathrm{CN}=1.6$ is of the order of 0.6 , whereas it reaches a value of 1 as $\mathrm{Re}$ and $\mathrm{CN}$ increase.

An additional quantity suitable for the characterization of the string dynamical behaviour is the lifetime period, i.e. the time interval between the string onset and collapse. Similar to the break-up frequency, the lifetime period was determined for each visualization location by assigning an appropriate control window and recording the average cavitation projected area within it. It is essential to clarify that for the low $\mathrm{CN}$ cases $(\mathrm{CN} \leq 2.0)$ examined, a plentiful of diverse structures emerge in the nozzle apart from coherent elongated, cavitating vortices. Hence, a conventional limit was set equal to $40 \%$ of the average projected string area (see Fig. 4), below which the string was considered to have lost its coherence. The limit specified was confirmed by observation of the actual radiographies, since once the area decreased below the limit, only ligaments or bubble clusters could be discerned in the control window.

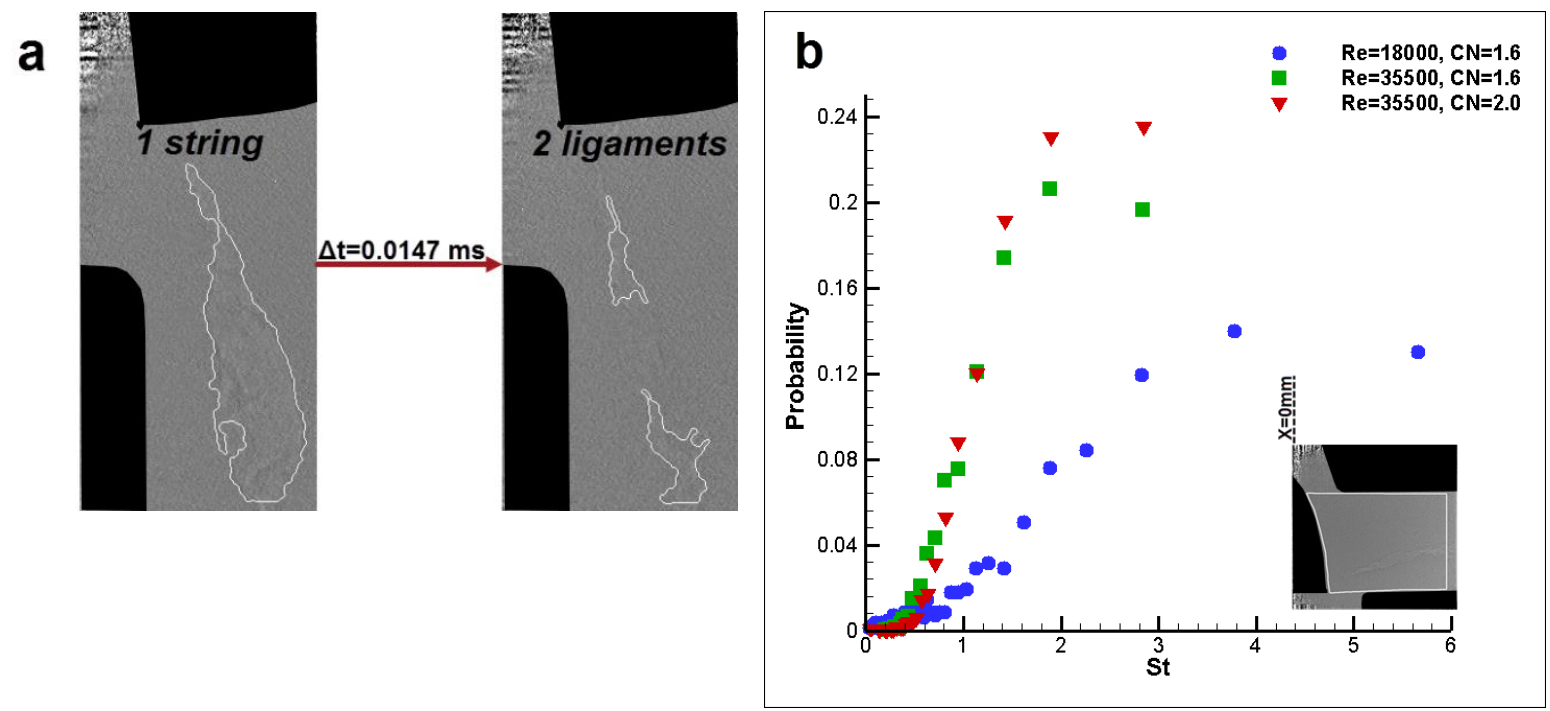

Figure 5. (a) Detection of string break-up and (b) effect of the flow conditions on the string break-up behaviour.

As shown by the data of Fig. 6a, increase of the Reynolds and cavitation number leads to increase of the string lifetime. For $\mathrm{Re}=18000$ and $\mathrm{CN}=1.6$ the maximum string life is, in essence, equal to $0.1 \mathrm{~ms}$, while the cumulative probability of a string to exhibit a lifetime lower or equal to $0.03 \mathrm{~ms}$ is of the order of 0.73 . It must be pointed that there is a 0.3 probability the string life to be even lower than the minimum lifetime that can be visualized by the method employed in this study. As the Reynolds number increases to 30000 , while the $\mathrm{CN}$ is maintained constant, the distribution of possible lifetimes is shifted towards larger values approximately up to $0.3 \mathrm{~ms}$, where the cumulative probability reaches a values approximately equal to 1 . Yet once again about $30 \%$ of the strings recorded have a lifetime smaller than $0.03 \mathrm{~ms}$. Increase of the $\mathrm{CN}$ value to 2.0 has a considerable effect on string stability, since the average lifetime is increased compared to $\mathrm{CN}=1.6$, while the probability distribution becomes more even across the range $0.02-0.2 \mathrm{~ms}$. The maximum probability is of the order of 0.09 for a lifetime approximately equal to $0.059 \mathrm{~ms}$. The effect of the flow conditions on the string lifetime is justifiable since, increase of the Reynolds number, i.e. of the mean flow velocity, enhances the secondary-flow vorticity magnitude, which is responsible for the string formation. Furthermore, increase of the $\mathrm{CN}$ number, in other words, decrease of the back pressure leads to a pressure distribution in the injector-hole, which is closer to the cavitation threshold and thus a more insensitive cavity to flow perturbations establishes.

Besides, Fig. $6 \mathrm{~b}$ indicates the string coherence does not decay simultaneously along the orifice length. It must be noted that the control windows were properly placed at each region of interest, so as not to include any overlapping areas of flow visualization. The string exhibits a similar behaviour in the first two regions downstream the needle tip $(\mathrm{X}=0.0$ and $1.0 \mathrm{~mm})$ with a relatively long lifetime, as the cumulative lifetime probability reaches a value of 1 for $\mathrm{t}=0.4 \mathrm{~ms}$. On the contrary, at a further downstream location the string lifetime is much shorter, since its cumulative probability becomes equal to 1 at $\mathrm{t}=0.2 \mathrm{~ms}$, and is indicative of a "flapping" behaviour at the string closure region. The inset of Fig. $6 \mathrm{~b}$ depicting the string mean image at two different regions downstream the nozzle entrance verifies the string lifetime data. At the region between $X=1.0$ and $2.0 \mathrm{~mm}$, the string existence probability is in general high 
$(>80 \%)$ almost at the entire length of the control window, whereas at a further downstream location $(3.0 \mathrm{~mm} \leq X \leq 4.0 \mathrm{~mm})$ the lower half of the image, corresponding to the area where the flapping occurs, presents probability values in the range of $30-50 \%$. Nevertheless, as also elucidated by Fig. 3, it must be emphasized that the actual cavitation topology in both regions is significantly different than the one inferred by the mean image.

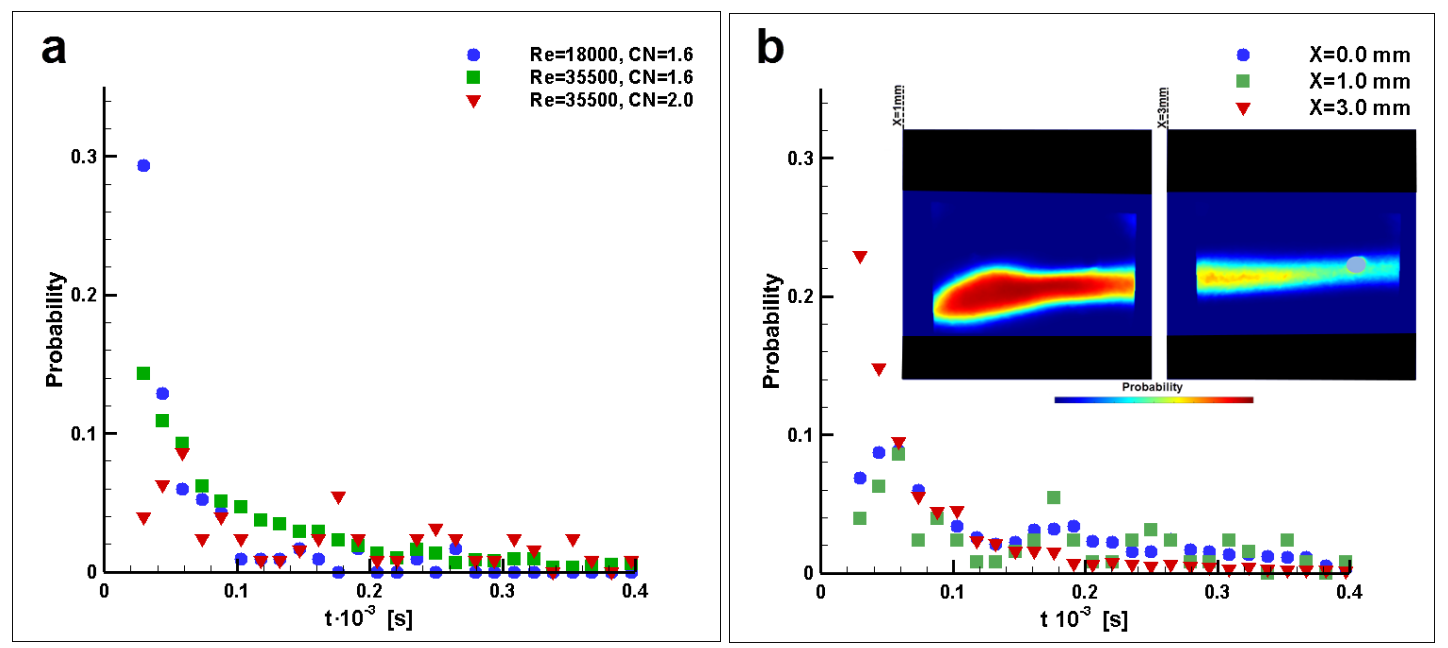

Figure 6. String lifetime: (a) effect of the flow conditions $(X=1.0 \mathrm{~mm})(\mathrm{b})$ probability for different positions along the channe length for $\mathrm{Re}=30000$ and $\mathrm{CN}=2.0$. For $\mathrm{CN}=2.0$ a small artefact evident at the right part of the inset was detected and the respective area (shown in grey) was excluded from the control window.

$\mathrm{XPCl}$ employed in the present study enabled the resolution of scarce cavitating structures (small ligaments or bubble clusters) in reference to the low $\mathrm{CN}$ cases and of fine features of the string interface, in reference to high $\mathrm{CN}$ values. Such features served as points of reference, the trajectories of which allowed the derivation of the local, two-dimensional velocity field presented in Fig. 7 for two extreme cases in terms of Re and CN. The experimental uncertainty associated with the velocity values, stemming from the limitations posed by the spatial discretization and the exposure time, lies in the range of $3.5 \%-5.3 \%$ and $3.4 \%-4.0 \%$ for $\mathrm{CN}=1.6$ (Fig $7 \mathrm{a}$ ) and $\mathrm{CN}=7.7$ (Fig. $7 \mathrm{~b}$ ), respectively. The maximum uncertainties are of course associated with the minimum velocity values for each case. Referring to both low and high-CN cases, regions where adequate points of reference could be identified have been selected to produce the respective velocity-vector plots, however a comparison between Figs 7a-b and 7c-d makes clear that the vector plot is much more thorough for $\mathrm{CN}=7.7$. This is attributed to the fact that for $\mathrm{Re}=18000$ and $\mathrm{CN}=1.6$ only few scarce structures were identified, since for the specific flow conditions cavitating vortices are highly fluctuating and short-lived. On the contrary for $\mathrm{Re}=35500$ and $\mathrm{CN}=7.7$, a plentiful of distinct features could be identified at the string interface due to the high spatial resolution of XPCI and, therefore, a better representation of the local velocity field could be obtained. The average measured velocities for $R e=18000$ and 35500 are equal 28.8 and $60.2 \mathrm{~m} / \mathrm{s}$, while the respective nominal velocities based on the imposed flow rate and orifice cross section were calculated equal to 30.0 and $59.9 \mathrm{~m} / \mathrm{s}$. Hence, there is a strong indication that XPCI can be employed as a velocimetry method in cavitating flows, especially since conventional methods such as PIV, fail to produce measurements in regions of vapour.

a

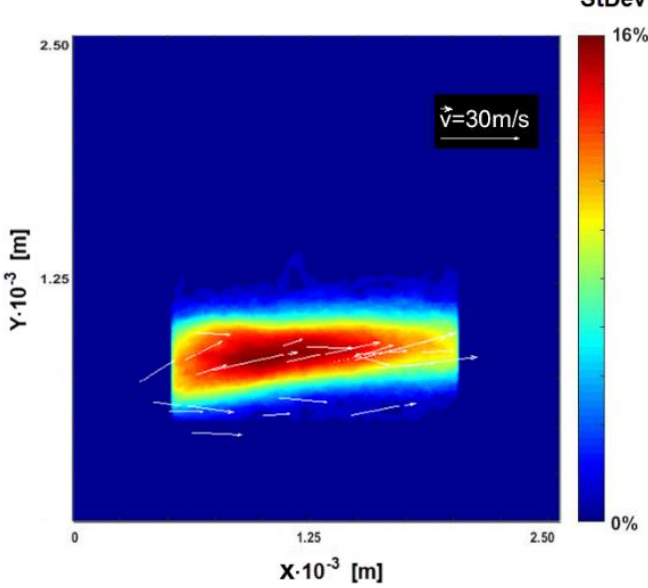

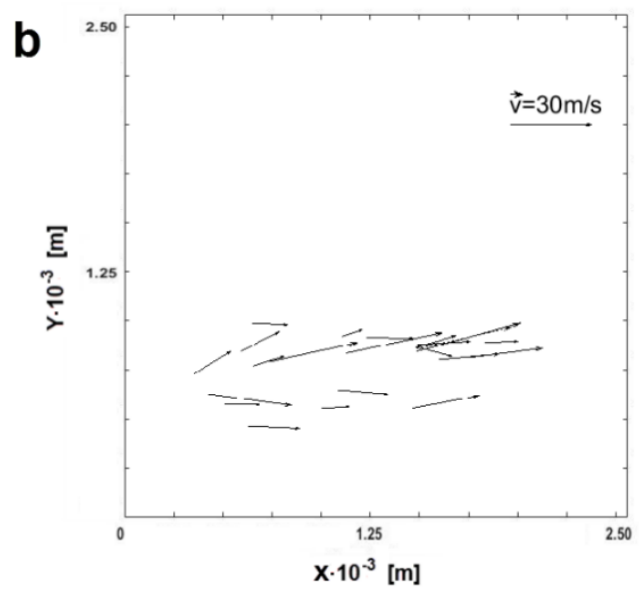

This work is licensed under a Creative Commons 4.0 International License (CC BY-NC-ND 4.0). 

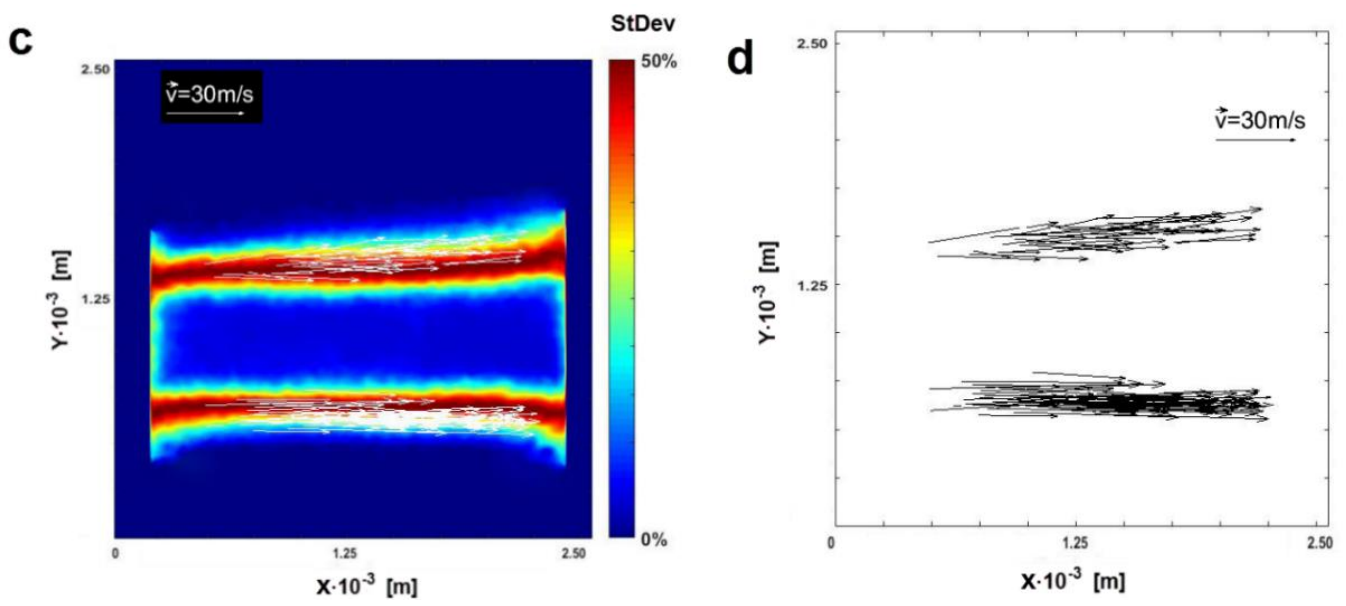

Figure 7. Local velocity field: (a-b) $\mathrm{Re}=18000, \mathrm{CN}=1.6, \mathrm{X}=1.0$ and (c-d) $\mathrm{Re}=35500, \mathrm{CN}=7.7, \mathrm{X}=2.0$.

\section{Conclusions}

The present study elucidated the spatial and temporal evolution of the cavitating strings emerging in an enlarged injector orifice with the use of XPCI. Post processing of the side-view radiographies obtained for the examined flow conditions and needle lift demonstrated that cavitation primarily emerges in the form of vortical structures of highly fluctuating and irregular shape for low $\mathrm{Re}$ and $\mathrm{CN}$, while stable strings set in for high $\mathrm{CN}$ values with their interphase exhibiting high morphological variance. Average and standard deviation images of the string-shaped cavities were obtained for a wide range of conditions, while the spatial resolution of the present flow visualization technique enabled the capturing of fine features that cannot be resolved using optical imaging techniques, e.g. shadowgraphy or Schlieren. The temporally-resolved nature of the measurements allowed the characterization of the string dynamical behaviour and it was verified that the string breakup frequency decreases, while its lifetime increases, as the Re and $\mathrm{CN}$ increase. Finally, local velocity fields were measured in the string region for cases of low and high cavitation extent; the obtained average velocities compared well with the respective nominal values.

\section{Acknowledgements}

The research leading to these results has received funding from the MSCA-ITN-ETN of the EU H2020 programme, under REA grant agreement n. 642536. This research used resources of the Advanced Photon Source, an Office of Science User Facility operated for the U.S. Department of Energy (DOE) by Argonne National Laboratory under Contract No. 53697.

\section{Nomenclature}

CN cavitation number

D nozzle internal diameter [m]

f frequency $[\mathrm{Hz}]$

$\mathrm{L} \quad$ nozzle length [m]

$\begin{array}{ll}\text { Re } & \text { Reynolds number } \\ \text { St } & \text { Strouhal number } \\ \text { u } & \text { axial velocity }\left[\mathrm{m} \mathrm{s}^{-1}\right] \\ \mathrm{v} & \text { kinematic viscosity }\left[\mathrm{m}^{2} \mathrm{~s}^{-}\right.\end{array}$

\section{References}

[1] Hult. J., Simmank, P., Matlok, S., Mayer, S., Falgout, Z., Linne, M., 2016, Experiment in Fluids 57, pp. 49.

[2] Arcoumanis, C., Gavaises, M., Flora, H., Roth, H., 2001, Mécanique \& Industries 2, pp. 375-381.

[3] Mitroglou, N., Gavaises, M., Nouri J. M., Arcoumanis C., May 27. 2011, DIPSI Workshop.

[4] Petkovšek, M., Dular, M., 2013, Wear 300(1), pp. 55-64.

[5] Gavaises, M., Andriotis, A., Papoulias, D., Mitroglou, N., Theodorakakos, A., 2009, Physics of Fluids 21(5), pp. 052107.

[6] Mitroglou, N., McLorn, M., Gavaises, M., Soteriou, C., Winterbourne, M., 2014, Fuel 116, pp. 736-742.

[7] Moon S., 2016, Energy 115, pp. 615-625.

[8] Kastengren, A. L., Tilocco, F. Z., Duke, D., Powell, C. F., Zhang, X., Moon S., 2014, Atomization and Sprays, 24(3), pp. 251-272.

[9] Duke, D., Kastengren, A. L., Tilocco, F. Z., Swantek, A. B., Powell, C. F., 2013, Atomization and Sprays, 23(9), pp. 841-860.

[10] Mitroglou, N., Lorenzi, M., Santini, M., Gavaises, M., 2016, Experiments in Fluids, 57, pp. 175.

[11] Moffat, R.J., 1988, Experimental Thermal and Fluid Science 1, pp. 3-17.

[12] Kolev, N. I., 2007, "Multiphase Flow Dynamics 3: Turbulence, Gas Absorption and Release, Diesel Fuel Properties.", Springer, pp. 269-302.

[13] Kovesi P., 1995, Technical Report 95/4, The University of Western Australia.

[14] Koukouvinis, P., Mitroglou, N., Gavaises, M., 2017, Journal of Fluid Mechanics, 819, pp. 21-57. 


\title{
High Speed Shadowgraphy of Transparent Nozzles as an Evaluation Tool for In-Nozzle Cavitation Behavior of GDI Injectors
}

\author{
Dmitrii Mamaikin¹, Tobias Knorsch¹, Philipp Rogler ${ }^{1}$, Philippe Leick ${ }^{1}$, Michael Wensing ${ }^{2}$ \\ ${ }^{1}$ Robert Bosch GmbH, Germany \\ ${ }^{2}$ Department of Engineering Thermodynamics, FAU Erlangen-Nuremberg, Germany \\ ${ }^{*}$ Corresponding author: Dmitrii.Mamaikin@de.bosch.com
}

\begin{abstract}
Gasoline Direct Injection (GDI) systems have become a rapidly developing technology taking up a considerable and rapidly growing share in the Gasoline Engine market due to the thermodynamic advantages of direct injection. The process of spray formation and propagation from a fuel injector is very crucial in optimizing the air-fuel mixture of DI engines. Previous studies have shown that the presence of some cavitation in high-pressure fuel nozzles can lead to better atomization of the fluid. However, under some very specific circumstances, high levels of cavitation can also delay the atomization process; spray stabilization due to hydraulic flip is the most well-known example. Therefore, a better understanding of cavitation behavior is of vital importance for further optimization of next generation fuel injectors.

In contrast to the abundance of investigations conducted on the inner flow and cavitation patterns of diesel injectors, corresponding in-depth research on the inner flow of gasoline direct-injection nozzles is still relatively scarce. In this study, the results of an experiment performed on real-size GDI injector nozzles made of acrylic glass are presented. The inner flow of the nozzle is visualized using a high-power pulsed laser, a long-distance microscope and a highspeed camera. The ambiguity of dark areas on the images, which may represent cavitation regions as well as ambient air drawn into the nozzle holes, is resolved by injecting the fuel both into a fuel or gas filled environment. In addition, the influence of backpressure on the transient flow characteristics of the internal flow is investigated. In good agreement with observations made in previous studies, higher backpressure levels decrease the amount of cavitation inside the nozzles. Due to the high temporal and spatial resolution of the experiment, the transient cavitation behavior during the opening, quasi-steady and closing phases of the injector needle motion can be analyzed. For example, it is found that cavitation patterns oscillate with a characteristic frequency that depends on the backpressure. The link between cavitation and air drawn into the nozzle at the beginning of injection is also revealed.
\end{abstract}

\section{Keywords}

Direct injection, transparent nozzle, internal flow, cavitation, high-speed imaging

\section{Introduction}

The performance of GDI engines depends significantly on the fuel injection system. The injector plays a dominant role in delivery of the fuel into the combustion chamber in the form of spray patterns. In past years, many studies have been dedicated to the processes of spray formation with the main goal of achieving better control of spray propagation and improved atomization characteristics [1-3]. These factors consequently influence the processes of fuel evaporation, ignition and combustion to a great extent.

The technology development trend towards higher injection pressures makes the investigations of two-phase internal flows highly important, in part due to the possibility of more intense pressure fluctuations inside the nozzle. In addition, it is known that the velocity profile at the nozzle outlet, as well as turbulence intensity and liquid-cavitation interaction inside the nozzle provoke the first perturbations of the liquid stream [4,5]. These perturbations directly lead to droplet formation via primary break-up processes but also contribute indirectly to secondary atomization due to increased aerodynamic resistance.

The high speed cavitating flow that occurs inside real-size injector nozzles is quite challenging for experimental studies due to the small relevant scales and the complexity of the highly transient and turbulent flow. Therefore, the cavitating flow is still not fully understood, which in turn obstructs the understanding of some crucial processes such as primary break-up and hole-to-hole spray variations.

Most of the experimental work conducted on internal nozzle phenomena has relied on shadowgraphy. For instance, some studies were carried out on enlarged transparent nozzles [6-8]. The large scale significantly improves the optical accessibility to the flow inside the nozzle. For these measurements, however, the relevant dimensionless parameters such as Reynolds and cavitation numbers should ideally be identical to those of the real-size nozzles 
in order to maintain equivalent flow conditions. Moreover, the Ohnesorge number should be preserved in order to obtain similar break-up of the liquid leaving the nozzle. In most of the cases, it is not possible to meet all these conditions simultaneously and therefore, large scale experiments cannot be fully representative. Other experimental studies have used two-dimensional (2D) nozzles for internal flow analysis [5,9]. Optical measurements are simplified due to minimization of the optical aberrations by virtue of the 2D nozzle geometry. This approach facilitates deep and profound studies of cavitation but the results are not fully transferable to real three dimensional nozzles. Another method for internal flow investigation uses real-size transparent nozzles. This avoids scaling effects and thus comes closer to the inner flow of commercial injector nozzles. However, manufacturing such nozzles out of transparent materials is challenging, since in addition to the small size of the nozzle, some resistance to high-pressure conditions is needed. A number of experimental studies were conducted using single hole axial nozzles [10-12] which simplifies both the manufacturing of the nozzle and the resulting flow. However, it is known that sharp turn the liquid needs to take to enter the spray hole is an important driving factor for the subsequent spray breakup. Additionally, in multi-hole injectors, the individual holes can 'communicate'. Only a few experimental studies have compared flows inside single hole and two-hole nozzles [10,13]. Different string cavitation behavior has been found and cavitation strings from one hole can extend to the neighboring hole. The string cavitation, in turn, highly influences the internal flow dynamics as well as the atomization properties [14,15].

Overall, the simplifications that are often made for internal flow measurements, do not provide fully representative results that can be used for further development of injector nozzles. The relevant boundary conditions, needle movement, time scales of measurements, geometries are not or only partially considered. Therefore, experiments, which are performed as close as possible to real conditions, are of significant interest to the research and engineering communities. In this work, the measurement results from high-speed imaging of the internal flow of two-hole real-size transparent nozzles are presented.

\section{Material and methods}

The experimental setup used for this study is schematically depicted in Figure1. The overall setup consists of the injection and the imaging system. The fuel is stored in a vessel which is connected to a high pressure nitrogen supply on one side and the fuel line feeding the injector from the other side. A Bosch HDEV5 gasoline injector is used for injecting the fuel. Such an injector facilitates the mounting of the acrylic parts and the adjustment of maximum needle lift using carefully selected spacer rings. An in-line filter is mounted right upstream of the injector in order to remove contaminants from the fuel, which could affect cavitation inception, deteriorate image quality or even damage the acrylic components. The injection system works without a fuel pump whose operation might provoke the release of dissolved gases upstream the injector and thereby influence the internal flow. The visualization system is composed of a light source, an optical diffusor and a high-speed camera equipped with a long-distance microscope (LDM). The Cavilux HF laser of $810 \mathrm{~nm}$ wavelength is used to illuminate the flow field. Ultra-short pulses of only 50 ns duration are used instead of continuous illumination in order to avoid motion blur in the images. Additionally, the low-coherence of the light provided by the laser avoids formation of any speckle patterns. The laser light is guided by an optical waveguide towards the acrylic nozzle, but first passes through an optical diffusor in order to illuminate the nozzle homogeneously. A high-speed camera (FASTCAM SA-Z) equipped with a long-distance microscope (Infinity K2 with CF4 lens) is used for the image acquisition and operated at $120,000 \mathrm{fps}$. In this way, a magnification of 7.4 is achieved at an image resolution of $640 \times 216$ pixels. A trigger is used to synchronize the injector, camera gate pulses and the laser illumination.

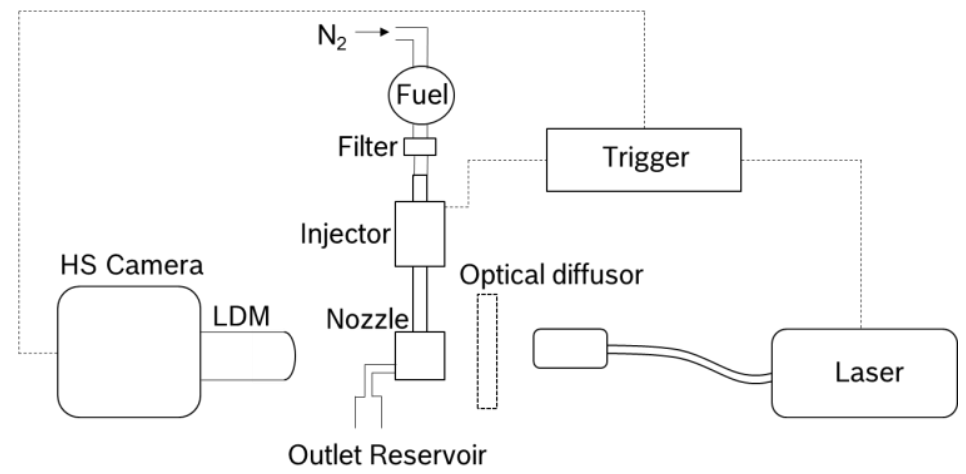

Figure 1. Scheme of the experimental setup. 
The optically accessible nozzle is made of acrylic glass. Figure $2 a$ illustrates the nozzle, where the injector needle and O-ring can also be seen. The spray holes and counterbores are followed by a "multi-step" configuration that allows the fuel to leave the acrylic block and is relatively straightforward to manufacture. The nozzle was tested at maximum injection pressure of 200 bar and showed reliable performance under high pressure load conditions. Due to the configuration of the nozzle, the visualization of the internal nozzle flow is focused on the spray holes area. The spray hole and the counterbore are followed by a "multi-step" outlet that facilitates the withdrawal of fuel from the nozzle exit region before the start of the next injection but does not affect flow and cavitation within the spray hole. The region of interest is outlined with a rectangle in Figure 2a. A typical shadowgraph image captured during a quasi-steady-state of the injection phase is shown in Figure $2 \mathrm{~b}$. As can be seen, the nozzle contains two holes with different inclination angles (I-angles). The I-angle is given with respect to the vertical nozzle axis. A mixture of calibration fluid (ISO 4113 norm) and Bromonaphthalene is used for investigating internal phenomena. This mixture has the same refractive index $(n=1.49)$ as the acrylic glass, which allows an undisturbed view through curved structures. The dark round area on the top of the image is the injector needle, which is opaque to the incident light. The dark areas inside the nozzle holes are gas-phase structures and can either contain fuel vapor (cavitation) or ambient air drawn into the nozzle holes. The refractive index of either of these gases $(n \approx 1)$ is significantly different from the refractive indices of the liquid fuel and the transparent nozzle. Therefore, the incident light is strongly refracted making the corresponding areas to appear as dark regions.

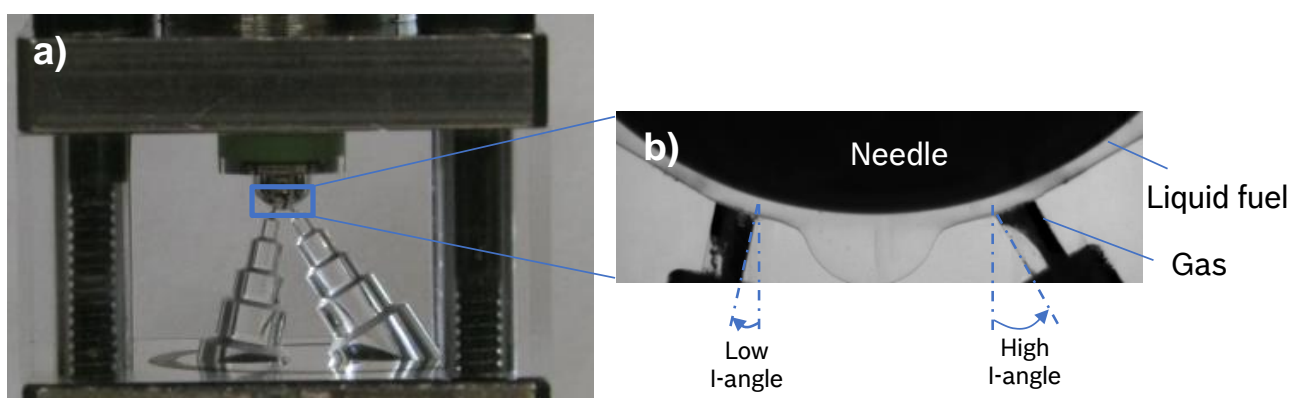

Figure 2. a) Acrylic nozzle configuration, b) Typical shadowgraph image of in-nozzle flow.

In order to resolve the ambiguity of dark areas inside the nozzle holes on shadowgraph images, visualizations are performed while injecting into the fuel into reservoirs filled with air or fuel; in the latter case, no ambient air can be drawn into the system. However, the release of gases dissolved in the fuel still can take place during the fuel injection. It should be pointed out that cavitation and released gases cannot be distinguished in the images. Keeping in mind both possibilities, dark areas will be referred to as 'cavitation' in this paper for the sake of simplicity and easier reading. Figure 3 shows the acrylic nozzle in both air-filled and liquid-filled environments.
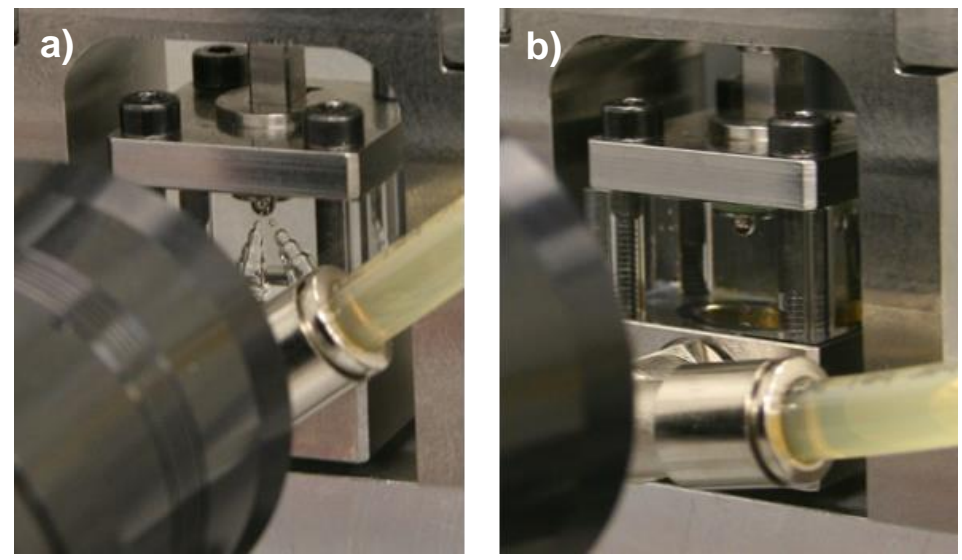

Figure 3. Acrylic nozzle for: a) air-filled nozzle holes, b) fuel-filled nozzle holes

The measurement results of two different acrylic nozzles are presented and discussed in the following sections. One nozzle has two cylindrical holes, whereas another one has two divergent holes. The corresponding holes have identical inclination angles (low I-angle, high I-angle in Figure $2 \mathrm{~b}$ ) but different length to diameter ratios. All the measurements are carried out at room temperature. 


\section{Results and discussion}

Figure 4 shows typical images taken during the measurements while injecting into an air-filled environment. A nozzle with two cylindrical holes was used for this case. The operating conditions for these measurements were as follows: injection pressure - 100 bar, back pressure - 1bar. On the first image (Figure 4a), the needle is closed and one can see stagnant residual air bubbles inside the nozzle holes as well as inside the sac volume. The succeeding image Figure $4 \mathrm{~b}$ is taken during the needle opening phase. The needle lift at this time is still low and as a result, highly turbulent flow occurs inside the nozzle. The holes are filled with gas and one can observe gas pockets, which extend from the nozzle holes and reach the needle. This phenomenon was also observed by Gilles-Birth et al. [10]. It is commonly attributed to strong vorticities in the flow field, which result in a radial pressure drop from the wall to the middle of the hole. In addition, the residual air inside the sac volume is being forced out by the build-up of the liquid flow as can be observed in Figure 4b. The next two images (Figure 4c,d) reveal that the developed cavitation structures inside the nozzle holes maintain their shapes during the steady maximum lift needle position. As it can be seen, cavitation is not formed in the needle seat area. Rather, the cavitation starts at the sharp inlet edges of the nozzle holes. According to the terminology used in [16], 'partial supercavitation' is observed during this stage. It is obvious that the inclination of the nozzle holes affects the location and structure of the in-nozzle cavitation during the steady-state phase of the injection (Figure 4c,d). From the flow point of view, the liquid flow undergoes deflection while it enters the nozzle holes. The flow deflection angle determines the onset of cavitation. In fact, a greater flow deflection angle leads to a higher pressure drop. This, in turn, leads to the cavitation and release of air induced by the nozzle geometry. Therefore, the cavitation is dominant on the right side of both nozzle holes. Figure $4 \mathrm{e}$ represents the nozzle flow during the closing phase of the needle. The amount of cavitation is increased in comparison to the quasi steady state of injection (Figure $4 \mathrm{c}, \mathrm{d}$ ) due to the increased nozzle flow turbulence caused by the needle motion. After the needle has completely closed (Figure 4f), significant amount of gas remains inside the nozzle holes and some gas amount is drawn into the sac volume, which is observed in the form of large bubbles.

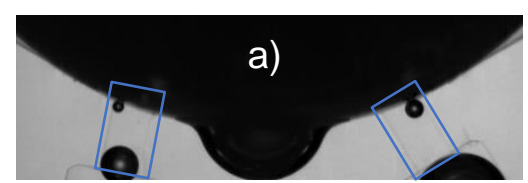

to

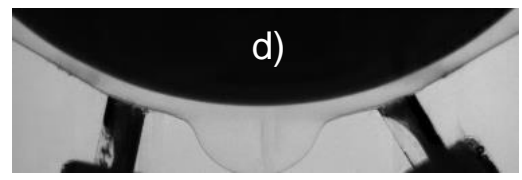

$\mathrm{t}_{0}+1 \mathrm{~ms}$

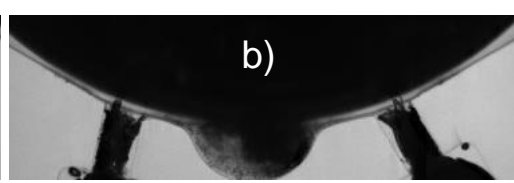

$\mathrm{t}_{0}+0.066 \mathrm{~ms}$

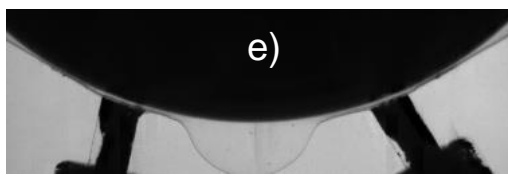

$\mathrm{t}_{0}+1.3 \mathrm{~ms}$

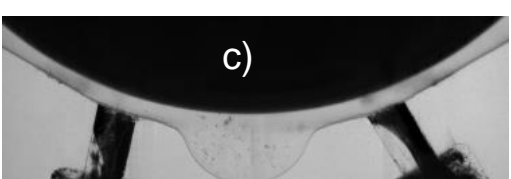

$\mathrm{t}_{0}+0.6 \mathrm{~ms}$

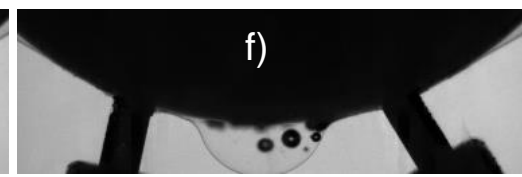

$\mathrm{t}_{0}+1.6 \mathrm{~ms}$

Figure 4. In-nozzle transient gas structures during an injection

\section{Injection into air and fuel}

In the following, we present the internal flow data focusing on the nozzle holes as highlighted by two rectangles in Figure 4a. As mentioned in the previous section, experiments were performed injecting fuel into both air and fuel environments at 1 bar backpressure in order to evaluate the impact of outside air entrained into the nozzle holes. Figure 5 shows exemplary single images before the injection and the probability of cavitation occurrence (calculated during the quasi steady phase of the injection according to Eq. 1) inside the holes in false color. As we see on the single images for the case of injection into liquid, no air bubbles are observed inside the nozzle holes before the injection phase.

$$
P=\frac{\sum_{i=1}^{N} p_{i}}{N}
$$

The average images for injection into liquid and gas exhibit a high similarity meaning that the air entrainment inside the holes is negligible at least during the steady-state injection phase evaluated here. Further internal flow measurements are carried out by injection into the liquid environment. 


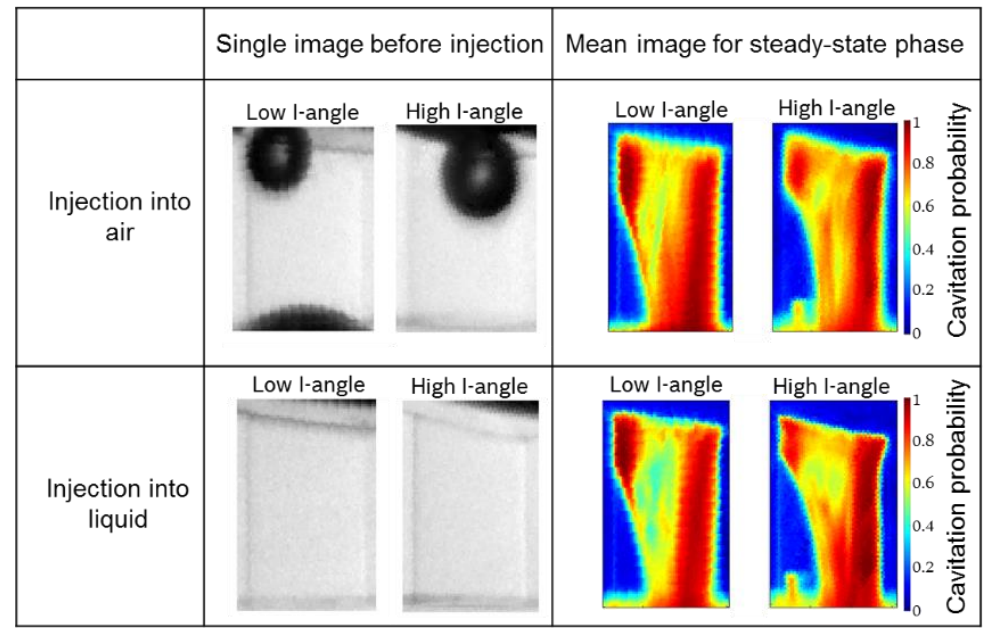

Figure 5. Comparison of injection into air and liquid fuel environment

\section{Effect of backpressure}

Figure 6 shows the development of cavitation for the nozzle with cylindrical holes against different backpressure levels. The injection pressure for these measurements was maintained at 100 bar. Only one nozzle hole (low Iangle) is presented in the pictures. The time gap between the sequential images is $8.3 \mu \mathrm{s}$. As can be observed, the gas structure inside the holes maintain their shape for the case of 1 bar backpressure, while rapid changes are seen for the higher backpressure conditions. The cavitation fraction seems to fluctuate and the rate of fluctuation tends to depend on the backpressure applied. The corresponding cavitation probability as well as the level and region of fluctuation in the images are presented on the right of Figure 6 . The cavitation probability is calculated according to Eq. 1; the fluctuation information is calculated based on the corresponding to steady-state injection phase (135 images) according to Eq. 2. Fluctuations are low where cavitation is almost always present and that the level of fluctuation is increased for higher backpressures. The region of cavitation fluctuation occupies a larger area for increasing backpressure. Possible reasons for this scenario could be that the cavitation film is thicker for lower backpressures. Due to the line-of-sight nature of shadowgraph imaging, the fluctuation can be observed only on the outer sides of the film. For higher backpressure, however, the cavitation film is thinner. Thus, the observable fluctuation area is larger. In addition, shear cavitation, which forms inside the counterbore, is inhibited for increased backpressure conditions to a high extent.

$$
R M S D=\sqrt{\frac{\sum_{i=1}^{N}\left(p_{i}-\bar{p}_{l}\right)^{2}}{N}}
$$

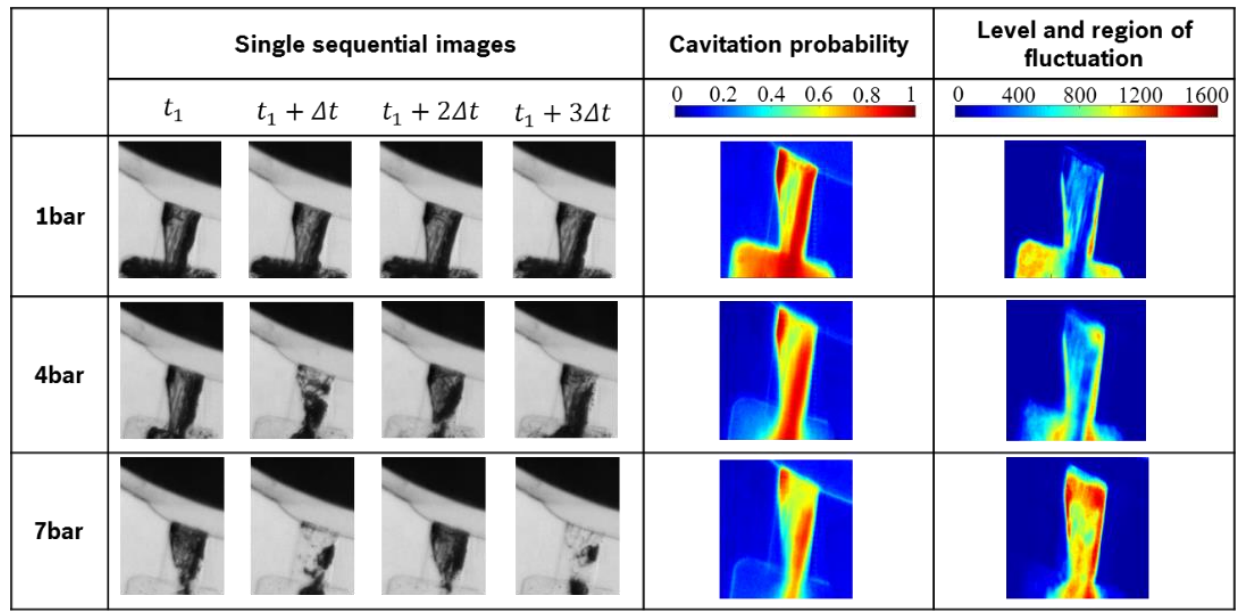

Figure 6. Cavitation under different backpressure levels during steady-state injection phase (cylindrical hole). Injection pressure -100 bar. 
Figure 7 shows the cavitation behavior in the nozzle with divergent holes under the same boundary conditions as Figure 6. From line-of-sight imaging, the holes appear to be relatively more affected by cavitation. From the sequential raw images, it can be observed that the fluctuations of the cavitation again increase with rising backpressure levels, although the cavitation seems to be more resistant against backpressure than for the case of cylindrical holes. Based on the mean images, it can be noticed that the average cavitation distribution and intensity is hardly changed for higher backpressures. There is also a relatively small change in the level and region of cavitation fluctuations. Evidentially, the divergent holes induce significantly higher levels of cavitation. The shear cavitation inside the counterbore, in turn, is also inhibited by increasing backpressures, but to a smaller degree than for cylindrical holes.

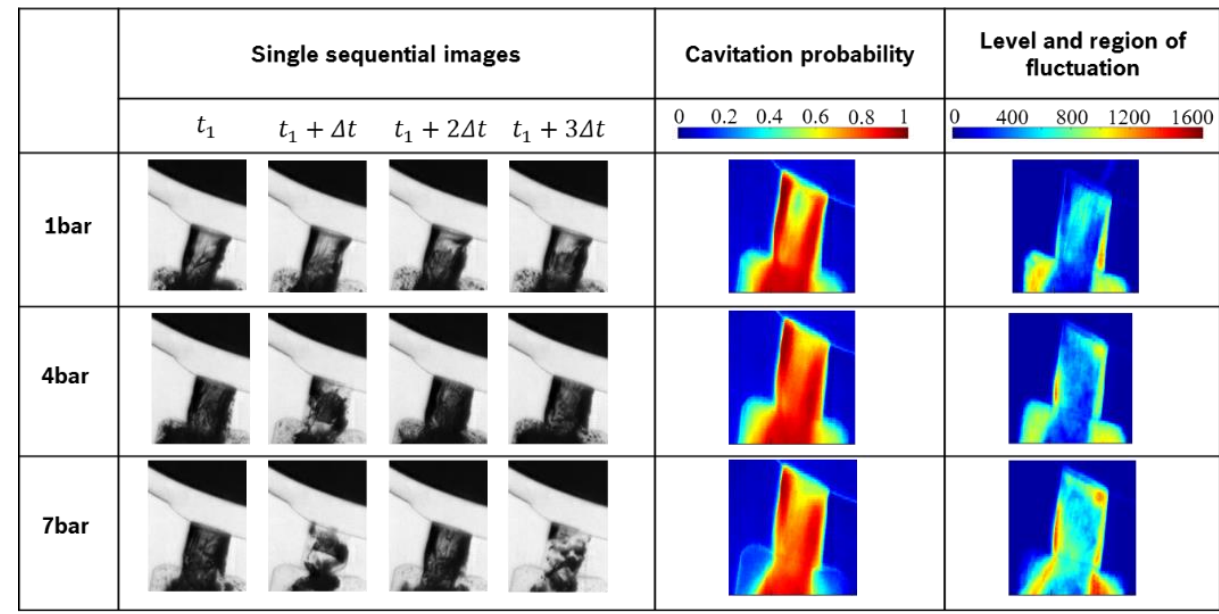

Figure 7. Cavitation under different backpressure levels during steady-state injection phase (divergent hole). Injection pressure - 100 bar.

In the following, the transient behavior of cavitation inside the nozzle holes under different backpressure conditions is analyzed and presented. Each nozzle image is cropped and rotated to obtain a vertical nozzle hole image for further analysis (see Figure 5). As a next step, the image is binarized by a fixed threshold in order to extract the cavitation contour and corresponding cavitation area in each nozzle hole. These 2D cavitation area changes are then tracked as shown in Figure 8. Afterwards, the resulting projected cavitation data is plotted over time. Figure 9 shows typical cavitating behavior of two cylindrical holes (low and high I-angle) over time after start of energization (SOE) for the case of back pressure of 7 bar. The data that corresponds to the quasi steady state of the injection is used for further evaluation. Within this quasi steady-state interval, the cavitation data is fitted with to a third order polynomial that is then subtracted from the data. This procedure is used to remove the long-term evolution of the cavitation pattern, while conserving all fast fluctuations for further Fast Fourier Transformation (FFT) analysis. However, before the FFT is applied, the data is multiplied by a Tukey windowing function in order to minimize spectral leakage. Figure 10 shows the result obtained by FFT transform for the measurement data depicted in Figure 9. Based on the FFT result, the dominant frequencies (depicted as 'peaks' in Figure 10) that correspond to the fluctuations of cavitation during the steady-state injection phase can be found. It should be kept in mind that the maximum frequency that can be recognized is determined by the Nyquist-Shannon sampling theorem and is $60 \mathrm{kHz}$ for the frame rate used for the measurements.

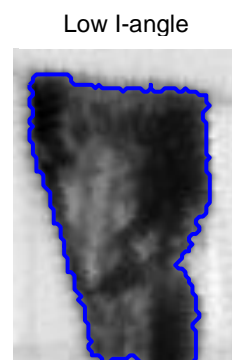

$\mathrm{t}_{1}$
Low I-angle

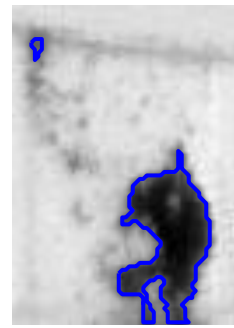

$\mathrm{t}_{1}+8.3 \mu \mathrm{s}$

Figure 8. Tracking of projected cavitation structures for two sequential images (cylindrical holes). Injection pressure - 100 bar, back pressure -7 bar 

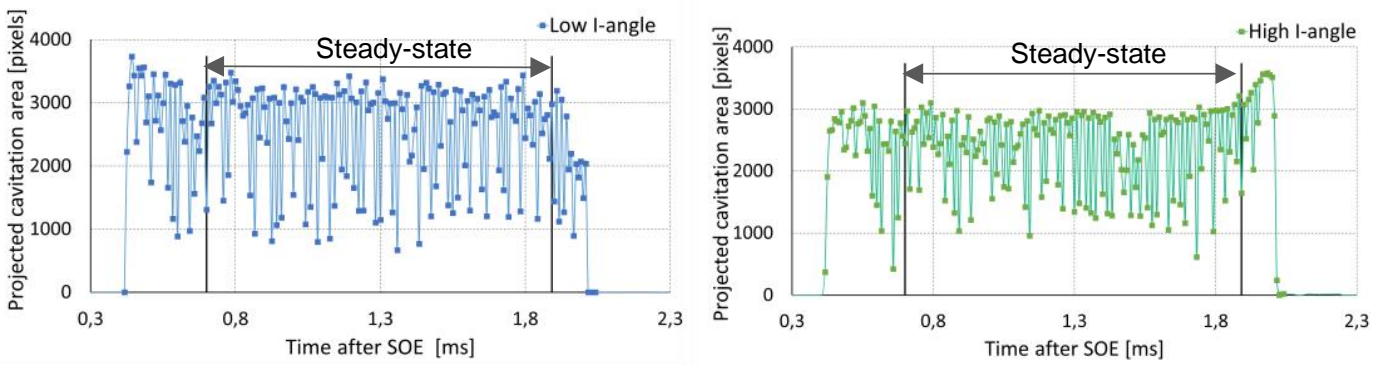

Figure 9. Projected cavitation area over time (cylindrical holes). Injection pressure -100 bar, backpressure -7 bar.
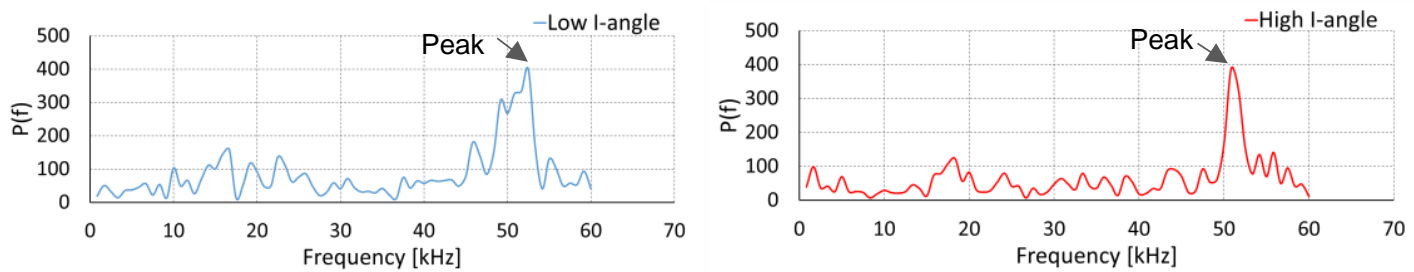

Figure 10. Spectral amplitude over frequency (cylindrical holes). Injection pressure -100 bar, backpressure -7 bar.

This procedure was performed for each backpressure condition and nozzle type. The results are combined and shown in Figure 11, where the dominant frequencies of cavitation fluctuations are depicted over the corresponding backpressure levels for each nozzle type and nozzle hole. For backpressures below 3 bar, no distinct dominant frequencies can be found; as a result, the lines on the graph start at 3 bar and continue up to 7 bar. Due to the fact that the experimental technique is based on the transmitted light principle, the cavitation analysis was performed for cavitation changes projected in $2 \mathrm{D}$ image plane over the injection process. It can be observed that the backpressure influences the frequency of cavitation fluctuations to a high extent for all nozzle holes measured. This graph confirms that divergent holes provide more robust cavitation structures (lower frequencies of cavitation fluctuations for the corresponding holes) than cylindrical ones. It can be observed that the holes under the low inclination angles are found to be subjected to cavitation fluctuations of higher frequencies than the holes under high inclination angles.

It is known that elevated backpressure inhibits the cavitation appearance [17]. Thus, the cavitation structures that appear, for instance, at the highly transient needle opening phase have more potential to collapse for higher back pressures. These collapses in turn generate pressure waves, which result in high pressure oscillations inside the nozzle and lead to cavitation instabilities. Further detailed theoretical interpretation of the observed cavitation fluctuations, however, would have to be performed as a continuation of this experimental study. Furthermore, the analysis and discussions of transient effects at the beginning and end of injection is to be carried out.

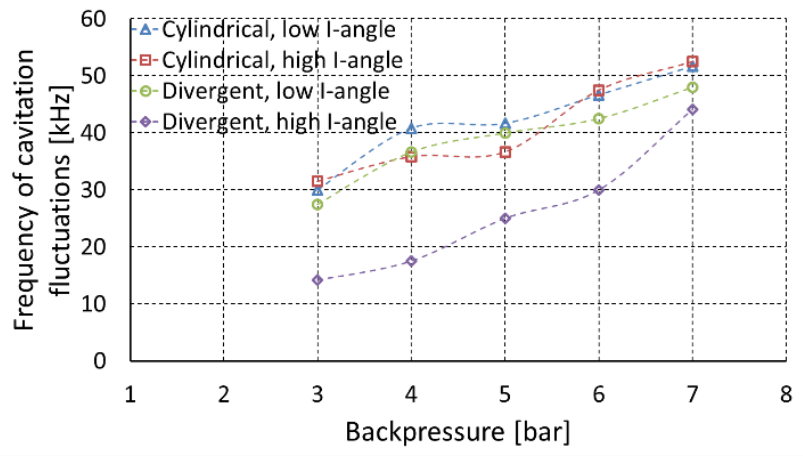

Figure 11. Dominant frequency of cavitation fluctuations vs. backpressure.

\section{Conclusions}

In this work, the internal cavitation behavior was visualized by means of optically accessible GDI nozzles and highspeed shadowgraphy. The detailed analysis of the transient cavitation characteristics was possible due to high temporal and spatial resolution of the images. The ambiguity between cavitation and ambient air drawn inside the nozzle holes was resolved by injecting into liquid fuel environment. The influence of drawn air was found to be 
negligible during the steady-state injection phase for the nozzle configuration used for this work. In addition, the cavitation behavior was investigated at elevated backpressures. It was found that the cavitation tends to fluctuate and the intensity of fluctuations is highly dependent on the backpressure being applied. It was shown that the divergent holes form cavitation structures that are more sustainable at elevated backpressure levels. The holes at low inclination angles, in turn, are subjected to higher fluctuations of cavitation.

\section{Acknowledgements}

The authors gratefully acknowledge the fruitful collaboration with Robert Bosch Corporate Research in Renningen. The invaluable advice of Dr. Kai Gartung, the expert support of Dr. Roman Grzeszik on the use of the high-speed shadowgraph imaging setup and the generous assistance of Andreas Herzig in the laboratory are greatly appreciated. This work could not have been completed without their support.

\section{Nomenclature}

$p_{i} \quad$ intensity of a, ' $i$ ' pixel [-]

$\overline{p_{l}} \quad$ average intensity of ' $\mathrm{c}$ ' pixel over the steady-state phase of an injection [-]

$\mathrm{N} \quad$ number of samples in a dataset [-]

$P \quad$ Probability [-]

RMSD Root-mean-square deviation [-]

\section{References}

[1] Payri, R., Molina, S., Salvador, F.J., Gimeno J., 2004, KSME International Journal, Vol. 18 No. 7, pp. 1222-1235. [2] Kufferath, A., Wiese, W., Samenfink, W., Dageförde, H., Knorsch, T., and Jochmann, P., March 10-11 2015, Fuel systems for IC engines.

[3] Knorsch, T., Rogler, P., Miller, M., and Wiese, W., Oct. 24-26 2016, SAE International Powertrains, Fuels \& Lubricants Meeting.

[4] Tamaki, N., Nishida K., and Hiroyasu, H., Jul. 6-8 1998, 14th International Conference on Liquid Atomization and Spray Systems.

[5] Sou, A., Hosokawa, S., Tomiyama, A., 2007, International Journal of Heat and Mass Transfer 50, pp. 35753582.

[6] Arcoumanis, C., Gavaises, M., Nouri, J. M., Abdul-Wahab, E. and Horrocks, R. W., 1998, SAE Technical Paper Series, 980811.

[7] Arcoumanis, C., Flora, H., Gavaises, M. and Badami, M., 2000, SAE Technical Paper Series, 200-01-1249.

[8] Aleiferis, P.G., Hardalupas, Y., Kolokotronis, D. and Taylor, A., Arioka, A., Saito, M., 2006, SAE Technical Paper Series, 2006-01-3374.

[9] Henry, M.E., Collicott, S.H., 2000, Atomization Sprays 10, pp. 545-563.

[10] Gilles-Birth, I., Rechs M. Spicher U., Bernhardt S., 2005, SAE Technical paper, 2005-01-3684.

[11] Walther, J., Schaller, J. K., Wirth, R., and Tropea, C., 2000, Eighth International Conference on Liquid Atomization and Spray Systems, Pasadena, CA, USA.

[12] Miranda R., Chaves H., and Obermeier F., Sep. 9.-11. 2002, 18th European Conference on Liquid Atomization and Spray Systems.

[13] He, Z., Zhang, Z., Guo, G., Wang, Q., Leng, X., and Sun, S., 2016, International Communications in Heat and Mass Transfer 78 13-20.

[14] Gavaises, M., Andriotis, A., 2006, SAE Technical Paper Series 2006-01-1114.

[15] Balewski, B., Heine, B., Tropea, C., 2010, Atomization and Sprays, 20 (1) , pp. 57-50.

[16] Gilles-Birth, A.I., 2008, „Optische Untersuchung der Düseninnenströmung von Sitzlochinjektoren für die Benzindirekteinspritzung", Dissertation, Universität Karlsruhe.

[17] Tao, Q., Lei, Y., 2016, Fuel 173, pp. 79-89. 


\author{
Binary cavitation in a transparent three hole GDI nozzle \\ Humberto Chaves ${ }^{* 1}$, Sebastian Donath ${ }^{1}$ \\ ${ }^{1}$ Institute for Mechanics and Fluiddynamics, Technical University of Freiberg, Germany \\ *Corresponding author: Humberto.Chaves@imfd.tu-freiberg.de
}

\begin{abstract}
A more or less real size three hole $\left(0.1 \mathrm{~mm}\right.$ diameter) transparent injection nozzle was made with $120^{\circ}$ between the orifices and an inclination of $20^{\circ}$ to the injector axis. The geometry is similar to that of a multi hole GDI injector. Experiments are performed using n-pentane and $\alpha$-methyl-naphtalene mixtures in varying composition. The flow in the orifices is observed under submerged injection conditions from downstream looking in direction of the injector using a beam splitter plate for illumination with the light from a Minilite NdYag laser that was made incoherent by fluorescence in a cuvette filled with a dilute rhodamine-ethanol mixture. The images show the appearance of cavitation depending on the cavitation number as well as on the composition of the mixture. The behaviour is not what can be expected from equilibrium thermodynamics. Due to the transient nature of the flow the $n$-pentane concentration cannot attain the equilibrium value one would expect and cavitation occurs at higher cavitation values. Diffusion appears to play a role in the onset appearance of cavitation for binary mixtures.
\end{abstract}

\title{
Keywords
}

Binary cavitation, real size transparent nozzle, fuel injection.

\section{Introduction}

The internal flow of gasoline direct injection nozzles has been investigated in the last years intensively. X-ray phase contrast imaging [1] allows visualizing the density gradients in the nozzle orifices of aluminium nozzles using very short high energy x-ray pulses from the APS Electron Storage Ring, Argonne National Laboratory. These images have shown that the flow can show hydraulic flip and cavitation in real size geometries. These results corroborate the results obtained in large scaled up transparent acrylic models of GDI multi-hole injectors, [2]. Characteristic for multi-hole injectors is the small length to diameter ratio of the orifices. The counter bore with a larger diameter does not have a direct contact with the fluid, [1] and is therefore mainly important for reducing the L/D ratio. Already in the early work with cavitating nozzles Lichtarowicz [3] noted "The function of the orifice bore, which must be at least one diameter long, is to stabilize the cavitation bubble. If the bore length is insufficient and the orifice tends to be a plate orifice cavitation occurs in bursts." This result is found in new results for GDI nozzles [4] that show flapping in timescales "in the order of a hundredth of a millisecond ". In the case of longer orifices the length of the cavitation zone in the orifice also fluctuates [5] with frequencies of about $29 \mathrm{kHz}$. Therefore, when taking instantaneous images of cavitation there will be large scatter of the cavitation length within the nozzle. There have been some real size transparent nozzles for Diesel injector geometries, e.g. [6-8]. However, the geometry of multi-hole GDI injectors is more complex and what is more demanding is that gasoline is an aggressive medium for acrylics. This is more so when ethanol is contained in the fuel. Therefore, transparent nozzles for gasoline fuels have to be made of glass. To date no real size transparent nozzle for GDI multi-hole injectors is known to the authors. This is why measurable spray and nozzle properties are compared with the results of CFD simulations, e.g. $[9,10]$. Glass is a material that imposes limitations to the length to diameter ratio that can be achieved. Due to the brittleness of glass very short nozzles would require some kind of support as was done by [6] for acrylic nozzles for diesel injection. In the present paper a first step to develop a nozzle type and methodology that allows to study real size GDI nozzle flow. The length of the orifices in the present case is not yet realistic.

Gasoline fuels are never pure liquids except for a few comparison tests. They are composed of many different components that have a large range of fuel properties. The most important one for cavitation in the orifices of injectors is the vapour pressure. In the case of a mixture of pure liquids an equilibrium boiling line can be derived using thermodynamics. In mixture thermodynamics the concentration of each component is calculated for each phase. Except for azeotropic mixtures it does not have the same value in each phase. This is the basis for distillation. However, the flow in the orifices of injection nozzles is a very transient process and there is no time for establishing equilibrium. This is the basic question behind this work. 


\section{Material and methods}

The core of the work presented here is the development of a transparent multi-hole real size nozzle similar to a gasoline direct injection (GDI) nozzle. There have been various concepts for transparent nozzles [e.g. 11,12], they have in common that they use acrylics as a nozzle material. This material can be used when looking into diesel injection nozzles, however is has the drawback that when using ethanol as a fuel, the nozzle will only survive a couple of injections. Many of the modern gasoline blends contain ethanol and other components that will sooner or later damage the nozzle. Therefore, glass was chosen as a material for the nozzle. Glass is a material that requires a completely different handling than acrylics. Most of the work was performed on a diamond wire saw controlled with a microscope coupled to a CCD camera.
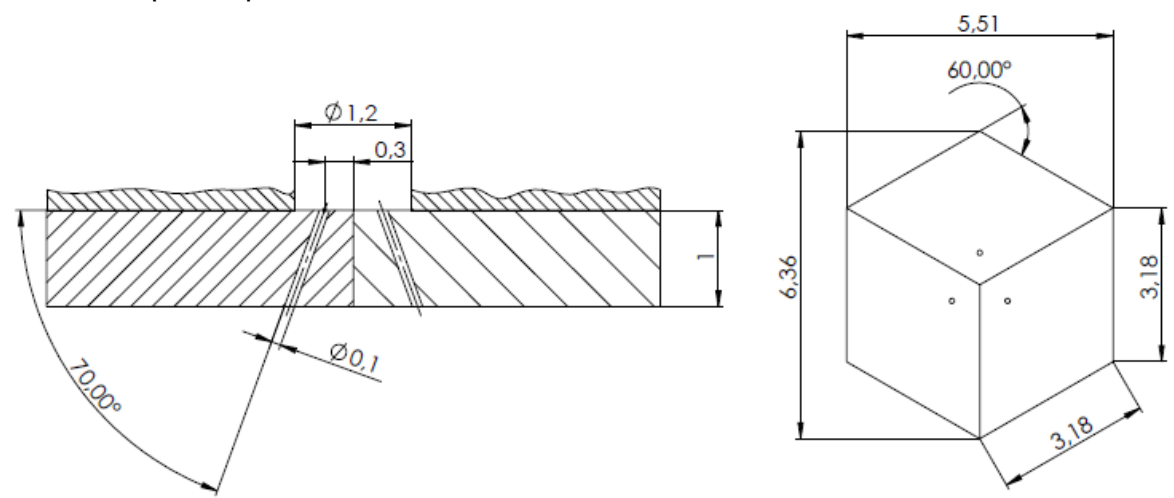

Figure 1. side view and top view of the glass nozzle. It is composed of three rhombic slides of glass each with one orifice.

Once the three rhombic slides, Figure 1 had been cut they were polished and then glued onto a diesel injector which had been ground down to the tip of the needle as can be seen on Figure 2. The first crucial part of this procedure is the positioning of the three rhombi relative to shortened sac hole of the injector, which is performed under a binocular microscope. The second difficulty was placing minute amounts of epoxy glue between the rhombi using a needle tip to seal the contact lines between them. Capillary forces let the glue flow into the thin crevice between the rhombi and form a smooth surface. Once the right position and amount of glue had been achieved the whole injector was put in an oven for curing the epoxy glue, after many trials.

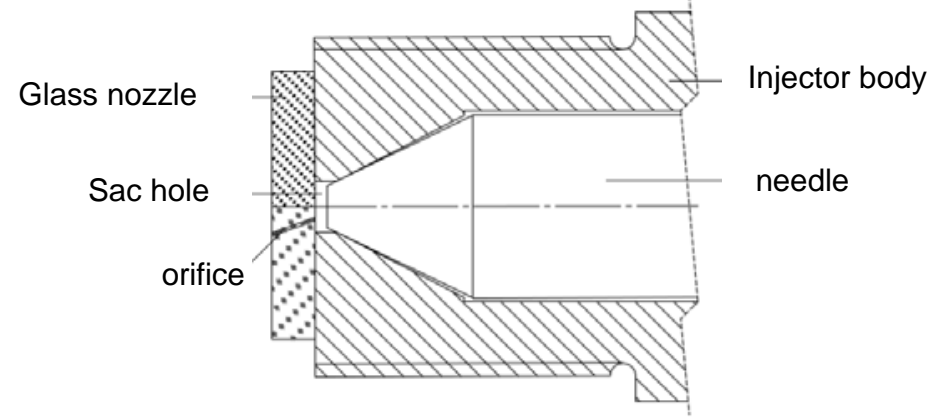

Figure 2. side view of the tip of the injector with the glass nozzle each with one orifice.

Figure 3 shows the experimental set-up. The injector tip is inserted into the injection chamber and pressed to seal the system. The fuel is filled by gravity into the not yet pressurized reservoir, then the appropriate valves are closed and the reservoir is pressurized using nitrogen from a bottle. A Bourdon pressure gauge is used to preset the injection pressure. Both the pressure histories of injection and back pressure are measured using Kistler absolute pressure sensors attached to the corresponding bridge amplifiers. Both these signals as well as the trigger signals for the Nd-YAG pulse laser are recorded simultaneously on a LeCroy digital oscilloscope, not shown here. This allows measuring the exact pressure levels present at every instant of image acquisition. The images are recorded at 14 images per second. The camera delivers the appropriate trigger signals and is started with the same signal that opens the magnetic valve that starts the injection. The back pressure can be preset using compressed air but usually it starts at atmospheric pressure and due to the displacement of air in the injection chamber by the injected fuel the air contained in the compression vessel is compressed. This leads to a slow variation of back pressure whilst the injection pressure remains constant. Every new run will give a series of images for an increasing back pressure and therefore cavitation number. 


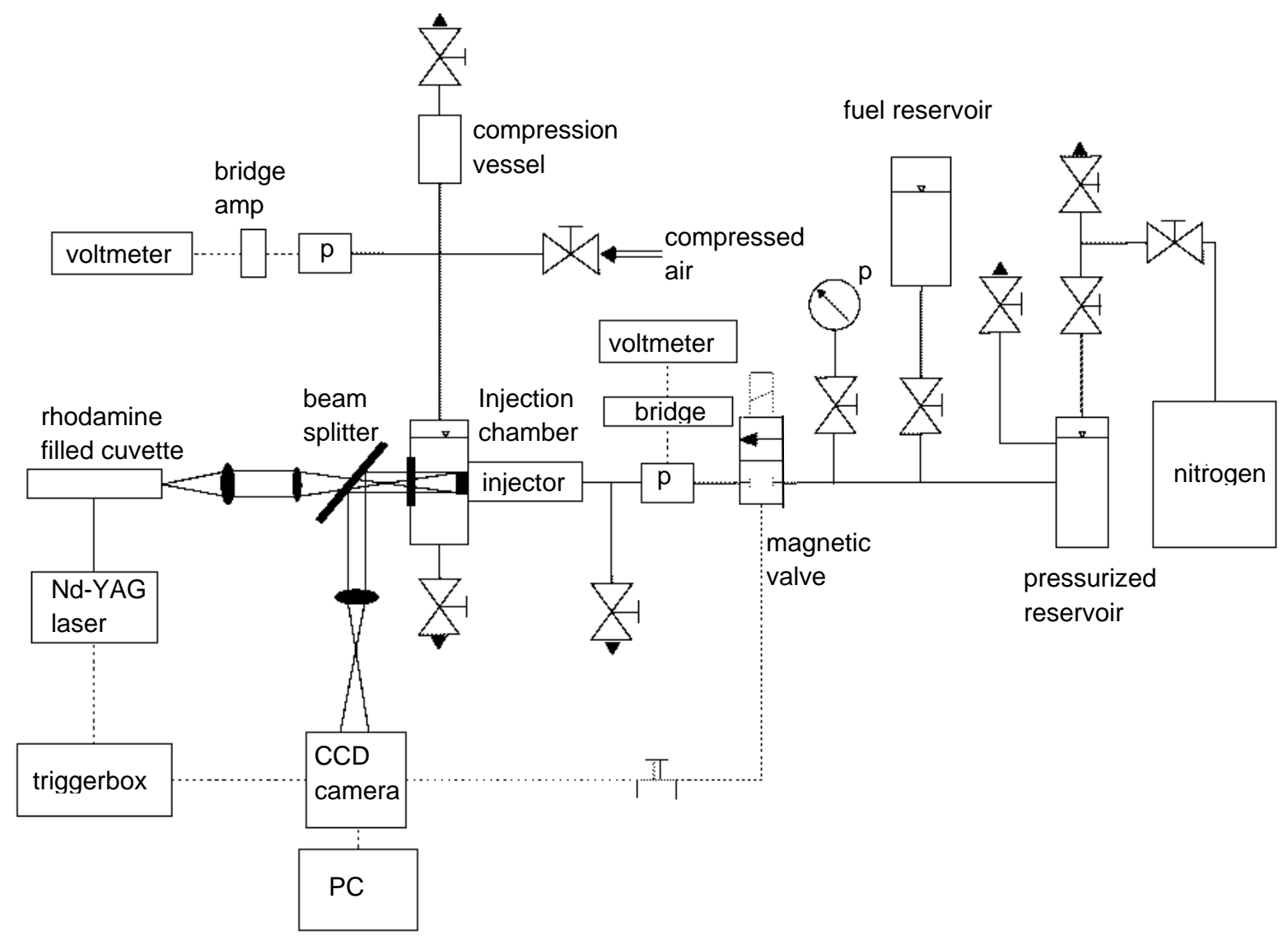

Figure 3. Schematic diagram of the experimental set-up.

The optical set-up results from the nozzle geometry and the limited depth of field of microscopic imaging. It is not possible to image all the orifices with an acceptable resolution when looking from the side as has been previously done for single orifices or for orifices that are aligned in one plane, see e.g. [13]. Here, a different approach was chosen. Both the top surface of the injector on which the nozzle is glued as well as the tip of the injector needle has been polished. This allows illuminating from the downstream side. Simultaneous observation is achieved with a beam splitter plate positioned behind the injection chamber window opposite to the injector tip. Figure 4 shows four images taken with no flow but a different fuel filling of the injection chamber. The volume fraction $\varphi_{2}$ of $\mathrm{n}$ pentane in the mixture with $\alpha$-methyl-naphtalene has been varied. The resulting difference of refractive index relative to the refractive index of the glass of the nozzle is shown above each image.

On Figure 4 various observations can be made. First one sees the polished but scratched metallic surface of the tip of the injector body and the needle tip. We decided to leave the scratches since they allow a simple focusing on the plane of the orifice inlets. The second obvious features are the glue joints between the rhombi that compose the nozzle. They appear dark due to the strong refractive index mismatch relative to the glass. The inner dark circle is the shadow of the sac hole on the needle. One can also see that the needle is at different rotation angles using e.g. the scratches or a dark feature at the rim. However, the most important features are the nozzle orifices. They can be clearly seen on image a). There are to be some dark shadows on the image of the orifices in this case. The refractive index mismatch between the glass and the fluid causes reflections and refractions at the interface leading to these intensity modulations. With decreasing volume fraction of $n$-pentane in the mixture the refractive index increases. In the case of image $c$ ) there is a match between the fluid and the glass. The orifices seem to disappear. In the case d) where the index of the fluid is higher than that of the glass the spurious reflections disappear. It requires some practice and comparison with these images with no flow to distinguish the reflections from cavitation voids. One can also observe that the orifice inlets are sharply imaged whereas the outlets are out of focus. This is due to the limited depth of field of the microscope objective that was used, about $0.3 \mathrm{~mm}$. It is a compromise between depth of field, optical resolution, optical sensitivity and working distance. It has a working distance of $50 \mathrm{~mm}$, which may appear large but keep in mind that a beam splitter plate and the injection chamber with its window are installed between the nozzle and the front lens of the objective. Its aperture gives both a good resolution and a moderate depth of field. However, even with a fully open aperture and the 
a) $n=1.454 \quad \varphi_{2}=0.64 \quad \Delta n_{r e l}=-0.036$

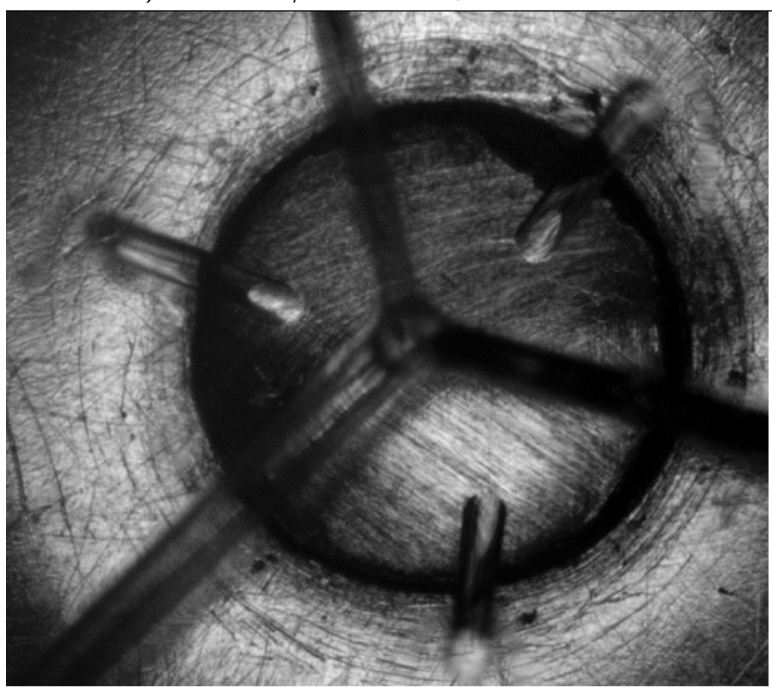

c) $n=1.509 \quad \varphi_{2}=0.45 \quad \Delta n_{r e l}=0$

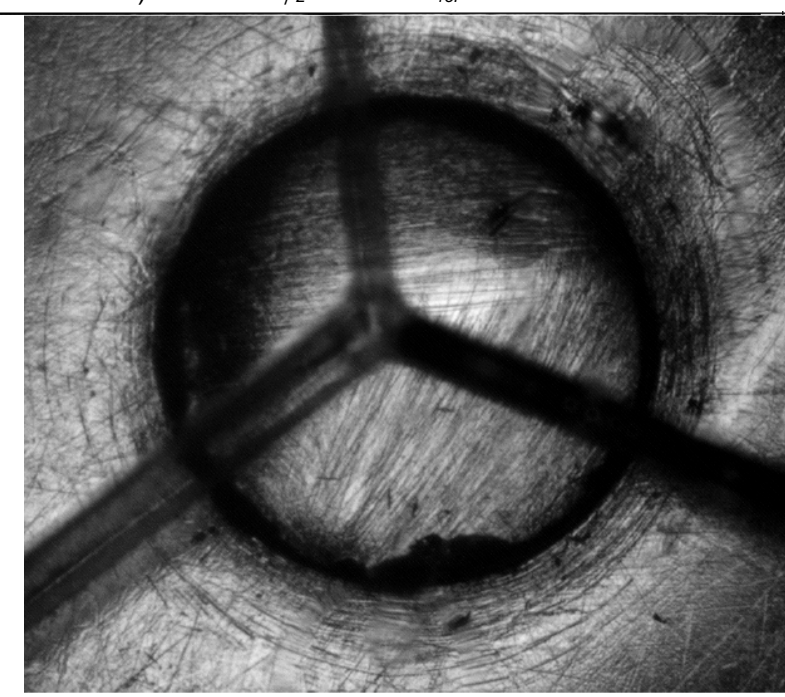

Figure 4. Optical image quality depending on the volume fraction $\varphi_{2}$ of $n$-pentane in the mixture with $\alpha$-methyl-naphtalene, the

dark circle in the middle is the shadow of the sac hole of $1.2 \mathrm{~mm}$ in diameter

b) $n=1.474 \quad \varphi_{2}=0.58 \quad \Delta n_{\text {rel }}=-0,023$

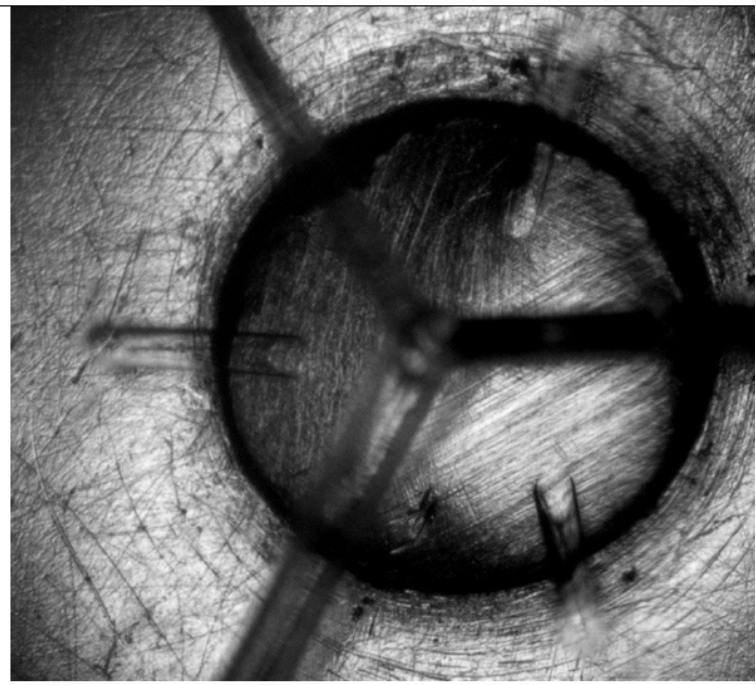

d) $n=1.522 \quad \varphi_{2}=0.4 \quad \Delta n_{r e l}=0.0009$

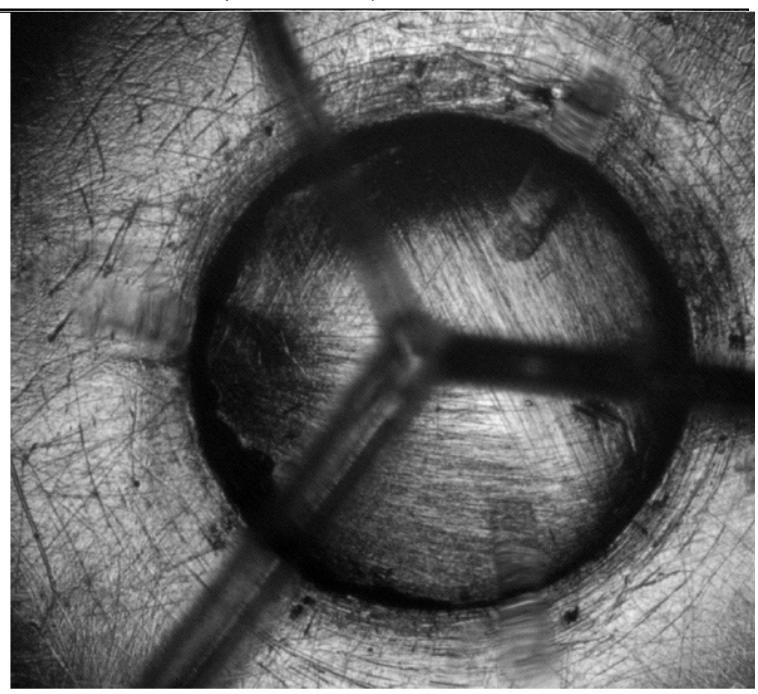

sensitivity is relatively low also due to the illumination from the view direction. This is the reason for the use of a more energetic pulsed light source. For back lit images LEDs or a Nanolite flash lamp are sufficient, see [e.g. 13,14]. Here, a frequency doubled Nd-YAG laser, MiniLite is used. To get rid of the coherence the laser light it is pointed at a small cuvette with a dilute solution of Rhodamine in ethanol. The fluorescence light from the fluorophore is collected with lenses at right angle to the incidence direction of the laser light and focused onto a plastic optical fibre, not shown on Figure 3. The other end of the fibre is the incoherent pulsed light source with $5 \mathrm{~ns}$ pulse width and sufficient energy to modulate the images. Due to the fluorescence the peak intensity is at wavelength of $590 \mathrm{~nm}$ vs. $532 \mathrm{~nm}$ of the laser.

Table 1 shows a list of the properties of the binary mixtures used for the experiments. Also two vapour pressure values are included; one based on an ideal mixture and one using a molecular simulation model (COSMOThermX). The later value is used to calculate the cavitation number for each image acquired since little data is available on this binary mixture. Here the definition of the cavitation number introduced by Bergwerk [15] is used:

$$
C a=\left(p_{i n j}-p_{c h}\right) /\left(p_{c h}-p_{v m}\right)
$$


Table 1. List of the binary mixtures of n-pentane with $\alpha$-methyl-naphtalene used for the experiments

\begin{tabular}{c|ccc}
\hline \hline $\begin{array}{c}\text { Refractive index } \\
\text { measured: } n\end{array}$ & $\begin{array}{c}\text { Volume fraction } \\
\text { n-pentane: } \varphi_{2}\end{array}$ & $\begin{array}{c}\text { Vapor pressure: } p_{v i} \\
\text { Ideal mixture }[\mathrm{hPa}]\end{array}$ & $\begin{array}{c}\text { Vapor pressure: } p_{v m} \\
\text { Molecular simulation }[\mathrm{hPa}]\end{array}$ \\
\hline \hline 1.454 & 0.65 & 386.2 & 431.1 \\
1.474 & 0.58 & 354.1 & 409.8 \\
1.509 & 0.45 & 281.9 & 357.8 \\
1.522 & 0.4 & 253.1 & 334.4
\end{tabular}

\section{Results and discussion}

Every experiment is initiated by opening of the magnetic valve. It last for 2 to 3 seconds giving a sequence of 30 to 40 images with increasing cavitation number. Once the image and oscilloscope data have been stored a next run can be initiated provided there is still enough fuel in the pressurized reservoir. Due to the way the nozzle has been produced and to the wish of having complete sets of data for all mixtures before the nozzle breaks the maximum injection pressure used up to now has been limited to ca. 1.5 MPa. This is however sufficient to cover a range of cavitation numbers which are characteristic for the onset and extent of cavitation to the orifice exit. At much higher cavitation numbers there a few visible differences in the nozzle flow; cavitation always reaches up to the exit of of the orifice, i.e. supercavitation. Figure 5 shows exemplarily four images taken from one run with increasing chamber pressure, constant injection pressure and thus a variation of the cavitation number. One can see that the cavitation behaviour is different for all three orifices. This is due to the differences of the inlet corner of each orifice. Glass has the disadvantage that it can chip and standard microscope images with a large magnification have shown that the left orifice inlet is chipped. This disturbance promotes cavitation. The right orifice is also slightly chipped whereas the middle one has a smooth edge. It is not oriented to the injector axis. The other orifices also show a deviation from symmetry. The nozzle is "handmade". This is occurs however also in series injectors.

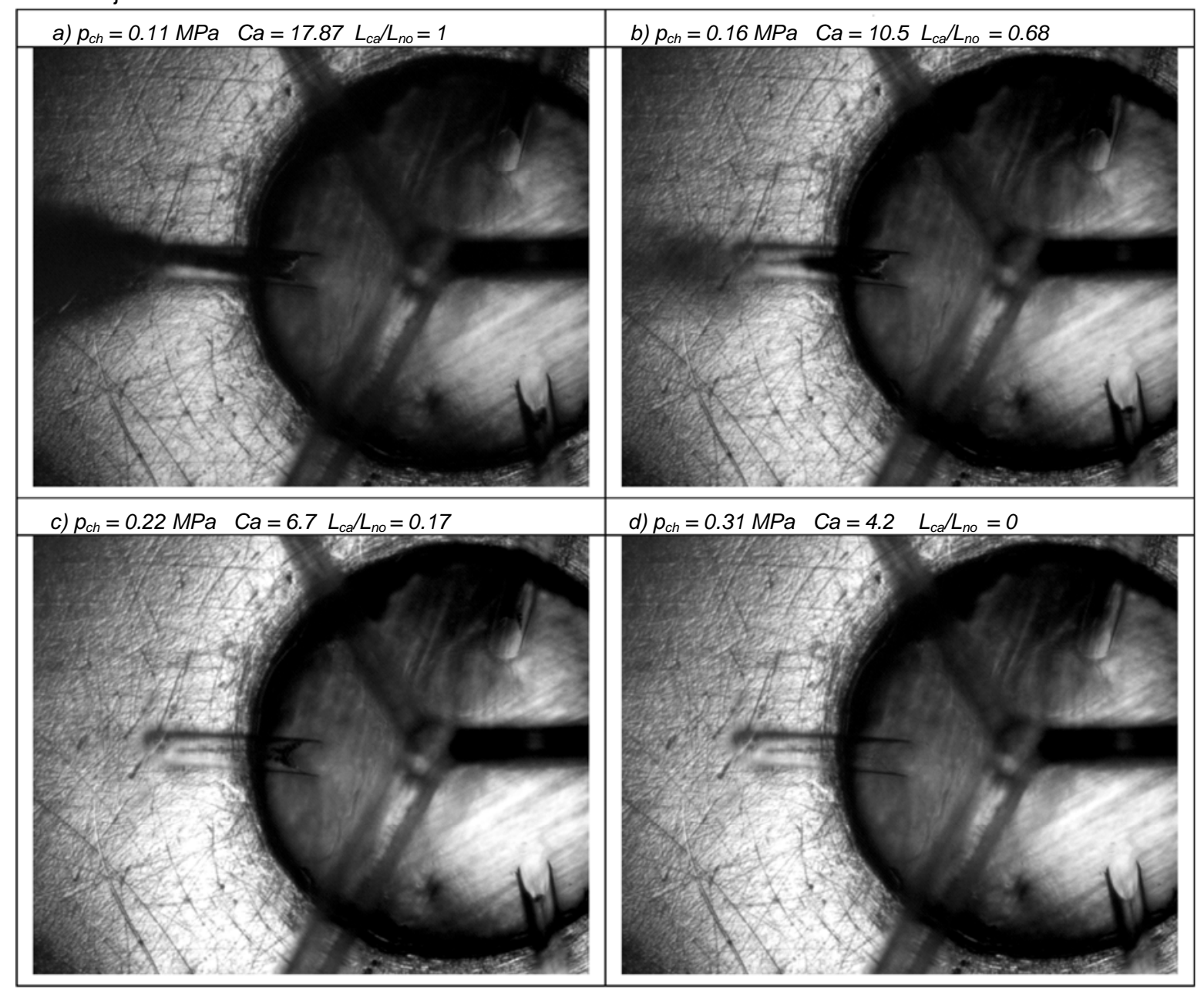

Figure 5. Development of cavitation during a run for a volume fraction of $n$-pentane $\varphi_{2}=0.58$, an injection pressure $p_{i n j}=1.42 \mathrm{MPa}$ and a relative index of refraction $\Delta n_{r e}=-0,023$, the cavitation length shown is for the left orifice. 
In the following the length of the cavitation voids normalized with the orifice length $L_{c a} / L_{n o}$ will be discussed. In the plots each point corresponds to one image and the orifice on the left of the images in figure 5. Firstly, we look at the dependence on the injection pressure shown in Figure 6.

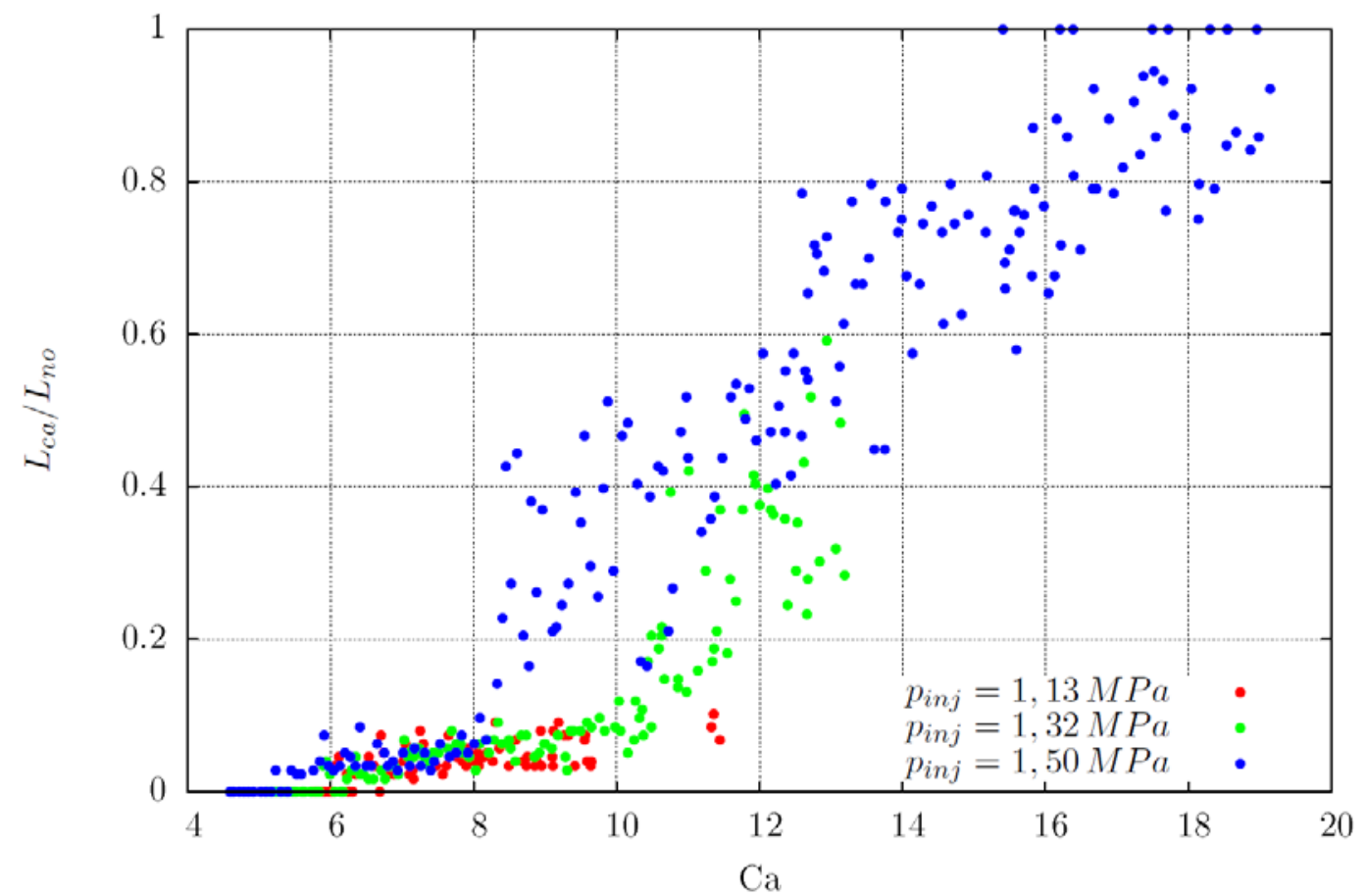

Figure 6. Normalized cavitation length vs. cavitation number for different injection pressures for a mixture with a volume fraction of $n$-pentane $\varphi_{2}=0.45$.

When comparing these results with those for pure liquids [4] the transition of the length of the cavitation voids from a localized area near the inlet of the orifice to supercavitating conditions occurs over a larger span of cavitation numbers. The results for pure n-pentane would range from 3 to 10 independent of the inlet radius. Here it is almost doubled.

It is therefore interesting to look at the dependence of cavitation length for the same injection conditions but for a variation of composition shown in Figure 7. The behaviour of the mixture with the highest $n$-pentane content comes close to what had been found in [4] for pure n-pentane. With decreasing n-pentane content however the extent of cavitation decreases rapidly. Please bear in mind that the dependence on vapour pressure of the cavitation number has been taken into account by using the vapour pressure of the corresponding mixture. Obviously something is happening here which can not be accounted for by equilibrium thermodynamics. The resulting hypothesis is that a concentration gradient of $n$-pentane appears in the liquid at the interface to the void because $n$-pentane evaporates at the interface, the aromatic component has a much lower vapour pressure and fugacity. Therefore the concentration of $n$-pentane is reduced and diffusion is now limiting since is a relatively slow process. One could argue that the length scales are small. However, the transit time of the liquid through the nozzle is very short also, a few microseconds, and even shorter near the inlet. This could explain why with reduced $n$-pentane volume fraction in the liquid the voids do not grow to the same extent. 


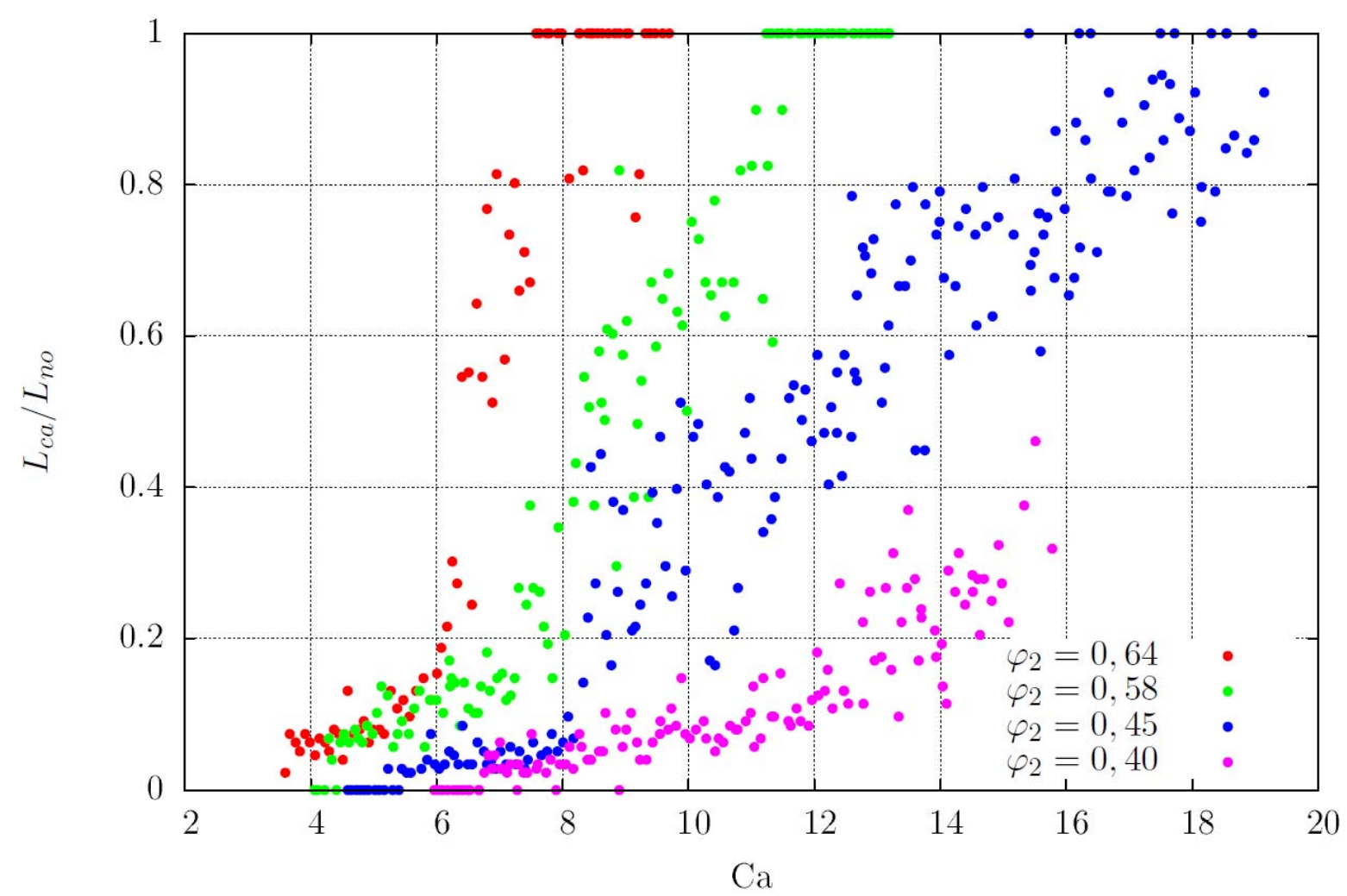

Figure 7. Normalized cavitation length vs. cavitation number for different for various mixtures (volume fraction of $\mathrm{n}$-pentane $\varphi_{2}$ ) at an injection pressure of about $1.5 \mathrm{MPa}$.

\section{Conclusions}

The main conclusion of this paper is that nozzle cavitation depends strongly on the concentration of the components with higher vapour pressure of a mixture. A thermodynamic equilibrium of the concentration of each component at the interface between the phases is not reached within the nozzle.

\section{Acknowledgements}

The authors would like to thank Ricard Miranda. He conceived the idea to build a multi-hole nozzle in the way shown here and to Antonio Ortega Planes for his endurance, patience and precision in cutting and polishing the rhombi for the nozzle.

\section{Nomenclature}

Ca cavitation number

$L_{c a} \quad$ length of cavitation zone in the orifice

$L_{n o} \quad$ orifice length

$n \quad$ refractive index [-]

$n_{f l} \quad$ refractive index of fluid mixture [- ]

$n_{g l} \quad$ refractive index of glass of the nozzle [-]

$\Delta n_{\text {rel }} \quad=\left(n_{f l}-n_{g l}\right) / n_{g l}$, relative difference in refractive index between fluid and glass [-]

$p_{i n j} \quad$ injection pressure [Pa]

$p_{c h} \quad$ chamber or back pressure $[\mathrm{Pa}]$

$p_{v m} \quad$ vapour pressure from molecular simulations[Pa]

$p_{v i} \quad$ ideal mixture vapour pressure [Pa] 


\section{References}

[1] Moon, S., Komada, K., Li, Z. , Wang, J., Kimijima, T., Arima, T., Maeda, Y., 2015, Proc. ICLASS 2015, 13th Triennial International Conference on Liquid Atomization and Spray Systems

[2] Mirshahi, M., Nouri, J., Yan, Y., Gavaises, M., Link between in-nozzle cavitation and jet spray in a gasoline multi-hole injector, 2013, Proc. 25th European Conference on Liquid Atomization and Spray Systems

[3 Lichtarowicz, A., 1972, Nature Physical Science Vol. 239

[4] Rewse-Davies, Z., Nouri, J., Gavaises, M. and Arcoumanis, C., Proc. 25th European Conference on Liquid Atomization and Spray Systems, 2013

[5] Marcer, R., Le Cottier, P., Chaves, H., Argueyrolles, B., Habchi, C., Barbeau, B., 2000, SAE Paper 2000-012932

[6] Falgout, Z., Linne, M., 2015, Journal of Physics: Conference Series 656012082

[7] Miranda, R., Chaves, H., Martin, U. and Obermeier, F., 2003, Proc. 9th International Conference on Liquid Atomization and Spray Systems

[8] Payri, R., Salvador, F.J., Gimeno, J., Venegas, O., 2013, Experimental Thermal and Fluid Science 44, pp. 235-244

[9] Payri, R., Gimeno, J., Marti-Aldaravi, P.\& Vaquerizo, D., 2015, Atomization and Sprays, 26(9), pp. 889-919 [10] Bode, M., Falkenstein, T., Davidovic, M., Pitsch, H. et al., 2017, SAE Int. J. Fuels Lubr. 10(2), doi:10.4271/2017-01-0848.

[11] Falgout, Z. and Linne M., 2016, Review of Scientific Instruments 87, 085108.

[12] Serras-Pereira, J., van Romunde, Z., Aleiferis, P.G., Richardson, D., Wallace, S., Cracknell, R.F., 2010, Fuel 89, pp. 2592-2607.

[13] Miranda, R., Chaves, H., Martin, U. and Obermeier, F., July 13.-17. 2003, 9th International Conference on Liquid Atomization and Spray Systems.

[14] Chaves, H., Bauz, F., Martinez Lopez, E., Sep. 4.-7. 2016, 27th Annual Conference on Liquid Atomization and Spray Systems.

[15] Bergwerk, W., 1959, Proceedings of the Institution of Mechanical Engineers, 173(1), pp. $655^{\perp} 660$. 
$\underset{\text { Droplet } 7}{10 Y}$ 


\title{
Models for automotive fuel droplets heating and evaporation

\author{
Mansour al Qubeissi ${ }^{1, *}$, Sergei S Sazhin ${ }^{2}$,Nawar Al-Esawi ${ }^{1}$ \\ ${ }^{1}$ Centre for Mobility \& Transport, Faculty of Engineering, Environment and Computing, \\ Coventry University, Coventry, UK \\ ${ }^{2}$ Advanced Engineering Centre, School of Computing, Engineering and Mathematics, \\ University of Brighton, Brighton BN2 4GJ, UK
}

${ }^{*}$ Corresponding author: mansour.qubeissi@coventry.ac.uk

\begin{abstract}
The paper presents recent approaches to the modelling of heating and evaporation of automotive fuel droplets with application to biodiesel, diesel, gasoline, and blended fuels in conditions representative of internal combustion engines. The evolutions of droplet radii and temperatures for gasoline, diesel, and a broad range of biodiesel fuels and their selective diesel fuel blends have been predicted using the Discrete Component model (DCM). These mixtures combine up to 112 components of hydrocarbons and methyl esters. The results are compared with the predictions of the case when blended diesel-biodiesel fuel are represented by pure fossil and biodiesel fuels. In contrast to previous studies, it is shown that droplet evaporation time and surface temperature predicted for $100 \%$ biodiesel (B100) are not always close to those predicted for pure diesel fuel. Also, the previously introduced MultiDimensional Quasi-Discrete model and its application to these fuels and their mixtures are discussed. The previous application of this model has resulted in up to $96 \%$ reduction in CPU time compared to the case when all fuel components are considered using the DCM.
\end{abstract}

\section{Keywords}

Biodiesel, Diesel, Fuel droplet, Fuel blends, Gasoline

\section{Introduction}

There have been several attempts to simulate fuel droplets heating and evaporation. These have been either based on the analysis of individual components, the discrete component model (DCM) [1-3], or on the probabilistic analysis of a large number of components (the continuous thermodynamics [4-6] and the distillation curve ([7-9] models]. In most studies, several simplifying assumptions have been made, e.g. species inside droplets mix infinitely quickly (infinite diffusivity (ID) model) or do not mix at all (the single component (SC) model). Also, the temperature gradients inside droplets have been ignored in most cases with the assumption that the liquid thermal conductivity is infinitely large (infinite thermal conductivity (ITC) model). This paper will present our recent approaches to address these gaps in literature.

Based on recent research findings (e.g. [3,10-13]), the drawbacks in modelling fuel droplets heating and evaporation processes (computationally expensive models, ignoring temperature gradient and transient species diffusion) are partially addressed using the MDQD model. This paper summarises some comparisons between the results, referring to fuel droplet evaporation times and time evolution of droplet surface temperatures and radii, predicted by the previously suggested simplified models, the recently developed version of the DCM and the multidimensional quasi-discrete model (MDQDM) [14-16]. The latter two models take into account the recirculation, temperature gradient, and diffusion of species inside the droplets, based on the Effective Thermal Conductivity and Effective Diffusivity (ETC/ED) models.

In the following section (Models), the main principles of the DCM and MDQDM are summarised. The results of using these models for the analysis of heating and evaporation of biodiesel, diesel and gasoline fuel droplets, and their blends are reviewed in the Sections: Biodiesel fuel droplets, Diesel Fuel Droplets, Gasoline fuel droplets, and Blended diesel-biodiesel fuel droplets, respectively. The impact of in-cylinder conditions on these fuel droplets' lifetimes are presented in the Section of 'Impact of in-cylinder conditions'. The results are summarised in the Section of Conclusions.

\section{Models}

In the case of small number of components (e.g. biodiesel fuels), DCM approach (described in $[2,10,13,17]$ ) is easily implemented, where the number of components (up to 14 components) are relatively small. In the DCM analyses, we assume that droplets are spherically symmetric but temperature gradients and species diffusions in the liquid phase and the effect of internal recirculation due to relative velocity between ambient gas and droplets are all accounted for, using the Effective Thermal Conductivity and Effective Diffusivity (ETC/ED) models. 
In the cases of diesel, gasoline, and diesel-biodiesel blended fuels, the MDQDM approach, in which the actual composition of fuel is reduced to a much smaller number of representative components/quasi-components (C/QC), is used. These components/quasi-components are formed within groups of components (e.g. 9 groups of diesel fuel species, 6 groups of gasoline fuel species, and 4 groups of FAME (Fatty Acid Methyl Ester) biodiesel fuel species). Some components within groups form quasi-components, while other components are considered separately described as characteristic components. Thus, a mixture of components/quasi-components (C/QC) is formed in such a way that molar fractions of these $\mathrm{C} / \mathrm{QC}$ are as close as possible (for further details about this approach, see [12,14-16]).

We assume droplets with several initial homogeneous temperatures and radii within the ranges of $300-360 \mathrm{~K}$ and 10-15 $\mu \mathrm{m}$, respectively, are moving through air at relative velocities of $U_{d}=0-35 \mathrm{~m} / \mathrm{s}$, under ambient temperatures and pressures of 500-800 K and 5-50 bar, respectively.

The verification of the selected droplet SMD and velocities have been made using ANSYS-Fluent 17.2 simulation; typical examples of which are shown in Figures 1 and 2 using realizable $k-\varepsilon$ model and air-blast atomizer for injection.
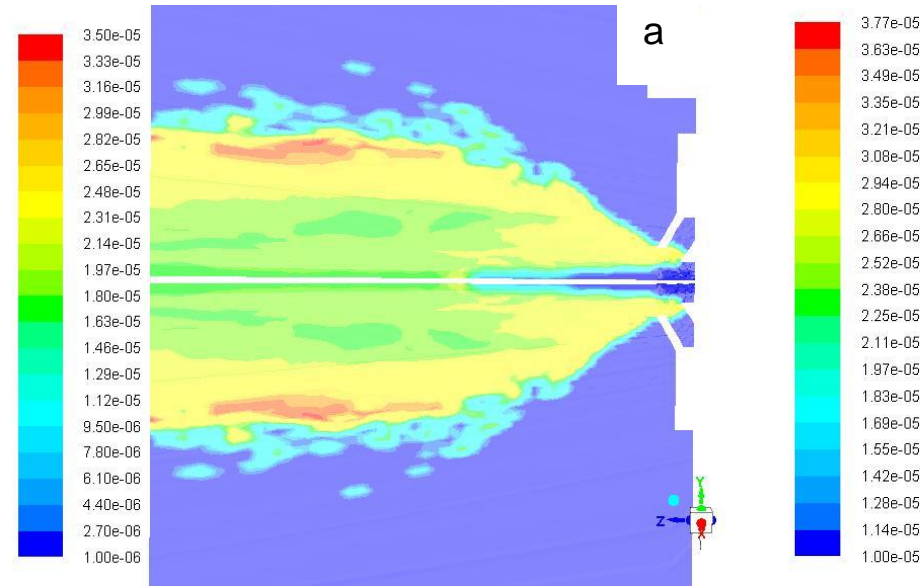

Figure 1. SMD and droplets distribution of a diesel fuel spray in conditions averaged from those presented in this paper, showing the contour gradient of SMD (a) and space distribution (b), produced using ANSYS-Fluent 17.2.
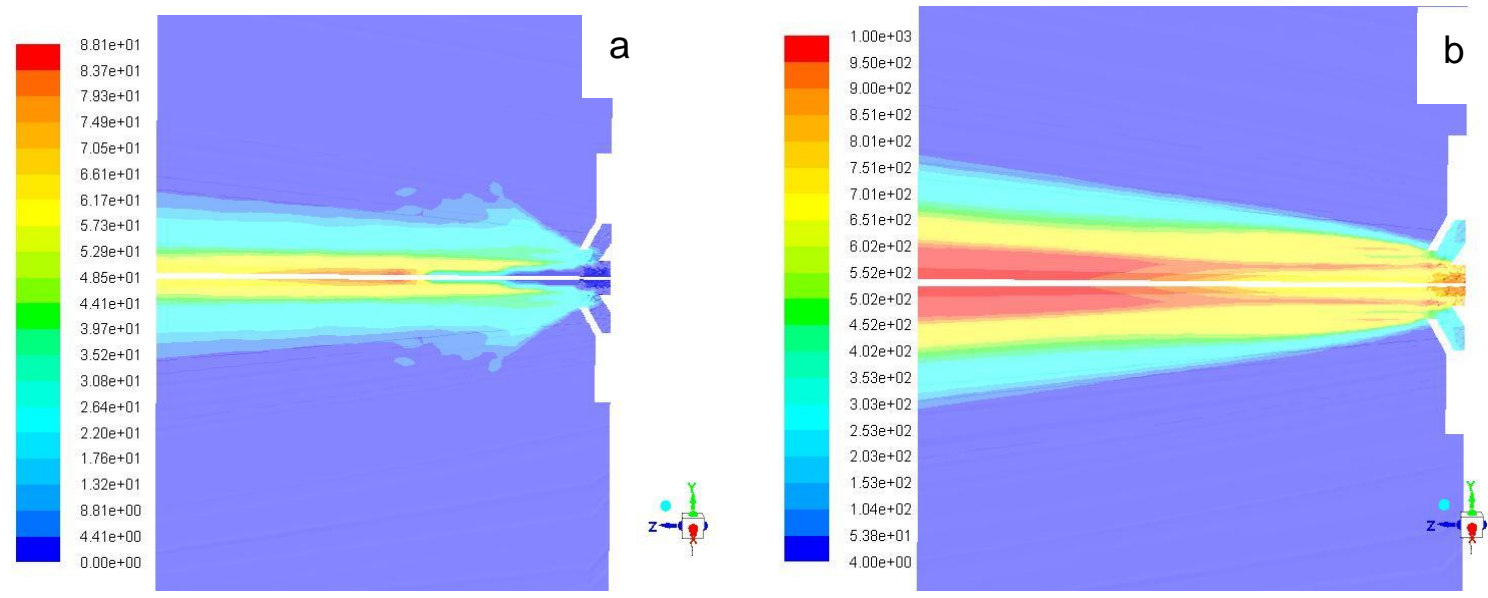

Figure 2. Droplets velocity gradient (a) and turbulence intensity (b) of diesel fuel spray in conditions averaged from those presented in this paper, produced using ANSYS-Fluent 17.2.

\section{Biodiesel fuel droplets}

The interest in biodiesel fuels has been mainly stimulated by depletion of fossil fuels and the need to reduce emissions that contribute toward climate change [18]. Biodiesel fuel can be blended with fossil fuels and used in many different concentrations. For instance, a mixture of $95 \%$ diesel and $5 \%$ biodiesel (B5) fuel can be called diesel fuel, with no separate labelling required at the pump [19]. Hence, it is essential to investigate the validity of this assumption based on estimated droplets lifetimes of both, fossil and its FAME biodiesel blended, fuels. 
Both, the DCM and MDQDM, approaches are applied to 22 types of FAME biodiesel fuel droplets. These methyl esters are: tallow (TME), lard (LME), butter (BME), coconut (CME), palm kernel (PMK), palm (PME), safflower (SFE), peanut (PTE), cottonseed (CSE), corn (CNE), sunflower (SNE), soybean (SME), rapeseed (RME), linseed (LNE), tung (TGE), hemp-oil, produced from hemp seed oil in the Ukraine (HME1), hemp-oil, produced in European Union (HME2), canola (CAN), waste cooking-oil (WCO), yellow grease oil (YME), camelina (CML), and jatropha (JME). A typical example of applying the MDQDM to FAME biodiesel fuel is shown for waste cooking oil (WCO) methyl esters in Figure 3; in which 14 methyl esters of the fuel are reduced to 5, 4 and 3 C/QC [20].

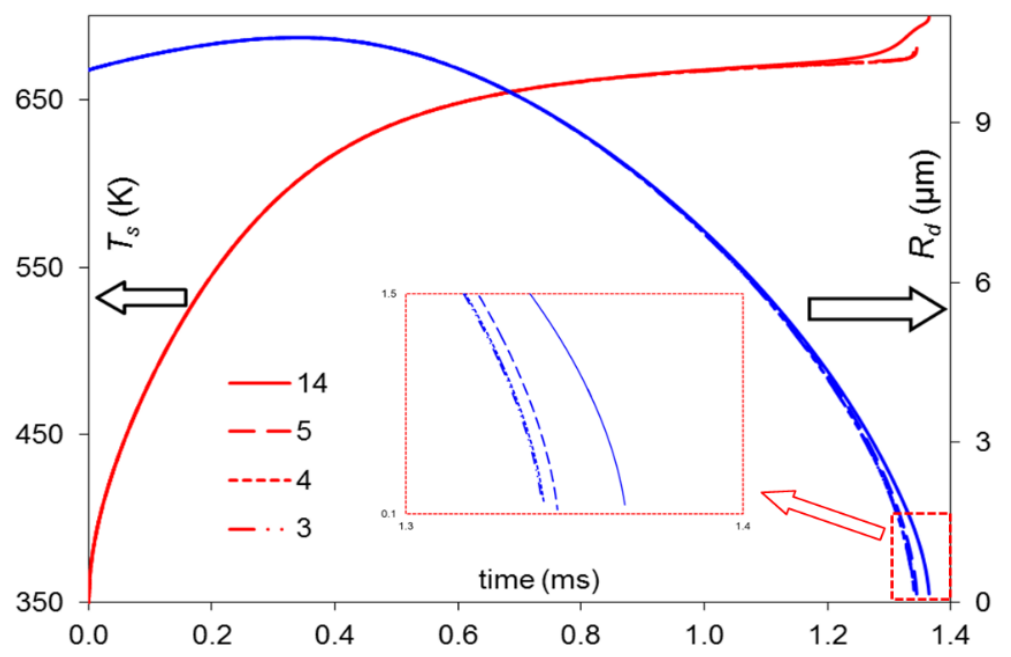

Figure 3. The plots of droplet surface temperatures $\left(T_{s}\right)$ and radii $\left(R_{d}\right)$ versus time for various WCO fuel using the MDQDM. The initial droplet radius and temperature were $10 \mu \mathrm{m}$ and $350 \mathrm{~K}$ respectively. The ambient gas temperature and pressure were 800 $\mathrm{K}$ and 30 bar respectively. The droplet was moving at constant velocity $10 \mathrm{~m} / \mathrm{s}$ in still air.

As can be seen from Figure 3, replacing the full composition of WCO methyl esters with 5, 4 and 3 C/QC produces almost identical plots for the evolutions of $T_{s}$ and $R_{d}$. The predicted errors in estimating $T_{s}$ evolutions of these C/QC approximations compared to those for 14 components are less than about $2 \%$, while the same errors for the prediction of droplet lifetimes are less than about $1.8 \%$. Hence, there is no noticeable reduction in the accuracy of predicting the droplet lifetimes or surface temperatures due to the implementation of the MDQD model. At the same time, using the MDQD model improves computational efficiency significantly. For B100 WCO, the MDQD model resulted in a reduction of up to $96 \%$ in computational time compared with the case when the original 14 components are considered using the DCM.

\section{Diesel fuel droplets}

The plots of typical evolutions of droplet surface temperatures $T_{s}$ and radii $R_{d}$ versus time for diesel fuel droplet as predicted by the DCM and MDQDM approaches, accounting for the contributions of all 98 components, are shown in Figure 4. The plots in this figure illustrate 4 cases as presented in [14,15]: the contributions of all 98 components are taken into account using the DCM (labelled as "(98)"); the contributions of only 20 alkane components are taken into account using the ID/ITC model (following the approximation used in [21,22]) (labelled "(20A)"); the contributions of all 98 components are approximated by $15 \mathrm{C} / \mathrm{QC}$ using the MDQDM; and the contribution of only $\mathrm{n}$-dodecane is taken into account using the SC model. As can be seen from this figure, the approximation of Diesel fuel by classical approaches, such as 20 alkane components, SC (e.g. n-dodecane), ITC and ID models, lead to under-estimation of droplet evaporation time by up to $50 \%$, which are not acceptable in many engineering applications. At the same time, the approximation of 98 components of Diesel fuel by $15 \mathrm{C} / \mathrm{QC}$ leads to acceptable prediction of less than $3 \%$ error compared to the droplet lifetime predicted by the DCM, which can be acceptable in most applications. 


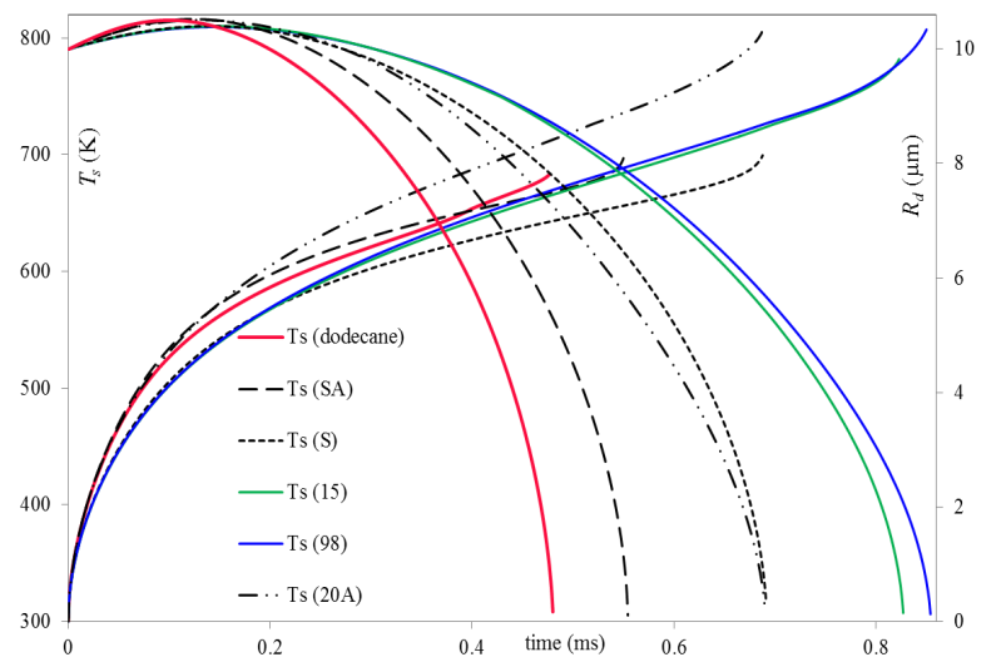

Figure 4. The plots of $T_{s}$ and $R_{d}$ versus time for a diesel fuel droplets, predicted using the MDQDM (indicated as15), $\mathrm{SC} / \mathrm{ETC} / \mathrm{ED}$ model (indicated as S, SA and dodecane), ITC/ID model (indicated as 20A), and DCM (indicated as 98). The droplet of $12.66 \mu \mathrm{m}$ initial radius and $360 \mathrm{~K}$ temperature is moving at $10 \mathrm{~m} / \mathrm{s}$ in still air of pressure and temperature equal to 32 bar and $700 \mathrm{~K}$, respectively.

Using the MDQD model (shown in Figure 4) has contributed to reducing the CPU time to $1 / 6$ th compared with the model considering the contributions of all 98 components, as shown in Figure 5.

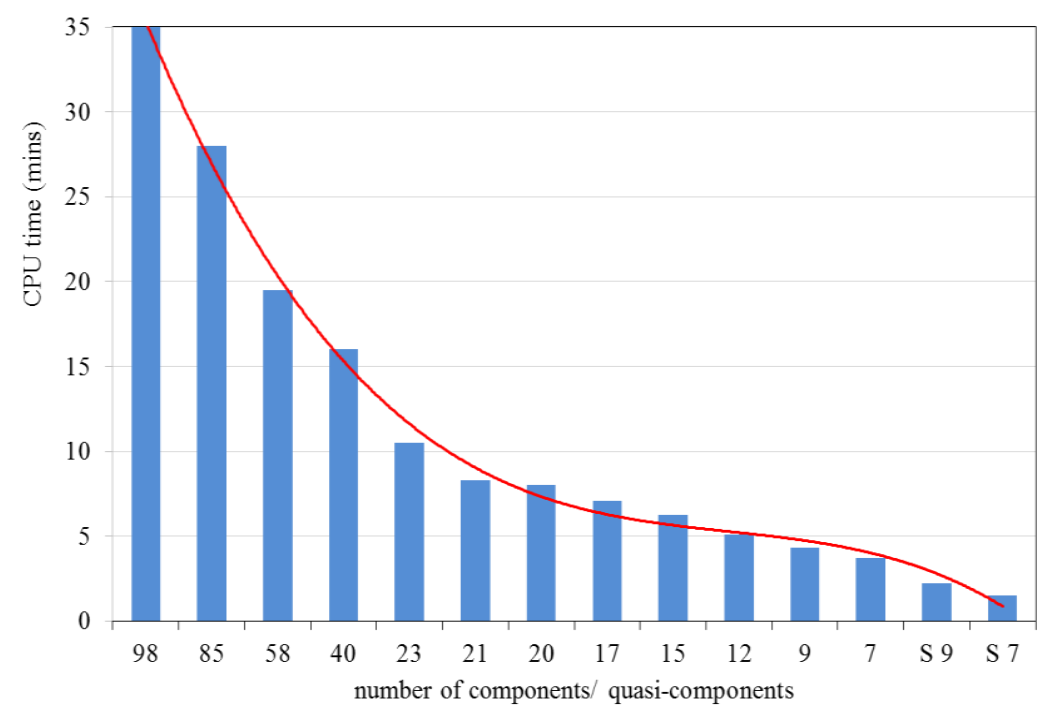

Figure 5. The plot of CPU time required for calculations of stationary diesel fuel droplet heating and evaporation for Intel Xeon (core duo) E8400, $2 \mathrm{GHz}$ and 3 GB RAM for $1 \mu$ s time-step.

\section{Gasoline fuel droplets}

The evolutions of the droplet surface temperatures $T_{s}$ and radii $R_{d}$ versus time for gasoline FACE C fuel are presented in Figure 6. In this figure, four cases have been examined [16]: (1) the contributions of all 20 components are taken into account using the DCM (indicated as ME); (2) the contributions of 20 components are taken into account, but represented by $6 \mathrm{C} / \mathrm{QC}$ using the MDQDM (indicated as 6); (3) the thermodynamic and transport properties of 20 components are averaged to form a single component and temperature gradient is ignored (SC and ITC models) (indicated as SI); and (4) the ITC model in which gasoline fuel is approximated with iso-octane (indicated as $\mathrm{IO}$ ) is used. As one can see from this figure, the errors in droplet lifetimes predicted by the classical approaches, such as $\mathrm{SI}, \mathrm{IO}$, or $\mathrm{MI}$ models, are up to about $68 \%$, which cannot be tolerated in any engineering application. However, the errors in droplet surface temperatures and evaporation times predicted by the MDQDM using $6 \mathrm{QC} / \mathrm{C}$ are $0.8 \%$ and $6.6 \%$, respectively, which can be tolerated in many applications. 


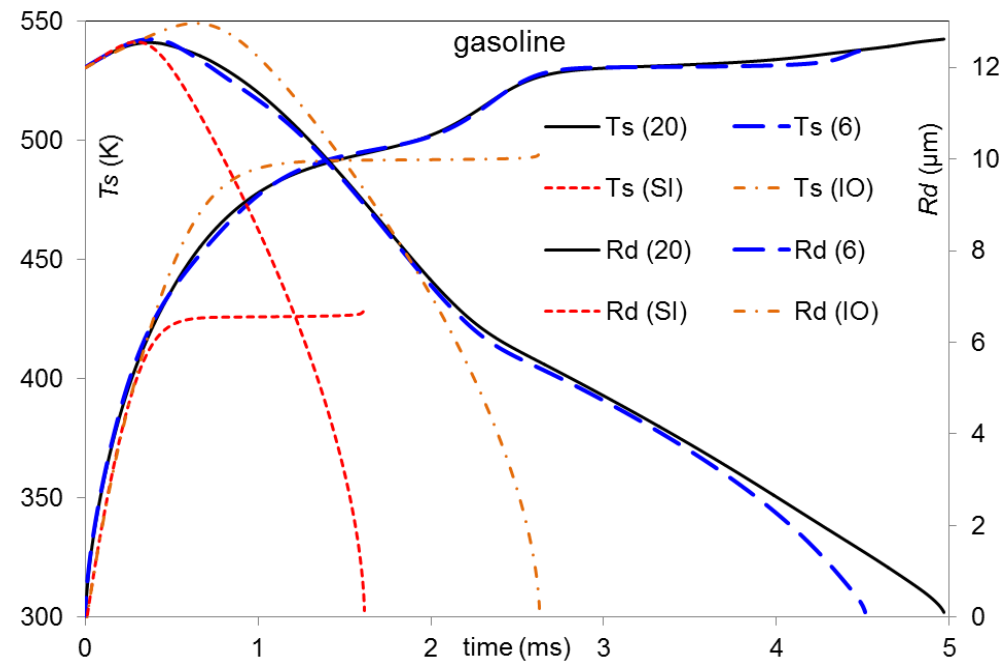

Figure 6. The plots of $T_{s}$ and $R_{d}$ versus time for gasoline fuel droplets, predicted using the MDQDM (indicated as 6), SC/IT/ID model (indicated as SI), SC/ET/ED model (indicated as IO), and DCM (indicated as 20). The droplet with initial radius $12 \mu \mathrm{m}$ and initial temperature $296 \mathrm{~K}$ is assumed to be moving with relative velocity $24 \mathrm{~m} / \mathrm{s}$ in air. Ambient pressure and temperature are equal to $0.9 \mathrm{MPa}$ and $545 \mathrm{~K}$ respectively.

The use of the MDQDM (shown in Figure 6) has contributed to significant increase of CPU efficiency by reducing $70 \%$ of the CPU time spent to run the DCM for the same fuel (See Figure 7 ).

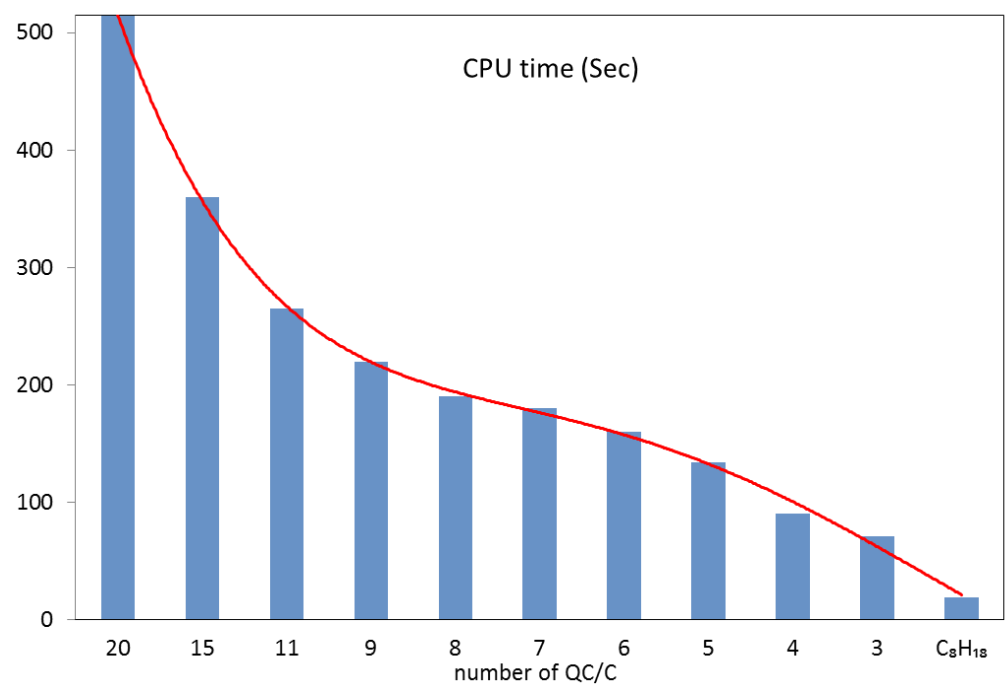

Figure 7. The plot of CPU time required for calculations of stationary gasoline fuel droplet heating and evaporation for Intel Xeon (core duo) E8400, $2 \mathrm{GHz}$ and 3 GB RAM for $1 \mu$ s time-step.

\section{Blended diesel-biodiesel fuel droplets}

The blended fuel droplet heating and evaporation for diesel-biodiesel fuels and their blends, taking into account the contributions of all (up to 114) components of hydrocarbons and methyl esters have been investigated [12]. Both, DCM and MDQDM, approaches are considered. As inferred from previous analyses, the blends of all 22 types of biodiesel fuel with commercial used diesel fuel in certain conditions like aforementioned are tested (see Figure 8). 


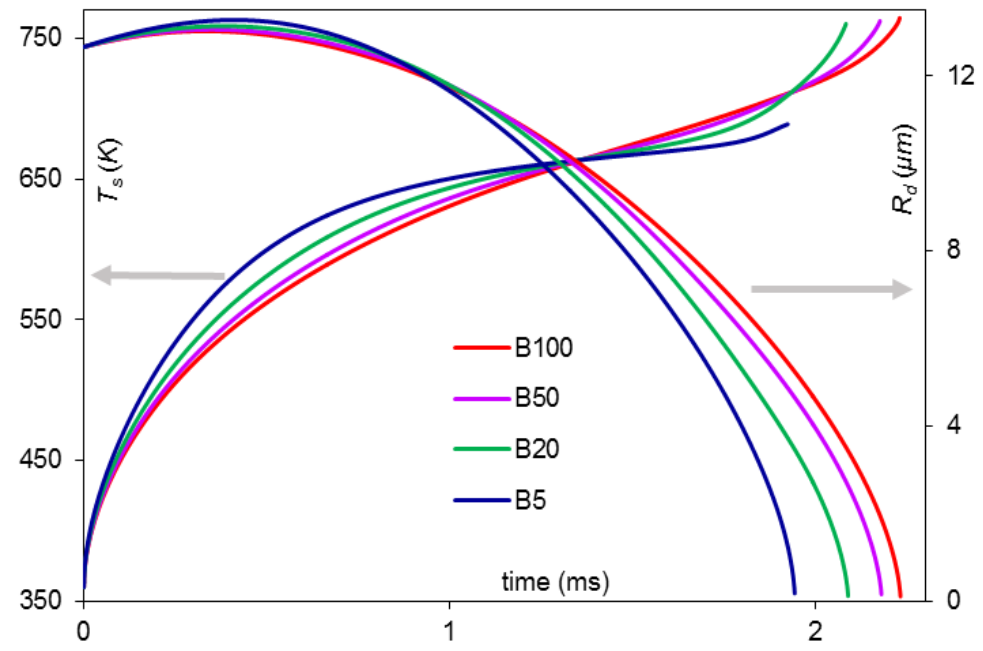

Figure 8. The plots of $T_{s}$ and $R_{d}$ versus time predicted by the DCM, for a diesel-BME blended fuel droplets moving at $10 \mathrm{~m} / \mathrm{s}$, with $12.66 \mu \mathrm{m}$ initial radius, $360 \mathrm{~K}$ initial temperature, and ambient pressure and temperature of $30 \mathrm{bar}$ and $800 \mathrm{~K}$, respectively.

A typical example of the time evolutions of droplet surface temperatures $T_{s}$ and radii $R_{d}$ of diesel-biodiesel blended fuel droplets, using DCM, was given for Butter Methyl Ester (BME) droplets in Figure 8. In this figure, four mixtures of diesel-biodiesel fuels were shown; B100 (pure biodiesel fuel), B50 (50\% biodiesel and 50\% diesel fuels), B20 (20\% biodiesel and $80 \%$ diesel fuels) and B5 (5\% biodiesel and $95 \%$ diesel fuels).

A typical example of the evolutions of $T_{s}$ and $R_{d}$ over time for a B5 diesel-biodiesel fuel blend (5\% biodiesel and $95 \%$ diesel) droplet is shown in Figure 9 . The approximations of the blended fuel of 105 hydrocarbons and methyl esters with $25 \mathrm{C} / \mathrm{QCs}$ lead to underestimation of droplet lifetime by less than $3.2 \%$. This underestimation increases to arrange between $4 \%-15 \%$ for a selection range between 21 to $10 \mathrm{C} / \mathrm{QCs}$, respectively. The errors in predicted droplet surface temperatures for all MDQDM approximations were up to $2 \%$. Using the MDQDM for this analysis has made significant contribution to the CPU efficiency of the code (See Figure 10).

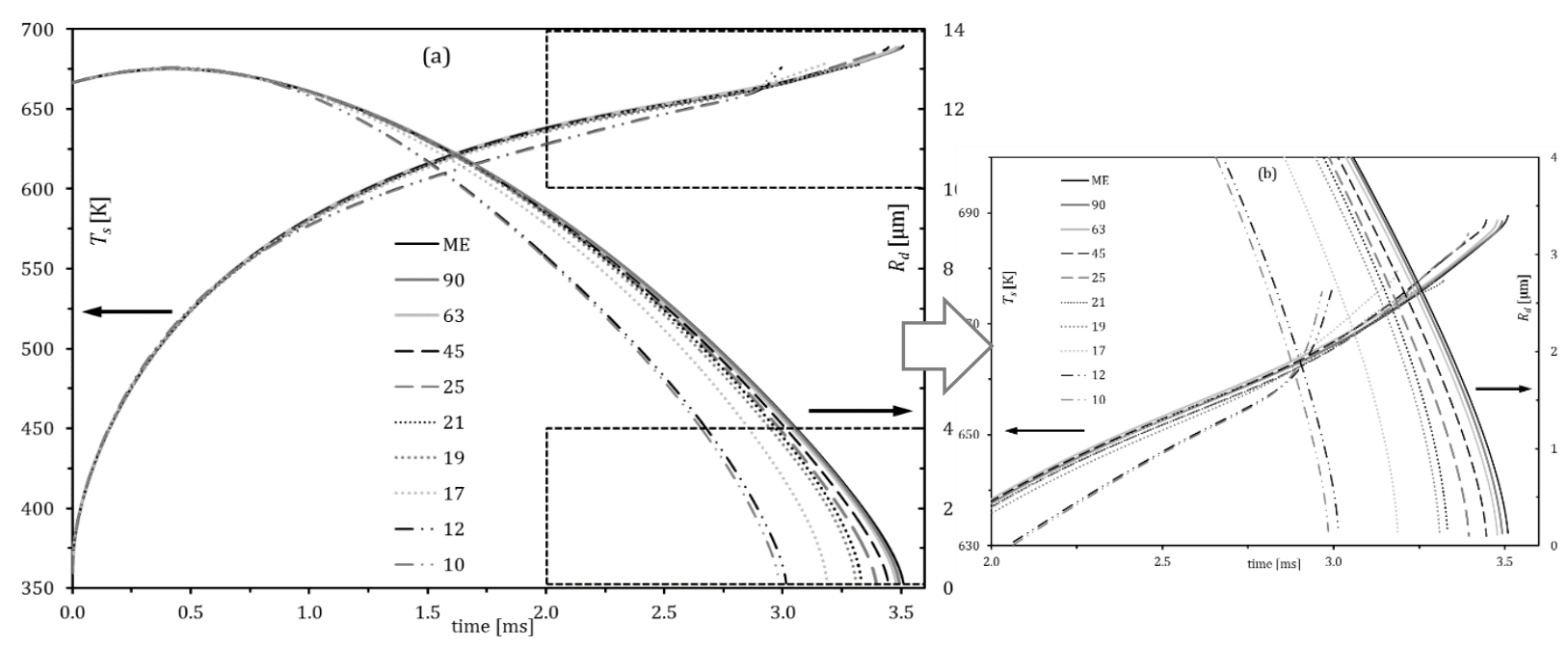

Figure 9. The plots of $T_{s}$ and $R_{d}$ versus time for various Diesel/SME blends, using the DCM and MDQDM approaches. The initial droplet radius is taken equal to $12.66 \mu \mathrm{m}$, its axial velocity in still air (assumed constant) and initial temperature are assumed equal to $U_{\mathrm{drop}}=10 \mathrm{~m} / \mathrm{s}$ and $T_{d}=360 \mathrm{~K}$, respectively; ambient air (gas) pressure and temperature are assumed equal to $p_{g}=32$ bar and $T_{g}=700 \mathrm{~K}$, respectively. The data and compositions of these approximations are inferred from [12]. 


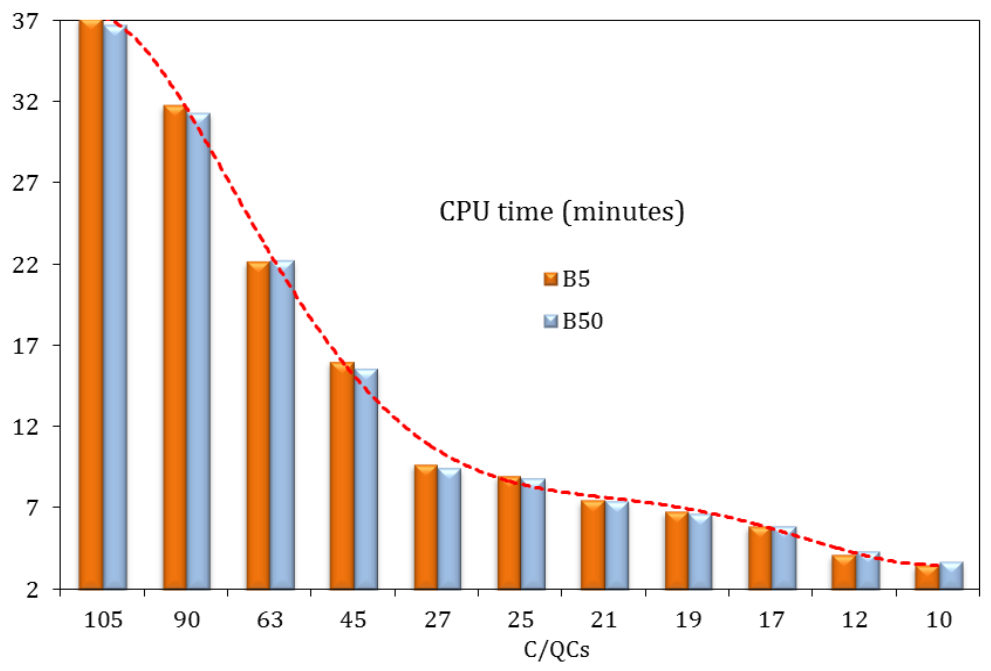

Figure 10. The plot of CPU time required for calculations of droplet heating and evaporation versus the number of C/QCs for B5 and B50 fuel droplets, using the same parameters as in Figure 8. The specifications of the workstation used were: Z210, Intel core, 64-bit, $3.10 \mathrm{GHz}$ and $8 \mathrm{~GB}$ RAM.

An example of the impact of using the MDQDM approach on the computational costs among these several approximations is illustrated in Figure 10. As can be seen from this figure, approximating 105 components of the blended fuel with $17 \mathrm{C} / \mathrm{QCs}$ reduces the required CPU time by more than $83 \%$ compared with the DCM approach considering the contributions of all 105 components. Also, it has been found that the droplet lifetime predicted for $17 \mathrm{C} / \mathrm{QC}$ is about $4 \%$ for $\mathrm{B} 50$, and $9 \%$ for $\mathrm{B} 5$, less than that predicted by the model considering the contributions of full blended fuel composition (all 105 components). Such an option can ensure a good compromise between CPU efficiency of the model and its accuracy when small errors in predicted droplet evaporation times can be tolerated for commonly used fuel blends.

\section{Conclusions}

The modelling of the heating and evaporation of multi-component biodiesel, diesel, gasoline, and blended dieselbiodiesel fuel droplets has been performed using the Discrete Component model (DCM) and the Multi-Dimensional Quasi-Discrete Model (MDQDM). It can be concluded that ignoring the effects of species diffusion, temperature gradient and recirculation inside droplets can lead to noticeable errors in the predictions of droplet surface temperatures and evaporation times.

The influence of increasing the fraction of biodiesel in the diesel-biodiesel mixture on droplet surface temperature and evaporation time is shown to be noticeable, and it needs to be accounted for in engineering modelling. Smaller fractions of biodiesel fuel (up to $5 \%$ ) have very small effects on the evolutions of droplet surface temperatures $\left(T_{s}\right)$ and radii $\left(R_{d}\right)$.

The application of the models to diesel, gasoline, and several types of biodiesel fuel is investigated. It is shown that the errors in the estimated $T_{s}$ and $R_{d}$ for selected number of components/quasi-components of fuels, using the MDQD model, are negligible compared to those predicted by the model considering the contributions of all components of these fuels (i.e. using the DCM). For instance, replacing the full composition of WCO methyl esters with 5,4 and $3 \mathrm{C} / \mathrm{QC}$ produces almost identical plots for the evolutions of $T_{s}$ and $R_{d}$. It can be concluded that the predictions of the MDQDM are very close to those of the DCM. At the same time, using the MDQDM has resulted in a reduction of up to $96 \%$ in computational time compared to the cases when the original full compositions of fuels are considered using the DCM.

\section{Acknowledgment}

The authors are grateful to the Centre for Mobility and Transport - Coventry University for financial support.

\section{Nomenclature}

$\begin{array}{llll}\text { B\# } & \# \% \text { biodiesel/Diesel fraction } & \text { CML } & \text { camelina methyl ester } \\ \text { BME } & \text { butter methyl ester } & \text { CNE } & \text { corn methyl ester } \\ \text { C/QC } & \text { components/quasi-components } & \text { CPU } & \text { central processing unit } \\ \text { CAN } & \text { canola methyl ester } & \text { CSE } & \text { cottonseed methyl ester } \\ \text { CME } & \text { coconut methyl ester } & \text { DCM } & \text { discrete component model }\end{array}$




$\begin{array}{ll}\text { ED } & \text { effective diffusivity } \\ \text { ETC } & \text { effective thermal conductivity } \\ \text { FAME } & \text { fatty acid methyl ester } \\ \text { HME1 } & \text { hemp-oil, Ukrainian hemp seed oil } \\ \text { HME2 } & \text { hemp-oil, produced in the European Union } \\ \text { ID } & \text { infinite diffusivity } \\ \text { ITC } & \text { infinite thermal conductivity } \\ \text { JME } & \text { jatropha methyl ester } \\ \text { LME } & \text { lard methyl ester } \\ \text { LNE } & \text { linseed methyl ester } \\ \text { MDQDM multi-dimensional-quasi-discrete model } \\ \text { PME } & \text { palm methyl ester } \\ \text { PMK } & \text { palm kernel methyl ester } \\ \text { PTE } & \text { peanut methyl ester } \\ \text { RME } & \text { rapeseed methyl ester } \\ \text { SC } & \text { single component } \\ \text { SFE } & \text { safflower methyl ester } \\ \text { SME } & \text { soybean methyl ester }\end{array}$

$\begin{array}{ll}\text { SNE } & \text { sunflower methyl ester } \\ \text { TGE } & \text { tung methyl ester } \\ \text { TME } & \text { tallow methyl ester } \\ \text { WCO } & \text { waste cooking oil } \\ \text { YME } & \text { yellow grass oil methyl ester }\end{array}$

\section{Symbols}

$p \quad$ gas pressure

$R \quad$ radius

$T \quad$ temperature

velocity

$\begin{array}{ll}\text { Subscripts } \\ g & \text { gas } \\ d & \text { droplet } \\ s & \text { droplet surface }\end{array}$

\section{References}

[1] Ra, Y., and Reitz, R. D., 2009, Int. J. Multiph. Flow, 35(2), pp. 101-117.

[2] Sazhin, S. S., Al Qubeissi, M., Kolodnytska, R., Elwardany, A. E., Nasiri, R., and Heikal, M. R., 2014, Fuel, 115, pp. 559-572.

[3] Al Qubeissi, M., 2015, Heating and Evaporation of Multi-Component Fuel Droplets, WiSa, Stuttgart.

[4] Zhu, G.-S., and Reitz, R. D., 2002, Int. J. Heat Mass Transf., 45(3), pp. 495-507.

[5] Laurent, C., Lavergne, G., and Villedieu, P., 2009, Comptes Rendus Mécanique, 337(6-7), pp. 449-457.

[6] Grote, M., Lucka, K., and Köhne, H., 2010, V ECCOMAS CFD, Lisbon, Portugal.

[7] Burger, M., Schmehl, R., Prommersberger, K., Schäfer, O., Koch, R., and Wittig, S., 2003, Int. J. Heat Mass Transf., 46(23), pp. 4403-4412.

[8] Smith, B. L., and Bruno, T. J., 2006, Int. J. Thermophys., 27(5), pp. 1419-1434.

[9] Aleiferis, P. G., and van Romunde, Z. R., 2013, Fuel, 105, pp. 143-168.

[10] Sazhin, S. S., 2014, Droplets and Sprays, Springer, London.

[11] Al Qubeissi, M., Sazhin, S. S., Crua, C., Turner, J., and Heikal, M. R., 2015, Fuel, 154, pp. 308-318.

[12] Al Qubeissi, M., Sazhin, S. S., and Elwardany, A. E., 2017, Fuel, 187, pp. 349-355.

[13] Sazhin, S. S., 2017, Fuel, 196, pp. 69-101.

[14] Sazhin, S. S., Al Qubeissi, M., Nasiri, R., Gun'ko, V. M., Elwardany, A. E., Lemoine, F., Grisch, F., and Heikal, M. R., 2014, Fuel, 129, pp. 238-266.

[15] Al Qubeissi, M., Sazhin, S. S., de Sercey, G., and Crua, C., 2014, 26th European Conference on Liquid Atomization and Spray Systems, University of Bremen, Bremen, Germany.

[16] Al Qubeissi, M., Sazhin, S. S., Turner, J., Begg, S., Crua, C., and Heikal, M. R., 2015, Fuel, 159, pp. $373-384$.

[17] Al Qubeissi, M., Kolodnytska, R., and Sazhin, S. S., 2013, 25th European Conference on Liquid Atomization and Spray Systems, Crete, Greece.

[18] Lapuerta, M., Armas, O., and Rodriguezfernandez, J., 2008, Prog. Energy Combust. Sci., 34(2), pp. 198-223.

[19] US Department of Energy: Energy Efficiency and Renewable Energy, http://www.afdc.energy.gov/fuels/biodiesel_blends.html. [Accessed: 13-Mar-2017].

[20] Al Qubeissi, M., and Sazhin, S. S., 2016, 26th European Conference on Liquid Atomization and Spray Systems, Brighton, UK, p. 179.

[21] Elwardany, A. E., and Sazhin, S. S., 2012, Fuel, 97, pp. 685-694.

[22] Sazhin, S. S., Elwardany, A. E., Sazhina, E. M., and Heikal, M. R., 2011, Int. J. Heat Mass Transf., 54(19-20), pp. 4325-4332. 


\title{
Numerical investigation of the aerodynamic breakup of Diesel droplets under various gas pressures
}

\author{
Dionisis Stefanitsis ${ }^{* 1,2,}$, Ilias Malgarinos ${ }^{1,2}$, George Strotos ${ }^{3}$, Nikolaos Nikolopoulos ${ }^{1}$, \\ Emmanouil Kakaras ${ }^{1}$, Manolis Gavaises ${ }^{2}$ \\ ${ }^{1}$ Centre for Research and Technology Hellas/Chemical Process and Energy Resources \\ Institute (CERTH/CPERI), Egialeias 52, Marousi, Greece \\ ${ }^{2}$ City University London, School of Engineering and Mathematical Sciences, Northampton \\ Square, EC1V OHB London, UK \\ ${ }^{3}$ Piraeus University of Applied Sciences, Mechanical Engineering Department, 250 Thivon \\ and P. Ralli str., Aegaleo 12244, Greece \\ *Corresponding author: stefanitsis@certh.gr
}

\begin{abstract}
The present study investigates numerically the aerodynamic breakup of Diesel droplets for a wide range of ambient pressures encountered in engineering applications relevant to oil burners and internal combustion engines. The numerical model solves the Navier-Stokes equations coupled with the Volume of Fluid (VOF) methodology utilized for capturing the interface between the liquid and the surrounding gas. An adaptive local grid refinement technique is used to increase the accuracy of the numerical results around the interface. The Weber $(W e)$ numbers examined are in the range of 14 to 279 which correspond to bag, multimode and sheet-thinning breakup regimes. Model results are initially compared against published experimental data and show a good agreement in predicting the drop deformation and the different breakup modes. The predicted breakup initiation times for all cases lie within the theoretical limits given by empirical correlations based on the We number. Following the model validation, the effect of density ratio on the breakup process is examined by varying the gas density (or equivalently the ambient pressure), while the We number is kept almost constant equal to 270; ambient gas pressure varies from 1 up to $146 \mathrm{bar}$ and the corresponding density ratios $(\varepsilon)$ range from 700 down to 5 . Results indicate that the predicted breakup mode of sheet-thinning remains unchanged for changing the density ratio. Useful information about the instantaneous drag coefficient $\left(C_{d}\right)$ and surface area as functions of the selected non-dimensional time is given. It is shown that the density ratio is affecting the drag coefficient, in agreement with previous numerical studies.
\end{abstract}

\section{Keywords}

Droplet breakup, Diesel, VOF, density ratio, breakup initiation time.

\section{Introduction}

Droplet motion, deformation and breakup are observed in a wide variety of engineering applications such as in the injection systems of oil burners and internal combustion engines. Droplet deformation and subsequent breakup are caused by aerodynamic forces exerted on the drop by the surrounding gas, while surface tension and viscosity of the drop hinder deformation and tend to restore it to a spherical shape. The non-dimensional numbers that account for these effects are the Weber $(W e)$, Ohnesorge $(\mathrm{Oh})$ and Reynolds $(R e)$ numbers as well as the density $(\varepsilon)$ and viscosity $(N)$ ratios of the two phases [1]; the timescale used to non-dimensionalise the time is the shear breakup timescale [2]. For low $O h$ numbers $(O h<0.1)$ the droplet breakup is mainly controlled by the We number and according to [1] four different breakup regimes are observed as the We number increases. For We numbers in the range of 11 up to 35 the bag breakup mode is encountered during which the drop deforms into a bag resembling shape. As the We number is further increased up to 80 , the multimode regime starts to appear which is essentially a combination of the bag and sheet-thinning modes. In the sheet-thinning breakup mode $(80<W e<350)$ a sheet is formed at the periphery of the drop, which eventually breaks into ligaments. The final breakup mode is called catastrophic (We>350) and according to [1] is attributed to Rayleigh-Taylor and Kelvin-Helmholtz instabilities.

To the authors' best knowledge, a limited number of publications exist in the open literature regarding the experiments of droplet breakup utilizing Diesel as test fluid, which is the subject of the present work. The work of [3] investigated the breakup of Diesel droplets for We numbers ranging from 14 up to 10000 and Oh number equal to 0.027 . They produced photographic sequences of the breakup process and measured the breakup time and deformation. The series of works [4-6] have also examined Diesel fuel for conditions of $W e=56-532, R e=509-8088$, $O h=0.038-0.065$ and $\varepsilon=79-700$, corresponding to three breakup regimes, i.e. bag, sheet-thinning and catastrophic. 
They generated photographs of the breakup process and presented graphs for the drop deformation and trajectory. It was concluded that the breakup process in the three breakup regimes is independent of the $R e$ number and it is only a function of the We number for the range of $O h$ and $\varepsilon$ examined. The work of $[7,8]$ investigated the microscopic (droplet scale) and macroscopic (spray scale) breakup characteristics of Diesel droplets by introducing monodispersed droplets into a gas stream ejected from a nozzle. They examined We numbers from 4.3 up to 383 in three breakup regimes (vibrational, bag and sheet-thinning) and produced images of the breakup process and graphs for the droplet mean velocities and diameters.

Regarding the numerical modelling of droplet breakup, there are no studies (at least to the best of our knowledge) that investigate the breakup of Diesel fuel droplets corresponding to a range of We and Oh numbers, which are of practical interest. On the other hand, a few studies examine droplet breakup in Diesel engine conditions (i.e. low density ratios) without referring to specific fluid, while there are also some studies which examined the effect of density ratio on the breakup process. In [9] a front-tracking scheme in 2-D axisymmetric coordinates was used to study the breakup of impulsively accelerated drops. They investigated Diesel engine conditions (density ratios 1.15 and 10) and provided maps in the We-Re plane for the breakup outcome. They found that by increasing the $R e$ number the critical We number, that separates the different breakup regimes, decreases. The work of [10] used the Level-Set method in a 2-D axisymmetric domain to investigate the deformation of droplets at small Re numbers (25-200) and density ratios (2-32); they found that for density ratios above 32 the boundaries of the breakup regimes are almost unaffected by the density ratio. Furthermore, [11] simulated impulsively accelerated drops using a moving mesh interface tracking scheme and found that the drag coefficient is not affected much by the density ratio although it is larger than those of solid spheres at the same Re numbers. In [12] they used the Level Set method to simulate in a 2-D axisymmetric domain the deformation and breakup of liquid drops under the effect of gas flow. They performed three test simulations (free fall of droplet, bubble rising in liquid and droplet fall into a water pool) in order to validate their model and following this, they investigated the effect of the non-dimensional parameters (We, $R e, R e /$ and $\varepsilon$ ) on the breakup process. They concluded that the most influential parameter in droplet breakup phenomena is the We number followed by the $R e$ and $R e$, while the change in the density ratio from 10 to 100 led to alternation of the breakup mode. In [13] they studied droplet deformation and breakup on the bag and shear breakup regimes using a 3-D VOF method. They made parametric studies with different $W e, R e$ and $\varepsilon$ numbers and identified 5 new breakup modes in the multimode breakup regime. Moreover, they proposed a new breakup map in the $R e-N / \sqrt{\varepsilon}$ plane and concluded that any breakup regime can be represented in the proposed map, without any dependence on the We number. In the work of [14] they performed 3-D simulations of droplet breakup using a variant of the Coupled Level Set-VOF (CLSVOF) method called S-CLSVOF in the interFOAM solver of the OpenFOAM software package. They examined the sheet-thinning breakup regime under Diesel-like engine conditions and found that the density ratio affects the shape and size of the fragments and also the recirculation region of the gas flow.

To the author's best knowledge, the present work is the first one investigating the aerodynamic droplet breakup mechanism of Diesel fuel droplets under conditions of practical interest. The gas pressure and density are increased parametrically to cover a wide range of conditions, from atmospheric up to those corresponding to Diesel engine conditions. The following sections include initially a short description of the numerical model and the computational setup, followed by the CFD model validation against experimental data. After that, a parametric study is performed for the effect of density ratio on the breakup phenomenon while the most important conclusions are summarized at the end.

\section{Numerical model and computational setup}

The numerical model solves the Navier-Stokes equations coupled with the Volume of Fluid (VOF) [15] method for capturing the interface between liquid and gas. Surface tension forces are included in the momentum equation by using the Continuum Surface Stress (CSS) model of [16]. The CFD model has been validated and used for many applications including the aerodynamic breakup of droplets with high density ratios as described in [17-20].

The simulations are performed in a 2-D axisymmetric domain with the commercial CFD tool ANSYS FLUENT v16 [21], along with various user defined functions (UDFs) for the implementation of the adaptive local grid refinement [22] and the adaptive time-step for the implicit VOF solver. With the current grid resolution employed, drop radius is resolved by 192 cells per Radius $(\mathrm{cpR})$ with minimum cell size ranging from $0.48 \mu \mathrm{m}$ up to $6.25 \mu \mathrm{m}$ depending on the droplet radius; systematic runs with $48,96,192$ and $384 \mathrm{cpR}$ have revealed that the resolution of $192 \mathrm{cpR}$ is adequate for achieving a grid independent solution.

\section{Model validation}

In order to evaluate the performance of the model the results are compared against the experimental data reported in the publications of [3-5]; in all these works, Diesel fuel was used as test fluid, along with the continuous air jet 
experimental setup. In [3] the droplets were introduced to the air jet with a syringe and a needle ensuring that they enter with almost zero initial velocity, while in [4] and [5] the droplets were introduced with a small initial velocity. The We number of interest in [3] is 14, which corresponds to the bag breakup regime and the droplet diameter is equal to $2.4 \mathrm{~mm}$. In [4] and [5] the examined We number ranges from 54 to 279, density ratios from 79 to 700 and drop diameters $184 \mu \mathrm{m}$ and $198 \mu \mathrm{m}$. The breakup modes observed in the experiments for these conditions were the bag and sheet-thinning.

The simulated cases for the model validation are presented in Table 1. Case 1 corresponds to the experimental study of [3], while cases 2-3 correspond to the experimental study of [4]. Cases 1-3 have a high density ratio equal to 695 ( $\left.P_{g}=1 \mathrm{bar}\right)$ and We numbers equal to 14,54 and 254 respectively. Case 4 corresponds to the experiment of [5] and have a small density ratio equal to $79\left(P_{g}=9.2 \mathrm{bar}\right)$ and We number equal to 264 . The Oh number for all cases is less than 0.1 so its effect on the phenomenon is considered to be insignificant [1].

Table 1: Validation cases.

\begin{tabular}{lllllllllll}
\hline Case & $P_{g}(\mathrm{bar})$ & $D_{0}(\mu \mathrm{m})$ & $U_{g}(\mathrm{~m} / \mathrm{s})$ & We & $R e$ & Oh & $\varepsilon$ & Breakup regime & $\begin{array}{l}\text { Reference } \\
\text { publication }\end{array}$ \\
\hline 1 & 1 & 2400 & 10 & 14 & 1540 & 0.027 & 695 & Bag & {$[3]$} \\
2 & 1 & 198 & 68 & 54 & 864 & 0.038 & 695 & Multi-bag & {$[4]$} \\
3 & 1 & 198 & 147 & 254 & 1867 & 0.038 & 695 & Sheet-thinning & {$[4]$} \\
4 & 9.2 & 184 & 52 & 264 & 5761 & 0.039 & 79 & Sheet-thinning & {$[5]$} \\
\hline
\end{tabular}

The temporal evolution of droplet shape and deformation for case 1 are presented in Figure 1 both for the simulation (denoted with the VOF iso-value 0.5 ) and the experiment. The droplet initially deforms into an ellipsoid shape $\left(t=1.4 t_{r e f}\right)$ which results in an increase of cross-stream deformation and a decrease in the streamwise one. Following that, a bag is formed $\left(t=1.75 t_{r e f}\right)$ and the deformation in both axes increases with the eventual breakup occurring at $t=1.85 t_{\text {ref. }}$ There is a good agreement between the simulation and the experiment as both predict bag breakup mode and a similar evolution for the droplet shape and deformation. The breakup initiation occurs earlier in the simulation at $t=1.85 t_{\text {ref }}$ instead of $2.93 t_{\text {ref }}$ in the experiment. Nevertheless, the predicted breakup initiation time of $t=1.85 t_{\text {ref }}$ is within the limits proposed in [23] and [24] (see Figure 5 of this section).

(a)

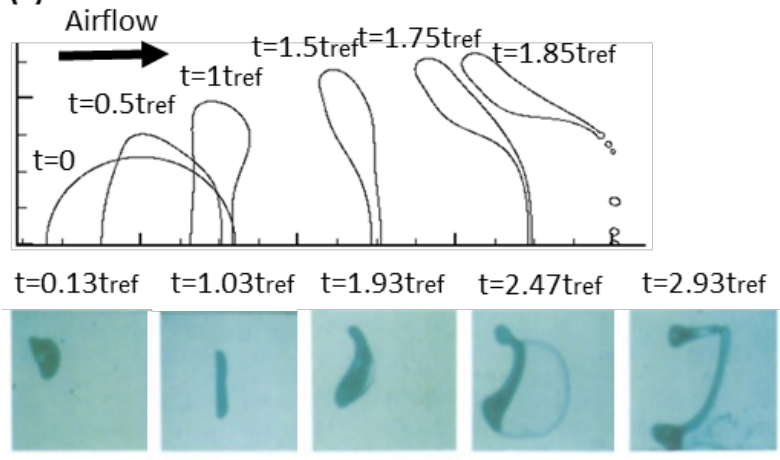

(b)

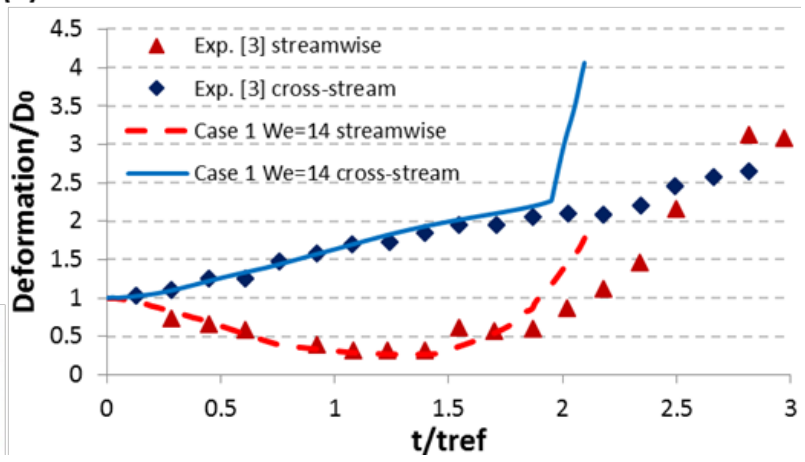

Figure 1. Temporal evolution of a) droplet shape and b) deformation from the simulation of case 1 and the experiment of [3].

The temporal evolution of droplet shape for cases 2-4 is shown in Figure 2. The sheet-thinning breakup mode predicted for cases 3 and 4 is in agreement with the experimental observations of [4] and [5] respectively. Nevertheless, for case 2, the present simulations predict a multi-bag (multimode) breakup mode characterized by a bag formed at the periphery of the droplet, while in the experiment of [4] a bag breakup was reported. The predicted multimode breakup for the examined range of We and Oh numbers is in accordance with the results reported in [25] and [1], which both report multimode breakup for the conditions of case 2. It should be mentioned that case 2 has been also simulated in a 3-D domain (additional to the 2-D axisymmetric) and the qualitative performance of this case, remained unchanged; the results of the 3-D simulation are not presented in this work. 


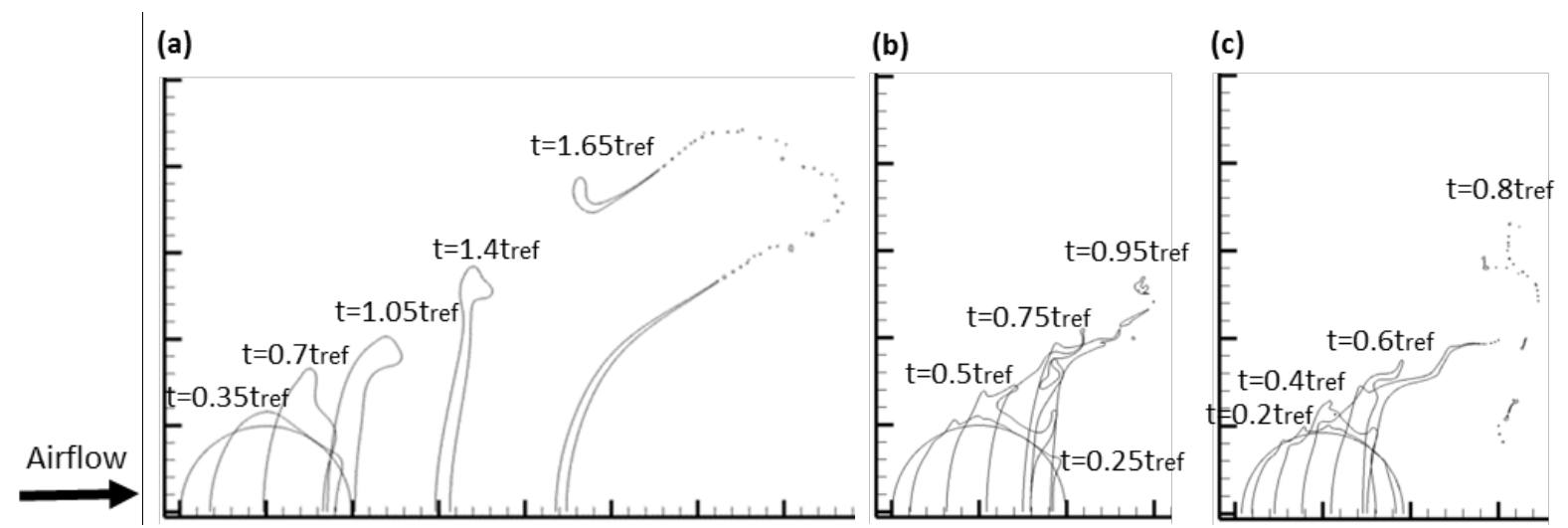

Figure 2. Temporal evolution of droplet shape for the cases 2, 3 and 4 (shown in a, b and c respectively).

Turning now to the quantitative comparison between the simulations and the experiments, the experimental measurements of [4] for the droplet deformation are initially provided as a function of the distance travelled along the direction of the cross-stream droplet motion (the reader is referred to the relevant paper [4] for details on the experimental setup). In the present 2-D axisymmetric approach, this secondary motion has been neglected. On the other hand, a comparison can still be made based on the corresponding experimental time needed for the droplet to travel along the cross-stream direction. This is estimated as $t=$ (cross-stream distance)/ $U_{d, 0}$; it has been assumed that the cross-stream velocity remains unchanged. The temporal variation of the droplet cross-stream deformation is presented in Figure 3, for cases 2 and 3. As it can be seen, there is a very good agreement between the results of the simulation and those of the experiment. Moreover, it is observed that the temporal variation of the deformation for the two cases is similar, although the We numbers differ significantly (54 compared to 254). This reveals the dimensionless character of the phenomenon when using the appropriate timescale.

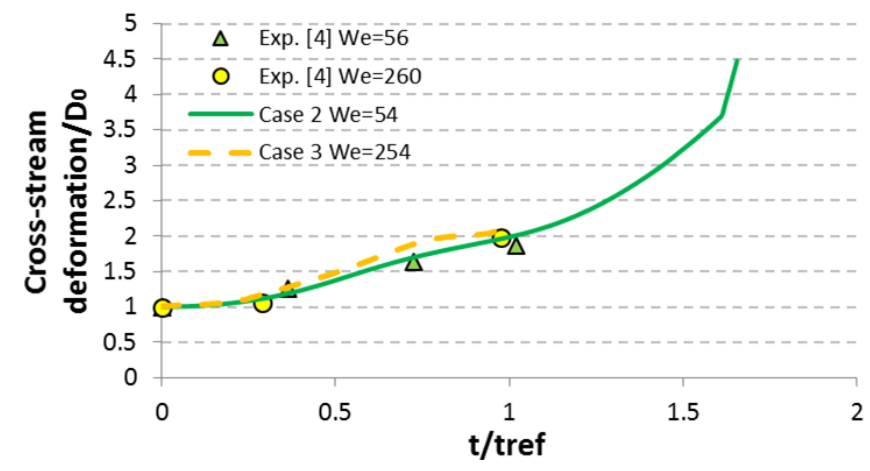

Figure 3. Drop deformation as function of non-dimensional time (cases 2-3 and experiment).

The temporal evolution of the non-dimensional droplet velocity $\left(U_{d} /\left(U_{g}-U_{d}\right)\right)$ versus a modified time $\left(\boldsymbol{t} /\left(\boldsymbol{t}_{\boldsymbol{r} e f} \sqrt{\boldsymbol{\varepsilon}}\right)\right)$ for all cases of Table 1 are compared against the experimental data of [24] in Figure 4; the axes have been nondimensionalised with the same fashion as in [24]. The dimensionless character of droplet velocity is confirmed for all cases; only case 4 shows a small deviation from the experimental data but it can be attributed to its low density ratio and high $R e$ number, compared to those examined in the experimental data. 


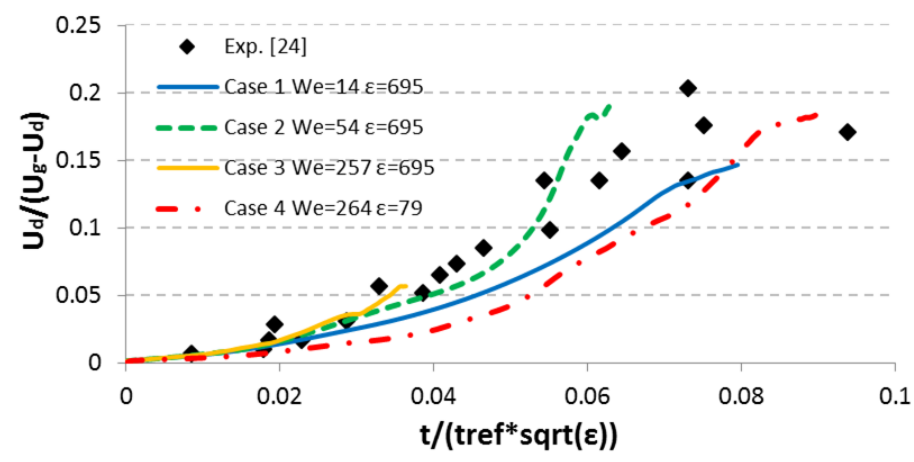

Figure 4. Non-dimensional droplet velocity as function of modified time for cases 1-4 and the experiment of [24]

The predicted breakup initiation time as a function of the We number is presented in Figure 5 for all cases of Table 1 along with the correlations proposed in [23] and [24]. In all cases, the breakup initiation time lies within the proposed limits and decreases with increasing We number. Cases 1-2 (bag and multi-bag breakup modes) give breakup initiation times closer to the correlation of [24], while cases 3-4 are closer to the correlation of [23].

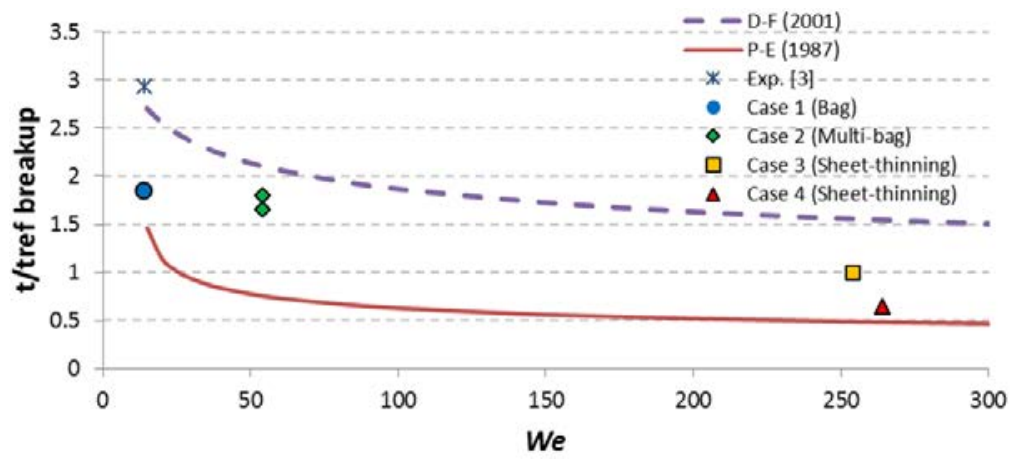

Figure 5. Breakup initiation time as function of We number (simulation and predictions in [23] and [24]).

\section{Parametric study}

Following the model validation, the numerical model is utilized to study the effect of density ratio (or equivalently the gas pressure) on the sheet-thinning breakup mode, for We numbers in the range of 265-279. The Oh number is constant and equal to 0.039 while the density ratio varies from 5 up to 700 (gas pressure varies from 146 down to 1 bar). The cases examined are presented in Table 2. Cases 5 and 6 have the smallest density ratios equal to 5 and 10 respectively. Case 8 is identical to case 4 of the validation part and cases $9-11$ correspond to the experimental conditions of [5] (not presented in the validation part since case 4, with the lowest density ratio of 79 , is sufficient for validation purposes).

Table 2. Cases of parametric study.

\begin{tabular}{llllllll}
\hline Case & $P_{g}(\mathrm{bar})$ & $D_{0}(\mu \mathrm{m})$ & $U_{g}(\mathrm{~m} / \mathrm{s})$ & $W e$ & $R e$ & $\varepsilon$ & $\begin{array}{l}\text { Reference } \\
\text { publication }\end{array}$ \\
\hline 5 & 146 & 184 & 13.2 & 270 & 21503 & 5 & - \\
6 & 73 & 184 & 18.7 & 270 & 15205 & 10 & - \\
7 & 24 & 184 & 32.4 & 270 & 9297 & 30 & - \\
8 & 9.2 & 184 & 52 & 264 & 5761 & 79 & {$[5]$} \\
9 & 6.4 & 184 & 62 & 265 & 4829 & $112[5]$ \\
10 & 3.7 & 184 & 82 & 266 & 3688 & $195[5]$ \\
11 & 1 & 184 & 159 & 279 & 1920 & $700[5]$ \\
\hline
\end{tabular}

Starting from the breakup initiation time, this quantity slightly increases with the density ratio, being in the range of $0.45 t_{\text {ref }}$ to $0.70 t_{\text {ref. }}$ Regarding droplet deformation, the temporal evolution of liquid surface area (instead of the crossstream deformation presented so far) for all parametric cases is presented in Figure 6; this quantity is difficult to be measured experimentally and plays a very important role in combustion systems. After a short non-deforming period 
(0.25tref), the liquid surface area starts to increase. Up to the point of breakup initiation the liquid surface is not affected much by the density ratio while after that point a small deviation appears; nevertheless, the 2-D axisymmetric solution is not reliable after the breakup initiation, since 3-D effects become important.

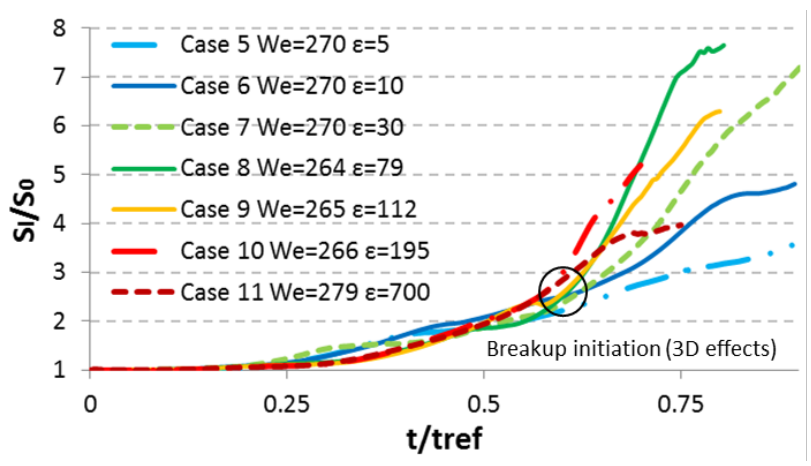

Figure 6. Liquid surface area as function of non-dimensional time (cases 5-11).

Another important parameter especially for spray codes, based on Lagrangian approaches, is the drag coefficient $\left(C_{d}\right)$. In order to calculate the instantaneous drag coefficient, the momentum equation for the droplet motion is employed:

$$
C_{d}(t)=\frac{\frac{4}{3} D_{0} \varepsilon \frac{d U_{l}(t)}{d t} \frac{A_{f}(0)}{A_{f}(t)}}{\left(U_{g}-U_{l}(t)\right)^{2}}
$$

The temporal evolution of drag coefficient is presented in Figure 7 a for selected cases. These are: case 6 with $\varepsilon=10$ (representative case for low density ratios), case 9 with $\varepsilon=112$ (representative case for moderate density ratios) and case 11 with $\varepsilon=700$ (representative case for high density ratios). The drag coefficient starts from a value close to 0.4 , which is the drag coefficient of solid spheres with Re number in the range 2000-20000 [26], and increases with time reaching values close to 1.17, which is the drag coefficient of solid disks with Re number greater than 100 [26]. In addition, it is observed that the overall drag coefficient increases with decreasing density ratio. This trend can be explained by examining each term of eq. (1) separately: $D_{0}$ is the same for all cases, $A_{f, 0} / A_{f}$ is almost constant in all cases due to similar droplet shape and the term $U_{d}$ is small compared to $U_{g}$ for high We numbers so it can be ignored. Therefore, the ratio $\varepsilon / U_{g}=\rho /\left(\rho_{g} U_{g}{ }^{2}\right)$ appearing in the equation, which is constant for constant We number, results in making the term $\mathrm{d} U_{d}(t) / d t$ for droplet acceleration the one that is the most influential among all, when changing the density ratio. The droplet acceleration is larger for lower density ratios (relatively lighter drops accelerate faster) thus making the drag coefficient higher as well. This is in agreement with the numerical study of [14] in which they state that generally, a lower density ratio results in a larger drag coefficient.

Due to the short duration of the phenomenon, it is interesting to examine the time-averaged drag coefficient for each case (calculated as the area under the curve of Figure 7a divided by the breakup time). This is shown as a function of the Re number in Figure $7 \mathrm{~b}$. The drag coefficient for all cases lies within the one of disk (purple dotted line) and the one of sphere (blue line) as taken from [26]. Again, we notice the same trend for the drag coefficient which increases with decreasing density ratio.

(a)

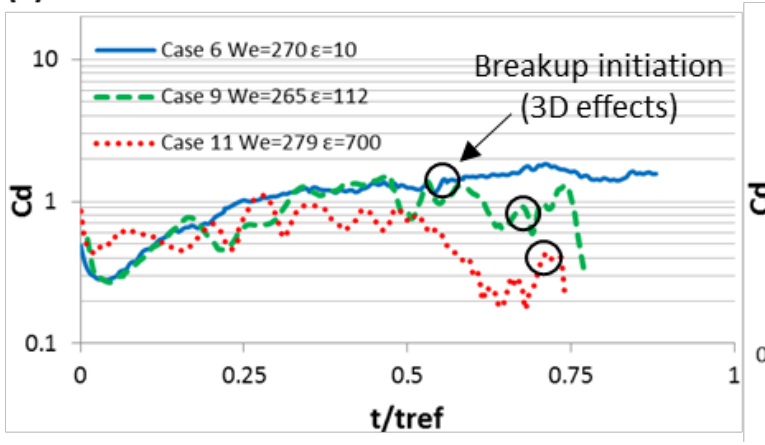

(b)

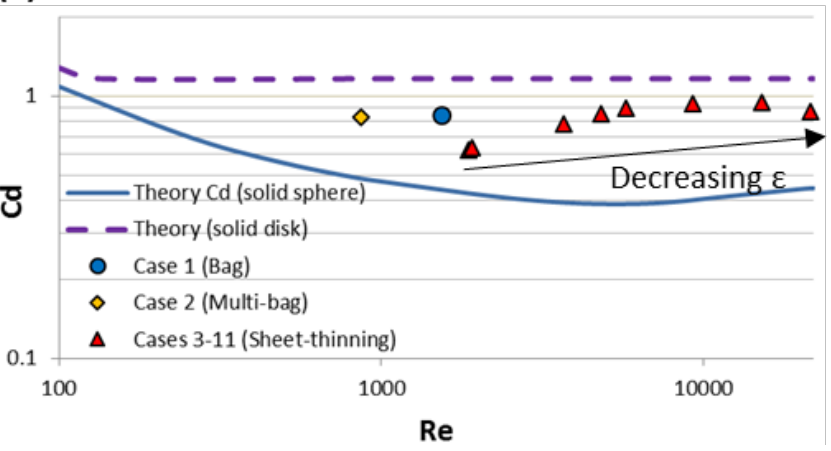

Figure 7. a) Temporal evolution of drag coefficient and b) time-averaged drag coefficient as function of $\operatorname{Re}$ (cases 1-11). 
Finally, a closer inspection of the breakup initiation time, reveals that decreasing the density ratio results in a decrease of the breakup initiation time (35\% for the lower density ratio examined). To account for this effect, the breakup initiation time proposed in [19] can be multiplied by the factor $1 /\left(1+\varepsilon^{-0.5}\right)$ which approaches unity for large density ratios.

\section{Conclusions}

In the present study the aerodynamic breakup of Diesel droplets under various density ratios was numerically investigated. The CFD model was initially validated against published experimental data for Diesel fuel and conditions of We numbers from 14 up to 264 and density ratios from 79 up to 695 . The predicted breakup regimes of bag and sheet-thinning as well as the temporal evolution of drop deformation are in close agreement with the experimental data. In one case the model predicts multi-bag breakup regime instead of bag as suggested by the experiments, but this is in accordance with the relevant experimental data. The dimensionless character of droplet velocity was confirmed for all the validation cases and also the breakup initiation times lie within the limits given by the experiments.

The parametric study performed for the density ratio (or equivalently the gas pressure) in the range of 5-700 has shown that the breakup regime and the temporal variation of the surface area of drop remain unaffected by the change in density ratio. On the other hand, the drag coefficient showed a decreasing trend with increasing density ratio in agreement with previous numerical studies. Finally, a correction factor has been proposed for the equation of breakup initiation time that accounts for the effect of density ratio.

\section{Acknowledgements}

Financial support from the MSCA-ITN-ETN of the European Union's H2020 programme, under REA grant agreement n. 675676 is acknowledged.

\begin{tabular}{|c|c|c|c|}
\hline \multicolumn{4}{|c|}{ Nomenclature } \\
\hline \multicolumn{2}{|c|}{ Roman symbols } & $U$ & Velocity [m/s] \\
\hline$A$ & Area $\left[\mathrm{m}^{2}\right]$ & We & Weber number [-] $W e=\rho_{g} U_{r e l, 0}^{2} D_{0} / \sigma$ \\
\hline$C_{d}$ & Drag coefficient [-] & \multicolumn{2}{|c|}{ Greek symbols } \\
\hline$D$ & Droplet diameter [m] & $\varepsilon$ & Density ratio $[-] \varepsilon=\rho_{l} / \rho_{g}$ \\
\hline$N$ & Viscosity ratio [-] $N=\mu_{l} / \mu_{g}$ & $\mu$ & Dynamic viscosity $[\mathrm{kg} /(\mathrm{s} \cdot \mathrm{m})]$ \\
\hline$P$ & Pressure [bar] & $\rho$ & Density $\left[\mathrm{kg} / \mathrm{m}^{3}\right]$ \\
\hline Oh & Ohnesorge number [-] $O h=\mu_{l} \sqrt{\rho_{l} \sigma \mathrm{D}_{0}}$ & \multicolumn{2}{|c|}{ Subscripts } \\
\hline $\operatorname{Re}$ & Reynolds number [-] $R e=\rho_{g} U_{r e l, 0} D_{0} / \mu_{g}$ & 0 & Initial \\
\hline$R e_{l}$ & Reynolds number for liquid [-] $R e_{l}=\rho_{l} U_{r e l, 0} D_{0} / \mu_{l}$ & $f$ & Frontal \\
\hline$S$ & Surface $\left[\mathrm{m}^{2}\right]$ & $g$ & Gas phase \\
\hline$t$ & Time $[s]$ & I & Liquid phase \\
\hline$t_{\text {ref }}$ & Shear breakup timescale $[\mathrm{s}] t_{r e f}=\left(\mathrm{D}_{0} / U_{g, 0}\right) \sqrt{\varepsilon}$ & rel & Relative \\
\hline
\end{tabular}

\section{References}

[1] Guildenbecher, D. R., López-Rivera, C., and Sojka, P. E., 2009, "Secondary atomization," Experiments in Fluids, 46(3), pp. 371-402.

[2] Nicholls, J. A., and Ranger, A. A., 1969, "Aerodynamic shattering of liquid drops," AIAA Journal, 7(2), pp. 285290.

[3] Arcoumanis, C., Khezzar, L., Whitelaw, D. S., and Warren, B. C. H., 1994, "Breakup of Newtonian and nonNewtonian fluids in air jets," Experiments in Fluids, 17(6), pp. 405-414.

[4] Liu, Z., and Reitz, R. D., 1997, "An analysis of the distortion and breakup mechanisms of high speed liquid drops," International Journal of Multiphase Flow, 23(4), pp. 631-650.

[5] Lee, C. H., and Reitz, R. D., 2000, "An experimental study of the effect of gas density on the distortion and breakup mechanism of drops in high speed gas stream," International Journal of Multiphase Flow, 26(2), pp. 229244.

[6] Lee, C. S., and Reitz, R. D., 2001, "EFFECT OF LIQUID PROPERTIES ON THE BREAKUP MECHANISM OF HIGH-SPEED LIQUID DROPS," 11(1), pp. 1-19.

[7] Park, S. W., Kim, S., and Lee, C. S., 2006, "Breakup and atomization characteristics of mono-dispersed diesel droplets in a cross-flow air stream," International Journal of Multiphase Flow, 32(7), pp. 807-822.

[8] Kim, S., Hwang, J. W., and Lee, C. S., 2010, "Experiments and modeling on droplet motion and atomization of diesel and bio-diesel fuels in a cross-flowed air stream," International Journal of Heat and Fluid Flow, 31(4), pp. 667-679. 
[9] Han, J., and Tryggvason, G., 2001, "Secondary breakup of axisymmetric liquid drops. II. Impulsive acceleration," Physics of Fluids, 13(6), pp. 1554-1565.

[10] Aalburg, C., 2002, Deformation and breakup of round drops and nonturbulent liquid jets in uniform crossflows.

[11] Quan, S., and Schmidt, D. P., 2006, "Direct numerical study of a liquid droplet impulsively accelerated by gaseous flow," Physics of Fluids (1994-present), 18(10), p. 102103.

[12] Jing, L., and Xu, X., 2010, "Direct Numerical Simulation of Secondary Breakup of Liquid Drops," Chinese Journal of Aeronautics, 23(2), pp. 153-161.

[13] Kékesi, T., Amberg, G., and Prahl Wittberg, L., 2014, "Drop deformation and breakup," International Journal of Multiphase Flow, 66, pp. 1-10.

[14] Yang, W., Jia, M., Sun, K., and Wang, T., 2016, "Influence of density ratio on the secondary atomization of liquid droplets under highly unstable conditions," Fuel, 174, pp. 25-35.

[15] Hirt, C. W., and Nichols, B. D., 1981, "Volume of fluid (VOF) method for the dynamics of free boundaries," Journal of Computational Physics, 39(1), pp. 201-225.

[16] Lafaurie, B., Nardone, C., Scardovelli, R., Zaleski, S., and Zanetti, G., 1994, "Modelling Merging and Fragmentation in Multiphase Flows with SURFER," Journal of Computational Physics, 113(1), pp. 134-147.

[17] G. Strotos, I. M., N. Nikolopoulos, K. Papadopoulos, A. Theodorakakos, M.Gavaises, 2015, "Performance of VOF methodology in predicting the deformation and breakup of impulsively accelerated droplets," ICLASS 2015, 13th Triennial International Conference on Liquid Atomization and Spray Systems, August 23-27Tainan, Taiwan.

[18] Strotos, G., Malgarinos, I., Nikolopoulos, N., and Gavaises, M., 2016, "Predicting droplet deformation and breakup for moderate Weber numbers," International Journal of Multiphase Flow, 85, pp. 96-109.

[19] Strotos, G., Malgarinos, I., Nikolopoulos, N., and Gavaises, M., 2016, "Aerodynamic breakup of an n-decane droplet in a high temperature gas environment," Fuel, 185, pp. 370-380.

[20] Strotos, G., Malgarinos, I., Nikolopoulos, N., and Gavaises, M., 2016, "Numerical investigation of aerodynamic droplet breakup in a high temperature gas environment," Fuel, 181, pp. 450-462.

[21] "ANSYS®FLUENT, Release 16.0.."

[22] Malgarinos, I., Nikolopoulos, N., and Gavaises, M., 2015, "Coupling a local adaptive grid refinement technique with an interface sharpening scheme for the simulation of two-phase flow and free-surface flows using VOF methodology," Journal of Computational Physics, 300, pp. 732-753.

[23] Pilch, M., and Erdman, C. A., 1987, "Use of breakup time data and velocity history data to predict the maximum size of stable fragments for acceleration-induced breakup of a liquid drop," International Journal of Multiphase Flow, 13(6), pp. 741-757.

[24] Dai, Z., and Faeth, G. M., 2001, "Temporal properties of secondary drop breakup in the multimode breakup regime," International Journal of Multiphase Flow, 27(2), pp. 217-236.

[25] Hsiang, L. P., and Faeth, G. M., 1995, "Drop deformation and breakup due to shock wave and steady disturbances," International Journal of Multiphase Flow, 21(4), pp. 545-560.

[26] Clift, R., Grace, J. R., and Weber, M. E., 2005, Bubbles, drops, and particles, Courier Corporation. 


\title{
Droplets heating and evaporation: an application to diesel-biodiesel fuel mixtures
}

\author{
Mansour al Qubeissi¹ ${ }^{\star 1}$ Nawar Al-Esawi ${ }^{1}$, Sergei S. Sazhin ${ }^{2}$ \\ ${ }^{1}$ Centre for Mobility and Transport, Faculty of Engineering, Environment and Computing, \\ Coventry University, Coventry CV1 2JH, UK \\ ${ }^{2}$ Advanced Engineering Centre, School of Computing, Engineering and Mathematics, \\ University of Brighton, Brighton BN2 4GJ, UK \\ ${ }^{*}$ Corresponding author: mansour.qubeissi@coventry.ac.uk
}

\begin{abstract}
The heating and evaporation of automotive fuel droplets are crucial to the design of internal combustion engines and to ensuring their good performance. Accurate modelling is essential to the understanding of these processes and ultimately improving engine design. The interest in fossil-biodiesel fuel blends has been mainly stimulated by depletion of fossil fuels and the need to reduce carbon dioxide emissions that contribute towards climate change. This paper presents an analytical investigation into the application of discrete component model for the heating and evaporation of multi-component fuel droplets to several blended diesel-biodiesel fuels. The model considers the contribution of all groups of hydrocarbons in diesel fuel and methyl esters in biodiesel fuels. The main features of new application to the analysis of blended-fuel droplets in engine-like conditions is described. The model is applied to several blends of diesel, combining 98 components of hydrocarbons, and 19 types biodiesel fuels, combining up to 17 species of methyl ester, considering the differences in their chemical levels of saturation, and thermodynamic and transport properties. One important finding is that some fuel blends, e.g. B5 (5\% biodiesel fuel and 95\% diesel fuel), can give almost identical droplet lifetimes to the one predicted for pure diesel fuel; i.e. such mixtures can be directly used in conventional diesel engines with minimal, or no, modification to the droplet break-up process.
\end{abstract}

\section{Keywords}

Biodiesel, Diesel, Fuel droplet, Fuel mixture, Heating and evaporation

\section{Introduction}

Renewable sources of energy, such as biodiesel fuels, have been of great interest to scientists and public in the last decades due to depletion of fossil fuels and impact on global warming [1,2]. Also, compared to fossil fuel, biodiesel fuel has several advantages: it has less carbon dioxide emissions, higher flash point, higher lubricity and it is cost effective; in addition, the blend of diesel-biodiesel fuels can be used in diesel engines with minimal/no modifications $[3,4]$.

The delay in processes preceding the onset of combustion (mainly the heating and evaporation of fuel droplets) in the internal combustion engines is crucial to the design and performance of these engines $[5,6]$. However, the complexity of modelling these processes should be taken into account as it involves detailed physics of heat transfer, mass transfer and fluid dynamics associated processes.

In this paper, the discrete component model (DCM) (see [5-7]) is utilised to analyse the droplets heating and evaporation of diesel-biodiesel fuel blends.

These blends are represented by a mixture of 19 types of biodiesel fuels with up to 17 species of methyl ester (see [8] for more details) and diesel fuel, formed of 98 hydrocarbons (see [7] for more details). The thermodynamic and transport properties of diesel fuel are inferred from [7]; while for properties of biodiesel fuel are taken from [9]. The contribution of species and average droplet temperatures are taken into account in the calculation of all properties in the liquid phase. The vapour mixtures are treated as ideal gases.

\section{Model and fuel compositions}

Following $[10,11]$, the analysis of droplets heating and evaporation processes is made in application to several blends of diesel and biodiesel fuels. In contrast to previous approaches, the discrete component model (DCM) (described in [5,12] and validated experimentally in [13]) is used for this analysis; and the model is applied to a broad range of diesel-biodiesel fuel fractions. The mixture of EU diesel fuel with 19 types of widely used biodiesel fuels have been investigated. These are: Tallow Methyl Ester (TME), Lard Methyl Ester (LME), Butter Methyl Ester (BME), Coconut Methyl Ester (CME), Palm Kernel Methyl Ester (PMK), Palm Methyl Ester (PME), Safflower Methyl Ester (SFE), Peanut Methyl Ester (PTE), Cottonseed Methyl Ester (CSE), Corn Methyl Ester (CNE), Sunflower 
Methyl Ester (SNE), Soybean Methyl Ester (SME), Rapeseed Methyl Ester (RME), Linseed Methyl Ester (LNE), Tung Methyl Ester (TGE), Hemp-oil Methyl Ester, produced from Hemp seed oil in Ukraine (HME1), Hemp-oil Methyl Ester, produced in European Union (HME2), Canola seed methyl ester (CAN) and Waste cooking-oil Methyl Ester (WCO). Droplets with four fractions of diesel-biodiesel blends have been investigated in the DCM; these are 5\% biodiesel with $95 \%$ diesel fuels (B5), $20 \%$ biodiesel with $80 \%$ diesel fuels (B20), $50 \%$ biodiesel with $50 \%$ diesel fuels (B50), pure biodiesel (B100) and pure diesel fuels (PD).

The DCM is based on the analytical solutions to the heat transfer and species diffusion equations via the Effective thermal conductivity model (ETC) and Effective Diffusivity model (ED). The importance of these models can be attributed to the fact that they take into account the recirculation, temperature gradients and species diffusion inside droplets.

The heat conduction equation for the temperature $T=T(t, R)$ in the liquid phase in a spherical droplet can be presented as:

$$
\frac{\partial T}{\partial t}=\kappa\left(\frac{\partial^{2} T}{\partial R^{2}}+\frac{2}{R} \frac{\partial T}{\partial R}\right)
$$

where $\kappa=k_{e f f} / \rho_{l} c_{l}$ is liquid thermal diffusivity, $k_{e f f}, \rho_{l}$ and $c_{l}$ are the effective thermal conductivity, liquid density, and liquid specific heat capacity, respectively, $R$ is the distance from the centre of the droplet (assumed to be spherical), $t$ is time. $k_{\text {eff }}$ is linked with the liquid thermal conductivity $k_{l}$ via the following equation [14]:

$$
k_{\text {eff }}=\chi k_{l} \text {, }
$$

where $\chi$ is the coefficient of recirculation, which varies between 1 and 2.72 (see [15] for details about the calculation of this coefficient).

The diffusion of mass fractions $\left(Y_{l i}\right)$ of liquid species $i$ in a spherical droplet is described by the following equation:

$$
\frac{\partial Y_{l i}}{\partial t}=D_{\text {eff }}\left(\frac{\partial^{2} Y_{l i}}{\partial R^{2}}+\frac{2}{R} \frac{\partial Y_{l i}}{\partial R}\right)
$$

where the diffusion coefficient of liquid species is linked with the liquid diffusion coefficient by the following equation of the Effective Diffusivity (ED) model [6,11]:

$$
D_{\text {eff }}=\chi_{Y} D_{l}
$$

where $\chi$ is the recirculation coefficient for species diffusion. The analysis of droplets evaporation is based on the following expression:

$$
\dot{m}_{d}=-2 \pi R_{d} D_{v} \rho_{\text {total }} B_{M} \mathrm{Sh}_{\mathrm{iso}},
$$

where $D_{v}$ is the binary diffusion coefficient of vapour in gas (air), $\rho_{\text {total }}=\rho_{g}+\rho_{v}$ is the total density of mixture of gas and vapour, $B_{M}$ is the Spalding mass transfer number:

$$
B_{M}=\frac{\rho_{v s}-\rho_{v \infty}}{1-\rho v s}=\frac{Y_{v S}-Y_{v \infty}}{1-Y v s},
$$

$Y_{v}$ are the vapour mass fractions and $\rho_{v}$ are densities of vapour, in the vicinity of droplet surfaces $(s)$ and at a large distance from them $(\infty)$, respectively, $\mathrm{Sh}_{\mathrm{iso}}$ is the Sherwood number for isolated droplets defined as $[15,16]$.

$$
\mathrm{Sh}_{\mathrm{iso}}=2 \frac{\ln \left(1+B_{M}\right)}{B_{M}}\left[1+\frac{\left(1+\mathrm{Re}_{\mathrm{d}} \mathrm{Sc}_{d}\right)^{\frac{1}{3}} \max \left\{1, \mathrm{Re}_{d}^{0.077}\right\}-1}{2 F\left(B_{M}\right)}\right] \text {, }
$$

where $\operatorname{Re}_{d}=\frac{2 \rho_{l} u_{s} R_{d}}{\mu_{l}}$ is the Reynolds number, $\mathrm{S}_{\mathrm{C}_{\mathrm{d}}}=\frac{v_{l}}{D_{l}}$ is the liquid Schmidt number, $v_{l}$ is the kinematic viscosity of liquid, and $u_{s}$ is the maximal liquid velocity near the surface of the droplet, calculated using the Abramzon and Sirignano [15] approach as:

$$
u_{S}=\frac{1}{32} \Delta u\left(\frac{\mu_{g}}{\mu_{l}}\right) \operatorname{Re}_{d} C_{F}
$$

where $\Delta u=\left|u_{g}-u_{d}\right|$ and $C_{F}=\frac{12.69}{\operatorname{Re}_{\mathrm{d}}^{2 / 3}\left(1+B_{M}\right)}$ is the drag coefficient, $u_{g}$ and $u_{d}$ are the gas and droplet velocities, respectively. The partial vapour pressure of species $i$ at the surface of the droplet is calculated based the following expression: 


$$
p_{v i s}=X_{l i s} \gamma_{i} p_{v i s}^{*}
$$

where $X_{\text {lis }}$ is the molar fraction of $i^{\text {th }}$ species in the liquid near the droplet surface, $\gamma$ is the activity coefficient and $p_{v i s}^{*}$ is the saturated vapour pressure of species (in the absence of other species).

The thermodynamic and transport properties are inferred from [7] for diesel fuel; while those for biodiesel fuel are inferred from previous calculations in [9]. All properties are averaged based on molar contribution of species and average droplet temperatures. The mixtures are treated as ideal gases. As in previous studies $[7,10,11]$, blended fuel vapour is assumed to diffuse from the surface of the droplet, without changing its composition, based on averaged binary diffusion of diesel fuel vapour into dry air.

\section{Results}

The discrete component model (DCM) described in the previous section is facilitated for the analysis of heating and evaporation of diesel-biodiesel fuel droplets of initial radius $R_{d 0}=12.66 \mu \mathrm{m}$ and temperature $T_{0}=360 \mathrm{~K}$. The droplets are moving at $10 \mathrm{~m} \mathrm{~s}^{-1}$ in still air of pressure and temperature equal to $30 \mathrm{bar}$ and $800 \mathrm{~K}$, respectively. This assumption is compatible with those used in [17].

The evolutions of droplet surface temperatures $\left(T_{s}\right)$ and radii $\left(R_{d}\right)$ for three mixtures of diesel-biodiesel fuels (B5, B20, B50) and pure biodiesel fuel (B100) of 19 types of biodiesel fuels are analysed. Figures 1-6 show some typical examples of the analysed blends of diesel-biodiesel.

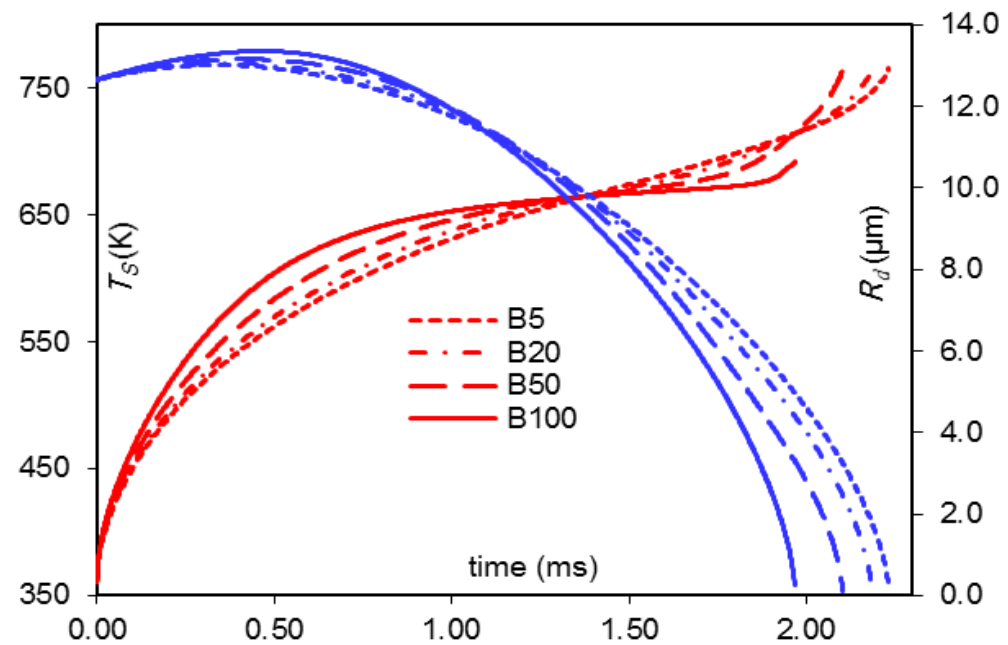

Figure 1. Droplet surface temperatures $T_{s}$ and radii $R_{d}$ versus time for four fractions of TME biodiesel fuel: B5, B20, B50 and $\mathrm{B} 100$. The droplet, of $12.66 \mu \mathrm{m}$ initial radius and $360 \mathrm{~K}$ initial temperature, is moving at $10 \mathrm{~m} / \mathrm{s}$ in still and dry air. The air pressure and temperature are 30 bar and $800 \mathrm{~K}$, respectively.

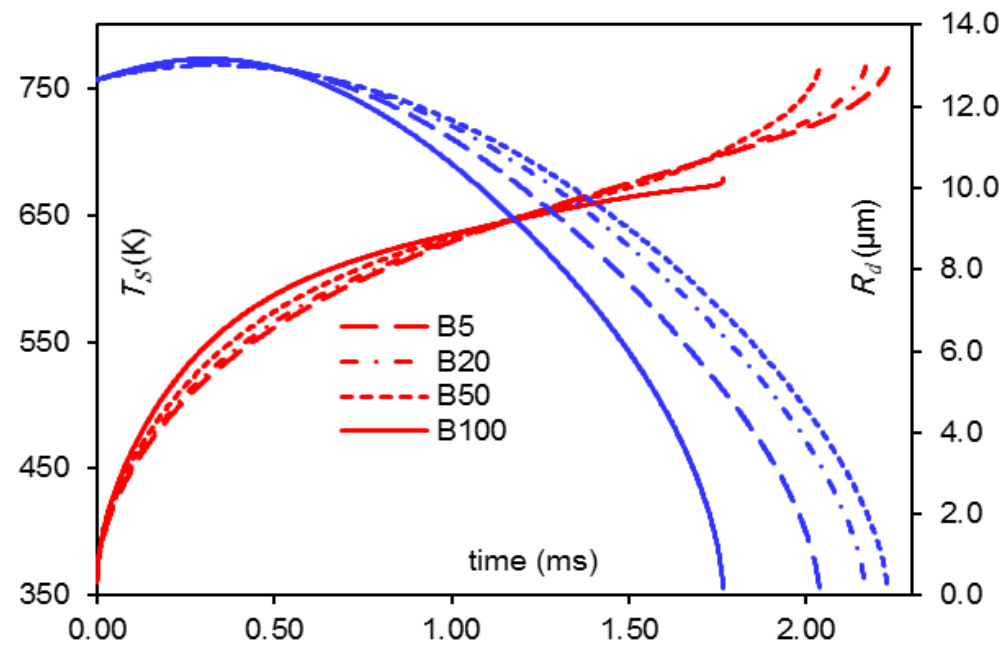

Figure 2. Droplet surface temperatures $T_{s}$ and radii $R_{d}$ versus time for four fractions of CME biodiesel fuel: B5, B20, B50 and B100, using the same ambient conditions and input parameters as in Figure 1. 


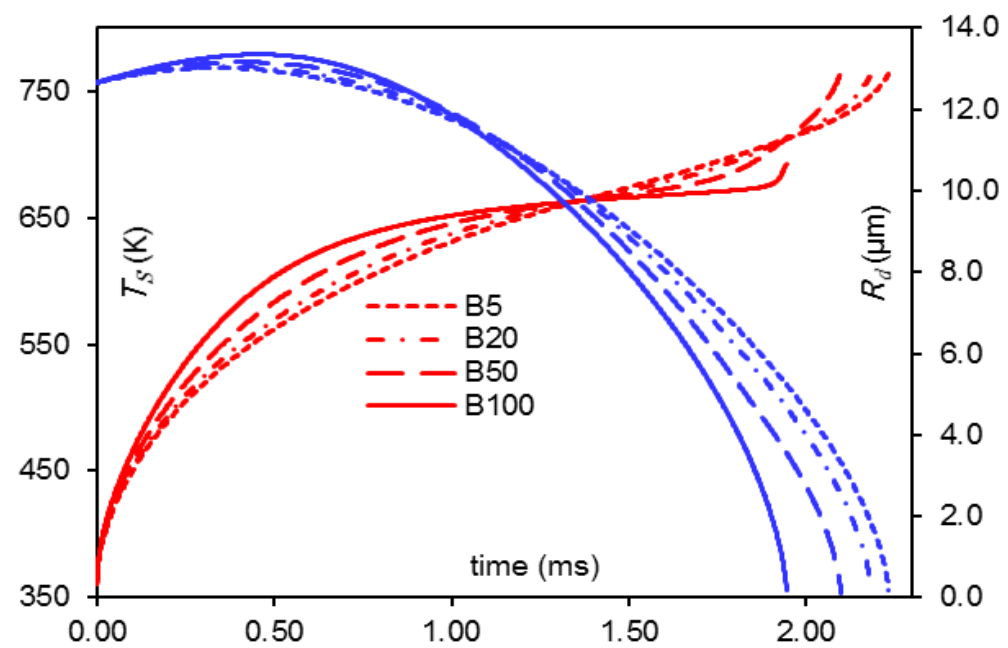

Figure 3. Droplet surface temperatures $T_{s}$ and radii $R_{d}$ versus time for four fractions of PME biodiesel fuel: B5, B20, B50 and B100, using the same ambient conditions and input parameters as in Figures 1 and 2.

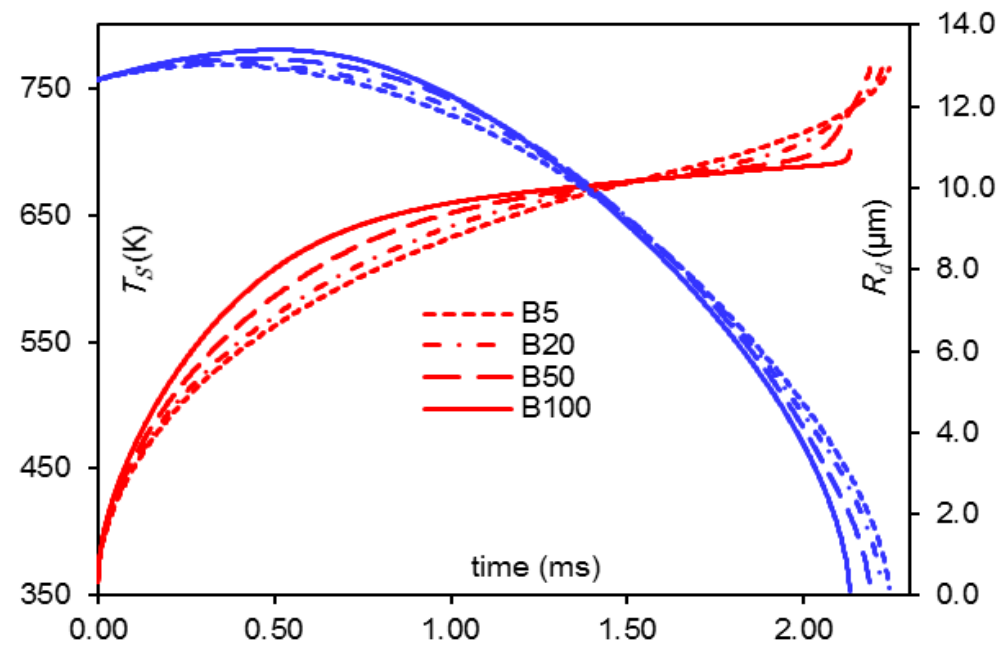

Figure 4. Droplet surface temperatures $T_{s}$ and radii $R_{d}$ versus time for four fractions of RME biodiesel fuel: B5, B20, B50 and B100, using the same ambient conditions and input parameters as in Figures 1-3.

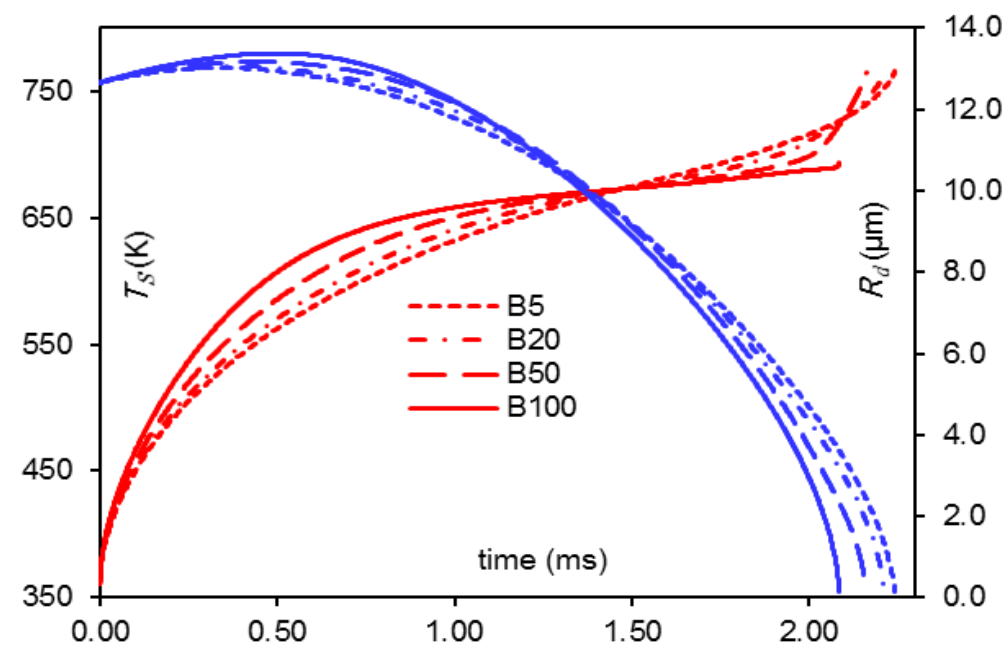

Figure 5. Droplet surface temperatures $T_{s}$ and radii $R_{d}$ versus time for four fractions of TGE biodiesel fuel: B5, B20, B50 and B100, using the same ambient conditions and input parameters as in Figures 1-4. 


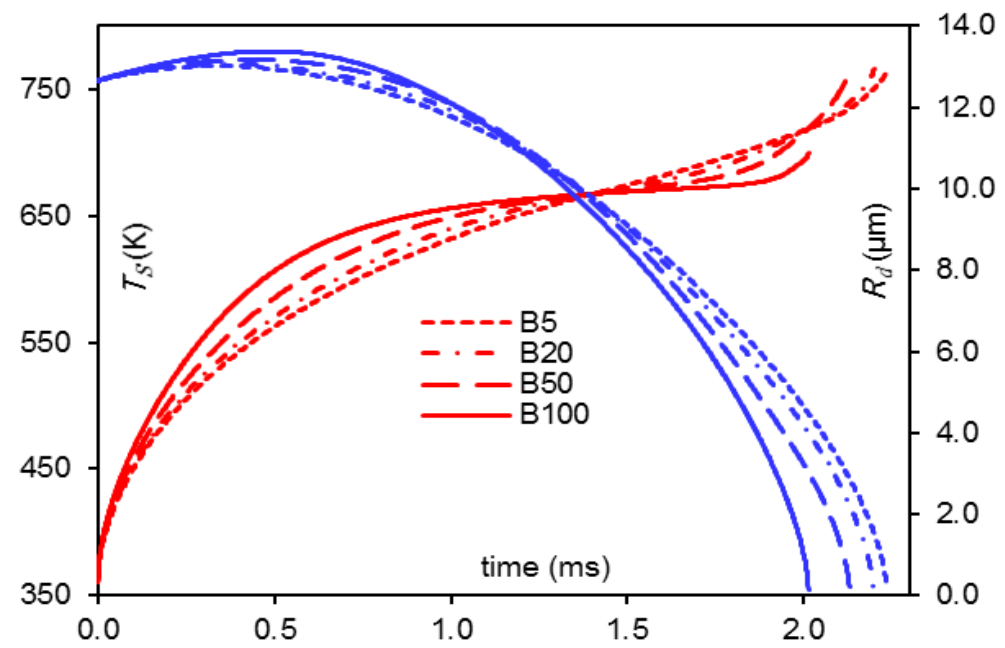

Figure 6. Droplet surface temperatures $T_{s}$ and radii $R_{d}$ versus time for four fractions of CAN biodiesel fuel: B5, B20, B50 and B100, using the same ambient conditions and input parameters as in Figures 1-5.

In Figures 1-6, one can see that increasing the concentration of biodiesel from B5 to B100 has a noticeable effect on the evolution of $R_{d}$ and $T_{s}$ for TME, CME, PME, RME, TGE, and CAN biodiesel fuels. In addition, the predicted surface temperature of the droplet for B100 is higher than that of B5 during the initial heating period. According to [10], the droplet break-up process can be enhanced as a result of the increase in droplet surface temperature. This can be attributed to the decrease in droplet surface tension. A full illustration of the results provided in Figure 1-6 are shown in Table 1. The droplet lifetimes of 19 types of biodiesel fuels mixtures with PD and their deviations from the one predicted for PD $(2.25 \mathrm{~ms})$ are presented in this table.

Table 1. Estimation of biodiesel fuel droplets lifetimes and their deviations compared with those of PD (2.25 ms) under the same conditions shown in Figures 1-8.

\begin{tabular}{|c|c|c|c|c|c|c|c|c|}
\hline \multirow{2}{*}{$\begin{array}{l}\text { Biodiesel } \\
\text { fuels }\end{array}$} & \multicolumn{2}{|c|}{ B100 } & \multicolumn{2}{|c|}{ B50 } & \multicolumn{2}{|c|}{ B20 } & \multicolumn{2}{|c|}{ B5 } \\
\hline & $\begin{array}{l}\text { Lifetime } \\
\text { (ms) }\end{array}$ & $\begin{array}{c}\text { Deviation } \\
(\%)\end{array}$ & $\begin{array}{l}\text { Lifetime } \\
\text { (ms) }\end{array}$ & $\begin{array}{c}\text { Deviation } \\
(\%)\end{array}$ & $\begin{array}{l}\text { Lifetime } \\
\text { (ms) }\end{array}$ & $\begin{array}{c}\text { Deviation } \\
(\%)\end{array}$ & $\begin{array}{l}\text { Lifetime } \\
\text { (ms) }\end{array}$ & $\begin{array}{c}\text { Deviation } \\
(\%)\end{array}$ \\
\hline TME & 1.967 & 12.58 & 2.102 & 6.58 & 2.184 & 2.93 & 2.232 & 0.80 \\
\hline LME & 1.995 & 11.33 & 2.114 & 6.04 & 2.190 & 2.67 & 2.234 & 0.71 \\
\hline BME & 1.943 & 13.64 & 2.089 & 7.16 & 2.180 & 3.11 & 2.232 & 0.80 \\
\hline CME & 1.765 & 21.56 & 2.036 & 9.51 & 2.166 & 3.73 & 2.229 & 0.93 \\
\hline PMK & 1.846 & 17.96 & 2.050 & 8.89 & 2.169 & 3.60 & 2.230 & 0.89 \\
\hline PME & 1.944 & 13.60 & 2.097 & 6.80 & 2.183 & 2.98 & 2.232 & 0.80 \\
\hline SFE & 1.980 & 12.00 & 2.122 & 5.69 & 2.195 & 2.44 & 2.235 & 0.67 \\
\hline PTE & 2.052 & 8.80 & 2.138 & 4.98 & 2.199 & 2.27 & 2.236 & 0.62 \\
\hline CSE & 2.014 & 10.49 & 2.128 & 5.42 & 2.197 & 2.36 & 2.236 & 0.62 \\
\hline CNE & 2.002 & 11.02 & 2.128 & 5.42 & 2.197 & 2.36 & 2.236 & 0.62 \\
\hline SNE & 2.011 & 10.62 & 2.132 & 5.24 & 2.200 & 2.22 & 2.237 & 0.58 \\
\hline SME & 1.981 & 11.96 & 2.127 & 5.47 & 2.198 & 2.31 & 2.236 & 0.62 \\
\hline RME & 2.131 & 5.29 & 2.188 & 2.76 & 2.222 & 1.24 & 2.242 & 0.36 \\
\hline LNE & 1.991 & 11.51 & 2.141 & 4.84 & 2.206 & 1.96 & 2.239 & 0.49 \\
\hline TGE & 2.085 & 7.33 & 2.160 & 4.00 & 2.211 & 1.73 & 2.240 & 0.44 \\
\hline HME1 & 2.022 & 10.13 & 2.138 & 4.98 & 2.203 & 2.09 & 2.237 & 0.58 \\
\hline HME2 & 1.994 & 11.38 & 2.135 & 5.11 & 2.202 & 2.13 & 2.238 & 0.53 \\
\hline CAN & 2.014 & 10.49 & 2.130 & 5.33 & 2.199 & 2.27 & 2.236 & 0.62 \\
\hline WCO & 2.002 & 11.02 & 2.121 & 5.73 & 2.194 & 2.49 & 2.235 & 0.67 \\
\hline
\end{tabular}


As can be seen from Table 1, the droplet lifetime for B100 of RME fuel is $6 \%$ less than that of PD. This reduction does not exceed $0.4 \%$ for the B5 fuel blend for the same fuel. Also, droplet lifetime of TGE biodiesel fuel droplet are noticeably close to that of PD droplet; it is less than $8 \%$ and $0.5 \%$ for B100 and B5 mixtures, respectively. The maximum deviation in droplet lifetimes for these fuels are up to 21. 6\% (B100 CME), which cannot be sacrificed in any engineering application, and it is always higher than $5.29 \%$ (RME) compared to PD, which may be tolerated in some limited engineering applications.

A typical example of time evolutions of mass fractions at the surface of droplets $\left(Y_{\text {lis }}\right)$ of selected nine species of B50 fuel mixture of diesel with RME and CME are shown in Figures 7 and 8, respectively.

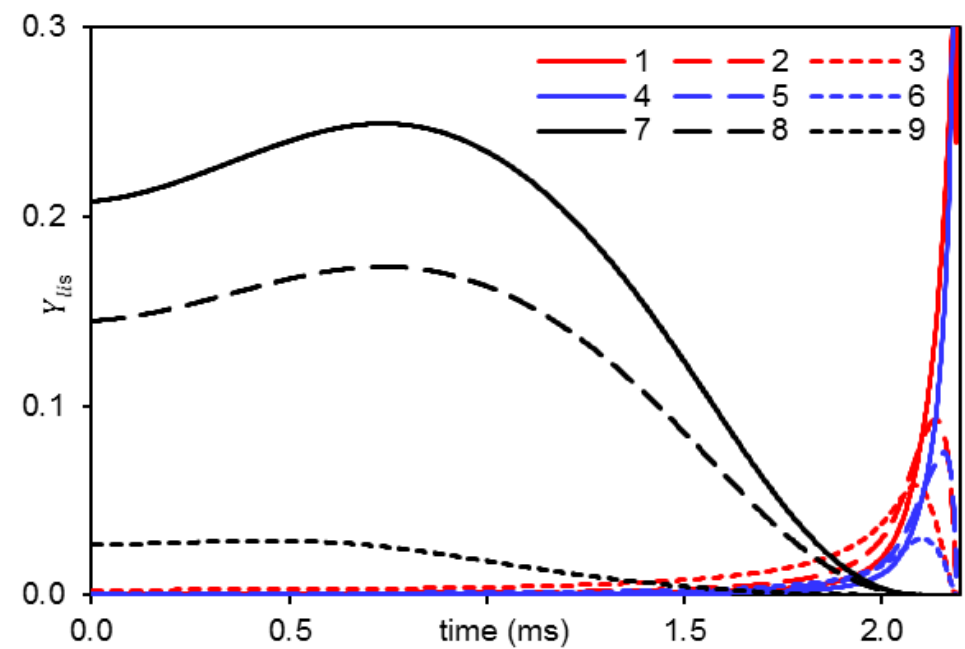

Figure 7. The liquid mass fractions at the surface of droplet $\left(Y_{l i s}\right)$ versus time for selected 9 components of 106 components of B50 (50\% diesel hydrocarbons and 50\% rapeseed methyl ester (RME)) fuel mixture. The red coloured curves, 1, 2 and 3, refer to alkane hydrocarbons of $\mathrm{C}_{23} \mathrm{H}_{48}, \mathrm{C}_{25} \mathrm{H}_{52}$ and $\mathrm{C}_{27} \mathrm{H}_{56}$, respectively; the blue curves, 4,5 and 6 , refer to cycloalkane hydrocarbons of $\mathrm{C}_{23} \mathrm{H}_{46}, \mathrm{C}_{25} \mathrm{H}_{50}$ and $\mathrm{C}_{27} \mathrm{H}_{54}$, respectively; and the black curves, 7,8 and 9 , refer to rapeseed methyl esters of $\mathrm{C}_{19} \mathrm{H}_{36} \mathrm{O}_{2}, \mathrm{C}_{19} \mathrm{H}_{34} \mathrm{O}_{2}$ and $\mathrm{C}_{17} \mathrm{H}_{34} \mathrm{O}_{2}$, respectively, under the same conditions used in Figures 1-6.

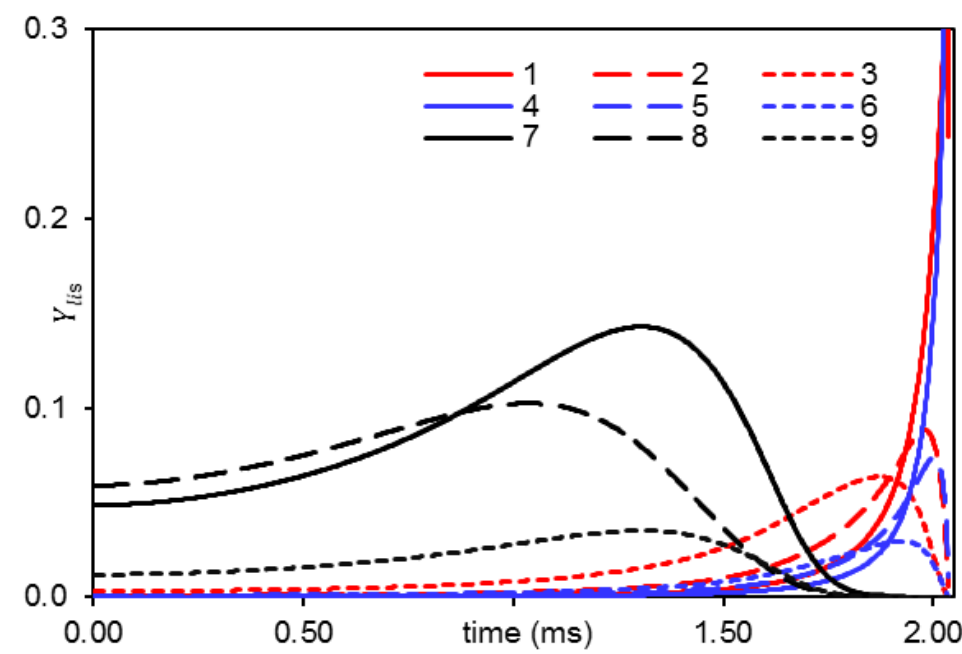

Figure 8. The liquid mass fractions at the surface of droplet $\left(Y_{l i s}\right)$ versus time for selected 9 components of 106 components of B50 (50\% diesel hydrocarbons and 50\% coconut methyl ester (CME)) fuel mixture. The red coloured curves 1, 2 and 3, refer to alkane hydrocarbons of $\mathrm{C}_{27} \mathrm{H}_{56}, \mathrm{C}_{25} \mathrm{H}_{52}$ and $\mathrm{C}_{23} \mathrm{H}_{48}$, respectively; and the blue curves 4,5 and 6 , refer to cycloalkane

hydrocarbons of $\mathrm{C}_{27} \mathrm{H}_{54}, \mathrm{C}_{25} \mathrm{H}_{50}$ and $\mathrm{C}_{23} \mathrm{H}_{46}$, respectively; and the black curves, 7, 8 and 9, refer to rapeseed methyl esters of $\mathrm{C}_{19} \mathrm{H}_{36} \mathrm{O}_{2}, \mathrm{C}_{19} \mathrm{H}_{34} \mathrm{O}_{2}$ and $\mathrm{C}_{17} \mathrm{H}_{34} \mathrm{O}_{2}$, respectively, under the same conditions used in Figures 1-7.

As can be seen from Figures 7 and 8, the diffusion of mass fractions of components at the surface of droplets are typical and similar to those presented in previous studies. The mass fractions of the heavy components, e.g. $\mathrm{C}_{27} \mathrm{H}_{56}$, $\mathrm{C}_{27} \mathrm{H}_{54}(1,4)$, increase with time at the expense of the lighter ones. This result is compatible with the findings presented in $[7,11,18]$.

In some previous studies (e.g. [10,17]) the heating and evaporation of PD droplets and their comparison to the results of diesel-biodiesel blends were analysed. For instance, in [17] the droplet lifetime for B100 of WCO was 
shown to be $11 \%$ less than that of PD. While in [10], the droplet lifetime for B100 of SME fuel was shown to be $6 \%$ less than that for PD. In this study, similar trends were predicted for the same fuels. This prediction, however, was different for the other types of biodiesel fuel presented in this paper. For example, the B100 droplet lifetimes for CME and PMK biodiesel fuels showed deviations of $21.6 \%$ and $18 \%$, respectively, from that of PD.

A general trend shows that droplets' lifetimes of all 19 types of B5 diesel-biodiesel blends that are used in this study deviate with less than $1 \%$ from the one predicated for PD droplets. This concludes the possibility of labelling dieselbiodiesel blends, with up to about $5 \%$ biodiesel concentration, without modifying the automotive system achievable. For some fuel blends (e.g. B20 RME, TGE, LNE, and HME1), this deviation (up to $2 \%$ ) is still relatively negligible to mix higher biodiesel concentrations (e.g. $20 \%$ biodiesel and $80 \%$ diesel fuels) without losing the main feature of these processes (i.e. droplet lifetime).

The difference in thermodynamic and transport properties between hydrocarbons and methyl esters is the main reason for the influence of biodiesel fuel fractions on the heating and evaporation of diesel fuel droplets. For instance, when increasing the biodiesel fractions, the droplet surface temperature tends to reach a plateau during the evaporation process, which is similar to the case of single component model (see [7,13]). Also, the significance of such behaviour can change depending on the input parameters and ambient conditions.

\section{Conclusion}

In this analysis, the discrete component model was used to analyse the heating and evaporation of blended dieselbiodiesel fuel droplets using 19 types of biodiesel fuel. The full compositions of diesel and biodiesel fuels were considered. The diesel fuel consisted of 98 hydrocarbons and the 19 biodiesel fuels, TME, LME, BME, CME, PMK, PME, SFE, PTE, CSE, CNE, SNE, SME, RME, LNE, TGE, HME1, HME2, CAN and WCO, consisted of 11, 8, 14, $8,11,10,4,8,7,8,7,7,8,6,7,13,7,12$ and 14 components of methyl esters, respectively.

The effect of increasing biodiesel fuel concentration on the evolutions of droplet surface temperature and evaporation time was clearly illustrated. The predicted B5 fuel droplet lifetimes for the 19 types of biodiesel fuel were only $1 \%$ less than that of pure diesel fuel (PD). The RME biodiesel fuel droplets were observed to have lifetimes close to that of PD, where their predicted lifetimes for B5 and B100 droplets were up to $6 \%$ and $0.4 \%$, respectively, less than the one estimated for PD droplet.

To conclude, the B5 fuel droplet lifetimes for all 19 types of biodiesel fuels used in this study are almost identical to the one predicted for PD; i.e. diesel fuel can be possibly blended with up to $5 \%$ biodiesel fuel without any noticeable effect on the evolutions of their radii or temperatures.

\section{Acknowledgment}

The authors are grateful to the Centre for Mobility and Transport - Coventry University for financial support.

$\begin{array}{ll}\text { Nomenclature } \\ \text { B\# } & \text { \#\% biodiesel/diesel fraction } \\ \text { BME } & \text { butter methyl ester } \\ \text { CAN } & \text { canola methyl ester } \\ \text { CME } & \text { coconut methyl ester } \\ \text { CNE } & \text { corn methyl ester } \\ \text { CSE } & \text { cottonseed methyl ester } \\ \text { DCM } & \text { discrete component model } \\ \text { ED } & \text { effective diffusivity } \\ \text { ETC } & \text { effective thermal conductivity } \\ \text { FAME } & \text { fatty acid methyl ester } \\ \text { HME1 } & \text { hempseed-oil, produced in Ukraine } \\ \text { HME2 } & \text { hempseed-oil, produced in the EU } \\ \text { LME } & \text { lard methyl ester } \\ \text { LNE } & \text { linseed methyl ester } \\ \text { PD } & \text { pure diesel fuel } \\ \text { PME } & \text { palm methyl ester } \\ \text { PMK } & \text { palm kernel methyl ester } \\ \text { PTE } & \text { peanut methyl ester } \\ \text { Re } & \text { Reynolds number } \\ \text { RME } & \text { rapeseed methyl ester } \\ \text { Sc } & \text { Schmidt number } \\ \text { SFE } & \text { safflower methyl ester } \\ & \end{array}$

$\begin{array}{ll}\text { Sh } & \text { Sherwood number } \\ \text { SME } & \text { soybean methyl ester } \\ \text { SNE } & \text { sunflower methyl ester } \\ \text { TGE } & \text { tung methyl ester } \\ \text { TME } & \text { tallow methyl ester } \\ \text { WCO } & \text { waste cooking oil }\end{array}$

\section{Symbols}

$B_{M} \quad$ Spalding mass transfer number

$c \quad$ heat capacity [J. $\mathrm{kg}^{-1} \mathrm{~K}^{-1}$ ]

$C_{F} \quad$ friction drag coefficient

$D \quad$ diffusion coefficient $\left[\mathrm{m}^{2} \mathrm{~s}^{-1}\right]$

$k \quad$ thermal conductivity [W. $\mathrm{m}^{-1} \mathrm{~K}^{-1}$ ]

$\dot{m} \quad$ evaporation rate $\left[\mathrm{kg} \cdot \mathrm{s}^{-1}\right]$

$p \quad$ gas pressure [bar]

$R \quad$ radius $[\mu \mathrm{m}]$

$\rho \quad$ density $\left[\mathrm{kg} \cdot \mathrm{m}^{-3}\right]$

$t \quad$ time [ms]

$T \quad$ temperature $[\mathrm{K}]$

$u \quad$ velocity $\left[\mathrm{m} \cdot \mathrm{s}^{-1}\right]$

$v \quad$ kinematic viscosity $\left[\mathrm{m}^{2} \mathrm{~s}^{-1}\right]$

$X \quad$ molar fraction 


$\begin{array}{llll}\chi & \text { recirculation coefficient } & i & \text { liquid species } \\ Y & \text { mass fraction } & \text { iso } & \text { isothermal process } \\ & & g & \text { gas } \\ \text { Subscripts } & l & \text { liquid } \\ \text { eff } & \text { effective thermal conductivity } & s & \text { droplet surface } \\ d \quad & \text { droplet } & v & \text { vapour }\end{array}$

\section{References}

[1] Lapuerta, M., Armas, O., and Rodríguez-Fernández, J., 2008, Prog. Energy Combust. Sci., 34(2), pp. $198-223$.

[2] Meher, L. C., Vidya Sagar, D., and Naik, S. N., 2006, Renew. Sustain. Energy Rev., 10(3), pp. $248-268$.

[3] Pan, K.-L., Li, J.-W., Chen, C.-P., and Wang, C.-H., 2009, Combust. Flame, 156(10), pp. 1926-1936.

[4] US Department of Energy: Energy Efficiency and Renewable Energy, "Biodiesel Blends" [Online]. Available: http://www.afdc.energy.gov/fuels/biodiesel_blends.html. [Accessed: 19-Mar-2017].

[5] Sazhin, S. S., 2017, Fuel, 196, pp. 69-101.

[6] Al Qubeissi, M., 2015, Heating and Evaporation of Multi-Component Fuel Droplets, WiSa, Germany.

[7] Sazhin, S. S., Al Qubeissi, M., Nasiri, R., Gun'ko, V. M., Elwardany, A. E., Lemoine, F., Grisch, F., and Heikal, M. R., 2014, Fuel, 129, pp. 238-266.

[8] Al Qubeissi, M., Sazhin, S. S., Crua, C., Turner, J., and Heikal, M. R., 2015, Fuel, 154, pp. 308-318.

[9] Sazhin, S. S., Al Qubeissi, M., Kolodnytska, R., Elwardany, A. E., Nasiri, R., and Heikal, M. R., 2014, Fuel, 115, pp. 559-572.

[10] Al Qubeissi, M., Sazhin, S. S., and Elwardany, A. E., 2017, Fuel, 187, pp. 349-355.

[11] Al Qubeissi, M., and Sazhin, S. S., 2016, 27th European Conference on Liquid Atomization and Spray Systems, Brighton, UK, p. 179.

[12] Sazhin, S. S., 2014, Droplets and Sprays, Springer, London.

[13] Elwardany, A. E., Gusev, I. G., Castanet, G., Lemoine, F., and Sazhin, S. S., 2011, At. Sprays, 21(11), pp. $907-931$.

[14] Abramzon, B., and Sazhin, S. S., 2006, Fuel, 85(1), pp. 32-46.

[15] Abramzon, B., and Sirignano, W. A., 1989, Int. J. Heat Mass Transf., 32(9), pp. 1605-1618.

[16] Sirignano, W. A., 1999, Fluid Dynamics and Transport of Droplets and Sprays, Cambridge University Press, Cambridge, U.K.

[17] Al Qubeissi, M., Sazhin, S. S., de Sercey, G., and Crua, C., 2014, 26th European Conference on Liquid Atomization and Spray Systems, University of Bremen, Bremen, Germany. 


\title{
Expansion rates of bubble clusters in superheated liquids
}

\author{
Dirk Dietzel ${ }^{1}$, Timon Hitz ${ }^{2}$, Claus-Dieter Munz ${ }^{2}$, Andreas Kronenburg*,1 \\ ${ }^{1}$ Institute of Combustion Technology, University of Stuttgart, Germany \\ ${ }^{2}$ Institute of Aerodynamcis and Gas Dynamics, University of Stuttgart, Germany \\ *Corresponding author: kronenburg@itv.uni-stuttgart.de
}

\begin{abstract}
The present work analyses the growth of multiple bubbles in superheated liquid jets by means of direct numerical simulations (DNS). A discontinuous Galerkin approach is used to solve the Euler equations and an adequate interface resolution is ensured by applying finite-volume sub-cells in cells with interfaces. An approximate Riemann solver has been adapted to account for evaporation and provides consistency of all conserved quantities across the interface. The setup mimics conditions typical for orbital manoeuvring systems when liquid oxygen (LOX) is injected into the combustion chamber prior to ignition. The liquid oxygen will then be in a superheated state, bubble nucleation will occur and the growth of the bubbles will determine the break-up of the liquid jet. The expansion rates of bubble groups under such conditions are not known and standard models rely on single bubble assumptions. This is a first DNS study on bubble-bubble interactions in flash boiling sprays and on the effects of these interactions on the growth rates of the individual bubbles. The present simulations resolve a small section of the jet close to the nozzle exit and the growth of bubble groups inside of the jet is analysed. The results suggest that an individual bubble within the group grows more slowly than conventional models for single bubble growth would predict. The reduction in bubble growth can amount to up to $30 \%$ and depends on the distances between the bubbles and the number of bubbles within the bubble group. In the present case, the volume expansion of the superheated liquid decreases by approximately $50 \%$ if the distance between the bubbles is doubled.
\end{abstract}

\section{Keywords}

Vapour bubble growth, compressible flow, Riemann solver, jet expansion

\section{Introduction}

The attitude control of rockets, space probes and satellites is usually accomplished by utilizing small thrusters. One of the major challenges is the ignition of the mixture of fuel and oxidizer under vacuum conditions. Fuel and oxidizer are injected as liquids and the jet atomization determines the prospects of successful ignition events and of the efficiency of the subsequent combustion process. Due to the low pressure being below saturation conditions, nucleation and growth of the vapour bubbles enhances the disintegration of the liquid jet into a disperse spray. Any current modelling strategies of the so-called flash evaporation process do not resolve bubble nucleation and growth of single bubbles and will require closures for the jet expansion and atomization at sub-grid scale. Models for single bubble growth exist, however, nucleation rates in superheated liquids can be very high and bubbles will interact during their growth process. These interactions are not yet fully understood, but quantifying these small scale processes will be necessary for finding suitable closure models for flash-boiling sprays. One particular aspect are the expansion rates of the bubble groups within the superheated liquid jets that have not been investigated before.

A great effort has been spent on the investigation of flash evaporation, both, experimentally and numerically. The morphology of flashing sprays and the resulting jet angles were investigated experimentally e.g. in [1, 2]. Here, the influence of the ambient temperature and pressure as well as the injection pressure were investigated for different fluids. It was found that, depending on the degree of superheat, spray angles up to $160^{\circ}$ with a homogeneous distribution of fine droplets can be realized. The liquid jets rapidly expand in radial direction immediately after exiting the nozzle. Similar observations were made in various other studies $[3,4,5,6]$. Generally, the experimental results show that models for mechanical break-up are not valid for flash-boiling sprays. Examples for numerical studies on flash evaporation are found in [7, 8, 9, 10, 11]. Different modelling strategies are presented there, ranging from single fluid approaches with additional transport equations for the vapour mass fraction to Euler-Lagrangian simulations, where droplets are explicitly tracked as Lagrangian particles. These are promising approaches for the simulation of the entire spray process, but the initial bubble dynamics in the superheated liquid core cannot be resolved. However, the growth and dynamic behaviour of vapour bubbles in the liquid jet have a significant influence on the jet spreading, break-up and droplet formation. The underlying processes, e.g. the pressure wave dynamics and its effect on the velocity and temperature fields, are not entirely understood and an improved understanding is necessary to obtain further improvements of suitable simulation procedures.

The present work analyses the expansion rates of bubble groups in superheated liquids with the aid of fully resolved direct numerical simulations. The dependency of the growth rates on the bubble number density is investigated for cryogenic oxygen. We use a discontinuous Galerkin (DG) approach to solve for the Euler equations. Special approximate Riemann solvers are applied at the interface between liquid and vapour capturing compressible wave 
dynamics as well as phase change. The interface is accounted for using the Level-set method, and we apply a kinetic relation for determining the evaporation rates. The kinetic relation is calibrated by computations of single vapour bubble growth that correspond to conditions given in the vacuum chamber. A subdivision of a Galerkin cell into finite-volume cells close to the interface allows for a better representation of the interface for a fixed Galerkin resolution. The 3D computations are conducted for one set of initial conditions with a different number of bubble nuclei. The paper is organized as follows: First, the numerical methods for the solution of the conservation equations and the treatment of the phase interface are outlined. Then we give the initial conditions of all physical quantities and detail the calibration of the evaporation model. The geometrical configuration of the three-dimensional simulation is given thereafter. Results of two three-dimenensional DNS are presented and discussed, and the paper is concluded with a summary and outlines future work.

\section{Numerical Methods}

The DNS of multiphase flows with phase change requires a numerical approximation of the conservation equations for mass, momentum and energy. Here, we use a discontinuous Galerkin spectral element method (DGSEM). In contrast to conventional finite-volume approaches, this allows for high order accuracy in the bulk phases without applying computationally expensive numerical schemes. Details of the algorithm are found in [12]. Finite-volume sub-cells are used only in the vicinity of the liquid-vapour interface to improve its representation within the Galerkin cells as described in [13]. To keep track of the interface, we apply the level-set algorithm introduced in [14]. A detailed description and validation of the algorithm consisting of the DGSEM for the compressible Euler equations, including the finite-volume sub-cells and the Level-set algorithm, is given in [15]. The phase change is accounted for by using approximate Riemann solvers along with a kinetic relation for the mass flux estimation [16]. This approximate Riemann solver has been newly developed in [16] and for the first time, it is now applied to problems of single or multiple bubble growth. For coupling the thermodynamics to the hydrodynamics the open-source library CoolProp is used, which is based on a Helmholtz energy functional [17]. In the following sections we give a brief introduction to the different algorithms.

\section{Discontinuous Galerkin spectral element method}

Within the discontinuous Galerkin approach the simulation domain is discretized with a set of control volumes $\Omega$. Each cell can be handled separately and in a first step, a control volume is mapped from physical space, $\mathbf{x}$, to a reference space, $\xi$. The computational cells are spanned to a unit reference element $\mathcal{E}$ of the size $[-1 ; 1]$ in each direction. After multiplying the resulting system of equations by a test function $\phi$, the governing equations read [12]

$$
\int_{\mathcal{E}} J U_{t} \phi \mathrm{d} \boldsymbol{\xi}+\int_{\mathcal{E}} \nabla_{\xi} \cdot \hat{\mathbf{F}}(U) \phi \mathrm{d} \boldsymbol{\xi}=0
$$

with the vector of conservative variables $U$, the flux function $\hat{\mathbf{F}}(U)$ and the Jacobi determinant $J$. An integration by parts of the second term in Eq. (1) gives the weak formulation of the conservation laws,

$$
\frac{\partial}{\partial t} \int_{\mathcal{E}} J U \phi d \boldsymbol{\xi}+\int_{\partial \mathcal{E}}(\hat{\mathbf{F}} \cdot \mathbf{N}) \phi \mathrm{d} S-\int_{\mathcal{E}} \hat{F}(U) \cdot\left(\nabla_{\xi} \phi\right) \mathrm{d} \boldsymbol{\xi}=0 .
$$

The first and the second terms on the left hand side contribute to the time derivative of the conserved quantity and the fluxes across the surface of the DG cell. Each cell additionally provides an inner cell resolution which is accounted for by a continuous in-cell ansatz function. For the numerical integration of the fluxes Lagrange polynomials are used, where the polynomial degree $N$ determines the accuracy of integration. As polynomials are local for one cell only, discontinuities may arise at the cell face and Riemann solvers are used to determine consistent fluxes across the cell boundaries. For the present investigation an HLLC-Riemann solver is applied at single phase DG cell boundaries. As the in-cell polynomials are continuous, the interface between liquid and vapour cannot be accounted for and a DG cell is sub-divided into finite-volume cells in the vicinity of the interface [13]. The time integration is accomplished by a third order Runge-Kutta method.

\section{Interface treatment and phase change modeling}

The interface between gas and liquid is tracked using the level-set approach introduced by Sussman et al. [14]. The signed distance function $\Phi(\mathbf{x}, t)$ is transported as

$$
\frac{\partial \Phi}{\partial t}+\mathbf{u}_{\llcorner S} \cdot(\nabla \Phi)=0
$$

The transport velocity of the level-set, $\mathbf{u}_{\mathrm{LS}}$, accounts for the movement of the zero-isosurface of $\Phi$. In the interface cells $\mathbf{u}_{\mathrm{LS}}$ is obtained from the liquid-vapour jump conditions for the mass and the momentum balance. This velocity is then extended to several neighbouring cells. The level-set determines the distance to the interface at every position in the flow field and the zero isocontour of $\Phi$ defines the position of the interface. Since the transport further away from the interface must not necessarily account for the displacement of the nearest interface, the Hamilton-Jacobi 


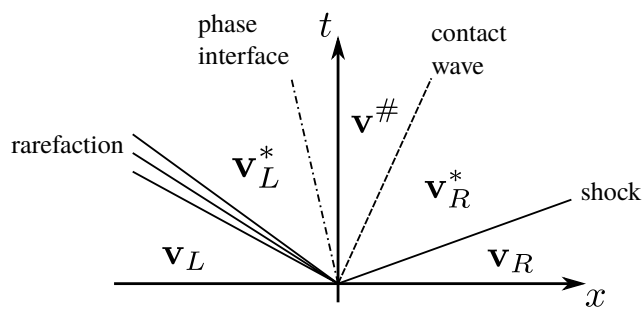

Figure 1. Riemann wave fan of a two-phase Riemann problem including phase change. State vector $\mathbf{v}=(\rho, \rho u, \rho e, p)^{t}$. Here, the different state vectors describe the initial liquid state $\left(\mathbf{v}_{L}\right)$, rarefied liquid $\left(\mathbf{v}_{L}^{*}\right)$, evaporated fluid $\left(\mathbf{v}^{\#}\right)$, compressed vapour $\left(\mathbf{v}_{R}^{*}\right)$ and the initial vapour state $\left(\mathbf{v}_{R}\right)$.

equation

$$
\frac{\partial \Phi}{\partial \tau}=S_{\epsilon}\left(\Phi_{\tau=0}\right)(|\nabla \cdot \Phi|-1)
$$

is iterated for a pseudo time $\tau$. When Eq. (4) has converged to a steady state the distance property of $\Phi$ is recovered fulfilling the condition $|\nabla \cdot \Phi|=1$.

At the phase interface between liquid and vapour, a strong contact discontinuity usually appears, particularly for density and pressure, and fluxes across the interface cannot be determined in the same manner as in the bulk phases. In addition, phase change shall be considered within the present investigation which complicates the Riemann problem at the phase interface. In order to guarantee thermo- and hydrodynamically consistent fluxes across the phase interface including the modeling of phase transition, special approximate Riemann solvers are applied as introduced in [16]. Figure 1 depicts a typical two-phase Riemann problem including rarefaction, shock and contact waves as well as the phase interface as an additional discontinuity. The different fluid states describe the initial liquid state, rarefied liquid, evaporated fluid, compressed vapour and the initial vapour state from the left to the right, respectively. An exact solution of the Riemann problem requires an iterative solution of the RankineHugoniot jump conditions across the different waves. Within the rarefaction fan, an isentropic flow is assumed, and the phase interface is modeled as under-compressive shock wave. Given a suitable expression that determines the evaporating mass, the solution of the resulting system of equations provides physically exact interface fluxes but its iterative solution procedure is computationally expensive. Thus, an approximate Riemann solver is introduced, where the complexity of the mathematical problem is reduced. The simplifications include an estimation of the outer wave speeds (rarefaction and shock waves) as well as omission of the contact wave. The iteration procedure now simplifies to finding the interfacial mass flux where we use the kinetic relation [18]

$$
\frac{c_{\text {ent }} \dot{m}^{\prime \prime 2}}{T_{\text {ref }}}=\llbracket \rho\left(u-a_{\text {if }}\right)\left(s-s_{\text {sat }}\left(T_{\text {ref }}\right)\right) \rrbracket .
$$

Here, the square brackets indicate the jump across the interface, i.e. the difference between liquid and vapour state. The mass flux $\dot{m}^{\prime \prime}$ is estimated comparing the deviation of the local entropy, $s$, to the saturation entropy, $s$ sat, between the liquid and the vapour phases. The coefficient $c_{\text {ent }}$ guarantees a consistent scaling of the entropy production with mass flux. Details of the Riemann solver are found in [16]. The calibration of $c_{\text {ent }}$ is presented in the next section. As continuous polynomials are used within one DG cell, it is not possible to capture the interface discontinuity between polynomial nodes, and the liquid-vapour interface must lie on DG cell interfaces. To enhance the interface resolution, the polynomial nodes are treated as finite volume (FV) cells in the vicinity of the interface by subdividing each DG cell into $N+1$ FV sub-cells per spatial direction [13]. These cells are decoupled from their polynomial ansatz function and discontinuities may arise between them. For the present investigation a sharp interface approach is used. Every computational cell is considered as either liquid or gaseous and there are no mixed cells. Thus, interfacial fluxes can be determined directly across FV cell faces in the refined region and the interface is shifted from one cell face to the next whenever the sign of $\Phi$ changes. It has to be noted that this approach is not strictly conservative but is needed to avoid a smearing of the interface over several computational cells.

\section{Setup}

\section{Physical initial conditions}

The physical initial conditions are given in Tab. 1. We have chosen an ambient temperature of $T=120 \mathrm{~K}$ and an ambient pressure of $p_{\infty}=2.0 \cdot 10^{5} \mathrm{~Pa}$. The conditions inside of the bubble are typically estimated assuming saturation conditions at the ambient temperature with $p_{\text {bub }}=p_{\text {sat }}(T)$. The initial bubble radius results from the Young-Laplace law as [19]

$$
r_{0}=\frac{2 \sigma}{p_{\text {sat }}-p_{\infty}}
$$


Table 1. Initial conditions for liquid oxygen. The initial velocity is zero everywhere.

\begin{tabular}{c|ccc}
\hline & vapour bubble & liquid & ambient vapour \\
\hline$\rho\left[\mathrm{kg} / \mathrm{m}^{3}\right]$ & 39.308 & 969.208 & 6.752 \\
$p[\mathrm{~Pa}]$ & $1.022 \cdot 10^{6}$ & $2.045 \cdot 10^{5}$ & $2.045 \cdot 10^{5}$ \\
$T[\mathrm{~K}]$ & 120 & 120 & 120
\end{tabular}
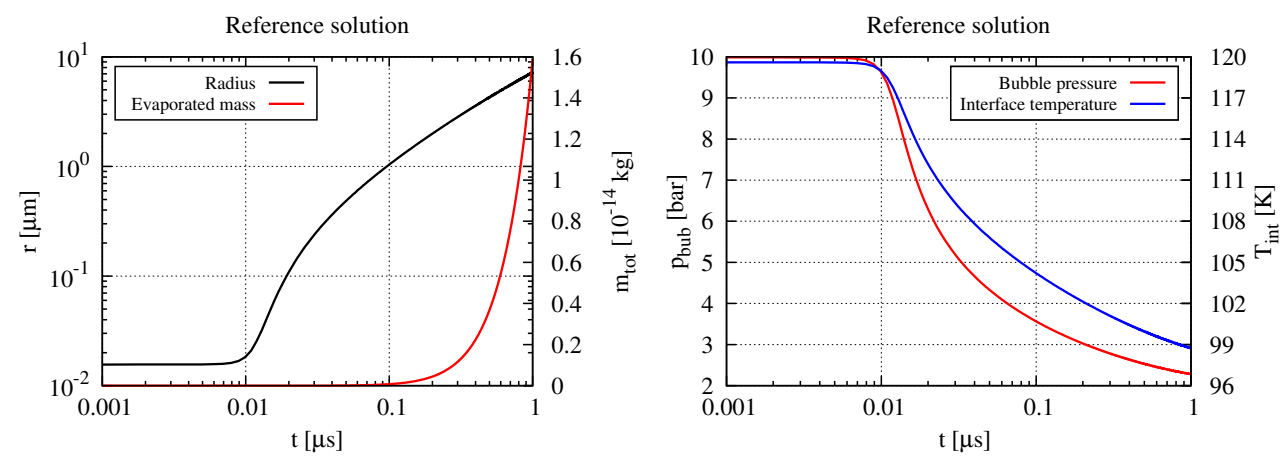

Figure 2. Left: Time evolution of the bubble radius (black line) and the accumulated mass (red line). Right: Time evolution of the bubble pressure (red line) and the interface temperature (blue line). Results are obtained from the solution of the Rayleigh-Plesset equation coupled to the temperature equation in the liquid.

where $\sigma$ is the surface tension coefficient. It is $\sigma=0.006 \mathrm{~N} / \mathrm{m}$ for liquid oxygen at $T=120 \mathrm{~K}$ giving $r_{0}=0.015 \mu \mathrm{m}$. The saturation pressure inside the bubble and the ambient pressure give a pressure ratio of $R_{p}=p_{\text {sat }} / p_{\infty}=5$. Note that flash-boiling with liquid jets containing numerous bubbles occurs typically at much higher pressure ratios (e.g. [2]). However, the growth of single vapour bubbles is found to be relatively insensitive to $R_{p}$ for the time range considered and in the present investigation, the bubble number density does not depend on the choice of $R_{p}$.

\section{Scaling of the evaporation model}

As a reference case for calibrating the entropy production coefficient, $c_{\text {ent }}$ (see Eq. (5)), the growth of a single bubble is used. Figure 2 depicts the reference solution obtained by solving the Rayleigh-Plesset equation (see [20]) including a coupling with the temperature equation in the liquid as given in [21]. The black line in Fig. 2 (left) shows the bubble radius over time, and it is seen that it has grown to approximately $r=10 \mu \mathrm{m}$ after $t=1 \mu \mathrm{s}$. This radius corresponds to the average bubble size when bubbles coalesce for conditions given in our first reference case (see below). The red line in the left sub-figure in Fig. 2 shows the accumulated evaporated mass over time. Only at time scales of the order of a micro-second, we observe a significant rise in the evaporated mass. This indicates that bubble expansion at time scales one or several orders smaller than the final time (here one micro-second) will not affect the volume expansion of the liquid jet and the dynamics related to the bubble expansion at these scales do not need to be considered. In Fig. 2 (right), the pressure inside of the bubble (red line) and the interface temperature (blue line) are depicted. We observe a fast decrease in the interface temperature due to the latent heat of vaporization. As the bulk liquid remains at a high temperature, a thermal boundary layer develops. The pressure inside of the bubble decreases with the interface temperature and it quickly reaches pressures close to the ambient pressure after $1 \mu \mathrm{s}$. Typically, bubble growth is divided in an early inertia driven stage and a subsequent stage driven by heat diffusion (see e.g. [19]). However, the decreased pressure seen in Fig. 2 starts to affect the bubble growth even at early times. Thus, despite an expectancy of a slow heat diffusion process, it needs to be included at the small time scales if the effects of evaporative cooling and pressure drop are to be considered. In order to account for this effect the evaporation model in Eq. (5) is scaled to match the total evaporated mass after $t=1 \mu \mathrm{s}$. In Fig. 3 the solution from the DG solver with $c_{\mathrm{ent}}=540$ is compared to the reference solution. The initial radius in the DG solution is increased to $r_{0, D G}=1.0 \mu \mathrm{m}$. This corresponds to the radius at $t=0.1 \mu \mathrm{s}$ for the reference solution, where significant mass accumulation starts. The increase in the initial radius allows for a coarser mesh resolution and thus, a coarser time step such that the computational effort for the three-dimensional computations stays acceptable. The left subfigure of Fig. 3 shows the bubble surface over time. Differences are only observed at early times, when the difference in the initial radii is not compensated. The middle and the right sub-figures of Fig. 3 depict the volume change of the bubble and the total evaporated mass, respectively. The results from the DG solver match very well with the reference solution at all times, and the difference in initial bubble radius should therefore not unduly affect the dynamics of jet expansion and breakup. Note, that the calibration is specific for the present ambient conditions and needs to be adapted accordingly if conditions change. 

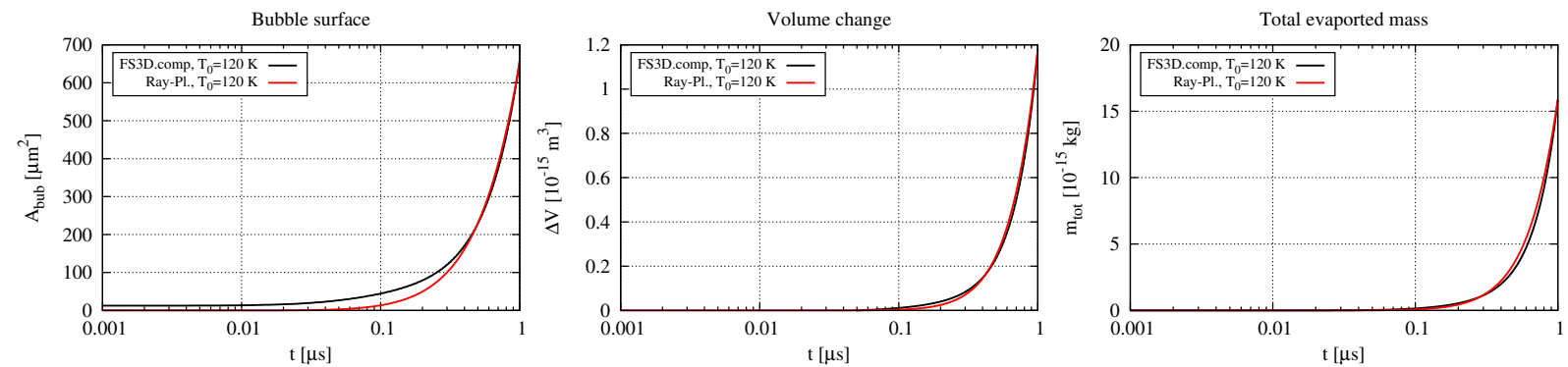

Figure 3. Bubble surface (left), volume change (middle) and total evaporated mass (right) of the scaled computations for single bubble growth. The red and the black lines represent the reference solution and the solution obtained from the DG solver, respectively.
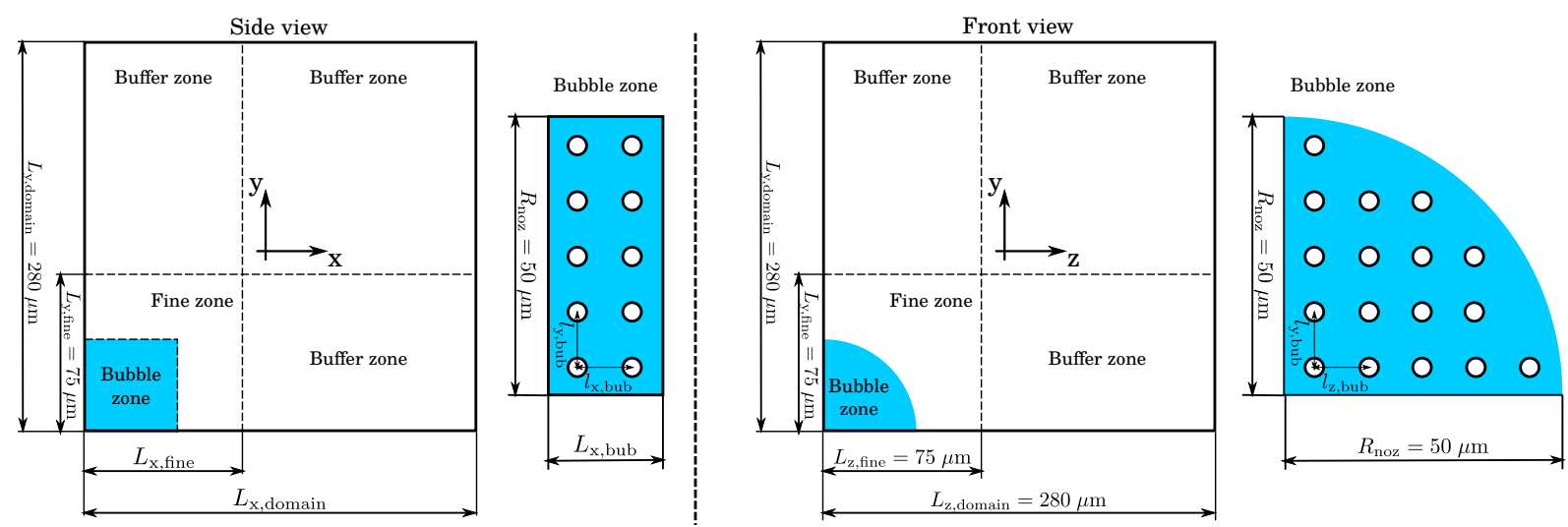

Figure 4. Geometrical setup of the three-dimensional simulations. The bubble zone represents the tip of a liquid jet immediately after the nozzle exit.

\section{Geometry and Numerics}

In the present investigation we focus on the small scale processes close to the nozzle exit, where the growth of vapour bubbles begins to affect the dynamics of the liquid jet. The computational domain is depicted in Fig. 4. A small liquid core is considered where a fixed number of bubbles is prescribed (bubble zone). The lengths of the bubble zone in $y$ - and $z$-directions are defined by the nozzle radius. Typical nozzle radii in corresponding experimental investigation of the German national aeronautics and space research centre (DLR Lampoldshausen) lie in between $R_{\mathrm{noz}}=50 \mu \mathrm{m}$ and $R_{\mathrm{noz}}=500 \mu \mathrm{m}$ and we choose $R_{\mathrm{noz}}=50 \mu \mathrm{m}$ for the present case. The fine zone in Fig. 4 has the same equidistant mesh resolution as the bubble zone, and therefore guarantees a sufficient resolution during liquid core expansion. The grid spacing in the fine zone is chosen such that a bubble diameter is initially resolved with eight FV-cells. In the buffer zone the cells are stretched, and this zone is needed to reduce the influence of the outflow boundary conditions applied in the positive $x-, y-$ and $z$-directions. At all other boundaries symmetry conditions are applied imposing some degree of rotational symmetry of the expansion process. The mesh dimensions in $y$ - and $z$-directions are the same for all simulations and they are depicted in Fig. 4 . We set up two cases with a different bubble spacing. The cases are labelled Case 1 and Case 2. The number of bubbles in the $y$ - and $z$-direction is $N_{\text {bub,y }}=N_{\text {bub,z }}=5$ for Case 1 and $N_{\text {bub,y }}=N_{\text {bub,z }}=3$ for Case 2 . The distance of the bubbles is $l_{\mathrm{bub}}=10 \mu \mathrm{m}$ and $l_{\mathrm{bub}}=20 \mu \mathrm{m}$, respectively, and equal in all three spatial directions. The number of bubbles in $x$-direction is the same in Case 1 and 2 but the length of the bubble zone is extended such that we have a consistent spacing of the bubbles according to the other directions. In that way we resolve two bubble layers in the $x$-direction in both cases allowing for a comparison between bubbles located in the center and at the edges of the bubble group. The total number of bubbles resolved is 34 in Case 1 and twelve in Case 2. The axial extension of the initial liquid core corresponds to the estimated penetration of the liquid into the vacuum chamber during the time needed for jet break-up. All the geometric data are given in Tab. 2.

\section{Results and discussion}

Three-dimensional results are shown in Fig. 5 for Case 1. The curved iso-surfaces indicate the bubbles and the liquid jet, respectively. The $x-$ and the $z$-normal plane show the velocity fields. The inner bubbles grow isotropically while the outer bubbles expand stronger towards the jet surface (e.g. top right bubbles in Fig. 5). In the direction of the jet surface, bubbles are permanently facing the low-pressure environment being the driving force of bubble expansion. This is different in the jet center, where the expansion of the bubbles is more sensitive to the local pressure fluctuations and the resistance effectuated by the neighbouring bubbles decreases the expansion rates. 
Table 2. Geometrical specifications for Case 1 and Case 2.

\begin{tabular}{c|cc}
\hline & Case 1 & Case 2 \\
\hline$N_{\text {bub }, \mathrm{x}}$ & 2 & 2 \\
$N_{\text {bub,yz }}$ & 5 & 3 \\
$l_{\text {bub }}[\mu \mathrm{m}]$ & 10 & 20 \\
$L_{\mathrm{x}, \text { bub }}[\mu \mathrm{m}]$ & 20 & 30 \\
$L_{\mathrm{x}, \text { fine }}[\mu \mathrm{m}]$ & 30 & 45 \\
$L_{\mathrm{x}, \text { domain }}[\mu \mathrm{m}]$ & 235 & 250
\end{tabular}

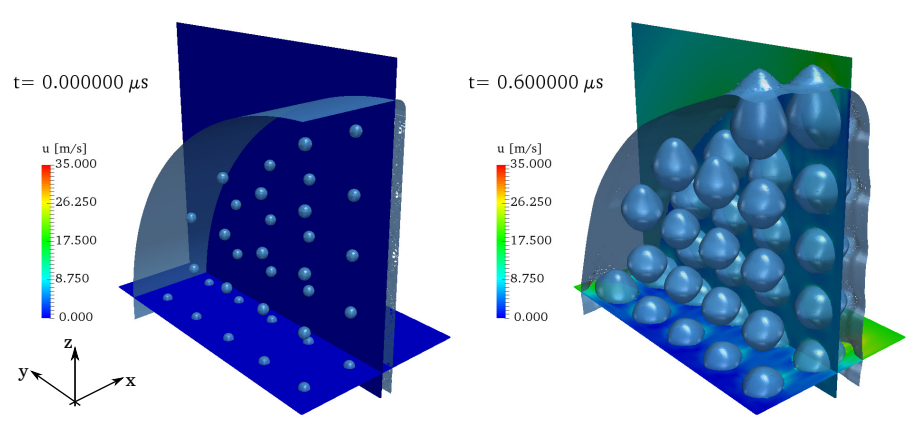

Figure 5. Expansion of vapour bubbles in a liquid jet. The curved iso-surfaces represent the bubble and jet surfaces, while the velocity field is shown in the $\mathrm{x}$ - and $\mathrm{z}$-normal slices. Left: Initial conditions. Right: Solution after $t=0.6 \mu \mathrm{s}$.

Furthermore, we observe an expansion of the liquid jet despite the rather short time interval $(t=0.6 \mu \mathrm{s})$. Twodimensional plots of density, velocity and pressure fields are depicted in Fig. 6 . The initial conditions in the top row are compared to the solution after $t=0.6 \mu \mathrm{s}$ in the bottom row. The density fields in the left column qualitatively confirm the impression from the three-dimensional plots. The outer bubbles grow faster and become non-spherical while inner bubbles expand isotropically at a lower rate. All inner bubbles grow approximately at the same rate. The velocity field is depicted in the middle column of Fig. 6 . The expansion velocity of $u \approx 15 \mathrm{~m} / \mathrm{s}$ is roughly of the order of typical jet velocities for the corresponding DLR experiments. Thus, the bubble expansion can have a considerable influence on the jet break-up. Peak velocities of up to $u=30 \mathrm{~m} / \mathrm{s}$ at the jet surface arise locally where the bubbles grow at the highest rate and where deformation of the jet surface is observed and jet break-up imminent. Note that the high velocities in the surrounding vapour phase do not only result from jet expansion but also from the evaporation at the jet surface. It is also seen that the velocities decrease rapidly towards the jet center. This may change when the outer bubbles merge and the jet surface breaks up by forming droplets, which will be investigated in future studies. In the right column of Fig. 6 the pressure fields are depicted. The pressure in the center is slightly higher than in the environment and at the jet surface. The evaporation rate in Eq. (5) is determined by the deviation of the local entropy from the entropy at saturation conditions. The increase in pressure decreases the degree of superheat in the liquid and the difference of the local entropy to the saturation entropy in Eq. (5) is reduced resulting in lower evaporation rates. In addition, the mechanical resistance against the bubble expansion is higher for higher liquid pressures. The pressure increase originates from pressure fluctuations travelling continuously between the jet center and the jet surface. The temporal average of the local pressures determines the deviations of the growth rates between the inner and outer bubbles. Here, the growth rate is defined as the time derivative of the bubble radius $\mathrm{d} r_{\mathrm{bub}} / \mathrm{d} t$, i.e. it corresponds to the gradient of the functions plotted in the $r-t$-diagrams. The density, velocity and pressure fields of Case 2 are similar (not shown here).

Figures 5 and 6 reveal a variation of growth rates dependent on the position of the bubble within the bubble array. It is apparent that single bubble growth cannot capture the average growth process accurately and a quantification of characteristic growth rates is required. For the present case the growth rates of all inner bubbles and all outer bubbles are averaged. Here, we use the bubble volume and calculate an equivalent radius of the corresponding spherical bubble. All bubbles directly facing the jet surface to the positive $x-, y-$ or $z$-direction are marked as outer bubbles, while the bubbles that only face neighbouring bubbles are inner bubbles. The left subfigure in Fig. 7 depicts the radii over time for the outer and inner bubbles for Case 1. The solution for the single bubble growth is given as reference and the growth rate for the bubble located at the lowest $x-, y$ - and $z$-coordinate is also plotted as it was expected to present a limiting case. It seems, however, that the exact position in the array is not so important. The innermost bubble grows approximately at the same rate as the average of the inner bubbles as indicated in the figure by the red and black solid lines, respectively. This is consistent with the qualitative impression from the twoand three-dimensional plots. The growth rate of the inner bubbles is approximately $30 \%$ lower than the growth rate of a single bubble (dash-dot line). A jet expansion based on growth rates of single bubbles would then be prone to model inaccuracies. The outer bubbles (dashed lines in Fig. 7) grow faster than the inner bubbles by approximately $20 \%$ but the expansion is still lower than for single bubbles. As a result, the outer bubbles will coalesce first and the subsequent jet disintegration will propagate from the outside towards the jet center. The right subfigure shows 

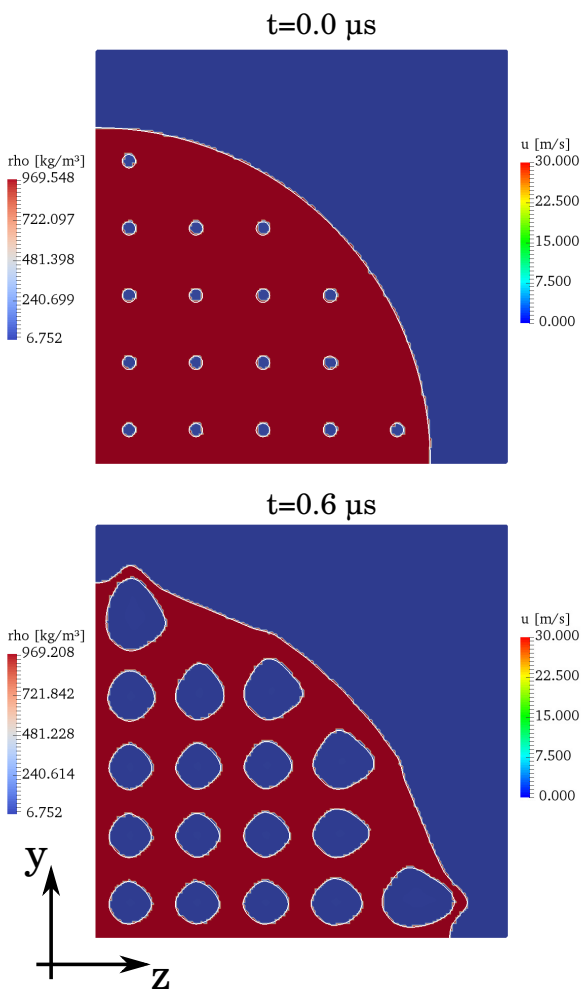
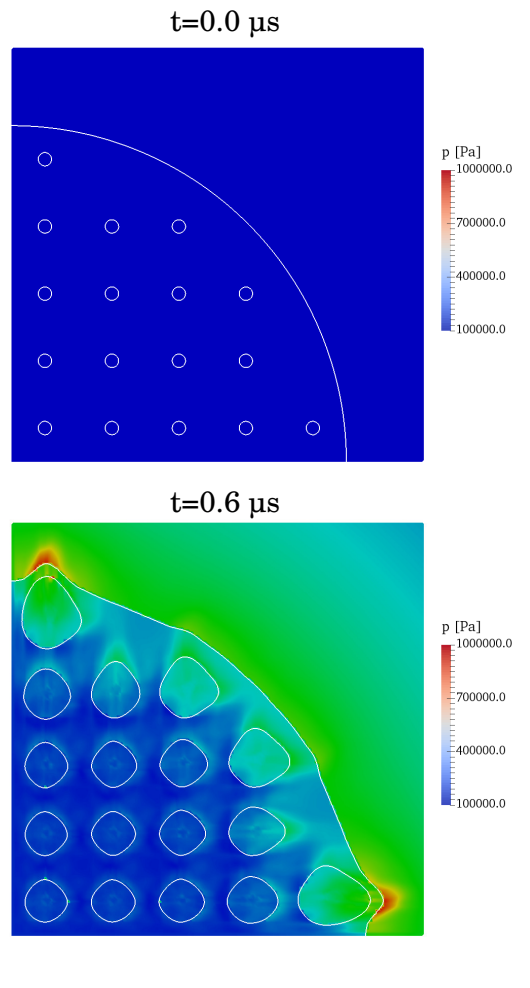

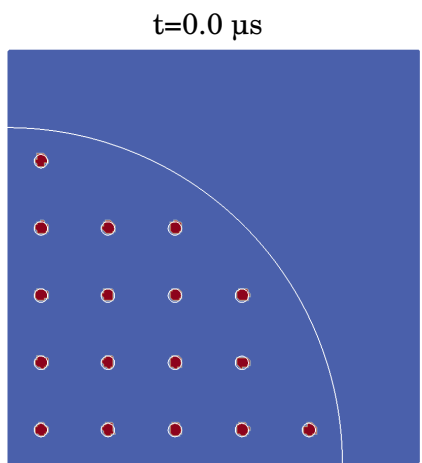

$\mathrm{t}=0.6 \mu \mathrm{s}$

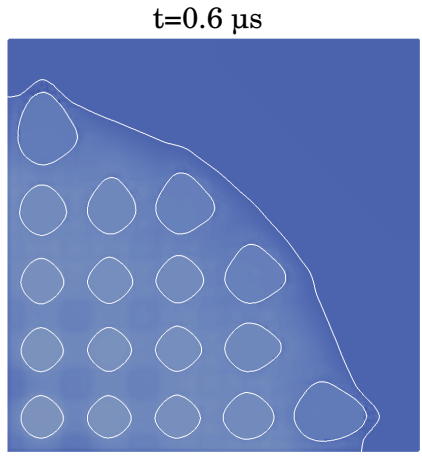

Figure 6. Density (left), velocity (middle) and pressure (right) fields in an $y-z-$ plane. Top row: Initial conditons. Bottom row: Solution after $t=0.6 \mu \mathrm{s}$.

results of Case 2. Qualitatively, there is little difference between the two cases. However, the absolute growth rates of Case 1 and Case 2 deviate. Both, the inner and the outer bubbles grow faster by $15 \%$ in Case 2 compared to Case 1. For the present test condition, we illustrate the dependency of individual bubble growth rates on the bubble density (i.e. the number of bubbles per volume) and their position within the liquid jet. The expansion of the liquid jet will be determined by the total expansion of all bubbles. The bubbles grow faster for Case 2, but less bubbles are contained in the liquid and the total expansion of the jet is smaller. Table 3 shows the volume of the jet as the sum of the liquid and the bubble volume. The volume change compared to the initial solution is $20 \%$ in Case 1 and $7 \%$ in Case 2. The absolute change in the jet volume in Case 2 is $54 \%$ of the absolute change in Case 1 (consult the fourth column in Tab. 3). This demonstrates the sensitivity of the jet expansion to the bubble growth and its dependence on both, the local growth rates and the bubble density. The volume change based on the solution of the growth model for a single bubble multiplied with the number of bubbles in the liquid jet would be doubled in Case 1 and would be overpredicted by $25 \%$ in Case 2 . Yet, the parameter range in our investigation is small and a more comprehensive study for wide parameter ranges and at simulation times including droplet formation will be needed to derive models for flash-boiling that are valid for a wider range of conditions with varying nucleation rates.

Table 3. Volume expansion of the liquid jet for Case 1 and Case 2.

\begin{tabular}{c|cccc}
\hline & $V(t=0.0 \mu \mathrm{s})\left[\mathrm{m}^{3}\right]$ & $V(t=0.6 \mu \mathrm{s})\left[\mathrm{m}^{3}\right]$ & $\Delta V / \Delta V_{\text {Case } 1}$ & $\Delta V[\%]$ \\
\hline Case 1 & $3.927 \cdot 10^{-14}$ & $4.705 \cdot 10^{-14}$ & 1 & 20 \\
Case 2 & $5.890 \cdot 10^{-14}$ & $6.307 \cdot 10^{-14}$ & 0.54 & 7
\end{tabular}

\section{Conclusions}

In the present paper the expansion rates of multiple bubbles in a superheated liquid jet are investigated. The numerical simulations are conducted using a discontinuous Galerkin solver. The fully compressible approach requires a modified approximate Riemann solver that accounts for phase change and captures pressure wave dynamics. The evaporation model requires closure which is realized by the calibration of DG calculations with the RayleighPlesset equation for cases of single bubble growth. We show that the dynamics of early bubble growth becomes unimportant at larger time scales, and the total volume expansion obtained with the DG solver matches well with the reference solution throughout the entire simulation time.

The calibrated model is used to investigate the growth rates in a three-dimensional liquid jet containing multiple 

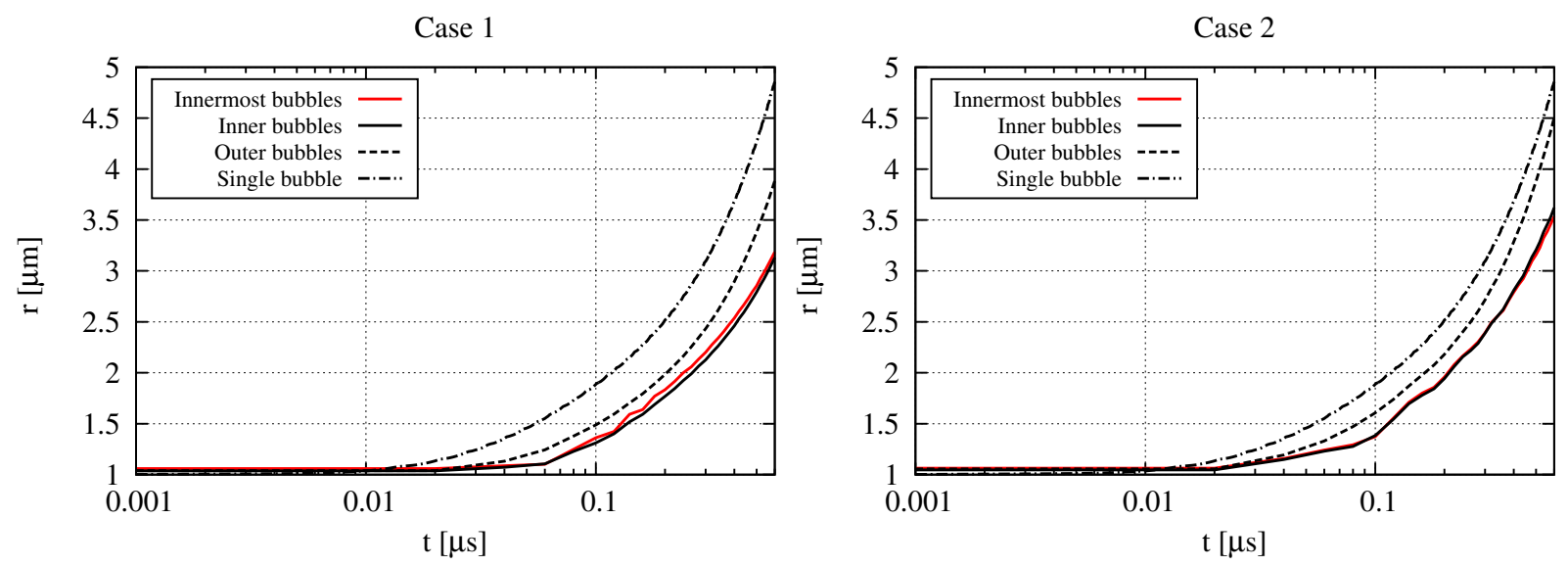

Figure 7. Comparison of bubble growth rates of inner bubbles, outer bubbles and single bubble growth. Left: Case 1. Right: Case 2:

bubbles. We observe a strong growth of the bubbles resulting in high expansion velocities for the liquid jet in the axial and radial direction. The velocities are of the order of the characteristic mean flow velocities and they are reached at very short time scales. Moreover, we observed non-homogeneous growth of the bubbles and have quantified the deviation to single bubble growth. It is found that bubbles in the center expand more slowly than bubbles at the edges of the jet, however, even bubbles right at the jet interface do not reach expansion rates as were predicted by existing expressions for single bubble growth. The present investigation is a first approximation and will be extended to include a wider parameter range. Particularly, the influence of the bubble number density has to be investigated and computations that capture the entire jet break-up due to bubble growth will be attempted. The bubble number density is a function of the ambient temperature and the pressure ratio $R_{p}$. It may not be possible to achieve realistic bubble number densities for high $R_{p}$, however, the current studies show that there is little influence of the exact position within the array on bubble growth rates (as long as the bubble does not face the jet interface). This may allow for future computations of a reduced domain size located in the immediate vicinity of the jet surface where much higher nucleation rates could be realized.

\section{Acknowledgements}

The authors acknowledge the financial support by the Deutsche Forschungsgemeinschaft (DFG) as part of the Collaborative Research Center SFB TRR 75 "Droplet dynamics under extreme ambient conditions" held by University of Stuttgart and University of Technology Darmstadt.

\section{References}

[1] Lamanna, G. et al., 2014, International Journal of Multiphase Flow, 58, pp. 168-184.

[2] Lamanna, G. et al., 2015, Atomization and Sprays, 25 (10), pp. 837-856.

[3] Günther, A. and Wirth, K.E., 2013, International Journal of Heat and Mass Transfer, 64, pp. 952-965.

[4] Huang, Y., et al., 2016, Energy Conversion and Management, 108, pp. 68-77.

[5] Ju, D. et al., 2015, International Journal of Multiphase Flow, 75, pp. 224-236.

[6] Thompson, A.S. and Heister, S.D., 2016, Atomization and Sprays, 26 (7), pp. 633-658.

[7] Neroorkar, K. and Schmidt, D., 2011, Fuel, 90, pp. 665-673.

[8] Calay, R.K. and Holdo, A.E., 2008, Journal of Hazardous Materials, 154, pp. 1198-1209.

[9] Downar-Zapolski, P. et al., 1996, International Journal of Multiphase Flow, 22 (3), pp. 473-483.

[10] Zeng, Y. and Lee, C.-F.F., 2001, Combustion, Science and Technology, 169, pp. 45-67.

[11] Saha, K. et al., 2016, Journal of Energy Resources Technology, 138 (052208), pp. 1-11.

[12] Hindenlang, F. et al., 2012, Computers \& Fluids, 61, pp. 86-93.

[13] Fechter, S. et al., 2012, In: 17th International Conference in on Computational Fluid Dyanmics, Big Island, Hawaii, 9-13 July 2012.

[14] Sussman M. et al., 1994, Journal of Computational Physics, 114, pp. 146-159.

[15] Fechter S. et al., 2015, International Journal in Numerical Methods in Fluids, 78 (7), pp. 413-435.

[16] Fechter S., 2015, Compressible Multiphase Simulation using a discontinuous Galerkin scheme, PhD. thesis, Universität Stuttgart.

[17] Bell, H. et al., 2014, Industrial \& Engineering Chemistry Research, 53, pp. 2498-2508.

[18] Fechter S. et al., 2017, Journal of Computational Physics, 336, pp. 347-374.

[19] Brennen, C. E., 1995, "Cavitation and bubble dynamics". Oxford University Press.

[20] Plesset, M.S. and Prosperetti, A., 1977, Annual Review of Fluid Mechanics, 9, pp. 145-185.

[21] Lee, M. and Merte, H. Jr., 1996, International Journal of Heat and Mass Transfer, 39 (12), pp. 2427-2447. 


\title{
Kinetic and MD modelling of automotive fuel droplet heating and evaporation: recent results
}

\author{
S.S. Sazhin ${ }^{1, *}$, I.N. Shishkova ${ }^{2}$ \\ ${ }^{1}$ Sir Harry Ricardo Laboratories, Advanced Engineering Centre, School of Computing, \\ Engineering and Mathematics, University of Brighton, Brighton BN2 4GJ, UK \\ ${ }^{2}$ Low Temperature Department, Moscow Power Engineering Institute, Krasnokazarmennaya \\ 14, Moscow 111250, Russia \\ *Corresponding author: S.Sazhin@brighton.ac.uk
}

\begin{abstract}
Recent results of the investigation of kinetic and molecular dynamics (MD) models for automotive fuel droplet heating and evaporation are summarised. The kinetic model is based on the consideration of the kinetic region in the close vicinity of the surface of the heated and evaporating droplets, where the motion of molecules is described in terms of the Boltzmann equations for vapour components and air, and the hydrodynamic region away from this surface. The effects of finite thermal conductivity and species diffusivity inside the droplets and inelastic collisions in the kinetic region are taken into account. A new self-consistent kinetic model for heating and evaporation of Diesel fuel droplets is briefly described. The values of temperature and vapour densities at the outer boundary of the kinetic region are inferred from the requirement that both heat flux and mass flux of vapour components in the kinetic and hydrodynamic regions in the vicinity of the interface between these regions are equal. At first, the heat and mass fluxes in the hydrodynamic region are calculated based on the values of temperature and vapour density at the surface of the droplet. Then the values of temperature and vapour density at the outer boundary of the kinetic region, obtained following this procedure, are used to calculate the corrected values of hydrodynamic heat and species mass fluxes. The latter in their turn lead to new corrected values of temperature and vapour density at the outer boundary of the kinetic region. It is shown that this process quickly converges and leads to self-consistent values for both heat and mass fluxes. Boundary conditions at the surface of the droplet for kinetic calculations are inferred from the MD calculations. These calculations are based on the observation that methyl $\left(\mathrm{CH}_{3}\right)$ or methylene $\left(\mathrm{CH}_{2}\right)$ groups in n-dodecane (approximation of Diesel fuel) molecules can be regarded as separate atom-like structures in a relatively simple United Atom Model. Some results of the application of quantum chemical methods to the estimation of the evaporation/condensation coefficient are discussed.
\end{abstract}

\section{Keywords}

Diesel fuel, droplets, heating, evaporation, kinetic modelling.

\section{Introduction}

In most applications, including those focused on automotive fuel droplets, the modelling of heating and evaporation processes has been based on the assumption that vapour at the liquid surface is saturated and the problem of evaporation reduces to the analysis of diffusion of vapour from the liquid (droplet) surface to the ambient gas [1]. The limitations of this approximation have been well known for over a century (see the references in [2]). In a number of studies, summarised in [1], the evaporation of n-dodecane $\mathrm{C}_{12} \mathrm{H}_{26}$ or a mixture of $n$-dodecane and $p$ dipropylbenzene $\mathrm{C}_{12} \mathrm{H}_{18}$ (approximating alkanes and aromatics in Diesel fuel) was studied and a new model based on a combination of the kinetic and hydrodynamic approaches was developed. In the close vicinity of droplet surfaces (up to one hundred molecular mean free paths), the vapour and ambient gas dynamics were studied based on the kinetic Boltzmann equations for vapour and air (kinetic region), while at larger distances the analysis was based on the conventional hydrodynamic equations (hydrodynamic region). These regions alongside the liquid region are schematically shown in Figure 1. Mass fluxes of components, momentum and energy fluxes were conserved at the interface between kinetic and hydrodynamic regions. The boundary conditions at the droplet surface were specified in terms of the evaporation coefficient which was estimated using molecular dynamics (MD) or quantum chemical (QC) methods.

The purpose of this paper is to provide an overview of the most important results in kinetic, MD and QC analysis of automotive fuel droplet heating and evaporation, mainly obtained since the publication of [1]. This overview is complementary to the more general review [3] recently published in Fuel.

The fundamentals of the most advanced self-consistent kinetic model for automotive fuel droplet heating and evaporation are summarised in Section 2, following [4]. Basic principles of the United Atom Model, used for the MD estimation of the evaporation coefficient (used as the boundary condition at the surface of the droplet in kinetic modelling), are briefly described in Section 3 , following $[5,6]$. The feasibility of using quantum chemical methods for studies of the processes at the surface of droplets and estimation of the evaporation coefficient is discussed in Section 4, following [7]. The main results of the paper are summarised in Section 5. 


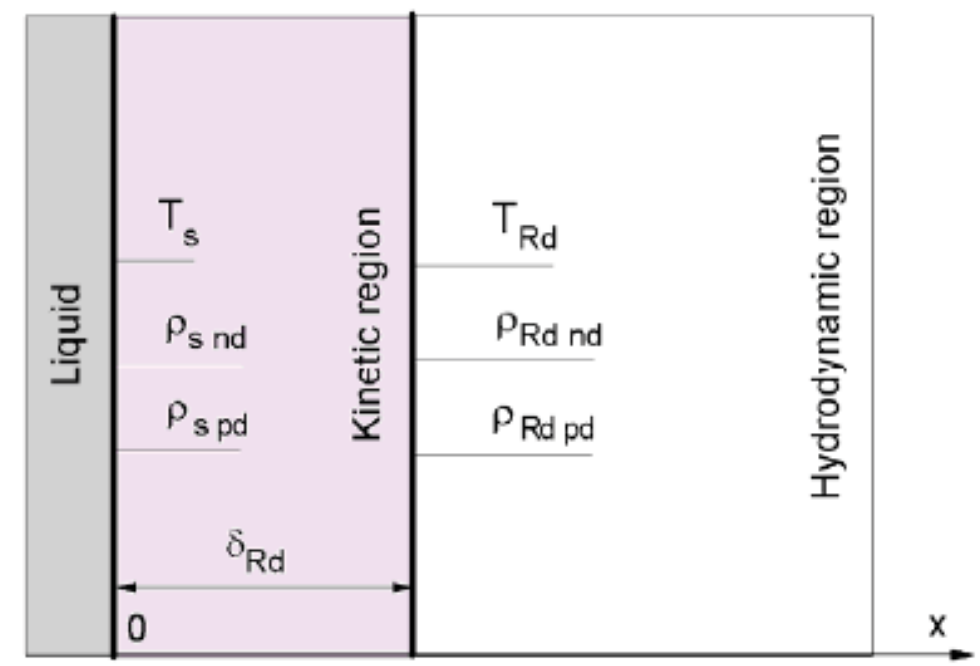

Figure 1. Liquid, kinetic and hydrodynamic regions near the surface of the droplet. $T_{s}$ is the droplet surface temperature, $\rho_{s(n d, p d)}$ are n-dodecane $\left(_{n d}\right)$ and p-dipropylbenzene $\left({ }_{p d}\right)$ vapour densities in the immediate vicinity of the droplet surface, $T_{R d}$ and $\rho_{R d(n d, p d)}$ are the temperature and n-dodecane $\left.{ }_{n d}\right)$ and p-dipropylbenzene $\left(_{p d}\right)$ vapour densities at the outer boundary of the kinetic region. Reprinted from International Journal of Heat and Mass Transfer, Volume 93, Sazhin et al., A self-consistent kinetic model for droplet heating and evaporation, Pages 1206-1217, Copyright Elsevier (2016).

\section{A self-consistent kinetic model}

As already mentioned, the boundary condition at the interface between the kinetic and hydrodynamic regions has been inferred from the requirement of the conservation of heat and mass (or species mass) fluxes at this interface. In all papers published before [4], hydrodynamic heat and mass fluxes were calculated based on the simplifying assumptions that the temperature at the outer boundary of the kinetic region is the same as the droplet surface temperature and vapour pressure at this boundary is the same as the saturated vapour pressure at the droplet surface. This approach allows one to find the corrected values of temperature and vapour density at the interface between the kinetic and hydrodynamic regions.

The main problem with this approach is that the heat and mass (or species mass in the case of multi-component droplets) fluxes in the hydrodynamic region, calculated based on these corrected values of temperature and vapour density, are not necessarily equal to the heat and mass fluxes in the hydrodynamic region used to find these corrected values. The authors of [4] suggested that this problem can be overcome if an iterative process is used. In this case, the corrected values of temperature and vapour density (or species densities) at the outer boundary of the kinetic region were used to calculate the corrected values of hydrodynamic heat and mass fluxes. The latter leads to new corrected values of temperature and vapour density at the outer boundary of the kinetic region. If this process converges then self-consistent values for both heat and mass fluxes are obtained.

This iterative procedure is illustrated in Figures 2 and 3 in which the process of calculation of density and temperature at the interface between the kinetic and hydrodynamic regions is shown. Diesel fuel is approximated by $\mathrm{n}$-dodecane $\left(\mathrm{C}_{12} \mathrm{H}_{26}\right)$, a droplet is assumed to be moving with constant relative velocity equal to $10 \mathrm{~m} / \mathrm{s}$ and its surface temperature is equal to $600 \mathrm{~K}$; gas temperature and pressure are taken equal to $1000 \mathrm{~K}$ and 30 bar respectively. The calculations are based on the Effective Thermal Conductivity (ETC) model (see [1] for the details). The plots of $\tilde{j}_{\mathrm{k}} \equiv j_{\mathrm{kn}} /\left(\rho_{0} \sqrt{R_{v} T_{0}}\right)$ versus $\alpha_{\rho}$ and $\tilde{q}_{\mathrm{k}} \equiv q_{\mathrm{k}} /\left(p_{0} \sqrt{R_{v} T_{0}}\right)$ versus $\alpha_{T}$ are shown in these figures (lines ' $\mathrm{k}$ '). Also, the plots of $\tilde{j}_{\mathrm{h}} \equiv j_{\mathrm{h} \mathrm{n}} /\left(\rho_{0} \sqrt{R_{v} T_{0}}\right)$ versus $\alpha_{\rho}$ and $\tilde{q}_{\mathrm{h}} \equiv q_{\mathrm{h}} /\left(p_{0} \sqrt{R_{v} T_{0}}\right)$ versus $\alpha_{T}$ (assuming that $\alpha_{\rho} \equiv \rho_{R d} / \rho_{s}=1$ and $\alpha_{T} \equiv T_{R d} / T_{s}=1$ ) are shown in the same figures (lines ' $h$ ', iteration 1). The intersection between these two pairs of lines gives the updated values $\alpha_{\rho}=0.979$ and $\alpha_{T}=1.031$.

In the kinetic models used before [4], these corrections were directly used for calculation of mass and heat fluxes (see [1] for the details). In the new model these corrections are used to update the values of $\tilde{j}_{\mathrm{h}}$ and $\tilde{q}_{\mathrm{h}}$. The updated values of these fluxes are shown in Figures 2 and 3 (lines ' $h$ ', iteration 2). The intersections of these lines with lines $\tilde{j}_{\mathrm{k}}$ and $\tilde{q}_{\mathrm{k}}$ give us new updated values $\alpha_{\rho}=0.969$ and $\alpha_{T}=1.029$. The following iterations, up to iteration 50 , lead to relatively small changes in these corrections, as shown in Figures 2 and 3 . The difference between the values of $\alpha_{\rho}$ and $\alpha_{T}$ decreases with increasing iteration number; the differences between the values inferred from the 49th iteration are almost indistinguishable from those inferred from the 50th iteration. Note that, in contrast to the previously used non-self-consistent models, the approach described above does not rely on the observation that $\tilde{q}_{\mathrm{k}}$ is almost independent of $\alpha_{\rho}$ and $\tilde{j}_{\mathrm{k}}$ is almost independent of $\alpha_{T}$.

The analysis as presented in Figures 2 and 3 was repeated for other droplet surface temperatures in the range $300-650 \mathrm{~K}$ and gas temperatures $800 \mathrm{~K}, 1000 \mathrm{~K}$ and $1200 \mathrm{~K}$. The same analysis was performed for bi-component droplets ( $80 \% \mathrm{n}$-dodecane and $20 \% \mathrm{p}$-dipropylbenzene mixture) for gas temperature equal to $1000 \mathrm{~K}$.

The new model was applied to the analysis of heating and evaporation of $n$-dodecane droplets with initial radii and 


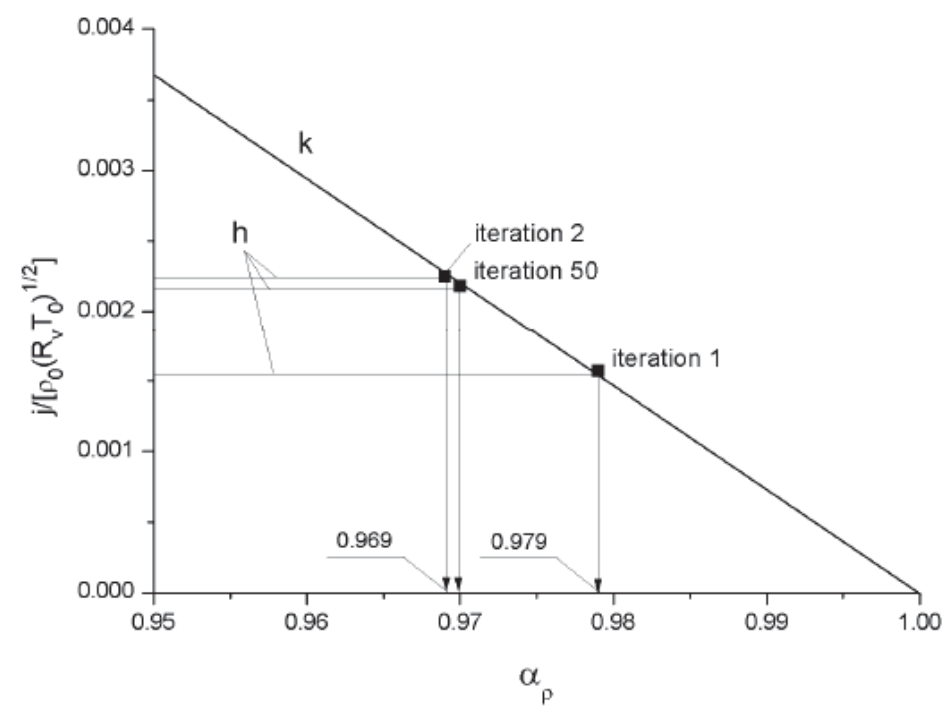

Figure 2. The plots of normalised mass fluxes $\tilde{j} \equiv j /\left(\rho_{0} \sqrt{R_{v} T_{0}}\right)$ predicted by the kinetic (line 'k') and hydrodynamic (lines ' $\mathrm{h}$ ') models for an n-dodecane droplet moving with constant velocity $10 \mathrm{~m} / \mathrm{s}$ versus $\alpha_{\rho} \equiv \rho_{R d} / \rho_{s}$ (normalised vapour density at the outer boundary of the kinetic region). Droplet surface and gas temperatures are assumed equal to $600 \mathrm{~K}$ and $1000 \mathrm{~K}$ respectively. Reprinted from International Journal of Heat and Mass Transfer, Volume 93, Sazhin et al., A self-consistent kinetic model for droplet heating and evaporation, Pages 1206-1217, Copyright Elsevier (2016).

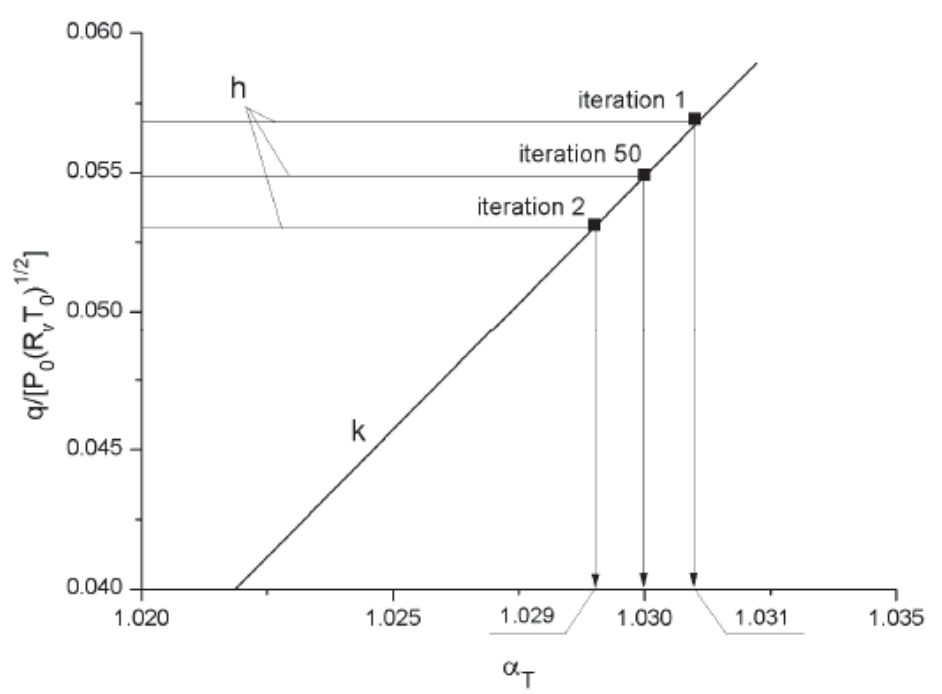

Figure 3. The plots of normalised heat fluxes $\tilde{q} \equiv q /\left(p_{0} \sqrt{R_{v} T_{0}}\right)$ predicted by the kinetic (line ' $\mathrm{k}$ ') and hydrodynamic (lines ' $\mathrm{h}$ ') models for an n-dodecane droplet moving with constant velocity $10 \mathrm{~m} / \mathrm{s}$ versus $\alpha_{T} \equiv T_{R d} / T_{s}$ (normalised temperature at the outer boundary of the kinetic region). Droplet surface and gas temperatures are assumed equal to $600 \mathrm{~K}$ and $1000 \mathrm{~K}$ respectively. Reprinted from International Journal of Heat and Mass Transfer, Volume 93, Sazhin et al., A self-consistent kinetic model for droplet heating and evaporation, Pages 1206-1217, Copyright Elsevier (2016). 
temperature equal to $5 \mu \mathrm{m}$ and $300 \mathrm{~K}$, immersed into gas with temperatures equal to $800 \mathrm{~K}, 1000 \mathrm{~K}$ and $1200 \mathrm{~K}$ and pressure equal to 30 bar. Droplets were either stationary or moving with constant velocity equal to $10 \mathrm{~m} / \mathrm{s}$. It was shown that in all cases the kinetic effects result in a decrease in droplet surface temperature and an increase in the evaporation time. This increase was shown to be particularly important for higher gas temperatures and moving droplets $[1,3,4]$. The contribution of $p$-dipropylbenzene was shown to decrease the kinetic effects on droplet evaporation time [4].

The analysis presented in this section is based on the assumption that droplets are perfect spheres. The kinetic model for non-spherical droplets has not yet been developed, to the best of our knowledge. The results of development of a hydrodynamic model of spheroidal droplet heating and evaporation are described in [8].

\section{United Atom Model}

As already mentioned, the solution to the Boltzmann equation in the kinetic region requires the formulation of the boundary condition at the liquid/gas interface. This boundary condition is essentially controlled by the evaporation coefficient (see review [9] for the details). A theoretical estimation of this coefficient requires the application of molecular dynamics (MD) methods [1]. A number of models have been developed to describe the molecular dynamics of complex hydrocarbon molecules, such as n-dodecane. Most of these models are based on the observation that the $\mathrm{C}-\mathrm{H}$ bond in complex hydrocarbon molecules is much shorter and much stronger than the $\mathrm{C}-\mathrm{C}$ bond, and also stronger than the van der Waals forces between molecules. Thus the methyl $\left(\mathrm{CH}_{3}\right)$ or methylene $\left(\mathrm{CH}_{2}\right)$ groups in the n-dodecane molecule can be regarded as separate atom-like structures in a relatively simple United Atom Model. In $[5,6]$ this model was used for the estimation of the evaporation coefficient at the surface of $n$-dodecane droplets based on MD calculations.

The contributions of the Lennard-Jones, bond bending and torsion potentials with the bond length constrained were taken into account. The analysis of [5] was based on 400 molecules, while the analysis of [6] was based on 720 molecules. The results of MD calculations of the evaporation coefficient $\beta$, which are presented in the latter paper, were approximated by the following simple formula (see [1] for the details):

$$
\beta\left(T_{s}\right)=7 \times 10^{-6} T_{s}^{2}-9.8 \times 10^{-3} T_{s}+3.7215,
$$

where $T_{s}$ is the droplet surface temperature (in $\mathrm{K}$ ). The values of $\beta$ predicted by Expression (1) turned out to be compatible with results reported by a number of other authors (see Figure 4).

Note that the United Atom Model is not valid at temperatures close to the critical temperature. In this case, a much more complex All Atom Model, taking into account the contributions of all atoms should be used. Also, the United Atom Model does not explicitly take into account quantum chemical effects. The importance of these effects is discussed in the next section.

\section{Quantum chemical effects}

The analysis summarised in the previous section did not explicitly take into account quantum chemical (mechanical) effects when analysing molecular dynamics in the vicinity of the droplet surface. In a series of our previous papers, the results of which are reviewed in [7, 3], the applicability of this approach has been investigated. In this section, the main findings of this investigation are summarised.

\section{Quantum chemical methods}

Although the solution to the Schrödinger equation for the wave function $\psi$ in some simple cases (e.g. an isolated hydrogen atom) is well known, its general solution in the case when many atoms need to be analysed simultaneously is still a challenge for quantum mechanical modelling. Perhaps the most widely used simplified method for solving this equation is the Hartree-Fock (HF) method. Two strategies for application of the HF method for practical calculations have been suggested. In the semi-empirical methods the integrals used in the HF method are estimated based on experimental data or based on a series of rules which allow one to set certain integrals to zero. In the $a b$ initio methods an attempt is made to calculate all these integrals.

Although this method is widely used in practical computations, it is still an approximate one and demands considerable computational effort. This stimulated the development of alternative approaches to the calculation of electronic systems, including the Density Functional Theory (DFT) which is focused on the electron density $\left(\rho_{e}\right)$ rather than on the wave function $\psi$. In this approach it is assumed that the energy of a molecule is a function of the electron density. Since the electron density is a function of position $\rho_{e}(\mathbf{r})$, this energy appears to be a function of a function (functional) of density

On some occasions various approximations of the energy functional in the DFT, that incorporate parts of the exact exchange from the HF theory, have been used. One such approach is known as B3LYP (Becke, 3-parameter, Lee-Yang-Parr). Various semi-empirical quantum chemistry methods are important for dealing with large molecules where the HF method without the approximations ( $a b$ initio approach) and DFT are too expensive. In these cases a range of fitting parameters are typically used to produce the results. The parameters in the PM7 method were calibrated to obtain results consistent with experimental and ab initio data for more than 9000 compounds. The accuracy of the PM7 method is close to that of the ab initio and DFT methods used with the 6-31G(d) basis set.

The fundamental differences between the classical molecular mechanics/molecular dynamics (MM/MD), semiempirical PM7, ab initio and DFT methods are in the way in which the contributions of electrons are taken into account. The contribution of all electrons is taken into account in ab initio and DFT with a self-consistent field 
(SCF); only valence electrons are considered in semi-empirical quantum-chemical methods (QCMs) with SCF, and no electrons are considered in classical MM/MD methods without SCF. A new continuum solvation model based on the quantum mechanical charge density of a solute molecule interacting with a continuum description of a solvent is known as the SMD. The term continuum indicates that the solvent is represented as a dielectric medium with surface tension at the solute-solvent boundary.

In the case of modelling of the transient processes, the Dynamic Reaction Coordinates (DRC) method has been widely used. The key concept of this method is the Dynamic Reaction Coordinate which is the path followed by all the atoms in a system assuming the conservation of energy. In contrast to conventional molecular dynamic (MD) approaches, in this approach the contributions of the processes at the electronic level are taken into account. These models have been implemented in a number of known programs, including Gaussian 09, WinGAMESS 2013 R1, and MOPAC2012.

\section{Interaction between molecules and droplets}

In this section, the results of the analysis of the collision processes between n-dodecane molecules and clusters/nanodroplets, based on the Dynamic Reaction Coordinate (DRC) method, are briefly summarised, following $[7,10]$. In the DRC approach, the total kinetic energy includes the kinetic energy of random thermal bond vibrations and rotations and the kinetic energy of the translational motion of the whole molecule. In our previous analysis, this approach was applied to study the dependence of sticking/scattering of $n$-dodecane molecules on their angles of attack, temperature, and cluster/nanodroplet size. The calculations were performed for molecules interacting with a cluster (7 molecules) or a nanodroplet (64 or 128 molecules) of n-dodecane molecules.

It was shown that at large angles of attack, molecules are absorbed by a cluster or nanodroplet of relatively small size (diameters in the range 2-7 nm) if the kinetic energy of attacking molecules is low and they are not oriented exactly towards one of the surface molecules. At angles close to $\approx 1^{\circ}$ almost perfectly elastic collisions were observed if the molecules had high kinetic energy ( $\sim 10 \mathrm{~kJ} / \mathrm{mol}$ or larger) and were oriented directly towards one of the surface molecules. It was assumed that the kinetic energy of the molecules in the clusters or nanodroplets was low and thermal vibrations and bond rotations corresponded to 300-400 K. The kinetic energy of the attacking molecule was high with their effective temperatures being in the range 500-1200 K.

It was concluded that the probability of the attacking molecule sticking to a droplet is maximal if the molecular plane is parallel or almost parallel to the droplet surface (multi-point interactions of long n-dodecane molecules with the droplet surfaces are expected). If the kinetic energy of the attacking molecules is greater than that of boiling temperature then it is expected that their scattering and removal from the cluster/nanodroplet surface will take place. It was shown that molecule-nanodroplet interaction results depend on the kinetic energy and orientations of the attacking and surface molecules. The mechanisms of evaporation of microdroplets and nanodroplets were shown to involve different processes. In the case of microdroplets, individual carbon molecules were evaporated from their surfaces, while nanodroplets disintegrated into clusters and individual molecules.

The predicted decrease in the lifetime of the "surface" molecules with temperature when the temperature of nanodroplets increases agrees with the prediction of the classical theory based on MD simulations of $n$-dodecane molecules using the United Atom Model (see Equation (1)). The analysis described above, however, does not allow us to predict the evaporation coefficient, as we did in the MD analysis (see Equation (1)). To obtain the values of this coefficient, using this analysis, one would need to repeat these calculations for a wide range of angles of attack, orientation of molecules and energies for various conformers and various conditions of clusters and nanodroplets. Since this is not currently feasible, an alternative approach to calculating this coefficient, taking into account quantum chemical effects, is described in the next section, following $[11,7]$.

\section{Evaporation/condensation coefficient}

In the analysis originally presented in [11] and summarised in [7] the transition state theory (TST) was used. This theory was based on a QC DFT approach taking into account the conformerisation of n-dodecane molecules (approximation of Diesel fuel). It was shown that the most accurate expression for the condensation coefficient (assumed to be equal to the evaporation coefficient) is the one averaged over the states of various conformers transferred between two phases. This was given by the following formula [11]:

$$
\left\langle\beta_{V}\right\rangle=\left\{1-\left[\frac{\rho_{g}}{\rho_{l}} \exp \frac{\left\langle\Delta G_{g \rightarrow l}\right\rangle}{R_{u} T}\right]^{1 / 3}\right\} \exp \left\{-0.5\left[\left[\frac{\rho_{g}}{\rho_{l}} \exp \frac{\left\langle\Delta G_{g \rightarrow l}\right\rangle}{R_{u} T}\right]^{1 / 3}-1\right]^{-1}\right\},
$$

where $R_{u}$ is the universal gas constant, $\rho_{g(l)}$ is the gas (liquid) density, $\Delta G_{g \rightarrow l}$ is the change in the Gibbs free energy during the condensation process, subscript ${ }_{V}$ shows that the expression $\beta_{V}$ depends on specific volumes, \langle\rangle shows the averaging over the states of various conformers transferred between two phases. The effects of both the conformerisation and cross-conformerisation of molecules were taken into account. Ninety-five stable conformers were selected based on the changes in the Gibbs free energy.

A comparison between the results of calculations of $\beta=\beta_{V}$ based on Expression (2) and those obtained previously is shown in Figure 4, reproduced from [7]. As can be seen from this figure, taking into account QC effects leads to marginal modification of the predicted evaporation/condensation coefficient unless the temperature approaches the critical temperature. Thus, although the analysis of QC effects takes into account many new effects ignored in the conventional force fields (FF) approach, the contribution of these effects to the values of the evaporation coefficient turned out to be marginal. 


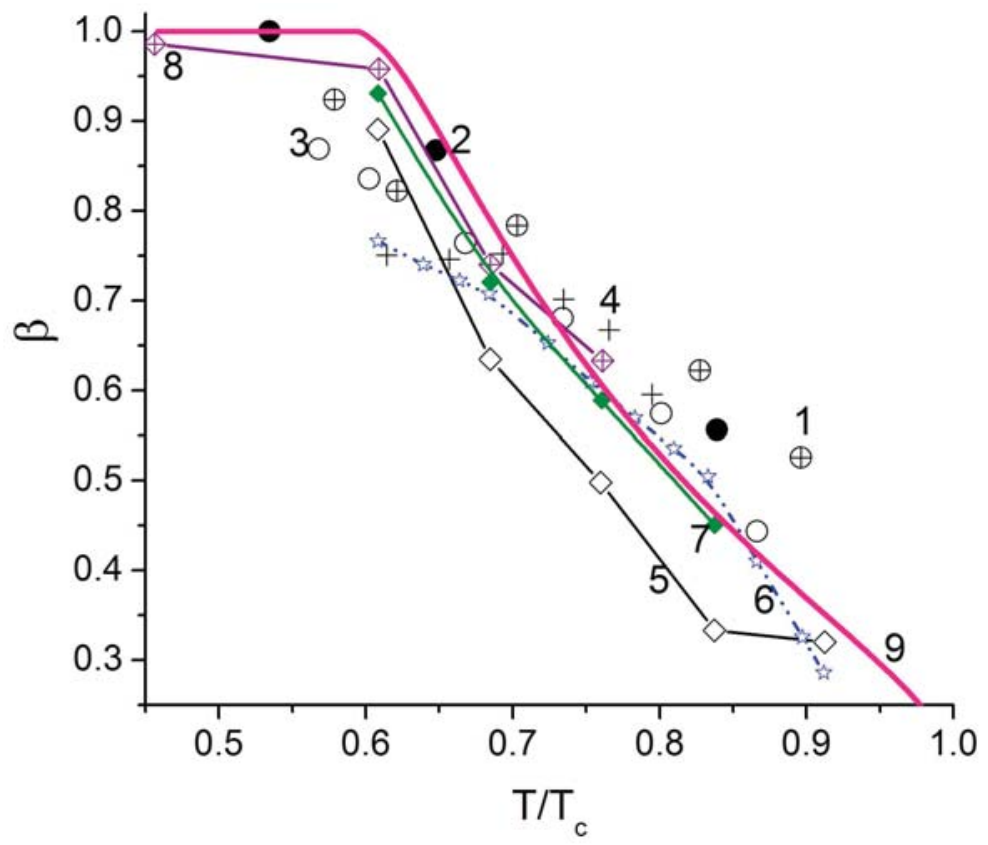

Figure 4. Comparison of the values of the evaporation coefficient $\beta$, predicted by MD, FF (symbols 1-4, curves 5-8) and Expression (2) (curve 9), versus normalised temperature $\left(T / T_{c}\right.$ ( $T_{c}$ is the critical temperature)). Symbols $(1-4)$ refer to the models for structureless LJ fluids with various input parameters (see [7] for the details), curves 5 and 7 refer to the results obtained from the United Atom Model, curve 6 refers to the results of calculations based on the TST model reproduced from [5], curve 8 is based on the results of calculations using the model described by Mizuguchi et al. [12]. QC calculations were performed using DFT $\omega$ B97X-D/cc-pVTZ and SMD/ $\omega$ B97X-D/cc-pVTZ. Reprinted from Fuel, Volume 165, Sazhin et al., Quantum-chemical analysis of the processes at the surfaces of Diesel fuel droplets, Pages 405-412, Copyright Elsevier (2016).

\section{Conclusions}

The results of several recent developments of kinetic and molecular dynamics (MD) models for automotive fuel droplet heating and evaporation are described, based on previously published journal papers (mainly from 20152017). The kinetic models, developed in a series of our recent papers, are based on consideration of the kinetic region in the immediate vicinity of the surface of the heated and evaporating droplets and the hydrodynamic region away from this surface. In the kinetic region the motion of molecules was described in terms of the Boltzmann equations for vapour components and air. The effects of finite thermal conductivity, recirculation and species diffusivity inside the liquid droplets were taken into account, alongside the effects of inelastic collisions in the kinetic region. A self-consistent kinetic model for heating and evaporation of Diesel fuel (approximated by $n$-dodecane $\mathrm{C}_{12} \mathrm{H}_{26}$ or a mixture of $\mathrm{n}$-dodecane and $\mathrm{p}$-dipropylbenzene $\mathrm{C}_{12} \mathrm{H}_{18}$ (approximating alkanes and aromatics in Diesel fuel)) droplets is described. In this model, the values of temperature and vapour density at the outer boundary of the kinetic region are inferred from the requirement that the heat flux and mass flux of vapour components in the kinetic and hydrodynamic regions in the vicinity of the interface between these regions are equal. At first, the heat and mass fluxes in the hydrodynamic region were calculated based on the values of temperature and vapour density at the surface of the droplet. Then the values of temperature and vapour density at the outer boundary of the kinetic region, obtained following this procedure, were used to calculate the corrected values of hydrodynamic heat and species mass fluxes. The latter in their turn led to new corrected values of temperature and vapour density at the outer boundary of the kinetic region. It was shown that this process quickly converges and leads to self-consistent values for both heat and mass fluxes.

Boundary conditions at the surface of the droplet for kinetic calculations were formulated in terms of the evaporation coefficient, the values of which were inferred from the molecular dynamics (MD) calculations. These calculations were based on the United Atom Model. The latter model is based on the observation that methyl $\left(\mathrm{CH}_{3}\right)$ or methylene $\left(\mathrm{CH}_{2}\right)$ groups in $\mathrm{n}$-dodecane (approximation of Diesel fuel) molecules can be regarded as separate atom-like structures. Some recent results of the application of quantum chemical methods to the analysis of the interaction between molecules and the surfaces of molecular clusters and nanodroplets are summarised. It is shown that quantum-chemical (QC) analysis leads to marginal modifications of the predicted evaporation coefficient, using the United Atom Model, unless the temperature approaches to the critical temperature for $\mathrm{n}$-dodecane.

\section{Acknowledgements}

The authors are grateful to the EPSRC (UK) (grants EP/K005758/1, EP/K020528/1 and EP/M002608/1), the Royal Society (UK) (grant IE 160014) and the Russian Foundation for Basic Research (grant No. 17-08-01274) for their financial support. 


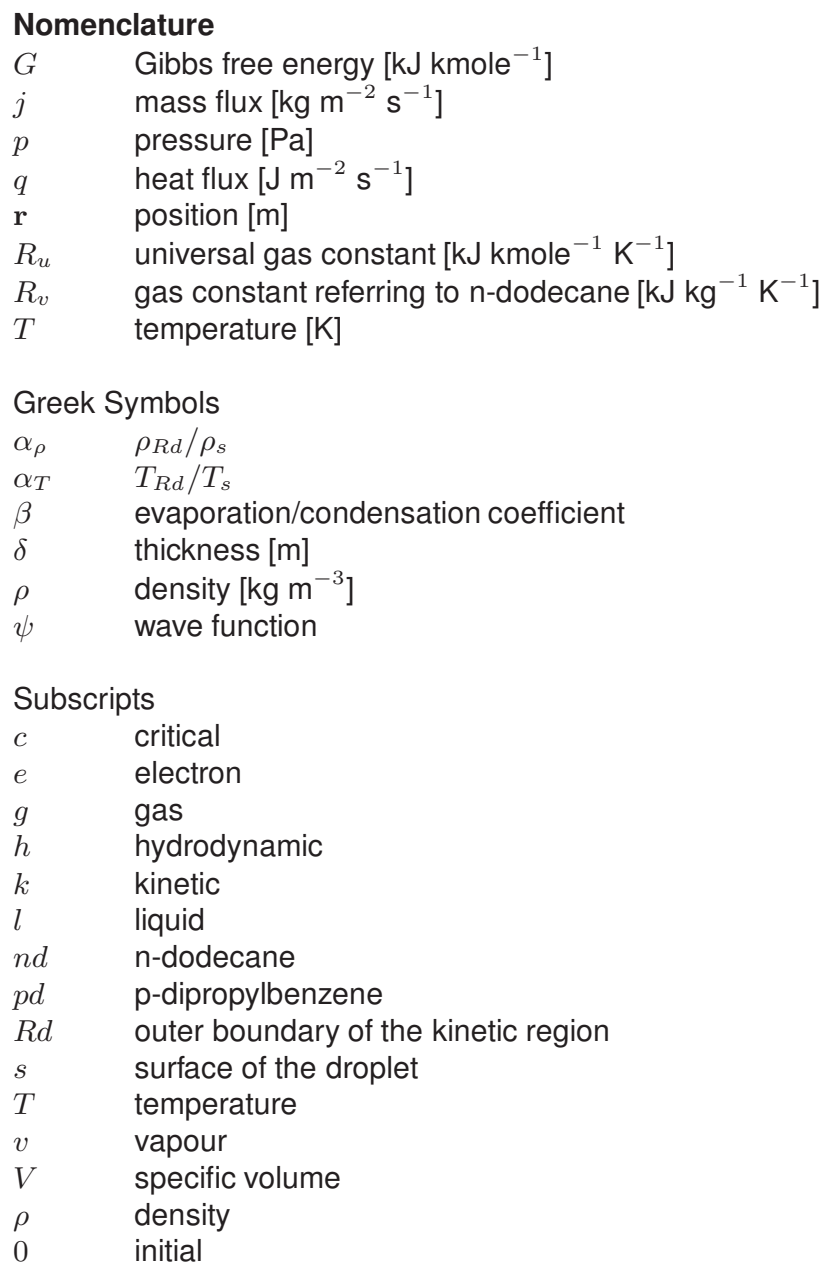

\section{References}

[1] Sazhin, S.S., 2014, "Droplets and Sprays". Springer.

[2] Fuchs, N.A., 1959, "Evaporation and Droplet Growth in Gaseous Media". London: Pergamon Press.

[3] Sazhin, S.S., 2017, Fuel, 196, pp. 69-101.

[4] Sazhin, S.S., Shishkova, I.N., Al Qubeissi, M., 2016, International Journal of Heat and Mass Transfer, 93, pp. 1206-1217.

[5] Cao, B.-Y., Xie, J.-F., Sazhin, S.S., 2011, Journal of Chemical Physics, 134, 164309.

[6] Xie, J.-F., Sazhin, S.S., Cao, B.-Y., 2011, Physics of Fluids, 23 (11), 112104.

[7] Sazhin, S.S., Gun'ko, V.M., Nasiri, R., 2016, Fuel, 165, pp. 405-412.

[8] Zubkov, V.S., Cossali, G.E., Tonini, S., Rybdylova, O., Crua, C., Heikal, M. and Sazhin, S.S., 2017, International Journal of Heat and Mass Transfer, 108, pp. 2181-2190.

[9] Persad, A.H., Ward, C.A., 2016, Chemical Reviews, 116 (14), pp. 7727-7767.

[10] Gun'ko, V.M., Nasiri, R., Sazhin, S.S., 2015, Fluid Phase Equilibria, 366, pp. 99-107.

[11] Gun'ko, V.M., Nasiri, R., Sazhin, S.S., 2015, Journal of Chemical Physics, 142 (3), 034502.

[12] Mizuguchi, H., Nagayama, G., Tsuruta, T., 2010, Seventh International Conference on Flow Dynamics, November 1st-3rd, 2010, Sendai International Center, p. 386. 


\title{
Dilute polymer solution drops impacting on heated surfaces: New impact morphologies and impact regime maps
}

\author{
Chetan Lakhanpal ${ }^{1}$, Volfango Bertola*1 \\ ${ }^{1}$ School of Engineering, University of Liverpool, United Kingdom \\ ${ }^{*}$ Corresponding author: Volfango.Bertola@liverpool.ac.uk
}

\begin{abstract}
The impact morphology of dilute polymer solution drops on a heated surface is studied experimentally by means of high-speed imaging, with respect to the following parameters: surface temperature; impact Weber number; polymer concentration; polymer molecular weight. In addition to impact morphologies observed in Newtonian drops (deposition, rebound, secondary atomisation and breakup/splashing), three new impact regimes have been identified: (i) a single satellite droplet ejected in the direction of bouncing but tethered to the main drop by a thin liquid filament; (ii) a splashing-like behaviour (semi-splashing), where the rim instability generates satellite droplets tethered to the lamella by thin liquid filaments; (iii) a spray-like behaviour (semi-spray), where a fine secondary atomisation generated upon impact is quickly absorbed back into the drop globule. Experiments were carried out using drops of aqueous polyethylene oxide (PEO) solutions, with mass concentrations of $100 \mathrm{ppm}$, $200 \mathrm{ppm}$ and $400 \mathrm{ppm}$, and PEO molecular weights of $2 \mathrm{MDa}, 4 \mathrm{MDa}$, and $8 \mathrm{MDa}$. The impact morphology on a polished aluminium surface with temperatures ranging between $160^{\circ} \mathrm{C}$ and $400^{\circ} \mathrm{C}$ was investigated for impact Weber numbers between 20 and 170, taking side view images of impacting drops at a rate of 1,000 frames per second.
\end{abstract}

\section{Keywords}

Drop Impact; Heated Surfaces; Polymer Solutions; Impact Regime Map.

\section{Introduction}

The dynamics of liquid drops impinging on a heated surface is a phenomenon of interest in many areas of engineering, including spray cooling, inkjet printing for advanced manufacturing applications, quenching of alloys in the steel industry and nuclear reactor safety. Despite several decades of research, this phenomenon is understood only to a limited extent and requires further research; specifically, the development of impact regime maps (IRMs) is of practical interest because they provide a consolidated understanding of the impact behaviour and represent a tool of practical use in the design of nozzles, print heads, etc. A quantitative and comprehensive IRM also presents an important test case for the development and validation of theoretical models describing specific features, such as transitions between different impact regimes.

Currently, an extensive body of literature on the behaviour of Newtonian drops impacting on heated surfaces exists. The first IRM produced [1] identified most of the impact regimes, however lacked clear quantitative description on the initiation and extent of these regimes. This work was soon followed by a detailed investigation that allowed production of three distinct IRMs corresponding to Weber numbers of 20, 60 and 220 and for surface temperatures ranging from $100^{\circ}$ to $300^{\circ} \mathrm{C}$ [2]. Although this work included substantial information on the spreading behaviour of a drop and its heat transfer characteristics, the IRMs were far from being fully comprehensive, particularly lacking information on all possible impact regimes that can be realised as well as the transitional boundaries. This was partly because of the limitations in photographic techniques available for analysis at the time. Moreover, the photographic results reported were not based on studying the lifetime of a single drop but rather based upon an ensemble principle of several drops, with one photograph taken per drop. Other semi-qualitative maps were proposed in the following years $[3,4]$

Drop impact was investigated for liquid drops impacting upon a nickel surface with angle of incidence ranging between $10^{\circ}$ and $80^{\circ}$ [5]. The impact regime map developed as a result uses as coordinates the dimensionless temperature, $\mathrm{T}^{*}$, defined as

$$
T^{*}=\frac{T_{\mathrm{w}}-T_{\mathrm{b}}}{T_{1}-T_{\mathrm{b}}}
$$

and the K-number $[6,7]$ 


$$
\mathrm{K}=\mathrm{We} \mathrm{Oh}^{-0.4}
$$

where $T_{w}-T_{b}$ is the wall superheat temperature, i.e. the difference between wall temperature and the boiling point of the fluid, $T_{1}$ is the Leidenfrost temperature .

For cases where the wall temperature is lower than the Leidenfrost temperature, the proposal was to replace $T_{w}$ in equation (2) by effective contact temperature as described in the expression (3) below [8].

$$
T_{w}=\frac{\varepsilon_{\mathrm{w}} T_{\mathrm{TH}}+\varepsilon_{1} T_{1}}{\varepsilon_{w}+\varepsilon_{1}}
$$

where $T_{H}$ and $T_{1}$ denote the wall and liquid temperatures respectively and $\varepsilon_{w}$ and $\varepsilon_{1}$ are the respective thermal effusivities of the wall and the liquid. This map identifies three impact regimes: deposition, splashing and rebound and displays qualitative transition boundaries; the Leidenfrost temperature is assumed to be $220^{\circ} \mathrm{C}$, however this quantity depends on the Weber number and, for water drops interacting with polished aluminium surfaces, has a minimum value of approximately $160^{\circ} \mathrm{C}$ when We $\rightarrow 0$ [9-11]. A comprehensive review on single droplet impact research and its relevance to understanding fuel sprays impingement can be found in [12].

To rationalise the rich variety of impact morphologies observed for Newtonian drops impinging on heated surfaces, it was proposed to identify "simple" impact regimes, displaying one distinctive feature (deposition, rebound, splashing/breakup) and "mixed" regimes, resulting from the combination of simple regimes with secondary atomisation [13]. Such unifying classification on one hand embraces the different impact morphologies reported in the existing literature, and on the other hand is simple enough to be used for practical purposes; in addition, it allows one to derive simple models for transition boundaries.

Results about Newtonian drop impact on heated surfaces reported in the literature are generally consistent with one another, to the exception of one recent work presenting an IRM based upon the impact of water and FC-72 drops upon silicon and sapphire surfaces, for temperatures between $200^{\circ} \mathrm{C}$ and $600^{\circ} \mathrm{C}$ and the Weber numbers between 0.5 and 600 [14]. Whilst one may argue the differences between these results and the rest of the literature are due to the high smoothness of the surfaces used, a more careful analysis suggests they may be caused by the design of the experimental setup, which leads to overestimate the surface temperature [13].

The impact of non-Newtonian drops on heated surfaces received comparatively less attention. Early studies focused primarily on dilute polymer solution drops, finding significant differences with respect to Newtonian drops [15]; in particular, it was observed that adding small amounts of a flexible polymer to the aqueous phase, secondary atomisation can be suppressed completely [16], and droplet rebound in the Leidenfrost regime is significantly enhanced [17]. More recently, it was shown that Leidenfrost rebounds are only weakly affected by the polymer concentration (i.e., by the fluid rheology) [18], but can be related to the symmetry of the rebound process [19]. Finally, the study of non-Newtonian drop impact in the Leidenfrost regime was extended to viscoplastic gels [20], showing that the fluid yield stress can suppress drop rebound.

The aim of the present work is to provide a systematic analysis of the impact morphology of dilute polymer solution drops, to produce impact regime maps for different polymer molecular weights and concentrations.

\section{Material and methods}

Test fluids were prepared by dissolving polyethylene oxide (PEO) in de-ionized water with mass concentrations of 100,200 , and $400 \mathrm{ppm}$; the polymer had average molecular weights of $2 \mathrm{MDa}, 4 \mathrm{MDa}$, and $8 \mathrm{MDa}$, and typical density of $1210 \mathrm{~kg} / \mathrm{m}^{3}$. In total, five distinct batches were produced:

- $\quad \mathrm{S} 1$ : Molecular weight $=4 \mathrm{MDa}$; Concentration $=100 \mathrm{ppm}$

- S2: Molecular weight $=4 \mathrm{MDa}$; Concentration $=200 \mathrm{ppm}$

- S3: Molecular weight $=4 \mathrm{MDa}$; Concentration $=400 \mathrm{ppm}$

- S4: Molecular weight $=2 \mathrm{MDa} ;$ Concentration $=200 \mathrm{ppm}$

- S5: Molecular weight $=8 \mathrm{MDa}$; Concentration $=200 \mathrm{ppm}$

In all cases, the polymer concentration was smaller than the overlap concentration, which can be estimated through the Mark-Houwink equation [18], therefore all test fluids are dilute solutions. The test fluid viscosities ranged between $1.1 \mathrm{mPa} \mathrm{s}$ and $1.5 \mathrm{mPa} \mathrm{s}$, while their surface tension was almost the same as that of the pure solvent $(\sim 70 \mathrm{mN} / \mathrm{m})[18]$.

Drops of test fluids were released from a blunt hypodermic needle (gauge 21, i.d. $0.495 \mathrm{~mm}$ ) and impacted upon the polished top surface of a square aluminium block $(40 \mathrm{~mm} \times 40 \mathrm{~mm})$ containing two $100 \mathrm{~W}$ cartridge heaters. The surface temperature was controlled by a PID controller driven by a thermocouple inserted into the aluminium block, approximately $1 \mathrm{~mm}$ below the impact point, and was varied in increments of $20^{\circ} \mathrm{C}$ between $160^{\circ} \mathrm{C}$ and $400^{\circ} \mathrm{C}$, with an accuracy of $\pm 1^{\circ} \mathrm{C}$. A schematic of the experimental setup is displayed in Figure 1. 
The drop diameter at equilibrium was calculated from the drop mass, $m$, measured with a precision scale (Mettler Toledo) as $D_{0}=\sqrt[3]{6 m / \pi \rho}$, where $\rho$ is the fluid density. Averages over 50 samples returned a value of $D_{0}=2.93 \pm$ $0.1 \mathrm{~mm}$.

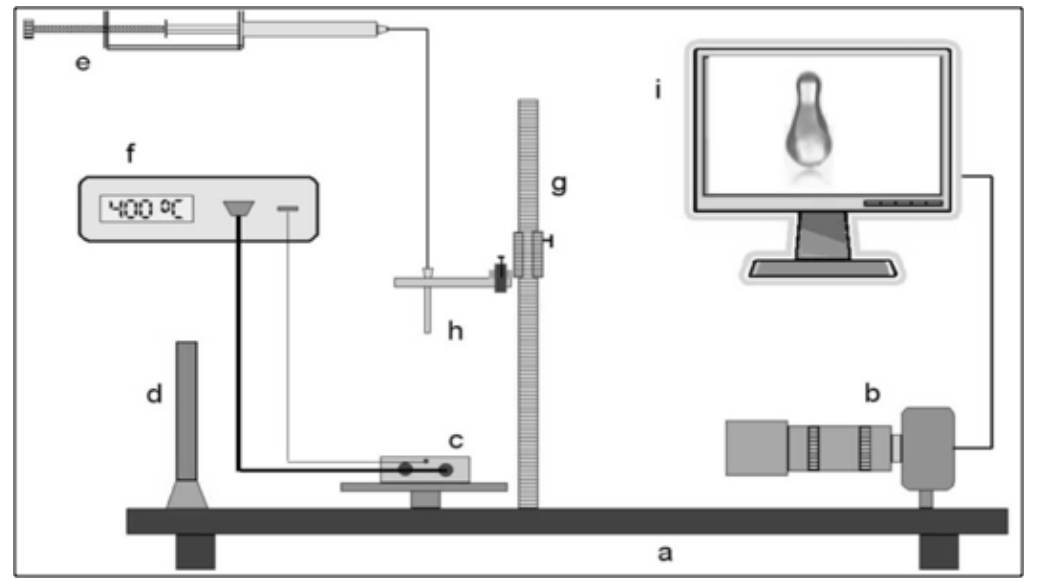

Figure 1. Schematic of experimental setup: a) optical breadboard; b) high-speed camera; c) heated aluminium block; d) LED backlight; e) drop dispensing system; f) temperature controller; g) digital vernier callipers; h) needle; i) computer.

The drop falling height, $\mathrm{H}$, was adjusted using a digital height gauge, and the free-fall impact velocity, $\mathrm{u}$, was calculated as $\sqrt{2 g\left(H-D_{0}\right)}$; previous experiments showed this is almost identical to the measured impact velocity, for falling heights up to $30 \mathrm{~cm}$ [17]. In the present study, the drop falling height was varied within the range from $30 \mathrm{~mm}$ to $200 \mathrm{~mm}$, corresponding to Weber numbers, between 23 and 166 .

The impact of single drops was recorded using a high-speed CMOS camera (Mikrotron MC1310) at the rate of 1000 frames per second, with back-to-front illumination. For each surface temperature and Weber number, the experiment was repeated 10 times for the sake of statistical coherence.

The recorded videos were processed in Labview environment. The processed frames obtained as a result were used to produce pictorial maps for each of the five specified combinations. These pictorial maps use surface temperature and the Weber number as the coordinates and apart from showing the impact behaviour, also allow one to make a qualitative assessment of the boundaries between the various impact regimes. The data was also processed in Matlab environment to generate impact regime maps, showing the impact behaviour as a function of surface temperature and Weber number. More details about the experimental apparatus and procedure can be found in previous works $[13,17,18]$.

\section{Results and discussion}

The impact regimes observed for water drops impacting on a heated surface, based on the simplified classification proposed in [13], i.e., secondary atomisation (SA), rebound, (R), breakup (B) and/or splashing (S), rebound with secondary atomisation (RSA), and breakup with secondary atomisation (BSA), are also observed in case of dilute polymer solution drops, and will not be discussed further in the present work. Besides these conventional impact regimes, dilute polymer solution drops may exhibit other impact morphologies, depending on the Weber number, the impact surface temperature, the polymer concentration and molecular weight.

At low polymer concentrations, there is a range of Weber numbers where a single satellite drop separates in the vertical direction during rebound, shortly after the drop has bounced off the surface; this drop is tethered to the main drop body by a thin liquid filament, which is subject to uniaxial stretching, and does not break up until the two droplets re-coalesce into a single drop, as shown in Figure 2. The diameter of the single satellite drop is between $40 \%$ and $50 \%$ of the equilibrium drop diameter, $D_{0}$; this means that the mass of the satellite drop is about $10 \%$ of the total mass of the drop, therefore the equivalent drop diameter of the drop after the satellite droplet separation is about $96 \%$ of the initial equilibrium diameter.

A second impact morphology peculiar of polymer solution drops can be observed at high Weber numbers. When the drop reaches maximum spreading, satellite droplets are formed around the disk perimeter due to the rim instability; in Newtonian drops, this instability eventually evolves into drop splashing. In the case of polymer solutions, a liquid bridge prevents the separation of the satellite droplets from the lamella, as shown in Figure 3; although the stretching of the liquid bridge is less than in the case of the single satellite drop filament, its elasticity is sufficient to recall the satellite droplets and prevent splashing or breakup. This impact morphology is not observed in Newtonian drops, and can be labelled as partial splashing (or semi-splashing). 


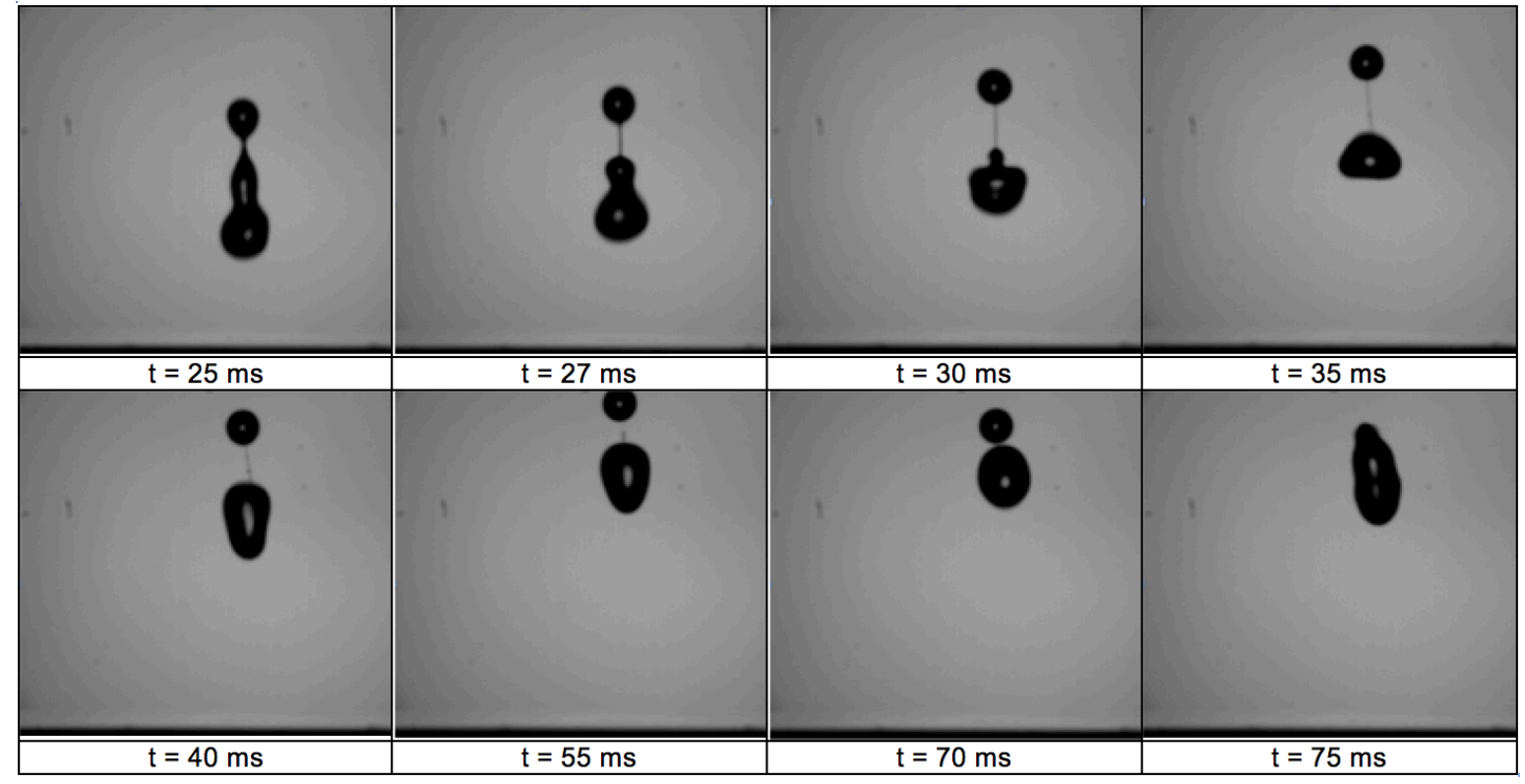

Figure 2. Single satellite drop morphology observed during the impact of a PEO solution drop (concentration: 100ppm; molecular weight: $4 \mathrm{MDa}$ ) impacting on a surface at $\mathrm{T}=400^{\circ} \mathrm{C}$ with $\mathrm{We}=80$; the time origin is the moment of impact.

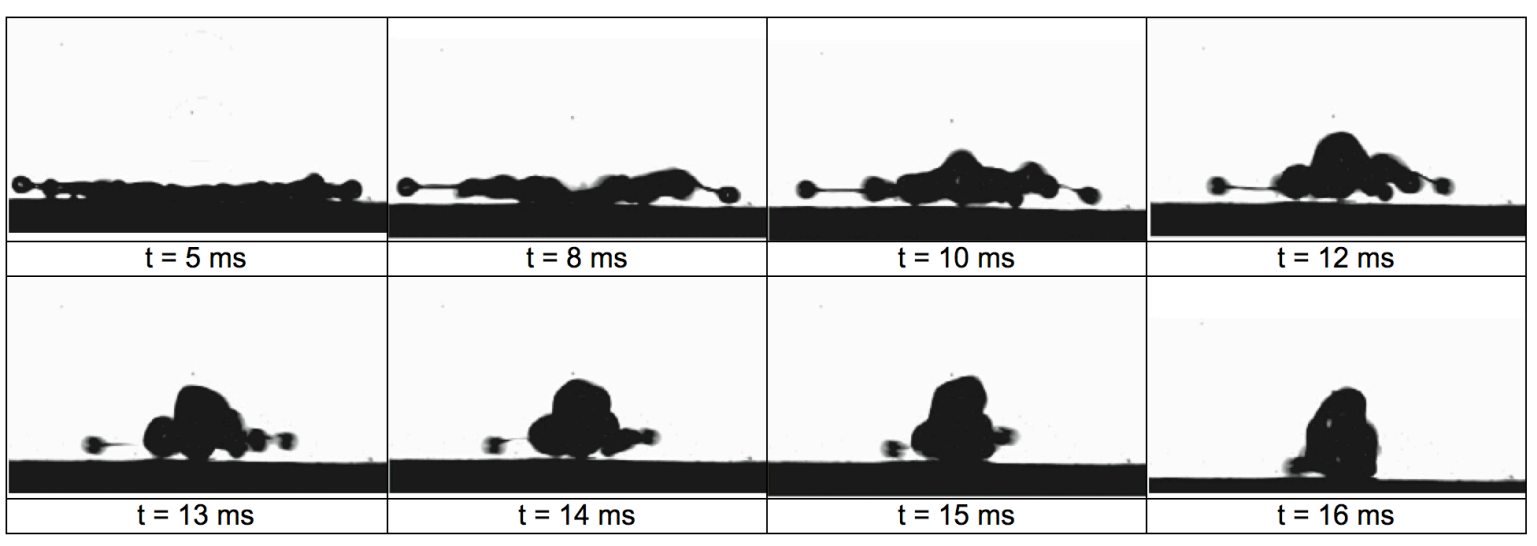

Figure 3. Semi-splash morphology observed during the impact of a PEO solution drop (concentration: 300ppm; molecular weight: $4 \mathrm{MDa}$ ) impacting on a surface at $\mathrm{T}=400^{\circ} \mathrm{C}$ with $\mathrm{We}=200$; the time origin is the moment of impact.

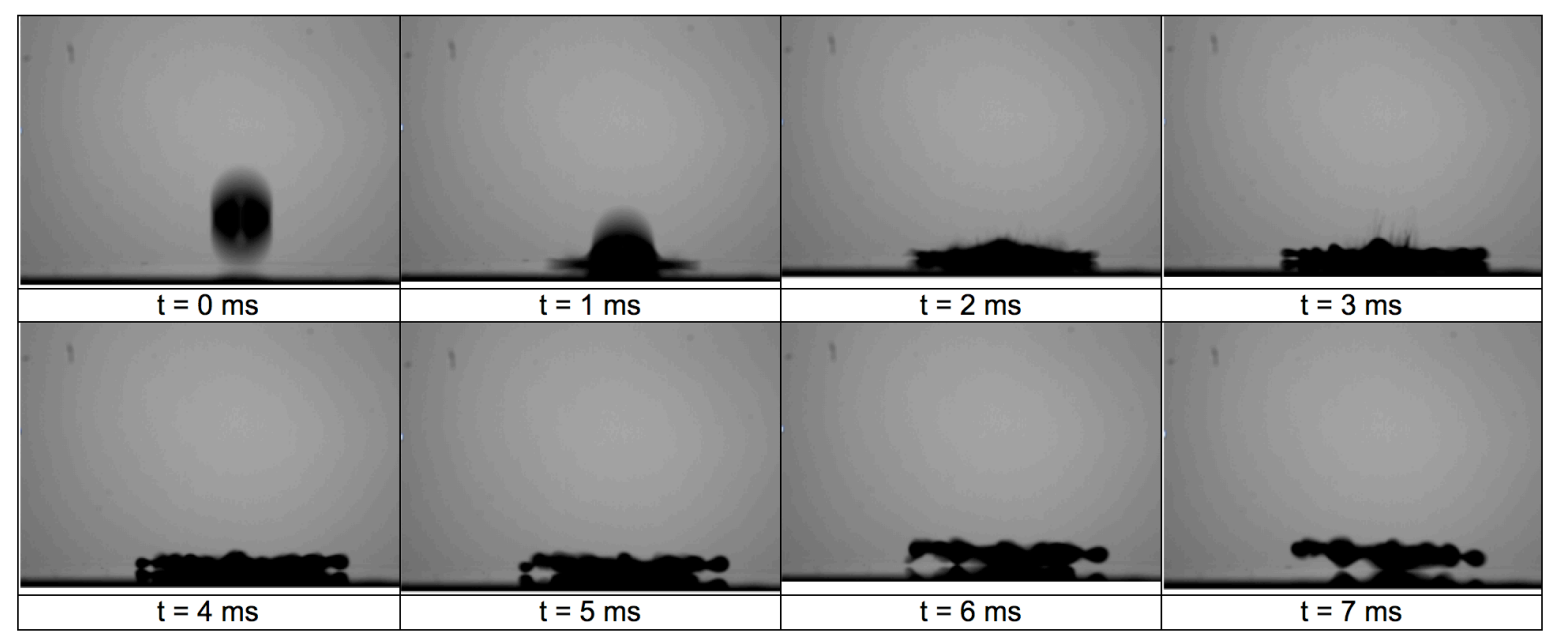

Figure 4. Semi-spray morphology observed during the impact of a PEO solution drop (concentration: 200ppm; molecular weight: $4 \mathrm{MDa}$ ) impacting on a surface at $\mathrm{T}=250^{\circ} \mathrm{C}$ with $\mathrm{We}=100$; the time origin is the moment of impact.

This work is licensed under a Creative Commons 4.0 International License (CC BY-NC-ND 4.0).

EDITORIAL UNIVERSITAT POLITĖCNICA DE VALĖNCIA 


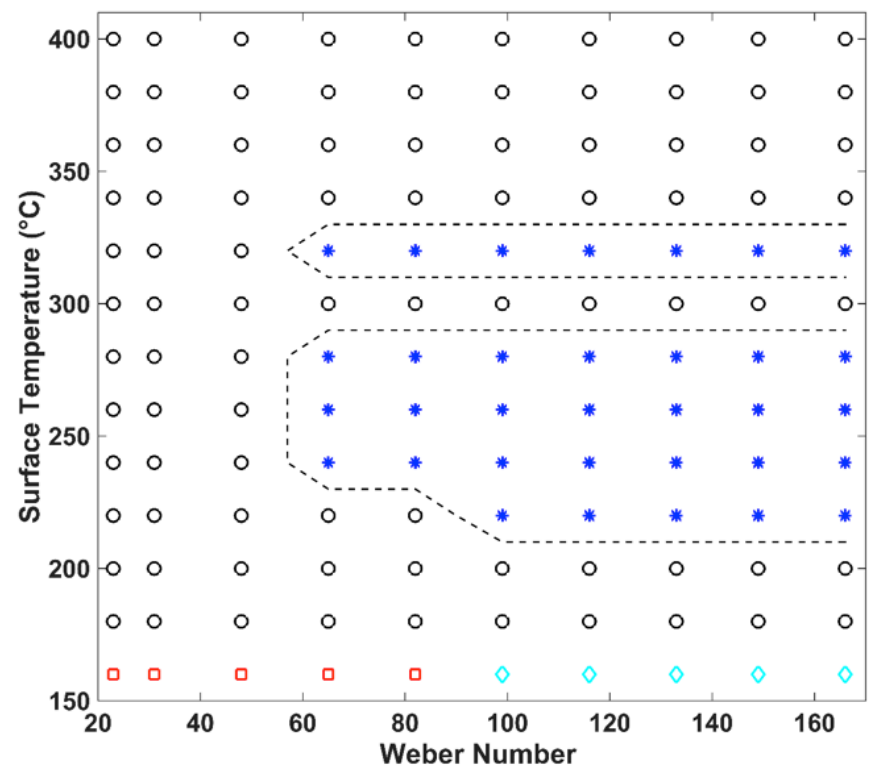

Figure 5. Impact regime map obtained for a concentration of $200 \mathrm{ppm}$ and a molecular weight of $4 \mathrm{MDa}$; regimes shown: SA $(\square), \operatorname{RSA}(\diamond), \mathrm{R}(0)$, and $\mathrm{R}^{*}\left({ }^{*}\right)$.

The formation of liquid bridges preventing the separation of satellite droplets also affects the secondary atomization regime, as shown in Figure 4. In this case, satellite droplets are sprayed out of the spreading drop free surface due to the bursting of vapour bubbles produced at nucleation sites on the impact surface; however, shortly after their ejection all satellite droplets forming the spray are pulled back into the main drop, hence this morphology can be labelled "semi-spray". The phenomenon has an overall duration of a few milliseconds therefore it is very difficult to detect and analyse.

Figure 5 shows the impact regime map relative to a dilute PEO solution with concentration of 200 ppm and molecular weight of the $\mathrm{PEO}$ of $4 \mathrm{MDa}$. In the range of parameters considered, the impact regimes observed are secondary atomization (SA), rebound with secondary atomisation (RSA), dry rebound (R), and semi-spray $\left(R^{*}\right)$. Thus, the map is significantly different with respect to the map obtained for drops of pure water [13]; the dominant impact morphology, observed for most combinations of surface temperature and Weber number, is dry rebound, meaning that the polymer additive strongly inhibits both secondary atomization and splashing.

The effect of polymer concentration is clearly seen upon comparing the map in Figure 5 with the impact regime maps for a molecular weight of 4MDa and PEO concentrations of $100 \mathrm{ppm}$ and $400 \mathrm{ppm}$, displayed in Figure 6 . Reducing the polymer concentration increases the number of different impact morphologies, while for the higher polymer concentration dry rebound is observes almost everywhere, with the semi-spray regime confined to a small region. Upon keeping the molecular weight constant at 4MDa and gradually increasing the concentration from 100 to 200 ppm, all break-up and splashing is completely overcome; with the exception of small scale secondary atomisation which is prevalent at a surface temperature of $160^{\circ} \mathrm{C}$. However, upon increasing the concentration to $400 \mathrm{ppm}$, no secondary atomisation is observed even at surface temperature of $160^{\circ} \mathrm{C}$. For all surface temperatures considered, rebound is the primary impact outcome.

Similarly, the effect of the molecular weight of the polymer can be seen upon comparing the map in Figure 5 with the impact regime maps for a concentration of $200 \mathrm{ppm}$ and molecular weights of $2 \mathrm{MDa}$ and $8 \mathrm{MDa}$, displayed in Figure 7. Within the $2 \mathrm{MDa}(200 \mathrm{ppm})$ impact regime map, secondary atomisation (SA), rebound with secondary atomisation $(R S A)$, rebound $(R)$, semi-spray $\left(R^{*}\right)$ and drop break-up $(B)$ regimes are observed. Upon gradual increase of molecular weight from $2 \mathrm{MDa}$ to $4 \mathrm{MDa}$, the break up regime is completely suppressed; however some secondary atomisation is still present at a surface temperature of $160^{\circ} \mathrm{C}$. Increasing the molecular weight to $8 \mathrm{MDa}$, all secondary atomisation is completely suppressed.

Thus, from a qualitative standpoint, the effect of molecular weight is similar to that of the polymer concentration; low molecular weights enable the development of different impact morphologies, while increasing the molecular weight progressively suppresses secondary atomization and breakup/splashing, until only the dry rebound regime can be observed. 

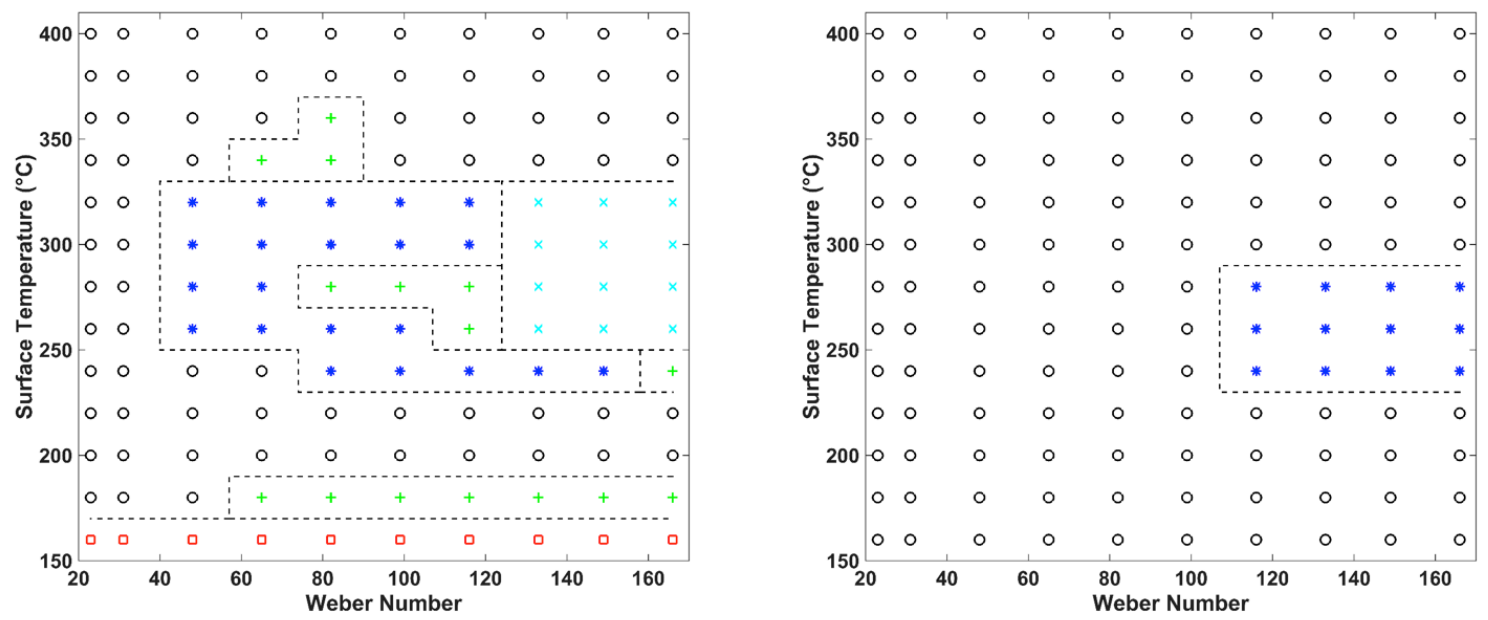

Figure 6. Impact regime maps obtained for a molecular weight of $4 \mathrm{MDa}$ at concentrations of $100 \mathrm{ppm}$ (left) and $400 \mathrm{ppm}$ (right); regimes shown: SA ( $\square), \mathrm{B}(+), \mathrm{R}(0), \mathrm{R}^{*}\left(^{*}\right)$ and $\mathrm{S}(\mathrm{x})$.

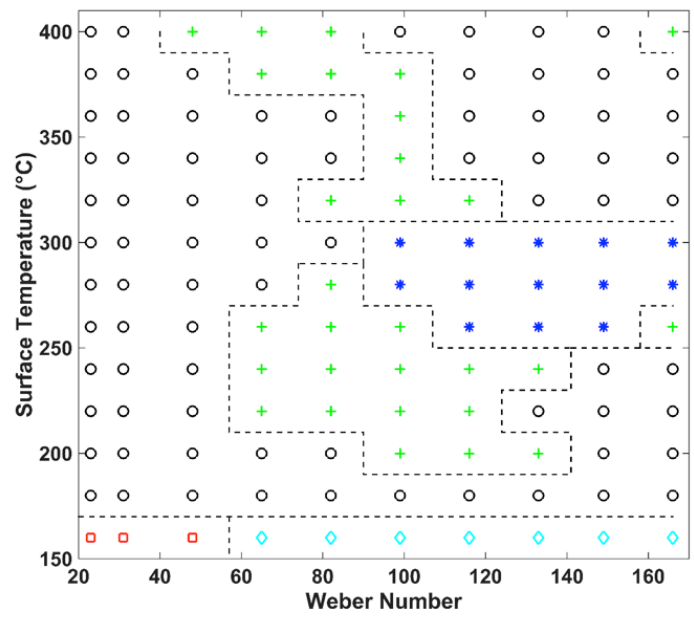

\begin{tabular}{|c|c|c|c|c|c|c|c|c|c|}
\hline \multirow[t]{2}{*}{$40 \mathrm{C}$} & $\begin{array}{lll}0 & 0\end{array}$ & o & 0 & 0 & 0 & 0 & 0 & 0 & o- \\
\hline & $\circ \quad 0$ & 0 & 0 & 0 & 0 & 0 & 0 & 0 & 0 \\
\hline \multirow{2}{*}{350} & 00 & o & 0 & 0 & 0 & 0 & 0 & 0 & 0 \\
\hline & 00 & 0 & 0 & 0 & 0 & 0 & 0 & $\circ$ & 0 \\
\hline \multirow{4}{*}{ 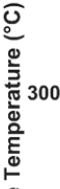 } & 00 & 0 & 0 & 0 & 0 & 0 & 0 & 0 & 0 \\
\hline & $\circ 0$ & $\circ$ & 0 & 0 & $\circ$ & 0 & 0 & 0 & o- \\
\hline & 00 & 0 & $\circ$ & $\circ$ & $\circ$ & $\circ$ & $\circ$ & * & * \\
\hline & $\circ 0$ & $\circ$ & 0 & 0 & 0 & 0 & $\circ$ & * & * \\
\hline \multirow{2}{*}{ 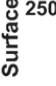 } & 00 & $\circ$ & 0 & 0 & $\circ$ & 0 & $\circ$ & 0 & 0 \\
\hline & 00 & 0 & 0 & 0 & 0 & 0 & 0 & 0 & 0 \\
\hline \multirow[t]{3}{*}{200} & $\circ 0$ & 0 & 0 & 0 & $\circ$ & $\circ$ & 0 & $\circ$ & 0 \\
\hline & $\circ \quad 0$ & 0 & 0 & 0 & 0 & 0 & 0 & 0 & 0 \\
\hline & $\begin{array}{ll}0 & 0\end{array}$ & 0 & 0 & 0 & $\circ$ & 0 & 0 & 0 & 0 \\
\hline 150 & & & & 80 & 10 & 120 & & & \\
\hline
\end{tabular}

Figure 7. Impact regime maps obtained for a concentration of $200 \mathrm{ppm}$ molecular weights of $2 \mathrm{MDa}$ (left) and $8 \mathrm{MDa}$ (right); regimes shown: $S A(\square), B(+), R(0), R^{*}\left({ }^{*}\right)$ and $S(x)$.

The similarity between the effects of the molecular weight and of the polymer concentration on the impact morphology is justified because both of these parameters affect the relaxation time of polymer solutions [21-23]. When the relaxation time is shorter than the characteristic hydrodynamic time scales corresponding to the various impact morphologies, the effect of the polymer additive is negligible, however when the relaxation time and the hydrodynamic time scales are of the same order the the behaviour of polymer solutions becomes significantly different from that of the pure solvent.

\section{Conclusions}

The impact of drops of dilute polyethylene oxide aqueous solutions on a heated polished aluminium surface was investigated by high-speed imaging, for different values of the polymer molecular weight and concentration, with the purpose to evaluate the influence of these parameters on the impact morphology. In addition to impact morphologies observed in Newtonian drops (deposition, rebound, secondary atomisation and breakup/splashing), three new impact regimes have been identified: (i) a single satellite droplet ejected in the direction of bouncing but tethered to the main drop by a thin liquid filament; (ii) a splashing-like behaviour (semi-splashing), where the rim instability generates satellite droplets tethered to the lamella by thin liquid filaments; (iii) a spray-like behaviour (semi-spray), where a fine secondary atomisation generated upon impact is quickly absorbed back into the drop 
globule. Based on experimental observations, five impact regime maps were produced to demonstrate the correlation between varying polymer molecular weight and concentration in suppressing the production of secondary droplets and splashing/breakup.

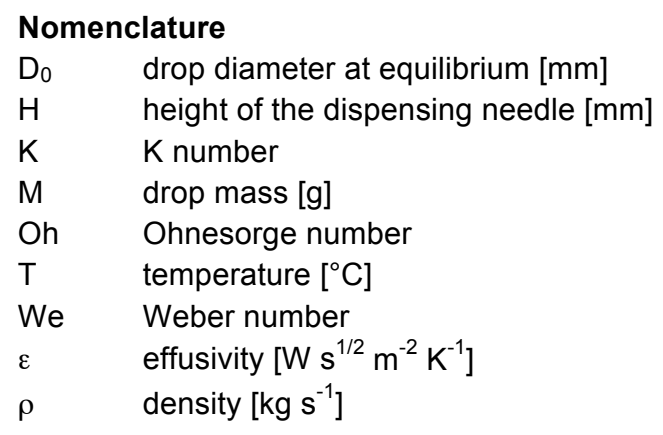

\section{References}

[1] Bai, C., 1995, "Development of methodology for spray impingement simulation".

[2] Bernadin, J.D., Stebbins, C.J. and Mudawar, I., 1997. International Journal of Heat and Mass Transfer, 40(2), pp. 247-267.

[3] Rein, M., 2002, "Interactions between drops and hot surfaces". Springer Vienna.

[4] Lee, S.Y. and Ryu, S.U., 2006. Journal of mechanical science and technology, 20(8), pp. 1101-1117.

[5] Castanet, G., Lienart, T. and Lemoine, F., 2009. International Journal of Heat and Mass Transfer, 52(3), pp. 670-679.

[6] Mundo, C, Sommerfield, M, Tropea, C, 1995. Int. J. Multiphase Flow, 21 (2) 151-173

[7] C. Amiel, 2002, "Application de techniques optiques à l'étude du comportement dynamique et thermique de gouttes en interaction avec une paroi chauffée" Ph.D. Thesis, École Nationale Supérieure de l'Aéronautique et de l'Espace.

[8] Cossali, G.E., Coghe, A., and Marengo, M., 1997. Experiments in Fluids, 22, pp. 463-472.

[9] J.D. Bernardin, I. Mudawar, 1999. The Leidenfrost point: experimental study and assessment of existing models, J. Heat Transfer (Trans. ASME) 121, pp. 894-903.

[10] J.D. Bernardin, I. Mudawar, 1999. A Leidenfrost point model for impinging droplets and sprays, J. Heat Transfer (Trans. ASME) 126, pp. 272-278.

[11] Wang, A.B., Lin, C.H. and Chen, C.C., 2000. Physics of Fluids, 12(6), pp.1622-1625.

[12] Moreira, A.L.N., Moita, A.S. and Panao, M.R., 2010. Progress in energy and combustion science,36(5), pp.554-580.

[13] V. Bertola, 2015, An impact regime map for water drops impacting on heated surfaces, International Journal of Heat and Mass Transfer, vol. 85, pp. 430-437.

[14] Tran, T., Staat, H.J.J., Prosperetti, A., Sun, C. and Lohse, D., 2012. Physical Review Letters, 108(3), 036101

[15] V. Bertola, 2004. Drop impact on a hot surface: effect of a polymer additive, Experiments in Fluids, vol. 37(5), pp. 653-664.

[16] V. Bertola, K. Sefiane, 2005. Controlling secondary atomization during drop impact on hot surfaces by polymer additives, Physics of Fluids, vol. 17(10), 108104.

[17] V. Bertola, 2009. An experimental study of bouncing Leidenfrost drops: comparison between Newtonian and viscoelastic liquids, International Journal of Heat and Mass Transfer, vol. 52, pp. 1786-1793.

[18] V. Bertola, 2014. Effect of polymer concentration on the dynamics of dilute polymer solution drops impacting on heated surfaces in the Leidenfrost regime, Experimental Thermal and Fluid Science, vol. 52, pp. 259-269.

[19] S. Chen, V. Bertola, 2016. Jumps, somersaults and symmetry breaking in Leidenfrost drops, Physical Review E, vol. 94, 021102(R).

[20] S. Chen, V. Bertola, 2016. The impact of viscoplastic drops on a heated surface in the Leidenfrost regime, Soft Matter, vol. 12, pp. 7624-7631.

[21] F.E. Beiley, J.V. Koleske, 1976. Poly(ethylene oxide), New York: Academic Press.

[22] N. P. Cheremisinoff, 1997. Handbook of engineering polymeric materials, New York: Marcel Dekker.

[23] V. N. Kalashnikov, A. N. Askarov, 1989. Relaxation time of elastic stresses in liquids with small additions of soluble polymers of high molecular weights, J Eng Phys Thermophys 57, pp. 874-878. 